\title{
Enantioselective, Catalytic Multicomponent Synthesis of Homoallylic Amines Enabled by Hydrogen-Bonding and Dispersive Interactions
}

\author{
Elisabetta Ronchi ${ }^{\ddagger}$ Shauna M. Paradine $*$, and Eric N. Jacobsen* \\ Department of Chemistry and Chemical Biology, Harvard University, 12 Oxford St., \\ Cambridge, MA 02138
}

\section{Supporting Information}

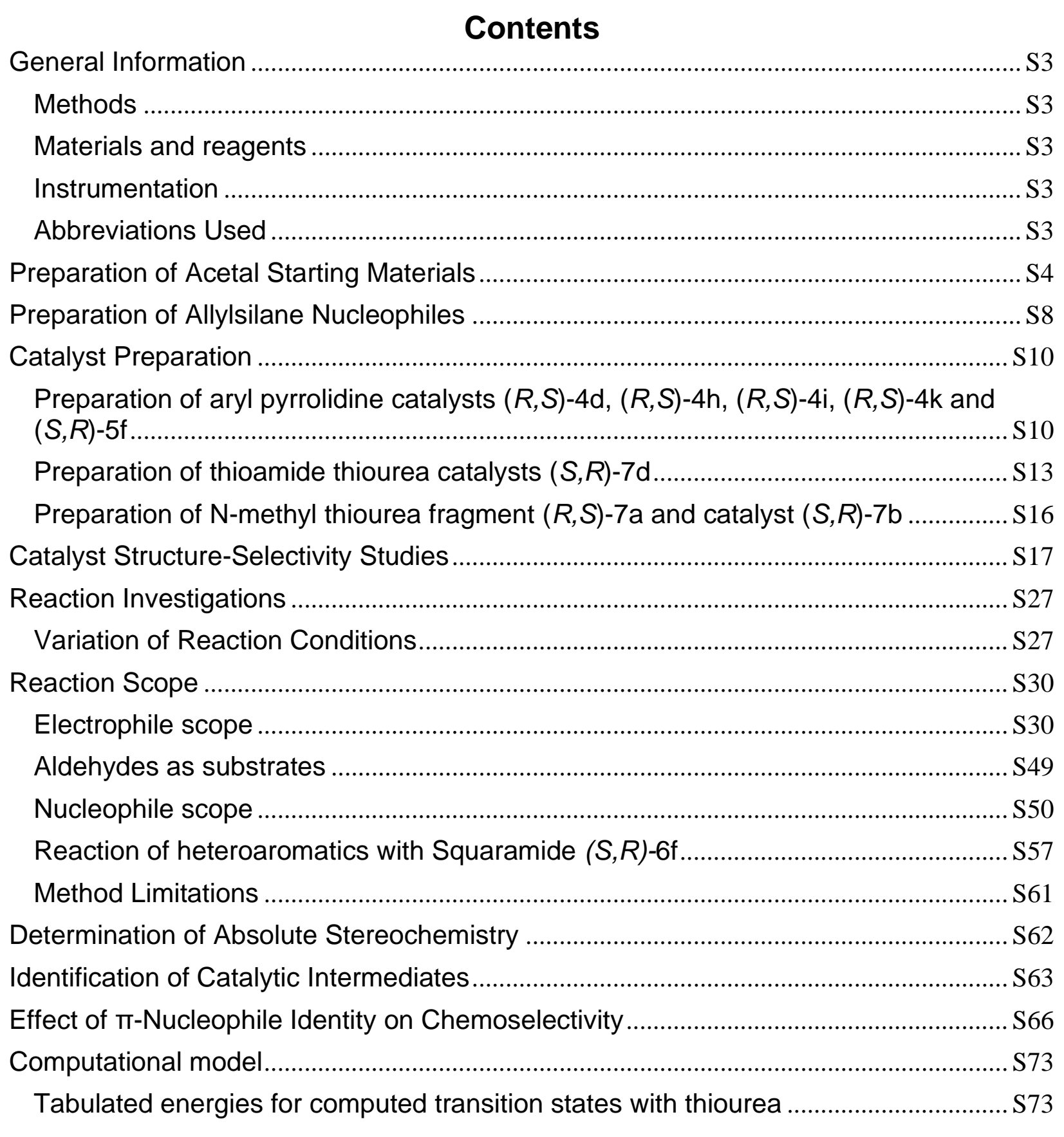


Tabulated energies for computed transition states with squaramide ........................... S77

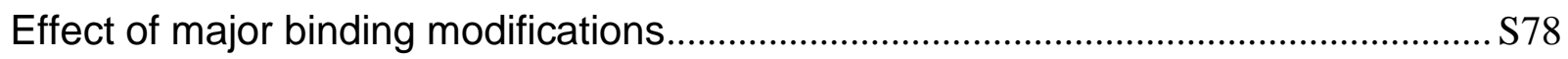

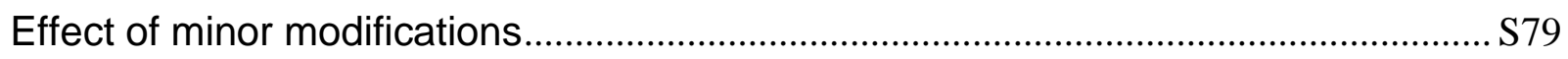

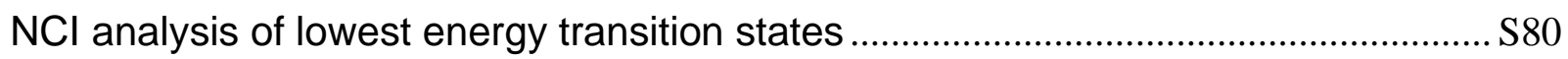

Comparison of Thiourea and Squaramide (with dispersion correction)....................... S84

Coordinates of computed transition states - Dispersion................................................. 85

Coordinates of computed transition states - No Dispersion ...................................... S228

Coordinates of computed transition states with squaramide $(\mathrm{R}, \mathrm{S})$-ent-6f..................S385

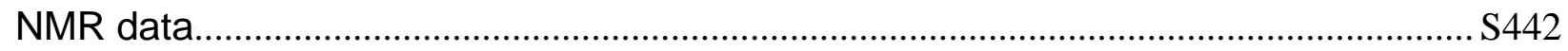

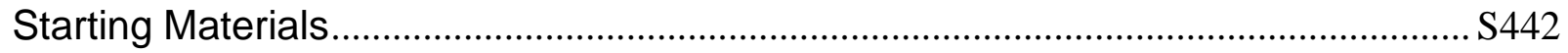

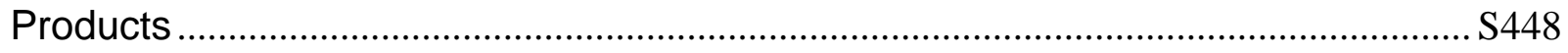

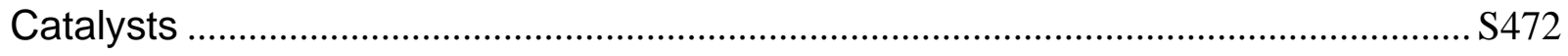




\title{
General Information
}

\begin{abstract}
Methods
All reactions were performed in round bottom flasks under a nitrogen $\left(\mathrm{N}_{2}\right)$ atmosphere unless otherwise noted. Catalytic experiments were performed under $\mathrm{N}_{2}$, in oven-dried 2-dram vials with septum-lined caps. Gas-tight syringes were used to transfer air- and moisture-sensitive liquids. Reactions were monitored by thin-layer chromatography (TLC) on Silica Gel 60 F254 plates (EMD) and visualized under UV light $(254 \mathrm{~nm})$ or with $\mathrm{KMnO}_{4}$ or anisaldehyde upon heating. Flash chromatography was conducted on a Biotage Isolera automated chromatography system. Reported product yields were determined based on material isolated after column purification.
\end{abstract}

\section{Materials and reagents}

Commercial reagents were purchased from Sigma-Aldrich, Alfa Aesar, Strem, Oakwood, Matrix Scientific, or $\mathrm{TCl}$ and used as received unless otherwise noted. tert-Butyl carbamate was recrystallized from hot TBME. Acetals, and allylsilanes were stored at $4^{\circ} \mathrm{C}$, and were generally stable under these storage conditions, with a few exceptions (noted where relevant). Reaction solvents $\left(\mathrm{Et}_{2} \mathrm{O}, \mathrm{CH}_{2} \mathrm{Cl}_{2}\right.$, toluene, THF, TBME, and CPME) were dried by passing through columns of activated alumina. Deuterated solvents $\mathrm{CDCl}_{3}$ and $\mathrm{CD}_{3} \mathrm{OD}$ (Cambridge Isotope Laboratories), and HPLC solvents (EMD) were used without purification. Note: We observed that catalyst from multiple runs could be recovered, combined, purified via column chromatography and its use yielded the same results as fresh catalyst. See Run 2 of substrate 3k for the head-to-head comparison. Recovered and purified catalyst was routinely employed in the experiments here described.

\section{Instrumentation}

Proton nuclear magnetic resonance $\left({ }^{1} \mathrm{H}\right.$ NMR) spectra and proton-decoupled carbon nuclear magnetic resonance $\left({ }^{13} \mathrm{C}\left\{{ }^{1} \mathrm{H}\right\}\right.$ NMR) spectra were recorded on a Bruker AVANCE NEO $400(400 \mathrm{MHz})$, Bruker AVANCE NEO 400B (400 MHz), Varian Inova 500 (500 MHz), Inova 600 (600 MHz) spectrometer at ambient temperature unless otherwise noted. All chemical shifts $(\delta)$ are reported in parts per million (ppm) downfield from tetramethylsilane. Proton resonances are referenced to residual protium in the NMR solvent. Carbon resonances are referenced to the carbon resonances of the NMR solvent. Data are represented as follows: chemical shift, multiplicity $(b r=$ broad, $s=$ singlet, $d=$ doublet, $t=$ triplet, $q=$ quartet, $m=$ multiplet, app = apparent), coupling constants $(\mathcal{J})$ in Hertz $(\mathrm{Hz})$, integration. Infrared (IR) spectra were obtained using a Bruker Alpha FTIR spectrometer equipped with an attenuated total reflectance (ATR) single reflection unit. Optical rotations ([a]) were measured using a $1 \mathrm{~mL}$ cell with a 0.5 $\mathrm{dm}$ path length on a Jasco DIP 370 digital polarimeter at $589 \mathrm{~nm}$ (sodium D line) at ambient temperature. Mass spectral (MS) data were obtained on an Agilent 6210 TOF LC/MS spectrometer (Harvard FAS Division of Science Small Molecule Mass Spectrometry). High-performance liquid chromatography (HPLC) analysis was performed using an Agilent 1200 series quaternary HPLC system with commercially available ChiralPak and ChiralCel columns. Enantioenriched and racemic samples were injected as solutions in $30 \% \mathrm{P} \mathrm{PrOH} /$ hexanes.

\section{Abbreviations Used}

acac $=$ acetoacetonate, $\mathrm{aq}=$ aqueous, $\mathrm{Boc}=$ tert-butyloxycarbonyl, $\mathrm{c}=$ concentration (in grams $/ 100 \mathrm{~mL}$ ), $\mathrm{ca}=$ circa, calc $=$ calculated, $\mathrm{cm}^{-1}=$ wavenumber, $\mathrm{COD}=$ cyclooctadiene, $\mathrm{CPME}=$ cyclopentyl methyl ether, DMAP $=N, N$-dimethylaminopyridine, e.e. $=$ enantiomeric excess, equiv $=$ equivalents, $\mathrm{h}=$ hours, $\mathrm{LDA}=$ lithium(diisopropylamide), $\mathrm{min}=$ minutes, $\mathrm{m} / \mathrm{z}=$ mass to charge ratio, $\mathrm{n} / \mathrm{d}=$ not determined, $\mathrm{NB}=$ nota bene, $\mathrm{NMM}=\mathrm{N}$-Methyl morpholine, $\mathrm{p}$-Ts $=$ para-toluenesulfonyl, $\mathrm{rbf}=$ round bottom flask, $\mathrm{R}_{\mathrm{f}}=$ retention factor, $\mathrm{rt}=$ room temperature, sat $=$ saturated, TBME $=$ tert-butyl methyl ether, TES $=$ triethylsilyl, $\mathrm{Tf}=$ trifluoromethanesulfonate, $\mathrm{THF}=$ tetrahydrofuran, $\mathrm{TMEDA}=\mathrm{N}, \mathrm{N}$-tetramethylethylenediamine, $\mathrm{TMS}=$ trimethylsilyl, $t_{R}=$ retention time, $\mathrm{v} / \mathrm{v}=$ volume/volume. 


\section{Preparation of Acetal Starting Materials}

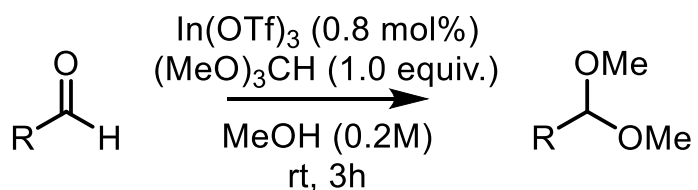

\section{General Procedure for Acetal Preparation:}

Modified from a previously reported procedure. ${ }^{1}$ An oven-dried $50 \mathrm{~mL}$ rbf equipped with stir bar and septum was charged with aldehyde (1.0 equiv.), trimethyl orthoformate (1.0 equiv.), anhydrous $\mathrm{MeOH}$ $(0.2 \mathrm{M})$ and, lastly, $\operatorname{In}(\mathrm{OTf})_{3}(0.008$ equiv.). The solution was stirred at rt until completion as determined by TLC analysis (2-3h) (In cases where conversion was problematic, additional trimethyl orthoformate and/or $\ln (\mathrm{OTf})_{3}$ could be added and the reaction times could be extended to several days.). Upon completion, the solution was diluted with $\mathrm{CH}_{2} \mathrm{Cl}_{2}$, transferred to a separatory funnel and washed with sat. aq. $\mathrm{NaHCO}_{3}$, then the aqueous layer was extracted with $\mathrm{CH}_{2} \mathrm{Cl}_{2}$ twice. The combined organic layers were washed with brine and dried over $\mathrm{Na}_{2} \mathrm{SO}_{4}$. Note: when reaction mixture was concentrated without work-up (as described in literature procedure), partial reversion to aldehyde, which can be challenging to separate from acetal via chromatography, was observed. Solvent was removed under reduced pressure, then the crude residue was purified via flash column chromatography on Davisil or silica basified with $1 \% \mathrm{Et}_{3} \mathrm{~N}$. Pure acetals were stored at $4^{\circ} \mathrm{C}$ and were generally stable at this temperature (exceptions noted below). Commercial acetals were also stored at $4^{\circ} \mathrm{C}$ and were used as received.

\section{4-Fluorobenzaldehyde dimethyl acetal [1c]}<smiles>COC(OC)c1ccc(F)cc1</smiles>

Prepared according to the general procedure for acetal preparation. 4Fluorobenzaldehyde (536 $\mu \mathrm{L}, 5.00 \mathrm{mmol}, 1.0$ equiv.), trimethyl orthoformate (548 $\mu \mathrm{L}, 5.00 \mathrm{mmol}, 1.0$ equiv.), $\mathrm{MeOH}(25 \mathrm{~mL}, 0.2 \mathrm{M})$, and $\ln (\mathrm{OTf})_{3}(22.5 \mathrm{mg}, 0.040$ $\mathrm{mmol}, 0.008$ equiv.) were used. Flash column chromatography on Davisil (Biotage, $25 \mathrm{~g}$ column) using a gradient of $1 \%$ EtOAc/hexanes $\rightarrow 10 \%$ EtOAc/hexanes as eluent gave pure product as a colorless oil.

${ }^{1} \mathrm{H}-\mathrm{NMR}\left(600 \mathrm{MHz}, \mathrm{CDCl}_{3}\right) \delta 7.43(\mathrm{dd}, J=8.5,5.7 \mathrm{~Hz}, 2 \mathrm{H}), 7.05(\mathrm{t}, J=8.7 \mathrm{~Hz}, 2 \mathrm{H}), 5.37(\mathrm{~s}, 1 \mathrm{H}), 3.32(\mathrm{~s}$, $6 \mathrm{H})$. All spectral data agree with that reported in the literature. ${ }^{2}$

\section{4-Methylbenzaldehyde dimethyl acetal [1d]}<smiles>COC(OC)c1ccc(C)cc1</smiles>

Prepared according to the general procedure for acetal preparation. $p$ Tolualdehyde $(590 \mu \mathrm{L}, 5.00 \mathrm{mmol}, 1.0$ equiv.), trimethyl orthoformate $(548 \mu \mathrm{L}$, $5.00 \mathrm{mmol}, 1.0$ equiv.), $\mathrm{MeOH}(25 \mathrm{~mL}, 0.2 \mathrm{M})$, and $\ln (\mathrm{OTf})_{3}(22.5 \mathrm{mg}, 0.040 \mathrm{mmol}$, 0.008 equiv.) were used. Flash column chromatography on Davisil (Biotage, $25 \mathrm{~g}$ column) using a gradient of $1 \%$ EtOAc/hexanes $\rightarrow 10 \%$ EtOAc/hexanes as eluent gave pure product as a colorless oil.

${ }^{1} \mathrm{H}-\mathrm{NMR}\left(500 \mathrm{MHz}, \mathrm{CDCl}_{3}\right) \delta 7.33(\mathrm{~d}, \mathrm{~J}=8.2 \mathrm{~Hz}, 2 \mathrm{H}), 7.21-7.15(\mathrm{~m}, 2 \mathrm{H}), 5.37(\mathrm{~s}, 1 \mathrm{H}), 3.33(\mathrm{~s}, 6 \mathrm{H}), 2.36$ $(\mathrm{s}, 3 \mathrm{H})$. All spectral data agree with that reported in the literature. ${ }^{2}$

\section{2-Naphthaldehyde dimethyl acetal [1h]}


<smiles>COC(OC)c1ccc2ccccc2c1</smiles>

Prepared according to the general procedure for acetal preparation. 2Naphthaldehyde $(700 \mu \mathrm{L}, 5.00 \mathrm{mmol}, 1.0$ equiv.), trimethyl orthoformate $(548 \mu \mathrm{L}$, $5.00 \mathrm{mmol}, 1.0$ equiv.), $\mathrm{MeOH}(25 \mathrm{~mL}, 0.2 \mathrm{M})$, and $\ln (\mathrm{OTf})_{3}(22.5 \mathrm{mg}, 0.040$ mmol, 0.008 equiv.) were used. Flash column chromatography on Davisil (Biotage, 25g column) using a gradient of $1 \%$ EtOAc/hexanes $\rightarrow 10 \%$ EtOAc/hexanes as eluent gave pure product as a colorless oil.

${ }^{1} \mathrm{H}-\mathrm{NMR}\left(500 \mathrm{MHz}, \mathrm{CDCl}_{3}\right) \delta 7.95(\mathrm{~d}, J=1.6 \mathrm{~Hz}, 1 \mathrm{H}), 7.88-7.84(\mathrm{~m}, 3 \mathrm{H}), 7.57(\mathrm{~d}, J=9.0 \mathrm{~Hz}, 1 \mathrm{H}), 7.51$ $-7.49(\mathrm{~m}, 2 \mathrm{H}), 5.57(\mathrm{~s}, 1 \mathrm{H}), 3.38(\mathrm{~s}, 6 \mathrm{H})$. All spectral data agree with that reported in the literature. ${ }^{3}$

\section{1-Naphthaldehyde dimethyl acetal [1i]}<smiles>COC(OC)c1cccc2ccccc12</smiles>

Prepared according to the general procedure for acetal preparation. 1-Naphthaldehyde (700 $\mu \mathrm{L}, 5.00 \mathrm{mmol}, 1.0$ equiv.), trimethyl orthoformate (548 $\mu \mathrm{L}, 5.00 \mathrm{mmol}, 1.0$ equiv.), $\mathrm{MeOH}(25 \mathrm{~mL}, 0.2 \mathrm{M})$, and $\ln (\mathrm{OTf})_{3}(22.5 \mathrm{mg}, 0.040 \mathrm{mmol}, 0.008$ equiv.) were used. Flash column chromatography on Davisil (Biotage, $25 \mathrm{~g}$ column) using a gradient of $1 \%$ EtOAc/hexanes $\rightarrow 10 \% \mathrm{EtOAc/hexanes} \mathrm{as} \mathrm{eluent} \mathrm{gave} \mathrm{pure} \mathrm{product} \mathrm{as} \mathrm{a} \mathrm{colorless} \mathrm{oil.}$

${ }^{1} \mathrm{H}-\mathrm{NMR}\left(500 \mathrm{MHz}, \mathrm{CDCl}_{3}\right) \delta 8.28(\mathrm{~d}, J=8.3 \mathrm{~Hz}, 1 \mathrm{H}), 7.88-7.84(\mathrm{~m}, 2 \mathrm{H}), 7.73(\mathrm{~d}, J=$ $7.1 \mathrm{~Hz}, 1 \mathrm{H}), 7.55-7.46(\mathrm{~m}, 3 \mathrm{H}), 5.93(\mathrm{~s}, 1 \mathrm{H}), 3.39(\mathrm{~s}, 6 \mathrm{H})$. All spectral data agree with that reported in the literature. ${ }^{2}$

\section{Methyl 3-(dimethoxymethyl)benzoate [1j]}<smiles>COC(=O)c1cccc(C(OC)OC)c1</smiles>

Prepared according to the general procedure for acetal preparation. Methyl 3formylbenzoate (821 mg, $5.00 \mathrm{mmol}, 1.0$ equiv.), trimethyl orthoformate $(548 \mu \mathrm{L}$, $5.00 \mathrm{mmol}, 1.0$ equiv.), $\mathrm{MeOH}(25 \mathrm{~mL}, 0.2 \mathrm{M})$, and $\ln (\mathrm{OTf}) 3(22.5 \mathrm{mg}, 0.040 \mathrm{mmol}$, 0.008 equiv.) were used. Flash column chromatography on Davisil (Biotage, $25 \mathrm{~g}$ column) using a gradient of $1 \% \mathrm{EtOAc} /$ hexanes $\rightarrow 10 \% \mathrm{EtOAc} /$ hexanes as eluent afforded pure product as a colorless oil in $84 \%$ isolated yield.

${ }^{1} \mathrm{H}-\mathrm{NMR}\left(500 \mathrm{MHz}, \mathrm{CDCl}_{3}\right) \delta 8.13-8.12(\mathrm{~m}, 1 \mathrm{H}), 8.02$ (dd, $\left.J=7.7,1.5 \mathrm{~Hz}, 1 \mathrm{H}\right), 7.65$ (dd, $J=7.7,1.5 \mathrm{~Hz}$, $1 \mathrm{H}), 7.45(\mathrm{t}, J=7.7 \mathrm{~Hz}, 1 \mathrm{H}), 5.43(\mathrm{~s}, 1 \mathrm{H}), 3.92(\mathrm{~s}, 3 \mathrm{H}), 3.33(\mathrm{~s}, 6 \mathrm{H}) ;{ }^{3} \mathrm{C}-\mathrm{NMR}\left(125 \mathrm{MHz}, \mathrm{CDCl}_{3}\right) \delta 167.1$, 138.7, 131.3, 130.3, 129.8, 128.5, 128.1, 102.6, 52.8, 52.2; IR (ATR, $\left.\mathrm{cm}^{-1}\right)$ 2952, 2831, 1720, 1433, 1354, 1283, 1269, 1198, 1098, 1053, 977; HRMS (ESI) $\mathrm{m} / \mathrm{z}$ calculated for $\mathrm{C}_{11} \mathrm{H}_{14} \mathrm{O}{ }_{4} \mathrm{Na}[\mathrm{M}+\mathrm{Na}]^{+}:$233.0784, found 233.0775. All spectral data agree with that reported in the literature. ${ }^{4}$

\section{Piperonal dimethyl acetal [1k]}<smiles>COC(OC)c1ccc2c(c1)OCO2</smiles>

Prepared according to the general procedure for acetal preparation. Piperonal $(1.501 \mathrm{~g}, 10.0 \mathrm{mmol}, 1.0$ equiv.), trimethyl orthoformate $(1.1 \mathrm{~mL}, 10.0 \mathrm{mmol}, 1.0$ equiv.), $\mathrm{MeOH}(50 \mathrm{~mL}, 0.2 \mathrm{M})$, and $\ln (\mathrm{OTf})_{3}(45.0 \mathrm{mg}, 0.080 \mathrm{mmol}, 0.008$ equiv.) were used. Flash column chromatography on Davisil (Biotage, $50 \mathrm{~g}$ column) using a gradient of $1 \% \mathrm{EtOAc/hexanes} \rightarrow 10 \% \mathrm{EtOAc/hexanes}$ as eluent gave pure product as a colorless oil in $88 \%$ isolated yield.

${ }^{1} \mathrm{H}-\mathrm{NMR}\left(600 \mathrm{MHz}, \mathrm{CDCl}_{3}\right) \delta 6.94(\mathrm{~d}, J=1.6 \mathrm{~Hz}, 1 \mathrm{H}), 6.92(\mathrm{dd}, J=7.9,1.5 \mathrm{~Hz}, 1 \mathrm{H}), 6.79$ (dd, $J=7.9,1.1$ $\mathrm{Hz}, 1 \mathrm{H}), 5.95(\mathrm{~s}, 2 \mathrm{H}), 5.28(\mathrm{~s}, 1 \mathrm{H}), 3.31(\mathrm{~s}, 6 \mathrm{H})$. All spectral data agree with that reported in the literature. ${ }^{5}$

\section{2-Bromo-3-hydroxy-4-methoxybenzaldehyde dimethyl acetal [1I]}

Prepared according to the general procedure for acetal preparation. 2-Bromo-3-hydroxy-4methoxybenzaldehyde (924 mg, $4.00 \mathrm{mmol}, 1.0$ equiv.), trimethyl orthoformate (440 $\mu \mathrm{L}, 4.00 \mathrm{mmol}, 1.0$ 
<smiles>COc1ccc(C(OC)OC)c(Br)c1O</smiles>

equiv.), $\mathrm{MeOH}(20 \mathrm{~mL}, 0.2 \mathrm{M})$, and $\ln (\mathrm{OTf})_{3}(18.0 \mathrm{mg}, 0.032 \mathrm{mmol}, 0.008$ equiv.) were used. Flash column chromatography on Davisil (Biotage, $25 \mathrm{~g}$ column) using a gradient of $5 \%$ EtOAc/hexanes $\rightarrow 20 \%$ EtOAc/hexanes as eluent afforded pure product as a white solid in $85 \%$ isolated yield. This acetal reverted to aldehyde within $\sim 6$ months while storing at $4^{\circ} \mathrm{C}$, but can be recovered by resubjecting the material to the acetal-forming reaction.

${ }^{1} \mathrm{H}-\mathrm{NMR}\left(500 \mathrm{MHz}, \mathrm{CDCl}_{3}\right) \delta 7.15(\mathrm{dd}, J=8.5,1.3 \mathrm{~Hz}, 1 \mathrm{H}), 6.84(\mathrm{dd}, J=8.5,1.3 \mathrm{~Hz}, 1 \mathrm{H}$ ), 5.98 (app. d, $J$ $=1.4 \mathrm{~Hz}, 1 \mathrm{H}$ ), 5.52 (app. d, $J=1.3 \mathrm{~Hz}, 1 \mathrm{H}$ ), 3.92 (app. d, $J=1.3 \mathrm{~Hz}, 3 \mathrm{H}$ ), 3.37 (app. d, $J=1.3 \mathrm{~Hz}, 6 \mathrm{H}$ ); ${ }^{13} \mathrm{C}-\mathrm{NMR}\left(126 \mathrm{MHz}, \mathrm{CDCl}_{3}\right) \delta 147.4,143.1,129.9,119.1,109.5,109.2,103.0,56.5,53.9$; IR $\left(\mathrm{ATR}, \mathrm{cm}^{-1}\right)$ 3347, 2949, 2909, 2825, 1669, 1594, 1492, 1363, 1329, 1278, 1232, 1200, 1167, 1034, 957 805; HRMS (ESI) $\mathrm{m} / \mathrm{z}$ calculated for $\mathrm{C}_{10} \mathrm{H}_{14} \mathrm{BrO}_{4}[\mathrm{M}+\mathrm{H}]^{+}: 276.9904$, found 276.9903.

\section{1-(Dimethoxymethyl)cyclohex-1-ene [1 $\mathrm{m}]$}<smiles>COC(OC)C1=CCCCC1</smiles>

Prepared according to the general procedure for acetal preparation. Cyclohex-1-ene-1carbaldehyde $(1.00 \mathrm{~g}, 8.00 \mathrm{mmol}, 1.0$ equiv. $)$, trimethyl orthoformate $(880 \mu \mathrm{L}, 8.00$ mmol, 1.0 equiv.), $\mathrm{MeOH}(40 \mathrm{~mL}, 0.2 \mathrm{M})$, and $\mathrm{In}(\mathrm{OTf})_{3}(36.0 \mathrm{mg}, 0.064 \mathrm{mmol}, 0.008$ equiv.) were used. Crude material was concentrated under reduced pressure in an ice bath (product is volatile). Flash column chromatography on Davisil (Biotage, $25 \mathrm{~g}$ column) using a gradient of $1 \% \mathrm{Et}_{2} \mathrm{O} /$ pentane $\rightarrow 15 \% \mathrm{Et}_{2} \mathrm{O} /$ pentane as eluent gave pure product as a colorless oil in $42 \%$ isolated yield. This acetal decomposed to a complex mixture within $\sim 6$ months while storing at $4^{\circ} \mathrm{C}$.

${ }^{1} \mathrm{H}-\mathrm{NMR}\left(600 \mathrm{MHz}, \mathrm{CDCl}_{3}\right) \delta 5.86-5.80(\mathrm{~m}, 1 \mathrm{H}), 4.47(\mathrm{~s}, 1 \mathrm{H}), 3.29(\mathrm{~s}, 6 \mathrm{H}), 2.14-1.90(\mathrm{~m}, 4 \mathrm{H}), 1.71-$ $1.46(\mathrm{~m}, 4 \mathrm{H}) ;{ }^{13} \mathrm{C}-\mathrm{NMR}\left(126 \mathrm{MHz}, \mathrm{CDCl}_{3}\right) \delta 134.6,126.4,106.8,53.6,24.9,23.3,22.6,22.4$; IR (ATR, $\left.\mathrm{cm}^{-1}\right)$ 2934, 2861, 2834, 1685, 1448, 1437, 1191, 1136; HRMS (ESI) $\mathrm{m} / z$ calculated for $\mathrm{C}_{9} \mathrm{H}_{17} \mathrm{O}_{2}[\mathrm{M}+\mathrm{H}]^{+}$: 157.1178, found 157.1224 .

\section{tert-Butyl 2-(dimethoxymethyl)-1H-pyrrole-1-carboxylate [1n]}<smiles>COC(OC)c1cccn1C(=O)OC(C)(C)C</smiles>

Prepared according to the general procedure for acetal preparation. $\mathrm{N}$-Boc-pyrrole-2carbaldehyde ( $976 \mathrm{mg}, 5.00 \mathrm{mmol}, 1.0$ equiv.), trimethyl orthoformate $(548 \mu \mathrm{L}, 5.00$ mmol, 1.0 equiv.), $\mathrm{MeOH}(25 \mathrm{~mL}, 0.2 \mathrm{M})$, and $\ln (\mathrm{OTf}) 3(22.5 \mathrm{mg}, 0.040 \mathrm{mmol}, 0.008$ equiv.) were used. Flash column chromatography on Davisil (Biotage, $25 \mathrm{~g}$ column) using a gradient of $1 \% \mathrm{EtOAc} /$ hexanes $\rightarrow 10 \% \mathrm{EtOAc} /$ hexanes as eluent afforded pure product as a colorless oil in $85 \%$ isolated yield.

${ }^{1} \mathrm{H}-\mathrm{NMR}\left(600 \mathrm{MHz}, \mathrm{CDCl}_{3}\right) \delta 7.22(\mathrm{dd}, J=3.3,1.8 \mathrm{~Hz}, 1 \mathrm{H}), 6.45-6.44(\mathrm{~m}, 1 \mathrm{H}), 6.12(\mathrm{t}$, $J=3.3 \mathrm{~Hz}, 1 \mathrm{H}), 5.90(\mathrm{~s}, 1 \mathrm{H}), 3.35(\mathrm{~s}, 6 \mathrm{H}), 1.59(\mathrm{~s}, 9 \mathrm{H}) ;{ }^{13} \mathrm{C}-\mathrm{NMR}\left(125 \mathrm{MHz}, \mathrm{CDCl}_{3}\right) \delta$ 149.0, 131.6, 122.6, 113.5, 109.9, 98.5, 84.0, 53.5, 28.1; IR (ATR, $\left.\mathrm{cm}^{-1}\right)$ 2981, 2935, 2828, 1741, 1416, 1395, 1327, 1299, 1165, 1131, 1097, 971; HRMS (ESI) $\mathrm{m} / \mathrm{z}$ calculated for $\mathrm{C}_{12} \mathrm{H}_{20} \mathrm{NO}_{4}[\mathrm{M}+\mathrm{H}]^{+}:$242.1342, found 242.1342.

\section{tert-Butyl 2-(dimethoxymethyl)-1H-indole-1-carboxylate [10]}<smiles>COC(OC)c1cc2ccccc2n1C(=O)OC(C)(C)C</smiles>

N-Boc protection: Prepared from a modified literature procedure. ${ }^{6}$ Indole-2carboxaldehyde $(580 \mathrm{mg}, 4.00 \mathrm{mmol}, 1.0$ equiv.) was taken up in THF (16 mL, $0.25 \mathrm{M})$ in a $50 \mathrm{~mL}$ rbf. The flask was cooled to $0^{\circ} \mathrm{C}$, then DMAP $(48.9 \mathrm{mg}, 0.400$ mmol, 0.10 equiv.), $\mathrm{Boc}_{2} \mathrm{O}$ (1.048 g, 4.80 mmol, 1.2 equiv.), and $\mathrm{Et}_{3} \mathrm{~N}(669 \mu \mathrm{L}, 4.80$ mmol, 1.2 equiv.) were added sequentially. The reaction was warmed to $\mathrm{rt}$ and stirred until completion as determined by TLC analysis ( $3 \mathrm{~h})$. Upon completion, the reaction mixture was partitioned between EtOAc $(30 \mathrm{~mL})$ and $\mathrm{H}_{2} \mathrm{O}(20 \mathrm{~mL})$, and the aqueous layer was extracted three times with EtOAc. The combined organic 
layers were dried over $\mathrm{Na}_{2} \mathrm{SO}_{4}$ and concentrated under reduced pressure. Crude $\mathrm{N}$-Boc-indole-2carboxaldehyde was carried forward to the next step.

Acetal formation: Prepared according to the general procedure for acetal preparation. $\mathrm{N}$-Boc-indole-2carboxaldehyde (903 mg, $3.68 \mathrm{mmol}, 1.0$ equiv.), trimethyl orthoformate (403 $\mu \mathrm{L}, 3.68 \mathrm{mmol}, 1.0$ equiv.), $\mathrm{MeOH}(18 \mathrm{~mL}, 0.2 \mathrm{M})$, and $\ln (\mathrm{OTf})_{3}(16.6 \mathrm{mg}, 0.029 \mathrm{mmol}, 0.008$ equiv.) were used. Flash column chromatography on Davisil (Biotage, $25 \mathrm{~g}$ column) using a gradient of $1 \%$ EtOAc/hexanes $\rightarrow 15 \%$ $\mathrm{EtOAc/hexanes} \mathrm{as} \mathrm{eluent} \mathrm{gave} \mathrm{pure} \mathrm{product} \mathrm{as} \mathrm{a} \mathrm{colorless} \mathrm{oil.}$

${ }^{1} \mathrm{H}-\mathrm{NMR}\left(600 \mathrm{MHz}, \mathrm{CDCl}_{3}\right) \delta 8.07(\mathrm{~d}, J=8.4 \mathrm{~Hz}, 1 \mathrm{H}), 7.55$ (dd, $\left.J=8.0,1.4 \mathrm{~Hz}, 1 \mathrm{H}\right), 7.30$ (ddd, $J=8.5$, 7.1, $1.5 \mathrm{~Hz}, 1 \mathrm{H}), 7.22(\mathrm{t}, J=7.5 \mathrm{~Hz}, 1 \mathrm{H}), 6.86(\mathrm{~s}, 1 \mathrm{H}), 6.06(\mathrm{~s}, 1 \mathrm{H}), 3.38(\mathrm{~s}, 6 \mathrm{H}), 1.69(\mathrm{~s}, 9 \mathrm{H}) ;{ }^{13} \mathrm{C}-\mathrm{NMR}$ (126 MHz, $\left.\mathrm{CDCl}_{3}\right) \delta 150.2,137.2,137.0,128.5,124.6,122.9,121.2,115.6,109.6,98.8,84.3,53.5,28.3$; IR (ATR, $\left.\mathrm{cm}^{-1}\right)$ 2988, 2944, 2828, 1729, 1564, 1436, 1356, 1193, 1054, 990; HRMS (ESI) $\mathrm{m} / \mathrm{z}$ calculated for $\mathrm{C}_{16} \mathrm{H}_{22} \mathrm{NO}_{4}[\mathrm{M}+\mathrm{H}]^{+}:$292.1499, found 292.1497.

\section{tert-Butyl 5-(dimethoxymethyl)-1H-indole-1-carboxylate [1p]}<smiles>COC(OC)c1ccc2c(ccn2C(=O)OC(C)(C)C)c1</smiles>

$N$-Boc protection: Prepared according to the procedure described for 10 . Indole-5-carboxaldehyde (580 mg, $4.00 \mathrm{mmol}, 1.0$ equiv.), THF (16 mL, $0.25 \mathrm{M}$ ), DMAP (48.9 mg, $0.400 \mathrm{mmol}, 0.10$ equiv.), Boc $2 \mathrm{O}$ (1.048 g, 4.80 mmol, 1.2 equiv.), and $\mathrm{Et}_{3} \mathrm{~N}(669 \mu \mathrm{L}, 4.80$ mmol, 1.2 equiv.) were used.

Acetal formation: Prepared according to the general procedure for acetal preparation. $\mathrm{N}$-Boc-indole-5-carboxaldehyde (491 mg, $2.00 \mathrm{mmol}, 1.0$ equiv.), trimethyl orthoformate $(220 \mu \mathrm{L}, 2.00 \mathrm{mmol}, 1.0$ equiv.), $\mathrm{MeOH}(10 \mathrm{~mL}, 0.2 \mathrm{M})$, and $\ln (\mathrm{OTf})_{3}(9.0 \mathrm{mg}, 0.040 \mathrm{mmol}, 0.008$ equiv.) were used. Flash column chromatography on Davisil (Biotage, $25 \mathrm{~g}$ column) using a gradient of $1 \%$ EtOAc/hexanes $\rightarrow 10 \%$ EtOAc/hexanes as eluent afforded pure product as a colorless oil in $84 \%$ isolated yield. This acetal reverted to aldehyde within $\sim 6$ months while storing at $4^{\circ} \mathrm{C}$, but can be recovered by resubjecting the material to the acetal-forming reaction.

${ }^{1} \mathrm{H}-\mathrm{NMR}\left(500 \mathrm{MHz}, \mathrm{CDCl}_{3}\right) \delta 8.14(\mathrm{~d}, J=8.5 \mathrm{~Hz}, 1 \mathrm{H}), 7.67(\mathrm{~d}, J=1.7 \mathrm{~Hz}, 1 \mathrm{H}), 7.60(\mathrm{~d}, J=3.7 \mathrm{~Hz}, 1 \mathrm{H})$, $7.40(\mathrm{dd}, J=8.6,1.7 \mathrm{~Hz}, 1 \mathrm{H}), 6.57(\mathrm{~d}, J=3.7 \mathrm{~Hz}, 1 \mathrm{H}), 5.51(\mathrm{~s}, 1 \mathrm{H}), 3.35(\mathrm{~s}, 6 \mathrm{H}), 1.67(\mathrm{~s}, 9 \mathrm{H}) .{ }^{13} \mathrm{C}-\mathrm{NMR}$ $\left(126 \mathrm{MHz}, \mathrm{CDCl}_{3}\right) \delta 149.8,135.3,132.7,130.5,126.4,123.1,119.4,114.9,107.6,103.5,83.8,52.7$, 28.3; IR (ATR, $\left.\mathrm{cm}^{-1}\right)$ 2984, 2935, 2828, 1730, 1471, 1216, 1157, 1095, 1080, 1052, 1022, 987; HRMS (ESI) $\mathrm{m} / \mathrm{z}$ calculated for $\mathrm{C}_{16} \mathrm{H}_{22} \mathrm{NO}_{4}[\mathrm{M}+\mathrm{H}]^{+}:$292.1499, found 292.1500 .

\section{3-(Dimethoxymethyl)benzo[b]thiophene [1q]}<smiles>COC(OC)c1csc2ccccc12</smiles>

Prepared according to the general procedure for acetal preparation. Benzo[b]thiophene-3-carbaldehyde (324 $\mathrm{mg}, 2.00 \mathrm{mmol}, 1.0$ equiv), trimethyl orthoformate $\left(220 \mu \mathrm{L}, 2.00 \mathrm{mmol}, 1.0\right.$ equiv.), $\mathrm{MeOH}(10 \mathrm{~mL}, 0.2 \mathrm{M})$, and $\mathrm{In}(\mathrm{OTf})_{3}$ $(9.0 \mathrm{mg}, 0.016 \mathrm{mmol}, 0.008$ equiv.) were used. Flash column chromatography on Davisil (Biotage, 25g column) using a gradient of 1\% EtOAc/hexanes $\rightarrow 10 \%$ EtOAc/hexanes as eluent afforded pure product as a colorless oil in $90 \%$ isolated yield.

${ }^{1} \mathrm{H}-\mathrm{NMR}\left(500 \mathrm{MHz}, \mathrm{CDCl}_{3}\right) \delta 8.00-7.98(\mathrm{~m}, 1 \mathrm{H}), 7.87-7.85(\mathrm{~m}, 1 \mathrm{H}), 7.57(\mathrm{~d}, J=1.2 \mathrm{~Hz}, 1 \mathrm{H}), 7.43-7.33$ $(\mathrm{m}, 2 \mathrm{H}), 5.72(\mathrm{~d}, J=1.1 \mathrm{~Hz}, 1 \mathrm{H}), 3.36$ (app. d, $J=1.8 \mathrm{~Hz}, 6 \mathrm{H}) ;{ }^{13} \mathrm{C}-\mathrm{NMR}\left(126 \mathrm{MHz}, \mathrm{CDCl}_{3}\right) \delta 140.8$, 137.2, 133.6, 125.6, 124.6, 124.4, 122.9, 122.8, 100.0, 52.6; IR (ATR, $\left.\mathrm{cm}^{-1}\right)$ 2934, 2897, 2827, 1528, 1459, 1356, 1259, 1116, 1073, 1050, 978; HRMS (ESI) $m / z$ calculated for $\mathrm{C}_{11} \mathrm{H}_{13} \mathrm{O}_{2} \mathrm{~S}[\mathrm{M}+\mathrm{H}]^{+}: 209.8586$, found 209.0588 .

\section{2-Benzo[b]furan dimethyl acetal [1r]}


<smiles>COC(OC)c1cc2ccccc2o1</smiles>

Prepared according to the general procedure for acetal preparation. Benzofuran-2carbaldehyde ( $485 \mu \mathrm{L}, 4.00 \mathrm{mmol}, 1.0$ equiv.), trimethyl orthoformate $(440 \mu \mathrm{L}, 4.00$ mmol, 1.0 equiv.), $\mathrm{MeOH}(20 \mathrm{~mL}, 0.2 \mathrm{M})$, and $\mathrm{In}(\mathrm{OTf})_{3}(18.0 \mathrm{mg}, 0.032 \mathrm{mmol}, 0.008$ equiv.) were used. Flash column chromatography on Davisil (Biotage, $25 \mathrm{~g}$ column) using a gradient of $1 \% \mathrm{EtOAc/hexanes} \rightarrow 10 \% \mathrm{EtOAc/hexanes} \mathrm{as} \mathrm{eluent} \mathrm{gave}$ pure product as a colorless oil.

${ }^{1} \mathrm{H}-\mathrm{NMR}\left(600 \mathrm{MHz}, \mathrm{CDCl}_{3}\right) \delta 7.58(\mathrm{~d}, J=7.6 \mathrm{~Hz}, 1 \mathrm{H}), 7.51$ (d, $\left.J=8.5 \mathrm{~Hz}, 1 \mathrm{H}\right), 7.30(\mathrm{dt}, J=7.2,1.4 \mathrm{~Hz}$, $1 \mathrm{H}), 7.24(\mathrm{td}, J=7.5,1.1 \mathrm{~Hz}, 1 \mathrm{H}), 6.82(\mathrm{~s}, 1 \mathrm{H}), 5.58(\mathrm{~s}, 1 \mathrm{H}), 3.42(\mathrm{~s}, 6 \mathrm{H})$. All spectral data agree with that reported in the literature. ${ }^{7}$

\section{2-Bromo-5-(dimethoxymethyl)furan [1s]}<smiles>COC(OC)c1ccc(Br)o1</smiles>

Prepared according to the general procedure for acetal preparation. 2-bromo-furan-5carbaldehyde $(700 \mathrm{mg}, 4.00 \mathrm{mmol}, 1.0$ equiv), trimethyl orthoformate $(440 \mu \mathrm{L}, 4.00$ mmol, 1.0 equiv.), $\mathrm{MeOH}(20 \mathrm{~mL}, 0.2 \mathrm{M})$, and $\ln (\mathrm{OTf})_{3}(18.0 \mathrm{mg}, 0.032 \mathrm{mmol}, 0.008$ equiv.) were used. Flash column chromatography on Davisil (Biotage, $25 \mathrm{~g}$ column) using a gradient of $1 \% \mathrm{EtOAc/hexanes} \rightarrow 10 \% \mathrm{EtOAc/hexanes}$ as eluent afforded pure product as a colorless oil in $87 \%$ isolated yield.

${ }^{1} \mathrm{H}-\mathrm{NMR}\left(600 \mathrm{MHz}, \mathrm{CDCl}_{3}\right) \delta 6.39(\mathrm{~d}, J=3.3 \mathrm{~Hz}, 1 \mathrm{H}), 6.28(\mathrm{~d}, J=3.2 \mathrm{~Hz}, 1 \mathrm{H}), 5.38(\mathrm{~s}, 1 \mathrm{H}), 3.35(\mathrm{~s}, 6 \mathrm{H})$. All spectral data agree with that reported in the literature. ${ }^{8}$

\section{Preparation of Allylsilane Nucleophiles}

Note: All allylsilane nucleophiles were stored for extended periods at $4^{\circ} \mathrm{C}$, whether commercial or synthesized.

1) TMEDA, $n$-BuLi $3: 2 \mathrm{Et}_{2} \mathrm{O} / \mathrm{THF}$<smiles>C=C(C)CCO</smiles>
TMSCl

2) $\mathrm{K}_{2} \mathrm{CO}_{3}, \mathrm{MeOH}$<smiles>C=C(CCO)CC[As]</smiles>

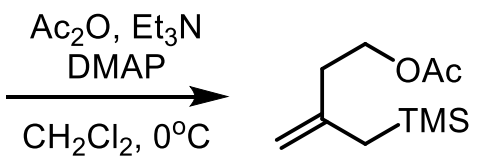

\section{3-((trimethylsilyl)methyl)but-3-en-1-yl acetate [2b]}

Prepared according to a modified literature procedure. ${ }^{9}$ TMEDA was taken up in $\mathrm{Et}_{2} \mathrm{O}(20 \mathrm{~mL})$ in a flamedried $250 \mathrm{~mL}$ rbf equipped with a large stir bar and septum, and flask was cooled to $0^{\circ} \mathrm{C}$. A $2.5 \mathrm{M}$ solution of $n$-BuLi in pentane (20.0 mL, $50.0 \mathrm{mmol}, 2.5$ equiv.) was added dropwise, then the solution was stirred for $10 \mathrm{~min}$ before adding 3-methyl-3-buten-1-ol (2.0 mL, $20.0 \mathrm{mmol}, 1.0$ equiv.) dropwise. THF (14 mL) was added at this point, and reaction mixture was stirred at $0^{\circ} \mathrm{C}$ for $1 \mathrm{~h}$, then warmed to rt, and stirred vigorously for $\sim 24 \mathrm{~h}$ (the dilithium salt of the alkenol forms a very sticky oil that settles to the bottom of the flask and tends to seize stir bars). The reaction mixture was then cooled to $0^{\circ} \mathrm{C}$ again, and $\mathrm{TMSCl}(12.8$ $\mathrm{mL}, 100 \mathrm{mmol}, 5.0$ equiv.) was added via syringe in a single portion. THe reaction mixture stirred at $0^{\circ} \mathrm{C}$ for $10 \mathrm{~min}$, then warmed to rt and stirred $30 \mathrm{~min}$. Upon completion, the mixture was diluted with $\mathrm{Et}_{2} \mathrm{O}(60$ $\mathrm{mL})$, washed with sat. aq. $\mathrm{NaHCO}_{3}(30 \mathrm{~mL})$, then the aqueous layer was extracted with $\mathrm{Et}_{2} \mathrm{O}(2 \times 60 \mathrm{~mL})$. The combined organic layers were washed with $\mathrm{H}_{2} \mathrm{O}(1 \times 30 \mathrm{~mL})$, sat. aq. $\mathrm{CuSO}_{4}(2 \times 30 \mathrm{~mL})$, and $\mathrm{H}_{2} \mathrm{O}$ again $(1 \times 30 \mathrm{~mL})$, then organic layer was dried over $\mathrm{K}_{2} \mathrm{CO}_{3}$ and concentrated under reduced pressure. The remaining residue was taken up in $\mathrm{MeOH}(100 \mathrm{~mL})$, cooled to $0^{\circ} \mathrm{C}$, then $\mathrm{K}_{2} \mathrm{CO}_{3}(3.455 \mathrm{~g}, 25.0 \mathrm{mmol}$, 1.2 equiv.) was added, and reaction was stirred at $0^{\circ} \mathrm{C}$ until disappearance of the silanol by TLC (3-4h). Note: extended reaction times can lead to proto-disilylation. Upon completion, the reaction was diluted 
with hexanes, washed with $\mathrm{H}_{2} \mathrm{O}$, and the aqueous layer was extracted with hexanes $(3 \times 50 \mathrm{~mL})$. Combined organic layers were dried over $\mathrm{K}_{2} \mathrm{CO}_{3}$ and carefully concentrated under reduced pressure (material is somewhat volatile). Crude material could be further purified by flash chromatography or carried forward directly. The product was isolated as a colorless oil in $58 \%$ yield $(1.814 \mathrm{~g}, 6.16 \mathrm{mmol})$.<smiles>C=C(CCOC(C)=O)COC(C)=O</smiles>
The product from the previous step was taken up in $\mathrm{CH}_{2} \mathrm{Cl}_{2}(24 \mathrm{~mL}, 0.25 \mathrm{M})$ in a $50 \mathrm{~mL}$ $\mathrm{rbf}$, then the reaction mixture was cooled to $0^{\circ} \mathrm{C}$. $\mathrm{Ac}_{2} \mathrm{O}(697 \mu \mathrm{L}, 7.39 \mathrm{mmol}, 1.2$ equiv.), $\mathrm{Et}_{3} \mathrm{~N}(1.0 \mathrm{~mL}, 7.39 \mathrm{mmol}, 1.2$ equiv.), and DMAP (37.6 mg, $0.308 \mathrm{mmol}, 0.05$ equiv.) were added, and the reaction mixture was stirred at $0^{\circ} \mathrm{C}$ until completion (1-2h). Upon completion, the mixture was diluted with $\mathrm{CH}_{2} \mathrm{Cl}_{2}(40 \mathrm{~mL})$ and washed with sat. aq. $\mathrm{NH}_{4} \mathrm{Cl}$ $(20 \mathrm{~mL})$, sat. aq. $\mathrm{NaHCO}_{3}(20 \mathrm{~mL})$, and brine $(20 \mathrm{~mL})$. The organic layer was dried over $\mathrm{Na}_{2} \mathrm{SO}_{4}$ and carefully concentrated under reduced pressure (material is somewhat volatile). The crude product was purified via flash column chromatography on $\mathrm{SiO}_{2}$ (Biotage, $25 \mathrm{~g}$ column, $10 \% \mathrm{Et}_{2} \mathrm{O} /$ pentane). The product was isolated as a colorless oil in $79 \%$ yield $(972 \mathrm{mg}, 4.85 \mathrm{mmol})$.

${ }^{1} \mathrm{H}-\mathrm{NMR}\left(600 \mathrm{MHz}, \mathrm{CDCl}_{3}\right) \delta 4.63(\mathrm{q}, J=1.4 \mathrm{~Hz}, 1 \mathrm{H}), 4.60(\mathrm{~m}, 1 \mathrm{H}), 4.17(\mathrm{dd}, J=7.6,6.5 \mathrm{~Hz}, 2 \mathrm{H}), 2.28$ (td, $J=7.1,1.4 \mathrm{~Hz}, 2 \mathrm{H}), 2.04(\mathrm{~s}, 3 \mathrm{H}), 1.55(\mathrm{~s}, 2 \mathrm{H}), 0.02(\mathrm{~s}, 9 \mathrm{H}) ;{ }^{13} \mathrm{C}-\mathrm{NMR}\left(125 \mathrm{MHz}, \mathrm{CDCl}_{3}\right) \delta 171.1$, $143.4,109.1,63.0,37.1,27.1,-1.3$. All spectral data agree with that reported in the literature. ${ }^{10}$

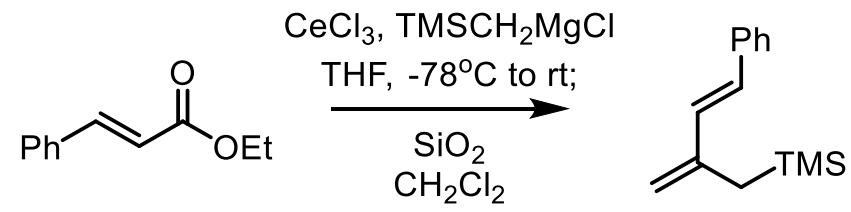

\section{(E)-trimethyl(2-methylene-4-phenylbut-3-en-1-yl)silane [2c]}

Prepared according to a modified literature procedure. ${ }^{11}$ Anhydrous $\mathrm{CeCl}_{3}(6.160 \mathrm{~g}, 25.0 \mathrm{mmol}, 5.0$ equiv.) was flame dried in a $250 \mathrm{~mL}$ rbf equipped with stir bar and septum. After the flask cooled to rt, THF $(50 \mathrm{~mL}, 0.1 \mathrm{M})$ was added via syringe, and the suspension was vigorously stirred for $3 \mathrm{~h}$. At this point, the solution was cooled to $-78^{\circ} \mathrm{C}$, then $\mathrm{TMSCH}_{2} \mathrm{MgCl}(25.0 \mathrm{~mL}, 25.0 \mathrm{mmol}, 5.0$ equiv., $1 \mathrm{M}$ solution in $\mathrm{Et}_{2} \mathrm{O}$ ) was added dropwise, and the reaction mixture was stirred at this temperature for $1 \mathrm{~h}$. Ethyl transcinnamate $(840 \mu \mathrm{L}, 5.00 \mathrm{mmol}, 1.0$ equiv.) was added dropwise over $10 \mathrm{~min}$, then the reaction mixture was stirred at $-78^{\circ} \mathrm{C}$ for $2 \mathrm{~h}$ and warmed to rt overnight. Upon completion, the reaction was quenched with sat. aq. $\mathrm{NH}_{4} \mathrm{Cl}(30 \mathrm{~mL})$, then the mixture was transferred to a separatory funnel and the aqueous layer was extracted with $\mathrm{Et}_{2} \mathrm{O}(3 \times 30 \mathrm{~mL})$. The combined organic layers were dried over $\mathrm{MgSO}_{4}$ and concentrated under reduced pressure. The remaining residue was taken up in $\mathrm{CH}_{2} \mathrm{Cl}_{2}(50 \mathrm{~mL})$ in a 250 $\mathrm{mL} \mathrm{rbf}$, then $\mathrm{SiO}_{2}(5.00 \mathrm{~g})$ was added, and the reaction mixture stirred at $\mathrm{rt}$ for $3 \mathrm{~h}$, with no precautions taken to exclude air or moisture. The mixture was filtered, the filtrate was concentrated under reduced pressure, and the crude material was purified via flash column chromatography on $\mathrm{SiO}_{2}$ (Biotage, $50 \mathrm{~g}$ column, $100 \%$ hexanes) to afford pure product as a colorless oil in $85 \%$ yield ( $925 \mathrm{mg}, 4.27 \mathrm{mmol})$.<smiles>C=C(/C=C/c1ccccc1)C[SiH3]C</smiles>

${ }^{1} \mathrm{H}-\mathrm{NMR}\left(600 \mathrm{MHz}, \mathrm{CDCl}_{3}\right) \delta 7.43-7.32(\mathrm{~m}, 2 \mathrm{H}), 7.32(\mathrm{t}, J=7.7 \mathrm{~Hz}, 2 \mathrm{H}), 7.24-7.21$ $(\mathrm{m}, 1 \mathrm{H}), 6.81(\mathrm{~d}, J=16.1 \mathrm{~Hz}, 1 \mathrm{H}), 6.47(\mathrm{~d}, J=16.2 \mathrm{~Hz}, 1 \mathrm{H}), 5.04(\mathrm{~d}, J=1.9 \mathrm{~Hz}, 1 \mathrm{H})$, $4.87(\mathrm{~d}, J=1.2 \mathrm{~Hz}, 1 \mathrm{H}), 1.85(\mathrm{~s}, 2 \mathrm{H}), 0.05(\mathrm{~s}, 9 \mathrm{H})$. All spectral data agree with that reported in the literature. ${ }^{11}$<smiles>C=C(O[Na])c1ccccc1</smiles>
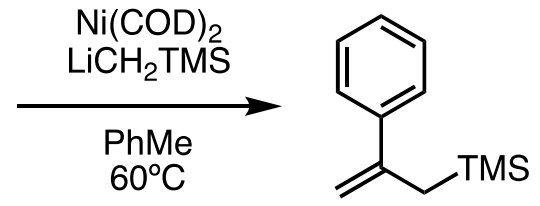


\section{(2-phenylallyl)trimethylsilane [2d]}<smiles>C=C(CSCCCCCC)c1ccccc1</smiles>

Prepared according to a modified literature procedure. ${ }^{12} \mathrm{Ni}(\mathrm{COD})_{2}(138 \mathrm{mg}, 0.500$ mmol, 0.05 equiv.) was weighed into a $250 \mathrm{~mL}$ rbf in a glovebox, then a stir bar was added and the flask was sealed with a septum before removing it from the glovebox. 1phenyl-1-trimethylsiloxyethylene $(2.1 \mathrm{~mL}, 10.0 \mathrm{mmol}, 1.0$ equiv.) and toluene $(60 \mathrm{~mL}$, $0.17 \mathrm{M})$ were added via syringe, followed by $\mathrm{LiCH}_{2} \mathrm{TMS}(13.0 \mathrm{~mL}, 13.0 \mathrm{mmol}, 1.3$ equiv., $1 \mathrm{M}$ in pentane). The reaction mixture was heated to $60^{\circ} \mathrm{C}$ until complete consumption of silyl enol ether as determined by TLC analysis ( $\sim \mathrm{h})$. Upon completion, the reaction mixture was cooled to rt, concentrated under reduced pressure, and filtered through a $\mathrm{SiO}_{2}$ plug with hexanes. No further purification was required. The product was isolated as a colorless oil in $90 \%$ yield $(1.716 \mathrm{~g}, 9.01 \mathrm{mmol})$.

${ }^{1} \mathrm{H}-N M R\left(600 \mathrm{MHz}, \mathrm{CDCl}_{3}\right) \delta 7.41-7.39(\mathrm{~m}, 2 \mathrm{H}), 7.32-7.29(\mathrm{~m}, 2 \mathrm{H}), 7.26-7.24(\mathrm{~m}, 1 \mathrm{H}), 5.13(\mathrm{~d}, J=$ $1.6 \mathrm{~Hz}, 1 \mathrm{H}), 4.87(\mathrm{~m}, 1 \mathrm{H}), 2.03(\mathrm{~m}, 3 \mathrm{H}),-0.10(\mathrm{~s}, 9 \mathrm{H})$. All spectral data agree with that reported in the literature. ${ }^{12}$

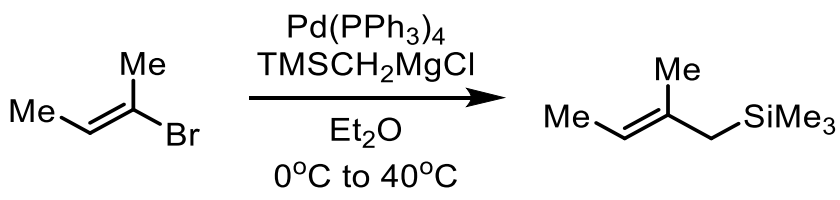

\section{(E)-trimethyl(2-methylbut-2-en-1-yl)silane [2e]}<smiles>CC=C(C)C[SiH3]</smiles>

Prepared according to a modified literature procedure. ${ }^{13} \mathrm{Pd}\left(\mathrm{PPh}_{3}\right)_{4}(867 \mathrm{mg}, 0.750$ $\mathrm{mmol}, 0.05$ equiv.) was taken up in $\mathrm{Et}_{2} \mathrm{O}(15 \mathrm{~mL}, 1.0 \mathrm{M})$ in a $100 \mathrm{~mL}$ multi-neck rbf equipped with condenser, stir bar, and septum, then $(E)$-2-bromo-2-butene $(1.5 \mathrm{~mL}$, $15.0 \mathrm{mmol}, 1.0$ equiv.) was added and the suspension was stirred until homogeneous ( 15 min). The reaction mixture was then cooled to $0^{\circ} \mathrm{C}$ and $\mathrm{TMSCH}_{2} \mathrm{MgCl}\left(15.0 \mathrm{~mL}, 15.0 \mathrm{mmol}, 1.0\right.$ equiv., $1 \mathrm{M}$ in $\left.\mathrm{Et}_{2} \mathrm{O}\right)$ was added dropwise. The reaction mixture was heated to reflux $\left(40^{\circ} \mathrm{C}\right)$ and stirred overnight. After cooling to $\mathrm{rt}$, the reaction was quenched with $\mathrm{H}_{2} \mathrm{O}(10 \mathrm{~mL})$, then the mixture was transferred to a separatory funnel and the aqueous layer was extracted with $\mathrm{Et}_{2} \mathrm{O}(3 \times 20 \mathrm{~mL})$. The combined organic layers were dried over $\mathrm{MgSO}_{4}$ and filtered through Celite. The crude material was concentrated under reduced pressure in an ice bath (product is volatile) and purified via flash column chromatography on $\mathrm{SiO}_{2}$ (Biotage, $25 \mathrm{~g}$ column, $100 \%$ pentane). Product was isolated as a colorless oil in $41 \%$ yield $(881 \mathrm{mg}, 6.19$ $\mathrm{mmol})$.

${ }^{1} \mathrm{H}-\mathrm{NMR}\left(600 \mathrm{MHz}, \mathrm{CDCl}_{3}\right) \delta 5.02-4.98(\mathrm{~m}, 1 \mathrm{H}), 1.59(\mathrm{~d}, J=1.2 \mathrm{~Hz}, 3 \mathrm{H}), 1.56(\mathrm{~d}, J=6.8 \mathrm{~Hz}, 3 \mathrm{H}), 1.46$ $(\mathrm{d}, J=2.4 \mathrm{~Hz}, 2 \mathrm{H}),-0.01$ (s, 9H); ${ }^{13} \mathrm{C}-\mathrm{NMR}\left(125 \mathrm{MHz}, \mathrm{CDCl}_{3}\right) \delta 133.5,116.3,30.0,18.5,13.7,-1.1$. All spectral data agree with that reported in the literature. ${ }^{13}$

\section{Catalyst Preparation}

\section{Preparation of aryl pyrrolidine catalysts $(R, S)-4 \mathrm{~d},(R, S)-4 \mathrm{~h},(R, S)-4 \mathrm{i},(R, S)-4 \mathrm{k}$ and $(S, R)-5 f$}

All catalysts used in the present study have been reported and fully characterized, except where indicated below.

Thioureas: $(R, S)-\mathbf{4 a},{ }^{14}(R, S)-\mathbf{4 b},{ }^{15}(R, S)-\mathbf{4 c},,^{15}(R, S)-\mathbf{4 e},,^{15}(R, S)-\mathbf{4 f},{ }^{15}(R, S)-\mathbf{4 g},{ }^{14}(R, S)-\mathbf{4 j} .{ }^{16}$ Squaramide: $(S, R)-6 f .{ }^{17}$

Note on Optical Rotations of H-Bond Donor Catalysts: These catalysts are effective anion-binding agents, and are sensitive to the presence of small amounts of $\mathrm{HCl}$ in $\mathrm{CHCl}_{3}$. For accurate measurements, 
care should be taken to use a new bottle of $\mathrm{CHCl} 3$ or to neutralize any potential acid generated upon storage of $\mathrm{CHCl}_{3}$, by inclusion of molecular sieves and/or anhydrous $\mathrm{K}_{2} \mathrm{CO}_{3}$.

$(-)-(\boldsymbol{R}, \boldsymbol{S})-4 d$ : Synthesized according to previously published procedures using (-)-sparteine (to set $\alpha-$ stereocenter of pyrrolidine) and Boc-L-tert-leucine (to set stereocenter of tert-butyl group). ${ }^{15}$ The catalyst exists as a mixture of rotamers in $\mathrm{CDCl}_{3}\left(\sim 3: 1\right.$ ratio). ${ }^{1} \mathrm{H}$ NMR peaks reported are those of the major rotamer. ${ }^{13} \mathrm{C}$ NMR peaks reported are those of both rotamers.

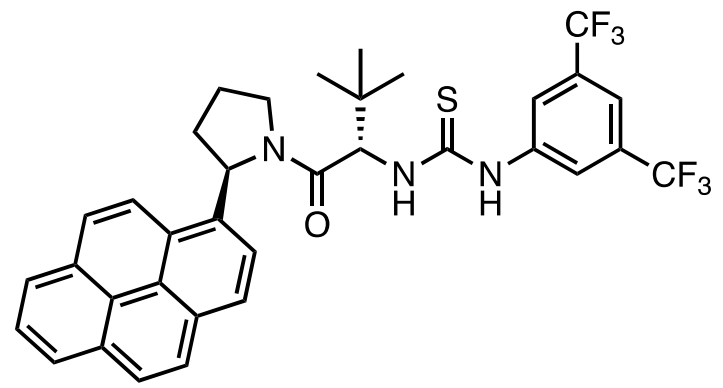

${ }^{1} \mathrm{H}-\mathrm{NMR}\left(500 \mathrm{MHz}, \mathrm{CDCl}_{3}\right) \delta 8.52(\mathrm{~s}, 1 \mathrm{H}), 8.15(\mathrm{dd}, \mathrm{J}=$ 8.5, 3.8 Hz, 3H), $8.09-8.02(\mathrm{~m}, 2 \mathrm{H}), 8.01-7.93(\mathrm{~m}$, $2 \mathrm{H}), 7.85(\mathrm{dd}, J=8.6,4.6 \mathrm{~Hz}, 2 \mathrm{H}), 7.80-7.72(\mathrm{~m}, 2 \mathrm{H})$, $7.51(\mathrm{~s}, 1 \mathrm{H}), 6.18(\mathrm{~d}, J=7.8 \mathrm{~Hz}, 1 \mathrm{H}), 5.57(\mathrm{~d}, J=9.0$ $\mathrm{Hz}, 1 \mathrm{H}), 4.73(\mathrm{~d}, J=6.8 \mathrm{~Hz}, 1 \mathrm{H}), 4.08-3.93(\mathrm{~m}, 1 \mathrm{H})$, $2.66-2.48(\mathrm{~m}, 1 \mathrm{H}), 2.13-1.84(\mathrm{~m}, 4 \mathrm{H}), 1.16(\mathrm{~s}, 9 \mathrm{H})$. ${ }^{13} \mathrm{C}$ NMR $\left(126 \mathrm{MHz}, \mathrm{CDCl}_{3}\right) \delta 181.7,181.2,172.9$, $171.0,140.4,139.7,135.5,134.8,131.7(q, J=32.5$, $31.2 \mathrm{~Hz}), 131.4,131.3,130.9,130.5,130.2,128.3$, $127.6,127.6,127.4,127.23,127.17,127.15,127.09$, $126.8,126.4,126.3,126.1,125.53,125.50,125.4,125.1,125.0,124.9,124.8,124.7,124.5,124.24$, 124.15, 124.08, 124.0, 123.3, 122.8, 122.3, 122.2, 122.1, 123.0 (q, $J=272.9 \mathrm{~Hz}$ ), 118.4, 118.1, 63.5, $62.4,60.2,59.4,49.1,48.2,35.8,35.7,34.9,34.0,27.5,27.3,27.2,23.5,21.4$. Only RHS of the $\mathrm{CF}_{3}$ major quartet could be identified due to overlap (121.9 and 119.7). Overlap is the reason for missing peaks, especially in the aromatic region. IR $\left(\right.$ ATR $\left.\mathrm{cm}^{-1}\right) 3285,3047,2960,1709,1621,1534,1471$, $1385,1328,1277,1178,1134,1108,962,887,846,819,799,762,700,682,652,633,612 .[\alpha]^{22} \mathrm{D}=$ $-2.9^{\circ}\left(c=1.0, \mathrm{CDCl}_{3}\right)$. HRMS (ESI) $m / z$ calculated for $\mathrm{C}_{35} \mathrm{H}_{31} \mathrm{~F}_{6} \mathrm{~N}_{3} \mathrm{OS}[\mathrm{M}+\mathrm{H}]^{+}: 656.2165$, found 656.2155.

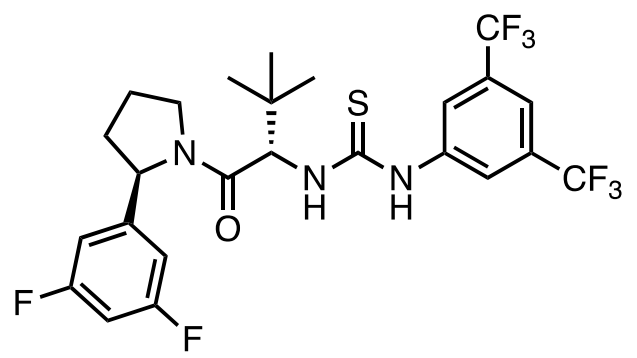

$(-)-(R, S)-4 h:$ Synthesized according to previously published procedures using (-)-sparteine (to set $\alpha$ stereocenter of pyrrolidine) and Boc-L-tert-leucine (to set stereocenter of tert-butyl group). ${ }^{15}$ The catalyst exists as a mixture of rotamers in $\mathrm{CDCl}_{3} \quad(\sim 7: 1)$. Unless otherwise specified the resonance of the major rotamer are reported. We thank Prof. Dave Stuart for the synthesis of this catalyst. H) $1.88-1.78(\mathrm{~m}, 1 \mathrm{H}), 1.12(\mathrm{~s}, 9 \mathrm{H}){ }^{13} \mathrm{C} \mathrm{NMR}(126.68(\mathrm{mH}, 1 \mathrm{H})$ $2 \mathrm{H}), 1.88-1.78(\mathrm{~m}, 1 \mathrm{H}), 1.12(\mathrm{~s}, 9 \mathrm{H}) .{ }^{3} \mathrm{C}$ NMR $\left(126 \mathrm{MHz}, \mathrm{CDCl}_{3}\right) \delta 181.5,170.6,163.0$ (dd, J = 248.8, $13.0 \mathrm{~Hz}$ ), 146.2, 139.4, 139.4, 132.4 (q, $J=33.6 \mathrm{~Hz}), 124.2,123.1$ (q, $J=274.0 \mathrm{~Hz}), 119.1,108.4(\mathrm{~d}, J=$ $26.0 \mathrm{~Hz}), 102.1(\mathrm{t}, J=25.4 \mathrm{~Hz}), 63.4,60.7,48.7,35.5,34.1,26.9,23.4-{ }^{1} \mathrm{H}$ decoupled, not ${ }^{19} \mathrm{~F}$ decoupled. ${ }^{19} \mathrm{~F} \mathrm{NMR}\left(471 \mathrm{MHz}, \mathrm{CDCl}_{3}\right) \delta-62.8(6 \mathrm{~F}),-109.3(2 \mathrm{~F}) ;[\alpha]^{21} \mathrm{D}=-22.7^{\circ}\left(c=1.0, \mathrm{CDCl}_{3}\right)$.

HRMS (ESI) $\mathrm{m} / \mathrm{z}$ calculated for $\mathrm{C}_{25} \mathrm{H}_{25} \mathrm{~F}_{8} \mathrm{~N}_{3} \mathrm{OS}[\mathrm{M}+\mathrm{H}]^{+}: 568.1663$, found 568.1666 .

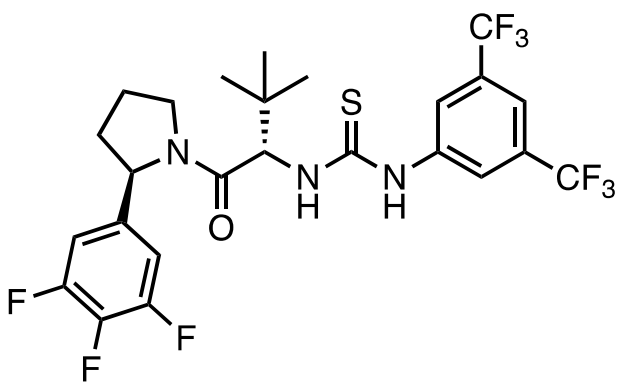

$(+)-(\boldsymbol{R}, \boldsymbol{S})-4 \mathrm{i}: \quad$ Synthesized according to previously published procedures using (-)-sparteine (to set astereocenter of pyrrolidine) and Boc-L-tert-leucine (to set stereocenter of tert-butyl group). ${ }^{15}$ The catalyst exists as a mixture of rotamers in $\mathrm{CDCl}_{3}(\sim 7: 1)$. Unless otherwise specified the resonance of the major rotamer are reported. We thank Prof. Dave Stuart for the synthesis of this catalyst.

${ }^{1} \mathrm{H}-\mathrm{NMR}\left(600 \mathrm{MHz}, \mathrm{CDCl}_{3}\right) \delta 9.63(\mathrm{~s}, 1 \mathrm{H}), 7.85(\mathrm{~s}, 2 \mathrm{H}), 7.64$ (s, 1H), $7.40(\mathrm{~s}, 1 \mathrm{H}), 6.68(\mathrm{t}, J=7.0 \mathrm{~Hz}, 2 \mathrm{H}), 5.30(\mathrm{~d}, J=9.3$ $\mathrm{Hz}, 1 \mathrm{H}$ ), 4.98 (dd, $J=8.0,2.3 \mathrm{~Hz}, 1 \mathrm{H}), 4.56(\mathrm{ddd}, J=10.8,8.0,3.3 \mathrm{~Hz} 1 \mathrm{H}), 3.76(\mathrm{td}, J=9.8,7.1 \mathrm{~Hz} 1 \mathrm{H}$ ), $2.36-2.19(\mathrm{~m}, 1 \mathrm{H}), 2.08-1.86(\mathrm{~m}, 3 \mathrm{H}$, expected $2 \mathrm{H}), 1.86-1.74(\mathrm{~m}, 1 \mathrm{H}), 1.11(\mathrm{~s}, 9 \mathrm{H})$. 
${ }^{13} \mathrm{C}$ NMR $\left(126 \mathrm{MHz}, \mathrm{CDCl}_{3}\right) \delta 181.3,170.6,151.0(\mathrm{dd}, J=250.0,9.8 \mathrm{~Hz}), 139.5,138.5,132.5(\mathrm{q}, J=$ 33.8, 33.3 Hz), 123.9, 123.0 (q, $J=272.7 \mathrm{~Hz}), 119.0,109.6$ (d, $J=18.5 \mathrm{~Hz}), 63.5,60.5,48.7,35.4,34.2$, 26.9, $23.3-{ }^{1} \mathrm{H}$ decoupled, not ${ }^{19} \mathrm{~F}$ decoupled, one carbon resonance could not be located. ${ }^{19} \mathrm{~F}$ NMR (471 $\left.\mathrm{MHz}, \mathrm{CDCl}_{3}\right) \delta-63.3(6 \mathrm{~F}),-133.8(\mathrm{~d}, \mathrm{~J}=18.9 \mathrm{~Hz}, 2 \mathrm{~F}),-162.8(\mathrm{t}, \mathrm{J}=18.9 \mathrm{~Hz}, 1 \mathrm{~F}),[\alpha]^{21} \mathrm{D}=+4.4^{\circ}(c=1.0$, $\left.\mathrm{CDCl}_{3}\right)$. HRMS (ESI) $\mathrm{m} / z$ calculated for $\mathrm{C}_{25} \mathrm{H}_{24} \mathrm{~F}_{9} \mathrm{~N}_{3} \mathrm{OS}[\mathrm{M}+\mathrm{H}]^{+}: 586.1569$, found 586.1558 .<smiles>CC(C)(C)[C@H](NC(=S)Nc1cc(C(F)(F)F)cc(C(F)(F)F)c1)C(=O)N1CCC[C@H]1c1cc(F)c(F)c(F)c1F</smiles>

$(+)-(R, S)-4 k$ : Synthesized according to previously published procedures using (-)-sparteine (to set $\alpha$-stereocenter of pyrrolidine) and Boc-L-tert-leucine (to set stereocenter of tertbutyl group)..$^{15}$ The catalyst exists as a mixture of rotamers in $\mathrm{CDCl}_{3}(\sim 5 / 6: 1)$, measured by ${ }^{19} \mathrm{~F} \mathrm{NMR}$. Unless otherwise specified the resonance of the major rotamer are reported. We thank Prof. Dave Stuart for the synthesis of this catalyst.

${ }^{1} \mathrm{H}-\mathrm{NMR}\left(400 \mathrm{MHz}, \mathrm{CDCl}_{3}\right) \delta 8.81(\mathrm{~s}, 1 \mathrm{H}), 7.81(\mathrm{~s}, 2 \mathrm{H}), 7.69$ $(\mathrm{s}, 1 \mathrm{H}), 7.03(\mathrm{~d}, J=9.1 \mathrm{~Hz}, 1 \mathrm{H}), 6.93-6.81(\mathrm{~m}, 1 \mathrm{H}), 5.28(\mathrm{~d}, J$ $=8.1 \mathrm{~Hz}, 1 \mathrm{H}), 5.24(\mathrm{~d}, J=9.2 \mathrm{~Hz}, 1 \mathrm{H}), 4.64-4.46(\mathrm{~m}, 1 \mathrm{H}), 3.81-3.66(\mathrm{~m}, 1 \mathrm{H}), 2.39-2.22(\mathrm{~m}, 1 \mathrm{H})$, $2.08-1.79(\mathrm{~m}, 4 \mathrm{H}$ - expected $3 \mathrm{H}), 1.09(\mathrm{~s}, 10 \mathrm{H}$ - expected $9 \mathrm{H})$. Over-integration was attributed to impurities. ${ }^{13} \mathrm{C}$ NMR $\left(101 \mathrm{MHz}, \mathrm{CDCl}_{3}\right) \delta 181.3,170.4,148.1(\mathrm{~m}), 145.7,143.0,141.7,140.4,139.2$, 138.0,132.8 (q, $J=33.8 \mathrm{~Hz}$ ), 125.8, 123.9, 123.0 (q, $J=273.2 \mathrm{~Hz}), 122.8,119.3,108.5(\mathrm{~d}, J=20.0 \mathrm{~Hz}$ ), 63.6, 55.4, 48.5, 35.3, 32.8, 26.8, $23.6-{ }^{1} \mathrm{H}$ decoupled, not ${ }^{19} \mathrm{~F}$ decoupled. Due to the extensive ${ }^{13} \mathrm{C}-{ }^{19} \mathrm{~F}$ coupling and the lower intensity signal observed with carbons not bonded to any protons, some resonances couldn't be assigned. ${ }^{19} \mathrm{~F}$ NMR $\left(376 \mathrm{MHz}, \mathrm{CDCl}_{3}\right) \delta-63.1(\mathrm{~s}, 6 \mathrm{~F}),-139.4$ (dt, $J=22.2,11.7$ $\mathrm{Hz}, 1 \mathrm{~F}),-144.1--144.2(\mathrm{~m}, 1 \mathrm{~F}),-155.8(\mathrm{t}, J=20.0 \mathrm{~Hz}, 1 \mathrm{~F}),-158.0(\mathrm{td}, J=20.5,7.8 \mathrm{~Hz}, 1 \mathrm{~F}) .[\alpha]^{22} \mathrm{D}=+3.0^{\circ}$ $\left(c=0.4, \mathrm{CDCl}_{3}\right)$. HRMS (ESI) $\mathrm{m} / z$ calculated for $\mathrm{C}_{25} \mathrm{H}_{23} \mathrm{~F}_{10} \mathrm{~N}_{3} \mathrm{OS}[\mathrm{M}+\mathrm{H}]^{+}: 604.1475$, found 604.1472 .

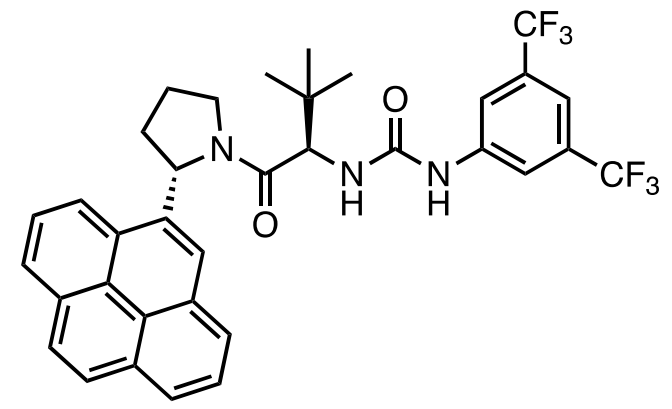

$(-)-(S, R)-5 f: \quad$ Synthesized according to previously published procedures using (+)-sparteine (to set $\alpha$ stereocenter of pyrrolidine)c and Boc-D-tert-leucine (to set stereocenter of tert-butyl group)..$^{15}$ The catalyst exists as a mixture of rotamers in $\mathrm{CDCl}_{3}(\sim 2: 1)$. Due to the significant overlap of the minor and major rotamers in the aromatic region, the resonances reported are those of the mixture of rotamers (noth for ${ }^{1} \mathrm{H}$ and for ${ }^{13} \mathrm{C}$, missing peaks are to be attributed to overlap). We thank Jesus Castro-Esteban for the synthesis of this catalyst.

1H NMR $\left(600 \mathrm{MHz}, \mathrm{CDCl}_{3}\right) \delta 8.40-7.28(\mathrm{~m}, 12 \mathrm{H}), 6.10(\mathrm{~d}, \mathrm{~J}=8.0 \mathrm{~Hz}, 1.4 \mathrm{H}$, major $)$ and $4.94(\mathrm{~d}, \mathrm{~J}=5.6$ $\mathrm{Hz}, 0.6 \mathrm{H}$, minor), $4.67-4.09(\mathrm{~m}, 1 \mathrm{H}), 4.07-3.51(\mathrm{~m}, 1 \mathrm{H}), 3.30-2.39(\mathrm{~m}, 1 \mathrm{H}), 2.30-1.95(\mathrm{~m}, 2 \mathrm{H}), 1.66$ $(\mathrm{s}, 2 \mathrm{H}), 1.38-0.57(\mathrm{~m}, 9 \mathrm{H})$. One proton resonance could not be observed (likely acidic $\mathrm{NH}){ }^{13} \mathrm{C}$ NMR $\left(100 \mathrm{MHz}, \mathrm{CDCl}_{3}\right) \delta 153.1,139.9,139.2,128.2,124.5,123.3,121.0$ (q, $\left.J=26.8 \mathrm{~Hz}\right) ., 120.7,120.6$, $120.3,120.0,119.6,119.2,118.3,117.8,117.6,117.4,117.33,117.28,116.5,116.3,116.1,116.0$, $115.94,115.87,115.7,115.6,115.54,115.50,115.46,115.2,114.8,114.4,113.6,113.0,112.8,111.9$, $110.7,109.8,108.2,107.7,62.8,62.6,62.5,61.4,54.7,53.3,43.6,43.1,42.0,41.2,36.8,34.3,32.6-{ }^{1} \mathrm{H}$ decoupled, not ${ }^{19} \mathrm{~F}$ decoupled. The $\mathrm{CF}_{3}$ quartets could not be located. $[\alpha]^{22} \mathrm{D}=-75.7^{\circ}\left(c=0.47, \mathrm{CDCl}_{3}\right)$. IR (ATR, $\left.\mathrm{cm}^{-1}\right)$ 3449, 3116, 3052, 2961, 1702, ,1648,1612, 1571, 1514, 1474, 1443, 1388, 1340, 1276, 1244, 1222, 1178, 1132, 1065, 1013, 950, 909, 880, 859, 849, 827, 801, 763, 731, 723, 702, 682, 647. HRMS (ESI) $m / z$ calculated for $\mathrm{C}_{35} \mathrm{H}_{32} \mathrm{~F}_{6} \mathrm{~N}_{3} \mathrm{O}_{2}[\mathrm{M}+\mathrm{H}]^{+}: 640.2393$, found 640.2394. 


\section{Preparation of thioamide thiourea catalysts $(S, R)-7 \mathrm{~d}$}

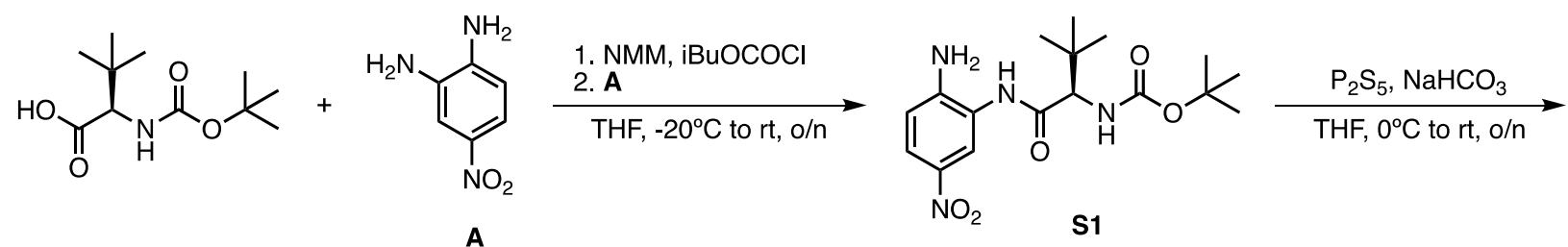

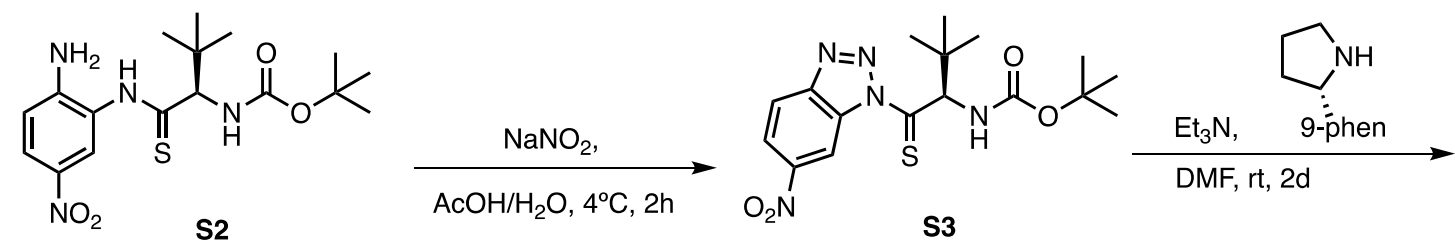<smiles>CC(C)(C)OC(=O)NC(C(=S)N1CCC[C@H]1c1cc2ccccc2c2ccccc12)C(C)(C)C</smiles>

S4<smiles>FC(F)(F)c1cc(N=C=S)cc(C(F)(F)F)c1</smiles>

1. $\mathrm{HCl}(4 \mathrm{M}$ in dioxane) 2. B, $\mathrm{Et}_{3} \mathrm{~N}$

DCM, rt, o/n<smiles>CC(C)(C)[C@H](NC(=S)Nc1cc(C(F)(F)F)cc(C(F)(F)F)c1)C(=S)N1CCC[C@H]1c1cc2ccccc2c2ccccc12</smiles>

\section{tert-Butyl-(R)-(1-((2-amino-5-nitrophenyl)amino)-3,3-dimethyl-1-oxobutan-2-}

yl)carbamate [S1]<smiles>CC(C)(C)OC(=O)NC(C(=O)Nc1cc([N+](=O)[O-])ccc1N)C(C)(C)C</smiles>

Synthesised following a modified literature procedure ${ }^{18}(R)-2-(($ tertbutoxycarbonyl)amino)-3,3-dimethylbutanoic acid (3.0 g, $13 \mathrm{mmol}, 1.0$ equiv) was dissolved in tetrahydrofuran (approx. $130 \mathrm{~mL}$ ) and put under an inert atmosphere. The solution was cooled to around $-20^{\circ} \mathrm{C}$ then $\mathrm{N}$ methylmorpholine $(2.9 \mathrm{~mL}, 25.9 \mathrm{mmol}, 2.0$ equiv) was added in one portion. Isobutyl chloroformate $(2.0 \mathrm{~mL}, 13 \mathrm{mmol}, 1.0$ equiv) was added over $10 \mathrm{~min}$ with a syringe pump $(200 \mu \mathrm{L} / \mathrm{min})$ and the mixture was left to stir for approx. 20 minutes. 4-Nitrobenzene-1,2-diamine $(2.0 \mathrm{~g}, 13 \mathrm{mmol}$, 1.0 equiv) was added in one portion, and the mixture was left to stir in the bath, slowly warming up to room temperature overnight. The reaction mixture was then diluted with EtOAc (ca. $50 \mathrm{~mL}$ ) and washed with a saturated $\mathrm{NaH}_{2} \mathrm{PO}_{4}$ (ca. $50 \mathrm{~mL}$ ). The aqueous layer was extracted twice with EtOAc (ca. $50 \mathrm{~mL}$ ), then the combined organic layers were washed with brine (ca. $50 \mathrm{~mL}$ ), saturated sodium bicarbonate (ca. $50 \mathrm{~mL}$ ), and brine (ca. $50 \mathrm{~mL}$ ), then dried over $\mathrm{Na}_{2} \mathrm{SO}_{4}$. Purification via column chromatography afforded the product as a dark yellow solid.

${ }^{1} \mathrm{H}-\mathrm{NMR}\left(500 \mathrm{MHz}, \mathrm{CDCl}_{3}\right) \delta 8.07(\mathrm{~s}, 1 \mathrm{H}), 7.99(\mathrm{~d}, J=2.5 \mathrm{~Hz}, 1 \mathrm{H}), 7.96(\mathrm{dd}, J=8.9,2.6 \mathrm{~Hz}, 1 \mathrm{H}), 6.66(\mathrm{~d}$, $J=8.9 \mathrm{~Hz}, 1 \mathrm{H}), 5.32(\mathrm{~d}, J=7.6 \mathrm{~Hz}, 1 \mathrm{H}), 4.86(\mathrm{~s}, 2 \mathrm{H}), 3.98(\mathrm{~d}, J=7.6 \mathrm{~Hz}, 1 \mathrm{H}), 1.42(\mathrm{~s}, 9 \mathrm{H}), 1.12(\mathrm{~s}, 9 \mathrm{H})$. N.B. ${ }^{1} \mathrm{H}$ NMR shifts were found to be highly dependent on concentration. ${ }^{13} \mathrm{C}-\mathrm{NMR}\left(126 \mathrm{MHz}, \mathrm{CDCl}_{3}\right) \delta$ 170.8 (Amide), 157.0 (Carbamate), 149.1, 138.4, 124.6, 124.0, 121.0, 115.2, 81.1, 63.7, 33.8, 28.5, 26.9. IR (ATR, $\left.\mathrm{cm}^{-1}\right)$ 3442, 3356, 3257, 2970, 1661, 1510, 1311, 1244, 1164, 1093, 1065, 908, 736;

HRMS (ESI) $m / z$ calculated for $\mathrm{C}_{12} \mathrm{H}_{20} \mathrm{NO}_{4}[\mathrm{M}+\mathrm{H}]^{+}:$: 367.1976, found 367.1973 . 


\section{tert-Butyl-( $R)$-(1-((2-amino-5-nitrophenyl)amino)-3,3-dimethyl-1-thioxobutan-2- yl)carbamate [S2]}<smiles>CC(C)(C)OC(=O)N[C@H](C(=S)Nc1cc([N+](=O)[O-])ccc1N)C(C)(C)C</smiles>

Synthesised following a modified literature procedure, ${ }^{18}$ a $100 \mathrm{~mL}$ roundbottomed flask was charged with $\mathrm{P}_{2} \mathrm{~S}_{5}$ (650 mg, $2.92 \mathrm{mmol}, 1.1$ equiv) and sodium bicarbonate (144 $\mathrm{mg}, 1.36 \mathrm{mmol}, 0.5$ equiv) and the solids were dissolved in tetrahydrofuran (ca. $50 \mathrm{~mL}$ ). The mixture was left to stir at room temperature under a $\mathrm{CO}_{2}$ atmosphere for ca. $1 \mathrm{~h}$ then it was cooled to $0^{\circ} \mathrm{C}$ and a solution of tert-butyl- $(R)-(1-((2-$ amino-5nitrophenyl)amino)-3,3-dimethyl-1-oxobutan-2-yl)carbamate S1 (950 mg, $2.59 \mathrm{mmol}, 1.0$ equiv) in THF (ca. $5 \mathrm{~mL}$ ) was added dropwise. The mixture was left to stir at $0^{\circ} \mathrm{C}$ and then allowed to warm to room temperature overnight. The solvent was removed under reduced pressure, then the residue was dissolved in 2:1 EtOAc:Heptane and filtered through a celite plug. The organic layer was washed with saturated bicarbonate (ca. $50 \mathrm{~mL}$ ) and the aqueous layer was extracted with 2:1 EtOAc:Heptane. The combined organic layers were washed with saturated bicarbonate (ca. $50 \mathrm{~mL}$ ), brine (ca. $50 \mathrm{~mL}$ ) and dried over $\mathrm{Na}_{2} \mathrm{SO}_{4}$. Purification via column chromatography afforded the product as a yellow solid (452 mg, $1.18 \mathrm{mmol}, 46 \%$ yield).

${ }^{1} \mathrm{H}-\mathrm{NMR}\left(500 \mathrm{MHz}, \mathrm{CDCl}_{3}\right) \delta 9.84(\mathrm{~s}, 1 \mathrm{H}), 8.06(\mathrm{dd}, J=9.0,2.6 \mathrm{~Hz}, 1 \mathrm{H}), 8.01(\mathrm{~d}, J=2.5 \mathrm{~Hz}, 1 \mathrm{H}), 6.74(\mathrm{~d}$, $J=9.0 \mathrm{~Hz}, 1 \mathrm{H}), 5.59(\mathrm{~d}, J=8.9 \mathrm{~Hz}, 1 \mathrm{H}), 4.81(\mathrm{~s}, 2 \mathrm{H}), 4.44(\mathrm{~d}, J=8.9 \mathrm{~Hz}, 1 \mathrm{H}), 1.34(\mathrm{~s}, 9 \mathrm{H}), 1.17(\mathrm{~s}, 9 \mathrm{H})$. ${ }^{13} \mathrm{C}$ NMR (126 MHz, CDCl 3 ) $\delta 204.9$ (Thioamide), 157.5 (Carbamate), 149.0, 138.5, 125.8, 125.0, 122.8, 115.4, 81.4, 69.1, 34.7, 28.4, 27.2; IR (ATR, $\left.\mathrm{cm}^{-1}\right)$ 3273, 2959, 2925, 2856, 1519, 1469, 1447, 1380, 1275, 1176, 1134, 958, 906, 854, 729, 679; HRMS (ESI) $\mathrm{m} / z$ calculated for $\mathrm{C}_{17} \mathrm{H}_{26} \mathrm{~N}_{4} \mathrm{O}_{4} \mathrm{~S}[\mathrm{M}+\mathrm{H}]^{+}$: 383.1748, found 383.1747.

\section{tert-butyl-(R)-(3,3-dimethyl-1-(6-nitro-1H-benzo[d][1,2,3]triazol-1-yl)-1-thioxobutan- 2-yl)carbamate [S3]}<smiles>CC(C)(C)OC(=O)N[C@H](C(=S)n1nnc2ccc([N+](=O)[O-])cc21)C(C)(C)C</smiles>

Synthesised following a modified literature procedure, ${ }^{18}$ a $20 \mathrm{~mL}$ vial was charged with tert-Butyl-( $R)-(1-((2-a m i n o-5-n i t r o p h e n y l) a m i n o)-3,3-$ dimethyl-1-thioxobutan-2-yl)carbamate $\mathbf{S 2}(420 \mathrm{mg}, 1.10 \mathrm{mmol}, 1.0$ equiv) and the solid was dissolved in $\mathrm{AcOH}: \mathrm{H}_{2} \mathrm{O}(95: 5,11.48 \mathrm{~mL})$. The solution was cooled in a water/ice bath being careful to avoid solvent freezing. $\mathrm{NaNO}_{2}$ (135 mg, $1.96 \mathrm{mmol}, 1.8$ equiv) was added in one portion and the mixture was left to stir at $4^{\circ} \mathrm{C}$ for ca. $2 \mathrm{~h}$. The reaction was then diluted with EtOAc:Hexanes $(3: 1$, ca. $30 \mathrm{~mL})$ and washed with water (ca. $30 \mathrm{~mL}$ ). The aqueous layer was extracted with EtOAc:Hexanes (3:1, ca. $30 \mathrm{~mL}$ ) then the combined organic layers were washed with saturated sodium bicarbonate (ca. $50 \mathrm{~mL}, 2 \mathrm{x}$ ), brine (ca. 50 $\mathrm{mL}$ ) and dried over $\mathrm{Na}_{2} \mathrm{SO}_{4}$. Purification via column chromatography on Davisil afforded the product as an orange solid with a minor impurity $(327.7 \mathrm{mg}, 0.833 \mathrm{mmol}, 76 \%$ yield).

${ }^{1} \mathrm{H}-\mathrm{NMR}\left(500 \mathrm{MHz}, \mathrm{CDCl}_{3}\right) \delta$ 9.73-9.69 (m, 1H), 8.54-8.40 (m, 1H), $8.32(\mathrm{dd}, J=9.2,3.5 \mathrm{~Hz}, 1 \mathrm{H}), 6.51$ $(\mathrm{d}, J=9.8 \mathrm{~Hz}, 1 \mathrm{H}), 5.59(\mathrm{~d}, J=9.9 \mathrm{~Hz}, 1 \mathrm{H}), 1.44$ (app. d, $J=3.3 \mathrm{~Hz}, 9 \mathrm{H}), 0.99$ (app. d, $J=3.9 \mathrm{~Hz}, 9 \mathrm{H}$ ). ${ }^{13} \mathrm{C}$ NMR (125 MHz, CDCl 3 ) $\delta 210.3$ (Thioamide), 155.4 (Carbamate), 149.39, 149.36, 131.4, 122.2, 121.6, 112.8, 80.3, 66.2, 37.0, 28.4, 26.6. IR (ATR, $\left.\mathrm{cm}^{-1}\right)$ 3361, 3231, 2967, 1677, 1628, 1494, 1394, 1367, 1311, 1260, 1154, 1091, 1063, 907, 826, 729; HRMS (ESI) $\mathrm{m} / z$ calculated for $\mathrm{C}_{17} \mathrm{H}_{27} \mathrm{~N}_{6} \mathrm{O}_{4} \mathrm{~S}$ $\left[\mathrm{M}+\mathrm{NH}_{4}\right]^{+}:$:11.1809, found 411.1817. 


\section{tert-Butyl-((R)-3,3-dimethyl-1-((S)-2-(phenanthren-9-yl)pyrrolidin-1-yl)-1- thioxobutan-2-yl)carbamate [S4]}

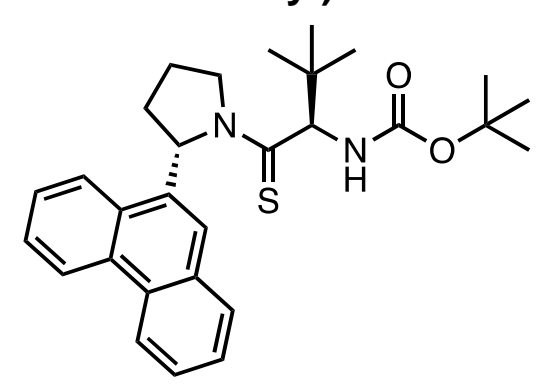

Synthesised following a modified literature procedure. ${ }^{19}$

tert-Butyl-( $R$ )-(3,3-dimethyl-1-(6-nitro-1H-benzo[d][1,2,3]triazol-1-yl)1-thioxobutan-2-yl)carbamate derivative $\mathbf{S} 3(315 \mathrm{mg}, 0.86 \mathrm{mmol}, 1.0$ equiv) was dissolved in DMF (ca. $4.0 \mathrm{~mL}$ ). (S)-2-(phenanthren-9yl)pyrrolidine (213 mg, $0.86 \mathrm{mmol}, 1.0$ equiv) was solubilized in DMF $(4.4 \mathrm{~mL})$ then $4 \mathrm{~mL}$ of the solution were added to S3, followed by triethylamine (170 $\mu \mathrm{L}, 1.3 \mathrm{mmol}, 1.5$ equiv). The reaction was stirred at room temperature and monitored by TLC. The solution was diluted with diethyl ether (ca. $30 \mathrm{~mL}$ ) and the organic layer was washed with brine (ca. $50 \mathrm{~mL}, 5 \mathrm{x}$ ), dried over anhydrous $\mathrm{MgSO}_{4}$ and the solvent evaporated to afford the corresponding crude compound. Purification via column chromatography afforded the product as a white solid (128.9 $\mathrm{mg}, 0.270 \mathrm{mmol}, 30 \%$ yield).

${ }^{1} \mathrm{H}-\mathrm{NMR}\left(500 \mathrm{MHz}, \mathrm{CDCl}_{3}\right)$ ठ 8.79-8.72 $(\mathrm{m}, 1 \mathrm{H}), 8.65(\mathrm{~d}, \mathrm{~J}=8.2 \mathrm{~Hz}, 1 \mathrm{H}), 8.10-8.03(\mathrm{~m}, 1 \mathrm{H}), 7.89-7.81$ $(\mathrm{m}, 1 \mathrm{H}), 7.66$ (ddt, J = 5.9, 3.5, $1.9 \mathrm{~Hz}, 2 \mathrm{H}), 7.61$ (ddt, $\mathrm{J}=8.5,7.0,1.5 \mathrm{~Hz}, 1 \mathrm{H}), 7.55$ (ddt, J = 8.4, 7.0, 1.4 $\mathrm{Hz}, 1 \mathrm{H}), 7.29(\mathrm{~s}, 1 \mathrm{H}), 6.63(\mathrm{~d}, \mathrm{~J}=7.9 \mathrm{~Hz}, 1 \mathrm{H}), 5.54(\mathrm{~d}, \mathrm{~J}=10.3 \mathrm{~Hz}, 1 \mathrm{H}), 4.88(\mathrm{dd}, \mathrm{J}=10.3,1.5 \mathrm{~Hz}, 1 \mathrm{H})$, $4.64(\mathrm{t}, J=9.0 \mathrm{~Hz}, 1 \mathrm{H}), 4.18-3.99(\mathrm{~m}, 1 \mathrm{H}), 2.52-2.34(\mathrm{~m}, 1 \mathrm{H}), 2.22-1.99(\mathrm{~m}, 3 \mathrm{H}), 1.54(\mathrm{~s}, 9 \mathrm{H}), 1.21(\mathrm{~s}$, 9H). ${ }^{13} \mathrm{C}$ NMR (125 MHz, CDCl 3 ) $\delta 201.6$ (Thioamide), 156.6 (Carbamate), 132.4, 131.3, 130.0, 129.5, 129.0, 126.7, 126.5, 126.4, 126.3, 123.9, 123.6, 122.7, 122.4, 79.8, 65.2, 62.9, 52.5, 35.7, 31.8, 28.6, 27.2, 23.6. One carbon corresponding to a 9-Phenanthryl signal could not be located. Major rotamer reported. IR (ATR, $\mathrm{cm}^{-1}$ ) 2960, 2925, 2870, 2855, 1707, 1491, 1437, 1392, 1305, 1245, 1166, 748, 726; HRMS (ESI) $\mathrm{m} / \mathrm{z}$ calculated for $\mathrm{C}_{29} \mathrm{H}_{36} \mathrm{~N}_{2} \mathrm{O}_{2} \mathrm{~S}[\mathrm{M}+\mathrm{H}]^{+}:$477.2570, found 477.2570.

\section{1-(3,5-Bis(trifluoromethyl)phenyl)-3-(3,3-dimethyl-1-(2-(phenanthren-9- yl)pyrrolidin-1-yl)-1-thioxobutan-2-yl)thiourea $[(-)-(S, R)-7 \mathrm{~d}]$}

Synthesised according to a reported procedure. ${ }^{16}$ tert-Butyl-((R)-3,3-dimethyl-1-((S)-2-(phenanthren-9yl)pyrrolidin-1-yl)-1-thioxobutan-2-yl)carbamate $\mathbf{S 4}$ (34.4 $\mathrm{mg}, 0.072 \mathrm{mmol}, 1.0$ equiv) was dissolved in $\mathrm{HCl}$ $(0.130 \mathrm{~mL}, 0.52 \mathrm{mmol}, 10$ equiv, $4 \mathrm{M}$ in dioxane). The reaction was stirred at room temperature until deprotection was complete (determined by TLC analysis). The solvent was removed and the deprotected $\mathrm{HCl}$ salt was dissolved in DCM $(0.720 \mathrm{~mL})$. To this solution, 3,5-Bis(trifluoromethyl)phenyl isothiocyanate $(0.013 \mathrm{~mL}, 0.052 \mathrm{mmol}, 1.0$ equiv) was added, followed by triethylamine ( $40 \mu \mathrm{L}, 0.29 \mathrm{mmol}, 4.0$ equiv). The reaction was stirred overnight at room temperature. The solvent was removed under reduced pressure and purification via column chromatography afforded the product as a white solid (24.5 mg, $0.038 \mathrm{mmol}, 53 \%$ yield).<smiles>CC(C)(C)[C@H](NC(=S)Nc1cc(C(F)(F)F)cc(C(F)(F)F)c1)C(=S)N1CCC[C@H]1c1cc2ccccc2c2ccccc12</smiles>

${ }^{1} \mathrm{H}-\mathrm{NMR}\left(500 \mathrm{MHz}, \mathrm{CDCl}_{3}\right) \delta 8.94(\mathrm{~s}, 1 \mathrm{H}), 8.71$ (dd, $J=7.9$, $1.9 \mathrm{~Hz}, 1 \mathrm{H}), 8.58(\mathrm{~d}, J=8.3 \mathrm{~Hz}, 1 \mathrm{H}), 8.00(\mathrm{dd}, J=7.7,1.9$ $\mathrm{Hz}, 1 \mathrm{H}), 7.88(\mathrm{dd}, J=8.0,1.3 \mathrm{~Hz}, 3 \mathrm{H}), 7.86(\mathrm{~s}, 2 \mathrm{H}), 7.67-$ $7.62(\mathrm{~m}, 2 \mathrm{H}), 7.58-7.53(\mathrm{~m}, 1 \mathrm{H}), 7.51(\mathrm{~s}, 1 \mathrm{H}), 7.49-7.43(\mathrm{~m}$, $1 \mathrm{H}), 7.35(\mathrm{~d}, J=9.5 \mathrm{~Hz}, 1 \mathrm{H}), 6.59(\mathrm{~d}, J=7.9 \mathrm{~Hz}, 1 \mathrm{H}), 5.84$ $(\mathrm{d}, J=9.6 \mathrm{~Hz}, 1 \mathrm{H}), 5.20-5.06(\mathrm{~m}, 1 \mathrm{H}), 4.20-4.05(\mathrm{~m}, 1 \mathrm{H})$, $2.62-2.39(\mathrm{~m}, 1 \mathrm{H}), 2.25-2.08(\mathrm{~m}, 3 \mathrm{H}), 1.23(\mathrm{~s}, 9 \mathrm{H})$.

${ }^{13} \mathrm{C}$ NMR (126 MHz, $\left.\mathrm{CDCl}_{3}\right) \delta 199.4$ (Thioamide), 180.9 (Thiourea), 138.6, 133.0 (q, $J=35.4,34.4 \mathrm{~Hz}$ ), 131.7, 131.5, $131.3,130.0,129.6,129.1,126.9,126.8,126.6,126.3$,

124.0, 123.8, 123.7, 123.4, 122.4, 119.6, 67.4, 65.8, 53.2, 36.4, 31.9, 27.5, 23.7. Major rotamer reported. The $\mathrm{CF}_{3}$ quartet couldn't be located, likely under the 9-phenantryl resonances. $[\alpha]^{24} \mathrm{D}=-38.4^{\circ}(c=1.0$, $\mathrm{CDCl}_{3}$ ); IR (ATR, $\mathrm{cm}^{-1}$ ) 3270, 2965, 1520, 1472, 1452, 1382, 1277, 1179, 1136; HRMS (ESI) $\mathrm{m} / \mathrm{z}$ calculated for $\mathrm{C}_{33} \mathrm{H}_{31} \mathrm{~F}_{6} \mathrm{~N}_{3} \mathrm{~S}_{2}[\mathrm{M}+\mathrm{H}]^{+}:$648.1936, found 648.1929. 


\section{Preparation of $\mathrm{N}$-methyl thiourea fragment $(R, S)-7 \mathrm{a}$ and catalyst $(S, R)-7 \mathrm{~b}$}

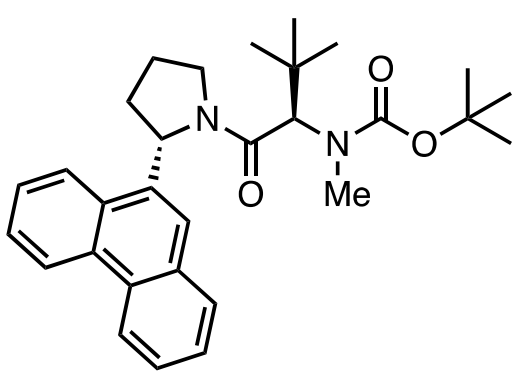

$(-)-(R, S)-7 a:$ The 9-phenanthryl-aryl-pyrrolidine-boc-D-tert-leucine starting material was synthesized according to previously published procedures using $(+)$-sparteine (to set $\alpha$-stereocenter of pyrrolidine) and Boc-D-tert-leucine (to set stereocenter of tert-butyl group). ${ }^{15}$ The starting material (116.7 $\mathrm{mg}, 0.253 \mathrm{mmol}, 1.0$ equiv.) was added to an oven dried 2-dram vial, followed by DMF (anhydrous, $1.3 \mathrm{~mL}$ ). The vial was put under an inert atmosphere, cooled to $0^{\circ} \mathrm{C}$ and $\mathrm{NaH}(60 \%$ $\mathrm{w} / \mathrm{t}, 105.0 \mathrm{mg}, 2.63 \mathrm{mmol}, 10$ equiv.) was added in one portion. The mixture was left to stir at $0^{\circ} \mathrm{C}$ for $1.5 \mathrm{~h}$, then Mel was added in one portion $(80.0 \mu \mathrm{L}, 1.29 \mathrm{mmol}, 5.0$ equiv). The reaction was allowed to warm to room temperature and left to stir overnight. The residual Mel was quenched with $1 \mathrm{~mL} 5 \%$ $\mathrm{NH}_{4} \mathrm{OH}$, then the solution was diluted with diethyl ether and the organic layer was washed with brine $(5 x)$, dried over anhydrous $\mathrm{Na}_{2} \mathrm{SO}_{4}$ and the solvent evaporated to afford the corresponding crude compound. Purification via column chromatography afforded the product as an off white solid. NB. Well defined rotamers are present at room temperature in $\mathrm{CDCl}_{3}$ in a roughly 1:1:2:2 mixture - $1: 1.4: 1.7: 1.8$ based on $\mathrm{N}$-Me signals in ${ }^{1} \mathrm{H}$ NMR). The peaks reported are those of all rotameric species, where possible more detailed assignment is reported.

${ }^{1} \mathrm{H}-\mathrm{NMR}\left(600 \mathrm{MHz}, \mathrm{CDCl}_{3}\right) \delta 8.97-8.47(\mathrm{~m}, 2 \mathrm{H}), 8.14-7.94(\mathrm{~m}, 1 \mathrm{H}), 7.82-7.51(\mathrm{~m}, 5 \mathrm{H}), 7.43-7.18$ (s, $1 \mathrm{H},(7.40,7.37,7.21,7.19 ; \sim 1: 1: 1: 1)), 6.16-5.48(\mathrm{~d}, 1 \mathrm{H},(6.05, \mathrm{~J}=8.5 \mathrm{~Hz} ; 5.83, \mathrm{~J}=8.5 \mathrm{~Hz} ; 5.60 \mathrm{~J}=$ $7.9 \mathrm{~Hz} ; 2: 1: 1$ ), $5.10-4.14(\mathrm{~s}, 1 \mathrm{H}(5.06,4.75,4.49,4.20 ;)), 4.13-3.65(\mathrm{~m}, 2 \mathrm{H}), 3.04-2.95(\mathrm{~s}, 3 \mathrm{H}$, $(3.02,2.99,2.98,2.96)), 2.54-2.07(\mathrm{~m}, 2 \mathrm{H}), 2.07-1.70(\mathrm{~m}, 2 \mathrm{H}), 1.70-0.78(\mathrm{~s}, 18 \mathrm{H}(1.64,1.61,1.51$, $1.11,0.95,0.91,0.88)) .{ }^{13} \mathrm{C}$ NMR $\left(126 \mathrm{MHz}, \mathrm{CDCl}_{3}\right) \delta 170.2,169.5,169.0,168.5,157.2,156.6,156.11$, 156.05, 136.4, 136.3, 135.8, 135.6, 131.4, 131.3, 131.27, 131.2, 131.00,130.95, 130.4, 130.20, 130.18, $130.1129 .8,129.7,129.5,129.4,128.8,128.7,128.6,128.2,127.3,127.01,126.97,126.9,126.8$, $126.7,126.63,126.57,126.5,126.43,126.37,125.6,125.0,124.7,124.2,124.1,124.0,123.7,123.59$, $123.49,123.45,123.4,122.70,122.65,122.59,122.5,122.0,121.8,80.7,80.6,80.1,79.8,63.2,63.1$, $61.8,61.5,58.3,58.1,58.0,57.7,48.1,47.6,47.2,46.9,37.1,36.6,36.5,36.4,34.5,34.0,33.2,33.0$, 32.9, 31.94, 30.5, 29.8, 28.72, 28.70, 28.5, 27.9, 27.79, 27.76, 27.7, 27.6, 24.00, 23.96, 21.5, 21.4. Due to the highly rotameric nature of $7 \mathbf{a}$ and to peak overlap, not all carbon signals could be located.

IR (ATR, $\left.\mathrm{cm}^{-1}\right)$ 2959, 2931, 2872, 2246, 1685, 1642, 1481, 1432, 1393, 1336, 1303, 1172, 1139, 907, $764,724,646 .[\alpha]^{21} \mathrm{D}=-21.2^{\circ}\left(c=1.0, \mathrm{CDCl}_{3}\right)$. HRMS (ESI) $\mathrm{m} / z$ calculated for $\mathrm{C}_{30} \mathrm{H}_{38} \mathrm{~N}_{2} \mathrm{O}_{3}[\mathrm{M}+\mathrm{H}]^{+}$: 475.2955, found 475.2958 .

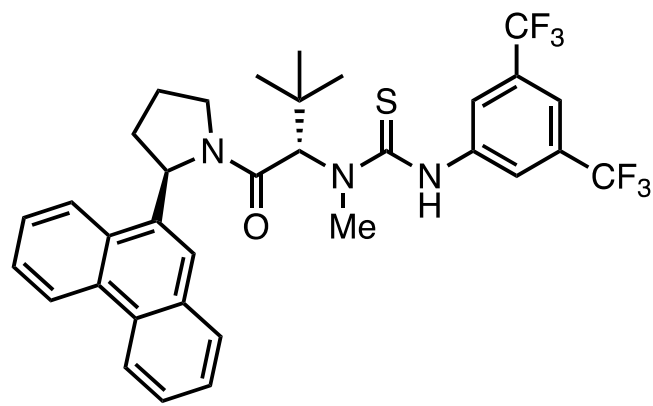

$(+)-(S, R)-7 b$ : Synthesized according to previously published procedures using methylated fragment (+)$(\boldsymbol{S}, \boldsymbol{R})$-ent-7a as precursor. NB. Broad rotameric peaks are observed at room temperature in $\mathrm{CDCl}_{3}$, in DMSO 2:1 rotamers can be observed.

${ }^{1} \mathrm{H}-\mathrm{NMR}\left(600 \mathrm{MHz}, \mathrm{DMSO}-\mathrm{d}_{6}\right) \delta 9.71(\mathrm{~s}, 1 \mathrm{H}), 8.93-8.83$ $(\mathrm{m}, 1 \mathrm{H}), 8.76(\mathrm{~d}, J=8.4 \mathrm{~Hz}, 1 \mathrm{H}), 8.25(\mathrm{~s}, 2 \mathrm{H}), 8.21-8.16$ $(\mathrm{m}, 1 \mathrm{H}), 7.88(\mathrm{~s}, 1 \mathrm{H}), 7.70(\mathrm{~m}, 2 \mathrm{H}), 7.65-7.58(\mathrm{~m}, 2 \mathrm{H})$, $7.50(\mathrm{t}, J=7.5 \mathrm{~Hz}, 1 \mathrm{H}), 7.35(\mathrm{~s}, 1 \mathrm{H}), 6.36(\mathrm{~s}, 1 \mathrm{H}), 5.91(\mathrm{~d}$, $J=8.8 \mathrm{~Hz}, 1 \mathrm{H}, 4.58(\mathrm{~s}, 1 \mathrm{H}), 3.99-3.79(\mathrm{~m}, 1 \mathrm{H}), 3.40(\mathrm{~s}$, $3 \mathrm{H}), 2.42(\mathrm{~m}, 1 \mathrm{H}), 2.01-1.90(\mathrm{~m}, 1 \mathrm{H}), 1.85(\mathrm{~m}, 1 \mathrm{H}), 1.76$

$(\mathrm{m}, 1 \mathrm{H}), 1.13(\mathrm{~s}, 9 \mathrm{H})$. Major rotamer reported. (Distinctive minor signals: $\delta 9.55(\mathrm{~s}, 1 \mathrm{H}), 6.54(\mathrm{~s}, 1 \mathrm{H}), 5.85$ (s, 1H), $3.43(\mathrm{~s}, 3 \mathrm{H}), 0.86(\mathrm{~s}, 9 \mathrm{H})) .{ }^{13} \mathrm{C}$ NMR (126 MHz, DMSO-d 6 ) $\delta 183.6,167.9,143.6,143.5,137.0$, $131.3,130.7,130.4,130.1,129.8,129.7,128.4,127.4,127.1,127.0,124.5,124.0,123.8$ (q, $J=272.5$ $\mathrm{Hz}), 123.3,121.8,118.0,65.7,58.0,48.8,38.2,37.6,32.1,28.3,23.9$. Major rotamer reported. One peak of the $\mathrm{CF}_{3}$ quartet couldn't be located due to overlap with larger peaks. IR (ATR, $\left.\mathrm{cm}^{-1}\right) 3295,2962,2928$, $2874,1620,1531,1471,1382,1310,1276,1175,1132,908,748,726,699,682 .[\alpha]^{22} \mathrm{D}=+60.7^{\circ}(c=1.0$, $\left.\mathrm{CDCl}_{3}\right)$.

HRMS (ESI) $m / z$ calculated for $\mathrm{C}_{34} \mathrm{H}_{33} \mathrm{~F}_{6} \mathrm{~N}_{3} \mathrm{OS}[\mathrm{M}+\mathrm{H}]^{+}:$646.2321, found 646.2316. 


\section{Catalyst Structure-Selectivity Studies}

Catalyst Screening Procedure: An oven-dried 3-dram vial was charged with catalyst $(0.03 \mathrm{mmol}, 0.10$ equiv), and stir bar. The vial was sealed with a septum-lined cap, evacuated, and refilled with $\mathrm{N}_{2}(3 \mathrm{x})$. Benzaldehyde dimethyl acetal $(45.0 \mu \mathrm{L}, 0.300 \mathrm{mmol}, 1.0$ equiv), 2-methylallyl)trimethylsilane $(53.0 \mu \mathrm{L}$, $0.300 \mathrm{mmol}, 1.0$ equiv), tert-butyl carbamate (stock solution of $0.3 \mathrm{M}^{2} \mathrm{Et}_{2} \mathrm{O}, 1.00 \mathrm{~mL}, 0.300 \mathrm{mmol}, 1.0$ equiv), and $\mathrm{Et}_{2} \mathrm{O}(2.0 \mathrm{~mL}$, total of $0.1 \mathrm{M})$ were then added via syringe. The vial was carefully sealed with electrical tape, covering the septum. The reaction mixture was cooled to $-78^{\circ} \mathrm{C}$ in a dry ice/acetone bath, then TESOTf ( $6.8 \mu \mathrm{L}, 0.030 \mathrm{mmol}, 0.10$ equiv) was added. Vial was transferred to $\mathrm{a}-50^{\circ} \mathrm{C}$ cryocool and stirred for $18 \mathrm{~h}$. The reaction was quenched with addition of $1: 1 \mathrm{Et} 3 \mathrm{~N} / \mathrm{MeOH}(200 \mu \mathrm{L})$, stirred for $5 \mathrm{~min}$, then warmed to rt. The solvent was removed under reduced pressure, then $100 \mu \mathrm{L}$ of a stock solution of internal standard $\left(70 \mu \mathrm{L}\right.$ of mesitylene in $1 \mathrm{~mL}$ of $\left.\mathrm{CDCl}_{3}\right)$ was added. Conversions were calculated with respect to the mesitylene aromatic peak at $6.80 \mathrm{ppm},(\mathrm{s}, 3 \mathrm{H})$ integrating the starting material peaks at $5.39(\mathrm{~s}, 1 \mathrm{H})$ and $3.33(\mathrm{~s}, 6 \mathrm{H})$ and averaging the resulting conversions. The product was purified via column chromatography then dissolved in $30 \%$ PrOH/hexanes for HPLC analysis. Enantiomeric excess was determined by chiral HPLC.

Table S1. Catalyst structure-selectivity studies: variation of arylpyrrolidine.
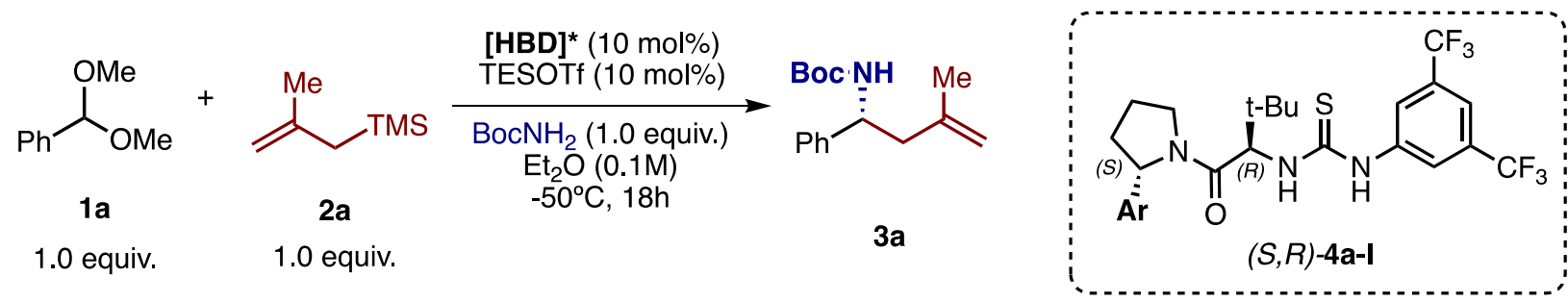<smiles>CC(C)(C)c1ccccc1</smiles>

$(R, S)-e n t-4 a$ $-60 \%$ e.e.<smiles>CC(C)(C)c1ccc(F)cc1</smiles><smiles>CC(C)(C)c1cc(F)cc(F)c1</smiles>
(R,S)-ent-4h:
$-68 \%$ ee<smiles>CC(C)(C)c1cccc2ccccc12</smiles><smiles>CC(C)(C)c1ccc2ccc3cccc4ccc1c2c34</smiles>

\section{$(R, S)$-ent-4d} $-89 \%$ e.e.<smiles>CC(C)c1cc2ccccc2c2ccccc12</smiles>

(R,S)-ent-4e $-91 \%$ e.e.<smiles>CC(C)(C)c1cc(F)c(F)c(F)c1F</smiles>

$(R, S)$-ent-4k: $-92 \%$ ee<smiles>CC(C)(C)c1cc2cccc3ccc4cccc1c4c32</smiles>

$(S, R)-\mathbf{4 f}$ 95\% e.e.<smiles>CC(C)(C)c1ccccc1</smiles>

(R,S)-ent-4I $-18 \%$ e.e. 


\section{(+)-(R)-N-Boc-3-methyl-1-phenylbut-3-en-1-amine [3a]}<smiles>C=C(C)C[C@H](NC(=O)OC(C)(C)C)c1ccccc1</smiles>

Chiral HPLC conditions: CHIRALPAK AD-H, $3 \%{ }^{\mathrm{P} P O H} /$ hexanes, $1 \mathrm{~mL} / \mathrm{min}$ Racemic product: $\mathrm{t}_{\mathrm{R}}(R)=11.4 \mathrm{~min}, \mathrm{t}_{\mathrm{R}}(S)=12.6 \mathrm{~min}$

${ }^{1} \mathrm{H}-\mathrm{NMR}\left(600 \mathrm{MHz}, \mathrm{CDCl}_{3}\right)$ ठ 7.36-7.27 (m, 4H), 7.26-7.24 (m, 1H), $4.85(\mathrm{~s}, 1 \mathrm{H})$, 4.82 (br. s, 2H), $4.74(\mathrm{~s}, 1 \mathrm{H}), 2.61-2.21(\mathrm{~m}, 2 \mathrm{H}), 1.73(\mathrm{~s}, 3 \mathrm{H}), 1.41(\mathrm{~s}, 9 \mathrm{H}) ;{ }^{13} \mathrm{C}-$ NMR $\left(126 \mathrm{MHz}, \mathrm{CDCl}_{3}\right) \delta 155.4,143.2,142.0,128.6,127.2,126.2,113.9,79.5$, 52.9, 45.9, 28.4, 22.1; IR (ATR, $\left.\mathrm{cm}^{-1}\right)$ 3380, 2975, 2935, 2915, 1681, 1519, 1389, $1248,1167,1015,906 ;[\alpha]^{24} \mathrm{D}=+28.6^{\circ}\left(c=1.0, \mathrm{CHCl}_{3}\right) ; \mathrm{HRMS}(\mathrm{ESI}) \mathrm{m} / \mathrm{z}$ calculated for $\mathrm{C}_{16} \mathrm{H}_{24} \mathrm{NO}_{2}$ $[\mathrm{M}+\mathrm{H}]^{+}:$262.1802, found 262.1800 .

\section{racemic standard}

$86 \%$ conversion, $61.5 \mathrm{mg}, 0.234 \mathrm{mmol}, 78 \%$ yield

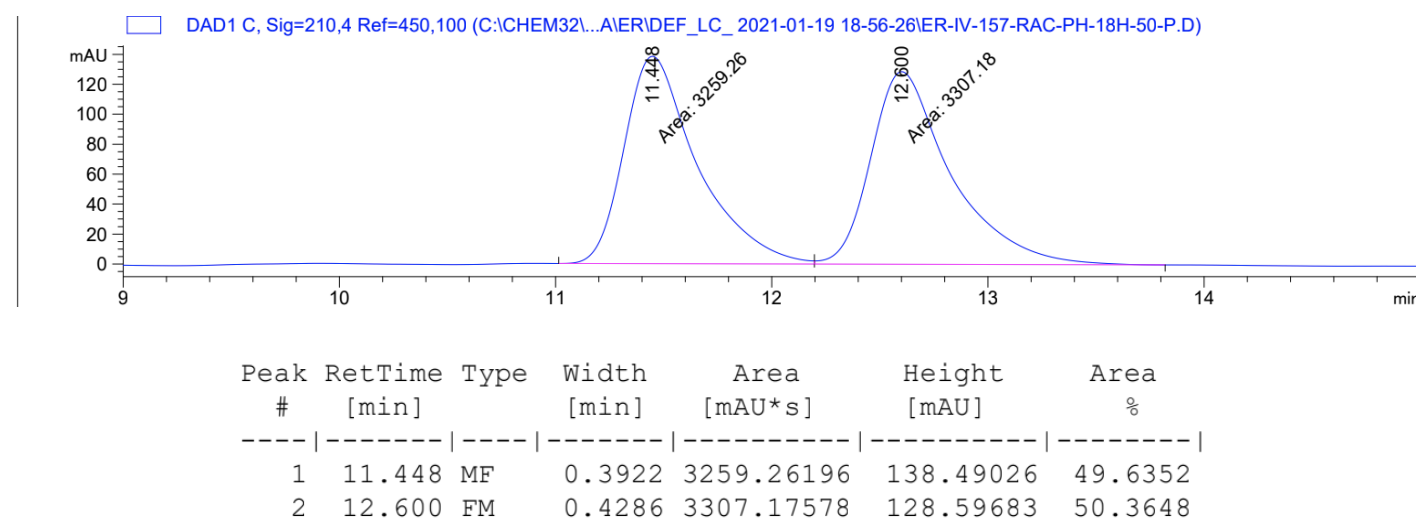

Thiourea 4a. tert-Butyl carbamate $(35.1 \mathrm{mg}, 0.300 \mathrm{mmol}, 1.0$ equiv), Ph-pyrrolidino thiourea $(R, S)-4 a$ (15.9 mg, $0.030 \mathrm{mmol}, 0.10$ equiv), benzaldehyde dimethyl acetal (45.0 $\mu \mathrm{L}, 0.300 \mathrm{mmol}, 1.0$ equiv), (2methylallyl)trimethylsilane $(53.0 \mu \mathrm{L}, 0.300 \mathrm{mmol}, 1.0$ equiv), TESOTf $(6.8 \mu \mathrm{L}, 0.030 \mathrm{mmol}, 0.10$ equiv), and $\mathrm{Et}_{2} \mathrm{O}(3.0 \mathrm{~mL}, 0.1 \mathrm{M})$ were used.

$90 \%$ conversion, $-60 \%$ e.e.

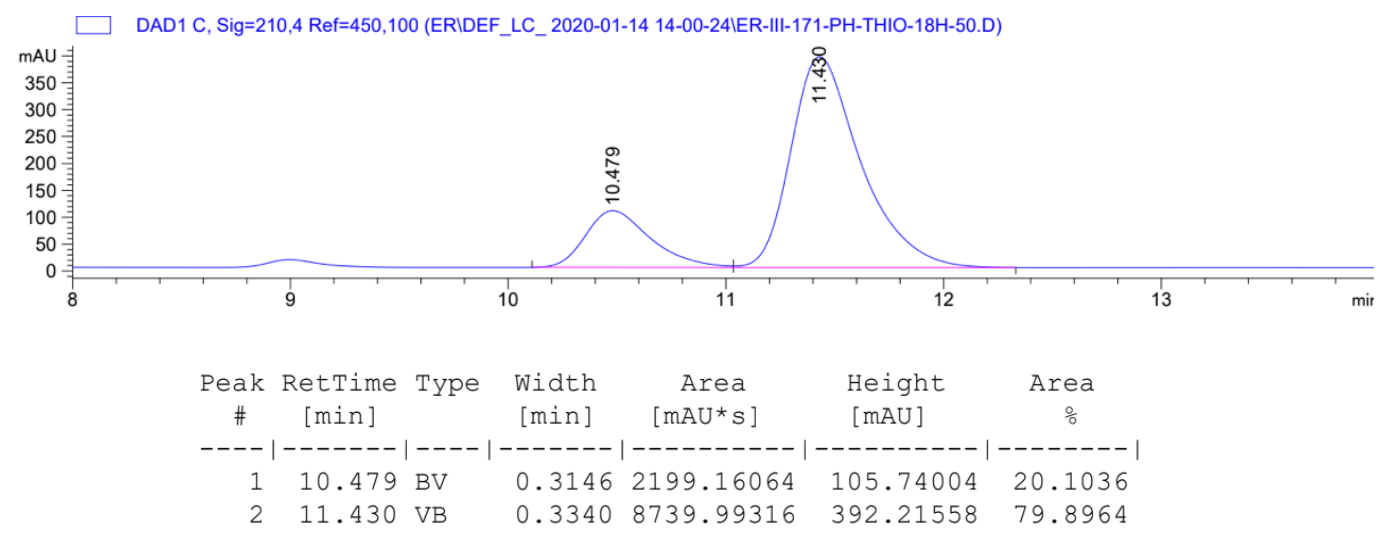


Thiourea 4b. tert-Butyl carbamate $(35.1 \mathrm{mg}, 0.300 \mathrm{mmol}, 1.0$ equiv), 2-Napthyl-pyrrolidino thiourea $(R, S)$ 4c (17.4 mg, $0.030 \mathrm{mmol}, 0.10$ equiv), benzaldehyde dimethyl acetal $(45.0 \mu \mathrm{L}, 0.300 \mathrm{mmol}, 1.0$ equiv), (2methylallyl)trimethylsilane (53.0 $\mu \mathrm{L}, 0.300 \mathrm{mmol}, 1.0$ equiv), TESOTf $(6.8 \mu \mathrm{L}, 0.030 \mathrm{mmol}, 0.10$ equiv), and $\mathrm{Et}_{2} \mathrm{O}(3.0 \mathrm{~mL}, 0.1 \mathrm{M})$ were used.

Run 1: $86 \%$ conversion, $-66 \%$ e.e.

Run 2: $83 \%$ conversion, $-66 \%$ e.e.

Average: $84 \%$ conversion, $-66 \%$ e.e.

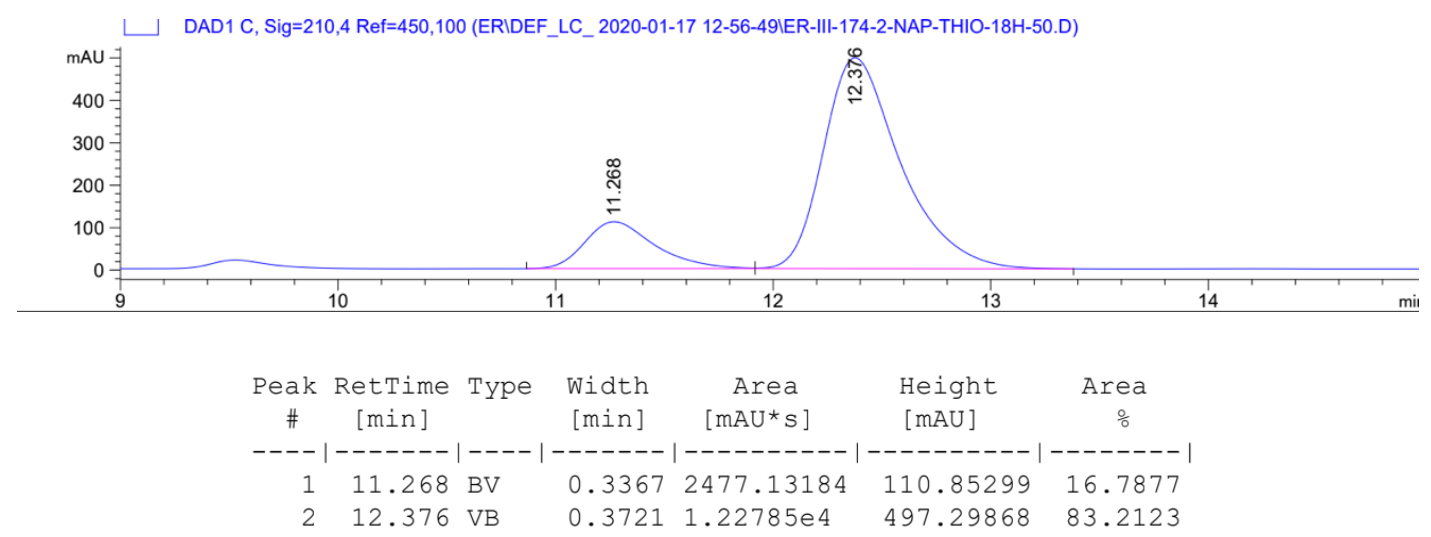

Thiourea 4c. tert-Butyl carbamate $(35.1 \mathrm{mg}, 0.300 \mathrm{mmol}, 1.0$ equiv), 1 -Napthyl-pyrrolidino thiourea $(R, S)$ 4c $(17.4 \mathrm{mg}, 0.030 \mathrm{mmol}, 0.10$ equiv), benzaldehyde dimethyl acetal ( $45.0 \mu \mathrm{L}, 0.300 \mathrm{mmol}, 1.0$ equiv), (2methylallyl)trimethylsilane $(53.0 \mu \mathrm{L}, 0.300 \mathrm{mmol}, 1.0$ equiv), TESOTf $(6.8 \mu \mathrm{L}, 0.030 \mathrm{mmol}, 0.10$ equiv), and $\mathrm{Et}_{2} \mathrm{O}(3.0 \mathrm{~mL}, 0.1 \mathrm{M})$ were used. Only peak at $6.80 \mathrm{ppm}$ used due to overlap at $3.33 \mathrm{ppm}$.

90\% conversion, $-\mathbf{8 1} \%$ e.e.

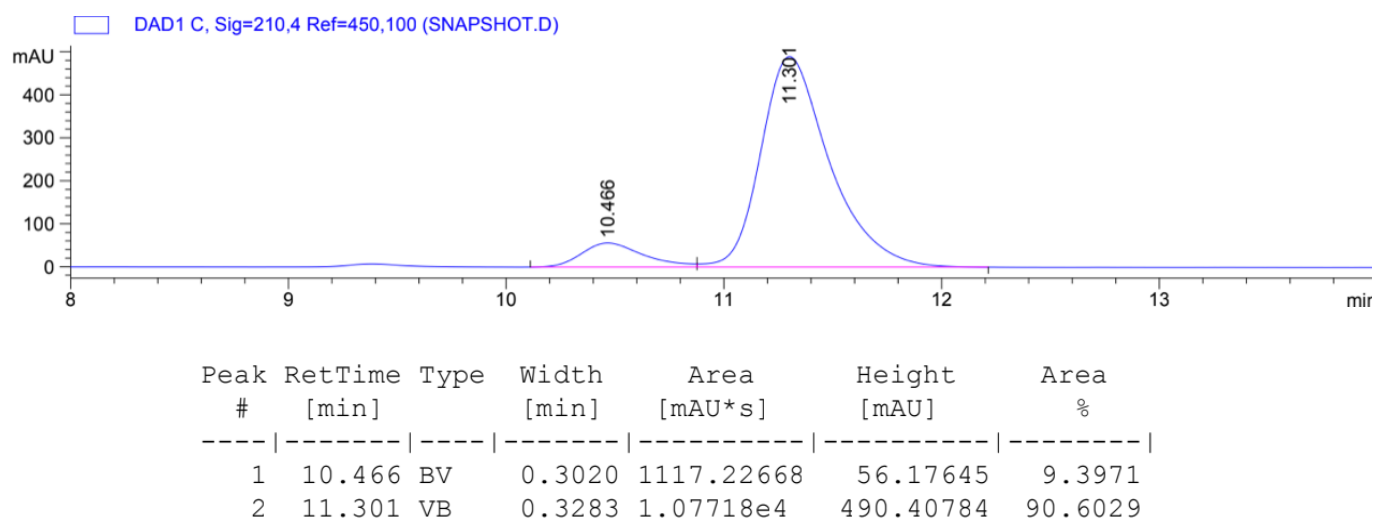

Thiourea 4d. tert-Butyl carbamate ( $35.1 \mathrm{mg}, 0.300 \mathrm{mmol}, 1.0$ equiv), 1 -Pyrenyl-pyrrolidino thiourea $(R, S)$ 4d $(19.7 \mathrm{mg}, 0.030 \mathrm{mmol}, 0.10$ equiv), benzaldehyde dimethyl acetal $(45.0 \mu \mathrm{L}, 0.300 \mathrm{mmol}, 1.0$ equiv), (2-methylallyl)trimethylsilane $(53.0 \mu \mathrm{L}, 0.300 \mathrm{mmol}, 1.0$ equiv), TESOTf (6.8 $\mu \mathrm{L}, 0.030 \mathrm{mmol}, 0.10$ equiv), and $\mathrm{Et}_{2} \mathrm{O}(3.0 \mathrm{~mL}, 0.1 \mathrm{M})$ were used.

91\% conversion, $-89 \%$ e.e. 


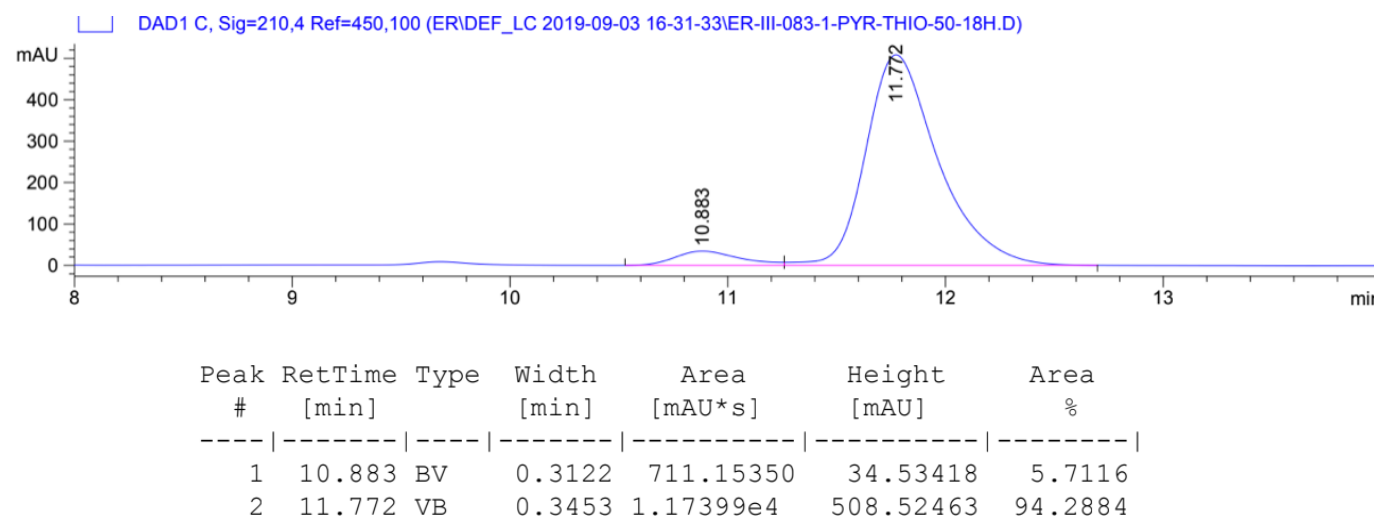

Thiourea 4 e. tert-Butyl carbamate $(35.1 \mathrm{mg}, 0.300 \mathrm{mmol}, 1.0$ equiv), 9-Phenanthryl-pyrrolidino thiourea $(R, S)-4 \mathrm{e}(19,0 \mathrm{mg}, 0.030 \mathrm{mmol}, 0.10$ equiv), benzaldehyde dimethyl acetal $(45.0 \mu \mathrm{L}, 0.300 \mathrm{mmol}, 1.0$ equiv), (2-methylallyl)trimethylsilane $(53.0 \mu \mathrm{L}, 0.300 \mathrm{mmol}, 1.0$ equiv), TESOTf $(6.8 \mu \mathrm{L}, 0.030 \mathrm{mmol}, 0.10$ equiv), and $\mathrm{Et}_{2} \mathrm{O}(3.0 \mathrm{~mL}, 0.1 \mathrm{M})$ were used. Trace of hemiaminal side product $(<6 \%)$ was observed and accounted for in the yield calculation.

90\% conversion, $58.9 \mathrm{mg}, 0.225 \mathrm{mmol}, 75 \%$ yield, $-91 \%$ e.e.

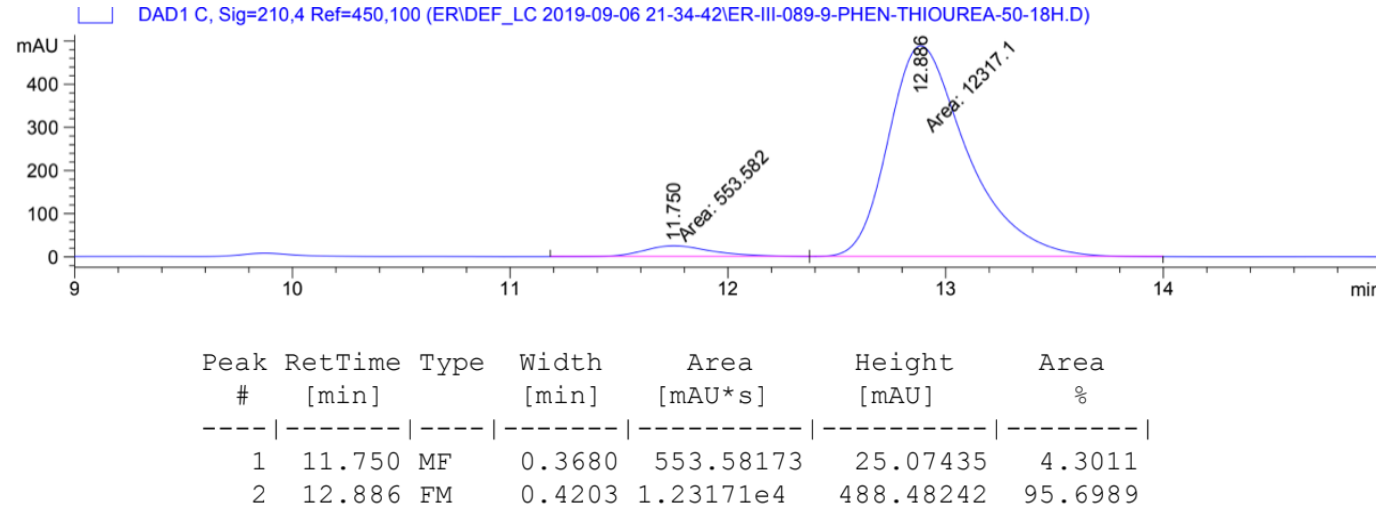

Thiourea 4f. Benzaldehyde dimethyl acetal (45.0 $\mu \mathrm{L}, \quad 0.300 \mathrm{mmol}, \quad 1.0$ equiv), (2methylallyl)trimethylsilane $(53.0 \mu \mathrm{L}, 0.300 \mathrm{mmol}, 1.0$ equiv), tert-butyl carbamate $(35.1 \mathrm{mg}, 0.300 \mathrm{mmol}$, 1.0 equiv), 4-Pyrenyl-pyrrolidino thiourea $4 \mathrm{f}(19.7 \mathrm{mg}, 0.030 \mathrm{mmol}, 0.10$ equiv), TESOTf $(6.8 \mu \mathrm{L}, 0.030$ mmol, 0.10 equiv), and $\mathrm{Et}_{2} \mathrm{O}(3.0 \mathrm{~mL}, 0.1 \mathrm{M})$ were used. Reaction was run at $-50^{\circ} \mathrm{C}$ for $18 \mathrm{~h}$. A second chromatographic purification (100\% DCM) was necessary to remove hemiaminal impurities.

91\% conversion, $61.7 \mathrm{mg}, 0.236 \mathrm{mmol}, 79 \%$ yield, $95 \%$ e.e.

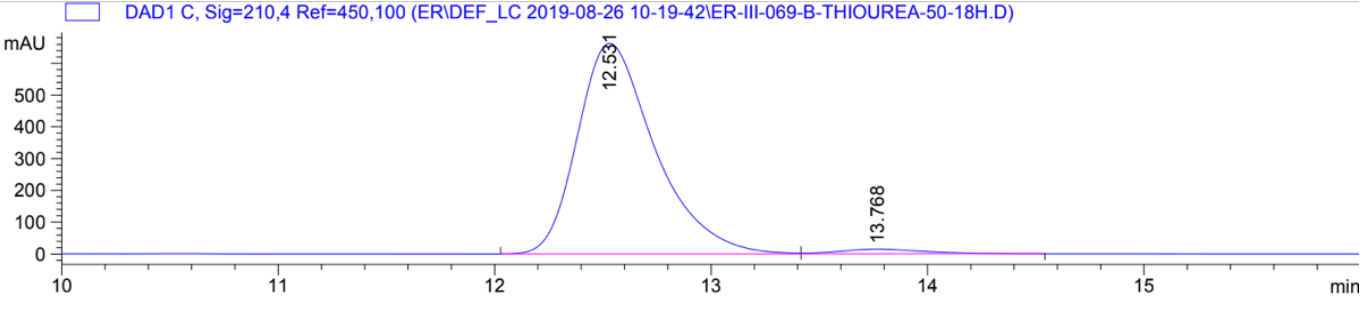




\begin{tabular}{|c|c|c|c|c|c|c|}
\hline $\begin{array}{c}\text { Peak } \\
\#\end{array}$ & $\begin{array}{c}\text { RetTime } \\
\text { [min] }\end{array}$ & Type & $\begin{array}{l}\text { Width } \\
\text { [min] }\end{array}$ & $\begin{array}{c}\text { Area } \\
{\left[\mathrm{mAU}^{*} \mathrm{~s}\right]}\end{array}$ & $\begin{array}{l}\text { Height } \\
\text { [mAU] }\end{array}$ & $\begin{array}{c}\text { Area } \\
\quad \%\end{array}$ \\
\hline & & & & & & \\
\hline 1 & & & & $1.64611 \mathrm{e} 4$ & & 303 \\
\hline 2 & 13. & $\mathrm{~B}$ & 932 & 399.54666 & 610 & 3697 \\
\hline
\end{tabular}

Thiourea $4 \mathrm{~g}$. tert-Butyl carbamate $(35.1 \mathrm{mg}, 0.300 \mathrm{mmol}, 1.0$ equiv), 4-F-Phenyl-pyrrolidino thiourea $(R, S)-\mathbf{4 g}(16.5 \mathrm{mg}, 0.030 \mathrm{mmol}, 0.10$ equiv), benzaldehyde dimethyl acetal $(45.0 \mu \mathrm{L}, 0.300 \mathrm{mmol}, 1.0$ equiv), (2-methylallyl)trimethylsilane $(53.0 \mu \mathrm{L}, 0.300 \mathrm{mmol}, 1.0$ equiv), TESOTf $(6.8 \mu \mathrm{L}, 0.030 \mathrm{mmol}, 0.10$ equiv), and $\mathrm{Et}_{2} \mathrm{O}(3.0 \mathrm{~mL}, 0.1 \mathrm{M})$ were used.

Run 1: $84 \%$ conversion, $-68 \%$ e.e.

Run 2: $86 \%$ conversion, $-68 \%$ e.e.

Average: $85 \%$ conversion, $-68 \%$ e.e.

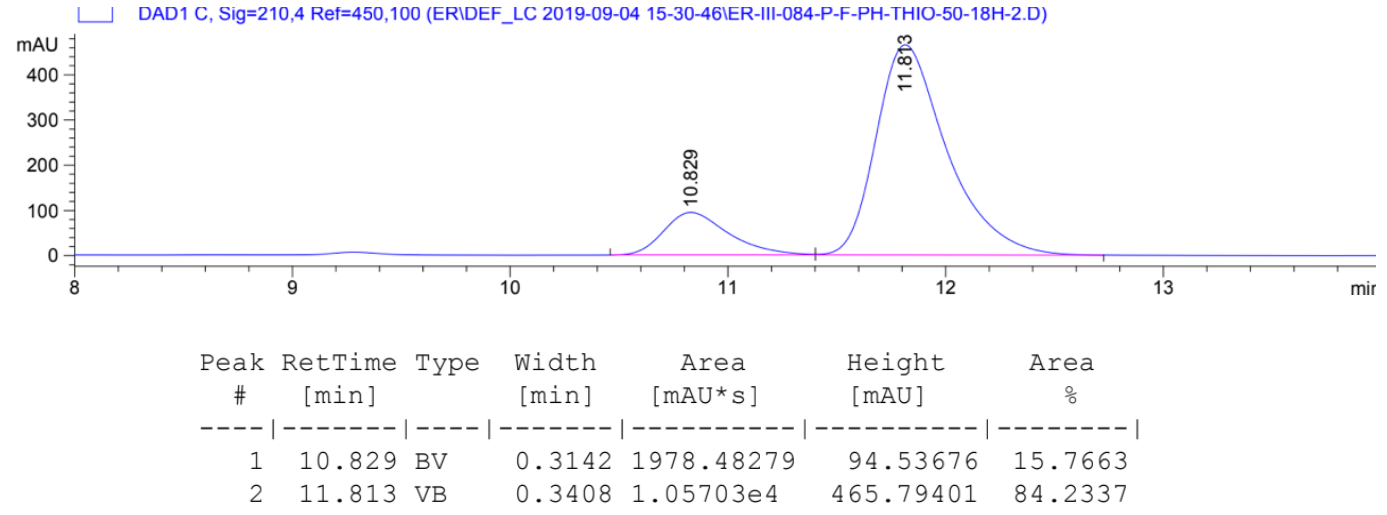

Thiourea $4 \mathrm{~h}$. tert-Butyl carbamate $\left(35.1 \mathrm{mg}, 0.300 \mathrm{mmol}, 1.0\right.$ equiv), 3,5- $\mathrm{F}_{2}$-Phenyl-pyrrolidino thiourea $(R, S)$-4h $(16.5 \mathrm{mg}, 0.030 \mathrm{mmol}, 0.10$ equiv), benzaldehyde dimethyl acetal $(45.0 \mu \mathrm{L}, 0.300 \mathrm{mmol}, 1.0$ equiv), (2-methylallyl)trimethylsilane ( $53.0 \mu \mathrm{L}, 0.300 \mathrm{mmol}, 1.0$ equiv), TESOTf ( $6.8 \mu \mathrm{L}, 0.030 \mathrm{mmol}, 0.10$ equiv), and $\mathrm{Et}_{2} \mathrm{O}(3.0 \mathrm{~mL}, 0.1 \mathrm{M})$ were used.

Run 1: $83 \%$ conversion, $-68 \%$ e.e. (reaction run on a $0.1 \mathrm{mmol}$ scale)

Run 2: $88 \%$ conversion, $-68 \%$ e.e.

Average: $86 \%$ conversion, $-68 \%$ e.e.

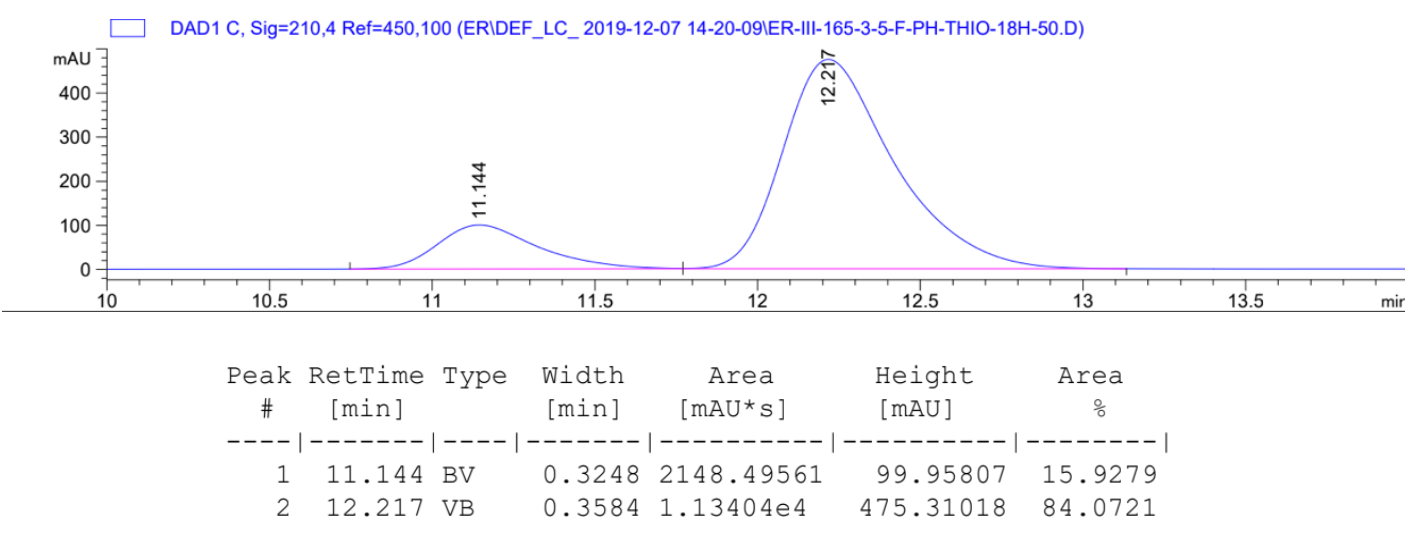


Thiourea 4 i. tert-Butyl carbamate $\left(35.1 \mathrm{mg}, 0.300 \mathrm{mmol}, 1.0\right.$ equiv), 3,4,5- $\mathrm{F}_{3}$-Phenyl-pyrrolidino thiourea $(R, S)-4 \mathrm{i}(18.1 \mathrm{mg}, 0.030 \mathrm{mmol}, 0.10$ equiv), benzaldehyde dimethyl acetal $(45.0 \mu \mathrm{L}, 0.300 \mathrm{mmol}, 1.0$ equiv), (2-methylallyl)trimethylsilane $(53.0 \mu \mathrm{L}, 0.300 \mathrm{mmol}, 1.0$ equiv), TESOTf $(6.8 \mu \mathrm{L}, 0.030 \mathrm{mmol}, 0.10$ equiv), and $\mathrm{Et}_{2} \mathrm{O}(3.0 \mathrm{~mL}, 0.1 \mathrm{M})$ were used.

Run 1: $88 \%$ conversion, $-71 \%$ e.e.

Run 2: $90 \%$ conversion, $-71 \%$ e.e.

Average: $89 \%$ conversion, $-\mathbf{7 1} \%$ e.e.

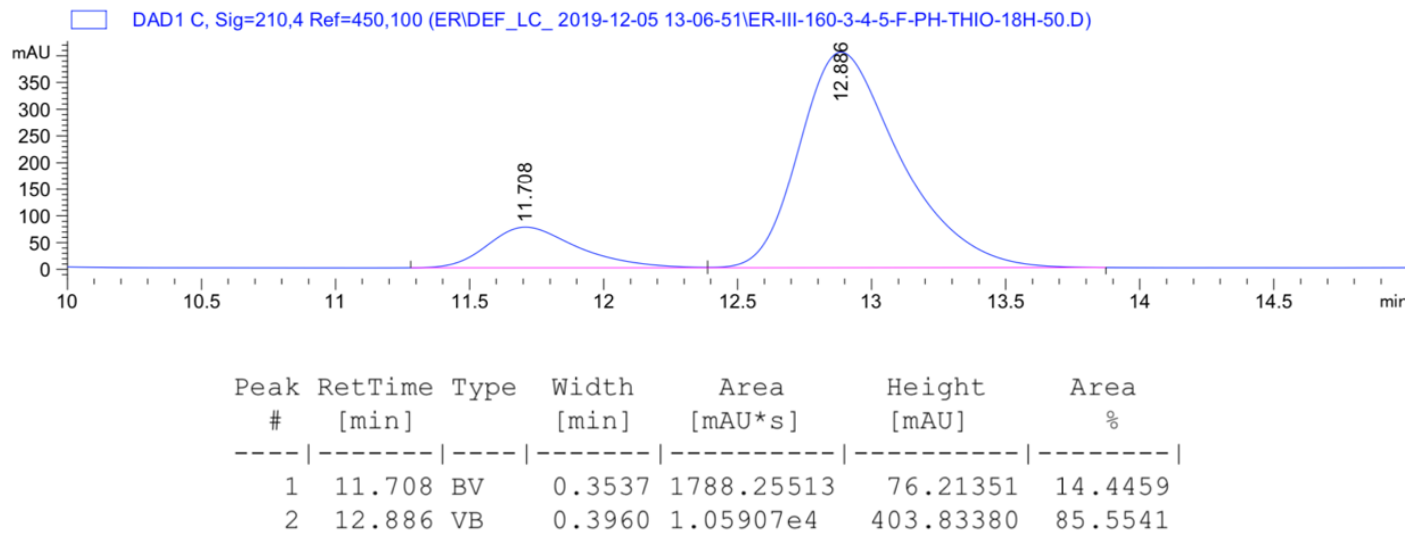

Thiourea 4 j. tert-Butyl carbamate $(11.7 \mathrm{mg}, 0.100 \mathrm{mmol}, 1.0$ equiv), 2-F-Phenyl-pyrrolidino thiourea $(R, S)-4 \mathrm{j}(5.5 \mathrm{mg}, 0.010 \mathrm{mmol}, 0.10$ equiv), benzaldehyde dimethyl acetal $(15.0 \mu \mathrm{L}, 0.100 \mathrm{mmol}, 1.0$ equiv), (2-methylallyl)trimethylsilane $(17.5 \mu \mathrm{L}, 0.100 \mathrm{mmol}, 1.0$ equiv), TESOTf (2.3 $\mu \mathrm{L}, 0.010 \mathrm{mmol}, 0.10$ equiv), and $\mathrm{Et}_{2} \mathrm{O}(1.0 \mathrm{~mL}, 0.1 \mathrm{M})$ were used. The reaction was carried out in a 1 -dram vial.

85\% conversion, $-89 \%$ e.e.

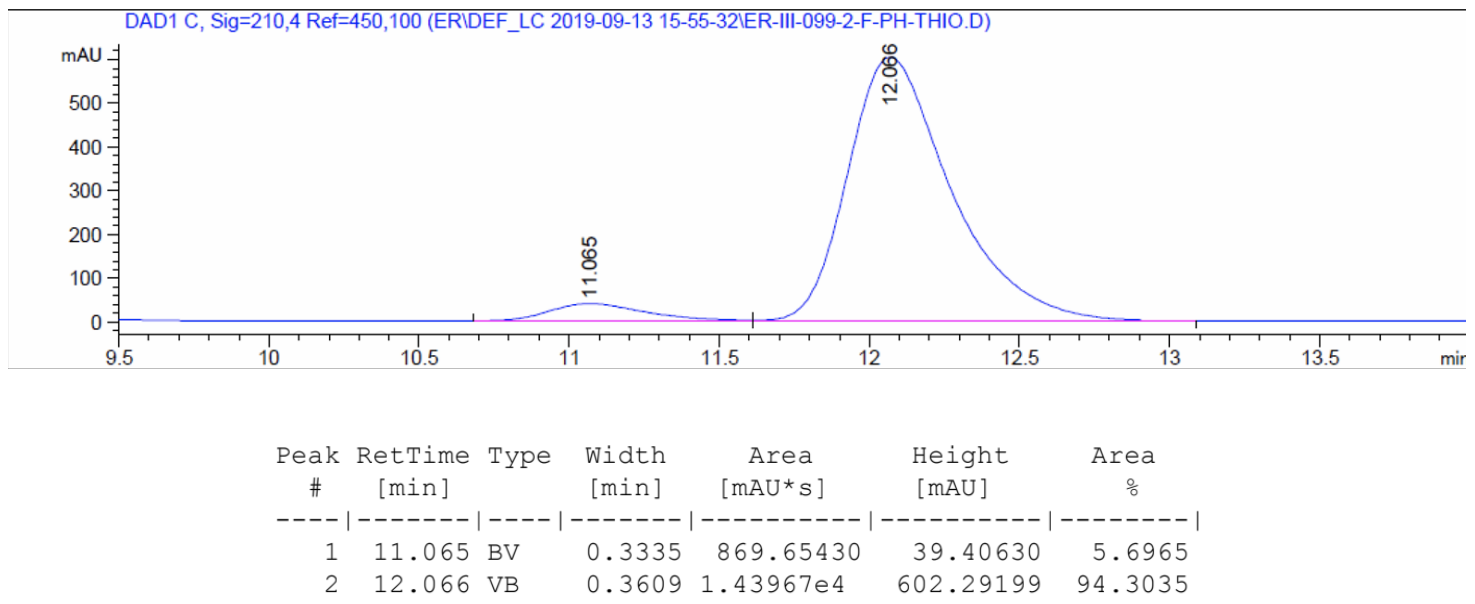

Thiourea 4k. tert-Butyl carbamate $\left(11.7 \mathrm{mg}, 0.100 \mathrm{mmol}, 1.0\right.$ equiv), 2,3,4,5- $\mathrm{F}_{4}-\mathrm{Phenyl}_{\text {-pyrrolidino }}$ thiourea $(R, S)-4 \mathbf{k}(6.0 \mathrm{mg}, 0.010 \mathrm{mmol}, 0.10$ equiv), benzaldehyde dimethyl acetal $(15.0 \mu \mathrm{L}, 0.100 \mathrm{mmol}$, 1.0 equiv), (2-methylallyl)trimethylsilane $(17.5 \mu \mathrm{L}, 0.100 \mathrm{mmol}, 1.0$ equiv), TESOTf $(2.3 \mu \mathrm{L}, 0.010 \mathrm{mmol}$, 0.10 equiv), and $\mathrm{Et}_{2} \mathrm{O}(1.0 \mathrm{~mL}, 0.1 \mathrm{M})$ were used. The reaction was carried out in a 1 -dram vial. 
Run 1: $90 \%$ conversion, $-92 \%$ e.e.

Run 2: $90 \%$ conversion, $\mathbf{- 9 2} \%$ e.e. (ran on a $0.3 \mathrm{mmol}$ scale)

Average: $90 \%$ conversion, $-92 \%$ e.e.

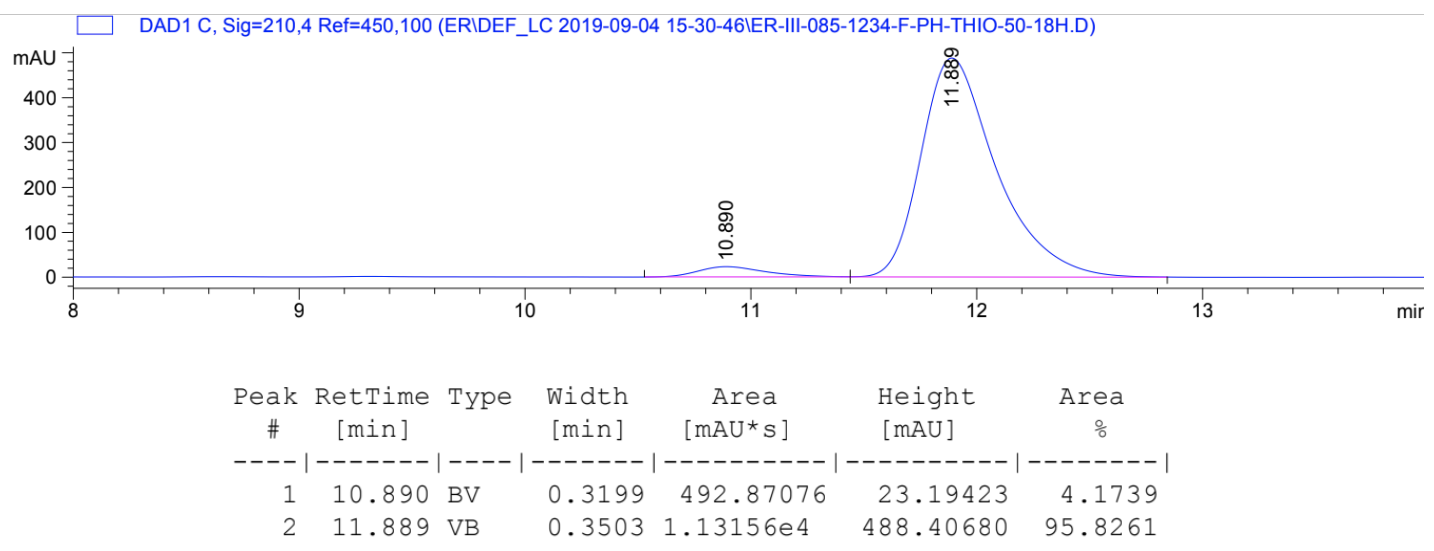

Thiourea 4l. tert-Butyl carbamate $(11.7 \mathrm{mg}, 0.100 \mathrm{mmol}, 1.0$ equiv), 2-Methyl-2-(9-Phenanthryl)pyrrolidino thiourea $(R, S)-4 \mathrm{j}(6.5 \mathrm{mg}, 0.010 \mathrm{mmol}, 0.10$ equiv), benzaldehyde dimethyl acetal $(15.0 \mu \mathrm{L}$, $0.100 \mathrm{mmol}, 1.0$ equiv), (2-methylallyl)trimethylsilane (17.5 $\mu \mathrm{L}, 0.100 \mathrm{mmol}, 1.0$ equiv), TESOTf $(2.3 \mu \mathrm{L}$, $0.010 \mathrm{mmol}, 0.10$ equiv), and $\mathrm{Et}_{2} \mathrm{O}(1.0 \mathrm{~mL}, 0.1 \mathrm{M})$ were used. The reaction was carried out in a 1 -dram vial.

$-18 \%$ e.e.

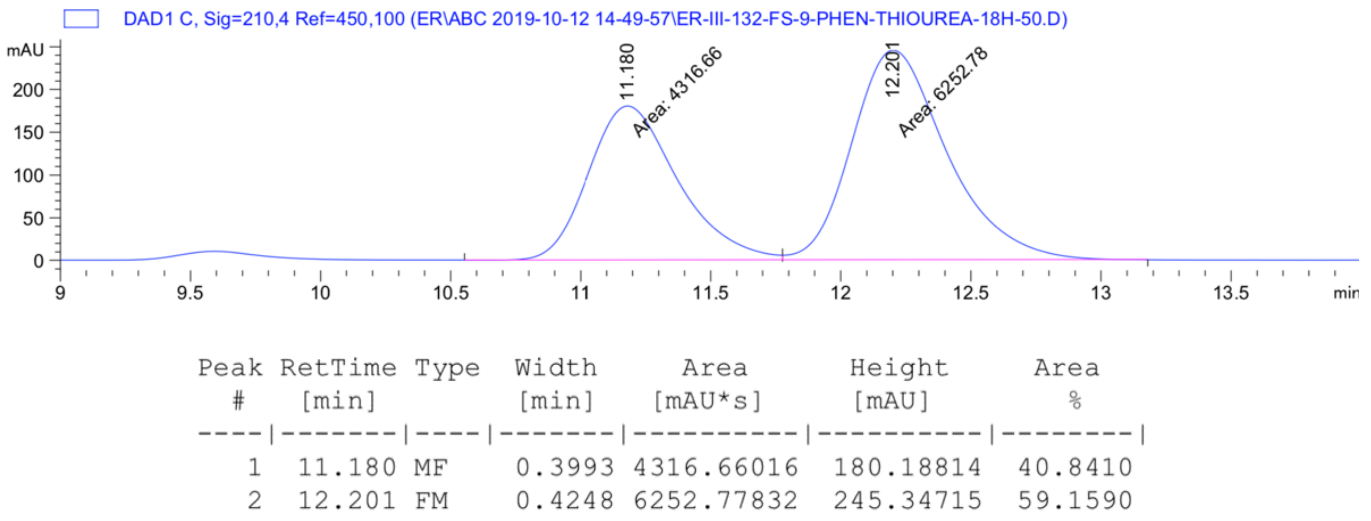

Urea 5a. Benzaldehyde dimethyl acetal $(45.0 \mu \mathrm{L}, 0.300 \mathrm{mmol}, 1.0$ equiv), (2-methylallyl)trimethylsilane $(53.0 \mu \mathrm{L}, 0.300 \mathrm{mmol}, 1.0$ equiv), tert-butyl carbamate $(35.1 \mathrm{mg}, 0.300 \mathrm{mmol}, 1.0$ equiv), 4-Pyrenylpyrrolidino urea $(S, R)-5 a(19.2 \mathrm{mg}, 0.030 \mathrm{mmol}, 0.10$ equiv), TESOTf $(6.8 \mu \mathrm{L}, 0.030 \mathrm{mmol}, 0.10$ equiv), and $\mathrm{Et}_{2} \mathrm{O}(3.0 \mathrm{~mL}, 0.1 \mathrm{M})$ were used. Reaction was run at $-50^{\circ} \mathrm{C}$ for $18 \mathrm{~h}$. After the second chromatographic purification (100\% DCM) 6\% residual hemiaminal was left. Measured weight $56.7 \mathrm{mg}$, calculated weight accounting for impurity, $53.6 \mathrm{mg}$.

$53.6 \mathrm{mg}, 0.205 \mathrm{mmol}, 68 \%$ yield, $95 \%$ e.e. 


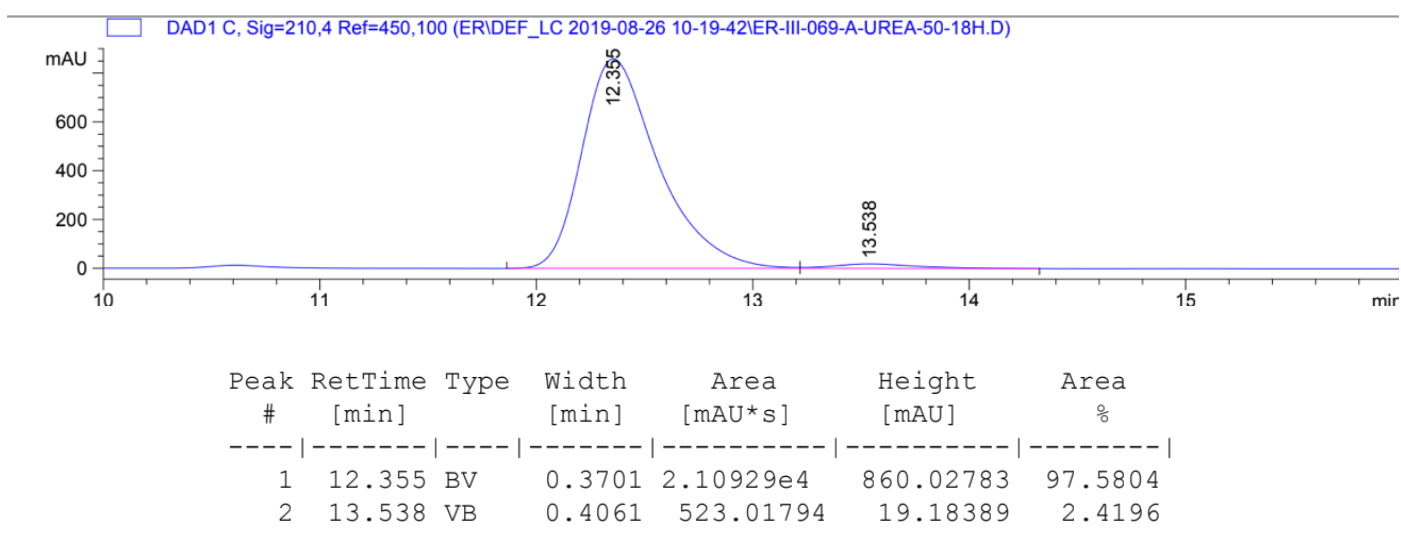

Squaramide 5b. Benzaldehyde dimethyl acetal $(45.0 \mu \mathrm{L}, \quad 0.300 \mathrm{mmol}, 1.0$ equiv), (2methylallyl)trimethylsilane $(53.0 \mu \mathrm{L}, 0.300 \mathrm{mmol}, 1.0$ equiv), tert-butyl carbamate $(35.1 \mathrm{mg}, 0.300 \mathrm{mmol}$, 1.0 equiv), 4-Pyrenyl-pyrrolidino squaramide $(S, R)-5 \mathbf{b}(20.8 \mathrm{mg}, 0.030 \mathrm{mmol}, 0.10$ equiv), TESOTf $(6.8$ $\mu \mathrm{L}, 0.030 \mathrm{mmol}, 0.10$ equiv), and $\mathrm{Et}_{2} \mathrm{O}(3.0 \mathrm{~mL}, 0.1 \mathrm{M})$ were used. A second chromatographic purification $(100 \%$ DCM) was necessary to remove hemiaminal impurities.

$65.9 \mathrm{mg}, 0.252 \mathrm{mmol}, \mathbf{8 4} \%$ yield, $\mathbf{9 3} \%$ e.e.

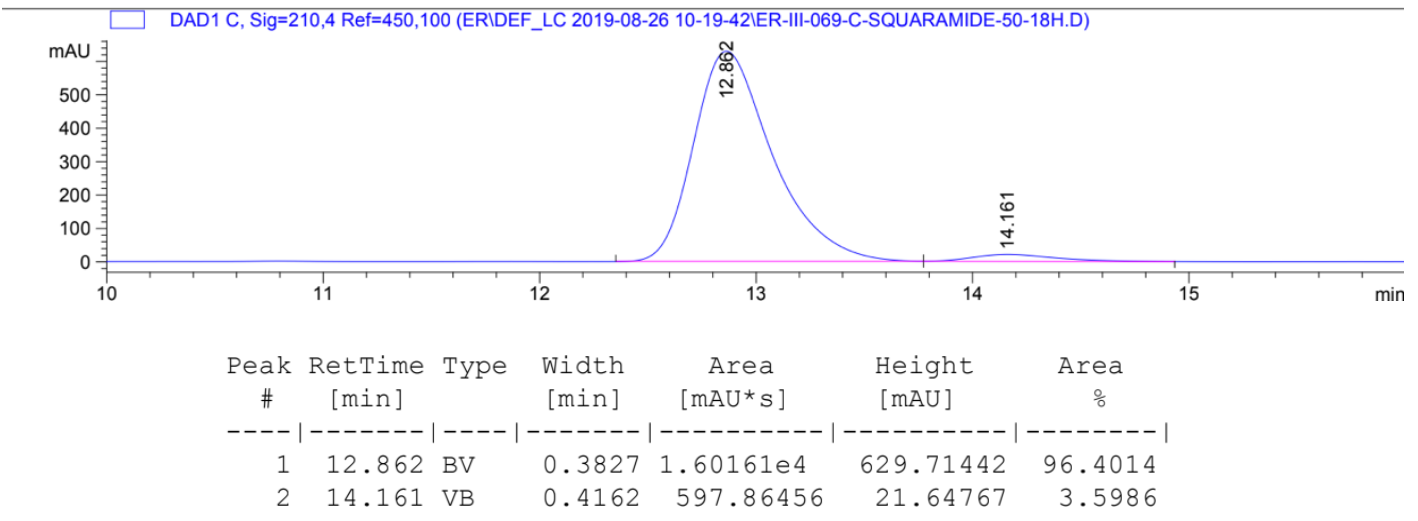

N-Methyl 9-phen-thiourea 7b. Benzaldehyde dimethyl acetal (45.0 $\mu \mathrm{L}, 0.300 \mathrm{mmol}, 1.0$ equiv), (2methylallyl)trimethylsilane $(53.0 \mu \mathrm{L}, 0.300 \mathrm{mmol}, 1.0$ equiv), tert-butyl carbamate $(35.1 \mathrm{mg}, 0.300 \mathrm{mmol}$, 1.0 equiv), ( $S, R)-7 \mathrm{~b}$ 9-Phenanthryl-N-Methyl-pyrrolidine thiourea (19.4 mg, $0.030 \mathrm{mmol}, 0.1$ equiv), TESOTf $\left(6.8 \mu \mathrm{L}, 0.030 \mathrm{mmol}, 0.10\right.$ equiv), and $\mathrm{Et}_{2} \mathrm{O}(3.0 \mathrm{~mL}, 0.1 \mathrm{M})$ were used. Reaction was run at $-50^{\circ} \mathrm{C}$ for $18 \mathrm{~h}$. Purified with flash column chromatography on silica (Biotage, $25 \mathrm{~g}$ column).

Run1: $62.1 \mathrm{mg}, 0.178 \mathrm{mmol}, 59 \%$ yield, $7 \%$ e.e.

Run2: $56.3 \mathrm{mg}, 0.161 \mathrm{mmol}, 54 \%$ yield, $7 \%$ e.e.

Average: $57 \%$ yield, $7 \%$ e.e. 


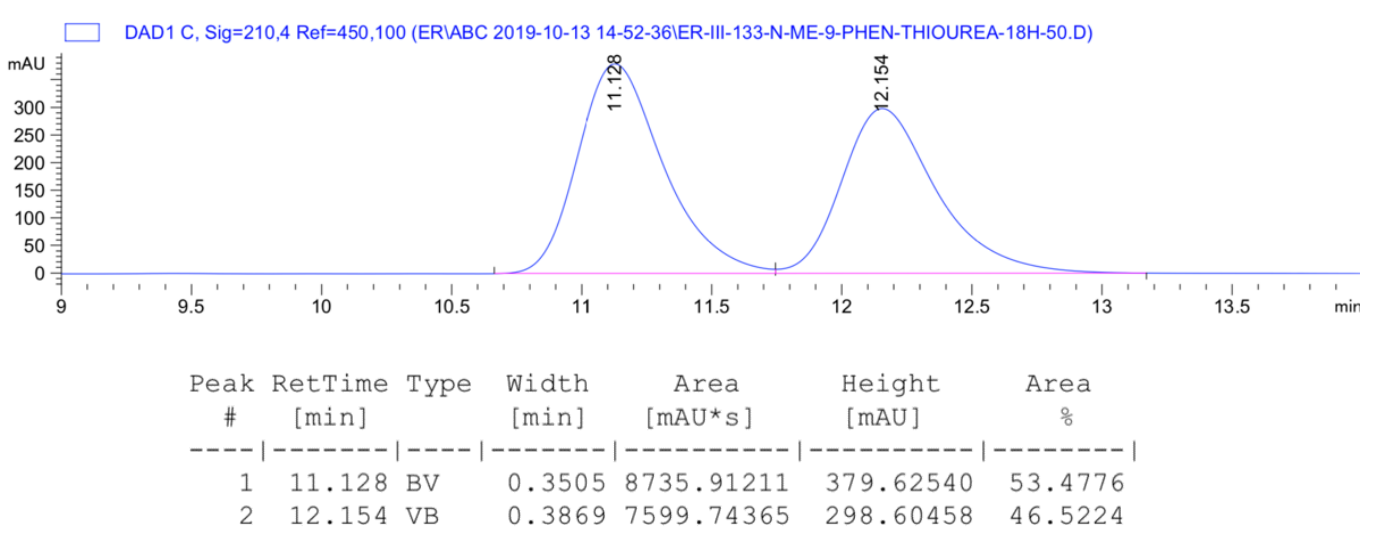

Chiral Fragment 7c. Benzaldehyde dimethyl acetal $(45.0 \mu \mathrm{L}, 0.300 \mathrm{mmol}, 1.0$ equiv), (2methylallyl)trimethylsilane $(53.0 \mu \mathrm{L}, 0.300 \mathrm{mmol}, 1.0$ equiv), tert-butyl carbamate $(35.1 \mathrm{mg}, 0.300 \mathrm{mmol}$, 1.0 equiv), 9-Phenantryl-pyrrolidine- $N$-Boc-tert-leucine, $(S, R)-7 \mathrm{c}(13.8 \mathrm{mg}, 0.030 \mathrm{mmol}, 0.10$ equiv), TESOTf $\left(6.8 \mu \mathrm{L}, 0.030 \mathrm{mmol}, 0.10\right.$ equiv), and $\mathrm{Et}_{2} \mathrm{O}(3.0 \mathrm{~mL}, 0.1 \mathrm{M})$ were used. Reaction was run at $-50^{\circ} \mathrm{C}$ for $18 \mathrm{~h}$. Purified with flash column chromatography on silica (Biotage, $10 \mathrm{~g}$ column) using an $\mathrm{Et}_{2} \mathrm{O} /$ hexanes gradient.

Trace of hemiaminal side product $(<3 \%)$ were observed in Run 1 and accounted for in the yield calculation.

Run 1: $50.7 \mathrm{mg}, 0.194 \mathrm{mmol}, 65 \%$ yield, $-31 \%$ e.e.

Run 2: $54.7 \mathrm{mg}, 0.209 \mathrm{mmol}, 70 \%$ yield, $26 \%$ e.e., [(R,S)-ent-7c used as catalyst]

\section{Average: $68 \%$ yield, $28 \%$ e.e.}

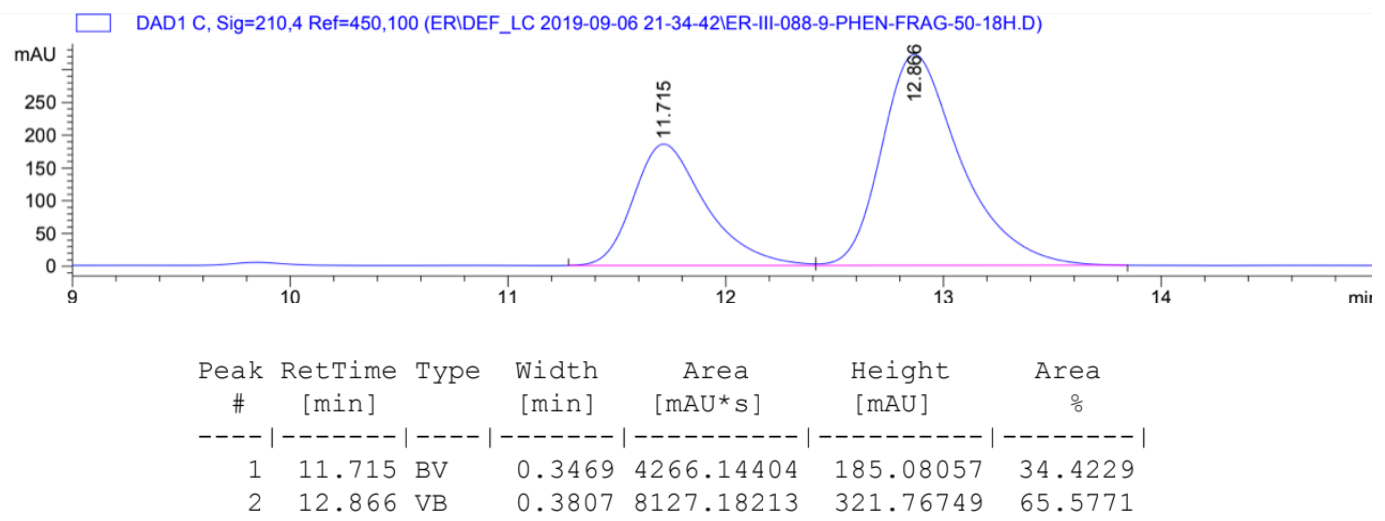

N-Methyl Chiral Fragment 7a. Benzaldehyde dimethyl acetal $(45.0 \mu \mathrm{L}, 0.300 \mathrm{mmol}, 1.0$ equiv), (2methylallyl)trimethylsilane $(53.0 \mu \mathrm{L}, 0.300 \mathrm{mmol}, 1.0$ equiv), tert-butyl carbamate $(35.1 \mathrm{mg}, 0.300 \mathrm{mmol}$, 1.0 equiv), 9-Phenantryl-pyrrolidine- $N$-Methyl- $N$-Boc-tert-leucine, $(R, S)-7 a(12.4 \mathrm{mg}, 0.026 \mathrm{mmol}, 0.09$ equiv), TESOTf $\left(6.8 \mu \mathrm{L}, 0.030 \mathrm{mmol}, 0.10\right.$ equiv), and $\mathrm{Et}_{2} \mathrm{O}(3.0 \mathrm{~mL}, 0.1 \mathrm{M})$ were used. Reaction was run at $-50^{\circ} \mathrm{C}$ for $18 \mathrm{~h}$. Purified with flash column chromatography on silica (Biotage, $25 \mathrm{~g}$ column).

Run1: $57.6 \mathrm{mg}, 0.165 \mathrm{mmol}, 55 \%$ yield, $-1 \%$ e.e.

Run2: $57.8 \mathrm{mg}, 0.165 \mathrm{mmol}, 55 \%$ yield, $-\mathbf{- 1} \%$ e.e.

Average: $55 \%$ yield, $-1 \%$ e.e. 


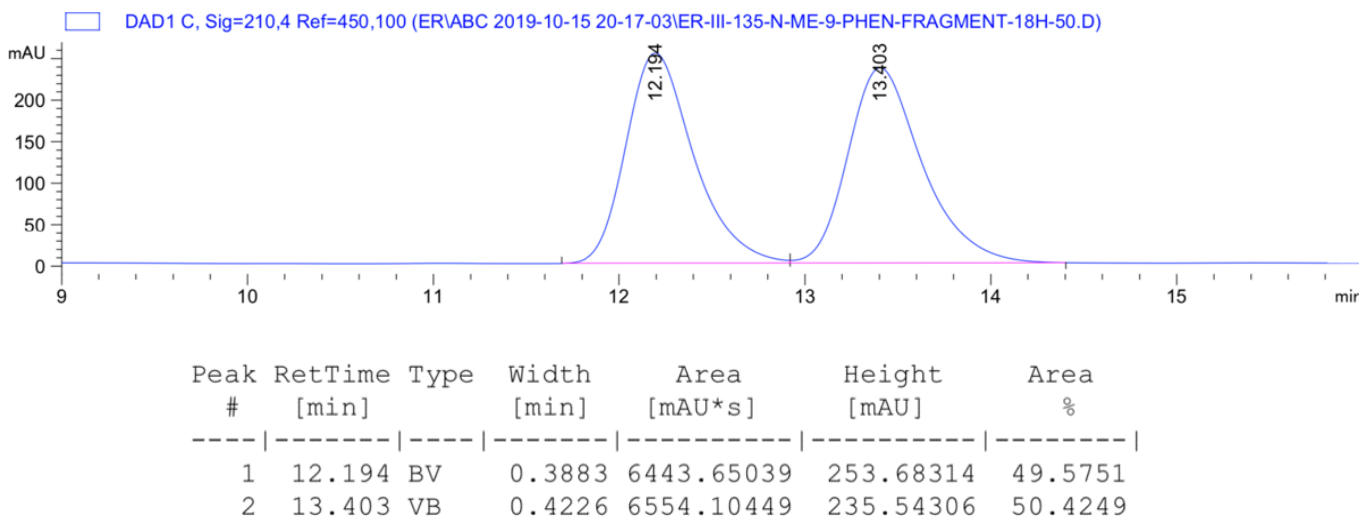

Thioamide 7d. Benzaldehyde dimethyl acetal $(45.0 \mu \mathrm{L}, \quad 0.300 \mathrm{mmol}, 1.0$ equiv), (2methylallyl)trimethylsilane $(53.0 \mu \mathrm{L}, 0.300 \mathrm{mmol}, 1.0$ equiv), tert-butyl carbamate $(35.1 \mathrm{mg}, 0.300 \mathrm{mmol}$, 1.0 equiv), 9-Phenanthryl-pyrrolidine-thioamide thiourea $(S, R)-7 \mathrm{~d}(19.4 \mathrm{mg}, 0.030 \mathrm{mmol}, 0.10$ equiv), TESOTf $\left(6.8 \mu \mathrm{L}, 0.030 \mathrm{mmol}, 0.10\right.$ equiv), and $\mathrm{Et}_{2} \mathrm{O}(3.0 \mathrm{~mL}, 0.1 \mathrm{M})$ were used. Reaction was run at $-50^{\circ} \mathrm{C}$ for $18 \mathrm{~h}$. Purified with flash column chromatography on silica (Biotage, $10 \mathrm{~g}$ column then $25 \mathrm{~g}$ column) using a gradient of $\mathrm{Et}_{2} \mathrm{O} /$ hexanes followed by a $100 \%$ DCM column.

Run 1: $64.7 \mathrm{mg}, 0.248 \mathrm{mmol}, 83 \%$ yield, $4 \%$ e.e. Run 2: $54.1 \mathrm{mg}, 0.207 \mathrm{mmol}, 69 \%$ yield, $8 \%$ e.e.

Average: $76 \%$ yield, $6 \%$ e.e.

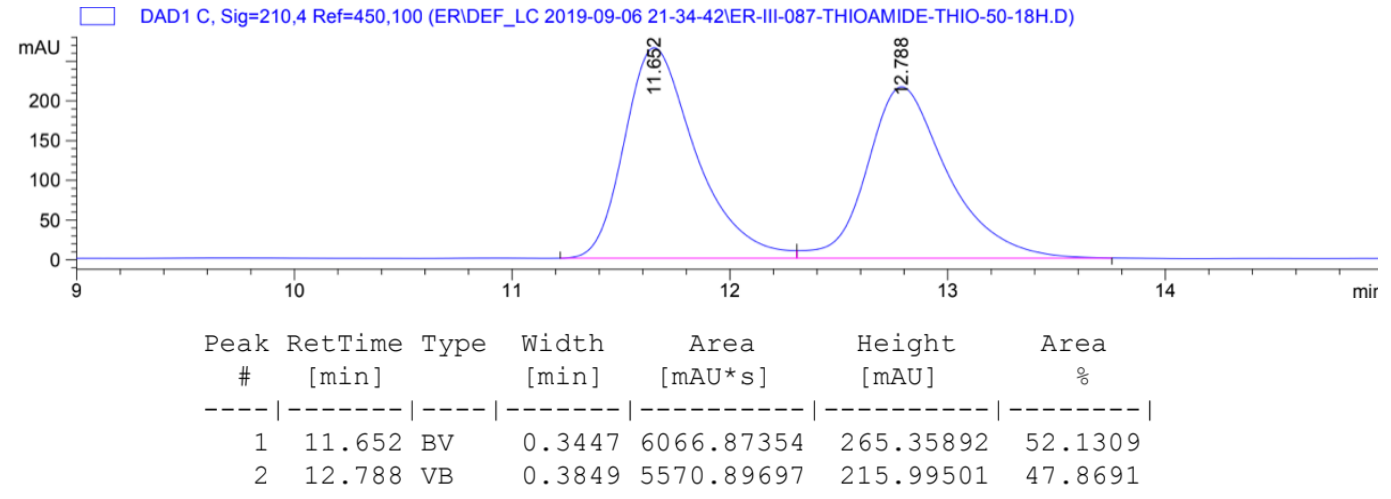




\section{Reaction Investigations}

\section{Variation of Reaction Conditions}

Method A: An oven-dried vial was charged with tert-butyl carbamate (1.0 equiv), sub-optimal squaramide catalyst $(S, R)-6 \mathbf{f}$, and a stir bar. The vial was sealed with a septum-lined cap and double thickness parafilm, evacuated, and refilled with $\mathrm{N}_{2}(2 \mathrm{x})$. Benzaldehyde dimethyl acetal (1.0 equiv), (2methylallyl)trimethylsilane ( 1.0 equiv), and $\mathrm{Et}_{2} \mathrm{O}(0.1 \mathrm{M})$ were then added via syringe. The reaction mixture was cooled to $-78^{\circ} \mathrm{C}$ in a dry ice/acetone bath, then TESOTf ( 0.10 equiv) was added dropwise. The vial was transferred to a cryocool and stirred for the reported reaction time. The reaction was quenched by dropwise addition via syringe of a solution of $1: 1 \mathrm{Et} 3 \mathrm{~N} / \mathrm{MeOH}$, stirred for $5 \mathrm{~min}$ at the reaction temperature, and then warmed to rt. Solvent was removed under reduced pressure, then the remaining residue was dissolved in minimal $\mathrm{CH}_{2} \mathrm{Cl}_{2}$ and applied to a silica column for purification. Flash column chromatography on silica (Biotage, $10 \mathrm{~g}$ column) using a gradient of $1 \%$ EtOAc/hexanes $\rightarrow 10 \%$ EtOAc/hexanes as eluent gave pure product as a white solid. Enantiomeric excess was determined by chiral HPLC analysis of purified product. NB: All the reactions run according to Method A have been run at a measured temperature of $-60^{\circ} \mathrm{C}$, further investigation led us to conclude that, due to the positioning of the thermometer in the cryocool and the presence of a temperature gradient in the cryocool bath, the temperature of the reactions was actually closer to $-\mathbf{5 0} \mathbf{0}$. Careful temperature measurement was employed for the scope reactions.

All the reactions in this section were run following Method $A$, in the screening tables for reaction conditions, e.e.'s are reported for each run (\% e.e. run 1, \% e.e. run 2). Entries indicated in bold are the standard reaction conditions.

Table S2. Amine source screen.

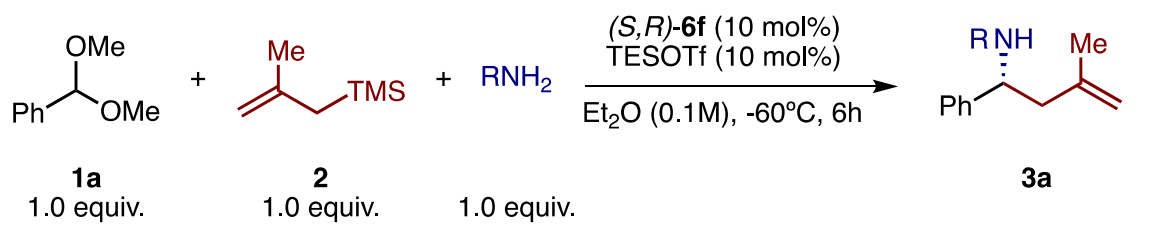

carbamates<smiles>C=CCOC(=O)NC(CC(=C)O)c1ccccc1</smiles><smiles>C=C(C)CC(NC(=O)OCc1ccccc1)c1ccccc1</smiles><smiles>C=C(C)CC(NC(=O)OC(C)(C)C)c1ccccc1</smiles>

other amines<smiles>C=C(C)CC(NCc1ccccc1)c1ccccc1</smiles>

N.R.<smiles>[R]C(=O)N[C@H](CC(=C)N)c1ccccc1</smiles>

$\mathrm{R}=\mathrm{Ph}$
$\mathrm{R}=\mathrm{CC}$<smiles>[Y4]C(=C)CC(NS(=O)(=O)[OH+])c1ccccc1</smiles>

1:1 amine/ether 
Table S3. Solvent screen.

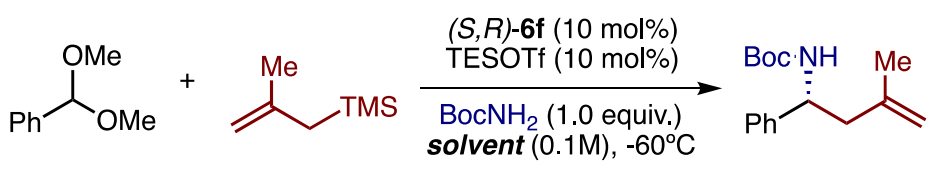

$1 a$

2

$3 a$

\begin{tabular}{|c|c|c|}
\hline entry & solvent & e.e. (\%) \\
\hline $\mathbf{1}$ & $\mathrm{Et}_{2} \mathbf{O}$ & $\mathbf{9 2 ,} \mathbf{9 2}$ \\
\hline 2 & $\mathrm{TBME}$ & 92,90 \\
\hline 3 & $\mathrm{CPME}$ & 86,84 \\
\hline 4 & $\mathrm{THF}$ & 46,41 \\
\hline 5 & Toluene & 84,84 \\
\hline 6 & $\mathrm{CH}_{2} \mathrm{Cl}_{2}$ & 56,56 \\
\hline
\end{tabular}

Table S4. Concentration screen.

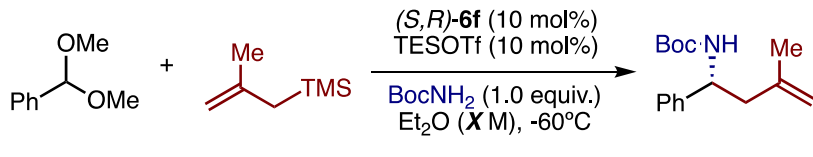

$1 a$

2

$3 \mathbf{a}$

\begin{tabular}{|c|c|c|}
\hline entry & concentration (M) & e.e. (\%) \\
\hline 1 & 0.05 & $92,92,90$ \\
\hline 2 & $\mathbf{0 . 1}$ & $\mathbf{9 2 ,} \mathbf{9 2 , 8 8}$ \\
\hline 3 & 0.25 & $90,89,88$ \\
\hline 4 & 0.5 & $88,90,86$ \\
\hline 5 & 1.0 & $86,81,85$ \\
\hline
\end{tabular}

Table S5. TESOTf co-catalyst loading screen.

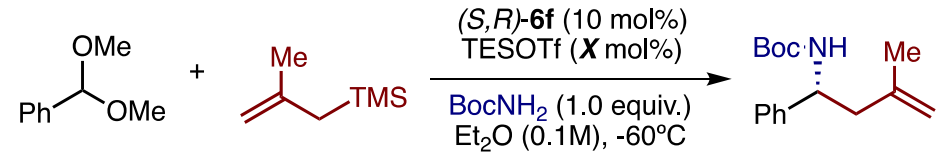

$1 \mathbf{a}$

2

$3 a$

\begin{tabular}{|c|c|c|}
\hline entry & TESOTf (mol\%) & e.e. (\%) \\
\hline 1 & 5 & 92,93 \\
\hline 2 & $\mathbf{1 0}$ & $\mathbf{9 2 ,} \mathbf{9 4 , 9 4 , 9 2}$ \\
\hline 3 & 25 & $88,88,75,88$ \\
\hline 4 & 50 & $74,78,57,80$ \\
\hline 5 & 100 & $38,44,32$ \\
\hline
\end{tabular}




\section{Variation of Silyl Lewis acid}

Table S6. R3SiOTf screen.
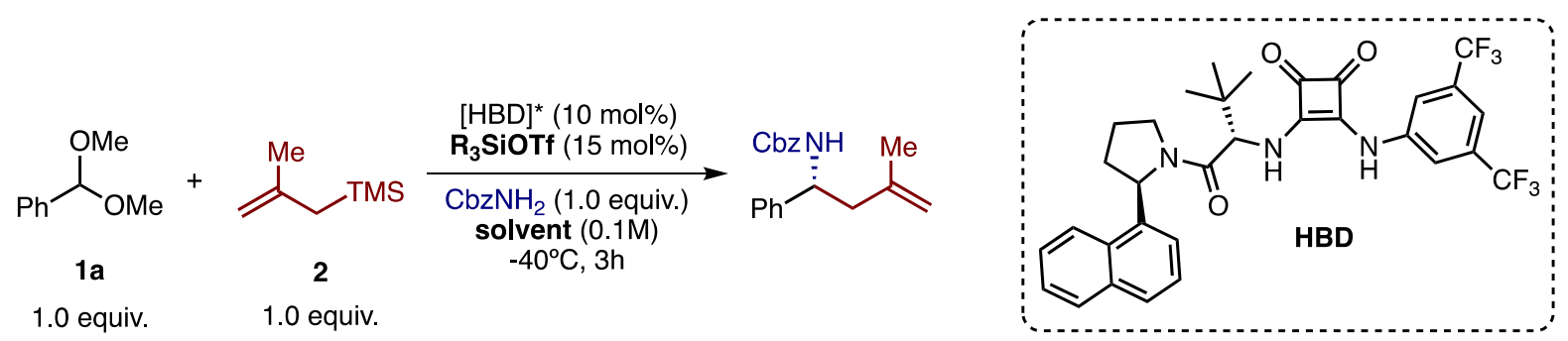

\begin{tabular}{|c|c|c|c|}
\hline entry & Solvent & $\mathbf{R}_{3}$ SiOTf & e.e. (\%) \\
\hline 1 & $\mathrm{Et}_{2} \mathrm{O}$ & TMSOTf & 40 \\
\hline 2 & $\mathrm{Et}_{2} \mathrm{O}$ & TESOTf & 45 \\
\hline 3 & $\mathrm{Et}_{2} \mathrm{O}$ & TBSOTf & 42 \\
\hline 4 & $\mathrm{TBME}$ & TMSOTf & 42 \\
\hline 5 & TBME & TESOTf & 44 \\
\hline 6 & TBME & TBSOTf & 43 \\
\hline
\end{tabular}




\section{Electrophile scope}

\section{Reaction Scope}

Table S7. Electrophile Scope.

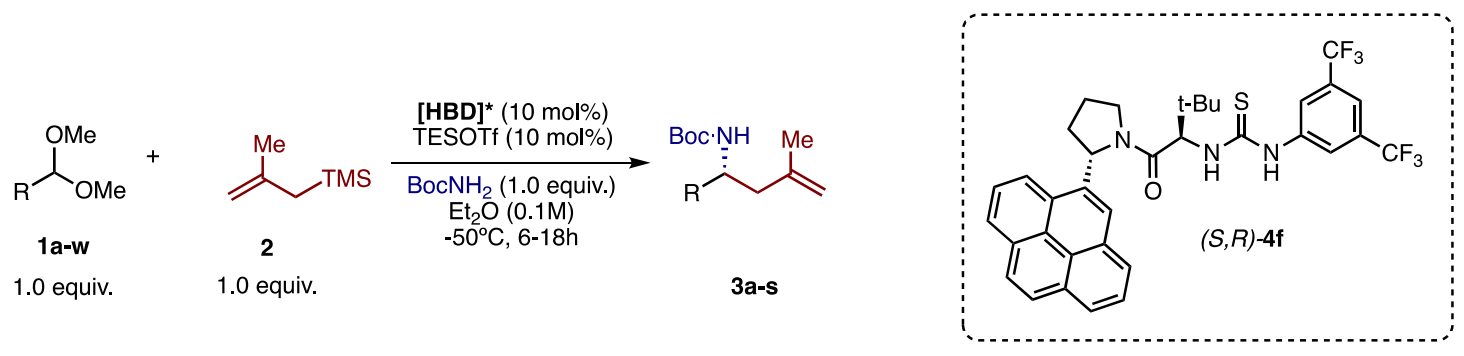

Electrophile scope
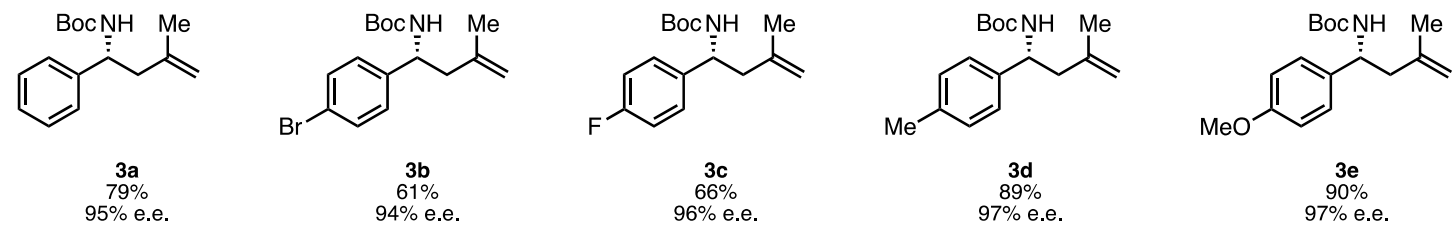

$3 \mathbf{d}$
$89 \%$
$97 \%$ e.e.
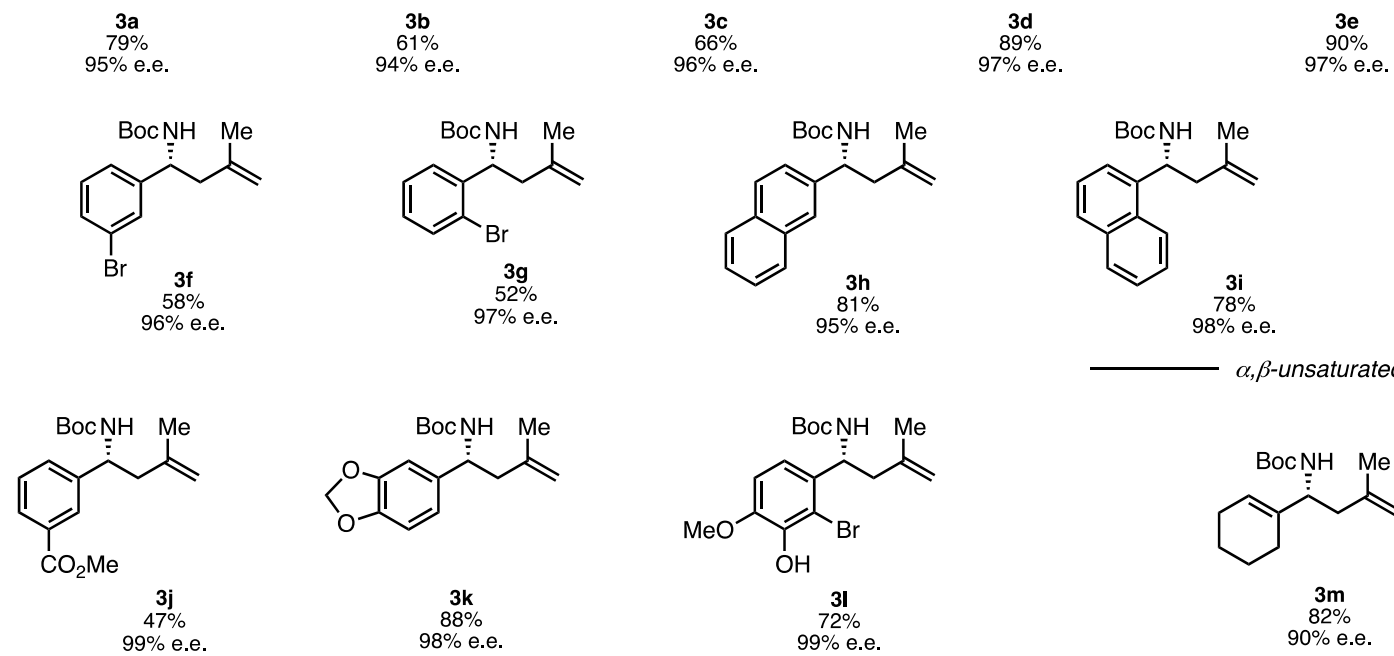

$\alpha, \beta$-unsaturated

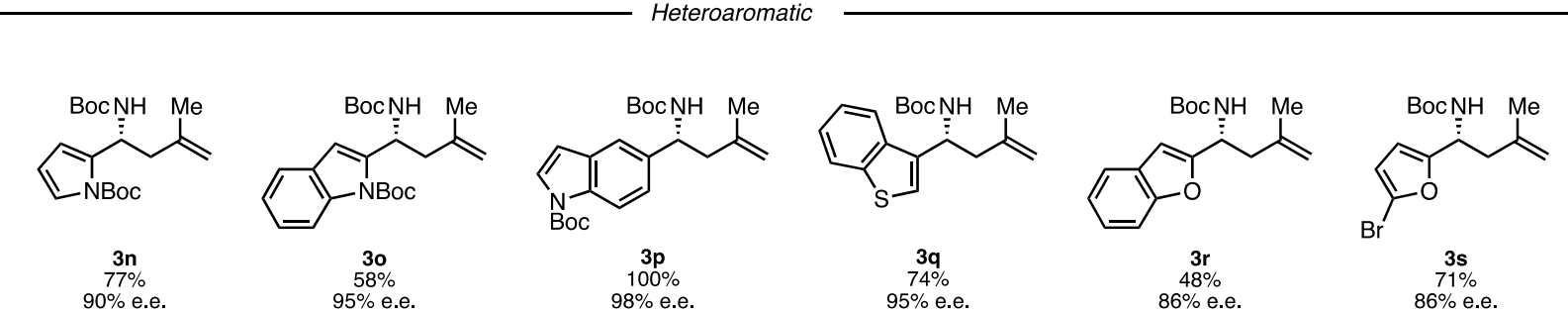

Method B: An oven-dried 2-dram vial equipped with a stir bar was sealed with a septum-lined cap, then evacuated and refilled with $\mathrm{N}_{2}(3 \mathrm{x})$. It was then charged with tert-butyl carbamate (stock solution of $0.3 \mathrm{M}$ in $\mathrm{Et}_{2} \mathrm{O}, 1.00 \mathrm{~mL}, 0.300 \mathrm{mmol}, 1.0$ equiv, otherwise noted), catalyst $(S, R)-\mathbf{4 f}$ (stock solution of $0.03 \mathrm{M}$ in $\mathrm{Et}_{2} \mathrm{O}, 1.00 \mathrm{~mL}, 0.030 \mathrm{mmol}, 0.10$ equiv, otherwise noted), acetal $(0.300 \mathrm{mmol}, 1.0$ equiv), nucleophile $\left(0.300 \mathrm{mmol}, 1.0\right.$ equiv) and the remaining volume of $\mathrm{Et}_{2} \mathrm{O}$ (to achieve a total acetal concentration of $0.1 \mathrm{M})$. The vial was carefully sealed with electrical tape, covering the septum with three layers of tape. The reaction mixture was cooled to $-78^{\circ} \mathrm{C}$ in a dry ice/acetone bath, then TESOTf $(6.8 \mu \mathrm{L}, 0.030 \mathrm{mmol}$, 
0.10 equiv) was added. The vial was transferred to a cooling bath (cryocool) at the indicated reaction temperature and the mixture was stirred for the reported time. In many cases, the initially homogeneous mixture became heterogeneous over the course of the reaction. Upon completion, the reaction was quenched by addition via syringe of a solution of $1: 1 \mathrm{Et}{ }_{3} \mathrm{~N} / \mathrm{MeOH}(200 \mu \mathrm{L})$, stirred for $5 \mathrm{~min}$ at the reaction temperature, then warmed to rt. Solvent was removed under reduced pressure, then the remaining residue was applied to a silica column for purification. Enantiomeric excess was determined by chiral HPLC analysis of the purified product.

The observed variations in isolated product yield are ascribed to incomplete conversion and recovery of starting material (with 1c, 1j, and 1s), hydrolysis to the corresponding aldehyde (e.g. for 1b), and/or formation the hemiaminal (identified as doublet around 6.0 ppm, e.g. for 1a, 1f, 1g, 1j). In no cases was the homoallylic ether identified as a by-product.

Note: All the scope reactions were run in a CC-100 Thermo NES-LAB cryocool and monitored with an $\mathrm{H}$ $B$ Instrument Thermometer, Digital w/Probe $\left(-100 / 400^{\circ} \mathrm{C}\right.$ range, $0.01^{\circ} \mathrm{C}$ resolution). The thermometer probe was positioned in the pie-block containing the reaction vial(s). A stir bar ensured circulation at the bottom of the cooling bath solution to minimize the formation of a temperature gradient.

\section{(+)-(R)-N-Boc-3-methyl-1-(4-bromophenyl)but-3-en-1-amine [3b]}<smiles>C=C(C)C[C@H](NC(=O)OC(C)(C)C)c1ccc(Br)cc1</smiles>

Prepared according to Method B. 4-bromobenzaldehyde dimethyl acetal (40.0 $\mu \mathrm{L}, 0.300 \mathrm{mmol}, 1.0$ equiv), (2-methylallyl)trimethylsilane $(53.0 \mu \mathrm{L}, 0.300 \mathrm{mmol}$, 1.0 equiv), tert-butyl carbamate ( $35.1 \mathrm{mg}, 0.300 \mathrm{mmol}, 1.0$ equiv), catalyst $(S, R)$ $4 f(19.7 \mathrm{mg}, 0.030 \mathrm{mmol}, 0.10$ equiv), TESOTf $(6.8 \mu \mathrm{L}, 0.030 \mathrm{mmol}, 0.10$ equiv), and $\mathrm{Et}_{2} \mathrm{O}(3.0 \mathrm{~mL}, 0.1 \mathrm{M})$ were used. The reaction was run at $-25^{\circ} \mathrm{C}$ for $24 \mathrm{~h}$. Flash column chromatography on silica (Biotage, $25 \mathrm{~g}$ column, Diethyl ether:Hexanes gradient followed by a second purification in $100 \%$ DCM) gave pure product as a white solid.

Chiral HPLC conditions: CHIRALPAK AS-H, $2 \%$ PrOH/hexanes, $1 \mathrm{~mL} / \mathrm{min}$

Racemic product: $\mathrm{t}_{\mathrm{R}}(R)=11.3 \mathrm{~min}, \mathrm{t}_{\mathrm{R}}(S)=14.6 \mathrm{~min}$

Enantioenriched product: $\mathrm{t}_{\mathrm{R}}(R)=10.8 \mathrm{~min}$

\section{$62.0 \mathrm{mg}, 0.182 \mathrm{mmol}, 61 \%$ yield, $94 \%$ e.e.}

${ }^{1} \mathrm{H}-\mathrm{NMR}\left(600 \mathrm{MHz}, \mathrm{CDCl}_{3}\right) \delta 7.44(\mathrm{~d}, J=8.4 \mathrm{~Hz}, 2 \mathrm{H}), 7.16(\mathrm{~d}, J=8.1 \mathrm{~Hz}, 2 \mathrm{H}), 4.84(\mathrm{~s}, 1 \mathrm{H}), 4.79$ (br. s, $2 \mathrm{H}), 4.73(\mathrm{~s}, 1 \mathrm{H}), 2.39-2.34(\mathrm{~m}, 2 \mathrm{H}), 1.72(\mathrm{~s}, 3 \mathrm{H}), 1.40$ (br. s, 9H); ${ }^{13} \mathrm{C}-\mathrm{NMR}\left(126 \mathrm{MHz}, \mathrm{CDCl}_{3}\right) \delta 155.3$, 142.5, 141.6, 131.7, 128.0, 120.9, 114.3, 79.8, 52.4, 45.8, 28.4, 22.0; IR (ATR, $\left.\mathrm{cm}^{-1}\right) 3380,2970,1679$, $1519,1487,1286,1249,1170,1071,1041 ;[\alpha]^{22} \mathrm{D}=+37.7^{\circ}\left(c=1.0, \mathrm{CDCl}_{3}\right) ; \mathrm{HRMS}(\mathrm{ESI}) \mathrm{m} / \mathrm{z}$ calculated for $\mathrm{C}_{16} \mathrm{H}_{22} \mathrm{BrNO}_{2} \mathrm{Na}[\mathrm{M}+\mathrm{Na}]^{+}:$364.0707, found 364.0719.

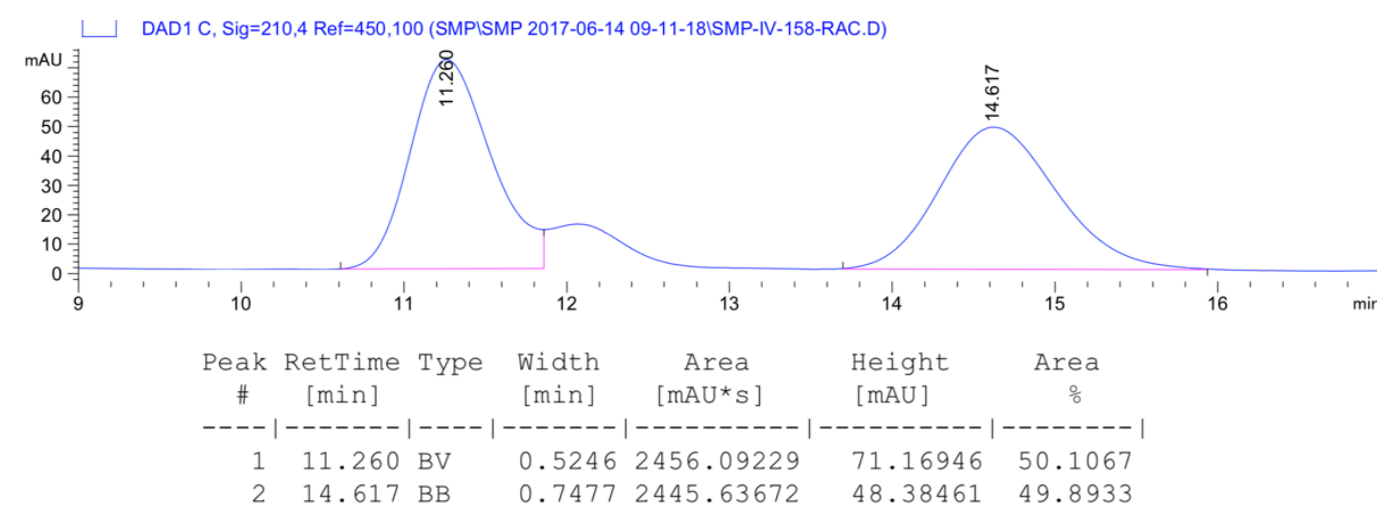




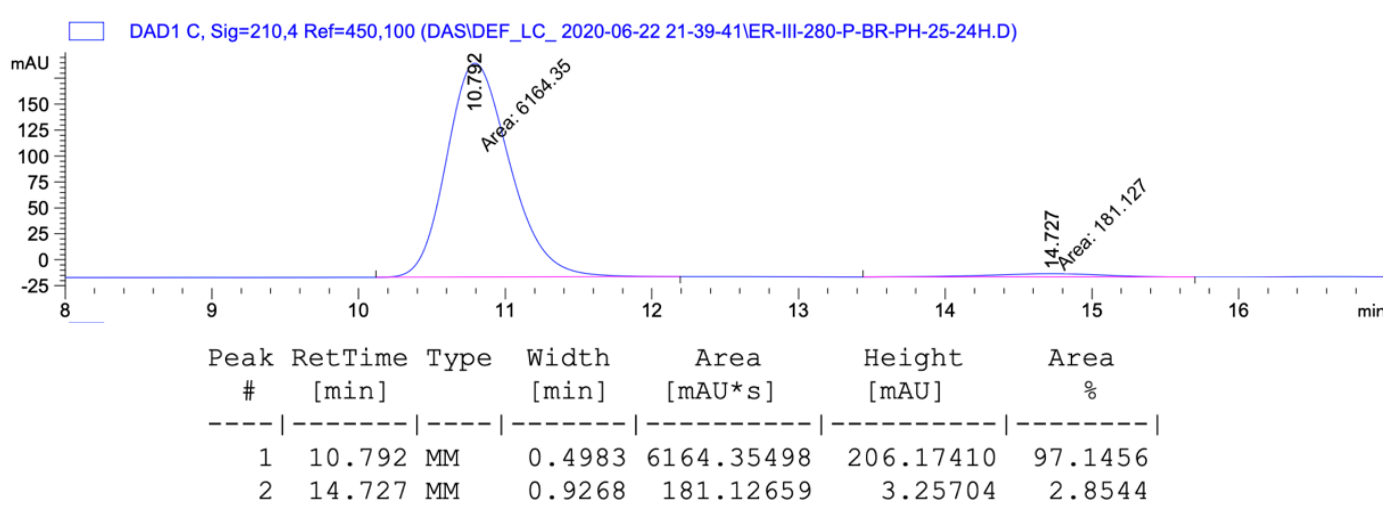

\section{(+)-(R)-N-Boc-3-methyl-1-(4-fluorophenyl)but-3-en-1-amine [3c]}<smiles>C=C(C)C[C@H](NC(=O)OC(C)(C)C)c1ccc(F)cc1</smiles>

Racemic product: $\mathrm{t}_{\mathrm{R}}(R)=10.8 \mathrm{~min}, \mathrm{t}_{\mathrm{R}}(S)=11.7 \mathrm{~min}$

Enantioenriched product: $\mathrm{t}_{\mathrm{R}}(R)=10.6 \mathrm{~min}$

$55.2 \mathrm{mg}, 0.198 \mathrm{mmol}, 66 \%$ yield, $96 \%$ e.e.

${ }^{1} \mathrm{H}-\mathrm{NMR}\left(500 \mathrm{MHz}, \mathrm{CDCl}_{3}\right) \delta 7.26-7.23(\mathrm{~m}, 2 \mathrm{H}), 7.00$ (t, $\left.J=8.6 \mathrm{~Hz}, 2 \mathrm{H}\right), 4.82(\mathrm{~s}, 1 \mathrm{H}), 4.77$ (br. s, 2H), $4.72(\mathrm{~s}, 1 \mathrm{H}), 2.42-2.37(\mathrm{~m}, 2 \mathrm{H}), 1.72(\mathrm{~s}, 3 \mathrm{H}), 1.39$ (br. s, 9H); ${ }^{13} \mathrm{C}-\mathrm{NMR}\left(126 \mathrm{MHz}, \mathrm{CDCl}_{3}\right) \delta 163.0$, 161.0, 155.3, 141.8, $127.8(\mathrm{JC}-\mathrm{F}=8.0 \mathrm{~Hz}), 115.4(\mathrm{JC}-\mathrm{F}=21.3 \mathrm{~Hz}), 114.2,79.7,52.4,45.9,28.5,22.1$; IR $\left(\right.$ ATR, $\left.\mathrm{cm}^{-1}\right) 3384,2978,1681,1509,1364,1249,1217,1171,1158,1051 ;[\alpha]^{24} \mathrm{D}=+28.0^{\circ}(c=0.5$, $\mathrm{CDCl}_{3}$ ); HRMS (ESI) $\mathrm{m} / 2$ calculated for $\mathrm{C}_{16} \mathrm{H}_{22} \mathrm{FNO}_{2} \mathrm{Na}[\mathrm{M}+\mathrm{Na}]^{+}: 302.1527$, found 302.1530 .
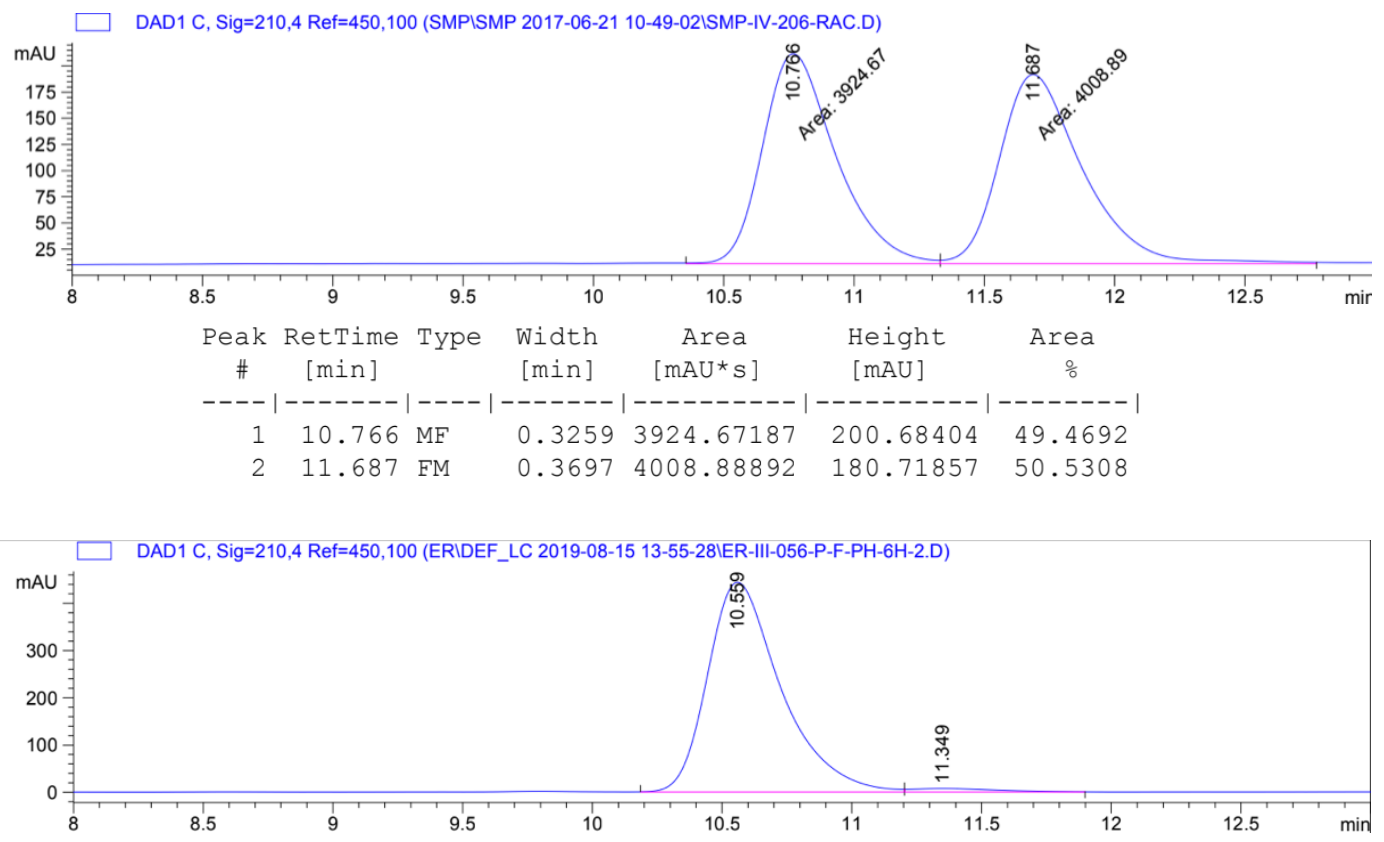


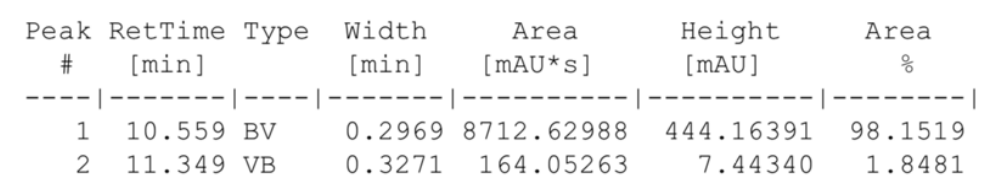

\section{(+)-(R)-N-Boc-3-methyl-1-(4-methylphenyl)but-3-en-1-amine [3d]}<smiles>C=C(C)C[C@H](NC(=O)OC(C)(C)C)c1ccc(C)cc1</smiles>

Prepared according to Method B. 4-methylbenzaldehyde dimethyl acetal 1d (49.9 mg, $0.300 \mathrm{mmol}, 1.0$ equiv), (2-methylallyl) trimethylsilane $(53.0 \mu \mathrm{L}, 0.300$ mmol, 1.0 equiv), tert-butyl carbamate $(35.1 \mathrm{mg}, 0.300 \mathrm{mmol}, 1.0$ equiv), catalyst $(S, R)-\mathbf{4 f} \quad(19.7 \mathrm{mg}, 0.030 \mathrm{mmol}, 0.10$ equiv $)$, TESOTf $(6.8 \mu \mathrm{L}, 0.030$ mmol, 0.10 equiv), and $\mathrm{Et}_{2} \mathrm{O}(3.0 \mathrm{~mL}, 0.1 \mathrm{M})$ were used. The reaction was run at $-50^{\circ} \mathrm{C}$ for $6 \mathrm{~h}$ or $48 \mathrm{~h}$. Flash column chromatography afforded pure product as a white solid.

Chiral HPLC conditions: CHIRALPAK AS-H, $3 \%{ }^{\mathrm{i} P \mathrm{POH}} / \mathrm{hexanes} 1 \mathrm{~mL} / \mathrm{min}$

Racemic product: $\mathrm{tR}_{\mathrm{R}}(R)=6.5 \mathrm{~min}, \mathrm{tR}_{\mathrm{R}}(S)=9.1 \mathrm{~min}$

Enantioenriched product: $\operatorname{tR}_{\mathrm{R}}(R)=6.3 \mathrm{~min}$

6h: $73.2 \mathrm{mg}, 0.266 \mathrm{mmol}, 89 \%$ yield, $97 \%$ e.e.

48h: $78.4 \mathrm{mg}, 0.285 \mathrm{mmol}, 95 \%$ yield, $98 \%$ e.e.

${ }^{1} \mathrm{H}-\mathrm{NMR}\left(500 \mathrm{MHz}, \mathrm{CDCl}_{3}\right) \delta 7.18(\mathrm{~d}, J=7.9 \mathrm{~Hz}, 2 \mathrm{H}), 7.12(\mathrm{~d}, J=7.1 \mathrm{~Hz}, 2 \mathrm{H}), 4.81(\mathrm{~s}, 1 \mathrm{H}), 4.77$ (br. s, $2 \mathrm{H}$ ), $4.73(\mathrm{~s}, 1 \mathrm{H}), 2.43(\mathrm{~m}, 2 \mathrm{H}), 2.33(\mathrm{~s}, 3 \mathrm{H}), 1.73$ (s, 3H), 1.41 (br. s, 9H); ${ }^{33} \mathrm{C}-\mathrm{NMR}\left(126 \mathrm{MHz}, \mathrm{CDCl}_{3}\right) \delta$ 155.4, 142.2, 140.1, 136.8, 129.3, 126.2, 113.8, 79.5, 52.7, 45.9, 28.5, 22.1, 21.2; IR (ATR, $\left.\mathrm{cm}^{-1}\right) 3373$, $2971,2919,1682,1650,1514,1442,1390,1362,1287,1250,1166,1062,1014 ;[\alpha]^{24} \mathrm{D}=+24.3^{\circ}(c=1.0$, $\mathrm{CDCl}_{3}$ ); HRMS (ESI) $\mathrm{m} / z$ calculated for $\mathrm{C}_{17} \mathrm{H}_{26} \mathrm{NO}_{2}[\mathrm{M}+\mathrm{H}]^{+}: 276.1958$, found 276.1961 .
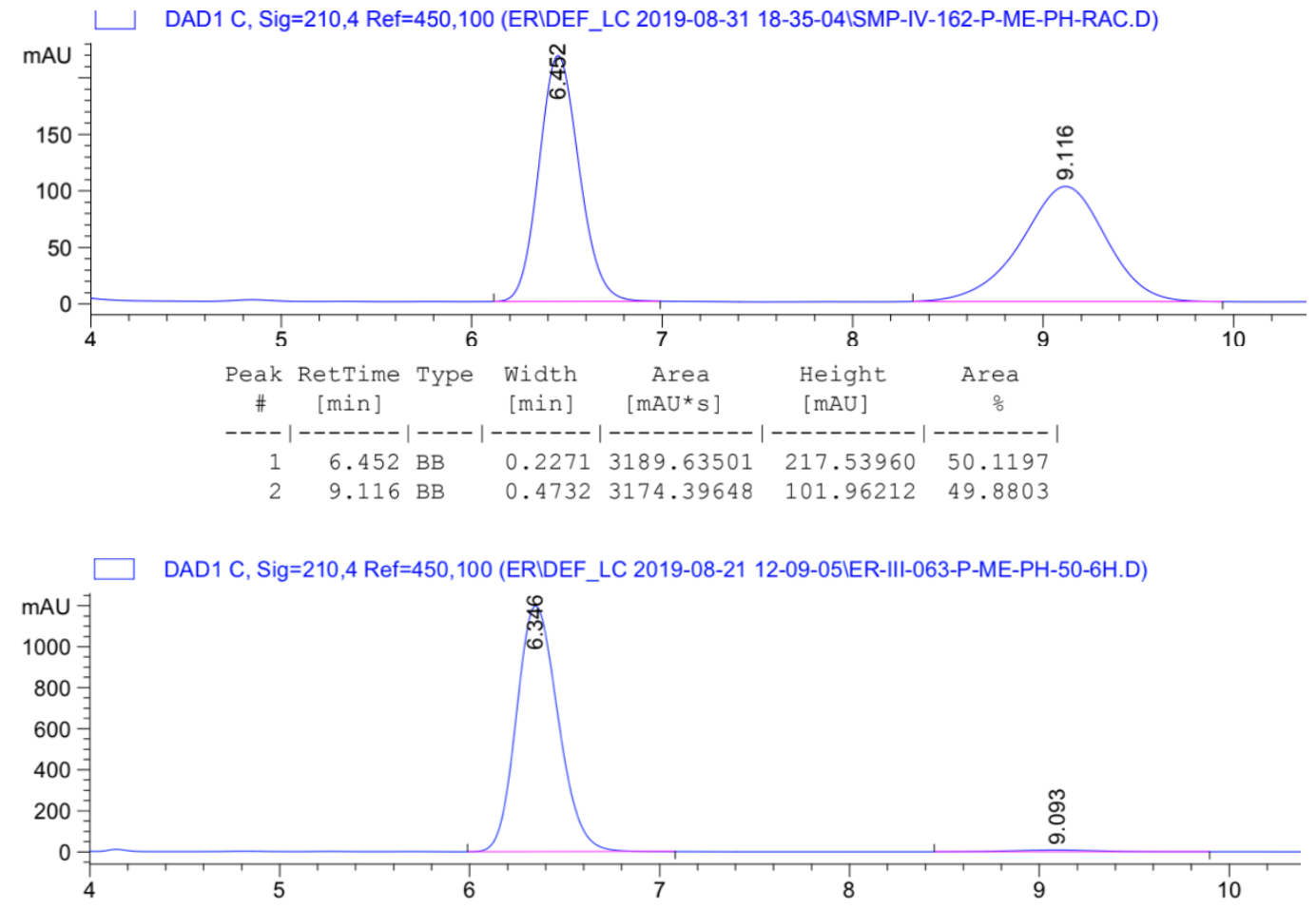


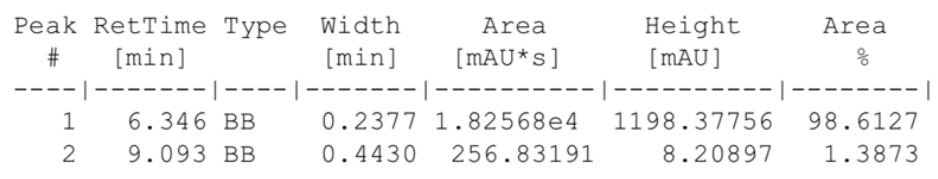

\section{(+)-(R)-N-Boc-3-methyl-1-(4-methoxyphenyl)but-3-en-1-amine [3e]}<smiles>C=C(C)C[C@H](NC(=O)OC(C)(C)C)c1ccc(OC)cc1</smiles>

Prepared according to Method B. 4-methoxybenzaldehyde dimethyl acetal $(51.0 \mu \mathrm{L}, 0.300 \mathrm{mmol}, 1.0$ equiv), (2-methylallyl)trimethylsilane $(53.0 \mu \mathrm{L}, 0.300$ mmol, 1.0 equiv), tert-butyl carbamate $(35.1 \mathrm{mg}, 0.300 \mathrm{mmol}, 1.0$ equiv), catalyst $(S, R)-\mathbf{4 f}(19.7 \mathrm{mg}, 0.030 \mathrm{mmol}, 0.10$ equiv), TESOTf $(6.8 \mu \mathrm{L}, 0.030$ mmol, 0.10 equiv), and $\mathrm{Et}_{2} \mathrm{O}(3.0 \mathrm{~mL}, 0.1 \mathrm{M})$ were used. The reaction was run at $-50^{\circ} \mathrm{C}$ for $6 \mathrm{~h}$ or $48 \mathrm{~h}$ Flash column chromatography afforded pure product as a white solid.

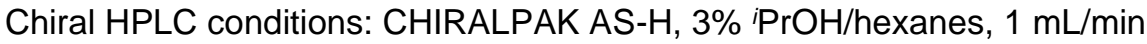

Racemic product: $\mathrm{tR}_{\mathrm{R}}(R)=11.4 \mathrm{~min}, \mathrm{t}_{\mathrm{R}}(S)=14.2 \mathrm{~min}$

Enantioenriched product: $\mathrm{t}_{\mathrm{R}}(R)=11.6 \mathrm{~min}$

6h: $79.0 \mathrm{mg}, 0.271 \mathrm{mmol}, \mathbf{9 0 \%}$ yield, $97 \%$ e.e.

48h: $83.2 \mathrm{mg}, 0.286 \mathrm{mmol}, 95 \%$ yield, $97 \%$ e.e.

${ }^{1} \mathrm{H}-\mathrm{NMR}\left(500 \mathrm{MHz}, \mathrm{CDCl}_{3}\right) \delta 7.20(\mathrm{~d}, J=8.7 \mathrm{~Hz}, 2 \mathrm{H}), 6.85(\mathrm{~d}, J=8.7 \mathrm{~Hz}, 2 \mathrm{H}), 4.80(\mathrm{~s}, 1 \mathrm{H}), 4.75$ (br. s, 2H), $4.72(\mathrm{~s}, 1 \mathrm{H}), 3.78(\mathrm{~s}, 3 \mathrm{H}), 2.53-226(\mathrm{~m}, 2 \mathrm{H}), 1.71(\mathrm{~s}, 3 \mathrm{H}), 1.39(\mathrm{~s}, 9 \mathrm{H}) ;{ }^{13} \mathrm{C}-\mathrm{NMR}\left(126 \mathrm{MHz}, \mathrm{CDCl}_{3}\right) \delta$ 158.7, 155.4, 142.1, 135.3, 127.4, 114.0, 113.8, 79.4, 55.3, 52.4, 45.8, 28.5, 22.1; IR (ATR, $\left.\mathrm{cm}^{-1}\right) 3379$, $3005,2974,2934,1680,1588,1512,1458,1442,1365,1241,1168,1028 ;[\alpha]^{24} \mathrm{D}=+48.1^{\circ}(c=1.0$, $\mathrm{CDCl}_{3}$ ); HRMS (ESI) $\mathrm{m} / z$ calculated for $\mathrm{C}_{17} \mathrm{H}_{26} \mathrm{NO}_{3}[\mathrm{M}+\mathrm{H}]^{+}: 292.1907$, found 292.1902.
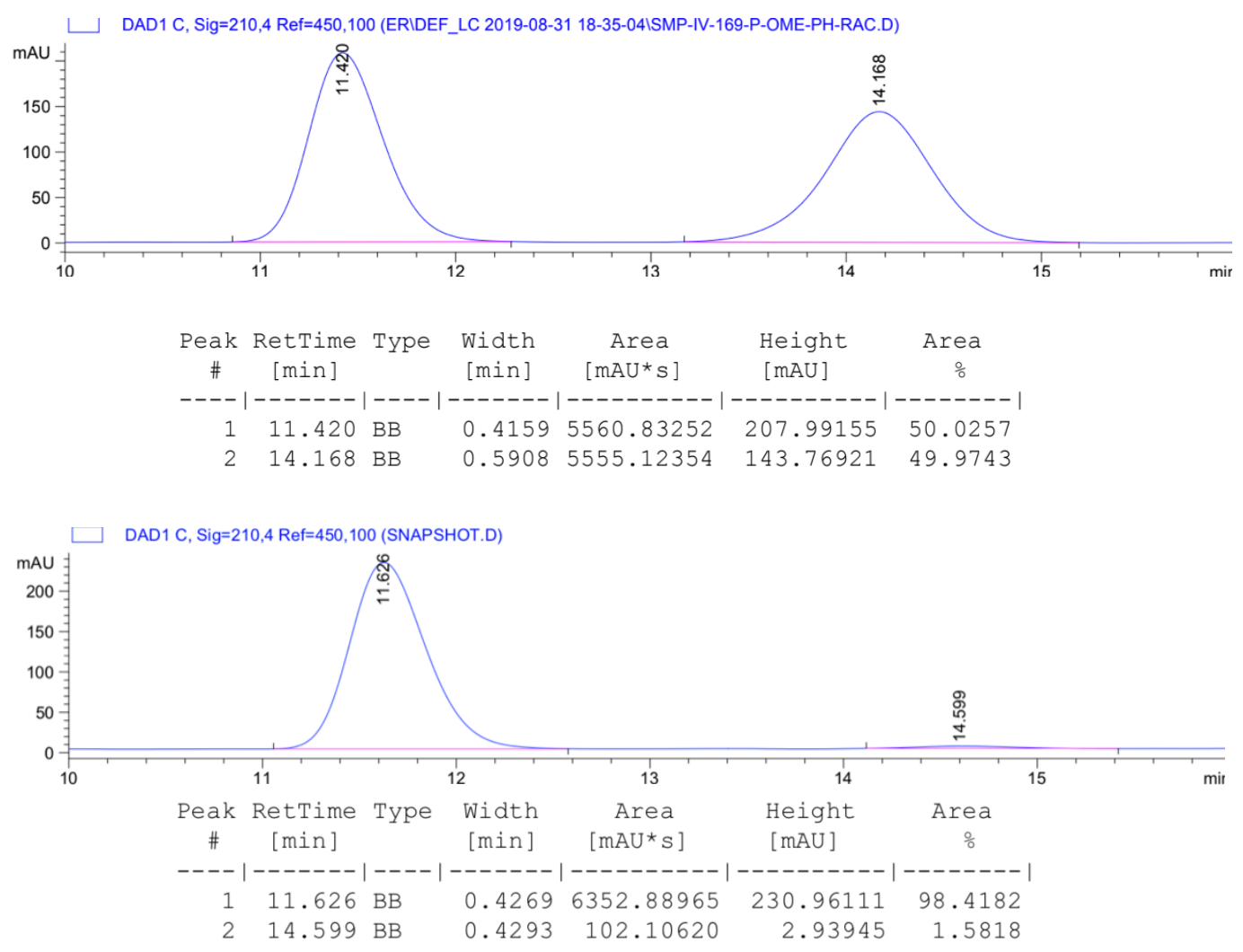


\section{(+)-(R)-N-Boc-3-methyl-1-(3-bromophenyl)but-3-en-1-amine [3f]}<smiles>C=C(C)C[C@H](NC(=O)OC(C)(C)C)c1cccc(Br)c1</smiles>

Prepared according to Method B. 3-Bromobenzaldehyde diethyl acetal $(60.0 \mu \mathrm{L}$, $0.300 \mathrm{mmol}, 1.0$ equiv), (2-methylallyl)trimethylsilane $(53.0 \mu \mathrm{L}, 0.300 \mathrm{mmol}, 1.0$ equiv), tert-butyl carbamate ( $35.1 \mathrm{mg}, 0.300 \mathrm{mmol}, 1.0$ equiv), catalyst $(S, R)-\mathbf{4 f}$ (19.7 mg, $0.030 \mathrm{mmol}, 0.10$ equiv), TESOTf ( $6.8 \mu \mathrm{L}, 0.030 \mathrm{mmol}, 0.10$ equiv), and $\mathrm{Et}_{2} \mathrm{O}(3.0 \mathrm{~mL}, 0.1 \mathrm{M})$ were used. The reaction was run at $-25^{\circ} \mathrm{C}$ for $6 \mathrm{~h}$ or $18 \mathrm{~h}$. Flash column chromatography on silica (Biotage, $10 \mathrm{~g}$ column) using a EtOAc/hexanes gradient followed by a $100 \%$ DCM run, gave pure product as a white solid.

Chiral HPLC conditions: CHIRALPAK AS-H, 3\% ${ }^{\mathrm{P} P O H} / \mathrm{hexanes} 1 \mathrm{~mL} / \mathrm{min}$

Racemic product: $\mathrm{t}_{\mathrm{R}}(R)=9.0 \mathrm{~min}, \mathrm{t}_{\mathrm{R}}(S)=11.0 \mathrm{~min}$

Enantioenriched product: $\mathrm{t}_{\mathrm{R}}(R)=7.3 \mathrm{~min}$

6h: $54.3 \mathrm{mg}, 0.160 \mathrm{mmol}, 53 \%$ yield, $95 \%$ e.e.

18h: $59.4 \mathrm{mg}, 0.175 \mathrm{mmol}, 58 \%$ yield, $96 \%$ e.e.

${ }^{1} \mathrm{H}-\mathrm{NMR}\left(500 \mathrm{MHz}, \mathrm{CDCl}_{3}\right) \delta 7.48-7.40(\mathrm{~m}, 1 \mathrm{H}), 7.38-7.36(\mathrm{~m}, 1 \mathrm{H}), 7.23-7.15(\mathrm{~m}, 2 \mathrm{H}), 4.84(\mathrm{~s}, 1 \mathrm{H})$, 4.79 (br. s, 2H), $4.74(\mathrm{~s}, 1 \mathrm{H}), 2.51-2.23(\mathrm{~m}, 2 \mathrm{H}), 1.73(\mathrm{~s}, 3 \mathrm{H}), 1.41(\mathrm{~s}, 9 \mathrm{H}) ;{ }^{13} \mathrm{C}-\mathrm{NMR}\left(126 \mathrm{MHz}, \mathrm{CDCl}_{3}\right)$ $\delta$ 155.3, 145.8, 141.5, 130.3, 130.2, 129.3, 124.9, 122.7, 114.3, 79.9, 52.5, 45.9, 28.4, 22.0; IR (ATR, cm 1) $3376,2973,2920,1682,1518,1249,1165,996 ;[\alpha]^{24} \mathrm{D}=+28.7^{\circ}\left(c=0.5, \mathrm{CDCl}_{3}\right)$; HRMS $(\mathrm{ESI}) \mathrm{m} / \mathrm{z}$ calculated for $\mathrm{C}_{16} \mathrm{H}_{23} \mathrm{BrNO}_{2}[\mathrm{M}+\mathrm{H}]^{+}: 340.0907$, found 340.0904 .
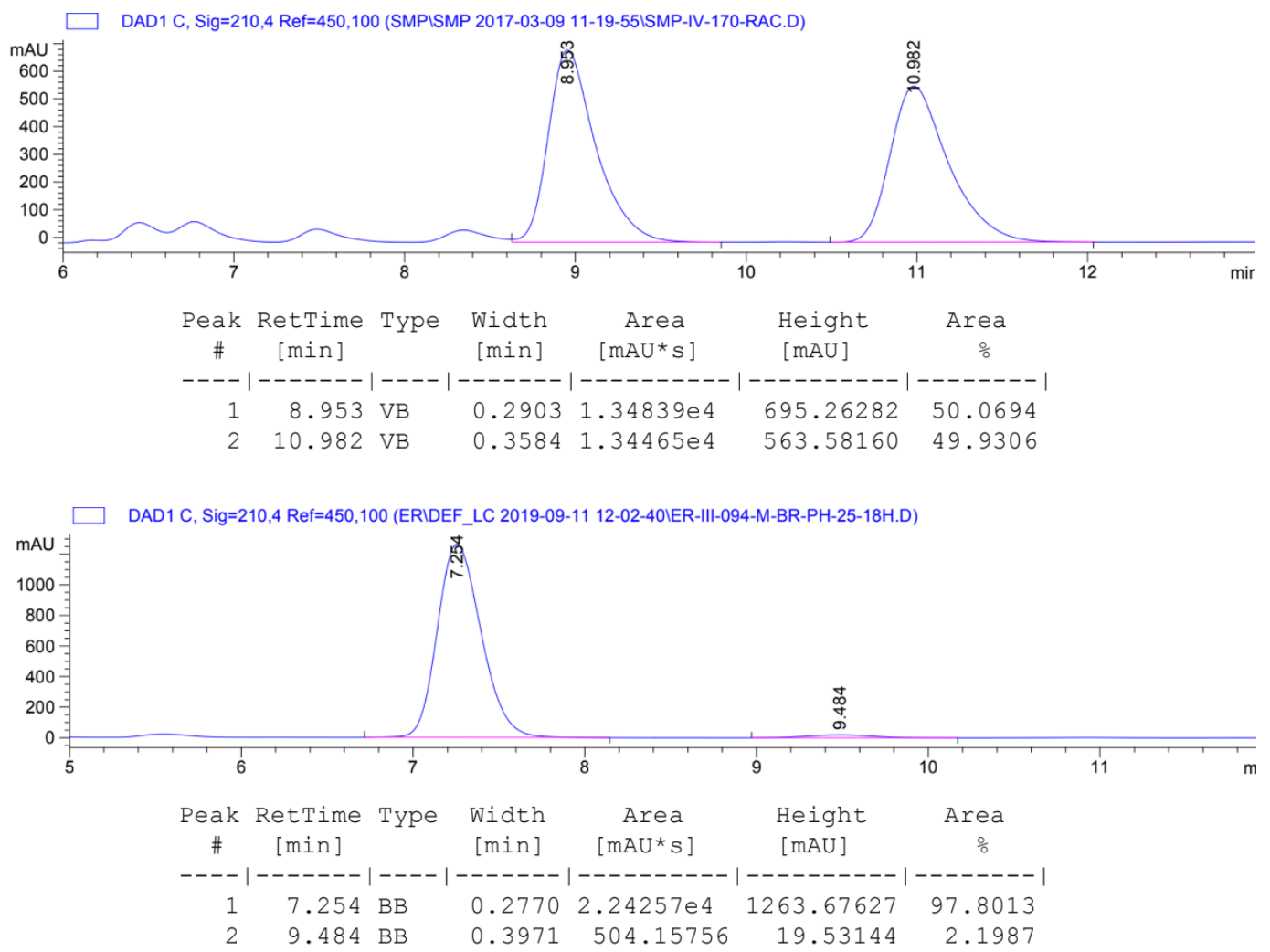


\section{$(-)-(R)-N-B o c-3-m e t h y l-1-(2-b r o m o p h e n y l) b u t-3-e n-1-a m i n e ~[3 g]$}<smiles>C=C(C)C[C@H](NC(=O)OC(C)(C)C)c1ccccc1Br</smiles>

Prepared according to Method B. 2-bromobenzaldehyde diethyl acetal $(60.0 \mu \mathrm{L}$, $0.300 \mathrm{mmol}, 1.0$ equiv), (2-methylallyl)trimethylsilane $(53.0 \mu \mathrm{L}, 0.300 \mathrm{mmol}, 1.0$ equiv), tert-butyl carbamate (35.1 $\mathrm{mg}, 0.300 \mathrm{mmol}, 1.0$ equiv), catalyst $(S, R)-\mathbf{4 f}$ (19.7 mg, $0.030 \mathrm{mmol}, 0.10$ equiv), TESOTf ( $6.8 \mu \mathrm{L}, 0.030 \mathrm{mmol}, 0.10$ equiv), and $\mathrm{Et}_{2} \mathrm{O}(3.0 \mathrm{~mL}, 0.1 \mathrm{M})$ were used. The reaction was run at $-25^{\circ} \mathrm{C}$ for $18 \mathrm{~h}$. Flash column chromatography on silica (Biotage, $10 \mathrm{~g}$ column) gave pure product as a white solid.

Chiral HPLC conditions: CHIRALPAK AS-H, 15\% 'PrOH/hexanes, $1 \mathrm{~mL} / \mathrm{min}$

Racemic product: $\mathrm{t}_{\mathrm{R}}(R)=4.1 \mathrm{~min}, \mathrm{t}_{\mathrm{R}}(S)=5.8 \mathrm{~min}$

Enantioenriched product: $\operatorname{tR}_{\mathrm{R}}(R)=4.1 \mathrm{~min}$

$53.5 \mathrm{mg}, 0.157 \mathrm{mmol}, \mathbf{5 2} \%$ yield, $\mathbf{9 7} \%$ e.e.

The reported ${ }^{1} \mathrm{H}-\mathrm{NMR}$ was run at $50^{\circ} \mathrm{C} .{ }^{1} \mathrm{H}-\mathrm{NMR}\left(500 \mathrm{MHz}, \mathrm{CDCl}_{3}\right) \delta 7.53(\mathrm{~d}, J=8.0 \mathrm{~Hz}, 1 \mathrm{H}), 7.33(\mathrm{~d}, J=$ $8.0 \mathrm{~Hz}, 1 \mathrm{H}$ ), 7.28 (t, $J=7.6 \mathrm{~Hz}, 1 \mathrm{H}$ ), 7.09 (td, $J=7.6,1.8 \mathrm{~Hz}, 1 \mathrm{H}), 5.12$ (br. s, $1 \mathrm{H}$ ), 4.91 (br. s, $1 \mathrm{H}$ ), 4.87 $(\mathrm{s}, 1 \mathrm{H}), 4.80(\mathrm{~s}, 1 \mathrm{H}), 2.71-2.42(\mathrm{~m}, 1 \mathrm{H}), 2.19(\mathrm{t}, J=12.3 \mathrm{~Hz}, 1 \mathrm{H}), 1.80(\mathrm{~s}, 3 \mathrm{H}), 1.37(\mathrm{~s}, 9 \mathrm{H})$; Rotamers are observed at $25{ }^{\circ} \mathrm{C}$ in the ${ }^{13} \mathrm{C}-\mathrm{NMR}$ spectrum. ${ }^{13} \mathrm{C}-\mathrm{NMR}\left(126 \mathrm{MHz}, \mathrm{CDCl}_{3}\right) \delta 155.2,142.3,142.1$, 133.3, 128.5, 127.7, 127.0, 122.6, 114.0, 79.7, 52.5, 44.4, 28.4, 21.7; IR (ATR, $\left.\mathrm{cm}^{-1}\right)$ 3366, 2979, 2940, 2917, 1683, 1522, 1440, 1354, 1281, 1248, 1164, 1014, 753; $[\alpha]^{24} \mathrm{D}=-10.3^{\circ}\left(c=1.0, \mathrm{CDCl}_{3}\right)$; HRMS (ESI) $\mathrm{m} / \mathrm{z}$ calculated for $\mathrm{C}_{16} \mathrm{H}_{23} \mathrm{BrNO}_{2}[\mathrm{M}+\mathrm{H}]^{+}: 340.0907$, found 340.0904 .
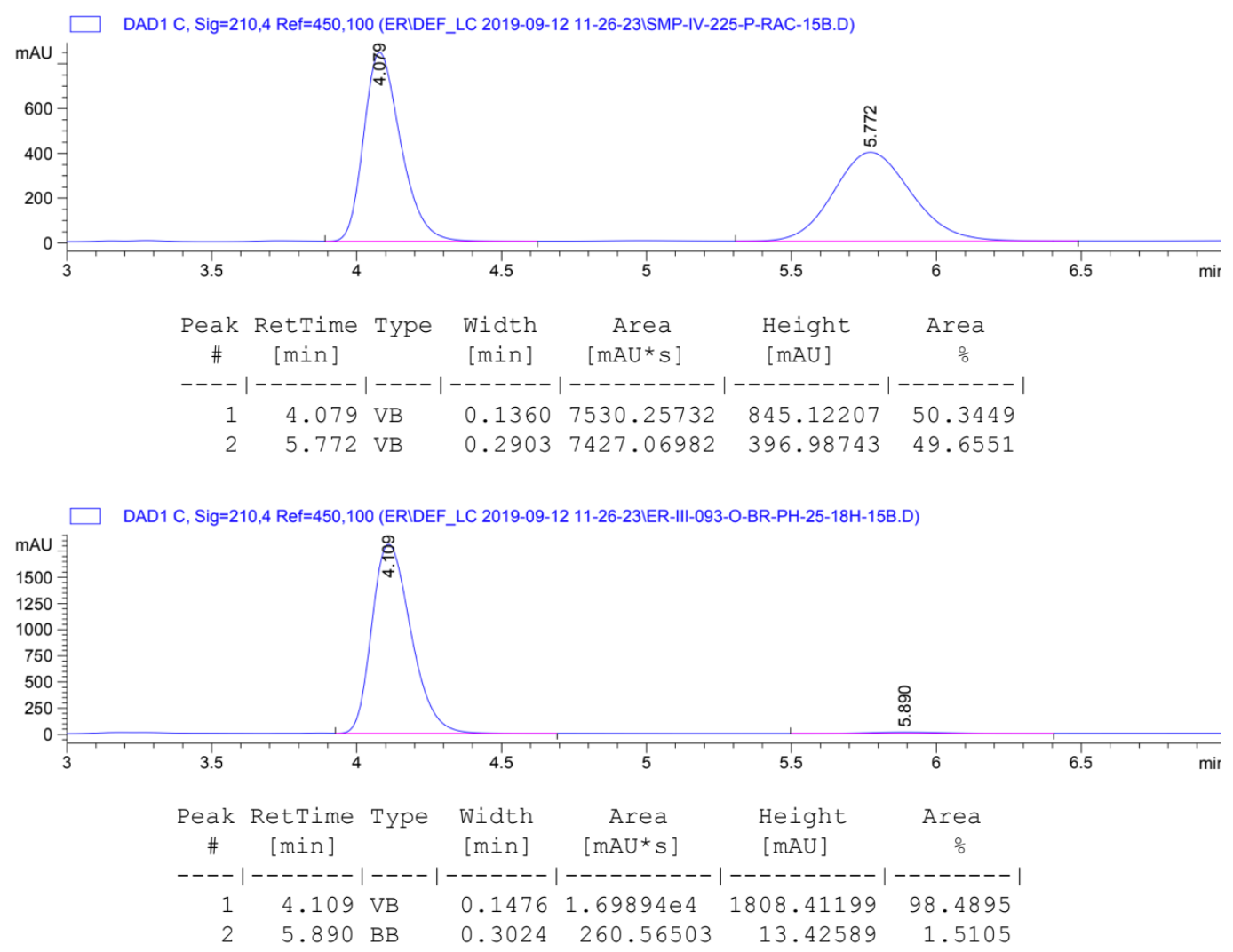


\section{(+)-(R)-N-Boc-3-methyl-1-(2-naphthyl)but-3-en-1-amine [3h]}<smiles>C=C(C)C[C@H](NC(=O)OC(C)(C)C)c1ccc2ccccc2c1</smiles>

Prepared according to Method B. 2-Naphthaldehyde dimethyl acetal $\mathbf{1 h}$ (60.6 $\mathrm{mg}, 0.300 \mathrm{mmol}, 1.0$ equiv), (2-methylallyl)trimethylsilane $(53.0 \mu \mathrm{L}, 0.300 \mathrm{mmol}$, 1.0 equiv), tert-butyl carbamate $(35.1 \mathrm{mg}, 0.300 \mathrm{mmol}, 1.0$ equiv), catalyst $(S, R)$-4f $(19.7 \mathrm{mg}, 0.030 \mathrm{mmol}, 0.10$ equiv), TESOTf $(6.8 \mu \mathrm{L}, 0.030 \mathrm{mmol}, 0.10$ equiv), and $\mathrm{Et}_{2} \mathrm{O}(3.0 \mathrm{~mL}, 0.1 \mathrm{M})$ were used. The reaction was run at $-50^{\circ} \mathrm{C}$ for $6 \mathrm{~h}$ or $48 \mathrm{~h}$. Flash column chromatography afforded pure product as a white solid.

Chiral HPLC conditions: CHIRALPAK AD-H, 3\% $\mathrm{PrOH} / \mathrm{hexanes,} 1 \mathrm{~mL} / \mathrm{min}$

Racemic product: $\mathrm{t}_{\mathrm{R}}(R)=13.2 \mathrm{~min}, \mathrm{t}_{\mathrm{R}}(S)=15.5 \mathrm{~min}$

Enantioenriched product: $\mathrm{t}_{\mathrm{R}}(R)=14.2 \mathrm{~min}$

6h: $75.5 \mathrm{mg}, 0.242 \mathrm{mmol}, \mathbf{8 1} \%$ yield, $95 \%$ e.e.

48h: $87.3 \mathrm{mg}, 0.280 \mathrm{mmol}, 93 \%$ yield, $94 \%$ e.e.

${ }^{1} \mathrm{H}-\mathrm{NMR}\left(600 \mathrm{MHz}, \mathrm{CDCl}_{3}\right) \delta 7.83$ (dd, $\left.J=7.8,1.8 \mathrm{~Hz}, 3 \mathrm{H}\right), 7.76(\mathrm{~s}, 1 \mathrm{H}), 7.46$ (tdd, $J=16.6,7.6,1.7 \mathrm{~Hz}$, 3H), 5.02 (br. s, 2H), $4.86(\mathrm{~s}, 1 \mathrm{H}), 4.79(\mathrm{~s}, 1 \mathrm{H}), 2.70-2.38(\mathrm{~m}, 2 \mathrm{H}), 1.78(\mathrm{~s}, 3 \mathrm{H}), 1.41(\mathrm{~s}, 9 \mathrm{H}) ;{ }^{13} \mathrm{C}-\mathrm{NMR}$ $\left(126 \mathrm{MHz}, \mathrm{CDCl}_{3}\right) \delta 155.4,141.9,140.6,133.5,132.8,128.4,127.9,127.7,126.1,125.7,124.8,124.6$, 114.0, 79.5, 53.0, 45.7, 28.4, 22.1; IR (ATR, $\mathrm{cm}^{-1}$ ) 3395, 2979, 2935, 2909, 1684, 1505, 1245, 1167, 1042, 892, 858, 825; $[\mathrm{\alpha}]^{24} \mathrm{D}=+49.5^{\circ}\left(c=1.0, \mathrm{CDCl}_{3}\right)$; HRMS $(\mathrm{ESI}) \mathrm{m} / z$ calculated for $\mathrm{C}_{20} \mathrm{H}_{26} \mathrm{NO}_{2}[\mathrm{M}+\mathrm{H}]^{+}$: 312.1958, found 312.1955.
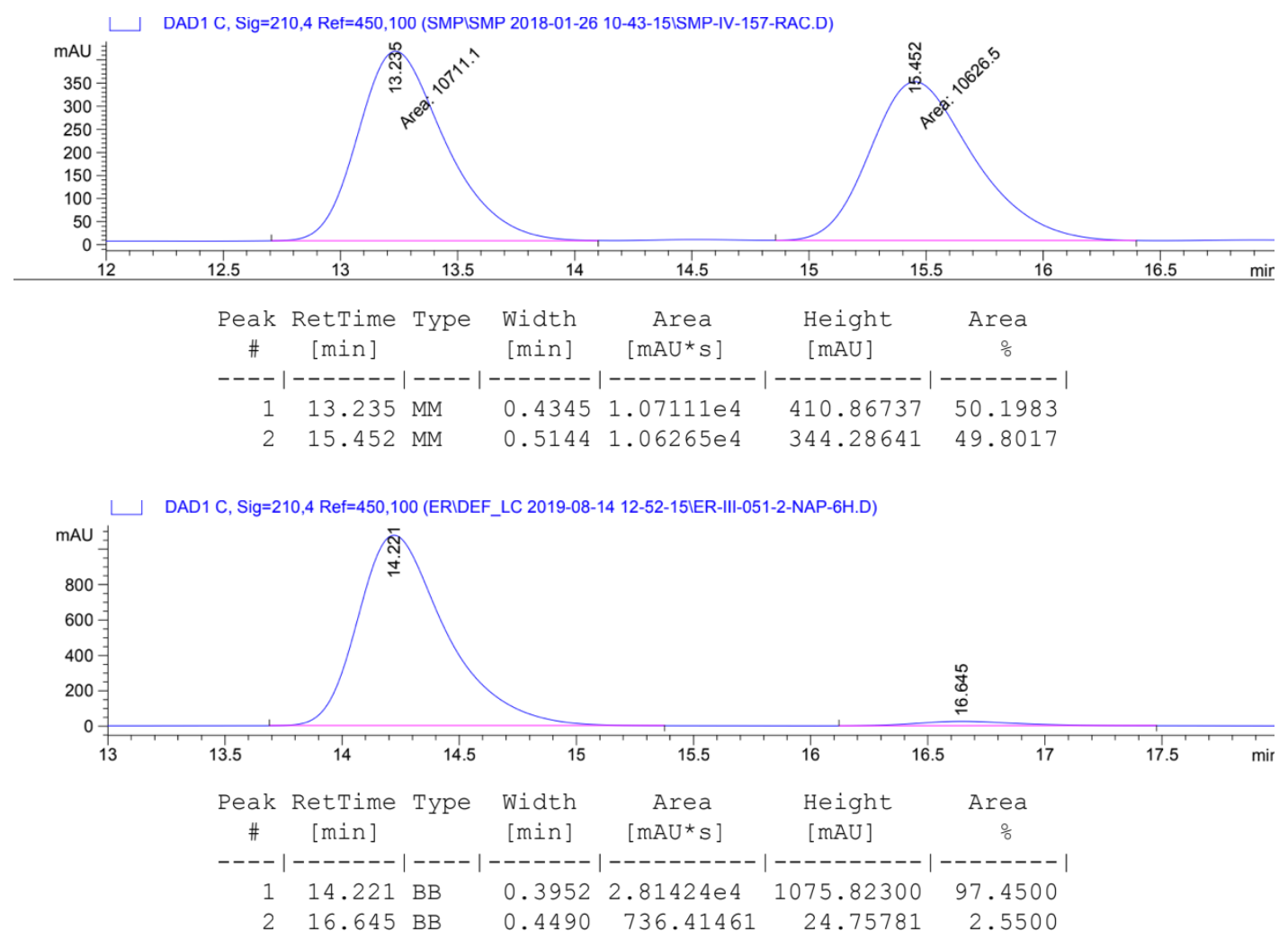


\section{(+)-(R)-N-Boc-3-methyl-1-(1-naphthyl)but-3-en-1-amine [3i]}<smiles>C=C(C)C[C@H](NC(=O)OC(C)(C)C)c1cccc2ccccc12</smiles>

Prepared according to Method B. 1-Naphthaldehyde dimethyl acetal 1i $(60.7 \mathrm{mg}$, $0.300 \mathrm{mmol}, 1.0$ equiv), (2-methylallyl)trimethylsilane $(53.0 \mu \mathrm{L}, 0.300 \mathrm{mmol}, 1.0$ equiv), tert-butyl carbamate (35.1 $\mathrm{mg}, 0.300 \mathrm{mmol}, 1.0$ equiv), catalyst $(S, R)-\mathbf{4 f}$ (19.7 mg, $0.030 \mathrm{mmol}, 0.10$ equiv), TESOTf ( $6.8 \mu \mathrm{L}, 0.030 \mathrm{mmol}, 0.10$ equiv), and $\mathrm{Et}_{2} \mathrm{O}(3.0 \mathrm{~mL}, 0.1 \mathrm{M})$ were used. The reaction was run at $-50^{\circ} \mathrm{C}$ for $6 \mathrm{~h}$. Flash column chromatography afforded pure product as a white solid.

Chiral HPLC conditions: CHIRALPAK AD-H, $4 \%$ PrOH/hexanes, $1 \mathrm{~mL} / \mathrm{min}$

Racemic product: $\mathrm{t}_{\mathrm{R}}(R)=10.2 \mathrm{~min}, \mathrm{t}_{\mathrm{R}}(S)=16.2 \mathrm{~min}$

Enantioenriched product: $\mathrm{t}_{\mathrm{R}}(R)=9.5 \mathrm{~min}$

$73.3 \mathrm{mg}, 0.235 \mathrm{mmol}, 78 \%$ yield, $98 \%$ e.e.

${ }^{1} \mathrm{H}-\mathrm{NMR}\left(500 \mathrm{MHz}, \mathrm{CDCl}_{3}\right) \delta 8.19(\mathrm{~d}, J=8.5 \mathrm{~Hz}, 1 \mathrm{H}), 7.89(\mathrm{~d}, J=8.1 \mathrm{~Hz}, 1 \mathrm{H}), 7.78(\mathrm{~d}, J=8.1 \mathrm{~Hz}, 1 \mathrm{H})$, 7.56 (ddd, $J=8.5,6.8,1.5 \mathrm{~Hz}, 1 \mathrm{H}$ ), $7.53-7.42$ (m, 3H), 5.72 (br. s, $1 \mathrm{H}$ ), 4.96 (br. d, $J=7.6 \mathrm{~Hz}, 1 \mathrm{H}$ ), 4.89 $(\mathrm{s}, 1 \mathrm{H}), 4.85(\mathrm{~s}, 1 \mathrm{H}), 2.73(\mathrm{~d}, J=14.9 \mathrm{~Hz}, 1 \mathrm{H}), 2.50-2.45(\mathrm{~m}, 1 \mathrm{H}), 1.84(\mathrm{~s}, 3 \mathrm{H}), 1.37$ (br. s, 9H); ${ }^{13} \mathrm{C}-$ NMR $\left(126 \mathrm{MHz}, \mathrm{CDCl}_{3}\right) \delta 155.4,142.3,138.8,134.1,130.9,129.0,127.9,126.4,125.7,125.4,123.0$, 122.6, 113.7, 79.6, 48.7, 45.0, 28.5, 22.2; IR (ATR, $\left.\mathrm{cm}^{-1}\right)$ 3358, 2981, 2963, 2938, 1679, 1526, 1441, 1387, 1363, 1336, 1274, 1250, 1164, 1069, 1018; $[\alpha]^{24} \mathrm{D}=+2.9^{\circ}\left(c=1.0, \mathrm{CDCl}_{3}\right) ; \mathrm{HRMS}(\mathrm{ESI}) \mathrm{m} / \mathrm{z}$ calculated for $\mathrm{C}_{20} \mathrm{H}_{26} \mathrm{NO}_{2}[\mathrm{M}+\mathrm{H}]^{+}: 312.1937$, found 312.1947.
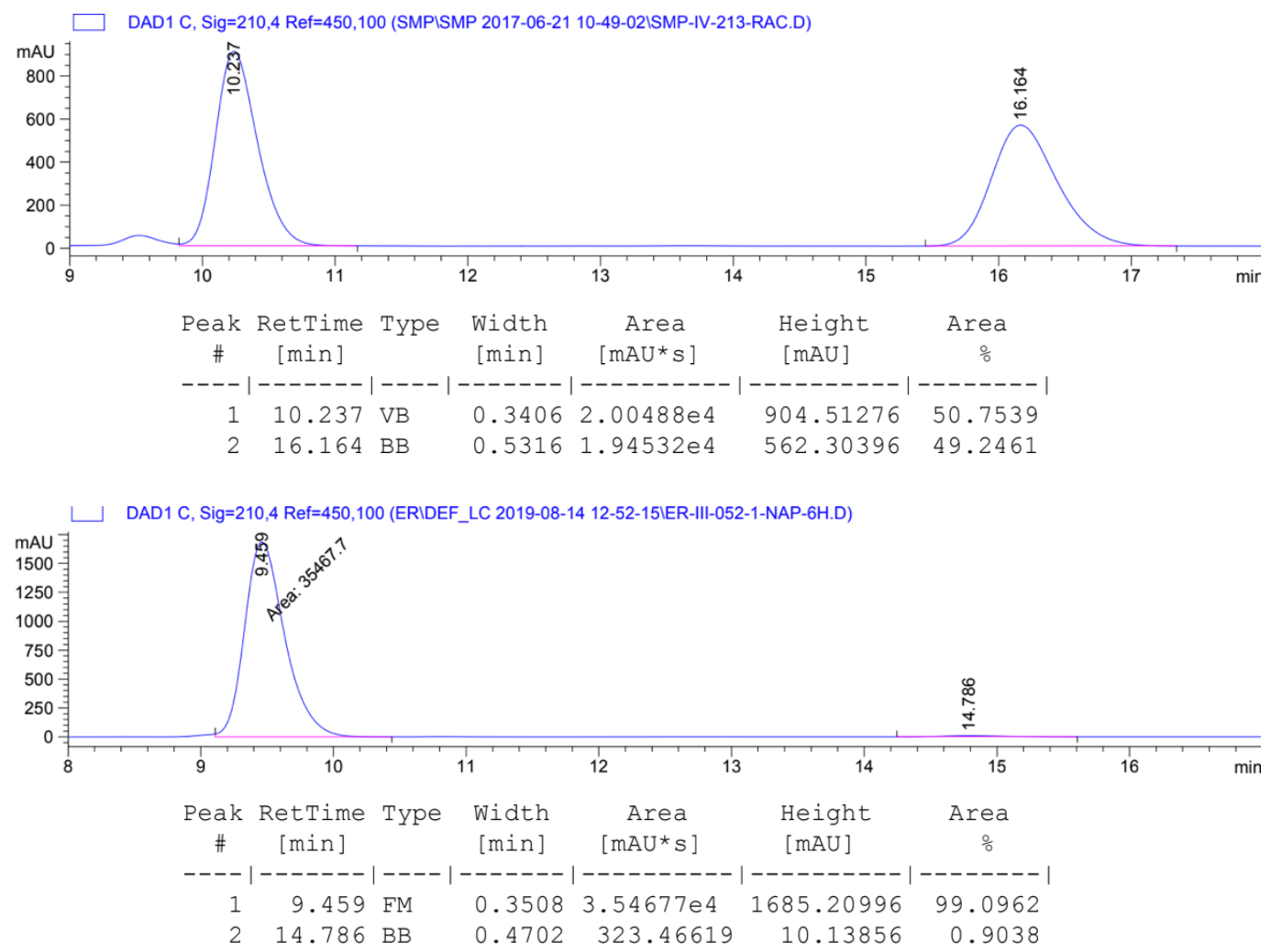


\section{(+)-(R)-methyl-1-(1-((tert-butoxycarbonyl)amino)-3-methylbut-3-en-1-yl)benzoate}

[3j]<smiles>C=C(C)C[C@H](NC(=O)OC(C)(C)C)c1cccc(C(=O)OC)c1</smiles>

Prepared according to Method B. Methyl 3-(dimethoxymethyl) benzoate 1j (63.1 $\mathrm{mg}, 0.300 \mathrm{mmol}, 1.0$ equiv), (2-methylallyl) trimethylsilane $(53.0 \mu \mathrm{L}, 0.300 \mathrm{mmol}$, 1.0 equiv), tert-butyl carbamate ( $35.1 \mathrm{mg}, 0.300 \mathrm{mmol}, 1.0$ equiv), catalyst $(S, R)$ $4 f(19.7 \mathrm{mg}, 0.030 \mathrm{mmol}, 0.10$ equiv), TESOTf $(6.8 \mu \mathrm{L}, 0.030 \mathrm{mmol}, 0.10$ equiv), and $\mathrm{Et}_{2} \mathrm{O}(3.0 \mathrm{~mL}, 0.1 \mathrm{M})$ were used. The reaction was run at $-50^{\circ} \mathrm{C}$ for $48 \mathrm{~h}$ or at $-25^{\circ} \mathrm{C}$ for $18 \mathrm{~h}$. Flash column chromatography on silica (Biotage, $10 \mathrm{~g}$ column) using a $\mathrm{Et}_{2} \mathrm{O} /$ hexanes gradient followed by $100 \% \mathrm{DCM}$ as eluent gave pure product as a white solid (the main impurity prior to the second chromatography is the corresponding hemiaminal).

Chiral HPLC conditions: CHIRALPAK AS-H, 3\% ${ }^{\mathrm{P} P O H} /$ hexanes, $1 \mathrm{~mL} / \mathrm{min}^{2}$

Racemic product: $\mathrm{t}_{\mathrm{R}}(R)=11.9 \mathrm{~min}, \mathrm{t}_{\mathrm{R}}(S)=15.2 \mathrm{~min}$

Enantioenriched product: $\mathrm{t}_{\mathrm{R}}(R)=12.2 \mathrm{~min}$

$-50^{\circ} \mathrm{C}, 48 \mathrm{~h}: 45.3 \mathrm{mg}, 0.142 \mathrm{mmol}, 47 \%$ yield, $99 \%$ e.e.

$-25^{\circ} \mathrm{C}, 18 \mathrm{~h}: 42.4 \mathrm{mg}, 0.133 \mathrm{mmol}, 44 \%$ yield, $98 \%$ e.e.

${ }^{1} \mathrm{H}-\mathrm{NMR}\left(500 \mathrm{MHz}, \mathrm{CDCl}_{3}\right) \delta 7.96(\mathrm{~s}, 1 \mathrm{H}), 7.91(\mathrm{~d}, J=7.7 \mathrm{~Hz}, 1 \mathrm{H}), 7.48(\mathrm{~d}, J=7.7 \mathrm{~Hz}, 1 \mathrm{H}), 7.38(\mathrm{t}, J=$ $7.7 \mathrm{~Hz}, 1 \mathrm{H}), 4.83(\mathrm{~s}, 1 \mathrm{H}), 4.80$ (br. s, 2H), $4.74(\mathrm{~s}, 1 \mathrm{H}), 3.91(\mathrm{~s}, 3 \mathrm{H}), 2.44-2.38(\mathrm{~m}, 2 \mathrm{H}), 1.73(\mathrm{~s}, 3 \mathrm{H})$, $1.38(\mathrm{~s}, 9 \mathrm{H}) ;{ }^{13} \mathrm{C}-\mathrm{NMR}\left(126 \mathrm{MHz}, \mathrm{CDCl}_{3}\right) \delta 167.1,155.3,143.9,141.6,131.0,130.5,128.6,128.4,127.2$, 114.2, 79.7, 52.6, 52.2, 45.8, 28.4, 22.0; IR (ATR, $\left.\mathrm{cm}^{-1}\right)$ 3380, 2978, 2944, 1721, 1684, 1588, 1507, 1460, $1390,1367,1333,1288,1249,1065,1020 ;[\alpha]^{24} \mathrm{D}=+32.8^{\circ}\left(c=1.0, \mathrm{CDCl}_{3}\right) ; \mathrm{HRMS}(\mathrm{ESI}) \mathrm{m} / \mathrm{z}$ calculated for $\mathrm{C}_{18} \mathrm{H}_{26} \mathrm{NO}_{4}[\mathrm{M}+\mathrm{H}]^{+}:$320.1856, found 320.1833.
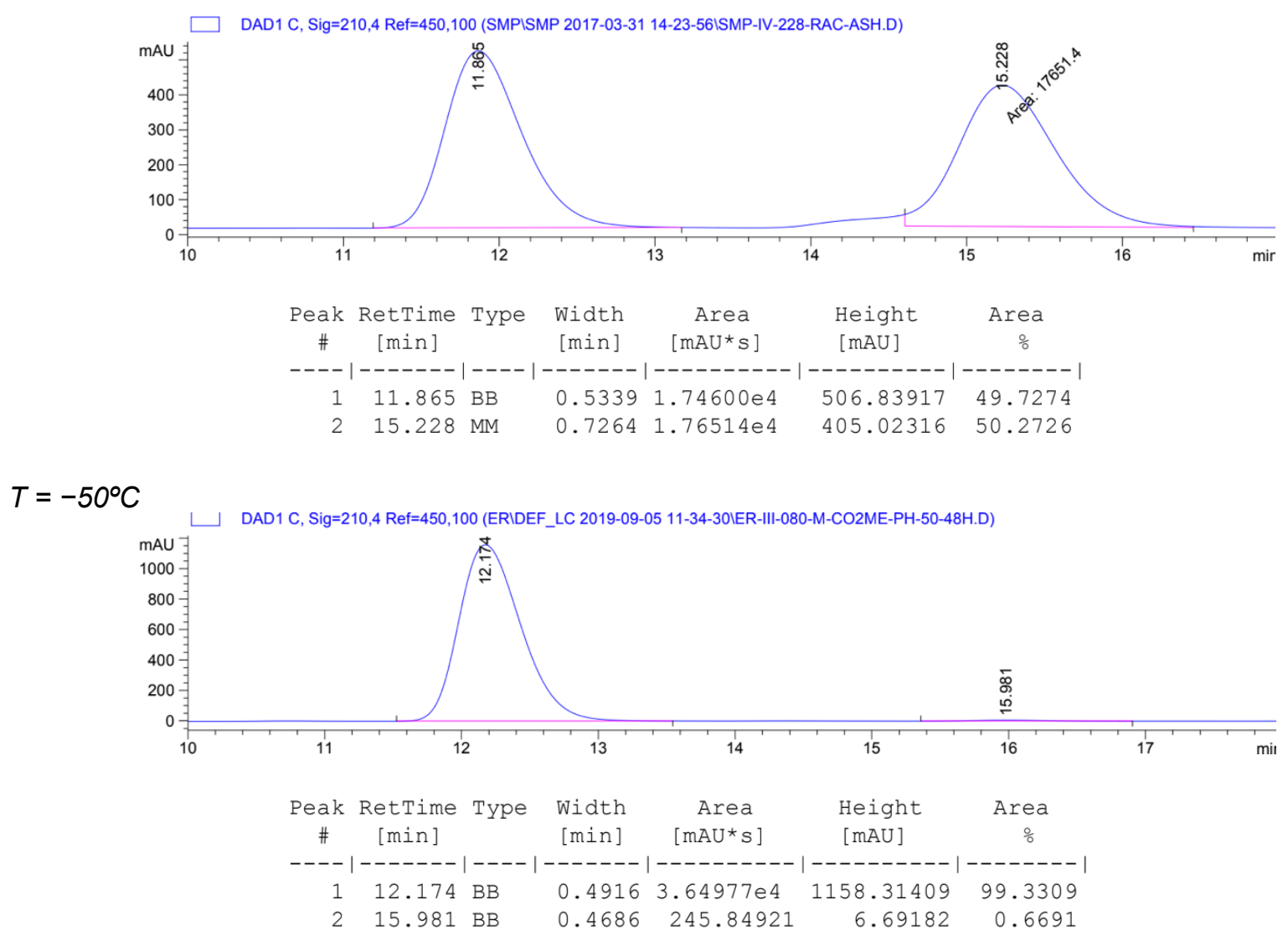
$T=-25^{\circ} \mathrm{C}$

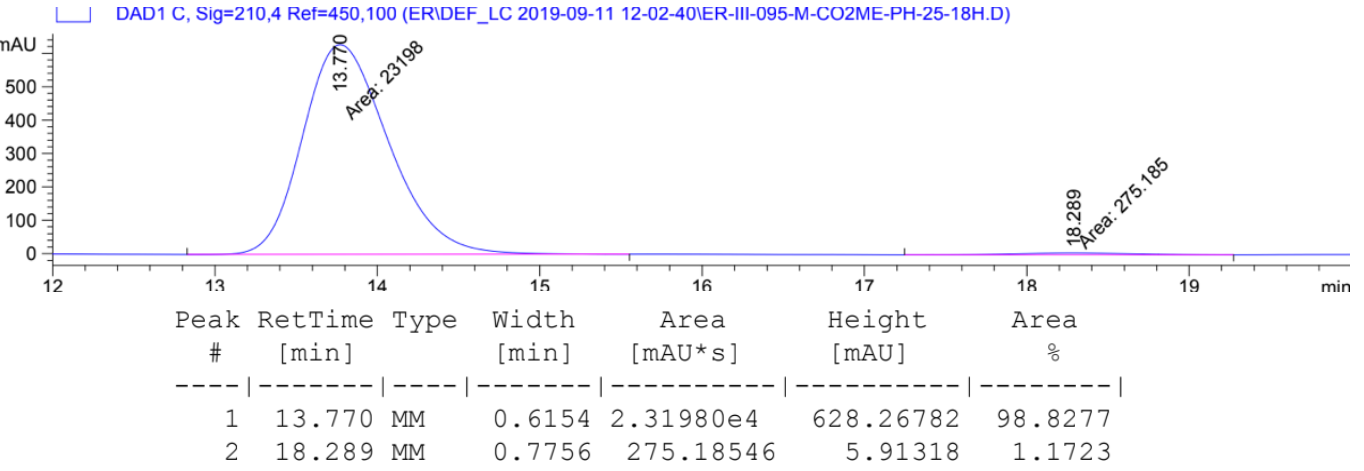

\section{(+)-(R)-N-Boc-1-(benzo[d][1,3]dioxol-5-yl)-3-methylbut-3-en-1-amine [3k]}<smiles>C=C(C)C[C@H](NC(=O)OC(C)(C)C)c1ccc2c(c1)OCO2</smiles>

Prepared according to Method B. Piperonal dimethyl acetal 1k (58.9 mg, 0.300 $\mathrm{mmol}, 1.0$ equiv), (2-methylallyl)trimethylsilane (53.0 $\mu \mathrm{L}, 0.300 \mathrm{mmol}, 1.0$ equiv), tert-butyl carbamate $(35.1 \mathrm{mg}, 0.300 \mathrm{mmol}, 1.0$ equiv), catalyst $(S, R)-\mathbf{4 f}(19.7$ $\mathrm{mg}, 0.030 \mathrm{mmol}, 0.10$ equiv), TESOTf $(6.8 \mu \mathrm{L}, 0.030 \mathrm{mmol}, 0.10$ equiv), and $\mathrm{Et}_{2} \mathrm{O}(3.0 \mathrm{~mL}, 0.1 \mathrm{M})$ were used. The reaction was run at $-50^{\circ} \mathrm{C}$ for $6 \mathrm{~h}$. Flash column chromatography afforded pure product as a white solid.

Chiral HPLC conditions: CHIRALPAK AD-H, 3\% ${ }^{\mathrm{P} P O H} / \mathrm{hexanes} 1 \mathrm{~mL} / \mathrm{min}$

Racemic product: $\mathrm{t}_{\mathrm{R}}(R)=16.3 \mathrm{~min}, \mathrm{t}_{\mathrm{R}}(S)=18.6 \mathrm{~min}$

Enantioenriched product: $\mathrm{t}_{\mathrm{R}}(R)=14.7 \mathrm{~min}$

Run 1: $81.1 \mathrm{mg}, 0.266 \mathrm{mmol}, 89 \%$ yield, $98 \%$ e.e.

Run 2: $82.2 \mathrm{mg}, 0.269 \mathrm{mmol}, \mathbf{8 7} \%$ yield, $98 \%$ e.e. (Ran with $60.8 \mathrm{mg}, 0.310 \mathrm{mmol}$ of acetal and with catalyst recovered from multiple runs and purified by column chromatography)

\section{Average: $88 \%$ yield, $98 \%$ e.e.}

${ }^{1} \mathrm{H}-\mathrm{NMR}\left(500 \mathrm{MHz}, \mathrm{CDCl}_{3}\right) \delta 6.76(\mathrm{~s}, 1 \mathrm{H}), 6.74(\mathrm{~s}, 2 \mathrm{H}), 5.91$ (s, 2H), 4.81 (s, 1H), 4.80 (br. s, 1H), 4.72 (s, 1H), 4.69 (br. s, 1H), $2.43-2.30(\mathrm{~m}, 2 \mathrm{H}), 1.71$ (s, 3H), 1.39 (s, 9H); ${ }^{3} \mathrm{C}-\mathrm{NMR}\left(126 \mathrm{MHz}, \mathrm{CDCl}_{3}\right) \delta 155.3$, 147.8, 146.6, 141.9, 137.3, 119.4, 113.9, 108.3, 106.7, 101.1, 79.6, 53.0, 46.0, 28.5, 22.1; IR (ATR, cm $\left.{ }^{-1}\right)$ $3368,2979,2835,2875,1679,1520,1505,1488,1447,1335,1269,1210,1169,1123,1070,1017$; $[\alpha]^{24} \mathrm{D}=+46.5^{\circ}\left(c=1.0, \mathrm{CDCl}_{3}\right)$; HRMS (ESI) $\mathrm{m} / \mathrm{z}$ calculated for $\mathrm{C}_{17} \mathrm{H}_{24} \mathrm{NO}_{4}[\mathrm{M}+\mathrm{H}]^{+}: 306.1700$, found 306.1707.

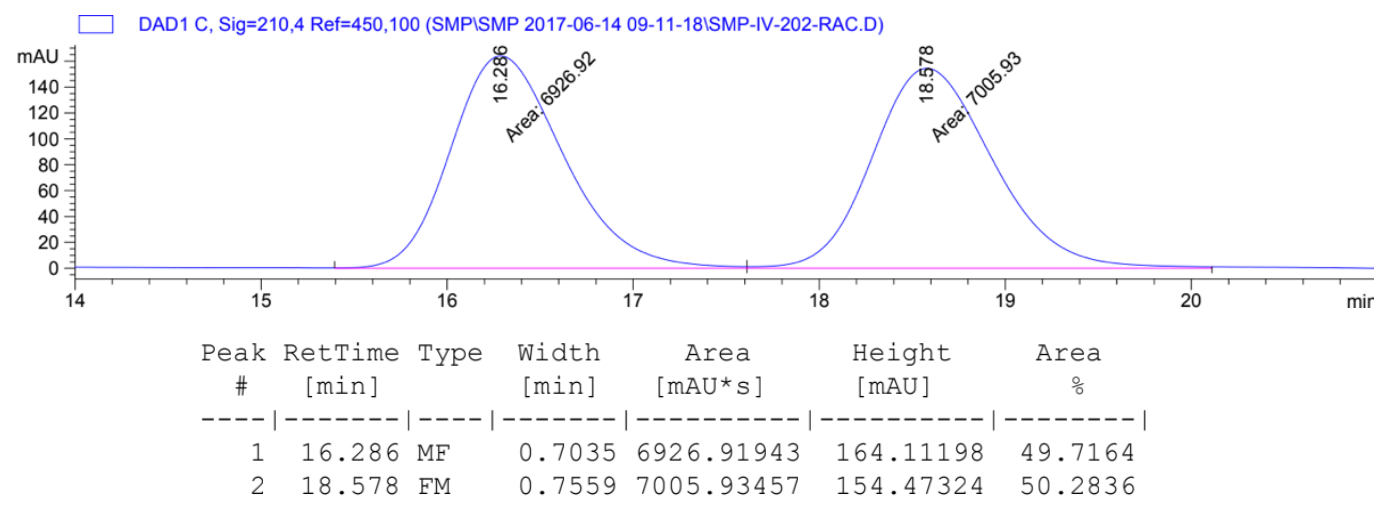




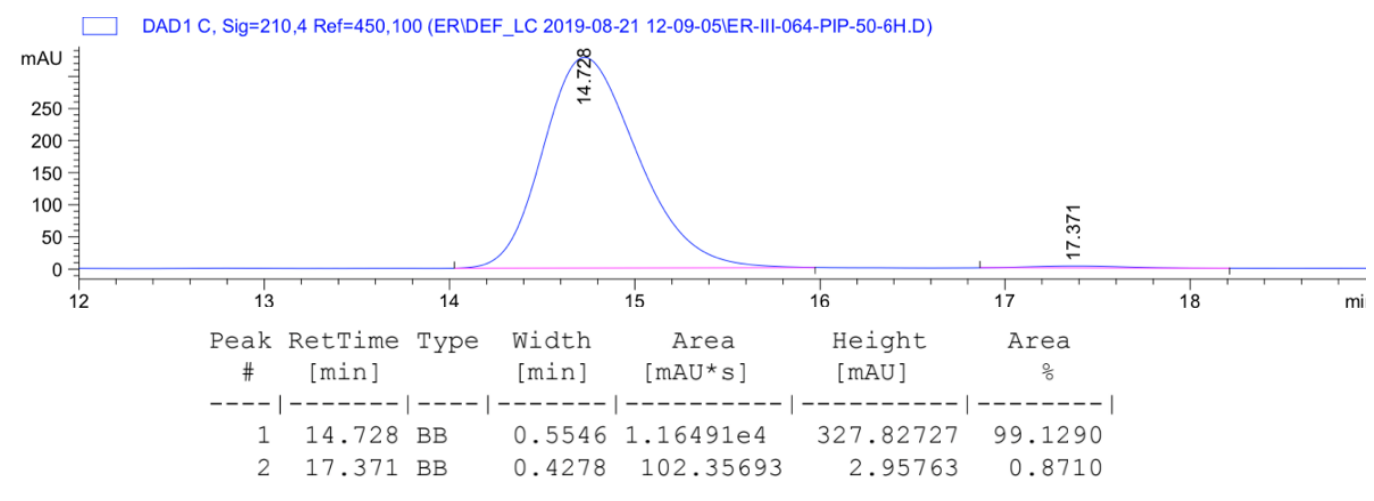

(+)-(R)-N-Boc-3-methyl-1-(2-bromo-3-hydroxy-4-methoxyphenyl)but-3-en-1-amine [3I]<smiles>C=C(C)C[C@H](NC(=O)OC(C)(C)C)c1ccc(OC)c(O)c1Br</smiles>

Prepared according to Method B. (2-Bromo-3-hydroxy-4-methoxy) benzaldehyde dimethyl acetal 11 (83.1 $\mathrm{mg}, 0.300 \mathrm{mmol}, 1.0$ equiv), (2methylallyl)trimethylsilane $(53.0 \mu \mathrm{L}, 0.300 \mathrm{mmol}, 1.0$ equiv), tert-butyl carbamate $(35.1 \mathrm{mg}, 0.300 \mathrm{mmol}, 1.0$ equiv), catalyst $(S, R)-\mathbf{4 f}(19.7 \mathrm{mg}$, $0.030 \mathrm{mmol}, 0.10$ equiv), TESOTf $\left(6.8 \mu \mathrm{L}, 0.030 \mathrm{mmol}, 0.10\right.$ equiv), and $\mathrm{Et}_{2} \mathrm{O}$ $(3.0 \mathrm{~mL}, 0.1 \mathrm{M})$ were used. The reaction was run at $-50^{\circ} \mathrm{C}$ for $18 \mathrm{~h}$. Flash column chromatography on silica (Biotage, $25 \mathrm{~g}$ column) gave pure product as a white solid. NB. Due to the high polarity of the product, separation from the thiourea catalyst proved challenging, requiring multiple rounds of chromatography for purification (100\% DCM was the most successful eluent).

Chiral HPLC conditions: CHIRALPAK AS-H, 20\% 'PrOH/hexanes, $1 \mathrm{~mL} / \mathrm{min}$

Racemic product: $\mathrm{t}_{\mathrm{R}}(R)=7.7 \mathrm{~min}, \mathrm{t}_{\mathrm{R}}(S)=10.5 \mathrm{~min}$

Enantioenriched product: $\mathrm{t}_{\mathrm{R}}(R)=7.1 \mathrm{~min}$

\section{$83.3 \mathrm{mg}, 0.216 \mathrm{mmol}, 72 \%$ yield, $99 \%$ e.e.}

${ }^{1} \mathrm{H}-\mathrm{NMR}\left(500 \mathrm{MHz}, \mathrm{CDCl}_{3}\right) \delta 6.83(\mathrm{~d}, J=8.5 \mathrm{~Hz}, 1 \mathrm{H}), 6.77(\mathrm{~d}, J=8.4 \mathrm{~Hz}, 1 \mathrm{H}), 6.11(\mathrm{~s}, 1 \mathrm{H}), 5.07$ (br. s, $1 \mathrm{H}), 4.93$ (br. s, 1H), $4.83(\mathrm{~s}, 1 \mathrm{H}), 4.76(\mathrm{~s}, 1 \mathrm{H}), 3.85(\mathrm{~s}, 3 \mathrm{H}), 2.66-2.34(\mathrm{~m}, 1 \mathrm{H}), 2.27-1.93(\mathrm{~m}, 1 \mathrm{H}), 1.77$ (s, 3H), $1.40(\mathrm{~s}, 9 \mathrm{H}) ;{ }^{13} \mathrm{C}-\mathrm{NMR}\left(126 \mathrm{MHz}, \mathrm{CDCl}_{3}\right) \delta 155.1,146.0,143.1,142.1,135.0,117.2,113.6$, 109.7, 109.1, 79.4, 56.3, 52.0, 44.4, 28.3, 21.6; IR (ATR, $\left.\mathrm{cm}^{-1}\right)$ 3474, 3436, 3372, 2970, 2930, 2915, 2838, 1687, 1530, 1503, 1492, 1270, 1232, 1037, 1014; [a $]^{24} \mathrm{D}=+9.5^{\circ}\left(c=1.0, \mathrm{CHDCl}_{3}\right) ; \mathrm{HRMS}(\mathrm{ESI}) \mathrm{m} / \mathrm{z}$ calculated for $\mathrm{C}_{17} \mathrm{H}_{25} \mathrm{BrNO}_{4}[\mathrm{M}+\mathrm{H}]^{+}: 386.0961$, found 386.0959.

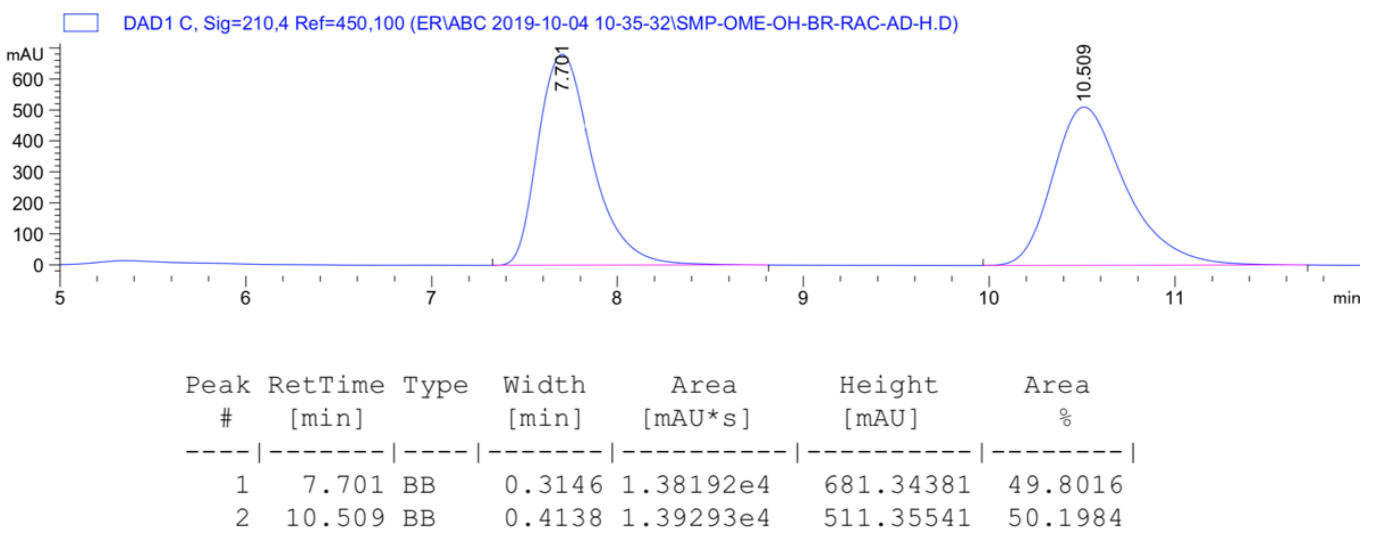




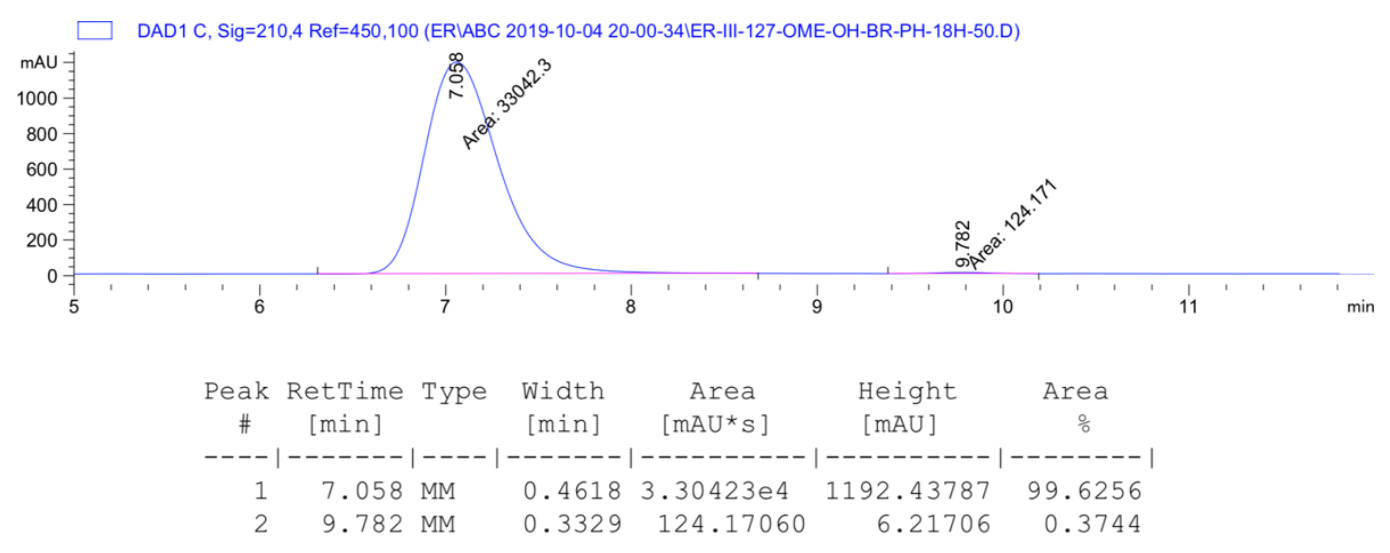

\section{(+)-(R)-N-Boc-3-methyl-1-(cyclohex-1-en-1-yl)but-3-en-1-amine [3m]}<smiles>C=C(C)C[C@H](NC(=O)OC(C)(C)C)C1=CCCCC1</smiles>

Prepared according to Method B. Cyclohex-1-ene-1-carbaldehyde dimethyl acetal $1 \mathrm{~m}$ - containing 18\% Cyclohex-1-ene-1-carbaldehyde according to integration at 2.40-2.17 ppm (aldehyde) vs 2.14-1.93 ppm (acetal)- (46.9 mg of mixture, 0.317 mmol, 1.1 equiv), (2-methylallyl)trimethylsilane $(53.0 \mu \mathrm{L}, 0.300 \mathrm{mmol}, 1.0$ equiv), tert-butyl carbamate $(35.1 \mathrm{mg}, 0.300 \mathrm{mmol}, 1.0$ equiv), catalyst $(S, R)-4 \mathrm{f} \quad(19.7 \mathrm{mg}$, $0.030 \mathrm{mmol}, 0.10$ equiv), TESOTf $\left(6.8 \mu \mathrm{L}, 0.030 \mathrm{mmol}, 0.10\right.$ equiv), and $\mathrm{Et}_{2} \mathrm{O}$ (3.0 $\mathrm{mL}, 0.1 \mathrm{M})$ were used. The reaction was run at $-50^{\circ} \mathrm{C}$ for $18 \mathrm{~h}$. Flash column chromatography on silica (Biotage, $10 \mathrm{~g}$ column) gave pure product as a white solid.

Chiral HPLC conditions: CHIRALPAK AS-H, 3\% $\mathrm{PrOH} / \mathrm{hexanes,} 1 \mathrm{~mL} / \mathrm{min}$

Racemic product: $\mathrm{tR}_{\mathrm{R}}(R)=5.1 \mathrm{~min}, \mathrm{t}_{\mathrm{R}}(S)=5.8 \mathrm{~min}$

Enantioenriched product: $\mathrm{t}_{\mathrm{R}}(R)=4.7 \mathrm{~min}$

$68.7 \mathrm{mg}, 0.259 \mathrm{mmol}, \mathbf{8 2} \%$ yield, $90 \%$ e.e.

${ }^{1} \mathrm{H}-\mathrm{NMR}\left(500 \mathrm{MHz}, \mathrm{CDCl}_{3}\right) \delta 5.57(\mathrm{td}, J=3.8,2.1 \mathrm{~Hz}, 1 \mathrm{H}), 4.76(\mathrm{~s}, 1 \mathrm{H}), 4.68(\mathrm{~s}, 1 \mathrm{H}), 4.45$ (br. s, $\left.1 \mathrm{H}\right)$, 4.06 (br. s, 1H), 2.24 (dd, $J=14.1,5.9 \mathrm{~Hz}, 2 \mathrm{H}), 2.12$ (dd, $J=14.0,9.0 \mathrm{~Hz}, 1 \mathrm{H}), 2.03-1.95(\mathrm{~m}, 2 \mathrm{H}), 1.95$ - $1.87(\mathrm{~m}, 2 \mathrm{H}), 1.71(\mathrm{~s}, 3 \mathrm{H}), 1.64-1.56(\mathrm{~m}, 2 \mathrm{H}), 1.56-1.48(\mathrm{~m}, 2 \mathrm{H}), 1.40(\mathrm{~s}, 9 \mathrm{H}) ;{ }^{13} \mathrm{C}-\mathrm{NMR}(126 \mathrm{MHz}$, $\left.\mathrm{CDCl}_{3}\right) \delta 155.5,142.6,137.7,122.2,113.2,79.1,54.3,42.6,28.5,25.12,25.07,22.8,22.6,22.1$; IR $\left(\right.$ ATR, $\left.\mathrm{cm}^{-1}\right)$ 3351, 2975, 2935, 2919, 2855, 2834, 1680, 1514, 1447, 1364, 1166, 1061, 1040, 918; [a] ${ }^{24} \mathrm{D}$ $=+11.0^{\circ}\left(c=1.0, \mathrm{CDCl}_{3}\right)$; HRMS $(\mathrm{ESI}) \mathrm{m} / z$ calculated for $\mathrm{C}_{16} \mathrm{H}_{28} \mathrm{NO}_{2}[\mathrm{M}+\mathrm{H}]^{+}:$266.2115, found 266.2111.

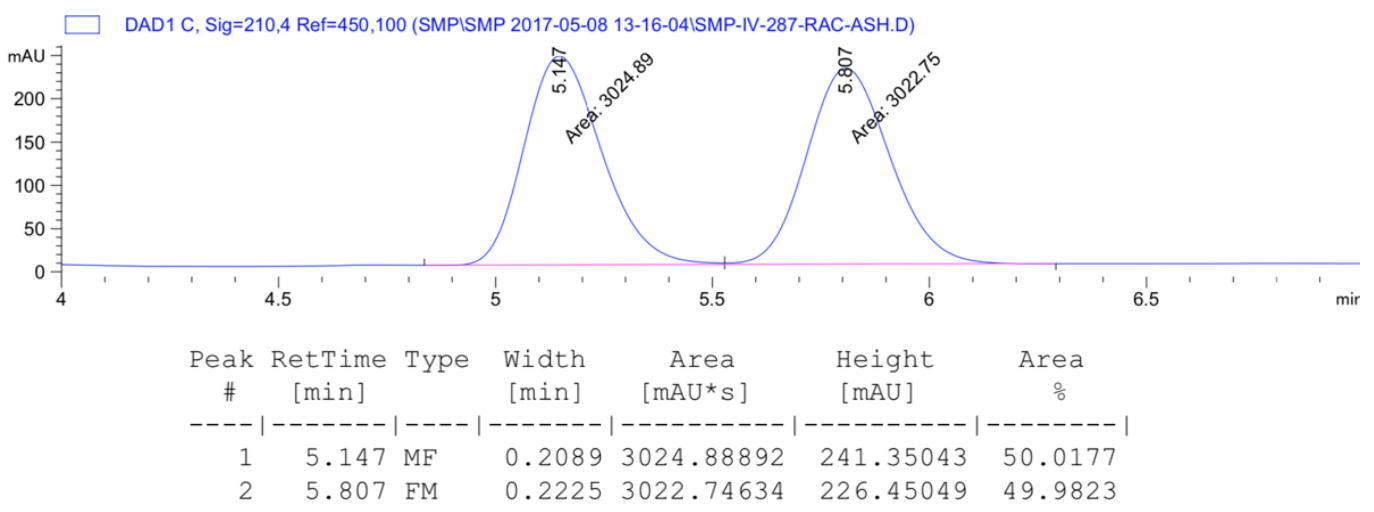




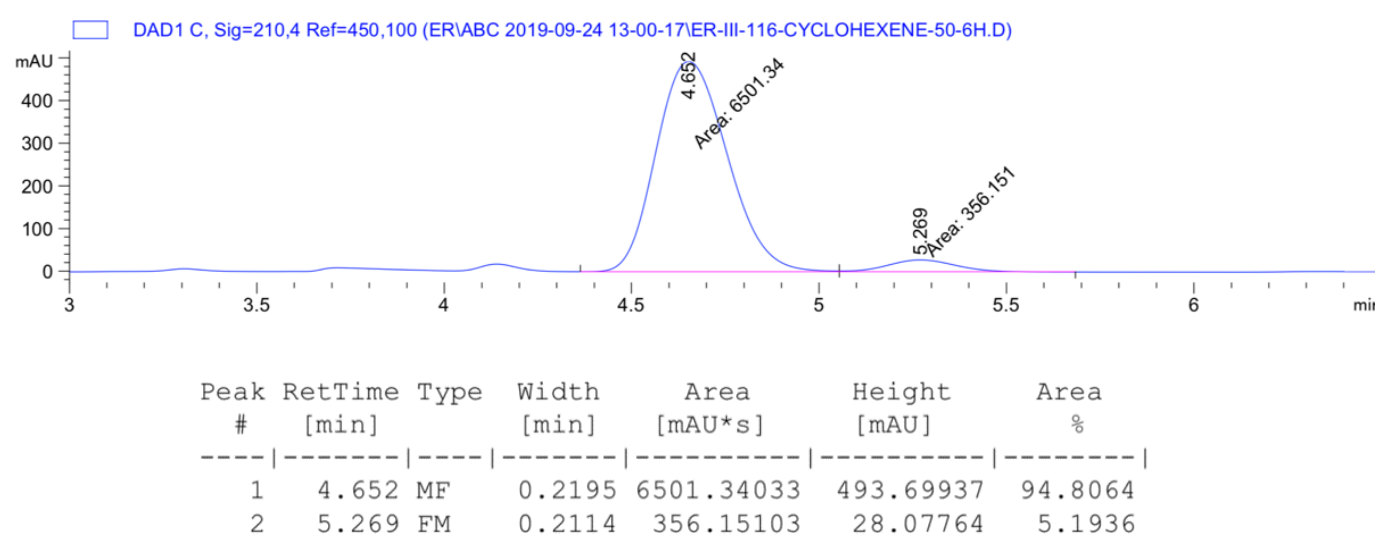

\section{(+)-(R)-N-Boc-3-methyl-1-(N-Boc-2-pyrryl)-but-3-en-1-amine [3n]}<smiles>C=C(C)C[C@H](NC(=O)OC(C)(C)C)c1cccn1C(=O)OC(C)(C)C</smiles>

Prepared according to Method B. N-Boc-pyrrole-2-carbaldehyde dimethyl acetal 1n - containing $14 \% \mathrm{~N}$-Boc-pyrrole-2-carbaldehyde, from integration of peaks at $1.64 \mathrm{ppm}$ (aldehyde) vs $1.59 \mathrm{ppm}$ (acetal) - (72.4 $\mathrm{mg}$ of mixture, $0.308 \mathrm{mmol}, 1.0$ equiv), (2-methylallyl)trimethylsilane $(53.0 \mu \mathrm{L}, 0.300 \mathrm{mmol}, 1.0$ equiv), tert-butyl carbamate $(35.1 \mathrm{mg}, 0.300 \mathrm{mmol}, 1.0$ equiv), catalyst $(S, R)-4 \mathrm{f}(19.7 \mathrm{mg}, 0.030$ mmol, 0.10 equiv), TESOTf $\left(6.8 \mu \mathrm{L}, 0.030 \mathrm{mmol}, 0.10\right.$ equiv), and $\mathrm{Et}_{2} \mathrm{O}$ ( $3.0 \mathrm{~mL}$, $0.1 \mathrm{M})$ were used. The reaction was run at $-50^{\circ} \mathrm{C}$ for $6 \mathrm{~h}$. Flash column chromatography on silica (Biotage, $10 \mathrm{~g}$ column) using a gradient of $1 \%$ EtOAc/hexanes $\rightarrow 10 \%$ EtOAc/hexanes followed by $100 \%$ DCM as eluent gave pure product as a pale yellow oil.

Chiral HPLC conditions: CHIRALPAK AS-H, $3 \%$ PrOH/hexanes, $1 \mathrm{~mL} / \mathrm{min}$

Racemic product: $\mathrm{t}_{\mathrm{R}}(S)=7.1 \mathrm{~min}, \mathrm{t}_{\mathrm{R}}(R)=9.5 \mathrm{~min}$

Enantioenriched product: $\mathrm{t}_{\mathrm{R}}(R)=8.5 \mathrm{~min}$

\section{$83.7 \mathrm{mg}, 0.239 \mathrm{mmol}, 77 \%$ yield, $90 \%$ e.e.}

${ }^{1} \mathrm{H}-\mathrm{NMR}\left(500 \mathrm{MHz}, \mathrm{CDCl}_{3}\right) \delta 7.13(\mathrm{dt}, J=3.1,1.4 \mathrm{~Hz}, 1 \mathrm{H}), 6.11$ (dd, $\left.J=3.3,1.8 \mathrm{~Hz} 1 \mathrm{H}\right), 6.04(\mathrm{t}, J=3.4$ $\mathrm{Hz}, 1 \mathrm{H}), 5.48$ (br. s, $1 \mathrm{H}), 5.36-5.25$ (br. $\mathrm{m}, 1 \mathrm{H}), 4.73(\mathrm{~s}, 1 \mathrm{H}), 4.67(\mathrm{~s}, 1 \mathrm{H}), 2.55$ (dd, $J=13.9,6.6 \mathrm{~Hz}, 1 \mathrm{H})$, $2.41(\mathrm{dd}, J=13.9,8.2 \mathrm{~Hz}, 1 \mathrm{H}), 1.69(\mathrm{~s}, 3 \mathrm{H}), 1.59(\mathrm{~s}, 9 \mathrm{H}), 1.41(\mathrm{~s}, 9 \mathrm{H}) ;{ }^{13} \mathrm{C}-\mathrm{NMR}\left(126 \mathrm{MHz}, \mathrm{CDCl}_{3}\right) \delta$ 155.2, 149.5, 142.6, 135.8, 122.2, 113.1, 112.6, 110.0, 84.0, 79.1, 47.6, 43.7, 28.5, 28.1, 22.1; IR (ATR, $\left.\mathrm{cm}^{-1}\right)$ 3435, 2978, 2934, 1736, 1702, 1490, 1456, 1392, 1367, 1327, 1160, 1138, 1119, 1055, 1013; [a] ${ }^{24} \mathrm{D}$ $=+36.5^{\circ}\left(c=1.0, \mathrm{CHCl}_{3}, 94 \%\right.$ e.e. sample); HRMS (ESI) $\mathrm{m} / \mathrm{z}$ calculated for $\mathrm{C}_{19} \mathrm{H}_{31} \mathrm{~N}_{2} \mathrm{O}_{4} \mathrm{~N}[\mathrm{M}+\mathrm{H}]^{+}$: 351.2278 , found 351.2269 .

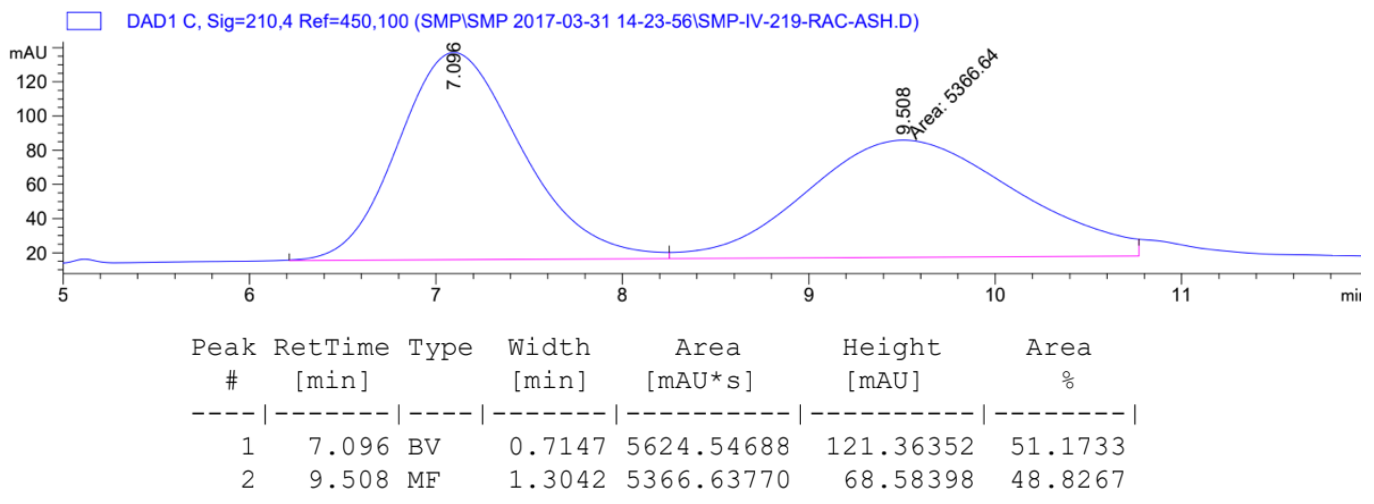




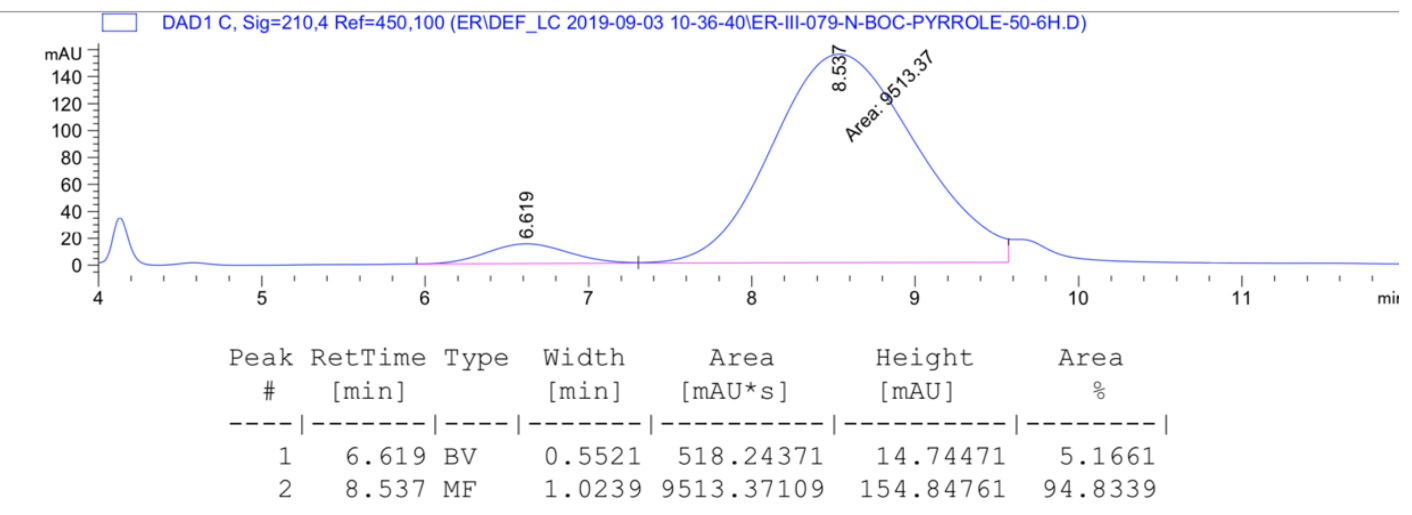

\section{(+)-(R)-N-Boc-3-methyl-1-(N-Boc-2-indolyl)-but-3-en-1-amine [3o]}<smiles>C=C(C)C[C@H](NC(=O)OC(C)(C)C)c1cc2ccccc2n1C(=O)OC(C)(C)C</smiles>

Prepared according to Method B. tert-Butyl 2-(dimethoxymethyl)-1H-indole-1carboxylate $10(87.4 \mathrm{mg}, 0.300 \mathrm{mmol}, 1.0$ equiv), (2-methylallyl)trimethylsilane (53.0 $\mu \mathrm{L}, 0.300 \mathrm{mmol}, 1.0$ equiv), tert-butyl carbamate $(35.1 \mathrm{mg}, 0.300 \mathrm{mmol}, 1.0$ equiv), catalyst $(S, R)-4 \mathbf{f}(19.7 \mathrm{mg}, 0.030 \mathrm{mmol}, 0.10$ equiv), TESOTf $(6.8 \mu \mathrm{L}$, $0.030 \mathrm{mmol}, 0.10$ equiv), and $\mathrm{Et}_{2} \mathrm{O}(3.0 \mathrm{~mL}, 0.1 \mathrm{M})$ were used. The reaction was run at $-50^{\circ} \mathrm{C}$ for $18 \mathrm{~h}$. Purification by flash column chromatography on silica (Biotage, $25 \mathrm{~g}$ column) afforded $90.7 \mathrm{mg}$ of a white solid contaminated with $29 \%$ starting material, identified by NMR. The which was taken into account in the yield calculation. The mass reported in the manuscript is the calculated product mass, the measured mass of the mixture was $90.7 \mathrm{mg}$.

Chiral HPLC conditions: CHIRALPAK OD-H, $2 \%{ }^{\top} \mathrm{PrOH} /$ hexanes, $^{1} \mathrm{~mL} / \mathrm{min}$

Racemic product: $\mathrm{t}_{\mathrm{R}}(S)=6.6 \mathrm{~min}, \mathrm{t}_{\mathrm{R}}(R)=7.4 \mathrm{~min}$

Enantioenriched product: $\mathrm{t}_{\mathrm{R}}(R)=7.2 \mathrm{~min}$

$70.3 \mathrm{mg}, 0.175 \mathrm{mmol}, 58 \%$ yield, $\mathbf{9 5 \%}$ e.e.

${ }^{1} \mathrm{H}-\mathrm{NMR}\left(500 \mathrm{MHz}, \mathrm{CDCl}_{3}\right) \delta 7.99(\mathrm{~d}, J=8.3 \mathrm{~Hz}, 1 \mathrm{H}), 7.47(\mathrm{~d}, J=7.6 \mathrm{~Hz}, 1 \mathrm{H}), 7.24(\mathrm{t}, J=7.9 \mathrm{~Hz}, 1 \mathrm{H})$, 7.19 (t, $J=7.4 \mathrm{~Hz}, 1 \mathrm{H}), 6.56$ (s, 1H), 5.55 (br. s, 1H), 5.47 (br. s, 1H), 4.79 (s, 1H), $4.74(\mathrm{~s}, 1 \mathrm{H}$ ), 2.68 (dd, $J=14.4,5.8 \mathrm{~Hz}, 1 \mathrm{H}), 2.44(\mathrm{dd}, J=11.3,5.7 \mathrm{~Hz}, 1 \mathrm{H}), 1.73(\mathrm{~s}, 3 \mathrm{H}), 1.71(\mathrm{~s}, 9 \mathrm{H}), 1.43(\mathrm{~s}, 9 \mathrm{H})$; ${ }^{13} \mathrm{C}-\mathrm{NMR}$ $\left(126 \mathrm{MHz}, \mathrm{CDCl}_{3}\right) \delta 155.3,150.7,142.3,142.2,136.9,128.9,124.0,122.9,120.6,115.7,113.5,108.5$, 84.5, 79.4, 48.4, 43.7, 28.5, 28.3, 22.2; IR (ATR, $\left.\mathrm{cm}^{-1}\right)$ 3423, 2975, 2932, 1728, 1703, 1488, 1453, 1367, $1324,1248,1155,1116,1084,742 ;[\alpha]^{24} \mathrm{D}=+32.7^{\circ}\left(c=1.0, \mathrm{CDCl}_{3}\right)$; HRMS (ESI) $\mathrm{m} / z$ calculated for $\mathrm{C}_{23} \mathrm{H}_{33} \mathrm{~N}_{2} \mathrm{O}_{4}[\mathrm{M}+\mathrm{H}]^{+}:$401.2435, found 401.2431 .

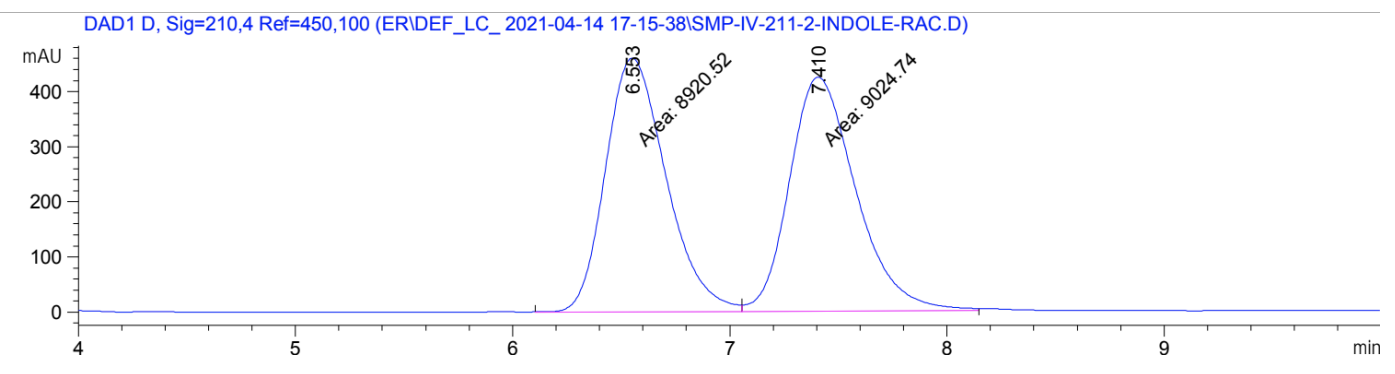



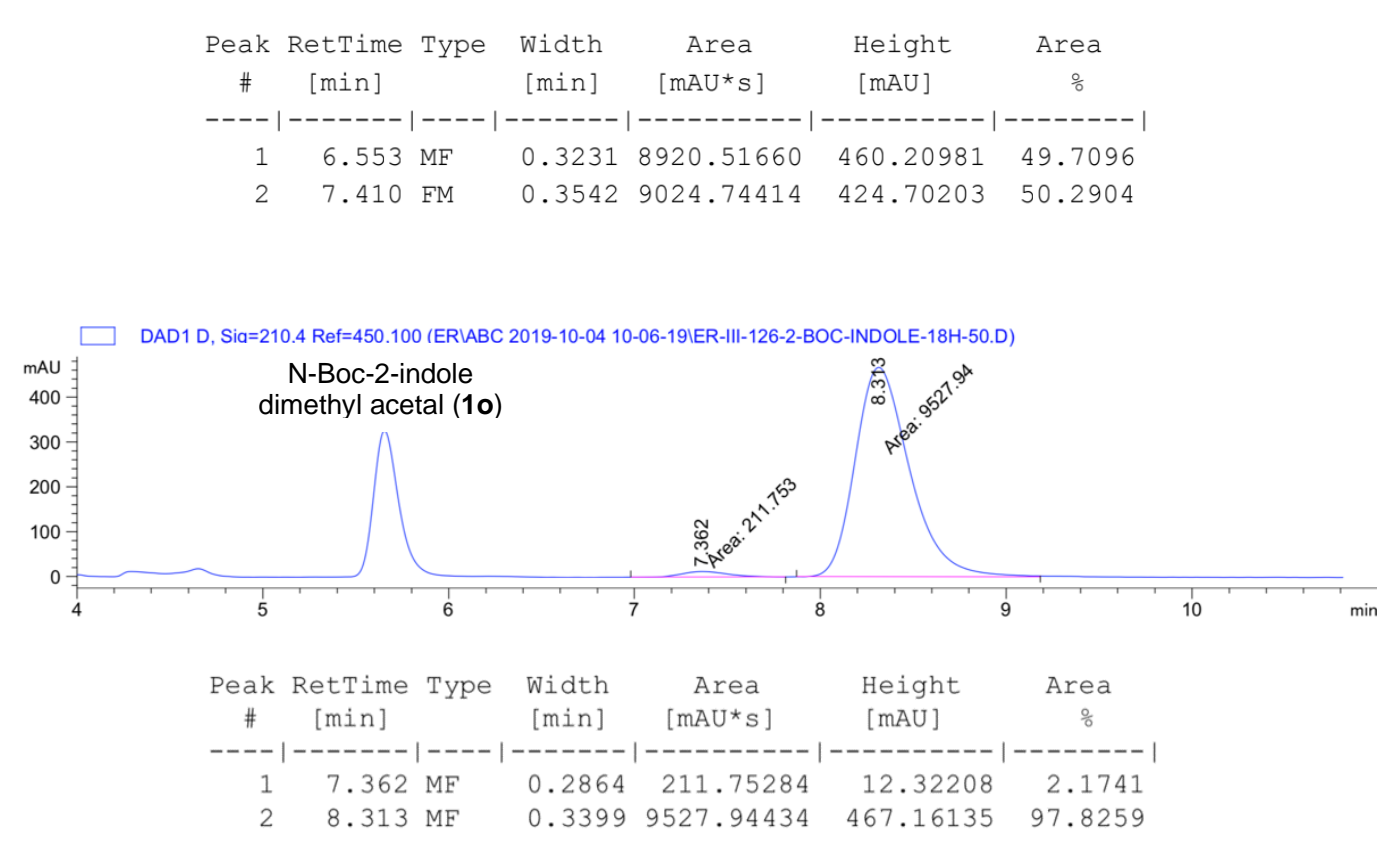

\section{(+)-(R)-N-Boc-3-methyl-1-(N-Boc-5-indolyl)-but-3-en-1-amine [3p]}<smiles>C=C(C)C[C@H](NC(=O)OC(C)(C)C)c1ccc2c(ccn2C(=O)OC(C)(C)C)c1</smiles>

Prepared according to Method B. tert-Butyl 5-(dimethoxymethyl)-1 $\mathrm{H}$ indole-1-carboxylate 1p (87.4 $\mathrm{mg}, 0.300 \mathrm{mmol}, 1.0$ equiv), (2methylallyl)trimethylsilane $(53.0 \mu \mathrm{L}, 0.300 \mathrm{mmol}, 1.0$ equiv), tert-butyl carbamate $(35.1 \mathrm{mg}, 0.300 \mathrm{mmol}, 1.0$ equiv), catalyst $(S, R)-4 f \quad(19.7$ $\mathrm{mg}, 0.030 \mathrm{mmol}, 0.10$ equiv), TESOTf $(6.8 \mu \mathrm{L}, 0.030 \mathrm{mmol}, 0.10$ equiv), and $\mathrm{Et}_{2} \mathrm{O}(3.0 \mathrm{~mL}, 0.1 \mathrm{M})$ were used. The reaction was run at $-50^{\circ} \mathrm{C}$ for $6 \mathrm{~h}$. Flash column chromatography on silica (Biotage, $10 \mathrm{~g}$ column) gave pure product as a tacky colorless oil.

Chiral HPLC conditions: CHIRALPAK AS-H, 5\% $\mathrm{PrOH} / \mathrm{hexanes,} 1 \mathrm{~mL} / \mathrm{min}$

Racemic product: $\mathrm{t}_{\mathrm{R}}(S)=7.0 \mathrm{~min}, \mathrm{t}_{\mathrm{R}}(R)=8.6 \mathrm{~min}$

Enantioenriched product: $\operatorname{tR}_{\mathrm{R}}(R)=8.5 \mathrm{~min}$

$120.5 \mathrm{mg}, 0.301 \mathrm{mmol}, \mathbf{1 0 0} \%$ yield, $\mathbf{9 8 \%}$ e.e.

${ }^{1} \mathrm{H}-\mathrm{NMR}\left(500 \mathrm{MHz}, \mathrm{CDCl}_{3}\right) \delta 8.09(\mathrm{~d}, J=8.6 \mathrm{~Hz}, 1 \mathrm{H}), 7.58(\mathrm{~d}, J=3.7 \mathrm{~Hz}, 1 \mathrm{H}), 7.48(\mathrm{~s}, 1 \mathrm{H}), 7.25(\mathrm{~d}, J=$ $8.7 \mathrm{~Hz}, 1 \mathrm{H}), 6.53$ (d, $J=3.8 \mathrm{~Hz}, 1 \mathrm{H}), 4.93$ (br. s, 2H), 4.80 (d, $J=2.4 \mathrm{~Hz}, 1 \mathrm{H}), 4.74(\mathrm{~s}, 1 \mathrm{H}), 2.49(\mathrm{~d}, J=$ $7.2 \mathrm{~Hz}, 2 \mathrm{H}), 1.74$ (s, 3H), 1.66 (s, 9H), 1.40 (br. s, 9H); ${ }^{13} \mathrm{C}-\mathrm{NMR}\left(126 \mathrm{MHz}, \mathrm{CDCl}_{3}\right) \delta$ 155.3, 149.7, 142.0, 137.6, 134.3, 130.7, 126.2, 122.6, 118.5, 115.2, 113.7, 107.4, 83.6, 79.2, 53.0, 46.0, 28.4, 28.2, 22.1; IR $\left(\right.$ ATR, $\left.\mathrm{cm}^{-1}\right) 3362,2977,2933,1730,1699,1496,1367,1355,1248,1159,1133,1083,1083 ;[\alpha]^{24} \mathrm{D}=$ $+41.5^{\circ}\left(c=1.0, \mathrm{CDCl}_{3}\right)$; HRMS (ESI) $\mathrm{m} / z$ calculated for $\mathrm{C}_{23} \mathrm{H}_{33} \mathrm{~N}_{2} \mathrm{O}_{4}[\mathrm{M}+\mathrm{H}]^{+}: 401.2435$, found 401.2431.

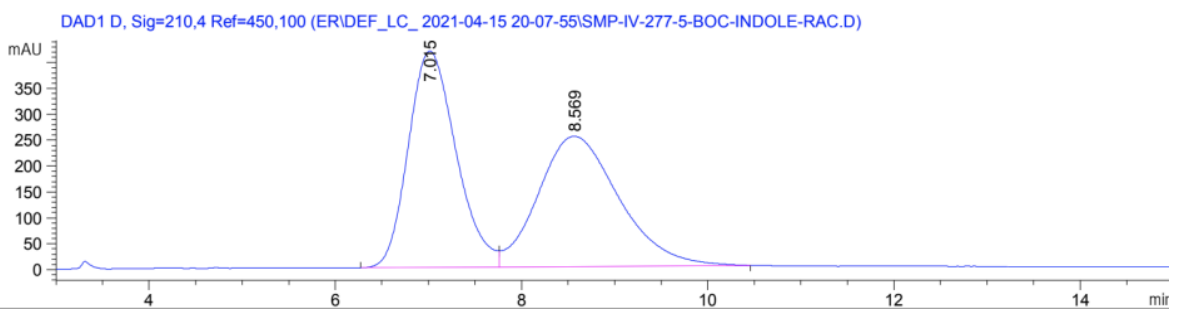



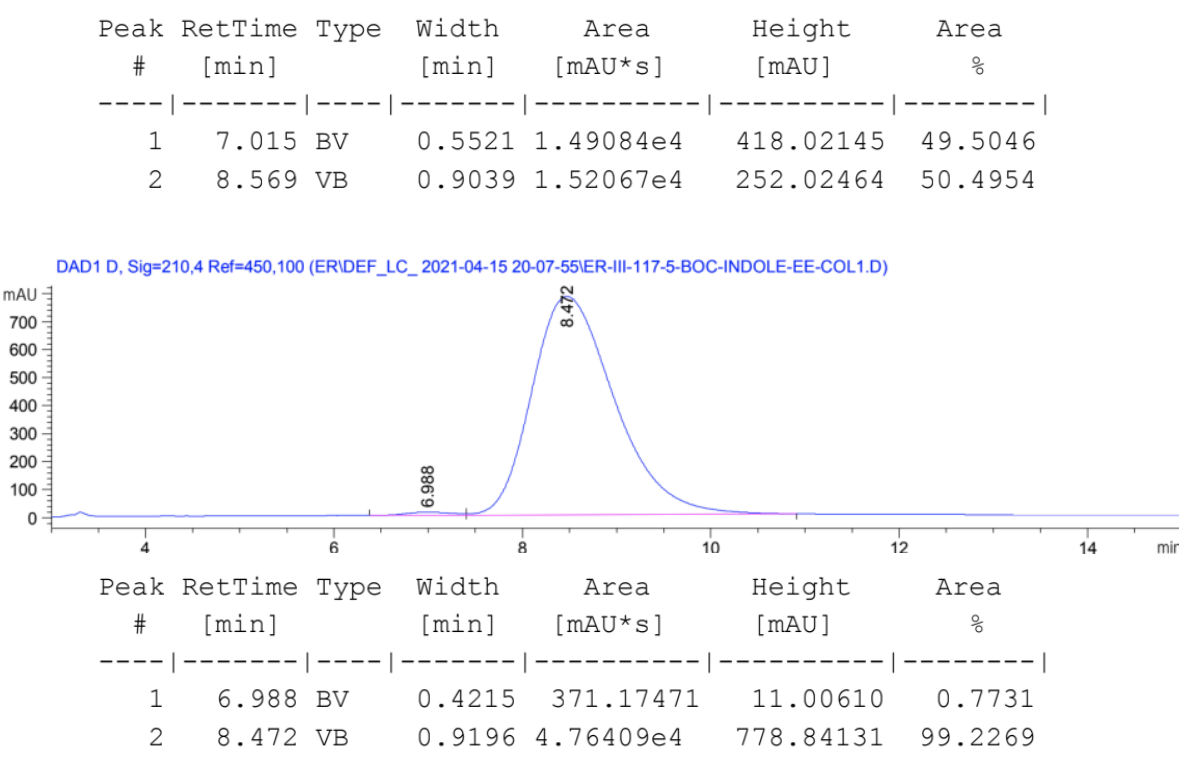

\section{(+)-(R)-N-Boc-3-methyl-1-(3-benzo[b]thiophene)-but-3-en-1-amine [3q]}<smiles>C=C(C)C[C@H](NC(=O)OC(C)(C)C)c1csc2ccccc12</smiles>

Prepared according to Method B. 3-(dimethoxymethyl)benzo[ $b]$ thiophene 1q (62.5 mg, $0.300 \mathrm{mmol}, 1.0$ equiv), (2-methylallyl) trimethylsilane (53.0 $\mu \mathrm{L}, 0.300$ mmol, 1.0 equiv), tert-butyl carbamate ( $35.1 \mathrm{mg}, 0.300 \mathrm{mmol}, 1.0$ equiv), catalyst $(S, R)-4 f \quad(19.7 \mathrm{mg}, 0.030 \mathrm{mmol}, 0.10$ equiv), TESOTf $(6.8 \mu \mathrm{L}, 0.030 \mathrm{mmol}, 0.10$ equiv), and $\mathrm{Et}_{2} \mathrm{O}(3.0 \mathrm{~mL}, 0.1 \mathrm{M})$ were used. The reaction was run at $-50^{\circ} \mathrm{C}$ for $18 \mathrm{~h}$ or $48 \mathrm{~h}$. Flash column chromatography on silica (Biotage, $10 \mathrm{~g}$ column) gave pure product as a white solid. Chiral HPLC conditions: CHIRALPAK AS-H, 5\% $\mathrm{PrOH} / \mathrm{hexanes,} 1 \mathrm{~mL} / \mathrm{min}$

Racemic product: $\mathrm{t}_{\mathrm{R}}(R)=6.7 \mathrm{~min}, \mathrm{t}_{\mathrm{R}}(S)=11.4 \mathrm{~min}$ Enantioenriched product: $\mathrm{t}_{\mathrm{R}}(R)=6.8 \mathrm{~min}$

18h: $70.4 \mathrm{mg}, 0.222 \mathrm{mmol}, 74 \%$ yield, $95 \%$ e.e.

48h: $77.7 \mathrm{mg}, 0.245 \mathrm{mmol}, 82 \%$ yield, $95 \%$ e.e.

${ }^{1} \mathrm{H}-\mathrm{NMR}\left(500 \mathrm{MHz}, \mathrm{CDCl}_{3}\right) \delta 7.90(\mathrm{~d}, J=8.0 \mathrm{~Hz}, 1 \mathrm{H}), 7.86(\mathrm{~d}, J=7.9 \mathrm{~Hz}, 1 \mathrm{H}), 7.44-7.32(\mathrm{~m}, 2 \mathrm{H}), 7.28$ (s, 1H), 5.33 (br. s, 1H), 4.87 (s, 1H), 4.82 (s, 1H), 4.81 (br. s, 1H), 2.73 (dd, J = 14.5, $5.9 \mathrm{~Hz}, 1 \mathrm{H}$ ), 2.59 (dd, $J=14.2,9.0 \mathrm{~Hz}, 1 \mathrm{H}), 1.80(\mathrm{~s}, 3 \mathrm{H}), 1.44(\mathrm{~s}, 9 \mathrm{H}) ;{ }^{13} \mathrm{C}-\mathrm{NMR}\left(126 \mathrm{MHz}, \mathrm{CDCl}_{3}\right) \delta 155.4,142.0,140.8$, 137.8, 124.6, 124.3, 123.0, 122.1, 122.0, 113.9, 79.6, 47.3, 43.9, 28.5, 22.2; One carbon resonance could not be located. IR (ATR, $\left.\mathrm{cm}^{-1}\right) 3355,2981,2941,1677,1530,1368,1164,1081,902 ;[\alpha]^{24} \mathrm{D}=+33.3^{\circ}(c=$ 1.0, $\mathrm{CDCl}_{3}$ ); HRMS (ESI) $\mathrm{m} / z$ calculated for $\mathrm{C}_{17} \mathrm{H}_{24} \mathrm{NO}_{2}[\mathrm{M}+\mathrm{H}]^{+}: 318.1522$, found 318.1509. 

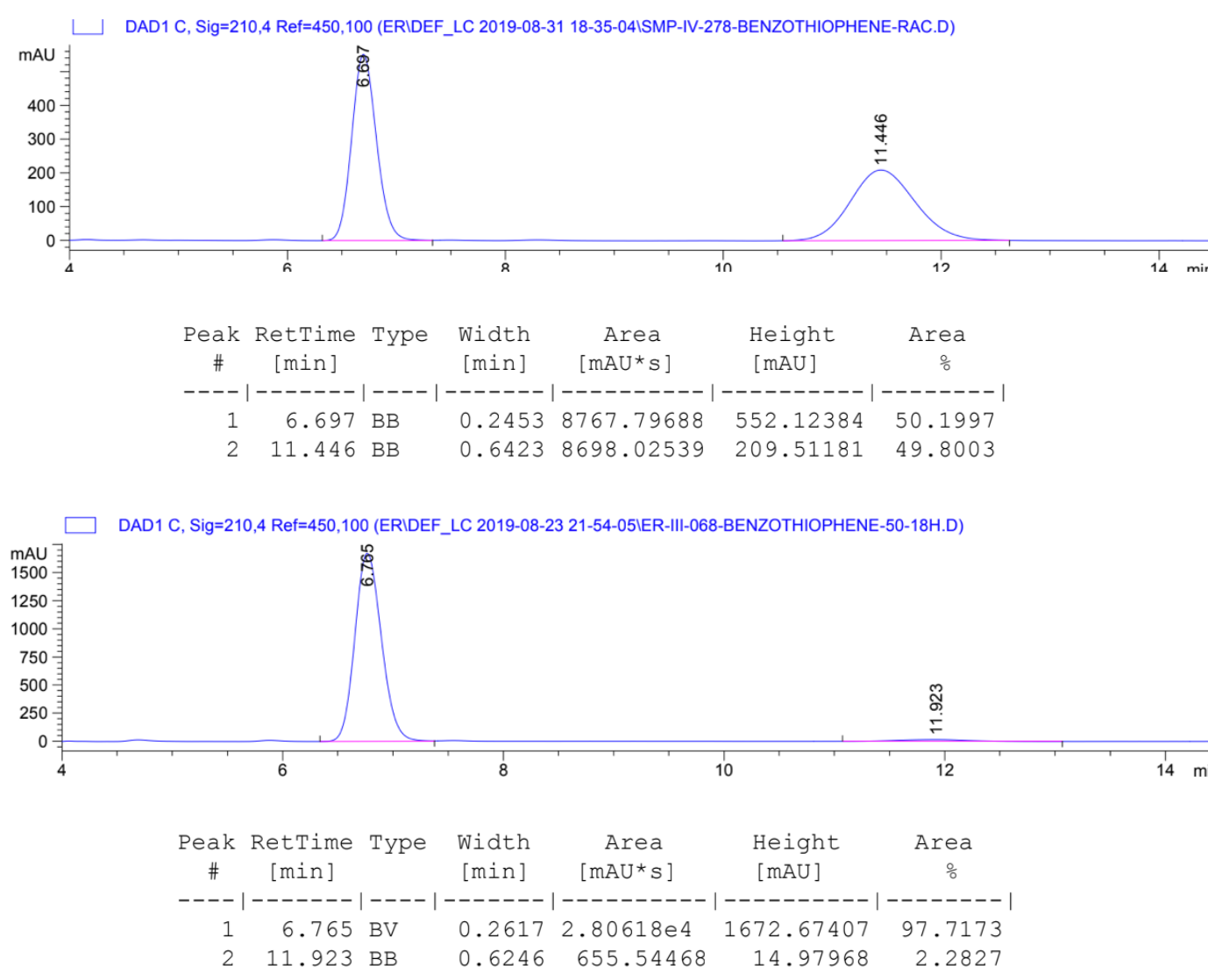

\section{$(+)-(R)-N-B o c-3-m e t h y l-1-(2-b e n z o[b]$ furanyl)-but-3-en-1-amine [3r]}<smiles>C=C(C)C[C@H](NC(=O)OC(C)(C)C)c1cc2ccccc2o1</smiles>

Prepared according to Method B. 2-Benzo[b]furan dimethyl acetal 1r (57.7 mg, $0.300 \mathrm{mmol}, 1.0$ equiv), (2-methylallyl)trimethylsilane $(53.0 \mu \mathrm{L}, 0.300 \mathrm{mmol}, 1.0$ equiv), tert-butyl carbamate (35.1 $\mathrm{mg}, 0.300 \mathrm{mmol}, 1.0$ equiv), catalyst $(S, R)-\mathbf{4 f}$ (19.7 mg, $0.030 \mathrm{mmol}, 0.10$ equiv), TESOTf $(6.8 \mu \mathrm{L}, 0.030 \mathrm{mmol}, 0.10$ equiv), and $\mathrm{Et}_{2} \mathrm{O}(3.0 \mathrm{~mL}, 0.1 \mathrm{M})$ were used. The reaction was run at $-50^{\circ} \mathrm{C}$ for $18 \mathrm{~h}$. Flash column chromatography on silica (Biotage, $10 \mathrm{~g}$ column) using a gradient of $1 \% \mathrm{EtOAc/hexanes} \rightarrow 10 \% \mathrm{EtOAc} /$ hexanes as eluent gave pure product as a white solid.

Racemic product: $t_{R}(R)=6.3 \mathrm{~min}, \mathrm{t}_{\mathrm{R}}(S)=11.1 \mathrm{~min}$ Enantioenriched product: $\operatorname{tR}_{\mathrm{R}}(R)=5.8 \mathrm{~min}$

\section{$43.1 \mathrm{mg}, 0.143 \mathrm{mmol}, 48 \%$ yield, $86 \%$ e.e.}

${ }^{1} \mathrm{H}-\mathrm{NMR}\left(600 \mathrm{MHz}, \mathrm{CDCl}_{3}\right) \delta 7.51(\mathrm{~d}, J=7.7,1 \mathrm{H}), 7.43(\mathrm{~d}, J=8.1,1 \mathrm{H}), 7.24(\mathrm{t}, J=7.4,1 \mathrm{H}), 7.20(\mathrm{t}, J=$ 7.4, 1H), 6.56 (s, 1H), 5.07 (br. s, 1H), 4.93 (br. s, 1H), 4.83 (s, 1H), 4.77 (s, 1H), 2.66 (dd, J = 14.0, 6.4 $\mathrm{Hz}, 1 \mathrm{H}), 2.56(\mathrm{dd}, J=14.1,8.4 \mathrm{~Hz}, 1 \mathrm{H}), 1.73(\mathrm{~s}, 3 \mathrm{H}), 1.44(\mathrm{~s}, 9 \mathrm{H}) ;{ }^{13} \mathrm{C}-\mathrm{NMR}\left(126 \mathrm{MHz}, \mathrm{CDCl}_{3}\right) \delta 157.8$, 155.2, 154.9, 141.2, 128.4, 124.0, 122.8, 121.1, 114.2, 111.2, 102.8, 79.9, 47.6, 42.7, 28.5, 22.2; IR $\left(\right.$ ATR, $\left.\mathrm{cm}^{-1}\right) 3392,2975,2927,1688,1510,1474,1393,1279,1247,1166,775 ;[\alpha]^{24} \mathrm{D}=+107.8^{\circ}(c=1.0$, $\mathrm{CHCl}_{3}$, for $87 \%$ e.e. sample); HRMS (ESI) $\mathrm{m} / 2$ calculated for $\mathrm{C}_{18} \mathrm{H}_{24} \mathrm{NO}_{3}[\mathrm{M}+\mathrm{H}]^{+}: 302.1751$, found 302.1726 . 

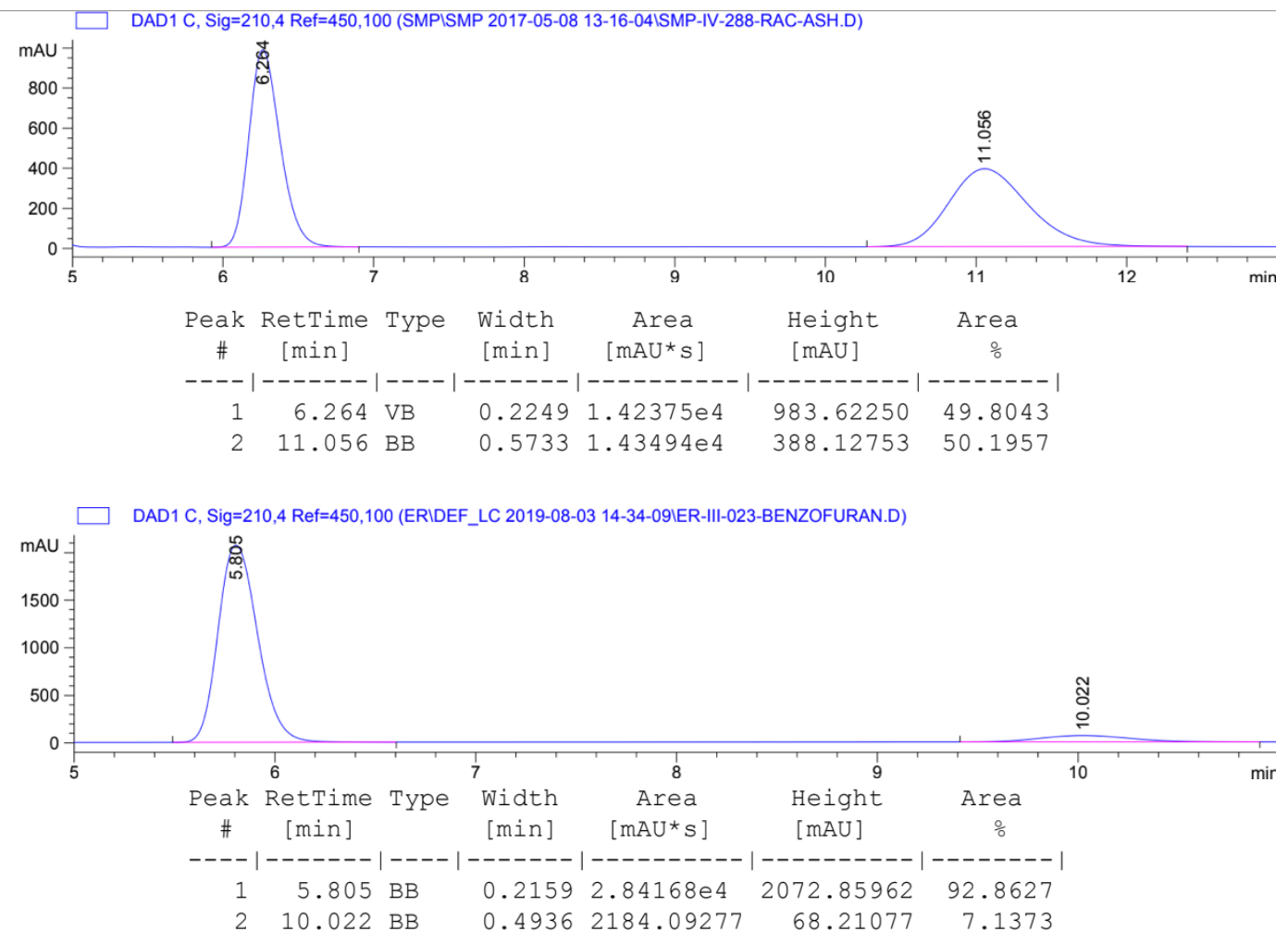

\section{(+)-(R)-N-Boc-3-methyl-1-(5-bromo-2-furanyl)-but-3-en-1-amine [3s]}<smiles>C=C(C)C[C@H](NC(=O)OC(C)(C)C)c1ccc(Br)o1</smiles>

Prepared according to Method B. 2-Bromo-5-(dimethoxymethyl) furan 1s (66.3 $\mu \mathrm{L}$, $0.300 \mathrm{mmol}, 1.0$ equiv), (2-methylallyl)trimethylsilane $(53.0 \mu \mathrm{L}, 0.300 \mathrm{mmol}, 1.0$ equiv), tert-butyl carbamate (35.1 mg, $0.300 \mathrm{mmol}, 1.0$ equiv), catalyst $(S, R)-\mathbf{4 f}$ (19.7 mg, $0.030 \mathrm{mmol}, 0.10$ equiv), TESOTf ( $6.8 \mu \mathrm{L}, 0.030 \mathrm{mmol}, 0.10$ equiv), and $\mathrm{Et}_{2} \mathrm{O}(3.0 \mathrm{~mL}, 0.1 \mathrm{M})$ were used. In the $18 \mathrm{~h}$ reaction, the solids were weighed out directly and no stock solution was used. The reaction was run at $-50^{\circ} \mathrm{C}$ for $6 \mathrm{~h}$ or $18 \mathrm{~h}$. Flash column chromatography on silica (Biotage, $10 \mathrm{~g}$ column) gave pure product as a white solid.

Chiral HPLC conditions: CHIRALPAK AS-H, $3 \%$ PrOH/hexanes, $1 \mathrm{~mL} / \mathrm{min}$

Racemic product: $\mathrm{t}_{\mathrm{R}}(R)=6.1 \mathrm{~min}, \mathrm{t}_{\mathrm{R}}(S)=9.2 \mathrm{~min}$

Enantioenriched product: $\mathrm{t}_{\mathrm{R}}(R)=5.8 \mathrm{~min}$

6h: $30.8 \mathrm{mg}, 0.093 \mathrm{mmol}, \mathbf{3 1} \%$ yield, $87 \%$ e.e

18h: $70.8 \mathrm{mg}, 0.214 \mathrm{mmol}, 71 \%$ yield, $86 \%$ e.e.

${ }^{1} \mathrm{H}-\mathrm{NMR}\left(500 \mathrm{MHz}, \mathrm{CDCl}_{3}\right) \delta 6.19$ (dd, $\left.J=3.3,1.8 \mathrm{~Hz}, 1 \mathrm{H}\right), 6.13(\mathrm{~d}, J=2.9 \mathrm{~Hz}, 1 \mathrm{H}), 4.85$ (br. s, $\left.1 \mathrm{H}\right), 4.81$ (s, 2H), 4.72 (br. s, $1 \mathrm{H}$ ), 2.51 (ddt, $J=13.9,6.5,1.5 \mathrm{~Hz}, 1 \mathrm{H}$ ), 2.44 (dd, $J=14.1,8.2 \mathrm{~Hz}, 1 \mathrm{H}), 1.69(\mathrm{~s}, 3 \mathrm{H}$ ), $1.42(\mathrm{~d}, J=2.1 \mathrm{~Hz}, 9 \mathrm{H}) ;{ }^{13} \mathrm{C}-\mathrm{NMR}\left(126 \mathrm{MHz}, \mathrm{CDCl}_{3}\right) \delta 157.0,155.1,141.1,120.6,114.2,111.9,108.7$, 79.9, 47.2, 42.6, 28.4, 22.1; IR (ATR, $\left.\mathrm{cm}^{-1}\right)$ 3372, 2981, 2970, 2931, 1685, 1512, 1265, 1245, 1162, 1124, 1025; $[\alpha]^{22} \mathrm{D}=+74.4^{\circ}\left(c=1.0, \mathrm{CDCl}_{3}\right)$; HRMS (ESI) $\mathrm{m} / z$ calculated for $\mathrm{C}_{14} \mathrm{H}_{21} \mathrm{BrNO}_{3}[\mathrm{M}+\mathrm{H}]^{+}: 330.0699$, found 330.0695 . 


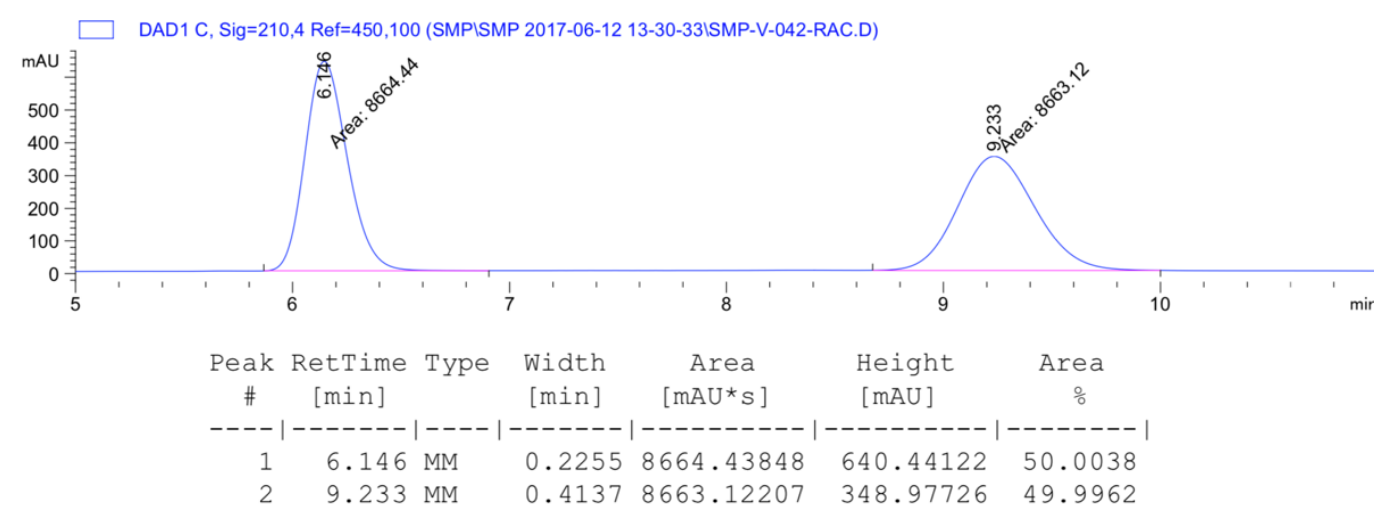

$t=6 h$

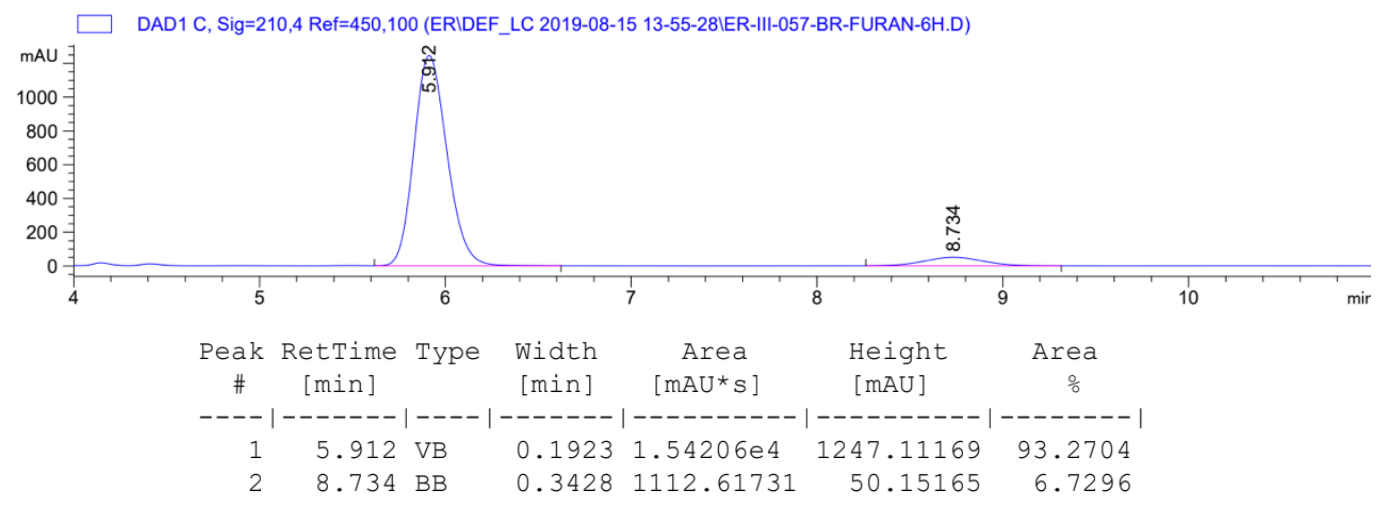

$t=18 h$

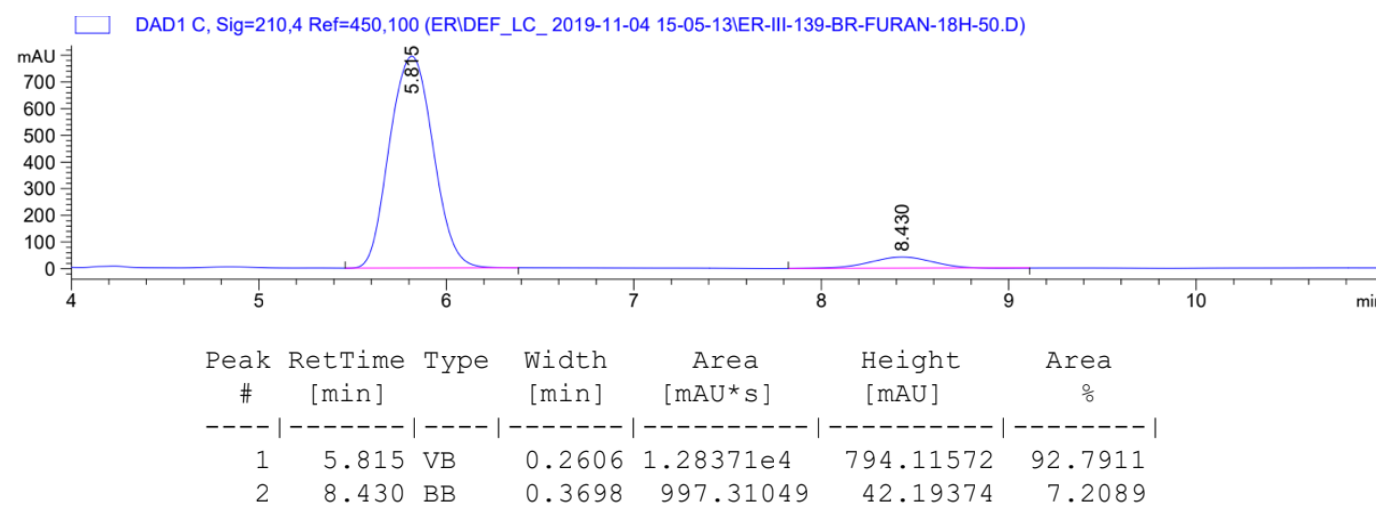

\section{Aldehydes as substrates}

Method C: An oven-dried 2-dram vial equipped with a stir bar was sealed with a septum-lined cap, then evacuated and refilled with $\mathrm{N}_{2}(3 \mathrm{x})$. It was then charged with tert-butyl carbamate (stock solution of $0.2 \mathrm{M}$ in $\mathrm{Et}_{2} \mathrm{O}, 1.00 \mathrm{~mL}, 0.200 \mathrm{mmol}, 1.0$ equiv, unless otherwise noted), catalyst $(S, R)-\mathbf{4 f}$ (stock solution of 0.02 $\mathrm{M}$ in $\mathrm{Et}_{2} \mathrm{O}, 1.00 \mathrm{~mL}, 0.020 \mathrm{mmol}, 0.10$ equiv, unless otherwise noted), aldehyde $(0.200 \mathrm{mmol}, 1.0$ equiv) and nucleophile $(0.200 \mathrm{mmol}, 1.0$ equiv). The vial was carefully sealed with electrical tape, covering the septum $\times 3$. The reaction mixture was cooled to $-78^{\circ} \mathrm{C}$ in a dry ice/acetone bath, then TESOTf $(4.5 \mu \mathrm{L}$, $0.020 \mathrm{mmol}, 0.10$ equiv) was added. The vial was transferred to a cooling bath (cryocool) at the indicated temperature and the mixture was stirred for the reported time. In many cases, the initially homogeneous mixture became heterogeneous over the course of the reaction. After the corresponding time, the reaction 
was quenched by addition via syringe of a solution of $1: 1 \mathrm{Et}_{3} \mathrm{~N} / \mathrm{MeOH}(160 \mu \mathrm{L})$, stirred for 5 min at the reaction temperature, then warmed to rt. The solvent was removed under reduced pressure, then $100 \mu \mathrm{L}$ of a stock solution of internal standard $\left(70 \mu \mathrm{L}\right.$ of mesitylene in $1 \mathrm{~mL}$ of $\left.\mathrm{CDCl}_{3}\right)$ was added. Conversions were calculated with respect to the mesitylene aromatic peak at $6.80 \mathrm{ppm},(\mathrm{s}, 3 \mathrm{H})$ integrating the starting material peaks at $10.01(\mathrm{~s}, 1 \mathrm{H})$ for benzaldehyde and $9.80(\mathrm{~s}, 1 \mathrm{H})$ for piperonal. The remaining residue was applied to a silica column for purification and enantiomeric excess was determined by chiral HPLC analysis of purified product. Note: Both reactions reported here were run in a CC-100 Thermo NES-LAB cryocool and monitored with an $\mathrm{H}-\mathrm{B}$ Instrument Thermometer, Digital w/Probe $\left(-100 / 400^{\circ} \mathrm{C}\right.$ range, $0.01^{\circ} \mathrm{C}$ resolution). The thermometer probe was positioned in the pie-block together with the reaction vials. A stir bar ensured circulation at the bottom of the cooling bath solution to minimize the formation of temperature gradients in the cooling bath.

\section{(+)-(R)-N-Boc-3-methyl-1-phenylbut-3-en-1-amine [3a]}<smiles>C=C(C)C[C@H](NC(=O)OC(C)(C)C)c1ccccc1</smiles>

Prepared according to Method C. Benzaldehyde (20.5 $\mu \mathrm{L}, 0.200 \mathrm{mmol}, 1.0$ equiv), (2-methylallyl)trimethylsilane $(35.0 \mu \mathrm{L}, 0.200 \mathrm{mmol}, 1.0$ equiv), tert- butyl carbamate $(23.4 \mathrm{mg}, 0.200 \mathrm{mmol}, 1.0$ equiv), catalyst $(S, R)-\mathbf{4 f}(13.2 \mathrm{mg}, 0.020$ mmol, 0.10 equiv), TESOTf $\left(4.5 \mu \mathrm{L}, 0.020 \mathrm{mmol}, 0.10\right.$ equiv), and $\mathrm{Et}_{2} \mathrm{O}$ ( $2.0 \mathrm{~mL}$, $0.1 \mathrm{M})$ were used. The reaction was run at $-50^{\circ} \mathrm{C}$ for $48 \mathrm{~h}$. Flash column chromatography on silica gel ( $\mathrm{Et}_{2} \mathrm{O}: \mathrm{Hexanes}$ and then $100 \% \mathrm{DCM}$ ) gave the pure product as a white solid. See acetal reaction for full characterization. NMR analysis showed residual starting material ( $20 \%)$ as main additional component of the crude reaction mixture.

$35.9 \mathrm{mg}, 0.137 \mathrm{mmol}, 68 \%$ yield, $96 \%$ e.e

\section{$(+)-(R)-N-B o c-1-($ benzo[d][1,3]dioxol-5-yl)-3-methylbut-3-en-1-amine [3k]}<smiles>C=C(C)C[C@H](NC(=O)OC(C)(C)C)c1ccc2c(c1)OCO2</smiles>

Prepared according to Method C. Piperonal (30.0 mg, $0.200 \mathrm{mmol}, 1.0$ equiv), (2-methylallyl)trimethylsilane $(35.0 \mu \mathrm{L}, 0.200 \mathrm{mmol}, 1.0$ equiv), tert- butyl carbamate (23.4 mg, $0.200 \mathrm{mmol}, 1.0$ equiv), catalyst $(S, R)-\mathbf{4 f}(13.2 \mathrm{mg}, 0.020$ mmol, 0.10 equiv), TESOTf $\left(4.5 \mu \mathrm{L}, 0.020 \mathrm{mmol}, 0.10\right.$ equiv), and $\mathrm{Et}_{2} \mathrm{O}(2.0 \mathrm{~mL}$, $0.1 \mathrm{M}$ ) were used. The reaction was run at $-50^{\circ} \mathrm{C}$ for $26 \mathrm{~h}$. Flash column chromatography ( $\mathrm{Et}_{2} \mathrm{O}:$ Hexanes and then $100 \% \mathrm{DCM}$ ) afforded pure product as a white solid. See acetal reaction for full characterization. NMR analysis showed residual starting material ( $37 \%)$ as main additional component of the crude reaction mixture.

$34.6 \mathrm{mg}, 0.113 \mathrm{mmol}, 57 \%$ yield, $98 \%$ e.e.

\section{Nucleophile scope}

Table S8. Nucleophile Scope.

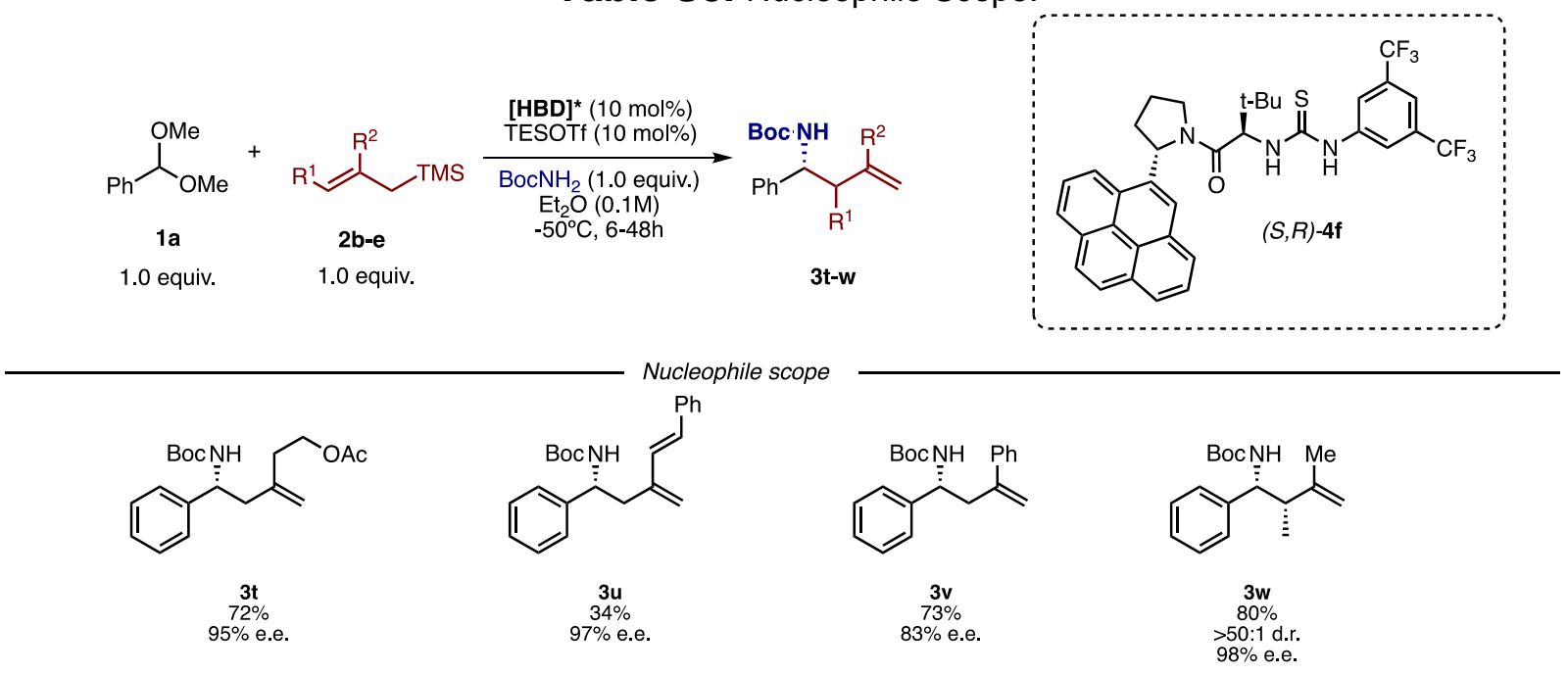




\section{(+)-(R)-5-((tert-butoxycarbonyl)amino)-3-methylene-5-phenylpentyl acetate [3t]}<smiles>C=C(CCOC(C)=O)C[C@H](NC(=O)OC(C)(C)C)c1ccccc1</smiles>

product as a white solid.

Chiral HPLC conditions: CHIRALPAK AS-H, 3\% $\mathrm{PrOH} /$ hexanes, $1 \mathrm{~mL} / \mathrm{min}$

Racemic product: $\mathrm{t}_{\mathrm{R}}(R)=17.8 \mathrm{~min}, \mathrm{t}_{\mathrm{R}}(S)=24.1 \mathrm{~min}$

Enantioenriched product: $\mathrm{t}_{\mathrm{R}}(R)=17.7 \mathrm{~min}$

\section{$73.2 \mathrm{mg}, 0.220 \mathrm{mmol}, 72$ yield, $95 \%$ e.e.}

${ }^{1} \mathrm{H}-\mathrm{NMR}\left(500 \mathrm{MHz}, \mathrm{CDCl}_{3}\right) \delta 7.39-7.17$ (m, 5H), 4.93 (br. d, J = $\left.7.5 \mathrm{~Hz}, 1 \mathrm{H}\right), 4.89$ (br. s, 2H), 4.82 (br. s, $1 \mathrm{H}$ ), 4.28-4.07 (m, 2H), 2.48 (td, $J=15.1,8.0 \mathrm{~Hz}, 2 \mathrm{H}$ ), 2.35 (hept, $J=7.6 \mathrm{~Hz}, 2 \mathrm{H}), 2.04(\mathrm{~s}, 3 \mathrm{H}), 1.41$ (s, 9H); ${ }^{13} \mathrm{C}-\mathrm{NMR}\left(126 \mathrm{MHz}, \mathrm{CDCl}_{3}\right) \delta 171.1,155.3,142.9,141.8,128.6,127.3,126.2,114.9,79.5,62.6$, 52.9, 44.0, 34.6, 28.4, 21.0. IR (ATR, $\left.\mathrm{cm}^{-1}\right)$ 3381, 2976, 1728, 1684, 1519, 1361, 1238, 1170, 1015, 909; $[\alpha]^{24} \mathrm{D}=+25.1^{\circ}\left(c=1.0, \mathrm{CDCl}_{3}\right)$; HRMS $(\mathrm{ESI}) \mathrm{m} / \mathrm{z}$ calculated for $\mathrm{C}_{19} \mathrm{H}_{27} \mathrm{NO}_{4} \mathrm{Na}[\mathrm{M}+\mathrm{Na}]^{+}: 356.1832$, found 356.1836.
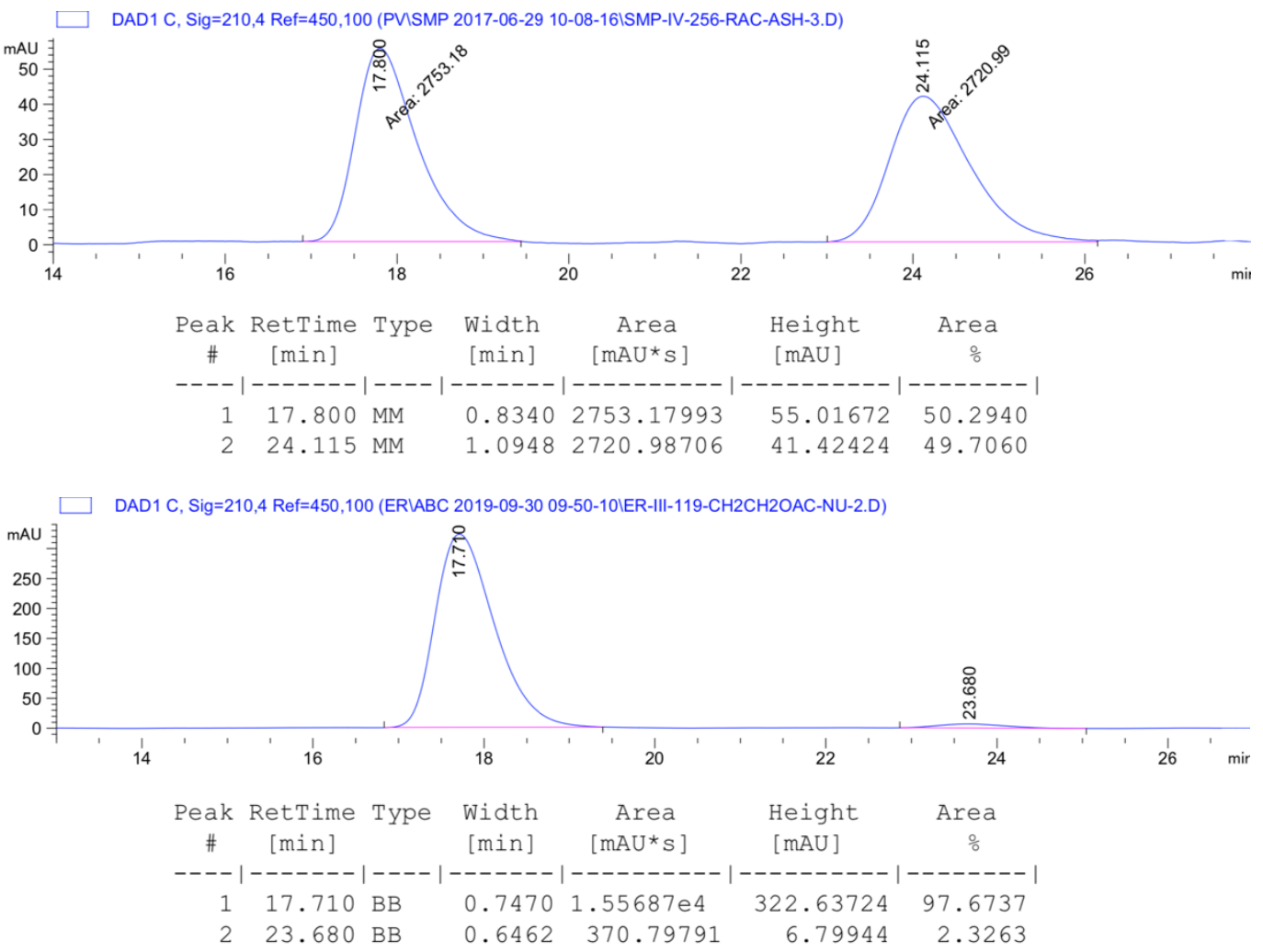


\section{(-)-(R)-tert-butyl (E)-(3-methylene-1,5-diphenylpent-4-en-1-yl)carbamate [3u]}<smiles>C=C(/C=C/c1ccccc1)C[C@H](NC(=O)OC(C)(C)C)c1ccccc1</smiles>

Prepared according to Method B. Benzaldehyde dimethyl acetal $(45.0 \mu \mathrm{L}$, $0.300 \mathrm{mmol}, 1.0$ equiv), (E)-trimethyl(2-methylene-4-phenylbut-3-en-1-yl)silane 2c $(64.9 \mathrm{mg}, 0.300 \mathrm{mmol}, 1.0$ equiv), tert-butyl carbamate $(35.1 \mathrm{mg}, 0.300$ mmol, 1.0 equiv), catalyst $(S, R)-\mathbf{4 f}$ (19.7 $\mathrm{mg}, 0.030 \mathrm{mmol}, 0.10$ equiv), TESOTf $\left(6.8 \mu \mathrm{L}, 0.030 \mathrm{mmol}, 0.10\right.$ equiv), and $\mathrm{Et}_{2} \mathrm{O}(3.0 \mathrm{~mL}, 0.1 \mathrm{M})$ were used. The reaction was run at $-50^{\circ} \mathrm{C}$ for $48 \mathrm{~h}$. Flash column chromatography on silica (Biotage, $25 \mathrm{~g}$ column) gave the product as a white solid mixed with a small amount of an unknown impurity $(<5 \%)$.

Chiral HPLC conditions: CHIRALPAK IB, 3\% $\mathrm{PrOH} /$ hexanes, $1 \mathrm{~mL} / \mathrm{min}$

Racemic product: $\mathrm{t}_{\mathrm{R}}(S)=6.5 \mathrm{~min}, \mathrm{t}_{\mathrm{R}}(R)=7.0 \mathrm{~min}$

Enantioenriched product: $\mathrm{t}_{\mathrm{R}}(R)=8.3 \mathrm{~min}$

$35.9 \mathrm{mg}, 0.103 \mathrm{mmol}, \mathbf{3 4} \%$ yield, $97 \%$ e.e.

When run with Method A:

Run 1: ( $80.2 \mathrm{mg}, 0.230 \mathrm{mmol}, 77 \%), 94 \%$ e.e.

Run 2: (78.1 mg, $0.224 \mathrm{mmol}, 75 \%), 94 \%$ e.e.

Average: $76 \%$ yield, $94 \%$ e.e.

${ }^{1} \mathrm{H}-\mathrm{NMR}\left(600 \mathrm{MHz}, \mathrm{CDCl}_{3}\right) \delta 7.41(\mathrm{~d}, J=7.8 \mathrm{~Hz}, 2 \mathrm{H}), 7.35-7.27(\mathrm{~m}, 6 \mathrm{H}), 7.27-7.20(\mathrm{~m}, 2 \mathrm{H}), 6.75(\mathrm{~d}, J$ $=16.2 \mathrm{~Hz}, 1 \mathrm{H}), 6.67(\mathrm{~d}, J=16.6 \mathrm{~Hz}, 1 \mathrm{H}), 5.18(\mathrm{~s}, 1 \mathrm{H}), 4.96$ (br. s, $1 \mathrm{H}), 4.92($ br. $\mathrm{s}, 2 \mathrm{H}), 2.86-2.65(\mathrm{~m}$, 2H), 1.40 (s, 9H). ${ }^{13} \mathrm{C}-\mathrm{NMR}\left(126 \mathrm{MHz}, \mathrm{CDCl}_{3}\right) \delta 155.3,142.9,142.1,137.3,130.5,128.9,128.7,128.6$, 127.7, 127.4, 126.7, 126.4, 118.9, 79.6, 53.8, 40.2, 28.5; IR (ATR, $\left.\mathrm{cm}^{-1}\right)$ 3387, 3027, 2976, 2927, 1679, $1507,1445,1364,1264,1247,1170,1021,956 ;[\alpha]^{24} \mathrm{D}=4.3^{\circ}\left(c=1.0, \mathrm{CDCl}_{3}\right) ; \mathrm{HRMS}(\mathrm{ESI}) \mathrm{m} / \mathrm{z}$ calculated for $\mathrm{C}_{23} \mathrm{H}_{28} \mathrm{NO}_{2}[\mathrm{M}+\mathrm{H}]+: 350.2115$, found 350.2111 .
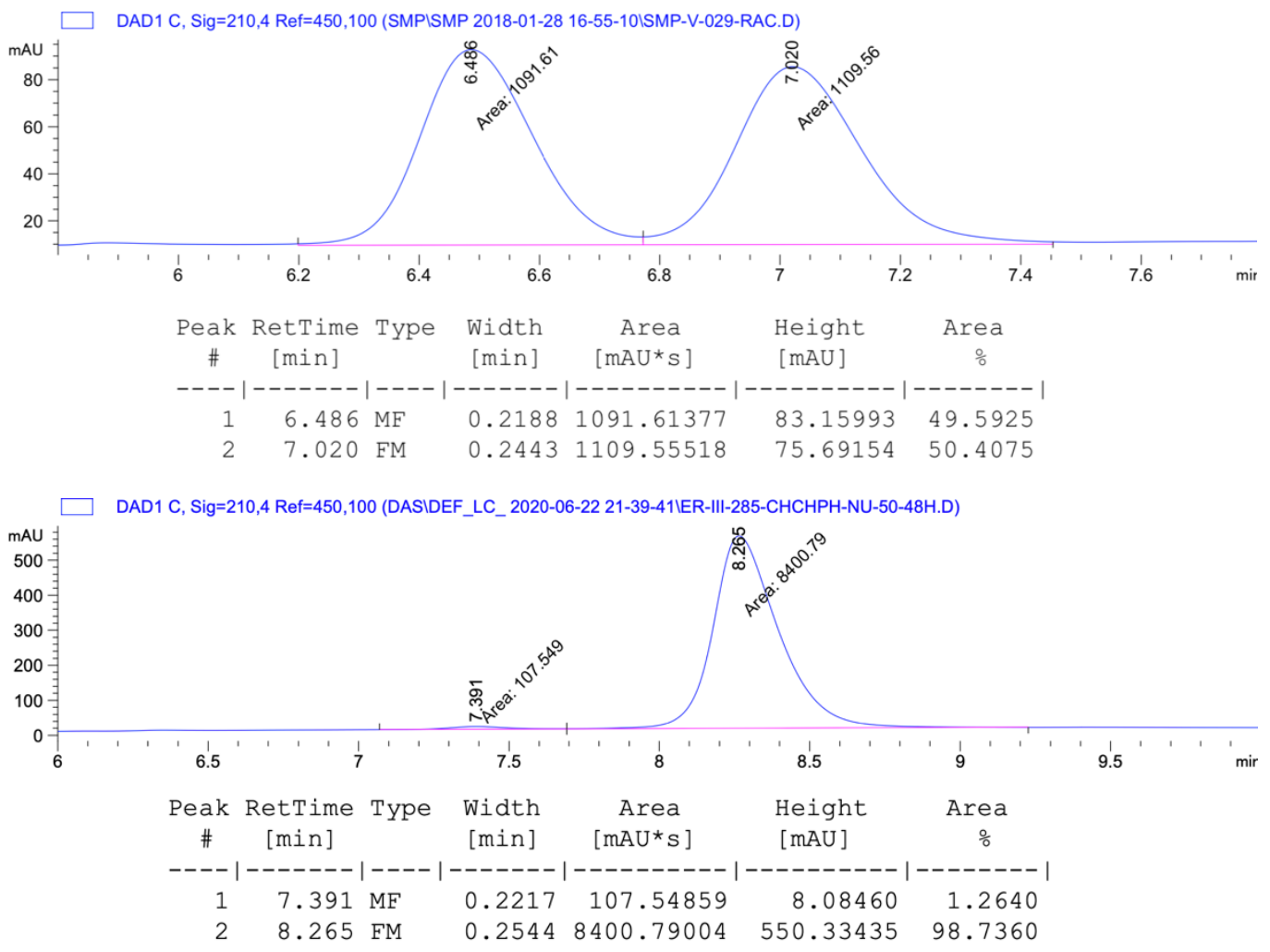


\section{$(-)-(R)-N-B o c-3-p h e n y l-1-p h e n y l b u t-3-e n-1-a m i n e ~[3 v]$}<smiles>C=C(C[C@H](NC(=O)OC(C)(C)C)c1ccccc1)c1ccccc1</smiles>

Prepared according to Method B. Benzaldehyde dimethyl acetal $(45.0 \mu \mathrm{L}, 0.300$ mmol, 1.0 equiv), (2-phenylallyl)trimethylsilane $2 \mathrm{~d}(57.1 \mathrm{mg}, 0.300 \mathrm{mmol}, 1.0$ equiv), tert-butyl carbamate (35.1 $\mathrm{mg}, 0.300 \mathrm{mmol}, 1.0$ equiv), catalyst $(S, R)-\mathbf{4 f}$ (19.7 mg, $0.030 \mathrm{mmol}, 0.10$ equiv), TESOTf ( $6.8 \mu \mathrm{L}, 0.030 \mathrm{mmol}, 0.10$ equiv), and $\mathrm{Et}_{2} \mathrm{O}(3.0 \mathrm{~mL}, 0.1 \mathrm{M})$ were used. The reaction was run at $-50^{\circ} \mathrm{C}$ for $18 \mathrm{~h}$. Flash column chromatography on silica (Biotage, $10 \mathrm{~g}$ column) gave pure product as a white solid.

Chiral HPLC conditions: AD-H, $3 \%$ PrOH/hexanes, $1 \mathrm{~mL} / \mathrm{min}$

Racemic product: $\mathrm{t}_{\mathrm{R}}(S)=17.1 \mathrm{~min}, \mathrm{t}_{\mathrm{R}}(R)=20.2 \mathrm{~min}$

Enantioenriched product: $\mathrm{t}_{\mathrm{R}}(R)=21.3 \mathrm{~min}$

$70.9 \mathrm{mg}, 0.219 \mathrm{mmol}, 73 \%$ yield, $83 \%$ e.e.

${ }^{1} \mathrm{H}-\mathrm{NMR}\left(500 \mathrm{MHz}, \mathrm{CDCl}_{3}\right) \delta 7.41-7.26(\mathrm{~m}, 7 \mathrm{H}), 7.25-7.14(\mathrm{~m}, 3 \mathrm{H}), 5.28(\mathrm{~s}, 1 \mathrm{H}), 5.02(\mathrm{~s}, 1 \mathrm{H}), 4.87$ (br. $\mathrm{d}, J=7.2 \mathrm{~Hz}, 1 \mathrm{H}$ ), 4.71 (br. s, $1 \mathrm{H}), 2.93(\mathrm{~d}, J=6.7 \mathrm{~Hz}, 1 \mathrm{H}), 1.39(\mathrm{~s}, 9 \mathrm{H}) ;{ }^{13} \mathrm{C}-\mathrm{NMR}\left(126 \mathrm{MHz}, \mathrm{CDCl}_{3}\right) \delta$ 155.2, 145.0, 142.8, 140.5, 128.6, 127.8, 127.2, 126.41, 126.36, 115.7, 79.4, 53.7, 43.4, 28.4; One carbon resonance could not be located. IR (ATR, $\left.\mathrm{cm}^{-1}\right) 3362,2973,2932,1682,1522,1497,1362,1264$, $1250,1171,1014,904 ;[\alpha]^{24} \mathrm{D}=-4.2^{\circ}\left(c=1.0, \mathrm{CDCl}_{3}\right)$; HRMS $(\mathrm{ESI}) \mathrm{m} / z$ calculated for $\mathrm{C}_{21} \mathrm{H}_{26} \mathrm{NO}_{2}[\mathrm{M}+\mathrm{H}]^{+}$: 324.1958, found 324.1956.
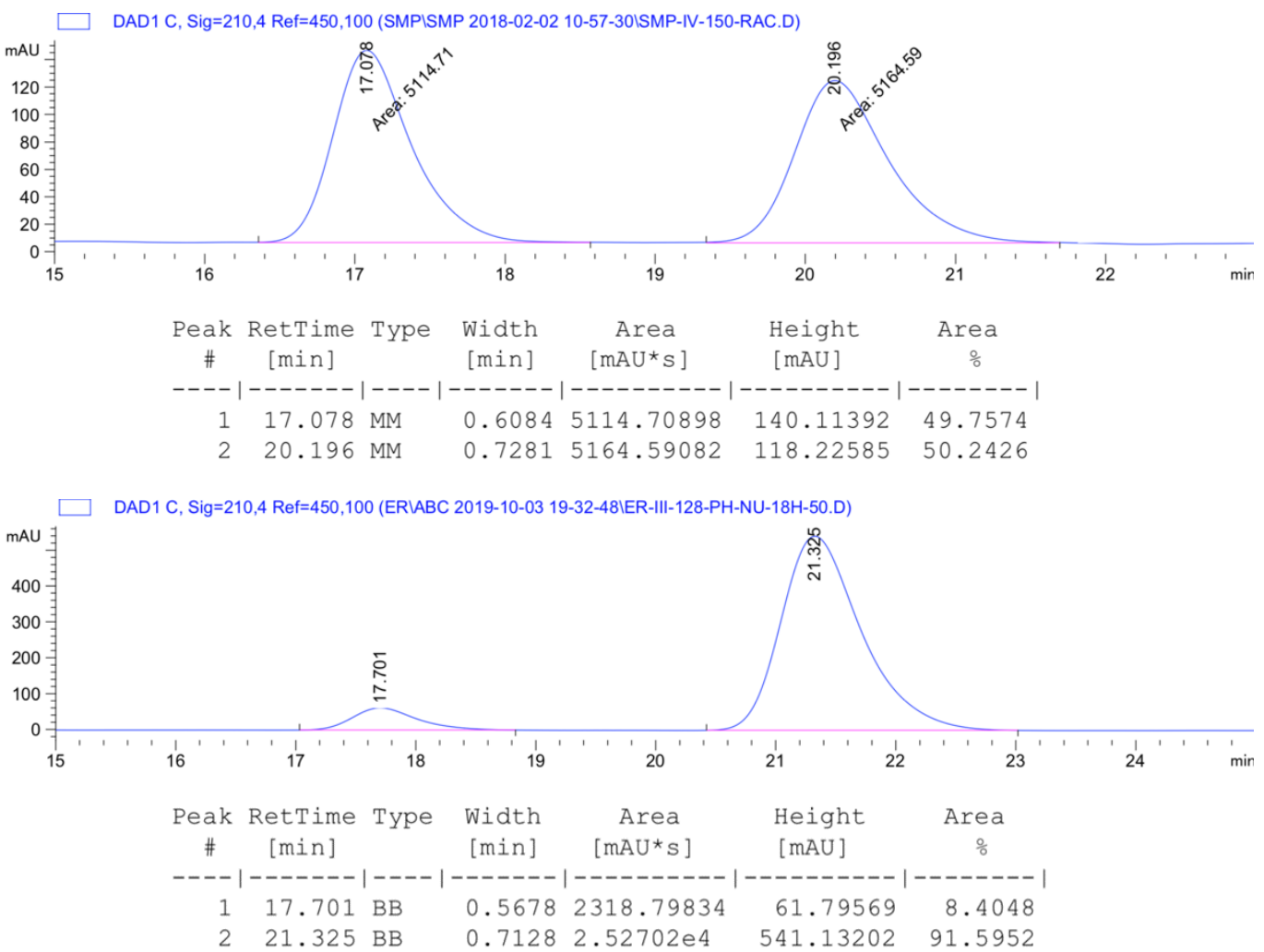


\section{(+)-(1R,2S)-N-Boc-2,3-dimethyl-1-phenylbut-3-en-1-amine [3w]}<smiles>C=C(C)[C@H](C)[C@H](NC(=O)OC(C)(C)C)c1ccccc1</smiles>

Prepared according to Method B. Benzaldehyde dimethyl acetal $(45.0 \mu \mathrm{L}, 0.300$ mmol, 1.0 equiv), ( $E$ )-trimethyl(2-methylbut-2-en-1-yl)silane 2 e $(42.7 \mathrm{mg}, 0.300$ mmol, 1.0 equiv), tert-butyl carbamate $(35.1 \mathrm{mg}, 0.300 \mathrm{mmol}, 1.0$ equiv), catalyst $(S, R)-4 f(19.7 \mathrm{mg}, 0.030 \mathrm{mmol}, 0.10$ equiv), TESOTf $(6.8 \mu \mathrm{L}, 0.030 \mathrm{mmol}, 0.10$ equiv), and $\mathrm{Et}_{2} \mathrm{O}(3.0 \mathrm{~mL}, 0.1 \mathrm{M})$ were used. The reaction was run at $-50^{\circ} \mathrm{C}$ for 18h. Flash column chromatography on silica (Biotage, $10 \mathrm{~g}$ column) gave the product as an inseparable mixture of diastereomers (the yield reported is of the diastereomeric mixture). Diastereomeric ratio was determined by chiral GC assuming identical response factors for the two diastereomers. Assignment of relative stereochemistry was made in analogy to that of related compounds reported in the literature, based on diagnostic ${ }^{1} \mathrm{H}-\mathrm{NMR}$ shifts. ${ }^{20}$

Chiral GC conditions: Cyclodex $\beta, 60 \mathrm{~m} \times 0.25 \mathrm{~mm} \times 0.25 \mathrm{~mm}, 140^{\circ} \mathrm{C}, 80 \mathrm{~min}$.

anti: $\mathrm{t}=35.1 \mathrm{~min}$,

syn: $\mathrm{t}=42.6 \mathrm{~min}$;

dr in absence of catalyst: $5: 1$;

dr with catalyst: $179: 1$

Racemic product $(s y n): \mathrm{t}_{\mathrm{R}}(1 S, 2 R)=41.7 \mathrm{~min}, \mathrm{t}_{\mathrm{R}}(1 R, 2 S)=42.6 \mathrm{~min}$

Enantioenriched product $(s y n): \mathrm{t}_{\mathrm{R}}(1 R, 2 S)=42.4 \mathrm{~min}$

Chiral HPLC conditions: OJ-H, $3 \%$ PrOH/hexanes, $1 \mathrm{~mL} / \mathrm{min}$

Racemic product:

syn: $\mathrm{t}_{\mathrm{R}}(1 \mathrm{~S}, 2 R)=6.0 \mathrm{~min}, \mathrm{t}_{\mathrm{R}}(1 R, 2 S)=8.2 \mathrm{~min}$

anti: $t_{R}\left(e_{\min }\right)=4.8 \mathrm{~min}, t_{R}\left(e_{m a j}\right)=9.0 \mathrm{~min}$

Enantioenriched product $(s y n): t_{R}(1 R, 2 S)=8.3 \mathrm{~min}$

\section{$65.8 \mathrm{mg}, 0.239 \mathrm{mmol}, \mathbf{8 0} \%$ yield, $98 \%$ e.e., $>50: 1$ syn/anti.}

${ }^{1} \mathrm{H}-\mathrm{NMR}\left(500 \mathrm{MHz}, \mathrm{CDCl}_{3}\right) \delta 7.29(\mathrm{t}, J=6.9 \mathrm{~Hz}, 2 \mathrm{H}), 7.22(\mathrm{~d}, J=7.2 \mathrm{~Hz}, 3 \mathrm{H}), 4.86(\mathrm{~d}, J=8.7,1 \mathrm{H}), 4.77$ (br. s, 1H), 4.73 (br. s, 1H), 4.67 (br. s, 1H), $2.55(\mathrm{~m}, 1 \mathrm{H}), 1.69(\mathrm{~s}, 3 \mathrm{H}$, ant $), 1.66(\mathrm{~s}, 3 \mathrm{H}, \mathrm{syn}), 1.41$ (s, $9 \mathrm{H}), 1.05(\mathrm{~d}, J=7.0 \mathrm{~Hz}, 3 \mathrm{H}, s y n), 0.84\left(\mathrm{~d}, J=6.9 \mathrm{~Hz}, 3 \mathrm{H}\right.$, anti); ${ }^{3} \mathrm{C}-\mathrm{NMR}\left(126 \mathrm{MHz}, \mathrm{CDCl}_{3}\right) \delta 155.6$, 146.8, 142.1, 128.3, 127.0, 126.8, 112.4, 79.4, 57.2, 46.7, 28.5, 20.7, 15.1; IR (ATR, cm $\left.{ }^{-1}\right)$ 3384, 2975, $1678,1514,1360,1249,1169,1009,890 ;[\alpha]^{21} \mathrm{D}=+36.5^{\circ}\left(c=1.0, \mathrm{CDCl}_{3}\right) ; \mathrm{HRMS}(\mathrm{ESI}) \mathrm{m} / \mathrm{z}$ calculated for $\mathrm{C}_{17} \mathrm{H}_{26} \mathrm{NO}_{2}[\mathrm{M}+\mathrm{H}]^{+}:$276.1958, found 276.1956.

\section{Diastereomeric ratio - reaction in absence of catalyst}

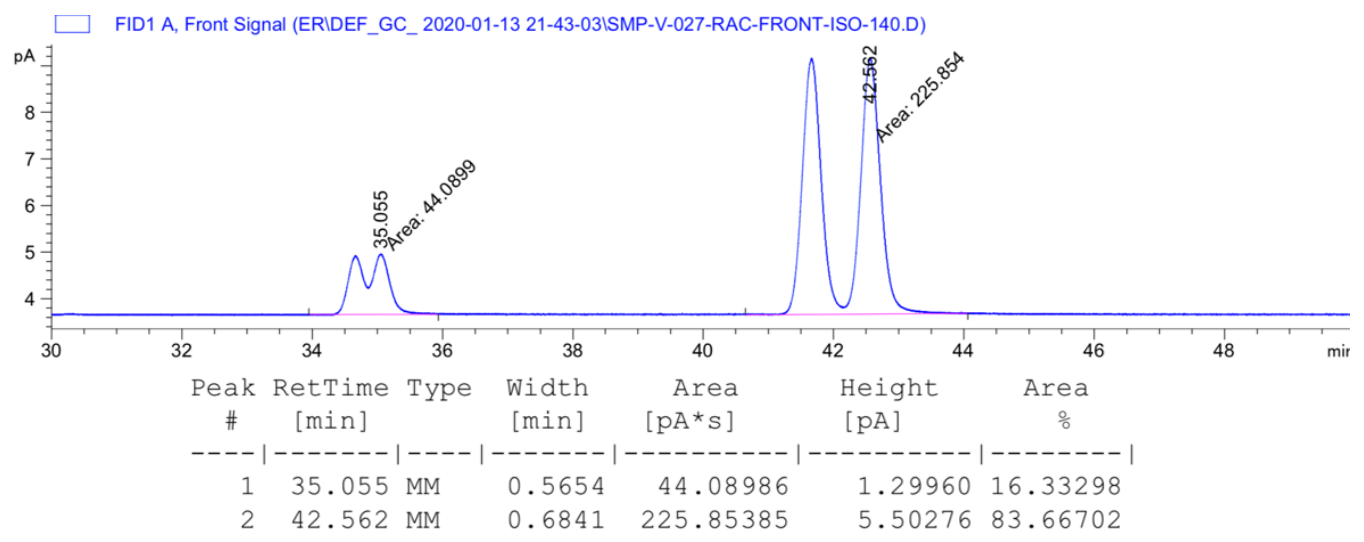


Diastereomeric ratio - reaction in presence of catalyst $(S, R)-\mathbf{4 f}$
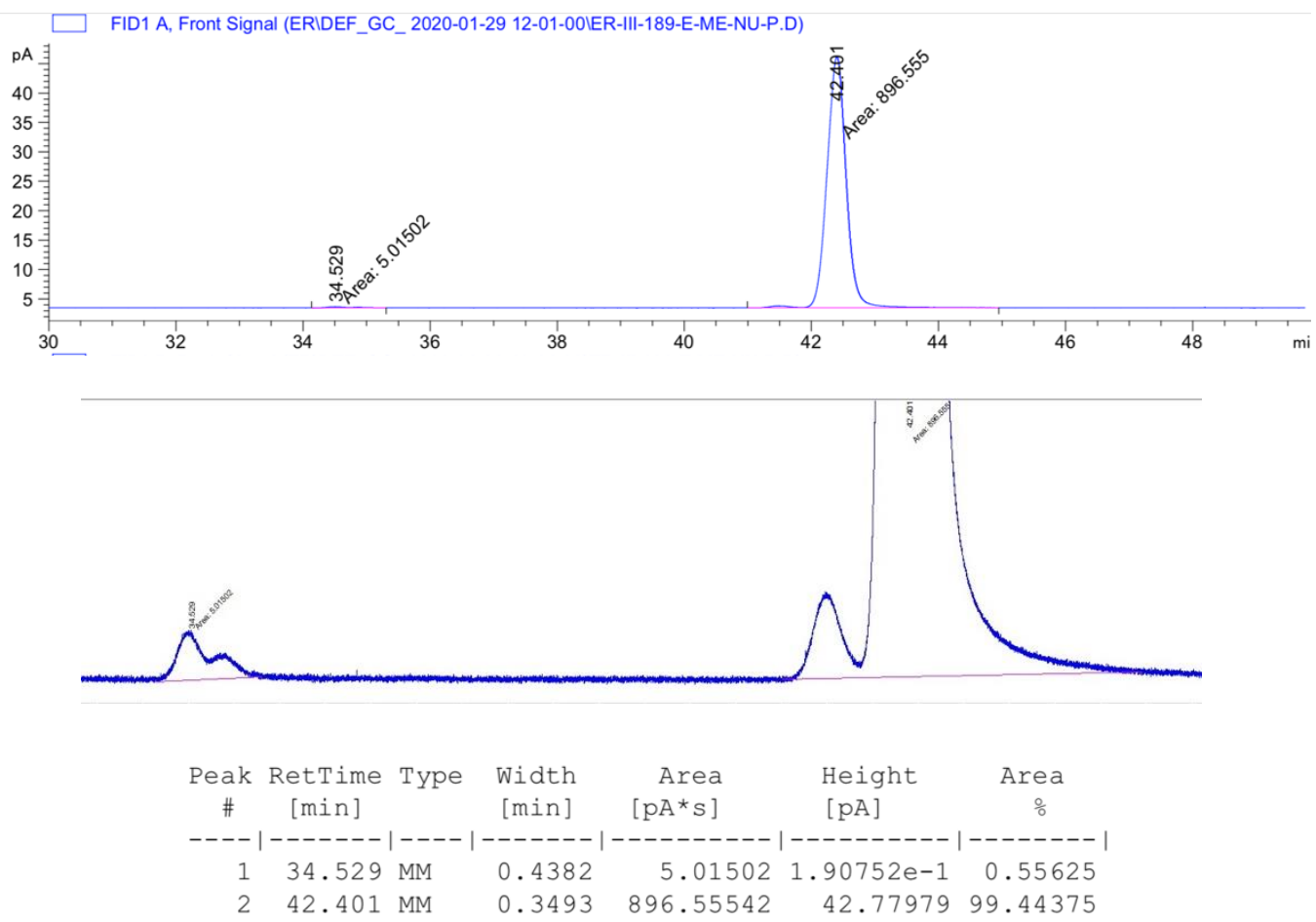

Similar results can be obtained via ${ }^{1} \mathrm{H}$ NMR integration of related peaks in the racemic sample.

Diastereomeric ratio - reaction in absence of catalyst

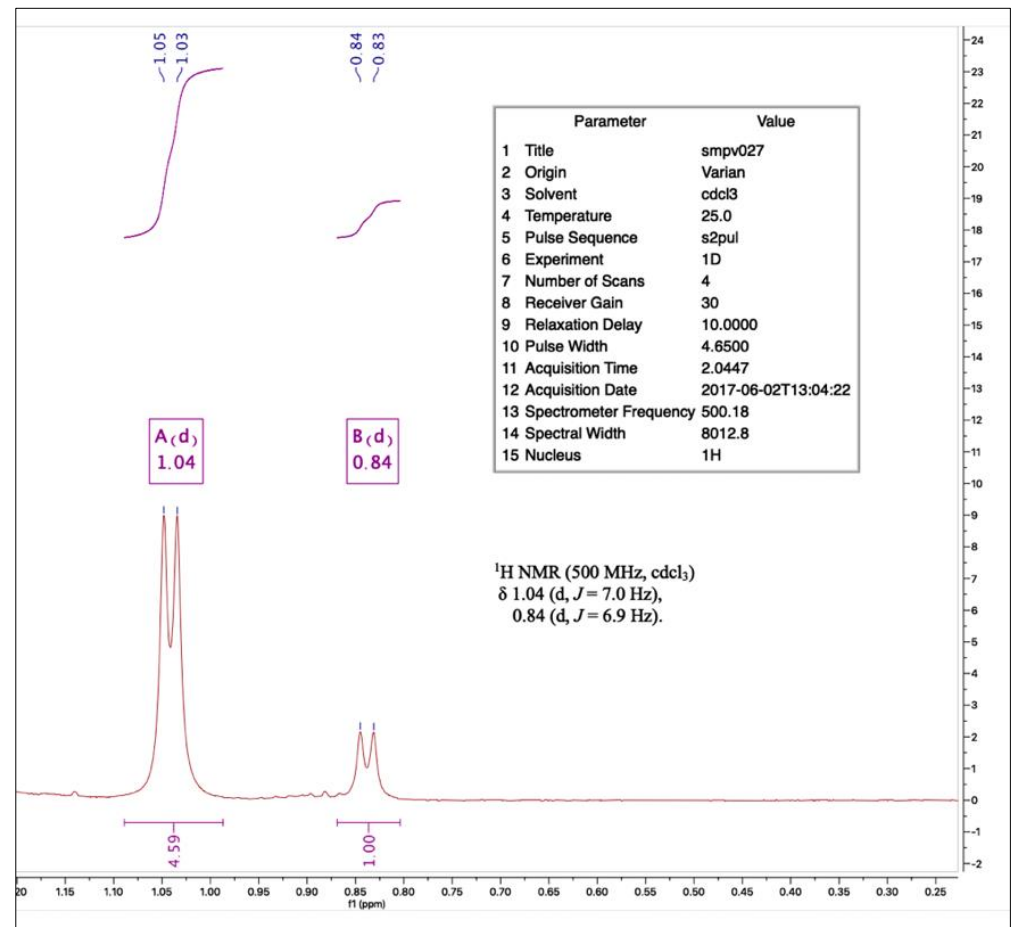

The high diastereoselectivity observed in presence of catalyst leads to difficulties in reliable integration of the minor diastereomer doublet. 


\section{Enantiomeric ratio}

\section{Chiral HPLC:}
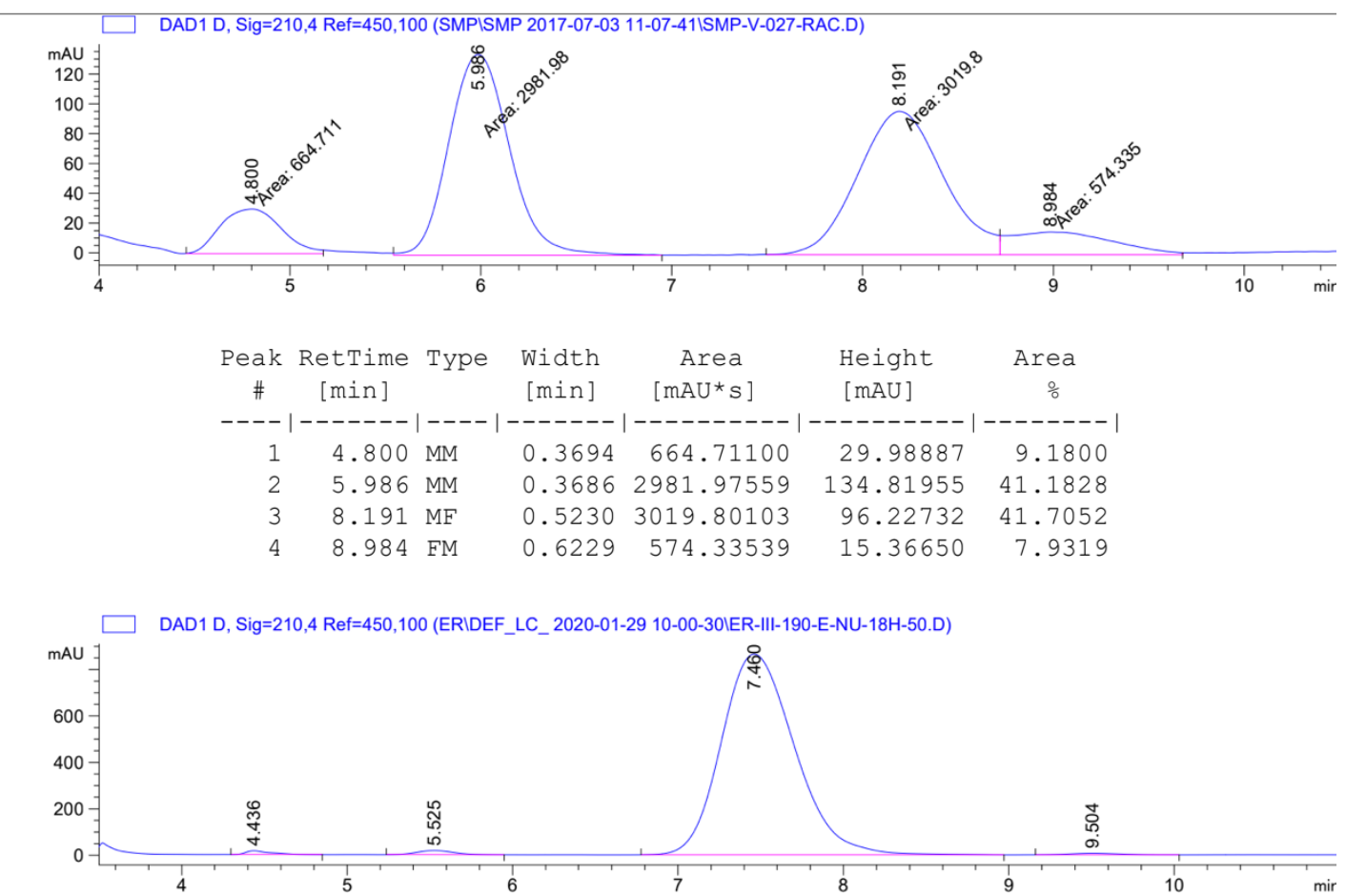

\begin{tabular}{|c|c|c|c|c|c|c|}
\hline $\begin{array}{c}\text { Peak } \\
\quad \#\end{array}$ & $\begin{array}{c}\text { RetTime } \\
\text { [min] }\end{array}$ & Type & $\begin{array}{l}\text { Width } \\
\text { [min] }\end{array}$ & $\begin{array}{c}\text { Area } \\
{\left[\mathrm{mAU}^{\star} \mathrm{s}\right]}\end{array}$ & $\begin{array}{l}\text { Height } \\
{[\mathrm{mAU}]}\end{array}$ & $\begin{array}{c}\text { Area } \\
\frac{\circ}{0}\end{array}$ \\
\hline & & & & --------- & ---------- & -------- \\
\hline 1 & 4.436 & BB & 0.1506 & 176.05539 & 16.48475 & 0.6467 \\
\hline 2 & 5.525 & BB & 0.2385 & 280.11356 & 18.30489 & 290 \\
\hline 3 & 7.460 & BB & 0.4856 & $2.66346 \mathrm{e} 4$ & 864.22162 & 97.8379 \\
\hline 4 & 9.504 & $\mathrm{BB}$ & 0.3037 & 132.43559 & 6.66726 & 0.4865 \\
\hline
\end{tabular}

Major only:

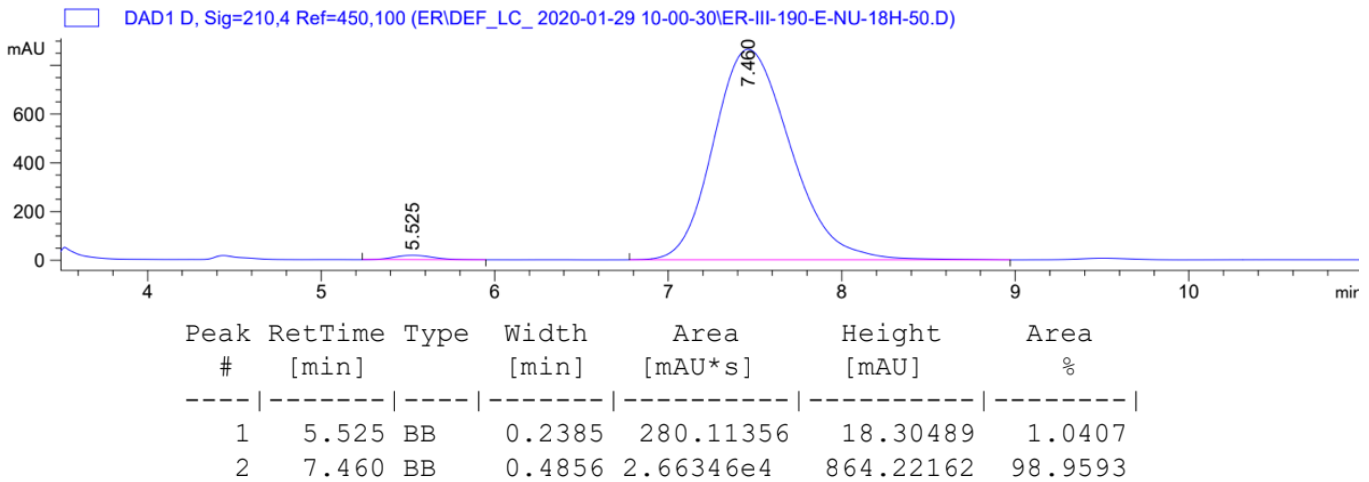

\section{Chiral GC, Major only:}



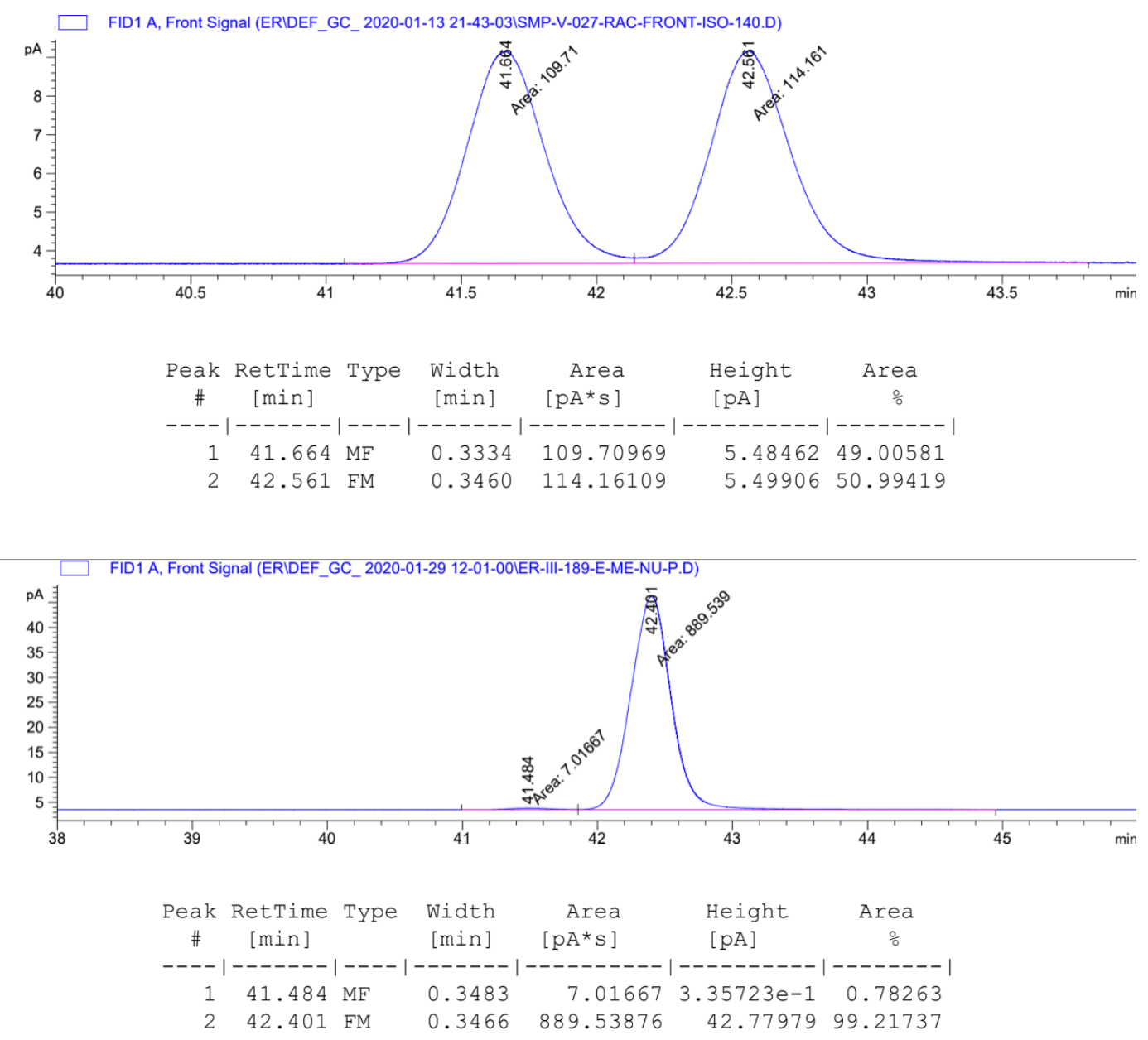

\section{Reaction of heteroaromatics with Squaramide $(S, R)-6 f$}

In contrast to other classes of substrates where thiourea catalyst $(S, R)-\mathbf{4 f}$ gave optimal enantioselectivity, heteroaromatic compounds afforded comparable or better e.e. and yields with squaramide $(S, R)-6 \mathbf{f}$, making it an effective alternative catalyst. Note: All the reactions in this section have been run at a measured temperature of $-60^{\circ} \mathrm{C}$, further investigation led us to conclude that, due to the positioning of the thermometer in the cryocool and the presence of a temperature gradient in the cryocool bath, the temperature of the reactions was actually closer to $-\mathbf{5 0}$ ․ C. Careful temperature measurement was employed for the scope reactions.

\section{(+)-(R)-N-Boc-3-methyl-1-(N-Boc-2-pyrryl)-but-3-en-1-amine [3n]}

Prepared according to Method A, with catalyst $(S, R)-6 \mathbf{f}(20.8 \mathrm{mg}, 0.030 \mathrm{mmol}$, 0.10 equiv). Run for $6 \mathrm{~h}$. See main scope result for full characterization.

Chiral HPLC conditions: CHIRALPAK AS-H, $3 \%$ PrOH/hexanes, $1 \mathrm{~mL} / \mathrm{min}$ Racemic product: $\mathrm{tR}_{\mathrm{R}}(S)=7.1 \mathrm{~min}, \mathrm{tR}_{\mathrm{R}}(R)=9.5 \mathrm{~min}$ Enantioenriched product: $\mathrm{t}_{\mathrm{R}}(R)=10.1 \mathrm{~min}$

Run 1: (104.4 mg, $0.298 \mathrm{mmol}, 99 \%), 94 \%$ e.e.<smiles>C=C(C)C[C@H](NC(=O)OC(C)(C)C)c1cccn1C(=O)OC(C)(C)C</smiles>
Run 2: (103.2 mg, $0.295 \mathrm{mmol}, 98 \%), 94 \%$ e.e. 
Average: $99 \%$ yield, $94 \%$ e.e.
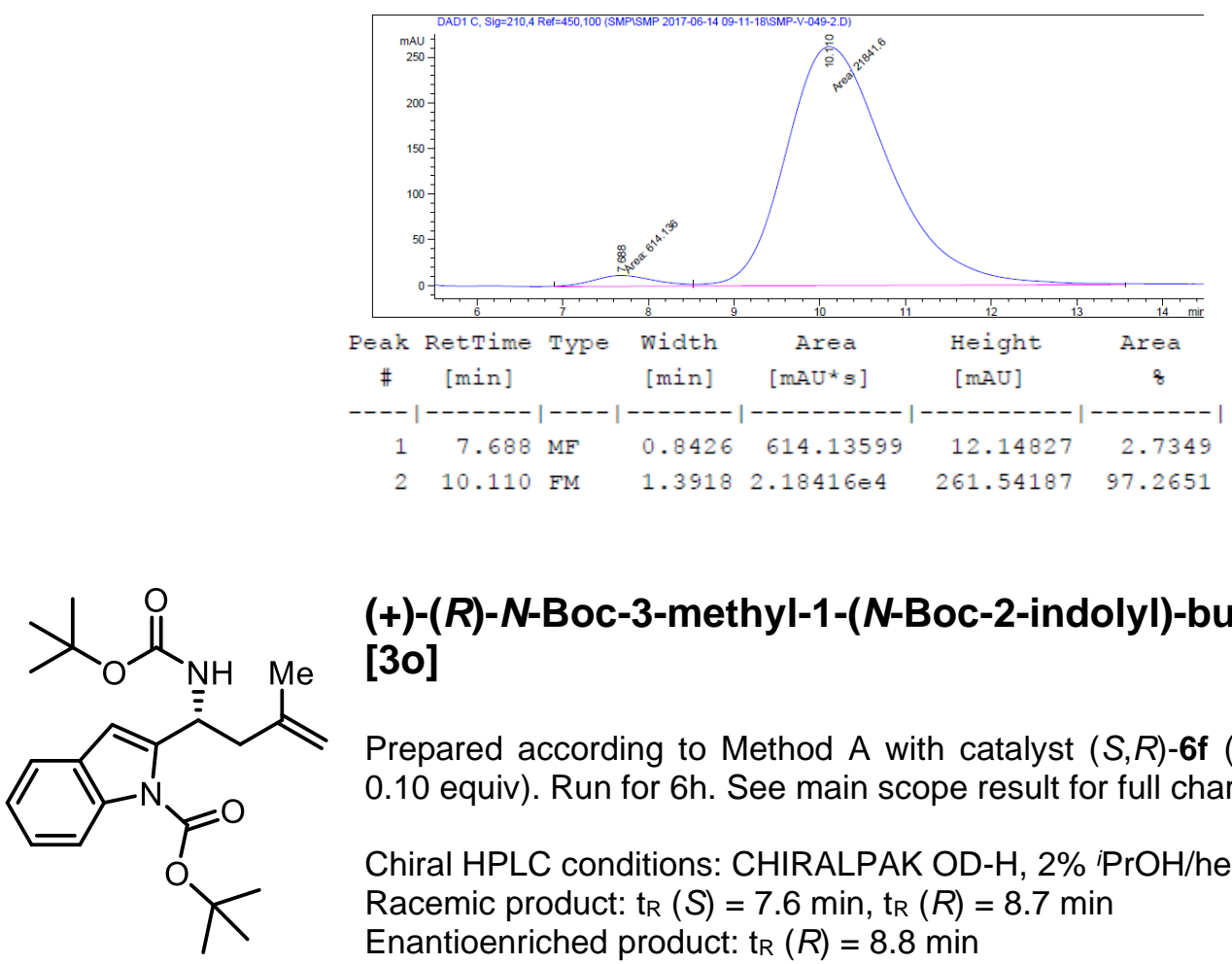

(+)-(R)-N-Boc-3-methyl-1-(N-Boc-2-indolyl)-but-3-en-1-amine [30]

Prepared according to Method A with catalyst $(S, R)-6 f(20.8 \mathrm{mg}, 0.030 \mathrm{mmol}$, 0.10 equiv). Run for $6 \mathrm{~h}$. See main scope result for full characterization.

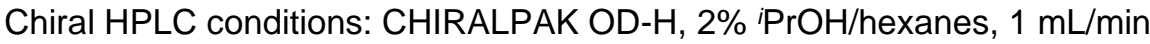
Racemic product: $\mathrm{t}_{\mathrm{R}}(S)=7.6 \mathrm{~min}, \mathrm{t}_{\mathrm{R}}(R)=8.7 \mathrm{~min}$ Enantioenriched product: $\mathrm{t}_{\mathrm{R}}(R)=8.8 \mathrm{~min}$

Run 1: (83.8 mg, $0.209 \mathrm{mmol}, 70 \%), 96 \%$ e.e.

Run 2: (83.4 mg, $0.209 \mathrm{mmol}, 69 \%), 96 \%$ e.e.

Average: $70 \%$ yield, $96 \%$ e.e.

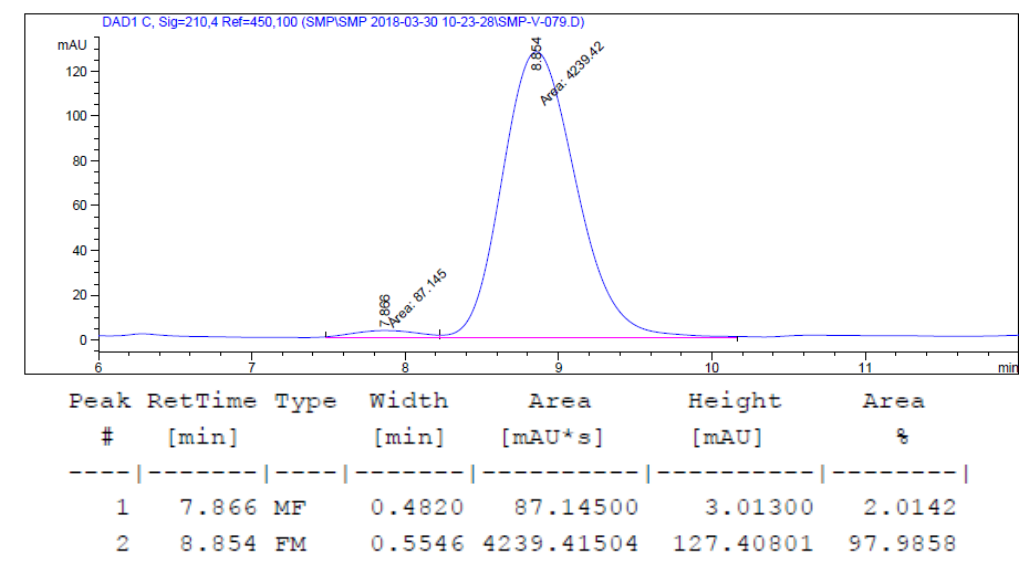

\section{$(+)-(R)-N-B o c-3-m e t h y l-1-(N-B o c-5-i n d o l y l)-b u t-3-e n-1-a m i n e$ [3p]}

Prepared according to Method A, with catalyst $(S, R)-6 \mathbf{f}(20.8 \mathrm{mg}, 0.030 \mathrm{mmol}, 0.10$ equiv). Run for $6 \mathrm{~h}$. See main scope result for full characterization.

Chiral HPLC conditions: CHIRALPAK AS-H, 5\% $\mathrm{PrOH} / \mathrm{h}^{2}$ exanes, $1 \mathrm{~mL} / \mathrm{min}$ Racemic product: $\mathrm{t}_{\mathrm{R}}(S)=8.9 \mathrm{~min}, \mathrm{t}_{\mathrm{R}}(R)=11.7 \mathrm{~min}$ 
<smiles>C=C(C)C[C@H](NC(=O)OC(C)(C)C)c1ccc2c(ccn2C(=O)OC(C)(C)C)c1</smiles>

Enantioenriched product: $\mathrm{t}_{\mathrm{R}}(R)=11.8 \mathrm{~min}$

Run 1: (113.8 mg, $0.284 \mathrm{mmol}, 95 \%), 95 \%$ e.e.

Run 2: (117.6 mg, $0.294 \mathrm{mmol}, 98 \%), 95 \%$ e.e.

Average: $97 \%$ yield, $95 \%$ e.e.
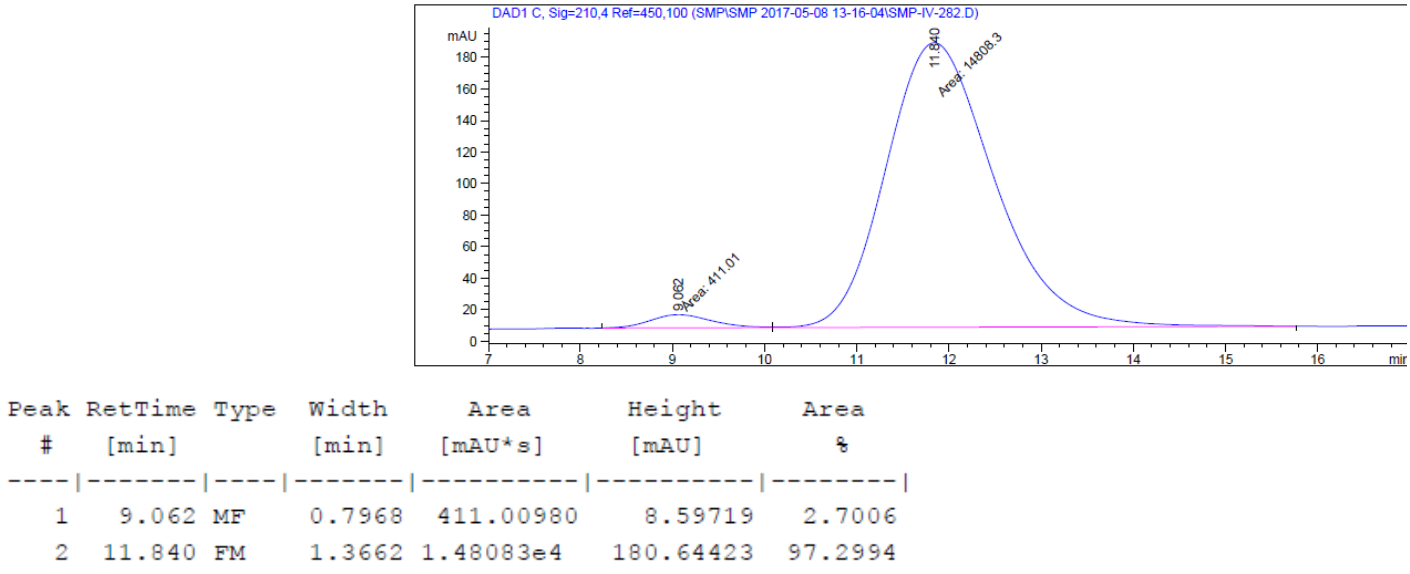

\section{(+)-(R)-N-Boc-3-methyl-1-(3-benzo[b]thiophene)-but-3-en-1-amine [3q]}<smiles>C=C(C)C[C@H](NC(=O)OC(C)(C)C)c1csc2ccccc12</smiles>

Prepared according to Method A, with catalyst $(S, R)-6 f(20.8 \mathrm{mg}, 0.030 \mathrm{mmol}$, 0.10 equiv). Run for $18 \mathrm{~h}$. See main scope result for full characterization.

Chiral HPLC conditions: CHIRALPAK AS-H, $5 \%$ PrOH/hexanes, $1 \mathrm{~mL} / \mathrm{min}$

Racemic product: $t_{R}(R)=8.1 \mathrm{~min}, t_{R}(S)=16.0 \mathrm{~min}$

Enantioenriched product: $\mathrm{t}_{\mathrm{R}}(R)=8.2 \mathrm{~min}$

Run 1: (76.4 mg, $0.241 \mathrm{mmol}, 80 \%), 96 \%$ e.e.

Run 2: (79.2 mg, $0.250 \mathrm{mmol}, 83 \%$ ), $96 \%$ e.e.

Average: $82 \%$ yield, $96 \%$ e.e.

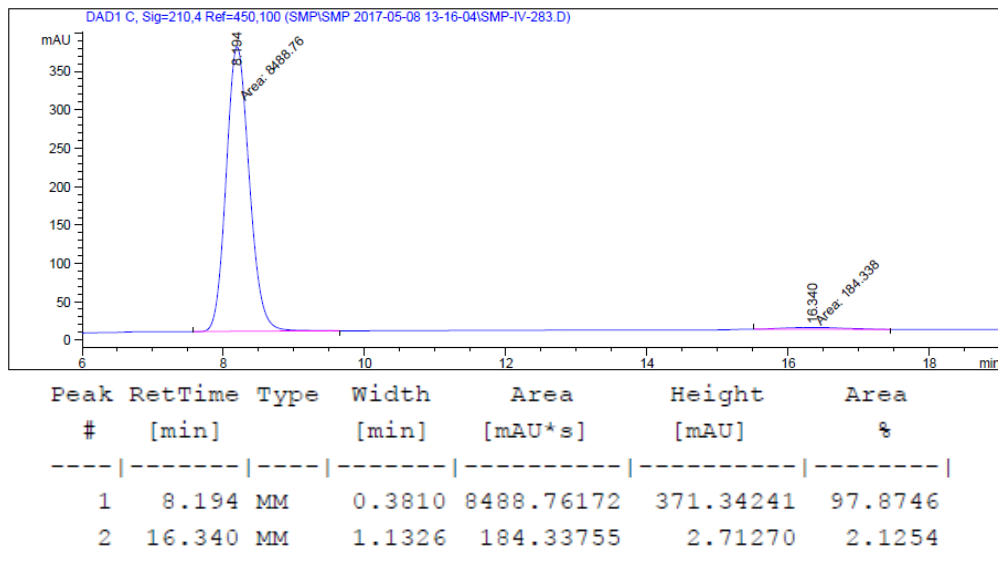




\section{(+)-(R)-N-Boc-3-methyl-1-(2-benzo[b]furanyl)-but-3-en-1-amine [3r]}

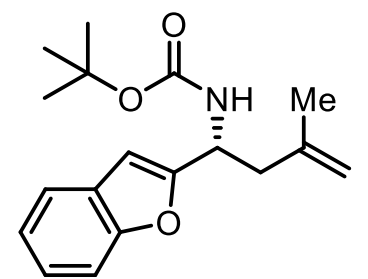

Prepared according to Method A, with catalyst $(S, R)-6 \mathbf{f}(20.8 \mathrm{mg}, 0.030 \mathrm{mmol}$, 0.10 equiv). Run for $18 \mathrm{~h}$. See main scope result for full characterization.

Chiral HPLC conditions: CHIRALPAK AS-H, $5 \%$ PrOH/hexanes, $1 \mathrm{~mL} / \mathrm{min}$

Racemic product: $\mathrm{t}_{\mathrm{R}}(R)=6.3 \mathrm{~min}, \mathrm{t}_{\mathrm{R}}(S)=11.1 \mathrm{~min}$

Enantioenriched product: $\mathrm{t}_{\mathrm{R}}(R)=6.2 \mathrm{~min}$

Run 1: (47.1 mg, $0.156 \mathrm{mmol}, 52 \%), 87 \%$ e.e.

Run 2: (49.8 mg, 0.165 mmol, 55\%), 87\% e.e.

Average: $54 \%$ yield, $87 \%$ e.e.

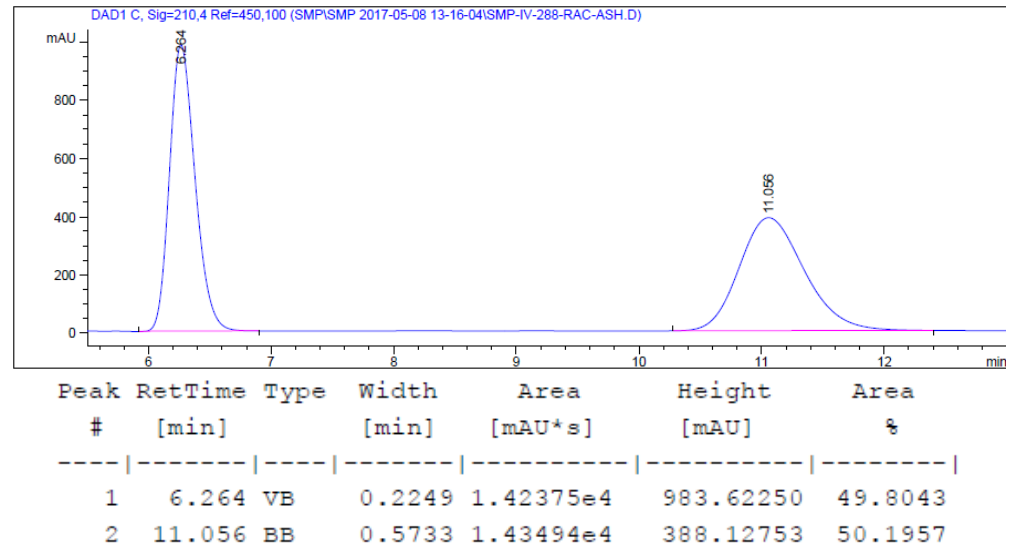

\section{(+)-(R)-N-Boc-3-methyl-1-(5-bromo-2-furanyl)-but-3-en-1-amine [3s]}<smiles>C=C(C)C[C@H](NC(=O)OC(C)(C)C)c1ccc(Br)o1</smiles>

Prepared according to Method A, with catalyst $(S, R)-6 \mathbf{f}(20.8 \mathrm{mg}, 0.030 \mathrm{mmol}, 0.10$ equiv). Run for $18 \mathrm{~h}$. See main scope result for full characterization.

Chiral HPLC conditions: CHIRALPAK AS-H, 3\% $\mathrm{PrOH} / \mathrm{h}$ exanes, $1 \mathrm{~mL} / \mathrm{min}$

Racemic product: $\mathrm{t}_{\mathrm{R}}(R)=6.1 \mathrm{~min}, \mathrm{t}_{\mathrm{R}}(S)=9.2 \mathrm{~min}$

Enantioenriched product: $\mathrm{t}_{\mathrm{R}}(R)=6.2 \mathrm{~min}$

Run 1: (82.3 mg, $0.246 \mathrm{mmol}, 83 \%), 89 \%$ e.e.

Run 2: (81.1 mg, $0.249 \mathrm{mmol}, 82 \%), 89 \%$ e.e.

Average: $83 \%$ yield, $89 \%$ e.e.

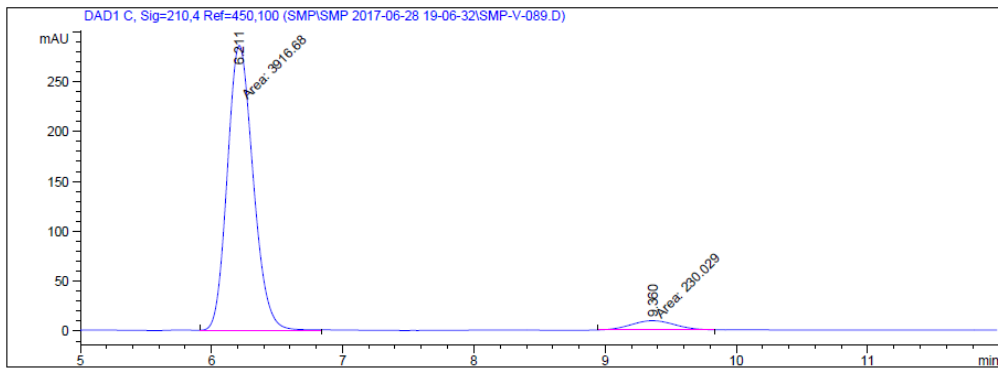




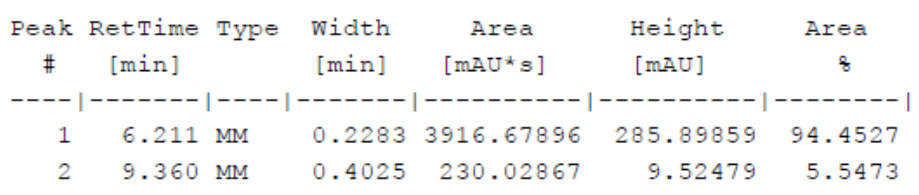

\section{Method Limitations}

Note: All the reactions in this section have been run with sub-optimal squaramide catalyst $(S, R)-6 \mathbf{f}$, according to Method $A$, at a measured temperature of $-60^{\circ} \mathrm{C}$. further investigation led us to conclude that, due to the positioning of the thermometer in the cryocool and the presence of a temperature gradient in the cryocool bath, the temperature of the reactions was actually closer to $-\mathbf{5 0} 0^{\circ} \mathbf{C}$. Careful temperature measurement was employed for the scope reactions.

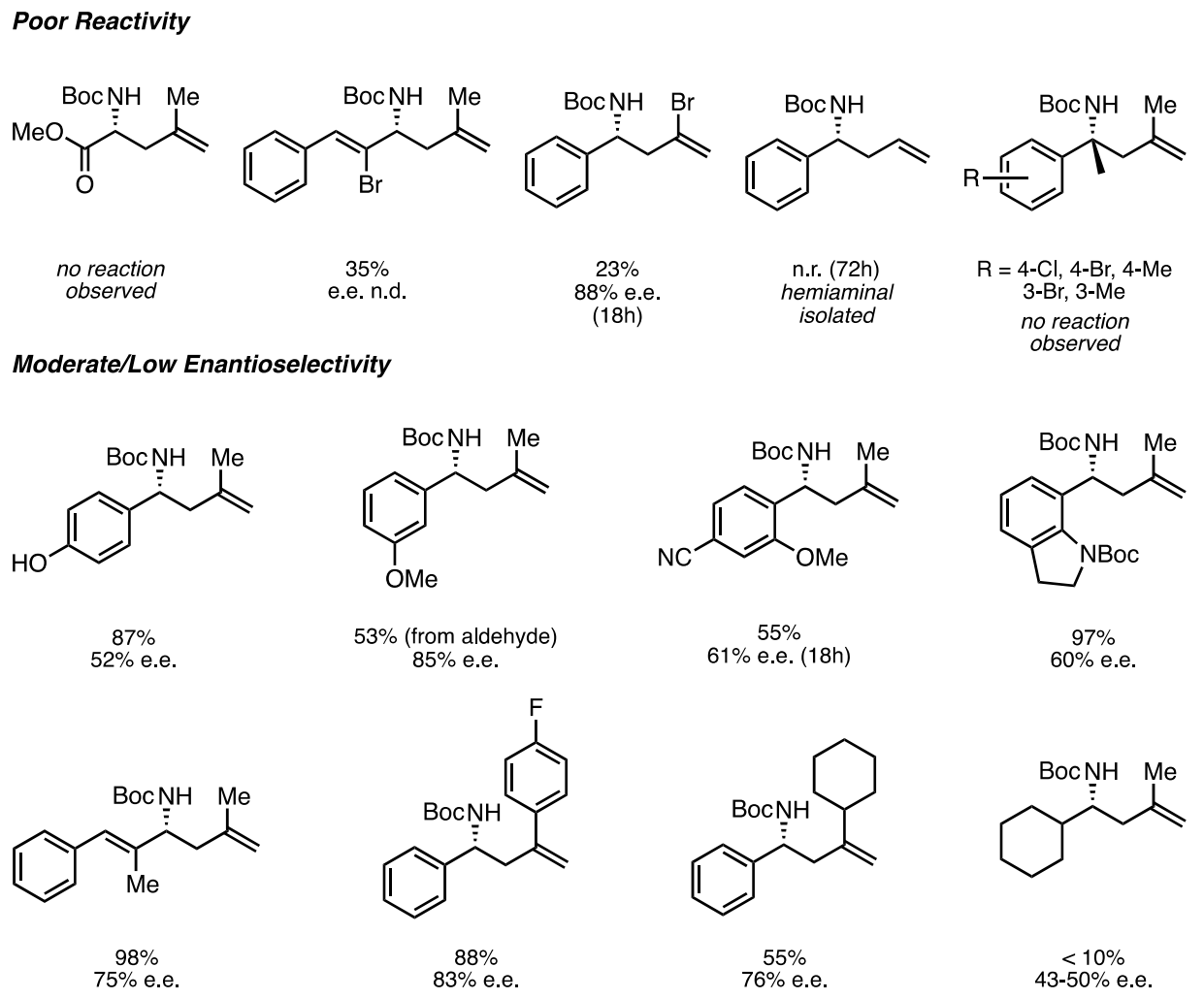

Figure S1. Less successful substrates, due to poor reactivity, lower enantioselectivity, or both. 


\section{Determination of Absolute Stereochemistry}<smiles>C=C(C)C[C@H](NC(=O)OCc1ccccc1)c1ccccc1</smiles>

$3 a$

\author{
$\mathrm{RuCl}_{3} \cdot \mathrm{xH}_{2} \mathrm{O}(3 \mathrm{~mol} \%)$ \\ $\mathrm{NaIO}_{4}$ (4 equiv.) \\ $\mathrm{CCl}_{4} / \mathrm{MeCN} / \mathrm{H}_{2} \mathrm{O}$ \\ $(2: 2: 3)$ \\ $\mathrm{rt}, 2 \mathrm{~h}$
}<smiles>CC(=O)C[C@H](NC(C)=O)c1ccccc1</smiles>

S5

absolute configuration matched to literature

Prepared according to a modified literature procedure. ${ }^{21}$ Homoallylic amine $3 a(450 \mathrm{mg}, 1.72 \mathrm{mmol}, 1.0$ equiv., $92 \%$ e.e.) was taken up in a $2: 2: 3$ mixture of $\mathrm{CCl}_{4} / \mathrm{MeCN} / \mathrm{H}_{2} \mathrm{O}(24 \mathrm{~mL}$ total) in a $50 \mathrm{~mL} \mathrm{rbf}$ equipped with stir bar. $\mathrm{RuCl}_{3} \cdot \mathrm{xH}_{2} \mathrm{O}\left(10.8 \mathrm{mg}, 0.052 \mathrm{mmol}, 0.03\right.$ equiv.) was added, followed by $\mathrm{NalO}_{4}$ (1.472 g, $6.88 \mathrm{mmol}, 4.0$ equiv.). No precautions were taken to exclude air or moisture. The biphasic reaction mixture was stirred vigorously at rt until complete consumption of starting material, as determined by TLC analysis [note: reaction should be closely monitored, as extended reaction times can lead to oxidative cleavage of arene]. Upon completion, the reaction was quenched by addition of $1 \mathrm{M} \mathrm{aq.} \mathrm{HCl}(12$ $\mathrm{mL})$. The product was extracted with $\mathrm{CH}_{2} \mathrm{Cl}_{2}(3 \times 10 \mathrm{~mL})$, then the combined organic layers were dried over $\mathrm{MgSO}_{4}$, filtered, and concentrated. The crude material was purified via flash column chromatography on $\mathrm{SiO}_{2}$ (Biotage, $25 \mathrm{~g}$ column, $5 \% \mathrm{EtOAc} /$ hexanes $\rightarrow 25 \% \mathrm{EtOAc} /$ hexanes). The product was isolated as a white solid in $92 \%$ e.e. and $79 \%$ yield $(360 \mathrm{mg}, 1.37 \mathrm{mmol})$. Enantiomeric excess was determined by chiral HPLC analysis.

Chiral HPLC conditions: CHIRALPAK AD-H, $5 \%$ PrOH/hexanes, $1 \mathrm{~mL} / \mathrm{min}$

Racemic product: $\mathrm{t}_{\mathrm{R}}(R)=16.1 \mathrm{~min}, \mathrm{t}_{\mathrm{R}}(S)=18.9 \mathrm{~min}$

Enantioenriched product: $\mathrm{t}_{\mathrm{R}}(R)=16.1 \mathrm{~min}$

Optical rotation was found to agree, both in sign and value, with that reported in the literature for an identical compound. The absolute stereochemistry of all other homoallylic amines was assigned by analogy.

\section{(+)-(R)-tert-butyl (3-oxo-1-phenylbutyl)carbamate [S5]}
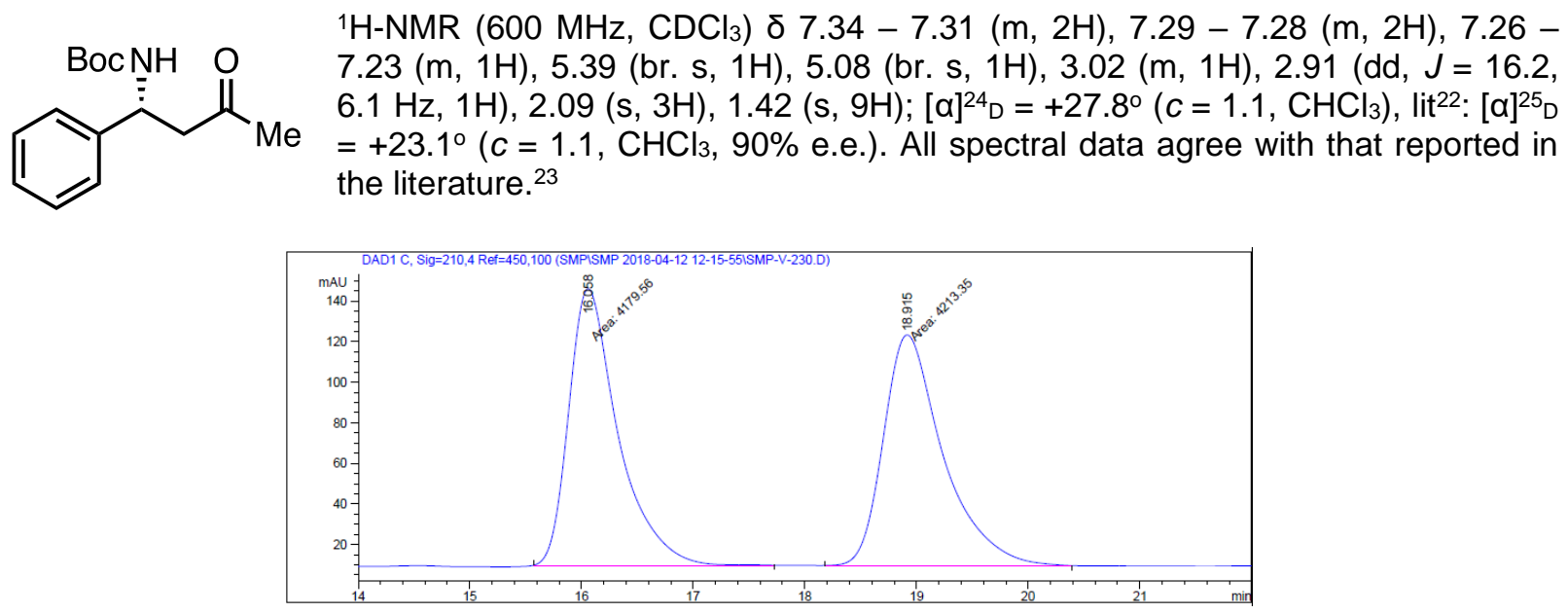

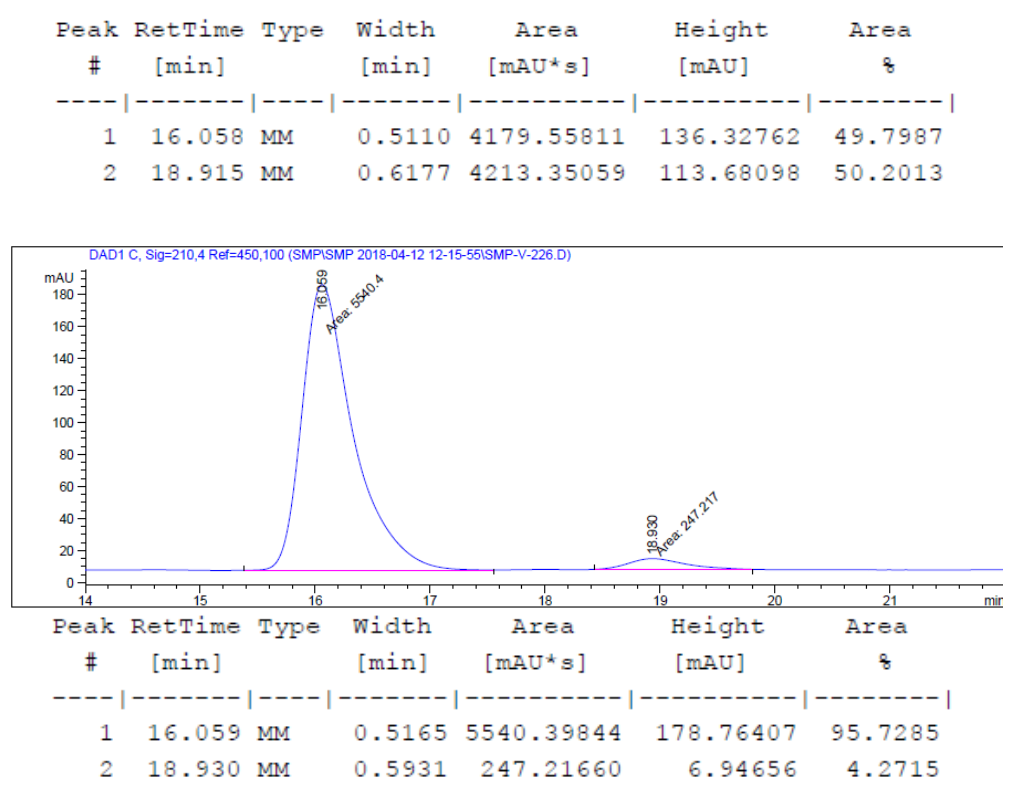

Identification of Catalytic Intermediates

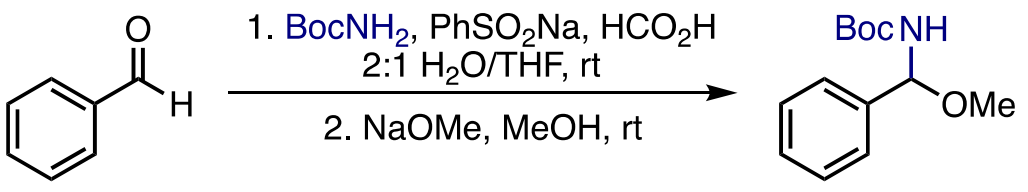

\section{tert-Butyl (methoxy(phenyl)methyl)carbamate [S6]}

S6

Prepared according to a modified literature procedure. ${ }^{24,25} \mathrm{~A} 100 \mathrm{~mL}$ rbf equipped with stir bar and septum was charged with tert-butyl carbamate (1.172 g, $10.0 \mathrm{mmol}, 1.0$ equiv.), $2: 1 \mathrm{H} 2 \mathrm{O} / \mathrm{THF}(30 \mathrm{~mL}$, $0.33 \mathrm{M})$, sodium benzenesulfinate $(3.282 \mathrm{~g}, 20.0 \mathrm{mmol}, 2.0$ equiv.), benzaldehyde $(1.3 \mathrm{~mL}, 13.0 \mathrm{mmol}, 1.3$ equiv.), and, lastly, formic acid $(1.0 \mathrm{~mL}$. $26.5 \mathrm{mmol}, 2.65$ equiv.). The reaction mixture was stirred at $\mathrm{rt}$ for $72 \mathrm{~h}$, during which a precipitate formed. Upon completion, the resulting suspension was filtered through a fritted glass funnel, and the solid was washed with $\mathrm{H}_{2} \mathrm{O}$ and dried on the filter for 10 min. The solid was then copiously washed with hexanes to remove any excess benzaldehyde. The crude product was dried under vacuum overnight.

Material from the previous step was taken up in methanol (40 mL, $0.25 \mathrm{M})$ in a $200 \mathrm{~mL}$ rbf, then sodium methoxide $(4.3 \mathrm{~mL}, 20.0 \mathrm{mmol}, 2.0$ equiv., $25 \mathrm{wt} \%)$ was added dropwise. Reaction mixture was stirred at $\mathrm{rt}$ for $14 \mathrm{~h}$, then it was diluted with $\mathrm{H}_{2} \mathrm{O}(120 \mathrm{~mL})$ and cooled to $0^{\circ} \mathrm{C}$. The resulting precipitate was filtered on a glass frit. The collected solid was dissolved in $\mathrm{CH}_{2} \mathrm{Cl}_{2}$, dried over $\mathrm{MgSO}_{4}$, filtered, and concentrated. No further purification was performed. The product was isolated as a white solid (37\% yield over two steps).<smiles>[M]OC(NC(=O)OCc1ccccc1)c1ccccc1</smiles>

${ }^{1} \mathrm{H}-\mathrm{NMR}\left(600 \mathrm{MHz}, \mathrm{CDCl}_{3}\right) \delta 7.42(\mathrm{~d}, J=7.1 \mathrm{~Hz}, 2 \mathrm{H}), 7.38-7.36(\mathrm{~m}, 2 \mathrm{H}), 7.34-7.31$ $(\mathrm{m}, 1 \mathrm{H}), 5.82(\mathrm{~d}, J=9.9 \mathrm{~Hz}, 1 \mathrm{H}), 5.10(\mathrm{~d}, J=9.9 \mathrm{~Hz}, 1 \mathrm{H}), 3.45(\mathrm{~s}, 3 \mathrm{H}), 1.48(\mathrm{~s}, 9 \mathrm{H})$. All spectral data agree with that reported in the literature. ${ }^{25}$ 
<smiles>O=Cc1ccccc1</smiles>

1. $\mathrm{BocNH}_{2}, \mathrm{PhSO}_{2} \mathrm{Na}, \mathrm{HCO}_{2} \mathrm{H}$

$\underset{\text { 2. } \mathrm{K}_{2} \mathrm{CO}_{3}, \mathrm{Na}_{2} \mathrm{SO}_{4} \text {, THF, reflux }}{\stackrel{2: 1 \mathrm{H}_{2} \mathrm{O} / \mathrm{THF}, \mathrm{rt}}{\longrightarrow}}$<smiles>CC(C)(C)OC(=O)N=Cc1ccccc1</smiles>

S7

\section{Benzaldehyde $\mathrm{N}$-(tert-butoxycarbonyl)imine [S7]}<smiles>CC(C)(C)OC(=O)N=Cc1ccccc1</smiles>

The first step was carried out identically to that described for the first step in the preparation of $\mathbf{S 6}$ (vide supra).

The crude material from the previous step was taken up in THF $(60 \mathrm{~mL}, 0.17 \mathrm{M})$ in a multi-neck rbf equipped with stir bar, septa, and condenser. Potassium carbonate ( $8.293 \mathrm{~g}, 60.0 \mathrm{mmol}, 6.0$ equiv.) and sodium sulfate $(10.0 \mathrm{~g})$ were added, and reaction mixture was heated to reflux overnight. Upon completion, reaction mixture was cooled to $\mathrm{rt}$ and filtered through alternating layers of celite/ $\mathrm{Na} 2 \mathrm{SO}_{4} / \mathrm{celite}$. The filter cake was washed with THF, then the filtrate was concentrated and dried under vacuum to afford the product as a colorless oil in $57 \%$ yield (two steps).

${ }^{1} \mathrm{H}-\mathrm{NMR}\left(500 \mathrm{MHz}, \mathrm{CDCl}_{3}\right) \delta 8.88(\mathrm{~s}, 1 \mathrm{H}), 7.91(\mathrm{~d}, J=7.0 \mathrm{~Hz}, 2 \mathrm{H}), 7.58-7.54(\mathrm{td}, J=7.3,1.5 \mathrm{~Hz}, 1 \mathrm{H})$, $7.47(\mathrm{t}, J=7.7 \mathrm{~Hz}, 2 \mathrm{H}), 1.59(\mathrm{~s}, 9 \mathrm{H})$. All spectral data agree with that reported in the literature. ${ }^{24}$

Note: All the optimization reactions in this section have been run at a measured temperature of $-60^{\circ} \mathrm{C}$, further investigation led us to conclude that, due to the positioning of the thermometer in the cryocool and the presence of a temperature gradient in the cryocool bath, the temperature of the reactions was actually closer to $-\mathbf{5 0}=\mathbf{C}$. Careful temperature measurement was employed for the scope reactions.

\section{Table S9. Reactivity of Model Electrophiles}

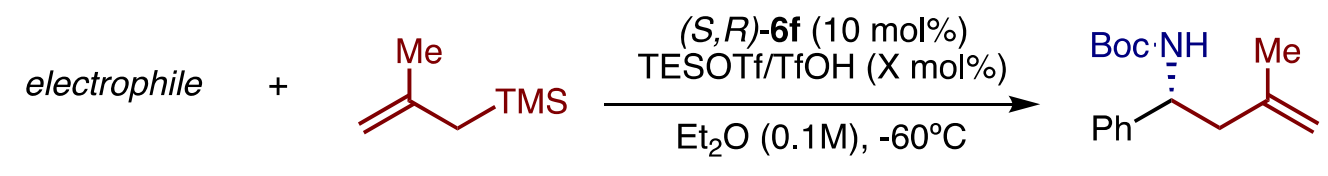

2

$3 a$

\begin{tabular}{|c|c|c|c|c|}
\hline entry & electrophile & co-catalyst & $\%$ yield & \% e.e. \\
\hline 1 & & TESOTf & 86 & 90 \\
\hline 2 & $\mathrm{OMe}$ & $\mathrm{TfOH}$ & 81 & 92 \\
\hline 3 & $\mathrm{NHBoc}$ & TESOTfa & 98 & 92 \\
\hline 4 & $\mathrm{OMe}$ & TfOH ${ }^{\mathrm{a}}$ & 98 & 92 \\
\hline 5 & $\mathrm{NBoc}$ & TESOTf $f^{a, b}$ & 83 & 89 \\
\hline 6 & $\mathrm{Ph}^{\prime}$ & $\mathrm{TfOH}^{\mathrm{a}, \mathrm{b}}$ & 85 & 90 \\
\hline
\end{tabular}


Isolated yields are an average of two runs at $0.3 \mathrm{mmol}$; enantiomeric eccess determined by chiral HPLC analysis of purified product on commercial columns, a $2.5 \mathrm{~mol} \%$ of co-catalyst was used. ${ }^{b} \mathrm{MeOH}(1.0$ equiv. was added).

Method D: An oven-dried 2 dram vial was charged with catalyst $(S, R)-6 f(20.8 \mathrm{mg}, 0.030 \mathrm{mmol}, 0.10$ equiv), hemiacetal $\mathbf{S} 6(0.300 \mathrm{mmol}, 1.0$ equiv), and a stir bar. The vial was sealed with a septum-lined cap, evacuated, and refilled with $\mathrm{N}_{2}(2 \mathrm{x})$. (Methallyl)trimethylsilane $2(0.300 \mathrm{mmol}, 1.0$ equiv) was added via syringe after this step, followed by $\mathrm{Et}_{2} \mathrm{O}(3.0 \mathrm{~mL}, 0.1 \mathrm{M})$. The reaction mixture was cooled to $-78^{\circ} \mathrm{C}$ in a dry ice/acetone bath, then a freshly prepared stock solution of TESOTf or TfOH in $\mathrm{Et}_{2} \mathrm{O}(0.0075 \mathrm{mmol}$, 0.025 equiv, $0.25 \mathrm{M}$ in $\mathrm{Et}_{2} \mathrm{O}$ ) was added dropwise. Vial was transferred to a $-60^{\circ} \mathrm{C}$ cooling bath and stirred for $6 \mathrm{~h}$. Upon completion, the reaction was quenched with dropwise addition of $1: 1 \mathrm{Et}_{3} \mathrm{~N} / \mathrm{MeOH}(300 \mu \mathrm{L})$, stirred for $5 \mathrm{~min}$ at $-60^{\circ} \mathrm{C}$, then warmed to rt. Solvent was removed under reduced pressure, then the remaining residue was dissolved in minimal $\mathrm{CH}_{2} \mathrm{Cl}_{2}$ and applied to a silica column for purification. Enantiomeric excess was determined by chiral HPLC analysis of purified product.

Method E: An oven-dried 2 dram vial was charged with catalyst $(S, R)-6 f(20.8 \mathrm{mg}, 0.030 \mathrm{mmol}, 0.10$ equiv), imine $\mathbf{S} 7(0.300 \mathrm{mmol}, 1.0$ equiv), and a stir bar. The vial was sealed with a septum-lined cap, evacuated, and refilled with $\mathrm{N}_{2}(2 \mathrm{x})$. (Methallyl)trimethylsilane $2(0.300 \mathrm{mmol}, 1.0$ equiv) and methanol (12.6 $\mu \mathrm{L}, 0.300 \mathrm{mmol}, 1.0$ equiv.) were added via syringe after this step, followed by $\mathrm{Et}_{2} \mathrm{O}(3.0 \mathrm{~mL}, 0.1 \mathrm{M})$. The reaction mixture was cooled to $-78^{\circ} \mathrm{C}$ in a dry ice/acetone bath, then a freshly prepared stock solution of TESOTf or $\mathrm{TfOH}$ in $\mathrm{Et}_{2} \mathrm{O}\left(0.0075 \mathrm{mmol}, 0.025\right.$ equiv, $0.25 \mathrm{M}$ in $\left.\mathrm{Et}_{2} \mathrm{O}\right)$ was added dropwise. The vial was transferred to a $-60^{\circ} \mathrm{C}$ cooling bath and stirred for $6 \mathrm{~h}$. Upon completion, the reaction was quenched with dropwise addition of $1: 1 \mathrm{Et}{ }_{3} \mathrm{~N} / \mathrm{MeOH}(300 \mu \mathrm{L})$, stirred for $5 \mathrm{~min}$ at $-60^{\circ} \mathrm{C}$, then warmed to rt. Solvent was removed under reduced pressure, then the remaining residue was dissolved in minimal $\mathrm{CH}_{2} \mathrm{Cl}_{2}$ and applied to a silica column for purification. Enantiomeric excess was determined by chiral HPLC analysis of purified product.

Entry 1. Reaction run according to Method A. Benzaldehyde dimethyl acetal $(45.0 \mu \mathrm{L}, 0.300 \mathrm{mmol}, 1.0$ equiv), (2-methylallyl)trimethylsilane $(52.7 \mu \mathrm{L}, 0.300 \mathrm{mmol}, 1.0$ equiv), tert-butyl carbamate (35.1 $\mathrm{mg}$, $0.300 \mathrm{mmol}, 1.0$ equiv), catalyst $(S, R)-6 f(20.8 \mathrm{mg}, 0.030 \mathrm{mmol}, 0.10$ equiv), TESOTf $(6.8 \mu \mathrm{L}, 0.030$ mmol, 0.10 equiv), and $\mathrm{Et}_{2} \mathrm{O}(3.0 \mathrm{~mL}, 0.1 \mathrm{M})$ were used.

Run 1: (68.1 mg, $0.261 \mathrm{mmol}, 87 \%), 92 \%$ e.e.

Run 2: (66.3 mg, $0.254 \mathrm{mmol}, 85 \%)$, $92 \%$ e.e.

Average: $86 \%$ yield, $92 \%$ e.e.

Entry 2. Reaction run according to Method A. Benzaldehyde dimethyl acetal $(45.0 \mu \mathrm{L}, 0.300 \mathrm{mmol}, 1.0$ equiv), (2-methylallyl)trimethylsilane $(52.7 \mu \mathrm{L}, 0.300 \mathrm{mmol}, 1.0$ equiv), tert-butyl carbamate (35.1 $\mathrm{mg}$, $0.300 \mathrm{mmol}, 1.0$ equiv), catalyst $(S, R)-6 \mathrm{f}(20.8 \mathrm{mg}, 0.030 \mathrm{mmol}, 0.10$ equiv), TfOH $(2.7 \mu \mathrm{L}, 0.030 \mathrm{mmol}$, 0.10 equiv), and $\mathrm{Et}_{2} \mathrm{O}(3.0 \mathrm{~mL}, 0.1 \mathrm{M})$ were used.

Run 1: (64.3 mg, $0.246 \mathrm{mmol}, 82 \%), 90 \%$ e.e.

Run 2: (63.1 mg, $0.241 \mathrm{mmol}, 80 \%), 90 \%$ e.e.

Average: $81 \%$ yield, $90 \%$ e.e.

Entry 3. Reaction run according to Method D. Benzaldehyde $N$-Boc hemiaminal S6 $(71.2 \mathrm{mg}, 0.300$ mmol, 1.0 equiv), (2-methylallyl)trimethylsilane (52.7 $\mu \mathrm{L}, 0.300 \mathrm{mmol}, 1.0$ equiv), tert-butyl carbamate (35.1 mg, $0.300 \mathrm{mmol}, 1.0$ equiv), catalyst $(S, R)-6 f(20.8 \mathrm{mg}, 0.030 \mathrm{mmol}, 0.10$ equiv), TESOTf $(30 \mu \mathrm{L}$, $0.0075 \mathrm{mmol}, 0.025$ equiv, $0.25 \mathrm{M}$ in $\left.\mathrm{Et}_{2} \mathrm{O}\right)$, and $\mathrm{Et}_{2} \mathrm{O}(3.0 \mathrm{~mL}, 0.1 \mathrm{M})$ were used.

Run 1: (76.8 mg, $0.294 \mathrm{mmol}, 98 \%), 92 \%$ e.e.

Run 2: (77.6 mg, $0.297 \mathrm{mmol}, 99 \%), 92 \%$ e.e.

Average: $98 \%$ yield, $92 \%$ e.e.

Entry 4. Reaction run according to Method D. Benzaldehyde $N$-Boc hemiaminal S6 $(71.2 \mathrm{mg}, 0.300$ mmol, 1.0 equiv), (2-methylallyl)trimethylsilane (52.7 $\mu \mathrm{L}, 0.300 \mathrm{mmol}, 1.0$ equiv), tert-butyl carbamate (35.1 mg, $0.300 \mathrm{mmol}, 1.0$ equiv), catalyst $(S, R)-6 f(20.8 \mathrm{mg}, 0.030 \mathrm{mmol}, 0.10$ equiv), TfOH (30 $\mu \mathrm{L}$, $0.0075 \mathrm{mmol}, 0.025$ equiv, $0.25 \mathrm{M}$ in $\left.\mathrm{Et}_{2} \mathrm{O}\right)$, and $\mathrm{Et}_{2} \mathrm{O}(3.0 \mathrm{~mL}, 0.1 \mathrm{M})$ were used. 
Run 1: (77.9 mg, $0.298 \mathrm{mmol}, 99 \%), 92 \%$ e.e.

Run 2: (76.4 mg, $0.292 \mathrm{mmol}, 97 \%)$, 92\% e.e.

Average: $98 \%$ yield, $92 \%$ e.e.

Entry 5. Reaction run according to Method E. Benzaldehyde $N$-Boc imine S7 (61.6 mg, $0.300 \mathrm{mmol}, 1.0$ equiv), (2-methylallyl)trimethylsilane $(52.7 \mu \mathrm{L}, 0.300 \mathrm{mmol}, 1.0$ equiv), $\mathrm{MeOH}$ (12.6 $\mu \mathrm{L}, 0.300 \mathrm{mmol}, 1.0$ equiv.), tert-butyl carbamate $(35.1 \mathrm{mg}, 0.300 \mathrm{mmol}, 1.0$ equiv), catalyst $(S, R)-6 \mathbf{f}(20.8 \mathrm{mg}, 0.030 \mathrm{mmol}$, 0.10 equiv), TESOTf ( $30 \mu \mathrm{L}, 0.0075 \mathrm{mmol}, 0.025$ equiv, $0.25 \mathrm{M}$ in $\left.\mathrm{Et}_{2} \mathrm{O}\right)$, and $\mathrm{Et}_{2} \mathrm{O}(3.0 \mathrm{~mL}, 0.1 \mathrm{M})$ were used. Note: when $\mathrm{MeOH}$ is left out of reaction, the e.e. remains the same, but conversion is considerably lower after $6 \mathrm{~h}(<50 \%)$. While $\mathrm{MeOH}$ improved the reactivity of the aldimine S7, it inhibited reactions with the acetal $\mathbf{1 a}$ and hemiaminal ether $\mathbf{S 6}$.

Run 1: $(64.1 \mathrm{mg}, 0.245 \mathrm{mmol}, 82 \%), 89 \%$ e.e.

Run 2: (66.2 mg, $0.253 \mathrm{mmol}, 84 \%), 89 \%$ e.e.

Average: $83 \%$ yield, $89 \%$ e.e.

Entry 6. Reaction run according to Method E. Benzaldehyde $N$-Boc imine S7 (61.6 mg, $0.300 \mathrm{mmol}, 1.0$ equiv), (2-methylallyl)trimethylsilane $(52.7 \mu \mathrm{L}, 0.300 \mathrm{mmol}, 1.0$ equiv), $\mathrm{MeOH}$ (12.6 $\mu \mathrm{L}, 0.300 \mathrm{mmol}, 1.0$ equiv.), tert-butyl carbamate $(35.1 \mathrm{mg}, 0.300 \mathrm{mmol}, 1.0$ equiv), catalyst $(S, R)-6 \mathrm{f}(20.8 \mathrm{mg}, 0.030 \mathrm{mmol}$, 0.10 equiv), $\mathrm{TfOH}\left(30 \mu \mathrm{L}, 0.0075 \mathrm{mmol}, 0.025\right.$ equiv, $0.25 \mathrm{M}$ in $\left.\mathrm{Et}_{2} \mathrm{O}\right)$, and $\mathrm{Et}_{2} \mathrm{O}(3.0 \mathrm{~mL}, 0.1 \mathrm{M})$ were used. Run 1: (67.4 mg, $0.258 \mathrm{mmol}, 86 \%), 90 \%$ e.e.

Run 2: (65.9 mg, $0.252 \mathrm{mmol}, 84 \%), 90 \%$ e.e.

Average: $85 \%$ yield, $90 \%$ e.e.

\section{Effect of m-Nucleophile Identity on Chemoselectivity}

Table S10. Effect of m-nucleophile on product chemoselectivity.

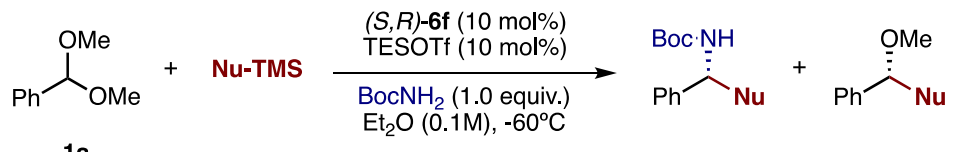

1a

nucleophiles


Product ratios determined by HPLC or ${ }^{1} \mathrm{H}-\mathrm{NMR}$ analysis of crude reaction mixtures; enantiomeric excess determined by chiral HPLC analysis of purified product on commercial columns. ${ }^{a}$ Reported nucleophilicity value as determined by $\mathrm{H}$. Mayr and co-workers. ${ }^{26}$

Note: All the optimization reactions in this section have been run at a measured temperature of $-60^{\circ} \mathrm{C}$, further investigation led us to conclude that, due to the positioning of the thermometer in the cryocool and the presence of a temperature gradient in the cryocool bath, the temperature of the reactions was actually closer to $-50^{\circ} \mathrm{C}$. Careful temperature measurement was employed for the scope reactions.

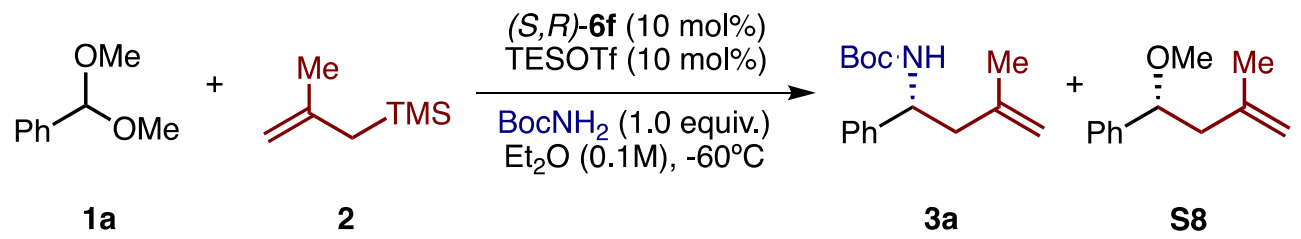

In the presence of $\mathrm{BocNH}_{2}$, no ether-containing product (S8) was observed; amine/ether $>50: 1$. See reaction scope for full reaction details and characterization of amine-containing product $\mathbf{3 a}$.

\section{(+)-(R)-N-Boc-3-methyl-1-phenylbut-3-en-1-amine [S8]}<smiles>C=C(C)C[C@@H](OC)c1ccccc1</smiles>

Prepared according to Method $\mathrm{A}$, in the absence of $\mathrm{BocNH}_{2}$. Benzaldehyde dimethyl acetal $(45.0 \mu \mathrm{L}, 0.300 \mathrm{mmol}, 1.0$ equiv), (2-methylallyl)trimethylsilane $(53.0 \mu \mathrm{L}, 0.300$ mmol, 1.0 equiv), catalyst $(S, R)-6 f(20.8 \mathrm{mg}, 0.030 \mathrm{mmol}, 0.10$ equiv), TESOTf (6.8 $\mu \mathrm{L}, 0.030 \mathrm{mmol}, 0.10$ equiv), and $\mathrm{Et}_{2} \mathrm{O}(3.0 \mathrm{~mL}, 0.1 \mathrm{M})$ were used. The reaction was run at $-60^{\circ} \mathrm{C}$ for $6 \mathrm{~h}$. Flash column chromatography on silica (Biotage, $10 \mathrm{~g}$ column) using a gradient of $1 \% \mathrm{EtOAc/hexanes} \rightarrow 10 \% \mathrm{EtOAc} /$ hexanes as eluent gave pure product as a colorless oil; product is volatile and care should be taken to minimize product loss during isolation. In both runs, starting material conversion was $100 \%$ and HPLC yield of product was $100 \%$ (from crude reaction mixture).

Chiral HPLC conditions: AD-H, $1 \%{ }^{\mathrm{i}} \mathrm{PrOH} /$ hexanes, $1 \mathrm{~mL} / \mathrm{min}$

Racemic product: $\operatorname{tr}_{\mathrm{R}}(R)=3.9 \mathrm{~min}, \mathrm{t}_{\mathrm{R}}(S)=4.1 \mathrm{~min}$

Enantioenriched product: $\operatorname{tR}_{\mathrm{R}}(R)=4.1 \mathrm{~min}$

Run 1: (35.8 mg, $0.203 \mathrm{mmol}, 68 \%), 50 \%$ e.e.

Run 2: (37.9 mg, $0.215 \mathrm{mmol}, 72 \%), 50 \%$ e.e.

Average: $70 \%$ yield, $50 \%$ e.e.

${ }^{1} \mathrm{H}-\mathrm{NMR}\left(500 \mathrm{MHz}, \mathrm{CDCl}_{3}\right) \delta 7.39-7.26$ (m, 5H), 4.79 (app. t, $J=1.9 \mathrm{~Hz}, 1 \mathrm{H}$ ), 4.71 (app. dt, $J=2.2,1.1$ $\mathrm{Hz}, 1 \mathrm{H}), 4.29(\mathrm{dd}, J=8.4,5.3 \mathrm{~Hz}, 1 \mathrm{H}), 3.22(\mathrm{~s}, 3 \mathrm{H}), 2.55$ (ddd, $J=14.4,8.4,1.1 \mathrm{~Hz}, 1 \mathrm{H}), 2.31(\mathrm{dd}, J=$ 14.4, $5.3 \mathrm{~Hz}, 1 \mathrm{H}), 1.74(\mathrm{~s}, 3 \mathrm{H}) ;{ }^{13} \mathrm{C}-\mathrm{NMR}\left(125 \mathrm{MHz}, \mathrm{CDCl}_{3}\right) \delta 142.6,142.2,128.5,127.7,126.8,112.7$, 82.7, 56.8, 46.7, 22.9; IR (ATR, $\left.\mathrm{cm}^{-1}\right) 3075,3029,2935,2821,1492,1098,889,775$; $[\alpha]^{24} \mathrm{D}=+45.5^{\circ}(c=$ 1.0, $\left.\mathrm{CHCl}_{3}\right)$; HRMS (ESI) $\mathrm{m} / \mathrm{z}$ calculated for $\mathrm{C}_{12} \mathrm{H}_{16} \mathrm{ONa}[\mathrm{M}+\mathrm{Na}]^{+}:$199.1093, found 199.1094.

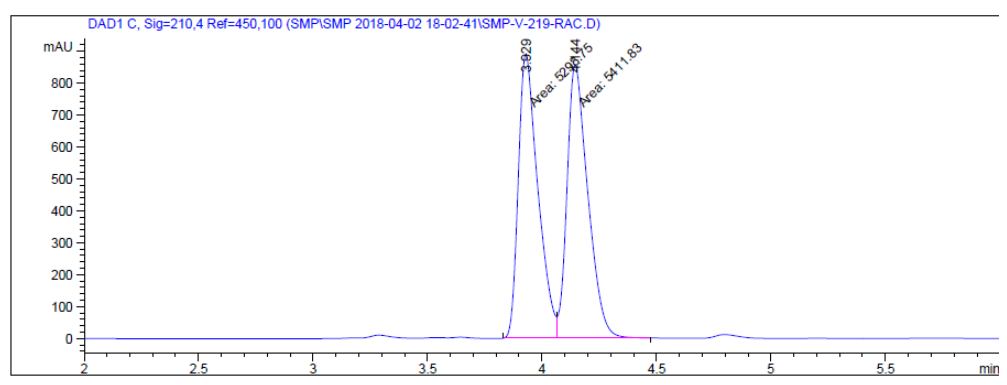



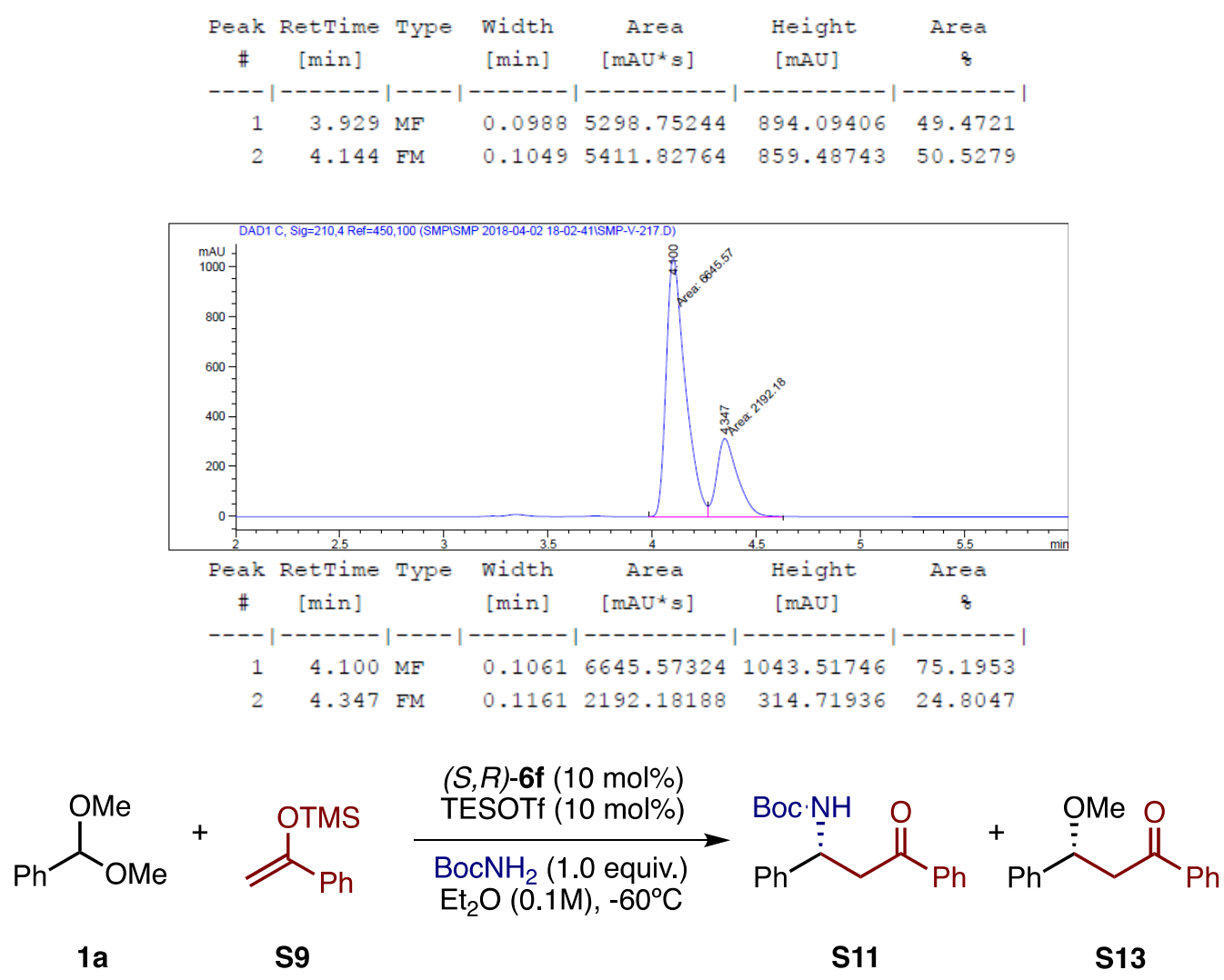

Reaction run according to Method A. Benzaldehyde dimethyl acetal (45.0 $\mu \mathrm{L}, 0.300 \mathrm{mmol}, 1.0$ equiv), 1 phenyl-1-trimethylsilyloxyethylene $\mathbf{S 9}(61.5 \mu \mathrm{L}, 0.300 \mathrm{mmol}, 1.0$ equiv), tert-butyl carbamate (35.1 $\mathrm{mg}$, $0.300 \mathrm{mmol}, 1.0$ equiv), catalyst $(S, R)-6 f(20.8 \mathrm{mg}, 0.030 \mathrm{mmol}, 0.10$ equiv), TESOTf $(6.8 \mu \mathrm{L}, 0.030$ mmol, 0.10 equiv), and $\mathrm{Et}_{2} \mathrm{O}(3.0 \mathrm{~mL}, 0.1 \mathrm{M})$ were used. The reaction was run at $-60^{\circ} \mathrm{C}$ for $6 \mathrm{~h}$. Product ratios were obtained by ${ }^{1} \mathrm{H}-\mathrm{NMR}$ of the crude reaction mixture after concentrating under reduced pressure. Products were separated by flash column chromatography on silica (Biotage, $10 \mathrm{~g}$ column) using a gradient of $1 \% \mathrm{EtOAc/hexanes} \rightarrow 15 \% \mathrm{EtOAc} /$ hexanes as eluent. Ether product was not isolated quantitatively due to volatility. Note: The enantiomeric excess of ethereal product was found to be the same when reaction was run with or without $\mathrm{BocNH}_{2}$.

Run 1:

amine: (52.4 mg, $0.161 \mathrm{mmol}, 54 \%), 81 \%$ e.e.

ether: $62 \%$ e.e.

amine/ether: $4: 1$

Run 2:

amine: (50.4 mg, $0.155 \mathrm{mmol}, 52 \%), 83 \%$ e.e.

ether: $62 \%$ e.e.

amine/ether: $4: 1$

Average: 4:1 amine/ether; amine: $53 \%$ yield, $82 \%$ e.e.; ether: $62 \%$ e.e.

\section{$(-)-(R)-N-B o c-3-a m i n o-1,3-d i p h e n y l p r o p a n-1-o n e ~[S 11]$}

Isolated as a white solid.

Chiral HPLC conditions: CHIRALPAK AD-H, $10 \%$ PrOH/hexanes, $1 \mathrm{~mL} / \mathrm{min}$

Racemic product: $\mathrm{t}_{\mathrm{R}}(S)=15.9 \mathrm{~min}, \mathrm{t}_{\mathrm{R}}(R)=19.4 \mathrm{~min}$

Enantioenriched product: $\mathrm{t}_{\mathrm{R}}(R)=19.5 \mathrm{~min}$<smiles>O=C(N[C@@H](CC(=O)c1ccccc1)c1ccccc1)OCc1ccccc1</smiles> 
${ }^{1} \mathrm{H}-\mathrm{NMR}\left(500 \mathrm{MHz}, \mathrm{CDCl}_{3}\right) \delta 7.91-7.89(\mathrm{~m}, 2 \mathrm{H}), 7.57-7.54(\mathrm{~m}, 1 \mathrm{H}), 7.44(\mathrm{t}, J=7.8 \mathrm{~Hz}, 2 \mathrm{H}), 7.35-$ $7.29(\mathrm{~m}, 4 \mathrm{H}), 7.24-7.21(\mathrm{~m}, 1 \mathrm{H}), 5.58(\mathrm{~s}, 1 \mathrm{H}), 5.25(\mathrm{~d}, J=7.4 \mathrm{~Hz}, 1 \mathrm{H}), 3.66(\mathrm{~s}, 1 \mathrm{H}), 3.44(\mathrm{dd}, J=16.6$, $6.1 \mathrm{~Hz}, 1 \mathrm{H}), 1.41(\mathrm{~s}, 9 \mathrm{H}) ;[\alpha]^{25} \mathrm{D}=-5.5^{\circ}\left(c=1.0, \mathrm{CHCl}_{3}\right)$, lit $^{27 \mathrm{a}}:[\alpha]^{25 \mathrm{D}}=-3.6^{\circ}\left(c=1.02, \mathrm{CHCl}_{3}, 67 \%\right.$ e.e. $)$. lit ${ }^{27 b}:[\alpha]^{27} \mathrm{D}=-11.9^{\circ}\left(c=1.02, \mathrm{CHCl}_{3}, 73 \%\right.$ e.e. $)$. Opposite enantiomer, lit ${ }^{27 c}:[\alpha]^{25} \mathrm{D}=+2.0^{\circ}(c=1.0$, $\mathrm{CHCl}_{3}, 72 \%$ e.e.).

All spectral data agree with that reported in the literature. ${ }^{27}$
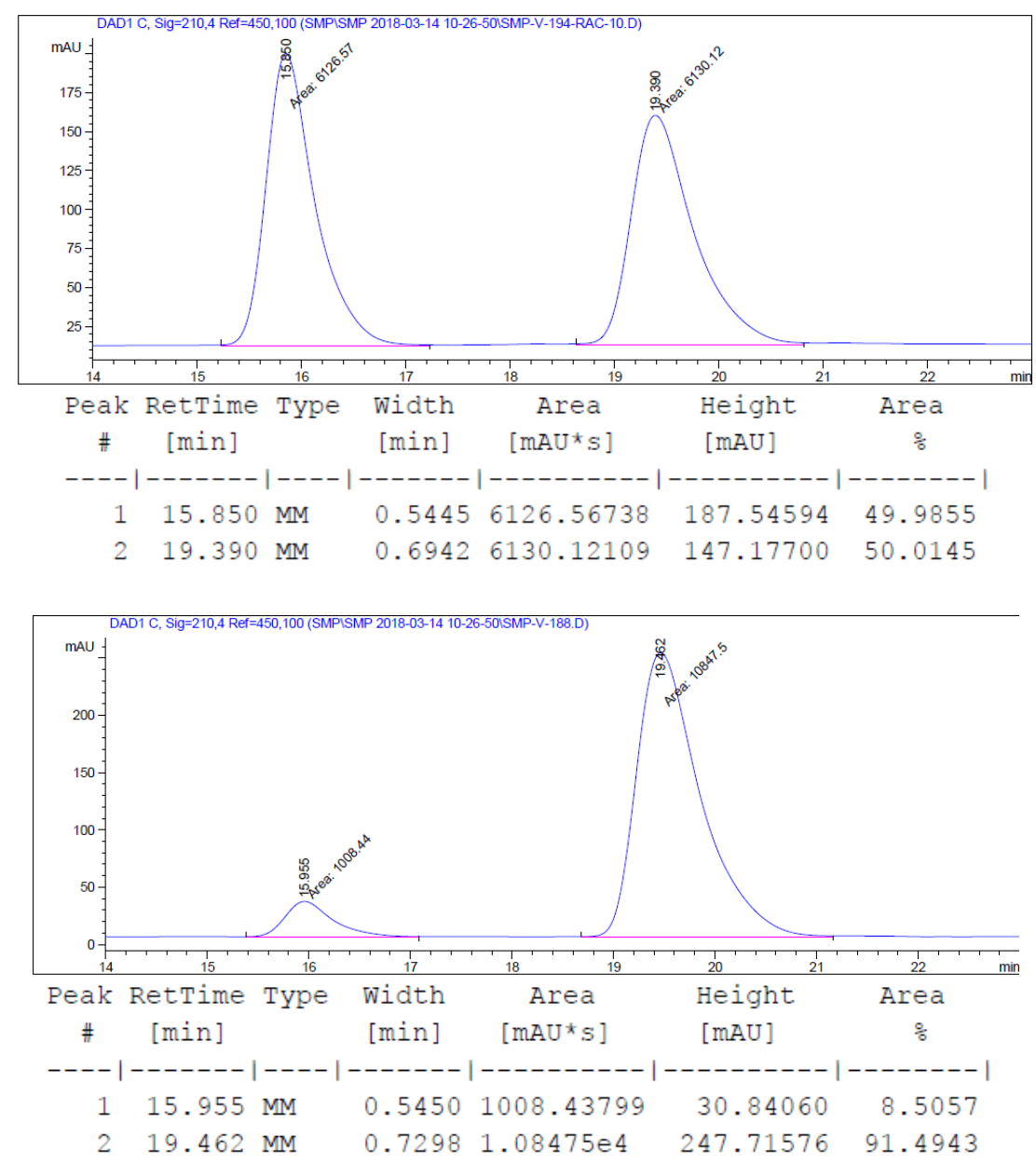

\section{(+)-(R)-1,3-diphenylpropan-1-one [S13]}

Isolated as a colorless oil.

Chiral HPLC conditions: CHIRALPAK IC, $10 \% \mathrm{PrOH} /$ hexanes, $1 \mathrm{~mL} / \mathrm{min}$

Racemic product: $\mathrm{t}_{\mathrm{R}}(S)=5.7 \mathrm{~min}, \mathrm{t}_{\mathrm{R}}(R)=7.1 \mathrm{~min}$

Enantioenriched product: $\mathrm{t}_{\mathrm{R}}(R)=6.9 \mathrm{~min}$

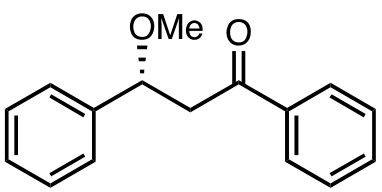

${ }^{1} \mathrm{H}-\mathrm{NMR}\left(500 \mathrm{MHz}, \mathrm{CDCl}_{3}\right) \delta 7.96-7.94(\mathrm{~m}, 2 \mathrm{H}), 7.57-7.53(\mathrm{~m}, 1 \mathrm{H}), 7.46-7.36(\mathrm{~m}, 6 \mathrm{H}), 7.32-7.29$ (m, 1H), 4.89 (dd, $J=8.4,4.4 \mathrm{~Hz}, 1 \mathrm{H}), 3.60(\mathrm{dd}, J=16.5,8.4 \mathrm{~Hz}, 1 \mathrm{H}), 3.24(\mathrm{~s}, 3 \mathrm{H}), 3.09$ (dd, $J=16.5$, $4.4 \mathrm{~Hz}, 1 \mathrm{H}) ;[\alpha]^{24} \mathrm{D}=+35.7^{\circ}\left(c=1.0, \mathrm{CHCl}_{3}\right)$. All spectral data agree with that reported in the literature. ${ }^{28}$ 

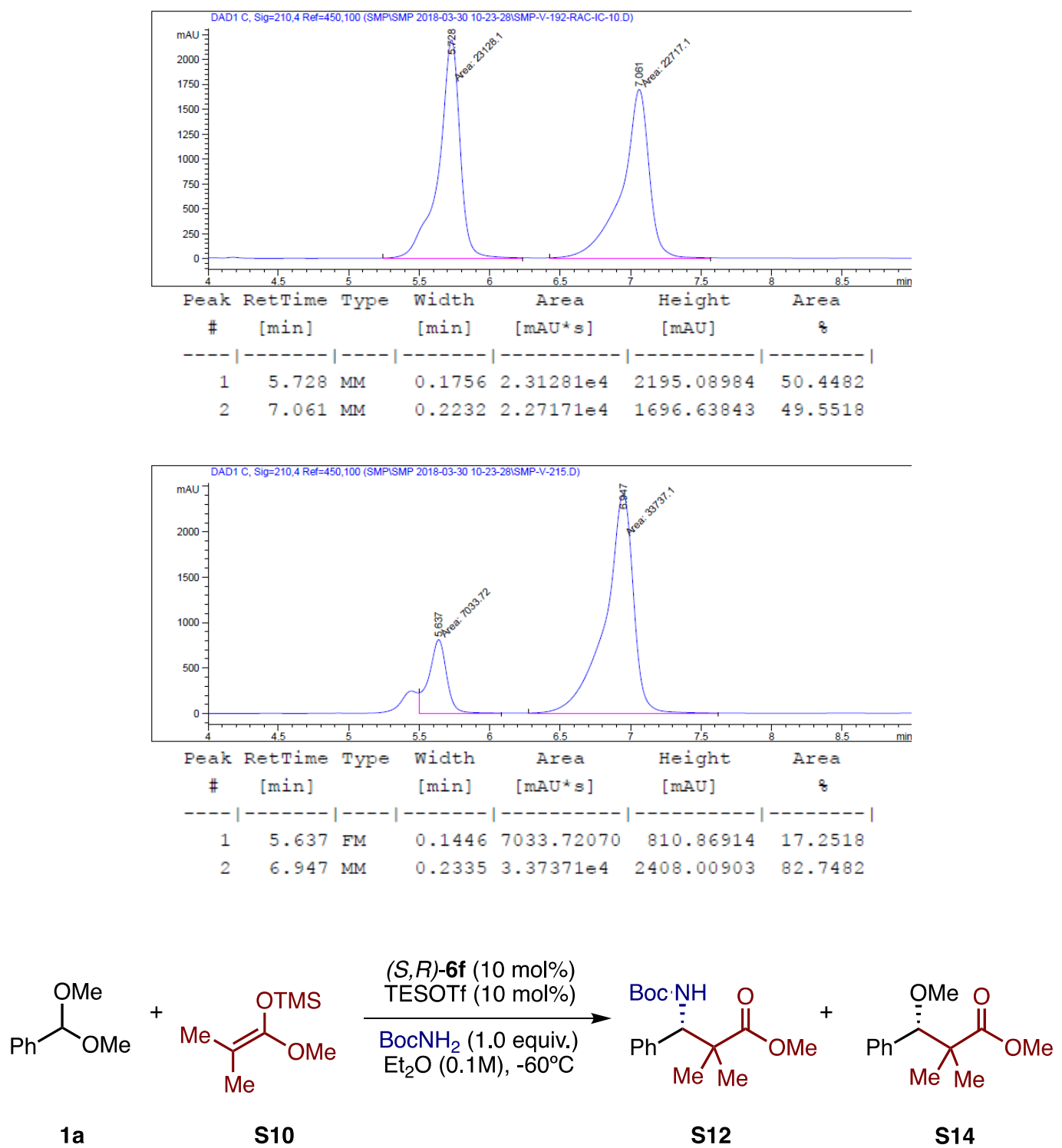

Reaction run according to Method A. Benzaldehyde dimethyl acetal $(45.0 \mu \mathrm{L}, 0.300 \mathrm{mmol}, 1.0$ equiv), methyl trimethylsilyl dimethylketene acetal $\mathbf{S} 10(60.9 \mu \mathrm{L}, 0.300 \mathrm{mmol}, 1.0$ equiv), tert-butyl carbamate (35.1 mg, $0.300 \mathrm{mmol}, 1.0$ equiv), catalyst $(S, R)-6 \mathrm{f}(20.8 \mathrm{mg}, 0.030 \mathrm{mmol}, 0.10$ equiv), TESOTf $(6.8 \mu \mathrm{L}$, $0.030 \mathrm{mmol}, 0.10$ equiv), and $\mathrm{Et}_{2} \mathrm{O}(3.0 \mathrm{~mL}, 0.1 \mathrm{M})$ were used. The reaction was run at $-60^{\circ} \mathrm{C}$ for $6 \mathrm{~h}$. Note: silyl ketene acetal S10 was distilled prior to use; without this step, even material that appeared clean by ${ }^{1} \mathrm{H}$-NMR gave poor reactivity. Product ratios were obtained by ${ }^{1} \mathrm{H}$-NMR of the crude reaction mixture after concentrating under reduced pressure. The products were separated by flash column chromatography on silica (Biotage, $10 \mathrm{~g}$ column) using a gradient of $1 \%$ EtOAc/hexanes $\rightarrow 10 \%$ EtOAc/hexanes as eluent. The ether product was not isolated quantitatively due to volatility. The remaining mass balance is due to unreacted starting material. Note: Enantiomeric excess of ethereal product was found to be the same when reaction was run with or without $\mathrm{BocNH}_{2}$.

Run 1:

amine: (26.8 mg, $0.087 \mathrm{mmol}, 29 \%), 25 \%$ e.e.

ether: $58 \%$ e.e.

amine/ether: $1: 1$

Run 2: 
amine: (27.2 $\mathrm{mg}, 0.089 \mathrm{mmol}, 30 \%), 25 \%$ e.e.

ether: $58 \%$ e.e.

amine/ether: 1:1

Average: 1:1 amine/ether; amine: $30 \%$ yield, $25 \%$ e.e.; ether: $58 \%$ e.e.

\section{(+)-(R)-Methyl N-Boc-3-amino-2,2-dimethyl-3-phenylpropanoate [S12]}<smiles>COC(=O)C(C)(C)[C@H](NC(=O)OC(C)(C)C)c1ccccc1</smiles>

Preparation of racemic standard: An oven-dried 2-dram vial was charged with $\mathrm{N}$-Boc imine $\mathbf{S 7}(103 \mathrm{mg}, 0.500 \mathrm{mmol}, 1.0$ equiv) and a stir bar. The vial was sealed with a septum-lined cap, then evacuated and refilled with $\mathrm{N}_{2}(2 \mathrm{x})$. (2methylallyl)trimethylsilane (102 $\mu \mathrm{L}, 0.500 \mathrm{mmol}, 1.0$ equiv.) was next added via syringe, followed by $\mathrm{Et}_{2} \mathrm{O}(4.0 \mathrm{~mL}, 0.13 \mathrm{M})$. The reaction mixture was cooled to $-78^{\circ} \mathrm{C}$ in a dry ice/acetone bath, then TESOTf $(57.0 \mu \mathrm{L}, 0.250$ mmol, 0.50 equiv) was added dropwise. The vial was transferred to a $-60^{\circ} \mathrm{C}$ cryocool and the mixture was stirred until completion, as determined by TLC analysis. Upon completion, the reaction was quenched by dropwise addition via syringe of a solution of $1: 1 \mathrm{Et}_{3} \mathrm{~N} / \mathrm{MeOH}(200 \mu \mathrm{L})$, then warmed to rt. Solvent was removed under reduced pressure, then the remaining residue was dissolved in minimal $\mathrm{CH}_{2} \mathrm{Cl}_{2}$ and applied to a silica column for purification. The product was isolated as a white solid.

Chiral HPLC conditions: CHIRALPAK AD-H, 3\% $\mathrm{PrOH} / \mathrm{hexanes,} 1 \mathrm{~mL} / \mathrm{min}$

Racemic product: $\mathrm{tR}_{\mathrm{R}}(R)=11.9 \mathrm{~min}, \mathrm{t}_{\mathrm{R}}(S)=20.1 \mathrm{~min}$

Enantioenriched product: $\mathrm{t}_{\mathrm{R}}(R)=12.3 \mathrm{~min}$

${ }^{1} \mathrm{H}-\mathrm{NMR}\left(500 \mathrm{MHz}, \mathrm{CDCl}_{3}\right) \delta 7.30-7.23(\mathrm{~m}, 3 \mathrm{H}), 7.18(\mathrm{~d}, J=7.5 \mathrm{~Hz}, 2 \mathrm{H}), 5.96(\mathrm{~d}, J=9.8 \mathrm{~Hz}, 1 \mathrm{H}), 4.70$ $(\mathrm{d}, J=9.7 \mathrm{~Hz}, 1 \mathrm{H}), 3.65(\mathrm{~s}, 3 \mathrm{H}), 1.40(\mathrm{~s}, 9 \mathrm{H}), 1.30(\mathrm{~s}, 3 \mathrm{H}), 1.11(\mathrm{~s}, 3 \mathrm{H}) .[\alpha]^{25} \mathrm{D}=+10.9^{\circ}\left(c=1.0, \mathrm{CHCl}_{3}\right)$. All spectral data agree with that reported in the literature. ${ }^{29}$
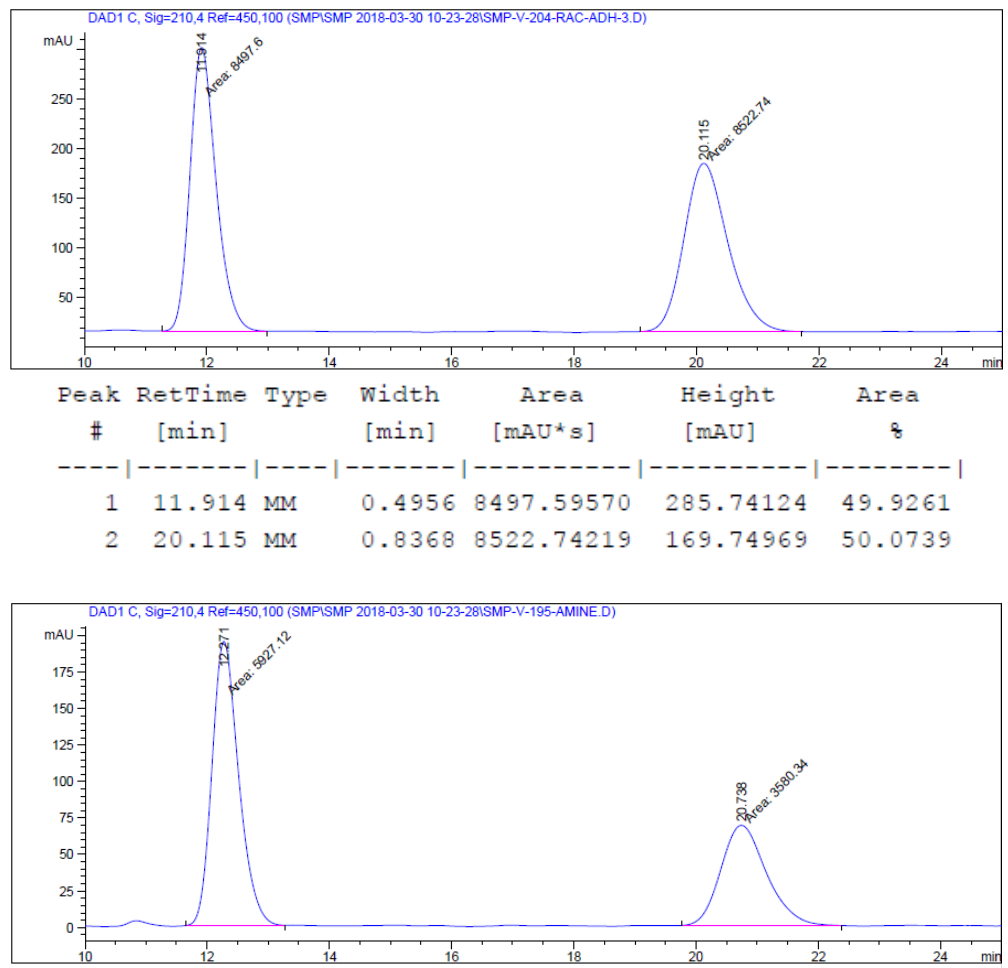


\begin{tabular}{|c|c|c|c|c|c|c|}
\hline $\begin{array}{c}\text { Peak } \\
\#\end{array}$ & $\begin{array}{c}\text { RetTime } \\
\text { [min] }\end{array}$ & Type & $\begin{array}{l}\text { Width } \\
\text { [min] }\end{array}$ & $\begin{array}{c}\text { Area } \\
{\left[\mathrm{mAU}{ }^{\star} \mathrm{s}\right]}\end{array}$ & $\begin{array}{l}\text { Height } \\
\text { [mAU] }\end{array}$ & $\begin{array}{c}\text { Area } \\
\&\end{array}$ \\
\hline 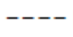 & -- & & ----- & -------- & -------- & $------\mid$ \\
\hline 1 & 12.271 & MM & 0.5073 & 5927.11572 & 194.74649 & 62.3418 \\
\hline 2 & 20.738 & MM & 0.8677 & 3580.33618 & 68.77320 & 37.6582 \\
\hline
\end{tabular}

\section{(+)-(R)-Methyl 3-methoxy-2,2-dimethyl-3-phenylpropanoate [S14]}<smiles>COC(=O)C(C)(C)[C@H](OC)c1ccccc1</smiles>

Isolated as a colorless oil; product is volatile, and care should be taken to minimize product loss during isolation.

Chiral HPLC conditions: CHIRALPAK IC, $3 \% \mathrm{PrOH} /$ hexanes, $1 \mathrm{~mL} / \mathrm{min}$

Racemic product: $\mathrm{t}_{\mathrm{R}}(S)=5.6 \mathrm{~min}, \mathrm{t}_{\mathrm{R}}(R)=6.3 \mathrm{~min}$

Enantioenriched product: $\mathrm{t}_{\mathrm{R}}(R)=6.4 \mathrm{~min}$

${ }^{1} \mathrm{H}-\mathrm{NMR}\left(500 \mathrm{MHz}, \mathrm{CDCl}_{3}\right) \delta 7.36-7.26(\mathrm{~m}, 5 \mathrm{H}), 4.48(\mathrm{~s}, 1 \mathrm{H}), 3.72(\mathrm{~s}, 3 \mathrm{H}), 3.19(\mathrm{~s}, 3 \mathrm{H}), 1.11(\mathrm{~s}, 3 \mathrm{H})$, $1.01(\mathrm{~s}, 3 \mathrm{H}) ;[\alpha]^{25} \mathrm{D}=+23.0^{\circ}\left(c=1.0, \mathrm{CHCl}_{3}\right)$. All spectral data agree with that reported in the literature. ${ }^{30}$
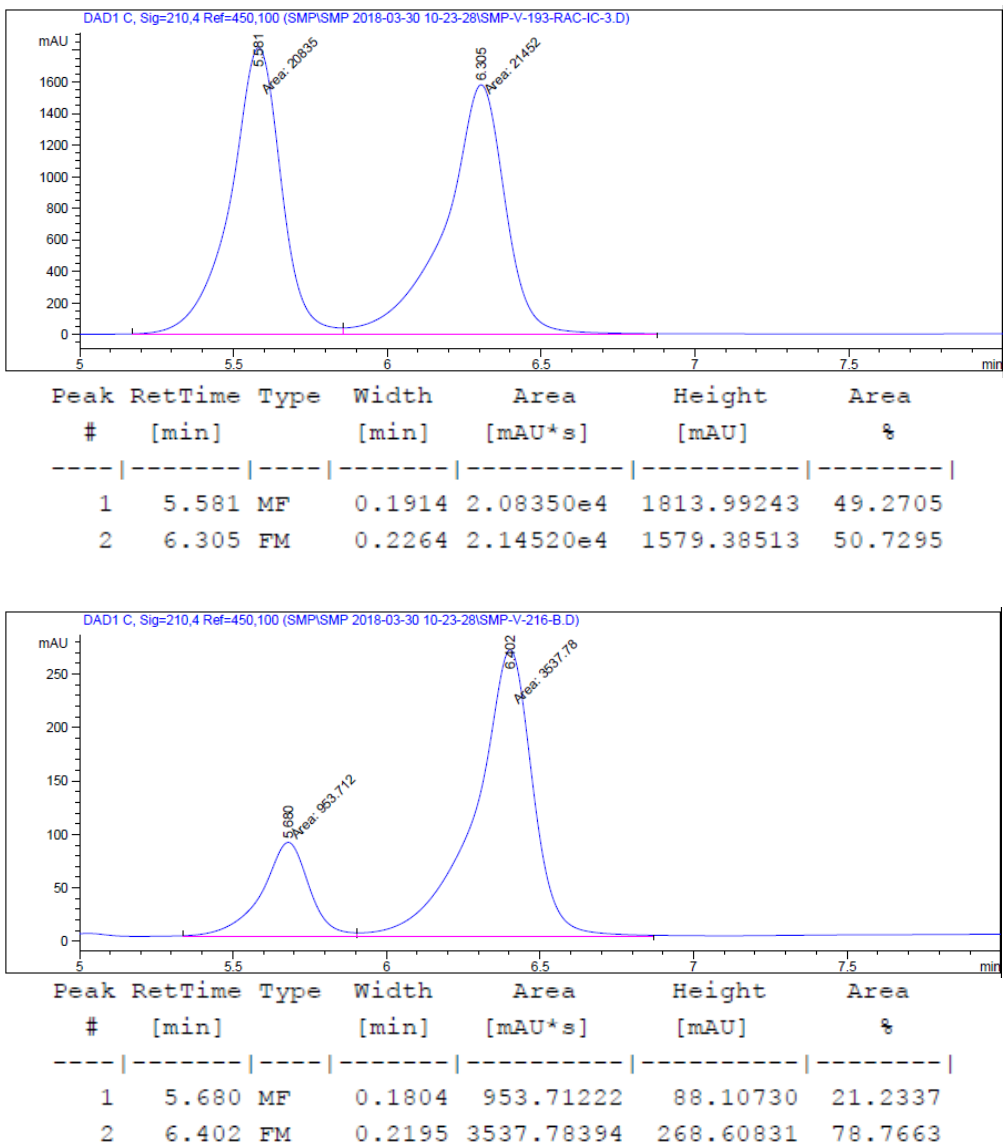


\section{Computational model}

Density functional theory (DFT) calculations were performed with Gaussian $16^{31}$ using Harvard University's Odyssey and Cannon computer clusters. Careful searches of catalyst and substrate conformations, nucleophile approaches and substrate-catalyst orientations were performed. The first set of geometry optimizations and frequency calculations were obtained using gas-phase B3LYP/6$31 \mathrm{G}(\mathrm{d})^{32,33,34}$ and the D3 version of Grimme's dispersion with Becke-Johnson damping as dispersion correction $(\mathrm{D} 3(\mathrm{BJ})) .^{35}$ Transition state structures were identified by the presence of a single imaginary frequency which was visually inspected to ensure it corresponded to the formation of the desired carboncarbon bond.

The combination of dispersion-corrected DFT with the small basis sets employed could, potentially, cause the model to over-emphasize non-covalent stabilizing interactions. ${ }^{36}$ To address this issue, a second round of geometry optimizations with catalyst ent-4f was performed without a dispersion correction (B3LPY/6-31G(d)), which predicted the correct sense of enantioinduction (compare tables S11 and S12), and led to similar transition structures to those optimized with a dispersion correction (see page S76 for overlayed structures). The energy of all the transition structures obtained was then calculated at a higher level of theory (B3LYP/6-311G(d,p), pcm=DiethylEther, $T=223.15$, D3(BJ)), ${ }^{32,34,35,37}$ using the PCM continuous surface charge polarizable continuum model of solvation for $\mathrm{Et}_{2} \mathrm{O} .{ }^{38}$ The energy differences for the thiourea catalyst ent-4f with substrate 1 a were found to be $5.62 \mathrm{kcal} / \mathrm{mol}$ with dispersion correction and $3.60 \mathrm{kcal} / \mathrm{mol}$ without dispersion correction. Unless otherwise specified, computational pictures were rendered in CYLview. ${ }^{39}$

Tabulated energies for computed transition states with thiourea catalyst $(R, S)$ -
ent-4f - geometries optimized with dispersion correction

Table S11. Energies of thiourea geometries optimized with dispersion correction

\begin{tabular}{|c|c|c|c|c|c|}
\hline Name & $\begin{array}{c}\mathrm{E}\left[\mathrm{PCM}\left(\mathrm{Et}_{2} \mathrm{O}\right)-\mathrm{B} 3 \mathrm{LYP} / 6-\right. \\
311 \mathrm{G}(\mathrm{d}, \mathrm{p}) / \mathrm{D} 3(\mathrm{BJ})] \text { (Hartree) }\end{array}$ & $\begin{array}{c}\Delta \mathrm{E}_{\mathrm{rel}} \\
(\mathrm{kcal} / \mathrm{mol})\end{array}$ & Name & $\begin{array}{c}\mathrm{E}\left[\mathrm{PCM}\left(\mathrm{Et}_{2} \mathrm{O}\right)-\mathrm{B} 3 \mathrm{LYP} / 6-\right. \\
311 \mathrm{G}(\mathrm{d}, \mathrm{p}) / \mathrm{D} 3(\mathrm{BJ})] \text { (Hartree) }\end{array}$ & $\begin{array}{c}\Delta \mathrm{E}_{\text {rel }} \\
(\mathrm{kcal} / \mathrm{mol})\end{array}$ \\
\hline TS-S-4f-1 & -4790.242621 & 0.00 & TS- $R-4 \mathrm{f}-1$ & -4790.233671 & 5.62 \\
\hline TS-S-4f-2 & -4790.241663 & 0.60 & TS- $R-4 \mathrm{f}-2$ & -4790.232586 & 6.30 \\
\hline TS-S-4f-3 & -4790.239074 & 2.23 & TS-R-4f-3 & -4790.229891 & 7.99 \\
\hline TS-S-4f-4 & -4790.236742 & 3.69 & TS-R-4f-4 & -4790.229413 & 8.29 \\
\hline TS-S-4f-5 & -4790.236586 & 3.79 & TS- $R-4 \mathrm{f}-5$ & -4790.229048 & 8.52 \\
\hline TS-S-4f-6 & -4790.235347 & 4.56 & TS-R-4f-6 & -4790.228307 & 8.98 \\
\hline TS-S-4f-7 & -4790.234868 & 4.87 & TS- $R-4 f-7$ & -4790.228003 & 9.17 \\
\hline TS-S-4f-8 & -4790.234098 & 5.35 & TS- $R-4 f-8$ & -4790.227243 & 9.65 \\
\hline TS-S-4f-9 & -4790.234060 & 5.37 & TS- $R-4 f-9$ & -4790.227190 & 9.68 \\
\hline TS-S-4f-10 & -4790.233830 & 5.52 & TS- $R-4 \mathrm{f}-10$ & -4790.227118 & 9.73 \\
\hline TS-S-4f-11 & -4790.233802 & 5.53 & TS- $R-4 \mathrm{f}-11$ & -4790.226955 & 9.83 \\
\hline TS-S-4f-12 & -4790.233683 & 5.61 & TS- $R-4 \mathrm{f}-12$ & 4790.226463 & 10.14 \\
\hline TS-S-4f-13 & -4790.233477 & 5.74 & TS- $R-4 \mathrm{f}-13$ & -4790.225989 & 10.44 \\
\hline TS-S-4f-14 & -4790.231952 & 6.69 & TS- $R-4 \mathrm{f}-14$ & -4790.225616 & 10.67 \\
\hline TS-S-4f-15 & -4790.231046 & 7.26 & TS- $R-4 \mathrm{f}-15$ & -4790.225582 & 10.69 \\
\hline TS-S-4f-16 & -4790.230677 & 7.49 & TS-R-4f-16 & -4790.225223 & 10.92 \\
\hline TS-S-4f-17 & -4790.230203 & 7.79 & TS-R-4f-17 & -4790.225220 & 10.92 \\
\hline TS-S-4f-18 & -4790.229126 & 8.47 & TS-R-4f-18 & -4790.224955 & 11.09 \\
\hline TS-S-4f-19 & -4790.228517 & 8.85 & TS-R-4f-19 & -4790.224510 & 11.36 \\
\hline TS-S-4f-20 & -4790.228203 & 9.05 & TS- $R-4 \mathrm{f}-20$ & -4790.224490 & 11.38 \\
\hline TS-S-4f-21 & -4790.227957 & 9.20 & TS- $R-4 f-21$ & -4790.223806 & 11.81 \\
\hline TS-S-4f-22 & -4790.227021 & 9.79 & TS- $R-4 f-22$ & -4790.223699 & 11.87 \\
\hline TS-S-4f-23 & -4790.226106 & 10.36 & TS- $R-4 \mathrm{f}-23$ & -4790.223640 & 11.91 \\
\hline
\end{tabular}




\begin{tabular}{|c|c|c|c|c|c|}
\hline TS-S-4f-24 & -4790.225487 & 10.75 & TS- $R-4 \mathrm{f}-24$ & -4790.223351 & 12.09 \\
\hline TS-S-4f-25 & -4790.225025 & 11.04 & TS-R-4f-25 & -4790.223317 & 12.11 \\
\hline TS-S-4f-26 & -4790.225022 & 11.04 & TS-R-4f-26 & -4790.223087 & 12.26 \\
\hline TS-S-4f-27 & -4790.224510 & 11.36 & TS-R-4f-27 & -4790.222285 & 12.76 \\
\hline TS-S-4f-28 & -4790.224144 & 11.59 & TS- $R-4 f-28$ & -4790.222036 & 12.92 \\
\hline TS-S-4f-29 & -4790.223711 & 11.87 & TS- $R-4 \mathrm{f}-29$ & -4790.222006 & 12.94 \\
\hline TS-S-4f-30 & -4790.223649 & 11.91 & TS- $R-4 \mathrm{f}-30$ & -4790.221170 & 13.46 \\
\hline TS-S-4f-31 & -4790.223620 & 11.92 & TS-R-4f-31 & -4790.220760 & 13.72 \\
\hline TS-S-4f-32 & -4790.223176 & 12.20 & TS- $R-4 \mathrm{f}-32$ & -4790.220038 & 14.17 \\
\hline TS-S-4f-33 & -4790.222999 & 12.31 & TS- $R-4 \mathrm{f}-33$ & -4790.219668 & 14.40 \\
\hline TS-S-4f-34 & -4790.222549 & 12.60 & TS- $R-4 \mathrm{f}-34$ & -4790.219156 & 14.72 \\
\hline TS-S-4f-35 & -4790.221783 & 13.08 & TS- $R-4 \mathrm{f}-35$ & -4790.218924 & 14.87 \\
\hline TS-S-4f-36 & -4790.221649 & 13.16 & TS- $R-4 f-36$ & -4790.218355 & 15.23 \\
\hline TS-S-4f-37 & -4790.221141 & 13.48 & TS-R-4f-37 & -4790.218323 & 15.25 \\
\hline TS-S-4f-38 & -4790.221057 & 13.53 & TS- $R-4 f-38$ & -4790.218162 & 15.35 \\
\hline TS-S-4f-39 & -4790.220877 & 13.64 & TS- $R-4 f-39$ & -4790.216871 & 16.16 \\
\hline TS-S-4f-40 & -4790.220859 & 13.66 & TS- $R-4 \mathrm{f}-40$ & -4790.216449 & 16.42 \\
\hline TS-S-4f-41 & -4790.220215 & 14.06 & TS-R-4f-41 & -4790.212045 & 19.19 \\
\hline TS-S-4f-42 & -4790.219978 & 14.21 & TS- $R-4 \mathrm{f}-42$ & -4790.203500 & 24.55 \\
\hline TS-S-4f-43 & -4790.219400 & 14.57 & & & \\
\hline TS-S-4f-44 & -4790.217814 & 15.57 & & & \\
\hline TS-S-4f-45 & -4790.217494 & 15.77 & & & \\
\hline TS-S-4f-46 & -4790.216947 & 16.11 & & & \\
\hline TS-S-4f-47 & -4790.215113 & 17.26 & & & \\
\hline TS-S-4f-48 & -4790.214938 & 17.37 & & & \\
\hline TS-S-4f-49 & -4790.214108 & 17.89 & & & \\
\hline TS-S-4f-50 & -4790.213586 & 18.22 & & & \\
\hline TS-S-4f-51 & -4790.213559 & 18.24 & & & \\
\hline TS-S-4f-52 & -4790.211286 & 19.66 & & & \\
\hline TS-S-4f-53 & -4790.210859 & 19.93 & & & \\
\hline
\end{tabular}

Due to the importance of dispersion in the system, the energies of TS-S-4f-1 and TS- $R-4 f-1$ were also computed using M06-2 $\mathrm{x}^{40}$ as functional, yielding comparable results.

\begin{tabular}{|l|c|c|l|l|c|c|}
\hline Name & $\begin{array}{c}\text { E[PCM(Et } 2 \text { O)-M06-2x/6- } \\
\text { 311G(d,p)] (Hartree) }\end{array}$ & $\begin{array}{l}\Delta \mathrm{E}_{\text {rel }} \\
(\mathbf{k c a l} / \mathbf{m o l})\end{array}$ & Name & $\begin{array}{c}\text { E[PCM(Et } 2 \text { O)-M06-2x/6- } \\
\mathbf{3 1 1 G}(\mathrm{d}, \mathbf{p})] \text { (Hartree) }\end{array}$ & $\begin{array}{l}\Delta \mathrm{E}_{\text {rel }} \\
(\mathrm{kcal} / \mathrm{mol})\end{array}$ \\
\hline TS-S-4f-1 & -4788.364972 & $\mathbf{0 . 0 0}$ & TS-R-4f-1 & -4788.355923 & $\mathbf{5 . 6 8}$ \\
\hline
\end{tabular}

Tabulated energies for computed transition states with thiourea catalyst $(R, S)$ ent-4f - geometries optimized without dispersion correction

Table S12. Energies of thiourea geometries optimized without dispersion correction

\begin{tabular}{|c|c|c|c|c|c|}
\hline Name & $\begin{array}{c}\mathrm{E}\left[\mathrm{PCM}\left(\mathrm{Et} \mathrm{t}_{2} \mathrm{O}\right)-\mathrm{B} 3 \mathrm{LYP} / 6-\right. \\
311 \mathrm{G}(\mathrm{d}, \mathrm{p}) / \mathrm{D} 3(\mathrm{BJ})] \text { (Hartree) }\end{array}$ & $\begin{array}{c}\Delta \mathrm{E}_{\text {rel }} \\
(\mathrm{kcal} / \mathrm{mol})\end{array}$ & Name & 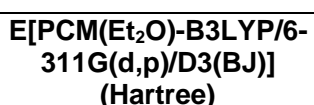 & $\begin{array}{c}\Delta \mathrm{E}_{\text {rel }} \\
\text { (kcal/mol) }\end{array}$ \\
\hline TS-S-4f-nd-1 & -4790.231770 & 0.00 & TS- $R-4 \mathrm{f}-\mathrm{nd}-1$ & -4790.226028 & 3.60 \\
\hline TS-S-4f-nd-2 & -4790.230050 & 1.08 & TS- $R-4 f-n d-2$ & -4790.224436 & 4.60 \\
\hline TS-S-4f-nd-3 & -4790.227991 & 2.37 & TS-R-4f-nd-3 & -4790.224394 & 4.63 \\
\hline TS-S-4f-nd-4 & -4790.227775 & 2.51 & TS-R-4f-nd-4 & -4790.222779 & 5.64 \\
\hline TS-S-4f-nd-5 & -4790.226725 & 3.17 & TS- $R-4 f-n d-5$ & -4790.221999 & 6.13 \\
\hline TS-S-4f-nd-6 & -4790.224784 & 4.38 & TS- $R-4 f-n d-6$ & -4790.221811 & 6.25 \\
\hline TS-S-4f-nd-7 & -4790.223886 & 4.95 & TS- $R-4 \mathrm{f}-\mathrm{nd}-7$ & -4790.221195 & 6.64 \\
\hline
\end{tabular}




\begin{tabular}{|c|c|c|c|c|c|}
\hline TS-S-4f-nd-8 & -4790.223420 & 5.24 & TS- $R-4 \mathrm{f}-\mathrm{nd}-8$ & -4790.220456 & 7.10 \\
\hline TS-S-4f-nd-9 & -4790.222351 & 5.91 & TS-R-4f-nd-9 & -4790.218917 & 8.07 \\
\hline TS-S-4f-nd-10 & -4790.222224 & 5.99 & TS-R-4f-nd-10 & -4790.218117 & 8.57 \\
\hline TS-S-4f-nd-11 & -4790.219987 & 7.39 & TS-R-4f-nd-11 & -4790.217344 & 9.05 \\
\hline TS-S-4f-nd-12 & -4790.219973 & 7.40 & TS-R-4f-nd-12 & -4790.217258 & 9.11 \\
\hline TS-S-4f-nd-13 & -4790.219045 & 7.99 & TS-R-4f-nd-13 & -4790.217007 & 9.26 \\
\hline TS-S-4f-nd-14 & -4790.218919 & 8.06 & TS-R-4f-nd-14 & -4790.216899 & 9.33 \\
\hline TS-S-4f-nd-15 & -4790.218904 & 8.07 & TS-R-4f-nd-15 & -4790.216665 & 9.48 \\
\hline TS-S-4f-nd-16 & -4790.218281 & 8.46 & TS-R-4f-nd-16 & -4790.216372 & 9.66 \\
\hline TS-S-4f-nd-17 & -4790.217647 & 8.86 & TS-R-4f-nd-17 & -4790.216230 & 9.75 \\
\hline TS-S-4f-nd-18 & -4790.216935 & 9.31 & TS-R-4f-nd-18 & -4790.215926 & 9.94 \\
\hline TS-S-4f-nd-19 & -4790.216878 & 9.34 & TS-R-4f-nd-19 & -4790.215557 & 10.17 \\
\hline TS-S-4f-nd-20 & -4790.216739 & 9.43 & TS-R-4f-nd-20 & -4790.215442 & 10.25 \\
\hline TS-S-4f-nd-21 & -4790.216631 & 9.50 & TS-R-4f-nd-21 & -4790.215368 & 10.29 \\
\hline TS-S-4f-nd-22 & -4790.216419 & 9.63 & TS-R-4f-nd-22 & -4790.215072 & 10.48 \\
\hline TS-S-4f-nd-23 & -4790.216353 & 9.67 & TS-R-4f-nd-23 & -4790.214553 & 10.80 \\
\hline TS-S-4f-nd-24 & -4790.216043 & 9.87 & TS-R-4f-nd-24 & -4790.214359 & 10.93 \\
\hline TS-S-4f-nd-25 & -4790.215615 & 10.14 & TS-R-4f-nd-25 & -4790.214309 & 10.96 \\
\hline TS-S-4f-nd-26 & -4790.214367 & 10.92 & TS-R-4f-nd-26 & -4790.214289 & 10.97 \\
\hline TS-S-4f-nd-27 & -4790.214148 & 11.06 & TS-R-4f-nd-27 & -4790.214199 & 11.03 \\
\hline TS-S-4f-nd-28 & -4790.214093 & 11.09 & TS-R-4f-nd-28 & -4790.214031 & 11.13 \\
\hline TS-S-4f-nd-29 & -4790.213690 & 11.35 & TS-R-4f-nd-29 & -4790.213984 & 11.16 \\
\hline TS-S-4f-nd-30 & -4790.213685 & 11.35 & TS- $R-4 f-n d-30$ & -4790.213232 & 11.63 \\
\hline TS-S-4f-nd-31 & -4790.213669 & 11.36 & TS-R-4f-nd-31 & -4790.212950 & 11.81 \\
\hline TS-S-4f-nd-32 & -4790.213459 & 11.49 & TS-R-4f-nd-32 & -4790.212405 & 12.15 \\
\hline TS-S-4f-nd-33 & -4790.213422 & 11.51 & TS- $R-4 f-n d-33$ & -4790.212127 & 12.33 \\
\hline TS-S-4f-nd-34 & -4790.212870 & 11.86 & TS-R-4f-nd-34 & -4790.212067 & 12.36 \\
\hline TS-S-4f-nd-35 & -4790.212481 & 12.10 & TS-R-4f-nd-35 & -4790.211996 & 12.41 \\
\hline TS-S-4f-nd-36 & -4790.212306 & 12.21 & TS-R-4f-nd-36 & -4790.211443 & 12.76 \\
\hline TS-S-4f-nd-37 & -4790.212091 & 12.35 & TS-R-4f-nd-37 & -4790.211418 & 12.77 \\
\hline TS-S-4f-nd-38 & -4790.211568 & 12.68 & TS-R-4f-nd-38 & -4790.211206 & 12.90 \\
\hline TS-S-4f-nd-39 & -4790.211101 & 12.97 & TS-R-4f-nd-39 & -4790.210228 & 13.52 \\
\hline TS-S-4f-nd-40 & -4790.210653 & 13.25 & TS-R-4f-nd-40 & -4790.210036 & 13.64 \\
\hline TS-S-4f-nd-41 & -4790.209966 & 13.68 & TS-R-4f-nd-41 & -4790.209692 & 13.85 \\
\hline TS-S-4f-nd-42 & -4790.209550 & 13.94 & TS-R-4f-nd-42 & -4790.209123 & 14.21 \\
\hline TS-S-4f-nd-43 & -4790.209020 & 14.28 & TS-R-4f-nd-43 & -4790.208605 & 14.54 \\
\hline TS-S-4f-nd-44 & -4790.208519 & 14.59 & TS-R-4f-nd-44 & -4790.207466 & 15.25 \\
\hline TS-S-4f-nd-45 & -4790.207836 & 15.02 & TS-R-4f-nd-45 & -4790.206109 & 16.10 \\
\hline TS-S-4f-nd-46 & -4790.207594 & 15.17 & TS-R-4f-nd-46 & -4790.204949 & 16.83 \\
\hline TS-S-4f-nd-47 & -4790.207297 & 15.36 & TS-R-4f-nd-47 & -4790.204911 & 16.85 \\
\hline TS-S-4f-nd-48 & -4790.207085 & 15.49 & TS-R-4f-nd-48 & -4790.201599 & 18.93 \\
\hline TS-S-4f-nd-49 & -4790.206308 & 15.98 & & & \\
\hline TS-S-4f-nd-50 & -4790.205835 & 16.27 & & & \\
\hline TS-S-4f-nd-51 & -4790.204556 & 17.08 & & & \\
\hline TS-S-4f-nd-52 & -4790.204281 & 17.25 & & & \\
\hline TS-S-4f-nd-53 & -4790.204107 & 17.36 & & & \\
\hline TS-S-4f-nd-54 & -4790.202299 & 18.49 & & & \\
\hline TS-S-4f-nd-55 & -4790.201298 & 19.12 & & & \\
\hline TS-S-4f-nd-56 & -4790.201108 & 19.24 & & & \\
\hline
\end{tabular}

The main differences observed between lowest energy transition states with or without a dispersion correction (the D3 version of Grimme's dispersion with Becke-Johnson damping - D3(BJ)) were in the positioning on the aromatic rings on the catalysts, with both the 4-pyrenyl and the 3,5-bis-trifluoromethyl 
phenyl moieties closer to the substrate when a dispersion correction was employed. PyMOL was used to overlap of TS-S-4f-1 with TS-S-4f-nd-1 and TS- $R-4 f-1$ with TS- $R-4 f-n d-1$.

Major transition states, in yellow TS-S-4f-1, in blue TS-S-4f-nd-1, front view (left) and side view (right).
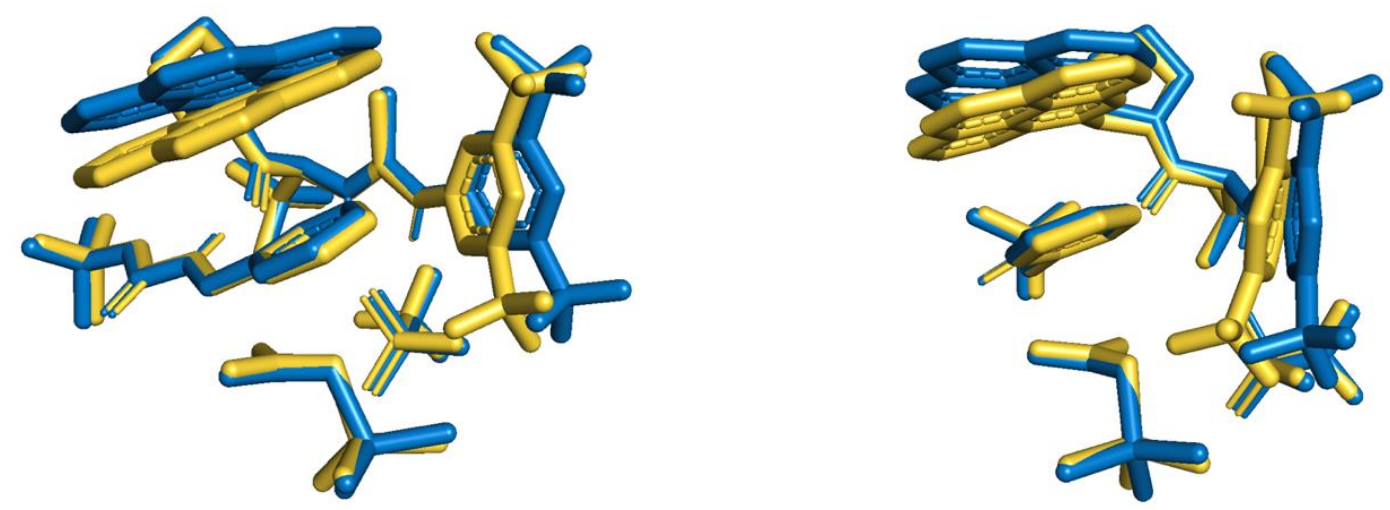

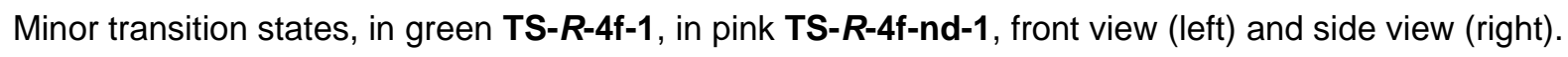
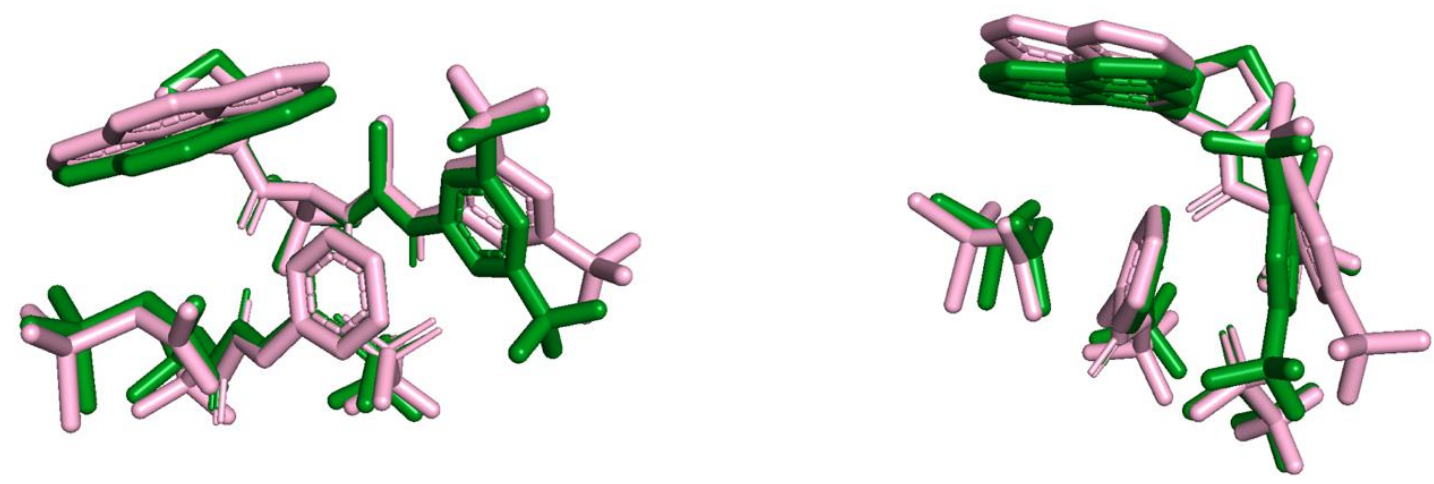

Application of the Gaussian thermal correction to the energies of TS-S-4f-1, TS- $R-4 f-1, T S-S-4 f-n d-1$ and TS- $\boldsymbol{R}-\mathbf{4 f - n d - 1}$ led to results indicating the same direction of enantioinduction, albeit a smaller energy gap (more so for the dispersion corrected structures TS-S-4f-1 and TS-R-4f-1).

\begin{tabular}{|c|c|c|c|c|}
\hline Name & $\begin{array}{c}\left.\text { E[PCM(Et }{ }_{2} \mathrm{O}\right)-\mathrm{B} 3 \mathrm{LYP} / 6- \\
311 \mathrm{G}(\mathrm{d}, \mathrm{p}) / \mathrm{D} 3(\mathrm{BJ})] \text { (Hartree) }\end{array}$ & $\begin{array}{c}\text { Thermal correction } \\
\text { (Hartree) }\end{array}$ & $\begin{array}{c}\mathrm{G}\left[\mathrm{PCM}\left(\mathrm{Et}_{2} \mathrm{O}\right)-\mathrm{B} 3 \mathrm{LYP} / 6-\right. \\
311 \mathrm{G}(\mathrm{d}, \mathrm{p}) / \mathrm{D} 3(\mathrm{BJ})] \text { (Hartree) }\end{array}$ & $\Delta \mathbf{G}_{\text {rel }}(\mathrm{kcal} / \mathrm{mol})$ \\
\hline TS-S-4f-1 & -4790.242621 & 1.045375 & -4789.197246 & 0.00 \\
\hline TS- $R-4 \mathrm{f}-1$ & -4790.233671 & 1.04354 & -4789.190131 & 4.46 \\
\hline
\end{tabular}

\begin{tabular}{|c|c|c|c|c|}
\hline Name & $\begin{array}{c}\left.\text { E[PCM(Et }{ }_{2} \mathrm{O}\right)-\mathrm{B} 3 \mathrm{LYP} / 6- \\
311 \mathrm{G}(\mathrm{d}, \mathrm{p}) / \mathrm{D} 3(\mathrm{BJ})] \text { (Hartree) }\end{array}$ & $\begin{array}{c}\text { Thermal correction } \\
\text { (Hartree) }\end{array}$ & $\begin{array}{c}\left.\text { G[PCM(Et }{ }_{2} \mathrm{O}\right)-\mathrm{B} 3 \mathrm{LYP} / 6- \\
\text { 311G(d,p)/D3(BJ)] (Hartree) }\end{array}$ & $\Delta \mathbf{G}_{\mathrm{rel}}(\mathrm{kcal} / \mathrm{mol})$ \\
\hline TS-S-4f-nd-1 & -4790.231770 & 1.033232 & -4789.198538 & 0.00 \\
\hline TS- $R-4 f-n d-1$ & -4790.226028 & 1.032859 & -4789.193169 & 3.37 \\
\hline
\end{tabular}


Tabulated energies for computed transition states with squaramide catalyst $(R, S)$ ent-6f - geometries optimized with dispersion correction

Table S13. Energies of squaramide geometries optimized with dispersion

\begin{tabular}{|c|c|c|c|c|c|}
\hline Name & $\begin{array}{c}\mathrm{E}\left[\mathrm{PCM}\left(\mathrm{Et}_{2} \mathrm{O}\right)-\mathrm{B} 3 \mathrm{LYP} / 6-\right. \\
311 \mathrm{G}(\mathrm{d}, \mathrm{p}) / \mathrm{D} 3(\mathrm{BJ})] \text { (Hartree) }\end{array}$ & $\begin{array}{l}\Delta \mathrm{E}_{\mathrm{rel}} \\
\text { (kcal/mol) }\end{array}$ & Name & $\begin{array}{c}E\left[P C M\left(E t_{2} O\right)-B 3 L Y P / 6-\right. \\
311 G(d, p) / D 3(B J)] \text { (Hartree) } \\
\end{array}$ & $\begin{array}{l}\Delta \mathrm{E}_{\mathrm{rel}} \\
\text { (kcal/mol) }\end{array}$ \\
\hline TS-S-6f-1 & -4656.843048 & 0.00 & TS-R-6f-1 & -4656.835192 & 4.93 \\
\hline TS-S-6f-2 & -4656.839866 & 2.00 & TS- $R-6 f-2$ & -4656.828048 & 9.41 \\
\hline TS-S-6f-3 & -4656.838857 & 2.63 & TS-R-6f-3 & -4656.826141 & 10.61 \\
\hline TS-S-6f-4 & -4656.837448 & 3.51 & TS- $R-6 f-4$ & -4656.825889 & 10.77 \\
\hline TS-S-6f-5 & -4656.837110 & 3.73 & TS- $R-6 \mathrm{f}-5$ & -4656.825173 & 11.22 \\
\hline TS-S-6f-6 & -4656.835274 & 4.88 & TS-R-6f-6 & -4656.824841 & 11.43 \\
\hline TS-S-6f-7 & -4656.835256 & 4.89 & TS- $R-6 f-7$ & -4656.824583 & 11.59 \\
\hline TS-S-6f-8 & -4656.833697 & 5.87 & TS- $R-6 \mathrm{f}-8$ & -4656.824582 & 11.59 \\
\hline TS-S-6f-9 & -4656.821113 & 13.76 & TS- $R-6 f-9$ & -4656.823502 & 12.27 \\
\hline TS-S-6f-10 & -4656.820696 & 14.03 & TS- $R-6 \mathrm{f}-10$ & -4656.823325 & 12.38 \\
\hline TS-S-6f-11 & -4656.819804 & 14.59 & TS-R-6f-11 & -4656.822292 & 13.02 \\
\hline TS-S-6f-12 & -4656.819658 & 14.68 & TS- $R-6 \mathrm{f}-12$ & -4656.821816 & 13.32 \\
\hline TS-S-6f-13 & -4656.817910 & 15.77 & TS- $R-6 f-13$ & -4656.820110 & 14.39 \\
\hline TS-S-6f-14 & -4656.816844 & 16.44 & TS- $R-6 f-14$ & -4656.819420 & 14.83 \\
\hline TS-S-6f-15 & -4656.816636 & 16.57 & TS-R-6f-15 & -4656.816777 & 16.49 \\
\hline TS-S-6f-16 & -4656.815557 & 17.25 & TS-R-6f-16 & -4656.816359 & 16.75 \\
\hline TS-S-6f-17 & -4656.815038 & 17.58 & TS-R-6f-17 & -4656.815657 & 17.19 \\
\hline TS-S-6f-18 & -4656.812186 & 19.37 & TS-R-6f-18 & -4656.815418 & 17.34 \\
\hline & & & TS-R-6f-19 & -4656.801409 & 26.13 \\
\hline
\end{tabular}




\section{Effect of major binding modifications}

Experimental data suggested the presence of non-covalent stabilizing interactions that are dispersive in nature involving the aryl pyrrolidine portion of the catalyst (Figure 5). Various substrate-catalyst orientations that were consistent with such interactions were investigated both with and without an empirical dispersion correction. For dispersion corrected geometries, the face-face $\pi$ stacking orientation was strongly favored for the major enantiomer $\left.\Delta \mathrm{E}_{\text {rel(S) }}=11.04 \mathrm{kcal} / \mathrm{mol}\right)$, with TS-S-4f-25 being the lowest energy TS that visually did not have such an interaction. For geometries obtained without a dispersion correction the same is true although with a smaller energy gap (see TS-S-4f-nd-11, $\Delta \mathrm{E}_{\mathrm{rel}(\mathrm{S})}=\mathbf{7 . 3 9}$ $\mathrm{kcal} / \mathrm{mol}$ ). In contrast, the minor transition state was found to give a geometry closer to an edge-face interaction in the lowest energy TS, although with smaller energy differences (first face-face $\pi$ stacking transition states being TS- $\boldsymbol{R}-\mathbf{4 f}-\mathbf{5}, \Delta \mathrm{E}_{\mathrm{rel}(\mathrm{R})}=2.90 \mathrm{kcal} / \mathrm{mol}$ with dispersion and $\mathbf{T S}-\boldsymbol{R}-\mathbf{4 f}-\mathbf{n d}-\mathbf{9}, \Delta \mathrm{E}_{\mathrm{rel}}(\mathrm{R})=4.46$ $\mathrm{kcal} / \mathrm{mol}$ without).

\section{Major TS (with dispersion correction)}

interaction between 4-pyrenyl and substrate.




\section{Minor TS (with dispersion)}

\begin{tabular}{|c|c|}
\hline Amide-Iminium $\mathrm{H}$-bonding $(\mathrm{NH})$ and $\mathrm{CH}-\pi$ & Amide-Iminium $\mathrm{H}$-bonding $(\mathrm{NH})$ and some \\
interactions between 4-pyrenyl, substrate \\
and possibly silane.
\end{tabular}

\section{Effect of minor modifications}

\section{Catalyst conformations}

The following table summarizes the main effect of each conformational change on the transition state energy (everything else equal). Because of that, comparison in the major transition state is with respect to TS-S-4f-2 and TS- $\boldsymbol{R - 4 f - 2}$, which differ from TS-S-4f-1 and TS- $\boldsymbol{R}-\mathbf{4 f - 1}$ for the binding mode with triflate (2xO vs $1 \times 0$ binding) and are $0.60 \mathrm{kcal} / \mathrm{mol}$ and $0.68 \mathrm{kcal} / \mathrm{mol}$ higher in energy, respectively. The structure variations were carried out before the optimization of the geometries (with dispersion) and the reported energies are of the single point calculations (PCM(Et $2 \mathrm{O})-\mathrm{B} 3 \mathrm{LYP} / 6-311 \mathrm{G}(\mathrm{d}, \mathrm{p})-\mathrm{D} 3(\mathrm{BJ})$ ) of the obtained transition states. NB More hindered conformers of Boc-Imine were considered but they tended to slowly converge to the two conformers depicted below upon optimization, see TS-R-4f-22 for an exception.

Similar conformational differences were taken into account when the transition states with squaramide catalyst ent-6f were computed. 


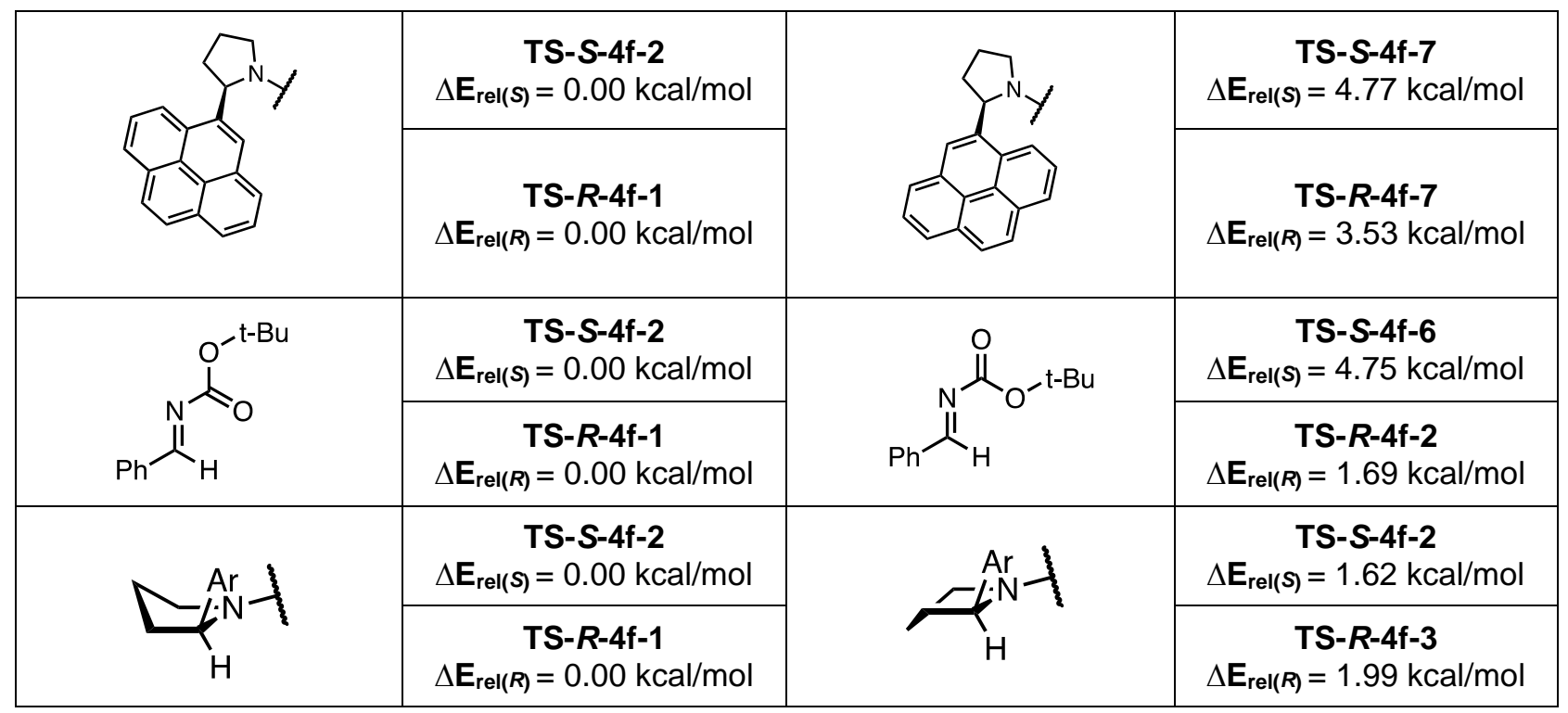

\section{Nucleophile approach}

\begin{tabular}{|c|c|c|}
\hline NBoc & NBoc & NBoc \\
\hline $\begin{array}{c}\text { TS-S-4f-20 } \\
\Delta \mathrm{E}_{\mathrm{rel}(\mathrm{S})}=8.45 \mathrm{kcal} / \mathrm{mol}\end{array}$ & $\begin{array}{c}\text { TS-S-4f-7 } \\
\Delta \mathrm{E}_{\mathrm{rel}(\boldsymbol{S})}=4.26 \mathrm{kcal} / \mathrm{mol}\end{array}$ & $\begin{array}{c}\text { TS-S-4f-2 } \\
\Delta \mathrm{E}_{\mathrm{rel}(\boldsymbol{S})}=0.00 \mathrm{kcal} / \mathrm{mol}\end{array}$ \\
\hline n.d. & Only low frequency TS observed & $\begin{array}{c}\text { TS- } \boldsymbol{R}-\mathbf{4 f - 1} \\
\Delta \mathrm{E}_{\mathrm{rel}(\boldsymbol{R})}=0.00 \mathrm{kcal} / \mathrm{mol}\end{array}$ \\
\hline
\end{tabular}

NB. Eclipsed conformations (e.g. $120^{\circ}$ trajectory) were found to optimize to staggered structures (mainly into $180^{\circ}$ trajectories).

\section{$\mathrm{NCl}$ analysis of lowest energy transition states}

The obtained lowest energy transition states were then subjected to a non-covalent interaction analysis to attempt to visualize and categorize the proposed enantiodiscriminating interactions. The $\mathrm{NCl}$ analysis ${ }^{41}$ was carried out with Multiwfn and rendered with VMD. ${ }^{42}$ Both a lower resolution analysis of the full structure and a zoomed in, higher resolution, analysis of the arene region were carried out. Note: All exponential functions $\exp (\mathrm{x})$ with $\mathrm{x}<-40.000$ were ignored.

\section{TS-S-4f-1 - Full structure analysis}

Coordinate of origin in $X, Y, Z$ is $\quad-14.074444-10.775201-9.614868$ Bohr

Coordinate of end point in $X, Y, Z$ is 10.62555512 .23479811 .965131 Bohr

Grid spacing in $X, Y, Z$ is $0.130000 \quad 0.130000 \quad 0.130000$ Bohr

The number of points in X,Y,Z is 191178167 Total: 5677666 

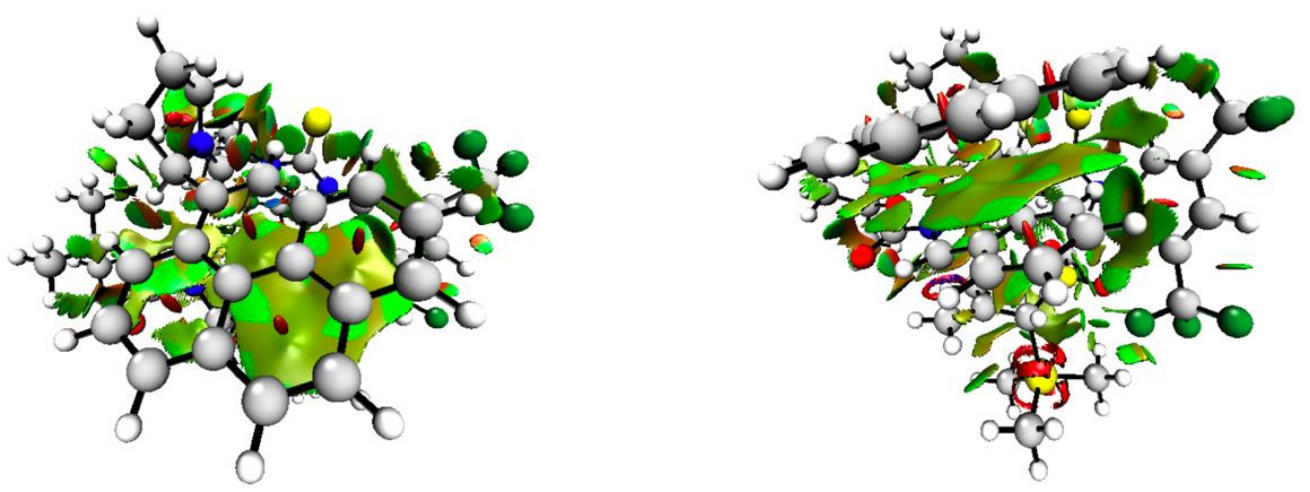

\section{TS-S-4f-1 - Arene localized analysis}

Coordinate of origin in $X, Y, Z$ is $\quad-5.350000-11.725000-5.885000$ Bohr Coordinate of end point in $X, Y, Z$ is $10.540000-0.805000 \quad 8.535000$ Bohr Grid spacing in $X, Y, Z$ is $0.070000 \quad 0.070000 \quad 0.070000$ Bohr

The number of points in $\mathrm{X}, \mathrm{Y}, \mathrm{Z}$ is 228157207 Total: 7409772
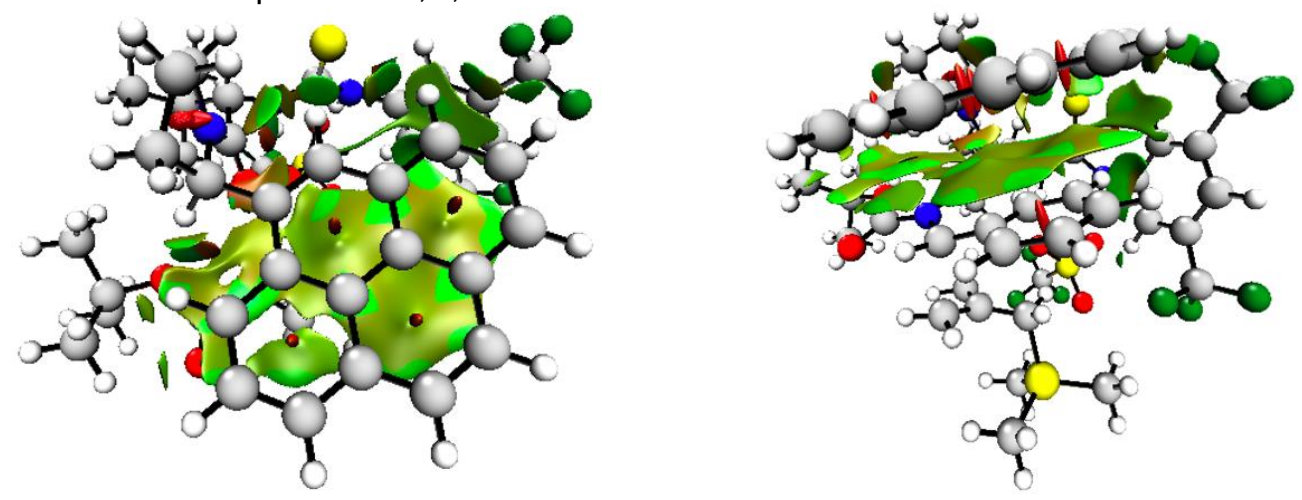

\section{TS-S-4f-nd-1 - Full structure analysis}

Coordinate of origin in $X, Y, Z$ is

Coordinate of end point in $X, Y, Z$ is

Grid spacing in $X, Y, Z$ is

The number of points in $X, Y, Z$ is

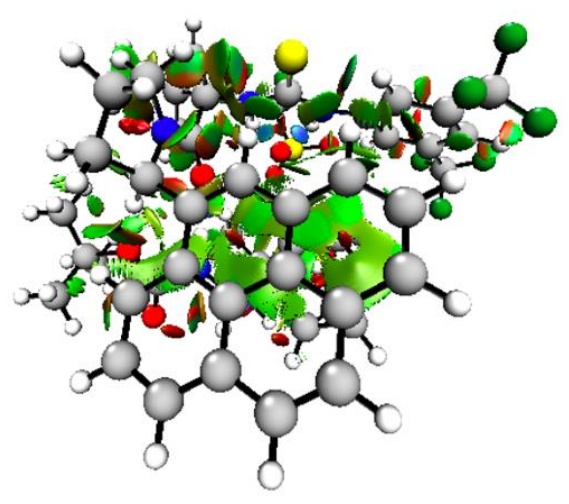

$-13.592900-11.491541-10.392827$ Bohr $12.66709912 .558458 \quad 12.227173 \mathrm{Bohr}$ $0.130000 \quad 0.130000 \quad 0.130000$ Bohr

203186175 Total: $\mathbf{6 6 0 7 6 5 0}$

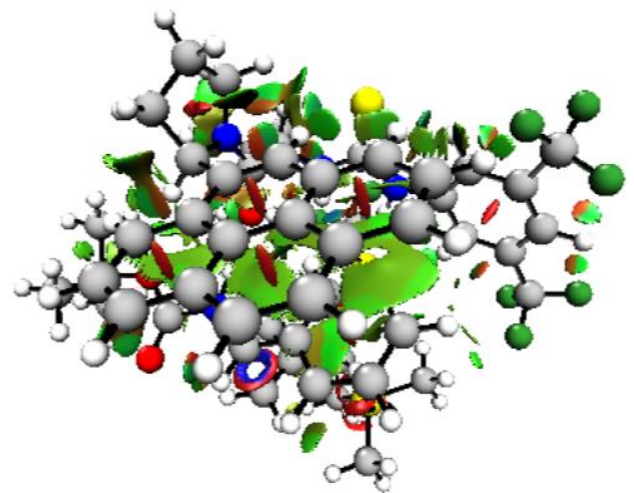




\section{TS-S-4f-nd-1 - Arene localized analysis}

The box of grid data was set visually using a GUI window.

Coordinate of origin in $X, Y, Z$ is $\quad-13.590000-3.390000-3.795000$ Bohr

Coordinate of end point in $\mathrm{X}, \mathrm{Y}, \mathrm{Z}$ is $\quad 2.340001 \quad 13.260001 \quad 8.895000 \mathrm{Bohr}$

Grid spacing in $X, Y, Z$ is

$0.090000 \quad 0.090000 \quad 0.090000$ Bohr

The number of points in $X, Y, Z$ is

178186142 Total: $\mathbf{4 7 0 1 3 3 6}$
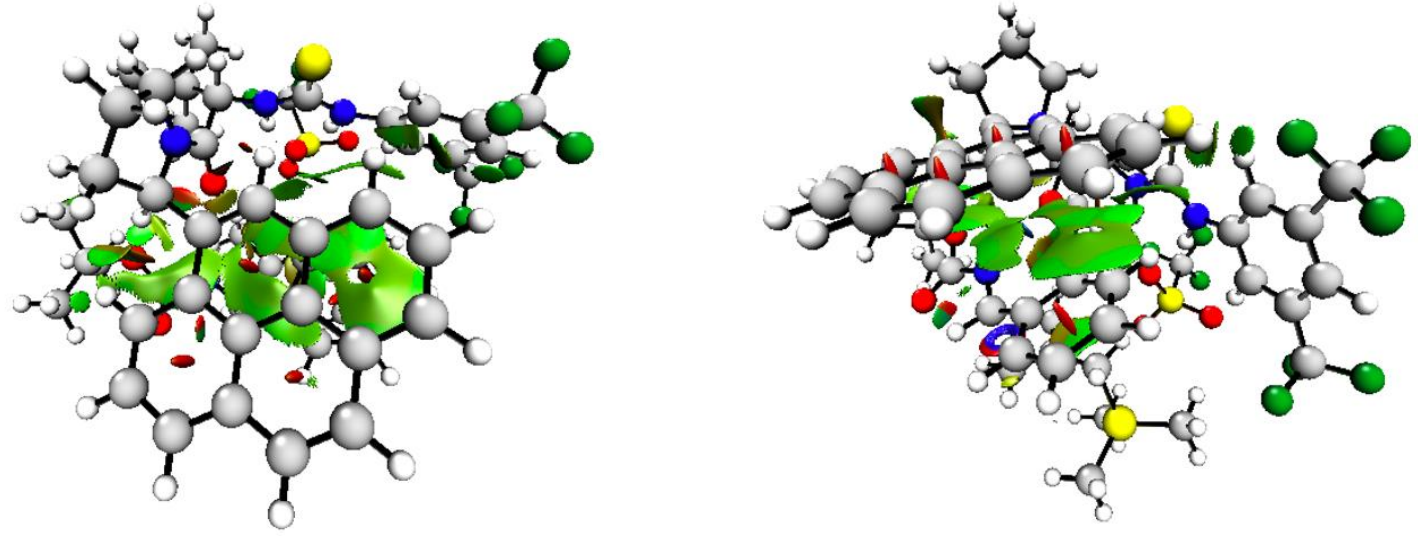

\section{TS- $R-4 f-1$ - Full structure analysis}

Coordinate of origin in $X, Y, Z$ is

Coordinate of end point in $X, Y, Z$ is

Grid spacing in $X, Y, Z$ is

The number of points in $X, Y, Z$ is

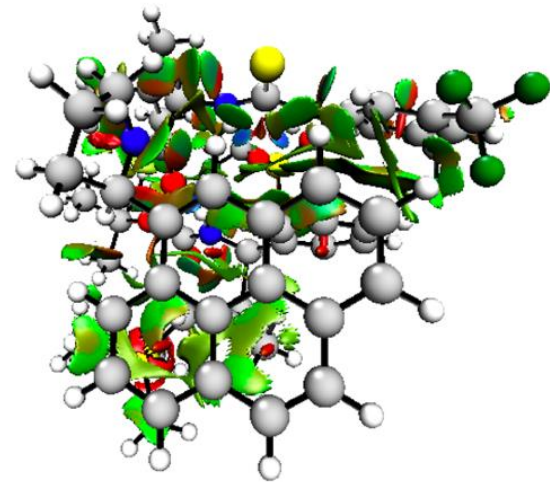

$-14.003811-12.198543-8.806203$ Bohr

13.556188 12.111456 11.213796 Bohr

$0.130000 \quad 0.130000 \quad 0.130000$ Bohr

213188155 Total: $\mathbf{6 2 0 6 8 2 0}$

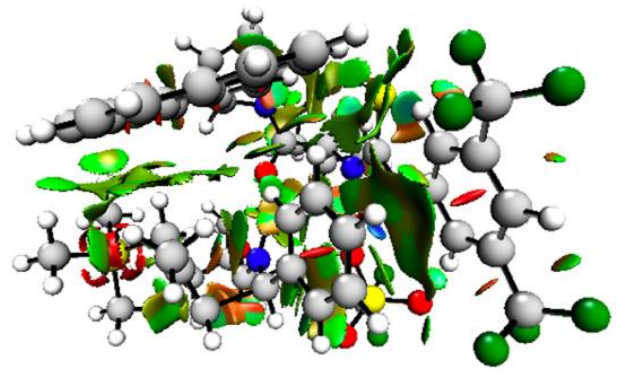

\section{TS- $R-4 f-1$ - Arene localized analysis}

The box of grid data was set visually using a GUI window.

Coordinate of origin in $X, Y, Z$ is

in $X, Y, Z$ is

$10.800001 \quad 12.650001$

Grid spacing in $X, Y, Z$ is

The number of points in $X, Y, Z$ is
$-7.200000-3.100000-6.910000$ Bohr Coordinate of end point

$5.240001 \mathrm{Bohr}$

$0.090000 \quad 0.090000 \quad 0.090000$ Bohr

201176136 Total: $\mathbf{4 8 1 1 1 3 6}$ 

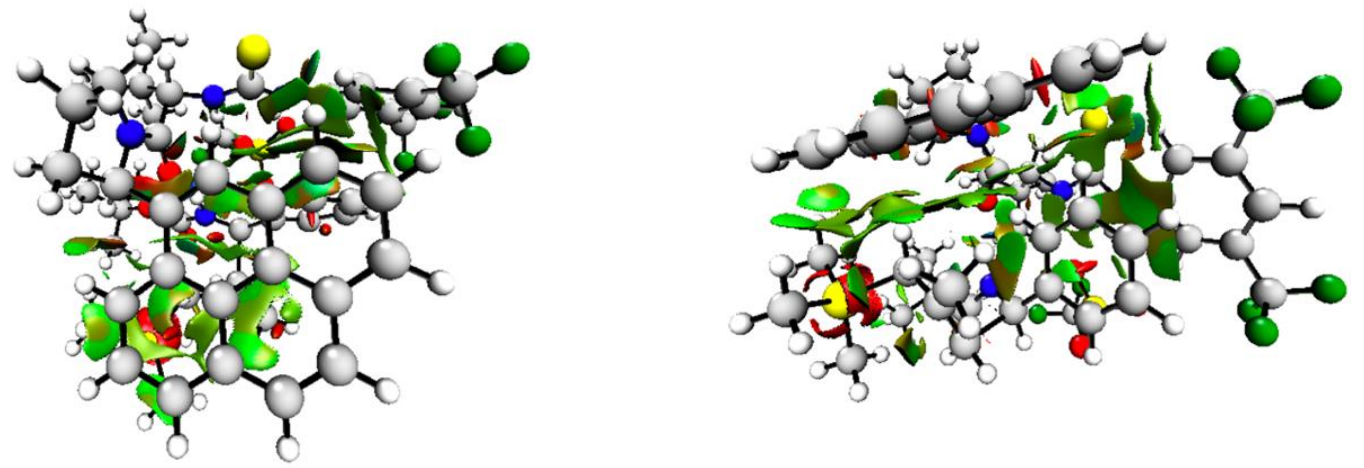

\section{TS-R-4f-nd-1 - Full structure analysis}

Coordinate of origin in $X, Y, Z$ is

Coordinate of end point in $X, Y, Z$ is

Grid spacing in $X, Y, Z$ is

The number of points in $X, Y, Z$ is

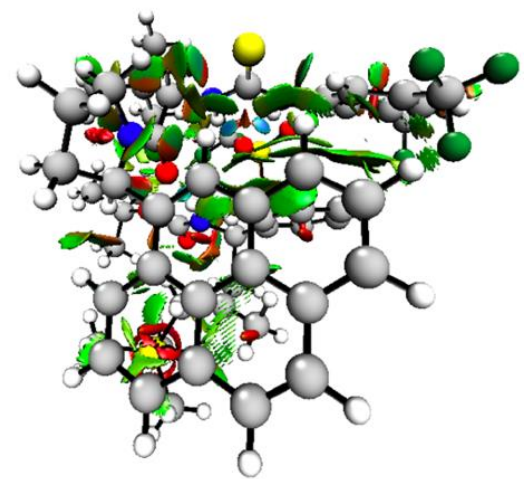

$-14.350501-12.303338-9.177251$ Bohr

$14.59950013 .64666311 .372750 \mathrm{Bohr}$

$0.150000 \quad 0.150000 \quad 0.150000$ Bohr

194174138 Total: 4658328

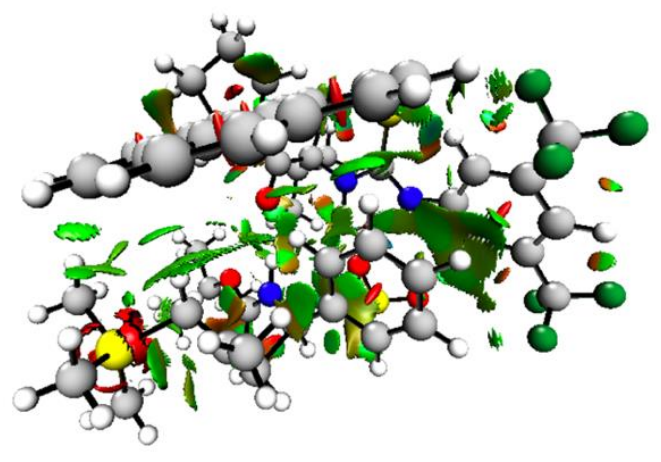

\section{TS- $R-4 f-n d-1$ - Arene localized analysis}

The box of grid data was set visually using a GUI window.

Coordinate of origin in $X, Y, Z$ is $\quad-6.450001-2.7000$

in $X, Y, Z$ is $\quad 11.58999914 .900000 \quad 5.180000$ Bohr

Grid spacing in $X, Y, Z$ is $\quad 0.110000 \quad 0.110000 \quad 0.110000$ Bohr

The number of points in $X, Y, Z$ is

165161147 Total: $\mathbf{3 9 0 5 0 5 5}$
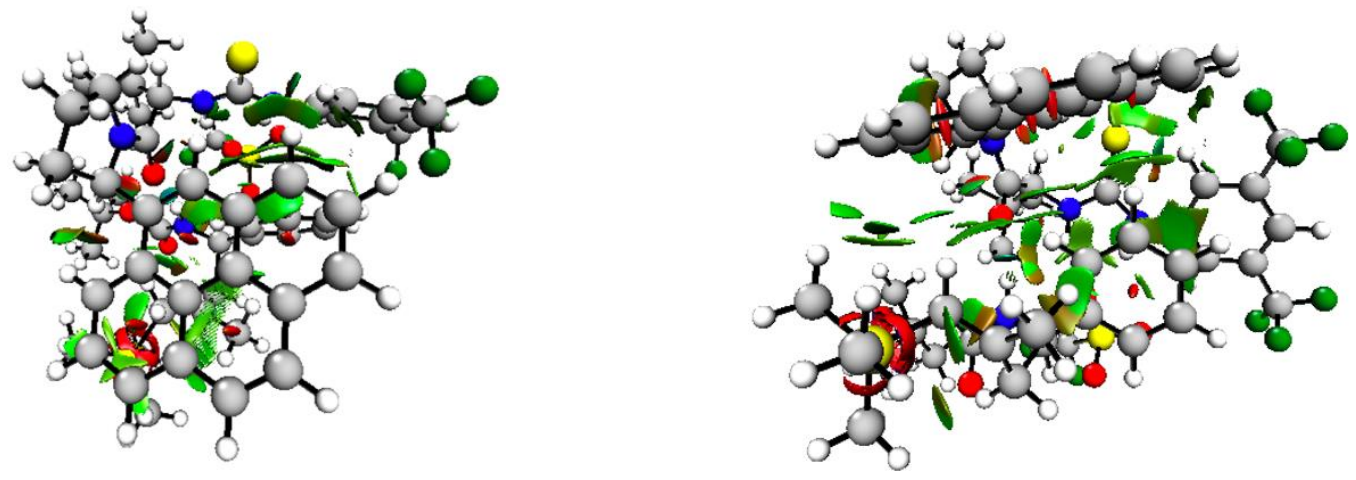


\section{Comparison of Thiourea and Squaramide (with dispersion correction)}

PyMOL was employed to overlap TS-S-4f-1 (yellow) with TS-S-6f-1 (blue) and TS- $\boldsymbol{R}-\mathbf{4 f - 1}$ (green) with TS$\boldsymbol{R}$-6f-1 (pink). This analysis revealed a significant resemblance of the lowest energy transition states for the two catalysts. The main difference observed is in the positioning of the bis-trifluoromethyl-phenyl moiety and of the triflate anion. The energetic gap between TS-S-4f-1 and TS- $R-4 f-1(\Delta E=5.62 \mathrm{kcal} / \mathrm{mol})$ is larger than that between TS-S-6f-1 and TS-R-6f-1 $(\Delta \mathrm{E}=4.93 \mathrm{kcal} / \mathrm{mol})$, consistent with the observed higher selectivity of the thiourea catalyst with substrate 1a (see S26, Entry 12 for the squaramide data).

Major transition states, front, side and top view.
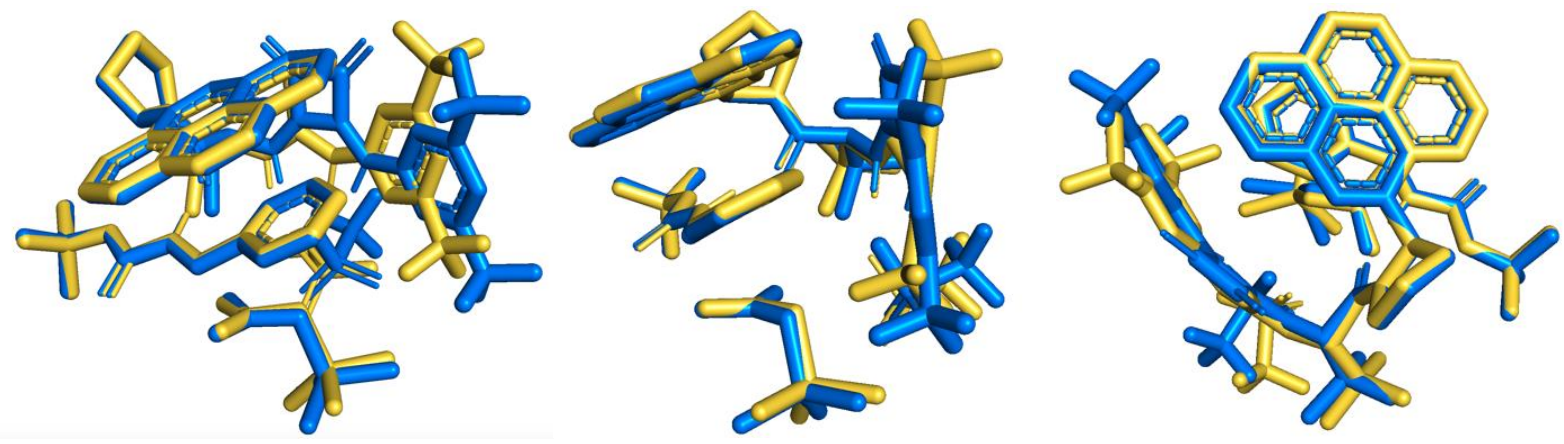

Minor transition states, front, side and top view
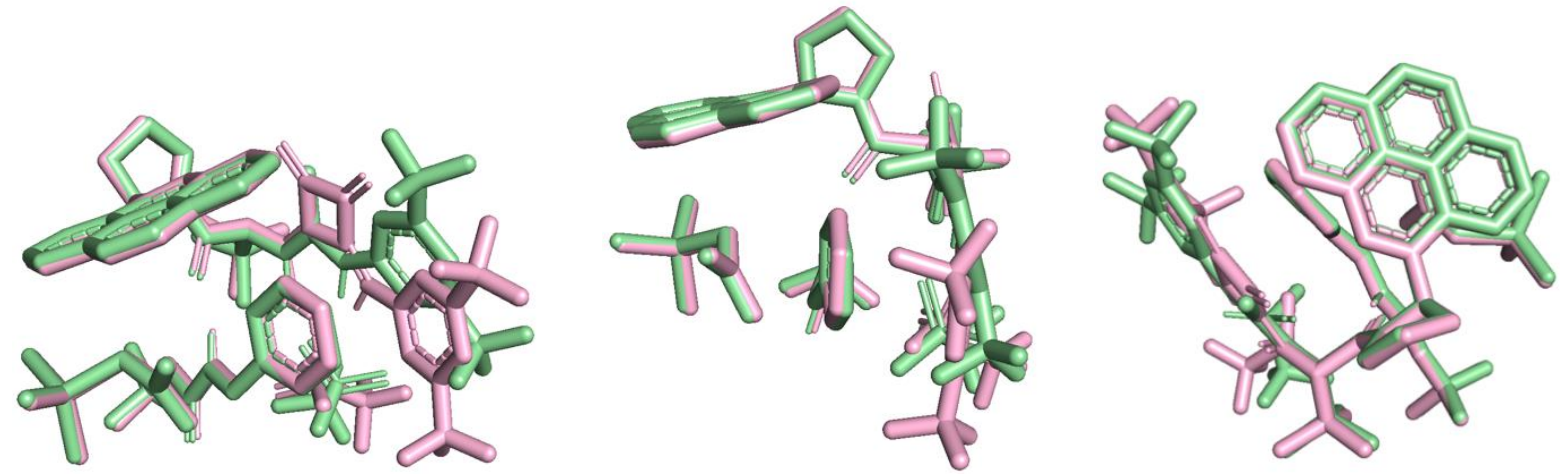


\section{Coordinates of computed transition states - Dispersion}

\section{TS-S-4f-1}

Imaginary frequency: $-198.36 \mathrm{~cm}^{-1}$

E[B3LYP/6-31G(d)-D3(BJ)]: -4789.106996 Hartree

E[PCM(Et $2 \mathrm{O})-B 3 L Y P / 6-311 G(d, p)-D 3(B J)]:-4790.242621$ Hartree

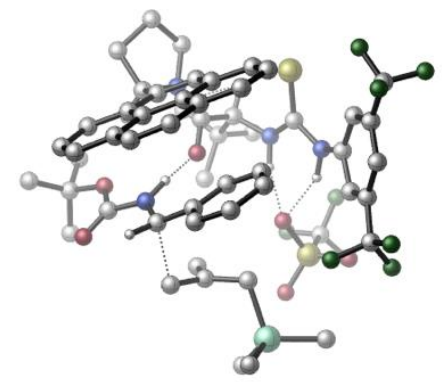

\begin{tabular}{|c|c|c|c|c|c|c|c|}
\hline 01 & & & & $\mathrm{~N}$ & 2.14091300 & -0.57321200 & -2.25284900 \\
\hline $\mathrm{H}$ & 2.21730100 & -1.40073400 & -1.65518800 & C & 2.94036800 & 0.48136400 & -1.79214600 \\
\hline $\mathrm{C}$ & 3.62873200 & 0.25233800 & -0.58888500 & $\mathrm{C}$ & 3.04887800 & 1.73245700 & -2.41595200 \\
\hline $\mathrm{C}$ & 4.32113900 & 1.29190900 & 0.02360000 & $\mathrm{H}$ & 3.59014300 & -0.72775000 & -0.12259300 \\
\hline C & 3.75210300 & 2.75543400 & -1.78173300 & $\mathrm{H}$ & 2.55189500 & 1.90963500 & -3.35682400 \\
\hline $\mathrm{C}$ & 4.38063500 & 2.55917500 & -0.55346300 & $\mathrm{H}$ & 4.90034900 & 3.36919800 & -0.05877200 \\
\hline $\mathrm{C}$ & 4.90557900 & 1.05005800 & 1.38612300 & $\mathrm{C}$ & 3.86099400 & 4.09259800 & -2.45919000 \\
\hline $\mathrm{F}$ & 5.68478100 & -0.04837500 & 1.41620500 & $\mathrm{~F}$ & 3.92162400 & 0.85527900 & 2.30985200 \\
\hline $\mathrm{F}$ & 5.64587700 & 2.09086300 & 1.82213700 & $\mathrm{~F}$ & 2.79435300 & 4.34819000 & -3.25394400 \\
\hline $\mathrm{F}$ & 3.93528500 & 5.10369600 & -1.55764600 & $\mathrm{~F}$ & 4.96030700 & 4.18156100 & -3.23722800 \\
\hline C & -1.17137300 & -1.74741000 & -3.13291900 & $\mathrm{H}$ & -1.27557800 & -1.35460000 & -4.14440100 \\
\hline $\mathrm{C}$ & -1.66324200 & -3.23135300 & -3.13613800 & C & -1.63622900 & -3.87200900 & -1.73521300 \\
\hline $\mathrm{H}$ & -2.30430300 & -3.35428500 & -1.04187900 & $\bar{H}$ & -0.63704800 & -3.87484300 & -1.29410600 \\
\hline $\mathrm{H}$ & -1.95851800 & -4.91662200 & -1.81418100 & C & -0.75692300 & -4.03128000 & -4.08917200 \\
\hline $\mathrm{H}$ & -1.13313500 & -5.05602400 & -4.18554000 & $\mathrm{H}$ & 0.27090900 & -4.08226400 & -3.72529200 \\
\hline $\mathrm{H}$ & -0.74085800 & -3.57962500 & -5.08796900 & $\mathrm{C}$ & -3.10499300 & -3.26954600 & -3.67158600 \\
\hline $\mathrm{H}$ & -3.47039300 & -4.30201100 & -3.68266500 & $\mathrm{H}$ & -3.16002200 & -2.88850700 & -4.69785600 \\
\hline $\mathrm{H}$ & -3.78789600 & -2.68280000 & -3.04776500 & $\mathrm{C}$ & -1.95443700 & -0.87632800 & -2.13710400 \\
\hline $\mathrm{C}$ & -3.77367000 & 0.66917600 & -1.61125800 & $\mathrm{H}$ & -4.08619700 & -0.02997300 & -0.83638600 \\
\hline $\mathrm{N}$ & -2.94739300 & -0.08350700 & -2.57377300 & C & -3.04577000 & 1.84074800 & -0.97247900 \\
\hline C & -1.94897400 & 2.39970800 & -1.55915700 & $\mathrm{C}$ & -3.54595600 & 2.36973100 & 0.28040100 \\
\hline C & -1.25743900 & 3.51622500 & -0.98605500 & $\mathrm{H}$ & -1.54014900 & 1.97163200 & -2.46830300 \\
\hline C & -2.84593200 & 3.45447600 & 0.89593700 & C & -4.66553200 & 1.83768400 & 0.94321500 \\
\hline$C$ & -0.12290700 & 4.07750400 & -1.59362200 & $\mathrm{C}$ & -1.71604400 & 4.03995000 & 0.25573000 \\
\hline $\mathrm{C}$ & -3.26817400 & 3.94743300 & 2.16911200 & $\mathrm{H}$ & -5.22923400 & 1.02976500 & 0.49316200 \\
\hline C & -5.05777200 & 2.30984000 & 2.19394100 & C & 0.54092400 & 5.14801300 & -0.99751400 \\
\hline $\mathrm{H}$ & 0.24649600 & 3.65327700 & -2.52216700 & C & -1.02385700 & 5.12749900 & 0.86490700 \\
\hline $\mathrm{C}$ & -4.36557400 & 3.34976500 & 2.80716600 & $\mathrm{C}$ & -2.54926600 & 5.04065200 & 2.76130500 \\
\hline $\mathrm{H}$ & -5.91087800 & 1.85829300 & 2.69165200 & C & 0.09541300 & 5.66958100 & 0.21540100 \\
\hline $\mathrm{H}$ & 1.42049500 & 5.56544000 & -1.47341100 & C & -1.48358800 & 5.61002000 & 2.13517900 \\
\hline $\mathrm{H}$ & -4.67708200 & 3.71685000 & 3.78157700 & $\mathrm{H}$ & -2.88307900 & 5.40983500 & 3.72771500 \\
\hline $\mathrm{H}$ & 0.62376400 & 6.49910700 & 0.67858700 & $\mathrm{H}$ & -0.95227200 & 6.43935700 & 2.59516700 \\
\hline $\mathrm{C}$ & 0.95777600 & -0.53310800 & -2.94912900 & $\mathrm{~S}$ & 0.42916000 & 0.73544600 & -3.93492000 \\
\hline $\mathrm{N}$ & 0.23181800 & -1.66940900 & -2.77403600 & $\mathrm{H}$ & 0.50714900 & -2.24455000 & -1.97770000 \\
\hline $\mathrm{S}$ & 2.58111500 & -3.67583200 & -0.08749800 & $\mathrm{O}$ & 3.93856400 & -3.10240400 & -0.08892000 \\
\hline $\mathrm{O}$ & 2.20757600 & -4.53160900 & 1.05184300 & 0 & 1.52669700 & -2.66037500 & -0.44189600 \\
\hline $\mathrm{C}$ & 2.53670400 & -4.79881000 & -1.56822200 & $\mathrm{~F}$ & 2.67102900 & -4.06799300 & -2.69199500 \\
\hline $\mathrm{F}$ & 1.35832300 & -5.44296100 & -1.63254300 & $\mathrm{~F}$ & 3.51619400 & -5.70199100 & -1.52118800 \\
\hline C & -3.62287600 & -1.08876400 & 2.00338700 & 0 & -4.03208300 & -1.16947500 & 3.14751600 \\
\hline $\mathrm{O}$ & -4.17279900 & -1.62373400 & 0.90266700 & $\mathrm{C}$ & -5.36726200 & -2.48570900 & 0.99063000 \\
\hline $\mathrm{C}$ & -5.04148700 & -3.71658100 & 1.83668500 & $\mathrm{H}$ & -5.87799900 & -4.42169200 & 1.79241800 \\
\hline $\mathrm{H}$ & -4.86838000 & -3.44437600 & 2.87884600 & $\mathrm{H}$ & -4.15078800 & -4.21832500 & 1.44398900 \\
\hline
\end{tabular}




\begin{tabular}{|c|c|c|c|c|c|c|c|}
\hline $\mathrm{C}$ & -5.60059100 & -2.87140900 & -0.46865900 & $\mathrm{H}$ & -5.76835400 & -1.97983600 & -1.08212300 \\
\hline $\mathrm{H}$ & -6.48169400 & -3.51556700 & -0.54761600 & $\mathrm{H}$ & -4.73741800 & -3.41028300 & -0.86891900 \\
\hline C & -6.54632100 & -1.68481400 & 1.54340200 & $\mathrm{H}$ & -7.44787500 & -2.30603900 & 1.52337100 \\
\hline $\mathrm{H}$ & -6.73187300 & -0.80060000 & 0.92409600 & $\mathrm{H}$ & -6.35976300 & -1.36803400 & 2.57009000 \\
\hline C & -1.73635800 & 0.23902000 & 2.60085800 & $\mathrm{H}$ & -2.33715000 & 0.52534300 & 3.45820200 \\
\hline C & -0.66416000 & 1.16235200 & 2.22661800 & C & -0.27641000 & 2.12518100 & 3.17562400 \\
\hline C & 0.01099900 & 1.09571700 & 0.99760900 & C & 0.76324600 & 3.00461300 & 2.90405800 \\
\hline $\mathrm{H}$ & -0.80611500 & 2.18306100 & 4.12274600 & C & 1.03301100 & 1.99931400 & 0.72391400 \\
\hline $\mathrm{H}$ & -0.26213200 & 0.35940500 & 0.25025700 & C & 1.42054200 & 2.94239200 & 1.67336900 \\
\hline $\mathrm{H}$ & 1.04680800 & 3.75003800 & 3.64024800 & $\mathrm{H}$ & 1.51821400 & 1.97593000 & -0.24039600 \\
\hline $\mathrm{H}$ & 2.22155100 & 3.63655600 & 1.44395700 & $\mathrm{~N}$ & -2.47146900 & -0.40634200 & 1.64620900 \\
\hline $\mathrm{H}$ & -2.16255000 & -0.48673800 & 0.66529100 & C & -0.82377200 & -1.16756300 & 3.82775000 \\
\hline $\mathrm{H}$ & -0.38199500 & -0.47937600 & 4.54274500 & $\mathrm{H}$ & -1.74550100 & -1.65233100 & 4.13632400 \\
\hline C & 0.00583600 & -1.80010600 & 2.90332200 & C & 1.38841200 & -1.39089900 & 2.70925000 \\
\hline $\mathrm{H}$ & 1.55738400 & -0.33803500 & 2.95599100 & $\mathrm{H}$ & 1.74537800 & -1.61380400 & 1.70259800 \\
\hline C & -0.55439200 & -2.85857500 & 2.01720400 & $\mathrm{H}$ & 0.17515500 & -3.65383900 & 1.83830000 \\
\hline $\mathrm{H}$ & -0.73894500 & -2.41411000 & 1.02717900 & $\mathrm{H}$ & -1.49028000 & -3.27194700 & 2.40156400 \\
\hline C & 4.26616600 & -2.42456800 & 3.31900300 & $\mathrm{H}$ & 4.29720200 & -2.89409200 & 2.33160800 \\
\hline $\mathrm{H}$ & 4.89151600 & -3.01689400 & 3.99899700 & $\mathrm{H}$ & 4.70709300 & -1.42904300 & 3.23287600 \\
\hline C & 1.83572400 & -4.07710800 & 4.31266800 & $\mathrm{H}$ & 1.87093800 & -4.67404200 & 3.39712700 \\
\hline $\mathrm{H}$ & 0.80353800 & -4.05225700 & 4.68088500 & $\mathrm{H}$ & 2.44655700 & -4.58116400 & 5.07158500 \\
\hline $\mathrm{C}$ & 2.44693900 & -1.32247900 & 5.57364900 & $\mathrm{H}$ & 1.43273400 & -1.27312800 & 5.98659100 \\
\hline $\mathrm{H}$ & 2.79226800 & -0.29767100 & 5.39372200 & $\mathrm{H}$ & 3.09256500 & -1.76037500 & 6.34441400 \\
\hline $\mathrm{Si}$ & 2.51889500 & -2.35452300 & 3.99213600 & C & -3.30020300 & 0.20640000 & -3.97715900 \\
\hline $\mathrm{H}$ & -3.76356200 & -0.67107500 & -4.43925800 & $\mathrm{H}$ & -2.40229300 & 0.47503500 & -4.53981300 \\
\hline C & -4.30475100 & 1.36032800 & -3.85542300 & $\mathrm{H}$ & -5.01969000 & 1.37151900 & -4.68209400 \\
\hline $\mathrm{H}$ & -3.77600900 & 2.31848800 & -3.84932200 & C & -4.96040900 & 1.11988500 & -2.48876200 \\
\hline $\mathrm{H}$ & -5.45567600 & 2.00477800 & -2.08308400 & $\mathrm{H}$ & -5.69857300 & 0.31058400 & -2.55593600 \\
\hline 0 & -1.65708100 & -0.95267900 & -0.93067500 & & & & \\
\hline
\end{tabular}

\section{TS-S-4f-2}

Imaginary frequency: $-302.91 \mathrm{~cm}^{-1}$ E[B3LYP/6-31G(d)-D3(BJ)]: -4789.107505 Hartree E[PCM(Et 2 O)-B3LYP/6-311G(d,p)-D3(BJ)]: - 4790.241663 Hartree

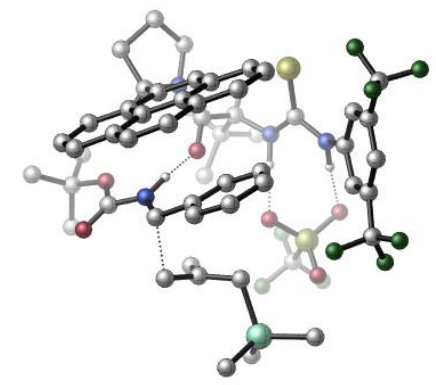

\begin{tabular}{|lrrr|lrrr|}
\hline 01 & & & & N & -2.25149600 & 0.52266100 & -2.66830800 \\
\hline $\mathrm{H}$ & -2.40145800 & 1.51250400 & -2.44349600 & $\mathrm{C}$ & -3.14146100 & -0.33864900 & -2.00753500 \\
\hline $\mathrm{C}$ & -3.80920300 & 0.17671800 & -0.88383200 & $\mathrm{C}$ & -3.35688300 & -1.67411300 & -2.37534300 \\
\hline $\mathrm{C}$ & -4.60490100 & -0.65488900 & -0.10283900 & $\mathrm{H}$ & -3.67391700 & 1.21223900 & -0.59476000 \\
\hline $\mathrm{C}$ & -4.15916700 & -2.48774800 & -1.57607700 & $\mathrm{H}$ & -2.87369800 & -2.07100000 & -3.25503000 \\
\hline $\mathrm{C}$ & -4.78134100 & -1.99874100 & -0.42939600 & $\mathrm{H}$ & -5.38724800 & -2.64458200 & 0.19345900 \\
\hline $\mathrm{C}$ & -5.15114300 & -0.12388300 & 1.19211700 & $\mathrm{C}$ & -4.40022600 & -3.91161500 & -1.99107300 \\
\hline $\mathrm{F}$ & -5.52216900 & 1.16654600 & 1.09920100 & $\mathrm{~F}$ & -4.21237300 & -0.19639200 & 2.17672000 \\
\hline $\mathrm{F}$ & -6.21797400 & -0.83090600 & 1.62392200 & $\mathrm{~F}$ & -3.37008600 & -4.41339300 & -2.71250000 \\
\hline $\mathrm{F}$ & -4.56569700 & -4.72294500 & -0.91575500 & $\mathrm{~F}$ & -5.50968800 & -4.03975100 & -2.74941100 \\
\hline $\mathrm{C}$ & 1.27264500 & 1.14661400 & -3.34836700 & $\mathrm{H}$ & 1.31775200 & 0.62510100 & -4.30465800 \\
\hline $\mathrm{C}$ & 1.92661900 & 2.55520200 & -3.53961700 & $\mathrm{C}$ & 2.08563400 & 3.33575000 & -2.22147700 \\
\hline $\mathrm{H}$ & 2.76244200 & 2.82608100 & -1.53035100 & $\mathrm{H}$ & 1.13463500 & 3.47823100 & -1.70681100 \\
\hline $\mathrm{H}$ & 2.49830100 & 4.32707000 & -2.44293700 & $\mathrm{C}$ & 1.04556000 & 3.36122800 & -4.51049200 \\
\hline
\end{tabular}




\begin{tabular}{|c|c|c|c|c|c|c|c|}
\hline$\overline{\mathrm{H}}$ & 1.53972600 & 4.30670100 & -4.76143500 & $\mathrm{H}$ & 0.07256400 & 3.59467700 & -4.07176100 \\
\hline $\mathrm{H}$ & 0.87429900 & 2.80887300 & -5.44182300 & C & 3.31855700 & 2.35879100 & -4.16576700 \\
\hline $\mathrm{H}$ & 3.79932800 & 3.33106700 & -4.31867500 & $\mathrm{H}$ & 3.25396600 & 1.86298600 & -5.14138400 \\
\hline $\mathrm{H}$ & 3.97315800 & 1.76449800 & -3.51825700 & C & 1.97825000 & 0.32654700 & -2.25831100 \\
\hline C & 3.60635100 & -1.35116000 & -1.54774100 & $\mathrm{H}$ & 4.02402100 & -0.60537400 & -0.87233500 \\
\hline $\mathrm{N}$ & 2.86243700 & -0.62799200 & -2.59645300 & C & 2.74627600 & -2.32321900 & -0.75582900 \\
\hline C & 1.56894000 & -2.79738300 & -1.25422000 & C & 3.20313800 & -2.74970000 & 0.55130500 \\
\hline C & 0.74965700 & -3.72682500 & -0.53446800 & $\mathrm{H}$ & 1.19821800 & -2.43891800 & -2.20865100 \\
\hline C & 2.37779500 & -3.63637400 & 1.31174900 & C & 4.40279200 & -2.29775000 & 1.12776000 \\
\hline C & -0.45883400 & -4.21202600 & -1.05890700 & C & 1.16405800 & -4.14103900 & 0.76280100 \\
\hline C & 2.76194400 & -4.01161800 & 2.63618700 & $\mathrm{H}$ & 5.05984500 & -1.64325500 & 0.56850000 \\
\hline C & 4.75931100 & -2.65085600 & 2.42749800 & C & -1.23852900 & -5.10506000 & -0.32636000 \\
\hline $\mathrm{H}$ & -0.78926500 & -3.86955900 & -2.03464300 & C & 0.35108500 & -5.04063000 & 1.51295000 \\
\hline C & 3.94699400 & -3.49352400 & 3.18040100 & C & 1.91696900 & -4.90886600 & 3.37375100 \\
\hline $\mathrm{H}$ & 5.67907400 & -2.26108900 & 2.85346600 & C & -0.83953100 & -5.51600600 & 0.94365100 \\
\hline $\mathrm{H}$ & -2.17038000 & -5.46922800 & -0.74184300 & C & 0.77161200 & -5.40819700 & 2.83421000 \\
\hline $\mathrm{H}$ & 4.23081100 & -3.76915100 & 4.19272300 & $\mathrm{H}$ & 2.22146800 & -5.18976000 & 4.37882500 \\
\hline $\mathrm{H}$ & -1.45885700 & -6.20435600 & 1.51328900 & $\mathrm{H}$ & 0.14681500 & -6.09225500 & 3.40299000 \\
\hline C & -0.99613800 & 0.22195500 & -3.14591400 & S & -0.56893600 & -1.23942000 & -3.88351000 \\
\hline $\mathrm{N}$ & -0.12955700 & 1.25323600 & -2.98661400 & $\mathrm{H}$ & -0.33787700 & 1.88980400 & -2.20978800 \\
\hline S & -2.08811400 & 3.56853900 & -0.78579200 & 0 & -2.90297500 & 3.67611400 & 0.43687900 \\
\hline 0 & -0.85648400 & 2.73692800 & -0.63218300 & $\mathrm{O}$ & -2.82535600 & 3.26939700 & -2.03770100 \\
\hline $\mathrm{C}$ & -1.42145600 & 5.28131200 & -1.04843300 & $\mathrm{~F}$ & -2.40955800 & 6.16377100 & -1.19750800 \\
\hline $\mathrm{F}$ & -0.63794400 & 5.31880700 & -2.13850900 & $\mathrm{~F}$ & -0.67428100 & 5.64931200 & 0.01292900 \\
\hline C & 3.75582700 & 0.81466800 & 1.81464300 & 0 & 4.20266400 & 0.95927600 & 2.93893900 \\
\hline 0 & 4.34759400 & 1.14667800 & 0.65600800 & C & 5.64811500 & 1.84101500 & 0.63422900 \\
\hline C & 5.51068400 & 3.19198200 & 1.33651900 & $\mathrm{H}$ & 6.43399100 & 3.76644700 & 1.20847600 \\
\hline $\mathrm{H}$ & 5.32409600 & 3.06327300 & 2.40379100 & $\mathrm{H}$ & 4.68816300 & 3.76399500 & 0.89427100 \\
\hline C & 5.89696900 & 2.02388900 & -0.86193700 & $\mathrm{H}$ & 5.93150900 & 1.05383600 & -1.36896600 \\
\hline $\mathrm{H}$ & 6.85343000 & 2.53118700 & -1.02145400 & $\mathrm{H}$ & 5.10331500 & 2.62353200 & -1.31626400 \\
\hline C & 6.72319100 & 0.95596300 & 1.26555800 & $\mathrm{H}$ & 7.69837400 & 1.44457400 & 1.16817500 \\
\hline $\mathrm{H}$ & 6.77615700 & -0.00850600 & 0.74924000 & $\mathrm{H}$ & 6.51937800 & 0.78324700 & 2.32282100 \\
\hline C & 1.72032300 & -0.18101400 & 2.56791500 & $\mathrm{H}$ & 2.30140900 & -0.51562600 & 3.42220700 \\
\hline C & 0.50693600 & -0.95002900 & 2.27489600 & C & 0.00037200 & -1.78065500 & 3.28999400 \\
\hline C & -0.18625300 & -0.84942300 & 1.05788000 & C & -1.17096300 & -2.50034700 & 3.09296800 \\
\hline $\mathrm{H}$ & 0.54281000 & -1.86907600 & 4.22743900 & C & -1.34392400 & -1.59617800 & 0.86003900 \\
\hline $\mathrm{H}$ & 0.16948900 & -0.20502200 & 0.26239400 & C & -1.84549300 & -2.41031300 & 1.87320700 \\
\hline $\mathrm{H}$ & -1.54441400 & -3.14907600 & 3.87892600 & $\mathrm{H}$ & -1.85018200 & -1.54659200 & -0.09339100 \\
\hline $\mathrm{H}$ & -2.74961600 & -2.98430500 & 1.70293000 & $\mathrm{~N}$ & 2.51236500 & 0.27244700 & 1.54545400 \\
\hline $\mathrm{H}$ & 2.19817200 & 0.27875800 & 0.56225500 & C & 1.09380700 & 1.40400600 & 3.70341900 \\
\hline $\mathrm{H}$ & 0.57176500 & 0.84406700 & 4.47413600 & $\mathrm{H}$ & 2.08597800 & 1.76158900 & 3.96336700 \\
\hline C & 0.33623300 & 2.11002300 & 2.76680600 & C & -1.09250900 & 1.89847400 & 2.63109700 \\
\hline $\mathrm{H}$ & -1.40989500 & 0.91675900 & 2.99907600 & $\mathrm{H}$ & -1.45535900 & 2.08090300 & 1.61739000 \\
\hline C & 1.03423500 & 3.01014000 & 1.80527100 & $\mathrm{H}$ & 0.41267400 & 3.85521400 & 1.50609200 \\
\hline $\mathrm{H}$ & 1.20725500 & 2.44245400 & 0.87795600 & $\mathrm{H}$ & 1.99745600 & 3.35774600 & 2.18773400 \\
\hline C & -3.89414300 & 2.85632200 & 3.44825000 & $\mathrm{H}$ & -4.13588900 & 3.05809000 & 2.40202600 \\
\hline $\mathrm{H}$ & -4.49891100 & 3.51665700 & 4.08200200 & $\mathrm{H}$ & -4.17342400 & 1.82170600 & 3.66746400 \\
\hline C & -1.55580900 & 4.92061700 & 3.35622800 & $\mathrm{H}$ & -1.70944500 & 5.13469900 & 2.29535800 \\
\hline $\mathrm{H}$ & -0.50518600 & 5.11084500 & 3.60400000 & $\mathrm{H}$ & -2.16395500 & 5.62774500 & 3.93342900 \\
\hline C & -1.62357900 & 2.74412100 & 5.54925200 & $\mathrm{H}$ & -0.56088100 & 2.91047500 & 5.75811600 \\
\hline $\mathrm{H}$ & -1.85660100 & 1.69963400 & 5.78902000 & $\mathrm{H}$ & -2.19850400 & 3.37442700 & 6.23840700 \\
\hline $\mathrm{Si}$ & -2.07037100 & 3.15798200 & 3.76018300 & C & 3.15045200 & -1.13457900 & -3.95209000 \\
\hline $\mathrm{H}$ & 3.71611100 & -0.38974000 & -4.52134900 & $\mathrm{H}$ & 2.21586900 & -1.35174400 & -4.47523800 \\
\hline
\end{tabular}




\begin{tabular}{|lrrr|llll|}
\hline $\mathrm{C}$ & 3.99725800 & -2.38628600 & -3.68615400 & $\mathrm{H}$ & 4.68792700 & -2.59764900 & -4.50656500 \\
\hline $\mathrm{H}$ & 3.34701000 & -3.25686500 & -3.55525900 & $\mathrm{C}$ & 4.70561700 & -2.06343100 & -2.36360600 \\
\hline $\mathrm{H}$ & 5.08850200 & -2.94747000 & -1.84895200 & $\mathrm{H}$ & 5.54237100 & -1.37463300 & -2.53652800 \\
\hline $\mathrm{O}$ & 1.72814200 & 0.59639700 & -1.06992100 & & & & \\
\hline
\end{tabular}

\section{TS-S-4f-3}

Imaginary frequency: $-325.20 \mathrm{~cm}^{-1}$

E[B3LYP/6-31G(d)-D3(BJ)]: -4789.104018 Hartree

E[PCM(Et $2 \mathrm{O})-B 3 L Y P / 6-311 \mathrm{G}(\mathrm{d}, \mathrm{p})-\mathrm{D} 3(\mathrm{BJ})]$ : - 4790.239074 Hartree

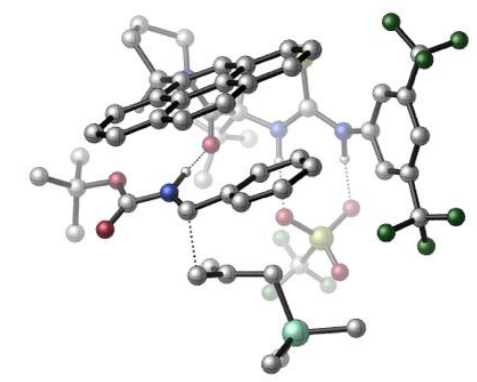

\begin{tabular}{|c|c|c|c|c|c|c|c|}
\hline 01 & & & & $\mathrm{~N}$ & -2.18593200 & 0.63035600 & -2.68309400 \\
\hline $\mathrm{H}$ & -2.28155200 & 1.62489800 & -2.45010400 & C & -3.14125700 & -0.18205700 & -2.05471400 \\
\hline $\mathrm{C}$ & -3.81639000 & 0.36916100 & -0.95171000 & $\mathrm{C}$ & -3.41908300 & -1.50255100 & -2.43344600 \\
\hline C & -4.68516700 & -0.41604800 & -0.20177900 & $\mathrm{H}$ & -3.63162300 & 1.39509000 & -0.65578700 \\
\hline C & -4.29547700 & -2.26926100 & -1.66593500 & $\mathrm{H}$ & -2.92982000 & -1.92420000 & -3.29833100 \\
\hline C & -4.92781500 & -1.74738000 & -0.53980300 & $\mathrm{H}$ & -5.59391000 & -2.35744800 & 0.05725400 \\
\hline C & -5.25122500 & 0.14054300 & 1.07416700 & C & -4.60986600 & -3.67231100 & -2.10255900 \\
\hline $\mathrm{F}$ & -5.48629000 & 1.46333700 & 0.99637000 & $\mathrm{~F}$ & -4.38565900 & -0.04825300 & 2.10888400 \\
\hline $\mathrm{F}$ & -6.40685500 & -0.46532900 & 1.42429100 & $\mathrm{~F}$ & -3.57926700 & -4.23987100 & -2.77288400 \\
\hline $\mathrm{F}$ & -4.88665400 & -4.47556100 & -1.04443400 & $\mathrm{~F}$ & -5.68337900 & -3.72140000 & -2.91967500 \\
\hline C & 1.36901500 & 1.06694200 & -3.32929500 & $\mathrm{H}$ & 1.37895200 & 0.56492500 & -4.29637600 \\
\hline $\mathrm{C}$ & 2.10480700 & 2.43912500 & -3.48238800 & C & 2.30259100 & 3.17351000 & -2.14288600 \\
\hline $\mathrm{H}$ & 2.94651000 & 2.60774900 & -1.46402900 & $\mathrm{H}$ & 1.35866800 & 3.35562600 & -1.62767600 \\
\hline $\mathrm{H}$ & 2.77143000 & 4.14562500 & -2.33595300 & $\mathrm{C}$ & 1.27654200 & 3.32038000 & -4.43433000 \\
\hline $\mathrm{H}$ & 1.82499200 & 4.24263200 & -4.65751100 & $\mathrm{H}$ & 0.31581800 & 3.59668400 & -3.99412900 \\
\hline $\mathrm{H}$ & 1.07970700 & 2.80364000 & -5.38093400 & C & 3.48694200 & 2.18425800 & -4.11011500 \\
\hline $\mathrm{H}$ & 4.02948700 & 3.13033600 & -4.21202200 & $\mathrm{H}$ & 3.40071100 & 1.74576500 & -5.11144500 \\
\hline $\mathrm{H}$ & 4.09874300 & 1.51837000 & -3.49095600 & C & 2.01533400 & 0.17670200 & -2.25494100 \\
\hline $\mathrm{O}$ & 1.76852600 & 0.43361600 & -1.06380000 & C & 3.21744500 & -1.26984500 & -3.94645300 \\
\hline C & 3.52256800 & -1.61990000 & -1.55420200 & C & 4.64277100 & -1.78123100 & -3.72907500 \\
\hline $\mathrm{H}$ & 2.53969200 & -2.06504900 & -4.28019100 & $\mathrm{H}$ & 3.16335400 & -0.45289600 & -4.66197900 \\
\hline $\mathrm{H}$ & 4.01787600 & -0.90193200 & -0.89905500 & $\mathrm{H}$ & 5.33444000 & -0.93048700 & -3.70354100 \\
\hline $\mathrm{H}$ & 4.96997400 & -2.46229700 & -4.51947400 & $\mathrm{~N}$ & 2.83799900 & -0.82914700 & -2.59988200 \\
\hline $\mathrm{C}$ & 2.58895200 & -2.49099600 & -0.73262400 & $\mathrm{C}$ & 1.38496600 & -2.89090100 & -1.23190900 \\
\hline C & 3.00993300 & -2.92520300 & 0.58256700 & C & 0.49814500 & -3.74503300 & -0.50006000 \\
\hline $\mathrm{H}$ & 1.05157200 & -2.53114700 & -2.20016600 & $\mathrm{C}$ & 2.12150600 & -3.74011700 & 1.35272100 \\
\hline $\mathrm{C}$ & 4.23680800 & -2.55077400 & 1.15719500 & $\mathrm{C}$ & -0.73930400 & -4.15374400 & -1.02272700 \\
\hline C & 0.87759200 & -4.16670300 & 0.80589900 & C & 2.47498600 & -4.12223600 & 2.68383400 \\
\hline $\mathrm{H}$ & 4.93914700 & -1.95232100 & 0.59039000 & C & 4.56353500 & -2.90768500 & 2.46334000 \\
\hline C & -1.58248400 & -4.97753200 & -0.27970700 & $\mathrm{H}$ & -1.03986700 & -3.80714000 & -2.00646900 \\
\hline $\mathrm{C}$ & 0.00144600 & -4.99662600 & 1.56542300 & $\mathrm{C}$ & 3.69149300 & -3.67998200 & 3.22535600 \\
\hline C & 1.56755800 & -4.94825400 & 3.43031100 & $\mathrm{H}$ & 5.50703800 & -2.57709200 & 2.88740100 \\
\hline C & -1.21773100 & -5.39576700 & 0.99839400 & $\mathrm{H}$ & -2.53600100 & -5.28267600 & -0.69388400 \\
\hline C & 0.39177100 & -5.37406200 & 2.89315100 & $\mathrm{H}$ & 3.95244800 & -3.96005300 & 4.24260700 \\
\hline $\mathrm{H}$ & 1.84888000 & -5.23579800 & 4.44026600 & $\mathrm{H}$ & -1.88557200 & -6.03044500 & 1.57549100 \\
\hline $\mathrm{H}$ & -0.28084000 & -6.00486800 & 3.46892800 & C & -0.94531200 & 0.26456500 & -3.15332400 \\
\hline $\mathrm{S}$ & -0.58585500 & -1.20476100 & -3.90854500 & $\mathrm{~N}$ & -0.02547500 & 1.24637600 & -2.96981400 \\
\hline $\mathrm{H}$ & -0.20293100 & 1.87832800 & -2.18203200 & S & -1.86987900 & 3.65037600 & -0.76965900 \\
\hline $\mathrm{O}$ & -2.69457800 & 3.79996400 & 0.44174800 & $\mathrm{O}$ & -0.68961200 & 2.74957200 & -0.60251300 \\
\hline
\end{tabular}




\begin{tabular}{|c|c|c|c|c|c|c|c|}
\hline $\mathrm{O}$ & -2.60460800 & 3.39966000 & -2.03351000 & C & -1.10452900 & 5.32414800 & -1.01563900 \\
\hline $\mathrm{F}$ & -2.03960400 & 6.25874900 & -1.18673500 & $\mathrm{~F}$ & -0.29487500 & 5.31887300 & -2.08708800 \\
\hline $\mathrm{F}$ & -0.36302400 & 5.65248900 & 0.06284100 & $\mathrm{C}$ & 3.79298700 & 0.58409500 & 1.82856600 \\
\hline 0 & 4.24664200 & 0.71521400 & 2.95192500 & 0 & 4.41012600 & 0.85585600 & 0.66672300 \\
\hline C & 5.75375200 & 1.46165600 & 0.64111400 & C & 5.70273500 & 2.83159300 & 1.31799600 \\
\hline $\mathrm{H}$ & 6.66246300 & 3.34166300 & 1.18506700 & $\mathrm{H}$ & 5.50314200 & 2.73504600 & 2.38632800 \\
\hline $\mathrm{H}$ & 4.92159300 & 3.44813200 & 0.86080500 & C & 6.02306800 & 1.60061600 & -0.85633500 \\
\hline $\mathrm{H}$ & 5.99481300 & 0.62153300 & -1.34609300 & $\mathrm{H}$ & 7.01259600 & 2.03913100 & -1.01797900 \\
\hline $\mathrm{H}$ & 5.27467400 & 2.24446200 & -1.32649200 & C & 6.76561000 & 0.51995800 & 1.29482200 \\
\hline $\mathrm{H}$ & 7.77083900 & 0.94302000 & 1.19602900 & $\mathrm{H}$ & 6.75945100 & -0.45475800 & 0.79531400 \\
\hline $\mathrm{H}$ & 6.54469000 & 0.37884500 & 2.35326100 & C & 1.69750200 & -0.27399600 & 2.59003500 \\
\hline $\mathrm{H}$ & 2.25729800 & -0.65086600 & 3.44114700 & C & 0.43442800 & -0.95955800 & 2.29733600 \\
\hline C & -0.13594800 & -1.73882500 & 3.31927900 & C & -0.24847500 & -0.81704300 & 1.07874000 \\
\hline C & -1.36114500 & -2.36422900 & 3.12871900 & $\mathrm{H}$ & 0.39729300 & -1.86059300 & 4.25824600 \\
\hline C & -1.46182000 & -1.47173400 & 0.88688000 & $\mathrm{H}$ & 0.15577900 & -0.20939100 & 0.27779700 \\
\hline C & -2.02710300 & -2.23240700 & 1.90783800 & $\mathrm{H}$ & -1.78530900 & -2.97287100 & 3.92103800 \\
\hline $\mathrm{H}$ & -1.96350000 & -1.38991600 & -0.06684000 & $\mathrm{H}$ & -2.97679500 & -2.72967200 & 1.74510900 \\
\hline $\mathrm{N}$ & 2.51585100 & 0.12632400 & 1.56446000 & $\mathrm{H}$ & 2.20774500 & 0.13014300 & 0.58006600 \\
\hline C & 1.17896300 & 1.33863900 & 3.72783500 & $\mathrm{H}$ & 0.62730100 & 0.80885700 & 4.49922300 \\
\hline $\mathrm{H}$ & 2.18972300 & 1.64006800 & 3.98711600 & C & 0.45946500 & 2.08966100 & 2.79533200 \\
\hline C & -0.97616000 & 1.94573400 & 2.65212700 & $\mathrm{H}$ & -1.33783000 & 0.97844600 & 3.01727900 \\
\hline $\mathrm{H}$ & -1.32328500 & 2.14378000 & 1.63572300 & C & 1.20522100 & 2.95548700 & 1.83888800 \\
\hline $\mathrm{H}$ & 0.62642300 & 3.82849400 & 1.53446900 & $\mathrm{H}$ & 1.35775600 & 2.37697400 & 0.91446000 \\
\hline $\mathrm{H}$ & 2.18164900 & 3.25663600 & 2.22718300 & C & -3.74747700 & 2.98511800 & 3.42881900 \\
\hline $\mathrm{H}$ & -3.96849900 & 3.21761100 & 2.38425000 & $\mathrm{H}$ & -4.34864300 & 3.64177100 & 4.06967100 \\
\hline $\mathrm{H}$ & -4.05375500 & 1.95143300 & 3.61607900 & $\mathrm{C}$ & -1.34077600 & 4.97944800 & 3.38115300 \\
\hline $\mathrm{H}$ & -1.47076500 & 5.20085300 & 2.31870400 & $\mathrm{H}$ & -0.28739900 & 5.13001400 & 3.64448200 \\
\hline $\bar{H}$ & -1.93065500 & 5.70729100 & 3.95147100 & $\mathrm{C}$ & -1.51008100 & 2.79927700 & 5.56486600 \\
\hline $\mathrm{H}$ & -0.44406500 & 2.92658300 & 5.78454100 & $\mathrm{H}$ & -1.78259900 & 1.76294800 & 5.79827200 \\
\hline $\mathrm{H}$ & -2.06855500 & 3.44742000 & 6.25097000 & $\mathrm{Si}$ & -1.92130100 & 3.23478900 & 3.77246700 \\
\hline $\mathrm{C}$ & 4.56127000 & -2.45605000 & -2.35171900 & $\bar{H}$ & 4.19346900 & -3.48132900 & -2.45448400 \\
\hline $\bar{H}$ & 5.52447100 & -2.50163800 & -1.83635100 & & & & \\
\hline
\end{tabular}

\section{TS-S-4f-4}

Imaginary frequency: $-393.98 \mathrm{~cm}^{-1}$

E[B3LYP/6-31G(d)-D3(BJ)]: -4789.096976 Hartree

E[PCM(Et2O)-B3LYP/6-311G(d,p)-D3(BJ)]: - 4790.236742 Hartree

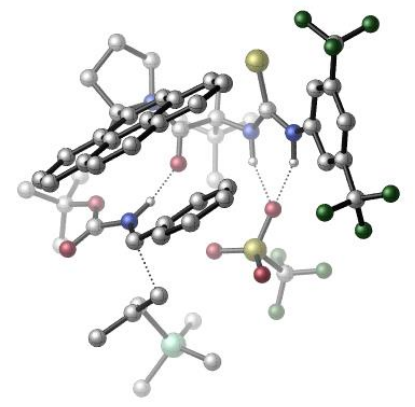

\begin{tabular}{|cccc|cccc|}
\hline 01 & & & $\mathrm{~N}$ & 2.38808200 & 1.68757200 & 1.95489000 \\
\hline $\mathrm{H}$ & 1.66702100 & 2.30551900 & 1.57921200 & $\mathrm{C}$ & 3.56980600 & 1.73591100 & 1.20648100 \\
\hline $\mathrm{C}$ & 3.54291900 & 2.58543300 & 0.08361100 & $\mathrm{C}$ & 4.72362300 & 0.98631200 & 1.46206300 \\
\hline $\mathrm{C}$ & 4.62618000 & 2.63497400 & -0.78411100 & $\mathrm{H}$ & 2.65102600 & 3.16591300 & -0.13129700 \\
\hline $\mathrm{C}$ & 5.79027900 & 1.03909500 & 0.56478900 & $\mathrm{H}$ & 4.76429700 & 0.34160600 & 2.32622900 \\
\hline $\mathrm{C}$ & 5.76127200 & 1.85295400 & -0.56236600 & $\mathrm{H}$ & 6.59269000 & 1.87535400 & -1.25573400 \\
\hline $\mathrm{C}$ & 4.53983500 & 3.44944600 & -2.04453800 & $\mathrm{C}$ & 7.00245900 & 0.19466200 & 0.84434000 \\
\hline $\mathrm{F}$ & 3.62478900 & 4.43218900 & -1.96030300 & $\mathrm{~F}$ & 4.19703000 & 2.67277300 & -3.10512000 \\
\hline $\mathrm{F}$ & 5.72794300 & 4.01602300 & -2.35311400 & $\mathrm{~F}$ & 7.65209800 & -0.13625300 & -0.29931000 \\
\hline $\mathrm{F}$ & 7.89616000 & 0.82150400 & 1.63793000 & $\mathrm{~F}$ & 6.67035900 & -0.96471800 & 1.45841600 \\
\hline $\mathrm{C}$ & -0.24724800 & -0.06308600 & 3.72745400 & $\mathrm{H}$ & 0.37442600 & -0.57623900 & 4.45912400 \\
\hline
\end{tabular}




\begin{tabular}{|c|c|c|c|c|c|c|c|}
\hline $\mathrm{C}$ & -1.45587200 & 0.56820000 & 4.48154000 & $\mathrm{C}$ & -2.35469500 & 1.41146600 & 3.56123600 \\
\hline $\mathrm{H}$ & -2.73343000 & 0.83721700 & 2.71362000 & $\mathrm{H}$ & -1.82612600 & 2.27721600 & 3.15547400 \\
\hline $\mathrm{H}$ & -3.20479900 & 1.79374800 & 4.13895600 & C & -0.89430300 & 1.45644300 & 5.60440300 \\
\hline $\mathrm{H}$ & -1.71419300 & 1.90591500 & 6.17603200 & $\mathrm{H}$ & -0.27965200 & 2.26336900 & 5.19543600 \\
\hline $\mathrm{H}$ & -0.27331500 & 0.87673900 & 6.29769100 & C & -2.28385300 & -0.56955100 & 5.10248900 \\
\hline $\mathrm{H}$ & -3.12773000 & -0.15565600 & 5.66510000 & $\mathrm{H}$ & -1.68365200 & -1.16767600 & 5.79838800 \\
\hline $\mathrm{H}$ & -2.69144900 & -1.23839600 & 4.33714300 & C & -0.66673400 & -1.02637200 & 2.60789300 \\
\hline C & 0.03180500 & -3.05588400 & 3.95172500 & C & -0.84031600 & -3.26697000 & 1.66605800 \\
\hline $\mathrm{H}$ & 0.97186600 & -2.59620100 & 4.26715100 & $\mathrm{H}$ & -0.68350000 & -3.01243400 & 4.78079300 \\
\hline $\mathrm{H}$ & -1.82084100 & -2.98703900 & 1.28460300 & $\mathrm{~N}$ & -0.51625100 & -2.35406100 & 2.77580000 \\
\hline C & 0.18185500 & -3.21204800 & 0.54032100 & C & 1.43395900 & -2.71166800 & 0.74461900 \\
\hline C & -0.18604400 & -3.72641300 & -0.76325600 & C & 2.42692800 & -2.68024800 & -0.28788400 \\
\hline $\mathrm{H}$ & 1.70448100 & -2.28932600 & 1.70684600 & C & 0.78460900 & -3.69852100 & -1.81414600 \\
\hline C & -1.46661700 & -4.22649600 & -1.05416500 & C & 3.70788000 & -2.14962400 & -0.06762100 \\
\hline C & 2.09254800 & -3.18870000 & -1.57446200 & C & 0.44032400 & -4.16676000 & -3.11935800 \\
\hline $\mathrm{H}$ & -2.22021800 & -4.27453900 & -0.27806000 & C & -1.80416000 & -4.65222900 & -2.33767500 \\
\hline C & 4.64724300 & -2.12082300 & -1.09698100 & $\mathrm{H}$ & 3.95626100 & -1.75638700 & 0.91278800 \\
\hline C & 3.06111400 & -3.16114700 & -2.62032300 & C & -0.86306100 & -4.62632900 & -3.36226700 \\
\hline C & 1.43953100 & -4.14008000 & -4.15054800 & $\mathrm{H}$ & -2.81200300 & -5.00615900 & -2.53318800 \\
\hline $\mathrm{C}$ & 4.33028300 & -2.62303600 & -2.35755900 & $\mathrm{H}$ & 5.63282700 & -1.70600200 & -0.91591300 \\
\hline C & 2.69256600 & -3.66602100 & -3.91144200 & $\mathrm{H}$ & -1.12708200 & -4.96923000 & -4.35943000 \\
\hline $\mathrm{H}$ & 1.16871000 & -4.51046400 & -5.13611800 & $\mathrm{H}$ & 5.06806300 & -2.59777400 & -3.15559300 \\
\hline $\mathrm{H}$ & 3.43703200 & -3.65078600 & -4.70352400 & $\mathrm{C}$ & 1.90365600 & 0.75442900 & 2.83801800 \\
\hline S & 2.80456100 & -0.48585900 & 3.55656500 & $\mathrm{~N}$ & 0.58281800 & 0.95299300 & 3.09987100 \\
\hline $\mathrm{H}$ & 0.08420200 & 1.54783800 & 2.43241800 & $\mathrm{~S}$ & -1.14581000 & 3.04845800 & 0.04965600 \\
\hline 0 & -0.74508400 & 3.35624300 & -1.33312600 & $\mathrm{O}$ & -2.28023800 & 2.10765700 & 0.19048900 \\
\hline 0 & -0.01281900 & 2.77724100 & 0.98624600 & C & -1.80106000 & 4.65109300 & 0.73407900 \\
\hline $\mathrm{F}$ & -0.82659700 & 5.56144100 & 0.81795400 & $\mathrm{~F}$ & -2.31550200 & 4.45793300 & 1.96176000 \\
\hline $\mathrm{F}$ & -2.77696700 & 5.14503900 & -0.04772200 & $\mathrm{C}$ & -3.58749100 & -1.75808000 & -0.67443300 \\
\hline 0 & -4.29531900 & -2.15794300 & -1.58494000 & $\mathrm{O}$ & -3.80634500 & -1.90212000 & 0.64118700 \\
\hline C & -5.02552900 & -2.54955100 & 1.15196100 & C & -6.25588900 & -1.76516000 & 0.69517600 \\
\hline $\mathrm{H}$ & -7.14637400 & -2.15965300 & 1.19551600 & $\mathrm{H}$ & -6.39558900 & -1.84667900 & -0.38378200 \\
\hline $\mathrm{H}$ & -6.15073500 & -0.70942600 & 0.96595700 & C & -4.83825300 & -2.44070900 & 2.66374300 \\
\hline $\mathrm{H}$ & -3.92559900 & -2.95884700 & 2.97529900 & $\mathrm{H}$ & -5.68943100 & -2.89333300 & 3.18175100 \\
\hline $\mathrm{H}$ & -4.75851900 & -1.39263500 & 2.96632700 & C & -5.06432700 & -4.01135700 & 0.70572300 \\
\hline $\mathrm{H}$ & -5.93392000 & -4.50597200 & 1.15117900 & $\mathrm{H}$ & -4.16642100 & -4.53838100 & 1.04597500 \\
\hline $\mathrm{H}$ & -5.13419600 & -4.08867400 & -0.38017000 & C & -1.92592600 & -0.85930800 & -2.13234200 \\
\hline $\mathrm{H}$ & -2.36472500 & -1.53823200 & -2.85593600 & C & -0.49718700 & -0.55907400 & -2.31914700 \\
\hline C & 0.06554200 & -0.80455500 & -3.58307600 & C & 0.29583900 & -0.00185600 & -1.30657900 \\
\hline C & 1.39360200 & -0.48821800 & -3.83624700 & $\mathrm{H}$ & -0.54583800 & -1.25472600 & -4.36068000 \\
\hline C & 1.62959500 & 0.29221700 & -1.56436600 & $\mathrm{H}$ & -0.10918900 & 0.20155000 & -0.32341700 \\
\hline C & 2.17991300 & 0.06931800 & -2.82514700 & $\mathrm{H}$ & 1.82040600 & -0.69186100 & -4.81278200 \\
\hline $\mathrm{H}$ & 2.24406500 & 0.69739200 & -0.77408800 & $\mathrm{H}$ & 3.21607400 & 0.32523900 & -3.01304000 \\
\hline $\mathrm{N}$ & -2.40192200 & -1.07891800 & -0.86323600 & $\mathrm{H}$ & -1.89089400 & -0.78494200 & -0.01957700 \\
\hline C & -2.68550600 & 0.84072500 & -2.88373500 & $\mathrm{H}$ & -2.17762600 & 1.51031300 & -2.19750100 \\
\hline $\mathrm{H}$ & -2.26376700 & 0.78957500 & -3.88341100 & C & -4.06482600 & 0.66756700 & -2.74381000 \\
\hline C & -4.72109700 & 1.03665500 & -1.50206000 & $\mathrm{H}$ & -4.02515200 & 1.02917100 & -0.65766600 \\
\hline $\mathrm{H}$ & -5.62094300 & 0.44959400 & -1.29915200 & C & -4.87750000 & 0.05814400 & -3.84417300 \\
\hline $\mathrm{H}$ & -5.80381500 & 0.62397100 & -3.99535900 & $\mathrm{H}$ & -5.16333800 & -0.95785500 & -3.54887200 \\
\hline $\mathrm{H}$ & -4.32856300 & 0.01290100 & -4.78815000 & $\mathrm{C}$ & -5.45051200 & 3.40832100 & 0.26311100 \\
\hline $\mathrm{H}$ & -6.23821300 & 2.82523600 & 0.75551600 & $\mathrm{H}$ & -5.71181100 & 4.46892500 & 0.35324600 \\
\hline $\mathrm{H}$ & -4.51138400 & 3.24100400 & 0.79565100 & C & -6.99056300 & 2.94394500 & -2.37383000 \\
\hline $\mathrm{H}$ & -7.70660200 & 2.28980700 & -1.86276200 & $\mathrm{H}$ & -6.94268200 & 2.63744300 & -3.42532600 \\
\hline
\end{tabular}




\begin{tabular}{|rrrr|lrrr|}
\hline $\mathrm{H}$ & -7.39961600 & 3.96159100 & -2.35245300 & $\mathrm{C}$ & -4.08998800 & 3.94140800 & -2.54845700 \\
\hline $\mathrm{H}$ & -4.15650800 & 3.67615000 & -3.61039400 & $\mathrm{H}$ & -3.05076300 & 3.82201700 & -2.23300200 \\
\hline $\mathrm{H}$ & -4.34414200 & 5.00381200 & -2.45556000 & $\mathrm{Si}$ & -5.29219200 & 2.90852400 & -1.54257600 \\
\hline $\mathrm{C}$ & -0.87028900 & -4.63889800 & 2.37114600 & $\mathrm{H}$ & -0.68454700 & -5.46118200 & 1.67664300 \\
\hline $\mathrm{H}$ & -1.85327000 & -4.79364800 & 2.83450000 & $\mathrm{C}$ & 0.21277100 & -4.49508200 & 3.44928800 \\
\hline $\mathrm{H}$ & 0.12131300 & -5.22725300 & 4.25577800 & $\mathrm{H}$ & 1.20257100 & -4.60894600 & 2.99641300 \\
\hline $\mathrm{O}$ & -1.10293800 & -0.54684700 & 1.55077300 & & & & \\
\hline
\end{tabular}

\section{TS-S-4f-5}

Imaginary frequency: $-300.35 \mathrm{~cm}^{-1}$

E[B3LYP/6-31G(d)-D3(BJ)]: -4789.097160 Hartree

E[PCM(Et $\left.\left.t_{2} \mathrm{O}\right)-B 3 L Y P / 6-311 \mathrm{G}(\mathrm{d}, \mathrm{p})-\mathrm{D} 3(\mathrm{BJ})\right]$ : - 4790.236586 Hartree

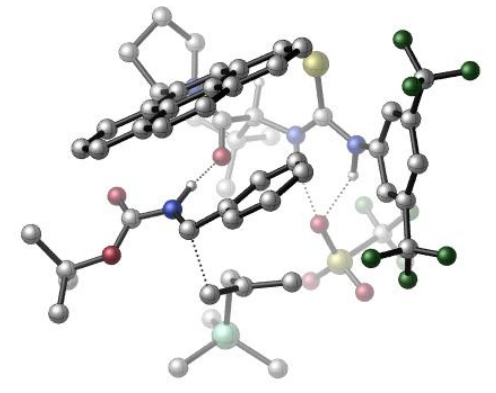

\begin{tabular}{|c|c|c|c|c|c|c|c|}
\hline 01 & & & & $\mathrm{~N}$ & 2.88824100 & 1.08246000 & 1.46001300 \\
\hline $\mathrm{H}$ & 2.57461200 & 1.95769900 & 1.03751700 & C & 3.58069000 & 0.29964000 & 0.52541100 \\
\hline $\mathrm{C}$ & 3.56594900 & 0.77506600 & -0.79754500 & $\mathrm{C}$ & 4.22549000 & -0.90906000 & 0.81611700 \\
\hline C & 4.12474400 & 0.01699200 & -1.81774200 & $\mathrm{H}$ & 3.07788400 & 1.71569100 & -1.02822400 \\
\hline $\mathrm{C}$ & 4.77248200 & -1.65598600 & -0.22898400 & $\mathrm{H}$ & 4.25038000 & -1.27646100 & 1.83056100 \\
\hline $\mathrm{C}$ & 4.72869200 & -1.21116400 & -1.54852700 & $\mathrm{H}$ & 5.13912500 & -1.81220200 & -2.35109500 \\
\hline C & 3.93592000 & 0.44634500 & -3.24598300 & C & 5.42256300 & -2.97944300 & 0.06052100 \\
\hline $\mathrm{F}$ & 3.69099800 & 1.76441200 & -3.35991700 & $\mathrm{~F}$ & 2.87769800 & -0.20076200 & -3.80656200 \\
\hline $\mathrm{F}$ & 5.01250600 & 0.15297800 & -4.00738800 & $\mathrm{~F}$ & 5.04658300 & -3.48642600 & 1.25644000 \\
\hline $\mathrm{F}$ & 5.09396700 & -3.90043700 & -0.88370500 & $\mathrm{~F}$ & 6.76994600 & -2.89859900 & 0.06807100 \\
\hline C & -0.08826900 & 1.27221400 & 3.53167500 & $\mathrm{H}$ & 0.26543800 & 0.85214400 & 4.47404100 \\
\hline $\mathrm{C}$ & -0.88139000 & 2.58489000 & 3.84730100 & $\mathrm{C}$ & -1.48362400 & 3.24161200 & 2.58915600 \\
\hline $\mathrm{H}$ & -2.19726200 & 2.58071100 & 2.09031100 & $\mathrm{H}$ & -0.72357200 & 3.53395500 & 1.85990400 \\
\hline $\mathrm{H}$ & -2.01030900 & 4.15599500 & 2.88493300 & C & 0.08675100 & 3.57191300 & 4.52443600 \\
\hline $\mathrm{H}$ & -0.45990500 & 4.46519500 & 4.84667700 & $\mathrm{H}$ & 0.87992900 & 3.89058700 & 3.84381400 \\
\hline $\mathrm{H}$ & 0.55654500 & 3.12335300 & 5.40779100 & C & -2.02206000 & 2.25070800 & 4.82347200 \\
\hline $\mathrm{H}$ & -2.57916700 & 3.16141900 & 5.06717700 & $\mathrm{H}$ & -1.63685800 & 1.83760700 & 5.76298400 \\
\hline $\mathrm{H}$ & -2.73074200 & 1.53531800 & 4.39195800 & C & -0.94719400 & 0.24468500 & 2.77474200 \\
\hline C & -2.44996300 & -1.68715000 & 2.75302200 & $\mathrm{H}$ & -3.15915300 & -1.07411400 & 2.19579400 \\
\hline $\mathrm{N}$ & -1.51931800 & -0.77155800 & 3.44168900 & C & -1.75032200 & -2.66171400 & 1.81786100 \\
\hline C & -0.42270800 & -2.94030800 & 1.95705100 & $\mathrm{C}$ & -2.52227400 & -3.32546200 & 0.78622900 \\
\hline C & 0.25969700 & -3.87809000 & 1.11680900 & $\mathrm{H}$ & 0.16840200 & -2.41025000 & 2.69532300 \\
\hline $\mathrm{C}$ & -1.85524000 & -4.23891300 & -0.09100400 & C & -3.89635200 & -3.09820500 & 0.59309900 \\
\hline $\mathrm{C}$ & 1.63089600 & -4.14302200 & 1.26192800 & $C$ & -0.47091700 & -4.52662900 & 0.08220600 \\
\hline C & -2.57496000 & -4.86687100 & -1.15397300 & $\mathrm{H}$ & -4.43272900 & -2.42176800 & 1.24741800 \\
\hline C & -4.58881900 & -3.71753300 & -0.44623400 & $\mathrm{C}$ & 2.27055700 & -5.04413800 & 0.41329300 \\
\hline $\mathrm{H}$ & 2.19356500 & -3.61616500 & 2.02668200 & $C$ & 0.19318100 & -5.44038000 & -0.78819300 \\
\hline C & -3.93886400 & -4.58476000 & -1.31890400 & C & -1.87903800 & -5.77604800 & -2.02117100 \\
\hline $\bar{H}$ & -5.65143500 & -3.52884600 & -0.56500300 & $\mathrm{C}$ & 1.56098600 & -5.68829600 & -0.59830100 \\
\hline $\mathrm{H}$ & 3.33205000 & -5.22822700 & 0.52952300 & $\mathrm{C}$ & -0.55935600 & -6.05549900 & -1.84313300 \\
\hline $\mathrm{H}$ & -4.48606200 & -5.06469600 & -2.12627500 & $\mathrm{H}$ & -2.43885600 & -6.24647200 & -2.82561000 \\
\hline $\bar{H}$ & 2.06864300 & -6.38343100 & -1.26224500 & $\mathrm{H}$ & -0.04803300 & -6.74968900 & -2.50524700 \\
\hline $\mathrm{C}$ & 2.05228600 & 0.66542400 & 2.47264300 & $\mathrm{~S}$ & 2.21808900 & -0.76920500 & 3.34711100 \\
\hline $\mathrm{N}$ & 1.07144800 & 1.57031900 & 2.71402800 & $\mathrm{H}$ & 0.91373600 & 2.25505300 & 1.97340700 \\
\hline $\mathrm{S}$ & 1.56302600 & 4.39315200 & -0.23559500 & $\mathrm{O}$ & 0.48962800 & 5.37048100 & 0.00605800 \\
\hline $\mathrm{O}$ & 1.24222800 & 3.01623500 & 0.28725900 & $\mathrm{O}$ & 2.16181400 & 4.36142100 & -1.58189900 \\
\hline
\end{tabular}




\begin{tabular}{|c|c|c|c|c|c|c|c|}
\hline $\mathrm{C}$ & 2.94490000 & 4.91304100 & 0.89349100 & $\mathrm{~F}$ & 3.41656900 & 6.11398400 & 0.55882600 \\
\hline $\mathrm{F}$ & 3.94762200 & 4.01666500 & 0.82400900 & $\mathrm{~F}$ & 2.51404500 & 4.95574100 & 2.16632400 \\
\hline $\mathrm{C}$ & -3.99605600 & 0.08291200 & -0.33306400 & 0 & -4.39602200 & 0.29859300 & 0.79715200 \\
\hline 0 & -4.71298000 & 0.16104200 & -1.46479400 & $\mathrm{C}$ & -6.15997600 & 0.47202500 & -1.42910500 \\
\hline C & -6.89764200 & -0.66193900 & -0.72013900 & $\mathrm{H}$ & -7.97663900 & -0.48324400 & -0.77112600 \\
\hline $\mathrm{H}$ & -6.60389800 & -0.72365100 & 0.32911000 & $\mathrm{H}$ & -6.68349700 & -1.61616200 & -1.21022400 \\
\hline C & -6.51642300 & 0.52247800 & -2.91303000 & $\mathrm{H}$ & -5.95922300 & 1.31769900 & -3.41853300 \\
\hline $\mathrm{H}$ & -7.58577200 & 0.72143600 & -3.03227700 & $\mathrm{H}$ & -6.28437500 & -0.43059600 & -3.39859000 \\
\hline C & -6.39850400 & 1.82675900 & -0.76164400 & $\mathrm{H}$ & -7.45243900 & 2.10030600 & -0.87808600 \\
\hline $\mathrm{H}$ & -5.79490300 & 2.60387500 & -1.24041300 & $\mathrm{H}$ & -6.15537600 & 1.79543300 & 0.30007800 \\
\hline C & -2.20284300 & -0.70144400 & -1.80310700 & $\mathrm{H}$ & -2.97961800 & -1.11112600 & -2.43980700 \\
\hline C & -0.90768200 & -1.39056000 & -1.87773100 & C & -0.69175700 & -2.25969200 & -2.96145300 \\
\hline C & 0.11870500 & -1.19076800 & -0.94159400 & C & 0.52555200 & -2.91115800 & -3.11210800 \\
\hline $\mathrm{H}$ & -1.49181000 & -2.42895700 & -3.67764000 & C & 1.32935500 & -1.85906600 & -1.09430400 \\
\hline $\mathrm{H}$ & -0.02154200 & -0.53401200 & -0.09031900 & C & 1.54366300 & -2.70686900 & -2.17796900 \\
\hline $\mathrm{H}$ & 0.67476200 & -3.59006400 & -3.94565500 & $\mathrm{H}$ & 2.10187400 & -1.72679600 & -0.35167800 \\
\hline $\mathrm{H}$ & 2.49333500 & -3.22178000 & -2.27289300 & $\mathrm{~N}$ & -2.68129700 & -0.27128900 & -0.59223100 \\
\hline $\mathrm{H}$ & -2.06007700 & -0.15769000 & 0.22695700 & C & -2.04517000 & 0.88698500 & -3.08725000 \\
\hline $\mathrm{H}$ & -1.70730300 & 0.36804600 & -3.97983200 & $\mathrm{H}$ & -3.10279100 & 1.12728600 & -3.04845600 \\
\hline $\mathrm{C}$ & -1.14810100 & 1.72738500 & -2.42154200 & $\mathrm{C}$ & -1.61796800 & 2.62502300 & -1.38117900 \\
\hline $\mathrm{H}$ & -0.85164100 & 2.84603800 & -0.63425600 & $\mathrm{H}$ & -2.53199300 & 2.25984200 & -0.89964900 \\
\hline $\mathrm{C}$ & 0.31343800 & 1.64026800 & -2.68578400 & $\mathrm{H}$ & 0.79079200 & 2.62544700 & -2.67287100 \\
\hline $\mathrm{H}$ & 0.55253000 & 1.10821200 & -3.60755700 & $\mathrm{H}$ & 0.77057600 & 1.08919400 & -1.85082600 \\
\hline $\mathrm{C}$ & -2.71389200 & 5.36061100 & -0.65166800 & $\mathrm{H}$ & -3.47179800 & 4.84381100 & -0.05092900 \\
\hline $\bar{H}$ & -3.13386000 & 6.32153900 & -0.97249700 & $\mathrm{H}$ & -1.83913500 & 5.55710800 & -0.02576000 \\
\hline $\mathrm{C}$ & -3.69891300 & 3.95890100 & -3.23403300 & $\mathrm{H}$ & -4.47123900 & 3.39351200 & -2.70085000 \\
\hline $\mathrm{H}$ & -3.41863500 & 3.38951000 & -4.12706600 & $\mathrm{H}$ & -4.15385800 & 4.89764800 & -3.57242400 \\
\hline $\mathrm{C}$ & -0.85712100 & 5.10811800 & -3.18484300 & $\mathrm{H}$ & -0.64519500 & 4.49920200 & -4.07174400 \\
\hline $\bar{H}$ & 0.07387000 & 5.24201700 & -2.62944200 & $\mathrm{H}$ & -1.18819100 & 6.09407900 & -3.53401000 \\
\hline $\mathrm{Si}$ & -2.20310200 & 4.33026900 & -2.13578500 & C & -1.27439900 & -1.15689700 & 4.84495300 \\
\hline $\bar{H}$ & -1.70859300 & -0.41496800 & 5.52205800 & $\mathrm{H}$ & -0.19912300 & -1.22439400 & 5.03180700 \\
\hline $\mathrm{C}$ & -1.99508700 & -2.50632800 & 4.97219100 & $\mathrm{H}$ & -2.35298300 & -2.68538800 & 5.98938700 \\
\hline $\mathrm{H}$ & -1.31657500 & -3.32128100 & 4.70183800 & C & -3.12652600 & -2.40579200 & 3.94011100 \\
\hline $\mathrm{H}$ & -3.53565000 & -3.37653100 & 3.65132700 & $\mathrm{H}$ & -3.94464300 & -1.78572100 & 4.32556900 \\
\hline $\mathrm{O}$ & -1.11485100 & 0.41165300 & 1.55293600 & & & & \\
\hline
\end{tabular}

\section{TS-S-4f-6}

Imaginary frequency: $-303.90 \mathrm{~cm}^{-1}$

E[B3LYP/6-31G(d)-D3(BJ)]: -4789.096698 Hartree

E[PCM(Et 2 O)-B3LYP/6-311G(d,p)-D3(BJ)]: - 4790.235347 Hartree

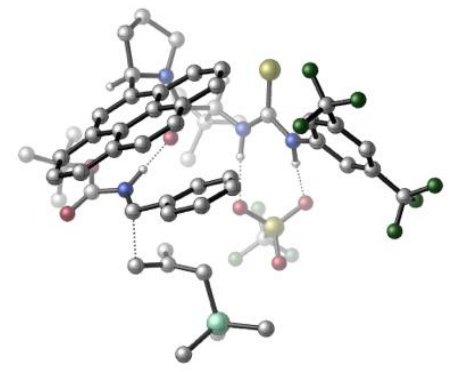

\begin{tabular}{|lrrr|lrrr|}
\hline 01 & & & $\mathrm{~N}$ & -2.63629000 & 0.01989400 & -1.89475400 \\
\hline $\mathrm{H}$ & -2.76928300 & 1.02416800 & -1.72965600 & $\mathrm{C}$ & -3.62965800 & -0.78341300 & -1.32918000 \\
\hline $\mathrm{C}$ & -4.82697600 & -0.15335900 & -0.95530700 & $\mathrm{C}$ & -3.44025700 & -2.13580500 & -1.01592400 \\
\hline $\mathrm{C}$ & -5.81715300 & -0.87403000 & -0.29452300 & $\mathrm{H}$ & -4.96625700 & 0.89971500 & -1.17338200 \\
\hline $\mathrm{C}$ & -4.44324400 & -2.83550200 & -0.34853600 & $\mathrm{H}$ & -2.51371000 & -2.62419600 & -1.27711100 \\
\hline $\mathrm{C}$ & -5.64163200 & -2.22269600 & 0.01369400 & $\mathrm{H}$ & -6.41966600 & -2.78031500 & 0.51910000 \\
\hline $\mathrm{C}$ & -7.06977100 & -0.17199400 & 0.15424700 & $\mathrm{C}$ & -4.17953300 & -4.25252900 & 0.07057200 \\
\hline $\mathrm{F}$ & -7.38357800 & 0.86690300 & -0.64616000 & $\mathrm{~F}$ & -6.93839700 & 0.31850300 & 1.40885600 \\
\hline $\mathrm{F}$ & -8.13164800 & -1.00995600 & 0.17200400 & $\mathrm{~F}$ & -3.43103700 & -4.92861600 & -0.83163000 \\
\hline
\end{tabular}




\begin{tabular}{|c|c|c|c|c|c|c|c|}
\hline $\mathrm{F}$ & -3.49004300 & -4.30216400 & 1.24748600 & $\mathrm{~F}$ & -5.31411600 & -4.95431000 & 0.26207300 \\
\hline C & 0.61920700 & 0.54521500 & -3.43794100 & $\mathrm{H}$ & 0.38811600 & -0.05809900 & -4.31457200 \\
\hline C & 1.12303700 & 1.94058400 & -3.92986700 & C & 1.55240900 & 2.87454500 & -2.78260300 \\
\hline $\mathrm{H}$ & 2.42237500 & 2.48241200 & -2.24934200 & $\mathrm{H}$ & 0.75503400 & 3.02890200 & -2.05401800 \\
\hline $\mathrm{H}$ & 1.81305200 & 3.85410400 & -3.20065600 & C & -0.01763000 & 2.59366900 & -4.72994800 \\
\hline $\mathrm{H}$ & 0.32839400 & 3.53348600 & -5.17483500 & $\mathrm{H}$ & -0.87640100 & 2.81782400 & -4.09368600 \\
\hline $\mathrm{H}$ & -0.35713300 & 1.93759500 & -5.54001400 & C & 2.32984500 & 1.72104100 & -4.86000000 \\
\hline $\mathrm{H}$ & 2.71523700 & 2.68676200 & -5.20458300 & $\mathrm{H}$ & 2.05385900 & 1.14200800 & -5.74919300 \\
\hline $\mathrm{H}$ & 3.14983100 & 1.20256100 & -4.34872800 & C & 1.65386900 & -0.16818300 & -2.55399000 \\
\hline 0 & 1.83720200 & 0.25662400 & -1.40380400 & C & 2.21571600 & -1.78903900 & -4.39817800 \\
\hline C & 3.39066900 & -1.89734800 & -2.24270500 & C & 3.51313000 & -2.58413800 & -4.56393300 \\
\hline $\mathrm{H}$ & 1.32482900 & -2.42362600 & -4.46192400 & $\mathrm{H}$ & 2.12001500 & -0.99799800 & -5.14036600 \\
\hline $\mathrm{H}$ & 4.22477100 & -1.18926600 & -2.16604300 & $\mathrm{H}$ & 4.31713100 & -1.92049200 & -4.90439800 \\
\hline $\mathrm{H}$ & 3.40969200 & -3.39205200 & -5.29310500 & $\mathrm{~N}$ & 2.33174100 & -1.22543400 & -3.03832100 \\
\hline C & 3.06658400 & -2.33685200 & -0.82690500 & C & 4.01617000 & -2.08430800 & 0.11963400 \\
\hline C & 1.90000300 & -3.12550400 & -0.47568400 & C & 3.92103000 & -2.55371100 & 1.46816200 \\
\hline $\mathrm{H}$ & 4.88548200 & -1.48873900 & -0.14557000 & C & 1.80877500 & -3.65802100 & 0.85144900 \\
\hline C & 0.85466900 & -3.39708000 & -1.37109000 & C & 4.86668400 & -2.21161500 & 2.44733100 \\
\hline $\mathrm{C}$ & 2.81506100 & -3.37831200 & 1.82039200 & C & 0.69030000 & -4.46462800 & 1.22248600 \\
\hline $\mathrm{H}$ & 0.86037300 & -2.97473700 & -2.36434600 & C & -0.24026300 & -4.17234900 & -0.99238200 \\
\hline C & 4.74057300 & -2.69261900 & 3.75032600 & $\mathrm{H}$ & 5.69118800 & -1.55507300 & 2.18343300 \\
\hline $\mathrm{C}$ & 2.70932100 & -3.89006700 & 3.14869900 & $\mathrm{C}$ & -0.32489900 & -4.70829100 & 0.28660500 \\
\hline $\mathrm{C}$ & 0.62882100 & -4.99870800 & 2.55348200 & $\mathrm{H}$ & -1.03542700 & -4.34646600 & -1.70903500 \\
\hline C & 3.68359200 & -3.53297000 & 4.09519900 & $\mathrm{H}$ & 5.47763900 & -2.41707300 & 4.49914800 \\
\hline $\mathrm{C}$ & 1.59269200 & -4.72923100 & 3.47508600 & $\mathrm{H}$ & -1.18719500 & -5.29912100 & 0.57610000 \\
\hline $\mathrm{H}$ & -0.22303100 & -5.62202200 & 2.81254700 & $\mathrm{H}$ & 3.60007900 & -3.91718300 & 5.10861000 \\
\hline $\mathrm{H}$ & 1.52549100 & -5.13388300 & 4.48172500 & C & -1.56057500 & -0.32663900 & -2.67427800 \\
\hline S & -1.45366600 & -1.75318700 & -3.57385500 & $\mathrm{~N}$ & -0.61549900 & 0.64656100 & -2.68443900 \\
\hline $\mathrm{H}$ & -0.65316200 & 1.34178600 & -1.93319000 & $\mathrm{~S}$ & -2.19101300 & 3.27743300 & -0.44144600 \\
\hline 0 & -2.66244100 & 3.64839100 & 0.90234400 & $\mathrm{O}$ & -0.92643200 & 2.48075300 & -0.45630200 \\
\hline 0 & -3.21344700 & 2.75237000 & -1.38157500 & C & -1.68712800 & 4.89653700 & -1.19974300 \\
\hline $\mathrm{F}$ & -2.72110700 & 5.73602700 & -1.26219900 & $\mathrm{~F}$ & -1.20258300 & 4.71313700 & -2.43831800 \\
\hline $\mathrm{F}$ & -0.71651100 & 5.46901100 & -0.45664800 & C & 4.28785500 & 1.11707900 & 0.97341300 \\
\hline 0 & 4.97816700 & 1.37582900 & 1.94493600 & $\mathrm{O}$ & 4.61714400 & 1.24103100 & -0.32077500 \\
\hline C & 5.89476300 & 1.84574500 & -0.73253600 & C & 5.96334400 & 3.28539000 & -0.22223300 \\
\hline $\mathrm{H}$ & 6.85206300 & 3.77707100 & -0.63127600 & $\mathrm{H}$ & 6.01836100 & 3.31427100 & 0.86718900 \\
\hline $\mathrm{H}$ & 5.08120800 & 3.84426000 & -0.55222000 & C & 5.79521900 & 1.80832800 & -2.25674900 \\
\hline $\mathrm{H}$ & 5.69420800 & 0.77692600 & -2.61030600 & $\mathrm{H}$ & 6.69672600 & 2.24070500 & -2.70174600 \\
\hline $\mathrm{H}$ & 4.92642400 & 2.37736000 & -2.60065400 & C & 7.05977000 & 0.98996500 & -0.23457900 \\
\hline $\mathrm{H}$ & 7.99948400 & 1.38339400 & -0.63636900 & $\mathrm{H}$ & 6.94948100 & -0.04301900 & -0.58234400 \\
\hline $\mathrm{H}$ & 7.11369500 & 0.99523900 & 0.85484400 & C & 2.41909300 & 0.32345200 & 2.25898700 \\
\hline $\mathrm{H}$ & 3.14048100 & 0.02587200 & 3.01432400 & C & 1.14141400 & -0.39638300 & 2.25296600 \\
\hline C & 0.80788700 & -1.18343000 & 3.36725800 & C & 0.23433600 & -0.29580900 & 1.18541400 \\
\hline C & -0.38971700 & -1.89012400 & 3.39400700 & $\mathrm{H}$ & 1.50957800 & -1.26714800 & 4.19225700 \\
\hline C & -0.95735400 & -1.01074800 & 1.21710200 & $\mathrm{H}$ & 0.44414100 & 0.32967300 & 0.32828400 \\
\hline C & -1.27031400 & -1.81639500 & 2.31105100 & $\mathrm{H}$ & -0.62344300 & -2.52038200 & 4.24534700 \\
\hline $\mathrm{H}$ & -1.64348300 & -0.92835600 & 0.38523000 & $\mathrm{H}$ & -2.18875300 & -2.39420600 & 2.30916300 \\
\hline $\mathrm{N}$ & 2.99459200 & 0.63797200 & 1.05419000 & $\mathrm{H}$ & 2.52726200 & 0.42695800 & 0.16273800 \\
\hline C & 2.13210300 & 2.04469900 & 3.33104400 & $\mathrm{H}$ & 1.84276900 & 1.59061900 & 4.27528500 \\
\hline $\mathrm{H}$ & 3.15812000 & 2.39537800 & 3.26739300 & $\mathrm{C}$ & 1.14116600 & 2.64964500 & 2.55798000 \\
\hline C & -0.26513800 & 2.43723600 & 2.83991600 & $\mathrm{H}$ & -0.43492600 & 1.53361100 & 3.43536700 \\
\hline $\mathrm{H}$ & -0.88262900 & 2.43825500 & 1.93881900 & C & 1.53611200 & 3.40871800 & 1.33718000 \\
\hline $\mathrm{H}$ & 0.78770000 & 4.14410800 & 1.04221700 & $\mathrm{H}$ & 1.59107900 & 2.69006900 & 0.50396000 \\
\hline
\end{tabular}




\begin{tabular}{|rrrr|rrrr|}
\hline $\mathrm{H}$ & 2.51905300 & 3.87649300 & 1.44093000 & $\mathrm{C}$ & -2.81175900 & 3.43748700 & 4.17298000 \\
\hline $\mathrm{H}$ & -3.31486200 & 3.40377200 & 3.20255500 & $\mathrm{H}$ & -3.31053200 & 4.18717900 & 4.79903900 \\
\hline $\mathrm{H}$ & -2.92767000 & 2.46262300 & 4.66061000 & $\mathrm{C}$ & -0.76542400 & 5.52800400 & 3.07737600 \\
\hline $\mathrm{H}$ & -1.19452500 & 5.50578000 & 2.07218500 & $\mathrm{H}$ & 0.29243000 & 5.80455000 & 3.00093400 \\
\hline $\mathrm{H}$ & -1.27279300 & 6.31851700 & 3.64356500 & $\mathrm{C}$ & -0.07282300 & 3.84371500 & 5.57906100 \\
\hline $\mathrm{H}$ & 0.99390200 & 4.05885200 & 5.44859900 & $\mathrm{H}$ & -0.16186500 & 2.86918800 & 6.07405700 \\
\hline $\mathrm{H}$ & -0.48136300 & 4.59747400 & 6.26281000 & $\mathrm{Si}$ & -1.00092900 & 3.87161800 & 3.93369200 \\
\hline $\mathrm{C}$ & 3.79778300 & -3.08154200 & -3.14477600 & $\mathrm{H}$ & 3.17283300 & -3.94889600 & -2.90989800 \\
\hline $\mathrm{H}$ & 4.83968700 & -3.36359500 & -2.97419900 & & & & \\
\hline
\end{tabular}

\section{TS-S-4f-7}

Imaginary frequency: $-398.87 \mathrm{~cm}^{-1}$

E[B3LYP/6-31G(d)-D3(BJ)]: -4789.098396 Hartree

E[PCM(Et $\left.\left.{ }_{2} \mathrm{O}\right)-\mathrm{B} 3 L Y P / 6-311 \mathrm{G}(\mathrm{d}, \mathrm{p})-\mathrm{D} 3(\mathrm{BJ})\right]:-4790.234868$ Hartree

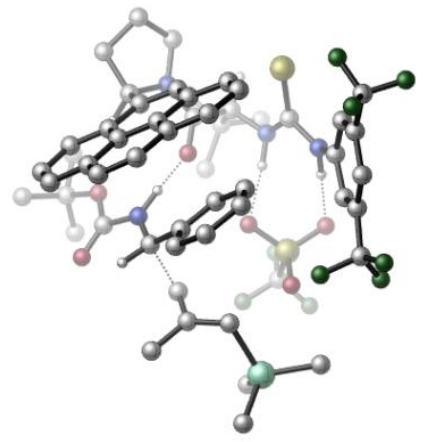

\begin{tabular}{|c|c|c|c|c|c|c|c|}
\hline 01 & & & & $\mathrm{~N}$ & -1.54228300 & -0.44762200 & -3.10723900 \\
\hline $\mathrm{H}$ & -1.60237000 & 0.57570900 & -3.16273600 & C & -2.62677100 & -1.02031100 & -2.42429200 \\
\hline $\mathrm{C}$ & -3.44727400 & -0.13958200 & -1.69892600 & $\mathrm{C}$ & -2.90094200 & -2.39495300 & -2.38173000 \\
\hline $\mathrm{C}$ & -4.45538300 & -0.63707800 & -0.88081900 & $\mathrm{H}$ & -3.26030300 & 0.92693500 & -1.73161500 \\
\hline C & -3.91524100 & -2.86874600 & -1.55017800 & $\mathrm{H}$ & -2.29961100 & -3.07992000 & -2.95900000 \\
\hline C & -4.69501000 & -2.00663200 & -0.78190900 & $\mathrm{H}$ & -5.46562100 & -2.38883800 & -0.12485400 \\
\hline $\mathrm{C}$ & -5.19493000 & 0.32445900 & 0.00550000 & C & -4.20125300 & -4.34362700 & -1.51662500 \\
\hline $\mathrm{F}$ & -5.51075800 & 1.46594900 & -0.63773900 & $\mathrm{~F}$ & -4.44338000 & 0.67568400 & 1.08438700 \\
\hline $\mathrm{F}$ & -6.34092700 & -0.20383800 & 0.48900400 & $\mathrm{~F}$ & -3.10204800 & -5.08289300 & -1.79708200 \\
\hline $\mathrm{F}$ & -4.64464800 & -4.73795400 & -0.29642200 & $\mathrm{~F}$ & -5.15067600 & -4.69851700 & -2.40904000 \\
\hline C & 2.07510200 & -0.29727600 & -3.45702700 & $\mathrm{H}$ & 2.18819100 & -1.14745300 & -4.12957200 \\
\hline $\mathrm{C}$ & 2.90686700 & 0.89215500 & -4.03827000 & $C$ & 2.91092400 & 2.13184500 & -3.12535000 \\
\hline $\mathrm{H}$ & 3.33155400 & 1.91087300 & -2.14073700 & $\mathrm{H}$ & 1.90985100 & 2.53516100 & -2.96513800 \\
\hline $\mathrm{H}$ & 3.51522600 & 2.91723500 & -3.59488400 & C & 2.30759400 & 1.26526300 & -5.40493300 \\
\hline $\mathrm{H}$ & 2.91679300 & 2.04176200 & -5.88143700 & $\mathrm{H}$ & 1.28833500 & 1.64625800 & -5.29972500 \\
\hline $\mathrm{H}$ & 2.27667900 & 0.39781500 & -6.07486900 & $\mathrm{C}$ & 4.35733600 & 0.41791700 & -4.23587300 \\
\hline $\mathrm{H}$ & 4.95886000 & 1.22606400 & -4.66587500 & $\mathrm{H}$ & 4.41057300 & -0.43620400 & -4.92108800 \\
\hline $\mathrm{H}$ & 4.82158600 & 0.12791500 & -3.28654800 & $C$ & 2.50998200 & -0.66940700 & -2.03403300 \\
\hline $\mathrm{C}$ & 3.79817000 & -1.95749900 & -0.43073200 & $\mathrm{H}$ & 4.16867800 & -1.01476000 & -0.02917400 \\
\hline $\mathrm{N}$ & 3.34733700 & -1.69757000 & -1.80950900 & C & 2.69594800 & -2.48969400 & 0.47166600 \\
\hline $\mathrm{C}$ & 1.61242500 & -3.13681900 & -0.04545400 & $\mathrm{C}$ & 2.81943600 & -2.31843900 & 1.90519900 \\
\hline $\mathrm{C}$ & 0.58541300 & -3.69644500 & 0.78131300 & $\mathrm{H}$ & 1.48354800 & -3.21039700 & -1.12026300 \\
\hline C & 1.79227200 & -2.84380700 & 2.75152100 & C & 3.88810900 & -1.63002700 & 2.50560700 \\
\hline $\mathrm{C}$ & -0.52572800 & -4.36064500 & 0.23749100 & $\mathrm{C}$ & 0.69135500 & -3.55489300 & 2.19385200 \\
\hline $\mathrm{C}$ & 1.86085900 & -2.64984700 & 4.16570400 & $\mathrm{H}$ & 4.68793400 & -1.23136400 & 1.89459000 \\
\hline C & 3.93196100 & -1.42042600 & 3.88226100 & C & -1.50288600 & -4.90214800 & 1.07041500 \\
\hline $\mathrm{H}$ & -0.62443200 & -4.43341200 & -0.84124100 & $\mathrm{C}$ & -0.31790100 & -4.10357300 & 3.03869500 \\
\hline $\mathrm{C}$ & 2.93003000 & -1.92132200 & 4.70777300 & $\mathrm{C}$ & 0.82832100 & -3.20986000 & 4.99227100 \\
\hline $\mathrm{H}$ & 4.75954800 & -0.86253200 & 4.31106800 & C & -1.39802200 & -4.78212100 & 2.45478700 \\
\hline $\mathrm{H}$ & -2.35780500 & -5.40710900 & 0.63608800 & C & -0.20511200 & -3.91436700 & 4.45616500 \\
\hline $\mathrm{H}$ & 2.97330700 & -1.76293900 & 5.78230000 & $\mathrm{H}$ & 0.89701100 & -3.06325000 & 6.06733500 \\
\hline $\mathrm{H}$ & -2.16751300 & -5.20183100 & 3.09801400 & $\mathrm{H}$ & -0.97564600 & -4.33694600 & 5.09622100 \\
\hline C & -0.28408700 & -0.96012700 & -3.31136000 & $S$ & 0.08568000 & -2.60248600 & -3.47714200 \\
\hline
\end{tabular}




\begin{tabular}{|c|c|c|c|c|c|c|c|}
\hline $\mathrm{N}$ & 0.65664200 & 0.02192000 & -3.39539800 & $\mathrm{H}$ & 0.45109400 & 0.86350000 & -2.84792800 \\
\hline S & -1.22061400 & 2.89317900 & -1.80216500 & 0 & -2.15148600 & 3.14730400 & -0.68411900 \\
\hline $\mathrm{O}$ & 0.00094900 & 2.12849400 & -1.42544400 & 0 & -1.84735500 & 2.41345900 & -3.06006000 \\
\hline $\mathrm{C}$ & -0.56498800 & 4.57259100 & -2.24341800 & $\mathrm{~F}$ & -1.56015700 & 5.40462500 & -2.56296600 \\
\hline $\mathrm{F}$ & 0.28012500 & 4.48958700 & -3.28137000 & $\mathrm{~F}$ & 0.10311200 & 5.08922100 & -1.19486300 \\
\hline C & 3.34561200 & 1.62272100 & 1.73472400 & 0 & 3.50130900 & 2.35178300 & 2.70105000 \\
\hline $\mathrm{O}$ & 4.25292200 & 1.31598700 & 0.79383800 & C & 5.58537200 & 1.94359500 & 0.78390100 \\
\hline C & 5.43390300 & 3.45645800 & 0.62359600 & $\mathrm{H}$ & 6.42149700 & 3.90997600 & 0.48886400 \\
\hline $\mathrm{H}$ & 4.96203400 & 3.89967300 & 1.50173700 & $\mathrm{H}$ & 4.82867700 & 3.68291600 & -0.26031400 \\
\hline C & 6.22959200 & 1.32538800 & -0.45621600 & $\mathrm{H}$ & 6.29250500 & 0.23650000 & -0.35788500 \\
\hline $\mathrm{H}$ & 7.24245600 & 1.71902300 & -0.58615100 & $\mathrm{H}$ & 5.64582300 & 1.55974000 & -1.35103000 \\
\hline $\mathrm{C}$ & 6.35054400 & 1.55915600 & 2.05081300 & $\mathrm{H}$ & 7.37238000 & 1.94862100 & 1.99114200 \\
\hline $\mathrm{H}$ & 6.40841800 & 0.46950700 & 2.14607000 & $\mathrm{H}$ & 5.86797500 & 1.96803300 & 2.93920700 \\
\hline C & 1.08735300 & 1.09480000 & 2.29785600 & $\mathrm{H}$ & 1.40391100 & 1.31209300 & 3.31321200 \\
\hline $\mathrm{C}$ & -0.03062900 & 0.14551400 & 2.17322100 & $C$ & -0.70117500 & -0.23167100 & 3.34787000 \\
\hline $\mathrm{C}$ & -0.46790300 & -0.34766700 & 0.93637000 & $\mathrm{C}$ & -1.79951800 & -1.08241300 & 3.29017200 \\
\hline $\mathrm{H}$ & -0.34312400 & 0.13093100 & 4.30785500 & C & -1.55388700 & -1.21711700 & 0.89220500 \\
\hline $\mathrm{H}$ & 0.02496200 & -0.05065800 & 0.01776700 & C & -2.23137300 & -1.57164400 & 2.05743200 \\
\hline $\mathrm{H}$ & -2.30141100 & -1.37950100 & 4.20558500 & $\mathrm{H}$ & -1.86539000 & -1.63126300 & -0.05501100 \\
\hline $\mathrm{H}$ & -3.07765300 & -2.24726200 & 2.00098800 & $\mathrm{~N}$ & 2.16304300 & 0.96901700 & 1.45175200 \\
\hline $\mathrm{H}$ & 2.08682000 & 0.50599200 & 0.53364500 & C & 0.38550500 & 2.89781100 & 1.85292100 \\
\hline $\mathrm{H}$ & 0.13631700 & 2.74565700 & 0.80654700 & $\mathrm{H}$ & 1.31652500 & 3.41992000 & 2.06084700 \\
\hline $\mathrm{C}$ & -0.64441600 & 3.01511100 & 2.78886200 & $C$ & -2.02085900 & 2.69882100 & 2.46321100 \\
\hline $\mathrm{H}$ & -2.52788000 & 2.14556100 & 3.26139000 & $\mathrm{H}$ & -2.12680000 & 2.19285600 & 1.50184300 \\
\hline $\mathrm{C}$ & -0.29641600 & 3.40712500 & 4.19398300 & $\mathrm{H}$ & -0.80762300 & 2.76632200 & 4.92130600 \\
\hline $\mathrm{H}$ & -0.63754100 & 4.43268800 & 4.38487500 & $\mathrm{H}$ & 0.78225500 & 3.37816900 & 4.36910600 \\
\hline C & -4.57533600 & 3.95402400 & 1.25248800 & $\mathrm{H}$ & -4.27731800 & 3.65730600 & 0.24493300 \\
\hline $\bar{H}$ & -5.22513600 & 4.83542200 & 1.18564000 & $\mathrm{H}$ & -5.15435600 & 3.13576400 & 1.69077200 \\
\hline $\mathrm{C}$ & -2.00039200 & 5.71288000 & 1.54780500 & $\mathrm{H}$ & -1.58873400 & 5.40234900 & 0.58538100 \\
\hline $\mathrm{H}$ & -1.16695400 & 5.98658800 & 2.20577200 & $\mathrm{H}$ & -2.60138500 & 6.61568100 & 1.38530100 \\
\hline $\mathrm{C}$ & -3.61220400 & 4.84224200 & 4.03398300 & $\mathrm{H}$ & -2.77864400 & 5.09587400 & 4.69790300 \\
\hline $\mathrm{H}$ & -4.18784000 & 4.03878500 & 4.50855800 & $\mathrm{H}$ & -4.26288300 & 5.72395400 & 3.98069800 \\
\hline $\mathrm{Si}$ & -3.06656200 & 4.35365900 & 2.28853300 & C & 3.81980400 & -2.70634100 & -2.77249400 \\
\hline $\bar{H}$ & 4.56502700 & -2.26998700 & -3.44618900 & $\bar{H}$ & 2.98097000 & -3.08200400 & -3.36463700 \\
\hline $\mathrm{C}$ & 4.45090700 & -3.77749300 & -1.87064300 & $\bar{H}$ & 5.25364500 & -4.32081500 & -2.37599200 \\
\hline $\mathrm{H}$ & 3.69074100 & -4.50217800 & -1.56285100 & C & 4.93315800 & -2.97967200 & -0.64994400 \\
\hline $\mathrm{H}$ & 5.10243100 & -3.59902100 & 0.23381200 & $\mathrm{H}$ & 5.86547400 & -2.45061100 & -0.88464100 \\
\hline $\mathrm{O}$ & 2.08252400 & 0.02656100 & -1.10231700 & & & & \\
\hline
\end{tabular}

\section{TS-S-4f-8}

Imaginary frequency: $-119.87 \mathrm{~cm}^{-1}$

E[B3LYP/6-31G(d)-D3(BJ)]: -4789.094107 Hartree

E[PCM(Et 2 O)-B3LYP/6-311G(d,p)-D3(BJ)]: - 4790.234098 Hartree

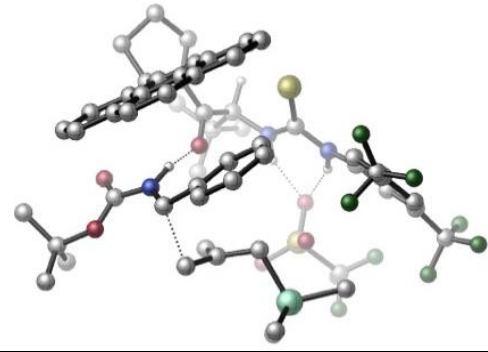

\begin{tabular}{|lrrr|rrrr|}
\hline 01 & & & & N & 2.70150400 & -0.06403000 & -2.22316800 \\
\hline $\mathrm{H}$ & 3.22105700 & -0.93063700 & -2.05786000 & $\mathrm{C}$ & 3.30026700 & 1.03797400 & -1.60927000 \\
\hline $\mathrm{C}$ & 4.66764600 & 0.90954000 & -1.32386200 & $\mathrm{C}$ & 2.62456400 & 2.18731200 & -1.19007900 \\
\hline $\mathrm{C}$ & 5.33708000 & 1.90186400 & -0.62060200 & $\mathrm{H}$ & 5.19306300 & 0.01041300 & -1.62174300 \\
\hline $\mathrm{C}$ & 3.31466700 & 3.17181200 & -0.48381400 & $\mathrm{H}$ & 1.57365400 & 2.29401000 & -1.40364500 \\
\hline $\mathrm{C}$ & 4.67172700 & 3.05022600 & -0.19373000 & $\mathrm{H}$ & 5.19366700 & 3.81818200 & 0.36187000 \\
\hline $\mathrm{C}$ & 6.76684300 & 1.66187700 & -0.22510900 & $\mathrm{C}$ & 2.54255200 & 4.31975800 & 0.09640600 \\
\hline
\end{tabular}




\begin{tabular}{|c|c|c|c|c|c|c|c|}
\hline $\mathrm{F}$ & 7.47061700 & 1.06809100 & -1.21299400 & $\mathrm{~F}$ & 6.84583900 & 0.84835700 & 0.85458400 \\
\hline $\mathrm{F}$ & 7.40404400 & 2.80956300 & 0.09667900 & $\mathrm{~F}$ & 1.50964400 & 4.70380900 & -0.69240000 \\
\hline $\mathrm{F}$ & 2.00042400 & 3.98457800 & 1.30065800 & $\mathrm{~F}$ & 3.31151600 & 5.40731100 & 0.30889900 \\
\hline C & -0.12331700 & -2.15937800 & -3.14376000 & $\mathrm{H}$ & -0.21800400 & -1.88556200 & -4.19907200 \\
\hline C & -0.04030100 & -3.72739800 & -3.04958900 & C & 0.04086500 & -4.22369000 & -1.59281900 \\
\hline $\bar{H}$ & -0.85330900 & -3.95518900 & -1.02413400 & $\mathrm{H}$ & 0.91475600 & -3.84189100 & -1.05860800 \\
\hline $\bar{H}$ & 0.12831300 & -5.31595300 & -1.59714100 & $\mathrm{C}$ & 1.20821600 & -4.19563100 & -3.82547600 \\
\hline $\mathrm{H}$ & 1.20238100 & -5.28896800 & -3.89570100 & $\mathrm{H}$ & 2.14072900 & -3.90771400 & -3.33333100 \\
\hline $\mathrm{H}$ & 1.21477100 & -3.79187400 & -4.84509200 & $\mathrm{C}$ & -1.27971100 & -4.35337200 & -3.71141700 \\
\hline $\bar{H}$ & -1.22102900 & -5.44370000 & -3.63148100 & $\mathrm{H}$ & -1.33393400 & -4.10671600 & -4.77793000 \\
\hline $\mathrm{H}$ & -2.20968600 & -4.03691600 & -3.22853600 & C & -1.32741600 & -1.61352600 & -2.36344300 \\
\hline C & -3.73607900 & -1.16516200 & -2.27619700 & $\mathrm{H}$ & -3.76090000 & -1.87454700 & -1.44780600 \\
\hline $\mathrm{N}$ & -2.48480700 & -1.39922700 & -3.01920500 & C & -3.87601200 & 0.25702900 & -1.75980400 \\
\hline C & -3.18137300 & 1.27804100 & -2.33797800 & C & -4.78582500 & 0.53168800 & -0.66583700 \\
\hline C & -3.31066700 & 2.63680000 & -1.90710700 & $\mathrm{H}$ & -2.45869500 & 1.06545600 & -3.11720900 \\
\hline $\mathrm{C}$ & -4.88495800 & 1.87235000 & -0.17585500 & C & -5.55283400 & -0.46636100 & -0.03989900 \\
\hline $\mathrm{C}$ & -2.59353700 & 3.67805400 & -2.51530100 & C & -4.16005100 & 2.92616400 & -0.80297500 \\
\hline C & -5.71107600 & 2.16341900 & 0.95296700 & $\mathrm{H}$ & -5.50861100 & -1.48917200 & -0.39385800 \\
\hline $\mathrm{C}$ & -6.35599100 & -0.17155100 & 1.06033800 & C & -2.70825900 & 4.98454800 & -2.04592100 \\
\hline $\mathrm{H}$ & -1.93153900 & 3.44809700 & -3.34539600 & C & -4.26170500 & 4.26366300 & -0.31930100 \\
\hline C & -6.42945200 & 1.12428500 & 1.56236600 & C & -5.78384300 & 3.51696100 & 1.42815900 \\
\hline $\mathrm{H}$ & -6.93649300 & -0.96395600 & 1.52343800 & C & -3.53066000 & 5.27624700 & -0.95916300 \\
\hline $\mathrm{H}$ & -2.14373800 & 5.77984300 & -2.52376700 & C & -5.09727400 & 4.52102700 & 0.81820800 \\
\hline $\mathrm{H}$ & -7.05718800 & 1.34616300 & 2.42157500 & $\mathrm{H}$ & -6.41351100 & 3.72420300 & 2.28972900 \\
\hline $\mathrm{H}$ & -3.60796800 & 6.29613000 & -0.59130200 & $\mathrm{H}$ & -5.16789500 & 5.54109200 & 1.18707000 \\
\hline C & 1.41702400 & -0.27703900 & -2.65140100 & $\mathrm{~S}$ & 0.35569700 & 0.91014200 & -3.22524600 \\
\hline $\mathrm{N}$ & 1.09940900 & -1.58879200 & -2.62680500 & $\mathrm{H}$ & 1.76031900 & -2.21134700 & -2.16358700 \\
\hline S & 3.58303800 & -2.62719300 & 0.01781200 & $\mathrm{O}$ & 2.97743000 & -3.76612300 & 0.72738000 \\
\hline 0 & 3.51625900 & -2.70770100 & -1.48019700 & $\mathrm{O}$ & 3.23117900 & -1.28292800 & 0.53118200 \\
\hline C & 5.40303000 & -2.75865000 & 0.37184100 & $\mathrm{~F}$ & 5.61350900 & -2.63812500 & 1.69073300 \\
\hline $\mathrm{F}$ & 6.07915700 & -1.77820300 & -0.25263200 & $\mathrm{~F}$ & 5.88602400 & -3.93491100 & -0.03725000 \\
\hline C & -3.31089400 & -2.32793000 & 1.44543600 & $\mathrm{O}$ & -3.52813700 & -3.16138800 & 0.58422600 \\
\hline 0 & -3.64881100 & -2.40147700 & 2.74105500 & C & -4.33089800 & -3.59642400 & 3.28211400 \\
\hline $\mathrm{C}$ & -5.68953500 & -3.76770600 & 2.60398200 & $\mathrm{H}$ & -6.23560900 & -4.58404000 & 3.08813800 \\
\hline $\mathrm{H}$ & -5.57448400 & -4.00086100 & 1.54498700 & $\mathrm{H}$ & -6.28040300 & -2.85229000 & 2.70794500 \\
\hline C & -4.49671600 & -3.23656400 & 4.75676200 & $\mathrm{H}$ & -3.52214500 & -3.07636300 & 5.22884000 \\
\hline $\mathrm{H}$ & -5.00563800 & -4.04956100 & 5.28344500 & $\mathrm{H}$ & -5.09216000 & -2.32474900 & 4.86683900 \\
\hline C & -3.42754200 & -4.81720900 & 3.11007600 & $\mathrm{H}$ & -3.87867300 & -5.67700700 & 3.61626900 \\
\hline $\mathrm{H}$ & -2.44754000 & -4.63238000 & 3.56291700 & $\mathrm{H}$ & -3.29060000 & -5.06167900 & 2.05612900 \\
\hline $\mathrm{C}$ & -2.43586100 & -0.13476600 & 2.06324300 & $\mathrm{H}$ & -3.13831900 & -0.14255600 & 2.88840200 \\
\hline $\mathrm{C}$ & -2.00259600 & 1.18840200 & 1.60977700 & $\mathrm{C}$ & -2.29919400 & 2.28678900 & 2.43696500 \\
\hline C & -1.26726600 & 1.39282400 & 0.43071000 & C & -1.86837200 & 3.56228000 & 2.09746400 \\
\hline $\mathrm{H}$ & -2.87749100 & 2.13011800 & 3.34394500 & $\mathrm{C}$ & -0.85060000 & 2.67675700 & 0.09388900 \\
\hline $\mathrm{H}$ & -1.02046300 & 0.56818200 & -0.22838500 & $\mathrm{C}$ & -1.13511100 & 3.75858900 & 0.92466700 \\
\hline $\mathrm{H}$ & -2.11258800 & 4.40370900 & 2.73828200 & $\mathrm{H}$ & -0.31712500 & 2.83101600 & -0.83398300 \\
\hline $\mathrm{H}$ & -0.78865800 & 4.74845900 & 0.65267600 & $\mathrm{~N}$ & -2.65335400 & -1.14016800 & 1.16565400 \\
\hline $\mathrm{H}$ & -2.22183000 & -1.12866400 & 0.22636600 & $\mathrm{C}$ & -0.94208000 & -0.69787800 & 3.42891800 \\
\hline $\mathrm{H}$ & -0.94652900 & 0.20807700 & 4.02802900 & $\mathrm{H}$ & -1.47618500 & -1.54878900 & 3.84037100 \\
\hline $\mathrm{C}$ & 0.11441400 & -0.92334000 & 2.54828600 & $\mathrm{C}$ & 1.06760900 & 0.12249000 & 2.21712000 \\
\hline $\mathrm{H}$ & 0.65105000 & 1.12812300 & 2.33242100 & $\mathrm{H}$ & 1.52453100 & -0.01616200 & 1.23563600 \\
\hline C & 0.21552100 & -2.22908100 & 1.84064900 & $\mathrm{H}$ & 1.24526800 & -2.59633700 & 1.80238500 \\
\hline $\mathrm{H}$ & -0.07541900 & -2.07768000 & 0.78985500 & $\mathrm{H}$ & -0.43152600 & -2.99449300 & 2.27674400 \\
\hline $\mathrm{C}$ & 3.93910800 & 1.08774700 & 2.68395400 & $\mathrm{H}$ & 4.35601900 & 0.53615700 & 1.83870900 \\
\hline
\end{tabular}




\begin{tabular}{|c|c|c|c|c|c|c|c|}
\hline $\mathrm{H}$ & 4.74471000 & 1.28197300 & 3.40222400 & $\mathrm{H}$ & 3.56957800 & 2.05153200 & 2.31895400 \\
\hline C & 3.05471800 & -1.64740000 & 3.94575600 & $\mathrm{H}$ & 3.38901800 & -2.22165000 & 3.07914300 \\
\hline $\mathrm{H}$ & 2.22587700 & -2.18716200 & 4.41880300 & $\mathrm{H}$ & 3.88067500 & -1.61596700 & 4.66709300 \\
\hline $\mathrm{C}$ & 1.91289400 & 0.99727400 & 5.02119900 & $\mathrm{H}$ & 1.07885600 & 0.45946100 & 5.48695800 \\
\hline $\mathrm{H}$ & 1.57115600 & 2.01095300 & 4.78040500 & $\mathrm{H}$ & 2.70612500 & 1.08493000 & 5.77345700 \\
\hline $\mathrm{Si}$ & 2.55663000 & 0.10495000 & 3.48233000 & C & -2.67226200 & -1.32752100 & -4.48054800 \\
\hline $\mathrm{H}$ & -2.45786800 & -2.29424500 & -4.94190700 & $\mathrm{H}$ & -1.99806500 & -0.57868600 & -4.90816800 \\
\hline C & -4.15841300 & -0.96467200 & -4.63737000 & $\mathrm{H}$ & -4.59119900 & -1.40076600 & -5.54137800 \\
\hline $\mathrm{H}$ & -4.27964500 & 0.12104700 & -4.69413400 & C & -4.79874100 & -1.49386500 & -3.34613800 \\
\hline $\mathrm{H}$ & -5.76350900 & -1.03258700 & -3.12279600 & $\mathrm{H}$ & -4.93732900 & -2.58002700 & -3.40112100 \\
\hline 0 & -1.23977100 & -1.48784700 & -1.13007000 & & & & \\
\hline
\end{tabular}

\section{TS-S-4f-9}

Imaginary frequency: $-290.29 \mathrm{~cm}^{-1}$

E[B3LYP/6-31G(d)-D3(BJ)]: -4789.095757 Hartree

E[PCM(Et 2 O)-B3LYP/6-311G(d,p)-D3(BJ)]: - 4790.234060 Hartree

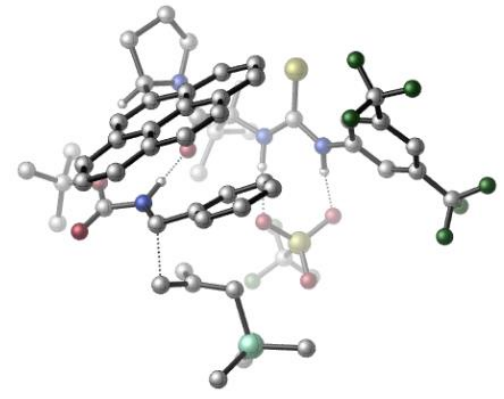

\begin{tabular}{|c|c|c|c|c|c|c|c|}
\hline 01 & & & & $\mathrm{~N}$ & -2.67592200 & 0.08250100 & -1.86435000 \\
\hline $\mathrm{H}$ & -2.80847800 & 1.08029500 & -1.66229700 & C & -3.65179500 & -0.74367600 & -1.30032100 \\
\hline C & -4.84247500 & -0.13278200 & -0.87704600 & C & -3.44868500 & -2.10433000 & -1.03607200 \\
\hline $\mathrm{C}$ & -5.81290300 & -0.88061500 & -0.21689700 & $\mathrm{H}$ & -4.99206800 & 0.92610600 & -1.05673600 \\
\hline $\mathrm{C}$ & -4.43216800 & -2.83201000 & -0.36985600 & $\mathrm{H}$ & -2.52663300 & -2.57882700 & -1.33494700 \\
\hline C & -5.62427400 & -2.23807800 & 0.04111900 & $\mathrm{H}$ & -6.38786000 & -2.81651200 & 0.54517900 \\
\hline C & -7.05726200 & -0.20040900 & 0.28545200 & $\mathrm{C}$ & -4.15277400 & -4.26234500 & -0.00986400 \\
\hline $\mathrm{F}$ & -7.39670400 & 0.86112900 & -0.47368100 & $\mathrm{~F}$ & -6.89604700 & 0.25140800 & 1.55104100 \\
\hline $\mathrm{F}$ & -8.11399000 & -1.04475200 & 0.30394500 & $\mathrm{~F}$ & -3.42118300 & -4.89939600 & -0.95352800 \\
\hline $\mathrm{F}$ & -3.43733000 & -4.35369500 & 1.14879600 & $\mathrm{~F}$ & -5.27983900 & -4.97663600 & 0.17934200 \\
\hline C & 0.55345400 & 0.65627300 & -3.44730100 & $\mathrm{H}$ & 0.33394200 & 0.03801200 & -4.31600300 \\
\hline C & 1.02955300 & 2.04911600 & -3.97126600 & C & 1.46477500 & 3.01238400 & -2.85140200 \\
\hline $\mathrm{H}$ & 2.35361100 & 2.64806700 & -2.32973000 & $\mathrm{H}$ & 0.68035900 & 3.16295600 & -2.10805300 \\
\hline $\mathrm{H}$ & 1.69636400 & 3.98902900 & -3.29263600 & $\mathrm{C}$ & -0.13381900 & 2.67148800 & -4.76277200 \\
\hline $\mathrm{H}$ & 0.19533600 & 3.60059100 & -5.24161900 & $\mathrm{H}$ & -0.97789300 & 2.90819700 & -4.11075200 \\
\hline $\mathrm{H}$ & -0.48923000 & 1.99135600 & -5.54562100 & C & 2.22244400 & 1.81618900 & -4.91563100 \\
\hline $\mathrm{H}$ & 2.57693700 & 2.77256300 & -5.31499200 & $\mathrm{H}$ & 1.94383800 & 1.18302700 & -5.76618500 \\
\hline $\mathrm{H}$ & 3.06460200 & 1.34384700 & -4.39541200 & C & 1.60885300 & -0.01591400 & -2.56150100 \\
\hline $\mathrm{C}$ & 2.14897000 & -1.71740100 & -4.34459300 & C & 3.41078400 & -1.64087200 & -2.23028100 \\
\hline $\mathrm{H}$ & 1.09094600 & -1.88404800 & -4.56049800 & $\mathrm{H}$ & 2.57855700 & -1.07999100 & -5.12636100 \\
\hline $\mathrm{H}$ & 4.10110400 & -0.82071000 & -2.01494900 & $\mathrm{~N}$ & 2.30205600 & -1.07194900 & -3.02529700 \\
\hline $\mathrm{C}$ & 3.07345700 & -2.25202600 & -0.87257100 & $C$ & 4.02218600 & -2.06212700 & 0.09136200 \\
\hline $\mathrm{C}$ & 1.91382500 & -3.07359000 & -0.57353700 & $\mathrm{C}$ & 3.93997000 & -2.62622900 & 1.40174200 \\
\hline $\mathrm{H}$ & 4.88411500 & -1.43790400 & -0.12951100 & C & 1.84014900 & -3.70791700 & 0.71264400 \\
\hline C & 0.84667200 & -3.27548400 & -1.46351100 & C & 4.88175400 & -2.33450800 & 2.40120400 \\
\hline $\mathrm{C}$ & 2.84617400 & -3.48754700 & 1.69676700 & $C$ & 0.73153400 & -4.54989600 & 1.03412900 \\
\hline $\mathrm{H}$ & 0.81690400 & -2.76336700 & -2.41073800 & $C$ & -0.23326700 & -4.09255400 & -1.13463700 \\
\hline C & 4.76518400 & -2.90406700 & 3.66848400 & $\mathrm{H}$ & 5.69488500 & -1.64834800 & 2.18012800 \\
\hline $\mathrm{C}$ & 2.74980300 & -4.08716600 & 2.98892300 & $C$ & -0.29261300 & -4.73431300 & 0.09532400 \\
\hline $\mathrm{C}$ & 0.68128100 & -5.17574200 & 2.32506300 & $\mathrm{H}$ & -1.03780200 & -4.21222100 & -1.85199600 \\
\hline C & 3.72079400 & -3.78112600 & 3.95619100 & $\mathrm{H}$ & 5.49880700 & -2.66871100 & 4.43422700 \\
\hline $\mathrm{C}$ & 1.64386300 & -4.95849100 & 3.26138100 & $\mathrm{H}$ & -1.14464800 & -5.35583100 & 0.34882200 \\
\hline
\end{tabular}




\begin{tabular}{|c|c|c|c|c|c|c|c|}
\hline $\mathrm{H}$ & -0.16351000 & -5.82405800 & 2.54251000 & $\mathrm{H}$ & 3.64340400 & -4.23286500 & 4.94181700 \\
\hline $\mathrm{H}$ & 1.58368900 & -5.43048200 & 4.23868800 & $\mathrm{C}$ & -1.61895100 & -0.23379200 & -2.68032400 \\
\hline $\mathrm{S}$ & -1.53337300 & -1.63445900 & -3.62524200 & $\mathrm{~N}$ & -0.67756600 & 0.74242300 & -2.68499100 \\
\hline $\mathrm{H}$ & -0.70285400 & 1.41866600 & -1.91521700 & S & -2.20851900 & 3.29927800 & -0.33672900 \\
\hline 0 & -2.64127600 & 3.63940800 & 1.02810000 & 0 & -0.94176000 & 2.50912100 & -0.40656400 \\
\hline 0 & -3.25759500 & 2.78909400 & -1.25571600 & C & -1.73390000 & 4.93560400 & -1.07549600 \\
\hline $\mathrm{F}$ & -2.76882800 & 5.77636900 & -1.07772700 & $\mathrm{~F}$ & -1.29924900 & 4.78061700 & -2.33640800 \\
\hline $\mathrm{F}$ & -0.73462200 & 5.48938400 & -0.35729700 & C & 4.32398600 & 1.13085500 & 0.94490100 \\
\hline $\mathrm{O}$ & 5.02752100 & 1.34507100 & 1.91763700 & $\mathrm{O}$ & 4.63733800 & 1.31052000 & -0.34699800 \\
\hline $\mathrm{C}$ & 5.91345000 & 1.92601900 & -0.74952400 & $\mathrm{C}$ & 5.99955000 & 3.33991700 & -0.17413700 \\
\hline $\mathrm{H}$ & 6.88598600 & 3.84381100 & -0.57308600 & $\mathrm{H}$ & 6.07029200 & 3.31817700 & 0.91449600 \\
\hline $\mathrm{H}$ & 5.11708300 & 3.91952700 & -0.46515200 & C & 5.79149600 & 1.96018300 & -2.27222300 \\
\hline $\mathrm{H}$ & 5.69023700 & 0.94651500 & -2.67383900 & $\mathrm{H}$ & 6.68467200 & 2.41742700 & -2.70903200 \\
\hline $\mathrm{H}$ & 4.91543200 & 2.54103300 & -2.57594700 & C & 7.07832300 & 1.03965400 & -0.30850000 \\
\hline $\mathrm{H}$ & 8.01550100 & 1.44421200 & -0.70509200 & $\mathrm{H}$ & 6.95459500 & 0.02477600 & -0.70207100 \\
\hline $\mathrm{H}$ & 7.14755800 & 0.99409100 & 0.77914800 & $\mathrm{C}$ & 2.47296900 & 0.28577100 & 2.21913300 \\
\hline $\mathrm{H}$ & 3.20714200 & -0.02384300 & 2.95697200 & C & 1.20815700 & -0.45568700 & 2.20449200 \\
\hline C & 0.91743700 & -1.30828200 & 3.28209200 & C & 0.27612300 & -0.32096500 & 1.16223500 \\
\hline $\mathrm{C}$ & -0.26316300 & -2.04337500 & 3.29620500 & $\mathrm{H}$ & 1.63963200 & -1.42037500 & 4.08585800 \\
\hline $\mathrm{C}$ & -0.89782000 & -1.06514500 & 1.18028800 & $\mathrm{H}$ & 0.45262100 & 0.35515300 & 0.33631400 \\
\hline C & -1.16836400 & -1.93401300 & 2.23681300 & $\mathrm{H}$ & -0.46334100 & -2.72401500 & 4.11661000 \\
\hline $\mathrm{H}$ & -1.60371400 & -0.95617200 & 0.36788300 & $\mathrm{H}$ & -2.07347300 & -2.53214300 & 2.22361700 \\
\hline $\mathrm{N}$ & 3.03163400 & 0.64858300 & 1.02108500 & $\mathrm{H}$ & 2.54680600 & 0.48519100 & 0.12773700 \\
\hline C & 2.17282400 & 1.97467900 & 3.34508500 & $\mathrm{H}$ & 1.89779600 & 1.49155900 & 4.27915700 \\
\hline $\mathrm{H}$ & 3.19470700 & 2.33731100 & 3.28094100 & C & 1.16852400 & 2.58898300 & 2.59766600 \\
\hline C & -0.23288500 & 2.35809600 & 2.89076300 & $\mathrm{H}$ & -0.38900600 & 1.43761300 & 3.46368400 \\
\hline $\mathrm{H}$ & -0.86204100 & 2.37994300 & 1.99810000 & C & 1.54083300 & 3.38499000 & 1.39283300 \\
\hline $\mathrm{H}$ & 0.78710600 & 4.12983900 & 1.13680700 & $\mathrm{H}$ & 1.57685900 & 2.69529500 & 0.53467400 \\
\hline $\mathrm{H}$ & 2.52549200 & 3.85000600 & 1.49212100 & $\mathrm{C}$ & -2.76713600 & 3.31507900 & 4.28271200 \\
\hline $\mathrm{H}$ & -3.28394000 & 3.30945100 & 3.31901600 & $\mathrm{H}$ & -3.25815100 & 4.04469600 & 4.93796100 \\
\hline $\mathrm{H}$ & -2.87442700 & 2.32593100 & 4.74278700 & C & -0.73756200 & 5.44059800 & 3.21973300 \\
\hline $\mathrm{H}$ & -1.17493200 & 5.44480600 & 2.21781300 & $\mathrm{H}$ & 0.31874900 & 5.72293900 & 3.14321700 \\
\hline $\mathrm{H}$ & -1.24312700 & 6.21311100 & 3.81181100 & C & -0.01290600 & 3.68990700 & 5.66505100 \\
\hline $\mathrm{H}$ & 1.05190500 & 3.91019800 & 5.52790000 & $\mathrm{H}$ & -0.09486200 & 2.70255400 & 6.13523900 \\
\hline $\mathrm{H}$ & -0.41443700 & 4.42472700 & 6.37314600 & $\mathrm{Si}$ & -0.96047600 & 3.76023300 & 4.03200400 \\
\hline C & 4.06364900 & -2.64089000 & -3.21875500 & $\mathrm{H}$ & 4.49032200 & -3.49954800 & -2.69652100 \\
\hline $\mathrm{H}$ & 4.87499100 & -2.13463800 & -3.75509800 & C & 2.94337000 & -3.01792800 & -4.19596700 \\
\hline $\mathrm{H}$ & 3.31902900 & -3.37750400 & -5.15768400 & $\mathrm{H}$ & 2.31105400 & -3.79991800 & -3.76634200 \\
\hline 0 & 1.80457200 & 0.44320600 & -1.42484200 & & & & \\
\hline
\end{tabular}

\section{TS-S-4f-10}

Imaginary frequency: $-396.39 \mathrm{~cm}^{-1}$

E[B3LYP/6-31G(d)-D3(BJ)]: -4789.093494 Hartree

E[PCM(Et $\left.\left.{ }_{2} \mathrm{O}\right)-\mathrm{B} 3 \mathrm{LYP} / 6-311 \mathrm{G}(\mathrm{d}, \mathrm{p})-\mathrm{D} 3(\mathrm{BJ})\right]$ : -4790.233830 Hartree

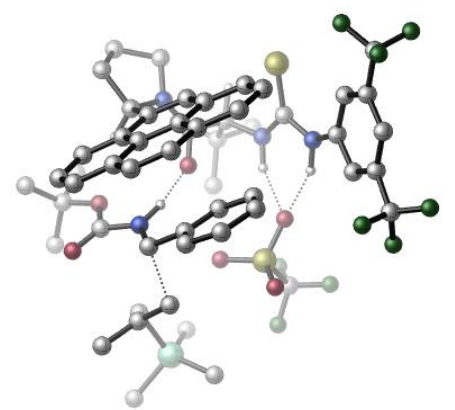

\begin{tabular}{|llll|lrrr|}
\hline 01 & & & & N & 2.41406900 & 1.59593100 & 1.99900800 \\
\hline $\mathrm{H}$ & 1.71051500 & 2.23506100 & 1.62621100 & $\mathrm{C}$ & 3.60785700 & 1.63824400 & 1.27035200 \\
\hline $\mathrm{C}$ & 3.61709700 & 2.51780200 & 0.17052600 & $\mathrm{C}$ & 4.74133500 & 0.85738200 & 1.52349100 \\
\hline $\mathrm{C}$ & 4.71498500 & 2.56728500 & -0.67857800 & $\mathrm{H}$ & 2.74189600 & 3.12422300 & -0.04123400 \\
\hline
\end{tabular}




\begin{tabular}{|c|c|c|c|c|c|c|c|}
\hline$C$ & 5.82326600 & 0.91151400 & 0.64478400 & $\mathrm{H}$ & 4.75363700 & 0.18925400 & 2.37074000 \\
\hline C & 5.82968500 & 1.75561900 & -0.46029600 & $\mathrm{H}$ & 6.67257200 & 1.77876700 & -1.13965000 \\
\hline C & 4.66723600 & 3.41811800 & -1.91672000 & C & 7.01251500 & 0.03348500 & 0.91925400 \\
\hline $\mathrm{F}$ & 3.76784900 & 4.41434600 & -1.82221900 & $\mathrm{~F}$ & 4.33172800 & 2.67784200 & -3.00559300 \\
\hline $\mathrm{F}$ & 5.87065200 & 3.97175700 & -2.18682600 & $\mathrm{~F}$ & 7.67187300 & -0.28102900 & -0.22370300 \\
\hline $\mathrm{F}$ & 7.90784500 & 0.61959800 & 1.74172600 & $\mathrm{~F}$ & 6.64651100 & -1.13388800 & 1.49714400 \\
\hline C & -0.27374400 & -0.13390900 & 3.71011900 & $\mathrm{H}$ & 0.33618600 & -0.65357600 & 4.44741400 \\
\hline C & -1.49169300 & 0.50863500 & 4.44019900 & C & -2.38023400 & 1.33472000 & 3.49457800 \\
\hline $\mathrm{H}$ & -2.75827300 & 0.74225800 & 2.65922300 & $\mathrm{H}$ & -1.84532700 & 2.18685700 & 3.07027500 \\
\hline $\mathrm{H}$ & -3.23115800 & 1.73567300 & 4.05831900 & C & -0.94546400 & 1.41748500 & 5.55424700 \\
\hline $\mathrm{H}$ & -1.77328600 & 1.86441200 & 6.11639700 & $\mathrm{H}$ & -0.33797600 & 2.22614100 & 5.13855400 \\
\hline $\mathrm{H}$ & -0.32106400 & 0.85355600 & 6.25741000 & C & -2.33223200 & -0.61385900 & 5.07289200 \\
\hline $\mathrm{H}$ & -3.20322000 & -0.18704400 & 5.58195800 & $\mathrm{H}$ & -1.75881800 & -1.17428000 & 5.82098000 \\
\hline $\mathrm{H}$ & -2.70125800 & -1.31926300 & 4.32087100 & C & -0.67414500 & -1.08930900 & 2.57453500 \\
\hline 0 & -1.07032300 & -0.60030200 & 1.50682900 & C & -0.14735900 & -3.16033300 & 3.93587600 \\
\hline C & -0.84554400 & -3.32449700 & 1.60637300 & C & -0.92374800 & -4.46947200 & 3.77297900 \\
\hline $\mathrm{H}$ & 0.93740600 & -3.32289100 & 3.94114800 & $\mathrm{H}$ & -0.41672700 & -2.61613500 & 4.83880000 \\
\hline $\mathrm{H}$ & -1.84029700 & -3.05664700 & 1.24900400 & $\mathrm{H}$ & -1.96341400 & -4.31626600 & 4.08687800 \\
\hline $\mathrm{H}$ & -0.50481700 & -5.28378300 & 4.37064100 & $\mathrm{~N}$ & -0.53969200 & -2.41991300 & 2.73234200 \\
\hline $\mathrm{C}$ & 0.15337100 & -3.21948700 & 0.46620100 & C & 1.41059200 & -2.73794800 & 0.68174700 \\
\hline C & -0.22534800 & -3.69241800 & -0.84848500 & $\mathrm{C}$ & 2.39836700 & -2.68292000 & -0.35399000 \\
\hline $\mathrm{H}$ & 1.68990500 & -2.35741000 & 1.65930900 & $\mathrm{C}$ & 0.74142300 & -3.64237200 & -1.90250400 \\
\hline C & -1.51007600 & -4.17667700 & -1.14730800 & $\mathrm{C}$ & 3.68662200 & -2.17444600 & -0.12344900 \\
\hline C & 2.05436800 & -3.15090000 & -1.65370100 & $\mathrm{C}$ & 0.38872200 & -4.07404700 & -3.21812700 \\
\hline $\mathrm{H}$ & -2.26001500 & -4.24035800 & -0.36882600 & $\mathrm{C}$ & -1.85603100 & -4.56502900 & -2.44003300 \\
\hline C & 4.62279200 & -2.12693700 & -1.15476000 & $\mathrm{H}$ & 3.94375600 & -1.81529000 & 0.86755600 \\
\hline C & 3.02031600 & -3.10543700 & -2.70154600 & C & -0.91878300 & -4.51770800 & -3.46774300 \\
\hline $\mathrm{C}$ & 1.38487700 & -4.02900700 & -4.25163500 & $\mathrm{H}$ & -2.86698700 & -4.90688600 & -2.64081400 \\
\hline $\mathrm{C}$ & 4.29614700 & -2.58908800 & -2.42831000 & $\mathrm{H}$ & 5.61419800 & -1.72998900 & -0.96494500 \\
\hline C & 2.64283100 & -3.57255400 & -4.00425500 & $\mathrm{H}$ & -1.18911000 & -4.83249700 & -4.47246800 \\
\hline $\mathrm{H}$ & 1.10784700 & -4.37130400 & -5.24561300 & $\mathrm{H}$ & 5.03186300 & -2.55003800 & -3.22767900 \\
\hline $\mathrm{H}$ & 3.38503300 & -3.54350000 & -4.79809300 & C & 1.90045700 & 0.65654100 & 2.85893700 \\
\hline S & 2.76332500 & -0.61244600 & 3.57100900 & $\mathrm{~N}$ & 0.57823400 & 0.87607500 & 3.10303100 \\
\hline $\mathrm{H}$ & 0.09981800 & 1.48273700 & 2.43187600 & $\mathrm{~S}$ & -1.07602700 & 3.06401400 & 0.07346000 \\
\hline 0 & -0.67150200 & 3.39948300 & -1.30141100 & $\mathrm{O}$ & -2.22550500 & 2.13802100 & 0.19228800 \\
\hline 0 & 0.05095700 & 2.75036100 & 1.00381800 & C & -1.70602500 & 4.65991800 & 0.79639700 \\
\hline $\mathrm{F}$ & -0.71835200 & 5.55411700 & 0.89727200 & $\mathrm{~F}$ & -2.21913900 & 4.44682800 & 2.02155500 \\
\hline $\mathrm{F}$ & -2.67737800 & 5.18516300 & 0.02957000 & C & -3.58415700 & -1.70007200 & -0.73600100 \\
\hline 0 & -4.30144900 & -2.06694800 & -1.65295100 & $\mathrm{O}$ & -3.80007600 & -1.87554700 & 0.57697200 \\
\hline C & -5.02997400 & -2.51245500 & 1.07436100 & C & -6.24729700 & -1.69598400 & 0.63906500 \\
\hline $\mathrm{H}$ & -7.14347000 & -2.08595300 & 1.13274900 & $\mathrm{H}$ & -6.39101700 & -1.74996300 & -0.44103400 \\
\hline $\mathrm{H}$ & -6.12253000 & -0.64885700 & 0.93381800 & C & -4.83981400 & -2.44258200 & 2.58814200 \\
\hline $\mathrm{H}$ & -3.93651800 & -2.98442100 & 2.88641600 & $\mathrm{H}$ & -5.69863400 & -2.89187300 & 3.09633100 \\
\hline $\mathrm{H}$ & -4.74090100 & -1.40363400 & 2.91529700 & C & -5.09620100 & -3.96253200 & 0.59438700 \\
\hline $\mathrm{H}$ & -5.97575500 & -4.45078800 & 1.02709800 & $\mathrm{H}$ & -4.20906200 & -4.51439800 & 0.92358400 \\
\hline $\mathrm{H}$ & -5.16523500 & -4.01337800 & -0.49308600 & C & -1.91520400 & -0.78491700 & -2.17694600 \\
\hline $\mathrm{H}$ & -2.35830900 & -1.44376900 & -2.91637300 & C & -0.48441200 & -0.48881300 & -2.35499900 \\
\hline C & 0.07904200 & -0.70508300 & -3.62392300 & C & 0.30942000 & 0.04070600 & -1.32835300 \\
\hline C & 1.40860900 & -0.38764200 & -3.86725200 & $\mathrm{H}$ & -0.53282100 & -1.13360400 & -4.41327000 \\
\hline C & 1.64453500 & 0.33630900 & -1.57680500 & $\mathrm{H}$ & -0.09596700 & 0.22288700 & -0.34146300 \\
\hline C & 2.19588700 & 0.14179000 & -2.84173500 & $\mathrm{H}$ & 1.83596100 & -0.56869400 & -4.84801700 \\
\hline $\mathrm{H}$ & 2.25867800 & 0.72091500 & -0.77593100 & $\mathrm{H}$ & 3.23321700 & 0.39862600 & -3.02190900 \\
\hline $\mathrm{N}$ & -2.39214300 & -1.03024600 & -0.91259900 & $\mathrm{H}$ & -1.87090000 & -0.77168100 & -0.06443300 \\
\hline
\end{tabular}




\begin{tabular}{|llll|llll|}
\hline $\mathrm{C}$ & -2.66191700 & 0.93539300 & -2.89033600 & $\mathrm{H}$ & -2.14501200 & 1.58552000 & -2.19243500 \\
\hline $\mathrm{H}$ & -2.24420100 & 0.90032800 & -3.89239100 & $\mathrm{C}$ & -4.04289200 & 0.77634200 & -2.74938300 \\
\hline $\mathrm{C}$ & -4.69023000 & 1.12828600 & -1.49798100 & $\mathrm{H}$ & -3.99197100 & 1.09228700 & -0.65627700 \\
\hline $\mathrm{H}$ & -5.59798800 & 0.55023500 & -1.30487100 & $\mathrm{C}$ & -4.86691500 & 0.20073300 & -3.85939500 \\
\hline $\mathrm{H}$ & -5.78933800 & 0.77755300 & -3.99139800 & $\mathrm{H}$ & -5.15936700 & -0.81976100 & -3.58688900 \\
\hline $\mathrm{H}$ & -4.32363300 & 0.17358700 & -4.80733400 & $\mathrm{C}$ & -5.37554000 & 3.47424900 & 0.31933000 \\
\hline $\mathrm{H}$ & -6.16686400 & 2.89027700 & 0.80484100 & $\mathrm{H}$ & -5.62441500 & 4.53568600 & 0.43209300 \\
\hline $\mathrm{H}$ & -4.43522500 & 3.28593800 & 0.84265700 & $\mathrm{C}$ & -6.93495300 & 3.08649400 & -2.31856500 \\
\hline $\mathrm{H}$ & -7.65804800 & 2.43211500 & -1.81784300 & $\mathrm{H}$ & -6.89642700 & 2.80161800 & -3.37650700 \\
\hline $\mathrm{H}$ & -7.32904900 & 4.10926900 & -2.27379500 & $\mathrm{C}$ & -4.02172200 & 4.04576400 & -2.48758100 \\
\hline $\mathrm{H}$ & -4.09636500 & 3.80286100 & -3.55431300 & $\mathrm{H}$ & -2.98275700 & 3.90683800 & -2.17951400 \\
\hline $\mathrm{H}$ & -4.26169000 & 5.10927700 & -2.37221600 & $\mathrm{Si}$ & -5.23324500 & 3.00899800 & -1.49694100 \\
\hline $\mathrm{C}$ & -0.84732700 & -4.73371200 & 2.26129800 & $\mathrm{H}$ & 0.08680900 & -5.24653200 & 2.01367600 \\
\hline $\mathrm{H}$ & -1.67004800 & -5.35144500 & 1.89075800 & & & & \\
\hline
\end{tabular}

\section{TS-S-4f-11}

Imaginary frequency: $-398.16 \mathrm{~cm}^{-1}$

E[B3LYP/6-31G(d)-D3(BJ)]: -4789.092613 Hartree

E[PCM(Et2O)-B3LYP/6-311G(d,p)-D3(BJ)]: - 4790.233802 Hartree

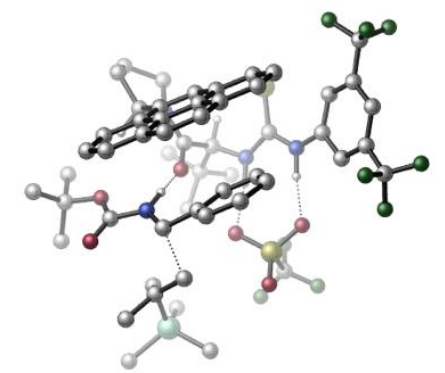

\begin{tabular}{|c|c|c|c|c|c|c|c|}
\hline 01 & & & & $\mathrm{~N}$ & 2.26197500 & 1.70189700 & 1.57206500 \\
\hline $\mathrm{H}$ & 1.67687300 & 2.33763100 & 1.01554800 & C & 3.55796000 & 1.57477700 & 1.04111000 \\
\hline C & 3.79373800 & 2.25067500 & -0.17319600 & C & 4.58703800 & 0.79746000 & 1.58677900 \\
\hline $\mathrm{C}$ & 5.00214900 & 2.09389800 & -0.83980300 & $\mathrm{H}$ & 3.01334100 & 2.86433200 & -0.60704200 \\
\hline $\mathrm{C}$ & 5.78511800 & 0.64467100 & 0.88892800 & $\mathrm{H}$ & 4.43508800 & 0.28841200 & 2.52499600 \\
\hline C & 6.01246400 & 1.28006100 & -0.32611400 & $\mathrm{H}$ & 6.94180600 & 1.14108700 & -0.86466900 \\
\hline $\mathrm{C}$ & 5.19469100 & 2.71605700 & -2.19381200 & $\mathrm{C}$ & 6.85398700 & -0.23415900 & 1.47554400 \\
\hline $\mathrm{F}$ & 4.35011500 & 3.73708800 & -2.42132200 & $\mathrm{~F}$ & 4.99261200 & 1.80247700 & -3.18358100 \\
\hline $\mathrm{F}$ & 6.45516800 & 3.17690900 & -2.35760600 & $\mathrm{~F}$ & 6.33042500 & -1.22125600 & 2.23701400 \\
\hline $\mathrm{F}$ & 7.58890000 & -0.83138500 & 0.50236200 & $\mathrm{~F}$ & 7.71923000 & 0.45117100 & 2.25295400 \\
\hline $\mathrm{C}$ & -0.63742400 & 0.42043900 & 3.36448900 & $\mathrm{H}$ & -0.06497900 & 0.10403500 & 4.23438200 \\
\hline C & -1.89496800 & 1.18232900 & 3.87959700 & C & -2.87910700 & 1.58567300 & 2.76932700 \\
\hline $\bar{H}$ & -3.27885000 & 0.71358700 & 2.24340400 & $\mathrm{H}$ & -2.40819900 & 2.22718500 & 2.02389400 \\
\hline $\mathrm{H}$ & -3.71618200 & 2.13498700 & 3.21644300 & $\mathrm{C}$ & -1.41368100 & 2.44492000 & 4.61433200 \\
\hline $\mathrm{H}$ & -2.26184500 & 2.94463600 & 5.09633400 & $\mathrm{H}$ & -0.95199900 & 3.15062500 & 3.91930000 \\
\hline $\mathrm{H}$ & -0.67693900 & 2.19922000 & 5.38792600 & $\mathrm{C}$ & -2.62480200 & 0.25985700 & 4.87333200 \\
\hline $\bar{H}$ & -3.52482100 & 0.75490000 & 5.25374600 & $\bar{H}$ & -1.99325200 & 0.01471600 & 5.73561400 \\
\hline $\mathrm{H}$ & -2.94003200 & -0.67767800 & 4.39820000 & C & -0.96626100 & -0.78482000 & 2.47624400 \\
\hline $\mathrm{O}$ & -1.34303300 & -0.56242200 & 1.31561400 & $\mathrm{C}$ & -0.43865700 & -2.49278800 & 4.27239200 \\
\hline $\mathrm{C}$ & -1.00121500 & -3.17044700 & 1.99220400 & $\mathrm{C}$ & -1.12347300 & -3.86104400 & 4.33515600 \\
\hline $\mathrm{H}$ & 0.64973500 & -2.57038600 & 4.37767900 & $\mathrm{H}$ & -0.80610800 & -1.79643700 & 5.02414700 \\
\hline $\mathrm{H}$ & -2.00388500 & -3.04851200 & 1.58188200 & $\mathrm{H}$ & -2.19100400 & -3.72618200 & 4.54776900 \\
\hline $\mathrm{H}$ & -0.70048300 & -4.50622700 & 5.11010100 & $\mathrm{~N}$ & -0.78249300 & -2.04003300 & 2.91993300 \\
\hline $\mathrm{C}$ & 0.00863200 & -3.20820800 & 0.85688200 & $\mathrm{C}$ & 1.26717300 & -2.71415300 & 1.03832000 \\
\hline C & -0.35779800 & -3.80845400 & -0.40839700 & C & 2.26508400 & -2.76422600 & 0.01280800 \\
\hline $\mathrm{H}$ & 1.53528400 & -2.23525900 & 1.97579600 & $\mathrm{C}$ & 0.61120800 & -3.83891300 & -1.46131600 \\
\hline $\mathrm{C}$ & -1.63193500 & -4.34423300 & -0.66289500 & $\mathrm{C}$ & 3.56047100 & -2.26143100 & 0.21369400 \\
\hline C & 1.92443200 & -3.33246500 & -1.24736700 & C & 0.26422500 & -4.37838500 & -2.73839800 \\
\hline $\mathrm{H}$ & -2.37790800 & -4.35699100 & 0.12120800 & $\mathrm{C}$ & -1.97091600 & -4.84321200 & -1.91820200 \\
\hline $\mathrm{C}$ & 4.50481800 & -2.30910300 & -0.80954400 & $\mathrm{H}$ & 3.81964400 & -1.83532700 & 1.17678500 \\
\hline
\end{tabular}




\begin{tabular}{|c|c|c|c|c|c|c|c|}
\hline C & 2.89709400 & -3.37956300 & -2.28914000 & C & -1.03696900 & -4.85739800 & -2.95030200 \\
\hline $\mathrm{C}$ & 1.26346700 & -4.41384700 & -3.76943900 & $\mathrm{H}$ & -2.97312100 & -5.22612300 & -2.08808200 \\
\hline C & 4.17826700 & -2.86091200 & -2.04730000 & $\mathrm{H}$ & 5.50215800 & -1.91743600 & -0.63904300 \\
\hline C & 2.52201400 & -3.94408000 & -3.55338200 & $\mathrm{H}$ & -1.30329500 & -5.25518700 & -3.92619000 \\
\hline $\mathrm{H}$ & 0.98911700 & -4.83257200 & -4.73443100 & $\mathrm{H}$ & 4.91941800 & -2.89347200 & -2.84180000 \\
\hline $\mathrm{H}$ & 3.26737800 & -3.97970300 & -4.34387500 & C & 1.61031700 & 0.99955800 & 2.54909400 \\
\hline $\mathrm{S}$ & 2.34606600 & -0.05727000 & 3.65285300 & $\mathrm{~N}$ & 0.26580400 & 1.25308400 & 2.57416800 \\
\hline $\mathrm{H}$ & -0.16261600 & 1.63663800 & 1.72588200 & $S$ & -0.51232100 & 3.38997200 & -0.53775400 \\
\hline 0 & -0.93037800 & 3.65858600 & -1.92207900 & 0 & -1.21228100 & 2.25020300 & 0.12182500 \\
\hline 0 & 0.95155700 & 3.39878000 & -0.28132700 & $\mathrm{C}$ & -1.09457600 & 4.85958400 & 0.43887000 \\
\hline $\mathrm{F}$ & -0.46888700 & 5.97297600 & 0.05507000 & $\mathrm{~F}$ & -0.86087500 & 4.66097800 & 1.74878300 \\
\hline $\mathrm{F}$ & -2.42002700 & 5.04135800 & 0.27834000 & C & -3.67807700 & -1.78838900 & -0.93864200 \\
\hline 0 & -4.44430600 & -1.98372200 & -1.86944100 & $\mathrm{O}$ & -3.89669300 & -2.06197500 & 0.35869500 \\
\hline C & -5.18362100 & -2.59711300 & 0.82795800 & C & -6.29952700 & -1.59537200 & 0.52787600 \\
\hline $\mathrm{H}$ & -7.23420200 & -1.94176500 & 0.98079900 & $\mathrm{H}$ & -6.45301400 & -1.49033600 & -0.54754400 \\
\hline $\mathrm{H}$ & -6.05541700 & -0.61817800 & 0.95799000 & C & -4.95610400 & -2.71731100 & 2.33475200 \\
\hline $\mathrm{H}$ & -4.14418500 & -3.41960100 & 2.54862600 & $\mathrm{H}$ & -5.86503700 & -3.08104500 & 2.82377000 \\
\hline $\mathrm{H}$ & -4.69219400 & -1.74457400 & 2.76115300 & C & -5.44866200 & -3.96488500 & 0.19787200 \\
\hline $\mathrm{H}$ & -6.36757000 & -4.38718200 & 0.61818200 & $\mathrm{H}$ & -4.62818900 & -4.65497600 & 0.41948100 \\
\hline $\mathrm{H}$ & -5.56063700 & -3.88242500 & -0.88388800 & $\mathrm{C}$ & -1.95586700 & -0.89391800 & -2.33007500 \\
\hline $\mathrm{H}$ & -2.44692800 & -1.45931500 & -3.11627400 & C & -0.50595400 & -0.68463100 & -2.49461000 \\
\hline $\mathrm{C}$ & 0.06745700 & -0.95196900 & -3.74729200 & C & 0.29389200 & -0.17618500 & -1.46068200 \\
\hline $\mathrm{C}$ & 1.41804300 & -0.70611200 & -3.96797600 & $\mathrm{H}$ & -0.55031600 & -1.36027400 & -4.54308100 \\
\hline C & 1.64365700 & 0.05851800 & -1.68893100 & $\mathrm{H}$ & -0.13301600 & 0.05690000 & -0.49404800 \\
\hline C & 2.20911600 & -0.18986400 & -2.93977300 & $\mathrm{H}$ & 1.85474700 & -0.92447600 & -4.93736000 \\
\hline $\mathrm{H}$ & 2.26113100 & 0.43353200 & -0.88630600 & $\mathrm{H}$ & 3.25884400 & 0.02345700 & -3.10582700 \\
\hline $\mathrm{N}$ & -2.42782800 & -1.23119200 & -1.08379400 & $\mathrm{H}$ & -1.89856700 & -1.04066400 & -0.22135500 \\
\hline C & -2.58991500 & 0.90227000 & -2.90674300 & $\mathrm{H}$ & -1.93512900 & 1.51353800 & -2.29382900 \\
\hline $\mathrm{H}$ & -2.31852300 & 0.84846900 & -3.95860400 & C & -3.94582700 & 0.87035100 & -2.56970100 \\
\hline C & -4.35558700 & 1.24453600 & -1.22743500 & $\mathrm{H}$ & -3.50985800 & 1.30674100 & -0.53508300 \\
\hline $\mathrm{H}$ & -5.14871300 & 0.60134500 & -0.83567000 & C & -4.96961000 & 0.45601200 & -3.58161900 \\
\hline $\mathrm{H}$ & -5.25501000 & 1.33626200 & -4.17549700 & $\mathrm{H}$ & -5.86874800 & 0.04770700 & -3.11786500 \\
\hline $\mathrm{H}$ & -4.55988500 & -0.28369400 & -4.27428200 & C & -5.19870300 & 3.50066900 & 0.61668400 \\
\hline $\mathrm{H}$ & -5.71187600 & 2.74219900 & 1.21928300 & $\mathrm{H}$ & -5.72525900 & 4.45252500 & 0.75552200 \\
\hline $\mathrm{H}$ & -4.18435600 & 3.61673500 & 1.00294900 & $\mathrm{C}$ & -6.96267700 & 2.80958100 & -1.80773400 \\
\hline $\mathrm{H}$ & -7.51027400 & 2.07164100 & -1.20957400 & $\mathrm{H}$ & -7.01447900 & 2.49880100 & -2.85660400 \\
\hline $\mathrm{H}$ & -7.49754700 & 3.76357800 & -1.72400800 & C & -4.24142300 & 4.19392900 & -2.30951700 \\
\hline $\mathrm{H}$ & -4.40522600 & 3.94584900 & -3.36527700 & $\mathrm{H}$ & -3.16428600 & 4.16694200 & -2.12221300 \\
\hline $\mathrm{H}$ & -4.58973500 & 5.22228800 & -2.15562900 & $\mathrm{Si}$ & -5.18541000 & 3.01546600 & -1.19956300 \\
\hline $\mathrm{C}$ & -0.92036600 & -4.41344100 & 2.91603700 & $\mathrm{H}$ & 0.07247700 & -4.86444400 & 2.82533800 \\
\hline $\mathrm{H}$ & -1.65544700 & -5.17330800 & 2.63642100 & & & & \\
\hline
\end{tabular}

\section{TS-S-4f-12}

Imaginary frequency: $-411.89 \mathrm{~cm}^{-1}$

E[B3LYP/6-31G(d)-D3(BJ)]: -4789.097454 Hartree

$\mathrm{E}\left[\mathrm{PCM}\left(\mathrm{Et}_{2} \mathrm{O}\right)-\mathrm{B} 3 \mathrm{LYP} / 6-311 \mathrm{G}(\mathrm{d}, \mathrm{p})-\mathrm{D} 3(\mathrm{BJ})\right]$ : -4790.233683 Hartree

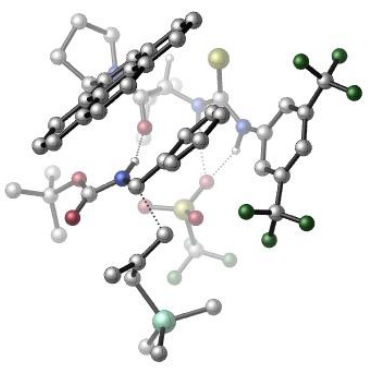

\begin{tabular}{|lrll|llll|}
\hline 01 & & & $\mathrm{~N}$ & -0.73034300 & 1.24587300 & -3.18789300 \\
\hline $\mathrm{H}$ & 0.29246300 & 1.30143700 & -3.17725300 & $\mathrm{C}$ & -1.35403700 & 2.29556600 & -2.49172500 \\
\hline $\mathrm{C}$ & -0.57780800 & 2.98609600 & -1.55113300 & $\mathrm{C}$ & -2.69291700 & 2.64951000 & -2.68329300 \\
\hline
\end{tabular}




\begin{tabular}{|c|c|c|c|c|c|c|c|}
\hline$C$ & -1.15605900 & 3.98863200 & -0.77933500 & $\mathrm{H}$ & 0.45626100 & 2.69713600 & -1.38794300 \\
\hline C & -3.25832000 & 3.63651100 & -1.87542300 & $\mathrm{H}$ & -3.29153400 & 2.13344100 & -3.41914100 \\
\hline C & -2.50411900 & 4.31315200 & -0.91953800 & $\mathrm{H}$ & -2.95846600 & 5.07088500 & -0.29361000 \\
\hline C & -0.35720800 & 4.61523300 & 0.32544600 & C & -4.73878900 & 3.87100100 & -1.94287500 \\
\hline $\mathrm{F}$ & 0.96195300 & 4.66658800 & 0.03942300 & $\mathrm{~F}$ & -0.47579700 & 3.90884200 & 1.48185700 \\
\hline $\mathrm{F}$ & -0.76550300 & 5.87331300 & 0.60107300 & $\mathrm{~F}$ & -5.23879200 & 3.66689700 & -3.17797300 \\
\hline $\mathrm{F}$ & -5.39857900 & 3.02051800 & -1.10819100 & $\mathrm{~F}$ & -5.07790800 & 5.12272200 & -1.56407700 \\
\hline C & -0.32863400 & -2.31111200 & -3.58165500 & $\mathrm{H}$ & -1.27060100 & -2.66515100 & -4.00484100 \\
\hline C & 0.85485300 & -3.02365700 & -4.32557300 & C & 2.22463400 & -2.51447800 & -3.84193800 \\
\hline $\mathrm{H}$ & 2.30723600 & -2.53010800 & -2.75425200 & $\mathrm{H}$ & 2.42790400 & -1.48915700 & -4.15881000 \\
\hline $\mathrm{H}$ & 3.01513700 & -3.14564400 & -4.26390100 & C & 0.69900900 & -2.74317200 & -5.83113100 \\
\hline $\mathrm{H}$ & 1.50841400 & -3.22919400 & -6.38789500 & $\mathrm{H}$ & 0.73446600 & -1.67116300 & -6.04195700 \\
\hline $\mathrm{H}$ & -0.25436100 & -3.13074800 & -6.21014400 & C & 0.80180000 & -4.54446800 & -4.10826700 \\
\hline $\mathrm{H}$ & 1.64543800 & -5.01262600 & -4.62626300 & $\mathrm{H}$ & -0.11343200 & -4.98111700 & -4.52215100 \\
\hline $\mathrm{H}$ & 0.87566500 & -4.81161800 & -3.04878500 & C & -0.33875400 & -2.52070700 & -2.05000300 \\
\hline C & -0.90764300 & -3.85701400 & -0.08755500 & $\mathrm{H}$ & 0.12434900 & -3.67958600 & 0.21344400 \\
\hline $\mathrm{N}$ & -1.00315400 & -3.57469600 & -1.52717000 & C & -1.85730500 & -3.02177100 & 0.75517000 \\
\hline C & -2.95778500 & -2.44067800 & 0.19789900 & C & -1.62159300 & -2.89893200 & 2.17904400 \\
\hline C & -3.93406400 & -1.73966300 & 0.97470600 & $\mathrm{H}$ & -3.10048000 & -2.46635500 & -0.87635400 \\
\hline $\mathrm{C}$ & -2.55722800 & -2.16068200 & 2.97046000 & C & -0.49042100 & -3.43826500 & 2.81491700 \\
\hline C & -5.07438000 & -1.16926000 & 0.39032800 & $\mathrm{C}$ & -3.72490900 & -1.60345000 & 2.37506300 \\
\hline $\mathrm{C}$ & -2.31815900 & -1.96658700 & 4.36542200 & $\mathrm{H}$ & 0.23128900 & -4.01021600 & 2.24494400 \\
\hline C & -0.25159800 & -3.22257300 & 4.17086900 & $\mathrm{C}$ & -6.00121500 & -0.48255600 & 1.17111800 \\
\hline $\mathrm{H}$ & -5.21448400 & -1.25397400 & -0.68345600 & $\mathrm{C}$ & -4.66951500 & -0.88526600 & 3.16532000 \\
\hline C & -1.15194400 & -2.49299600 & 4.94156800 & $\mathrm{C}$ & -3.28055700 & -1.22927600 & 5.13603200 \\
\hline $\mathrm{H}$ & 0.65019100 & -3.62432900 & 4.62249200 & $\mathrm{C}$ & -5.80389900 & -0.34170200 & 2.54351500 \\
\hline $\mathrm{H}$ & -6.87698600 & -0.04171600 & 0.70396300 & C & -4.40789900 & -0.72216800 & 4.56663300 \\
\hline $\mathrm{H}$ & -0.96463500 & -2.33269400 & 6.00027100 & $\mathrm{H}$ & -3.08956000 & -1.09137300 & 6.19736400 \\
\hline $\mathrm{H}$ & -6.52464600 & 0.20584500 & 3.14545300 & $\mathrm{H}$ & -5.12875500 & -0.17188600 & 5.16609900 \\
\hline C & -1.20710400 & -0.03086400 & -3.36529300 & $\mathrm{~S}$ & -2.79297500 & -0.53504100 & -3.07091500 \\
\hline $\mathrm{N}$ & -0.23709900 & -0.87691500 & -3.80772600 & $\mathrm{H}$ & 0.70998500 & -0.51693800 & -3.68036400 \\
\hline $\mathrm{S}$ & 2.81975400 & 0.66131500 & -1.80796100 & 0 & 2.22488600 & 1.53477100 & -0.76817500 \\
\hline 0 & 3.26532300 & -0.66145900 & -1.34105800 & $\mathrm{O}$ & 2.07917500 & 0.69788200 & -3.10557800 \\
\hline C & 4.40707100 & 1.50718200 & -2.26771900 & $\mathrm{~F}$ & 4.20486600 & 2.80376400 & -2.54277300 \\
\hline $\mathrm{F}$ & 4.96839500 & 0.92417300 & -3.33040300 & $\mathrm{~F}$ & 5.27663500 & 1.43023800 & -1.23759100 \\
\hline C & 2.02926000 & -1.78043000 & 1.75611100 & $\mathrm{O}$ & 2.54779400 & -1.67772800 & 2.85837300 \\
\hline 0 & 2.29394000 & -2.69261200 & 0.82235300 & C & 3.50415600 & -3.53233000 & 0.89290900 \\
\hline C & 4.74249600 & -2.64357700 & 1.01407600 & $\mathrm{H}$ & 5.63917900 & -3.25602500 & 0.87071100 \\
\hline $\mathrm{H}$ & 4.79786200 & -2.17398700 & 1.99779300 & $\mathrm{H}$ & 4.71599800 & -1.86974700 & 0.24187500 \\
\hline C & 3.48177200 & -4.24261200 & -0.45816200 & $\mathrm{H}$ & 2.54825300 & -4.80205100 & -0.58364300 \\
\hline $\mathrm{H}$ & 4.31870800 & -4.94511800 & -0.52450700 & $\mathrm{H}$ & 3.56315100 & -3.51269300 & -1.26620900 \\
\hline C & 3.36497200 & -4.52085500 & 2.05036100 & $\mathrm{H}$ & 4.22665100 & -5.19696100 & 2.06087200 \\
\hline $\mathrm{H}$ & 2.46061600 & -5.12778600 & 1.92940100 & $\mathrm{H}$ & 3.31706900 & -3.99664100 & 3.00626100 \\
\hline C & 0.45537200 & -0.03780600 & 2.17731800 & $\mathrm{H}$ & 0.58201900 & -0.33559100 & 3.21343500 \\
\hline C & -0.86006700 & 0.54755200 & 1.85495100 & C & -1.56878800 & 1.16588500 & 2.90111400 \\
\hline C & -1.41662800 & 0.52474000 & 0.57080600 & $\mathrm{C}$ & -2.79060100 & 1.77668700 & 2.66376100 \\
\hline $\mathrm{H}$ & -1.15075700 & 1.15933200 & 3.90446200 & C & -2.66417900 & 1.10428100 & 0.34957900 \\
\hline $\mathrm{H}$ & -0.90785400 & 0.04129200 & -0.25487100 & C & -3.33910600 & 1.75384700 & 1.37845500 \\
\hline $\mathrm{H}$ & -3.32567800 & 2.24972100 & 3.48113200 & $\mathrm{H}$ & -3.10474400 & 1.03886700 & -0.63589600 \\
\hline $\mathrm{H}$ & -4.29782700 & 2.21881500 & 1.17731300 & $\mathrm{~N}$ & 1.02281500 & -0.94153700 & 1.31476600 \\
\hline $\mathrm{H}$ & 0.73119100 & -1.05315700 & 0.33764400 & C & 1.55022700 & 1.63724100 & 2.29840700 \\
\hline $\mathrm{H}$ & 1.58483300 & 1.90004900 & 1.24678300 & $\mathrm{H}$ & 0.84266800 & 2.19174000 & 2.90365800 \\
\hline $\mathrm{C}$ & 2.73985200 & 1.23740000 & 2.91884000 & C & 3.95721000 & 1.01299100 & 2.15488500 \\
\hline
\end{tabular}




\begin{tabular}{|c|c|c|c|c|c|c|c|}
\hline $\mathrm{H}$ & 3.75659600 & 0.75217600 & 1.11136100 & $\mathrm{H}$ & 4.62482700 & 0.28330700 & 2.62160400 \\
\hline C & 2.76374900 & 1.02625700 & 4.40069600 & $\mathrm{H}$ & 3.74271800 & 1.25525500 & 4.82825600 \\
\hline $\mathrm{H}$ & 2.57408400 & -0.04062800 & 4.57746000 & $\mathrm{H}$ & 1.99520500 & 1.60985600 & 4.91546900 \\
\hline C & 6.69653800 & 2.23246700 & 1.51557600 & $\mathrm{H}$ & 7.17652800 & 1.57798600 & 2.25255900 \\
\hline $\mathrm{H}$ & 7.31200400 & 3.13558400 & 1.42245900 & $\mathrm{H}$ & 6.69988500 & 1.72286900 & 0.54951200 \\
\hline $\mathrm{C}$ & 5.05871000 & 3.46876000 & 3.78688400 & $\mathrm{H}$ & 5.53493500 & 2.80510500 & 4.51788800 \\
\hline $\mathrm{H}$ & 4.07489300 & 3.75429500 & 4.17526700 & $\mathrm{H}$ & 5.66542300 & 4.38112000 & 3.73355000 \\
\hline C & 4.08995800 & 3.88882000 & 0.90656000 & $\mathrm{H}$ & 3.07253700 & 4.10988000 & 1.24291100 \\
\hline $\bar{H}$ & 4.01615700 & 3.50296300 & -0.11153800 & $\mathrm{H}$ & 4.64309400 & 4.83588100 & 0.87558200 \\
\hline $\mathrm{Si}$ & 4.95439300 & 2.69474900 & 2.06539500 & $\mathrm{C}$ & -2.40581500 & -5.44060000 & -1.09916900 \\
\hline $\mathrm{H}$ & -2.55839500 & -6.45585900 & -1.47449800 & $\mathrm{H}$ & -3.34988500 & -5.09421200 & -0.66960300 \\
\hline $\mathrm{C}$ & -1.29162700 & -5.35030000 & -0.04464800 & $\mathrm{H}$ & -1.61404300 & -5.66802300 & 0.94964700 \\
\hline $\mathrm{H}$ & -0.42783700 & -5.95759800 & -0.34132000 & C & -1.94151700 & -4.48204100 & -2.21318900 \\
\hline $\mathrm{H}$ & -1.43422100 & -5.02871500 & -3.00746600 & $\mathrm{H}$ & -2.76557200 & -3.91393000 & -2.65781500 \\
\hline 0 & 0.28378900 & -1.72682000 & -1.33178100 & & & & \\
\hline
\end{tabular}

\section{TS-S-4f-13}

Imaginary frequency: $-312.21 \mathrm{~cm}^{-1}$

E[B3LYP/6-31G(d)-D3(BJ)]: -4789.093331 Hartree

E[PCM(Et $2 \mathrm{O})-B 3 L Y P / 6-311 \mathrm{G}(\mathrm{d}, \mathrm{p})-\mathrm{D} 3(\mathrm{BJ})]$ : - 4790.233477 Hartree

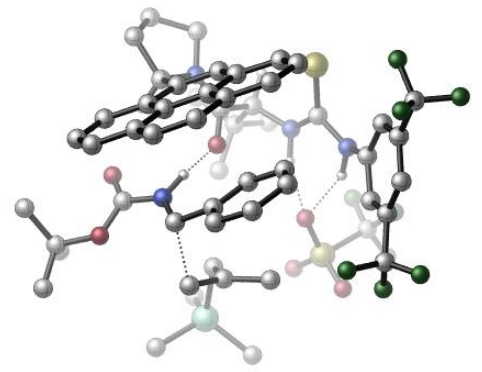

\begin{tabular}{|c|c|c|c|c|c|c|c|}
\hline 01 & & & & $\mathrm{~N}$ & 2.68056300 & 1.45707900 & 1.51242500 \\
\hline $\mathrm{H}$ & 2.25717300 & 2.27796300 & 1.07708700 & $\mathrm{C}$ & 3.49817300 & 0.77603500 & 0.60046400 \\
\hline $\mathrm{C}$ & 3.45288800 & 1.24255200 & -0.72506300 & $\mathrm{C}$ & 4.29789100 & -0.33001800 & 0.91453900 \\
\hline $\mathrm{C}$ & 4.14060900 & 0.56936200 & -1.72588100 & $\mathrm{H}$ & 2.84450100 & 2.10536700 & -0.97352800 \\
\hline C & 4.97389000 & -0.99307400 & -0.11141700 & $\mathrm{H}$ & 4.34617500 & -0.68863300 & 1.93137500 \\
\hline $\mathrm{C}$ & 4.90429000 & -0.56083600 & -1.43412300 & $\mathrm{H}$ & 5.41818500 & -1.09845800 & -2.22188300 \\
\hline $\mathrm{C}$ & 3.93212600 & 0.96749300 & -3.16035300 & $C$ & 5.80022400 & -2.20761500 & 0.20468000 \\
\hline $\mathrm{F}$ & 3.52449700 & 2.24342900 & -3.28698200 & $\mathrm{~F}$ & 2.97786200 & 0.19000500 & -3.74156000 \\
\hline $\mathrm{F}$ & 5.05470200 & 0.81154300 & -3.89555100 & $\mathrm{~F}$ & 5.45884200 & -2.76793200 & 1.38729700 \\
\hline $\mathrm{F}$ & 5.64663100 & -3.16382800 & -0.74863800 & $\mathrm{~F}$ & 7.11982200 & -1.92893500 & 0.26292500 \\
\hline C & -0.33049600 & 1.24050800 & 3.52747900 & $\mathrm{H}$ & 0.06876000 & 0.90329300 & 4.48436300 \\
\hline C & -1.32507500 & 2.42294800 & 3.78569400 & C & -1.99006600 & 2.93939800 & 2.49354300 \\
\hline $\mathrm{H}$ & -2.58604500 & 2.16291000 & 2.00655800 & $\mathrm{H}$ & -1.26537200 & 3.31808700 & 1.76825800 \\
\hline $\mathrm{H}$ & -2.65377100 & 3.77392200 & 2.74663000 & C & -0.53882900 & 3.56871800 & 4.44844900 \\
\hline $\mathrm{H}$ & -1.22528200 & 4.37483400 & 4.73061800 & $\mathrm{H}$ & 0.21167700 & 3.98741500 & 3.77395000 \\
\hline $\mathrm{H}$ & -0.02673600 & 3.22494700 & 5.35500200 & $\mathrm{C}$ & -2.42883700 & 1.95198600 & 4.74845200 \\
\hline $\mathrm{H}$ & -3.12988300 & 2.77242600 & 4.93412200 & $\mathrm{H}$ & -2.01700600 & 1.64848200 & 5.71821000 \\
\hline $\mathrm{H}$ & -3.00289400 & 1.11597000 & 4.33426300 & $\mathrm{C}$ & -1.00122600 & 0.07424000 & 2.77736200 \\
\hline 0 & -1.13705900 & 0.18416000 & 1.54609900 & $\mathrm{C}$ & -1.33410300 & -1.29391400 & 4.87701500 \\
\hline C & -2.22320600 & -2.04941500 & 2.73954600 & C & -2.63021200 & -2.05461300 & 5.16134200 \\
\hline $\mathrm{H}$ & -0.44688800 & -1.91503600 & 5.05411700 & $\mathrm{H}$ & -1.23655500 & -0.38413900 & 5.46383200 \\
\hline $\mathrm{H}$ & -3.01085000 & -1.53553100 & 2.18522500 & $\mathrm{H}$ & -3.45275600 & -1.33923100 & 5.27513100 \\
\hline $\mathrm{H}$ & -2.57234300 & -2.65651100 & 6.07251000 & $\mathrm{~N}$ & -1.45330700 & -1.00070000 & 3.44514000 \\
\hline C & -1.38068100 & -2.88836000 & 1.79421400 & C & -0.03134100 & -2.98325900 & 1.96405200 \\
\hline C & -2.03232200 & -3.64015800 & 0.74194700 & C & 0.79147800 & -3.80797900 & 1.13176500 \\
\hline $\mathrm{H}$ & 0.46112500 & -2.39128300 & 2.72794200 & $\mathrm{C}$ & -1.22800700 & -4.44879100 & -0.12314700 \\
\hline C & -3.41980700 & -3.59936300 & 0.51775100 & C & 2.18332400 & -3.88233400 & 1.30357900 \\
\hline C & 0.17827400 & -4.54574900 & 0.08006800 & C & -1.83280500 & -5.16308100 & -1.20322400 \\
\hline $\mathrm{H}$ & -4.05621800 & -3.00551800 & 1.16256200 & C & -3.99865800 & -4.29782400 & -0.54033000 \\
\hline
\end{tabular}




\begin{tabular}{|c|c|c|c|c|c|c|c|}
\hline $\mathrm{C}$ & 2.95728600 & -4.68410600 & 0.46736000 & $\mathrm{H}$ & 2.65326300 & -3.28641800 & 2.08013000 \\
\hline C & 0.97892900 & -5.35736000 & -0.77669400 & C & -3.21792400 & -5.06510000 & -1.39974500 \\
\hline $\mathrm{C}$ & -1.00154900 & -5.96708800 & -2.05493200 & $\mathrm{H}$ & -5.07391800 & -4.25215600 & -0.68435600 \\
\hline C & 2.36343100 & -5.41669600 & -0.55866300 & $\mathrm{H}$ & 4.03138200 & -4.72193300 & 0.60544000 \\
\hline C & 0.33953600 & -6.06597500 & -1.84753100 & $\mathrm{H}$ & -3.67703300 & -5.60945600 & -2.22104000 \\
\hline $\mathrm{H}$ & -1.47471100 & -6.50609500 & -2.87189400 & $\mathrm{H}$ & 2.97489300 & -6.03402600 & -1.21193900 \\
\hline $\mathrm{H}$ & 0.95455500 & -6.68237400 & -2.49836800 & C & 1.89216000 & 0.93372200 & 2.51409100 \\
\hline S & 2.23646800 & -0.45429200 & 3.40876800 & $\mathrm{~N}$ & 0.78809300 & 1.69437300 & 2.72519900 \\
\hline $\mathrm{H}$ & 0.54733400 & 2.33717900 & 1.97001300 & $S$ & 0.94769600 & 4.54446400 & -0.24209200 \\
\hline 0 & -0.25796900 & 5.36364100 & -0.03869500 & $\mathrm{O}$ & 0.80842600 & 3.13999500 & 0.28689100 \\
\hline 0 & 1.58047900 & 4.58752100 & -1.57256400 & C & 2.21291000 & 5.26124200 & 0.91536300 \\
\hline$F$ & 2.52205900 & 6.51313800 & 0.57749800 & $\mathrm{~F}$ & 3.33170000 & 4.51255300 & 0.88284600 \\
\hline $\mathrm{F}$ & 1.74604700 & 5.25544500 & 2.17606200 & C & -3.92229900 & -0.46704100 & -0.38919900 \\
\hline 0 & -4.35617100 & -0.29939200 & 0.73701600 & $\mathrm{O}$ & -4.63500200 & -0.48819400 & -1.52623600 \\
\hline C & -6.10877000 & -0.35413100 & -1.50200900 & C & -6.71031600 & -1.56179300 & -0.78604200 \\
\hline $\mathrm{H}$ & -7.80255000 & -1.51470300 & -0.84604600 & $\mathrm{H}$ & -6.41932100 & -1.57761100 & 0.26557000 \\
\hline $\mathrm{H}$ & -6.37937200 & -2.48813400 & -1.26462700 & C & -6.45862300 & -0.36090800 & -2.98838900 \\
\hline $\mathrm{H}$ & -5.99918000 & 0.49161900 & -3.49877300 & $\mathrm{H}$ & -7.54340300 & -0.29508300 & -3.11570600 \\
\hline $\mathrm{H}$ & -6.10863200 & -1.28293600 & -3.46306000 & C & -6.51215900 & 0.96868300 & -0.84966100 \\
\hline $\mathrm{H}$ & -7.58931700 & 1.11676500 & -0.97982600 & $\mathrm{H}$ & -5.99711600 & 1.80689700 & -1.32894900 \\
\hline $\mathrm{H}$ & -6.27938800 & 0.97457400 & 0.21486600 & C & -2.02907100 & -1.01518500 & -1.84258700 \\
\hline $\mathrm{H}$ & -2.73655500 & -1.53254100 & -2.48183200 & C & -0.65071400 & -1.52023500 & -1.90023600 \\
\hline C & -0.30355600 & -2.35184100 & -2.97932100 & C & 0.32676000 & -1.17948700 & -0.95260100 \\
\hline C & 0.99398700 & -2.82822700 & -3.11390600 & $\mathrm{H}$ & -1.06379600 & -2.63106700 & -3.70438200 \\
\hline $\mathrm{C}$ & 1.61982700 & -1.67474800 & -1.08826200 & $\mathrm{H}$ & 0.08660500 & -0.54877900 & -0.10427500 \\
\hline $\mathrm{C}$ & 1.96237600 & -2.48506100 & -2.16748700 & $\mathrm{H}$ & 1.24605100 & -3.48034500 & -3.94406800 \\
\hline $\mathrm{H}$ & 2.35692900 & -1.43724200 & -0.33595600 & $\mathrm{H}$ & 2.97518600 & -2.86382200 & -2.25034700 \\
\hline $\mathrm{N}$ & -2.57152500 & -0.64740600 & -0.63741200 & $\mathrm{H}$ & -1.97953100 & -0.46672600 & 0.19043500 \\
\hline C & -2.07605500 & 0.56586600 & -3.13842100 & $\mathrm{H}$ & -1.65355300 & 0.09118700 & -4.01946500 \\
\hline $\mathrm{H}$ & -3.15730100 & 0.65769800 & -3.12034600 & C & -1.31765100 & 1.53155900 & -2.46958400 \\
\hline C & -1.92957200 & 2.36545600 & -1.45070300 & $\mathrm{H}$ & -1.21746600 & 2.69893100 & -0.69212200 \\
\hline $\mathrm{H}$ & -2.79458600 & 1.88245000 & -0.98291200 & C & 0.14655000 & 1.64667200 & -2.70615300 \\
\hline $\mathrm{H}$ & 0.48394200 & 2.68792300 & -2.68667000 & $\mathrm{H}$ & 0.47436200 & 1.15167500 & -3.62130900 \\
\hline $\mathrm{H}$ & 0.65910200 & 1.16325500 & -1.86120600 & $\mathrm{C}$ & -3.41579500 & 4.92486500 & -0.77755700 \\
\hline $\mathrm{H}$ & -4.11104000 & 4.31090900 & -0.19262300 & $\mathrm{H}$ & -3.95610400 & 5.81626600 & -1.11834800 \\
\hline $\mathrm{H}$ & -2.59442800 & 5.24427500 & -0.13024600 & C & -4.12244900 & 3.38679400 & -3.37465600 \\
\hline $\mathrm{H}$ & -4.83240300 & 2.73227700 & -2.85701000 & $\mathrm{H}$ & -3.73923500 & 2.84700800 & -4.24758800 \\
\hline $\mathrm{H}$ & -4.68442600 & 4.25275800 & -3.74486400 & C & -1.47032800 & 4.91904400 & -3.25619500 \\
\hline $\mathrm{H}$ & -1.15592000 & 4.34377800 & -4.13511000 & $\mathrm{H}$ & -0.58038300 & 5.17681500 & -2.67768000 \\
\hline $\mathrm{H}$ & -1.92171400 & 5.85151500 & -3.61726100 & $\mathrm{Si}$ & -2.72545000 & 3.96778500 & -2.23796500 \\
\hline C & -2.82079800 & -2.90299000 & 3.89463000 & $\mathrm{H}$ & -2.25988900 & -3.83867800 & 3.97807500 \\
\hline $\mathrm{H}$ & -3.86622900 & -3.15942100 & 3.70554000 & & & & \\
\hline
\end{tabular}

\section{TS-S-4f-14}

Imaginary frequency: $-402.79 \mathrm{~cm}^{-1}$

E[B3LYP/6-31G(d)-D3(BJ)]: -4789.089358 Hartree

E[PCM(Et $2 \mathrm{O})-B 3 L Y P / 6-311 \mathrm{G}(\mathrm{d}, \mathrm{p})-\mathrm{D} 3(\mathrm{BJ})]$ : - 4790.231952 Hartree

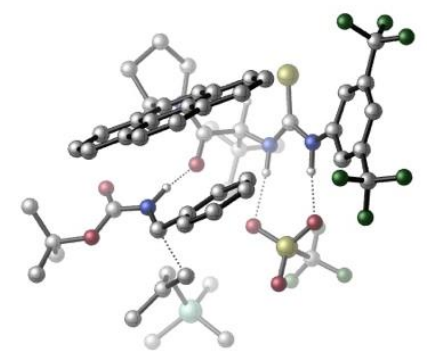

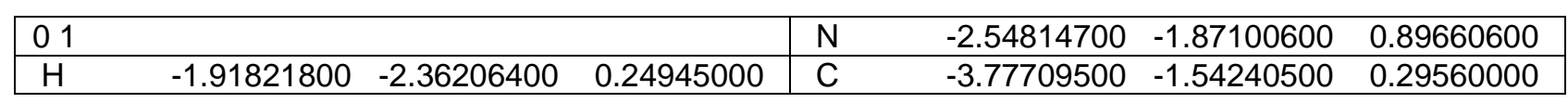




\begin{tabular}{|c|c|c|c|c|c|c|c|}
\hline C & -3.83172500 & -1.67204400 & -1.10627200 & C & -4.91699500 & -1.09692500 & 0.97577700 \\
\hline $\mathrm{C}$ & -4.97222700 & -1.29167900 & -1.80155900 & $\mathrm{H}$ & -2.97380000 & -2.05920200 & -1.64298200 \\
\hline C & -6.04982700 & -0.71926600 & 0.25268600 & $\mathrm{H}$ & -4.91357100 & -1.02994100 & 2.05202100 \\
\hline C & -6.09400000 & -0.79792200 & -1.13434800 & $\mathrm{H}$ & -6.97928700 & -0.49847900 & -1.68166200 \\
\hline C & -4.97619100 & -1.31757900 & -3.30348800 & C & -7.20831900 & -0.12023000 & 0.99833400 \\
\hline $\mathrm{F}$ & -4.02775000 & -2.12297600 & -3.81285300 & $\mathrm{~F}$ & -4.75528400 & -0.07180900 & -3.81140600 \\
\hline $\mathrm{F}$ & -6.16920200 & -1.71820600 & -3.79593400 & $\mathrm{~F}$ & -7.36800200 & -0.66298700 & 2.22151400 \\
\hline $\mathrm{F}$ & -7.02663700 & 1.21543800 & 1.18377400 & $\mathrm{~F}$ & -8.37350500 & -0.26325800 & 0.33020300 \\
\hline $\mathrm{C}$ & 0.15043500 & -1.30438200 & 3.28900500 & $\mathrm{H}$ & -0.53111300 & -1.07720900 & 4.10549300 \\
\hline $\mathrm{C}$ & 1.16991100 & -2.35013000 & 3.82438000 & $\mathrm{C}$ & 2.23980700 & -2.74718700 & 2.79719900 \\
\hline $\mathrm{H}$ & 2.84401000 & -1.89008600 & 2.48880700 & $\mathrm{H}$ & 1.79442400 & -3.17652200 & 1.89877600 \\
\hline $\mathrm{H}$ & 2.90391600 & -3.49815500 & 3.24108200 & C & 0.37954000 & -3.60112600 & 4.24357500 \\
\hline $\mathrm{H}$ & 1.04866200 & -4.32565500 & 4.72166800 & $\mathrm{H}$ & -0.07945500 & -4.08206400 & 3.37536800 \\
\hline $\mathrm{H}$ & -0.41712700 & -3.35076200 & 4.95366400 & C & 1.85661100 & -1.73543400 & 5.05703400 \\
\hline $\mathrm{H}$ & 2.57221200 & -2.44659300 & 5.48370800 & $\mathrm{H}$ & 1.12893300 & -1.48516700 & 5.83812500 \\
\hline $\mathrm{H}$ & 2.41038700 & -0.82517000 & 4.79722400 & $\mathrm{C}$ & 0.78811900 & -0.01468600 & 2.76032600 \\
\hline $\mathrm{C}$ & 1.06714200 & 2.40263700 & 2.80882300 & $\mathrm{H}$ & 2.07651600 & 2.26944300 & 2.41903100 \\
\hline $\mathrm{N}$ & 0.64943100 & 1.13564200 & 3.44113600 & C & 0.11221400 & 2.80982400 & 1.69177800 \\
\hline $\mathrm{C}$ & -1.16390700 & 2.32742300 & 1.65593900 & $\mathrm{C}$ & 0.55308300 & 3.74766300 & 0.67834300 \\
\hline $\mathrm{C}$ & -2.10538500 & 2.70759300 & 0.64686900 & $\mathrm{H}$ & -1.49869900 & 1.60389300 & 2.39136100 \\
\hline C & -0.36288100 & 4.12522400 & -0.35632700 & C & 1.84623900 & 4.29915700 & 0.65697900 \\
\hline $\mathrm{C}$ & -3.41047600 & 2.19056600 & 0.62216400 & C & -1.69241800 & 3.61523600 & -0.36714600 \\
\hline $\mathrm{C}$ & 0.05184800 & 5.01843200 & -1.39161500 & $\mathrm{H}$ & 2.55943600 & 4.04177400 & 1.43101800 \\
\hline C & 2.23919100 & 5.16957100 & -0.35854000 & C & -4.29671600 & 2.55697800 & -0.38868900 \\
\hline $\mathrm{H}$ & -3.71772000 & 1.49525500 & 1.39683700 & C & -2.60647100 & 3.98931700 & -1.39588800 \\
\hline C & 1.36007700 & 5.52254800 & -1.37734400 & C & -0.88889800 & 5.38075300 & -2.41488100 \\
\hline $\mathrm{H}$ & 3.23996300 & 5.59084700 & -0.34317700 & C & -3.90075600 & 3.44623600 & -1.38643700 \\
\hline $\mathrm{H}$ & -5.30211400 & 2.15105400 & -0.38715300 & C & -2.15941300 & 4.89389600 & -2.41526700 \\
\hline $\mathrm{H}$ & 1.67449400 & 6.20472000 & -2.16317600 & $\mathrm{H}$ & -0.55955400 & 6.06342800 & -3.19423600 \\
\hline $\mathrm{H}$ & -4.59609100 & 3.72707800 & -2.17345800 & $\mathrm{H}$ & -2.85943000 & 5.17830600 & -3.19685600 \\
\hline $\mathrm{C}$ & -2.00113800 & -1.47870300 & 2.08827200 & $S$ & -2.84507100 & -0.67885400 & 3.32610100 \\
\hline $\mathrm{N}$ & -0.68253300 & -1.80883000 & 2.20068600 & $\mathrm{H}$ & -0.16312500 & -2.04115700 & 1.34821000 \\
\hline $\mathrm{S}$ & 0.36617900 & -3.26169300 & -1.21310200 & 0 & 0.99426200 & -3.28183900 & -2.54279500 \\
\hline $\mathrm{O}$ & 1.04001700 & -2.38609900 & -0.21063700 & $\mathrm{O}$ & -1.11514200 & -3.13723700 & -1.19150100 \\
\hline $\mathrm{C}$ & 0.64004400 & -4.96345300 & -0.51973000 & $\mathrm{~F}$ & 0.01558700 & -5.89075100 & -1.24671900 \\
\hline $\mathrm{F}$ & 0.18090700 & -5.02121700 & 0.74380400 & $\mathrm{~F}$ & 1.95516200 & -5.25699400 & -0.49615200 \\
\hline $\mathrm{C}$ & 3.93454200 & 1.71706800 & 0.21168600 & $\mathrm{O}$ & 4.15240600 & 1.77935200 & 1.40915200 \\
\hline $\mathrm{O}$ & 4.77999800 & 2.09263400 & -0.76929000 & C & 6.08083800 & 2.71121400 & -0.43528800 \\
\hline C & 5.87013200 & 3.97150000 & 0.40444700 & $\mathrm{H}$ & 6.82916400 & 4.48601300 & 0.52537800 \\
\hline $\mathrm{H}$ & 5.47109600 & 3.73205300 & 1.38963200 & $\mathrm{H}$ & 5.18122000 & 4.65015400 & -0.10616600 \\
\hline $\mathrm{C}$ & 6.64336800 & 3.07870400 & -1.80706300 & $\mathrm{H}$ & 6.78850600 & 2.19045100 & -2.42738700 \\
\hline $\mathrm{H}$ & 7.61077900 & 3.57613700 & -1.68841300 & $\mathrm{H}$ & 5.96501900 & 3.76103400 & -2.32874000 \\
\hline $\mathrm{C}$ & 6.96597200 & 1.68591300 & 0.27407400 & $\mathrm{H}$ & 7.95661100 & 2.11811100 & 0.44968600 \\
\hline $\mathrm{H}$ & 7.09557100 & 0.79163700 & -0.34477300 & $\mathrm{H}$ & 6.53383200 & 1.39755900 & 1.23333000 \\
\hline C & 2.40214700 & 1.21311500 & -1.63164100 & $\mathrm{H}$ & 3.03232700 & 1.85742700 & -2.23448300 \\
\hline $\mathrm{C}$ & 0.96930000 & 1.22894000 & -1.99085700 & $\mathrm{C}$ & 0.58637700 & 1.88843000 & -3.16837400 \\
\hline $\mathrm{C}$ & 0.00530200 & 0.55784200 & -1.22497900 & $\mathrm{C}$ & -0.74238800 & 1.87264400 & -3.58050200 \\
\hline $\mathrm{H}$ & 1.33307000 & 2.41948300 & -3.75355200 & C & -1.31819400 & 0.54960400 & -1.64415300 \\
\hline $\mathrm{H}$ & 0.28766900 & 0.02649200 & -0.32517900 & C & -1.69723800 & 1.19068600 & -2.82441600 \\
\hline $\mathrm{H}$ & -1.03336800 & 2.39574700 & -4.48576900 & $\mathrm{H}$ & -2.06313300 & 0.05106300 & -1.04159500 \\
\hline $\mathrm{H}$ & -2.73192600 & 1.15553600 & -3.14519800 & $\mathrm{~N}$ & 2.73966100 & 1.25028400 & -0.29696400 \\
\hline $\mathrm{H}$ & 2.10846600 & 0.87099800 & 0.42798200 & $\mathrm{C}$ & 2.87291700 & -0.51892000 & -2.46968200 \\
\hline $\mathrm{H}$ & 2.10217600 & -1.13194400 & -2.01591200 & $\mathrm{H}$ & 2.70821700 & -0.26986900 & -3.51536700 \\
\hline
\end{tabular}




\begin{tabular}{|c|c|c|c|c|c|c|c|}
\hline C & 4.18294400 & -0.71223000 & -2.02812700 & $\mathrm{C}$ & 4.42210300 & -1.33114500 & -0.73916100 \\
\hline $\mathrm{H}$ & 3.50893500 & -1.42800000 & -0.14484700 & $\mathrm{H}$ & 5.22219100 & -0.84607300 & -0.16953000 \\
\hline C & 5.33427400 & -0.27622800 & -2.88074000 & $\mathrm{H}$ & 5.55575200 & -1.07141900 & -3.60643100 \\
\hline $\mathrm{H}$ & 6.23901200 & -0.09316200 & -2.29903800 & $\mathrm{H}$ & 5.08319800 & 0.61984100 & -3.45499500 \\
\hline C & 4.75293800 & -4.03756200 & 0.63605300 & $\mathrm{H}$ & 5.15241300 & -3.47533500 & 1.48720800 \\
\hline $\mathrm{H}$ & 5.21963100 & -5.03009400 & 0.63685200 & $\mathrm{H}$ & 3.67958100 & -4.16839400 & 0.78505100 \\
\hline C & 6.96670400 & -3.00718600 & -1.25046300 & $\mathrm{H}$ & 7.45014400 & -2.48195800 & -0.41815600 \\
\hline $\mathrm{H}$ & 7.21798100 & -2.47901900 & -2.17671900 & $\mathrm{H}$ & 7.41604800 & -4.00572700 & -1.31444900 \\
\hline C & 4.28986200 & -3.96622800 & -2.46974800 & $\mathrm{H}$ & 4.65181400 & -3.52600100 & -3.40678500 \\
\hline $\bar{H}$ & 3.20022100 & -3.87101000 & -2.45242200 & $\mathrm{H}$ & 4.53680300 & -5.03452200 & -2.49444800 \\
\hline $\mathrm{Si}$ & 5.10136700 & -3.15940000 & -0.98534200 & C & 0.01890700 & 1.33626100 & 4.76021600 \\
\hline $\mathrm{H}$ & 0.67214000 & 0.94085600 & 5.54617600 & $\mathrm{H}$ & -0.94594700 & 0.82555900 & 4.80751100 \\
\hline $\mathrm{C}$ & -0.11507400 & 2.86233900 & 4.86027000 & $\mathrm{H}$ & -0.07282900 & 3.21041600 & 5.89566500 \\
\hline $\mathrm{H}$ & -1.07187800 & 3.17956900 & 4.43431000 & C & 1.04122300 & 3.38623200 & 3.99701100 \\
\hline $\mathrm{H}$ & 0.90197800 & 4.41892000 & 3.66893000 & $\mathrm{H}$ & 1.98934000 & 3.32245300 & 4.54411600 \\
\hline 0 & 1.35958700 & -0.05373100 & 1.65696500 & & & & \\
\hline
\end{tabular}

\section{TS-S-4f-15}

Imaginary frequency: $-416.95 \mathrm{~cm}^{-1}$

E[B3LYP/6-31G(d)-D3(BJ)]: -4789.094037 Hartree

E[PCM(Et $\left.\left.{ }_{2} \mathrm{O}\right)-B 3 L Y P / 6-311 G(d, p)-D 3(B J)\right]:-4790.231046$ Hartree

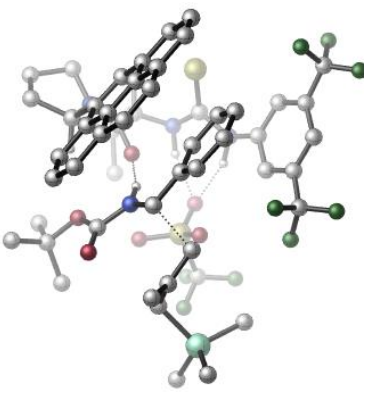

\begin{tabular}{|c|c|c|c|c|c|c|c|}
\hline 01 & & & & $\mathrm{~N}$ & 0.78387000 & -1.15176900 & -3.21367500 \\
\hline $\mathrm{H}$ & -0.23728600 & -1.23279200 & -3.20241800 & C & 1.43500600 & -2.19799900 & -2.53829900 \\
\hline $\mathrm{C}$ & 0.67946600 & -2.92324100 & -1.60668200 & C & 2.78109900 & -2.51626200 & -2.74093500 \\
\hline C & 1.28465700 & -3.92553400 & -0.85572400 & $\mathrm{H}$ & -0.35975600 & -2.66019600 & -1.43380100 \\
\hline C & 3.37344000 & -3.50383400 & -1.95315100 & $\mathrm{H}$ & 3.36404800 & -1.97298600 & -3.46971800 \\
\hline C & 2.63956000 & -4.21545200 & -1.00723700 & $\mathrm{H}$ & 3.11435900 & -4.97365200 & -0.39709300 \\
\hline C & 0.50948300 & -4.59460700 & 0.24059800 & C & 4.85876900 & -3.70195500 & -2.03084700 \\
\hline $\mathrm{F}$ & -0.81713800 & -4.62530600 & -0.01344400 & $\mathrm{~F}$ & 0.66259000 & -3.94140700 & 1.42390500 \\
\hline $\mathrm{F}$ & 0.91545500 & -5.86609200 & 0.45029900 & $\mathrm{~F}$ & 5.34777400 & -3.46594600 & -3.26467500 \\
\hline $\mathrm{F}$ & 5.50216400 & -2.85015400 & -1.18520200 & $\mathrm{~F}$ & 5.22893900 & -4.95153900 & -1.67442500 \\
\hline C & 0.28803600 & 2.39899800 & -3.53515700 & $\mathrm{H}$ & 1.23090100 & 2.77924400 & -3.93138100 \\
\hline $\mathrm{C}$ & -0.89932500 & 3.09812300 & -4.28637500 & C & -2.26055300 & 2.51944300 & -3.85981100 \\
\hline $\mathrm{H}$ & -2.37223500 & 2.49188100 & -2.77483900 & $\mathrm{H}$ & -2.41613700 & 1.49991000 & -4.21952500 \\
\hline $\mathrm{H}$ & -3.06431300 & 3.13539000 & -4.27899700 & C & -0.69067300 & 2.88004300 & -5.79564700 \\
\hline $\mathrm{H}$ & -1.50836900 & 3.34491800 & -6.35827400 & $\mathrm{H}$ & -0.66630100 & 1.81550300 & -6.04284300 \\
\hline $\mathrm{H}$ & 0.25173900 & 3.32726700 & -6.13443100 & C & -0.91935500 & 4.61072600 & -4.01118100 \\
\hline $\mathrm{H}$ & -1.76452400 & 5.06062800 & -4.54250400 & $\mathrm{H}$ & -0.01292700 & 5.10883100 & -4.37161800 \\
\hline $\mathrm{H}$ & -1.04527500 & 4.83169300 & -2.94662300 & C & 0.26702400 & 2.56814800 & -1.99635800 \\
\hline $\mathrm{O}$ & -0.31724500 & 1.71900300 & -1.31012500 & C & 1.67223000 & 4.69721100 & -2.02463300 \\
\hline C & 0.73316500 & 3.85855900 & 0.02404700 & C & 1.22241300 & 5.93319400 & -1.23917300 \\
\hline $\mathrm{H}$ & 2.73733900 & 4.49094000 & -1.86022800 & $\mathrm{H}$ & 1.50525800 & 4.77436900 & -3.09445400 \\
\hline $\mathrm{H}$ & -0.28131700 & 3.56890400 & 0.29677600 & $\mathrm{H}$ & 0.29872600 & 6.32373800 & -1.67868900 \\
\hline $\mathrm{H}$ & 1.96845900 & 6.73250200 & -1.25776400 & $\mathrm{~N}$ & 0.86492100 & 3.63644700 & -1.42311700 \\
\hline $\mathrm{C}$ & 1.73686800 & 3.05909200 & 0.83779000 & $\mathrm{C}$ & 2.85457700 & 2.53756500 & 0.25804500 \\
\hline C & 1.51970600 & 2.89763500 & 2.25980800 & $C$ & 3.86672800 & 1.86309300 & 1.01267700 \\
\hline $\mathrm{H}$ & 2.98624500 & 2.59084200 & -0.81674100 & C & 2.49120500 & 2.18196900 & 3.02796000 \\
\hline $\mathrm{C}$ & 0.37153600 & 3.37483100 & 2.91381900 & $\mathrm{C}$ & 5.02567800 & 1.35479100 & 0.40793400 \\
\hline
\end{tabular}




\begin{tabular}{|c|c|c|c|c|c|c|c|}
\hline C & 3.67573300 & 1.68726700 & 2.41138100 & C & 2.27100700 & 1.94768100 & 4.41963200 \\
\hline $\mathrm{H}$ & -0.37684100 & 3.92811200 & 2.35974500 & $\mathrm{C}$ & 0.15210400 & 3.12033500 & 4.26636900 \\
\hline C & 5.98726800 & 0.69144600 & 1.16679500 & $\mathrm{H}$ & 5.15342100 & 1.46874900 & -0.66465600 \\
\hline C & 4.65593200 & 0.99242400 & 3.17871900 & C & 1.08808100 & 2.41263800 & 5.01477100 \\
\hline C & 3.26923700 & 1.23411300 & 5.16664700 & $\mathrm{H}$ & -0.76226000 & 3.47390200 & 4.73274900 \\
\hline C & 5.80738000 & 0.51149400 & 2.53696300 & $\mathrm{H}$ & 6.87750800 & 0.29944600 & 0.68366500 \\
\hline C & 4.41217400 & 0.78743000 & 4.57779500 & $\mathrm{H}$ & 0.91565200 & 2.22195300 & 6.07098300 \\
\hline $\mathrm{H}$ & 3.09281300 & 1.06472900 & 6.22595500 & $\mathrm{H}$ & 6.55561300 & -0.01801400 & 3.12125600 \\
\hline $\mathrm{H}$ & 5.16007400 & 0.25463100 & 5.15970100 & C & 1.22977800 & 0.13922900 & -3.36788200 \\
\hline $\mathrm{S}$ & 2.80170900 & 0.67598300 & -3.06342800 & $\mathrm{~N}$ & 0.23782500 & 0.96873600 & -3.79651000 \\
\hline $\mathrm{H}$ & -0.69867100 & 0.57947900 & -3.67851700 & $\mathrm{~S}$ & -2.78495300 & -0.67886400 & -1.84366900 \\
\hline 0 & -2.13308000 & -1.48047500 & -0.77976500 & 0 & -3.33127900 & 0.61786800 & -1.41308500 \\
\hline $\mathrm{O}$ & -2.03797900 & -0.69062800 & -3.13796700 & C & -4.30016900 & -1.65513900 & -2.28666500 \\
\hline $\mathrm{F}$ & -3.99351700 & -2.93445500 & -2.54732000 & $\mathrm{~F}$ & -4.91145100 & -1.13351700 & -3.35335200 \\
\hline $\mathrm{F}$ & -5.16945700 & -1.63753600 & -1.25333400 & $\mathrm{C}$ & -2.06773800 & 1.64582100 & 1.78494500 \\
\hline 0 & -2.57153400 & 1.52029500 & 2.89165700 & 0 & -2.37720900 & 2.54751600 & 0.85481900 \\
\hline $\mathrm{C}$ & -3.61327000 & 3.34678200 & 0.93742500 & C & -4.82040800 & 2.41873000 & 1.07614000 \\
\hline $\mathrm{H}$ & -5.73822600 & 3.00220500 & 0.94637000 & $\mathrm{H}$ & -4.84624900 & 1.94734300 & 2.06020400 \\
\hline $\mathrm{H}$ & -4.78046900 & 1.64780900 & 0.30164200 & $\mathrm{C}$ & -3.62968000 & 4.05197500 & -0.41648500 \\
\hline $\mathrm{H}$ & -2.71753300 & 4.64323000 & -0.55319000 & $\mathrm{H}$ & -4.49130900 & 4.72456900 & -0.47688200 \\
\hline $\mathrm{H}$ & -3.69288500 & 3.31487700 & -1.21951700 & C & -3.49192700 & 4.34219500 & 2.09084700 \\
\hline $\mathrm{H}$ & -4.37576100 & 4.98874300 & 2.11248400 & $\mathrm{H}$ & -2.61047300 & 4.97938900 & 1.95597300 \\
\hline $\mathrm{H}$ & -3.41247800 & 3.82229400 & 3.04712400 & $\mathrm{C}$ & -0.42823100 & -0.04403600 & 2.17858400 \\
\hline $\mathrm{H}$ & -0.55995200 & 0.23651000 & 3.21894000 & C & 0.90805300 & -0.57251900 & 1.84282400 \\
\hline C & 1.64370000 & -1.18034600 & 2.87648500 & C & 1.45968700 & -0.50446200 & 0.55817000 \\
\hline C & 2.88839300 & -1.73728100 & 2.62594000 & $\mathrm{H}$ & 1.22860700 & -1.20793900 & 3.88070500 \\
\hline C & 2.72870200 & -1.03009500 & 0.32408200 & $\mathrm{H}$ & 0.93002500 & -0.02735800 & -0.25797600 \\
\hline $\mathrm{C}$ & 3.43180500 & -1.67006700 & 1.33996700 & $\mathrm{H}$ & 3.44431300 & -2.20259200 & 3.43376900 \\
\hline $\mathrm{H}$ & 3.16324300 & -0.93029400 & -0.66124100 & $\mathrm{H}$ & 4.40746200 & -2.09349600 & 1.12855400 \\
\hline $\mathrm{N}$ & -1.03270100 & 0.84999600 & 1.33072600 & $\mathrm{H}$ & -0.75404400 & 0.97755600 & 0.35255800 \\
\hline $\mathrm{C}$ & -1.45881600 & -1.75548300 & 2.27607000 & $\mathrm{H}$ & -1.49104700 & -2.00264300 & 1.22071700 \\
\hline $\mathrm{H}$ & -0.73053300 & -2.29787700 & 2.86759800 & C & -2.65745800 & -1.40401800 & 2.90897500 \\
\hline C & -3.88331900 & -1.20036100 & 2.15352800 & $\mathrm{H}$ & -3.69180200 & -0.92104300 & 1.11284900 \\
\hline $\mathrm{H}$ & -4.56763600 & -0.49486000 & 2.63283100 & C & -2.68038900 & -1.21807100 & 4.39407500 \\
\hline $\mathrm{H}$ & -3.64937400 & -1.48573300 & 4.82205400 & $\mathrm{H}$ & -2.52487300 & -0.14854400 & 4.58767300 \\
\hline $\mathrm{H}$ & -1.89065700 & -1.78457700 & 4.89564000 & C & -6.58724500 & -2.47419600 & 1.48062000 \\
\hline $\mathrm{H}$ & -7.08993500 & -1.84884700 & 2.22770800 & $\mathrm{H}$ & -7.17873500 & -3.39020300 & 1.36184000 \\
\hline $\mathrm{H}$ & -6.59546700 & -1.94302400 & 0.52630700 & $C$ & -4.93661000 & -3.70223000 & 3.74760000 \\
\hline $\mathrm{H}$ & -5.43269400 & -3.05869700 & 4.48343100 & $\mathrm{H}$ & -3.94902000 & -3.96991400 & 4.13908000 \\
\hline $\mathrm{H}$ & -5.52189200 & -4.62749300 & 3.67910400 & C & -3.93066700 & -4.05469700 & 0.86929000 \\
\hline $\mathrm{H}$ & -2.92697600 & -4.28610000 & 1.23830200 & $\mathrm{H}$ & -3.81870200 & -3.63162900 & -0.13059200 \\
\hline $\mathrm{H}$ & -4.47865300 & -5.00101200 & 0.78104300 & $\mathrm{Si}$ & -4.83905200 & -2.90319800 & 2.03743900 \\
\hline C & 0.96003000 & 5.39551000 & 0.18579000 & $\mathrm{H}$ & 1.81975400 & 5.57396300 & 0.83633200 \\
\hline $\mathrm{H}$ & 0.09435300 & 5.87782700 & 0.64877400 & & & & \\
\hline
\end{tabular}

\section{TS-S-4f-16}

Imaginary frequency: $-132.31 \mathrm{~cm}^{-1}$ E[B3LYP/6-31G(d)-D3(BJ)]: -4789.090006 Hartree

E[PCM(Et $\left.\left.{ }_{2} \mathrm{O}\right)-B 3 L Y P / 6-311 G(d, p)-D 3(B J)\right]:-4790.230677$ Hartree

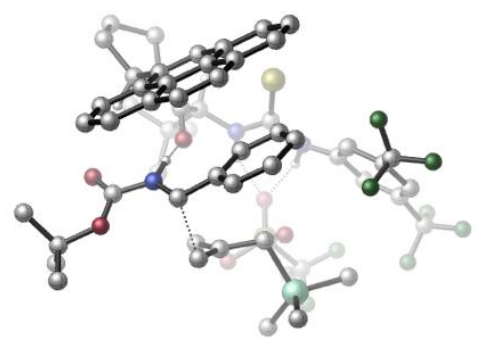




\begin{tabular}{|c|c|c|c|c|c|c|}
\hline 01 & & & $\mathrm{~N}$ & 2.68130500 & -0.15589600 & -2.21156000 \\
\hline $\mathrm{H}$ & 3.18999500 & -1.02345900 & C & 3.30012000 & 0.95877100 & -1.64273500 \\
\hline $\mathrm{C}$ & 4.66961800 & $0.82519800 \quad-1.37003300$ & C & 2.64136200 & 2.12798300 & -1.25287500 \\
\hline C & 5.35782700 & $1.83285300-0.70795400$ & $\mathrm{H}$ & 5.18180400 & -0.08895600 & -1.64430400 \\
\hline C & 3.35048800 & $3.12825000-0.58876100$ & $\mathrm{H}$ & 1.58844300 & 2.23768500 & -1.45468100 \\
\hline $\mathrm{C}$ & 4.70977800 & $3.00196800-0.31167300$ & $\mathrm{H}$ & 5.24648200 & 3.78220900 & 0.21174400 \\
\hline $\mathrm{C}$ & 6.78921600 & -0.32027100 & $\mathrm{C}$ & 2.59629500 & 4.30251100 & -0.03855300 \\
\hline $\mathrm{F}$ & 7.47473400 & -1.29363500 & $\mathrm{~F}$ & 6.87133600 & 0.81552500 & 0.78743000 \\
\hline $\mathrm{F}$ & 7.44336100 & -0.04741500 & $\mathrm{~F}$ & 1.57140700 & 4.68346200 & -0.83908900 \\
\hline $\mathrm{F}$ & 2.04641900 & 1.17242900 & $\mathrm{~F}$ & 3.38214800 & 5.38255400 & 0.14883400 \\
\hline C & -0.16510800 & -3.05950900 & $\mathrm{H}$ & -0.22712700 & -2.01311800 & -4.12524300 \\
\hline C & -0.09085500 & -2.89918400 & C & -0.01742700 & -4.24446300 & -1.42094400 \\
\hline $\mathrm{H}$ & -0.90629300 & -3.93311300 & $\mathrm{H}$ & 0.86402500 & -3.85581800 & -0.90470000 \\
\hline $\mathrm{H}$ & 0.04948700 & -5.33716000 & $\mathrm{C}$ & 1.15724700 & -4.32132700 & -3.65039300 \\
\hline $\mathrm{H}$ & 1.15229000 & -5.41681900 & $\mathrm{H}$ & 2.09002900 & -4.00772900 & -3.17499800 \\
\hline $\mathrm{H}$ & 1.16229500 & -3.97117500 & C & -1.33161300 & -4.46728700 & -3.53131800 \\
\hline $\mathrm{H}$ & -1.26322500 & -5.55409600 & $\mathrm{H}$ & -1.40307100 & -4.25550500 & -4.60438400 \\
\hline $\mathrm{H}$ & -2.25760000 & -4.14474400 & $\mathrm{C}$ & -1.38327700 & -1.66514100 & -2.32659600 \\
\hline $\mathrm{O}$ & -1.30144800 & $-1.46471000-1.10337100$ & $\mathrm{C}$ & -2.81265800 & -1.61572600 & -4.42445000 \\
\hline C & -3.78856000 & $-1.20211700-2.24349200$ & $\mathrm{C}$ & -4.21554600 & -2.22580100 & -4.44591200 \\
\hline $\mathrm{H}$ & -2.79676900 & -0.62235800 & $\mathrm{H}$ & -2.07061400 & -2.23454600 & -4.92111900 \\
\hline $\mathrm{H}$ & -3.80313000 & -1.88367300 & $\mathrm{H}$ & -4.14210900 & -3.30652800 & -4.28517200 \\
\hline $\mathrm{H}$ & -4.72993300 & $-2.05772800 \quad-5.39618400$ & $\mathrm{~N}$ & -2.54485600 & -1.48889500 & -2.98906200 \\
\hline C & -3.88845600 & -1.76714100 & $\mathrm{C}$ & -3.18883200 & 1.22139000 & -2.39725000 \\
\hline $\mathrm{C}$ & -4.77957300 & -0.67463200 & $\mathrm{C}$ & -3.29065600 & 2.59715200 & -2.01650400 \\
\hline $\mathrm{H}$ & -2.48851600 & -3.18470300 & $\mathrm{C}$ & -4.85442500 & 1.92569300 & -0.23744600 \\
\hline C & -5.55351500 & -0.39618800 & C & -2.56685500 & 3.60387400 & -2.67351600 \\
\hline $\mathrm{C}$ & -4.12276800 & -0.91474900 & $\mathrm{C}$ & -5.66410400 & 2.27271100 & 0.88747400 \\
\hline $\mathrm{H}$ & -5.52655900 & -1.43235000 & C & -6.33941600 & -0.04729300 & 1.09448500 \\
\hline C & -2.65868800 & -2.25535800 & $\mathrm{H}$ & -1.91781600 & 3.33218400 & -3.50115700 \\
\hline C & -4.20180800 & -0.48463900 & C & -6.38910300 & 1.26847600 & 1.54557400 \\
\hline $\mathrm{C}$ & -5.71412000 & 1.30800200 & $\mathrm{H}$ & -6.92554400 & -0.81237300 & 1.59493800 \\
\hline C & -3.46491400 & -1.17250800 & $\mathrm{H}$ & -2.08908000 & 5.69705100 & -2.77093500 \\
\hline C & -5.02139900 & 0.65024500 & $\mathrm{H}$ & -7.00406400 & 1.53295200 & 2.40197900 \\
\hline $\mathrm{H}$ & -6.33137800 & 2.16706100 & $\mathrm{H}$ & -3.52468800 & 6.31090100 & -0.84543300 \\
\hline $\mathrm{H}$ & -5.07458900 & 5.64930700 & C & 1.39061600 & -0.36319300 & -2.62557300 \\
\hline$S$ & 0.35077300 & -3.24980600 & $\mathrm{~N}$ & 1.04950800 & -1.66715300 & -2.53771700 \\
\hline $\mathrm{H}$ & 1.70551600 & -2.28015100 & $S$ & 3.55963300 & -2.64837700 & 0.10766900 \\
\hline 0 & 2.94829900 & -3.75360200 & $\mathrm{O}$ & 3.47390200 & -2.78160900 & -1.38546000 \\
\hline $\mathrm{O}$ & 3.22890600 & -1.28255800 & C & 5.38162700 & -2.78862100 & 0.44735600 \\
\hline $\mathrm{F}$ & 5.60701700 & -2.62256700 & $\mathrm{~F}$ & 6.06221500 & -1.83979700 & -0.21954600 \\
\hline $\mathrm{F}$ & 5.84653200 & -3.98479000 & C & -3.32515300 & -2.20606000 & 1.53947100 \\
\hline $\mathrm{O}$ & -3.55593600 & -3.06979200 & $\mathrm{O}$ & -3.65210800 & -2.22915700 & 2.83992000 \\
\hline C & -4.33658400 & -3.39861300 & C & -5.70227400 & -3.58632000 & 2.77163100 \\
\hline $\mathrm{H}$ & -6.24856700 & -4.38122000 & $\mathrm{H}$ & -5.59792800 & -3.85848000 & 1.72088300 \\
\hline $\mathrm{H}$ & -6.28702600 & -2.66423700 & C & -4.48717000 & -2.98315700 & 4.89263700 \\
\hline $\mathrm{H}$ & -3.50752900 & -2.81155400 & $\mathrm{H}$ & -4.99616500 & -3.77283000 & 5.45362600 \\
\hline $\mathrm{H}$ & -5.07622200 & -2.06420000 & C & -3.44234200 & -4.63084400 & 3.29687800 \\
\hline $\mathrm{H}$ & -3.89468600 & -5.46852400 & $\mathrm{H}$ & -2.45752200 & -4.43603500 & 3.73485700 \\
\hline $\mathrm{H}$ & -3.31554100 & -4.91467500 & C & -2.42316400 & -0.00013700 & 2.06955100 \\
\hline $\mathrm{H}$ & -3.11867000 & 0.03139400 & C & -1.97863600 & 1.30018700 & 1.56327900 \\
\hline C & -2.25436500 & 2.43141400 & C & -1.25226500 & 1.45264900 & 0.37078900 \\
\hline C & -1.81244400 & 3.68853800 & $\mathrm{H}$ & -2.82551000 & 2.31472400 & 3.27001400 \\
\hline
\end{tabular}




\begin{tabular}{|c|c|c|c|c|c|c|c|}
\hline $\mathrm{C}$ & -0.82524300 & 2.71865200 & -0.01708500 & $\mathrm{H}$ & -1.02171800 & 0.60252000 & -0.26136400 \\
\hline C & -1.08936600 & 3.83334400 & 0.77614500 & $\mathrm{H}$ & -2.04049500 & 4.55555000 & 2.57462700 \\
\hline $\bar{H}$ & -0.30048500 & 2.83264200 & -0.95578500 & $\mathrm{H}$ & -0.73472800 & 4.80859100 & 0.46479500 \\
\hline $\mathrm{N}$ & -2.66154500 & -1.03486700 & 1.21073600 & $\mathrm{H}$ & -2.24962300 & -1.05585700 & 0.26382000 \\
\hline C & -0.92721000 & -0.53256900 & 3.43946700 & $\mathrm{H}$ & -0.91700800 & 0.39118100 & 4.01072700 \\
\hline $\mathrm{H}$ & -1.46692200 & -1.36450900 & 3.88121900 & C & 0.12131200 & -0.79721000 & 2.55982800 \\
\hline C & 1.08706200 & 0.22517100 & 2.19456600 & $\mathrm{H}$ & 0.68466100 & 1.23953400 & 2.28019100 \\
\hline $\mathrm{H}$ & 1.54078200 & 0.05093700 & 1.21743600 & C & 0.19970200 & -2.12403200 & 1.88959000 \\
\hline $\mathrm{H}$ & 1.22356900 & -2.50778900 & 1.85845700 & $\mathrm{H}$ & -0.09495400 & -1.99792600 & 0.83641900 \\
\hline $\mathrm{H}$ & -0.45653900 & -2.86692100 & 2.35009700 & $\mathrm{C}$ & 3.97798700 & 1.15290000 & 2.63552300 \\
\hline $\mathrm{H}$ & 4.38609600 & 0.56792100 & 1.80863300 & $\mathrm{H}$ & 4.78551600 & 1.35380900 & 3.34982000 \\
\hline $\mathrm{H}$ & 3.62730600 & 2.11178600 & 2.24058600 & C & 3.04104300 & -1.52110000 & 3.98754800 \\
\hline $\mathrm{H}$ & 3.36831700 & -2.13117400 & 3.14306600 & $\mathrm{H}$ & 2.20118200 & -2.02952300 & 4.47565000 \\
\hline $\mathrm{H}$ & 3.86456500 & -1.47858000 & 4.71112400 & C & 1.94635600 & 1.17873000 & 4.96818700 \\
\hline $\mathrm{H}$ & 1.10359000 & 0.67144000 & 5.45209500 & $\mathrm{H}$ & 1.62162600 & 2.18902500 & 4.69223600 \\
\hline $\mathrm{H}$ & 2.74102300 & 1.27910400 & 5.71735500 & $\mathrm{Si}$ & 2.57583200 & 0.22276900 & 3.46183000 \\
\hline C & -4.92014200 & -1.54627800 & -3.25884500 & $\overline{\mathrm{H}}$ & -5.40814600 & -0.62271700 & -3.58246100 \\
\hline $\mathrm{H}$ & -5.68471500 & -2.18173100 & -2.80467000 & & & & \\
\hline
\end{tabular}

\section{TS-S-4f-17}

Imaginary frequency: $-348.30 \mathrm{~cm}^{-1}$

E[B3LYP/6-31G(d)-D3(BJ)]: -4789.090567 Hartree

E[PCM(Et $2 \mathrm{O})-B 3 L Y P / 6-311 \mathrm{G}(\mathrm{d}, \mathrm{p})-\mathrm{D} 3(\mathrm{BJ})]$ : - 4790.230203 Hartree

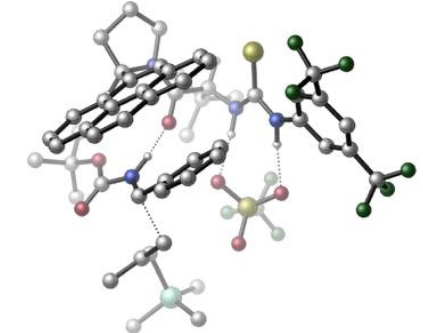

\begin{tabular}{|c|c|c|c|c|c|c|c|}
\hline 01 & & & & $\mathrm{~N}$ & 2.34690000 & 1.48263400 & 1.60271600 \\
\hline $\mathrm{H}$ & 1.75543500 & 2.26277800 & 1.29576800 & C & 3.60012700 & 1.49293500 & 0.97083700 \\
\hline $\mathrm{C}$ & 3.98554200 & 2.70348000 & 0.37324900 & $\mathrm{C}$ & 4.41771800 & 0.36521000 & 0.81278500 \\
\hline $\mathrm{C}$ & 5.16681000 & 2.77989300 & -0.35787500 & $\mathrm{H}$ & 3.34591000 & 3.57283200 & 0.46374400 \\
\hline C & 5.58424500 & 0.46076800 & 0.05807900 & $\mathrm{H}$ & 4.13332200 & -0.57313300 & 1.26192400 \\
\hline $\mathrm{C}$ & 5.98118200 & 1.66168200 & -0.52643100 & $\mathrm{H}$ & 6.90014700 & 1.72596700 & -1.09603100 \\
\hline $\mathrm{C}$ & 5.53475600 & 4.07103900 & -1.03696100 & C & 6.37643000 & -0.78107400 & -0.23005800 \\
\hline $\mathrm{F}$ & 5.05348700 & 5.14524500 & -0.37844200 & $\mathrm{~F}$ & 5.04562800 & 4.12473600 & -2.29701900 \\
\hline $\mathrm{F}$ & 6.87649500 & 4.21762100 & -1.13211800 & $\mathrm{~F}$ & 6.15596600 & -1.76241700 & 0.67373200 \\
\hline $\mathrm{F}$ & 6.04911700 & -1.29602200 & -1.44879300 & $\mathrm{~F}$ & 7.70476100 & -0.54621500 & -0.26050500 \\
\hline C & -0.32320500 & 0.05202700 & 3.61373200 & $\mathrm{H}$ & 0.31721300 & -0.37648900 & 4.38367000 \\
\hline $\mathrm{C}$ & -1.48426700 & 0.81841100 & 4.32542800 & C & -2.54153000 & 1.35498500 & 3.34524900 \\
\hline $\mathrm{H}$ & -3.02427300 & 0.54713200 & 2.78899000 & $\mathrm{H}$ & -2.11934100 & 2.03773500 & 2.60932200 \\
\hline $\mathrm{H}$ & -3.30947200 & 1.89910600 & 3.90801400 & C & -0.87008300 & 1.98764400 & 5.11336900 \\
\hline $\mathrm{H}$ & -1.64320800 & 2.47930200 & 5.71505400 & $\mathrm{H}$ & -0.43645200 & 2.73176200 & 4.44191000 \\
\hline $\mathrm{H}$ & -0.08139800 & 1.63913800 & 5.79033700 & C & -2.17034100 & -0.14808900 & 5.30686100 \\
\hline $\mathrm{H}$ & -2.97395200 & 0.37091700 & 5.84052300 & $\mathrm{H}$ & -1.46575300 & -0.52882200 & 6.05547300 \\
\hline $\mathrm{H}$ & -2.61789700 & -1.00220700 & 4.78579200 & $\mathrm{C}$ & -0.83860300 & -1.03500100 & 2.66908600 \\
\hline $\mathrm{O}$ & -1.22179100 & -0.69100400 & 1.54358500 & $\mathrm{C}$ & -1.41341800 & -3.31228100 & 2.09821300 \\
\hline $\mathrm{H}$ & -2.37007500 & -2.93580100 & 1.73634000 & $\mathrm{~N}$ & -0.88559400 & -2.32368500 & 3.05201200 \\
\hline C & -0.47935000 & -3.54509500 & 0.92104700 & C & 0.86369900 & -3.33977100 & 1.04575700 \\
\hline $\mathrm{C}$ & -1.02312300 & -4.02040100 & -0.33441600 & $\mathrm{C}$ & 1.78636900 & -3.63202900 & -0.00889300 \\
\hline $\mathrm{H}$ & 1.26913300 & -2.91300700 & 1.95774000 & $\mathrm{C}$ & -0.12432200 & -4.29231500 & -1.41429200 \\
\hline C & -2.40017500 & -4.19515700 & -0.55642700 & C & 3.16901800 & -3.44190000 & 0.14039300 \\
\hline $\mathrm{C}$ & 1.27988100 & -4.12887800 & -1.24274600 & $\mathrm{C}$ & -0.63305600 & -4.72429100 & -2.67790700 \\
\hline $\bar{H}$ & -3.10547900 & -4.00996100 & 0.24341700 & $\mathrm{C}$ & -2.89032400 & -4.58068300 & -1.80236900 \\
\hline C & 4.04312600 & -3.75837300 & -0.89722400 & $\bar{H}$ & 3.54980900 & -3.04319500 & 1.07633500 \\
\hline
\end{tabular}




\begin{tabular}{|c|c|c|c|c|c|c|c|}
\hline $\mathrm{C}$ & 2.17989900 & -4.43814400 & -2.30465900 & $\mathrm{C}$ & -2.01949100 & -4.84415400 & -2.85549600 \\
\hline C & 0.29561300 & -5.02280800 & -3.73241900 & $\mathrm{H}$ & -3.96229200 & -4.67872000 & -1.94687600 \\
\hline C & 3.55617800 & -4.25467500 & -2.10483000 & $\mathrm{H}$ & 5.10685000 & -3.59983600 & -0.76789300 \\
\hline C & 1.63867500 & -4.90017600 & -3.55043500 & $\mathrm{H}$ & -2.40545300 & -5.15768400 & -3.82208000 \\
\hline $\mathrm{H}$ & -0.10307500 & -5.36157400 & -4.68542500 & $\mathrm{H}$ & 4.24348200 & -4.49067700 & -2.91330000 \\
\hline $\mathrm{H}$ & 2.32844300 & -5.13766300 & -4.35648000 & C & 1.80733600 & 0.63165300 & 2.52827500 \\
\hline S & 2.64133000 & -0.63291300 & 3.28920100 & $\mathrm{~N}$ & 0.50866400 & 0.93726400 & 2.81115400 \\
\hline $\mathrm{H}$ & -0.00649200 & 1.42634900 & 2.07118600 & $S$ & -0.45366000 & 3.40369300 & 0.05296100 \\
\hline 0 & -1.03310400 & 3.83991200 & -1.22984300 & $\mathrm{O}$ & -0.89753400 & 2.05245000 & 0.49896300 \\
\hline 0 & 0.99969100 & 3.63738700 & 0.23942300 & C & -1.22181400 & 4.52967400 & 1.31987300 \\
\hline $\mathrm{F}$ & -1.14575100 & 5.80909100 & 0.93796300 & $\mathrm{~F}$ & -0.59564600 & 4.39350200 & 2.50110500 \\
\hline $\mathrm{F}$ & -2.51884800 & 4.21916800 & 1.50116300 & C & -3.72931500 & -1.14356200 & -0.81422900 \\
\hline 0 & -4.47871000 & -1.19192100 & -1.77764100 & $\mathrm{O}$ & -4.05205500 & -1.33325300 & 0.47270100 \\
\hline C & -5.45146700 & -1.52623400 & 0.88776400 & C & -6.26782600 & -0.28808700 & 0.51510700 \\
\hline $\mathrm{H}$ & -7.26598100 & -0.35954400 & 0.95948700 & $\mathrm{H}$ & -6.37433800 & -0.19968800 & -0.56745300 \\
\hline $\mathrm{H}$ & -5.78266900 & 0.61305000 & 0.90449600 & C & -5.32818300 & -1.66346200 & 2.40452600 \\
\hline $\mathrm{H}$ & -4.68740700 & -2.51306900 & 2.66250100 & $\mathrm{H}$ & -6.31594600 & -1.82822300 & 2.84608000 \\
\hline $\mathrm{H}$ & -4.89478700 & -0.75956100 & 2.84151400 & C & -6.01287400 & -2.80561600 & 0.26573900 \\
\hline $\mathrm{H}$ & -7.02583500 & -2.97893200 & 0.64425800 & $\mathrm{H}$ & -5.39736700 & -3.66797900 & 0.54236600 \\
\hline $\mathrm{H}$ & -6.05064000 & -2.72982500 & -0.82148700 & C & -1.78894800 & -0.78877200 & -2.15061300 \\
\hline $\mathrm{H}$ & -2.35359200 & -1.30599900 & -2.92016900 & C & -0.32380100 & -0.89102500 & -2.24048700 \\
\hline $\mathrm{C}$ & 0.23654100 & -1.40388900 & -3.42206000 & C & 0.51665300 & -0.44846700 & -1.20881900 \\
\hline C & 1.61645500 & -1.47963100 & -3.56997600 & $\mathrm{H}$ & -0.41678100 & -1.76017500 & -4.21401300 \\
\hline C & 1.89463500 & -0.54063300 & -1.36368400 & $\mathrm{H}$ & 0.10540500 & -0.01603100 & -0.30522200 \\
\hline $\mathrm{C}$ & 2.45134000 & -1.04525200 & -2.53818100 & $\mathrm{H}$ & 2.03889000 & -1.89632000 & -4.47841600 \\
\hline $\mathrm{H}$ & 2.54045100 & -0.21371300 & -0.56316900 & $\mathrm{H}$ & 3.53017100 & -1.11548100 & -2.63054100 \\
\hline $\mathrm{N}$ & -2.37967900 & -0.88229100 & -0.91853500 & $\mathrm{H}$ & -1.84867700 & -0.79299800 & -0.04156700 \\
\hline $\mathrm{C}$ & -1.99979400 & 1.05590500 & -2.96342800 & $\mathrm{H}$ & -1.35342800 & 1.62411200 & -2.30159200 \\
\hline $\mathrm{H}$ & -1.57937200 & 0.79660000 & -3.93167000 & C & -3.37311100 & 1.29226300 & -2.88448800 \\
\hline C & -3.92024200 & 2.05687000 & -1.77375000 & $\mathrm{H}$ & -3.21479500 & 2.15539300 & -0.94394300 \\
\hline $\mathrm{H}$ & -4.89109500 & 1.68344000 & -1.43400900 & C & -4.27776000 & 0.81578600 & -3.98145900 \\
\hline $\mathrm{H}$ & -4.33270000 & 1.58930900 & -4.76026000 & $\mathrm{H}$ & -5.28806700 & 0.61731200 & -3.62129500 \\
\hline $\mathrm{H}$ & -3.88540300 & -0.09028400 & -4.45040000 & C & -4.29750400 & 5.04440000 & -0.95367800 \\
\hline $\mathrm{H}$ & -4.79900200 & 4.61611000 & -0.07958900 & $\mathrm{H}$ & -4.82209000 & 5.96939300 & -1.22331900 \\
\hline $\mathrm{H}$ & -3.27553000 & 5.29970700 & -0.66871900 & C & -6.03537400 & 3.80653400 & -3.17209600 \\
\hline $\mathrm{H}$ & -6.77792300 & 3.49637600 & -2.42718300 & $\mathrm{H}$ & -6.10546500 & 3.11891400 & -4.02142300 \\
\hline $\mathrm{H}$ & -6.32551600 & 4.80139700 & -3.53159200 & C & -3.03834500 & 4.39293000 & -3.71548000 \\
\hline $\mathrm{H}$ & -3.05960700 & 3.75665800 & -4.60751300 & $\mathrm{H}$ & -2.03368700 & 4.36126200 & -3.28507700 \\
\hline $\mathrm{H}$ & -3.23956200 & 5.42119100 & -4.03979400 & $\mathrm{Si}$ & -4.29958900 & 3.87541400 & -2.42352900 \\
\hline $\mathrm{C}$ & -0.35043400 & -2.93146200 & 4.28212800 & $\mathrm{H}$ & -0.98205300 & -2.67389600 & 5.13864900 \\
\hline $\mathrm{H}$ & 0.66507100 & -2.57169500 & 4.47095600 & $\mathrm{C}$ & -0.41190900 & -4.43788700 & 3.98194500 \\
\hline $\mathrm{H}$ & -0.55557300 & -5.03180200 & 4.88833000 & $\mathrm{H}$ & 0.51852200 & -4.76379000 & 3.50732900 \\
\hline $\mathrm{C}$ & -1.57597700 & -4.56305700 & 2.98643500 & $\mathrm{H}$ & -1.54923600 & -5.48655400 & 2.40338300 \\
\hline $\mathrm{H}$ & -2.53647700 & -4.51610300 & 3.51465200 & & & & \\
\hline
\end{tabular}

\section{TS-S-4f-18}

Imaginary frequency: $-403.86 \mathrm{~cm}^{-1}$

E[B3LYP/6-31G(d)-D3(BJ)]: -4789.085990 Hartree

E[PCM(Et $\left.\left.t_{2} \mathrm{O}\right)-B 3 L Y P / 6-311 G(d, p)-D 3(B J)\right]:-4790.229126$ Hartree

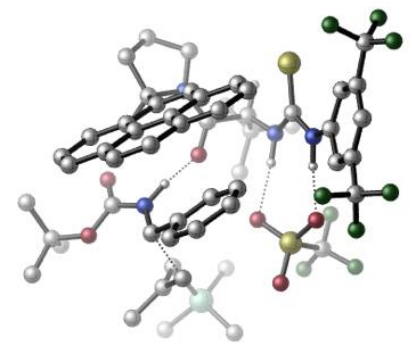




\begin{tabular}{|c|c|c|c|c|c|c|c|}
\hline 01 & & & & $\mathrm{~N}$ & -2.56105200 & -1.84731100 & 0.93248200 \\
\hline $\mathrm{H}$ & -1.93993100 & -2.34182100 & 0.27988700 & C & -3.79558000 & -1.51718400 & 0.34520100 \\
\hline C & -3.87102400 & -1.66131800 & -1.05427800 & $\mathrm{C}$ & -4.92271200 & -1.05659000 & 1.03667900 \\
\hline C & -5.01865200 & -1.28037200 & -1.73746300 & $\mathrm{H}$ & -3.02353900 & -2.06012200 & -1.59904200 \\
\hline C & -6.06305400 & -0.67911500 & 0.32557900 & $\mathrm{H}$ & -4.90215600 & -0.97694900 & 2.11198900 \\
\hline C & -6.12769200 & -0.77240900 & -1.05982100 & $\mathrm{H}$ & -7.01855400 & -0.47247200 & -1.59771400 \\
\hline C & -5.04253000 & -1.32023300 & -3.23884600 & C & -7.20524500 & -0.06084100 & 1.08048700 \\
\hline $\mathrm{F}$ & -4.11211100 & -2.14375200 & -3.75267900 & $\mathrm{~F}$ & -4.81043100 & -0.08252900 & -3.76147600 \\
\hline $\mathrm{F}$ & -6.24725600 & -1.70839500 & -3.71240000 & $\mathrm{~F}$ & -7.35162400 & -0.58643000 & 2.31277700 \\
\hline $\mathrm{F}$ & -7.00883900 & 1.27533300 & 1.24554700 & $\mathrm{~F}$ & -8.38151800 & -0.20203800 & 0.43146500 \\
\hline C & 0.16677300 & -1.29538000 & 3.29199900 & $\mathrm{H}$ & -0.50651900 & -1.07963700 & 4.11942100 \\
\hline C & 1.20824200 & -2.33806200 & 3.79138200 & C & 2.28001100 & -2.68414500 & 2.74717000 \\
\hline $\mathrm{H}$ & 2.87594200 & -1.80962800 & 2.47213000 & $\mathrm{H}$ & 1.83841900 & -3.08171800 & 1.83265600 \\
\hline $\mathrm{H}$ & 2.95214700 & -3.44464900 & 3.16161300 & $\mathrm{C}$ & 0.44439900 & -3.61614600 & 4.17753500 \\
\hline $\mathrm{H}$ & 1.12824600 & -4.33624800 & 4.64138500 & $\mathrm{H}$ & -0.00084800 & -4.08694200 & 3.29714200 \\
\hline $\mathrm{H}$ & -0.35999900 & -3.40014200 & 4.89021500 & C & 1.89696800 & -1.75590700 & 5.03870600 \\
\hline $\mathrm{H}$ & 2.64948100 & -2.45778100 & 5.41415500 & $\mathrm{H}$ & 1.17921000 & -1.57807800 & 5.84854000 \\
\hline $\mathrm{H}$ & 2.40960100 & -0.81245600 & 4.81471600 & C & 0.77469100 & 0.00746400 & 2.75639200 \\
\hline $\mathrm{O}$ & 1.31684900 & -0.01589300 & 1.63895600 & C & 0.12541700 & 1.34272200 & 4.80712700 \\
\hline C & 1.02120100 & 2.43478000 & 2.81562700 & $\mathrm{C}$ & 0.89624300 & 2.58584000 & 5.25958300 \\
\hline $\mathrm{H}$ & -0.95801700 & 1.51177400 & 4.79056100 & $\mathrm{H}$ & 0.32734800 & 0.46842700 & 5.42334900 \\
\hline $\mathrm{H}$ & 2.04685800 & 2.31937200 & 2.46015800 & $\mathrm{H}$ & 1.90789500 & 2.29522800 & 5.56557000 \\
\hline $\mathrm{H}$ & 0.41613300 & 3.09167100 & 6.10195300 & $\mathrm{~N}$ & 0.62961700 & 1.15349900 & 3.44217800 \\
\hline C & 0.09515500 & 2.81135800 & 1.66688000 & $\mathrm{C}$ & -1.18323100 & 2.33558000 & 1.63626400 \\
\hline $\mathrm{C}$ & 0.54568000 & 3.73094800 & 0.64319500 & $\mathrm{C}$ & -2.11760900 & 2.70244200 & 0.61668100 \\
\hline $\mathrm{H}$ & -1.52476700 & 1.63445100 & 2.39092200 & $\mathrm{C}$ & -0.36513400 & 4.09853400 & -0.40003900 \\
\hline C & 1.84036300 & 4.27879000 & 0.62088800 & C & -3.42637000 & 2.19405800 & 0.59544800 \\
\hline $\mathrm{C}$ & -1.69668000 & 3.59456200 & -0.40844400 & $\mathrm{C}$ & 0.05659100 & 4.97864300 & -1.44378800 \\
\hline $\mathrm{H}$ & 2.54848100 & 4.02910200 & 1.40214200 & $\mathrm{C}$ & 2.24071400 & 5.13411600 & -0.40402700 \\
\hline C & -4.30741600 & 2.55260000 & -0.42243700 & $\mathrm{H}$ & -3.74099700 & 1.51308400 & 1.37964200 \\
\hline C & -2.60646000 & 3.96183200 & -1.44365300 & C & 1.36669000 & 5.47744400 & -1.43087700 \\
\hline $\mathrm{C}$ & -0.87981000 & 5.33417700 & -2.47338600 & $\mathrm{H}$ & 3.24264500 & 5.55258900 & -0.39002900 \\
\hline C & -3.90367100 & 3.42637300 & -1.43091900 & $\mathrm{H}$ & -5.31563700 & 2.15383400 & -0.41755600 \\
\hline C & -2.15247700 & 4.85313800 & -2.47165300 & $\mathrm{H}$ & 1.68660000 & 6.14915300 & -2.22349300 \\
\hline $\mathrm{H}$ & -0.54537800 & 6.00691300 & -3.25920500 & $\mathrm{H}$ & -4.59562000 & 3.70153300 & -2.22290100 \\
\hline $\mathrm{H}$ & -2.84928500 & 5.13227900 & -3.25801000 & C & -1.99935000 & -1.45643800 & 2.11811200 \\
\hline$S$ & -2.81765500 & -0.64732200 & 3.36507800 & $\mathrm{~N}$ & -0.68119100 & -1.79855200 & 2.21479800 \\
\hline $\mathrm{H}$ & -0.17324400 & -2.02305600 & 1.35373500 & $\mathrm{~S}$ & 0.33316500 & -3.24234900 & -1.21787500 \\
\hline 0 & 0.94372100 & -3.26435200 & -2.55574500 & $\mathrm{O}$ & 1.02407600 & -2.37001800 & -0.22427600 \\
\hline $\mathrm{O}$ & -1.14702700 & -3.11242200 & -1.17558900 & C & 0.61055500 & -4.94554600 & -0.52940500 \\
\hline $\mathrm{F}$ & -0.02447400 & -5.87044400 & -1.25026200 & $\mathrm{~F}$ & 0.16617200 & -5.00404900 & 0.73918500 \\
\hline $\mathrm{F}$ & 1.92529000 & -5.24258700 & -0.52150300 & C & 3.90981800 & 1.70593200 & 0.19087900 \\
\hline $\mathrm{O}$ & 4.12215100 & 1.76505800 & 1.38959400 & $\mathrm{O}$ & 4.76175100 & 2.08109700 & -0.78520500 \\
\hline C & 6.06463500 & 2.69022000 & -0.44273000 & C & 5.85993200 & 3.94621100 & 0.40505700 \\
\hline $\mathrm{H}$ & 6.82226500 & 4.45301500 & 0.53227000 & $\mathrm{H}$ & 5.45616100 & 3.70275000 & 1.38724800 \\
\hline $\mathrm{H}$ & 5.17759300 & 4.63340500 & -0.10280700 & C & 6.63411300 & 3.06412600 & -1.80992100 \\
\hline $\mathrm{H}$ & 6.77686100 & 2.17955700 & -2.43596400 & $\mathrm{H}$ & 7.60367600 & 3.55566700 & -1.68440500 \\
\hline $\mathrm{H}$ & 5.96113300 & 3.75357500 & -2.32917600 & C & 6.94141000 & 1.65512200 & 0.26284000 \\
\hline $\mathrm{H}$ & 7.93301300 & 2.08126000 & 0.44759100 & $\mathrm{H}$ & 7.07045900 & 0.76538400 & -0.36256800 \\
\hline $\mathrm{H}$ & 6.50252600 & 1.36104300 & 1.21733900 & $\mathrm{C}$ & 2.38389900 & 1.20559900 & -1.66082200 \\
\hline $\mathrm{H}$ & 3.01386000 & 1.85233300 & -2.26152200 & C & 0.95144300 & 1.21804200 & -2.02322600 \\
\hline C & 0.57070800 & 1.86487200 & -3.20842200 & C & -0.01336600 & 0.55082700 & -1.25499900 \\
\hline C & -0.75625000 & 1.84012800 & -3.62566100 & $\mathrm{H}$ & 1.31776200 & 2.39302100 & -3.79580400 \\
\hline
\end{tabular}




\begin{tabular}{|c|c|c|c|c|c|c|c|}
\hline $\mathrm{C}$ & -1.33517100 & 0.53345600 & -1.67929800 & $\overline{\mathrm{H}}$ & 0.26636600 & 0.02945900 & -0.34879200 \\
\hline C & -1.71180900 & 1.16187900 & -2.86696400 & $\mathrm{H}$ & -1.04545300 & 2.35370700 & -4.53694700 \\
\hline $\mathrm{H}$ & -2.08052700 & 0.03762500 & -1.07489400 & $\mathrm{H}$ & -2.74535600 & 1.12041000 & -3.19081000 \\
\hline $\mathrm{N}$ & 2.71692800 & 1.24313400 & -0.32467800 & $\mathrm{H}$ & 2.07687400 & 0.87859700 & 0.39909000 \\
\hline C & 2.85811300 & -0.51973000 & -2.49796300 & $\mathrm{H}$ & 2.08796000 & -1.13629500 & -2.04787000 \\
\hline $\mathrm{H}$ & 2.69447600 & -0.26892600 & -3.54340100 & C & 4.16860600 & -0.71486700 & -2.05757400 \\
\hline C & 4.40835600 & -1.34157300 & -0.77278400 & $\mathrm{H}$ & 3.49606800 & -1.43886200 & -0.17712900 \\
\hline $\mathrm{H}$ & 5.21212700 & -0.86413500 & -0.20206000 & C & 5.31876000 & -0.27406800 & -2.90916100 \\
\hline $\mathrm{H}$ & 5.53402100 & -1.06144000 & -3.64516700 & $\mathrm{H}$ & 6.22643000 & -0.10131700 & -2.32895000 \\
\hline $\mathrm{H}$ & 5.06898900 & 0.62969800 & -3.47185000 & C & 4.74812900 & -4.05893100 & 0.58313800 \\
\hline $\mathrm{H}$ & 5.15669300 & -3.50098400 & 1.43277900 & $\mathrm{H}$ & 5.21684100 & -5.05047800 & 0.57242200 \\
\hline $\mathrm{H}$ & 3.67693100 & -4.19301100 & 0.74398400 & C & 6.94011800 & -3.02602300 & -1.32454200 \\
\hline $\mathrm{H}$ & 7.43601300 & -2.50605000 & -0.49626700 & $\mathrm{H}$ & 7.18312100 & -2.49606100 & -2.25191500 \\
\hline $\mathrm{H}$ & 7.38380300 & -4.02652000 & -1.39706800 & C & 4.24323400 & -3.96475300 & -2.51613300 \\
\hline $\mathrm{H}$ & 4.59581300 & -3.52017400 & -3.45468200 & $\mathrm{H}$ & 3.15436900 & -3.86411400 & -2.48495500 \\
\hline $\mathrm{H}$ & 4.48415900 & -5.03412800 & -2.55079500 & $\mathrm{Si}$ & 5.07751600 & -3.17125500 & -1.03718800 \\
\hline C & 0.94436500 & 3.45007700 & 3.98954800 & $\mathrm{H}$ & 0.02629400 & 4.03817700 & 3.89808600 \\
\hline $\mathrm{H}$ & 1.78736800 & 4.14573000 & 3.97623600 & & & & \\
\hline
\end{tabular}

\section{TS-S-4f-19}

Imaginary frequency: $-294.81 \mathrm{~cm}^{-1}$

E[B3LYP/6-31G(d)-D3(BJ)]: -4789.087981 Hartree

$\mathrm{E}\left[\mathrm{PCM}\left(\mathrm{Et}_{2} \mathrm{O}\right)-\mathrm{B} 3 \mathrm{LYP} / 6-311 \mathrm{G}(\mathrm{d}, \mathrm{p})-\mathrm{D} 3(\mathrm{BJ})\right]$ : -4790.228517 Hartree

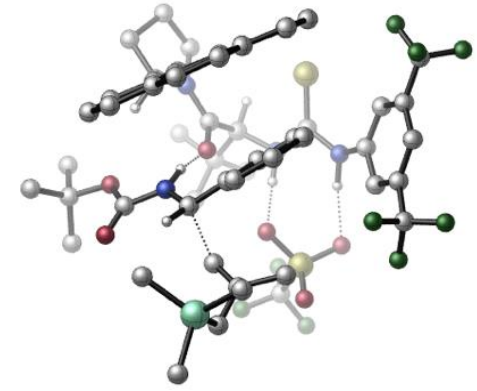

\begin{tabular}{|c|c|c|c|c|c|c|c|}
\hline 01 & & & & $\mathrm{~N}$ & 1.91172900 & -3.09364200 & 0.53752300 \\
\hline $\mathrm{H}$ & 1.53440000 & -3.17610800 & 1.48906400 & C & 3.06409800 & -2.29254200 & 0.48652600 \\
\hline $\mathrm{C}$ & 3.31414500 & -1.48705600 & 1.61240500 & C & 3.92461500 & -2.20873600 & -0.61587900 \\
\hline $\mathrm{C}$ & 4.35012000 & -0.56343800 & 1.59443600 & $\mathrm{H}$ & 2.67554600 & -1.57090000 & 2.48263400 \\
\hline C & 4.95097300 & -1.26151100 & -0.61517400 & $\mathrm{H}$ & 3.75803300 & -2.83669900 & -1.47710600 \\
\hline $\mathrm{C}$ & 5.17447000 & -0.42715800 & 0.47633200 & $\bar{H}$ & 5.96180700 & 0.31683700 & 0.45612100 \\
\hline $\mathrm{C}$ & 4.50045700 & 0.40881400 & 2.73011300 & $\mathrm{C}$ & 5.84566300 & -1.14382000 & -1.81682200 \\
\hline $\mathrm{F}$ & 3.87257000 & 0.00229200 & 3.84952200 & $\mathrm{~F}$ & 3.97400600 & 1.62175200 & 2.40268900 \\
\hline $\mathrm{F}$ & 5.79779700 & 0.62875400 & 3.03776300 & $\mathrm{~F}$ & 5.24661500 & -1.58822300 & -2.94625400 \\
\hline $\mathrm{F}$ & 6.20344000 & 0.14742600 & -2.03932400 & $\mathrm{~F}$ & 6.99078700 & -1.84488700 & -1.67547500 \\
\hline C & -1.36735700 & -3.71040300 & -0.91372100 & $\mathrm{H}$ & -1.06309700 & -4.39360600 & -1.70719100 \\
\hline $\mathrm{C}$ & -2.61183700 & -4.32805200 & -0.19161800 & C & -3.24901400 & -3.37516400 & 0.83747300 \\
\hline $\mathrm{H}$ & -3.64173900 & -2.47214800 & 0.36117400 & $\mathrm{H}$ & -2.54585700 & -3.05281300 & 1.60536600 \\
\hline $\mathrm{H}$ & -4.07803100 & -3.89229600 & 1.33485700 & C & -2.16128600 & -5.62232400 & 0.50776400 \\
\hline $\mathrm{H}$ & -3.03058700 & -6.13695500 & 0.93278400 & $\mathrm{H}$ & -1.45967400 & -5.41608200 & 1.31836500 \\
\hline $\mathrm{H}$ & -1.67367600 & -6.30404200 & -0.19897000 & C & -3.67024800 & -4.67561300 & -1.25316300 \\
\hline $\mathrm{H}$ & -4.54182700 & -5.13272100 & -0.77229700 & $\mathrm{H}$ & -3.28372800 & -5.39065000 & -1.98883300 \\
\hline $\mathrm{H}$ & -4.01710100 & -3.78303200 & -1.78599000 & C & -1.67387300 & -2.31946100 & -1.48399500 \\
\hline $\mathrm{C}$ & -2.42748000 & -0.82306800 & -3.24543100 & $\mathrm{H}$ & -3.18032200 & -0.44747700 & -2.55279900 \\
\hline $\mathrm{N}$ & -2.02585600 & -2.16014700 & -2.77219300 & C & -1.27067700 & 0.16138800 & -3.31922500 \\
\hline $\mathrm{C}$ & 0.01281900 & -0.27579000 & -3.46694200 & $\mathrm{C}$ & -1.54675300 & 1.58219900 & -3.24895900 \\
\hline $\mathrm{C}$ & 1.12151900 & 0.62221900 & -3.58771700 & $\mathrm{H}$ & 0.23058400 & -1.33812900 & -3.44948800 \\
\hline C & -0.45138900 & 2.49962500 & -3.33021100 & C & -2.83957600 & 2.10473300 & -3.06922200 \\
\hline C & 2.43934000 & 0.15905000 & -3.73183300 & C & 0.87646100 & 2.02308800 & -3.52719800 \\
\hline C & -0.68285800 & 3.90416100 & -3.20389600 & $\mathrm{H}$ & -3.69170000 & 1.43876400 & -3.01949600 \\
\hline C & -3.05260900 & 3.47362900 & -2.92194700 & C & 3.49677300 & 1.05927300 & -3.84470000 \\
\hline
\end{tabular}




\begin{tabular}{|c|c|c|c|c|c|c|c|}
\hline$\overline{\mathrm{H}}$ & 2.62876800 & -0.91011300 & -3.72979500 & C & 1.96545700 & 2.93776800 & -3.63313700 \\
\hline C & -1.98818000 & 4.36774900 & -2.98288300 & C & 0.43322900 & 4.80218800 & -3.30912200 \\
\hline $\mathrm{H}$ & -4.06141400 & 3.84259200 & -2.75959200 & $\mathrm{C}$ & 3.26328800 & 2.43273300 & -3.80407200 \\
\hline $\mathrm{H}$ & 4.50955800 & 0.68604300 & -3.94338300 & $\mathrm{C}$ & 1.69552100 & 4.34264200 & -3.52662000 \\
\hline $\mathrm{H}$ & -2.16070900 & 5.43536300 & -2.87352300 & $\mathrm{H}$ & 0.24256000 & 5.86868400 & -3.21879900 \\
\hline $\mathrm{H}$ & 4.09417200 & 3.12916800 & -3.88483600 & $\mathrm{H}$ & 2.52857100 & 5.03617400 & -3.60978900 \\
\hline C & 1.02119500 & -3.36778300 & -0.47285500 & S & 1.41724300 & -3.47805700 & -2.11498400 \\
\hline $\mathrm{N}$ & -0.24045800 & -3.56691800 & -0.00512400 & $\mathrm{H}$ & -0.46019000 & -3.06407800 & 0.86251200 \\
\hline $\mathrm{S}$ & 0.02112200 & -2.19630500 & 3.53166000 & $\mathrm{O}$ & 0.24885300 & -1.08370700 & 4.46796100 \\
\hline $\mathrm{O}$ & -0.62418400 & -1.80691400 & 2.23970500 & $\mathrm{O}$ & 1.15874800 & -3.13130600 & 3.32961200 \\
\hline C & -1.26798900 & -3.24463100 & 4.36082000 & $\mathrm{~F}$ & -0.86625700 & -3.62312800 & 5.57630200 \\
\hline$F$ & -1.51083800 & -4.34659000 & 3.62701600 & $\mathrm{~F}$ & -2.41607400 & -2.56059800 & 4.47638200 \\
\hline C & -3.64165200 & 1.38467400 & 0.16523100 & $\mathrm{O}$ & -4.11839600 & 2.29505700 & 0.82122100 \\
\hline $\mathrm{O}$ & -4.30382300 & 0.43456600 & -0.51194700 & C & -5.77422100 & 0.34332200 & -0.46555900 \\
\hline C & -6.22125100 & 0.09171100 & 0.97442800 & $\mathrm{H}$ & -7.29914900 & -0.10034200 & 0.99337900 \\
\hline $\mathrm{H}$ & -6.00829300 & 0.95352900 & 1.60863700 & $\mathrm{H}$ & -5.70873400 & -0.78634000 & 1.38071200 \\
\hline C & -6.05475800 & -0.87361200 & -1.34612900 & $\mathrm{H}$ & -5.69092600 & -0.70642200 & -2.36545900 \\
\hline $\mathrm{H}$ & -7.13207600 & -1.06061300 & -1.39110900 & $\mathrm{H}$ & -5.56257100 & -1.76343200 & -0.94268800 \\
\hline C & -6.38945600 & 1.60904800 & -1.06322400 & $\mathrm{H}$ & -7.47681200 & 1.49264200 & -1.12111200 \\
\hline $\mathrm{H}$ & -6.01135100 & 1.77421600 & -2.07794700 & $\mathrm{H}$ & -6.16012400 & 2.48298100 & -0.45244000 \\
\hline C & -1.37735000 & 2.00897500 & 0.60201700 & $\mathrm{H}$ & -1.79768800 & 2.98001400 & 0.82510100 \\
\hline $\mathrm{C}$ & 0.02340200 & 1.98454200 & 0.16508700 & C & 0.71465700 & 3.20470800 & 0.09705100 \\
\hline C & 0.69546400 & 0.79180800 & -0.14160700 & C & 2.05951000 & 3.23594700 & -0.25472200 \\
\hline $\mathrm{H}$ & 0.18813400 & 4.12988100 & 0.30470100 & C & 2.03597400 & 0.83562400 & -0.51049800 \\
\hline $\mathrm{H}$ & 0.18224800 & -0.16142700 & -0.07793900 & C & 2.72567400 & 2.04647400 & -0.55079300 \\
\hline $\mathrm{H}$ & 2.57945200 & 4.18677500 & -0.31397800 & $\mathrm{H}$ & 2.54281500 & -0.07966100 & -0.77583100 \\
\hline $\mathrm{H}$ & 3.77229000 & 2.05566900 & -0.83304800 & $\mathrm{~N}$ & -2.28264200 & 1.18095900 & -0.00068100 \\
\hline $\mathrm{H}$ & -1.99145200 & 0.28313300 & -0.41979700 & C & -1.37892700 & 1.28812600 & 2.52340800 \\
\hline $\mathrm{H}$ & -1.03232000 & 0.30197800 & 2.22265700 & $\mathrm{H}$ & -2.44795100 & 1.38511700 & 2.69558500 \\
\hline C & -0.53014100 & 2.05615000 & 3.31728700 & C & -1.04449800 & 3.14121600 & 4.16792400 \\
\hline $\mathrm{H}$ & -0.46688500 & 3.17972500 & 5.10086500 & $\mathrm{H}$ & -2.10108500 & 2.99013300 & 4.41642400 \\
\hline C & 0.93153600 & 1.77637700 & 3.33009400 & $\mathrm{H}$ & 1.08438300 & 0.87884100 & 3.94885100 \\
\hline $\mathrm{H}$ & 1.52037000 & 2.59960900 & 3.73938000 & $\mathrm{H}$ & 1.30306700 & 1.51655000 & 2.33529300 \\
\hline C & -1.51818700 & 6.00725100 & 4.96775600 & $\mathrm{H}$ & -2.55217800 & 5.76755600 & 5.24179300 \\
\hline $\mathrm{H}$ & -1.48187200 & 7.07341200 & 4.71368500 & $\mathrm{H}$ & -0.89193600 & 5.85517500 & 5.85423400 \\
\hline C & -2.09580500 & 5.22951000 & 2.04779400 & $\mathrm{H}$ & -2.93709800 & 4.52733400 & 2.04647500 \\
\hline $\mathrm{H}$ & -1.58910800 & 5.14071600 & 1.08117900 & $\mathrm{H}$ & -2.51264100 & 6.24280800 & 2.09544400 \\
\hline C & 0.85459100 & 5.38923300 & 3.08964000 & $\mathrm{H}$ & 1.27392100 & 4.72515600 & 2.32821800 \\
\hline $\mathrm{H}$ & 1.49401000 & 5.32371000 & 3.97762500 & $\mathrm{H}$ & 0.91934500 & 6.41764600 & 2.71353600 \\
\hline $\mathrm{Si}$ & -0.93015700 & 4.96003500 & 3.51308300 & C & -1.95217000 & -3.15711100 & -3.85441000 \\
\hline $\mathrm{H}$ & -2.74500400 & -3.90313900 & -3.74015600 & $\mathrm{H}$ & -0.98270300 & -3.66256400 & -3.83100400 \\
\hline C & -2.16553100 & -2.31437300 & -5.12106400 & $\mathrm{H}$ & -2.63651600 & -2.88905000 & -5.92280400 \\
\hline $\mathrm{H}$ & -1.20513100 & -1.94120800 & -5.48964800 & C & -3.02639400 & -1.14010600 & -4.63156800 \\
\hline $\mathrm{H}$ & -2.99767500 & -0.27465000 & -5.29755200 & $\mathrm{H}$ & -4.07148300 & -1.45442800 & -4.51777000 \\
\hline $\mathrm{O}$ & -1.63119500 & -1.35590600 & -0.70339000 & & & & \\
\hline
\end{tabular}

\section{TS-S-4f-20}

Imaginary frequency: $-296.93 \mathrm{~cm}^{-1}$

E[B3LYP/6-31G(d)-D3(BJ)]: -4789.086549 Hartree

E[PCM(Et $2 \mathrm{O})-B 3 L Y P / 6-311 G(d, p)-D 3(B J)]:-4790.228203$ Hartree

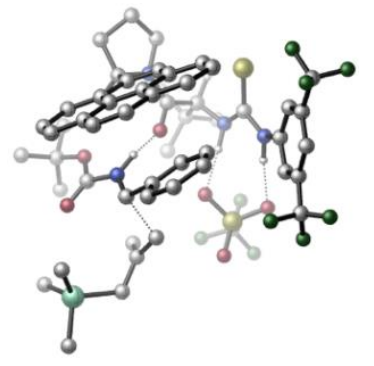




\begin{tabular}{|c|c|c|c|c|c|c|c|}
\hline 01 & & & & $\mathrm{~N}$ & 2.48978100 & 2.25846900 & 1.27138000 \\
\hline $\mathrm{H}$ & 1.87836400 & 2.98968100 & 0.88977300 & $\mathrm{C}$ & 3.51022100 & 1.89401400 & 0.37743400 \\
\hline C & 3.38792800 & 2.38132000 & -0.93753500 & C & 4.58855500 & 1.05703400 & 0.69329400 \\
\hline C & 4.28199100 & 1.97268200 & -1.91868200 & $\mathrm{H}$ & 2.57387400 & 3.05330900 & -1.18504300 \\
\hline C & 5.46393500 & 0.64953800 & -0.31439400 & $\mathrm{H}$ & 4.71527700 & 0.70048500 & 1.70286700 \\
\hline C & 5.32190800 & 1.08817400 & -1.62683300 & $\mathrm{H}$ & 6.00135100 & 0.75670000 & -2.40248600 \\
\hline $\mathrm{C}$ & 4.05922500 & 2.38108000 & -3.34823200 & C & 6.61420100 & -0.24871200 & 0.04380600 \\
\hline $\mathrm{F}$ & 3.32865700 & 3.50514200 & -3.45575900 & $\mathrm{~F}$ & 3.40472100 & 1.41003300 & -4.03538300 \\
\hline $\mathrm{F}$ & 5.23005900 & 2.58558600 & -3.99578200 & $\mathrm{~F}$ & 6.32959000 & -1.04347400 & 1.10368200 \\
\hline $\mathrm{F}$ & 6.94840000 & -1.06746500 & -0.98540100 & $\mathrm{~F}$ & 7.72564500 & 0.44754000 & 0.36454300 \\
\hline C & -0.02544100 & 1.21023200 & 3.67140800 & $\mathrm{H}$ & 0.63457900 & 0.99780900 & 4.51207900 \\
\hline C & -1.24998300 & 2.01460100 & 4.21748200 & C & -2.34440500 & 2.25044100 & 3.16020200 \\
\hline $\mathrm{H}$ & -2.76222100 & 1.30684800 & 2.79745400 & $\mathrm{H}$ & -1.97669000 & 2.79451500 & 2.29067400 \\
\hline $\mathrm{H}$ & -3.15386900 & 2.83804900 & 3.60953400 & C & -0.73331200 & 3.36523300 & 4.74168200 \\
\hline $\mathrm{H}$ & -1.55100400 & 3.91796800 & 5.21836200 & $\mathrm{H}$ & -0.33260500 & 3.97899500 & 3.93304900 \\
\hline $\mathrm{H}$ & 0.05979100 & 3.22214700 & 5.48491700 & C & -1.86435800 & 1.22617500 & 5.38779000 \\
\hline $\mathrm{H}$ & -2.71037600 & 1.78162300 & 5.80688400 & $\mathrm{H}$ & -1.13743300 & 1.07047600 & 6.19337800 \\
\hline $\mathrm{H}$ & -2.23768500 & 0.24755300 & 5.06550100 & C & -0.44952600 & -0.08919000 & 2.97537600 \\
\hline C & -0.94188900 & -2.46737800 & 2.93492800 & $\mathrm{H}$ & -1.92957200 & -2.21977500 & 2.54831200 \\
\hline $\mathrm{N}$ & -0.43117800 & -1.26732400 & 3.62253600 & $\mathrm{C}$ & -0.05680900 & -2.93022200 & 1.78855100 \\
\hline C & 1.27583900 & -2.64068600 & 1.76665000 & C & -0.64606100 & -3.70560300 & 0.71516800 \\
\hline $\mathrm{C}$ & 2.14475200 & -3.10189700 & 0.72567600 & $\mathrm{H}$ & 1.70691900 & -2.00307200 & 2.53135900 \\
\hline C & 0.19514800 & -4.15082900 & -0.35350700 & $\mathrm{C}$ & -2.01633300 & -4.01482600 & 0.65467200 \\
\hline C & 3.51357400 & -2.78949900 & 0.70962600 & C & 1.59189100 & -3.87292600 & -0.33569500 \\
\hline $\mathrm{C}$ & -0.36526300 & -4.86885600 & -1.45472900 & $\mathrm{H}$ & -2.67601600 & -3.71186500 & 1.45770600 \\
\hline $\mathrm{C}$ & -2.55886800 & -4.69043800 & -0.43637700 & $\mathrm{C}$ & 4.33129800 & -3.24832500 & -0.32117500 \\
\hline $\mathrm{H}$ & 3.92518800 & -2.16669900 & 1.49769300 & C & 2.43415000 & -4.33334300 & -1.39012200 \\
\hline C & -1.74651300 & -5.11175900 & -1.48452000 & C & 0.50665700 & -5.32087000 & -2.50264200 \\
\hline $\mathrm{H}$ & -3.62613300 & -4.89172400 & -0.46300500 & $\mathrm{C}$ & 3.80074800 & -4.01732200 & -1.35534700 \\
\hline $\mathrm{H}$ & 5.38397900 & -2.98976100 & -0.32587900 & $\mathrm{C}$ & 1.84455600 & -5.07563600 & -2.46659100 \\
\hline $\mathrm{H}$ & -2.17319100 & -5.64466500 & -2.33030200 & $\mathrm{H}$ & 0.06909400 & -5.87307400 & -3.33049700 \\
\hline $\mathrm{H}$ & 4.44356900 & -4.36683000 & -2.15938000 & $\mathrm{H}$ & 2.49154000 & -5.42672900 & -3.26651500 \\
\hline C & 2.01116900 & 1.59568300 & 2.37157800 & $\mathrm{~S}$ & 2.86844700 & 0.44302400 & 3.27193200 \\
\hline $\mathrm{N}$ & 0.74248900 & 1.97525900 & 2.69807200 & $\mathrm{H}$ & 0.18065000 & 2.32312900 & 1.91195200 \\
\hline $\mathrm{S}$ & -0.38518600 & 3.94583900 & -0.37830400 & $\mathrm{O}$ & -0.89214400 & 4.07297500 & -1.75107700 \\
\hline $\mathrm{O}$ & -0.71926300 & 2.65154400 & 0.29600300 & $\mathrm{O}$ & 1.02243400 & 4.35635900 & -0.13385000 \\
\hline $\mathrm{C}$ & -1.37403100 & 5.18757100 & 0.58789000 & $\mathrm{~F}$ & -1.24185200 & 6.41300200 & 0.07788400 \\
\hline $\mathrm{F}$ & -0.97852900 & 5.21169100 & 1.87314900 & $\mathrm{~F}$ & -2.68120000 & 4.85462000 & 0.56768600 \\
\hline C & -3.51878100 & -1.08684700 & -0.09571100 & $\mathrm{O}$ & -4.36969900 & -1.21260900 & -0.96189800 \\
\hline $\mathrm{O}$ & -3.70528900 & -1.12150900 & 1.23018600 & $\mathrm{C}$ & -5.05030900 & -1.26098200 & 1.81976000 \\
\hline C & -5.89013800 & -0.03701500 & 1.45586400 & $\mathrm{H}$ & -6.83598300 & -0.06750100 & 2.00656900 \\
\hline $\mathrm{H}$ & -6.11379900 & -0.01091700 & 0.38853900 & $\mathrm{H}$ & -5.36148700 & 0.88058300 & 1.73287800 \\
\hline C & -4.74973200 & -1.28635800 & 3.31781000 & $\mathrm{H}$ & -4.11331900 & -2.14041900 & 3.57105600 \\
\hline $\mathrm{H}$ & -5.68292300 & -1.37279300 & 3.88273200 & $\mathrm{H}$ & -4.23908500 & -0.36940500 & 3.62495200 \\
\hline C & -5.69241700 & -2.57289100 & 1.36784600 & $\mathrm{H}$ & -6.64282200 & -2.70894200 & 1.89450600 \\
\hline $\mathrm{H}$ & -5.04480600 & -3.42069200 & 1.61450700 & $\mathrm{H}$ & -5.88088700 & -2.57214100 & 0.29404000 \\
\hline C & -1.72443600 & -0.81940700 & -1.64957000 & $\mathrm{H}$ & -2.41169500 & -1.25911900 & -2.36017500 \\
\hline C & -0.29394500 & -1.01291200 & -1.92846200 & C & 0.05546400 & -1.63206300 & -3.13930400 \\
\hline C & 0.71252200 & -0.53580400 & -1.07368900 & C & 1.39190700 & -1.76932100 & -3.49661300 \\
\hline $\mathrm{H}$ & -0.72763100 & -2.00700800 & -3.79321800 & C & 2.04564300 & -0.68565900 & -1.43611100 \\
\hline $\mathrm{H}$ & 0.45702500 & -0.03568700 & -0.14545100 & C & 2.38862900 & -1.28524000 & -2.64888900 \\
\hline $\mathrm{H}$ & 1.65484900 & -2.25620300 & -4.43030800 & $\mathrm{H}$ & 2.82302800 & -0.34259000 & -0.76920000 \\
\hline $\mathrm{H}$ & 3.43332800 & -1.37839300 & -2.92291900 & $\mathrm{~N}$ & -2.18047000 & -0.88230100 & -0.36328900 \\
\hline
\end{tabular}




\begin{tabular}{|c|c|c|c|c|c|c|c|}
\hline $\mathrm{H}$ & -1.56841600 & -0.68338600 & 0.44538500 & C & -1.83160300 & 1.11935700 & -2.31782300 \\
\hline $\mathrm{H}$ & -1.21571900 & 1.46389200 & -1.49384900 & $\mathrm{H}$ & -1.32906900 & 1.01303900 & -3.27454700 \\
\hline $\mathrm{C}$ & -3.15298300 & 1.55176700 & -2.31573200 & C & -3.93857100 & 1.67491500 & -3.55397400 \\
\hline $\mathrm{H}$ & -3.27642800 & 1.79794900 & -4.41852400 & $\mathrm{H}$ & -4.58206500 & 2.56262000 & -3.48943100 \\
\hline C & -3.76369300 & 2.00357700 & -1.03094200 & $\mathrm{H}$ & -4.84445800 & 1.84332900 & -1.00454500 \\
\hline $\mathrm{H}$ & -3.57009000 & 3.08189600 & -0.94411400 & $\mathrm{H}$ & -3.27777200 & 1.55063300 & -0.16369300 \\
\hline $\mathrm{C}$ & -5.81826400 & 0.88551300 & -5.75257000 & $\mathrm{H}$ & -6.29686500 & 1.86861000 & -5.67415000 \\
\hline $\mathrm{H}$ & -6.56205400 & 0.18664800 & -6.15375100 & $\mathrm{H}$ & -5.00881400 & 0.96527600 & -6.48755000 \\
\hline C & -6.60262900 & 0.17596400 & -2.85120200 & $\mathrm{H}$ & -7.07453900 & 1.15265600 & -2.69257000 \\
\hline $\mathrm{H}$ & -6.24890700 & -0.21132100 & -1.89290800 & $\mathrm{H}$ & -7.37506800 & -0.50676500 & -3.22594900 \\
\hline C & -4.30601800 & -1.37071200 & -4.28709900 & $\mathrm{H}$ & -4.18225400 & -1.86187200 & -3.31836400 \\
\hline $\mathrm{H}$ & -3.32262100 & -1.25317500 & -4.75811700 & $\mathrm{H}$ & -4.89843800 & -2.03310200 & -4.92970000 \\
\hline $\mathrm{Si}$ & -5.18214800 & 0.28538900 & -4.07892400 & $\mathrm{C}$ & 0.17390600 & -3.09787200 & 4.97709600 \\
\hline $\mathrm{H}$ & 0.09682800 & -3.48793900 & 5.99532600 & $\mathrm{H}$ & 1.10600900 & -3.46944500 & 4.54039200 \\
\hline $\mathrm{C}$ & -1.01257300 & -3.49320300 & 4.08532500 & $\mathrm{H}$ & -0.95737500 & -4.52131400 & 3.72028500 \\
\hline $\mathrm{H}$ & -1.95647400 & -3.37077000 & 4.63158900 & C & 0.15219900 & -1.56267500 & 4.94262800 \\
\hline $\mathrm{H}$ & -0.48319900 & -1.16058000 & 5.73848200 & $\mathrm{H}$ & 1.14656800 & -1.11491900 & 5.01984300 \\
\hline 0 & -0.83861800 & -0.00643800 & 1.80003600 & & & & \\
\hline
\end{tabular}

\section{TS-S-4f-21}

Imaginary frequency: $-421.94 \mathrm{~cm}^{-1}$

E[B3LYP/6-31G(d)-D3(BJ)]: -4789.092929 Hartree

E[PCM(Et $\left.\left.{ }_{2} \mathrm{O}\right)-\mathrm{B} 3 L Y P / 6-311 \mathrm{G}(\mathrm{d}, \mathrm{p})-\mathrm{D} 3(\mathrm{BJ})\right]$ : -4790.227957 Hartree

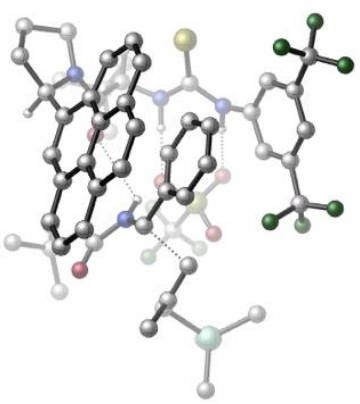

\begin{tabular}{|c|c|c|c|c|c|c|c|}
\hline 01 & & & & $\mathrm{~N}$ & -1.19599300 & 0.87881900 & -3.13959700 \\
\hline $\mathrm{H}$ & -0.19571000 & 0.82847000 & -3.34497800 & C & -1.58168500 & 2.10844500 & -2.57307300 \\
\hline $\mathrm{C}$ & -0.57397600 & 2.87162700 & -1.96453400 & $\mathrm{C}$ & -2.89801800 & 2.58234200 & -2.56391300 \\
\hline $\mathrm{C}$ & -0.89328300 & 4.04536500 & -1.29224700 & $\mathrm{H}$ & 0.45538200 & 2.53755000 & -1.99087400 \\
\hline C & -3.19792400 & 3.75676800 & -1.87258600 & $\mathrm{H}$ & -3.67550100 & 2.02131800 & -3.06066700 \\
\hline $\mathrm{C}$ & -2.21161800 & 4.49330800 & -1.22117500 & $\mathrm{H}$ & -2.46031800 & 5.39954500 & -0.68423500 \\
\hline $\mathrm{C}$ & 0.19170400 & 4.76616200 & -0.54415500 & $\mathrm{C}$ & -4.63493000 & 4.17940700 & -1.76142900 \\
\hline $\mathrm{F}$ & 1.37200100 & 4.73215400 & -1.19757600 & $\mathrm{~F}$ & 0.41059800 & 4.20492000 & 0.67648100 \\
\hline $\mathrm{F}$ & -0.11631400 & 6.06231800 & -0.32132300 & $\mathrm{~F}$ & -5.33799400 & 3.89899900 & -2.87702800 \\
\hline $\mathrm{F}$ & -5.26118600 & 3.53716300 & -0.73721600 & $\mathrm{~F}$ & -4.76059100 & 5.50271500 & -1.51918400 \\
\hline C & -1.30190000 & -2.75524200 & -2.83837300 & $\mathrm{H}$ & -2.31719200 & -2.86302700 & -3.21544100 \\
\hline $\mathrm{C}$ & -0.35798900 & -3.70980500 & -3.64113700 & $\mathrm{C}$ & 1.12065600 & -3.32764200 & -3.45955500 \\
\hline $\bar{H}$ & 1.38235500 & -3.22416100 & -2.40477100 & $\bar{H}$ & 1.35027900 & -2.38344600 & -3.95890900 \\
\hline $\mathrm{H}$ & 1.75876500 & -4.09681700 & -3.91015100 & C & -0.73540500 & -3.59355400 & -5.12686900 \\
\hline$\overline{\mathrm{H}}$ & -0.05629500 & -4.19379300 & -5.74332100 & $\mathrm{H}$ & -0.66963400 & -2.55339100 & -5.46118300 \\
\hline $\bar{H}$ & -1.75841000 & -3.94514200 & -5.30792800 & $\mathrm{C}$ & -0.55783700 & -5.16155400 & -3.17219200 \\
\hline $\mathrm{H}$ & 0.07196700 & -5.83133500 & -3.76777700 & $\mathrm{H}$ & -1.59396200 & -5.49644700 & -3.29587700 \\
\hline $\mathrm{H}$ & -0.27546000 & -5.28881000 & -2.12116600 & $\mathrm{C}$ & -1.26407200 & -3.02560200 & -1.32335800 \\
\hline $\mathrm{O}$ & -0.27406900 & -2.70933000 & -0.65724300 & $\mathrm{C}$ & -3.48224900 & -4.25439200 & -1.40159700 \\
\hline C & -2.21878600 & -4.01727500 & 0.68505500 & C & -3.78174000 & -5.45686000 & -0.49972200 \\
\hline $\mathrm{H}$ & -4.32892000 & -3.55961600 & -1.45854600 & $\mathrm{H}$ & -3.23626300 & -4.55033700 & -2.41967600 \\
\hline $\mathrm{H}$ & -1.29578300 & -4.60215200 & 0.77259200 & $\mathrm{H}$ & -3.12047400 & -6.28863400 & -0.77029800 \\
\hline $\mathrm{H}$ & -4.81570000 & -5.80106500 & -0.59258400 & $\mathrm{~N}$ & -2.33525400 & -3.62029800 & -0.73719100 \\
\hline $\mathrm{C}$ & -2.10864400 & -2.89362800 & 1.70230700 & C & -1.02602300 & -2.89761900 & 2.53266700 \\
\hline $\mathrm{C}$ & -3.15399300 & -1.90694500 & 1.90217200 & $\mathrm{C}$ & -0.89095300 & -2.00381700 & 3.64235800 \\
\hline
\end{tabular}




\begin{tabular}{|c|c|c|c|c|c|c|c|}
\hline$\overline{\mathrm{H}}$ & -0.22530300 & -3.61155700 & 2.35726500 & $\mathrm{C}$ & -3.08311700 & -1.05340800 & 3.04930600 \\
\hline C & -4.23117500 & -1.73440400 & 1.01755400 & C & 0.24449400 & -2.00223400 & 4.46768100 \\
\hline C & -1.95480500 & -1.09748800 & 3.91714400 & C & -4.13195000 & -0.12119400 & 3.31499600 \\
\hline $\mathrm{H}$ & -4.26458100 & -2.29501500 & 0.09574300 & $\mathrm{C}$ & -5.23886100 & -0.80708700 & 1.27373900 \\
\hline C & 0.33631100 & -1.12103700 & 5.54396200 & $\mathrm{H}$ & 1.06552500 & -2.67487300 & 4.24344400 \\
\hline C & -1.86437400 & -0.21716100 & 5.03675700 & C & -5.20787600 & -0.02406600 & 2.42176500 \\
\hline C & -4.03793900 & 0.71886500 & 4.47525100 & $\mathrm{H}$ & -6.04874300 & -0.69213300 & 0.55961500 \\
\hline C & -0.70699200 & -0.24228800 & 5.83130700 & $\mathrm{H}$ & 1.22660100 & -1.12557500 & 6.16660300 \\
\hline C & -2.95468600 & 0.67809500 & 5.29709400 & $\mathrm{H}$ & -6.00043200 & 0.69238900 & 2.62097400 \\
\hline $\mathrm{H}$ & -4.85333100 & 1.41018900 & 4.67244100 & $\mathrm{H}$ & -0.63441900 & 0.43438500 & 6.67911300 \\
\hline $\mathrm{H}$ & -2.89142400 & 1.33317400 & 6.16230700 & C & -1.82222600 & -0.34326500 & -3.01057300 \\
\hline$S$ & -3.48755000 & -0.57177000 & -2.84403500 & $\mathrm{~N}$ & -0.92830000 & -1.36669500 & -3.05251400 \\
\hline $\mathrm{H}$ & 0.01305200 & -1.14097400 & -2.72247900 & $S$ & 2.30495400 & 0.49538700 & -2.29534700 \\
\hline 0 & 2.74669000 & 1.56591600 & -1.37580900 & $\mathrm{O}$ & 1.45719500 & -0.54819400 & -1.64940800 \\
\hline $\mathrm{O}$ & 1.77528000 & 0.93042400 & -3.60394900 & C & 3.88379000 & -0.40489800 & -2.68542900 \\
\hline$F$ & 4.73245600 & 0.39912200 & -3.33829700 & $\mathrm{~F}$ & 3.66321700 & -1.49213100 & -3.43139700 \\
\hline $\mathrm{F}$ & 4.47539500 & -0.79781900 & -1.53699600 & C & 2.45642800 & -1.58439100 & 1.59052500 \\
\hline 0 & 2.92590700 & -1.58518700 & 2.72013200 & $\mathrm{O}$ & 2.75947400 & -2.40104400 & 0.58308800 \\
\hline C & 3.71592500 & -3.50536300 & 0.74274300 & C & 5.09915000 & -2.94914800 & 1.08432500 \\
\hline $\mathrm{H}$ & 5.82984300 & -3.76497500 & 1.08376800 & $\mathrm{H}$ & 5.10029000 & -2.47892500 & 2.06913500 \\
\hline $\mathrm{H}$ & 5.40442700 & -2.21516900 & 0.33292300 & C & 3.70476100 & -4.14486700 & -0.64435400 \\
\hline $\mathrm{H}$ & 2.70207900 & -4.50876800 & -0.88529700 & $\mathrm{H}$ & 4.40438200 & -4.98635900 & -0.67428800 \\
\hline $\mathrm{H}$ & 3.99433300 & -3.41479300 & -1.40418600 & C & 3.19417400 & -4.47832800 & 1.80064800 \\
\hline $\mathrm{H}$ & 3.82386700 & -5.37423400 & 1.81800600 & $\mathrm{H}$ & 2.17131800 & -4.78245700 & 1.55376900 \\
\hline $\mathrm{H}$ & 3.20295300 & -4.02489100 & 2.79331600 & C & 1.01655800 & 0.29918600 & 1.98472300 \\
\hline $\mathrm{H}$ & 1.20343500 & 0.08835200 & 3.03232700 & C & -0.31097300 & 0.87727400 & 1.67385300 \\
\hline C & -0.84857100 & 1.85041800 & 2.53421100 & C & -1.06683200 & 0.44585600 & 0.57587700 \\
\hline $\mathrm{C}$ & -2.08395000 & 2.42175800 & 2.27196200 & $\mathrm{H}$ & -0.29330400 & 2.15270900 & 3.41723100 \\
\hline $\mathrm{C}$ & -2.32384400 & 0.99824600 & 0.33865500 & $\mathrm{H}$ & -0.71254400 & -0.35431700 & -0.06191200 \\
\hline C & -2.82309400 & 2.00302600 & 1.16192900 & $\mathrm{H}$ & -2.48195500 & 3.17621200 & 2.94281100 \\
\hline $\mathrm{H}$ & -2.91649100 & 0.62857900 & -0.48791600 & $\mathrm{H}$ & -3.78966300 & 2.44344000 & 0.94638300 \\
\hline $\mathrm{N}$ & 1.49548300 & -0.68737700 & 1.16207900 & $\mathrm{H}$ & 1.27860500 & -0.70802100 & 0.15901700 \\
\hline $\mathrm{C}$ & 2.16888900 & 1.91751200 & 1.83517900 & $\mathrm{H}$ & 2.04659400 & 2.09895500 & 0.77251200 \\
\hline $\mathrm{H}$ & 1.61186900 & 2.57783000 & 2.48902000 & C & 3.41076000 & 1.46258700 & 2.28788400 \\
\hline $\mathrm{C}$ & 4.40482300 & 0.97946200 & 1.34851400 & $\mathrm{H}$ & 3.95811100 & 0.62146000 & 0.41764300 \\
\hline $\mathrm{H}$ & 5.09218600 & 0.24435000 & 1.77674000 & C & 3.70633800 & 1.41136000 & 3.75420100 \\
\hline $\mathrm{H}$ & 3.53859900 & 0.37964400 & 4.08987200 & $\mathrm{H}$ & 3.05959400 & 2.07682300 & 4.33232000 \\
\hline $\mathrm{H}$ & 4.75426500 & 1.65132200 & 3.95786600 & C & 6.57171300 & 1.78066900 & -0.64690300 \\
\hline $\mathrm{H}$ & 7.12980000 & 0.89732100 & -0.31572700 & $\mathrm{H}$ & 7.29976600 & 2.53148700 & -0.97694800 \\
\hline $\mathrm{H}$ & 5.96517500 & 1.49619700 & -1.51000700 & C & 6.58510300 & 3.02939600 & 2.18218200 \\
\hline $\mathrm{H}$ & 5.99517000 & 3.48833300 & 2.98367700 & $\mathrm{H}$ & 7.31103700 & 3.77883600 & 1.84401200 \\
\hline $\mathrm{H}$ & 7.15135300 & 2.19445800 & 2.61162700 & C & 4.41825900 & 3.89380900 & 0.14859500 \\
\hline $\mathrm{H}$ & 3.81343400 & 4.31177000 & 0.96076400 & $\mathrm{H}$ & 3.73776700 & 3.56616400 & -0.64131900 \\
\hline $\mathrm{H}$ & 5.04393100 & 4.70234900 & -0.24907800 & $\mathrm{Si}$ & 5.50295800 & 2.48017100 & 0.73400600 \\
\hline C & -3.44149600 & -4.93850500 & 0.90393500 & $\mathrm{H}$ & -4.27750600 & -4.36441600 & 1.31215300 \\
\hline $\mathrm{H}$ & -3.20857700 & -5.73429500 & 1.61613200 & & & & \\
\hline
\end{tabular}

\section{TS-S-4f-22}

Imaginary frequency: $-349.14 \mathrm{~cm}^{-1}$

E[B3LYP/6-31G(d)-D3(BJ)]: -4789.087294 Hartree

E[PCM(Et $\left.\left.t_{2} \mathrm{O}\right)-B 3 L Y P / 6-311 G(d, p)-D 3(B J)\right]:-4790.227021$ Hartree

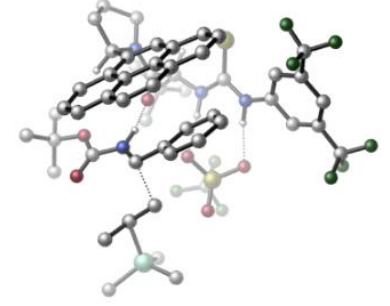




\begin{tabular}{|c|c|c|c|c|c|c|c|}
\hline 01 & & & & $\mathrm{~N}$ & 2.27590000 & 1.47092100 & 1.76889000 \\
\hline $\mathrm{H}$ & 1.65552600 & 2.24864100 & 1.51844700 & $\mathrm{C}$ & 3.51989700 & 1.56027400 & 1.12718600 \\
\hline C & 3.82351200 & 2.79668900 & 0.53409100 & C & 4.40800500 & 0.48811200 & 0.95595600 \\
\hline C & 4.98901600 & 2.95099700 & -0.20975400 & $\mathrm{H}$ & 3.13164800 & 3.62395000 & 0.63609100 \\
\hline C & 5.55420400 & 0.66038800 & 0.18446400 & $\mathrm{H}$ & 4.19089100 & -0.46765600 & 1.40602200 \\
\hline C & 5.86824500 & 1.88659600 & -0.39824600 & $\mathrm{H}$ & 6.77267800 & 2.01061900 & -0.98131700 \\
\hline $\mathrm{C}$ & 5.26545300 & 4.26659300 & -0.88532500 & C & 6.42258400 & -0.52425200 & -0.12476500 \\
\hline $\mathrm{F}$ & 4.73203800 & 5.30527000 & -0.21052200 & $\mathrm{~F}$ & 4.75077700 & 4.29843400 & -2.13618900 \\
\hline $\mathrm{F}$ & 6.59384100 & 4.49530200 & -1.00374800 & $\mathrm{~F}$ & 6.21509900 & -1.56163200 & 0.71647300 \\
\hline $\mathrm{F}$ & 6.17841600 & -0.98884900 & -1.38284100 & $\mathrm{~F}$ & 7.73693600 & -0.22082200 & -0.08120300 \\
\hline C & -0.36163300 & -0.19591500 & 3.63447900 & $\mathrm{H}$ & 0.28066700 & -0.60505600 & 4.41337000 \\
\hline C & -1.59792300 & 0.47311000 & 4.31856900 & C & -2.64044800 & 0.97990100 & 3.30726800 \\
\hline $\mathrm{H}$ & -3.05178700 & 0.16418700 & 2.70760600 & $\mathrm{H}$ & -2.22602500 & 1.70537500 & 2.60989300 \\
\hline $\mathrm{H}$ & -3.46126200 & 1.46467900 & 3.84933900 & C & -1.09096500 & 1.64718800 & 5.17305900 \\
\hline $\mathrm{H}$ & -1.92014500 & 2.07715000 & 5.74670500 & $\mathrm{H}$ & -0.66325600 & 2.43467100 & 4.54968200 \\
\hline $\mathrm{H}$ & -0.32126300 & 1.31799500 & 5.88093700 & C & -2.27388600 & -0.55981400 & 5.23776000 \\
\hline $\mathrm{H}$ & -3.14586600 & -0.10867600 & 5.72361900 & $\mathrm{H}$ & -1.59706200 & -0.90046700 & 6.03026200 \\
\hline $\mathrm{H}$ & -2.62507800 & -1.43461000 & 4.67828700 & C & -0.76471700 & -1.28498100 & 2.63575800 \\
\hline $\mathrm{O}$ & -1.13265100 & -0.93440400 & 1.50757000 & C & -0.32375000 & -3.21862400 & 4.22462800 \\
\hline C & -1.18719500 & -3.57413000 & 1.96879700 & C & -1.19871100 & -4.47567600 & 4.24707000 \\
\hline $\mathrm{H}$ & 0.74532800 & -3.46393700 & 4.19370500 & $\mathrm{H}$ & -0.49704200 & -2.55979000 & 5.07286000 \\
\hline $\mathrm{H}$ & -2.18582600 & -3.26068300 & 1.65899500 & $\mathrm{H}$ & -2.20454200 & -4.21200800 & 4.59534700 \\
\hline $\mathrm{H}$ & -0.80262500 & -5.25234300 & 4.90713400 & $\mathrm{~N}$ & -0.73107100 & -2.58802800 & 2.96756400 \\
\hline C & -0.27473000 & -3.64878600 & 0.75678200 & C & 1.06380500 & -3.42964000 & 0.90331400 \\
\hline $\mathrm{C}$ & -0.81242300 & -4.00319300 & -0.53871100 & $\mathrm{C}$ & 1.98905200 & -3.58247200 & -0.17669300 \\
\hline $\mathrm{H}$ & 1.45920500 & -3.10323000 & 1.86068700 & $\mathrm{C}$ & 0.09099800 & -4.14815900 & -1.63991000 \\
\hline C & -2.18573900 & -4.18439200 & -0.77822100 & C & 3.36629600 & -3.36425600 & -0.01306000 \\
\hline C & 1.49111500 & -3.96884400 & -1.45344600 & C & -0.41031500 & -4.46779400 & -2.93957900 \\
\hline $\mathrm{H}$ & -2.89334000 & -4.09161900 & 0.03558900 & C & -2.66924600 & -4.45617500 & -2.05590300 \\
\hline C & 4.24411400 & -3.55170900 & -1.07803600 & $\mathrm{H}$ & 3.73966800 & -3.04277900 & 0.95494300 \\
\hline $\mathrm{C}$ & 2.39578000 & -4.15129400 & -2.54078900 & C & -1.79403900 & -4.59813600 & -3.12905100 \\
\hline$C$ & 0.52301400 & -4.64548300 & -4.01691300 & $\mathrm{H}$ & -3.73901900 & -4.56098900 & -2.21086000 \\
\hline C & 3.76688000 & -3.94750100 & -2.32636200 & $\mathrm{H}$ & 5.30291700 & -3.37018300 & -0.93706300 \\
\hline $\mathrm{C}$ & 1.86309600 & -4.50845000 & -3.82408200 & $\mathrm{H}$ & -2.17466200 & -4.82512900 & -4.12162300 \\
\hline $\mathrm{H}$ & 0.13037400 & -4.90227400 & -4.99766300 & $\mathrm{H}$ & 4.45726900 & -4.08510100 & -3.15469000 \\
\hline $\mathrm{H}$ & 2.55656100 & -4.65190000 & -4.64895400 & C & 1.75683700 & 0.53525000 & 2.62263500 \\
\hline $\mathrm{S}$ & 2.62068700 & -0.74387800 & 3.31848400 & $\mathrm{~N}$ & 0.43875600 & 0.76879800 & 2.89401700 \\
\hline $\mathrm{H}$ & -0.08280800 & 1.26441900 & 2.16354300 & $S$ & -0.59075900 & 3.34807800 & 0.27626100 \\
\hline $\mathrm{O}$ & -1.08897000 & 3.82812600 & -1.02492000 & $\mathrm{O}$ & -0.94919900 & 1.93573700 & 0.58988300 \\
\hline $\mathrm{O}$ & 0.81130300 & 3.68971600 & 0.62031400 & $\mathrm{C}$ & -1.57763300 & 4.31118600 & 1.52551300 \\
\hline $\mathrm{F}$ & -1.58871500 & 5.61640300 & 1.23085800 & $\mathrm{~F}$ & -1.06117000 & 4.15373700 & 2.75506100 \\
\hline $\mathrm{F}$ & -2.85053000 & 3.87193600 & 1.55183600 & C & -3.61328900 & -1.19117600 & -0.92381700 \\
\hline $\mathrm{O}$ & -4.34104700 & -1.20019300 & -1.90517900 & 0 & -3.95556900 & -1.47785300 & 0.33959100 \\
\hline C & -5.35617800 & -1.75159700 & 0.70283300 & C & -6.20761500 & -0.51676600 & 0.40513100 \\
\hline $\mathrm{H}$ & -7.21358300 & -0.65691600 & 0.81422300 & $\mathrm{H}$ & -6.28950800 & -0.34758000 & -0.66996800 \\
\hline $\mathrm{H}$ & -5.76535300 & 0.36725100 & 0.87621100 & C & -5.26451800 & -2.00321300 & 2.20709400 \\
\hline $\mathrm{H}$ & -4.59410600 & -2.84356200 & 2.41532800 & $\mathrm{H}$ & -6.25499600 & -2.24323300 & 2.60606300 \\
\hline $\mathrm{H}$ & -4.88211100 & -1.12023300 & 2.72605100 & $\mathrm{C}$ & -5.85803200 & -2.99793400 & -0.02825900 \\
\hline $\mathrm{H}$ & -6.87036000 & -3.23601600 & 0.31515500 & $\mathrm{H}$ & -5.21586200 & -3.85659000 & 0.19371900 \\
\hline $\mathrm{H}$ & -5.87931800 & -2.83957000 & -1.10683500 & C & -1.66204800 & -0.69484000 & -2.19588600 \\
\hline $\mathrm{H}$ & -2.19374900 & -1.18824200 & -3.00348100 & $\mathrm{C}$ & -0.19316000 & -0.74010000 & -2.26430300 \\
\hline C & 0.40379800 & -1.09727300 & -3.48531200 & $\mathrm{C}$ & 0.61676000 & -0.39873000 & -1.17211700 \\
\hline C & 1.78687100 & -1.11117900 & -3.61516300 & $\mathrm{H}$ & -0.22471300 & -1.37877500 & -4.32610100 \\
\hline
\end{tabular}




\begin{tabular}{|c|c|c|c|c|c|c|c|}
\hline $\mathrm{C}$ & 1.99940900 & -0.43563500 & -1.30826200 & $\overline{\mathrm{H}}$ & 0.18008400 & -0.09423000 & -0.23005500 \\
\hline C & 2.59122700 & -0.77708100 & -2.52307800 & $\mathrm{H}$ & 2.23691000 & -1.40440500 & -4.55810000 \\
\hline $\mathrm{H}$ & 2.62109900 & -0.19751900 & -0.45910800 & $\mathrm{H}$ & 3.67306400 & -0.80264000 & -2.60173800 \\
\hline $\mathrm{N}$ & -2.27262300 & -0.87657400 & -0.98335600 & $\mathrm{H}$ & -1.75980900 & -0.83042400 & -0.09486300 \\
\hline C & -1.93345300 & 1.17919200 & -2.91778800 & $\mathrm{H}$ & -1.33009200 & 1.74050500 & -2.21110300 \\
\hline $\mathrm{H}$ & -1.47618100 & 0.98358000 & -3.88414200 & C & -3.31716200 & 1.35727100 & -2.87489900 \\
\hline C & -3.93382500 & 2.05965700 & -1.75857700 & $\mathrm{H}$ & -3.26631600 & 2.14854600 & -0.89735700 \\
\hline $\mathrm{H}$ & -4.90370100 & 1.64143400 & -1.47223000 & C & -4.16367900 & 0.89100800 & -4.02174000 \\
\hline $\mathrm{H}$ & -4.21377800 & 1.68789700 & -4.77690300 & $\mathrm{H}$ & -5.18003200 & 0.64880500 & -3.70829300 \\
\hline $\mathrm{H}$ & -3.72450400 & 0.01370300 & -4.50378100 & C & -4.46190000 & 5.00859900 & -0.85791200 \\
\hline $\mathrm{H}$ & -5.00535900 & 4.54177500 & -0.03009400 & $\mathrm{H}$ & -4.99491500 & 5.92647600 & -1.13554600 \\
\hline $\mathrm{H}$ & -3.46872100 & 5.28534400 & -0.50020800 & C & -6.03174400 & 3.79497400 & -3.20572000 \\
\hline $\mathrm{H}$ & -6.80214000 & 3.43167200 & -2.51507600 & $\mathrm{H}$ & -6.03407800 & 3.14119600 & -4.08402100 \\
\hline $\mathrm{H}$ & -6.33513200 & 4.79442700 & -3.54070200 & C & -3.02840500 & 4.49352800 & -3.55889800 \\
\hline $\mathrm{H}$ & -2.98005000 & 3.89206000 & -4.47370300 & $\mathrm{H}$ & -2.04821100 & 4.47324800 & -3.07439200 \\
\hline $\mathrm{H}$ & -3.24313000 & 5.52747800 & -3.85515000 & $\mathrm{Si}$ & -4.34196900 & 3.88805600 & -2.36069700 \\
\hline C & -1.23134800 & -4.90269700 & 2.77011900 & $\mathrm{H}$ & -0.34400700 & -5.49591100 & 2.52980800 \\
\hline $\mathrm{H}$ & -2.10918800 & -5.50137500 & 2.51235800 & & & & \\
\hline
\end{tabular}

\section{TS-S-4f-23}

Imaginary frequency: $-129.61 \mathrm{~cm}^{-1}$

E[B3LYP/6-31G(d)-D3(BJ)]: -4789.085671 Hartree

$\mathrm{E}\left[\mathrm{PCM}\left(\mathrm{Et}_{2} \mathrm{O}\right)-\mathrm{B} 3 \mathrm{LYP} / 6-311 \mathrm{G}(\mathrm{d}, \mathrm{p})-\mathrm{D} 3(\mathrm{BJ})\right]$ : -4790.226106 Hartree

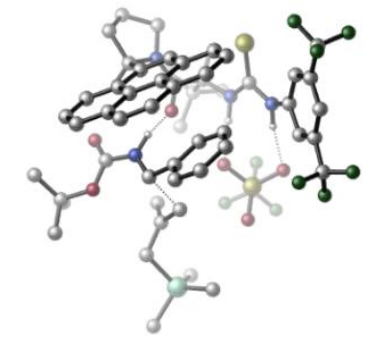

\begin{tabular}{|c|c|c|c|c|c|c|c|}
\hline 01 & & & & $\mathrm{~N}$ & -2.18718300 & -2.09042900 & 1.68391600 \\
\hline $\mathrm{H}$ & -1.43060700 & -2.75241000 & 1.48536500 & C & -3.21503700 & -2.15248500 & 0.72843900 \\
\hline $\mathrm{C}$ & -2.93976600 & -2.89846400 & -0.43381500 & $\mathrm{C}$ & -4.44664300 & -1.49433500 & 0.83502700 \\
\hline $\mathrm{C}$ & -3.84842700 & -2.91481200 & -1.48360000 & $\mathrm{H}$ & -2.00085300 & -3.43398400 & -0.51564100 \\
\hline C & -5.33665200 & -1.51600500 & -0.23996200 & $\mathrm{H}$ & -4.68620600 & -0.94495200 & 1.73144500 \\
\hline $\mathrm{C}$ & -5.05247800 & -2.21109200 & -1.41013800 & $\mathrm{H}$ & -5.74670200 & -2.21151500 & -2.24157700 \\
\hline $\mathrm{C}$ & -3.49229600 & -3.59913300 & -2.77352500 & $\mathrm{C}$ & -6.65376200 & -0.80627300 & -0.09922500 \\
\hline $\mathrm{F}$ & -2.50890700 & -4.50428400 & -2.62563400 & $\mathrm{~F}$ & -3.07303400 & -2.69964700 & -3.70211500 \\
\hline $\mathrm{F}$ & -4.55978700 & -4.23269300 & -3.31215200 & $\mathrm{~F}$ & -6.56170800 & 0.27805900 & 0.70758600 \\
\hline $\mathrm{F}$ & -7.11739300 & -0.36843000 & -1.29721200 & $\mathrm{~F}$ & -7.61108800 & -1.60239200 & 0.42238600 \\
\hline C & -0.05994100 & -0.09241100 & 3.85655800 & $\mathrm{H}$ & -0.82829500 & 0.16813400 & 4.58300300 \\
\hline $\mathrm{C}$ & 1.21099200 & -0.55050100 & 4.64116900 & $\mathrm{C}$ & 2.42213100 & -0.82100700 & 3.73089800 \\
\hline $\bar{H}$ & 2.75438400 & 0.09027400 & 3.22521000 & $\mathrm{H}$ & 2.20660000 & -1.56598300 & 2.96457700 \\
\hline $\mathrm{H}$ & 3.25213800 & -1.19488400 & 4.34197200 & C & 0.84982400 & -1.82743300 & 5.41936100 \\
\hline $\mathrm{H}$ & 1.68666300 & -2.12074400 & 6.06352500 & $\mathrm{H}$ & 0.63246300 & -2.65715700 & 4.74432400 \\
\hline $\mathrm{H}$ & -0.02925200 & -1.66753800 & 6.05467000 & $\mathrm{C}$ & 1.59306800 & 0.55372600 & 5.64329300 \\
\hline $\mathrm{H}$ & 2.48836900 & 0.25583600 & 6.19948000 & $\mathrm{H}$ & 0.79529600 & 0.72844500 & 6.37501300 \\
\hline $\mathrm{H}$ & 1.81957800 & 1.50048400 & 5.13962100 & C & 0.21240500 & 1.09331200 & 2.91728700 \\
\hline $\mathrm{O}$ & 0.74917600 & 0.84937800 & 1.82225800 & $\mathrm{C}$ & -0.76597400 & 2.81238100 & 4.49680700 \\
\hline $\mathrm{C}$ & 0.19470400 & 3.46778500 & 2.35317200 & $\mathrm{C}$ & -0.16537700 & 4.20998200 & 4.66369400 \\
\hline $\mathrm{H}$ & -1.85354900 & 2.84544500 & 4.35740700 & $\mathrm{H}$ & -0.54904700 & 2.15053500 & 5.33186900 \\
\hline $\mathrm{H}$ & 1.25575100 & 3.38165200 & 2.10773800 & $\mathrm{H}$ & 0.83615200 & 4.12566500 & 5.10142900 \\
\hline $\mathrm{H}$ & -0.76872600 & 4.85092000 & 5.31255200 & $\mathrm{~N}$ & -0.12183500 & 2.34645900 & 3.26490500 \\
\hline C & -0.63426100 & 3.45065000 & 1.07861800 & C & -1.85867900 & 2.84981400 & 1.05833700 \\
\hline $\mathrm{C}$ & -0.13964900 & 4.12564200 & -0.10324700 & C & -2.69566700 & 2.85088500 & -0.10348400 \\
\hline $\mathrm{H}$ & -2.21654700 & 2.31445000 & 1.93209900 & C & -0.93777100 & 4.10133500 & -1.29175400 \\
\hline
\end{tabular}




\begin{tabular}{|c|c|c|c|c|c|c|c|}
\hline $\mathrm{C}$ & 1.09585700 & 4.79527800 & -0.14279200 & $\mathrm{C}$ & -3.95509100 & 2.22945100 & -0.11205800 \\
\hline C & -2.21834300 & 3.47823100 & -1.28911400 & C & -0.45478100 & 4.70977600 & -2.49124600 \\
\hline $\mathrm{H}$ & 1.71801700 & 4.84099500 & 0.74231900 & $\mathrm{C}$ & 1.55404400 & 5.39050300 & -1.31669700 \\
\hline $\mathrm{C}$ & -4.73558600 & 2.23008200 & -1.26638700 & $\mathrm{H}$ & -4.30619100 & 1.72972300 & 0.78511100 \\
\hline C & -3.01968800 & 3.46625000 & -2.46868700 & C & 0.79761600 & 5.34089900 & -2.48348100 \\
\hline C & -1.27555700 & 4.66803900 & -3.66909900 & $\mathrm{H}$ & 2.50856700 & 5.90818500 & -1.31327700 \\
\hline C & -4.27493300 & 2.84074800 & -2.43134400 & $\mathrm{H}$ & -5.70235300 & 1.73979000 & -1.26157400 \\
\hline C & -2.50251700 & 4.08004100 & -3.65736600 & $\mathrm{H}$ & 1.16197100 & 5.80719200 & -3.39538600 \\
\hline $\mathrm{H}$ & -0.89422300 & 5.13078400 & -4.57592600 & $\mathrm{H}$ & -4.88627500 & 2.83084300 & -3.33024500 \\
\hline $\mathrm{H}$ & -3.11458500 & 4.06221300 & -4.55560200 & $\mathrm{C}$ & -1.90881800 & -1.12287000 & 2.61728600 \\
\hline $\mathrm{S}$ & -3.02206100 & -0.00392400 & 3.23379000 & $\mathrm{~N}$ & -0.61204800 & -1.15999500 & 3.03362500 \\
\hline $\mathrm{H}$ & 0.05443000 & -1.57603900 & 2.37379500 & S & 1.03855900 & -3.61741900 & 0.57289400 \\
\hline $\mathrm{O}$ & 1.72301700 & -3.93845100 & -0.68933400 & 0 & 1.10965800 & -2.17831200 & 0.97043400 \\
\hline $\mathrm{O}$ & -0.31393400 & -4.19325900 & 0.77548500 & $\mathrm{C}$ & 2.07188200 & -4.45825700 & 1.86754800 \\
\hline $\mathrm{F}$ & 2.14563000 & -5.77168700 & 1.65017200 & $\mathrm{~F}$ & 1.56013500 & -4.24967700 & 3.09109700 \\
\hline $\mathrm{F}$ & 3.32686100 & -3.95505100 & 1.85578000 & $\mathrm{C}$ & 3.24621300 & 2.25692300 & -0.06999400 \\
\hline $\mathrm{O}$ & 3.42940800 & 2.75443600 & 1.02543100 & 0 & 4.11246900 & 2.21871500 & -1.09403800 \\
\hline C & 5.41840400 & 2.90636000 & -1.01323700 & C & 5.20316400 & 4.39844500 & -0.76302200 \\
\hline $\mathrm{H}$ & 6.16362100 & 4.91889900 & -0.83823300 & $\mathrm{H}$ & 4.78174200 & 4.57761100 & 0.22656600 \\
\hline $\mathrm{H}$ & 4.52813100 & 4.81118800 & -1.51854700 & $\mathrm{C}$ & 6.00139800 & 2.66986900 & -2.40476400 \\
\hline $\mathrm{H}$ & 6.10086700 & 1.59977200 & -2.60751700 & $\mathrm{H}$ & 6.99130400 & 3.13082400 & -2.47612600 \\
\hline $\mathrm{H}$ & 5.35722000 & 3.11058600 & -3.17210100 & C & 6.27712000 & 2.24978400 & 0.06662200 \\
\hline $\mathrm{H}$ & 7.26768100 & 2.71640500 & 0.07639700 & $\mathrm{H}$ & 6.40721700 & 1.18348300 & -0.14345400 \\
\hline $\mathrm{H}$ & 5.82349700 & 2.36596100 & 1.05170900 & C & 1.77383100 & 1.09004900 & -1.62514600 \\
\hline $\bar{H}$ & 2.44006000 & 1.42939600 & -2.40857100 & $\mathrm{C}$ & 0.37881200 & 0.84651600 & -2.02234000 \\
\hline $\mathrm{C}$ & 0.04454200 & 1.02676700 & -3.37402600 & C & -0.59223300 & 0.38088300 & -1.12109900 \\
\hline C & -1.24137100 & 0.74516900 & -3.82241900 & $\mathrm{H}$ & 0.79680000 & 1.39316500 & -4.06783000 \\
\hline $\mathrm{C}$ & -1.87406100 & 0.10351900 & -1.57897300 & $\bar{H}$ & -0.34099400 & 0.21706900 & -0.07875800 \\
\hline $\mathrm{C}$ & -2.19962100 & 0.27123600 & -2.92629200 & $\bar{H}$ & -1.49475900 & 0.89595200 & -4.86704700 \\
\hline $\mathrm{H}$ & -2.62804700 & -0.23556700 & -0.88388300 & $\mathrm{H}$ & -3.19903300 & 0.03135800 & -3.26970400 \\
\hline $\mathrm{N}$ & 2.05086300 & 1.63610400 & -0.40743800 & $\bar{H}$ & 1.40822700 & 1.51065300 & 0.39316100 \\
\hline $\mathrm{C}$ & 2.35627600 & -0.91720700 & -1.66820900 & $\bar{H}$ & 1.60754700 & -1.22409900 & -0.94837200 \\
\hline $\mathrm{H}$ & 2.13566100 & -1.12180000 & -2.71202000 & C & 3.67881700 & -0.93547500 & -1.24860400 \\
\hline $\mathrm{C}$ & 4.78818700 & -1.05892700 & -2.19554000 & $\mathrm{H}$ & 5.71665400 & -0.61090900 & -1.82760600 \\
\hline $\bar{H}$ & 4.54328000 & -0.64672300 & -3.18128100 & $\mathrm{C}$ & 3.96506400 & -0.90485000 & 0.21657400 \\
\hline $\mathrm{H}$ & 4.99163800 & -0.60493300 & 0.43813800 & $\mathrm{H}$ & 3.78824700 & -1.89992300 & 0.63908300 \\
\hline $\mathrm{H}$ & 3.25729200 & -0.25542800 & 0.74060100 & C & 6.70855200 & -2.92561200 & -3.61877700 \\
\hline $\mathrm{H}$ & 6.54731800 & -2.36179600 & -4.54497200 & $\mathrm{H}$ & 6.99354900 & -3.94685600 & -3.89832800 \\
\hline $\mathrm{H}$ & 7.55927700 & -2.47623100 & -3.09328600 & C & 3.68856300 & -3.67936500 & -3.44770000 \\
\hline $\mathrm{H}$ & 3.41607200 & -3.09340500 & -4.33360400 & $\mathrm{H}$ & 2.82012500 & -3.74536100 & -2.78457600 \\
\hline $\mathrm{H}$ & 3.92517800 & -4.69569900 & -3.78551600 & $\mathrm{C}$ & 5.47724500 & -3.84807500 & -0.92221600 \\
\hline $\mathrm{H}$ & 4.55276000 & -3.97116900 & -0.35118300 & $\mathrm{H}$ & 6.21003400 & -3.33014000 & -0.29265100 \\
\hline $\mathrm{H}$ & 5.87041400 & -4.85096700 & -1.12857500 & $\mathrm{Si}$ & 5.15825900 & -2.94626800 & -2.54001600 \\
\hline $\mathrm{C}$ & -0.08304400 & 4.72809200 & 3.21908300 & $\mathrm{H}$ & -1.04034400 & 5.16538800 & 2.91992900 \\
\hline $\mathrm{H}$ & 0.68777500 & 5.49035800 & 3.07962700 & & & & \\
\hline
\end{tabular}

\section{TS-S-4f-24}

Imaginary frequency: $-422.98 \mathrm{~cm}^{-1}$

E[B3LYP/6-31G(d)-D3(BJ)]: -4789.091165 Hartree

E[PCM(Et2O)-B3LYP/6-311G(d,p)-D3(BJ)]: - 4790.225487 Hartree

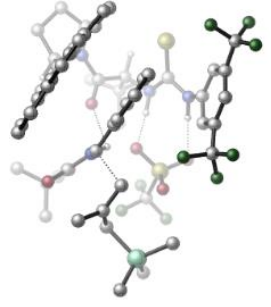




\begin{tabular}{|c|c|c|c|c|c|c|c|}
\hline 01 & & & & $\mathrm{~N}$ & -1.14041700 & 0.85870900 & -3.18522600 \\
\hline $\mathrm{H}$ & -0.13585000 & 0.84162000 & -3.37520200 & C & -1.57240200 & 2.07278600 & -2.61620700 \\
\hline $\mathrm{C}$ & -0.60187000 & 2.85743800 & -1.97596900 & C & -2.90210200 & 2.50684100 & -2.63097500 \\
\hline C & -0.97163400 & 4.01021400 & -1.29338900 & $\mathrm{H}$ & 0.43751800 & 2.55605200 & -1.98416500 \\
\hline C & -3.25342000 & 3.65999400 & -1.92790300 & $\mathrm{H}$ & -3.65166000 & 1.93017600 & -3.15204100 \\
\hline $\mathrm{C}$ & -2.30449200 & 4.41567000 & -1.24374600 & $\mathrm{H}$ & -2.59309800 & 5.30357400 & -0.69613300 \\
\hline $\mathrm{C}$ & 0.07177900 & 4.74593500 & -0.50202600 & $\mathrm{C}$ & -4.70559400 & 4.03149900 & -1.83472800 \\
\hline $\mathrm{F}$ & 1.27455600 & 4.74230900 & -1.11362100 & $\mathrm{~F}$ & 0.25796000 & 4.17633300 & 0.72007100 \\
\hline $\mathrm{F}$ & -0.27098000 & 6.03316300 & -0.27808000 & $\mathrm{~F}$ & -5.38075400 & 3.74422900 & -2.96574400 \\
\hline $\mathrm{F}$ & -5.32417100 & 3.35120000 & -0.83059000 & $\mathrm{~F}$ & -4.88120800 & 5.34550100 & -1.57445000 \\
\hline C & -1.12393900 & -2.78095500 & -2.88776200 & $\mathrm{H}$ & -2.13618200 & -2.93747700 & -3.25748900 \\
\hline $\mathrm{C}$ & -0.14732000 & -3.68905200 & -3.70472700 & C & 1.31707900 & -3.26074400 & -3.51184700 \\
\hline $\mathrm{H}$ & 1.57057600 & -3.15901200 & -2.45502600 & $\mathrm{H}$ & 1.51818700 & -2.30432300 & -4.00041000 \\
\hline $\mathrm{H}$ & 1.98243800 & -4.00364300 & -3.96691900 & C & -0.52478300 & -3.55886900 & -5.18942200 \\
\hline $\mathrm{H}$ & 0.17307300 & -4.12953000 & -5.81298300 & $\mathrm{H}$ & -0.48798700 & -2.51220900 & -5.50723300 \\
\hline $\mathrm{H}$ & -1.53662700 & -3.93712600 & -5.37866700 & C & -0.30596400 & -5.15329600 & -3.26369900 \\
\hline $\mathrm{H}$ & 0.34822500 & -5.79399200 & -3.86497500 & $\mathrm{H}$ & -1.33186500 & -5.51111400 & -3.40506900 \\
\hline $\mathrm{H}$ & -0.03099000 & -5.29264300 & -2.21211600 & C & -1.06170400 & -3.05163100 & -1.37419300 \\
\hline $\mathrm{C}$ & -3.25524200 & -4.32342000 & -1.43987900 & C & -1.94837200 & -4.07055300 & 0.63781600 \\
\hline $\mathrm{H}$ & -3.81007800 & -3.57398600 & -2.01312900 & $\mathrm{H}$ & -2.93028400 & -5.11081200 & -2.12825200 \\
\hline $\mathrm{H}$ & -0.96143300 & -4.52882000 & 0.75202900 & $\mathrm{~N}$ & -2.09345300 & -3.70543100 & -0.78190400 \\
\hline $\mathrm{C}$ & -1.97977200 & -2.92441700 & 1.64249700 & C & -0.96000600 & -2.87693000 & 2.54879600 \\
\hline $\mathrm{C}$ & -3.08098300 & -1.98625600 & 1.76776100 & C & -0.95198100 & -1.99062100 & 3.67247000 \\
\hline $\mathrm{H}$ & -0.11436100 & -3.55037800 & 2.43287500 & C & -3.15163000 & -1.16490300 & 2.93978900 \\
\hline $\mathrm{C}$ & -4.06724100 & -1.80835400 & 0.78376500 & C & 0.12814600 & -1.92499500 & 4.56701600 \\
\hline $\mathrm{C}$ & -2.09011100 & -1.16262600 & 3.88924100 & C & -4.26828600 & -0.29737500 & 3.14109800 \\
\hline $\mathrm{H}$ & -3.96070800 & -2.28827800 & -0.17551100 & C & -5.14336700 & -0.94524300 & 0.98018800 \\
\hline C & 0.08825900 & -1.06199400 & 5.66129800 & $\mathrm{H}$ & 1.00784300 & -2.53277000 & 4.38200700 \\
\hline $\mathrm{C}$ & -2.13343000 & -0.30192600 & 5.02689100 & C & -5.26722900 & -0.22229700 & 2.16024300 \\
\hline $\mathrm{C}$ & -4.31435800 & 0.51362200 & 4.32468100 & $\mathrm{H}$ & -5.87741700 & -0.82579600 & 0.18896600 \\
\hline C & -1.03158600 & -0.26579500 & 5.89621200 & $\mathrm{H}$ & 0.93489800 & -1.01689000 & 6.34079000 \\
\hline $\mathrm{C}$ & -3.29433700 & 0.51714700 & 5.22485900 & $\mathrm{H}$ & -6.11219500 & 0.44405300 & 2.31260900 \\
\hline $\mathrm{H}$ & -5.18238800 & 1.15092200 & 4.47327800 & $\mathrm{H}$ & -1.06090000 & 0.39658500 & 6.75774100 \\
\hline $\mathrm{H}$ & -3.33517000 & 1.15364400 & 6.10515900 & C & -1.72694600 & -0.38343900 & -3.05754400 \\
\hline$S$ & -3.38569500 & -0.66249600 & -2.89310800 & $\mathrm{~N}$ & -0.80289600 & -1.37771400 & -3.09920800 \\
\hline $\mathrm{H}$ & 0.12914900 & -1.12124100 & -2.76442600 & $\mathrm{~S}$ & 2.35682700 & 0.60049700 & -2.26043600 \\
\hline $\mathrm{O}$ & 2.75635600 & 1.66900200 & -1.31937500 & $\mathrm{O}$ & 1.51553500 & -0.46667900 & -1.64594000 \\
\hline $\mathrm{O}$ & 1.84752700 & 1.03889100 & -3.57624800 & C & 3.96177300 & -0.26461000 & -2.62385500 \\
\hline $\mathrm{F}$ & 4.81086300 & 0.56479600 & -3.24391700 & $\mathrm{~F}$ & 3.78025800 & -1.34393100 & -3.39156500 \\
\hline $\mathrm{F}$ & 4.53270100 & -0.66461600 & -1.46739100 & C & 2.44118900 & -1.56040100 & 1.60846300 \\
\hline $\mathrm{O}$ & 2.86344000 & -1.58691400 & 2.75676000 & $\mathrm{O}$ & 2.80451200 & -2.33809400 & 0.59102400 \\
\hline $\mathrm{C}$ & 3.76927600 & -3.43324400 & 0.76151300 & C & 5.12874900 & -2.86399300 & 1.17021300 \\
\hline $\mathrm{H}$ & 5.87312500 & -3.66728300 & 1.17949300 & $\mathrm{H}$ & 5.08342800 & -2.41712800 & 2.16487800 \\
\hline $\mathrm{H}$ & 5.44986100 & -2.10729600 & 0.44814000 & C & 3.82271900 & -4.03875500 & -0.63966400 \\
\hline $\mathrm{H}$ & 2.83704000 & -4.41513800 & -0.92658400 & $\mathrm{H}$ & 4.53915600 & -4.86620600 & -0.66272100 \\
\hline $\mathrm{H}$ & 4.12629000 & -3.28528000 & -1.37061500 & C & 3.22093100 & -4.44029900 & 1.77303300 \\
\hline $\mathrm{H}$ & 3.86131800 & -5.32857900 & 1.79086400 & $\mathrm{H}$ & 2.21244100 & -4.74963700 & 1.47802200 \\
\hline $\mathrm{H}$ & 3.18466900 & -4.01374200 & 2.77680100 & $\mathrm{C}$ & 0.96578100 & 0.29153800 & 2.00144700 \\
\hline $\mathrm{H}$ & 1.13887700 & 0.06102800 & 3.04725300 & C & -0.36857400 & 0.84985600 & 1.68768900 \\
\hline $\mathrm{C}$ & -0.94619200 & 1.76378900 & 2.58644300 & C & -1.08796200 & 0.46389500 & 0.54982400 \\
\hline $\mathrm{C}$ & -2.18701200 & 2.32394100 & 2.32572500 & $\mathrm{H}$ & -0.41690600 & 2.02665400 & 3.49786000 \\
\hline $\mathrm{C}$ & -2.35023700 & 1.00374000 & 0.31209300 & $\mathrm{H}$ & -0.69900000 & -0.29037000 & -0.12300200 \\
\hline
\end{tabular}




\begin{tabular}{|crrr|rrrr|}
\hline $\mathrm{C}$ & -2.89001100 & 1.95160500 & 1.17618500 & $\mathrm{H}$ & -2.61788800 & 3.03097900 & 3.02707500 \\
\hline $\mathrm{H}$ & -2.91532000 & 0.66767800 & -0.54689400 & $\mathrm{H}$ & -3.86141600 & 2.38163400 & 0.96071300 \\
\hline $\mathrm{N}$ & 1.48067000 & -0.66837000 & 1.16871600 & $\mathrm{H}$ & 1.28788700 & -0.67624700 & 0.16078000 \\
\hline $\mathrm{C}$ & 2.07867700 & 1.93512600 & 1.89965000 & $\mathrm{H}$ & 1.97658200 & 2.13094500 & 0.83716500 \\
\hline $\mathrm{H}$ & 1.49335200 & 2.57429800 & 2.55001300 & $\mathrm{C}$ & 3.31895400 & 1.49655200 & 2.37297100 \\
\hline $\mathrm{C}$ & 4.34059500 & 1.04365100 & 1.44885600 & $\mathrm{H}$ & 3.92096200 & 0.69627300 & 0.50145700 \\
\hline $\mathrm{H}$ & 5.02922800 & 0.31140300 & 1.88008000 & $\mathrm{C}$ & 3.58243000 & 1.42513000 & 3.84455000 \\
\hline $\mathrm{H}$ & 3.43032400 & 0.38410800 & 4.15842500 & $\mathrm{H}$ & 2.90856800 & 2.06592000 & 4.41949800 \\
\hline $\mathrm{H}$ & 4.62004000 & 1.68369200 & 4.07619000 & $\mathrm{C}$ & 6.53181700 & 1.92279200 & -0.49031500 \\
\hline $\mathrm{H}$ & 7.08340300 & 1.02847600 & -0.17838600 & $\mathrm{H}$ & 7.26607400 & 2.68570100 & -0.77595000 \\
\hline $\mathrm{H}$ & 5.94561200 & 1.66915800 & -1.37650000 & $\mathrm{C}$ & 6.48259700 & 3.09719700 & 2.36869300 \\
\hline $\mathrm{H}$ & 5.87521800 & 3.52949200 & 3.17194100 & $\mathrm{H}$ & 7.20491500 & 3.86299000 & 2.06068600 \\
\hline $\mathrm{H}$ & 7.05195100 & 2.25827800 & 2.78610000 & $\mathrm{C}$ & 4.33772500 & 3.98770700 & 0.32387000 \\
\hline $\mathrm{H}$ & 3.72304600 & 4.38222500 & 1.14042100 & $\mathrm{H}$ & 3.66743300 & 3.67130400 & -0.47932200 \\
\hline $\mathrm{H}$ & 4.95912800 & 4.81044000 & -0.05071200 & $\mathrm{Si}$ & 5.43100000 & 2.57241200 & 0.88920100 \\
\hline $\mathrm{C}$ & -3.05171200 & -5.13936900 & 0.84485200 & $\mathrm{H}$ & -3.50026400 & -5.06919600 & 1.83834300 \\
\hline $\mathrm{H}$ & -2.59927900 & -6.13287000 & 0.75409900 & $\mathrm{C}$ & -4.07442300 & -4.91824900 & -0.28279200 \\
\hline $\mathrm{H}$ & -4.56997600 & -5.84615000 & -0.58196000 & $\mathrm{H}$ & -4.85002300 & -4.22051500 & 0.03910900 \\
\hline $\mathrm{O}$ & -0.08719000 & -2.68182400 & -0.71246300 & & & & \\
\hline
\end{tabular}

\section{TS-S-4f-25}

Imaginary frequency: $-362.14 \mathrm{~cm}^{-1}$

E[B3LYP/6-31G(d)-D3(BJ)]: -4789.076425 Hartree

E[PCM(Et $2 \mathrm{O})-B 3 L Y P / 6-311 G(d, p)-D 3(B J)]:-4790.225025$ Hartree

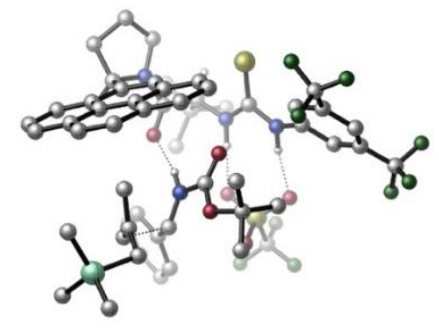

\begin{tabular}{|c|c|c|c|c|c|c|c|}
\hline 01 & & & & $\mathrm{~N}$ & 3.09355300 & -0.15240100 & 1.25140000 \\
\hline $\mathrm{H}$ & 3.32349100 & 0.63738200 & 0.63990500 & C & 3.57461700 & -1.36617600 & 0.74435700 \\
\hline C & 4.73074400 & -1.30645500 & -0.04763300 & $\mathrm{C}$ & 2.91271000 & -2.58971200 & 0.89596800 \\
\hline C & 5.21129400 & -2.45345800 & -0.67248400 & $\mathrm{H}$ & 5.21880600 & -0.35361500 & -0.20854400 \\
\hline C & 3.41675200 & -3.72753000 & 0.27140700 & $\mathrm{H}$ & 2.00414200 & -2.63345700 & 1.47312400 \\
\hline C & 4.56646600 & -3.67895800 & -0.51537500 & $\mathrm{H}$ & 4.94623200 & -4.57031000 & -0.99917500 \\
\hline C & 6.47761100 & -2.38824000 & -1.48003000 & C & 2.65196400 & -5.01408600 & 0.36510100 \\
\hline $\mathrm{F}$ & 7.56132700 & -2.70936200 & -0.73368600 & $\mathrm{~F}$ & 6.69691300 & -1.15880100 & -1.98661700 \\
\hline $\mathrm{F}$ & 6.45050800 & -3.25841600 & -2.51756200 & $\mathrm{~F}$ & 1.93063800 & -5.10329100 & 1.50744200 \\
\hline $\mathrm{F}$ & 1.76194100 & -5.14550800 & -0.65886800 & $\mathrm{~F}$ & 3.45716100 & -6.09584500 & 0.30975600 \\
\hline $\mathrm{C}$ & 0.95184900 & 1.87849600 & 3.38241700 & $\mathrm{H}$ & 1.23430800 & 1.31652700 & 4.27165700 \\
\hline $\mathrm{C}$ & 1.26076800 & 3.38277100 & 3.67398400 & $\mathrm{C}$ & 0.76079500 & 4.33582200 & 2.57695400 \\
\hline $\mathrm{H}$ & -0.32948600 & 4.32558800 & 2.51088100 & $\mathrm{H}$ & 1.15831500 & 4.08787400 & 1.59097400 \\
\hline $\mathrm{H}$ & 1.07600200 & 5.35820600 & 2.81857900 & $\mathrm{C}$ & 2.78455900 & 3.53046700 & 3.82732500 \\
\hline $\mathrm{H}$ & 3.02704600 & 4.54266700 & 4.17054600 & $\mathrm{H}$ & 3.29765600 & 3.36440100 & 2.87629300 \\
\hline $\mathrm{H}$ & 3.18358100 & 2.81681500 & 4.55759200 & C & 0.58055500 & 3.75654100 & 5.00313500 \\
\hline $\mathrm{H}$ & 0.77332800 & 4.80851500 & 5.24002900 & $\mathrm{H}$ & 0.96247300 & 3.15169900 & 5.83394000 \\
\hline $\mathrm{H}$ & -0.50684800 & 3.62476200 & 4.95113500 & $\mathrm{C}$ & -0.52621600 & 1.61489700 & 3.06579600 \\
\hline $\mathrm{O}$ & -1.01424300 & 1.92916100 & 1.96576200 & C & -0.90363100 & 0.56841800 & 5.34762300 \\
\hline C & -2.71022900 & 0.75036600 & 3.69823800 & $\mathrm{C}$ & -2.13923700 & -0.20187900 & 5.83179100 \\
\hline $\mathrm{H}$ & -0.00892300 & -0.05556800 & 5.28107200 & $\mathrm{H}$ & -0.69576800 & 1.42730400 & 5.99437100 \\
\hline C & -3.30035800 & 0.48537700 & 5.10017000 & $\mathrm{H}$ & -3.13548800 & 1.63962100 & 3.22799700 \\
\hline $\mathrm{H}$ & -2.23923900 & -0.17628800 & 6.91999200 & $\mathrm{H}$ & -2.07187100 & -1.24916400 & 5.52094100 \\
\hline $\mathrm{H}$ & -3.54601400 & 1.43952200 & 5.58201200 & $\mathrm{H}$ & -4.20652200 & -0.12291100 & 5.05829000 \\
\hline $\mathrm{N}$ & -1.30013700 & 1.03335100 & 4.00360600 & C & -2.88733900 & -0.44416000 & 2.77209700 \\
\hline $\mathrm{C}$ & -1.87443200 & -1.33244700 & 2.55800300 & $\mathrm{C}$ & -4.17901200 & -0.65566200 & 2.14799400 \\
\hline
\end{tabular}




\begin{tabular}{|c|c|c|c|c|c|c|c|}
\hline $\mathrm{C}$ & -2.04387700 & -2.50536600 & 1.75152100 & $\mathrm{H}$ & -0.89178400 & -1.16138300 & 2.98722400 \\
\hline C & -4.37018000 & -1.81468100 & 1.32931800 & C & -5.24921600 & 0.24729900 & 2.27962900 \\
\hline $\mathrm{C}$ & -1.00545300 & -3.43001900 & 1.56379700 & C & -3.30386900 & -2.73878400 & 1.13140900 \\
\hline $\mathrm{C}$ & -5.61908300 & -2.02939600 & 0.66863400 & $\mathrm{H}$ & -5.13967500 & 1.13246600 & 2.89537100 \\
\hline C & -6.46121500 & 0.03432000 & 1.62318700 & C & -1.19599800 & -4.55918100 & 0.76930800 \\
\hline $\mathrm{H}$ & -0.05177500 & -3.25382100 & 2.05011800 & $\mathrm{C}$ & -3.48341500 & -3.88359500 & 0.29822800 \\
\hline $\mathrm{C}$ & -6.65038400 & -1.09081000 & 0.82636400 & C & -5.77227100 & -3.18837600 & -0.16620400 \\
\hline $\mathrm{H}$ & -7.26603200 & 0.75448300 & 1.74101800 & C & -2.41588800 & -4.77895800 & 0.13374400 \\
\hline $\mathrm{H}$ & -0.38130600 & -5.25830500 & 0.62988800 & C & -4.75311600 & -4.07349600 & -0.34395300 \\
\hline $\mathrm{H}$ & -7.59896000 & -1.25497500 & 0.32170200 & $\mathrm{H}$ & -6.72932900 & -3.34238200 & -0.65774500 \\
\hline $\mathrm{H}$ & -2.55222500 & -5.65398300 & -0.49664800 & $\mathrm{H}$ & -4.88620000 & -4.94802300 & -0.97596500 \\
\hline C & 2.25530200 & 0.08488500 & 2.30183800 & S & 1.92885400 & -1.04216500 & 3.53148400 \\
\hline $\mathrm{N}$ & 1.76185800 & 1.34601000 & 2.30429700 & $\mathrm{H}$ & 1.83296700 & 1.89804400 & 1.44349500 \\
\hline C & -0.08535600 & 0.03366900 & -0.90867700 & $\mathrm{O}$ & 0.39879800 & -0.61314100 & -0.00298200 \\
\hline $\mathrm{O}$ & -0.23165800 & -0.33665200 & -2.18922400 & C & 0.51066300 & -1.50880300 & -2.72632700 \\
\hline C & -0.04423200 & -2.78069500 & -2.09268400 & $\mathrm{H}$ & 0.42405700 & -3.65598700 & -2.55356700 \\
\hline $\mathrm{H}$ & 0.16030800 & -2.80693300 & -1.02410300 & $\mathrm{H}$ & -1.12802800 & -2.85042700 & -2.24157000 \\
\hline C & 0.20768100 & -1.44101900 & -4.22085400 & $\mathrm{H}$ & 0.52564800 & -0.47718100 & -4.62975900 \\
\hline $\mathrm{H}$ & 0.75456900 & -2.23476100 & -4.73874700 & $\mathrm{H}$ & -0.85926600 & -1.57747000 & -4.42190100 \\
\hline C & 2.00475300 & -1.32474200 & -2.48063500 & $\mathrm{H}$ & 2.55186000 & -2.12885800 & -2.98392800 \\
\hline $\mathrm{H}$ & 2.34272300 & -0.36110900 & -2.86814600 & $\mathrm{H}$ & 2.23564000 & -1.37163000 & -1.42014700 \\
\hline C & -0.97665700 & 2.15713800 & -1.71422400 & $\mathrm{H}$ & -0.56573100 & 1.85047800 & -2.66760600 \\
\hline C & -0.89001000 & 3.61930900 & -1.45210500 & C & -0.33240100 & 4.43268500 & -2.44447700 \\
\hline C & -1.35758600 & 4.19223500 & -0.26299100 & C & -0.22474900 & 5.80663300 & -2.24210900 \\
\hline $\mathrm{H}$ & 0.06949200 & 3.97848000 & -3.34381900 & C & -1.25396300 & 5.56592600 & -0.06924400 \\
\hline $\mathrm{H}$ & -1.77151200 & 3.56496900 & 0.51952200 & C & -0.68936000 & 6.37645000 & -1.05721000 \\
\hline $\mathrm{H}$ & 0.23760300 & 6.42720700 & -3.00346600 & $\mathrm{H}$ & -1.60379700 & 6.00448300 & 0.86043200 \\
\hline $\bar{H}$ & -0.60440300 & 7.44760900 & -0.89846000 & $\mathrm{~N}$ & -0.62080600 & 1.30233500 & -0.69926100 \\
\hline $\mathrm{H}$ & -0.56532700 & 1.63066300 & 0.27452200 & $S$ & 2.70806900 & 2.43662400 & -1.31713000 \\
\hline $\mathrm{O}$ & 1.91923600 & 2.08979700 & -2.51810300 & $\mathrm{O}$ & 1.90036100 & 2.85605000 & -0.13762600 \\
\hline $\mathrm{O}$ & 3.79483800 & 1.48714900 & -0.96830700 & $\mathrm{C}$ & 3.61705700 & 3.98535900 & -1.78939100 \\
\hline $\mathrm{F}$ & 4.45139100 & 3.74233900 & -2.80750200 & $\mathrm{~F}$ & 4.32739400 & 4.44077000 & -0.74927000 \\
\hline $\mathrm{F}$ & 2.75510900 & 4.94378900 & -2.16657800 & C & -2.84667600 & 2.02685100 & -2.10866100 \\
\hline $\mathrm{H}$ & -3.22394600 & 2.44783500 & -1.18079600 & $\mathrm{H}$ & -2.90641200 & 2.69726800 & -2.96248600 \\
\hline $\mathrm{C}$ & -3.10590400 & 0.66576900 & -2.32701500 & $\mathrm{C}$ & -3.14501100 & 0.08479200 & -3.65953700 \\
\hline $\mathrm{H}$ & -2.60482500 & 0.68261500 & -4.40141400 & $\mathrm{H}$ & -2.77935600 & -0.94562200 & -3.66929600 \\
\hline $\mathrm{C}$ & -3.33974700 & -0.19276000 & -1.14115000 & $\mathrm{H}$ & -3.37094700 & -1.25710200 & -1.37259500 \\
\hline $\mathrm{H}$ & -2.55983200 & -0.00233700 & -0.38973700 & $\mathrm{H}$ & -4.27677400 & 0.09318900 & -0.64953800 \\
\hline C & -4.87762800 & -0.83499100 & -5.98142200 & $\mathrm{H}$ & -4.46093500 & -1.84567000 & -5.90481300 \\
\hline $\mathrm{H}$ & -5.87266700 & -0.91867000 & -6.43414100 & $\mathrm{H}$ & -4.24588100 & -0.26128600 & -6.66878200 \\
\hline $\mathrm{C}$ & -6.01265800 & -1.03862100 & -3.09748900 & $\mathrm{H}$ & -5.55165200 & -2.01032200 & -2.89012100 \\
\hline $\mathrm{H}$ & -6.15726900 & -0.53656500 & -2.13527300 & $\mathrm{H}$ & -7.00520500 & -1.22583800 & -3.52513900 \\
\hline C & -5.64987000 & 1.74602100 & -4.42142500 & $\mathrm{H}$ & -5.67401900 & 2.23943600 & -3.44346500 \\
\hline $\mathrm{H}$ & -5.03839400 & 2.36010800 & -5.09266800 & $\mathrm{H}$ & -6.67257500 & 1.74336100 & -4.81632400 \\
\hline $\mathrm{Si}$ & -4.98611800 & -0.01033300 & -4.29207600 & & & & \\
\hline
\end{tabular}

\section{TS-S-4f-26}

Imaginary frequency: $-230.65 \mathrm{~cm}^{-1}$

E[B3LYP/6-31G(d)-D3(BJ)]: -4789.082437 Hartree

E[PCM(Et $2 \mathrm{O})-B 3 L Y P / 6-311 G(d, p)-D 3(B J)]:-4790.225022$ Hartree

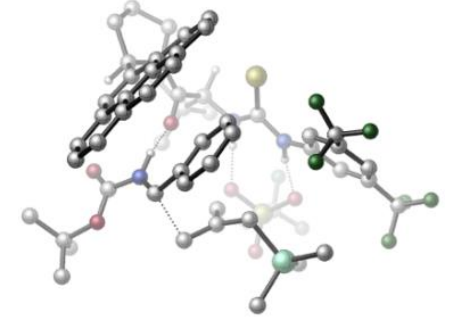




\begin{tabular}{|c|c|c|c|c|c|c|c|}
\hline 01 & & & & $\mathrm{~N}$ & 2.48457700 & -0.09515100 & -1.56519400 \\
\hline $\mathrm{H}$ & 3.12021100 & -0.80879900 & -1.18302300 & C & 2.93682600 & 1.20720300 & -1.32895300 \\
\hline C & 4.28980700 & 1.35649100 & -0.97642400 & C & 2.11149400 & 2.33989200 & -1.33802100 \\
\hline C & 4.79380400 & 2.61146400 & -0.65075000 & $\mathrm{H}$ & 4.93043000 & 0.48407100 & -0.93716200 \\
\hline C & 2.64172200 & 3.58815500 & -1.01716900 & $\mathrm{H}$ & 1.07134400 & 2.23981200 & -1.59787400 \\
\hline C & 3.98300800 & 3.74528100 & -0.67710000 & $\mathrm{H}$ & 4.38484200 & 4.72034600 & -0.43313600 \\
\hline $\mathrm{C}$ & 6.21530800 & 2.73410700 & -0.17840700 & C & 1.72155700 & 4.77132200 & -0.93203400 \\
\hline $\mathrm{F}$ & 7.01588600 & 1.79079600 & -0.70764200 & $\mathrm{~F}$ & 6.29345100 & 2.60322900 & 1.17429500 \\
\hline $\mathrm{F}$ & 6.74323400 & 3.94097900 & -0.47870200 & $\mathrm{~F}$ & 0.67967000 & 4.68304200 & -1.78974700 \\
\hline $\mathrm{F}$ & 1.18143600 & 4.88728600 & 0.31477700 & $\mathrm{~F}$ & 2.35685600 & 5.93413300 & -1.18346600 \\
\hline C & 0.04357700 & -2.55291900 & -2.69528000 & $\mathrm{H}$ & 0.12464000 & -2.37379200 & -3.76946200 \\
\hline C & 0.12729800 & -4.10332800 & -2.44782900 & C & -0.13338400 & -4.48881100 & -0.97851300 \\
\hline $\mathrm{H}$ & -1.14581500 & -4.22610100 & -0.65988300 & $\mathrm{H}$ & 0.57515400 & -4.02063900 & -0.29541100 \\
\hline $\mathrm{H}$ & -0.01472100 & -5.57383200 & -0.87591500 & C & 1.52182800 & -4.59249900 & -2.88871100 \\
\hline $\mathrm{H}$ & 1.54452400 & -5.68797200 & -2.86665500 & $\mathrm{H}$ & 2.31879800 & -4.23786500 & -2.23379000 \\
\hline $\mathrm{H}$ & 1.74378200 & -4.27233900 & -3.91405200 & C & -0.91922800 & -4.82174400 & -3.31972900 \\
\hline $\mathrm{H}$ & -0.85858000 & -5.89939300 & -3.13634700 & $\mathrm{H}$ & -0.73914600 & -4.66422800 & -4.38966700 \\
\hline $\mathrm{H}$ & -1.94104500 & -4.50835700 & -3.08265600 & C & -1.28141000 & -1.97448800 & -2.16351500 \\
\hline $\mathrm{O}$ & -1.36715200 & -1.72427900 & -0.95200300 & C & -2.37994000 & -2.07092800 & -4.43375100 \\
\hline $\mathrm{C}$ & -3.71737800 & -1.72040300 & -2.40867600 & $\mathrm{C}$ & -3.79721100 & -2.58806800 & -4.68782700 \\
\hline $\mathrm{H}$ & -2.19008400 & -1.12128200 & -4.94965300 & $\mathrm{H}$ & -1.61662600 & -2.77404100 & -4.75328200 \\
\hline $\mathrm{H}$ & -3.86818600 & -2.58598600 & -1.75411100 & $\mathrm{H}$ & -3.83257700 & -3.66743200 & -4.49984900 \\
\hline $\mathrm{H}$ & -4.12665400 & -2.41170500 & -5.71549500 & $\mathrm{~N}$ & -2.35235600 & -1.86831700 & -2.97851900 \\
\hline C & -4.02518700 & -0.49431500 & -1.56722700 & C & -4.58166200 & -0.71292300 & -0.34024900 \\
\hline $\mathrm{C}$ & -3.89209100 & 0.86476300 & -2.05765500 & $\mathrm{C}$ & -5.04277500 & 0.34658800 & 0.50409100 \\
\hline $\mathrm{H}$ & -4.66026700 & -1.73036800 & 0.03303600 & $\mathrm{C}$ & -4.43917400 & 1.93311700 & -1.27743700 \\
\hline C & -3.23197600 & 1.18941000 & -3.25283400 & C & -5.51864200 & 0.11169500 & 1.80318300 \\
\hline C & -5.00015400 & 1.67954400 & 0.00706500 & C & -4.37040600 & 3.27708100 & -1.75495100 \\
\hline $\mathrm{H}$ & -2.73681600 & 0.41573300 & -3.81885500 & C & -3.15231800 & 2.50655700 & -3.70210000 \\
\hline C & -5.95411700 & 1.16928500 & 2.60007600 & $\mathrm{H}$ & -5.54614800 & -0.90752700 & 2.17972800 \\
\hline C & -5.47653200 & 2.75324300 & 0.81689700 & C & -3.73300600 & 3.54021100 & -2.97585200 \\
\hline C & -4.91408500 & 4.33153800 & -0.94618400 & $\mathrm{H}$ & -2.62280900 & 2.72191400 & -4.62525700 \\
\hline C & -5.94342600 & 2.47441100 & 2.11101300 & $\mathrm{H}$ & -6.31706400 & 0.97357000 & 3.60534600 \\
\hline C & -5.43765700 & 4.08517200 & 0.28491600 & $\mathrm{H}$ & -3.67307800 & 4.56473100 & -3.33345500 \\
\hline $\mathrm{H}$ & -4.87418900 & 5.34590300 & -1.33477100 & $\mathrm{H}$ & -6.29601400 & 3.29323400 & 2.73277400 \\
\hline $\mathrm{H}$ & -5.82410700 & 4.89771000 & 0.89466100 & C & 1.37181400 & -0.54369000 & -2.23366900 \\
\hline $\mathrm{S}$ & 0.37638900 & 0.40687800 & -3.22481900 & $\mathrm{~N}$ & 1.14799100 & -1.86301200 & -2.05517800 \\
\hline $\mathrm{H}$ & 1.68046900 & -2.36377900 & -1.33513800 & $S$ & 3.94946900 & -2.84512500 & 0.48849800 \\
\hline $\mathrm{O}$ & 4.12481600 & -2.90591700 & 1.94531500 & $\mathrm{O}$ & 2.60614800 & -3.26253500 & -0.00921300 \\
\hline $\mathrm{O}$ & 4.42271400 & -1.59473500 & -0.17587200 & $\mathrm{C}$ & 5.07351100 & -4.15062600 & -0.20270100 \\
\hline $\mathrm{F}$ & 6.35060700 & -3.87269200 & 0.08029300 & $\mathrm{~F}$ & 4.93474300 & -4.21163400 & -1.53869200 \\
\hline $\mathrm{F}$ & 4.76725600 & -5.34849100 & 0.30976300 & C & -2.75087100 & -2.34647800 & 2.12031600 \\
\hline $\mathrm{O}$ & -3.05955900 & -3.30193300 & 1.43518500 & 0 & -2.85793500 & -2.23541000 & 3.45437900 \\
\hline C & -3.41168500 & -3.34142900 & 4.26538300 & C & -4.85935800 & -3.60389200 & 3.85089400 \\
\hline $\mathrm{H}$ & -5.30044000 & -4.34686000 & 4.52347700 & $\mathrm{H}$ & -4.91423700 & -3.98060000 & 2.82884500 \\
\hline $\mathrm{H}$ & -5.44991900 & -2.68437200 & 3.92828300 & C & -3.34035000 & -2.77396000 & 5.68110600 \\
\hline $\mathrm{H}$ & -2.30554100 & -2.54496200 & 5.95435000 & $\mathrm{H}$ & -3.73277200 & -3.50445300 & 6.39503500 \\
\hline $\mathrm{H}$ & -3.93382300 & -1.85750400 & 5.76080000 & C & -2.52314300 & -4.57620800 & 4.12003500 \\
\hline $\mathrm{H}$ & -2.86596400 & -5.35237200 & 4.81223100 & $\mathrm{H}$ & -1.48606600 & -4.33032800 & 4.37111700 \\
\hline $\mathrm{H}$ & -2.55727000 & -4.97010400 & 3.10378900 & C & -2.05067400 & -0.00752600 & 2.24579300 \\
\hline $\mathrm{H}$ & -2.62189100 & 0.04475400 & 3.16515200 & C & -1.93370000 & 1.25387400 & 1.50404700 \\
\hline C & -2.26621600 & 2.43952700 & 2.17855500 & C & -1.46892900 & 1.32021200 & 0.18097900 \\
\hline C & -2.14255800 & 3.67011200 & 1.54229800 & $\mathrm{H}$ & -2.63964100 & 2.38805400 & 3.19784700 \\
\hline
\end{tabular}




\begin{tabular}{|c|c|c|c|c|c|c|c|}
\hline $\mathrm{C}$ & -1.36094700 & 2.55365900 & -0.45195700 & $\mathrm{H}$ & -1.20963700 & 0.42386200 & -0.37113300 \\
\hline $\mathrm{C}$ & -1.68698200 & 3.72843800 & 0.22480300 & $\mathrm{H}$ & -2.41589400 & 4.58018000 & 2.06706700 \\
\hline $\mathrm{H}$ & -1.04501400 & 2.58775600 & -1.48771900 & $\mathrm{H}$ & -1.58873900 & 4.68342800 & -0.27812800 \\
\hline $\mathrm{N}$ & -2.23634100 & -1.18053700 & 1.56776000 & $\mathrm{H}$ & -2.04627700 & -1.26157100 & 0.56006300 \\
\hline C & -0.33186900 & -0.16097700 & 3.38558000 & $\mathrm{H}$ & -0.53590400 & 0.64023100 & 4.09154000 \\
\hline $\mathrm{H}$ & -0.51817300 & -1.16647900 & 3.74981700 & $\mathrm{C}$ & 0.64763100 & 0.04861100 & 2.41366900 \\
\hline C & 1.17489500 & 1.37547500 & 2.15252100 & $\mathrm{H}$ & 0.50354400 & 2.17632600 & 2.47949300 \\
\hline $\mathrm{H}$ & 1.45907400 & 1.52065800 & 1.10595500 & C & 1.10261700 & -1.07967400 & 1.55904800 \\
\hline $\mathrm{H}$ & 1.99047400 & -0.81521500 & 0.98907800 & $\mathrm{H}$ & 0.31267600 & -1.32699600 & 0.83014000 \\
\hline $\mathrm{H}$ & 1.30265700 & -1.99138200 & 2.12658100 & C & 3.30991300 & 3.46331400 & 2.79606400 \\
\hline $\mathrm{H}$ & 2.62039900 & 3.94006600 & 2.09371900 & $\mathrm{H}$ & 4.30966000 & 3.49201800 & 2.35384600 \\
\hline $\mathrm{H}$ & 3.32555600 & 4.06361000 & 3.71291000 & C & 4.13460100 & 0.44311900 & 2.67362000 \\
\hline $\mathrm{H}$ & 4.33426200 & 0.46553800 & 1.59927700 & $\mathrm{H}$ & 3.88102700 & -0.59134700 & 2.92463800 \\
\hline $\mathrm{H}$ & 5.07655500 & 0.68936200 & 3.17933700 & C & 2.36740800 & 1.48495700 & 4.99634800 \\
\hline $\mathrm{H}$ & 2.05949400 & 0.46057300 & 5.23250300 & $\mathrm{H}$ & 1.55439100 & 2.16154000 & 5.28676300 \\
\hline $\mathrm{H}$ & 3.23360900 & 1.72285000 & 5.62539900 & $\mathrm{Si}$ & 2.81997300 & 1.67615700 & 3.17419500 \\
\hline $\mathrm{C}$ & -4.63488800 & -1.83250700 & -3.64963000 & $\mathrm{H}$ & -4.88380200 & -0.83268800 & -4.01651800 \\
\hline $\mathrm{H}$ & -5.57173000 & -2.33425500 & -3.39513800 & & & & \\
\hline
\end{tabular}

\section{TS-S-4f-27}

Imaginary frequency: $-338.08 \mathrm{~cm}^{-1}$

E[B3LYP/6-31G(d)-D3(BJ)]: -4789.082874 Hartree

$\mathrm{E}\left[\mathrm{PCM}\left(\mathrm{Et}_{2} \mathrm{O}\right)-\mathrm{B} 3 \mathrm{LYP} / 6-311 \mathrm{G}(\mathrm{d}, \mathrm{p})-\mathrm{D} 3(\mathrm{BJ})\right]$ : -4790.224510 Hartree

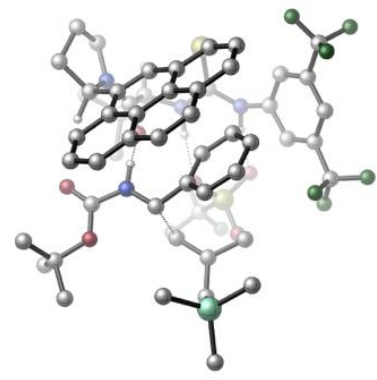

\begin{tabular}{|lrrl|lrrr|}
\hline O & & & & $\mathrm{N}$ & -2.98269100 & 2.21506600 & 0.56105800 \\
\hline $\mathrm{H}$ & -2.51768700 & 2.63658100 & 1.37353700 & $\mathrm{C}$ & -3.65438200 & 1.02568900 & 0.88571300 \\
\hline $\mathrm{C}$ & -3.32027000 & 0.43552000 & 2.11828500 & $\mathrm{C}$ & -4.57055900 & 0.37784100 & 0.04638300 \\
\hline $\mathrm{C}$ & -3.82678100 & -0.81228800 & 2.45506300 & $\mathrm{H}$ & -2.64207600 & 0.94804300 & 2.78885600 \\
\hline $\mathrm{C}$ & -5.05337700 & -0.88370600 & 0.40074700 & $\mathrm{H}$ & -4.85338500 & 0.83006000 & -0.89124800 \\
\hline $\mathrm{C}$ & -4.68799700 & -1.49645200 & 1.59573900 & $\mathrm{H}$ & -5.05357800 & -2.48443800 & 1.84911200 \\
\hline $\mathrm{C}$ & -3.33305300 & -1.51175700 & 3.68923500 & $\mathrm{C}$ & -5.99620100 & -1.60016800 & -0.52448000 \\
\hline $\mathrm{F}$ & -2.73340300 & -0.67524300 & 4.55811300 & $\mathrm{~F}$ & -2.41694600 & -2.46836200 & 3.36926000 \\
\hline $\mathrm{F}$ & -4.32991300 & -2.14575300 & 4.34476600 & $\mathrm{~F}$ & -5.87561600 & -1.17948900 & -1.80481000 \\
\hline $\mathrm{F}$ & -5.76274300 & -2.93860800 & -0.52284200 & $\mathrm{~F}$ & -7.28882800 & -1.43184700 & -0.17296200 \\
\hline $\mathrm{C}$ & -0.62302800 & 3.79033600 & -1.72135900 & $\mathrm{H}$ & -1.33492400 & 4.09651300 & -2.48820800 \\
\hline $\mathrm{C}$ & 0.30673200 & 5.00931600 & -1.40583700 & $\mathrm{C}$ & 1.47733300 & 4.64751500 & -0.47225400 \\
\hline $\mathrm{H}$ & 2.16569000 & 3.94036000 & -0.94447900 & $\mathrm{H}$ & 1.14626600 & 4.20205300 & 0.46606400 \\
\hline $\mathrm{H}$ & 2.03605700 & 5.55984500 & -0.23313100 & $\mathrm{C}$ & -0.55712800 & 6.10968100 & -0.76506500 \\
\hline $\mathrm{H}$ & 0.04160600 & 7.01703300 & -0.62604500 & $\mathrm{H}$ & -0.94022200 & 5.80374100 & 0.21008500 \\
\hline $\mathrm{H}$ & -1.41301800 & 6.36091200 & -1.40272700 & $\mathrm{C}$ & 0.88666000 & 5.53993500 & -2.72850300 \\
\hline $\mathrm{H}$ & 1.51913600 & 6.41217200 & -2.53134500 & $\mathrm{H}$ & 0.09436500 & 5.85289700 & -3.41884500 \\
\hline $\mathrm{H}$ & 1.50903600 & 4.78891600 & -3.22707600 & $\mathrm{C}$ & 0.17569100 & 2.56420900 & -2.18256200 \\
\hline $\mathrm{C}$ & 1.21302500 & 1.17706000 & -3.89094000 & $\mathrm{H}$ & 2.15908800 & 1.29025900 & -3.35920600 \\
\hline $\mathrm{N}$ & 0.34383700 & 2.29914000 & -3.48950000 & $\mathrm{C}$ & 0.60435900 & -0.18387600 & -3.58541600 \\
\hline $\mathrm{C}$ & -0.74513300 & -0.33237300 & -3.45318600 & $\mathrm{C}$ & 1.47240800 & -1.33818400 & -3.46373100 \\
\hline $\mathrm{C}$ & -1.36068100 & -1.60347100 & -3.21822700 & $\mathrm{H}$ & -1.39300800 & 0.53683400 & -3.48143200 \\
\hline $\mathrm{C}$ & 0.88436400 & -2.61446900 & -3.19039800 & $\mathrm{C}$ & 2.87186300 & -1.25932800 & -3.57580200 \\
\hline $\mathrm{C}$ & -2.75151500 & -1.74032000 & -3.08066500 & $\mathrm{C}$ & -0.53011400 & -2.75228100 & -3.09172300 \\
\hline $\mathrm{C}$ & 1.71596100 & -3.76257300 & -3.00967200 & $\mathrm{H}$ & 3.34866000 & -0.31034800 & -3.78668800 \\
\hline $\mathrm{C}$ & 3.67390800 & -2.38344700 & -3.38703200 & $\mathrm{C}$ & -3.31942600 & -2.99129200 & -2.84853800 \\
\hline
\end{tabular}




\begin{tabular}{|c|c|c|c|c|c|c|c|}
\hline $\mathrm{H}$ & -3.37864100 & -0.85541700 & -3.13253400 & C & -1.11839600 & -4.02721700 & -2.84174800 \\
\hline $\mathrm{C}$ & 3.10855100 & -3.62200900 & -3.09984400 & $C$ & 1.09732800 & -5.03212200 & -2.74716500 \\
\hline $\mathrm{H}$ & 4.75237200 & -2.28841400 & -3.47586000 & C & -2.51424300 & -4.12330300 & -2.73620300 \\
\hline $\mathrm{H}$ & -4.39359500 & -3.07875000 & -2.73349400 & C & -0.25558000 & -5.16150000 & -2.67791300 \\
\hline $\mathrm{H}$ & 3.73998800 & -4.49563200 & -2.95861700 & $\mathrm{H}$ & 1.74145500 & -5.89801300 & -2.61517200 \\
\hline $\mathrm{H}$ & -2.96355500 & -5.09494300 & -2.54644000 & $\mathrm{H}$ & -0.70820400 & -6.13125100 & -2.48638000 \\
\hline C & -2.49561100 & 2.60713200 & -0.66438100 & $S$ & -3.18277400 & 2.20257900 & -2.15777000 \\
\hline $\mathrm{N}$ & -1.39011400 & 3.38911100 & -0.55147700 & $\mathrm{H}$ & -0.82305000 & 3.21674400 & 0.28614000 \\
\hline $\mathrm{S}$ & -0.39145900 & 2.83297300 & 3.08322800 & 0 & 0.09684800 & 1.98908800 & 4.18734700 \\
\hline 0 & 0.11652200 & 2.44986600 & 1.73002900 & $\mathrm{O}$ & -1.84859900 & 3.12566700 & 3.07191900 \\
\hline C & 0.39130200 & 4.48795400 & 3.39587400 & $\mathrm{~F}$ & 0.09357700 & 4.93028900 & 4.62002000 \\
\hline $\mathrm{F}$ & -0.04707100 & 5.38744000 & 2.49694500 & $\mathrm{~F}$ & 1.72436900 & 4.39752100 & 3.27945600 \\
\hline C & 3.78779600 & 0.43086100 & -0.84329700 & 0 & 4.00657100 & 1.27952300 & -1.68911700 \\
\hline 0 & 4.69831400 & -0.19893800 & -0.08167100 & C & 6.13821500 & 0.08873300 & -0.24061100 \\
\hline C & 6.58295400 & -0.28241500 & -1.65532100 & $\mathrm{H}$ & 7.67233500 & -0.19821100 & -1.72801800 \\
\hline $\mathrm{H}$ & 6.12872800 & 0.37640600 & -2.39605000 & $\mathrm{H}$ & 6.30261300 & -1.31709600 & -1.87762600 \\
\hline C & 6.77590300 & -0.83967400 & 0.79054300 & $\mathrm{H}$ & 6.40495500 & -0.61171200 & 1.79526700 \\
\hline $\mathrm{H}$ & 7.86255000 & -0.71106100 & 0.78686000 & $\mathrm{H}$ & 6.54951300 & -1.88552000 & 0.56142500 \\
\hline C & 6.40995700 & 1.55380800 & 0.10017100 & $\mathrm{H}$ & 7.49029100 & 1.73314100 & 0.09441200 \\
\hline $\mathrm{H}$ & 6.03055700 & 1.78802400 & 1.10046400 & $\mathrm{H}$ & 5.93786900 & 2.22026900 & -0.62209300 \\
\hline C & 2.18027500 & -0.98243900 & 0.34346700 & $\mathrm{H}$ & 3.00672700 & -1.64487700 & 0.56418400 \\
\hline C & 0.85299300 & -1.61239400 & 0.25599900 & C & 0.75853800 & -2.99570100 & 0.47380100 \\
\hline C & -0.31254400 & -0.87301600 & 0.00567200 & C & -0.47886600 & -3.63084800 & 0.46054200 \\
\hline $\mathrm{H}$ & 1.65955200 & -3.57767200 & 0.63425000 & C & -1.54367900 & -1.52086800 & -0.02311800 \\
\hline $\mathrm{H}$ & -0.26223000 & 0.19909600 & -0.14905500 & C & -1.63624000 & -2.88966200 & 0.22222100 \\
\hline $\mathrm{H}$ & -0.53545500 & -4.70331800 & 0.61729100 & $\mathrm{H}$ & -2.43471600 & -0.95421400 & -0.24622800 \\
\hline $\mathrm{H}$ & -2.60691000 & -3.37170200 & 0.20172600 & $\mathrm{~N}$ & 2.50603900 & -0.01277800 & -0.56798300 \\
\hline $\mathrm{H}$ & 1.76995100 & 0.57812300 & -0.99147400 & C & 2.19870800 & 0.04155100 & 2.10332100 \\
\hline $\mathrm{H}$ & 1.45451600 & 0.76641400 & 1.77752400 & $\mathrm{H}$ & 3.23327100 & 0.37189500 & 2.05343900 \\
\hline C & 1.84517300 & -0.79722100 & 3.16146500 & C & 2.85650500 & -1.44070100 & 4.01565200 \\
\hline $\mathrm{H}$ & 2.50924200 & -1.41095700 & 5.05763500 & $\mathrm{H}$ & 3.82260600 & -0.92624600 & 3.95903800 \\
\hline C & 0.41171900 & -1.03872300 & 3.46958300 & $\mathrm{H}$ & 0.04113700 & -0.13955600 & 3.98714300 \\
\hline $\mathrm{H}$ & 0.24838400 & -1.90991600 & 4.10532600 & $\mathrm{H}$ & -0.18882300 & -1.12707800 & 2.56023500 \\
\hline C & 4.30949700 & -3.77877000 & 5.24640900 & $\mathrm{H}$ & 5.22586300 & -3.17811800 & 5.28194500 \\
\hline $\bar{H}$ & 4.60640800 & -4.83323600 & 5.19901500 & $\mathrm{H}$ & 3.77321900 & -3.62737700 & 6.19018400 \\
\hline C & 4.25253200 & -3.58610600 & 2.19215000 & $\mathrm{H}$ & 5.04480100 & -2.83495700 & 2.10804100 \\
\hline $\bar{H}$ & 3.66011700 & -3.54880700 & 1.27393700 & $\mathrm{H}$ & 4.73398600 & -4.57102200 & 2.22666400 \\
\hline $\mathrm{C}$ & 1.65751900 & -4.34450100 & 3.76589900 & $\mathrm{H}$ & 0.96265000 & -4.04586300 & 2.97608500 \\
\hline $\mathrm{H}$ & 1.13306200 & -4.25475500 & 4.72416400 & $\mathrm{H}$ & 1.89777900 & -5.40520800 & 3.62222900 \\
\hline $\mathrm{Si}$ & 3.23766400 & -3.32284500 & 3.76253800 & C & -0.34507000 & 2.93055500 & -4.62919200 \\
\hline $\mathrm{H}$ & 0.03892300 & 3.94200700 & -4.79331000 & $\mathrm{H}$ & -1.41946700 & 2.98641400 & -4.43276300 \\
\hline C & 0.00794800 & 2.00872900 & -5.80625100 & $\mathrm{H}$ & 0.03362700 & 2.54826200 & -6.75664000 \\
\hline $\mathrm{H}$ & -0.73234300 & 1.20720600 & -5.89048500 & C & 1.36958300 & 1.42114000 & -5.40691500 \\
\hline $\mathrm{H}$ & 1.61935600 & 0.50392100 & -5.94525400 & $\mathrm{H}$ & 2.16837800 & 2.15212100 & -5.57985100 \\
\hline 0 & 0.69231300 & 1.85214200 & -1.30638900 & & & & \\
\hline
\end{tabular}

\section{TS-S-4f-28}

Imaginary frequency: $-411.16 \mathrm{~cm}^{-1}$

E[B3LYP/6-31G(d)-D3(BJ)]: -4789.081142 Hartree

E[PCM(Et $\left.\left.{ }_{2} \mathrm{O}\right)-\mathrm{B} 3 L Y P / 6-311 \mathrm{G}(\mathrm{d}, \mathrm{p})-\mathrm{D} 3(\mathrm{BJ})\right]$ : -4790.224144 Hartree

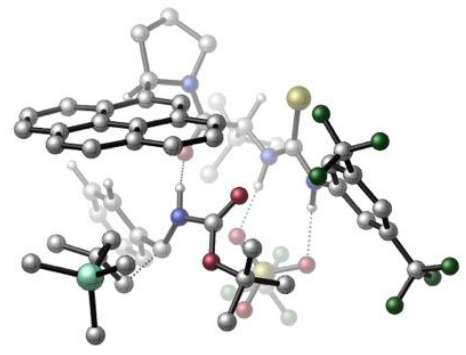




\begin{tabular}{|c|c|c|c|c|c|c|c|}
\hline 01 & & & & $\mathrm{~N}$ & 3.08426700 & 0.96461300 & 1.10895900 \\
\hline $\mathrm{H}$ & 3.25660000 & 1.38970500 & 0.19315000 & C & 3.69673200 & -0.28469900 & 1.25257100 \\
\hline C & 4.83618500 & -0.52572000 & 0.47299300 & C & 3.17626000 & -1.32092100 & 2.03896300 \\
\hline C & 5.43482200 & -1.78400400 & 0.47736000 & $\mathrm{H}$ & 5.22014100 & 0.25827700 & -0.16921600 \\
\hline C & 3.79320600 & -2.56637400 & 2.03478800 & $\mathrm{H}$ & 2.29092500 & -1.14594600 & 2.62618200 \\
\hline C & 4.92784800 & -2.81689800 & 1.26102900 & $\mathrm{H}$ & 5.40312500 & -3.79042100 & 1.26944700 \\
\hline $\mathrm{C}$ & 6.57971700 & -2.06159700 & -0.45377800 & C & 3.18581000 & -3.70110300 & 2.80660600 \\
\hline $\mathrm{F}$ & 7.30262800 & -0.95613800 & -0.72285100 & $\mathrm{~F}$ & 6.13893800 & -2.54388900 & -1.64513300 \\
\hline $\mathrm{F}$ & 7.42413300 & -2.99138600 & 0.04784800 & $\mathrm{~F}$ & 4.11541700 & -4.42660000 & 3.46353800 \\
\hline $\mathrm{F}$ & 2.27315500 & -3.28906400 & 3.71411000 & $\mathrm{~F}$ & 2.53801300 & -4.57332300 & 1.98015000 \\
\hline C & 0.62344100 & 3.49619100 & 1.97351500 & $\mathrm{H}$ & 0.94561500 & 3.57011500 & 3.01146400 \\
\hline C & 0.58808300 & 4.94099500 & 1.37646500 & C & 0.01656900 & 5.00409900 & -0.05138400 \\
\hline $\mathrm{H}$ & -1.02842300 & 4.68927300 & -0.08806600 & $\mathrm{H}$ & 0.57058200 & 4.37361200 & -0.74773300 \\
\hline $\mathrm{H}$ & 0.07662400 & 6.03834000 & -0.41184300 & C & 2.02662200 & 5.48709900 & 1.37361400 \\
\hline $\mathrm{H}$ & 2.02229200 & 6.54595100 & 1.09021100 & $\mathrm{H}$ & 2.65303200 & 4.94828700 & 0.65839600 \\
\hline $\mathrm{H}$ & 2.48916000 & 5.40045800 & 2.36390200 & C & -0.28246500 & 5.81603200 & 2.29651800 \\
\hline $\mathrm{H}$ & -0.33669700 & 6.83616700 & 1.90129300 & $\mathrm{H}$ & 0.13426200 & 5.87601000 & 3.30932400 \\
\hline $\mathrm{H}$ & -1.30858100 & 5.43552900 & 2.36598800 & C & -0.73506500 & 2.79479400 & 1.88608200 \\
\hline 0 & -1.12736300 & 2.36146000 & 0.79435600 & C & -1.25376300 & 3.11180700 & 4.35472400 \\
\hline C & -2.85938000 & 2.09469200 & 2.82007900 & C & -2.66475100 & 3.39650000 & 4.87656400 \\
\hline $\mathrm{H}$ & -0.75379400 & 2.31551700 & 4.91856300 & $\mathrm{H}$ & -0.61235500 & 3.99092900 & 4.36832600 \\
\hline $\mathrm{H}$ & -3.36469300 & 2.70039300 & 2.06098500 & $\mathrm{H}$ & -2.98978300 & 4.38630100 & 4.53433100 \\
\hline $\mathrm{H}$ & -2.72014200 & 3.37858900 & 5.96851500 & $\mathrm{~N}$ & -1.51156700 & 2.66401200 & 2.98186200 \\
\hline C & -2.87564800 & 0.64093100 & 2.38620500 & C & -1.80071800 & -0.17072600 & 2.60088100 \\
\hline C & -4.08849800 & 0.10003000 & 1.80638300 & C & -1.83230100 & -1.57204300 & 2.30488400 \\
\hline $\mathrm{H}$ & -0.87740700 & 0.24193800 & 2.99891600 & C & -4.15586300 & -1.30439200 & 1.53320000 \\
\hline C & -5.21230400 & 0.88881100 & 1.49742800 & C & -0.72157100 & -2.40060200 & 2.52410400 \\
\hline$C$ & -3.03172800 & -2.14146300 & 1.79069300 & C & -5.35386600 & -1.87495900 & 1.00062500 \\
\hline $\mathrm{H}$ & -5.19689700 & 1.95453300 & 1.69340500 & C & -6.36301100 & 0.32833200 & 0.94406100 \\
\hline C & -0.78527700 & -3.76505700 & 2.25162500 & $\mathrm{H}$ & 0.19186600 & -1.96344300 & 2.90851500 \\
\hline C & -3.09777800 & -3.54400200 & 1.53158100 & C & -6.44245300 & -1.04005000 & 0.70418400 \\
\hline$C$ & -5.40632400 & -3.29510200 & 0.78677100 & $\mathrm{H}$ & -7.21025300 & 0.96767300 & 0.71290100 \\
\hline C & -1.96143800 & -4.33420800 & 1.76580000 & $\mathrm{H}$ & 0.09090700 & -4.38191300 & 2.41597600 \\
\hline C & -4.33104200 & -4.09145500 & 1.04152100 & $\mathrm{H}$ & -7.35012300 & -1.47577800 & 0.29473500 \\
\hline $\mathrm{H}$ & -6.33657900 & -3.72419800 & 0.42334900 & $\mathrm{H}$ & -2.00993900 & -5.40228800 & 1.56901900 \\
\hline $\mathrm{H}$ & -4.39280900 & -5.16468100 & 0.87965900 & C & 2.19442600 & 1.60686400 & 1.92589100 \\
\hline$S$ & 1.91786000 & 1.21401800 & 3.55249600 & $\mathrm{~N}$ & 1.59743500 & 2.65552700 & 1.30512300 \\
\hline $\mathrm{H}$ & 1.50950100 & 2.59428000 & 0.28421700 & C & 0.12265500 & -0.67866100 & -0.78067800 \\
\hline $\mathrm{O}$ & 0.68828200 & -0.78576200 & 0.28599100 & $\mathrm{O}$ & 0.30994200 & -1.42340600 & -1.88488500 \\
\hline C & 1.39465600 & -2.43320800 & -1.97468000 & C & 1.26379800 & -3.45060100 & -0.84289500 \\
\hline $\mathrm{H}$ & 1.95363500 & -4.28084600 & -1.02697900 & $\mathrm{H}$ & 1.50491800 & -3.00870500 & 0.12069800 \\
\hline $\mathrm{H}$ & 0.24615200 & -3.85381000 & -0.79421400 & C & 1.12246300 & -3.07856500 & -3.33110200 \\
\hline $\mathrm{H}$ & 1.12688300 & -2.32112800 & -4.12121400 & $\mathrm{H}$ & 1.90340100 & -3.81212000 & -3.55307100 \\
\hline $\mathrm{H}$ & 0.15670500 & -3.59539000 & -3.33570800 & C & 2.73381600 & -1.71174000 & -1.97241100 \\
\hline $\mathrm{H}$ & 3.55112000 & -2.43536800 & -2.04529000 & $\mathrm{H}$ & 2.79831800 & -1.01217900 & -2.80807900 \\
\hline $\mathrm{H}$ & 2.86233800 & -1.15471500 & -1.04784600 & C & -1.15879400 & 0.80840300 & -2.22711900 \\
\hline $\mathrm{H}$ & -0.30139100 & 0.80102600 & -2.89572700 & C & -1.91767200 & 2.07819200 & -2.20703000 \\
\hline C & -1.50522900 & 3.11462800 & -3.05337200 & C & -3.01602900 & 2.27016500 & -1.35793200 \\
\hline C & -2.17894700 & 4.33368000 & -3.04215300 & $\mathrm{H}$ & -0.63296600 & 2.97493300 & -3.68269800 \\
\hline C & -3.69301500 & 3.48526100 & -1.35694500 & $\mathrm{H}$ & -3.33012600 & 1.47740900 & -0.68742300 \\
\hline C & -3.27564800 & 4.51996100 & -2.19815300 & $\mathrm{H}$ & -1.83890600 & 5.14129100 & -3.68313800 \\
\hline $\mathrm{H}$ & -4.54311100 & 3.62526300 & -0.69506800 & $\mathrm{H}$ & -3.80107200 & 5.47074500 & -2.19205100 \\
\hline $\mathrm{N}$ & -0.90766600 & 0.23352300 & -0.99133000 & $\mathrm{H}$ & -1.08034100 & 0.83917800 & -0.17969200 \\
\hline
\end{tabular}




\begin{tabular}{|c|c|c|c|c|c|c|c|}
\hline S & 2.34271400 & 1.98910600 & -2.39063600 & $\mathrm{O}$ & 1.95688500 & 1.21021600 & -3.58397600 \\
\hline 0 & 1.21391300 & 2.22632100 & -1.44577900 & $\mathrm{O}$ & 3.60230800 & 1.60227200 & -1.70936400 \\
\hline $\mathrm{C}$ & 2.69131800 & 3.69398000 & -3.03934800 & $\mathrm{~F}$ & 3.70843900 & 3.68586500 & -3.90452600 \\
\hline $\mathrm{F}$ & 2.99522700 & 4.52085000 & -2.02401500 & $\mathrm{~F}$ & 1.60289100 & 4.18446000 & -3.66243400 \\
\hline C & -2.12309700 & -0.35744700 & -3.43613800 & $\mathrm{H}$ & -2.36725700 & 0.35841300 & -4.21651500 \\
\hline $\mathrm{H}$ & -1.28618300 & -1.01352300 & -3.64918000 & C & -3.17449000 & -0.90574000 & -2.69362400 \\
\hline C & -2.94770900 & -2.08150900 & -1.87690900 & $\mathrm{H}$ & -1.89210100 & -2.20577200 & -1.62537600 \\
\hline $\mathrm{H}$ & -3.57183300 & -2.11948100 & -0.98266500 & C & -4.52940000 & -0.27542100 & -2.71029400 \\
\hline $\mathrm{H}$ & -5.14325300 & -0.77886900 & -3.47188300 & $\mathrm{H}$ & -5.04468200 & -0.38561000 & -1.75135300 \\
\hline $\mathrm{H}$ & -4.47459900 & 0.78119000 & -2.98312400 & C & -2.48747500 & -5.10224600 & -2.02131300 \\
\hline $\mathrm{H}$ & -2.70141600 & -5.10407000 & -0.94950700 & $\mathrm{H}$ & -2.79404000 & -6.06969100 & -2.43634000 \\
\hline $\mathrm{H}$ & -1.40268900 & -5.01334400 & -2.14356500 & C & -5.24625600 & -3.93121200 & -2.88209300 \\
\hline $\mathrm{H}$ & -5.62429100 & -4.00581300 & -1.85856100 & $\mathrm{H}$ & -5.76873800 & -3.10501500 & -3.37715200 \\
\hline $\mathrm{H}$ & -5.51423200 & -4.85280800 & -3.41253400 & C & -2.76169500 & -3.50965700 & -4.66149900 \\
\hline $\mathrm{H}$ & -3.28512500 & -2.71043500 & -5.19780700 & $\mathrm{H}$ & -1.68936300 & -3.29244700 & -4.70285800 \\
\hline $\mathrm{H}$ & -2.92970500 & -4.44299300 & -5.21225100 & $\mathrm{Si}$ & -3.37892600 & -3.69384800 & -2.89151700 \\
\hline $\mathrm{C}$ & -3.50549700 & 2.29504200 & 4.21584200 & $\mathrm{H}$ & -3.42057200 & 1.36363300 & 4.78487500 \\
\hline $\mathrm{H}$ & -4.56781100 & 2.54156800 & 4.14139300 & & & & \\
\hline
\end{tabular}

\section{TS-S-4f-29}

Imaginary frequency: $-339.74 \mathrm{~cm}^{-1}$

E[B3LYP/6-31G(d)-D3(BJ)]: -4789.079062 Hartree

$\mathrm{E}\left[\mathrm{PCM}\left(\mathrm{Et}_{2} \mathrm{O}\right)-\mathrm{B} 3 \mathrm{LYP} / 6-311 \mathrm{G}(\mathrm{d}, \mathrm{p})-\mathrm{D} 3(\mathrm{BJ})\right]$ : -4790.223711 Hartree

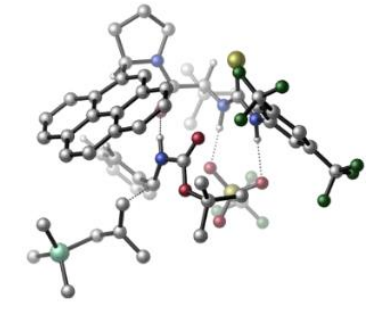

\begin{tabular}{|c|c|c|c|c|c|c|c|}
\hline 01 & & & & $\mathrm{~N}$ & -3.13748400 & 0.22883000 & 1.40624400 \\
\hline $\mathrm{H}$ & -2.84838300 & -0.68882200 & 1.75420100 & C & -4.06177200 & 0.15012300 & 0.35675600 \\
\hline $\mathrm{C}$ & -4.92197200 & -0.95383300 & 0.34715900 & C & -4.07455000 & 1.04194000 & -0.72348200 \\
\hline $\mathrm{C}$ & -5.77539700 & -1.16555300 & -0.73464500 & $\mathrm{H}$ & -4.88255800 & -1.66955100 & 1.15992100 \\
\hline C & -4.94473300 & 0.82167000 & -1.78430100 & $\mathrm{H}$ & -3.38232200 & 1.86664100 & -0.74075000 \\
\hline$C$ & -5.80558500 & -0.27780500 & -1.80591200 & $\mathrm{H}$ & -6.47747100 & -0.44033900 & -2.63996600 \\
\hline$C$ & -6.58658300 & -2.42695700 & -0.79804200 & $\mathrm{C}$ & -4.89176900 & 1.71705600 & -2.98705000 \\
\hline $\mathrm{F}$ & -6.92794300 & -2.87965800 & 0.42589900 & $\mathrm{~F}$ & -5.89398200 & -3.42358400 & -1.40721100 \\
\hline $\mathrm{F}$ & -7.72731600 & -2.26106300 & -1.50541100 & $\mathrm{~F}$ & -6.12056400 & 1.98089800 & -3.48093700 \\
\hline $\mathrm{F}$ & -4.29265800 & 2.90220400 & -2.73333100 & $\mathrm{~F}$ & -4.18929100 & 1.14031500 & -4.00408700 \\
\hline C & -0.65738100 & 1.97591900 & 3.41482100 & $\mathrm{H}$ & -1.30480000 & 2.82536900 & 3.63018500 \\
\hline $\mathrm{C}$ & -0.10555800 & 1.44864500 & 4.77897600 & $\mathrm{C}$ & 0.89198400 & 0.28581700 & 4.63066600 \\
\hline $\mathrm{H}$ & 1.77427700 & 0.57336700 & 4.05538000 & $\mathrm{H}$ & 0.44654300 & -0.57869500 & 4.13518300 \\
\hline $\mathrm{H}$ & 1.21731600 & -0.03219600 & 5.62882600 & C & -1.30431100 & 0.98355100 & 5.62378500 \\
\hline $\mathrm{H}$ & -0.96877100 & 0.71451300 & 6.63199200 & $\mathrm{H}$ & -1.78220100 & 0.10569200 & 5.18045600 \\
\hline $\mathrm{H}$ & -2.06016000 & 1.77259200 & 5.71461800 & $\mathrm{C}$ & 0.59281500 & 2.62195400 & 5.48930900 \\
\hline $\mathrm{H}$ & 0.96847600 & 2.29962200 & 6.46650500 & $\mathrm{H}$ & -0.09724600 & 3.45783200 & 5.65594500 \\
\hline $\mathrm{H}$ & 1.44798800 & 2.99259200 & 4.91224800 & C & 0.46350100 & 2.39128500 & 2.46041500 \\
\hline $\mathrm{O}$ & 1.13462400 & 1.52000900 & 1.88793500 & $\mathrm{C}$ & -0.00186600 & 4.87254600 & 2.75446400 \\
\hline $\mathrm{C}$ & 1.89126500 & 4.04898400 & 1.42222000 & $\mathrm{C}$ & 0.52204100 & 5.99781200 & 1.85383200 \\
\hline $\mathrm{H}$ & -1.07668200 & 4.70657500 & 2.65160600 & $\mathrm{H}$ & 0.23691900 & 5.06575500 & 3.80780400 \\
\hline C & 1.97544700 & 5.58396300 & 1.57662000 & $\mathrm{H}$ & 2.76499300 & 3.55287800 & 1.84943900 \\
\hline $\mathrm{H}$ & 0.43737000 & 6.98077200 & 2.32490500 & $\mathrm{H}$ & -0.04758600 & 6.01938400 & 0.91900400 \\
\hline $\mathrm{H}$ & 2.60942100 & 5.83425600 & 2.43575800 & $\mathrm{H}$ & 2.39984000 & 6.06015100 & 0.68941700 \\
\hline $\mathrm{N}$ & 0.73787200 & 3.69827700 & 2.26738600 & C & 1.73022900 & 3.57725200 & -0.01362400 \\
\hline $\mathrm{C}$ & 0.49887200 & 3.50276900 & -0.59645000 & C & 2.90566900 & 3.16314200 & -0.75266800 \\
\hline C & 0.31084000 & 3.01176500 & -1.92847900 & $\mathrm{H}$ & -0.39041900 & 3.75425500 & -0.02512400 \\
\hline
\end{tabular}




\begin{tabular}{|c|c|c|c|c|c|c|c|}
\hline C & 2.73178000 & 2.57109800 & -2.04447100 & C & 4.21444300 & 3.28193400 & -0.24804100 \\
\hline C & -0.95191900 & 2.97985700 & -2.53825900 & C & 1.43664600 & 2.50193900 & -2.63451700 \\
\hline C & 3.86091500 & 2.06285600 & -2.75941500 & $\mathrm{H}$ & 4.38693800 & 3.76042300 & 0.70881400 \\
\hline C & 5.31134200 & 2.79865400 & -0.95952800 & C & -1.10936200 & 2.45804200 & -3.81899800 \\
\hline $\mathrm{H}$ & -1.81288800 & 3.35170700 & -1.99278800 & C & 1.26276700 & 1.93546600 & -3.93280100 \\
\hline C & 5.14103400 & 2.18366400 & -2.19604300 & C & 3.65084800 & 1.45879400 & -4.04681500 \\
\hline $\mathrm{H}$ & 6.30886000 & 2.90900100 & -0.54344100 & C & -0.01706600 & 1.93296100 & -4.50688600 \\
\hline $\mathrm{H}$ & -2.09537400 & 2.43124700 & -4.26765500 & C & 2.41121500 & 1.39923200 & -4.60667800 \\
\hline $\mathrm{H}$ & 6.00068800 & 1.81967800 & -2.75195100 & $\mathrm{H}$ & 4.51539200 & 1.06578200 & -4.57544800 \\
\hline $\bar{H}$ & -0.15024900 & 1.51110900 & -5.49979400 & $\mathrm{H}$ & 2.27148800 & 0.95447900 & -5.58891200 \\
\hline C & -2.49938600 & 1.32413800 & 1.91713300 & $S$ & -2.97674500 & 2.92638800 & 1.62731100 \\
\hline $\mathrm{N}$ & -1.47829800 & 0.98730200 & 2.74312600 & $\mathrm{H}$ & -1.04882500 & 0.06840700 & 2.59411600 \\
\hline C & -0.25340600 & -0.41880900 & -1.00996000 & $\mathrm{O}$ & -1.16746300 & 0.37672300 & -0.99118500 \\
\hline 0 & -0.18179400 & -1.57085300 & -1.69754600 & C & -1.30956500 & -2.09365300 & -2.50534400 \\
\hline C & -1.63582600 & -1.10650200 & -3.62147700 & $\mathrm{H}$ & -2.35878100 & -1.56314200 & -4.30592200 \\
\hline $\mathrm{H}$ & -2.06797600 & -0.18918800 & -3.22493200 & $\mathrm{H}$ & -0.73353100 & -0.85269800 & -4.18727000 \\
\hline C & -0.72975500 & -3.39420300 & -3.05567200 & $\mathrm{H}$ & -0.45057100 & -4.05681100 & -2.23105900 \\
\hline $\mathrm{H}$ & -1.47954300 & -3.90190500 & -3.67007700 & $\mathrm{H}$ & 0.15012100 & -3.19911300 & -3.68022300 \\
\hline C & -2.50311900 & -2.37214100 & -1.60480700 & $\mathrm{H}$ & -3.31234300 & -2.82183900 & -2.18881900 \\
\hline $\mathrm{H}$ & -2.23266600 & -3.05497800 & -0.79761700 & $\mathrm{H}$ & -2.87310900 & -1.44720500 & -1.17314700 \\
\hline C & 1.76425100 & -1.27044700 & 0.03029500 & $\mathrm{H}$ & 1.23920500 & -2.21855600 & 0.07528100 \\
\hline C & 2.72364400 & -1.03490300 & 1.13599800 & C & 2.82752500 & -1.98666900 & 2.15573800 \\
\hline C & 3.55910400 & 0.08765100 & 1.14507300 & C & 3.74566700 & -1.80247600 & 3.18849700 \\
\hline $\mathrm{H}$ & 2.16425200 & -2.84338100 & 2.15881000 & C & 4.48339800 & 0.26254000 & 2.17058900 \\
\hline $\mathrm{H}$ & 3.48136200 & 0.82891600 & 0.35917800 & C & 4.57687300 & -0.68100400 & 3.19714800 \\
\hline $\mathrm{H}$ & 3.80179900 & -2.53158300 & 3.99127700 & $\mathrm{H}$ & 5.12347500 & 1.14023500 & 2.16789000 \\
\hline $\mathrm{H}$ & 5.29265300 & -0.54077000 & 4.00231200 & $\mathrm{~N}$ & 0.93238500 & -0.21654600 & -0.30708400 \\
\hline $\mathrm{H}$ & 0.94878500 & 0.60686300 & 0.30577000 & $\mathrm{~S}$ & -1.00399900 & -2.78379300 & 1.90539300 \\
\hline 0 & -0.35785200 & -3.72650600 & 0.96583500 & $\mathrm{O}$ & -0.28837400 & -1.48385200 & 2.03409500 \\
\hline 0 & -2.47442200 & -2.63456700 & 1.79863800 & C & -0.75130300 & -3.55311200 & 3.57732000 \\
\hline $\mathrm{F}$ & -1.38751300 & -4.72345900 & 3.67376500 & $\mathrm{~F}$ & -1.21095900 & -2.73043300 & 4.53457400 \\
\hline $\mathrm{F}$ & 0.56075000 & -3.76145900 & 3.80218800 & C & 2.81664100 & -1.56168600 & -1.51659900 \\
\hline $\mathrm{H}$ & 2.00908900 & -1.58845400 & -2.23983000 & $\mathrm{H}$ & 3.42623900 & -0.66272700 & -1.53689100 \\
\hline C & 3.44034300 & -2.77590100 & -1.19669800 & C & 4.75758200 & -2.81111000 & -0.58389600 \\
\hline $\mathrm{H}$ & 4.98750500 & -1.88101100 & -0.05202900 & $\mathrm{H}$ & 4.89565900 & -3.67194900 & 0.07908100 \\
\hline C & 2.67248800 & -4.04738000 & -1.33097000 & $\mathrm{H}$ & 3.32645200 & -4.92219700 & -1.36157500 \\
\hline $\mathrm{H}$ & 2.02444000 & -4.15154500 & -0.44312400 & $\mathrm{H}$ & 2.00587100 & -4.03994400 & -2.19599100 \\
\hline C & 7.76012200 & -3.01338500 & -1.02427000 & $\mathrm{H}$ & 7.80729800 & -3.89838300 & -0.37986000 \\
\hline $\mathrm{H}$ & 8.60430900 & -3.06342200 & -1.72210400 & $\mathrm{H}$ & 7.90020400 & -2.12892300 & -0.39272000 \\
\hline $\mathrm{C}$ & 5.84917000 & -4.48017700 & -2.99060600 & $\mathrm{H}$ & 5.83932700 & -5.38392700 & -2.37039900 \\
\hline $\mathrm{H}$ & 4.90162500 & -4.43697300 & -3.53920100 & $\mathrm{H}$ & 6.65151700 & -4.59733700 & -3.72863700 \\
\hline C & 6.02015200 & -1.37741300 & -3.01400200 & $\mathrm{H}$ & 5.07403900 & -1.32229400 & -3.56261100 \\
\hline $\mathrm{H}$ & 6.09168400 & -0.47710500 & -2.39464800 & $\mathrm{H}$ & 6.83589500 & -1.34836600 & -3.74608800 \\
\hline $\mathrm{Si}$ & 6.12947800 & -2.92944300 & -1.96071200 & & & & \\
\hline
\end{tabular}

\section{TS-S-4f-30}

Imaginary frequency: $-331.51 \mathrm{~cm}^{-1}$ E[B3LYP/6-31G(d)-D3(BJ)]: -4789.080988 Hartree $\mathrm{E}\left[\mathrm{PCM}\left(\mathrm{Et}_{2} \mathrm{O}\right)-\mathrm{B} 3 \mathrm{LYP} / 6-311 \mathrm{G}(\mathrm{d}, \mathrm{p})-\mathrm{D} 3(\mathrm{BJ})\right]$ : -4790.223649 Hartree

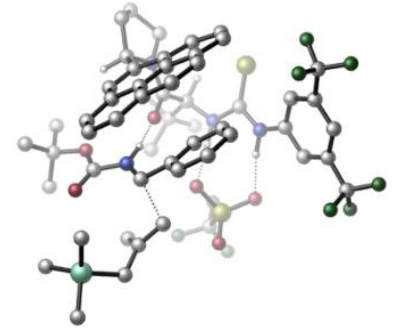




\begin{tabular}{|c|c|c|c|c|c|c|c|}
\hline 01 & & & & $\mathrm{~N}$ & 2.78345500 & 1.59277400 & 1.38293600 \\
\hline $\mathrm{H}$ & 2.23291600 & 2.43287300 & 1.16745400 & C & 3.82912900 & 1.38241600 & 0.47699000 \\
\hline $\mathrm{C}$ & 4.08664800 & 2.42699400 & -0.42765600 & $\mathrm{C}$ & 4.54306000 & 0.18171900 & 0.34801900 \\
\hline C & 5.02650500 & 2.26143300 & -1.43963000 & $\mathrm{H}$ & 3.52913800 & 3.35358600 & -0.34623600 \\
\hline C & 5.45722800 & 0.03086200 & -0.69161600 & $\mathrm{H}$ & 4.36303300 & -0.62781100 & 1.03807000 \\
\hline $\mathrm{C}$ & 5.71986400 & 1.06143900 & -1.59175800 & $\mathrm{H}$ & 6.44608000 & 0.93704300 & -2.38553200 \\
\hline $\mathrm{C}$ & 5.23855300 & 3.36583000 & -2.43909100 & $\mathrm{C}$ & 6.08737200 & -1.31137000 & -0.92428500 \\
\hline $\mathrm{F}$ & 6.50620700 & 3.37107000 & -2.91333200 & $\mathrm{~F}$ & 4.99551900 & 4.58236000 & -1.91255100 \\
\hline$F$ & 4.42290100 & 3.22522600 & -3.51044700 & $F$ & 6.08898300 & -2.09164500 & 0.17806500 \\
\hline$F$ & 5.41047200 & -2.00724100 & -1.88618100 & $F$ & 7.36138000 & -1.21301900 & -1.35699800 \\
\hline C & 0.30776700 & 0.40252300 & 3.76399500 & $\mathrm{H}$ & 1.02709900 & 0.03676400 & 4.49501400 \\
\hline C & -0.75869900 & 1.25866400 & 4.51914700 & C & -1.88480200 & 1.77655700 & 3.60593000 \\
\hline $\mathrm{H}$ & -2.45972300 & 0.95545800 & 3.17087900 & $\mathrm{H}$ & -1.50720400 & 2.37850300 & 2.78039700 \\
\hline $\mathrm{H}$ & -2.56250800 & 2.40409000 & 4.19725100 & C & -0.02927800 & 2.44648800 & 5.16936900 \\
\hline $\mathrm{H}$ & -0.73038000 & 3.02949700 & 5.77747600 & $\mathrm{H}$ & 0.39965000 & 3.10855800 & 4.41498000 \\
\hline $\mathrm{H}$ & 0.78259400 & 2.10211100 & 5.82076700 & $\mathrm{C}$ & -1.38832400 & 0.39036700 & 5.62366300 \\
\hline $\mathrm{H}$ & -2.14854500 & 0.96691900 & 6.16184000 & $\mathrm{H}$ & -0.64166800 & 0.06708600 & 6.35858800 \\
\hline $\mathrm{H}$ & -1.87990100 & -0.49986300 & 5.21292300 & C & -0.31929800 & -0.76582300 & 2.99068800 \\
\hline $\mathrm{O}$ & -0.91123000 & -0.51820200 & 1.93210700 & C & 0.42381000 & -2.46907700 & 4.70043200 \\
\hline C & -0.91747800 & -3.13333000 & 2.74998300 & $\mathrm{C}$ & -0.25103800 & -3.81499100 & 4.98218700 \\
\hline $\mathrm{H}$ & 1.50443100 & -2.56839600 & 4.54656300 & $\mathrm{H}$ & 0.26463700 & -1.74850800 & 5.50091000 \\
\hline $\mathrm{H}$ & -1.99336400 & -2.94841200 & 2.85793100 & $\mathrm{H}$ & -1.19515400 & -3.65188600 & 5.51581000 \\
\hline $\mathrm{H}$ & 0.37439200 & -4.47043700 & 5.59415200 & $\mathrm{~N}$ & -0.22331600 & -2.02602600 & 3.45414600 \\
\hline C & -0.65662800 & -3.29544600 & 1.26339700 & C & -1.74575100 & -3.37856700 & 0.44590500 \\
\hline $\mathrm{C}$ & 0.66961100 & -3.48553300 & 0.70619900 & $\mathrm{C}$ & -1.65114800 & -3.66696700 & -0.95236100 \\
\hline $\mathrm{H}$ & -2.73463600 & -3.20320200 & 0.86065500 & $\mathrm{C}$ & 0.78406100 & -3.88427700 & -0.66446300 \\
\hline C & 1.85041100 & -3.27213100 & 1.43487400 & C & -2.77016700 & -3.64520400 & -1.79949900 \\
\hline C & -0.37077400 & -3.97559200 & -1.49352400 & C & 2.07074600 & -4.13503200 & -1.23064900 \\
\hline $\mathrm{H}$ & 1.80503400 & -2.89288400 & 2.44479500 & $\mathrm{C}$ & 3.10342000 & -3.47214500 & 0.85710900 \\
\hline C & -2.63752800 & -3.94744900 & -3.15413500 & $\mathrm{H}$ & -3.74203600 & -3.38081900 & -1.39056800 \\
\hline $\mathrm{C}$ & -0.24975900 & -4.32108000 & -2.87266900 & C & 3.21824800 & -3.92299600 & -0.45278700 \\
\hline C & 2.15669300 & -4.54885800 & -2.60280100 & $\mathrm{H}$ & 3.99512000 & -3.25873200 & 1.43702900 \\
\hline C & -1.39656400 & -4.29929800 & -3.68234000 & $\mathrm{H}$ & -3.51064200 & -3.91800200 & -3.79997900 \\
\hline $\mathrm{C}$ & 1.05005800 & -4.64056900 & -3.38895600 & $\mathrm{H}$ & 4.19761700 & -4.06837000 & -0.89665700 \\
\hline $\mathrm{H}$ & 3.14140000 & -4.76361200 & -3.00962800 & $\mathrm{H}$ & -1.30298600 & -4.54895800 & -4.73611700 \\
\hline $\mathrm{H}$ & 1.13519300 & -4.93247300 & -4.43251800 & C & 2.32537900 & 0.83024800 & 2.42545600 \\
\hline $\mathrm{S}$ & 3.21426600 & -0.37337100 & 3.21642100 & $\mathrm{~N}$ & 1.06361900 & 1.18516000 & 2.79943100 \\
\hline $\mathrm{H}$ & 0.49790000 & 1.66998900 & 2.09401200 & $\mathrm{~S}$ & 0.02618900 & 3.81489300 & 0.26258700 \\
\hline 0 & -0.44034200 & 4.26659000 & -1.05469300 & $\mathrm{O}$ & -0.46245100 & 2.45682200 & 0.66547500 \\
\hline 0 & 1.46480500 & 4.01432400 & 0.57684400 & $\mathrm{C}$ & -0.83679300 & 4.94951600 & 1.45572000 \\
\hline $\mathrm{F}$ & -0.58941000 & 6.22757100 & 1.16346700 & $\mathrm{~F}$ & -0.43698000 & 4.71214000 & 2.71668300 \\
\hline $\mathrm{F}$ & -2.16943400 & 4.74830700 & 1.40267300 & C & -3.73437700 & -0.54683800 & -0.07909100 \\
\hline $\mathrm{O}$ & -4.61276400 & -0.50749800 & -0.92798200 & $\mathrm{O}$ & -3.88490400 & -0.75392400 & 1.23269700 \\
\hline $\mathrm{C}$ & -5.21691100 & -0.90180800 & 1.84604000 & $\mathrm{C}$ & -5.99426900 & 0.40404400 & 1.68358700 \\
\hline $\mathrm{H}$ & -6.92424700 & 0.35005000 & 2.25900300 & $\mathrm{H}$ & -6.24590200 & 0.58956600 & 0.63822200 \\
\hline $\mathrm{H}$ & -5.40413700 & 1.24415600 & 2.06349800 & $\mathrm{C}$ & -4.87229100 & -1.15396000 & 3.31312200 \\
\hline $\mathrm{H}$ & -4.26328500 & -2.05810100 & 3.41382700 & $\mathrm{H}$ & -5.79009300 & -1.28669400 & 3.89420200 \\
\hline $\mathrm{H}$ & -4.31222400 & -0.31191800 & 3.72900200 & C & -5.94483000 & -2.10282000 & 1.24132500 \\
\hline $\mathrm{H}$ & -6.87619900 & -2.27546100 & 1.79080000 & $\mathrm{H}$ & -5.32894200 & -3.00460300 & 1.32827000 \\
\hline $\mathrm{H}$ & -6.18236200 & -1.93541000 & 0.19036700 & $\mathrm{C}$ & -1.95403400 & -0.24906000 & -1.65206300 \\
\hline $\mathrm{H}$ & -2.68533100 & -0.55866200 & -2.38582000 & C & -0.55202900 & -0.55751700 & -1.95982300 \\
\hline $\mathrm{C}$ & -0.23195200 & -0.95203400 & -3.26857000 & C & 0.46501000 & -0.44031200 & -1.00054700 \\
\hline C & 1.08474200 & -1.23745000 & -3.61217300 & $\bar{H}$ & -1.02453800 & -1.05521300 & -4.00453200 \\
\hline
\end{tabular}




\begin{tabular}{|c|c|c|c|c|c|c|c|}
\hline $\mathrm{C}$ & 1.77239400 & -0.74951200 & -1.34846100 & $\mathrm{H}$ & 0.24438100 & -0.11212700 & 0.00720000 \\
\hline C & 2.09081800 & -1.13902000 & -2.64972700 & $\mathrm{H}$ & 1.32279100 & -1.55876600 & -4.62113300 \\
\hline $\mathrm{H}$ & 2.54736600 & -0.70398900 & -0.59806000 & $\mathrm{H}$ & 3.11958200 & -1.37913100 & -2.89657900 \\
\hline $\mathrm{N}$ & -2.39477000 & -0.38921400 & -0.36405700 & $\mathrm{H}$ & -1.74913400 & -0.42193600 & 0.43445900 \\
\hline C & -1.90745000 & 1.72714700 & -2.16195400 & $\mathrm{H}$ & -1.22027800 & 1.94432000 & -1.35217900 \\
\hline $\mathrm{H}$ & -1.46574900 & 1.65613500 & -3.15166100 & C & -3.18699300 & 2.25893700 & -2.05236700 \\
\hline C & -4.01632300 & 2.54961800 & -3.23319300 & $\mathrm{H}$ & -3.38438500 & 2.71252600 & -4.11380100 \\
\hline $\mathrm{H}$ & -4.59609300 & 3.46549300 & -3.05650600 & C & -3.69610000 & 2.63704000 & -0.70110500 \\
\hline $\mathrm{H}$ & -4.78560500 & 2.58012900 & -0.63389400 & $\mathrm{H}$ & -3.38872900 & 3.67618300 & -0.51694900 \\
\hline $\mathrm{H}$ & -3.22265600 & 2.05247400 & 0.09169500 & C & -6.00117500 & 2.10286600 & -5.43082400 \\
\hline $\mathrm{H}$ & -6.39622700 & 3.10935500 & -5.25013400 & $\mathrm{H}$ & -6.80976700 & 1.50412400 & -5.86706500 \\
\hline $\mathrm{H}$ & -5.20772900 & 2.18484700 & -6.18286200 & C & -6.77121200 & 1.19441000 & -2.58575300 \\
\hline $\mathrm{H}$ & -7.16771600 & 2.18673100 & -2.34089700 & $\mathrm{H}$ & -6.42826700 & 0.70883300 & -1.66952400 \\
\hline $\mathrm{H}$ & -7.59833700 & 0.60016100 & -2.99317900 & C & -4.63528000 & -0.38608600 & -4.23319100 \\
\hline $\mathrm{H}$ & -4.51474400 & -0.97853100 & -3.32290300 & $\mathrm{H}$ & -3.66306600 & -0.29874900 & -4.73303500 \\
\hline $\mathrm{H}$ & -5.30048400 & -0.93548300 & -4.91045000 & $\mathrm{Si}$ & -5.37326900 & 1.30373100 & -3.83865500 \\
\hline $\mathrm{C}$ & -0.51985800 & -4.37268900 & 3.57989300 & $\mathrm{H}$ & 0.38926200 & -4.81716800 & 3.16438200 \\
\hline $\mathrm{H}$ & -1.30522200 & -5.13219700 & 3.55202900 & & & & \\
\hline
\end{tabular}

\section{TS-S-4f-31}

Imaginary frequency: $-329.60 \mathrm{~cm}^{-1}$

E[B3LYP/6-31G(d)-D3(BJ)]: -4789.081006 Hartree

$\mathrm{E}\left[\mathrm{PCM}\left(\mathrm{Et}_{2} \mathrm{O}\right)-\mathrm{B} 3 \mathrm{LYP} / 6-311 \mathrm{G}(\mathrm{d}, \mathrm{p})-\mathrm{D} 3(\mathrm{BJ})\right]$ : -4790.223620 Hartree

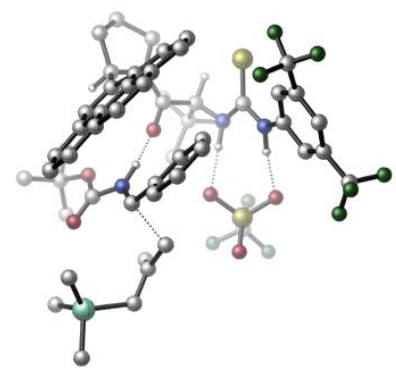

\begin{tabular}{|lrrr|rrrr|}
\hline 01 & & & & $\mathrm{~N}$ & 2.78988600 & 1.58239800 & 1.35274300 \\
\hline $\mathrm{H}$ & 2.24625000 & 2.42495200 & 1.12947800 & $\mathrm{C}$ & 3.83459500 & 1.35542900 & 0.44952400 \\
\hline $\mathrm{C}$ & 4.09349200 & 2.38517700 & -0.47164400 & $\mathrm{C}$ & 4.54258700 & 0.14965700 & 0.33590000 \\
\hline $\mathrm{C}$ & 5.02877000 & 2.20023100 & -1.48462700 & $\mathrm{H}$ & 3.53094700 & 3.31032800 & -0.41056700 \\
\hline $\mathrm{C}$ & 5.45078400 & -0.02136200 & -0.70586300 & $\mathrm{H}$ & 4.35900400 & -0.64993900 & 1.03650300 \\
\hline $\mathrm{C}$ & 5.71465400 & 0.99419500 & -1.62253300 & $\mathrm{H}$ & 6.42511300 & 0.84881000 & -2.42684700 \\
\hline $\mathrm{C}$ & 5.33699400 & 3.33506100 & -2.42318800 & $\mathrm{C}$ & 6.06533500 & -1.37270800 & -0.92702700 \\
\hline $\mathrm{F}$ & 5.69562100 & 2.88348200 & -3.64835500 & $\mathrm{~F}$ & 6.36656200 & 4.08717700 & -1.96890600 \\
\hline $\mathrm{F}$ & 4.28445600 & 4.16022200 & -2.58609100 & $\mathrm{~F}$ & 6.06746000 & -2.13926600 & 0.18486500 \\
\hline $\mathrm{F}$ & 5.37442800 & -2.07365000 & -1.87525600 & $\mathrm{~F}$ & 7.33697000 & -1.29262800 & -1.37046400 \\
\hline $\mathrm{C}$ & 0.31034200 & 0.44089100 & 3.75335900 & $\mathrm{H}$ & 1.02808100 & 0.07830000 & 4.48752100 \\
\hline $\mathrm{C}$ & -0.74833500 & 1.31389700 & 4.50015900 & $\mathrm{C}$ & -1.87228300 & 1.82914400 & 3.58279200 \\
\hline $\mathrm{H}$ & -2.45444100 & 1.00715000 & 3.15918500 & $\mathrm{H}$ & -1.49181600 & 2.41779600 & 2.74902400 \\
\hline $\mathrm{H}$ & -2.54390100 & 2.46920100 & 4.16758800 & $\mathrm{C}$ & -0.00882800 & 2.50386600 & 5.13489500 \\
\hline $\mathrm{H}$ & -0.70443300 & 3.09941300 & 5.73713400 & $\mathrm{H}$ & 0.42385000 & 3.15360700 & 4.37199400 \\
\hline $\mathrm{H}$ & 0.80152200 & 2.16110200 & 5.78903000 & $\mathrm{C}$ & -1.38237000 & 0.46359800 & 5.61608800 \\
\hline $\mathrm{H}$ & -2.13743100 & 1.05215500 & 6.14849900 & $\mathrm{H}$ & -0.63682500 & 0.14375000 & 6.35363700 \\
\hline $\mathrm{H}$ & -1.88115300 & -0.42788500 & 5.21692100 & $\mathrm{C}$ & -0.32689300 & -0.73162800 & 2.99476500 \\
\hline $\mathrm{O}$ & -0.91815700 & -0.49175100 & 1.93400300 & $\mathrm{C}$ & 0.40441200 & -2.42051500 & 4.72368600 \\
\hline $\mathrm{C}$ & -0.94446300 & -3.09691600 & 2.78266500 & $\mathrm{C}$ & -0.28128600 & -3.75731600 & 5.02222600 \\
\hline $\mathrm{H}$ & 1.48398300 & -2.53069000 & 4.56977000 & $\mathrm{H}$ & 0.25224600 & -1.68910300 & 5.51563000 \\
\hline $\mathrm{H}$ & -2.01868900 & -2.90191400 & 2.88933500 & $\mathrm{H}$ & -1.22344100 & -3.57997600 & 5.55477800 \\
\hline $\mathrm{H}$ & 0.33933900 & -4.41056000 & 5.64139400 & $\mathrm{~N}$ & -0.24054400 & -1.98700300 & 3.47294000 \\
\hline $\mathrm{C}$ & -0.68635000 & -3.27889900 & 1.29788300 & $\mathrm{C}$ & -1.77682100 & -3.36250400 & 0.48222800 \\
\hline $\mathrm{C}$ & 0.63775200 & -3.48680800 & 0.74199100 & $\mathrm{C}$ & -1.68581500 & -3.66814300 & -0.91263400 \\
\hline $\mathrm{H}$ & -2.76385500 & -3.17398200 & 0.89560100 & $\mathrm{C}$ & 0.74770200 & -3.90260300 & -0.62395700 \\
\hline
\end{tabular}

S130 


\begin{tabular}{|c|c|c|c|c|c|c|c|}
\hline C & 1.82089800 & -3.27482300 & 1.46724600 & C & -2.80530600 & -3.64694600 & -1.75916600 \\
\hline $\mathrm{C}$ & -0.40852600 & -3.99383700 & -1.45106900 & C & 2.03175100 & -4.17092700 & -1.18804300 \\
\hline $\mathrm{H}$ & 1.77951500 & -2.88319300 & 2.47258400 & C & 3.07170500 & -3.49225300 & 0.89100900 \\
\hline C & -2.67631500 & -3.96612700 & -3.11027000 & $\mathrm{H}$ & -3.77461100 & -3.36972400 & -1.35266100 \\
\hline C & -0.29152700 & -4.35640800 & -2.82618100 & C & 3.18162500 & -3.95960000 & -0.41350200 \\
\hline C & 2.11312600 & -4.60137200 & -2.55534600 & $\mathrm{H}$ & 3.96566600 & -3.27941300 & 1.46766500 \\
\hline C & -1.43876700 & -4.33445800 & -3.63523000 & $\mathrm{H}$ & -3.54970400 & -3.93699500 & -3.75575900 \\
\hline C & 1.00513400 & -4.69283800 & -3.33962500 & $\mathrm{H}$ & 4.15934700 & -4.11858500 & -0.85630000 \\
\hline $\mathrm{H}$ & 3.09566100 & -4.82919400 & -2.96030900 & $\mathrm{H}$ & -1.34815500 & -4.59724200 & -4.68607300 \\
\hline $\mathrm{H}$ & 1.08696500 & -4.99762200 & -4.37976400 & C & 2.32797900 & 0.83612600 & 2.40518200 \\
\hline $\mathrm{S}$ & 3.20785700 & -0.36637300 & 3.20792800 & $\mathrm{~N}$ & 1.07039900 & 1.20651500 & 2.77849500 \\
\hline $\mathrm{H}$ & 0.50703800 & 1.68813000 & 2.06900800 & $S$ & 0.04941800 & 3.81616100 & 0.21600300 \\
\hline $\mathrm{O}$ & -0.41360200 & 4.25698600 & -1.10618800 & $\mathrm{O}$ & -0.44925700 & 2.46599500 & 0.63315300 \\
\hline $\mathrm{O}$ & 1.48929400 & 4.00829500 & 0.52900600 & C & -0.80542200 & 4.96993600 & 1.39664900 \\
\hline $\mathrm{F}$ & -0.54856800 & 6.24287100 & 1.09057000 & $\mathrm{~F}$ & -0.40757500 & 4.74332700 & 2.66019100 \\
\hline $\mathrm{F}$ & -2.13953600 & 4.77804300 & 1.34556000 & C & -3.74418600 & -0.52135400 & -0.07299000 \\
\hline $\mathrm{O}$ & -4.62298700 & -0.48471300 & -0.92155000 & $\mathrm{O}$ & -3.89505700 & -0.71203500 & 1.24120600 \\
\hline C & -5.22760400 & -0.84258600 & 1.85742800 & C & -5.99498500 & 0.46733300 & 1.68091800 \\
\hline $\mathrm{H}$ & -6.92475700 & 0.42700100 & 2.25778200 & $\mathrm{H}$ & -6.24628300 & 0.64299000 & 0.63377000 \\
\hline $\mathrm{H}$ & -5.39803900 & 1.30710700 & 2.05079900 & C & -4.88346900 & -1.08078500 & 3.32695100 \\
\hline $\mathrm{H}$ & -4.28130000 & -1.98837100 & 3.43732200 & $\mathrm{H}$ & -5.80169300 & -1.19992400 & 3.91029900 \\
\hline $\mathrm{H}$ & -4.31659600 & -0.23840700 & 3.73281500 & C & -5.96536600 & -2.04468300 & 1.26699600 \\
\hline $\mathrm{H}$ & -6.89747400 & -2.20391500 & 1.81925500 & $\mathrm{H}$ & -5.35634600 & -2.95014000 & 1.36353800 \\
\hline $\mathrm{H}$ & -6.20267400 & -1.88732000 & 0.21443800 & C & -1.96300100 & -0.25606200 & -1.65072100 \\
\hline $\mathrm{H}$ & -2.69766200 & -0.56754800 & -2.38030500 & C & -0.56396900 & -0.57966600 & -1.95657300 \\
\hline C & -0.24923900 & -0.99195600 & -3.26113200 & C & 0.45546200 & -0.46030200 & -1.00006600 \\
\hline C & 1.06439600 & -1.29292600 & -3.60315800 & $\mathrm{H}$ & -1.04379000 & -1.09684600 & -3.99473200 \\
\hline C & 1.75968400 & -0.78473700 & -1.34618700 & $\mathrm{H}$ & 0.23913500 & -0.11898200 & 0.00424300 \\
\hline C & 2.07272900 & -1.19217400 & -2.64329700 & $\mathrm{H}$ & 1.29807600 & -1.62838500 & -4.60854600 \\
\hline $\mathrm{H}$ & 2.53615300 & -0.73729600 & -0.59737500 & $\mathrm{H}$ & 3.09888600 & -1.44441400 & -2.88873000 \\
\hline $\mathrm{N}$ & -2.40354800 & -0.37793300 & -0.36091300 & $\mathrm{H}$ & -1.75743300 & -0.40679400 & 0.43741400 \\
\hline C & -1.90054500 & 1.71436700 & -2.18288700 & $\mathrm{H}$ & -1.21112400 & 1.93486700 & -1.37587900 \\
\hline $\mathrm{H}$ & -1.46017700 & 1.62854000 & -3.17201700 & C & -3.17575200 & 2.25743900 & -2.07855300 \\
\hline $\mathrm{C}$ & -4.00357300 & 2.54134400 & -3.26210000 & $\mathrm{H}$ & -3.37109100 & 2.68824700 & -4.14512700 \\
\hline $\bar{H}$ & -4.57496600 & 3.46440900 & -3.09578800 & C & -3.68091200 & 2.65488500 & -0.73139600 \\
\hline $\mathrm{H}$ & -4.77084900 & 2.60874600 & -0.66332200 & $\mathrm{H}$ & -3.36387700 & 3.69306600 & -0.55866000 \\
\hline $\bar{H}$ & -3.21261900 & 2.07469200 & 0.06766500 & C & -5.99549400 & 2.08684900 & -5.45173100 \\
\hline $\bar{H}$ & -6.38260900 & 3.09828200 & -5.28178900 & $\mathrm{H}$ & -6.80910100 & 1.48959000 & -5.88061800 \\
\hline $\mathrm{H}$ & -5.20219500 & 2.15441600 & -6.20535500 & C & -6.76926300 & 1.21733400 & -2.59559400 \\
\hline $\mathrm{H}$ & -7.15559400 & 2.21594300 & -2.36021400 & $\mathrm{H}$ & -6.42987400 & 0.73764600 & -1.67495300 \\
\hline $\mathrm{H}$ & -7.60279500 & 0.62727900 & -2.99599300 & C & -4.64899900 & -0.39984600 & -4.22802600 \\
\hline $\mathrm{H}$ & -4.53187100 & -0.98307000 & -3.31137500 & $\mathrm{H}$ & -3.67690800 & -0.32623000 & -4.73032300 \\
\hline $\mathrm{H}$ & -5.31985300 & -0.95124600 & -4.89804200 & $\mathrm{Si}$ & -5.37216500 & 1.30046000 & -3.85143900 \\
\hline C & -0.55616800 & -4.32949200 & 3.62697800 & $\mathrm{H}$ & 0.34886000 & -4.78631900 & 3.21598500 \\
\hline $\mathrm{H}$ & -1.34775800 & -5.08280600 & 3.60893000 & & & & \\
\hline
\end{tabular}

\section{TS-S-4f-32}

Imaginary frequency: $-328.27 \mathrm{~cm}-1$

E[B3LYP/6-31G(d)-D3(BJ)]: -4789.076605 Hartree

E[PCM(Et $\left.\left.{ }_{2} \mathrm{O}\right)-\mathrm{B} 3 L Y P / 6-311 \mathrm{G}(\mathrm{d}, \mathrm{p})-\mathrm{D} 3(\mathrm{BJ})\right]$ : -4790.223176 Hartree

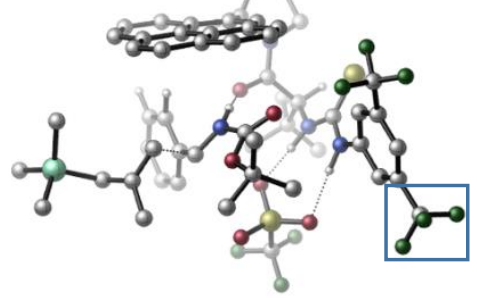




\begin{tabular}{|c|c|c|c|c|c|c|c|}
\hline 01 & & & & $\mathrm{~N}$ & -3.00756600 & 0.17646100 & 1.34708000 \\
\hline $\mathrm{H}$ & -2.69102800 & -0.72101700 & 1.71985100 & C & -4.00178800 & 0.05375300 & 0.36728700 \\
\hline C & -4.89453600 & -1.01570400 & 0.48713900 & C & -4.05177800 & 0.87262400 & -0.76803300 \\
\hline C & -5.82839300 & -1.26322700 & -0.51904100 & $\mathrm{H}$ & -4.82388000 & -1.67749500 & 1.34266700 \\
\hline C & -4.99158400 & 0.61084000 & -1.75837100 & $\mathrm{H}$ & -3.32712100 & 1.66106300 & -0.88797300 \\
\hline C & -5.89238000 & -0.45094800 & -1.64728300 & $\mathrm{H}$ & -6.61510000 & -0.64762500 & -2.42923100 \\
\hline $\mathrm{C}$ & -6.80567000 & -2.39248000 & -0.35299100 & C & -4.95489100 & 1.39982400 & -3.03341000 \\
\hline $\mathrm{F}$ & -7.89045400 & -2.01553300 & 0.36418400 & $\mathrm{~F}$ & -6.25537200 & -3.44280600 & 0.29233800 \\
\hline $\mathrm{F}$ & -7.26218000 & -2.84210000 & -1.54509300 & $\mathrm{~F}$ & -4.42712900 & 2.63440700 & -2.86877100 \\
\hline $\mathrm{F}$ & -4.19287800 & 0.77908900 & -3.97847900 & $\mathrm{~F}$ & -6.18140500 & 1.55348100 & -3.57709800 \\
\hline C & -0.55304900 & 2.07438200 & 3.24777600 & $\mathrm{H}$ & -1.25965700 & 2.86341500 & 3.50485600 \\
\hline C & 0.09062900 & 1.55689600 & 4.57503400 & C & 1.18506000 & 0.49818000 & 4.35383700 \\
\hline $\mathrm{H}$ & 2.02300000 & 0.89078700 & 3.77486400 & $\mathrm{H}$ & 0.80831100 & -0.38321500 & 3.83198500 \\
\hline $\mathrm{H}$ & 1.56460900 & 0.17006800 & 5.32932600 & C & -1.02840900 & 0.95339800 & 5.44255900 \\
\hline $\mathrm{H}$ & -0.63739600 & 0.70932800 & 6.43712500 & $\mathrm{H}$ & -1.42103100 & 0.03356300 & 5.00135000 \\
\hline $\mathrm{H}$ & -1.86184600 & 1.65492500 & 5.56626900 & C & 0.70553300 & 2.76070800 & 5.31112300 \\
\hline $\mathrm{H}$ & 1.18086400 & 2.42792900 & 6.24020200 & $\mathrm{H}$ & -0.05726900 & 3.50205700 & 5.57808900 \\
\hline $\mathrm{H}$ & 1.47417300 & 3.25665900 & 4.70672000 & C & 0.48597400 & 2.60675700 & 2.25252000 \\
\hline 0 & 1.20711700 & 1.81375700 & 1.62858800 & C & -0.14830800 & 5.01957600 & 2.71036400 \\
\hline C & 1.63131900 & 4.44392800 & 1.12088800 & C & 0.74528700 & 6.24375500 & 2.48609800 \\
\hline $\mathrm{H}$ & -1.12617200 & 5.11807600 & 2.22537100 & $\mathrm{H}$ & -0.31081400 & 4.81410900 & 3.76806100 \\
\hline $\mathrm{H}$ & 2.60745900 & 4.22733500 & 1.56147000 & $\mathrm{H}$ & 1.51971600 & 6.28641800 & 3.26146400 \\
\hline $\mathrm{H}$ & 0.18172200 & 7.18024500 & 2.51824100 & $\mathrm{~N}$ & 0.61486200 & 3.93712200 & 2.07132400 \\
\hline C & 1.53357700 & 3.79645500 & -0.24847600 & C & 0.31065300 & 3.68618900 & -0.84328000 \\
\hline C & 2.71052500 & 3.27508900 & -0.91114300 & C & 0.12663900 & 3.04754400 & -2.10862800 \\
\hline $\mathrm{H}$ & -0.57743800 & 4.03450100 & -0.32180000 & C & 2.54137700 & 2.55784800 & -2.14004600 \\
\hline C & 4.01543100 & 3.41603400 & -0.40284800 & C & -1.12943000 & 2.96939200 & -2.72829600 \\
\hline $\mathrm{C}$ & 1.25341700 & 2.44887200 & -2.73986600 & C & 3.67086200 & 1.96335300 & -2.78619200 \\
\hline $\mathrm{H}$ & 4.18700100 & 3.97748400 & 0.50767400 & C & 5.11208700 & 2.84948600 & -1.04907400 \\
\hline C & -1.27897900 & 2.32058300 & -3.95015200 & $\mathrm{H}$ & -1.99170400 & 3.40624200 & -2.23483400 \\
\hline C & 1.08579200 & 1.75581800 & -3.97612200 & C & 4.94622100 & 2.11873200 & -2.22169400 \\
\hline$C$ & 3.46691600 & 1.23744300 & -4.01029100 & $\mathrm{H}$ & 6.10593400 & 2.98252400 & -0.63072100 \\
\hline C & -0.18575500 & 1.71449900 & -4.56590100 & $\mathrm{H}$ & -2.25909300 & 2.26062900 & -4.40781400 \\
\hline C & 2.23340700 & 1.13817600 & -4.57755200 & $\mathrm{H}$ & 5.80628300 & 1.69094000 & -2.72940900 \\
\hline $\mathrm{H}$ & 4.33208100 & 0.78342600 & -4.48637500 & $\mathrm{H}$ & -0.31259600 & 1.19489600 & -5.51204300 \\
\hline $\mathrm{H}$ & 2.09853600 & 0.59980600 & -5.51254200 & C & -2.39207600 & 1.31042600 & 1.79930800 \\
\hline$S$ & -2.94550000 & 2.88147400 & 1.48476300 & $\mathrm{~N}$ & -1.32512500 & 1.04038800 & 2.58882400 \\
\hline $\mathrm{H}$ & -0.88797700 & 0.12065700 & 2.48726400 & C & -0.20196000 & -0.46639300 & -1.04327800 \\
\hline $\mathrm{O}$ & -1.10685200 & 0.33491000 & -1.13054600 & $\mathrm{O}$ & -0.13183200 & -1.68599500 & -1.60267200 \\
\hline C & -1.22406700 & -2.26263100 & -2.41913600 & C & -1.42333100 & -1.40664400 & -3.66640900 \\
\hline $\mathrm{H}$ & -2.11104500 & -1.91753200 & -4.34897100 & $\mathrm{H}$ & -1.84599300 & -0.43484100 & -3.41327800 \\
\hline $\mathrm{H}$ & -0.47101400 & -1.25286000 & -4.18505800 & C & -0.66674900 & -3.64294300 & -2.75878200 \\
\hline $\mathrm{H}$ & -0.45946700 & -4.19613100 & -1.83808800 & $\mathrm{H}$ & -1.39859300 & -4.20331700 & -3.34854400 \\
\hline $\mathrm{H}$ & 0.25384800 & -3.55891700 & -3.34857700 & C & -2.49931200 & -2.38390700 & -1.59719600 \\
\hline $\mathrm{H}$ & -3.25052100 & -2.92671200 & -2.18096700 & $\mathrm{H}$ & -2.32147600 & -2.92792400 & -0.66767800 \\
\hline $\mathrm{H}$ & -2.89644600 & -1.40068900 & -1.36366100 & C & 1.82771500 & -1.22199400 & 0.05379800 \\
\hline $\mathrm{H}$ & 1.32059900 & -2.17332300 & 0.16367600 & C & 2.79969200 & -0.90695800 & 1.12886500 \\
\hline C & 2.97322600 & -1.82118200 & 2.17407100 & C & 3.59746100 & 0.24087900 & 1.07101400 \\
\hline C & 3.92746200 & -1.57832100 & 3.16082100 & $\mathrm{H}$ & 2.33745200 & -2.69696300 & 2.23134400 \\
\hline C & 4.55577900 & 0.47606600 & 2.05276200 & $\mathrm{H}$ & 3.46668100 & 0.95352800 & 0.26672400 \\
\hline C & 4.72302900 & -0.43273000 & 3.10077600 & $\mathrm{H}$ & 4.04013400 & -2.28025200 & 3.98174400 \\
\hline $\mathrm{H}$ & 5.16440800 & 1.37403000 & 1.99818100 & $\mathrm{H}$ & 5.46682400 & -0.24609300 & 3.87035900 \\
\hline $\mathrm{N}$ & 0.97089800 & -0.20661100 & -0.33803400 & $\mathrm{H}$ & 0.98750400 & 0.66885900 & 0.19796500 \\
\hline
\end{tabular}




\begin{tabular}{|c|c|c|c|c|c|c|c|}
\hline $\mathrm{S}$ & -0.85700500 & -2.80709500 & 1.95502900 & $\mathrm{O}$ & -0.18340300 & -3.76652500 & 1.05290300 \\
\hline 0 & -0.17177100 & -1.48677200 & 2.04326300 & $\mathrm{O}$ & -2.32852500 & -2.69280100 & 1.83158600 \\
\hline $\mathrm{C}$ & -0.58976100 & -3.50575000 & 3.65478900 & $\mathrm{~F}$ & -1.18527200 & -4.69338000 & 3.79025000 \\
\hline $\mathrm{F}$ & -1.08183100 & -2.66616200 & 4.57961100 & $\mathrm{~F}$ & 0.72804300 & -3.65962400 & 3.88945600 \\
\hline C & 2.86640500 & -1.59725300 & -1.47461400 & $\mathrm{H}$ & 2.05465400 & -1.68926300 & -2.18777300 \\
\hline $\mathrm{H}$ & 3.45908100 & -0.69125900 & -1.56381400 & C & 3.51981900 & -2.77697300 & -1.08628000 \\
\hline C & 4.84651500 & -2.74530500 & -0.49522200 & $\mathrm{H}$ & 5.07074400 & -1.77468400 & -0.03895800 \\
\hline $\mathrm{H}$ & 5.00962100 & -3.55232500 & 0.22705300 & C & 2.77724500 & -4.06913700 & -1.12605800 \\
\hline $\mathrm{H}$ & 3.44619800 & -4.93161000 & -1.07906900 & $\mathrm{H}$ & 2.11531600 & -4.11345000 & -0.24328200 \\
\hline $\mathrm{H}$ & 2.12146000 & -4.14321600 & -1.99638000 & C & 7.84417100 & -2.94015700 & -0.97114000 \\
\hline $\mathrm{H}$ & 7.91548400 & -3.77287700 & -0.26248900 & $\mathrm{H}$ & 8.67770300 & -3.03088300 & -1.67761300 \\
\hline $\mathrm{H}$ & 7.98042800 & -2.00837300 & -0.41083200 & C & 5.92347500 & -4.57993500 & -2.78621400 \\
\hline $\mathrm{H}$ & 5.94038500 & -5.43383000 & -2.09917400 & $\mathrm{H}$ & 4.96500800 & -4.59299500 & -3.31695500 \\
\hline $\mathrm{H}$ & 6.71352400 & -4.74071400 & -3.52927900 & C & 6.04677900 & -1.48646700 & -3.04980600 \\
\hline $\mathrm{H}$ & 5.08860600 & -1.48718700 & -3.57985800 & $\mathrm{H}$ & 6.11761200 & -0.54179100 & -2.50046200 \\
\hline $\mathrm{H}$ & 6.84689000 & -1.50185700 & -3.79909700 & $\mathrm{Si}$ & 6.19760300 & -2.95158100 & -1.88284300 \\
\hline $\mathrm{C}$ & 1.37420800 & 5.96509400 & 1.11586600 & $\mathrm{H}$ & 0.66455100 & 6.20681400 & 0.31798300 \\
\hline $\mathrm{H}$ & 2.29196700 & 6.52918100 & 0.92772500 & & & & \\
\hline
\end{tabular}

TS-S-4f-33 - $\mathrm{CF}_{3}$ rotamer of TS-S-4f-32

Imaginary frequency: $-325.01 \mathrm{~cm}^{-1}$

E[B3LYP/6-31G(d)-D3(BJ)]: -4789.077479 Hartree

$\mathrm{E}\left[\mathrm{PCM}\left(\mathrm{Et}_{2} \mathrm{O}\right)-\mathrm{B} 3 \mathrm{LYP} / 6-311 \mathrm{G}(\mathrm{d}, \mathrm{p})-\mathrm{D} 3(\mathrm{BJ})\right]$ : -4790.222999 Hartree

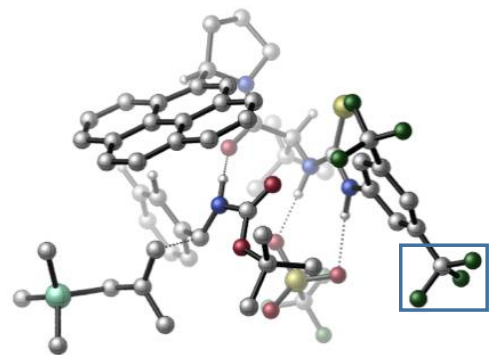

\begin{tabular}{|crrr|rrrr|}
\hline 01 & & & & $\mathrm{~N}$ & -3.03217700 & 0.43265800 & 1.38389600 \\
\hline $\mathrm{H}$ & -2.71670600 & -0.40350100 & 1.87981500 & $\mathrm{C}$ & -4.00949300 & 0.16377200 & 0.41785300 \\
\hline $\mathrm{C}$ & -4.86047100 & -0.92120700 & 0.65624800 & $\mathrm{C}$ & -4.08166400 & 0.84144400 & -0.80607000 \\
\hline $\mathrm{C}$ & -5.76400100 & -1.32864500 & -0.32381400 & $\mathrm{H}$ & -4.77491100 & -1.47400800 & 1.58434800 \\
\hline $\mathrm{C}$ & -5.00121700 & 0.42871300 & -1.76333800 & $\mathrm{H}$ & -3.39325100 & 1.64403800 & -1.01227300 \\
\hline $\mathrm{C}$ & -5.85347700 & -0.65436700 & -1.53792000 & $\mathrm{H}$ & -6.56240700 & -0.96890300 & -2.29404700 \\
\hline $\mathrm{C}$ & -6.56427000 & -2.58098300 & -0.11370800 & $\mathrm{C}$ & -5.00262600 & 1.07507000 & -3.11698300 \\
\hline $\mathrm{F}$ & -6.84493000 & -2.79844200 & 1.18778600 & $\mathrm{~F}$ & -5.88936000 & -3.67193700 & -0.55842400 \\
\hline $\mathrm{F}$ & -7.73784000 & -2.55186300 & -0.78544000 & $\mathrm{~F}$ & -6.24815700 & 1.19356600 & -3.62547300 \\
\hline $\mathrm{F}$ & -4.44827400 & 2.30829000 & -3.11065900 & $\mathrm{~F}$ & -4.29242700 & 0.33742800 & -4.01777400 \\
\hline $\mathrm{C}$ & -0.52236300 & 2.54456000 & 2.95726300 & $\mathrm{H}$ & -1.21031700 & 3.37685000 & 3.10489400 \\
\hline $\mathrm{C}$ & 0.13810400 & 2.21805500 & 4.33623300 & $\mathrm{C}$ & 1.20578600 & 1.11271300 & 4.25865800 \\
\hline $\mathrm{H}$ & 2.03575700 & 1.39088400 & 3.60651200 & $\mathrm{H}$ & 0.79875000 & 0.16996700 & 3.88923500 \\
\hline $\mathrm{H}$ & 1.60370300 & 0.93158500 & 5.26470900 & $\mathrm{C}$ & -0.97520900 & 1.77926300 & 5.30405200 \\
\hline $\mathrm{H}$ & -0.56783800 & 1.67219200 & 6.31608900 & $\mathrm{H}$ & -1.40070200 & 0.81646300 & 5.00938000 \\
\hline $\mathrm{H}$ & -1.78782300 & 2.51447000 & 5.33933100 & $\mathrm{C}$ & 0.79306800 & 3.50409300 & 4.87134200 \\
\hline $\mathrm{H}$ & 1.27878900 & 3.30422100 & 5.83258400 & $\mathrm{H}$ & 0.05181800 & 4.29563200 & 5.03487400 \\
\hline $\mathrm{H}$ & 1.56047900 & 3.88411800 & 4.18673000 & $\mathrm{C}$ & 0.50732200 & 2.90091100 & 1.87714900 \\
\hline $\mathrm{O}$ & 1.20016000 & 2.00808900 & 1.36689000 & $\mathrm{C}$ & -0.06724200 & 5.36990500 & 1.98216100 \\
\hline $\mathrm{C}$ & 1.68343700 & 4.52569000 & 0.48393200 & $\mathrm{C}$ & 0.85833000 & 6.52452100 & 1.58515100 \\
\hline $\mathrm{H}$ & -1.04515500 & 5.42712300 & 1.49075300 & $\mathrm{H}$ & -0.22890000 & 5.32040600 & 3.05852600 \\
\hline $\mathrm{H}$ & 2.65630900 & 4.34303800 & 0.94686800 & $\mathrm{H}$ & 1.63883400 & 6.65369900 & 2.34467700 \\
\hline $\mathrm{H}$ & 0.32127700 & 7.47190800 & 1.48666800 & $\mathrm{~N}$ & 0.66140400 & 4.18712800 & 1.50022300 \\
\hline $\mathrm{C}$ & 1.55956500 & 3.69688100 & -0.78134500 & $\mathrm{C}$ & 0.32801600 & 3.51410600 & -1.33924700 \\
\hline $\mathrm{C}$ & 2.72507100 & 3.08372100 & -1.38264900 & $\mathrm{C}$ & 0.12413000 & 2.70911400 & -2.50272400 \\
\hline $\mathrm{H}$ & -0.55091800 & 3.93616700 & -0.85814400 & $\mathrm{C}$ & 2.53608600 & 2.20484800 & -2.49837700 \\
\hline
\end{tabular}




\begin{tabular}{|c|c|c|c|c|c|c|c|}
\hline C & 4.03759800 & 3.28690400 & -0.91647800 & $\mathrm{C}$ & -1.14074700 & 2.55351000 & -3.08895300 \\
\hline C & 1.23943600 & 2.02201200 & -3.06016700 & C & 3.65407500 & 1.52006800 & -3.07087200 \\
\hline $\mathrm{H}$ & 4.22389800 & 3.96916500 & -0.09571700 & $\mathrm{C}$ & 5.12274600 & 2.62919500 & -1.49187800 \\
\hline C & -1.31023800 & 1.74247800 & -4.20675800 & $\mathrm{H}$ & -1.99407600 & 3.05988500 & -2.64973100 \\
\hline C & 1.05173700 & 1.16564500 & -4.18633800 & C & 4.93759900 & 1.74386600 & -2.54951500 \\
\hline $\mathrm{C}$ & 3.43012800 & 0.63349800 & -4.18027100 & $\mathrm{H}$ & 6.12285300 & 2.81294600 & -1.10921000 \\
\hline $\mathrm{C}$ & -0.22830100 & 1.05037800 & -4.74730800 & $\mathrm{H}$ & -2.29716600 & 1.62541900 & -4.63812800 \\
\hline C & 2.18841900 & 0.46421200 & -4.71193400 & $\mathrm{H}$ & 5.78868500 & 1.24387700 & -3.00359600 \\
\hline $\bar{H}$ & 4.28672700 & 0.11310800 & -4.60058200 & $\mathrm{H}$ & -0.37060000 & 0.40610500 & -5.61099400 \\
\hline $\bar{H}$ & 2.03836700 & -0.19735000 & -5.56166500 & $\mathrm{C}$ & -2.39686100 & 1.61142100 & 1.66281600 \\
\hline $\mathrm{S}$ & -2.93159000 & 3.13095700 & 1.13599300 & $\mathrm{~N}$ & -1.32394900 & 1.43915100 & 2.47231200 \\
\hline $\bar{H}$ & -0.89319200 & 0.51048000 & 2.48461800 & $\mathrm{C}$ & -0.25886800 & -0.59925200 & -0.96259800 \\
\hline $\mathrm{O}$ & -1.16710000 & 0.18582600 & -1.12789500 & $\mathrm{O}$ & -0.19751300 & -1.87884600 & -1.36862500 \\
\hline C & -1.31748700 & -2.55966200 & -2.05988900 & C & -1.58473500 & -1.86497500 & -3.39167900 \\
\hline$\overline{\mathrm{H}}$ & -2.29358700 & -2.46343000 & -3.97412800 & $\mathrm{H}$ & -2.01117900 & -0.87378100 & -3.24088200 \\
\hline $\bar{H}$ & -0.65866700 & -1.76615400 & -3.96770500 & $\mathrm{C}$ & -0.75856700 & -3.96675300 & -2.25528900 \\
\hline $\bar{H}$ & -0.51129700 & -4.40639100 & -1.28454500 & $\mathrm{H}$ & -1.50729500 & -4.59738600 & -2.74426800 \\
\hline $\mathrm{H}$ & 0.13796900 & -3.94860500 & -2.88647800 & C & -2.54838700 & -2.58632200 & -1.16692000 \\
\hline $\mathrm{H}$ & -3.33274400 & -3.18812000 & -1.63705400 & $\mathrm{H}$ & -2.31818400 & -3.01908400 & -0.19170200 \\
\hline $\bar{H}$ & -2.93210800 & -1.58071400 & -1.02635800 & $\mathrm{C}$ & 1.78270300 & -1.21322300 & 0.19760200 \\
\hline $\mathrm{H}$ & 1.26792100 & -2.13446200 & 0.44485900 & C & 2.76905900 & -0.76130300 & 1.20908700 \\
\hline $\mathrm{C}$ & 2.94582000 & -1.52223000 & 2.37008000 & $\mathrm{C}$ & 3.57581400 & 0.35928500 & 0.98446100 \\
\hline $\mathrm{C}$ & 3.91102000 & -1.15241900 & 3.30535100 & $\mathrm{H}$ & 2.30521700 & -2.37668100 & 2.55371300 \\
\hline C & 4.54515200 & 0.72087500 & 1.91564300 & $\mathrm{H}$ & 3.44360200 & 0.95308300 & 0.08913500 \\
\hline $\mathrm{C}$ & 4.71484800 & -0.03360400 & 3.07931700 & $\mathrm{H}$ & 4.02567200 & -1.73382400 & 4.21537000 \\
\hline $\mathrm{H}$ & 5.16048400 & 1.59656300 & 1.73051200 & $\mathrm{H}$ & 5.46710600 & 0.25216800 & 3.80922800 \\
\hline $\mathrm{N}$ & 0.92902000 & -0.25337400 & -0.32171300 & $\mathrm{H}$ & 0.95547000 & 0.68483300 & 0.09368100 \\
\hline $\mathrm{S}$ & -0.88274300 & -2.45389200 & 2.36485600 & $\mathrm{O}$ & -0.23765100 & -3.54425000 & 1.60117300 \\
\hline $\mathrm{O}$ & -0.18809200 & -1.14209800 & 2.23519100 & $\mathrm{O}$ & -2.35632500 & -2.34593600 & 2.26286200 \\
\hline C & -0.57884700 & -2.89222200 & 4.14402500 & $\mathrm{~F}$ & -1.18479500 & -4.03619800 & 4.47191000 \\
\hline $\mathrm{F}$ & -1.03719600 & -1.91469800 & 4.94244800 & $\mathrm{~F}$ & 0.74242000 & -3.02934700 & 4.36938800 \\
\hline C & 2.79992400 & -1.80546700 & -1.27309300 & $\mathrm{H}$ & 1.98053800 & -1.98347900 & -1.96081900 \\
\hline $\mathrm{H}$ & 3.40278500 & -0.92839000 & -1.49061700 & C & 3.44382400 & -2.92936200 & -0.73250600 \\
\hline $\mathrm{C}$ & 4.77573200 & -2.83383100 & -0.16072700 & $\mathrm{H}$ & 5.01593500 & -1.81227600 & 0.15417500 \\
\hline $\mathrm{H}$ & 4.93450100 & -3.53433700 & 0.66617200 & C & 2.68581200 & -4.20498300 & -0.58814600 \\
\hline $\mathrm{H}$ & 3.34528900 & -5.06179000 & -0.43149300 & $\mathrm{H}$ & 2.03557900 & -4.11988000 & 0.30039300 \\
\hline $\mathrm{H}$ & 2.01820600 & -4.38780100 & -1.43301200 & C & 7.76656400 & -3.13789200 & -0.61662000 \\
\hline $\mathrm{H}$ & 7.83061700 & -3.86736700 & 0.19852500 & $\mathrm{H}$ & 8.59458000 & -3.33591200 & -1.30748500 \\
\hline $\mathrm{H}$ & 7.91891000 & -2.14058900 & -0.18893400 & C & 5.81271800 & -4.98211900 & -2.18269100 \\
\hline $\mathrm{H}$ & 5.81854700 & -5.73364400 & -1.38481100 & $\mathrm{H}$ & 4.85270100 & -5.05323500 & -2.70600400 \\
\hline $\mathrm{H}$ & 6.59816000 & -5.25570200 & -2.89712700 & $\mathrm{C}$ & 5.97876100 & -1.95603500 & -2.86849100 \\
\hline $\mathrm{H}$ & 5.01584600 & -2.01196900 & -3.38690500 & $\mathrm{H}$ & 6.07099100 & -0.94621100 & -2.45489600 \\
\hline $\mathrm{H}$ & 6.77156100 & -2.08914900 & -3.61388400 & $\mathrm{Si}$ & 6.11476300 & -3.24944400 & -1.51194800 \\
\hline C & 1.47000100 & 6.03805900 & 0.26603500 & $\mathrm{H}$ & 0.76224700 & 6.18560300 & -0.55619200 \\
\hline $\mathrm{H}$ & 2.40210900 & 6.54331600 & -0.00190600 & & & & \\
\hline
\end{tabular}

\section{TS-S-4f-34}

Imaginary frequency: $-425.86 \mathrm{~cm}^{-1}$

E[B3LYP/6-31G(d)-D3(BJ)]: -4789.082536 Hartree

E[PCM(Et $\left.\left.{ }_{2} \mathrm{O}\right)-B 3 L Y P / 6-311 G(d, p)-D 3(B J)\right]:-4790.222549$ Hartree

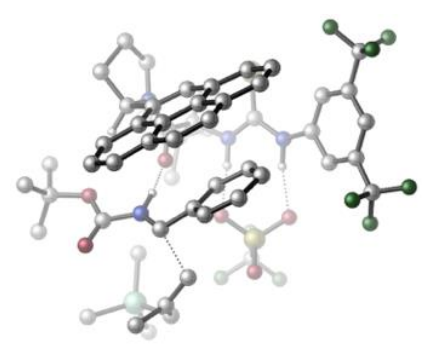




\begin{tabular}{|c|c|c|c|c|c|c|c|}
\hline 01 & & & & $\mathrm{~N}$ & -2.19896900 & -2.02606300 & 1.53547800 \\
\hline $\mathrm{H}$ & -1.55945700 & -2.66275300 & 1.04488100 & C & -3.44021500 & -1.90974600 & 0.88285700 \\
\hline $\mathrm{C}$ & -3.52110900 & -2.49029600 & -0.39806700 & $\mathrm{C}$ & -4.55157200 & -1.22003900 & 1.38176000 \\
\hline $\mathrm{C}$ & -4.65996800 & -2.31562700 & -1.17376600 & $\mathrm{H}$ & -2.67300200 & -3.03975300 & -0.79111200 \\
\hline C & -5.67870600 & -1.04864800 & 0.57720400 & $\mathrm{H}$ & -4.51829700 & -0.79005800 & 2.36974600 \\
\hline C & -5.75040600 & -1.58049200 & -0.70479200 & $\mathrm{H}$ & -6.62440200 & -1.42714200 & -1.32546500 \\
\hline C & -4.67571800 & -2.81175000 & -2.59226900 & C & -6.84555000 & -0.28376200 & 1.13531100 \\
\hline $\mathrm{F}$ & -3.81662700 & -3.82641400 & -2.79488700 & $\mathrm{~F}$ & -4.33380500 & -1.82013600 & -3.45760200 \\
\hline $\mathrm{F}$ & -5.90714200 & -3.23266500 & -2.96142100 & $\mathrm{~F}$ & -6.44401500 & 0.69955100 & 1.97669900 \\
\hline $\mathrm{F}$ & -7.56982000 & 0.31034300 & 0.15401600 & $\mathrm{~F}$ & -7.69522000 & -1.07080800 & 1.82859600 \\
\hline C & 0.52773400 & -0.59943100 & 3.49413500 & $\mathrm{H}$ & -0.12887600 & -0.23051800 & 4.27949400 \\
\hline C & 1.68765600 & -1.37015000 & 4.18735800 & C & 2.69259600 & -1.96474200 & 3.19282700 \\
\hline $\mathrm{H}$ & 3.14694100 & -1.18933600 & 2.57466300 & $\mathrm{H}$ & 2.22471500 & -2.68884900 & 2.52361300 \\
\hline $\mathrm{H}$ & 3.48690300 & -2.48338900 & 3.74337200 & C & 1.06416700 & -2.49654800 & 5.02783600 \\
\hline $\mathrm{H}$ & 1.84466400 & -3.02926800 & 5.58298600 & $\mathrm{H}$ & 0.54576900 & -3.21779400 & 4.38972900 \\
\hline $\mathrm{H}$ & 0.33981500 & -2.10193300 & 5.75000100 & C & 2.42244000 & -0.38554100 & 5.11418700 \\
\hline $\mathrm{H}$ & 3.23822900 & -0.89768000 & 5.63579200 & $\mathrm{H}$ & 1.75026000 & 0.02845300 & 5.87484600 \\
\hline $\mathrm{H}$ & 2.86135600 & 0.44767100 & 4.55138800 & C & 0.97921200 & 0.56653000 & 2.60422900 \\
\hline C & 0.24868000 & 2.30547600 & 4.30442900 & C & 1.03125800 & 2.93371700 & 2.06478900 \\
\hline $\mathrm{H}$ & -0.69120100 & 1.80256500 & 4.54753300 & $\mathrm{H}$ & 0.97736000 & 2.09670700 & 5.09478300 \\
\hline $\mathrm{H}$ & 1.98194300 & 2.72989400 & 1.57549200 & $\mathrm{~N}$ & 0.76901700 & 1.83460600 & 3.00733600 \\
\hline C & -0.07235000 & 3.07595900 & 1.02508400 & C & -1.33445800 & 2.62098200 & 1.27544900 \\
\hline C & 0.21349500 & 3.75133500 & -0.22420300 & C & -2.41926100 & 2.81886200 & 0.36081800 \\
\hline $\mathrm{H}$ & -1.54580300 & 2.05881700 & 2.17908000 & C & -0.85294300 & 3.96526700 & -1.15371000 \\
\hline C & 1.50236200 & 4.17967500 & -0.58242800 & C & -3.70734100 & 2.32393300 & 0.62031900 \\
\hline C & -2.17115400 & 3.51906000 & -0.85342100 & C & -0.59498000 & 4.60786300 & -2.40355000 \\
\hline $\mathrm{H}$ & 2.32712300 & 4.03389700 & 0.10133200 & C & 1.75262900 & 4.77430600 & -1.81782000 \\
\hline C & -4.73913200 & 2.52575800 & -0.29509200 & $\mathrm{H}$ & -3.88824200 & 1.76852800 & 1.53508400 \\
\hline C & -3.23383200 & 3.72811500 & -1.78082400 & C & 0.71633200 & 4.99326500 & -2.72028000 \\
\hline C & -1.68852400 & 4.82676500 & -3.30840700 & $\mathrm{H}$ & 2.76603100 & 5.07382500 & -2.06991600 \\
\hline $\mathrm{C}$ & -4.50868000 & 3.22576200 & -1.47787100 & $\mathrm{H}$ & -5.72748900 & 2.13159600 & -0.08706600 \\
\hline C & -2.95004100 & 4.41384200 & -3.00850200 & $\mathrm{H}$ & 0.91246200 & 5.46944600 & -3.67753900 \\
\hline $\mathrm{H}$ & -1.48226700 & 5.33145800 & -4.24892100 & $\mathrm{H}$ & -5.31845100 & 3.37906200 & -2.18680800 \\
\hline $\mathrm{H}$ & -3.76630900 & 4.58229300 & -3.70646400 & C & -1.64198700 & -1.26807700 & 2.53268500 \\
\hline $\mathrm{S}$ & -2.50047800 & -0.22473100 & 3.56084800 & $\mathrm{~N}$ & -0.29522600 & -1.45236200 & 2.64211900 \\
\hline $\mathrm{H}$ & 0.19832600 & -1.82208900 & 1.82113000 & $\mathrm{~S}$ & 0.66362600 & -3.47938100 & -0.51542200 \\
\hline $\mathrm{O}$ & 0.98420100 & -3.51202800 & -1.95429600 & $\mathrm{O}$ & 1.14388200 & -2.26916500 & 0.20813500 \\
\hline $\mathrm{O}$ & -0.71970800 & -3.86944400 & -0.13708400 & C & 1.70303200 & -4.84078700 & 0.20174200 \\
\hline $\mathrm{F}$ & 1.38838100 & -6.02410800 & -0.32694200 & $\mathrm{~F}$ & 1.54148700 & -4.90541100 & 1.53312300 \\
\hline $\mathrm{F}$ & 3.00939000 & -4.59924600 & -0.04494500 & C & 3.72714900 & 1.61967100 & -0.89315800 \\
\hline $\mathrm{O}$ & 4.44285800 & 1.91317100 & -1.83821200 & $\mathrm{O}$ & 3.97421700 & 1.84937000 & 0.40494500 \\
\hline C & 5.19412300 & 2.53458500 & 0.87666400 & C & 6.44320500 & 1.76298100 & 0.45635800 \\
\hline $\mathrm{H}$ & 7.31541800 & 2.19911400 & 0.95463200 & $\mathrm{H}$ & 6.59664900 & 1.81346900 & -0.62110700 \\
\hline $\mathrm{H}$ & 6.36730700 & 0.71459400 & 0.75728200 & C & 5.01310400 & 2.49190000 & 2.39336900 \\
\hline $\mathrm{H}$ & 4.09877000 & 3.01507700 & 2.68924600 & $\mathrm{H}$ & 5.86370200 & 2.97414900 & 2.88477000 \\
\hline $\mathrm{H}$ & 4.94539800 & 1.45682900 & 2.74180300 & C & 5.21017500 & 3.97112700 & 0.35473700 \\
\hline $\mathrm{H}$ & 6.10122800 & 4.48354300 & 0.73219900 & $\mathrm{H}$ & 4.33246300 & 4.52401400 & 0.70311100 \\
\hline $\mathrm{H}$ & 5.23564900 & 3.98813400 & -0.73648300 & C & 1.86252900 & 0.92231100 & -2.22664300 \\
\hline $\mathrm{H}$ & 2.33282300 & 1.57146300 & -2.95831000 & C & 0.38954000 & 0.87902000 & -2.25478500 \\
\hline $\mathrm{C}$ & -0.26161000 & 1.35992600 & -3.40320900 & C & -0.36410400 & 0.29621600 & -1.22649200 \\
\hline C & -1.63816600 & 1.23621700 & -3.53723600 & $\mathrm{H}$ & 0.32146600 & 1.83073900 & -4.19093300 \\
\hline C & -1.74448000 & 0.19526600 & -1.36164400 & $\mathrm{H}$ & 0.11642100 & -0.09975100 & -0.34099900 \\
\hline C & -2.38367000 & 0.63804300 & -2.51851700 & $\mathrm{H}$ & -2.13134700 & 1.61676800 & -4.42536800 \\
\hline
\end{tabular}




\begin{tabular}{|c|c|c|c|c|c|c|c|}
\hline $\mathrm{H}$ & -2.32718100 & -0.22868700 & -0.55802600 & $\mathrm{H}$ & -3.45668400 & 0.52535100 & -2.61776000 \\
\hline $\mathrm{N}$ & 2.49996400 & 0.99294300 & -1.01005300 & $\mathrm{H}$ & 2.02719300 & 0.73577200 & -0.13181800 \\
\hline $\mathrm{C}$ & 2.14776200 & -0.77929200 & -3.26703300 & $\mathrm{H}$ & 1.51421900 & -1.47886100 & -2.72950300 \\
\hline $\mathrm{H}$ & 1.68959800 & -0.31632600 & -4.13572600 & C & 3.49071600 & -1.14168500 & -3.44123200 \\
\hline C & 4.05567800 & -2.30909200 & -2.77593300 & $\mathrm{H}$ & 4.67625800 & -2.85165600 & -3.50323000 \\
\hline $\mathrm{H}$ & 3.27307800 & -2.96905800 & -2.38915300 & C & 4.35871900 & -0.32427600 & -4.35107900 \\
\hline $\mathrm{H}$ & 5.27000400 & -0.85132100 & -4.64210000 & $\mathrm{H}$ & 4.64831700 & 0.59334800 & -3.82217200 \\
\hline $\mathrm{H}$ & 3.81168700 & -0.02032800 & -5.24975800 & C & 4.51352000 & -1.60926300 & 0.25902100 \\
\hline $\mathrm{H}$ & 4.38830000 & -0.53151800 & 0.36661900 & $\mathrm{H}$ & 5.10545200 & -1.96258700 & 1.11241400 \\
\hline $\mathrm{H}$ & 3.51864600 & -2.05540100 & 0.33275100 & C & 6.69867700 & -0.91962700 & -1.89677900 \\
\hline $\mathrm{H}$ & 6.29874300 & 0.08047300 & -2.08548700 & $\mathrm{H}$ & 7.18656900 & -1.27449400 & -2.81213900 \\
\hline $\mathrm{H}$ & 7.47336800 & -0.82940300 & -1.12619900 & C & 6.07372800 & -3.84932400 & -1.16637300 \\
\hline $\mathrm{H}$ & 6.56335300 & -4.17942500 & -2.08991700 & $\mathrm{H}$ & 5.29551200 & -4.57591100 & -0.91305400 \\
\hline $\mathrm{H}$ & 6.82337700 & -3.87155400 & -0.36605100 & $\mathrm{Si}$ & 5.35320200 & -2.11517100 & -1.33625400 \\
\hline C & 1.10710300 & 4.15512900 & 3.00307700 & $\mathrm{H}$ & 0.89403000 & 5.08984200 & 2.47922200 \\
\hline $\mathrm{H}$ & 2.11284900 & 4.22393300 & 3.43680200 & C & 0.07713300 & 3.81823600 & 4.09177500 \\
\hline $\mathrm{H}$ & 0.22814500 & 4.38138600 & 5.01640700 & $\mathrm{H}$ & -0.93145200 & 4.03228400 & 3.72614000 \\
\hline $\mathrm{O}$ & 1.46995300 & 0.31726200 & 1.49272000 & & & & \\
\hline
\end{tabular}

\section{TS-S-4f-35}

Imaginary frequency: $-306.09 \mathrm{~cm}^{-1}$

E[B3LYP/6-31G(d)-D3(BJ)]: -4789.079598 Hartree

$\mathrm{E}\left[\mathrm{PCM}\left(\mathrm{Et}_{2} \mathrm{O}\right)-\mathrm{B} 3 \mathrm{LYP} / 6-311 \mathrm{G}(\mathrm{d}, \mathrm{p})-\mathrm{D} 3(\mathrm{BJ})\right]$ : -4790.221783 Hartree

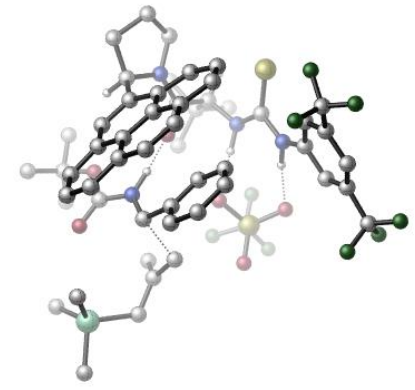

\begin{tabular}{|lrrr|rrrr|}
\hline 01 & & & N & 2.79613600 & 1.75225600 & 1.21082600 \\
\hline $\mathrm{H}$ & 2.24329000 & 2.56332100 & 0.90723100 & $\mathrm{C}$ & 3.83132000 & 1.43920400 & 0.32169600 \\
\hline $\mathrm{C}$ & 4.08914200 & 2.38132900 & -0.68910900 & $\mathrm{C}$ & 4.53280300 & 0.22469000 & 0.31191700 \\
\hline $\mathrm{C}$ & 5.01853700 & 2.10230300 & -1.68568000 & $\mathrm{H}$ & 3.53978200 & 3.31615800 & -0.70075600 \\
\hline $\mathrm{C}$ & 5.43766900 & -0.04202100 & -0.71271100 & $\mathrm{H}$ & 4.35119800 & -0.50734900 & 1.08299700 \\
\hline $\mathrm{C}$ & 5.70110400 & 0.88685400 & -1.71702500 & $\mathrm{H}$ & 6.41965600 & 0.67417800 & -2.49890500 \\
\hline $\mathrm{C}$ & 5.23123400 & 3.09463100 & -2.79648900 & $\mathrm{C}$ & 6.05405200 & -1.40741400 & -0.80707800 \\
\hline $\mathrm{F}$ & 6.49549300 & 3.04037500 & -3.27649800 & $\mathrm{~F}$ & 5.00046700 & 4.36126000 & -2.39828100 \\
\hline $\mathrm{F}$ & 4.40702600 & 2.84905200 & -3.84194500 & $\mathrm{~F}$ & 6.07528100 & -2.05858100 & 0.37609800 \\
\hline $\mathrm{F}$ & 5.35157700 & -2.20155700 & -1.66907200 & $\mathrm{~F}$ & 7.31862700 & -1.36912300 & -1.27502300 \\
\hline $\mathrm{C}$ & 0.33783600 & 0.80618200 & 3.71250200 & $\mathrm{H}$ & 1.05311000 & 0.48950400 & 4.46944700 \\
\hline $\mathrm{C}$ & -0.71504100 & 1.72346000 & 4.41258700 & $\mathrm{C}$ & -1.84836600 & 2.18034100 & 3.47666700 \\
\hline $\mathrm{H}$ & -2.42928800 & 1.33320500 & 3.10368300 & $\mathrm{H}$ & -1.47650100 & 2.72301200 & 2.60847700 \\
\hline $\mathrm{H}$ & -2.51946400 & 2.84885800 & 4.02928400 & $\mathrm{C}$ & 0.02931200 & 2.95122300 & 4.96383900 \\
\hline $\mathrm{H}$ & -0.66028200 & 3.58107300 & 5.53736700 & $\mathrm{H}$ & 0.44980100 & 3.55357100 & 4.15607200 \\
\hline $\mathrm{H}$ & 0.84922600 & 2.65133100 & 5.62695700 & $\mathrm{C}$ & -1.33135100 & 0.93716100 & 5.58345800 \\
\hline $\mathrm{H}$ & -2.05636700 & 1.56409900 & 6.11378300 & $\mathrm{H}$ & -0.56790000 & 0.62772300 & 6.30670200 \\
\hline $\mathrm{H}$ & -1.86127200 & 0.04195100 & 5.23653500 & $\mathrm{C}$ & -0.31128400 & -0.40590400 & 3.03543400 \\
\hline $\mathrm{C}$ & 0.43686200 & -1.99898200 & 4.84760400 & $\mathrm{C}$ & -1.02560400 & -2.73750500 & 3.00560400 \\
\hline $\mathrm{H}$ & 1.43566300 & -1.55567600 & 4.86711000 & $\mathrm{H}$ & -0.12825200 & -1.64300900 & 5.71673600 \\
\hline $\mathrm{H}$ & -2.07620800 & -2.43117400 & 3.00565900 & $\mathrm{~N}$ & -0.25478300 & -1.62588900 & 3.60190600 \\
\hline $\mathrm{C}$ & -0.71467300 & -3.10953700 & 1.56123100 & $\mathrm{C}$ & -1.79718400 & -3.31865900 & 0.75606300 \\
\hline $\mathrm{C}$ & 0.61648100 & -3.36330500 & 1.03982300 & $\mathrm{C}$ & -1.69588400 & -3.80517500 & -0.58467500 \\
\hline $\mathrm{H}$ & -2.79079100 & -3.09766300 & 1.13657500 & $\mathrm{C}$ & 0.73756900 & -3.96546800 & -0.25618600 \\
\hline $\mathrm{C}$ & 1.79749400 & -3.01317300 & 1.71392100 & $\mathrm{C}$ & -2.81087800 & -3.90755900 & -1.43086700 \\
\hline $\mathrm{C}$ & -0.41237500 & -4.18645300 & -1.06761200 & $\mathrm{C}$ & 2.02743200 & -4.28936600 & -0.77715500 \\
\hline & & & & & & & \\
\hline
\end{tabular}

S136 


\begin{tabular}{|c|c|c|c|c|c|c|c|}
\hline $\mathrm{H}$ & 1.75353000 & -2.45669700 & 2.63639300 & C & 3.05256600 & -3.29482200 & 1.17614100 \\
\hline C & -2.67153400 & -4.40635000 & -2.72538900 & $\mathrm{H}$ & -3.78449500 & -3.58705900 & -1.06840700 \\
\hline C & -0.28447900 & -4.72991600 & -2.38083400 & C & 3.17292100 & -3.95297700 & -0.04153900 \\
\hline C & 2.12072600 & -4.90367700 & -2.07155500 & $\mathrm{H}$ & 3.94071500 & -2.97869100 & 1.71300200 \\
\hline C & -1.42771200 & -4.83039100 & -3.18986600 & $\mathrm{H}$ & -3.54138800 & -4.47447800 & -3.37271000 \\
\hline C & 1.01830900 & -5.11672500 & -2.83979300 & $\mathrm{H}$ & 4.15376300 & -4.16055700 & -0.45661200 \\
\hline $\mathrm{H}$ & 3.10831400 & -5.17143200 & -2.43799900 & $\mathrm{H}$ & -1.32865800 & -5.23242400 & -4.19489600 \\
\hline $\mathrm{H}$ & 1.10921900 & -5.56001400 & -3.82808700 & C & 2.34909400 & 1.10996600 & 2.33522400 \\
\hline $\mathrm{S}$ & 3.24927400 & -0.00339500 & 3.24108800 & $\mathrm{~N}$ & 1.09353300 & 1.50584600 & 2.68565800 \\
\hline $\mathrm{H}$ & 0.52249000 & 1.91737800 & 1.93844800 & $\mathrm{~S}$ & 0.03020500 & 3.84061900 & -0.11685400 \\
\hline $\mathrm{O}$ & -0.47769000 & 4.15647300 & -1.45847100 & $\mathrm{O}$ & -0.44331700 & 2.53237900 & 0.43841200 \\
\hline $\mathrm{O}$ & 1.47775700 & 4.06879000 & 0.13057200 & C & -0.79678600 & 5.09215600 & 0.98064800 \\
\hline $\mathrm{F}$ & -0.55380800 & 6.33420800 & 0.55840200 & $\mathrm{~F}$ & -0.36278700 & 4.97687500 & 2.24784300 \\
\hline $\mathrm{F}$ & -2.13074700 & 4.89385700 & 0.98311300 & C & -3.74356500 & -0.54067300 & 0.00324100 \\
\hline $\mathrm{O}$ & -4.62844100 & -0.57286800 & -0.83878900 & $\mathrm{O}$ & -3.88669600 & -0.60419700 & 1.33104900 \\
\hline C & -5.21767700 & -0.64893600 & 1.96415900 & C & -5.96135400 & 0.65254500 & 1.66587300 \\
\hline $\mathrm{H}$ & -6.88862000 & 0.68486800 & 2.24723300 & $\mathrm{H}$ & -6.21515500 & 0.73221600 & 0.60777000 \\
\hline $\mathrm{H}$ & -5.34716400 & 1.51243500 & 1.95178400 & C & -4.86938900 & -0.75000300 & 3.44841800 \\
\hline $\mathrm{H}$ & -4.29230700 & -1.65893200 & 3.64691400 & $\mathrm{H}$ & -5.78654900 & -0.78525800 & 4.04439500 \\
\hline $\mathrm{H}$ & -4.27755300 & 0.11271700 & 3.76645300 & C & -5.98116800 & -1.88825700 & 1.49633200 \\
\hline $\mathrm{H}$ & -6.91114500 & -1.97731400 & 2.06763600 & $\mathrm{H}$ & -5.38673500 & -2.79092700 & 1.67504300 \\
\hline $\mathrm{H}$ & -6.22434500 & -1.82805000 & 0.43515200 & C & -1.97175000 & -0.44686900 & -1.60362000 \\
\hline $\mathrm{H}$ & -2.71636300 & -0.81161100 & -2.29782200 & C & -0.57862600 & -0.81190300 & -1.88934800 \\
\hline C & -0.29051500 & -1.40765600 & -3.12705300 & C & 0.46061100 & -0.54834400 & -0.98377200 \\
\hline C & 1.01767900 & -1.75095500 & -3.45089900 & $\mathrm{H}$ & -1.10038300 & -1.62335600 & -3.81836300 \\
\hline C & 1.76041500 & -0.90739100 & -1.31180900 & $\mathrm{H}$ & 0.26083800 & -0.06050500 & -0.03742000 \\
\hline C & 2.04638700 & -1.50206000 & -2.54149200 & $\mathrm{H}$ & 1.23125700 & -2.23086700 & -4.40050400 \\
\hline $\mathrm{H}$ & 2.55582200 & -0.73762100 & -0.60156600 & $\mathrm{H}$ & 3.06928300 & -1.77954000 & -2.77269300 \\
\hline $\mathrm{N}$ & -2.40304900 & -0.44443300 & -0.30602200 & $\mathrm{H}$ & -1.74964000 & -0.40247600 & 0.48737700 \\
\hline C & -1.87854700 & 1.47576600 & -2.30742900 & $\mathrm{H}$ & -1.18753900 & 1.75573700 & -1.52045900 \\
\hline $\mathrm{H}$ & -1.43858200 & 1.29513100 & -3.28387400 & C & -3.14582300 & 2.04349600 & -2.25235900 \\
\hline C & -3.97223900 & 2.22916500 & -3.45639000 & $\mathrm{H}$ & -3.34043600 & 2.27846800 & -4.35065800 \\
\hline $\mathrm{H}$ & -4.52343400 & 3.17589300 & -3.37734000 & C & -3.64152200 & 2.56992300 & -0.94671100 \\
\hline $\bar{H}$ & -4.73201000 & 2.55337900 & -0.87531600 & $\mathrm{H}$ & -3.30100300 & 3.61208500 & -0.86784000 \\
\hline $\bar{H}$ & -3.18406200 & 2.05406000 & -0.09860700 & $\mathrm{C}$ & -5.99324200 & 1.61258000 & -5.57970800 \\
\hline $\mathrm{H}$ & -6.36089200 & 2.64231100 & -5.50101700 & $\mathrm{H}$ & -6.82099800 & 0.99330200 & -5.94566300 \\
\hline $\bar{H}$ & -5.20563600 & 1.59586100 & -6.34208900 & $\mathrm{C}$ & -6.75384200 & 1.02695700 & -2.64775000 \\
\hline $\bar{H}$ & -7.12046900 & 2.05000200 & -2.50351000 & $\mathrm{H}$ & -6.41213700 & 0.62976300 & -1.68941900 \\
\hline $\mathrm{H}$ & -7.60172300 & 0.41677300 & -2.98240600 & C & -4.67936000 & -0.77451200 & -4.13936300 \\
\hline $\mathrm{H}$ & -4.56615300 & -1.27158200 & -3.17270200 & $\mathrm{H}$ & -3.70968100 & -0.76496700 & -4.65156600 \\
\hline $\mathrm{H}$ & -5.36426700 & -1.37399700 & -4.75126600 & $\mathrm{Si}$ & -5.36902800 & 0.96649300 & -3.91828100 \\
\hline C & -0.81315600 & -3.89376500 & 4.01849700 & $\mathrm{H}$ & -0.74217300 & -4.85921800 & 3.51314300 \\
\hline $\mathrm{H}$ & -1.67212000 & -3.93456900 & 4.69773600 & C & 0.46019400 & -3.53084300 & 4.79744700 \\
\hline $\mathrm{H}$ & 0.48077900 & -3.96825500 & 5.79934300 & $\mathrm{H}$ & 1.34617000 & -3.87960300 & 4.26121400 \\
\hline 0 & -0.89523900 & -0.22783400 & 1.95776800 & & & & \\
\hline
\end{tabular}

\section{TS-S-4f-36}

Imaginary frequency: $-353.21 \mathrm{~cm}^{-1}$

E[B3LYP/6-31G(d)-D3(BJ)]: -4789.079574 Hartree

E[PCM(Et $\left.\left.{ }_{2} \mathrm{O}\right)-\mathrm{B} 3 L Y P / 6-311 \mathrm{G}(\mathrm{d}, \mathrm{p})-\mathrm{D} 3(\mathrm{BJ})\right]$ : -4790.221649 Hartree

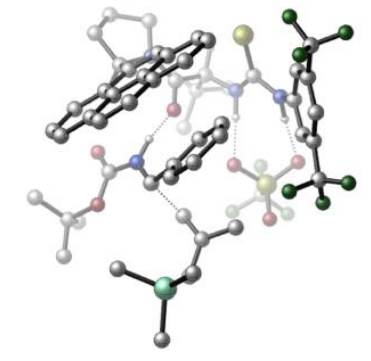




\begin{tabular}{|c|c|c|c|c|c|c|c|}
\hline 01 & & & & $\mathrm{~N}$ & -2.90778100 & 2.17900100 & 1.02296500 \\
\hline $\mathrm{H}$ & -2.43699300 & 2.38608000 & 1.91124400 & C & -3.63491900 & 0.97946700 & 1.06698500 \\
\hline $\mathrm{C}$ & -3.35008900 & 0.11375500 & 2.13887700 & $\mathrm{C}$ & -4.56377200 & 0.57961600 & 0.09691500 \\
\hline$C$ & -3.92020800 & -1.15105500 & 2.18559600 & $\mathrm{H}$ & -2.66060100 & 0.42881300 & 2.91185700 \\
\hline C & -5.11287600 & -0.70295900 & 0.15904400 & $\mathrm{H}$ & -4.80792100 & 1.24290200 & -0.71822100 \\
\hline C & -4.79849000 & -1.58310500 & 1.19036500 & $\mathrm{H}$ & -5.21592100 & -2.58260100 & 1.21576200 \\
\hline C & -3.48121200 & -2.13075800 & 3.23620700 & C & -6.07655600 & -1.14461900 & -0.90610900 \\
\hline $\mathrm{F}$ & -2.85707200 & -1.53929300 & 4.27270500 & $\mathrm{~F}$ & -2.60668700 & -3.03773800 & 2.71703700 \\
\hline $\mathrm{F}$ & -4.51833700 & -2.84342400 & 3.72836300 & $\mathrm{~F}$ & -5.90477600 & -0.46693700 & -2.06464300 \\
\hline $\mathrm{F}$ & -5.92373100 & -2.46387900 & -1.19148100 & $\mathrm{~F}$ & -7.36396800 & -0.97457700 & -0.53627500 \\
\hline C & -0.43811000 & 4.10363700 & -0.83079800 & $\mathrm{H}$ & -1.12341400 & 4.63716100 & -1.48947500 \\
\hline C & 0.58268900 & 5.13142700 & -0.23533900 & C & 1.71634500 & 4.45763000 & 0.56175600 \\
\hline $\mathrm{H}$ & 2.35548600 & 3.84676900 & -0.08294900 & $\mathrm{H}$ & 1.34576800 & 3.81245000 & 1.35859800 \\
\hline $\mathrm{H}$ & 2.33838800 & 5.23525800 & 1.02010400 & C & -0.19577800 & 6.10051800 & 0.67198400 \\
\hline $\mathrm{H}$ & 0.47132700 & 6.89591900 & 1.02332500 & $\mathrm{H}$ & -0.60683300 & 5.59251500 & 1.54587800 \\
\hline $\mathrm{H}$ & -1.02620100 & 6.56745100 & 0.12917900 & C & 1.21463900 & 5.93414400 & -1.38625600 \\
\hline $\mathrm{H}$ & 1.92651100 & 6.66082200 & -0.98029200 & $\mathrm{H}$ & 0.46024600 & 6.49432100 & -1.95182800 \\
\hline $\mathrm{H}$ & 1.76590600 & 5.28823400 & -2.07832700 & C & 0.26784900 & 2.96481200 & -1.58238000 \\
\hline $\mathrm{O}$ & 0.70837000 & 2.02326900 & -0.90458100 & C & -0.06286900 & 3.98213500 & -3.87799600 \\
\hline C & 1.25993500 & 1.95755000 & -3.56729100 & C & 1.03011000 & 3.97337700 & -4.94936900 \\
\hline $\mathrm{H}$ & -1.02873600 & 3.64547900 & -4.27608500 & $\mathrm{H}$ & -0.20552200 & 4.95683500 & -3.41743800 \\
\hline $\mathrm{H}$ & 2.21205100 & 1.92809900 & -3.03272200 & $\mathrm{H}$ & 1.87028000 & 4.59362300 & -4.61617900 \\
\hline $\mathrm{H}$ & 0.67998700 & 4.36076200 & -5.91024200 & $\mathrm{~N}$ & 0.44457800 & 3.00348700 & -2.91393300 \\
\hline C & 0.60847200 & 0.58500000 & -3.54121200 & C & -0.74786500 & 0.46866000 & -3.45952200 \\
\hline C & 1.43120900 & -0.60084100 & -3.65507700 & C & -1.41382300 & -0.79728300 & -3.49963400 \\
\hline $\mathrm{H}$ & -1.36050900 & 1.35373600 & -3.32299900 & C & 0.79088800 & -1.88133800 & -3.66950300 \\
\hline $\mathrm{C}$ & 2.83473000 & -0.55621600 & -3.72910000 & C & -2.81088900 & -0.90531300 & -3.40284300 \\
\hline C & -0.62906300 & -1.97945500 & -3.61679400 & C & 1.57607000 & -3.07383700 & -3.73318100 \\
\hline $\mathrm{H}$ & 3.34942000 & 0.39653300 & -3.72377000 & C & 3.59102900 & -1.72558300 & -3.77590900 \\
\hline C & -3.42871500 & -2.15308800 & -3.45007200 & $\mathrm{H}$ & -3.40352900 & -0.00565900 & -3.26716300 \\
\hline $\mathrm{C}$ & -1.26904700 & -3.25352200 & -3.65556700 & C & 2.97441600 & -2.97331700 & -3.77199500 \\
\hline C & 0.90564500 & -4.34387200 & -3.76254300 & $\mathrm{H}$ & 4.67400600 & -1.65735700 & -3.82707100 \\
\hline C & -2.66883300 & -3.31411300 & -3.58276700 & $\mathrm{H}$ & -4.50703200 & -2.22027800 & -3.36292200 \\
\hline $\mathrm{C}$ & -0.45225200 & -4.43005500 & -3.73712400 & $\mathrm{H}$ & 3.57034900 & -3.88153600 & -3.81568100 \\
\hline $\mathrm{H}$ & 1.51429600 & -5.24315600 & -3.81690600 & $\mathrm{H}$ & -3.15748700 & -4.28484900 & -3.61237200 \\
\hline $\mathrm{H}$ & -0.94432700 & -5.39905200 & -3.76733300 & C & -2.38314700 & 2.81314300 & -0.07992200 \\
\hline $\mathrm{S}$ & -3.06082600 & 2.79700400 & -1.62918100 & $\mathrm{~N}$ & -1.24106100 & 3.49016700 & 0.21596000 \\
\hline $\mathrm{H}$ & -0.69472200 & 3.09092400 & 0.98671800 & $\mathrm{~S}$ & -0.33030000 & 2.04669300 & 3.61984200 \\
\hline $\mathrm{O}$ & 0.05283700 & 0.92696500 & 4.49691400 & $\mathrm{O}$ & 0.17426400 & 1.94318400 & 2.21574500 \\
\hline $\mathrm{O}$ & -1.75379100 & 2.46870300 & 3.67320300 & C & 0.59438300 & 3.50055500 & 4.31292300 \\
\hline $\mathrm{F}$ & 0.30720200 & 3.67384500 & 5.60535800 & $\mathrm{~F}$ & 0.26727500 & 4.62240400 & 3.64726900 \\
\hline $\mathrm{F}$ & 1.91590600 & 3.30943300 & 4.18404100 & C & 3.76068900 & 0.46230700 & -0.69695100 \\
\hline $\mathrm{O}$ & 4.01139700 & 1.47697400 & -1.32306500 & $\mathrm{O}$ & 4.64645400 & -0.34962100 & -0.09465000 \\
\hline $\mathrm{C}$ & 6.09383800 & -0.06523200 & -0.16637300 & C & 6.55441600 & -0.10617200 & -1.62350000 \\
\hline $\mathrm{H}$ & 7.64618500 & -0.03070700 & -1.66216600 & $\mathrm{H}$ & 6.12400000 & 0.71661100 & -2.19507600 \\
\hline $\mathrm{H}$ & 6.25917700 & -1.05443300 & -2.08425000 & C & 6.69757400 & -1.22214200 & 0.62669200 \\
\hline $\mathrm{H}$ & 6.31505200 & -1.22677400 & 1.65271000 & $\mathrm{H}$ & 7.78635800 & -1.11998500 & 0.66527600 \\
\hline $\mathrm{H}$ & 6.45562400 & -2.18052700 & 0.15710000 & C & 6.38781900 & 1.27374200 & 0.50988300 \\
\hline $\mathrm{H}$ & 7.47127900 & 1.42553900 & 0.55865400 & $\mathrm{H}$ & 5.99639200 & 1.27688500 & 1.53270700 \\
\hline $\mathrm{H}$ & 5.94021100 & 2.10037900 & -0.04239200 & $\mathrm{C}$ & 2.10259000 & -1.14290300 & 0.12100300 \\
\hline $\mathrm{H}$ & 2.90465300 & -1.86686800 & 0.17787300 & C & 0.75481000 & -1.68721500 & -0.10996100 \\
\hline $\mathrm{C}$ & 0.60755200 & -3.07949200 & -0.20836100 & C & -0.38096000 & -0.86721000 & -0.18596800 \\
\hline C & -0.65243800 & -3.64834300 & -0.36039100 & $\mathrm{H}$ & 1.48515000 & -3.71616700 & -0.18431200 \\
\hline
\end{tabular}




\begin{tabular}{|c|c|c|c|c|c|c|c|}
\hline $\mathrm{C}$ & -1.63522500 & -1.44504200 & -0.35749300 & $\mathrm{H}$ & -0.29065300 & 0.20960200 & -0.09719400 \\
\hline $\mathrm{C}$ & -1.78003300 & -2.82950700 & -0.42424600 & $\mathrm{H}$ & -0.74984100 & -4.72575300 & -0.44779500 \\
\hline $\mathrm{H}$ & -2.50287900 & -0.80925000 & -0.44674900 & $\mathrm{H}$ & -2.76761000 & -3.25850000 & -0.54951200 \\
\hline $\mathrm{N}$ & 2.46598900 & 0.00220700 & -0.53875300 & $\mathrm{H}$ & 1.75082000 & 0.68871500 & -0.83236800 \\
\hline C & 2.14197500 & -0.56593700 & 2.06826200 & $\mathrm{H}$ & 1.42381200 & 0.23896100 & 1.92118700 \\
\hline $\mathrm{H}$ & 3.18660500 & -0.26637900 & 2.09891700 & $\mathrm{C}$ & 1.75353800 & -1.61507900 & 2.90374400 \\
\hline C & 2.73808000 & -2.46963000 & 3.58648500 & $\mathrm{H}$ & 2.38197700 & -2.67776200 & 4.60476500 \\
\hline $\mathrm{H}$ & 3.71845300 & -1.98450200 & 3.65532800 & C & 0.31061200 & -1.87706700 & 3.14310000 \\
\hline $\mathrm{H}$ & -0.04575800 & -1.10035100 & 3.83822700 & $\mathrm{H}$ & 0.11710700 & -2.86056000 & 3.57377900 \\
\hline $\mathrm{H}$ & -0.27988600 & -1.75008300 & 2.23164800 & C & 4.13075200 & -5.06713200 & 4.22306600 \\
\hline $\mathrm{H}$ & 5.06356600 & -4.51648300 & 4.39161300 & $\mathrm{H}$ & 4.39805100 & -6.08887400 & 3.92826500 \\
\hline $\mathrm{H}$ & 3.59934700 & -5.12627200 & 5.17979200 & C & 4.07971700 & -4.15878000 & 1.29990100 \\
\hline $\mathrm{H}$ & 4.87955300 & -3.41568300 & 1.38359200 & $\mathrm{H}$ & 3.48508800 & -3.90926600 & 0.41693200 \\
\hline $\mathrm{H}$ & 4.55080300 & -5.13060500 & 1.10844000 & C & 1.46453000 & -5.19947200 & 2.66665900 \\
\hline $\mathrm{H}$ & 0.77810500 & -4.70277300 & 1.97534300 & $\mathrm{H}$ & 0.94352000 & -5.32495000 & 3.62279600 \\
\hline $\mathrm{H}$ & 1.67443100 & -6.20198900 & 2.27386400 & $\mathrm{Si}$ & 3.07172300 & -4.24798000 & 2.89414500 \\
\hline C & 1.44203700 & 2.49337600 & -5.01495900 & $\mathrm{H}$ & 0.77484800 & 1.94568200 & -5.68738200 \\
\hline $\mathrm{H}$ & 2.46482800 & 2.34816300 & -5.37212400 & & & & \\
\hline
\end{tabular}

\section{TS-S-4f-37}

Imaginary frequency: $-390.21 \mathrm{~cm}^{-1}$

E[B3LYP/6-31G(d)-D3(BJ)]: -4789.076692 Hartree

$\mathrm{E}\left[\mathrm{PCM}\left(\mathrm{Et}_{2} \mathrm{O}\right)-\mathrm{B} 3 \mathrm{LYP} / 6-311 \mathrm{G}(\mathrm{d}, \mathrm{p})-\mathrm{D} 3(\mathrm{BJ})\right]$ : -4790.221141 Hartree

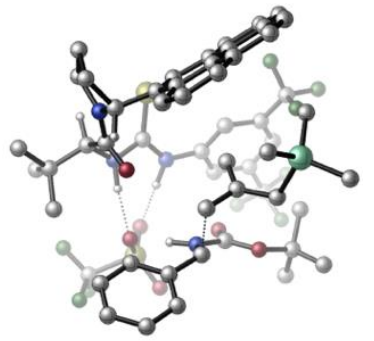

\begin{tabular}{|c|c|c|c|c|c|c|c|}
\hline 01 & & & & $\mathrm{~N}$ & 2.13777300 & 0.29974100 & -1.97697100 \\
\hline $\mathrm{H}$ & 3.02943400 & -0.03145400 & -1.58886100 & $\mathrm{C}$ & 2.00900600 & 1.69069000 & -1.97662600 \\
\hline C & 3.18209600 & 2.43789900 & -1.77192600 & C & 0.78460900 & 2.36052500 & -2.05575200 \\
\hline C & 3.11736200 & 3.82130000 & -1.66949700 & $\mathrm{H}$ & 4.12697100 & 1.92016300 & -1.65135700 \\
\hline $\mathrm{C}$ & 0.74394000 & 3.75078300 & -1.95036200 & $\mathrm{H}$ & -0.12791700 & 1.80040400 & -2.19042000 \\
\hline $\mathrm{C}$ & 1.90039100 & 4.50105100 & -1.77054900 & $\mathrm{H}$ & 1.85914800 & 5.57924900 & -1.68589500 \\
\hline C & 4.35086400 & 4.59696700 & -1.30688600 & C & -0.60412400 & 4.40500300 & -1.93659100 \\
\hline $\mathrm{F}$ & 4.42660200 & 4.78443600 & 0.03892900 & $\mathrm{~F}$ & 4.35207100 & 5.83000600 & -1.86372300 \\
\hline $\mathrm{F}$ & 5.48332700 & 3.97591400 & -1.68255000 & $\mathrm{~F}$ & -1.38244300 & 3.99177700 & -2.96462100 \\
\hline $\mathrm{F}$ & -1.28954100 & 4.09315300 & -0.79478000 & $\mathrm{~F}$ & -0.53931600 & 5.74884200 & -1.99269700 \\
\hline $\mathrm{C}$ & 0.75281000 & -3.05609000 & -2.25208900 & $\mathrm{H}$ & 0.43418600 & -2.97596800 & -3.29148700 \\
\hline C & 1.56254000 & -4.38791800 & -2.11179100 & C & 1.99737600 & -4.67890000 & -0.66549400 \\
\hline $\mathrm{H}$ & 1.12792000 & -4.78595000 & -0.00986900 & $\mathrm{H}$ & 2.64292200 & -3.90094200 & -0.25565400 \\
\hline $\mathrm{H}$ & 2.55963600 & -5.61979400 & -0.64278300 & C & 2.79788700 & -4.28287200 & -3.02372300 \\
\hline $\mathrm{H}$ & 3.35742700 & -5.22495300 & -3.00319000 & $\mathrm{H}$ & 3.47047700 & -3.48523400 & -2.70606000 \\
\hline $\mathrm{H}$ & 2.50164300 & -4.08532100 & -4.06071400 & C & 0.69828500 & -5.56557000 & -2.59527300 \\
\hline $\mathrm{H}$ & 1.25815300 & -6.50022500 & -2.48320700 & $\mathrm{H}$ & 0.43785000 & -5.46609400 & -3.65485000 \\
\hline $\mathrm{H}$ & -0.22525500 & -5.66226400 & -2.01344200 & C & -0.48210800 & -2.97020300 & -1.32987300 \\
\hline $\mathrm{O}$ & -0.35314400 & -2.62687000 & -0.14689800 & C & -1.99486200 & -3.59495400 & -3.26511400 \\
\hline C & -2.89612600 & -3.31386600 & -1.01449500 & C & -3.49745300 & -3.92468400 & -3.25564400 \\
\hline $\mathrm{H}$ & -1.74542900 & -2.73894400 & -3.89898000 & $\mathrm{H}$ & -1.41268900 & -4.45502800 & -3.59705800 \\
\hline $\mathrm{C}$ & -3.77713900 & -4.31405700 & -1.79750300 & $\mathrm{H}$ & -2.61423400 & -3.67820800 & -0.02373800 \\
\hline $\mathrm{H}$ & -3.74471500 & -4.71858000 & -3.96517100 & $\mathrm{H}$ & -4.08296600 & -3.03992400 & -3.52173800 \\
\hline $\mathrm{H}$ & -3.42961400 & -5.33414500 & -1.59535200 & $\mathrm{H}$ & -4.83345700 & -4.24796400 & -1.53262000 \\
\hline $\mathrm{N}$ & -1.69027700 & -3.28824100 & -1.85182600 & $\mathrm{C}$ & -3.58605000 & -1.95874800 & -0.91201100 \\
\hline $\mathrm{C}$ & -3.16025600 & -0.89667400 & -1.65419600 & $\mathrm{C}$ & -4.78684900 & -1.83826300 & -0.10610600 \\
\hline $\mathrm{C}$ & -3.89034100 & 0.33715300 & -1.72097600 & $\mathrm{H}$ & -2.24901600 & -0.97258100 & -2.23899300 \\
\hline
\end{tabular}




\begin{tabular}{|c|c|c|c|c|c|c|c|}
\hline $\mathrm{C}$ & -5.56615800 & -0.64174000 & -0.20468500 & $\mathrm{C}$ & -5.22600700 & -2.84858800 & 0.76687900 \\
\hline C & -3.43115700 & 1.43227900 & -2.47021200 & C & -5.12798600 & 0.43918000 & -1.02305900 \\
\hline $\mathrm{C}$ & -6.78218100 & -0.51602700 & 0.53434400 & $\mathrm{H}$ & -4.64336000 & -3.75602200 & 0.87949800 \\
\hline $\mathrm{C}$ & -6.40287500 & -2.70705400 & 1.50125800 & $\mathrm{C}$ & -4.19378100 & 2.59671900 & -2.55925000 \\
\hline $\mathrm{H}$ & -2.47824300 & 1.35856400 & -2.98615900 & C & -5.90528100 & 1.63041300 & -1.12754200 \\
\hline C & -7.18269000 & -1.56212000 & 1.37885100 & $\mathrm{C}$ & -7.54926700 & 0.69188600 & 0.40567800 \\
\hline $\mathrm{H}$ & -6.71430300 & -3.50403400 & 2.17031200 & C & -5.42223400 & 2.69074400 & -1.91003000 \\
\hline $\mathrm{H}$ & -3.81630700 & 3.43838800 & -3.12898100 & C & -7.13478600 & 1.71433600 & -0.39198900 \\
\hline $\mathrm{H}$ & -8.10541400 & -1.46330000 & 1.94454700 & $\mathrm{H}$ & -8.47760900 & 0.77126800 & 0.96555800 \\
\hline $\mathrm{H}$ & -6.01013400 & 3.60191300 & -1.98467400 & $\mathrm{H}$ & -7.72742000 & 2.62154200 & -0.47813800 \\
\hline C & 1.27836400 & -0.66203600 & -2.43896500 & $\mathrm{~S}$ & -0.00079000 & -0.37368300 & -3.51770200 \\
\hline $\mathrm{N}$ & 1.58614400 & -1.89769700 & -1.98255400 & $\mathrm{H}$ & 2.20605600 & -1.94932800 & -1.16779800 \\
\hline C & 2.15930400 & 1.02815200 & 1.89339300 & $\mathrm{O}$ & 2.85624300 & 1.35307700 & 0.95774000 \\
\hline $\mathrm{O}$ & 1.66819200 & 1.82916600 & 2.85172400 & C & 2.04818700 & 3.26490300 & 2.88233400 \\
\hline $\mathrm{C}$ & 1.46818200 & 3.96498500 & 1.65619900 & $\mathrm{H}$ & 1.71327400 & 5.03122600 & 1.69659300 \\
\hline $\mathrm{H}$ & 1.89246400 & 3.55425800 & 0.74339300 & $\mathrm{H}$ & 0.37797100 & 3.86928100 & 1.61434900 \\
\hline C & 1.39466800 & 3.75173700 & 4.17358300 & $\mathrm{H}$ & 1.79245400 & 3.20844300 & 5.03652400 \\
\hline $\mathrm{H}$ & 1.60116200 & 4.81753700 & 4.31029000 & $\mathrm{H}$ & 0.30945800 & 3.61345000 & 4.14592200 \\
\hline $\mathrm{C}$ & 3.56931700 & 3.39933400 & 2.96059100 & $\mathrm{H}$ & 3.82258400 & 4.44566800 & 3.16110800 \\
\hline $\mathrm{H}$ & 3.96244000 & 2.78740300 & 3.77889500 & $\mathrm{H}$ & 4.04763600 & 3.09907700 & 2.02970200 \\
\hline C & 0.91638900 & -0.73775300 & 3.06363800 & $\mathrm{H}$ & 0.77992100 & -0.01702900 & 3.86285700 \\
\hline $\mathrm{C}$ & 1.06223000 & -2.14588100 & 3.50465500 & $C$ & 0.95651500 & -2.43193000 & 4.87247000 \\
\hline $\mathrm{C}$ & 1.31282100 & -3.18026900 & 2.59264500 & $C$ & 1.12976300 & -3.73595200 & 5.33069300 \\
\hline $\mathrm{H}$ & 0.75820800 & -1.62836700 & 5.57752400 & C & 1.48613100 & -4.48017100 & 3.05672000 \\
\hline $\bar{H}$ & 1.34132900 & -2.97498400 & 1.53058300 & $\mathrm{C}$ & 1.39907700 & -4.76128900 & 4.42256300 \\
\hline $\mathrm{H}$ & 1.06157500 & -3.94928700 & 6.39326600 & $\mathrm{H}$ & 1.68983600 & -5.27543700 & 2.34662900 \\
\hline $\mathrm{H}$ & 1.53784000 & -5.77811300 & 4.77831900 & $\mathrm{~N}$ & 1.76169300 & -0.29515700 & 2.08301500 \\
\hline $\bar{H}$ & 2.25148900 & -0.95701000 & 1.45537800 & $\mathrm{~S}$ & 4.63317400 & -1.22382300 & 0.21376500 \\
\hline $\mathrm{O}$ & 5.14844800 & -0.60906700 & 1.43737900 & $\mathrm{O}$ & 3.25219400 & -1.82738900 & 0.35458900 \\
\hline 0 & 4.76499800 & -0.47765500 & -1.06224200 & C & 5.67635200 & -2.74022800 & -0.03589100 \\
\hline $\mathrm{F}$ & 6.96004800 & -2.40429100 & -0.18818100 & $\mathrm{~F}$ & 5.27734800 & -3.41474000 & -1.12918200 \\
\hline $\mathrm{F}$ & 5.56182400 & -3.55515300 & 1.02092400 & $\mathrm{C}$ & -0.98017000 & -0.79797700 & 2.45850700 \\
\hline $\mathrm{H}$ & -0.91784700 & -1.57925500 & 1.70402600 & $\mathrm{H}$ & -1.42200800 & -1.08152200 & 3.41076300 \\
\hline $\mathrm{C}$ & -1.15539300 & 0.51829100 & 2.02251200 & C & -1.56972500 & 1.57719800 & 2.93525400 \\
\hline $\bar{H}$ & -1.40915400 & 1.30440100 & 3.98413900 & $\bar{H}$ & -1.06413300 & 2.52136800 & 2.71657900 \\
\hline C & -0.83464100 & 0.83244100 & 0.60449700 & $\mathrm{H}$ & -1.49066100 & 0.25601000 & -0.05919200 \\
\hline $\bar{H}$ & -0.90582300 & 1.89249300 & 0.36406900 & $\bar{H}$ & 0.17743000 & 0.46981700 & 0.37301900 \\
\hline $\mathrm{C}$ & -3.81519700 & 3.14733600 & 4.22981400 & $\bar{H}$ & -3.19271300 & 4.04834500 & 4.18185500 \\
\hline $\mathrm{H}$ & -4.86343500 & 3.46908800 & 4.21643000 & $\mathrm{H}$ & -3.63032400 & 2.65857600 & 5.19325100 \\
\hline $\mathrm{C}$ & -3.72324700 & 2.87956200 & 1.15277700 & $\mathrm{H}$ & -3.12060600 & 3.79345500 & 1.10506500 \\
\hline $\mathrm{H}$ & -3.45069000 & 2.26378000 & 0.29271300 & $\mathrm{H}$ & -4.77380100 & 3.16023100 & 1.02865200 \\
\hline C & -4.42413400 & 0.38785500 & 2.95781700 & $\mathrm{H}$ & -4.14305000 & -0.34584900 & 2.19671000 \\
\hline $\mathrm{H}$ & -4.24904400 & -0.06270200 & 3.94230500 & $\mathrm{H}$ & -5.49999900 & 0.56075600 & 2.85606300 \\
\hline Si & -3.46437300 & 1.99130000 & 2.78100100 & & & & \\
\hline
\end{tabular}

\section{TS-S-4f-38}

Imaginary frequency: $-310.42 \mathrm{~cm}^{-1}$

E[B3LYP/6-31G(d)-D3(BJ)]: -4789.075165 Hartree

$\mathrm{E}\left[\mathrm{PCM}\left(\mathrm{Et}_{2} \mathrm{O}\right)-\mathrm{B} 3 \mathrm{LYP} / 6-311 \mathrm{G}(\mathrm{d}, \mathrm{p})-\mathrm{D} 3(\mathrm{BJ})\right]$ : -4790.221057 Hartree

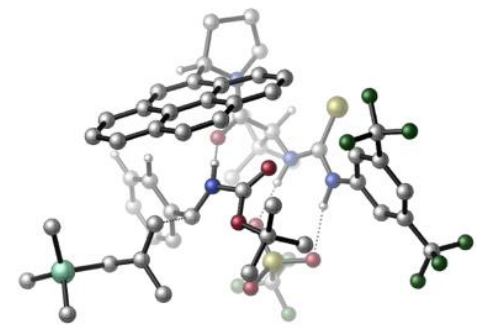




\begin{tabular}{|c|c|c|c|c|c|c|c|}
\hline 01 & & & & $\mathrm{~N}$ & -3.00047600 & -0.58023800 & 1.27817600 \\
\hline $\mathrm{H}$ & -2.52960300 & -1.48608700 & 1.32970100 & C & -3.95507300 & -0.50636600 & 0.25418100 \\
\hline $\mathrm{C}$ & -4.68068800 & -1.66589000 & -0.03932500 & C & -4.10703500 & 0.62820600 & -0.55100200 \\
\hline C & -5.54913300 & -1.68730000 & -1.12940200 & $\mathrm{H}$ & -4.52636200 & -2.55760100 & 0.55673200 \\
\hline C & -4.98839700 & 0.59105200 & -1.62755700 & $\mathrm{H}$ & -3.50146800 & 1.50001100 & -0.36040200 \\
\hline C & -5.71876800 & -0.55949700 & -1.92914400 & $\mathrm{H}$ & -6.39420100 & -0.57971800 & -2.77495100 \\
\hline C & -6.35312500 & -2.92471200 & -1.41328900 & C & -5.06791500 & 1.77620700 & -2.54470300 \\
\hline $\mathrm{F}$ & -7.54030500 & -2.91156000 & -0.76237500 & $\mathrm{~F}$ & -5.70744500 & -4.04611600 & -1.03110100 \\
\hline $\mathrm{F}$ & -6.63398100 & -3.04673300 & -2.73221500 & $\mathrm{~F}$ & -4.93485500 & 2.95018500 & -1.88499200 \\
\hline $\mathrm{F}$ & -4.07853500 & 1.75380000 & -3.48281100 & $\mathrm{~F}$ & -6.23401900 & 1.82498700 & -3.22246500 \\
\hline C & -0.79508500 & 0.97896100 & 3.72012700 & $\mathrm{H}$ & -1.58272100 & 1.59505200 & 4.14957700 \\
\hline $\mathrm{C}$ & -0.11951700 & 0.20478100 & 4.89580800 & $\mathrm{C}$ & 1.02526500 & -0.72183200 & 4.45061000 \\
\hline $\mathrm{H}$ & 1.83296800 & -0.17110900 & 3.96607600 & $\mathrm{H}$ & 0.68492700 & -1.49000000 & 3.75366000 \\
\hline $\mathrm{H}$ & 1.43351200 & -1.23124000 & 5.33216800 & C & -1.20800800 & -0.63491800 & 5.58647300 \\
\hline $\mathrm{H}$ & -0.79773400 & -1.11747100 & 6.48102200 & $\mathrm{H}$ & -1.58035200 & -1.41792700 & 4.92001300 \\
\hline $\mathrm{H}$ & -2.05924100 & -0.01529100 & 5.89236200 & C & 0.43121800 & 1.24501800 & 5.88730500 \\
\hline $\mathrm{H}$ & 0.88111100 & 0.74175000 & 6.75000400 & $\mathrm{H}$ & -0.36206200 & 1.90331500 & 6.26132400 \\
\hline $\mathrm{H}$ & 1.20790100 & 1.86860100 & 5.42829200 & C & 0.19811400 & 1.84762100 & 2.93925700 \\
\hline $\mathrm{O}$ & 1.04124100 & 1.30399100 & 2.21122200 & C & -0.74177100 & 3.98907000 & 3.90328300 \\
\hline C & 1.17567400 & 4.01677600 & 2.37514400 & C & -0.67176500 & 5.39289500 & 3.29258500 \\
\hline $\mathrm{H}$ & -1.75196700 & 3.57586200 & 3.87758500 & $\mathrm{H}$ & -0.37479900 & 3.98724900 & 4.93783300 \\
\hline $\mathrm{C}$ & 0.73233900 & 5.47972200 & 2.66527000 & $\mathrm{H}$ & 2.12925900 & 3.82087400 & 2.87919000 \\
\hline $\mathrm{H}$ & -0.84252800 & 6.16856500 & 4.04404800 & $\mathrm{H}$ & -1.44230700 & 5.50485000 & 2.52553200 \\
\hline $\mathrm{H}$ & 1.44032000 & 5.93676300 & 3.36361000 & $\mathrm{H}$ & 0.73893500 & 6.08033100 & 1.75291000 \\
\hline $\mathrm{N}$ & 0.14905600 & 3.19308500 & 3.04743700 & C & 1.41900300 & 3.70657600 & 0.90609700 \\
\hline C & 2.71929800 & 3.55953100 & 0.51625100 & C & 0.36366000 & 3.63908700 & -0.08521100 \\
\hline C & 3.10533800 & 3.29703200 & -0.83589200 & $\mathrm{H}$ & 3.50791800 & 3.61411200 & 1.26391800 \\
\hline C & 0.72418500 & 3.41891900 & -1.45454000 & C & -0.99885700 & 3.76661600 & 0.22558900 \\
\hline C & 4.44521200 & 3.10457700 & -1.21102700 & C & 2.08549400 & 3.22551700 & -1.82883700 \\
\hline C & -0.28796000 & 3.37907700 & -2.46078800 & $\mathrm{H}$ & -1.31794800 & 3.83955700 & 1.25268700 \\
\hline $\mathrm{C}$ & -1.97610700 & 3.73808400 & -0.76583800 & C & 4.77990800 & 2.85201100 & -2.54060000 \\
\hline $\mathrm{H}$ & 5.22168000 & 3.16994200 & -0.45261000 & C & 2.43829800 & 2.96727600 & -3.18873500 \\
\hline C & -1.63029100 & 3.55748500 & -2.09849100 & C & 0.09634800 & 3.14005100 & -3.82258300 \\
\hline $\mathrm{H}$ & -3.01887200 & 3.82987700 & -0.48309700 & C & 3.79024700 & 2.77684000 & -3.51877100 \\
\hline $\mathrm{H}$ & 5.82127000 & 2.72281600 & -2.81874300 & C & 1.39494100 & 2.93114900 & -4.17311900 \\
\hline $\mathrm{H}$ & -2.39929000 & 3.51171700 & -2.86217600 & $\mathrm{H}$ & -0.68728300 & 3.11603500 & -4.57493700 \\
\hline $\mathrm{H}$ & 4.05923900 & 2.58847400 & -4.55508400 & $\mathrm{H}$ & 1.66584900 & 2.74486700 & -5.20923600 \\
\hline C & -2.57733600 & 0.41575400 & 2.11343500 & $\mathrm{~S}$ & -3.42879700 & 1.86218900 & 2.35801600 \\
\hline $\mathrm{N}$ & -1.44387800 & 0.08808800 & 2.77910000 & $\mathrm{H}$ & -0.86903300 & -0.65867200 & 2.37878600 \\
\hline C & 0.01045300 & -0.03061400 & -1.08043900 & 0 & -0.98731400 & 0.62617800 & -0.87585300 \\
\hline $\mathrm{O}$ & 0.21219300 & -0.93026200 & -2.05970900 & C & -0.84928700 & -1.29902000 & -3.02518600 \\
\hline $\mathrm{C}$ & -1.27034900 & -0.06531200 & -3.81826800 & $\mathrm{H}$ & -1.94635700 & -0.36981300 & -4.62450100 \\
\hline $\mathrm{H}$ & -1.79091300 & 0.65143600 & -3.18461700 & $\mathrm{H}$ & -0.39681000 & 0.42234000 & -4.26129400 \\
\hline C & -0.13671100 & -2.31694400 & -3.91208400 & $\mathrm{H}$ & 0.19784300 & -3.16849600 & -3.31227800 \\
\hline $\mathrm{H}$ & -0.82455100 & -2.68312300 & -4.68036000 & $\mathrm{H}$ & 0.72647300 & -1.86343200 & -4.41319900 \\
\hline $\mathrm{C}$ & -2.01484200 & -1.94612400 & -2.29132900 & $\mathrm{H}$ & -2.75737800 & -2.28758700 & -3.02048400 \\
\hline $\mathrm{H}$ & -1.68316300 & -2.80313500 & -1.70217300 & $\mathrm{H}$ & -2.49387700 & -1.22910200 & -1.63131400 \\
\hline C & 2.08721800 & -0.94092400 & -0.22297600 & $\mathrm{H}$ & 1.65180100 & -1.90268400 & -0.46659800 \\
\hline $\mathrm{C}$ & 2.96703400 & -0.95825400 & 0.97251700 & $\mathrm{C}$ & 3.15815300 & -2.17150700 & 1.64331000 \\
\hline $\mathrm{C}$ & 3.64489600 & 0.18711000 & 1.40507800 & $C$ & 4.00233300 & -2.23326700 & 2.75021300 \\
\hline $\mathrm{H}$ & 2.61995700 & -3.05282800 & 1.31616300 & C & 4.49736100 & 0.11959800 & 2.50415800 \\
\hline $\mathrm{H}$ & 3.49377600 & 1.12813400 & 0.88954700 & $\mathrm{C}$ & 4.67624900 & -1.08962800 & 3.18103400 \\
\hline
\end{tabular}




\begin{tabular}{|c|c|c|c|c|c|c|c|}
\hline $\mathrm{H}$ & 4.12192100 & -3.17313500 & 3.28071600 & $\mathrm{H}$ & 5.01378700 & 1.01445200 & 2.84019600 \\
\hline $\mathrm{H}$ & 5.33399100 & -1.13760300 & 4.04428700 & $\mathrm{~N}$ & 1.16218000 & 0.08889100 & -0.30635900 \\
\hline $\mathrm{H}$ & 1.07802600 & 0.71647400 & 0.50167800 & $S$ & -0.45788700 & -3.26923600 & 0.99798700 \\
\hline 0 & 0.36236800 & -3.85119200 & -0.08797300 & 0 & 0.03844700 & -1.94980600 & 1.47834900 \\
\hline $\mathrm{O}$ & -1.92731600 & -3.31244300 & 0.82027100 & C & -0.15077800 & -4.37632000 & 2.45878300 \\
\hline $\mathrm{F}$ & -0.60873400 & -5.61045900 & 2.23385100 & $F$ & -0.76325200 & -3.87731100 & 3.54477600 \\
\hline $\mathrm{F}$ & 1.16791600 & -4.45275200 & 2.72314400 & C & 3.22189700 & -0.71046300 & -1.70147400 \\
\hline $\mathrm{H}$ & 2.46371900 & -0.54884700 & -2.46038100 & $\mathrm{H}$ & 3.78547000 & 0.17537000 & -1.41981000 \\
\hline C & 3.89146200 & -1.94556300 & -1.71519600 & C & 5.15859700 & -2.13116700 & -1.02930300 \\
\hline $\mathrm{H}$ & 5.32552200 & -1.37359800 & -0.25625600 & $\mathrm{H}$ & 5.26028000 & -3.13552000 & -0.60295500 \\
\hline C & 3.20481300 & -3.14073300 & -2.28363200 & $\mathrm{H}$ & 3.90370200 & -3.95022400 & -2.50646800 \\
\hline $\mathrm{H}$ & 2.49178700 & -3.51915200 & -1.52975600 & $\mathrm{H}$ & 2.61278400 & -2.89551100 & -3.16778600 \\
\hline C & 8.18140600 & -2.34249700 & -1.24599700 & $\mathrm{H}$ & 8.15403100 & -3.38068400 & -0.89645900 \\
\hline $\mathrm{H}$ & 9.08986700 & -2.21672500 & -1.84684900 & $\mathrm{H}$ & 8.26949800 & -1.69349200 & -0.36749000 \\
\hline $\mathrm{C}$ & 6.45278400 & -3.09123100 & -3.71639800 & $\mathrm{H}$ & 6.36875300 & -4.13464200 & -3.39153600 \\
\hline $\mathrm{H}$ & 5.56684900 & -2.85128600 & -4.31462500 & $\mathrm{H}$ & 7.32482800 & -3.02329000 & -4.37765100 \\
\hline C & 6.67746200 & -0.12602100 & -2.82476200 & $\mathrm{H}$ & 5.76021900 & 0.14446100 & -3.35767400 \\
\hline $\mathrm{H}$ & 6.77071700 & 0.55421600 & -1.97118000 & $\mathrm{H}$ & 7.52507200 & 0.05877000 & -3.49522600 \\
\hline Si & 6.65348700 & -1.91645600 & -2.25931400 & & & & \\
\hline
\end{tabular}

\section{TS-S-4f-39}

Imaginary frequency: $-307.89 \mathrm{~cm}^{-1}$ E[B3LYP/6-31G(d)-D3(BJ)]: -4789.076166 Hartree E[PCM(Et $\left.\left.{ }_{2} \mathrm{O}\right)-\mathrm{B} 3 \mathrm{LYP} / 6-311 \mathrm{G}(\mathrm{d}, \mathrm{p})-\mathrm{D} 3(\mathrm{BJ})\right]$ : -4790.220877 Hartree

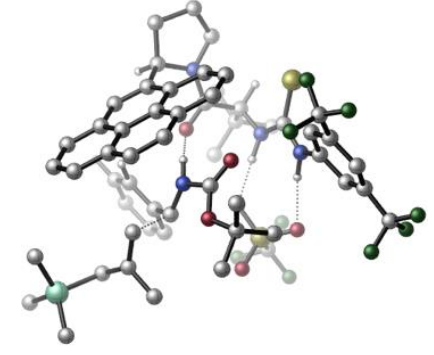

\begin{tabular}{|c|c|c|c|c|c|c|c|}
\hline 01 & & & & $\mathrm{~N}$ & -3.02164500 & -0.37441800 & 1.42453300 \\
\hline $\mathrm{H}$ & -2.53803200 & -1.25024900 & 1.63511300 & $\mathrm{C}$ & -3.94996200 & -0.49488200 & 0.38284400 \\
\hline$C$ & -4.58572500 & -1.73309900 & 0.23060600 & $\mathrm{C}$ & -4.16286500 & 0.51077500 & -0.56766100 \\
\hline C & -5.41608700 & -1.96029900 & -0.86473000 & $\mathrm{H}$ & -4.38562800 & -2.52711200 & 0.94017500 \\
\hline C & -5.01778900 & 0.27244600 & -1.63943500 & $\mathrm{H}$ & -3.63256500 & 1.44510200 & -0.48093400 \\
\hline C & -5.65279400 & -0.95958600 & -1.80361600 & $\mathrm{H}$ & -6.31254800 & -1.13551200 & -2.64398200 \\
\hline C & -5.97509000 & -3.33399900 & -1.09587900 & $\mathrm{C}$ & -5.18112600 & 1.32413100 & -2.69779500 \\
\hline $\mathrm{F}$ & -6.16931700 & -4.01032400 & 0.05451400 & $\mathrm{~F}$ & -5.13355200 & -4.08240600 & -1.85552500 \\
\hline $\mathrm{F}$ & -7.15678800 & -3.29663300 & -1.75290400 & $\mathrm{~F}$ & -6.38292700 & 1.25281600 & -3.30846600 \\
\hline$F$ & -5.04946300 & 2.57829600 & -2.20903700 & $F$ & -4.24459100 & 1.19819200 & -3.68142000 \\
\hline C & -0.80311500 & 1.59629600 & 3.53478900 & $\mathrm{H}$ & -1.59105400 & 2.27180300 & 3.86223100 \\
\hline C & -0.10773100 & 1.03917000 & 4.81714600 & C & 1.05202500 & 0.07163500 & 4.52310800 \\
\hline $\mathrm{H}$ & 1.85185000 & 0.55108600 & 3.95646400 & $\mathrm{H}$ & 0.72529600 & -0.80173400 & 3.95617100 \\
\hline $\mathrm{H}$ & 1.46672000 & -0.28519100 & 5.47389800 & C & -1.17747700 & 0.30792200 & 5.64651400 \\
\hline $\mathrm{H}$ & -0.75560400 & -0.00623200 & 6.60813800 & $\mathrm{H}$ & -1.53466200 & -0.58483200 & 5.12587100 \\
\hline $\mathrm{H}$ & -2.04078300 & 0.95352500 & 5.84653800 & C & 0.42888400 & 2.23915600 & 5.61761100 \\
\hline $\mathrm{H}$ & 0.89439200 & 1.89389400 & 6.54715100 & $\mathrm{H}$ & -0.37470900 & 2.93568000 & 5.88532300 \\
\hline $\mathrm{H}$ & 1.19013900 & 2.79233600 & 5.05430600 & C & 0.17555500 & 2.32438100 & 2.60551000 \\
\hline 0 & 1.01459300 & 1.66947000 & 1.97053000 & C & -0.77156200 & 4.59362000 & 3.20015700 \\
\hline C & 1.13310800 & 4.37145000 & 1.67164100 & C & -0.71619900 & 5.87300700 & 2.35833400 \\
\hline $\mathrm{H}$ & -1.77885600 & 4.17602000 & 3.25414800 & $\mathrm{H}$ & -0.39699900 & 4.77044900 & 4.21670400 \\
\hline C & 0.68351300 & 5.86035300 & 1.71586700 & $\mathrm{H}$ & 2.09310700 & 4.26759700 & 2.19061700 \\
\hline $\mathrm{H}$ & -0.88864600 & 6.76452100 & 2.96741900 & $\mathrm{H}$ & -1.49177300 & 5.84717000 & 1.58857400 \\
\hline $\mathrm{H}$ & 1.39284200 & 6.43072200 & 2.32363400 & $\mathrm{H}$ & 0.68176800 & 6.30008600 & 0.71596600 \\
\hline $\mathrm{N}$ & 0.11867500 & 3.66862100 & 2.48476200 & C & 1.36359200 & 3.81910500 & 0.27322800 \\
\hline C & 2.66149300 & 3.62465300 & -0.10367800 & $\mathrm{C}$ & 0.29838800 & 3.57037200 & -0.67782300 \\
\hline
\end{tabular}




\begin{tabular}{|c|c|c|c|c|c|c|c|}
\hline C & 3.03633800 & 3.14015300 & -1.39638100 & $\mathrm{H}$ & 3.45768700 & 3.81663400 & 0.61267600 \\
\hline $\mathrm{C}$ & 0.64671200 & 3.12059800 & -1.99316200 & $C$ & -1.06214100 & 3.73666000 & -0.37674500 \\
\hline C & 4.37492700 & 2.90660600 & -1.75215300 & C & 2.00649400 & 2.88325300 & -2.34736200 \\
\hline C & -0.37600600 & 2.89423600 & -2.96337700 & $\mathrm{H}$ & -1.37126500 & 3.98557600 & 0.62554100 \\
\hline C & -2.04952200 & 3.52585500 & -1.33543600 & C & 4.69856400 & 2.43368900 & -3.02302100 \\
\hline $\mathrm{H}$ & 5.15886300 & 3.11370700 & -1.02766400 & C & 2.34783100 & 2.39818500 & -3.64684000 \\
\hline C & -1.71650100 & 3.11774400 & -2.62011300 & C & -0.00354200 & 2.42720800 & -4.26834100 \\
\hline $\mathrm{H}$ & -3.08992400 & 3.65788100 & -1.06049000 & C & 3.69898600 & 2.17441800 & -3.95873600 \\
\hline $\mathrm{H}$ & 5.73875300 & 2.27502900 & -3.29003000 & C & 1.29392100 & 2.17693400 & -4.59519100 \\
\hline $\mathrm{H}$ & -2.49336600 & 2.93014500 & -3.35359200 & $\mathrm{H}$ & -0.79503500 & 2.26648700 & -4.99523500 \\
\hline $\mathrm{H}$ & 3.95912700 & 1.81365500 & -4.95068000 & $\mathrm{H}$ & 1.55590300 & 1.81769700 & -5.58710300 \\
\hline C & -2.60055000 & 0.75502900 & 2.07225400 & $S$ & -3.46496600 & 2.21398100 & 2.08534800 \\
\hline $\mathrm{N}$ & -1.45477300 & 0.55425600 & 2.76647700 & $\mathrm{H}$ & -0.87379700 & -0.24110200 & 2.48528300 \\
\hline C & -0.03069500 & -0.20033100 & -1.04165300 & 0 & -1.04762200 & 0.44167000 & -0.89283200 \\
\hline $\mathrm{O}$ & 0.18373800 & -1.20903700 & -1.90609900 & C & -0.90512600 & -1.77827400 & -2.73773900 \\
\hline C & -1.50664200 & -0.69409300 & -3.62688800 & $\mathrm{H}$ & -2.21332700 & -1.15611100 & -4.32454400 \\
\hline $\mathrm{H}$ & -2.03861400 & 0.05209900 & -3.03989400 & $\mathrm{H}$ & -0.72188400 & -0.19647100 & -4.20437200 \\
\hline C & -0.16659300 & -2.82396900 & -3.56926800 & $\mathrm{H}$ & 0.27829500 & -3.58172600 & -2.91764200 \\
\hline $\mathrm{H}$ & -0.87068000 & -3.32206900 & -4.24281000 & $\mathrm{H}$ & 0.61936000 & -2.35918300 & -4.17576300 \\
\hline C & -1.93213800 & -2.43474400 & -1.82797700 & $\mathrm{H}$ & -2.72495900 & -2.89593100 & -2.42523100 \\
\hline $\mathrm{H}$ & -1.46797000 & -3.20406900 & -1.20781200 & $\mathrm{H}$ & -2.38937900 & -1.69322800 & -1.17925900 \\
\hline C & 2.07184200 & -0.93545900 & -0.08887200 & $\mathrm{H}$ & 1.64480600 & -1.92824400 & -0.17117800 \\
\hline C & 2.96349700 & -0.75362900 & 1.08398000 & C & 3.17748400 & -1.84262100 & 1.93633100 \\
\hline C & 3.62690900 & 0.45508000 & 1.32326000 & C & 4.02928000 & -1.71631100 & 3.03180900 \\
\hline $\mathrm{H}$ & 2.64932900 & -2.77158600 & 1.75824400 & C & 4.48799200 & 0.57416100 & 2.41116500 \\
\hline $\mathrm{H}$ & 3.45696000 & 1.30064200 & 0.66728400 & C & 4.68914500 & -0.50976600 & 3.26965700 \\
\hline $\mathrm{H}$ & 4.16540700 & -2.55806300 & 3.70409100 & $\mathrm{H}$ & 4.99326100 & 1.51794000 & 2.59658200 \\
\hline $\mathrm{H}$ & 5.35296500 & -0.41110100 & 4.12391100 & $\mathrm{~N}$ & 1.13255300 & 0.05842000 & -0.31985400 \\
\hline $\mathrm{H}$ & 1.04423400 & 0.79756700 & 0.38743400 & $\mathrm{~S}$ & -0.42889900 & -3.03454500 & 1.59973600 \\
\hline 0 & 0.37708100 & -3.80109200 & 0.62306800 & 0 & 0.05655300 & -1.64211100 & 1.80832200 \\
\hline 0 & -1.90121900 & -3.12827400 & 1.47335900 & C & -0.07310300 & -3.84545400 & 3.23403600 \\
\hline $\mathrm{F}$ & -0.50890300 & -5.10776600 & 3.24986600 & $\mathrm{~F}$ & -0.67858600 & -3.16567200 & 4.22166200 \\
\hline $\mathrm{F}$ & 1.25072000 & -3.84857700 & 3.48238700 & C & 3.18742800 & -0.92800100 & -1.59790500 \\
\hline $\bar{H}$ & 2.41919000 & -0.89183300 & -2.36308200 & $\mathrm{H}$ & 3.74560300 & -0.00485800 & -1.46410100 \\
\hline $\mathrm{C}$ & 3.86898700 & -2.14449600 & -1.42646900 & $\mathrm{C}$ & 5.14753500 & -2.21020500 & -0.73981800 \\
\hline $\mathrm{H}$ & 5.32228500 & -1.33473100 & -0.10550100 & $\mathrm{H}$ & 5.26125200 & -3.12995400 & -0.15482400 \\
\hline $\mathrm{C}$ & 3.18531100 & -3.41938000 & -1.78846200 & $\mathrm{H}$ & 3.88650400 & -4.25154100 & -1.88217400 \\
\hline $\bar{H}$ & 2.47953500 & -3.67437800 & -0.97808900 & $\mathrm{H}$ & 2.58716000 & -3.32232700 & -2.69698100 \\
\hline C & 8.16792000 & -2.44479700 & -0.96687100 & $\mathrm{H}$ & 8.15140300 & -3.40738500 & -0.44350000 \\
\hline $\mathrm{H}$ & 9.06627000 & -2.42207900 & -1.59503600 & $\mathrm{H}$ & 8.26628400 & -1.65407200 & -0.21466400 \\
\hline $\mathrm{C}$ & 6.40592800 & -3.61103300 & -3.24404300 & $\mathrm{H}$ & 6.33291800 & -4.58355600 & -2.74388700 \\
\hline $\mathrm{H}$ & 5.50992800 & -3.48002900 & -3.86069300 & $\mathrm{H}$ & 7.26769000 & -3.65473200 & -3.92066300 \\
\hline $\mathrm{C}$ & 6.62674000 & -0.53609900 & -2.87714500 & $\mathrm{H}$ & 5.69725300 & -0.36145500 & -3.42856900 \\
\hline $\mathrm{H}$ & 6.73472900 & 0.27986600 & -2.15431300 & $\mathrm{H}$ & 7.45947300 & -0.46797300 & -3.58707000 \\
\hline $\mathrm{Si}$ & 6.62213600 & -2.20330300 & -2.01335400 & & & & \\
\hline
\end{tabular}

\section{TS-S-4f-40}

Imaginary frequency: $-425.51 \mathrm{~cm}^{-1}$

E[B3LYP/6-31G(d)-D3(BJ)]: -4789.077531Hartree

E[PCM(Et $\left.\left.{ }_{2} \mathrm{O}\right)-\mathrm{B} 3 L Y P / 6-311 \mathrm{G}(\mathrm{d}, \mathrm{p})-\mathrm{D} 3(\mathrm{BJ})\right]$ : -4790.220859 Hartree

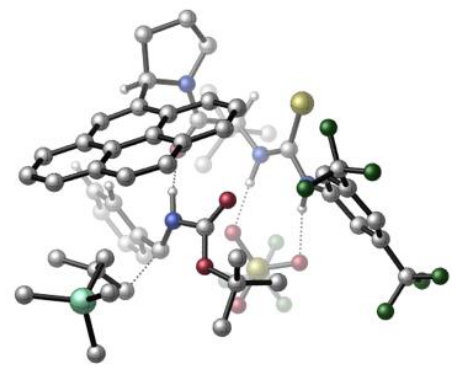




\begin{tabular}{|c|c|c|c|c|c|c|c|}
\hline 01 & & & & $\mathrm{~N}$ & 3.17812300 & 0.69455400 & 0.75648400 \\
\hline $\mathrm{H}$ & 3.19268100 & 1.12442800 & -0.17224800 & C & 3.73847700 & -0.58769200 & 0.77454500 \\
\hline C & 4.75465100 & -0.85944700 & -0.15102500 & C & 3.23783200 & -1.62396100 & 1.57035200 \\
\hline C & 5.25172800 & -2.15398500 & -0.27810900 & $\mathrm{H}$ & 5.11024600 & -0.06950200 & -0.80142300 \\
\hline C & 3.76832900 & -2.90496900 & 1.44729500 & $\mathrm{H}$ & 2.41762100 & -1.42824200 & 2.24109900 \\
\hline C & 4.77715000 & -3.18775500 & 0.52635500 & $\mathrm{H}$ & 5.17954300 & -4.18866400 & 0.43424000 \\
\hline $\mathrm{C}$ & 6.23021500 & -2.46712100 & -1.37287100 & C & 3.16893900 & -4.02343800 & 2.24847500 \\
\hline $\mathrm{F}$ & 6.99211000 & -1.40483200 & -1.70173800 & $\mathrm{~F}$ & 5.58624400 & -2.85733400 & -2.50319500 \\
\hline $\mathrm{F}$ & 7.06210500 & -3.47859000 & -1.03432100 & $\mathrm{~F}$ & 4.03589700 & -5.03915800 & 2.44194000 \\
\hline $\mathrm{F}$ & 2.74400300 & -3.61667100 & 3.46776500 & $\mathrm{~F}$ & 2.08005200 & -4.56015600 & 1.62754700 \\
\hline C & 0.99215600 & 3.25934700 & 2.13373800 & $\mathrm{H}$ & 1.46368900 & 3.24480000 & 3.11490800 \\
\hline C & 0.99236400 & 4.74203700 & 1.64016700 & C & 0.30406000 & 4.93924200 & 0.27853900 \\
\hline $\mathrm{H}$ & -0.75133500 & 4.66278000 & 0.30987700 & $\mathrm{H}$ & 0.77575100 & 4.35103800 & -0.51039700 \\
\hline $\mathrm{H}$ & 0.37432100 & 5.99628600 & -0.00569000 & C & 2.45809400 & 5.19817600 & 1.53740000 \\
\hline $\mathrm{H}$ & 2.50075400 & 6.26932000 & 1.30902900 & $\mathrm{H}$ & 2.98137900 & 4.66171100 & 0.74128400 \\
\hline $\mathrm{H}$ & 2.99832800 & 5.02671700 & 2.47586400 & C & 0.26590900 & 5.59070300 & 2.69872500 \\
\hline $\mathrm{H}$ & 0.26712500 & 6.64485100 & 2.40103400 & $\mathrm{H}$ & 0.75682100 & 5.51965600 & 3.67673200 \\
\hline $\mathrm{H}$ & -0.78013700 & 5.28285100 & 2.81571200 & C & -0.41947900 & 2.66806900 & 2.21528500 \\
\hline 0 & -1.03025900 & 2.37090000 & 1.17934000 & C & -0.41350900 & 2.73567000 & 4.74969700 \\
\hline C & -2.39814900 & 2.02780800 & 3.48009600 & C & -1.33574100 & 1.95507200 & 5.69160200 \\
\hline $\mathrm{H}$ & 0.62017100 & 2.38236400 & 4.77872300 & $\mathrm{H}$ & -0.43257100 & 3.80896600 & 4.97572100 \\
\hline C & -2.70569600 & 2.02830900 & 5.00247000 & $\mathrm{H}$ & -3.00920900 & 2.78575700 & 2.97847600 \\
\hline $\mathrm{H}$ & -1.34492700 & 2.37993700 & 6.69903000 & $\mathrm{H}$ & -1.00296400 & 0.91627100 & 5.76787300 \\
\hline $\mathrm{H}$ & -3.20966500 & 2.96561400 & 5.26346100 & $\mathrm{H}$ & -3.36886800 & 1.20547700 & 5.27791100 \\
\hline $\mathrm{N}$ & -0.99805100 & 2.48394900 & 3.42118000 & C & -2.73914400 & 0.71078800 & 2.78972400 \\
\hline$C$ & -3.96164900 & 0.66810500 & 2.17797700 & C & -1.93614300 & -0.49578000 & 2.86024300 \\
\hline C & -4.53289600 & -0.53118100 & 1.64710100 & $\mathrm{H}$ & -4.56020300 & 1.57551400 & 2.12279400 \\
\hline$C$ & -2.48016100 & -1.71106400 & 2.32537900 & C & -0.65034400 & -0.54587200 & 3.42233100 \\
\hline$C$ & -5.81533600 & -0.56332800 & 1.07358700 & C & -3.78070000 & -1.73790300 & 1.74318400 \\
\hline C & -1.72567400 & -2.92232700 & 2.39339000 & $\mathrm{H}$ & -0.16787700 & 0.35076900 & 3.77367200 \\
\hline C & 0.06974600 & -1.73504000 & 3.49154500 & C & -6.36319600 & -1.76521900 & 0.62720800 \\
\hline $\mathrm{H}$ & -6.38830000 & 0.35806300 & 1.00642900 & C & -4.34412200 & -2.96305400 & 1.26876700 \\
\hline C & -0.45416800 & -2.91486000 & 2.98230200 & C & -2.30281600 & -4.13326900 & 1.88353600 \\
\hline $\mathrm{H}$ & 1.05904500 & -1.72279200 & 3.93445700 & C & -5.63963800 & -2.95335200 & 0.72520500 \\
\hline $\mathrm{H}$ & -7.36504100 & -1.77725600 & 0.20677300 & C & -3.55932300 & -4.16085800 & 1.36174800 \\
\hline $\mathrm{H}$ & 0.12091500 & -3.83324900 & 3.02208600 & $\mathrm{H}$ & -1.71190700 & -5.04320700 & 1.94500500 \\
\hline $\mathrm{H}$ & -6.07442500 & -3.88800500 & 0.38038400 & $\mathrm{H}$ & -3.99245600 & -5.09440200 & 1.01242900 \\
\hline C & 2.52326200 & 1.36217000 & 1.75599200 & $S$ & 2.69113500 & 0.99446100 & 3.40406500 \\
\hline $\mathrm{N}$ & 1.78945400 & 2.39723500 & 1.28607900 & $\mathrm{H}$ & 1.54551200 & 2.38117200 & 0.29048100 \\
\hline C & -0.27694300 & -0.60997900 & -0.75424000 & $\mathrm{O}$ & 0.30722200 & -0.87073300 & 0.27490500 \\
\hline $\mathrm{O}$ & -0.23157500 & -1.28729300 & -1.91560500 & C & 0.68571500 & -2.43616200 & -2.11032700 \\
\hline C & 0.40806800 & -3.51151100 & -1.05967900 & $\mathrm{H}$ & 0.96650500 & -4.41701600 & -1.31986900 \\
\hline $\mathrm{H}$ & 0.71380300 & -3.18985300 & -0.06589800 & $\mathrm{H}$ & -0.65882800 & -3.76311300 & -1.03361400 \\
\hline C & 0.30665200 & -2.91737900 & -3.50849100 & $\mathrm{H}$ & 0.42322300 & -2.10638900 & -4.23425000 \\
\hline $\mathrm{H}$ & 0.96073300 & -3.74288400 & -3.80499300 & $\mathrm{H}$ & -0.72847200 & -3.27524800 & -3.53373200 \\
\hline C & 2.11934700 & -1.92738100 & -2.07591200 & $\mathrm{H}$ & 2.81730400 & -2.74394400 & -2.28523600 \\
\hline $\mathrm{H}$ & 2.26792300 & -1.13998900 & -2.81857400 & $\mathrm{H}$ & 2.35205200 & -1.52801200 & -1.09247000 \\
\hline C & -1.38290500 & 1.14518600 & -2.03941100 & $\mathrm{H}$ & -0.55846000 & 1.07229700 & -2.74234000 \\
\hline C & -1.96547800 & 2.49645300 & -1.91354900 & C & -1.53625700 & 3.48491100 & -2.81003800 \\
\hline C & -2.91646600 & 2.81705800 & -0.93311100 & C & -2.04120600 & 4.77812700 & -2.72035600 \\
\hline $\mathrm{H}$ & -0.77791600 & 3.24083700 & -3.54597900 & C & -3.42480100 & 4.11168000 & -0.85196700 \\
\hline $\mathrm{H}$ & -3.23183100 & 2.06661400 & -0.21606400 & C & -2.98997300 & 5.09326100 & -1.74421400 \\
\hline $\mathrm{H}$ & -1.68359200 & 5.54334600 & -3.40245100 & $\mathrm{H}$ & -4.15235700 & 4.35621100 & -0.08332100 \\
\hline
\end{tabular}




\begin{tabular}{|c|c|c|c|c|c|c|c|}
\hline $\mathrm{H}$ & -3.38208900 & 6.10376100 & -1.67356700 & $\mathrm{~N}$ & -1.17999100 & 0.44704600 & -0.85883300 \\
\hline $\mathrm{H}$ & -1.24128400 & 0.99813200 & 0.00480600 & S & 2.11777200 & 1.92297300 & -2.54944000 \\
\hline 0 & 1.49174000 & 1.28742700 & -3.72626000 & $\mathrm{O}$ & 1.15537800 & 2.20172400 & -1.44485900 \\
\hline 0 & 3.39716100 & 1.34329600 & -2.07661100 & C & 2.58348500 & 3.62894600 & -3.11823000 \\
\hline $\mathrm{F}$ & 3.49872900 & 3.58125800 & -4.08912000 & $\mathrm{~F}$ & 3.08028300 & 4.33412900 & -2.08818900 \\
\hline $\mathrm{F}$ & 1.49971100 & 4.27888400 & -3.58265500 & C & -2.51335800 & 0.22219200 & -3.33184000 \\
\hline $\mathrm{H}$ & -2.66065600 & 1.02958700 & -4.04392400 & $\mathrm{H}$ & -1.77327400 & -0.51798900 & -3.61468900 \\
\hline C & -3.61946000 & -0.23716000 & -2.61088900 & C & -3.54993200 & -1.49850400 & -1.90331500 \\
\hline $\mathrm{H}$ & -2.52091000 & -1.80681200 & -1.70629400 & $\mathrm{H}$ & -4.15575500 & -1.52543600 & -0.99637300 \\
\hline $\mathrm{C}$ & -4.85982100 & 0.59006900 & -2.52507800 & $\mathrm{H}$ & -5.59449300 & 0.16273600 & -1.84000900 \\
\hline $\mathrm{H}$ & -4.59657300 & 1.60105800 & -2.18404500 & $\mathrm{H}$ & -5.31024200 & 0.71107300 & -3.51848800 \\
\hline $\mathrm{C}$ & -3.67081400 & -4.52621300 & -2.32116400 & $\mathrm{H}$ & -3.94587300 & -4.61214700 & -1.26707500 \\
\hline $\mathrm{H}$ & -4.10186500 & -5.37722500 & -2.86176600 & $\mathrm{H}$ & -2.57990900 & -4.60465200 & -2.38615900 \\
\hline C & -6.14980600 & -2.77668200 & -3.05449100 & $\mathrm{H}$ & -6.54982400 & -2.83568800 & -2.03810200 \\
\hline $\mathrm{H}$ & -6.49104900 & -1.83444800 & -3.49785700 & $\mathrm{H}$ & -6.58711100 & -3.59354400 & -3.64086500 \\
\hline $\mathrm{C}$ & -3.61229400 & -2.68399200 & -4.80463600 & $\mathrm{H}$ & -3.97484800 & -1.75899100 & -5.26618100 \\
\hline $\mathrm{H}$ & -2.51789700 & -2.66376500 & -4.83309300 & $\mathrm{H}$ & -3.94258000 & -3.51960600 & -5.43339400 \\
\hline $\mathrm{Si}$ & -4.27138100 & -2.90274000 & -3.05385100 & & & & \\
\hline
\end{tabular}

\section{TS-S-4f-41}

Imaginary frequency: $-202.39 \mathrm{~cm}^{-1}$

E[B3LYP/6-31G(d)-D3(BJ)]: -4789.081654 Hartree

$\mathrm{E}\left[\mathrm{PCM}\left(\mathrm{Et}_{2} \mathrm{O}\right)-\mathrm{B} 3 \mathrm{LYP} / 6-311 \mathrm{G}(\mathrm{d}, \mathrm{p})-\mathrm{D} 3(\mathrm{BJ})\right]$ : -4790.220215 Hartree

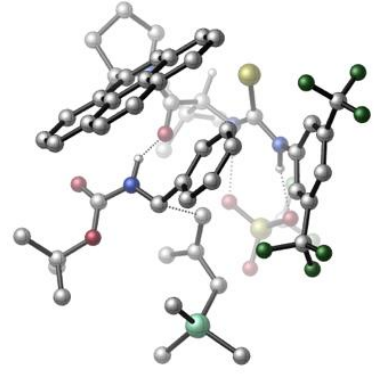

\begin{tabular}{|c|c|c|c|c|c|c|c|}
\hline 01 & & & & $\mathrm{~N}$ & -1.46659900 & 2.43994500 & 1.53499100 \\
\hline $\mathrm{H}$ & -2.40211700 & 2.04562000 & 1.38596200 & C & -1.03350900 & 3.20212500 & 0.43279800 \\
\hline $\mathrm{C}$ & -1.63984800 & 2.92674800 & -0.80101500 & $\mathrm{C}$ & -0.04075600 & 4.18656400 & 0.49639600 \\
\hline C & -1.16643000 & 3.52080700 & -1.96580700 & $\mathrm{H}$ & -2.47624200 & 2.23938000 & -0.83301000 \\
\hline C & 0.40463000 & 4.78870100 & -0.68157200 & $\mathrm{H}$ & 0.39713800 & 4.45278000 & 1.44630600 \\
\hline C & -0.12638900 & 4.44773800 & -1.92404600 & $\mathrm{H}$ & 0.25419000 & 4.89677700 & -2.83212700 \\
\hline C & -1.70549100 & 3.02956800 & -3.27751300 & $\mathrm{C}$ & 1.56758500 & 5.73550200 & -0.60471100 \\
\hline $\mathrm{F}$ & -3.04466700 & 2.83777800 & -3.24935800 & $\mathrm{~F}$ & -1.16814400 & 1.81321700 & -3.60079100 \\
\hline $\mathrm{F}$ & -1.42874200 & 3.85900200 & -4.30089400 & $\mathrm{~F}$ & 1.53490600 & 6.49777700 & 0.50602300 \\
\hline $\mathrm{F}$ & 2.75335100 & 5.05988300 & -0.57358600 & $\mathrm{~F}$ & 1.62702600 & 6.56179600 & -1.67083900 \\
\hline C & -0.53260800 & -0.13137800 & 3.95230200 & $\mathrm{H}$ & -0.06517600 & 0.48614700 & 4.72267500 \\
\hline C & -1.49712600 & -1.14977000 & 4.66086600 & $\mathrm{C}$ & -1.98311200 & -2.27196500 & 3.72365200 \\
\hline $\mathrm{H}$ & -1.15124500 & -2.88755200 & 3.36756200 & $\mathrm{H}$ & -2.52106900 & -1.88666600 & 2.85809000 \\
\hline $\mathrm{H}$ & -2.66893900 & -2.92197100 & 4.27951300 & C & -2.70189800 & -0.37147700 & 5.22312300 \\
\hline $\mathrm{H}$ & -3.31364100 & -1.04217000 & 5.83707100 & $\mathrm{H}$ & -3.34201900 & 0.03221800 & 4.43718600 \\
\hline $\mathrm{H}$ & -2.37283600 & 0.46096100 & 5.85632100 & $\mathrm{C}$ & -0.75668800 & -1.80619700 & 5.83921800 \\
\hline $\mathrm{H}$ & -1.43872700 & -2.48160000 & 6.36635800 & $\mathrm{H}$ & -0.40286700 & -1.06216500 & 6.56258000 \\
\hline $\mathrm{H}$ & 0.09533200 & -2.40372600 & 5.49918100 & C & 0.55179200 & -0.84997500 & 3.13056100 \\
\hline C & 2.67682700 & -2.02509800 & 2.94985400 & $\mathrm{H}$ & 2.10344200 & -2.85361000 & 2.53218000 \\
\hline $\mathrm{N}$ & 1.73210800 & -1.17081000 & 3.69274000 & $\mathrm{C}$ & 3.40428000 & -1.28537200 & 1.83666800 \\
\hline C & 3.66773500 & 0.04838700 & 1.95160500 & C & 3.84951300 & -2.00711000 & 0.66035100 \\
\hline C & 4.38061000 & 0.78379100 & 0.95239300 & $\mathrm{H}$ & 3.28353200 & 0.60683000 & 2.79662700 \\
\hline C & 4.50016800 & -1.27758900 & -0.38676500 & C & 3.64958700 & -3.38691000 & 0.48491400 \\
\hline C & 4.65064100 & 2.15352900 & 1.09185600 & C & 4.77724200 & 0.11132300 & -0.23693100 \\
\hline C & 4.87887800 & -1.94107000 & -1.59367800 & $\mathrm{H}$ & 3.16919700 & -3.97399800 & 1.25675200 \\
\hline C & 4.03266200 & -4.02642900 & -0.69290800 & C & 5.29177300 & 2.85670000 & 0.07475800 \\
\hline
\end{tabular}




\begin{tabular}{|c|c|c|c|c|c|c|c|}
\hline $\mathrm{H}$ & 4.32517600 & 2.66627100 & 1.99262800 & $\mathrm{C}$ & 5.42556200 & 0.83919300 & -1.27807900 \\
\hline C & 4.62909800 & -3.31485200 & -1.72813200 & C & 5.51578400 & -1.18097000 & -2.63277500 \\
\hline $\mathrm{H}$ & 3.85910000 & -5.09296800 & -0.79772300 & $\mathrm{C}$ & 5.67420800 & 2.20870100 & -1.09838900 \\
\hline $\mathrm{H}$ & 5.47439600 & 3.92095100 & 0.18842200 & $\mathrm{C}$ & 5.78036400 & 0.14512100 & -2.48230800 \\
\hline $\mathrm{H}$ & 4.92012900 & -3.81894700 & -2.64626200 & $\mathrm{H}$ & 5.79049800 & -1.69708200 & -3.54922000 \\
\hline $\mathrm{H}$ & 6.16376000 & 2.76465500 & -1.89382500 & $\mathrm{H}$ & 6.26601500 & 0.70500200 & -3.27740800 \\
\hline C & -0.66957200 & 1.81466700 & 2.46541400 & $\mathrm{~S}$ & 0.90857400 & 2.29664200 & 2.85805400 \\
\hline $\mathrm{N}$ & -1.26385300 & 0.75531600 & 3.06023700 & $\mathrm{H}$ & -2.09863900 & 0.36522300 & 2.60982500 \\
\hline S & -4.59268100 & 0.39038800 & 1.01775600 & 0 & -5.37070800 & -0.38557500 & 0.03870400 \\
\hline $\mathrm{O}$ & -3.53304000 & -0.38643700 & 1.72563100 & $\mathrm{O}$ & -4.10524200 & 1.72160300 & 0.56571300 \\
\hline C & -5.79219100 & 0.79516100 & 2.37495400 & $\mathrm{~F}$ & -6.79997200 & 1.54068500 & 1.91340700 \\
\hline $\mathrm{F}$ & -5.16167100 & 1.47868800 & 3.34755000 & $\mathrm{~F}$ & -6.29006300 & -0.32873600 & 2.90495800 \\
\hline C & 0.41624100 & -3.68455200 & -0.50313800 & $\mathrm{O}$ & 0.60112400 & -4.39152900 & 0.46930200 \\
\hline $\mathrm{O}$ & 0.00337600 & -4.07284400 & -1.72103400 & C & -0.16679000 & -5.50594500 & -2.03890700 \\
\hline $\mathrm{C}$ & 1.16056400 & -6.23694900 & -1.83597500 & $\mathrm{H}$ & 1.07127800 & -7.26030700 & -2.21517400 \\
\hline $\mathrm{H}$ & 1.43248800 & -6.27452100 & -0.78066700 & $\mathrm{H}$ & 1.95727800 & -5.73148400 & -2.39106400 \\
\hline C & -0.54527100 & -5.47320900 & -3.51781600 & $\mathrm{H}$ & -1.46583500 & -4.90314200 & -3.67143100 \\
\hline $\mathrm{H}$ & -0.70577800 & -6.49201800 & -3.88352800 & $\mathrm{H}$ & 0.25142600 & -5.01285500 & -4.11060400 \\
\hline $\mathrm{C}$ & -1.29483700 & -6.09308000 & -1.19175100 & $\mathrm{H}$ & -1.47121900 & -7.13140700 & -1.49177700 \\
\hline $\mathrm{H}$ & -2.22220600 & -5.53287700 & -1.34631500 & $\mathrm{H}$ & -1.04005300 & -6.07137100 & -0.13153900 \\
\hline C & 0.31696200 & -1.42594400 & -1.44673600 & $\mathrm{H}$ & 0.02086100 & -1.90981500 & -2.36600100 \\
\hline $\mathrm{C}$ & 1.08379600 & -0.17709200 & -1.62332300 & $C$ & 1.49234500 & 0.15514700 & -2.92373000 \\
\hline $\mathrm{C}$ & 1.36415500 & 0.69697700 & -0.56353900 & $C$ & 2.17928100 & 1.34270300 & -3.16106600 \\
\hline $\mathrm{H}$ & 1.27836800 & -0.52277700 & -3.74395300 & C & 2.01828200 & 1.89397600 & -0.81376200 \\
\hline $\bar{H}$ & 1.05051300 & 0.45893200 & 0.44615200 & $\mathrm{C}$ & 2.42851300 & 2.22129300 & -2.10811800 \\
\hline $\mathrm{H}$ & 2.50370300 & 1.58524700 & -4.16853900 & $\mathrm{H}$ & 2.20558800 & 2.57293300 & 0.00477100 \\
\hline $\mathrm{H}$ & 2.93855500 & 3.16172200 & -2.28211200 & $\mathrm{~N}$ & 0.65486400 & -2.31808700 & -0.46613900 \\
\hline $\bar{H}$ & 0.86760700 & -1.96151000 & 0.47451400 & $\mathrm{C}$ & -1.44080500 & -0.53910300 & -0.78898900 \\
\hline $\bar{H}$ & -1.14318700 & -0.68210300 & 0.24565500 & $\mathrm{H}$ & -1.30855400 & 0.45783800 & -1.18516700 \\
\hline C & -2.50976700 & -1.28093900 & -1.27573800 & C & -3.33126000 & -0.77592700 & -2.38628900 \\
\hline $\bar{H}$ & -3.18240500 & 0.29981500 & -2.51516300 & $\mathrm{H}$ & -4.38678200 & -0.93205000 & -2.12759900 \\
\hline $\mathrm{C}$ & -2.89895000 & -2.56168100 & -0.61810900 & $\mathrm{H}$ & -3.13663300 & -3.33749800 & -1.35219300 \\
\hline $\mathrm{H}$ & -3.81419200 & -2.35603400 & -0.04754200 & $\mathrm{H}$ & -2.14207700 & -2.91811300 & 0.08301500 \\
\hline $\mathrm{C}$ & -4.07658300 & -0.28347900 & -5.24419300 & $\mathrm{H}$ & -5.13673000 & -0.22498000 & -4.97225900 \\
\hline $\bar{H}$ & -4.01580500 & -0.56148400 & -6.30280900 & $\mathrm{H}$ & -3.64990700 & 0.71945300 & -5.12963700 \\
\hline C & -3.98126100 & -3.21400700 & -4.21968900 & $\mathrm{H}$ & -5.03457500 & -3.15411300 & -3.92282400 \\
\hline $\mathrm{H}$ & -3.49193100 & -3.93814400 & -3.55893700 & $\mathrm{H}$ & -3.94473100 & -3.62158800 & -5.23723500 \\
\hline $\mathrm{C}$ & -1.35908000 & -1.63468100 & -4.69082600 & $\mathrm{H}$ & -0.82522800 & -2.43588700 & -4.16873500 \\
\hline $\mathrm{H}$ & -0.84067000 & -0.68734000 & -4.51326500 & $\mathrm{H}$ & -1.30150900 & -1.85035600 & -5.76454600 \\
\hline $\mathrm{Si}$ & -3.16155700 & -1.51530600 & -4.15239800 & $\mathrm{C}$ & 2.29778800 & -0.65844500 & 4.95417600 \\
\hline $\mathrm{H}$ & 1.71346900 & -1.00819100 & 5.80696300 & $\mathrm{H}$ & 2.28499100 & 0.43631700 & 4.95020300 \\
\hline C & 3.72029100 & -1.24894800 & 4.98156800 & $\mathrm{H}$ & 4.03953400 & -1.49204100 & 5.99834300 \\
\hline $\mathrm{H}$ & 4.43729300 & -0.53483400 & 4.56717400 & $\mathrm{C}$ & 3.62832400 & -2.48514600 & 4.07257800 \\
\hline $\mathrm{H}$ & 4.59763200 & -2.80603600 & 3.68376700 & $\mathrm{H}$ & 3.17578900 & -3.32566300 & 4.61171200 \\
\hline 0 & 0.28041300 & -1.20277100 & 1.96920500 & & & & \\
\hline
\end{tabular}

\section{TS-S-4f-42}

Imaginary frequency: $-405.29 \mathrm{~cm}-1$

E[B3LYP/6-31G(d)-D3(BJ)]: -4789.069944 Hartree

E[PCM(Et $\left.\left.{ }_{2} \mathrm{O}\right)-B 3 L Y P / 6-311 G(d, p)-D 3(B J)\right]: ~-4790.219978$ Hartree

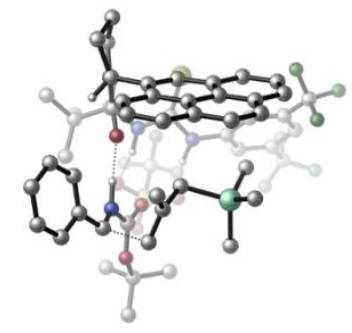




\begin{tabular}{|c|c|c|c|c|c|c|c|}
\hline 01 & & & & $\mathrm{~N}$ & -2.46406700 & 0.29135500 & 0.69959000 \\
\hline $\mathrm{H}$ & -2.81645700 & -0.54036700 & 0.20033500 & C & -3.16922500 & 1.46411600 & 0.36789600 \\
\hline C & -4.49946600 & 1.33533700 & -0.05589100 & C & -2.56944600 & 2.72658700 & 0.36395500 \\
\hline C & -5.20864100 & 2.46120600 & -0.46870900 & $\mathrm{H}$ & -4.96505300 & 0.35828800 & -0.07574200 \\
\hline C & -3.29610800 & 3.84065300 & -0.04962600 & $\mathrm{H}$ & -1.54095200 & 2.83027300 & 0.66926400 \\
\hline C & -4.61988200 & 3.72546700 & -0.46743300 & $\mathrm{H}$ & -5.17730300 & 4.59505300 & -0.79147100 \\
\hline C & -6.65554600 & 2.32248400 & -0.85561300 & C & -2.58857000 & 5.16033700 & -0.12102500 \\
\hline $\mathrm{F}$ & -7.47319400 & 2.49490200 & 0.20935000 & $\mathrm{~F}$ & -6.92833100 & 1.10908300 & -1.37274900 \\
\hline $\mathrm{F}$ & -7.01554800 & 3.24964300 & -1.77562500 & $\mathrm{~F}$ & -1.79430500 & 5.37069400 & 0.95985800 \\
\hline $\mathrm{F}$ & -1.76693300 & 5.23359100 & -1.20564700 & $\mathrm{~F}$ & -3.43696300 & 6.20392100 & -0.20578700 \\
\hline C & -0.16826000 & -1.56333000 & 2.84636800 & $\mathrm{H}$ & -0.41890200 & -1.03030700 & 3.76406400 \\
\hline C & -0.24225600 & -3.08969700 & 3.17626100 & C & 0.04958500 & -3.98683900 & 1.96122500 \\
\hline $\mathrm{H}$ & 1.06421800 & -3.82971500 & 1.58382700 & $\mathrm{H}$ & -0.66395700 & -3.82840700 & 1.15146100 \\
\hline $\mathrm{H}$ & -0.03641500 & -5.03622400 & 2.26545000 & $\mathrm{C}$ & -1.65130200 & -3.38348600 & 3.72534600 \\
\hline $\mathrm{H}$ & -1.71345700 & -4.43307700 & 4.03472600 & $\mathrm{H}$ & -2.42494800 & -3.21003900 & 2.97629800 \\
\hline $\mathrm{H}$ & -1.86353100 & -2.75923200 & 4.60176600 & C & 0.78746300 & -3.40537000 & 4.27639000 \\
\hline $\mathrm{H}$ & 0.73653500 & -4.46934400 & 4.53007400 & $\mathrm{H}$ & 0.58235000 & -2.83971500 & 5.19215800 \\
\hline $\mathrm{H}$ & 1.81433200 & -3.19055400 & 3.95797900 & C & 1.23087000 & -1.11934100 & 2.39025100 \\
\hline $\mathrm{O}$ & 1.64091600 & -1.34906800 & 1.23609300 & C & 1.71187500 & -0.05428100 & 4.64178500 \\
\hline $\mathrm{C}$ & 3.39761200 & -0.10369700 & 2.86137200 & C & 2.90410200 & 0.83213500 & 5.02294200 \\
\hline $\mathrm{H}$ & 0.75940600 & 0.48108900 & 4.64611400 & $\mathrm{H}$ & 1.64231000 & -0.92286000 & 5.30389900 \\
\hline $\mathrm{C}$ & 4.06311400 & 0.23732700 & 4.21236400 & $\mathrm{H}$ & 3.86570300 & -0.96912300 & 2.38901100 \\
\hline $\mathrm{H}$ & 3.08803500 & 0.83243400 & 6.10031700 & $\mathrm{H}$ & 2.72008300 & 1.86442400 & 4.70936200 \\
\hline $\mathrm{H}$ & 4.42743200 & -0.68263800 & 4.68548100 & $\mathrm{H}$ & 4.90661600 & 0.92116700 & 4.09300700 \\
\hline $\mathrm{N}$ & 2.03683700 & -0.48978400 & 3.26895900 & $\mathrm{C}$ & 3.42166300 & 1.07436300 & 1.90038600 \\
\hline C & 2.36402600 & 1.93168700 & 1.82080700 & $\mathrm{C}$ & 4.60906900 & 1.31482800 & 1.10537100 \\
\hline $\mathrm{C}$ & 2.37922300 & 3.09377100 & 0.98251400 & $\mathrm{H}$ & 1.45552900 & 1.73160200 & 2.37933800 \\
\hline $\mathrm{C}$ & 4.63393300 & 2.44700100 & 0.22843500 & C & 5.74186800 & 0.48299400 & 1.14624400 \\
\hline $\mathrm{C}$ & 1.29862600 & 3.99001200 & 0.93686000 & $\mathrm{C}$ & 3.52430700 & 3.34004200 & 0.17238400 \\
\hline $\mathrm{C}$ & 5.77920100 & 2.69540100 & -0.59012000 & $\mathrm{H}$ & 5.76624400 & -0.36545200 & 1.81848300 \\
\hline $\mathrm{C}$ & 6.85135900 & 0.72845900 & 0.33769100 & C & 1.33736800 & 5.10415500 & 0.09897200 \\
\hline $\mathrm{H}$ & 0.42745200 & 3.79946900 & 1.55667400 & $\mathrm{C}$ & 3.55510100 & 4.48187500 & -0.68312000 \\
\hline C & 6.87329000 & 1.81840400 & -0.52579100 & C & 5.77563700 & 3.84180400 & -1.45671300 \\
\hline $\mathrm{H}$ & 7.70579800 & 0.05966300 & 0.38707100 & C & 2.44857300 & 5.34516600 & -0.70573800 \\
\hline $\mathrm{H}$ & 0.48618300 & 5.77304900 & 0.06369200 & C & 4.71755000 & 4.69740000 & -1.49775000 \\
\hline $\mathrm{H}$ & 7.74190400 & 2.00723100 & -1.15089600 & $\mathrm{H}$ & 6.65037300 & 4.01770700 & -2.07728600 \\
\hline $\mathrm{H}$ & 2.46880600 & 6.21148000 & -1.36167800 & $\mathrm{H}$ & 4.73481400 & 5.56381600 & -2.15363400 \\
\hline $\mathrm{C}$ & -1.59372100 & 0.10181800 & 1.73174900 & $\mathrm{~S}$ & -1.12601600 & 1.33133600 & 2.81575100 \\
\hline $\mathrm{N}$ & -1.14366600 & -1.16770200 & 1.84678500 & $\mathrm{H}$ & -1.50006700 & -1.87770500 & 1.20502700 \\
\hline $\mathrm{C}$ & 0.07378600 & -2.86755600 & -1.60197800 & $\mathrm{O}$ & -0.81811000 & -2.29755600 & -1.01483200 \\
\hline $\mathrm{O}$ & 0.00433500 & -3.54534300 & -2.74835800 & C & -1.32121100 & -4.00249200 & -3.28515700 \\
\hline $\mathrm{C}$ & -2.23240500 & -2.81142900 & -3.56714800 & $\mathrm{H}$ & -3.12508200 & -3.17590400 & -4.08598100 \\
\hline $\mathrm{H}$ & -2.55649800 & -2.30869500 & -2.65668600 & $\mathrm{H}$ & -1.72846600 & -2.09663900 & -4.22787900 \\
\hline $\mathrm{C}$ & -0.90866800 & -4.69708400 & -4.57950400 & $\mathrm{H}$ & -0.19984000 & -5.50705600 & -4.37921300 \\
\hline $\mathrm{H}$ & -1.79346300 & -5.12437500 & -5.06059800 & $\mathrm{H}$ & -0.44740800 & -3.98741700 & -5.27477600 \\
\hline $\mathrm{C}$ & -1.90921400 & -4.98753100 & -2.28052700 & $\mathrm{H}$ & -2.88233400 & -5.33688200 & -2.63614200 \\
\hline $\mathrm{H}$ & -1.24503400 & -5.85104500 & -2.16307200 & $\mathrm{H}$ & -2.07554200 & -4.52355500 & -1.30826100 \\
\hline $\mathrm{C}$ & 2.48238000 & -3.15643700 & -1.86357800 & $\mathrm{H}$ & 2.24395800 & -3.86958100 & -2.64589600 \\
\hline $\mathrm{C}$ & 3.80417700 & -3.36303100 & -1.23264700 & C & 4.75868600 & -4.10680800 & -1.94440900 \\
\hline $\mathrm{C}$ & 4.13670500 & -2.84644600 & 0.02770500 & C & 6.02074700 & -4.33870900 & -1.40744600 \\
\hline $\mathrm{H}$ & 4.50316500 & -4.50988000 & -2.92137900 & C & 5.39816700 & -3.09551500 & 0.56653700 \\
\hline $\mathrm{H}$ & 3.41634200 & -2.25736300 & 0.58511400 & C & 6.34280100 & -3.83508700 & -0.14481500 \\
\hline $\mathrm{H}$ & 6.74777900 & -4.92020400 & -1.96614400 & $\mathrm{H}$ & 5.64196900 & -2.71211200 & 1.55267300 \\
\hline
\end{tabular}




\begin{tabular}{|c|c|c|c|c|c|c|c|}
\hline $\mathrm{H}$ & 7.32339600 & -4.02317500 & 0.28221400 & $\mathrm{~N}$ & 1.38950300 & -2.83895100 & -1.10720500 \\
\hline $\mathrm{H}$ & 1.46081300 & -2.32338300 & -0.21621500 & S & -4.07800700 & -2.91849800 & -0.05659300 \\
\hline 0 & -4.77080900 & -3.95011200 & -0.83628700 & $\mathrm{O}$ & -2.89034200 & -3.36794600 & 0.71955400 \\
\hline 0 & -3.85071700 & -1.62426800 & -0.77063700 & $\mathrm{C}$ & -5.25709200 & -2.41327300 & 1.28780500 \\
\hline $\mathrm{F}$ & -6.34561100 & -1.82022400 & 0.77417300 & $\mathrm{~F}$ & -4.64905600 & -1.52745700 & 2.10502200 \\
\hline $\mathrm{F}$ & -5.64400200 & -3.46326200 & 2.02030800 & C & 2.70294200 & -1.73059500 & -3.24084000 \\
\hline $\mathrm{H}$ & 3.48194600 & -2.13968800 & -3.87912900 & $\mathrm{H}$ & 1.69251200 & -1.84121800 & -3.62242000 \\
\hline C & 3.01156900 & -0.60367500 & -2.46867300 & C & 1.97931000 & 0.13739400 & -1.77484800 \\
\hline $\mathrm{H}$ & 1.13054600 & -0.48581600 & -1.47336700 & $\mathrm{H}$ & 2.35220500 & 0.69889100 & -0.91432200 \\
\hline $\mathrm{C}$ & 4.43188200 & -0.21010800 & -2.25434100 & $\mathrm{H}$ & 4.54849700 & 0.87656800 & -2.30933700 \\
\hline $\mathrm{H}$ & 4.72534000 & -0.48875100 & -1.23210000 & $\mathrm{H}$ & 5.11635700 & -0.69344800 & -2.95438700 \\
\hline $\mathrm{C}$ & -0.17505700 & 2.23850200 & -1.86683900 & $\mathrm{H}$ & 0.29747800 & 2.70323300 & -0.99868900 \\
\hline $\mathrm{H}$ & -0.73551100 & 3.01879500 & -2.39249400 & $\mathrm{H}$ & -0.89108300 & 1.49310700 & -1.50487700 \\
\hline C & 2.39242600 & 2.58733500 & -3.65989900 & $\mathrm{H}$ & 2.97494900 & 3.06654900 & -2.86815600 \\
\hline $\mathrm{H}$ & 3.08668300 & 2.06225800 & -4.32656800 & $\mathrm{H}$ & 1.91202500 & 3.38218700 & -4.24277500 \\
\hline $\mathrm{C}$ & 0.25388900 & 0.45107200 & -4.34155200 & $\mathrm{H}$ & 0.96340600 & -0.02459600 & -5.02675900 \\
\hline $\mathrm{H}$ & -0.40667100 & -0.32128200 & -3.93223400 & $\mathrm{H}$ & -0.37129200 & 1.13001100 & -4.93388000 \\
\hline $\mathrm{Si}$ & 1.09573800 & 1.41793700 & -2.96254200 & & & & \\
\hline
\end{tabular}

\section{TS-S-4f-43}

Imaginary frequency: $-420.61 \mathrm{~cm}^{-1}$

E[B3LYP/6-31G(d)-D3(BJ)]: -4789.078831 Hartree

$\mathrm{E}\left[\mathrm{PCM}\left(\mathrm{Et}_{2} \mathrm{O}\right)-\mathrm{B} 3 \mathrm{LYP} / 6-311 \mathrm{G}(\mathrm{d}, \mathrm{p})-\mathrm{D} 3(\mathrm{BJ})\right]$ : -4790.219400 Hartree

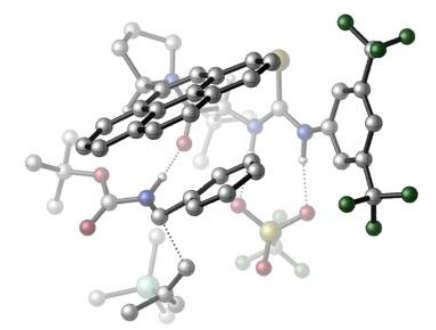

\begin{tabular}{|c|c|c|c|c|c|c|c|}
\hline 01 & & & & $\mathrm{~N}$ & -2.20738500 & -2.01622100 & 1.52497900 \\
\hline $\mathrm{H}$ & -1.57996300 & -2.65616200 & 1.02368500 & C & -3.45410900 & -1.88305100 & 0.88799200 \\
\hline C & -3.55787300 & -2.46034300 & -0.39275000 & C & -4.54994500 & -1.17915500 & 1.40147200 \\
\hline C & -4.70381500 & -2.26995600 & -1.15408000 & $\mathrm{H}$ & -2.72106000 & -3.01867000 & -0.79740400 \\
\hline C & -5.68479100 & -0.99275400 & 0.61125600 & $\mathrm{H}$ & -4.49768800 & -0.75101100 & 2.38959600 \\
\hline $\mathrm{C}$ & -5.77916000 & -1.52208100 & -0.67039100 & $\mathrm{H}$ & -6.65896600 & -1.35723900 & -1.27980600 \\
\hline C & -4.74321200 & -2.76284300 & -2.57318600 & $\mathrm{C}$ & -6.83599200 & -0.21576700 & 1.18493500 \\
\hline $\mathrm{F}$ & -3.89961200 & -3.78809300 & -2.78824700 & $\mathrm{~F}$ & -4.39903100 & -1.77405600 & -3.44082000 \\
\hline $\mathrm{F}$ & -5.98422600 & -3.16734500 & -2.92825400 & $\mathrm{~F}$ & -6.41415100 & 0.75887100 & 2.02637700 \\
\hline$F$ & -7.56351000 & 0.39126900 & 0.21381100 & $\mathrm{~F}$ & -7.68802100 & -0.99557400 & 1.88357800 \\
\hline C & 0.55336900 & -0.64404300 & 3.47002400 & $\mathrm{H}$ & -0.09286000 & -0.30352200 & 4.27716900 \\
\hline C & 1.73450100 & -1.43085900 & 4.10911500 & $\mathrm{C}$ & 2.76612800 & -1.90805700 & 3.07954500 \\
\hline $\mathrm{H}$ & 3.21827700 & -1.06914400 & 2.54776600 & $\mathrm{H}$ & 2.32171300 & -2.56842200 & 2.33442200 \\
\hline $\mathrm{H}$ & 3.56051400 & -2.46530600 & 3.59090900 & C & 1.14672400 & -2.64443600 & 4.84850600 \\
\hline $\mathrm{H}$ & 1.93747100 & -3.16938300 & 5.39662300 & $\mathrm{H}$ & 0.69390200 & -3.34839800 & 4.14504500 \\
\hline $\mathrm{H}$ & 0.37639900 & -2.34012800 & 5.56669800 & C & 2.43523700 & -0.50601600 & 5.12035700 \\
\hline $\mathrm{H}$ & 3.28461000 & -1.02517400 & 5.57737400 & $\mathrm{H}$ & 1.75918900 & -0.20596600 & 5.92989000 \\
\hline $\mathrm{H}$ & 2.82386900 & 0.40015400 & 4.63875300 & $\mathrm{C}$ & 0.97285600 & 0.55063000 & 2.60034200 \\
\hline $\mathrm{O}$ & 1.42828900 & 0.33639500 & 1.46718200 & $\mathrm{C}$ & 0.38669500 & 2.27368000 & 4.37335700 \\
\hline C & 1.01918400 & 2.93264800 & 2.11957200 & C & 1.17714600 & 3.58165500 & 4.48308600 \\
\hline $\mathrm{H}$ & -0.69572500 & 2.44262500 & 4.42466900 & $\mathrm{H}$ & 0.65585700 & 1.54564900 & 5.13545300 \\
\hline $\mathrm{H}$ & 1.97912700 & 2.73774900 & 1.64210000 & $\mathrm{H}$ & 2.21778300 & 3.35350000 & 4.74278100 \\
\hline $\mathrm{H}$ & 0.77508000 & 4.25344300 & 5.24658400 & $\mathrm{~N}$ & 0.76414100 & 1.80752700 & 3.03690300 \\
\hline C & -0.06707400 & 3.07690200 & 1.06458000 & C & -1.33492700 & 2.63984400 & 1.31590000 \\
\hline $\mathrm{C}$ & 0.23023000 & 3.74818400 & -0.18244900 & $\mathrm{C}$ & -2.41436200 & 2.84769500 & 0.39797600 \\
\hline $\mathrm{H}$ & -1.55366400 & 2.09005400 & 2.22590400 & $\mathrm{C}$ & -0.83168900 & 3.97480800 & -1.11431300 \\
\hline $\mathrm{C}$ & 1.52423300 & 4.16464800 & -0.53544800 & C & -3.70947800 & 2.36926900 & 0.65459200 \\
\hline
\end{tabular}




\begin{tabular}{|c|c|c|c|c|c|c|c|}
\hline $\mathrm{C}$ & -2.15548600 & 3.54493200 & -0.81624100 & C & -0.56337600 & 4.61733200 & -2.36208100 \\
\hline $\mathrm{H}$ & 2.34379400 & 4.00815300 & 0.15226800 & $\mathrm{C}$ & 1.78458800 & 4.75791800 & -1.76899100 \\
\hline $\mathrm{C}$ & -4.73685700 & 2.58509000 & -0.26230500 & $\mathrm{H}$ & -3.89931300 & 1.81582400 & 1.56865400 \\
\hline $\mathrm{C}$ & -3.21436100 & 3.76994100 & -1.74446100 & $\mathrm{C}$ & 0.75292800 & 4.98858600 & -2.67448700 \\
\hline C & -1.65291700 & 4.85207400 & -3.26785100 & $\mathrm{H}$ & 2.80152000 & 5.04823500 & -2.01773700 \\
\hline C & -4.49594600 & 3.28397300 & -1.44393700 & $\mathrm{H}$ & -5.73040400 & 2.20290500 & -0.05633400 \\
\hline C & -2.91998800 & 4.45482000 & -2.97017600 & $\mathrm{H}$ & 0.95694400 & 5.46450700 & -3.63023700 \\
\hline $\mathrm{H}$ & -1.43922000 & 5.35645000 & -4.20688900 & $\mathrm{H}$ & -5.30287700 & 3.44903600 & -2.15347900 \\
\hline $\mathrm{H}$ & -3.73319700 & 4.63550700 & -3.66867300 & C & -1.63170700 & -1.27326100 & 2.52325000 \\
\hline $\mathrm{S}$ & -2.46140600 & -0.23045600 & 3.57309900 & $\mathrm{~N}$ & -0.28485400 & -1.47454300 & 2.61215300 \\
\hline $\mathrm{H}$ & 0.19376000 & -1.82744900 & 1.77539600 & S & 0.64050700 & -3.50199300 & -0.52990300 \\
\hline 0 & 1.00340900 & -3.60769300 & -1.95486600 & $\mathrm{O}$ & 1.10609100 & -2.25750500 & 0.14487000 \\
\hline 0 & -0.75468000 & -3.86728000 & -0.17236300 & C & 1.65170100 & -4.82623900 & 0.29052500 \\
\hline $\mathrm{F}$ & 1.36185100 & -6.03355100 & -0.19617400 & $\mathrm{~F}$ & 1.43145000 & -4.82982400 & 1.61606600 \\
\hline $\mathrm{F}$ & 2.96677000 & -4.59158200 & 0.09208200 & C & 3.69997200 & 1.59075900 & -0.94554100 \\
\hline $\mathrm{O}$ & 4.41806100 & 1.84258000 & -1.90085100 & $\mathrm{O}$ & 3.95817800 & 1.85052000 & 0.34494800 \\
\hline C & 5.19479300 & 2.52499900 & 0.78869300 & C & 6.42129300 & 1.69469400 & 0.41577300 \\
\hline $\mathrm{H}$ & 7.30743600 & 2.13792400 & 0.88216500 & $\mathrm{H}$ & 6.57078100 & 1.67359300 & -0.66325400 \\
\hline $\mathrm{H}$ & 6.31969300 & 0.66947300 & 0.78207300 & C & 5.00513300 & 2.56728000 & 2.30458700 \\
\hline $\mathrm{H}$ & 4.11427400 & 3.14580100 & 2.56810500 & $\mathrm{H}$ & 5.87430000 & 3.03534300 & 2.77678900 \\
\hline $\mathrm{H}$ & 4.88966400 & 1.55512900 & 2.70414100 & C & 5.26094100 & 3.93257000 & 0.19556000 \\
\hline $\mathrm{H}$ & 6.15975200 & 4.43729000 & 0.56495100 & $\mathrm{H}$ & 4.39392400 & 4.52718500 & 0.49885800 \\
\hline $\mathrm{H}$ & 5.30568100 & 3.89477400 & -0.89418100 & C & 1.81104900 & 0.91279700 & -2.25340400 \\
\hline $\mathrm{H}$ & 2.28316700 & 1.54704000 & -2.99687700 & C & 0.33743300 & 0.88736800 & -2.26854900 \\
\hline C & -0.31700400 & 1.37221900 & -3.41355800 & C & -0.41498100 & 0.31745800 & -1.23233400 \\
\hline C & -1.69576300 & 1.26403100 & -3.53666300 & $\mathrm{H}$ & 0.26549900 & 1.83316500 & -4.20760200 \\
\hline C & -1.79764100 & 0.23249900 & -1.35646000 & $\mathrm{H}$ & 0.06783500 & -0.08019300 & -0.34906800 \\
\hline C & -2.44035800 & 0.67789200 & -2.51020200 & $\mathrm{H}$ & -2.19124600 & 1.64693200 & -4.42254900 \\
\hline $\mathrm{H}$ & -2.37909900 & -0.18080100 & -0.54653900 & $\mathrm{H}$ & -3.51525300 & 0.57698600 & -2.60107300 \\
\hline $\mathrm{N}$ & 2.45928700 & 0.98826500 & -1.04330700 & $\mathrm{H}$ & 1.98782500 & 0.75146700 & -0.15929400 \\
\hline C & 2.06700100 & -0.80578100 & -3.28235300 & $\mathrm{H}$ & 1.45159600 & -1.50101100 & -2.71929100 \\
\hline $\mathrm{H}$ & 1.58001600 & -0.34903400 & -4.13828800 & C & 3.40301800 & -1.16999100 & -3.50159300 \\
\hline C & 3.98613400 & -2.34273700 & -2.86297600 & $\mathrm{H}$ & 4.59805600 & -2.87385400 & -3.60546200 \\
\hline $\mathrm{H}$ & 3.21302000 & -3.01003300 & -2.46880700 & C & 4.24340800 & -0.34846900 & -4.43331800 \\
\hline $\mathrm{H}$ & 5.14054800 & -0.87817400 & -4.76146100 & $\mathrm{H}$ & 4.55740500 & 0.56124200 & -3.90457800 \\
\hline $\mathrm{H}$ & 3.66759200 & -0.03124500 & -5.30913100 & C & 4.47305200 & -1.62299200 & 0.15835000 \\
\hline $\mathrm{H}$ & 4.40744300 & -0.54123800 & 0.27605100 & $\mathrm{H}$ & 5.03821600 & -2.01606000 & 1.01213300 \\
\hline $\mathrm{H}$ & 3.45512100 & -2.01472400 & 0.22234200 & C & 6.65012800 & -0.97684700 & -2.01470200 \\
\hline $\mathrm{H}$ & 6.25511400 & 0.02489400 & -2.20647300 & $\mathrm{H}$ & 7.12888600 & -1.34072100 & -2.93133400 \\
\hline $\mathrm{H}$ & 7.43024000 & -0.88734200 & -1.24945400 & $\mathrm{C}$ & 5.98930900 & -3.89880500 & -1.25750600 \\
\hline $\mathrm{H}$ & 6.45687300 & -4.25058600 & -2.18437900 & $\mathrm{H}$ & 5.20028300 & -4.60583100 & -0.98211600 \\
\hline $\mathrm{H}$ & 6.75096900 & -3.92619600 & -0.46876500 & $\mathrm{Si}$ & 5.29669900 & -2.15444800 & -1.43642100 \\
\hline C & 1.08984600 & 4.16756300 & 3.06287400 & $\mathrm{H}$ & 0.17707800 & 4.75970900 & 2.95193500 \\
\hline $\mathrm{H}$ & 1.93495200 & 4.81670200 & 2.81631100 & & & & \\
\hline
\end{tabular}

\section{TS-S-4f-44}

Imaginary frequency: $-407.36 \mathrm{~cm}^{-1}$

E[B3LYP/6-31G(d)-D3(BJ)]: -4789.072081 Hartree

$\mathrm{E}\left[\mathrm{PCM}\left(\mathrm{Et}_{2} \mathrm{O}\right)-\mathrm{B} 3 \mathrm{LYP} / 6-311 \mathrm{G}(\mathrm{d}, \mathrm{p})-\mathrm{D} 3(\mathrm{BJ})\right]$ : -4790.217814 Hartree

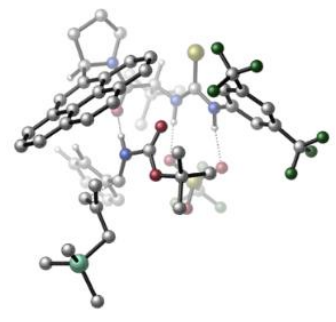




\begin{tabular}{|c|c|c|c|c|c|c|c|}
\hline 01 & & & & $\mathrm{~N}$ & 3.25156200 & 0.79469100 & -0.24528900 \\
\hline $\mathrm{H}$ & 2.95161000 & 0.90676200 & -1.21865200 & C & 3.87156500 & -0.43636400 & -0.00268800 \\
\hline $\mathrm{C}$ & 4.58033100 & -1.01831700 & -1.06181300 & $\mathrm{C}$ & 3.69825600 & -1.15532800 & 1.18551600 \\
\hline$C$ & 5.09825100 & -2.30409600 & -0.92933200 & $\mathrm{H}$ & 4.67352600 & -0.48363000 & -1.99941000 \\
\hline C & 4.24917100 & -2.42768200 & 1.30798000 & $\mathrm{H}$ & 3.10818000 & -0.73007700 & 1.98067900 \\
\hline C & 4.95286100 & -3.01822000 & 0.25800200 & $\mathrm{H}$ & 5.37389700 & -4.01042600 & 0.36101000 \\
\hline C & 5.72196100 & -2.97672100 & -2.11767600 & C & 3.99369700 & -3.22084900 & 2.55637000 \\
\hline $\mathrm{F}$ & 6.67779000 & -3.86249600 & -1.75485600 & $\mathrm{~F}$ & 6.28337100 & -2.09764800 & -2.97152300 \\
\hline $\mathrm{F}$ & 4.79429400 & -3.67534400 & -2.82228400 & $\mathrm{~F}$ & 3.89094600 & -2.43840400 & 3.65585900 \\
\hline $\mathrm{F}$ & 2.82598000 & -3.92077600 & 2.47858700 & $\mathrm{~F}$ & 4.96459100 & -4.12719400 & 2.79549700 \\
\hline C & 1.41099400 & 3.73220600 & 0.87040900 & $\mathrm{H}$ & 2.14163700 & 3.97543700 & 1.63970800 \\
\hline C & 1.23559400 & 5.00336900 & -0.01887100 & C & 0.22029700 & 4.82189100 & -1.15916100 \\
\hline $\mathrm{H}$ & -0.77980100 & 4.60105900 & -0.78424200 & $\mathrm{H}$ & 0.50321300 & 4.01428900 & -1.83620300 \\
\hline $\mathrm{H}$ & 0.17340800 & 5.74740800 & -1.74602500 & C & 2.61198000 & 5.34445800 & -0.61518600 \\
\hline $\mathrm{H}$ & 2.55752200 & 6.29079300 & -1.16538900 & $\mathrm{H}$ & 2.94001800 & 4.56694700 & -1.31071300 \\
\hline $\mathrm{H}$ & 3.37445200 & 5.44597500 & 0.16610600 & C & 0.77232800 & 6.15324300 & 0.89304100 \\
\hline $\mathrm{H}$ & 0.66943900 & 7.07635800 & 0.31240000 & $\mathrm{H}$ & 1.49299000 & 6.34381800 & 1.69749700 \\
\hline $\mathrm{H}$ & -0.20280900 & 5.94053600 & 1.34789500 & C & 0.10018800 & 3.27254200 & 1.51600200 \\
\hline $\mathrm{O}$ & -0.79083900 & 2.75917400 & 0.82322900 & C & 0.84022000 & 4.03158100 & 3.81947700 \\
\hline C & -1.37997800 & 3.04363100 & 3.43087700 & C & 0.27265800 & 3.58099200 & 5.16962000 \\
\hline $\mathrm{H}$ & 1.85851100 & 3.67443000 & 3.64729400 & $\mathrm{H}$ & 0.82289300 & 5.12463600 & 3.72672300 \\
\hline C & -1.23849900 & 3.47226500 & 4.91680000 & $\mathrm{H}$ & -2.15018100 & 3.64949200 & 2.94228000 \\
\hline $\mathrm{H}$ & 0.51748000 & 4.28513500 & 5.96948200 & $\mathrm{H}$ & 0.68420400 & 2.60609600 & 5.44416700 \\
\hline $\mathrm{H}$ & -1.71542800 & 4.44935200 & 5.05157200 & $\mathrm{H}$ & -1.73343400 & 2.76567000 & 5.58658800 \\
\hline $\mathrm{N}$ & -0.08234900 & 3.42548500 & 2.84416500 & C & -1.82505900 & 1.59600400 & 3.24851200 \\
\hline C & -3.15522900 & 1.41115400 & 2.99209800 & C & -0.97322700 & 0.43926800 & 3.45591600 \\
\hline C & -3.78162400 & 0.12443700 & 2.97830400 & $\mathrm{H}$ & -3.78944800 & 2.27931600 & 2.82347400 \\
\hline C & -1.57711300 & -0.86264400 & 3.45918300 & C & 0.41561400 & 0.51714800 & 3.64494900 \\
\hline C & -5.15546200 & -0.04126800 & 2.73666700 & C & -2.97816500 & -1.02124700 & 3.24981100 \\
\hline C & -0.77328600 & -2.02333800 & 3.67855100 & $\mathrm{H}$ & 0.92902900 & 1.46108600 & 3.56847000 \\
\hline C & 1.18639800 & -0.62099100 & 3.86643500 & C & -5.74016800 & -1.30586300 & 2.79827800 \\
\hline $\mathrm{H}$ & -5.76541900 & 0.83402200 & 2.52773400 & C & -3.58290900 & -2.31507700 & 3.29668300 \\
\hline C & 0.60594500 & -1.88149900 & 3.88557700 & C & -1.40154900 & -3.31383300 & 3.69169500 \\
\hline $\mathrm{H}$ & 2.25584500 & -0.51052200 & 4.00477400 & C & -4.96703300 & -2.43028700 & 3.08431500 \\
\hline $\mathrm{H}$ & -6.80812600 & -1.41412300 & 2.62967400 & C & -2.74460200 & -3.45710800 & 3.52110700 \\
\hline $\mathrm{H}$ & 1.21683400 & -2.76485100 & 4.03663300 & $\mathrm{H}$ & -0.77071500 & -4.18402800 & 3.85262600 \\
\hline $\mathrm{H}$ & -5.42802000 & -3.41367600 & 3.13291300 & $\mathrm{H}$ & -3.20474500 & -4.44153800 & 3.54613400 \\
\hline $\mathrm{C}$ & 2.87079100 & 1.75526700 & 0.65137200 & $S$ & 3.54013300 & 1.91175200 & 2.20360100 \\
\hline $\mathrm{N}$ & 1.95972400 & 2.61148700 & 0.13311000 & $\mathrm{H}$ & 1.42860100 & 2.29442100 & -0.68536100 \\
\hline C & -0.43646900 & -0.67763300 & -0.21477800 & $\mathrm{O}$ & 0.48431700 & -0.55453000 & 0.56291100 \\
\hline $\mathrm{O}$ & -0.70021700 & -1.72013900 & -1.01720200 & C & 0.23492000 & -2.87701900 & -1.08718300 \\
\hline C & 0.42936200 & -3.48108500 & 0.30293800 & $\mathrm{H}$ & 0.98763900 & -4.41825400 & 0.20786800 \\
\hline $\mathrm{H}$ & 0.98678500 & -2.81399600 & 0.95644200 & $\mathrm{H}$ & -0.53702500 & -3.70274100 & 0.76946600 \\
\hline C & -0.49079800 & -3.85254900 & -2.00886500 & $\mathrm{H}$ & -0.73664100 & -3.36820600 & -2.95850700 \\
\hline $\mathrm{H}$ & 0.16248900 & -4.70528000 & -2.21688500 & $\mathrm{H}$ & -1.40641100 & -4.23521600 & -1.54501700 \\
\hline C & 1.53430300 & -2.41141500 & -1.72547600 & $\mathrm{H}$ & 2.23537300 & -3.24789900 & -1.80746300 \\
\hline $\mathrm{H}$ & 1.35149700 & -2.00471800 & -2.72196400 & $\mathrm{H}$ & 1.99799700 & -1.64084800 & -1.11769900 \\
\hline C & -2.16704700 & 0.48560400 & -1.48295200 & $\mathrm{H}$ & -1.81679800 & -0.10308800 & -2.32447500 \\
\hline C & -2.57191400 & 1.87472500 & -1.82576200 & C & -2.38154000 & 2.30571100 & -3.14317200 \\
\hline C & -3.14050300 & 2.74012800 & -0.88319300 & $C$ & -2.73268600 & 3.60341500 & -3.50827300 \\
\hline $\mathrm{H}$ & -1.91195000 & 1.64054200 & -3.85959400 & C & -3.50582800 & 4.02911400 & -1.25696100 \\
\hline $\mathrm{H}$ & -3.27216800 & 2.41459900 & 0.14332400 & C & -3.30219100 & 4.46404200 & -2.56943600 \\
\hline $\mathrm{H}$ & -2.54853100 & 3.94229600 & -4.52290300 & $\mathrm{H}$ & -3.93912800 & 4.70067000 & -0.52138700 \\
\hline
\end{tabular}




\begin{tabular}{|c|c|c|c|c|c|c|c|}
\hline $\mathrm{H}$ & -3.57940900 & 5.47465600 & -2.85534300 & $\mathrm{~N}$ & -1.42414700 & 0.30699300 & -0.33884900 \\
\hline $\mathrm{H}$ & -1.21676200 & 1.14523500 & 0.22054600 & S & 1.13010200 & 0.95458700 & -3.27631800 \\
\hline 0 & 0.23602900 & 0.01168000 & -3.97716400 & $\mathrm{O}$ & 0.51126800 & 1.59431100 & -2.08109600 \\
\hline 0 & 2.52455800 & 0.50557100 & -3.03967600 & C & 1.34122900 & 2.37628300 & -4.45539300 \\
\hline $\mathrm{F}$ & 1.97725200 & 1.98614600 & -5.56381700 & $\mathrm{~F}$ & 2.05619200 & 3.35272200 & -3.86998600 \\
\hline $\mathrm{F}$ & 0.14827300 & 2.88856300 & -4.80811400 & C & -3.95682200 & -0.26258000 & -1.25725700 \\
\hline $\mathrm{H}$ & -4.21043600 & 0.19936300 & -0.30693700 & $\mathrm{H}$ & -4.43447900 & 0.17588700 & -2.12793800 \\
\hline C & -3.73124200 & -1.64039600 & -1.27086300 & C & -3.79199000 & -2.39646500 & -2.51728200 \\
\hline $\mathrm{H}$ & -3.66387700 & -1.75606000 & -3.39745400 & $\mathrm{H}$ & -3.06520400 & -3.21141600 & -2.54717500 \\
\hline $\mathrm{C}$ & -3.44812400 & -2.32316500 & 0.01657900 & $\mathrm{H}$ & -3.03279200 & -3.32376800 & -0.10433000 \\
\hline $\mathrm{H}$ & -2.77071800 & -1.71269400 & 0.62178900 & $\mathrm{H}$ & -4.37267000 & -2.38683200 & 0.60287200 \\
\hline $\mathrm{C}$ & -5.49098700 & -4.07633200 & -4.40451700 & $\mathrm{H}$ & -4.70054900 & -4.83422100 & -4.44603400 \\
\hline $\mathrm{H}$ & -6.44418400 & -4.57717100 & -4.61094400 & $\mathrm{H}$ & -5.30990100 & -3.35786200 & -5.21177300 \\
\hline C & -5.80313500 & -4.47902100 & -1.34392600 & $\mathrm{H}$ & -4.98632200 & -5.20842300 & -1.29891400 \\
\hline $\mathrm{H}$ & -5.88089900 & -4.00099600 & -0.36177100 & $\mathrm{H}$ & -6.73258200 & -5.03535900 & -1.51427500 \\
\hline $\mathrm{C}$ & -6.84556700 & -1.87475700 & -2.66860300 & $\mathrm{H}$ & -6.85409500 & -1.36429500 & -1.69911200 \\
\hline $\mathrm{H}$ & -6.67637600 & -1.11835200 & -3.44374300 & $\mathrm{H}$ & -7.84424100 & -2.29655200 & -2.83145200 \\
\hline $\mathrm{Si}$ & -5.53701500 & -3.22655800 & -2.72546300 & & & & \\
\hline
\end{tabular}

\section{TS-S-4f-45}

Imaginary frequency: $-260.82 \mathrm{~cm}^{-1}$

E[B3LYP/6-31G(d)-D3(BJ)]: -4789.078254 Hartree

$\mathrm{E}\left[\mathrm{PCM}\left(\mathrm{Et}_{2} \mathrm{O}\right)-\mathrm{B} 3 \mathrm{LYP} / 6-311 \mathrm{G}(\mathrm{d}, \mathrm{p})-\mathrm{D} 3(\mathrm{BJ})\right]$ : -4790.217494 Hartree

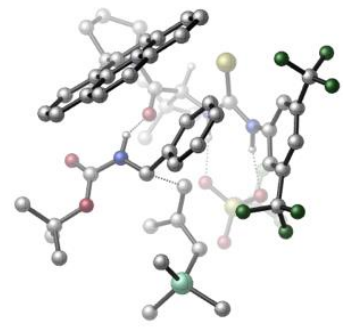

\begin{tabular}{|c|c|c|c|c|c|c|c|}
\hline 01 & & & & $\mathrm{~N}$ & -1.44697600 & 2.22933100 & 1.78129600 \\
\hline $\mathrm{H}$ & -2.37644200 & 1.83738000 & 1.59480000 & C & -1.03942900 & 3.12384400 & 0.77335400 \\
\hline C & -1.66048700 & 2.99010700 & -0.47656700 & C & -0.05678300 & 4.10647500 & 0.94065900 \\
\hline C & -1.21051700 & 3.72422300 & -1.56844300 & $\mathrm{H}$ & -2.49021800 & 2.30166300 & -0.57973300 \\
\hline C & 0.36494800 & 4.84959600 & -0.16303300 & $\mathrm{H}$ & 0.39262500 & 4.26223600 & 1.90952100 \\
\hline $\mathrm{C}$ & -0.17999600 & 4.65236000 & -1.43041400 & $\mathrm{H}$ & 0.18287600 & 5.21067600 & -2.28345000 \\
\hline C & -1.76444000 & 3.38462300 & -2.92123000 & $\mathrm{C}$ & 1.51972700 & 5.79352000 & 0.01124300 \\
\hline $\mathrm{F}$ & -3.10315700 & 3.18699600 & -2.89869700 & $\mathrm{~F}$ & -1.22983400 & 2.21472400 & -3.38752000 \\
\hline $\mathrm{F}$ & -1.50197900 & 4.32647200 & -3.84634500 & $\mathrm{~F}$ & 1.49264900 & 6.42135900 & 1.20330100 \\
\hline $\mathrm{F}$ & 2.71191100 & 5.13150400 & -0.04867900 & $\mathrm{~F}$ & 1.55907600 & 6.73876300 & -0.95217400 \\
\hline C & -0.45374400 & -0.57606800 & 3.89832300 & $\mathrm{H}$ & -0.02058000 & -0.02549400 & 4.73582300 \\
\hline C & -1.39901600 & -1.69185600 & 4.47333300 & $\mathrm{C}$ & -1.84321800 & -2.70946700 & 3.40469500 \\
\hline $\mathrm{H}$ & -0.99164100 & -3.25832400 & 2.99094500 & $\mathrm{H}$ & -2.38161800 & -2.23863200 & 2.58284900 \\
\hline $\mathrm{H}$ & -2.51925400 & -3.43636800 & 3.86980700 & $\mathrm{C}$ & -2.63015300 & -1.01376100 & 5.10483100 \\
\hline $\mathrm{H}$ & -3.23074900 & -1.76586200 & 5.62887000 & $\mathrm{H}$ & -3.27246000 & -0.53753900 & 4.36306900 \\
\hline $\mathrm{H}$ & -2.32877900 & -0.25313800 & 5.83483600 & C & -0.66121800 & -2.46372100 & 5.58186600 \\
\hline $\mathrm{H}$ & -1.32967000 & -3.22384300 & 5.99949900 & $\mathrm{H}$ & -0.35847300 & -1.80623700 & 6.40557000 \\
\hline $\mathrm{H}$ & 0.22262700 & -2.98374700 & 5.19924100 & $\mathrm{C}$ & 0.66804400 & -1.17165300 & 3.02465100 \\
\hline $\mathrm{O}$ & 0.42403000 & -1.40464600 & 1.82816500 & $\mathrm{C}$ & 2.36626000 & -1.27054900 & 4.90994600 \\
\hline C & 2.82550000 & -2.27600900 & 2.74438600 & C & 3.21709700 & -2.51720500 & 5.16503500 \\
\hline $\mathrm{H}$ & 2.97418800 & -0.35661200 & 4.91510400 & $\mathrm{H}$ & 1.56213900 & -1.14322400 & 5.62909800 \\
\hline $\mathrm{H}$ & 2.26793900 & -3.09194000 & 2.28098800 & $\mathrm{H}$ & 2.56610200 & -3.34374000 & 5.47132400 \\
\hline $\mathrm{H}$ & 3.96206600 & -2.36318200 & 5.95061500 & $\mathrm{~N}$ & 1.85245900 & -1.51913300 & 3.56196400 \\
\hline C & 3.48718000 & -1.42783500 & 1.67000000 & C & 3.76471600 & -0.11643800 & 1.92299300 \\
\hline $\mathrm{C}$ & 3.87159700 & -2.01497400 & 0.40309900 & $\mathrm{C}$ & 4.42179200 & 0.72922500 & 0.97524600 \\
\hline $\mathrm{H}$ & 3.44335600 & 0.33422900 & 2.85535500 & $\mathrm{C}$ & 4.47559200 & -1.17301000 & -0.58649000 \\
\hline $\mathrm{C}$ & 3.66404800 & -3.36860000 & 0.08830100 & C & 4.69747600 & 2.07784400 & 1.24864100 \\
\hline
\end{tabular}




\begin{tabular}{|c|c|c|c|c|c|c|c|}
\hline C & 4.76144800 & 0.19177900 & -0.29828900 & C & 4.79869200 & -1.70024900 & -1.87412400 \\
\hline $\mathrm{H}$ & 3.21826100 & -4.03793400 & 0.81270900 & C & 3.99311500 & -3.87495500 & -1.16756900 \\
\hline $\mathrm{C}$ & 5.29022900 & 2.88977700 & 0.28487400 & $\mathrm{H}$ & 4.41505200 & 2.48867900 & 2.21386500 \\
\hline C & 5.36290000 & 1.03085700 & -1.28246700 & C & 4.54176000 & -3.05223900 & -2.14546000 \\
\hline C & 5.38911700 & -0.82951000 & -2.85191300 & $\mathrm{H}$ & 3.81420000 & -4.92440100 & -1.37962100 \\
\hline C & 5.61999100 & 2.37381600 & -0.96728300 & $\mathrm{H}$ & 5.47741300 & 3.93661900 & 0.50408600 \\
\hline C & 5.66193000 & 0.47316900 & -2.56980700 & $\mathrm{H}$ & 4.79067600 & -3.45258100 & -3.12506200 \\
\hline $\mathrm{H}$ & 5.62127800 & -1.24175700 & -3.83062400 & $\mathrm{H}$ & 6.07303400 & 3.01475700 & -1.71920600 \\
\hline $\mathrm{H}$ & 6.11148600 & 1.11824700 & -3.32033000 & $\mathrm{C}$ & -0.62930600 & 1.51539200 & 2.62744300 \\
\hline $\mathrm{S}$ & 0.93977800 & 1.97811800 & 3.07056700 & $\mathrm{~N}$ & -1.19735800 & 0.37933800 & 3.09286300 \\
\hline $\mathrm{H}$ & -2.03690800 & 0.03214000 & 2.61787800 & $\mathrm{~S}$ & -4.57581500 & 0.21209600 & 1.10291000 \\
\hline 0 & -5.38390700 & -0.47214000 & 0.08057600 & $\mathrm{O}$ & -3.50957000 & -0.63111800 & 1.71903100 \\
\hline $\mathrm{O}$ & -4.08365700 & 1.57161500 & 0.75143100 & $\mathrm{C}$ & -5.74001000 & 0.51368500 & 2.51655100 \\
\hline $\mathrm{F}$ & -6.74967500 & 1.30392400 & 2.14186100 & $\mathrm{~F}$ & -5.07963700 & 1.10807700 & 3.52684800 \\
\hline $\mathrm{F}$ & -6.23782600 & -0.64583400 & 2.96324700 & C & 0.44946000 & -3.59991400 & -0.93146000 \\
\hline 0 & 0.65554200 & -4.41750800 & -0.05492200 & $\mathrm{O}$ & 0.00876700 & -3.84241300 & -2.17800000 \\
\hline C & -0.16479500 & -5.22807800 & -2.66020000 & $\mathrm{C}$ & 1.16884900 & -5.97103300 & -2.57644500 \\
\hline $\mathrm{H}$ & 1.07381900 & -6.94196400 & -3.07375700 & $\mathrm{H}$ & 1.46479000 & -6.13352300 & -1.53960900 \\
\hline $\mathrm{H}$ & 1.95128000 & -5.39827700 & -3.08454200 & C & -0.57748200 & -5.02186800 & -4.11580500 \\
\hline $\mathrm{H}$ & -1.50270500 & -4.44232600 & -4.17969300 & $\mathrm{H}$ & -0.74398500 & -5.99076700 & -4.59641400 \\
\hline $\mathrm{H}$ & 0.20410400 & -4.49011100 & -4.66752800 & C & -1.27082600 & -5.91987100 & -1.86440600 \\
\hline $\mathrm{H}$ & -1.44818400 & -6.91686700 & -2.28131900 & $\mathrm{H}$ & -2.20452300 & -5.35307000 & -1.93179600 \\
\hline $\mathrm{H}$ & -0.99263100 & -6.02152600 & -0.81480700 & C & 0.31143400 & -1.24449500 & -1.59319500 \\
\hline $\mathrm{H}$ & -0.00542400 & -1.61932700 & -2.55550000 & C & 1.06801100 & 0.02333900 & -1.64456000 \\
\hline C & 1.43425700 & 0.51017800 & -2.90811000 & C & 1.37161500 & 0.77169000 & -0.49837100 \\
\hline C & 2.09941200 & 1.72778400 & -3.02479500 & $\mathrm{H}$ & 1.20369700 & -0.07070900 & -3.79548200 \\
\hline C & 2.00412200 & 1.99938000 & -0.62555600 & $\mathrm{H}$ & 1.09252800 & 0.41268500 & 0.48542300 \\
\hline C & 2.36962500 & 2.48184200 & -1.88430500 & $\mathrm{H}$ & 2.38999700 & 2.09081800 & -4.00606900 \\
\hline $\mathrm{H}$ & 2.20977000 & 2.58031500 & 0.26141800 & $\mathrm{H}$ & 2.86263100 & 3.44393200 & -1.96228000 \\
\hline $\mathrm{N}$ & 0.68774900 & -2.24694600 & -0.73878400 & $\mathrm{H}$ & 0.93928100 & -2.00466300 & 0.22699800 \\
\hline C & -1.42034000 & -0.47005100 & -0.78903800 & $\mathrm{H}$ & -1.10409600 & -0.74125900 & 0.21399800 \\
\hline $\mathrm{H}$ & -1.30606000 & 0.57234400 & -1.05135300 & C & -2.49519500 & -1.15062400 & -1.35029400 \\
\hline C & -3.33550500 & -0.51265000 & -2.37389400 & $\mathrm{H}$ & -3.18327600 & 0.57019500 & -2.37714300 \\
\hline $\mathrm{H}$ & -4.38629700 & -0.69565900 & -2.11211800 & C & -2.86837600 & -2.50783900 & -0.85769300 \\
\hline $\mathrm{H}$ & -3.10643700 & -3.18589300 & -1.68254100 & $\mathrm{H}$ & -3.78060200 & -2.38524500 & -0.25909500 \\
\hline $\mathrm{H}$ & -2.10246600 & -2.94437900 & -0.21411100 & C & -4.16108600 & 0.31218200 & -5.13098500 \\
\hline $\mathrm{H}$ & -5.21390600 & 0.33274300 & -4.82695300 & $\mathrm{H}$ & -4.12740500 & 0.16325400 & -6.21650900 \\
\hline $\mathrm{H}$ & -3.73294700 & 1.29578700 & -4.90764200 & C & -4.04519900 & -2.71721600 & -4.46371000 \\
\hline $\mathrm{H}$ & -5.08555900 & -2.69567600 & -4.11968100 & $\mathrm{H}$ & -3.53291100 & -3.51827000 & -3.91957500 \\
\hline $\mathrm{H}$ & -4.05107900 & -2.99392700 & -5.52497900 & C & -1.43143600 & -1.09282600 & -4.81977800 \\
\hline $\mathrm{H}$ & -0.88074800 & -1.94224700 & -4.40189800 & $\mathrm{H}$ & -0.90982000 & -0.16731800 & -4.55728300 \\
\hline $\mathrm{H}$ & -1.40515700 & -1.19239700 & -5.91163000 & $\mathrm{Si}$ & -3.21736200 & -1.03883100 & -4.21988500 \\
\hline C & 3.84938100 & -2.80413100 & 3.79100300 & $\mathrm{H}$ & 4.78857000 & -2.25458500 & 3.68356200 \\
\hline $\mathrm{H}$ & 4.06777900 & -3.86422700 & 3.63793300 & & & & \\
\hline
\end{tabular}

\section{TS-S-4f-46}

Imaginary frequency: $-403.50 \mathrm{~cm}^{-1}$

E[B3LYP/6-31G(d)-D3(BJ)]: -4789.066112 Hartree

$\mathrm{E}\left[\mathrm{PCM}\left(\mathrm{Et}_{2} \mathrm{O}\right)-\mathrm{B} 3 \mathrm{LYP} / 6-311 \mathrm{G}(\mathrm{d}, \mathrm{p})-\mathrm{D} 3(\mathrm{BJ})\right]$ : -4790.216947 Hartree

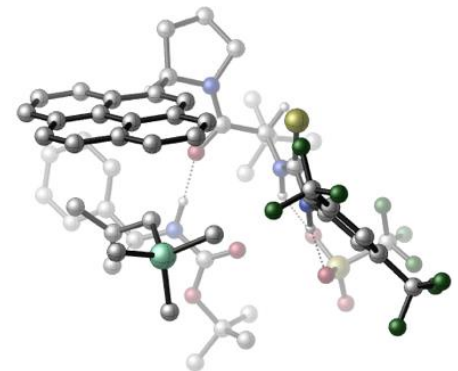

S152 


\begin{tabular}{|c|c|c|c|c|c|c|c|}
\hline 01 & & & & $\mathrm{~N}$ & -2.46276500 & 0.27298100 & 0.73949900 \\
\hline $\mathrm{H}$ & -2.81656100 & -0.54669600 & 0.22216500 & C & -3.17390300 & 1.45054000 & 0.44343900 \\
\hline C & -4.50746900 & 1.32645500 & 0.02897300 & C & -2.58049900 & 2.71575000 & 0.46928000 \\
\hline C & -5.22699900 & 2.45922200 & -0.34446300 & $\mathrm{H}$ & -4.97133200 & 0.34909000 & -0.00170000 \\
\hline C & -3.31760500 & 3.83689400 & 0.09510900 & $\mathrm{H}$ & -1.55082900 & 2.81600200 & 0.77197900 \\
\hline C & -4.64544300 & 3.72628000 & -0.31176000 & $\mathrm{H}$ & -5.21536300 & 4.60351000 & -0.59028600 \\
\hline C & -6.63832800 & 2.30658200 & -0.84115700 & C & -2.62088800 & 5.16375400 & 0.05940500 \\
\hline $\mathrm{F}$ & -6.67531700 & 2.14304200 & -2.18511300 & $\mathrm{~F}$ & -7.38609200 & 3.40069700 & -0.56294300 \\
\hline $\mathrm{F}$ & -7.25660000 & 1.24100400 & -0.29557600 & $\mathrm{~F}$ & -1.80558900 & 5.34134300 & 1.13025100 \\
\hline $\mathrm{F}$ & -1.82277600 & 5.28237400 & -1.03885900 & $\mathrm{~F}$ & -3.47811700 & 6.20323500 & 0.02993700 \\
\hline C & -0.14186900 & -1.62613200 & 2.81269400 & $\mathrm{H}$ & -0.40917500 & -1.12090100 & 3.74055300 \\
\hline C & -0.19054200 & -3.16337700 & 3.09145600 & C & 0.09537600 & -4.01430400 & 1.84216100 \\
\hline $\mathrm{H}$ & 1.10142800 & -3.82871800 & 1.45511300 & $\mathrm{H}$ & -0.63354000 & -3.83956000 & 1.04973800 \\
\hline $\mathrm{H}$ & 0.02985200 & -5.07467700 & 2.11140900 & C & -1.58827100 & -3.49467200 & 3.64794500 \\
\hline $\mathrm{H}$ & -1.63631600 & -4.55653200 & 3.91505200 & $\mathrm{H}$ & -2.37372800 & -3.29843800 & 2.91681600 \\
\hline $\mathrm{H}$ & -1.79444600 & -2.90861300 & 4.55181400 & C & 0.85998600 & -3.50920400 & 4.16367600 \\
\hline $\mathrm{H}$ & 0.82904200 & -4.58410400 & 4.36987800 & $\mathrm{H}$ & 0.66174300 & -2.99200100 & 5.10937600 \\
\hline $\mathrm{H}$ & 1.87891000 & -3.26458900 & 3.84140900 & C & 1.25088000 & -1.14054100 & 2.37539000 \\
\hline $\mathrm{O}$ & 1.66680700 & -1.32769300 & 1.21590200 & C & 1.77741800 & -0.20176500 & 4.67819000 \\
\hline C & 3.40335600 & -0.09984300 & 2.87513700 & C & 3.16688300 & -0.32104200 & 5.30577400 \\
\hline $\mathrm{H}$ & 1.37038100 & 0.81307100 & 4.75752900 & $\mathrm{H}$ & 1.05649000 & -0.88981700 & 5.11088300 \\
\hline $\mathrm{H}$ & 3.88513600 & -0.96642700 & 2.41512000 & $\mathrm{H}$ & 3.39751400 & -1.37826800 & 5.48104300 \\
\hline $\mathrm{H}$ & 3.24303700 & 0.20764500 & 6.25980400 & $\mathrm{~N}$ & 2.04575500 & -0.51893400 & 3.27017700 \\
\hline C & 3.41512000 & 1.06767600 & 1.90393700 & C & 2.35438200 & 1.92233300 & 1.84411600 \\
\hline $\mathrm{C}$ & 4.59547100 & 1.31990100 & 1.10472100 & C & 2.35876800 & 3.09179600 & 1.01708300 \\
\hline $\mathrm{H}$ & 1.45508600 & 1.71663000 & 2.41624000 & C & 4.61072900 & 2.46219000 & 0.24039800 \\
\hline C & 5.73206800 & 0.49305100 & 1.13075700 & C & 1.27665000 & 3.98720200 & 0.99137700 \\
\hline $\mathrm{C}$ & 3.49733600 & 3.35091300 & 0.20111200 & C & 5.75135200 & 2.72522600 & -0.58001100 \\
\hline $\mathrm{H}$ & 5.76232200 & -0.36186100 & 1.79447900 & $\mathrm{C}$ & 6.83630900 & 0.75202700 & 0.31975700 \\
\hline C & 1.30684400 & 5.11161800 & 0.16718300 & $\mathrm{H}$ & 0.41182900 & 3.78893000 & 1.61746800 \\
\hline C & 3.51897100 & 4.50313300 & -0.64086600 & C & 6.84941900 & 1.85246500 & -0.53091500 \\
\hline $\mathrm{C}$ & 5.73783400 & 3.88078200 & -1.43426300 & $\mathrm{H}$ & 7.69373100 & 0.08628400 & 0.35695900 \\
\hline C & 2.41067800 & 5.36431500 & -0.64436900 & $\mathrm{H}$ & 0.45458500 & 5.77983400 & 0.14770300 \\
\hline C & 4.67553800 & 4.73181800 & -1.46038600 & $\mathrm{H}$ & 7.71426400 & 2.05225600 & -1.15784000 \\
\hline $\mathrm{H}$ & 6.60846200 & 4.06762400 & -2.05743500 & $\mathrm{H}$ & 2.42395700 & 6.23870400 & -1.28968000 \\
\hline $\mathrm{H}$ & 4.68557100 & 5.60560800 & -2.10660100 & C & -1.57873100 & 0.05983500 & 1.75559000 \\
\hline$S$ & -1.10298600 & 1.25371500 & 2.87351700 & $\mathrm{~N}$ & -1.11918700 & -1.21048000 & 1.82468200 \\
\hline $\mathrm{H}$ & -1.47605000 & -1.90287800 & 1.16492700 & C & 0.07412100 & -2.79655100 & -1.65599900 \\
\hline 0 & -0.81950900 & -2.24736800 & -1.05201000 & $\mathrm{O}$ & 0.00269600 & -3.45656200 & -2.81238100 \\
\hline C & -1.32123800 & -3.92539400 & -3.34426100 & C & -2.25219600 & -2.74396400 & -3.60022800 \\
\hline $\mathrm{H}$ & -3.14730400 & -3.11493500 & -4.11010500 & $\mathrm{H}$ & -2.57023700 & -2.25555100 & -2.67993800 \\
\hline $\mathrm{H}$ & -1.76741100 & -2.01520200 & -4.25993700 & C & -0.91002800 & -4.59433500 & -4.65245600 \\
\hline $\mathrm{H}$ & -0.18869800 & -5.39760800 & -4.47056900 & $\mathrm{H}$ & -1.79299700 & -5.02615800 & -5.13285500 \\
\hline $\mathrm{H}$ & -0.46431500 & -3.86780700 & -5.34038900 & C & -1.88494600 & -4.93416700 & -2.34941500 \\
\hline $\mathrm{H}$ & -2.85439600 & -5.29512900 & -2.70345100 & $\mathrm{H}$ & -1.20518800 & -5.78766900 & -2.24891500 \\
\hline $\mathrm{H}$ & -2.05264700 & -4.48678700 & -1.36961700 & C & 2.48166800 & -3.05607100 & -1.94517900 \\
\hline $\mathrm{H}$ & 2.23947700 & -3.75718900 & -2.73700400 & C & 3.81181600 & -3.26534400 & -1.33370900 \\
\hline C & 4.76027000 & -3.99223800 & -2.07092500 & C & 4.15730300 & -2.77202200 & -0.06753500 \\
\hline C & 6.02890000 & -4.23137800 & -1.55332600 & $\mathrm{H}$ & 4.49433900 & -4.37699100 & -3.05248900 \\
\hline C & 5.42528300 & -3.02950200 & 0.45176800 & $\mathrm{H}$ & 3.44268900 & -2.19574700 & 0.51041000 \\
\hline C & 6.36357000 & -3.75255500 & -0.28436000 & $\mathrm{H}$ & 6.75098800 & -4.79958100 & -2.13175500 \\
\hline $\mathrm{H}$ & 5.67946400 & -2.66557100 & 1.44247400 & $\mathrm{H}$ & 7.34913700 & -3.94690300 & 0.12819700 \\
\hline $\mathrm{N}$ & 1.39423800 & -2.75953700 & -1.17277700 & $\mathrm{H}$ & 1.47026100 & -2.26055000 & -0.27277400 \\
\hline
\end{tabular}




\begin{tabular}{|c|c|c|c|c|c|c|c|}
\hline $\mathrm{S}$ & -4.06979800 & -2.92344300 & -0.09459300 & $\mathrm{O}$ & -4.75826800 & -3.93763700 & -0.90079200 \\
\hline 0 & -2.87689600 & -3.38710600 & 0.66529400 & $\mathrm{O}$ & -3.85267400 & -1.60885800 & -0.77353800 \\
\hline C & -5.24838300 & -2.46244400 & 1.26613700 & $\mathrm{~F}$ & -6.33700400 & -1.85331200 & 0.77199000 \\
\hline $\mathrm{F}$ & -4.64013500 & -1.60363200 & 2.11122800 & $\mathrm{~F}$ & -5.63510600 & -3.53580600 & 1.96425000 \\
\hline C & 2.67333400 & -1.60577100 & -3.30344600 & $\mathrm{H}$ & 3.45030300 & -1.99677600 & -3.95536300 \\
\hline $\mathrm{H}$ & 1.66032700 & -1.72185300 & -3.67623500 & C & 2.97793400 & -0.48907200 & -2.51556400 \\
\hline C & 1.94526300 & 0.23027100 & -1.79909200 & $\mathrm{H}$ & 1.10845600 & -0.40803500 & -1.49540000 \\
\hline $\mathrm{H}$ & 2.32213400 & 0.78463200 & -0.93574000 & C & 4.39657000 & -0.08481700 & -2.30990000 \\
\hline $\mathrm{H}$ & 4.50238900 & 1.00320800 & -2.35925000 & $\mathrm{H}$ & 4.70180600 & -0.36752100 & -1.29231600 \\
\hline $\mathrm{H}$ & 5.07898100 & -0.55675700 & -3.01967400 & C & -0.23885100 & 2.30735600 & -1.83870400 \\
\hline $\mathrm{H}$ & 0.23802200 & 2.77367900 & -0.97387300 & $\mathrm{H}$ & -0.81725300 & 3.08266000 & -2.35246300 \\
\hline $\mathrm{H}$ & -0.93996300 & 1.54987500 & -1.47270600 & C & 2.29894700 & 2.70671500 & -3.66243800 \\
\hline $\mathrm{H}$ & 2.88200800 & 3.19027400 & -2.87369300 & $\mathrm{H}$ & 2.99435500 & 2.19644600 & -4.33934500 \\
\hline $\mathrm{H}$ & 1.79979400 & 3.49693200 & -4.23580500 & C & 0.17800900 & 0.55075400 & -4.33594400 \\
\hline $\mathrm{H}$ & 0.88264000 & 0.09513400 & -5.03953400 & $\mathrm{H}$ & -0.46375500 & -0.23676300 & -3.92570200 \\
\hline $\mathrm{H}$ & -0.46765600 & 1.22760600 & -4.90835400 & $\mathrm{Si}$ & 1.02784900 & 1.51312800 & -2.95862500 \\
\hline C & 4.08728300 & 0.26394900 & 4.22419500 & $\mathrm{H}$ & 4.13696200 & 1.35273100 & 4.31814500 \\
\hline $\mathrm{H}$ & 5.11031800 & -0.11806400 & 4.27619500 & & & & \\
\hline
\end{tabular}

\section{TS-S-4f-47}

Imaginary frequency: $-436.69 \mathrm{~cm}^{-1}$

E[B3LYP/6-31G(d)-D3(BJ)]: -4789.066242 Hartree

$\mathrm{E}\left[\mathrm{PCM}\left(\mathrm{Et}_{2} \mathrm{O}\right)-\mathrm{B} 3 \mathrm{LYP} / 6-311 \mathrm{G}(\mathrm{d}, \mathrm{p})-\mathrm{D} 3(\mathrm{BJ})\right]$ : -4790.215113 Hartree

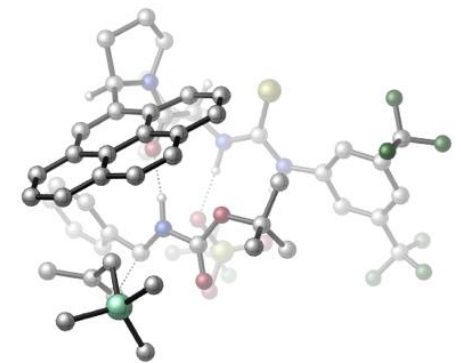

\begin{tabular}{|crrr|rrrr|}
\hline $\mathrm{O} 1$ & & & & $\mathrm{~N}$ & 3.26474900 & 0.45966900 & 0.80212100 \\
\hline $\mathrm{H}$ & 3.17500700 & 1.16344000 & 0.06286000 & $\mathrm{C}$ & 4.23950400 & -0.50332500 & 0.49507100 \\
\hline $\mathrm{C}$ & 5.03346800 & -0.23193600 & -0.63504400 & $\mathrm{C}$ & 4.42353200 & -1.71288200 & 1.17436200 \\
\hline $\mathrm{C}$ & 5.96171100 & -1.16390100 & -1.08157800 & $\mathrm{H}$ & 4.88516300 & 0.68922400 & -1.18578600 \\
\hline $\mathrm{C}$ & 5.34608600 & -2.64153800 & 0.69297000 & $\mathrm{H}$ & 3.83970500 & -1.93244800 & 2.05458800 \\
\hline $\mathrm{C}$ & 6.12490700 & -2.38782700 & -0.43088900 & $\mathrm{H}$ & 6.83370500 & -3.12055400 & -0.79511500 \\
\hline $\mathrm{C}$ & 6.83815700 & -0.83568500 & -2.25895000 & $\mathrm{C}$ & 5.39590900 & -3.99178700 & 1.34850100 \\
\hline $\mathrm{F}$ & 8.02471200 & -0.31633600 & -1.86333000 & $\mathrm{~F}$ & 6.26811000 & 0.06219300 & -3.08564200 \\
\hline $\mathrm{F}$ & 7.12234700 & -1.94052000 & -2.98840400 & $\mathrm{~F}$ & 5.22763800 & -3.92026300 & 2.68501300 \\
\hline $\mathrm{F}$ & 4.40593600 & -4.79861100 & 0.87614300 & $\mathrm{~F}$ & 6.56116200 & -4.63366000 & 1.11638300 \\
\hline $\mathrm{C}$ & 0.75196300 & 2.17157200 & 2.79930800 & $\mathrm{H}$ & 1.19906500 & 1.85487800 & 3.74031600 \\
\hline $\mathrm{C}$ & 0.66914400 & 3.73984100 & 2.83434600 & $\mathrm{C}$ & -0.10078500 & 4.33744200 & 1.64587800 \\
\hline $\mathrm{H}$ & -1.14720000 & 4.02427300 & 1.64341800 & $\mathrm{H}$ & 0.32783400 & 4.05358200 & 0.68505000 \\
\hline $\mathrm{H}$ & -0.07612600 & 5.43140200 & 1.71740400 & $\mathrm{C}$ & 2.10889700 & 4.28292100 & 2.84591200 \\
\hline $\mathrm{H}$ & 2.09359600 & 5.36708000 & 3.00612000 & $\mathrm{H}$ & 2.61582000 & 4.09036900 & 1.89833100 \\
\hline $\mathrm{H}$ & 2.70039100 & 3.82753300 & 3.64889700 & $\mathrm{C}$ & -0.03147200 & 4.16670800 & 4.13620300 \\
\hline $\mathrm{H}$ & -0.07811600 & 5.25981800 & 4.18863100 & $\mathrm{H}$ & 0.51157200 & 3.81469200 & 5.02101000 \\
\hline $\mathrm{H}$ & -1.05980400 & 3.79145000 & 4.18650900 & $\mathrm{C}$ & -0.62556500 & 1.52518800 & 2.63285100 \\
\hline $\mathrm{O}$ & -1.14786800 & 1.42664000 & 1.51671200 & $\mathrm{C}$ & -0.75819000 & 0.96395500 & 5.10250500 \\
\hline $\mathrm{C}$ & -2.72963100 & 0.80240500 & 3.64143500 & $\mathrm{C}$ & -1.79302400 & 0.06161400 & 5.78194400 \\
\hline $\mathrm{H}$ & 0.24027900 & 0.52073400 & 5.06133700 & $\mathrm{H}$ & -0.69448700 & 1.93321500 & 5.60898900 \\
\hline $\mathrm{C}$ & -3.11224100 & 0.46317600 & 5.10827900 & $\mathrm{H}$ & -3.24401600 & 1.71722100 & 3.32721600 \\
\hline $\mathrm{H}$ & -1.80988300 & 0.20449700 & 6.86582600 & $\mathrm{H}$ & -1.56912800 & -0.99016900 & 5.58371700 \\
\hline $\mathrm{H}$ & -3.52120100 & 1.36033900 & 5.58639500 & $\mathrm{H}$ & -3.87476800 & -0.31725600 & 5.15450200 \\
\hline $\mathrm{N}$ & -1.29548400 & 1.12269700 & 3.73915500 & $\mathrm{C}$ & -3.16235900 & -0.26725400 & 2.64770200 \\
\hline $\mathrm{C}$ & -4.34937700 & -0.03256700 & 2.01176000 & $\mathrm{C}$ & -2.49916500 & -1.54515200 & 2.46802300 \\
\hline & & & & & & & \\
\hline
\end{tabular}




\begin{tabular}{|c|c|c|c|c|c|c|c|}
\hline C & -5.01064700 & -1.00423100 & 1.19686300 & $\mathrm{H}$ & -4.84587300 & 0.92591700 & 2.15075800 \\
\hline C & -3.16669800 & -2.55759700 & 1.70222300 & C & -1.23484400 & -1.84875400 & 3.00243800 \\
\hline C & -6.22871900 & -0.74013200 & 0.54933800 & C & -4.41706100 & -2.29313700 & 1.06987800 \\
\hline $\mathrm{C}$ & -2.58106900 & -3.85449100 & 1.56753200 & $\mathrm{H}$ & -0.65311800 & -1.08832400 & 3.49849600 \\
\hline $\mathrm{C}$ & -0.67439300 & -3.11594100 & 2.86504400 & C & -6.86120000 & -1.72853000 & -0.20359300 \\
\hline $\bar{H}$ & -6.67931600 & 0.24383500 & 0.65049400 & $\mathrm{C}$ & -5.08144400 & -3.30545700 & 0.31287200 \\
\hline $\mathrm{C}$ & -1.34452400 & -4.11827100 & 2.17353500 & $\mathrm{C}$ & -3.28000400 & -4.86061200 & 0.81808200 \\
\hline $\mathrm{H}$ & 0.30922600 & -3.30203200 & 3.28440900 & C & -6.29818500 & -2.99901500 & -0.31889400 \\
\hline $\bar{H}$ & -7.80596000 & -1.51151600 & -0.69426900 & $\mathrm{C}$ & -4.47617300 & -4.60404200 & 0.22320800 \\
\hline $\bar{H}$ & -0.90457800 & -5.10695000 & 2.07320300 & $\bar{H}$ & -2.82680200 & -5.84521000 & 0.73916600 \\
\hline $\mathrm{H}$ & -6.80241300 & -3.76979300 & -0.89579800 & $\mathrm{H}$ & -4.99413200 & -5.38025200 & -0.33386700 \\
\hline $\mathrm{C}$ & 2.44724400 & 0.63274000 & 1.88989800 & $\mathrm{~S}$ & 2.44424200 & -0.33267200 & 3.28921200 \\
\hline $\bar{N}$ & 1.63200800 & 1.70487600 & 1.75003200 & $\bar{H}$ & 1.44051200 & 2.05432300 & 0.80298100 \\
\hline C & -0.44318900 & -0.34335800 & -1.80500300 & $\mathrm{O}$ & -0.33422200 & -0.43832200 & -3.01762300 \\
\hline $\bar{O}$ & 0.09323200 & -1.13078800 & -0.87976300 & $\mathrm{C}$ & 0.99172800 & -2.24465100 & -1.28516300 \\
\hline $\mathrm{C}$ & 2.16106900 & -1.69402100 & -2.10180000 & $\bar{H}$ & 2.96190600 & -2.43957700 & -2.13503600 \\
\hline $\bar{H}$ & 1.86113800 & -1.44432000 & -3.11948000 & $\bar{H}$ & 2.55870100 & -0.79209100 & -1.63450600 \\
\hline C & 1.44398900 & -2.79571900 & 0.06120300 & $\mathrm{H}$ & 0.60451600 & -3.25897500 & 0.58215300 \\
\hline $\mathrm{H}$ & 2.23404900 & -3.53947200 & -0.07372800 & $\mathrm{H}$ & 1.82725200 & -1.99340600 & 0.69222900 \\
\hline $\mathrm{C}$ & 0.17369300 & -3.28609700 & -2.04808800 & $\bar{H}$ & 0.79683800 & -4.16774800 & -2.23285800 \\
\hline $\mathrm{H}$ & -0.68969600 & -3.59915900 & -1.45011000 & $\mathrm{H}$ & -0.16819000 & -2.89596800 & -3.00871600 \\
\hline C & -1.62050000 & 1.73001000 & -1.93247800 & $\mathrm{H}$ & -0.89218100 & 2.01937600 & -2.68739700 \\
\hline $\mathrm{C}$ & -2.26353200 & 2.84052900 & -1.20978300 & $\mathrm{C}$ & -1.96421100 & 4.15098600 & -1.60570200 \\
\hline C & -3.16300800 & 2.62308900 & -0.15541800 & C & -2.55644300 & 5.22992600 & -0.95514300 \\
\hline $\mathrm{H}$ & -1.23586200 & 4.31500800 & -2.39327300 & $\mathrm{C}$ & -3.75749200 & 3.70532300 & 0.48803500 \\
\hline $\mathrm{H}$ & -3.37139100 & 1.61337400 & 0.17924000 & $\mathrm{C}$ & -3.45963900 & 5.00967700 & 0.08736300 \\
\hline $\mathrm{H}$ & -2.30363000 & 6.24273000 & -1.25337700 & $\mathrm{H}$ & -4.44776500 & 3.53061300 & 1.30898100 \\
\hline $\mathrm{H}$ & -3.92102200 & 5.85267400 & 0.59363600 & $\bar{N}$ & -1.24695700 & 0.62071900 & -1.20479100 \\
\hline $\bar{H}$ & -1.22380900 & 0.70391200 & -0.18266100 & $\mathrm{~S}$ & 1.86773400 & 2.71935700 & -1.93646500 \\
\hline 0 & 1.27142100 & 2.61484500 & -3.27746000 & $\mathrm{O}$ & 0.89963400 & 2.48631800 & -0.82487700 \\
\hline $\mathrm{O}$ & 3.16600400 & 2.03812500 & -1.70624600 & $C$ & 2.27084500 & 4.52194700 & -1.74646000 \\
\hline $\mathrm{F}$ & 3.12288300 & 4.93207000 & -2.68846500 & $\mathrm{~F}$ & 2.81974200 & 4.75260400 & -0.54041300 \\
\hline $\mathrm{F}$ & 1.14571300 & 5.25683900 & -1.83562900 & C & -2.85892800 & 1.20252600 & -3.37446600 \\
\hline $\mathrm{H}$ & -3.04925700 & 2.16884600 & -3.83417800 & $\mathrm{H}$ & -2.09241400 & 0.59492200 & -3.84485900 \\
\hline $\mathrm{C}$ & -3.92783300 & 0.54156000 & -2.76724300 & $\mathrm{C}$ & -3.79383700 & -0.83709800 & -2.32351600 \\
\hline $\mathrm{H}$ & -2.77230100 & -1.07318500 & -2.01386400 & $\mathrm{H}$ & -4.50036900 & -1.10220500 & -1.53457100 \\
\hline $\mathrm{C}$ & -5.19868700 & 1.26863400 & -2.47559100 & $\mathrm{H}$ & -5.43671600 & 2.01085700 & -3.24256300 \\
\hline $\mathrm{H}$ & -6.03717600 & 0.58180400 & -2.33744600 & $\mathrm{H}$ & -5.06262200 & 1.81745200 & -1.52998400 \\
\hline C & -3.63804400 & -3.77545000 & -3.18836400 & $\mathrm{H}$ & -4.17290800 & -4.05049700 & -2.27669000 \\
\hline $\bar{H}$ & -3.85585900 & -4.52800600 & -3.95592000 & $\bar{H}$ & -2.56565700 & -3.81873000 & -2.97197500 \\
\hline $\mathrm{C}$ & -5.96477600 & -1.97380000 & -4.20082300 & $\bar{H}$ & -6.56830800 & -2.18883300 & -3.31184000 \\
\hline $\mathrm{H}$ & -6.24809900 & -0.98193000 & -4.57158300 & $\mathrm{H}$ & -6.23289800 & -2.70343200 & -4.97389100 \\
\hline $\mathrm{C}$ & -3.06278800 & -1.58800100 & -5.27262900 & $\overline{\mathrm{H}}$ & -3.37249200 & -0.64012300 & -5.72455800 \\
\hline $\bar{H}$ & -2.00980800 & -1.49687900 & -4.98298100 & $\overline{\mathrm{H}}$ & -3.13131700 & -2.36285600 & -6.04571500 \\
\hline Si & -4.12587500 & -2.06718800 & -3.79802400 & & & & \\
\hline
\end{tabular}

\section{TS-S-4f-48}

Imaginary frequency: $-374.19 \mathrm{~cm}^{-1}$

E[B3LYP/6-31G(d)-D3(BJ)]: -4789.064090 Hartree

E[PCM(Et $2 \mathrm{O})-B 3 L Y P / 6-311 G(d, p)-D 3(B J)]:-4790.214938$ Hartree

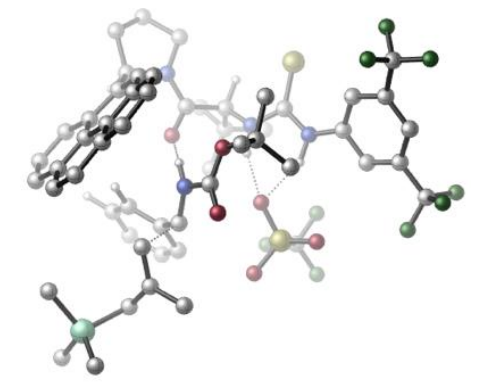




\begin{tabular}{|c|c|c|c|c|c|c|c|}
\hline 01 & & & & $\mathrm{~N}$ & -3.31104600 & 0.17941500 & 1.00341800 \\
\hline $\mathrm{H}$ & -2.70590100 & -0.64311600 & 1.03636400 & C & -4.49819600 & -0.01540300 & 0.28147600 \\
\hline C & -4.94348000 & -1.34151100 & 0.16815600 & C & -5.17127300 & 0.99582600 & -0.40864500 \\
\hline $\mathrm{C}$ & -6.02636400 & -1.64571100 & -0.64789900 & $\mathrm{H}$ & -4.40955400 & -2.13494000 & 0.67718200 \\
\hline C & -6.24416800 & 0.66520600 & -1.23562800 & $\mathrm{H}$ & -4.84623600 & 2.02148700 & -0.32077200 \\
\hline $\mathrm{C}$ & -6.68577900 & -0.64788900 & -1.36791300 & $\mathrm{H}$ & -7.51434200 & -0.89190000 & -2.02050300 \\
\hline $\mathrm{C}$ & -6.52673300 & -3.06193500 & -0.72794500 & C & -6.83539200 & 1.75428800 & -2.08371300 \\
\hline $\mathrm{F}$ & -7.56602200 & -3.26496300 & 0.11900500 & $F$ & -5.57633700 & -3.95935800 & -0.41312900 \\
\hline$F$ & -6.97850000 & -3.35731600 & -1.97002100 & $F$ & -6.99364500 & 2.90566800 & -1.39853700 \\
\hline$F$ & -6.02463400 & 2.04184700 & -3.13933300 & $F$ & -8.03687500 & 1.41496600 & -2.59726000 \\
\hline C & -0.72272400 & 1.96998900 & 2.78153300 & $\mathrm{H}$ & -1.38772100 & 2.80923100 & 2.97793900 \\
\hline $\mathrm{C}$ & -0.41886000 & 1.26156100 & 4.14706200 & C & 0.51098500 & 0.04879300 & 3.98808300 \\
\hline $\mathrm{H}$ & 1.50416200 & 0.33374300 & 3.63822300 & $\mathrm{H}$ & 0.10757100 & -0.68522700 & 3.28675700 \\
\hline $\mathrm{H}$ & 0.61771200 & -0.45520800 & 4.95593600 & C & -1.76051400 & 0.79055300 & 4.73731200 \\
\hline $\mathrm{H}$ & -1.60453200 & 0.40852000 & 5.75264800 & $\mathrm{H}$ & -2.19248300 & -0.01875300 & 4.14121100 \\
\hline $\mathrm{H}$ & -2.49107500 & 1.60531200 & 4.78299500 & $\mathrm{C}$ & 0.22915000 & 2.28051800 & 5.09976600 \\
\hline $\mathrm{H}$ & 0.46210000 & 1.80285000 & 6.05767700 & $\mathrm{H}$ & -0.44352800 & 3.12148700 & 5.30704700 \\
\hline $\mathrm{H}$ & 1.16734800 & 2.67850700 & 4.69468100 & C & 0.54807500 & 2.44416900 & 2.08050200 \\
\hline $\mathrm{O}$ & 1.31599700 & 1.62461500 & 1.55535600 & C & 0.05205800 & 4.86708000 & 2.64725300 \\
\hline C & 2.17086800 & 4.21244200 & 1.58855900 & $\mathrm{C}$ & 1.09535900 & 5.97030000 & 2.85675100 \\
\hline $\mathrm{H}$ & -0.73407900 & 5.16903900 & 1.94514400 & $\mathrm{H}$ & -0.42671500 & 4.57016800 & 3.57943000 \\
\hline $\mathrm{H}$ & 2.90251000 & 3.83338200 & 2.30597000 & $\mathrm{H}$ & 1.59858400 & 5.82465400 & 3.81990400 \\
\hline $\mathrm{H}$ & 0.64909500 & 6.96829800 & 2.85614300 & $\mathrm{~N}$ & 0.84298100 & 3.76297300 & 2.07415700 \\
\hline C & 2.51694600 & 3.70911300 & 0.19990600 & C & 1.62882500 & 3.89766600 & -0.81691300 \\
\hline C & 3.77292600 & 3.03102200 & -0.05607800 & $\mathrm{C}$ & 1.86728900 & 3.42773900 & -2.14677000 \\
\hline $\mathrm{H}$ & 0.68272700 & 4.39523800 & -0.61796200 & $C$ & 3.99066500 & 2.46302000 & -1.35291700 \\
\hline C & 4.79086000 & 2.88920400 & 0.90398300 & C & 0.99315100 & 3.70539700 & -3.20762000 \\
\hline$C$ & 3.04170700 & 2.66177400 & -2.39743300 & C & 5.18348500 & 1.72114500 & -1.62197900 \\
\hline $\mathrm{H}$ & 4.67979400 & 3.33081800 & 1.88668500 & C & 5.95934500 & 2.18254600 & 0.62608700 \\
\hline C & 1.25607500 & 3.22634600 & -4.48875000 & $\mathrm{H}$ & 0.10721700 & 4.30493400 & -3.01921600 \\
\hline$C$ & 3.27962100 & 2.13653900 & -3.70286100 & C & 6.15047000 & 1.58452500 & -0.61505800 \\
\hline C & 5.37144300 & 1.15803400 & -2.93089500 & $\mathrm{H}$ & 6.72459000 & 2.09464500 & 1.39196600 \\
\hline C & 2.37366200 & 2.43103800 & -4.73248600 & $\mathrm{H}$ & 0.57610700 & 3.46289100 & -5.30171400 \\
\hline $\mathrm{C}$ & 4.46229000 & 1.35366500 & -3.92404300 & $\mathrm{H}$ & 7.06125500 & 1.03077800 & -0.82578000 \\
\hline $\mathrm{H}$ & 6.27762900 & 0.58844400 & -3.11668400 & $\mathrm{H}$ & 2.56070800 & 2.04250500 & -5.72979500 \\
\hline $\mathrm{H}$ & 4.62898100 & 0.93878900 & -4.91467400 & C & -2.75218000 & 1.30943700 & 1.53310100 \\
\hline $\mathrm{S}$ & -3.55837600 & 2.77615600 & 1.77332500 & $\mathrm{~N}$ & -1.45261500 & 1.10736200 & 1.87941200 \\
\hline $\mathrm{H}$ & -1.01783300 & 0.22520700 & 1.61771800 & $C$ & 0.37909100 & -0.35193400 & -1.63684200 \\
\hline 0 & 0.38523300 & -1.35027400 & -2.34092500 & $\mathrm{O}$ & -0.42796200 & 0.69827700 & -1.73454600 \\
\hline $\mathrm{C}$ & -1.61695200 & 0.62425600 & -2.63338300 & C & -2.44199300 & -0.62250800 & -2.31220400 \\
\hline $\mathrm{H}$ & -3.45839700 & -0.49021500 & -2.69590800 & $\mathrm{H}$ & -2.00853000 & -1.51924500 & -2.75399500 \\
\hline $\mathrm{H}$ & -2.49830600 & -0.77816300 & -1.23795500 & C & -2.36077600 & 1.90594400 & -2.26873700 \\
\hline $\mathrm{H}$ & -1.76021000 & 2.78348500 & -2.52757400 & $\mathrm{H}$ & -3.31280000 & 1.95798400 & -2.80483000 \\
\hline $\mathrm{H}$ & -2.56643400 & 1.93877100 & -1.19674700 & $\mathrm{C}$ & -1.16349100 & 0.63347600 & -4.09036400 \\
\hline $\mathrm{H}$ & -2.04905900 & 0.65872100 & -4.73522700 & $\mathrm{H}$ & -0.55356200 & 1.51266700 & -4.30254500 \\
\hline $\mathrm{H}$ & -0.58910200 & -0.26495300 & -4.32246000 & C & 1.90691600 & -1.34326400 & -0.13464800 \\
\hline $\mathrm{H}$ & 1.28263500 & -2.21699900 & -0.27180800 & C & 2.61299200 & -1.29300400 & 1.16486400 \\
\hline C & 2.38741700 & -2.32311000 & 2.08872700 & C & 3.54329700 & -0.28878100 & 1.45884100 \\
\hline$C$ & 3.07546500 & -2.33191900 & 3.30198700 & $\mathrm{H}$ & 1.65852500 & -3.09230300 & 1.85804300 \\
\hline$C$ & 4.23631200 & -0.30932100 & 2.66412900 & $\mathrm{H}$ & 3.72089000 & 0.50696000 & 0.74822400 \\
\hline C & 4.00335200 & -1.33082300 & 3.59000300 & $\mathrm{H}$ & 2.88176000 & -3.12206500 & 4.02131300 \\
\hline $\mathrm{H}$ & 4.95419300 & 0.47613300 & 2.87910600 & $\mathrm{H}$ & 4.54257600 & -1.34303300 & 4.53321600 \\
\hline $\mathrm{N}$ & 1.29315800 & -0.19354900 & -0.59907500 & $\mathrm{H}$ & 1.26546700 & 0.63373700 & 0.00488400 \\
\hline
\end{tabular}




\begin{tabular}{|c|c|c|c|c|c|c|c|}
\hline$S$ & -1.24455100 & -3.03844900 & 0.76422300 & 0 & -0.01933100 & -3.83410700 & 0.51300500 \\
\hline 0 & -0.99473000 & -1.56040600 & 0.82541700 & 0 & -2.45103600 & -3.41590200 & 0.01703900 \\
\hline $\mathrm{C}$ & -1.66420900 & -3.39379100 & 2.53933200 & $\mathrm{~F}$ & -2.02756000 & -4.66534800 & 2.71514400 \\
\hline $\mathrm{F}$ & -2.68251000 & -2.59639900 & 2.92491400 & $\mathrm{~F}$ & -0.61266400 & -3.13627000 & 3.33931700 \\
\hline C & 3.27154300 & -1.70039800 & -1.44478600 & $\mathrm{H}$ & 2.72017300 & -1.39943700 & -2.32821400 \\
\hline $\mathrm{H}$ & 4.05284900 & -1.02166300 & -1.11774900 & C & 3.43898300 & -3.07323200 & -1.23388400 \\
\hline C & 4.43422300 & -3.57484300 & -0.29287900 & $\mathrm{H}$ & 4.65195500 & -2.84252700 & 0.49309900 \\
\hline $\mathrm{H}$ & 4.14108500 & -4.52904200 & 0.15827500 & C & 2.47753000 & -4.03363200 & -1.84420700 \\
\hline $\mathrm{H}$ & 2.94931000 & -5.00041600 & -2.04501000 & $\mathrm{H}$ & 1.66998300 & -4.21439500 & -1.11308200 \\
\hline $\mathrm{H}$ & 2.00369100 & -3.63536400 & -2.74282200 & C & 7.27174900 & -4.58185000 & 0.14741000 \\
\hline $\mathrm{H}$ & 6.89247300 & -5.54562300 & 0.50534300 & $\mathrm{H}$ & 8.27873300 & -4.74788100 & -0.25322900 \\
\hline $\mathrm{H}$ & 7.36147600 & -3.91402400 & 1.01168100 & C & 5.93044000 & -5.01896000 & -2.62456500 \\
\hline $\mathrm{H}$ & 5.50639400 & -5.97978300 & -2.31070200 & $\mathrm{H}$ & 5.27167800 & -4.59497300 & -3.39073000 \\
\hline $\mathrm{H}$ & 6.89785000 & -5.22519100 & -3.09754400 & C & 6.76816700 & -2.16427100 & -1.73802700 \\
\hline $\mathrm{H}$ & 6.12685600 & -1.74445500 & -2.51963400 & $\mathrm{H}$ & 6.79089600 & -1.44833900 & -0.90844000 \\
\hline $\mathrm{H}$ & 7.78419100 & -2.24397000 & -2.14223700 & $\mathrm{Si}$ & 6.14369500 & -3.84585200 & -1.16807400 \\
\hline C & 2.07891300 & 5.74591100 & 1.70292000 & $\mathrm{H}$ & 1.67469100 & 6.15695300 & 0.77187800 \\
\hline $\mathrm{H}$ & 3.06171600 & 6.19643800 & 1.86605500 & & & & \\
\hline
\end{tabular}

\section{TS-S-4f-49}

Imaginary frequency: $-411.06 \mathrm{~cm}^{-1}$

E[B3LYP/6-31G(d)-D3(BJ)]: -4789.078557 Hartree

$\mathrm{E}\left[\mathrm{PCM}\left(\mathrm{Et}_{2} \mathrm{O}\right)-\mathrm{B} 3 \mathrm{LYP} / 6-311 \mathrm{G}(\mathrm{d}, \mathrm{p})-\mathrm{D} 3(\mathrm{BJ})\right]$ : -4790.214108 Hartree

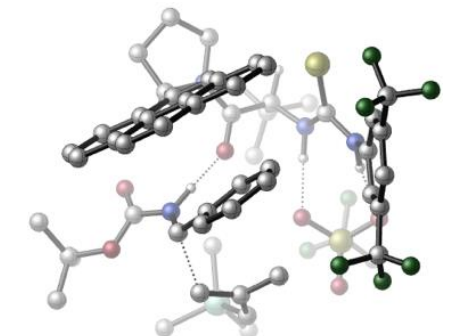

\begin{tabular}{|c|c|c|c|c|c|c|c|}
\hline 01 & & & & $\mathrm{~N}$ & -1.70270400 & -2.87539900 & 1.31612800 \\
\hline $\mathrm{H}$ & -0.97598700 & -3.49867300 & 0.94945900 & C & -2.74544400 & -2.67139500 & 0.39504100 \\
\hline C & -2.46294100 & -2.97852000 & -0.94680100 & C & -3.99585000 & -2.13203400 & 0.72384400 \\
\hline C & -3.37959000 & -2.66886400 & -1.94249000 & $\mathrm{H}$ & -1.51525900 & -3.43528400 & -1.19988800 \\
\hline C & -4.89551900 & -1.81836400 & -0.29632000 & $\mathrm{H}$ & -4.23546100 & -1.91613300 & 1.75308400 \\
\hline $\mathrm{C}$ & -4.60167200 & -2.07208200 & -1.63306300 & $\mathrm{H}$ & -5.30053000 & -1.80346800 & -2.41659800 \\
\hline C & -3.00352300 & -2.83522900 & -3.38674600 & $\mathrm{C}$ & -6.22018300 & -1.19479900 & 0.03975500 \\
\hline $\mathrm{F}$ & -1.93748900 & -3.64305000 & -3.55715700 & $\mathrm{~F}$ & -2.68131800 & -1.63449200 & -3.94178300 \\
\hline $\mathrm{F}$ & -4.02123300 & -3.33335900 & -4.12110600 & $\mathrm{~F}$ & -6.23450600 & -0.64890900 & 1.27649000 \\
\hline$F$ & -6.52727700 & -0.20315100 & -0.83826100 & $\mathrm{~F}$ & -7.23455500 & -2.08419600 & -0.01461600 \\
\hline C & 0.67612100 & -1.18207800 & 3.50650600 & $\mathrm{H}$ & -0.01905100 & -1.05100800 & 4.33508700 \\
\hline C & 1.98622300 & -1.81130500 & 4.07895900 & $\mathrm{C}$ & 3.08369600 & -1.95069900 & 3.01490800 \\
\hline $\mathrm{H}$ & 3.40584800 & -0.97487900 & 2.64407100 & $\mathrm{H}$ & 2.75768700 & -2.53668000 & 2.15659400 \\
\hline $\mathrm{H}$ & 3.95261100 & -2.45673400 & 3.45169700 & C & 1.63047300 & -3.19260400 & 4.65550600 \\
\hline $\mathrm{H}$ & 2.50953500 & -3.63022200 & 5.14213000 & $\mathrm{H}$ & 1.29791000 & -3.87801500 & 3.87390100 \\
\hline $\mathrm{H}$ & 0.83144100 & -3.11497200 & 5.40237000 & C & 2.51938400 & -0.92120700 & 5.21420000 \\
\hline $\mathrm{H}$ & 3.43824800 & -1.35574800 & 5.62241200 & $\mathrm{H}$ & 1.79860000 & -0.84135300 & 6.03605600 \\
\hline $\mathrm{H}$ & 2.76241600 & 0.08684500 & 4.85985000 & $\mathrm{C}$ & 0.89246800 & 0.16413100 & 2.78983400 \\
\hline C & 0.81026700 & 2.60838200 & 2.76399600 & $\mathrm{H}$ & 1.74060000 & 2.57108700 & 2.19571200 \\
\hline $\mathrm{N}$ & 0.64007000 & 1.31219600 & 3.44496500 & C & -0.36622500 & 2.94546600 & 1.85705300 \\
\hline C & -1.56818300 & 2.31884100 & 2.01041400 & C & -0.22447900 & 3.99409600 & 0.86723400 \\
\hline $\mathrm{C}$ & -2.72077800 & 2.65779700 & 1.23154300 & $\mathrm{H}$ & -1.67223900 & 1.50164100 & 2.71482600 \\
\hline C & -1.35430900 & 4.33180000 & 0.05465100 & $\mathrm{C}$ & 0.97535000 & 4.69810200 & 0.66617100 \\
\hline C & -3.94697500 & 1.99373500 & 1.39420800 & C & -2.60289600 & 3.67347000 & 0.24285400 \\
\hline $\mathrm{C}$ & -1.23843300 & 5.33752600 & -0.95344700 & $\mathrm{H}$ & 1.84585500 & 4.47056300 & 1.26984700 \\
\hline $\mathrm{C}$ & 1.06888800 & 5.69421900 & -0.30552800 & $\mathrm{C}$ & -5.04438400 & 2.33215300 & 0.60508900 \\
\hline $\mathrm{H}$ & -4.02298100 & 1.19341500 & 2.12377000 & $\mathrm{C}$ & -3.72826400 & 4.00803000 & -0.56674900 \\
\hline
\end{tabular}




\begin{tabular}{|c|c|c|c|c|c|c|c|}
\hline $\mathrm{C}$ & -0.01664700 & 6.00564700 & -1.11696100 & $\mathrm{C}$ & -2.38395700 & 5.64685700 & -1.76269500 \\
\hline $\mathrm{H}$ & 1.99728100 & 6.24344100 & -0.41691600 & C & -4.93845200 & 3.32786200 & -0.36378400 \\
\hline $\mathrm{H}$ & -5.98073600 & 1.80289400 & 0.73461300 & $\mathrm{C}$ & -3.57468000 & 5.01563700 & -1.57604200 \\
\hline $\mathrm{H}$ & 0.06723300 & 6.78163900 & -1.87356100 & $\mathrm{H}$ & -2.27866700 & 6.41148900 & -2.52826900 \\
\hline $\mathrm{H}$ & -5.79642600 & 3.58047500 & -0.98172200 & $\mathrm{H}$ & -4.43464800 & 5.26482200 & -2.19264000 \\
\hline C & -1.31439100 & -2.03657300 & 2.33610300 & $S$ & -2.37341400 & -1.08347900 & 3.25064300 \\
\hline $\mathrm{N}$ & 0.02525900 & -2.07072200 & 2.55404100 & $\mathrm{H}$ & 0.59799900 & -2.34185400 & 1.74788000 \\
\hline $\mathrm{S}$ & 1.27326000 & -4.14426100 & -0.43899000 & 0 & 1.72010300 & -4.34354300 & -1.82844600 \\
\hline $\mathrm{O}$ & 1.34078600 & -2.72953600 & 0.04208200 & 0 & 0.00487800 & -4.80834800 & -0.04807900 \\
\hline C & 2.55845600 & -5.02012600 & 0.57943100 & $\mathrm{~F}$ & 2.71549800 & -6.27847700 & 0.16650700 \\
\hline $\mathrm{F}$ & 2.20228600 & -5.03280100 & 1.87472900 & $\mathrm{~F}$ & 3.74266700 & -4.38523700 & 0.48277000 \\
\hline $\mathrm{C}$ & 3.25432600 & 2.34121600 & -0.39432300 & $\mathrm{O}$ & 3.68330800 & 2.46961200 & 0.73831900 \\
\hline $\mathrm{O}$ & 3.81135900 & 2.81796800 & -1.51986200 & C & 4.99344700 & 3.71452600 & -1.46523500 \\
\hline C & 4.59264000 & 5.02449600 & -0.79300400 & $\mathrm{H}$ & 5.44172000 & 5.71558100 & -0.80820800 \\
\hline $\mathrm{H}$ & 4.29988100 & 4.85847200 & 0.24544500 & $\mathrm{H}$ & 3.76253800 & 5.48667400 & -1.33280800 \\
\hline $\mathrm{C}$ & 5.30391200 & 3.93364500 & -2.94446000 & $\mathrm{H}$ & 5.57801400 & 2.99250500 & -3.42996900 \\
\hline $\mathrm{H}$ & 6.14156500 & 4.63029300 & -3.04700400 & $\mathrm{H}$ & 4.43681300 & 4.35603900 & -3.46204600 \\
\hline C & 6.16535500 & 3.04133200 & -0.75024600 & $\mathrm{H}$ & 7.05035100 & 3.67808800 & -0.85612000 \\
\hline $\mathrm{H}$ & 6.39085200 & 2.07227500 & -1.20004800 & $\mathrm{H}$ & 5.95545000 & 2.90019900 & 0.30935600 \\
\hline $\mathrm{C}$ & 1.34404500 & 1.77308400 & -1.81305100 & $\mathrm{H}$ & 1.64092700 & 2.65101800 & -2.37739100 \\
\hline C & -0.11334900 & 1.54575500 & -1.75806500 & C & -0.90486900 & 2.16125400 & -2.74444700 \\
\hline $\mathrm{C}$ & -0.72225400 & 0.70071600 & -0.82042000 & $C$ & -2.26754800 & 1.90777000 & -2.81845400 \\
\hline $\mathrm{H}$ & -0.44165800 & 2.84381700 & -3.45313000 & $C$ & -2.09345500 & 0.46579500 & -0.89190900 \\
\hline $\mathrm{H}$ & -0.14241000 & 0.21958300 & -0.04129900 & C & -2.86473900 & 1.04322200 & -1.89634100 \\
\hline $\bar{H}$ & -2.86775600 & 2.39689300 & -3.57873800 & $\mathrm{H}$ & -2.56109000 & -0.16329600 & -0.14870300 \\
\hline $\mathrm{H}$ & -3.93041500 & 0.84813100 & -1.93275100 & $\mathrm{~N}$ & 2.08654800 & 1.64105300 & -0.65964700 \\
\hline $\mathrm{H}$ & 1.72119900 & 1.13462500 & 0.16090400 & C & 1.94393200 & 0.62608000 & -3.35049100 \\
\hline $\bar{H}$ & 1.16944300 & 1.05618600 & -3.97825300 & $\mathrm{H}$ & 2.94579000 & 0.99882400 & -3.55006900 \\
\hline $\mathrm{C}$ & 1.80920800 & -0.75539900 & -3.09866100 & $\mathrm{C}$ & 2.96026800 & -1.65977800 & -3.05113800 \\
\hline $\mathrm{H}$ & 3.46350000 & -1.58658100 & -4.02867700 & $\mathrm{H}$ & 2.63611000 & -2.69062200 & -2.89365900 \\
\hline $\mathrm{C}$ & 0.46588200 & -1.35485700 & -2.89586100 & $\mathrm{H}$ & 0.40349900 & -2.34319500 & -3.35951100 \\
\hline $\bar{H}$ & -0.35860400 & -0.72594400 & -3.22474600 & $\mathrm{H}$ & 0.36123000 & -1.54275000 & -1.81424600 \\
\hline C & 4.13532400 & -1.02642900 & -0.12707500 & $\mathrm{H}$ & 3.09119400 & -1.23032200 & 0.11584100 \\
\hline $\mathrm{H}$ & 4.35591800 & 0.01416500 & 0.12583600 & $\mathrm{H}$ & 4.74528900 & -1.66183600 & 0.52439600 \\
\hline $\mathrm{C}$ & 5.58889900 & -0.09849100 & -2.72158600 & $\bar{H}$ & 5.10257600 & 0.87990800 & -2.67750500 \\
\hline $\mathrm{H}$ & 5.80042300 & -0.33245100 & -3.77181700 & $\mathrm{H}$ & 6.55120200 & -0.01608400 & -2.20215400 \\
\hline $\mathrm{C}$ & 5.37193400 & -3.10055300 & -2.09207900 & $\mathrm{H}$ & 5.59726400 & -3.32888500 & -3.14032100 \\
\hline $\bar{H}$ & 4.74035700 & -3.90097500 & -1.69721600 & $\bar{H}$ & 6.31735900 & -3.10908000 & -1.53619300 \\
\hline $\mathrm{Si}$ & 4.51777400 & -1.43165700 & -1.91696100 & C & 0.09556100 & 1.46272400 & 4.81009700 \\
\hline $\mathrm{H}$ & 0.81413900 & 1.08893000 & 5.54269000 & $\mathrm{H}$ & -0.83605300 & 0.89812900 & 4.91099700 \\
\hline C & -0.10292700 & 2.98039100 & 4.95802800 & $\mathrm{H}$ & -1.12207600 & 3.25696900 & 4.67309900 \\
\hline $\mathrm{H}$ & 0.06341700 & 3.31390000 & 5.98562900 & C & 0.89792200 & 3.57959500 & 3.95953400 \\
\hline $\mathrm{H}$ & 0.65911200 & 4.60676400 & 3.67500300 & $\mathrm{H}$ & 1.91427500 & 3.55999100 & 4.37025800 \\
\hline $\mathrm{O}$ & 1.28553700 & 0.15474300 & 1.60959600 & & & & \\
\hline
\end{tabular}

\section{TS-S-4f-50}

Imaginary frequency: $-402.90 \mathrm{~cm}^{-1}$ E[B3LYP/6-31G(d)-D3(BJ)]: -4789.064769 Hartree $\mathrm{E}\left[\mathrm{PCM}\left(\mathrm{Et}_{2} \mathrm{O}\right)-\mathrm{B} 3 \mathrm{LYP} / 6-311 \mathrm{G}(\mathrm{d}, \mathrm{p})-\mathrm{D} 3(\mathrm{BJ})\right]$ : -4790.213586 Hartree

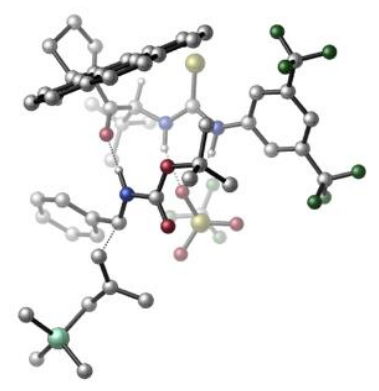




\begin{tabular}{|c|c|c|c|c|c|c|c|}
\hline 01 & & & & $\mathrm{~N}$ & -1.71878500 & -1.80132900 & 1.45767500 \\
\hline $\mathrm{H}$ & -0.78517500 & -2.11918200 & 1.18583900 & C & -2.75304800 & -2.43511100 & 0.75662400 \\
\hline C & -2.42842100 & -3.66168100 & 0.15782100 & $\mathrm{C}$ & -4.01568900 & -1.87330100 & 0.52948500 \\
\hline C & -3.34461400 & -4.29347300 & -0.67748900 & $\mathrm{H}$ & -1.44287500 & -4.09430800 & 0.30477800 \\
\hline C & -4.91544100 & -2.52566400 & -0.30839700 & $\mathrm{H}$ & -4.27456800 & -0.92991000 & 0.98329500 \\
\hline C & -4.59828000 & -3.73686300 & -0.92133900 & $\mathrm{H}$ & -5.30656900 & -4.23214000 & -1.57385400 \\
\hline C & -2.92168700 & -5.53120600 & -1.41810600 & C & -6.20632500 & -1.84778000 & -0.66586200 \\
\hline $\mathrm{F}$ & -2.27581700 & -5.21058100 & -2.56608000 & $\mathrm{~F}$ & -3.98239500 & -6.29588000 & -1.76771800 \\
\hline $\mathrm{F}$ & -2.08612700 & -6.29925700 & -0.69383500 & $\mathrm{~F}$ & -6.58747600 & -0.93509100 & 0.25674000 \\
\hline $\mathrm{F}$ & -6.09782400 & -1.18156200 & -1.84732600 & $\mathrm{~F}$ & -7.22143100 & -2.72594700 & -0.81314700 \\
\hline C & -0.13076000 & 0.74035500 & 3.51467000 & $\mathrm{H}$ & -1.01654400 & 0.93125100 & 4.11840200 \\
\hline C & 1.04411800 & 0.41828700 & 4.48516400 & C & 2.35180200 & 0.14636800 & 3.73444800 \\
\hline $\mathrm{H}$ & 2.68750000 & 1.02606200 & 3.18485800 & $\mathrm{H}$ & 2.25436400 & -0.67797800 & 3.02613400 \\
\hline $\mathrm{H}$ & 3.13563500 & -0.13261500 & 4.44762800 & $\mathrm{C}$ & 0.65354400 & -0.82446300 & 5.30400600 \\
\hline $\mathrm{H}$ & 1.40940300 & -1.01515600 & 6.07465800 & $\mathrm{H}$ & 0.58678400 & -1.71133200 & 4.66849600 \\
\hline $\mathrm{H}$ & -0.31422600 & -0.68839100 & 5.80045700 & C & 1.24683300 & 1.61226000 & 5.43186300 \\
\hline $\mathrm{H}$ & 2.07909200 & 1.41086400 & 6.11518600 & $\mathrm{H}$ & 0.35465700 & 1.79679200 & 6.04130300 \\
\hline $\mathrm{H}$ & 1.48689900 & 2.52913300 & 4.88043600 & C & 0.11526500 & 1.92666200 & 2.56940900 \\
\hline $\mathrm{O}$ & 0.81642900 & 1.78967900 & 1.55544300 & C & -1.35501100 & 3.43657900 & 3.98903000 \\
\hline C & -0.42133300 & 4.18879800 & 1.85391300 & C & -1.88066600 & 4.84219200 & 3.64989000 \\
\hline $\mathrm{H}$ & -2.15860100 & 2.70194000 & 4.09779600 & $\mathrm{H}$ & -0.76311700 & 3.44660600 & 4.90889900 \\
\hline C & -0.81820500 & 5.41543200 & 2.69994000 & $\mathrm{H}$ & 0.60476900 & 4.24531700 & 1.48781000 \\
\hline $\mathrm{H}$ & -2.02800800 & 5.44986400 & 4.54649500 & $\mathrm{H}$ & -2.84016200 & 4.76775700 & 3.13021700 \\
\hline $\mathrm{H}$ & 0.05307100 & 5.76898200 & 3.26456700 & $\mathrm{H}$ & -1.18918900 & 6.23851200 & 2.08503100 \\
\hline $\mathrm{N}$ & -0.49219300 & 3.10141200 & 2.83928100 & $\mathrm{C}$ & -1.37824500 & 3.96458400 & 0.69180300 \\
\hline C & -2.44417000 & 3.12273600 & 0.82037100 & $\mathrm{C}$ & -1.18761900 & 4.70594600 & -0.53789000 \\
\hline C & -3.42228500 & 2.95719600 & -0.21179300 & $\mathrm{H}$ & -2.57213000 & 2.52743400 & 1.71825100 \\
\hline $\mathrm{C}$ & -2.12934400 & 4.52741700 & -1.60135100 & C & -0.11291400 & 5.58865500 & -0.74154600 \\
\hline $\mathrm{C}$ & -4.53027600 & 2.11046800 & -0.05321600 & $\mathrm{C}$ & -3.25109900 & 3.66433200 & -1.43512900 \\
\hline C & -1.95405400 & 5.22107000 & -2.83774400 & $\mathrm{H}$ & 0.60856000 & 5.75716000 & 0.05022100 \\
\hline C & 0.04860800 & 6.26209100 & -1.95165500 & C & -5.45480800 & 1.95441200 & -1.08393900 \\
\hline $\mathrm{H}$ & -4.64522900 & 1.56455500 & 0.87868000 & $\mathrm{C}$ & -4.19694800 & 3.49169600 & -2.48991400 \\
\hline C & -0.85633800 & 6.08091900 & -2.99205900 & C & -2.91441800 & 5.01974800 & -3.88757800 \\
\hline $\mathrm{H}$ & 0.89089000 & 6.93640000 & -2.07993600 & C & -5.28924600 & 2.63347400 & -2.28965100 \\
\hline $\mathrm{H}$ & -6.29465500 & 1.28244000 & -0.95303200 & C & -3.98550500 & 4.19672200 & -3.72221200 \\
\hline $\mathrm{H}$ & -0.72457800 & 6.60856600 & -3.93312100 & $\mathrm{H}$ & -2.76606000 & 5.54907800 & -4.82535700 \\
\hline $\mathrm{H}$ & -6.00702100 & 2.49668400 & -3.09432100 & $\mathrm{H}$ & -4.70406000 & 4.05815300 & -4.52596500 \\
\hline C & -1.71741500 & -0.76605500 & 2.35059400 & $\mathrm{~S}$ & -3.09328700 & -0.04784300 & 3.03315900 \\
\hline $\mathrm{N}$ & -0.45217800 & -0.39658700 & 2.67683300 & $\mathrm{H}$ & 0.29925400 & -0.74740800 & 2.08515600 \\
\hline C & 0.96622700 & -0.12382800 & -1.77100500 & $\mathrm{O}$ & 1.47021600 & -0.88740300 & -2.58238000 \\
\hline $\mathrm{O}$ & -0.31164900 & 0.20927600 & -1.66668600 & C & -1.30436600 & -0.41553400 & -2.58823900 \\
\hline C & -1.19471500 & -1.94094000 & -2.53737100 & $\mathrm{H}$ & -2.13086600 & -2.38470800 & -2.88898200 \\
\hline $\mathrm{H}$ & -0.37286300 & -2.31451000 & -3.14669500 & $\mathrm{H}$ & -1.02954800 & -2.27585500 & -1.51291200 \\
\hline C & -2.62235500 & 0.07259600 & -2.00567000 & $\mathrm{H}$ & -2.70092300 & 1.15625400 & -2.09354100 \\
\hline $\mathrm{H}$ & -3.46753100 & -0.38389600 & -2.52798800 & $\mathrm{H}$ & -2.69186700 & -0.19222600 & -0.95016300 \\
\hline C & -1.08179500 & 0.14725000 & -3.99047300 & $\mathrm{H}$ & -1.86189700 & -0.23421100 & -4.65828900 \\
\hline $\mathrm{H}$ & -1.14752500 & 1.24044000 & -3.97860000 & $\mathrm{H}$ & -0.10980000 & -0.15558100 & -4.38561600 \\
\hline C & 3.00726300 & 0.03324200 & -0.59487100 & $\mathrm{H}$ & 3.03880100 & -1.04501800 & -0.68034900 \\
\hline C & 3.79198400 & 0.58680300 & 0.52556700 & C & 4.58829000 & -0.29227300 & 1.27417900 \\
\hline C & 3.82767500 & 1.96171000 & 0.80185200 & C & 5.41801000 & 0.20251200 & 2.28063200 \\
\hline $\mathrm{H}$ & 4.52486300 & -1.35832000 & 1.07821700 & C & 4.65888000 & 2.44979000 & 1.80427900 \\
\hline $\mathrm{H}$ & 3.20191600 & 2.64082100 & 0.23148700 & C & 5.46192200 & 1.57193900 & 2.54043700 \\
\hline $\mathrm{H}$ & 6.02232900 & -0.48461200 & 2.86523700 & $\mathrm{H}$ & 4.68200800 & 3.51486800 & 2.01563300 \\
\hline
\end{tabular}




\begin{tabular}{|c|c|c|c|c|c|c|c|}
\hline $\mathrm{H}$ & 6.11045100 & 1.95709400 & 3.32207500 & $\mathrm{~N}$ & 1.74653300 & 0.55245600 & -0.83862500 \\
\hline $\mathrm{H}$ & 1.29615000 & 1.12654900 & -0.11872100 & S & 1.73782900 & -3.22708800 & 0.49158600 \\
\hline 0 & 3.10261100 & -3.10271300 & -0.07591700 & $\mathrm{O}$ & 1.06605000 & -1.90474300 & 0.71885600 \\
\hline 0 & 0.85731400 & -4.24755400 & -0.09393800 & $\mathrm{C}$ & 2.03844000 & -3.79847500 & 2.23459500 \\
\hline $\mathrm{F}$ & 2.48280500 & -5.05616800 & 2.26491400 & $\mathrm{~F}$ & 0.89399300 & -3.72491200 & 2.93924000 \\
\hline $\mathrm{F}$ & 2.94951700 & -3.01103100 & 2.83773500 & $\mathrm{C}$ & 4.05822900 & 0.50720900 & -2.16476300 \\
\hline $\mathrm{H}$ & 3.25280100 & 0.55010700 & -2.88788700 & $\mathrm{H}$ & 4.47650400 & 1.45854400 & -1.85068700 \\
\hline C & 4.91975700 & -0.59676600 & -2.24507200 & C & 6.21948800 & -0.58346900 & -1.58658700 \\
\hline $\mathrm{H}$ & 6.24160500 & 0.09973800 & -0.73012300 & $\mathrm{H}$ & 6.53980100 & -1.58427700 & -1.27617300 \\
\hline $\mathrm{C}$ & 4.45677200 & -1.86054300 & -2.87931700 & $\mathrm{H}$ & 5.29661100 & -2.43819600 & -3.27631700 \\
\hline $\mathrm{H}$ & 3.98534000 & -2.47403700 & -2.09087300 & $\mathrm{H}$ & 3.69466600 & -1.69408000 & -3.64079800 \\
\hline $\mathrm{C}$ & 9.20837800 & -0.07299900 & -1.81996100 & $\mathrm{H}$ & 9.42080800 & -1.12068900 & -1.57911200 \\
\hline $\mathrm{H}$ & 10.05018800 & 0.31088000 & -2.40826000 & $\mathrm{H}$ & 9.17468400 & 0.48881400 & -0.87965100 \\
\hline C & 7.62715900 & -0.92480600 & -4.35722100 & $\mathrm{H}$ & 7.78822700 & -1.98854300 & -4.14797600 \\
\hline $\mathrm{H}$ & 6.69163200 & -0.83055800 & -4.91958100 & $\mathrm{H}$ & 8.44133000 & -0.59136300 & -5.01156500 \\
\hline $\mathrm{C}$ & 7.21130000 & 1.90299100 & -3.14610400 & $\mathrm{H}$ & 6.27563300 & 2.01168600 & -3.70495900 \\
\hline $\mathrm{H}$ & 7.12404900 & 2.49016400 & -2.22447300 & $\mathrm{H}$ & 8.01099700 & 2.34974300 & -3.74852200 \\
\hline $\mathrm{Si}$ & 7.59691100 & 0.09809400 & -2.77820700 & & & & \\
\hline
\end{tabular}

\section{TS-S-4f-51}

Imaginary frequency: $-399.41 \mathrm{~cm}^{-1}$

E[B3LYP/6-31G(d)-D3(BJ)]: -4789.064922 Hartree

$\mathrm{E}\left[\mathrm{PCM}\left(\mathrm{Et}_{2} \mathrm{O}\right)-\mathrm{B} 3 \mathrm{LYP} / 6-311 \mathrm{G}(\mathrm{d}, \mathrm{p})-\mathrm{D} 3(\mathrm{BJ})\right]$ : -4790.213559 Hartree

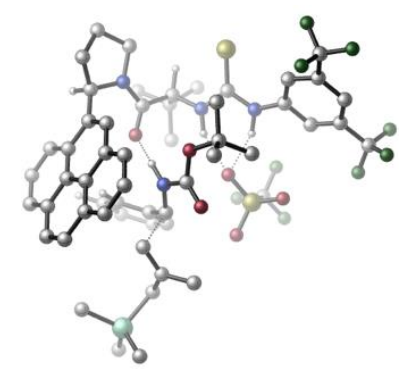

\begin{tabular}{|c|c|c|c|c|c|c|c|}
\hline 01 & & & & $\mathrm{~N}$ & -3.37352100 & 0.31768100 & 1.06818200 \\
\hline $\mathrm{H}$ & -2.75396900 & -0.48870500 & 1.17230500 & C & -4.55013600 & 0.03864300 & 0.35372000 \\
\hline$C$ & -5.00562100 & -1.28696800 & 0.39907100 & C & -5.19500700 & 0.95700200 & -0.47785500 \\
\hline C & -6.06818400 & -1.68716200 & -0.40229300 & $\mathrm{H}$ & -4.49718900 & -2.01077900 & 1.02277700 \\
\hline C & -6.24685500 & 0.53028000 & -1.28705900 & $\mathrm{H}$ & -4.86036200 & 1.98252700 & -0.51597200 \\
\hline C & -6.69692700 & -0.78657200 & -1.26355800 & $\mathrm{H}$ & -7.50723800 & -1.10801400 & -1.90543300 \\
\hline C & -6.57719500 & -3.09975600 & -0.31491900 & C & -6.79417500 & 1.50541300 & -2.28866100 \\
\hline$F$ & -7.64393400 & -3.18720600 & 0.51789500 & $\mathrm{~F}$ & -5.64402300 & -3.95224300 & 0.14495600 \\
\hline$F$ & -6.99185000 & -3.55221100 & -1.52137800 & $\mathrm{~F}$ & -6.98579800 & 2.73056400 & -1.75719800 \\
\hline $\mathrm{F}$ & -5.92774900 & 1.66812700 & -3.32820600 & $\mathrm{~F}$ & -7.96685200 & 1.10066800 & -2.81935700 \\
\hline C & -0.75136300 & 2.35823400 & 2.51083400 & $\mathrm{H}$ & -1.42753600 & 3.20195800 & 2.62672800 \\
\hline $\mathrm{C}$ & -0.37334700 & 1.85570800 & 3.94414300 & C & 0.36392400 & 0.50706500 & 3.92348300 \\
\hline $\mathrm{H}$ & 1.29869800 & 0.56879700 & 3.36664000 & $\mathrm{H}$ & -0.24417000 & -0.28777100 & 3.48083800 \\
\hline $\mathrm{H}$ & 0.59288000 & 0.20212700 & 4.95139200 & C & -1.68180400 & 1.70685900 & 4.73898900 \\
\hline $\mathrm{H}$ & -1.46490000 & 1.36671500 & 5.75804200 & $\mathrm{H}$ & -2.34372200 & 0.97003100 & 4.27363100 \\
\hline $\mathrm{H}$ & -2.22755100 & 2.65471700 & 4.80055000 & $\mathrm{C}$ & 0.51923600 & 2.91530700 & 4.61317000 \\
\hline $\mathrm{H}$ & 0.72555100 & 2.63195100 & 5.65107800 & $\mathrm{H}$ & 0.03457100 & 3.89961300 & 4.63029000 \\
\hline $\mathrm{H}$ & 1.48231300 & 3.01071900 & 4.09899700 & C & 0.47983800 & 2.77150800 & 1.70227700 \\
\hline $\mathrm{O}$ & 1.33996900 & 1.92898700 & 1.40219500 & C & -0.35848900 & 5.15011400 & 1.64823800 \\
\hline$C$ & 1.85067300 & 4.60006000 & 0.73143100 & C & 0.38440000 & 6.43370300 & 1.24850600 \\
\hline $\mathrm{H}$ & -1.27156900 & 4.98994400 & 1.06635600 & $\mathrm{H}$ & -0.63546900 & 5.15917600 & 2.70489100 \\
\hline $\mathrm{H}$ & 2.55729800 & 4.79132100 & 1.54326100 & $\mathrm{H}$ & 0.91629700 & 6.84446400 & 2.11438800 \\
\hline $\mathrm{H}$ & -0.29958000 & 7.20330500 & 0.88175900 & $\mathrm{~N}$ & 0.61279800 & 4.07062500 & 1.36108000 \\
\hline C & 2.50132900 & 3.69762400 & -0.30098000 & C & 1.84974500 & 3.43450200 & -1.46905800 \\
\hline $\mathrm{C}$ & 3.82140800 & 3.13418000 & -0.07261700 & C & 2.40891600 & 2.61356500 & -2.49827500 \\
\hline $\mathrm{H}$ & 0.84836100 & 3.82370300 & -1.62955800 & $\mathrm{C}$ & 4.39860900 & 2.30048200 & -1.08590800 \\
\hline
\end{tabular}




\begin{tabular}{|c|c|c|c|c|c|c|c|}
\hline $\mathrm{C}$ & 4.55679000 & 3.32886400 & 1.10923700 & $\mathrm{C}$ & 1.72789100 & 2.35944400 & -3.69573100 \\
\hline C & 3.69155200 & 2.03309900 & -2.29338700 & C & 5.68642900 & 1.71011700 & -0.88777000 \\
\hline $\mathrm{H}$ & 4.14686900 & 3.91992300 & 1.91804600 & $\mathrm{C}$ & 5.81301700 & 2.75359600 & 1.29135600 \\
\hline C & 2.27464100 & 1.52328400 & -4.66507700 & $\mathrm{H}$ & 0.75807100 & 2.81864600 & -3.84990700 \\
\hline C & 4.25456300 & 1.18797600 & -3.29684700 & C & 6.37810700 & 1.95084900 & 0.30720100 \\
\hline $\mathrm{C}$ & 6.23644500 & 0.87512300 & -1.91914400 & $\mathrm{H}$ & 6.35031100 & 2.93234300 & 2.21842900 \\
\hline $\mathrm{C}$ & 3.52385700 & 0.93843100 & -4.46863600 & $\mathrm{H}$ & 1.72161400 & 1.32325300 & -5.57762000 \\
\hline C & 5.55313000 & 0.62182000 & -3.06827000 & $\mathrm{H}$ & 7.35634800 & 1.50154600 & 0.45550600 \\
\hline $\bar{H}$ & 7.22017800 & 0.44386100 & -1.75642400 & $\mathrm{H}$ & 3.94860700 & 0.28769300 & -5.22836900 \\
\hline $\bar{H}$ & 5.98426800 & -0.01210900 & -3.83902000 & $\mathrm{C}$ & -2.81582900 & 1.50941100 & 1.43872800 \\
\hline $\mathrm{S}$ & -3.64627900 & 2.97993600 & 1.52892900 & $\mathrm{~N}$ & -1.50203700 & 1.37060600 & 1.76474700 \\
\hline $\bar{H}$ & -1.05588900 & 0.47412100 & 1.58371400 & $\mathrm{C}$ & 0.37686400 & -0.24405200 & -1.63004900 \\
\hline $\mathrm{O}$ & 0.36499600 & -1.27485300 & -2.28368500 & $\mathrm{O}$ & -0.40839800 & 0.82019700 & -1.79510500 \\
\hline C & -1.62984200 & 0.70206800 & -2.64571100 & C & -2.45349200 & -0.50977100 & -2.21069400 \\
\hline$\overline{\mathrm{H}}$ & -3.47681900 & -0.39895600 & -2.58189900 & $\mathrm{H}$ & -2.03609900 & -1.44187200 & -2.59009500 \\
\hline $\bar{H}$ & -2.48535100 & -0.57878800 & -1.12648600 & $\mathrm{C}$ & -2.34952100 & 2.01270900 & -2.34203700 \\
\hline $\bar{H}$ & -1.74509300 & 2.86829500 & -2.66394700 & $\mathrm{H}$ & -3.30856400 & 2.04465400 & -2.86720400 \\
\hline $\mathrm{H}$ & -2.53792700 & 2.11398600 & -1.27076400 & C & -1.25215700 & 0.60082200 & -4.12289200 \\
\hline $\mathrm{H}$ & -2.16858000 & 0.45578400 & -4.70517600 & $\mathrm{H}$ & -0.76888200 & 1.51239200 & -4.48087600 \\
\hline $\bar{H}$ & -0.59247600 & -0.25082100 & -4.29532500 & $\mathrm{C}$ & 1.85981000 & -1.19420900 & -0.05210600 \\
\hline $\mathrm{H}$ & 1.21657300 & -2.05765400 & -0.15333600 & C & 2.53223400 & -1.09936700 & 1.26399100 \\
\hline $\mathrm{C}$ & 2.24591200 & -2.08526700 & 2.22046300 & $\mathrm{C}$ & 3.46612400 & -0.09783400 & 1.56174200 \\
\hline $\mathrm{C}$ & 2.87371000 & -2.05391400 & 3.46551900 & $\mathrm{H}$ & 1.51961200 & -2.85484500 & 1.98597400 \\
\hline C & 4.09663700 & -0.07729900 & 2.80080300 & $\mathrm{H}$ & 3.69177000 & 0.66626300 & 0.83052100 \\
\hline $\mathrm{C}$ & 3.80152800 & -1.05424600 & 3.75649200 & $\mathrm{H}$ & 2.63122500 & -2.81024500 & 4.20606200 \\
\hline $\mathrm{H}$ & 4.81440500 & 0.70687500 & 3.01771200 & $\mathrm{H}$ & 4.29264900 & -1.03195700 & 4.72544700 \\
\hline $\mathrm{N}$ & 1.27839200 & -0.05764000 & -0.58804900 & $\mathrm{H}$ & 1.29072200 & 0.81216400 & -0.04593800 \\
\hline $\mathrm{S}$ & -1.29803500 & -2.87360800 & 0.80358900 & $\mathrm{O}$ & -0.06205800 & -3.65292100 & 0.54591400 \\
\hline $\mathrm{O}$ & -1.05813000 & -1.39753400 & 0.92481600 & $\mathrm{O}$ & -2.48676300 & -3.23492100 & 0.02219200 \\
\hline C & -1.73619100 & -3.29868300 & 2.55799300 & $\mathrm{~F}$ & -1.99937800 & -4.59889600 & 2.69979700 \\
\hline $\mathrm{F}$ & -2.82365800 & -2.59415100 & 2.93238700 & $\mathrm{~F}$ & -0.72902100 & -2.97284900 & 3.39042500 \\
\hline C & 3.26506200 & -1.66041000 & -1.30354300 & $\mathrm{H}$ & 2.84328300 & -1.24897200 & -2.21309200 \\
\hline $\mathrm{H}$ & 4.09946900 & -1.10878600 & -0.88313100 & C & 3.23123800 & -3.05158800 & -1.15223300 \\
\hline $\mathrm{C}$ & 4.02885800 & -3.72017700 & -0.12856900 & $\mathrm{H}$ & 4.23371300 & -3.05938900 & 0.72157800 \\
\hline $\mathrm{H}$ & 3.55341400 & -4.64114000 & 0.22653500 & C & 2.24694800 & -3.86251300 & -1.92120100 \\
\hline $\mathrm{H}$ & 2.63802900 & -4.86331200 & -2.13010200 & $\mathrm{H}$ & 1.35443800 & -3.99210400 & -1.28502500 \\
\hline $\mathrm{H}$ & 1.91927900 & -3.36916600 & -2.83649500 & C & 6.57382000 & -5.23152600 & 0.57305600 \\
\hline $\mathrm{H}$ & 6.00323300 & -6.14831100 & 0.75955200 & $\mathrm{H}$ & 7.59435500 & -5.52373200 & 0.29869100 \\
\hline $\mathrm{H}$ & 6.62801400 & -4.67214600 & 1.51392400 & C & 5.62210200 & -5.18211400 & -2.38598200 \\
\hline $\mathrm{H}$ & 5.01854300 & -6.08602300 & -2.24435100 & $\mathrm{H}$ & 5.15558600 & -4.58778900 & -3.17971000 \\
\hline $\mathrm{H}$ & 6.60853500 & -5.49834900 & -2.74534800 & $\mathrm{C}$ & 6.74678400 & -2.60500900 & -1.07577100 \\
\hline $\mathrm{H}$ & 6.29369900 & -1.99822200 & -1.86553900 & $\mathrm{H}$ & 6.77783100 & -1.99150600 & -0.16763000 \\
\hline $\mathrm{H}$ & 7.78072500 & -2.82297700 & -1.36874800 & $\mathrm{Si}$ & 5.78694000 & -4.19743300 & -0.78958700 \\
\hline C & 1.38738200 & 5.96243600 & 0.19168500 & $\mathrm{H}$ & 0.89277300 & 5.83272900 & -0.77596000 \\
\hline $\mathrm{H}$ & 2.23044500 & 6.64367000 & 0.04880500 & & & & \\
\hline
\end{tabular}

\section{TS-S-4f-52}

Imaginary frequency: $-364.92 \mathrm{~cm}^{-1}$

E[B3LYP/6-31G(d)-D3(BJ)]: -4789.060595 Hartree

E[PCM(Et $\left.\left.{ }_{2} \mathrm{O}\right)-\mathrm{B} 3 L Y P / 6-311 \mathrm{G}(\mathrm{d}, \mathrm{p})-\mathrm{D} 3(\mathrm{BJ})\right]$ : -4790.211286 Hartree

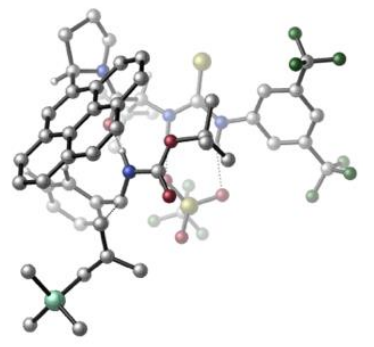




\begin{tabular}{|c|c|c|c|c|c|c|c|}
\hline 01 & & & & $\mathrm{~N}$ & -3.36521900 & -0.35860700 & 0.98450300 \\
\hline $\mathrm{H}$ & -2.79465300 & -1.20455200 & 1.01153700 & C & -4.50099500 & -0.50662100 & 0.16994600 \\
\hline $\mathrm{C}$ & -4.88196500 & -1.82320500 & -0.13855900 & $\mathrm{C}$ & -5.19580300 & 0.55565000 & -0.41719200 \\
\hline$C$ & -5.92341600 & -2.06092000 & -1.02839200 & $\mathrm{H}$ & -4.32935900 & -2.65369000 & 0.28247800 \\
\hline C & -6.22396900 & 0.29306800 & -1.32132100 & $\mathrm{H}$ & -4.92700600 & 1.57400000 & -0.18405800 \\
\hline C & -6.60455600 & -1.00696600 & -1.63859000 & $\mathrm{H}$ & -7.40204900 & -1.19656500 & -2.34555200 \\
\hline C & -6.36009000 & -3.47347500 & -1.30244800 & C & -6.83886800 & 1.45821800 & -2.04187800 \\
\hline $\mathrm{F}$ & -7.38217300 & -3.84163400 & -0.49277300 & $\mathrm{~F}$ & -5.36570800 & -4.36243700 & -1.11252900 \\
\hline $\mathrm{F}$ & -6.80195900 & -3.61957300 & -2.57395700 & $\mathrm{~F}$ & -7.04577700 & 2.51259900 & -1.22622900 \\
\hline $\mathrm{F}$ & -6.02464900 & 1.89721600 & -3.04120600 & $\mathrm{~F}$ & -8.02113900 & 1.14521600 & -2.61421500 \\
\hline C & -0.97824400 & 1.43840900 & 3.07077800 & $\mathrm{H}$ & -1.73322900 & 2.16295800 & 3.36662400 \\
\hline C & -0.49882100 & 0.71370000 & 4.37595700 & C & 0.59278100 & -0.33858800 & 4.12470500 \\
\hline $\mathrm{H}$ & 1.51431100 & 0.10986400 & 3.75026300 & $\mathrm{H}$ & 0.27935700 & -1.09310600 & 3.40264500 \\
\hline $\mathrm{H}$ & 0.81880400 & -0.85256900 & 5.06678900 & C & -1.73022800 & 0.03275700 & 4.99888200 \\
\hline $\mathrm{H}$ & -1.46942300 & -0.38456200 & 5.97821800 & $\mathrm{H}$ & -2.08591300 & -0.78648200 & 4.36921200 \\
\hline $\mathrm{H}$ & -2.55529600 & 0.74102700 & 5.13531500 & C & 0.04091000 & 1.77412300 & 5.35113500 \\
\hline $\mathrm{H}$ & 0.36676800 & 1.29491100 & 6.28075700 & $\mathrm{H}$ & -0.72751300 & 2.51196900 & 5.60952700 \\
\hline $\mathrm{H}$ & 0.90663600 & 2.30517500 & 4.93669100 & C & 0.17819000 & 2.13219300 & 2.34619600 \\
\hline 0 & 1.02049300 & 1.45537300 & 1.74442400 & C & -0.62058200 & 4.39720000 & 3.12361800 \\
\hline C & 1.57102200 & 4.14644900 & 2.07748900 & C & -0.23098500 & 5.78049000 & 2.59167800 \\
\hline $\mathrm{H}$ & -1.65915400 & 4.13746800 & 2.90676800 & $\mathrm{H}$ & -0.46094800 & 4.33801700 & 4.20753000 \\
\hline C & 1.26478000 & 5.66566900 & 2.23017300 & $\mathrm{H}$ & 2.29417200 & 3.85210700 & 2.84848800 \\
\hline $\mathrm{H}$ & -0.42222000 & 6.56203900 & 3.33170200 & $\mathrm{H}$ & -0.82565400 & 6.02383600 & 1.70660800 \\
\hline $\mathrm{H}$ & 1.89298100 & 6.07978200 & 3.02389100 & $\mathrm{H}$ & 1.50312500 & 6.20770300 & 1.31230100 \\
\hline $\mathrm{N}$ & 0.29026600 & 3.48257300 & 2.41885700 & C & 2.19733100 & 3.74534400 & 0.75444000 \\
\hline C & 3.49214300 & 3.31513600 & 0.77374200 & C & 1.50602500 & 3.86473200 & -0.51258600 \\
\hline C & 4.18655500 & 2.87804300 & -0.39942800 & $\mathrm{H}$ & 4.01468900 & 3.24347400 & 1.72506800 \\
\hline C & 2.18112400 & 3.45208800 & -1.70534600 & C & 0.21068000 & 4.39061300 & -0.63136800 \\
\hline C & 5.49637300 & 2.37346200 & -0.35293700 & C & 3.50266100 & 2.92285200 & -1.64757400 \\
\hline C & 1.53654100 & 3.58017500 & -2.97268500 & $\mathrm{H}$ & -0.34661100 & 4.65264600 & 0.25523600 \\
\hline C & -0.38189900 & 4.57488800 & -1.87801500 & C & 6.12125900 & 1.91361100 & -1.51049200 \\
\hline $\mathrm{H}$ & 6.01787800 & 2.34158000 & 0.60023700 & C & 4.15236000 & 2.45745200 & -2.83047400 \\
\hline C & 0.26967400 & 4.17639000 & -3.03944900 & C & 2.20821300 & 3.09709900 & -4.14662200 \\
\hline $\mathrm{H}$ & -1.37222000 & 5.01625000 & -1.93648000 & C & 5.45734600 & 1.94809700 & -2.73584700 \\
\hline $\mathrm{H}$ & 7.13563600 & 1.53004200 & -1.45946800 & C & 3.45008400 & 2.54550200 & -4.07933500 \\
\hline $\mathrm{H}$ & -0.20607900 & 4.30647400 & -4.00762000 & $\mathrm{H}$ & 1.69322000 & 3.17801900 & -5.10009500 \\
\hline $\mathrm{H}$ & 5.95372700 & 1.59506900 & -3.63612300 & $\mathrm{H}$ & 3.94151600 & 2.17959700 & -4.97693900 \\
\hline $\mathrm{C}$ & -2.89758600 & 0.72387000 & 1.68977800 & $S$ & -3.79033600 & 2.13356600 & 1.99349400 \\
\hline $\mathrm{N}$ & -1.64006200 & 0.53272900 & 2.15424200 & $\mathrm{H}$ & -1.09584900 & -0.25806900 & 1.79588400 \\
\hline C & 0.63311900 & -0.16517600 & -1.64143000 & $\mathrm{O}$ & 0.78525300 & -1.04828900 & -2.47379500 \\
\hline $\mathrm{O}$ & -0.32905200 & 0.75072700 & -1.60938600 & C & -1.50218900 & 0.60098300 & -2.51875800 \\
\hline C & -2.12109600 & -0.78642800 & -2.34404900 & $\mathrm{H}$ & -3.13090500 & -0.78351800 & -2.76639100 \\
\hline $\mathrm{H}$ & -1.53252300 & -1.55928500 & -2.83790700 & $\mathrm{H}$ & -2.19732800 & -1.04215400 & -1.28750600 \\
\hline C & -2.44249100 & 1.69006600 & -2.01604400 & $\mathrm{H}$ & -2.00523300 & 2.67664400 & -2.17279600 \\
\hline $\mathrm{H}$ & -3.39827900 & 1.63736500 & -2.54451200 & $\mathrm{H}$ & -2.62853600 & 1.56900100 & -0.94745400 \\
\hline C & -1.06574700 & 0.86091100 & -3.95894500 & $\mathrm{H}$ & -1.94930700 & 0.84654300 & -4.60663200 \\
\hline $\mathrm{H}$ & -0.59172300 & 1.84163800 & -4.04343600 & $\mathrm{H}$ & -0.36809400 & 0.09445400 & -4.29981600 \\
\hline C & 2.25116000 & -1.14160100 & -0.23757000 & $\mathrm{H}$ & 1.73204900 & -2.05490100 & -0.49892700 \\
\hline C & 2.91139100 & -1.17930900 & 1.08475700 & C & 2.86328900 & -2.37257300 & 1.81753800 \\
\hline C & 3.60938300 & -0.08047700 & 1.59822000 & C & 3.48971400 & -2.45556700 & 3.05978400 \\
\hline $\mathrm{H}$ & 2.30415000 & -3.21462200 & 1.42581400 & C & 4.24672600 & -0.17112600 & 2.83122200 \\
\hline $\mathrm{H}$ & 3.64098600 & 0.84069700 & 1.03404900 & C & 4.18717500 & -1.35844700 & 3.56659200 \\
\hline $\mathrm{H}$ & 3.42225100 & -3.37502700 & 3.63320100 & $\mathrm{H}$ & 4.78227500 & 0.68837800 & 3.22522700 \\
\hline
\end{tabular}




\begin{tabular}{|c|c|c|c|c|c|c|c|}
\hline $\mathrm{H}$ & 4.67695300 & -1.42424200 & 4.53405800 & $\mathrm{~N}$ & 1.51620200 & -0.01584600 & -0.57893400 \\
\hline $\mathrm{H}$ & 1.31386700 & 0.67369700 & 0.15291100 & S & -0.68886000 & -3.04362400 & 0.80325300 \\
\hline 0 & 0.28969100 & -3.87470000 & 0.07204100 & $\mathrm{O}$ & -0.21006800 & -1.66134500 & 1.09009300 \\
\hline $\mathrm{O}$ & -2.09454500 & -3.07976300 & 0.34248700 & C & -0.78146700 & -3.81325400 & 2.49328200 \\
\hline $\mathrm{F}$ & -1.21235200 & -5.07503900 & 2.42452600 & $\mathrm{~F}$ & -1.63108500 & -3.10925400 & 3.26368900 \\
\hline $\mathrm{F}$ & 0.42352500 & -3.80649900 & 3.09261400 & C & 3.67071400 & -1.17719700 & -1.52669800 \\
\hline $\mathrm{H}$ & 3.12931400 & -0.75977700 & -2.36774400 & $\mathrm{H}$ & 4.40475100 & -0.52234200 & -1.06631900 \\
\hline C & 3.91611000 & -2.55598500 & -1.54946700 & C & 4.92258700 & -3.15916200 & -0.68548700 \\
\hline $\mathrm{H}$ & 5.12142800 & -2.54635600 & 0.20081000 & $\mathrm{H}$ & 4.65781300 & -4.17860700 & -0.38285900 \\
\hline $\mathrm{C}$ & 3.01857800 & -3.45109500 & -2.33364700 & $\mathrm{H}$ & 3.53951000 & -4.35508000 & -2.66193600 \\
\hline $\mathrm{H}$ & 2.20038800 & -3.77676500 & -1.66728600 & $\mathrm{H}$ & 2.54797500 & -2.93957800 & -3.17399400 \\
\hline $\mathrm{C}$ & 7.76760000 & -4.20219300 & -0.40941700 & $\mathrm{H}$ & 7.39717300 & -5.21470700 & -0.21341100 \\
\hline $\mathrm{H}$ & 8.77685700 & -4.29187500 & -0.82833800 & $\mathrm{H}$ & 7.84997100 & -3.68205400 & 0.55161500 \\
\hline C & 6.43357800 & -4.20912300 & -3.21543400 & $\mathrm{H}$ & 6.01074300 & -5.20803500 & -3.05799500 \\
\hline $\mathrm{H}$ & 5.78160900 & -3.67363900 & -3.91445600 & $\mathrm{H}$ & 7.40662900 & -4.33804900 & -3.70412300 \\
\hline $\mathrm{C}$ & 7.25043200 & -1.52203700 & -1.89403300 & $\mathrm{H}$ & 6.57398300 & -0.96198300 & -2.54772600 \\
\hline $\mathrm{H}$ & 7.33240000 & -0.96686100 & -0.95288500 & $\mathrm{H}$ & 8.24117100 & -1.53391100 & -2.36347600 \\
\hline $\mathrm{Si}$ & 6.63677500 & -3.27415200 & -1.59445500 & & & & \\
\hline
\end{tabular}

\section{TS-S-4f-53}

Imaginary frequency: $-411.72 \mathrm{~cm}^{-1}$

E[B3LYP/6-31G(d)-D3(BJ)]: -4789.074472 Hartree

$\mathrm{E}\left[\mathrm{PCM}\left(\mathrm{Et}_{2} \mathrm{O}\right)-\mathrm{B} 3 \mathrm{LYP} / 6-311 \mathrm{G}(\mathrm{d}, \mathrm{p})-\mathrm{D} 3(\mathrm{BJ})\right]$ : -4790.210859 Hartree

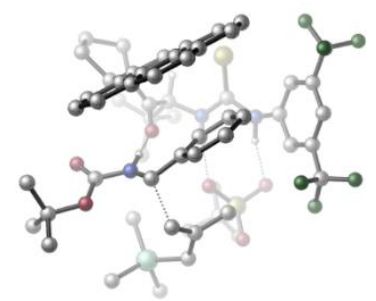

\begin{tabular}{|c|c|c|c|c|c|c|c|}
\hline 01 & & & & $\mathrm{~N}$ & -1.80321900 & -2.80565200 & 1.35253700 \\
\hline $\mathrm{H}$ & -1.10729700 & -3.45638000 & 0.97434000 & C & -2.85367200 & -2.56310400 & 0.45019600 \\
\hline $\mathrm{C}$ & -2.60826600 & -2.88373400 & -0.89583500 & $\mathrm{C}$ & -4.07612700 & -1.97540200 & 0.80058400 \\
\hline $\mathrm{C}$ & -3.53093300 & -2.54167600 & -1.87519400 & $\mathrm{H}$ & -1.68350100 & -3.37664800 & -1.16527800 \\
\hline C & -4.98259300 & -1.63079700 & -0.20354900 & $\mathrm{H}$ & -4.28717800 & -1.74759900 & 1.83355100 \\
\hline $\mathrm{C}$ & -4.72375700 & -1.89850000 & -1.54481100 & $\mathrm{H}$ & -5.42673700 & -1.60577300 & -2.31589800 \\
\hline C & -3.18825800 & -2.72576600 & -3.32560400 & C & -6.27716400 & -0.95921300 & 0.15661500 \\
\hline $\mathrm{F}$ & -2.15563300 & -3.57249600 & -3.51274100 & $\mathrm{~F}$ & -2.83270000 & -1.53935800 & -3.89109900 \\
\hline $\mathrm{F}$ & -4.23689600 & -3.18856800 & -4.03927900 & $\mathrm{~F}$ & -6.24382200 & -0.40231600 & 1.38814200 \\
\hline $\mathrm{F}$ & -6.57131200 & 0.03442800 & -0.72336600 & $\mathrm{~F}$ & -7.32199600 & -1.81416500 & 0.13475900 \\
\hline C & 0.67276200 & -1.20343900 & 3.49942600 & $\mathrm{H}$ & -0.00770000 & -1.05229500 & 4.33645000 \\
\hline $\mathrm{C}$ & 1.97515000 & -1.87238100 & 4.04451000 & $\mathrm{C}$ & 3.04341700 & -2.04234800 & 2.95511900 \\
\hline $\mathrm{H}$ & 3.38647700 & -1.07600400 & 2.57787800 & $\mathrm{H}$ & 2.68014200 & -2.61665000 & 2.10389700 \\
\hline $\mathrm{H}$ & 3.90663900 & -2.57513900 & 3.37082100 & C & 1.59112200 & -3.24375900 & 4.62655700 \\
\hline $\mathrm{H}$ & 2.46716500 & -3.70903700 & 5.09255500 & $\mathrm{H}$ & 1.22014500 & -3.91653100 & 3.85149600 \\
\hline $\mathrm{H}$ & 0.81170600 & -3.14364900 & 5.39126000 & $\mathrm{C}$ & 2.56478100 & -1.00413500 & 5.16964300 \\
\hline $\mathrm{H}$ & 3.48965600 & -1.46032800 & 5.53858500 & $\mathrm{H}$ & 1.87926900 & -0.92262000 & 6.02135000 \\
\hline $\mathrm{H}$ & 2.81375200 & 0.00409800 & 4.82020700 & $\mathrm{C}$ & 0.91656100 & 0.13558300 & 2.77579300 \\
\hline $\mathrm{O}$ & 1.26809600 & 0.11063000 & 1.58325000 & $\mathrm{C}$ & 0.32857600 & 1.51903100 & 4.82105000 \\
\hline $\mathrm{C}$ & 0.94694900 & 2.58293000 & 2.73969700 & $\mathrm{C}$ & 1.14386200 & 2.75913900 & 5.19418900 \\
\hline $\mathrm{H}$ & -0.75019100 & 1.71305700 & 4.86687000 & $\mathrm{H}$ & 0.54732400 & 0.65701000 & 5.44439000 \\
\hline $\mathrm{H}$ & 1.86014100 & 2.48556600 & 2.14997100 & $\mathrm{H}$ & 2.16649100 & 2.45549000 & 5.44457600 \\
\hline $\mathrm{H}$ & 0.72720100 & 3.29068000 & 6.05427000 & $\bar{N}$ & 0.73413700 & 1.29717000 & 3.43056800 \\
\hline C & -0.22824900 & 2.95877400 & 1.84843200 & C & -1.44807500 & 2.37437800 & 2.02112900 \\
\hline C & -0.06431100 & 4.00460500 & 0.86076600 & $\mathrm{C}$ & -2.59952400 & 2.75605300 & 1.26046300 \\
\hline $\mathrm{H}$ & -1.57008900 & 1.56250400 & 2.72924700 & C & -1.19313500 & 4.38342500 & 0.06515600 \\
\hline C & 1.15596100 & 4.66775500 & 0.64615500 & $\mathrm{C}$ & -3.84702700 & 2.13800400 & 1.44229200 \\
\hline
\end{tabular}




\begin{tabular}{|c|c|c|c|c|c|c|c|}
\hline $\mathrm{C}$ & -2.46090900 & 3.76901100 & 0.27124400 & $\mathrm{C}$ & -1.05660700 & 5.38818800 & -0.94116500 \\
\hline $\mathrm{H}$ & 2.02531100 & 4.40747900 & 1.23825800 & C & 1.27040100 & 5.66394100 & -0.32317600 \\
\hline $\mathrm{C}$ & -4.94385000 & 2.51865200 & 0.67173300 & $\mathrm{H}$ & -3.94100200 & 1.34006600 & 2.17236100 \\
\hline $\mathrm{C}$ & -3.58608000 & 4.14650700 & -0.51955900 & $\mathrm{C}$ & 0.18511400 & 6.01483200 & -1.11892000 \\
\hline C & -2.20232700 & 5.73969600 & -1.73271900 & $\mathrm{H}$ & 2.21508200 & 6.18239000 & -0.44515500 \\
\hline C & -4.81718400 & 3.51136300 & -0.29774300 & $\mathrm{H}$ & -5.89713800 & 2.02473500 & 0.81642100 \\
\hline C & -3.41171700 & 5.15042600 & -1.52918900 & $\mathrm{H}$ & 0.28517400 & 6.79057600 & -1.87382300 \\
\hline $\mathrm{H}$ & -2.08176000 & 6.50237400 & -2.49800300 & $\mathrm{H}$ & -5.67530500 & 3.79688900 & -0.90098800 \\
\hline $\mathrm{H}$ & -4.27156300 & 5.43177000 & -2.13200300 & C & -1.36593500 & -1.98185700 & 2.36496600 \\
\hline $\mathrm{S}$ & -2.36696200 & -0.98456500 & 3.29532000 & $\mathrm{~N}$ & -0.02387400 & -2.07059200 & 2.56047500 \\
\hline $\mathrm{H}$ & 0.52279000 & -2.35673300 & 1.74167700 & S & 1.09143800 & -4.18431700 & -0.45699400 \\
\hline $\mathrm{O}$ & 1.50593700 & -4.40155900 & -1.85380400 & 0 & 1.21751300 & -2.77207300 & 0.01947300 \\
\hline $\mathrm{O}$ & -0.19193200 & -4.80296100 & -0.04164400 & C & 2.36359800 & -5.10320700 & 0.53968900 \\
\hline $\mathrm{F}$ & 2.46732600 & -6.36753800 & 0.12818100 & $\mathrm{~F}$ & 2.03241000 & -5.09954500 & 1.84167700 \\
\hline $\mathrm{F}$ & 3.56803100 & -4.51189800 & 0.41839800 & C & 3.30526900 & 2.20660500 & -0.45356600 \\
\hline $\mathrm{O}$ & 3.75693900 & 2.31423100 & 0.67257700 & $\mathrm{O}$ & 3.86433500 & 2.66303000 & -1.58642400 \\
\hline C & 5.08201000 & 3.51142100 & -1.54819100 & C & 4.74414100 & 4.83461000 & -0.86738300 \\
\hline $\mathrm{H}$ & 5.61899300 & 5.49230800 & -0.89657600 & $\mathrm{H}$ & 4.46313600 & 4.67811800 & 0.17577500 \\
\hline $\mathrm{H}$ & 3.92311600 & 5.32939500 & -1.39180600 & $C$ & 5.37827900 & 3.72208100 & -3.03155900 \\
\hline $\mathrm{H}$ & 5.60720500 & 2.77220200 & -3.52336000 & $\mathrm{H}$ & 6.24135600 & 4.38511400 & -3.14544200 \\
\hline $\mathrm{H}$ & 4.52087800 & 4.18014300 & -3.53474300 & C & 6.23691600 & 2.79028400 & -0.85254800 \\
\hline $\mathrm{H}$ & 7.14502500 & 3.39119200 & -0.97144700 & $\mathrm{H}$ & 6.41627700 & 1.81377700 & -1.30690500 \\
\hline $\mathrm{H}$ & 6.03800000 & 2.65574200 & 0.21003200 & $C$ & 1.35396000 & 1.71829300 & -1.84594700 \\
\hline $\mathrm{H}$ & 1.67837500 & 2.58398700 & -2.41401100 & C & -0.11040800 & 1.54948100 & -1.77144000 \\
\hline $\mathrm{C}$ & -0.88948400 & 2.19906900 & -2.74592500 & $\mathrm{C}$ & -0.74018000 & 0.72596000 & -0.82842300 \\
\hline $\mathrm{C}$ & -2.26183500 & 1.99963000 & -2.80325700 & $\mathrm{H}$ & -0.40867700 & 2.86527200 & -3.45845600 \\
\hline C & -2.12032800 & 0.54483500 & -0.88354000 & $\mathrm{H}$ & -0.17023800 & 0.22016900 & -0.05775000 \\
\hline $\mathrm{C}$ & -2.88070500 & 1.15558500 & -1.87638200 & $\mathrm{H}$ & -2.85196800 & 2.51472900 & -3.55422500 \\
\hline $\bar{H}$ & -2.60308900 & -0.06874300 & -0.13709500 & $\mathrm{H}$ & -3.95349400 & 1.00200100 & -1.90018900 \\
\hline $\mathrm{N}$ & 2.10604200 & 1.55567700 & -0.70247000 & $\mathrm{H}$ & 1.72988700 & 1.06592000 & 0.12295900 \\
\hline $\mathrm{C}$ & 1.88673900 & 0.54983500 & -3.39153100 & $\mathrm{H}$ & 1.11893300 & 1.00876800 & -4.00695100 \\
\hline $\bar{H}$ & 2.89847000 & 0.88485400 & -3.60760100 & $C$ & 1.70468500 & -0.82596900 & -3.13816500 \\
\hline C & 2.82181400 & -1.77284700 & -3.11095300 & $\mathrm{H}$ & 3.30990300 & -1.71742900 & -4.09732200 \\
\hline $\mathrm{H}$ & 2.46179900 & -2.79086900 & -2.94828000 & C & 0.34338600 & -1.37503500 & -2.91367000 \\
\hline $\bar{H}$ & 0.23667700 & -2.36036200 & -3.37559600 & $\bar{H}$ & -0.46213200 & -0.71569800 & -3.22970400 \\
\hline $\mathrm{H}$ & 0.24878800 & -1.55889100 & -1.83040900 & C & 4.07021500 & -1.18435600 & -0.20852200 \\
\hline $\bar{H}$ & 3.02309200 & -1.34842200 & 0.05124000 & $\mathrm{H}$ & 4.33441800 & -0.15272300 & 0.03899200 \\
\hline $\bar{H}$ & 4.66602300 & -1.84200900 & 0.43386100 & $\mathrm{C}$ & 5.51386500 & -0.31446200 & -2.82801900 \\
\hline $\mathrm{H}$ & 5.06623700 & 0.68176500 & -2.77425200 & $\mathrm{H}$ & 5.69653500 & -0.55508000 & -3.88213900 \\
\hline $\mathrm{H}$ & 6.48815600 & -0.26969200 & -2.32665800 & $\mathrm{C}$ & 5.19160100 & -3.30633300 & -2.19239900 \\
\hline $\mathrm{H}$ & 5.38977200 & -3.54322800 & -3.24421300 & $\mathrm{H}$ & 4.53571000 & -4.08113200 & -1.78629300 \\
\hline $\mathrm{H}$ & 6.14529300 & -3.35204700 & -1.65270200 & $\mathrm{Si}$ & 4.40644000 & -1.60545300 & -2.00413100 \\
\hline C & 1.13049500 & 3.60400400 & 3.90554600 & $\mathrm{H}$ & 0.28994100 & 4.30312600 & 3.91281600 \\
\hline $\mathrm{H}$ & 2.04551000 & 4.18940100 & 3.78416200 & & & & \\
\hline
\end{tabular}

\section{TS- $R-4 f-1$}

Imaginary frequency: $-378.77 \mathrm{~cm}^{-1}$

E[B3LYP/6-31G(d)-D3(BJ)]: -4789.089043 Hartree

E[PCM(Et $\left.\left.{ }_{2} \mathrm{O}\right)-\mathrm{B} 3 \mathrm{LYP} / 6-311 \mathrm{G}(\mathrm{d}, \mathrm{p})-\mathrm{D} 3(\mathrm{BJ})\right]$ : -4790.233671 Hartree

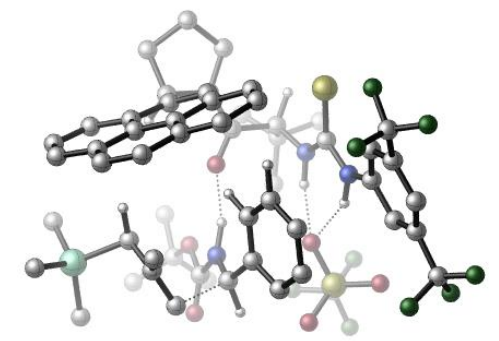




\begin{tabular}{|c|c|c|c|c|c|c|c|}
\hline 01 & & & & $\mathrm{~N}$ & -2.60003400 & -1.09354800 & 1.36259300 \\
\hline $\mathrm{H}$ & -2.20771000 & -1.86879800 & 0.81778000 & C & -3.76143700 & -0.56121400 & 0.79245400 \\
\hline C & -4.38112900 & -1.36195200 & -0.18195800 & $\mathrm{C}$ & -4.27439300 & 0.71351000 & 1.06217100 \\
\hline C & -5.48216400 & -0.87889800 & -0.88053100 & $\mathrm{H}$ & -3.98605800 & -2.35001500 & -0.40438800 \\
\hline C & -5.37259100 & 1.17534400 & 0.34179600 & $\mathrm{H}$ & -3.80416800 & 1.33559600 & 1.80671800 \\
\hline C & -5.99318100 & 0.39400600 & -0.63051800 & $\mathrm{H}$ & -6.85010500 & 0.76521900 & -1.17989400 \\
\hline C & -6.07822300 & -1.70058900 & -1.99012400 & C & -5.82414700 & 2.59515100 & 0.51317500 \\
\hline $\mathrm{F}$ & -5.88227600 & -3.01752100 & -1.81911300 & $\mathrm{~F}$ & -5.53496200 & -1.36779300 & -3.19250700 \\
\hline $\mathrm{F}$ & -7.41049800 & -1.49054000 & -2.10462200 & $\mathrm{~F}$ & -5.33963100 & 3.17031600 & 1.63689700 \\
\hline $\mathrm{F}$ & -5.39538800 & 3.36638100 & -0.52894700 & $\mathrm{~F}$ & -7.16774100 & 2.71076200 & 0.54155300 \\
\hline C & 0.44028100 & -1.15889200 & 3.34978700 & $\mathrm{H}$ & 0.02734400 & -0.69983800 & 4.24672600 \\
\hline C & 1.15762300 & -2.48019800 & 3.77801600 & C & 1.45322600 & -3.38953100 & 2.57497900 \\
\hline $\mathrm{H}$ & 2.00088200 & -2.86290800 & 1.79268100 & $\mathrm{H}$ & 0.54230700 & -3.80304400 & 2.13921900 \\
\hline $\mathrm{H}$ & 2.05944300 & -4.24148800 & 2.90531200 & $\mathrm{C}$ & 0.22303800 & -3.21648900 & 4.75253700 \\
\hline $\mathrm{H}$ & 0.65852200 & -4.17943400 & 5.04234700 & $\mathrm{H}$ & -0.74912700 & -3.40887800 & 4.28871900 \\
\hline $\mathrm{H}$ & 0.05537800 & -2.63058100 & 5.66408000 & C & 2.47723000 & -2.14263000 & 4.49101200 \\
\hline $\mathrm{H}$ & 2.95982000 & -3.06527700 & 4.83131200 & $\mathrm{H}$ & 2.31224600 & -1.51696600 & 5.37514000 \\
\hline $\mathrm{H}$ & 3.17855300 & -1.62573500 & 3.82677000 & C & 1.35799800 & -0.14384900 & 2.64892100 \\
\hline $\mathrm{O}$ & 1.69094200 & -0.28247100 & 1.46000300 & C & 1.45786300 & 1.27708800 & 4.75085500 \\
\hline C & 2.60781600 & 1.94933600 & 2.69426900 & C & 2.06158200 & 2.67997300 & 4.91986600 \\
\hline $\mathrm{H}$ & 0.37548200 & 1.26016000 & 4.90641900 & $\mathrm{H}$ & 1.92534500 & 0.56326900 & 5.43646600 \\
\hline C & 3.20460700 & 2.71162700 & 3.89526700 & $\mathrm{H}$ & 3.36945800 & 1.43801500 & 2.10187500 \\
\hline $\mathrm{H}$ & 2.39739200 & 2.86039800 & 5.94419200 & $\mathrm{H}$ & 1.31686400 & 3.44143200 & 4.66945800 \\
\hline $\mathrm{H}$ & 4.07813400 & 2.16931000 & 4.27640400 & $\mathrm{H}$ & 3.51583500 & 3.72457000 & 3.62947700 \\
\hline $\mathrm{N}$ & 1.78297300 & 0.92650300 & 3.35445000 & $\mathrm{C}$ & 1.78379100 & 2.84906100 & 1.78543000 \\
\hline C & 0.43527500 & 2.96692600 & 1.95553900 & $\mathrm{C}$ & 2.44810500 & 3.61269300 & 0.74969700 \\
\hline C & -0.37486300 & 3.80200800 & 1.12036800 & $\mathrm{H}$ & -0.07629900 & 2.37834800 & 2.70957600 \\
\hline $\mathrm{C}$ & 1.64957300 & 4.38510300 & -0.15360900 & C & 3.84498500 & 3.62857500 & 0.58466800 \\
\hline $\mathrm{C}$ & -1.75792500 & 3.93840100 & 1.32417000 & $\mathrm{C}$ & 0.23783200 & 4.46779000 & 0.02219100 \\
\hline C & 2.26662300 & 5.09846400 & -1.22702700 & $\mathrm{H}$ & 4.47782000 & 3.07361200 & 1.26796200 \\
\hline C & 4.44066400 & 4.36109600 & -0.44202000 & C & -2.53362200 & 4.68429000 & 0.43805700 \\
\hline $\mathrm{H}$ & -2.22112400 & 3.41958200 & 2.15803400 & $\mathrm{C}$ & -0.56316000 & 5.22423400 & -0.88390300 \\
\hline C & 3.66465600 & 5.07622100 & -1.34938500 & C & 1.43397900 & 5.83004000 & -2.14201300 \\
\hline $\mathrm{H}$ & 5.52287400 & 4.36937000 & -0.53552200 & C & -1.94646100 & 5.30810500 & -0.66141500 \\
\hline $\mathrm{H}$ & -3.60333600 & 4.75634100 & 0.59260600 & C & 0.08300000 & 5.88332300 & -1.98341900 \\
\hline $\mathrm{H}$ & 4.13575800 & 5.63985200 & -2.15037700 & $\mathrm{H}$ & 1.91329600 & 6.35279400 & -2.96567000 \\
\hline $\mathrm{H}$ & -2.55967700 & 5.87604100 & -1.35592500 & $\mathrm{H}$ & -0.53144600 & 6.44585800 & -2.68135700 \\
\hline C & -1.78922700 & -0.67104600 & 2.37577400 & $\mathrm{~S}$ & -2.10059500 & 0.60922500 & 3.44597400 \\
\hline $\mathrm{N}$ & -0.67526200 & -1.44406300 & 2.47364000 & $\mathrm{H}$ & -0.49248200 & -2.07388300 & 1.69327700 \\
\hline$S$ & -1.28903100 & -3.88149900 & -0.91921400 & $\mathrm{O}$ & -0.79390800 & -3.74764900 & -2.30235400 \\
\hline $\mathrm{O}$ & -0.79539900 & -2.80721600 & 0.00611800 & $\mathrm{O}$ & -2.71859100 & -4.18595300 & -0.7432900 \\
\hline C & -0.38350200 & -5.36693700 & -0.27310900 & $\mathrm{~F}$ & -0.64579500 & -6.45519100 & -0.99992300 \\
\hline $\mathrm{F}$ & -0.72430800 & -5.61216300 & 1.00427900 & $\mathrm{~F}$ & 0.94614600 & -5.13852700 & -0.30802400 \\
\hline C & 2.71162000 & -2.46732200 & -1.20981600 & $\mathrm{O}$ & 2.94592200 & -3.05257400 & -2.25371200 \\
\hline $\mathrm{O}$ & 3.42725600 & -2.52153000 & -0.07707900 & C & 4.55324300 & -3.46796400 & 0.07077800 \\
\hline C & 4.03010000 & -4.90067300 & -0.00657700 & $\mathrm{H}$ & 4.85548500 & -5.59616500 & 0.18004700 \\
\hline $\mathrm{H}$ & 3.61036400 & -5.11225900 & -0.99126800 & $\mathrm{H}$ & 3.25643200 & -5.06996000 & 0.74583400 \\
\hline C & 5.07706000 & -3.13802200 & 1.46758200 & $\mathrm{H}$ & 5.38449600 & -2.08846500 & 1.52393800 \\
\hline $\mathrm{H}$ & 5.94286700 & -3.76731300 & 1.69601400 & $\mathrm{H}$ & 4.30882800 & -3.31454800 & 2.22339400 \\
\hline C & 5.62808600 & -3.18984100 & -0.98109900 & $\mathrm{H}$ & 6.51940200 & -3.78107200 & -0.74578500 \\
\hline $\mathrm{H}$ & 5.91316100 & -2.13265000 & -0.96934500 & $\mathrm{H}$ & 5.28486400 & -3.45663600 & -1.98035400 \\
\hline C & 0.85421900 & -1.28124300 & -2.13900000 & $\mathrm{H}$ & 0.71198300 & -2.15557700 & -2.76863300 \\
\hline C & -0.36750300 & -0.47509800 & -1.94375400 & C & -1.41951500 & -0.67549100 & -2.85073800 \\
\hline
\end{tabular}




\begin{tabular}{|c|c|c|c|c|c|c|c|}
\hline $\mathrm{C}$ & -0.48218400 & 0.52166700 & -0.96384400 & C & -2.55844700 & 0.12280000 & -2.80124300 \\
\hline $\mathrm{H}$ & -1.35160100 & -1.48756300 & -3.56675400 & C & -1.62707400 & 1.31444800 & -0.92077600 \\
\hline $\mathrm{H}$ & 0.30679900 & 0.68152400 & -0.23479600 & C & -2.65489500 & 1.13430100 & -1.84549300 \\
\hline $\bar{H}$ & -3.38167200 & -0.06742400 & -3.48092500 & $\mathrm{H}$ & -1.72191600 & 2.07108700 & -0.15671000 \\
\hline $\mathrm{H}$ & -3.53962200 & 1.75950100 & -1.78965600 & $\mathrm{~N}$ & 1.68199900 & -1.55207900 & -1.06609400 \\
\hline $\mathrm{H}$ & 1.52431600 & -1.14586800 & -0.13689500 & C & 1.88635500 & -0.39340500 & -3.47743700 \\
\hline $\mathrm{H}$ & 1.20858200 & -0.40101300 & -4.32710300 & $\mathrm{H}$ & 2.65248100 & -1.16355700 & -3.50595400 \\
\hline C & 2.21117000 & 0.85543300 & -2.92988200 & C & 3.24109400 & 0.97472700 & -1.90903400 \\
\hline $\mathrm{H}$ & 3.25774900 & 0.09004800 & -1.25732500 & $\mathrm{H}$ & 3.12651200 & 1.87628900 & -1.30111800 \\
\hline $\mathrm{C}$ & 1.40777500 & 2.06420400 & -3.25956500 & $\mathrm{H}$ & 2.02029600 & 2.97044300 & -3.23600400 \\
\hline $\mathrm{H}$ & 0.64858100 & 2.18211700 & -2.46902900 & $\mathrm{H}$ & 0.88378400 & 1.97945200 & -4.21389000 \\
\hline C & 6.17575600 & 1.04093300 & -1.15678800 & $\mathrm{H}$ & 5.99866600 & 1.92674100 & -0.53956200 \\
\hline $\mathrm{H}$ & 7.22745900 & 1.04556900 & -1.46601500 & $\mathrm{H}$ & 6.01460100 & 0.15730500 & -0.52863600 \\
\hline C & 5.21314900 & 2.56691300 & -3.70814500 & $\mathrm{H}$ & 4.96245700 & 3.46860700 & -3.14208900 \\
\hline $\mathrm{H}$ & 4.55514300 & 2.52300600 & -4.58402500 & $\mathrm{H}$ & 6.24170200 & 2.66871700 & -4.07414000 \\
\hline $\mathrm{C}$ & 5.32014800 & -0.51256600 & -3.70668900 & $\mathrm{H}$ & 4.78464300 & -0.44872000 & -4.66004200 \\
\hline $\mathrm{H}$ & 5.00337700 & -1.43248300 & -3.20706400 & $\mathrm{H}$ & 6.38837600 & -0.60881100 & -3.93604600 \\
\hline $\mathrm{Si}$ & 5.03025000 & 1.01924700 & -2.65262600 & & & & \\
\hline
\end{tabular}

\section{TS- $R-4 \mathrm{f}-2$}

Imaginary frequency: $-400.36 \mathrm{~cm}^{-1}$ E[B3LYP/6-31G(d)-D3(BJ)]: -4789.090016 Hartree $\mathrm{E}\left[\mathrm{PCM}\left(\mathrm{Et}_{2} \mathrm{O}\right)-\mathrm{B} 3 \mathrm{LYP} / 6-311 \mathrm{G}(\mathrm{d}, \mathrm{p})-\mathrm{D} 3(\mathrm{BJ})\right]$ : -4790.232586 Hartree

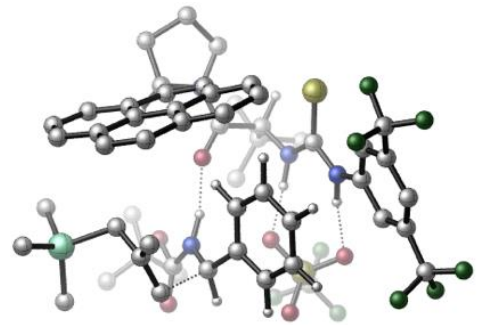

\begin{tabular}{|c|c|c|c|c|c|c|c|}
\hline 01 & & & & $\mathrm{~N}$ & 2.76837600 & 1.19572300 & 1.24893000 \\
\hline $\mathrm{H}$ & 2.51826400 & 2.07776000 & 0.78746400 & C & 3.89498200 & 0.59622800 & 0.67387700 \\
\hline C & 4.54315100 & 1.33896000 & -0.32862900 & C & 4.35746500 & -0.69389000 & 0.96704600 \\
\hline C & 5.61414900 & 0.79028200 & -1.02453200 & $\mathrm{H}$ & 4.17783200 & 2.32790900 & -0.58153200 \\
\hline $\mathrm{C}$ & 5.42529500 & -1.22334500 & 0.24814800 & $\mathrm{H}$ & 3.87251800 & -1.27707900 & 1.73249700 \\
\hline C & 6.07264000 & -0.49729100 & -0.74898500 & $\mathrm{H}$ & 6.90509500 & -0.92093400 & -1.29808400 \\
\hline C & 6.22117500 & 1.55180800 & -2.16914600 & C & 5.81268200 & -2.65796000 & 0.45278700 \\
\hline $\mathrm{F}$ & 6.06961100 & 2.88225500 & -2.04276000 & $\mathrm{~F}$ & 5.64792600 & 1.19778300 & -3.35211500 \\
\hline $\mathrm{F}$ & 7.54305400 & 1.29678700 & -2.29645000 & $\mathrm{~F}$ & 5.29188100 & -3.19007200 & 1.58120600 \\
\hline $\mathrm{F}$ & 5.36174800 & -3.42896600 & -0.57974500 & $\mathrm{~F}$ & 7.14977900 & -2.83088200 & 0.49864900 \\
\hline$C$ & -0.28088200 & 1.28221700 & 3.23874200 & $\mathrm{H}$ & 0.19802400 & 0.87259400 & 4.12726900 \\
\hline C & -0.98538100 & 2.60897800 & 3.66851400 & C & -1.79975200 & 3.24817400 & 2.53624100 \\
\hline $\mathrm{H}$ & -2.54547800 & 2.55641200 & 2.14200400 & $\mathrm{H}$ & -1.17187300 & 3.56187000 & 1.70182500 \\
\hline $\mathrm{H}$ & -2.31594100 & 4.13594000 & 2.92220800 & C & 0.09894800 & 3.59243700 & 4.14169000 \\
\hline $\mathrm{H}$ & -0.37155700 & 4.48917100 & 4.56094500 & $\mathrm{H}$ & 0.74045900 & 3.90565900 & 3.31428700 \\
\hline $\mathrm{H}$ & 0.73301700 & 3.14594200 & 4.91666500 & C & -1.93059200 & 2.29692600 & 4.84193500 \\
\hline $\mathrm{H}$ & -2.43130800 & 3.21431700 & 5.16947800 & $\mathrm{H}$ & -1.38453100 & 1.89255900 & 5.70198900 \\
\hline $\mathrm{H}$ & -2.70956300 & 1.58034100 & 4.55600700 & C & -1.23572800 & 0.22807900 & 2.65250700 \\
\hline $\mathrm{O}$ & -1.62583600 & 0.29344800 & 1.47476300 & C & -1.23369900 & -1.08903600 & 4.82801800 \\
\hline C & -2.50240200 & -1.83877500 & 2.86882500 & C & -1.81324400 & -2.48939700 & 5.08046800 \\
\hline $\mathrm{H}$ & -0.14483000 & -1.05610000 & 4.92241300 & $\mathrm{H}$ & -1.67177300 & -0.35414200 & 5.50952900 \\
\hline C & -3.01744700 & -2.56148800 & 4.13046200 & $\mathrm{H}$ & -3.30190300 & -1.34517300 & 2.31285700 \\
\hline $\mathrm{H}$ & -2.08391500 & -2.63743800 & 6.12905900 & $\mathrm{H}$ & -1.07960700 & -3.25471300 & 4.80968300 \\
\hline $\mathrm{H}$ & -3.86903500 & -2.01043300 & 4.54719700 & $\mathrm{H}$ & -3.33871200 & -3.58410300 & 3.91872200 \\
\hline $\mathrm{N}$ & -1.63633700 & -0.79527700 & 3.43868700 & C & -1.74309800 & -2.76882500 & 1.93452700 \\
\hline C & -0.38927400 & -2.90518900 & 2.03335900 & C & -2.47474200 & -3.54077500 & 0.95168700 \\
\hline $\mathrm{C}$ & 0.36092300 & -3.77109800 & 1.17375400 & $\mathrm{H}$ & 0.17167300 & -2.30708300 & 2.74369600 \\
\hline
\end{tabular}




\begin{tabular}{|c|c|c|c|c|c|c|c|}
\hline $\mathrm{C}$ & -1.73918900 & -4.34742900 & 0.02489100 & $\mathrm{C}$ & -3.87822400 & -3.53385500 & 0.86049600 \\
\hline C & 1.75076200 & -3.92722500 & 1.30519500 & C & -0.32127900 & -4.45111000 & 0.12625400 \\
\hline $\mathrm{C}$ & -2.42535400 & -5.07476200 & -0.99604900 & $\mathrm{H}$ & -4.46433000 & -2.95219500 & 1.56281900 \\
\hline $\mathrm{C}$ & -4.54082200 & -4.27807700 & -0.11546800 & $\mathrm{C}$ & 2.46483900 & -4.70719400 & 0.39686900 \\
\hline $\mathrm{H}$ & 2.26805500 & -3.39814200 & 2.10005300 & C & 0.41671100 & -5.24355000 & -0.80262800 \\
\hline C & -3.82720600 & -5.02948800 & -1.04446800 & $\mathrm{C}$ & -1.65588000 & -5.84431700 & -1.93512100 \\
\hline $\mathrm{H}$ & -5.62643300 & -4.26628200 & -0.15197800 & C & 1.80852700 & -5.34696100 & -0.65316400 \\
\hline $\mathrm{H}$ & 3.54034100 & -4.79317400 & 0.49467900 & C & -0.29925800 & -5.91809600 & -1.84836200 \\
\hline $\mathrm{H}$ & -4.34990500 & -5.60278900 & -1.80553100 & $\mathrm{H}$ & -2.18811300 & -6.37970200 & -2.71723100 \\
\hline $\mathrm{H}$ & 2.37328700 & -5.94296900 & -1.36507700 & $\mathrm{H}$ & 0.26679400 & -6.50955100 & -2.56317000 \\
\hline C & 1.90972100 & 0.77104200 & 2.22723800 & $\mathrm{~S}$ & 2.21100700 & -0.49704400 & 3.32036200 \\
\hline $\mathrm{N}$ & 0.77890400 & 1.51489900 & 2.27668500 & $\mathrm{H}$ & 0.54185600 & 2.08526300 & 1.45519600 \\
\hline $\mathrm{S}$ & 1.02951300 & 3.80846600 & -0.77752800 & $\mathrm{O}$ & 0.84337100 & 3.81930000 & -2.23616700 \\
\hline $\mathrm{O}$ & 0.06924400 & 2.94062700 & -0.03420700 & 0 & 2.42191000 & 3.66120500 & -0.27834400 \\
\hline $\mathrm{C}$ & 0.52544200 & 5.50181800 & -0.20858400 & $\mathrm{~F}$ & 1.28486700 & 6.45022800 & -0.76161000 \\
\hline $\mathrm{F}$ & 0.63614000 & 5.58632500 & 1.12996900 & $\mathrm{~F}$ & -0.75865900 & 5.73159300 & -0.53286400 \\
\hline C & -2.87743500 & 2.31675400 & -1.32054200 & $\mathrm{O}$ & -3.04117800 & 2.89822100 & -2.38074900 \\
\hline $\mathrm{O}$ & -3.64280100 & 2.41028300 & -0.22705600 & C & -4.65843900 & 3.48589100 & -0.12524200 \\
\hline $\mathrm{C}$ & -3.94752400 & 4.83793500 & -0.16529000 & $\mathrm{H}$ & -4.67443500 & 5.63609300 & 0.01958800 \\
\hline $\mathrm{H}$ & -3.48190300 & 5.00959000 & -1.13724900 & $\mathrm{H}$ & -3.17253100 & 4.88823800 & 0.60370800 \\
\hline C & -5.29433200 & 3.22313700 & 1.23849400 & $\mathrm{H}$ & -5.72999200 & 2.21890900 & 1.27186800 \\
\hline $\mathrm{H}$ & -6.09163000 & 3.95142700 & 1.41657300 & $\mathrm{H}$ & -4.55871600 & 3.31065700 & 2.03995800 \\
\hline $\mathrm{C}$ & -5.70919000 & 3.34449500 & -1.22879500 & $\mathrm{H}$ & -6.52857800 & 4.04322500 & -1.02869100 \\
\hline $\mathrm{H}$ & -6.12549500 & 2.33149000 & -1.23502700 & $\mathrm{H}$ & -5.29160300 & 3.56479000 & -2.21036000 \\
\hline $\mathrm{C}$ & -0.99202900 & 1.15121000 & -2.16901400 & $\mathrm{H}$ & -0.89252200 & 2.02467400 & -2.80846300 \\
\hline $\mathrm{C}$ & 0.26877800 & 0.42368600 & -1.94197800 & $\mathrm{C}$ & 1.32341600 & 0.68775900 & -2.83106400 \\
\hline C & 0.42324800 & -0.56687600 & -0.96069800 & C & 2.49973300 & -0.05245200 & -2.77320900 \\
\hline $\bar{H}$ & 1.22213700 & 1.49848700 & -3.54486500 & $\mathrm{C}$ & 1.60611700 & -1.30085600 & -0.90938100 \\
\hline $\bar{H}$ & -0.36387300 & -0.76463200 & -0.23967900 & $\mathrm{C}$ & 2.63129200 & -1.06719900 & -1.82519400 \\
\hline $\mathrm{H}$ & 3.32255800 & 0.18202300 & -3.43977400 & $\mathrm{H}$ & 1.73133200 & -2.05572200 & -0.14785700 \\
\hline $\bar{H}$ & 3.54118500 & -1.65475900 & -1.77018700 & $\mathrm{~N}$ & -1.88085900 & 1.36933400 & -1.13482200 \\
\hline $\bar{H}$ & -1.67503000 & 1.06268300 & -0.17579200 & $C$ & -1.94178300 & 0.19032600 & -3.54037300 \\
\hline $\mathrm{H}$ & -1.23621100 & 0.21137800 & -4.36681100 & $\mathrm{H}$ & -2.73225700 & 0.93174700 & -3.60548200 \\
\hline $\mathrm{C}$ & -2.23367900 & -1.05978500 & -2.97679900 & C & -3.26037400 & -1.18899600 & -1.95351200 \\
\hline $\bar{H}$ & -3.20690900 & -0.31973200 & -1.27620700 & $\bar{H}$ & -3.15599600 & -2.10857100 & -1.37221500 \\
\hline C & -1.37565100 & -2.24168100 & -3.26633500 & $\mathrm{H}$ & -1.95856500 & -3.16781600 & -3.26277900 \\
\hline $\bar{H}$ & -0.64679500 & -2.33352100 & -2.44459500 & $\mathrm{H}$ & -0.81673300 & -2.14477400 & -4.19947000 \\
\hline $\mathrm{C}$ & -6.10550400 & -0.90448800 & -0.99319600 & $\bar{H}$ & -5.95259600 & -1.74850000 & -0.31372300 \\
\hline $\mathrm{H}$ & -7.17690400 & -0.83217100 & -1.21317900 & $\mathrm{H}$ & -5.80555500 & 0.00843600 & -0.46501700 \\
\hline $\mathrm{C}$ & -5.49409300 & -2.69758600 & -3.48952900 & $\mathrm{H}$ & -5.30844200 & -3.57611800 & -2.86513800 \\
\hline $\mathrm{H}$ & -4.88093100 & -2.79055800 & -4.39388800 & $\mathrm{H}$ & -6.54535200 & -2.71476800 & -3.80053200 \\
\hline C & -5.34911300 & 0.37035300 & -3.70818300 & $\mathrm{H}$ & -4.85163400 & 0.22814800 & -4.67370600 \\
\hline $\mathrm{H}$ & -4.98549400 & 1.30768400 & -3.27711100 & $\mathrm{H}$ & -6.42111000 & 0.49164300 & -3.90738200 \\
\hline Si & -5.09185100 & -1.10540600 & -2.56856700 & & & & \\
\hline
\end{tabular}

\section{TS- $R-4 f-3$}

Imaginary frequency: $-359.35 \mathrm{~cm}^{-1}$

E[B3LYP/6-31G(d)-D3(BJ)]: -4789.090459 Hartree

$\mathrm{E}\left[\mathrm{PCM}\left(\mathrm{Et}_{2} \mathrm{O}\right)-\mathrm{B} 3 \mathrm{LYP} / 6-311 \mathrm{G}(\mathrm{d}, \mathrm{p})-\mathrm{D} 3(\mathrm{BJ})\right]$ : -4790.229891 Hartree

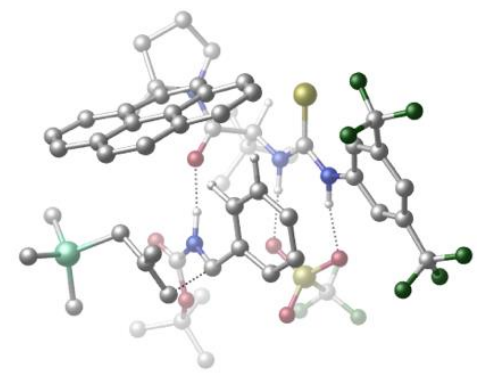




\begin{tabular}{|c|c|c|c|c|c|c|c|}
\hline 01 & & & & $\mathrm{~N}$ & 2.66667100 & 0.65330800 & 1.26017400 \\
\hline $\mathrm{H}$ & 2.47746100 & 1.58979600 & 0.88551300 & C & 3.76267500 & 0.04490700 & 0.63277500 \\
\hline $\mathrm{C}$ & 4.51959600 & 0.86483100 & -0.22089200 & $\mathrm{C}$ & 4.08808300 & -1.31535100 & 0.72464000 \\
\hline $\mathrm{C}$ & 5.55691000 & 0.32701500 & -0.97460900 & $\mathrm{H}$ & 4.26234100 & 1.91135500 & -0.32498200 \\
\hline C & 5.13396200 & -1.82828600 & -0.03721600 & $\mathrm{H}$ & 3.51384800 & -1.96334100 & 1.36570000 \\
\hline $\mathrm{C}$ & 5.88351500 & -1.02513500 & -0.89395400 & $\mathrm{H}$ & 6.69332400 & -1.43881400 & -1.48256800 \\
\hline C & 6.26090400 & 1.19699900 & -1.97681600 & C & 5.37782000 & -3.30782400 & -0.04921800 \\
\hline $\mathrm{F}$ & 6.29685100 & 2.48950500 & -1.60239500 & $\mathrm{~F}$ & 5.63584200 & 1.15412400 & -3.18558900 \\
\hline$F$ & 7.53348500 & 0.79420400 & -2.19042400 & $F$ & 4.82974900 & -3.94173500 & 1.01203500 \\
\hline$F$ & 4.83100000 & -3.87825000 & -1.16295600 & $F$ & 6.69139300 & -3.61443100 & -0.07348700 \\
\hline C & -0.28914200 & 0.72326000 & 3.39858200 & $\mathrm{H}$ & 0.22277700 & 0.22763700 & 4.22319100 \\
\hline $\mathrm{C}$ & -0.93746400 & 2.02188000 & 3.98307200 & $\mathrm{C}$ & -1.90231100 & 2.71233200 & 3.01110700 \\
\hline $\mathrm{H}$ & -2.73628300 & 2.06790900 & 2.72685500 & $\mathrm{H}$ & -1.39757600 & 3.03244200 & 2.10031100 \\
\hline $\mathrm{H}$ & -2.31527700 & 3.60851000 & 3.48984800 & C & 0.19367500 & 2.99402400 & 4.36602800 \\
\hline $\mathrm{H}$ & -0.22230300 & 3.83279900 & 4.93589400 & $\mathrm{H}$ & 0.68786000 & 3.40684700 & 3.48262800 \\
\hline $\mathrm{H}$ & 0.95166700 & 2.50324200 & 4.98772700 & $\mathrm{C}$ & -1.71667600 & 1.63209500 & 5.25182000 \\
\hline $\mathrm{H}$ & -2.16581300 & 2.52528600 & 5.69880000 & $\mathrm{H}$ & -1.06352300 & 1.17397200 & 6.00386700 \\
\hline $\mathrm{H}$ & -2.53126200 & 0.93500900 & 5.02462300 & C & -1.30386000 & -0.25456300 & 2.78430000 \\
\hline $\mathrm{O}$ & -1.73238300 & -0.09822600 & 1.62827500 & C & -1.28172200 & -1.75810800 & 4.83761200 \\
\hline C & -2.69032400 & -2.24099200 & 2.88468600 & $\mathrm{C}$ & -1.88379700 & -3.16637200 & 4.96050000 \\
\hline $\mathrm{H}$ & -0.18929600 & -1.75600000 & 4.88240900 & $\mathrm{H}$ & -1.67105600 & -1.09508300 & 5.61595400 \\
\hline C & -3.14191000 & -3.10233300 & 4.08147200 & $\mathrm{H}$ & -3.50696700 & -1.64907800 & 2.46849800 \\
\hline $\mathrm{H}$ & -2.09968600 & -3.43030700 & 5.99906200 & $\mathrm{H}$ & -1.18717900 & -3.91004000 & 4.56173600 \\
\hline $\mathrm{H}$ & -3.95432900 & -2.59350300 & 4.61377800 & $\mathrm{H}$ & -3.49974000 & -4.08606300 & 3.76782700 \\
\hline $\mathrm{N}$ & -1.73619100 & -1.30790500 & 3.50912900 & $\mathrm{C}$ & -2.04802200 & -3.05450400 & 1.77030800 \\
\hline C & -0.71408500 & -3.33797000 & 1.80106900 & $\mathrm{C}$ & -2.86043100 & -3.53751100 & 0.67268000 \\
\hline C & -0.05995500 & -4.08088700 & 0.76658200 & $\mathrm{H}$ & -0.08921400 & -2.94045700 & 2.59287900 \\
\hline C & -2.21493800 & -4.20484100 & -0.41823600 & C & -4.25668300 & -3.37861400 & 0.62108800 \\
\hline C & 1.30847400 & -4.39276100 & 0.82525200 & $\mathrm{C}$ & -0.81464200 & -4.46706200 & -0.37686200 \\
\hline C & -2.97605100 & -4.63547700 & -1.54871400 & $\mathrm{H}$ & -4.77945600 & -2.90442700 & 1.44370100 \\
\hline $\mathrm{C}$ & -4.99593700 & -3.83754300 & -0.46896400 & C & 1.93143900 & -5.04898800 & -0.23532400 \\
\hline $\mathrm{H}$ & 1.88440300 & -4.08253600 & 1.69176200 & C & -0.16671800 & -5.12463500 & -1.46471200 \\
\hline C & -4.36643200 & -4.44545800 & -1.55103500 & C & -2.29370200 & -5.26785200 & -2.64442800 \\
\hline $\mathrm{H}$ & -6.07512000 & -3.71399800 & -0.47292300 & C & 1.20686600 & -5.39783000 & -1.37370200 \\
\hline $\mathrm{H}$ & 2.99187100 & -5.26231800 & -0.18434700 & C & -0.95207900 & -5.49502600 & -2.60801700 \\
\hline $\mathrm{H}$ & -4.94788000 & -4.79668700 & -2.39942300 & $\mathrm{H}$ & -2.88177000 & -5.57557800 & -3.50540700 \\
\hline $\mathrm{H}$ & 1.70309800 & -5.89415300 & -2.20339100 & $\mathrm{H}$ & -0.45272300 & -5.98176200 & -3.44165300 \\
\hline $\mathrm{C}$ & 1.80403200 & 0.19442300 & 2.21726200 & $\mathrm{~S}$ & 2.04085800 & -1.20918600 & 3.14917500 \\
\hline $\mathrm{N}$ & 0.73211400 & 1.00271400 & 2.40242500 & $\mathrm{H}$ & 0.56171400 & 1.77069600 & 1.74564500 \\
\hline $\mathrm{S}$ & 1.23600100 & 3.84206800 & -0.25085500 & $\mathrm{O}$ & 0.81424200 & 4.07765300 & -1.64500800 \\
\hline $\mathrm{O}$ & 0.19215700 & 3.24328800 & 0.62888100 & $\mathrm{O}$ & 2.56632000 & 3.20933700 & -0.06828900 \\
\hline C & 1.46296800 & 5.54000300 & 0.46344900 & $\mathrm{~F}$ & 2.47294000 & 6.17574200 & -0.13578200 \\
\hline $\mathrm{F}$ & 1.72107800 & 5.45637300 & 1.77658900 & $\mathrm{~F}$ & 0.34374500 & 6.26985000 & 0.29889300 \\
\hline $\mathrm{C}$ & -2.75760500 & 2.54699000 & -0.41936600 & 0 & -3.64469900 & 2.47176300 & 0.41144900 \\
\hline $\mathrm{O}$ & -2.56934300 & 3.53243600 & -1.30288400 & C & -3.15152800 & 4.88019000 & -1.08957900 \\
\hline $\mathrm{C}$ & -4.64484900 & 4.83503100 & -1.40348400 & $\mathrm{H}$ & -5.07058500 & 5.83856100 & -1.29674400 \\
\hline $\mathrm{H}$ & -5.16360600 & 4.16176600 & -0.71766600 & $\mathrm{H}$ & -4.81440100 & 4.50423400 & -2.43343900 \\
\hline C & -2.37808100 & 5.72243100 & -2.10088000 & $\mathrm{H}$ & -1.30809500 & 5.69105100 & -1.88085400 \\
\hline $\mathrm{H}$ & -2.72439800 & 6.76017200 & -2.06000400 & $\mathrm{H}$ & -2.53668000 & 5.34471900 & -3.11655800 \\
\hline $\mathrm{C}$ & -2.87365400 & 5.34895300 & 0.33845100 & $\mathrm{H}$ & -3.13762600 & 6.40903600 & 0.41646700 \\
\hline $\mathrm{H}$ & -1.81347900 & 5.24096200 & 0.57364600 & $\mathrm{H}$ & -3.46021700 & 4.78710100 & 1.06599700 \\
\hline $\mathrm{C}$ & -0.98369400 & 1.46493500 & -1.69164500 & $\mathrm{H}$ & -0.76430800 & 2.44709100 & -2.10172400 \\
\hline C & 0.19176900 & 0.56452200 & -1.67367100 & $\mathrm{C}$ & 1.27186000 & 0.91127500 & -2.50150300 \\
\hline
\end{tabular}




\begin{tabular}{|c|c|c|c|c|c|c|c|}
\hline $\mathrm{C}$ & 0.22456500 & -0.64877300 & -0.96894400 & C & 2.34519900 & 0.04038900 & -2.66728000 \\
\hline $\mathrm{H}$ & 1.27109300 & 1.87901700 & -2.99260900 & C & 1.30266600 & -1.51461700 & -1.14260800 \\
\hline $\mathrm{H}$ & -0.57822100 & -0.92347100 & -0.29153600 & C & 2.34743300 & -1.18800400 & -2.00692100 \\
\hline $\mathrm{H}$ & 3.19081300 & 0.33292600 & -3.28070100 & $\mathrm{H}$ & 1.33182100 & -2.44456700 & -0.59500900 \\
\hline $\mathrm{H}$ & 3.17771800 & -1.87612600 & -2.12454400 & $\mathrm{~N}$ & -1.82188900 & 1.53002400 & -0.59641500 \\
\hline $\mathrm{H}$ & -1.71443500 & 0.90783200 & 0.21817600 & C & -2.04557400 & 0.95627200 & -3.16598100 \\
\hline $\mathrm{H}$ & -1.38486300 & 1.10241900 & -4.01719300 & $\mathrm{H}$ & -2.77362500 & 1.75117300 & -3.03836100 \\
\hline C & -2.46112900 & -0.36486200 & -2.90386100 & C & -3.44339500 & -0.64066600 & -1.87376300 \\
\hline $\mathrm{H}$ & -3.29009100 & 0.04599300 & -1.02544400 & $\mathrm{H}$ & -3.40901300 & -1.67662500 & -1.52643000 \\
\hline $\mathrm{C}$ & -1.75558600 & -1.51598400 & -3.53183500 & $\mathrm{H}$ & -2.42711600 & -2.36925500 & -3.66671200 \\
\hline $\mathrm{H}$ & -0.97262700 & -1.84643500 & -2.82999300 & $\mathrm{H}$ & -1.27007600 & -1.25626300 & -4.47507700 \\
\hline $\mathrm{C}$ & -6.17297600 & -0.23993300 & -0.64821000 & $\mathrm{H}$ & -6.04405200 & -1.18892100 & -0.11952000 \\
\hline $\mathrm{H}$ & -7.24717000 & -0.05574800 & -0.76618600 & $\mathrm{H}$ & -5.75450500 & 0.55670300 & -0.02145500 \\
\hline C & -5.91868900 & -1.59187500 & -3.47968800 & $\mathrm{H}$ & -5.79617700 & -2.58815500 & -3.04527800 \\
\hline $\mathrm{H}$ & -5.37306400 & -1.56820600 & -4.43059600 & $\mathrm{H}$ & -6.98186800 & -1.44652500 & -3.70491100 \\
\hline $\mathrm{C}$ & -5.43788800 & 1.43365000 & -3.12424500 & $\mathrm{H}$ & -4.94355900 & 1.47420300 & -4.10061100 \\
\hline $\mathrm{H}$ & -5.02048600 & 2.22215100 & -2.49138700 & $\mathrm{H}$ & -6.49748200 & 1.67044600 & -3.28076300 \\
\hline $\mathrm{Si}$ & -5.29361600 & -0.25605500 & -2.30911200 & & & & \\
\hline
\end{tabular}

\section{TS- $R-4 f-4$}

Imaginary frequency: $-400.99 \mathrm{~cm}^{-1}$

E[B3LYP/6-31G(d)-D3(BJ)]: -4789.086070 Hartree

$\mathrm{E}\left[\mathrm{PCM}\left(\mathrm{Et}_{2} \mathrm{O}\right)-\mathrm{B} 3 \mathrm{LYP} / 6-311 \mathrm{G}(\mathrm{d}, \mathrm{p})-\mathrm{D} 3(\mathrm{BJ})\right]$ : -4790.229413 Hartree

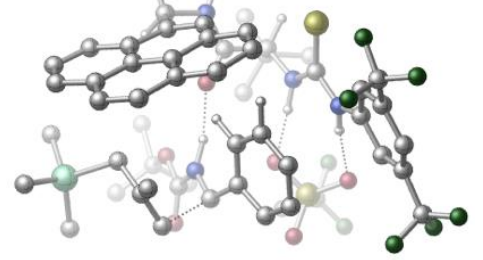

\begin{tabular}{|c|c|c|c|c|c|c|c|}
\hline 01 & & & & $\mathrm{~N}$ & 2.76520900 & 1.20303600 & 1.25682900 \\
\hline $\mathrm{H}$ & 2.51847400 & 2.08117200 & 0.78661300 & C & 3.89769000 & 0.60021300 & 0.69807700 \\
\hline C & 4.55372200 & 1.33398700 & -0.30588800 & C & 4.35988800 & -0.68584400 & 1.00931600 \\
\hline C & 5.63253800 & 0.78049900 & -0.98573900 & $\mathrm{H}$ & 4.18879000 & 2.31962100 & -0.57219800 \\
\hline C & 5.43558300 & -1.22031100 & 0.30607300 & $\mathrm{H}$ & 3.86851100 & -1.26146100 & 1.77654600 \\
\hline $\mathrm{C}$ & 6.09097400 & -0.50315600 & -0.69235000 & $\mathrm{H}$ & 6.92963600 & -0.93061100 & -1.22890900 \\
\hline $\mathrm{C}$ & 6.24911100 & 1.53141700 & -2.13217000 & C & 5.82463600 & -2.65168400 & 0.52926100 \\
\hline $\mathrm{F}$ & 6.09361500 & 2.86282300 & -2.02110700 & $\mathrm{~F}$ & 5.68821000 & 1.16408300 & -3.31708300 \\
\hline $\mathrm{F}$ & 7.57271100 & 1.27800500 & -2.24408900 & $\mathrm{~F}$ & 5.29367400 & -3.17399800 & 1.65743200 \\
\hline $\mathrm{F}$ & 5.38684600 & -3.43434600 & -0.50022200 & $\mathrm{~F}$ & 7.16174000 & -2.82034900 & 0.59079600 \\
\hline $\mathrm{C}$ & -0.29596500 & 1.30988900 & 3.22308800 & $\mathrm{H}$ & 0.18941500 & 0.92152500 & 4.11765100 \\
\hline $\mathrm{C}$ & -1.01422400 & 2.64130800 & 3.61554200 & $\mathrm{C}$ & -1.83804000 & 3.23432200 & 2.46513700 \\
\hline $\mathrm{H}$ & -2.58509900 & 2.52626600 & 2.10368400 & $\mathrm{H}$ & -1.21707900 & 3.51721100 & 1.61466600 \\
\hline $\mathrm{H}$ & -2.35388000 & 4.13504800 & 2.82022200 & C & 0.05965900 & 3.65177500 & 4.05494100 \\
\hline $\mathrm{H}$ & -0.42047200 & 4.55348300 & 4.45212600 & $\mathrm{H}$ & 0.69275200 & 3.95072500 & 3.21620700 \\
\hline $\mathrm{H}$ & 0.70346700 & 3.23489000 & 4.83833000 & $\mathrm{C}$ & -1.95649200 & 2.36346500 & 4.80061800 \\
\hline $\mathrm{H}$ & -2.48666000 & 3.28185000 & 5.07450200 & $\mathrm{H}$ & -1.40499500 & 2.02858400 & 5.68702600 \\
\hline $\mathrm{H}$ & -2.71256700 & 1.60882100 & 4.55345700 & $\mathrm{C}$ & -1.23705400 & 0.23124400 & 2.65550100 \\
\hline $\mathrm{O}$ & -1.61636200 & 0.26332100 & 1.47322600 & $\mathrm{C}$ & -1.33967200 & -1.01491900 & 4.87526500 \\
\hline $\mathrm{C}$ & -2.49179000 & -1.84441100 & 2.90625000 & $\mathrm{C}$ & -2.61807000 & -1.70521300 & 5.35632500 \\
\hline $\mathrm{H}$ & -0.46001700 & -1.66299900 & 4.97453900 & $\mathrm{H}$ & -1.13737000 & -0.08491900 & 5.39916700 \\
\hline $\mathrm{H}$ & -3.30385100 & -1.35384100 & 2.36378900 & $\mathrm{H}$ & -3.39002300 & -0.94919000 & 5.54058400 \\
\hline $\mathrm{H}$ & -2.46780900 & -2.27043700 & 6.28024800 & $\mathrm{~N}$ & -1.63477200 & -0.78040300 & 3.45839800 \\
\hline C & -1.73444100 & -2.75836900 & 1.95718900 & C & -0.38264300 & -2.89911400 & 2.07201100 \\
\hline $\mathrm{C}$ & -2.45888500 & -3.52590200 & 0.96785400 & $\mathrm{C}$ & 0.37435500 & -3.76194300 & 1.21640300 \\
\hline $\mathrm{H}$ & 0.16922300 & -2.30989500 & 2.79720100 & $\mathrm{C}$ & -1.71613400 & -4.33390800 & 0.04751300 \\
\hline $\mathrm{C}$ & -3.86158400 & -3.51981500 & 0.86734500 & C & 1.76266700 & -3.92223500 & 1.36085900 \\
\hline
\end{tabular}




\begin{tabular}{|c|c|c|c|c|c|c|c|}
\hline $\mathrm{C}$ & -0.29947900 & -4.43931600 & 0.16125900 & C & -2.39521700 & -5.06414800 & -0.97609400 \\
\hline $\mathrm{H}$ & -4.45165500 & -2.93723400 & 1.56573000 & $\mathrm{C}$ & -4.51723600 & -4.26756900 & -0.11032200 \\
\hline $\mathrm{C}$ & 2.48317000 & -4.70292100 & 0.45843700 & $\mathrm{H}$ & 2.27388600 & -3.39525800 & 2.16097000 \\
\hline $\mathrm{C}$ & 0.44534700 & -5.23363300 & -0.76072400 & $\mathrm{C}$ & -3.79674100 & -5.02085200 & -1.03284500 \\
\hline C & -1.61891200 & -5.83545400 & -1.90813300 & $\mathrm{H}$ & -5.60262500 & -4.25835100 & -0.15324000 \\
\hline C & 1.83538500 & -5.34019500 & -0.59858700 & $\mathrm{H}$ & 3.55748300 & -4.79167600 & 0.56628300 \\
\hline C & -0.26291500 & -5.90869700 & -1.81145300 & $\mathrm{H}$ & -4.31411200 & -5.59788800 & -1.79471300 \\
\hline $\mathrm{H}$ & -2.14541100 & -6.37266800 & -2.69290300 & $\mathrm{H}$ & 2.40541700 & -5.93730000 & -1.30535300 \\
\hline $\mathrm{H}$ & 0.30837500 & -6.50141700 & -2.52104500 & C & 1.89830600 & 0.78562000 & 2.23142100 \\
\hline $\mathrm{S}$ & 2.18694100 & -0.46845300 & 3.34180400 & $\mathrm{~N}$ & 0.76522500 & 1.52890000 & 2.26039700 \\
\hline $\mathrm{H}$ & 0.53248100 & 2.08491400 & 1.42832900 & S & 1.03956600 & 3.79428700 & -0.81251900 \\
\hline 0 & 0.87381900 & 3.79072100 & -2.27367700 & $\mathrm{O}$ & 0.07428600 & 2.92651800 & -0.07525700 \\
\hline 0 & 2.42545000 & 3.66090300 & -0.29225000 & C & 0.51406100 & 5.48885000 & -0.26681500 \\
\hline $\mathrm{F}$ & 1.27022700 & 6.43841900 & -0.82232200 & $\mathrm{~F}$ & 0.60976700 & 5.58936000 & 1.07175700 \\
\hline $\mathrm{F}$ & -0.76875300 & 5.70273300 & -0.60687900 & C & -2.86156700 & 2.27134900 & -1.35796600 \\
\hline 0 & -3.01114700 & 2.84862500 & -2.42250000 & $\mathrm{O}$ & -3.64379500 & 2.36784500 & -0.27676900 \\
\hline C & -4.66652200 & 3.43921900 & -0.19912300 & C & -3.96356900 & 4.79544400 & -0.24141500 \\
\hline $\mathrm{H}$ & -4.69842000 & 5.59020400 & -0.07408500 & $\mathrm{H}$ & -3.48534500 & 4.96145900 & -1.20813700 \\
\hline $\mathrm{H}$ & -3.20003100 & 4.85820800 & 0.53796400 & C & -5.32070700 & 3.18501400 & 1.15756900 \\
\hline $\mathrm{H}$ & -5.75074600 & 2.17847000 & 1.19385500 & $\mathrm{H}$ & -6.12493000 & 3.90994600 & 1.31722000 \\
\hline $\mathrm{H}$ & -4.59748200 & 3.28456700 & 1.96887300 & C & -5.70035900 & 3.28230500 & -1.31649300 \\
\hline $\mathrm{H}$ & -6.52621600 & 3.97835400 & -1.13445300 & $\mathrm{H}$ & -6.11124400 & 2.26716300 & -1.32014400 \\
\hline $\mathrm{H}$ & -5.26968700 & 3.49665100 & -2.29370200 & C & -0.96128000 & 1.11135800 & -2.17726200 \\
\hline $\mathrm{H}$ & -0.85694100 & 1.98492100 & -2.81579900 & C & 0.29893500 & 0.38798100 & -1.93641300 \\
\hline C & 1.35972500 & 0.65179800 & -2.81856100 & C & 0.44845700 & -0.59861200 & -0.95050300 \\
\hline C & 2.53753400 & -0.08482300 & -2.74915000 & $\mathrm{H}$ & 1.26162100 & 1.45973600 & -3.53594200 \\
\hline C & 1.63331800 & -1.32852900 & -0.88732600 & $\mathrm{H}$ & -0.34334600 & -0.79635300 & -0.23464000 \\
\hline C & 2.66473600 & -1.09544900 & -1.79610200 & $\mathrm{H}$ & 3.36470800 & 0.14948600 & -3.41036300 \\
\hline $\mathrm{H}$ & 1.75521800 & -2.07947000 & -0.12163000 & $\mathrm{H}$ & 3.57603500 & -1.67984000 & -1.73134900 \\
\hline $\mathrm{N}$ & -1.86511200 & 1.32696800 & -1.15493200 & $\mathrm{H}$ & -1.67030100 & 1.02323300 & -0.19274100 \\
\hline C & -1.89050800 & 0.14803300 & -3.56244500 & $\mathrm{H}$ & -1.17282400 & 0.17112700 & -4.37827800 \\
\hline $\mathrm{H}$ & -2.68184800 & 0.88733700 & -3.63878500 & C & -2.18592900 & -1.10225900 & -3.00169900 \\
\hline C & -3.22739800 & -1.23410400 & -1.99346600 & $\mathrm{H}$ & -3.18555900 & -0.36433000 & -1.31608000 \\
\hline $\mathrm{H}$ & -3.12840200 & -2.15277500 & -1.41006200 & C & -1.31822500 & -2.28048600 & -3.27622200 \\
\hline $\mathrm{H}$ & -1.89681500 & -3.20924300 & -3.28161600 & $\mathrm{H}$ & -0.60280600 & -2.36873700 & -2.44233100 \\
\hline $\mathrm{H}$ & -0.74420100 & -2.18161100 & -4.19991800 & C & -6.08650300 & -0.94144200 & -1.07479700 \\
\hline $\mathrm{H}$ & -5.94589000 & -1.78114600 & -0.38751900 & $\mathrm{H}$ & -7.15429200 & -0.86765300 & -1.31127900 \\
\hline $\mathrm{H}$ & -5.79181300 & -0.02597000 & -0.54817600 & C & -5.44040800 & -2.75548000 & -3.54636800 \\
\hline $\mathrm{H}$ & -5.26873000 & -3.62812700 & -2.90982500 & $\mathrm{H}$ & -4.81124800 & -2.85944900 & -4.43845100 \\
\hline $\mathrm{H}$ & -6.48593100 & -2.77294600 & -3.87609400 & $\mathrm{C}$ & -5.29238000 & 0.31083700 & -3.78881100 \\
\hline $\mathrm{H}$ & -4.77808300 & 0.16314200 & -4.74463800 & $\mathrm{H}$ & -4.93879900 & 1.25224100 & -3.35822400 \\
\hline $\mathrm{H}$ & -6.36118400 & 0.42755100 & -4.00696000 & $\mathrm{Si}$ & -5.05021200 & -1.15548800 & -2.63375200 \\
\hline C & -3.00819700 & -2.59834300 & 4.16617000 & $\mathrm{H}$ & -2.50507200 & -3.56695300 & 4.23776300 \\
\hline $\mathrm{H}$ & -4.08350100 & -2.78895100 & 4.11331400 & & & & \\
\hline
\end{tabular}

\section{TS- $R-4 f-5$}

Imaginary frequency: $-388.74 \mathrm{~cm}^{-1}$

E[B3LYP/6-31G(d)-D3(BJ)]: -4789.087113 Hartree

$\mathrm{E}\left[\mathrm{PCM}\left(\mathrm{Et}_{2} \mathrm{O}\right)-\mathrm{B} 3 \mathrm{LYP} / 6-311 \mathrm{G}(\mathrm{d}, \mathrm{p})-\mathrm{D} 3(\mathrm{BJ})\right]$ : -4790.229048 Hartree

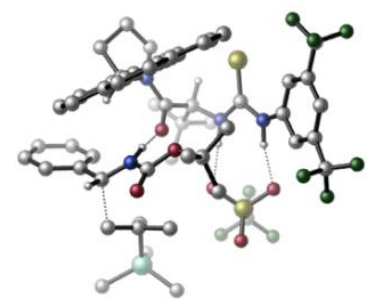




\begin{tabular}{|c|c|c|c|c|c|c|c|}
\hline 01 & & & & & & & \\
\hline $\mathrm{N}$ & -1.54699700 & -2.36500600 & 1.54581900 & $\mathrm{H}$ & -0.86785500 & -2.96233700 & 1.06105900 \\
\hline C & -2.82242500 & -2.42504200 & 0.96095200 & C & -2.90769200 & -3.11988400 & -0.26016300 \\
\hline C & -3.97446100 & -1.81581000 & 1.47486600 & C & -4.10430600 & -3.14786400 & -0.96537400 \\
\hline $\mathrm{H}$ & -2.02768800 & -3.61126700 & -0.65970300 & C & -5.16030800 & -1.85209700 & 0.74314900 \\
\hline $\mathrm{H}$ & -3.93333100 & -1.30296100 & 2.42197000 & C & -5.24531300 & -2.50671100 & -0.48142300 \\
\hline $\mathrm{H}$ & -6.16940500 & -2.51634900 & -1.04683200 & C & -4.14738600 & -3.76208200 & -2.33529500 \\
\hline C & -6.39105500 & -1.20563300 & 1.31411500 & $\mathrm{~F}$ & -3.15517200 & -4.64543000 & -2.54131600 \\
\hline $\mathrm{F}$ & -4.03319200 & -2.80431900 & -3.30045000 & $\mathrm{~F}$ & -5.31969800 & -4.39240800 & -2.56788800 \\
\hline $\mathrm{F}$ & -6.09226900 & -0.24985700 & 2.22646400 & $\mathrm{~F}$ & -7.13537300 & -0.61122700 & 0.34826200 \\
\hline $\mathrm{F}$ & -7.19641400 & -2.09825900 & 1.92983100 & C & 1.08222600 & -0.51198200 & 3.23238400 \\
\hline $\mathrm{H}$ & 0.52285500 & -0.32185700 & 4.14700600 & C & 2.50032800 & -1.00664900 & 3.66156500 \\
\hline $\mathrm{C}$ & 3.44973000 & -1.26617300 & 2.48134500 & $\mathrm{H}$ & 3.66037100 & -0.34968000 & 1.92378700 \\
\hline $\mathrm{H}$ & 3.04536100 & -1.99619000 & 1.78114200 & $\mathrm{H}$ & 4.40234900 & -1.65262900 & 2.86294400 \\
\hline $\mathrm{C}$ & 2.31518600 & -2.30763300 & 4.46176800 & $\mathrm{H}$ & 3.27909300 & -2.64172600 & 4.86262200 \\
\hline $\mathrm{H}$ & 1.91184500 & -3.10321700 & 3.83226600 & $\mathrm{H}$ & 1.62881700 & -2.15928300 & 5.30342400 \\
\hline $\mathrm{C}$ & 3.13586800 & 0.05794500 & 4.57277400 & $\mathrm{H}$ & 4.13222400 & -0.26952000 & 4.88894500 \\
\hline $\mathrm{H}$ & 2.53911000 & 0.22219100 & 5.47686900 & $\mathrm{H}$ & 3.25006400 & 1.01849700 & 4.05565200 \\
\hline $\mathrm{C}$ & 1.09071700 & 0.75396800 & 2.35991800 & $\mathrm{O}$ & 1.31195800 & 0.63634100 & 1.14132800 \\
\hline $\mathrm{N}$ & 0.84841900 & 1.95279500 & 2.92394200 & $\mathrm{C}$ & -0.39953300 & 3.27931800 & 1.21573400 \\
\hline C & -1.54397800 & 2.60515400 & 1.52338000 & C & -0.35939400 & 4.15496600 & 0.06256300 \\
\hline $\mathrm{C}$ & -2.74875200 & 2.75393200 & 0.76324700 & $\mathrm{H}$ & -1.56333800 & 1.90892100 & 2.35525700 \\
\hline $\mathrm{C}$ & -1.53523300 & 4.28240600 & -0.74224900 & C & 0.78980700 & 4.86963000 & -0.31714100 \\
\hline C & -3.93124400 & 2.07973000 & 1.10721000 & C & -2.73432200 & 3.60267000 & -0.37997400 \\
\hline C & -1.51561600 & 5.10030500 & -1.91336400 & $\mathrm{H}$ & 1.69131300 & 4.81314800 & 0.28069100 \\
\hline C & 0.80406300 & 5.65033400 & -1.47167500 & C & -5.08591100 & 2.24364600 & 0.34361200 \\
\hline $\mathrm{H}$ & -3.93014700 & 1.41072600 & 1.96182400 & C & -3.91768100 & 3.75686100 & -1.16198500 \\
\hline C & -0.33098000 & 5.76309200 & -2.26799100 & C & -2.71810300 & 5.22798000 & -2.68949200 \\
\hline $\mathrm{H}$ & 1.71257500 & 6.18047100 & -1.74463500 & $\mathrm{C}$ & -5.08069500 & 3.07148600 & -0.77760000 \\
\hline $\mathrm{H}$ & -5.98720600 & 1.70493000 & 0.61183900 & C & -3.86719200 & 4.59534300 & -2.32646400 \\
\hline $\mathrm{H}$ & -0.31495700 & 6.37545600 & -3.16558500 & $\mathrm{H}$ & -2.68994900 & 5.85193900 & -3.57891300 \\
\hline $\mathrm{H}$ & -5.98157000 & 3.18523900 & -1.37499800 & $\mathrm{H}$ & -4.76949000 & 4.70603400 & -2.92222400 \\
\hline C & -1.02795500 & -1.51414300 & 2.48326700 & $\mathrm{~S}$ & -1.93555100 & -0.57105300 & 3.56282100 \\
\hline $\mathrm{N}$ & 0.33721700 & -1.52084000 & 2.49288900 & $\mathrm{H}$ & 0.79314300 & -1.82441300 & 1.62525600 \\
\hline $\mathrm{S}$ & 1.35646400 & -3.75713200 & -0.43458000 & $\mathrm{O}$ & 1.71926800 & -4.03086200 & -1.83406300 \\
\hline 0 & 1.64338600 & -2.36524200 & 0.02776500 & $\mathrm{O}$ & 0.03046000 & -4.24655100 & 0.01890600 \\
\hline $\mathrm{C}$ & 2.55120700 & -4.77879000 & 0.55387100 & $\mathrm{~F}$ & 2.43524200 & -6.07504300 & 0.26894500 \\
\hline $\mathrm{F}$ & 2.34717700 & -4.60390100 & 1.86993800 & $\mathrm{~F}$ & 3.81797000 & -4.39449600 & 0.28031400 \\
\hline C & 0.45982200 & 1.54531600 & -2.43676500 & $\mathrm{O}$ & 0.67318100 & 1.75231500 & -3.61951300 \\
\hline $\mathrm{O}$ & -0.63614300 & 1.06779400 & -1.86415500 & $\mathrm{C}$ & -1.71685800 & 0.43917100 & -2.67896900 \\
\hline $\mathrm{C}$ & -1.16174900 & -0.83763400 & -3.30669200 & $\mathrm{H}$ & -1.97722500 & -1.38965400 & -3.78185900 \\
\hline $\mathrm{H}$ & -0.40638600 & -0.61459900 & -4.06344500 & $\mathrm{H}$ & -0.72485300 & -1.48685700 & -2.54084000 \\
\hline $\mathrm{C}$ & -2.77297500 & 0.11849100 & -1.63190900 & $\mathrm{H}$ & -3.23360800 & 1.02764900 & -1.25155700 \\
\hline $\mathrm{H}$ & -3.54898200 & -0.50862500 & -2.07664200 & $\mathrm{H}$ & -2.32757400 & -0.42234300 & -0.79532700 \\
\hline C & -2.25715900 & 1.42257700 & -3.71435000 & $\mathrm{H}$ & -3.17290600 & 1.00288600 & -4.14533900 \\
\hline $\mathrm{H}$ & -2.51166200 & 2.37311800 & -3.23990100 & $\mathrm{H}$ & -1.53915500 & 1.60611700 & -4.51337400 \\
\hline$C$ & 2.64097400 & 2.30871100 & -1.86089700 & $\mathrm{H}$ & 2.51954900 & 2.92417800 & -2.74678500 \\
\hline C & 3.56777400 & 2.86136200 & -0.84770300 & C & 4.26551500 & 4.03732600 & -1.16350800 \\
\hline $\mathrm{C}$ & 3.78219000 & 2.25414200 & 0.39792700 & C & 5.13789100 & 4.61108100 & -0.24241200 \\
\hline $\mathrm{H}$ & 4.10672000 & 4.50966800 & -2.12922800 & $\mathrm{C}$ & 4.66109700 & 2.82767300 & 1.31598100 \\
\hline $\mathrm{H}$ & 3.23634200 & 1.35760500 & 0.65808800 & C & 5.33737200 & 4.00741300 & 1.00201200 \\
\hline $\mathrm{H}$ & 5.65907600 & 5.53011900 & -0.49292100 & $\mathrm{H}$ & 4.81247500 & 2.34831900 & 2.27904700 \\
\hline $\mathrm{H}$ & 6.01800900 & 4.45454700 & 1.72043800 & $\mathrm{~N}$ & 1.43773000 & 1.78329000 & -1.46359900 \\
\hline
\end{tabular}




\begin{tabular}{|c|c|c|c|c|c|c|c|}
\hline $\mathrm{H}$ & 1.27751600 & 1.44432600 & -0.50505700 & C & 3.79507000 & 1.03627200 & -2.87898200 \\
\hline $\mathrm{H}$ & 3.36975500 & 1.20023100 & -3.86489500 & $\mathrm{H}$ & 4.76287100 & 1.49577300 & -2.69948600 \\
\hline C & 3.52406900 & -0.20102800 & -2.27129500 & C & 4.29236800 & -0.70124700 & -1.14800600 \\
\hline $\mathrm{H}$ & 4.82885300 & 0.09375700 & -0.61911600 & $\mathrm{H}$ & 3.66585500 & -1.28916100 & -0.46850500 \\
\hline C & 2.32878000 & -0.96224900 & -2.69503600 & $\mathrm{H}$ & 2.49086800 & -2.04351400 & -2.68570600 \\
\hline $\mathrm{H}$ & 1.56385100 & -0.81272900 & -1.91649500 & $\mathrm{H}$ & 1.93496400 & -0.62732900 & -3.65389900 \\
\hline C & 6.49064400 & -2.48126300 & -0.08743500 & $\mathrm{H}$ & 5.76345100 & -3.02655700 & 0.52078600 \\
\hline $\mathrm{H}$ & 7.33849700 & -3.14942100 & -0.27975700 & $\mathrm{H}$ & 6.86032000 & -1.63199700 & 0.49879300 \\
\hline C & 5.03652100 & -3.31526300 & -2.73434400 & $\mathrm{H}$ & 4.19440100 & -3.81493600 & -2.24699700 \\
\hline $\mathrm{H}$ & 4.69506900 & -2.96455900 & -3.71490400 & $\mathrm{H}$ & 5.82084300 & -4.06235400 & -2.90765800 \\
\hline C & 6.94851400 & -0.87701100 & -2.70137400 & $\mathrm{H}$ & 6.49547200 & -0.48775800 & -3.62026000 \\
\hline $\mathrm{H}$ & 7.32541000 & -0.02497800 & -2.12315400 & $\mathrm{H}$ & 7.81229500 & -1.48627100 & -2.99277600 \\
\hline $\mathrm{Si}$ & 5.71957700 & -1.90432300 & -1.70485400 & C & 0.07237600 & 3.70918900 & 4.30679300 \\
\hline $\mathrm{H}$ & 0.26815600 & 4.19605200 & 5.26568800 & $\mathrm{H}$ & -0.99392900 & 3.81226400 & 4.08452400 \\
\hline C & 0.90215400 & 4.28669400 & 3.15238800 & $\mathrm{H}$ & 0.51656900 & 5.23503700 & 2.77178000 \\
\hline $\mathrm{H}$ & 1.94422700 & 4.43486600 & 3.46176800 & C & 0.46583000 & 2.22501600 & 4.32492300 \\
\hline $\mathrm{H}$ & 1.31983200 & 2.05503200 & 4.98687200 & $\mathrm{H}$ & -0.35464700 & 1.56825900 & 4.62552400 \\
\hline C & 0.83564200 & 3.16913000 & 2.09199000 & $\mathrm{H}$ & 1.72815200 & 3.15877700 & 1.46942600 \\
\hline
\end{tabular}

\section{TS- $R-4 f-6$}

Imaginary frequency: $-396.56 \mathrm{~cm}^{-1}$

E[B3LYP/6-31G(d)-D3(BJ)]: -4789.085110 Hartree

$\mathrm{E}\left[\mathrm{PCM}\left(\mathrm{Et}_{2} \mathrm{O}\right)-\mathrm{B} 3 \mathrm{LYP} / 6-311 \mathrm{G}(\mathrm{d}, \mathrm{p})-\mathrm{D} 3(\mathrm{BJ})\right]:-4790.228307$ Hartree

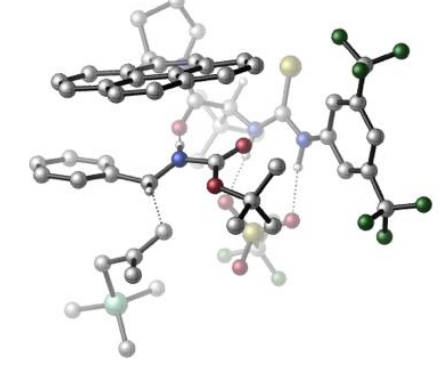

\begin{tabular}{|lrrr|rrrr|}
\hline O & & & & $\mathrm{N}$ & -2.47745700 & -1.37946000 & 1.71064000 \\
\hline $\mathrm{H}$ & -1.87059100 & -2.15290700 & 1.42042300 & $\mathrm{C}$ & -3.59129900 & -1.24285900 & 0.87421200 \\
\hline $\mathrm{C}$ & -3.84754500 & -2.31536100 & 0.00079700 & $\mathrm{C}$ & -4.41752600 & -0.11244800 & 0.81022700 \\
\hline $\mathrm{C}$ & -4.88329200 & -2.23629800 & -0.92364000 & $\mathrm{H}$ & -3.21017400 & -3.19075000 & 0.02764300 \\
\hline $\mathrm{C}$ & -5.44535500 & -0.05552700 & -0.12729000 & $\mathrm{H}$ & -4.24982100 & 0.71005900 & 1.48556800 \\
\hline $\mathrm{C}$ & -5.69752500 & -1.10743600 & -1.00370200 & $\mathrm{H}$ & -6.49729200 & -1.04875200 & -1.73270800 \\
\hline $\mathrm{C}$ & -5.08770700 & -3.33688100 & -1.92373100 & $\mathrm{C}$ & -6.32115300 & 1.16273700 & -0.19507600 \\
\hline $\mathrm{F}$ & -4.44858300 & -4.47280000 & -1.59044000 & $\mathrm{~F}$ & -4.62738400 & -2.97214500 & -3.15626100 \\
\hline $\mathrm{F}$ & -6.39669900 & -3.63188300 & -2.08744600 & $\mathrm{~F}$ & -5.80538000 & 2.21171000 & 0.48514400 \\
\hline $\mathrm{F}$ & -6.49324500 & 1.57404100 & -1.48013200 & $\mathrm{~F}$ & -7.55625200 & 0.93421000 & 0.30122400 \\
\hline $\mathrm{C}$ & 0.15711100 & -0.03457600 & 3.84297800 & $\mathrm{H}$ & -0.55419200 & 0.43058100 & 4.52280900 \\
\hline $\mathrm{C}$ & 1.11739200 & -0.90483800 & 4.71118200 & $\mathrm{C}$ & 2.26891900 & -1.54054200 & 3.91433100 \\
\hline $\mathrm{H}$ & 2.94739200 & -0.78115700 & 3.51617300 & $\mathrm{H}$ & 1.91195900 & -2.13305900 & 3.07215600 \\
\hline $\mathrm{H}$ & 2.84229600 & -2.19972500 & 4.57717000 & $\mathrm{C}$ & 0.27609800 & -2.00997100 & 5.37154000 \\
\hline $\mathrm{H}$ & 0.89003100 & -2.57191100 & 6.08476100 & $\mathrm{H}$ & -0.10724900 & -2.71016500 & 4.62629800 \\
\hline $\mathrm{H}$ & -0.57840500 & -1.58844100 & 5.91372100 & $\mathrm{C}$ & 1.70916700 & 0.00555200 & 5.80254500 \\
\hline $\mathrm{H}$ & 2.38641900 & -0.56736300 & 6.44530600 & $\mathrm{H}$ & 0.92406200 & 0.43062600 & 6.43909300 \\
\hline $\mathrm{H}$ & 2.28593500 & 0.83221400 & 5.36990500 & $\mathrm{C}$ & 0.88205700 & 1.03808300 & 3.02853700 \\
\hline $\mathrm{O}$ & 1.53996100 & 0.70185600 & 2.02690200 & $\mathrm{~N}$ & 0.78025700 & 2.33219000 & 3.37972400 \\
\hline $\mathrm{C}$ & 0.70508800 & 3.53107200 & 1.20117900 & $\mathrm{C}$ & -0.59853000 & 3.16015000 & 1.04487400 \\
\hline $\mathrm{C}$ & 1.42582400 & 4.12981100 & 0.09554000 & $\mathrm{C}$ & -1.30375900 & 3.34455400 & -0.18868200 \\
\hline $\mathrm{H}$ & -1.13559700 & 2.67906300 & 1.85685700 & $\mathrm{C}$ & 0.76329500 & 4.26173700 & -1.16559200 \\
\hline $\mathrm{C}$ & 2.75137900 & 4.58547800 & 0.20107500 & $\mathrm{C}$ & -2.66016300 & 3.01221700 & -0.32247200 \\
\hline $\mathrm{C}$ & -0.60112100 & 3.87481800 & -1.30670500 & $\mathrm{C}$ & 1.45901800 & 4.80904800 & -2.28735900 \\
\hline $\mathrm{H}$ & 3.27870200 & 4.51151400 & 1.14456900 & $\mathrm{C}$ & 3.41468800 & 5.13532300 & -0.89320100 \\
\hline $\mathrm{C}$ & -3.31962500 & 3.20110900 & -1.53420300 & $\mathrm{H}$ & -3.18855200 & 2.60200500 & 0.53043300 \\
\hline
\end{tabular}




\begin{tabular}{|c|c|c|c|c|c|c|c|}
\hline C & -1.27504400 & 4.03731700 & -2.55424800 & C & 2.78448200 & 5.23846400 & -2.12846000 \\
\hline C & 0.76832400 & 4.92191200 & -3.54278500 & $\mathrm{H}$ & 4.44020600 & 5.47296000 & -0.77951300 \\
\hline $\mathrm{C}$ & -2.63420600 & 3.70024600 & -2.64083500 & $\mathrm{H}$ & -4.36801500 & 2.93918100 & -1.62207900 \\
\hline $\mathrm{C}$ & -0.53641800 & 4.55655800 & -3.67026900 & $\mathrm{H}$ & 3.31054300 & 5.66197500 & -2.98040200 \\
\hline $\mathrm{H}$ & 1.31261800 & 5.32756900 & -4.39212300 & $\mathrm{H}$ & -3.15225000 & 3.83511300 & -3.58717300 \\
\hline $\bar{H}$ & -1.04880800 & 4.66412900 & -4.62306700 & $\mathrm{C}$ & -1.93371900 & -0.52075400 & 2.62592700 \\
\hline $\mathrm{S}$ & -2.78133400 & 0.72562000 & 3.40931600 & $\mathrm{~N}$ & -0.63473400 & -0.81416100 & 2.90408600 \\
\hline $\mathrm{H}$ & -0.09708000 & -1.31345600 & 2.18645400 & $S$ & 0.46415900 & -3.46511200 & 0.40938400 \\
\hline $\mathrm{O}$ & 1.06078600 & -3.92740000 & -0.85811000 & $\mathrm{O}$ & 0.97382000 & -2.14786100 & 0.88956600 \\
\hline $\mathrm{O}$ & -1.01044400 & -3.59870300 & 0.53082300 & $\mathrm{C}$ & 1.08322900 & -4.66734600 & 1.68758200 \\
\hline $\mathrm{F}$ & 0.82091200 & -5.92554700 & 1.32429200 & $\mathrm{~F}$ & 0.48869400 & -4.42856200 & 2.86938700 \\
\hline $\mathrm{F}$ & 2.41070900 & -4.53962500 & 1.86059700 & $\mathrm{C}$ & -0.21937400 & 0.26344000 & -1.12118200 \\
\hline $\mathrm{O}$ & -1.11093200 & 0.12492200 & -0.31424100 & $\mathrm{O}$ & -0.25593000 & 0.02882500 & -2.43959200 \\
\hline C & -1.38270500 & -0.71330200 & -3.06844600 & C & -2.70244000 & 0.01891100 & -2.84977900 \\
\hline $\mathrm{H}$ & -3.49524000 & -0.52555400 & -3.37156200 & $\mathrm{H}$ & -2.95708300 & 0.07146300 & -1.79307100 \\
\hline $\bar{H}$ & -2.64744700 & 1.03565600 & -3.24630100 & $\mathrm{C}$ & -0.99031100 & -0.69565600 & -4.54332200 \\
\hline $\bar{H}$ & -0.03223300 & -1.20399900 & -4.69829400 & $\mathrm{H}$ & -1.75176900 & -1.21594600 & -5.13213200 \\
\hline $\mathrm{H}$ & -0.90713400 & 0.33271100 & -4.90941500 & C & -1.39505900 & -2.13092400 & -2.51146700 \\
\hline $\mathrm{H}$ & -2.19207300 & -2.70344000 & -2.99244500 & $\mathrm{H}$ & -0.44505600 & -2.64264300 & -2.69030500 \\
\hline $\bar{H}$ & -1.58269200 & -2.13150000 & -1.43942500 & $\mathrm{C}$ & 2.13830300 & 0.67525000 & -1.57074200 \\
\hline $\mathrm{H}$ & 1.86817000 & 0.82534300 & -2.61019700 & C & 3.39204900 & 1.35032900 & -1.15903100 \\
\hline $\mathrm{C}$ & 4.13981500 & 2.00788000 & -2.14354100 & $\mathrm{C}$ & 3.89656500 & 1.26767300 & 0.14729900 \\
\hline $\mathrm{C}$ & 5.37246700 & 2.57861100 & -1.83306400 & $\mathrm{H}$ & 3.74194300 & 2.09179200 & -3.15104300 \\
\hline C & 5.12823100 & 1.84331100 & 0.45284000 & $\mathrm{H}$ & 3.32624800 & 0.76680200 & 0.92060800 \\
\hline $\mathrm{C}$ & 5.87181300 & 2.49489200 & -0.53395900 & $\mathrm{H}$ & 5.93489000 & 3.09776900 & -2.60340900 \\
\hline $\bar{H}$ & 5.50991900 & 1.77715100 & 1.46775900 & $\mathrm{H}$ & 6.83325100 & 2.93756600 & -0.29000500 \\
\hline $\mathrm{N}$ & 1.04501000 & 0.72836100 & -0.74217700 & $\mathrm{H}$ & 1.16996400 & 0.78747700 & 0.27794400 \\
\hline C & 2.56181500 & -1.21651900 & -1.64438700 & $\mathrm{H}$ & 2.72410800 & -1.40175300 & -0.58725300 \\
\hline $\bar{H}$ & 1.60213600 & -1.56527200 & -2.00731200 & $\mathrm{C}$ & 3.64364400 & -1.29605100 & -2.52235000 \\
\hline C & 5.01546700 & -1.31129000 & -2.04597000 & $\mathrm{H}$ & 5.73143100 & -0.91243800 & -2.77200700 \\
\hline $\mathrm{H}$ & 5.12218800 & -0.79683100 & -1.08461700 & C & 3.40180400 & -1.26817400 & -3.99701000 \\
\hline $\mathrm{H}$ & 3.66115500 & -0.26748100 & -4.37588100 & $\mathrm{H}$ & 4.05603100 & -1.97448000 & -4.51844900 \\
\hline $\mathrm{H}$ & 2.36048300 & -1.47601300 & -4.25227700 & C & 7.36935700 & -2.99240200 & -1.13904600 \\
\hline $\mathrm{H}$ & 7.48273600 & -2.34258400 & -0.26386700 & $\mathrm{H}$ & 7.77887600 & -3.97539400 & -0.87771000 \\
\hline $\mathrm{H}$ & 7.98220900 & -2.58024100 & -1.94880900 & C & 4.48101100 & -3.79368700 & -0.25799800 \\
\hline $\mathrm{H}$ & 4.47372300 & -3.12238500 & 0.60803500 & $\mathrm{H}$ & 3.44290400 & -3.92536900 & -0.57989100 \\
\hline $\mathrm{H}$ & 4.84620100 & -4.76818900 & 0.08791200 & C & 5.38032300 & -4.19424900 & -3.19875400 \\
\hline $\mathrm{H}$ & 4.32827500 & -4.31034300 & -3.48206000 & $\mathrm{H}$ & 5.92411700 & -3.77005900 & -4.05114900 \\
\hline $\mathrm{H}$ & 5.78088000 & -5.19966400 & -3.02252900 & $\mathrm{Si}$ & 5.55935800 & -3.14858300 & -1.64240200 \\
\hline $\mathrm{C}$ & 0.03780300 & 2.91224600 & 4.51516600 & $\mathrm{H}$ & 0.56381500 & 2.69836300 & 5.45267500 \\
\hline $\mathrm{H}$ & -0.96890000 & 2.49077500 & 4.56377100 & $\mathrm{C}$ & 0.04465600 & 4.41442100 & 4.20343600 \\
\hline $\mathrm{H}$ & -0.81136700 & 4.66612300 & 3.56947200 & $\mathrm{H}$ & -0.01271800 & 5.02347000 & 5.10934000 \\
\hline $\mathrm{C}$ & 1.35059700 & 4.61343500 & 3.42112500 & $\mathrm{H}$ & 1.36601700 & 5.53156400 & 2.82955900 \\
\hline $\bar{H}$ & 2.20734200 & 4.62953000 & 4.10586200 & $\mathrm{C}$ & 1.41939200 & 3.35331700 & 2.53238700 \\
\hline $\mathrm{H}$ & 2.44795500 & 3.03768900 & 2.34577200 & & & & \\
\hline
\end{tabular}

TS- $R-4 f-7$

Imaginary frequency: $-366.45 \mathrm{~cm}^{-1}$

E[B3LYP/6-31G(d)-D3(BJ)]: -4789.087711 Hartree

$\mathrm{E}\left[\mathrm{PCM}\left(\mathrm{Et}_{2} \mathrm{O}\right)-\mathrm{B} 3 \mathrm{LYP} / 6-311 \mathrm{G}(\mathrm{d}, \mathrm{p})-\mathrm{D} 3(\mathrm{BJ})\right]$ : -4790.228003 Hartree

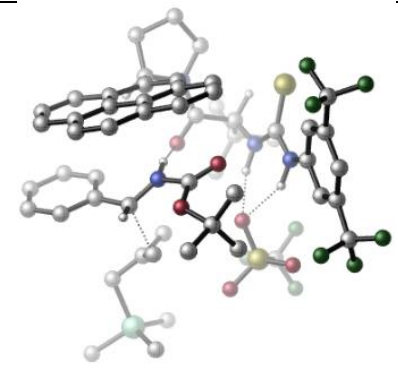




\begin{tabular}{|c|c|c|c|c|c|c|c|}
\hline 01 & & & & $\mathrm{~N}$ & -2.20787500 & -1.30762400 & 1.68852900 \\
\hline $\mathrm{H}$ & -1.49101700 & -1.92538800 & 1.30366300 & C & -3.36816400 & -1.27402100 & 0.90361200 \\
\hline C & -3.57189500 & -2.37613800 & 0.05636600 & $\mathrm{C}$ & -4.27732300 & -0.20929800 & 0.86725300 \\
\hline $\mathrm{C}$ & -4.65351800 & -2.39060200 & -0.81915700 & $\mathrm{H}$ & -2.86949000 & -3.20435700 & 0.06695000 \\
\hline C & -5.35015100 & -0.24748200 & -0.01861100 & $\mathrm{H}$ & -4.13557200 & 0.63742300 & 1.51802600 \\
\hline $\mathrm{C}$ & -5.55754200 & -1.33068500 & -0.86897500 & $\mathrm{H}$ & -6.39398300 & -1.34724100 & -1.55796000 \\
\hline $\mathrm{C}$ & -4.81504100 & -3.51721200 & -1.79906800 & $\mathrm{C}$ & -6.31573300 & 0.90181700 & -0.06940300 \\
\hline $\mathrm{F}$ & -4.12542800 & -4.61555400 & -1.45097800 & $\mathrm{~F}$ & -4.37924000 & -3.15066700 & -3.04029000 \\
\hline $\mathrm{F}$ & -6.11184000 & -3.87009100 & -1.94864400 & $\mathrm{~F}$ & -5.87617400 & 1.98097300 & 0.61805000 \\
\hline $\mathrm{F}$ & -6.52721300 & 1.31043400 & -1.34875600 & $\mathrm{~F}$ & -7.52616600 & 0.57490700 & 0.43234500 \\
\hline C & 0.36194300 & 0.16427000 & 3.80049000 & $\mathrm{H}$ & -0.34702400 & 0.61472300 & 4.49266500 \\
\hline C & 1.37990800 & -0.65499400 & 4.64912500 & C & 2.45615100 & -1.34704300 & 3.79861200 \\
\hline $\mathrm{H}$ & 3.07930500 & -0.62302500 & 3.26891700 & $\bar{H}$ & 2.01881000 & -2.02192200 & 3.06025000 \\
\hline $\mathrm{H}$ & 3.10122900 & -1.95001500 & 4.44829500 & C & 0.58867500 & -1.72034100 & 5.42743100 \\
\hline $\mathrm{H}$ & 1.25316300 & -2.24739400 & 6.12160500 & $\mathrm{H}$ & 0.15154300 & -2.45740500 & 4.74901600 \\
\hline $\mathrm{H}$ & -0.22361000 & -1.26842300 & 6.00807100 & $\mathrm{C}$ & 2.05734000 & 0.30855200 & 5.63857000 \\
\hline $\mathrm{H}$ & 2.77384200 & -0.23432300 & 6.26439700 & $\mathrm{H}$ & 1.32319200 & 0.77793500 & 6.30386700 \\
\hline $\mathrm{H}$ & 2.60741600 & 1.10250100 & 5.11898800 & $\mathrm{C}$ & 1.01136400 & 1.25222800 & 2.93992200 \\
\hline $\mathrm{O}$ & 1.66369500 & 0.92355700 & 1.92784100 & $\mathrm{~N}$ & 0.84438700 & 2.54432400 & 3.26435600 \\
\hline C & 0.61405400 & 3.71673300 & 1.08381200 & C & -0.69412000 & 3.33462500 & 1.01379700 \\
\hline C & 1.27520400 & 4.25778400 & -0.08748100 & C & -1.46545500 & 3.45858600 & -0.18718200 \\
\hline $\bar{H}$ & -1.18238200 & 2.87971100 & 1.87018200 & $\mathrm{C}$ & 0.55166500 & 4.30500500 & -1.32115900 \\
\hline C & 2.60507900 & 4.71051600 & -0.08014000 & C & -2.82447700 & 3.11415400 & -0.23417800 \\
\hline C & -0.81984300 & 3.92018800 & -1.36818500 & $\mathrm{C}$ & 1.19860700 & 4.74969300 & -2.51437000 \\
\hline $\mathrm{H}$ & 3.17444500 & 4.71287900 & 0.84174400 & $\mathrm{C}$ & 3.22649800 & 5.14922400 & -1.24777000 \\
\hline C & -3.54376600 & 3.23145600 & -1.42071600 & $\mathrm{H}$ & -3.30793800 & 2.74731100 & 0.66470000 \\
\hline C & -1.55349300 & 4.00184200 & -2.58960400 & $\mathrm{C}$ & 2.54034500 & 5.15585700 & -2.45630000 \\
\hline $\mathrm{C}$ & 0.44764200 & 4.78076000 & -3.73919100 & $\mathrm{H}$ & 4.26265300 & 5.47066600 & -1.21159200 \\
\hline C & -2.91554100 & 3.66549700 & -2.58697800 & $\mathrm{H}$ & -4.59258300 & 2.95824000 & -1.44272200 \\
\hline C & -0.86710700 & 4.43108400 & -3.77482600 & $\mathrm{H}$ & 3.03330300 & 5.48900500 & -3.36593300 \\
\hline $\mathrm{H}$ & 0.95493000 & 5.11026600 & -4.64252600 & $\mathrm{H}$ & -3.47925000 & 3.74031500 & -3.51360800 \\
\hline $\mathrm{H}$ & -1.42516100 & 4.47657500 & -4.70664900 & $\mathrm{C}$ & -1.73219400 & -0.44257700 & 2.63218900 \\
\hline $\mathrm{S}$ & -2.64481900 & 0.73336200 & 3.44532200 & $\mathrm{~N}$ & -0.41981200 & -0.67308700 & 2.91189200 \\
\hline $\mathrm{H}$ & 0.09932100 & -1.22733400 & 2.23160500 & $\mathrm{~S}$ & 0.43009300 & -3.74971500 & 0.20949000 \\
\hline 0 & 1.51904200 & -4.00060300 & -0.75737600 & 0 & 0.35521600 & -2.32076700 & 0.66289400 \\
\hline $\mathrm{O}$ & -0.88516700 & -4.34154700 & -0.08182500 & $\mathrm{C}$ & 0.99900700 & -4.62514100 & 1.74908900 \\
\hline $\mathrm{F}$ & 0.94420300 & -5.94932500 & 1.60318600 & $\mathrm{~F}$ & 0.23433000 & -4.27078600 & 2.79595300 \\
\hline $\mathrm{F}$ & 2.27673600 & -4.28663200 & 2.03899100 & C & -0.16555300 & 0.50108200 & -1.15684400 \\
\hline $\mathrm{O}$ & -1.01156400 & 0.37439100 & -0.30076300 & 0 & -0.26690400 & 0.25601000 & -2.46690700 \\
\hline C & -1.36389800 & -0.57352000 & -3.03566800 & C & -2.72522300 & 0.04795900 & -2.74514000 \\
\hline $\bar{H}$ & -3.49754400 & -0.56712800 & -3.21678000 & $\mathrm{H}$ & -2.92239100 & 0.09388900 & -1.67609700 \\
\hline$\overline{\mathrm{H}}$ & -2.77865000 & 1.06032900 & -3.15195600 & $\mathrm{C}$ & -1.05182100 & -0.53026000 & -4.52869200 \\
\hline $\mathrm{H}$ & -0.05968300 & -0.94771000 & -4.73070600 & $\mathrm{H}$ & -1.79234000 & -1.12188500 & -5.07552500 \\
\hline $\mathrm{H}$ & -1.08337200 & 0.49950500 & -4.89898000 & C & -1.22884600 & -1.98429800 & -2.47667500 \\
\hline$\overline{\mathrm{H}}$ & -2.01613200 & -2.62115300 & -2.88540700 & $\mathrm{H}$ & -0.26187500 & -2.42538800 & -2.73728600 \\
\hline$\overline{\mathrm{H}}$ & -1.32818100 & -1.98452800 & -1.39348300 & $\mathrm{C}$ & 2.17335700 & 0.82577600 & -1.71859600 \\
\hline $\mathrm{H}$ & 1.85678200 & 0.87998800 & -2.75325900 & C & 3.46303500 & 1.49993400 & -1.44410200 \\
\hline $\mathrm{C}$ & 4.24462100 & 1.87932300 & -2.54362100 & $\mathrm{C}$ & 3.94469400 & 1.73438700 & -0.14811600 \\
\hline C & 5.48897600 & 2.47774700 & -2.35829600 & $\mathrm{H}$ & 3.86840700 & 1.71067300 & -3.54933100 \\
\hline C & 5.19289200 & 2.32535000 & 0.03211200 & $\mathrm{H}$ & 3.34744100 & 1.46507000 & 0.71500600 \\
\hline $\mathrm{C}$ & 5.96902200 & 2.69819900 & -1.06700100 & $\mathrm{H}$ & 6.07959800 & 2.77369600 & -3.22022000 \\
\hline
\end{tabular}




\begin{tabular}{|crrr|lrrr|}
\hline $\mathrm{H}$ & 5.55766900 & 2.50268100 & 1.04003500 & $\mathrm{H}$ & 6.94039200 & 3.16091700 & -0.91811700 \\
\hline $\mathrm{N}$ & 1.12360600 & 0.96572500 & -0.85098300 & $\mathrm{H}$ & 1.27752200 & 1.11090900 & 0.15340100 \\
\hline $\mathrm{C}$ & 2.48123000 & -1.16092700 & -1.89570300 & $\mathrm{H}$ & 1.51890500 & -1.47809800 & -1.51739100 \\
\hline $\mathrm{H}$ & 2.62289700 & -1.27853900 & -2.96785700 & $\mathrm{C}$ & 3.58960900 & -1.37701600 & -1.07286500 \\
\hline $\mathrm{C}$ & 4.92094000 & -1.52602900 & -1.63368000 & $\mathrm{H}$ & 5.02065500 & -1.07018000 & -2.62432800 \\
\hline $\mathrm{H}$ & 5.71465000 & -1.17028000 & -0.96973100 & $\mathrm{C}$ & 3.41555400 & -1.47373900 & 0.40043300 \\
\hline $\mathrm{H}$ & 3.19141100 & -2.51481000 & 0.65466200 & $\mathrm{H}$ & 4.32108200 & -1.17491600 & 0.93483500 \\
\hline $\mathrm{H}$ & 2.56006300 & -0.89698200 & 0.75375400 & $\mathrm{C}$ & 6.98836300 & -3.49505900 & -2.64898600 \\
\hline $\mathrm{H}$ & 7.72222800 & -3.09824000 & -1.93818300 & $\mathrm{H}$ & 7.27336300 & -4.52859600 & -2.87910300 \\
\hline $\mathrm{H}$ & 7.06627000 & -2.91459800 & -3.57536400 & $\mathrm{C}$ & 5.13229100 & -4.38823700 & -0.32191600 \\
\hline $\mathrm{H}$ & 5.70423100 & -3.91072500 & 0.48176300 & $\mathrm{H}$ & 4.09337600 & -4.49601100 & 0.00401900 \\
\hline $\mathrm{H}$ & 5.53956500 & -5.39721100 & -0.46131200 & $\mathrm{C}$ & 3.96121500 & -4.05778900 & -3.16842300 \\
\hline $\mathrm{H}$ & 2.96871000 & -4.07570700 & -2.70607700 & $\mathrm{H}$ & 3.92119800 & -3.44021500 & -4.07352000 \\
\hline $\mathrm{H}$ & 4.20278700 & -5.08149000 & -3.47912100 & $\mathrm{Si}$ & 5.23981500 & -3.44062400 & -1.94115400 \\
\hline $\mathrm{C}$ & 0.11181500 & 3.10079200 & 4.41973200 & $\mathrm{H}$ & 0.67522200 & 2.91346100 & 5.34078300 \\
\hline $\mathrm{H}$ & -0.87448500 & 2.63768500 & 4.50141800 & $\mathrm{C}$ & 0.04507000 & 4.60102700 & 4.10354000 \\
\hline $\mathrm{H}$ & -0.84678700 & 4.81666600 & 3.50724500 & $\mathrm{H}$ & 0.00203700 & 5.20994300 & 5.01019800 \\
\hline $\mathrm{C}$ & 1.30732800 & 4.85108600 & 3.26619600 & $\mathrm{H}$ & 1.25699200 & 5.76387200 & 2.66827200 \\
\hline $\mathrm{H}$ & 2.19022000 & 4.91078100 & 3.91422800 & $\mathrm{C}$ & 1.39577900 & 3.58946200 & 2.38256800 \\
\hline $\mathrm{H}$ & 2.42985700 & 3.32346100 & 2.15712100 & & & & \\
\hline
\end{tabular}

\section{TS- $R-4 \mathrm{f}-8$}

Imaginary frequency: $-361.88 \mathrm{~cm}^{-1}$

E[B3LYP/6-31G(d)-D3(BJ)]: -4789.087202 Hartree

E[PCM(Et $\left.\left.{ }_{2} \mathrm{O}\right)-\mathrm{B} 3 \mathrm{LYP} / 6-311 \mathrm{G}(\mathrm{d}, \mathrm{p})-\mathrm{D} 3(\mathrm{BJ})\right]$ : -4790.227243 Hartree

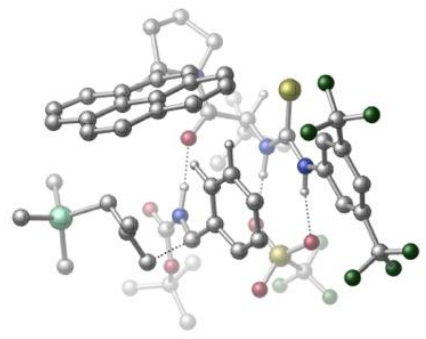

\begin{tabular}{|lrrr|rrrr|}
\hline 01 & & & & N & 2.65208200 & 0.71734300 & 1.26960500 \\
\hline $\mathrm{H}$ & 2.44353700 & 1.64806800 & 0.89169400 & $\mathrm{C}$ & 3.76485300 & 0.13210100 & 0.65089300 \\
\hline $\mathrm{C}$ & 4.50477400 & 0.96406300 & -0.20595800 & $\mathrm{C}$ & 4.12390000 & -1.21889000 & 0.75552800 \\
\hline $\mathrm{C}$ & 5.55853000 & 0.44638200 & -0.95105100 & $\mathrm{H}$ & 4.22180400 & 2.00305700 & -0.31926700 \\
\hline $\mathrm{C}$ & 5.18490900 & -1.71155400 & 0.00149700 & $\mathrm{H}$ & 3.56345600 & -1.87496200 & 1.40069800 \\
\hline $\mathrm{C}$ & 5.91794700 & -0.89664500 & -0.85866200 & $\mathrm{H}$ & 6.74019800 & -1.29466900 & -1.44081900 \\
\hline $\mathrm{C}$ & 6.24506100 & 1.32607800 & -1.95679700 & $\mathrm{C}$ & 5.46761200 & -3.18423500 & 0.00289500 \\
\hline $\mathrm{F}$ & 6.25035300 & 2.62139500 & -1.59022900 & $\mathrm{~F}$ & 5.62442600 & 1.26187900 & -3.16705700 \\
\hline $\mathrm{F}$ & 7.52710800 & 0.95144300 & -2.16488900 & $\mathrm{~F}$ & 4.91881900 & -3.82653600 & 1.05857900 \\
\hline $\mathrm{F}$ & 4.95537800 & -3.77512900 & -1.11660700 & $\mathrm{~F}$ & 6.78918100 & -3.45609300 & 0.00247100 \\
\hline $\mathrm{C}$ & -0.31167800 & 0.73075400 & 3.39461900 & $\mathrm{H}$ & 0.22144900 & 0.27150100 & 4.22688400 \\
\hline $\mathrm{C}$ & -1.00979200 & 2.01907600 & 3.94391100 & $\mathrm{C}$ & -1.99835100 & 2.64259800 & 2.95140100 \\
\hline $\mathrm{H}$ & -2.81014300 & 1.96104300 & 2.69090500 & $\mathrm{H}$ & -1.50431500 & 2.94820200 & 2.03021000 \\
\hline $\mathrm{H}$ & -2.44089500 & 3.54063300 & 3.39912000 & $\mathrm{C}$ & 0.08166100 & 3.04574300 & 4.30000100 \\
\hline $\mathrm{H}$ & -0.36732800 & 3.88031400 & 4.85076000 & $\mathrm{H}$ & 0.55756200 & 3.45746900 & 3.40644400 \\
\hline $\mathrm{H}$ & 0.85961600 & 2.60109000 & 4.93165400 & $\mathrm{C}$ & -1.77793200 & 1.64505500 & 5.22454600 \\
\hline $\mathrm{H}$ & -2.28690800 & 2.53040500 & 5.61990700 & $\mathrm{H}$ & -1.10606600 & 1.27415000 & 6.00796300 \\
\hline $\mathrm{H}$ & -2.54571300 & 0.88700700 & 5.03069600 & $\mathrm{C}$ & -1.28413100 & -0.30354000 & 2.79837400 \\
\hline $\mathrm{O}$ & -1.72140800 & -0.18684400 & 1.64110900 & $\mathrm{C}$ & -1.28175800 & -1.73479000 & 4.90539400 \\
\hline $\mathrm{C}$ & -2.58370900 & -2.35491900 & 2.94117600 & $\mathrm{C}$ & -2.51746700 & -2.49901700 & 5.38704800 \\
\hline $\mathrm{H}$ & -0.38619800 & -2.36831800 & 4.88735000 & $\mathrm{H}$ & -1.06414900 & -0.85731300 & 5.50849100 \\
\hline $\mathrm{H}$ & -3.44910100 & -1.79927300 & 2.57324900 & $\mathrm{H}$ & -3.29152700 & -1.78482800 & 5.69119100 \\
\hline $\mathrm{H}$ & -2.30206300 & -3.15115700 & 6.23796500 & $\mathrm{~N}$ & -1.66339000 & -1.36586900 & 3.53879300 \\
\hline $\mathrm{C}$ & -1.94284900 & -3.11345200 & 1.79033700 & $\mathrm{C}$ & -0.60666900 & -3.38514300 & 1.82707700 \\
\hline
\end{tabular}




\begin{tabular}{|c|c|c|c|c|c|c|c|}
\hline $\mathrm{C}$ & -2.74396600 & -3.58313000 & 0.68102100 & C & 0.06419800 & -4.09284000 & 0.78044900 \\
\hline $\mathrm{H}$ & 0.00208300 & -3.00967600 & 2.64265000 & C & -2.08493900 & -4.23014200 & -0.41471500 \\
\hline $\mathrm{C}$ & -4.14222500 & -3.44350600 & 0.62619100 & C & 1.43765900 & -4.38375500 & 0.83805800 \\
\hline $\mathrm{C}$ & -0.68123100 & -4.47233000 & -0.37195100 & C & -2.83736200 & -4.66481100 & -1.54967800 \\
\hline $\mathrm{H}$ & -4.67341000 & -2.98237900 & 1.45099900 & C & -4.87203900 & -3.90879200 & -0.46704300 \\
\hline C & 2.07362900 & -5.01338400 & -0.23050200 & $\mathrm{H}$ & 2.00650000 & -4.07757600 & 1.71064600 \\
\hline $\mathrm{C}$ & -0.02022300 & -5.10653900 & -1.46608100 & $\mathrm{C}$ & -4.23074100 & -4.50056800 & -1.55157800 \\
\hline C & -2.14287300 & -5.27538800 & -2.65017200 & $\mathrm{H}$ & -5.95322100 & -3.80447400 & -0.47180000 \\
\hline C & 1.35721300 & -5.35861700 & -1.37544700 & $\mathrm{H}$ & 3.13747600 & -5.20938800 & -0.18038100 \\
\hline $\mathrm{C}$ & -0.79737400 & -5.47812100 & -2.61468100 & $\mathrm{H}$ & -4.80508600 & -4.85839300 & -2.40199000 \\
\hline $\mathrm{H}$ & -2.72465700 & -5.58595800 & -3.51444300 & $\mathrm{H}$ & 1.86319400 & -5.83601400 & -2.21033000 \\
\hline $\mathrm{H}$ & -0.28841400 & -5.94751000 & -3.45245900 & C & 1.79335100 & 0.24056500 & 2.22225900 \\
\hline $\mathrm{S}$ & 2.05156500 & -1.15100000 & 3.16370800 & $\mathrm{~N}$ & 0.70061400 & 1.02487600 & 2.39418700 \\
\hline $\mathrm{H}$ & 0.51321700 & 1.78160500 & 1.72948800 & $\mathrm{~S}$ & 1.14493900 & 3.86143000 & -0.27137900 \\
\hline $\mathrm{O}$ & 0.72792600 & 4.07826900 & -1.67021600 & $\mathrm{O}$ & 0.11097000 & 3.23522900 & 0.60106200 \\
\hline $\mathrm{O}$ & 2.49194600 & 3.27038900 & -0.07406300 & $\mathrm{C}$ & 1.31165800 & 5.56907000 & 0.43634000 \\
\hline $\mathrm{F}$ & 2.30015500 & 6.23711200 & -0.16341400 & $\mathrm{~F}$ & 1.56964200 & 5.50028300 & 1.75047000 \\
\hline $\mathrm{F}$ & 0.16786300 & 6.25863000 & 0.26653700 & C & -2.83247300 & 2.42846500 & -0.43754700 \\
\hline $\mathrm{O}$ & -3.72995800 & 2.32485000 & 0.37881200 & $\mathrm{O}$ & -2.66155200 & 3.42041000 & -1.31776500 \\
\hline $\mathrm{C}$ & -3.28073700 & 4.75244200 & -1.10893600 & C & -4.77105300 & 4.66873700 & -1.42927400 \\
\hline $\mathrm{H}$ & -5.22358700 & 5.66011600 & -1.31929800 & $\mathrm{H}$ & -5.27449000 & 3.97819100 & -0.74938200 \\
\hline $\mathrm{H}$ & -4.92762900 & 4.33949400 & -2.46174300 & C & -2.52535100 & 5.61408400 & -2.11750200 \\
\hline $\mathrm{H}$ & -1.45570200 & 5.61112200 & -1.89379800 & $\mathrm{H}$ & -2.89886500 & 6.64242200 & -2.07837400 \\
\hline $\mathrm{H}$ & -2.67035600 & 5.23208900 & -3.13360800 & C & -3.02099400 & 5.22807000 & 0.32008500 \\
\hline $\bar{H}$ & -3.31585600 & 6.27993100 & 0.39837100 & $\mathrm{H}$ & -1.95879900 & 5.15028100 & 0.55835800 \\
\hline $\mathrm{H}$ & -3.59380200 & 4.64887100 & 1.04510500 & $\mathrm{C}$ & -1.00298200 & 1.41175800 & -1.68259400 \\
\hline $\mathrm{H}$ & -0.80434100 & 2.40391800 & -2.07896100 & C & 0.19490000 & 0.54258600 & -1.65855400 \\
\hline $\mathrm{C}$ & 1.26897900 & 0.91932200 & -2.48172300 & $\mathrm{C}$ & 0.25635300 & -0.67122700 & -0.95684600 \\
\hline $\mathrm{C}$ & 2.36483900 & 0.07703300 & -2.64596200 & $\mathrm{H}$ & 1.24471600 & 1.88775800 & -2.97083900 \\
\hline C & 1.35803400 & -1.50755000 & -1.12791000 & $\mathrm{H}$ & -0.54115800 & -0.96897900 & -0.28307500 \\
\hline $\mathrm{C}$ & 2.39688200 & -1.15213200 & -1.98767300 & $\mathrm{H}$ & 3.20453800 & 0.39264400 & -3.25599700 \\
\hline $\bar{H}$ & 1.41007600 & -2.43702500 & -0.58173000 & $\mathrm{H}$ & 3.24551400 & -1.81791500 & -2.10306500 \\
\hline $\mathrm{N}$ & -1.86230000 & 1.44155800 & -0.60143500 & $\mathrm{H}$ & -1.74898700 & 0.81738900 & 0.21080300 \\
\hline $\mathrm{C}$ & -2.02512600 & 0.89643500 & -3.18541900 & $\mathrm{H}$ & -1.34925700 & 1.06860200 & -4.01955900 \\
\hline $\bar{H}$ & -2.77379600 & 1.67265200 & -3.06332600 & $\mathrm{C}$ & -2.41252200 & -0.43655300 & -2.94479800 \\
\hline C & -3.41119800 & -0.74606000 & -1.93983300 & $\mathrm{H}$ & -3.29112200 & -0.06537400 & -1.08153900 \\
\hline $\bar{H}$ & -3.35962400 & -1.78419200 & -1.60203200 & $\mathrm{C}$ & -1.66149100 & -1.56286700 & -3.56443000 \\
\hline $\bar{H}$ & -2.30564700 & -2.43299600 & -3.72232900 & $\mathrm{H}$ & -0.88726800 & -1.87785600 & -2.84585300 \\
\hline $\mathrm{H}$ & -1.15998700 & -1.28213000 & -4.49315400 & C & -6.17050100 & -0.37905200 & -0.76677200 \\
\hline $\mathrm{H}$ & -6.03115800 & -1.31759800 & -0.22228400 & $\mathrm{H}$ & -7.24601000 & -0.21969800 & -0.90694700 \\
\hline $\mathrm{H}$ & -5.78034900 & 0.43424800 & -0.14321800 & C & -5.84194100 & -1.76085800 & -3.57485300 \\
\hline $\mathrm{H}$ & -5.72373300 & -2.74841400 & -3.11990900 & $\mathrm{H}$ & -5.27092800 & -1.74948700 & -4.51095000 \\
\hline $\mathrm{H}$ & -6.89972900 & -1.62828100 & -3.83116300 & $\mathrm{C}$ & -5.42177600 & 1.27601400 & -3.25157000 \\
\hline $\mathrm{H}$ & -4.90731600 & 1.31384300 & -4.21759700 & $\mathrm{H}$ & -5.03403400 & 2.08148500 & -2.62122000 \\
\hline $\mathrm{H}$ & -6.48245700 & 1.48856800 & -3.43338600 & $\mathrm{Si}$ & -5.26024500 & -0.39971800 & -2.41094100 \\
\hline $\mathrm{C}$ & -2.95790400 & -3.27467000 & 4.13507100 & $\mathrm{H}$ & -2.39826900 & -4.21148300 & 4.05616200 \\
\hline $\mathrm{H}$ & -4.02238600 & -3.52441500 & 4.13570800 & & & & \\
\hline
\end{tabular}

\section{TS- $R-4 f-9$}

Imaginary frequency: $-417.02 \mathrm{~cm}^{-1}$

E[B3LYP/6-31G(d)-D3(BJ)]: -4789.085732 Hartree

$\mathrm{E}\left[\mathrm{PCM}\left(\mathrm{Et}_{2} \mathrm{O}\right)-\mathrm{B} 3 \mathrm{LYP} / 6-311 \mathrm{G}(\mathrm{d}, \mathrm{p})-\mathrm{D} 3(\mathrm{BJ})\right]:-4790.227190$ Hartree

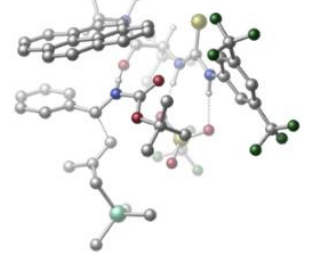

S176 


\begin{tabular}{|c|c|c|c|c|c|c|c|}
\hline 01 & & & & $\mathrm{~N}$ & 2.54715000 & -0.84111700 & 1.91086400 \\
\hline $\mathrm{H}$ & 2.60736700 & 0.17777500 & 1.99248900 & C & 3.45599600 & -1.35958400 & 0.98398500 \\
\hline $\mathrm{C}$ & 4.55722200 & -0.54895200 & 0.66821700 & $\mathrm{C}$ & 3.28298000 & -2.57020000 & 0.29933800 \\
\hline$C$ & 5.45633600 & -0.94043300 & -0.31969000 & $\mathrm{H}$ & 4.67645500 & 0.40702700 & 1.16385900 \\
\hline C & 4.20191600 & -2.94648000 & -0.67459200 & $\mathrm{H}$ & 2.43419100 & -3.19388700 & 0.52531500 \\
\hline C & 5.29703200 & -2.14538200 & -0.99874400 & $\mathrm{H}$ & 6.00630200 & -2.45286900 & -1.75776900 \\
\hline C & 6.55455200 & -0.00295300 & -0.73189200 & C & 3.98999700 & -4.20716700 & -1.46059600 \\
\hline $\mathrm{F}$ & 6.94484200 & 0.80613700 & 0.27267100 & $\mathrm{~F}$ & 6.15623900 & 0.80140900 & -1.75322500 \\
\hline $\mathrm{F}$ & 7.64734300 & -0.66719600 & -1.17158100 & $\mathrm{~F}$ & 3.03590500 & -5.00344300 & -0.92883100 \\
\hline $\mathrm{F}$ & 3.60198300 & -3.93322000 & -2.73774400 & $\mathrm{~F}$ & 5.12040900 & -4.93971500 & -1.55930100 \\
\hline C & -0.52048000 & -0.87471800 & 3.86680500 & $\mathrm{H}$ & -0.28150900 & -1.82569500 & 4.34057600 \\
\hline C & -0.79587900 & 0.15898400 & 5.00412100 & C & -1.28247800 & 1.52463800 & 4.48830700 \\
\hline $\mathrm{H}$ & -2.27118700 & 1.45192200 & 4.02829900 & $\mathrm{H}$ & -0.60425100 & 1.95122800 & 3.74821600 \\
\hline $\mathrm{H}$ & -1.34589000 & 2.22349600 & 5.33089600 & C & 0.51135100 & 0.35023300 & 5.79232600 \\
\hline $\mathrm{H}$ & 0.32361500 & 0.95888400 & 6.68427400 & $\mathrm{H}$ & 1.26605400 & 0.85888700 & 5.18682500 \\
\hline $\mathrm{H}$ & 0.92700800 & -0.61124300 & 6.11558500 & C & -1.86964500 & -0.43890800 & 5.93037700 \\
\hline $\mathrm{H}$ & -2.09560700 & 0.25656800 & 6.74602700 & $\mathrm{H}$ & -1.53243600 & -1.38131900 & 6.37816800 \\
\hline $\mathrm{H}$ & -2.80560500 & -0.63026500 & 5.39144800 & C & -1.70658400 & -1.04128000 & 2.91562000 \\
\hline 0 & -1.97203800 & -0.13036300 & 2.10895300 & C & -3.55553900 & -2.32153200 & 1.99911800 \\
\hline $\mathrm{H}$ & -4.14582300 & -1.40332000 & 2.02563200 & $\mathrm{~N}$ & -2.44074300 & -2.16612200 & 2.94830600 \\
\hline C & -3.08164500 & -2.56946000 & 0.57544600 & C & -1.83515800 & -3.06300800 & 0.32233100 \\
\hline C & -3.99534000 & -2.30972600 & -0.51956700 & C & -1.38610800 & -3.35904900 & -1.00600900 \\
\hline $\mathrm{H}$ & -1.13087800 & -3.22415300 & 1.13309900 & C & -3.54483700 & -2.53331200 & -1.85876000 \\
\hline C & -5.30785600 & -1.84481000 & -0.32944900 & C & -0.13028600 & -3.93350300 & -1.25275600 \\
\hline C & -2.24539500 & -3.06530500 & -2.10187500 & C & -4.40833100 & -2.25319400 & -2.96228200 \\
\hline $\mathrm{H}$ & -5.68579300 & -1.67648400 & 0.67175300 & C & -6.14655800 & -1.58705400 & -1.41120500 \\
\hline C & 0.27463400 & -4.22038600 & -2.55403100 & $\mathrm{H}$ & 0.52164700 & -4.15427400 & -0.41425000 \\
\hline C & -1.81048000 & -3.33111100 & -3.43521000 & C & -5.70407800 & -1.77797600 & -2.71521800 \\
\hline C & -3.92789300 & -2.48922200 & -4.29582200 & $\mathrm{H}$ & -7.15283400 & -1.22237800 & -1.22958600 \\
\hline C & -0.55005200 & -3.91370100 & -3.63469300 & $\mathrm{H}$ & 1.24827700 & -4.66164400 & -2.72739400 \\
\hline C & -2.69004400 & -3.00685100 & -4.52232100 & $\mathrm{H}$ & -6.36203400 & -1.57001800 & -3.55522300 \\
\hline $\mathrm{H}$ & -4.59034000 & -2.26002500 & -5.12693200 & $\mathrm{H}$ & -0.22139500 & -4.12773000 & -4.64866100 \\
\hline $\mathrm{H}$ & -2.34808100 & -3.19634700 & -5.53674800 & C & 1.50594700 & -1.43519100 & 2.56882000 \\
\hline $\mathrm{S}$ & 1.35653100 & -3.10443400 & 2.82702000 & $\mathrm{~N}$ & 0.63424500 & -0.51881500 & 3.06367200 \\
\hline $\mathrm{H}$ & 0.57995600 & 0.37887800 & 2.57066000 & $S$ & 1.70130700 & 2.72868500 & 1.34893400 \\
\hline $\mathrm{O}$ & 1.71411400 & 3.55519200 & 0.12412000 & 0 & 0.49028400 & 1.87267200 & 1.49825300 \\
\hline $\mathrm{O}$ & 2.96334400 & 2.02530600 & 1.68143800 & C & 1.50954100 & 3.97534800 & 2.71064500 \\
\hline $\mathrm{F}$ & 2.49938400 & 4.87137700 & 2.68570800 & $\mathrm{~F}$ & 1.50831900 & 3.36723500 & 3.90823000 \\
\hline $\mathrm{F}$ & 0.33853900 & 4.62548600 & 2.56996500 & C & -0.11464800 & -0.08284600 & -0.96817900 \\
\hline $\mathrm{O}$ & 0.54907700 & -0.85419900 & -0.31198800 & $\mathrm{O}$ & 0.22124000 & 0.52960200 & -2.11076800 \\
\hline C & 1.56311400 & 0.33908700 & -2.72761300 & C & 1.78833000 & -1.13768400 & -3.03183500 \\
\hline $\mathrm{H}$ & 2.72392600 & -1.25199000 & -3.58864000 & $\mathrm{H}$ & 1.86098400 & -1.72356400 & -2.11811400 \\
\hline $\mathrm{H}$ & 0.97041000 & -1.53729300 & -3.63814900 & C & 1.44312400 & 1.15327100 & -4.01303400 \\
\hline $\mathrm{H}$ & 1.25323700 & 2.20741900 & -3.78662000 & $\mathrm{H}$ & 2.37992600 & 1.09076400 & -4.57511900 \\
\hline $\mathrm{H}$ & 0.63308000 & 0.77150800 & -4.64323700 & C & 2.62037400 & 0.92160000 & -1.79987200 \\
\hline $\mathrm{H}$ & 3.61104600 & 0.81416900 & -2.25110400 & $\mathrm{H}$ & 2.43639300 & 1.98013300 & -1.60550400 \\
\hline $\mathrm{H}$ & 2.62872800 & 0.39526600 & -0.84807800 & C & -2.15572800 & 1.23036700 & -1.22032800 \\
\hline $\mathrm{H}$ & -1.90224300 & 1.31657800 & -2.27089600 & C & -3.60238400 & 1.35171700 & -0.91661100 \\
\hline C & -4.48493800 & 1.54977500 & -1.98612700 & C & -4.09881100 & 1.35144900 & 0.39528200 \\
\hline $\mathrm{C}$ & -5.84525600 & 1.75162600 & -1.75258900 & $\mathrm{H}$ & -4.10481100 & 1.53573000 & -3.00407400 \\
\hline C & -5.45568700 & 1.56167000 & 0.62234100 & $\mathrm{H}$ & -3.42703100 & 1.18201400 & 1.22902100 \\
\hline C & -6.33154700 & 1.76865700 & -0.44658200 & $\mathrm{H}$ & -6.52061700 & 1.89193600 & -2.59120100 \\
\hline $\mathrm{H}$ & -5.83133200 & 1.56739800 & 1.64160500 & $\mathrm{H}$ & -7.38869300 & 1.93558700 & -0.26118900 \\
\hline
\end{tabular}




\begin{tabular}{|c|c|c|c|c|c|c|c|}
\hline $\mathrm{N}$ & -1.41629900 & 0.26905900 & -0.58698200 & $\mathrm{H}$ & -1.65277400 & -0.01491400 & 0.37438700 \\
\hline C & -1.36316600 & 2.93011700 & -0.59166700 & $\mathrm{H}$ & -1.67262500 & 2.88654500 & 0.44736900 \\
\hline $\mathrm{H}$ & -0.32689800 & 2.66185900 & -0.75732400 & C & -1.89956400 & 3.91360500 & -1.41987700 \\
\hline $\mathrm{C}$ & -1.24041700 & 4.26253100 & -2.67247000 & $\mathrm{H}$ & -0.61107300 & 3.44915900 & -3.04549500 \\
\hline $\mathrm{H}$ & -1.94010000 & 4.59374000 & -3.44703700 & C & -3.18412000 & 4.59404000 & -1.07745300 \\
\hline $\mathrm{H}$ & -3.40871800 & 4.54284200 & -0.01042900 & $\mathrm{H}$ & -3.18099300 & 5.63802300 & -1.40723800 \\
\hline $\mathrm{H}$ & -4.00139100 & 4.08866200 & -1.61339900 & C & 1.72391800 & 5.15851200 & -2.76477600 \\
\hline $\mathrm{H}$ & 1.77840300 & 4.71057400 & -3.76363700 & $\mathrm{H}$ & 2.43909500 & 5.98946000 & -2.73212700 \\
\hline $\mathrm{H}$ & 2.03947900 & 4.42055400 & -2.02093200 & C & -0.56485100 & 7.11947300 & -3.56138600 \\
\hline $\mathrm{H}$ & -0.52356400 & 6.77456700 & -4.60114300 & $\mathrm{H}$ & -1.59226600 & 7.44175100 & -3.35357900 \\
\hline $\mathrm{H}$ & 0.08086400 & 8.00177700 & -3.47764900 & C & -0.11818500 & 6.37623600 & -0.58690400 \\
\hline $\mathrm{H}$ & -1.12380800 & 6.72069500 & -0.32049100 & $\mathrm{H}$ & 0.18567200 & 5.59300900 & 0.11314100 \\
\hline $\mathrm{H}$ & 0.56483000 & 7.22333500 & -0.44868900 & $\mathrm{Si}$ & -0.00188500 & 5.77397000 & -2.36275900 \\
\hline C & -3.20774800 & -4.38059000 & 3.20209000 & $\mathrm{H}$ & -2.69153900 & -4.93950000 & 2.41532100 \\
\hline $\mathrm{H}$ & -3.57193300 & -5.09602100 & 3.94396300 & C & -2.25604000 & -3.34519500 & 3.81599600 \\
\hline $\mathrm{H}$ & -2.54496400 & -3.10026900 & 4.84451000 & $\mathrm{H}$ & -1.21042800 & -3.66232700 & 3.80250000 \\
\hline $\mathrm{C}$ & -4.32410400 & -3.52426400 & 2.58731100 & $\mathrm{H}$ & -4.90439800 & -4.05012900 & 1.82588800 \\
\hline $\mathrm{H}$ & -5.01242300 & -3.17644800 & 3.36728900 & & & & \\
\hline
\end{tabular}

\section{TS- $R-4 f-10$}

Imaginary frequency: $-319.57 \mathrm{~cm}^{-1}$ E[B3LYP/6-31G(d)-D3(BJ)]: -4789.085734 Hartree $\mathrm{E}\left[\mathrm{PCM}\left(\mathrm{Et}_{2} \mathrm{O}\right)-\mathrm{B} 3 \mathrm{LYP} / 6-311 \mathrm{G}(\mathrm{d}, \mathrm{p})-\mathrm{D} 3(\mathrm{BJ})\right]$ : -4790.227118 Hartree

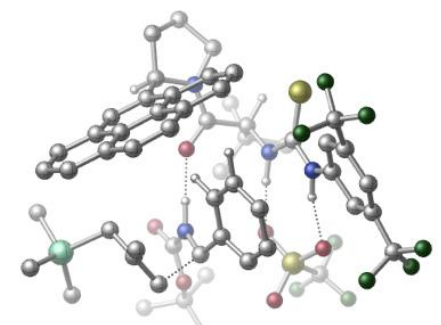

\begin{tabular}{|c|c|c|c|c|c|c|c|}
\hline 01 & & & & $\mathrm{~N}$ & 2.55668300 & 1.29475800 & 1.06140900 \\
\hline $\mathrm{H}$ & 2.10440300 & 2.10420600 & 0.62245000 & $\mathrm{C}$ & 3.70144500 & 0.87661400 & 0.37305500 \\
\hline C & 4.22014000 & 1.76273300 & -0.58388500 & C & 4.26707400 & -0.40025300 & 0.48992700 \\
\hline $\mathrm{C}$ & 5.26337400 & 1.36452800 & -1.41411300 & $\mathrm{H}$ & 3.76888500 & 2.73939800 & -0.70791500 \\
\hline $\mathrm{C}$ & 5.31573300 & -0.77227100 & -0.34545600 & $\mathrm{H}$ & 3.86478900 & -1.10177500 & 1.20216400 \\
\hline C & 5.83087400 & 0.09627200 & -1.30656300 & $\mathrm{H}$ & 6.64327100 & -0.20669000 & -1.95528600 \\
\hline $\mathrm{C}$ & 5.70107900 & 2.27789100 & -2.52324500 & $\mathrm{C}$ & 5.79161100 & -2.19409300 & -0.32352000 \\
\hline $\mathrm{F}$ & 5.57246500 & 3.57879500 & -2.20051600 & $\mathrm{~F}$ & 4.95494300 & 2.07508300 & -3.64391700 \\
\hline $\mathrm{F}$ & 6.98846500 & 2.06672400 & -2.87686100 & $\mathrm{~F}$ & 5.59359600 & -2.79905400 & 0.86933300 \\
\hline $\mathrm{F}$ & 5.11836900 & -2.94500900 & -1.24730800 & $\mathrm{~F}$ & 7.10090100 & -2.30731700 & -0.62533100 \\
\hline $\mathrm{C}$ & -0.18627200 & 0.72428800 & 3.40270600 & $\mathrm{H}$ & 0.50904700 & 0.41883900 & 4.18298500 \\
\hline C & -1.07338600 & 1.86196800 & 4.00019700 & C & -2.23474300 & 2.28047700 & 3.09099000 \\
\hline $\mathrm{H}$ & -2.92838000 & 1.46061300 & 2.89874100 & $\mathrm{H}$ & -1.87747800 & 2.65825000 & 2.13320600 \\
\hline $\mathrm{H}$ & -2.79457000 & 3.09129800 & 3.57277600 & C & -0.16713800 & 3.07797900 & 4.26734000 \\
\hline $\mathrm{H}$ & -0.71889700 & 3.82719700 & 4.84671600 & $\mathrm{H}$ & 0.15531100 & 3.54984100 & 3.33544600 \\
\hline $\mathrm{H}$ & 0.72560900 & 2.79566300 & 4.83764600 & $\mathrm{C}$ & -1.65271900 & 1.36154200 & 5.33598000 \\
\hline $\mathrm{H}$ & -2.30226100 & 2.12942400 & 5.76933400 & $\mathrm{H}$ & -0.86269800 & 1.14763500 & 6.06588900 \\
\hline $\mathrm{H}$ & -2.26093800 & 0.45842100 & 5.20224700 & C & -0.96830700 & -0.51561200 & 2.92287000 \\
\hline $\mathrm{O}$ & -1.63698500 & -0.50244900 & 1.87607200 & C & -0.25644000 & -1.82433900 & 4.97292000 \\
\hline C & -1.61483800 & -2.86681900 & 3.21474000 & C & -1.00463100 & -3.02512400 & 5.55801900 \\
\hline $\mathrm{H}$ & 0.81747800 & -2.01335200 & 4.86549100 & $\mathrm{H}$ & -0.37789000 & -0.92879700 & 5.57777000 \\
\hline $\mathrm{H}$ & -2.68230000 & -2.61852000 & 3.23751500 & $\mathrm{H}$ & -1.94230400 & -2.69058900 & 6.01748800 \\
\hline $\mathrm{H}$ & -0.41898800 & -3.54387500 & 6.32176800 & $\mathrm{~N}$ & -0.87883800 & -1.65441500 & 3.64612100 \\
\hline C & -1.32632200 & -3.41105200 & 1.82514200 & C & -2.40287400 & -3.77534900 & 1.06853700 \\
\hline C & 0.01365500 & -3.69465100 & 1.34152900 & C & -2.28195700 & -4.43149400 & -0.19881200 \\
\hline $\mathrm{H}$ & -3.40543000 & -3.56563200 & 1.43461700 & C & 0.16381200 & -4.34781200 & 0.07409200 \\
\hline $\mathrm{C}$ & 1.17774500 & -3.35773900 & 2.05097500 & $C$ & -3.40072200 & -4.82224700 & -0.95320800 \\
\hline
\end{tabular}




\begin{tabular}{|c|c|c|c|c|c|c|c|}
\hline C & -0.97683800 & -4.69597500 & -0.70558200 & C & 1.46806100 & -4.65378300 & -0.42409000 \\
\hline $\mathrm{H}$ & 1.11172400 & -2.80519600 & 2.97443300 & C & 2.44495700 & -3.67157600 & 1.56109100 \\
\hline $\mathrm{C}$ & -3.24327000 & -5.43202600 & -2.19701500 & $\mathrm{H}$ & -4.39548900 & -4.65172800 & -0.55068300 \\
\hline C & -0.82347300 & -5.31837400 & -1.98212200 & C & 2.59583800 & -4.32054800 & 0.34140900 \\
\hline C & 1.59377400 & -5.26588700 & -1.71647500 & $\mathrm{H}$ & 3.31946000 & -3.37413900 & 2.12909300 \\
\hline C & -1.97041900 & -5.66645100 & -2.71427500 & $\mathrm{H}$ & -4.11925500 & -5.73818300 & -2.76201500 \\
\hline C & 0.50223900 & -5.57762700 & -2.46637800 & $\mathrm{H}$ & 3.58613300 & -4.53125500 & -0.04719400 \\
\hline $\mathrm{H}$ & 2.59439900 & -5.47134500 & -2.08694500 & $\mathrm{H}$ & -1.85362300 & -6.14279400 & -3.68400500 \\
\hline $\mathrm{H}$ & 0.61543100 & -6.04002800 & -3.44324300 & $\mathrm{C}$ & 1.93327200 & 0.74238700 & 2.14888200 \\
\hline $\mathrm{S}$ & 2.68518100 & -0.31505900 & 3.24383100 & $\mathrm{~N}$ & 0.65519400 & 1.16743400 & 2.30360200 \\
\hline $\mathrm{H}$ & 0.24720700 & 1.79339500 & 1.60342300 & $\mathrm{~S}$ & 0.33750400 & 3.98703900 & -0.43274300 \\
\hline 0 & -0.27455700 & 4.12056600 & -1.76976700 & $\mathrm{O}$ & -0.45973600 & 3.19002400 & 0.54246100 \\
\hline $\mathrm{O}$ & 1.78149000 & 3.64570000 & -0.40986800 & $\mathrm{C}$ & 0.27842100 & 5.69853900 & 0.28251400 \\
\hline $\mathrm{F}$ & 1.05421300 & 6.53216500 & -0.41502800 & $\mathrm{~F}$ & 0.69663000 & 5.67996000 & 1.55626100 \\
\hline $\mathrm{F}$ & -0.98223500 & 6.17107200 & 0.25910000 & $\mathrm{C}$ & -3.39779200 & 1.91735100 & -0.12950200 \\
\hline 0 & -4.21013300 & 1.67935200 & 0.74577800 & $\mathrm{O}$ & -3.44476700 & 2.91619700 & -1.01840500 \\
\hline C & -4.26736400 & 4.12453000 & -0.77783600 & $\mathrm{C}$ & -3.97848500 & 4.67333100 & 0.61904200 \\
\hline $\mathrm{H}$ & -4.45558800 & 5.65412900 & 0.71863100 & $\mathrm{H}$ & -2.90315300 & 4.79777600 & 0.76075300 \\
\hline $\mathrm{H}$ & -4.36832700 & 4.01385300 & 1.39538700 & C & -5.74114100 & 3.77685800 & -0.97459500 \\
\hline $\mathrm{H}$ & -5.90997300 & 3.36985100 & -1.97714400 & $\mathrm{H}$ & -6.34719900 & 4.68328000 & -0.87065300 \\
\hline $\mathrm{H}$ & -6.07004400 & 3.04910900 & -0.22997000 & C & -3.75663100 & 5.07681200 & -1.85627000 \\
\hline $\mathrm{H}$ & -4.29224700 & 6.02946100 & -1.79236800 & $\mathrm{H}$ & -3.92238200 & 4.65330500 & -2.85262600 \\
\hline $\mathrm{H}$ & -2.68626200 & 5.25624600 & -1.72914000 & $\mathrm{C}$ & -1.51836600 & 1.20207300 & -1.50377300 \\
\hline $\mathrm{H}$ & -1.49837500 & 2.21693000 & -1.89368000 & C & -0.19543000 & 0.53760600 & -1.55528300 \\
\hline C & 0.73707400 & 1.03968300 & -2.47820100 & C & 0.12396400 & -0.60299700 & -0.80487000 \\
\hline C & 1.94772000 & 0.38592900 & -2.68695700 & $\mathrm{H}$ & 0.51355300 & 1.95956400 & -3.00901600 \\
\hline C & 1.34644500 & -1.24032700 & -1.00710200 & $\mathrm{H}$ & -0.56351300 & -0.99497900 & -0.06328500 \\
\hline C & 2.24368900 & -0.77120800 & -1.96549400 & $\mathrm{H}$ & 2.67447600 & 0.79845300 & -3.37907000 \\
\hline $\mathrm{H}$ & 1.60247700 & -2.10035200 & -0.40790100 & $\mathrm{H}$ & 3.18582900 & -1.28683500 & -2.11424200 \\
\hline $\mathrm{N}$ & -2.29862100 & 1.09675200 & -0.36461400 & $\mathrm{H}$ & -2.04507000 & 0.48399400 & 0.42100300 \\
\hline C & -2.52894300 & 0.55406600 & -2.93526800 & $\mathrm{H}$ & -1.94251200 & 0.83468400 & -3.80677900 \\
\hline $\mathrm{H}$ & -3.39033900 & 1.19362100 & -2.76361600 & C & -2.67660400 & -0.82454600 & -2.68309700 \\
\hline C & -3.58151000 & -1.29078000 & -1.65246100 & $\mathrm{H}$ & -3.64728100 & -0.56558100 & -0.82935300 \\
\hline $\mathrm{H}$ & -3.30126100 & -2.27639400 & -1.27374100 & C & -1.77010200 & -1.81436000 & -3.32444800 \\
\hline $\mathrm{H}$ & -2.25664700 & -2.78725800 & -3.44394900 & $\mathrm{H}$ & -0.92325200 & -1.97339700 & -2.63626800 \\
\hline $\mathrm{H}$ & -1.36317900 & -1.46593300 & -4.27609500 & C & -6.34658200 & -2.05782400 & -0.69980700 \\
\hline $\mathrm{H}$ & -5.98720500 & -3.04238700 & -0.38283400 & $\mathrm{H}$ & -7.42085100 & -2.14308200 & -0.90133300 \\
\hline $\mathrm{H}$ & -6.21555500 & -1.36349000 & 0.13805500 & C & -5.52528600 & -2.63038200 & -3.66508100 \\
\hline $\mathrm{H}$ & -5.05615400 & -3.58806000 & -3.41699300 & $\mathrm{H}$ & -5.01571200 & -2.22648700 & -4.54772400 \\
\hline $\mathrm{H}$ & -6.56708200 & -2.82601700 & -3.94464600 & C & -6.05812700 & 0.27487700 & -2.69559500 \\
\hline $\mathrm{H}$ & -5.56768600 & 0.66535000 & -3.59363200 & $\mathrm{H}$ & -5.90395100 & 0.99032200 & -1.88135800 \\
\hline $\mathrm{H}$ & -7.13472500 & 0.23451700 & -2.90035400 & $\mathrm{Si}$ & -5.42842600 & -1.43103100 & -2.21799500 \\
\hline C & -1.29256900 & -3.88978800 & 4.32706300 & $\mathrm{H}$ & -0.40581000 & -4.46833600 & 4.05070000 \\
\hline $\mathrm{H}$ & -2.11849500 & -4.59212300 & 4.46457500 & & & & \\
\hline
\end{tabular}

\section{TS- $R-4 f-11$}

Imaginary frequency: $-388.72 \mathrm{~cm}^{-1}$

E[B3LYP/6-31G(d)-D3(BJ)]: -4789.083091 Hartree

$\mathrm{E}\left[\mathrm{PCM}\left(\mathrm{Et}_{2} \mathrm{O}\right)-\mathrm{B} 3 \mathrm{LYP} / 6-311 \mathrm{G}(\mathrm{d}, \mathrm{p})-\mathrm{D} 3(\mathrm{BJ})\right]$ : -4790.226955 Hartree

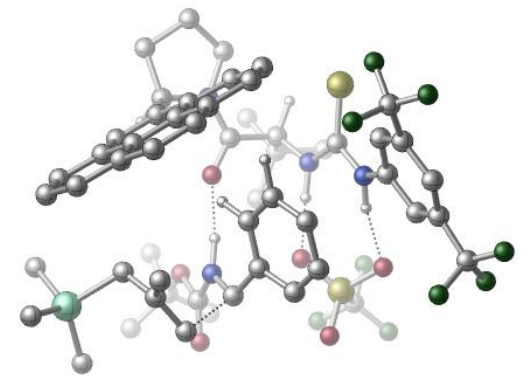




\begin{tabular}{|c|c|c|c|c|c|c|c|}
\hline 01 & & & & $\mathrm{~N}$ & -2.70592100 & -1.58316400 & 0.98519400 \\
\hline $\mathrm{H}$ & -2.29607100 & -2.35409700 & 0.44576200 & C & -3.82847500 & -1.02338500 & 0.36946600 \\
\hline $\mathrm{C}$ & -4.36045400 & -1.72491400 & -0.72501700 & $\mathrm{C}$ & -4.36687300 & 0.22685600 & 0.70079500 \\
\hline $\mathrm{C}$ & -5.39870500 & -1.17380200 & -1.46903200 & $\mathrm{H}$ & -3.92761800 & -2.67730200 & -1.00985700 \\
\hline C & -5.40531700 & 0.75658900 & -0.05853100 & $\mathrm{H}$ & -3.95544000 & 0.78596500 & 1.52479600 \\
\hline C & -5.94021400 & 0.07044100 & -1.14736900 & $\mathrm{H}$ & -6.74891200 & 0.49261800 & -1.73116100 \\
\hline C & -5.87213600 & -1.87926200 & -2.70824700 & C & -5.85090800 & 2.16453100 & 0.20355100 \\
\hline $\mathrm{F}$ & -5.70072100 & -3.21216100 & -2.64086900 & $\mathrm{~F}$ & -5.19174000 & -1.44857500 & -3.80655900 \\
\hline $\mathrm{F}$ & -7.18028600 & -1.64327600 & -2.95448200 & $\mathrm{~F}$ & -5.60281300 & 2.56968000 & 1.46937700 \\
\hline $\mathrm{F}$ & -5.19118000 & 3.04157900 & -0.61202500 & $\mathrm{~F}$ & -7.16624200 & 2.34410300 & -0.03241900 \\
\hline C & 0.16444200 & -1.38906500 & 3.22095500 & $\mathrm{H}$ & -0.44804600 & -1.10874200 & 4.07545600 \\
\hline C & 1.00274700 & -2.62993800 & 3.65694500 & C & 1.97558400 & -3.11564200 & 2.57551800 \\
\hline $\mathrm{H}$ & 2.67219100 & -2.32953800 & 2.28226900 & $\mathrm{H}$ & 1.45745300 & -3.44945300 & 1.67530900 \\
\hline $\mathrm{H}$ & 2.55075000 & -3.96420700 & 2.96687100 & C & 0.01918700 & -3.75992200 & 4.00647800 \\
\hline $\mathrm{H}$ & 0.56499300 & -4.60966700 & 4.43233800 & $\mathrm{H}$ & -0.50881300 & -4.11179900 & 3.11647500 \\
\hline $\mathrm{H}$ & -0.72656800 & -3.43010700 & 4.73907500 & C & 1.80448900 & -2.24356000 & 4.91242400 \\
\hline $\mathrm{H}$ & 2.40126700 & -3.09677700 & 5.25245900 & $\mathrm{H}$ & 1.14445700 & -1.95082500 & 5.73718800 \\
\hline $\mathrm{H}$ & 2.49739900 & -1.41684900 & 4.71260600 & C & 1.00755300 & -0.17788500 & 2.78351400 \\
\hline 0 & 1.59908300 & -0.15371900 & 1.69150800 & C & 0.48974100 & 1.01362600 & 4.96310100 \\
\hline C & 1.90093200 & 2.05000500 & 3.23372000 & C & 0.70773000 & 2.48765200 & 5.32083800 \\
\hline $\mathrm{H}$ & -0.56545200 & 0.72794600 & 4.95544500 & $\mathrm{H}$ & 1.02745900 & 0.35898700 & 5.65694100 \\
\hline C & 1.98173900 & 2.86001400 & 4.55241500 & $\mathrm{H}$ & 2.88851500 & 1.66348800 & 2.96699900 \\
\hline $\mathrm{H}$ & 0.80540600 & 2.63140900 & 6.40027200 & $\mathrm{H}$ & -0.13526200 & 3.09299300 & 4.97695100 \\
\hline $\mathrm{H}$ & 2.86658800 & 2.53678900 & 5.11277400 & $\mathrm{H}$ & 2.07605000 & 3.93149500 & 4.36383700 \\
\hline $\mathrm{N}$ & 1.06559600 & 0.89428600 & 3.60710100 & C & 1.45308100 & 2.88607400 & 2.03504300 \\
\hline C & 2.46817200 & 3.39922900 & 1.27711500 & C & 0.08208700 & 3.26561900 & 1.73388100 \\
\hline $\mathrm{C}$ & 2.26264000 & 4.31454600 & 0.19710000 & $\mathrm{H}$ & 3.49394100 & 3.11485700 & 1.50346200 \\
\hline C & -0.15331300 & 4.18070500 & 0.65118000 & C & -1.03840300 & 2.77533700 & 2.42667700 \\
\hline C & 3.32667400 & 4.84700800 & -0.54983800 & C & 0.92757100 & 4.69105500 & -0.12493600 \\
\hline C & -1.48658400 & 4.58732300 & 0.33156100 & $\mathrm{H}$ & -0.92763200 & 2.01453400 & 3.18000000 \\
\hline $\mathrm{C}$ & -2.33195400 & 3.19417100 & 2.11802500 & C & 3.08382100 & 5.71636000 & -1.61187000 \\
\hline $\mathrm{H}$ & 4.34633600 & 4.57862200 & -0.28457000 & C & 0.68739700 & 5.57853100 & -1.21857900 \\
\hline C & -2.56133400 & 4.09770000 & 1.08835300 & C & -1.70066200 & 5.47111400 & -0.77936200 \\
\hline $\mathrm{H}$ & -3.16493300 & 2.77468600 & 2.67119700 & C & 1.77936300 & 6.07048000 & -1.95168400 \\
\hline $\mathrm{H}$ & 3.91714400 & 6.12820000 & -2.17409800 & $\mathrm{C}$ & -0.66582600 & 5.94251200 & -1.52541200 \\
\hline $\mathrm{H}$ & -3.57356500 & 4.39083700 & 0.83156000 & $\mathrm{H}$ & -2.72346000 & 5.75289700 & -1.01412200 \\
\hline $\mathrm{H}$ & 1.59570900 & 6.74722300 & -2.78178400 & $\mathrm{H}$ & -0.84575400 & 6.60987900 & -2.36387900 \\
\hline $\mathrm{C}$ & -2.03219800 & -1.20148400 & 2.11698900 & $\mathrm{~S}$ & -2.71033800 & -0.29321600 & 3.38240600 \\
\hline $\mathrm{N}$ & -0.76822200 & -1.68351000 & 2.15103800 & $\mathrm{H}$ & -0.38718900 & -2.11177300 & 1.29866100 \\
\hline $\mathrm{S}$ & -0.51530700 & -3.84773700 & -1.01488000 & $\mathrm{O}$ & -0.15605000 & -3.79746900 & -2.44124500 \\
\hline $\mathrm{O}$ & 0.27165600 & -2.92447600 & -0.14540800 & $\mathrm{O}$ & -1.96522700 & -3.83258300 & -0.69159000 \\
\hline C & 0.07135900 & -5.50588200 & -0.42208800 & $\mathrm{~F}$ & -0.51636700 & -6.50103500 & -1.09028900 \\
\hline $\mathrm{F}$ & -0.20071300 & -5.64535600 & 0.88789900 & $\mathrm{~F}$ & 1.40245900 & -5.60313100 & -0.58165000 \\
\hline $\mathrm{C}$ & 3.26809700 & -2.01395300 & -1.07137000 & $\mathrm{O}$ & 3.54757500 & -2.57128800 & -2.12029200 \\
\hline $\mathrm{O}$ & 3.95828100 & -2.06418200 & 0.07371500 & C & 5.07149500 & -3.02861100 & 0.22384600 \\
\hline C & 4.52461300 & -4.44898500 & 0.08295700 & $\mathrm{H}$ & 5.32020400 & -5.16772500 & 0.30691000 \\
\hline $\mathrm{H}$ & 4.16138600 & -4.63366500 & -0.92914400 & $\mathrm{H}$ & 3.70071900 & -4.61380300 & 0.78229200 \\
\hline C & 5.56608200 & -2.75596000 & 1.64314600 & $\mathrm{H}$ & 5.88001500 & -1.71189200 & 1.74574900 \\
\hline $\mathrm{H}$ & 6.42414900 & -3.39898700 & 1.86238800 & $\mathrm{H}$ & 4.78351700 & -2.95773800 & 2.37700800 \\
\hline $\mathrm{C}$ & 6.17800500 & -2.72193000 & -0.78782600 & $\mathrm{H}$ & 7.05848900 & -3.32851600 & -0.55060000 \\
\hline $\mathrm{H}$ & 6.46932800 & -1.66715900 & -0.72572200 & $\mathrm{H}$ & 5.86126400 & -2.94625000 & -1.80590900 \\
\hline $\mathrm{C}$ & 1.34223800 & -1.00158000 & -2.02619800 & $\mathrm{H}$ & 1.31627400 & -1.89455300 & -2.64674000 \\
\hline C & 0.03280500 & -0.34732500 & -1.85551700 & C & -0.96625100 & -0.65956600 & -2.79227800 \\
\hline
\end{tabular}




\begin{tabular}{|c|c|c|c|c|c|c|c|}
\hline C & -0.22364700 & 0.61215700 & -0.86532400 & C & -2.18818200 & 0.00465900 & -2.76844600 \\
\hline $\mathrm{H}$ & -0.78552300 & -1.44834600 & -3.51517300 & C & -1.45521200 & 1.26280200 & -0.84099100 \\
\hline $\bar{H}$ & 0.52232900 & 0.84971700 & -0.11436000 & C & -2.42497700 & 0.98478500 & -1.80349700 \\
\hline $\mathrm{H}$ & -2.96619000 & -0.26435800 & -3.47482400 & $\mathrm{H}$ & -1.66164400 & 1.98383400 & -0.06517000 \\
\hline $\mathrm{H}$ & -3.37221800 & 1.51079700 & -1.77590700 & $\mathrm{~N}$ & 2.19801600 & -1.14116300 & -0.94911500 \\
\hline $\mathrm{H}$ & 1.92049800 & -0.84474900 & -0.00613800 & C & 2.28114000 & -0.02918800 & -3.39208500 \\
\hline $\mathrm{H}$ & 1.60423800 & -0.11611800 & -4.23786200 & $\mathrm{H}$ & 3.11996900 & -0.71966900 & -3.40953400 \\
\hline C & 2.46929300 & 1.24527300 & -2.84316900 & C & 3.47370600 & 1.46213100 & -1.81332700 \\
\hline $\mathrm{H}$ & 3.55274000 & 0.57758600 & -1.16409000 & $\mathrm{H}$ & 3.25294800 & 2.34542700 & -1.21041400 \\
\hline $\mathrm{C}$ & 1.53470000 & 2.35975200 & -3.15867500 & $\mathrm{H}$ & 2.04119100 & 3.32965200 & -3.13106100 \\
\hline $\mathrm{H}$ & 0.77255200 & 2.38526000 & -2.36245500 & $\mathrm{H}$ & 1.01641500 & 2.22372600 & -4.11023500 \\
\hline C & 6.33649400 & 1.84945700 & -0.94641700 & $\mathrm{H}$ & 6.03264200 & 2.71106600 & -0.34083700 \\
\hline $\mathrm{H}$ & 7.39273200 & 1.98139500 & -1.20815400 & $\mathrm{H}$ & 6.25606900 & 0.95447700 & -0.31807900 \\
\hline C & 5.33199600 & 3.22775100 & -3.55923400 & $\mathrm{H}$ & 4.96772800 & 4.10416200 & -3.01426600 \\
\hline $\mathrm{H}$ & 4.71183900 & 3.11120200 & -4.45574100 & $\mathrm{H}$ & 6.35627400 & 3.43494600 & -3.89057300 \\
\hline C & 5.76127700 & 0.16243100 & -3.48861200 & $\mathrm{H}$ & 5.24315400 & 0.13112800 & -4.45317500 \\
\hline $\mathrm{H}$ & 5.53837200 & -0.77006300 & -2.96070000 & $\mathrm{H}$ & 6.83825100 & 0.18083600 & -3.69478300 \\
\hline Si & 5.26925100 & 1.67917600 & -2.49072900 & & & & \\
\hline
\end{tabular}

\section{TS-R-4f-12}

Imaginary frequency: $-192.47 \mathrm{~cm}^{-1}$

E[B3LYP/6-31G(d)-D3(BJ)]: -4789.083957 Hartree

$\mathrm{E}\left[\mathrm{PCM}\left(\mathrm{Et}_{2} \mathrm{O}\right)-\mathrm{B} 3 \mathrm{LYP} / 6-311 \mathrm{G}(\mathrm{d}, \mathrm{p})-\mathrm{D} 3(\mathrm{BJ})\right]$ : -4790.226463 Hartree

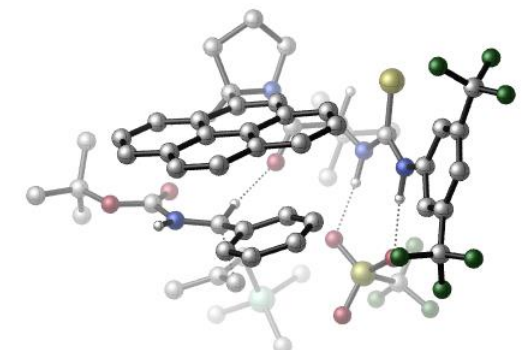

\begin{tabular}{|c|c|c|c|c|c|c|c|}
\hline 01 & & & & $\mathrm{~N}$ & 2.78114900 & 1.59515100 & 0.95988900 \\
\hline $\mathrm{H}$ & 2.27636300 & 2.16499900 & 0.26987000 & C & 3.98957100 & 1.09306500 & 0.44486200 \\
\hline C & 4.18327500 & 1.25668000 & -0.94043200 & $\mathrm{C}$ & 4.98455900 & 0.44314600 & 1.18813100 \\
\hline $\mathrm{C}$ & 5.29359400 & 0.70387400 & -1.56695300 & $\mathrm{H}$ & 3.45211700 & 1.80420700 & -1.52328200 \\
\hline $\mathrm{C}$ & 6.09365000 & -0.09642200 & 0.53550400 & $\mathrm{H}$ & 4.88356000 & 0.34476300 & 2.25722300 \\
\hline C & 6.26052600 & 0.00914100 & -0.84156500 & $\mathrm{H}$ & 7.12262800 & -0.42294700 & -1.33395000 \\
\hline C & 5.38932400 & 0.75630800 & -3.06515900 & C & 7.07461900 & -0.90625300 & 1.33517100 \\
\hline $\mathrm{F}$ & 4.77842800 & 1.83613400 & -3.58488000 & $\mathrm{~F}$ & 4.80134800 & -0.33825400 & -3.62642100 \\
\hline $\mathrm{F}$ & 6.67109400 & 0.75959400 & -3.49136000 & $\mathrm{~F}$ & 7.19368400 & -0.46638600 & 2.60315000 \\
\hline $\mathrm{F}$ & 6.68316300 & -2.20710700 & 1.40556200 & $\mathrm{~F}$ & 8.30715600 & -0.90296500 & 0.78180200 \\
\hline C & -0.13922800 & 1.46302800 & 3.15963700 & $\mathrm{H}$ & 0.44781200 & 1.10608800 & 4.00239900 \\
\hline C & -0.94133100 & 2.69608100 & 3.66321300 & C & -1.86394800 & 3.30614900 & 2.59969600 \\
\hline $\mathrm{H}$ & -2.61391200 & 2.58855100 & 2.25908300 & $\mathrm{H}$ & -1.30377700 & 3.64112500 & 1.72518500 \\
\hline $\mathrm{H}$ & -2.38084300 & 4.17458600 & 3.02433800 & C & 0.07154200 & 3.75753200 & 4.12481400 \\
\hline $\mathrm{H}$ & -0.45266000 & 4.60280200 & 4.58530900 & $\mathrm{H}$ & 0.65112100 & 4.13741000 & 3.27848100 \\
\hline $\mathrm{H}$ & 0.77370400 & 3.34776900 & 4.86002600 & $\mathrm{C}$ & -1.78866400 & 2.23465400 & 4.86230700 \\
\hline $\mathrm{H}$ & -2.36474000 & 3.07543000 & 5.26386500 & $\mathrm{H}$ & -1.15948800 & 1.84457500 & 5.67111200 \\
\hline $\mathrm{H}$ & -2.50172000 & 1.45295300 & 4.57323200 & C & -0.99787300 & 0.31765200 & 2.59855600 \\
\hline C & -1.63514500 & -2.03656300 & 2.64428100 & $\mathrm{H}$ & -2.56905300 & -1.74786500 & 2.15640500 \\
\hline $\mathrm{N}$ & -1.06051200 & -0.84524300 & 3.28960900 & $\mathrm{C}$ & -0.68158500 & -2.62773700 & 1.61000000 \\
\hline C & 0.63774500 & -2.28123300 & 1.59180900 & C & -1.18156500 & -3.58673800 & 0.64596900 \\
\hline $\mathrm{C}$ & 1.56016600 & -2.80503400 & 0.62994200 & $\mathrm{H}$ & 1.02575000 & -1.56169000 & 2.30437800 \\
\hline $\mathrm{C}$ & -0.29187200 & -4.08446000 & -0.36012200 & $C$ & -2.50816000 & -4.04910100 & 0.64758000 \\
\hline C & 2.91560100 & -2.44093000 & 0.63285900 & C & 1.07790700 & -3.69681400 & -0.36710000 \\
\hline $\mathrm{C}$ & -0.77196200 & -4.98099500 & -1.36335100 & $\mathrm{H}$ & -3.19640600 & -3.70732300 & 1.41146100 \\
\hline C & -2.96399000 & -4.93733300 & -0.32614100 & C & 3.78227300 & -2.93366600 & -0.34071500 \\
\hline
\end{tabular}




\begin{tabular}{|c|c|c|c|c|c|c|c|}
\hline $\mathrm{H}$ & 3.27827900 & -1.76735800 & 1.40232500 & $\mathrm{C}$ & 1.96860700 & -4.19337100 & -1.36317300 \\
\hline C & -2.11614600 & -5.38578900 & -1.33375500 & C & 0.14141500 & -5.45277500 & -2.36615900 \\
\hline $\mathrm{H}$ & -3.99449500 & -5.27643200 & -0.30122200 & C & 3.31429000 & -3.79453000 & -1.33196400 \\
\hline $\mathrm{H}$ & 4.82837700 & -2.64962600 & -0.31472600 & C & 1.45054000 & -5.07968500 & -2.36453000 \\
\hline $\mathrm{H}$ & -2.47964900 & -6.07523500 & -2.09156400 & $\mathrm{H}$ & -0.23784900 & -6.13042200 & -3.12693900 \\
\hline $\mathrm{H}$ & 3.99406700 & -4.17178200 & -2.09170800 & $\mathrm{H}$ & 2.13041300 & -5.45158000 & -3.12689900 \\
\hline C & 2.09373500 & 1.28650100 & 2.10533200 & $S$ & 2.72629100 & 0.38655500 & 3.40070900 \\
\hline $\mathrm{N}$ & 0.82883100 & 1.78662600 & 2.11574300 & $\mathrm{H}$ & 0.41727000 & 2.12368600 & 1.23814700 \\
\hline S & 0.26680600 & 3.35835800 & -1.34899800 & $\mathrm{O}$ & -0.32316000 & 3.39019600 & -2.69440700 \\
\hline 0 & -0.55231200 & 2.65528000 & -0.31897400 & $\mathrm{O}$ & 1.70926100 & 3.00764500 & -1.26739300 \\
\hline C & 0.24318300 & 5.11941300 & -0.75755400 & $\mathrm{~F}$ & 1.06548100 & 5.88350400 & -1.47780100 \\
\hline$F$ & 0.62106100 & 5.17147900 & 0.53287200 & $\mathrm{~F}$ & -0.99942700 & 5.63067000 & -0.84666900 \\
\hline C & -2.75943000 & 0.94390500 & -2.31441300 & $\mathrm{H}$ & -1.84707000 & 1.50101900 & -2.13465800 \\
\hline $\mathrm{H}$ & -2.85090300 & 0.47718400 & -3.29239900 & C & -3.91649600 & 1.32774100 & -1.64299800 \\
\hline C & -3.86437500 & 2.22552300 & -0.49558700 & $\mathrm{H}$ & -2.84887300 & 2.31487100 & -0.10043300 \\
\hline $\mathrm{H}$ & -4.55714600 & 1.91480400 & 0.29392900 & C & -5.24115400 & 0.82985600 & -2.13842300 \\
\hline $\mathrm{H}$ & -5.55510400 & 1.44752400 & -2.99112800 & $\mathrm{H}$ & -6.01880900 & 0.87979800 & -1.37525700 \\
\hline $\mathrm{H}$ & -5.16060200 & -0.19558500 & -2.51546600 & C & -3.95397600 & 5.14791400 & 0.42652000 \\
\hline $\mathrm{H}$ & -4.39800000 & 4.79557700 & 1.36403400 & $\mathrm{H}$ & -4.32290100 & 6.16386900 & 0.23986600 \\
\hline $\mathrm{H}$ & -2.87185100 & 5.20101600 & 0.55949900 & C & -6.29798000 & 4.02931500 & -1.20339000 \\
\hline $\mathrm{H}$ & -6.79936900 & 3.67052900 & -0.29659600 & $\mathrm{H}$ & -6.63931100 & 3.41459500 & -2.04308300 \\
\hline $\mathrm{H}$ & -6.64626700 & 5.05301500 & -1.38784600 & C & -3.59298900 & 4.49333700 & -2.62544600 \\
\hline $\mathrm{H}$ & -4.00571400 & 3.91036000 & -3.45789700 & $\mathrm{H}$ & -2.51247900 & 4.32353500 & -2.60641500 \\
\hline $\mathrm{H}$ & -3.76801500 & 5.55277800 & -2.84811000 & $\mathrm{Si}$ & -4.41600700 & 4.02006800 & -1.00560600 \\
\hline C & -0.52562200 & -1.13053700 & 4.63492300 & $\mathrm{H}$ & -1.11919500 & -0.60276000 & 5.39008000 \\
\hline $\mathrm{H}$ & 0.51625200 & -0.81071500 & 4.71665100 & C & -0.68136200 & -2.65247400 & 4.76596600 \\
\hline $\mathrm{H}$ & -0.84317800 & -2.96154700 & 5.80197400 & $\mathrm{H}$ & 0.22191300 & -3.15083100 & 4.40066100 \\
\hline C & -1.86406900 & -2.97949900 & 3.84379100 & $\mathrm{H}$ & -1.89721700 & -4.02967900 & 3.54499900 \\
\hline $\mathrm{H}$ & -2.81384000 & -2.72777400 & 4.33111300 & $\mathrm{O}$ & -1.54135600 & 0.43957700 & 1.49431100 \\
\hline C & -4.36394100 & -1.73309700 & -0.56179400 & $\mathrm{O}$ & -4.53898100 & -0.93934400 & 0.34258400 \\
\hline 0 & -5.17599100 & -2.72422200 & -0.95059800 & C & -6.44721100 & -2.98332100 & -0.23956600 \\
\hline $\mathrm{C}$ & -7.35985000 & -1.76194000 & -0.35425000 & $\mathrm{H}$ & -8.34806800 & -2.01085500 & 0.04610100 \\
\hline $\mathrm{H}$ & -6.95879000 & -0.91629200 & 0.20567000 & $\mathrm{H}$ & -7.48121800 & -1.47388300 & -1.40352200 \\
\hline C & -7.02013700 & -4.16727200 & -1.01496400 & $\mathrm{H}$ & -6.33907800 & -5.02275100 & -0.97601300 \\
\hline $\mathrm{H}$ & -7.97865600 & -4.46713100 & -0.58062800 & $\mathrm{H}$ & -7.18149300 & -3.90045600 & -2.06387100 \\
\hline C & -6.16768900 & -3.35956700 & 1.21561900 & $\mathrm{H}$ & -7.11220100 & -3.61161100 & 1.70895900 \\
\hline $\mathrm{H}$ & -5.51476700 & -4.23566200 & 1.27057100 & $\mathrm{H}$ & -5.70450500 & -2.53161900 & 1.75429400 \\
\hline C & -2.26058500 & -0.75850200 & -1.24171800 & $\mathrm{H}$ & -2.24501000 & -0.28724700 & -0.26319300 \\
\hline C & -0.95789400 & -1.01141500 & -1.86686600 & C & 0.16747300 & -0.41015800 & -1.28260300 \\
\hline C & -0.80474500 & -1.78808400 & -3.02824600 & C & 1.42178100 & -0.58834200 & -1.85263400 \\
\hline $\mathrm{H}$ & 0.03790000 & 0.19173600 & -0.39234200 & $\mathrm{C}$ & 0.45340800 & -1.95954700 & -3.59175200 \\
\hline $\mathrm{H}$ & -1.66497100 & -2.24852100 & -3.50831600 & C & 1.57005500 & -1.35335200 & -3.00777200 \\
\hline $\mathrm{H}$ & 2.29029300 & -0.14508900 & -1.38815500 & $\mathrm{H}$ & 0.56584100 & -2.56624400 & -4.48441300 \\
\hline $\mathrm{H}$ & 2.55477200 & -1.47110700 & -3.44607800 & $\mathrm{~N}$ & -3.25347700 & -1.70087500 & -1.39484100 \\
\hline $\mathrm{H}$ & -3.13139600 & -2.47945900 & -2.02961500 & & & & \\
\hline
\end{tabular}

\section{TS- $R-4 f-13$}

Imaginary frequency: $-393.12 \mathrm{~cm}^{-1}$

E[B3LYP/6-31G(d)-D3(BJ)]: -4789.083194 Hartree

E[PCM(Et $\left.\left.{ }_{2} \mathrm{O}\right)-B 3 L Y P / 6-311 G(d, p)-D 3(B J)\right]:-4790.225989$ Hartree

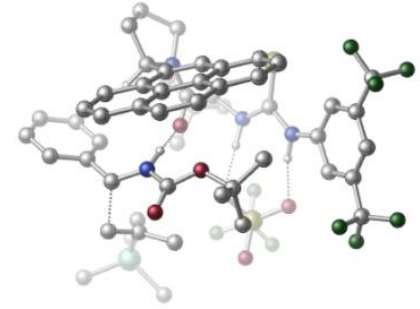

S182 


\begin{tabular}{|c|c|c|c|c|c|c|c|}
\hline 01 & & & & $\mathrm{~N}$ & -1.64119400 & -2.28719900 & 1.58011100 \\
\hline $\mathrm{H}$ & -0.98828800 & -2.91271900 & 1.09526100 & C & -2.92420100 & -2.30978600 & 1.01032100 \\
\hline $\mathrm{C}$ & -3.05064900 & -3.02794000 & -0.19358100 & $\mathrm{C}$ & -4.04511800 & -1.64272300 & 1.52169500 \\
\hline$C$ & -4.25532400 & -3.02416400 & -0.88548900 & $\mathrm{H}$ & -2.19495000 & -3.56169600 & -0.59162500 \\
\hline C & -5.23955100 & -1.64850200 & 0.80328100 & $\mathrm{H}$ & -3.97221100 & -1.11043300 & 2.45621800 \\
\hline C & -5.36446200 & -2.32727000 & -0.40471000 & $\mathrm{H}$ & -6.29461700 & -2.31313100 & -0.95994800 \\
\hline C & -4.33688500 & -3.66640200 & -2.24066400 & C & -6.43765600 & -0.94054000 & 1.37070500 \\
\hline $\mathrm{F}$ & -3.39022100 & -4.60178600 & -2.43127200 & $\mathrm{~F}$ & -4.18159700 & -2.73766100 & -3.22826000 \\
\hline $\mathrm{F}$ & -5.53991200 & -4.24377400 & -2.45309000 & $\mathrm{~F}$ & -6.09267500 & 0.02057900 & 2.26055500 \\
\hline $\mathrm{F}$ & -7.16580300 & -0.33606100 & 0.39853200 & $\mathrm{~F}$ & -7.27346800 & -1.78687400 & 2.01089300 \\
\hline C & 1.06650100 & -0.52673500 & 3.23748000 & $\mathrm{H}$ & 0.51868000 & -0.33276200 & 4.15796400 \\
\hline C & 2.47722400 & -1.06437600 & 3.63890000 & C & 3.39513800 & -1.34398300 & 2.43832000 \\
\hline $\mathrm{H}$ & 3.63072000 & -0.42856100 & 1.88911600 & $\mathrm{H}$ & 2.95123000 & -2.04842900 & 1.73611100 \\
\hline $\mathrm{H}$ & 4.33909500 & -1.77119100 & 2.79731200 & C & 2.26909800 & -2.36429500 & 4.43523900 \\
\hline $\mathrm{H}$ & 3.23028200 & -2.73062600 & 4.81390000 & $\mathrm{H}$ & 1.82783900 & -3.14270300 & 3.81022100 \\
\hline $\mathrm{H}$ & 1.60541600 & -2.19985500 & 5.29199600 & C & 3.16843400 & -0.02842600 & 4.54387900 \\
\hline $\mathrm{H}$ & 4.17153200 & -0.37932900 & 4.80945300 & $\mathrm{H}$ & 2.61855700 & 0.12478100 & 5.47939800 \\
\hline $\mathrm{H}$ & 3.27778300 & 0.94188800 & 4.04420400 & C & 1.09387500 & 0.74716300 & 2.37469200 \\
\hline 0 & 1.29035100 & 0.63401600 & 1.15216500 & C & 0.63141000 & 2.26533400 & 4.35494700 \\
\hline C & 0.91263100 & 3.17446200 & 2.12174500 & C & 1.30231500 & 3.63200600 & 4.50343700 \\
\hline $\mathrm{H}$ & -0.44983200 & 2.31763000 & 4.53090800 & $\mathrm{H}$ & 1.04701500 & 1.50988200 & 5.01596000 \\
\hline $\mathrm{H}$ & 1.82363600 & 3.14010700 & 1.52504400 & $\mathrm{H}$ & 2.38390800 & 3.49425700 & 4.61955300 \\
\hline $\mathrm{H}$ & 0.93482200 & 4.18848800 & 5.37005400 & $\mathrm{~N}$ & 0.89086100 & 1.94985800 & 2.94601500 \\
\hline C & -0.29291400 & 3.30271000 & 1.21001000 & C & -1.46532200 & 2.67996600 & 1.51936000 \\
\hline C & -0.20440700 & 4.14977800 & 0.04027200 & C & -2.65291100 & 2.85401100 & 0.73894600 \\
\hline $\mathrm{H}$ & -1.52118900 & 2.01149200 & 2.37251300 & C & -1.36305100 & 4.29795300 & -0.78635100 \\
\hline $\mathrm{C}$ & 0.97316400 & 4.81877400 & -0.33474300 & C & -3.86616000 & 2.23768500 & 1.08491300 \\
\hline C & -2.59122900 & 3.67248600 & -0.42492300 & C & -1.29666800 & 5.08588600 & -1.97603700 \\
\hline $\mathrm{H}$ & 1.86139300 & 4.74732700 & 0.28118000 & C & 1.03262400 & 5.57018400 & -1.50690800 \\
\hline C & -5.00481700 & 2.43042500 & 0.30448900 & $\mathrm{H}$ & -3.90241800 & 1.59164300 & 1.95618300 \\
\hline $\mathrm{C}$ & -3.75853900 & 3.85465000 & -1.22502100 & C & -0.08438500 & 5.69919400 & -2.32631800 \\
\hline C & -2.48256500 & 5.23792200 & -2.77299600 & $\mathrm{H}$ & 1.96214600 & 6.06477800 & -1.77559000 \\
\hline C & -4.95333400 & 3.22858900 & -0.83716400 & $\mathrm{H}$ & -5.93062100 & 1.93640600 & 0.57566300 \\
\hline $\mathrm{C}$ & -3.66007800 & 4.65992100 & -2.40976500 & $\mathrm{H}$ & -0.03309300 & 6.28880700 & -3.23773700 \\
\hline $\mathrm{H}$ & -2.41882700 & 5.83734000 & -3.67733400 & $\mathrm{H}$ & -5.84226700 & 3.36425800 & -1.44772300 \\
\hline $\mathrm{H}$ & -4.54974400 & 4.79045600 & -3.02036300 & C & -1.08294700 & -1.44830500 & 2.50608100 \\
\hline $\mathrm{S}$ & -1.93904200 & -0.46156300 & 3.58654500 & $\mathrm{~N}$ & 0.28267100 & -1.50738300 & 2.50106400 \\
\hline $\mathrm{H}$ & 0.71571900 & -1.81834300 & 1.62464800 & $\mathrm{~S}$ & 1.19374000 & -3.77886000 & -0.43363600 \\
\hline $\mathrm{O}$ & 1.53392600 & -4.07824600 & -1.83347500 & $\mathrm{O}$ & 1.52072600 & -2.38909900 & 0.00889000 \\
\hline $\mathrm{O}$ & -0.13805200 & -4.22968500 & 0.04175400 & C & 2.37480200 & -4.81986800 & 0.55142300 \\
\hline $\mathrm{F}$ & 2.23360600 & -6.11435500 & 0.27002700 & $\mathrm{~F}$ & 2.18249700 & -4.63891200 & 1.86823100 \\
\hline $\mathrm{F}$ & 3.64736300 & -4.45970700 & 0.26970500 & C & 0.98408100 & 4.32393900 & 3.16788000 \\
\hline $\mathrm{H}$ & 0.01217500 & 4.82267000 & 3.22101700 & $\mathrm{H}$ & 1.72706100 & 5.07555800 & 2.88845700 \\
\hline $\mathrm{C}$ & 0.46926800 & 1.48290800 & -2.46059000 & $\mathrm{O}$ & 0.68308400 & 1.66331200 & -3.64763800 \\
\hline $\mathrm{O}$ & -0.63698400 & 1.04838300 & -1.87408500 & C & -1.73529000 & 0.42221700 & -2.66693700 \\
\hline C & -1.21715000 & -0.89491800 & -3.24032700 & $\mathrm{H}$ & -2.04804300 & -1.44299200 & -3.69286100 \\
\hline $\mathrm{H}$ & -0.45626500 & -0.72559100 & -4.00553400 & $\mathrm{H}$ & -0.79782600 & -1.52335300 & -2.44784400 \\
\hline C & -2.80229500 & 0.17657400 & -1.61096800 & $\mathrm{H}$ & -3.23472900 & 1.11434800 & -1.26926500 \\
\hline $\mathrm{H}$ & -3.59700500 & -0.44285100 & -2.03279100 & $\mathrm{H}$ & -2.37556900 & -0.34362000 & -0.75202400 \\
\hline C & -2.24518200 & 1.37756800 & -3.74329300 & $\mathrm{H}$ & -3.17430700 & 0.96862300 & -4.15565500 \\
\hline $\mathrm{H}$ & -2.46864200 & 2.35522000 & -3.30979300 & $\mathrm{H}$ & -1.52307600 & 1.50449000 & -4.54949100 \\
\hline $\mathrm{C}$ & 2.67239400 & 2.20069600 & -1.90607900 & $\mathrm{H}$ & 2.56049600 & 2.81506500 & -2.79403000 \\
\hline C & 3.61959300 & 2.73370600 & -0.90129000 & C & 4.36098200 & 3.87809000 & -1.23306000 \\
\hline
\end{tabular}




\begin{tabular}{|c|c|c|c|c|c|c|c|}
\hline C & 3.81262300 & 2.13502400 & 0.35151600 & C & 5.25461100 & 4.43110800 & -0.31992000 \\
\hline $\mathrm{H}$ & 4.21918800 & 4.34311500 & -2.20490900 & C & 4.71269900 & 2.68761300 & 1.26204100 \\
\hline $\mathrm{H}$ & 3.23442000 & 1.26283400 & 0.62379500 & $\mathrm{C}$ & 5.43188500 & 3.83727500 & 0.93277900 \\
\hline $\bar{H}$ & 5.80979600 & 5.32649000 & -0.58280100 & $\mathrm{H}$ & 4.84673000 & 2.21556400 & 2.23131700 \\
\hline $\mathrm{H}$ & 6.12906000 & 4.26834000 & 1.64517100 & $\mathrm{~N}$ & 1.45872000 & 1.70855900 & -1.49642700 \\
\hline $\mathrm{H}$ & 1.28978600 & 1.39838100 & -0.53059300 & C & 3.78727200 & 0.89840800 & -2.92282800 \\
\hline $\mathrm{H}$ & 3.36490800 & 1.06860600 & -3.90889200 & $\mathrm{H}$ & 4.76721100 & 1.33302300 & -2.74750300 \\
\hline C & 3.48737100 & -0.33042000 & -2.31098200 & C & 4.24262800 & -0.84321300 & -1.18457100 \\
\hline $\mathrm{H}$ & 4.79846000 & -0.05903900 & -0.65946000 & $\mathrm{H}$ & 3.60104600 & -1.41105800 & -0.50208100 \\
\hline $\mathrm{C}$ & 2.27506600 & -1.06454800 & -2.73172200 & $\mathrm{H}$ & 2.40964700 & -2.14935000 & -2.71549500 \\
\hline $\mathrm{H}$ & 1.51441900 & -0.89263400 & -1.95360200 & $\mathrm{H}$ & 1.88928600 & -0.72494500 & -3.69210200 \\
\hline C & 6.40007100 & -2.66915000 & -0.11600500 & $\mathrm{H}$ & 5.66157200 & -3.18833200 & 0.50145700 \\
\hline $\mathrm{H}$ & 7.22843800 & -3.36198500 & -0.30589500 & $\mathrm{H}$ & 6.79582500 & -1.82444500 & 0.45968600 \\
\hline C & 4.90876500 & -3.49124900 & -2.74524200 & $\mathrm{H}$ & 4.05209800 & -3.95722600 & -2.24986900 \\
\hline $\mathrm{H}$ & 4.57747200 & -3.14422700 & -3.73051900 & $\mathrm{H}$ & 5.66877900 & -4.26529400 & -2.90808400 \\
\hline C & 6.88608400 & -1.10415600 & -2.74906700 & $\mathrm{H}$ & 6.43786500 & -0.71169000 & -3.66892900 \\
\hline $\mathrm{H}$ & 7.28934700 & -0.25704200 & -2.18151400 & $\mathrm{H}$ & 7.73129200 & -1.73931400 & -3.03958700 \\
\hline Si & 5.63608100 & -2.08780000 & -1.73526800 & & & & \\
\hline
\end{tabular}

\section{TS-R-4f-14}

Imaginary frequency: $-390.35 \mathrm{~cm}^{-1}$

E[B3LYP/6-31G(d)-D3(BJ)]: -4789.081781 Hartree

$\mathrm{E}\left[\mathrm{PCM}\left(\mathrm{Et}_{2} \mathrm{O}\right)-\mathrm{B} 3 \mathrm{LYP} / 6-311 \mathrm{G}(\mathrm{d}, \mathrm{p})-\mathrm{D} 3(\mathrm{BJ})\right]$ : -4790.225616 Hartree

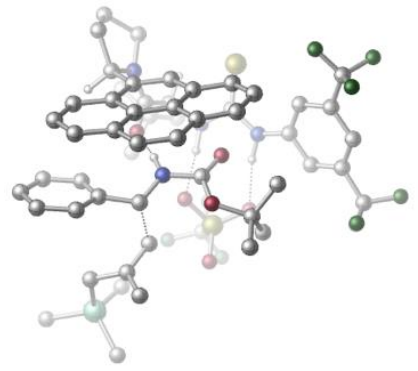

\begin{tabular}{|c|c|c|c|c|c|c|c|}
\hline 01 & & & & $\mathrm{~N}$ & -2.48174000 & -1.27875400 & 1.78106900 \\
\hline $\mathrm{H}$ & -1.88224100 & -2.06865800 & 1.52237200 & C & -3.59896000 & -1.17023200 & 0.94577500 \\
\hline$C$ & -3.87119600 & -2.27973300 & 0.12520300 & C & -4.41412600 & -0.03540400 & 0.83439700 \\
\hline C & -4.91273900 & -2.23404400 & -0.79503100 & $\mathrm{H}$ & -3.24189500 & -3.15911100 & 0.18915400 \\
\hline C & -5.44762500 & -0.01228500 & -0.09838900 & $\mathrm{H}$ & -4.23266500 & 0.81561400 & 1.46999000 \\
\hline C & -5.71626900 & -1.10196500 & -0.92251300 & $\mathrm{H}$ & -6.52083600 & -1.06958200 & -1.64784000 \\
\hline C & -5.13520300 & -3.37905300 & -1.73984700 & C & -6.31325900 & 1.20917600 & -0.21878200 \\
\hline$F$ & -4.51197200 & -4.50652500 & -1.35188400 & $\mathrm{~F}$ & -4.67253300 & -3.08288100 & -2.98974900 \\
\hline $\mathrm{F}$ & -6.44875600 & -3.66267300 & -1.88674800 & $\mathrm{~F}$ & -5.78488600 & 2.28512300 & 0.40726100 \\
\hline $\mathrm{F}$ & -6.49076400 & 1.56006300 & -1.52105100 & $\mathrm{~F}$ & -7.54704500 & 1.01514500 & 0.29539600 \\
\hline C & 0.17159500 & 0.13101300 & 3.84259300 & $\mathrm{H}$ & -0.53979900 & 0.59381100 & 4.52432500 \\
\hline $\mathrm{C}$ & 1.16673800 & -0.70884700 & 4.70132000 & C & 2.31323400 & -1.33350500 & 3.88794000 \\
\hline $\mathrm{H}$ & 2.97261700 & -0.56615500 & 3.47285200 & $\mathrm{H}$ & 1.95135500 & -1.93809500 & 3.05653400 \\
\hline $\mathrm{H}$ & 2.90892200 & -1.97817300 & 4.54539300 & C & 0.36162900 & -1.81978100 & 5.39633800 \\
\hline $\mathrm{H}$ & 1.00241200 & -2.36086300 & 6.10208300 & $\mathrm{H}$ & -0.02757100 & -2.53661300 & 4.67092700 \\
\hline $\mathrm{H}$ & -0.48661000 & -1.40518400 & 5.95356300 & $\mathrm{C}$ & 1.76989900 & 0.22127500 & 5.76993600 \\
\hline $\mathrm{H}$ & 2.49067300 & -0.32961000 & 6.38396100 & $\mathrm{H}$ & 0.99827800 & 0.61635200 & 6.44165100 \\
\hline $\mathrm{H}$ & 2.30190100 & 1.06776300 & 5.31829300 & C & 0.85378300 & 1.20623700 & 2.99252500 \\
\hline $\mathrm{O}$ & 1.50153100 & 0.86976200 & 1.98530200 & $\mathrm{C}$ & 0.08647300 & 3.10047300 & 4.49674800 \\
\hline$C$ & 1.32559900 & 3.52573800 & 2.43457000 & C & 0.89689700 & 4.38597800 & 4.68335100 \\
\hline $\mathrm{H}$ & -0.97188500 & 3.30104000 & 4.29485300 & $\mathrm{H}$ & 0.14939900 & 2.43078800 & 5.35233500 \\
\hline $\mathrm{H}$ & 2.37972200 & 3.25922800 & 2.31852600 & $\mathrm{H}$ & 1.83906000 & 4.15438000 & 5.19435900 \\
\hline $\mathrm{H}$ & 0.36314700 & 5.13409200 & 5.27597400 & $\mathrm{~N}$ & 0.71859600 & 2.50566900 & 3.31220900 \\
\hline C & 0.66982900 & 3.59263000 & 1.06634000 & C & -0.63534000 & 3.22765600 & 0.91046200 \\
\hline $\mathrm{C}$ & 1.42009100 & 4.10733000 & -0.05958600 & C & -1.31337200 & 3.33486800 & -0.34587000 \\
\hline $\mathrm{H}$ & -1.19404400 & 2.81849200 & 1.74777100 & C & 0.78293700 & 4.16299400 & -1.33996800 \\
\hline
\end{tabular}




\begin{tabular}{|c|c|c|c|c|c|c|c|}
\hline $\mathrm{C}$ & 2.74732600 & 4.55935700 & 0.04235400 & $\mathrm{C}$ & -2.67242100 & 3.01443200 & -0.48392900 \\
\hline C & -0.58291600 & 3.78262500 & -1.48239900 & C & 1.50542900 & 4.63189000 & -2.48030300 \\
\hline $\mathrm{H}$ & 3.25484600 & 4.54284200 & 0.99955300 & $\mathrm{C}$ & 3.43565200 & 5.03468000 & -1.07056500 \\
\hline $\mathrm{C}$ & -3.30757700 & 3.13567200 & -1.71662700 & $\mathrm{H}$ & -3.22290500 & 2.66895000 & 0.38327100 \\
\hline C & -1.23228500 & 3.87336000 & -2.75033600 & C & 2.83017800 & 5.06282400 & -2.32241200 \\
\hline C & 0.84050200 & 4.66797800 & -3.75420000 & $\mathrm{H}$ & 4.46137400 & 5.37216000 & -0.95849300 \\
\hline C & -2.59493700 & 3.55253700 & -2.84036200 & $\mathrm{H}$ & -4.35848500 & 2.88367900 & -1.80611400 \\
\hline C & -0.46580800 & 4.30884700 & -3.88336800 & $\mathrm{H}$ & 3.37615200 & 5.42767200 & -3.18886300 \\
\hline $\mathrm{H}$ & 1.40546600 & 5.01189700 & -4.61723000 & $\mathrm{H}$ & -3.09409900 & 3.63440400 & -3.80279800 \\
\hline $\mathrm{H}$ & -0.95866000 & 4.35941900 & -4.85111600 & $\mathrm{C}$ & -1.92578100 & -0.38476000 & 2.65455900 \\
\hline $\mathrm{S}$ & -2.75206600 & 0.90070700 & 3.39257400 & $\mathrm{~N}$ & -0.62549000 & -0.67828600 & 2.93446400 \\
\hline $\mathrm{H}$ & -0.09534900 & -1.19867200 & 2.22685200 & $S$ & 0.44377400 & -3.42837100 & 0.53657000 \\
\hline $\mathrm{O}$ & 1.02065100 & -3.94517500 & -0.71893500 & $\mathrm{O}$ & 0.95970700 & -2.09037800 & 0.94943100 \\
\hline $\mathrm{O}$ & -1.02791500 & -3.55748700 & 0.68885800 & C & 1.08886700 & -4.57479800 & 1.85320400 \\
\hline $\mathrm{F}$ & 0.84572800 & -5.84877000 & 1.53425200 & $\mathrm{~F}$ & 0.49725200 & -4.30644700 & 3.02961200 \\
\hline $\mathrm{F}$ & 2.41519700 & -4.41938700 & 2.01368300 & C & 1.16183400 & 4.84102300 & 3.24074200 \\
\hline $\mathrm{H}$ & 0.29803100 & 5.39049900 & 2.85423400 & $\mathrm{H}$ & 2.03711200 & 5.48863400 & 3.14289000 \\
\hline C & -0.24882900 & 0.21511700 & -1.14486700 & 0 & -1.14030200 & 0.11502500 & -0.33225700 \\
\hline $\mathrm{O}$ & -0.28816400 & -0.07686800 & -2.45209900 & $C$ & -1.41711400 & -0.84573600 & -3.04447400 \\
\hline $\mathrm{C}$ & -2.73548100 & -0.10200200 & -2.86035700 & $\mathrm{H}$ & -3.53094900 & -0.67445100 & -3.34681600 \\
\hline $\mathrm{H}$ & -2.98428800 & 0.00927000 & -1.80679600 & $\mathrm{H}$ & -2.68227700 & 0.89082000 & -3.31305900 \\
\hline $\mathrm{C}$ & -1.02580000 & -0.89881200 & -4.51876100 & $\mathrm{H}$ & -0.07144900 & -1.42038900 & -4.65094300 \\
\hline $\mathrm{H}$ & -1.79129200 & -1.44045200 & -5.08257400 & $\mathrm{H}$ & -0.93607500 & 0.11108800 & -4.93169000 \\
\hline C & -1.43387300 & -2.23561100 & -2.42150100 & $\mathrm{H}$ & -2.23228700 & -2.82739400 & -2.87618600 \\
\hline $\bar{H}$ & -0.48539100 & -2.75811500 & -2.57449200 & $\mathrm{H}$ & -1.62351300 & -2.18517200 & -1.35094200 \\
\hline $\mathrm{C}$ & 2.11055900 & 0.60112000 & -1.61409800 & $\mathrm{H}$ & 1.84176100 & 0.72637500 & -2.65740600 \\
\hline C & 3.37113700 & 1.27393300 & -1.21917000 & C & 4.13377600 & 1.88697400 & -2.22072100 \\
\hline $\mathrm{C}$ & 3.86990000 & 1.22284200 & 0.09085600 & $\mathrm{C}$ & 5.37607800 & 2.44374600 & -1.92382800 \\
\hline $\bar{H}$ & 3.74061500 & 1.94643800 & -3.23176700 & C & 5.11047100 & 1.78587200 & 0.38321400 \\
\hline $\mathrm{H}$ & 3.28980700 & 0.75426500 & 0.87668300 & C & 5.86929200 & 2.39216700 & -0.62073500 \\
\hline $\bar{H}$ & 5.95065200 & 2.92777100 & -2.70799700 & $\mathrm{H}$ & 5.48717500 & 1.74490200 & 1.40129200 \\
\hline $\bar{H}$ & 6.83799900 & 2.82449300 & -0.38709800 & $\mathrm{~N}$ & 1.01776600 & 0.68932000 & -0.78690000 \\
\hline $\mathrm{H}$ & 1.14052800 & 0.80171500 & 0.22853700 & C & 2.52136400 & -1.28905700 & -1.63684500 \\
\hline $\mathrm{H}$ & 2.69144600 & -1.44617100 & -0.57647000 & $\mathrm{H}$ & 1.55749000 & -1.64377600 & -1.98220500 \\
\hline $\mathrm{C}$ & 3.59614700 & -1.40320300 & -2.52050000 & $\mathrm{C}$ & 4.97135200 & -1.41445400 & -2.05480600 \\
\hline $\mathrm{H}$ & 5.68368600 & -1.03949400 & -2.79697000 & $\mathrm{H}$ & 5.08903400 & -0.87594900 & -1.10803100 \\
\hline $\mathrm{C}$ & 3.34280500 & -1.41430100 & -3.99332100 & $\mathrm{H}$ & 3.59836200 & -0.42321000 & -4.39934000 \\
\hline $\mathrm{H}$ & 3.99325100 & -2.13318300 & -4.50200200 & $\mathrm{H}$ & 2.29949500 & -1.62797200 & -4.23503300 \\
\hline C & 7.32777600 & -3.08550100 & -1.13644700 & $\mathrm{H}$ & 7.45815500 & -2.41127900 & -0.28235600 \\
\hline $\mathrm{H}$ & 7.73463400 & -4.06331100 & -0.85248100 & $\mathrm{H}$ & 7.93138100 & -2.70133200 & -1.96665600 \\
\hline C & 4.44835300 & -3.84140100 & -0.19047600 & $\mathrm{H}$ & 4.46261900 & -3.14670200 & 0.65675100 \\
\hline $\mathrm{H}$ & 3.40363100 & -3.97075900 & -0.49128200 & $\mathrm{H}$ & 4.80995600 & -4.80981900 & 0.17572200 \\
\hline $\mathrm{C}$ & 5.30101800 & -4.33095300 & -3.13237100 & $\mathrm{H}$ & 4.24432100 & -4.44677900 & -3.39788800 \\
\hline $\mathrm{H}$ & 5.83586700 & -3.93491200 & -4.00375400 & $\mathrm{H}$ & 5.69678500 & -5.33405800 & -2.93360800 \\
\hline $\mathrm{Si}$ & 5.50960700 & -3.24356300 & -1.60870900 & & & & \\
\hline
\end{tabular}

\section{TS-R-4f-15}

Imaginary frequency: $-417.73 \mathrm{~cm}^{-1}$

E[B3LYP/6-31G(d)-D3(BJ)]: -4789.083772 Hartree

E[PCM(Et $\left.\left.{ }_{2} \mathrm{O}\right)-\mathrm{B} 3 L Y P / 6-311 \mathrm{G}(\mathrm{d}, \mathrm{p})-\mathrm{D} 3(\mathrm{BJ})\right]$ : -4790.225582 Hartree

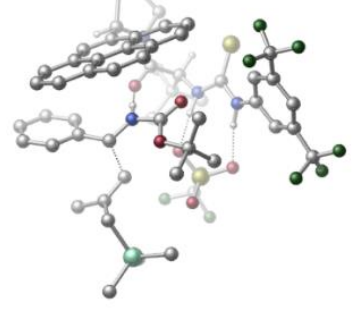




\begin{tabular}{|c|c|c|c|c|c|c|c|}
\hline 01 & & & & $\mathrm{~N}$ & 2.67167100 & -0.28102300 & 1.78682400 \\
\hline $\mathrm{H}$ & 2.38526900 & 0.68174000 & 1.98097800 & C & 3.68667100 & -0.37507300 & 0.83285000 \\
\hline $\mathrm{C}$ & 4.49449000 & 0.75648800 & 0.64801000 & C & 3.85646700 & -1.48188500 & -0.00912300 \\
\hline $\mathrm{C}$ & 5.44712100 & 0.77625600 & -0.36713100 & $\mathrm{H}$ & 4.33893000 & 1.63066000 & 1.26946800 \\
\hline C & 4.83095200 & -1.44863800 & -1.00194500 & $\mathrm{H}$ & 3.21367300 & -2.33941400 & 0.10362300 \\
\hline $\mathrm{C}$ & 5.63698300 & -0.32635600 & -1.19670600 & $\mathrm{H}$ & 6.39220900 & -0.31250900 & -1.97264400 \\
\hline $\mathrm{C}$ & 6.20759600 & 2.04169200 & -0.63922700 & C & 4.96086600 & -2.60268400 & -1.95312400 \\
\hline $\mathrm{F}$ & 6.37941700 & 2.78830300 & 0.46967600 & $\mathrm{~F}$ & 5.55277700 & 2.82196000 & -1.54067400 \\
\hline $\mathrm{F}$ & 7.43035600 & 1.79651500 & -1.16177100 & $\mathrm{~F}$ & 4.51783000 & -3.76614500 & -1.42587700 \\
\hline $\mathrm{F}$ & 4.23864300 & -2.39770300 & -3.09181700 & $\mathrm{~F}$ & 6.23774300 & -2.79695000 & -2.34644000 \\
\hline C & -0.20487300 & -1.60476900 & 3.58105200 & $\mathrm{H}$ & 0.37782900 & -2.42353400 & 4.00051700 \\
\hline $\mathrm{C}$ & -0.83706200 & -0.81237200 & 4.76904500 & $\mathrm{C}$ & -1.77013500 & 0.32622700 & 4.31899700 \\
\hline $\mathrm{H}$ & -2.67576900 & -0.06168800 & 3.84607100 & $\mathrm{H}$ & -1.28660100 & 0.99935000 & 3.60992600 \\
\hline $\mathrm{H}$ & -2.06455200 & 0.91601100 & 5.19522700 & C & 0.31543500 & -0.23003100 & 5.60585300 \\
\hline $\mathrm{H}$ & -0.08026400 & 0.22602600 & 6.52056200 & $\mathrm{H}$ & 0.85851000 & 0.53906100 & 5.05193000 \\
\hline $\mathrm{H}$ & 1.03042700 & -1.00955400 & 5.89359500 & $\mathrm{C}$ & -1.64010900 & -1.80139200 & 5.63241500 \\
\hline $\mathrm{H}$ & -2.11932800 & -1.27282700 & 6.46373400 & $\mathrm{H}$ & -0.99337900 & -2.57558200 & 6.06239000 \\
\hline $\mathrm{H}$ & -2.43268700 & -2.29368000 & 5.05542300 & C & -1.25138300 & -2.15140300 & 2.60029900 \\
\hline 0 & -1.88928200 & -1.36766400 & 1.87598200 & C & -0.84545300 & -4.50919600 & 3.40136600 \\
\hline C & -2.42816800 & -4.05547700 & 1.57499900 & C & -1.75505700 & -5.72519400 & 3.19737000 \\
\hline $\mathrm{H}$ & 0.18804000 & -4.69856700 & 3.09107100 & $\mathrm{H}$ & -0.82949700 & -4.17741000 & 4.43938600 \\
\hline $\mathrm{H}$ & -3.42923300 & -3.77764600 & 1.92829200 & $\mathrm{H}$ & -2.60896000 & -5.67173400 & 3.88321500 \\
\hline $\mathrm{H}$ & -1.23007700 & -6.66556300 & 3.38526000 & $\mathrm{~N}$ & -1.45180700 & -3.48051900 & 2.53282300 \\
\hline C & -2.32925800 & -3.59532200 & 0.13355400 & C & -3.49283300 & -3.24599600 & -0.48700200 \\
\hline $\mathrm{C}$ & -1.09199100 & -3.62594500 & -0.62364400 & C & -3.56131800 & -2.88439500 & -1.86970900 \\
\hline $\mathrm{H}$ & -4.41741600 & -3.22271900 & 0.08393400 & C & -1.13277800 & -3.27149500 & -2.01126200 \\
\hline C & 0.14550700 & -3.98919200 & -0.07082400 & C & -4.76622900 & -2.52420600 & -2.48881900 \\
\hline C & -2.35768800 & -2.88656000 & -2.63045400 & C & 0.06135400 & -3.30915600 & -2.79293700 \\
\hline $\mathrm{H}$ & 0.23403100 & -4.20884800 & 0.98192800 & C & 1.30079000 & -4.03558800 & -0.84712800 \\
\hline C & -4.79002900 & -2.15058200 & -3.83057000 & $\mathrm{H}$ & -5.67931500 & -2.51596800 & -1.90164000 \\
\hline $\mathrm{C}$ & -2.39181100 & -2.51199300 & -4.00794400 & C & 1.26642700 & -3.70286400 & -2.19485300 \\
\hline C & -0.00358400 & -2.94106400 & -4.17953900 & $\mathrm{H}$ & 2.23723800 & -4.32079300 & -0.38124700 \\
\hline C & -3.61765200 & -2.13910700 & -4.58282700 & $\mathrm{H}$ & -5.73093200 & -1.86483700 & -4.29285300 \\
\hline C & -1.17002600 & -2.54818200 & -4.76002200 & $\mathrm{H}$ & 2.17395000 & -3.72236100 & -2.78819800 \\
\hline $\mathrm{H}$ & 0.91465800 & -2.98202300 & -4.75942700 & $\mathrm{H}$ & -3.64258600 & -1.85302200 & -5.63135300 \\
\hline $\mathrm{H}$ & -1.19970000 & -2.27026100 & -5.81052100 & $\mathrm{C}$ & 1.92476100 & -1.27229500 & 2.36948100 \\
\hline $\mathrm{S}$ & 2.46390900 & -2.86162800 & 2.58881200 & $\mathrm{~N}$ & 0.73713900 & -0.80470000 & 2.82520100 \\
\hline $\mathrm{H}$ & 0.38675300 & 0.06150800 & 2.40672500 & $\mathrm{~S}$ & 0.76220200 & 2.87459600 & 1.71161600 \\
\hline 0 & 0.52665600 & 3.86767000 & 0.64406000 & $\mathrm{O}$ & -0.08011900 & 1.64852800 & 1.60488400 \\
\hline 0 & 2.17562600 & 2.58368400 & 2.04610300 & C & 0.08977800 & 3.71679400 & 3.22378500 \\
\hline $\mathrm{F}$ & 0.67750700 & 4.90007100 & 3.41608800 & $\mathrm{~F}$ & 0.27544200 & 2.96014200 & 4.31631200 \\
\hline $\mathrm{F}$ & -1.23581800 & 3.91676400 & 3.07429600 & $\mathrm{C}$ & -2.22803000 & -5.57578600 & 1.74797400 \\
\hline $\mathrm{H}$ & -1.45901800 & -5.92645100 & 1.05347600 & $\mathrm{H}$ & -3.14992800 & -6.11985200 & 1.52786900 \\
\hline C & -0.21337200 & -0.02214700 & -1.02259800 & 0 & 0.64334600 & -0.68610900 & -0.48429000 \\
\hline 0 & -0.07749100 & 0.83167800 & -2.04556800 & C & 1.25722700 & 1.13995300 & -2.62752200 \\
\hline $\mathrm{C}$ & 1.91656700 & -0.13630900 & -3.13846900 & $\mathrm{H}$ & 2.85209900 & 0.12281600 & -3.64444800 \\
\hline $\mathrm{H}$ & 2.14585900 & -0.81818600 & -2.32243700 & $\mathrm{H}$ & 1.26235200 & -0.64451400 & -3.85207700 \\
\hline C & 0.89318200 & 2.07018200 & -3.78139800 & $\mathrm{H}$ & 0.38607200 & 2.96607600 & -3.40887400 \\
\hline $\mathrm{H}$ & 1.80342100 & 2.38554400 & -4.30053400 & $\mathrm{H}$ & 0.24026000 & 1.56270800 & -4.49922000 \\
\hline $\mathrm{C}$ & 2.09572700 & 1.85673200 & -1.57834700 & $\mathrm{H}$ & 3.07671500 & 2.10817700 & -1.99198900 \\
\hline $\mathrm{H}$ & 1.61129300 & 2.77635800 & -1.24465400 & $\mathrm{H}$ & 2.25294200 & 1.21637900 & -0.71317400 \\
\hline $\mathrm{C}$ & -2.53580000 & 0.73483100 & -1.10029500 & $\mathrm{H}$ & -2.33223800 & 1.06286300 & -2.11337200 \\
\hline
\end{tabular}




\begin{tabular}{|c|c|c|c|c|c|c|c|}
\hline $\mathrm{C}$ & -3.95436800 & 0.40969300 & -0.82723600 & $\mathrm{C}$ & -4.89845600 & 0.71230000 & -1.81845400 \\
\hline C & -4.38408100 & -0.14372300 & 0.38659000 & C & -6.25399000 & 0.46662800 & -1.60619000 \\
\hline $\mathrm{H}$ & -4.56362400 & 1.12433900 & -2.76655400 & $\mathrm{C}$ & -5.73978300 & -0.38803500 & 0.59328200 \\
\hline $\mathrm{H}$ & -3.66612000 & -0.40049200 & 1.15738600 & $\mathrm{C}$ & -6.67821800 & -0.08281900 & -0.39605700 \\
\hline $\mathrm{H}$ & -6.97282600 & 0.69406900 & -2.38776100 & $\mathrm{H}$ & -6.06514300 & -0.81942200 & 1.53556200 \\
\hline $\mathrm{H}$ & -7.73351900 & -0.27435100 & -0.22469300 & $\mathrm{~N}$ & -1.55446500 & -0.08897100 & -0.61725700 \\
\hline $\mathrm{H}$ & -1.68312300 & -0.61216100 & 0.25837800 & C & -2.21663600 & 2.45844200 & -0.20771500 \\
\hline $\mathrm{H}$ & -2.39620900 & 2.16452900 & 0.82090600 & $\mathrm{H}$ & -1.16806600 & 2.52230400 & -0.47265800 \\
\hline C & -3.06975500 & 3.39068900 & -0.79614200 & $\mathrm{C}$ & -2.67695500 & 4.09912000 & -2.00742800 \\
\hline $\mathrm{H}$ & -1.91357600 & 3.55899200 & -2.57621200 & $\mathrm{H}$ & -3.52877400 & 4.35032400 & -2.64830000 \\
\hline C & -4.42675700 & 3.64675800 & -0.22959800 & $\mathrm{H}$ & -4.47083700 & 3.43939000 & 0.84175000 \\
\hline $\mathrm{H}$ & -4.75813900 & 4.67116500 & -0.42406800 & $\mathrm{H}$ & -5.14114300 & 2.97307300 & -0.72708800 \\
\hline C & -0.02614500 & 5.71144800 & -2.00941800 & $\mathrm{H}$ & 0.09824400 & 5.42464000 & -3.05987900 \\
\hline $\mathrm{H}$ & 0.46111500 & 6.68414200 & -1.86944600 & $\mathrm{H}$ & 0.49229900 & 4.98835500 & -1.37295600 \\
\hline $\mathrm{C}$ & -2.75227000 & 7.11582700 & -2.54503200 & $\mathrm{H}$ & -2.67132000 & 6.91025000 & -3.61880100 \\
\hline $\mathrm{H}$ & -3.81756100 & 7.15306300 & -2.28775300 & $\mathrm{H}$ & -2.33387900 & 8.11338200 & -2.36532400 \\
\hline $\mathrm{C}$ & -2.03195600 & 6.16291900 & 0.31545000 & $\mathrm{H}$ & -3.08129600 & 6.24262700 & 0.62076400 \\
\hline $\mathrm{H}$ & -1.54629800 & 5.38680900 & 0.91348900 & $\mathrm{H}$ & -1.54640500 & 7.11561000 & 0.55947400 \\
\hline Si & -1.83347700 & 5.82280200 & -1.52169600 & & & & \\
\hline
\end{tabular}

\section{TS-R-4f-16}

Imaginary frequency: $-330.06 \mathrm{~cm}^{-1}$

E[B3LYP/6-31G(d)-D3(BJ)]: -4789.084511 Hartree

E[PCM(Et $\left.\left.{ }_{2} \mathrm{O}\right)-\mathrm{B} 3 \mathrm{LYP} / 6-311 \mathrm{G}(\mathrm{d}, \mathrm{p})-\mathrm{D} 3(\mathrm{BJ})\right]$ : -4790.225223 Hartree

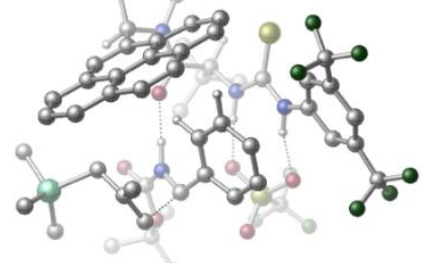

\begin{tabular}{|lrrr|rrrr|}
\hline 01 & & & N & 2.57043700 & 1.24974400 & 1.07871500 \\
\hline $\mathrm{H}$ & 2.13962600 & 2.06669800 & 0.63134900 & $\mathrm{C}$ & 3.70921900 & 0.80022700 & 0.39953200 \\
\hline $\mathrm{C}$ & 4.26585000 & 1.67376500 & -0.54731500 & $\mathrm{C}$ & 4.23154600 & -0.49459700 & 0.51563100 \\
\hline $\mathrm{C}$ & 5.30537300 & 1.24611100 & -1.36766000 & $\mathrm{H}$ & 3.84735100 & 2.66485600 & -0.67127900 \\
\hline $\mathrm{C}$ & 5.27808600 & -0.89615900 & -0.30858600 & $\mathrm{H}$ & 3.79767700 & -1.18672100 & 1.21816400 \\
\hline $\mathrm{C}$ & 5.83151200 & -0.03981100 & -1.25921600 & $\mathrm{H}$ & 6.64184400 & -0.36518300 & -1.89959800 \\
\hline $\mathrm{C}$ & 5.78564000 & 2.15008800 & -2.46688700 & $\mathrm{C}$ & 5.70853500 & -2.33240600 & -0.28572700 \\
\hline $\mathrm{F}$ & 5.69192600 & 3.45298300 & -2.14024000 & $\mathrm{~F}$ & 5.04929900 & 1.97420100 & -3.59838300 \\
\hline $\mathrm{F}$ & 7.07080300 & 1.90134100 & -2.80361300 & $\mathrm{~F}$ & 5.48974900 & -2.92941000 & 0.90777700 \\
\hline $\mathrm{F}$ & 5.01283800 & -3.06261600 & -1.20901300 & $\mathrm{~F}$ & 7.01395200 & -2.48717300 & -0.58540800 \\
\hline $\mathrm{C}$ & -0.20490600 & 0.73405500 & 3.39436200 & $\mathrm{H}$ & 0.46550600 & 0.38984500 & 4.17952000 \\
\hline $\mathrm{C}$ & -1.06092600 & 1.88300900 & 4.01451900 & $\mathrm{C}$ & -2.19364200 & 2.37302500 & 3.10440500 \\
\hline $\mathrm{H}$ & -2.91095900 & 1.58449000 & 2.87174100 & $\mathrm{H}$ & -1.81024800 & 2.77262100 & 2.16523100 \\
\hline $\mathrm{H}$ & -2.73408100 & 3.18370200 & 3.60816600 & $\mathrm{C}$ & -0.11456900 & 3.05521800 & 4.33181000 \\
\hline $\mathrm{H}$ & -0.64803600 & 3.80956300 & 4.92144600 & $\mathrm{H}$ & 0.24283900 & 3.53799300 & 3.41802900 \\
\hline $\mathrm{H}$ & 0.75636700 & 2.72378000 & 4.90928300 & $\mathrm{C}$ & -1.67196600 & 1.35157200 & 5.32358200 \\
\hline $\mathrm{H}$ & -2.27354900 & 2.13362000 & 5.79881700 & $\mathrm{H}$ & -0.89720300 & 1.04546500 & 6.03639300 \\
\hline $\mathrm{H}$ & -2.33410300 & 0.49643100 & 5.14075700 & $\mathrm{C}$ & -1.02772000 & -0.46021800 & 2.87962100 \\
\hline $\mathrm{O}$ & -1.67333600 & -0.39922500 & 1.81937600 & $\mathrm{C}$ & -0.37336000 & -1.86729700 & 4.88750000 \\
\hline $\mathrm{C}$ & -1.84379000 & -2.74510400 & 3.11730400 & $\mathrm{C}$ & -0.49299800 & -3.38529600 & 5.06339400 \\
\hline $\mathrm{H}$ & 0.66327600 & -1.52142400 & 4.86928600 & $\mathrm{H}$ & -0.91009300 & -1.33704800 & 5.68131800 \\
\hline $\mathrm{C}$ & -1.77639100 & -3.74404000 & 4.30151200 & $\mathrm{H}$ & -2.86567300 & -2.37299200 & 3.00056200 \\
\hline $\mathrm{H}$ & -0.53693300 & -3.66620600 & 6.11913700 & $\mathrm{H}$ & 0.36859000 & -3.89109900 & 4.62007600 \\
\hline $\mathrm{H}$ & -2.65072300 & -3.59016900 & 4.94368300 & $\mathrm{H}$ & -1.79444700 & -4.77988700 & 3.95587300 \\
\hline $\mathrm{N}$ & -1.01856900 & -1.61365700 & 3.58413700 & $\mathrm{C}$ & -1.48129300 & -3.36053700 & 1.76665100 \\
\hline
\end{tabular}




\begin{tabular}{|c|c|c|c|c|c|c|c|}
\hline $\mathrm{C}$ & -2.54044900 & -3.73620100 & 0.98913900 & $\mathrm{C}$ & -0.13215700 & -3.67078100 & 1.32368500 \\
\hline C & -2.39872900 & -4.42994700 & -0.25470900 & $\mathrm{H}$ & -3.55007000 & -3.50144700 & 1.31965300 \\
\hline $\mathrm{C}$ & 0.03865300 & -4.37734400 & 0.08480100 & $\mathrm{C}$ & 1.02605700 & -3.30174000 & 2.02820900 \\
\hline $\mathrm{C}$ & -3.50381300 & -4.80957200 & -1.03473600 & $\mathrm{C}$ & -1.08563800 & -4.73484600 & -0.71430600 \\
\hline C & 1.34979200 & -4.71984200 & -0.37148100 & $\mathrm{H}$ & 0.95728400 & -2.68248600 & 2.90606900 \\
\hline C & 2.29840700 & -3.65727600 & 1.58287600 & $\mathrm{C}$ & -3.32368900 & -5.45231400 & -2.25844600 \\
\hline $\mathrm{H}$ & -4.50602800 & -4.60228900 & -0.66960800 & C & -0.90972700 & -5.39676300 & -1.96819300 \\
\hline C & 2.46618800 & -4.37084600 & 0.40299200 & C & 1.49836200 & -5.38363400 & -1.63571900 \\
\hline $\mathrm{H}$ & 3.16338700 & -3.33778500 & 2.15344500 & $\mathrm{C}$ & -2.04186000 & -5.73454200 & -2.72725200 \\
\hline $\mathrm{H}$ & -4.18907700 & -5.74589200 & -2.84606700 & $\mathrm{C}$ & 0.42252900 & -5.70084300 & -2.40508700 \\
\hline $\mathrm{H}$ & 3.46097200 & -4.61506900 & 0.04683100 & $\mathrm{H}$ & 2.50483300 & -5.62019400 & -1.97001500 \\
\hline $\mathrm{H}$ & -1.90708800 & -6.23751000 & -3.68103600 & $\mathrm{H}$ & 0.55313900 & -6.19837500 & -3.36229700 \\
\hline C & 1.93063700 & 0.72026400 & 2.16708000 & $\mathrm{~S}$ & 2.65891600 & -0.34572300 & 3.27243000 \\
\hline $\mathrm{N}$ & 0.66274100 & 1.17473600 & 2.31507600 & $\mathrm{H}$ & 0.27362900 & 1.80920600 & 1.61140500 \\
\hline $\mathrm{S}$ & 0.43503300 & 3.99113800 & -0.43093400 & $\mathrm{O}$ & -0.16809100 & 4.14448600 & -1.76965800 \\
\hline $\mathrm{O}$ & -0.38871600 & 3.21931400 & 0.54247000 & $\mathrm{O}$ & 1.86764800 & 3.60398200 & -0.40504500 \\
\hline C & 0.43024400 & 5.70326500 & 0.28547000 & $\mathrm{~F}$ & 1.24636800 & 6.50744100 & -0.40059200 \\
\hline $\mathrm{F}$ & 0.83024000 & 5.66756500 & 1.56462200 & $\mathrm{~F}$ & -0.81106200 & 6.22311800 & 0.24540700 \\
\hline $\mathrm{C}$ & -3.32932200 & 2.04713200 & -0.18307400 & $\mathrm{O}$ & -4.14373800 & 1.83939400 & 0.69817400 \\
\hline $\mathrm{O}$ & -3.35372800 & 3.03660100 & -1.08196400 & $\mathrm{C}$ & -4.14560000 & 4.26783400 & -0.85212000 \\
\hline C & -5.62643600 & 3.95661900 & -1.05585000 & $\mathrm{H}$ & -6.20894500 & 4.88014100 & -0.96927200 \\
\hline $\mathrm{H}$ & -5.98041200 & 3.24887100 & -0.30338100 & $\mathrm{H}$ & -5.79866400 & 3.53914600 & -2.05347500 \\
\hline $\mathrm{C}$ & -3.60287400 & 5.20014100 & -1.93233400 & $\mathrm{H}$ & -2.52885700 & 5.35093600 & -1.79824300 \\
\hline $\mathrm{H}$ & -4.11314700 & 6.16712600 & -1.87722600 & $\mathrm{H}$ & -3.77309600 & 4.77557300 & -2.92748100 \\
\hline $\mathrm{C}$ & -3.85195800 & 4.81764300 & 0.54345000 & $\mathrm{H}$ & -4.30225000 & 5.81208000 & 0.63216100 \\
\hline $\mathrm{H}$ & -2.77489800 & 4.91343700 & 0.69321700 & $\mathrm{H}$ & -4.26579700 & 4.17502600 & 1.32141100 \\
\hline C & -1.47656600 & 1.25818100 & -1.55533500 & $\mathrm{H}$ & -1.43399900 & 2.26327200 & -1.96785300 \\
\hline $\mathrm{C}$ & -0.17079400 & 0.55912600 & -1.59400700 & $\mathrm{C}$ & 0.78943200 & 1.04197100 & -2.49781800 \\
\hline $\mathrm{C}$ & 0.10436100 & -0.59669400 & -0.84849200 & C & 1.98541800 & 0.35652700 & -2.69157000 \\
\hline $\mathrm{H}$ & 0.59982400 & 1.97149500 & -3.02499200 & C & 1.30910300 & -1.26991800 & -1.03991400 \\
\hline $\bar{H}$ & -0.60692100 & -0.97470700 & -0.12214200 & $\mathrm{C}$ & 2.23591600 & -0.81581000 & -1.97781900 \\
\hline $\bar{H}$ & 2.73484500 & 0.75460300 & -3.36778300 & $\mathrm{H}$ & 1.52763400 & -2.14733600 & -0.45042400 \\
\hline $\mathrm{H}$ & 3.16475100 & -1.35741500 & -2.11737300 & $\mathrm{~N}$ & -2.25277800 & 1.19486200 & -0.41191900 \\
\hline $\mathrm{H}$ & -2.00582100 & 0.59557300 & 0.38681200 & C & -2.51667000 & 0.59724500 & -2.96564300 \\
\hline $\bar{H}$ & -1.94231100 & 0.85475000 & -3.85239400 & $\mathrm{H}$ & -3.36451800 & 1.25341500 & -2.78941900 \\
\hline C & -2.67996300 & -0.77515900 & -2.68918000 & C & -3.55316200 & -1.21272000 & -1.61959100 \\
\hline $\mathrm{H}$ & -3.56053300 & -0.47896900 & -0.80039300 & $\mathrm{H}$ & -3.27765600 & -2.20091200 & -1.24480000 \\
\hline $\mathrm{C}$ & -1.81306300 & -1.79079300 & -3.34662300 & $\mathrm{H}$ & -2.32677100 & -2.75236000 & -3.44526000 \\
\hline $\mathrm{H}$ & -0.95194000 & -1.96576500 & -2.68087500 & $\mathrm{H}$ & -1.42578900 & -1.45987200 & -4.31264200 \\
\hline $\mathrm{C}$ & -6.26833100 & -1.86979200 & -0.48467700 & $\mathrm{H}$ & -5.90396400 & -2.84780300 & -0.15273700 \\
\hline $\mathrm{H}$ & -7.35395600 & -1.94468900 & -0.61730900 & $\mathrm{H}$ & -6.07571900 & -1.14716000 & 0.31671900 \\
\hline C & -5.64613100 & -2.54405800 & -3.48002200 & $\mathrm{H}$ & -5.18695400 & -3.50706000 & -3.23433400 \\
\hline $\mathrm{H}$ & -5.18002400 & -2.17665700 & -4.40183100 & $\mathrm{H}$ & -6.70743500 & -2.71845500 & -3.69217200 \\
\hline $\mathrm{C}$ & -6.04377300 & 0.39898500 & -2.56030600 & $\bar{H}$ & -5.58924800 & 0.75821000 & -3.48980000 \\
\hline $\mathrm{H}$ & -5.83418700 & 1.12703100 & -1.77019500 & $\mathrm{H}$ & -7.12974400 & 0.37986700 & -2.71191300 \\
\hline $\mathrm{Si}$ & -5.43117300 & -1.31060100 & -2.07469600 & & & & \\
\hline
\end{tabular}

\section{TS-R-4f-17}

Imaginary frequency: $-381.13 \mathrm{~cm}^{-1}$

E[B3LYP/6-31G(d)-D3(BJ)]: -4789.084225 Hartree

$\mathrm{E}\left[\mathrm{PCM}\left(\mathrm{Et}_{2} \mathrm{O}\right)-\mathrm{B} 3 \mathrm{LYP} / 6-311 \mathrm{G}(\mathrm{d}, \mathrm{p})-\mathrm{D} 3(\mathrm{BJ})\right]:-4790.225220$ Hartree

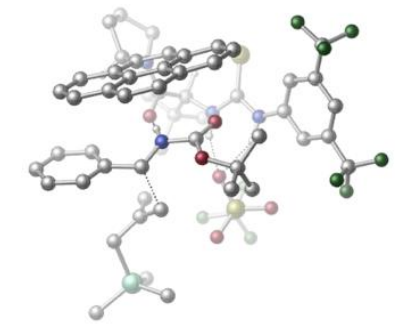

S188 


\begin{tabular}{|c|c|c|c|c|c|c|c|}
\hline 01 & & & & $\mathrm{~N}$ & 2.23144400 & 1.20174900 & 1.73491700 \\
\hline $\mathrm{H}$ & 1.53658900 & 1.85197900 & 1.36396200 & C & 3.39772600 & 1.16056500 & 0.96033500 \\
\hline C & 3.63765100 & 2.28034000 & 0.14633600 & C & 4.28097600 & 0.07505800 & 0.90422100 \\
\hline C & 4.72904300 & 2.29261300 & -0.71696100 & $\mathrm{H}$ & 2.95569300 & 3.12517100 & 0.17275300 \\
\hline C & 5.36347300 & 0.11097300 & 0.03004600 & $\mathrm{H}$ & 4.11096000 & -0.78469200 & 1.53093400 \\
\hline C & 5.60686000 & 1.21205700 & -0.78741100 & $\mathrm{H}$ & 6.45094800 & 1.22678800 & -1.46700500 \\
\hline C & 4.92942600 & 3.44276900 & -1.66164200 & C & 6.30190800 & -1.05939700 & -0.04304100 \\
\hline $\mathrm{F}$ & 4.26855200 & 4.54944400 & -1.28490300 & $\mathrm{~F}$ & 4.49254500 & 3.12696300 & -2.91638300 \\
\hline $\mathrm{F}$ & 6.23673100 & 3.76307800 & -1.79202800 & $\mathrm{~F}$ & 5.82810300 & -2.14765900 & 0.60595300 \\
\hline $\mathrm{F}$ & 6.52091700 & -1.43463400 & -1.33147300 & $\mathrm{~F}$ & 7.51309700 & -0.77688200 & 0.48346300 \\
\hline C & -0.39666800 & -0.25261500 & 3.78360800 & $\mathrm{H}$ & 0.30120100 & -0.70753500 & 4.48447800 \\
\hline $\mathrm{C}$ & -1.42466800 & 0.57927800 & 4.60744900 & $\mathrm{C}$ & -2.47562300 & 1.27543300 & 3.72928300 \\
\hline $\mathrm{H}$ & -3.09516300 & 0.55293400 & 3.19329600 & $\mathrm{H}$ & -2.01567100 & 1.93892500 & 2.99462600 \\
\hline $\mathrm{H}$ & -3.12736300 & 1.89122200 & 4.36001200 & C & -0.64213100 & 1.64285100 & 5.39722800 \\
\hline $\mathrm{H}$ & -1.31650400 & 2.17530000 & 6.07768600 & $\mathrm{H}$ & -0.18841100 & 2.37595900 & 4.72584400 \\
\hline $\mathrm{H}$ & 0.15662700 & 1.18795500 & 5.99414300 & $\mathrm{C}$ & -2.13739400 & -0.36623000 & 5.58987000 \\
\hline $\mathrm{H}$ & -2.88443200 & 0.18659900 & 6.16956100 & $\mathrm{H}$ & -1.43202500 & -0.81033800 & 6.30215600 \\
\hline $\bar{H}$ & -2.65908600 & -1.17804600 & 5.06839200 & $\mathrm{C}$ & -1.03111100 & -1.34035700 & 2.90738000 \\
\hline $\mathrm{O}$ & -1.65844000 & -1.01292500 & 1.88019900 & $\mathrm{C}$ & -0.27007100 & -3.19948500 & 4.44970200 \\
\hline C & -1.39199000 & -3.68527300 & 2.33438900 & C & -1.04944300 & -4.50678200 & 4.61315400 \\
\hline $\mathrm{H}$ & 0.80192400 & -3.36928900 & 4.29792900 & $\mathrm{H}$ & -0.39289800 & -2.52541700 & 5.29500100 \\
\hline $\mathrm{H}$ & -2.44663000 & -3.45604200 & 2.16211200 & $\mathrm{H}$ & -2.02078100 & -4.29824600 & 5.07710800 \\
\hline $\mathrm{H}$ & -0.52159900 & -5.23131600 & 5.23924600 & $\mathrm{~N}$ & -0.86506500 & -2.63363600 & 3.23038200 \\
\hline $\mathrm{C}$ & -0.65824700 & -3.74418800 & 1.00560200 & C & 0.65882000 & -3.39409300 & 0.93851400 \\
\hline $\mathrm{C}$ & -1.34698700 & -4.21459300 & -0.17810200 & C & 1.41132200 & -3.47609100 & -0.27657100 \\
\hline $\mathrm{H}$ & 1.16897700 & -3.00642300 & 1.81589600 & C & -0.63930000 & -4.22041700 & -1.42252200 \\
\hline $\mathrm{C}$ & -2.68431100 & -4.64475200 & -0.17353900 & C & 2.77877200 & -3.16602500 & -0.32520500 \\
\hline $\mathrm{C}$ & 0.74001100 & -3.86582700 & -1.46961100 & C & -1.31040200 & -4.59702500 & -2.62580500 \\
\hline $\mathrm{H}$ & -3.24122900 & -4.67895200 & 0.75559100 & C & -3.32810800 & -5.02018900 & -1.35062200 \\
\hline C & 3.48094500 & -3.24771600 & -1.52448500 & $\mathrm{H}$ & 3.28254000 & -2.85480100 & 0.58322400 \\
\hline $\mathrm{C}$ & 1.45729800 & -3.90887200 & -2.70287100 & C & -2.65834500 & -4.98161900 & -2.56782500 \\
\hline C & -0.57603900 & -4.58524000 & -3.86104400 & $\mathrm{H}$ & -4.36916100 & -5.32535700 & -1.31519300 \\
\hline C & 2.82787000 & -3.61010300 & -2.70179700 & $\mathrm{H}$ & 4.53669800 & -3.00197100 & -1.54672600 \\
\hline C & 0.74592600 & -4.26397100 & -3.89789400 & $\mathrm{H}$ & -3.16951300 & -5.26292000 & -3.48484100 \\
\hline $\mathrm{H}$ & -1.10194400 & -4.86034500 & -4.77193600 & $\mathrm{H}$ & 3.37886100 & -3.65716500 & -3.63786900 \\
\hline $\mathrm{H}$ & 1.29116800 & -4.27794000 & -4.83829800 & C & 1.72100800 & 0.31969700 & 2.64485100 \\
\hline$S$ & 2.58540600 & -0.90477900 & 3.43574700 & $\mathrm{~N}$ & 0.41012800 & 0.57724200 & 2.91191100 \\
\hline $\mathrm{H}$ & -0.08716800 & 1.15576400 & 2.23606400 & S & -0.34081800 & 3.74668100 & 0.28378500 \\
\hline $\mathrm{O}$ & -1.41602400 & 4.03684100 & -0.68779500 & $\mathrm{O}$ & -0.29790000 & 2.30793400 & 0.70881400 \\
\hline 0 & 0.98774200 & 4.31956700 & 0.01673500 & C & -0.90893600 & 4.60293900 & 1.83403900 \\
\hline $\mathrm{F}$ & -0.84727900 & 5.92880700 & 1.70647500 & $\mathrm{~F}$ & -0.14885400 & 4.23087000 & 2.87783300 \\
\hline $\mathrm{F}$ & -2.18929600 & 4.26687300 & 2.11564900 & C & -1.23184300 & -4.98523400 & 3.16569500 \\
\hline $\mathrm{H}$ & -0.33659200 & -5.51573600 & 2.82793200 & $\mathrm{H}$ & -2.08435500 & -5.65684200 & 3.03415000 \\
\hline C & 0.19137400 & -0.47179600 & -1.19069300 & $\mathrm{O}$ & 1.03429000 & -0.38426200 & -0.32695900 \\
\hline 0 & 0.30425800 & -0.18793100 & -2.49222800 & C & 1.41771400 & 0.64118000 & -3.02915700 \\
\hline $\mathrm{C}$ & 2.76708200 & -0.01260700 & -2.75464200 & $\mathrm{H}$ & 3.55191100 & 0.60612500 & -3.19993300 \\
\hline $\mathrm{H}$ & 2.95741800 & -0.09995300 & -1.68688600 & $\mathrm{H}$ & 2.80597500 & -1.01038300 & -3.19703600 \\
\hline $\mathrm{C}$ & 1.11074400 & 0.65287500 & -4.52379000 & $\mathrm{H}$ & 0.12742400 & 1.09541000 & -4.71527100 \\
\hline $\mathrm{H}$ & 1.86439700 & 1.24796400 & -5.04847500 & $\mathrm{H}$ & 1.12427500 & -0.36462200 & -4.92759800 \\
\hline C & 1.30597800 & 2.03467900 & -2.42401200 & $\mathrm{H}$ & 2.10702200 & 2.67003500 & -2.80765700 \\
\hline $\mathrm{H}$ & 0.34885100 & 2.50277500 & -2.67343700 & $\mathrm{H}$ & 1.40064200 & 1.99652700 & -1.34114000 \\
\hline
\end{tabular}




\begin{tabular}{|c|c|c|c|c|c|c|c|}
\hline $\mathrm{C}$ & -2.14746100 & -0.74499900 & -1.77895400 & $\mathrm{H}$ & -1.82305100 & -0.78416500 & -2.81196700 \\
\hline C & -3.45371200 & -1.39774400 & -1.53042700 & C & -4.24505300 & -1.70956400 & -2.64447600 \\
\hline $\mathrm{C}$ & -3.94496900 & -1.66684500 & -0.24515800 & $\mathrm{C}$ & -5.50954400 & -2.27110300 & -2.48370200 \\
\hline $\mathrm{H}$ & -3.86108700 & -1.51477900 & -3.64256000 & $\mathrm{C}$ & -5.21385300 & -2.22009200 & -0.08914300 \\
\hline $\mathrm{H}$ & -3.34006700 & -1.45632600 & 0.62877400 & C & -6.00045600 & -2.52251300 & -1.20193400 \\
\hline $\mathrm{H}$ & -6.10782400 & -2.51389600 & -3.35690500 & $\mathrm{H}$ & -5.58588600 & -2.42463500 & 0.91093700 \\
\hline $\mathrm{H}$ & -6.98789200 & -2.95596000 & -1.07167100 & $\mathrm{~N}$ & -1.10712400 & -0.92411600 & -0.90629400 \\
\hline $\mathrm{H}$ & -1.26759800 & -1.10652300 & 0.09057100 & C & -2.41785100 & 1.24433600 & -1.91565900 \\
\hline $\mathrm{H}$ & -1.45394700 & 1.53811800 & -1.52292100 & $\mathrm{H}$ & -2.54912500 & 1.39171200 & -2.98547600 \\
\hline C & -3.52897600 & 1.45945200 & -1.09566700 & $\mathrm{C}$ & -4.85256800 & 1.65044900 & -1.66025700 \\
\hline $\mathrm{H}$ & -4.95305600 & 1.23038300 & -2.66637300 & $\mathrm{H}$ & -5.65762200 & 1.28787700 & -1.01390000 \\
\hline C & -3.36252300 & 1.50711500 & 0.38057600 & $\mathrm{H}$ & -3.10478500 & 2.53174500 & 0.66709600 \\
\hline $\mathrm{H}$ & -4.28000600 & 1.22154000 & 0.90124700 & $\mathrm{H}$ & -2.52809300 & 0.89195800 & 0.71987500 \\
\hline C & -6.87703400 & 3.68961300 & -2.62023300 & $\mathrm{H}$ & -7.62238200 & 3.28365200 & -1.92676100 \\
\hline $\mathrm{H}$ & -7.14178500 & 4.73505800 & -2.81855500 & $\mathrm{H}$ & -6.95960300 & 3.14076400 & -3.56528300 \\
\hline C & -5.02001500 & 4.46936300 & -0.25323800 & $\mathrm{H}$ & -5.60233300 & 3.97230100 & 0.53089300 \\
\hline $\mathrm{H}$ & -3.98118900 & 4.55044800 & 0.08047400 & $\mathrm{H}$ & -5.41215300 & 5.48839200 & -0.35870300 \\
\hline C & -3.83707300 & 4.21377000 & -3.10387500 & $\mathrm{H}$ & -2.84723300 & 4.19833900 & -2.63579500 \\
\hline $\mathrm{H}$ & -3.80302100 & 3.62526600 & -4.02837700 & $\mathrm{H}$ & -4.05804600 & 5.25131200 & -3.38232300 \\
\hline $\mathrm{Si}$ & -5.13416200 & 3.58038700 & -1.90480800 & & & & \\
\hline
\end{tabular}

\section{TS- $R-4 f-18$}

Imaginary frequency: $-355.07 \mathrm{~cm}^{-1}$

E[B3LYP/6-31G(d)-D3(BJ)]: -4789.083246 Hartree

E[PCM(Et $\left.\left.{ }_{2} \mathrm{O}\right)-\mathrm{B} 3 \mathrm{LYP} / 6-311 \mathrm{G}(\mathrm{d}, \mathrm{p})-\mathrm{D} 3(\mathrm{BJ})\right]$ : -4790.224955 Hartree

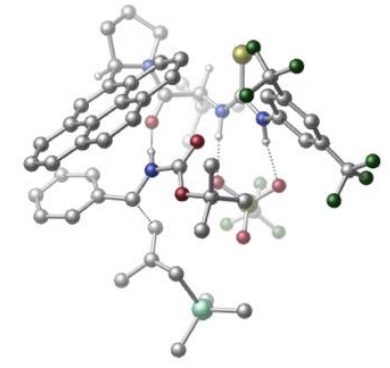

\begin{tabular}{|c|c|c|c|c|c|c|c|}
\hline 01 & & & & $\mathrm{~N}$ & 2.44472300 & -1.04143600 & 1.73408400 \\
\hline $\mathrm{H}$ & 2.49320000 & -0.02822700 & 1.85995100 & C & 3.34047400 & -1.51717100 & 0.77412700 \\
\hline C & 4.46367900 & -0.72205800 & 0.50191400 & C & 3.11591900 & -2.66753800 & 0.00704600 \\
\hline C & 5.33472900 & -1.07082900 & -0.52635600 & $\mathrm{H}$ & 4.62231700 & 0.18899200 & 1.06616800 \\
\hline C & 4.01252500 & -3.00876300 & -1.00135100 & $\mathrm{H}$ & 2.23651300 & -3.26351900 & 0.18751000 \\
\hline C & 5.12905800 & -2.22132600 & -1.28373500 & $\mathrm{H}$ & 5.81893300 & -2.49758000 & -2.07136800 \\
\hline $\mathrm{C}$ & 6.45253400 & -0.13802400 & -0.89228500 & $C$ & 3.72734300 & -4.19092000 & -1.88096900 \\
\hline $\mathrm{F}$ & 6.89661400 & 0.57344600 & 0.16287600 & $\mathrm{~F}$ & 6.05221500 & 0.76128800 & -1.83150300 \\
\hline$F$ & 7.51066300 & -0.79510500 & -1.41818100 & $\mathrm{~F}$ & 2.94804400 & -5.11593700 & -1.27706000 \\
\hline $\mathrm{F}$ & 3.07318100 & -3.81973200 & -3.01882600 & $\mathrm{~F}$ & 4.85697600 & -4.81277600 & -2.28115600 \\
\hline C & -0.65050100 & -1.23163200 & 3.64424500 & $\mathrm{H}$ & -0.35771200 & -2.17792200 & 4.09633500 \\
\hline C & -0.92545400 & -0.21896100 & 4.80008900 & C & -1.40225500 & 1.15910100 & 4.30792600 \\
\hline $\mathrm{H}$ & -2.38931100 & 1.09847000 & 3.84339600 & $\mathrm{H}$ & -0.71921900 & 1.59646200 & 3.57864800 \\
\hline $\mathrm{H}$ & -1.46358000 & 1.84331700 & 5.16266200 & $\mathrm{C}$ & 0.38149800 & -0.05686300 & 5.59554200 \\
\hline $\mathrm{H}$ & 0.20544700 & 0.56072000 & 6.48369600 & $\mathrm{H}$ & 1.15343800 & 0.42797300 & 4.99393700 \\
\hline $\mathrm{H}$ & 0.76859500 & -1.02805800 & 5.92520600 & C & -2.00492900 & -0.81954300 & 5.71817200 \\
\hline $\mathrm{H}$ & -2.23891800 & -0.12218100 & 6.52996900 & $\bar{H}$ & -1.66693300 & -1.75681300 & 6.17618800 \\
\hline $\mathrm{H}$ & -2.93727800 & -1.01622500 & 5.17476300 & C & -1.86485000 & -1.44533900 & 2.72830900 \\
\hline 0 & -2.25843500 & -0.52766900 & 1.98744500 & C & -2.20570500 & -3.76614700 & 3.65846800 \\
\hline C & -3.61466400 & -2.92606000 & 1.82724500 & C & -3.46681600 & -4.62872300 & 3.54641700 \\
\hline $\mathrm{H}$ & -1.30117200 & -4.29935600 & 3.34587400 & $\mathrm{H}$ & -2.04687800 & -3.40111800 & 4.67294200 \\
\hline $\mathrm{H}$ & -4.46089300 & -2.31648500 & 2.16830500 & $\mathrm{H}$ & -4.23352300 & -4.26039000 & 4.23843300 \\
\hline $\mathrm{H}$ & -3.26926400 & -5.67644800 & 3.78830800 & $\mathrm{~N}$ & -2.47659700 & -2.64490600 & 2.73606600 \\
\hline C & -3.41393700 & -2.61556900 & 0.35639200 & C & -4.43358400 & -1.97649200 & -0.28579000 \\
\hline
\end{tabular}




\begin{tabular}{|c|c|c|c|c|c|c|c|}
\hline $\mathrm{C}$ & -2.26677400 & -3.07910800 & -0.40148000 & $\mathrm{C}$ & -4.42527600 & -1.70989600 & -1.69186200 \\
\hline $\mathrm{H}$ & -5.29531300 & -1.63845600 & 0.28354300 & C & -2.23176900 & -2.82320800 & -1.81079500 \\
\hline $\mathrm{C}$ & -1.19488700 & -3.78244500 & 0.16840300 & $\mathrm{C}$ & -5.48539900 & -1.05675300 & -2.33513900 \\
\hline $\mathrm{C}$ & -3.29603300 & -2.12532200 & -2.45276900 & $\mathrm{C}$ & -1.13219600 & -3.28784600 & -2.59355200 \\
\hline $\mathrm{H}$ & -1.15313300 & -3.95337600 & 1.23276400 & C & -0.13822500 & -4.24888600 & -0.60961300 \\
\hline C & -5.42945700 & -0.78875900 & -3.70089700 & $\mathrm{H}$ & -6.34269700 & -0.73999100 & -1.75003400 \\
\hline C & -3.25130800 & -1.85793500 & -3.85475100 & C & -0.10026400 & -4.00809500 & -1.97644700 \\
\hline C & -1.11472400 & -3.01075500 & -4.00258200 & $\mathrm{H}$ & 0.66631900 & -4.79528200 & -0.13070400 \\
\hline C & -4.32421400 & -1.17817400 & -4.45368800 & $\mathrm{H}$ & -6.25509500 & -0.27121600 & -4.18163800 \\
\hline $\mathrm{C}$ & -2.11783300 & -2.31674500 & -4.60585500 & $\mathrm{H}$ & 0.73444900 & -4.35811600 & -2.57314300 \\
\hline $\mathrm{H}$ & -0.26905400 & -3.37338800 & -4.58102300 & $\mathrm{H}$ & -4.28853700 & -0.97331000 & -5.52077600 \\
\hline $\mathrm{H}$ & -2.08781000 & -2.11542800 & -5.67369500 & $C$ & 1.43283000 & -1.69420000 & 2.38980800 \\
\hline $\mathrm{S}$ & 1.43743100 & -3.35393900 & 2.71913000 & $\mathrm{~N}$ & 0.47324400 & -0.83709300 & 2.81794200 \\
\hline $\mathrm{H}$ & 0.41649700 & 0.07100400 & 2.35119100 & $\mathrm{~S}$ & 1.74097300 & 2.58780900 & 1.54593900 \\
\hline $\mathrm{O}$ & 1.77478200 & 3.60342800 & 0.47419600 & 0 & 0.50899400 & 1.74653100 & 1.53100100 \\
\hline $\mathrm{O}$ & 2.98264600 & 1.81365400 & 1.76504000 & $\mathrm{C}$ & 1.55846800 & 3.59210300 & 3.09810400 \\
\hline $\mathrm{F}$ & 2.54015400 & 4.49195100 & 3.19920300 & $\mathrm{~F}$ & 1.58299900 & 2.80067000 & 4.18029300 \\
\hline $\mathrm{F}$ & 0.37984800 & 4.24384300 & 3.08223500 & C & -3.91304600 & -4.41625800 & 2.09682000 \\
\hline $\mathrm{H}$ & -3.32203500 & -5.03710100 & 1.41704500 & $\mathrm{H}$ & -4.96811900 & -4.64207400 & 1.92309400 \\
\hline $\mathrm{C}$ & -0.30622700 & 0.10489600 & -0.97641200 & $\mathrm{O}$ & 0.30306100 & -0.77194800 & -0.40358700 \\
\hline $\mathrm{O}$ & 0.07407900 & 0.79177600 & -2.06425700 & C & 1.41128300 & 0.57186000 & -2.68008600 \\
\hline $\mathrm{C}$ & 1.59528000 & -0.89473700 & -3.05807400 & $\mathrm{H}$ & 2.54030000 & -1.00650700 & -3.59936900 \\
\hline $\mathrm{H}$ & 1.62477700 & -1.53379600 & -2.17876400 & $\mathrm{H}$ & 0.78054700 & -1.22445300 & -3.70826700 \\
\hline C & 1.34156700 & 1.44270700 & -3.93237800 & $\mathrm{H}$ & 1.18846600 & 2.49439200 & -3.67250600 \\
\hline $\bar{H}$ & 2.28189900 & 1.36101100 & -4.48610300 & $\mathrm{H}$ & 0.52422900 & 1.11764700 & -4.58445500 \\
\hline $\mathrm{C}$ & 2.47852700 & 1.06769500 & -1.71290700 & $\mathrm{H}$ & 3.47263100 & 0.92929300 & -2.14790900 \\
\hline $\mathrm{H}$ & 2.34610700 & 2.12701500 & -1.48089400 & $\mathrm{H}$ & 2.44118900 & 0.50568200 & -0.78207800 \\
\hline $\mathrm{C}$ & -2.27399500 & 1.57016200 & -1.07589600 & $\mathrm{H}$ & -1.96465900 & 1.76374200 & -2.09596900 \\
\hline $\mathrm{C}$ & -3.74868600 & 1.62388200 & -0.87585800 & $\mathrm{C}$ & -4.54109700 & 2.12844700 & -1.91416900 \\
\hline C & -4.35545700 & 1.24719300 & 0.33115300 & C & -5.91755100 & 2.27843900 & -1.74955200 \\
\hline $\bar{H}$ & -4.07763300 & 2.39080800 & -2.86118900 & $\mathrm{C}$ & -5.73075600 & 1.39380100 & 0.48939700 \\
\hline $\mathrm{H}$ & -3.76135800 & 0.82793000 & 1.13627900 & $\mathrm{C}$ & -6.51489600 & 1.91786700 & -0.54258600 \\
\hline $\mathrm{H}$ & -6.51982000 & 2.66494900 & -2.56631600 & $\mathrm{H}$ & -6.19363000 & 1.09960100 & 1.42705300 \\
\hline $\bar{H}$ & -7.58600200 & 2.03690500 & -0.40714800 & $\bar{N}$ & -1.57474600 & 0.50585600 & -0.54833500 \\
\hline $\bar{H}$ & -1.83578000 & 0.09887800 & 0.35810200 & $\mathrm{C}$ & -1.67904300 & 3.15295000 & -0.15645500 \\
\hline $\mathrm{H}$ & -2.47397500 & 3.27444100 & 0.57474400 & $\mathrm{H}$ & -0.74597000 & 2.78011300 & 0.25328200 \\
\hline $\mathrm{C}$ & -1.59645800 & 4.11017100 & -1.18221900 & $\mathrm{C}$ & -0.37117500 & 4.29024400 & -1.93346700 \\
\hline $\bar{H}$ & 0.36053600 & 3.51013200 & -1.72465500 & $\bar{H}$ & -0.54439600 & 4.40447600 & -3.01048400 \\
\hline C & -2.78881300 & 4.95033800 & -1.51536700 & $\mathrm{H}$ & -2.75868000 & 5.85602500 & -0.89271200 \\
\hline $\mathrm{H}$ & -2.79352700 & 5.27213100 & -2.56073800 & $\mathrm{H}$ & -3.72274400 & 4.43407800 & -1.27955900 \\
\hline C & 2.32009200 & 5.74509000 & -1.82510600 & $\mathrm{H}$ & 2.47240400 & 5.40122900 & -2.85491400 \\
\hline $\mathrm{H}$ & 2.86642300 & 6.68829300 & -1.70374800 & $\mathrm{H}$ & 2.74322400 & 5.00559200 & -1.14029000 \\
\hline $\mathrm{C}$ & -0.24366300 & 7.30065900 & -2.59613200 & $\mathrm{H}$ & -0.08661900 & 7.04873200 & -3.65153400 \\
\hline $\mathrm{H}$ & -1.31930400 & 7.43689100 & -2.43761700 & $\mathrm{H}$ & 0.23673700 & 8.26991400 & -2.41469600 \\
\hline C & 0.15006400 & 6.44611700 & 0.33725800 & $\mathrm{H}$ & -0.90905000 & 6.67300400 & 0.50745200 \\
\hline $\bar{H}$ & 0.44801500 & 5.64108800 & 1.01082200 & $\mathrm{H}$ & 0.72106300 & 7.34282900 & 0.60713100 \\
\hline Si & 0.49882600 & 5.99238200 & -1.45079000 & & & & \\
\hline
\end{tabular}

\section{TS- $R-4 f-19$}

Imaginary frequency: $-425.90 \mathrm{~cm}^{-1}$

E[B3LYP/6-31G(d)-D3(BJ)]: -4789.084168 Hartree

$\mathrm{E}\left[\mathrm{PCM}\left(\mathrm{Et}_{2} \mathrm{O}\right)-\mathrm{B} 3 \mathrm{LYP} / 6-311 \mathrm{G}(\mathrm{d}, \mathrm{p})-\mathrm{D} 3(\mathrm{BJ})\right]:-4790.224510$ Hartree

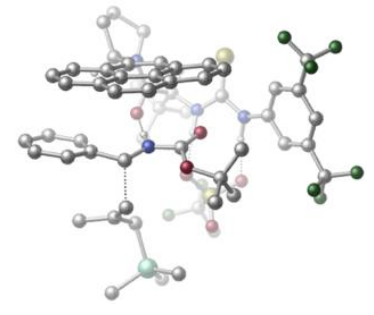

S191 


\begin{tabular}{|c|c|c|c|c|c|c|c|}
\hline 01 & & & & $\mathrm{~N}$ & 2.61876900 & 0.12500700 & 1.95206100 \\
\hline $\mathrm{H}$ & 2.51183800 & 1.12377000 & 1.75174100 & C & 3.58614000 & -0.48231800 & 1.14705300 \\
\hline $\mathrm{C}$ & 4.32759900 & 0.37624500 & 0.31368500 & $\mathrm{C}$ & 3.83388700 & -1.86045500 & 1.08392100 \\
\hline $\mathrm{C}$ & 5.25393600 & -0.14374600 & -0.58237800 & $\mathrm{H}$ & 4.15233400 & 1.44507400 & 0.35743400 \\
\hline C & 4.76150500 & -2.35689500 & 0.17075000 & $\mathrm{H}$ & 3.30231300 & -2.52883600 & 1.74207900 \\
\hline $\mathrm{C}$ & 5.48209500 & -1.51677800 & -0.67307300 & $\mathrm{H}$ & 6.19595100 & -1.91645800 & -1.38372600 \\
\hline C & 5.96554300 & 0.76341400 & -1.54385000 & C & 5.01577600 & -3.83593900 & 0.10602000 \\
\hline $\mathrm{F}$ & 5.86574600 & 2.06252100 & -1.20977900 & $\mathrm{~F}$ & 5.45451200 & 0.63988700 & -2.80419600 \\
\hline$F$ & 7.27908300 & 0.46117400 & -1.64336400 & $F$ & 4.03718400 & -4.55462600 & 0.70065900 \\
\hline$F$ & 5.09278400 & -4.26571500 & -1.18217000 & $F$ & 6.18011500 & -4.18300300 & 0.69553900 \\
\hline C & -0.53001100 & 0.14790100 & 3.78177300 & $\mathrm{H}$ & -0.15786900 & -0.56750400 & 4.51285100 \\
\hline $\mathrm{C}$ & -1.04739700 & 1.39506000 & 4.56170700 & $\mathrm{C}$ & -1.71001600 & 2.45156200 & 3.66003900 \\
\hline $\mathrm{H}$ & -2.65754900 & 2.08903700 & 3.25280200 & $\mathrm{H}$ & -1.07973900 & 2.73198200 & 2.81652200 \\
\hline $\mathrm{H}$ & -1.91114800 & 3.35360600 & 4.24996800 & C & 0.15285300 & 2.01537700 & 5.29709700 \\
\hline $\mathrm{H}$ & -0.18636900 & 2.83158100 & 5.94512200 & $\mathrm{H}$ & 0.88306000 & 2.42052500 & 4.59400700 \\
\hline $\mathrm{H}$ & 0.66061900 & 1.27147800 & 5.92229600 & $\mathrm{C}$ & -2.07844100 & 0.91064400 & 5.59732300 \\
\hline $\mathrm{H}$ & -2.48484900 & 1.76376200 & 6.15146400 & $\mathrm{H}$ & -1.62602400 & 0.22812600 & 6.32695000 \\
\hline $\mathrm{H}$ & -2.92193700 & 0.39647600 & 5.12008700 & C & -1.60384300 & -0.50824900 & 2.90679000 \\
\hline $\mathrm{O}$ & -1.99891700 & 0.07812300 & 1.88359600 & C & -1.83087000 & -2.50070100 & 4.45527400 \\
\hline C & -3.03109400 & -2.40541400 & 2.33306300 & $\mathrm{C}$ & -3.14268100 & -3.27014000 & 4.62182300 \\
\hline $\mathrm{H}$ & -0.97294800 & -3.16562200 & 4.30586300 & $\mathrm{H}$ & -1.61404300 & -1.84651300 & 5.29755300 \\
\hline $\mathrm{H}$ & -3.84443000 & -1.70347200 & 2.12585500 & $\mathrm{H}$ & -3.89514600 & -2.61706900 & 5.07971500 \\
\hline $\mathrm{H}$ & -3.03042800 & -4.15553700 & 5.25365900 & $\mathrm{~N}$ & -2.08081200 & -1.72348400 & 3.23358800 \\
\hline C & -2.39755800 & -2.84278500 & 1.02284100 & C & -1.04710200 & -3.00097100 & 0.92042100 \\
\hline $\mathrm{C}$ & -3.25414600 & -3.17127400 & -0.09774800 & $\mathrm{C}$ & -0.42428200 & -3.48656200 & -0.27471700 \\
\hline $\mathrm{H}$ & -0.39934700 & -2.75462300 & 1.75644000 & $\mathrm{C}$ & -2.64912700 & -3.58672700 & -1.32629100 \\
\hline C & -4.65687500 & -3.11499900 & -0.03457900 & C & 0.95585100 & -3.72947200 & -0.34327600 \\
\hline C & -1.23586600 & -3.74810000 & -1.41429300 & C & -3.46383300 & -3.88920100 & -2.46106800 \\
\hline $\mathrm{H}$ & -5.14664500 & -2.81368600 & 0.88341300 & $\mathrm{C}$ & -5.44284900 & -3.43526000 & -1.13827200 \\
\hline C & 1.53138900 & -4.22070500 & -1.51219300 & $\mathrm{H}$ & 1.56735900 & -3.53245000 & 0.52923400 \\
\hline $\mathrm{C}$ & -0.63370500 & -4.21515600 & -2.62123000 & C & -4.85845700 & -3.80664000 & -2.34445400 \\
\hline C & -2.82542800 & -4.29885700 & -3.68198100 & $\mathrm{H}$ & -6.52399700 & -3.37853000 & -1.05656600 \\
\hline C & 0.74957600 & -4.44809800 & -2.64383200 & $\mathrm{H}$ & 2.59841400 & -4.41001700 & -1.54772500 \\
\hline $\mathrm{C}$ & -1.47578700 & -4.45687500 & -3.75849300 & $\mathrm{H}$ & -5.47812700 & -4.04734300 & -3.20459600 \\
\hline $\mathrm{H}$ & -3.45431500 & -4.50555600 & -4.54447200 & $\mathrm{H}$ & 1.20768700 & -4.81928400 & -3.55728100 \\
\hline $\mathrm{H}$ & -1.01114900 & -4.78967200 & -4.68329300 & C & 1.63513800 & -0.41426100 & 2.73731400 \\
\hline $\mathrm{S}$ & 1.70254500 & -1.93875200 & 3.47663100 & $\mathrm{~N}$ & 0.60133100 & 0.45002800 & 2.92166100 \\
\hline $\mathrm{H}$ & 0.44276100 & 1.15042900 & 2.18983500 & $\mathrm{~S}$ & 1.32104500 & 3.42691400 & 0.69714700 \\
\hline 0 & 1.37798500 & 4.08949800 & -0.61890900 & $\mathrm{O}$ & 0.21312500 & 2.44014400 & 0.83924500 \\
\hline 0 & 2.61185100 & 2.94869000 & 1.24990500 & $\mathrm{C}$ & 0.81565500 & 4.79803900 & 1.85078000 \\
\hline $\mathrm{F}$ & 1.54786000 & 5.89682400 & 1.63787600 & $\mathrm{~F}$ & 0.95695400 & 4.42942500 & 3.13186300 \\
\hline $\mathrm{F}$ & -0.48167500 & 5.10672800 & 1.64704000 & C & -3.53045000 & -3.61135500 & 3.17628800 \\
\hline $\mathrm{H}$ & -3.00857200 & -4.51538800 & 2.84824900 & $\mathrm{H}$ & -4.60186400 & -3.78351600 & 3.04654200 \\
\hline $\mathrm{C}$ & -0.07498000 & -0.15288900 & -1.12451900 & $\mathrm{O}$ & 0.71685700 & -0.37016900 & -0.23552500 \\
\hline $\mathrm{O}$ & 0.17634000 & -0.03083800 & -2.43580600 & C & 1.57244300 & 0.12923900 & -2.93185400 \\
\hline $\mathrm{C}$ & 2.38156100 & -1.13321000 & -2.66306200 & $\mathrm{H}$ & 3.38643700 & -1.00098100 & -3.07487800 \\
\hline $\mathrm{H}$ & 2.46950300 & -1.32512900 & -1.59490900 & $\mathrm{H}$ & 1.91303000 & -1.99896900 & -3.13682400 \\
\hline C & 1.35829900 & 0.33622600 & -4.42871200 & $\mathrm{H}$ & 0.77356600 & 1.24254300 & -4.61996900 \\
\hline $\mathrm{H}$ & 2.32731200 & 0.44386900 & -4.92536200 & $\mathrm{H}$ & 0.83595800 & -0.52051700 & -4.86664400 \\
\hline $\mathrm{C}$ & 2.17951100 & 1.36674300 & -2.28400600 & $\mathrm{H}$ & 3.16475900 & 1.55739000 & -2.71638800 \\
\hline $\mathrm{H}$ & 1.55205900 & 2.24425600 & -2.44750900 & $\mathrm{H}$ & 2.30072800 & 1.22688400 & -1.21158000 \\
\hline $\mathrm{C}$ & -2.40752400 & 0.11854400 & -1.83158500 & $\mathrm{H}$ & -2.14055200 & -0.42005800 & -2.73639900 \\
\hline $\mathrm{C}$ & -3.83605900 & -0.00759000 & -1.45010900 & C & -4.75258800 & -0.35232400 & -2.45281300 \\
\hline
\end{tabular}




\begin{tabular}{|c|c|c|c|c|c|c|c|}
\hline $\mathrm{C}$ & -4.31201700 & 0.28463300 & -0.16281300 & C & -6.11776000 & -0.38578000 & -2.18729900 \\
\hline $\mathrm{H}$ & -4.38905000 & -0.60168800 & -3.44609100 & $\mathrm{C}$ & -5.68058600 & 0.23367700 & 0.10228400 \\
\hline $\mathrm{H}$ & -3.62522400 & 0.53217100 & 0.63736500 & $\mathrm{C}$ & -6.58789900 & -0.08791500 & -0.90736800 \\
\hline $\bar{H}$ & -6.81280800 & -0.66013400 & -2.97510200 & $\mathrm{H}$ & -6.03559300 & 0.45606600 & 1.10462800 \\
\hline $\mathrm{H}$ & -7.65318100 & -0.11559700 & -0.69745700 & $\mathrm{~N}$ & -1.43946000 & 0.01623000 & -0.86665100 \\
\hline $\mathrm{H}$ & -1.65569900 & 0.07003100 & 0.13663100 & C & -2.29099300 & 1.80687300 & -2.82086400 \\
\hline $\mathrm{H}$ & -1.22168700 & 1.77315100 & -3.00309700 & $\mathrm{H}$ & -2.90740900 & 1.51609200 & -3.66817000 \\
\hline C & -2.77998900 & 2.85850800 & -2.02313700 & C & -1.89854800 & 3.66301300 & -1.21492100 \\
\hline $\mathrm{H}$ & -2.38296600 & 4.10859200 & -0.34026000 & $\mathrm{H}$ & -0.96883900 & 3.16494400 & -0.92760100 \\
\hline $\mathrm{C}$ & -4.24793100 & 3.12431800 & -1.96329400 & $\mathrm{H}$ & -4.65124700 & 2.56883100 & -1.10379300 \\
\hline $\mathrm{H}$ & -4.45706600 & 4.18239400 & -1.79075100 & $\mathrm{H}$ & -4.77983100 & 2.77714300 & -2.85144200 \\
\hline C & -0.67856100 & 6.54183100 & -1.18001800 & $\mathrm{H}$ & 0.27764200 & 6.25857600 & -0.73744800 \\
\hline $\mathrm{H}$ & -0.53881300 & 7.46300500 & -1.75968000 & $\mathrm{H}$ & -1.38532100 & 6.75698400 & -0.37203700 \\
\hline C & 0.00238500 & 4.58873100 & -3.52507100 & $\mathrm{H}$ & 0.89072600 & 4.27491800 & -2.97192300 \\
\hline $\mathrm{H}$ & -0.35001500 & 3.75429700 & -4.14158900 & $\mathrm{H}$ & 0.28826800 & 5.40139400 & -4.20393600 \\
\hline $\mathrm{C}$ & -2.79383200 & 5.85292400 & -3.29000800 & $\mathrm{H}$ & -3.22263200 & 5.10859400 & -3.97028000 \\
\hline $\mathrm{H}$ & -3.59378500 & 6.21584700 & -2.63425500 & $\mathrm{H}$ & -2.46803300 & 6.70412500 & -3.90047300 \\
\hline $\mathrm{Si}$ & -1.30683200 & 5.19310600 & -2.32425100 & & & & \\
\hline
\end{tabular}

\section{TS- $R-4 f-20$}

Imaginary frequency: $-418.37 \mathrm{~cm}^{-1}$

E[B3LYP/6-31G(d)-D3(BJ)]: -4789.082211 Hartree

$\mathrm{E}\left[\mathrm{PCM}\left(\mathrm{Et}_{2} \mathrm{O}\right)-\mathrm{B} 3 \mathrm{LYP} / 6-311 \mathrm{G}(\mathrm{d}, \mathrm{p})-\mathrm{D} 3(\mathrm{BJ})\right]$ : -4790.224490 Hartree

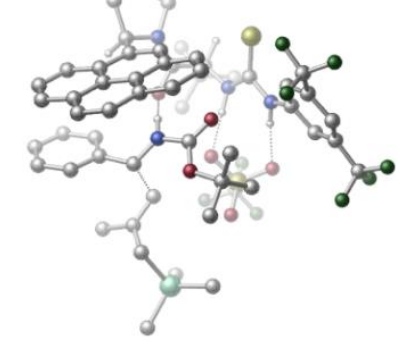

\begin{tabular}{|c|c|c|c|c|c|c|c|}
\hline 01 & & & & $\mathrm{~N}$ & 2.51112900 & -0.85579000 & 1.94382000 \\
\hline $\mathrm{H}$ & 2.55148600 & 0.16073000 & 2.05774500 & C & 3.45432500 & -1.33276900 & 1.03003100 \\
\hline$C$ & 4.54624300 & -0.49235200 & 0.76341500 & $\mathrm{C}$ & 3.32547400 & -2.53061800 & 0.31363800 \\
\hline C & 5.48024900 & -0.84204400 & -0.20766000 & $\mathrm{H}$ & 4.63193900 & 0.45368800 & 1.28471000 \\
\hline C & 4.27747600 & -2.86380900 & -0.64405200 & $\mathrm{H}$ & 2.48435300 & -3.17670900 & 0.50286100 \\
\hline C & 5.36411200 & -2.03310900 & -0.91909100 & $\mathrm{H}$ & 6.09968700 & -2.30787300 & -1.66554900 \\
\hline C & 6.57118300 & 0.12559200 & -0.56588200 & C & 4.11450200 & -4.10832900 & -1.46664100 \\
\hline$F$ & 6.91324900 & 0.92112800 & 0.46672200 & $\mathrm{~F}$ & 6.18918500 & 0.94339100 & -1.58300400 \\
\hline $\mathrm{F}$ & 7.69058500 & -0.50743400 & -0.98415800 & $\mathrm{~F}$ & 3.15443700 & -4.93184200 & -0.99051300 \\
\hline $\mathrm{F}$ & 3.77029100 & -3.80858800 & -2.75063800 & $\mathrm{~F}$ & 5.25939000 & -4.82126500 & -1.54054800 \\
\hline C & -0.60239800 & -0.98696900 & 3.81492400 & $\mathrm{H}$ & -0.33058700 & -1.92206100 & 4.30246200 \\
\hline$C$ & -0.95416300 & 0.04986700 & 4.92809900 & C & -1.49308300 & 1.38247900 & 4.37753200 \\
\hline $\mathrm{H}$ & -2.47226100 & 1.25556400 & 3.90856300 & $\mathrm{H}$ & -0.82594800 & 1.82340100 & 3.63601300 \\
\hline $\mathrm{H}$ & -1.59805900 & 2.09499400 & 5.20434500 & C & 0.32180000 & 0.31459200 & 5.74603200 \\
\hline $\mathrm{H}$ & 0.08262900 & 0.92819000 & 6.62217300 & $\mathrm{H}$ & 1.06904000 & 0.84699000 & 5.15268700 \\
\hline $\mathrm{H}$ & 0.77126300 & -0.62176300 & 6.09671200 & $\mathrm{C}$ & -2.02196900 & -0.57824000 & 5.84157500 \\
\hline $\mathrm{H}$ & -2.31375200 & 0.13085000 & 6.62391300 & $\mathrm{H}$ & -1.64590800 & -1.48141600 & 6.33721600 \\
\hline $\mathrm{H}$ & -2.92822700 & -0.84273600 & 5.28285300 & C & -1.75105400 & -1.22030600 & 2.82979400 \\
\hline $\mathrm{O}$ & -2.03792600 & -0.33134800 & 2.00741200 & $\mathrm{C}$ & -2.30927800 & -3.50291600 & 3.78251800 \\
\hline$C$ & -3.50861000 & -2.59127700 & 1.85889000 & $\mathrm{C}$ & -3.73642500 & -4.05714000 & 3.80435800 \\
\hline $\mathrm{H}$ & -1.58361400 & -4.23245000 & 3.40507500 & $\mathrm{H}$ & -1.97227300 & -3.16114400 & 4.75942200 \\
\hline $\mathrm{H}$ & -4.18109800 & -1.73311600 & 1.94170000 & $\mathrm{H}$ & -4.35521000 & -3.44828300 & 4.47431700 \\
\hline $\mathrm{H}$ & -3.77714000 & -5.09327200 & 4.15151000 & $\mathrm{~N}$ & -2.42675400 & -2.38212100 & 2.84124000 \\
\hline C & -3.00380100 & -2.70106500 & 0.43099100 & C & -1.74415200 & -3.16035300 & 0.17953200 \\
\hline $\mathrm{C}$ & -3.89606200 & -2.38676500 & -0.66516300 & C & -1.25888400 & -3.36514200 & -1.15207200 \\
\hline $\mathrm{H}$ & -1.06262500 & -3.36853800 & 0.99989300 & C & -3.41058900 & -2.52074300 & -2.00471900 \\
\hline
\end{tabular}

S193 


\begin{tabular}{|c|c|c|c|c|c|c|c|}
\hline $\mathrm{C}$ & -5.22129200 & -1.95710300 & -0.47965600 & $\mathrm{C}$ & 0.01004900 & -3.90837100 & -1.40390300 \\
\hline C & -2.09790200 & -3.01797800 & -2.24855700 & C & -4.25419300 & -2.18933600 & -3.10956400 \\
\hline $\mathrm{H}$ & -5.62505700 & -1.85689800 & 0.52076400 & $\mathrm{C}$ & -6.04004200 & -1.65014600 & -1.56319500 \\
\hline $\mathrm{C}$ & 0.44691200 & -4.11401300 & -2.70978900 & $\mathrm{H}$ & 0.64653800 & -4.17034300 & -0.56560000 \\
\hline C & -1.62929500 & -3.19869300 & -3.58500100 & C & -5.56402400 & -1.75377000 & -2.86551800 \\
\hline C & -3.73925900 & -2.33572200 & -4.44314300 & $\mathrm{H}$ & -7.05692600 & -1.31478600 & -1.38440100 \\
\hline C & -0.35774500 & -3.75418200 & -3.78953600 & $\mathrm{H}$ & 1.43023100 & -4.53237600 & -2.88610100 \\
\hline C & -2.48854400 & -2.82070300 & -4.67106800 & $\mathrm{H}$ & -6.20649700 & -1.50677000 & -3.70693100 \\
\hline $\mathrm{H}$ & -4.38633500 & -2.06646300 & -5.27440100 & $\mathrm{H}$ & -0.00411600 & -3.90460700 & -4.80655200 \\
\hline $\mathrm{H}$ & -2.12067100 & -2.94350600 & -5.68672400 & $\mathrm{C}$ & 1.46045600 & -1.48431300 & 2.55430600 \\
\hline $\mathrm{S}$ & 1.32620100 & -3.15864600 & 2.77345800 & $\mathrm{~N}$ & 0.55867200 & -0.59159800 & 3.04155700 \\
\hline $\mathrm{H}$ & 0.49668100 & 0.31042500 & 2.55815600 & $S$ & 1.60520900 & 2.71486900 & 1.45153500 \\
\hline $\mathrm{O}$ & 1.65017700 & 3.57995200 & 0.25480200 & 0 & 0.41372000 & 1.82099500 & 1.51767200 \\
\hline $\mathrm{O}$ & 2.86926400 & 2.03590700 & 1.82336500 & C & 1.30945600 & 3.91464100 & 2.83705300 \\
\hline $\mathrm{F}$ & 2.26780800 & 4.84326500 & 2.88268600 & $\mathrm{~F}$ & 1.27246800 & 3.27489500 & 4.01738700 \\
\hline $\mathrm{F}$ & 0.12420200 & 4.52960400 & 2.65799900 & $\mathrm{C}$ & -4.19490900 & -3.89429100 & 2.34774100 \\
\hline $\mathrm{H}$ & -3.83689300 & -4.73345100 & 1.74358900 & $\mathrm{H}$ & -5.28140200 & -3.84647800 & 2.23708000 \\
\hline C & -0.06701700 & -0.07336400 & -0.99916200 & 0 & 0.59627100 & -0.85002000 & -0.34914500 \\
\hline $\mathrm{O}$ & 0.28616900 & 0.58288700 & -2.11210500 & $C$ & 1.65170400 & 0.44963800 & -2.69073200 \\
\hline $\mathrm{C}$ & 1.93025700 & -1.00914600 & -3.03433800 & $\mathrm{H}$ & 2.88552800 & -1.07745800 & -3.56479200 \\
\hline $\mathrm{H}$ & 1.99215300 & -1.62278500 & -2.13815900 & $\mathrm{H}$ & 1.14358600 & -1.41148600 & -3.67856500 \\
\hline $\mathrm{C}$ & 1.54810300 & 1.30127000 & -3.95312400 & $\mathrm{H}$ & 1.31781700 & 2.34108600 & -3.69986100 \\
\hline $\mathrm{H}$ & 2.50404600 & 1.28562600 & -4.48545700 & $\mathrm{H}$ & 0.77111300 & 0.91575000 & -4.62151400 \\
\hline C & 2.66250500 & 1.03041300 & -1.71165900 & $\mathrm{H}$ & 3.66964300 & 0.96133300 & -2.13280800 \\
\hline $\bar{H}$ & 2.44511600 & 2.07748100 & -1.49204200 & $\mathrm{H}$ & 2.65396600 & 0.47522500 & -0.77628600 \\
\hline $\mathrm{C}$ & -2.12931800 & 1.20454900 & -1.26588600 & $\mathrm{H}$ & -1.85723900 & 1.32311500 & -2.30871600 \\
\hline C & -3.58313700 & 1.29482000 & -0.98663100 & C & -4.44815600 & 1.52279600 & -2.06450000 \\
\hline $\mathrm{C}$ & -4.10324800 & 1.24309900 & 0.31490900 & $\mathrm{C}$ & -5.81412300 & 1.70548300 & -1.84899800 \\
\hline $\bar{H}$ & -4.04932300 & 1.54851600 & -3.07503500 & $\mathrm{C}$ & -5.46575400 & 1.43390000 & 0.52436600 \\
\hline $\mathrm{H}$ & -3.44561700 & 1.05024900 & 1.15458000 & C & -6.32395800 & 1.67209000 & -0.55226200 \\
\hline $\bar{H}$ & -6.47551400 & 1.87009000 & -2.69434900 & $\mathrm{H}$ & -5.85971400 & 1.39939100 & 1.53609700 \\
\hline $\bar{H}$ & -7.38568900 & 1.82385600 & -0.38044200 & $\mathrm{~N}$ & -1.38603500 & 0.23854300 & -0.64342000 \\
\hline $\mathrm{H}$ & -1.64538300 & -0.09171000 & 0.29662200 & C & -1.38139900 & 2.89915600 & -0.58404700 \\
\hline $\mathrm{H}$ & -1.69476000 & 2.81736300 & 0.45133800 & $\mathrm{H}$ & -0.33871400 & 2.65789600 & -0.75131900 \\
\hline $\mathrm{C}$ & -1.93008900 & 3.90316700 & -1.37952700 & $\mathrm{C}$ & -1.27080100 & 4.31093500 & -2.61386100 \\
\hline $\mathrm{H}$ & -0.62621800 & 3.52370700 & -3.01612900 & $\mathrm{H}$ & -1.97186600 & 4.66252000 & -3.37808500 \\
\hline $\mathrm{C}$ & -3.22761700 & 4.54844400 & -1.01908600 & $\mathrm{H}$ & -3.45087500 & 4.46340100 & 0.04607100 \\
\hline $\mathrm{H}$ & -3.24645800 & 5.60068000 & -1.32046300 & $\mathrm{H}$ & -4.03409400 & 4.04023900 & -1.56862400 \\
\hline C & 1.68626600 & 5.24339800 & -2.60032000 & $\mathrm{H}$ & 1.77676900 & 4.82252400 & -3.60821500 \\
\hline $\mathrm{H}$ & 2.38858900 & 6.08252000 & -2.52479900 & $\mathrm{H}$ & 1.99039100 & 4.49100300 & -1.86632500 \\
\hline $\mathrm{C}$ & -0.60274000 & 7.19409200 & -3.41835200 & $\mathrm{H}$ & -0.53057500 & 6.87240500 & -4.46385000 \\
\hline $\mathrm{H}$ & -1.63841400 & 7.50197500 & -3.23091900 & $\mathrm{H}$ & 0.03189600 & 8.08024300 & -3.29838000 \\
\hline $\mathrm{C}$ & -0.22976000 & 6.38736200 & -0.44926500 & $\mathrm{H}$ & -1.24453500 & 6.71975900 & -0.20323600 \\
\hline $\mathrm{H}$ & 0.05969000 & 5.58997500 & 0.24095200 & $\mathrm{H}$ & 0.44381500 & 7.23475400 & -0.27206500 \\
\hline $\mathrm{Si}$ & -0.05811600 & 5.82803800 & -2.23477700 & & & & \\
\hline
\end{tabular}

\section{TS- $R-4 f-21$}

Imaginary frequency: $-304.78 \mathrm{~cm}^{-1}$

E[B3LYP/6-31G(d)-D3(BJ)]: -4789.075552 Hartree

E[PCM(Et $2 \mathrm{O})-B 3 L Y P / 6-311 G(d, p)-D 3(B J)]:-4790.223806$ Hartree

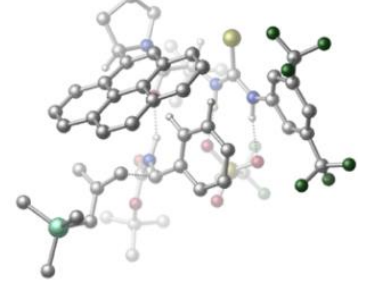




\begin{tabular}{|c|c|c|c|c|c|c|c|}
\hline 01 & & & & $\mathrm{~N}$ & 3.01665100 & 1.36061400 & 0.56399800 \\
\hline $\mathrm{H}$ & 2.44565900 & 2.08576900 & 0.11851400 & C & 3.94065000 & 0.80226000 & -0.33062900 \\
\hline $\mathrm{C}$ & 4.02621400 & 1.41639300 & -1.59258300 & $\mathrm{C}$ & 4.70490400 & -0.34848700 & -0.08885400 \\
\hline C & 4.82848800 & 0.86747700 & -2.58654200 & $\mathrm{H}$ & 3.41733300 & 2.28610000 & -1.80722900 \\
\hline C & 5.49562600 & -0.88163500 & -1.10316000 & $\mathrm{H}$ & 4.66224400 & -0.82606900 & 0.87582900 \\
\hline $\mathrm{C}$ & 5.57169100 & -0.29074100 & -2.36252500 & $\mathrm{H}$ & 6.18265900 & -0.72039300 & -3.14750800 \\
\hline $\mathrm{C}$ & 4.77107000 & 1.44153700 & -3.97294100 & $\mathrm{C}$ & 6.29046600 & -2.12987100 & -0.84415000 \\
\hline $\mathrm{F}$ & 4.47869700 & 2.75431800 & -3.97978600 & $\mathrm{~F}$ & 3.81326600 & 0.81772200 & -4.71492300 \\
\hline$F$ & 5.93612700 & 1.27440000 & -4.63865300 & $F$ & 5.96231000 & -2.72260600 & 0.32791100 \\
\hline$F$ & 6.08538700 & -3.05457200 & -1.81994600 & $F$ & 7.61937400 & -1.89407900 & -0.81391000 \\
\hline C & 0.92063300 & 1.40082300 & 3.55249300 & $\mathrm{H}$ & 1.71777100 & 1.06943300 & 4.21634100 \\
\hline C & 0.29388200 & 2.67966600 & 4.19505300 & C & -0.97799400 & 3.17167100 & 3.48947700 \\
\hline $\mathrm{H}$ & -1.77147200 & 2.42192100 & 3.49663500 & $\mathrm{H}$ & -0.80197800 & 3.44202000 & 2.44798000 \\
\hline $\mathrm{H}$ & -1.35161100 & 4.06282100 & 4.00827300 & C & 1.35750200 & 3.79123900 & 4.16862600 \\
\hline $\mathrm{H}$ & 1.00636100 & 4.65273500 & 4.74813600 & $\mathrm{H}$ & 1.55004400 & 4.13040200 & 3.14724200 \\
\hline $\mathrm{H}$ & 2.30608600 & 3.45177900 & 4.60138500 & $\mathrm{C}$ & -0.05091600 & 2.34002000 & 5.65659800 \\
\hline $\mathrm{H}$ & -0.47802200 & 3.21781300 & 6.15309400 & $\mathrm{H}$ & 0.83800200 & 2.03471400 & 6.22171900 \\
\hline $\mathrm{H}$ & -0.79389800 & 1.53573900 & 5.71725300 & C & -0.07858000 & 0.25382300 & 3.34513700 \\
\hline $\mathrm{O}$ & -0.87381300 & 0.26081300 & 2.39219000 & C & 0.81587500 & -1.04209800 & 5.34742600 \\
\hline C & -0.96294500 & -1.93106200 & 3.90543600 & $\mathrm{C}$ & 0.56053700 & -2.51921000 & 5.68466300 \\
\hline $\mathrm{H}$ & 1.85727500 & -0.84262700 & 5.08142500 & $\mathrm{H}$ & 0.53013100 & -0.39103400 & 6.18029700 \\
\hline C & -0.87806300 & -2.76140300 & 5.20249300 & $\mathrm{H}$ & -1.96197200 & -1.52525400 & 3.74127100 \\
\hline $\mathrm{H}$ & 0.69531300 & -2.72622500 & 6.74956100 & $\mathrm{H}$ & 1.25419800 & -3.15692900 & 5.12821700 \\
\hline $\mathrm{H}$ & -1.59555300 & -2.37101800 & 5.93402500 & $\mathrm{H}$ & -1.10368100 & -3.81683800 & 5.03064500 \\
\hline $\mathrm{N}$ & -0.06344700 & -0.80102800 & 4.18996900 & $\mathrm{C}$ & -0.52779400 & -2.69946500 & 2.66426800 \\
\hline C & 0.79282300 & -2.77236500 & 2.32592700 & $\mathrm{C}$ & -1.51772600 & -3.35744100 & 1.83573100 \\
\hline C & 1.25436500 & -3.45901800 & 1.15739500 & $\mathrm{H}$ & 1.53602900 & -2.23900500 & 2.90878800 \\
\hline C & -1.09020100 & -3.98975600 & 0.62225800 & C & -2.88631800 & -3.40095200 & 2.15996100 \\
\hline C & 2.61778300 & -3.52942600 & 0.82641700 & $\mathrm{C}$ & 0.29203100 & -4.03065400 & 0.27844700 \\
\hline C & -2.04836600 & -4.59679700 & -0.24827100 & $\mathrm{H}$ & -3.23848300 & -2.96200600 & 3.08620400 \\
\hline $\mathrm{C}$ & -3.80788000 & -4.02868500 & 1.32151100 & C & 3.02701200 & -4.12533600 & -0.36544400 \\
\hline $\mathrm{H}$ & 3.34733800 & -3.07513800 & 1.48962900 & C & 0.71871600 & -4.63668300 & -0.93998800 \\
\hline C & -3.40227900 & -4.60770800 & 0.12251700 & C & -1.59244700 & -5.19025700 & -1.47504900 \\
\hline $\mathrm{H}$ & -4.85324500 & -4.07396800 & 1.61671500 & C & 2.08843500 & -4.66394000 & -1.24351700 \\
\hline $\mathrm{H}$ & 4.07995800 & -4.14811900 & -0.62111800 & C & -0.27299900 & -5.20190800 & -1.80921200 \\
\hline $\mathrm{H}$ & -4.12614600 & -5.09491600 & -0.52534900 & $\mathrm{H}$ & -2.33127200 & -5.64093900 & -2.13261900 \\
\hline $\mathrm{H}$ & 2.41327300 & -5.11619900 & -2.17678200 & $\mathrm{H}$ & 0.05512600 & -5.65521800 & -2.74088800 \\
\hline $\mathrm{C}$ & 2.64596800 & 0.99994300 & 1.83582700 & $\mathrm{~S}$ & 3.47147700 & -0.11852800 & 2.81933200 \\
\hline $\mathrm{N}$ & 1.54941900 & 1.66377100 & 2.26854100 & $\mathrm{H}$ & 0.96911600 & 2.14958400 & 1.57248700 \\
\hline $\mathrm{S}$ & 0.21685500 & 3.55056600 & -0.83399300 & $\mathrm{O}$ & -0.80213500 & 3.51815500 & -1.89791200 \\
\hline $\mathrm{O}$ & -0.15115800 & 2.79742300 & 0.39770200 & $\mathrm{O}$ & 1.62375600 & 3.31394700 & -1.24076300 \\
\hline C & 0.21981500 & 5.30366600 & -0.22383900 & $\mathrm{~F}$ & 0.54232300 & 6.15702500 & -1.20138900 \\
\hline $\mathrm{F}$ & 1.11088500 & 5.43781700 & 0.77444700 & $\mathrm{~F}$ & -0.99213700 & 5.63433300 & 0.25160100 \\
\hline $\mathrm{C}$ & -3.25109800 & 2.15850900 & 0.34451500 & $\mathrm{O}$ & -3.35673700 & 2.76793200 & 1.38681200 \\
\hline $\mathrm{O}$ & -4.01807200 & 2.31004800 & -0.75527100 & C & -4.82707900 & 3.53308100 & -0.93283400 \\
\hline $\mathrm{C}$ & -5.98811300 & 3.51852000 & 0.06066200 & $\mathrm{H}$ & -6.64535300 & 4.37388000 & -0.12940600 \\
\hline $\mathrm{H}$ & -5.61896700 & 3.57600000 & 1.08520900 & $\mathrm{H}$ & -6.57972600 & 2.60262400 & -0.05506400 \\
\hline C & -5.32410100 & 3.39020100 & -2.36999500 & $\mathrm{H}$ & -4.47906000 & 3.35739400 & -3.06437100 \\
\hline $\mathrm{H}$ & -5.95662300 & 4.24383800 & -2.63227000 & $\mathrm{H}$ & -5.91438400 & 2.47510500 & -2.49008100 \\
\hline $\mathrm{C}$ & -3.93202800 & 4.76350800 & -0.78924000 & $\mathrm{H}$ & -4.49379900 & 5.65354900 & -1.09311900 \\
\hline $\mathrm{H}$ & -3.04976900 & 4.66403200 & -1.42743500 & $\mathrm{H}$ & -3.59783000 & 4.89395200 & 0.24010700 \\
\hline $\mathrm{C}$ & -2.10252400 & 0.55342100 & -1.07557000 & $\mathrm{H}$ & -2.61834100 & 1.08455000 & -1.86585100 \\
\hline $\mathrm{C}$ & -0.72623100 & 0.15145300 & -1.46236300 & $\mathrm{C}$ & -0.32063100 & 0.43963700 & -2.77328500 \\
\hline
\end{tabular}




\begin{tabular}{|c|c|c|c|c|c|c|c|}
\hline C & 0.13306000 & -0.54095900 & -0.60156200 & C & 0.93208300 & 0.03554000 & -3.22583500 \\
\hline $\mathrm{H}$ & -0.97275700 & 1.01972600 & -3.41847700 & C & 1.37612000 & -0.95269000 & -1.06589800 \\
\hline $\mathrm{H}$ & -0.16324200 & -0.75280800 & 0.42014000 & C & 1.77230100 & -0.68294400 & -2.37683800 \\
\hline $\mathrm{H}$ & 1.26159400 & 0.29513600 & -4.22626800 & $\mathrm{H}$ & 2.04528100 & -1.48066600 & -0.40306600 \\
\hline $\mathrm{H}$ & 2.74380400 & -1.01472500 & -2.72549400 & $\mathrm{~N}$ & -2.32935500 & 1.13770800 & 0.15408900 \\
\hline $\mathrm{H}$ & -1.65102600 & 1.02884000 & 0.91594600 & C & -2.94409900 & -1.12444700 & -1.13835800 \\
\hline $\mathrm{H}$ & -2.46751100 & -1.59721200 & -0.28370100 & $\mathrm{H}$ & -2.59419900 & -1.48765100 & -2.10008000 \\
\hline C & -4.31027400 & -0.82405700 & -1.02777200 & C & -5.14172100 & -0.62553500 & -2.20810500 \\
\hline $\mathrm{H}$ & -4.55836900 & -0.30279600 & -3.07816100 & $\mathrm{H}$ & -5.96261200 & 0.07566000 & -2.03059300 \\
\hline $\mathrm{C}$ & -4.88785400 & -0.67154600 & 0.33206500 & $\mathrm{H}$ & -5.89176400 & -0.24325200 & 0.33009500 \\
\hline $\mathrm{H}$ & -4.21349800 & -0.04682500 & 0.93538600 & $\mathrm{H}$ & -4.89633100 & -1.64493700 & 0.83674500 \\
\hline $\mathrm{C}$ & -7.16516300 & -1.88084500 & -4.11268800 & $\mathrm{H}$ & -7.95726500 & -1.22145400 & -3.74028200 \\
\hline $\mathrm{H}$ & -7.64292900 & -2.78573300 & -4.50642400 & $\mathrm{H}$ & -6.67083000 & -1.37495800 & -4.94967700 \\
\hline C & -6.80517000 & -3.10500000 & -1.27890700 & $\mathrm{H}$ & -7.53748300 & -2.42700100 & -0.82612600 \\
\hline $\mathrm{H}$ & -6.08751100 & -3.39587900 & -0.50527500 & $\mathrm{H}$ & -7.34144800 & -4.00901000 & -1.59079200 \\
\hline $\mathrm{C}$ & -4.55932800 & -3.42420100 & -3.39619700 & $\mathrm{H}$ & -3.80658300 & -3.61944200 & -2.62622100 \\
\hline $\mathrm{H}$ & -4.05216300 & -2.97547900 & -4.25808200 & $\mathrm{H}$ & -4.96429100 & -4.39083800 & -3.71928800 \\
\hline $\mathrm{Si}$ & -5.93838600 & -2.31928400 & -2.75332600 & & & & \\
\hline
\end{tabular}

\section{TS- $R-4 f-22$}

Imaginary frequency: $-107.66 \mathrm{~cm}^{-1}$

E[B3LYP/6-31G(d)-D3(BJ)]: -4789.088485 Hartree

E[PCM(Et $\left.\left.{ }_{2} \mathrm{O}\right)-\mathrm{B} 3 L Y P / 6-311 \mathrm{G}(\mathrm{d}, \mathrm{p})-\mathrm{D} 3(\mathrm{BJ})\right]:-4790.223699$ Hartree

\begin{tabular}{|c|c|c|c|c|c|c|c|}
\hline 01 & & & & $\mathrm{~N}$ & 2.40833700 & 0.94339400 & -2.13809300 \\
\hline $\mathrm{H}$ & 3.17921400 & 0.30075000 & -1.91944400 & C & 2.50777300 & 2.16944200 & -1.46412500 \\
\hline C & 3.38103000 & 2.20379500 & -0.36082800 & C & 1.77983100 & 3.32419700 & -1.78884700 \\
\hline C & 3.49192900 & 3.35642300 & 0.40902600 & $\mathrm{H}$ & 3.95142600 & 1.32319400 & -0.09154100 \\
\hline C & 1.90163500 & 4.46213000 & -0.99369200 & $\mathrm{H}$ & 1.12073900 & 3.31923800 & -2.64249100 \\
\hline C & 2.75811400 & 4.50115500 & 0.10404900 & $\mathrm{H}$ & 2.84525900 & 5.39296800 & 0.71284400 \\
\hline C & 4.30693200 & 3.31546400 & 1.67157000 & C & 1.10327800 & 5.69061700 & -1.32727600 \\
\hline $\mathrm{F}$ & 3.54257300 & 2.89405900 & 2.71805500 & $\mathrm{~F}$ & 4.77615700 & 4.53706000 & 2.00852900 \\
\hline $\mathrm{F}$ & 5.35253500 & 2.47827000 & 1.58461600 & $\mathrm{~F}$ & 0.07106400 & 5.42335100 & -2.15810500 \\
\hline $\mathrm{F}$ & 0.57364100 & 6.24629900 & -0.20369300 & $\mathrm{~F}$ & 1.85237700 & 6.64724400 & -1.91295200 \\
\hline C & 0.31320000 & -1.73324100 & -3.47235000 & $\mathrm{H}$ & 0.18426700 & -1.30146600 & -4.46589700 \\
\hline C & 0.76040700 & -3.22225000 & -3.66143600 & C & 0.76292900 & -4.02825700 & -2.35020100 \\
\hline $\mathrm{H}$ & -0.24030900 & -4.11139300 & -1.92372100 & $\mathrm{H}$ & 1.41575300 & -3.58518900 & -1.59815900 \\
\hline $\mathrm{H}$ & 1.13218800 & -5.03964800 & -2.55627800 & C & 2.17557400 & -3.23035600 & -4.26990400 \\
\hline $\mathrm{H}$ & 2.44182500 & -4.25176200 & -4.56423300 & $\mathrm{H}$ & 2.92748200 & -2.88460000 & -3.55578300 \\
\hline $\mathrm{H}$ & 2.23043700 & -2.59492900 & -5.16149800 & $\mathrm{C}$ & -0.21149600 & -3.89163700 & -4.64926500 \\
\hline $\mathrm{H}$ & 0.08120200 & -4.93504300 & -4.80684100 & $\mathrm{H}$ & -0.20159500 & -3.39188700 & -5.62499100 \\
\hline $\mathrm{H}$ & -1.23911000 & -3.89132500 & -4.26895500 & C & -1.00416200 & -1.62152200 & -2.68010700 \\
\hline $\mathrm{O}$ & -0.97708200 & -1.74272000 & -1.44349300 & C & -2.32598300 & -1.12776100 & -4.77713300 \\
\hline C & -3.45188900 & -1.47086100 & -2.62174600 & $\mathrm{C}$ & -3.79933400 & -0.71858600 & -4.88031800 \\
\hline $\mathrm{H}$ & -1.63906900 & -0.32603200 & -5.05754600 & $\mathrm{H}$ & -2.11893200 & -2.01030300 & -5.39098700 \\
\hline C & -4.47279600 & -1.54540200 & -3.77816900 & $\mathrm{H}$ & -3.46952000 & -2.36959600 & -1.99808300 \\
\hline $\mathrm{H}$ & -4.21106000 & -0.90699600 & -5.87515000 & $\mathrm{H}$ & -3.90718400 & 0.34871800 & -4.66204900 \\
\hline $\mathrm{H}$ & -4.59358400 & -2.58689900 & -4.10008700 & $\mathrm{H}$ & -5.45118300 & -1.15847900 & -3.48655400 \\
\hline $\mathrm{N}$ & -2.16492800 & -1.45048600 & -3.34554000 & C & -3.66609400 & -0.26042700 & -1.72633100 \\
\hline $\mathrm{C}$ & -2.89855800 & 0.85828800 & -1.85433400 & $\mathrm{C}$ & -4.69888200 & -0.31714800 & -0.71090800 \\
\hline C & -3.02465600 & 1.98288100 & -0.97467800 & $\mathrm{H}$ & -2.11633500 & 0.89596000 & -2.60405800 \\
\hline
\end{tabular}




\begin{tabular}{|c|c|c|c|c|c|c|c|}
\hline C & -4.82445900 & 0.77891200 & 0.20041800 & C & -5.58432000 & -1.39980100 & -0.57751900 \\
\hline $\mathrm{C}$ & -2.20338000 & 3.11550100 & -1.09450100 & $\mathrm{C}$ & -3.98211400 & 1.92176800 & 0.07710500 \\
\hline C & -5.80089900 & 0.73550500 & 1.24249100 & $\mathrm{H}$ & -5.52120700 & -2.24014600 & -1.25926100 \\
\hline C & -6.56113400 & -1.41437400 & 0.41706400 & C & -2.31610000 & 4.16817400 & -0.18827200 \\
\hline $\mathrm{H}$ & -1.46638800 & 3.15370600 & -1.89038800 & C & -4.09026900 & 3.00377000 & 0.99875500 \\
\hline C & -6.66497200 & -0.36664800 & 1.32657000 & C & -5.87491400 & 1.83109400 & 2.16938800 \\
\hline $\mathrm{H}$ & -7.24147300 & -2.25863600 & 0.48624200 & C & -3.24515700 & 4.11303800 & 0.84727800 \\
\hline $\mathrm{H}$ & -1.66318000 & 5.02806200 & -0.28018900 & C & -5.05588300 & 2.91184200 & 2.05605200 \\
\hline $\mathrm{H}$ & -7.41940900 & -0.39070600 & 2.10853900 & $\mathrm{H}$ & -6.60993500 & 1.77707100 & 2.96826000 \\
\hline $\bar{H}$ & -3.31439700 & 4.92971500 & 1.56013200 & $\mathrm{H}$ & -5.12330500 & 3.73246400 & 2.76548200 \\
\hline C & 1.35397700 & 0.40376800 & -2.83599300 & $S$ & 0.20572600 & 1.30225300 & -3.69927000 \\
\hline $\mathrm{N}$ & 1.33370800 & -0.94720800 & -2.79919500 & $\mathrm{H}$ & 1.91738500 & -1.42131800 & -2.10272300 \\
\hline $\mathrm{S}$ & 4.20591900 & -1.49197600 & -0.34768400 & $\mathrm{O}$ & 4.16590300 & -0.90483600 & 1.00649200 \\
\hline 0 & 2.96172400 & -2.21036200 & -0.75689300 & 0 & 4.70848000 & -0.58648100 & -1.41313200 \\
\hline C & 5.47163300 & -2.84692000 & -0.25314700 & $\mathrm{~F}$ & 6.67319300 & -2.34328400 & 0.03943500 \\
\hline $\mathrm{F}$ & 5.54189900 & -3.49497900 & -1.42134800 & $\mathrm{~F}$ & 5.12914300 & -3.72623300 & 0.70438600 \\
\hline $\mathrm{C}$ & -2.31974200 & -1.39182800 & 2.01750700 & $\mathrm{O}$ & -2.18891600 & -1.13807100 & 3.19965500 \\
\hline 0 & -2.97907100 & -2.43294200 & 1.48955400 & C & -3.57177800 & -3.47511100 & 2.35071900 \\
\hline $\mathrm{C}$ & -2.45706400 & -4.19806100 & 3.10702800 & $\mathrm{H}$ & -2.88099700 & -5.04495900 & 3.65678000 \\
\hline $\mathrm{H}$ & -1.96825000 & -3.53338900 & 3.82062000 & $\mathrm{H}$ & -1.70991300 & -4.58668800 & 2.40702900 \\
\hline C & -4.22696500 & -4.40211900 & 1.32886500 & $\mathrm{H}$ & -4.99665100 & -3.86531400 & 0.76875500 \\
\hline $\mathrm{H}$ & -4.69001900 & -5.25216600 & 1.83957200 & $\mathrm{H}$ & -3.48145500 & -4.78201600 & 0.62310400 \\
\hline $\mathrm{C}$ & -4.61269300 & -2.86734600 & 3.29049500 & $\mathrm{H}$ & -5.15400300 & -3.67571600 & 3.79426000 \\
\hline $\mathrm{H}$ & -5.33046300 & -2.27095200 & 2.72199800 & $\mathrm{H}$ & -4.14392200 & -2.23402300 & 4.04391300 \\
\hline $\mathrm{C}$ & -0.86244600 & 0.35510000 & 0.97122300 & $\mathrm{H}$ & -0.63646000 & 0.62545900 & -0.05530300 \\
\hline C & -0.83012700 & 1.51905100 & 1.87796000 & C & 0.07750800 & 2.54444000 & 1.54699100 \\
\hline C & -1.67528000 & 1.70262200 & 2.98389300 & C & 0.16370900 & 3.69773500 & 2.31359800 \\
\hline $\mathrm{H}$ & 0.71396600 & 2.43637700 & 0.67647800 & C & -1.59129000 & 2.86789500 & 3.74449200 \\
\hline $\mathrm{H}$ & -2.39713100 & 0.94464600 & 3.24371200 & C & -0.66979700 & 3.86189700 & 3.42216300 \\
\hline $\mathrm{H}$ & 0.86167400 & 4.47614400 & 2.03026600 & $\mathrm{H}$ & -2.25893600 & 2.99449300 & 4.59161500 \\
\hline $\mathrm{H}$ & -0.60586200 & 4.76514100 & 4.02218300 & $\mathrm{~N}$ & -1.79418700 & -0.65699400 & 0.95935800 \\
\hline $\mathrm{H}$ & -1.83826600 & -1.13624200 & 0.05247900 & C & 1.01682700 & -0.54621600 & 0.96284900 \\
\hline $\mathrm{H}$ & 1.60854900 & 0.29999300 & 0.63461300 & $\mathrm{H}$ & 0.76505900 & -1.27188700 & 0.19752800 \\
\hline C & 1.14188100 & -0.95654400 & 2.28438700 & C & 0.65121200 & -2.26025900 & 2.73095000 \\
\hline $\mathrm{H}$ & -0.06140700 & -2.68762100 & 2.01829000 & $\mathrm{H}$ & 0.17905000 & -2.17566100 & 3.71429500 \\
\hline C & 1.68889200 & -0.01667800 & 3.30601500 & $\mathrm{H}$ & 2.38744200 & 0.70334000 & 2.87856300 \\
\hline $\mathrm{H}$ & 2.17261000 & -0.55491200 & 4.12491600 & $\mathrm{H}$ & 0.84303200 & 0.54442100 & 3.73481900 \\
\hline C & 1.23391300 & -4.78563100 & 4.28639400 & $\mathrm{H}$ & 1.11501500 & -4.25312900 & 5.23743000 \\
\hline $\mathrm{H}$ & 1.88332800 & -5.65023400 & 4.46960800 & $\mathrm{H}$ & 0.25040700 & -5.16702900 & 3.98922700 \\
\hline $\mathrm{C}$ & 3.62837700 & -2.98535100 & 3.54277400 & $\mathrm{H}$ & 3.54411100 & -2.53627500 & 4.53950000 \\
\hline $\mathrm{H}$ & 4.01941000 & -2.23553200 & 2.84971300 & $\mathrm{H}$ & 4.36222900 & -3.79762800 & 3.60983500 \\
\hline C & 2.10522600 & -4.61503600 & 1.33687800 & $\mathrm{H}$ & 2.43100600 & -3.96404800 & 0.52295600 \\
\hline $\mathrm{H}$ & 1.13308200 & -5.04637000 & 1.06803700 & $\mathrm{H}$ & 2.82527700 & -5.43720100 & 1.42386700 \\
\hline $\mathrm{Si}$ & 1.98308700 & -3.67153500 & 2.95423900 & & & & \\
\hline
\end{tabular}

\section{TS- $R-4 f-23$}

Imaginary frequency: $-416.72 \mathrm{~cm}^{-1}$

E[B3LYP/6-31G(d)-D3(BJ)]: -4789.081952 Hartree

$\mathrm{E}\left[\mathrm{PCM}\left(\mathrm{Et}_{2} \mathrm{O}\right)-\mathrm{B} 3 \mathrm{LYP} / 6-311 \mathrm{G}(\mathrm{d}, \mathrm{p})-\mathrm{D} 3(\mathrm{BJ})\right]:-4790.223640$ Hartree

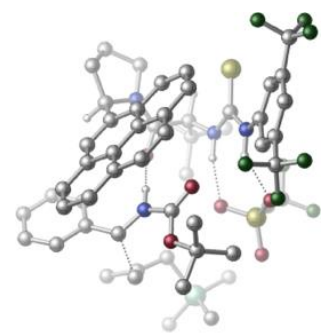




\begin{tabular}{|c|c|c|c|c|c|c|c|}
\hline 01 & & & & $\mathrm{~N}$ & -1.99877900 & -1.75096400 & 0.73453500 \\
\hline $\mathrm{H}$ & -1.26134000 & -1.89635800 & 0.04458000 & C & -3.22910000 & -1.49554900 & 0.11653400 \\
\hline $\mathrm{C}$ & -3.16986300 & -1.22365700 & -1.26264100 & $\mathrm{C}$ & -4.47106900 & -1.47407500 & 0.75837200 \\
\hline $\mathrm{C}$ & -4.32246100 & -0.90093300 & -1.96475500 & $\mathrm{H}$ & -2.21325700 & -1.25571300 & -1.76418400 \\
\hline C & -5.60971100 & -1.10818500 & 0.03883000 & $\mathrm{H}$ & -4.53809400 & -1.70178900 & 1.81109800 \\
\hline C & -5.55923100 & -0.82082700 & -1.32038900 & $\mathrm{H}$ & -6.45495200 & -0.55062300 & -1.86553700 \\
\hline C & -4.23746800 & -0.54545800 & -3.42072000 & C & -6.88547400 & -0.89693400 & 0.79953700 \\
\hline $\mathrm{F}$ & -3.15296100 & -1.07940800 & -4.02345300 & $\mathrm{~F}$ & -4.15854000 & 0.80180700 & -3.60346400 \\
\hline $\mathrm{F}$ & -5.32721500 & -0.95686000 & -4.10537100 & $\mathrm{~F}$ & -6.88475400 & 0.32933500 & 1.39370800 \\
\hline $\mathrm{F}$ & -7.97633100 & -0.94181800 & 0.00425900 & $\mathrm{~F}$ & -7.05865900 & -1.80243900 & 1.78299200 \\
\hline C & 0.45007000 & -1.20465700 & 3.35280600 & $\mathrm{H}$ & -0.29342800 & -1.24048900 & 4.14730100 \\
\hline C & 1.64277400 & -2.09675600 & 3.79795000 & C & 2.77138900 & -2.19659300 & 2.75787100 \\
\hline $\mathrm{H}$ & 3.20729000 & -1.21702400 & 2.54657600 & $\mathrm{H}$ & 2.41880300 & -2.61725000 & 1.81502700 \\
\hline $\mathrm{H}$ & 3.55943900 & -2.85295300 & 3.14818200 & C & 1.08877800 & -3.50323900 & 4.08241100 \\
\hline $\mathrm{H}$ & 1.88385300 & -4.14837400 & 4.47401700 & $\mathrm{H}$ & 0.69460700 & -3.96041200 & 3.17266600 \\
\hline $\mathrm{H}$ & 0.27963900 & -3.46934300 & 4.82085800 & C & 2.20921500 & -1.49698700 & 5.09775000 \\
\hline $\mathrm{H}$ & 3.04982000 & -2.10018600 & 5.45766600 & $\mathrm{H}$ & 1.45459900 & -1.47653300 & 5.89328000 \\
\hline $\mathrm{H}$ & 2.57841300 & -0.47469400 & 4.94571300 & C & 0.81629000 & 0.25398900 & 3.04531500 \\
\hline 0 & 1.56083800 & 0.51407700 & 2.08309200 & C & -0.43363600 & 1.07961900 & 5.06985400 \\
\hline C & 0.37588900 & 2.65665900 & 3.36189400 & C & -0.34941900 & 2.48607700 & 5.67221000 \\
\hline $\mathrm{H}$ & -1.46715400 & 0.74552600 & 4.92919500 & $\mathrm{H}$ & 0.08029400 & 0.33493500 & 5.67830600 \\
\hline $\mathrm{H}$ & 1.43610700 & 2.92407100 & 3.44748900 & $\mathrm{H}$ & 0.61322000 & 2.61667400 & 6.18077700 \\
\hline $\mathrm{H}$ & -1.14387400 & 2.66841000 & 6.40075400 & $\mathrm{~N}$ & 0.24035000 & 1.23436800 & 3.76501900 \\
\hline C & -0.05326500 & 2.99687400 & 1.94455600 & C & 0.78775300 & 3.78550400 & 1.21353000 \\
\hline C & -1.34625100 & 2.62729000 & 1.39709400 & C & 0.47288200 & 4.25057900 & -0.10301500 \\
\hline $\mathrm{H}$ & 1.74589400 & 4.07698000 & 1.63289700 & C & -1.69513100 & 3.09692300 & 0.08921200 \\
\hline $\mathrm{C}$ & -2.29313200 & 1.86001600 & 2.09370100 & C & 1.36142900 & 5.04008900 & -0.85005900 \\
\hline C & -0.78755400 & 3.89630000 & -0.66387700 & C & -2.97268100 & 2.78199800 & -0.46803900 \\
\hline $\mathrm{H}$ & -2.06092900 & 1.45171700 & 3.06415200 & C & -3.54425000 & 1.57814800 & 1.55073200 \\
\hline C & 1.02388500 & 5.46494900 & -2.13400600 & $\mathrm{H}$ & 2.31448600 & 5.31942900 & -0.41217900 \\
\hline $\mathrm{C}$ & -1.13987900 & 4.36559200 & -1.96600400 & C & -3.88551600 & 2.02965900 & 0.28315200 \\
\hline C & -3.30085500 & 3.26480400 & -1.77955500 & $\mathrm{H}$ & -4.24743700 & 0.98275500 & 2.11999100 \\
\hline C & -0.21242400 & 5.13535400 & -2.68652400 & $\mathrm{H}$ & 1.72292500 & 6.07286200 & -2.70224500 \\
\hline $\mathrm{C}$ & -2.42970500 & 4.02530800 & -2.49489400 & $\mathrm{H}$ & -4.85923700 & 1.79237700 & -0.13334500 \\
\hline $\mathrm{H}$ & -4.26527300 & 2.99170000 & -2.19574400 & $\mathrm{H}$ & -0.47686100 & 5.48330900 & -3.68182300 \\
\hline $\mathrm{H}$ & -2.69167300 & 4.37913400 & -3.48851100 & C & -1.59484900 & -1.62744000 & 2.03274800 \\
\hline $\mathrm{S}$ & -2.62643400 & -1.47124300 & 3.37016500 & $\mathrm{~N}$ & -0.23671100 & -1.67458600 & 2.15900800 \\
\hline $\mathrm{H}$ & 0.32380500 & -1.67573000 & 1.30208000 & $\mathrm{~S}$ & 0.80840600 & -3.28564000 & -1.08234400 \\
\hline $\mathrm{O}$ & 1.71549100 & -3.74222800 & -2.14984800 & $\mathrm{O}$ & 1.38802700 & -2.22506800 & -0.20069400 \\
\hline $\mathrm{O}$ & -0.59603700 & -3.02100700 & -1.46684100 & C & 0.64647100 & -4.74802000 & 0.05993500 \\
\hline $\mathrm{F}$ & 0.33362900 & -5.85207700 & -0.62119500 & $\mathrm{~F}$ & -0.31241600 & -4.51611700 & 0.97132800 \\
\hline $\mathrm{F}$ & 1.80081400 & -4.96709200 & 0.72126300 & C & -0.43256200 & 3.40212900 & 4.44627600 \\
\hline $\mathrm{H}$ & -1.47027700 & 3.51495900 & 4.12009900 & $\mathrm{H}$ & -0.02693600 & 4.40231600 & 4.61787700 \\
\hline $\mathrm{C}$ & 0.86070500 & 0.68200900 & -1.49479800 & $\mathrm{O}$ & -0.17453100 & 0.22204000 & -1.06908100 \\
\hline $\mathrm{O}$ & 1.23312600 & 0.85652600 & -2.76886800 & C & 0.35466600 & 0.50101300 & -3.91509700 \\
\hline C & -0.94461000 & 1.28890500 & -3.81858600 & $\mathrm{H}$ & -1.57505000 & 1.06197100 & -4.68238900 \\
\hline $\mathrm{H}$ & -1.50077200 & 1.03874500 & -2.91707100 & $\mathrm{H}$ & -0.73367600 & 2.36064900 & -3.80951100 \\
\hline C & 1.18941000 & 0.96734400 & -5.10574900 & $\mathrm{H}$ & 2.14261900 & 0.42972100 & -5.14740800 \\
\hline $\mathrm{H}$ & 0.64485700 & 0.77239200 & -6.03464400 & $\mathrm{H}$ & 1.39087200 & 2.04177100 & -5.04065800 \\
\hline $\mathrm{C}$ & 0.15111100 & -1.00775800 & -3.94201200 & $\mathrm{H}$ & -0.41115500 & -1.27842800 & -4.84138000 \\
\hline $\mathrm{H}$ & 1.11212600 & -1.53125600 & -3.96669100 & $\mathrm{H}$ & -0.40147600 & -1.36414400 & -3.07583100 \\
\hline $\mathrm{C}$ & 2.96715200 & 1.83788000 & -1.07800800 & $\mathrm{H}$ & 2.76913900 & 2.33314600 & -2.02253300 \\
\hline C & 3.76552800 & 2.65565500 & -0.13540400 & C & 4.51875000 & 3.71116300 & -0.67214600 \\
\hline
\end{tabular}




\begin{tabular}{|c|c|c|c|c|c|c|c|}
\hline C & 3.81888000 & 2.41381700 & 1.24422500 & C & 5.29963800 & 4.51879900 & 0.14859500 \\
\hline $\mathrm{H}$ & 4.47513000 & 3.90730200 & -1.74041900 & C & 4.59856700 & 3.23056600 & 2.06543700 \\
\hline $\mathrm{H}$ & 3.23057200 & 1.61881600 & 1.68666400 & C & 5.33948300 & 4.28163500 & 1.52529900 \\
\hline $\bar{H}$ & 5.86860500 & 5.33735000 & -0.28196000 & $\mathrm{H}$ & 4.62220100 & 3.04018000 & 3.13468900 \\
\hline $\mathrm{H}$ & 5.94368800 & 4.91333100 & 2.16962800 & $\mathrm{~N}$ & 1.88268500 & 1.12692900 & -0.64164200 \\
\hline $\mathrm{H}$ & 1.73273200 & 0.89622200 & 0.34746200 & C & 4.25779000 & 0.64699700 & -2.00072700 \\
\hline $\mathrm{H}$ & 3.51541700 & 0.11769700 & -2.58953800 & $\mathrm{H}$ & 4.82766600 & 1.39698300 & -2.54417500 \\
\hline C & 4.92110300 & -0.05338200 & -0.98316600 & C & 4.41570500 & -1.30601400 & -0.47884300 \\
\hline $\mathrm{H}$ & 4.72125900 & -1.51579200 & 0.55126400 & $\mathrm{H}$ & 3.33475000 & -1.45531400 & -0.60272900 \\
\hline $\mathrm{C}$ & 6.14035500 & 0.53372900 & -0.34797900 & $\mathrm{H}$ & 5.83704700 & 1.01451100 & 0.59324000 \\
\hline $\mathrm{H}$ & 6.86453200 & -0.24359400 & -0.09133200 & $\mathrm{H}$ & 6.61766400 & 1.29422200 & -0.96944100 \\
\hline C & 4.74482300 & -4.35433300 & -0.67817400 & $\mathrm{H}$ & 3.67117800 & -4.53450400 & -0.74030000 \\
\hline $\mathrm{H}$ & 5.26112700 & -5.19745300 & -1.15365400 & $\mathrm{H}$ & 5.03719200 & -4.33272800 & 0.37764800 \\
\hline C & 4.52785700 & -2.64493300 & -3.30055700 & $\mathrm{H}$ & 3.44007000 & -2.75964600 & -3.28807800 \\
\hline $\mathrm{H}$ & 4.79548800 & -1.69949900 & -3.78524900 & $\mathrm{H}$ & 4.94082900 & -3.45772800 & -3.91046600 \\
\hline C & 7.07952300 & -2.55510300 & -1.56309800 & $\mathrm{H}$ & 7.40225600 & -1.64557900 & -2.08191200 \\
\hline $\mathrm{H}$ & 7.50213400 & -2.54093800 & -0.55131500 & $\mathrm{H}$ & 7.52999200 & -3.40652100 & -2.08800600 \\
\hline Si & 5.20000500 & -2.76168800 & -1.55422900 & & & & \\
\hline
\end{tabular}

\section{TS-R-4f-24}

Imaginary frequency: $-417.00 \mathrm{~cm}^{-1}$

E[B3LYP/6-31G(d)-D3(BJ)]: -4789.081763 Hartree

$\mathrm{E}\left[\mathrm{PCM}\left(\mathrm{Et}_{2} \mathrm{O}\right)-\mathrm{B} 3 \mathrm{LYP} / 6-311 \mathrm{G}(\mathrm{d}, \mathrm{p})-\mathrm{D} 3(\mathrm{BJ})\right]$ : -4790.223351 Hartree

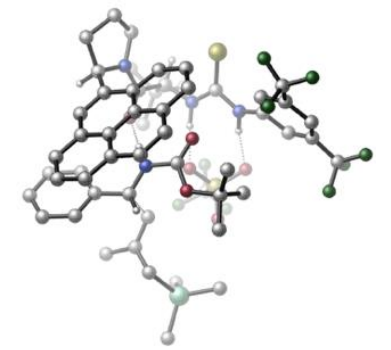

\begin{tabular}{|c|c|c|c|c|c|c|c|}
\hline 01 & & & & $\mathrm{~N}$ & 2.70536500 & -0.13612100 & 1.78435900 \\
\hline $\mathrm{H}$ & 2.39698300 & 0.82754000 & 1.93822800 & C & 3.70524700 & -0.24687800 & 0.81534300 \\
\hline C & 4.48271700 & 0.89424800 & 0.56940400 & C & 3.88462400 & -1.38247800 & 0.01508500 \\
\hline $\mathrm{C}$ & 5.41540500 & 0.89417000 & -0.46420700 & $\mathrm{H}$ & 4.31795200 & 1.78941300 & 1.15750600 \\
\hline $\mathrm{C}$ & 4.83998100 & -1.36823100 & -0.99672700 & $\mathrm{H}$ & 3.26346600 & -2.24848600 & 0.17378600 \\
\hline C & 5.61577100 & -0.23696000 & -1.25194000 & $\mathrm{H}$ & 6.35623100 & -0.23773300 & -2.04211300 \\
\hline $\mathrm{C}$ & 6.14002500 & 2.16449600 & -0.80329200 & $\mathrm{C}$ & 4.98091100 & -2.55830600 & -1.90102000 \\
\hline $\mathrm{F}$ & 6.31097200 & 2.96245300 & 0.26934400 & $\mathrm{~F}$ & 5.45174600 & 2.88839200 & -1.72631800 \\
\hline $\mathrm{F}$ & 7.36003100 & 1.92605500 & -1.33536200 & $\mathrm{~F}$ & 4.57949700 & -3.70880700 & -1.31496700 \\
\hline $\mathrm{F}$ & 4.23107300 & -2.42152100 & -3.03170000 & $\mathrm{~F}$ & 6.25450100 & -2.73667600 & -2.31220100 \\
\hline $\mathrm{C}$ & -0.10973100 & -1.46381000 & 3.67304700 & $\mathrm{H}$ & 0.48511300 & -2.27137800 & 4.09508800 \\
\hline C & -0.73368500 & -0.67886400 & 4.86946100 & C & -1.68510500 & 0.45109900 & 4.43831400 \\
\hline $\bar{H}$ & -2.58977700 & 0.05740800 & 3.96893900 & $\bar{H}$ & -1.21506600 & 1.13628600 & 3.73154900 \\
\hline $\mathrm{H}$ & -1.97871700 & 1.02923100 & 5.32261800 & $\mathrm{C}$ & 0.42487000 & -0.08400300 & 5.68862200 \\
\hline $\bar{H}$ & 0.03887700 & 0.36347500 & 6.61163800 & $\mathrm{H}$ & 0.94578400 & 0.69506800 & 5.12636900 \\
\hline $\mathrm{H}$ & 1.15683800 & -0.85319200 & 5.96090200 & $\mathrm{C}$ & -1.50933800 & -1.68651400 & 5.73625100 \\
\hline $\mathrm{H}$ & -1.96006200 & -1.17853100 & 6.59581900 & $\mathrm{H}$ & -0.85040200 & -2.47396200 & 6.12104400 \\
\hline $\mathrm{H}$ & -2.32119500 & -2.16296500 & 5.17285400 & $\mathrm{C}$ & -1.16675600 & -2.02628500 & 2.71730000 \\
\hline $\mathrm{O}$ & -1.83333100 & -1.24932600 & 2.00991800 & $\mathrm{~N}$ & -1.35848600 & -3.35679400 & 2.64903900 \\
\hline C & -2.26566300 & -3.56985400 & 0.27311400 & C & -3.43070100 & -3.27627700 & -0.37572800 \\
\hline $\mathrm{C}$ & -1.02669900 & -3.63844100 & -0.48071400 & $\mathrm{C}$ & -3.50504700 & -3.01870000 & -1.77995600 \\
\hline $\mathrm{H}$ & -4.35635200 & -3.21670300 & 0.19066700 & $\mathrm{C}$ & -1.07466900 & -3.39304600 & -1.89431900 \\
\hline C & 0.22401000 & -3.92085700 & 0.09074100 & C & -4.71381900 & -2.70888100 & -2.41922700 \\
\hline $\mathrm{C}$ & -2.30317800 & -3.06906100 & -2.54043500 & $\mathrm{C}$ & 0.11866600 & -3.47154800 & -2.67527200 \\
\hline $\mathrm{H}$ & 0.33033300 & -4.02648600 & 1.15705800 & $\mathrm{C}$ & 1.37675400 & -4.01352900 & -0.68469200 \\
\hline C & -4.74303900 & -2.43363300 & -3.78426200 & $\mathrm{H}$ & -5.62510000 & -2.66419300 & -1.83084100 \\
\hline C & -2.34327100 & -2.79615100 & -3.94196100 & $C$ & 1.33219500 & -3.79796300 & -2.05504900 \\
\hline
\end{tabular}




\begin{tabular}{|c|c|c|c|c|c|c|c|}
\hline $\mathrm{C}$ & 0.04851000 & -3.20800900 & -4.08531300 & $\mathrm{H}$ & 2.31954100 & -4.23815400 & -0.19881300 \\
\hline C & -3.57243000 & -2.47304100 & -4.53886000 & $\mathrm{H}$ & -5.68643300 & -2.18730000 & -4.26395800 \\
\hline $\mathrm{C}$ & -1.12265200 & -2.87432600 & -4.69217100 & $\mathrm{H}$ & 2.23754400 & -3.85012100 & -2.64965800 \\
\hline $\mathrm{H}$ & 0.96812100 & -3.27683600 & -4.66046000 & $\mathrm{H}$ & -3.60121700 & -2.26349700 & -5.60519100 \\
\hline $\mathrm{H}$ & -1.15622400 & -2.67343600 & -5.75993900 & C & 1.99515800 & -1.11727500 & 2.42451600 \\
\hline $\mathrm{S}$ & 2.57670000 & -2.68652200 & 2.68938200 & $\mathrm{~N}$ & 0.80961600 & -0.65829100 & 2.89426000 \\
\hline $\mathrm{H}$ & 0.42882700 & 0.18345100 & 2.45147300 & S & 0.71186000 & 2.95616500 & 1.60353400 \\
\hline $\mathrm{O}$ & 0.42208700 & 3.89180800 & 0.49801100 & 0 & -0.10734100 & 1.71037300 & 1.58359900 \\
\hline $\mathrm{O}$ & 2.14206300 & 2.71117400 & 1.90310800 & C & 0.07476600 & 3.85375500 & 3.09856400 \\
\hline $\mathrm{F}$ & 0.65225000 & 5.05104100 & 3.22359300 & $\mathrm{~F}$ & 0.30604600 & 3.14422500 & 4.21420000 \\
\hline $\mathrm{F}$ & -1.25711300 & 4.03062700 & 2.98382900 & C & -0.24737200 & -0.07008700 & -0.99213800 \\
\hline $\mathrm{O}$ & 0.63276500 & -0.68535300 & -0.43391100 & $\mathrm{O}$ & -0.14574400 & 0.73829900 & -2.05494200 \\
\hline C & 1.17240800 & 1.04086400 & -2.67568300 & C & 1.83871800 & -0.24863100 & -3.14295100 \\
\hline $\mathrm{H}$ & 2.75894700 & -0.00108300 & -3.68168800 & $\mathrm{H}$ & 2.09624900 & -0.88917900 & -2.30208700 \\
\hline $\mathrm{H}$ & 1.17626700 & -0.79886100 & -3.81723000 & C & 0.77234600 & 1.91460400 & -3.86135500 \\
\hline $\mathrm{H}$ & 0.25978100 & 2.81903400 & -3.51785500 & $\mathrm{H}$ & 1.66759600 & 2.21920000 & -4.41201400 \\
\hline $\mathrm{H}$ & 0.11291900 & 1.36751100 & -4.54321200 & C & 2.01969300 & 1.81526300 & -1.67534100 \\
\hline $\mathrm{H}$ & 2.98610000 & 2.06863600 & -2.12102100 & $\mathrm{H}$ & 1.52420500 & 2.73857500 & -1.36836800 \\
\hline $\mathrm{H}$ & 2.20805900 & 1.21416500 & -0.78860600 & C & -2.58679000 & 0.63008000 & -1.06992100 \\
\hline $\mathrm{H}$ & -2.40368700 & 0.92264000 & -2.09762800 & C & -3.99426300 & 0.28638000 & -0.76418600 \\
\hline C & -4.95493800 & 0.51034900 & -1.76026800 & C & -4.39820800 & -0.20863600 & 0.48328300 \\
\hline $\mathrm{C}$ & -6.30169200 & 0.24232600 & -1.52007800 & $\mathrm{H}$ & -4.63978800 & 0.87783800 & -2.73305600 \\
\hline $\mathrm{C}$ & -5.74514800 & -0.47545800 & 0.71791600 & $\mathrm{H}$ & -3.66595100 & -0.40122500 & 1.25945500 \\
\hline C & -6.70017200 & -0.25041300 & -0.27718200 & $\mathrm{H}$ & -7.03352500 & 0.40819500 & -2.30506800 \\
\hline $\bar{H}$ & -6.05086600 & -0.86080300 & 1.68638300 & $\bar{H}$ & -7.74846200 & -0.45905400 & -0.08425600 \\
\hline $\mathrm{N}$ & -1.58183100 & -0.15246900 & -0.56817900 & $\mathrm{H}$ & -1.68779400 & -0.63558000 & 0.33333800 \\
\hline C & -2.29080400 & 2.39427800 & -0.24574300 & $\mathrm{H}$ & -2.44897100 & 2.13419000 & 0.79554800 \\
\hline $\bar{H}$ & -1.24787700 & 2.47170800 & -0.52868000 & $\mathrm{C}$ & -3.17504900 & 3.27979100 & -0.85912700 \\
\hline $\mathrm{C}$ & -2.81497100 & 3.94939800 & -2.10258300 & $\bar{H}$ & -2.04676600 & 3.40461700 & -2.66036400 \\
\hline $\mathrm{H}$ & -3.68076900 & 4.15468400 & -2.74105400 & C & -4.53262700 & 3.52233500 & -0.28767200 \\
\hline$\overline{\mathrm{H}}$ & -4.56610800 & 3.33663300 & 0.78791200 & $\bar{H}$ & -4.88276800 & 4.53691500 & -0.50071600 \\
\hline $\bar{H}$ & -5.23883300 & 2.82737400 & -0.76704300 & $\mathrm{C}$ & -0.20328400 & 5.61931100 & -2.21367500 \\
\hline $\mathrm{H}$ & -0.09072100 & 5.29372700 & -3.25417400 & $\mathrm{H}$ & 0.26372300 & 6.60748600 & -2.12122900 \\
\hline $\mathrm{H}$ & 0.34275700 & 4.93383800 & -1.55889400 & C & -2.96979800 & 6.93984200 & -2.75465300 \\
\hline$\overline{\mathrm{H}}$ & -2.90103000 & 6.69476100 & -3.82096000 & $\bar{H}$ & -4.03146400 & 6.96200000 & -2.48113400 \\
\hline $\mathrm{H}$ & -2.57273900 & 7.95313400 & -2.62036700 & C & -2.18070000 & 6.11621300 & 0.12753000 \\
\hline$\overline{\mathrm{H}}$ & -3.22661400 & 6.18330900 & 0.44734500 & $\bar{H}$ & -1.66685100 & 5.37571300 & 0.74681400 \\
\hline$\overline{\mathrm{H}}$ & -1.71450100 & 7.08894300 & 0.32602200 & $\mathrm{Si}$ & -2.00423600 & 5.70892400 & -1.69823200 \\
\hline C & -1.04549400 & -5.69958800 & 2.73389400 & $\mathrm{H}$ & -1.07538600 & -6.54987000 & 3.42065500 \\
\hline $\mathrm{H}$ & -0.29637900 & -5.91630100 & 1.96815200 & C & -0.69809600 & -4.39645500 & 3.46171000 \\
\hline$\overline{\mathrm{H}}$ & -1.10665200 & -4.38916400 & 4.47954700 & $\mathrm{H}$ & 0.37798400 & -4.21246400 & 3.50749900 \\
\hline C & -2.40767900 & -5.41347500 & 2.08527300 & $\mathrm{H}$ & -2.58889600 & -6.01426200 & 1.19155500 \\
\hline $\mathrm{H}$ & -3.21517200 & -5.62092200 & 2.79620100 & C & -2.40352500 & -3.89721100 & 1.75558200 \\
\hline $\mathrm{H}$ & -3.35471800 & -3.45192300 & 2.06446700 & & & & \\
\hline
\end{tabular}

\section{TS- $R-4 f-25$}

Imaginary frequency: $-402.70 \mathrm{~cm}^{-1}$

E[B3LYP/6-31G(d)-D3(BJ)]: -4789.079582 Hartree

E[PCM(Et $2 \mathrm{O})-B 3 L Y P / 6-311 G(d, p)-D 3(B J)]:-4790.223317$ Hartree

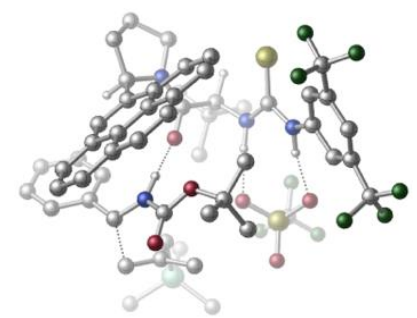




\begin{tabular}{|c|c|c|c|c|c|c|c|}
\hline 01 & & & & $\mathrm{~N}$ & -1.95219300 & -2.15962200 & 1.47417800 \\
\hline $\mathrm{H}$ & -1.33907900 & -2.79296600 & 0.94993900 & C & -3.23511800 & -2.06840700 & 0.91441400 \\
\hline $\mathrm{C}$ & -3.48657700 & -2.89603900 & -0.19280300 & $\mathrm{C}$ & -4.21558800 & -1.14534600 & 1.30686300 \\
\hline$C$ & -4.67439900 & -2.76852800 & -0.90563900 & $\mathrm{H}$ & -2.73138400 & -3.60247000 & -0.51767900 \\
\hline C & -5.39357300 & -1.03623200 & 0.57527600 & $\mathrm{H}$ & -4.04235500 & -0.50944000 & 2.15897300 \\
\hline C & -5.64494200 & -1.83950400 & -0.53568600 & $\mathrm{H}$ & -6.56460500 & -1.74168700 & -1.10001100 \\
\hline C & -4.84718500 & -3.54582700 & -2.17919100 & C & -6.39350700 & 0.02823100 & 0.92806600 \\
\hline $\mathrm{F}$ & -4.20034400 & -4.72587200 & -2.15645400 & $\mathrm{~F}$ & -4.35770500 & -2.84670300 & -3.24082200 \\
\hline $\mathrm{F}$ & -6.14756200 & -3.79287700 & -2.45069000 & $\mathrm{~F}$ & -6.12589900 & 0.63169800 & 2.10664500 \\
\hline $\mathrm{F}$ & -6.41749100 & 1.00766900 & -0.01671500 & $\mathrm{~F}$ & -7.65028000 & -0.46138400 & 0.99852000 \\
\hline C & 0.83646400 & -0.62983700 & 3.24016900 & $\mathrm{H}$ & 0.26567100 & -0.47694900 & 4.15343100 \\
\hline C & 2.18860900 & -1.29971300 & 3.64468000 & C & 3.09819200 & -1.61262200 & 2.44680000 \\
\hline $\mathrm{H}$ & 3.40923200 & -0.70156500 & 1.92918900 & $\mathrm{H}$ & 2.60764500 & -2.25793900 & 1.71922700 \\
\hline $\mathrm{H}$ & 4.00015600 & -2.12567200 & 2.80108700 & C & 1.85179500 & -2.60305200 & 4.38899900 \\
\hline $\mathrm{H}$ & 2.77112500 & -3.08366900 & 4.74296500 & $\mathrm{H}$ & 1.32879700 & -3.30375400 & 3.73649100 \\
\hline $\mathrm{H}$ & 1.21338400 & -2.40617800 & 5.25799300 & C & 2.95075000 & -0.36260200 & 4.59946900 \\
\hline $\mathrm{H}$ & 3.91727700 & -0.80738000 & 4.86007800 & $\mathrm{H}$ & 2.40387800 & -0.20380300 & 5.53554600 \\
\hline $\mathrm{H}$ & 3.14867100 & 0.61570800 & 4.14428100 & C & 1.00452000 & 0.70958000 & 2.50117000 \\
\hline 0 & 1.32728800 & 0.70119200 & 1.30222500 & C & 0.40110300 & 1.97753800 & 4.59913300 \\
\hline C & 1.00976300 & 3.18414600 & 2.55114000 & C & 0.82183600 & 3.40334900 & 4.95878800 \\
\hline $\mathrm{H}$ & -0.67833300 & 1.82410600 & 4.70763500 & $\mathrm{H}$ & 0.90714300 & 1.23222600 & 5.20798400 \\
\hline $\mathrm{H}$ & 2.08923900 & 3.27840900 & 2.38383000 & $\mathrm{H}$ & 1.88666900 & 3.42316100 & 5.22105700 \\
\hline $\mathrm{H}$ & 0.25623200 & 3.79748200 & 5.80735800 & $\mathrm{~N}$ & 0.78576800 & 1.85596100 & 3.17795600 \\
\hline C & 0.33535900 & 3.47907400 & 1.22641200 & C & 1.09986600 & 4.10043200 & 0.28228600 \\
\hline C & -1.08643500 & 3.29832000 & 0.99630400 & C & 0.57130000 & 4.57212200 & -0.96066100 \\
\hline $\mathrm{H}$ & 2.16004300 & 4.24781900 & 0.46684800 & C & -1.65445000 & 3.83396500 & -0.20462400 \\
\hline $\mathrm{C}$ & -1.94027100 & 2.64118300 & 1.89669300 & C & 1.38312600 & 5.15243200 & -1.94691600 \\
\hline C & -0.82830300 & 4.44826500 & -1.19055900 & C & -3.06222300 & 3.74570900 & -0.42984000 \\
\hline $\mathrm{H}$ & -1.54116800 & 2.16285400 & 2.77822900 & C & -3.31070200 & 2.54609000 & 1.65888900 \\
\hline C & 0.83071600 & 5.60051200 & -3.14574700 & $\mathrm{H}$ & 2.44867300 & 5.25315700 & -1.75901800 \\
\hline $\mathrm{C}$ & -1.38986800 & 4.93973700 & -2.40704200 & C & -3.87486300 & 3.10816400 & 0.52049000 \\
\hline C & -3.60575000 & 4.27830400 & -1.64784800 & $\mathrm{H}$ & -3.93667800 & 2.01448400 & 2.36701400 \\
\hline C & -0.53966200 & 5.50053100 & -3.37323400 & $\mathrm{H}$ & 1.47138400 & 6.04026700 & -3.90494200 \\
\hline $\mathrm{C}$ & -2.80932500 & 4.84177700 & -2.59691200 & $\mathrm{H}$ & -4.93991500 & 3.01216300 & 0.33503200 \\
\hline $\mathrm{H}$ & -4.67884500 & 4.20357800 & -1.80266000 & $\mathrm{H}$ & -0.96599400 & 5.85992600 & -4.30591000 \\
\hline $\mathrm{H}$ & -3.23560400 & 5.22518000 & -3.52015800 & C & -1.35382200 & -1.43294500 & 2.47147300 \\
\hline $\mathrm{S}$ & -2.17703600 & -0.61535300 & 3.70502500 & $\mathrm{~N}$ & 0.00796200 & -1.48003100 & 2.39996800 \\
\hline $\mathrm{H}$ & 0.41793800 & -1.73901600 & 1.49697500 & $\mathrm{~S}$ & 0.80639000 & -3.79159200 & -0.55346500 \\
\hline $\mathrm{O}$ & 1.23790700 & -4.14927300 & -1.91327200 & $\mathrm{O}$ & 1.21523800 & -2.42597500 & -0.10037300 \\
\hline $\mathrm{O}$ & -0.59501700 & -4.11157500 & -0.18831200 & C & 1.80129900 & -4.91910200 & 0.53699500 \\
\hline $\mathrm{F}$ & 1.60139300 & -6.19749500 & 0.21949900 & $\mathrm{~F}$ & 1.48142700 & -4.73877300 & 1.82785700 \\
\hline $\mathrm{F}$ & 3.11920200 & -4.64807500 & 0.39772700 & C & 0.57652200 & 4.17010900 & 3.65773000 \\
\hline $\mathrm{H}$ & -0.48744800 & 4.39994800 & 3.54475800 & $\mathrm{H}$ & 1.13165900 & 5.10842200 & 3.58330400 \\
\hline $\mathrm{C}$ & 0.63873700 & 1.31044800 & -2.43855300 & $\mathrm{O}$ & 0.84084000 & 1.42547700 & -3.63506900 \\
\hline $\mathrm{O}$ & -0.48223800 & 0.99062300 & -1.80806700 & C & -1.63971700 & 0.37705900 & -2.51808500 \\
\hline C & -1.23108400 & -1.02455600 & -2.96489600 & $\mathrm{H}$ & -2.10874400 & -1.55468600 & -3.34646000 \\
\hline $\mathrm{H}$ & -0.47745200 & -0.99022000 & -3.75551600 & $\mathrm{H}$ & -0.83993900 & -1.59593100 & -2.11768700 \\
\hline C & -2.68190200 & 0.31050500 & -1.41336800 & $\mathrm{H}$ & -3.02189800 & 1.30666500 & -1.13472300 \\
\hline $\mathrm{H}$ & -3.54188200 & -0.27503100 & -1.74711500 & $\mathrm{H}$ & -2.25430500 & -0.16466500 & -0.53052700 \\
\hline C & -2.11144500 & 1.25684600 & -3.67204100 & $\mathrm{H}$ & -3.06019900 & 0.85511400 & -4.04517000 \\
\hline $\mathrm{H}$ & -2.28858500 & 2.27754000 & -3.32426000 & $\mathrm{H}$ & -1.38857400 & 1.27883500 & -4.48724900 \\
\hline $\mathrm{C}$ & 2.88855800 & 1.92890700 & -1.93060400 & $\mathrm{H}$ & 2.79656100 & 2.51851600 & -2.83791600 \\
\hline C & 3.84614700 & 2.46153400 & -0.93548700 & C & 4.71190400 & 3.49186300 & -1.33457100 \\
\hline
\end{tabular}




\begin{tabular}{|llll|llll|}
\hline $\mathrm{C}$ & 3.92437000 & 1.97341000 & 0.37629900 & $\mathrm{C}$ & 5.62183700 & 4.04056700 & -0.43519800 \\
\hline $\mathrm{H}$ & 4.66002000 & 3.86871900 & -2.35281400 & $\mathrm{C}$ & 4.84160500 & 2.52097800 & 1.27253700 \\
\hline $\mathrm{H}$ & 3.24494000 & 1.20090900 & 0.71037700 & $\mathrm{C}$ & 5.68935800 & 3.55524400 & 0.87361200 \\
\hline $\mathrm{H}$ & 6.27533200 & 4.84741400 & -0.75287400 & $\mathrm{H}$ & 4.88790500 & 2.13598200 & 2.28752900 \\
\hline $\mathrm{H}$ & 6.39933100 & 3.98196000 & 1.57589000 & $\mathrm{~N}$ & 1.65680700 & 1.50136900 & -1.49911000 \\
\hline $\mathrm{H}$ & 1.46224500 & 1.28215400 & -0.51740200 & $\mathrm{C}$ & 3.96553200 & 0.56593400 & -2.88456200 \\
\hline $\mathrm{H}$ & 3.63707200 & 0.75297500 & -3.90285900 & $\mathrm{H}$ & 4.96043800 & 0.92563400 & -2.64001600 \\
\hline $\mathrm{C}$ & 3.53275700 & -0.63318500 & -2.29213700 & $\mathrm{C}$ & 4.13646300 & -1.15714000 & -1.08322400 \\
\hline $\mathrm{H}$ & 4.68653300 & -0.39240800 & -0.52373500 & $\mathrm{H}$ & 3.39212600 & -1.65925600 & -0.45564600 \\
\hline $\mathrm{C}$ & 2.32605100 & -1.30399600 & -2.81725600 & $\mathrm{H}$ & 2.02253500 & -0.93029000 & -3.79431700 \\
\hline $\mathrm{H}$ & 2.41855500 & -2.39356000 & -2.81435700 & $\mathrm{H}$ & 1.52063400 & -1.12545100 & -2.08918500 \\
\hline $\mathrm{C}$ & 6.04773900 & -3.08859800 & 0.24649400 & $\mathrm{H}$ & 5.22239400 & -3.55451000 & 0.79211300 \\
\hline $\mathrm{H}$ & 6.85359200 & -3.82673400 & 0.15669800 & $\mathrm{H}$ & 6.42508100 & -2.25181000 & 0.84548200 \\
\hline $\mathrm{C}$ & 4.76526800 & -3.89672800 & -2.49047100 & $\mathrm{H}$ & 3.83020600 & -4.28054800 & -2.07215400 \\
\hline $\mathrm{H}$ & 4.56407700 & -3.56883400 & -3.51654400 & $\mathrm{H}$ & 5.47777100 & -4.72894100 & -2.54562000 \\
\hline $\mathrm{C}$ & 6.89639800 & -1.64755700 & -2.36588600 & $\mathrm{H}$ & 6.56915400 & -1.25993100 & -3.33739900 \\
\hline $\mathrm{H}$ & 7.29893000 & -0.80872800 & -1.78563500 & $\mathrm{H}$ & 7.72047500 & -2.34673200 & -2.55089800 \\
\hline $\mathrm{Si}$ & 5.48771400 & -2.50919600 & -1.45434000 & & & & \\
\hline
\end{tabular}

\section{TS-R-4f-26}

Imaginary frequency: $-214.66 \mathrm{~cm}^{-1}$

E[B3LYP/6-31G(d)-D3(BJ)]: -4789.085600 Hartree

$\mathrm{E}\left[\mathrm{PCM}\left(\mathrm{Et}_{2} \mathrm{O}\right)-\mathrm{B} 3 \mathrm{LYP} / 6-311 \mathrm{G}(\mathrm{d}, \mathrm{p})-\mathrm{D} 3(\mathrm{BJ})\right]$ : -4790.223087 Hartree

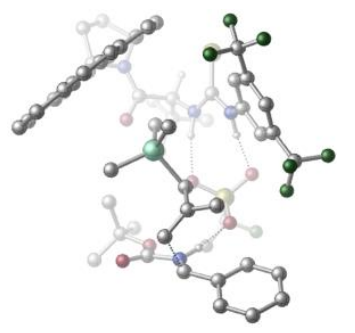

\begin{tabular}{|c|c|c|c|c|c|c|c|}
\hline 01 & & & & $\mathrm{~N}$ & 0.19529100 & 0.04636300 & -2.79901800 \\
\hline $\mathrm{H}$ & -0.59181100 & 0.69894500 & -2.74956400 & C & -0.18111600 & -1.28920900 & -2.73976300 \\
\hline C & -1.56566900 & -1.54249400 & -2.69829900 & C & 0.70808100 & -2.37283700 & -2.64924000 \\
\hline C & -2.03947100 & -2.84233300 & -2.55790000 & $\mathrm{H}$ & -2.25980000 & -0.71148800 & -2.76047700 \\
\hline C & 0.20681100 & -3.66634000 & -2.52706600 & $\mathrm{H}$ & 1.77259700 & -2.19836400 & -2.68316200 \\
\hline $\mathrm{C}$ & -1.16356100 & -3.92524300 & -2.47628200 & $\mathrm{H}$ & -1.53556900 & -4.93758800 & -2.37909600 \\
\hline C & -3.52225200 & -3.08331200 & -2.55020200 & $\mathrm{C}$ & 1.16390100 & -4.81758900 & -2.37820900 \\
\hline $\mathrm{F}$ & -4.20539800 & -2.01775300 & -2.05450800 & $\mathrm{~F}$ & -3.85218300 & -4.15297600 & -1.78485900 \\
\hline $\mathrm{F}$ & -4.01289700 & -3.31956500 & -3.78375900 & $\mathrm{~F}$ & 2.38615600 & -4.54245200 & -2.88237000 \\
\hline$F$ & 1.34683500 & -5.15418000 & -1.07375500 & $\mathrm{~F}$ & 0.70931900 & -5.92888000 & -2.99784900 \\
\hline C & 2.57139400 & 2.72053700 & -2.09287500 & $\mathrm{H}$ & 3.23246700 & 2.44678600 & -2.91400000 \\
\hline C & 2.20119900 & 4.22606500 & -2.25187300 & $\mathrm{C}$ & 1.29760600 & 4.73855100 & -1.11986300 \\
\hline $\mathrm{H}$ & 1.79104700 & 4.67727100 & -0.14811100 & $\mathrm{H}$ & 0.37195000 & 4.16560200 & -1.05915600 \\
\hline $\mathrm{H}$ & 1.02678400 & 5.78306600 & -1.31510400 & C & 1.47474300 & 4.39893200 & -3.59767800 \\
\hline $\mathrm{H}$ & 1.29807800 & 5.46265000 & -3.79397200 & $\mathrm{H}$ & 0.50586000 & 3.89147100 & -3.59432600 \\
\hline $\mathrm{H}$ & 2.06730600 & 3.99301600 & -4.42606200 & C & 3.50234000 & 5.04665800 & -2.26614200 \\
\hline $\mathrm{H}$ & 3.27133000 & 6.11442800 & -2.34660300 & $\mathrm{H}$ & 4.13599400 & 4.78181400 & -3.12104900 \\
\hline $\mathrm{H}$ & 4.08217600 & 4.90335100 & -1.34649100 & $\mathrm{C}$ & 3.23163500 & 2.38505900 & -0.73887600 \\
\hline $\mathrm{O}$ & 2.57147700 & 2.41485500 & 0.30121700 & $\mathrm{C}$ & 5.49791000 & 2.12852000 & -1.84929700 \\
\hline C & 5.19642400 & 1.66645900 & 0.54134000 & C & 6.84537100 & 2.27333900 & -1.13368700 \\
\hline $\mathrm{H}$ & 5.46085200 & 1.23531600 & -2.48471800 & $\mathrm{H}$ & 5.27147600 & 2.99081800 & -2.47498400 \\
\hline $\mathrm{H}$ & 5.13612300 & 2.54911500 & 1.18927100 & $\mathrm{H}$ & 7.00945000 & 3.32352700 & -0.86424300 \\
\hline $\mathrm{H}$ & 7.68306700 & 1.94890000 & -1.75739600 & $\mathrm{~N}$ & 4.53947400 & 2.01450400 & -0.73822600 \\
\hline C & 4.60389800 & 0.51037400 & 1.32899500 & C & 4.34994000 & 0.71933100 & 2.65317700 \\
\hline $\mathrm{C}$ & 4.40799100 & -0.81353500 & 0.76659700 & $\mathrm{C}$ & 3.93639000 & -0.32252500 & 3.54387700 \\
\hline $\mathrm{H}$ & 4.46761800 & 1.71845600 & 3.06547700 & $\mathrm{C}$ & 4.01974400 & -1.88120800 & 1.63971400 \\
\hline $\mathrm{C}$ & 4.54214600 & -1.09754400 & -0.60108000 & $\mathrm{C}$ & 3.67777600 & -0.08853800 & 4.90315200 \\
\hline
\end{tabular}




\begin{tabular}{|c|c|c|c|c|c|c|c|}
\hline $\mathrm{C}$ & 3.79671800 & -1.64266500 & 3.02657500 & C & 3.82509300 & -3.19728000 & 1.11928300 \\
\hline $\mathrm{H}$ & 4.76361700 & -0.30571000 & -1.29843000 & $\mathrm{C}$ & 4.31708600 & -2.37841200 & -1.10342000 \\
\hline $\mathrm{C}$ & 3.30213400 & -1.13312200 & 5.74553700 & $\mathrm{H}$ & 3.77569500 & 0.92104800 & 5.29330500 \\
\hline $\mathrm{C}$ & 3.41238600 & -2.70823500 & 3.89656100 & C & 3.97399100 & -3.42458600 & -0.25625800 \\
\hline C & 3.45703900 & -4.25495700 & 2.01893100 & $\mathrm{H}$ & 4.39379300 & -2.54829500 & -2.17234600 \\
\hline C & 3.17519700 & -2.42985200 & 5.25188100 & $\mathrm{H}$ & 3.10533400 & -0.93454200 & 6.79517400 \\
\hline C & 3.26295300 & -4.02508300 & 3.34617900 & $\mathrm{H}$ & 3.79392800 & -4.41699000 & -0.65405400 \\
\hline $\mathrm{H}$ & 3.32352000 & -5.25118000 & 1.60538000 & $\mathrm{H}$ & 2.87956100 & -3.24018400 & 5.91307500 \\
\hline $\mathrm{H}$ & 2.97767100 & -4.83515400 & 4.01231100 & $\mathrm{C}$ & 1.44229000 & 0.63813300 & -2.80733400 \\
\hline $\mathrm{S}$ & 2.81326600 & -0.03075700 & -3.52196400 & $\mathrm{~N}$ & 1.40625300 & 1.86149400 & -2.22606100 \\
\hline $\mathrm{H}$ & 0.62012300 & 2.06092800 & -1.60567500 & S & -2.36294800 & 2.16842800 & -1.42170800 \\
\hline 0 & -3.53157300 & 1.54669500 & -0.74442200 & $\mathrm{O}$ & -1.13124100 & 2.22774500 & -0.59474200 \\
\hline 0 & -2.13449900 & 1.68614900 & -2.80500500 & C & -2.89964300 & 3.93261300 & -1.65474700 \\
\hline $\mathrm{F}$ & -3.89216900 & 3.99411100 & -2.54506800 & $\mathrm{~F}$ & -1.87265100 & 4.67295100 & -2.09810300 \\
\hline $\mathrm{F}$ & -3.32984200 & 4.44421500 & -0.49429600 & C & -3.00578000 & 2.07990400 & 2.84836000 \\
\hline 0 & -2.79584200 & 1.74651400 & 4.00234000 & $\mathrm{O}$ & -2.47100100 & 3.07982700 & 2.16549700 \\
\hline C & -1.29260400 & 3.83200800 & 2.68657400 & C & -1.61585800 & 4.44674000 & 4.04812700 \\
\hline $\mathrm{H}$ & -0.80344300 & 5.12679600 & 4.32592100 & $\mathrm{H}$ & -1.72249400 & 3.68745600 & 4.82233400 \\
\hline $\mathrm{H}$ & -2.54088800 & 5.03049700 & 3.99221200 & C & -1.09836200 & 4.91833300 & 1.63558100 \\
\hline $\mathrm{H}$ & -0.92381500 & 4.47181500 & 0.65843300 & $\mathrm{H}$ & -0.22913000 & 5.52705800 & 1.90303600 \\
\hline $\mathrm{H}$ & -1.97732800 & 5.56677400 & 1.57845100 & C & -0.09879800 & 2.88071400 & 2.72322500 \\
\hline $\mathrm{H}$ & 0.80076900 & 3.43530100 & 3.00876900 & $\mathrm{H}$ & 0.08036800 & 2.44702700 & 1.73633000 \\
\hline $\mathrm{H}$ & -0.25322900 & 2.08716800 & 3.45842000 & C & -4.64772100 & 0.36185300 & 2.54005700 \\
\hline $\mathrm{H}$ & -4.82473900 & 0.47562200 & 3.60515100 & C & -5.73919600 & -0.25778200 & 1.77107400 \\
\hline C & -6.78298800 & -0.86028600 & 2.49341700 & C & -5.75563300 & -0.31191500 & 0.36713500 \\
\hline C & -7.82923300 & -1.49248900 & 1.83001000 & $\mathrm{H}$ & -6.77046700 & -0.82637100 & 3.58002600 \\
\hline C & -6.80568000 & -0.94949700 & -0.29105900 & $\mathrm{H}$ & -4.95589000 & 0.12859800 & -0.21632600 \\
\hline C & -7.84221300 & -1.53892500 & 0.43364000 & $\mathrm{H}$ & -8.63361700 & -1.94764900 & 2.39996200 \\
\hline $\mathrm{H}$ & -6.79771900 & -0.99618200 & -1.37526900 & $\mathrm{H}$ & -8.65574000 & -2.03653700 & -0.08587900 \\
\hline $\mathrm{N}$ & -3.91122400 & 1.38737800 & 2.02630500 & $\mathrm{H}$ & -3.84111700 & 1.55581000 & 1.01106400 \\
\hline C & -3.35450900 & -1.18378300 & 3.04568100 & $\mathrm{H}$ & -4.01794500 & -1.82898900 & 3.61580600 \\
\hline $\mathrm{H}$ & -2.73267300 & -0.51027500 & 3.62680000 & C & -2.92691800 & -1.61120100 & 1.79228700 \\
\hline C & -1.90351800 & -0.89555700 & 1.05813800 & $\mathrm{H}$ & -1.86594700 & 0.17552700 & 1.28096800 \\
\hline $\mathrm{H}$ & -1.96604300 & -1.04949100 & -0.02253700 & C & -3.57744600 & -2.78225100 & 1.13327700 \\
\hline $\mathrm{H}$ & -2.84387500 & -3.38543400 & 0.59128200 & $\mathrm{H}$ & -4.28696800 & -2.41138500 & 0.38240100 \\
\hline $\mathrm{H}$ & -4.12443000 & -3.41146500 & 1.83912200 & C & 1.04121000 & -0.47442200 & 0.52772900 \\
\hline $\mathrm{H}$ & 0.46954000 & 0.21009300 & -0.10152200 & $\mathrm{H}$ & 1.65079100 & -1.10858700 & -0.12181200 \\
\hline $\mathrm{H}$ & 1.71515200 & 0.14026000 & 1.12888800 & C & -0.02380000 & -3.34286900 & 1.27831100 \\
\hline $\mathrm{H}$ & -0.26653200 & -3.58756700 & 0.24046600 & $\mathrm{H}$ & -0.70919700 & -3.89444300 & 1.93270900 \\
\hline $\mathrm{H}$ & 0.98789100 & -3.71045700 & 1.47193800 & C & 0.05943500 & -1.12130800 & 3.43163700 \\
\hline $\mathrm{H}$ & -0.55271000 & -1.79256900 & 4.04359400 & $\mathrm{H}$ & -0.23378200 & -0.09189300 & 3.66269000 \\
\hline $\mathrm{H}$ & 1.10000600 & -1.24492200 & 3.74337900 & $\mathrm{Si}$ & -0.11426400 & -1.49377400 & 1.59550600 \\
\hline C & 6.66508800 & 1.42200100 & 0.12777000 & $\mathrm{H}$ & 6.81343100 & 0.36271200 & -0.10228500 \\
\hline $\mathrm{H}$ & 7.35078600 & 1.68545800 & 0.93712500 & & & & \\
\hline
\end{tabular}

\section{TS- $R-4 f-27$}

Imaginary frequency: $-158.18 \mathrm{~cm}^{-1}$

E[B3LYP/6-31G(d)-D3(BJ)]: -4789.076471 Hartree

$\mathrm{E}\left[\mathrm{PCM}\left(\mathrm{Et}_{2} \mathrm{O}\right)-\mathrm{B} 3 \mathrm{LYP} / 6-311 \mathrm{G}(\mathrm{d}, \mathrm{p})-\mathrm{D} 3(\mathrm{BJ})\right]$ : -4790.222285 Hartree

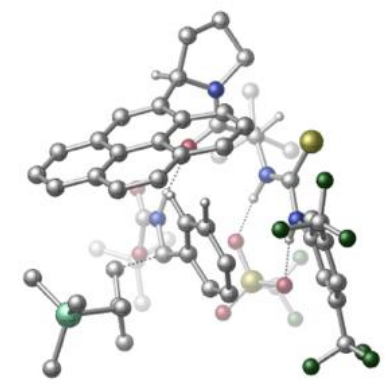




\begin{tabular}{|c|c|c|c|c|c|c|c|}
\hline 01 & & & & $\mathrm{~N}$ & -0.30930800 & 2.74640400 & 0.93412700 \\
\hline $\mathrm{H}$ & -1.17979900 & 2.63328400 & 0.40386700 & C & 0.65940500 & 3.48851600 & 0.25217100 \\
\hline $\mathrm{C}$ & 0.22189800 & 4.25168100 & -0.83981200 & $\mathrm{C}$ & 2.03336100 & 3.40542400 & 0.51618100 \\
\hline $\mathrm{C}$ & 1.14630900 & 4.89846400 & -1.65573100 & $\mathrm{H}$ & -0.83530400 & 4.28962400 & -1.07474800 \\
\hline C & 2.93721400 & 4.07476600 & -0.30240300 & $\mathrm{H}$ & 2.38775400 & 2.79210100 & 1.32901500 \\
\hline C & 2.51397700 & 4.82753900 & -1.39761100 & $\mathrm{H}$ & 3.22672800 & 5.33687400 & -2.03371900 \\
\hline C & 0.66057800 & 5.57697200 & -2.90344700 & C & 4.40463500 & 3.84416700 & -0.09264700 \\
\hline $\mathrm{F}$ & -0.55388100 & 6.13762900 & -2.74655700 & $\mathrm{~F}$ & 0.54962900 & 4.68478600 & -3.92811800 \\
\hline $\mathrm{F}$ & 1.50791800 & 6.54106900 & -3.32511000 & $\mathrm{~F}$ & 4.72226800 & 3.62660300 & 1.20296600 \\
\hline $\mathrm{F}$ & 4.82985300 & 2.73961500 & -0.77740300 & $\mathrm{~F}$ & 5.15787300 & 4.87441100 & -0.52912400 \\
\hline C & -1.32428700 & 0.48241600 & 3.60724300 & $\mathrm{H}$ & -0.84999200 & 1.09927400 & 4.36918500 \\
\hline C & -2.79667600 & 0.23625500 & 4.06235300 & C & -3.55444300 & -0.76310500 & 3.17998900 \\
\hline $\mathrm{H}$ & -3.09823500 & -1.75360200 & 3.18654500 & $\mathrm{H}$ & -3.61190800 & -0.42649200 & 2.14451000 \\
\hline $\mathrm{H}$ & -4.58253100 & -0.86096500 & 3.54983500 & C & -3.52852900 & 1.59084700 & 4.04773500 \\
\hline $\mathrm{H}$ & -4.51117400 & 1.48362600 & 4.52144200 & $\mathrm{H}$ & -3.68839100 & 1.94747700 & 3.02660500 \\
\hline $\mathrm{H}$ & -2.96741200 & 2.35724300 & 4.59540600 & C & -2.76348600 & -0.30506300 & 5.50307000 \\
\hline $\mathrm{H}$ & -3.78298000 & -0.50997800 & 5.84665900 & $\mathrm{H}$ & -2.31959400 & 0.41833900 & 6.19781900 \\
\hline $\mathrm{H}$ & -2.20202700 & -1.24497200 & 5.56975000 & C & -0.49316100 & -0.79861200 & 3.40611300 \\
\hline $\mathrm{O}$ & -0.66147900 & -1.52627700 & 2.41800000 & C & 0.74679900 & -0.44354900 & 5.59130700 \\
\hline C & 1.25006200 & -2.35396600 & 4.13318300 & C & 1.41267700 & -1.54967300 & 6.41597500 \\
\hline $\mathrm{H}$ & 1.40826000 & 0.41628500 & 5.43539700 & $\mathrm{H}$ & -0.16963100 & -0.07977300 & 6.05172500 \\
\hline $\mathrm{H}$ & 0.54307200 & -3.18886000 & 4.20384800 & $\mathrm{H}$ & 0.64436500 & -2.17329000 & 6.88821900 \\
\hline $\mathrm{H}$ & 2.05451200 & -1.14768700 & 7.20466000 & $\mathrm{~N}$ & 0.46564500 & -1.10852700 & 4.30758500 \\
\hline C & 1.99143400 & -2.52911000 & 2.81810900 & C & 1.79742800 & -3.69846400 & 2.14143600 \\
\hline C & 2.96704500 & -1.57431400 & 2.32407900 & C & 2.53561100 & -4.05293800 & 0.96746400 \\
\hline $\mathrm{H}$ & 1.04712300 & -4.39998500 & 2.49738500 & C & 3.76828000 & -1.93296600 & 1.19098400 \\
\hline $\mathrm{C}$ & 3.14676500 & -0.29812400 & 2.88197000 & C & 2.30788800 & -5.25236200 & 0.27344100 \\
\hline C & 3.54633600 & -3.16158200 & 0.50429500 & C & 4.76335600 & -1.02903000 & 0.70278600 \\
\hline $\mathrm{H}$ & 2.50130700 & 0.04146100 & 3.67718100 & C & 4.10128600 & 0.58583400 & 2.38134500 \\
\hline C & 3.06642100 & -5.57698000 & -0.84984900 & $\mathrm{H}$ & 1.53926200 & -5.93233400 & 0.63102700 \\
\hline $\mathrm{C}$ & 4.31157100 & -3.49641900 & -0.65437400 & $\mathrm{C}$ & 4.91976000 & 0.22250200 & 1.31795300 \\
\hline $\mathrm{C}$ & 5.52909900 & -1.39808300 & -0.45464400 & $\mathrm{H}$ & 4.18346600 & 1.57686900 & 2.81441100 \\
\hline C & 4.05550600 & -4.71005400 & -1.31272400 & $\mathrm{H}$ & 2.88779000 & -6.51527500 & -1.36737000 \\
\hline $\mathrm{C}$ & 5.31501400 & -2.57378600 & -1.10556900 & $\mathrm{H}$ & 5.64405100 & 0.92584500 & 0.92072400 \\
\hline $\mathrm{H}$ & 6.28209800 & -0.70009500 & -0.81071300 & $\mathrm{H}$ & 4.64437500 & -4.97071800 & -2.18830300 \\
\hline $\mathrm{H}$ & 5.90056300 & -2.83436300 & -1.98358400 & C & -0.24184300 & 2.15423900 & 2.16830800 \\
\hline $\mathrm{S}$ & 0.91105600 & 2.54829600 & 3.35042500 & $\mathrm{~N}$ & -1.23031700 & 1.25384900 & 2.37912600 \\
\hline $\mathrm{H}$ & -1.84729700 & 0.99833800 & 1.60354400 & $\mathrm{~S}$ & -3.49637100 & 1.65005100 & -0.84295900 \\
\hline $\mathrm{O}$ & -3.71114200 & 0.98629200 & -2.14876900 & $\mathrm{O}$ & -3.22104800 & 0.72184600 & 0.28825500 \\
\hline $\mathrm{O}$ & -2.59244000 & 2.82786600 & -0.86045200 & C & -5.15407500 & 2.36501800 & -0.41316100 \\
\hline $\mathrm{F}$ & -5.49695400 & 3.32113300 & -1.28110900 & $\mathrm{~F}$ & -5.12255100 & 2.89031300 & 0.81983900 \\
\hline $\mathrm{F}$ & -6.09637900 & 1.40590200 & -0.44227500 & C & -3.20632400 & -2.57485800 & 0.18923100 \\
\hline $\mathrm{O}$ & -3.50804100 & -3.16558000 & 1.20779500 & $\mathrm{O}$ & -3.96826000 & -2.43998200 & -0.91413300 \\
\hline $\mathrm{C}$ & -5.42464200 & -2.66315900 & -0.86601000 & C & -6.04154400 & -1.90348400 & 0.30922600 \\
\hline $\mathrm{H}$ & -7.13258500 & -1.93364000 & 0.21587200 & $\mathrm{H}$ & -5.72405700 & -0.85877300 & 0.29316000 \\
\hline $\mathrm{H}$ & -5.75727300 & -2.34675500 & 1.26371400 & C & -5.68974800 & -4.16577600 & -0.79356300 \\
\hline $\mathrm{H}$ & -5.21703500 & -4.67944800 & -1.63834400 & $\mathrm{H}$ & -6.76773000 & -4.35514000 & -0.83889600 \\
\hline $\mathrm{H}$ & -5.29779800 & -4.57849500 & 0.13805800 & C & -5.88135700 & -2.06103100 & -2.19399500 \\
\hline $\mathrm{H}$ & -6.96563600 & -2.16713800 & -2.30076200 & $\mathrm{H}$ & -5.40133500 & -2.57534300 & -3.03456800 \\
\hline $\mathrm{H}$ & -5.61889300 & -0.99994200 & -2.23691900 & $\mathrm{C}$ & -1.62312400 & -1.35581700 & -1.20061100 \\
\hline $\mathrm{H}$ & -2.48955300 & -0.94975100 & -1.71335100 & C & -0.45898100 & -0.42577700 & -1.20847600 \\
\hline $\mathrm{C}$ & -0.47654000 & 0.62900000 & -2.13704500 & C & 0.70464300 & -0.66447900 & -0.46746600 \\
\hline C & 0.66372800 & 1.39317000 & -2.36855900 & $\mathrm{H}$ & -1.39420500 & 0.85642800 & -2.66707000 \\
\hline
\end{tabular}




\begin{tabular}{|c|c|c|c|c|c|c|c|}
\hline C & 1.83714100 & 0.11951700 & -0.69053900 & $\mathrm{H}$ & 0.73840500 & -1.45566700 & 0.27456700 \\
\hline $\mathrm{C}$ & 1.83321900 & 1.12105800 & -1.66015600 & $\mathrm{H}$ & 0.62888900 & 2.21419500 & -3.07711400 \\
\hline $\mathrm{H}$ & 2.72872800 & -0.05255200 & -0.10872200 & $\mathrm{H}$ & 2.72917900 & 1.70742200 & -1.82735500 \\
\hline $\mathrm{N}$ & -1.97723700 & -1.96836500 & 0.00014900 & $\mathrm{H}$ & -1.40942000 & -1.86449100 & 0.84846100 \\
\hline C & -1.19350600 & -2.73885100 & -2.26820800 & $\mathrm{H}$ & -2.13093900 & -3.28969000 & -2.25153100 \\
\hline $\mathrm{H}$ & -0.38799400 & -3.21010700 & -1.70917700 & C & -0.82766800 & -2.18008000 & -3.51854800 \\
\hline C & 0.55107000 & -1.88341500 & -3.81189700 & $\mathrm{H}$ & 1.14805800 & -1.75552200 & -2.90216600 \\
\hline $\mathrm{H}$ & 0.68126500 & -1.03209300 & -4.48628400 & C & -1.90275400 & -1.75407600 & -4.45626700 \\
\hline $\mathrm{H}$ & -1.52800500 & -1.56596000 & -5.46501100 & $\mathrm{H}$ & -2.32172800 & -0.80774300 & -4.07426400 \\
\hline $\mathrm{H}$ & -2.73230000 & -2.46686100 & -4.48251400 & C & 3.24582400 & -2.98347900 & -4.62383600 \\
\hline $\mathrm{H}$ & 3.46593000 & -2.04220000 & -5.13959700 & $\mathrm{H}$ & 3.84360100 & -3.77253400 & -5.09557200 \\
\hline $\mathrm{H}$ & 3.57392600 & -2.88689400 & -3.58343700 & C & 0.77818900 & -3.46541900 & -6.47693900 \\
\hline $\mathrm{H}$ & 0.95139500 & -2.52055500 & -7.00454400 & $\mathrm{H}$ & -0.29538300 & -3.68265500 & -6.51402200 \\
\hline $\mathrm{H}$ & 1.29158400 & -4.25530200 & -7.03812600 & C & 1.00382700 & -4.97928500 & -3.77912600 \\
\hline $\mathrm{H}$ & -0.06438500 & -5.21630100 & -3.83274800 & $\mathrm{H}$ & 1.28996100 & -4.91312600 & -2.72477900 \\
\hline $\mathrm{H}$ & 1.55363900 & -5.82260700 & -4.21432200 & $\mathrm{Si}$ & 1.41474800 & -3.39976200 & -4.70671300 \\
\hline C & 2.18201400 & -2.35764200 & 5.36462900 & $\mathrm{H}$ & 3.12610400 & -1.86280500 & 5.11820400 \\
\hline $\mathrm{H}$ & 2.41344400 & -3.37855500 & 5.67871600 & & & & \\
\hline
\end{tabular}

\section{TS- $R-4 f-28$}

Imaginary frequency: $-328.98 \mathrm{~cm}^{-1}$ E[B3LYP/6-31G(d)-D3(BJ)]: -4789.076031 Hartree $\mathrm{E}\left[\mathrm{PCM}\left(\mathrm{Et}_{2} \mathrm{O}\right)-\mathrm{B} 3 \mathrm{LYP} / 6-311 \mathrm{G}(\mathrm{d}, \mathrm{p})-\mathrm{D} 3(\mathrm{BJ})\right]$ : -4790.222036 Hartree

\begin{tabular}{|c|c|c|c|c|c|c|c|}
\hline 01 & & & & $\mathrm{~N}$ & 0.93400200 & 1.85416900 & -1.91494300 \\
\hline $\mathrm{H}$ & 1.87419200 & 1.53751600 & -1.66078900 & $\mathrm{C}$ & 0.66082600 & 3.15546900 & -1.47896100 \\
\hline C & 1.75983000 & 3.95002600 & -1.11900900 & C & -0.63281900 & 3.65781700 & -1.28526100 \\
\hline C & 1.56160800 & 5.21910400 & -0.57974400 & $\mathrm{H}$ & 2.76288900 & 3.55500500 & -1.22461400 \\
\hline $\mathrm{C}$ & -0.80707100 & 4.93339000 & -0.75697100 & $\mathrm{H}$ & -1.48678800 & 3.04516500 & -1.52432200 \\
\hline C & 0.27853900 & 5.73173200 & -0.39818600 & $\mathrm{H}$ & 0.12945500 & 6.72135400 & 0.01452200 \\
\hline C & 2.75691000 & 6.06936900 & -0.24845500 & C & -2.19916600 & 5.41674500 & -0.47413400 \\
\hline $\mathrm{F}$ & 3.19042700 & 6.76381700 & -1.32514800 & $\mathrm{~F}$ & 3.79698000 & 5.32774800 & 0.18738100 \\
\hline $\mathrm{F}$ & 2.47367700 & 6.97689300 & 0.71542700 & $\mathrm{~F}$ & -3.10680800 & 4.90616300 & -1.33701000 \\
\hline $\mathrm{F}$ & -2.61240100 & 5.05162300 & 0.77495500 & $\mathrm{~F}$ & -2.29770000 & 6.76065100 & -0.52728500 \\
\hline $\mathrm{C}$ & 0.06451600 & -1.32121200 & -3.48653800 & $\mathrm{H}$ & -0.39431300 & -0.83371900 & -4.34641600 \\
\hline C & 1.12376900 & -2.32811100 & -4.04290000 & C & 1.71070300 & -3.24336100 & -2.96167500 \\
\hline $\mathrm{H}$ & 0.95025500 & -3.86872300 & -2.49148600 & $\mathrm{H}$ & 2.21246000 & -2.67501400 & -2.17843200 \\
\hline $\mathrm{H}$ & 2.45862600 & -3.90482400 & -3.41530600 & C & 2.25488900 & -1.52121300 & -4.70699600 \\
\hline $\mathrm{H}$ & 2.91862700 & -2.20137500 & -5.25315200 & $\mathrm{H}$ & 2.86084500 & -0.99035100 & -3.96799200 \\
\hline $\mathrm{H}$ & 1.85951400 & -0.78742500 & -5.41923900 & $\mathrm{C}$ & 0.44059800 & -3.20309700 & -5.10843300 \\
\hline $\mathrm{H}$ & 1.16242600 & -3.91754600 & -5.51839400 & $\mathrm{H}$ & 0.05724200 & -2.60093200 & -5.94048800 \\
\hline $\mathrm{H}$ & -0.38818000 & -3.78040600 & -4.68369000 & C & -1.03516900 & -1.98268200 & -2.63737300 \\
\hline $\mathrm{O}$ & -0.84451500 & -2.27007500 & -1.44781900 & C & -2.69347700 & -1.87067200 & -4.56412100 \\
\hline C & -3.27817000 & -2.89276300 & -2.41366700 & C & -4.19353600 & -2.19899500 & -4.52630000 \\
\hline $\mathrm{H}$ & -2.49344600 & -0.81218700 & -4.75098800 & $\mathrm{H}$ & -2.18104400 & -2.46847300 & -5.32376800 \\
\hline $\mathrm{C}$ & -4.28869100 & -3.33144900 & -3.49414700 & $\mathrm{H}$ & -2.82730600 & -3.74015400 & -1.89360900 \\
\hline $\mathrm{H}$ & -4.57689200 & -2.47946200 & -5.51091600 & $\mathrm{H}$ & -4.75904800 & -1.32988600 & -4.17646800 \\
\hline $\mathrm{H}$ & -3.96541800 & -4.28174500 & -3.93584800 & $\mathrm{H}$ & -5.29436100 & -3.46364800 & -3.08861700 \\
\hline $\mathrm{N}$ & -2.22767200 & -2.24859300 & -3.21617700 & C & -3.90160900 & -1.95148200 & -1.39500300 \\
\hline C & -3.79455900 & -0.59929100 & -1.53274800 & C & -4.64993800 & -2.50987700 & -0.28690000 \\
\hline $\mathrm{C}$ & -4.39853900 & 0.31584600 & -0.61166200 & $\mathrm{H}$ & -3.19823700 & -0.17625200 & -2.33434500 \\
\hline
\end{tabular}




\begin{tabular}{|c|c|c|c|c|c|c|c|}
\hline C & -5.24141500 & -1.61776500 & 0.66332100 & C & -4.81461500 & -3.89241700 & -0.09274200 \\
\hline $\mathrm{C}$ & -4.26409800 & 1.70637000 & -0.75188000 & $\mathrm{C}$ & -5.11639700 & -0.20707300 & 0.50229000 \\
\hline C & -5.95970000 & -2.13700100 & 1.78391400 & $\mathrm{H}$ & -4.38684100 & -4.59458900 & -0.79897300 \\
\hline C & -5.52353000 & -4.38912600 & 1.00003300 & $\mathrm{C}$ & -4.81645700 & 2.56998500 & 0.19262300 \\
\hline $\mathrm{H}$ & -3.70906200 & 2.09966000 & -1.59831200 & C & -5.68172500 & 0.68406900 & 1.46363600 \\
\hline C & -6.08900400 & -3.52579800 & 1.93231300 & C & -6.52829700 & -1.21665200 & 2.72954100 \\
\hline $\mathrm{H}$ & -5.63217400 & -5.46307200 & 1.12230900 & C & -5.51382100 & 2.06679300 & 1.28949400 \\
\hline $\mathrm{H}$ & -4.68995700 & 3.63960700 & 0.07786200 & C & -6.39356900 & 0.12905000 & 2.57999800 \\
\hline $\mathrm{H}$ & -6.63848900 & -3.91934400 & 2.78357000 & $\mathrm{H}$ & -7.07355800 & -1.62508600 & 3.57672500 \\
\hline $\bar{H}$ & -5.93858400 & 2.74658500 & 2.02378200 & $\mathrm{H}$ & -6.82786000 & 0.81168000 & 3.30607000 \\
\hline C & 0.16531900 & 0.99352800 & -2.65455500 & $S$ & -1.23904400 & 1.44831700 & -3.49420300 \\
\hline $\mathrm{N}$ & 0.67197700 & -0.25882800 & -2.70280100 & $\mathrm{H}$ & 1.44043900 & -0.50695500 & -2.07589300 \\
\hline $\mathrm{S}$ & 3.95806600 & 0.07950400 & -0.52512900 & 0 & 4.29896600 & -0.08507200 & 0.90619300 \\
\hline $\mathrm{O}$ & 3.00706600 & -0.93758200 & -1.05205700 & 0 & 3.62463500 & 1.46305800 & -0.94300700 \\
\hline C & 5.53706500 & -0.29254500 & -1.42615800 & $\mathrm{~F}$ & 6.47460400 & 0.60855300 & -1.12433300 \\
\hline $\mathrm{F}$ & 5.32290000 & -0.27470800 & -2.74793700 & $\mathrm{~F}$ & 5.99111500 & -1.51212700 & -1.08518500 \\
\hline C & 1.79966700 & -3.70782200 & 0.46025600 & 0 & 1.48074000 & -4.68217300 & -0.19078100 \\
\hline $\mathrm{O}$ & 2.95677400 & -3.51950200 & 1.11972900 & C & 4.12140800 & -4.39553500 & 0.88480600 \\
\hline C & 3.83643600 & -5.77341400 & 1.48013600 & $\mathrm{H}$ & 4.72842100 & -6.40308400 & 1.39261700 \\
\hline $\mathrm{H}$ & 3.01027700 & -6.25734600 & 0.95648700 & $\mathrm{H}$ & 3.58390800 & -5.68674600 & 2.54297800 \\
\hline C & 5.22876400 & -3.66713200 & 1.64455700 & $\mathrm{H}$ & 5.35573100 & -2.65319700 & 1.25652600 \\
\hline $\mathrm{H}$ & 6.17297900 & -4.21041200 & 1.53784200 & $\mathrm{H}$ & 4.98804100 & -3.60722400 & 2.71202300 \\
\hline C & 4.43780100 & -4.45710500 & -0.60966800 & $\mathrm{H}$ & 5.39648800 & -4.96830500 & -0.74895200 \\
\hline $\mathrm{H}$ & 4.52246500 & -3.44846900 & -1.01877500 & $\mathrm{H}$ & 3.66660200 & -5.00056500 & -1.15587900 \\
\hline C & 1.31914500 & -1.49841800 & 1.37644400 & $\mathrm{H}$ & 2.37830700 & -1.28214400 & 1.31780600 \\
\hline C & 0.42933100 & -0.31550400 & 1.29800400 & C & 1.00505700 & 0.96223200 & 1.37935700 \\
\hline C & -0.96795200 & -0.44218600 & 1.31222700 & C & 0.19627000 & 2.09260400 & 1.47597300 \\
\hline $\mathrm{H}$ & 2.08444300 & 1.06773100 & 1.36922500 & C & -1.76992300 & 0.69082100 & 1.43050000 \\
\hline $\mathrm{H}$ & -1.43196500 & -1.42126000 & 1.24086200 & C & -1.19160200 & 1.95944100 & 1.51087000 \\
\hline $\mathrm{H}$ & 0.65182100 & 3.07664300 & 1.51572400 & $\mathrm{H}$ & -2.84591200 & 0.58548600 & 1.46084900 \\
\hline $\mathrm{H}$ & -1.82271700 & 2.83893100 & 1.57525000 & $\mathrm{~N}$ & 0.95392700 & -2.62379000 & 0.65343000 \\
\hline $\mathrm{H}$ & 0.15319800 & -2.58357900 & 0.01256800 & C & 1.22998900 & -2.06342800 & 3.17114900 \\
\hline $\mathrm{H}$ & 1.82842800 & -2.96478100 & 3.08569500 & $\mathrm{H}$ & 0.16025700 & -2.22190400 & 3.27450600 \\
\hline C & 1.79670900 & -0.99896000 & 3.89542900 & C & 0.96808000 & 0.05939900 & 4.43785500 \\
\hline $\mathrm{H}$ & 0.01867100 & 0.15905800 & 3.90056600 & $\mathrm{H}$ & 1.48425100 & 1.02371800 & 4.48410800 \\
\hline C & 3.27814000 & -0.87428400 & 3.96649700 & $\mathrm{H}$ & 3.59843500 & -0.22955700 & 4.78864600 \\
\hline $\mathrm{H}$ & 3.62704900 & -0.41249000 & 3.02574500 & $\mathrm{H}$ & 3.77294900 & -1.84707900 & 4.03031800 \\
\hline C & -0.56170800 & 1.14312400 & 6.82319200 & $\mathrm{H}$ & 0.05416600 & 2.04935800 & 6.82202700 \\
\hline $\mathrm{H}$ & -0.94137500 & 0.99839900 & 7.84153200 & $\mathrm{H}$ & -1.42144000 & 1.31941400 & 6.16715000 \\
\hline C & 1.95427200 & -0.61921200 & 7.32634200 & $\mathrm{H}$ & 2.61607900 & 0.25419600 & 7.30340000 \\
\hline $\mathrm{H}$ & 2.53327600 & -1.48993900 & 6.99890800 & $\mathrm{H}$ & 1.66857900 & -0.78724800 & 8.37147600 \\
\hline C & -0.64694800 & -1.90446800 & 6.21373800 & $\mathrm{H}$ & -0.08070800 & -2.78219100 & 5.88414000 \\
\hline $\mathrm{H}$ & -1.49994200 & -1.77942900 & 5.53675200 & $\mathrm{H}$ & -1.04662900 & -2.12322600 & 7.21097900 \\
\hline $\mathrm{Si}$ & 0.42308400 & -0.35871200 & 6.26351100 & & & & \\
\hline
\end{tabular}

\section{TS- $R-4 f-29$}

Imaginary frequency: $-408.70 \mathrm{~cm}^{-1}$

E[B3LYP/6-31G(d)-D3(BJ)]: -4789.078185 Hartree

$\mathrm{E}\left[\mathrm{PCM}\left(\mathrm{Et}_{2} \mathrm{O}\right)-\mathrm{B} 3 \mathrm{LYP} / 6-311 \mathrm{G}(\mathrm{d}, \mathrm{p})-\mathrm{D} 3(\mathrm{BJ})\right]$ : -4790.222006 Hartree

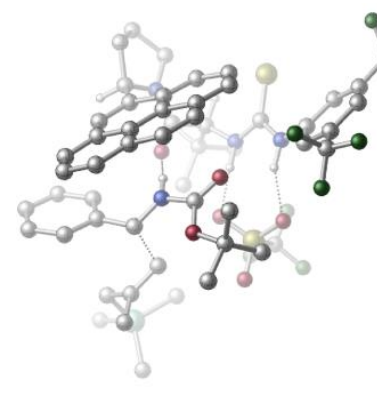




\begin{tabular}{|c|c|c|c|c|c|c|c|}
\hline 01 & & & & $\mathrm{~N}$ & -2.44698700 & -1.72131800 & 0.16133000 \\
\hline $\mathrm{H}$ & -1.71588200 & -1.72880600 & -0.55544400 & C & -3.67484100 & -1.23134700 & -0.31858300 \\
\hline $\mathrm{C}$ & -3.61481700 & -0.26253800 & -1.32596800 & $\mathrm{C}$ & -4.91955200 & -1.69272900 & 0.12450000 \\
\hline $\mathrm{C}$ & -4.78953300 & 0.24965900 & -1.87251400 & $\mathrm{H}$ & -2.64680400 & 0.10661800 & -1.63833400 \\
\hline C & -6.08384700 & -1.15244200 & -0.42069200 & $\mathrm{H}$ & -4.97365700 & -2.46072300 & 0.88150700 \\
\hline C & -6.03523800 & -0.18052900 & -1.41989300 & $\mathrm{H}$ & -6.94710300 & 0.21862300 & -1.84494400 \\
\hline C & -4.69075000 & 1.32262300 & -2.91672500 & C & -7.41683200 & -1.59730300 & 0.11333200 \\
\hline $\mathrm{F}$ & -3.74699300 & 1.04314600 & -3.84555500 & $\mathrm{~F}$ & -4.34142600 & 2.52521300 & -2.37539300 \\
\hline $\mathrm{F}$ & -5.85456600 & 1.51896100 & -3.56926100 & $\mathrm{~F}$ & -7.80018300 & -0.85224800 & 1.17699600 \\
\hline $\mathrm{F}$ & -8.39484200 & -1.48229800 & -0.81510600 & $\mathrm{~F}$ & -7.39568900 & -2.88363900 & 0.52238700 \\
\hline C & -0.06268800 & -2.21044100 & 2.85543800 & $\mathrm{H}$ & -0.83760300 & -2.35804200 & 3.60583000 \\
\hline C & 0.97841500 & -3.35623500 & 3.03230900 & C & 2.18587200 & -3.25592500 & 2.08369400 \\
\hline $\mathrm{H}$ & 2.77845200 & -2.35778500 & 2.27985200 & $\mathrm{H}$ & 1.88351500 & -3.23192000 & 1.03606700 \\
\hline $\mathrm{H}$ & 2.82984000 & -4.13140300 & 2.23369500 & C & 0.24976100 & -4.68824100 & 2.78599000 \\
\hline $\mathrm{H}$ & 0.91372400 & -5.52813200 & 3.02180600 & $\mathrm{H}$ & -0.05876800 & -4.77811800 & 1.74233600 \\
\hline $\mathrm{H}$ & -0.64570600 & -4.77236100 & 3.41231300 & C & 1.48342900 & -3.31220400 & 4.48582300 \\
\hline $\mathrm{H}$ & 2.23066800 & -4.09637000 & 4.64974800 & $\mathrm{H}$ & 0.66760400 & -3.47943000 & 5.19906000 \\
\hline $\mathrm{H}$ & 1.95715900 & -2.35098600 & 4.72211900 & C & 0.53073800 & -0.80055900 & 2.97597300 \\
\hline 0 & 1.25667300 & -0.36318500 & 2.06681700 & C & -0.59750700 & -0.45933600 & 5.20348200 \\
\hline C & 0.63179200 & 1.37840300 & 4.12435600 & $\mathrm{C}$ & -0.34696200 & 0.65845200 & 6.22039800 \\
\hline $\mathrm{H}$ & -1.65484500 & -0.54370500 & 4.92871400 & $\mathrm{H}$ & -0.26241700 & -1.43195000 & 5.56454900 \\
\hline $\mathrm{H}$ & 1.70282700 & 1.37606100 & 4.36406100 & $\mathrm{H}$ & 0.57062200 & 0.45379100 & 6.78493600 \\
\hline $\mathrm{H}$ & -1.16898300 & 0.75335200 & 6.93477300 & $\mathrm{~N}$ & 0.19699100 & -0.03706400 & 4.03158500 \\
\hline C & 0.45795500 & 2.24890800 & 2.89389500 & C & 1.50276800 & 3.06427800 & 2.56539500 \\
\hline C & -0.78886700 & 2.34585200 & 2.15725600 & C & 1.42983400 & 4.04128300 & 1.52269600 \\
\hline $\mathrm{H}$ & 2.43985100 & 2.97942400 & 3.10951400 & C & -0.89943500 & 3.34004000 & 1.13186000 \\
\hline $\mathrm{C}$ & -1.89438400 & 1.51182200 & 2.38874100 & C & 2.52093800 & 4.85495100 & 1.18277900 \\
\hline C & 0.20217900 & 4.18635100 & 0.81509000 & C & -2.12135000 & 3.48450900 & 0.40583800 \\
\hline $\mathrm{H}$ & -1.84746400 & 0.72486800 & 3.12552300 & C & -3.06915500 & 1.63867800 & 1.65126000 \\
\hline C & 2.41043500 & 5.79928000 & 0.16370900 & $\mathrm{H}$ & 3.45611600 & 4.73470200 & 1.72148400 \\
\hline $\mathrm{C}$ & 0.08933500 & 5.17439300 & -0.21025400 & C & -3.19139500 & 2.62017100 & 0.67656900 \\
\hline C & -2.22015800 & 4.51097900 & -0.59261800 & $\mathrm{H}$ & -3.88052300 & 0.94442800 & 1.84210500 \\
\hline C & 1.20862400 & 5.96290500 & -0.52242600 & $\mathrm{H}$ & 3.26463600 & 6.42164000 & -0.08954800 \\
\hline $\mathrm{C}$ & -1.16784100 & 5.32210900 & -0.88587600 & $\mathrm{H}$ & -4.10457200 & 2.71141600 & 0.09859400 \\
\hline $\mathrm{H}$ & -3.15856200 & 4.60855200 & -1.13059600 & $\mathrm{H}$ & 1.12397300 & 6.71099800 & -1.30663000 \\
\hline $\mathrm{H}$ & -1.25710200 & 6.08851000 & -1.65168100 & C & -2.07301900 & -1.94902000 & 1.45200800 \\
\hline $\mathrm{S}$ & -3.13970700 & -1.89368300 & 2.77351000 & $\mathrm{~N}$ & -0.74859500 & -2.23835300 & 1.57402200 \\
\hline $\mathrm{H}$ & -0.14587600 & -2.06593800 & 0.76219100 & $\mathrm{~S}$ & 0.55093100 & -2.48833800 & -1.96183800 \\
\hline $\mathrm{O}$ & 1.48961300 & -2.30266300 & -3.08611600 & $\mathrm{O}$ & 1.03062500 & -1.91820500 & -0.66598700 \\
\hline $\mathrm{O}$ & -0.87654700 & -2.19667000 & -2.22810100 & C & 0.54833800 & -4.32036900 & -1.63877000 \\
\hline $\mathrm{F}$ & 0.26641500 & -5.00267700 & -2.75140000 & $\mathrm{~F}$ & -0.37683600 & -4.61270500 & -0.70763500 \\
\hline $\mathrm{F}$ & 1.74449300 & -4.72927900 & -1.17792100 & C & -0.16450600 & 1.89787900 & 5.33977100 \\
\hline $\mathrm{H}$ & -1.13487300 & 2.27546100 & 5.00276600 & $\mathrm{H}$ & 0.36210200 & 2.71825800 & 5.83361100 \\
\hline $\mathrm{C}$ & 0.46563900 & 1.17555800 & -1.27105700 & $\mathrm{O}$ & -0.65894200 & 0.84990500 & -0.96289000 \\
\hline $\mathrm{O}$ & 0.91010700 & 1.59468700 & -2.46131100 & C & 0.02331400 & 1.75827300 & -3.64408000 \\
\hline C & -1.05554100 & 2.78447800 & -3.32421700 & $\mathrm{H}$ & -1.67527000 & 2.94650800 & -4.21138900 \\
\hline $\mathrm{H}$ & -1.70140700 & 2.44779500 & -2.51584700 & $\mathrm{H}$ & -0.60305200 & 3.73530200 & -3.03136400 \\
\hline C & 1.00012400 & 2.29676600 & -4.68792600 & $\mathrm{H}$ & 1.79319000 & 1.56766100 & -4.88652600 \\
\hline $\mathrm{H}$ & 0.46973900 & 2.48832200 & -5.62563600 & $\mathrm{H}$ & 1.45454300 & 3.23341100 & -4.34830200 \\
\hline $\mathrm{C}$ & -0.53395700 & 0.40547300 & -4.06770500 & $\mathrm{H}$ & -1.08416400 & 0.53076100 & -5.00623500 \\
\hline $\mathrm{H}$ & 0.27080600 & -0.31713100 & -4.22568300 & $\mathrm{H}$ & -1.21192500 & -0.01173900 & -3.32733600 \\
\hline $\mathrm{C}$ & 2.81672000 & 1.42639800 & -0.70097000 & $\mathrm{H}$ & 2.87207600 & 2.10542700 & -1.54335000 \\
\hline C & 3.84132900 & 1.67478100 & 0.33916100 & C & 4.80610600 & 2.65824800 & 0.07628100 \\
\hline
\end{tabular}




\begin{tabular}{|c|c|c|c|c|c|c|c|}
\hline C & 3.91925700 & 0.93912100 & 1.52904100 & C & 5.82417900 & 2.91614600 & 0.99145800 \\
\hline $\mathrm{H}$ & 4.73569400 & 3.24528000 & -0.83514800 & C & 4.93854000 & 1.20290200 & 2.44365200 \\
\hline $\mathrm{H}$ & 3.17348400 & 0.18589700 & 1.75143600 & C & 5.89199800 & 2.18838100 & 2.18098200 \\
\hline $\mathrm{H}$ & 6.55548400 & 3.69107600 & 0.78128500 & $\mathrm{H}$ & 4.98474700 & 0.63286300 & 3.36726900 \\
\hline $\mathrm{H}$ & 6.68302800 & 2.38862500 & 2.89778300 & $\mathrm{~N}$ & 1.51840300 & 1.16399200 & -0.34460500 \\
\hline $\mathrm{H}$ & 1.30469100 & 0.67163300 & 0.53122200 & C & 3.35878900 & -0.20331700 & -1.64178000 \\
\hline $\mathrm{H}$ & 3.09919800 & -0.92435200 & -0.87369200 & $\mathrm{H}$ & 2.63097500 & -0.13580300 & -2.44295100 \\
\hline C & 4.70009300 & -0.01434500 & -1.96362900 & C & 5.75993600 & -0.54297500 & -1.11867900 \\
\hline $\mathrm{H}$ & 6.70413200 & 0.00409000 & -1.20831400 & $\mathrm{H}$ & 5.45335300 & -0.59657100 & -0.06781100 \\
\hline $\mathrm{C}$ & 5.07576300 & 0.80086700 & -3.15866400 & $\mathrm{H}$ & 5.49020300 & 1.76108900 & -2.81660100 \\
\hline $\mathrm{H}$ & 5.86931200 & 0.31080500 & -3.73349100 & $\mathrm{H}$ & 4.22281000 & 0.99830200 & -3.81197400 \\
\hline C & 7.48028500 & -2.94922300 & -0.42962300 & $\mathrm{H}$ & 7.16529100 & -2.87226200 & 0.61725200 \\
\hline $\mathrm{H}$ & 7.75321800 & -3.99434400 & -0.61789500 & $\mathrm{H}$ & 8.38255900 & -2.34042700 & -0.55771000 \\
\hline C & 4.53043800 & -3.39576100 & -1.34395500 & $\mathrm{H}$ & 4.12400300 & -3.27515600 & -0.33367800 \\
\hline $\mathrm{H}$ & 3.74430200 & -3.10653900 & -2.04861800 & $\mathrm{H}$ & 4.72789300 & -4.46450900 & -1.49065500 \\
\hline C & 6.67255700 & -2.47646000 & -3.39067900 & $\mathrm{H}$ & 5.86616400 & -2.19565900 & -4.07742900 \\
\hline $\mathrm{H}$ & 7.53022900 & -1.81954500 & -3.57800200 & $\mathrm{H}$ & 6.97813200 & -3.49693700 & -3.65060300 \\
\hline Si & 6.10160800 & -2.40944900 & -1.59636100 & & & & \\
\hline
\end{tabular}

\section{TS- $R-4 f-30$}

Imaginary frequency: $-341.70 \mathrm{~cm}^{-1}$

E[B3LYP/6-31G(d)-D3(BJ)]: -4789.078049 Hartree

$\mathrm{E}\left[\mathrm{PCM}\left(\mathrm{Et}_{2} \mathrm{O}\right)-\mathrm{B} 3 \mathrm{LYP} / 6-311 \mathrm{G}(\mathrm{d}, \mathrm{p})-\mathrm{D} 3(\mathrm{BJ})\right]$ : -4790.221170 Hartree

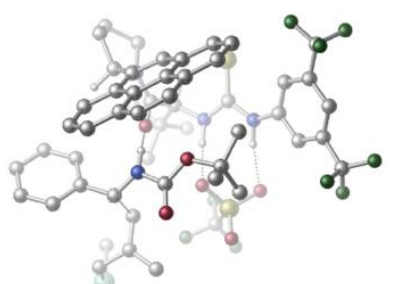

\begin{tabular}{|c|c|c|c|c|c|c|c|}
\hline 01 & & & & $\mathrm{~N}$ & -2.01623900 & -1.85679800 & 1.77082600 \\
\hline $\mathrm{H}$ & -1.32876100 & -2.53741300 & 1.43341200 & C & -3.26530300 & -1.99567700 & 1.14855200 \\
\hline C & -3.36236500 & -3.01593700 & 0.18561100 & $\mathrm{C}$ & -4.37104500 & -1.16149800 & 1.36682000 \\
\hline $\mathrm{C}$ & -4.52080700 & -3.15477900 & -0.56991200 & $\mathrm{H}$ & -2.51669600 & -3.67236800 & 0.01196100 \\
\hline $\mathrm{C}$ & -5.51924200 & -1.32200300 & 0.59569600 & $\mathrm{H}$ & -4.32076400 & -0.38960500 & 2.11725500 \\
\hline C & -5.61308900 & -2.30818000 & -0.38264900 & $\mathrm{H}$ & -6.50796300 & -2.41582700 & -0.98388800 \\
\hline C & -4.55950000 & -4.15126000 & -1.69329200 & C & -6.70587000 & -0.43218200 & 0.83806300 \\
\hline $\mathrm{F}$ & -3.68950900 & -5.16230200 & -1.51973600 & $\mathrm{~F}$ & -4.24887000 & -3.55714400 & -2.87877700 \\
\hline $\mathrm{F}$ & -5.79085800 & -4.68717300 & -1.84883300 & $\mathrm{~F}$ & -6.39334800 & 0.66629800 & 1.56481300 \\
\hline $\mathrm{F}$ & -7.24349700 & 0.00568900 & -0.32891200 & $\mathrm{~F}$ & -7.69386900 & -1.07447600 & 1.49912300 \\
\hline C & 0.58614600 & 0.09783200 & 3.39982500 & $\mathrm{H}$ & -0.02344900 & 0.33709800 & 4.26985000 \\
\hline C & 1.95381400 & -0.44851900 & 3.91416100 & C & 2.95868200 & -0.73608000 & 2.78532400 \\
\hline $\mathrm{H}$ & 3.28875000 & 0.18659000 & 2.30075800 & $\mathrm{H}$ & 2.54048900 & -1.38345200 & 2.01541300 \\
\hline $\mathrm{H}$ & 3.84116300 & -1.23349800 & 3.20461800 & C & 1.67513400 & -1.73904800 & 4.70355600 \\
\hline $\mathrm{H}$ & 2.60047700 & -2.10481500 & 5.16317900 & $\mathrm{H}$ & 1.28232400 & -2.52396200 & 4.05486100 \\
\hline $\mathrm{H}$ & 0.94463500 & -1.56189000 & 5.50148700 & C & 2.56906200 & 0.59718000 & 4.86135000 \\
\hline $\mathrm{H}$ & 3.54526900 & 0.25099600 & 5.21804200 & $\mathrm{H}$ & 1.93738200 & 0.76417900 & 5.74170800 \\
\hline $\mathrm{H}$ & 2.72439900 & 1.55986700 & 4.35889800 & C & 0.72153600 & 1.33263000 & 2.49936100 \\
\hline 0 & 1.13378000 & 1.18677900 & 1.33533000 & C & -0.04764700 & 2.91670300 & 4.32200800 \\
\hline C & 0.44140200 & 3.71589700 & 2.06030800 & C & 0.38600200 & 4.38248400 & 4.40872000 \\
\hline $\mathrm{H}$ & -1.13264000 & 2.79713400 & 4.42160000 & $\mathrm{H}$ & 0.43442100 & 2.28876200 & 5.06905500 \\
\hline $\mathrm{H}$ & 1.47092200 & 3.78865900 & 1.70745200 & $\mathrm{H}$ & 1.45734600 & 4.43645600 & 4.63723400 \\
\hline $\mathrm{H}$ & -0.15491300 & 4.93265400 & 5.18357100 & $\mathrm{~N}$ & 0.37541100 & 2.54573700 & 2.96515000 \\
\hline C & -0.51076900 & 3.60197200 & 0.88171300 & C & -1.76881500 & 3.11748700 & 1.09467600 \\
\hline C & -0.11928800 & 4.04804100 & -0.43866400 & C & -2.76275400 & 3.07988800 & 0.06652600 \\
\hline $\mathrm{H}$ & -2.04364000 & 2.73163700 & 2.07226400 & C & -1.08215900 & 3.97457200 & -1.49737700 \\
\hline
\end{tabular}




\begin{tabular}{|c|c|c|c|c|c|c|c|}
\hline $\mathrm{C}$ & 1.16400900 & 4.53277600 & -0.74413200 & $\mathrm{C}$ & -4.06848400 & 2.62436100 & 0.30892100 \\
\hline C & -2.41023900 & 3.53052400 & -1.23765300 & C & -0.71850000 & 4.36210100 & -2.82348500 \\
\hline $\mathrm{H}$ & 1.91133700 & 4.63136500 & 0.03181500 & $\mathrm{C}$ & 1.51764700 & 4.88052000 & -2.04594400 \\
\hline $\mathrm{C}$ & -5.02475400 & 2.64305900 & -0.70414900 & $\mathrm{H}$ & -4.32432300 & 2.24818100 & 1.29414400 \\
\hline C & -3.38979800 & 3.52523600 & -2.27591500 & C & 0.59217200 & 4.79156900 & -3.07973800 \\
\hline C & -1.71487700 & 4.31539300 & -3.85753600 & $\mathrm{H}$ & 2.52871100 & 5.22277400 & -2.24723300 \\
\hline C & -4.69293300 & 3.09521000 & -1.98049100 & $\mathrm{H}$ & -6.02547900 & 2.27767500 & -0.50319900 \\
\hline C & -2.99403300 & 3.93175000 & -3.59437400 & $\mathrm{H}$ & 0.86967300 & 5.06974200 & -4.09311600 \\
\hline $\mathrm{H}$ & -1.42382800 & 4.60978900 & -4.86253500 & $\mathrm{H}$ & -5.43916900 & 3.09232700 & -2.77071000 \\
\hline $\mathrm{H}$ & -3.73914800 & 3.91506200 & -4.38554000 & $\mathrm{C}$ & -1.52460100 & -0.88779700 & 2.60694400 \\
\hline $\mathrm{S}$ & -2.45840400 & 0.17197600 & 3.54274900 & $\mathrm{~N}$ & -0.16205600 & -0.89498200 & 2.64594000 \\
\hline $\mathrm{H}$ & 0.31201200 & -1.27513000 & 1.81911300 & $S$ & 0.87228800 & -3.43390200 & -0.02571200 \\
\hline $\mathrm{O}$ & 1.14981000 & -3.84258300 & -1.41017500 & $\mathrm{O}$ & 0.98807400 & -1.96487900 & 0.23292300 \\
\hline $\mathrm{O}$ & -0.31819300 & -4.02036800 & 0.63695800 & C & 2.31116400 & -4.14395700 & 0.91443500 \\
\hline $\mathrm{F}$ & 2.42510300 & -5.45614600 & 0.71178400 & $\mathrm{~F}$ & 2.19846500 & -3.92007600 & 2.23258300 \\
\hline $\mathrm{F}$ & 3.45764400 & -3.55059200 & 0.49554000 & $\mathrm{C}$ & 0.10622300 & 4.90616100 & 2.99356200 \\
\hline $\mathrm{H}$ & -0.95364800 & 5.15602700 & 2.88788000 & $\mathrm{H}$ & 0.68467400 & 5.79657000 & 2.73305800 \\
\hline C & 0.67127600 & 0.46672200 & -2.34470800 & 0 & 1.09580600 & -0.04018800 & -3.36904800 \\
\hline $\mathrm{O}$ & -0.57037500 & 0.49137600 & -1.89685600 & $C$ & -1.62399800 & -0.31943700 & -2.58564600 \\
\hline $\mathrm{C}$ & -1.24361500 & -1.79210800 & -2.49660400 & $\mathrm{H}$ & -2.04501900 & -2.39592400 & -2.93076700 \\
\hline $\mathrm{H}$ & -0.31189600 & -2.01443100 & -3.01585600 & $\mathrm{H}$ & -1.13479800 & -2.07689400 & -1.44992900 \\
\hline $\mathrm{C}$ & -2.86126800 & -0.03210100 & -1.74956300 & $\mathrm{H}$ & -3.20247800 & 0.99038500 & -1.89191600 \\
\hline $\mathrm{H}$ & -3.66529100 & -0.71294200 & -2.04251700 & $\mathrm{H}$ & -2.64816700 & -0.18459400 & -0.69121700 \\
\hline C & -1.80152900 & 0.17993400 & -4.01761200 & $\mathrm{H}$ & -2.69717400 & -0.28774400 & -4.44109500 \\
\hline $\bar{H}$ & -1.94723100 & 1.26474800 & -4.02532700 & $\mathrm{H}$ & -0.94368100 & -0.07274200 & -4.64141400 \\
\hline $\mathrm{C}$ & 2.86143700 & 1.13040700 & -1.71571300 & $\mathrm{H}$ & 3.03810900 & 0.91257500 & -2.76252500 \\
\hline C & 3.78267600 & 2.13422200 & -1.13808300 & C & 4.78361600 & 2.65748600 & -1.96806200 \\
\hline $\mathrm{C}$ & 3.70766800 & 2.55740400 & 0.19760800 & $\mathrm{C}$ & 5.68128500 & 3.60918900 & -1.48141300 \\
\hline $\bar{H}$ & 4.84576100 & 2.33146000 & -3.00259900 & C & 4.60847300 & 3.50023600 & 0.68074700 \\
\hline $\mathrm{H}$ & 2.93934500 & 2.14329100 & 0.84091100 & C & 5.59492000 & 4.03305200 & -0.15601400 \\
\hline $\bar{H}$ & 6.44427600 & 4.01648900 & -2.13800300 & $\mathrm{H}$ & 4.54340100 & 3.82209900 & 1.71648600 \\
\hline $\bar{H}$ & 6.29439700 & 4.77047400 & 0.22660800 & $\mathrm{~N}$ & 1.51933600 & 1.15661500 & -1.45849500 \\
\hline $\mathrm{H}$ & 1.16922900 & 1.35458800 & -0.51612300 & C & 3.52819600 & -0.57391900 & -0.83943000 \\
\hline $\mathrm{H}$ & 4.07466300 & -0.12660400 & -0.01679700 & $\mathrm{H}$ & 2.57092200 & -1.01200600 & -0.57674100 \\
\hline $\mathrm{C}$ & 4.27018200 & -1.14415000 & -1.87319300 & $\mathrm{C}$ & 5.69582600 & -0.85010300 & -2.01215800 \\
\hline $\mathrm{H}$ & 6.05281600 & -0.94303300 & -3.04363300 & $\mathrm{H}$ & 5.96056800 & 0.13368200 & -1.60542700 \\
\hline $\mathrm{C}$ & 3.61609200 & -2.04495000 & -2.87099700 & $\mathrm{H}$ & 3.06061400 & -1.43126500 & -3.59268900 \\
\hline $\mathrm{H}$ & 4.35358200 & -2.63665500 & -3.41934400 & $\mathrm{H}$ & 2.87501500 & -2.70018000 & -2.40089000 \\
\hline C & 8.52983000 & -1.72381000 & -1.33031000 & $\mathrm{H}$ & 8.78125600 & -0.68547900 & -1.08529500 \\
\hline $\mathrm{H}$ & 9.19674200 & -2.37319500 & -0.75083400 & $\mathrm{H}$ & 8.75081800 & -1.88353500 & -2.39182200 \\
\hline $\mathrm{C}$ & 6.34573500 & -1.77796500 & 0.87788100 & $\mathrm{H}$ & 6.45990600 & -0.71770500 & 1.13209700 \\
\hline $\mathrm{H}$ & 5.32791700 & -2.08472000 & 1.13496500 & $\mathrm{H}$ & 7.03411000 & -2.34626900 & 1.51501600 \\
\hline $\mathrm{C}$ & 6.27964200 & -3.87025800 & -1.41421900 & $\mathrm{H}$ & 5.24258000 & -4.10242800 & -1.15300700 \\
\hline $\mathrm{H}$ & 6.41262000 & -4.05063000 & -2.48691500 & $\mathrm{H}$ & 6.92179500 & -4.58007800 & -0.87896900 \\
\hline $\mathrm{Si}$ & 6.72646400 & -2.10821600 & -0.93533700 & & & & \\
\hline
\end{tabular}

\section{TS-R-4f-31}

Imaginary frequency: $-154.37 \mathrm{~cm}^{-1}$

E[B3LYP/6-31G(d)-D3(BJ)]: -4789.074965 Hartree

E[PCM(Et $2 \mathrm{O})-B 3 L Y P / 6-311 G(d, p)-D 3(B J)]:-4790.220760$ Hartree

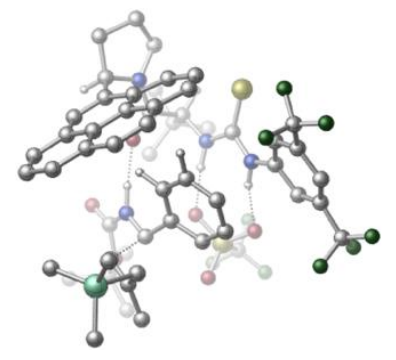




\begin{tabular}{|c|c|c|c|c|c|c|c|}
\hline 01 & & & & $\mathrm{~N}$ & -0.63094700 & 2.71626200 & 0.91617400 \\
\hline $\mathrm{H}$ & -1.48910900 & 2.49634900 & 0.39877000 & C & 0.23819300 & 3.55315200 & 0.20932400 \\
\hline $\mathrm{C}$ & -0.29916200 & 4.25187900 & -0.88116700 & C & 1.61731700 & 3.62243900 & 0.44779900 \\
\hline $\mathrm{C}$ & 0.53502500 & 4.98573900 & -1.72035500 & $\mathrm{H}$ & -1.35807700 & 4.17241900 & -1.09651100 \\
\hline C & 2.42844600 & 4.37811700 & -0.39254200 & $\mathrm{H}$ & 2.05053600 & 3.05913600 & 1.25832700 \\
\hline $\mathrm{C}$ & 1.90636500 & 5.06767300 & -1.48664600 & $\mathrm{H}$ & 2.54800700 & 5.64482800 & -2.14031200 \\
\hline $\mathrm{C}$ & -0.04274100 & 5.59147300 & -2.96634800 & C & 3.91571600 & 4.31598800 & -0.20604200 \\
\hline $\mathrm{F}$ & -1.30942400 & 6.01534600 & -2.79473000 & $\mathrm{~F}$ & -0.06912500 & 4.68018100 & -3.97964900 \\
\hline $\mathrm{F}$ & 0.68536500 & 6.63878800 & -3.41160500 & $\mathrm{~F}$ & 4.27585400 & 4.14531500 & 1.08572700 \\
\hline $\mathrm{F}$ & 4.45302600 & 3.26207100 & -0.89140000 & $\mathrm{~F}$ & 4.54028200 & 5.42179000 & -0.66002500 \\
\hline C & -1.32198700 & 0.36443800 & 3.61680700 & $\mathrm{H}$ & -0.89110000 & 1.02225600 & 4.36927200 \\
\hline $\mathrm{C}$ & -2.74215800 & -0.03112300 & 4.12762200 & $\mathrm{C}$ & -3.43587900 & -1.09273000 & 3.26534700 \\
\hline $\mathrm{H}$ & -2.88136800 & -2.03133100 & 3.23424100 & $\mathrm{H}$ & -3.57750400 & -0.75288500 & 2.23864100 \\
\hline $\mathrm{H}$ & -4.42916400 & -1.30042200 & 3.68192700 & C & -3.60207400 & 1.24534900 & 4.16023100 \\
\hline $\mathrm{H}$ & -4.55146600 & 1.03719800 & 4.66687100 & $\mathrm{H}$ & -3.83265600 & 1.59563400 & 3.15021100 \\
\hline $\mathrm{H}$ & -3.09831600 & 2.05687700 & 4.69854100 & $\mathrm{C}$ & -2.58488600 & -0.57760200 & 5.55796700 \\
\hline $\mathrm{H}$ & -3.56457000 & -0.85505200 & 5.96128800 & $\mathrm{H}$ & -2.14469000 & 0.16864000 & 6.23010000 \\
\hline $\mathrm{H}$ & -1.95709300 & -1.47666700 & 5.57801300 & C & -0.37850500 & -0.82730900 & 3.38464900 \\
\hline 0 & -0.50155400 & -1.56237800 & 2.39474600 & C & 0.91490600 & -0.34042600 & 5.51451300 \\
\hline C & 1.48027300 & -2.24402800 & 4.05708800 & C & 2.30161100 & -0.85681500 & 5.91614900 \\
\hline $\mathrm{H}$ & 0.89756600 & 0.73896000 & 5.34407700 & $\mathrm{H}$ & 0.16665200 & -0.59213700 & 6.27440300 \\
\hline $\mathrm{C}$ & 2.35167700 & -2.28045200 & 5.34047000 & $\mathrm{H}$ & 0.82028000 & -3.11619800 & 4.03136000 \\
\hline $\mathrm{H}$ & 2.44047100 & -0.83708000 & 7.00058600 & $\mathrm{H}$ & 3.08309600 & -0.23677000 & 5.46993600 \\
\hline $\mathrm{H}$ & 1.90918000 & -2.98977800 & 6.04828400 & $\mathrm{H}$ & 3.36865800 & -2.61658200 & 5.12623200 \\
\hline $\mathrm{N}$ & 0.62661200 & -1.05696600 & 4.25976800 & C & 2.28157300 & -2.30596300 & 2.75990200 \\
\hline C & 2.27211400 & -3.50549700 & 2.10662900 & C & 3.12442900 & -1.23399600 & 2.25851500 \\
\hline C & 3.08707400 & -3.78489400 & 0.96416600 & $\mathrm{H}$ & 1.62115900 & -4.29945200 & 2.46466300 \\
\hline C & 4.00329900 & -1.50840400 & 1.15699400 & C & 3.09636900 & 0.07722100 & 2.76146900 \\
\hline C & 3.04969300 & -5.02252200 & 0.30159900 & C & 3.97825800 & -2.77401000 & 0.50265900 \\
\hline C & 4.87583800 & -0.48742400 & 0.66416600 & $\mathrm{H}$ & 2.36667700 & 0.35941000 & 3.50239600 \\
\hline $\mathrm{C}$ & 3.93845800 & 1.06933500 & 2.26172600 & C & 3.87921800 & -5.26748100 & -0.79135800 \\
\hline $\mathrm{H}$ & 2.37238800 & -5.79353200 & 0.65927600 & C & 4.81758600 & -3.02982800 & -0.62463200 \\
\hline C & 4.83931500 & 0.79124400 & 1.24099400 & C & 5.72197300 & -0.77718500 & -0.45939100 \\
\hline $\mathrm{H}$ & 3.86199700 & 2.07505800 & 2.66044000 & C & 4.75168900 & -4.28361600 & -1.25361400 \\
\hline $\mathrm{H}$ & 3.84545800 & -6.23354500 & -1.28701500 & C & 5.69575100 & -1.98867900 & -1.07810200 \\
\hline $\mathrm{H}$ & 5.47426400 & 1.57559100 & 0.84246400 & $\mathrm{H}$ & 6.38041200 & 0.01014100 & -0.81654600 \\
\hline $\mathrm{H}$ & 5.39548700 & -4.48060800 & -2.10684300 & $\mathrm{H}$ & 6.33845200 & -2.18837700 & -1.93169400 \\
\hline C & -0.47914800 & 2.15178800 & 2.15504100 & $\mathrm{~S}$ & 0.63365700 & 2.70010900 & 3.31651700 \\
\hline $\mathrm{N}$ & -1.34959000 & 1.14310400 & 2.39002200 & $\mathrm{H}$ & -1.94875600 & 0.81421200 & 1.62789100 \\
\hline $\mathrm{S}$ & -3.69866600 & 1.26451700 & -0.79232600 & $\mathrm{O}$ & -3.86971200 & 0.56494900 & -2.08520700 \\
\hline 0 & -3.31077300 & 0.38557100 & 0.34508000 & 0 & -2.91625900 & 2.52582700 & -0.84352200 \\
\hline $\mathrm{C}$ & -5.40892800 & 1.82242300 & -0.33628600 & $\mathrm{~F}$ & -5.85418900 & 2.73829200 & -1.20126400 \\
\hline $\mathrm{F}$ & -5.40503000 & 2.35422900 & 0.89429500 & $\mathrm{~F}$ & -6.25815600 & 0.77996800 & -0.34598600 \\
\hline C & -2.98439800 & -2.87448600 & 0.22560300 & 0 & -3.22309700 & -3.47149400 & 1.25729400 \\
\hline 0 & -3.75917100 & -2.83611500 & -0.87578200 & C & -5.18275000 & -3.21504400 & -0.81810400 \\
\hline $\mathrm{C}$ & -5.28222400 & -4.73671700 & -0.72766000 & $\mathrm{H}$ & -6.33328200 & -5.04293300 & -0.76586300 \\
\hline $\mathrm{H}$ & -4.84482600 & -5.09352700 & 0.20688400 & $\mathrm{H}$ & -4.75918800 & -5.20572800 & -1.56875500 \\
\hline C & -5.70670500 & -2.68159600 & -2.15057000 & $\mathrm{H}$ & -5.55936700 & -1.59909700 & -2.20650000 \\
\hline $\mathrm{H}$ & -6.77374800 & -2.90465100 & -2.25049500 & $\mathrm{H}$ & -5.17751000 & -3.15131200 & -2.98772000 \\
\hline $\mathrm{C}$ & -5.87561900 & -2.51419000 & 0.35141200 & $\mathrm{H}$ & -6.95722800 & -2.66251400 & 0.26109900 \\
\hline $\mathrm{H}$ & -5.67283100 & -1.44165900 & 0.32434100 & $\mathrm{H}$ & -5.54368600 & -2.91417900 & 1.30955900 \\
\hline $\mathrm{C}$ & -1.52920000 & -1.53005800 & -1.19098800 & $\mathrm{H}$ & -2.43112800 & -1.21996300 & -1.71030800 \\
\hline
\end{tabular}




\begin{tabular}{|c|c|c|c|c|c|c|c|}
\hline $\mathrm{C}$ & -0.46413000 & -0.48749400 & -1.21026300 & $\mathrm{C}$ & -0.59546600 & 0.55913500 & -2.13851000 \\
\hline C & 0.72710300 & -0.61253500 & -0.48453100 & C & 0.46167500 & 1.43243900 & -2.38070200 \\
\hline $\mathrm{H}$ & -1.53758300 & 0.69524800 & -2.65734200 & $\mathrm{C}$ & 1.77587500 & 0.27718900 & -0.72064300 \\
\hline $\mathrm{H}$ & 0.84804200 & -1.39831500 & 0.25444600 & $\mathrm{C}$ & 1.66069300 & 1.27637600 & -1.68621600 \\
\hline $\mathrm{H}$ & 0.33769700 & 2.24624700 & -3.08765000 & $\mathrm{H}$ & 2.68948800 & 0.18794700 & -0.15415200 \\
\hline $\mathrm{H}$ & 2.49243900 & 1.94849300 & -1.86252200 & $\mathrm{~N}$ & -1.82099900 & -2.15545500 & 0.01941500 \\
\hline $\mathrm{H}$ & -1.26110500 & -1.98453000 & 0.86203200 & C & -0.95730900 & -2.87731900 & -2.23359600 \\
\hline $\mathrm{H}$ & -1.84033400 & -3.51218900 & -2.22553600 & $\mathrm{H}$ & -0.12380900 & -3.26596100 & -1.65309000 \\
\hline C & -0.61587400 & -2.30874900 & -3.48692900 & $\mathrm{C}$ & 0.73591700 & -1.89149700 & -3.75812800 \\
\hline $\mathrm{H}$ & 1.29003400 & -1.66963800 & -2.83899800 & $\mathrm{H}$ & 0.80343400 & -1.05883900 & -4.46415000 \\
\hline C & -1.70297600 & -1.99846300 & -4.45530100 & $\mathrm{H}$ & -1.32501100 & -1.82518200 & -5.46567200 \\
\hline $\mathrm{H}$ & -2.18787100 & -1.06655800 & -4.11886200 & $\mathrm{H}$ & -2.48202400 & -2.76611900 & -4.46320400 \\
\hline C & 3.54713100 & -2.71851500 & -4.50117400 & $\mathrm{H}$ & 3.65749100 & -1.78853100 & -5.06985300 \\
\hline $\mathrm{H}$ & 4.23225600 & -3.45855500 & -4.93181900 & $\mathrm{H}$ & 3.86277800 & -2.52850900 & -3.46981900 \\
\hline $\mathrm{C}$ & 1.16406600 & -3.60918400 & -6.31285500 & $\mathrm{H}$ & 1.22838700 & -2.68806700 & -6.90330100 \\
\hline $\mathrm{H}$ & 0.12346300 & -3.95271200 & -6.33296600 & $\mathrm{H}$ & 1.76972400 & -4.36908700 & -6.82087700 \\
\hline $\mathrm{C}$ & 1.55158500 & -4.90147700 & -3.51902200 & $\mathrm{H}$ & 0.51784900 & -5.26286600 & -3.54599700 \\
\hline $\mathrm{H}$ & 1.83400300 & -4.73755700 & -2.47424300 & $\mathrm{H}$ & 2.19362400 & -5.70222600 & -3.90559200 \\
\hline Si & 1.77786600 & -3.35050600 & -4.55208600 & & & & \\
\hline
\end{tabular}

\section{TS- $R-4 f-32$}

Imaginary frequency: $-287.02 \mathrm{~cm}^{-1}$

E[B3LYP/6-31G(d)-D3(BJ)]: -4789.071240 Hartree

$\mathrm{E}\left[\mathrm{PCM}\left(\mathrm{Et}_{2} \mathrm{O}\right)-\mathrm{B} 3 \mathrm{LYP} / 6-311 \mathrm{G}(\mathrm{d}, \mathrm{p})-\mathrm{D} 3(\mathrm{BJ})\right]$ : -4790.220038 Hartree

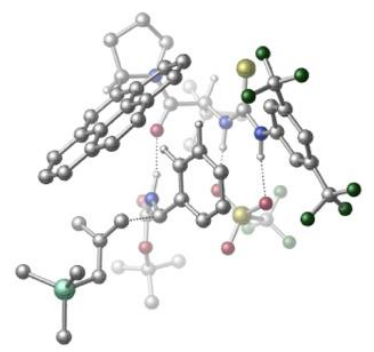

\begin{tabular}{|rrrr|rrrr|}
\hline O & & & & N & 2.91526900 & 1.54432300 & 0.21366500 \\
\hline $\mathrm{H}$ & 2.20847400 & 2.17056100 & -0.18798800 & $\mathrm{C}$ & 3.76551300 & 1.01378700 & -0.76308200 \\
\hline $\mathrm{C}$ & 3.78051100 & 1.67175700 & -2.00311300 & $\mathrm{C}$ & 4.48176500 & -0.18329000 & -0.62326800 \\
\hline $\mathrm{C}$ & 4.48009400 & 1.12847500 & -3.07616900 & $\mathrm{H}$ & 3.19437000 & 2.57265600 & -2.13665900 \\
\hline $\mathrm{C}$ & 5.17211500 & -0.70795600 & -1.71084400 & $\mathrm{H}$ & 4.46715400 & -0.70915800 & 0.31729900 \\
\hline $\mathrm{C}$ & 5.18916400 & -0.06465600 & -2.94821000 & $\mathrm{H}$ & 5.72882200 & -0.48407500 & -3.78848400 \\
\hline $\mathrm{C}$ & 4.36121000 & 1.77355700 & -4.42741100 & $\mathrm{C}$ & 5.78268600 & -2.07400200 & -1.59875800 \\
\hline $\mathrm{F}$ & 4.18792100 & 3.10659000 & -4.34896400 & $\mathrm{~F}$ & 3.29518500 & 1.27682400 & -5.11224400 \\
\hline $\mathrm{F}$ & 5.44993100 & 1.54475000 & -5.19568700 & $\mathrm{~F}$ & 6.01021500 & -2.44745000 & -0.32031600 \\
\hline $\mathrm{F}$ & 4.95293700 & -3.01768700 & -2.13308700 & $\mathrm{~F}$ & 6.95031400 & -2.17334000 & -2.26743900 \\
\hline $\mathrm{C}$ & 1.23666500 & 1.42176600 & 3.45101700 & $\mathrm{H}$ & 2.16490800 & 1.21451600 & 3.97911700 \\
\hline $\mathrm{C}$ & 0.58457600 & 2.65047600 & 4.15924200 & $\mathrm{C}$ & -0.82411400 & 2.99432800 & 3.65568000 \\
\hline $\mathrm{H}$ & -1.52883800 & 2.17435200 & 3.80158300 & $\mathrm{H}$ & -0.83402400 & 3.24621500 & 2.59455800 \\
\hline $\mathrm{H}$ & -1.19680900 & 3.86476500 & 4.20977100 & $\mathrm{C}$ & 1.51425500 & 3.85776900 & 3.94462400 \\
\hline $\mathrm{H}$ & 1.17087800 & 4.70243400 & 4.55287800 & $\mathrm{H}$ & 1.51313300 & 4.17547300 & 2.89834600 \\
\hline $\mathrm{H}$ & 2.54723900 & 3.62713900 & 4.23101100 & $\mathrm{C}$ & 0.50832600 & 2.32430600 & 5.66207600 \\
\hline $\mathrm{H}$ & 0.07269700 & 3.16867700 & 6.20684600 & $\mathrm{H}$ & 1.50095300 & 2.12850500 & 6.08515700 \\
\hline $\mathrm{H}$ & -0.12800800 & 1.45092500 & 5.85163000 & $\mathrm{C}$ & 0.36111500 & 0.16037500 & 3.44132000 \\
\hline $\mathrm{O}$ & -0.62869800 & 0.06991600 & 2.69939600 & $\mathrm{C}$ & 1.77670600 & -0.93231800 & 5.24052500 \\
\hline $\mathrm{C}$ & -0.14309700 & -2.09034800 & 4.22372000 & $\mathrm{C}$ & 1.87274100 & -2.41730500 & 5.61268500 \\
\hline $\mathrm{H}$ & 2.71007900 & -0.54210600 & 4.82732000 & $\mathrm{H}$ & 1.49222300 & -0.32658900 & 6.10826200 \\
\hline $\mathrm{C}$ & 0.46472100 & -2.97514700 & 5.34497900 & $\mathrm{H}$ & -1.14962800 & -1.77214500 & 4.51269100 \\
\hline $\mathrm{H}$ & 2.18258000 & -2.54981900 & 6.65285900 & $\mathrm{H}$ & 2.61104500 & -2.92213500 & 4.98508300 \\
\hline $\mathrm{H}$ & -0.15624300 & -2.88420500 & 6.24225100 & $\mathrm{H}$ & 0.47792700 & -4.02951000 & 5.05941600 \\
\hline $\mathrm{N}$ & 0.71267000 & -0.88872900 & 4.22193500 & $\mathrm{C}$ & -0.31338900 & -2.78955400 & 2.87905200 \\
\hline $\mathrm{C}$ & -1.59069800 & -3.13117500 & 2.53741000 & $\mathrm{C}$ & 0.78277300 & -3.17217400 & 2.00549100 \\
\hline
\end{tabular}




\begin{tabular}{|c|c|c|c|c|c|c|c|}
\hline $\mathrm{C}$ & -1.91993500 & -3.84948000 & 1.34559600 & $\mathrm{H}$ & -2.41045700 & -2.83288000 & 3.18676400 \\
\hline C & 0.48459500 & -3.91991200 & 0.81730100 & C & 2.12343800 & -2.82123300 & 2.23770900 \\
\hline $\mathrm{C}$ & -3.24312600 & -4.18582200 & 1.01296800 & $\mathrm{C}$ & -0.86206200 & -4.23206400 & 0.47086700 \\
\hline $\mathrm{C}$ & 1.53906000 & -4.33132100 & -0.05483600 & $\mathrm{H}$ & 2.37913300 & -2.16277300 & 3.05094800 \\
\hline C & 3.14682100 & -3.24425700 & 1.38959800 & C & -3.52834600 & -4.85425200 & -0.17653400 \\
\hline $\mathrm{H}$ & -4.04090600 & -3.93167000 & 1.70639900 & $\mathrm{C}$ & -1.16264100 & -4.92035500 & -0.74413000 \\
\hline C & 2.86674700 & -4.00760800 & 0.26270700 & C & 1.20960200 & -5.02693100 & -1.26647300 \\
\hline $\mathrm{H}$ & 4.16620300 & -2.93620400 & 1.59595900 & C & -2.50263300 & -5.20882500 & -1.05190100 \\
\hline $\mathrm{H}$ & -4.55519600 & -5.11618400 & -0.41749600 & C & -0.07934300 & -5.30152800 & -1.60386800 \\
\hline $\mathrm{H}$ & 3.66431200 & -4.29948200 & -0.41238400 & $\mathrm{H}$ & 2.02499200 & -5.31916900 & -1.92255800 \\
\hline $\mathrm{H}$ & -2.73026700 & -5.73425900 & -1.97560500 & $\mathrm{H}$ & -0.31065800 & -5.82018000 & -2.53038000 \\
\hline C & 2.78038500 & 1.22597100 & 1.54078500 & $S$ & 3.95821600 & 0.40667900 & 2.45376000 \\
\hline $\mathrm{N}$ & 1.62421400 & 1.68176200 & 2.07521900 & $\mathrm{H}$ & 0.89977200 & 2.05743200 & 1.45167300 \\
\hline S & -0.18154800 & 3.49604700 & -0.82736700 & 0 & -1.33242600 & 3.47007600 & -1.74955000 \\
\hline $\mathrm{O}$ & -0.41252400 & 2.79589700 & 0.46724400 & $\mathrm{O}$ & 1.14692900 & 3.19247300 & -1.41647200 \\
\hline C & -0.02209900 & 5.26763500 & -0.29399200 & $\mathrm{~F}$ & 0.16972100 & 6.07177400 & -1.34461300 \\
\hline $\mathrm{F}$ & 1.02344700 & 5.39607000 & 0.54133900 & $\mathrm{~F}$ & -1.12734600 & 5.66735100 & 0.35601500 \\
\hline C & -3.32818500 & 1.72103400 & 0.93822900 & 0 & -3.45101700 & 2.12400200 & 2.07531000 \\
\hline 0 & -4.10308000 & 2.04444400 & -0.11655900 & C & -4.96091900 & 3.24677500 & -0.07536000 \\
\hline C & -6.12310000 & 3.00000400 & 0.88517800 & $\mathrm{H}$ & -6.81792500 & 3.84584700 & 0.84771000 \\
\hline $\mathrm{H}$ & -5.76041300 & 2.88420100 & 1.90735800 & $\mathrm{H}$ & -6.67304800 & 2.09610600 & 0.59706100 \\
\hline $\mathrm{C}$ & -5.43845600 & 3.35902200 & -1.52169900 & $\mathrm{H}$ & -4.58034900 & 3.46268100 & -2.19234700 \\
\hline $\mathrm{H}$ & -6.07924200 & 4.23906500 & -1.63416100 & $\mathrm{H}$ & -6.01507400 & 2.47570200 & -1.81640400 \\
\hline C & -4.12094000 & 4.46585300 & 0.30037900 & $\mathrm{H}$ & -4.73904200 & 5.36608600 & 0.21240400 \\
\hline $\bar{H}$ & -3.27107800 & 4.55540500 & -0.37930200 & $\mathrm{H}$ & -3.74941500 & 4.39559600 & 1.32318600 \\
\hline $\mathrm{C}$ & -2.15779800 & 0.43139800 & -0.75644300 & $\mathrm{H}$ & -2.64614900 & 1.11765300 & -1.43764200 \\
\hline C & -0.79354600 & 0.05347900 & -1.19671700 & C & -0.41971500 & 0.38328100 & -2.50813900 \\
\hline $\mathrm{C}$ & 0.07467400 & -0.68703600 & -0.38830600 & $\mathrm{C}$ & 0.80132400 & -0.04730100 & -3.01743500 \\
\hline $\bar{H}$ & -1.07295300 & 1.01016600 & -3.10686000 & $\mathrm{C}$ & 1.29403100 & -1.10920900 & -0.90239600 \\
\hline $\mathrm{H}$ & -0.19531500 & -0.93127000 & 0.63240500 & C & 1.64702200 & -0.81876300 & -2.22116200 \\
\hline $\bar{H}$ & 1.11028500 & 0.24196000 & -4.01651000 & $\mathrm{H}$ & 1.97752100 & -1.65712300 & -0.27085800 \\
\hline $\bar{H}$ & 2.59475700 & -1.17249300 & -2.61193800 & $\mathrm{~N}$ & -2.37749800 & 0.77847400 & 0.56635800 \\
\hline $\mathrm{H}$ & -1.66715800 & 0.59912400 & 1.28562900 & C & -3.04036300 & -1.16671200 & -1.14519700 \\
\hline $\mathrm{H}$ & -2.56318200 & -1.81919500 & -0.41889700 & $\mathrm{H}$ & -2.71543200 & -1.33222700 & -2.16804200 \\
\hline $\mathrm{C}$ & -4.40666800 & -0.89631500 & -0.95714700 & $\mathrm{C}$ & -5.25613400 & -0.49204500 & -2.06645900 \\
\hline $\mathrm{H}$ & -4.68950500 & -0.01227700 & -2.87253400 & $\mathrm{H}$ & -6.08333800 & 0.15246200 & -1.75547900 \\
\hline $\mathrm{C}$ & -4.96945100 & -1.02565200 & 0.41221300 & $\mathrm{H}$ & -5.96153700 & -0.58264100 & 0.51481100 \\
\hline $\mathrm{H}$ & -4.27660200 & -0.56854300 & 1.13179000 & $\mathrm{H}$ & -5.00951300 & -2.08693700 & 0.68613300 \\
\hline C & -7.19214500 & -1.40253800 & -4.23275900 & $\mathrm{H}$ & -7.98152300 & -0.77200400 & -3.80833200 \\
\hline $\mathrm{H}$ & -7.67640600 & -2.22579100 & -4.77109200 & $\mathrm{H}$ & -6.64050900 & -0.80420000 & -4.96649500 \\
\hline $\mathrm{C}$ & -7.01352500 & -3.03496200 & -1.59517600 & $\mathrm{H}$ & -7.74205400 & -2.40459800 & -1.07289400 \\
\hline $\mathrm{H}$ & -6.34811600 & -3.47647900 & -0.84584200 & $\mathrm{H}$ & -7.56648700 & -3.85559600 & -2.06740500 \\
\hline $\mathrm{C}$ & -4.66562000 & -3.11878700 & -3.61651600 & $\mathrm{H}$ & -3.99894500 & -3.50221500 & -2.83679700 \\
\hline $\mathrm{H}$ & -4.05963900 & -2.55197700 & -4.33280200 & $\mathrm{H}$ & -5.08369100 & -3.98077700 & -4.15032900 \\
\hline $\mathrm{Si}$ & -6.04971100 & -2.07149500 & -2.89438600 & & & & \\
\hline
\end{tabular}

\section{TS-R-4f-33}

Imaginary frequency: $-222.07 \mathrm{~cm}^{-1}$

E[B3LYP/6-31G(d)-D3(BJ)]: -4789.074953 Hartree

E[PCM(Et $\left.\left.t_{2} \mathrm{O}\right)-B 3 L Y P / 6-311 G(d, p)-D 3(B J)\right]:-4790.219668$ Hartree

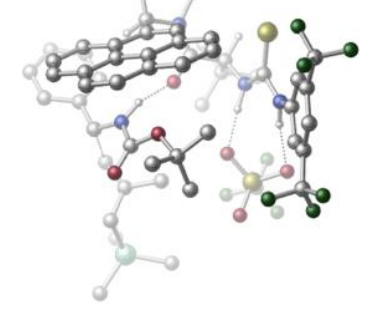




\begin{tabular}{|c|c|c|c|c|c|c|c|}
\hline 01 & & & & $\mathrm{~N}$ & 2.41139200 & 1.53013900 & 1.96305300 \\
\hline $\mathrm{H}$ & 1.80686000 & 2.34441900 & 1.82389600 & C & 3.54532100 & 1.56005200 & 1.13554500 \\
\hline $\mathrm{C}$ & 3.56956900 & 2.57182200 & 0.15985700 & C & 4.60199700 & 0.64182100 & 1.18530200 \\
\hline $\mathrm{C}$ & 4.58662300 & 2.60721600 & -0.78639800 & $\mathrm{H}$ & 2.77256000 & 3.30680000 & 0.13063100 \\
\hline C & 5.61295000 & 0.70191200 & 0.22704900 & $\mathrm{H}$ & 4.61640300 & -0.11756900 & 1.95122000 \\
\hline $\mathrm{C}$ & 5.61702200 & 1.66732500 & -0.77605900 & $\mathrm{H}$ & 6.39665800 & 1.68951000 & -1.52701600 \\
\hline $\mathrm{C}$ & 4.48403500 & 3.58808200 & -1.91934600 & C & 6.75211400 & -0.27352200 & 0.31272900 \\
\hline $\mathrm{F}$ & 3.91849500 & 4.74958300 & -1.54492100 & $\mathrm{~F}$ & 3.71700300 & 3.08572700 & -2.92943300 \\
\hline $\mathrm{F}$ & 5.68985500 & 3.86954500 & -2.45989300 & $\mathrm{~F}$ & 6.38952700 & -1.43136800 & 0.91569900 \\
\hline $\mathrm{F}$ & 7.22428600 & -0.60151400 & -0.91744700 & $\mathrm{~F}$ & 7.79822500 & 0.22000700 & 1.00975000 \\
\hline C & -0.30306100 & -0.31095900 & 3.55385900 & $\mathrm{H}$ & 0.32840700 & -0.75329300 & 4.32320200 \\
\hline $\mathrm{C}$ & -1.52861700 & 0.34678000 & 4.26255200 & $\mathrm{C}$ & -2.53424400 & 0.98515000 & 3.28698100 \\
\hline $\mathrm{H}$ & -3.03180300 & 0.23021800 & 2.67212500 & $\mathrm{H}$ & -2.06139700 & 1.70127200 & 2.61483900 \\
\hline $\mathrm{H}$ & -3.30142400 & 1.51719000 & 3.86173000 & C & -0.99050200 & 1.42562500 & 5.21869600 \\
\hline $\mathrm{H}$ & -1.81142500 & 1.84576300 & 5.81083300 & $\mathrm{H}$ & -0.51654400 & 2.24109000 & 4.66877500 \\
\hline $\mathrm{H}$ & -0.25056700 & 1.00654500 & 5.91055100 & $\mathrm{C}$ & -2.25494200 & -0.73601100 & 5.08077100 \\
\hline $\mathrm{H}$ & -3.13622500 & -0.30773000 & 5.57029000 & $\mathrm{H}$ & -1.61067800 & -1.14854700 & 5.86603900 \\
\hline $\mathrm{H}$ & -2.59852700 & -1.56367800 & 4.44750400 & C & -0.70497500 & -1.38562400 & 2.53023000 \\
\hline $\mathrm{O}$ & -1.21572800 & -1.02943100 & 1.45300200 & C & 0.00800500 & -3.27576100 & 4.05826600 \\
\hline C & -0.80048400 & -3.72013700 & 1.79683600 & C & -0.65118700 & -4.65688200 & 4.04796600 \\
\hline $\mathrm{H}$ & 1.10207600 & -3.33680400 & 4.03629100 & $\mathrm{H}$ & -0.28323600 & -2.67633500 & 4.91847400 \\
\hline $\mathrm{H}$ & -1.84841700 & -3.59605200 & 1.52483700 & $\mathrm{H}$ & -1.69046300 & -4.56992200 & 4.38798000 \\
\hline $\mathrm{H}$ & -0.13843700 & -5.36947600 & 4.69987800 & $\mathrm{~N}$ & -0.48654300 & -2.68491600 & 2.80699200 \\
\hline C & 0.06706200 & -3.62223300 & 0.55585600 & C & 1.35187100 & -3.17750300 & 0.66065900 \\
\hline $\mathrm{C}$ & -0.45198900 & -4.05632000 & -0.72354600 & C & 2.23953200 & -3.12822900 & -0.46034600 \\
\hline $\mathrm{H}$ & 1.72823000 & -2.81808700 & 1.61373200 & C & 0.39894300 & -3.96228000 & -1.87214900 \\
\hline C & -1.75409900 & -4.55599200 & -0.89968700 & C & 3.56952300 & -2.69497900 & -0.33682100 \\
\hline C & 1.74564000 & -3.51701400 & -1.73855300 & C & -0.09459200 & -4.33217300 & -3.16078000 \\
\hline $\mathrm{H}$ & -2.41633500 & -4.67146600 & -0.05028600 & C & -2.22762900 & -4.90664700 & -2.16260300 \\
\hline C & 4.40228300 & -2.64268400 & -1.45304600 & $\mathrm{H}$ & 3.93680900 & -2.38115700 & 0.63451100 \\
\hline $\mathrm{C}$ & 2.60383200 & -3.45373700 & -2.87702700 & C & -1.41594100 & -4.78732500 & -3.28538600 \\
\hline C & 0.78578800 & -4.24360500 & -4.29268800 & $\mathrm{H}$ & -3.24298200 & -5.27883900 & -2.26306600 \\
\hline C & 3.92574000 & -3.01399600 & -2.70894600 & $\mathrm{H}$ & 5.42336400 & -2.29543200 & -1.34663900 \\
\hline $\mathrm{C}$ & 2.07597800 & -3.83221500 & -4.15722900 & $\mathrm{H}$ & -1.79056800 & -5.06313000 & -4.26767900 \\
\hline $\mathrm{H}$ & 0.39612100 & -4.52371300 & -5.26790000 & $\mathrm{H}$ & 4.57813900 & -2.96112600 & -3.57667600 \\
\hline $\mathrm{H}$ & 2.73114000 & -3.77643700 & -5.02268600 & C & 1.85689800 & 0.49492700 & 2.67556100 \\
\hline $\mathrm{S}$ & 2.70786400 & -0.82979400 & 3.30232200 & $\mathrm{~N}$ & 0.52277200 & 0.66736400 & 2.86940100 \\
\hline $\mathrm{H}$ & 0.04376400 & 1.28896000 & 2.20763900 & $\mathrm{~S}$ & -0.24434300 & 3.83728200 & 0.81437200 \\
\hline 0 & -0.57189900 & 4.44132100 & -0.48474200 & $\mathrm{O}$ & -0.57466600 & 2.38406900 & 0.92271200 \\
\hline 0 & 1.08299600 & 4.15080400 & 1.39968400 & C & -1.44520500 & 4.63024100 & 1.99306300 \\
\hline $\mathrm{F}$ & -1.30460600 & 5.95599200 & 2.00839300 & $\mathrm{~F}$ & -1.26990100 & 4.16295700 & 3.24098500 \\
\hline $\mathrm{F}$ & -2.71804400 & 4.34422300 & 1.62882400 & $\mathrm{C}$ & -0.59762100 & -5.05082700 & 2.56592800 \\
\hline $\mathrm{H}$ & 0.38701200 & -5.45970100 & 2.32005700 & $\mathrm{H}$ & -1.34689300 & -5.79557700 & 2.28434100 \\
\hline C & -1.55228300 & -0.53432500 & -2.11096700 & 0 & -2.10845900 & -0.11635700 & -3.11300700 \\
\hline 0 & -0.28603300 & -0.45152200 & -1.76374400 & C & 0.68581800 & 0.35611000 & -2.56852000 \\
\hline $\mathrm{C}$ & 0.23309700 & 1.81192600 & -2.59732600 & $\mathrm{H}$ & 1.03715900 & 2.42117900 & -3.02214700 \\
\hline $\mathrm{H}$ & -0.66330200 & 1.94591800 & -3.20689500 & $\mathrm{H}$ & 0.03990800 & 2.17598000 & -1.58753000 \\
\hline C & 1.96323000 & 0.20635200 & -1.75788100 & $\mathrm{H}$ & 2.30762000 & -0.82579600 & -1.75434600 \\
\hline $\mathrm{H}$ & 2.74430100 & 0.83747300 & -2.18656900 & $\mathrm{H}$ & 1.78733500 & 0.52060000 & -0.72695300 \\
\hline $\mathrm{C}$ & 0.81890700 & -0.25855400 & -3.95787100 & $\mathrm{H}$ & 1.63935500 & 0.24088700 & -4.48447400 \\
\hline $\mathrm{H}$ & 1.05679900 & -1.32241900 & -3.88771000 & $\mathrm{H}$ & -0.09707300 & -0.13332700 & -4.53798600 \\
\hline $\mathrm{C}$ & -3.60710800 & -1.40992100 & -1.28103000 & $\mathrm{H}$ & -3.88807100 & -1.40252900 & -2.32863900 \\
\hline
\end{tabular}




\begin{tabular}{|c|c|c|c|c|c|c|c|}
\hline $\mathrm{C}$ & -4.29614600 & -2.39366200 & -0.41591200 & C & -5.19103100 & -3.29353600 & -1.00738000 \\
\hline C & -4.06760300 & -2.45603800 & 0.96640600 & C & -5.81798300 & -4.27147600 & -0.23625000 \\
\hline $\mathrm{H}$ & -5.37448900 & -3.24234800 & -2.07698600 & C & -4.70036700 & -3.42888900 & 1.73458300 \\
\hline $\mathrm{H}$ & -3.38030200 & -1.75636600 & 1.42916400 & C & -5.57059500 & -4.34412500 & 1.13531000 \\
\hline $\mathrm{H}$ & -6.49741600 & -4.97575300 & -0.70663900 & $\mathrm{H}$ & -4.51318900 & -3.47272900 & 2.80372100 \\
\hline $\mathrm{H}$ & -6.06011400 & -5.10428200 & 1.73670600 & $\mathrm{~N}$ & -2.27779400 & -1.19943600 & -1.10441100 \\
\hline $\mathrm{H}$ & -1.80702900 & -1.36231100 & -0.20248500 & C & -4.74487900 & 0.24568300 & -0.78975600 \\
\hline $\mathrm{H}$ & -5.69863900 & -0.07113400 & -1.20532200 & $\mathrm{H}$ & -4.64182600 & 0.12358100 & 0.28440400 \\
\hline $\mathrm{C}$ & -4.06094100 & 1.30979700 & -1.38116300 & $\mathrm{C}$ & -4.46188100 & 1.90372700 & -2.65177300 \\
\hline $\bar{H}$ & -3.59572000 & 2.11885200 & -3.28523000 & $\mathrm{H}$ & -5.17834800 & 1.28508400 & -3.20234900 \\
\hline C & -2.90358300 & 1.87594500 & -0.64536400 & $\mathrm{H}$ & -2.25857000 & 1.09018700 & -0.23803800 \\
\hline $\mathrm{H}$ & -2.29840400 & 2.56337800 & -1.23250900 & $\mathrm{H}$ & -3.25657500 & 2.41328700 & 0.24275700 \\
\hline C & -6.03344100 & 4.12844900 & -4.00246700 & $\mathrm{H}$ & -6.77253000 & 3.40045100 & -4.35580200 \\
\hline $\mathrm{H}$ & -6.53164000 & 5.10248700 & -3.93010800 & $\mathrm{H}$ & -5.25070400 & 4.21235700 & -4.76493800 \\
\hline $\mathrm{C}$ & -6.66754200 & 3.38701100 & -1.05207900 & $\mathrm{H}$ & -7.38992400 & 2.62548900 & -1.36880500 \\
\hline $\mathrm{H}$ & -6.25378000 & 3.07562100 & -0.08620800 & $\mathrm{H}$ & -7.21893000 & 4.32032100 & -0.88914300 \\
\hline C & -4.03225900 & 4.88407000 & -1.75601100 & $\mathrm{H}$ & -3.65782000 & 4.68955900 & -0.74734700 \\
\hline $\mathrm{H}$ & -3.15953200 & 4.91376800 & -2.41765800 & $\mathrm{H}$ & -4.47890200 & 5.88630000 & -1.75459800 \\
\hline $\mathrm{Si}$ & -5.30813900 & 3.63158400 & -2.33484200 & & & & \\
\hline
\end{tabular}

\section{TS- $R-4 f-34$}

Imaginary frequency: $-397.38 \mathrm{~cm}-1$

E[B3LYP/6-31G(d)-D3(BJ)]: -4789.077484 Hartree

E[PCM(Et $\left.\left.{ }_{2} \mathrm{O}\right)-\mathrm{B} 3 \mathrm{LYP} / 6-311 \mathrm{G}(\mathrm{d}, \mathrm{p})-\mathrm{D} 3(\mathrm{BJ})\right]$ : -4790.219156 Hartree

\begin{tabular}{|lrrr|rrrr|}
\hline 01 & & & N & 2.15993600 & 1.13481000 & 2.11220200 \\
\hline $\mathrm{H}$ & 1.39338100 & 1.81098400 & 2.03638200 & $\mathrm{C}$ & 3.30419900 & 1.54289200 & 1.41175800 \\
\hline $\mathrm{C}$ & 3.24181400 & 2.81832000 & 0.82156400 & $\mathrm{C}$ & 4.44267900 & 0.75308600 & 1.19885000 \\
\hline $\mathrm{C}$ & 4.27351000 & 3.25972200 & 0.00190600 & $\mathrm{H}$ & 2.36461900 & 3.43688600 & 0.97196800 \\
\hline $\mathrm{C}$ & 5.46302800 & 1.22031800 & 0.37446600 & $\mathrm{H}$ & 4.51411400 & -0.21901400 & 1.65828900 \\
\hline $\mathrm{C}$ & 5.39651600 & 2.46833100 & -0.23843000 & $\mathrm{H}$ & 6.19074600 & 2.81480300 & -0.88898700 \\
\hline $\mathrm{C}$ & 4.13220800 & 4.55630100 & -0.74275400 & $\mathrm{C}$ & 6.68277000 & 0.37032100 & 0.15381000 \\
\hline $\mathrm{F}$ & 3.23613200 & 5.38574500 & -0.17790900 & $\mathrm{~F}$ & 3.72144600 & 4.33750500 & -2.02288800 \\
\hline $\mathrm{F}$ & 5.30855600 & 5.21685100 & -0.83073400 & $\mathrm{~F}$ & 6.48622100 & -0.92145400 & 0.50774100 \\
\hline $\mathrm{F}$ & 7.05653300 & 0.36958700 & -1.15111000 & $\mathrm{~F}$ & 7.74592000 & 0.81555700 & 0.85849600 \\
\hline $\mathrm{C}$ & -0.06964700 & -1.35778100 & 3.54068000 & $\mathrm{H}$ & 0.70725800 & -1.83977900 & 4.13221700 \\
\hline $\mathrm{C}$ & -1.28382700 & -1.08767300 & 4.48162500 & $\mathrm{C}$ & -2.51784100 & -0.53496400 & 3.74735300 \\
\hline $\mathrm{H}$ & -2.99137800 & -1.30484200 & 3.13090200 & $\mathrm{H}$ & -2.27695600 & 0.30562000 & 3.09798300 \\
\hline $\mathrm{H}$ & -3.25695400 & -0.19811300 & 4.48355100 & $\mathrm{C}$ & -0.82787200 & -0.08374800 & 5.55441200 \\
\hline $\mathrm{H}$ & -1.61254800 & 0.03891500 & 6.30982800 & $\mathrm{H}$ & -0.61853900 & 0.89380500 & 5.11619800 \\
\hline $\mathrm{H}$ & 0.08142300 & -0.43067600 & 6.05875900 & $\mathrm{C}$ & -1.67329400 & -2.41116300 & 5.16351700 \\
\hline $\mathrm{H}$ & -2.54285100 & -2.25755300 & 5.81196100 & $\mathrm{H}$ & -0.85946400 & -2.79720200 & 5.78868500 \\
\hline $\mathrm{H}$ & -1.94203700 & -3.18093300 & 4.42942000 & $\mathrm{C}$ & -0.40396100 & -2.21424600 & 2.31410200 \\
\hline $\mathrm{O}$ & -0.99584200 & -1.69135000 & 1.35643600 & $\mathrm{C}$ & 0.63643900 & -4.31428800 & 3.28979300 \\
\hline $\mathrm{C}$ & -0.28855600 & -4.26881800 & 1.02452800 & $\mathrm{C}$ & 0.16246500 & -5.72693800 & 2.93523400 \\
\hline $\mathrm{H}$ & 1.72546000 & -4.21146500 & 3.21169400 & $\mathrm{H}$ & 0.33550700 & -4.00296500 & 4.28849400 \\
\hline $\mathrm{H}$ & -1.36379000 & -4.21933900 & 0.84616500 & $\mathrm{H}$ & -0.84747300 & -5.88464300 & 3.33257300 \\
\hline $\mathrm{H}$ & 0.81447900 & -6.50260800 & 3.34627600 & $\mathrm{~N}$ & -0.02175200 & -3.50232400 & 2.26030600 \\
\hline $\mathrm{C}$ & 0.46678100 & -3.71947300 & -0.17437000 & $\mathrm{C}$ & 1.73991700 & -3.25864000 & -0.00272700 \\
\hline $\mathrm{C}$ & -0.12411500 & -3.74287600 & -1.49576900 & $\mathrm{C}$ & 2.56264400 & -2.84395000 & -1.09735800 \\
\hline $\mathrm{H}$ & 2.16373900 & -3.19051200 & 0.99524400 & $\mathrm{C}$ & 0.66956100 & -3.30437700 & -2.60600000 \\
\hline & & & & & & & \\
\hline
\end{tabular}




\begin{tabular}{|c|c|c|c|c|c|c|c|}
\hline $\mathrm{C}$ & -1.44237100 & -4.16159300 & -1.74711500 & $\mathrm{C}$ & 3.88755700 & -2.41675700 & -0.91368100 \\
\hline C & 2.01914600 & -2.89071900 & -2.41285900 & C & 0.11521600 & -3.29366000 & -3.92267900 \\
\hline $\mathrm{H}$ & -2.07218000 & -4.50796800 & -0.93717500 & $\mathrm{C}$ & -1.97910300 & -4.12709800 & -3.03253100 \\
\hline $\mathrm{C}$ & 4.67929500 & -2.07307300 & -2.00746800 & $\mathrm{H}$ & 4.28868700 & -2.35244200 & 0.09266400 \\
\hline C & 2.83137800 & -2.51910500 & -3.52641400 & C & -1.21553100 & -3.69501900 & -4.11115000 \\
\hline C & 0.94568900 & -2.88764600 & -5.02258600 & $\mathrm{H}$ & -3.00639500 & -4.44375800 & -3.18533100 \\
\hline C & 4.16106700 & -2.13038400 & -3.30030700 & $\mathrm{H}$ & 5.69737200 & -1.73632200 & -1.84781100 \\
\hline C & 2.24624300 & -2.53185700 & -4.83687400 & $\mathrm{H}$ & -1.63709100 & -3.67764500 & -5.11291800 \\
\hline $\mathrm{H}$ & 0.51015100 & -2.88315900 & -6.01847100 & $\mathrm{H}$ & 4.77943600 & -1.84801700 & -4.14839300 \\
\hline $\mathrm{H}$ & 2.86427700 & -2.23829000 & -5.68144200 & $\mathrm{C}$ & 1.84869300 & -0.04431500 & 2.73727500 \\
\hline $\mathrm{S}$ & 2.95480100 & -1.25268200 & 3.17336700 & $\mathrm{~N}$ & 0.51768400 & -0.13130100 & 3.02210400 \\
\hline $\mathrm{H}$ & -0.11134100 & 0.41206700 & 2.41902200 & $S$ & -1.15435200 & 2.78073500 & 1.24467300 \\
\hline $\mathrm{O}$ & -1.83656400 & 3.44286400 & 0.11893200 & 0 & -1.21615200 & 1.29178500 & 1.21611400 \\
\hline $\mathrm{O}$ & 0.18985800 & 3.29302400 & 1.61197400 & C & -2.20650600 & 3.22872200 & 2.70966200 \\
\hline $\mathrm{F}$ & -2.36330100 & 4.55044900 & 2.80614000 & $\mathrm{~F}$ & -1.65902600 & 2.77462800 & 3.84882100 \\
\hline $\mathrm{F}$ & -3.42686600 & 2.66380900 & 2.58398300 & $\mathrm{C}$ & 0.14361800 & -5.70985500 & 1.39927500 \\
\hline $\mathrm{H}$ & 1.14886100 & -5.89492500 & 1.00909200 & $\mathrm{H}$ & -0.52451500 & -6.45738000 & 0.96335700 \\
\hline C & -1.13548700 & 0.26165100 & -1.96540300 & 0 & -1.73784400 & 0.99210000 & -2.73758400 \\
\hline $\mathrm{O}$ & 0.16304300 & 0.21007300 & -1.73978500 & $C$ & 1.07275500 & 1.19336200 & -2.40704900 \\
\hline $\mathrm{C}$ & 0.73217000 & 2.59695700 & -1.91856800 & $\mathrm{H}$ & 1.44380400 & 3.30529400 & -2.35230800 \\
\hline $\mathrm{H}$ & -0.27908400 & 2.89243700 & -2.19820400 & $\mathrm{H}$ & 0.82485600 & 2.65744200 & -0.83309100 \\
\hline $\mathrm{C}$ & 2.44266100 & 0.75488800 & -1.91355600 & $\mathrm{H}$ & 2.73383600 & -0.19320700 & -2.36028200 \\
\hline $\mathrm{H}$ & 3.18679400 & 1.51142300 & -2.17716300 & $\mathrm{H}$ & 2.43550400 & 0.63986700 & -0.83037200 \\
\hline C & 0.96975800 & 1.04397100 & -3.92401300 & $\mathrm{H}$ & 1.78455300 & 1.61357700 & -4.38441200 \\
\hline $\bar{H}$ & 1.08521200 & -0.00553500 & -4.21136400 & $\mathrm{H}$ & 0.01924900 & 1.41909900 & -4.30409100 \\
\hline $\mathrm{C}$ & -3.17134000 & -0.76135900 & -1.34387600 & $\mathrm{H}$ & -3.45510000 & -0.42969400 & -2.33622800 \\
\hline C & -3.93103900 & -1.93010700 & -0.84820900 & C & -4.86179700 & -2.51965500 & -1.71559800 \\
\hline $\mathrm{C}$ & -3.75694300 & -2.46226900 & 0.43543600 & $\mathrm{C}$ & -5.58619700 & -3.64370300 & -1.31832800 \\
\hline $\bar{H}$ & -4.99432300 & -2.11327400 & -2.71479300 & $\mathrm{C}$ & -4.48448300 & -3.58117500 & 0.83089900 \\
\hline $\mathrm{H}$ & -3.03266300 & -2.01323400 & 1.10214300 & C & -5.39678600 & -4.17859500 & -0.04379300 \\
\hline $\bar{H}$ & -6.29381600 & -4.10025200 & -2.00399400 & $\mathrm{H}$ & -4.33951400 & -3.98769000 & 1.82794800 \\
\hline $\bar{H}$ & -5.96063100 & -5.05260200 & 0.26879700 & $\mathrm{~N}$ & -1.81369200 & -0.69261100 & -1.18974900 \\
\hline $\mathrm{H}$ & -1.34674000 & -1.08438500 & -0.36453500 & C & -3.86284500 & 0.81390200 & -0.31986400 \\
\hline $\mathrm{H}$ & -3.91667600 & 0.41042700 & 0.68620400 & $\bar{H}$ & -3.01415200 & 1.46010100 & -0.50864700 \\
\hline $\mathrm{C}$ & -5.03950000 & 1.08991000 & -1.01227500 & $\mathrm{C}$ & -5.00655300 & 1.90388900 & -2.22185400 \\
\hline $\mathrm{H}$ & -4.03318700 & 1.84200700 & -2.71865200 & $\mathrm{H}$ & -5.81728600 & 1.66354200 & -2.91741800 \\
\hline $\mathrm{C}$ & -6.33786300 & 0.48584100 & -0.58537300 & $\mathrm{H}$ & -6.33416000 & 0.19342000 & 0.46687000 \\
\hline $\mathrm{H}$ & -7.17316500 & 1.16718700 & -0.77849400 & $\mathrm{H}$ & -6.51909000 & -0.42147100 & -1.18189000 \\
\hline C & -6.72890500 & 4.39651800 & -2.71250400 & $\mathrm{H}$ & -7.62897600 & 3.86120400 & -2.38671700 \\
\hline $\mathrm{H}$ & -6.90087100 & 5.46602300 & -2.54190000 & $\mathrm{H}$ & -6.61946900 & 4.24586400 & -3.79279700 \\
\hline C & -5.43699900 & 4.03922300 & 0.07484200 & $\mathrm{H}$ & -6.32073900 & 3.50940600 & 0.44964700 \\
\hline $\mathrm{H}$ & -4.56252200 & 3.69497000 & 0.63262900 & $\mathrm{H}$ & -5.57282300 & 5.10398100 & 0.29997500 \\
\hline $\mathrm{C}$ & -3.63693500 & 4.66391300 & -2.38008000 & $\mathrm{H}$ & -2.77398700 & 4.31496300 & -1.80443900 \\
\hline $\mathrm{H}$ & -3.45628000 & 4.46477500 & -3.44259600 & $\mathrm{H}$ & -3.71397700 & 5.74993600 & -2.24759900 \\
\hline $\mathrm{Si}$ & -5.19545700 & 3.81564300 & -1.77600500 & & & & \\
\hline
\end{tabular}

\section{TS-R-4f-35}

Imaginary frequency: $-330.32 \mathrm{~cm}^{-1}$

E[B3LYP/6-31G(d)-D3(BJ)]: -4789.072240 Hartree

E[PCM(Et $\left.\left.{ }_{2} \mathrm{O}\right)-\mathrm{B} 3 L Y P / 6-311 \mathrm{G}(\mathrm{d}, \mathrm{p})-\mathrm{D} 3(\mathrm{BJ})\right]$ : -4790.218924 Hartree

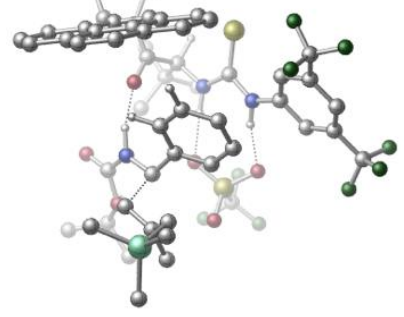




\begin{tabular}{|c|c|c|c|c|c|c|c|}
\hline 01 & & & & $\mathrm{~N}$ & 0.97416000 & 1.76028600 & -2.00564200 \\
\hline $\mathrm{H}$ & 1.90770200 & 1.44334400 & -1.72858300 & C & 0.71317600 & 3.08202500 & -1.62981600 \\
\hline $\mathrm{C}$ & 1.81759000 & 3.87200900 & -1.27650500 & C & -0.57560900 & 3.61447500 & -1.48918900 \\
\hline $\mathrm{C}$ & 1.62993300 & 5.16599100 & -0.79550500 & $\mathrm{H}$ & 2.81556100 & 3.45525500 & -1.34042900 \\
\hline C & -0.73860600 & 4.91383800 & -1.01909800 & $\mathrm{H}$ & -1.43426900 & 3.00718700 & -1.72506000 \\
\hline $\mathrm{C}$ & 0.35270700 & 5.70798500 & -0.66730300 & $\mathrm{H}$ & 0.21200900 & 6.71676800 & -0.30058300 \\
\hline $\mathrm{C}$ & 2.83378100 & 6.00558900 & -0.46841600 & C & -2.12673400 & 5.43594700 & -0.79061300 \\
\hline $\mathrm{F}$ & 3.32751700 & 6.62209300 & -1.56629600 & $\mathrm{~F}$ & 3.83593100 & 5.26547800 & 0.05184400 \\
\hline $\mathrm{F}$ & 2.53940900 & 6.97627400 & 0.42807400 & $\mathrm{~F}$ & -3.03169300 & 4.88040900 & -1.62767200 \\
\hline $\mathrm{F}$ & -2.56129700 & 5.16681300 & 0.47560100 & $\mathrm{~F}$ & -2.20256700 & 6.77443600 & -0.93892800 \\
\hline C & 0.09218200 & -1.46670000 & -3.45222200 & $\mathrm{H}$ & -0.33588200 & -1.00688900 & -4.34244300 \\
\hline $\mathrm{C}$ & 1.13885200 & -2.52649300 & -3.92824100 & $\mathrm{C}$ & 1.66869600 & -3.40136000 & -2.78554800 \\
\hline $\mathrm{H}$ & 0.87904500 & -3.98520900 & -2.30990200 & $\mathrm{H}$ & 2.15865800 & -2.80641800 & -2.01494200 \\
\hline $\mathrm{H}$ & 2.41446400 & -4.10093200 & -3.18196000 & C & 2.31113000 & -1.78378700 & -4.59610100 \\
\hline $\mathrm{H}$ & 2.96901000 & -2.50722200 & -5.09144400 & $\mathrm{H}$ & 2.91219400 & -1.23628700 & -3.86563800 \\
\hline $\mathrm{H}$ & 1.95686100 & -1.07318500 & -5.35210500 & $\mathrm{C}$ & 0.46933000 & -3.43899800 & -4.97156100 \\
\hline $\mathrm{H}$ & 1.18018500 & -4.20376400 & -5.30209000 & $\mathrm{H}$ & 0.15331500 & -2.87733200 & -5.85884500 \\
\hline $\mathrm{H}$ & -0.40146600 & -3.95884100 & -4.55645800 & C & -1.04201500 & -2.05458400 & -2.59246600 \\
\hline 0 & -0.88182000 & -2.27178400 & -1.38430300 & C & -2.66766000 & -2.12562200 & -4.55641800 \\
\hline C & -3.30148400 & -2.92434000 & -2.35307600 & C & -3.64610900 & -3.28491300 & -4.75896300 \\
\hline $\mathrm{H}$ & -3.16196000 & -1.15048400 & -4.65051300 & $\mathrm{H}$ & -1.83078700 & -2.15018000 & -5.24928600 \\
\hline $\mathrm{H}$ & -2.86176300 & -3.76981200 & -1.81824700 & $\mathrm{H}$ & -3.08296800 & -4.20122400 & -4.97091600 \\
\hline $\mathrm{H}$ & -4.33785800 & -3.11099000 & -5.58800300 & $\mathrm{~N}$ & -2.23029500 & -2.33399000 & -3.17371300 \\
\hline C & -3.88527500 & -1.94455200 & -1.35031900 & C & -3.77968100 & -0.60126200 & -1.55620200 \\
\hline $\mathrm{C}$ & -4.60710500 & -2.44912600 & -0.20165600 & C & -4.35945000 & 0.35752900 & -0.66559200 \\
\hline $\mathrm{H}$ & -3.20494500 & -0.22070500 & -2.39447600 & C & -5.17635700 & -1.51152300 & 0.71831600 \\
\hline C & -4.76863800 & -3.82053500 & 0.06100500 & C & -4.22659300 & 1.73990300 & -0.87373900 \\
\hline C & -5.05508000 & -0.11062000 & 0.48667700 & C & -5.87024800 & -1.97618300 & 1.87757300 \\
\hline $\mathrm{H}$ & -4.35751800 & -4.55522500 & -0.62175600 & C & -5.45239800 & -4.26389000 & 1.19175700 \\
\hline C & -4.76026500 & 2.64821800 & 0.03879600 & $\mathrm{H}$ & -3.68653400 & 2.09192100 & -1.74751200 \\
\hline $\mathrm{C}$ & -5.60156800 & 0.82635400 & 1.41506900 & C & -5.99626100 & -3.35615600 & 2.09464300 \\
\hline C & -6.41906700 & -1.01093400 & 2.78958600 & $\mathrm{H}$ & -5.55901200 & -5.33061800 & 1.36734600 \\
\hline C & -5.43725700 & 2.19904400 & 1.17158500 & $\mathrm{H}$ & -4.63431200 & 3.71103100 & -0.12860600 \\
\hline $\mathrm{C}$ & -6.28912200 & 0.32592400 & 2.57183600 & $\mathrm{H}$ & -6.52697100 & -3.70815500 & 2.97551200 \\
\hline $\mathrm{H}$ & -6.94589300 & -1.37764100 & 3.66701200 & $\mathrm{H}$ & -5.84746000 & 2.91372400 & 1.88059000 \\
\hline $\mathrm{H}$ & -6.70877800 & 1.04299900 & 3.27292000 & C & 0.20562800 & 0.87626900 & -2.71805000 \\
\hline $\mathrm{S}$ & -1.18732800 & 1.29769000 & -3.59102300 & $\mathrm{~N}$ & 0.70654500 & -0.38042300 & -2.70932100 \\
\hline $\mathrm{H}$ & 1.45452400 & -0.61230000 & -2.05225100 & $\mathrm{~S}$ & 3.94424500 & -0.00688100 & -0.48276200 \\
\hline 0 & 4.25872200 & -0.09400100 & 0.96139300 & $\mathrm{O}$ & 2.97732400 & -1.03147300 & -0.96429600 \\
\hline 0 & 3.65176200 & 1.35646500 & -0.98843800 & $\mathrm{C}$ & 5.52784800 & -0.47025700 & -1.33171500 \\
\hline $\mathrm{F}$ & 6.48485900 & 0.42059900 & -1.06241400 & $\mathrm{~F}$ & 5.33809700 & -0.52063800 & -2.65651100 \\
\hline $\mathrm{F}$ & 5.94166000 & -1.68085200 & -0.91523700 & $\mathrm{C}$ & 1.66071900 & -3.67364900 & 0.66053400 \\
\hline $\mathrm{O}$ & 1.31924200 & -4.66898200 & 0.05423200 & $\mathrm{O}$ & 2.81500600 & -3.48841400 & 1.32589100 \\
\hline C & 3.95231500 & -4.41379700 & 1.15789700 & C & 3.61043000 & -5.74869400 & 1.81784700 \\
\hline $\mathrm{H}$ & 4.48193600 & -6.41113500 & 1.78013200 & $\mathrm{H}$ & 2.77758300 & -6.23178900 & 1.30412600 \\
\hline $\mathrm{H}$ & 3.34238200 & -5.59845000 & 2.86975700 & $\mathrm{C}$ & 5.07110500 & -3.68431700 & 1.89971300 \\
\hline $\mathrm{H}$ & 5.23827900 & -2.69614500 & 1.46335300 & $\mathrm{H}$ & 5.99825400 & -4.26273400 & 1.83726600 \\
\hline $\mathrm{H}$ & 4.81536900 & -3.56232400 & 2.95835200 & C & 4.29178900 & -4.56364400 & -0.32512500 \\
\hline $\mathrm{H}$ & 5.23170400 & -5.11808100 & -0.41992800 & $\mathrm{H}$ & 4.42321500 & -3.58127900 & -0.78296700 \\
\hline $\mathrm{H}$ & 3.50913100 & -5.10435400 & -0.85756900 & $\mathrm{C}$ & 1.23959600 & -1.40861100 & 1.46241900 \\
\hline $\mathrm{H}$ & 2.30575700 & -1.22874600 & 1.40773800 & C & 0.38966500 & -0.20290200 & 1.31838500 \\
\hline $\mathrm{C}$ & 1.00666000 & 1.05786500 & 1.34584200 & $\mathrm{C}$ & -1.01104600 & -0.28334000 & 1.32959500 \\
\hline
\end{tabular}




\begin{tabular}{|c|c|c|c|c|c|c|c|}
\hline C & 0.23516800 & 2.21725400 & 1.38759900 & $\mathrm{H}$ & 2.08891200 & 1.12760600 & 1.33819400 \\
\hline C & -1.77544700 & 0.87962900 & 1.39484000 & $\mathrm{H}$ & -1.50711800 & -1.24902000 & 1.30079400 \\
\hline C & -1.15623800 & 2.13083400 & 1.42257600 & $\mathrm{H}$ & 0.72261800 & 3.18665300 & 1.38519200 \\
\hline $\mathrm{H}$ & -2.85396400 & 0.81149900 & 1.42712500 & $\mathrm{H}$ & -1.75906700 & 3.03191900 & 1.44540700 \\
\hline $\mathrm{N}$ & 0.84740100 & -2.55559800 & 0.78895100 & $\mathrm{H}$ & 0.05711400 & -2.52271200 & 0.13555100 \\
\hline C & 1.11081000 & -1.88481900 & 3.28048300 & $\mathrm{H}$ & 1.68498900 & -2.80514000 & 3.24688300 \\
\hline $\mathrm{H}$ & 0.03580600 & -2.00917700 & 3.37509900 & C & 1.69689200 & -0.80279700 & 3.96213600 \\
\hline C & 0.89071700 & 0.30247300 & 4.44097900 & $\mathrm{H}$ & -0.04533900 & 0.40507600 & 3.88120200 \\
\hline $\mathrm{H}$ & 1.43443000 & 1.25248200 & 4.45412800 & C & 3.18010100 & -0.71487300 & 4.04888000 \\
\hline $\mathrm{H}$ & 3.50695300 & -0.04270700 & 4.84615200 & $\mathrm{H}$ & 3.55326800 & -0.30466700 & 3.09359700 \\
\hline $\mathrm{H}$ & 3.64745400 & -1.69660700 & 4.16270900 & C & -0.65103100 & 1.53859300 & 6.74236100 \\
\hline $\mathrm{H}$ & -0.00685800 & 2.42450900 & 6.71503800 & $\mathrm{H}$ & -1.05641600 & 1.45128000 & 7.75732400 \\
\hline $\mathrm{H}$ & -1.49074300 & 1.71169100 & 6.06005200 & C & 1.79774400 & -0.27633900 & 7.37630800 \\
\hline $\mathrm{H}$ & 2.48624700 & 0.57525400 & 7.33019100 & $\mathrm{H}$ & 2.35621300 & -1.17707300 & 7.09791900 \\
\hline $\mathrm{H}$ & 1.48672400 & -0.39091700 & 8.42142800 & $C$ & -0.82136600 & -1.52862000 & 6.26696800 \\
\hline $\mathrm{H}$ & -0.27658100 & -2.43855900 & 5.99344700 & $\mathrm{H}$ & -1.65365800 & -1.40922500 & 5.56368400 \\
\hline $\mathrm{H}$ & -1.25134500 & -1.68666600 & 7.26308700 & $\mathrm{Si}$ & 0.29679700 & -0.01645100 & 6.27099900 \\
\hline C & -4.35667800 & -3.38833200 & -3.39878300 & $\mathrm{H}$ & -5.21533200 & -2.71121300 & -3.36752200 \\
\hline $\mathrm{H}$ & -4.72378000 & -4.39569200 & -3.18550400 & & & & \\
\hline
\end{tabular}

\section{TS- $R-4 f-36$}

Imaginary frequency: $-396.82 \mathrm{~cm}^{-1}$

E[B3LYP/6-31G(d)-D3(BJ)]: -4789.076558 Hartree

E[PCM(Et $\left.\left.{ }_{2} \mathrm{O}\right)-\mathrm{B} 3 \mathrm{LYP} / 6-311 \mathrm{G}(\mathrm{d}, \mathrm{p})-\mathrm{D} 3(\mathrm{BJ})\right]$ : -4790.218355 Hartree

\begin{tabular}{|c|c|c|c|c|c|c|c|}
\hline 01 & & & & $\mathrm{~N}$ & 2.43045300 & 0.97815400 & 1.95003700 \\
\hline $\mathrm{H}$ & 1.67777300 & 1.67352200 & 1.93585200 & $\mathrm{C}$ & 3.53829400 & 1.36318400 & 1.18530700 \\
\hline $\mathrm{C}$ & 3.51689700 & 2.67962400 & 0.68696400 & $\mathrm{C}$ & 4.58706800 & 0.51482100 & 0.81038900 \\
\hline C & 4.50360600 & 3.10953900 & -0.19009100 & $\mathrm{H}$ & 2.70179300 & 3.34106300 & 0.95741800 \\
\hline C & 5.56985900 & 0.97363200 & -0.06643400 & $\mathrm{H}$ & 4.61899500 & -0.49509100 & 1.18740800 \\
\hline C & 5.54638200 & 2.26597900 & -0.57881100 & $\mathrm{H}$ & 6.30943000 & 2.60765100 & -1.26637400 \\
\hline$C$ & 4.39166900 & 4.46115700 & -0.83442900 & C & 6.68509400 & 0.03511800 & -0.43396800 \\
\hline $\mathrm{F}$ & 3.61875000 & 5.31004700 & -0.13267500 & $F$ & 3.84463300 & 4.36044700 & -2.07823300 \\
\hline $\mathrm{F}$ & 5.60061800 & 5.04315500 & -0.99889600 & $\mathrm{~F}$ & 6.22184500 & -1.21339900 & -0.71030600 \\
\hline $\mathrm{F}$ & 7.36602200 & 0.45231200 & -1.52305000 & $F$ & 7.57904800 & -0.10427500 & 0.56817800 \\
\hline C & 0.24093400 & -1.50634500 & 3.45431800 & $\mathrm{H}$ & 1.05316500 & -2.02005200 & 3.96579300 \\
\hline C & -0.86908200 & -1.19935900 & 4.50945800 & C & -2.14039500 & -0.59084600 & 3.89191100 \\
\hline $\mathrm{H}$ & -2.70360700 & -1.33867800 & 3.32518700 & $\mathrm{H}$ & -1.92173100 & 0.23709200 & 3.21883800 \\
\hline $\mathrm{H}$ & -2.79133200 & -0.21924100 & 4.69172800 & C & -0.27159100 & -0.22388900 & 5.53819100 \\
\hline $\mathrm{H}$ & -0.97737500 & -0.07132500 & 6.36279100 & $\mathrm{H}$ & -0.05917000 & 0.74626400 & 5.08581800 \\
\hline $\mathrm{H}$ & 0.66340300 & -0.61518400 & 5.95541600 & C & -1.25033100 & -2.50813000 & 5.22293900 \\
\hline $\mathrm{H}$ & -2.04955700 & -2.31924000 & 5.94813100 & $\mathrm{H}$ & -0.40037100 & -2.93000200 & 5.77203600 \\
\hline $\mathrm{H}$ & -1.61838700 & -3.26309800 & 4.51736100 & C & -0.25473900 & -2.33888300 & 2.26456100 \\
\hline 0 & -0.91426500 & -1.78233100 & 1.37420600 & C & 0.85073000 & -4.41891300 & 3.17029000 \\
\hline C & -0.41326000 & -4.48342700 & 1.06063100 & C & 0.55003400 & -5.87403300 & 2.79770200 \\
\hline $\mathrm{H}$ & 1.91348200 & -4.17255400 & 3.06126400 & $\mathrm{H}$ & 0.55486600 & -4.18408700 & 4.19175100 \\
\hline $\mathrm{H}$ & -1.48951100 & -4.64601100 & 1.19883200 & $\mathrm{H}$ & -0.36802300 & -6.20418300 & 3.29850000 \\
\hline $\mathrm{H}$ & 1.35726000 & -6.54912500 & 3.09396600 & $\mathrm{~N}$ & 0.05134300 & -3.64916900 & 2.19821900 \\
\hline C & -0.23267600 & -3.91449500 & -0.33289400 & C & -1.30530700 & -3.97882600 & -1.17428200 \\
\hline C & 1.04248500 & -3.42901600 & -0.82646700 & C & -1.24276700 & -3.57244100 & -2.54477100 \\
\hline $\mathrm{H}$ & -2.25950400 & -4.33722200 & -0.79825300 & C & 1.14871000 & -3.08186600 & -2.21190100 \\
\hline
\end{tabular}




\begin{tabular}{|c|c|c|c|c|c|c|c|}
\hline $\mathrm{C}$ & 2.18246400 & -3.28194500 & -0.01896600 & $\mathrm{C}$ & -2.36697400 & -3.59587700 & -3.38398400 \\
\hline C & 0.00830100 & -3.13484300 & -3.06520100 & C & 2.40522200 & -2.66854500 & -2.74967000 \\
\hline $\mathrm{H}$ & 2.13020200 & -3.47055200 & 1.04243900 & $\mathrm{C}$ & 3.39634100 & -2.84884300 & -0.54856500 \\
\hline $\mathrm{C}$ & -2.26795100 & -3.18875200 & -4.71301100 & $\mathrm{H}$ & -3.31689300 & -3.93446400 & -2.98144000 \\
\hline C & 0.10724300 & -2.74936600 & -4.43597600 & C & 3.51788100 & -2.56370100 & -1.90238800 \\
\hline C & 2.48612700 & -2.32881400 & -4.14288600 & $\mathrm{H}$ & 4.24878500 & -2.72655600 & 0.10983600 \\
\hline C & -1.04551700 & -2.77317400 & -5.23662000 & $\mathrm{H}$ & -3.14816500 & -3.20604500 & -5.34992900 \\
\hline C & 1.38931400 & -2.35602900 & -4.94790100 & $\mathrm{H}$ & 4.46719100 & -2.22018900 & -2.30197100 \\
\hline $\mathrm{H}$ & 3.45236400 & -2.02735600 & -4.53916900 & $\mathrm{H}$ & -0.97162600 & -2.46751400 & -6.27695600 \\
\hline $\mathrm{H}$ & 1.46582800 & -2.08061100 & -5.99655600 & $\mathrm{C}$ & 2.14413100 & -0.19723000 & 2.59700700 \\
\hline $\mathrm{S}$ & 3.28284800 & -1.36679800 & 3.04997700 & $\mathrm{~N}$ & 0.81987500 & -0.29903000 & 2.89115500 \\
\hline $\mathrm{H}$ & 0.17393100 & 0.26534800 & 2.32757700 & $S$ & -0.86899200 & 2.76142600 & 1.40260800 \\
\hline $\mathrm{O}$ & -1.59140400 & 3.50908800 & 0.35835000 & $\mathrm{O}$ & -0.98504000 & 1.27884000 & 1.29255900 \\
\hline $\mathrm{O}$ & 0.51150700 & 3.20747100 & 1.71680800 & C & -1.82331000 & 3.16376200 & 2.94643600 \\
\hline $\mathrm{F}$ & -1.93846000 & 4.48352700 & 3.10824400 & $\mathrm{~F}$ & -1.22611100 & 2.64674600 & 4.03159100 \\
\hline $\mathrm{F}$ & -3.06394400 & 2.63590000 & 2.86529400 & C & 0.33731400 & -5.81350600 & 1.28150700 \\
\hline $\mathrm{H}$ & 1.29858700 & -5.78267000 & 0.75996200 & $\mathrm{H}$ & -0.23244400 & -6.65665100 & 0.88306800 \\
\hline C & -1.13990600 & 0.42329600 & -1.87802400 & 0 & -1.77335700 & 1.21794800 & -2.55587400 \\
\hline 0 & 0.17145200 & 0.31318700 & -1.77420900 & C & 1.04976100 & 1.31924500 & -2.45643600 \\
\hline C & 0.81408200 & 2.68618900 & -1.82492400 & $\mathrm{H}$ & 1.50236600 & 3.40814200 & -2.27372100 \\
\hline $\mathrm{H}$ & -0.20885600 & 3.03215200 & -1.97146900 & $\mathrm{H}$ & 1.02067700 & 2.65389700 & -0.75426500 \\
\hline C & 2.45017700 & 0.80727200 & -2.14864600 & $\mathrm{H}$ & 2.67485500 & -0.08718300 & -2.72795900 \\
\hline $\mathrm{H}$ & 3.18129600 & 1.58272100 & -2.39252300 & $\mathrm{H}$ & 2.54419300 & 0.56329800 & -1.09065900 \\
\hline C & 0.79203100 & 1.30394100 & -3.96240400 & $\mathrm{H}$ & 1.58584400 & 1.87806100 & -4.45308500 \\
\hline $\bar{H}$ & 0.83134400 & 0.27856500 & -4.34144200 & $\mathrm{H}$ & -0.17135400 & 1.74621400 & -4.21470000 \\
\hline $\mathrm{C}$ & -3.16551600 & -0.55912700 & -1.17337300 & $\mathrm{H}$ & -3.49246700 & -0.17708300 & -2.13351300 \\
\hline C & -3.95442700 & -1.70713000 & -0.67546600 & C & -4.97033800 & -2.20429300 & -1.50573500 \\
\hline $\mathrm{C}$ & -3.74581400 & -2.29421900 & 0.57859200 & $\mathrm{C}$ & -5.74775300 & -3.28978000 & -1.10178100 \\
\hline $\bar{H}$ & -5.13017800 & -1.75485500 & -2.48214400 & $\mathrm{C}$ & -4.52751700 & -3.37344200 & 0.98155800 \\
\hline $\mathrm{H}$ & -2.95485000 & -1.92111100 & 1.21532000 & C & -5.52656000 & -3.87766900 & 0.14421800 \\
\hline $\bar{H}$ & -6.52195300 & -3.67413400 & -1.75917500 & $\mathrm{H}$ & -4.35557000 & -3.82258800 & 1.95577400 \\
\hline $\bar{H}$ & -6.13244400 & -4.72070600 & 0.46321500 & $\mathrm{~N}$ & -1.79832500 & -0.54517100 & -1.09950500 \\
\hline $\mathrm{H}$ & -1.30013400 & -0.99309400 & -0.32373900 & C & -3.73729100 & 1.00377600 & -0.05532100 \\
\hline $\mathrm{H}$ & -3.73339100 & 0.56810500 & 0.93849600 & $\mathrm{H}$ & -2.88295900 & 1.62820700 & -0.28620200 \\
\hline $\mathrm{C}$ & -4.95016400 & 1.34091700 & -0.65069100 & $\mathrm{C}$ & -4.97569700 & 2.19269300 & -1.83442900 \\
\hline $\mathrm{H}$ & -4.04695700 & 2.10745700 & -2.40748500 & $\mathrm{H}$ & -5.84761400 & 2.00986900 & -2.47082500 \\
\hline $\mathrm{C}$ & -6.23556800 & 0.77097400 & -0.14552200 & $\mathrm{H}$ & -6.16178000 & 0.43306600 & 0.89031800 \\
\hline $\mathrm{H}$ & -7.05303500 & 1.49329000 & -0.24305100 & $\mathrm{H}$ & -6.50240700 & -0.10091400 & -0.76219000 \\
\hline C & -3.48082200 & 4.88773600 & -1.98959600 & $\mathrm{H}$ & -3.37661700 & 4.71836300 & -3.06739400 \\
\hline $\mathrm{H}$ & -3.49481200 & 5.97071400 & -1.81676400 & $\mathrm{H}$ & -2.60242600 & 4.47740900 & -1.48199000 \\
\hline $\mathrm{C}$ & -6.59687400 & 4.78194600 & -2.13206700 & $\mathrm{H}$ & -6.56361800 & 4.66362500 & -3.22137700 \\
\hline $\mathrm{H}$ & -7.50077700 & 4.28049300 & -1.76564600 & $\mathrm{H}$ & -6.70255000 & 5.85206300 & -1.91709800 \\
\hline $\mathrm{C}$ & -5.15235300 & 4.26224700 & 0.55370100 & $\mathrm{H}$ & -6.03513200 & 3.76052700 & 0.96733600 \\
\hline $\mathrm{H}$ & -4.26192500 & 3.85796800 & 1.04172400 & $\mathrm{H}$ & -5.22189200 & 5.32331500 & 0.82219900 \\
\hline $\mathrm{Si}$ & -5.03942900 & 4.09413000 & -1.31538100 & & & & \\
\hline
\end{tabular}

\section{TS-R-4f-37}

Imaginary frequency: $-362.22 \mathrm{~cm}^{-1}$

E[B3LYP/6-31G(d)-D3(BJ)]: -4789.079453 Hartree

E[PCM(Et $\left.\left.{ }_{2} \mathrm{O}\right)-\mathrm{B} 3 L Y P / 6-311 \mathrm{G}(\mathrm{d}, \mathrm{p})-\mathrm{D} 3(\mathrm{BJ})\right]$ : -4790.218323 Hartree

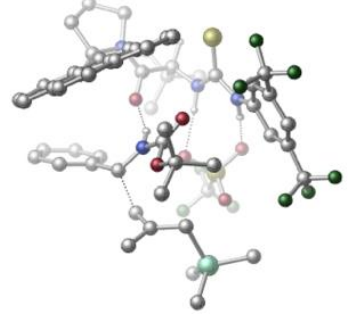




\begin{tabular}{|c|c|c|c|c|c|c|c|}
\hline 01 & & & & $\mathrm{~N}$ & 1.23948300 & 0.08076800 & -2.86677100 \\
\hline $\mathrm{H}$ & 1.90364900 & -0.67349100 & -2.66999400 & C & 1.74286600 & 1.35036700 & -2.59085500 \\
\hline $\mathrm{C}$ & 3.06365700 & 1.39568900 & -2.10778500 & $\mathrm{C}$ & 1.02013200 & 2.54509500 & -2.70762900 \\
\hline $\mathrm{C}$ & 3.64556800 & 2.61305500 & -1.77442800 & $\mathrm{H}$ & 3.61872600 & 0.47379300 & -1.97269700 \\
\hline C & 1.62321400 & 3.74977300 & -2.35712200 & $\mathrm{H}$ & -0.00176500 & 2.51927200 & -3.05136200 \\
\hline C & 2.94102700 & 3.80952100 & -1.90863100 & $\mathrm{H}$ & 3.39653400 & 4.75571200 & -1.64216700 \\
\hline C & 5.01018900 & 2.64922100 & -1.15068900 & C & 0.79550000 & 5.00172300 & -2.32774300 \\
\hline $\mathrm{F}$ & 5.69241000 & 1.49969000 & -1.31223800 & $\mathrm{~F}$ & 4.92472300 & 2.87166500 & 0.19428400 \\
\hline $\mathrm{F}$ & 5.76756500 & 3.65178800 & -1.64734300 & $\mathrm{~F}$ & -0.29061200 & 4.93074500 & -3.12913200 \\
\hline $\mathrm{F}$ & 0.33587400 & 5.25192100 & -1.07101000 & $\mathrm{~F}$ & 1.50350800 & 6.09160000 & -2.69839700 \\
\hline C & -1.41482800 & -2.39082300 & -3.01577000 & $\mathrm{H}$ & -1.87813300 & -2.00586800 & -3.92418800 \\
\hline C & -1.16938900 & -3.92592800 & -3.19462800 & C & -0.73764300 & -4.61695200 & -1.88932100 \\
\hline $\mathrm{H}$ & -1.53285300 & -4.58242100 & -1.13914800 & $\mathrm{H}$ & 0.15206400 & -4.16050800 & -1.45384300 \\
\hline $\mathrm{H}$ & -0.50837400 & -5.66856500 & -2.09749200 & C & -0.07444800 & -4.11545700 & -4.25945300 \\
\hline $\mathrm{H}$ & 0.02639700 & -5.17965400 & -4.50202000 & $\mathrm{H}$ & 0.89417600 & -3.75232900 & -3.90589400 \\
\hline $\mathrm{H}$ & -0.32062700 & -3.57691700 & -5.18197200 & C & -2.47194900 & -4.58130000 & -3.68435400 \\
\hline $\mathrm{H}$ & -2.32113900 & -5.65822700 & -3.81660000 & $\mathrm{H}$ & -2.79134400 & -4.17162600 & -4.65008500 \\
\hline $\mathrm{H}$ & -3.28638600 & -4.45044900 & -2.96237000 & C & -2.27188300 & -2.05663700 & -1.78800100 \\
\hline $\mathrm{O}$ & -1.73585000 & -2.01728100 & -0.67214300 & C & -4.43342400 & -1.78632400 & -3.10107600 \\
\hline C & -4.35950400 & -1.49915600 & -0.69101400 & C & -5.77283800 & -2.28477500 & -2.55051500 \\
\hline $\mathrm{H}$ & -4.50615300 & -0.76030900 & -3.48390500 & $\mathrm{H}$ & -4.02849300 & -2.41781100 & -3.88874500 \\
\hline $\mathrm{H}$ & -4.08631800 & -2.24288000 & 0.06136000 & $\mathrm{H}$ & -5.74840500 & -3.37834800 & -2.47800300 \\
\hline $\mathrm{H}$ & -6.61874300 & -2.00536500 & -3.18477800 & $\mathrm{~N}$ & -3.59052200 & -1.81496800 & -1.90612700 \\
\hline C & -4.07884100 & -0.10143700 & -0.16315000 & C & -3.51835100 & 0.84693100 & -0.96585000 \\
\hline C & -4.51504200 & 0.24753800 & 1.17263500 & C & -3.41463800 & 2.22024800 & -0.57176400 \\
\hline $\mathrm{H}$ & -3.13919100 & 0.58150700 & -1.94691600 & C & -4.42521000 & 1.61458400 & 1.58999400 \\
\hline $\mathrm{C}$ & -5.02265100 & -0.69506100 & 2.08246500 & C & -2.87000300 & 3.19651300 & -1.42105600 \\
\hline C & -3.91207600 & 2.60647700 & 0.70457700 & C & -4.86099200 & 1.99484200 & 2.89621800 \\
\hline $\mathrm{H}$ & -5.09238900 & -1.73870800 & 1.80012100 & C & -5.42175000 & -0.31776300 & 3.36411300 \\
\hline C & -2.85233300 & 4.53670700 & -1.03928700 & $\mathrm{H}$ & -2.46165000 & 2.89011300 & -2.37906600 \\
\hline $\mathrm{C}$ & -3.88002200 & 3.97916300 & 1.09285500 & $\mathrm{C}$ & -5.34705000 & 1.01056400 & 3.77072600 \\
\hline $\mathrm{C}$ & -4.79779200 & 3.38127400 & 3.26967500 & $\mathrm{H}$ & -5.80189200 & -1.07204400 & 4.04789000 \\
\hline C & -3.36111500 & 4.92732300 & 0.19810900 & $\mathrm{H}$ & -2.41740500 & 5.27476700 & -1.70379400 \\
\hline $\mathrm{C}$ & -4.34505900 & 4.33013300 & 2.40461800 & $\mathrm{H}$ & -5.67381900 & 1.30034600 & 4.76620300 \\
\hline $\mathrm{H}$ & -5.13641200 & 3.65966300 & 4.26456000 & $\mathrm{H}$ & -3.33592200 & 5.97329800 & 0.49300500 \\
\hline $\mathrm{H}$ & -4.31453400 & 5.37580300 & 2.70046500 & C & -0.02164900 & -0.36014100 & -3.17501800 \\
\hline $\mathrm{S}$ & -1.22868800 & 0.53776200 & -3.94387900 & $\mathrm{~N}$ & -0.16611600 & -1.67368400 & -2.84880200 \\
\hline $\mathrm{H}$ & 0.41213400 & -2.00619500 & -2.07594000 & $\mathrm{~S}$ & 2.83316600 & -2.40326800 & -0.81301700 \\
\hline $\mathrm{O}$ & 3.75005200 & -1.69765900 & 0.10326000 & $\mathrm{O}$ & 1.40510400 & -2.40006400 & -0.38226400 \\
\hline $\mathrm{O}$ & 3.01391800 & -2.09873400 & -2.25399300 & C & 3.32126700 & -4.18690800 & -0.66047100 \\
\hline $\mathrm{F}$ & 4.61259000 & -4.35504500 & -0.95316300 & $\mathrm{~F}$ & 2.58717700 & -4.94987500 & -1.48243100 \\
\hline $\mathrm{F}$ & 3.10975600 & -4.60217000 & 0.60367500 & C & -5.84164700 & -1.65016100 & -1.14865700 \\
\hline $\mathrm{H}$ & -6.30471700 & -0.66082400 & -1.20187300 & $\mathrm{H}$ & -6.42436100 & -2.24782500 & -0.44295500 \\
\hline $\mathrm{C}$ & -0.06028600 & 0.75966400 & 1.00447400 & $\mathrm{O}$ & -0.11071200 & 1.22094000 & -0.11472500 \\
\hline $\mathrm{O}$ & 0.27444100 & 1.39309400 & 2.14228700 & C & 0.58821800 & 2.84706900 & 2.12628300 \\
\hline C & -0.65196800 & 3.61277500 & 1.68680300 & $\mathrm{H}$ & -0.46627500 & 4.68811100 & 1.77165500 \\
\hline $\mathrm{H}$ & -0.90407400 & 3.39545500 & 0.65072200 & $\mathrm{H}$ & -1.50251300 & 3.35981800 & 2.32486200 \\
\hline C & 0.91626300 & 3.14088300 & 3.58763000 & $\mathrm{H}$ & 1.80128800 & 2.58845600 & 3.91784500 \\
\hline $\mathrm{H}$ & 1.12112800 & 4.20916900 & 3.70608100 & $\mathrm{H}$ & 0.07276800 & 2.88003700 & 4.23563200 \\
\hline C & 1.78559600 & 3.11543300 & 1.22151800 & $\mathrm{H}$ & 2.02509500 & 4.18303600 & 1.25212100 \\
\hline $\mathrm{H}$ & 2.67134900 & 2.56056700 & 1.53952100 & $\mathrm{H}$ & 1.55613700 & 2.84899700 & 0.19390800 \\
\hline $\mathrm{C}$ & -0.49111800 & -1.15231600 & 2.48096800 & $\mathrm{H}$ & -0.61693200 & -0.43474900 & 3.28401500 \\
\hline C & -1.35061300 & -2.36057800 & 2.58116300 & C & -2.36976800 & -2.36868000 & 3.54065500 \\
\hline
\end{tabular}




\begin{tabular}{|c|c|c|c|c|c|c|c|}
\hline C & -1.17917800 & -3.46439500 & 1.73407500 & C & -3.23035000 & -3.46137100 & 3.63868000 \\
\hline $\mathrm{H}$ & -2.51347000 & -1.50582900 & 4.18458700 & C & -2.03502700 & -4.55548100 & 1.84320200 \\
\hline $\mathrm{H}$ & -0.38825800 & -3.45956200 & 0.99295200 & C & -3.06341000 & -4.55622800 & 2.79055700 \\
\hline $\mathrm{H}$ & -4.02938300 & -3.45305200 & 4.37414300 & $\mathrm{H}$ & -1.89909600 & -5.40807000 & 1.18484400 \\
\hline $\mathrm{H}$ & -3.72862300 & -5.41141900 & 2.86971000 & $\mathrm{~N}$ & -0.38172200 & -0.57255400 & 1.25195000 \\
\hline $\mathrm{H}$ & -0.64145400 & -1.10951500 & 0.41518400 & C & 1.27363200 & -1.95298900 & 3.04900500 \\
\hline $\mathrm{H}$ & 0.87338100 & -2.63559900 & 3.79227000 & $\mathrm{H}$ & 1.56826100 & -2.39857200 & 2.10280800 \\
\hline C & 1.97920100 & -0.83924200 & 3.51612500 & C & 2.95359600 & -0.14947600 & 2.69852700 \\
\hline $\mathrm{H}$ & 2.84334800 & -0.35259900 & 1.62993800 & $\mathrm{H}$ & 3.00929300 & 0.92136400 & 2.90853600 \\
\hline $\mathrm{C}$ & 1.67198700 & -0.30792600 & 4.88367200 & $\mathrm{H}$ & 2.55554300 & 0.12781100 & 5.35651300 \\
\hline $\mathrm{H}$ & 0.93967500 & 0.50443900 & 4.77921900 & $\mathrm{H}$ & 1.24382900 & -1.07027800 & 5.54004800 \\
\hline C & 5.86040900 & -0.02024900 & 1.76127000 & $\mathrm{H}$ & 5.75177500 & 1.06855400 & 1.73373100 \\
\hline $\mathrm{H}$ & 6.91427700 & -0.24986100 & 1.96126500 & $\mathrm{H}$ & 5.59823600 & -0.40791400 & 0.77420900 \\
\hline C & 5.25920800 & -0.14154700 & 4.78480700 & $\mathrm{H}$ & 5.10273800 & 0.94035200 & 4.87177300 \\
\hline $\mathrm{H}$ & 4.71325900 & -0.63335100 & 5.59777900 & $\mathrm{H}$ & 6.32677200 & -0.32890700 & 4.95438200 \\
\hline C & 4.79968100 & -2.67098400 & 3.09187500 & $\mathrm{H}$ & 4.19719800 & -3.07517500 & 3.91377200 \\
\hline $\mathrm{H}$ & 4.41968300 & -3.07667100 & 2.15203000 & $\mathrm{H}$ & 5.82714000 & -3.03001700 & 3.22695400 \\
\hline Si & 4.76992700 & -0.79333500 & 3.07658300 & & & & \\
\hline
\end{tabular}

\section{TS- $R-4 f-38$}

Imaginary frequency: $-418.35 \mathrm{~cm}^{-1}$

E[B3LYP/6-31G(d)-D3(BJ)]: -4789.074209 Hartree

$\mathrm{E}\left[\mathrm{PCM}\left(\mathrm{Et}_{2} \mathrm{O}\right)-\mathrm{B} 3 \mathrm{LYP} / 6-311 \mathrm{G}(\mathrm{d}, \mathrm{p})-\mathrm{D} 3(\mathrm{BJ})\right]$ : -4790.218162 Hartree

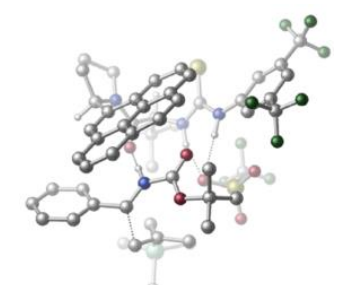

\begin{tabular}{|c|c|c|c|c|c|c|c|}
\hline 01 & & & & $\mathrm{~N}$ & -2.19450200 & -1.02224000 & 0.55488100 \\
\hline $\mathrm{H}$ & -1.49086500 & -0.92635600 & -0.17335400 & C & -3.44398300 & -0.50745800 & 0.19108900 \\
\hline C & -3.45217900 & 0.44394700 & -0.83456500 & C & -4.65349200 & -0.90944500 & 0.76921900 \\
\hline C & -4.65313500 & 1.00542200 & -1.25861600 & $\mathrm{H}$ & -2.51028300 & 0.75418500 & -1.26769300 \\
\hline C & -5.84423100 & -0.32853200 & 0.33659200 & $\mathrm{H}$ & -4.65958800 & -1.65365600 & 1.55146600 \\
\hline C & -5.86146900 & 0.63436200 & -0.67266400 & $\mathrm{H}$ & -6.79347400 & 1.08164000 & -0.99284700 \\
\hline C & -4.61193700 & 2.07471400 & -2.30967900 & C & -7.14705100 & -0.80281800 & 0.91929500 \\
\hline $\mathrm{F}$ & -3.85296100 & 1.71515300 & -3.37251300 & $\mathrm{~F}$ & -4.06329200 & 3.22770400 & -1.83114300 \\
\hline $\mathrm{F}$ & -5.83523000 & 2.39236600 & -2.77737000 & $\mathrm{~F}$ & -7.00745400 & -1.23687900 & 2.18883600 \\
\hline $\mathrm{F}$ & -8.07850900 & 0.18000500 & 0.92538000 & $\mathrm{~F}$ & -7.66865600 & -1.82822000 & 0.20595900 \\
\hline $\mathrm{C}$ & 0.42870200 & -2.02302400 & 2.81719000 & $\mathrm{H}$ & -0.21181100 & -1.97548900 & 3.69612300 \\
\hline C & 1.20419800 & -3.36763100 & 2.88141800 & C & 2.06633800 & -3.62181100 & 1.63584400 \\
\hline $\mathrm{H}$ & 2.83415700 & -2.85371000 & 1.51927600 & $\mathrm{H}$ & 1.45800300 & -3.64245800 & 0.72918000 \\
\hline $\mathrm{H}$ & 2.55780500 & -4.59811200 & 1.72863200 & $\mathrm{C}$ & 0.16590800 & -4.49490100 & 3.01552600 \\
\hline $\mathrm{H}$ & 0.67190100 & -5.46129700 & 3.12398400 & $\mathrm{H}$ & -0.47623800 & -4.54201800 & 2.13321900 \\
\hline $\mathrm{H}$ & -0.47513700 & -4.34204100 & 3.89078600 & $\mathrm{C}$ & 2.10646400 & -3.34324100 & 4.12684700 \\
\hline $\mathrm{H}$ & 2.66985100 & -4.27953800 & 4.20473500 & $\mathrm{H}$ & 1.51809400 & -3.23748900 & 5.04603100 \\
\hline $\mathrm{H}$ & 2.83358000 & -2.52247700 & 4.08569200 & $\mathrm{C}$ & 1.30378100 & -0.76185100 & 2.73580800 \\
\hline $\mathrm{O}$ & 1.98828200 & -0.54349100 & 1.71909600 & $\mathrm{C}$ & 0.60634600 & -0.11397300 & 5.06315800 \\
\hline $\mathrm{C}$ & 1.83246000 & 1.47740900 & 3.64409900 & $\mathrm{C}$ & 1.23752600 & 0.96806800 & 5.94267300 \\
\hline $\mathrm{H}$ & -0.48393900 & -0.01707200 & 5.00662800 & $\mathrm{H}$ & 0.83959900 & -1.12044300 & 5.41022100 \\
\hline $\mathrm{H}$ & 2.92064600 & 1.34121900 & 3.62815900 & $\mathrm{H}$ & 2.21289500 & 0.62866900 & 6.31120200 \\
\hline $\mathrm{H}$ & 0.61342100 & 1.20952400 & 6.80710900 & $\mathrm{~N}$ & 1.21553300 & 0.13176100 & 3.73816900 \\
\hline C & 1.47413900 & 2.34203500 & 2.44696900 & C & 2.49160100 & 3.07028400 & 1.89959800 \\
\hline C & 0.11134900 & 2.56252800 & 1.99561500 & C & 2.28521400 & 4.06429400 & 0.89080200 \\
\hline $\mathrm{H}$ & 3.50873100 & 2.91204800 & 2.24552600 & C & -0.12613900 & 3.57740200 & 1.01151000 \\
\hline C & -0.99468000 & 1.84823300 & 2.48171600 & C & 3.34540200 & 4.79632000 & 0.33460900 \\
\hline C & 0.95315900 & 4.32739300 & 0.46134800 & C & -1.45863400 & 3.85602500 & 0.57711500 \\
\hline
\end{tabular}




\begin{tabular}{|c|c|c|c|c|c|c|c|}
\hline $\mathrm{H}$ & -0.86494400 & 1.05605500 & 3.20172200 & C & -2.28610100 & 2.10468700 & 2.02747300 \\
\hline C & 3.10357000 & 5.77115900 & -0.63142700 & $\mathrm{H}$ & 4.35775100 & 4.59409400 & 0.66954600 \\
\hline $\mathrm{C}$ & 0.71080900 & 5.35163300 & -0.50482200 & $\mathrm{C}$ & -2.52286500 & 3.10012200 & 1.08866300 \\
\hline $\mathrm{C}$ & -1.67761100 & 4.91115800 & -0.37219400 & $\mathrm{H}$ & -3.10035700 & 1.49834300 & 2.40949100 \\
\hline C & 1.80238600 & 6.05066100 & -1.04446000 & $\mathrm{H}$ & 3.93472600 & 6.32864500 & -1.05479700 \\
\hline C & -0.64414400 & 5.63031700 & -0.88653000 & $\mathrm{H}$ & -3.52868700 & 3.29162500 & 0.72963000 \\
\hline $\mathrm{H}$ & -2.69780400 & 5.10961200 & -0.68563700 & $\mathrm{H}$ & 1.61821200 & 6.82443400 & -1.78536800 \\
\hline $\mathrm{H}$ & -0.82617500 & 6.42117900 & -1.60962500 & C & -1.73323900 & -1.43889100 & 1.76823900 \\
\hline S & -2.59995600 & -1.35108100 & 3.22161400 & $\mathrm{~N}$ & -0.46785800 & -1.92941700 & 1.68234800 \\
\hline $\mathrm{H}$ & -0.04083400 & -1.94959400 & 0.75327800 & $S$ & -0.66890600 & -2.98801700 & -2.00701300 \\
\hline 0 & 0.00960800 & -3.56712900 & -3.18225800 & 0 & 0.27548000 & -2.29913700 & -1.05797000 \\
\hline 0 & -1.90441800 & -2.22310200 & -2.24908900 & C & -1.22712500 & -4.46536500 & -1.02186400 \\
\hline $\mathrm{F}$ & -1.97660100 & -5.27762600 & -1.76997100 & $\mathrm{~F}$ & -1.94309400 & -4.07951600 & 0.04630900 \\
\hline $\mathrm{F}$ & -0.16270900 & -5.16309500 & -0.57219400 & C & 1.40949700 & 2.14039900 & 4.97429200 \\
\hline $\mathrm{H}$ & 0.45648900 & 2.65919500 & 4.83070300 & $\mathrm{H}$ & 2.14776300 & 2.87868300 & 5.29660600 \\
\hline C & 0.92668100 & 1.03599700 & -1.41785700 & $\mathrm{O}$ & -0.13040000 & 0.79431800 & -0.87015100 \\
\hline 0 & 1.12266600 & 1.47040700 & -2.66877300 & C & -0.01531500 & 1.77469000 & -3.58014800 \\
\hline C & -0.84823900 & 2.89301500 & -2.96855200 & $\mathrm{H}$ & -1.63714900 & 3.18802000 & -3.66564800 \\
\hline $\mathrm{H}$ & -1.31796400 & 2.58591400 & -2.03619200 & $\mathrm{H}$ & -0.21760000 & 3.76128000 & -2.76406000 \\
\hline C & 0.70601600 & 2.26249700 & -4.83429900 & $\mathrm{H}$ & 1.36211900 & 1.48258000 & -5.23452100 \\
\hline $\mathrm{H}$ & -0.02888300 & 2.51948000 & -5.60321200 & $\mathrm{H}$ & 1.30671200 & 3.15167000 & -4.61676400 \\
\hline C & -0.81615800 & 0.50755000 & -3.86714700 & $\mathrm{H}$ & -1.66672200 & 0.76206500 & -4.50598500 \\
\hline $\mathrm{H}$ & -0.20965700 & -0.23335000 & -4.39566700 & $\mathrm{H}$ & -1.20634300 & 0.04314000 & -2.96367700 \\
\hline C & 3.36794700 & 1.23189500 & -1.30085000 & $\mathrm{H}$ & 3.25153000 & 1.97412300 & -2.08196400 \\
\hline C & 4.49317500 & 1.49676500 & -0.36764900 & C & 5.46410100 & 2.42905600 & -0.76230400 \\
\hline C & 4.64667700 & 0.82186400 & 0.85155700 & C & 6.56296000 & 2.69557700 & 0.04933400 \\
\hline $\mathrm{H}$ & 5.34427700 & 2.95801800 & -1.70388800 & C & 5.75186000 & 1.08895500 & 1.65905000 \\
\hline $\mathrm{H}$ & 3.89419900 & 0.12187600 & 1.19482400 & C & 6.71063400 & 2.02367500 & 1.26540000 \\
\hline $\mathrm{H}$ & 7.29984100 & 3.42951500 & -0.26312800 & $\mathrm{H}$ & 5.85831100 & 0.56539600 & 2.60493000 \\
\hline $\mathrm{H}$ & 7.56750900 & 2.22870700 & 1.90032900 & $\mathrm{~N}$ & 2.14649300 & 0.87803800 & -0.76139400 \\
\hline $\mathrm{H}$ & 2.06804500 & 0.45279200 & 0.16803900 & C & 4.22781200 & -0.08738300 & -2.48800900 \\
\hline $\mathrm{H}$ & 4.28149700 & 0.44077700 & -3.43723700 & $\mathrm{H}$ & 5.17782400 & -0.18380100 & -1.97419600 \\
\hline C & 3.35814800 & -1.19343500 & -2.41204900 & C & 3.41213900 & -2.09019400 & -1.28450900 \\
\hline $\mathrm{H}$ & 4.01921500 & -1.69775600 & -0.46442200 & $\mathrm{H}$ & 2.40143900 & -2.35972900 & -0.95314600 \\
\hline C & 2.27358700 & -1.36407000 & -3.40579700 & $\mathrm{H}$ & 2.18167000 & -2.40567000 & -3.72787600 \\
\hline $\mathrm{H}$ & 1.32157600 & -1.17473400 & -2.90073500 & $\mathrm{H}$ & 2.37892300 & -0.70145400 & -4.26528100 \\
\hline C & 5.32789900 & -4.25931400 & -0.32708400 & $\mathrm{H}$ & 4.75653600 & -4.31022300 & 0.60539800 \\
\hline $\mathrm{H}$ & 5.79572300 & -5.23777900 & -0.48919300 & $\mathrm{H}$ & 6.12952400 & -3.52316200 & -0.19571200 \\
\hline C & 2.83887800 & -5.05790800 & -2.03169400 & $\mathrm{H}$ & 2.34768500 & -5.28700200 & -1.08101900 \\
\hline $\mathrm{H}$ & 2.05767000 & -4.69111400 & -2.70581400 & $\mathrm{H}$ & 3.24161500 & -5.99263100 & -2.44082700 \\
\hline C & 5.25101100 & -3.58825200 & -3.33978500 & $\mathrm{H}$ & 4.63427000 & -3.32457400 & -4.20618300 \\
\hline $\mathrm{H}$ & 6.01098400 & -2.80811700 & -3.21567100 & $\mathrm{H}$ & 5.77206100 & -4.52215500 & -3.58182200 \\
\hline $\mathrm{Si}$ & 4.21408000 & -3.81346800 & -1.77945800 & & & & \\
\hline
\end{tabular}

\section{TS-R-4f-39}

Imaginary frequency: $-386.69 \mathrm{~cm}^{-1}$

E[B3LYP/6-31G(d)-D3(BJ)]: -4789.073840 Hartree

$\mathrm{E}\left[\mathrm{PCM}\left(\mathrm{Et}_{2} \mathrm{O}\right)-\mathrm{B} 3 \mathrm{LYP} / 6-311 \mathrm{G}(\mathrm{d}, \mathrm{p})-\mathrm{D} 3(\mathrm{BJ})\right]:-4790.216871$ Hartree

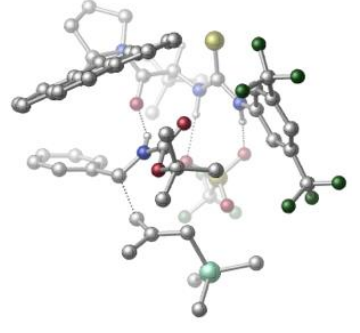

\begin{tabular}{|cccc|cccc|}
\hline 01 & & & & & 2.34646600 & 1.12152100 & 1.92874300 \\
\hline $\mathrm{H}$ & 1.66924500 & 1.89142800 & 1.91161500 & $\mathrm{C}$ & 3.47503300 & 1.36459000 & 1.13753200 \\
\hline
\end{tabular}




\begin{tabular}{|c|c|c|c|c|c|c|c|}
\hline$C$ & 3.61572100 & 2.67043800 & 0.64072700 & $\mathrm{C}$ & 4.38158300 & 0.37725400 & 0.72476100 \\
\hline C & 4.62623200 & 2.96861800 & -0.26786000 & $\mathrm{H}$ & 2.90729600 & 3.43528900 & 0.93841700 \\
\hline C & 5.38729900 & 0.70261600 & -0.18000300 & $\mathrm{H}$ & 4.28032900 & -0.63309300 & 1.08734100 \\
\hline C & 5.52791300 & 1.99338400 & -0.68825000 & $\mathrm{H}$ & 6.31590500 & 2.23213300 & -1.39192200 \\
\hline C & 4.67662900 & 4.33753700 & -0.88514200 & C & 6.27580900 & -0.37872500 & -0.72344100 \\
\hline $\mathrm{F}$ & 4.20579000 & 5.29114100 & -0.05846300 & $\mathrm{~F}$ & 3.92633000 & 4.39013500 & -2.01739500 \\
\hline $\mathrm{F}$ & 5.93376800 & 4.68541100 & -1.24053400 & $\mathrm{~F}$ & 6.29641800 & -1.48391800 & 0.05423500 \\
\hline $\mathrm{F}$ & 5.85942900 & -0.77751800 & -1.95688500 & $\mathrm{~F}$ & 7.55247300 & 0.03757000 & -0.86947500 \\
\hline $\mathrm{C}$ & -0.01592400 & -1.09978000 & 3.58745500 & $\mathrm{H}$ & 0.76477700 & -1.58857800 & 4.16814100 \\
\hline C & -1.11824400 & -0.62197900 & 4.58074600 & C & -2.32304300 & 0.03498300 & 3.88658300 \\
\hline $\mathrm{H}$ & -2.88593200 & -0.68938900 & 3.29243600 & $\mathrm{H}$ & -2.02122100 & 0.84589800 & 3.22489800 \\
\hline $\mathrm{H}$ & -2.99419900 & 0.45272800 & 4.64622900 & C & -0.46687000 & 0.38353000 & 5.54569900 \\
\hline $\mathrm{H}$ & -1.18128800 & 0.67339000 & 6.32462200 & $\mathrm{H}$ & -0.15133900 & 1.28683200 & 5.01967000 \\
\hline $\mathrm{H}$ & 0.41379200 & -0.05031300 & 6.03330900 & C & -1.61127100 & -1.84275700 & 5.37759800 \\
\hline $\mathrm{H}$ & -2.40627300 & -1.54403000 & 6.06950300 & $\mathrm{H}$ & -0.80520400 & -2.28614100 & 5.97411400 \\
\hline $\mathrm{H}$ & -2.02292700 & -2.61859500 & 4.71976100 & C & -0.53298000 & -2.04822900 & 2.49784900 \\
\hline 0 & -1.26991300 & -1.60074800 & 1.60232500 & C & 0.56682500 & -4.04009300 & 3.58989400 \\
\hline C & -0.56587700 & -4.25402400 & 1.41654200 & C & 0.19176500 & -5.50701600 & 3.35451100 \\
\hline $\mathrm{H}$ & 1.64552500 & -3.86493400 & 3.51344800 & $\mathrm{H}$ & 0.23768300 & -3.68000900 & 4.56365100 \\
\hline $\mathrm{H}$ & -1.65856100 & -4.33375000 & 1.46544800 & $\mathrm{H}$ & -0.77099300 & -5.72418200 & 3.83250800 \\
\hline $\mathrm{H}$ & 0.93584900 & -6.19330100 & 3.76755000 & $\mathrm{~N}$ & -0.14370900 & -3.33705200 & 2.50556400 \\
\hline $\mathrm{C}$ & -0.21969800 & -3.82016200 & 0.00376000 & $\mathrm{C}$ & -1.23363600 & -3.78310000 & -0.90850100 \\
\hline C & 1.14154600 & -3.55947300 & -0.42746600 & $\mathrm{C}$ & -1.02741200 & -3.47966800 & -2.29142400 \\
\hline $\mathrm{H}$ & -2.25104100 & -3.97880400 & -0.58513400 & $\mathrm{C}$ & 1.38883700 & -3.37491200 & -1.82604400 \\
\hline $\mathrm{C}$ & 2.23378100 & -3.48206000 & 0.45148100 & $\mathrm{C}$ & -2.09280300 & -3.34953900 & -3.19513500 \\
\hline C & 0.30842800 & -3.30994400 & -2.75288100 & $\mathrm{C}$ & 2.72635100 & -3.21201800 & -2.29950700 \\
\hline $\mathrm{H}$ & 2.07908500 & -3.52784800 & 1.51824000 & C & 3.53146100 & -3.28905300 & -0.01888200 \\
\hline C & -1.84845200 & -3.06704600 & -4.53753500 & $\mathrm{H}$ & -3.11097100 & -3.46585300 & -2.83307600 \\
\hline $\mathrm{C}$ & 0.55142800 & -3.05510000 & -4.13596000 & $\mathrm{C}$ & 3.78507400 & -3.18207300 & -1.38077700 \\
\hline C & 2.94675000 & -3.01425400 & -3.70545600 & $\mathrm{H}$ & 4.34644400 & -3.20728800 & 0.69233300 \\
\hline C & -0.54307100 & -2.93099400 & -5.00687500 & $\mathrm{H}$ & -2.68136400 & -2.96042300 & -5.22685300 \\
\hline $\mathrm{C}$ & 1.90997400 & -2.92615600 & -4.58242800 & $\mathrm{H}$ & 4.79615500 & -3.02300700 & -1.73846400 \\
\hline $\mathrm{H}$ & 3.97126600 & -2.89943600 & -4.04901100 & $\mathrm{H}$ & -0.35962100 & -2.72444200 & -6.05803600 \\
\hline $\mathrm{H}$ & 2.09281800 & -2.74833500 & -5.63890000 & C & 1.97657200 & 0.01158700 & 2.64475900 \\
\hline S & 3.04590400 & -1.16803700 & 3.21755800 & $\mathrm{~N}$ & 0.63954900 & -0.00115100 & 2.89841600 \\
\hline $\mathrm{H}$ & 0.04344000 & 0.57838700 & 2.29796400 & $\mathrm{~S}$ & -0.68264400 & 3.21664800 & 1.28158000 \\
\hline 0 & -1.21204500 & 4.00987800 & 0.16143200 & $\mathrm{O}$ & -0.94749300 & 1.74600000 & 1.19205100 \\
\hline 0 & 0.70351100 & 3.51005800 & 1.72312400 & C & -1.72864900 & 3.75404200 & 2.72250300 \\
\hline $\mathrm{F}$ & -1.70212100 & 5.08058200 & 2.86302700 & $\mathrm{~F}$ & -1.29835000 & 3.19372900 & 3.86489600 \\
\hline $\mathrm{F}$ & -3.00740300 & 3.36951600 & 2.52897000 & $\mathrm{C}$ & 0.06430900 & -5.60196700 & 1.82960500 \\
\hline $\mathrm{H}$ & 1.04982200 & -5.70721700 & 1.36815300 & $\mathrm{H}$ & -0.55125500 & -6.44027200 & 1.49385600 \\
\hline C & -1.32353500 & 0.38062200 & -1.63331600 & $\mathrm{O}$ & -1.85939900 & 1.25756700 & -2.28987700 \\
\hline 0 & -0.04042500 & 0.08565700 & -1.55422000 & $\mathrm{C}$ & 0.96869300 & 0.90971600 & -2.29091900 \\
\hline C & 0.98472000 & 2.31375200 & -1.70209100 & $\mathrm{H}$ & 1.77931300 & 2.89725800 & -2.17683700 \\
\hline $\mathrm{H}$ & 0.03540100 & 2.83078100 & -1.83778900 & $\mathrm{H}$ & 1.20297100 & 2.26673100 & -0.63529900 \\
\hline C & 2.26635300 & 0.17155000 & -1.99444600 & $\mathrm{H}$ & 2.31685300 & -0.76451500 & -2.54709600 \\
\hline $\mathrm{H}$ & 3.12188000 & 0.79043300 & -2.27972800 & $\mathrm{H}$ & 2.34262800 & -0.04921000 & -0.92926700 \\
\hline C & 0.66154500 & 0.88581800 & -3.78735400 & $\mathrm{H}$ & 1.52122700 & 1.30163900 & -4.32401000 \\
\hline $\mathrm{H}$ & 0.51388200 & -0.14403100 & -4.12717000 & $\mathrm{H}$ & -0.22309000 & 1.47471300 & -4.03048100 \\
\hline C & -3.43655200 & -0.41179600 & -0.88317400 & $\mathrm{H}$ & -3.75619900 & 0.17022800 & -1.73534700 \\
\hline C & -4.29630800 & -1.58521800 & -0.59523300 & C & -5.38217000 & -1.81891700 & -1.45073900 \\
\hline C & -4.06555100 & -2.45700800 & 0.47936500 & C & -6.21517100 & -2.92066100 & -1.25099500 \\
\hline $\mathrm{H}$ & -5.55976800 & -1.14693400 & -2.28578900 & C & -4.90286000 & -3.55075900 & 0.67739900 \\
\hline
\end{tabular}




\begin{tabular}{|c|c|c|c|c|c|c|c|}
\hline $\mathrm{H}$ & -3.22443000 & -2.27703400 & 1.14063500 & $\mathrm{C}$ & -5.97684200 & -3.78874000 & -0.18607100 \\
\hline $\mathrm{H}$ & -7.04623400 & -3.09815500 & -1.92694600 & $\mathrm{H}$ & -4.71747900 & -4.22309400 & 1.51043400 \\
\hline $\mathrm{H}$ & -6.62552900 & -4.64480100 & -0.02582600 & $\mathrm{~N}$ & -2.07612200 & -0.52477400 & -0.86825100 \\
\hline $\mathrm{H}$ & -1.59679100 & -1.06911800 & -0.14425900 & $\mathrm{C}$ & -3.89827300 & 0.84497400 & 0.62208000 \\
\hline $\mathrm{H}$ & -4.33996700 & 0.11189000 & 1.28959700 & $\mathrm{H}$ & -2.88939600 & 1.16108600 & 0.86663900 \\
\hline $\mathrm{C}$ & -4.75977300 & 1.78031400 & 0.04787300 & $\mathrm{C}$ & -4.27541300 & 3.02978100 & -0.53711700 \\
\hline $\mathrm{H}$ & -4.86827200 & 3.85452000 & -0.11225300 & $\mathrm{H}$ & -3.21958100 & 3.20786400 & -0.32258000 \\
\hline C & -6.23437800 & 1.50201700 & 0.04182300 & $\mathrm{H}$ & -6.75495200 & 2.01661900 & -0.76998100 \\
\hline $\mathrm{H}$ & -6.43717900 & 0.42716600 & -0.01300500 & $\mathrm{H}$ & -6.66399600 & 1.86263800 & 0.98729100 \\
\hline $\mathrm{C}$ & -2.99272300 & 4.23775300 & -3.03044700 & $\mathrm{H}$ & -2.14121400 & 3.55433300 & -2.98313600 \\
\hline $\mathrm{H}$ & -3.10755200 & 4.59151300 & -4.06212400 & $\mathrm{H}$ & -2.76506000 & 5.09951500 & -2.39375900 \\
\hline $\mathrm{C}$ & -4.88448700 & 1.79042800 & -3.44269100 & $\mathrm{H}$ & -3.97538000 & 1.19324500 & -3.55078000 \\
\hline $\mathrm{H}$ & -5.67489700 & 1.17361700 & -2.99790200 & $\mathrm{H}$ & -5.22422700 & 2.07170900 & -4.44700800 \\
\hline C & -6.05398000 & 4.48035600 & -2.58291900 & $\mathrm{H}$ & -6.96590700 & 4.00021200 & -2.20675700 \\
\hline $\mathrm{H}$ & -5.91243500 & 5.41158000 & -2.02169500 & $\mathrm{H}$ & -6.23618900 & 4.75091800 & -3.63000200 \\
\hline $\mathrm{Si}$ & -4.53858100 & 3.35575100 & -2.44199700 & & & & \\
\hline
\end{tabular}

\section{TS-R-4f-40}

Imaginary frequency: $-394.78 \mathrm{~cm}^{-1}$

E[B3LYP/6-31G(d)-D3(BJ)]: -4789.073189 Hartree

$\mathrm{E}\left[\mathrm{PCM}\left(\mathrm{Et}_{2} \mathrm{O}\right)-\mathrm{B} 3 \mathrm{LYP} / 6-311 \mathrm{G}(\mathrm{d}, \mathrm{p})-\mathrm{D} 3(\mathrm{BJ})\right]$ : -4790.216449 Hartree

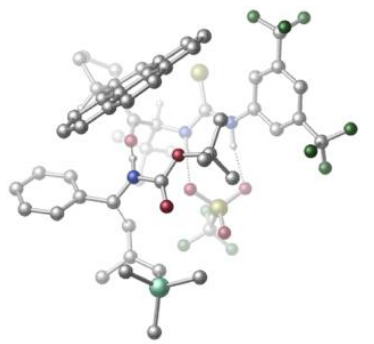

\begin{tabular}{|c|c|c|c|c|c|c|c|}
\hline 01 & & & & $\mathrm{~N}$ & 2.37974200 & 0.99902500 & 2.02347900 \\
\hline $\mathrm{H}$ & 1.73366400 & 1.79521200 & 2.00607700 & C & 3.52996200 & 1.21939100 & 1.25198200 \\
\hline $\mathrm{C}$ & 3.62252100 & 2.47834100 & 0.63219900 & $\mathrm{C}$ & 4.52664300 & 0.26569800 & 1.00019100 \\
\hline C & 4.65515200 & 2.74405400 & -0.25948500 & $\mathrm{H}$ & 2.86271400 & 3.22760700 & 0.82390300 \\
\hline $\mathrm{C}$ & 5.55358500 & 0.55965100 & 0.10702900 & $\mathrm{H}$ & 4.48444000 & -0.69520100 & 1.48616000 \\
\hline $\mathrm{C}$ & 5.63208200 & 1.78963000 & -0.54089000 & $\mathrm{H}$ & 6.42865000 & 2.00136500 & -1.24410500 \\
\hline C & 4.65771900 & 4.03432000 & -1.02865900 & C & 6.62790900 & -0.45938200 & -0.14954200 \\
\hline $\mathrm{F}$ & 3.97907600 & 5.01264000 & -0.40235800 & $\mathrm{~F}$ & 4.08233200 & 3.87027000 & -2.25182900 \\
\hline $\mathrm{F}$ & 5.91266300 & 4.48240700 & -1.25663600 & $F$ & 6.28091100 & -1.69885000 & 0.26975200 \\
\hline $\mathrm{F}$ & 6.91354900 & -0.55589400 & -1.47343300 & $F$ & 7.78532100 & -0.14256600 & 0.47092200 \\
\hline C & -0.13256500 & -1.12583000 & 3.58570200 & $\mathrm{H}$ & 0.59948200 & -1.66223700 & 4.18718700 \\
\hline $\mathrm{C}$ & -1.26317400 & -0.63845000 & 4.54115100 & C & -2.41697400 & 0.06924900 & 3.81128500 \\
\hline $\mathrm{H}$ & -2.97412600 & -0.62637900 & 3.17834100 & $\mathrm{H}$ & -2.06591100 & 0.88423600 & 3.17992400 \\
\hline $\mathrm{H}$ & -3.10926700 & 0.48919300 & 4.55039700 & C & -0.62944200 & 0.32103500 & 5.56283400 \\
\hline $\mathrm{H}$ & -1.37205800 & 0.61378100 & 6.31381800 & $\mathrm{H}$ & -0.25913000 & 1.22622400 & 5.07796100 \\
\hline $\mathrm{H}$ & 0.21131600 & -0.15437700 & 6.08138000 & C & -1.82698800 & -1.86456400 & 5.28146500 \\
\hline $\mathrm{H}$ & -2.64244200 & -1.55975700 & 5.94635800 & $\mathrm{H}$ & -1.06158700 & -2.34866600 & 5.89951400 \\
\hline $\mathrm{H}$ & -2.23125200 & -2.60956100 & 4.58480800 & $\mathrm{C}$ & -0.62986400 & -2.02380200 & 2.44728100 \\
\hline 0 & -1.27025700 & -1.51568200 & 1.50998700 & C & 0.28762500 & -4.13241100 & 3.51414600 \\
\hline C & -0.72434400 & -4.16234800 & 1.28522300 & C & -0.31338300 & -5.52024500 & 3.27372700 \\
\hline $\mathrm{H}$ & 1.37753100 & -4.12521400 & 3.39931300 & $\mathrm{H}$ & 0.04559200 & -3.73068600 & 4.49661300 \\
\hline $\mathrm{H}$ & -1.80105800 & -4.04608500 & 1.15532500 & $\mathrm{H}$ & -1.31813200 & -5.56662100 & 3.71083300 \\
\hline $\mathrm{H}$ & 0.28787300 & -6.31810000 & 3.71825300 & $\mathrm{~N}$ & -0.33601400 & -3.33552300 & 2.44948500 \\
\hline C & 0.00524100 & -3.75669500 & 0.01481800 & C & 1.33226900 & -3.44521400 & 0.08720100 \\
\hline C & -0.67015800 & -3.75566300 & -1.26567700 & C & 2.12178400 & -3.16845500 & -1.07346100 \\
\hline $\mathrm{H}$ & 1.82643600 & -3.39312700 & 1.05335600 & C & 0.08728200 & -3.43131700 & -2.43807700 \\
\hline C & -2.03692200 & -4.04230300 & -1.42005600 & C & 3.49752700 & -2.90085900 & -0.99212600 \\
\hline C & 1.48581400 & -3.17776500 & -2.34788300 & C & -0.55514100 & -3.37795300 & -3.71315200 \\
\hline $\mathrm{H}$ & -2.63672300 & -4.32237000 & -0.56503800 & C & -2.66033200 & -3.96124800 & -2.66338300 \\
\hline
\end{tabular}




\begin{tabular}{|c|c|c|c|c|c|c|c|}
\hline $\mathrm{C}$ & 4.24517600 & -2.67584500 & -2.14613800 & $\mathrm{H}$ & 3.97360100 & -2.86357300 & -0.01779100 \\
\hline C & 2.25480800 & -2.92429500 & -3.52369600 & C & -1.93323300 & -3.62577800 & -3.80002700 \\
\hline $\mathrm{C}$ & 0.23616900 & -3.08656200 & -4.87649800 & $\mathrm{H}$ & -3.72500800 & -4.16269800 & -2.73600400 \\
\hline $\mathrm{C}$ & 3.63368800 & -2.69331400 & -3.39871000 & $\mathrm{H}$ & 5.30459300 & -2.46080000 & -2.06497300 \\
\hline C & 1.57950400 & -2.88473300 & -4.78952700 & $\mathrm{H}$ & -2.42131100 & -3.56967400 & -4.76961300 \\
\hline $\mathrm{H}$ & -0.26578700 & -3.04490800 & -5.83973400 & $\mathrm{H}$ & 4.21955000 & -2.50140000 & -4.29383900 \\
\hline $\mathrm{H}$ & 2.16560200 & -2.67845300 & -5.68134400 & C & 1.91961500 & -0.12195100 & 2.66356500 \\
\hline $\mathrm{S}$ & 2.86391500 & -1.45922200 & 3.10285800 & $\mathrm{~N}$ & 0.59279600 & -0.02895500 & 2.96312800 \\
\hline $\mathrm{H}$ & 0.03151900 & 0.60320900 & 2.38149200 & $S$ & -0.58145700 & 3.23391300 & 1.33979200 \\
\hline 0 & -1.07556700 & 4.06108600 & 0.22832000 & 0 & -0.88843200 & 1.77353700 & 1.22483400 \\
\hline $\mathrm{O}$ & 0.81003700 & 3.47440300 & 1.79794300 & C & -1.62760700 & 3.78160900 & 2.77579600 \\
\hline $\mathrm{F}$ & -1.55416400 & 5.10288100 & 2.94523100 & $\mathrm{~F}$ & -1.23804900 & 3.18196000 & 3.91327500 \\
\hline $\mathrm{F}$ & -2.91590500 & 3.44909300 & 2.55270700 & C & -0.38381900 & -5.60330200 & 1.74247400 \\
\hline $\mathrm{H}$ & 0.59151300 & -5.88677800 & 1.33601300 & $\mathrm{H}$ & -1.11838800 & -6.32570500 & 1.37661900 \\
\hline C & -1.31259600 & 0.44283300 & -1.69855600 & 0 & -1.83684000 & 1.30762200 & -2.38208300 \\
\hline $\mathrm{O}$ & -0.02872600 & 0.16754500 & -1.57500200 & C & 0.98736500 & 0.99500400 & -2.29859500 \\
\hline C & 0.93789200 & 2.42567000 & -1.77910400 & $\mathrm{H}$ & 1.71777300 & 3.01049900 & -2.27494800 \\
\hline $\mathrm{H}$ & -0.02639900 & 2.90125600 & -1.94938900 & $\mathrm{H}$ & 1.14530900 & 2.43914700 & -0.70913200 \\
\hline C & 2.29526500 & 0.32554000 & -1.90606900 & $\mathrm{H}$ & 2.39563000 & -0.64662900 & -2.38164800 \\
\hline $\mathrm{H}$ & 3.13587000 & 0.95462800 & -2.21256600 & $\mathrm{H}$ & 2.34146500 & 0.19115300 & -0.82563800 \\
\hline C & 0.74878400 & 0.88660000 & -3.80347200 & $\mathrm{H}$ & 1.60372900 & 1.33097700 & -4.32452900 \\
\hline $\mathrm{H}$ & 0.67815300 & -0.16415900 & -4.10224700 & $\mathrm{H}$ & -0.15793700 & 1.41026600 & -4.10861100 \\
\hline C & -3.43927000 & -0.29983800 & -0.94204300 & $\mathrm{H}$ & -3.75101500 & 0.27880200 & -1.79958100 \\
\hline C & -4.33590600 & -1.43666900 & -0.62225900 & C & -5.42947100 & -1.65877900 & -1.46970300 \\
\hline C & -4.13688000 & -2.27971800 & 0.48050700 & C & -6.29957300 & -2.72480000 & -1.23622300 \\
\hline $\mathrm{H}$ & -5.58352900 & -1.00863000 & -2.32646100 & C & -5.01213600 & -3.33499300 & 0.71534400 \\
\hline $\mathrm{H}$ & -3.28864900 & -2.10698700 & 1.13402500 & C & -6.09207800 & -3.56544600 & -0.14318000 \\
\hline $\mathrm{H}$ & -7.13652700 & -2.89494500 & -1.90685800 & $\mathrm{H}$ & -4.85195900 & -3.98406500 & 1.57185600 \\
\hline $\mathrm{H}$ & -6.77064100 & -4.39234100 & 0.04446700 & $\mathrm{~N}$ & -2.08098700 & -0.45578800 & -0.94217000 \\
\hline $\mathrm{H}$ & -1.61145700 & -0.99868400 & -0.21013300 & C & -3.83960000 & 0.99039800 & 0.54729500 \\
\hline $\bar{H}$ & -4.30698000 & 0.28415500 & 1.22577000 & $\mathrm{H}$ & -2.81885900 & 1.26900800 & 0.78874300 \\
\hline $\mathrm{C}$ & -4.66846000 & 1.95886500 & -0.02122800 & $C$ & -4.14166700 & 3.18753000 & -0.61249200 \\
\hline $\mathrm{H}$ & -4.69823300 & 4.03486500 & -0.18286800 & $\mathrm{H}$ & -3.07776800 & 3.32533700 & -0.40626500 \\
\hline $\mathrm{C}$ & -6.15244700 & 1.73563800 & -0.01283400 & $\mathrm{H}$ & -6.67009600 & 2.31352100 & -0.78234600 \\
\hline $\bar{H}$ & -6.39272100 & 0.67248500 & -0.12585500 & $\mathrm{H}$ & -6.55097400 & 2.05009800 & 0.96206100 \\
\hline C & -2.85505400 & 4.32467300 & -3.14158000 & $\mathrm{H}$ & -2.03771500 & 3.59923700 & -3.13053800 \\
\hline $\mathrm{H}$ & -2.98403600 & 4.69668100 & -4.16513300 & $\mathrm{H}$ & -2.56616500 & 5.16703000 & -2.50355800 \\
\hline $\mathrm{C}$ & -4.85272900 & 1.95459200 & -3.49430400 & $\mathrm{H}$ & -3.96953000 & 1.32151700 & -3.61229800 \\
\hline $\mathrm{H}$ & -5.65736400 & 1.37296800 & -3.02828800 & $\mathrm{H}$ & -5.20020100 & 2.23941500 & -4.49493200 \\
\hline $\mathrm{C}$ & -5.89134900 & 4.70187000 & -2.63781300 & $\mathrm{H}$ & -6.81702600 & 4.26545600 & -2.24257500 \\
\hline $\mathrm{H}$ & -5.69876800 & 5.62964700 & -2.08614000 & $\mathrm{H}$ & -6.07960000 & 4.97313000 & -3.68364800 \\
\hline $\mathrm{Si}$ & -4.42474400 & 3.51248600 & -2.51507800 & & & & \\
\hline
\end{tabular}

\section{TS-R-4f-41}

Imaginary frequency: $-407.23 \mathrm{~cm}^{-1}$

E[B3LYP/6-31G(d)-D3(BJ)]: -4789.067464 Hartree

$\mathrm{E}\left[\mathrm{PCM}\left(\mathrm{Et}_{2} \mathrm{O}\right)-\mathrm{B} 3 \mathrm{LYP} / 6-311 \mathrm{G}(\mathrm{d}, \mathrm{p})-\mathrm{D} 3(\mathrm{BJ})\right]$ : -4790.212045 Hartree

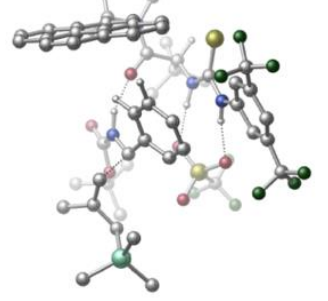

\begin{tabular}{|llll|llll|}
\hline 01 & & & & N & -0.25243900 & 1.78643300 & -2.12010100 \\
\hline $\mathrm{H}$ & 0.76016200 & 1.64261500 & -2.07938100 & $\mathrm{C}$ & -0.63744500 & 3.05009900 & -1.66014600 \\
\hline $\mathrm{C}$ & 0.39137800 & 3.98696300 & -1.47697300 & $\mathrm{C}$ & -1.94246900 & 3.39461900 & -1.28197800 \\
\hline
\end{tabular}




\begin{tabular}{|c|c|c|c|c|c|c|c|}
\hline $\mathrm{C}$ & 0.11854600 & 5.22689200 & -0.90790300 & $\mathrm{H}$ & 1.40929000 & 3.72084200 & -1.73460900 \\
\hline C & -2.19345100 & 4.65008200 & -0.73646600 & $\mathrm{H}$ & -2.74103600 & 2.68015300 & -1.39681500 \\
\hline C & -1.17595500 & 5.58167100 & -0.53591400 & $\mathrm{H}$ & -1.38438800 & 6.55161900 & -0.10202500 \\
\hline C & 1.26494400 & 6.13430000 & -0.56940800 & C & -3.57210500 & 4.96944700 & -0.23771600 \\
\hline $\mathrm{F}$ & 2.26975600 & 6.05305100 & -1.46327000 & $\mathrm{~F}$ & 1.79280100 & 5.80791400 & 0.64340400 \\
\hline $\mathrm{F}$ & 0.88827000 & 7.42901700 & -0.49040100 & $\mathrm{~F}$ & -4.53055000 & 4.24000400 & -0.85110300 \\
\hline $\mathrm{F}$ & -3.68549500 & 4.71146300 & 1.09727600 & $\mathrm{~F}$ & -3.88591200 & 6.27209200 & -0.39916300 \\
\hline C & -0.86944200 & -1.62624800 & -3.23684200 & $\mathrm{H}$ & -1.61634100 & -1.31974000 & -3.96841700 \\
\hline C & 0.17426300 & -2.52658300 & -3.97290900 & C & 1.15243700 & -3.20986700 & -3.01052700 \\
\hline $\mathrm{H}$ & 0.64861000 & -3.88767200 & -2.31861100 & $\mathrm{H}$ & 1.71041300 & -2.48325400 & -2.42048000 \\
\hline $\mathrm{H}$ & 1.87891500 & -3.79221700 & -3.58966800 & C & 0.95731800 & -1.64823300 & -4.96613300 \\
\hline $\mathrm{H}$ & 1.57477500 & -2.28399400 & -5.61127500 & $\mathrm{H}$ & 1.62324200 & -0.95130000 & -4.45107600 \\
\hline $\mathrm{H}$ & 0.28223800 & -1.06782300 & -5.60578400 & C & -0.57862400 & -3.61532800 & -4.75781700 \\
\hline $\mathrm{H}$ & 0.14045600 & -4.26599900 & -5.26696200 & $\mathrm{H}$ & -1.23298000 & -3.18377400 & -5.52472900 \\
\hline $\mathrm{H}$ & -1.18117300 & -4.24801000 & -4.09649800 & C & -1.56793000 & -2.32349600 & -2.05520400 \\
\hline $\mathrm{O}$ & -1.02191600 & -2.38253500 & -0.94611600 & C & -3.64049900 & -2.88494200 & -3.43122500 \\
\hline C & -3.43741000 & -3.55418700 & -1.10612700 & C & -4.38281700 & -4.21466700 & -3.27604200 \\
\hline $\mathrm{H}$ & -4.32704400 & -2.02895200 & -3.41305800 & $\mathrm{H}$ & -3.05279900 & -2.82888900 & -4.34361100 \\
\hline $\mathrm{H}$ & -2.70169100 & -4.24740700 & -0.69185800 & $\mathrm{H}$ & -3.73333400 & -5.03274500 & -3.60866900 \\
\hline $\mathrm{H}$ & -5.30507300 & -4.25214700 & -3.86265600 & $\mathrm{~N}$ & -2.78949900 & -2.87155600 & -2.23911700 \\
\hline C & -3.88863600 & -2.58985100 & -0.02304200 & C & -4.16092800 & -1.29021300 & -0.33233800 \\
\hline $\mathrm{C}$ & -4.08264700 & -3.07086800 & 1.32804600 & C & -4.63888900 & -0.35499400 & 0.63969600 \\
\hline $\mathrm{H}$ & -3.97554100 & -0.91668700 & -1.33413500 & C & -4.52932300 & -2.14805700 & 2.32750000 \\
\hline C & -3.84271700 & -4.40203800 & 1.71074500 & C & -4.90565400 & 0.98485800 & 0.31363600 \\
\hline C & -4.80828400 & -0.79313700 & 1.98491900 & C & -4.70011800 & -2.58180800 & 3.67794000 \\
\hline $\mathrm{H}$ & -3.51561800 & -5.12742900 & 0.97459800 & C & -4.01824900 & -4.81669700 & 3.02973700 \\
\hline C & -5.32527100 & 1.88176800 & 1.29416200 & $\mathrm{H}$ & -4.75880700 & 1.31800600 & -0.70939100 \\
\hline $\mathrm{C}$ & -5.23758600 & 0.13243400 & 2.98327200 & C & -4.43825300 & -3.91992500 & 4.00675600 \\
\hline C & -5.13749400 & -1.62989100 & 4.66139700 & $\mathrm{H}$ & -3.82342800 & -5.85216900 & 3.29444200 \\
\hline C & -5.48607000 & 1.46344700 & 2.61420000 & $\mathrm{H}$ & -5.51160300 & 2.91527600 & 1.02976300 \\
\hline $\mathrm{C}$ & -5.39146100 & -0.33444900 & 4.33205400 & $\mathrm{H}$ & -4.57127700 & -4.24911300 & 5.03409500 \\
\hline $\mathrm{H}$ & -5.26190700 & -1.97256800 & 5.68554000 & $\mathrm{H}$ & -5.80685400 & 2.17098700 & 3.37444700 \\
\hline $\mathrm{H}$ & -5.71992700 & 0.37348700 & 5.08871400 & C & -0.99814700 & 0.74560900 & -2.60943400 \\
\hline S & -2.62388800 & 0.85365900 & -3.08255800 & $\mathrm{~N}$ & -0.27977300 & -0.39546300 & -2.73941400 \\
\hline $\mathrm{H}$ & 0.62745600 & -0.46173200 & -2.27354000 & $\mathrm{~S}$ & 3.32312300 & 0.62933800 & -1.43986800 \\
\hline 0 & 4.08352100 & 0.68447800 & -0.16897700 & $\mathrm{O}$ & 2.44133600 & -0.56197500 & -1.58008300 \\
\hline $\mathrm{O}$ & 2.66551700 & 1.89344800 & -1.84726100 & C & 4.62337600 & 0.36173400 & -2.73701400 \\
\hline $\mathrm{F}$ & 5.46341300 & 1.39824600 & -2.78442400 & $\mathrm{~F}$ & 4.04966000 & 0.20980500 & -3.93779900 \\
\hline $\mathrm{F}$ & 5.33223600 & -0.74953400 & -2.46260200 & C & 2.07151000 & -3.24648700 & 0.39882800 \\
\hline $\mathrm{O}$ & 1.69003300 & -4.31205100 & -0.04121900 & $\mathrm{O}$ & 3.34748700 & -2.88063700 & 0.62743000 \\
\hline C & 4.45835500 & -3.66739400 & 0.03427200 & C & 4.54185300 & -5.03520500 & 0.71116700 \\
\hline $\mathrm{H}$ & 5.41518400 & -5.57393100 & 0.32788500 & $\mathrm{H}$ & 3.64677100 & -5.62388800 & 0.50718800 \\
\hline $\mathrm{H}$ & 4.66041000 & -4.93071600 & 1.79439600 & C & 5.68487600 & -2.81113400 & 0.34040000 \\
\hline $\mathrm{H}$ & 5.54722900 & -1.79426200 & -0.03556900 & $\mathrm{H}$ & 6.56467300 & -3.24951800 & -0.14064100 \\
\hline $\mathrm{H}$ & 5.87759800 & -2.76972500 & 1.41671200 & C & 4.26194200 & -3.78688600 & -1.47622000 \\
\hline $\mathrm{H}$ & 5.16320400 & -4.22945600 & -1.91394000 & $\mathrm{H}$ & 4.11211900 & -2.80043000 & -1.91759600 \\
\hline $\mathrm{H}$ & 3.40963200 & -4.42135100 & -1.71592500 & C & 1.57090500 & -0.99491500 & 1.21714000 \\
\hline $\mathrm{H}$ & 2.55204200 & -0.69566400 & 0.87374800 & C & 0.56760000 & 0.09853700 & 1.22697700 \\
\hline C & 1.06042500 & 1.41729400 & 1.20930400 & C & -0.79996900 & -0.12193100 & 1.43611100 \\
\hline C & 0.20969200 & 2.49321400 & 1.43215600 & $\mathrm{H}$ & 2.11573400 & 1.58880600 & 1.02342900 \\
\hline C & -1.64874900 & 0.96315000 & 1.65338300 & $\mathrm{H}$ & -1.21055200 & -1.12633800 & 1.44421900 \\
\hline C & -1.14909100 & 2.26510000 & 1.66702700 & $\mathrm{H}$ & 0.60272700 & 3.50388200 & 1.40576900 \\
\hline $\mathrm{H}$ & -2.70053100 & 0.79101700 & 1.82988700 & $\mathrm{H}$ & -1.82402900 & 3.09737600 & 1.83453300 \\
\hline
\end{tabular}




\begin{tabular}{|c|c|c|c|c|c|c|c|}
\hline $\mathrm{N}$ & 1.18578700 & -2.24237100 & 0.77004700 & $\mathrm{H}$ & 0.24579300 & -2.37755500 & 0.38679000 \\
\hline C & 1.96473400 & -1.07620800 & 3.09138300 & $\mathrm{H}$ & 1.18008300 & -1.71861600 & 3.48468600 \\
\hline $\mathrm{H}$ & 1.80440200 & -0.02194800 & 3.27146900 & C & 3.29569500 & -1.51185400 & 3.21852100 \\
\hline $\mathrm{C}$ & 4.39695500 & -0.62096200 & 2.88087500 & $\mathrm{H}$ & 4.18416000 & -0.03676200 & 1.97457900 \\
\hline $\mathrm{H}$ & 5.33294600 & -1.16230400 & 2.72737100 & C & 3.57822900 & -2.88717800 & 3.72631900 \\
\hline $\mathrm{H}$ & 4.52967300 & -3.28025000 & 3.36513200 & $\mathrm{H}$ & 2.77036600 & -3.57888400 & 3.47083500 \\
\hline $\mathrm{H}$ & 3.62945600 & -2.84289600 & 4.82501100 & C & 6.22672800 & 1.69964700 & 3.45654400 \\
\hline $\mathrm{H}$ & 7.10648500 & 1.05804000 & 3.33280200 & $\mathrm{H}$ & 6.51372600 & 2.54265500 & 4.09575300 \\
\hline $\mathrm{H}$ & 5.96086400 & 2.09724500 & 2.47045900 & C & 5.23896000 & -0.08765000 & 5.82059400 \\
\hline $\mathrm{H}$ & 6.07842200 & -0.78081700 & 5.69241000 & $\mathrm{H}$ & 4.39419300 & -0.65199500 & 6.23346000 \\
\hline $\mathrm{H}$ & 5.53466500 & 0.65355400 & 6.57234700 & C & 3.28396100 & 1.87079100 & 4.42304400 \\
\hline $\mathrm{H}$ & 2.47848400 & 1.38329300 & 4.98274100 & $\mathrm{H}$ & 2.87288200 & 2.20911500 & 3.46536700 \\
\hline $\mathrm{H}$ & 3.57667200 & 2.76452600 & 4.98741600 & $\mathrm{Si}$ & 4.78084000 & 0.75264200 & 4.19657500 \\
\hline C & -4.63353800 & -4.30406800 & -1.76067300 & $\mathrm{H}$ & -5.56454700 & -3.79223400 & -1.50017700 \\
\hline $\mathrm{H}$ & -4.71191400 & -5.33374100 & -1.40180400 & & & & \\
\hline
\end{tabular}

\section{TS- $R-4 f-42$}

Imaginary frequency: $-322.06 \mathrm{~cm}^{-1}$ E[B3LYP/6-31G(d)-D3(BJ)]: -4789.065415 Hartree $\mathrm{E}\left[\mathrm{PCM}\left(\mathrm{Et}_{2} \mathrm{O}\right)-\mathrm{B} 3 \mathrm{LYP} / 6-311 \mathrm{G}(\mathrm{d}, \mathrm{p})-\mathrm{D} 3(\mathrm{BJ})\right]$ : -4790.203500 Hartree

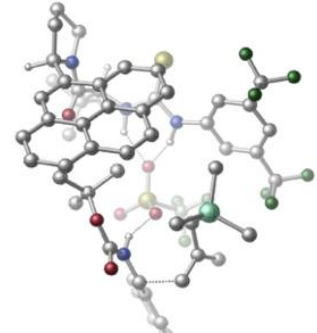

\begin{tabular}{|c|c|c|c|c|c|c|c|}
\hline 01 & & & & $\mathrm{~N}$ & -0.37535300 & 1.22449900 & -2.22162000 \\
\hline $\mathrm{H}$ & 0.36519300 & 0.52170800 & -2.29180800 & C & 0.04801700 & 2.46719500 & -1.76593900 \\
\hline $\mathrm{C}$ & 1.43451000 & 2.67246800 & -1.67771600 & $\mathrm{C}$ & -0.81437000 & 3.47791800 & -1.32009600 \\
\hline C & 1.93548300 & 3.87145900 & -1.17863300 & $\mathrm{H}$ & 2.11254700 & 1.89610300 & -2.00927300 \\
\hline $\mathrm{C}$ & -0.29153500 & 4.66400100 & -0.81808700 & $\mathrm{H}$ & -1.87857400 & 3.32457000 & -1.36730700 \\
\hline $\mathrm{C}$ & 1.08314700 & 4.89020300 & -0.75347200 & $\mathrm{H}$ & 1.47723600 & 5.82195900 & -0.36651500 \\
\hline C & 3.41353600 & 4.03015400 & -0.98794500 & C & -1.22869800 & 5.66707000 & -0.20723800 \\
\hline $\mathrm{F}$ & 4.13669100 & 3.22666600 & -1.79231900 & $\mathrm{~F}$ & 3.77302700 & 3.70168400 & 0.29646900 \\
\hline $\mathrm{F}$ & 3.82782300 & 5.29600800 & -1.17998400 & $\mathrm{~F}$ & -2.48141600 & 5.57033700 & -0.69613900 \\
\hline $\mathrm{F}$ & -1.32060800 & 5.48256600 & 1.14000800 & $\mathrm{~F}$ & -0.80823400 & 6.93563300 & -0.39224600 \\
\hline C & -2.79869300 & -1.32801600 & -3.19076500 & $\mathrm{H}$ & -3.39730500 & -0.65211600 & -3.79984600 \\
\hline C & -2.35924000 & -2.52355000 & -4.08849000 & C & -1.42863300 & -3.51635100 & -3.36691900 \\
\hline $\mathrm{H}$ & -1.90854100 & -3.94606300 & -2.48581600 & $\mathrm{H}$ & -0.48528200 & -3.05360000 & -3.05925200 \\
\hline $\mathrm{H}$ & -1.16830400 & -4.32793100 & -4.05622100 & C & -1.62817200 & -1.94826900 & -5.31455600 \\
\hline $\mathrm{H}$ & -1.37425500 & -2.75445200 & -6.01200800 & $\mathrm{H}$ & -0.69825100 & -1.45192500 & -5.02195400 \\
\hline $\mathrm{H}$ & -2.25245900 & -1.22060700 & -5.84637200 & C & -3.62817900 & -3.26373300 & -4.54514600 \\
\hline $\mathrm{H}$ & -3.36039300 & -4.10705100 & -5.19062500 & $\mathrm{H}$ & -4.29057800 & -2.60558200 & -5.12038600 \\
\hline $\mathrm{H}$ & -4.19170100 & -3.66385300 & -3.69425900 & C & -3.58457100 & -1.75477400 & -1.93315900 \\
\hline $\mathrm{O}$ & -3.02766200 & -2.40494700 & -1.04332400 & $\mathrm{C}$ & -5.68664000 & -0.70273600 & -2.86828800 \\
\hline C & -5.65672300 & -1.63840400 & -0.59768200 & C & -7.12463400 & -0.90158600 & -2.37558000 \\
\hline $\mathrm{H}$ & -5.41992800 & 0.35670000 & -2.95795000 & $\mathrm{H}$ & -5.51885100 & -1.17517700 & -3.83748800 \\
\hline $\mathrm{H}$ & -5.85013600 & -2.71834300 & -0.57725700 & $\mathrm{H}$ & -7.50302400 & -1.87407700 & -2.71183000 \\
\hline $\mathrm{H}$ & -7.79884000 & -0.12825700 & -2.75364400 & $\mathrm{~N}$ & -4.87266500 & -1.34838200 & -1.82127700 \\
\hline C & -5.00184500 & -1.28956400 & 0.72573800 & $\mathrm{C}$ & -5.09186200 & -2.21272900 & 1.72561600 \\
\hline $\mathrm{C}$ & -4.36880000 & -0.01029000 & 0.98835700 & C & -4.53796800 & -2.00896600 & 3.02869900 \\
\hline $\mathrm{H}$ & -5.56831000 & -3.16998500 & 1.52602900 & C & -3.76929900 & 0.20551900 & 2.27242400 \\
\hline $\mathrm{C}$ & -4.31874400 & 1.03640400 & 0.05416700 & $\mathrm{C}$ & -4.60221600 & -2.98869100 & 4.03017700 \\
\hline $\mathrm{C}$ & -3.83950800 & -0.79457000 & 3.28508200 & $\mathrm{C}$ & -3.09708800 & 1.43594600 & 2.55254200 \\
\hline $\mathrm{H}$ & -4.73843500 & 0.91120300 & -0.93213400 & $\mathrm{C}$ & -3.71233300 & 2.25321400 & 0.36048500 \\
\hline $\mathrm{C}$ & -3.97437000 & -2.79121100 & 5.25826100 & $\mathrm{H}$ & -5.13231900 & -3.91596300 & 3.82934000 \\
\hline
\end{tabular}




\begin{tabular}{|c|c|c|c|c|c|c|c|}
\hline C & -3.19987800 & -0.59964200 & 4.54618500 & C & -3.08913300 & 2.45296800 & 1.58572100 \\
\hline $\mathrm{C}$ & -2.45689700 & 1.60300900 & 3.82749600 & $\mathrm{H}$ & -3.72650900 & 3.04779400 & -0.37675900 \\
\hline C & -3.27588700 & -1.61358000 & 5.51396700 & $\mathrm{H}$ & -4.02281000 & -3.56473500 & 6.01921900 \\
\hline C & -2.49592000 & 0.62932700 & 4.77717900 & $\mathrm{H}$ & -2.59382600 & 3.39500800 & 1.80020600 \\
\hline $\mathrm{H}$ & -1.93602200 & 2.53718300 & 4.01740100 & $\mathrm{H}$ & -2.77977700 & -1.46874500 & 6.46988200 \\
\hline $\mathrm{H}$ & -2.00083500 & 0.76972900 & 5.73435700 & C & -1.60442100 & 0.79639900 & -2.67665200 \\
\hline $\mathrm{S}$ & -2.86745100 & 1.80576100 & -3.16070100 & $\mathrm{~N}$ & -1.65648200 & -0.55316600 & -2.74373200 \\
\hline $\mathrm{H}$ & -0.83237200 & -1.07192700 & -2.45728600 & $S$ & 2.40425500 & -1.60577200 & -2.15550500 \\
\hline 0 & 2.56544100 & -1.17080900 & -0.73631500 & $\mathrm{O}$ & 2.59828900 & -3.04214800 & -2.39713100 \\
\hline 0 & 1.21548600 & -1.00316200 & -2.82058300 & C & 3.82838600 & -0.76122600 & -3.00515700 \\
\hline $\mathrm{F}$ & 3.73291700 & 0.57432600 & -2.86356300 & $\mathrm{~F}$ & 3.84468500 & -1.05509900 & -4.30463600 \\
\hline $\mathrm{F}$ & 4.99216400 & -1.14555700 & -2.45715000 & C & 1.79252800 & -2.57489200 & 2.87850600 \\
\hline 0 & 2.19729200 & -2.61826800 & 4.02732000 & $\mathrm{O}$ & 0.50083200 & -2.53394700 & 2.61988200 \\
\hline C & -0.31037100 & -2.65118100 & 1.36083300 & C & -0.48244600 & -1.24998700 & 0.78124900 \\
\hline $\mathrm{H}$ & -1.24462900 & -1.29472400 & -0.00112600 & $\mathrm{H}$ & 0.44317700 & -0.86981000 & 0.34313300 \\
\hline $\mathrm{H}$ & -0.83662200 & -0.56170800 & 1.55529800 & C & -1.62705700 & -3.17750300 & 1.91859600 \\
\hline $\mathrm{H}$ & -1.50732900 & -4.20265000 & 2.28397600 & $\mathrm{H}$ & -2.38915800 & -3.15294300 & 1.13696500 \\
\hline $\mathrm{H}$ & -1.95130100 & -2.55344300 & 2.75057600 & C & 0.26958300 & -3.65086200 & 0.36529300 \\
\hline $\mathrm{H}$ & -0.50980200 & -3.85520100 & -0.37485300 & $\mathrm{H}$ & 0.52600800 & -4.59231600 & 0.86160800 \\
\hline $\mathrm{H}$ & 1.13596500 & -3.28925500 & -0.18685100 & C & 4.04888400 & -2.46193300 & 2.13670000 \\
\hline $\mathrm{H}$ & 4.21795600 & -2.82840300 & 3.14417500 & C & 5.08648500 & -2.81097000 & 1.14572200 \\
\hline C & 6.44239400 & -2.73068300 & 1.51353800 & C & 4.75793300 & -3.31381600 & -0.11888800 \\
\hline C & 7.43978200 & -3.10703000 & 0.62327700 & $\mathrm{H}$ & 6.71475000 & -2.37460900 & 2.50286200 \\
\hline C & 5.76035100 & -3.69113700 & -1.01052000 & $\mathrm{H}$ & 3.72864700 & -3.43377400 & -0.42187100 \\
\hline C & 7.10064300 & -3.58183400 & -0.64855700 & $\mathrm{H}$ & 8.48196100 & -3.03549800 & 0.91978000 \\
\hline $\mathrm{H}$ & 5.47470500 & -4.06107900 & -1.98952300 & $\mathrm{H}$ & 7.88118600 & -3.87166700 & -1.34584100 \\
\hline $\mathrm{N}$ & 2.71864100 & -2.52940900 & 1.81057700 & $\mathrm{H}$ & 2.43853200 & -2.23603200 & 0.87124700 \\
\hline C & 4.42328200 & -0.59765600 & 2.86837900 & $\mathrm{H}$ & 5.47955800 & -0.68645900 & 3.09905800 \\
\hline $\mathrm{H}$ & 3.74780400 & -0.71977800 & 3.70966100 & C & 4.04738500 & 0.23819600 & 1.81678600 \\
\hline C & 2.65768100 & 0.64406800 & 1.64154500 & $\mathrm{H}$ & 1.98518700 & -0.20578400 & 1.80460800 \\
\hline $\mathrm{H}$ & 2.46691600 & 1.05385600 & 0.64893400 & C & 5.04107900 & 0.63499800 & 0.77951900 \\
\hline $\mathrm{H}$ & 4.89817400 & 1.67554400 & 0.47699400 & $\mathrm{H}$ & 4.84711700 & 0.02078100 & -0.11219600 \\
\hline $\mathrm{H}$ & 6.07322100 & 0.47186100 & 1.09596800 & C & 0.59464400 & 2.82232700 & 2.17005900 \\
\hline $\mathrm{H}$ & 0.97396100 & 3.44057000 & 1.35432000 & $\mathrm{H}$ & 0.09928900 & 3.48922000 & 2.88507000 \\
\hline $\mathrm{H}$ & -0.16689600 & 2.15039500 & 1.76121700 & C & 3.36582000 & 3.02294400 & 3.56655700 \\
\hline $\mathrm{H}$ & 3.75685000 & 3.61495800 & 2.73327000 & $\mathrm{H}$ & 4.19766400 & 2.46281700 & 4.00883200 \\
\hline $\mathrm{H}$ & 2.99418700 & 3.71961100 & 4.32731800 & C & 1.32325700 & 0.83442600 & 4.42000300 \\
\hline $\bar{H}$ & 2.08570800 & 0.21348300 & 4.90103300 & $\mathrm{H}$ & 0.52377700 & 0.16722000 & 4.07941700 \\
\hline $\mathrm{H}$ & 0.89760800 & 1.49003900 & 5.18977000 & $\mathrm{Si}$ & 1.98600200 & 1.87477900 & 2.99630200 \\
\hline C & -6.98171100 & -0.88895500 & -0.85040900 & $\mathrm{H}$ & -6.90471200 & 0.13692300 & -0.47767800 \\
\hline $\mathrm{H}$ & -7.81135700 & -1.37138500 & -0.32748700 & & & & \\
\hline
\end{tabular}




\title{
Coordinates of computed transition states - No Dispersion
}

\author{
TS-S-4f-nd-1
}

Imaginary frequency: $-206.03 \mathrm{~cm}^{-1}$

E[B3LYP/6-31G(d)-D3(BJ)]: -4788.700247 Hartree

$\mathrm{E}\left[\mathrm{PCM}\left(\mathrm{Et}_{2} \mathrm{O}\right)-\mathrm{B} 3 \mathrm{LYP} / 6-311 \mathrm{G}(\mathrm{d}, \mathrm{p})-\mathrm{D} 3(\mathrm{BJ})\right]:-4790.231770$ Hartree

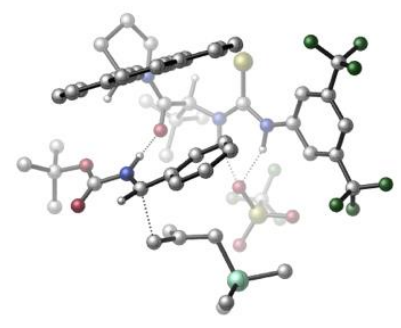

\begin{tabular}{|c|c|c|c|c|c|c|c|}
\hline 01 & & & & $\mathrm{~N}$ & 2.26763400 & 0.33427600 & -1.91060400 \\
\hline $\mathrm{H}$ & 2.50596000 & -0.54614700 & -1.43950300 & $\mathrm{C}$ & 3.01111500 & 1.42552400 & -1.43371600 \\
\hline C & 4.00470300 & 1.11428600 & -0.48253500 & C & 2.80808400 & 2.76714900 & -1.78757000 \\
\hline C & 4.73988600 & 2.12936900 & 0.11953900 & $\mathrm{H}$ & 4.19876400 & 0.07708000 & -0.21897800 \\
\hline$C$ & 3.55812600 & 3.76836200 & -1.16538700 & $\mathrm{H}$ & 2.06803100 & 3.01957800 & -2.53238000 \\
\hline C & 4.52388500 & 3.47053200 & -0.20849400 & $\mathrm{H}$ & 5.09655800 & 4.25629300 & 0.26844900 \\
\hline C & 5.72087500 & 1.78651600 & 1.21060600 & C & 3.33154100 & 5.19719000 & -1.58393900 \\
\hline $\mathrm{F}$ & 6.24201100 & 0.55651700 & 1.06711400 & $\mathrm{~F}$ & 5.12227200 & 1.82445300 & 2.43294500 \\
\hline $\mathrm{F}$ & 6.74478200 & 2.66828300 & 1.25918000 & $\mathrm{~F}$ & 2.02102500 & 5.44248400 & -1.84690200 \\
\hline $\mathrm{F}$ & 3.71430600 & 6.07208100 & -0.62725900 & $\mathrm{~F}$ & 4.01377200 & 5.51236900 & -2.70556800 \\
\hline C & -0.66713600 & -1.35293300 & -3.26813600 & $\mathrm{H}$ & -0.75515300 & -0.78226300 & -4.19284200 \\
\hline C & -0.72779800 & -2.87756100 & -3.64339200 & C & -0.61723800 & -3.80576700 & -2.41668500 \\
\hline $\mathrm{H}$ & -1.43422700 & -3.63944200 & -1.70839600 & $\mathrm{H}$ & 0.32778400 & -3.68110800 & -1.88096400 \\
\hline $\mathrm{H}$ & -0.65804500 & -4.84924600 & -2.75129400 & C & 0.42909700 & -3.17541400 & -4.62022900 \\
\hline $\mathrm{H}$ & 0.36246900 & -4.21293700 & -4.96815100 & $\mathrm{H}$ & 1.40633200 & -3.04341200 & -4.15017200 \\
\hline $\mathrm{H}$ & 0.38468200 & -2.52166300 & -5.49964700 & C & -2.06097600 & -3.16733600 & -4.36174600 \\
\hline $\mathrm{H}$ & -2.11302300 & -4.22818700 & -4.63103500 & $\mathrm{H}$ & -2.15325400 & -2.59089700 & -5.28983800 \\
\hline $\mathrm{H}$ & -2.92902400 & -2.94677700 & -3.72884200 & C & -1.77071500 & -0.91196000 & -2.27577100 \\
\hline C & -3.92893600 & 0.18473400 & -1.82333600 & $\mathrm{H}$ & -4.13426100 & -0.65892200 & -1.15964000 \\
\hline $\mathrm{N}$ & -2.83557100 & -0.21945800 & -2.73383900 & C & -3.60961500 & 1.42427300 & -0.98914400 \\
\hline C & -2.56595000 & 2.24494600 & -1.30666700 & C & -4.47032000 & 1.75580300 & 0.13533800 \\
\hline C & -2.27453300 & 3.44429400 & -0.57435000 & $\mathrm{H}$ & -1.90308800 & 1.99160300 & -2.12921800 \\
\hline C & -4.20109300 & 2.95000600 & 0.88138900 & C & -5.55967000 & 0.95831000 & 0.53246800 \\
\hline C & -1.18826100 & 4.27049000 & -0.90671300 & C & -3.10714000 & 3.79449800 & 0.52666900 \\
\hline C & -5.02911100 & 3.30446800 & 1.99230000 & $\mathrm{H}$ & -5.79634000 & 0.05052500 & -0.01226500 \\
\hline C & -6.35932700 & 1.31106500 & 1.62007300 & C & -0.92423500 & 5.42559800 & -0.17128000 \\
\hline $\mathrm{H}$ & -0.54683400 & 3.99520000 & -1.73938800 & C & -2.83450200 & 4.98199300 & 1.27139300 \\
\hline C & -6.10226500 & 2.47040900 & 2.34376300 & C & -4.73670500 & 4.50992200 & 2.71945200 \\
\hline $\mathrm{H}$ & -7.19305300 & 0.67268200 & 1.90019800 & C & -1.73894700 & 5.78075600 & 0.90280000 \\
\hline $\mathrm{H}$ & -0.07224100 & 6.04320500 & -0.43889400 & C & -3.69086100 & 5.31089700 & 2.37650200 \\
\hline $\mathrm{H}$ & -6.73189300 & 2.74159300 & 3.18766400 & $\mathrm{H}$ & -5.37763400 & 4.77066300 & 3.55835100 \\
\hline $\mathrm{H}$ & -1.53046200 & 6.68519200 & 1.46963900 & $\mathrm{H}$ & -3.48556500 & 6.21919400 & 2.93816500 \\
\hline C & 1.16311000 & 0.24170100 & -2.71956000 & $\mathrm{~S}$ & 0.53056100 & 1.48108300 & -3.69091500 \\
\hline $\mathrm{N}$ & 0.61759500 & -1.00655500 & -2.68278800 & $\mathrm{H}$ & 0.97567100 & -1.64106200 & -1.96950400 \\
\hline $\mathrm{S}$ & 3.47513100 & -3.06737600 & -0.36222500 & 0 & 4.68901400 & -2.26191900 & -0.13555100 \\
\hline $\mathrm{O}$ & 3.22270400 & -4.18550300 & 0.56489200 & $\mathrm{O}$ & 2.25242900 & -2.22202300 & -0.61912800 \\
\hline C & 3.74904700 & -3.88445500 & -2.01340300 & $\mathrm{~F}$ & 3.82459600 & -2.95354900 & -2.98258200 \\
\hline $\mathrm{F}$ & 2.72379200 & -4.70443100 & -2.30706300 & $\mathrm{~F}$ & 4.87718300 & -4.59751200 & -2.01473600 \\
\hline C & -3.54474000 & -2.45005300 & 1.76403200 & $\mathrm{O}$ & -3.92640400 & -2.80647600 & 2.86233100 \\
\hline $\mathrm{O}$ & -3.95743200 & -2.88370200 & 0.57067100 & C & -4.95955400 & -3.97656700 & 0.45676000 \\
\hline C & -4.40368700 & -5.24937400 & 1.10061700 & $\mathrm{H}$ & -5.08973200 & -6.08106200 & 0.90727200 \\
\hline $\mathrm{H}$ & -4.29411500 & -5.13552600 & 2.18043300 & $\mathrm{H}$ & -3.43142500 & -5.50500200 & 0.66600800 \\
\hline $\mathrm{C}$ & -5.09236300 & -4.14272500 & -1.05750000 & $\mathrm{H}$ & -5.45296700 & -3.21835800 & -1.52077800 \\
\hline
\end{tabular}




\begin{tabular}{|c|c|c|c|c|c|c|c|}
\hline $\mathrm{H}$ & -5.80727200 & -4.94092900 & -1.28137900 & $\mathrm{H}$ & -4.12900800 & -4.40380100 & -1.50614800 \\
\hline C & -6.28397200 & -3.53025700 & 1.08108000 & $\mathrm{H}$ & -7.04850300 & -4.29080900 & 0.88865700 \\
\hline $\mathrm{H}$ & -6.62234100 & -2.59021300 & 0.63156800 & $\mathrm{H}$ & -6.19069900 & -3.39440200 & 2.15961900 \\
\hline C & -2.01965500 & -0.85904800 & 2.67304300 & $\mathrm{H}$ & -2.64894000 & -0.95160200 & 3.55079100 \\
\hline C & -1.20130300 & 0.34610900 & 2.57179800 & C & -1.14980900 & 1.18998900 & 3.69920700 \\
\hline C & -0.46415300 & 0.68742100 & 1.42227100 & C & -0.39484100 & 2.35667700 & 3.67407500 \\
\hline $\mathrm{H}$ & -1.71595200 & 0.92860100 & 4.59007900 & C & 0.29156600 & 1.85734800 & 1.40611600 \\
\hline $\mathrm{H}$ & -0.48030400 & 0.04935500 & 0.54459700 & C & 0.32737200 & 2.69226500 & 2.52501600 \\
\hline $\mathrm{H}$ & -0.37278900 & 3.00610300 & 4.54413500 & $\mathrm{H}$ & 0.85232200 & 2.12088500 & 0.51592100 \\
\hline $\mathrm{H}$ & 0.91576700 & 3.60438800 & 2.49925100 & $\mathrm{~N}$ & -2.55377200 & -1.47270400 & 1.58572700 \\
\hline $\mathrm{H}$ & -2.23789700 & -1.28386700 & 0.62099700 & C & -0.75339900 & -2.27603500 & 3.77862600 \\
\hline $\mathrm{H}$ & -0.57719300 & -1.61237300 & 4.62082000 & $\mathrm{H}$ & -1.55563800 & -2.99861100 & 3.89718400 \\
\hline $\mathrm{C}$ & 0.26826600 & -2.51589500 & 2.87499000 & C & 1.50624300 & -1.72958300 & 2.87266700 \\
\hline $\mathrm{H}$ & 1.34353800 & -0.71719000 & 3.26219500 & $\mathrm{H}$ & 1.94217100 & -1.67689100 & 1.86986500 \\
\hline $\mathrm{C}$ & 0.08274600 & -3.53684600 & 1.79682500 & $\mathrm{H}$ & 1.02084400 & -4.04876200 & 1.55889800 \\
\hline $\mathrm{H}$ & -0.20170800 & -3.01479900 & 0.86904100 & $\mathrm{H}$ & -0.69768600 & -4.26356100 & 2.04010500 \\
\hline $\mathrm{C}$ & 4.50535100 & -1.59394200 & 3.49189300 & $\mathrm{H}$ & 4.77448100 & -1.89237500 & 2.47234600 \\
\hline $\mathrm{H}$ & 5.33414400 & -1.87571700 & 4.15378900 & $\mathrm{H}$ & 4.42805800 & -0.50076900 & 3.50668500 \\
\hline $\mathrm{C}$ & 3.03254300 & -4.30878800 & 3.94010800 & $\mathrm{H}$ & 3.26519200 & -4.62343800 & 2.91795900 \\
\hline $\mathrm{H}$ & 2.10412100 & -4.80022800 & 4.25544300 & $\mathrm{H}$ & 3.83412400 & -4.66826000 & 4.59779400 \\
\hline C & 2.45356700 & -1.91094400 & 5.81122200 & $\mathrm{H}$ & 1.50238900 & -2.34979700 & 6.13590800 \\
\hline $\mathrm{H}$ & 2.37407000 & -0.82122200 & 5.90628100 & $\mathrm{H}$ & 3.22348700 & -2.24597200 & 6.51734200 \\
\hline $\mathrm{Si}$ & 2.91289300 & -2.43202900 & 4.04744000 & C & -3.08179500 & 0.20671800 & -4.13242900 \\
\hline $\mathrm{H}$ & -3.21651500 & -0.67064600 & -4.77238900 & $\mathrm{H}$ & -2.23515100 & 0.79008300 & -4.50540400 \\
\hline C & -4.38107700 & 1.02125000 & -4.04191000 & $\mathrm{H}$ & -4.97334800 & 0.94709200 & -4.95824400 \\
\hline $\mathrm{H}$ & -4.15511000 & 2.07860300 & -3.87183800 & C & -5.09170300 & 0.43219500 & -2.81505900 \\
\hline $\mathrm{H}$ & -5.85601800 & 1.09376900 & -2.40133500 & $\mathrm{H}$ & -5.56656800 & -0.52389000 & -3.07063200 \\
\hline 0 & -1.64091400 & -1.19676600 & -1.07021800 & & & & \\
\hline
\end{tabular}

\section{TS-S-4f-nd-2}

Imaginary frequency: $-236.04 \mathrm{~cm}^{-1}$ E[B3LYP/6-31G(d)]: -4788.702588 Hartree E[PCM(Et $\left.\left.{ }_{2} \mathrm{O}\right)-\mathrm{B} 3 L Y P / 6-311 \mathrm{G}(\mathrm{d}, \mathrm{p})-\mathrm{D} 3(\mathrm{BJ})\right]$ : -4790.230050 Hartree

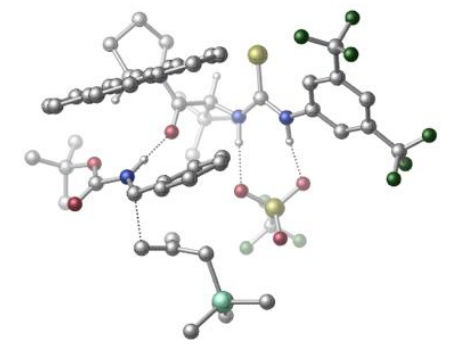

\begin{tabular}{|c|c|c|c|c|c|c|c|}
\hline 01 & & & & $\mathrm{~N}$ & 2.84551400 & -0.18516600 & 1.61924300 \\
\hline $\mathrm{H}$ & 2.92993700 & 0.82610200 & 1.46054300 & C & 3.91110700 & -0.91585400 & 1.06262800 \\
\hline C & 5.03014000 & -0.17678400 & 0.64011700 & C & 3.88853900 & -2.30009200 & 0.83739100 \\
\hline C & 6.10004600 & -0.81663100 & 0.01877100 & $\mathrm{H}$ & 5.05064900 & 0.89715500 & 0.79222900 \\
\hline C & 4.97133300 & -2.92072400 & 0.21591500 & $\mathrm{H}$ & 3.03861800 & -2.88303200 & 1.15586400 \\
\hline C & 6.08726400 & -2.19455400 & -0.19772100 & $\mathrm{H}$ & 6.92453600 & -2.68698200 & -0.67743300 \\
\hline C & 7.26078400 & 0.00066700 & -0.48557000 & C & 4.95852400 & -4.41461100 & 0.03241900 \\
\hline $\mathrm{F}$ & 7.46045000 & 1.10999500 & 0.25455600 & $\mathrm{~F}$ & 7.06492100 & 0.40584300 & -1.76265700 \\
\hline $\mathrm{F}$ & 8.41264800 & -0.70946500 & -0.47517600 & $\mathrm{~F}$ & 3.69602500 & -4.91173600 & -0.02844900 \\
\hline $\mathrm{F}$ & 5.58652200 & -4.78592700 & -1.10651100 & $\mathrm{~F}$ & 5.57776600 & -5.05582100 & 1.04718000 \\
\hline C & -0.31431700 & 0.24607700 & 3.41765700 & $\mathrm{H}$ & -0.06640500 & -0.50994300 & 4.16193800 \\
\hline C & -0.68277200 & 1.55848000 & 4.19919800 & C & -1.17386100 & 2.70362600 & 3.29187900 \\
\hline $\mathrm{H}$ & -2.10217300 & 2.44093900 & 2.77580300 & $\mathrm{H}$ & -0.43515100 & 2.97773300 & 2.53554700 \\
\hline $\mathrm{H}$ & -1.36303400 & 3.59166600 & 3.90764900 & C & 0.56814600 & 2.02175500 & 4.97378400 \\
\hline $\mathrm{H}$ & 0.30733400 & 2.85830600 & 5.63283200 & $\mathrm{H}$ & 1.35785600 & 2.36208200 & 4.29882500 \\
\hline $\mathrm{H}$ & 0.97614800 & 1.21615900 & 5.59585200 & C & -1.79558100 & 1.22174300 & 5.21323800 \\
\hline
\end{tabular}




\begin{tabular}{|c|c|c|c|c|c|c|c|}
\hline $\mathrm{H}$ & -2.07006900 & 2.12026900 & 5.77741900 & $\mathrm{H}$ & -1.46814300 & 0.46612300 & 5.93745700 \\
\hline $\mathrm{H}$ & -2.70416600 & 0.85581100 & 4.71913400 & C & -1.45120700 & -0.27086300 & 2.50971800 \\
\hline C & -3.23876100 & -1.88728500 & 2.02432300 & $\mathrm{H}$ & -3.83095400 & -1.03080300 & 1.69384600 \\
\hline $\mathrm{N}$ & -2.14414400 & -1.37221600 & 2.87483400 & C & -2.75138800 & -2.66528500 & 0.80311600 \\
\hline C & -1.47291700 & -3.13721000 & 0.72322900 & C & -3.68791300 & -2.93748600 & -0.27566000 \\
\hline C & -1.00151800 & -3.90590300 & -0.39332100 & $\mathrm{H}$ & -0.76069500 & -2.91894200 & 1.51469000 \\
\hline C & -3.23800800 & -3.69429600 & -1.40685800 & C & -5.02199000 & -2.48928800 & -0.26799600 \\
\hline C & 0.31572600 & -4.38765300 & -0.45944300 & C & -1.89808400 & -4.18013700 & -1.46552200 \\
\hline $\mathrm{C}$ & -4.13270400 & -3.97085800 & -2.48800300 & $\mathrm{H}$ & -5.40032000 & -1.92313600 & 0.57623000 \\
\hline $\mathrm{C}$ & -5.88629400 & -2.76154000 & -1.32896500 & C & 0.74945100 & -5.12435000 & -1.56101200 \\
\hline $\mathrm{H}$ & 0.99726900 & -4.18283200 & 0.36117300 & C & -1.44801700 & -4.93614600 & -2.59027100 \\
\hline C & -5.45153500 & -3.49335300 & -2.42873900 & C & -3.65330900 & -4.73504500 & -3.60766600 \\
\hline $\mathrm{H}$ & -6.91007400 & -2.39896800 & -1.29109900 & C & -0.12106200 & -5.39659400 & -2.61501000 \\
\hline $\mathrm{H}$ & 1.77395600 & -5.48205800 & -1.59163400 & C & -2.37361800 & -5.19655900 & -3.65717900 \\
\hline $\mathrm{H}$ & -6.13117400 & -3.70545100 & -3.25052200 & $\mathrm{H}$ & -4.34496100 & -4.94175000 & -4.42100700 \\
\hline $\mathrm{H}$ & 0.22202600 & -5.97411300 & -3.47030700 & $\mathrm{H}$ & -2.02991700 & -5.77643200 & -4.51060700 \\
\hline $\mathrm{C}$ & 1.81233100 & -0.58040500 & 2.43275200 & $\mathrm{~S}$ & 1.71448200 & -2.09274800 & 3.20034800 \\
\hline $\mathrm{N}$ & 0.87928900 & 0.39851000 & 2.59463400 & $\mathrm{H}$ & 0.88579000 & 1.17903500 & 1.92961300 \\
\hline $\mathrm{S}$ & 2.28133600 & 3.28479200 & 0.40492300 & $\mathrm{O}$ & 2.55420900 & 3.76700000 & -0.96024900 \\
\hline 0 & 0.98522100 & 2.55562600 & 0.56128400 & $\mathrm{O}$ & 3.40408000 & 2.61056400 & 1.10647300 \\
\hline C & 2.02347100 & 4.84554900 & 1.38551000 & $\mathrm{~F}$ & 3.12331800 & 5.60112800 & 1.38103700 \\
\hline $\mathrm{F}$ & 1.70161500 & 4.56070800 & 2.65843900 & $\mathrm{~F}$ & 1.01161600 & 5.56301400 & 0.85367400 \\
\hline $\mathrm{C}$ & -4.55615800 & 1.37035900 & -0.54006200 & $\mathrm{O}$ & -5.31768200 & 1.73476900 & -1.41686200 \\
\hline 0 & -4.77393600 & 1.35898500 & 0.77897900 & C & -6.02589600 & 1.91422000 & 1.35522400 \\
\hline C & -6.13370600 & 3.40085300 & 1.00477000 & $\mathrm{H}$ & -6.98921800 & 3.83589500 & 1.53255600 \\
\hline $\mathrm{H}$ & -6.27731300 & 3.54857500 & -0.06701000 & $\mathrm{H}$ & -5.23235000 & 3.93513400 & 1.32392800 \\
\hline C & -5.80934600 & 1.72128200 & 2.85675600 & $\mathrm{H}$ & -5.69440500 & 0.65996000 & 3.10041000 \\
\hline $\mathrm{H}$ & -6.67134100 & 2.10933100 & 3.40884800 & $\mathrm{H}$ & -4.91369100 & 2.25318700 & 3.19212600 \\
\hline C & -7.22975200 & 1.10678300 & 0.86323700 & $\mathrm{H}$ & -8.13218100 & 1.45371500 & 1.37838300 \\
\hline $\mathrm{H}$ & -7.09893400 & 0.04362000 & 1.09266900 & $\mathrm{H}$ & -7.37465200 & 1.22289600 & -0.21185100 \\
\hline C & -2.84416400 & 0.76956100 & -2.09263800 & $\mathrm{H}$ & -3.66679600 & 0.68427400 & -2.79434000 \\
\hline C & -1.61707800 & 0.04595500 & -2.42878800 & C & -1.55231600 & -0.57200700 & -3.69269800 \\
\hline C & -0.51370600 & -0.04572700 & -1.55909700 & C & -0.42162100 & -1.28787300 & -4.07076500 \\
\hline $\mathrm{H}$ & -2.40158400 & -0.50227800 & -4.36828500 & C & 0.61585300 & -0.76193300 & -1.94730400 \\
\hline $\mathrm{H}$ & -0.52654900 & 0.43679900 & -0.58795000 & C & 0.66463200 & -1.38506300 & -3.19629700 \\
\hline $\mathrm{H}$ & -0.38933800 & -1.77555600 & -5.04044400 & $\mathrm{H}$ & 1.46190600 & -0.82898700 & -1.27066300 \\
\hline $\mathrm{H}$ & 1.54732400 & -1.94663800 & -3.48730300 & $\mathrm{~N}$ & -3.26701500 & 0.89436800 & -0.80346400 \\
\hline $\mathrm{H}$ & -2.67114900 & 0.64442400 & 0.00316800 & C & -2.62747400 & 2.73048900 & -2.95614000 \\
\hline $\mathrm{H}$ & -2.50435400 & 2.39539400 & -3.98305000 & $\mathrm{H}$ & -3.63151100 & 3.03131500 & -2.67201200 \\
\hline $\mathrm{C}$ & -1.52783800 & 3.24048000 & -2.28187300 & $\mathrm{C}$ & -0.17436500 & 3.12291400 & -2.82282400 \\
\hline $\mathrm{H}$ & -0.10112800 & 2.30969200 & -3.55508600 & $\mathrm{H}$ & 0.57953900 & 2.99544400 & -2.03771700 \\
\hline C & -1.70724500 & 3.82929900 & -0.91697400 & $\mathrm{H}$ & -0.98983800 & 4.62938900 & -0.71838000 \\
\hline $\bar{H}$ & -1.48188900 & 3.05330500 & -0.16945000 & $\mathrm{H}$ & -2.72546100 & 4.19204300 & -0.75056100 \\
\hline $\mathrm{C}$ & 2.20915300 & 4.28664300 & -4.32754000 & $\mathrm{H}$ & 2.82745400 & 4.09005200 & -3.44572600 \\
\hline $\mathrm{H}$ & 2.65408300 & 5.12251300 & -4.88150000 & $\mathrm{H}$ & 2.24282200 & 3.40299900 & -4.97592400 \\
\hline $\mathrm{C}$ & 0.40155200 & 6.22712000 & -2.69551800 & $\mathrm{H}$ & 0.94722000 & 6.05358100 & -1.76241800 \\
\hline $\bar{H}$ & -0.62180000 & 6.53259700 & -2.44745700 & $\mathrm{H}$ & 0.87900400 & 7.07177100 & -3.20796200 \\
\hline C & -0.67218000 & 4.94123800 & -5.30743100 & $\mathrm{H}$ & -1.71284700 & 5.13282300 & -5.02057900 \\
\hline $\mathrm{H}$ & -0.66146000 & 4.06425500 & -5.96613600 & $\mathrm{H}$ & -0.33173300 & 5.79869600 & -5.90110700 \\
\hline Si & 0.44843200 & 4.69824900 & -3.79965300 & $\mathrm{C}$ & -1.96750100 & -2.16933500 & 4.11250100 \\
\hline $\mathrm{H}$ & -2.25377700 & -1.57219700 & 4.98504600 & $\mathrm{H}$ & -0.92420700 & -2.47776100 & 4.22013300 \\
\hline $\mathrm{C}$ & -2.92770000 & -3.35144200 & 3.92018400 & $\mathrm{H}$ & -3.30818700 & -3.72819700 & 4.87379900 \\
\hline $\mathrm{H}$ & -2.41625600 & -4.17557900 & 3.41248800 & C & -4.02722200 & -2.77796000 & 3.01534700 \\
\hline
\end{tabular}




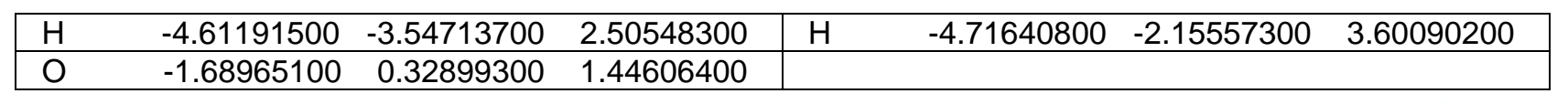

\section{TS-S-4f-nd-3}

Imaginary frequency: $-239.08 \mathrm{~cm}^{-1}$ E[B3LYP/6-31G(d)]: -4788.699155 Hartree

E[PCM(Et $\left.\left.{ }_{2} \mathrm{O}\right)-\mathrm{B} 3 L \mathrm{YP} / 6-311 \mathrm{G}(\mathrm{d}, \mathrm{p})-\mathrm{D} 3(\mathrm{BJ})\right]$ : -4790.227991 Hartree

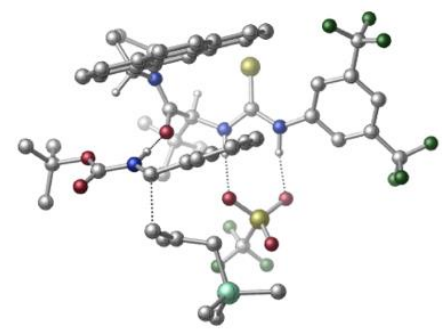

\begin{tabular}{|c|c|c|c|c|c|c|c|}
\hline 01 & & & & $\mathrm{~N}$ & 2.83441500 & -0.10516800 & 1.65096700 \\
\hline $\mathrm{H}$ & 2.86433100 & 0.90895800 & 1.49240100 & C & 3.94002400 & -0.77576300 & 1.09738900 \\
\hline C & 5.00333900 & 0.02912400 & 0.64950300 & C & 4.01139500 & -2.16255500 & 0.90079900 \\
\hline C & 6.10863100 & -0.54833800 & 0.03009200 & $\mathrm{H}$ & 4.95274700 & 1.10476100 & 0.78165300 \\
\hline C & 5.12830300 & -2.71969300 & 0.27850200 & $\mathrm{H}$ & 3.20770500 & -2.79581000 & 1.24266200 \\
\hline $\mathrm{C}$ & 6.18790600 & -1.92816700 & -0.16095800 & $\mathrm{H}$ & 7.05208400 & -2.37180700 & -0.64035500 \\
\hline C & 7.20692100 & 0.33493900 & -0.50185200 & C & 5.21224500 & -4.21476000 & 0.12582100 \\
\hline $\mathrm{F}$ & 7.32680500 & 1.47806700 & 0.20295900 & $\mathrm{~F}$ & 6.98189900 & 0.68516600 & -1.79061500 \\
\hline $\mathrm{F}$ & 8.40702200 & -0.28983200 & -0.47314300 & $\mathrm{~F}$ & 3.98407700 & -4.78769700 & 0.03001500 \\
\hline $\mathrm{F}$ & 5.90255700 & -4.56959700 & -0.98159700 & $\mathrm{~F}$ & 5.82875100 & -4.79888000 & 1.17605900 \\
\hline C & -0.35466000 & 0.16162900 & 3.42439900 & $\mathrm{H}$ & -0.05915000 & -0.57700900 & 4.16839800 \\
\hline C & -0.80384600 & 1.45429300 & 4.19538800 & C & -1.36061400 & 2.56021200 & 3.27694200 \\
\hline $\mathrm{H}$ & -2.27172500 & 2.23870900 & 2.76327500 & $\mathrm{H}$ & -0.63832200 & 2.86910100 & 2.51820300 \\
\hline $\mathrm{H}$ & -1.60179700 & 3.44175300 & 3.88383800 & C & 0.41413200 & 1.99832200 & 4.97014500 \\
\hline $\mathrm{H}$ & 0.10121500 & 2.82274900 & 5.62178400 & $\mathrm{H}$ & 1.18477400 & 2.37987200 & 4.29558400 \\
\hline $\mathrm{H}$ & 0.86715300 & 1.22324700 & 5.59982600 & C & -1.89783200 & 1.06301800 & 5.21080400 \\
\hline $\mathrm{H}$ & -2.23822900 & 1.95290400 & 5.75227400 & $\mathrm{H}$ & -1.52368000 & 0.35151000 & 5.95718500 \\
\hline $\mathrm{H}$ & -2.77586000 & 0.62269700 & 4.72201900 & C & -1.45118300 & -0.43008000 & 2.50992400 \\
\hline $\mathrm{O}$ & -1.71355400 & 0.14632600 & 1.44001500 & C & -1.96485100 & -2.32295000 & 4.12816200 \\
\hline C & -3.11467800 & -2.16371900 & 1.98220000 & C & -3.38916800 & -2.84679500 & 4.33059900 \\
\hline $\mathrm{H}$ & -1.23938300 & -3.13792300 & 4.01860300 & $\mathrm{H}$ & -1.63199900 & -1.68046300 & 4.94037200 \\
\hline $\mathrm{H}$ & -3.78646300 & -1.34820600 & 1.69991300 & $\mathrm{H}$ & -4.01595600 & -2.05368400 & 4.75635800 \\
\hline $\mathrm{H}$ & -3.42578900 & -3.70554600 & 5.00749500 & $\mathrm{~N}$ & -2.07755600 & -1.57577200 & 2.86323800 \\
\hline C & -2.55837200 & -2.82054000 & 0.72239800 & C & -1.25652800 & -3.22523800 & 0.65773800 \\
\hline C & -3.45242000 & -3.08375700 & -0.39229100 & C & -0.71813900 & -3.91064000 & -0.48178500 \\
\hline $\mathrm{H}$ & -0.57986400 & -3.01905200 & 1.48338300 & C & -2.93496800 & -3.75686300 & -1.54767800 \\
\hline C & -4.80857100 & -2.70779300 & -0.39828900 & C & 0.62217000 & -4.32659100 & -0.53338600 \\
\hline C & -1.57151000 & -4.17299600 & -1.59192800 & C & -3.78666100 & -4.02170200 & -2.66597100 \\
\hline $\mathrm{H}$ & -5.23727300 & -2.20893400 & 0.46403500 & C & -5.63020100 & -2.96659100 & -1.49553400 \\
\hline C & 1.11991100 & -4.98636000 & -1.65621200 & $\mathrm{H}$ & 1.27110500 & -4.13154700 & 0.31535000 \\
\hline C & -1.05542100 & -4.84975800 & -2.73874600 & C & -5.12948300 & -3.61514600 & -2.61954600 \\
\hline$C$ & -3.24043300 & -4.70306300 & -3.80824800 & $\mathrm{H}$ & -6.67247100 & -2.66020800 & -1.46731800 \\
\hline C & 0.29220300 & -5.24593600 & -2.74742700 & $\mathrm{H}$ & 2.16057900 & -5.29497000 & -1.67325700 \\
\hline C & -1.93859400 & -5.09943400 & -3.84349700 & $\mathrm{H}$ & -5.77616400 & -3.81809900 & -3.46977000 \\
\hline $\mathrm{H}$ & -3.89944800 & -4.90171500 & -4.65023100 & $\mathrm{H}$ & 0.68535500 & -5.76373900 & -3.61926900 \\
\hline $\mathrm{H}$ & -1.54440200 & -5.61850900 & -4.71411100 & C & 1.81399000 & -0.55468100 & 2.45279500 \\
\hline$S$ & 1.77500600 & -2.06956700 & 3.21795500 & $\mathrm{~N}$ & 0.83200100 & 0.37837100 & 2.60690700 \\
\hline $\mathrm{H}$ & 0.80124700 & 1.15679600 & 1.94058800 & $S$ & 2.08903500 & 3.35208600 & 0.44649300 \\
\hline $\mathrm{O}$ & 2.34006100 & 3.87067200 & -0.90955200 & $\mathrm{O}$ & 0.83108800 & 2.55573400 & 0.58640600 \\
\hline $\mathrm{O}$ & 3.24263300 & 2.72450600 & 1.14075600 & C & 1.75005600 & 4.88178700 & 1.45134700 \\
\hline $\mathrm{F}$ & 2.81022000 & 5.69193800 & 1.46250200 & $\mathrm{~F}$ & 1.43945300 & 4.56116500 & 2.71852100 \\
\hline $\mathrm{F}$ & 0.70441200 & 5.55614700 & 0.92793600 & C & -4.61720800 & 1.10919300 & -0.53874900 \\
\hline $\mathrm{O}$ & -5.39931000 & 1.44324700 & -1.40969000 & $\mathrm{O}$ & -4.83541700 & 1.06018300 & 0.77973000 \\
\hline
\end{tabular}




\begin{tabular}{|c|c|c|c|c|c|c|c|}
\hline $\mathrm{C}$ & -6.11903800 & 1.52937600 & 1.36193800 & $\mathrm{C}$ & -6.31719000 & 3.01231000 & 1.03572200 \\
\hline $\mathrm{H}$ & -7.19744600 & 3.38580900 & 1.56994200 & $\mathrm{H}$ & -6.46959200 & 3.16870600 & -0.03356600 \\
\hline $\mathrm{H}$ & -5.44992000 & 3.59504800 & 1.36444300 & $\mathrm{C}$ & -5.89326300 & 1.32624100 & 2.86081700 \\
\hline $\mathrm{H}$ & -5.71245500 & 0.27046000 & 3.08772100 & $\mathrm{H}$ & -6.77867800 & 1.65075800 & 3.41684800 \\
\hline $\mathrm{H}$ & -5.03313400 & 1.90758700 & 3.20692800 & C & -7.27138600 & 0.65844900 & 0.85495200 \\
\hline $\mathrm{H}$ & -8.19423900 & 0.94316900 & 1.37206800 & $\mathrm{H}$ & -7.07764800 & -0.39820900 & 1.06961800 \\
\hline $\mathrm{H}$ & -7.42045200 & 0.78113800 & -0.21883500 & C & -2.87076600 & 0.64649300 & -2.10061700 \\
\hline $\mathrm{H}$ & -3.68634100 & 0.52030600 & -2.80445800 & C & -1.60047500 & 0.00685100 & -2.44729700 \\
\hline $\mathrm{C}$ & -1.49076400 & -0.57014200 & -3.72742500 & C & -0.49799800 & -0.04004000 & -1.57320000 \\
\hline $\mathrm{C}$ & -0.31480600 & -1.20101600 & -4.11824500 & $\mathrm{H}$ & -2.33983300 & -0.53602200 & -4.40597000 \\
\hline C & 0.67653200 & -0.67238200 & -1.97361600 & $\mathrm{H}$ & -0.54543100 & 0.41215100 & -0.58876400 \\
\hline $\mathrm{C}$ & 0.77134300 & -1.25400600 & -3.23970700 & $\mathrm{H}$ & -0.24704300 & -1.65798400 & -5.10095100 \\
\hline $\mathrm{H}$ & 1.52099900 & -0.70627900 & -1.29260800 & $\mathrm{H}$ & 1.68927300 & -1.75004300 & -3.54054600 \\
\hline $\mathrm{N}$ & -3.30146700 & 0.71968000 & -0.80905100 & $\mathrm{H}$ & -2.69616500 & 0.47888700 & -0.00801700 \\
\hline $\mathrm{C}$ & -2.77734600 & 2.62645200 & -2.92395400 & $\mathrm{H}$ & -2.63799000 & 2.32054600 & -3.95788200 \\
\hline $\mathrm{H}$ & -3.79641500 & 2.86322000 & -2.63283900 & $\mathrm{C}$ & -1.70751800 & 3.18968300 & -2.24316600 \\
\hline $\mathrm{C}$ & -0.35154300 & 3.16173100 & -2.78856100 & $\mathrm{H}$ & -0.23424100 & 2.37128900 & -3.53976000 \\
\hline $\mathrm{H}$ & 0.41094400 & 3.05916100 & -2.00792800 & C & -1.91772500 & 3.74020900 & -0.86696200 \\
\hline $\mathrm{H}$ & -1.24417400 & 4.57311800 & -0.65105600 & $\mathrm{H}$ & -1.65119200 & 2.96136600 & -0.13611100 \\
\hline $\mathrm{H}$ & -2.95396400 & 4.04382400 & -0.69363900 & $\mathrm{C}$ & 1.96092300 & 4.49076000 & -4.26483300 \\
\hline $\mathrm{H}$ & 2.58924000 & 4.30373900 & -3.38796300 & $\mathrm{H}$ & 2.36008100 & 5.36404000 & -4.79543600 \\
\hline $\mathrm{H}$ & 2.04209400 & 3.62798900 & -4.93667200 & $C$ & 0.05233600 & 6.28865800 & -2.58619600 \\
\hline $\mathrm{H}$ & 0.60869300 & 6.12302100 & -1.65797400 & $\mathrm{H}$ & -0.98568000 & 6.53102900 & -2.32954600 \\
\hline $\mathrm{H}$ & 0.48128300 & 7.17065300 & -3.07820600 & C & -0.95476100 & 5.00929000 & -5.22799600 \\
\hline $\mathrm{H}$ & -2.00358700 & 5.13646200 & -4.93514300 & $\mathrm{H}$ & -0.89766200 & 4.15004200 & -5.90733300 \\
\hline $\mathrm{H}$ & -0.66355700 & 5.89800500 & -5.80154300 & $\mathrm{Si}$ & 0.18080900 & 4.79214300 & -3.72775400 \\
\hline C & -3.84084000 & -3.19230100 & 2.90302900 & $\mathrm{H}$ & -3.51752100 & -4.20403600 & 2.63925800 \\
\hline $\bar{H}$ & -4.92666400 & -3.15385500 & 2.77772300 & & & & \\
\hline
\end{tabular}

\section{TS-S-4f-nd-4}

Imaginary frequency: $-243.84 \mathrm{~cm}^{-1}$ E[B3LYP/6-31G(d)]: -4788.695661 Hartree

E[PCM(Et $\left.\left.{ }_{2} \mathrm{O}\right)-\mathrm{B} 3 L Y P / 6-311 \mathrm{G}(\mathrm{d}, \mathrm{p})-\mathrm{D} 3(\mathrm{BJ})\right]$ : -4790.227775 Hartree

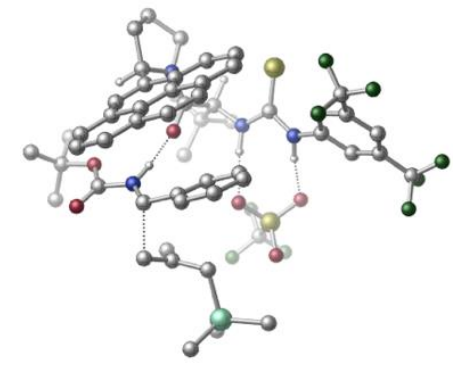

\begin{tabular}{|c|c|c|c|c|c|c|c|}
\hline 01 & & & & $\mathrm{~N}$ & 2.79067000 & -0.19314500 & 1.71124600 \\
\hline $\mathrm{H}$ & 2.92072800 & 0.81942400 & 1.59252000 & C & 3.77193300 & -0.97715300 & 1.08425400 \\
\hline C & 5.02366700 & -0.39262000 & 0.83703600 & C & 3.51210400 & -2.26617800 & 0.59984900 \\
\hline C & 5.99757100 & -1.09843800 & 0.13281400 & $\mathrm{H}$ & 5.22234900 & 0.61351800 & 1.19015800 \\
\hline C & 4.49643800 & -2.95384700 & -0.10903200 & $\mathrm{H}$ & 2.54425400 & -2.71941600 & 0.76698000 \\
\hline C & 5.74842700 & -2.38608900 & -0.34420200 & $\mathrm{H}$ & 6.51192100 & -2.93239200 & -0.88370200 \\
\hline C & 7.31872200 & -0.44125000 & -0.17077200 & C & 4.14811800 & -4.29262500 & -0.69893900 \\
\hline $\mathrm{F}$ & 7.66367600 & 0.46224300 & 0.76977000 & $\mathrm{~F}$ & 7.28801600 & 0.21229200 & -1.35562500 \\
\hline $\mathrm{F}$ & 8.31977000 & -1.34791500 & -0.25177200 & $\mathrm{~F}$ & 3.45907800 & -5.07311300 & 0.16758800 \\
\hline $\mathrm{F}$ & 3.35354800 & -4.15925700 & -1.79796000 & $\mathrm{~F}$ & 5.23645600 & -4.98655500 & -1.08804800 \\
\hline $\mathrm{C}$ & -0.38177000 & 0.19407300 & 3.49475700 & $\mathrm{H}$ & -0.09147900 & -0.51472200 & 4.26961200 \\
\hline C & -0.79520500 & 1.53220800 & 4.20701600 & C & -1.29581800 & 2.61997500 & 3.23540400 \\
\hline $\mathrm{H}$ & -2.20285900 & 2.30748100 & 2.70994900 & $\mathrm{H}$ & -0.54432100 & 2.88234700 & 2.48698000 \\
\hline $\mathrm{H}$ & -1.52344600 & 3.52958800 & 3.80508900 & C & 0.43199800 & 2.06278100 & 4.97699600 \\
\hline $\mathrm{H}$ & 0.14342500 & 2.92901200 & 5.58412400 & $\mathrm{H}$ & 1.22829600 & 2.38143200 & 4.29944900 \\
\hline $\mathrm{H}$ & 0.84287900 & 1.30059300 & 5.64990000 & C & -1.91718800 & 1.22711800 & 5.22128300 \\
\hline
\end{tabular}




\begin{tabular}{|c|c|c|c|c|c|c|c|}
\hline $\mathrm{H}$ & -2.23161900 & 2.15272100 & 5.71663400 & $\mathrm{H}$ & -1.58006900 & 0.53828600 & 6.00547800 \\
\hline $\mathrm{H}$ & -2.80436400 & 0.79745700 & 4.73974000 & C & -1.50896300 & -0.41559700 & 2.62328500 \\
\hline 0 & -1.80630600 & 0.12763300 & 1.54867700 & $\mathrm{C}$ & -1.95109600 & -2.21978200 & 4.34599100 \\
\hline C & -3.26919500 & -2.12621000 & 2.27220600 & $\mathrm{C}$ & -3.28669700 & -2.93607000 & 4.56784000 \\
\hline $\mathrm{H}$ & -1.10988300 & -2.92065000 & 4.29406400 & $\mathrm{H}$ & -1.73688500 & -1.49942500 & 5.13351400 \\
\hline $\mathrm{H}$ & -4.06184300 & -1.36761400 & 2.26782600 & $\mathrm{H}$ & -4.01458500 & -2.24444500 & 5.00985800 \\
\hline $\mathrm{H}$ & -3.18693800 & -3.79393900 & 5.23922600 & $\mathrm{~N}$ & -2.13228300 & -1.54438000 & 3.04073100 \\
\hline C & -3.06300900 & -2.53223400 & 0.81615000 & C & -4.07163400 & -2.20448500 & -0.04650100 \\
\hline C & -1.97076200 & -3.36995900 & 0.34492800 & C & -4.12638300 & -2.65626100 & -1.40527900 \\
\hline $\mathrm{H}$ & -4.88863600 & -1.57742200 & 0.30526500 & C & -2.00889000 & -3.85258500 & -1.00649900 \\
\hline C & -0.87089700 & -3.72894600 & 1.14242200 & C & -5.16788100 & -2.29300200 & -2.27562500 \\
\hline C & -3.08427500 & -3.50553000 & -1.87778800 & C & -0.95505200 & -4.68422100 & -1.50010500 \\
\hline $\mathrm{H}$ & -0.78043700 & -3.35045600 & 2.15073500 & C & 0.15851100 & -4.52851900 & 0.64540300 \\
\hline C & -5.19325500 & -2.76507600 & -3.58762200 & $\mathrm{H}$ & -5.95807700 & -1.63831100 & -1.91578000 \\
\hline C & -3.12022000 & -3.98790100 & -3.22249900 & C & 0.12216600 & -5.00500200 & -0.65996900 \\
\hline C & -1.02272200 & -5.16559500 & -2.85281100 & $\mathrm{H}$ & 1.00340500 & -4.76257000 & 1.28537800 \\
\hline C & -4.18515800 & -3.60616900 & -4.05604800 & $\mathrm{H}$ & -6.00773800 & -2.47893100 & -4.24775100 \\
\hline C & -2.05629600 & -4.83913700 & -3.67600300 & $\mathrm{H}$ & 0.93962300 & -5.60952300 & -1.04254000 \\
\hline $\mathrm{H}$ & -0.21846000 & -5.80599800 & -3.20695300 & $\mathrm{H}$ & -4.21456100 & -3.97574200 & -5.07834200 \\
\hline $\mathrm{H}$ & -2.09155100 & -5.21570200 & -4.69558800 & C & 1.76653000 & -0.59732600 & 2.53652400 \\
\hline $\mathrm{S}$ & 1.74846300 & -2.07934600 & 3.35618300 & $\mathrm{~N}$ & 0.79953200 & 0.35127700 & 2.65836600 \\
\hline $\mathrm{H}$ & 0.80560300 & 1.12316600 & 1.98520300 & $S$ & 2.30741100 & 3.25152500 & 0.53003800 \\
\hline $\mathrm{O}$ & 2.65162300 & 3.71526700 & -0.82584600 & $\mathrm{O}$ & 1.00043900 & 2.52934400 & 0.62771100 \\
\hline $\mathrm{O}$ & 3.39145400 & 2.58471100 & 1.29577800 & $\mathrm{C}$ & 2.00028000 & 4.82671900 & 1.47267000 \\
\hline $\mathrm{F}$ & 3.10839800 & 5.56730600 & 1.54093500 & $\mathrm{~F}$ & 1.58183500 & 4.56138600 & 2.72141300 \\
\hline $\mathrm{F}$ & 1.04109900 & 5.55103700 & 0.85966600 & $\mathrm{C}$ & -4.40395000 & 1.43197700 & -0.66303700 \\
\hline 0 & -5.12303200 & 1.75162900 & -1.59289700 & $\mathrm{O}$ & -4.68818900 & 1.47286500 & 0.64212100 \\
\hline C & -5.97073200 & 2.03807700 & 1.13128000 & $\mathrm{C}$ & -6.07393500 & 3.50817900 & 0.71562800 \\
\hline $\mathrm{H}$ & -6.95916300 & 3.95515000 & 1.18078900 & $\mathrm{H}$ & -6.16319900 & 3.61065400 & -0.36731800 \\
\hline $\mathrm{H}$ & -5.19472400 & 4.06448200 & 1.05808600 & C & -5.82849300 & 1.90735300 & 2.64838200 \\
\hline $\mathrm{H}$ & -5.71180100 & 0.85799300 & 2.93818500 & $\mathrm{H}$ & -6.72211600 & 2.30393400 & 3.14102500 \\
\hline $\mathrm{H}$ & -4.95730900 & 2.46426800 & 3.00663000 & C & -7.14257300 & 1.19930700 & 0.61422100 \\
\hline $\mathrm{H}$ & -8.07217700 & 1.55591000 & 1.07121500 & $\mathrm{H}$ & -7.01413600 & 0.14719000 & 0.89184000 \\
\hline $\mathrm{H}$ & -7.23700500 & 1.27300000 & -0.47030300 & C & -2.61529000 & 0.75289200 & -2.10038900 \\
\hline $\mathrm{H}$ & -3.39887100 & 0.61160500 & -2.83694300 & C & -1.37125100 & 0.01141300 & -2.30742600 \\
\hline C & -1.22304800 & -0.71211400 & -3.50664100 & C & -0.33128500 & -0.00607400 & -1.35988400 \\
\hline C & -0.07814100 & -1.46887900 & -3.73543400 & $\mathrm{H}$ & -2.02441800 & -0.70443200 & -4.24132100 \\
\hline C & 0.81272400 & -0.76099900 & -1.60014300 & $\mathrm{H}$ & -0.40423000 & 0.56127400 & -0.43939600 \\
\hline C & 0.93970100 & -1.50162500 & -2.77725100 & $\mathrm{H}$ & 0.01298300 & -2.04680800 & -4.64991900 \\
\hline $\mathrm{H}$ & 1.60854700 & -0.76673900 & -0.86385200 & $\mathrm{H}$ & 1.82540300 & -2.11007700 & -2.93590000 \\
\hline $\mathrm{N}$ & -3.10172800 & 0.95743300 & -0.84123800 & $\mathrm{H}$ & -2.57647300 & 0.67885600 & -0.00108600 \\
\hline C & -2.36453400 & 2.64620900 & -3.07347700 & $\mathrm{H}$ & -2.18954900 & 2.24528900 & -4.06885800 \\
\hline $\mathrm{H}$ & -3.38179900 & 2.96137100 & -2.86077700 & C & -1.30058100 & 3.20680200 & -2.38194700 \\
\hline C & 0.07840700 & 3.05535100 & -2.84286300 & $\mathrm{H}$ & 0.18288400 & 2.20938300 & -3.53284500 \\
\hline $\mathrm{H}$ & 0.78604500 & 2.95507800 & -2.01156800 & C & -1.55013200 & 3.88375600 & -1.07008800 \\
\hline $\mathrm{H}$ & -0.84830500 & 4.70203000 & -0.89108400 & $\mathrm{H}$ & -1.35645000 & 3.16045800 & -0.26346500 \\
\hline $\mathrm{H}$ & -2.57730500 & 4.24710200 & -0.97720200 & C & 2.56326600 & 4.11416200 & -4.24982300 \\
\hline $\mathrm{H}$ & 3.12555300 & 3.93656000 & -3.32725500 & $\mathrm{H}$ & 3.05875000 & 4.91969600 & -4.80575100 \\
\hline $\mathrm{H}$ & 2.61376200 & 3.20687200 & -4.86347300 & C & 0.71664100 & 6.14840100 & -2.78926800 \\
\hline $\mathrm{H}$ & 1.19866200 & 5.99025400 & -1.81889800 & $\mathrm{H}$ & -0.30977100 & 6.49256300 & -2.61581700 \\
\hline $\mathrm{H}$ & 1.25117900 & 6.96062000 & -3.29779500 & C & -0.24468900 & 4.79637700 & -5.41303500 \\
\hline $\mathrm{H}$ & -1.29453900 & 5.02040800 & -5.19004900 & $\mathrm{H}$ & -0.21952200 & 3.89663500 & -6.03981600 \\
\hline $\mathrm{H}$ & 0.14746600 & 5.62484100 & -6.01588000 & $\mathrm{Si}$ & 0.78527900 & 4.58132600 & -3.83809300 \\
\hline
\end{tabular}




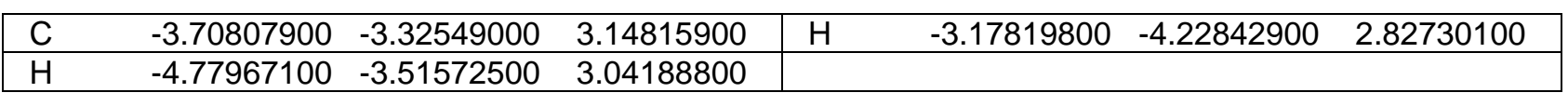

\section{TS-S-4f-nd-5}

Imaginary frequency: $-219.67 \mathrm{~cm}^{-1}$ E[B3LYP/6-31G(d)]: -4788.698086 Hartree

E[PCM(Et $\left.\left.{ }_{2} \mathrm{O}\right)-\mathrm{B} 3 L \mathrm{YP} / 6-311 \mathrm{G}(\mathrm{d}, \mathrm{p})-\mathrm{D} 3(\mathrm{BJ})\right]$ : -4790.226725 Hartree

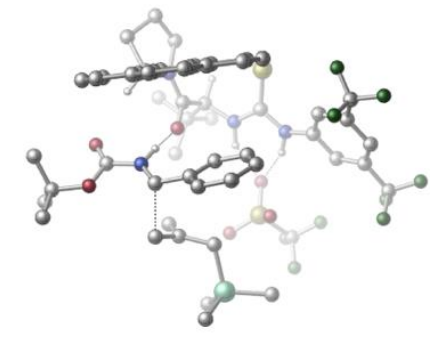

\begin{tabular}{|c|c|c|c|c|c|c|c|}
\hline 01 & & & & $\mathrm{~N}$ & -2.72640200 & 0.96843400 & 1.83666300 \\
\hline $\mathrm{H}$ & -3.13362600 & 0.02672200 & 1.83168400 & C & -3.36731100 & 1.86955000 & 0.97779000 \\
\hline C & -4.64704900 & 1.50049300 & 0.52344100 & C & -2.79330500 & 3.05165500 & 0.49028100 \\
\hline C & -5.32754100 & 2.29920900 & -0.38858300 & $\mathrm{H}$ & -5.09844700 & 0.57975200 & 0.87525400 \\
\hline $\mathrm{C}$ & -3.48482800 & 3.82828200 & -0.44094400 & $\mathrm{H}$ & -1.82043800 & 3.36073700 & 0.84261100 \\
\hline $\mathrm{C}$ & -4.75654600 & 3.47399700 & -0.88442200 & $\mathrm{H}$ & -5.29321700 & 4.09491300 & -1.59168300 \\
\hline C & -6.67645600 & 1.86948300 & -0.90435900 & C & -2.78925900 & 5.01696800 & -1.04576100 \\
\hline $\mathrm{F}$ & -7.28592900 & 0.99584100 & -0.07813300 & $\mathrm{~F}$ & -6.57710000 & 1.27269400 & -2.11485000 \\
\hline $\mathrm{F}$ & -7.50457800 & 2.92867800 & -1.06178300 & $\mathrm{~F}$ & -1.92435500 & 5.60385900 & -0.18601200 \\
\hline $\mathrm{F}$ & -2.05327600 & 4.65766600 & -2.13596500 & $\mathrm{~F}$ & -3.65466900 & 5.96359200 & -1.46202200 \\
\hline C & 0.10785200 & -0.35597100 & 3.73229100 & $\mathrm{H}$ & 0.12281900 & 0.42067400 & 4.49900900 \\
\hline C & 0.03515800 & -1.74446400 & 4.46947100 & C & 0.02720400 & -2.94659300 & 3.50244200 \\
\hline $\mathrm{H}$ & 0.94091900 & -2.98838600 & 2.90352800 & $\mathrm{H}$ & -0.83148200 & -2.94194300 & 2.82378000 \\
\hline $\mathrm{H}$ & -0.04010800 & -3.87210100 & 4.08662400 & C & -1.25118300 & -1.76943800 & 5.32372800 \\
\hline $\mathrm{H}$ & -1.26539000 & -2.67606300 & 5.93984700 & $\mathrm{H}$ & -2.15376800 & -1.77606900 & 4.70635400 \\
\hline $\mathrm{H}$ & -1.30073300 & -0.90475800 & 5.99683500 & $\mathrm{C}$ & 1.24992500 & -1.87824900 & 5.40968100 \\
\hline $\mathrm{H}$ & 1.20973300 & -2.84269400 & 5.92784400 & $\mathrm{H}$ & 1.25411400 & -1.09536200 & 6.17742400 \\
\hline $\mathrm{H}$ & 2.20095700 & -1.83848400 & 4.86617900 & C & 1.36052200 & -0.20855300 & 2.83034300 \\
\hline C & 3.66314900 & 0.62640900 & 2.50457200 & $\mathrm{H}$ & 3.91087000 & -0.38102900 & 2.16061400 \\
\hline $\mathrm{N}$ & 2.39553500 & 0.54096700 & 3.26758400 & $\mathrm{C}$ & 3.60436000 & 1.57839700 & 1.30955000 \\
\hline C & 2.56741100 & 2.45051500 & 1.14494800 & C & 4.70255700 & 1.58024000 & 0.35474200 \\
\hline C & 2.50576800 & 3.38422400 & 0.05671400 & $\mathrm{H}$ & 1.73530400 & 2.44330700 & 1.84287200 \\
\hline C & 4.66637700 & 2.50733900 & -0.73874300 & C & 5.80846800 & 0.71506300 & 0.45329800 \\
\hline C & 1.42532800 & 4.26724900 & -0.10228500 & C & 3.56900600 & 3.40654200 & -0.89008500 \\
\hline C & 5.73448600 & 2.53968200 & -1.68971600 & $\mathrm{H}$ & 5.86471500 & -0.00554000 & 1.26140100 \\
\hline $\mathrm{C}$ & 6.84866100 & 0.76049400 & -0.47636000 & $\mathrm{C}$ & 1.38434200 & 5.15476500 & -1.17689700 \\
\hline $\mathrm{H}$ & 0.61729500 & 4.25036600 & 0.62383000 & C & 3.52590900 & 4.32503000 & -1.98276000 \\
\hline $\mathrm{C}$ & 6.81757900 & 1.65988900 & -1.53692700 & C & 5.66851700 & 3.47848500 & -2.77688200 \\
\hline $\mathrm{H}$ & 7.69596000 & 0.08943700 & -0.36137200 & $\mathrm{C}$ & 2.42300200 & 5.18638900 & -2.10588300 \\
\hline $\mathrm{H}$ & 0.52975000 & 5.81436400 & -1.29027400 & C & 4.61552000 & 4.32946700 & -2.91839800 \\
\hline $\mathrm{H}$ & 7.63379600 & 1.69216700 & -2.25471800 & $\mathrm{H}$ & 6.48901400 & 3.49470100 & -3.49050200 \\
\hline $\mathrm{H}$ & 2.38741300 & 5.88303100 & -2.94023700 & $\mathrm{H}$ & 4.58344900 & 5.03300000 & -3.74707900 \\
\hline C & -1.56603100 & 1.07342700 & 2.56549700 & $\mathrm{~S}$ & -0.83214000 & 2.53057300 & 3.03030700 \\
\hline $\mathrm{N}$ & -1.08268900 & -0.13995000 & 2.92780900 & $\mathrm{H}$ & -1.59561400 & -0.95937500 & 2.60246500 \\
\hline $\mathrm{S}$ & -3.50320400 & -2.57410700 & 0.88670600 & 0 & -2.85161200 & -3.89840100 & 0.85354500 \\
\hline $\mathrm{O}$ & -3.31711500 & -1.82544000 & 2.17411300 & $\mathrm{O}$ & -3.31233300 & -1.71814100 & -0.30781000 \\
\hline C & -5.32666500 & -2.94609800 & 0.87929900 & $\mathrm{~F}$ & -5.65800600 & -3.57043100 & -0.26166400 \\
\hline $\mathrm{F}$ & -6.03797200 & -1.80803900 & 0.96022200 & $\mathrm{~F}$ & -5.66129400 & -3.72991800 & 1.90862900 \\
\hline $\mathrm{C}$ & 3.90441000 & -2.46284900 & -0.16357400 & $\mathrm{O}$ & 4.25210100 & -2.55086900 & 0.99650000 \\
\hline $\mathrm{O}$ & 4.44344600 & -3.08081800 & -1.22210200 & C & 5.61152200 & -3.99217800 & -1.07571900 \\
\hline C & 6.80228900 & -3.22129400 & -0.50236000 & $\mathrm{H}$ & 7.69453700 & -3.85542400 & -0.54473200 \\
\hline $\mathrm{H}$ & 6.62771100 & -2.93372800 & 0.53538800 & $\mathrm{H}$ & 6.99418900 & -2.32003300 & -1.09353900 \\
\hline C & 5.87772000 & -4.41457400 & -2.52123800 & $\mathrm{H}$ & 5.00698200 & -4.92338700 & -2.94740100 \\
\hline
\end{tabular}




\begin{tabular}{|c|c|c|c|c|c|c|c|}
\hline $\mathrm{H}$ & 6.72753200 & -5.10360700 & -2.55499900 & $\mathrm{H}$ & 6.11401200 & -3.54567300 & -3.14390100 \\
\hline C & 5.21472000 & -5.19159200 & -0.21230200 & $\mathrm{H}$ & 6.04213000 & -5.90902500 & -0.18816800 \\
\hline $\mathrm{H}$ & 4.34077100 & -5.69924900 & -0.63487700 & $\mathrm{H}$ & 4.98910600 & -4.88686100 & 0.81095700 \\
\hline C & 2.42936000 & -1.43589300 & -1.82646600 & $\mathrm{H}$ & 3.18579700 & -1.68897300 & -2.55832900 \\
\hline C & 1.52592900 & -0.34197800 & -2.18100300 & C & 1.60288300 & 0.16653500 & -3.49291600 \\
\hline C & 0.60001500 & 0.21719200 & -1.28020500 & C & 0.79469000 & 1.22709000 & -3.88845600 \\
\hline $\mathrm{H}$ & 2.31361000 & -0.26449900 & -4.19443900 & C & -0.20831500 & 1.27454900 & -1.68749600 \\
\hline $\mathrm{H}$ & 0.51236000 & -0.15855300 & -0.26588300 & C & -0.11177800 & 1.78654000 & -2.98293000 \\
\hline $\mathrm{H}$ & 0.87620100 & 1.62216500 & -4.89681800 & $\mathrm{H}$ & -0.92057000 & 1.70232400 & -0.99081200 \\
\hline $\mathrm{H}$ & -0.74062000 & 2.62196700 & -3.27493600 & $\mathrm{~N}$ & 2.82501000 & -1.65057000 & -0.54358600 \\
\hline $\mathrm{H}$ & 2.33383000 & -1.22555000 & 0.26212300 & C & 1.45043800 & -3.25241900 & -2.58626300 \\
\hline $\mathrm{H}$ & 1.42778000 & -2.95075300 & -3.63045600 & $\mathrm{H}$ & 2.27932900 & -3.88775200 & -2.29128900 \\
\hline C & 0.27212100 & -3.26667000 & -1.85796900 & C & -0.96277100 & -2.68301200 & -2.38149500 \\
\hline $\mathrm{H}$ & -0.76382100 & -1.92920700 & -3.15332700 & $\mathrm{H}$ & -1.60173500 & -2.26214800 & -1.59504400 \\
\hline $\mathrm{C}$ & 0.27467300 & -3.79717100 & -0.45737800 & $\mathrm{H}$ & -0.70600400 & -4.17166000 & -0.15220800 \\
\hline $\mathrm{H}$ & 0.49867000 & -2.96326300 & 0.22863900 & $\mathrm{H}$ & 1.04025500 & -4.56351100 & -0.30231000 \\
\hline $\mathrm{C}$ & -3.74892700 & -3.07840700 & -3.57955200 & $\mathrm{H}$ & -4.20422000 & -2.78782800 & -2.62729800 \\
\hline $\mathrm{H}$ & -4.45523500 & -3.72235400 & -4.11823500 & $\mathrm{H}$ & -3.60567700 & -2.16923700 & -4.17535400 \\
\hline $\mathrm{C}$ & -2.37612100 & -5.51850700 & -2.22091800 & $\overline{\mathrm{H}}$ & -2.77548200 & -5.25859600 & -1.23466700 \\
\hline $\bar{H}$ & -1.44518000 & -6.07979000 & -2.07619600 & $\mathrm{H}$ & -3.09136500 & -6.19188800 & -2.71022100 \\
\hline C & -1.29427100 & -4.44398900 & -4.92119600 & $\mathrm{H}$ & -0.31792300 & -4.91915900 & -4.76822800 \\
\hline $\mathrm{H}$ & -1.14568200 & -3.56717400 & -5.56322000 & $\mathrm{H}$ & -1.91987900 & -5.15371000 & -5.47661700 \\
\hline $\mathrm{Si}$ & -2.12185500 & -3.98063100 & -3.28011900 & C & 2.44001800 & 1.33419500 & 4.51977000 \\
\hline $\mathrm{H}$ & 2.40273800 & 0.66789400 & 5.38727700 & $\mathrm{H}$ & 1.58938800 & 2.02000200 & 4.56109000 \\
\hline C & 3.79265800 & 2.05534400 & 4.44728400 & $\mathrm{H}$ & 4.21172500 & 2.23690000 & 5.44109100 \\
\hline $\mathrm{H}$ & 3.68078300 & 3.02251400 & 3.94662200 & C & 4.65221900 & 1.11582500 & 3.59143300 \\
\hline $\mathrm{H}$ & 5.52902200 & 1.60678000 & 3.16394300 & $\mathrm{H}$ & 4.99396400 & 0.25975700 & 4.18656900 \\
\hline 0 & 1.38965600 & -0.79695800 & 1.73238500 & & & & \\
\hline
\end{tabular}

\section{TS-S-4f-nd-6}

Imaginary frequency: $-227.49 \mathrm{~cm}^{-1}$ E[B3LYP/6-31G(d)]: -4788.694851 Hartree

$\mathrm{E}\left[\mathrm{PCM}\left(\mathrm{Et}_{2} \mathrm{O}\right)-\mathrm{B} 3 \mathrm{LYP} / 6-311 \mathrm{G}(\mathrm{d}, \mathrm{p})-\mathrm{D} 3(\mathrm{BJ})\right]$ : -4790.224784 Hartree

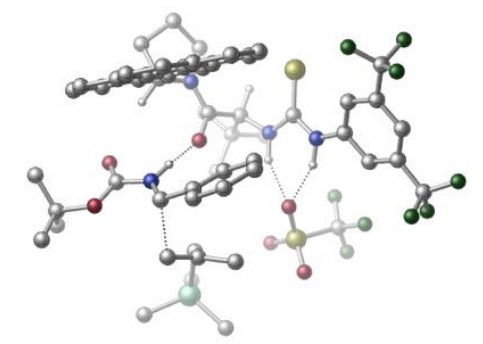

\begin{tabular}{|crrr|rrrr|}
\hline 01 & & & & N & 2.98123000 & 0.09838200 & 1.05168600 \\
\hline $\mathrm{H}$ & 2.82685500 & 1.02451600 & 0.64065000 & $\mathrm{C}$ & 3.99390700 & -0.63622900 & 0.41290900 \\
\hline $\mathrm{C}$ & 4.87261900 & 0.09787300 & -0.40392100 & $\mathrm{C}$ & 4.13571200 & -2.03036400 & 0.47642100 \\
\hline $\mathrm{C}$ & 5.86133400 & -0.55099600 & -1.13798900 & $\mathrm{H}$ & 4.78567400 & 1.17810100 & -0.45224500 \\
\hline $\mathrm{C}$ & 5.13356800 & -2.66085300 & -0.26718100 & $\mathrm{H}$ & 3.47788900 & -2.60787300 & 1.10749500 \\
\hline $\mathrm{C}$ & 6.00514900 & -1.93794800 & -1.07920300 & $\mathrm{H}$ & 6.77722300 & -2.43837000 & -1.65092200 \\
\hline $\mathrm{C}$ & 6.74863300 & 0.24647200 & -2.05845400 & $\mathrm{C}$ & 5.29693400 & -4.15345900 & -0.14970200 \\
\hline $\mathrm{F}$ & 6.92070300 & 1.51188300 & -1.62674100 & $\mathrm{~F}$ & 6.22725200 & 0.31957800 & -3.30615100 \\
\hline $\mathrm{F}$ & 7.97283200 & -0.31500100 & -2.18000400 & $\mathrm{~F}$ & 4.12046000 & -4.77232800 & 0.12679000 \\
\hline $\mathrm{F}$ & 5.77435800 & -4.69772100 & -1.29161900 & $\mathrm{~F}$ & 6.15406600 & -4.49307500 & 0.83662900 \\
\hline $\mathrm{C}$ & 0.16579500 & 0.68060900 & 3.31117400 & $\mathrm{H}$ & 0.54488300 & 0.05127800 & 4.11605200 \\
\hline $\mathrm{C}$ & -0.19417700 & 2.07545000 & 3.93757500 & $\mathrm{C}$ & -0.79655400 & 3.06376500 & 2.92084900 \\
\hline $\mathrm{H}$ & -1.71728100 & 2.67590000 & 2.47576000 & $\mathrm{H}$ & -0.10003400 & 3.31799700 & 2.11718800 \\
\hline $\mathrm{H}$ & -1.03485200 & 4.00304200 & 3.43450200 & $\mathrm{C}$ & 1.09362000 & 2.67887000 & 4.53576600 \\
\hline $\mathrm{H}$ & 0.85343400 & 3.60470300 & 5.07136900 & $\mathrm{H}$ & 1.82623000 & 2.92363100 & 3.76208300 \\
\hline $\mathrm{H}$ & 1.56592300 & 1.99011800 & 5.24683100 & $\mathrm{C}$ & -1.21106000 & 1.85659300 & 5.07641000 \\
\hline
\end{tabular}




\begin{tabular}{|c|c|c|c|c|c|c|c|}
\hline $\mathrm{H}$ & -1.46684800 & 2.81924200 & 5.53276000 & $\mathrm{H}$ & -0.80111000 & 1.21763800 & 5.86785300 \\
\hline $\mathrm{H}$ & -2.14470600 & 1.40873300 & 4.71557600 & C & -1.02876400 & -0.01808700 & 2.62169100 \\
\hline C & -2.80115300 & -1.72679700 & 2.62021200 & $\mathrm{H}$ & -3.45965700 & -0.95293000 & 2.21786300 \\
\hline $\mathrm{N}$ & -1.63902200 & -1.05141800 & 3.23979200 & C & -2.41575600 & -2.70301400 & 1.50854300 \\
\hline C & -1.12714700 & -3.12312300 & 1.34433000 & C & -3.45784400 & -3.22735600 & 0.63935100 \\
\hline C & -0.74385200 & -4.07087100 & 0.33705500 & $\mathrm{H}$ & -0.33948300 & -2.72749500 & 1.97967900 \\
\hline C & -3.10054100 & -4.17645200 & -0.37422900 & C & -4.80966500 & -2.85070400 & 0.74796900 \\
\hline C & 0.58779500 & -4.48821500 & 0.17950600 & C & -1.74584200 & -4.59674400 & -0.52748300 \\
\hline C & -4.10327900 & -4.71443300 & -1.24084000 & $\mathrm{H}$ & -5.11543300 & -2.13142000 & 1.49946100 \\
\hline C & -5.78089000 & -3.38741400 & -0.09864900 & C & 0.93320200 & -5.40405400 & -0.81363600 \\
\hline $\mathrm{H}$ & 1.34971400 & -4.08895700 & 0.84315800 & C & -1.38626200 & -5.53846900 & -1.53900700 \\
\hline C & -5.43759700 & -4.30797000 & -1.08328800 & C & -3.71404700 & -5.66313600 & -2.24879100 \\
\hline $\mathrm{H}$ & -6.81846700 & -3.08712300 & 0.02248400 & C & -0.04134000 & -5.92480200 & -1.66344400 \\
\hline $\mathrm{H}$ & 1.97042500 & -5.70663500 & -0.92106000 & C & -2.41876700 & -6.05615400 & -2.39266500 \\
\hline $\mathrm{H}$ & -6.20065400 & -4.72484200 & -1.73629500 & $\mathrm{H}$ & -4.48694000 & -6.06635700 & -2.89896700 \\
\hline $\mathrm{H}$ & 0.23325300 & -6.64183100 & -2.43362600 & $\mathrm{H}$ & -2.14365100 & -6.77629900 & -3.15970700 \\
\hline C & 2.15839300 & -0.19877400 & 2.11121800 & $S$ & 2.26788600 & -1.59538700 & 3.07007900 \\
\hline $\mathrm{N}$ & 1.24229200 & 0.78107500 & 2.33526400 & $\mathrm{H}$ & 1.18065800 & 1.53012600 & 1.64317400 \\
\hline$S$ & 2.13112300 & 3.99387800 & -0.11461000 & $\mathrm{O}$ & 1.17504800 & 4.81145100 & 0.66037300 \\
\hline 0 & 1.88702400 & 2.51450100 & 0.03370900 & $\mathrm{O}$ & 2.39059800 & 4.42158400 & -1.49989100 \\
\hline C & 3.75448300 & 4.22321800 & 0.76897300 & $\mathrm{~F}$ & 4.08876800 & 5.51379300 & 0.82766500 \\
\hline $\mathrm{F}$ & 4.73515900 & 3.55687300 & 0.13082500 & $\mathrm{~F}$ & 3.67589900 & 3.74062400 & 2.02263000 \\
\hline C & -4.46103800 & 0.82090400 & -0.25247400 & $\mathrm{O}$ & -4.75117600 & 0.86798400 & 0.92552100 \\
\hline 0 & -5.26249300 & 1.06521800 & -1.29791100 & $\mathrm{C}$ & -6.69761400 & 1.41358900 & -1.10929800 \\
\hline $\mathrm{C}$ & -7.41788500 & 0.26898200 & -0.39356400 & $\mathrm{H}$ & -8.49566500 & 0.46474200 & -0.39684300 \\
\hline $\mathrm{H}$ & -7.08400500 & 0.17355500 & 0.64067000 & $\mathrm{H}$ & -7.24060600 & -0.67817500 & -0.91350800 \\
\hline C & -7.19044900 & 1.55543400 & -2.55000000 & $\mathrm{H}$ & -6.63930600 & 2.34124200 & -3.07664300 \\
\hline $\mathrm{H}$ & -8.25239000 & 1.82066600 & -2.55354800 & $\mathrm{H}$ & -7.06874800 & 0.61553300 & -3.09806700 \\
\hline C & -6.80383700 & 2.74036400 & -0.35463500 & $\mathrm{H}$ & -7.85505300 & 3.04389200 & -0.30282300 \\
\hline $\mathrm{H}$ & -6.25148600 & 3.52745400 & -0.87974700 & $\mathrm{H}$ & -6.41730700 & 2.65054000 & 0.66190500 \\
\hline C & -2.79567200 & 0.29462600 & -1.96798100 & $\mathrm{H}$ & -3.63738800 & 0.16079800 & -2.63574000 \\
\hline $\mathrm{C}$ & -1.57939700 & -0.44153400 & -2.32449700 & C & -1.55688100 & -1.07795700 & -3.58061800 \\
\hline C & -0.46141900 & -0.55058400 & -1.47665700 & C & -0.45104100 & -1.82117600 & -3.97634700 \\
\hline $\mathrm{H}$ & -2.41892600 & -0.99715700 & -4.23890700 & C & 0.64324600 & -1.29677400 & -1.88189400 \\
\hline $\mathrm{H}$ & -0.44951400 & -0.06222100 & -0.50745400 & C & 0.65199000 & -1.93249900 & -3.12502400 \\
\hline $\mathrm{H}$ & -0.44998200 & -2.31801100 & -4.94201200 & $\mathrm{H}$ & 1.50090600 & -1.38157100 & -1.22287700 \\
\hline $\mathrm{H}$ & 1.51648700 & -2.51526000 & -3.42873600 & $\mathrm{~N}$ & -3.16551600 & 0.48416900 & -0.67307500 \\
\hline $\mathrm{H}$ & -2.50180700 & 0.36374100 & 0.11156200 & C & -2.61989900 & 2.22167200 & -2.94803600 \\
\hline $\mathrm{H}$ & -2.51147000 & 1.83562000 & -3.95792100 & $\mathrm{H}$ & -3.62378000 & 2.51420000 & -2.65489700 \\
\hline C & -1.52700900 & 2.81147600 & -2.32966900 & C & -1.67882900 & 3.58403700 & -1.09343100 \\
\hline $\mathrm{H}$ & -0.79240300 & 3.52446600 & -0.45370700 & $\mathrm{H}$ & -2.56670100 & 3.27735400 & -0.52520900 \\
\hline C & -0.15094800 & 2.60543100 & -2.87366800 & $\mathrm{H}$ & 0.42125900 & 3.53892000 & -2.88916500 \\
\hline $\mathrm{H}$ & -0.15698700 & 2.15458000 & -3.86891000 & $\mathrm{H}$ & 0.41227500 & 1.95391200 & -2.19000100 \\
\hline C & -1.89952400 & 6.28510900 & 0.32646700 & $\mathrm{H}$ & -2.65790500 & 5.85712100 & 0.99291800 \\
\hline $\mathrm{H}$ & -2.08127200 & 7.36527700 & 0.26363600 & $\mathrm{H}$ & -0.91404500 & 6.12577200 & 0.77573300 \\
\hline C & -3.65783000 & 5.72481900 & -2.17591500 & $\mathrm{H}$ & -4.45500300 & 5.33031000 & -1.53320100 \\
\hline $\mathrm{H}$ & -3.73044100 & 5.22807600 & -3.15055600 & $\mathrm{H}$ & -3.86648800 & 6.78989000 & -2.33596600 \\
\hline C & -0.61275900 & 6.20823400 & -2.51254000 & $\mathrm{H}$ & -0.69882000 & 5.83491900 & -3.54001200 \\
\hline $\mathrm{H}$ & 0.39097100 & 5.96975900 & -2.14403700 & $\mathrm{H}$ & -0.70406300 & 7.30110000 & -2.55396000 \\
\hline $\mathrm{Si}$ & -1.94784600 & 5.50414000 & -1.38624100 & C & -1.28615400 & -1.63167700 & 4.55794500 \\
\hline $\mathrm{H}$ & -1.48259100 & -0.90332000 & 5.35097100 & $\mathrm{H}$ & -0.22763500 & -1.90516100 & 4.58188500 \\
\hline C & -2.22277000 & -2.84147100 & 4.68402300 & $\mathrm{H}$ & -2.47638400 & -3.05368700 & 5.72650900 \\
\hline $\mathrm{H}$ & -1.74762900 & -3.73410500 & 4.26448900 & C & -3.43858400 & -2.44382300 & 3.83568100 \\
\hline
\end{tabular}




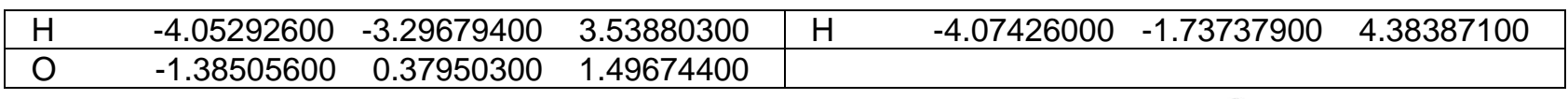

\section{TS-S-4f-nd-7}

Imaginary frequency: $-222.86 \mathrm{~cm}^{-1}$ E[B3LYP/6-31G(d)]: -4788.694300 Hartree

$\mathrm{E}\left[\mathrm{PCM}\left(\mathrm{Et}_{2} \mathrm{O}\right)-\mathrm{B} 3 \mathrm{LYP} / 6-311 \mathrm{G}(\mathrm{d}, \mathrm{p})-\mathrm{D} 3(\mathrm{BJ})\right]$ : -4790.223886 Hartree

\begin{tabular}{|c|c|c|c|c|c|c|c|}
\hline 01 & & & & $\mathrm{~N}$ & -2.72825200 & 0.91634100 & 1.85376100 \\
\hline $\mathrm{H}$ & -3.11592500 & -0.03327300 & 1.83798400 & C & -3.39591800 & 1.81656000 & 1.01499000 \\
\hline $\mathrm{C}$ & -4.66958400 & 1.42467700 & 0.56248300 & $\mathrm{C}$ & -2.85383500 & 3.02071600 & 0.54512800 \\
\hline $\mathrm{C}$ & -5.37536500 & 2.22219900 & -0.33103000 & $\mathrm{H}$ & -5.09638700 & 0.48745900 & 0.90116100 \\
\hline $\mathrm{C}$ & -3.57039300 & 3.79609600 & -0.36812200 & $\mathrm{H}$ & -1.88649500 & 3.34703300 & 0.89727800 \\
\hline $\mathrm{C}$ & -4.83612500 & 3.41881600 & -0.80984300 & $\mathrm{H}$ & -5.39221400 & 4.03879800 & -1.50280000 \\
\hline C & -6.71704100 & 1.76916700 & -0.84555400 & C & -2.90878300 & 5.01188700 & -0.95688300 \\
\hline $\mathrm{F}$ & -7.29692000 & 0.86181800 & -0.03444700 & $\mathrm{~F}$ & -6.61266100 & 1.20226600 & -2.06998000 \\
\hline $\mathrm{F}$ & -7.57351400 & 2.80975000 & -0.97271900 & $\mathrm{~F}$ & -2.04941300 & 5.60276600 & -0.09447000 \\
\hline $\mathrm{F}$ & -2.17593600 & 4.69042600 & -2.06084900 & $\mathrm{~F}$ & -3.80027900 & 5.94561600 & -1.34679600 \\
\hline$C$ & 0.13516200 & -0.37479500 & 3.72215200 & $\mathrm{H}$ & 0.11501200 & 0.39740000 & 4.49226400 \\
\hline C & 0.08904500 & -1.77378500 & 4.44073600 & C & 0.11840200 & -2.96145000 & 3.45610100 \\
\hline $\mathrm{H}$ & 1.03721800 & -2.97166400 & 2.86341300 & $\mathrm{H}$ & -0.73542100 & -2.96736100 & 2.77136900 \\
\hline $\mathrm{H}$ & 0.06975900 & -3.89723900 & 4.02554200 & C & -1.20396000 & -1.84168500 & 5.28254400 \\
\hline $\mathrm{H}$ & -1.20258500 & -2.75742700 & 5.88519900 & $\mathrm{H}$ & -2.10047000 & -1.86013300 & 4.65673400 \\
\hline $\mathrm{H}$ & -1.27979300 & -0.98847200 & 5.96776700 & C & 1.29812900 & -1.89523900 & 5.39048800 \\
\hline $\mathrm{H}$ & 1.27436400 & -2.86865800 & 5.89254400 & $\mathrm{H}$ & 1.27936800 & -1.12681500 & 6.17292100 \\
\hline $\mathrm{H}$ & 2.25313700 & -1.82610900 & 4.85719400 & C & 1.39337100 & -0.18481100 & 2.83342000 \\
\hline 0 & 1.43247200 & -0.74369400 & 1.72140900 & C & 2.53600800 & 1.27630900 & 4.56853700 \\
\hline $\mathrm{C}$ & 3.67811400 & 0.69653400 & 2.49998900 & C & 4.01703900 & 1.10826500 & 4.91169300 \\
\hline $\mathrm{H}$ & 2.26389200 & 2.33066400 & 4.43384300 & $\mathrm{H}$ & 1.87821300 & 0.85045200 & 5.32190100 \\
\hline $\mathrm{H}$ & 3.94428900 & -0.30897900 & 2.16172900 & $\mathrm{H}$ & 4.18139400 & 0.11590800 & 5.34755500 \\
\hline $\mathrm{H}$ & 4.36599800 & 1.85535600 & 5.63088200 & $\mathrm{~N}$ & 2.42282300 & 0.56747300 & 3.28266400 \\
\hline C & 3.56860800 & 1.63126200 & 1.29688900 & C & 2.51323500 & 2.48474800 & 1.15764800 \\
\hline $\mathrm{C}$ & 4.64960600 & 1.65849300 & 0.32453100 & C & 2.41521400 & 3.42261800 & 0.07576200 \\
\hline $\mathrm{H}$ & 1.69728600 & 2.46301400 & 1.87449500 & C & 4.57526100 & 2.58796800 & -0.76523700 \\
\hline $\mathrm{C}$ & 5.77564400 & 0.81804500 & 0.40249400 & C & 1.31912800 & 4.29081800 & -0.05641200 \\
\hline $\mathrm{C}$ & 3.45926900 & 3.46751500 & -0.89172100 & C & 5.62596300 & 2.64504400 & -1.73422600 \\
\hline $\mathrm{H}$ & 5.86022600 & 0.09760900 & 1.20799700 & C & 6.79882100 & 0.88779600 & -0.54417700 \\
\hline $\mathrm{C}$ & 1.24364300 & 5.18392000 & -1.12453400 & $\mathrm{H}$ & 0.52646700 & 4.25786100 & 0.68588200 \\
\hline $\mathrm{C}$ & 3.38022100 & 4.39095800 & -1.97830300 & C & 6.72986800 & 1.78801900 & -1.60233900 \\
\hline $\mathrm{C}$ & 5.52228200 & 3.58610200 & -2.81645200 & $\mathrm{H}$ & 7.66279100 & 0.23579100 & -0.44463100 \\
\hline $\mathrm{C}$ & 2.26222600 & 5.23594600 & -2.07460500 & $\mathrm{H}$ & 0.37789300 & 5.83208500 & -1.21647800 \\
\hline C & 4.45145800 & 4.41823400 & -2.93451800 & $\mathrm{H}$ & 7.53294000 & 1.83947900 & -2.33373700 \\
\hline $\mathrm{H}$ & 6.32911500 & 3.62051400 & -3.54487200 & $\mathrm{H}$ & 2.19939700 & 5.93671600 & -2.90388600 \\
\hline $\mathrm{H}$ & 4.39130900 & 5.12469200 & -3.75916700 & C & -1.56342000 & 1.03426300 & 2.57418500 \\
\hline$S$ & -0.85424300 & 2.49440400 & 3.06333100 & $\mathrm{~N}$ & -1.04982400 & -0.17610000 & 2.90681700 \\
\hline $\mathrm{H}$ & -1.54695900 & -1.00094400 & 2.57157000 & $S$ & -3.43870300 & -2.63294700 & 0.85649900 \\
\hline $\mathrm{O}$ & -2.76223500 & -3.94409200 & 0.80077900 & 0 & -3.25655900 & -1.89798400 & 2.15218700 \\
\hline $\mathrm{O}$ & -3.27327100 & -1.75817600 & -0.32809200 & $\mathrm{C}$ & -5.25475400 & -3.03972500 & 0.85786100 \\
\hline $\mathrm{F}$ & -5.58288200 & -3.65616600 & -0.28832000 & $\mathrm{~F}$ & -5.98700000 & -1.91667100 & 0.95830500 \\
\hline $\mathrm{F}$ & -5.56644500 & -3.84256500 & 1.87975200 & C & 3.94557900 & -2.37369900 & -0.20973500 \\
\hline $\mathrm{O}$ & 4.29845200 & -2.47126000 & 0.94808000 & $\mathrm{O}$ & 4.49027700 & -2.97034600 & -1.27771900 \\
\hline
\end{tabular}




\begin{tabular}{|c|c|c|c|c|c|c|c|}
\hline C & 5.66960500 & -3.86914800 & -1.14595200 & $\mathrm{C}$ & 6.85263800 & -3.09128200 & -0.56608600 \\
\hline $\mathrm{H}$ & 7.75227400 & -3.71413800 & -0.61831400 & $\mathrm{H}$ & 6.67715000 & -2.81844800 & 0.47547500 \\
\hline $\mathrm{H}$ & 7.03211700 & -2.18071400 & -1.14676100 & C & 5.93701600 & -4.27006100 & -2.59737400 \\
\hline $\mathrm{H}$ & 5.07162300 & -4.78457800 & -3.02757200 & $\mathrm{H}$ & 6.79541700 & -4.94773700 & -2.64209400 \\
\hline $\mathrm{H}$ & 6.16040000 & -3.39048700 & -3.20971700 & C & 5.28986200 & -5.08415600 & -0.29675300 \\
\hline $\mathrm{H}$ & 6.12600000 & -5.79169600 & -0.28384600 & $\mathrm{H}$ & 4.42101000 & -5.59712500 & -0.72342100 \\
\hline $\mathrm{H}$ & 5.06334100 & -4.79512200 & 0.73085000 & C & 2.44870900 & -1.34772500 & -1.85510100 \\
\hline $\mathrm{H}$ & 3.20839800 & -1.57210500 & -2.59300500 & C & 1.52121100 & -0.26702100 & -2.18764200 \\
\hline C & 1.58563600 & 0.27005600 & -3.48876600 & C & 0.58251600 & 0.25135700 & -1.27571400 \\
\hline C & 0.75270100 & 1.31949300 & -3.86214500 & $\mathrm{H}$ & 2.30623300 & -0.12928600 & -4.19891800 \\
\hline C & -0.25100100 & 1.29721800 & -1.66113400 & $\mathrm{H}$ & 0.50460300 & -0.14593800 & -0.26892700 \\
\hline C & -0.16669700 & 1.83836000 & -2.94549500 & $\mathrm{H}$ & 0.82495500 & 1.73760300 & -4.86187900 \\
\hline $\mathrm{H}$ & -0.97285400 & 1.69316200 & -0.95557000 & $\mathrm{H}$ & -0.81453800 & 2.66527900 & -3.21986100 \\
\hline $\mathrm{N}$ & 2.85249400 & -1.57406600 & -0.57625000 & $\mathrm{H}$ & 2.36260200 & -1.16046600 & 0.23538200 \\
\hline C & 1.50735600 & -3.16880800 & -2.64144800 & $\mathrm{H}$ & 1.47382700 & -2.85009100 & -3.68028900 \\
\hline $\mathrm{H}$ & 2.35038900 & -3.79190500 & -2.36095300 & C & 0.33206400 & -3.22187300 & -1.90971200 \\
\hline C & -0.91703800 & -2.65674000 & -2.41878000 & $\mathrm{H}$ & -0.73786900 & -1.88797100 & -3.18057300 \\
\hline $\mathrm{H}$ & -1.56115700 & -2.26096900 & -1.62352200 & C & 0.35260700 & -3.77539600 & -0.51816100 \\
\hline $\mathrm{H}$ & -0.62046100 & -4.16742900 & -0.21100600 & $\mathrm{H}$ & 0.57249800 & -2.94972900 & 0.17919000 \\
\hline $\mathrm{H}$ & 1.12970400 & -4.53370500 & -0.38181500 & C & -3.70180900 & -3.09473900 & -3.60610500 \\
\hline $\mathrm{H}$ & -4.15773500 & -2.82916100 & -2.64691300 & $\mathrm{H}$ & -4.39687600 & -3.74565700 & -4.15100900 \\
\hline $\mathrm{H}$ & -3.58218400 & -2.17335500 & -4.18818000 & C & -2.26727200 & -5.52457100 & -2.29365900 \\
\hline $\mathrm{H}$ & -2.66587200 & -5.28838500 & -1.30114900 & $\mathrm{H}$ & -1.32343700 & -6.06756300 & -2.16339900 \\
\hline $\mathrm{H}$ & -2.97092600 & -6.20581500 & -2.78883100 & C & -1.22647200 & -4.38476800 & -4.98362200 \\
\hline $\mathrm{H}$ & -0.23823000 & -4.83908700 & -4.84427000 & $\mathrm{H}$ & -1.10281700 & -3.49502900 & -5.61301500 \\
\hline $\mathrm{H}$ & -1.83911400 & -5.10045000 & -5.54580000 & $\mathrm{Si}$ & -2.05354300 & -3.96539800 & -3.33050500 \\
\hline C & 4.71265100 & 1.22584500 & 3.54601600 & $\mathrm{H}$ & 4.94737300 & 2.27206400 & 3.32776400 \\
\hline $\bar{H}$ & 5.65034800 & 0.66532300 & 3.50587800 & & & & \\
\hline
\end{tabular}

\section{TS-S-4f-nd-8}

Imaginary frequency: $-247.45 \mathrm{~cm}^{-1}$ E[B3LYP/6-31G(d)]: -4788.691536 Hartree $\mathrm{E}\left[\mathrm{PCM}\left(\mathrm{Et}_{2} \mathrm{O}\right)-\mathrm{B} 3 \mathrm{~L} Y \mathrm{P} / 6-311 \mathrm{G}(\mathrm{d}, \mathrm{p})-\mathrm{D} 3(\mathrm{BJ})\right]$ : -4790.223420 Hartree

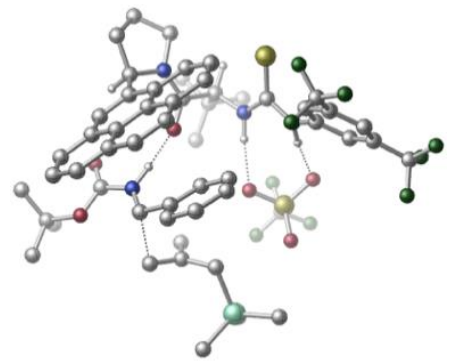

\begin{tabular}{|c|c|c|c|c|c|c|c|}
\hline 01 & & & & $\mathrm{~N}$ & 3.07379600 & -0.21661000 & 1.45264300 \\
\hline $\mathrm{H}$ & 3.17250300 & 0.80390700 & 1.38652100 & C & 3.96502400 & -0.93297300 & 0.63952000 \\
\hline C & 5.15910900 & -0.30168100 & 0.25643700 & C & 3.65941700 & -2.19393900 & 0.11004400 \\
\hline C & 6.03252100 & -0.93455800 & -0.62584600 & $\mathrm{H}$ & 5.39005700 & 0.68422200 & 0.64500800 \\
\hline C & 4.54300400 & -2.80839000 & -0.77693900 & $\mathrm{H}$ & 2.73339400 & -2.68218100 & 0.38231400 \\
\hline C & 5.73855100 & -2.19469500 & -1.14909600 & $\mathrm{H}$ & 6.42528100 & -2.68499500 & -1.82762400 \\
\hline C & 7.28531400 & -0.22583900 & -1.07019900 & C & 4.13648700 & -4.11619400 & -1.39792200 \\
\hline $\mathrm{F}$ & 7.74085600 & 0.63412600 & -0.13616200 & $\mathrm{~F}$ & 7.07721700 & 0.48989200 & -2.20019200 \\
\hline $\mathrm{F}$ & 8.28271700 & -1.09930500 & -1.34119900 & $\mathrm{~F}$ & 3.61051300 & -4.97439800 & -0.49120100 \\
\hline $\mathrm{F}$ & 3.17546200 & -3.93789900 & -2.34762000 & $\mathrm{~F}$ & 5.16499300 & -4.74702000 & -1.99943600 \\
\hline $\mathrm{C}$ & 0.15123400 & -0.02510800 & 3.64736700 & $\mathrm{H}$ & 0.54960700 & -0.76489300 & 4.34086300 \\
\hline C & -0.21309600 & 1.25915200 & 4.47759900 & C & -0.86147900 & 2.37436300 & 3.63256100 \\
\hline $\mathrm{H}$ & -1.82148800 & 2.05915500 & 3.21370000 & $\mathrm{H}$ & -0.21714300 & 2.69629700 & 2.81079600 \\
\hline $\mathrm{H}$ & -1.03908700 & 3.24789100 & 4.27197000 & C & 1.08242300 & 1.79267500 & 5.12355700 \\
\hline $\mathrm{H}$ & 0.84409300 & 2.61729500 & 5.80575400 & $\mathrm{H}$ & 1.78313000 & 2.17126200 & 4.37520900 \\
\hline $\mathrm{H}$ & 1.59180200 & 1.01258500 & 5.70210800 & C & -1.19504400 & 0.86608600 & 5.60095600 \\
\hline
\end{tabular}




\begin{tabular}{|c|c|c|c|c|c|c|c|}
\hline $\mathrm{H}$ & -1.47277500 & 1.75583300 & 6.17713400 & $\mathrm{H}$ & -0.74555100 & 0.15281000 & 6.30274300 \\
\hline $\mathrm{H}$ & -2.12215200 & 0.43223600 & 5.20733400 & C & -1.05392500 & -0.62082800 & 2.87713900 \\
\hline 0 & -1.45900700 & -0.04183300 & 1.85717200 & C & -1.28422100 & -2.50139500 & 4.55558400 \\
\hline C & -2.85263200 & -2.31698500 & 2.67087100 & $\mathrm{C}$ & -2.58675300 & -3.22081000 & 4.91678400 \\
\hline $\mathrm{H}$ & -0.46586000 & -3.20485100 & 4.36117200 & $\mathrm{H}$ & -0.95985000 & -1.82026600 & 5.33970400 \\
\hline $\mathrm{H}$ & -3.63295100 & -1.55621000 & 2.79075400 & $\mathrm{H}$ & -3.24677900 & -2.54447800 & 5.47362700 \\
\hline $\mathrm{H}$ & -2.41052600 & -4.10588400 & 5.53515300 & $\mathrm{~N}$ & -1.62661700 & -1.76608800 & 3.31713900 \\
\hline C & -2.83153200 & -2.67581100 & 1.18732600 & C & -3.96814600 & -2.37364300 & 0.48925300 \\
\hline C & -1.78378700 & -3.44773600 & 0.53709600 & C & -4.19515300 & -2.78698000 & -0.86402100 \\
\hline $\mathrm{H}$ & -4.75201900 & -1.79804500 & 0.97737500 & C & -1.98571200 & -3.87616500 & -0.81839700 \\
\hline C & -0.57488800 & -3.79450400 & 1.16387300 & C & -5.37335700 & -2.46680200 & -1.55937000 \\
\hline C & -3.18684500 & -3.55315600 & -1.51727100 & C & -0.97533000 & -4.63524500 & -1.48814000 \\
\hline $\mathrm{H}$ & -0.36483000 & -3.45899000 & 2.16933600 & C & 0.40719300 & -4.52744500 & 0.49730800 \\
\hline C & -5.56116800 & -2.89033300 & -2.87453000 & $\mathrm{H}$ & -6.14774500 & -1.89514600 & -1.05302500 \\
\hline C & -3.38732700 & -3.98459500 & -2.86509400 & C & 0.21583600 & -4.94519600 & -0.81453800 \\
\hline C & -1.20454400 & -5.05982600 & -2.84198700 & $\mathrm{H}$ & 1.33679700 & -4.75452900 & 1.00960100 \\
\hline C & -4.58083800 & -3.64037500 & -3.52194900 & $\mathrm{H}$ & -6.48122500 & -2.64028100 & -3.39654100 \\
\hline C & -2.35529200 & -4.75407600 & -3.50127200 & $\mathrm{H}$ & 0.99639800 & -5.49639200 & -1.33081500 \\
\hline $\mathrm{H}$ & -0.42764300 & -5.64168700 & -3.33214000 & $\mathrm{H}$ & -4.73507300 & -3.97195400 & -4.54604500 \\
\hline $\mathrm{H}$ & -2.51288600 & -5.08894700 & -4.52383600 & C & 2.17349100 & -0.69737900 & 2.37615800 \\
\hline $\mathrm{S}$ & 2.28338000 & -2.22993900 & 3.08861800 & $\mathrm{~N}$ & 1.21981200 & 0.21974300 & 2.68894200 \\
\hline $\mathrm{H}$ & 1.12161800 & 1.02719700 & 2.06702700 & $S$ & 2.40306700 & 3.27930800 & 0.56423900 \\
\hline 0 & 2.55671500 & 3.85464500 & -0.78412400 & $\mathrm{O}$ & 1.13673500 & 2.50994700 & 0.76991800 \\
\hline $\mathrm{O}$ & 3.59255600 & 2.59359600 & 1.13015600 & $\mathrm{C}$ & 2.18589900 & 4.76941500 & 1.65858300 \\
\hline $\mathrm{F}$ & 3.27561700 & 5.53974300 & 1.63976000 & $\mathrm{~F}$ & 1.94097900 & 4.39992300 & 2.92628300 \\
\hline $\mathrm{F}$ & 1.13763400 & 5.50585700 & 1.23273000 & $\mathrm{C}$ & -4.40862500 & 1.20477300 & 0.34829000 \\
\hline 0 & -4.63417300 & 1.09997500 & 1.53647500 & $\mathrm{O}$ & -5.24846800 & 1.64582600 & -0.60244500 \\
\hline C & -6.62221900 & 2.10219300 & -0.26236600 & $\mathrm{C}$ & -7.42129900 & 0.94522500 & 0.34215100 \\
\hline $\mathrm{H}$ & -8.46065900 & 1.26039800 & 0.48482200 & $\mathrm{H}$ & -7.01372000 & 0.64190300 & 1.30749200 \\
\hline $\mathrm{H}$ & -7.42084300 & 0.08422300 & -0.33504000 & C & -7.18397700 & 2.49879600 & -1.62852000 \\
\hline $\mathrm{H}$ & -6.59378400 & 3.30504500 & -2.07625100 & $\mathrm{H}$ & -8.21480900 & 2.85001500 & -1.51791300 \\
\hline $\mathrm{H}$ & -7.18259400 & 1.64446900 & -2.31308700 & C & -6.54224300 & 3.31068600 & 0.67366600 \\
\hline $\mathrm{H}$ & -7.54893800 & 3.71167500 & 0.83380800 & $\mathrm{H}$ & -5.93118500 & 4.10303300 & 0.22748100 \\
\hline $\mathrm{H}$ & -6.11819300 & 3.03666600 & 1.64090900 & C & -2.87096900 & 0.79596500 & -1.52544900 \\
\hline $\mathrm{H}$ & -3.74352300 & 0.70516900 & -2.16145600 & C & -1.66201100 & 0.10152500 & -1.97813400 \\
\hline C & -1.69157400 & -0.52525100 & -3.23871400 & C & -0.49004700 & 0.03217100 & -1.20214900 \\
\hline C & -0.58878500 & -1.24031700 & -3.69591100 & $\mathrm{H}$ & -2.59485700 & -0.47781800 & -3.84224100 \\
\hline C & 0.61017900 & -0.67982600 & -1.67059200 & $\mathrm{H}$ & -0.42733900 & 0.52360300 & -0.23818900 \\
\hline C & 0.56290800 & -1.32586300 & -2.90772900 & $\mathrm{H}$ & -0.63312300 & -1.74509600 & -4.65608300 \\
\hline $\mathrm{H}$ & 1.50858400 & -0.72733400 & -1.06499800 & $\mathrm{H}$ & 1.41974900 & -1.90176800 & -3.24534600 \\
\hline $\mathrm{N}$ & -3.16597200 & 0.85795200 & -0.19441300 & $\mathrm{H}$ & -2.50244200 & 0.51215500 & 0.51562000 \\
\hline C & -2.74573500 & 2.77793700 & -2.34234600 & $\mathrm{H}$ & -2.71246800 & 2.47216500 & -3.38514700 \\
\hline $\mathrm{H}$ & -3.72012500 & 3.06971000 & -1.96433700 & C & -1.59760700 & 3.29440700 & -1.75801900 \\
\hline $\mathrm{C}$ & -0.29781000 & 3.20561400 & -2.41847900 & $\mathrm{H}$ & -0.28236300 & 2.41506800 & -3.17839900 \\
\hline $\mathrm{H}$ & 0.52388200 & 3.06623700 & -1.70704800 & C & -1.66781700 & 3.85297600 & -0.37112500 \\
\hline $\mathrm{H}$ & -0.92476000 & 4.63560600 & -0.20272000 & $\mathrm{H}$ & -1.40705800 & 3.05228200 & 0.33782300 \\
\hline $\mathrm{H}$ & -2.66580000 & 4.22372400 & -0.12055500 & C & 1.93071200 & 4.43547600 & -4.09671800 \\
\hline $\mathrm{H}$ & 1.90908400 & 3.57251500 & -4.77282600 & $\mathrm{H}$ & 2.62741600 & 4.21657700 & -3.28110500 \\
\hline $\mathrm{H}$ & 2.31925300 & 5.29154500 & -4.66213100 & C & 0.26992800 & 6.30969100 & -2.24417900 \\
\hline $\mathrm{H}$ & 0.89837100 & 6.10880400 & -1.37058400 & $\mathrm{H}$ & -0.72799200 & 6.59998200 & -1.89490100 \\
\hline $\mathrm{H}$ & 0.69605800 & 7.17363100 & -2.76956900 & C & -1.03197400 & 5.09758900 & -4.78694000 \\
\hline $\mathrm{H}$ & -2.04215800 & 5.27496500 & -4.39939900 & $\mathrm{H}$ & -1.07962200 & 4.24040700 & -5.46963200 \\
\hline $\mathrm{H}$ & -0.75055600 & 5.97408100 & -5.38372700 & $\mathrm{Si}$ & 0.22449300 & 4.81735900 & -3.39681700 \\
\hline
\end{tabular}




\begin{tabular}{|llll|llll|}
\hline $\mathrm{C}$ & -3.18613400 & -3.55017900 & 3.54695700 & $\mathrm{H}$ & -2.70663400 & -4.44036800 & 3.12565200 \\
\hline $\mathrm{H}$ & -4.26389400 & -3.73337300 & 3.56873000 & & & & \\
\hline
\end{tabular}

\section{TS-S-4f-nd-9}

Imaginary frequency: $-230.41 \mathrm{~cm}^{-1}$ E[B3LYP/6-31G(d)]: -4788.691427 Hartree

E[PCM(Et $\left.\left.{ }_{2} \mathrm{O}\right)-\mathrm{B} 3 L \mathrm{YP} / 6-311 \mathrm{G}(\mathrm{d}, \mathrm{p})-\mathrm{D} 3(\mathrm{BJ})\right]$ : -4790.222351 Hartree

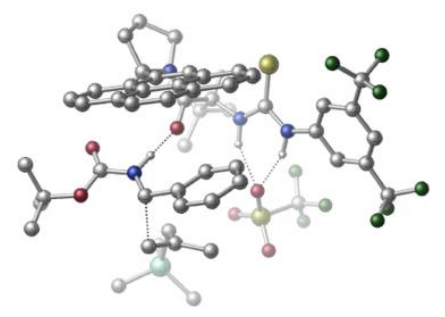

\begin{tabular}{|c|c|c|c|c|c|c|c|}
\hline 01 & & & & $\mathrm{~N}$ & 2.96391800 & 0.22902300 & 1.08562100 \\
\hline $\mathrm{H}$ & 2.75829700 & 1.13766500 & 0.65874800 & C & 4.01444400 & -0.45749100 & 0.45571200 \\
\hline $\mathrm{C}$ & 4.82083500 & 0.30544100 & -0.40906700 & $\mathrm{C}$ & 4.26471800 & -1.83261500 & 0.57429500 \\
\hline C & 5.84262300 & -0.29710600 & -1.13647900 & $\mathrm{H}$ & 4.65196300 & 1.37319400 & -0.49921800 \\
\hline $\mathrm{C}$ & 5.29329900 & -2.41678200 & -0.16588500 & $\mathrm{H}$ & 3.66597800 & -2.43008500 & 1.24462000 \\
\hline $\mathrm{C}$ & 6.09217300 & -1.66599300 & -1.02541200 & $\mathrm{H}$ & 6.88903600 & -2.13070600 & -1.59307600 \\
\hline C & 6.64956600 & 0.52560900 & -2.10712700 & C & 5.56920900 & -3.88684700 & 0.01020600 \\
\hline $\mathrm{F}$ & 6.73261600 & 1.81747400 & -1.73116500 & $\mathrm{~F}$ & 6.10134500 & 0.50670000 & -3.34555900 \\
\hline$F$ & 7.91055600 & 0.05343800 & -2.23236200 & $F$ & 4.43877400 & -4.58350800 & 0.29493400 \\
\hline $\mathrm{F}$ & 6.10456800 & -4.43539900 & -1.10344200 & $\mathrm{~F}$ & 6.43302800 & -4.12296300 & 1.02062300 \\
\hline $\mathrm{C}$ & 0.10800600 & 0.69128300 & 3.31863500 & $\mathrm{H}$ & 0.52801500 & 0.09963700 & 4.13137700 \\
\hline C & -0.33788000 & 2.07478200 & 3.91389000 & C & -1.00564400 & 2.99635300 & 2.87527600 \\
\hline $\mathrm{H}$ & -1.90446600 & 2.54207400 & 2.44831500 & $\mathrm{H}$ & -0.32963700 & 3.26902300 & 2.06043400 \\
\hline $\mathrm{H}$ & -1.29638500 & 3.93386000 & 3.36455500 & $\mathrm{C}$ & 0.91034900 & 2.77463100 & 4.49069700 \\
\hline $\mathrm{H}$ & 0.61252100 & 3.69451600 & 5.00726800 & $\mathrm{H}$ & 1.62203000 & 3.04929200 & 3.70778500 \\
\hline $\mathrm{H}$ & 1.42995900 & 2.13415600 & 5.21382100 & $\mathrm{C}$ & -1.33603200 & 1.82422600 & 5.06314500 \\
\hline $\mathrm{H}$ & -1.66173900 & 2.78215700 & 5.48335600 & $\mathrm{H}$ & -0.88109900 & 1.25005200 & 5.87970200 \\
\hline $\mathrm{H}$ & -2.23476400 & 1.29588400 & 4.72308500 & C & -1.03816100 & -0.09385000 & 2.63629300 \\
\hline $\mathrm{O}$ & -1.39893300 & 0.25319200 & 1.49698500 & C & -1.32972000 & -1.65282800 & 4.62081100 \\
\hline C & -2.70044200 & -1.91078000 & 2.62268600 & $\mathrm{C}$ & -2.71179100 & -2.12424500 & 5.07878200 \\
\hline $\mathrm{H}$ & -0.61044700 & -2.47998900 & 4.57512100 & $\mathrm{H}$ & -0.91441700 & -0.87608300 & 5.25828300 \\
\hline $\mathrm{H}$ & -3.41280900 & -1.17493000 & 2.23880100 & $\mathrm{H}$ & -3.29870700 & -1.26305900 & 5.41925200 \\
\hline $\mathrm{H}$ & -2.65603400 & -2.84271500 & 5.90203900 & $\mathrm{~N}$ & -1.60033900 & -1.14992900 & 3.26329700 \\
\hline C & -2.23592100 & -2.81858800 & 1.48618200 & C & -0.92208900 & -3.16184600 & 1.34920600 \\
\hline $\mathrm{C}$ & -3.22525000 & -3.38352200 & 0.58359100 & C & -0.46111400 & -4.06481700 & 0.33390100 \\
\hline $\mathrm{H}$ & -0.17546900 & -2.73897200 & 2.01621600 & C & -2.78941300 & -4.28710700 & -0.44115500 \\
\hline C & -4.59926000 & -3.09161900 & 0.66953800 & C & 0.89505400 & -4.40666200 & 0.20466500 \\
\hline $\mathrm{C}$ & -1.40974700 & -4.62639900 & -0.56799200 & $\mathrm{C}$ & -3.73892500 & -4.86334400 & -1.34243000 \\
\hline $\mathrm{H}$ & -4.96326500 & -2.41003100 & 1.43002800 & C & -5.51782000 & -3.66532800 & -0.21058300 \\
\hline C & 1.31580700 & -5.28118800 & -0.79654300 & $\mathrm{H}$ & 1.61633900 & -3.98107600 & 0.89659800 \\
\hline $\mathrm{C}$ & -0.97261400 & -5.52496000 & -1.58834300 & $\mathrm{C}$ & -5.09823900 & -4.54019900 & -1.20744500 \\
\hline $\mathrm{C}$ & -3.27161000 & -5.76527400 & -2.36008400 & $\mathrm{H}$ & -6.57417000 & -3.43137100 & -0.10629000 \\
\hline C & 0.39416900 & -5.83505300 & -1.68380100 & $\mathrm{H}$ & 2.37027800 & -5.52618500 & -0.87979700 \\
\hline $\mathrm{C}$ & -1.95276000 & -6.08064800 & -2.47901900 & $\mathrm{H}$ & -5.82050800 & -4.98657500 & -1.88683500 \\
\hline $\mathrm{H}$ & -4.00418900 & -6.19777200 & -3.03757800 & $\mathrm{H}$ & 0.72773700 & -6.51990400 & -2.45998800 \\
\hline $\mathrm{H}$ & -1.61835900 & -6.76724600 & -3.25326900 & $\mathrm{C}$ & 2.15077800 & -0.09719600 & 2.14426000 \\
\hline $\mathrm{S}$ & 2.32311900 & -1.46727900 & 3.12967700 & $\mathrm{~N}$ & 1.17937300 & 0.83545700 & 2.34347100 \\
\hline $\mathrm{H}$ & 1.07530400 & 1.56190200 & 1.63311800 & $\mathrm{~S}$ & 1.91220400 & 4.05224900 & -0.13896100 \\
\hline $\mathrm{O}$ & 0.92069500 & 4.81403800 & 0.64875700 & $\mathrm{O}$ & 1.74621600 & 2.56130800 & 0.00027300 \\
\hline $\mathrm{O}$ & 2.13890000 & 4.50314400 & -1.52278300 & C & 3.52766200 & 4.35972700 & 0.73512100 \\
\hline $\mathrm{F}$ & 3.79175000 & 5.66580000 & 0.80724900 & $\mathrm{~F}$ & 4.53775600 & 3.75491800 & 0.08247400 \\
\hline $\mathrm{F}$ & 3.48440900 & 3.85872200 & 1.98355900 & C & -4.49198100 & 0.58052500 & -0.24806200 \\
\hline 0 & -4.77826800 & 0.61375100 & 0.93145900 & $\mathrm{O}$ & -5.30946600 & 0.78766100 & -1.28938300 \\
\hline
\end{tabular}




\begin{tabular}{|c|c|c|c|c|c|c|c|}
\hline $\mathrm{C}$ & -6.75767600 & 1.07050700 & -1.09327800 & $\mathrm{C}$ & -7.42212300 & -0.10681400 & -0.37658800 \\
\hline $\mathrm{H}$ & -8.50745600 & 0.04132300 & -0.37159400 & $\mathrm{H}$ & -7.07698200 & -0.19078500 & 0.65494700 \\
\hline $\mathrm{H}$ & -7.20726800 & -1.04338700 & -0.90150200 & $\mathrm{C}$ & -7.26360600 & 1.19259000 & -2.53128200 \\
\hline $\mathrm{H}$ & -6.75220300 & 2.00438100 & -3.05860900 & $\mathrm{H}$ & -8.33674300 & 1.40813200 & -2.52914000 \\
\hline $\mathrm{H}$ & -7.10091800 & 0.26064000 & -3.08227100 & C & -6.92069700 & 2.38972600 & -0.33541000 \\
\hline $\mathrm{H}$ & -7.98427200 & 2.64581600 & -0.27965200 & $\mathrm{H}$ & -6.40589600 & 3.20175900 & -0.86071400 \\
\hline $\mathrm{H}$ & -6.52707600 & 2.31544500 & 0.67964300 & C & -2.81250800 & 0.12906100 & -1.97236400 \\
\hline $\mathrm{H}$ & -3.64968700 & -0.05390700 & -2.63424300 & C & -1.56132100 & -0.54594300 & -2.32947400 \\
\hline C & -1.51130700 & -1.19091000 & -3.58042800 & C & -0.43463300 & -0.58758900 & -1.48715700 \\
\hline C & -0.36924400 & -1.87713100 & -3.97611700 & $\mathrm{H}$ & -2.37999300 & -1.16225700 & -4.23430100 \\
\hline C & 0.70642100 & -1.27687300 & -1.89235400 & $\mathrm{H}$ & -0.44372500 & -0.09234300 & -0.52152100 \\
\hline C & 0.74270700 & -1.92178300 & -3.13020200 & $\mathrm{H}$ & -0.34666400 & -2.38177100 & -4.93745400 \\
\hline $\mathrm{H}$ & 1.57015100 & -1.31092200 & -1.23673600 & $\mathrm{H}$ & 1.63538700 & -2.46036400 & -3.43378800 \\
\hline $\mathrm{N}$ & -3.18459000 & 0.30615500 & -0.67565700 & $\mathrm{H}$ & -2.51355900 & 0.21120200 & 0.10528200 \\
\hline $\mathrm{C}$ & -2.74229700 & 2.05207700 & -2.96417100 & $\mathrm{H}$ & -2.61934300 & 1.66531800 & -3.97213100 \\
\hline $\mathrm{H}$ & -3.75831000 & 2.29579400 & -2.66820100 & C & -1.67808200 & 2.70348200 & -2.35701500 \\
\hline C & -1.86374200 & 3.47654700 & -1.12606600 & $\mathrm{H}$ & -0.97366300 & 3.46536400 & -0.48875300 \\
\hline $\mathrm{H}$ & -2.73396200 & 3.13135500 & -0.55267000 & C & -0.29618000 & 2.56390700 & -2.90686200 \\
\hline $\mathrm{H}$ & 0.23010400 & 3.52383500 & -2.92655800 & $\mathrm{H}$ & -0.28456000 & 2.11025400 & -3.90076600 \\
\hline $\mathrm{H}$ & 0.30065500 & 1.94184000 & -2.22413000 & $\mathrm{C}$ & -2.21068400 & 6.17012200 & 0.28007800 \\
\hline $\mathrm{H}$ & -2.94941100 & 5.71205600 & 0.94861500 & $\mathrm{H}$ & -2.43989800 & 7.24092800 & 0.21249800 \\
\hline $\mathrm{H}$ & -1.21918800 & 6.05630200 & 0.73010300 & C & -3.94360100 & 5.51545900 & -2.21774600 \\
\hline $\mathrm{H}$ & -4.72060300 & 5.08680400 & -1.57220300 & $\mathrm{H}$ & -3.99348000 & 5.01086400 & -3.18977700 \\
\hline $\mathrm{H}$ & -4.20268000 & 6.56862000 & -2.38283600 & C & -0.92449700 & 6.14122700 & -2.55908100 \\
\hline $\mathrm{H}$ & -0.99352200 & 5.76064000 & -3.58515800 & $\mathrm{H}$ & 0.08944700 & 5.95092100 & -2.19034900 \\
\hline $\mathrm{H}$ & -1.06672400 & 7.22849100 & -2.60434300 & $\mathrm{Si}$ & -2.22443500 & 5.37992100 & -1.42906300 \\
\hline C & -3.31955100 & -2.72671000 & 3.80164400 & $\mathrm{H}$ & -3.03295100 & -3.77853400 & 3.70542600 \\
\hline $\bar{H}$ & -4.41194000 & -2.68271100 & 3.79390300 & & & & \\
\hline
\end{tabular}

\section{TS-S-4f-nd-10}

Imaginary frequency: $-249.18 \mathrm{~cm}^{-1}$ E[B3LYP/6-31G(d)]: -4788.692509 Hartree

$\mathrm{E}\left[\mathrm{PCM}\left(\mathrm{Et}_{2} \mathrm{O}\right)-\mathrm{B} 3 \mathrm{LYP} / 6-311 \mathrm{G}(\mathrm{d}, \mathrm{p})-\mathrm{D} 3(\mathrm{BJ})\right]$ : -4790.222224 Hartree

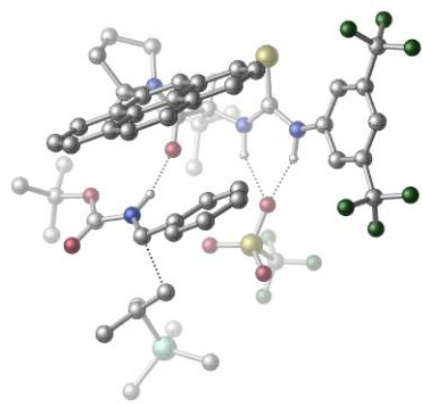

\begin{tabular}{|c|c|c|c|c|c|c|c|}
\hline 01 & & & & $\mathrm{~N}$ & -2.39585400 & -1.85562800 & 1.81415400 \\
\hline $\mathrm{H}$ & -1.60570400 & -2.38372300 & 1.43996900 & $\mathrm{C}$ & -3.56619100 & -2.04011200 & 1.06034400 \\
\hline $\mathrm{C}$ & -3.41648800 & -2.77682900 & -0.13044400 & $C$ & -4.83740900 & -1.55450900 & 1.39931600 \\
\hline C & -4.49981800 & -2.97258600 & -0.98145400 & $\mathrm{H}$ & -2.44192900 & -3.17780400 & -0.39520700 \\
\hline $\mathrm{C}$ & -5.90779100 & -1.75607700 & 0.52505700 & $\mathrm{H}$ & -4.97946200 & -1.01714200 & 2.32525700 \\
\hline $\mathrm{C}$ & -5.75849000 & -2.45667400 & -0.66903800 & $\mathrm{H}$ & -6.59922400 & -2.60720800 & -1.33451300 \\
\hline C & -4.28031700 & -3.65473500 & -2.30524400 & C & -7.23282000 & -1.12444000 & 0.85962700 \\
\hline $\mathrm{F}$ & -3.33134400 & -4.60816700 & -2.23699300 & $\mathrm{~F}$ & -3.87818200 & -2.76515600 & -3.25134800 \\
\hline $\mathrm{F}$ & -5.41018900 & -4.23068700 & -2.77058100 & $\mathrm{~F}$ & -7.25405500 & 0.18856500 & 0.50294100 \\
\hline $\mathrm{F}$ & -8.25930500 & -1.71848500 & 0.21372400 & $\mathrm{~F}$ & -7.50039700 & -1.16867500 & 2.18015300 \\
\hline $\mathrm{C}$ & 0.00877400 & 0.20077600 & 3.63644300 & $\mathrm{H}$ & -0.72098200 & 0.62224700 & 4.32715100 \\
\hline $\mathrm{C}$ & 1.21094800 & -0.35173400 & 4.47769400 & $\mathrm{C}$ & 2.26882800 & -1.06274100 & 3.61293600 \\
\hline $\mathrm{H}$ & 2.66742100 & -0.41383000 & 2.82943500 & $\mathrm{H}$ & 1.87196300 & -1.95628600 & 3.12538700 \\
\hline $\mathrm{H}$ & 3.09881100 & -1.38832500 & 4.25249000 & $\mathrm{C}$ & 0.64757100 & -1.34567900 & 5.51340300 \\
\hline $\mathrm{H}$ & 1.45758900 & -1.73094700 & 6.14392900 & $\mathrm{H}$ & 0.16494400 & -2.19732000 & 5.02527000 \\
\hline
\end{tabular}




\begin{tabular}{|c|c|c|c|c|c|c|c|}
\hline $\mathrm{H}$ & -0.09225400 & -0.86835800 & 6.16748400 & C & 1.88012200 & 0.81658700 & 5.22930800 \\
\hline $\mathrm{H}$ & 2.72394200 & 0.44275900 & 5.82031900 & $\mathrm{H}$ & 1.18740000 & 1.30226900 & 5.92757600 \\
\hline $\mathrm{H}$ & 2.27147800 & 1.57796400 & 4.54423000 & C & 0.39771900 & 1.25868900 & 2.57377800 \\
\hline 0 & 0.94597700 & 0.89150500 & 1.52449000 & $\mathrm{C}$ & -0.47550400 & 3.18664400 & 3.99021800 \\
\hline C & 0.34192700 & 3.56598100 & 1.72782200 & C & 0.20224500 & 4.56022000 & 3.97960800 \\
\hline $\mathrm{H}$ & -1.56712400 & 3.27091700 & 3.91624100 & $\mathrm{H}$ & -0.23914600 & 2.60542000 & 4.87920300 \\
\hline $\mathrm{H}$ & 1.36133900 & 3.38854300 & 1.37348100 & $\mathrm{H}$ & 1.21925900 & 4.46886100 & 4.37988200 \\
\hline $\mathrm{H}$ & -0.33397500 & 5.29627400 & 4.58612700 & $\mathrm{~N}$ & 0.06955500 & 2.55814600 & 2.77704200 \\
\hline C & -0.62546000 & 3.49111000 & 0.54904100 & C & -1.82732500 & 2.85631400 & 0.66896300 \\
\hline C & -0.28918800 & 4.16850200 & -0.69099300 & C & -2.79370400 & 2.82423700 & -0.39028600 \\
\hline $\mathrm{H}$ & -2.08104900 & 2.33631900 & 1.58843500 & C & -1.24024100 & 4.15479600 & -1.76421000 \\
\hline C & 0.92955100 & 4.84136300 & -0.89494100 & C & -4.02238500 & 2.15746800 & -0.25287800 \\
\hline C & -2.49193700 & 3.48754500 & -1.61379000 & C & -0.94001400 & 4.81299500 & -2.99772200 \\
\hline $\mathrm{H}$ & 1.66865700 & 4.87866100 & -0.10160200 & C & 1.21546500 & 5.47583800 & -2.10409200 \\
\hline C & -4.94313800 & 2.14325400 & -1.29990400 & $\mathrm{H}$ & -4.24697000 & 1.64641800 & 0.67947500 \\
\hline C & -3.44136800 & 3.47246800 & -2.68030000 & C & 0.29397000 & 5.46591200 & -3.14603600 \\
\hline C & -1.91634200 & 4.78765700 & -4.05310200 & $\mathrm{H}$ & 2.16698000 & 5.98623000 & -2.22827400 \\
\hline C & -4.65884200 & 2.79481600 & -2.49942400 & $\mathrm{H}$ & -5.88435800 & 1.61685300 & -1.17233000 \\
\hline C & -3.10964600 & 4.14972600 & -3.90266200 & $\mathrm{H}$ & 0.52038500 & 5.96765900 & -4.08384100 \\
\hline $\mathrm{H}$ & -1.67847800 & 5.29764200 & -4.98378500 & $\mathrm{H}$ & -5.38271900 & 2.78354200 & -3.31106600 \\
\hline $\mathrm{H}$ & -3.83643800 & 4.14333900 & -4.71174300 & C & -2.03261500 & -0.86702500 & 2.70141500 \\
\hline S & -3.09821700 & 0.20920700 & 3.46049300 & $\mathrm{~N}$ & -0.68723900 & -0.86282000 & 2.92276400 \\
\hline $\mathrm{H}$ & -0.11500500 & -1.39436300 & 2.26198300 & S & 1.39773100 & -3.13506400 & 0.12271800 \\
\hline 0 & 1.20324400 & -3.56852700 & -1.27172500 & $\mathrm{O}$ & 2.52090600 & -2.19627100 & 0.35402100 \\
\hline 0 & 0.14186000 & -2.74710500 & 0.83938500 & C & 1.90977200 & -4.67843000 & 1.03064100 \\
\hline $\mathrm{F}$ & 0.98555600 & -5.63604400 & 0.90221500 & $\mathrm{~F}$ & 2.08302000 & -4.42581600 & 2.33870700 \\
\hline $\mathrm{F}$ & 3.07362500 & -5.14366900 & 0.53937500 & C & 3.81091300 & 1.81208000 & -0.54769800 \\
\hline 0 & 4.60946200 & 2.11148800 & -1.41877300 & $\mathrm{O}$ & 3.90938200 & 2.05316300 & 0.76288700 \\
\hline C & 5.10555800 & 2.71901100 & 1.33327900 & C & 6.35118200 & 1.87027800 & 1.06410000 \\
\hline $\mathrm{H}$ & 7.20156900 & 2.29271300 & 1.61038700 & $\mathrm{H}$ & 6.59756500 & 1.85100500 & 0.00114800 \\
\hline $\bar{H}$ & 6.19697800 & 0.84491000 & 1.41642000 & $\mathrm{C}$ & 4.78261000 & 2.74953400 & 2.82767800 \\
\hline $\bar{H}$ & 3.86741900 & 3.32049300 & 3.01492900 & $\mathrm{H}$ & 5.60337800 & 3.22291200 & 3.37621300 \\
\hline $\mathrm{H}$ & 4.64299200 & 1.73590600 & 3.21499800 & C & 5.22872400 & 4.13652600 & 0.76772200 \\
\hline $\mathrm{H}$ & 6.04838400 & 4.65906400 & 1.27300800 & $\mathrm{H}$ & 4.30751800 & 4.70198100 & 0.94689900 \\
\hline $\bar{H}$ & 5.43275700 & 4.12105400 & -0.30405600 & $\mathrm{C}$ & 2.25075800 & 0.90675100 & -2.11086200 \\
\hline $\mathrm{H}$ & 2.80402400 & 1.51795400 & -2.81462500 & C & 0.85220200 & 0.61654100 & -2.45428200 \\
\hline $\mathrm{C}$ & 0.40963000 & 0.96781200 & -3.74351500 & C & -0.03404700 & -0.02023700 & -1.56919200 \\
\hline $\mathrm{C}$ & -0.89233000 & 0.68824000 & -4.14312400 & $\mathrm{H}$ & 1.09283700 & 1.46559500 & -4.42783400 \\
\hline C & -1.33440000 & -0.29801500 & -1.97886300 & $\mathrm{H}$ & 0.28472200 & -0.31368800 & -0.57505100 \\
\hline $\mathrm{C}$ & -1.76545200 & 0.04420400 & -3.26217600 & $\mathrm{H}$ & -1.22621800 & 0.97145600 & -5.13679300 \\
\hline $\mathrm{H}$ & -2.01542600 & -0.79191800 & -1.29569900 & $\mathrm{H}$ & -2.77744900 & -0.19872800 & -3.57059700 \\
\hline $\mathrm{N}$ & 2.61078900 & 1.15395100 & -0.81695300 & $\mathrm{H}$ & 2.00554000 & 0.92156000 & -0.01936400 \\
\hline C & 3.08459800 & -0.88944000 & -2.83211600 & $\mathrm{H}$ & 2.54052200 & -1.54949700 & -2.16303100 \\
\hline $\mathrm{H}$ & 2.67563400 & -0.78718400 & -3.83309300 & C & 4.45729700 & -0.74349700 & -2.66616000 \\
\hline C & 5.11450800 & -1.21620400 & -1.44560000 & $\mathrm{H}$ & 4.40204800 & -1.30090100 & -0.61676600 \\
\hline $\mathrm{H}$ & 5.97353400 & -0.59564900 & -1.16500400 & C & 5.28723800 & -0.08158800 & -3.72927000 \\
\hline $\mathrm{H}$ & 6.15558500 & -0.70126900 & -3.98258600 & $\mathrm{H}$ & 5.67266200 & 0.87227100 & -3.35137300 \\
\hline $\mathrm{H}$ & 4.71560600 & 0.10668200 & -4.64239900 & C & 6.12462100 & -3.58738900 & 0.18742200 \\
\hline $\mathrm{H}$ & 6.77335800 & -2.88448400 & 0.72379900 & $\mathrm{H}$ & 6.60955000 & -4.57107500 & 0.21156700 \\
\hline $\mathrm{H}$ & 5.17773500 & -3.66542000 & 0.72990500 & $C$ & 7.47403400 & -2.97044700 & -2.52732400 \\
\hline $\mathrm{H}$ & 8.17740900 & -2.26548600 & -2.06801800 & $\mathrm{H}$ & 7.35228100 & -2.68765500 & -3.57965800 \\
\hline $\mathrm{H}$ & 7.94835700 & -3.95999900 & -2.51490100 & C & 4.61663600 & -4.16345100 & -2.49703300 \\
\hline $\mathrm{H}$ & 4.51513000 & -3.87876100 & -3.55111600 & $\mathrm{H}$ & 3.61766200 & -4.14996000 & -2.04920600 \\
\hline
\end{tabular}




\begin{tabular}{|lrrr|rrrr|}
\hline $\mathrm{H}$ & 4.98149400 & -5.19807200 & -2.47099500 & $\mathrm{Si}$ & 5.82523800 & -3.03901300 & -1.59256500 \\
\hline $\mathrm{C}$ & 0.23465600 & 4.92676800 & 2.48602400 & $\mathrm{H}$ & -0.69425200 & 5.42891000 & 2.19914400 \\
\hline $\mathrm{H}$ & 1.05945300 & 5.59935000 & 2.23365400 & & & & \\
\hline
\end{tabular}

\section{TS-S-4f-nd-11}

Imaginary frequency: $-259.35 \mathrm{~cm}-1$ E[B3LYP/6-31G(d)]: -4788.681916 Hartree $\mathrm{E}\left[\mathrm{PCM}\left(\mathrm{Et}_{2} \mathrm{O}\right)-\mathrm{B} 3 \mathrm{LYP} / 6-311 \mathrm{G}(\mathrm{d}, \mathrm{p})-\mathrm{D} 3(\mathrm{BJ})\right]$ : -4790.219987 Hartree

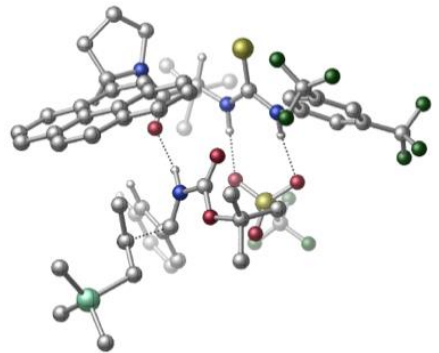

\begin{tabular}{|c|c|c|c|c|c|c|c|}
\hline 01 & & & & $\mathrm{~N}$ & 3.27137200 & -0.22089200 & 0.97750600 \\
\hline $\mathrm{H}$ & 3.39251700 & 0.67750200 & 0.49643900 & C & 3.80882900 & -1.30486000 & 0.25906400 \\
\hline C & 5.00055500 & -1.09644200 & -0.44806300 & C & 3.15433200 & -2.53732600 & 0.14764700 \\
\hline $\mathrm{C}$ & 5.53803400 & -2.11818800 & -1.23137500 & $\mathrm{H}$ & 5.48838800 & -0.12960900 & -0.39923000 \\
\hline $\mathrm{C}$ & 3.70471600 & -3.54818300 & -0.63783900 & $\mathrm{H}$ & 2.21226100 & -2.68415200 & 0.65403200 \\
\hline C & 4.90231100 & -3.35578600 & -1.32812900 & $\mathrm{H}$ & 5.32425300 & -4.14683100 & -1.93593300 \\
\hline $\mathrm{C}$ & 6.85679500 & -1.90891900 & -1.92660900 & C & 2.93307600 & -4.82513600 & -0.81671700 \\
\hline $\mathrm{F}$ & 7.89742800 & -2.29474500 & -1.14992500 & $\mathrm{~F}$ & 7.06376800 & -0.61512300 & -2.24629100 \\
\hline $\mathrm{F}$ & 6.93955100 & -2.62931200 & -3.07022100 & $\mathrm{~F}$ & 2.29084800 & -5.20015700 & 0.31655600 \\
\hline $\mathrm{F}$ & 1.96834800 & -4.70167300 & -1.77023100 & $\mathrm{~F}$ & 3.71895800 & -5.85523400 & -1.19436200 \\
\hline $\mathrm{C}$ & 1.17490900 & 1.17780200 & 3.63187000 & $\mathrm{H}$ & 1.53845500 & 0.45017800 & 4.35723300 \\
\hline C & 1.44336900 & 2.60230100 & 4.24041500 & C & 0.89330000 & 3.75465700 & 3.38012700 \\
\hline $\mathrm{H}$ & -0.19667200 & 3.71229900 & 3.30538300 & $\mathrm{H}$ & 1.29791900 & 3.75259000 & 2.36515900 \\
\hline $\mathrm{H}$ & 1.16365200 & 4.71083800 & 3.84591900 & C & 2.96794100 & 2.76941700 & 4.40416500 \\
\hline $\mathrm{H}$ & 3.18439400 & 3.70536600 & 4.93285000 & $\mathrm{H}$ & 3.47415500 & 2.80595400 & 3.43560400 \\
\hline $\mathrm{H}$ & 3.40296000 & 1.94525600 & 4.98223500 & C & 0.78899600 & 2.67414700 & 5.63546000 \\
\hline $\mathrm{H}$ & 0.96262900 & 3.66205300 & 6.07720000 & $\mathrm{H}$ & 1.21160900 & 1.92917800 & 6.32035200 \\
\hline $\mathrm{H}$ & -0.29671200 & 2.52491000 & 5.58818800 & C & -0.31486900 & 0.89046900 & 3.33345200 \\
\hline $\mathrm{O}$ & -0.88336700 & 1.39778800 & 2.35144300 & $\mathrm{C}$ & -0.48297000 & -0.61732800 & 5.37813800 \\
\hline $\mathrm{C}$ & -2.42191300 & -0.22071700 & 3.92108700 & $\mathrm{C}$ & -1.66870600 & -1.47819200 & 5.83814200 \\
\hline $\mathrm{H}$ & 0.40842000 & -1.20484400 & 5.14074700 & $\mathrm{H}$ & -0.22465000 & 0.12556900 & 6.13979100 \\
\hline $\mathrm{C}$ & -2.89288700 & -0.71619000 & 5.31038900 & $\mathrm{H}$ & -2.89944900 & 0.72052600 & 3.63660600 \\
\hline $\mathrm{H}$ & -1.68048200 & -1.61123700 & 6.92371500 & $\mathrm{H}$ & -1.61776800 & -2.47056200 & 5.37844300 \\
\hline $\mathrm{H}$ & -3.11147400 & 0.14699000 & 5.95176500 & $\mathrm{H}$ & -3.79233900 & -1.33360200 & 5.25091400 \\
\hline $\mathrm{N}$ & -0.99343000 & 0.05838600 & 4.16213900 & C & -2.67058100 & -1.25739600 & 2.82635400 \\
\hline C & -1.66756500 & -2.06018200 & 2.36424700 & C & -4.01847100 & -1.41814000 & 2.30366600 \\
\hline C & -1.88963400 & -3.08828600 & 1.38740800 & $\mathrm{H}$ & -0.65114200 & -1.92485900 & 2.72403800 \\
\hline C & -4.26597700 & -2.43707500 & 1.32512900 & C & -5.09965600 & -0.60993800 & 2.70374100 \\
\hline $\mathrm{C}$ & -0.84985000 & -3.91449700 & 0.93105800 & C & -3.20374900 & -3.27437500 & 0.86994300 \\
\hline $\mathrm{C}$ & -5.58047700 & -2.61582700 & 0.78835400 & $\mathrm{H}$ & -4.95113300 & 0.16878400 & 3.44368700 \\
\hline $\mathrm{C}$ & -6.37622500 & -0.78624500 & 2.16843900 & C & -1.09215400 & -4.90324000 & -0.02205000 \\
\hline $\mathrm{H}$ & 0.14989300 & -3.77916900 & 1.33270700 & C & -3.44532600 & -4.28926700 & -0.10602300 \\
\hline C & -6.62053100 & -1.77769100 & 1.22286800 & $\mathrm{C}$ & -5.79644000 & -3.64858200 & -0.18917600 \\
\hline $\mathrm{H}$ & -7.18811800 & -0.14510200 & 2.50183100 & $\mathrm{C}$ & -2.37437100 & -5.08980400 & -0.53611100 \\
\hline $\mathrm{H}$ & -0.26942500 & -5.51904400 & -0.36963200 & C & -4.77930700 & -4.44758200 & -0.61508000 \\
\hline $\mathrm{H}$ & -7.62056800 & -1.91794700 & 0.81917800 & $\mathrm{H}$ & -6.80228600 & -3.78169500 & -0.58090400 \\
\hline $\mathrm{H}$ & -2.55676600 & -5.86249800 & -1.27932800 & $\mathrm{H}$ & -4.96273400 & -5.22659900 & -1.35156500 \\
\hline C & 2.54495200 & -0.24880300 & 2.13629600 & $\mathrm{~S}$ & 2.46994600 & -1.60783300 & 3.15989300 \\
\hline $\mathrm{N}$ & 1.94478000 & 0.93564200 & 2.42027300 & $\mathrm{H}$ & 1.88824500 & 1.63739600 & 1.67482300 \\
\hline $\mathrm{C}$ & -0.20806300 & 0.26771300 & -1.08724700 & $\mathrm{O}$ & 0.41522500 & -0.51224500 & -0.39973100 \\
\hline 0 & -0.43873900 & 0.18952700 & -2.40786800 & C & 0.31803400 & -0.77524100 & -3.26121600 \\
\hline C & -0.13035300 & -2.19923800 & -2.92920600 & $\mathrm{H}$ & 0.35564000 & -2.90243100 & -3.61427900 \\
\hline
\end{tabular}




\begin{tabular}{|c|c|c|c|c|c|c|c|}
\hline $\mathrm{H}$ & 0.14292300 & -2.47453900 & -1.91029800 & $\mathrm{H}$ & -1.21535000 & -2.30548500 & -3.04650100 \\
\hline C & -0.10976300 & -0.37007800 & -4.67282100 & $\mathrm{H}$ & 0.16656300 & 0.66939200 & -4.87538800 \\
\hline $\mathrm{H}$ & 0.39260000 & -1.00928300 & -5.40578200 & $\mathrm{H}$ & -1.19070000 & -0.48231700 & -4.81029100 \\
\hline $\mathrm{C}$ & 1.82274700 & -0.57055100 & -3.08478900 & $\mathrm{H}$ & 2.34873800 & -1.15382700 & -3.84909200 \\
\hline $\mathrm{H}$ & 2.08433200 & 0.48438400 & -3.20030600 & $\mathrm{H}$ & 2.16149600 & -0.90681500 & -2.10540300 \\
\hline C & -1.34335200 & 2.43703800 & -1.27755200 & $\mathrm{H}$ & -1.00731700 & 2.39116500 & -2.30743000 \\
\hline C & -1.28787100 & 3.81285800 & -0.69310500 & C & -0.84898800 & 4.85749300 & -1.51786400 \\
\hline C & -1.68420700 & 4.08965800 & 0.62280400 & C & -0.79643500 & 6.16222200 & -1.03016200 \\
\hline $\mathrm{H}$ & -0.50183900 & 4.63818500 & -2.52290900 & $\mathrm{C}$ & -1.63932300 & 5.39683100 & 1.10144300 \\
\hline $\mathrm{H}$ & -2.00098400 & 3.28597200 & 1.28114600 & $\mathrm{C}$ & -1.19968000 & 6.43593500 & 0.27694200 \\
\hline $\mathrm{H}$ & -0.43001800 & 6.96039800 & -1.66904000 & $\mathrm{H}$ & -1.94035800 & 5.60426500 & 2.12434800 \\
\hline $\mathrm{H}$ & -1.16273500 & 7.45322000 & 0.65685200 & $\mathrm{~N}$ & -0.84084600 & 1.38903400 & -0.53285600 \\
\hline $\mathrm{H}$ & -0.69065900 & 1.49019500 & 0.47937900 & $\mathrm{~S}$ & 2.57699100 & 2.85330400 & -1.00122000 \\
\hline $\mathrm{O}$ & 1.73355200 & 2.75308400 & -2.21336600 & 0 & 1.81412300 & 2.93198500 & 0.27746400 \\
\hline $\mathrm{O}$ & 3.72815800 & 1.91654800 & -0.93511800 & $C$ & 3.37187400 & 4.53037800 & -1.14000600 \\
\hline $\mathrm{F}$ & 4.14671000 & 4.59306800 & -2.23081600 & $\mathrm{~F}$ & 4.12851300 & 4.78304200 & -0.06316300 \\
\hline $\mathrm{F}$ & 2.43415900 & 5.48901500 & -1.23179200 & C & -3.21616100 & 2.27594000 & -1.52347400 \\
\hline $\mathrm{H}$ & -3.51402100 & 2.36482200 & -0.48125800 & $\mathrm{H}$ & -3.40604000 & 3.17031900 & -2.11291700 \\
\hline $\mathrm{C}$ & -3.49062400 & 1.03908800 & -2.14071800 & $C$ & -3.66339800 & 0.92166800 & -3.58377400 \\
\hline $\mathrm{H}$ & -3.19716600 & 1.75065500 & -4.12903700 & $\mathrm{H}$ & -3.28755800 & -0.03238600 & -3.96832900 \\
\hline C & -3.58241700 & -0.16588100 & -1.27253600 & $\mathrm{H}$ & -3.61650000 & -1.10521200 & -1.82692400 \\
\hline $\mathrm{H}$ & -2.72738700 & -0.17791300 & -0.58015700 & $\mathrm{H}$ & -4.46662200 & -0.10412100 & -0.62436900 \\
\hline $\mathrm{C}$ & -5.50754400 & 0.80325400 & -6.00740600 & $\mathrm{H}$ & -5.05041900 & -0.14331800 & -6.31791900 \\
\hline $\mathrm{H}$ & -6.52496800 & 0.82834100 & -6.41625400 & $\mathrm{H}$ & -4.94341800 & 1.61805600 & -6.47555000 \\
\hline $\mathrm{C}$ & -6.48241600 & -0.46669000 & -3.34231000 & $\mathrm{H}$ & -6.02465400 & -1.43213700 & -3.58705200 \\
\hline $\mathrm{H}$ & -6.53229200 & -0.39015800 & -2.25050200 & $\mathrm{H}$ & -7.51356700 & -0.48924300 & -3.71635200 \\
\hline C & -6.28839500 & 2.62918100 & -3.60990600 & $\mathrm{H}$ & -6.29459000 & 2.75569700 & -2.52139900 \\
\hline $\bar{H}$ & -5.73919300 & 3.47343800 & -4.04310000 & $\mathrm{H}$ & -7.32699100 & 2.70415000 & -3.95455500 \\
\hline Si & -5.54999900 & 0.97255000 & -4.12817600 & & & & \\
\hline
\end{tabular}

\section{TS-S-4f-nd-12}

Imaginary frequency: $-169.55 \mathrm{~cm}^{-1}$ E[B3LYP/6-31G(d)]: -4788.687171 Hartree E[PCM(Et $\left.\left.t_{2} \mathrm{O}\right)-B 3 L Y P / 6-311 G(d, p)-D 3(B J)\right]:-4790.219973$ Hartree

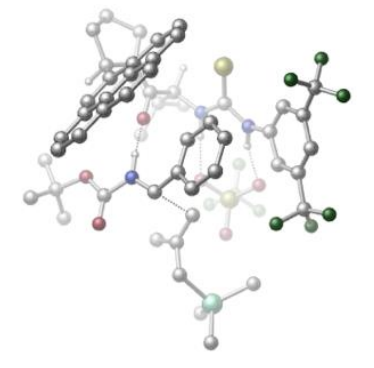

\begin{tabular}{|crrr|rrrc|}
\hline 01 & & & & $\mathrm{~N}$ & 1.33922900 & 1.55048300 & -2.30698600 \\
\hline $\mathrm{H}$ & 2.19474100 & 0.99634600 & -2.19025100 & $\mathrm{C}$ & 1.40407600 & 2.78089500 & -1.62129600 \\
\hline $\mathrm{C}$ & 2.37544100 & 2.88043700 & -0.60996200 & $\mathrm{C}$ & 0.57519800 & 3.88470500 & -1.87058300 \\
\hline $\mathrm{C}$ & 2.45957800 & 4.02410900 & 0.17879900 & $\mathrm{H}$ & 3.06267000 & 2.05656700 & -0.44970500 \\
\hline $\mathrm{C}$ & 0.67179800 & 5.01853200 & -1.06091100 & $\mathrm{H}$ & -0.14712900 & 3.84607300 & -2.67212200 \\
\hline $\mathrm{C}$ & 1.60015100 & 5.10321900 & -0.02535700 & $\mathrm{H}$ & 1.66114200 & 5.98740300 & 0.59651600 \\
\hline $\mathrm{C}$ & 3.43167600 & 4.02841400 & 1.32607600 & $\mathrm{C}$ & -0.32429100 & 6.12806100 & -1.25513900 \\
\hline $\mathrm{F}$ & 4.63945400 & 3.54494800 & 0.97654100 & $\mathrm{~F}$ & 2.98372800 & 3.24137500 & 2.34943600 \\
\hline $\mathrm{F}$ & 3.61566200 & 5.26178100 & 1.84255500 & $\mathrm{~F}$ & -0.66394200 & 6.29663600 & -2.54858600 \\
\hline $\mathrm{F}$ & -1.48159600 & 5.86947100 & -0.58150300 & $\mathrm{~F}$ & 0.13101800 & 7.31310700 & -0.79339900 \\
\hline $\mathrm{C}$ & -0.61645000 & -1.34145800 & -3.42401300 & $\mathrm{H}$ & -1.04473000 & -0.85549800 & -4.30212900 \\
\hline $\mathrm{C}$ & -0.01948300 & -2.72647400 & -3.88596700 & $\mathrm{C}$ & 0.49100000 & -3.59426100 & -2.71630200 \\
\hline $\mathrm{H}$ & -0.31205900 & -3.83868300 & -2.01407200 & $\mathrm{H}$ & 1.29628900 & -3.11182000 & -2.15898400 \\
\hline $\mathrm{H}$ & 0.88923300 & -4.53320800 & -3.12069400 & $\mathrm{C}$ & 1.13627300 & -2.45433100 & -4.87207100 \\
\hline
\end{tabular}




\begin{tabular}{|c|c|c|c|c|c|c|c|}
\hline $\mathrm{H}$ & 1.47446100 & -3.40045900 & -5.31123700 & $\mathrm{H}$ & 1.99708900 & -1.99128700 & -4.38658900 \\
\hline $\mathrm{H}$ & 0.81405800 & -1.80057200 & -5.69177200 & C & -1.10243400 & -3.52813500 & -4.63836900 \\
\hline $\mathrm{H}$ & -0.68798100 & -4.49290500 & -4.95117000 & $\mathrm{H}$ & -1.43718400 & -3.01184000 & -5.54619500 \\
\hline $\mathrm{H}$ & -1.97680000 & -3.73873500 & -4.01190900 & C & -1.70885400 & -1.49794800 & -2.32955600 \\
\hline 0 & -1.35688600 & -1.67567000 & -1.15359400 & C & -3.59945100 & -1.32038900 & -4.01285900 \\
\hline C & -4.05097900 & -1.94256100 & -1.68052700 & C & -4.86439700 & -2.18353700 & -3.97477700 \\
\hline $\mathrm{H}$ & -3.83184000 & -0.26216700 & -4.18594500 & $\mathrm{H}$ & -2.90626400 & -1.63344100 & -4.78914300 \\
\hline $\mathrm{H}$ & -3.75394400 & -2.95040200 & -1.36757600 & $\mathrm{H}$ & -4.60691500 & -3.22856000 & -4.18641100 \\
\hline $\mathrm{H}$ & -5.60719800 & -1.86432400 & -4.71193400 & $\mathrm{~N}$ & -3.02264200 & -1.50127100 & -2.66815400 \\
\hline C & -4.24651800 & -1.14781500 & -0.39157300 & C & -4.24436200 & -1.86391600 & 0.77310200 \\
\hline C & -4.59089600 & 0.26570100 & -0.35163200 & C & -4.59762400 & -1.30419300 & 2.04423800 \\
\hline $\mathrm{H}$ & -3.97158100 & -2.91684900 & 0.74833000 & C & -4.97778800 & 0.84563600 & 0.90264200 \\
\hline C & -4.56090100 & 1.10071600 & -1.48171000 & C & -4.59418900 & -2.06319300 & 3.22579200 \\
\hline C & -4.98415800 & 0.06587400 & 2.09729500 & C & -5.35559800 & 2.22349000 & 0.96975900 \\
\hline $\mathrm{H}$ & -4.21394100 & 0.71306300 & -2.42853600 & C & -4.92535800 & 2.44489400 & -1.40476600 \\
\hline C & -4.96568100 & -1.48981600 & 4.44054900 & $\mathrm{H}$ & -4.30272300 & -3.11002000 & 3.18349200 \\
\hline C & -5.36598500 & 0.64593700 & 3.34575500 & C & -5.32984000 & 3.00227400 & -0.19719400 \\
\hline C & -5.74586000 & 2.77888800 & 2.23684400 & $\mathrm{H}$ & -4.88169400 & 3.05969900 & -2.29918700 \\
\hline C & -5.34879900 & -0.15129800 & 4.50192400 & $\mathrm{H}$ & -4.95863100 & -2.09106900 & 5.34569800 \\
\hline C & -5.75197400 & 2.02885100 & 3.37200300 & $\mathrm{H}$ & -5.61625300 & 4.04971100 & -0.14393800 \\
\hline $\mathrm{H}$ & -6.03641700 & 3.82625800 & 2.26797000 & $\mathrm{H}$ & -5.63901200 & 0.28988000 & 5.45237400 \\
\hline $\mathrm{H}$ & -6.04767800 & 2.46681300 & 4.32225500 & C & 0.24132700 & 0.89238000 & -2.81399300 \\
\hline S & -1.21049800 & 1.63678400 & -3.27881000 & $\mathrm{~N}$ & 0.42401300 & -0.44901600 & -2.92820200 \\
\hline $\mathrm{H}$ & 1.22524300 & -0.87126100 & -2.44961500 & S & 4.12834800 & -0.93331900 & -1.50702600 \\
\hline 0 & 4.94291600 & -1.29602800 & -0.33191800 & $\mathrm{O}$ & 2.78223300 & -1.58270000 & -1.55838500 \\
\hline 0 & 4.06555000 & 0.51371500 & -1.83972200 & C & 5.04228700 & -1.68769600 & -2.94262900 \\
\hline $\mathrm{F}$ & 6.28751700 & -1.20722700 & -3.01039900 & $\mathrm{~F}$ & 4.41392200 & -1.40524000 & -4.09775200 \\
\hline $\mathrm{F}$ & 5.10034000 & -3.02005300 & -2.80947500 & C & -0.55015100 & -3.10468500 & 2.14469900 \\
\hline 0 & -0.05519200 & -3.32645700 & 3.23233800 & $\mathrm{O}$ & -1.09350500 & -3.97366000 & 1.29154200 \\
\hline C & -1.13024400 & -5.42890800 & 1.59035800 & C & 0.29625300 & -5.96562200 & 1.73011900 \\
\hline $\mathrm{H}$ & 0.26314500 & -7.05734500 & 1.81409800 & $\mathrm{H}$ & 0.78782000 & -5.56486300 & 2.61810700 \\
\hline $\mathrm{H}$ & 0.89028400 & -5.71139200 & 0.84627200 & C & -1.80952800 & -6.00057000 & 0.34519800 \\
\hline $\mathrm{H}$ & -2.81190000 & -5.57816200 & 0.22010400 & $\mathrm{H}$ & -1.90277100 & -7.08728400 & 0.43953300 \\
\hline $\mathrm{H}$ & -1.22434900 & -5.77835400 & -0.55233900 & C & -1.97409000 & -5.67652500 & 2.84325100 \\
\hline $\mathrm{H}$ & -2.07864100 & -6.75542400 & 3.00159100 & $\mathrm{H}$ & -2.97805400 & -5.25563000 & 2.72019400 \\
\hline $\mathrm{H}$ & -1.51014900 & -5.23838100 & 3.72854100 & C & -0.14996600 & -0.75796100 & 2.29585600 \\
\hline $\mathrm{H}$ & 0.11905800 & -1.02008600 & 3.31211400 & C & -0.57601000 & 0.61905200 & 2.05544500 \\
\hline C & -0.41905700 & 1.53820400 & 3.11252600 & C & -1.12126800 & 1.05744800 & 0.83563800 \\
\hline C & -0.79636400 & 2.86590400 & 2.95272300 & $\mathrm{H}$ & 0.00328100 & 1.20231300 & 4.05655800 \\
\hline C & -1.49741200 & 2.38872600 & 0.68337500 & $\mathrm{H}$ & -1.24606500 & 0.37113800 & 0.00468200 \\
\hline C & -1.33118700 & 3.29503000 & 1.73410500 & $\mathrm{H}$ & -0.67031300 & 3.56621700 & 3.77320200 \\
\hline $\mathrm{H}$ & -1.90869800 & 2.71864800 & -0.26406100 & $\mathrm{H}$ & -1.60603200 & 4.33538000 & 1.59081600 \\
\hline $\mathrm{N}$ & -0.64061100 & -1.81057500 & 1.60083600 & $\mathrm{H}$ & -1.01367500 & -1.71992800 & 0.64632100 \\
\hline C & 1.97828700 & -0.45346600 & 1.66756700 & $\mathrm{H}$ & 1.74136900 & -0.54524500 & 0.61194200 \\
\hline $\mathrm{H}$ & 2.06832700 & 0.55467800 & 2.05981800 & C & 2.63038900 & -1.49149000 & 2.30226600 \\
\hline C & 3.24913000 & -1.31520800 & 3.63135300 & $\mathrm{H}$ & 3.13308700 & -2.21429200 & 4.24948700 \\
\hline $\mathrm{H}$ & 2.81025100 & -0.46374400 & 4.16708700 & C & 2.76486600 & -2.81788500 & 1.61898300 \\
\hline $\mathrm{H}$ & 2.77945100 & -3.64844800 & 2.32985500 & $\mathrm{H}$ & 3.70700400 & -2.81288200 & 1.05494900 \\
\hline $\mathrm{H}$ & 1.97912000 & -2.96969900 & 0.87397400 & C & 5.60299500 & -0.70434000 & 5.44059600 \\
\hline $\mathrm{H}$ & 5.04318900 & 0.12194900 & 5.89492300 & $\mathrm{H}$ & 6.67045900 & -0.47876400 & 5.55418400 \\
\hline $\mathrm{H}$ & 5.39446100 & -1.61057500 & 6.02201800 & C & 5.47563300 & 0.65350300 & 2.63553100 \\
\hline $\mathrm{H}$ & 4.82657300 & 1.47887800 & 2.94638300 & $\mathrm{H}$ & 5.31810300 & 0.47762300 & 1.56579100 \\
\hline $\mathrm{H}$ & 6.51416000 & 0.98270500 & 2.76656300 & C & 6.13303900 & -2.36987800 & 2.87543700 \\
\hline
\end{tabular}




\begin{tabular}{|llll|llll|}
\hline $\mathrm{H}$ & 5.98665300 & -2.42630000 & 1.79173400 & $\mathrm{H}$ & 5.85377300 & -3.33279000 & 3.31959600 \\
\hline $\mathrm{H}$ & 7.20618200 & -2.22537500 & 3.05405100 & $\mathrm{Si}$ & 5.16568900 & -0.92533100 & 3.60955700 \\
\hline $\mathrm{C}$ & -5.34803800 & -2.02738900 & -2.52737400 & $\mathrm{H}$ & -5.92627200 & -1.10517800 & -2.41719900 \\
\hline $\mathrm{H}$ & -5.98006400 & -2.85264500 & -2.18761000 & & & & \\
\hline
\end{tabular}

\section{TS-S-4f-nd-13}

Imaginary frequency: $-247.56 \mathrm{~cm}^{-1}$ E[B3LYP/6-31G(d)]: -4788.689904 Hartree $\mathrm{E}\left[\mathrm{PCM}\left(\mathrm{Et}_{2} \mathrm{O}\right)-\mathrm{B} 3 \mathrm{~L} Y \mathrm{P} / 6-311 \mathrm{G}(\mathrm{d}, \mathrm{p})-\mathrm{D} 3(\mathrm{BJ})\right]$ : -4790.219045 Hartree

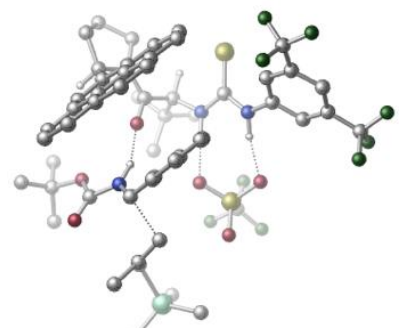

\begin{tabular}{|c|c|c|c|c|c|c|c|}
\hline 01 & & & & $\mathrm{~N}$ & 2.33965000 & 1.78941300 & 1.29757800 \\
\hline $\mathrm{H}$ & 1.64494600 & 2.47259900 & 0.97316200 & C & 3.57963800 & 1.90468700 & 0.64194100 \\
\hline$C$ & 3.95342700 & 3.16803000 & 0.16099700 & C & 4.38771300 & 0.79729200 & 0.35223600 \\
\hline C & 5.12429000 & 3.31536400 & -0.58155000 & $\mathrm{H}$ & 3.32026300 & 4.02516600 & 0.36070400 \\
\hline C & 5.54860300 & 0.96108900 & -0.40224200 & $\mathrm{H}$ & 4.10024400 & -0.18407700 & 0.70318400 \\
\hline C & 5.93566200 & 2.21717500 & -0.86884600 & $\mathrm{H}$ & 6.84704200 & 2.33866100 & -1.44111000 \\
\hline $\mathrm{C}$ & 5.49003200 & 4.66829300 & -1.13371900 & C & 6.32588900 & -0.26516000 & -0.79510200 \\
\hline $\mathrm{F}$ & 5.02486100 & 5.67321400 & -0.36263400 & $\mathrm{~F}$ & 4.98246300 & 4.85359300 & -2.37446100 \\
\hline $\mathrm{F}$ & 6.83084500 & 4.81950900 & -1.23479300 & $\mathrm{~F}$ & 6.40991700 & -1.16594000 & 0.21144000 \\
\hline $\mathrm{F}$ & 5.73128100 & -0.91492800 & -1.83507800 & $\mathrm{~F}$ & 7.58378000 & 0.02360500 & -1.18609500 \\
\hline $\mathrm{C}$ & -0.00272600 & -0.00398600 & 3.46760500 & $\mathrm{H}$ & 0.76962400 & -0.24917100 & 4.19576200 \\
\hline $\mathrm{C}$ & -1.19077000 & 0.66296600 & 4.24914600 & $C$ & -2.39303800 & 1.02704000 & 3.35595200 \\
\hline $\mathrm{H}$ & -2.81803100 & 0.14550700 & 2.86841000 & $\mathrm{H}$ & -2.13052400 & 1.74105400 & 2.57241300 \\
\hline $\mathrm{H}$ & -3.17162800 & 1.48821000 & 3.97715000 & $\mathrm{C}$ & -0.65687200 & 1.93952700 & 4.93022700 \\
\hline $\mathrm{H}$ & -1.43085600 & 2.36694100 & 5.57903200 & $\mathrm{H}$ & -0.37912100 & 2.69897600 & 4.19551700 \\
\hline $\mathrm{H}$ & 0.22318500 & 1.72649400 & 5.54873400 & C & -1.67286400 & -0.31165200 & 5.34412100 \\
\hline $\mathrm{H}$ & -2.51421700 & 0.13055500 & 5.88970500 & $\mathrm{H}$ & -0.88525700 & -0.52050300 & 6.07828700 \\
\hline $\mathrm{H}$ & -2.02012300 & -1.26474100 & 4.92644000 & $C$ & -0.40238700 & -1.27745400 & 2.68159000 \\
\hline 0 & -1.05707300 & -1.18570900 & 1.63434000 & C & 0.68742000 & -2.77425900 & 4.42002300 \\
\hline $\mathrm{C}$ & -0.41608300 & -3.73647600 & 2.44488200 & $C$ & 0.27160000 & -4.21591700 & 4.73117300 \\
\hline $\mathrm{H}$ & 1.77329400 & -2.67568200 & 4.30316700 & $\mathrm{H}$ & 0.37268100 & -2.07924800 & 5.19630300 \\
\hline $\mathrm{H}$ & -1.51183700 & -3.76727900 & 2.49493400 & $\mathrm{H}$ & -0.70542100 & -4.22401900 & 5.23009600 \\
\hline $\mathrm{H}$ & 0.98824100 & -4.71949700 & 5.38666100 & $\mathrm{~N}$ & 0.00554400 & -2.48885600 & 3.13896200 \\
\hline $\mathrm{C}$ & -0.05348500 & -3.92724300 & 0.97586400 & C & -1.04437900 & -4.40155300 & 0.16283600 \\
\hline C & 1.29614100 & -3.79704300 & 0.44785400 & C & -0.82130800 & -4.82247100 & -1.18841400 \\
\hline $\mathrm{H}$ & -2.05649500 & -4.48926200 & 0.55402000 & C & 1.55350500 & -4.23213900 & -0.89528100 \\
\hline C & 2.36698000 & -3.25880600 & 1.18278400 & $\mathrm{C}$ & -1.85495700 & -5.31587300 & -2.00149300 \\
\hline C & 0.50329600 & -4.75237000 & -1.70914800 & C & 2.87369100 & -4.13911400 & -1.43821400 \\
\hline $\mathrm{H}$ & 2.20408600 & -2.87294300 & 2.17926100 & $\mathrm{C}$ & 3.64593300 & -3.15403700 & 0.63573500 \\
\hline $\mathrm{C}$ & -1.59346400 & -5.74196500 & -3.30283000 & $\mathrm{H}$ & -2.86514600 & -5.36904700 & -1.60204600 \\
\hline C & 0.76328900 & -5.19322600 & -3.04325600 & C & 3.90403800 & -3.59222700 & -0.65822700 \\
\hline C & 3.10983400 & -4.60238400 & -2.77841600 & $\mathrm{H}$ & 4.44212600 & -2.71169300 & 1.22649300 \\
\hline C & -0.29979400 & -5.68512600 & -3.81878400 & $\mathrm{H}$ & -2.40314700 & -6.12617500 & -3.91770100 \\
\hline $\mathrm{C}$ & 2.10641400 & -5.11000000 & -3.54511100 & $\mathrm{H}$ & 4.89985700 & -3.49008600 & -1.08040700 \\
\hline $\mathrm{H}$ & 4.12241400 & -4.53774000 & -3.16961900 & $\mathrm{H}$ & -0.10162300 & -6.02385200 & -4.83298300 \\
\hline $\mathrm{H}$ & 2.30520300 & -5.45881100 & -4.55583000 & C & 1.98098800 & 0.93223400 & 2.31040500 \\
\hline $\mathrm{S}$ & 3.09814000 & 0.04782500 & 3.22980800 & $\mathrm{~N}$ & 0.63683100 & 0.90609400 & 2.52553300 \\
\hline $\mathrm{H}$ & 0.02843300 & 1.33275700 & 1.82004000 & $S$ & -0.75002100 & 3.52490100 & -0.01584400 \\
\hline 0 & -1.46636700 & 3.90931200 & -1.24440800 & 0 & -1.09235000 & 2.15829600 & 0.48690400 \\
\hline 0 & 0.70527000 & 3.81283400 & 0.02488200 & $\mathrm{C}$ & -1.44806000 & 4.64988000 & 1.29516400 \\
\hline $\mathrm{F}$ & -1.38940100 & 5.92745000 & 0.91210100 & $\mathrm{~F}$ & -0.76461300 & 4.51406200 & 2.44369200 \\
\hline
\end{tabular}




\begin{tabular}{|c|c|c|c|c|c|c|c|}
\hline $\mathrm{F}$ & -2.73632900 & 4.33725000 & 1.54393500 & C & -3.93582000 & -1.34631500 & -0.52542400 \\
\hline 0 & -4.73651800 & -1.50107300 & -1.43325200 & $\mathrm{O}$ & -4.13777600 & -1.53501200 & 0.77907100 \\
\hline $\mathrm{C}$ & -5.44456700 & -2.01020000 & 1.29443600 & C & -6.53291100 & -0.98134000 & 0.97670600 \\
\hline $\mathrm{H}$ & -7.46081800 & -1.26609500 & 1.48464700 & $\mathrm{H}$ & -6.72775300 & -0.92737500 & -0.09585000 \\
\hline $\mathrm{H}$ & -6.23813900 & 0.00899800 & 1.33980100 & C & -5.19398800 & -2.09257000 & 2.80084900 \\
\hline $\mathrm{H}$ & -4.37120600 & -2.78125100 & 3.01742300 & $\mathrm{H}$ & -6.09392400 & -2.45541600 & 3.30796300 \\
\hline $\mathrm{H}$ & -4.93671900 & -1.11000300 & 3.20704300 & C & -5.75726700 & -3.39294000 & 0.71536200 \\
\hline $\mathrm{H}$ & -6.66652600 & -3.78498900 & 1.18401100 & $\mathrm{H}$ & -4.93994500 & -4.09052600 & 0.92925600 \\
\hline $\mathrm{H}$ & -5.91334400 & -3.34780700 & -0.36368900 & C & -2.17319400 & -0.81265100 & -2.03594700 \\
\hline $\mathrm{H}$ & -2.80464500 & -1.34073200 & -2.74152200 & C & -0.73148500 & -0.81856400 & -2.32321900 \\
\hline C & -0.30450000 & -1.39980000 & -3.53139600 & C & 0.21593300 & -0.25686600 & -1.45202100 \\
\hline C & 1.04847300 & -1.43925000 & -3.85349400 & $\mathrm{H}$ & -1.03574800 & -1.83907500 & -4.20579100 \\
\hline $\mathrm{C}$ & 1.56574700 & -0.29430300 & -1.78587700 & $\mathrm{H}$ & -0.10096900 & 0.23147400 & -0.53766900 \\
\hline C & 1.98707500 & -0.88546000 & -2.97906300 & $\mathrm{H}$ & 1.37099000 & -1.90930300 & -4.77734800 \\
\hline $\mathrm{H}$ & 2.29407400 & 0.14597100 & -1.11579700 & $\mathrm{H}$ & 3.04672100 & -0.91480100 & -3.21591200 \\
\hline $\mathrm{N}$ & -2.62082600 & -0.93657200 & -0.75147900 & $\mathrm{H}$ & -2.00360000 & -0.82460900 & 0.06260700 \\
\hline $\mathrm{C}$ & -2.63108200 & 1.06250200 & -2.85707400 & $\mathrm{H}$ & -2.02673900 & 1.68121000 & -2.19882800 \\
\hline $\mathrm{H}$ & -2.19314900 & 0.82380800 & -3.82243000 & C & -4.01545400 & 1.15254800 & -2.77098500 \\
\hline C & -4.65372700 & 1.84568700 & -1.64696000 & $\mathrm{H}$ & -3.94985500 & 2.00231100 & -0.82211800 \\
\hline $\mathrm{H}$ & -5.54445500 & 1.31572200 & -1.28908400 & $\mathrm{C}$ & -4.87580000 & 0.56368900 & -3.85332100 \\
\hline $\mathrm{H}$ & -5.23433300 & 1.36502300 & -4.51418100 & $\mathrm{H}$ & -5.75255400 & 0.05886200 & -3.43949100 \\
\hline $\mathrm{H}$ & -4.31831200 & -0.14364400 & -4.47387400 & C & -5.56726300 & 4.52154900 & -0.46384200 \\
\hline $\mathrm{H}$ & -6.27326900 & 3.98262200 & 0.17959800 & $\mathrm{H}$ & -5.97459600 & 5.52641200 & -0.63092700 \\
\hline $\mathrm{H}$ & -4.62499600 & 4.62787700 & 0.08123800 & C & -6.99296600 & 3.44062300 & -2.97414000 \\
\hline $\mathrm{H}$ & -7.70478300 & 2.87975800 & -2.35646200 & $\mathrm{H}$ & -6.92006600 & 2.93466900 & -3.94343500 \\
\hline $\mathrm{H}$ & -7.43019100 & 4.42990900 & -3.15953300 & C & -4.09411800 & 4.53843300 & -3.22040800 \\
\hline $\mathrm{H}$ & -3.98101600 & 4.04650000 & -4.19404100 & $\mathrm{H}$ & -3.10833000 & 4.59168800 & -2.74772000 \\
\hline $\mathrm{H}$ & -4.44069800 & 5.56223700 & -3.40907300 & $\mathrm{Si}$ & -5.31650900 & 3.63755700 & -2.11049100 \\
\hline $\mathrm{C}$ & 0.17145100 & -4.85417100 & 3.34186900 & $\mathrm{H}$ & 1.16570700 & -5.13435600 & 2.97904700 \\
\hline $\mathrm{H}$ & -0.45535100 & -5.74981600 & 3.31269900 & & & & \\
\hline
\end{tabular}

\section{TS-S-4f-nd-14}

Imaginary frequency: $-243.07 \mathrm{~cm}^{-1}$ E[B3LYP/6-31G(d)]: -4788.690860 Hartree

E[PCM(Et $\left.\left.{ }_{2} \mathrm{O}\right)-\mathrm{B} 3 L Y P / 6-311 \mathrm{G}(\mathrm{d}, \mathrm{p})-\mathrm{D} 3(\mathrm{BJ})\right]$ : -4790.218919 Hartree

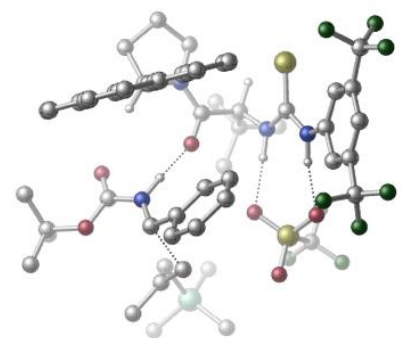

\begin{tabular}{|cccc|cccc|}
\hline 01 & & & N & -2.43313600 & -2.08788100 & 1.09487000 \\
\hline H & -1.75015200 & -2.67704600 & 0.60280900 & C & -3.64301900 & -1.96944700 & 0.37949600 \\
\hline C & -3.61744100 & -2.42598200 & -0.95498400 & C & -4.83769000 & -1.43396700 & 0.88158400 \\
\hline C & -4.73997000 & -2.29693000 & -1.76589200 & H & -2.71368300 & -2.88004700 & -1.34729300 \\
\hline C & -5.94744500 & -1.30147200 & 0.04207100 & H & -4.89587200 & -1.11705500 & 1.91166000 \\
\hline C & -5.91769400 & -1.72158000 & -1.28303900 & H & -6.78725400 & -1.61799000 & -1.92023900 \\
\hline C & -4.65884200 & -2.68876500 & -3.21657300 & C & -7.17092800 & -0.61564500 & 0.58715600 \\
\hline F & -3.73030300 & -3.63435300 & -3.44682000 & F & -4.33499400 & -1.61898300 & -3.99629300 \\
\hline F & -5.84180200 & -3.15042700 & -3.67984300 & F & -7.43876100 & -0.98180400 & 1.85703500 \\
\hline F & -7.01064800 & 0.73644100 & 0.60102700 & F & -8.27261000 & -0.86794800 & -0.15208000 \\
\hline C & 0.11728400 & -0.80170000 & 3.39273300 & H & -0.59985400 & -0.57400200 & 4.18013300 \\
\hline C & 1.27919900 & -1.62193500 & 4.05644100 & C & 2.45379900 & -1.91610300 & 3.10434400 \\
\hline H & 2.93281200 & -0.99466900 & 2.75868900 & H & 2.13773600 & -2.47863900 & 2.22330300 \\
\hline H & 3.20576700 & -2.51148300 & 3.63733400 & C & 0.69198200 & -2.95117700 & 4.57219700 \\
\hline H & 1.45010800 & -3.49270400 & 5.15064300 & H & 0.37353600 & -3.59494000 & 3.74861100 \\
\hline
\end{tabular}




\begin{tabular}{|c|c|c|c|c|c|c|c|}
\hline $\mathrm{H}$ & -0.17278700 & -2.78112300 & 5.22468800 & C & 1.81503200 & -0.81809900 & 5.25933900 \\
\hline $\mathrm{H}$ & 2.61997400 & -1.37671200 & 5.75036300 & $\mathrm{H}$ & 1.03381700 & -0.64003300 & 6.00807100 \\
\hline $\mathrm{H}$ & 2.23014800 & 0.14965800 & 4.95303200 & C & 0.57446800 & 0.50684200 & 2.70787800 \\
\hline $\mathrm{C}$ & 0.71205600 & 2.95801900 & 2.63130000 & $\mathrm{H}$ & 1.71165000 & 2.82765500 & 2.20973900 \\
\hline $\mathrm{N}$ & 0.33706100 & 1.69476200 & 3.30525700 & C & -0.26553500 & 3.35902300 & 1.52527000 \\
\hline C & -1.49630700 & 2.77748600 & 1.42076800 & C & 0.10955300 & 4.41400800 & 0.59713200 \\
\hline C & -2.45385500 & 3.16085900 & 0.42323000 & $\mathrm{H}$ & -1.78295500 & 1.98017600 & 2.10031100 \\
\hline C & -0.83118400 & 4.81857100 & -0.40676100 & C & 1.35604500 & 5.06576100 & 0.63916200 \\
\hline C & -3.71484400 & 2.54836200 & 0.33169200 & C & -2.11044800 & 4.19311500 & -0.49544100 \\
\hline C & -0.49484500 & 5.85832900 & -1.32940500 & $\mathrm{H}$ & 2.09403800 & 4.77969700 & 1.38032300 \\
\hline C & 1.67124700 & 6.08337800 & -0.26252300 & C & -4.62504500 & 2.94130600 & -0.64902400 \\
\hline $\mathrm{H}$ & -3.97583600 & 1.76199700 & 1.03486900 & C & -3.04860900 & 4.59206100 & -1.49537200 \\
\hline C & 0.76087100 & 6.47890000 & -1.23700600 & C & -1.45860200 & 6.24163700 & -2.32531300 \\
\hline $\mathrm{H}$ & 2.63785200 & 6.57594700 & -0.19426400 & C & -4.29748200 & 3.95109100 & -1.55260300 \\
\hline $\mathrm{H}$ & -5.59304100 & 2.45211100 & -0.70113500 & C & -2.67618400 & 5.63835400 & -2.40604200 \\
\hline $\mathrm{H}$ & 1.01233000 & 7.27517400 & -1.93364900 & $\mathrm{H}$ & -1.19041900 & 7.03479900 & -3.01939100 \\
\hline $\mathrm{H}$ & -5.01227400 & 4.25465700 & -2.31397200 & $\mathrm{H}$ & -3.39239900 & 5.94268800 & -3.16572100 \\
\hline C & -1.96902000 & -1.41520600 & 2.19914100 & S & -2.93923300 & -0.50180400 & 3.25507700 \\
\hline $\mathrm{N}$ & -0.62502800 & -1.56391600 & 2.38799600 & $\mathrm{H}$ & -0.06258300 & -1.90924100 & 1.60304100 \\
\hline $\mathrm{S}$ & 0.62581900 & -3.76331800 & -0.60589200 & $\mathrm{O}$ & 1.27902300 & -3.98863500 & -1.90490900 \\
\hline 0 & 1.12432700 & -2.56925000 & 0.14506600 & $\mathrm{O}$ & -0.85597700 & -3.88038800 & -0.57522800 \\
\hline C & 1.17804900 & -5.19760400 & 0.44555100 & $\mathrm{~F}$ & 0.80705600 & -6.36007300 & -0.09487500 \\
\hline $\mathrm{F}$ & 0.65247700 & -5.11049400 & 1.67939500 & $\mathrm{~F}$ & 2.52214800 & -5.19904400 & 0.56830900 \\
\hline C & 3.76472600 & 1.96016600 & -0.22219200 & $\mathrm{O}$ & 3.91567600 & 2.30191800 & 0.93422900 \\
\hline 0 & 4.59173700 & 2.21981100 & -1.25183600 & C & 5.75715800 & 3.12584000 & -1.08146400 \\
\hline C & 5.29033000 & 4.49417200 & -0.57745300 & $\mathrm{H}$ & 6.12937700 & 5.19722900 & -0.62136300 \\
\hline $\mathrm{H}$ & 4.93288400 & 4.44023100 & 0.45136500 & $\mathrm{H}$ & 4.48611700 & 4.88179800 & -1.21139300 \\
\hline C & 6.30404200 & 3.23777600 & -2.50574800 & $\mathrm{H}$ & 6.59929600 & 2.25802200 & -2.89386600 \\
\hline $\mathrm{H}$ & 7.18451400 & 3.88806600 & -2.51414300 & $\mathrm{H}$ & 5.55353500 & 3.66635900 & -3.17773700 \\
\hline C & 6.77797500 & 2.47622800 & -0.14402800 & $\mathrm{H}$ & 7.67185400 & 3.10678500 & -0.08447400 \\
\hline $\mathrm{H}$ & 7.08466500 & 1.49557800 & -0.52506100 & $\mathrm{H}$ & 6.36795000 & 2.35647800 & 0.86015300 \\
\hline C & 2.36041000 & 0.89403400 & -1.93057300 & $\mathrm{H}$ & 3.01508100 & 1.38462500 & -2.64040700 \\
\hline C & 0.95774900 & 0.75895800 & -2.37116900 & C & 0.67128500 & 1.02177700 & -3.72342200 \\
\hline $\mathrm{C}$ & -0.08119700 & 0.37754800 & -1.50626500 & C & -0.62828800 & 0.90609700 & -4.20530500 \\
\hline $\bar{H}$ & 1.47366700 & 1.31929900 & -4.39533700 & $\mathrm{C}$ & -1.38031000 & 0.27283400 & -1.99534200 \\
\hline $\mathrm{H}$ & 0.12085000 & 0.14873500 & -0.46551000 & C & -1.65779600 & 0.52380200 & -3.34132400 \\
\hline $\mathrm{H}$ & -0.83811100 & 1.11031700 & -5.25118700 & $\mathrm{H}$ & -2.18237500 & -0.01288000 & -1.32422400 \\
\hline $\mathrm{H}$ & -2.66997900 & 0.40125400 & -3.71348500 & $\mathrm{~N}$ & 2.64408400 & 1.23820700 & -0.63433000 \\
\hline $\mathrm{H}$ & 2.00820500 & 1.00281700 & 0.14307500 & C & 2.96566000 & -1.02499500 & -2.37023100 \\
\hline $\mathrm{H}$ & 2.27987600 & -1.54407000 & -1.70585400 & $\mathrm{H}$ & 2.68147600 & -1.01208100 & -3.41910900 \\
\hline $\mathrm{C}$ & 4.31983200 & -1.01886300 & -2.05523000 & C & 4.77306200 & -1.37377200 & -0.71154200 \\
\hline $\mathrm{H}$ & 3.93948400 & -1.41874600 & -0.00318500 & $\mathrm{H}$ & 5.55300300 & -0.69660500 & -0.34010400 \\
\hline C & 5.33409400 & -0.65128800 & -3.10107600 & $\mathrm{H}$ & 5.72048500 & -1.56914900 & -3.56663200 \\
\hline $\mathrm{H}$ & 6.19042600 & -0.11900100 & -2.67725700 & $\mathrm{H}$ & 4.89336100 & -0.04937100 & -3.90115200 \\
\hline C & 5.47661600 & -3.69621800 & 1.16317400 & $\mathrm{H}$ & 5.87457100 & -2.94081700 & 1.85085900 \\
\hline $\mathrm{H}$ & 6.03868400 & -4.62413400 & 1.32679900 & $\mathrm{H}$ & 4.43305900 & -3.88713500 & 1.42950200 \\
\hline C & 7.46121600 & -2.90998200 & -1.07128600 & $\mathrm{H}$ & 7.95367800 & -2.20675400 & -0.38881100 \\
\hline $\mathrm{H}$ & 7.60919100 & -2.54580700 & -2.09449400 & $\mathrm{H}$ & 7.99014700 & -3.86791700 & -0.99059700 \\
\hline C & 4.81681700 & -4.34085600 & -1.83753600 & $\mathrm{H}$ & 5.04382200 & -4.08268300 & -2.87917800 \\
\hline $\mathrm{H}$ & 3.72756200 & -4.36684800 & -1.73022100 & $\mathrm{H}$ & 5.19597100 & -5.35626300 & -1.66651800 \\
\hline $\mathrm{Si}$ & 5.63277100 & -3.14811500 & -0.63317200 & C & -0.29975000 & 1.92474100 & 4.62316400 \\
\hline $\mathrm{H}$ & 0.34810300 & 1.54860700 & 5.42152000 & $\mathrm{H}$ & -1.26318100 & 1.41034000 & 4.67798200 \\
\hline C & -0.43274100 & 3.45302600 & 4.70029400 & $\mathrm{H}$ & -0.37045000 & 3.81615800 & 5.73016700 \\
\hline
\end{tabular}




\begin{tabular}{|rrrr|rrrr|}
\hline $\mathrm{H}$ & -1.39674600 & 3.76966600 & 4.28943800 & $\mathrm{C}$ & 0.71074500 & 3.96074500 & 3.81029800 \\
\hline $\mathrm{H}$ & 0.57072700 & 4.99180400 & 3.47765900 & $\mathrm{H}$ & 1.66799900 & 3.89908800 & 4.34297600 \\
\hline $\mathrm{O}$ & 1.11460000 & 0.43480500 & 1.59049700 & & & & \\
\hline
\end{tabular}

\section{TS-S-4f-nd-15}

Imaginary frequency: $-269.92 \mathrm{~cm}-1$

E[B3LYP/6-31G(d)]: -4788.683661 Hartree

E[PCM(Et $\left.\left.t_{2} \mathrm{O}\right)-\mathrm{B} 3 L Y P / 6-311 \mathrm{G}(\mathrm{d}, \mathrm{p})-\mathrm{D} 3(\mathrm{BJ})\right]$ : -4790.218904 Hartree

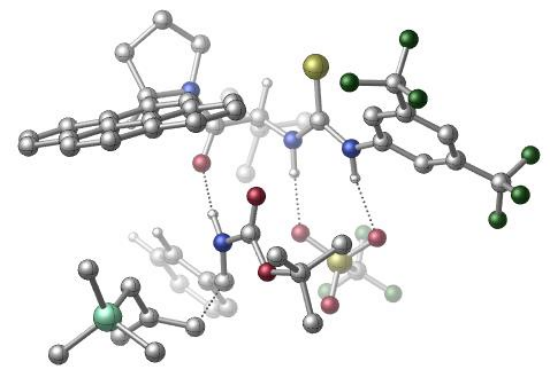

\begin{tabular}{|c|c|c|c|c|c|c|c|}
\hline 01 & & & & $\mathrm{~N}$ & 3.05745000 & -0.20595900 & 0.98653700 \\
\hline $\mathrm{H}$ & 3.14165700 & 0.69199100 & 0.50193000 & C & 3.84461200 & -1.22124300 & 0.41064800 \\
\hline $\mathrm{C}$ & 4.99790300 & -0.83245300 & -0.28652700 & $C$ & 3.47730100 & -2.57386500 & 0.41932300 \\
\hline C & 5.77252500 & -1.78638400 & -0.94674300 & $\mathrm{H}$ & 5.26494600 & 0.21713400 & -0.33623500 \\
\hline C & 4.26360100 & -3.51328400 & -0.24417800 & $\mathrm{H}$ & 2.57846100 & -2.88035300 & 0.93189700 \\
\hline C & 5.41918200 & -3.13528100 & -0.92874700 & $\mathrm{H}$ & 6.01772800 & -3.86977100 & -1.45376500 \\
\hline C & 7.04044400 & -1.36251300 & -1.63983700 & C & 3.86576500 & -4.96386300 & -0.19908400 \\
\hline $\mathrm{F}$ & 8.10583400 & -1.41308500 & -0.80537400 & $\mathrm{~F}$ & 6.96687800 & -0.09937300 & -2.10712700 \\
\hline $\mathrm{F}$ & 7.33047400 & -2.16797000 & -2.68887000 & $\mathrm{~F}$ & 4.40682500 & -5.60816900 & 0.85783400 \\
\hline $\mathrm{F}$ & 2.51991800 & -5.11819700 & -0.09975600 & $\mathrm{~F}$ & 4.26087500 & -5.62987600 & -1.30818600 \\
\hline C & 0.66335200 & 1.09162600 & 3.44063900 & $\mathrm{H}$ & 1.01350500 & 0.39148700 & 4.19800900 \\
\hline C & 0.83198000 & 2.53087900 & 4.04794200 & C & 0.27993000 & 3.65459800 & 3.15291100 \\
\hline $\mathrm{H}$ & -0.79913300 & 3.56368300 & 3.00954000 & $\mathrm{H}$ & 0.74882800 & 3.66842300 & 2.16577200 \\
\hline $\mathrm{H}$ & 0.47918800 & 4.62283600 & 3.62960100 & C & 2.33583600 & 2.77338300 & 4.29280200 \\
\hline $\mathrm{H}$ & 2.47357700 & 3.70348000 & 4.85716200 & $\mathrm{H}$ & 2.88426000 & 2.87174100 & 3.35137200 \\
\hline $\mathrm{H}$ & 2.78951100 & 1.95758500 & 4.86809400 & C & 0.09569000 & 2.57090000 & 5.40317200 \\
\hline $\mathrm{H}$ & 0.19203300 & 3.56641500 & 5.85137900 & $\mathrm{H}$ & 0.51478200 & 1.84779700 & 6.11334000 \\
\hline $\mathrm{H}$ & -0.97636000 & 2.36536400 & 5.29312500 & C & -0.78993800 & 0.72962100 & 3.05158000 \\
\hline $\mathrm{O}$ & -1.33710400 & 1.23856100 & 2.05942100 & C & -0.96796800 & -0.83305700 & 5.05229600 \\
\hline C & -2.83635000 & -0.56070400 & 3.47823300 & C & -2.10588500 & -1.79540600 & 5.41890300 \\
\hline $\mathrm{H}$ & -0.01753100 & -1.34220400 & 4.87142100 & $\mathrm{H}$ & -0.82393700 & -0.08421800 & 5.83882200 \\
\hline C & -3.35081300 & -1.11992500 & 4.82783200 & $\mathrm{H}$ & -3.36931800 & 0.34669000 & 3.18046700 \\
\hline $\mathrm{H}$ & -2.17426400 & -1.95549700 & 6.49874800 & $\mathrm{H}$ & -1.94623700 & -2.76869700 & 4.94349900 \\
\hline $\mathrm{H}$ & -3.67509200 & -0.28961000 & 5.46813200 & $\mathrm{H}$ & -4.19315100 & -1.80383400 & 4.70305900 \\
\hline $\mathrm{N}$ & -1.45046900 & -0.17786400 & 3.81299100 & C & -2.93298500 & -1.58964100 & 2.35193100 \\
\hline C & -1.83139400 & -2.25285100 & 1.89530700 & C & -4.23987300 & -1.89781200 & 1.79260200 \\
\hline C & -1.90611200 & -3.27497600 & 0.89119500 & $\mathrm{H}$ & -0.84841800 & -2.01155400 & 2.28864700 \\
\hline C & -4.34408400 & -2.93021900 & 0.80313000 & $\mathrm{C}$ & -5.41831600 & -1.23134400 & 2.17846500 \\
\hline C & -0.76314100 & -3.95057500 & 0.43542400 & C & -3.17879500 & -3.62124500 & 0.35495600 \\
\hline C & -5.61981400 & -3.27772100 & 0.25725000 & $\mathrm{H}$ & -5.37699300 & -0.44094000 & 2.92011800 \\
\hline C & -6.65651300 & -1.56794600 & 1.63031800 & $\mathrm{C}$ & -0.86504600 & -4.95325500 & -0.52796000 \\
\hline $\mathrm{H}$ & 0.20665400 & -3.68470700 & 0.84426500 & C & -3.27771500 & -4.65242700 & -0.62867800 \\
\hline C & -6.76343900 & -2.58388700 & 0.68562600 & C & -5.69245200 & -4.32958400 & -0.72099700 \\
\hline $\mathrm{H}$ & -7.54699800 & -1.03595100 & 1.95547900 & C & -2.10784300 & -5.30328800 & -1.05417100 \\
\hline $\mathrm{H}$ & 0.03398300 & -5.46201900 & -0.86290000 & $\mathrm{C}$ & -4.57647500 & -4.98476300 & -1.14473100 \\
\hline $\mathrm{H}$ & -7.73397800 & -2.85384500 & 0.27570600 & $\mathrm{H}$ & -6.66973600 & -4.59341900 & -1.11872800 \\
\hline $\mathrm{H}$ & -2.18228600 & -6.08984700 & -1.80155600 & $\mathrm{H}$ & -4.65176700 & -5.77829600 & -1.88471100 \\
\hline $\mathrm{C}$ & 2.26938400 & -0.25628900 & 2.10943600 & $S$ & 2.27626900 & -1.55822900 & 3.20414800 \\
\hline $\mathrm{N}$ & 1.52127700 & 0.86264300 & 2.28516500 & $\mathrm{H}$ & 1.47023400 & 1.53751500 & 1.51638700 \\
\hline $\mathrm{C}$ & -0.38621200 & 0.35037200 & -1.32368500 & $\mathrm{O}$ & -0.13939700 & -0.63078600 & -0.65603100 \\
\hline $\mathrm{O}$ & -0.07787000 & 0.55919300 & -2.61507400 & $C$ & 0.74823700 & -0.39969200 & -3.39807600 \\
\hline
\end{tabular}




\begin{tabular}{|c|c|c|c|c|c|c|c|}
\hline C & -0.03696500 & -1.70095200 & -3.57658700 & $\mathrm{H}$ & 0.52749500 & -2.38000900 & -4.22502600 \\
\hline $\mathrm{H}$ & -0.20448800 & -2.19634400 & -2.61794200 & $\mathrm{H}$ & -1.00556500 & -1.51023600 & -4.05428800 \\
\hline $\mathrm{C}$ & 0.92981100 & 0.34374000 & -4.72245000 & $\mathrm{H}$ & 1.42888600 & 1.30224000 & -4.55053500 \\
\hline $\mathrm{H}$ & 1.54474300 & -0.25456800 & -5.40245900 & $\mathrm{H}$ & -0.03542700 & 0.52810100 & -5.20847000 \\
\hline $\mathrm{C}$ & 2.09684700 & -0.62524700 & -2.71759200 & $\mathrm{H}$ & 2.73233700 & -1.22184700 & -3.38168300 \\
\hline $\mathrm{H}$ & 2.60219900 & 0.32528600 & -2.52586300 & $\mathrm{H}$ & 1.98144200 & -1.16382300 & -1.77731500 \\
\hline $\mathrm{C}$ & -1.12999900 & 2.69221500 & -1.41132000 & $\mathrm{H}$ & -0.24738200 & 2.87011000 & -2.02551600 \\
\hline C & -1.48552000 & 3.86333600 & -0.56151000 & C & -0.86895900 & 5.09076000 & -0.85131800 \\
\hline C & -2.41711000 & 3.79669700 & 0.48729600 & $\mathrm{C}$ & -1.18003500 & 6.22933000 & -0.11156600 \\
\hline $\mathrm{H}$ & -0.11175100 & 5.13550200 & -1.62875600 & $\mathrm{C}$ & -2.72658600 & 4.94084100 & 1.22450500 \\
\hline $\mathrm{H}$ & -2.87487900 & 2.84970000 & 0.75595900 & C & -2.11517400 & 6.15948300 & 0.92354700 \\
\hline $\mathrm{H}$ & -0.67981400 & 7.16737200 & -0.33416700 & $\mathrm{H}$ & -3.43984400 & 4.87559900 & 2.04161200 \\
\hline $\mathrm{H}$ & -2.35623500 & 7.04708200 & 1.50199100 & $\mathrm{~N}$ & -1.11749300 & 1.43337600 & -0.81531900 \\
\hline $\mathrm{H}$ & -1.27128400 & 1.35837900 & 0.19850900 & $\mathrm{~S}$ & 2.54538800 & 3.05016400 & -0.91277600 \\
\hline 0 & 2.02622400 & 3.36905400 & -2.26057100 & $\mathrm{O}$ & 1.47988000 & 2.77498600 & 0.09568700 \\
\hline $\mathrm{O}$ & 3.66424500 & 2.07725800 & -0.85122000 & C & 3.29327800 & 4.64484700 & -0.31203700 \\
\hline $\mathrm{F}$ & 4.32556500 & 5.00754100 & -1.07989900 & $\mathrm{~F}$ & 3.72361800 & 4.50714000 & 0.95183300 \\
\hline $\mathrm{F}$ & 2.37537200 & 5.62765200 & -0.33892300 & C & -2.25310200 & 2.69841800 & -2.90916100 \\
\hline $\mathrm{H}$ & -1.99403500 & 3.65078700 & -3.36787500 & $\mathrm{H}$ & -1.78917200 & 1.83884900 & -3.38614200 \\
\hline C & -3.58989900 & 2.54776700 & -2.48749900 & C & -4.11688900 & 1.23634700 & -2.15326000 \\
\hline $\mathrm{H}$ & -3.32197200 & 0.53694100 & -1.86397700 & $\mathrm{H}$ & -4.88988000 & 1.27549300 & -1.37580100 \\
\hline C & -4.44113100 & 3.75296700 & -2.26100300 & $\mathrm{H}$ & -5.50820000 & 3.51621100 & -2.27731000 \\
\hline $\mathrm{H}$ & -4.20277000 & 4.14224400 & -1.25690700 & $\mathrm{H}$ & -4.22448000 & 4.55583000 & -2.97137400 \\
\hline C & -5.49254500 & -1.34051900 & -3.05914100 & $\mathrm{H}$ & -6.12378200 & -1.28818100 & -2.16532000 \\
\hline $\mathrm{H}$ & -6.05296400 & -1.87990100 & -3.83295700 & $\mathrm{H}$ & -4.61572800 & -1.94532000 & -2.80399100 \\
\hline $\mathrm{C}$ & -6.53621200 & 1.38072400 & -4.14277400 & $\mathrm{H}$ & -7.21662800 & 1.50854400 & -3.29252300 \\
\hline $\mathrm{H}$ & -6.27921200 & 2.37525200 & -4.52480800 & $\mathrm{H}$ & -7.09736600 & 0.86521200 & -4.93202900 \\
\hline C & -3.81349600 & 0.26668200 & -5.13352400 & $\mathrm{H}$ & -3.53515400 & 1.25545400 & -5.51471200 \\
\hline $\mathrm{H}$ & -2.89253100 & -0.26347600 & -4.86552900 & $\mathrm{H}$ & -4.27850700 & -0.28496500 & -5.95985400 \\
\hline $\mathrm{Si}$ & -5.01039100 & 0.36769600 & -3.68077000 & & & & \\
\hline
\end{tabular}

\section{TS-S-4f-nd-16}

Imaginary frequency: $-252.59 \mathrm{~cm}^{-1}$ E[B3LYP/6-31G(d)]: -4788.697162 Hartree E[PCM(Et $\left.\left.{ }_{2} \mathrm{O}\right)-B 3 L Y P / 6-311 G(d, p)-D 3(B J)\right]:-4790.218281$ Hartree

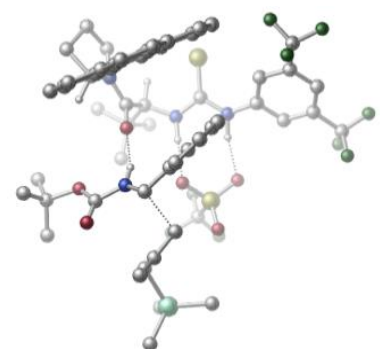

\begin{tabular}{|cccc|cccc|}
\hline 01 & & & & $\mathrm{~N}$ & 1.63060500 & 2.33011300 & 1.49083000 \\
\hline $\mathrm{H}$ & 0.75198700 & 2.82426500 & 1.29595700 & $\mathrm{C}$ & 2.73891800 & 2.90492300 & 0.84334800 \\
\hline $\mathrm{C}$ & 2.64182500 & 4.25566200 & 0.47283700 & $\mathrm{C}$ & 3.87515600 & 2.17642800 & 0.46564500 \\
\hline $\mathrm{C}$ & 3.67133400 & 4.86108000 & -0.24533600 & $\mathrm{H}$ & 1.75583600 & 4.82119000 & 0.73891700 \\
\hline $\mathrm{C}$ & 4.88861100 & 2.79587000 & -0.26414200 & $\mathrm{H}$ & 3.96198200 & 1.13597700 & 0.74189100 \\
\hline $\mathrm{C}$ & 4.80679100 & 4.14171600 & -0.61949500 & $\mathrm{H}$ & 5.60765800 & 4.61978000 & -1.17020900 \\
\hline $\mathrm{C}$ & 3.53154200 & 6.29801200 & -0.67614200 & $\mathrm{C}$ & 6.03682100 & 1.95995900 & -0.75961500 \\
\hline $\mathrm{F}$ & 2.95709700 & 6.39746800 & -1.89729600 & $\mathrm{~F}$ & 4.73421100 & 6.91219400 & -0.75978900 \\
\hline $\mathrm{F}$ & 2.76942300 & 7.01170000 & 0.17839200 & $\mathrm{~F}$ & 6.35617200 & 0.96248200 & 0.09844300 \\
\hline $\mathrm{F}$ & 5.73595100 & 1.35937000 & -1.94382000 & $\mathrm{~F}$ & 7.15193500 & 2.68957900 & -0.96776300 \\
\hline $\mathrm{C}$ & -0.03523500 & -0.26552700 & 3.46544300 & $\mathrm{H}$ & 0.79039900 & -0.36766100 & 4.16942600 \\
\hline $\mathrm{C}$ & -1.33747700 & -0.01900500 & 4.30904200 & $\mathrm{C}$ & -2.61189100 & 0.08544900 & 3.44997500 \\
\hline $\mathrm{H}$ & -2.78439100 & -0.82350600 & 2.86823300 & $\mathrm{H}$ & -2.57399400 & 0.92059900 & 2.74855600 \\
\hline $\mathrm{H}$ & -3.47551600 & 0.24532100 & 4.10813300 & $\mathrm{C}$ & -1.15035200 & 1.28675300 & 5.10826600 \\
\hline $\mathrm{H}$ & -1.99830800 & 1.43170400 & 5.78831000 & $\mathrm{H}$ & -1.09725600 & 2.15678900 & 4.44884500 \\
\hline
\end{tabular}




\begin{tabular}{|c|c|c|c|c|c|c|c|}
\hline $\mathrm{H}$ & -0.23454000 & 1.26051300 & 5.71094200 & $C$ & -1.51071600 & -1.18357100 & 5.30532700 \\
\hline $\mathrm{H}$ & -2.41440600 & -1.02724300 & 5.90533500 & $\mathrm{H}$ & -0.66484800 & -1.25137000 & 5.99968900 \\
\hline $\mathrm{H}$ & -1.61925100 & -2.14861800 & 4.79581700 & C & -0.09814300 & -1.51471300 & 2.55581400 \\
\hline 0 & -0.73379000 & -1.48464400 & 1.49241800 & C & 0.51131200 & -3.84626300 & 2.09595500 \\
\hline $\mathrm{H}$ & -0.51594300 & -3.94361700 & 1.73551900 & $\mathrm{~N}$ & 0.57520700 & -2.63106200 & 2.93099500 \\
\hline C & 1.46094300 & -3.82162000 & 0.90009600 & C & 2.49429200 & -2.93266600 & 0.83279200 \\
\hline C & 1.28532500 & -4.80255100 & -0.15855500 & C & 3.44101700 & -2.92811000 & -0.24571000 \\
\hline $\mathrm{H}$ & 2.61395900 & -2.17828000 & 1.60578600 & C & 2.21058800 & -4.81124100 & -1.25306200 \\
\hline C & 0.24462500 & -5.74954800 & -0.16441600 & C & 4.50786200 & -2.01613100 & -0.29443100 \\
\hline C & 3.28895500 & -3.87822400 & -1.29603900 & C & 2.06188100 & -5.76096500 & -2.31181200 \\
\hline $\mathrm{H}$ & -0.47081400 & -5.77640000 & 0.65050800 & C & 0.10657200 & -6.67054200 & -1.20314800 \\
\hline C & 5.40802500 & -2.02943700 & -1.35960900 & $\mathrm{H}$ & 4.63010300 & -1.29826700 & 0.51220900 \\
\hline C & 4.21525900 & -3.88801700 & -2.38298900 & C & 1.00238300 & -6.68071700 & -2.26678400 \\
\hline C & 3.00828200 & -5.74791400 & -3.39418900 & $\mathrm{H}$ & -0.70903400 & -7.38844900 & -1.17624100 \\
\hline C & 5.26450100 & -2.95382900 & -2.39309600 & $\mathrm{H}$ & 6.21603500 & -1.30559800 & -1.38385100 \\
\hline C & 4.03606500 & -4.85604800 & -3.42899700 & $\mathrm{H}$ & 0.89158600 & -7.40360000 & -3.07146800 \\
\hline $\mathrm{H}$ & 2.88529500 & -6.47728500 & -4.19145300 & $\mathrm{H}$ & 5.97029000 & -2.96048100 & -3.22051800 \\
\hline $\mathrm{H}$ & 4.74459500 & -4.86371800 & -4.25405300 & C & 1.57559000 & 1.27701100 & 2.37268000 \\
\hline$S$ & 2.93696500 & 0.58721700 & 3.11541800 & $\mathrm{~N}$ & 0.29983200 & 0.87475900 & 2.62056400 \\
\hline $\mathrm{H}$ & -0.42712200 & 1.17425000 & 1.96310800 & S & -1.84473400 & 3.15090700 & 0.30154800 \\
\hline 0 & -2.48476000 & 3.42279100 & -0.99772300 & 0 & -1.70665300 & 1.69683000 & 0.62152300 \\
\hline $\mathrm{O}$ & -0.62689200 & 3.93589200 & 0.62202700 & C & -3.08965300 & 3.74372600 & 1.55281700 \\
\hline $\mathrm{F}$ & -3.43619900 & 5.01271600 & 1.32406200 & $\mathrm{~F}$ & -2.59370700 & 3.64975600 & 2.79713400 \\
\hline $\mathrm{F}$ & -4.20155000 & 2.98105100 & 1.49456000 & C & -3.53832000 & -2.13070500 & -0.69511400 \\
\hline 0 & -4.24747500 & -2.45434400 & -1.63390300 & $\mathrm{O}$ & -3.72495500 & -2.40676500 & 0.59476500 \\
\hline C & -4.87404200 & -3.23542800 & 1.04029600 & C & -6.18644100 & -2.51931400 & 0.71106100 \\
\hline $\mathrm{H}$ & -7.02056700 & -3.06488000 & 1.16580300 & $\mathrm{H}$ & -6.35219400 & -2.46785800 & -0.36638000 \\
\hline $\mathrm{H}$ & -6.18043500 & -1.50465000 & 1.12312900 & C & -4.65978300 & -3.31466800 & 2.55217300 \\
\hline $\mathrm{H}$ & -3.69059700 & -3.76857600 & 2.78160300 & $\mathrm{H}$ & -5.44625500 & -3.92683900 & 3.00544200 \\
\hline $\mathrm{H}$ & -4.68977900 & -2.31896900 & 3.00352500 & C & -4.78403400 & -4.62538200 & 0.40375100 \\
\hline $\mathrm{H}$ & -5.55903200 & -5.26991400 & 0.83273100 & $\mathrm{H}$ & -3.80991600 & -5.07941900 & 0.61572200 \\
\hline $\mathrm{H}$ & -4.92650800 & -4.58132300 & -0.67705200 & C & -1.90058300 & -1.15931700 & -2.12895300 \\
\hline $\mathrm{H}$ & -2.36842300 & -1.80606700 & -2.86270400 & C & -0.47931500 & -0.85079600 & -2.35330600 \\
\hline C & 0.12927700 & -1.37189000 & -3.50968500 & C & 0.27146700 & -0.05462100 & -1.47316300 \\
\hline C & 1.47366900 & -1.12307900 & -3.77022300 & $\mathrm{H}$ & -0.45122600 & -1.98715400 & -4.19336400 \\
\hline C & 1.61045100 & 0.20155800 & -1.75062000 & $\mathrm{H}$ & -0.19610600 & 0.38399000 & -0.59822800 \\
\hline C & 2.21635400 & -0.33064000 & -2.89139300 & $\mathrm{H}$ & 1.94125600 & -1.54832300 & -4.65323100 \\
\hline $\mathrm{H}$ & 2.18507000 & 0.82769900 & -1.07798900 & $\mathrm{H}$ & 3.26562700 & -0.12824200 & -3.08418700 \\
\hline $\mathrm{N}$ & -2.36056600 & -1.39828300 & -0.86528400 & $\mathrm{H}$ & -1.80853900 & -1.16720600 & -0.02971800 \\
\hline C & -2.71758100 & 0.59156700 & -2.95023900 & $\mathrm{H}$ & -2.36707700 & 1.30236900 & -2.20584000 \\
\hline $\mathrm{H}$ & -2.12818100 & 0.52353500 & -3.85977300 & C & -4.07987800 & 0.32579300 & -3.03601800 \\
\hline C & -5.00789500 & 0.79008400 & -1.99915000 & $\mathrm{H}$ & -4.47868600 & 1.05537800 & -1.07707400 \\
\hline $\mathrm{H}$ & -5.79119100 & 0.05267200 & -1.78595800 & C & -4.61935800 & -0.47391900 & -4.18758100 \\
\hline $\mathrm{H}$ & -5.50687700 & 0.00165300 & -4.61834100 & $\mathrm{H}$ & -4.93006300 & -1.46033500 & -3.82245100 \\
\hline $\mathrm{H}$ & -3.87654800 & -0.60992600 & -4.97863900 & C & -6.72043100 & 3.06649700 & -0.88107700 \\
\hline $\mathrm{H}$ & -7.39338900 & 2.33310100 & -0.42036500 & $\mathrm{H}$ & -7.30311500 & 3.97716700 & -1.06718300 \\
\hline $\mathrm{H}$ & -5.93858700 & 3.31031300 & -0.15621100 & C & -7.40918100 & 1.93987500 & -3.66260400 \\
\hline $\mathrm{H}$ & -8.01987800 & 1.12373200 & -3.25820900 & $\mathrm{H}$ & -7.06317400 & 1.64146900 & -4.65891400 \\
\hline $\mathrm{H}$ & -8.07147100 & 2.80393700 & -3.80175100 & C & -4.85647400 & 3.67015000 & -3.32023200 \\
\hline $\mathrm{H}$ & -4.47322100 & 3.30284300 & -4.27978900 & $\mathrm{H}$ & -4.00164000 & 3.90515000 & -2.67866600 \\
\hline $\mathrm{H}$ & -5.40362800 & 4.60017500 & -3.51929100 & $\mathrm{Si}$ & -5.99211100 & 2.41862500 & -2.49624300 \\
\hline C & 1.37970500 & -2.83028000 & 4.15818500 & $\mathrm{H}$ & 0.74179600 & -2.75093900 & 5.04404800 \\
\hline $\mathrm{H}$ & 2.16916100 & -2.07597000 & 4.22623600 & C & 1.92473100 & -4.25971400 & 4.00703000 \\
\hline
\end{tabular}




\begin{tabular}{|lrrr|rrrr|}
\hline $\mathrm{H}$ & 2.05460300 & -4.75144000 & 4.97536900 & $\mathrm{H}$ & 2.89734800 & -4.24380000 & 3.50516000 \\
\hline $\mathrm{C}$ & 0.88461900 & -4.95157600 & 3.11328500 & $\mathrm{H}$ & 1.26570700 & -5.85330900 & 2.62829200 \\
\hline $\mathrm{H}$ & -0.00184500 & -5.22556000 & 3.69953300 & & & & \\
\hline
\end{tabular}

\section{TS-S-4f-nd-17}

Imaginary frequency: $-134.65 \mathrm{~cm}^{-1}$

E[B3LYP/6-31G(d)]: -4788.692630 Hartree

$\mathrm{E}\left[\mathrm{PCM}\left(\mathrm{Et}_{2} \mathrm{O}\right)-\mathrm{B} 3 \mathrm{LYP} / 6-311 \mathrm{G}(\mathrm{d}, \mathrm{p})-\mathrm{D} 3(\mathrm{BJ})\right]$ : -4790.217647 Hartree

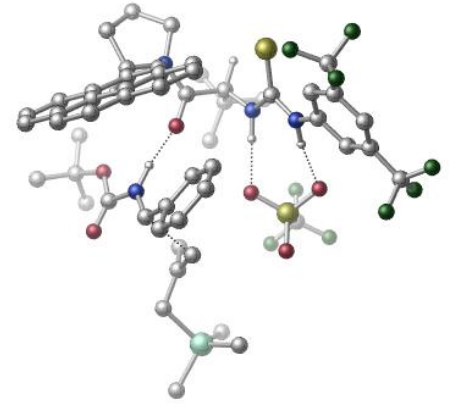

\begin{tabular}{|c|c|c|c|c|c|c|c|}
\hline 01 & & & & $\mathrm{~N}$ & 2.59162800 & 1.25666900 & 1.55548400 \\
\hline $\mathrm{H}$ & 2.05002900 & 2.12225900 & 1.44007200 & C & 3.83580300 & 1.30589600 & 0.90160800 \\
\hline $\mathrm{C}$ & 4.22759000 & 2.56011100 & 0.39695700 & $\mathrm{C}$ & 4.66024100 & 0.19546800 & 0.66546400 \\
\hline $\mathrm{C}$ & 5.41529100 & 2.69017300 & -0.31727700 & $\mathrm{H}$ & 3.59210800 & 3.42433200 & 0.55943000 \\
\hline C & 5.84496300 & 0.34924900 & -0.05513600 & $\mathrm{H}$ & 4.38369700 & -0.77176200 & 1.05507200 \\
\hline C & 6.24036700 & 1.58937500 & -0.55198500 & $\mathrm{H}$ & 7.16461600 & 1.69835700 & -1.10648500 \\
\hline C & 5.78542300 & 4.02711800 & -0.90521700 & C & 6.74319400 & -0.84258100 & -0.25124300 \\
\hline $\mathrm{F}$ & 5.29216800 & 5.05419300 & -0.18514500 & $\mathrm{~F}$ & 5.31255500 & 4.16246500 & -2.16684100 \\
\hline $\mathrm{F}$ & 7.12802800 & 4.18536400 & -0.97500100 & $\mathrm{~F}$ & 6.05128000 & -2.01168800 & -0.23987600 \\
\hline $\mathrm{F}$ & 7.40454400 & -0.78521100 & -1.42976700 & $\mathrm{~F}$ & 7.67880000 & -0.93887700 & 0.71824000 \\
\hline C & -0.11911100 & -0.33623500 & 3.42460000 & $\mathrm{H}$ & 0.56046100 & -0.80220400 & 4.13742800 \\
\hline C & -1.20434100 & 0.44662800 & 4.24992600 & $\mathrm{C}$ & -2.31421500 & 1.06591300 & 3.37759000 \\
\hline $\mathrm{H}$ & -2.87967700 & 0.29917800 & 2.83817500 & $\mathrm{H}$ & -1.91736900 & 1.77030300 & 2.64325100 \\
\hline $\mathrm{H}$ & -3.01184200 & 1.61422500 & 4.02295200 & C & -0.49204700 & 1.56343100 & 5.04069100 \\
\hline $\mathrm{H}$ & -1.20223400 & 2.04472500 & 5.72356600 & $\mathrm{H}$ & -0.08996800 & 2.33456400 & 4.37927900 \\
\hline $\mathrm{H}$ & 0.33487700 & 1.16400000 & 5.63997300 & $\mathrm{C}$ & -1.85781300 & -0.52384100 & 5.25588000 \\
\hline $\mathrm{H}$ & -2.62493900 & 0.00346100 & 5.83408100 & $\mathrm{H}$ & -1.12773600 & -0.92088900 & 5.97175400 \\
\hline $\mathrm{H}$ & -2.34869500 & -1.36815400 & 4.75638200 & C & -0.71031300 & -1.41566200 & 2.48788800 \\
\hline $\mathrm{O}$ & -1.22572900 & -1.05986000 & 1.41451300 & C & -0.12255800 & -3.32541300 & 4.06465300 \\
\hline C & -1.24277600 & -3.74964100 & 1.94212300 & C & -1.06910700 & -4.50936400 & 4.28058600 \\
\hline $\mathrm{H}$ & 0.91308400 & -3.65251300 & 3.91093400 & $\mathrm{H}$ & -0.13430100 & -2.61643600 & 4.88915200 \\
\hline $\mathrm{H}$ & -2.23770500 & -3.38596400 & 1.66775600 & $\mathrm{H}$ & -1.99725300 & -4.15732900 & 4.74671400 \\
\hline $\mathrm{H}$ & -0.63645200 & -5.27727200 & 4.92870500 & $\mathrm{~N}$ & -0.64593700 & -2.72300400 & 2.82778000 \\
\hline C & -0.44121100 & -4.01559100 & 0.67191000 & C & 0.87957300 & -3.68147100 & 0.59144200 \\
\hline C & -1.08275100 & -4.69924900 & -0.43787100 & C & 1.67993000 & -3.97580900 & -0.56238000 \\
\hline $\mathrm{H}$ & 1.35979500 & -3.15531200 & 1.41278200 & C & -0.30696200 & -4.99434600 & -1.60682500 \\
\hline C & -2.43429500 & -5.09109500 & -0.42420900 & C & 3.03877200 & -3.62667000 & -0.63269000 \\
\hline C & 1.07190600 & -4.63463800 & -1.66961500 & C & -0.91036700 & -5.65956800 & -2.71982200 \\
\hline $\mathrm{H}$ & -3.04705500 & -4.89750500 & 0.44966600 & C & -3.01339300 & -5.74003900 & -1.51480100 \\
\hline C & 3.78871600 & -3.91376200 & -1.77271100 & $\mathrm{H}$ & 3.50284900 & -3.12711300 & 0.21305200 \\
\hline C & 1.84700500 & -4.92912000 & -2.83243400 & C & -2.26494500 & -6.02140800 & -2.65283400 \\
\hline C & -0.10662900 & -5.94377300 & -3.87759500 & $\mathrm{H}$ & -4.05939400 & -6.03235800 & -1.47028400 \\
\hline C & 3.20147700 & -4.55776800 & -2.86081100 & $\mathrm{H}$ & 4.83514200 & -3.62659200 & -1.80700200 \\
\hline C & 1.20815600 & -5.59590500 & -3.93248200 & $\mathrm{H}$ & -2.72172200 & -6.52955100 & -3.49858400 \\
\hline $\mathrm{H}$ & -0.57730400 & -6.45101000 & -4.71645900 & $\mathrm{H}$ & 3.79242700 & -4.78199300 & -3.74579400 \\
\hline $\mathrm{H}$ & 1.80101800 & -5.82116300 & -4.81586800 & C & 2.02560800 & 0.29788800 & 2.35829100 \\
\hline $\mathrm{S}$ & 2.84766300 & -1.03549800 & 3.01655000 & $\mathrm{~N}$ & 0.71002100 & 0.54710500 & 2.61493000 \\
\hline $\mathrm{H}$ & 0.22092700 & 1.21522200 & 2.00914800 & $\mathrm{~S}$ & -0.01302900 & 3.80076100 & 0.58605600 \\
\hline $\mathrm{O}$ & -0.23280100 & 4.38691900 & -0.74324400 & 0 & -0.63711800 & 2.45497600 & 0.79582600 \\
\hline $\mathrm{O}$ & 1.36867700 & 3.86672100 & 1.13047600 & C & -0.97915200 & 4.90033600 & 1.73788100 \\
\hline
\end{tabular}




\begin{tabular}{|c|c|c|c|c|c|c|c|}
\hline $\mathrm{F}$ & -0.48594900 & 6.14009600 & 1.74418900 & $\mathrm{~F}$ & -0.95166100 & 4.41936100 & 2.99232800 \\
\hline $\mathrm{F}$ & -2.27421500 & 4.96450000 & 1.35333800 & C & -4.17565400 & -0.91714400 & -0.69356600 \\
\hline 0 & -4.99031000 & -0.59800800 & -1.53609700 & $\mathrm{O}$ & -4.39330500 & -1.44640400 & 0.51018600 \\
\hline $\mathrm{C}$ & -5.77005500 & -1.77067600 & 0.96903700 & C & -6.60414400 & -0.49022900 & 1.05262500 \\
\hline $\mathrm{H}$ & -7.57439500 & -0.72160500 & 1.50549200 & $\mathrm{H}$ & -6.77644300 & -0.06242800 & 0.06364900 \\
\hline $\mathrm{H}$ & -6.10432500 & 0.25262400 & 1.68298400 & C & -5.52211800 & -2.35312700 & 2.36097600 \\
\hline $\mathrm{H}$ & -4.89841200 & -3.25105000 & 2.30218000 & $\mathrm{H}$ & -6.47580500 & -2.62622000 & 2.82383400 \\
\hline $\mathrm{H}$ & -5.02069300 & -1.62289200 & 3.00366800 & C & -6.38464800 & -2.81507700 & 0.03374100 \\
\hline $\mathrm{H}$ & -7.33942200 & -3.15578700 & 0.44866300 & $\mathrm{H}$ & -5.72296800 & -3.68330900 & -0.05682600 \\
\hline $\mathrm{H}$ & -6.56497000 & -2.40324400 & -0.96072800 & C & -2.32852000 & -0.29601400 & -2.06153900 \\
\hline $\mathrm{H}$ & -3.08827300 & -0.25822300 & -2.83302000 & C & -0.93747400 & -0.43167500 & -2.47581200 \\
\hline $\mathrm{C}$ & -0.67986700 & -0.51496000 & -3.85799200 & C & 0.13453200 & -0.47331600 & -1.56423000 \\
\hline C & 0.62240000 & -0.66517100 & -4.32103200 & $\mathrm{H}$ & -1.50745900 & -0.47318800 & -4.56193600 \\
\hline C & 1.43491300 & -0.60848000 & -2.03774900 & $\mathrm{H}$ & -0.04673800 & -0.37629600 & -0.49873600 \\
\hline C & 1.68125000 & -0.71060400 & -3.41048800 & $\mathrm{H}$ & 0.81282300 & -0.74289400 & -5.38729300 \\
\hline $\mathrm{H}$ & 2.26191200 & -0.63344800 & -1.33634000 & $\mathrm{H}$ & 2.70052400 & -0.82160500 & -3.76820600 \\
\hline $\mathrm{N}$ & -2.78818000 & -0.77035600 & -0.88772100 & $\mathrm{H}$ & -2.16725300 & -0.99274700 & -0.09341700 \\
\hline C & -2.26713800 & 1.99012300 & -1.97668300 & $\mathrm{H}$ & -1.52827600 & 1.92792000 & -1.18513600 \\
\hline $\mathrm{H}$ & -1.89320500 & 2.12536200 & -2.98781900 & C & -3.54733000 & 2.38215000 & -1.66840900 \\
\hline $\mathrm{C}$ & -4.47818600 & 2.90150400 & -2.69573800 & $\mathrm{H}$ & -5.52257100 & 2.65884900 & -2.46594800 \\
\hline $\mathrm{H}$ & -4.24107300 & 2.49984700 & -3.68938100 & C & -3.99056600 & 2.37505900 & -0.23215200 \\
\hline $\mathrm{H}$ & -5.07430800 & 2.26035100 & -0.13673200 & $\mathrm{H}$ & -3.70247400 & 3.31724200 & 0.24922200 \\
\hline $\mathrm{H}$ & -3.48110300 & 1.59150100 & 0.33700400 & C & -5.64757600 & 5.27134300 & -4.22420200 \\
\hline $\mathrm{H}$ & -5.45399200 & 4.75157100 & -5.17014500 & $\mathrm{H}$ & -5.63878900 & 6.34814000 & -4.43279600 \\
\hline $\mathrm{H}$ & -6.66085300 & 5.00662500 & -3.89892700 & C & -2.61685900 & 5.27870000 & -3.48965100 \\
\hline $\mathrm{H}$ & -2.35081100 & 4.76223300 & -4.42020100 & $\mathrm{H}$ & -1.86266100 & 5.02991300 & -2.73389400 \\
\hline $\mathrm{H}$ & -2.54640300 & 6.35626500 & -3.68416000 & C & -4.75287400 & 5.72450500 & -1.29060600 \\
\hline $\mathrm{H}$ & -3.95661200 & 5.59745800 & -0.54949600 & $\mathrm{H}$ & -5.69457400 & 5.38161100 & -0.84553200 \\
\hline $\mathrm{H}$ & -4.85251600 & 6.80146600 & -1.47594400 & $\mathrm{Si}$ & -4.35457500 & 4.83556700 & -2.91009900 \\
\hline C & -1.33301600 & -5.01441500 & 2.85222200 & $\mathrm{H}$ & -0.55964800 & -5.72908800 & 2.55453500 \\
\hline $\bar{H}$ & -2.29830900 & -5.51887500 & 2.75259700 & & & & \\
\hline
\end{tabular}

\section{TS-S-4f-nd-18}

Imaginary frequency: $-169.55 \mathrm{~cm}-1$ E[B3LYP/6-31G(d)]: -4788.679742 Hartree $\mathrm{E}\left[\mathrm{PCM}\left(\mathrm{Et}_{2} \mathrm{O}\right)-\mathrm{B} 3 \mathrm{LYP} / 6-311 \mathrm{G}(\mathrm{d}, \mathrm{p})-\mathrm{D} 3(\mathrm{BJ})\right]$ : -4790.216935 Hartree

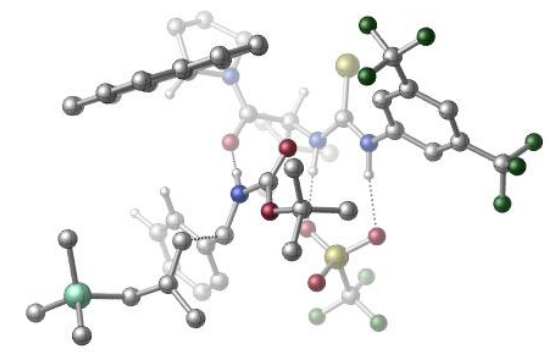

\begin{tabular}{|crrr|rrrr|}
\hline 01 & & & & $\mathrm{~N}$ & 3.23597500 & 0.54044700 & 1.03372500 \\
\hline $\mathrm{H}$ & 2.98880400 & 1.48674500 & 0.72919800 & $\mathrm{C}$ & 4.14979900 & -0.10777000 & 0.18113700 \\
\hline $\mathrm{C}$ & 5.17679900 & 0.65155700 & -0.39131400 & $\mathrm{C}$ & 4.00428800 & -1.45222900 & -0.18372400 \\
\hline $\mathrm{C}$ & 6.05970200 & 0.06259100 & -1.29897300 & $\mathrm{H}$ & 5.26849600 & 1.70266500 & -0.14130700 \\
\hline $\mathrm{C}$ & 4.89334700 & -2.02533800 & -1.08917800 & $\mathrm{H}$ & 3.18127700 & -2.02360100 & 0.21813600 \\
\hline $\mathrm{C}$ & 5.93341400 & -1.28052100 & -1.64994300 & $\mathrm{H}$ & 6.62167600 & -1.73379300 & -2.35297300 \\
\hline $\mathrm{C}$ & 7.20382700 & 0.86942100 & -1.85165500 & $\mathrm{C}$ & 4.65558200 & -3.43743500 & -1.54614800 \\
\hline $\mathrm{F}$ & 8.30038700 & 0.79044800 & -1.06047700 & $\mathrm{~F}$ & 6.89453600 & 2.17828800 & -1.96276000 \\
\hline $\mathrm{F}$ & 7.57848700 & 0.43483900 & -3.07800600 & $\mathrm{~F}$ & 4.13245000 & -4.21496100 & -0.56907500 \\
\hline $\mathrm{F}$ & 3.77231900 & -3.48813300 & -2.58213800 & $\mathrm{~F}$ & 5.78684200 & -4.03567000 & -1.97682100 \\
\hline $\mathrm{C}$ & 0.82452000 & 0.48364400 & 3.78639300 & $\mathrm{H}$ & 1.47988200 & -0.13616800 & 4.39809300 \\
\hline $\mathrm{C}$ & 0.46480400 & 1.76224700 & 4.62594200 & $\mathrm{C}$ & -0.51973900 & 2.71142000 & 3.91593600 \\
\hline $\mathrm{H}$ & -1.48410300 & 2.23187700 & 3.72953400 & $\mathrm{H}$ & -0.13586700 & 3.06877300 & 2.95721400 \\
\hline $\mathrm{H}$ & -0.69207300 & 3.58827700 & 4.55313100 & $\mathrm{C}$ & 1.77491800 & 2.51991500 & 4.92462800 \\
\hline
\end{tabular}




\begin{tabular}{|c|c|c|c|c|c|c|c|}
\hline $\mathrm{H}$ & 1.57454700 & 3.35579200 & 5.60558700 & $\mathrm{H}$ & 2.21812000 & 2.92911700 & 4.01240400 \\
\hline $\mathrm{H}$ & 2.51835900 & 1.86757400 & 5.39848600 & C & -0.15967700 & 1.31541100 & 5.96426100 \\
\hline $\mathrm{H}$ & -0.42816800 & 2.19451800 & 6.56112700 & $\mathrm{H}$ & 0.54095400 & 0.71837000 & 6.56113200 \\
\hline $\mathrm{H}$ & -1.07561500 & 0.73014200 & 5.81677300 & C & -0.40564900 & -0.34435300 & 3.35157300 \\
\hline 0 & -1.14129500 & 0.05537700 & 2.43519800 & C & -0.00748600 & -2.13210000 & 5.12876700 \\
\hline C & -1.89250900 & -2.27169300 & 3.57557500 & C & -1.15939700 & -2.84781500 & 5.84219200 \\
\hline $\bar{H}$ & 0.76612700 & -2.83262400 & 4.79143100 & $\mathrm{H}$ & 0.47005100 & -1.37902200 & 5.75260900 \\
\hline $\mathrm{H}$ & -2.72351000 & -1.56233100 & 3.62854800 & $\mathrm{H}$ & -1.71942600 & -2.12919200 & 6.45305600 \\
\hline $\mathrm{H}$ & -0.80975700 & -3.64976200 & 6.49939600 & $\mathrm{~N}$ & -0.67686200 & -1.52160800 & 3.96785400 \\
\hline $\mathrm{C}$ & -1.83789100 & -2.86942000 & 2.17448300 & C & -0.64266900 & -3.22140400 & 1.61706900 \\
\hline C & -3.07336400 & -3.13182500 & 1.45649100 & C & -0.54616500 & -3.86187900 & 0.33819900 \\
\hline $\mathrm{H}$ & 0.28507700 & -2.99576500 & 2.13727400 & C & -3.00281400 & -3.75531000 & 0.16603100 \\
\hline $\mathrm{C}$ & -4.34623400 & -2.80255500 & 1.96118700 & C & 0.68942300 & -4.23348200 & -0.21577700 \\
\hline C & -1.74223200 & -4.12733800 & -0.38868400 & C & -4.19987800 & -4.01774200 & -0.57250600 \\
\hline $\mathrm{H}$ & -4.44226000 & -2.35008700 & 2.94205600 & C & -5.50718600 & -3.05780200 & 1.23078600 \\
\hline $\mathrm{C}$ & 0.75353600 & -4.85530700 & -1.46145300 & $\mathrm{H}$ & 1.60225900 & -4.02862400 & 0.33522500 \\
\hline $\mathrm{C}$ & -1.66930200 & -4.76259400 & -1.66640600 & C & -5.44090100 & -3.65658200 & -0.02348100 \\
\hline C & -4.09686100 & -4.65414700 & -1.85784800 & $\mathrm{H}$ & -6.47313400 & -2.79519100 & 1.65498100 \\
\hline $\mathrm{C}$ & -0.41175100 & -5.11836500 & -2.18053100 & $\mathrm{H}$ & 1.72195000 & -5.11629500 & -1.87626500 \\
\hline $\mathrm{C}$ & -2.89102600 & -5.01177300 & -2.37896700 & $\mathrm{H}$ & -6.35018600 & -3.86482300 & -0.58270100 \\
\hline $\mathrm{H}$ & -5.01472700 & -4.85351100 & -2.40641800 & $\mathrm{H}$ & -0.35548800 & -5.60289000 & -3.15256200 \\
\hline $\mathrm{H}$ & -2.83330700 & -5.49851200 & -3.34993500 & C & 2.61791300 & 0.02442200 & 2.14248100 \\
\hline $\mathrm{S}$ & 3.12309800 & -1.40179500 & 2.91680800 & $\mathrm{~N}$ & 1.60079700 & 0.80194500 & 2.59687700 \\
\hline $\mathrm{H}$ & 1.23356400 & 1.52533100 & 1.97193400 & C & -0.04700500 & -0.05881700 & -1.14400800 \\
\hline 0 & 0.78296200 & -0.79322300 & -0.65056600 & $\mathrm{O}$ & -0.15395500 & 0.30061500 & -2.43811500 \\
\hline C & 0.88473800 & -0.04026200 & -3.44515400 & C & 0.96934700 & -1.55864400 & -3.60919100 \\
\hline $\mathrm{H}$ & 1.62336600 & -1.79288200 & -4.45671800 & $\mathrm{H}$ & 1.37783700 & -2.03564500 & -2.71841000 \\
\hline $\mathrm{H}$ & -0.02003600 & -1.98315200 & -3.81418600 & C & 0.33266500 & 0.62304700 & -4.70936000 \\
\hline $\mathrm{H}$ & 0.22692000 & 1.70224300 & -4.55823800 & $\mathrm{H}$ & 1.01980800 & 0.45852200 & -5.54544800 \\
\hline $\mathrm{H}$ & -0.64120300 & 0.19913800 & -4.98292800 & C & 2.21794000 & 0.58978800 & -3.04650300 \\
\hline $\mathrm{H}$ & 2.93552300 & 0.45801100 & -3.86426100 & $\mathrm{H}$ & 2.09803200 & 1.65892900 & -2.85287400 \\
\hline $\mathrm{H}$ & 2.62694700 & 0.11530300 & -2.15494900 & C & -1.86003400 & 1.57432900 & -0.84603700 \\
\hline $\mathrm{H}$ & -1.30503600 & 2.21935200 & -1.52528500 & C & -2.60923200 & 2.32969000 & 0.20818400 \\
\hline $\mathrm{C}$ & -2.50856000 & 3.72776700 & 0.23038400 & C & -3.44227700 & 1.68515900 & 1.13481900 \\
\hline $\mathrm{C}$ & -3.23432500 & 4.46986200 & 1.16259700 & $\mathrm{H}$ & -1.82332700 & 4.22605200 & -0.44833600 \\
\hline C & -4.17074500 & 2.43214500 & 2.06077400 & $\mathrm{H}$ & -3.50297700 & 0.60070700 & 1.15305800 \\
\hline $\mathrm{C}$ & -4.07294400 & 3.82582100 & 2.07354100 & $\mathrm{H}$ & -3.12931500 & 5.55089800 & 1.18474800 \\
\hline $\mathrm{H}$ & -4.80757900 & 1.92351300 & 2.77959600 & $\mathrm{H}$ & -4.63770100 & 4.40481700 & 2.79931400 \\
\hline $\mathrm{N}$ & -1.07943700 & 0.50850900 & -0.39867700 & $\mathrm{H}$ & -1.07119000 & 0.27667700 & 0.60294600 \\
\hline $\mathrm{S}$ & 1.38386500 & 3.52997000 & -0.35549400 & $\mathrm{O}$ & 0.62963200 & 3.57788500 & -1.63064800 \\
\hline $\mathrm{O}$ & 0.63910300 & 2.89034100 & 0.76602000 & $\mathrm{O}$ & 2.79750600 & 3.09101800 & -0.44688200 \\
\hline C & 1.48277000 & 5.31482900 & 0.16215600 & $\mathrm{~F}$ & 2.17388400 & 6.03328700 & -0.73023400 \\
\hline $\mathrm{F}$ & 2.07765100 & 5.42734000 & 1.35752000 & $\mathrm{~F}$ & 0.24422600 & 5.83803600 & 0.25784000 \\
\hline $\mathrm{C}$ & -3.08796700 & 0.84200200 & -1.97129700 & $\mathrm{H}$ & -2.39542400 & 0.33041600 & -2.63525100 \\
\hline $\mathrm{H}$ & -3.64766100 & 0.17855400 & -1.31501900 & C & -3.82029700 & 1.90557500 & -2.55439400 \\
\hline $\mathrm{C}$ & -5.11948500 & 2.30296700 & -2.04739700 & $\mathrm{H}$ & -5.24440400 & 2.03651300 & -0.99099700 \\
\hline $\bar{H}$ & -5.33068600 & 3.36763800 & -2.20103000 & $\mathrm{C}$ & -3.17499100 & 2.72454700 & -3.62240400 \\
\hline $\mathrm{H}$ & -3.90689000 & 3.23224700 & -4.25681800 & $\mathrm{H}$ & -2.58287100 & 3.51149000 & -3.12385200 \\
\hline $\mathrm{H}$ & -2.47651500 & 2.14658800 & -4.23271400 & C & -8.15148600 & 2.04178300 & -2.12076000 \\
\hline $\bar{H}$ & -8.24192400 & 3.12412000 & -2.26765600 & $\mathrm{H}$ & -9.04664300 & 1.57395800 & -2.54841600 \\
\hline $\mathrm{H}$ & -8.15914500 & 1.84672900 & -1.04237400 & C & -6.57171300 & 1.74972800 & -4.78467900 \\
\hline $\mathrm{H}$ & -6.61966400 & 2.82858200 & -4.97400500 & $\mathrm{H}$ & -5.67612700 & 1.35814000 & -5.28040700 \\
\hline $\mathrm{H}$ & -7.44024700 & 1.29499800 & -5.27686500 & C & -6.41865900 & -0.49800600 & -2.63840000 \\
\hline
\end{tabular}




\begin{tabular}{|llll|llll|}
\hline $\mathrm{H}$ & -5.52622900 & -0.91198800 & -3.12060300 & $\mathrm{H}$ & -6.36297300 & -0.74528600 & -1.57193500 \\
\hline $\mathrm{H}$ & -7.28759500 & -1.02551600 & -3.05146600 & $\mathrm{Si}$ & -6.59846900 & 1.35273800 & -2.94027200 \\
\hline $\mathrm{C}$ & -2.02157300 & -3.36015200 & 4.67893500 & $\mathrm{H}$ & -1.61992900 & -4.30610200 & 4.30114900 \\
\hline $\mathrm{H}$ & -3.06531100 & -3.53416700 & 4.95663700 & & & & \\
\hline
\end{tabular}

\section{TS-S-4f-nd-19}

Imaginary frequency: $-155.49 \mathrm{~cm}^{-1}$ E[B3LYP/6-31G(d)]: -4788.683546 Hartree

E[PCM(Et $\left.\left.{ }_{2} \mathrm{O}\right)-B 3 L Y P / 6-311 G(d, p)-D 3(B J)\right]:-4790.216878$ Hartree

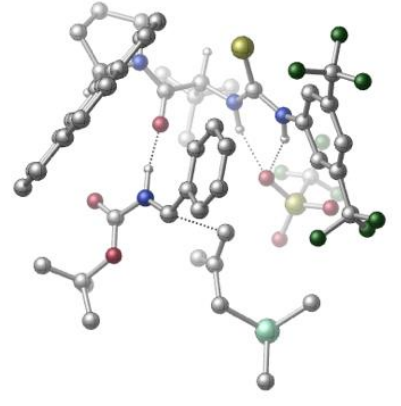

\begin{tabular}{|c|c|c|c|c|c|c|c|}
\hline 01 & & & & $\mathrm{~N}$ & -1.46175500 & 2.34956300 & 1.39529000 \\
\hline $\mathrm{H}$ & -2.16211700 & 1.60582300 & 1.48114200 & C & -1.62708900 & 3.11453700 & 0.22950700 \\
\hline $\mathrm{C}$ & -2.60944000 & 2.65742600 & -0.66831500 & C & -0.89270900 & 4.26426200 & -0.09455200 \\
\hline C & -2.79033300 & 3.29145000 & -1.89347900 & $\mathrm{H}$ & -3.22893200 & 1.80463700 & -0.40303500 \\
\hline C & -1.09348300 & 4.88318500 & -1.33036500 & $\mathrm{H}$ & -0.16471800 & 4.65301200 & 0.60266900 \\
\hline C & -2.02745300 & 4.40526600 & -2.24708800 & $\mathrm{H}$ & -2.16792900 & 4.89252100 & -3.20352900 \\
\hline C & -3.75584500 & 2.68781600 & -2.87709400 & C & -0.20282800 & 6.03537400 & -1.70608400 \\
\hline $\mathrm{F}$ & -4.90479700 & 2.29806300 & -2.29770800 & $\mathrm{~F}$ & -3.21926600 & 1.57580300 & -3.46298600 \\
\hline $\mathrm{F}$ & -4.06574600 & 3.53731300 & -3.88034400 & $\mathrm{~F}$ & 0.07668100 & 6.83311100 & -0.65606000 \\
\hline $\mathrm{F}$ & 0.99626600 & 5.59403200 & -2.18423900 & $\mathrm{~F}$ & -0.74131400 & 6.80680000 & -2.67483400 \\
\hline C & 0.56091800 & 0.50079500 & 3.80930600 & $\mathrm{H}$ & 0.94281100 & 1.34794000 & 4.38135100 \\
\hline $\mathrm{C}$ & -0.06682800 & -0.53574500 & 4.81890600 & C & -0.65565800 & -1.77562600 & 4.111118700 \\
\hline $\mathrm{H}$ & 0.10373800 & -2.31537000 & 3.53812600 & $\mathrm{H}$ & -1.47894100 & -1.52502300 & 3.43565600 \\
\hline $\mathrm{H}$ & -1.06208300 & -2.45730700 & 4.86767500 & C & -1.17992200 & 0.18188700 & 5.61310900 \\
\hline $\mathrm{H}$ & -1.57666700 & -0.49055900 & 6.38264800 & $\mathrm{H}$ & -2.01561900 & 0.48454100 & 4.97795700 \\
\hline $\mathrm{H}$ & -0.79424700 & 1.07758100 & 6.11530300 & C & 1.00372800 & -1.01435000 & 5.82034700 \\
\hline $\mathrm{H}$ & 0.56050300 & -1.74573200 & 6.50499400 & $\mathrm{H}$ & 1.38645600 & -0.19066000 & 6.43468500 \\
\hline $\mathrm{H}$ & 1.84961500 & -1.50427800 & 5.32487700 & C & 1.69968600 & -0.10807200 & 2.94538500 \\
\hline $\mathrm{O}$ & 1.39815700 & -0.80393700 & 1.96357100 & C & 3.49675200 & 0.87255600 & 4.43956700 \\
\hline C & 4.07857000 & -0.72275900 & 2.66423800 & C & 4.77962000 & 0.13821200 & 4.84031300 \\
\hline $\mathrm{H}$ & 3.69984900 & 1.90528600 & 4.12869000 & $\mathrm{H}$ & 2.77013600 & 0.90914100 & 5.24722700 \\
\hline $\mathrm{H}$ & 3.81762900 & -1.77112500 & 2.84912100 & $\mathrm{H}$ & 4.53381300 & -0.71250700 & 5.48737600 \\
\hline $\mathrm{H}$ & 5.47521600 & 0.78508100 & 5.38316500 & $\mathrm{~N}$ & 2.99450900 & 0.08731300 & 3.29512200 \\
\hline $\mathrm{C}$ & 4.33084800 & -0.61290900 & 1.16374800 & $\mathrm{C}$ & 4.47366400 & -1.79332500 & 0.48820000 \\
\hline C & 4.59563900 & 0.63880200 & 0.47057400 & C & 4.90966300 & -1.86976900 & -0.87496400 \\
\hline $\mathrm{H}$ & 4.25579100 & -2.72885400 & 0.99803500 & C & 5.04865500 & 0.58981900 & -0.89031800 \\
\hline $\mathrm{C}$ & 4.42630100 & 1.90179900 & 1.06376500 & $\mathrm{C}$ & 5.07276800 & -3.09614400 & -1.54024500 \\
\hline C & 5.20878000 & -0.65850500 & -1.56232800 & C & 5.34531300 & 1.80037000 & -1.59163900 \\
\hline $\mathrm{H}$ & 4.02876000 & 1.97769900 & 2.06570800 & C & 4.71413000 & 3.07691300 & 0.36938300 \\
\hline$C$ & 5.51835200 & -3.13422600 & -2.86070700 & $\mathrm{H}$ & 4.85816000 & -4.01884900 & -1.00737100 \\
\hline C & 5.66362600 & -0.70597900 & -2.91598500 & C & 5.17798400 & 3.03205900 & -0.94043200 \\
\hline C & 5.80521500 & 1.72361900 & -2.95142300 & $\mathrm{H}$ & 4.56282400 & 4.03423900 & 0.85974000 \\
\hline C & 5.80876000 & -1.95421300 & -3.54341800 & $\mathrm{H}$ & 5.64702900 & -4.09111000 & -3.36003000 \\
\hline C & 5.95822500 & 0.52968700 & -3.58584500 & $\mathrm{H}$ & 5.40385400 & 3.95130600 & -1.47524800 \\
\hline $\mathrm{H}$ & 6.02997200 & 2.65362500 & -3.46811300 & $\mathrm{H}$ & 6.15709700 & -1.99081600 & -4.57279800 \\
\hline $\mathrm{H}$ & 6.30739700 & 0.49151500 & -4.61487500 & $\mathrm{C}$ & -0.36034000 & 2.15426000 & 2.19788500 \\
\hline $\mathrm{S}$ & 0.98551900 & 3.17432200 & 2.31651000 & $\mathrm{~N}$ & -0.47137100 & 1.01547600 & 2.92987000 \\
\hline $\mathrm{H}$ & -1.22723800 & 0.37944700 & 2.68395000 & S & -4.32890000 & -0.34918700 & 1.67861200 \\
\hline $\mathrm{O}$ & -4.74494600 & -1.74893600 & 1.46069700 & $\mathrm{O}$ & -2.83486000 & -0.17140500 & 1.75777600 \\
\hline
\end{tabular}




\begin{tabular}{|c|c|c|c|c|c|c|c|}
\hline $\mathrm{O}$ & -4.98630900 & 0.67874100 & 0.85247800 & $\mathrm{C}$ & -4.85348000 & 0.01850700 & 3.42660800 \\
\hline $\mathrm{F}$ & -6.18357900 & -0.00541500 & 3.54524500 & $\mathrm{~F}$ & -4.40872100 & 1.23298700 & 3.79626100 \\
\hline $\mathrm{F}$ & -4.32989400 & -0.88814200 & 4.26966500 & $\mathrm{C}$ & 1.17886000 & -3.63966400 & -0.25242700 \\
\hline 0 & 1.73815200 & -4.05106000 & 0.74118300 & 0 & 0.71510000 & -4.34968300 & -1.28660200 \\
\hline C & 0.94246500 & -5.81828100 & -1.37815400 & C & 2.44643300 & -6.09997000 & -1.40542900 \\
\hline $\mathrm{H}$ & 2.61070100 & -7.16067600 & -1.62397100 & $\mathrm{H}$ & 2.91063400 & -5.86787900 & -0.44529800 \\
\hline $\mathrm{H}$ & 2.93265500 & -5.51024000 & -2.18969800 & C & 0.29610900 & -6.16408800 & -2.72008400 \\
\hline $\mathrm{H}$ & -0.76635000 & -5.90054700 & -2.71975700 & $\mathrm{H}$ & 0.38422400 & -7.23877100 & -2.90831300 \\
\hline $\mathrm{H}$ & 0.78701700 & -5.62896100 & -3.53937300 & C & 0.23629900 & -6.52989200 & -0.22245900 \\
\hline $\mathrm{H}$ & 0.34211800 & -7.61284000 & -0.34974700 & $\mathrm{H}$ & -0.83239600 & -6.29374000 & -0.21620200 \\
\hline $\mathrm{H}$ & 0.66734300 & -6.24818400 & 0.73937200 & C & 0.43686500 & -1.70259100 & -1.55876400 \\
\hline $\mathrm{H}$ & 0.23981500 & -2.41342200 & -2.34962500 & C & 0.73080300 & -0.33123200 & -1.97002400 \\
\hline C & 0.55761100 & -0.01475500 & -3.33263100 & C & 1.17408400 & 0.66481400 & -1.08136000 \\
\hline C & 0.81853800 & 1.26889000 & -3.79599400 & $\mathrm{H}$ & 0.21311300 & -0.78210600 & -4.02195100 \\
\hline C & 1.43231700 & 1.94820400 & -1.55302000 & $\mathrm{H}$ & 1.30732700 & 0.44964100 & -0.02629200 \\
\hline C & 1.24980900 & 2.25567200 & -2.90430700 & $\mathrm{H}$ & 0.68028300 & 1.50281300 & -4.84748600 \\
\hline $\mathrm{H}$ & 1.76430200 & 2.71099200 & -0.85761200 & $\mathrm{H}$ & 1.42771500 & 3.26856000 & -3.25119900 \\
\hline $\mathrm{N}$ & 0.96792000 & -2.25996300 & -0.44662300 & $\mathrm{H}$ & 1.27162200 & -1.68999700 & 0.35532500 \\
\hline C & -1.76501400 & -1.31226500 & -1.18066400 & $\mathrm{H}$ & -1.55558600 & -0.83341900 & -0.22959400 \\
\hline $\mathrm{H}$ & -1.92310100 & -0.65318200 & -2.02917000 & $\mathrm{C}$ & -2.30953100 & -2.57874200 & -1.18906700 \\
\hline C & -2.93605800 & -3.14234900 & -2.40258000 & $\mathrm{H}$ & -2.75055500 & -4.22070800 & -2.49141700 \\
\hline $\mathrm{H}$ & -2.56777300 & -2.64727100 & -3.31018600 & $C$ & -2.31427000 & -3.38640000 & 0.07257700 \\
\hline $\mathrm{H}$ & -2.37293700 & -4.46151200 & -0.11940800 & $\mathrm{H}$ & -3.18607500 & -3.08748400 & 0.67349800 \\
\hline $\mathrm{H}$ & -1.43761700 & -3.16754900 & 0.69191500 & C & -5.30904200 & -3.69652100 & -4.19697400 \\
\hline $\bar{H}$ & -4.81609600 & -3.16336900 & -5.01883400 & $\mathrm{H}$ & -6.39018200 & -3.64449300 & -4.37489400 \\
\hline $\mathrm{H}$ & -5.01715300 & -4.75205700 & -4.25803400 & C & -5.33926300 & -1.11697800 & -2.46086800 \\
\hline $\mathrm{H}$ & -4.75312400 & -0.51622200 & -3.16479000 & $\mathrm{H}$ & -5.20714700 & -0.68740200 & -1.46185800 \\
\hline $\bar{H}$ & -6.39676900 & -0.99319500 & -2.72734300 & $\mathrm{C}$ & -5.72692300 & -3.90569100 & -1.12910700 \\
\hline $\bar{H}$ & -5.57585900 & -3.41837700 & -0.16020900 & $\mathrm{H}$ & -5.37174500 & -4.94138300 & -1.06369000 \\
\hline $\mathrm{H}$ & -6.80824200 & -3.94202200 & -1.31346100 & $\mathrm{Si}$ & -4.88214900 & -2.94080500 & -2.51083800 \\
\hline $\mathrm{C}$ & 5.33260400 & -0.34714500 & 3.49536100 & $\mathrm{H}$ & 5.88079000 & 0.45847000 & 2.99677700 \\
\hline $\bar{H}$ & 6.01000300 & -1.20097400 & 3.58312600 & & & & \\
\hline
\end{tabular}

\section{TS-S-4f-nd-20}

Imaginary frequency: $-262.12 \mathrm{~cm}-1$

E[B3LYP/6-31G(d)]: -4788.678296 Hartree

E[PCM(Et $\left.\left.{ }_{2} \mathrm{O}\right)-B 3 L Y P / 6-311 G(d, p)-D 3(B J)\right]:-4790.216739$ Hartree

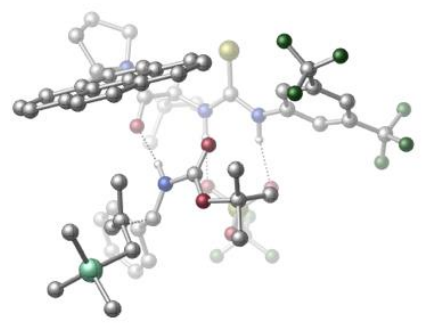

\begin{tabular}{|llll|lrrc|}
\hline $\mathrm{O} 1$ & & & $\mathrm{~N}$ & 3.26378800 & -0.13138900 & 0.98244600 \\
\hline $\mathrm{H}$ & 3.37286500 & 0.74138000 & 0.45410100 & $\mathrm{C}$ & 3.82494300 & -1.24321800 & 0.32881400 \\
\hline $\mathrm{C}$ & 5.00934100 & -1.04869500 & -0.39483600 & $\mathrm{C}$ & 3.20116200 & -2.49609800 & 0.29480600 \\
\hline $\mathrm{C}$ & 5.56941200 & -2.10194400 & -1.11819300 & $\mathrm{H}$ & 5.47338400 & -0.06903400 & -0.40637200 \\
\hline $\mathrm{C}$ & 3.77342800 & -3.53782500 & -0.43249300 & $\mathrm{H}$ & 2.26503500 & -2.63554600 & 0.81395500 \\
\hline $\mathrm{C}$ & 4.96371200 & -3.35809500 & -1.13866500 & $\mathrm{H}$ & 5.40255200 & -4.17340600 & -1.70073000 \\
\hline $\mathrm{C}$ & 6.88046900 & -1.90264800 & -1.83062300 & $\mathrm{C}$ & 3.03147200 & -4.84056800 & -0.53442600 \\
\hline $\mathrm{F}$ & 7.93262300 & -2.21949500 & -1.03839100 & $\mathrm{~F}$ & 7.05643300 & -0.62486400 & -2.22484400 \\
\hline $\mathrm{F}$ & 6.97581700 & -2.68520900 & -2.93166400 & $\mathrm{~F}$ & 2.39463500 & -5.16097300 & 0.61816800 \\
\hline $\mathrm{F}$ & 2.06720800 & -4.79658400 & -1.49550100 & $\mathrm{~F}$ & 3.84158000 & -5.87346900 & -0.84819400 \\
\hline $\mathrm{C}$ & 1.15738900 & 1.38721200 & 3.55676100 & $\mathrm{H}$ & 1.55735800 & 0.71492300 & 4.31518400 \\
\hline $\mathrm{C}$ & 1.39329300 & 2.85696500 & 4.06270800 & $\mathrm{C}$ & 0.76225600 & 3.92883700 & 3.15504200 \\
\hline
\end{tabular}




\begin{tabular}{|c|c|c|c|c|c|c|c|}
\hline $\mathrm{H}$ & -0.32779600 & 3.84433200 & 3.13476500 & $\mathrm{H}$ & 1.12167800 & 3.87136800 & 2.12493100 \\
\hline $\mathrm{H}$ & 1.01675900 & 4.92329400 & 3.54311100 & C & 2.91500200 & 3.09384000 & 4.15064500 \\
\hline $\mathrm{H}$ & 3.11276100 & 4.06473400 & 4.62040100 & $\mathrm{H}$ & 3.37851200 & 3.09967300 & 3.16036400 \\
\hline $\mathrm{H}$ & 3.40846800 & 2.32006400 & 4.75097100 & C & 0.79323700 & 2.99785300 & 5.47723500 \\
\hline $\mathrm{H}$ & 0.93144600 & 4.02321700 & 5.83870200 & $\mathrm{H}$ & 1.28381600 & 2.33020400 & 6.19627900 \\
\hline $\mathrm{H}$ & -0.28400700 & 2.79114200 & 5.49041000 & C & -0.32827200 & 1.03719000 & 3.30242000 \\
\hline $\mathrm{O}$ & -0.91657000 & 1.44797700 & 2.28827400 & $\mathrm{C}$ & -0.51997800 & -0.26154300 & 5.49243500 \\
\hline C & -2.40832300 & -0.07982000 & 3.97118900 & C & -1.78275800 & -0.17437200 & 6.35395600 \\
\hline $\mathrm{H}$ & -0.17044200 & -1.29535500 & 5.38120400 & $\mathrm{H}$ & 0.30209200 & 0.33121200 & 5.88588200 \\
\hline $\bar{H}$ & -2.90548200 & 0.84502300 & 3.66332200 & $\mathrm{H}$ & -1.91144600 & 0.85371500 & 6.71308500 \\
\hline $\mathrm{H}$ & -1.74086700 & -0.83367300 & 7.22620400 & $\mathrm{~N}$ & -0.98583600 & 0.25372400 & 4.19501000 \\
\hline C & -2.63417000 & -1.14696000 & 2.90389600 & C & -1.61737200 & -1.95740600 & 2.48909000 \\
\hline $\mathrm{C}$ & -3.97546200 & -1.34812700 & 2.38159800 & C & -1.81983300 & -3.03286800 & 1.56061700 \\
\hline $\mathrm{H}$ & -0.60664000 & -1.79585800 & 2.85497900 & C & -4.20350800 & -2.41560100 & 1.45102800 \\
\hline C & -5.06948500 & -0.53698500 & 2.73748100 & C & -0.76767600 & -3.86908400 & 1.15299600 \\
\hline $\mathrm{C}$ & -3.12803600 & -3.26034700 & 1.04396600 & C & -5.51258900 & -2.63750600 & 0.91705900 \\
\hline $\mathrm{H}$ & -4.93462300 & 0.27738700 & 3.44069900 & C & -6.34030000 & -0.75510500 & 2.20459900 \\
\hline C & -0.99189200 & -4.90690400 & 0.24903000 & $\mathrm{H}$ & 0.22724200 & -3.70239300 & 1.55473800 \\
\hline $\mathrm{C}$ & -3.35097000 & -4.32566800 & 0.11835000 & C & -6.56592500 & -1.79345300 & 1.30571300 \\
\hline $\mathrm{C}$ & -5.70942500 & -3.71955400 & -0.00997400 & $\mathrm{H}$ & -7.16256800 & -0.10991100 & 2.50291300 \\
\hline C & -2.26806900 & -5.13399200 & -0.26410300 & $\mathrm{H}$ & -0.15994800 & -5.53012800 & -0.06140800 \\
\hline $\mathrm{C}$ & -4.67964600 & -4.52543800 & -0.39011200 & $\mathrm{H}$ & -7.56177400 & -1.96621500 & 0.90420900 \\
\hline $\mathrm{H}$ & -6.71111000 & -3.88491300 & -0.40017100 & $\mathrm{H}$ & -2.43620100 & -5.94511000 & -0.96875500 \\
\hline $\mathrm{H}$ & -4.84874200 & -5.34221300 & -1.08816000 & C & 2.53430600 & -0.10498900 & 2.14001100 \\
\hline $\mathrm{S}$ & 2.47443400 & -1.39790000 & 3.24482200 & $\mathrm{~N}$ & 1.91346100 & 1.08475200 & 2.35136200 \\
\hline $\mathrm{H}$ & 1.84245600 & 1.74036200 & 1.56642700 & C & -0.21627800 & 0.16200100 & -1.09783100 \\
\hline 0 & 0.41535600 & -0.57247600 & -0.36950000 & $\mathrm{O}$ & -0.42791700 & 0.02184400 & -2.41635600 \\
\hline $\mathrm{C}$ & 0.35864600 & -0.96453100 & -3.21718200 & C & -0.06787200 & -2.38101000 & -2.82899700 \\
\hline $\mathrm{H}$ & 0.44019000 & -3.10365300 & -3.47685500 & $\mathrm{H}$ & 0.19701200 & -2.60677200 & -1.79569900 \\
\hline $\mathrm{H}$ & -1.14902300 & -2.51312100 & -2.95516600 & C & -0.05613900 & -0.62852600 & -4.65059700 \\
\hline $\mathrm{H}$ & 0.20294800 & 0.40678200 & -4.89317900 & $\mathrm{H}$ & 0.46963200 & -1.28797600 & -5.34836100 \\
\hline $\mathrm{H}$ & -1.13240100 & -0.76836300 & -4.79852400 & C & 1.85669200 & -0.72429700 & -3.03038100 \\
\hline $\mathrm{H}$ & 2.40347900 & -1.33049200 & -3.76153600 & $\mathrm{H}$ & 2.10088100 & 0.32913000 & -3.18920600 \\
\hline $\mathrm{H}$ & 2.18768400 & -1.01172600 & -2.03309800 & C & -1.39214900 & 2.29709400 & -1.39638100 \\
\hline $\mathrm{H}$ & -1.03679800 & 2.21691400 & -2.41762800 & C & -1.38194600 & 3.69658000 & -0.86885600 \\
\hline C & -0.94540900 & 4.71600100 & -1.72593100 & C & -1.81965600 & 4.01885800 & 0.42335400 \\
\hline C & -0.93642200 & 6.04094400 & -1.29309800 & $\mathrm{H}$ & -0.56482500 & 4.46270000 & -2.71059300 \\
\hline $\mathrm{C}$ & -1.81904900 & 5.34583700 & 0.84648200 & $\mathrm{H}$ & -2.13482000 & 3.23610100 & 1.10724800 \\
\hline C & -1.38185400 & 6.35973000 & -0.01009700 & $\mathrm{H}$ & -0.57111100 & 6.82006900 & -1.95566700 \\
\hline $\mathrm{H}$ & -2.15297600 & 5.58890800 & 1.85117800 & $\mathrm{H}$ & -1.37964000 & 7.39280500 & 0.32654300 \\
\hline $\mathrm{N}$ & -0.88072700 & 1.29264900 & -0.60019000 & $\mathrm{H}$ & -0.74484700 & 1.44107700 & 0.40835300 \\
\hline $\mathrm{S}$ & 2.51390500 & 2.82865700 & -1.15902300 & 0 & 1.67799600 & 2.64821500 & -2.36722400 \\
\hline $\mathrm{O}$ & 1.74325500 & 2.95907900 & 0.11071800 & $\mathrm{O}$ & 3.68304700 & 1.92015200 & -1.03969900 \\
\hline $\mathrm{C}$ & 3.27487900 & 4.51231200 & -1.38193800 & $\mathrm{~F}$ & 4.05262000 & 4.53521600 & -2.47211000 \\
\hline $\mathrm{F}$ & 4.02151500 & 4.83582300 & -0.31718000 & $\mathrm{~F}$ & 2.31758300 & 5.44515400 & -1.52583600 \\
\hline $\mathrm{C}$ & -3.25995500 & 2.08277800 & -1.67025400 & $\mathrm{H}$ & -3.57790900 & 2.20220000 & -0.63714700 \\
\hline $\mathrm{H}$ & -3.45668600 & 2.95128700 & -2.29494100 & $\mathrm{C}$ & -3.49520900 & 0.81938400 & -2.24737700 \\
\hline C & -3.63686000 & 0.64803500 & -3.68870900 & $\mathrm{H}$ & -3.17373200 & 1.46574500 & -4.25336900 \\
\hline $\mathrm{H}$ & -3.23600000 & -0.31161600 & -4.03163100 & C & -3.57939600 & -0.35596000 & -1.33847900 \\
\hline $\mathrm{H}$ & -3.58260500 & -1.31484800 & -1.85946500 & $\mathrm{H}$ & -2.73857100 & -0.32581300 & -0.62968800 \\
\hline $\mathrm{H}$ & -4.47772500 & -0.29093600 & -0.71038800 & C & -5.42604900 & 0.40538300 & -6.14417400 \\
\hline $\mathrm{H}$ & -4.94765400 & -0.54470400 & -6.40851200 & $\mathrm{H}$ & -6.43476100 & 0.39809200 & -6.57475800 \\
\hline $\mathrm{H}$ & -4.86484800 & 1.21083500 & -6.63162700 & C & -6.43679900 & -0.77853200 & -3.45334700 \\
\hline
\end{tabular}




\begin{tabular}{|c|c|c|c|c|c|c|c|}
\hline $\mathrm{H}$ & -5.95894900 & -1.74459000 & -3.65313400 & $\mathrm{H}$ & -6.50938300 & -0.66201200 & -2.36637400 \\
\hline $\mathrm{H}$ & -7.45986300 & -0.83210200 & -3.84590700 & C & -6.28911800 & 2.30759800 & -3.83636300 \\
\hline $\mathrm{H}$ & -6.31913200 & 2.47741300 & -2.75418100 & $\mathrm{H}$ & -5.74678000 & 3.14405600 & -4.29272700 \\
\hline $\mathrm{H}$ & -7.32189200 & 2.34929000 & -4.20344200 & $\mathrm{Si}$ & -5.51130600 & 0.64569200 & -4.27411200 \\
\hline C & -2.90594600 & -0.55346800 & 5.37423500 & $\mathrm{H}$ & -3.04733500 & -1.63861500 & 5.36177600 \\
\hline $\mathrm{H}$ & -3.86678200 & -0.10278100 & 5.63855200 & & & & \\
\hline
\end{tabular}

\section{TS-S-4f-nd-21}

Imaginary frequency: $-280.33 \mathrm{~cm}-1$

E[B3LYP/6-31G(d)]: -4788.680092 Hartree

E[PCM(Et $\left.\left.{ }_{2} \mathrm{O}\right)-\mathrm{B} 3 L Y P / 6-311 \mathrm{G}(\mathrm{d}, \mathrm{p})-\mathrm{D} 3(\mathrm{BJ})\right]$ : -4790.216631 Hartree

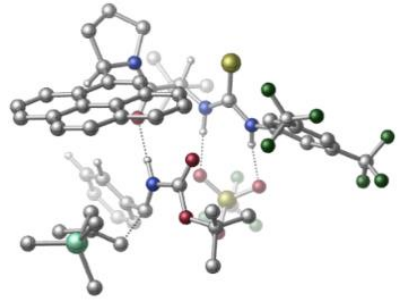

\begin{tabular}{|c|c|c|c|c|c|c|c|}
\hline 01 & & & & $\mathrm{~N}$ & 3.08028000 & -0.11573900 & 1.16546900 \\
\hline $\mathrm{H}$ & 3.32965800 & 0.66533200 & 0.55009000 & C & 3.65594400 & -1.34240800 & 0.78758500 \\
\hline C & 4.95116300 & -1.33183000 & 0.25448800 & C & 2.94349600 & -2.54652500 & 0.83456100 \\
\hline C & 5.52865000 & -2.51721200 & -0.20287800 & $\mathrm{H}$ & 5.49176600 & -0.39475400 & 0.18081800 \\
\hline C & 3.53462200 & -3.72170100 & 0.37598100 & $\mathrm{H}$ & 1.92583500 & -2.54525200 & 1.19607400 \\
\hline C & 4.83167400 & -3.72370000 & -0.13968200 & $\mathrm{H}$ & 5.28397200 & -4.64122400 & -0.49546800 \\
\hline C & 6.94676000 & -2.50804500 & -0.70816800 & C & 2.70958600 & -4.97782100 & 0.35091800 \\
\hline $\mathrm{F}$ & 7.83367000 & -2.74359100 & 0.28777300 & $\mathrm{~F}$ & 7.28132200 & -1.32334000 & -1.26013500 \\
\hline $\mathrm{F}$ & 7.15107200 & -3.46512500 & -1.64374300 & $\mathrm{~F}$ & 1.93509200 & -5.10290600 & 1.45615000 \\
\hline $\mathrm{F}$ & 1.85590500 & -4.99738000 & -0.70913200 & $\mathrm{~F}$ & 3.46600500 & -6.09170200 & 0.26051500 \\
\hline$C$ & 0.79610000 & 1.87843000 & 3.20787200 & $\mathrm{H}$ & 1.09493500 & 1.34787900 & 4.11157400 \\
\hline C & 1.03847800 & 3.41151500 & 3.46260000 & C & 0.51880800 & 4.31503300 & 2.32931300 \\
\hline $\mathrm{H}$ & -0.56692900 & 4.24713200 & 2.22369500 & $\mathrm{H}$ & 0.96506100 & 4.06977000 & 1.36246100 \\
\hline $\mathrm{H}$ & 0.77148500 & 5.35812700 & 2.55818500 & $C$ & 2.55521700 & 3.63757600 & 3.63472300 \\
\hline $\mathrm{H}$ & 2.73943200 & 4.66487000 & 3.97140700 & $\mathrm{H}$ & 3.09324300 & 3.49484000 & 2.69333900 \\
\hline $\mathrm{H}$ & 2.98364700 & 2.95561500 & 4.37894700 & $C$ & 0.33056100 & 3.81287100 & 4.77342400 \\
\hline $\mathrm{H}$ & 0.47076100 & 4.88390700 & 4.95870900 & $\mathrm{H}$ & 0.74003800 & 3.27596300 & 5.63805200 \\
\hline $\mathrm{H}$ & -0.74997200 & 3.62902800 & 4.73039700 & C & -0.66989800 & 1.51702500 & 2.86739200 \\
\hline $\mathrm{O}$ & -1.13310600 & 1.73963700 & 1.73730000 & C & -1.13000800 & 0.64931500 & 5.22374600 \\
\hline$C$ & -2.86089700 & 0.62372800 & 3.51266800 & $C$ & -2.46311400 & 0.91852100 & 5.92680100 \\
\hline $\mathrm{H}$ & -0.81534600 & -0.39642400 & 5.32779700 & $\mathrm{H}$ & -0.32439500 & 1.28139500 & 5.58943800 \\
\hline $\mathrm{H}$ & -3.28238900 & 1.50536100 & 3.01947800 & $\mathrm{H}$ & -2.58154900 & 1.99667600 & 6.08895700 \\
\hline $\mathrm{H}$ & -2.53160100 & 0.42158500 & 6.89923300 & $\mathrm{~N}$ & -1.44901800 & 0.93955300 & 3.81678700 \\
\hline C & -3.04977500 & -0.59191700 & 2.61058200 & C & -2.04320300 & -1.49256500 & 2.41813100 \\
\hline C & -4.35602300 & -0.83271400 & 2.02097200 & C & -2.22734800 & -2.70452800 & 1.67260400 \\
\hline $\mathrm{H}$ & -1.05694800 & -1.30615000 & 2.83504200 & C & -4.57021200 & -2.04520100 & 1.28571500 \\
\hline C & -5.43218100 & 0.06720300 & 2.13848500 & C & -1.18566900 & -3.62810600 & 1.48929100 \\
\hline C & -3.50978200 & -2.98507100 & 1.11950000 & C & -5.85413700 & -2.32531400 & 0.71942500 \\
\hline $\mathrm{H}$ & -5.30639300 & 0.99389700 & 2.68768900 & C & -6.67472700 & -0.20544000 & 1.56543700 \\
\hline C & -1.39648700 & -4.80789100 & 0.77669300 & $\mathrm{H}$ & -0.21016000 & -3.41771300 & 1.91704700 \\
\hline C & -3.72269000 & -4.20203300 & 0.40247900 & C & -6.89107800 & -1.38954900 & 0.86734800 \\
\hline C & -6.04599800 & -3.56674500 & 0.01830900 & $\mathrm{H}$ & -7.48408100 & 0.51135800 & 1.67836000 \\
\hline C & -2.65134500 & -5.09591800 & 0.24242900 & $\mathrm{H}$ & -0.57122800 & -5.49831600 & 0.63678700 \\
\hline $\mathrm{C}$ & -5.03063600 & -4.46301700 & -0.13083300 & $\mathrm{H}$ & -7.86797500 & -1.60611700 & 0.44113500 \\
\hline $\mathrm{H}$ & -7.03211400 & -3.77939200 & -0.38856700 & $\mathrm{H}$ & -2.81167300 & -6.02108000 & -0.30632500 \\
\hline $\mathrm{H}$ & -5.19608400 & -5.39829800 & -0.66078000 & C & 2.23056800 & 0.12418100 & 2.21390500 \\
\hline $\mathrm{S}$ & 1.98383700 & -0.97045300 & 3.49113700 & $\mathrm{~N}$ & 1.65418200 & 1.35132300 & 2.15911000 \\
\hline $\mathrm{H}$ & 1.68967700 & 1.86065600 & 1.27079500 & $\mathrm{C}$ & -0.07505200 & -0.02728900 & -1.35503100 \\
\hline $\mathrm{O}$ & 0.25232400 & -0.78851400 & -0.47050000 & 0 & 0.09642300 & -0.19214900 & -2.68031500 \\
\hline C & 0.94095200 & -1.28257300 & -3.24149800 & C & 0.33862000 & -2.64350700 & -2.88506700 \\
\hline
\end{tabular}




\begin{tabular}{|c|c|c|c|c|c|c|c|}
\hline $\mathrm{H}$ & 0.89294800 & -3.43013500 & -3.40865800 & $\mathrm{H}$ & 0.39181300 & -2.84072700 & -1.81414500 \\
\hline $\mathrm{H}$ & -0.70879100 & -2.69931800 & -3.20599200 & $\mathrm{C}$ & 0.85357800 & -1.01382400 & -4.74521600 \\
\hline $\mathrm{H}$ & 1.23521400 & -0.01509900 & -4.97883900 & $\mathrm{H}$ & 1.45562300 & -1.74929300 & -5.28823400 \\
\hline $\mathrm{H}$ & -0.18055800 & -1.09239800 & -5.10186400 & C & 2.37703900 & -1.12224900 & -2.74829200 \\
\hline $\mathrm{H}$ & 3.01816500 & -1.84335600 & -3.26753900 & $\mathrm{H}$ & 2.74601200 & -0.11396500 & -2.95422000 \\
\hline $\mathrm{H}$ & 2.44940000 & -1.31308500 & -1.67799600 & C & -0.80147000 & 2.23344100 & -1.98496700 \\
\hline $\bar{H}$ & 0.06232400 & 2.24473800 & -2.64870400 & $\mathrm{C}$ & -1.13492000 & 3.58072200 & -1.44847500 \\
\hline C & -0.49720900 & 4.68827400 & -2.03104400 & C & -2.06521400 & 3.79133300 & -0.41748100 \\
\hline $\mathrm{C}$ & -0.78848400 & 5.97919300 & -1.59859300 & $\mathrm{H}$ & 0.26180700 & 4.52658500 & -2.79106700 \\
\hline $\mathrm{C}$ & -2.35607200 & 5.08810000 & 0.00963000 & $\mathrm{H}$ & -2.53056000 & 2.94779600 & 0.08203500 \\
\hline C & -1.72620100 & 6.18357600 & -0.58326100 & $\mathrm{H}$ & -0.27198000 & 6.82439000 & -2.04415900 \\
\hline $\mathrm{H}$ & -3.07004100 & 5.23964800 & 0.81456900 & $\mathrm{H}$ & -1.95387400 & 7.19099500 & -0.24585400 \\
\hline $\mathrm{N}$ & -0.77263400 & 1.16009600 & -1.10118700 & $\mathrm{H}$ & -0.91870000 & 1.33542600 & -0.09894200 \\
\hline$S$ & 2.88858800 & 2.64154500 & -1.38914000 & 0 & 2.41205600 & 2.53823300 & -2.78669000 \\
\hline 0 & 1.78991300 & 2.74194000 & -0.38398700 & 0 & 3.96143000 & 1.69795000 & -0.98918700 \\
\hline $\mathrm{C}$ & 3.69247900 & 4.31698600 & -1.30005400 & $\mathrm{~F}$ & 4.75451300 & 4.38189600 & -2.11007300 \\
\hline $\mathrm{F}$ & 4.09254600 & 4.57453600 & -0.04530800 & $\mathrm{~F}$ & 2.81776900 & 5.27101500 & -1.66726700 \\
\hline C & -1.98242700 & 1.92209200 & -3.43364400 & $\mathrm{H}$ & -1.83175600 & 2.83000400 & -4.01386100 \\
\hline $\mathrm{H}$ & -1.44900700 & 1.05376700 & -3.80996700 & $\mathrm{C}$ & -3.28196400 & 1.68695300 & -2.94365200 \\
\hline $\mathrm{C}$ & -3.65434900 & 0.36764800 & -2.46385000 & $\mathrm{H}$ & -2.77942800 & -0.23507700 & -2.20030100 \\
\hline $\mathrm{H}$ & -4.36560200 & 0.39492100 & -1.62943000 & C & -4.25216000 & 2.81616700 & -2.82636100 \\
\hline $\mathrm{H}$ & -5.27326900 & 2.47526500 & -2.63899500 & $\mathrm{H}$ & -3.94132500 & 3.44223000 & -1.97350800 \\
\hline $\mathrm{H}$ & -4.23102600 & 3.46656700 & -3.70691800 & $\mathrm{C}$ & -6.40189700 & -0.18586000 & -3.83979600 \\
\hline $\mathrm{H}$ & -6.55821400 & 0.86195300 & -4.12102300 & $\mathrm{H}$ & -6.96705900 & -0.80054000 & -4.55131400 \\
\hline $\mathrm{H}$ & -6.84222000 & -0.34114000 & -2.84792200 & $\mathrm{C}$ & -3.80917200 & -0.31716100 & -5.55104200 \\
\hline $\mathrm{H}$ & -3.93925400 & 0.72619500 & -5.85962600 & $\mathrm{H}$ & -2.73597200 & -0.53983900 & -5.56716100 \\
\hline $\mathrm{H}$ & -4.28307700 & -0.94719400 & -6.31388300 & C & -4.34603900 & -2.48453600 & -3.40219900 \\
\hline $\mathrm{H}$ & -3.28549800 & -2.76093200 & -3.38453400 & $\mathrm{H}$ & -4.76788300 & -2.71793600 & -2.41916200 \\
\hline $\mathrm{H}$ & -4.84332200 & -3.12675600 & -4.14017800 & $\mathrm{Si}$ & -4.57883000 & -0.67698400 & -3.86571600 \\
\hline C & -3.50266900 & 0.40162700 & 4.91905900 & $\mathrm{H}$ & -3.67785200 & -0.66785000 & 5.07184000 \\
\hline $\mathrm{H}$ & -4.46864800 & 0.90618200 & 5.01011000 & & & & \\
\hline
\end{tabular}

\section{TS-S-4f-nd-22}

Imaginary frequency: $-225.00 \mathrm{~cm}^{-1}$ E[B3LYP/6-31G(d)]: -4788.697926 Hartree $\mathrm{E}\left[\mathrm{PCM}\left(\mathrm{Et}_{2} \mathrm{O}\right)-\mathrm{B} 3 \mathrm{LYP} / 6-311 \mathrm{G}(\mathrm{d}, \mathrm{p})-\mathrm{D} 3(\mathrm{BJ})\right]:-4790.216419$ Hartree

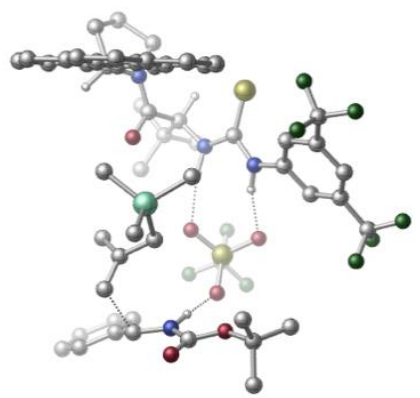

\begin{tabular}{|c|c|c|c|c|c|c|c|}
\hline 01 & & & & $\mathrm{~N}$ & 0.36321700 & 1.47275300 & -2.16957800 \\
\hline $\mathrm{H}$ & 1.29551400 & 1.05124700 & -2.15327200 & C & 0.36006700 & 2.82629300 & -1.78868500 \\
\hline $\mathrm{C}$ & 1.58528600 & 3.51146100 & -1.81393300 & C & -0.77907700 & 3.48977200 & -1.31130000 \\
\hline C & 1.65611000 & 4.83677700 & -1.38658400 & $\mathrm{H}$ & 2.47653100 & 3.00242100 & -2.16311600 \\
\hline C & -0.69048600 & 4.81877500 & -0.90129000 & $\mathrm{H}$ & -1.72465800 & 2.97127700 & -1.26901100 \\
\hline C & 0.52054100 & 5.50996400 & -0.93693400 & $\mathrm{H}$ & 0.57646100 & 6.54699300 & -0.62958400 \\
\hline C & 2.99048500 & 5.53148500 & -1.35692900 & C & -1.91435000 & 5.49038600 & -0.33815400 \\
\hline $\mathrm{F}$ & 3.62381600 & 5.33691900 & -0.16893500 & $\mathrm{~F}$ & 2.86816800 & 6.86845800 & -1.51110500 \\
\hline $\mathrm{F}$ & 3.82292600 & 5.08382400 & -2.31812900 & $\mathrm{~F}$ & -3.05435400 & 5.04748100 & -0.91989400 \\
\hline $\mathrm{F}$ & -2.04831300 & 5.25503600 & 0.99190000 & $\mathrm{~F}$ & -1.87553400 & 6.83115600 & -0.49408700 \\
\hline C & -1.26570900 & -1.61678100 & -3.29076300 & $\mathrm{H}$ & -1.83717900 & -1.10994800 & -4.06713000 \\
\hline C & -0.51032200 & -2.81554300 & -3.96822000 & C & 0.33356400 & -3.64288500 & -2.97805100 \\
\hline $\mathrm{H}$ & -0.28389800 & -4.07124800 & -2.18382900 & $\mathrm{H}$ & 1.12380200 & -3.05049400 & -2.50952600 \\
\hline
\end{tabular}




\begin{tabular}{|c|c|c|c|c|c|c|c|}
\hline $\mathrm{H}$ & 0.81735300 & -4.46560400 & -3.51935000 & $C$ & 0.40174200 & -2.24818100 & -5.07482800 \\
\hline $\mathrm{H}$ & 0.87175300 & -3.06959900 & -5.62884900 & $\mathrm{H}$ & 1.19866300 & -1.62475000 & -4.66335100 \\
\hline $\mathrm{H}$ & -0.16806000 & -1.64245900 & -5.78987200 & C & -1.54600000 & -3.75208200 & -4.62497500 \\
\hline $\mathrm{H}$ & -1.03172800 & -4.59529600 & -5.09968800 & $\mathrm{H}$ & -2.11970900 & -3.24031200 & -5.40706000 \\
\hline $\mathrm{H}$ & -2.24961500 & -4.16709200 & -3.89433900 & C & -2.21016800 & -2.05065600 & -2.13974600 \\
\hline 0 & -1.73703900 & -2.34052300 & -1.03508400 & C & -4.28976400 & -1.84598100 & -3.61473800 \\
\hline C & -4.46739200 & -2.58320300 & -1.31431200 & C & -5.36536600 & -2.93565900 & -3.59193500 \\
\hline $\mathrm{H}$ & -4.73050500 & -0.84115800 & -3.59112800 & $\mathrm{H}$ & -3.64310700 & -1.90715600 & -4.48670300 \\
\hline $\mathrm{H}$ & -3.97213000 & -3.42867900 & -0.82745800 & $\mathrm{H}$ & -4.93668200 & -3.87917300 & -3.94957100 \\
\hline $\mathrm{H}$ & -6.22179700 & -2.69245000 & -4.22829600 & $\mathrm{~N}$ & -3.55252300 & -2.10075300 & -2.36930700 \\
\hline C & -4.80373000 & -1.53371800 & -0.25814000 & C & -4.52247400 & -0.21391600 & -0.45788500 \\
\hline C & -5.48563800 & -1.95055600 & 0.95562500 & C & -4.87554800 & 0.80239300 & 0.49252300 \\
\hline $\mathrm{H}$ & -3.99629800 & 0.09944200 & -1.35569400 & C & -5.84608500 & -0.95553500 & 1.92243900 \\
\hline C & -5.80558400 & -3.29142900 & 1.23538900 & C & -4.57793400 & 2.15820600 & 0.27716600 \\
\hline C & -5.54058000 & 0.41937600 & 1.69327400 & C & -6.51425900 & -1.33472600 & 3.12878900 \\
\hline $\mathrm{H}$ & -5.54283300 & -4.07057000 & 0.52846300 & C & -6.45751100 & -3.65153300 & 2.41482000 \\
\hline C & -4.91455400 & 3.12144600 & 1.22800600 & $\mathrm{H}$ & -4.08177500 & 2.45279100 & -0.64341500 \\
\hline C & -5.89174600 & 1.41222800 & 2.65847100 & C & -6.81180900 & -2.68812600 & 3.35310700 \\
\hline $\mathrm{C}$ & -6.86124600 & -0.31383200 & 4.08000700 & $\mathrm{H}$ & -6.68968400 & -4.69715300 & 2.59916300 \\
\hline $\mathrm{C}$ & -5.56464800 & 2.75473100 & 2.40530000 & $\mathrm{H}$ & -4.65556600 & 4.15991100 & 1.04844000 \\
\hline C & -6.56426000 & 0.99585000 & 3.85758300 & $\mathrm{H}$ & -7.32162600 & -2.97510100 & 4.26966400 \\
\hline $\mathrm{H}$ & -7.37149000 & -0.61517600 & 4.99189200 & $\mathrm{H}$ & -5.82577900 & 3.51050600 & 3.14235400 \\
\hline $\mathrm{H}$ & -6.83379900 & 1.75388900 & 4.58931000 & C & -0.64739700 & 0.71839300 & -2.72609600 \\
\hline S & -2.08752200 & 1.36361400 & -3.34219400 & $\mathrm{~N}$ & -0.34690800 & -0.60731700 & -2.77179800 \\
\hline $\mathrm{H}$ & 0.43816100 & -0.93714100 & -2.20947900 & C & 4.44262200 & -0.15232000 & 3.23460100 \\
\hline 0 & 4.36799500 & -0.27801300 & 4.44296900 & $\mathrm{O}$ & 4.59284200 & 0.95482800 & 2.51932200 \\
\hline C & 4.76162000 & 2.28991700 & 3.15667000 & C & 6.02544900 & 2.28087900 & 4.01943000 \\
\hline $\mathrm{H}$ & 6.21057800 & 3.29220200 & 4.39712200 & $\mathrm{H}$ & 5.92462400 & 1.60544700 & 4.87132100 \\
\hline $\mathrm{H}$ & 6.89280300 & 1.97881700 & 3.42296200 & C & 4.91919400 & 3.20951900 & 1.94680100 \\
\hline $\mathrm{H}$ & 4.03193200 & 3.18274200 & 1.30929800 & $\mathrm{H}$ & 5.06771200 & 4.24099100 & 2.28185700 \\
\hline $\mathrm{H}$ & 5.78258800 & 2.91127900 & 1.34455600 & C & 3.50383900 & 2.63393000 & 3.95771800 \\
\hline $\mathrm{H}$ & 3.58514900 & 3.65953500 & 4.33352300 & $\mathrm{H}$ & 2.61672500 & 2.58224500 & 3.31693800 \\
\hline $\mathrm{H}$ & 3.37427800 & 1.96166300 & 4.80826200 & C & 4.15987900 & -2.49458800 & 2.85532900 \\
\hline $\mathrm{H}$ & 4.35176400 & -2.53688500 & 3.92230600 & C & 4.46929900 & -3.71376900 & 2.09534500 \\
\hline C & 4.59616200 & -4.91708500 & 2.81651400 & C & 4.67981000 & -3.72430600 & 0.70571600 \\
\hline C & 4.91942100 & -6.10192200 & 2.16568800 & $\mathrm{H}$ & 4.44617800 & -4.91540200 & 3.89367700 \\
\hline C & 5.00388100 & -4.91597500 & 0.05887300 & $\mathrm{H}$ & 4.61811300 & -2.80842400 & 0.13001500 \\
\hline C & 5.12089200 & -6.10413100 & 0.78144400 & $\mathrm{H}$ & 5.02084800 & -7.02135400 & 2.73491600 \\
\hline $\mathrm{H}$ & 5.16502400 & -4.90584900 & -1.01443100 & $\mathrm{H}$ & 5.37387700 & -7.02936500 & 0.27134000 \\
\hline $\mathrm{N}$ & 4.36651500 & -1.25166800 & 2.35518400 & $\mathrm{H}$ & 4.36425400 & -1.04585200 & 1.34335700 \\
\hline S & 3.48322500 & -0.57999900 & -1.52669500 & $\mathrm{O}$ & 4.46731000 & -0.62852000 & -0.41331800 \\
\hline 0 & 2.24837700 & -1.37541300 & -1.28315700 & $\mathrm{O}$ & 3.23004000 & 0.76006400 & -2.09790800 \\
\hline C & 4.36140800 & -1.50293600 & -2.88465200 & $\mathrm{~F}$ & 5.53900500 & -0.93820500 & -3.15562500 \\
\hline $\mathrm{F}$ & 3.62029800 & -1.51320300 & -4.00040300 & $\mathrm{~F}$ & 4.57339700 & -2.78009500 & -2.50981500 \\
\hline C & 2.09488700 & -2.69003700 & 3.42648800 & $\mathrm{H}$ & 2.14151200 & -3.71866400 & 3.77150200 \\
\hline $\mathrm{H}$ & 2.18390500 & -1.92433100 & 4.19302300 & C & 1.40908400 & -2.39599800 & 2.25577100 \\
\hline C & 1.07241000 & -1.01825100 & 1.89800700 & $\mathrm{H}$ & 1.78080300 & -0.29297300 & 2.31746100 \\
\hline $\mathrm{H}$ & 1.00680500 & -0.88667200 & 0.81369600 & C & 1.06689800 & -3.46919100 & 1.27196600 \\
\hline $\mathrm{H}$ & 0.09019700 & -3.29002900 & 0.80963200 & $\mathrm{H}$ & 1.79365600 & -3.41689500 & 0.44682400 \\
\hline $\mathrm{H}$ & 1.10123800 & -4.47073000 & 1.70807700 & C & -1.08010500 & 1.12977100 & 1.70690000 \\
\hline $\mathrm{H}$ & -1.20457700 & 0.92212300 & 0.63880100 & $\mathrm{H}$ & -2.01996300 & 1.56152800 & 2.06946900 \\
\hline $\mathrm{H}$ & -0.29867300 & 1.89130800 & 1.81385100 & C & -1.94865400 & -1.79114300 & 2.40320600 \\
\hline $\mathrm{H}$ & -2.08521300 & -2.03255700 & 1.34331800 & $\mathrm{H}$ & -1.68596100 & -2.71066500 & 2.94034000 \\
\hline
\end{tabular}




\begin{tabular}{|cccc|cccc|}
\hline $\mathrm{H}$ & -2.91541600 & -1.44953100 & 2.79154500 & $\mathrm{C}$ & -0.38996400 & -0.09452700 & 4.48143300 \\
\hline $\mathrm{H}$ & -0.10942000 & -0.99578800 & 5.03887500 & $\mathrm{H}$ & 0.38787200 & 0.66038600 & 4.64750100 \\
\hline $\mathrm{H}$ & -1.31818300 & 0.28651700 & 4.92492400 & $\mathrm{Si}$ & -0.65453700 & -0.44889400 & 2.64142500 \\
\hline $\mathrm{C}$ & -5.74078700 & -3.03828900 & -2.10226400 & $\mathrm{H}$ & -6.57263600 & -2.36667100 & -1.87030800 \\
\hline $\mathrm{H}$ & -6.05184700 & -4.04866000 & -1.82200200 & & & & \\
\hline
\end{tabular}

\section{TS-S-4f-nd-23}

Imaginary frequency: $-245.03 \mathrm{~cm}^{-1}$ E[B3LYP/6-31G(d)]: -4788.687657 Hartree E[PCM(Et $\left.\left.{ }_{2} \mathrm{O}\right)-\mathrm{B} 3 \mathrm{LYP} / 6-311 \mathrm{G}(\mathrm{d}, \mathrm{p})-\mathrm{D} 3(\mathrm{BJ})\right]$ : -4790.216353 Hartree

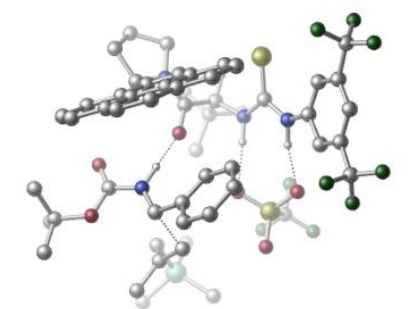

\begin{tabular}{|c|c|c|c|c|c|c|c|}
\hline 01 & & & & $\mathrm{~N}$ & -2.45561300 & -2.04855800 & 1.12309800 \\
\hline $\mathrm{H}$ & -1.78266100 & -2.63993700 & 0.62047300 & C & -3.67248400 & -1.91753300 & 0.42251400 \\
\hline $\mathrm{C}$ & -3.66593200 & -2.36529900 & -0.91497400 & $\mathrm{C}$ & -4.85765800 & -1.37829700 & 0.94306500 \\
\hline C & -4.79739900 & -2.22392200 & -1.71149400 & $\mathrm{H}$ & -2.76997000 & -2.82253800 & -1.32124000 \\
\hline $\mathrm{C}$ & -5.97665200 & -1.23394600 & 0.11808600 & $\mathrm{H}$ & -4.90034900 & -1.06733800 & 1.97579400 \\
\hline $\mathrm{C}$ & -5.96554300 & -1.64514400 & -1.21033800 & $\mathrm{H}$ & -6.84221600 & -1.53208500 & -1.83606900 \\
\hline C & -4.73446100 & -2.60580400 & -3.16570500 & C & -7.18924400 & -0.54295500 & 0.68051200 \\
\hline $\mathrm{F}$ & -3.82436800 & -3.56590100 & -3.41004000 & $\mathrm{~F}$ & -4.39728900 & -1.53621900 & -3.93987200 \\
\hline $\mathrm{F}$ & -5.92870800 & -3.04326400 & -3.62347400 & $\mathrm{~F}$ & -7.43590500 & -0.90077700 & 1.95694300 \\
\hline $\mathrm{F}$ & -7.02644300 & 0.80894900 & 0.68280300 & $\mathrm{~F}$ & -8.30404500 & -0.79766900 & -0.03816700 \\
\hline C & 0.12787400 & -0.79130900 & 3.39582500 & $\mathrm{H}$ & -0.58148800 & -0.57832600 & 4.19435600 \\
\hline $\mathrm{C}$ & 1.31058500 & -1.60972900 & 4.02514700 & $\mathrm{C}$ & 2.48323500 & -1.84972000 & 3.05471700 \\
\hline $\mathrm{H}$ & 2.94899800 & -0.90904600 & 2.74387400 & $\mathrm{H}$ & 2.16827200 & -2.38108500 & 2.15402200 \\
\hline $\mathrm{H}$ & 3.24624400 & -2.45580500 & 3.55927200 & $\mathrm{C}$ & 0.75510800 & -2.96633000 & 4.50364800 \\
\hline$\overline{\mathrm{H}}$ & 1.52605000 & -3.50494200 & 5.06778700 & $\mathrm{H}$ & 0.45193100 & -3.59524900 & 3.66326100 \\
\hline $\mathrm{H}$ & -0.11346600 & -2.83469300 & 5.15993000 & C & 1.84410600 & -0.83719200 & 5.24983000 \\
\hline $\mathrm{H}$ & 2.67750800 & -1.38756500 & 5.70098300 & $\bar{H}$ & 1.07484500 & -0.71966300 & 6.02302400 \\
\hline $\mathrm{H}$ & 2.22047400 & 0.15638200 & 4.97780300 & $\mathrm{C}$ & 0.56146900 & 0.52774500 & 2.71317500 \\
\hline $\mathrm{O}$ & 1.06981700 & 0.46855000 & 1.58114600 & C & -0.19063100 & 1.96986400 & 4.66873100 \\
\hline $\mathrm{C}$ & 0.69724000 & 2.98015400 & 2.63762500 & $\mathrm{C}$ & 0.58556700 & 3.22050500 & 5.09024100 \\
\hline $\mathrm{H}$ & -1.27051200 & 2.15748100 & 4.61974500 & $\bar{H}$ & -0.02271600 & 1.12194500 & 5.32895800 \\
\hline $\mathrm{H}$ & 1.70650300 & 2.84982200 & 2.23724400 & $\mathrm{H}$ & 1.58461500 & 2.93179400 & 5.43796100 \\
\hline $\mathrm{H}$ & 0.09058400 & 3.76644800 & 5.89906800 & $\mathrm{~N}$ & 0.33698300 & 1.71416600 & 3.31946300 \\
\hline C & -0.26034000 & 3.34970800 & 1.50624200 & $\mathrm{C}$ & -1.49535800 & 2.77623400 & 1.41459400 \\
\hline C & 0.12522000 & 4.38141000 & 0.55850600 & C & -2.44730900 & 3.14498200 & 0.40663500 \\
\hline $\mathrm{H}$ & -1.79065900 & 2.00042300 & 2.11543700 & $\mathrm{C}$ & -0.80878900 & 4.76892700 & -0.45853000 \\
\hline $\mathrm{C}$ & 1.37402000 & 5.02866400 & 0.59377500 & $\mathrm{C}$ & -3.71516700 & 2.54467000 & 0.32822900 \\
\hline $\mathrm{C}$ & -2.09259000 & 4.15189100 & -0.53605900 & $\mathrm{C}$ & -0.46195200 & 5.78600400 & -1.40227700 \\
\hline $\mathrm{H}$ & 2.10519400 & 4.75602600 & 1.34663800 & $\mathrm{C}$ & 1.69935100 & 6.02449300 & -0.32811500 \\
\hline C & -4.62008600 & 2.92396100 & -0.66261300 & $\mathrm{H}$ & -3.98585000 & 1.77880300 & 1.04997900 \\
\hline $\mathrm{C}$ & -3.02529400 & 4.53635100 & -1.54685700 & $\mathrm{C}$ & 0.79657400 & 6.40183100 & -1.31698200 \\
\hline $\mathrm{C}$ & -1.41932100 & 6.15321800 & -2.41031800 & $\mathrm{H}$ & 2.66724000 & 6.51517300 & -0.26435000 \\
\hline C & -4.28074000 & 3.90749800 & -1.59057700 & $\mathrm{H}$ & -5.59397000 & 2.44548700 & -0.70274200 \\
\hline $\mathrm{C}$ & -2.64136100 & 5.55768800 & -2.48080000 & $\mathrm{H}$ & 1.05580400 & 7.18129700 & -2.02962400 \\
\hline $\mathrm{H}$ & -1.14305700 & 6.92833400 & -3.12142900 & $\mathrm{H}$ & -4.99164400 & 4.20044400 & -2.35971400 \\
\hline $\mathrm{H}$ & -3.35305900 & 5.85024600 & -3.24933000 & C & -1.97352200 & -1.38276300 & 2.22406200 \\
\hline $\mathrm{S}$ & -2.91791900 & -0.45968400 & 3.29296700 & $\mathrm{~N}$ & -0.62819800 & -1.54719500 & 2.39756400 \\
\hline $\mathrm{H}$ & -0.07769700 & -1.89158200 & 1.60416800 & $\mathrm{~S}$ & 0.57453500 & -3.74439000 & -0.62396200 \\
\hline $\mathrm{O}$ & 1.21501600 & -3.96048200 & -1.93099400 & $\mathrm{O}$ & 1.09846200 & -2.56992800 & 0.14056100 \\
\hline $\mathrm{O}$ & -0.90848000 & -3.83706000 & -0.58457500 & $\mathrm{C}$ & 1.11051700 & -5.20398900 & 0.40069900 \\
\hline
\end{tabular}




\begin{tabular}{|c|c|c|c|c|c|c|c|}
\hline $\mathrm{F}$ & 0.72799100 & -6.35146400 & -0.16342600 & $\mathrm{~F}$ & 0.58369500 & -5.13648500 & 1.63502000 \\
\hline $\mathrm{F}$ & 2.45434300 & -5.22214000 & 0.52462000 & C & 3.76787600 & 1.91558500 & -0.21567800 \\
\hline $\mathrm{O}$ & 3.92225200 & 2.24019700 & 0.94525100 & $\mathrm{O}$ & 4.60035900 & 2.17613900 & -1.24102000 \\
\hline C & 5.77837100 & 3.06280700 & -1.05739600 & C & 5.33076500 & 4.43103900 & -0.53593300 \\
\hline $\mathrm{H}$ & 6.17980300 & 5.12255600 & -0.56982400 & $\mathrm{H}$ & 4.97130500 & 4.36875700 & 0.49171700 \\
\hline $\mathrm{H}$ & 4.53295100 & 4.83807900 & -1.16574100 & C & 6.32880400 & 3.18597600 & -2.47939700 \\
\hline $\mathrm{H}$ & 6.61099700 & 2.20746900 & -2.88012100 & $\mathrm{H}$ & 7.21822700 & 3.82403000 & -2.47809900 \\
\hline $\mathrm{H}$ & 5.58523100 & 3.63376700 & -3.14654800 & C & 6.78868000 & 2.38642300 & -0.12750000 \\
\hline $\mathrm{H}$ & 7.69071900 & 3.00414500 & -0.05733900 & $\mathrm{H}$ & 7.08319300 & 1.40733100 & -0.52190900 \\
\hline $\mathrm{H}$ & 6.37504200 & 2.25784200 & 0.87410800 & C & 2.35195900 & 0.88834300 & -1.93933600 \\
\hline $\mathrm{H}$ & 3.01100300 & 1.38163400 & -2.64329800 & C & 0.94747900 & 0.77266100 & -2.38050500 \\
\hline $\mathrm{C}$ & 0.66118300 & 1.06191300 & -3.72733600 & C & -0.09279200 & 0.38169800 & -1.52144900 \\
\hline $\mathrm{C}$ & -0.63986400 & 0.96383400 & -4.20934600 & $\mathrm{H}$ & 1.46459700 & 1.36668900 & -4.39475600 \\
\hline C & -1.39308700 & 0.29385100 & -2.01067900 & $\mathrm{H}$ & 0.10853100 & 0.13291500 & -0.48520600 \\
\hline $\mathrm{C}$ & -1.67068500 & 0.57200400 & -3.35123500 & $\mathrm{H}$ & -0.84972500 & 1.18929200 & -5.25086000 \\
\hline $\mathrm{H}$ & -2.19610500 & 0.00069500 & -1.34391800 & $\mathrm{H}$ & -2.68448200 & 0.46380200 & -3.72348500 \\
\hline $\mathrm{N}$ & 2.63817800 & 1.21476600 & -0.63869300 & $\mathrm{H}$ & 1.99302900 & 0.98858100 & 0.13303000 \\
\hline C & 2.93815700 & -1.02735900 & -2.40024500 & $\mathrm{H}$ & 2.24982300 & -1.54880300 & -1.74054000 \\
\hline $\mathrm{H}$ & 2.65131100 & -1.00091000 & -3.44817600 & $\mathrm{C}$ & 4.29380600 & -1.04054800 & -2.09008200 \\
\hline $\mathrm{C}$ & 4.74800300 & -1.41692700 & -0.75291500 & $\mathrm{H}$ & 3.91724800 & -1.45895700 & -0.04111600 \\
\hline $\mathrm{H}$ & 5.53929500 & -0.75548200 & -0.37740300 & C & 5.30797800 & -0.67254100 & -3.13580400 \\
\hline $\mathrm{H}$ & 5.67336600 & -1.58876300 & -3.62122000 & $\mathrm{H}$ & 6.17708200 & -0.16441800 & -2.70850100 \\
\hline $\mathrm{H}$ & 4.87319100 & -0.04776400 & -3.92151300 & $\mathrm{C}$ & 5.43266300 & -3.76583400 & 1.09436600 \\
\hline $\mathrm{H}$ & 5.84972800 & -3.02347200 & 1.78493700 & $\mathrm{H}$ & 5.98002800 & -4.70469900 & 1.24480700 \\
\hline $\mathrm{H}$ & 4.38835100 & -3.94112100 & 1.36831700 & $\mathrm{C}$ & 7.41049100 & -2.98904600 & -1.14953500 \\
\hline $\mathrm{H}$ & 7.91876000 & -2.29881300 & -0.46538800 & $\mathrm{H}$ & 7.55545200 & -2.61862200 & -2.17092100 \\
\hline $\mathrm{H}$ & 7.92597400 & -3.95523200 & -1.08107800 & C & 4.73852700 & -4.37329300 & -1.90666300 \\
\hline $\mathrm{H}$ & 4.96056100 & -4.10848700 & -2.94772400 & $\mathrm{H}$ & 3.64993200 & -4.38347600 & -1.79011300 \\
\hline $\mathrm{H}$ & 5.10357100 & -5.39592800 & -1.74854200 & $\mathrm{Si}$ & 5.58243400 & -3.20440500 & -0.69844100 \\
\hline C & 0.67748500 & 4.03561800 & 3.78866000 & $\mathrm{H}$ & -0.20289800 & 4.67642600 & 3.67974900 \\
\hline $\mathrm{H}$ & 1.55874300 & 4.68226900 & 3.75957300 & & & & \\
\hline
\end{tabular}

\section{TS-S-4f-nd-24}

Imaginary frequency: $-251.20 \mathrm{~cm}^{-1}$ E[B3LYP/6-31G(d)]: -4788.693585 Hartree $\mathrm{E}\left[\mathrm{PCM}\left(\mathrm{Et}_{2} \mathrm{O}\right)-\mathrm{B} 3 \mathrm{LYP} / 6-311 \mathrm{G}(\mathrm{d}, \mathrm{p})-\mathrm{D} 3(\mathrm{BJ})\right]$ : -4790.216043 Hartree

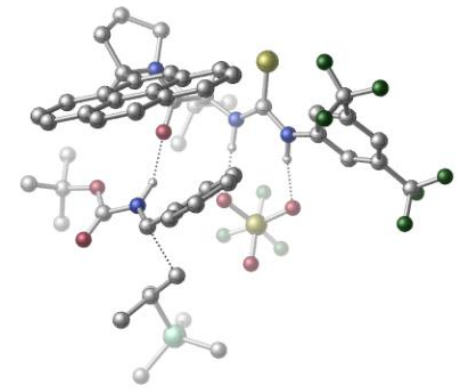

\begin{tabular}{|c|c|c|c|c|c|c|c|}
\hline 01 & & & & $\mathrm{~N}$ & -1.98734900 & -2.05702700 & 1.47872200 \\
\hline $\mathrm{H}$ & -1.19857500 & -2.67552700 & 1.25694700 & $\mathrm{C}$ & -3.18210900 & -2.44420900 & 0.84540000 \\
\hline C & -3.30455200 & -3.78939200 & 0.46303400 & C & -4.19474700 & -1.54183100 & 0.49201700 \\
\hline C & -4.42745900 & -4.21807300 & -0.24249000 & $\mathrm{H}$ & -2.51482100 & -4.49000500 & 0.70993700 \\
\hline C & -5.30397700 & -1.98660400 & -0.22561900 & $\mathrm{H}$ & -4.11137100 & -0.50344400 & 0.77678100 \\
\hline C & -5.44074800 & -3.32512100 & -0.59217000 & $\mathrm{H}$ & -6.31537100 & -3.66553900 & -1.13299700 \\
\hline C & -4.52278100 & -5.65469300 & -0.68605600 & C & -6.31349300 & -0.97651800 & -0.69794600 \\
\hline $\mathrm{F}$ & -3.86638700 & -6.48761400 & 0.14815500 & $\mathrm{~F}$ & -3.99386800 & -5.83083700 & -1.91908600 \\
\hline $\mathrm{F}$ & -5.80829000 & -6.07193000 & -0.75007400 & $\mathrm{~F}$ & -6.44521400 & 0.06174700 & 0.16061800 \\
\hline$F$ & -5.94953000 & -0.43628800 & -1.89371500 & $\mathrm{~F}$ & -7.53703800 & -1.51631700 & -0.87407500 \\
\hline C & 0.08052800 & 0.21143600 & 3.47179200 & $\mathrm{H}$ & -0.72142500 & 0.42597400 & 4.17739500 \\
\hline C & 1.33544000 & -0.24179900 & 4.30151000 & $\mathrm{C}$ & 2.57355000 & -0.52390200 & 3.42877400 \\
\hline $\mathrm{H}$ & 2.87851600 & 0.35825400 & 2.85970100 & $\mathrm{H}$ & 2.40420300 & -1.33092300 & 2.71374800 \\
\hline
\end{tabular}




\begin{tabular}{|c|c|c|c|c|c|c|c|}
\hline $\mathrm{H}$ & 3.40763100 & -0.82420600 & 4.07578100 & C & 0.95844300 & -1.51688700 & 5.08298900 \\
\hline $\mathrm{H}$ & 1.77950400 & -1.79974300 & 5.75260800 & $\mathrm{H}$ & 0.76887600 & -2.35759800 & 4.41120900 \\
\hline $\mathrm{H}$ & 0.06165800 & -1.36167800 & 5.69478100 & C & 1.69276300 & 0.86514500 & 5.31496200 \\
\hline $\mathrm{H}$ & 2.57371000 & 0.56618900 & 5.89424000 & $\mathrm{H}$ & 0.87966800 & 1.04069200 & 6.02979400 \\
\hline $\mathrm{H}$ & 1.93296900 & 1.81497000 & 4.82224100 & C & 0.32814400 & 1.44638400 & 2.57316200 \\
\hline 0 & 0.92434600 & 1.32074400 & 1.49470800 & C & -0.80169500 & 3.04153600 & 4.21717300 \\
\hline C & 0.11623800 & 3.84722400 & 2.11795000 & C & -0.20008000 & 4.42169500 & 4.49918500 \\
\hline $\mathrm{H}$ & -1.88758900 & 3.09561500 & 4.06863900 & $\mathrm{H}$ & -0.60538900 & 2.32193200 & 5.00830100 \\
\hline $\mathrm{H}$ & 1.15137800 & 3.76960000 & 1.77267900 & $\mathrm{H}$ & 0.78726500 & 4.30219100 & 4.96099500 \\
\hline $\mathrm{H}$ & -0.81880400 & 5.01774600 & 5.17680700 & $\mathrm{~N}$ & -0.14017100 & 2.66043400 & 2.96095200 \\
\hline C & -0.80579600 & 3.95194200 & 0.90702000 & C & -1.97710400 & 3.25449100 & 0.85174500 \\
\hline C & -0.45996800 & 4.86811300 & -0.16573000 & C & -2.90751400 & 3.39449500 & -0.23144700 \\
\hline $\mathrm{H}$ & -2.22932400 & 2.55077400 & 1.64073500 & C & -1.36658100 & 5.01647200 & -1.26594200 \\
\hline C & 0.72783200 & 5.62201200 & -0.18023800 & C & -4.11752100 & 2.68241300 & -0.26951600 \\
\hline C & -2.59057000 & 4.28468900 & -1.29774800 & C & -1.05269000 & 5.90745900 & -2.33950500 \\
\hline $\mathrm{H}$ & 1.43229600 & 5.54051200 & 0.64066100 & C & 1.02652300 & 6.48649000 & -1.23345800 \\
\hline C & -5.00040400 & 2.83489800 & -1.33822700 & $\mathrm{H}$ & -4.36421400 & 2.01064700 & 0.54829400 \\
\hline C & -3.49966000 & 4.43729200 & -2.38860800 & C & 0.14969600 & 6.63103900 & -2.30335100 \\
\hline C & -1.98466800 & 6.03976300 & -3.42637100 & $\mathrm{H}$ & 1.95307100 & 7.05432400 & -1.21328700 \\
\hline C & -4.69642500 & 3.70162400 & -2.38691800 & $\mathrm{H}$ & -5.92269000 & 2.26341200 & -1.35356200 \\
\hline C & -3.15225800 & 5.34026800 & -3.45039800 & $\mathrm{H}$ & 0.38619900 & 7.30971100 & -3.11935300 \\
\hline $\mathrm{H}$ & -1.73527100 & 6.72238500 & -4.23544200 & $\mathrm{H}$ & -5.38952400 & 3.81739400 & -3.21699100 \\
\hline $\mathrm{H}$ & -3.84737100 & 5.45697400 & -4.27867900 & C & -1.75795300 & -1.04120600 & 2.37604400 \\
\hline S & -2.98179000 & -0.15264800 & 3.14420900 & $\mathrm{~N}$ & -0.43111300 & -0.85182700 & 2.61642100 \\
\hline $\mathrm{H}$ & 0.23462400 & -1.24855200 & 1.94587600 & $S$ & 1.31964000 & -3.39812300 & 0.24515700 \\
\hline 0 & 1.95385300 & -3.74764400 & -1.03847100 & $\mathrm{O}$ & 1.42250200 & -1.94951100 & 0.60116500 \\
\hline 0 & -0.02728600 & -3.96629900 & 0.49986000 & C & 2.39089100 & -4.22865000 & 1.52197100 \\
\hline $\mathrm{F}$ & 2.51325200 & -5.53432400 & 1.27141200 & $\mathrm{~F}$ & 1.87025400 & -4.07266600 & 2.75028300 \\
\hline $\mathrm{F}$ & 3.62205600 & -3.67727700 & 1.52460800 & C & 3.75635400 & 1.62606100 & -0.72541800 \\
\hline 0 & 4.49863500 & 1.85597300 & -1.66604500 & $\mathrm{O}$ & 3.98305400 & 1.87037700 & 0.56487800 \\
\hline C & 5.23602900 & 2.53172000 & 1.00853400 & C & 6.43686800 & 1.64362300 & 0.67236400 \\
\hline $\mathrm{H}$ & 7.33968400 & 2.06867200 & 1.12451900 & $\mathrm{H}$ & 6.58970800 & 1.57291800 & -0.40591900 \\
\hline $\mathrm{H}$ & 6.29368200 & 0.63821700 & 1.08231200 & C & 5.03941200 & 2.63364500 & 2.52154000 \\
\hline $\mathrm{H}$ & 4.14389300 & 3.21765900 & 2.75624400 & $\mathrm{H}$ & 5.90498500 & 3.12769700 & 2.97476800 \\
\hline $\mathrm{H}$ & 4.93102800 & 1.64146900 & 2.96874500 & C & 5.33613300 & 3.92326300 & 0.37679800 \\
\hline $\mathrm{H}$ & 6.19009200 & 4.45596800 & 0.80929900 & $\mathrm{H}$ & 4.43149500 & 4.50380200 & 0.58822400 \\
\hline $\mathrm{H}$ & 5.47328900 & 3.86387300 & -0.70394700 & C & 1.99635700 & 0.88581300 & -2.15331800 \\
\hline $\mathrm{H}$ & 2.54321800 & 1.46572600 & -2.88829300 & C & 0.54544600 & 0.77194900 & -2.36984900 \\
\hline C & 0.00675900 & 1.36730500 & -3.52509500 & C & -0.30124100 & 0.08696500 & -1.48321500 \\
\hline C & -1.36021200 & 1.30150700 & -3.77827800 & $\mathrm{H}$ & 0.66150100 & 1.89671200 & -4.21354100 \\
\hline C & -1.66396600 & 0.01342300 & -1.75318000 & $\mathrm{H}$ & 0.10667500 & -0.40837400 & -0.60863100 \\
\hline C & -2.19839700 & 0.61926400 & -2.89290000 & $\mathrm{H}$ & -1.77073700 & 1.78390400 & -4.66023400 \\
\hline $\mathrm{H}$ & -2.31423900 & -0.52671200 & -1.07512200 & $\mathrm{H}$ & -3.26656500 & 0.56076000 & -3.07919600 \\
\hline $\mathrm{N}$ & 2.49117800 & 1.05723200 & -0.89158900 & $\mathrm{H}$ & 1.91883200 & 0.89794400 & -0.05314400 \\
\hline C & 2.56216800 & -0.95769300 & -2.98079800 & $\mathrm{H}$ & 2.11585400 & -1.61386800 & -2.23752400 \\
\hline $\mathrm{H}$ & 1.98956400 & -0.80895200 & -3.89146700 & C & 3.94825000 & -0.88244700 & -3.06198500 \\
\hline C & 4.79700600 & -1.46439400 & -2.01702900 & $\mathrm{H}$ & 4.22945100 & -1.64711600 & -1.09774900 \\
\hline $\mathrm{H}$ & 5.67386800 & -0.84169400 & -1.80252800 & C & 4.59861400 & -0.17212700 & -4.21473800 \\
\hline $\mathrm{H}$ & 5.40553600 & -0.77518500 & -4.64488800 & $\mathrm{H}$ & 5.05224800 & 0.75828800 & -3.85246200 \\
\hline $\mathrm{H}$ & 3.88356900 & 0.06991700 & -5.00601100 & C & 6.12829700 & -3.96906900 & -0.86927100 \\
\hline $\mathrm{H}$ & 6.88251600 & -3.33938400 & -0.38176900 & $\mathrm{H}$ & 6.58332100 & -4.95153700 & -1.04589500 \\
\hline $\mathrm{H}$ & 5.29737500 & -4.10443800 & -0.17104000 & C & 7.05000000 & -2.94276100 & -3.62328300 \\
\hline $\mathrm{H}$ & 7.76277600 & -2.22859700 & -3.19379100 & $\mathrm{H}$ & 6.77812100 & -2.58817300 & -4.62411000 \\
\hline
\end{tabular}




\begin{tabular}{|lrrr|lrrr|}
\hline $\mathrm{H}$ & 7.58174500 & -3.89358500 & -3.75659700 & $\mathrm{C}$ & 4.26726200 & -4.28921100 & -3.36396900 \\
\hline $\mathrm{H}$ & 3.96322800 & -3.86433100 & -4.32814700 & $\mathrm{H}$ & 3.37301500 & -4.40713800 & -2.74436800 \\
\hline $\mathrm{H}$ & 4.68302500 & -5.28543400 & -3.56065100 & $\mathrm{Si}$ & 5.54748400 & -3.21753100 & -2.49880200 \\
\hline $\mathrm{C}$ & -0.07491600 & 5.04927400 & 3.09892500 & $\mathrm{H}$ & -0.99164900 & 5.58735000 & 2.83992900 \\
\hline $\mathrm{H}$ & 0.75081200 & 5.76348300 & 3.03211500 & & & & \\
\hline
\end{tabular}

\section{TS-S-4f-nd-25}

Imaginary frequency: $-162.49 \mathrm{~cm}^{-1}$ E[B3LYP/6-31G(d)]: -4788.689641 Hartree

E[PCM(Et $\left.\left.{ }_{2} \mathrm{O}\right)-\mathrm{B} 3 \mathrm{LYP} / 6-311 \mathrm{G}(\mathrm{d}, \mathrm{p})-\mathrm{D} 3(\mathrm{BJ})\right]$ : -4790.215615 Hartree

\begin{tabular}{|c|c|c|c|c|c|c|c|}
\hline 01 & & & & $\mathrm{~N}$ & 2.87944100 & 1.25077300 & 1.33083900 \\
\hline $\mathrm{H}$ & 2.35550800 & 2.12538300 & 1.20826100 & C & 4.02870200 & 1.19270100 & 0.52273500 \\
\hline C & 4.38716200 & 2.38229600 & -0.13953000 & C & 4.78504300 & 0.03458700 & 0.28608900 \\
\hline C & 5.47510600 & 2.40326200 & -1.00744400 & $\mathrm{H}$ & 3.80217300 & 3.28161200 & 0.02196300 \\
\hline C & 5.86929700 & 0.07880800 & -0.59100600 & $\mathrm{H}$ & 4.53470500 & -0.88341800 & 0.79455400 \\
\hline $\mathrm{C}$ & 6.23140500 & 1.25452200 & -1.24473400 & $\mathrm{H}$ & 7.07803700 & 1.27851500 & -1.92013100 \\
\hline $\mathrm{C}$ & 5.80218200 & 3.66860300 & -1.75691100 & $\mathrm{C}$ & 6.69941800 & -1.16092400 & -0.79116800 \\
\hline $\mathrm{F}$ & 5.43922900 & 4.77307500 & -1.07490900 & $\mathrm{~F}$ & 5.16929500 & 3.71441800 & -2.95344200 \\
\hline $\mathrm{F}$ & 7.12740900 & 3.76581400 & -2.01530800 & $\mathrm{~F}$ & 5.97949000 & -2.29557200 & -0.59360900 \\
\hline $\mathrm{F}$ & 7.20908000 & -1.22782300 & -2.04252900 & $\mathrm{~F}$ & 7.74608200 & -1.21467800 & 0.06100200 \\
\hline $\mathrm{C}$ & 0.34858400 & -0.08369800 & 3.60961200 & $\mathrm{H}$ & 1.08011400 & -0.56384700 & 4.25876300 \\
\hline $\mathrm{C}$ & -0.58753700 & 0.79631800 & 4.51653200 & $\mathrm{C}$ & -1.76989400 & 1.42260400 & 3.75113900 \\
\hline $\mathrm{H}$ & -2.44358400 & 0.65719500 & 3.35293700 & $\mathrm{H}$ & -1.43872400 & 2.04709400 & 2.91872800 \\
\hline $\mathrm{H}$ & -2.34538800 & 2.05559700 & 4.43794900 & $\mathrm{C}$ & 0.26839000 & 1.91523500 & 5.14515000 \\
\hline $\mathrm{H}$ & -0.32614900 & 2.46441800 & 5.88493800 & $\mathrm{H}$ & 0.61335000 & 2.63090000 & 4.39501100 \\
\hline $\mathrm{H}$ & 1.14897000 & 1.50684700 & 5.65541700 & $\mathrm{C}$ & -1.15340000 & -0.08196000 & 5.65167000 \\
\hline $\mathrm{H}$ & -1.81538900 & 0.51702900 & 6.28709600 & $\mathrm{H}$ & -0.35769600 & -0.47885100 & 6.29412400 \\
\hline $\mathrm{H}$ & -1.74487900 & -0.92187000 & 5.26812700 & $\mathrm{C}$ & -0.40645400 & -1.15382400 & 2.78758600 \\
\hline $\mathrm{O}$ & -0.98463400 & -0.79664400 & 1.74598900 & $\mathrm{C}$ & 0.18546800 & -3.03844800 & 4.38908800 \\
\hline $\mathrm{C}$ & -1.17621400 & -3.45532600 & 2.41089300 & $\mathrm{C}$ & -0.82598300 & -4.12727500 & 4.75695000 \\
\hline $\mathrm{H}$ & 1.16865800 & -3.46086200 & 4.14666100 & $\mathrm{H}$ & 0.31914400 & -2.29779700 & 5.17389100 \\
\hline $\mathrm{H}$ & -2.16206600 & -3.02362100 & 2.21580800 & $\mathrm{H}$ & -1.67040500 & -3.67771900 & 5.29280900 \\
\hline $\mathrm{H}$ & -0.39238800 & -4.90105800 & 5.39753100 & $\mathrm{~N}$ & -0.41425400 & -2.44503200 & 3.18337100 \\
\hline $\mathrm{C}$ & -0.51940600 & -3.84157500 & 1.08857900 & $\mathrm{C}$ & 0.80526000 & -3.60105500 & 0.86368600 \\
\hline $\mathrm{C}$ & -1.30565600 & -4.54117400 & 0.08633500 & $\mathrm{C}$ & 1.47132100 & -4.01259900 & -0.33864500 \\
\hline $\mathrm{H}$ & 1.39487900 & -3.06464800 & 1.60289700 & $\mathrm{C}$ & -0.66285600 & -4.96289500 & -1.12403600 \\
\hline C & -2.67415700 & -4.82817900 & 0.2457 & $\mathrm{C}$ & 2.83410800 & -3.75297300 & -0.55929100 \\
\hline $\mathrm{C}$ & 0.72279700 & -4.69969400 & -1.33730500 & $\mathrm{C}$ & -1.40823000 & -5.65585900 & -2.12896800 \\
\hline $\mathrm{H}$ & -3.19341500 & -4.51871600 & 1.14 & $\mathrm{C}$ & -3.39125200 & -5.50637700 & -0.74048500 \\
\hline C & 3.45285300 & -4.15772200 & -1.741 & $\mathrm{H}$ & 3.40464600 & -3.22960700 & 0.20291000 \\
\hline $\mathrm{C}$ & 1.36444700 & -5.11633100 & -2.54330600 & $\mathrm{C}$ & -2.77060600 & -5.91810900 & -1.91547900 \\
\hline $\mathrm{C}$ & -0.73616000 & -6.06758700 & -3.33149800 & $\mathrm{H}$ & -4.44516800 & -5.72016000 & -0.58190100 \\
\hline $\mathrm{C}$ & 2.72853800 & -4.83304500 & -2.72273000 & $\mathrm{H}$ & 4.50511900 & -3.93763700 & -1.89326800 \\
\hline C & 0.58563400 & -5.81049800 & -3.53045100 & $\mathrm{H}$ & -3.33457800 & -6.45086000 & -2.67750200 \\
\hline $\mathrm{H}$ & -1.31356500 & -6.59672700 & -4.08604800 & $\mathrm{H}$ & 3.21768600 & -5.14953200 & -3.64104400 \\
\hline $\mathrm{H}$ & 1.07731500 & -6.13066300 & -4.44610400 & $\mathrm{C}$ & 2.37963700 & 0.37883700 & 2.26757700 \\
\hline $\mathrm{S}$ & 3.21701400 & -0.95175900 & 2.91070700 & $\mathrm{~N}$ & 1.12220100 & 0.71828700 & 2.66950400 \\
\hline $\mathrm{H}$ & 0.58612700 & 1.35297800 & 2.06768500 & $S$ & 0.23393800 & 3.80807400 & 0.45311600 \\
\hline $\mathrm{O}$ & -0.16975000 & 4.30802700 & -0.86916200 & $\mathrm{O}$ & -0.37879000 & 2.49934900 & 0.84617300 \\
\hline $\mathrm{O}$ & 1.68057000 & 3.87408400 & 0.78465100 & $\mathrm{C}$ & -0.53960300 & 5.00662200 & 1.64996100 \\
\hline
\end{tabular}




\begin{tabular}{|c|c|c|c|c|c|c|c|}
\hline $\mathrm{F}$ & -0.08320400 & 6.24543200 & 1.45675800 & $\mathrm{~F}$ & -0.28666900 & 4.64685900 & 2.91869400 \\
\hline $\mathrm{F}$ & -1.88425400 & 5.02396600 & 1.49125700 & C & -4.05183600 & -1.16720400 & 0.05423600 \\
\hline 0 & -4.24079300 & -1.64427000 & 1.15385300 & $\mathrm{O}$ & -4.96260800 & -0.89823200 & -0.88725100 \\
\hline $\mathrm{C}$ & -6.39131500 & -1.27449700 & -0.70890800 & C & -6.50369500 & -2.78858800 & -0.51498300 \\
\hline $\mathrm{H}$ & -7.56023600 & -3.07669700 & -0.54103000 & $\mathrm{H}$ & -6.08114700 & -3.10019500 & 0.44139800 \\
\hline $\mathrm{H}$ & -5.98536900 & -3.31829900 & -1.32126200 & C & -7.00923900 & -0.84499000 & -2.04013500 \\
\hline $\mathrm{H}$ & -6.86601500 & 0.22720300 & -2.20791300 & $\mathrm{H}$ & -8.08382200 & -1.05320400 & -2.03484500 \\
\hline $\mathrm{H}$ & -6.55676900 & -1.39122000 & -2.87413200 & C & -6.98782200 & -0.48682900 & 0.45930300 \\
\hline $\mathrm{H}$ & -8.06069300 & -0.69752800 & 0.52695400 & $\mathrm{H}$ & -6.86422500 & 0.58982600 & 0.30151300 \\
\hline $\mathrm{H}$ & -6.51800700 & -0.76345500 & 1.40447900 & C & -2.44672600 & -0.38546800 & -1.61844400 \\
\hline $\mathrm{H}$ & -3.27501200 & -0.41179400 & -2.31410000 & C & -1.10493800 & -0.53409000 & -2.18435800 \\
\hline $\mathrm{C}$ & -1.00779800 & -0.68830000 & -3.58019300 & C & 0.06487100 & -0.52685600 & -1.40251300 \\
\hline C & 0.23339200 & -0.86140000 & -4.18317600 & $\mathrm{H}$ & -1.91092700 & -0.68465300 & -4.18601300 \\
\hline C & 1.30281900 & -0.68810100 & -2.01566600 & $\mathrm{H}$ & 0.00814400 & -0.37288800 & -0.32978300 \\
\hline C & 1.39041100 & -0.86055100 & -3.40048600 & $\mathrm{H}$ & 0.29930600 & -0.99393900 & -5.25901100 \\
\hline $\mathrm{H}$ & 2.20507500 & -0.67773000 & -1.41353900 & $\mathrm{H}$ & 2.36223900 & -0.98916000 & -3.86769200 \\
\hline $\mathrm{N}$ & -2.75598300 & -0.83926800 & -0.38358300 & $\mathrm{H}$ & -2.03476800 & -0.97401200 & 0.34661800 \\
\hline C & -2.38017100 & 1.85390800 & -1.60889300 & $\mathrm{H}$ & -1.54722900 & 1.83005600 & -0.91376700 \\
\hline $\mathrm{H}$ & -2.13389900 & 1.96648800 & -2.66105200 & C & -3.60765600 & 2.28038300 & -1.15354700 \\
\hline $\mathrm{C}$ & -4.65175200 & 2.78217400 & -2.07104900 & $\mathrm{H}$ & -5.66495500 & 2.58083100 & -1.70145600 \\
\hline $\mathrm{H}$ & -4.54941600 & 2.35176600 & -3.07547700 & C & -3.86079400 & 2.31188800 & 0.32667400 \\
\hline $\mathrm{H}$ & -4.92396500 & 2.39375900 & 0.57010200 & $\mathrm{H}$ & -3.33342500 & 3.16228600 & 0.77534300 \\
\hline $\mathrm{H}$ & -3.43972900 & 1.42597200 & 0.81569800 & C & -5.98279100 & 5.12173900 & -3.50323100 \\
\hline $\mathrm{H}$ & -5.92112900 & 4.57210800 & -4.45010200 & $\mathrm{H}$ & -5.99181600 & 6.19157800 & -3.74490500 \\
\hline $\mathrm{H}$ & -6.94650000 & 4.88157200 & -3.03816700 & C & -2.88090800 & 5.10553000 & -3.18036400 \\
\hline $\mathrm{H}$ & -2.75137600 & 4.55828900 & -4.12227300 & $\mathrm{H}$ & -2.03338400 & 4.86749400 & -2.52703800 \\
\hline $\mathrm{H}$ & -2.82495500 & 6.17570500 & -3.41630500 & C & -4.70432800 & 5.64353300 & -0.72613300 \\
\hline $\mathrm{H}$ & -3.82360000 & 5.51662200 & -0.08819500 & $\mathrm{H}$ & -5.58846500 & 5.33000000 & -0.15830000 \\
\hline $\mathrm{H}$ & -4.80799300 & 6.71786100 & -0.92395300 & $\mathrm{Si}$ & -4.52916900 & 4.71277000 & -2.35955500 \\
\hline C & -1.27495200 & -4.66813900 & 3.38889600 & $\mathrm{H}$ & -0.59773600 & -5.46015700 & 3.05445700 \\
\hline $\bar{H}$ & -2.28429700 & -5.08800000 & 3.41124400 & & & & \\
\hline
\end{tabular}

\section{TS-S-4f-nd-26}

Imaginary frequency: $-241.89 \mathrm{~cm}-1$ E[B3LYP/6-31G(d)]: -4788.675566 Hartree $\mathrm{E}\left[\mathrm{PCM}\left(\mathrm{Et}_{2} \mathrm{O}\right)-\mathrm{B} 3 \mathrm{LYP} / 6-311 \mathrm{G}(\mathrm{d}, \mathrm{p})-\mathrm{D} 3(\mathrm{BJ})\right]$ : -4790.214367 Hartree

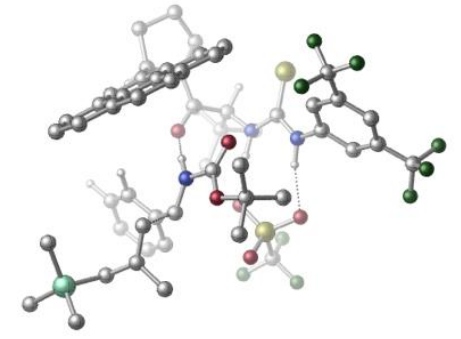

\begin{tabular}{|crrr|rrrr|}
\hline 01 & & & & N & -3.13679900 & -0.88112700 & 0.91273100 \\
\hline $\mathrm{H}$ & -2.67124800 & -1.76791000 & 0.70047500 & $\mathrm{C}$ & -4.07966400 & -0.47957900 & -0.05503800 \\
\hline $\mathrm{C}$ & -4.91382500 & -1.44886000 & -0.62315100 & $\mathrm{C}$ & -4.12727600 & 0.83559000 & -0.53195400 \\
\hline $\mathrm{C}$ & -5.79974700 & -1.09548900 & -1.64280100 & $\mathrm{H}$ & -4.85129200 & -2.47591000 & -0.28130400 \\
\hline $\mathrm{C}$ & -5.02012600 & 1.17402700 & -1.54667800 & $\mathrm{H}$ & -3.44218200 & 1.57047000 & -0.13294000 \\
\hline $\mathrm{C}$ & -5.86789300 & 0.21770000 & -2.10807100 & $\mathrm{H}$ & -6.55610500 & 0.48639000 & -2.89985600 \\
\hline $\mathrm{C}$ & -6.74159600 & -2.12806900 & -2.20179900 & $\mathrm{C}$ & -4.98619500 & 2.57598000 & -2.08903200 \\
\hline $\mathrm{F}$ & -7.91146500 & -2.15953400 & -1.52021600 & $\mathrm{~F}$ & -6.22171600 & -3.37204400 & -2.14652500 \\
\hline $\mathrm{F}$ & -7.05311200 & -1.87462600 & -3.49487000 & $\mathrm{~F}$ & -4.95564200 & 3.50428400 & -1.10319500 \\
\hline $\mathrm{F}$ & -3.87624200 & 2.79495300 & -2.84640200 & $\mathrm{~F}$ & -6.05105300 & 2.85458800 & -2.87001900 \\
\hline $\mathrm{C}$ & -1.01342000 & -0.07159000 & 3.78777500 & $\mathrm{H}$ & -1.80588800 & 0.49492900 & 4.27468600 \\
\hline $\mathrm{C}$ & -0.52506200 & -1.15186800 & 4.81698300 & $\mathrm{C}$ & 0.52904700 & -2.11658100 & 4.24172700 \\
\hline $\mathrm{H}$ & 1.42233500 & -1.58679700 & 3.90242000 & $\mathrm{H}$ & 0.14266400 & -2.69422800 & 3.39763900 \\
\hline $\mathrm{H}$ & 0.82601400 & -2.82946600 & 5.02150500 & $\mathrm{C}$ & -1.75869500 & -1.95992700 & 5.26817700 \\
\hline
\end{tabular}




\begin{tabular}{|c|c|c|c|c|c|c|c|}
\hline $\mathrm{H}$ & -1.46741700 & -2.69814500 & 6.02488400 & $\mathrm{H}$ & -2.20970200 & -2.49781900 & 4.42903400 \\
\hline $\mathrm{H}$ & -2.52757400 & -1.31163400 & 5.70546800 & C & 0.07134700 & -0.43039300 & 6.04272300 \\
\hline $\mathrm{H}$ & 0.38055700 & -1.16577700 & 6.79429600 & $\mathrm{H}$ & -0.65991500 & 0.23528900 & 6.51734500 \\
\hline $\mathrm{H}$ & 0.95763700 & 0.16026700 & 5.78070500 & $\mathrm{C}$ & 0.10164800 & 0.89228000 & 3.32521200 \\
\hline 0 & 1.00802300 & 0.49415000 & 2.57720400 & C & -0.87437400 & 2.79845800 & 4.69316000 \\
\hline $\mathrm{C}$ & 1.21046300 & 3.07704100 & 3.40618300 & $\mathrm{C}$ & -0.56512900 & 4.29773400 & 4.60429700 \\
\hline $\bar{H}$ & -1.90314900 & 2.55955400 & 4.41031700 & $\mathrm{H}$ & -0.68945900 & 2.42354200 & 5.70780000 \\
\hline C & 0.93173600 & 4.34759200 & 4.26063500 & $\mathrm{H}$ & 2.11916800 & 2.58707100 & 3.77163900 \\
\hline $\mathrm{H}$ & -0.80053400 & 4.81458800 & 5.53944200 & $\mathrm{H}$ & -1.15722900 & 4.76364700 & 3.81155500 \\
\hline $\bar{H}$ & 1.53028400 & 4.29644600 & 5.17764700 & $\mathrm{H}$ & 1.21764900 & 5.26018000 & 3.73198100 \\
\hline $\mathrm{N}$ & 0.07593800 & 2.18434900 & 3.74102000 & C & 1.48380800 & 3.36104000 & 1.92579300 \\
\hline $\mathrm{C}$ & 2.80390400 & 3.36771300 & 1.56544100 & $\mathrm{C}$ & 0.47896400 & 3.71797900 & 0.93595400 \\
\hline $\mathrm{C}$ & 3.26678000 & 3.72279700 & 0.25788400 & $\mathrm{H}$ & 3.55704500 & 3.09761800 & 2.30404100 \\
\hline C & 0.91597100 & 4.09717900 & -0.37944200 & C & -0.90486600 & 3.69526200 & 1.18400200 \\
\hline C & 4.62975300 & 3.71489500 & -0.08540300 & $\mathrm{C}$ & 2.30170200 & 4.10004500 & -0.72062200 \\
\hline $\mathrm{C}$ & -0.04301900 & 4.46651800 & -1.37473500 & $\bar{H}$ & -1.28232100 & 3.34207900 & 2.13162300 \\
\hline $\mathrm{C}$ & -1.82968800 & 4.05703300 & 0.20615700 & $\mathrm{C}$ & 5.04134300 & 4.07425400 & -1.36812000 \\
\hline $\mathrm{H}$ & 5.36412800 & 3.43763200 & 0.66782300 & C & 2.73321600 & 4.46747900 & -2.03340200 \\
\hline C & -1.40981800 & 4.44492200 & -1.06020600 & C & 0.41862500 & 4.84222100 & -2.68300900 \\
\hline $\bar{H}$ & -2.88984400 & 4.00925100 & 0.43381300 & $\mathrm{C}$ & 4.10579100 & 4.44626200 & -2.33250100 \\
\hline $\mathrm{H}$ & 6.09982300 & 4.07276500 & -1.61590400 & C & 1.74191500 & 4.84363700 & -3.00181300 \\
\hline $\bar{H}$ & -2.14031600 & 4.70051300 & -1.82277400 & $\mathrm{H}$ & -0.32536200 & 5.12700600 & -3.42310400 \\
\hline $\mathrm{H}$ & 4.43318500 & 4.73052000 & -3.32984300 & $\mathrm{H}$ & 2.07111400 & 5.13147000 & -3.99749500 \\
\hline C & -2.75694900 & -0.18092300 & 2.02777400 & $\mathrm{~S}$ & -3.66649100 & 1.10904500 & 2.65966300 \\
\hline $\mathrm{N}$ & -1.62579200 & -0.66246900 & 2.60536500 & $\mathrm{H}$ & -1.05633600 & -1.31568100 & 2.06060000 \\
\hline $\mathrm{C}$ & 0.27447300 & 0.11514700 & -1.05169200 & $\mathrm{O}$ & -0.66494500 & 0.79426900 & -0.69719100 \\
\hline $\mathrm{O}$ & 0.49032100 & -0.40961600 & -2.27254500 & C & -0.51028800 & -0.30458100 & -3.36777500 \\
\hline C & -0.76982600 & 1.16597500 & -3.70229300 & $\mathrm{H}$ & -1.36809700 & 1.22254300 & -4.61879300 \\
\hline $\mathrm{H}$ & -1.31545200 & 1.66807800 & -2.90311600 & $\mathrm{H}$ & 0.17191300 & 1.69829900 & -3.87690800 \\
\hline C & 0.20064200 & -1.01570600 & -4.52146300 & $\mathrm{H}$ & 0.42810200 & -2.05095800 & -4.24789400 \\
\hline $\mathrm{H}$ & -0.44671800 & -1.02801100 & -5.40410400 & $\mathrm{H}$ & 1.13015400 & -0.49934500 & -4.78975700 \\
\hline C & -1.78046900 & -1.05499900 & -2.97484300 & $\mathrm{H}$ & -2.46325900 & -1.08057700 & -3.83167200 \\
\hline $\mathrm{H}$ & -1.55081700 & -2.08233500 & -2.68036400 & $\mathrm{H}$ & -2.28913400 & -0.55764800 & -2.14968100 \\
\hline $\mathrm{C}$ & 2.20854700 & -1.25570000 & -0.40734400 & $\mathrm{H}$ & 1.74553400 & -2.06297200 & -0.97054900 \\
\hline $\mathrm{C}$ & 2.99466800 & -1.70900900 & 0.77886300 & C & 3.08477300 & -3.08304200 & 1.04414700 \\
\hline C & 3.67832700 & -0.80346000 & 1.60384000 & C & 3.84385000 & -3.54228500 & 2.12036800 \\
\hline $\mathrm{H}$ & 2.52575400 & -3.78509400 & 0.43455700 & C & 4.44189400 & -1.26773100 & 2.67439900 \\
\hline $\mathrm{H}$ & 3.59698900 & 0.26459500 & 1.42274400 & C & 4.52875400 & -2.63790600 & 2.93383700 \\
\hline $\mathrm{H}$ & 3.88649500 & -4.60749500 & 2.32926100 & $\mathrm{H}$ & 4.96140300 & -0.55796700 & 3.31251600 \\
\hline $\bar{H}$ & 5.11966500 & -2.99703300 & 3.77215000 & $\mathrm{~N}$ & 1.33736500 & -0.19254800 & -0.19761100 \\
\hline $\mathrm{H}$ & 1.23422200 & 0.17700900 & 0.75553100 & $\mathrm{~S}$ & -0.68342200 & -3.60692800 & -0.03967800 \\
\hline 0 & 0.18718400 & -3.77241900 & -1.22868900 & 0 & -0.15269600 & -2.64311600 & 0.96631900 \\
\hline $\mathrm{O}$ & -2.13242900 & -3.44033100 & -0.30342800 & $\mathrm{C}$ & -0.55532200 & -5.24739000 & 0.83180300 \\
\hline $\mathrm{F}$ & -1.08728400 & -6.22575600 & 0.09158700 & $\mathrm{~F}$ & -1.19285100 & -5.20283300 & 2.01028700 \\
\hline $\mathrm{F}$ & 0.73738200 & -5.55031900 & 1.06636500 & C & 3.44666300 & -0.65246400 & -1.66492100 \\
\hline $\bar{H}$ & 2.73231700 & -0.26937200 & -2.38775000 & $\mathrm{H}$ & 3.98191800 & 0.11228900 & -1.10627500 \\
\hline $\mathrm{C}$ & 4.18106500 & -1.79149900 & -2.05804000 & $\mathrm{C}$ & 5.46717400 & -2.11425600 & -1.46107200 \\
\hline $\mathrm{H}$ & 5.57914100 & -1.66903900 & -0.46529300 & $\mathrm{H}$ & 5.65147500 & -3.19413200 & -1.41563200 \\
\hline C & 3.55425400 & -2.76435100 & -3.00289200 & $\mathrm{H}$ & 4.28504600 & -3.44305400 & -3.44987600 \\
\hline$\overline{\mathrm{H}}$ & 2.83593300 & -3.38127000 & -2.43468400 & $\mathrm{H}$ & 2.97220900 & -2.26741500 & -3.78386900 \\
\hline C & 8.51030400 & -2.00440600 & -1.57399000 & $\mathrm{H}$ & 8.56108900 & -3.09927200 & -1.57605300 \\
\hline $\mathrm{H}$ & 9.42050700 & -1.63026500 & -2.05834100 & $\mathrm{H}$ & 8.52896500 & -1.66925200 & -0.53067400 \\
\hline $\mathrm{C}$ & 6.93005400 & -2.01969300 & -4.25198200 & $\mathrm{H}$ & 6.93640000 & -3.11531500 & -4.29136200 \\
\hline
\end{tabular}




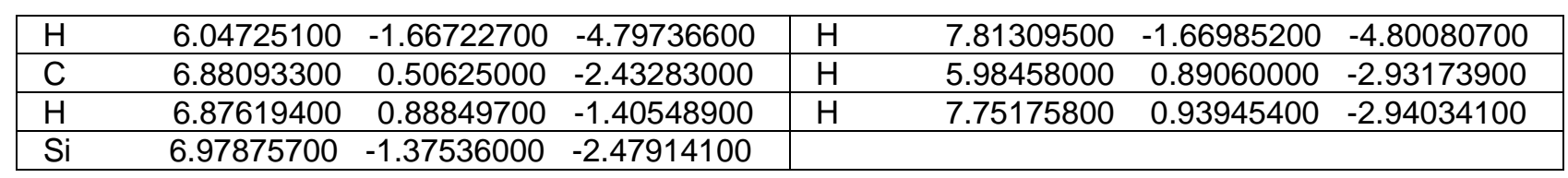

\section{TS-S-4f-nd-27}

Imaginary frequency: $-273.27 \mathrm{~cm}^{-1}$ E[B3LYP/6-31G(d)]: -4788.684129 Hartree $\mathrm{E}\left[\mathrm{PCM}\left(\mathrm{Et}_{2} \mathrm{O}\right)-\mathrm{B} 3 \mathrm{~L} Y \mathrm{P} / 6-311 \mathrm{G}(\mathrm{d}, \mathrm{p})-\mathrm{D} 3(\mathrm{BJ})\right]$ : -4790.214148 Hartree

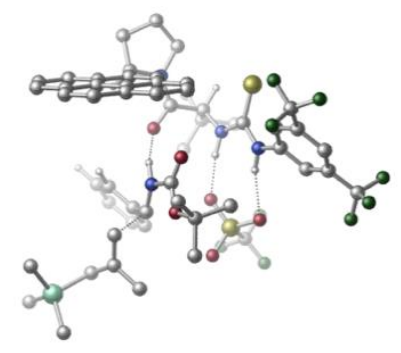

\begin{tabular}{|c|c|c|c|c|c|c|c|}
\hline 01 & & & & $\mathrm{~N}$ & -2.73792800 & -1.14897600 & 1.03597000 \\
\hline $\mathrm{H}$ & -2.14132800 & -1.96312400 & 0.88015400 & C & -3.87046700 & -1.10283600 & 0.19778600 \\
\hline C & -4.47870700 & -2.30827300 & -0.16967500 & C & -4.34175800 & 0.09816800 & -0.34780700 \\
\hline C & -5.56205000 & -2.30292300 & -1.05153100 & $\mathrm{H}$ & -4.09062500 & -3.24444800 & 0.21568900 \\
\hline C & -5.42434500 & 0.08781100 & -1.22261600 & $\mathrm{H}$ & -3.84756500 & 1.02483200 & -0.09756700 \\
\hline C & -6.05134100 & -1.10865800 & -1.57763900 & $\mathrm{H}$ & -6.89540100 & -1.11005400 & -2.25684300 \\
\hline C & -6.25447200 & -3.59869000 & -1.38080600 & C & -5.85749100 & 1.37528300 & -1.86912400 \\
\hline $\mathrm{F}$ & -7.22982800 & -3.88538000 & -0.48620400 & $\mathrm{~F}$ & -5.40080400 & -4.64370900 & -1.37839100 \\
\hline $\mathrm{F}$ & -6.84363400 & -3.55758500 & -2.59872700 & $\mathrm{~F}$ & -5.59501100 & 2.45274600 & -1.09426400 \\
\hline $\mathrm{F}$ & -5.21161100 & 1.58687300 & -3.04697000 & $\mathrm{~F}$ & -7.17907000 & 1.38575300 & -2.14690300 \\
\hline C & -0.58205700 & 0.27282400 & 3.64308000 & $\mathrm{H}$ & -1.42697500 & 0.74769400 & 4.14069400 \\
\hline C & 0.12367900 & -0.63593300 & 4.71030000 & C & 1.37660500 & -1.35627100 & 4.17914600 \\
\hline $\mathrm{H}$ & 2.14859600 & -0.65066200 & 3.86343700 & $\mathrm{H}$ & 1.14952000 & -2.00763400 & 3.33095700 \\
\hline $\mathrm{H}$ & 1.79257100 & -1.98587000 & 4.97600400 & C & -0.89640300 & -1.68906400 & 5.19051800 \\
\hline $\mathrm{H}$ & -0.47630500 & -2.25862000 & 6.02820000 & $\mathrm{H}$ & -1.14294700 & -2.39884700 & 4.39508700 \\
\hline $\mathrm{H}$ & -1.82882000 & -1.22341100 & 5.53126500 & C & 0.52704900 & 0.24893000 & 5.90765100 \\
\hline $\mathrm{H}$ & 1.01950800 & -0.36055000 & 6.67398800 & $\mathrm{H}$ & -0.34611400 & 0.72137100 & 6.37381800 \\
\hline $\mathrm{H}$ & 1.23105600 & 1.03760800 & 5.61521800 & C & 0.33088200 & 1.36374900 & 3.03581600 \\
\hline $\mathrm{O}$ & 1.24072600 & 1.06365600 & 2.24609300 & C & -0.87952800 & 3.18149900 & 4.34434800 \\
\hline C & 0.94936000 & 3.72310100 & 2.79229000 & C & -0.71860900 & 4.70490400 & 4.23536100 \\
\hline $\mathrm{H}$ & -1.88754000 & 2.83871400 & 4.09508200 & $\mathrm{H}$ & -0.62768200 & 2.83489500 & 5.35313000 \\
\hline C & 0.74225300 & 4.88240600 & 3.79681700 & $\mathrm{H}$ & 1.98049500 & 3.36090600 & 2.79122200 \\
\hline $\mathrm{H}$ & -0.95009900 & 5.20920100 & 5.17801400 & $\mathrm{H}$ & -1.39081300 & 5.10124300 & 3.46742600 \\
\hline $\mathrm{H}$ & 1.41630300 & 4.75194000 & 4.65315300 & $\mathrm{H}$ & 0.94299900 & 5.85919800 & 3.35031000 \\
\hline $\mathrm{N}$ & 0.10445100 & 2.65777400 & 3.36909100 & C & 0.55626400 & 4.10743900 & 1.36707600 \\
\hline C & -0.65701000 & 3.76202100 & 0.84699000 & C & 1.48560200 & 4.88885400 & 0.56707700 \\
\hline C & -1.06933400 & 4.14460700 & -0.47265700 & $\mathrm{H}$ & -1.34425800 & 3.15210500 & 1.42642900 \\
\hline C & 1.10236800 & 5.27251400 & -0.76005000 & C & 2.75310600 & 5.28752200 & 1.03067000 \\
\hline C & -2.32475800 & 3.78912000 & -0.98969600 & C & -0.17372200 & 4.90182700 & -1.27960200 \\
\hline C & 1.99757700 & 6.03400200 & -1.57485300 & $\mathrm{H}$ & 3.07415200 & 5.02176100 & 2.03214700 \\
\hline C & 3.61940900 & 6.03029600 & 0.22782200 & C & -2.69327200 & 4.15523000 & -2.28343500 \\
\hline $\mathrm{H}$ & -3.01325700 & 3.22499000 & -0.36771500 & C & -0.55835400 & 5.28032600 & -2.60202200 \\
\hline C & 3.25117200 & 6.40180400 & -1.06074500 & C & 1.58394800 & 6.40346500 & -2.90154200 \\
\hline $\mathrm{H}$ & 4.59060400 & 6.32335300 & 0.61852400 & C & -1.82004700 & 4.89198500 & -3.08268600 \\
\hline $\mathrm{H}$ & -3.66266200 & 3.85154900 & -2.66704300 & C & 0.36593400 & 6.04452000 & -3.39233900 \\
\hline $\mathrm{H}$ & 3.92955100 & 6.98378200 & -1.68021200 & $\mathrm{H}$ & 2.27307800 & 6.98462500 & -3.51007400 \\
\hline $\mathrm{H}$ & -2.11171200 & 5.17619400 & -4.09116800 & $\mathrm{H}$ & 0.07026000 & 6.33442400 & -4.39807200 \\
\hline C & -2.39815400 & -0.27127300 & 2.03618300 & $S$ & -3.44977300 & 0.93306200 & 2.61242500 \\
\hline $\mathrm{N}$ & -1.16262500 & -0.49689900 & 2.54945300 & $\mathrm{H}$ & -0.55530400 & -1.15692200 & 2.05964200 \\
\hline C & 0.53369600 & 0.05439200 & -1.16089000 & $\mathrm{O}$ & -0.36313200 & 0.83255200 & -0.91842300 \\
\hline
\end{tabular}




\begin{tabular}{|c|c|c|c|c|c|c|c|}
\hline $\mathrm{O}$ & 0.64786400 & -0.75642000 & -2.22865100 & $\mathrm{C}$ & -0.38765400 & -0.79619500 & -3.29623800 \\
\hline C & -0.43209900 & 0.56285300 & -3.99841000 & $\mathrm{H}$ & -1.11627200 & 0.50685700 & -4.85232600 \\
\hline $\mathrm{H}$ & -0.78438300 & 1.34617600 & -3.32448500 & $\mathrm{H}$ & 0.55955100 & 0.83734200 & -4.37681100 \\
\hline $\mathrm{C}$ & 0.14550500 & -1.89181100 & -4.22128900 & $\mathrm{H}$ & 0.25422500 & -2.83187700 & -3.67144200 \\
\hline $\mathrm{H}$ & -0.55264100 & -2.05027900 & -5.04942800 & $\mathrm{H}$ & 1.11617600 & -1.60979200 & -4.64680400 \\
\hline C & -1.74039400 & -1.19878300 & -2.71257800 & $\mathrm{H}$ & -2.44817500 & -1.35928000 & -3.53363700 \\
\hline $\mathrm{H}$ & -1.65895400 & -2.12972100 & -2.14350700 & $\mathrm{H}$ & -2.13780100 & -0.41802500 & -2.06511500 \\
\hline C & 2.53368200 & -1.14365600 & -0.37181300 & $\mathrm{H}$ & 2.07266700 & -2.06161100 & -0.72300200 \\
\hline C & 3.43339000 & -1.30466300 & 0.80549900 & $\mathrm{C}$ & 3.66016300 & -2.59188300 & 1.31548100 \\
\hline C & 4.09475100 & -0.21043100 & 1.38404600 & $\mathrm{C}$ & 4.53044600 & -2.77785400 & 2.38955600 \\
\hline $\mathrm{H}$ & 3.12612600 & -3.43764200 & 0.89490900 & C & 4.96924100 & -0.40206700 & 2.45268900 \\
\hline $\mathrm{H}$ & 3.91638000 & 0.79336200 & 1.00860000 & $C$ & 5.19085300 & -1.68640800 & 2.95666100 \\
\hline $\mathrm{H}$ & 4.68231700 & -3.77662400 & 2.78898800 & $\mathrm{H}$ & 5.47178900 & 0.45282700 & 2.89666600 \\
\hline $\mathrm{H}$ & 5.86927400 & -1.83344700 & 3.79266900 & $\mathrm{~N}$ & 1.64979300 & -0.07511100 & -0.32758400 \\
\hline $\mathrm{H}$ & 1.57929900 & 0.47673200 & 0.53739200 & $\mathrm{~S}$ & -0.04703600 & -3.74176400 & 0.29453400 \\
\hline 0 & 0.90254400 & -4.06527800 & -0.79701700 & 0 & 0.35664700 & -2.55995200 & 1.11074000 \\
\hline $\mathrm{O}$ & -1.48495700 & -3.74105400 & -0.06046000 & C & 0.14471700 & -5.15801200 & 1.48695200 \\
\hline $\mathrm{F}$ & -0.22616300 & -6.31206200 & 0.92410500 & $\mathrm{~F}$ & -0.60235700 & -4.95019400 & 2.58067700 \\
\hline $\mathrm{F}$ & 1.43143600 & -5.26567400 & 1.87069100 & $C$ & 3.67248700 & -0.81282100 & -1.85261000 \\
\hline $\mathrm{H}$ & 2.88857800 & -0.57804600 & -2.56603600 & $\mathrm{H}$ & 4.24556700 & 0.04064800 & -1.49772800 \\
\hline C & 4.37370400 & -2.01664300 & -2.06203100 & C & 5.70439500 & -2.22788300 & -1.50900800 \\
\hline $\mathrm{H}$ & 5.89937000 & -1.57629400 & -0.64919600 & $\mathrm{H}$ & 5.88054700 & -3.27486700 & -1.23409400 \\
\hline $\mathrm{C}$ & 3.68258300 & -3.15234900 & -2.74259500 & $\mathrm{H}$ & 4.37921800 & -3.92801600 & -3.07009400 \\
\hline $\mathrm{H}$ & 2.97919800 & -3.61577800 & -2.02765900 & $\mathrm{H}$ & 3.06975000 & -2.81713500 & -3.58430600 \\
\hline $\mathrm{C}$ & 8.73216100 & -2.19128400 & -1.85048800 & $\mathrm{H}$ & 8.78576200 & -3.25238300 & -1.58109400 \\
\hline $\mathrm{H}$ & 9.60512400 & -1.96415300 & -2.47446300 & $\mathrm{H}$ & 8.82316400 & -1.60573500 & -0.92849000 \\
\hline C & 6.97225500 & -2.84779600 & -4.32822800 & $\mathrm{H}$ & 6.97927300 & -3.91906400 & -4.09538700 \\
\hline $\bar{H}$ & 6.05364300 & -2.62968600 & -4.88448300 & $\mathrm{H}$ & 7.81543900 & -2.65627500 & -5.00333600 \\
\hline $\mathrm{C}$ & 7.03833000 & 0.04902900 & -3.19388900 & $\mathrm{H}$ & 6.10537400 & 0.30521400 & -3.70820700 \\
\hline $\mathrm{H}$ & 7.10966300 & 0.67461800 & -2.29643500 & $\mathrm{H}$ & 7.86624800 & 0.33129100 & -3.85572600 \\
\hline Si & 7.13863500 & -1.78706500 & -2.77689800 & & & & \\
\hline
\end{tabular}

\section{TS-S-4f-nd-28}

Imaginary frequency: $-237.42 \mathrm{~cm}^{-1}$ E[B3LYP/6-31G(d)]: -4788.682736 Hartree E[PCM(Et $\left.\left.{ }_{2} \mathrm{O}\right)-\mathrm{B} 3 \mathrm{LYP} / 6-311 \mathrm{G}(\mathrm{d}, \mathrm{p})-\mathrm{D} 3(\mathrm{BJ})\right]$ : -4790.214093 Hartree

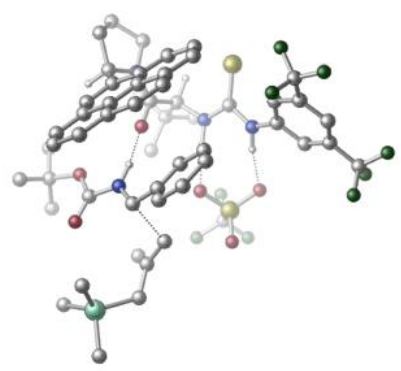

\begin{tabular}{|c|c|c|c|c|c|c|c|}
\hline 01 & & & & $\mathrm{~N}$ & -2.96563200 & -1.42284400 & 1.18619400 \\
\hline $\mathrm{H}$ & -2.46717800 & -2.30797600 & 1.02432900 & C & -4.05422600 & -1.22430600 & 0.32339900 \\
\hline C & -4.61944400 & -2.35845800 & -0.28299100 & C & -4.52234300 & 0.04533000 & -0.04128800 \\
\hline C & -5.63668100 & -2.21491400 & -1.22419600 & $\mathrm{H}$ & -4.24790700 & -3.34370900 & -0.02318200 \\
\hline C & -5.52772300 & 0.16894000 & -0.99951400 & $\mathrm{H}$ & -4.09778500 & 0.92785700 & 0.41659900 \\
\hline C & -6.10336600 & -0.95249100 & -1.59478600 & $\mathrm{H}$ & -6.89569300 & -0.84821100 & -2.32556500 \\
\hline C & -6.20058600 & -3.43607800 & -1.90278600 & C & -5.90600200 & 1.54930300 & -1.46005600 \\
\hline $\mathrm{F}$ & -6.11951800 & -4.53379400 & -1.12353000 & $\mathrm{~F}$ & -5.54218900 & -3.71527500 & -3.05193400 \\
\hline$F$ & -7.50232400 & -3.26555900 & -2.23238500 & $F$ & -5.96845200 & 2.43605400 & -0.43953700 \\
\hline $\mathrm{F}$ & -4.98408600 & 2.04675400 & -2.33448400 & $\mathrm{~F}$ & -7.09534000 & 1.57725200 & -2.09439600 \\
\hline $\mathrm{C}$ & -0.49505600 & -0.19460800 & 3.58909400 & $\bar{H}$ & -1.25567600 & 0.20699600 & 4.25790500 \\
\hline C & 0.43931200 & -1.12700600 & 4.44122100 & C & 1.60136200 & -1.73705700 & 3.63247000 \\
\hline $\mathrm{H}$ & 2.26339400 & -0.96489400 & 3.23010200 & $\mathrm{H}$ & 1.24982600 & -2.34402700 & 2.79545400 \\
\hline
\end{tabular}




\begin{tabular}{|c|c|c|c|c|c|c|c|}
\hline$\overline{\mathrm{H}}$ & 2.19014800 & -2.38911500 & 4.29004400 & C & -0.42722900 & -2.26056200 & 5.02736600 \\
\hline $\mathrm{H}$ & 0.17259200 & -2.87129700 & 5.71263900 & $\mathrm{H}$ & -0.81377500 & -2.91783700 & 4.24499900 \\
\hline $\mathrm{H}$ & -1.28045300 & -1.86162600 & 5.58904500 & C & 1.03127100 & -0.31013500 & 5.60865900 \\
\hline $\mathrm{H}$ & 1.69964900 & -0.94401000 & 6.20244800 & $\mathrm{H}$ & 0.25020300 & 0.05810200 & 6.28486300 \\
\hline $\mathrm{H}$ & 1.61969700 & 0.54700800 & 5.25858100 & C & 0.25275200 & 0.96982700 & 2.89292100 \\
\hline O & 0.95244200 & 0.74451400 & 1.89445100 & C & -0.61307700 & 2.64410100 & 4.59134800 \\
\hline C & 0.89058200 & 3.35615700 & 2.77979200 & C & 0.08871300 & 3.94259200 & 5.00239200 \\
\hline $\mathrm{H}$ & -1.67344000 & 2.80298700 & 4.36220500 & $\mathrm{H}$ & -0.55057600 & 1.88053500 & 5.36458500 \\
\hline $\mathrm{H}$ & 1.95022700 & 3.12636900 & 2.94930100 & $\mathrm{H}$ & 0.98191200 & 3.71365600 & 5.59677500 \\
\hline $\mathrm{H}$ & -0.55859400 & 4.58816200 & 5.60316300 & $\mathrm{~N}$ & 0.11476300 & 2.23009700 & 3.37181800 \\
\hline C & 0.75423200 & 3.65150900 & 1.29004200 & C & 1.91618700 & 3.88261100 & 0.60830400 \\
\hline C & -0.51794900 & 3.85742100 & 0.61478300 & C & 1.95610800 & 4.35650000 & -0.74326200 \\
\hline $\mathrm{H}$ & 2.86844600 & 3.71883900 & 1.10978300 & C & -0.50851100 & 4.35066700 & -0.73290200 \\
\hline C & -1.76271200 & 3.58675500 & 1.20945200 & C & 3.16342300 & 4.59667900 & -1.41944800 \\
\hline C & 0.72146700 & 4.60657200 & -1.40937500 & C & -1.74166000 & 4.58085100 & -1.42020300 \\
\hline $\mathrm{H}$ & -1.81167100 & 3.16419100 & 2.20321400 & C & -2.95858900 & 3.79543400 & 0.52197300 \\
\hline C & 3.16170300 & 5.07937100 & -2.72707800 & $\mathrm{H}$ & 4.10480500 & 4.40780500 & -0.90832400 \\
\hline C & 0.72834700 & 5.10130600 & -2.74985300 & C & -2.95484400 & 4.29273400 & -0.77639700 \\
\hline C & -1.70570600 & 5.09022900 & -2.76394500 & $\mathrm{H}$ & -3.89913200 & 3.54971900 & 1.00533000 \\
\hline C & 1.95953900 & 5.33101600 & -3.38591800 & $\mathrm{H}$ & 4.10439100 & 5.26435300 & -3.23532800 \\
\hline C & -0.52930700 & 5.34233600 & -3.39996800 & $\mathrm{H}$ & -3.89099300 & 4.43486400 & -1.30878800 \\
\hline $\mathrm{H}$ & -2.65288900 & 5.27089800 & -3.26659500 & $\mathrm{H}$ & 1.96511000 & 5.71026300 & -4.40503800 \\
\hline $\mathrm{H}$ & -0.52211100 & 5.72890500 & -4.41636400 & C & -2.50679100 & -0.62409700 & 2.20591900 \\
\hline S & -3.44239600 & 0.56152200 & 2.97678900 & $\mathrm{~N}$ & -1.22828800 & -0.92535000 & 2.56466400 \\
\hline $\mathrm{H}$ & -0.68115100 & -1.51424900 & 1.92802900 & $\mathrm{~S}$ & -0.38035400 & -3.94978900 & 0.25103400 \\
\hline $\mathrm{O}$ & 0.03388900 & -4.45234000 & -1.06507100 & $\mathrm{O}$ & 0.25686700 & -2.65097400 & 0.65292100 \\
\hline $\mathrm{O}$ & -1.83330800 & -3.98555000 & 0.56232600 & C & 0.34830000 & -5.16899700 & 1.45532400 \\
\hline $\mathrm{F}$ & -0.12489900 & -6.39813100 & 1.23573900 & $\mathrm{~F}$ & 0.06217900 & -4.82047200 & 2.72240700 \\
\hline $\mathrm{F}$ & 1.69077900 & -5.20188200 & 1.33293400 & $\mathrm{C}$ & 4.02600300 & 0.17204800 & 0.07979900 \\
\hline 0 & 4.92625000 & -0.05511000 & -0.71056600 & $\mathrm{O}$ & 4.13022400 & 0.45380100 & 1.37790300 \\
\hline $\mathrm{C}$ & 5.44937500 & 0.54922600 & 2.05297100 & C & 6.13889600 & -0.81671500 & 2.02879400 \\
\hline $\mathrm{H}$ & 7.04382200 & -0.77639900 & 2.64479700 & $\mathrm{H}$ & 6.42540700 & -1.10307200 & 1.01539600 \\
\hline $\mathrm{H}$ & 5.47940200 & -1.58537100 & 2.44483800 & C & 5.05850000 & 0.93824100 & 3.47959700 \\
\hline $\mathrm{H}$ & 4.51599600 & 1.88907400 & 3.48639000 & $\mathrm{H}$ & 5.95822900 & 1.04840000 & 4.09351500 \\
\hline $\mathrm{H}$ & 4.42082400 & 0.17230100 & 3.93071700 & C & 6.29109400 & 1.64732200 & 1.39735100 \\
\hline $\mathrm{H}$ & 7.20995600 & 1.79111300 & 1.97607100 & $\mathrm{H}$ & 5.74444500 & 2.59679600 & 1.39143400 \\
\hline $\mathrm{H}$ & 6.56186800 & 1.38720000 & 0.37296300 & C & 2.32878100 & 0.01383400 & -1.59616100 \\
\hline $\mathrm{H}$ & 3.16526600 & 0.12498900 & -2.27359100 & C & 1.01689600 & 0.44236400 & -2.08946400 \\
\hline C & 0.93239900 & 0.88186100 & -3.42396500 & C & -0.14313400 & 0.40886300 & -1.29582300 \\
\hline C & -0.28513000 & 1.29985000 & -3.95135800 & $\mathrm{H}$ & 1.82852700 & 0.90402700 & -4.03948200 \\
\hline C & -1.35695800 & 0.82175200 & -1.83400600 & $\mathrm{H}$ & -0.10169700 & 0.04578100 & -0.27468300 \\
\hline C & -1.43336300 & 1.26706600 & -3.15631500 & $\mathrm{H}$ & -0.33862600 & 1.65018800 & -4.97781300 \\
\hline $\mathrm{H}$ & -2.25279300 & 0.79580700 & -1.22521200 & $\mathrm{H}$ & -2.39137900 & 1.58509200 & -3.55665600 \\
\hline $\mathrm{N}$ & 2.67821400 & 0.18278900 & -0.29250900 & $\mathrm{H}$ & 1.98374900 & 0.34899400 & 0.44929900 \\
\hline C & 2.08565700 & -2.07865600 & -2.03596600 & $\mathrm{H}$ & 1.37224200 & -2.14554100 & -1.21931100 \\
\hline $\mathrm{H}$ & 1.66498300 & -1.96322900 & -3.03043700 & C & 3.27657800 & -2.76936800 & -1.90176900 \\
\hline C & 4.07567100 & -3.20826000 & -3.07124200 & $\mathrm{H}$ & 3.44878100 & -3.23572200 & -3.97099100 \\
\hline $\mathrm{H}$ & 4.42677600 & -4.23452700 & -2.88815400 & C & 3.71627200 & -3.21006900 & -0.53812000 \\
\hline $\mathrm{H}$ & 4.80503200 & -3.24862400 & -0.44145500 & $\mathrm{H}$ & 3.32532000 & -4.22378000 & -0.37067300 \\
\hline $\mathrm{H}$ & 3.28393700 & -2.59030900 & 0.25182900 & C & 6.29063000 & -3.30189100 & -5.11614800 \\
\hline $\mathrm{H}$ & 6.49657900 & -4.34516200 & -4.84857500 & $\mathrm{H}$ & 7.21661200 & -2.87833900 & -5.52401900 \\
\hline $\mathrm{H}$ & 5.54797600 & -3.30637900 & -5.92292300 & C & 7.00623500 & -2.34013200 & -2.26079100 \\
\hline $\mathrm{H}$ & 7.19837300 & -3.36024400 & -1.90752200 & $\mathrm{H}$ & 6.70028000 & -1.72466600 & -1.40927500 \\
\hline
\end{tabular}




\begin{tabular}{|c|c|c|c|c|c|c|c|}
\hline $\mathrm{H}$ & 7.95626400 & -1.93982900 & -2.63663200 & C & 5.34038500 & -0.51960200 & -4.15085700 \\
\hline $\mathrm{H}$ & 5.17401100 & 0.11864400 & -3.27734600 & $\mathrm{H}$ & 4.46651500 & -0.45582100 & -4.81079700 \\
\hline $\mathrm{H}$ & 6.19443300 & -0.10818300 & -4.70322900 & $\mathrm{Si}$ & 5.70076600 & -2.29848000 & -3.62315500 \\
\hline $\mathrm{C}$ & 0.48197300 & 4.56402600 & 3.65826000 & $\mathrm{H}$ & -0.37584300 & 5.07428400 & 3.20839600 \\
\hline $\mathrm{H}$ & 1.29744300 & 5.28907100 & 3.73005200 & & & & \\
\hline
\end{tabular}

\section{TS-S-4f-nd-29}

Imaginary frequency: $-273.95 \mathrm{~cm}^{-1}$ E[B3LYP/6-31G(d)]: -4788.677922 Hartree E[PCM(Et $\left.\left.{ }_{2} \mathrm{O}\right)-\mathrm{B} 3 \mathrm{LYP} / 6-311 \mathrm{G}(\mathrm{d}, \mathrm{p})-\mathrm{D} 3(\mathrm{BJ})\right]$ : -4790.213690 Hartree

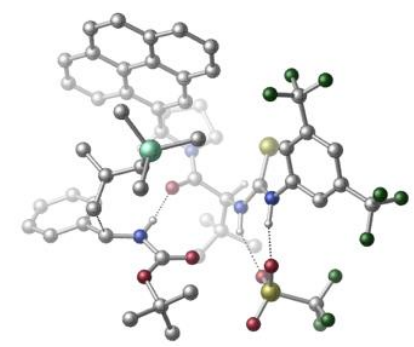

\begin{tabular}{|c|c|c|c|c|c|c|c|}
\hline 01 & & & & $\mathrm{~N}$ & -2.56671800 & 0.51230800 & 0.89866900 \\
\hline $\mathrm{H}$ & -3.00561400 & -0.31175100 & 0.46089100 & C & -3.15096300 & 1.73075700 & 0.48819900 \\
\hline C & -4.52859400 & 1.75163300 & 0.22711400 & $\mathrm{C}$ & -2.39456700 & 2.88518400 & 0.25937600 \\
\hline C & -5.13200000 & 2.92021000 & -0.23667700 & $\mathrm{H}$ & -5.11519100 & 0.85453700 & 0.38433500 \\
\hline $\mathrm{C}$ & -3.01576500 & 4.05023000 & -0.19159000 & $\mathrm{H}$ & -1.32904600 & 2.86870600 & 0.43645600 \\
\hline C & -4.38644400 & 4.08186700 & -0.44259300 & $\mathrm{H}$ & -4.86356400 & 4.98752900 & -0.79605200 \\
\hline C & -6.62301200 & 2.95616800 & -0.44777500 & C & -2.16355700 & 5.25750900 & -0.46362400 \\
\hline $\mathrm{F}$ & -7.27430100 & 3.36003700 & 0.66866200 & $\mathrm{~F}$ & -7.11874300 & 1.74867300 & -0.78022400 \\
\hline $\mathrm{F}$ & -6.96558800 & 3.82327500 & -1.43080200 & $\mathrm{~F}$ & -1.26253600 & 5.47642200 & 0.52924400 \\
\hline $\mathrm{F}$ & -1.43298600 & 5.11944400 & -1.60300600 & $\mathrm{~F}$ & -2.88941000 & 6.38533900 & -0.60074600 \\
\hline $\mathrm{C}$ & -0.26925300 & -1.39111300 & 3.03520400 & $\mathrm{H}$ & -0.42666200 & -0.77144700 & 3.91945700 \\
\hline C & -0.49146300 & -2.88313500 & 3.48520700 & C & -0.40511700 & -3.89214800 & 2.32295600 \\
\hline $\mathrm{H}$ & 0.58129000 & -3.87326200 & 1.84916900 & $\mathrm{H}$ & -1.16739100 & -3.71848900 & 1.56000200 \\
\hline $\mathrm{H}$ & -0.56893700 & -4.90237800 & 2.71713500 & $\mathrm{C}$ & -1.87946100 & -2.98102700 & 4.15532600 \\
\hline $\mathrm{H}$ & -2.01744000 & -3.98911100 & 4.56445700 & $\mathrm{H}$ & -2.69102700 & -2.79756400 & 3.44863600 \\
\hline $\mathrm{H}$ & -1.96672500 & -2.26863500 & 4.98527700 & $\mathrm{C}$ & 0.57428400 & -3.25989600 & 4.53587900 \\
\hline $\mathrm{H}$ & 0.41928100 & -4.29683500 & 4.85363200 & $\mathrm{H}$ & 0.50045500 & -2.63328500 & 5.43220400 \\
\hline $\mathrm{H}$ & 1.59589600 & -3.18777800 & 4.14389400 & C & 1.16246100 & -1.12296700 & 2.50465900 \\
\hline $\mathrm{O}$ & 1.52119700 & -1.50867700 & 1.37522400 & $\mathrm{C}$ & 1.76751500 & 0.05859200 & 4.67236800 \\
\hline C & 3.43791800 & -0.25481100 & 2.90068900 & $\mathrm{C}$ & 3.07579500 & 0.76207900 & 5.05723300 \\
\hline $\mathrm{H}$ & 0.90699800 & 0.73216900 & 4.64879500 & $\mathrm{H}$ & 1.55731000 & -0.76592700 & 5.36024400 \\
\hline $\mathrm{C}$ & 4.13958700 & -0.00186100 & 4.25818100 & $\mathrm{H}$ & 3.79518600 & -1.17434000 & 2.42996000 \\
\hline $\mathrm{H}$ & 3.25008800 & 0.73790300 & 6.13663900 & $\mathrm{H}$ & 3.05105300 & 1.81079900 & 4.74393600 \\
\hline $\mathrm{H}$ & 4.36232600 & -0.96234100 & 4.74003400 & $\mathrm{H}$ & 5.07568900 & 0.55019100 & 4.14683800 \\
\hline $\mathrm{N}$ & 2.03579200 & -0.47092700 & 3.31290800 & $\mathrm{C}$ & 3.63167500 & 0.91287300 & 1.93439200 \\
\hline $\mathrm{C}$ & 2.64853000 & 1.83977100 & 1.74010900 & $C$ & 4.91019600 & 1.06697100 & 1.25759700 \\
\hline C & 2.82584600 & 2.98886400 & 0.89752200 & $\mathrm{H}$ & 1.68150200 & 1.71668700 & 2.22008200 \\
\hline C & 5.10921300 & 2.20240600 & 0.40395400 & $\mathrm{C}$ & 5.97038400 & 0.15075100 & 1.39451700 \\
\hline $\mathrm{C}$ & 1.80800500 & 3.94184900 & 0.72028800 & $\mathrm{C}$ & 4.07082900 & 3.16560300 & 0.22759800 \\
\hline C & 6.35429600 & 2.38083500 & -0.27792700 & $\mathrm{H}$ & 5.86148400 & -0.71333200 & 2.04056800 \\
\hline $\mathrm{C}$ & 7.17898800 & 0.33037800 & 0.72005700 & $\mathrm{C}$ & 2.00785500 & 5.04945000 & -0.10451600 \\
\hline $\mathrm{H}$ & 0.86230700 & 3.81072900 & 1.23891400 & $\mathrm{C}$ & 4.26875200 & 4.30339300 & -0.61393200 \\
\hline $\mathrm{C}$ & 7.37438000 & 1.43095600 & -0.10731400 & $\mathrm{C}$ & 6.52599100 & 3.53417700 & -1.11990800 \\
\hline $\mathrm{H}$ & 7.97626100 & -0.39661600 & 0.84985500 & $\mathrm{C}$ & 3.22285800 & 5.22983200 & -0.76426300 \\
\hline $\mathrm{H}$ & 1.20277200 & 5.76536200 & -0.23466100 & $\mathrm{C}$ & 5.53371000 & 4.45279700 & -1.27912700 \\
\hline $\mathrm{H}$ & 8.32096000 & 1.56871300 & -0.62408100 & $\mathrm{H}$ & 7.47904600 & 3.66063200 & -1.62790700 \\
\hline $\mathrm{H}$ & 3.37223000 & 6.09626100 & -1.40411400 & $\mathrm{H}$ & 5.68466700 & 5.32154400 & -1.91543900 \\
\hline $\mathrm{C}$ & -1.62842700 & 0.32624900 & 1.87507200 & $S$ & -1.00675500 & 1.59606300 & 2.82986200 \\
\hline $\mathrm{N}$ & -1.24754000 & -0.96493400 & 2.04078500 & $\mathrm{H}$ & -1.78181900 & -1.68362900 & 1.54720100 \\
\hline C & -0.23883800 & -3.07204100 & -1.55953600 & $\mathrm{O}$ & -1.15267400 & -2.68030000 & -0.87394500 \\
\hline
\end{tabular}




\begin{tabular}{|c|c|c|c|c|c|c|c|}
\hline $\mathrm{O}$ & -0.28328200 & -3.60784200 & -2.78260600 & C & -1.58400400 & -3.99407100 & -3.43188100 \\
\hline C & -2.48107100 & -2.76689300 & -3.58921400 & $\mathrm{H}$ & -3.35378200 & -3.04782000 & -4.18865700 \\
\hline $\mathrm{H}$ & -2.84100700 & -2.38806800 & -2.63239900 & $\mathrm{H}$ & -1.95001100 & -1.97070000 & -4.12575800 \\
\hline $\mathrm{C}$ & -1.10726600 & -4.51090700 & -4.79003600 & $\mathrm{H}$ & -0.42399700 & -5.35876900 & -4.67091400 \\
\hline $\mathrm{H}$ & -1.96887900 & -4.84621800 & -5.37547100 & $\mathrm{H}$ & -0.59629900 & -3.72361100 & -5.35534000 \\
\hline $\mathrm{C}$ & -2.22751800 & -5.10598500 & -2.60652900 & $\mathrm{H}$ & -3.09265800 & -5.49740800 & -3.15219400 \\
\hline $\mathrm{H}$ & -1.51855100 & -5.92864300 & -2.45679800 & $\mathrm{H}$ & -2.59562800 & -4.74090100 & -1.64680000 \\
\hline C & 2.17500400 & -3.40540600 & -1.82668200 & $\mathrm{H}$ & 1.87723200 & -4.08803200 & -2.61475800 \\
\hline $\mathrm{C}$ & 3.45420000 & -3.77311800 & -1.17621500 & C & 4.29816400 & -4.66059100 & -1.86807600 \\
\hline $\mathrm{C}$ & 3.83533500 & -3.31785700 & 0.09584000 & C & 5.49502700 & -5.08967500 & -1.30334000 \\
\hline $\mathrm{H}$ & 4.00442600 & -5.02614300 & -2.84959200 & C & 5.03354600 & -3.75822800 & 0.65915500 \\
\hline $\mathrm{H}$ & 3.19504500 & -2.63781600 & 0.65012200 & C & 5.86562600 & -4.63877900 & -0.03362900 \\
\hline $\mathrm{H}$ & 6.13144500 & -5.78267800 & -1.84587400 & $\mathrm{H}$ & 5.31418300 & -3.41529600 & 1.65109200 \\
\hline $\mathrm{H}$ & 6.79526900 & -4.97808500 & 0.41442900 & $\mathrm{~N}$ & 1.09800700 & -2.97982500 & -1.10298000 \\
\hline $\mathrm{H}$ & 1.18563500 & -2.49465500 & -0.19748600 & $\mathrm{~S}$ & -4.37709400 & -2.60968900 & 0.12170600 \\
\hline 0 & -4.53111300 & -3.81022800 & -0.71540500 & $\mathrm{O}$ & -3.49606300 & -2.74758600 & 1.31451500 \\
\hline 0 & -4.13554300 & -1.33469500 & -0.61816400 & C & -6.06617500 & -2.35248400 & 0.86286500 \\
\hline $\mathrm{F}$ & -6.99516200 & -2.25945300 & -0.09712000 & $\mathrm{~F}$ & -6.09104600 & -1.21144500 & 1.58238300 \\
\hline $\mathrm{F}$ & -6.39251500 & -3.36501700 & 1.67682000 & C & 2.60226300 & -2.05293700 & -3.26654300 \\
\hline $\mathrm{H}$ & 3.39297600 & -2.58152300 & -3.79279600 & $\mathrm{H}$ & 1.61671600 & -2.12630600 & -3.71801100 \\
\hline C & 2.93248000 & -0.87655900 & -2.58907500 & C & 1.89620400 & 0.03747800 & -2.12666400 \\
\hline $\mathrm{H}$ & 0.94932500 & -0.47852800 & -1.92176500 & $\mathrm{H}$ & 2.19972900 & 0.61224600 & -1.24473800 \\
\hline C & 4.35634900 & -0.58428400 & -2.23938300 & $\mathrm{H}$ & 4.57655400 & 0.48475100 & -2.32176600 \\
\hline $\mathrm{H}$ & 4.52436300 & -0.84681700 & -1.18353800 & $\mathrm{H}$ & 5.06444600 & -1.15404700 & -2.84577700 \\
\hline C & 0.10345700 & 2.46031700 & -2.65997100 & $\mathrm{H}$ & 0.53855200 & 3.02399100 & -1.82855900 \\
\hline $\mathrm{H}$ & -0.30042000 & 3.18959100 & -3.37221500 & $\mathrm{H}$ & -0.74087300 & 1.88025300 & -2.27083100 \\
\hline C & 2.90066800 & 2.33707900 & -4.03609200 & $\mathrm{H}$ & 3.38817800 & 2.84001500 & -3.19337200 \\
\hline $\mathrm{H}$ & 3.64442800 & 1.70531300 & -4.53576800 & $\mathrm{H}$ & 2.60240500 & 3.11474300 & -4.75036700 \\
\hline C & 0.62814300 & 0.44045700 & -4.96063900 & $\mathrm{H}$ & 1.35190000 & -0.20611000 & -5.46939200 \\
\hline $\mathrm{H}$ & -0.22788800 & -0.17470300 & -4.65885300 & $\mathrm{H}$ & 0.26131100 & 1.16405300 & -5.69910500 \\
\hline Si & 1.37896100 & 1.36171700 & -3.49281300 & & & & \\
\hline
\end{tabular}

\section{TS-S-4f-nd-30}

Imaginary frequency: $-272.48 \mathrm{~cm}^{-1}$ E[B3LYP/6-31G(d)]: -4788.673461 Hartree $\mathrm{E}\left[\mathrm{PCM}\left(\mathrm{Et}_{2} \mathrm{O}\right)-\mathrm{B} 3 \mathrm{LYP} / 6-311 \mathrm{G}(\mathrm{d}, \mathrm{p})-\mathrm{D} 3(\mathrm{BJ})\right]$ : -4790.213685 Hartree

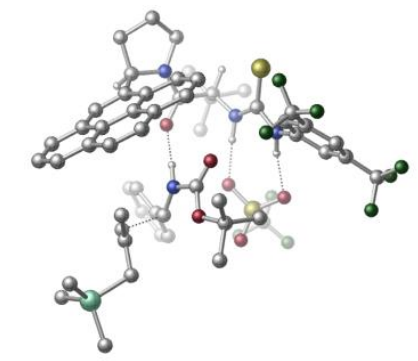

\begin{tabular}{|c|c|c|c|c|c|c|c|}
\hline 01 & & & & $\mathrm{~N}$ & 3.36689200 & 0.26190100 & 0.53832000 \\
\hline $\mathrm{H}$ & 3.17687200 & 1.19147500 & 0.14754500 & $\mathrm{C}$ & 3.95146100 & -0.62875000 & -0.38040700 \\
\hline C & 4.89074500 & -0.12641200 & -1.29044500 & $\mathrm{C}$ & 3.54855300 & -1.96561400 & -0.47975800 \\
\hline C & 5.43151200 & -0.96253800 & -2.26808600 & $\mathrm{H}$ & 5.18240000 & 0.91635200 & -1.23666500 \\
\hline C & 4.10506700 & -2.79023800 & -1.45522500 & $\mathrm{H}$ & 2.78579200 & -2.34078000 & 0.18698600 \\
\hline $\mathrm{C}$ & 5.05509600 & -2.30325500 & -2.35399600 & $\mathrm{H}$ & 5.49133800 & -2.95154100 & -3.10378300 \\
\hline C & 6.38749300 & -0.39894200 & -3.28467900 & $\mathrm{C}$ & 3.59629800 & -4.20007400 & -1.57154700 \\
\hline $\mathrm{F}$ & 7.09159100 & 0.64481400 & -2.79914400 & $\mathrm{~F}$ & 5.73659200 & 0.04327200 & -4.38783500 \\
\hline $\mathrm{F}$ & 7.27663500 & -1.32818000 & -3.70663400 & $\mathrm{~F}$ & 3.49234000 & -4.80864500 & -0.36554800 \\
\hline $\mathrm{F}$ & 2.34939700 & -4.24045900 & -2.11900100 & $\mathrm{~F}$ & 4.38535500 & -4.97394400 & -2.34550800 \\
\hline C & 1.53431800 & 0.86525000 & 3.65085800 & $\mathrm{H}$ & 2.17244600 & 0.18034600 & 4.20729000 \\
\hline $\mathrm{C}$ & 1.58915400 & 2.24364500 & 4.40204900 & $\mathrm{C}$ & 0.78944600 & 3.35840900 & 3.70404000 \\
\hline $\mathrm{H}$ & -0.27029000 & 3.10637000 & 3.62205500 & $\mathrm{H}$ & 1.15858300 & 3.56661200 & 2.69649500 \\
\hline
\end{tabular}




\begin{tabular}{|c|c|c|c|c|c|c|c|}
\hline $\mathrm{H}$ & 0.87779700 & 4.28330000 & 4.28817700 & C & 3.06966100 & 2.66516400 & 4.49798200 \\
\hline $\mathrm{H}$ & 3.15720400 & 3.59501700 & 5.07248600 & $\mathrm{H}$ & 3.49838500 & 2.84059900 & 3.50702800 \\
\hline $\mathrm{H}$ & 3.67574800 & 1.89961200 & 4.99723600 & C & 1.03654900 & 2.05024600 & 5.82902900 \\
\hline $\mathrm{H}$ & 1.09066700 & 2.99584200 & 6.38046200 & $\mathrm{H}$ & 1.61676300 & 1.30930400 & 6.39232800 \\
\hline $\mathrm{H}$ & -0.01437400 & 1.73571100 & 5.82439000 & C & 0.11372100 & 0.26368400 & 3.54493400 \\
\hline 0 & -0.73783300 & 0.75239000 & 2.78521600 & C & 0.67873100 & -1.49030100 & 5.29800700 \\
\hline $\mathrm{C}$ & -1.57558900 & -1.34269700 & 4.31177800 & C & -0.11063800 & -2.74515300 & 5.68625200 \\
\hline $\mathrm{H}$ & 1.65657200 & -1.71670900 & 4.86408200 & $\mathrm{H}$ & 0.82094500 & -0.83787600 & 6.16788200 \\
\hline $\mathrm{C}$ & -1.57328100 & -2.30825400 & 5.53047400 & $\mathrm{H}$ & -2.23307300 & -0.49178700 & 4.51586500 \\
\hline $\bar{H}$ & 0.12885800 & -3.07749500 & 6.70071200 & $\mathrm{H}$ & 0.11885100 & -3.56786600 & 5.00228100 \\
\hline $\mathrm{H}$ & -1.89632000 & -1.75603200 & 6.42132600 & $\mathrm{H}$ & -2.26278200 & -3.14380800 & 5.38885600 \\
\hline $\mathrm{N}$ & -0.18930100 & -0.82541200 & 4.29833200 & C & -2.13591700 & -1.98299700 & 3.03458300 \\
\hline C & -3.47210600 & -1.77007300 & 2.82811700 & C & -1.41587100 & -2.87358400 & 2.13540000 \\
\hline C & -4.22802500 & -2.41270100 & 1.79600400 & $\mathrm{H}$ & -4.01141700 & -1.09768200 & 3.49336600 \\
\hline C & -2.15111100 & -3.53787500 & 1.09306200 & C & -0.03219100 & -3.11397500 & 2.21185800 \\
\hline C & -5.60405700 & -2.18622300 & 1.62008200 & C & -3.55204800 & -3.31945100 & 0.92796900 \\
\hline C & -1.47962900 & -4.42775900 & 0.19546100 & $\mathrm{H}$ & 0.57545900 & -2.58879700 & 2.93276400 \\
\hline C & 0.61071300 & -3.98093300 & 1.32956000 & C & -6.30984700 & -2.84514700 & 0.61354300 \\
\hline $\mathrm{H}$ & -6.11809900 & -1.49961700 & 2.28879800 & C & -4.28234900 & -3.98660300 & -0.10547600 \\
\hline C & -0.09891600 & -4.63591900 & 0.33097000 & C & -2.23803600 & -5.08697900 & -0.83202000 \\
\hline $\mathrm{H}$ & 1.68246000 & -4.12635200 & 1.41833900 & C & -5.65887500 & -3.73574700 & -0.24061300 \\
\hline $\mathrm{H}$ & -7.37693300 & -2.67106400 & 0.50010300 & C & -3.57598000 & -4.88304600 & -0.97692900 \\
\hline $\mathrm{H}$ & 0.41746900 & -5.29042500 & -0.36575800 & $\mathrm{H}$ & -1.70999800 & -5.76451600 & -1.49865800 \\
\hline $\mathrm{H}$ & -6.21576900 & -4.25132100 & -1.01955900 & $\mathrm{H}$ & -4.13292900 & -5.39587400 & -1.75755400 \\
\hline $\mathrm{C}$ & 2.94590300 & -0.01397900 & 1.81261400 & $S$ & 3.49336400 & -1.36193300 & 2.69589200 \\
\hline $\mathrm{N}$ & 2.10917500 & 0.92934000 & 2.31510400 & $\mathrm{H}$ & 1.70057700 & 1.60325600 & 1.65922700 \\
\hline C & -0.50458800 & 0.14276200 & -0.85949700 & $\mathrm{O}$ & 0.31938300 & -0.59253900 & -0.36138500 \\
\hline 0 & -0.81385800 & 0.26501100 & -2.16128800 & C & 0.02302400 & -0.39560500 & -3.20984200 \\
\hline $\mathrm{C}$ & -0.04861100 & -1.91623700 & -3.05152100 & $\mathrm{H}$ & 0.44681000 & -2.38778800 & -3.90755400 \\
\hline $\mathrm{H}$ & 0.44921600 & -2.25075800 & -2.14177900 & $\mathrm{H}$ & -1.08976100 & -2.25902500 & -3.03273600 \\
\hline $\mathrm{C}$ & -0.65183900 & 0.05230400 & -4.50682900 & $\mathrm{H}$ & -0.69614600 & 1.14428200 & -4.56209600 \\
\hline $\mathrm{H}$ & -0.07531400 & -0.31242100 & -5.36277700 & $\mathrm{H}$ & -1.66617900 & -0.35423900 & -4.58845200 \\
\hline C & 1.45036800 & 0.14243800 & -3.14091000 & $\mathrm{H}$ & 2.02588800 & -0.25838500 & -3.98289800 \\
\hline $\mathrm{H}$ & 1.45614100 & 1.23368300 & -3.19663800 & $\mathrm{H}$ & 1.94313000 & -0.16256400 & -2.21881500 \\
\hline $\mathrm{C}$ & -1.99630900 & 2.07392300 & -0.54586900 & $\mathrm{H}$ & -1.68333800 & 2.35231200 & -1.54710300 \\
\hline C & -2.18046600 & 3.23689800 & 0.37514500 & C & -1.92931900 & 4.52054700 & -0.12902400 \\
\hline C & -2.62691400 & 3.08643400 & 1.69575400 & C & -2.11493500 & 5.63963700 & 0.68101800 \\
\hline $\mathrm{H}$ & -1.53292100 & 4.63346000 & -1.13339900 & C & -2.82188800 & 4.20929800 & 2.49657800 \\
\hline $\mathrm{H}$ & -2.79670600 & 2.09662600 & 2.10857100 & C & -2.57180700 & 5.48787600 & 1.99064800 \\
\hline $\mathrm{H}$ & -1.88968900 & 6.62747800 & 0.29011900 & $\mathrm{H}$ & -3.16395500 & 4.08605400 & 3.52038700 \\
\hline $\mathrm{H}$ & -2.72249500 & 6.36068200 & 2.62006400 & $\mathrm{~N}$ & -1.31346100 & 0.97397000 & -0.06804100 \\
\hline $\mathrm{H}$ & -1.08078800 & 0.92191000 & 0.93234800 & $\mathrm{~S}$ & 1.69365700 & 3.31835900 & -0.86087700 \\
\hline $\mathrm{O}$ & 0.77537500 & 3.38365800 & -2.01963500 & $\mathrm{O}$ & 1.04519600 & 2.87825700 & 0.40746500 \\
\hline 0 & 3.00615300 & 2.66580600 & -1.10115300 & $\mathrm{C}$ & 2.13253800 & 5.09667000 & -0.52627100 \\
\hline $\mathrm{F}$ & 2.84644700 & 5.60765300 & -1.53633300 & $\mathrm{~F}$ & 2.85310500 & 5.19510100 & 0.60105800 \\
\hline $\mathrm{F}$ & 1.01902300 & 5.83736100 & -0.37875200 & $\mathrm{C}$ & -3.83998000 & 1.65788700 & -0.85860600 \\
\hline $\mathrm{H}$ & -4.07962200 & 1.27554400 & 0.13080300 & $\mathrm{H}$ & -4.21519900 & 2.65932300 & -1.05303200 \\
\hline C & -3.91282600 & 0.74338500 & -1.92379800 & C & -4.10206100 & 1.20128000 & -3.29762100 \\
\hline $\mathrm{H}$ & -3.80392900 & 2.24857000 & -3.42782900 & $\mathrm{H}$ & -3.56675600 & 0.57693500 & -4.02015400 \\
\hline $\mathrm{C}$ & -3.79945000 & -0.71102200 & -1.62188900 & $\mathrm{H}$ & -3.59140700 & -1.31958200 & -2.50377800 \\
\hline $\mathrm{H}$ & -3.02121600 & -0.87761400 & -0.86739000 & $\mathrm{H}$ & -4.72942200 & -1.07048200 & -1.15916800 \\
\hline $\mathrm{C}$ & -5.95233000 & 1.85331500 & -5.63014200 & $\mathrm{H}$ & -5.32487600 & 1.25317500 & -6.29914100 \\
\hline $\mathrm{H}$ & -6.96519300 & 1.86633900 & -6.05071300 & $\mathrm{H}$ & -5.57379600 & 2.88174100 & -5.64866000 \\
\hline
\end{tabular}




\begin{tabular}{|llll|llll|}
\hline $\mathrm{C}$ & -6.59431600 & -0.63308100 & -3.88172600 & $\mathrm{H}$ & -5.97516600 & -1.27606200 & -4.51817600 \\
\hline $\mathrm{H}$ & -6.61426700 & -1.07393600 & -2.87893400 & $\mathrm{H}$ & -7.61724600 & -0.67119700 & -4.27634200 \\
\hline $\mathrm{C}$ & -7.00714500 & 2.22782700 & -2.72548200 & $\mathrm{H}$ & -7.01079300 & 1.84720500 & -1.69784900 \\
\hline $\mathrm{H}$ & -6.64186100 & 3.26123500 & -2.69907700 & $\mathrm{H}$ & -8.04869400 & 2.25852900 & -3.06797600 \\
\hline $\mathrm{Si}$ & -5.97495800 & 1.14954400 & -3.87914300 & & & & \\
\hline
\end{tabular}

\section{TS-S-4f-nd-31}

Imaginary frequency: $-256.16 \mathrm{~cm}^{-1}$ E[B3LYP/6-31G(d)]: -4788.685654 Hartree E[PCM(Et $\left.\left.{ }_{2} \mathrm{O}\right)-\mathrm{B} 3 \mathrm{LYP} / 6-311 \mathrm{G}(\mathrm{d}, \mathrm{p})-\mathrm{D} 3(\mathrm{BJ})\right]$ : -4790.213669 Hartree

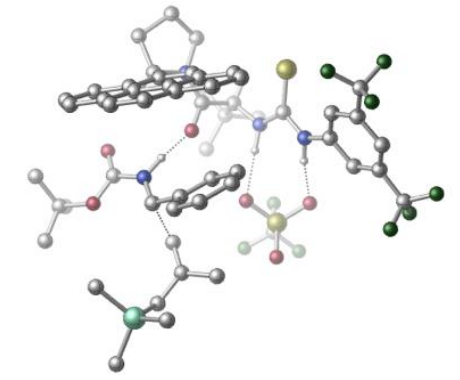

\begin{tabular}{|c|c|c|c|c|c|c|c|}
\hline 01 & & & & $\mathrm{~N}$ & -3.36075100 & -0.45224000 & 1.19995900 \\
\hline $\mathrm{H}$ & -3.12679700 & -1.45252600 & 1.24788500 & $\mathrm{C}$ & -4.33135900 & -0.16679000 & 0.22456600 \\
\hline C & -4.98924300 & -1.27226100 & -0.34681400 & C & -4.62438300 & 1.11775000 & -0.25848600 \\
\hline C & -5.91852800 & -1.08662200 & -1.36661300 & $\mathrm{H}$ & -4.75895100 & -2.27204300 & 0.00630300 \\
\hline C & -5.55840100 & 1.27964200 & -1.28193600 & $\mathrm{H}$ & -4.13602600 & 1.97790200 & 0.17245000 \\
\hline C & -6.21780200 & 0.18937600 & -1.84613400 & $\mathrm{H}$ & -6.94426500 & 0.32686400 & -2.63790900 \\
\hline C & -6.56269100 & -2.28606200 & -2.01147500 & C & -5.89914500 & 2.66901000 & -1.75043300 \\
\hline $\mathrm{F}$ & -6.66475600 & -3.32785200 & -1.16241400 & $\mathrm{~F}$ & -5.85238000 & -2.71463200 & -3.08242800 \\
\hline $\mathrm{F}$ & -7.80752300 & -2.00200300 & -2.46058900 & $\mathrm{~F}$ & -4.86973500 & 3.53627100 & -1.56909700 \\
\hline $\mathrm{F}$ & -6.21038600 & 2.69315800 & -3.06680700 & $\mathrm{~F}$ & -6.95837200 & 3.18307400 & -1.08860100 \\
\hline C & -0.86315100 & 0.36991500 & 3.74348400 & $\mathrm{H}$ & -1.48922500 & 1.10742100 & 4.24516800 \\
\hline C & -0.34652700 & -0.63146800 & 4.84027200 & $\mathrm{C}$ & 0.67380500 & -1.65597400 & 4.30467700 \\
\hline $\mathrm{H}$ & 1.59249900 & -1.17013300 & 3.96231200 & $\mathrm{H}$ & 0.27851200 & -2.24571900 & 3.47500600 \\
\hline $\mathrm{H}$ & 0.93768000 & -2.35108300 & 5.11121500 & C & -1.56748300 & -1.37161700 & 5.42387300 \\
\hline $\mathrm{H}$ & -1.25425900 & -1.98812600 & 6.27477700 & $\mathrm{H}$ & -2.03277600 & -2.03029500 & 4.68673700 \\
\hline $\mathrm{H}$ & -2.32934600 & -0.66738600 & 5.77950200 & $\mathrm{C}$ & 0.32227600 & 0.17868100 & 5.96977400 \\
\hline $\mathrm{H}$ & 0.67792400 & -0.50114300 & 6.75225000 & $\mathrm{H}$ & -0.38075200 & 0.87899700 & 6.43699500 \\
\hline $\mathrm{H}$ & 1.19049500 & 0.74393100 & 5.61084400 & $\mathrm{C}$ & 0.27521100 & 1.07577700 & 2.97852400 \\
\hline $\mathrm{C}$ & 1.74740200 & 2.99983500 & 2.59473200 & $\mathrm{H}$ & 2.57773200 & 2.28935800 & 2.59252100 \\
\hline $\mathrm{N}$ & 0.60835000 & 2.34897800 & 3.27870400 & $\mathrm{C}$ & 1.44037700 & 3.42351500 & 1.15934700 \\
\hline C & 0.15730300 & 3.52073200 & 0.70369300 & C & 2.54466400 & 3.76364800 & 0.27593100 \\
\hline $\mathrm{C}$ & -0.16028100 & 3.95371100 & -0.62709600 & $\mathrm{H}$ & -0.67205300 & 3.24368500 & 1.34903600 \\
\hline C & 2.25360700 & 4.20015700 & -1.05826600 & $\mathrm{C}$ & 3.89428700 & 3.68240600 & 0.66736200 \\
\hline $\mathrm{C}$ & -1.48585800 & 4.04282700 & -1.08239100 & $\mathrm{C}$ & 0.90378500 & 4.29575000 & -1.51018000 \\
\hline $\mathrm{C}$ & 3.31776900 & 4.54357200 & -1.95011800 & $\mathrm{H}$ & 4.15233800 & 3.34931600 & 1.66625400 \\
\hline $\mathrm{C}$ & 4.92467600 & 4.01956200 & -0.21115400 & $\mathrm{C}$ & -1.76480200 & 4.46018600 & -2.38336400 \\
\hline $\mathrm{H}$ & -2.29701400 & 3.78266300 & -0.40819000 & $\mathrm{C}$ & 0.61095900 & 4.72642500 & -2.83999000 \\
\hline $\mathrm{C}$ & 4.64560100 & 4.44744700 & -1.50496500 & $\mathrm{C}$ & 2.99417600 & 4.97993200 & -3.28145000 \\
\hline $\mathrm{H}$ & 5.95606700 & 3.95188900 & 0.12563600 & $\mathrm{C}$ & -0.72977100 & 4.80011100 & -3.25310400 \\
\hline $\mathrm{H}$ & -2.79737700 & 4.51478200 & -2.71443300 & C & 1.70410400 & 5.06746100 & -3.70702800 \\
\hline $\mathrm{H}$ & 5.45353700 & 4.71417600 & -2.18227200 & $\mathrm{H}$ & 3.81248900 & 5.24389600 & -3.94745800 \\
\hline $\mathrm{H}$ & -0.95359700 & 5.12937200 & -4.26522100 & $\mathrm{H}$ & 1.47870900 & 5.40167600 & -4.71715300 \\
\hline $\mathrm{C}$ & -2.75080500 & 0.34828000 & 2.13434800 & $S$ & -3.23848900 & 1.92700600 & 2.52886200 \\
\hline $\mathrm{N}$ & -1.71528600 & -0.28234000 & 2.75547000 & $\mathrm{H}$ & -1.31766900 & -1.10526700 & 2.29188900 \\
\hline $\mathrm{S}$ & -1.54729100 & -3.73073900 & 0.96170100 & $\mathrm{O}$ & -1.27962000 & -4.53130600 & -0.24576300 \\
\hline $\mathrm{O}$ & -0.57588600 & -2.61917100 & 1.20950500 & $\mathrm{O}$ & -2.95759000 & -3.30837000 & 1.17364000 \\
\hline C & -1.23442400 & -4.91423900 & 2.36338200 & $\mathrm{~F}$ & -2.04150300 & -5.97548100 & 2.27587400 \\
\hline $\mathrm{F}$ & -1.44834500 & -4.31243100 & 3.54729100 & $\mathrm{~F}$ & 0.03865300 & -5.33842500 & 2.33594200 \\
\hline C & 4.14113300 & -0.23629700 & 1.11905400 & $\mathrm{O}$ & 4.23301800 & 0.29478100 & 2.20774500 \\
\hline
\end{tabular}




\begin{tabular}{|c|c|c|c|c|c|c|c|}
\hline $\mathrm{O}$ & 5.10661700 & -0.88280200 & 0.44901100 & $\mathrm{C}$ & 6.48412100 & -0.97106500 & 1.00430800 \\
\hline C & 7.06478500 & 0.43547800 & 1.17302500 & $\mathrm{H}$ & 8.12525600 & 0.35821000 & 1.43655300 \\
\hline $\mathrm{H}$ & 6.54909400 & 0.98680400 & 1.96027600 & $\mathrm{H}$ & 6.98678500 & 0.99696900 & 0.23572100 \\
\hline C & 7.23135700 & -1.74097400 & -0.08506000 & $\mathrm{H}$ & 6.77997400 & -2.72593400 & -0.24423900 \\
\hline $\mathrm{H}$ & 8.27447900 & -1.88655100 & 0.21295000 & $\mathrm{H}$ & 7.21711500 & -1.19062000 & -1.03122000 \\
\hline C & 6.45908800 & -1.76157300 & 2.31473400 & $\mathrm{H}$ & 7.48614000 & -1.91622700 & 2.66311600 \\
\hline $\mathrm{H}$ & 6.00206200 & -2.74529000 & 2.16187400 & $\mathrm{H}$ & 5.90449100 & -1.22974900 & 3.08898400 \\
\hline C & 2.74769000 & -0.70550500 & -0.85341700 & $\mathrm{H}$ & 3.66950500 & -0.85489500 & -1.40124600 \\
\hline C & 1.58229600 & -0.25679100 & -1.63201700 & C & 1.77671400 & 0.05026400 & -2.99086300 \\
\hline C & 0.30438700 & -0.10447200 & -1.06463600 & C & 0.72191000 & 0.53120400 & -3.76298200 \\
\hline $\mathrm{H}$ & 2.76357600 & -0.05654000 & -3.43347800 & C & -0.74789600 & 0.36378700 & -1.84774600 \\
\hline $\mathrm{H}$ & 0.12872700 & -0.36787000 & -0.02731100 & C & -0.54263800 & 0.68809800 & -3.19131000 \\
\hline $\mathrm{H}$ & 0.88846000 & 0.78847800 & -4.80490000 & $\mathrm{H}$ & -1.73305600 & 0.47546300 & -1.40667600 \\
\hline $\mathrm{H}$ & -1.36736500 & 1.06306300 & -3.78971900 & $\mathrm{~N}$ & 2.93955700 & -0.21621800 & 0.40294400 \\
\hline $\mathrm{H}$ & 2.14899300 & 0.15853000 & 0.95745900 & $C$ & 2.42624600 & -2.77154500 & -0.50074300 \\
\hline $\mathrm{H}$ & 1.52766300 & -2.56973200 & 0.07972100 & $\mathrm{H}$ & 3.34540500 & -2.90313400 & 0.06380600 \\
\hline C & 2.28237100 & -3.48992300 & -1.68206000 & C & 3.41095600 & -4.22449800 & -2.30201800 \\
\hline $\mathrm{H}$ & 3.05161100 & -5.23156800 & -2.56236400 & $\mathrm{H}$ & 4.24528600 & -4.34375900 & -1.59988400 \\
\hline $\mathrm{C}$ & 0.94533500 & -3.56186300 & -2.34474400 & $\mathrm{H}$ & 0.20919600 & -4.00968400 & -1.65980100 \\
\hline $\mathrm{H}$ & 0.96747400 & -4.12976500 & -3.27722900 & $\mathrm{H}$ & 0.56755400 & -2.55150300 & -2.55243200 \\
\hline C & 5.25663300 & -5.08403400 & -4.55228200 & $\mathrm{H}$ & 6.01264700 & -5.35426000 & -3.80540900 \\
\hline $\mathrm{H}$ & 5.78538200 & -4.83660500 & -5.48075200 & $\mathrm{H}$ & 4.64868200 & -5.97533700 & -4.74739500 \\
\hline $\mathrm{C}$ & 5.30993100 & -2.12731800 & -3.69349800 & $\mathrm{H}$ & 6.04140800 & -2.31602400 & -2.89991500 \\
\hline $\mathrm{H}$ & 4.74992800 & -1.22508300 & -3.42439000 & $\mathrm{H}$ & 5.86987200 & -1.89795900 & -4.60868600 \\
\hline $\mathrm{C}$ & 2.87909800 & -3.23169900 & -5.27580000 & $\mathrm{H}$ & 2.22324400 & -2.41038000 & -4.96743300 \\
\hline $\mathrm{H}$ & 2.24617700 & -4.09922600 & -5.49495000 & $\mathrm{H}$ & 3.36309900 & -2.93604900 & -6.21517500 \\
\hline Si & 4.19399000 & -3.62837900 & -3.98026700 & C & -0.04944600 & 3.23812900 & 4.26311100 \\
\hline $\bar{H}$ & 0.13536500 & 2.87682000 & 5.28017800 & $\mathrm{H}$ & -1.12852900 & 3.26504900 & 4.08766700 \\
\hline $\mathrm{C}$ & 0.63418900 & 4.59352700 & 4.03257200 & $\mathrm{H}$ & 0.66171900 & 5.19775800 & 4.94385600 \\
\hline $\mathrm{H}$ & 0.09710100 & 5.16286100 & 3.26687100 & C & 2.02991400 & 4.20820900 & 3.52083000 \\
\hline $\bar{H}$ & 2.53637500 & 5.02185200 & 2.99623500 & $\mathrm{H}$ & 2.66780100 & 3.88894600 & 4.35446700 \\
\hline $\mathrm{O}$ & 0.86033000 & 0.44368100 & 2.08271600 & & & & \\
\hline
\end{tabular}

\section{TS-S-4f-nd-32}

Imaginary frequency: $-273.37 \mathrm{~cm}-1$

E[B3LYP/6-31G(d)]: -4788.683869 Hartree

E[PCM(Et $\left.\left.{ }_{2} \mathrm{O}\right)-B 3 L Y P / 6-311 G(d, p)-D 3(B J)\right]:-4790.213459$ Hartree

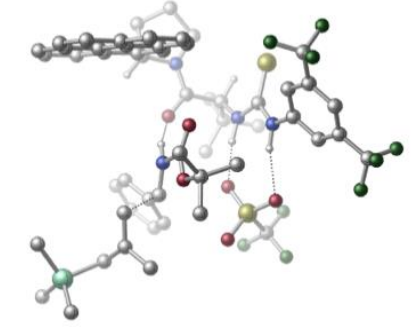

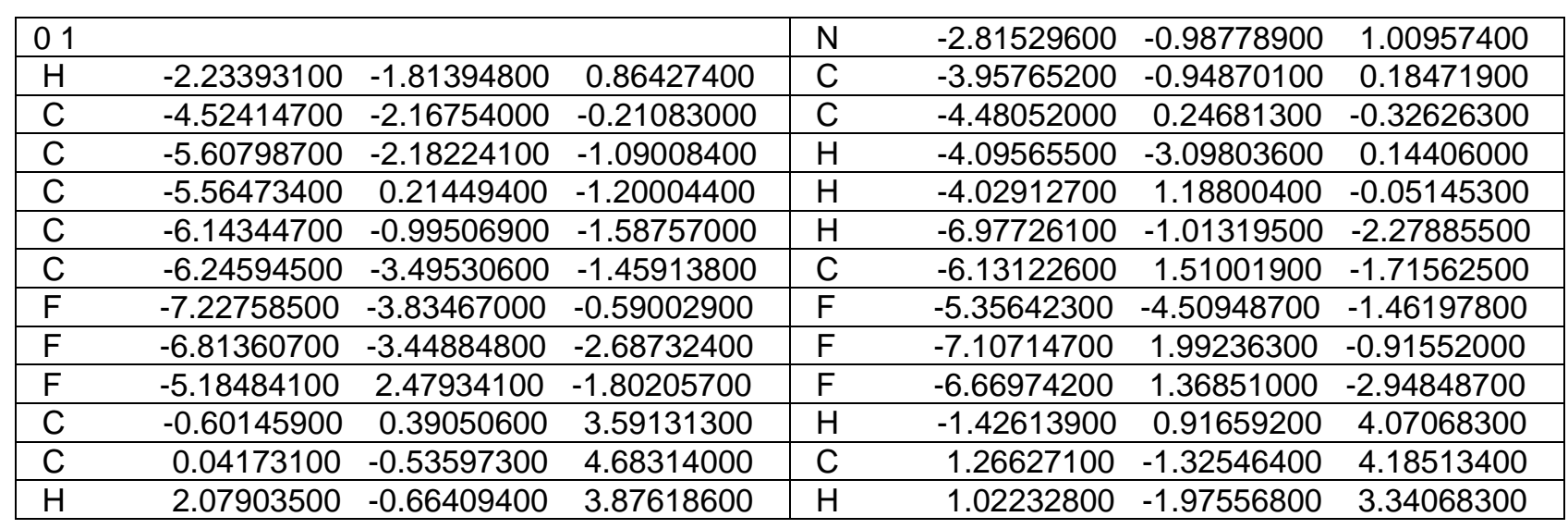




\begin{tabular}{|c|c|c|c|c|c|c|c|}
\hline $\mathrm{H}$ & 1.63433200 & -1.96484100 & 4.99763000 & C & -1.03680800 & -1.53027900 & 5.16107400 \\
\hline $\mathrm{H}$ & -0.66067900 & -2.10324500 & 6.01712900 & $\mathrm{H}$ & -1.30069800 & -2.24285000 & 4.37370800 \\
\hline $\mathrm{H}$ & -1.95263600 & -1.01502500 & 5.47415100 & C & 0.46798500 & 0.34743100 & 5.87373300 \\
\hline $\mathrm{H}$ & 0.91780500 & -0.27264100 & 6.65758000 & $\mathrm{H}$ & -0.38880900 & 0.86887200 & 6.31755300 \\
\hline $\mathrm{H}$ & 1.21411700 & 1.09651600 & 5.58153300 & C & 0.37602600 & 1.42242700 & 2.98180400 \\
\hline 0 & 1.28910100 & 1.06380400 & 2.22063200 & C & -0.77825200 & 3.31948800 & 4.22564600 \\
\hline C & 1.11809000 & 3.74498000 & 2.71866600 & C & -0.54038100 & 4.83064700 & 4.09671500 \\
\hline $\mathrm{H}$ & -1.79512800 & 3.02085000 & 3.95687500 & $\mathrm{H}$ & -0.56825100 & 2.97866600 & 5.24602500 \\
\hline C & 0.93976300 & 4.92837900 & 3.70099300 & $\mathrm{H}$ & 2.12952900 & 3.33180100 & 2.75397700 \\
\hline $\mathrm{H}$ & -0.77525500 & 5.36236200 & 5.02331000 & $\mathrm{H}$ & -1.16877500 & 5.24512100 & 3.30165500 \\
\hline $\mathrm{H}$ & 1.58024600 & 4.77666000 & 4.57930600 & $\mathrm{H}$ & 1.20284900 & 5.88722200 & 3.24840200 \\
\hline $\mathrm{N}$ & 0.20355700 & 2.73229500 & 3.28422900 & C & 0.78905800 & 4.12969400 & 1.27735100 \\
\hline C & -0.40998700 & 3.80980400 & 0.71051600 & C & 1.76690800 & 4.88717000 & 0.51252500 \\
\hline C & -0.76411300 & 4.20393100 & -0.62272200 & $\mathrm{H}$ & -1.13326700 & 3.21887700 & 1.26482600 \\
\hline C & 1.43884500 & 5.28940600 & -0.82369800 & C & 3.02930300 & 5.24650200 & 1.02015200 \\
\hline C & -2.00518700 & 3.87350300 & -1.18878700 & C & 0.17330800 & 4.95213600 & -1.38991300 \\
\hline C & 2.37928500 & 6.03530600 & -1.60115000 & $\mathrm{H}$ & 3.31081100 & 4.96239600 & 2.02843800 \\
\hline C & 3.94122400 & 5.97159100 & 0.25247400 & C & -2.32387500 & 4.26859000 & -2.48714500 \\
\hline $\mathrm{H}$ & -2.71988000 & 3.30313300 & -0.60323600 & C & -0.15815600 & 5.35380200 & -2.71993300 \\
\hline C & 3.62461500 & 6.36520900 & -1.04320000 & C & 2.01869900 & 6.42832300 & -2.93650400 \\
\hline $\mathrm{H}$ & 4.90722000 & 6.23363500 & 0.67659100 & C & -1.41180600 & 5.00070900 & -3.24623500 \\
\hline $\mathrm{H}$ & -3.29003800 & 3.99853600 & -2.90340700 & C & 0.80906500 & 6.10478000 & -3.47057200 \\
\hline $\mathrm{H}$ & 4.33781400 & 6.93453300 & -1.63464800 & $\mathrm{H}$ & 2.74146100 & 6.99834300 & -3.51582000 \\
\hline $\mathrm{H}$ & -1.66557200 & 5.30734000 & -4.25838900 & $\mathrm{H}$ & 0.55348700 & 6.41262800 & -4.48193400 \\
\hline C & -2.44284700 & -0.10645800 & 1.99591400 & $S$ & -3.44848300 & 1.13916700 & 2.56510700 \\
\hline $\mathrm{N}$ & -1.21047700 & -0.36407400 & 2.50264700 & $\mathrm{H}$ & -0.63388900 & -1.06243700 & 2.02949700 \\
\hline C & 0.55239500 & 0.01703800 & -1.15643000 & $\mathrm{O}$ & -0.30506800 & 0.84225200 & -0.92715500 \\
\hline 0 & 0.62508400 & -0.81565500 & -2.21087900 & C & -0.41354900 & -0.81921500 & -3.27623300 \\
\hline C & -0.38830100 & 0.52839000 & -4.00108600 & $\mathrm{H}$ & -1.07418100 & 0.49302100 & -4.85480900 \\
\hline $\mathrm{H}$ & -0.69949300 & 1.34022200 & -3.34081100 & $\mathrm{H}$ & 0.61606300 & 0.74507400 & -4.38338600 \\
\hline $\mathrm{C}$ & 0.05993400 & -1.95603800 & -4.18390000 & $\mathrm{H}$ & 0.12252000 & -2.89132700 & -3.61892400 \\
\hline $\bar{H}$ & -0.64800100 & -2.09259000 & -5.00761500 & $\mathrm{H}$ & 1.04240100 & -1.73111600 & -4.61623000 \\
\hline C & -1.78436800 & -1.14136200 & -2.68411500 & $\mathrm{H}$ & -2.50227700 & -1.27323800 & -3.50151300 \\
\hline $\mathrm{H}$ & -1.75081200 & -2.06960700 & -2.10604500 & $\mathrm{H}$ & -2.13699700 & -0.33391900 & -2.04297600 \\
\hline $\mathrm{C}$ & 2.48933100 & -1.26492800 & -0.34404500 & $\mathrm{H}$ & 1.98536100 & -2.16363700 & -0.68546700 \\
\hline C & 3.37159100 & -1.45492700 & 0.84190000 & C & 3.52988400 & -2.74540200 & 1.36936900 \\
\hline $\mathrm{C}$ & 4.07997200 & -0.38715000 & 1.41424600 & C & 4.37902700 & -2.96028700 & 2.45491000 \\
\hline $\mathrm{H}$ & 2.95959200 & -3.56912400 & 0.95251300 & C & 4.93331700 & -0.60815900 & 2.49419800 \\
\hline $\mathrm{H}$ & 3.95451800 & 0.61958500 & 1.02551100 & C & 5.08644600 & -1.89545300 & 3.01585900 \\
\hline $\mathrm{H}$ & 4.47751500 & -3.96019700 & 2.86797000 & $\mathrm{H}$ & 5.47276600 & 0.22652900 & 2.93327500 \\
\hline $\mathrm{H}$ & 5.74832100 & -2.06502600 & 3.86081300 & $\mathrm{~N}$ & 1.66046900 & -0.15284900 & -0.32034600 \\
\hline $\mathrm{H}$ & 1.61509100 & 0.41627800 & 0.53519100 & $\mathrm{~S}$ & -0.21059800 & -3.72539100 & 0.34524800 \\
\hline 0 & 0.75667200 & -4.12643300 & -0.70444500 & $\mathrm{O}$ & 0.21328700 & -2.52537400 & 1.12398600 \\
\hline 0 & -1.63650800 & -3.68864900 & -0.05110300 & C & -0.10370900 & -5.09648300 & 1.59961900 \\
\hline $\mathrm{F}$ & -0.49811100 & -6.25958800 & 1.07275700 & $\mathrm{~F}$ & -0.87572800 & -4.81772400 & 2.65990700 \\
\hline $\mathrm{F}$ & 1.16605100 & -5.23148000 & 2.02789900 & C & 3.65091300 & -1.01110000 & -1.82314100 \\
\hline $\mathrm{H}$ & 2.88323000 & -0.74638400 & -2.54373300 & $\mathrm{H}$ & 4.26393500 & -0.18294900 & -1.47516100 \\
\hline C & 4.29185400 & -2.25094700 & -2.01359100 & C & 5.60709800 & -2.52184100 & -1.44959600 \\
\hline $\mathrm{H}$ & 5.82933200 & -1.87084800 & -0.59598100 & $\mathrm{H}$ & 5.72932000 & -3.57302800 & -1.16179400 \\
\hline $\mathrm{C}$ & 3.54909300 & -3.35904100 & -2.68517700 & $\mathrm{H}$ & 4.20985700 & -4.16771500 & -3.00730000 \\
\hline $\mathrm{H}$ & 2.82726000 & -3.78517100 & -1.96549700 & $\mathrm{H}$ & 2.95105100 & -3.00295000 & -3.52890300 \\
\hline $\mathrm{C}$ & 8.63478400 & -2.64133200 & -1.77342200 & $\mathrm{H}$ & 8.63369000 & -3.70113400 & -1.49379300 \\
\hline $\mathrm{H}$ & 9.52151700 & -2.46419100 & -2.39416900 & $\mathrm{H}$ & 8.74982500 & -2.05219700 & -0.85640900 \\
\hline
\end{tabular}




\begin{tabular}{|llll|llll|}
\hline $\mathrm{C}$ & 6.85752500 & -3.23389600 & -4.25490200 & $\mathrm{H}$ & 6.80863300 & -4.30165600 & -4.01120600 \\
\hline $\mathrm{H}$ & 5.95438500 & -2.97511800 & -4.81901100 & $\mathrm{H}$ & 7.71322900 & -3.09255400 & -4.92661100 \\
\hline $\mathrm{C}$ & 7.06329500 & -0.33250900 & -3.14919700 & $\mathrm{H}$ & 6.14655500 & -0.03466700 & -3.67019700 \\
\hline $\mathrm{H}$ & 7.16274000 & 0.29781600 & -2.25774500 & $\mathrm{H}$ & 7.90706200 & -0.09935200 & -3.81013000 \\
\hline $\mathrm{Si}$ & 7.06872000 & -2.16697300 & -2.71326800 & & & & \\
\hline
\end{tabular}

\section{TS-S-4f-nd-33}

Imaginary frequency: $-175.03 \mathrm{~cm}^{-1}$

E[B3LYP/6-31G(d)]: -4788.682353 Hartree

E[PCM(Et $\left.\left.{ }_{2} \mathrm{O}\right)-\mathrm{B} 3 \mathrm{LYP} / 6-311 \mathrm{G}(\mathrm{d}, \mathrm{p})-\mathrm{D} 3(\mathrm{BJ})\right]$ : -4790.213422 Hartree

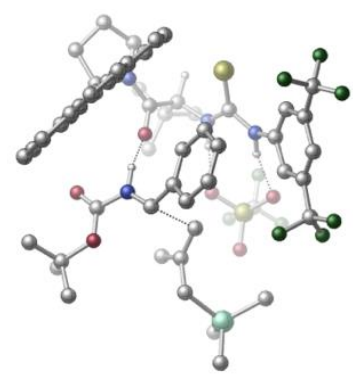

\begin{tabular}{|c|c|c|c|c|c|c|c|}
\hline 01 & & & & $\mathrm{~N}$ & -1.54752200 & 2.45223700 & 1.45646000 \\
\hline $\mathrm{H}$ & -2.38951900 & 1.87251400 & 1.53793900 & $\mathrm{C}$ & -1.55149200 & 3.23200200 & 0.28143800 \\
\hline C & -2.43173200 & 2.82378700 & -0.73541400 & C & -0.74622300 & 4.35988700 & 0.06366600 \\
\hline C & -2.44712200 & 3.48087300 & -1.96256800 & $\mathrm{H}$ & -3.10270400 & 1.99072000 & -0.55456100 \\
\hline C & -0.77185700 & 4.99770500 & -1.17832100 & $\mathrm{H}$ & -0.09553400 & 4.71758100 & 0.84734700 \\
\hline C & -1.60796400 & 4.56720000 & -2.20676500 & $\mathrm{H}$ & -1.61408900 & 5.06949300 & -3.16587700 \\
\hline C & -3.31894500 & 2.92684200 & -3.05507900 & $\mathrm{C}$ & 0.20680300 & 6.10905900 & -1.43889700 \\
\hline $\mathrm{F}$ & -4.56312100 & 2.64213500 & -2.62392100 & $\mathrm{~F}$ & -2.80616100 & 1.75817500 & -3.54527800 \\
\hline $\mathrm{F}$ & -3.42967700 & 3.76744100 & -4.10506900 & $\mathrm{~F}$ & 0.45723900 & 6.84630900 & -0.33904900 \\
\hline $\mathrm{F}$ & 1.40753400 & 5.61367000 & -1.85557600 & $\mathrm{~F}$ & -0.21735700 & 6.94924200 & -2.40792500 \\
\hline C & 0.32056700 & 0.40462300 & 3.85546300 & $\mathrm{H}$ & 0.75068300 & 1.22442400 & 4.43417000 \\
\hline C & -0.31119400 & -0.61970300 & 4.87849900 & $\mathrm{C}$ & -0.77124400 & -1.93864400 & 4.22308800 \\
\hline $\mathrm{H}$ & 0.06532900 & -2.48157700 & 3.77220600 & $\mathrm{H}$ & -1.53006000 & -1.78258400 & 3.45438300 \\
\hline $\mathrm{H}$ & -1.21170200 & -2.58134800 & 4.99531800 & $\mathrm{C}$ & -1.50929500 & 0.06433500 & 5.57100300 \\
\hline $\mathrm{H}$ & -1.87362700 & -0.57343600 & 6.38514000 & $\mathrm{H}$ & -2.34360500 & 0.23431300 & 4.88796700 \\
\hline $\mathrm{H}$ & -1.22122000 & 1.02993300 & 6.00430500 & $\mathrm{C}$ & 0.72630700 & -0.96642300 & 5.96585400 \\
\hline $\mathrm{H}$ & 0.27729500 & -1.65698800 & 6.68831300 & $\mathrm{H}$ & 1.04901900 & -0.07751400 & 6.52110900 \\
\hline $\mathrm{H}$ & 1.61024600 & -1.46359400 & 5.55103400 & $\mathrm{C}$ & 1.41685400 & -0.23929100 & 2.96755100 \\
\hline $\mathrm{C}$ & 3.27828600 & 0.68400500 & 4.41616100 & $\mathrm{C}$ & 3.72311800 & -1.06008100 & 2.71975300 \\
\hline $\mathrm{H}$ & 3.00059400 & 1.73211300 & 4.26549600 & $\mathrm{H}$ & 2.88764300 & 0.36143700 & 5.38406700 \\
\hline $\mathrm{H}$ & 3.28381900 & -2.06128500 & 2.74558400 & $\mathrm{~N}$ & 2.72131300 & -0.14801200 & 3.32549900 \\
\hline $\mathrm{C}$ & 4.16575000 & -0.86012900 & 1.26395700 & $\mathrm{C}$ & 4.37583300 & -2.02134800 & 0.56964700 \\
\hline C & 4.52064500 & 0.40708900 & 0.64048400 & $\mathrm{C}$ & 4.98552800 & -2.06950700 & -0.72537200 \\
\hline $\mathrm{H}$ & 4.08552800 & -2.96862400 & 1.01829300 & $\mathrm{C}$ & 5.15966900 & 0.38527200 & -0.64707100 \\
\hline $\mathrm{C}$ & 4.25326400 & 1.66233200 & 1.21579100 & $\mathrm{C}$ & 5.21165300 & -3.28000100 & -1.40174700 \\
\hline $\mathrm{C}$ & 5.39753300 & -0.84630000 & -1.32675300 & $\mathrm{C}$ & 5.55945200 & 1.60880300 & -1.27159600 \\
\hline $\mathrm{H}$ & 3.68838600 & 1.72629900 & 2.13318500 & $\mathrm{C}$ & 4.64128800 & 2.84943700 & 0.59436400 \\
\hline $\mathrm{C}$ & 5.83301200 & -3.29071700 & -2.64937900 & $\mathrm{H}$ & 4.90615700 & -4.21242300 & -0.93362500 \\
\hline $\mathrm{C}$ & 6.03315100 & -0.86607200 & -2.60661300 & $\mathrm{C}$ & 5.30258900 & 2.82851200 & -0.62771300 \\
\hline C & 6.20833900 & 1.55982700 & -2.55337700 & $\mathrm{H}$ & 4.40855200 & 3.79728500 & 1.07127400 \\
\hline $\mathrm{C}$ & 6.23927500 & -2.09881500 & -3.24715000 & $\mathrm{H}$ & 6.00747600 & -4.23536300 & -3.15789700 \\
\hline $\mathrm{C}$ & 6.43642200 & 0.38094100 & -3.19293400 & $\mathrm{H}$ & 5.60803800 & 3.75655900 & -1.10472200 \\
\hline $\mathrm{H}$ & 6.51262900 & 2.49983400 & -3.00749700 & $\mathrm{H}$ & 6.72407400 & -2.11386000 & -4.22031300 \\
\hline $\mathrm{H}$ & 6.92612200 & 0.36403800 & -4.16360400 & $\mathrm{C}$ & -0.49176300 & 2.12302000 & 2.27781100 \\
\hline$S$ & 0.93526600 & 3.03010000 & 2.43065000 & $\mathrm{~N}$ & -0.69802600 & 0.98572800 & 2.98835800 \\
\hline $\mathrm{H}$ & -1.46150000 & 0.36917500 & 2.69292300 & $S$ & -4.25354700 & -0.24468100 & 1.65879800 \\
\hline $\mathrm{O}$ & -4.96546500 & -1.16158400 & 0.74748000 & $\mathrm{O}$ & -2.90924600 & -0.72849400 & 2.09612500 \\
\hline $\mathrm{O}$ & -4.23589500 & 1.18814200 & 1.26528700 & C & -5.27011700 & -0.27250400 & 3.21786700 \\
\hline $\mathrm{F}$ & -6.51991700 & 0.13114900 & 2.96973200 & $\mathrm{~F}$ & -4.73294900 & 0.54676100 & 4.13904100 \\
\hline
\end{tabular}




\begin{tabular}{|c|c|c|c|c|c|c|c|}
\hline $\mathrm{F}$ & -5.30940700 & -1.51151900 & 3.72627200 & C & 0.99842600 & -3.63787400 & -0.31642600 \\
\hline $\mathrm{O}$ & 1.52077500 & -4.09657100 & 0.67726600 & $\mathrm{O}$ & 0.54083900 & -4.30641100 & -1.38277900 \\
\hline C & 0.74417800 & -5.77419600 & -1.51492100 & C & 2.24318200 & -6.08409100 & -1.51057500 \\
\hline $\mathrm{H}$ & 2.39406000 & -7.13993800 & -1.76023700 & $\mathrm{H}$ & 2.68427300 & -5.89261500 & -0.53090300 \\
\hline $\mathrm{H}$ & 2.76290700 & -5.47823000 & -2.26049400 & C & 0.12913000 & -6.06641000 & -2.88419300 \\
\hline $\mathrm{H}$ & -0.92847300 & -5.78414800 & -2.90407700 & $\mathrm{H}$ & 0.20364800 & -7.13609400 & -3.10414100 \\
\hline $\mathrm{H}$ & 0.65142400 & -5.51486700 & -3.67259500 & C & -0.00720600 & -6.50776700 & -0.40197800 \\
\hline $\mathrm{H}$ & 0.07995400 & -7.58825400 & -0.56066000 & $\mathrm{H}$ & -1.07086000 & -6.24927200 & -0.41667800 \\
\hline $\mathrm{H}$ & 0.40280100 & -6.26484900 & 0.57939900 & C & 0.33545300 & -1.64303900 & -1.57944200 \\
\hline $\mathrm{H}$ & 0.13703500 & -2.32563400 & -2.39505300 & C & 0.71252800 & -0.27848000 & -1.95836000 \\
\hline C & 0.60735600 & 0.06557900 & -3.32070600 & C & 1.16596600 & 0.68214500 & -1.03747700 \\
\hline C & 0.94852100 & 1.34151500 & -3.75360300 & $\mathrm{H}$ & 0.25467500 & -0.67423300 & -4.03562200 \\
\hline C & 1.50463000 & 1.95810100 & -1.47836700 & $\mathrm{H}$ & 1.24341700 & 0.44580400 & 0.01857900 \\
\hline C & 1.39309100 & 2.29277200 & -2.83065100 & $\mathrm{H}$ & 0.86418800 & 1.59580200 & -4.80611400 \\
\hline $\mathrm{H}$ & 1.84390700 & 2.69485700 & -0.75919700 & $\mathrm{H}$ & 1.63942600 & 3.29935400 & -3.15388900 \\
\hline $\mathrm{N}$ & 0.83457400 & -2.25072200 & -0.47564100 & $\mathrm{H}$ & 1.11776000 & -1.71372000 & 0.35744200 \\
\hline C & -1.80490300 & -1.16552300 & -1.20695600 & $\mathrm{H}$ & -1.61058000 & -0.78341600 & -0.20903000 \\
\hline $\mathrm{H}$ & -1.90229200 & -0.42983100 & -1.99905300 & C & -2.41391200 & -2.39706500 & -1.34559400 \\
\hline $\mathrm{C}$ & -2.97816600 & -2.83811000 & -2.63625500 & $\mathrm{H}$ & -2.82427900 & -3.91325300 & -2.79627600 \\
\hline $\mathrm{H}$ & -2.53215600 & -2.29080100 & -3.47642300 & C & -2.54091100 & -3.29463600 & -0.15372400 \\
\hline $\mathrm{H}$ & -2.60368200 & -4.35106200 & -0.43006900 & $\mathrm{H}$ & -3.45911500 & -3.01466900 & 0.38063500 \\
\hline $\mathrm{H}$ & -1.72494600 & -3.13840400 & 0.55858100 & C & -5.29650200 & -0.72536500 & -2.72674500 \\
\hline $\mathrm{H}$ & -5.20876300 & -0.39459100 & -1.68644500 & $\mathrm{H}$ & -6.32820500 & -0.53468300 & -3.04827100 \\
\hline $\mathrm{H}$ & -4.63898500 & -0.10366600 & -3.34336200 & C & -5.87329300 & -3.56763700 & -1.59961000 \\
\hline $\bar{H}$ & -5.76950700 & -3.13734900 & -0.59839200 & $\mathrm{H}$ & -5.55861000 & -4.61777700 & -1.56907400 \\
\hline $\mathrm{H}$ & -6.94064000 & -3.55312700 & -1.85440800 & C & -5.23710800 & -3.20348000 & -4.61548100 \\
\hline $\mathrm{H}$ & -4.98082900 & -4.26510800 & -4.71685300 & $\mathrm{H}$ & -4.66782700 & -2.64668900 & -5.36948300 \\
\hline $\mathrm{H}$ & -6.30021100 & -3.09756700 & -4.86419300 & $\mathrm{Si}$ & -4.90433400 & -2.55837100 & -2.86395800 \\
\hline $\mathrm{C}$ & 4.89830900 & -0.96987900 & 3.73122200 & $\mathrm{H}$ & 5.86216900 & -1.16157500 & 3.25416200 \\
\hline $\mathrm{H}$ & 4.75300400 & -1.72848800 & 4.50994900 & C & 4.79222500 & 0.43558900 & 4.34031900 \\
\hline $\bar{H}$ & 5.26028500 & 0.50491900 & 5.32680400 & $\mathrm{H}$ & 5.27523600 & 1.17065800 & 3.69076100 \\
\hline 0 & 1.06806700 & -0.88608100 & 1.96705200 & & & & \\
\hline
\end{tabular}

\section{TS-S-4f-nd-34}

Imaginary frequency: $-224.64 \mathrm{~cm}^{-1}$ E[B3LYP/6-31G(d)]: -4788.689665 Hartree E[PCM(Et $\left.\left.{ }_{2} \mathrm{O}\right)-B 3 L Y P / 6-311 G(d, p)-D 3(B J)\right]:-4790.212870$ Hartree

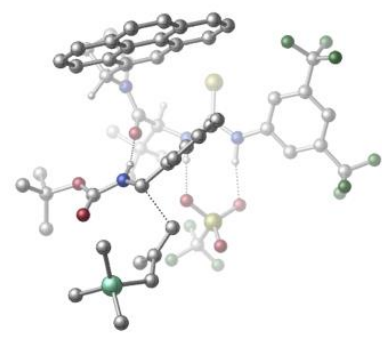

\begin{tabular}{|c|c|c|c|c|c|c|c|}
\hline 01 & & & & $\mathrm{~N}$ & -2.73825400 & -1.56555500 & 1.19846800 \\
\hline $\mathrm{H}$ & -2.14741300 & -2.39988000 & 1.09302200 & C & -3.90253300 & -1.62268300 & 0.41103900 \\
\hline C & -4.19501600 & -2.86176800 & -0.18752300 & C & -4.73036300 & -0.52489900 & 0.13205900 \\
\hline C & -5.29271600 & -2.99093400 & -1.03442100 & $\mathrm{H}$ & -3.55360800 & -3.71480600 & 0.00718100 \\
\hline $\mathrm{C}$ & -5.82479300 & -0.67733500 & -0.71900200 & $\mathrm{H}$ & -4.52751000 & 0.43150400 & 0.58802300 \\
\hline C & -6.12303700 & -1.90385100 & -1.30950600 & $\mathrm{H}$ & -6.97755300 & -2.01225100 & -1.96652300 \\
\hline C & -5.55131800 & -4.30889000 & -1.71709600 & C & -6.73532300 & 0.49657500 & -0.96133700 \\
\hline $\mathrm{F}$ & -5.11856400 & -5.35459800 & -0.98448900 & $\mathrm{~F}$ & -4.92596700 & -4.37808200 & -2.91609700 \\
\hline $\mathrm{F}$ & -6.87043800 & -4.49603700 & -1.95559000 & $\mathrm{~F}$ & -6.09275200 & 1.68266900 & -0.80399000 \\
\hline $\mathrm{F}$ & -7.24734400 & 0.48545200 & -2.21316900 & $\mathrm{~F}$ & -7.78425300 & 0.50936000 & -0.11015000 \\
\hline $\mathrm{C}$ & -0.34818200 & -0.00387100 & 3.49144200 & $\mathrm{H}$ & -1.12613900 & 0.41404500 & 4.12991300 \\
\hline C & 0.62570200 & -0.81331300 & 4.42135000 & C & 1.82822600 & -1.41614900 & 3.67055800 \\
\hline $\mathrm{H}$ & 2.44251800 & -0.64100100 & 3.20468600 & $\mathrm{H}$ & 1.52357900 & -2.11252600 & 2.88732800 \\
\hline
\end{tabular}




\begin{tabular}{|c|c|c|c|c|c|c|c|}
\hline $\mathrm{H}$ & 2.45280600 & -1.97132300 & 4.38193900 & $\mathrm{C}$ & -0.18304200 & -1.94233300 & 5.09242800 \\
\hline $\mathrm{H}$ & 0.44422900 & -2.46336700 & 5.82548700 & $\mathrm{H}$ & -0.52885400 & -2.67977500 & 4.36432700 \\
\hline $\mathrm{H}$ & -1.05987400 & -1.54676600 & 5.61907100 & C & 1.15652400 & 0.12820900 & 5.52180200 \\
\hline $\mathrm{H}$ & 1.83645100 & -0.42132900 & 6.18272900 & $\mathrm{H}$ & 0.34417100 & 0.52254200 & 6.14370600 \\
\hline $\mathrm{H}$ & 1.71689300 & 0.97426900 & 5.10587400 & C & 0.33649600 & 1.13758400 & 2.70424300 \\
\hline C & 0.82580500 & 3.52249300 & 2.39339300 & $\mathrm{H}$ & 1.83911400 & 3.19323600 & 2.14879300 \\
\hline $\mathrm{N}$ & 0.16057700 & 2.41689800 & 3.11264700 & C & 0.11283000 & 3.93879200 & 1.10836000 \\
\hline C & -1.17245500 & 3.55757900 & 0.85296700 & C & 0.80959400 & 4.79900400 & 0.16552400 \\
\hline C & -1.88470100 & 3.97852700 & -0.31986400 & $\mathrm{H}$ & -1.69090100 & 2.89453400 & 1.54038100 \\
\hline C & 0.12155200 & 5.22935900 & -1.01585700 & C & 2.13469300 & 5.23283500 & 0.35444100 \\
\hline C & -3.20757700 & 3.57749200 & -0.56892700 & C & -1.22384900 & 4.82152000 & -1.25867500 \\
\hline C & 0.77999100 & 6.07440100 & -1.96303400 & $\mathrm{H}$ & 2.68515900 & 4.93059600 & 1.23888300 \\
\hline C & 2.76906500 & 6.05581600 & -0.57643900 & C & -3.87173000 & 3.99314700 & -1.72287000 \\
\hline $\mathrm{H}$ & -3.71201300 & 2.93717100 & 0.14951700 & C & -1.91115800 & 5.24775500 & -2.43575200 \\
\hline C & 2.10365100 & 6.47544100 & -1.72313500 & C & 0.06300000 & 6.49188400 & -3.13731500 \\
\hline $\mathrm{H}$ & 3.79330600 & 6.37328100 & -0.39899500 & C & -3.23288800 & 4.81987600 & -2.64608000 \\
\hline $\mathrm{H}$ & -4.89117400 & 3.66351600 & -1.89861400 & C & -1.22029900 & 6.09875600 & -3.36395800 \\
\hline $\mathrm{H}$ & 2.60253700 & 7.11966700 & -2.44310100 & $\mathrm{H}$ & 0.57432200 & 7.13749300 & -3.84750400 \\
\hline $\mathrm{H}$ & -3.75738000 & 5.14479600 & -3.54171100 & $\mathrm{H}$ & -1.74724200 & 6.42605100 & -4.25721300 \\
\hline C & -2.32552200 & -0.65142100 & 2.13587700 & $\mathrm{~S}$ & -3.30041000 & 0.57985400 & 2.78462100 \\
\hline $\mathrm{N}$ & -1.03802300 & -0.85790000 & 2.53140500 & $\mathrm{H}$ & -0.45120800 & -1.46993900 & 1.95521100 \\
\hline $\mathrm{S}$ & 0.08469800 & -3.96239000 & 0.46392900 & $\mathrm{O}$ & 0.53661100 & -4.55204700 & -0.80361500 \\
\hline $\mathrm{O}$ & 0.61298700 & -2.58415400 & 0.73669300 & $\mathrm{O}$ & -1.36102200 & -4.08255500 & 0.78825600 \\
\hline C & 0.90834600 & -5.00795600 & 1.76685200 & $\mathrm{~F}$ & 0.55470400 & -6.28920200 & 1.64123800 \\
\hline $\mathrm{F}$ & 0.57270600 & -4.59036700 & 3.00027000 & $\mathrm{~F}$ & 2.24819200 & -4.92153300 & 1.65352700 \\
\hline C & 4.14571500 & 0.14784800 & -0.01163900 & $\mathrm{O}$ & 5.04219500 & -0.06589600 & -0.80944800 \\
\hline $\mathrm{O}$ & 4.25502100 & 0.43926600 & 1.28211100 & C & 5.58265000 & 0.59510800 & 1.93429000 \\
\hline $\mathrm{C}$ & 6.31811000 & -0.74649600 & 1.92209600 & $\mathrm{H}$ & 7.23201600 & -0.66356000 & 2.52036500 \\
\hline $\mathrm{H}$ & 6.59594500 & -1.04193600 & 0.90885500 & $\mathrm{H}$ & 5.69299100 & -1.52921300 & 2.36408200 \\
\hline C & 5.20310300 & 0.99911500 & 3.35948500 & $\mathrm{H}$ & 4.62205100 & 1.92678300 & 3.35658400 \\
\hline $\mathrm{H}$ & 6.10994600 & 1.15991100 & 3.95137400 & $\mathrm{H}$ & 4.60744700 & 0.21847900 & 3.84098600 \\
\hline C & 6.37122900 & 1.71101600 & 1.24353200 & $\mathrm{H}$ & 7.28909000 & 1.90474800 & 1.80909400 \\
\hline $\mathrm{H}$ & 5.78535600 & 2.63629400 & 1.22206100 & $\mathrm{H}$ & 6.64277600 & 1.43940800 & 0.22243100 \\
\hline $\mathrm{C}$ & 2.44863700 & -0.00497200 & -1.68312800 & $\mathrm{H}$ & 3.28405200 & 0.12656800 & -2.35803800 \\
\hline $\mathrm{C}$ & 1.13121700 & 0.41424100 & -2.16798100 & C & 1.05648200 & 0.94071700 & -3.47086800 \\
\hline C & -0.03820000 & 0.30214900 & -1.39510000 & C & -0.16094300 & 1.37837000 & -3.98207500 \\
\hline $\mathrm{H}$ & 1.95944400 & 1.01845600 & -4.07180300 & C & -1.25369000 & 0.72848300 & -1.91983400 \\
\hline $\mathrm{H}$ & 0.00034200 & -0.13213200 & -0.40227600 & C & -1.31784400 & 1.27185800 & -3.20649100 \\
\hline $\mathrm{H}$ & -0.20819800 & 1.80144600 & -4.98112100 & $\mathrm{H}$ & -2.15664500 & 0.63759400 & -1.32561300 \\
\hline $\mathrm{H}$ & -2.26996600 & 1.61504800 & -3.59937900 & $\mathrm{~N}$ & 2.79365600 & 0.13543400 & -0.37824100 \\
\hline $\mathrm{H}$ & 2.09412500 & 0.29303000 & 0.36201500 & C & 2.23670500 & -2.10813200 & -2.19039400 \\
\hline $\mathrm{H}$ & 1.53468000 & -2.20427400 & -1.36833500 & $\mathrm{H}$ & 1.80874200 & -1.96073400 & -3.17760800 \\
\hline $\mathrm{C}$ & 3.44214400 & -2.77523000 & -2.08402500 & $\mathrm{C}$ & 4.24798600 & -3.15110100 & -3.27216000 \\
\hline $\mathrm{H}$ & 3.62072900 & -3.15261700 & -4.17225700 & $\mathrm{H}$ & 4.61514500 & -4.17850400 & -3.13129100 \\
\hline C & 3.89565300 & -3.25664300 & -0.73915400 & $\mathrm{H}$ & 4.98529400 & -3.27236900 & -0.64565800 \\
\hline $\mathrm{H}$ & 3.52786800 & -4.28469000 & -0.61120500 & $\mathrm{H}$ & 3.44878500 & -2.67988700 & 0.07456000 \\
\hline $\mathrm{C}$ & 6.46627300 & -3.13212300 & -5.31471400 & $\mathrm{H}$ & 6.69008800 & -4.18071500 & -5.08492600 \\
\hline $\mathrm{H}$ & 7.38518000 & -2.67806500 & -5.70538900 & $\mathrm{H}$ & 5.72474600 & -3.12016700 & -6.12248900 \\
\hline C & 7.16296900 & -2.26124900 & -2.42607600 & $\mathrm{H}$ & 7.37478900 & -3.29012600 & -2.11166600 \\
\hline $\mathrm{H}$ & 6.84516600 & -1.68513200 & -1.55169800 & $\mathrm{H}$ & 8.10503100 & -1.82858000 & -2.78576900 \\
\hline C & 5.46595200 & -0.40435500 & -4.25333900 & $\mathrm{H}$ & 5.28988500 & 0.20058800 & -3.35831300 \\
\hline $\mathrm{H}$ & 4.59031400 & -0.33336000 & -4.91029800 & $\mathrm{H}$ & 6.31203200 & 0.04051200 & -4.79181000 \\
\hline $\mathrm{Si}$ & 5.85714900 & -2.19435100 & -3.78697800 & C & -0.51754300 & 4.42186000 & 4.18467800 \\
\hline
\end{tabular}




\begin{tabular}{|rrrr|rrrr|}
\hline $\mathrm{H}$ & -0.55401400 & 4.89209200 & 5.17147700 & $\mathrm{H}$ & -1.34153300 & 4.82657100 & 3.58844100 \\
\hline $\mathrm{C}$ & 0.81631500 & 4.64660300 & 3.45760700 & $\mathrm{H}$ & 0.90236500 & 5.64090700 & 3.01344900 \\
\hline $\mathrm{H}$ & 1.65708700 & 4.50539100 & 4.14878900 & $\mathrm{C}$ & -0.62660200 & 2.89188600 & 4.27423700 \\
\hline $\mathrm{H}$ & -0.18167100 & 2.52557900 & 5.20474300 & $\mathrm{H}$ & -1.65686800 & 2.53054300 & 4.21005500 \\
\hline $\mathrm{O}$ & 1.01901600 & 0.88203600 & 1.69965300 & & & & \\
\hline
\end{tabular}

\section{TS-S-4f-nd-35}

Imaginary frequency: $-284.99 \mathrm{~cm}^{-1}$ E[B3LYP/6-31G(d)]: -4788.674176 Hartree

E[PCM(Et $\left.\left.{ }_{2} \mathrm{O}\right)-\mathrm{B} 3 \mathrm{LYP} / 6-311 \mathrm{G}(\mathrm{d}, \mathrm{p})-\mathrm{D} 3(\mathrm{BJ})\right]$ : -4790.212481 Hartree

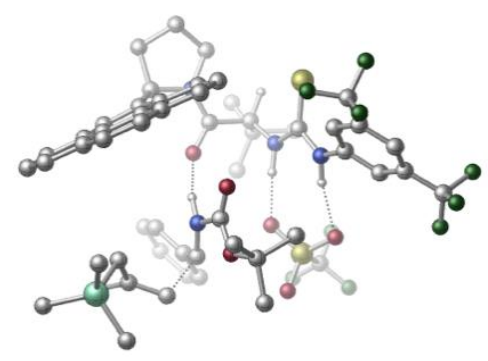

\begin{tabular}{|c|c|c|c|c|c|c|c|}
\hline 01 & & & & $\mathrm{~N}$ & 3.15597100 & 0.08928700 & 0.71872300 \\
\hline $\mathrm{H}$ & 3.08756400 & 0.94276500 & 0.15681500 & C & 3.95022400 & -0.91539300 & 0.13380400 \\
\hline C & 5.01944100 & -0.52583200 & -0.68139200 & C & 3.64466800 & -2.27770500 & 0.26092400 \\
\hline C & 5.78485400 & -1.49207500 & -1.33840100 & $\mathrm{H}$ & 5.23156700 & 0.52871900 & -0.81953100 \\
\hline C & 4.42383900 & -3.22857300 & -0.39145500 & $\mathrm{H}$ & 2.78931600 & -2.57975300 & 0.84618300 \\
\hline C & 5.50336600 & -2.84907100 & -1.19288500 & $\mathrm{H}$ & 6.09627300 & -3.59340600 & -1.71078500 \\
\hline C & 6.96773100 & -1.06164700 & -2.16457600 & C & 4.11455300 & -4.69030000 & -0.21239400 \\
\hline$F$ & 8.08829300 & -0.94971300 & -1.41277000 & $\mathrm{~F}$ & 6.76695800 & 0.13774800 & -2.74975500 \\
\hline $\mathrm{F}$ & 7.24501700 & -1.95134300 & -3.14640700 & $\mathrm{~F}$ & 4.86303600 & -5.25070200 & 0.76395700 \\
\hline $\mathrm{F}$ & 2.81675400 & -4.90263800 & 0.12016200 & $\mathrm{~F}$ & 4.35671500 & -5.39648800 & -1.34143200 \\
\hline $\mathrm{C}$ & 0.95866300 & 1.33313700 & 3.37401100 & $\mathrm{H}$ & 1.47059800 & 0.73330100 & 4.12481700 \\
\hline C & 1.02964100 & 2.82250100 & 3.86978200 & C & 0.28247300 & 3.81951200 & 2.96575300 \\
\hline $\mathrm{H}$ & -0.78885400 & 3.60917900 & 2.92713200 & $\mathrm{H}$ & 0.66205000 & 3.81352000 & 1.94092900 \\
\hline $\mathrm{H}$ & 0.41673500 & 4.83319400 & 3.36436300 & C & 2.51654000 & 3.22694100 & 3.94430200 \\
\hline $\mathrm{H}$ & 2.60997100 & 4.20862900 & 4.42402800 & $\mathrm{H}$ & 2.96042700 & 3.30028700 & 2.94722900 \\
\hline $\mathrm{H}$ & 3.10391800 & 2.50727500 & 4.52675700 & C & 0.42489700 & 2.88633100 & 5.28772000 \\
\hline $\mathrm{H}$ & 0.46660600 & 3.91408700 & 5.66607700 & $\mathrm{H}$ & 0.97716600 & 2.25293900 & 5.99257500 \\
\hline $\mathrm{H}$ & -0.62802800 & 2.57797700 & 5.29889800 & C & -0.48038100 & 0.79992400 & 3.18744800 \\
\hline $\mathrm{O}$ & -1.19239400 & 1.18517400 & 2.24755400 & C & -0.25910900 & -0.63996700 & 5.27379400 \\
\hline$C$ & -2.36422500 & -0.54653800 & 3.99540700 & C & -1.17905300 & -1.76618600 & 5.75645400 \\
\hline $\mathrm{H}$ & 0.74407900 & -0.98893100 & 5.01433000 & $\mathrm{H}$ & -0.17338200 & 0.14494300 & 6.03511600 \\
\hline$C$ & -2.57644700 & -1.28101100 & 5.34946900 & $\mathrm{H}$ & -2.97611000 & 0.36064700 & 3.96652600 \\
\hline $\mathrm{H}$ & -1.08294800 & -1.93674900 & 6.83283800 & $\mathrm{H}$ & -0.93487600 & -2.70261300 & 5.24546600 \\
\hline $\mathrm{H}$ & -2.95380600 & -0.56322700 & 6.08810100 & $\mathrm{H}$ & -3.30856100 & -2.08740100 & 5.26445400 \\
\hline $\mathrm{N}$ & -0.95211700 & -0.11444900 & 4.07445300 & C & -2.81793800 & -1.37493100 & 2.78634700 \\
\hline$C$ & -4.10788700 & -1.14308300 & 2.39136600 & C & -2.06503400 & -2.44499900 & 2.14805000 \\
\hline C & -4.79514300 & -1.93901600 & 1.42064100 & $\mathrm{H}$ & -4.66954000 & -0.33667300 & 2.86058000 \\
\hline$C$ & -2.73412400 & -3.26744100 & 1.17715100 & C & -0.71070400 & -2.71844300 & 2.41315700 \\
\hline$C$ & -6.13333100 & -1.69798300 & 1.06208000 & C & -4.09661100 & -3.02965800 & 0.82562900 \\
\hline C & -2.03592100 & -4.34544100 & 0.54647100 & $\mathrm{H}$ & -0.14734900 & -2.09025200 & 3.08645300 \\
\hline C & -0.03720200 & -3.76204900 & 1.78048400 & C & -6.78407300 & -2.52653000 & 0.14834100 \\
\hline $\mathrm{H}$ & -6.66584700 & -0.87110500 & 1.52678800 & C & -4.76895900 & -3.86926800 & -0.11674800 \\
\hline C & -0.68854700 & -4.57491200 & 0.86174100 & C & -2.73513400 & -5.17294900 & -0.39775200 \\
\hline $\mathrm{H}$ & 1.01137800 & -3.92899400 & 2.00610500 & C & -6.11303300 & -3.60290600 & -0.43241700 \\
\hline $\mathrm{H}$ & -7.82605600 & -2.34227700 & -0.10137700 & C & -4.04113100 & -4.95458200 & -0.71179400 \\
\hline $\mathrm{H}$ & -0.15319800 & -5.38406700 & 0.37141000 & $\mathrm{H}$ & -2.18928200 & -5.99294500 & -0.85835000 \\
\hline $\mathrm{H}$ & -6.63008200 & -4.25448500 & -1.13315800 & $\mathrm{H}$ & -4.55447700 & -5.59699400 & -1.42330600 \\
\hline $\mathrm{C}$ & 2.55170500 & 0.07247900 & 1.95083300 & $S$ & 2.89625300 & -1.09329900 & 3.14183000 \\
\hline $\mathrm{N}$ & 1.69662800 & 1.10844700 & 2.13941200 & $\mathrm{H}$ & 1.45743100 & 1.69200700 & 1.33230600 \\
\hline
\end{tabular}




\begin{tabular}{|c|c|c|c|c|c|c|c|}
\hline $\mathrm{C}$ & -0.58147100 & 0.14085000 & -1.23673800 & 0 & -0.20879200 & -0.81833300 & -0.59632800 \\
\hline $\mathrm{O}$ & -0.38106400 & 0.38007800 & -2.54526600 & C & 0.45022800 & -0.51357900 & -3.39483500 \\
\hline $\mathrm{C}$ & -0.26766600 & -1.85742500 & -3.54085200 & $\mathrm{H}$ & 0.29746500 & -2.50258300 & -4.22236700 \\
\hline $\mathrm{H}$ & -0.35374800 & -2.36201400 & -2.57598000 & $\mathrm{H}$ & -1.26993800 & -1.71844200 & -3.96449200 \\
\hline C & 0.49967500 & 0.25535500 & -4.71623900 & $\mathrm{H}$ & 0.94004400 & 1.24451900 & -4.55911500 \\
\hline $\mathrm{H}$ & 1.11010000 & -0.29155700 & -5.44210300 & $\mathrm{H}$ & -0.50426000 & 0.37848300 & -5.14055200 \\
\hline C & 1.85327900 & -0.66805800 & -2.81089100 & $\mathrm{H}$ & 2.47188600 & -1.22554800 & -3.52323600 \\
\hline $\mathrm{H}$ & 2.31855900 & 0.30811300 & -2.64882600 & $\mathrm{H}$ & 1.83342900 & -1.21598500 & -1.86907300 \\
\hline C & -1.56084200 & 2.39985100 & -1.25703400 & $\mathrm{H}$ & -0.74472000 & 2.68592100 & -1.91911500 \\
\hline C & -2.02002200 & 3.51018700 & -0.38011000 & $\mathrm{C}$ & -1.57898000 & 4.80825000 & -0.68557700 \\
\hline C & -2.88061000 & 3.31983000 & 0.71385600 & C & -1.99480100 & 5.89348100 & 0.08134000 \\
\hline $\mathrm{H}$ & -0.87721500 & 4.95382100 & -1.50190200 & C & -3.29528100 & 4.41131500 & 1.47864400 \\
\hline $\mathrm{H}$ & -3.19796000 & 2.32045300 & 0.99485000 & C & -2.86049100 & 5.69915300 & 1.16084500 \\
\hline $\mathrm{H}$ & -1.63124700 & 6.88927800 & -0.15516400 & $\mathrm{H}$ & -3.95229400 & 4.25185600 & 2.32922300 \\
\hline $\mathrm{H}$ & -3.18392000 & 6.54617100 & 1.75959400 & $\mathrm{~N}$ & -1.36867300 & 1.15207800 & -0.67301500 \\
\hline $\mathrm{H}$ & -1.44315200 & 1.07041600 & 0.34689300 & $\mathrm{~S}$ & 2.09704700 & 3.14716600 & -1.27381200 \\
\hline $\mathrm{O}$ & 1.41345600 & 3.37710700 & -2.56457900 & $\mathrm{O}$ & 1.17062100 & 2.80516600 & -0.15413900 \\
\hline 0 & 3.30524800 & 2.28540900 & -1.30785300 & C & 2.74115500 & 4.82393400 & -0.78728900 \\
\hline $\mathrm{F}$ & 3.63841400 & 5.26854400 & -1.67236300 & $\mathrm{~F}$ & 3.32079700 & 4.76663400 & 0.42235500 \\
\hline $\mathrm{F}$ & 1.72795300 & 5.70708800 & -0.72538000 & $\mathrm{C}$ & -2.76360900 & 2.32003400 & -2.71487400 \\
\hline $\mathrm{H}$ & -2.74582600 & 3.35930200 & -3.03570900 & $\mathrm{H}$ & -2.15149000 & 1.65501000 & -3.31769500 \\
\hline $\mathrm{C}$ & -4.00366300 & 1.80593400 & -2.28741600 & $C$ & -4.20893000 & 0.37143900 & -2.19334700 \\
\hline $\mathrm{H}$ & -3.26388200 & -0.17593300 & -2.11623800 & $\mathrm{H}$ & -4.88663500 & 0.07755700 & -1.38235500 \\
\hline C & -5.08221200 & 2.73581800 & -1.83786500 & $\mathrm{H}$ & -6.05258800 & 2.24169900 & -1.74815400 \\
\hline $\bar{H}$ & -4.80326500 & 3.11943200 & -0.84217100 & $\mathrm{H}$ & -5.16726200 & 3.61139000 & -2.48954400 \\
\hline $\mathrm{C}$ & -4.77079500 & -2.20326900 & -3.77361600 & $\mathrm{H}$ & -5.18130500 & -2.64564400 & -2.86014700 \\
\hline $\mathrm{H}$ & -5.25445100 & -2.68539500 & -4.63242700 & $\mathrm{H}$ & -3.70329900 & -2.44778500 & -3.81201800 \\
\hline $\mathrm{C}$ & -6.89913200 & 0.06661600 & -3.76564100 & $\mathrm{H}$ & -7.36614500 & -0.32850200 & -2.85600400 \\
\hline $\bar{H}$ & -7.09087500 & 1.14488300 & -3.80689900 & $\mathrm{H}$ & -7.41292900 & -0.38725800 & -4.62210900 \\
\hline C & -4.25121400 & 0.41684500 & -5.35522400 & $\mathrm{H}$ & -4.41169400 & 1.49869800 & -5.42051400 \\
\hline $\bar{H}$ & -3.17030700 & 0.23624600 & -5.38092200 & $\mathrm{H}$ & -4.67710600 & -0.03414200 & -6.26006400 \\
\hline Si & -5.05739300 & -0.34456000 & -3.82871700 & & & & \\
\hline
\end{tabular}

\section{TS-S-4f-nd-36}

Imaginary frequency: $-252.66 \mathrm{~cm}-1$ E[B3LYP/6-31G(d)]: -4788.677632 Hartree E[PCM(Et $\left.\left.{ }_{2} \mathrm{O}\right)-B 3 L Y P / 6-311 G(d, p)-D 3(B J)\right]:-4790.212306$ Hartree

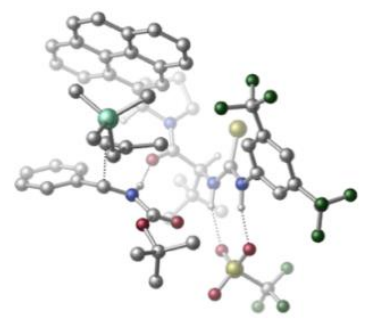

\begin{tabular}{|c|c|c|c|c|c|c|c|}
\hline 01 & & & & $\mathrm{~N}$ & 2.70188600 & -0.09727600 & -1.23352800 \\
\hline $\mathrm{H}$ & 3.37385500 & -0.46226300 & -0.54164600 & C & 2.86384000 & 1.27752600 & -1.48035200 \\
\hline C & 4.14166700 & 1.83377800 & -1.29554500 & C & 1.79813800 & 2.12575400 & -1.80407100 \\
\hline $\mathrm{C}$ & 4.33771300 & 3.20398800 & -1.45360700 & $\mathrm{H}$ & 4.96290200 & 1.19043600 & -1.00182000 \\
\hline $\mathrm{C}$ & 2.01387900 & 3.49592500 & -1.95721200 & $\mathrm{H}$ & 0.80777800 & 1.71430800 & -1.93604600 \\
\hline C & 3.28004100 & 4.05173000 & -1.79028500 & $\mathrm{H}$ & 3.43989900 & 5.11630400 & -1.90708800 \\
\hline $\mathrm{C}$ & 5.72280200 & 3.78201900 & -1.32172500 & C & 0.82388000 & 4.36721700 & -2.23683000 \\
\hline $\mathrm{F}$ & 6.34137200 & 3.86775000 & -2.52304700 & $\mathrm{~F}$ & 6.51169500 & 3.04193700 & -0.52028500 \\
\hline $\mathrm{F}$ & 5.68889600 & 5.03850700 & -0.81417800 & $\mathrm{~F}$ & 0.09489600 & 3.91841700 & -3.28954100 \\
\hline $\mathrm{F}$ & -0.03716800 & 4.39078600 & -1.17591600 & $\mathrm{~F}$ & 1.15636500 & 5.64635700 & -2.49518200 \\
\hline C & 0.91830200 & -3.27064100 & -1.69068200 & $\mathrm{H}$ & 1.03246100 & -3.31690200 & -2.77576500 \\
\hline $\mathrm{C}$ & 1.39455700 & -4.65945100 & -1.11671700 & $\mathrm{C}$ & 1.34407700 & -4.72502700 & 0.42409300 \\
\hline $\mathrm{H}$ & 0.32503400 & -4.58310800 & 0.79666800 & $\mathrm{H}$ & 1.99968800 & -3.99076400 & 0.89807300 \\
\hline
\end{tabular}




\begin{tabular}{|c|c|c|c|c|c|c|c|}
\hline $\mathrm{H}$ & 1.68775300 & -5.71471200 & 0.74808200 & $\mathrm{C}$ & 2.83560700 & -4.91588200 & -1.61052900 \\
\hline $\mathrm{H}$ & 3.15184600 & -5.92050700 & -1.30530100 & $\mathrm{H}$ & 3.54978500 & -4.20531500 & -1.19063400 \\
\hline $\mathrm{H}$ & 2.88948300 & -4.86718400 & -2.70555000 & C & 0.49643400 & -5.78118400 & -1.67969400 \\
\hline $\mathrm{H}$ & 0.83527900 & -6.74568100 & -1.28603700 & $\mathrm{H}$ & 0.55592600 & -5.83976200 & -2.77279900 \\
\hline $\mathrm{H}$ & -0.55527400 & -5.66326500 & -1.39392200 & C & -0.56839800 & -2.98197200 & -1.36513500 \\
\hline $\mathrm{O}$ & -0.92000600 & -2.61816000 & -0.22680800 & $\mathrm{C}$ & -1.24948500 & -3.54178000 & -3.74496400 \\
\hline C & -2.94213900 & -3.12429400 & -2.01224500 & C & -2.64806200 & -3.51895400 & -4.37527800 \\
\hline $\mathrm{H}$ & -0.56272000 & -2.81590200 & -4.18831700 & $\mathrm{H}$ & -0.80967700 & -4.54104000 & -3.82016900 \\
\hline C & -3.56935700 & -3.88872700 & -3.20531000 & $\mathrm{H}$ & -3.10332300 & -3.65205600 & -1.06774400 \\
\hline $\mathrm{H}$ & -2.72782700 & -4.20944400 & -5.21963500 & $\mathrm{H}$ & -2.88586100 & -2.51361500 & -4.73818700 \\
\hline $\mathrm{H}$ & -3.52619400 & -4.96805600 & -3.01248400 & $\mathrm{H}$ & -4.61286300 & -3.61314800 & -3.37343700 \\
\hline $\mathrm{N}$ & -1.49958100 & -3.19730700 & -2.32607500 & C & -3.49438000 & -1.70575700 & -1.88971500 \\
\hline C & -2.78147400 & -0.62823000 & -2.32928400 & C & -4.82826000 & -1.51302400 & -1.34141500 \\
\hline C & -3.30198600 & 0.70918600 & -2.28997400 & $\mathrm{H}$ & -1.77391400 & -0.76377900 & -2.71232100 \\
\hline C & -5.37057700 & -0.18668800 & -1.29231100 & C & -5.62115800 & -2.56892200 & -0.85471300 \\
\hline C & -2.55604400 & 1.80851100 & -2.74964200 & C & -4.61158900 & 0.92342700 & -1.77019600 \\
\hline C & -6.68169900 & 0.03477300 & -0.76580000 & $\mathrm{H}$ & -5.24656000 & -3.58630500 & -0.88042900 \\
\hline C & -6.89706700 & -2.34182000 & -0.33865500 & C & -3.09005700 & 3.09723100 & -2.70606400 \\
\hline $\mathrm{H}$ & -1.55884300 & 1.64467200 & -3.14903200 & C & -5.15244000 & 2.24512500 & -1.73106300 \\
\hline C & -7.42701400 & -1.05694300 & -0.29390000 & C & -7.20186000 & 1.37499300 & -0.73918600 \\
\hline $\mathrm{H}$ & -7.48233600 & -3.18098300 & 0.02758100 & C & -4.37399800 & 3.31459100 & -2.20636900 \\
\hline $\mathrm{H}$ & -2.49355300 & 3.93094400 & -3.06221900 & $\mathrm{C}$ & -6.47544600 & 2.42989300 & -1.20165100 \\
\hline $\mathrm{H}$ & -8.42450000 & -0.88761400 & 0.10409400 & $\mathrm{H}$ & -8.20264900 & 1.52852500 & -0.34258900 \\
\hline $\mathrm{H}$ & -4.78751200 & 4.32018700 & -2.18441300 & $\mathrm{H}$ & -6.88932000 & 3.43528800 & -1.18052000 \\
\hline C & 1.82372300 & -0.97048200 & -1.81886400 & $\mathrm{~S}$ & 0.92596800 & -0.60590900 & -3.22241400 \\
\hline $\mathrm{N}$ & 1.75578300 & -2.17755600 & -1.21181300 & $\mathrm{H}$ & 2.36585100 & -2.34720200 & -0.40672900 \\
\hline C & 0.84010100 & -0.64009100 & 2.41301900 & $\mathrm{O}$ & 1.78474500 & -1.00321800 & 1.75449300 \\
\hline $\mathrm{O}$ & 0.81210400 & 0.26078500 & 3.39925600 & $\mathrm{C}$ & 2.08350900 & 0.79668500 & 3.99650400 \\
\hline $\mathrm{C}$ & 2.89641900 & 1.54123200 & 2.93725100 & $\mathrm{H}$ & 3.71129700 & 2.07791500 & 3.43569300 \\
\hline $\mathrm{H}$ & 3.33842800 & 0.86445500 & 2.20519100 & $\mathrm{H}$ & 2.27381800 & 2.28278400 & 2.42259200 \\
\hline C & 1.54672300 & 1.75608400 & 5.05977400 & $\mathrm{H}$ & 0.91263200 & 1.22908000 & 5.78076600 \\
\hline $\mathrm{H}$ & 2.38406100 & 2.20399900 & 5.60351200 & $\mathrm{H}$ & 0.96420900 & 2.56545700 & 4.60587700 \\
\hline C & 2.85506700 & -0.35832700 & 4.63279900 & $\mathrm{H}$ & 3.70036900 & 0.05034600 & 5.19724800 \\
\hline $\mathrm{H}$ & 2.21410200 & -0.90591600 & 5.33363800 & $\mathrm{H}$ & 3.26643600 & -1.04269600 & 3.88819300 \\
\hline $\mathrm{C}$ & -1.55174300 & -0.88117200 & 2.91735100 & $\mathrm{H}$ & -1.31410000 & -0.26695300 & 3.77778600 \\
\hline C & -2.60397100 & -1.89764200 & 3.16879700 & C & -3.30028600 & -1.81760400 & 4.38718400 \\
\hline $\mathrm{C}$ & -2.90227800 & -2.93473000 & 2.27133500 & C & -4.26746600 & -2.76425900 & 4.71345100 \\
\hline $\mathrm{H}$ & -3.06949500 & -1.01804800 & 5.08758700 & C & -3.87168300 & -3.87848400 & 2.60482700 \\
\hline $\mathrm{H}$ & -2.38121500 & -3.00229700 & 1.32135600 & C & -4.55500600 & -3.79886700 & 3.82058600 \\
\hline $\mathrm{H}$ & -4.78920200 & -2.69928000 & 5.66383500 & $\mathrm{H}$ & -4.09306100 & -4.68428000 & 1.91084400 \\
\hline $\mathrm{H}$ & -5.30709200 & -4.54118700 & 4.07260400 & $\mathrm{~N}$ & -0.44369800 & -1.18903700 & 2.18790700 \\
\hline $\mathrm{H}$ & -0.48051100 & -1.82719900 & 1.37818200 & $\mathrm{~S}$ & 4.97050700 & -1.86240700 & 1.19572500 \\
\hline $\mathrm{O}$ & 5.08249200 & -1.90460200 & 2.66212800 & $\mathrm{O}$ & 4.02302000 & -2.83494400 & 0.58242500 \\
\hline $\mathrm{O}$ & 4.84939100 & -0.49732100 & 0.59921000 & C & 6.62926000 & -2.44576500 & 0.58509200 \\
\hline $\mathrm{F}$ & 7.60352200 & -1.63174000 & 1.01447400 & $\mathrm{~F}$ & 6.65408900 & -2.46374000 & -0.75782500 \\
\hline $\mathrm{F}$ & 6.88464600 & -3.68558400 & 1.02737200 & C & -2.69763700 & 0.50337300 & 1.92396400 \\
\hline $\mathrm{H}$ & -2.80992500 & -0.07680400 & 1.01111400 & $\mathrm{H}$ & -3.55463000 & 0.49443300 & 2.59130600 \\
\hline C & -1.89441900 & 1.64183300 & 1.89657500 & C & -1.96533700 & 2.66048100 & 2.95132200 \\
\hline $\mathrm{H}$ & -2.40607300 & 2.26209000 & 3.87366400 & $\mathrm{H}$ & -0.97449700 & 3.07119100 & 3.17708300 \\
\hline $\mathrm{C}$ & -0.91729800 & 1.80970100 & 0.77867700 & $\mathrm{H}$ & -0.11865400 & 2.51782600 & 1.00765500 \\
\hline $\mathrm{H}$ & -0.48423700 & 0.84293200 & 0.49389900 & $\mathrm{H}$ & -1.44170100 & 2.17232200 & -0.11687300 \\
\hline C & -2.85227400 & 5.35306600 & 4.03512000 & $\mathrm{H}$ & -1.80993100 & 5.67356400 & 4.14725900 \\
\hline $\mathrm{H}$ & -3.46368000 & 6.25699000 & 3.92524100 & $\mathrm{H}$ & -3.15501300 & 4.86241900 & 4.96762900 \\
\hline
\end{tabular}




\begin{tabular}{|llll|llll|}
\hline $\mathrm{C}$ & -2.48600500 & 5.06867600 & 0.96323900 & $\mathrm{H}$ & -1.41016000 & 5.27439400 & 0.97419700 \\
\hline $\mathrm{H}$ & -2.69968500 & 4.47605500 & 0.06743200 & $\mathrm{H}$ & -3.00169800 & 6.03068200 & 0.84891100 \\
\hline $\mathrm{C}$ & -4.87025600 & 3.65766900 & 2.38967300 & $\mathrm{H}$ & -5.02622100 & 2.98646400 & 1.53783200 \\
\hline $\mathrm{H}$ & -5.21101600 & 3.14181500 & 3.29552500 & $\mathrm{H}$ & -5.52540300 & 4.52566500 & 2.24451100 \\
\hline $\mathrm{Si}$ & -3.07581600 & 4.22142900 & 2.53802000 & & & & \\
\hline
\end{tabular}

\section{TS-S-4f-nd-37}

Imaginary frequency: $-277.78 \mathrm{~cm}^{-1}$ E[B3LYP/6-31G(d)]: -4788.673374 Hartree

E[PCM(Et $\left.\left.{ }_{2} \mathrm{O}\right)-B 3 L Y P / 6-311 G(d, p)-D 3(B J)\right]: ~-4790.212091$ Hartree

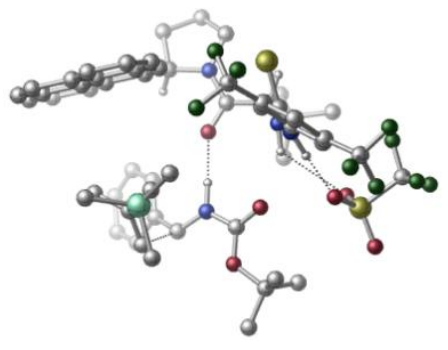

\begin{tabular}{|c|c|c|c|c|c|c|c|}
\hline 01 & & & & $\mathrm{~N}$ & -2.51857100 & 0.48749600 & 0.89001900 \\
\hline $\mathrm{H}$ & -3.00538100 & -0.31666400 & 0.45520100 & C & -3.07347900 & 1.73281600 & 0.53972600 \\
\hline $\mathrm{C}$ & -4.41372100 & 1.76660700 & 0.12125100 & C & -2.32115600 & 2.91345200 & 0.51327800 \\
\hline C & -4.98366800 & 2.97038400 & -0.29103300 & $\mathrm{H}$ & -4.99294300 & 0.84978700 & 0.10801900 \\
\hline C & -2.91085700 & 4.10954700 & 0.10524000 & $\mathrm{H}$ & -1.28360400 & 2.89254700 & 0.81251600 \\
\hline $\mathrm{C}$ & -4.24492900 & 4.15442800 & -0.29669200 & $\mathrm{H}$ & -4.69744400 & 5.08601800 & -0.61323800 \\
\hline $\mathrm{C}$ & -6.44105900 & 3.01240600 & -0.67115800 & C & -2.05793700 & 5.34429100 & 0.03191500 \\
\hline $\mathrm{F}$ & -7.22550300 & 3.29460100 & 0.39536600 & $\mathrm{~F}$ & -6.86820100 & 1.84100500 & -1.17952400 \\
\hline $\mathrm{F}$ & -6.68551500 & 3.97220300 & -1.59643700 & $\mathrm{~F}$ & -1.19792600 & 5.43367400 & 1.07859200 \\
\hline $\mathrm{F}$ & -1.28236200 & 5.36082900 & -1.08760800 & $\mathrm{~F}$ & -2.78728400 & 6.47805400 & 0.01367700 \\
\hline $\mathrm{C}$ & -0.27100500 & -1.63198200 & 2.84985200 & $\mathrm{H}$ & -0.48061500 & -1.08000200 & 3.76483000 \\
\hline C & -0.56522000 & -3.14806100 & 3.14584000 & C & -0.42857300 & -4.05107200 & 1.90475700 \\
\hline $\mathrm{H}$ & 0.57451900 & -3.99155000 & 1.46998400 & $\mathrm{H}$ & -1.17275500 & -3.81253200 & 1.14128500 \\
\hline $\mathrm{H}$ & -0.60451300 & -5.09210800 & 2.20093800 & $\mathrm{C}$ & -2.00259800 & -3.25220200 & 3.69789700 \\
\hline $\mathrm{H}$ & -2.21225000 & -4.28976800 & 3.98362500 & $\mathrm{H}$ & -2.74787200 & -2.96010100 & 2.95501400 \\
\hline $\mathrm{H}$ & -2.12975800 & -2.62603900 & 4.59001400 & $\mathrm{C}$ & 0.41433900 & -3.65045100 & 4.22748500 \\
\hline $\mathrm{H}$ & 0.20673700 & -4.70373700 & 4.44548800 & $\mathrm{H}$ & 0.30282000 & -3.10011800 & 5.16949200 \\
\hline $\mathrm{H}$ & 1.46179800 & -3.58386900 & 3.90842500 & C & 1.19131700 & -1.34392800 & 2.42974700 \\
\hline $\mathrm{O}$ & 1.59887000 & -1.61584700 & 1.28311500 & $\mathrm{C}$ & 1.78226600 & -0.44808100 & 4.74193300 \\
\hline $\mathrm{C}$ & 3.44374700 & -0.50614800 & 2.96787400 & $\mathrm{C}$ & 3.12953300 & -0.75829200 & 5.39773300 \\
\hline $\mathrm{H}$ & 1.51112500 & 0.61020100 & 4.83597400 & $\mathrm{H}$ & 0.96880300 & -1.04113600 & 5.15127900 \\
\hline $\mathrm{H}$ & 3.82359700 & -1.40506800 & 2.47314000 & $\mathrm{H}$ & 3.21438900 & -1.83778500 & 5.56967900 \\
\hline $\mathrm{H}$ & 3.25214200 & -0.25181200 & 6.35965100 & $\mathrm{~N}$ & 2.03349600 & -0.77488100 & 3.32684800 \\
\hline C & 3.63335300 & 0.69629200 & 2.04788300 & C & 2.66154200 & 1.64746800 & 1.93061200 \\
\hline C & 4.90131800 & 0.87069900 & 1.35957200 & C & 2.84210000 & 2.84145800 & 1.15436800 \\
\hline $\mathrm{H}$ & 1.70374600 & 1.51315900 & 2.42729500 & C & 5.10004800 & 2.04740000 & 0.56357000 \\
\hline C & 5.95345900 & -0.06157500 & 1.43307100 & C & 1.84237600 & 3.82518200 & 1.06224200 \\
\hline C & 4.07465200 & 3.03484900 & 0.46510700 & C & 6.33464200 & 2.24534200 & -0.13184200 \\
\hline $\mathrm{H}$ & 5.84658300 & -0.95242800 & 2.04211600 & $\mathrm{C}$ & 7.15189100 & 0.13783100 & 0.74670400 \\
\hline C & 2.04600100 & 4.97705500 & 0.30168700 & $\mathrm{H}$ & 0.90814400 & 3.68401600 & 1.59848100 \\
\hline C & 4.27551900 & 4.21680900 & -0.31276900 & C & 7.34501200 & 1.27564900 & -0.02958200 \\
\hline C & 6.50686900 & 3.43983700 & -0.91427900 & $\mathrm{H}$ & 7.94347200 & -0.60238900 & 0.82693900 \\
\hline C & 3.24691900 & 5.17160500 & -0.37981000 & $\mathrm{H}$ & 1.25492900 & 5.71681200 & 0.23696600 \\
\hline C & 5.52721600 & 4.38174300 & -0.99924600 & $\mathrm{H}$ & 8.28383100 & 1.42841800 & -0.55618700 \\
\hline $\mathrm{H}$ & 7.45101100 & 3.57994400 & -1.43519600 & $\mathrm{H}$ & 3.39910000 & 6.07236500 & -0.96960400 \\
\hline $\mathrm{H}$ & 5.67972300 & 5.28295000 & -1.58832300 & C & -1.60559700 & 0.21581900 & 1.87108000 \\
\hline $\mathrm{S}$ & -1.08301400 & 1.34190100 & 3.03586600 & $\mathrm{~N}$ & -1.17261600 & -1.07407100 & 1.85300900 \\
\hline $\mathrm{H}$ & -1.39502300 & -1.64183600 & 1.03768200 & C & -0.26456600 & -2.73866900 & -1.71121500 \\
\hline $\mathrm{O}$ & -1.10752500 & -2.26780900 & -0.98022300 & $\mathrm{O}$ & -0.42558100 & -3.26321500 & -2.92455300 \\
\hline
\end{tabular}




\begin{tabular}{|c|c|c|c|c|c|c|c|}
\hline C & -1.79932800 & -3.57264300 & -3.47262700 & C & -2.63225700 & -2.29510400 & -3.56436700 \\
\hline $\mathrm{H}$ & -3.55864300 & -2.52272400 & -4.10199100 & $\mathrm{H}$ & -2.90679000 & -1.90284000 & -2.58502500 \\
\hline $\mathrm{H}$ & -2.09255900 & -1.52767400 & -4.13363400 & C & -1.46120200 & -4.11249200 & -4.86211700 \\
\hline $\mathrm{H}$ & -0.80533400 & -4.98710500 & -4.79584600 & $\mathrm{H}$ & -2.38366400 & -4.41489400 & -5.36723400 \\
\hline $\mathrm{H}$ & -0.97033500 & -3.34869200 & -5.47544700 & C & -2.43033100 & -4.64896600 & -2.59293500 \\
\hline $\mathrm{H}$ & -3.41771200 & -4.90476900 & -2.98874800 & $\mathrm{H}$ & -1.80955400 & -5.55252700 & -2.59189200 \\
\hline $\mathrm{H}$ & -2.58123400 & -4.30754300 & -1.56776600 & C & 2.10939700 & -3.17424700 & -2.13124000 \\
\hline $\mathrm{H}$ & 1.74445000 & -3.79408300 & -2.94299900 & C & 3.40777800 & -3.62057900 & -1.57650800 \\
\hline C & 4.18145200 & -4.49112600 & -2.36534800 & C & 3.87635400 & -3.24832800 & -0.30611500 \\
\hline C & 5.39465600 & -4.98561100 & -1.89756300 & $\mathrm{H}$ & 3.81951100 & -4.79157200 & -3.34613400 \\
\hline C & 5.09170200 & -3.75245500 & 0.15811700 & $\mathrm{H}$ & 3.29093700 & -2.58753300 & 0.32663300 \\
\hline C & 5.85323100 & -4.61623100 & -0.63026000 & $\mathrm{H}$ & 5.97589500 & -5.66508000 & -2.51403700 \\
\hline $\mathrm{H}$ & 5.44238800 & -3.47096100 & 1.14677800 & $\mathrm{H}$ & 6.79684100 & -5.00534700 & -0.25839800 \\
\hline $\mathrm{N}$ & 1.09194000 & -2.75990800 & -1.31991500 & $\mathrm{H}$ & 1.24876200 & -2.34975000 & -0.38665900 \\
\hline $\mathrm{S}$ & -4.58250600 & -2.70716300 & -0.09054100 & $\mathrm{O}$ & -5.31424400 & -3.40689300 & -1.15734200 \\
\hline $\mathrm{O}$ & -3.55043800 & -3.49385600 & 0.62701100 & $\mathrm{O}$ & -4.13200600 & -1.32446200 & -0.46110500 \\
\hline $\mathrm{C}$ & -5.84878200 & -2.35641100 & 1.22828100 & $\mathrm{~F}$ & -6.77934700 & -1.49781400 & 0.78017800 \\
\hline $\mathrm{F}$ & -5.25350900 & -1.79738000 & 2.30114800 & $\mathrm{~F}$ & -6.46253300 & -3.47812600 & 1.62598300 \\
\hline $\mathrm{C}$ & 2.50466400 & -1.73798900 & -3.49341100 & $\mathrm{H}$ & 3.25985500 & -2.24487800 & -4.08860700 \\
\hline $\mathrm{H}$ & 1.50028400 & -1.75775600 & -3.90822200 & $\mathrm{C}$ & 2.88980200 & -0.61353900 & -2.75716000 \\
\hline C & 1.89585600 & 0.28991500 & -2.19408400 & $\mathrm{H}$ & 0.94433100 & -0.21445600 & -1.98350500 \\
\hline $\mathrm{H}$ & 2.24918000 & 0.80377600 & -1.29328900 & C & 4.33311200 & -0.37449300 & -2.44794600 \\
\hline $\mathrm{H}$ & 4.57180400 & 0.69357600 & -2.45389600 & $\mathrm{H}$ & 4.53895900 & -0.72616100 & -1.42486300 \\
\hline $\mathrm{H}$ & 5.00331000 & -0.90643000 & -3.12742700 & C & 0.12282200 & 2.76518500 & -2.49827600 \\
\hline $\mathrm{H}$ & 0.58578900 & 3.25042900 & -1.63327500 & $\mathrm{H}$ & -0.28139000 & 3.55779400 & -3.13929300 \\
\hline $\mathrm{H}$ & -0.72472200 & 2.17129300 & -2.13814100 & $\mathrm{C}$ & 2.87830300 & 2.70474000 & -3.96374200 \\
\hline $\mathrm{H}$ & 3.40036300 & 3.13110500 & -3.09967900 & $\mathrm{H}$ & 3.59441300 & 2.10188200 & -4.53445100 \\
\hline $\mathrm{H}$ & 2.57335100 & 3.54094700 & -4.60532000 & $\mathrm{C}$ & 0.54894400 & 0.90885400 & -4.95347600 \\
\hline $\mathrm{H}$ & 1.24758100 & 0.29033400 & -5.52789700 & $\mathrm{H}$ & -0.30730500 & 0.28537600 & -4.66980900 \\
\hline $\mathrm{H}$ & 0.17134500 & 1.68724900 & -5.62800800 & $\mathrm{Si}$ & 1.35609000 & 1.71512900 & -3.44886800 \\
\hline $\mathrm{C}$ & 4.14757100 & -0.29119700 & 4.34559300 & $\mathrm{H}$ & 4.36766300 & 0.77259700 & 4.47741600 \\
\hline $\mathrm{H}$ & 5.09726900 & -0.83042800 & 4.40508600 & & & & \\
\hline
\end{tabular}

\section{TS-S-4f-nd-38}

Imaginary frequency: $-259.56 \mathrm{~cm}-1$

E[B3LYP/6-31G(d)]: -4788.682572 Hartree

E[PCM(Et $\left.\left.{ }_{2} \mathrm{O}\right)-B 3 L Y P / 6-311 G(d, p)-D 3(B J)\right]:-4790.211568$ Hartree

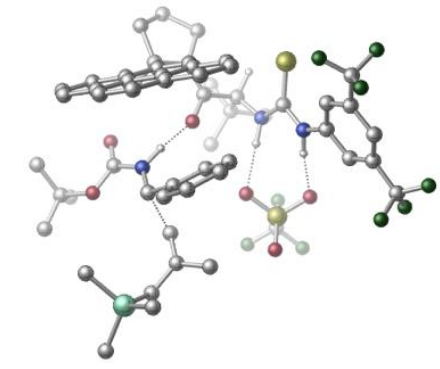

\begin{tabular}{|c|c|c|c|c|c|c|c|}
\hline 01 & & & & $\mathrm{~N}$ & 3.35351700 & 0.47359900 & 1.24394100 \\
\hline $\mathrm{H}$ & 3.09346600 & 1.46645800 & 1.30413900 & C & 4.33973900 & 0.22723200 & 0.27425900 \\
\hline C & 4.95948800 & 1.35915100 & -0.28875900 & C & 4.68645400 & -1.04355300 & -0.20960400 \\
\hline C & 5.90259900 & 1.21290600 & -1.30196700 & $\mathrm{H}$ & 4.68939600 & 2.34822100 & 0.06659600 \\
\hline $\mathrm{C}$ & 5.63292400 & -1.16554500 & -1.22735500 & $\mathrm{H}$ & 4.22947300 & -1.92305900 & 0.21685400 \\
\hline $\mathrm{C}$ & 6.25375600 & -0.04909600 & -1.78371800 & $\mathrm{H}$ & 6.99038100 & -0.15598500 & -2.57073700 \\
\hline C & 6.50516100 & 2.43826000 & -1.93808400 & C & 6.03005900 & -2.53971100 & -1.69622900 \\
\hline $\mathrm{F}$ & 6.55969900 & 3.48085600 & -1.08561500 & $\mathrm{~F}$ & 5.78789800 & 2.84227400 & -3.01408300 \\
\hline $\mathrm{F}$ & 7.76382300 & 2.20404300 & -2.37743300 & $\mathrm{~F}$ & 5.02512100 & -3.44111700 & -1.54572700 \\
\hline $\mathrm{F}$ & 6.37389500 & -2.54490900 & -3.00463500 & $\mathrm{~F}$ & 7.08856200 & -3.02439000 & -1.01137900 \\
\hline C & 0.84480300 & -0.44140300 & 3.74083800 & $\mathrm{H}$ & 1.49452300 & -1.15356000 & 4.24859700 \\
\hline $\mathrm{C}$ & 0.26643700 & 0.53948300 & 4.82524400 & $\mathrm{C}$ & -0.78725700 & 1.51728900 & 4.26655500 \\
\hline
\end{tabular}




\begin{tabular}{|c|c|c|c|c|c|c|c|}
\hline $\mathrm{H}$ & -1.67951500 & 0.99150700 & 3.91289200 & $\mathrm{H}$ & -0.40296500 & 2.11866400 & 3.43998900 \\
\hline $\mathrm{H}$ & -1.09327700 & 2.20529700 & 5.06428000 & C & 1.44349500 & 1.33331400 & 5.42854200 \\
\hline $\mathrm{H}$ & 1.08871500 & 1.93699900 & 6.27233000 & $\mathrm{H}$ & 1.89356100 & 2.01011900 & 4.69872800 \\
\hline $\mathrm{H}$ & 2.22841500 & 0.66306800 & 5.79939600 & C & -0.38885900 & -0.29040400 & 5.94875100 \\
\hline $\mathrm{H}$ & -0.80346200 & 0.38050800 & 6.70954500 & $\mathrm{H}$ & 0.33790700 & -0.94145900 & 6.45033100 \\
\hline $\mathrm{H}$ & -1.21448100 & -0.90884600 & 5.57704000 & C & -0.24784900 & -1.19087800 & 2.94728900 \\
\hline 0 & -0.82669500 & -0.58103400 & 2.03320400 & C & 0.01532700 & -3.33773700 & 4.28161300 \\
\hline C & -1.64913200 & -3.15658500 & 2.50311000 & C & -1.18449500 & -4.20040800 & 4.68230500 \\
\hline $\mathrm{H}$ & 0.83338000 & -3.94196100 & 3.86981300 & $\mathrm{H}$ & 0.41080000 & -2.75016000 & 5.10718300 \\
\hline $\mathrm{H}$ & -2.51155700 & -2.48504600 & 2.54445500 & $\mathrm{H}$ & -1.82787300 & -3.63890600 & 5.37023800 \\
\hline $\mathrm{H}$ & -0.88488800 & -5.12780900 & 5.17963900 & $\mathrm{~N}$ & -0.54952900 & -2.47665900 & 3.23023900 \\
\hline C & -1.32646400 & -3.47768500 & 1.04715300 & C & -0.03504000 & -3.54560600 & 0.61112100 \\
\hline C & -2.41352500 & -3.77995200 & 0.13134400 & C & 0.30727400 & -3.90612000 & -0.73439300 \\
\hline $\mathrm{H}$ & 0.78084300 & -3.30295100 & 1.28731900 & C & -2.09739700 & -4.14395700 & -1.21911900 \\
\hline C & -3.76979000 & -3.73253100 & 0.50488800 & C & 1.64088700 & -3.96539600 & -1.17141500 \\
\hline C & -0.73983700 & -4.20803200 & -1.65222800 & C & -3.14452400 & -4.44876200 & -2.14464200 \\
\hline $\mathrm{H}$ & -4.04580400 & -3.45580800 & 1.51607900 & C & -4.78313800 & -4.03170600 & -0.40614700 \\
\hline C & 1.94390200 & -4.31465200 & -2.48681400 & $\mathrm{H}$ & 2.43908100 & -3.73555600 & -0.47139700 \\
\hline C & -0.42208700 & -4.56900200 & -2.99712500 & C & -4.47988900 & -4.38727500 & -1.71653000 \\
\hline C & -2.79606700 & -4.81347600 & -3.49114800 & $\mathrm{H}$ & -5.82021000 & -3.99243600 & -0.08243500 \\
\hline C & 0.92568100 & -4.61506600 & -3.39056000 & $\mathrm{H}$ & 2.98230700 & -4.34756700 & -2.80215000 \\
\hline $\mathrm{C}$ & -1.49853200 & -4.87111000 & -3.89880700 & $\mathrm{H}$ & -5.27466000 & -4.62508700 & -2.41972300 \\
\hline $\mathrm{H}$ & -3.60140400 & -5.04831300 & -4.18340400 & $\mathrm{H}$ & 1.16852200 & -4.89175500 & -4.41398500 \\
\hline $\mathrm{H}$ & -1.25419400 & -5.15192300 & -4.92074500 & C & 2.74895400 & -0.35515000 & 2.15727700 \\
\hline S & 3.26060700 & -1.92756100 & 2.54029200 & $\mathrm{~N}$ & 1.69010100 & 0.24541100 & 2.77157100 \\
\hline $\mathrm{H}$ & 1.27450300 & 1.05821700 & 2.30681900 & $\mathrm{~S}$ & 1.45224700 & 3.70842400 & 1.01455100 \\
\hline 0 & 1.19835300 & 4.50950500 & -0.19556000 & $\mathrm{O}$ & 0.50315800 & 2.57047600 & 1.22790700 \\
\hline 0 & 2.86619500 & 3.32158800 & 1.26474700 & $\mathrm{C}$ & 1.06617400 & 4.87401900 & 2.41301300 \\
\hline $\mathrm{F}$ & 1.85170900 & 5.95352100 & 2.36032400 & $\mathrm{~F}$ & 1.25229900 & 4.26874600 & 3.59971500 \\
\hline $\mathrm{F}$ & -0.21458500 & 5.27034400 & 2.34437600 & C & -4.11840300 & 0.10609800 & 1.11207900 \\
\hline 0 & -4.18999100 & -0.44617900 & 2.19189900 & $\mathrm{O}$ & -5.10418300 & 0.74198400 & 0.46096100 \\
\hline $\mathrm{C}$ & -6.47940300 & 0.78504000 & 1.02623600 & $\mathrm{C}$ & -7.02386600 & -0.63872100 & 1.16997000 \\
\hline $\mathrm{H}$ & -8.08451900 & -0.59367800 & 1.44016400 & $\mathrm{H}$ & -6.49007300 & -1.19216200 & 1.94357500 \\
\hline $\mathrm{H}$ & -6.93657300 & -1.17937800 & 0.22128700 & C & -7.25290400 & 1.55752900 & -0.04284400 \\
\hline $\mathrm{H}$ & -6.82802500 & 2.55689500 & -0.18425400 & $\mathrm{H}$ & -8.29758800 & 1.67003300 & 0.26391000 \\
\hline $\mathrm{H}$ & -7.23026500 & 1.02715900 & -1.00018700 & C & -6.46609700 & 1.54950400 & 2.35222000 \\
\hline $\mathrm{H}$ & -7.49452900 & 1.67114700 & 2.70954100 & $\mathrm{H}$ & -6.03495200 & 2.54734700 & 2.21669300 \\
\hline $\mathrm{H}$ & -5.89336200 & 1.01644700 & 3.11225400 & C & -2.75040000 & 0.65021000 & -0.85981100 \\
\hline $\mathrm{H}$ & -3.67882500 & 0.78524200 & -1.40019200 & C & -1.57808100 & 0.24642100 & -1.65275500 \\
\hline $\mathrm{C}$ & -1.76866100 & -0.02867100 & -3.01902000 & $\mathrm{C}$ & -0.29571100 & 0.10819000 & -1.09198900 \\
\hline C & -0.70462800 & -0.46302300 & -3.80587100 & $\mathrm{H}$ & -2.75888700 & 0.06665700 & -3.45672700 \\
\hline C & 0.76539400 & -0.31404900 & -1.88921800 & $\mathrm{H}$ & -0.12337600 & 0.34683700 & -0.04828800 \\
\hline C & 0.56474000 & -0.60546900 & -3.24094100 & $\mathrm{H}$ & -0.86763600 & -0.69595400 & -4.85404800 \\
\hline $\mathrm{H}$ & 1.75344900 & -0.41637000 & -1.45233400 & $\mathrm{H}$ & 1.39648500 & -0.94471900 & -3.85077500 \\
\hline $\mathrm{N}$ & -2.92290300 & 0.12824200 & 0.38719300 & $\mathrm{H}$ & -2.12259300 & -0.24932800 & 0.92384400 \\
\hline C & -2.48356700 & 2.70936700 & -0.46732500 & $\mathrm{H}$ & -1.57647700 & 2.52194300 & 0.10479800 \\
\hline $\mathrm{H}$ & -3.40253700 & 2.80659800 & 0.10451500 & C & -2.36517400 & 3.45747300 & -1.63368900 \\
\hline C & -3.51607900 & 4.17491000 & -2.23156700 & $\mathrm{H}$ & -3.18525900 & 5.19647200 & -2.47238800 \\
\hline $\mathrm{H}$ & -4.34959900 & 4.25643300 & -1.52314300 & C & -1.03479100 & 3.57694600 & -2.30244500 \\
\hline $\mathrm{H}$ & -0.30052200 & 4.01376900 & -1.60870600 & $\mathrm{H}$ & -1.07461400 & 4.17462300 & -3.21554100 \\
\hline $\mathrm{H}$ & -0.64160100 & 2.58062100 & -2.54696400 & C & -5.39197900 & 5.03414200 & -4.45721200 \\
\hline $\mathrm{H}$ & -6.15111800 & 5.27074000 & -3.70215100 & $\mathrm{H}$ & -5.91855800 & 4.79244600 & -5.38842000 \\
\hline $\mathrm{H}$ & -4.80679400 & 5.94376600 & -4.63673600 & C & -5.36917700 & 2.06033400 & -3.65756500 \\
\hline
\end{tabular}




\begin{tabular}{|cccc|cccc|}
\hline $\mathrm{H}$ & -6.10127000 & 2.21555500 & -2.85730700 & $\mathrm{H}$ & -4.78624400 & 1.16685000 & -3.40867500 \\
\hline $\mathrm{H}$ & -5.92767900 & 1.83554500 & -4.57476600 & $\mathrm{C}$ & -2.97266800 & 3.25592200 & -5.22685600 \\
\hline $\mathrm{H}$ & -2.29544600 & 2.44525900 & -4.93716600 & $\mathrm{H}$ & -2.36232600 & 4.14311700 & -5.43074500 \\
\hline $\mathrm{H}$ & -3.45291700 & 2.96706800 & -6.17025500 & $\mathrm{Si}$ & -4.29170100 & 3.59370500 & -3.91898100 \\
\hline $\mathrm{C}$ & -1.90104400 & -4.44476700 & 3.34465100 & $\mathrm{H}$ & -1.45885400 & -5.30481700 & 2.83161800 \\
\hline $\mathrm{H}$ & -2.96891100 & -4.64735100 & 3.46413500 & & & & \\
\hline
\end{tabular}

\section{TS-S-4f-nd-39}

Imaginary frequency: $-271.48 \mathrm{~cm}-1$

E[B3LYP/6-31G(d)]: -4788.680405 Hartree

E[PCM(Et $\left.\left.{ }_{2} \mathrm{O}\right)-\mathrm{B} 3 L Y P / 6-311 \mathrm{G}(\mathrm{d}, \mathrm{p})-\mathrm{D} 3(\mathrm{BJ})\right]$ : -4790.211101 Hartree

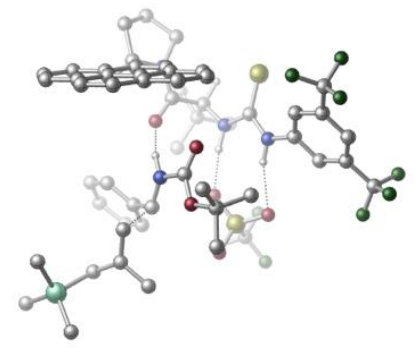

\begin{tabular}{|c|c|c|c|c|c|c|c|}
\hline 01 & & & & $\mathrm{~N}$ & -2.87722300 & -0.84254400 & 1.01695400 \\
\hline $\mathrm{H}$ & -2.33840400 & -1.69976100 & 0.88680300 & C & -4.01284500 & -0.75995300 & 0.18759300 \\
\hline $\mathrm{C}$ & -4.62391700 & -1.95778200 & -0.20683700 & C & -4.48920000 & 0.45313700 & -0.32790500 \\
\hline$C$ & -5.70486200 & -1.93438700 & -1.08925300 & $\mathrm{H}$ & -4.23192300 & -2.90290000 & 0.15199300 \\
\hline C & -5.57159500 & 0.45860100 & -1.20458700 & $\mathrm{H}$ & -4.00289800 & 1.37703500 & -0.05453800 \\
\hline $\mathrm{C}$ & -6.19433500 & -0.72929800 & -1.59101200 & $\mathrm{H}$ & -7.02646200 & -0.71788100 & -2.28446400 \\
\hline$C$ & -6.38980800 & -3.22384700 & -1.45698200 & $\mathrm{C}$ & -6.09001000 & 1.77235300 & -1.72453600 \\
\hline $\mathrm{F}$ & -7.38322700 & -3.52689800 & -0.58781700 & $\mathrm{~F}$ & -5.53739200 & -4.26960800 & -1.45848700 \\
\hline $\mathrm{F}$ & -6.95510100 & -3.15855100 & -2.68542200 & $\mathrm{~F}$ & -7.05781300 & 2.28561500 & -0.93366600 \\
\hline $\mathrm{F}$ & -5.11242100 & 2.71090400 & -1.80120700 & $\mathrm{~F}$ & -6.62105000 & 1.64902100 & -2.96273700 \\
\hline C & -0.61658800 & 0.44173500 & 3.60054500 & $\mathrm{H}$ & -1.43213400 & 0.98130700 & 4.08039700 \\
\hline $\mathrm{C}$ & 0.00848000 & -0.51129100 & 4.68017300 & C & 1.23998900 & -1.29005700 & 4.18246000 \\
\hline $\mathrm{H}$ & 2.06124400 & -0.62312200 & 3.90936200 & $\mathrm{H}$ & 1.01011600 & -1.91343100 & 3.31465400 \\
\hline $\mathrm{H}$ & 1.59048000 & -1.95443800 & 4.98258900 & C & -1.07706100 & -1.51586900 & 5.11994200 \\
\hline $\mathrm{H}$ & -0.71933400 & -2.09598100 & 5.97912800 & $\mathrm{H}$ & -1.31761400 & -2.22181700 & 4.31948400 \\
\hline $\mathrm{H}$ & -2.00277600 & -1.00812000 & 5.41584000 & C & 0.41998700 & 0.34023000 & 5.89927900 \\
\hline $\mathrm{H}$ & 0.87621200 & -0.29850000 & 6.66416300 & $\mathrm{H}$ & -0.44430400 & 0.83460800 & 6.35988600 \\
\hline $\mathrm{H}$ & 1.15717500 & 1.10819200 & 5.63416400 & C & 0.38036700 & 1.45706800 & 2.99252900 \\
\hline $\mathrm{O}$ & 1.26464200 & 1.08502500 & 2.20534400 & C & -0.60370300 & 3.39714000 & 4.32554900 \\
\hline C & 1.19481500 & 3.75203400 & 2.71540200 & C & 0.28525200 & 4.52163000 & 4.86546300 \\
\hline $\mathrm{H}$ & -1.51768300 & 3.78518400 & 3.85981500 & $\mathrm{H}$ & -0.89390500 & 2.68412800 & 5.09451200 \\
\hline $\mathrm{H}$ & 2.19835400 & 3.32822600 & 2.81851300 & $\mathrm{H}$ & 0.99345600 & 4.11198100 & 5.59603200 \\
\hline $\mathrm{H}$ & -0.29146100 & 5.30975400 & 5.35909600 & $\mathrm{~N}$ & 0.25700700 & 2.77109300 & 3.30768800 \\
\hline C & 0.93036900 & 4.05249500 & 1.24372700 & C & -0.29000900 & 3.80746500 & 0.68558800 \\
\hline C & 1.97456600 & 4.67719300 & 0.44991900 & C & -0.59952400 & 4.14963400 & -0.67219000 \\
\hline $\mathrm{H}$ & -1.06683000 & 3.32056900 & 1.26907900 & C & 1.69313000 & 5.02030700 & -0.91363300 \\
\hline C & 3.25694400 & 4.96526100 & 0.95287700 & C & -1.86271800 & 3.90011700 & -1.23110700 \\
\hline C & 0.40673000 & 4.76094200 & -1.47326700 & C & 2.70081100 & 5.63264600 & -1.72303200 \\
\hline $\mathrm{H}$ & 3.50227800 & 4.72838900 & 1.98256900 & C & 4.23377000 & 5.56050600 & 0.15453600 \\
\hline C & -2.13622800 & 4.24080900 & -2.55469100 & $\mathrm{H}$ & -2.62995200 & 3.43443700 & -0.62003500 \\
\hline C & 0.12106500 & 5.10713500 & -2.82947100 & C & 3.96436400 & 5.89212900 & -1.16908600 \\
\hline C & 2.38643300 & 5.96946900 & -3.08516400 & $\mathrm{H}$ & 5.21350800 & 5.77057100 & 0.57613900 \\
\hline C & -1.15632200 & 4.83728600 & -3.34756900 & $\mathrm{H}$ & -3.12044800 & 4.03369900 & -2.96463000 \\
\hline C & 1.15679600 & 5.72007600 & -3.61332000 & $\mathrm{H}$ & 4.72853700 & 6.36052000 & -1.78497200 \\
\hline $\mathrm{H}$ & 3.16065500 & 6.43679800 & -3.68945500 & $\mathrm{H}$ & -1.37516500 & 5.10204400 & -4.37951500 \\
\hline $\mathrm{H}$ & 0.93660100 & 5.98491500 & -4.64501100 & C & -2.46241000 & 0.03173800 & 1.99290600 \\
\hline $\mathrm{S}$ & -3.39494900 & 1.33824100 & 2.54711100 & $\mathrm{~N}$ & -1.24662200 & -0.28625200 & 2.50715400 \\
\hline $\mathrm{H}$ & -0.69918600 & -1.01108500 & 2.03895700 & C & 0.47858000 & -0.01638700 & -1.16706600 \\
\hline
\end{tabular}




\begin{tabular}{|c|c|c|c|c|c|c|c|}
\hline 0 & -0.37072700 & 0.81953200 & -0.94803800 & $\mathrm{O}$ & 0.54166700 & -0.86464600 & -2.20985200 \\
\hline C & -0.49368200 & -0.86845000 & -3.27845900 & C & -0.44057700 & 0.46588700 & -4.02580800 \\
\hline $\mathrm{H}$ & -1.12699800 & 0.43050700 & -4.87912700 & $\mathrm{H}$ & -0.73522000 & 1.29495100 & -3.37943000 \\
\hline $\mathrm{H}$ & 0.56806300 & 0.65577100 & -4.41111600 & C & -0.03771400 & -2.02944000 & -4.16429200 \\
\hline $\mathrm{H}$ & 0.00538600 & -2.95555700 & -3.58264600 & $\mathrm{H}$ & -0.74483600 & -2.16724000 & -4.98850300 \\
\hline $\mathrm{H}$ & 0.95035700 & -1.83017200 & -4.59652500 & $\mathrm{C}$ & -1.87283100 & -1.15517700 & -2.68752600 \\
\hline $\mathrm{H}$ & -2.58743000 & -1.29530400 & -3.50648200 & $\mathrm{H}$ & -1.85725800 & -2.06977900 & -2.08747800 \\
\hline $\mathrm{H}$ & -2.21675400 & -0.32760700 & -2.06782000 & C & 2.40374900 & -1.30696800 & -0.34188000 \\
\hline $\bar{H}$ & 1.88695400 & -2.20502000 & -0.66591300 & $\mathrm{C}$ & 3.29112700 & -1.48912100 & 0.84171500 \\
\hline $\mathrm{C}$ & 3.43590300 & -2.77271200 & 1.38943100 & $\mathrm{C}$ & 4.01951800 & -0.42199200 & 1.38956300 \\
\hline C & 4.29332800 & -2.98200600 & 2.46956800 & $\mathrm{H}$ & 2.84819600 & -3.59462100 & 0.99327500 \\
\hline $\mathrm{C}$ & 4.88082300 & -0.63764100 & 2.46436400 & $\bar{H}$ & 3.90371400 & 0.58010300 & 0.98602100 \\
\hline $\mathrm{C}$ & 5.02184100 & -1.91834800 & 3.00524200 & $\bar{H}$ & 4.38212600 & -3.97625800 & 2.89822300 \\
\hline $\mathrm{H}$ & 5.43636800 & 0.19640400 & 2.88421400 & $\mathrm{H}$ & 5.69045900 & -2.08359500 & 3.84574600 \\
\hline $\mathrm{N}$ & 1.58686500 & -0.18572200 & -0.33058800 & $\mathrm{H}$ & 1.55220600 & 0.39673900 & 0.51617900 \\
\hline $\mathrm{S}$ & -0.35669900 & -3.68723700 & 0.39829900 & $\mathrm{O}$ & 0.58137700 & -4.13003400 & -0.66069300 \\
\hline $\mathrm{O}$ & 0.12087900 & -2.49835700 & 1.16309200 & $\mathrm{O}$ & -1.78592200 & -3.60136700 & 0.02101700 \\
\hline C & -0.28241800 & -5.05161000 & 1.66206400 & $\mathrm{~F}$ & -0.71966600 & -6.20537100 & 1.14862700 \\
\hline $\mathrm{F}$ & -1.03481600 & -4.74040500 & 2.72791100 & $\mathrm{~F}$ & 0.98644000 & -5.22424700 & 2.07895600 \\
\hline $\mathrm{C}$ & 3.55874500 & -1.08977500 & -1.82870400 & $\bar{H}$ & 2.79005800 & -0.82955700 & -2.54984500 \\
\hline $\mathrm{H}$ & 4.18240600 & -0.26209500 & -1.49896200 & C & 4.18657400 & -2.33902800 & -2.00226800 \\
\hline $\mathrm{C}$ & 5.50369700 & -2.61248400 & -1.44445400 & $\mathrm{H}$ & 5.73915000 & -1.94887700 & -0.60420400 \\
\hline $\mathrm{H}$ & 5.61931400 & -3.65968800 & -1.13998800 & $\mathrm{C}$ & 3.42769000 & -3.45122000 & -2.64876100 \\
\hline $\mathrm{H}$ & 2.70204300 & -3.85295000 & -1.91898200 & $\mathrm{H}$ & 2.83150700 & -3.10473800 & -3.49780500 \\
\hline $\mathrm{H}$ & 4.07697700 & -4.27472600 & -2.95622000 & $\mathrm{C}$ & 8.52762700 & -2.76079500 & -1.79106600 \\
\hline $\mathrm{H}$ & 8.52068600 & -3.81513800 & -1.49159900 & $\mathrm{H}$ & 9.41037500 & -2.60232000 & -2.42245100 \\
\hline $\mathrm{H}$ & 8.65504300 & -2.15549800 & -0.88629900 & C & 6.72498000 & -3.38472400 & -4.24661100 \\
\hline $\mathrm{H}$ & 6.67085300 & -4.44751400 & -3.98309800 & $\mathrm{H}$ & 5.81847900 & -3.12971300 & -4.80704200 \\
\hline $\mathrm{H}$ & 7.57542100 & -3.26162700 & -4.92850500 & $\mathrm{C}$ & 6.96213600 & -0.46536100 & -3.19586600 \\
\hline $\mathrm{H}$ & 6.04392900 & -0.17025300 & -3.71581800 & $\mathrm{H}$ & 7.07244500 & 0.18049000 & -2.31690500 \\
\hline $\mathrm{H}$ & 7.80287000 & -0.25067200 & -3.86686000 & $\mathrm{Si}$ & 6.95744200 & -2.29149900 & -2.72659300 \\
\hline C & 1.02214000 & 5.01597600 & 3.61126700 & $\mathrm{H}$ & 0.41111700 & 5.75345100 & 3.08112100 \\
\hline $\mathrm{H}$ & 1.98214500 & 5.48866600 & 3.83739000 & & & & \\
\hline
\end{tabular}

\section{TS-S-4f-nd-40}

Imaginary frequency: $-229.44 \mathrm{~cm}^{-1}$

E[B3LYP/6-31G(d)]: -4788.681679 Hartree

$\mathrm{E}\left[\mathrm{PCM}\left(\mathrm{Et}_{2} \mathrm{O}\right)-\mathrm{B} 3 \mathrm{LYP} / 6-311 \mathrm{G}(\mathrm{d}, \mathrm{p})-\mathrm{D} 3(\mathrm{BJ})\right]:-4790.210653$ Hartree

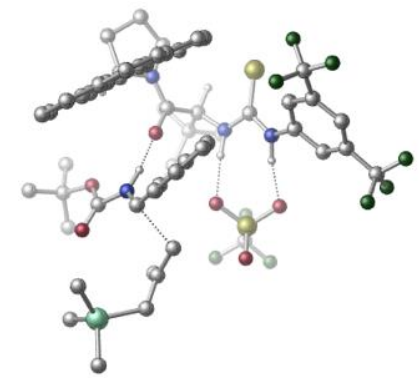

\begin{tabular}{|cccc|cccc|}
\hline 01 & & & N & -2.88455800 & -1.60730900 & 1.15539200 \\
\hline H & -2.34581800 & -2.46572800 & 0.97874800 & C & -3.96172400 & -1.42718700 & 0.27398400 \\
\hline C & -4.47348100 & -2.56481100 & -0.37200700 & C & -4.46762000 & -0.16657100 & -0.06913500 \\
\hline C & -5.47634400 & -2.43288200 & -1.33020000 & H & -4.07190200 & -3.54262300 & -0.12943900 \\
\hline C & -5.45874300 & -0.05340300 & -1.04320500 & H & -4.08353400 & 0.71843700 & 0.41846100 \\
\hline C & -5.98162900 & -1.17863700 & -1.67816600 & H & -6.76282700 & -1.08396400 & -2.42207100 \\
\hline C & -5.98209400 & -3.65492600 & -2.05179800 & C & -5.87992800 & 1.32598400 & -1.46819600 \\
\hline F & -7.28433400 & -3.52501400 & -2.39737300 & F & -5.87003900 & -4.77066800 & -1.30271000 \\
\hline F & -5.29616800 & -3.87512000 & -3.19770000 & F & -6.03249400 & 2.16567400 & -0.41752700 \\
\hline F & -4.94162800 & 1.90172700 & -2.27468400 & F & -7.03753100 & 1.32633100 & -2.15872200 \\
\hline C & -0.49052500 & -0.30244600 & 3.59574700 & H & -1.26629000 & 0.08329600 & 4.25617100 \\
\hline
\end{tabular}




\begin{tabular}{|c|c|c|c|c|c|c|c|}
\hline$C$ & 0.45678700 & -1.19831000 & 4.47211200 & $\mathrm{C}$ & 1.64162400 & -1.79236000 & 3.68596700 \\
\hline $\mathrm{H}$ & 2.28246700 & -1.01112300 & 3.26788900 & $\mathrm{H}$ & 1.31440500 & -2.42899900 & 2.86154800 \\
\hline $\mathrm{H}$ & 2.24518500 & -2.41101900 & 4.36215800 & C & -0.38984600 & -2.34263700 & 5.06589900 \\
\hline $\mathrm{H}$ & 0.21817200 & -2.93318600 & 5.76151000 & $\mathrm{H}$ & -0.75727900 & -3.01586600 & 4.28769400 \\
\hline $\mathrm{H}$ & -1.25480900 & -1.95553500 & 5.61775000 & C & 1.01106500 & -0.34433600 & 5.63090800 \\
\hline $\mathrm{H}$ & 1.66378600 & -0.95623700 & 6.26386000 & $\mathrm{H}$ & 0.20746400 & 0.04349300 & 6.26823600 \\
\hline $\mathrm{H}$ & 1.60669800 & 0.50364500 & 5.27098400 & C & 0.23702900 & 0.87330900 & 2.90334100 \\
\hline C & -0.73468700 & 2.53912600 & 4.55284000 & C & 0.88832700 & 3.24048000 & 2.82926800 \\
\hline $\mathrm{H}$ & -1.74821100 & 2.13592200 & 4.47363600 & $\mathrm{H}$ & -0.27821300 & 2.16490500 & 5.47619200 \\
\hline $\mathrm{H}$ & 1.93093900 & 2.90768100 & 2.85268700 & $\mathrm{~N}$ & 0.07411500 & 2.13109300 & 3.38090400 \\
\hline C & 0.64664200 & 3.69182900 & 1.38322100 & C & 1.77298700 & 4.07870100 & 0.70961200 \\
\hline C & -0.65054300 & 3.86402500 & 0.74440800 & C & 1.75350600 & 4.69768100 & -0.58100300 \\
\hline $\mathrm{H}$ & 2.74611800 & 3.93735000 & 1.17740600 & C & -0.70141100 & 4.49765000 & -0.54542400 \\
\hline C & -1.86393100 & 3.42170900 & 1.30244800 & $\mathrm{C}$ & 2.92748800 & 5.10100700 & -1.23820800 \\
\hline C & 0.49185300 & 4.92090200 & -1.20322700 & C & -1.95929300 & 4.69895900 & -1.19763800 \\
\hline $\mathrm{H}$ & -1.87037700 & 2.87702100 & 2.23431900 & C & -3.08207000 & 3.60885500 & 0.64911100 \\
\hline C & 2.86629900 & 5.71910700 & -2.48563200 & $\mathrm{H}$ & 3.88892200 & 4.93129800 & -0.75884200 \\
\hline C & 0.43849600 & 5.55231400 & -2.48435900 & C & -3.13731100 & 4.24754800 & -0.58388400 \\
\hline C & -1.98558200 & 5.34763000 & -2.48032800 & $\mathrm{H}$ & -3.99169000 & 3.23337400 & 1.10778300 \\
\hline C & 1.63702600 & 5.94396500 & -3.10297900 & $\mathrm{H}$ & 3.78300300 & 6.03005900 & -2.97972200 \\
\hline C & -0.84402300 & 5.75872900 & -3.09593000 & $\mathrm{H}$ & -4.08930500 & 4.37323600 & -1.09193800 \\
\hline $\mathrm{H}$ & -2.95250900 & 5.50245100 & -2.95314300 & $\mathrm{H}$ & 1.59564200 & 6.42713000 & -4.07624500 \\
\hline $\mathrm{H}$ & -0.88392400 & 6.24828400 & -4.06610700 & $\mathrm{C}$ & -2.47252100 & -0.81118400 & 2.19634200 \\
\hline S & -3.47249700 & 0.31552500 & 2.97847600 & $\mathrm{~N}$ & -1.18741800 & -1.06202600 & 2.56635900 \\
\hline $\mathrm{H}$ & -0.60741700 & -1.61209800 & 1.92341800 & $\mathrm{~S}$ & -0.18106900 & -4.00351100 & 0.21851400 \\
\hline 0 & 0.30328000 & -4.49735600 & -1.07769300 & $\mathrm{O}$ & 0.37868500 & -2.67259200 & 0.62882700 \\
\hline 0 & -1.64109000 & -4.10670400 & 0.47788800 & C & 0.56398800 & -5.17961800 & 1.45521900 \\
\hline $\mathrm{F}$ & 0.17621500 & -6.43450200 & 1.21445700 & $\mathrm{~F}$ & 0.19946800 & -4.85280400 & 2.70820600 \\
\hline $\mathrm{F}$ & 1.90931200 & -5.12935900 & 1.39261700 & $\mathrm{C}$ & 4.02990200 & 0.15440000 & 0.08037600 \\
\hline 0 & 4.92478800 & -0.04424300 & -0.72316400 & $\mathrm{O}$ & 4.13950600 & 0.36892300 & 1.38992000 \\
\hline C & 5.46332200 & 0.42216600 & 2.06284300 & C & 6.13926400 & -0.94765300 & 1.97257800 \\
\hline $\mathrm{H}$ & 7.04819400 & -0.94362200 & 2.58399100 & $\mathrm{H}$ & 6.41692900 & -1.19062500 & 0.94544300 \\
\hline $\mathrm{H}$ & 5.47477000 & -1.72805500 & 2.35752700 & C & 5.08318600 & 0.75032800 & 3.50739900 \\
\hline $\mathrm{H}$ & 4.55007800 & 1.70508000 & 3.55988100 & $\mathrm{H}$ & 5.98737700 & 0.82479800 & 4.12012000 \\
\hline $\mathrm{H}$ & 4.44113200 & -0.02906200 & 3.92780800 & C & 6.31095200 & 1.54209200 & 1.45333800 \\
\hline $\mathrm{H}$ & 7.23137500 & 1.65463200 & 2.03644400 & $\mathrm{H}$ & 5.77012900 & 2.49415000 & 1.48948300 \\
\hline $\mathrm{H}$ & 6.57899800 & 1.32519300 & 0.41827200 & C & 2.33144700 & 0.12255900 & -1.59969000 \\
\hline $\mathrm{H}$ & 3.17004200 & 0.25801800 & -2.26989500 & C & 1.02457600 & 0.58913300 & -2.06926400 \\
\hline C & 0.95305500 & 1.12681200 & -3.36818500 & C & -0.14271800 & 0.49981500 & -1.29029200 \\
\hline $\mathrm{C}$ & -0.25880000 & 1.58641600 & -3.87369600 & $\mathrm{H}$ & 1.85469500 & 1.19164600 & -3.97254500 \\
\hline C & -1.35134300 & 0.95253800 & -1.80804800 & $\mathrm{H}$ & -0.11022800 & 0.06007500 & -0.29908100 \\
\hline C & -1.41449200 & 1.49539700 & -3.09429700 & $\mathrm{H}$ & -0.30232700 & 2.01252900 & -4.87157000 \\
\hline $\mathrm{H}$ & -2.25391500 & 0.88208700 & -1.21304300 & $\mathrm{H}$ & -2.36864000 & 1.84118400 & -3.48010800 \\
\hline $\mathrm{N}$ & 2.68085000 & 0.20590400 & -0.29001400 & $\mathrm{H}$ & 1.98751300 & 0.33457100 & 0.46052900 \\
\hline C & 2.06300200 & -1.95291400 & -2.17375900 & $\mathrm{H}$ & 1.35874000 & -2.06268600 & -1.35467300 \\
\hline $\mathrm{H}$ & 1.63740200 & -1.76147300 & -3.15427400 & C & 3.24837400 & -2.65968500 & -2.09503000 \\
\hline $\mathrm{C}$ & 4.04039000 & -3.01588300 & -3.29813800 & $\mathrm{H}$ & 3.41127800 & -2.96719100 & -4.19557700 \\
\hline $\mathrm{H}$ & 4.37808200 & -4.05776900 & -3.19530200 & C & 3.68876700 & -3.20483400 & -0.77033100 \\
\hline $\mathrm{H}$ & 4.77754900 & -3.25709200 & -0.67951000 & $\mathrm{H}$ & 3.28987400 & -4.22546500 & -0.68157400 \\
\hline $\mathrm{H}$ & 3.26014300 & -2.64631300 & 0.06571600 & $\mathrm{C}$ & 6.25636100 & -2.98667600 & -5.34366200 \\
\hline $\mathrm{H}$ & 6.45128400 & -4.04846900 & -5.15125800 & $\mathrm{H}$ & 7.18700200 & -2.54463100 & -5.72008800 \\
\hline $\mathrm{H}$ & 5.51443800 & -2.92588000 & -6.14886000 & C & 6.97919800 & -2.23654800 & -2.42711400 \\
\hline $\mathrm{H}$ & 7.16269200 & -3.28092300 & -2.14801900 & $\mathrm{H}$ & 6.67726300 & -1.68172500 & -1.53351900 \\
\hline
\end{tabular}




\begin{tabular}{|c|c|c|c|c|c|c|c|}
\hline $\mathrm{H}$ & 7.93268400 & -1.81786400 & -2.77300000 & C & 5.33383200 & -0.27107900 & -4.18465900 \\
\hline $\mathrm{H}$ & 5.17583700 & 0.30676900 & -3.26855700 & $\mathrm{H}$ & 4.45994800 & -0.15175000 & -4.83690500 \\
\hline $\mathrm{H}$ & 6.19174600 & 0.16834900 & -4.70872600 & $\mathrm{Si}$ & 5.67479100 & -2.08683200 & -3.78281100 \\
\hline $\mathrm{C}$ & 0.67279700 & 4.37198700 & 3.87295500 & $\mathrm{H}$ & 0.72692600 & 5.36191000 & 3.41431300 \\
\hline $\mathrm{H}$ & 1.46231000 & 4.31144200 & 4.63205500 & C & -0.69069500 & 4.07109700 & 4.51001200 \\
\hline $\bar{H}$ & -0.79966700 & 4.50689100 & 5.50754200 & $\mathrm{H}$ & -1.49955200 & 4.46048000 & 3.88490200 \\
\hline $\mathrm{O}$ & 0.95382200 & 0.66134500 & 1.91352400 & & & & \\
\hline
\end{tabular}

\section{TS-S-4f-nd-41}

Imaginary frequency: $-229.15 \mathrm{~cm}^{-1}$ E[B3LYP/6-31G(d)]: -4788.680214 Hartree $\mathrm{E}\left[\mathrm{PCM}\left(\mathrm{Et}_{2} \mathrm{O}\right)-\mathrm{B} 3 \mathrm{LYP} / 6-311 \mathrm{G}(\mathrm{d}, \mathrm{p})-\mathrm{D} 3(\mathrm{BJ})\right]$ : -4790.209966 Hartree

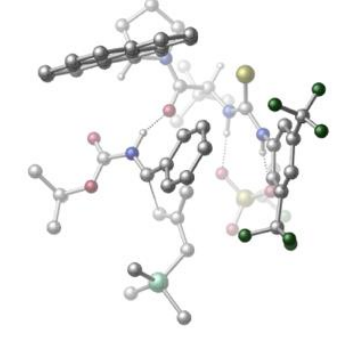

\begin{tabular}{|c|c|c|c|c|c|c|c|}
\hline 01 & & & & $\mathrm{~N}$ & -1.82539400 & 2.27519800 & 1.67877600 \\
\hline $\mathrm{H}$ & -2.68324000 & 1.71321700 & 1.72316400 & C & -1.80438200 & 3.11793800 & 0.54880000 \\
\hline $\mathrm{C}$ & -2.69311600 & 2.78975700 & -0.48917700 & $\mathrm{C}$ & -0.96803500 & 4.23344300 & 0.39277400 \\
\hline C & -2.68094900 & 3.50481700 & -1.68356600 & $\mathrm{H}$ & -3.39120800 & 1.97072200 & -0.34986000 \\
\hline C & -0.97462000 & 4.93800600 & -0.81315700 & $\mathrm{H}$ & -0.31172700 & 4.53175400 & 1.19664000 \\
\hline $\mathrm{C}$ & -1.81369700 & 4.58069100 & -1.86775800 & $\mathrm{H}$ & -1.80002800 & 5.13016200 & -2.80046200 \\
\hline C & -3.55893700 & 3.01862700 & -2.80300700 & C & 0.02194700 & 6.04783800 & -1.00448500 \\
\hline $\mathrm{F}$ & -4.83011200 & 2.81612700 & -2.40956500 & $\mathrm{~F}$ & -3.11147600 & 1.81791800 & -3.28481000 \\
\hline $\mathrm{F}$ & -3.58163300 & 3.86792500 & -3.85093500 & $\mathrm{~F}$ & 0.26361500 & 6.72613000 & 0.13453100 \\
\hline $\mathrm{F}$ & 1.22544900 & 5.56193600 & -1.42741100 & $\mathrm{~F}$ & -0.37716800 & 6.94004100 & -1.93664700 \\
\hline C & -0.00059200 & -0.01534600 & 3.88105300 & $\mathrm{H}$ & 0.41667800 & 0.71937100 & 4.57405600 \\
\hline C & -0.64498600 & -1.17684900 & 4.73840700 & $\mathrm{C}$ & -1.03889400 & -2.40685300 & 3.89372600 \\
\hline $\mathrm{H}$ & -0.16714400 & -2.86470100 & 3.41477500 & $\mathrm{H}$ & -1.77072900 & -2.16614900 & 3.12154400 \\
\hline $\mathrm{H}$ & -1.49157500 & -3.15792000 & 4.55261900 & $\mathrm{C}$ & -1.88764500 & -0.61867500 & 5.46661600 \\
\hline $\mathrm{H}$ & -2.25441100 & -1.36457200 & 6.18173800 & $\mathrm{H}$ & -2.70832000 & -0.38982900 & 4.78418100 \\
\hline $\mathrm{H}$ & -1.64388700 & 0.29166900 & 6.02778400 & $\mathrm{C}$ & 0.35432900 & -1.64572500 & 5.81563800 \\
\hline $\mathrm{H}$ & -0.11172200 & -2.42967100 & 6.42277400 & $\mathrm{H}$ & 0.63558000 & -0.83195000 & 6.49496900 \\
\hline $\mathrm{H}$ & 1.26299000 & -2.07335000 & 5.37862100 & $\mathrm{C}$ & 1.11746500 & -0.52006200 & 2.93168000 \\
\hline $\mathrm{C}$ & 3.43333000 & -1.27821700 & 2.59683600 & $\mathrm{H}$ & 2.96628800 & -2.18935200 & 2.21559900 \\
\hline $\mathrm{N}$ & 2.40249500 & -0.53892800 & 3.35504400 & $\mathrm{C}$ & 4.06147600 & -0.49841000 & 1.44441400 \\
\hline C & 3.95159700 & 0.86026300 & 1.36665600 & $\mathrm{C}$ & 4.86275100 & -1.21302200 & 0.46246600 \\
\hline $\mathrm{C}$ & 4.62771000 & 1.63513500 & 0.36540400 & $\mathrm{H}$ & 3.31870100 & 1.39217800 & 2.07103300 \\
\hline $\mathrm{C}$ & 5.54300100 & -0.46189200 & -0.55117600 & $\mathrm{C}$ & 5.00116100 & -2.61341000 & 0.45684100 \\
\hline C & 4.52662700 & 3.03524000 & 0.31511300 & $\mathrm{C}$ & 5.43301900 & 0.95999900 & -0.59634100 \\
\hline $\mathrm{C}$ & 6.34412600 & -1.13393500 & -1.52662700 & $\mathrm{H}$ & 4.48923100 & -3.21543000 & 1.19849300 \\
\hline $\mathrm{C}$ & 5.78145100 & -3.25899800 & -0.50272700 & $\mathrm{C}$ & 5.20312900 & 3.76134800 & -0.66433900 \\
\hline $\mathrm{H}$ & 3.91117700 & 3.54802900 & 1.049 & $\mathrm{C}$ & 6.11978100 & 1.71051700 & -1.59879600 \\
\hline C & 6.44975400 & -2.53282100 & -1.48274500 & C & 7.02288800 & -0.35368200 & -2.52543000 \\
\hline $\mathrm{H}$ & 5.86799000 & -4.34210000 & -0.47832400 & $\mathrm{C}$ & 5.99019700 & 3.10895000 & -1.61171000 \\
\hline $\mathrm{H}$ & 5.11358600 & 4.84386500 & -0.68970000 & $\mathrm{C}$ & 6.91848200 & 1.00284200 & -2.56025100 \\
\hline $\mathrm{H}$ & 7.06095100 & -3.04258700 & -2.22369300 & $\mathrm{H}$ & 7.63050100 & -0.87767200 & -3.25947400 \\
\hline $\mathrm{H}$ & 6.51347700 & 3.68143500 & -2.37381000 & $\mathrm{H}$ & 7.44094000 & 1.57666400 & -3.32196200 \\
\hline $\mathrm{C}$ & -0.77276200 & 1.85030000 & 2.45934500 & $S$ & 0.68329100 & 2.69953800 & 2.66819000 \\
\hline $\mathrm{N}$ & -1.00995400 & 0.66980800 & 3.08247200 & $\mathrm{H}$ & -1.80459300 & 0.10852400 & 2.75878900 \\
\hline$S$ & -4.58249700 & -0.38649100 & 1.63443900 & $\mathrm{O}$ & -5.22166500 & -1.19399600 & 0.57933600 \\
\hline $\mathrm{O}$ & -3.28562100 & -0.93523400 & 2.13397000 & $\mathrm{O}$ & -4.51672400 & 1.08003300 & 1.39410700 \\
\hline $\mathrm{C}$ & -5.71910600 & -0.54885500 & 3.09862700 & $\mathrm{~F}$ & -6.92708100 & -0.05028800 & 2.81611200 \\
\hline $\mathrm{F}$ & -5.21715400 & 0.11892200 & 4.15207400 & $\mathrm{~F}$ & -5.85465100 & -1.83715200 & 3.44233400 \\
\hline
\end{tabular}




\begin{tabular}{|c|c|c|c|c|c|c|c|}
\hline $\mathrm{C}$ & 1.11332800 & -3.47528400 & -0.62525200 & $\mathrm{O}$ & 1.64781600 & -3.97468900 & 0.34415600 \\
\hline $\mathrm{O}$ & 0.68086500 & -4.10043200 & -1.72917200 & C & 0.93055300 & -5.55512300 & -1.92169300 \\
\hline C & 2.43837300 & -5.82112000 & -1.91407500 & $\mathrm{H}$ & 2.61996400 & -6.86830600 & -2.17950200 \\
\hline $\mathrm{H}$ & 2.87205200 & -5.63002100 & -0.93162500 & $\mathrm{H}$ & 2.94288700 & -5.19033100 & -2.65375500 \\
\hline C & 0.34253800 & -5.81247900 & -3.30932200 & $\mathrm{H}$ & -0.72797600 & -5.58538000 & -3.33028200 \\
\hline $\mathrm{H}$ & 0.47393400 & -6.86569700 & -3.57717700 & $\mathrm{H}$ & 0.84423300 & -5.20007600 & -4.06551900 \\
\hline C & 0.18489300 & -6.35357700 & -0.84995900 & $\mathrm{H}$ & 0.27957600 & -7.42364400 & -1.06485800 \\
\hline $\mathrm{H}$ & -0.88083400 & -6.10114100 & -0.85389600 & $\mathrm{H}$ & 0.59075000 & -6.15932700 & 0.14381000 \\
\hline C & 0.28716000 & -1.42032500 & -1.70592300 & $\mathrm{H}$ & -0.00474800 & -2.05916300 & -2.52900200 \\
\hline C & 0.66547800 & -0.04729200 & -2.07113800 & C & 0.50950100 & 0.33137800 & -3.41735900 \\
\hline C & 1.14810700 & 0.88912900 & -1.14023100 & C & 0.83679900 & 1.61956800 & -3.82884500 \\
\hline $\mathrm{H}$ & 0.13527600 & -0.39060100 & -4.13825000 & C & 1.45806500 & 2.17971400 & -1.55655900 \\
\hline $\mathrm{H}$ & 1.25615900 & 0.62571300 & -0.09277100 & C & 1.30248800 & 2.54895700 & -2.89611600 \\
\hline $\mathrm{H}$ & 0.71871800 & 1.90007300 & -4.87152300 & $\mathrm{H}$ & 1.80916600 & 2.90607100 & -0.83341600 \\
\hline$\overline{\mathrm{H}}$ & 1.53251900 & 3.56533300 & -3.19983200 & $\mathrm{~N}$ & 0.91811400 & -2.09075500 & -0.70310200 \\
\hline $\mathrm{H}$ & 1.16623800 & -1.60748600 & 0.17343300 & C & -1.65723200 & -0.88959700 & -0.92104100 \\
\hline $\bar{H}$ & -1.21506400 & -0.86671700 & 0.07068200 & $\bar{H}$ & -1.78421500 & 0.07041200 & -1.40907300 \\
\hline C & -2.56836800 & -1.89119200 & -1.21441800 & C & -3.58166800 & -1.71579200 & -2.28314000 \\
\hline $\mathrm{H}$ & -3.59879700 & -0.67229100 & -2.61901400 & $\bar{H}$ & -4.56109600 & -1.91147900 & -1.81936300 \\
\hline $\mathrm{C}$ & -2.62436100 & -3.12259200 & -0.36730700 & $\mathrm{H}$ & -2.79241600 & -4.02840000 & -0.95811900 \\
\hline $\mathrm{H}$ & -3.48441400 & -3.00132200 & 0.30594900 & $\mathrm{H}$ & -1.73524100 & -3.23828700 & 0.25722200 \\
\hline $\mathrm{C}$ & -5.00916800 & -2.07015800 & -4.92591000 & $\mathrm{H}$ & -5.96914100 & -2.13967500 & -4.40101900 \\
\hline $\mathrm{H}$ & -5.11076200 & -2.60879900 & -5.87591400 & $\mathrm{H}$ & -4.83648700 & -1.01305100 & -5.15929100 \\
\hline C & -3.95964800 & -4.60922400 & -3.51240800 & $\mathrm{H}$ & -4.86236800 & -4.72406300 & -2.90102500 \\
\hline $\mathrm{H}$ & -3.13591800 & -5.10432100 & -2.98549300 & $\mathrm{H}$ & -4.12467200 & -5.15934800 & -4.44737300 \\
\hline $\mathrm{C}$ & -1.96933300 & -2.63129400 & -4.83086500 & $\mathrm{H}$ & -1.13113200 & -3.08011400 & -4.28482900 \\
\hline $\mathrm{H}$ & -1.72818500 & -1.58068800 & -5.03110700 & $\mathrm{H}$ & -2.03495500 & -3.14153100 & -5.79979000 \\
\hline $\mathrm{Si}$ & -3.60392800 & -2.78822400 & -3.88733200 & $\mathrm{C}$ & 2.95870000 & 0.12524700 & 4.55550100 \\
\hline $\mathrm{H}$ & 2.46200300 & -0.23311700 & 5.45791100 & $\mathrm{H}$ & 2.80959400 & 1.20789500 & 4.48825300 \\
\hline C & 4.44422400 & -0.28448900 & 4.55031000 & $\mathrm{H}$ & 4.82839100 & -0.43108600 & 5.56378200 \\
\hline $\mathrm{H}$ & 5.05243400 & 0.48868600 & 4.07117500 & C & 4.47041600 & -1.57036500 & 3.70857300 \\
\hline $\mathrm{H}$ & 5.46010100 & -1.79981900 & 3.30666000 & $\mathrm{H}$ & 4.13361600 & -2.42803400 & 4.30381600 \\
\hline $\mathrm{O}$ & 0.79715100 & -0.96772900 & 1.81662500 & & & & \\
\hline
\end{tabular}

\section{TS-S-4f-nd-42}

Imaginary frequency: $-314.38 \mathrm{~cm}^{-1}$

E[B3LYP/6-31G(d)]: -4788.676249 Hartree

$\mathrm{E}\left[\mathrm{PCM}\left(\mathrm{Et}_{2} \mathrm{O}\right)-\mathrm{B} 3 \mathrm{LYP} / 6-311 \mathrm{G}(\mathrm{d}, \mathrm{p})-\mathrm{D} 3(\mathrm{BJ})\right]:-4790.209550$ Hartree

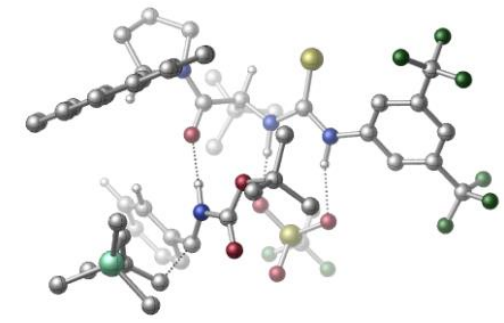

\begin{tabular}{|cccc|cccc|}
\hline 01 & & & & N & 3.34563500 & -0.27428800 & 0.63378500 \\
\hline H & 3.20507900 & 0.62571600 & 0.16440200 & C & 4.42906700 & -0.99929000 & 0.09310000 \\
\hline C & 5.37923600 & -0.26706700 & -0.63886200 & C & 4.56206600 & -2.39184800 & 0.17777800 \\
\hline C & 6.44795000 & -0.91778500 & -1.25067500 & H & 5.26113100 & 0.80516100 & -0.74753700 \\
\hline C & 5.64329400 & -3.02404800 & -0.43832900 & H & 3.82972400 & -2.97384800 & 0.71701500 \\
\hline C & 6.59711200 & -2.30152000 & -1.15171500 & H & 7.42472900 & -2.80441200 & -1.63665100 \\
\hline C & 7.49391700 & -0.11373400 & -1.97690600 & C & 5.78608900 & -4.51593400 & -0.29592100 \\
\hline F & 8.52373300 & 0.21525400 & -1.16036100 & F & 7.00001200 & 1.03868700 & -2.47184500 \\
\hline F & 8.02646500 & -0.80672700 & -3.01105100 & F & 6.39724800 & -4.85739900 & 0.86091400 \\
\hline F & 4.58416300 & -5.13921700 & -0.30032100 & F & 6.51866500 & -5.05186500 & -1.29943300 \\
\hline C & 0.83617600 & 0.51333800 & 3.17878000 & H & 1.22389300 & -0.27522800 & 3.82327100 \\
\hline C & 1.02746300 & 1.88255500 & 3.94691000 & C & 0.31751600 & 3.06847600 & 3.26986100 \\
\hline
\end{tabular}




\begin{tabular}{|c|c|c|c|c|c|c|c|}
\hline $\mathrm{H}$ & -0.76957500 & 2.94556600 & 3.27061100 & $\mathrm{H}$ & 0.63743700 & 3.20849800 & 2.23513500 \\
\hline $\mathrm{H}$ & 0.54968700 & 3.98808600 & 3.82177100 & C & 2.53999700 & 2.17663900 & 4.03142300 \\
\hline $\mathrm{H}$ & 2.71126700 & 3.02269300 & 4.70777400 & $\mathrm{H}$ & 2.95253900 & 2.44608000 & 3.05560900 \\
\hline $\mathrm{H}$ & 3.09934300 & 1.31577200 & 4.41516600 & C & 0.48026100 & 1.74229700 & 5.38211900 \\
\hline $\mathrm{H}$ & 0.61041900 & 2.68830400 & 5.92005000 & $\mathrm{H}$ & 1.01319900 & 0.96928300 & 5.94931100 \\
\hline $\mathrm{H}$ & -0.59127700 & 1.51014100 & 5.39420700 & C & -0.63005300 & 0.20559200 & 2.80763900 \\
\hline 0 & -1.16111400 & 0.71610600 & 1.81104200 & C & -0.96025100 & -1.30872000 & 4.84965200 \\
\hline C & -2.80832000 & -0.76386900 & 3.37349600 & C & -2.19457400 & -1.12453700 & 5.73745400 \\
\hline $\mathrm{H}$ & -0.76847500 & -2.37155400 & 4.65190100 & $\mathrm{H}$ & -0.05672300 & -0.88021700 & 5.27428800 \\
\hline $\mathrm{H}$ & -3.17396500 & 0.23792400 & 3.13337600 & $\mathrm{H}$ & -2.17735500 & -0.12491700 & 6.18679100 \\
\hline $\mathrm{H}$ & -2.24026100 & -1.85744300 & 6.54859100 & $\mathrm{~N}$ & -1.35456700 & -0.63232500 & 3.60337100 \\
\hline C & -3.18975100 & -1.71735900 & 2.24781300 & C & -2.33275600 & -2.69503300 & 1.83506200 \\
\hline C & -4.52697800 & -1.64738100 & 1.68354300 & C & -2.70586800 & -3.69642400 & 0.87774800 \\
\hline $\mathrm{H}$ & -1.32494000 & -2.73230600 & 2.23989500 & C & -4.92258200 & -2.62999300 & 0.71775600 \\
\hline C & -5.46158400 & -0.65792500 & 2.04159500 & C & -1.82831600 & -4.72303600 & 0.49072000 \\
\hline C & -4.01763400 & -3.65897400 & 0.32138300 & C & -6.23686700 & -2.59509300 & 0.15394300 \\
\hline $\mathrm{H}$ & -5.20102800 & 0.09466100 & 2.77753800 & C & -6.73599200 & -0.62277800 & 1.47473600 \\
\hline C & -2.22659600 & -5.69097300 & -0.43073800 & $\mathrm{H}$ & -0.83213200 & -4.75926000 & 0.92389400 \\
\hline C & -4.41790900 & -4.65413400 & -0.62193900 & C & -7.12541000 & -1.57956000 & 0.54255000 \\
\hline C & -6.61385100 & -3.61072300 & -0.79219600 & $\mathrm{H}$ & -7.43410100 & 0.15361700 & 1.77676000 \\
\hline C & -3.50516800 & -5.65835400 & -0.98397500 & $\mathrm{H}$ & -1.53448500 & -6.47793600 & -0.71690000 \\
\hline $\mathrm{C}$ & -5.74713800 & -4.59337800 & -1.16250500 & $\mathrm{H}$ & -8.12579900 & -1.55680200 & 0.11687000 \\
\hline $\mathrm{H}$ & -7.61994800 & -3.58069000 & -1.20405000 & $\mathrm{H}$ & -3.80947700 & -6.41715100 & -1.70067900 \\
\hline $\mathrm{H}$ & -6.05121100 & -5.35587600 & -1.87552600 & C & 2.60998400 & -0.50515800 & 1.77442900 \\
\hline S & 2.87406800 & -1.80282300 & 2.83758400 & $\mathrm{~N}$ & 1.64527300 & 0.43166500 & 1.97298400 \\
\hline $\mathrm{H}$ & 1.49731600 & 1.15677000 & 1.26335400 & $\mathrm{C}$ & -0.65871500 & 0.64213500 & -2.01057600 \\
\hline 0 & -0.62911100 & 0.94738000 & -3.19271800 & $\mathrm{O}$ & -0.20481900 & -0.47288100 & -1.45041100 \\
\hline C & 0.47630600 & -1.51664600 & -2.27136600 & $\mathrm{C}$ & 1.69583700 & -0.91848700 & -2.97699100 \\
\hline $\mathrm{H}$ & 2.28926400 & -1.73189900 & -3.40893500 & $\mathrm{H}$ & 1.40591500 & -0.23399800 & -3.77508900 \\
\hline $\mathrm{H}$ & 2.32767100 & -0.37535700 & -2.26871500 & C & 0.89176400 & -2.53679400 & -1.21364700 \\
\hline $\mathrm{H}$ & 0.01443600 & -2.93356400 & -0.69511900 & $\mathrm{H}$ & 1.42118800 & -3.36866100 & -1.68941700 \\
\hline $\mathrm{H}$ & 1.55646600 & -2.07888300 & -0.47772300 & C & -0.53322900 & -2.12212400 & -3.25025300 \\
\hline $\mathrm{H}$ & -0.06774100 & -2.96621500 & -3.77089500 & $\mathrm{H}$ & -1.41013700 & -2.50094700 & -2.71289700 \\
\hline $\mathrm{H}$ & -0.84966400 & -1.39109000 & -3.99718900 & C & -1.43094800 & 2.80581800 & -1.32717000 \\
\hline $\mathrm{H}$ & -0.67303800 & 3.19357000 & -2.00719800 & C & -1.78979500 & 3.72379900 & -0.22297800 \\
\hline C & -1.25814400 & 5.02328600 & -0.25450700 & C & -2.65048600 & 3.35838000 & 0.82543300 \\
\hline C & -1.58600200 & 5.94171400 & 0.73986200 & $\mathrm{H}$ & -0.55364900 & 5.29067300 & -1.03677600 \\
\hline C & -2.97953700 & 4.28407400 & 1.81516600 & $\mathrm{H}$ & -3.03873200 & 2.34621100 & 0.88339300 \\
\hline C & -2.45436900 & 5.57777300 & 1.77157100 & $\mathrm{H}$ & -1.15409900 & 6.93789600 & 0.71504600 \\
\hline $\mathrm{H}$ & -3.64136200 & 3.99247800 & 2.62622500 & $\mathrm{H}$ & -2.71170600 & 6.29542500 & 2.54571300 \\
\hline $\mathrm{N}$ & -1.25755600 & 1.46275700 & -1.04677400 & $\mathrm{H}$ & -1.21959900 & 1.15518800 & -0.06716200 \\
\hline S & 2.23094700 & 3.08767300 & -0.92164100 & $\mathrm{O}$ & 1.58925600 & 3.67092900 & -2.11597500 \\
\hline 0 & 1.26712500 & 2.60662100 & 0.11224200 & $\mathrm{O}$ & 3.33892200 & 2.12744700 & -1.15736100 \\
\hline C & 3.05055500 & 4.53457400 & -0.08775200 & $\mathrm{~F}$ & 3.96683300 & 5.09295800 & -0.88429600 \\
\hline $\mathrm{F}$ & 3.65367600 & 4.14004800 & 1.04685600 & $\mathrm{~F}$ & 2.13310800 & 5.46591000 & 0.22768300 \\
\hline C & -2.77628500 & 3.03660700 & -2.72876800 & $\mathrm{H}$ & -2.68507000 & 4.10569000 & -2.90651400 \\
\hline $\mathrm{H}$ & -2.21662000 & 2.40215600 & -3.41165700 & C & -4.00566300 & 2.56411200 & -2.25830900 \\
\hline C & -4.28898900 & 1.13221200 & -2.22140800 & $\mathrm{H}$ & -3.37083500 & 0.54880400 & -2.07764600 \\
\hline $\mathrm{H}$ & -5.02061400 & 0.86485100 & -1.44927800 & C & -5.01064700 & 3.51264800 & -1.68632600 \\
\hline $\mathrm{H}$ & -6.03113500 & 3.12747300 & -1.77287800 & $\mathrm{H}$ & -4.79471700 & 3.63099400 & -0.61188300 \\
\hline $\mathrm{H}$ & -4.94984200 & 4.50702400 & -2.13687100 & C & -5.05150300 & -1.42712900 & -3.72127300 \\
\hline $\mathrm{H}$ & -5.63794800 & -1.74668800 & -2.85332700 & $\mathrm{H}$ & -5.49396400 & -1.89042000 & -4.61199300 \\
\hline $\mathrm{H}$ & -4.04008500 & -1.83381000 & -3.60665900 & C & -6.75644500 & 1.14421800 & -4.13936400 \\
\hline
\end{tabular}




\begin{tabular}{|cccc|cccc|}
\hline $\mathrm{H}$ & -7.41067700 & 0.88876900 & -3.29733700 & $\mathrm{H}$ & -6.75343400 & 2.23522700 & -4.24694800 \\
\hline $\mathrm{H}$ & -7.21434100 & 0.73023200 & -5.04616700 & $\mathrm{C}$ & -3.89008600 & 0.95272200 & -5.33756000 \\
\hline $\mathrm{H}$ & -3.91918100 & 2.03099300 & -5.52917400 & $\mathrm{H}$ & -2.84693000 & 0.67451700 & -5.14852200 \\
\hline $\mathrm{H}$ & -4.20690300 & 0.44670700 & -6.25797100 & $\mathrm{Si}$ & -5.01454200 & 0.44675000 & -3.91241000 \\
\hline $\mathrm{C}$ & -3.36551800 & -1.25887600 & 4.74804000 & $\mathrm{H}$ & -3.67072500 & -2.30611800 & 4.66129600 \\
\hline $\mathrm{H}$ & -4.24630100 & -0.68801000 & 5.05594200 & & & & \\
\hline
\end{tabular}

\section{TS-S-4f-nd-43}

Imaginary frequency: $-274.02 \mathrm{~cm}-1$

E[B3LYP/6-31G(d)]: -4788.675627 Hartree

E[PCM(Et $\left.\left.{ }_{2} \mathrm{O}\right)-\mathrm{B} 3 L Y P / 6-311 \mathrm{G}(\mathrm{d}, \mathrm{p})-\mathrm{D} 3(\mathrm{BJ})\right]$ : -4790.209020 Hartree

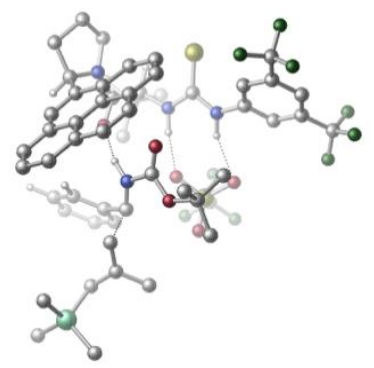

\begin{tabular}{|c|c|c|c|c|c|c|c|}
\hline 01 & & & & $\mathrm{~N}$ & -2.83048600 & -1.09146600 & 0.84660700 \\
\hline $\mathrm{H}$ & -2.17687200 & -1.85462900 & 0.66596100 & C & -3.94149400 & -1.07174000 & -0.02104300 \\
\hline C & -4.42719600 & -2.29062800 & -0.50760200 & C & -4.50479100 & 0.12640000 & -0.48167100 \\
\hline $\mathrm{C}$ & -5.48053100 & -2.30592100 & -1.42504800 & $\mathrm{H}$ & -3.96307200 & -3.21788200 & -0.19048000 \\
\hline $\mathrm{C}$ & -5.56079400 & 0.09352300 & -1.38795700 & $\mathrm{H}$ & -4.09964600 & 1.06994500 & -0.14772000 \\
\hline $\mathrm{C}$ & -6.06333200 & -1.11942700 & -1.86516100 & $\mathrm{H}$ & -6.87514200 & -1.13739700 & -2.58229900 \\
\hline $\mathrm{C}$ & -6.03099700 & -3.62644700 & -1.89412600 & C & -6.18890900 & 1.38318600 & -1.84291900 \\
\hline $\mathrm{F}$ & -6.97207700 & -4.10515500 & -1.04618100 & $\mathrm{~F}$ & -5.07109900 & -4.57064400 & -1.98657200 \\
\hline $\mathrm{F}$ & -6.61788600 & -3.52358900 & -3.10949000 & $F$ & -7.26979500 & 1.70973500 & -1.09965900 \\
\hline$F$ & -5.32885400 & 2.42862000 & -1.76131000 & $F$ & -6.60769900 & 1.31122300 & -3.12788300 \\
\hline C & -0.84612900 & 0.30433800 & 3.59925600 & $\mathrm{H}$ & -1.72626300 & 0.75909200 & 4.05087200 \\
\hline $\mathrm{C}$ & -0.20474500 & -0.61677500 & 4.69556900 & $\mathrm{C}$ & 1.07062500 & -1.34360900 & 4.23116000 \\
\hline $\mathrm{H}$ & 1.86459000 & -0.64290500 & 3.96323100 & $\mathrm{H}$ & 0.88472200 & -1.98736500 & 3.36720400 \\
\hline $\mathrm{H}$ & 1.43560900 & -1.98201800 & 5.04589300 & C & -1.25767300 & -1.66621600 & 5.10752700 \\
\hline $\mathrm{H}$ & -0.88718000 & -2.25160800 & 5.95764100 & $\mathrm{H}$ & -1.46662700 & -2.36197100 & 4.28915900 \\
\hline $\mathrm{H}$ & -2.20335000 & -1.19745000 & 5.40400200 & $\mathrm{C}$ & 0.13457600 & 0.25975800 & 5.91867900 \\
\hline $\mathrm{H}$ & 0.56847900 & -0.35743500 & 6.71371500 & $\mathrm{H}$ & -0.75926500 & 0.74520800 & 6.32929100 \\
\hline $\mathrm{H}$ & 0.86790800 & 1.03803700 & 5.67339100 & $\mathrm{C}$ & 0.09571400 & 1.41259400 & 3.07481100 \\
\hline $\mathrm{O}$ & 1.08947400 & 1.13119400 & 2.38761800 & $\mathrm{C}$ & -1.25666300 & 3.19944800 & 4.27501200 \\
\hline C & 0.72162100 & 3.77734200 & 2.91898900 & C & -1.21679600 & 4.72181700 & 4.09417800 \\
\hline $\mathrm{H}$ & -2.22566000 & 2.76588800 & 4.01250600 & $\mathrm{H}$ & -1.01819400 & 2.92415500 & 5.30995100 \\
\hline $\mathrm{C}$ & 0.24959200 & 5.00883600 & 3.74162200 & $\mathrm{H}$ & 1.72697300 & 3.48597100 & 3.23853600 \\
\hline $\mathrm{H}$ & -1.54667600 & 5.24451900 & 4.99699400 & $\mathrm{H}$ & -1.87424400 & 5.02632600 & 3.27477200 \\
\hline $\bar{H}$ & 0.84947500 & 5.07488200 & 4.65737900 & $\mathrm{H}$ & 0.38492000 & 5.94148900 & 3.18883000 \\
\hline $\mathrm{N}$ & -0.19950300 & 2.70614200 & 3.36262000 & $\mathrm{C}$ & 0.84226400 & 4.04198600 & 1.41320200 \\
\hline C & 2.10337000 & 4.35237100 & 0.98234100 & C & -0.26194800 & 4.09777900 & 0.46608900 \\
\hline C & 2.40555100 & 4.76764200 & -0.35370500 & $\mathrm{H}$ & 2.93205900 & 4.30339600 & 1.68720300 \\
\hline C & 0.01177700 & 4.51750700 & -0.88090300 & C & -1.58562200 & 3.74316300 & 0.78198100 \\
\hline C & 3.70947400 & 5.09405500 & -0.76226700 & C & 1.33585600 & 4.85635100 & -1.29061600 \\
\hline C & -1.04844000 & 4.59279000 & -1.83909000 & $\mathrm{H}$ & -1.82762400 & 3.35276000 & 1.75837300 \\
\hline C & -2.60972600 & 3.81819300 & -0.16047200 & $\mathrm{C}$ & 3.96255200 & 5.50348000 & -2.07005300 \\
\hline $\mathrm{H}$ & 4.52188300 & 5.03100400 & -0.04159700 & C & 1.60558900 & 5.27375600 & -2.63126700 \\
\hline C & -2.35194300 & 4.24282400 & -1.45752900 & $\mathrm{C}$ & -0.75083200 & 5.02123000 & -3.17861700 \\
\hline $\mathrm{H}$ & -3.61578200 & 3.52630400 & 0.12480000 & $C$ & 2.92421400 & 5.59232000 & -2.99573200 \\
\hline $\mathrm{H}$ & 4.97584100 & 5.75851400 & -2.36991100 & C & 0.51362500 & 5.34846900 & -3.56036900 \\
\hline $\mathrm{H}$ & -3.15542400 & 4.28677100 & -2.18841100 & $\mathrm{H}$ & -1.57198500 & 5.07833700 & -3.88935000 \\
\hline $\mathrm{H}$ & 3.12733300 & 5.91269200 & -4.01492900 & $\mathrm{H}$ & 0.71870300 & 5.67133000 & -4.57836100 \\
\hline C & -2.58883800 & -0.27261600 & 1.92306700 & $S$ & -3.74962900 & 0.79204500 & 2.56139400 \\
\hline
\end{tabular}




\begin{tabular}{|c|c|c|c|c|c|c|c|}
\hline $\mathrm{N}$ & -1.35038700 & -0.44052700 & 2.45252100 & $\mathrm{H}$ & -0.69117400 & -1.03875200 & 1.95071500 \\
\hline C & 0.67576400 & 0.25439100 & -1.10230700 & 0 & -0.20897500 & 1.06027900 & -0.91471500 \\
\hline $\mathrm{O}$ & 0.82588200 & -0.56581400 & -2.15820300 & $\mathrm{C}$ & -0.15554000 & -0.57906600 & -3.27683000 \\
\hline $\mathrm{C}$ & -0.11442900 & 0.77476800 & -3.98912500 & $\mathrm{H}$ & -0.75761500 & 0.73758800 & -4.87541300 \\
\hline $\mathrm{H}$ & -0.46744800 & 1.57602600 & -3.33727700 & $\mathrm{H}$ & 0.90403100 & 1.00916200 & -4.31997900 \\
\hline C & 0.38411000 & -1.70066100 & -4.16620300 & $\mathrm{H}$ & 0.43804200 & -2.63811400 & -3.60409600 \\
\hline $\mathrm{H}$ & -0.27956100 & -1.84533800 & -5.02470900 & $\mathrm{H}$ & 1.38204100 & -1.45360000 & -4.54810800 \\
\hline C & -1.55061300 & -0.92844700 & -2.76008900 & $\mathrm{H}$ & -2.22474700 & -1.05140300 & -3.61529700 \\
\hline $\mathrm{H}$ & -1.53384600 & -1.86932100 & -2.20219000 & $\mathrm{H}$ & -1.94498100 & -0.13895300 & -2.12036200 \\
\hline C & 2.60693300 & -0.98108500 & -0.20431300 & $\mathrm{H}$ & 2.15980900 & -1.88831200 & -0.59734800 \\
\hline C & 3.42370100 & -1.16691400 & 1.02785900 & C & 3.60648000 & -2.46331600 & 1.53197100 \\
\hline C & 4.04546500 & -0.08552100 & 1.67099000 & C & 4.39410100 & -2.67112900 & 2.66424700 \\
\hline $\mathrm{H}$ & 3.10151900 & -3.29918800 & 1.05896900 & C & 4.83735100 & -0.29897400 & 2.79815000 \\
\hline $\mathrm{H}$ & 3.90016300 & 0.92495500 & 1.29869400 & C & 5.01515000 & -1.59240100 & 3.29636300 \\
\hline $\mathrm{H}$ & 4.51153200 & -3.67687100 & 3.05768400 & $\mathrm{H}$ & 5.30963100 & 0.54567400 & 3.29217300 \\
\hline $\mathrm{H}$ & 5.62881000 & -1.75631500 & 4.17801500 & $\mathrm{~N}$ & 1.73655000 & 0.09822700 & -0.20380400 \\
\hline $\mathrm{H}$ & 1.62005400 & 0.64302900 & 0.65880000 & $S$ & -0.01434200 & -3.59880200 & 0.20343800 \\
\hline 0 & 1.02784100 & -3.93056900 & -0.79726800 & 0 & 0.30576200 & -2.39679200 & 1.02684800 \\
\hline 0 & -1.41690100 & -3.62345800 & -0.27085200 & C & 0.09090300 & -4.98965800 & 1.43636300 \\
\hline $\mathrm{F}$ & -0.21548100 & -6.15937600 & 0.86754100 & $\mathrm{~F}$ & -0.75068600 & -4.77088200 & 2.45713500 \\
\hline $\mathrm{F}$ & 1.34056000 & -5.07176500 & 1.93288600 & C & 3.84130000 & -0.64320800 & -1.60700900 \\
\hline $\mathrm{H}$ & 3.10651500 & -0.38562700 & -2.36366100 & $\mathrm{H}$ & 4.40141300 & 0.19644700 & -1.20214200 \\
\hline C & 4.53767200 & -1.85332100 & -1.79366800 & C & 5.82728100 & -2.09444100 & -1.16051200 \\
\hline $\mathrm{H}$ & 5.97499200 & -1.46302800 & -0.27656900 & $\mathrm{H}$ & 5.97165200 & -3.14905800 & -0.89650400 \\
\hline C & 3.87742100 & -2.96571600 & -2.53965000 & $\mathrm{H}$ & 4.58456300 & -3.74309000 & -2.83949900 \\
\hline $\mathrm{H}$ & 3.12530300 & -3.43500700 & -1.88010800 & $\mathrm{H}$ & 3.32244100 & -2.60555400 & -3.41073000 \\
\hline C & 8.87111900 & -2.10953300 & -1.31592800 & $\mathrm{H}$ & 8.88916700 & -3.17867600 & -1.07513500 \\
\hline $\mathrm{H}$ & 9.78458400 & -1.88207300 & -1.87881800 & $\mathrm{H}$ & 8.91601200 & -1.55224500 & -0.37323700 \\
\hline C & 7.25519900 & -2.66108000 & -3.91447700 & $\mathrm{H}$ & 7.23118800 & -3.73856100 & -3.71368800 \\
\hline $\mathrm{H}$ & 6.37482000 & -2.41082800 & -4.51707200 & $\mathrm{H}$ & 8.14007600 & -2.46498500 & -4.53247900 \\
\hline C & 7.30402600 & 0.20049500 & -2.69398800 & $\mathrm{H}$ & 6.40800400 & 0.49043800 & -3.25398500 \\
\hline $\bar{H}$ & 7.33427300 & 0.79897400 & -1.77594800 & $\mathrm{H}$ & 8.17475900 & 0.48382500 & -3.29790500 \\
\hline $\mathrm{Si}$ & 7.34478200 & -1.64826200 & -2.32521800 & & & & \\
\hline
\end{tabular}

\section{TS-S-4f-nd-44}

Imaginary frequency: $-303.92 \mathrm{~cm}^{-1}$

E[B3LYP/6-31G(d)]: -4788.683701 Hartree

$\mathrm{E}\left[\mathrm{PCM}\left(\mathrm{Et}_{2} \mathrm{O}\right)-\mathrm{B} 3 \mathrm{LYP} / 6-311 \mathrm{G}(\mathrm{d}, \mathrm{p})-\mathrm{D} 3(\mathrm{BJ})\right]:-4790.208519$ Hartree

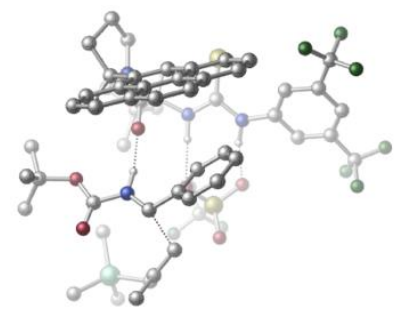

\begin{tabular}{|c|c|c|c|c|c|c|c|}
\hline 01 & & & & $\mathrm{~N}$ & -2.44604300 & -1.70306200 & 1.22337300 \\
\hline $\mathrm{H}$ & -1.83536700 & -2.46249100 & 0.89834200 & C & -3.74064300 & -1.77933700 & 0.67374300 \\
\hline C & -4.09200000 & -2.98997700 & 0.05104200 & $\mathrm{C}$ & -4.65092300 & -0.71246200 & 0.64105600 \\
\hline $\mathrm{C}$ & -5.32885100 & -3.12476400 & -0.57507600 & $\mathrm{H}$ & -3.38876600 & -3.81533900 & 0.04892800 \\
\hline C & -5.88479500 & -0.86963100 & 0.01042600 & $\mathrm{H}$ & -4.39957400 & 0.22346000 & 1.11485200 \\
\hline C & -6.24201700 & -2.07054400 & -0.60025200 & $\mathrm{H}$ & -7.20413500 & -2.18334000 & -1.08524400 \\
\hline $\mathrm{C}$ & -5.66033900 & -4.40785400 & -1.29165600 & $\mathrm{C}$ & -6.87237200 & 0.26614200 & 0.03377300 \\
\hline $\mathrm{F}$ & -5.05577800 & -5.47420100 & -0.72928800 & $\mathrm{~F}$ & -5.27043800 & -4.36857300 & -2.58743500 \\
\hline $\mathrm{F}$ & -6.99215100 & -4.64830200 & -1.29314300 & $\mathrm{~F}$ & -6.26035800 & 1.47316700 & 0.14777600 \\
\hline $\mathrm{F}$ & -7.62005600 & 0.30477900 & -1.09256700 & $\mathrm{~F}$ & -7.73239400 & 0.16940300 & 1.07091200 \\
\hline C & 0.21578500 & -0.20967400 & 3.25137900 & $\mathrm{H}$ & -0.47292400 & 0.13347300 & 4.02265600 \\
\hline $\mathrm{C}$ & 1.34439100 & -1.02764500 & 3.97490800 & $\mathrm{C}$ & 2.46643600 & -1.49479800 & 3.02977800 \\
\hline
\end{tabular}




\begin{tabular}{|c|c|c|c|c|c|c|c|}
\hline $\mathrm{H}$ & 2.98465100 & -0.65058100 & 2.56554100 & $\mathrm{H}$ & 2.08907900 & -2.13379800 & 2.22901000 \\
\hline $\mathrm{H}$ & 3.20119800 & -2.07426200 & 3.60269300 & C & 0.69316500 & -2.25637000 & 4.64236100 \\
\hline $\mathrm{H}$ & 1.43300100 & -2.77855200 & 5.26074200 & $\mathrm{H}$ & 0.31741300 & -2.96572100 & 3.90032400 \\
\hline $\mathrm{H}$ & -0.14345100 & -1.96592000 & 5.28900700 & C & 1.96483400 & -0.14174500 & 5.07472600 \\
\hline $\mathrm{H}$ & 2.75507700 & -0.69497500 & 5.59489200 & $\mathrm{H}$ & 1.22183200 & 0.15286300 & 5.82545100 \\
\hline $\mathrm{H}$ & 2.41986500 & 0.76688700 & 4.66192400 & C & 0.73184700 & 1.00358600 & 2.44334600 \\
\hline C & -0.06191100 & 2.61771400 & 4.24413900 & C & 1.04415000 & 3.42639500 & 2.20360200 \\
\hline $\mathrm{H}$ & -1.07197700 & 2.20487100 & 4.31867600 & $\mathrm{H}$ & 0.53947700 & 2.22707000 & 5.07115300 \\
\hline $\mathrm{H}$ & 2.01954200 & 3.16758100 & 1.78298600 & $\mathrm{~N}$ & 0.55493600 & 2.24951200 & 2.94847200 \\
\hline C & 0.11729300 & 3.86718500 & 1.07242200 & C & -1.17170800 & 3.42539600 & 0.99586100 \\
\hline C & 0.61386500 & 4.81219500 & 0.08545200 & C & -2.07864000 & 3.86192800 & -0.02710600 \\
\hline $\mathrm{H}$ & -1.54274700 & 2.70103200 & 1.71596800 & C & -0.26866900 & 5.25463300 & -0.95343300 \\
\hline C & 1.92653100 & 5.31957800 & 0.09881000 & C & -3.40473400 & 3.40373700 & -0.08854900 \\
\hline C & -1.61383200 & 4.78275400 & -1.00929300 & C & 0.19220500 & 6.17900900 & -1.94253900 \\
\hline $\mathrm{H}$ & 2.61768400 & 5.01851900 & 0.87886800 & C & 2.36710700 & 6.22060100 & -0.87121000 \\
\hline C & -4.26170200 & 3.83798400 & -1.09953600 & $\mathrm{H}$ & -3.76030700 & 2.70545900 & 0.66394700 \\
\hline C & -2.49664400 & 5.22476900 & -2.04112600 & C & 1.51412800 & 6.64754900 & -1.88318700 \\
\hline C & -0.71767600 & 6.60689600 & -2.97036000 & $\mathrm{H}$ & 3.38681400 & 6.59464200 & -0.83004700 \\
\hline C & -3.81451300 & 4.73826600 & -2.06565100 & $\mathrm{H}$ & -5.28100200 & 3.46526000 & -1.12985300 \\
\hline C & -2.00018500 & 6.15325100 & -3.01818600 & $\mathrm{H}$ & 1.86251100 & 7.35224700 & -2.63436700 \\
\hline $\mathrm{H}$ & -0.35516200 & 7.31182300 & -3.71486600 & $\mathrm{H}$ & -4.48818000 & 5.07608700 & -2.84977800 \\
\hline $\mathrm{H}$ & -2.67512300 & 6.49104600 & -3.80118100 & C & -1.92777400 & -0.87270900 & 2.18709200 \\
\hline S & -2.84698300 & 0.20834500 & 3.12172500 & $\mathrm{~N}$ & -0.58406000 & -1.03061800 & 2.34753400 \\
\hline $\mathrm{H}$ & -0.06789900 & -1.55146100 & 1.63175100 & S & 0.36478800 & -3.80356800 & -0.26104400 \\
\hline 0 & 0.72917400 & -4.15130600 & -1.64594000 & $\mathrm{O}$ & 0.92656600 & -2.50469000 & 0.22053600 \\
\hline 0 & -1.05794500 & -3.99231400 & 0.12040600 & C & 1.25100200 & -5.07733800 & 0.76942700 \\
\hline $\mathrm{F}$ & 0.77720200 & -6.30073200 & 0.52807700 & $\mathrm{~F}$ & 1.11322500 & -4.81091800 & 2.07838700 \\
\hline $\mathrm{F}$ & 2.57299200 & -5.07462000 & 0.48566500 & C & 4.11808900 & 0.79337600 & -0.93149800 \\
\hline 0 & 4.90144100 & 0.79942000 & -1.86618300 & $\mathrm{O}$ & 4.36442200 & 1.11024000 & 0.34106500 \\
\hline C & 5.62990400 & 1.77565400 & 0.74906300 & C & 6.82210300 & 0.84159800 & 0.53434000 \\
\hline $\mathrm{H}$ & 7.72109000 & 1.30818000 & 0.95235500 & $\mathrm{H}$ & 6.99244800 & 0.64988400 & -0.52578800 \\
\hline $\mathrm{H}$ & 6.66397500 & -0.11092700 & 1.04908800 & C & 5.39573600 & 2.02833700 & 2.23911500 \\
\hline $\mathrm{H}$ & 4.52464600 & 2.67316300 & 2.39227300 & $\mathrm{H}$ & 6.27220100 & 2.51918000 & 2.67438600 \\
\hline $\mathrm{H}$ & 5.22535400 & 1.08641800 & 2.76941100 & C & 5.77456700 & 3.09176400 & -0.01991100 \\
\hline $\mathrm{H}$ & 6.62499400 & 3.65390800 & 0.38075500 & $\mathrm{H}$ & 4.87432300 & 3.70521300 & 0.09491600 \\
\hline $\mathrm{H}$ & 5.94759800 & 2.91521800 & -1.08337900 & C & 2.22376900 & 0.37716800 & -2.32227600 \\
\hline $\mathrm{H}$ & 2.89514600 & 0.76612400 & -3.07965500 & C & 0.79119400 & 0.66673900 & -2.50624200 \\
\hline C & 0.41409600 & 1.38360300 & -3.65575700 & C & -0.19093400 & 0.25198000 & -1.59216800 \\
\hline C & -0.92101300 & 1.70778800 & -3.87416700 & $\mathrm{H}$ & 1.17341400 & 1.70119800 & -4.36698600 \\
\hline C & -1.52643100 & 0.56878100 & -1.82535000 & $\mathrm{H}$ & 0.08523500 & -0.33278900 & -0.72182800 \\
\hline C & -1.89376100 & 1.29808700 & -2.95899000 & $\mathrm{H}$ & -1.20215100 & 2.28087600 & -4.75270500 \\
\hline $\mathrm{H}$ & -2.28496300 & 0.24282300 & -1.12149500 & $\mathrm{H}$ & -2.93621400 & 1.55203400 & -3.12396200 \\
\hline $\mathrm{N}$ & 2.76878000 & 0.45458100 & -1.07386600 & $\mathrm{H}$ & 2.18369600 & 0.45829700 & -0.22727800 \\
\hline C & 2.22205100 & -1.54365600 & -3.18589800 & $\mathrm{H}$ & 1.52685400 & -2.05939600 & -2.53018700 \\
\hline $\mathrm{H}$ & 1.78688200 & -1.07851900 & -4.06447900 & C & 3.48892600 & -2.09497200 & -3.36589200 \\
\hline C & 3.94302500 & -3.26854000 & -2.59923700 & $\mathrm{H}$ & 4.34309500 & -3.98714600 & -3.33171800 \\
\hline $\mathrm{H}$ & 3.09907100 & -3.74391100 & -2.08779100 & C & 4.39828700 & -1.52764800 & -4.42057700 \\
\hline $\mathrm{H}$ & 5.02783100 & -2.29949100 & -4.87410600 & $\mathrm{H}$ & 5.06654100 & -0.78926400 & -3.95829800 \\
\hline $\mathrm{H}$ & 3.83745400 & -1.01670900 & -5.20911500 & C & 4.87173700 & -2.57670500 & 0.35067000 \\
\hline $\mathrm{H}$ & 4.63135200 & -1.51137000 & 0.33613500 & $\mathrm{H}$ & 5.67312200 & -2.72750600 & 1.08595700 \\
\hline $\mathrm{H}$ & 3.98653800 & -3.10923500 & 0.70821400 & C & 6.89666900 & -2.31004000 & -2.01480100 \\
\hline $\mathrm{H}$ & 6.67401800 & -1.24591500 & -2.14688100 & $\mathrm{H}$ & 7.22526800 & -2.71415800 & -2.97969300 \\
\hline $\mathrm{H}$ & 7.74521700 & -2.38789700 & -1.32341400 & C & 5.83927300 & -5.08718000 & -1.12390600 \\
\hline
\end{tabular}




\begin{tabular}{|c|c|c|c|c|c|c|c|}
\hline $\mathrm{H}$ & 6.17067600 & -5.53847800 & -2.06672000 & $\mathrm{H}$ & 4.97177200 & -5.65293100 & -0.76510300 \\
\hline $\mathrm{H}$ & 6.64567200 & -5.22013800 & -0.39226600 & $\mathrm{Si}$ & 5.41337000 & -3.25518200 & -1.32022600 \\
\hline C & 1.15045800 & 4.49127200 & 3.32199900 & $\mathrm{H}$ & 1.11765600 & 5.51099300 & 2.93147500 \\
\hline $\mathrm{H}$ & 2.09588700 & 4.36161000 & 3.86445300 & $\mathrm{C}$ & -0.03912000 & 4.15405200 & 4.23291100 \\
\hline $\mathrm{H}$ & 0.05964100 & 4.56833800 & 5.24031500 & $\mathrm{H}$ & -0.96503100 & 4.54399300 & 3.79827300 \\
\hline 0 & 1.27034100 & 0.83472100 & 1.33949500 & & & & \\
\hline
\end{tabular}

\section{TS-S-4f-nd-45}

Imaginary frequency: $-294.00 \mathrm{~cm}-1$

E[B3LYP/6-31G(d)]: -4788.683696 Hartree

E[PCM(Et $\left.\left.{ }_{2} \mathrm{O}\right)-B 3 L Y P / 6-311 G(d, p)-D 3(B J)\right]:-4790.207836$ Hartree

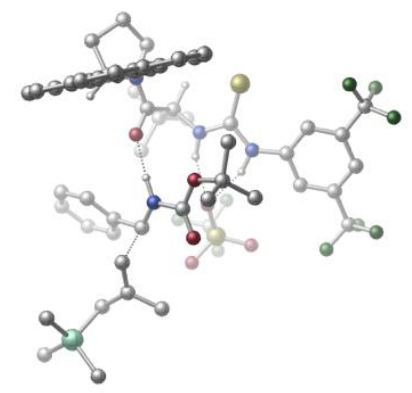

\begin{tabular}{|c|c|c|c|c|c|c|c|}
\hline 01 & & & & $\mathrm{~N}$ & 2.83551100 & -0.94354400 & 0.73865300 \\
\hline $\mathrm{H}$ & 2.70725200 & 0.07121900 & 0.68157600 & C & 3.99030600 & -1.38761100 & 0.06749500 \\
\hline C & 4.77913500 & -0.37609000 & -0.51689900 & C & 4.39051000 & -2.72463900 & -0.06895600 \\
\hline $\mathrm{C}$ & 5.93624600 & -0.70469100 & -1.21607300 & $\mathrm{H}$ & 4.48520100 & 0.66763000 & -0.42960800 \\
\hline $\mathrm{C}$ & 5.55702400 & -3.02747700 & -0.77460200 & $\mathrm{H}$ & 3.79439900 & -3.51306300 & 0.36598300 \\
\hline $\mathrm{C}$ & 6.34096300 & -2.03368800 & -1.35350100 & $\mathrm{H}$ & 7.23535700 & -2.28475400 & -1.90983100 \\
\hline $\mathrm{C}$ & 6.79501700 & 0.39410600 & -1.78836900 & $\mathrm{C}$ & 5.99477800 & -4.46621600 & -0.86120700 \\
\hline $\mathrm{F}$ & 7.71361700 & 0.82383300 & -0.89189400 & $\mathrm{~F}$ & 6.07048400 & 1.46539300 & -2.16060300 \\
\hline $\mathrm{F}$ & 7.48335600 & -0.03064000 & -2.87606000 & $\mathrm{~F}$ & 6.71103800 & -4.83790100 & 0.22549600 \\
\hline$F$ & 4.94365200 & -5.31179200 & -0.94364300 & $F$ & 6.77822800 & -4.69130400 & -1.94136400 \\
\hline C & 0.01346100 & -1.03239800 & 3.05136700 & $\mathrm{H}$ & 0.32962900 & -1.99027000 & 3.46478500 \\
\hline $\mathrm{C}$ & 0.05627600 & 0.02358400 & 4.22132900 & $\mathrm{C}$ & -0.38753800 & 1.42667800 & 3.77356200 \\
\hline $\mathrm{H}$ & -1.42633600 & 1.43611500 & 3.43548900 & $\mathrm{H}$ & 0.23635100 & 1.82387500 & 2.96876800 \\
\hline $\mathrm{H}$ & -0.29582700 & 2.12160800 & 4.61686400 & C & 1.50533900 & 0.09481100 & 4.74955600 \\
\hline $\mathrm{H}$ & 1.53725500 & 0.71256900 & 5.65490800 & $\mathrm{H}$ & 2.18429500 & 0.54572100 & 4.02044900 \\
\hline $\mathrm{H}$ & 1.89044800 & -0.89932200 & 5.00571800 & $\mathrm{C}$ & -0.85911800 & -0.43553300 & 5.37359100 \\
\hline $\mathrm{H}$ & -0.85156300 & 0.31642700 & 6.17076700 & $\mathrm{H}$ & -0.51435800 & -1.37839500 & 5.81391200 \\
\hline $\mathrm{H}$ & -1.89999800 & -0.55942500 & 5.05239600 & $\mathrm{C}$ & -1.38024800 & -1.19688200 & 2.40169500 \\
\hline $\mathrm{O}$ & -1.81669800 & -0.36249300 & 1.59415800 & $\mathrm{C}$ & -1.75776400 & -3.35004500 & 3.70801500 \\
\hline C & -3.52645400 & -2.38994200 & 2.30347700 & C & -3.03476000 & -4.20180600 & 3.81629900 \\
\hline $\mathrm{H}$ & -0.89419300 & -3.91064700 & 3.33694800 & $\mathrm{H}$ & -1.49868200 & -2.92408300 & 4.68006600 \\
\hline $\mathrm{C}$ & -4.15834000 & -3.22141400 & 3.44691600 & $\mathrm{H}$ & -3.93390400 & -1.37831500 & 2.24889900 \\
\hline $\mathrm{H}$ & -3.15240200 & -4.63185800 & 4.81502000 & $\mathrm{H}$ & -3.00687600 & -5.02680300 & 3.09785900 \\
\hline $\bar{H}$ & -4.38700500 & -2.56276900 & 4.29430500 & $\mathrm{H}$ & -5.08145900 & -3.71988000 & 3.14182200 \\
\hline $\mathrm{N}$ & -2.12758600 & -2.27433600 & 2.75736600 & $\mathrm{C}$ & -3.69779700 & -3.08183200 & 0.95431200 \\
\hline C & -2.70514600 & -3.85077300 & 0.41961600 & C & -4.97982500 & -2.98673900 & 0.27422600 \\
\hline C & -2.88240700 & -4.61540000 & -0.78255600 & $\mathrm{H}$ & -1.73447400 & -3.89599500 & 0.90603300 \\
\hline C & -5.17439700 & -3.72167900 & -0.94036900 & C & -6.04684200 & -2.20399000 & 0.75224900 \\
\hline C & -1.86907200 & -5.44232100 & -1.29505400 & C & -4.13187000 & -4.54304900 & -1.46365900 \\
\hline C & -6.42324600 & -3.64731700 & -1.63302200 & $\mathrm{H}$ & -5.93832300 & -1.63946300 & 1.67184700 \\
\hline C & -7.25936000 & -2.13328600 & 0.06626900 & $\mathrm{C}$ & -2.07563400 & -6.18076800 & -2.45953700 \\
\hline $\mathrm{H}$ & -0.91749000 & -5.49840000 & -0.77282300 & C & -4.33240600 & -5.29567400 & -2.66088300 \\
\hline C & -7.45072300 & -2.84488700 & -1.11306800 & $\mathrm{C}$ & -6.59321800 & -4.40590400 & -2.84244300 \\
\hline $\mathrm{H}$ & -8.06241000 & -1.51743700 & 0.46259200 & $C$ & -3.29053700 & -6.10737800 & -3.13839900 \\
\hline $\mathrm{H}$ & -1.28163500 & -6.81672200 & -2.84116100 & C & -5.59813500 & -5.19458300 & -3.33233100 \\
\hline $\bar{H}$ & -8.39989200 & -2.78926700 & -1.64048600 & $\mathrm{H}$ & -7.54733500 & -4.33884900 & -3.35977500 \\
\hline $\mathrm{H}$ & -3.44309000 & -6.68305600 & -4.04814300 & $\mathrm{H}$ & -5.74658200 & -5.76589800 & -4.24555400 \\
\hline C & 1.89178700 & -1.58176800 & 1.50445000 & $S$ & 1.80715200 & -3.25007700 & 1.80510900 \\
\hline
\end{tabular}




\begin{tabular}{|c|c|c|c|c|c|c|c|}
\hline $\mathrm{N}$ & 0.98932200 & -0.70468900 & 2.02598500 & $\mathrm{H}$ & 1.05266300 & 0.26845400 & 1.73621000 \\
\hline C & -0.30862600 & 0.88553000 & -1.68489500 & 0 & 0.16645700 & 1.63647800 & -2.52299900 \\
\hline $\mathrm{O}$ & -0.24353300 & -0.44267300 & -1.65482600 & $\mathrm{C}$ & 0.32134300 & -1.20232700 & -2.80661200 \\
\hline $\mathrm{C}$ & 1.78008700 & -0.81343600 & -3.05147900 & $\mathrm{H}$ & 2.20317300 & -1.48659100 & -3.80564600 \\
\hline $\mathrm{H}$ & 1.87301700 & 0.21381200 & -3.40460700 & $\mathrm{H}$ & 2.36487200 & -0.92715200 & -2.13570600 \\
\hline C & 0.21180700 & -2.64748000 & -2.32677200 & $\mathrm{H}$ & -0.82843900 & -2.90852000 & -2.11658600 \\
\hline $\mathrm{H}$ & 0.58763300 & -3.32286800 & -3.10249800 & $\mathrm{H}$ & 0.79942600 & -2.80294200 & -1.41659200 \\
\hline C & -0.56234000 & -0.95911400 & -4.03343400 & $\mathrm{H}$ & -0.22452700 & -1.59951600 & -4.85542800 \\
\hline $\mathrm{H}$ & -1.60492300 & -1.21299800 & -3.81296600 & $\mathrm{H}$ & -0.50614500 & 0.08054500 & -4.36379400 \\
\hline C & -1.06696300 & 2.71770700 & -0.34183900 & $\mathrm{H}$ & -0.09962500 & 3.18125600 & -0.50139500 \\
\hline C & -1.80698000 & 3.17539100 & 0.85727300 & C & -1.26965700 & 4.22641800 & 1.61967900 \\
\hline C & -3.04717700 & 2.62313600 & 1.22184500 & C & -1.96167800 & 4.70925800 & 2.73149900 \\
\hline $\mathrm{H}$ & -0.29616400 & 4.62919500 & 1.35626300 & C & -3.73548000 & 3.11516300 & 2.32701600 \\
\hline $\mathrm{H}$ & -3.46896800 & 1.80627400 & 0.64378500 & C & -3.19595500 & 4.16118800 & 3.08309000 \\
\hline $\mathrm{H}$ & -1.53047200 & 5.51048600 & 3.32496400 & $\mathrm{H}$ & -4.69349900 & 2.68239900 & 2.60156700 \\
\hline $\mathrm{H}$ & -3.73557700 & 4.54208900 & 3.94600900 & $\mathrm{~N}$ & -1.07306200 & 1.36260300 & -0.62045400 \\
\hline $\mathrm{H}$ & -1.44320400 & 0.69376400 & 0.06612600 & $S$ & 2.60644100 & 3.09414100 & 0.55137700 \\
\hline 0 & 1.75406000 & 4.20404900 & 0.05199400 & 0 & 1.84236400 & 1.82088400 & 0.77327100 \\
\hline 0 & 3.90526200 & 2.90877000 & -0.10923100 & C & 3.01654300 & 3.62310900 & 2.28935600 \\
\hline $\mathrm{F}$ & 3.79270800 & 4.71051300 & 2.28848200 & $\mathrm{~F}$ & 3.65570900 & 2.63708300 & 2.93927600 \\
\hline $\mathrm{F}$ & 1.88891400 & 3.90831500 & 2.97123400 & C & -2.03460300 & 3.57205400 & -1.84412800 \\
\hline $\mathrm{H}$ & -1.77788700 & 2.83127600 & -2.59289300 & $\mathrm{H}$ & -3.05262800 & 3.54298500 & -1.46568800 \\
\hline C & -1.41347300 & 4.82349200 & -1.96998400 & C & -1.95258700 & 6.01382200 & -1.31119900 \\
\hline $\mathrm{H}$ & -2.54806800 & 5.75493700 & -0.42705800 & $\mathrm{H}$ & -1.15985800 & 6.71661400 & -1.02635300 \\
\hline C & -0.10286900 & 4.93330700 & -2.67483600 & $\mathrm{H}$ & 0.02293900 & 5.91623300 & -3.13982600 \\
\hline $\mathrm{H}$ & 0.69625300 & 4.83318400 & -1.91855600 & $\mathrm{H}$ & 0.05086500 & 4.13478100 & -3.40223600 \\
\hline C & -3.65084500 & 8.54514100 & -1.39393800 & $\mathrm{H}$ & -2.77424100 & 9.15684400 & -1.15122000 \\
\hline $\mathrm{H}$ & -4.35842900 & 9.18290400 & -1.93759400 & $\mathrm{H}$ & -4.12671900 & 8.25227600 & -0.45089900 \\
\hline C & -2.31809200 & 7.57086000 & -4.02642200 & $\mathrm{H}$ & -1.40694400 & 8.14668000 & -3.82599900 \\
\hline $\mathrm{H}$ & -2.04395300 & 6.71482800 & -4.65324700 & $\mathrm{H}$ & -2.98380900 & 8.21033300 & -4.61918100 \\
\hline $\mathrm{C}$ & -4.69733800 & 5.98158400 & -2.81079200 & $\mathrm{H}$ & -4.44079500 & 5.09038700 & -3.39443700 \\
\hline $\bar{H}$ & -5.19897500 & 5.65027500 & -1.89385600 & $\mathrm{H}$ & -5.42678200 & 6.55726200 & -3.39356800 \\
\hline $\mathrm{Si}$ & -3.18162400 & 7.04099500 & -2.43429700 & & & & \\
\hline
\end{tabular}

\section{TS-S-4f-nd-46}

Imaginary frequency: $-318.06 \mathrm{~cm}^{-1}$

E[B3LYP/6-31G(d)]: -4788.672914 Hartree

E[PCM(Et $\left.\left.{ }_{2} \mathrm{O}\right)-B 3 L Y P / 6-311 G(d, p)-D 3(B J)\right]:-4790.207594$ Hartree

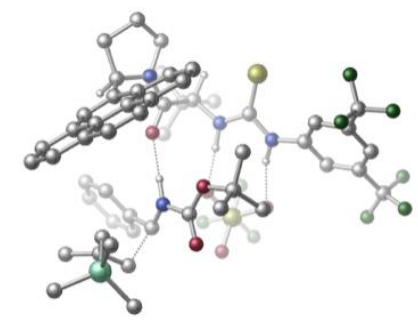

\begin{tabular}{|c|c|c|c|c|c|c|c|}
\hline 01 & & & & $\mathrm{~N}$ & 3.34826500 & -0.15377900 & 0.70169500 \\
\hline $\mathrm{H}$ & 3.25970200 & 0.76975800 & 0.26566600 & C & 4.31282600 & -0.95930300 & 0.06266600 \\
\hline C & 5.28489000 & -0.29434600 & -0.70579400 & $\mathrm{C}$ & 4.30224700 & -2.35926300 & 0.07694500 \\
\hline C & 6.22346000 & -1.02145800 & -1.43282600 & $\mathrm{H}$ & 5.28092700 & 0.78867700 & -0.75328500 \\
\hline C & 5.24248900 & -3.06938300 & -0.67119400 & $\mathrm{H}$ & 3.56608000 & -2.88905900 & 0.66321000 \\
\hline C & 6.21318800 & -2.41781400 & -1.42690400 & $\mathrm{H}$ & 6.93635900 & -2.97948400 & -2.00495800 \\
\hline C & 7.30445500 & -0.29876300 & -2.19267800 & $\mathrm{C}$ & 5.11760000 & -4.56781500 & -0.71451500 \\
\hline $\mathrm{F}$ & 8.43336200 & -0.17675800 & -1.45359100 & $\mathrm{~F}$ & 6.93236200 & 0.94508900 & -2.55343200 \\
\hline $\mathrm{F}$ & 7.65063700 & -0.96460000 & -3.31973900 & $\mathrm{~F}$ & 4.94031500 & -5.09937400 & 0.51388200 \\
\hline $\mathrm{F}$ & 4.04229900 & -4.94847600 & -1.45594300 & $\mathrm{~F}$ & 6.20004900 & -5.16081700 & -1.26206200 \\
\hline C & 0.89509300 & 0.67974800 & 3.28533600 & $\mathrm{H}$ & 1.31485100 & -0.06603300 & 3.95942600 \\
\hline $\mathrm{C}$ & 1.03226100 & 2.07574300 & 4.01148300 & C & 0.33653500 & 3.23078400 & 3.26976400 \\
\hline
\end{tabular}




\begin{tabular}{|c|c|c|c|c|c|c|c|}
\hline $\mathrm{H}$ & -0.74578800 & 3.08546200 & 3.21753100 & $\mathrm{H}$ & 0.70595700 & 3.35419800 & 2.24970400 \\
\hline $\mathrm{H}$ & 0.52449400 & 4.16705000 & 3.81042100 & C & 2.53626600 & 2.39172800 & 4.14998500 \\
\hline $\mathrm{H}$ & 2.66966000 & 3.27081300 & 4.79181600 & $\mathrm{H}$ & 2.99078200 & 2.61738600 & 3.18186700 \\
\hline $\mathrm{H}$ & 3.08553200 & 1.55662200 & 4.60043600 & C & 0.42569500 & 1.96983300 & 5.42574500 \\
\hline $\mathrm{H}$ & 0.51593400 & 2.93424000 & 5.93854200 & $\mathrm{H}$ & 0.94669400 & 1.22358400 & 6.03738700 \\
\hline $\mathrm{H}$ & -0.64116900 & 1.71650700 & 5.39916500 & C & -0.56224400 & 0.29854900 & 2.94510600 \\
\hline 0 & -1.15264900 & 0.79485700 & 1.97556600 & C & -0.62858100 & -1.29282000 & 4.93093200 \\
\hline C & -2.66742600 & -0.75224700 & 3.65066000 & C & -1.76305300 & -2.21394300 & 5.39717200 \\
\hline $\mathrm{H}$ & 0.27927800 & -1.83292500 & 4.64504500 & $\mathrm{H}$ & -0.36855800 & -0.57917800 & 5.71857400 \\
\hline C & -3.03193100 & -1.45237000 & 4.99005900 & $\mathrm{H}$ & -3.09858100 & 0.25352100 & 3.62992700 \\
\hline $\mathrm{H}$ & -1.71032900 & -2.40518400 & 6.47306300 & $\mathrm{H}$ & -1.71489000 & -3.17911200 & 4.88492100 \\
\hline $\mathrm{H}$ & -3.26427700 & -0.68471000 & 5.73815800 & $\mathrm{H}$ & -3.90942700 & -2.09544800 & 4.88939200 \\
\hline $\mathrm{N}$ & -1.20193300 & -0.58683700 & 3.76005800 & C & -3.25477400 & -1.46274800 & 2.42620100 \\
\hline C & -4.44299700 & -0.95242300 & 1.97883500 & C & -2.75074600 & -2.69410800 & 1.83612100 \\
\hline C & -5.26190500 & -1.59354800 & 0.99499300 & $\mathrm{H}$ & -4.82094200 & -0.02918900 & 2.41520200 \\
\hline C & -3.56880000 & -3.37869100 & 0.87294600 & C & -1.49883100 & -3.25532700 & 2.14849500 \\
\hline C & -6.49122700 & -1.05709100 & 0.57415600 & C & -4.82208200 & -2.83738600 & 0.45723600 \\
\hline C & -3.13219000 & -4.62148400 & 0.31421500 & $\mathrm{H}$ & -0.81771500 & -2.72845200 & 2.80018400 \\
\hline C & -1.08174700 & -4.46539900 & 1.59454800 & C & -7.28251000 & -1.73547400 & -0.35237100 \\
\hline $\mathrm{H}$ & -6.83136600 & -0.11482100 & 0.99794600 & C & -5.63828300 & -3.52561300 & -0.49306200 \\
\hline C & -1.89214700 & -5.15340300 & 0.69876800 & C & -3.97611200 & -5.29643500 & -0.63388400 \\
\hline $\mathrm{H}$ & -0.10567500 & -4.86017300 & 1.86118700 & C & -6.86390500 & -2.95680700 & -0.88006300 \\
\hline $\mathrm{H}$ & -8.23833900 & -1.31633000 & -0.65603500 & C & -5.17309900 & -4.77768600 & -1.02018900 \\
\hline $\mathrm{H}$ & -1.56459300 & -6.09943900 & 0.27490300 & $\mathrm{H}$ & -3.63048000 & -6.24347300 & -1.04119000 \\
\hline $\mathrm{H}$ & -7.48988100 & -3.48596100 & -1.59453800 & $\mathrm{H}$ & -5.79751300 & -5.30319500 & -1.73853500 \\
\hline C & 2.60422700 & -0.38424300 & 1.83500300 & $\mathrm{~S}$ & 2.80391100 & -1.73293200 & 2.85194900 \\
\hline $\mathrm{N}$ & 1.70192600 & 0.60058700 & 2.07790900 & $\mathrm{H}$ & 1.54404100 & 1.31814500 & 1.36153500 \\
\hline C & -0.64367000 & 0.60421500 & -1.90983200 & $\mathrm{O}$ & -0.55899700 & 0.93161200 & -3.08335700 \\
\hline 0 & -0.23708000 & -0.53226500 & -1.35629000 & C & 0.47119900 & -1.56445200 & -2.17161700 \\
\hline C & 1.72673800 & -0.96065800 & -2.80548800 & $\mathrm{H}$ & 2.33404800 & -1.76815900 & -3.22852700 \\
\hline $\bar{H}$ & 1.47981900 & -0.25395300 & -3.59852100 & $\mathrm{H}$ & 2.32855500 & -0.44170200 & -2.05498200 \\
\hline $\mathrm{C}$ & 0.82433500 & -2.61563000 & -1.12171200 & $\mathrm{H}$ & -0.07790900 & -3.07017500 & -0.70350300 \\
\hline $\mathrm{H}$ & 1.44066400 & -3.40202200 & -1.56972100 & $\mathrm{H}$ & 1.38890300 & -2.16211100 & -0.30386900 \\
\hline $\mathrm{C}$ & -0.49599500 & -2.13317400 & -3.21300900 & $\mathrm{H}$ & -0.02047200 & -2.98053800 & -3.71922700 \\
\hline $\bar{H}$ & -1.40861700 & -2.49960300 & -2.72901600 & $\mathrm{H}$ & -0.75635600 & -1.38553200 & -3.96512600 \\
\hline C & -1.43192600 & 2.76408800 & -1.23199000 & $\mathrm{H}$ & -0.64867500 & 3.16248300 & -1.87630100 \\
\hline $\mathrm{C}$ & -1.85032300 & 3.66821600 & -0.13853200 & C & -1.35156400 & 4.98125200 & -0.14935700 \\
\hline $\mathrm{C}$ & -2.73812900 & 3.27834700 & 0.87838500 & C & -1.73885000 & 5.88914500 & 0.83292200 \\
\hline $\mathrm{H}$ & -0.62883300 & 5.26952200 & -0.90725000 & C & -3.12571200 & 4.19349500 & 1.85684400 \\
\hline $\mathrm{H}$ & -3.09980600 & 2.25579800 & 0.92446300 & C & -2.63396900 & 5.50065400 & 1.83264300 \\
\hline $\mathrm{H}$ & -1.33285000 & 6.89642900 & 0.82421300 & $\mathrm{H}$ & -3.80718900 & 3.88281100 & 2.64419000 \\
\hline $\mathrm{H}$ & -2.93767400 & 6.21008900 & 2.59752200 & $\mathrm{~N}$ & -1.26796700 & 1.41795500 & -0.95696800 \\
\hline $\mathrm{H}$ & -1.25724300 & 1.10811300 & 0.02158500 & S & 2.24312700 & 3.18254700 & -0.86614800 \\
\hline 0 & 1.63117100 & 3.73195700 & -2.09041300 & $\mathrm{O}$ & 1.25195200 & 2.66224700 & 0.12346900 \\
\hline 0 & 3.40505200 & 2.27458500 & -1.03752500 & C & 2.95365800 & 4.66944200 & -0.00223900 \\
\hline $\mathrm{F}$ & 3.87713200 & 5.27058100 & -0.75805100 & $\mathrm{~F}$ & 3.52245300 & 4.30854300 & 1.16147100 \\
\hline $\mathrm{F}$ & 1.97884800 & 5.55675200 & 0.26831600 & $\mathrm{C}$ & -2.71406600 & 3.01348700 & -2.69391500 \\
\hline $\mathrm{H}$ & -2.63291900 & 4.08967100 & -2.82721700 & $\mathrm{H}$ & -2.11146700 & 2.41240600 & -3.37001700 \\
\hline $\mathrm{C}$ & -3.95674000 & 2.50305600 & -2.30445500 & C & -4.22126900 & 1.06857300 & -2.35082500 \\
\hline $\mathrm{H}$ & -3.30333800 & 0.48641400 & -2.20772500 & $\mathrm{H}$ & -4.97838100 & 0.74915300 & -1.62461000 \\
\hline C & -5.00068600 & 3.41124200 & -1.73690000 & $\mathrm{H}$ & -6.00953500 & 3.00883500 & -1.86782600 \\
\hline $\mathrm{H}$ & -4.81952900 & 3.50132600 & -0.65310400 & $\mathrm{H}$ & -4.94664800 & 4.42080000 & -2.15354500 \\
\hline C & -4.92776400 & -1.41381200 & -3.99622600 & $\mathrm{H}$ & -5.55724700 & -1.75900300 & -3.16889600 \\
\hline
\end{tabular}




\begin{tabular}{|c|c|c|c|c|c|c|c|}
\hline $\mathrm{H}$ & -5.33085000 & -1.83561200 & -4.92541000 & $\mathrm{H}$ & -3.92492000 & -1.83188700 & -3.85160600 \\
\hline C & -6.60142900 & 1.18199300 & -4.37830800 & $\mathrm{H}$ & -7.29386000 & 0.88956600 & -3.58002700 \\
\hline $\mathrm{H}$ & -6.59153000 & 2.27674800 & -4.43484100 & $\mathrm{H}$ & -7.01759200 & 0.81204100 & -5.32345700 \\
\hline $\mathrm{C}$ & -3.68082100 & 1.03088500 & -5.44857000 & $\mathrm{H}$ & -3.69742400 & 2.11681100 & -5.59240500 \\
\hline $\mathrm{H}$ & -2.64902500 & 0.73938300 & -5.22102200 & $\mathrm{H}$ & -3.95315100 & 0.56836200 & -6.40537000 \\
\hline $\mathrm{Si}$ & -4.87393400 & 0.46656200 & -4.10333600 & & & & \\
\hline
\end{tabular}

\section{TS-S-4f-nd-47}

Imaginary frequency: $-316.71 \mathrm{~cm}^{-1}$ E[B3LYP/6-31G(d)]: -4788.672886 Hartree E[PCM(Et $\left.\left.{ }_{2} \mathrm{O}\right)-\mathrm{B} 3 L Y P / 6-311 \mathrm{G}(\mathrm{d}, \mathrm{p})-\mathrm{D} 3(\mathrm{BJ})\right]$ : -4790.207297 Hartree

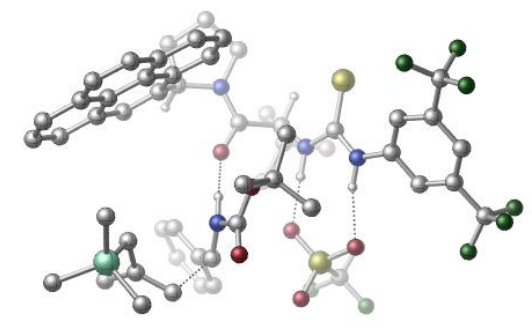

\begin{tabular}{|c|c|c|c|c|c|c|c|}
\hline 01 & & & & $\mathrm{~N}$ & 3.33685200 & -0.25451600 & 0.54438900 \\
\hline $\mathrm{H}$ & 3.18952400 & 0.68723900 & 0.16851200 & C & 4.39928500 & -0.92738400 & -0.09701300 \\
\hline C & 5.32491900 & -0.12845500 & -0.79043200 & C & 4.53530700 & -2.32112500 & -0.14471600 \\
\hline $\mathrm{C}$ & 6.37208800 & -0.71714500 & -1.49456900 & $\mathrm{H}$ & 5.20343800 & 0.94882300 & -0.79719900 \\
\hline $\mathrm{C}$ & 5.59474400 & -2.89104300 & -0.85301200 & $\mathrm{H}$ & 3.82252900 & -2.95276900 & 0.36374300 \\
\hline $\mathrm{C}$ & 6.52411000 & -2.10393100 & -1.52863300 & $\mathrm{H}$ & 7.33475500 & -2.55789200 & -2.08520100 \\
\hline $\mathrm{C}$ & 7.39375900 & 0.15227700 & -2.17909300 & $\mathrm{C}$ & 5.73837700 & -4.38964300 & -0.85286700 \\
\hline $\mathrm{F}$ & 8.45930200 & 0.39154100 & -1.37725000 & $\mathrm{~F}$ & 6.88995000 & 1.35090100 & -2.53365900 \\
\hline $\mathrm{F}$ & 7.87704100 & -0.43503600 & -3.29925000 & $F$ & 6.36195500 & -4.83769700 & 0.26016800 \\
\hline$F$ & 4.53532400 & -5.00954000 & -0.90097000 & $F$ & 6.45809700 & -4.82958500 & -1.91043800 \\
\hline C & 0.90397200 & 0.29724400 & 3.22216500 & $\mathrm{H}$ & 1.33702600 & -0.51205000 & 3.80871100 \\
\hline $\mathrm{C}$ & 1.06792000 & 1.61483700 & 4.08044000 & $\mathrm{C}$ & 0.34803500 & 2.83781100 & 3.48498900 \\
\hline $\mathrm{H}$ & -0.73598000 & 2.69785000 & 3.45782700 & $\mathrm{H}$ & 0.68103300 & 3.06406400 & 2.46993600 \\
\hline $\mathrm{H}$ & 0.55809200 & 3.71479100 & 4.11036100 & C & 2.57613400 & 1.91969600 & 4.19672600 \\
\hline $\mathrm{H}$ & 2.73283000 & 2.72615300 & 4.92309000 & $\mathrm{H}$ & 2.99430000 & 2.24979500 & 3.24215200 \\
\hline $\mathrm{H}$ & 3.14183600 & 1.04368900 & 4.53474100 & $\mathrm{C}$ & 0.51150100 & 1.36359200 & 5.49696900 \\
\hline $\mathrm{H}$ & 0.62026200 & 2.27087500 & 6.10212700 & $\mathrm{H}$ & 1.05313200 & 0.56019900 & 6.01039900 \\
\hline $\mathrm{H}$ & -0.55592900 & 1.11142900 & 5.48210100 & $\mathrm{C}$ & -0.56459100 & -0.04058500 & 2.89019400 \\
\hline $\mathrm{O}$ & -1.18471700 & 0.55819200 & 2.00017800 & $\mathrm{C}$ & -0.57789200 & -1.83093600 & 4.69933400 \\
\hline C & -2.65765200 & -1.13279600 & 3.56888400 & C & -1.70707400 & -2.78244900 & 5.11451500 \\
\hline $\mathrm{H}$ & 0.31090100 & -2.34836300 & 4.32527600 & $\mathrm{H}$ & -0.27711400 & -1.20461700 & 5.54464200 \\
\hline $\mathrm{C}$ & -2.97975600 & -1.96952200 & 4.83937500 & $\mathrm{H}$ & -3.06777800 & -0.12389400 & 3.67797900 \\
\hline $\mathrm{H}$ & -1.61508500 & -3.08315700 & 6.16235000 & $\mathrm{H}$ & -1.69366700 & -3.69106000 & 4.50585000 \\
\hline $\bar{H}$ & -3.17399000 & -1.28438000 & 5.67340300 & $\mathrm{H}$ & -3.86883400 & -2.59019100 & 4.70512900 \\
\hline $\mathrm{N}$ & -1.18522800 & -1.00291400 & 3.62970100 & $\mathrm{C}$ & -3.31282300 & -1.69238800 & 2.30162200 \\
\hline C & -4.50139900 & -1.10708100 & 1.95946800 & C & -2.86871900 & -2.86594300 & 1.56438000 \\
\hline C & -5.37509600 & -1.61803000 & 0.94684400 & $\mathrm{H}$ & -4.83492200 & -0.22618900 & 2.50558100 \\
\hline C & -3.74191200 & -3.42255800 & 0.56789100 & C & -1.62374600 & -3.48951600 & 1.76585800 \\
\hline C & -6.60213200 & -1.00750200 & 0.63409900 & C & -4.99335400 & -2.80823900 & 0.26324600 \\
\hline C & -3.36340500 & -4.60894900 & -0.13673600 & $\mathrm{H}$ & -0.90591100 & -3.05271300 & 2.44430600 \\
\hline C & -1.26355100 & -4.64381200 & 1.07045800 & $\mathrm{C}$ & -7.44711600 & -1.56187600 & -0.32687900 \\
\hline $\mathrm{H}$ & -6.89728600 & -0.10672500 & 1.16753100 & C & -5.86500100 & -3.37011000 & -0.72034700 \\
\hline C & -2.12607700 & -5.21005500 & 0.13878800 & $\mathrm{C}$ & -4.26241300 & -5.15748400 & -1.11536400 \\
\hline $\mathrm{H}$ & -0.29197400 & -5.09236100 & 1.25648000 & $C$ & -7.08614000 & -2.73109100 & -0.99612800 \\
\hline $\mathrm{H}$ & -8.39992800 & -1.08724700 & -0.54670500 & C & -5.45756700 & -4.57058700 & -1.39497700 \\
\hline $\bar{H}$ & -1.84279200 & -6.11348200 & -0.39549200 & $\mathrm{H}$ & -3.96077300 & -6.06420300 & -1.63409600 \\
\hline $\mathrm{H}$ & -7.75400400 & -3.16435700 & -1.73676000 & $\mathrm{H}$ & -6.12431300 & -5.00077500 & -2.13823800 \\
\hline C & 2.62327300 & -0.59310100 & 1.67114900 & $S$ & 2.89139800 & -2.00069700 & 2.58667100 \\
\hline
\end{tabular}




\begin{tabular}{|c|c|c|c|c|c|c|c|}
\hline $\mathrm{N}$ & 1.68050200 & 0.33045200 & 1.99268400 & $\mathrm{H}$ & 1.51196000 & 1.11504100 & 1.35375000 \\
\hline $\mathrm{C}$ & -0.72385200 & 0.74086400 & -1.85609100 & $\mathrm{O}$ & -0.68139800 & 1.15169900 & -3.00536600 \\
\hline $\mathrm{O}$ & -0.27529900 & -0.42148300 & -1.39568900 & C & 0.39493900 & -1.39339200 & -2.30902400 \\
\hline C & 1.61026200 & -0.74311800 & -2.97516800 & $\mathrm{H}$ & 2.20103200 & -1.52155200 & -3.47049900 \\
\hline $\mathrm{H}$ & 1.31692300 & 0.00078900 & -3.71652600 & $\mathrm{H}$ & 2.24647800 & -0.25670700 & -2.23078300 \\
\hline C & 0.81608600 & -2.50270300 & -1.34810900 & $\mathrm{H}$ & -0.05422400 & -2.94074300 & -0.85207700 \\
\hline $\mathrm{H}$ & 1.33793300 & -3.29138600 & -1.90000400 & $\mathrm{H}$ & 1.49086400 & -2.11344900 & -0.58217100 \\
\hline C & -0.62790500 & -1.90782000 & -3.32561500 & $\mathrm{H}$ & -0.17285000 & -2.70583500 & -3.92257300 \\
\hline $\mathrm{H}$ & -1.50162400 & -2.32647100 & -2.81310600 & $\mathrm{H}$ & -0.94786200 & -1.11334800 & -4.00328400 \\
\hline $\mathrm{C}$ & -1.50913200 & 2.83349000 & -0.98994500 & $\mathrm{H}$ & -0.74360400 & 3.28746000 & -1.61854200 \\
\hline C & -1.89985200 & 3.64264900 & 0.18506800 & $\mathrm{C}$ & -1.39797500 & 4.95171500 & 0.27295200 \\
\hline C & -2.76119200 & 3.16748700 & 1.18844000 & C & -1.75635100 & 5.77221300 & 1.33951800 \\
\hline $\mathrm{H}$ & -0.69405500 & 5.30259700 & -0.47627700 & C & -3.11966400 & 3.99561900 & 2.25183100 \\
\hline $\mathrm{H}$ & -3.12386600 & 2.14473000 & 1.15664000 & C & -2.62500400 & 5.29975700 & 2.32635500 \\
\hline $\mathrm{H}$ & -1.34806500 & 6.77641000 & 1.40620300 & $\mathrm{H}$ & -3.78045800 & 3.61899800 & 3.02794300 \\
\hline $\mathrm{H}$ & -2.90582700 & 5.94113300 & 3.15722800 & $\mathrm{~N}$ & -1.33462700 & 1.47071200 & -0.82973000 \\
\hline $\mathrm{H}$ & -1.30188800 & 1.08128300 & 0.12004400 & $\mathrm{~S}$ & 2.15677700 & 3.20965500 & -0.69632000 \\
\hline 0 & 1.49034200 & 3.89403200 & -1.82071300 & 0 & 1.21104400 & 2.59857800 & 0.28553700 \\
\hline 0 & 3.28955000 & 2.30967800 & -1.02829200 & $\mathrm{C}$ & 2.93858300 & 4.58734800 & 0.27951900 \\
\hline $\mathrm{F}$ & 3.82608900 & 5.25816800 & -0.46055700 & $\mathrm{~F}$ & 3.56810900 & 4.09640500 & 1.36134900 \\
\hline $\mathrm{F}$ & 1.99437300 & 5.44797400 & 0.70040200 & C & -2.82845600 & 3.19936200 & -2.39605200 \\
\hline $\mathrm{H}$ & -2.75281200 & 4.28329200 & -2.43968000 & $\mathrm{H}$ & -2.24091400 & 2.65975900 & -3.13420200 \\
\hline $\mathrm{C}$ & -4.05888900 & 2.65170000 & -2.01958500 & $\mathrm{C}$ & -4.31971800 & 1.22460200 & -2.18068600 \\
\hline $\mathrm{H}$ & -3.39593700 & 0.63863300 & -2.10723200 & $\mathrm{H}$ & -5.05854700 & 0.84146900 & -1.46658300 \\
\hline C & -5.08996800 & 3.50327600 & -1.34989700 & $\mathrm{H}$ & -6.09961800 & 3.10237800 & -1.47838000 \\
\hline $\mathrm{H}$ & -4.87325400 & 3.51188800 & -0.26889900 & $\mathrm{H}$ & -5.05685000 & 4.54244300 & -1.68888400 \\
\hline C & -5.02713900 & -1.11741800 & -4.02065500 & $\mathrm{H}$ & -5.63428400 & -1.54515300 & -3.21561000 \\
\hline $\mathrm{H}$ & -5.44175600 & -1.46331600 & -4.97576100 & $\mathrm{H}$ & -4.01469100 & -1.52788300 & -3.93206400 \\
\hline C & -6.75385000 & 1.46860500 & -4.13151200 & $\mathrm{H}$ & -7.41908500 & 1.09312300 & -3.34494600 \\
\hline $\mathrm{H}$ & -6.76567400 & 2.56412900 & -4.09184300 & $\mathrm{H}$ & -7.18789100 & 1.17413700 & -5.09498600 \\
\hline $\mathrm{C}$ & -3.86006300 & 1.46611900 & -5.28330400 & $\mathrm{H}$ & -3.89342000 & 2.56021300 & -5.32839100 \\
\hline $\mathrm{H}$ & -2.81885800 & 1.16976400 & -5.11167800 & $\mathrm{H}$ & -4.15452400 & 1.08728400 & -6.26990200 \\
\hline $\mathrm{Si}$ & -5.00749800 & 0.76588000 & -3.96227000 & & & & \\
\hline
\end{tabular}

\section{TS-S-4f-nd-48}

Imaginary frequency: $-230.54 \mathrm{~cm}^{-1}$ E[B3LYP/6-31G(d)]: -4788.673821 Hartree $\mathrm{E}\left[\mathrm{PCM}\left(\mathrm{Et}_{2} \mathrm{O}\right)-\mathrm{B} 3 \mathrm{LYP} / 6-311 \mathrm{G}(\mathrm{d}, \mathrm{p})-\mathrm{D} 3(\mathrm{BJ})\right]$ : -4790.207085 Hartree

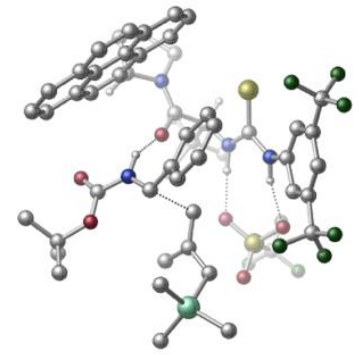

\begin{tabular}{|cccc|cccc|}
\hline 01 & & & N & -1.91418500 & 2.03587000 & 1.49989400 \\
\hline H & -2.69877900 & 1.38308100 & 1.61077700 & C & -2.07034700 & 2.86836300 & 0.37478600 \\
\hline C & -3.04897900 & 2.47558100 & -0.55899600 & C & -1.33292600 & 4.03441100 & 0.12103600 \\
\hline C & -3.22410000 & 3.18762400 & -1.74223600 & H & -3.67631900 & 1.61707900 & -0.34084300 \\
\hline C & -1.52411000 & 4.73061300 & -1.07474700 & H & -0.61285900 & 4.38261600 & 0.84667900 \\
\hline C & -2.45448700 & 4.31707400 & -2.02485400 & H & -2.58993700 & 4.86586600 & -2.94788500 \\
\hline C & -4.20381300 & 2.66195800 & -2.75540200 & C & -0.64627300 & 5.92242700 & -1.34544300 \\
\hline F & -5.35354300 & 2.24729000 & -2.19297800 & F & -3.68913300 & 1.57968300 & -3.41917400 \\
\hline F & -4.51051000 & 3.57538000 & -3.69921700 & F & -0.51582800 & 6.71481800 & -0.26184100 \\
\hline F & 0.61500000 & 5.54202000 & -1.69492100 & F & -1.11295300 & 6.68634000 & -2.35647300 \\
\hline C & 0.20951400 & 0.17637300 & 3.83908900 & H & 0.44773900 & 1.02399900 & 4.48429100 \\
\hline
\end{tabular}




\begin{tabular}{|c|c|c|c|c|c|c|c|}
\hline C & -0.26987900 & -1.01104100 & 4.76334000 & C & -0.49255400 & -2.33005000 & 3.99347700 \\
\hline $\mathrm{H}$ & 0.42858600 & -2.68461700 & 3.52114100 & $\mathrm{H}$ & -1.26319600 & -2.23952300 & 3.22630500 \\
\hline $\mathrm{H}$ & -0.82777400 & -3.09908300 & 4.70012400 & C & -1.57678700 & -0.58688000 & 5.46878400 \\
\hline $\mathrm{H}$ & -1.84376500 & -1.33753200 & 6.22215200 & $\mathrm{H}$ & -2.41923000 & -0.50311300 & 4.78012500 \\
\hline $\mathrm{H}$ & -1.45549900 & 0.37436200 & 5.98335800 & C & 0.78462400 & -1.27430600 & 5.85911900 \\
\hline $\mathrm{H}$ & 0.44753000 & -2.10340900 & 6.49079300 & $\mathrm{H}$ & 0.92146400 & -0.40554200 & 6.51431600 \\
\hline $\mathrm{H}$ & 1.75744200 & -1.55913100 & 5.44372200 & C & 1.46075700 & -0.19933600 & 2.99440600 \\
\hline 0 & 1.30263900 & -0.84496400 & 1.94546700 & C & 3.04521600 & 0.88840500 & 4.64954500 \\
\hline C & 3.90670000 & -0.56292200 & 2.86695600 & C & 4.35149100 & 0.25166800 & 5.13200800 \\
\hline $\mathrm{H}$ & 3.18110600 & 1.94460500 & 4.38428500 & $\mathrm{H}$ & 2.25834000 & 0.83958900 & 5.39691700 \\
\hline $\mathrm{H}$ & 3.72927100 & -1.63961700 & 2.96800900 & $\mathrm{H}$ & 4.13080100 & -0.63945900 & 5.73191100 \\
\hline $\mathrm{H}$ & 4.94305000 & 0.93478300 & 5.74893300 & $\mathrm{~N}$ & 2.70330600 & 0.10443900 & 3.44806400 \\
\hline C & 4.27353600 & -0.33893800 & 1.40235100 & C & 4.54345100 & -1.46152300 & 0.66907700 \\
\hline C & 4.51898000 & 0.96912800 & 0.81322100 & C & 5.09083400 & -1.42196500 & -0.65510300 \\
\hline $\mathrm{H}$ & 4.34006900 & -2.44021100 & 1.09652700 & C & 5.09507300 & 1.03653300 & -0.49939700 \\
\hline C & 4.21764700 & 2.17837600 & 1.46326900 & C & 5.36570300 & -2.59043900 & -1.38456100 \\
\hline C & 5.38301700 & -0.15327100 & -1.23246600 & C & 5.38273600 & 2.30577200 & -1.09251400 \\
\hline $\mathrm{H}$ & 3.72618700 & 2.16353300 & 2.42551300 & C & 4.49790500 & 3.41161000 & 0.87431800 \\
\hline C & 5.92285300 & -2.51524600 & -2.66023700 & $\mathrm{H}$ & 5.14330400 & -3.55600600 & -0.93764500 \\
\hline C & 5.95444300 & -0.08496000 & -2.53999700 & C & 5.08415800 & 3.48008300 & -0.38460800 \\
\hline C & 5.96669400 & 2.34542900 & -2.40555400 & $\mathrm{H}$ & 4.24659900 & 4.32460700 & 1.40668900 \\
\hline C & 6.21506100 & -1.27850600 & -3.23304700 & $\mathrm{H}$ & 6.13616800 & -3.42771200 & -3.21096600 \\
\hline C & 6.24043600 & 1.20684200 & -3.09844100 & $\mathrm{H}$ & 5.30453700 & 4.44404200 & -0.83663600 \\
\hline $\mathrm{H}$ & 6.18461700 & 3.31868900 & -2.83879600 & $\mathrm{H}$ & 6.65139000 & -1.22728700 & -4.22771300 \\
\hline $\mathrm{H}$ & 6.68030600 & 1.25683200 & -4.09158100 & C & -0.80556100 & 1.80295700 & 2.28414900 \\
\hline S & 0.51316500 & 2.85708000 & 2.45506100 & $\mathrm{~N}$ & -0.85552400 & 0.62064400 & 2.94862900 \\
\hline $\mathrm{H}$ & -1.59612600 & -0.04711300 & 2.71365800 & S & -4.38731600 & -0.88132900 & 1.81684200 \\
\hline 0 & -4.99108500 & -1.81001100 & 0.84231100 & $\mathrm{O}$ & -3.02956900 & -1.27804400 & 2.29570400 \\
\hline 0 & -4.46477500 & 0.56523800 & 1.47654000 & C & -5.45521600 & -1.04336000 & 3.33190000 \\
\hline $\mathrm{F}$ & -6.71319700 & -0.68006000 & 3.06190700 & $\mathrm{~F}$ & -4.98576600 & -0.25811900 & 4.31674100 \\
\hline $\mathrm{F}$ & -5.45614500 & -2.31052300 & 3.76684700 & $\mathrm{C}$ & 1.54489400 & -3.49115700 & -0.51300500 \\
\hline $\mathrm{O}$ & 2.19293900 & -3.97414900 & 0.39264900 & $\mathrm{O}$ & 1.00111200 & -4.13127000 & -1.55718800 \\
\hline C & 1.22484500 & -5.58800800 & -1.75969700 & C & 2.72355900 & -5.86323500 & -1.90869300 \\
\hline $\mathrm{H}$ & 2.86964800 & -6.90549900 & -2.21249700 & $\mathrm{H}$ & 3.25345600 & -5.69757300 & -0.96954200 \\
\hline $\bar{H}$ & 3.15662800 & -5.21939400 & -2.68180300 & $\mathrm{C}$ & 0.49057300 & -5.85348000 & -3.07445300 \\
\hline $\mathrm{H}$ & -0.57276100 & -5.60933200 & -2.98423500 & $\mathrm{H}$ & 0.57721800 & -6.91165400 & -3.34075400 \\
\hline $\mathrm{H}$ & 0.91774900 & -5.25763800 & -3.88761500 & C & 0.59533900 & -6.37287900 & -0.60661100 \\
\hline $\mathrm{H}$ & 0.67613300 & -7.44570300 & -0.81329800 & $\mathrm{H}$ & -0.46691000 & -6.12687600 & -0.50676500 \\
\hline $\mathrm{H}$ & 1.09879300 & -6.16112700 & 0.33790300 & C & 0.58445600 & -1.47014600 & -1.53602800 \\
\hline $\mathrm{H}$ & 0.27428000 & -2.12529300 & -2.33897200 & C & 0.87116900 & -0.08158000 & -1.92524400 \\
\hline $\mathrm{C}$ & 0.67901600 & 0.26123300 & -3.27619700 & C & 1.30039200 & 0.90123100 & -1.01621000 \\
\hline C & 0.91714400 & 1.56087700 & -3.71286200 & $\mathrm{H}$ & 0.35071200 & -0.49698500 & -3.98145000 \\
\hline C & 1.51809800 & 2.20267200 & -1.45677000 & $\mathrm{H}$ & 1.42895600 & 0.66468900 & 0.03502900 \\
\hline $\mathrm{C}$ & 1.32571000 & 2.53653900 & -2.80121100 & $\mathrm{H}$ & 0.77194100 & 1.81408500 & -4.75915300 \\
\hline $\mathrm{H}$ & 1.82177800 & 2.96078500 & -0.74367300 & $\mathrm{H}$ & 1.47571200 & 3.56084600 & -3.12655400 \\
\hline $\mathrm{N}$ & 1.31234000 & -2.11072800 & -0.57947800 & $\mathrm{H}$ & 1.63258800 & -1.60501900 & 0.25625800 \\
\hline C & -1.32896800 & -1.07324300 & -0.61044200 & $\mathrm{H}$ & -0.85350600 & -1.16887300 & 0.36026900 \\
\hline $\mathrm{H}$ & -1.47743700 & -0.05844800 & -0.96168400 & C & -2.24323100 & -2.03569100 & -1.00768400 \\
\hline C & -3.28318400 & -1.72807700 & -2.01811800 & $\mathrm{H}$ & -3.27728300 & -0.65891800 & -2.25763300 \\
\hline $\mathrm{H}$ & -4.25032800 & -1.93445300 & -1.53081300 & C & -2.28349000 & -3.36499800 & -0.32253300 \\
\hline $\mathrm{H}$ & -2.39982700 & -4.19201500 & -1.03052900 & $\mathrm{H}$ & -3.17101200 & -3.36005800 & 0.32568700 \\
\hline $\mathrm{H}$ & -1.40839500 & -3.53400500 & 0.30882900 & C & -4.83454400 & -1.71849300 & -4.59654700 \\
\hline $\mathrm{H}$ & -5.79263300 & -1.87787600 & -4.08768800 & $\mathrm{H}$ & -4.94711400 & -2.06289100 & -5.63163000 \\
\hline
\end{tabular}




\begin{tabular}{|llll|llll|}
\hline $\mathrm{H}$ & -4.64984400 & -0.63831400 & -4.61609900 & $\mathrm{C}$ & -3.88917700 & -4.45949700 & -3.50410400 \\
\hline $\mathrm{H}$ & -4.75237600 & -4.58524700 & -2.84025300 & $\mathrm{H}$ & -3.06566400 & -5.05805500 & -3.09749200 \\
\hline $\mathrm{H}$ & -4.15573300 & -4.89374500 & -4.47593700 & $\mathrm{C}$ & -1.83565800 & -2.48714700 & -4.71682600 \\
\hline $\mathrm{H}$ & -0.99514800 & -2.99845400 & -4.23149900 & $\mathrm{H}$ & -1.55993300 & -1.43739500 & -4.86871000 \\
\hline $\mathrm{H}$ & -1.96152800 & -2.93938700 & -5.70834600 & $\mathrm{Si}$ & -3.43678200 & -2.63351800 & -3.71529700 \\
\hline $\mathrm{C}$ & 5.05134400 & -0.13506100 & 3.82354300 & $\mathrm{H}$ & 5.58080000 & 0.72734600 & 3.40714400 \\
\hline $\mathrm{H}$ & 5.77991700 & -0.94210300 & 3.94018600 & & & & \\
\hline
\end{tabular}

\section{TS-S-4f-nd-49}

Imaginary frequency: $-293.65 \mathrm{~cm}^{-1}$ E[B3LYP/6-31G(d)]: -4788.680154 Hartree

$\mathrm{E}\left[\mathrm{PCM}\left(\mathrm{Et}_{2} \mathrm{O}\right)-\mathrm{B} 3 \mathrm{LYP} / 6-311 \mathrm{G}(\mathrm{d}, \mathrm{p})-\mathrm{D} 3(\mathrm{BJ})\right]$ : -4790.206308 Hartree

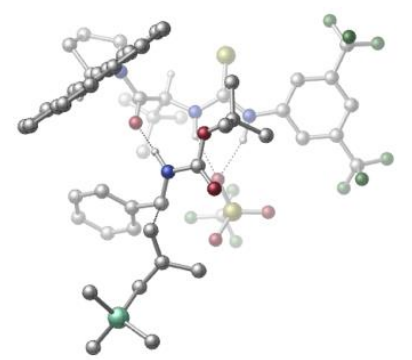

\begin{tabular}{|c|c|c|c|c|c|c|c|}
\hline 01 & & & & $\mathrm{~N}$ & 3.24661600 & -0.39428600 & 0.65506600 \\
\hline $\mathrm{H}$ & 2.77546300 & 0.51487100 & 0.61464900 & C & 4.47762200 & -0.41259000 & -0.02966700 \\
\hline C & 4.95632200 & 0.83845700 & -0.46424300 & C & 5.21814700 & -1.56529100 & -0.32924500 \\
\hline C & 6.15259300 & 0.92457300 & -1.17104000 & $\mathrm{H}$ & 4.39634300 & 1.74535100 & -0.24793400 \\
\hline C & 6.41605800 & -1.45205400 & -1.03718200 & $\mathrm{H}$ & 4.87185500 & -2.53175200 & 0.00544700 \\
\hline C & 6.89913600 & -0.21754500 & -1.46328800 & $\mathrm{H}$ & 7.83780100 & -0.14294200 & -1.99797000 \\
\hline C & 6.62158700 & 2.26632300 & -1.67255900 & C & 7.16194700 & -2.70809500 & -1.40340100 \\
\hline $\mathrm{F}$ & 6.27171600 & 3.26985700 & -0.84686200 & $\mathrm{~F}$ & 6.09993200 & 2.55006900 & -2.88976600 \\
\hline $\mathrm{F}$ & 7.96907800 & 2.30225800 & -1.81043300 & $\mathrm{~F}$ & 7.04717100 & -3.66422000 & -0.45785100 \\
\hline $\mathrm{F}$ & 6.69461800 & -3.24400800 & -2.55869000 & $\mathrm{~F}$ & 8.48162500 & -2.47697900 & -1.58888600 \\
\hline C & 0.63168600 & -1.50094500 & 2.94448000 & $\mathrm{H}$ & 1.20177200 & -2.38866000 & 3.21732100 \\
\hline C & 0.51626300 & -0.58583300 & 4.22760700 & C & -0.31583300 & 0.68508300 & 3.97719300 \\
\hline $\mathrm{H}$ & -1.35686900 & 0.45542500 & 3.73544900 & $\mathrm{H}$ & 0.09276500 & 1.29271300 & 3.16564900 \\
\hline $\mathrm{H}$ & -0.30469600 & 1.30813500 & 4.87978500 & C & 1.94276100 & -0.17702500 & 4.65275600 \\
\hline $\mathrm{H}$ & 1.90516800 & 0.33368800 & 5.62235100 & $\mathrm{H}$ & 2.39565800 & 0.51333100 & 3.93548900 \\
\hline $\mathrm{H}$ & 2.60144800 & -1.04719900 & 4.75168400 & C & -0.12853800 & -1.38128900 & 5.37990300 \\
\hline $\mathrm{H}$ & -0.21683100 & -0.74157600 & 6.26536000 & $\mathrm{H}$ & 0.47878700 & -2.24851500 & 5.66611200 \\
\hline $\mathrm{H}$ & -1.13771800 & -1.72902400 & 5.12985500 & C & -0.73935600 & -1.90683800 & 2.36788300 \\
\hline $\mathrm{O}$ & -1.39433200 & -1.10964600 & 1.68092600 & C & -0.69137900 & -4.20225400 & 3.49396100 \\
\hline C & -2.68562000 & -3.38707600 & 2.34494400 & C & -1.91331400 & -4.71358000 & 4.26362000 \\
\hline $\mathrm{H}$ & -0.26248600 & -4.98949400 & 2.85998800 & $\mathrm{H}$ & 0.09828000 & -3.83006200 & 4.14204800 \\
\hline $\mathrm{H}$ & -3.22529000 & -2.50002400 & 2.68501400 & $\mathrm{H}$ & -2.11621900 & -4.05240600 & 5.11423800 \\
\hline $\mathrm{H}$ & -1.77047400 & -5.72720100 & 4.65008300 & $\mathrm{~N}$ & -1.25619000 & -3.13348100 & 2.65246500 \\
\hline C & -2.99808900 & -3.62142200 & 0.87330800 & C & -2.08841900 & -4.23741500 & 0.06400800 \\
\hline C & -4.30862100 & -3.27501500 & 0.34998800 & C & -2.36829200 & -4.57347200 & -1.30136600 \\
\hline $\mathrm{H}$ & -1.10366800 & -4.48291000 & 0.45395900 & C & -4.60379900 & -3.57198800 & -1.02153100 \\
\hline C & -5.31319300 & -2.66247000 & 1.12335400 & C & -1.43841800 & -5.24514300 & -2.11275800 \\
\hline C & -3.63851700 & -4.22330200 & -1.84525800 & C & -5.87918900 & -3.23128500 & -1.57245600 \\
\hline $\mathrm{H}$ & -5.13822900 & -2.44533400 & 2.17122100 & C & -6.55359500 & -2.33439400 & 0.57632700 \\
\hline C & -1.74099100 & -5.55286200 & -3.43841600 & $\mathrm{H}$ & -0.47623200 & -5.52747100 & -1.69335000 \\
\hline C & -3.93877300 & -4.53760000 & -3.20619700 & C & -6.83716400 & -2.60960700 & -0.75710800 \\
\hline C & -6.14874100 & -3.54598100 & -2.94910200 & $\mathrm{H}$ & -7.30661100 & -1.86447700 & 1.20355100 \\
\hline C & -2.97407600 & -5.19918600 & -3.98325500 & $\mathrm{H}$ & -1.00938200 & -6.07256000 & -4.05083600 \\
\hline C & -5.22470000 & -4.16961600 & -3.72941100 & $\mathrm{H}$ & -7.80703100 & -2.35400900 & -1.17682600 \\
\hline $\mathrm{H}$ & -7.12103900 & -3.27758500 & -3.35532500 & $\mathrm{H}$ & -3.20273500 & -5.44008900 & -5.01849500 \\
\hline $\mathrm{H}$ & -5.44812800 & -4.40581300 & -4.76698800 & $\mathrm{C}$ & 2.60742200 & -1.32596500 & 1.43502100 \\
\hline S & 3.18899700 & -2.87279700 & 1.81106600 & $\mathrm{~N}$ & 1.41203200 & -0.85900400 & 1.90109500 \\
\hline
\end{tabular}




\begin{tabular}{|c|c|c|c|c|c|c|c|}
\hline $\mathrm{H}$ & 1.11046100 & 0.06425600 & 1.59866300 & $\mathrm{C}$ & -0.45937400 & 0.50987000 & -1.64816500 \\
\hline 0 & -0.29198100 & 1.38308900 & -2.48510000 & 0 & 0.05941600 & -0.71535500 & -1.63535300 \\
\hline $\mathrm{C}$ & 0.86385100 & -1.21670200 & -2.78577000 & $\mathrm{C}$ & 2.06311500 & -0.30563500 & -3.05619100 \\
\hline $\mathrm{H}$ & 2.72266700 & -0.79751100 & -3.77987300 & $\mathrm{H}$ & 1.75860000 & 0.66129900 & -3.45627100 \\
\hline $\mathrm{H}$ & 2.63489900 & -0.14259600 & -2.13958500 & C & 1.32219900 & -2.58707200 & -2.28917800 \\
\hline $\bar{H}$ & 0.46050900 & -3.21700600 & -2.05289200 & $\bar{H}$ & 1.91220600 & -3.08218100 & -3.06759100 \\
\hline $\bar{H}$ & 1.94268400 & -2.49269900 & -1.39321000 & $\mathrm{C}$ & -0.05848200 & -1.34471400 & -4.00101200 \\
\hline $\mathrm{H}$ & 0.49682000 & -1.79226200 & -4.83263600 & $\mathrm{H}$ & -0.91102900 & -1.99335800 & -3.77354600 \\
\hline $\mathrm{H}$ & -0.42399400 & -0.36634400 & -4.32221800 & C & -1.69224000 & 1.97188900 & -0.20314800 \\
\hline $\bar{H}$ & -0.91459500 & 2.70979200 & -0.36740100 & $\mathrm{C}$ & -2.46851600 & 2.13047300 & 1.04949800 \\
\hline C & -2.19708000 & 3.23616300 & 1.87295300 & C & -3.48733600 & 1.23281900 & 1.41302900 \\
\hline $\mathrm{C}$ & -2.92872500 & 3.43180400 & 3.04543900 & $\mathrm{H}$ & -1.38929700 & 3.91093500 & 1.60630400 \\
\hline C & -4.21912700 & 1.44014200 & 2.57846500 & $\mathrm{H}$ & -3.70518500 & 0.37136000 & 0.78854800 \\
\hline C & -3.94303200 & 2.54036100 & 3.39719700 & $\mathrm{H}$ & -2.69959800 & 4.27945500 & 3.68501400 \\
\hline $\mathrm{H}$ & -5.00655600 & 0.74207000 & 2.84919900 & $\mathrm{H}$ & -4.51562100 & 2.69787500 & 4.30736700 \\
\hline $\mathrm{N}$ & -1.30789300 & 0.69131100 & -0.55605200 & $\mathrm{H}$ & -1.43034500 & -0.07738700 & 0.11249100 \\
\hline$S$ & 1.75253300 & 3.34041400 & 0.60137600 & $\mathrm{O}$ & 0.59514700 & 4.18137600 & 0.20033300 \\
\hline 0 & 1.40192600 & 1.88902600 & 0.75539100 & $\mathrm{O}$ & 3.01966500 & 3.58363200 & -0.10167700 \\
\hline $\mathrm{C}$ & 2.07132300 & 3.86521000 & 2.35961600 & $F$ & 2.49876300 & 5.12997900 & 2.41187300 \\
\hline $\mathrm{F}$ & 2.99793700 & 3.07289900 & 2.92253800 & $\mathrm{~F}$ & 0.94253500 & 3.77031100 & 3.09101600 \\
\hline C & -2.94064300 & 2.55944200 & -1.62362000 & $\mathrm{H}$ & -2.50628100 & 1.96548000 & -2.41995200 \\
\hline $\mathrm{H}$ & -3.88402400 & 2.20579700 & -1.21684500 & $\mathrm{C}$ & -2.72492300 & 3.94305900 & -1.68803700 \\
\hline $\mathrm{C}$ & -3.55947200 & 4.88317000 & -0.93759500 & $\mathrm{H}$ & -4.00695100 & 4.41192200 & -0.05378300 \\
\hline $\mathrm{H}$ & -2.99619300 & 5.77343600 & -0.63156900 & C & -1.53891900 & 4.47465000 & -2.42191200 \\
\hline $\mathrm{H}$ & -1.73346300 & 5.47007500 & -2.83370100 & $\mathrm{H}$ & -0.71373500 & 4.58563700 & -1.69589000 \\
\hline $\mathrm{H}$ & -1.18623700 & 3.79390400 & -3.19820300 & C & -5.93156100 & 6.79558500 & -0.80580700 \\
\hline $\mathrm{H}$ & -5.26437000 & 7.62778000 & -0.55311000 & $\mathrm{H}$ & -6.82201100 & 7.21995000 & -1.28538800 \\
\hline $\mathrm{H}$ & -6.25236300 & 6.32686600 & 0.13172100 & C & -4.50170500 & 6.39718800 & -3.53559000 \\
\hline $\mathrm{H}$ & -3.79386300 & 7.20848300 & -3.32889900 & $\mathrm{H}$ & -4.01678900 & 5.69472300 & -4.22284600 \\
\hline $\mathrm{H}$ & -5.35541300 & 6.83694700 & -4.06593800 & C & -6.24169800 & 4.11207100 & -2.34730600 \\
\hline $\mathrm{H}$ & -5.76085500 & 3.36732100 & -2.99139400 & $\mathrm{H}$ & -6.57888100 & 3.60168700 & -1.43735900 \\
\hline $\mathrm{H}$ & -7.13527600 & 4.47251600 & -2.87145600 & $\mathrm{Si}$ & -5.08968700 & 5.55406200 & -1.95268500 \\
\hline C & -3.03892500 & -4.62496100 & 3.22134900 & $\mathrm{H}$ & -3.04397300 & -5.52275900 & 2.59496400 \\
\hline $\mathrm{H}$ & -4.03351300 & -4.53154500 & 3.66712000 & & & & \\
\hline
\end{tabular}

\section{TS-S-4f-nd-50}

Imaginary frequency: $-305.14 \mathrm{~cm}^{-1}$

E[B3LYP/6-31G(d)]: -4788.680115 Hartree

E[PCM(Et $\left.\left.t_{2} \mathrm{O}\right)-B 3 L Y P / 6-311 G(d, p)-D 3(B J)\right]:-4790.205835$ Hartree

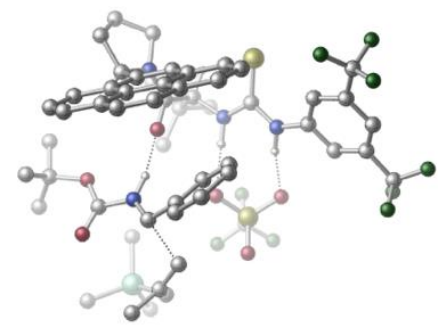

\begin{tabular}{|cccc|cccc|}
\hline 01 & & & N & -2.47672300 & -1.64615800 & 1.24910600 \\
\hline H & -1.87996300 & -2.41524400 & 0.92138500 & C & -3.77560300 & -1.70348600 & 0.70817000 \\
\hline C & -4.13960500 & -2.90225000 & 0.06908100 & C & -4.68028700 & -0.63132300 & 0.70042200 \\
\hline C & -5.38189500 & -3.01979000 & -0.54922000 & H & -3.44186600 & -3.73211300 & 0.04899000 \\
\hline C & -5.91952100 & -0.77116200 & 0.07588600 & H & -4.42036200 & 0.29451900 & 1.18917800 \\
\hline C & -6.28874900 & -1.95966500 & -0.55132400 & H & -7.25499200 & -2.05883500 & -1.03098900 \\
\hline C & -5.72673700 & -4.28936300 & -1.28328000 & C & -6.89941600 & 0.37043900 & 0.12558600 \\
\hline F & -5.12286100 & -5.36792400 & -0.74402800 & F & -5.34856000 & -4.23109200 & -2.58194200 \\
\hline F & -7.05980600 & -4.52280200 & -1.27649200 & F & -6.27785200 & 1.57261700 & 0.23936300 \\
\hline F & -7.66585900 & 0.42352400 & -0.98749600 & F & -7.74216300 & 0.27047100 & 1.17654100 \\
\hline C & 0.22342000 & -0.19627300 & 3.25353100 & H & -0.46273600 & 0.14374000 & 4.02789100 \\
\hline
\end{tabular}




\begin{tabular}{|c|c|c|c|c|c|c|c|}
\hline C & 1.35162200 & -1.02880900 & 3.96028300 & C & 2.46546900 & -1.48796300 & 3.00139100 \\
\hline $\mathrm{H}$ & 2.98826000 & -0.63974000 & 2.54949600 & $\mathrm{H}$ & 2.07877200 & -2.10943900 & 2.19128400 \\
\hline $\mathrm{H}$ & 3.19866200 & -2.08330900 & 3.55983600 & C & 0.69909800 & -2.26352600 & 4.61539600 \\
\hline $\mathrm{H}$ & 1.43959600 & -2.79542100 & 5.22467400 & $\mathrm{H}$ & 0.31805600 & -2.96274300 & 3.86671800 \\
\hline $\mathrm{H}$ & -0.13404600 & -1.97799900 & 5.26869400 & C & 1.98489800 & -0.16280100 & 5.06915200 \\
\hline $\mathrm{H}$ & 2.78609900 & -0.72294400 & 5.56446200 & $\mathrm{H}$ & 1.25426500 & 0.10981700 & 5.84053100 \\
\hline $\mathrm{H}$ & 2.42878200 & 0.75755000 & 4.67040100 & C & 0.74096100 & 1.02058900 & 2.44975900 \\
\hline 0 & 1.25798300 & 0.85552900 & 1.33555200 & C & 0.07903900 & 2.67394000 & 4.27867600 \\
\hline C & 1.07716800 & 3.43999900 & 2.20032700 & C & 0.98857200 & 3.85395600 & 4.63294100 \\
\hline $\mathrm{H}$ & -0.97152700 & 2.97969900 & 4.19845100 & $\mathrm{H}$ & 0.13952700 & 1.86076100 & 4.99791400 \\
\hline $\mathrm{H}$ & 2.05561700 & 3.16562100 & 1.79449200 & $\mathrm{H}$ & 1.94091600 & 3.47681800 & 5.02448000 \\
\hline $\mathrm{H}$ & 0.54844700 & 4.50871200 & 5.39107600 & $\mathrm{~N}$ & 0.58769000 & 2.26932000 & 2.95920200 \\
\hline C & 0.16316800 & 3.85384600 & 1.05070200 & C & -1.13204400 & 3.42923600 & 0.99160500 \\
\hline C & 0.66854500 & 4.77070200 & 0.04380500 & C & -2.03743500 & 3.85731600 & -0.03593400 \\
\hline $\mathrm{H}$ & -1.51065500 & 2.73002500 & 1.73267100 & C & -0.21202800 & 5.20241900 & -1.00145000 \\
\hline C & 1.98697500 & 5.26231700 & 0.04342000 & C & -3.37156000 & 3.42057300 & -0.07970900 \\
\hline C & -1.56404000 & 4.74948900 & -1.04056700 & C & 0.25757200 & 6.09957600 & -2.01120900 \\
\hline $\mathrm{H}$ & 2.67525100 & 4.96946900 & 0.82907300 & C & 2.43620100 & 6.13665000 & -0.94663200 \\
\hline C & -4.22761800 & 3.84780100 & -1.09439600 & $\mathrm{H}$ & -3.73384500 & 2.74456600 & 0.68964600 \\
\hline C & -2.44586500 & 5.18379300 & -2.07660900 & C & 1.58565400 & 6.55201700 & -1.96565100 \\
\hline C & -0.65075200 & 6.51838500 & -3.04413300 & $\mathrm{H}$ & 3.46037700 & 6.49934500 & -0.91588400 \\
\hline C & -3.77180500 & 4.71926900 & -2.08277000 & $\mathrm{H}$ & -5.25339100 & 3.49227800 & -1.10978400 \\
\hline C & -1.94015100 & 6.08309500 & -3.07600000 & $\mathrm{H}$ & 1.94063400 & 7.23621000 & -2.73255900 \\
\hline $\mathrm{H}$ & -0.28171100 & 7.20197500 & -3.80517200 & $\mathrm{H}$ & -4.44491400 & 5.05178000 & -2.86966200 \\
\hline $\mathrm{H}$ & -2.61399800 & 6.41449600 & -3.86269600 & C & -1.93547100 & -0.82092300 & 2.20482100 \\
\hline S & -2.82026700 & 0.28027800 & 3.14718200 & $\mathrm{~N}$ & -0.59176800 & -1.00166000 & 2.35081600 \\
\hline $\mathrm{H}$ & -0.08980900 & -1.52790900 & 1.62927900 & S & 0.29856300 & -3.79257100 & -0.25554400 \\
\hline 0 & 0.63915200 & -4.14752700 & -1.64463400 & $\mathrm{O}$ & 0.88703700 & -2.50204300 & 0.21674200 \\
\hline 0 & -1.12159900 & -3.95871600 & 0.14522100 & C & 1.17888300 & -5.07930400 & 0.76390400 \\
\hline $\mathrm{F}$ & 0.68274100 & -6.29521300 & 0.52993700 & $\mathrm{~F}$ & 1.06404200 & -4.81048300 & 2.07448400 \\
\hline $\mathrm{F}$ & 2.49676600 & -5.09748200 & 0.46148200 & C & 4.09596100 & 0.74043400 & -0.95519500 \\
\hline 0 & 4.87415900 & 0.73033700 & -1.89426000 & $\mathrm{O}$ & 4.35522400 & 1.05887800 & 0.31449500 \\
\hline C & 5.63244600 & 1.70927600 & 0.70995900 & C & 6.81031400 & 0.75749300 & 0.49411300 \\
\hline $\mathrm{H}$ & 7.71839300 & 1.21478400 & 0.90266000 & $\mathrm{H}$ & 6.97081700 & 0.55591000 & -0.56569900 \\
\hline $\mathrm{H}$ & 6.64276600 & -0.18907300 & 1.01677600 & C & 5.41163000 & 1.97497700 & 2.19977800 \\
\hline $\mathrm{H}$ & 4.55058000 & 2.63281600 & 2.35406800 & $\mathrm{H}$ & 6.29769000 & 2.45638800 & 2.62608300 \\
\hline $\mathrm{H}$ & 5.23169600 & 1.03901800 & 2.73742800 & C & 5.79065100 & 3.01834700 & -0.06837200 \\
\hline $\mathrm{H}$ & 6.64984200 & 3.57222800 & 0.32506600 & $\mathrm{H}$ & 4.89861800 & 3.64373900 & 0.04583900 \\
\hline $\mathrm{H}$ & 5.95691800 & 2.83263100 & -1.13132600 & C & 2.18984800 & 0.34677500 & -2.33560900 \\
\hline $\mathrm{H}$ & 2.86247600 & 0.72681300 & -3.09636600 & C & 0.76007700 & 0.65178700 & -2.51535100 \\
\hline C & 0.38626600 & 1.36341100 & -3.66925800 & C & -0.22320000 & 0.25344200 & -1.59532000 \\
\hline C & -0.94644500 & 1.69730100 & -3.88696600 & $\mathrm{H}$ & 1.14652100 & 1.66869700 & -4.38487400 \\
\hline C & -1.55650100 & 0.58008300 & -1.82749000 & $\mathrm{H}$ & 0.05002400 & -0.32677600 & -0.72109900 \\
\hline C & -1.92039400 & 1.30290600 & -2.96630600 & $\mathrm{H}$ & -1.22483600 & 2.26625200 & -4.76904300 \\
\hline $\mathrm{H}$ & -2.31592000 & 0.26738500 & -1.11866000 & $\mathrm{H}$ & -2.96099600 & 1.56445100 & -3.13070000 \\
\hline $\mathrm{N}$ & 2.74124600 & 0.42149700 & -1.08925900 & $\mathrm{H}$ & 2.15997700 & 0.43742300 & -0.24050200 \\
\hline C & 2.16434400 & -1.57354200 & -3.19367000 & $\mathrm{H}$ & 1.46722000 & -2.08052100 & -2.53322300 \\
\hline $\mathrm{H}$ & 1.72935700 & -1.10712800 & -4.07164700 & C & 3.42386500 & -2.13993700 & -3.38018500 \\
\hline C & 3.86911600 & -3.31700600 & -2.61371300 & $\mathrm{H}$ & 4.25174100 & -4.04318700 & -3.34811600 \\
\hline $\mathrm{H}$ & 3.02377100 & -3.77938200 & -2.09286300 & C & 4.33258100 & -1.58623200 & -4.44255800 \\
\hline $\mathrm{H}$ & 4.95438500 & -2.36538200 & -4.89417500 & $\mathrm{H}$ & 5.00814800 & -0.84990000 & -3.98768500 \\
\hline $\mathrm{H}$ & 3.77186200 & -1.07591200 & -5.23158900 & C & 4.83468200 & -2.63759900 & 0.32664200 \\
\hline $\mathrm{H}$ & 4.59955900 & -1.57102600 & 0.31404500 & $\mathrm{H}$ & 5.64386700 & -2.79211700 & 1.05254900 \\
\hline
\end{tabular}




\begin{tabular}{|llll|llll|}
\hline $\mathrm{H}$ & 3.95098700 & -3.16542200 & 0.69475000 & $\mathrm{C}$ & 6.83940300 & -2.39101500 & -2.05822200 \\
\hline $\mathrm{H}$ & 6.62630700 & -1.32483900 & -2.18927100 & $\mathrm{H}$ & 7.15570900 & -2.79917600 & -3.02551300 \\
\hline $\mathrm{H}$ & 7.69291900 & -2.47704100 & -1.37395600 & $\mathrm{C}$ & 5.76069700 & -5.15705000 & -1.15867600 \\
\hline $\mathrm{H}$ & 6.07952900 & -5.61092700 & -2.10458800 & $\mathrm{H}$ & 4.89014700 & -5.71391600 & -0.79337200 \\
\hline $\mathrm{H}$ & 6.57157300 & -5.29942800 & -0.43379400 & $\mathrm{Si}$ & 5.35269900 & -3.32042200 & -1.35007900 \\
\hline $\mathrm{C}$ & 1.19654300 & 4.56179200 & 3.28222300 & $\mathrm{H}$ & 0.41316000 & 5.30794700 & 3.11907600 \\
\hline $\mathrm{H}$ & 2.15712700 & 5.08187800 & 3.22529800 & & & & \\
\hline
\end{tabular}

\section{TS-S-4f-nd-51}

Imaginary frequency: $-216.95 \mathrm{~cm}-1$

E[B3LYP/6-31G(d)]: -4788.676364 Hartree

$\mathrm{E}\left[\mathrm{PCM}\left(\mathrm{Et}_{2} \mathrm{O}\right)-\mathrm{B} 3 \mathrm{LYP} / 6-311 \mathrm{G}(\mathrm{d}, \mathrm{p})-\mathrm{D} 3(\mathrm{BJ})\right]$ : -4790.204556 Hartree

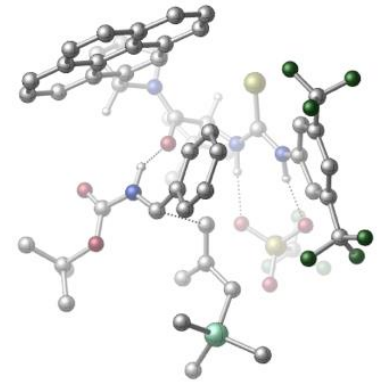

\begin{tabular}{|c|c|c|c|c|c|c|c|}
\hline 01 & & & & $\mathrm{~N}$ & 1.54838300 & 1.41806900 & -2.34455900 \\
\hline $\mathrm{H}$ & 2.45165700 & 0.94091700 & -2.24405700 & C & 1.50072500 & 2.62569200 & -1.62481300 \\
\hline C & 2.60897000 & 2.89940900 & -0.79850700 & $\mathrm{C}$ & 0.42915300 & 3.53365600 & -1.62989000 \\
\hline C & 2.60859800 & 4.01266700 & 0.03782600 & $\mathrm{H}$ & 3.46225900 & 2.22840500 & -0.81282100 \\
\hline C & 0.45259900 & 4.64137700 & -0.78198200 & $\mathrm{H}$ & -0.41833800 & 3.35622500 & -2.27449300 \\
\hline $\mathrm{C}$ & 1.52905300 & 4.89563800 & 0.06589100 & $\mathrm{H}$ & 1.52755800 & 5.75105600 & 0.73012400 \\
\hline C & 3.74696400 & 4.18914500 & 1.00554600 & C & -0.70737300 & 5.60110500 & -0.79821500 \\
\hline $\mathrm{F}$ & 4.93049800 & 3.82058100 & 0.48434500 & $\mathrm{~F}$ & 3.56015400 & 3.42294400 & 2.12476600 \\
\hline $\mathrm{F}$ & 3.86221400 & 5.46332500 & 1.43534600 & $\mathrm{~F}$ & -0.49668500 & 6.64946200 & -1.62259500 \\
\hline $\mathrm{F}$ & -1.85806800 & 5.00878400 & -1.20135000 & $\mathrm{~F}$ & -0.94329600 & 6.11834000 & 0.43542000 \\
\hline $\mathrm{C}$ & -0.16732900 & -1.62818700 & -3.44130400 & $\mathrm{H}$ & -0.58713900 & -1.24888400 & -4.37514000 \\
\hline $\mathrm{C}$ & 0.51105200 & -3.02017100 & -3.74563600 & $\mathrm{C}$ & 0.96649100 & -3.76094500 & -2.47047600 \\
\hline $\mathrm{H}$ & 0.12523400 & -3.97466000 & -1.80281400 & $\mathrm{H}$ & 1.72133000 & -3.20348900 & -1.91332700 \\
\hline $\mathrm{H}$ & 1.41754000 & -4.71833000 & -2.75859200 & $\mathrm{C}$ & 1.71770200 & -2.78452000 & -4.67983000 \\
\hline $\mathrm{H}$ & 2.11531300 & -3.75111200 & -5.01137900 & $\mathrm{H}$ & 2.53087500 & -2.24728300 & -4.18940100 \\
\hline $\mathrm{H}$ & 1.42219900 & -2.22029500 & -5.57290000 & C & -0.48490100 & -3.93081000 & -4.49252600 \\
\hline $\mathrm{H}$ & 0.00135700 & -4.88559800 & -4.72075400 & $\mathrm{H}$ & -0.80407600 & -3.49426700 & -5.44677000 \\
\hline $\mathrm{H}$ & -1.37222700 & -4.15492900 & -3.89196200 & $\mathrm{C}$ & -1.29085200 & -1.72383900 & -2.36981200 \\
\hline $\mathrm{O}$ & -0.97876100 & -1.64298600 & -1.16926500 & C & -3.16692600 & -1.97398400 & -4.07966700 \\
\hline C & -3.59564800 & -2.28468800 & -1.71864000 & C & -4.18548500 & -3.11480000 & -3.98603000 \\
\hline $\mathrm{H}$ & -3.65856000 & -1.01205600 & -4.27379400 & $\mathrm{H}$ & -2.41732400 & -2.12898300 & -4.85115300 \\
\hline $\mathrm{H}$ & -3.11445800 & -2.94025800 & -0.98756200 & $\mathrm{H}$ & -3.68384900 & -4.07061500 & -4.17171300 \\
\hline $\mathrm{H}$ & -4.98934600 & -3.01236200 & -4.72104800 & $\mathrm{~N}$ & -2.57310600 & -1.95920900 & -2.73652500 \\
\hline C & -4.17735400 & -1.06678500 & -1.00603400 & $\mathrm{C}$ & -4.00595100 & 0.19204200 & -1.50418300 \\
\hline C & -4.99657800 & -1.26927900 & 0.17758600 & C & -4.62773400 & 1.34535600 & -0.91790600 \\
\hline $\mathrm{H}$ & -3.36333600 & 0.35198100 & -2.36478200 & $\mathrm{C}$ & -5.63174700 & -0.13377800 & 0.77892000 \\
\hline $\mathrm{C}$ & -5.19391300 & -2.53120300 & 0.76732200 & $\mathrm{C}$ & -4.44328400 & 2.63567600 & -1.44244400 \\
\hline C & -5.45298700 & 1.17111900 & 0.23034000 & C & -6.45129400 & -0.30089600 & 1.93839000 \\
\hline $\mathrm{H}$ & -4.71534600 & -3.40912000 & 0.34792000 & C & -5.99199500 & -2.68233400 & 1.90144600 \\
\hline $\mathrm{C}$ & -5.06056000 & 3.73737100 & -0.85013700 & $\mathrm{H}$ & -3.80148100 & 2.76808900 & -2.30937900 \\
\hline $\mathrm{C}$ & -6.08503900 & 2.30368000 & 0.82838700 & $\mathrm{C}$ & -6.61905900 & -1.58450500 & 2.48113800 \\
\hline C & -7.08096700 & 0.85549300 & 2.51596700 & $\mathrm{H}$ & -6.12409300 & -3.67095500 & 2.33298400 \\
\hline C & -5.87448200 & 3.57534500 & 0.26997800 & $\mathrm{H}$ & -4.89665600 & 4.72929000 & -1.26150700 \\
\hline $\mathrm{C}$ & -6.90806100 & 2.09786400 & 1.98756500 & $\mathrm{H}$ & -7.24368600 & -1.71083700 & 3.36217200 \\
\hline $\mathrm{H}$ & -7.70519200 & 0.71373900 & 3.39505400 & $\mathrm{H}$ & -6.35276000 & 4.43881600 & 0.72613300 \\
\hline $\mathrm{H}$ & -7.39162200 & 2.96093700 & 2.43892400 & C & 0.53406500 & 0.68031700 & -2.91981100 \\
\hline $\mathrm{S}$ & -0.90970900 & 1.31981200 & -3.53910800 & $\mathrm{~N}$ & 0.81102100 & -0.64626500 & -2.98889500 \\
\hline
\end{tabular}




\begin{tabular}{|c|c|c|c|c|c|c|c|}
\hline$\overline{\mathrm{H}}$ & 1.63467500 & -0.99644200 & -2.49068200 & $\mathrm{~S}$ & 4.54491600 & -0.86170900 & -1.58884500 \\
\hline $\mathrm{O}$ & 5.32519800 & -1.10968000 & -0.36098500 & $\mathrm{O}$ & 3.28011500 & -1.64884900 & -1.69026500 \\
\hline $\mathrm{O}$ & 4.35432300 & 0.56444600 & -1.96746400 & C & 5.60644200 & -1.54932500 & -2.95358000 \\
\hline $\mathrm{F}$ & 6.77939000 & -0.90999800 & -3.00440200 & $\mathrm{~F}$ & 4.98981300 & -1.40577300 & -4.13906800 \\
\hline $\mathrm{F}$ & 5.83485100 & -2.85421500 & -2.75200600 & C & -0.92162500 & -3.19007000 & 1.94302700 \\
\hline $\mathrm{O}$ & -1.53725900 & -3.99905600 & 1.27933700 & $\mathrm{O}$ & -0.22581000 & -3.41085100 & 3.06505000 \\
\hline C & -0.19986700 & -4.75634500 & 3.69995100 & C & -1.62217200 & -5.15791500 & 4.09824300 \\
\hline $\mathrm{H}$ & -1.58698200 & -6.09226100 & 4.66889700 & $\mathrm{H}$ & -2.25234700 & -5.31013800 & 3.22055500 \\
\hline $\mathrm{H}$ & -2.07471900 & -4.38904000 & 4.73366700 & C & 0.66868400 & -4.51782900 & 4.93578100 \\
\hline $\mathrm{H}$ & 1.67179200 & -4.18526500 & 4.64930900 & $\mathrm{H}$ & 0.76586800 & -5.44702300 & 5.50612400 \\
\hline $\mathrm{H}$ & 0.22151900 & -3.75950100 & 5.58671800 & C & 0.45372400 & -5.76479700 & 2.75240200 \\
\hline $\mathrm{H}$ & 0.56750500 & -6.72380100 & 3.26962900 & $\mathrm{H}$ & 1.44969700 & -5.42262900 & 2.45244300 \\
\hline $\mathrm{H}$ & -0.15198800 & -5.92021400 & 1.85849900 & C & -0.21717300 & -0.84596800 & 2.22345300 \\
\hline $\mathrm{H}$ & 0.24673000 & -1.17481000 & 3.14420300 & C & -0.68478300 & 0.54673600 & 2.20363800 \\
\hline $\mathrm{C}$ & -0.40162700 & 1.34147100 & 3.32926700 & C & -1.38762700 & 1.10537600 & 1.12168400 \\
\hline $\mathrm{C}$ & -0.81100300 & 2.67070500 & 3.37485400 & $\mathrm{H}$ & 0.13472200 & 0.90954900 & 4.16904000 \\
\hline $\mathrm{C}$ & -1.78388400 & 2.43804400 & 1.16989300 & $\mathrm{H}$ & -1.59971200 & 0.51593800 & 0.23485700 \\
\hline C & -1.49282100 & 3.22427000 & 2.28939000 & $\mathrm{H}$ & -0.59011400 & 3.27462100 & 4.25023100 \\
\hline $\mathrm{H}$ & -2.30804400 & 2.87179800 & 0.32642600 & $\mathrm{H}$ & -1.78463100 & 4.26996800 & 2.30086000 \\
\hline $\mathrm{N}$ & -0.90746300 & -1.83331700 & 1.58967400 & $\mathrm{H}$ & -1.29708600 & -1.67068500 & 0.65177500 \\
\hline C & 1.55067200 & -0.54092900 & 0.98884400 & $\mathrm{H}$ & 1.07780500 & -1.11572800 & 0.19945400 \\
\hline $\mathrm{H}$ & 1.49260900 & 0.53658300 & 0.88155400 & C & 2.65261400 & -1.06909700 & 1.64104900 \\
\hline $\mathrm{C}$ & 3.63576300 & -0.18704200 & 2.31506100 & $\mathrm{H}$ & 3.38092800 & 0.86597900 & 2.15070400 \\
\hline $\mathrm{H}$ & 4.58922400 & -0.35885800 & 1.78563900 & C & 2.94571800 & -2.53463400 & 1.55878200 \\
\hline $\mathrm{H}$ & 3.17455700 & -2.96329700 & 2.54043900 & $\mathrm{H}$ & 3.84114400 & -2.65109600 & 0.93337000 \\
\hline $\mathrm{H}$ & 2.13195700 & -3.09359800 & 1.09239200 & C & 5.33848200 & 1.10076600 & 4.43250700 \\
\hline $\mathrm{H}$ & 6.27055400 & 0.94282000 & 3.87697400 & $\mathrm{H}$ & 5.59888900 & 1.21709400 & 5.49140700 \\
\hline $\mathrm{H}$ & 4.90691000 & 2.04572700 & 4.08274100 & C & 5.01835800 & -1.97875300 & 4.53167900 \\
\hline $\mathrm{H}$ & 5.80622000 & -2.18529600 & 3.79817700 & $\mathrm{H}$ & 4.33369200 & -2.83487100 & 4.53684700 \\
\hline $\mathrm{H}$ & 5.49170100 & -1.93760900 & 5.52081000 & C & 2.64825400 & -0.15976800 & 5.31900700 \\
\hline $\mathrm{H}$ & 1.90097100 & -0.94623800 & 5.15421900 & $\mathrm{H}$ & 2.15990600 & 0.81220200 & 5.18618000 \\
\hline $\mathrm{H}$ & 2.96288400 & -0.22670500 & 6.36784500 & $\mathrm{Si}$ & 4.14414200 & -0.33916300 & 4.16936400 \\
\hline C & -4.69913700 & -3.04329600 & -2.53170200 & $\mathrm{H}$ & -5.63992700 & -2.48897000 & -2.47914600 \\
\hline $\mathrm{H}$ & -4.88523800 & -4.03812700 & -2.11732000 & & & & \\
\hline
\end{tabular}

\section{TS-S-4f-nd-52}

Imaginary frequency: $-302.46 \mathrm{~cm}^{-1}$

E[B3LYP/6-31G(d)]: -4788.674607 Hartree

$\mathrm{E}\left[\mathrm{PCM}\left(\mathrm{Et}_{2} \mathrm{O}\right)-\mathrm{B} 3 \mathrm{LYP} / 6-311 \mathrm{G}(\mathrm{d}, \mathrm{p})-\mathrm{D} 3(\mathrm{BJ})\right]:-4790.204281$ Hartree

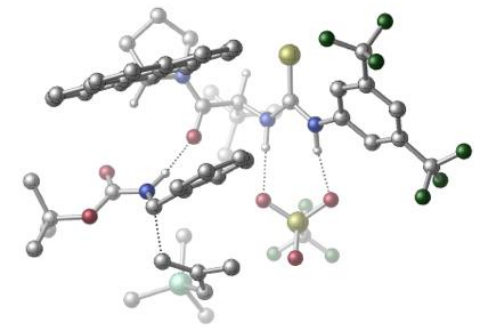

\begin{tabular}{|c|c|c|c|c|c|c|c|}
\hline 01 & & & & $\mathrm{~N}$ & -3.17622900 & -0.62203100 & 1.05063200 \\
\hline $\mathrm{H}$ & -2.98454600 & -1.57936200 & 0.73403400 & C & -4.29426500 & -0.05259400 & 0.41084700 \\
\hline C & -5.15510900 & -0.93246200 & -0.26708000 & C & -4.54396600 & 1.32630700 & 0.34313100 \\
\hline C & -6.24472600 & -0.43790500 & -0.98114400 & $\mathrm{H}$ & -4.95893200 & -1.99855000 & -0.24332600 \\
\hline C & -5.64220800 & 1.79977200 & -0.37290500 & $\mathrm{H}$ & -3.89176400 & 2.01642400 & 0.85478300 \\
\hline C & -6.50560100 & 0.93071300 & -1.03922200 & $\mathrm{H}$ & -7.35758600 & 1.30857900 & -1.59166200 \\
\hline $\mathrm{C}$ & -7.11706900 & -1.39156300 & -1.75490900 & $C$ & -5.93194900 & 3.27669000 & -0.39186100 \\
\hline $\mathrm{F}$ & -7.17277000 & -2.60988500 & -1.17912000 & $\mathrm{~F}$ & -6.65995900 & -1.57054000 & -3.01697100 \\
\hline $\mathrm{F}$ & -8.38560800 & -0.93294000 & -1.86212400 & $\mathrm{~F}$ & -4.82251500 & 4.01928400 & -0.14608900 \\
\hline $\mathrm{F}$ & -6.42019800 & 3.67578100 & -1.58900600 & $\mathrm{~F}$ & -6.84694300 & 3.62604700 & 0.53820900 \\
\hline C & -0.32649300 & -0.64825100 & 3.35887400 & $\mathrm{H}$ & -0.82389300 & 0.01600400 & 4.06416000 \\
\hline
\end{tabular}




\begin{tabular}{|c|c|c|c|c|c|c|c|}
\hline $\mathrm{C}$ & 0.10240500 & -1.92924000 & 4.15986500 & $\mathrm{C}$ & 0.88391400 & -2.94534000 & 3.31003400 \\
\hline $\mathrm{H}$ & 1.81038900 & -2.51885100 & 2.91747400 & $\mathrm{H}$ & 0.29586400 & -3.30787800 & 2.46471600 \\
\hline $\mathrm{H}$ & 1.14117900 & -3.81326300 & 3.92998100 & C & -1.17397000 & -2.59830200 & 4.70959900 \\
\hline $\mathrm{H}$ & -0.90229300 & -3.43056100 & 5.36979000 & $\mathrm{H}$ & -1.79486900 & -2.99962100 & 3.90370600 \\
\hline $\mathrm{H}$ & -1.78220400 & -1.89239500 & 5.28770300 & C & 0.98599300 & -1.49880300 & 5.34836900 \\
\hline $\mathrm{H}$ & 1.29843600 & -2.38213500 & 5.91676500 & $\mathrm{H}$ & 0.44473900 & -0.84033600 & 6.03823800 \\
\hline $\mathrm{H}$ & 1.89716000 & -0.98550000 & 5.01827400 & C & 0.83880300 & 0.10575700 & 2.67369200 \\
\hline C & 2.34282200 & 2.05832900 & 2.59208500 & $\mathrm{H}$ & 3.08900200 & 1.35594700 & 2.21120000 \\
\hline $\mathrm{N}$ & 1.25875000 & 1.27417800 & 3.21747100 & C & 1.85348600 & 2.96564900 & 1.46098200 \\
\hline C & 0.52221300 & 3.18625500 & 1.25365100 & C & 2.83008000 & 3.65464300 & 0.63156900 \\
\hline C & 0.03218600 & 4.08515500 & 0.24799500 & $\mathrm{H}$ & -0.21612300 & 2.66767700 & 1.85867600 \\
\hline C & 2.36638000 & 4.55951300 & -0.37957700 & C & 4.21905000 & 3.48441900 & 0.78024100 \\
\hline C & -1.34116200 & 4.30595200 & 0.05578300 & C & 0.96950300 & 4.77446600 & -0.57275700 \\
\hline C & 3.30313700 & 5.26273900 & -1.20050300 & $\mathrm{H}$ & 4.60515900 & 2.80266600 & 1.53002300 \\
\hline C & 5.12465500 & 4.18453000 & -0.01863100 & C & -1.78976100 & 5.18448800 & -0.93000800 \\
\hline $\mathrm{H}$ & -2.05459400 & 3.78577300 & 0.68883500 & C & 0.50404100 & 5.67527900 & -1.57854400 \\
\hline C & 4.67795100 & 5.06357300 & -0.99958600 & C & 2.80745300 & 6.16321300 & -2.20606700 \\
\hline $\mathrm{H}$ & 6.19118800 & 4.04585000 & 0.13704000 & C & -0.87906900 & 5.86183400 & -1.73933000 \\
\hline $\mathrm{H}$ & -2.85658900 & 5.33492100 & -1.06402000 & C & 1.47291300 & 6.36036900 & -2.38791500 \\
\hline $\mathrm{H}$ & 5.38972600 & 5.60862800 & -1.61504400 & $\mathrm{H}$ & 3.53100900 & 6.69286500 & -2.82145500 \\
\hline $\mathrm{H}$ & -1.23450800 & 6.54880400 & -2.50389900 & $\mathrm{H}$ & 1.11658600 & 7.04873700 & -3.15078800 \\
\hline $\mathrm{C}$ & -2.41065600 & -0.15613900 & 2.09476900 & $\mathrm{~S}$ & -2.79312900 & 1.21161500 & 3.02646500 \\
\hline $\mathrm{N}$ & -1.32053800 & -0.93624900 & 2.32902100 & $\mathrm{H}$ & -1.04196600 & -1.60511700 & 1.60429200 \\
\hline S & -1.60164000 & -3.71707400 & -0.44130000 & 0 & -1.34264400 & -4.13910300 & -1.83183000 \\
\hline $\mathrm{O}$ & -0.56676400 & -2.80032200 & 0.12716800 & $\mathrm{O}$ & -2.98656900 & -3.28664000 & -0.12612900 \\
\hline C & -1.39491800 & -5.27776200 & 0.55139100 & $\mathrm{~F}$ & -2.24347100 & -6.22240900 & 0.14039300 \\
\hline $\mathrm{F}$ & -1.61497200 & -5.03778500 & 1.85424200 & $\mathrm{~F}$ & -0.13778000 & -5.74854200 & 0.42478200 \\
\hline $\mathrm{C}$ & 4.46641700 & -0.51450700 & -0.17369100 & $\mathrm{O}$ & 4.73334900 & -0.54338000 & 1.01136000 \\
\hline $\mathrm{O}$ & 5.33810100 & -0.53026100 & -1.19921900 & C & 6.80282400 & -0.39468000 & -0.96754700 \\
\hline C & 7.08659600 & 0.94315000 & -0.28260300 & $\mathrm{H}$ & 8.16927900 & 1.10496800 & -0.24077100 \\
\hline $\mathrm{H}$ & 6.69466500 & 0.95615500 & 0.73607600 & $\mathrm{H}$ & 6.63791500 & 1.76706100 & -0.84621700 \\
\hline C & 7.36032100 & -0.41033700 & -2.39174900 & $\mathrm{H}$ & 7.11106000 & -1.34831800 & -2.89849200 \\
\hline $\mathrm{H}$ & 8.45051200 & -0.31556100 & -2.36436300 & $\mathrm{H}$ & 6.95749100 & 0.42185000 & -2.97830700 \\
\hline C & 7.32816800 & -1.58173600 & -0.15681700 & $\mathrm{H}$ & 8.41692500 & -1.49712800 & -0.06712600 \\
\hline $\mathrm{H}$ & 7.10472400 & -2.52780000 & -0.65893800 & $\mathrm{H}$ & 6.89511400 & -1.59940700 & 0.84403200 \\
\hline C & 2.77914900 & -0.14069600 & -1.91864400 & $\mathrm{H}$ & 3.59257900 & 0.35044600 & -2.44258800 \\
\hline C & 1.45739600 & 0.49182700 & -2.13394300 & C & 1.36128500 & 1.48394600 & -3.12486600 \\
\hline C & 0.31385900 & 0.13009300 & -1.40394000 & C & 0.14567200 & 2.11360200 & -3.37734500 \\
\hline $\mathrm{H}$ & 2.24661700 & 1.77299600 & -3.68748300 & C & -0.90013700 & 0.76307500 & -1.66383800 \\
\hline $\mathrm{H}$ & 0.35381800 & -0.65038800 & -0.65127100 & C & -0.98885800 & 1.75073800 & -2.64693900 \\
\hline $\mathrm{H}$ & 0.08576200 & 2.89230900 & -4.13167100 & $\mathrm{H}$ & -1.78024300 & 0.47567900 & -1.09810100 \\
\hline $\mathrm{H}$ & -1.93814500 & 2.24163000 & -2.83810500 & $\mathrm{~N}$ & 3.14786100 & -0.47042300 & -0.62924700 \\
\hline $\mathrm{H}$ & 2.45443300 & -0.47474100 & 0.13247700 & C & 2.92233000 & -1.63833300 & -3.19783700 \\
\hline $\mathrm{H}$ & 2.85216200 & -0.95339600 & -4.04069100 & $\mathrm{H}$ & 3.93101300 & -1.98002800 & -2.98184900 \\
\hline C & 1.88452800 & -2.58959000 & -3.14976900 & C & 2.05722400 & -3.98973700 & -2.69942500 \\
\hline $\mathrm{H}$ & 2.54753600 & -4.49154700 & -3.55508200 & $\mathrm{H}$ & 1.07290800 & -4.45849900 & -2.58779900 \\
\hline C & 0.53486200 & -2.23586500 & -3.66722500 & $\mathrm{H}$ & 0.28527000 & -2.92530700 & -4.48729400 \\
\hline $\mathrm{H}$ & 0.45360600 & -1.20890400 & -4.02300800 & $\mathrm{H}$ & -0.22193200 & -2.44921100 & -2.89897100 \\
\hline C & 2.80847600 & -3.87741700 & 0.40945200 & $\mathrm{H}$ & 1.75292100 & -3.60274300 & 0.49174700 \\
\hline $\mathrm{H}$ & 3.40691400 & -2.98136900 & 0.60057700 & $\mathrm{H}$ & 3.03094300 & -4.59478300 & 1.20910500 \\
\hline C & 4.99697400 & -4.44658900 & -1.74230100 & $\mathrm{H}$ & 5.29798200 & -3.39401200 & -1.76898600 \\
\hline $\mathrm{H}$ & 5.21572300 & -4.88878800 & -2.72202200 & $\mathrm{H}$ & 5.63808800 & -4.94888800 & -1.00692900 \\
\hline $\mathrm{C}$ & 2.76031400 & -6.50327300 & -1.24929800 & $\mathrm{H}$ & 2.92685900 & -6.96838500 & -2.22844900 \\
\hline
\end{tabular}




\begin{tabular}{|lrrr|rrrr|}
\hline $\mathrm{H}$ & 1.71573800 & -6.67104300 & -0.96739900 & $\mathrm{H}$ & 3.38874800 & -7.03205700 & -0.52206500 \\
\hline $\mathrm{Si}$ & 3.17629300 & -4.65973200 & -1.25847700 & $\mathrm{C}$ & 0.76951300 & 1.89797700 & 4.47129400 \\
\hline $\mathrm{H}$ & 0.96395100 & 1.23354800 & 5.31794700 & $\mathrm{H}$ & -0.30661200 & 2.08592800 & 4.41409800 \\
\hline $\mathrm{C}$ & 1.59751400 & 3.18722100 & 4.58970800 & $\mathrm{H}$ & 1.06566300 & 4.02474700 & 4.12734500 \\
\hline $\mathrm{H}$ & 1.79391900 & 3.44990700 & 5.63313000 & $\mathrm{C}$ & 2.87401900 & 2.87333600 & 3.79673300 \\
\hline $\mathrm{H}$ & 3.41826900 & 3.76935900 & 3.49067700 & $\mathrm{H}$ & 3.55079400 & 2.24499400 & 4.38899200 \\
\hline $\mathrm{O}$ & 1.33036100 & -0.34721900 & 1.62825600 & & & & \\
\hline
\end{tabular}

\section{TS-S-4f-nd-53}

Imaginary frequency: $-226.04 \mathrm{~cm}^{-1}$

E[B3LYP/6-31G(d)]: -4788.672810 Hartree

$\mathrm{E}\left[\mathrm{PCM}\left(\mathrm{Et}_{2} \mathrm{O}\right)-\mathrm{B} 3 \mathrm{LYP} / 6-311 \mathrm{G}(\mathrm{d}, \mathrm{p})-\mathrm{D} 3(\mathrm{BJ})\right]$ : -4790.204107 Hartree

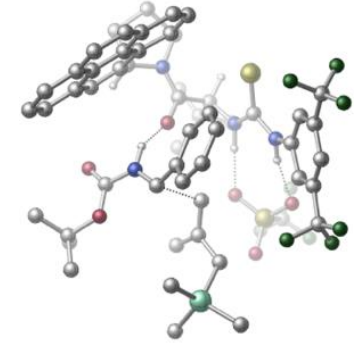

\begin{tabular}{|c|c|c|c|c|c|c|c|}
\hline 01 & & & & $\mathrm{~N}$ & -1.96725300 & 2.07421800 & 1.47366000 \\
\hline $\mathrm{H}$ & -2.76014700 & 1.42992800 & 1.57605100 & $\mathrm{C}$ & -2.08939300 & 2.88870200 & 0.33066300 \\
\hline $\mathrm{C}$ & -3.04936500 & 2.48823800 & -0.61900700 & $\mathrm{C}$ & -1.33542000 & 4.04347000 & 0.07444600 \\
\hline C & -3.18983100 & 3.18086500 & -1.81832400 & $\mathrm{H}$ & -3.68962200 & 1.63933600 & -0.40082000 \\
\hline $\mathrm{C}$ & -1.49100700 & 4.71967100 & -1.13789100 & $\mathrm{H}$ & -0.62991700 & 4.39897300 & 0.81059300 \\
\hline $\mathrm{C}$ & -2.40263700 & 4.29774200 & -2.10237000 & $\mathrm{H}$ & -2.51090100 & 4.83121600 & -3.03791700 \\
\hline C & -4.15140500 & 2.64740200 & -2.84494300 & C & -0.59371700 & 5.89637900 & -1.41071500 \\
\hline $\mathrm{F}$ & -5.31693100 & 2.25185600 & -2.30177600 & $\mathrm{~F}$ & -3.63212500 & 1.55058800 & -3.48034100 \\
\hline$F$ & -4.42871900 & 3.54872300 & -3.80933600 & $\mathrm{~F}$ & -0.46572600 & 6.69934500 & -0.33472500 \\
\hline $\mathrm{F}$ & 0.66663400 & 5.49470400 & -1.74017300 & $\mathrm{~F}$ & -1.03706500 & 6.65455700 & -2.43632900 \\
\hline C & 0.10897200 & 0.19665300 & 3.84271800 & $\mathrm{H}$ & 0.39477200 & 1.03640000 & 4.48048400 \\
\hline C & -0.40038700 & -0.96416600 & 4.78652800 & C & -0.65555300 & -2.29109400 & 4.04126700 \\
\hline $\mathrm{H}$ & 0.25845200 & -2.68006600 & 3.58193300 & $\mathrm{H}$ & -1.41861000 & -2.19584200 & 3.26736700 \\
\hline $\mathrm{H}$ & -1.01389100 & -3.03666000 & 4.76161100 & C & -1.69690700 & -0.49443600 & 5.48227700 \\
\hline $\mathrm{H}$ & -1.98091000 & -1.22277200 & 6.25112600 & $\mathrm{H}$ & -2.53704700 & -0.40590400 & 4.79117500 \\
\hline $\mathrm{H}$ & -1.55389200 & 0.47456100 & 5.97612100 & C & 0.64718400 & -1.23313200 & 5.88644000 \\
\hline $\mathrm{H}$ & 0.28165400 & -2.02789000 & 6.54594000 & $\mathrm{H}$ & 0.82126900 & -0.34793200 & 6.50967700 \\
\hline $\mathrm{H}$ & 1.60516200 & -1.56988800 & 5.47507200 & C & 1.32786100 & -0.23198700 & 2.98243700 \\
\hline C & 2.95958900 & 0.88139900 & 4.56798900 & C & 3.73605700 & -0.72111400 & 2.85114800 \\
\hline $\mathrm{H}$ & 2.55759600 & 1.88972700 & 4.42569300 & $\mathrm{H}$ & 2.55315500 & 0.47930800 & 5.49854300 \\
\hline $\mathrm{H}$ & 3.43665200 & -1.77254700 & 2.81963100 & $\mathrm{~N}$ & 2.58558000 & 0.02357100 & 3.42104100 \\
\hline C & 4.22966600 & -0.40930300 & 1.43232800 & C & 4.59852700 & -1.50756500 & 0.70253700 \\
\hline C & 4.48038400 & 0.91679500 & 0.88471900 & C & 5.27918700 & -1.42894300 & -0.55532600 \\
\hline $\mathrm{H}$ & 4.38434200 & -2.50080700 & 1.08989800 & C & 5.19752500 & 1.02396800 & -0.35637000 \\
\hline C & 4.03805300 & 2.10729600 & 1.48892000 & C & 5.65716500 & -2.57535400 & -1.27359000 \\
\hline C & 5.60191400 & -0.14210000 & -1.07223600 & C & 5.50373200 & 2.31129600 & -0.90036500 \\
\hline $\mathrm{H}$ & 3.40916800 & 2.06516800 & 2.36519500 & $\mathrm{C}$ & 4.33472800 & 3.35739100 & 0.94493800 \\
\hline C & 6.34845200 & -2.46105000 & -2.47844000 & $\mathrm{H}$ & 5.40985300 & -3.55536700 & -0.87294600 \\
\hline C & 6.31090400 & -0.03380000 & -2.30802600 & C & 5.07557500 & 3.46488700 & -0.22611200 \\
\hline C & 6.23407600 & 2.39193900 & -2.13597100 & $\mathrm{H}$ & 3.96979500 & 4.25185100 & 1.44190300 \\
\hline C & 6.67399700 & -1.20622100 & -2.99074700 & $\mathrm{H}$ & 6.63971900 & -3.35656300 & -3.02108200 \\
\hline C & 6.62121600 & 1.27522000 & -2.80975800 & $\mathrm{H}$ & 5.31056600 & 4.44182300 & -0.64145200 \\
\hline $\mathrm{H}$ & 6.46729900 & 3.37920900 & -2.52753100 & $\mathrm{H}$ & 7.21426000 & -1.12409700 & -3.93078900 \\
\hline $\mathrm{H}$ & 7.16874100 & 1.35620700 & -3.74567400 & $C$ & -0.87590900 & 1.83697800 & 2.27973500 \\
\hline$S$ & 0.45425700 & 2.87773800 & 2.45265800 & $\mathrm{~N}$ & -0.95480000 & 0.66831300 & 2.96382400 \\
\hline $\mathrm{H}$ & -1.69336700 & 0.00343200 & 2.71285200 & S & -4.46127900 & -0.82230400 & 1.75678600 \\
\hline $\mathrm{O}$ & -5.05350900 & -1.75402100 & 0.77845500 & 0 & -3.11125700 & -1.22001800 & 2.25620800 \\
\hline
\end{tabular}




\begin{tabular}{|c|c|c|c|c|c|c|c|}
\hline $\mathrm{O}$ & -4.52966100 & 0.62313400 & 1.40956600 & C & -5.54973200 & -0.97407600 & 3.25818300 \\
\hline$F$ & -6.80296200 & -0.60830600 & 2.97011400 & $\mathrm{~F}$ & -5.09086500 & -0.18546400 & 4.24548100 \\
\hline $\mathrm{F}$ & -5.56021700 & -2.23911600 & 3.69917500 & C & 1.51719800 & -3.50347300 & -0.47018800 \\
\hline $\mathrm{O}$ & 2.14870500 & -3.96949300 & 0.45592400 & $\mathrm{O}$ & 0.99433300 & -4.16113400 & -1.51386100 \\
\hline C & 1.22738300 & -5.61990300 & -1.69017200 & C & 2.72965400 & -5.88814100 & -1.81380800 \\
\hline $\mathrm{H}$ & 2.88702200 & -6.93728100 & -2.08668300 & $\mathrm{H}$ & 3.24693100 & -5.69239300 & -0.87332800 \\
\hline $\mathrm{H}$ & 3.16764600 & -5.26310200 & -2.59950600 & C & 0.51243600 & -5.90980600 & -3.01039600 \\
\hline $\mathrm{H}$ & -0.55375300 & -5.67209500 & -2.93765500 & $\mathrm{H}$ & 0.61012500 & -6.97110100 & -3.25994400 \\
\hline $\mathrm{H}$ & 0.94611900 & -5.32287000 & -3.82656700 & $\mathrm{C}$ & 0.58547700 & -6.38974000 & -0.53376800 \\
\hline $\mathrm{H}$ & 0.67006700 & -7.46517000 & -0.72475700 & $\mathrm{H}$ & -0.47826400 & -6.14382600 & -0.45035600 \\
\hline $\mathrm{H}$ & 1.07689800 & -6.16411600 & 0.41380300 & C & 0.57040500 & -1.49834700 & -1.53809500 \\
\hline $\mathrm{H}$ & 0.26654800 & -2.16428800 & -2.33470400 & $\mathrm{C}$ & 0.86350500 & -0.11560400 & -1.94249500 \\
\hline C & 0.68862600 & 0.20955800 & -3.30004100 & $\mathrm{C}$ & 1.28799800 & 0.87758500 & -1.04238100 \\
\hline C & 0.93918100 & 1.50170700 & -3.75203900 & $\mathrm{H}$ & 0.36448800 & -0.55666500 & -3.99850300 \\
\hline $\mathrm{C}$ & 1.51837600 & 2.17140500 & -1.49835500 & $\mathrm{H}$ & 1.40220700 & 0.65491100 & 0.01349900 \\
\hline $\mathrm{C}$ & 1.34321300 & 2.48754000 & -2.84951200 & $\mathrm{H}$ & 0.80746200 & 1.74100100 & -4.80336000 \\
\hline $\mathrm{H}$ & 1.81906500 & 2.93808300 & -0.79330300 & $\mathrm{H}$ & 1.50399500 & 3.50621000 & -3.18722000 \\
\hline $\mathrm{N}$ & 1.28619500 & -2.12446100 & -0.56414200 & $\mathrm{H}$ & 1.58067600 & -1.60919600 & 0.27580700 \\
\hline $\mathrm{C}$ & -1.35701400 & -1.07996200 & -0.63388600 & $\mathrm{H}$ & -0.89014100 & -1.16323300 & 0.34238400 \\
\hline $\mathrm{H}$ & -1.49605000 & -0.07036000 & -1.00373900 & C & -2.27056600 & -2.04434400 & -1.02629600 \\
\hline C & -3.29791100 & -1.74888100 & -2.05337100 & $\mathrm{H}$ & -3.28486000 & -0.68352600 & -2.30898600 \\
\hline $\mathrm{H}$ & -4.27184200 & -1.94386000 & -1.57508900 & C & -2.32462100 & -3.36204100 & -0.31949300 \\
\hline $\mathrm{H}$ & -2.43957100 & -4.20042000 & -1.01431100 & $\mathrm{H}$ & -3.21762300 & -3.34140400 & 0.32087600 \\
\hline $\mathrm{H}$ & -1.45600700 & -3.52518400 & 0.32242400 & C & -4.81024000 & -1.76885900 & -4.65394300 \\
\hline $\bar{H}$ & -5.77651200 & -1.91150400 & -4.15573100 & $\mathrm{H}$ & -4.91167700 & -2.12924500 & -5.68471100 \\
\hline $\mathrm{H}$ & -4.61576700 & -0.69067900 & -4.68873500 & C & -3.89763300 & -4.49963100 & -3.50500100 \\
\hline $\mathrm{H}$ & -4.77054400 & -4.61062800 & -2.85131000 & $\mathrm{H}$ & -3.08276400 & -5.09549700 & -3.07738800 \\
\hline $\mathrm{H}$ & -4.15278900 & -4.94806000 & -4.47343900 & C & -1.81604100 & -2.55922500 & -4.71916200 \\
\hline $\mathrm{H}$ & -0.98567000 & -3.06820300 & -4.21432400 & $\mathrm{H}$ & -1.53137200 & -1.51381400 & -4.88390100 \\
\hline $\mathrm{H}$ & -1.93120300 & -3.02634200 & -5.70507500 & $\mathrm{Si}$ & -3.43202600 & -2.67965300 & -3.73804100 \\
\hline $\mathrm{C}$ & 4.82743700 & -0.51650000 & 3.93697300 & $\mathrm{H}$ & 5.83418700 & -0.55395000 & 3.51448500 \\
\hline $\mathrm{H}$ & 4.74464300 & -1.32240900 & 4.67621300 & C & 4.49523800 & 0.83282400 & 4.59052600 \\
\hline $\mathrm{H}$ & 4.88354000 & 0.91382300 & 5.61017800 & $\mathrm{H}$ & 4.92009200 & 1.65434100 & 4.00768000 \\
\hline $\mathrm{O}$ & 1.13280800 & -0.87630700 & 1.93764700 & & & & \\
\hline
\end{tabular}

\section{TS-S-4f-nd-54}

Imaginary frequency: $-303.55 \mathrm{~cm}^{-1}$

E[B3LYP/6-31G(d)]: -4788.672732 Hartree

E[PCM(Et $\left.\left.t_{2} \mathrm{O}\right)-B 3 L Y P / 6-311 G(d, p)-D 3(B J)\right]:-4790.202299$ Hartree

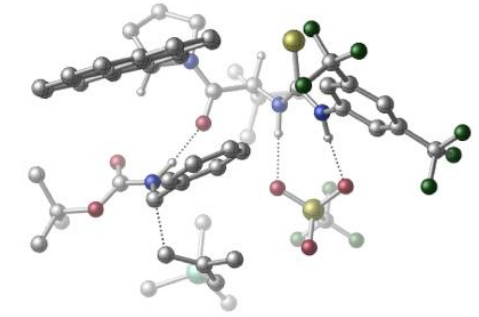

\begin{tabular}{|c|c|c|c|c|c|c|c|}
\hline 01 & & & & $\mathrm{~N}$ & -3.22710000 & -0.40644200 & 1.12609300 \\
\hline $\mathrm{H}$ & -3.18129400 & -1.39088500 & 0.83777800 & C & -4.24154700 & 0.31454900 & 0.47088500 \\
\hline C & -5.32840900 & -0.41201000 & -0.04103700 & C & -4.16483600 & 1.69074700 & 0.21973700 \\
\hline C & -6.32247600 & 0.23624300 & -0.77160700 & $\mathrm{H}$ & -5.38258400 & -1.48187700 & 0.12569200 \\
\hline C & -5.16442900 & 2.32022000 & -0.52085500 & $\mathrm{H}$ & -3.32919300 & 2.25994900 & 0.59958500 \\
\hline $\mathrm{C}$ & -6.25590600 & 1.60835700 & -1.01661100 & $\mathrm{H}$ & -7.03681200 & 2.10868200 & -1.57591900 \\
\hline C & -7.45727000 & -0.56390800 & -1.35611400 & $C$ & -4.99672900 & 3.77492300 & -0.86142100 \\
\hline $\mathrm{F}$ & -7.73813300 & -1.65886300 & -0.61995200 & $\mathrm{~F}$ & -7.17145500 & -0.99052200 & -2.60831400 \\
\hline $\mathrm{F}$ & -8.59070000 & 0.16998600 & -1.44395100 & $\mathrm{~F}$ & -4.17786700 & 3.94201700 & -1.93878600 \\
\hline $\mathrm{F}$ & -6.16530900 & 4.37213300 & -1.16950200 & $\mathrm{~F}$ & -4.43336700 & 4.47746000 & 0.14973900 \\
\hline $\mathrm{C}$ & -0.35066500 & -0.72296400 & 3.37170100 & $\mathrm{H}$ & -0.78304000 & -0.02991100 & 4.09209900 \\
\hline
\end{tabular}




\begin{tabular}{|c|c|c|c|c|c|c|c|}
\hline $\mathrm{C}$ & -0.02943700 & -2.05906000 & 4.13353600 & $\mathrm{C}$ & 0.64268000 & -3.11552400 & 3.23929100 \\
\hline $\mathrm{H}$ & 1.59676400 & -2.76259200 & 2.83928900 & $\mathrm{H}$ & 0.00882100 & -3.39909000 & 2.39664400 \\
\hline $\mathrm{H}$ & 0.83178800 & -4.02143200 & 3.82849900 & $\mathrm{C}$ & -1.35315300 & -2.62665900 & 4.68602900 \\
\hline $\mathrm{H}$ & -1.14804000 & -3.49784200 & 5.31949700 & $\mathrm{H}$ & -2.01945100 & -2.94844900 & 3.88083100 \\
\hline $\mathrm{H}$ & -1.88694700 & -1.88555300 & 5.29301900 & C & 0.90715500 & -1.75175000 & 5.31994200 \\
\hline $\mathrm{H}$ & 1.15146500 & -2.68058700 & 5.84756900 & $\mathrm{H}$ & 0.43616100 & -1.07995800 & 6.04793800 \\
\hline $\mathrm{H}$ & 1.85340900 & -1.30440300 & 4.99346900 & C & 0.87418900 & -0.06412600 & 2.68870100 \\
\hline 0 & 1.28062000 & -0.51249900 & 1.60612600 & C & 1.15025400 & 1.63128900 & 4.57389200 \\
\hline C & 2.60430400 & 1.69492700 & 2.64538500 & C & 2.54512100 & 1.96567600 & 5.10953600 \\
\hline $\mathrm{H}$ & 0.54536100 & 2.53485500 & 4.42722400 & $\mathrm{H}$ & 0.59734600 & 0.95571800 & 5.22096200 \\
\hline $\mathrm{H}$ & 3.22869800 & 0.92072000 & 2.19043300 & $\mathrm{H}$ & 2.98525700 & 1.06983000 & 5.56212700 \\
\hline $\mathrm{H}$ & 2.52153400 & 2.75030700 & 5.87180600 & $\mathrm{~N}$ & 1.45203600 & 1.01566800 & 3.27285100 \\
\hline C & 2.18887000 & 2.71419200 & 1.58310500 & C & 0.88468000 & 3.08392300 & 1.42571100 \\
\hline C & 3.21657300 & 3.34893300 & 0.77418400 & C & 0.47408300 & 4.09280500 & 0.49098800 \\
\hline $\mathrm{H}$ & 0.10666500 & 2.60864700 & 2.01666200 & C & 2.83162700 & 4.35476800 & -0.17236200 \\
\hline C & 4.58240000 & 3.02999100 & 0.88248300 & C & -0.87046600 & 4.47442100 & 0.35395900 \\
\hline C & 1.46200000 & 4.72591700 & -0.31585200 & C & 3.82176300 & 5.00432700 & -0.97433400 \\
\hline $\mathrm{H}$ & 4.90689600 & 2.26979600 & 1.58425700 & C & 5.54219000 & 3.67914300 & 0.10410800 \\
\hline C & -1.24285200 & 5.45149900 & -0.56903700 & $\mathrm{H}$ & -1.62087700 & 4.00272200 & 0.98278800 \\
\hline C & 1.07510800 & 5.72927200 & -1.25566500 & $\mathrm{C}$ & 5.17186900 & 4.65371100 & -0.81658400 \\
\hline C & 3.40441700 & 6.00818000 & -1.91541900 & $\mathrm{H}$ & 6.59116300 & 3.42322900 & 0.22725400 \\
\hline C & -0.28280900 & 6.07145500 & -1.36691200 & $\mathrm{H}$ & -2.28968000 & 5.71940000 & -0.66808800 \\
\hline C & 2.09483400 & 6.35413200 & -2.05114800 & $\mathrm{H}$ & 5.92560800 & 5.15769700 & -1.41694500 \\
\hline $\mathrm{H}$ & 4.16761400 & 6.49431000 & -2.51875600 & $\mathrm{H}$ & -0.57894900 & 6.83431000 & -2.08326200 \\
\hline $\mathrm{H}$ & 1.79830600 & 7.11916300 & -2.76502100 & C & -2.37683600 & -0.00436400 & 2.13015000 \\
\hline S & -2.57550900 & 1.42194000 & 3.02712300 & $\mathrm{~N}$ & -1.37815300 & -0.90058900 & 2.35292500 \\
\hline $\mathrm{H}$ & -1.18705600 & -1.59199400 & 1.62130700 & $\mathrm{~S}$ & -2.06941400 & -3.58826800 & -0.43788500 \\
\hline 0 & -1.92184000 & -3.98631400 & -1.85124100 & 0 & -0.92882600 & -2.78048600 & 0.09430600 \\
\hline 0 & -3.39419200 & -3.05477400 & -0.03303100 & C & -1.94107200 & -5.18943300 & 0.50213800 \\
\hline $\mathrm{F}$ & -2.88911200 & -6.04743000 & 0.11922600 & $\mathrm{~F}$ & -2.06603100 & -4.96996600 & 1.82149200 \\
\hline $\mathrm{F}$ & -0.73803300 & -5.76063400 & 0.29128600 & $\mathrm{C}$ & 4.31255100 & -0.91205600 & -0.37287500 \\
\hline $\mathrm{O}$ & 4.63364800 & -1.01802300 & 0.79430300 & $\mathrm{O}$ & 5.12610600 & -0.96552100 & -1.44364000 \\
\hline C & 6.60738700 & -0.96885800 & -1.28774900 & C & 7.04422000 & 0.31048500 & -0.57271600 \\
\hline $\mathrm{H}$ & 8.13779600 & 0.37510300 & -0.58443200 & $\mathrm{H}$ & 6.70770500 & 0.31658300 & 0.46561700 \\
\hline $\bar{H}$ & 6.64269900 & 1.19313200 & -1.08003100 & $\mathrm{C}$ & 7.08724300 & -0.97671000 & -2.73996900 \\
\hline $\mathrm{H}$ & 6.72849800 & -1.86791600 & -3.26508100 & $\mathrm{H}$ & 8.18150700 & -0.97988200 & -2.76873300 \\
\hline $\mathrm{H}$ & 6.73189800 & -0.08925500 & -3.27381300 & C & 7.06550900 & -2.22842300 & -0.54883600 \\
\hline $\mathrm{H}$ & 8.16059900 & -2.24104300 & -0.51357400 & $\overline{\mathrm{H}}$ & 6.73552100 & -3.13097600 & -1.07172800 \\
\hline $\mathrm{H}$ & 6.68246300 & -2.24879800 & 0.47215500 & C & 2.58261500 & -0.30431000 & -2.00866200 \\
\hline $\mathrm{H}$ & 3.41218400 & 0.12433100 & -2.56133900 & C & 1.32078200 & 0.46104200 & -2.12033400 \\
\hline $\mathrm{C}$ & 1.27316300 & 1.52564600 & -3.03697900 & $\mathrm{C}$ & 0.18283200 & 0.15422100 & -1.35812200 \\
\hline C & 0.11388400 & 2.28460900 & -3.17746500 & $\mathrm{H}$ & 2.15441200 & 1.77099800 & -3.62611200 \\
\hline C & -0.97495200 & 0.91248300 & -1.51185900 & $\mathrm{H}$ & 0.18218100 & -0.67921300 & -0.66314000 \\
\hline $\mathrm{C}$ & -1.01569200 & 1.97792900 & -2.41388200 & $\mathrm{H}$ & 0.09527600 & 3.11996800 & -3.87085900 \\
\hline $\mathrm{H}$ & -1.85180900 & 0.66022900 & -0.92722800 & $\mathrm{H}$ & -1.92138700 & 2.56934000 & -2.51006000 \\
\hline $\mathrm{N}$ & 2.98335400 & -0.72314700 & -0.75482400 & $\mathrm{H}$ & 2.33132800 & -0.69079500 & 0.04138000 \\
\hline C & 2.50142500 & -1.75805000 & -3.35013900 & $\mathrm{H}$ & 2.43897900 & -1.03309600 & -4.15943900 \\
\hline $\mathrm{H}$ & 3.48676600 & -2.19664300 & -3.21666000 & C & 1.38628900 & -2.61386600 & -3.26289800 \\
\hline C & 1.45912700 & -4.04026600 & -2.87244400 & $\mathrm{H}$ & 1.87043200 & -4.54854600 & -3.76475400 \\
\hline $\mathrm{H}$ & 0.44494900 & -4.43038000 & -2.73047600 & C & 0.04415500 & -2.12230600 & -3.67828400 \\
\hline $\mathrm{H}$ & -0.31100100 & -2.74798900 & -4.51059200 & $\mathrm{H}$ & 0.03259100 & -1.07651100 & -3.98480300 \\
\hline $\mathrm{H}$ & -0.68290500 & -2.30680000 & -2.87525600 & C & 2.34749800 & -4.11456000 & 0.20288000 \\
\hline $\mathrm{H}$ & 1.31965800 & -3.76945800 & 0.34705000 & $\mathrm{H}$ & 3.01797600 & -3.27103000 & 0.39361800 \\
\hline
\end{tabular}




\begin{tabular}{|llll|llll|}
\hline $\mathrm{H}$ & 2.55578900 & -4.87602700 & 0.96474800 & $\mathrm{C}$ & 4.38746600 & -4.77201000 & -2.06509800 \\
\hline $\mathrm{H}$ & 4.77136700 & -3.74658400 & -2.06743600 & $\mathrm{H}$ & 4.52779900 & -5.19150600 & -3.06888900 \\
\hline $\mathrm{H}$ & 5.01566200 & -5.35247800 & -1.37768800 & $\mathrm{C}$ & 2.01128800 & -6.65895300 & -1.54751800 \\
\hline $\mathrm{H}$ & 2.10004900 & -7.09643400 & -2.54927800 & $\mathrm{H}$ & 0.96855200 & -6.75288800 & -1.22736600 \\
\hline $\mathrm{H}$ & 2.62259200 & -7.26525400 & -0.86770600 & $\mathrm{Si}$ & 2.57764700 & -4.85648800 & -1.50756600 \\
\hline $\mathrm{C}$ & 3.33312200 & 2.38124900 & 3.85109700 & $\mathrm{H}$ & 3.31448400 & 3.46713200 & 3.72380600 \\
\hline $\mathrm{H}$ & 4.38230900 & 2.07832300 & 3.91051800 & & & & \\
\hline
\end{tabular}

\section{TS-S-4f-nd-55}

Imaginary frequency: $-303.11 \mathrm{~cm}^{-1}$

E[B3LYP/6-31G(d)]: -4788.674151 Hartree

$\mathrm{E}\left[\mathrm{PCM}\left(\mathrm{Et}_{2} \mathrm{O}\right)-\mathrm{B} 3 \mathrm{LYP} / 6-311 \mathrm{G}(\mathrm{d}, \mathrm{p})-\mathrm{D} 3(\mathrm{BJ})\right]$ : -4790.201298 Hartree

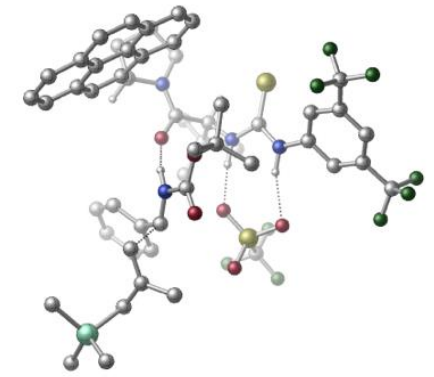

\begin{tabular}{|c|c|c|c|c|c|c|c|}
\hline 01 & & & & $\mathrm{~N}$ & -2.81222300 & -1.35884000 & 0.85113300 \\
\hline $\mathrm{H}$ & -2.14516700 & -2.12941800 & 0.77594200 & C & -3.94117900 & -1.52929700 & 0.02345500 \\
\hline C & -4.29118800 & -2.84359300 & -0.32537000 & $\mathrm{C}$ & -4.65090900 & -0.46319700 & -0.54129400 \\
\hline C & -5.33610100 & -3.07693400 & -1.21694800 & $\mathrm{H}$ & -3.72225300 & -3.67398900 & 0.07672600 \\
\hline C & -5.68004600 & -0.71644700 & -1.44787000 & $\mathrm{H}$ & -4.39131900 & 0.55417800 & -0.28732500 \\
\hline C & -6.04041800 & -2.01757500 & -1.79127000 & $\mathrm{H}$ & -6.83966300 & -2.20305700 & -2.49806200 \\
\hline C & -5.75302200 & -4.49067300 & -1.52584000 & C & -6.31406200 & 0.45807400 & -2.14050200 \\
\hline $\mathrm{F}$ & -6.74937000 & -4.90424300 & -0.70598800 & $\mathrm{~F}$ & -4.73765700 & -5.36550700 & -1.38439800 \\
\hline$F$ & -6.21989500 & -4.60792400 & -2.79121000 & $F$ & -6.58466600 & 1.46919000 & -1.28821400 \\
\hline $\mathrm{F}$ & -5.48442600 & 0.97014300 & -3.09201400 & $\mathrm{~F}$ & -7.46580900 & 0.13059500 & -2.76386700 \\
\hline C & -0.77095100 & 0.37692200 & 3.34890100 & $\mathrm{H}$ & -1.65043600 & 0.83531700 & 3.80043300 \\
\hline C & -0.06516400 & -0.47369400 & 4.47369600 & C & 1.26134900 & -1.11013200 & 4.02152300 \\
\hline $\mathrm{H}$ & 2.00433600 & -0.35482900 & 3.75237800 & $\mathrm{H}$ & 1.13235100 & -1.77446600 & 3.16439500 \\
\hline $\mathrm{H}$ & 1.66952100 & -1.70883000 & 4.84558400 & C & -1.04018800 & -1.58765000 & 4.90973800 \\
\hline $\mathrm{H}$ & -0.64186600 & -2.10166600 & 5.79276500 & $\mathrm{H}$ & -1.17488700 & -2.33596100 & 4.12443600 \\
\hline $\mathrm{H}$ & -2.02564900 & -1.18317600 & 5.16966100 & C & 0.21775400 & 0.42851500 & 5.69194100 \\
\hline $\mathrm{H}$ & 0.71479300 & -0.15552100 & 6.47490400 & $\mathrm{H}$ & -0.70626600 & 0.83214900 & 6.12254200 \\
\hline $\mathrm{H}$ & 0.88143500 & 1.26482500 & 5.44122000 & C & 0.12801200 & 1.48116300 & 2.74458500 \\
\hline $\mathrm{O}$ & 1.02045900 & 1.20611700 & 1.93042300 & C & -1.06058900 & 3.24593300 & 4.13838300 \\
\hline C & 0.91788600 & 3.80350600 & 2.77026300 & C & -0.82608700 & 4.76205500 & 4.20289500 \\
\hline $\mathrm{H}$ & -2.07559200 & 2.97790800 & 3.82841200 & $\mathrm{H}$ & -0.86977000 & 2.78958100 & 5.11367800 \\
\hline C & 0.65223000 & 4.91857900 & 3.81865100 & $\mathrm{H}$ & 1.90675700 & 3.36602100 & 2.93522500 \\
\hline $\mathrm{H}$ & -1.04851900 & 5.15984000 & 5.19763000 & $\mathrm{H}$ & -1.46457300 & 5.28495400 & 3.48593500 \\
\hline $\mathrm{H}$ & 1.28827500 & 4.73815800 & 4.69391500 & $\mathrm{H}$ & 0.89410700 & 5.91072400 & 3.43021300 \\
\hline $\mathrm{N}$ & -0.07137200 & 2.76778100 & 3.14322100 & C & 0.94528700 & 4.32257700 & 1.32812100 \\
\hline C & 2.19394800 & 4.55267500 & 0.81899200 & C & -0.21724700 & 4.73752000 & 0.55489900 \\
\hline C & 2.43455600 & 5.23684600 & -0.41577900 & $\mathrm{H}$ & 3.06557200 & 4.23774900 & 1.39045100 \\
\hline C & -0.00514300 & 5.47311200 & -0.66167200 & C & -1.54504400 & 4.45130600 & 0.92176400 \\
\hline C & 3.72978800 & 5.46906700 & -0.90658900 & C & 1.31345700 & 5.72394800 & -1.14678700 \\
\hline C & -1.12334600 & 5.95860500 & -1.41173400 & $\mathrm{H}$ & -1.74619300 & 3.80898100 & 1.76599600 \\
\hline C & -2.62732100 & 4.92681000 & 0.18166300 & C & 3.92412400 & 6.17162600 & -2.09432000 \\
\hline $\mathrm{H}$ & 4.58406300 & 5.09977100 & -0.34382800 & C & 1.52154100 & 6.44760500 & -2.36131000 \\
\hline $\mathrm{C}$ & -2.42504900 & 5.69093200 & -0.96143300 & $\mathrm{C}$ & -0.88484700 & 6.69914500 & -2.62083100 \\
\hline $\mathrm{H}$ & -3.63562200 & 4.67789700 & 0.49969700 & C & 2.83424000 & 6.65759400 & -2.81437300 \\
\hline $\mathrm{H}$ & 4.93266500 & 6.34542200 & -2.46007400 & C & 0.37524600 & 6.93268900 & -3.07741200 \\
\hline $\mathrm{H}$ & -3.27225300 & 6.06317900 & -1.53213700 & $\mathrm{H}$ & -1.74678900 & 7.06662100 & -3.17253500 \\
\hline $\mathrm{H}$ & 2.99231400 & 7.20694400 & -3.73927900 & $\mathrm{H}$ & 0.53550300 & 7.48956600 & -3.99744600 \\
\hline
\end{tabular}




\begin{tabular}{|c|c|c|c|c|c|c|c|}
\hline C & -2.53503600 & -0.36806700 & 1.76504500 & $S$ & -3.65194000 & 0.81796500 & 2.24623400 \\
\hline $\mathrm{N}$ & -1.28027800 & -0.46016300 & 2.27278900 & $\mathrm{H}$ & -0.62976200 & -1.12003200 & 1.83796500 \\
\hline C & 0.90774500 & -0.12818800 & -1.67524200 & 0 & 1.07143700 & -0.90443600 & -2.60490700 \\
\hline $\mathrm{O}$ & -0.03956400 & 0.79365500 & -1.54708300 & C & -1.06144400 & 0.98561900 & -2.61825900 \\
\hline C & -1.80601700 & -0.32410800 & -2.89010200 & $\mathrm{H}$ & -2.71377100 & -0.10562000 & -3.46280600 \\
\hline $\mathrm{H}$ & -1.19339900 & -1.03387700 & -3.44648700 & $\mathrm{H}$ & -2.11109800 & -0.78993600 & -1.94996100 \\
\hline C & -1.98692800 & 2.02771300 & -1.99474900 & $\mathrm{H}$ & -1.46068800 & 2.97174600 & -1.83426800 \\
\hline $\mathrm{H}$ & -2.84250700 & 2.20784700 & -2.65347100 & $\mathrm{H}$ & -2.36340200 & 1.67951300 & -1.02893100 \\
\hline C & -0.36855800 & 1.53655000 & -3.86654600 & $\mathrm{H}$ & -1.12504800 & 1.77753000 & -4.62163600 \\
\hline $\mathrm{H}$ & 0.17917100 & 2.45499100 & -3.62968300 & $\mathrm{H}$ & 0.32105600 & 0.80421000 & -4.29232100 \\
\hline C & 2.66331200 & -1.14603700 & -0.41063300 & $\mathrm{H}$ & 2.24173000 & -2.10165400 & -0.69893100 \\
\hline C & 3.48701300 & -1.15066800 & 0.81913100 & $\mathrm{C}$ & 3.76828900 & -2.37999600 & 1.43712700 \\
\hline C & 4.02839300 & 0.02939400 & 1.35552300 & $\mathrm{C}$ & 4.57373500 & -2.42499700 & 2.57492400 \\
\hline $\mathrm{H}$ & 3.32387700 & -3.28879100 & 1.04437500 & C & 4.83789300 & -0.02218700 & 2.48700700 \\
\hline $\mathrm{H}$ & 3.81096700 & 0.98614200 & 0.88960100 & $\mathrm{C}$ & 5.11467700 & -1.24924300 & 3.09796900 \\
\hline $\mathrm{H}$ & 4.76874800 & -3.37879700 & 3.05667500 & $\mathrm{H}$ & 5.25205000 & 0.89525200 & 2.89601400 \\
\hline $\mathrm{H}$ & 5.74481600 & -1.28588300 & 3.98249000 & $\mathrm{~N}$ & 1.78501100 & -0.09039300 & -0.59115400 \\
\hline $\mathrm{H}$ & 1.57047800 & 0.52943700 & 0.19787700 & $S$ & 0.13786900 & -3.77345700 & 0.44079100 \\
\hline $\mathrm{O}$ & 1.16125600 & -4.23537500 & -0.52236800 & $\mathrm{O}$ & 0.43254100 & -2.42960000 & 1.02322600 \\
\hline $\mathrm{O}$ & -1.28073100 & -3.93587900 & 0.05558600 & C & 0.35718500 & -4.91770000 & 1.89282400 \\
\hline $\mathrm{F}$ & 0.15266400 & -6.18934800 & 1.53732000 & $\mathrm{~F}$ & -0.50569200 & -4.60264600 & 2.87309100 \\
\hline $\mathrm{F}$ & 1.60609500 & -4.80932500 & 2.38690900 & C & 3.98329700 & -0.98123900 & -1.87091000 \\
\hline $\mathrm{H}$ & 3.31649600 & -0.50866700 & -2.58327400 & $\mathrm{H}$ & 4.72675400 & -0.33895500 & -1.40678000 \\
\hline C & 4.33557900 & -2.31271600 & -2.13751200 & C & 5.51625300 & -2.92697100 & -1.53062600 \\
\hline $\mathrm{H}$ & 5.79344700 & -2.43529500 & -0.59009600 & $\mathrm{H}$ & 5.37588400 & -4.00105200 & -1.35669900 \\
\hline $\mathrm{C}$ & 3.41813300 & -3.17295100 & -2.94126800 & $\mathrm{H}$ & 3.95978100 & -3.97394700 & -3.45355900 \\
\hline $\mathrm{H}$ & 2.70952000 & -3.65951200 & -2.24662400 & $\mathrm{H}$ & 2.81281300 & -2.59818200 & -3.64400000 \\
\hline $\mathrm{C}$ & 8.45728200 & -3.71908300 & -1.68255800 & $\mathrm{H}$ & 8.19367200 & -4.77429400 & -1.54634200 \\
\hline $\mathrm{H}$ & 9.41181700 & -3.68405800 & -2.22164900 & $\mathrm{H}$ & 8.62179600 & -3.28375900 & -0.69011900 \\
\hline C & 6.82058500 & -3.58795300 & -4.32134000 & $\mathrm{H}$ & 6.50838700 & -4.63459200 & -4.22647300 \\
\hline $\bar{H}$ & 6.05430000 & -3.05941600 & -4.89953600 & $\mathrm{H}$ & 7.74372500 & -3.57585700 & -4.91389400 \\
\hline C & 7.57678700 & -0.96573800 & -2.83181900 & $\bar{H}$ & 6.80075200 & -0.40309300 & -3.36270700 \\
\hline $\mathrm{H}$ & 7.73766100 & -0.48285600 & -1.86069700 & $\mathrm{H}$ & 8.50621200 & -0.86537400 & -3.40559200 \\
\hline Si & 7.12311200 & -2.78724300 & -2.63961700 & & & & \\
\hline
\end{tabular}

\section{TS-S-4f-nd-56}

Imaginary frequency: $-203.74 \mathrm{~cm}^{-1}$

E[B3LYP/6-31G(d)]: -4788.680070 Hartree

E[PCM(Et $\left.\left.t_{2} \mathrm{O}\right)-B 3 L Y P / 6-311 G(d, p)-D 3(B J)\right]:-4790.201108$ Hartree

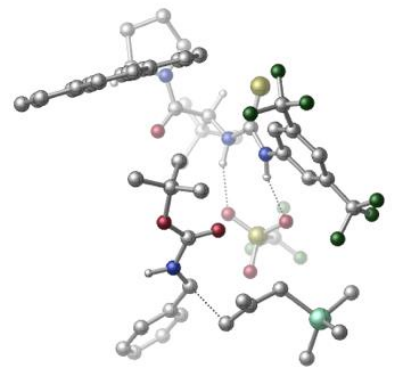

\begin{tabular}{|c|c|c|c|c|c|c|c|}
\hline 01 & & & & $\mathrm{~N}$ & 0.33849400 & 0.68622100 & -2.12888900 \\
\hline $\mathrm{H}$ & 1.19146100 & 0.14749200 & -1.96130300 & $\mathrm{C}$ & 0.45829900 & 2.05205000 & -1.83612500 \\
\hline $\mathrm{C}$ & 1.74599600 & 2.60883700 & -1.79306400 & $\mathrm{C}$ & -0.63994700 & 2.85698600 & -1.49572300 \\
\hline C & 1.91921900 & 3.94358500 & -1.42496400 & $\mathrm{H}$ & 2.60020400 & 1.98853400 & -2.04134600 \\
\hline $\mathrm{C}$ & -0.44643400 & 4.19232300 & -1.14612400 & $\bar{H}$ & -1.63603200 & 2.43760900 & -1.50174700 \\
\hline $\mathrm{C}$ & 0.82976600 & 4.75449100 & -1.10711300 & $\mathrm{H}$ & 0.97010200 & 5.79433600 & -0.84154300 \\
\hline C & 3.31212300 & 4.49526000 & -1.31882200 & C & -1.64456200 & 5.00848700 & -0.74222600 \\
\hline $\mathrm{F}$ & 4.13106500 & 4.01476500 & -2.28038200 & $\mathrm{~F}$ & 3.89059000 & 4.16124800 & -0.12426100 \\
\hline $\mathrm{F}$ & 3.34514500 & 5.84087300 & -1.39133700 & $\mathrm{~F}$ & -2.69031900 & 4.83247100 & -1.58239300 \\
\hline $\mathrm{F}$ & -2.09516500 & 4.66505100 & 0.49640000 & $\mathrm{~F}$ & -1.37687600 & 6.33066300 & -0.70341700 \\
\hline
\end{tabular}




\begin{tabular}{|c|c|c|c|c|c|c|c|}
\hline C & -1.74652000 & -2.22233100 & -2.96768300 & $\mathrm{H}$ & -2.16087500 & -1.77569100 & -3.87142300 \\
\hline $\mathrm{C}$ & -1.18509100 & -3.63475700 & -3.36301800 & C & -0.57949900 & -4.41126700 & -2.17582800 \\
\hline $\mathrm{H}$ & -1.31498900 & -4.57683600 & -1.38482100 & $\mathrm{H}$ & 0.27989900 & -3.89729200 & -1.73730400 \\
\hline $\mathrm{H}$ & -0.22751000 & -5.38769700 & -2.53228200 & C & -0.11135800 & -3.44096200 & -4.45266200 \\
\hline $\mathrm{H}$ & 0.22201100 & -4.41757500 & -4.82404300 & $\mathrm{H}$ & 0.76596600 & -2.91511800 & -4.06951200 \\
\hline $\mathrm{H}$ & -0.50413300 & -2.87328800 & -5.30520000 & C & -2.33833400 & -4.46931500 & -3.95809900 \\
\hline $\mathrm{H}$ & -1.96516100 & -5.45576600 & -4.25582900 & $\mathrm{H}$ & -2.76198100 & -3.99538900 & -4.85173600 \\
\hline $\mathrm{H}$ & -3.14671700 & -4.63032800 & -3.23528800 & C & -2.83314300 & -2.29880800 & -1.86290700 \\
\hline 0 & -2.51805600 & -2.54107200 & -0.69731900 & C & -4.67033400 & -1.83051100 & -3.56679800 \\
\hline C & -5.20414100 & -2.39993100 & -1.22993800 & C & -6.15874300 & -1.55499300 & -3.32281900 \\
\hline $\mathrm{H}$ & -4.14636100 & -0.98221500 & -4.01627300 & $\mathrm{H}$ & -4.54468500 & -2.70454000 & -4.21778800 \\
\hline C & -6.48634500 & -2.43560100 & -2.10931000 & $\mathrm{H}$ & -5.00312200 & -3.39847200 & -0.82857700 \\
\hline $\mathrm{H}$ & -6.76646500 & -1.79493700 & -4.20067000 & $\mathrm{H}$ & -6.31897800 & -0.49902900 & -3.08426000 \\
\hline $\mathrm{H}$ & -6.66628200 & -3.46768600 & -2.43432700 & $\mathrm{H}$ & -7.36852500 & -2.10235200 & -1.55747000 \\
\hline $\mathrm{N}$ & -4.14029600 & -2.10736800 & -2.21529000 & C & -5.34388400 & -1.50120800 & 0.00621500 \\
\hline C & -5.74247400 & -2.14814800 & 1.14368700 & C & -5.20985200 & -0.05205300 & 0.03295800 \\
\hline C & -6.07933700 & -1.47805200 & 2.36273200 & $\mathrm{H}$ & -5.82611300 & -3.23334600 & 1.13475800 \\
\hline C & -5.54081100 & 0.64878500 & 1.24452800 & C & -4.74243300 & 0.70920300 & -1.05378200 \\
\hline C & -6.50437700 & -2.17124100 & 3.50834500 & C & -5.98388600 & -0.05740400 & 2.40273900 \\
\hline C & -5.41402200 & 2.07355900 & 1.30842900 & $\mathrm{H}$ & -4.42052500 & 0.21999000 & -1.96018400 \\
\hline C & -4.61228800 & 2.09581600 & -0.97563500 & C & -6.83488900 & -1.48294500 & 4.67397900 \\
\hline $\mathrm{H}$ & -6.57576800 & -3.25575200 & 3.47465500 & C & -6.31734000 & 0.64009700 & 3.60510100 \\
\hline C & -4.94563200 & 2.77804000 & 0.18876100 & C & -5.75905700 & 2.75100600 & 2.52928500 \\
\hline $\mathrm{H}$ & -4.22583900 & 2.63941400 & -1.83214100 & C & -6.74320600 & -0.09296400 & 4.72473800 \\
\hline $\mathrm{H}$ & -7.16578000 & -2.03392700 & 5.55044600 & C & -6.19446800 & 2.07087900 & 3.62511400 \\
\hline $\mathrm{H}$ & -4.82210000 & 3.85594700 & 0.24591500 & $\mathrm{H}$ & -5.66494400 & 3.83414200 & 2.55514600 \\
\hline $\mathrm{H}$ & -6.99969400 & 0.43850100 & 5.63812800 & $\mathrm{H}$ & -6.45402700 & 2.60155400 & 4.53819600 \\
\hline C & -0.72721000 & 0.02689300 & -2.71424800 & $S$ & -1.91145900 & 0.81489700 & -3.63579700 \\
\hline $\mathrm{N}$ & -0.69477400 & -1.31201300 & -2.51921500 & $\mathrm{H}$ & 0.00425600 & -1.68207500 & -1.87473500 \\
\hline C & 1.06712500 & -0.28323000 & 2.51423800 & $\mathrm{O}$ & 1.48797500 & 0.26057400 & 1.51699600 \\
\hline 0 & -0.05704100 & -0.08505700 & 3.18624600 & C & -1.12455400 & 0.83846700 & 2.66285000 \\
\hline C & -1.59782100 & 0.32731100 & 1.30480000 & $\mathrm{H}$ & -2.44022300 & 0.94046200 & 0.97138000 \\
\hline $\mathrm{H}$ & -0.80829900 & 0.39401000 & 0.55423800 & $\mathrm{H}$ & -1.94075300 & -0.71004600 & 1.36067800 \\
\hline C & -2.21055100 & 0.70568400 & 3.72747600 & $\mathrm{H}$ & -1.83030800 & 1.00105200 & 4.71142300 \\
\hline $\mathrm{H}$ & -3.05450500 & 1.35357400 & 3.47376000 & $\mathrm{H}$ & -2.57630300 & -0.32383400 & 3.78527700 \\
\hline C & -0.56843300 & 2.26142000 & 2.60496000 & $\mathrm{H}$ & -1.36209800 & 2.94525600 & 2.28970700 \\
\hline $\mathrm{H}$ & -0.21225800 & 2.57959100 & 3.59159200 & $\mathrm{H}$ & 0.24603100 & 2.34067100 & 1.88276400 \\
\hline C & 2.92747500 & -1.83292200 & 2.64071700 & $\mathrm{H}$ & 3.06686900 & -1.65513200 & 1.57864000 \\
\hline C & 3.43898900 & -3.09524000 & 3.19291400 & C & 4.09479000 & -3.97661700 & 2.31392300 \\
\hline C & 3.27156700 & -3.45915800 & 4.54158000 & C & 4.54846700 & -5.20970100 & 2.77778000 \\
\hline $\mathrm{H}$ & 4.22758700 & -3.68507400 & 1.27569400 & C & 3.73383100 & -4.68908800 & 4.99789500 \\
\hline $\mathrm{H}$ & 2.80156400 & -2.77689200 & 5.24693900 & C & 4.36954700 & -5.56849500 & 4.11518100 \\
\hline $\mathrm{H}$ & 5.04135900 & -5.89176400 & 2.09138400 & $\mathrm{H}$ & 3.60283500 & -4.96243100 & 6.04079300 \\
\hline $\mathrm{H}$ & 4.72758800 & -6.52947500 & 4.47364100 & $\mathrm{~N}$ & 1.80336000 & -1.29792700 & 3.17240000 \\
\hline $\mathrm{H}$ & 1.41049100 & -1.66068300 & 4.03227900 & S & 3.19848500 & -1.69173000 & -1.36272900 \\
\hline 0 & 4.14520800 & -1.91848000 & -0.23485500 & $\mathrm{O}$ & 1.85179500 & -2.27680000 & -1.15416800 \\
\hline 0 & 3.18010400 & -0.30630100 & -1.89220000 & C & 3.95675000 & -2.69169400 & -2.73646100 \\
\hline $\mathrm{F}$ & 5.20655000 & -2.27184600 & -2.97530300 & $\mathrm{~F}$ & 3.24245600 & -2.56152500 & -3.86407500 \\
\hline $\mathrm{F}$ & 3.99648700 & -3.98913600 & -2.40266400 & C & 4.70146200 & -0.59897800 & 3.03328900 \\
\hline $\mathrm{H}$ & 4.70850000 & -0.74687200 & 4.10998000 & $\mathrm{H}$ & 5.31333900 & -1.27997400 & 2.45193700 \\
\hline C & 4.44277200 & 0.66504100 & 2.52440000 & C & 4.70392300 & 1.00917300 & 1.12208100 \\
\hline $\mathrm{H}$ & 4.53860300 & 0.14420600 & 0.47034200 & $\mathrm{H}$ & 4.07253800 & 1.83556900 & 0.78168900 \\
\hline C & 3.87844500 & 1.73147400 & 3.41955600 & $\mathrm{H}$ & 2.94482700 & 2.12424100 & 3.00212100 \\
\hline
\end{tabular}




\begin{tabular}{|llll|llll|}
\hline $\mathrm{H}$ & 3.70005700 & 1.37580500 & 4.43846100 & $\mathrm{H}$ & 4.57532600 & 2.57893700 & 3.46777000 \\
\hline $\mathrm{C}$ & 6.58130500 & 1.64603000 & -1.19058900 & $\mathrm{H}$ & 5.94685300 & 2.45869600 & -1.55774400 \\
\hline $\mathrm{H}$ & 7.60434400 & 1.83388600 & -1.53955500 & $\mathrm{H}$ & 6.22853200 & 0.71764300 & -1.65403900 \\
\hline $\mathrm{C}$ & 6.93585200 & 3.18657300 & 1.49332100 & $\mathrm{H}$ & 6.21538600 & 3.95318700 & 1.18624300 \\
\hline $\mathrm{H}$ & 6.93387400 & 3.13414000 & 2.58903200 & $\mathrm{H}$ & 7.93252600 & 3.52793400 & 1.18698000 \\
\hline $\mathrm{C}$ & 7.72201700 & 0.18560200 & 1.29407300 & $\mathrm{H}$ & 7.70962600 & 0.07486300 & 2.38446000 \\
\hline $\mathrm{H}$ & 7.48162400 & -0.78838900 & 0.85160400 & $\mathrm{H}$ & 8.74897600 & 0.43524400 & 0.99975600 \\
\hline $\mathrm{Si}$ & 6.52832700 & 1.52225600 & 0.68955600 & & & & \\
\hline
\end{tabular}

\section{TS-R-4f-nd-1}

Imaginary frequency: $-276.23 \mathrm{~cm}^{-1}$

E[B3LYP/6-31G(d)-D3(BJ)]: -4788.687560 Hartree

$\mathrm{E}\left[\mathrm{PCM}\left(\mathrm{Et}_{2} \mathrm{O}\right)-\mathrm{B} 3 \mathrm{LYP} / 6-311 \mathrm{G}(\mathrm{d}, \mathrm{p})-\mathrm{D} 3(\mathrm{BJ})\right]$ : -4790.226028 Hartree

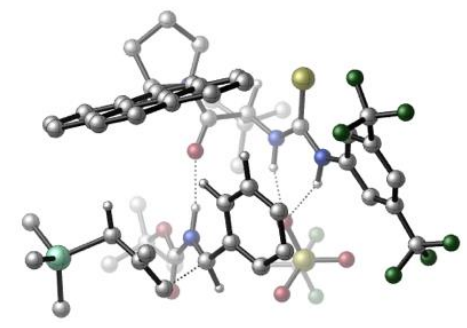

\begin{tabular}{|c|c|c|c|c|c|c|c|}
\hline 01 & & & & $\mathrm{~N}$ & -2.95188500 & -0.80667100 & 1.25616900 \\
\hline $\mathrm{H}$ & -2.61242800 & -1.63539000 & 0.75505700 & $\mathrm{C}$ & -4.00375900 & -0.15375500 & 0.59321900 \\
\hline C & -4.70059200 & -0.91287500 & -0.36407300 & C & -4.32600000 & 1.20110700 & 0.75520500 \\
\hline C & -5.68721000 & -0.31465000 & -1.14527700 & $\mathrm{H}$ & -4.45068700 & -1.96119600 & -0.51230000 \\
\hline C & -5.30971300 & 1.77893500 & -0.04467600 & $\mathrm{H}$ & -3.80166300 & 1.79314400 & 1.48931300 \\
\hline C & -6.00660900 & 1.03533500 & -0.99632500 & $\mathrm{H}$ & -6.77521200 & 1.49336300 & -1.60755500 \\
\hline C & -6.34990700 & -1.11078600 & -2.23859600 & C & -5.52506400 & 3.26514800 & 0.01894400 \\
\hline $\mathrm{F}$ & -6.45331100 & -2.41576300 & -1.93510000 & $\mathrm{~F}$ & -5.64688800 & -1.02498600 & -3.39936000 \\
\hline $\mathrm{F}$ & -7.59395800 & -0.65121100 & -2.50955500 & $\mathrm{~F}$ & -5.10705100 & 3.80819300 & 1.18392900 \\
\hline $\mathrm{F}$ & -4.82699700 & 3.89860400 & -0.96840100 & $\mathrm{~F}$ & -6.81946200 & 3.60675100 & -0.15066200 \\
\hline $\mathrm{C}$ & -0.04348000 & -1.09522700 & 3.44503200 & $\mathrm{H}$ & -0.47455700 & -0.53282500 & 4.27280200 \\
\hline C & 0.44816000 & -2.47399100 & 4.01382700 & C & 0.82355400 & -3.46604700 & 2.89777800 \\
\hline $\mathrm{H}$ & 1.56903200 & -3.04921500 & 2.21677700 & $\mathrm{H}$ & -0.04355900 & -3.77224600 & 2.30751900 \\
\hline $\mathrm{H}$ & 1.23754700 & -4.37704300 & 3.34814400 & $\mathrm{C}$ & -0.69829400 & -3.06960500 & 4.85573100 \\
\hline $\mathrm{H}$ & -0.40307000 & -4.04592000 & 5.25790800 & $\mathrm{H}$ & -1.59992800 & -3.21247900 & 4.25263500 \\
\hline $\mathrm{H}$ & -0.95568400 & -2.41993200 & 5.70106600 & $\mathrm{C}$ & 1.67323400 & -2.25722400 & 4.92336800 \\
\hline $\mathrm{H}$ & 2.00404600 & -3.21866500 & 5.33237200 & $\mathrm{H}$ & 1.43837100 & -1.60912100 & 5.77567200 \\
\hline $\mathrm{H}$ & 2.52032000 & -1.82258600 & 4.37951300 & C & 1.05505500 & -0.23456200 & 2.77259500 \\
\hline $\mathrm{O}$ & 1.48005400 & -0.50293900 & 1.63727900 & $\mathrm{C}$ & 1.14802000 & 1.28347900 & 4.81430000 \\
\hline $\mathrm{C}$ & 2.53108900 & 1.73040500 & 2.83726000 & $\mathrm{C}$ & 1.98830500 & 2.55217900 & 5.03889600 \\
\hline $\mathrm{H}$ & 0.07187800 & 1.46681300 & 4.88973200 & $\mathrm{H}$ & 1.42101600 & 0.50580000 & 5.53386800 \\
\hline $\mathrm{C}$ & 3.17941400 & 2.37657900 & 4.08488300 & $\mathrm{H}$ & 3.22964200 & 1.09446900 & 2.28849000 \\
\hline $\mathrm{H}$ & 2.28954200 & 2.66107600 & 6.08459000 & $\mathrm{H}$ & 1.41435300 & 3.44202600 & 4.76198800 \\
\hline $\mathrm{H}$ & 3.91527500 & 1.68439500 & 4.51282600 & $\mathrm{H}$ & 3.68708900 & 3.31650700 & 3.85489300 \\
\hline $\mathrm{N}$ & 1.51026300 & 0.85547400 & 3.44282300 & $\mathrm{C}$ & 1.94407200 & 2.77622800 & 1.88921000 \\
\hline C & 0.61143500 & 3.07476000 & 1.90705300 & $\mathrm{C}$ & 2.82928000 & 3.50224300 & 0.99252000 \\
\hline C & 0.03387000 & 4.09158200 & 1.07508800 & $\mathrm{H}$ & -0.06319200 & 2.51684800 & 2.55060100 \\
\hline C & 2.27422500 & 4.51290900 & 0.13928600 & C & 4.21470000 & 3.26150500 & 0.91944100 \\
\hline $\mathrm{C}$ & -1.34144200 & 4.37998800 & 1.10360600 & $\mathrm{C}$ & 0.87880500 & 4.80831900 & 0.18048100 \\
\hline C & 3.11741600 & 5.23813100 & -0.76064800 & $\mathrm{H}$ & 4.67098500 & 2.51584600 & 1.56150000 \\
\hline C & 5.03046100 & 3.98015200 & 0.04288400 & C & -1.88193500 & 5.34891000 & 0.25847600 \\
\hline $\mathrm{H}$ & -1.98409800 & 3.82622200 & 1.78307600 & C & 0.32071700 & 5.80906500 & -0.67182400 \\
\hline C & 4.49284200 & 4.95526400 & -0.79255200 & $C$ & 2.53033800 & 6.24441900 & -1.60386500 \\
\hline $\mathrm{H}$ & 6.09977000 & 3.78453200 & 0.02621400 & C & -1.06077200 & 6.05852100 & -0.61679400 \\
\hline $\mathrm{H}$ & -2.95156800 & 5.53308500 & 0.27341900 & C & 1.19708900 & 6.51686400 & -1.56288900 \\
\hline $\mathrm{H}$ & 5.13597700 & 5.51812700 & -1.46491400 & $\mathrm{H}$ & 3.18283700 & 6.79117400 & -2.28047700 \\
\hline
\end{tabular}




\begin{tabular}{|c|c|c|c|c|c|c|c|}
\hline $\mathrm{H}$ & -1.48793800 & 6.81296000 & -1.27291400 & $\overline{\mathrm{H}}$ & 0.77156700 & 7.28145300 & -2.20833800 \\
\hline C & -2.17757300 & -0.44526300 & 2.32762400 & $\mathrm{~S}$ & -2.49823300 & 0.83611600 & 3.39742500 \\
\hline $\mathrm{N}$ & -1.11272000 & -1.28006900 & 2.47867700 & $\mathrm{H}$ & -0.92227200 & -1.92010500 & 1.70750200 \\
\hline S & -1.75371000 & -3.86364000 & -0.89211300 & 0 & -1.07540200 & -3.86305200 & -2.20482000 \\
\hline 0 & -1.23563500 & -2.81700800 & 0.05226900 & 0 & -3.22293200 & -3.97048900 & -0.89635900 \\
\hline C & -1.15791300 & -5.43543000 & -0.09350300 & $\mathrm{~F}$ & -1.54147300 & -6.51064600 & -0.78775200 \\
\hline $\mathrm{F}$ & -1.63528100 & -5.54285800 & 1.15893400 & $\mathrm{~F}$ & 0.18977400 & -5.43504700 & -0.02022600 \\
\hline C & 2.58752800 & -2.95008300 & -1.01355400 & 0 & 2.80204500 & -3.59871500 & -2.02517200 \\
\hline 0 & 3.17453000 & -3.10939100 & 0.18080200 & C & 4.15248200 & -4.20402600 & 0.40240400 \\
\hline C & 3.46596200 & -5.55952300 & 0.21955300 & $\mathrm{H}$ & 4.16316500 & -6.35750100 & 0.49896100 \\
\hline $\mathrm{H}$ & 3.15659400 & -5.71225900 & -0.81564000 & $\mathrm{H}$ & 2.58327200 & -5.63308900 & 0.86061600 \\
\hline C & 4.57163500 & -3.98854800 & 1.85788100 & $\mathrm{H}$ & 5.00002500 & -2.99000900 & 1.99448200 \\
\hline $\mathrm{H}$ & 5.32624000 & -4.73074300 & 2.13825300 & $\mathrm{H}$ & 3.71613200 & -4.09340600 & 2.52979700 \\
\hline C & 5.35608000 & -4.02793600 & -0.53006000 & $\mathrm{H}$ & 6.12814800 & -4.75845200 & -0.26469200 \\
\hline $\mathrm{H}$ & 5.78837000 & -3.02692900 & -0.41190700 & $\mathrm{H}$ & 5.07751600 & -4.17978700 & -1.57363900 \\
\hline C & 0.99450200 & -1.55666800 & -2.13753600 & $\mathrm{H}$ & 0.73293400 & -2.46094400 & -2.68516500 \\
\hline C & -0.11848100 & -0.57833500 & -2.06417700 & C & -1.18423600 & -0.74281400 & -2.96605000 \\
\hline C & -0.12622700 & 0.51908200 & -1.18761900 & C & -2.23386200 & 0.17203700 & -2.99743700 \\
\hline $\mathrm{H}$ & -1.20950600 & -1.62063600 & -3.60368400 & C & -1.17706500 & 1.43627800 & -1.23001200 \\
\hline $\mathrm{H}$ & 0.66937300 & 0.65505700 & -0.46006200 & C & -2.22515100 & 1.27281500 & -2.13770700 \\
\hline $\mathrm{H}$ & -3.07455800 & 0.00613600 & -3.66430300 & $\mathrm{H}$ & -1.17990600 & 2.27805700 & -0.54743100 \\
\hline $\mathrm{H}$ & -3.04132500 & 1.98906500 & -2.14949700 & $\mathrm{~N}$ & 1.72477700 & -1.86055000 & -0.99006600 \\
\hline $\mathrm{H}$ & 1.54288200 & -1.39942900 & -0.09278300 & C & 2.18144300 & -0.98532300 & -3.48510500 \\
\hline $\mathrm{H}$ & 1.53905900 & -1.00452900 & -4.36311000 & $\mathrm{H}$ & 2.83830200 & -1.84961900 & -3.39935000 \\
\hline $\mathrm{C}$ & 2.69855200 & 0.27092900 & -3.11415500 & C & 3.76047300 & 0.38195100 & -2.12394000 \\
\hline $\mathrm{H}$ & 3.77262600 & -0.47863800 & -1.44290600 & $\mathrm{H}$ & 3.69950200 & 1.31422800 & -1.54847600 \\
\hline C & 2.06352300 & 1.51956500 & -3.62589200 & $\mathrm{H}$ & 2.73221100 & 2.38236500 & -3.56456100 \\
\hline $\mathrm{H}$ & 1.19731300 & 1.73244900 & -2.97570000 & $\mathrm{H}$ & 1.67940900 & 1.40863100 & -4.64361600 \\
\hline $\mathrm{C}$ & 6.72779400 & 0.39883000 & -1.42881500 & $\mathrm{H}$ & 6.53828600 & 1.25512300 & -0.77196500 \\
\hline $\mathrm{H}$ & 7.77097900 & 0.45701500 & -1.76246800 & $\mathrm{H}$ & 6.62153600 & -0.51439000 & -0.83186200 \\
\hline C & 5.76333200 & 1.97458100 & -3.92782500 & $\mathrm{H}$ & 5.55289100 & 2.86905200 & -3.32966700 \\
\hline $\mathrm{H}$ & 5.10505000 & 1.99003600 & -4.80401300 & $\mathrm{H}$ & 6.79424300 & 2.05691600 & -4.29366200 \\
\hline C & 5.79643600 & -1.14159700 & -3.96871100 & $\mathrm{H}$ & 5.15419200 & -1.13462300 & -4.85639200 \\
\hline $\mathrm{H}$ & 5.58191500 & -2.05931200 & -3.40906700 & $\mathrm{H}$ & 6.83534600 & -1.20233600 & -4.31561000 \\
\hline $\mathrm{Si}$ & 5.55741200 & 0.39906700 & -2.90927700 & & & & \\
\hline
\end{tabular}

\section{TS-R-4f-nd-2}

Imaginary frequency: $-289.64 \mathrm{~cm}^{-1}$

E[B3LYP/6-31G(d)]: -4788.689177 Hartree

E[PCM(Et $\left.\left.{ }_{2} \mathrm{O}\right)-B 3 L Y P / 6-311 G(d, p)-D 3(B J)\right]:-4790.224436$ Hartree

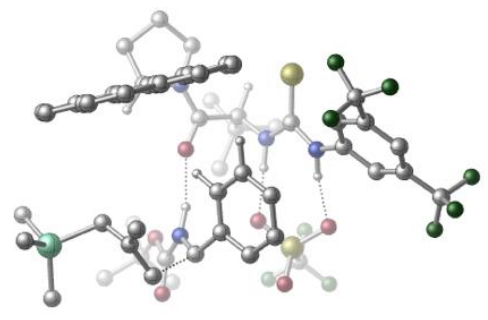

\begin{tabular}{|lrrr|lrrc|}
\hline 01 & & & & N & -3.15475300 & -0.59967300 & 0.94658300 \\
\hline H & -3.04899200 & -1.52383000 & 0.50718600 & C & -4.17735700 & 0.16962000 & 0.36671600 \\
\hline C & -5.19414800 & -0.51786100 & -0.31563600 & C & -4.17895500 & 1.57039100 & 0.35302400 \\
\hline C & -6.19848400 & 0.18970400 & -0.97445100 & H & -5.17636800 & -1.60161700 & -0.34976900 \\
\hline C & -5.18303800 & 2.26023300 & -0.32329900 & H & -3.39457200 & 2.11167800 & 0.85930500 \\
\hline C & -6.20800400 & 1.58503200 & -0.98555900 & H & -6.98617200 & 2.12915500 & -1.50683600 \\
\hline C & -7.32560400 & -0.55990600 & -1.63528800 & C & -5.07265700 & 3.75394600 & -0.44177300 \\
\hline F & -8.36500500 & -0.74359800 & -0.78701200 & F & -6.94416600 & -1.77979000 & -2.06261700 \\
\hline F & -7.81234800 & 0.11321700 & -2.70493200 & F & -4.51154700 & 4.32147900 & 0.65297000 \\
\hline
\end{tabular}




\begin{tabular}{|c|c|c|c|c|c|c|c|}
\hline $\mathrm{F}$ & -4.28125300 & 4.10954000 & -1.49509200 & $\mathrm{~F}$ & -6.26632300 & 4.34849500 & -0.64050200 \\
\hline C & -0.33314200 & -1.15389800 & 3.21099100 & $\mathrm{H}$ & -0.79997800 & -0.57955900 & 4.01088900 \\
\hline C & -0.01318500 & -2.57640300 & 3.79502000 & C & 0.72761100 & -3.48587000 & 2.80103100 \\
\hline $\mathrm{H}$ & 1.67989600 & -3.05018000 & 2.48969000 & $\mathrm{H}$ & 0.14147800 & -3.68028400 & 1.90046500 \\
\hline $\mathrm{H}$ & 0.92956600 & -4.45138600 & 3.28293900 & C & -1.34537700 & -3.24064700 & 4.19925500 \\
\hline $\mathrm{H}$ & -1.14514200 & -4.18210700 & 4.72466600 & $\mathrm{H}$ & -1.96047200 & -3.47150100 & 3.32517900 \\
\hline $\bar{H}$ & -1.93021900 & -2.59794200 & 4.86822600 & $\mathrm{C}$ & 0.85810600 & -2.41205700 & 5.05698200 \\
\hline $\mathrm{H}$ & 1.09547900 & -3.39724600 & 5.47418200 & $\mathrm{H}$ & 0.33871500 & -1.84314400 & 5.83735400 \\
\hline $\mathrm{H}$ & 1.81036300 & -1.91362800 & 4.83767200 & C & 0.89682300 & -0.37218100 & 2.68623100 \\
\hline $\mathrm{O}$ & 1.41136000 & -0.63869100 & 1.58771100 & C & 0.91105600 & 1.07641300 & 4.78417300 \\
\hline C & 2.51962000 & 1.45167200 & 2.96951500 & C & 1.82062100 & 2.26778500 & 5.12857600 \\
\hline $\mathrm{H}$ & -0.14975600 & 1.34312300 & 4.75750200 & $\mathrm{H}$ & 1.04954400 & 0.26348500 & 5.50235100 \\
\hline $\mathrm{C}$ & 3.08579600 & 2.01554100 & 4.29469100 & $\mathrm{H}$ & 3.21762000 & 0.77234400 & 2.47462800 \\
\hline $\mathrm{H}$ & 2.02091500 & 2.33003200 & 6.20198300 & $\mathrm{H}$ & 1.35045500 & 3.20731900 & 4.82145800 \\
\hline $\mathrm{H}$ & 3.71628000 & 1.25604800 & 4.77375800 & $\mathrm{H}$ & 3.68793100 & 2.91517100 & 4.14521700 \\
\hline $\mathrm{N}$ & 1.37497000 & 0.65175600 & 3.44196100 & C & 2.12112700 & 2.56334000 & 1.99846200 \\
\hline $\mathrm{C}$ & 0.82348700 & 2.97639700 & 1.89307300 & C & 3.15046300 & 3.22820300 & 1.21551500 \\
\hline C & 0.42083600 & 4.05449300 & 1.03534900 & $\mathrm{H}$ & 0.04313100 & 2.46780800 & 2.45291800 \\
\hline $\mathrm{C}$ & 2.77114500 & 4.29540300 & 0.33528300 & C & 4.51242500 & 2.87606000 & 1.28062200 \\
\hline $\mathrm{C}$ & -0.91940500 & 4.46758200 & 0.94202200 & C & 1.40830900 & 4.70837500 & 0.24445800 \\
\hline C & 3.75952700 & 4.96232500 & -0.45562000 & $\mathrm{H}$ & 4.83743700 & 2.08580800 & 1.94861500 \\
\hline $\mathrm{C}$ & 5.47097300 & 3.54005200 & 0.51211800 & C & -1.28771600 & 5.49573300 & 0.07452100 \\
\hline $\mathrm{H}$ & -1.67093300 & 3.97017800 & 1.54982800 & C & 1.02577700 & 5.76892500 & -0.63215100 \\
\hline C & 5.10388300 & 4.56848800 & -0.35131900 & C & 3.34694300 & 6.02738200 & -1.32959300 \\
\hline $\mathrm{H}$ & 6.51712200 & 3.25840300 & 0.60245800 & C & -0.32704700 & 6.14121900 & -0.70249900 \\
\hline $\mathrm{H}$ & -2.33266300 & 5.77916600 & -0.00129500 & C & 2.04375500 & 6.41203000 & -1.41509500 \\
\hline $\mathrm{H}$ & 5.85729600 & 5.08826300 & -0.93847000 & $\mathrm{H}$ & 4.10821900 & 6.52758800 & -1.92353500 \\
\hline $\mathrm{H}$ & -0.62020600 & 6.94267600 & -1.37621800 & $\mathrm{H}$ & 1.75134800 & 7.22089800 & -2.08032900 \\
\hline $\mathrm{C}$ & -2.33171000 & -0.31031300 & 2.00429200 & $S$ & -2.57660200 & 0.99121600 & 3.07661400 \\
\hline $\mathrm{N}$ & -1.31887100 & -1.20406900 & 2.13987000 & $\mathrm{H}$ & -1.12195700 & -1.83451300 & 1.35241800 \\
\hline $\mathrm{S}$ & -1.81776900 & -3.57650000 & -0.91584400 & $\mathrm{O}$ & -1.51680000 & -3.69365500 & -2.35153100 \\
\hline $\mathrm{O}$ & -0.73884100 & -2.93379400 & -0.10427500 & $\mathrm{O}$ & -3.17255800 & -3.08109200 & -0.55278000 \\
\hline C & -1.80898800 & -5.32554700 & -0.28046200 & $\mathrm{~F}$ & -2.75543700 & -6.06148800 & -0.87084900 \\
\hline $\mathrm{F}$ & -2.02385900 & -5.33800100 & 1.04802500 & $\mathrm{~F}$ & -0.61491200 & -5.90150700 & -0.51131300 \\
\hline $\mathrm{C}$ & 2.56803100 & -3.16695000 & -1.15705600 & $\mathrm{O}$ & 2.78793500 & -3.73498700 & -2.21643400 \\
\hline 0 & 3.02335700 & -3.51778200 & 0.04928500 & C & 3.77843400 & -4.78728000 & 0.21940300 \\
\hline C & 2.88864100 & -5.96930600 & -0.17460500 & $\mathrm{H}$ & 3.37975800 & -6.90450600 & 0.11690500 \\
\hline $\mathrm{H}$ & 2.70720500 & -5.99139300 & -1.25036800 & $\mathrm{H}$ & 1.92401200 & -5.91166900 & 0.33836400 \\
\hline C & 4.08554200 & -4.80317000 & 1.71736500 & $\mathrm{H}$ & 4.64590900 & -3.90826900 & 2.00808100 \\
\hline $\mathrm{H}$ & 4.69107300 & -5.68316700 & 1.95788000 & $\mathrm{H}$ & 3.16636200 & -4.84319000 & 2.30588200 \\
\hline $\mathrm{C}$ & 5.08005600 & -4.73397500 & -0.58822200 & $\mathrm{H}$ & 5.68527100 & -5.61687000 & -0.35470800 \\
\hline $\mathrm{H}$ & 5.66389000 & -3.84674000 & -0.31486100 & $\mathrm{H}$ & 4.88416400 & -4.72042300 & -1.66097600 \\
\hline $\mathrm{C}$ & 1.21783600 & -1.54187000 & -2.25392400 & $\mathrm{H}$ & 0.98741400 & -2.37700700 & -2.91275900 \\
\hline $\mathrm{C}$ & 0.14037000 & -0.52781000 & -2.20048100 & $\mathrm{C}$ & -0.82917300 & -0.56983000 & -3.21746500 \\
\hline C & 0.07747100 & 0.48986300 & -1.23434200 & C & -1.83328000 & 0.39211600 & -3.27894300 \\
\hline $\mathrm{H}$ & -0.81315000 & -1.38299600 & -3.93677800 & $\mathrm{C}$ & -0.93320600 & 1.44898600 & -1.30244100 \\
\hline $\mathrm{H}$ & 0.79567100 & 0.52737200 & -0.42029900 & $\mathrm{C}$ & -1.88205400 & 1.41215300 & -2.32643400 \\
\hline $\mathrm{H}$ & -2.58424900 & 0.33670500 & -4.06153300 & $\mathrm{H}$ & -0.97590600 & 2.23156800 & -0.55384800 \\
\hline $\mathrm{H}$ & -2.66145100 & 2.16704700 & -2.36468700 & $\mathrm{~N}$ & 1.83997700 & -1.98265400 & -1.09162200 \\
\hline $\mathrm{H}$ & 1.57862600 & -1.61282300 & -0.17199800 & $\mathrm{C}$ & 2.55376000 & -0.90965000 & -3.44713800 \\
\hline $\mathrm{H}$ & 1.98491600 & -0.78555100 & -4.36619200 & $\mathrm{H}$ & 3.13975900 & -1.82612900 & -3.40761900 \\
\hline $\mathrm{C}$ & 3.11750600 & 0.25382500 & -2.89121600 & C & 4.09703600 & 0.16788100 & -1.81752200 \\
\hline $\mathrm{H}$ & 3.96602400 & -0.74990200 & -1.22836000 & $\mathrm{H}$ & 4.07239600 & 1.04381600 & -1.15766600 \\
\hline
\end{tabular}




\begin{tabular}{|c|c|c|c|c|c|c|c|}
\hline C & 2.60949100 & 1.59699500 & -3.29404700 & $\mathrm{H}$ & 3.33054200 & 2.39302600 & -3.08921400 \\
\hline $\mathrm{H}$ & 1.71565900 & 1.80465000 & -2.68032100 & $\mathrm{H}$ & 2.29343800 & 1.63083400 & -4.34024700 \\
\hline C & 6.98273000 & -0.14664700 & -0.88948300 & $\mathrm{H}$ & 6.82508100 & 0.68497600 & -0.19374700 \\
\hline $\mathrm{H}$ & 8.05101800 & -0.17922200 & -1.13576700 & $\mathrm{H}$ & 6.73515500 & -1.07671000 & -0.36468600 \\
\hline C & 6.38120600 & 1.66779900 & -3.34446300 & $\mathrm{H}$ & 6.19895300 & 2.54077200 & -2.70679400 \\
\hline $\mathrm{H}$ & 5.80333000 & 1.79576900 & -4.26708400 & $\mathrm{H}$ & 7.44273300 & 1.67511000 & -3.62093800 \\
\hline C & 6.13624800 & -1.42819000 & -3.59412400 & $\mathrm{H}$ & 5.58540800 & -1.30163900 & -4.53274500 \\
\hline $\mathrm{H}$ & 5.78342900 & -2.35239600 & -3.12192300 & $\mathrm{H}$ & 7.19293100 & -1.57228000 & -3.85126100 \\
\hline $\mathrm{Si}$ & 5.94766300 & 0.06152400 & -2.45372700 & & & & \\
\hline
\end{tabular}

\section{TS- $R-4 f-n d-3$}

Imaginary frequency: $-172.92 \mathrm{~cm}^{-1}$

E[B3LYP/6-31G(d)]: -4788.689205 Hartree

E[PCM(Et $\left.\left.{ }_{2} \mathrm{O}\right)-\mathrm{B} 3 L Y P / 6-311 \mathrm{G}(\mathrm{d}, \mathrm{p})-\mathrm{D} 3(\mathrm{BJ})\right]:-4790.224394$ Hartree

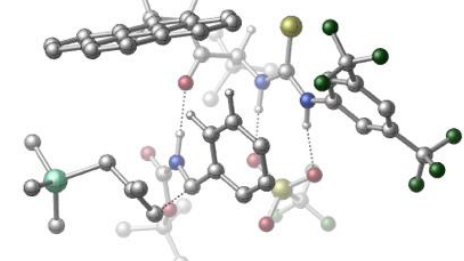

\begin{tabular}{|c|c|c|c|c|c|c|c|}
\hline 01 & & & & $\mathrm{~N}$ & 3.00674900 & -0.02537300 & 0.93518700 \\
\hline $\mathrm{H}$ & 3.01881500 & 0.93949300 & 0.58253800 & C & 3.91431900 & -0.86524600 & 0.26355800 \\
\hline C & 5.04569300 & -0.27081500 & -0.31526100 & C & 3.69224200 & -2.23448500 & 0.06942100 \\
\hline C & 5.94394100 & -1.04300500 & -1.05168800 & $\mathrm{H}$ & 5.20249400 & 0.79655500 & -0.20836600 \\
\hline C & 4.59671900 & -2.98938100 & -0.67447300 & $\mathrm{H}$ & 2.81215600 & -2.69796700 & 0.48801900 \\
\hline C & 5.73451500 & -2.40978200 & -1.23617500 & $\mathrm{H}$ & 6.43377800 & -3.00377700 & -1.81190800 \\
\hline C & 7.19301000 & -0.40662600 & -1.60205200 & C & 4.26393500 & -4.42486400 & -0.96816000 \\
\hline $\mathrm{F}$ & 8.21834200 & -0.47689900 & -0.72038100 & $\mathrm{~F}$ & 7.01303400 & 0.89730800 & -1.89397000 \\
\hline $\mathrm{F}$ & 7.61096900 & -1.02318200 & -2.73310100 & $\mathrm{~F}$ & 3.61889300 & -5.02724300 & 0.05973700 \\
\hline $\mathrm{F}$ & 3.43439600 & -4.53428600 & -2.04610300 & $\mathrm{~F}$ & 5.35684500 & -5.16304100 & -1.24914100 \\
\hline C & 0.37296800 & 0.62296800 & 3.40025800 & $\mathrm{H}$ & 0.81278900 & -0.06261300 & 4.12495000 \\
\hline C & 0.19120700 & 2.00398900 & 4.13126900 & $\mathrm{C}$ & -0.59271500 & 3.03819900 & 3.30664800 \\
\hline $\mathrm{H}$ & -1.60587800 & 2.70264100 & 3.07400100 & $\mathrm{H}$ & -0.09325800 & 3.27561400 & 2.36526500 \\
\hline $\mathrm{H}$ & -0.67037900 & 3.96985300 & 3.88154800 & C & 1.59176100 & 2.56435900 & 4.45767900 \\
\hline $\mathrm{H}$ & 1.49310600 & 3.44381100 & 5.10523500 & $\mathrm{H}$ & 2.12338900 & 2.87696100 & 3.55439300 \\
\hline $\mathrm{H}$ & 2.21113200 & 1.82666900 & 4.98211800 & C & -0.56240000 & 1.76434500 & 5.45557200 \\
\hline $\mathrm{H}$ & -0.68980200 & 2.71567600 & 5.98424100 & $\mathrm{H}$ & -0.01245400 & 1.08828400 & 6.12150000 \\
\hline $\mathrm{H}$ & -1.56405900 & 1.35127400 & 5.28677000 & C & -0.93643800 & 0.00030300 & 2.85641100 \\
\hline $\mathrm{O}$ & -1.43605100 & 0.38334900 & 1.78684600 & C & -1.06750800 & -1.61773800 & 4.82502400 \\
\hline C & -2.78276800 & -1.59491000 & 3.06592900 & C & -2.08143500 & -2.74792500 & 5.06727800 \\
\hline $\mathrm{H}$ & -0.03965400 & -1.97902700 & 4.72508800 & $\mathrm{H}$ & -1.10446400 & -0.88427900 & 5.63564900 \\
\hline$C$ & -3.34643200 & -2.26255800 & 4.34311900 & $\mathrm{H}$ & -3.41521700 & -0.77679000 & 2.71395000 \\
\hline $\mathrm{H}$ & -2.24168200 & -2.93049000 & 6.13372200 & $\mathrm{H}$ & -1.72619600 & -3.68071200 & 4.61760100 \\
\hline $\mathrm{H}$ & -3.86885300 & -1.51072600 & 4.94774900 & $\mathrm{H}$ & -4.04970700 & -3.06829300 & 4.11769900 \\
\hline $\mathrm{N}$ & -1.52424500 & -1.00314700 & 3.55776400 & C & -2.58353000 & -2.59838400 & 1.93081000 \\
\hline C & -1.36766900 & -3.17880800 & 1.70824600 & C & -3.72023300 & -2.97584000 & 1.10613000 \\
\hline C & -1.15590200 & -4.16544100 & 0.68772100 & $\mathrm{H}$ & -0.50442800 & -2.87530500 & 2.29439400 \\
\hline C & -3.53075900 & -3.94600800 & 0.06679700 & C & -5.00707200 & -2.43030000 & 1.27515100 \\
\hline C & 0.10365400 & -4.75102600 & 0.47118300 & C & -2.25115700 & -4.54225200 & -0.14158100 \\
\hline C & -4.62626700 & -4.32742700 & -0.77069700 & $\mathrm{H}$ & -5.19140000 & -1.70595800 & 2.06081000 \\
\hline C & -6.07057500 & -2.81201900 & 0.45517300 & C & 0.28796200 & -5.68420700 & -0.54899100 \\
\hline $\mathrm{H}$ & 0.93908300 & -4.46081900 & 1.10291700 & C & -2.05707900 & -5.50638700 & -1.17733500 \\
\hline C & -5.88710100 & -3.74620500 & -0.56042600 & C & -4.40380200 & -5.30330500 & -1.80345800 \\
\hline $\mathrm{H}$ & -7.05554800 & -2.38403600 & 0.62461600 & C & -0.77969100 & -6.06075200 & -1.36265700 \\
\hline $\mathrm{H}$ & 1.27528100 & -6.10364100 & -0.71427900 & C & -3.17962000 & -5.86600800 & -1.99827500 \\
\hline $\mathrm{H}$ & -6.72229600 & -4.04577200 & -1.18921000 & $\mathrm{H}$ & -5.24603000 & -5.58833300 & -2.42968900 \\
\hline
\end{tabular}




\begin{tabular}{|c|c|c|c|c|c|c|c|}
\hline $\mathrm{H}$ & -0.62960400 & -6.78914400 & -2.15589500 & $\mathrm{H}$ & -3.03131400 & -6.60361100 & -2.78339200 \\
\hline C & 2.18820400 & -0.31175300 & 1.99684500 & S & 2.27217300 & -1.75910200 & 2.89277500 \\
\hline $\mathrm{N}$ & 1.32850000 & 0.69319800 & 2.30050500 & $\mathrm{H}$ & 1.25081000 & 1.48524900 & 1.65472900 \\
\hline $\mathrm{S}$ & 2.25988900 & 3.43913800 & -0.45893400 & $\mathrm{O}$ & 1.76246000 & 3.85623400 & -1.78639300 \\
\hline 0 & 1.19284400 & 3.06011400 & 0.51322500 & $\mathrm{O}$ & 3.40574700 & 2.49351500 & -0.45543600 \\
\hline $\mathrm{C}$ & 2.97204100 & 4.99384200 & 0.27400300 & $\mathrm{~F}$ & 4.03463400 & 5.40693500 & -0.42416400 \\
\hline $\mathrm{F}$ & 3.34828400 & 4.78080100 & 1.54402700 & $\mathrm{~F}$ & 2.05479800 & 5.98066700 & 0.26771300 \\
\hline C & -2.10314500 & 3.39806300 & -0.08162200 & $\mathrm{O}$ & -2.75305700 & 3.52844900 & 0.93798300 \\
\hline 0 & -1.82381400 & 4.35347700 & -0.98421100 & C & -2.05133700 & 5.79157500 & -0.69677200 \\
\hline C & -3.55607300 & 6.06965600 & -0.71539600 & $\mathrm{H}$ & -3.73485800 & 7.14189400 & -0.57832300 \\
\hline $\mathrm{H}$ & -4.06056300 & 5.52805800 & 0.08805600 & $\mathrm{H}$ & -3.99216700 & 5.77778600 & -1.67820800 \\
\hline C & -1.33047000 & 6.46718900 & -1.86487600 & $\mathrm{H}$ & -0.27137700 & 6.19219200 & -1.86483000 \\
\hline $\mathrm{H}$ & -1.41447800 & 7.55536900 & -1.77640400 & $\mathrm{H}$ & -1.76963700 & 6.16560800 & -2.82237800 \\
\hline C & -1.40452100 & 6.18160900 & 0.63473600 & $\mathrm{H}$ & -1.44900700 & 7.27147400 & 0.74097700 \\
\hline $\mathrm{H}$ & -0.35450600 & 5.87961200 & 0.65289700 & $\mathrm{H}$ & -1.92096200 & 5.72636200 & 1.48053300 \\
\hline C & -0.96309700 & 1.96548400 & -1.72246000 & $\mathrm{H}$ & -0.44746400 & 2.86786600 & -2.05157700 \\
\hline C & -0.10962400 & 0.75189600 & -1.90317600 & C & 0.93343300 & 0.83262000 & -2.84115400 \\
\hline C & -0.34312900 & -0.46603900 & -1.24425800 & C & 1.71578000 & -0.28353200 & -3.12956300 \\
\hline $\mathrm{H}$ & 1.15399700 & 1.78599100 & -3.31144900 & C & 0.44532000 & -1.58009000 & -1.53836600 \\
\hline $\mathrm{H}$ & -1.11772300 & -0.55244200 & -0.48778700 & $\mathrm{C}$ & 1.46710700 & -1.49732100 & -2.48659500 \\
\hline $\mathrm{H}$ & 2.52922100 & -0.19857800 & -3.84425800 & $\mathrm{H}$ & 0.26084500 & -2.51506400 & -1.02119700 \\
\hline $\mathrm{H}$ & 2.07869000 & -2.36935200 & -2.69671900 & $\mathrm{~N}$ & -1.60145200 & 2.16312200 & -0.49213700 \\
\hline $\mathrm{H}$ & -1.53163700 & 1.47656000 & 0.26763400 & C & -2.21525800 & 2.00099700 & -3.02364100 \\
\hline $\mathrm{H}$ & -1.64037400 & 2.09555700 & -3.94380600 & $\mathrm{H}$ & -2.69854800 & 2.93310000 & -2.73593000 \\
\hline C & -3.00507900 & 0.82215600 & -2.91504800 & C & -3.99024600 & 0.68870300 & -1.86449500 \\
\hline $\mathrm{H}$ & -3.74953900 & 1.33031600 & -1.00500800 & $\mathrm{H}$ & -4.12615000 & -0.35246800 & -1.54595200 \\
\hline C & -2.69654200 & -0.35476300 & -3.77501700 & $\mathrm{H}$ & -3.53582800 & -1.05234600 & -3.84059700 \\
\hline $\mathrm{H}$ & -1.86118500 & -0.89281100 & -3.29434900 & $\mathrm{H}$ & -2.36075500 & -0.06818300 & -4.77534500 \\
\hline C & -6.78155000 & 1.14277600 & -0.75711400 & $\mathrm{H}$ & -6.73386900 & 0.13149600 & -0.33777800 \\
\hline $\mathrm{H}$ & -7.83705900 & 1.38709900 & -0.92822300 & $\mathrm{H}$ & -6.39483700 & 1.83861900 & -0.00392200 \\
\hline C & -6.46927400 & 0.06593000 & -3.66407400 & $\mathrm{H}$ & -6.44568100 & -0.97055600 & -3.30753800 \\
\hline $\mathrm{H}$ & -5.90395300 & 0.11561700 & -4.60182300 & $\mathrm{H}$ & -7.51302000 & 0.30767300 & -3.89984900 \\
\hline C & -5.75776400 & 3.02829100 & -3.01552600 & $\mathrm{H}$ & -5.18683200 & 3.11409600 & -3.94687800 \\
\hline $\mathrm{H}$ & -5.32200200 & 3.71878600 & -2.28438700 & $\mathrm{H}$ & -6.77759700 & 3.37498500 & -3.22349700 \\
\hline $\mathrm{Si}$ & -5.80555200 & 1.26075700 & -2.36347600 & & & & \\
\hline
\end{tabular}

\section{TS-R-4f-nd-4}

Imaginary frequency: $-199.41 \mathrm{~cm}^{-1}$

E[B3LYP/6-31G(d)]: -4788.682444 Hartree

E[PCM(Et $2 \mathrm{O})-B 3 L Y P / 6-311 G(d, p)-D 3(B J)]:-4790.222779$ Hartree

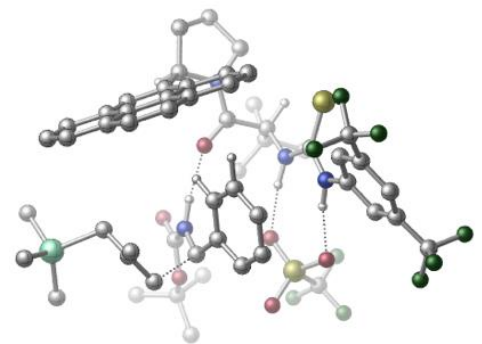

\begin{tabular}{|crrr|crrc|}
\hline 01 & & & & N & 3.05297800 & 0.53528100 & 0.74946800 \\
\hline $\mathrm{H}$ & 2.80735500 & 1.45127000 & 0.35525500 & $\mathrm{C}$ & 4.03033900 & -0.15069300 & 0.00733400 \\
\hline $\mathrm{C}$ & 4.94728900 & 0.60452600 & -0.73761800 & $\mathrm{C}$ & 4.05526900 & -1.54737100 & -0.09740100 \\
\hline $\mathrm{C}$ & 5.88212600 & -0.03548100 & -1.55247100 & $\mathrm{H}$ & 4.90695600 & 1.68725300 & -0.69582200 \\
\hline $\mathrm{C}$ & 4.99129400 & -2.16968200 & -0.91987000 & $\mathrm{H}$ & 3.33272500 & -2.13754200 & 0.44666200 \\
\hline $\mathrm{C}$ & 5.91943300 & -1.42670300 & -1.65047100 & $\mathrm{H}$ & 6.64419500 & -1.91689800 & -2.28871800 \\
\hline $\mathrm{C}$ & 6.90781800 & 0.78432600 & -2.28964700 & $\mathrm{C}$ & 4.90167400 & -3.65876500 & -1.09948600 \\
\hline $\mathrm{F}$ & 8.02344300 & 0.97459400 & -1.54647300 & $\mathrm{~F}$ & 6.44064900 & 2.00609900 & -2.61840100 \\
\hline $\mathrm{F}$ & 7.30306000 & 0.17871700 & -3.43487900 & $\mathrm{~F}$ & 4.60777100 & -4.30236500 & 0.05471800 \\
\hline
\end{tabular}




\begin{tabular}{|c|c|c|c|c|c|c|c|}
\hline $\mathrm{F}$ & 3.91084500 & -3.99612500 & -1.97698500 & $\mathrm{~F}$ & 6.04122500 & -4.19332400 & -1.58276100 \\
\hline C & 0.55730700 & 0.65882200 & 3.43139400 & $\mathrm{H}$ & 1.22531100 & 0.13237000 & 4.11211500 \\
\hline C & 0.12319500 & 1.98852700 & 4.14812900 & C & -0.95214100 & 2.77897300 & 3.38507200 \\
\hline $\mathrm{H}$ & -1.87996600 & 2.21494500 & 3.26877800 & $\mathrm{H}$ & -0.61013900 & 3.07586000 & 2.39155200 \\
\hline $\mathrm{H}$ & -1.18285600 & 3.69722500 & 3.94025200 & C & 1.37702900 & 2.87146300 & 4.32279500 \\
\hline $\mathrm{H}$ & 1.13618100 & 3.72929000 & 4.96217100 & $\mathrm{H}$ & 1.73210400 & 3.26222800 & 3.36501300 \\
\hline $\mathrm{H}$ & 2.19910800 & 2.31912800 & 4.79377200 & C & -0.42924400 & 1.63834000 & 5.54580100 \\
\hline $\mathrm{H}$ & -0.75950700 & 2.55261000 & 6.05153600 & $\mathrm{H}$ & 0.33342500 & 1.17186200 & 6.18176100 \\
\hline $\mathrm{H}$ & -1.29677100 & 0.96906400 & 5.49039200 & C & -0.61465600 & -0.28400200 & 3.04684700 \\
\hline 0 & -1.34927800 & -0.03998300 & 2.07876500 & C & -0.10007700 & -1.80713300 & 5.02052600 \\
\hline C & -1.95295400 & -2.31337700 & 3.49206100 & C & -1.13171900 & -2.66884900 & 5.75509500 \\
\hline $\mathrm{H}$ & 0.81210300 & -2.36870800 & 4.78570100 & $\mathrm{H}$ & 0.18926200 & -0.93229800 & 5.59822400 \\
\hline $\mathrm{H}$ & -2.85911200 & -1.71190500 & 3.63328300 & $\mathrm{H}$ & -1.82499200 & -2.02819200 & 6.31381300 \\
\hline $\mathrm{H}$ & -0.66435600 & -3.35888000 & 6.46385300 & $\mathrm{~N}$ & -0.79907000 & -1.41956400 & 3.77537000 \\
\hline C & -2.08074500 & -2.95110400 & 2.11125800 & C & -3.33382300 & -2.95935900 & 1.56448600 \\
\hline C & -1.01068300 & -3.67623200 & 1.44099000 & C & -3.66550100 & -3.66708000 & 0.36355600 \\
\hline $\mathrm{H}$ & -4.13415400 & -2.41686600 & 2.06387700 & C & -1.31618100 & -4.40070100 & 0.23908000 \\
\hline C & 0.31772700 & -3.69626200 & 1.90207400 & C & -4.96781100 & -3.67820700 & -0.16677400 \\
\hline C & -2.63728900 & -4.39720100 & -0.30114500 & C & -0.28923200 & -5.13081100 & -0.43955300 \\
\hline $\mathrm{H}$ & 0.60184800 & -3.11733800 & 2.76916300 & C & 1.31358800 & -4.40259000 & 1.22623900 \\
\hline C & -5.25479600 & -4.38549600 & -1.33509900 & $\mathrm{H}$ & -5.75765400 & -3.14706900 & 0.36007800 \\
\hline $\mathrm{C}$ & -2.93876500 & -5.11599700 & -1.49947900 & $\mathrm{C}$ & 1.01909700 & -5.11760100 & 0.07027300 \\
\hline C & -0.62112600 & -5.84589300 & -1.64155300 & $\mathrm{H}$ & 2.33286800 & -4.36902200 & 1.59801800 \\
\hline C & -4.25231300 & -5.09183200 & -1.99886300 & $\mathrm{H}$ & -6.27082300 & -4.39983500 & -1.72184200 \\
\hline $\mathrm{C}$ & -1.88362500 & -5.84179100 & -2.14957100 & $\mathrm{H}$ & 1.80792800 & -5.64344200 & -0.46119600 \\
\hline $\mathrm{H}$ & 0.17104000 & -6.39684700 & -2.14250300 & $\mathrm{H}$ & -4.48330700 & -5.64518400 & -2.90585700 \\
\hline $\mathrm{H}$ & -2.11498000 & -6.39084200 & -3.05905100 & C & 2.44543000 & 0.14356700 & 1.91607200 \\
\hline S & 3.03203900 & -1.11445400 & 2.90028000 & $\mathrm{~N}$ & 1.35722400 & 0.89042200 & 2.23481100 \\
\hline $\mathrm{H}$ & 1.02624700 & 1.58995500 & 1.56417000 & $\mathrm{~S}$ & 1.45621600 & 3.72382100 & -0.60321900 \\
\hline 0 & 0.76782400 & 4.03872300 & -1.87329400 & $\mathrm{O}$ & 0.57636700 & 3.13028400 & 0.44596400 \\
\hline 0 & 2.76431500 & 3.03071400 & -0.72278600 & C & 1.89908700 & 5.38807600 & 0.10089000 \\
\hline $\mathrm{F}$ & 2.78171500 & 6.01702800 & -0.68141800 & $\mathrm{~F}$ & 2.43010400 & 5.25133700 & 1.32523400 \\
\hline $\mathrm{F}$ & 0.80069600 & 6.16200000 & 0.20368400 & C & -2.79860400 & 2.79763800 & 0.22222800 \\
\hline 0 & -3.40844700 & 2.79303700 & 1.27413000 & $\mathrm{O}$ & -2.73802300 & 3.80061800 & -0.66935400 \\
\hline C & -3.20350900 & 5.17076500 & -0.33857300 & C & -2.55746300 & 5.65473500 & 0.96198200 \\
\hline $\mathrm{H}$ & -2.79531300 & 6.71564900 & 1.09951200 & $\mathrm{H}$ & -1.47077200 & 5.55284700 & 0.90896600 \\
\hline $\mathrm{H}$ & -2.92722500 & 5.09897500 & 1.82449200 & C & -4.73205500 & 5.17273300 & -0.26448500 \\
\hline $\mathrm{H}$ & -5.16541800 & 4.82005900 & -1.20800600 & $\mathrm{H}$ & -5.09126000 & 6.19344600 & -0.09256800 \\
\hline $\mathrm{H}$ & -5.08257700 & 4.53825600 & 0.55260000 & C & -2.68879200 & 5.97940400 & -1.53095500 \\
\hline $\mathrm{H}$ & -2.96149900 & 7.03323600 & -1.41183500 & $\mathrm{H}$ & -3.12590000 & 5.61578200 & -2.46762700 \\
\hline $\mathrm{H}$ & -1.59984600 & 5.90057600 & -1.60109500 & C & -1.47296600 & 1.62792700 & -1.48726800 \\
\hline $\mathrm{H}$ & -1.13910500 & 2.61510400 & -1.80703200 & C & -0.42955700 & 0.58959200 & -1.72863400 \\
\hline C & 0.51674700 & 0.85085100 & -2.73551300 & C & -0.38466300 & -0.63945600 & -1.05172000 \\
\hline C & 1.47578800 & -0.10121600 & -3.07087300 & $\mathrm{H}$ & 0.52163400 & 1.82097500 & -3.22316400 \\
\hline C & 0.58618700 & -1.58491200 & -1.38710200 & $\mathrm{H}$ & -1.08145800 & -0.86194400 & -0.24941400 \\
\hline C & 1.50796100 & -1.32714600 & -2.40301400 & $\mathrm{H}$ & 2.20951700 & 0.12349800 & -3.83947600 \\
\hline $\mathrm{H}$ & 0.62379600 & -2.52397200 & -0.84835300 & $\mathrm{H}$ & 2.26002800 & -2.07046500 & -2.64806700 \\
\hline $\mathrm{N}$ & -2.10524900 & 1.67858300 & -0.24040200 & $\mathrm{H}$ & -1.89487900 & 0.99679300 & 0.49447600 \\
\hline C & -2.74333400 & 1.48448800 & -2.78851600 & $\mathrm{H}$ & -2.17808500 & 1.66050000 & -3.70200900 \\
\hline $\mathrm{H}$ & -3.34870000 & 2.33894200 & -2.49185900 & C & -3.35680500 & 0.20955600 & -2.67589200 \\
\hline C & -4.36479200 & -0.03784500 & -1.66546300 & $\mathrm{H}$ & -4.27068700 & 0.65347600 & -0.81749300 \\
\hline $\mathrm{H}$ & -4.36543900 & -1.07927800 & -1.31973200 & C & -2.84943400 & -0.93222100 & -3.48650100 \\
\hline $\mathrm{H}$ & -3.56242800 & -1.75911100 & -3.53686100 & $\mathrm{H}$ & -1.94790700 & -1.31108000 & -2.97332100 \\
\hline
\end{tabular}




\begin{tabular}{|c|c|c|c|c|c|c|c|}
\hline $\mathrm{H}$ & -2.53928100 & -0.63058400 & -4.49084800 & $\mathrm{C}$ & -7.28518400 & -0.05881700 & -0.79660500 \\
\hline $\mathrm{H}$ & -7.16321700 & -1.07890700 & -0.41565300 & $\mathrm{H}$ & -8.34348700 & 0.07672000 & -1.05103400 \\
\hline $\mathrm{H}$ & -7.04684200 & 0.63564300 & 0.01692900 & C & -6.56650000 & -0.99188200 & -3.68015500 \\
\hline $\mathrm{H}$ & -6.37423600 & -2.02072900 & -3.35414000 & $\mathrm{H}$ & -5.96660100 & -0.80413400 & -4.57803100 \\
\hline $\mathrm{H}$ & -7.62158600 & -0.93027100 & -3.97397800 & C & -6.38431700 & 2.02238600 & -2.92304000 \\
\hline $\mathrm{H}$ & -5.75669600 & 2.22682700 & -3.79757300 & $\mathrm{H}$ & -6.12842400 & 2.75010600 & -2.14441100 \\
\hline $\mathrm{H}$ & -7.42486400 & 2.20946600 & -3.21581300 & $\mathrm{Si}$ & -6.20138000 & 0.25011200 & -2.30788900 \\
\hline C & -1.86114300 & -3.38059800 & 4.61159800 & $\mathrm{H}$ & -1.27437000 & -4.23627400 & 4.26087200 \\
\hline $\mathrm{H}$ & -2.85402700 & -3.74993500 & 4.88318100 & & & & \\
\hline
\end{tabular}

\section{TS-R-4f-nd-5}

Imaginary frequency: $-175.07 \mathrm{~cm}^{-1}$

E[B3LYP/6-31G(d)]: -4788.686050 Hartree

E[PCM(Et $\left.\left.{ }_{2} \mathrm{O}\right)-\mathrm{B} 3 L Y P / 6-311 \mathrm{G}(\mathrm{d}, \mathrm{p})-\mathrm{D} 3(\mathrm{BJ})\right]:-4790.221999$ Hartree

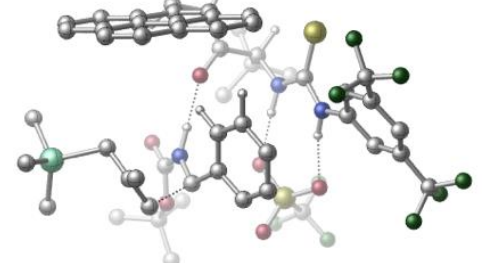

\begin{tabular}{|c|c|c|c|c|c|c|c|}
\hline 01 & & & & $\mathrm{~N}$ & 3.00814400 & 0.03591900 & 0.98838400 \\
\hline $\mathrm{H}$ & 3.00059100 & 0.99664400 & 0.62513400 & C & 3.93395300 & -0.79043200 & 0.32679700 \\
\hline C & 5.04120600 & -0.17224100 & -0.27443300 & C & 3.75713700 & -2.17070100 & 0.16607000 \\
\hline C & 5.95878000 & -0.93018700 & -1.00103200 & $\mathrm{H}$ & 5.17010000 & 0.90005000 & -0.18152300 \\
\hline C & 4.68215800 & -2.91147500 & -0.56683900 & $\mathrm{H}$ & 2.89893700 & -2.65485600 & 0.60609700 \\
\hline C & 5.79573500 & -2.30748000 & -1.15089500 & $\mathrm{H}$ & 6.51864900 & -2.89332300 & -1.70543000 \\
\hline C & 7.11163900 & -0.24454400 & -1.68522700 & C & 4.40049500 & -4.36546000 & -0.82006100 \\
\hline $\mathrm{F}$ & 8.18909700 & -1.05770100 & -1.78516400 & $\mathrm{~F}$ & 7.50272300 & 0.86879600 & -1.03194700 \\
\hline $\mathrm{F}$ & 6.79007000 & 0.12980000 & -2.94689200 & $\mathrm{~F}$ & 3.77171600 & -4.96140300 & 0.22120200 \\
\hline $\mathrm{F}$ & 3.58026600 & -4.53235300 & -1.89799100 & $\mathrm{~F}$ & 5.51943400 & -5.07304100 & -1.07682800 \\
\hline C & 0.34086500 & 0.66115400 & 3.41939700 & $\mathrm{H}$ & 0.80039500 & 0.00085000 & 4.15489300 \\
\hline C & 0.11281100 & 2.05213300 & 4.11830000 & $\mathrm{C}$ & -0.71123000 & 3.03535400 & 3.27054200 \\
\hline $\mathrm{H}$ & -1.71404000 & 2.65971500 & 3.05436000 & $\mathrm{H}$ & -0.22558100 & 3.26156600 & 2.31942700 \\
\hline $\mathrm{H}$ & -0.81675100 & 3.98025800 & 3.81870300 & C & 1.49301800 & 2.67197900 & 4.42391400 \\
\hline $\mathrm{H}$ & 1.36466700 & 3.55955800 & 5.05502800 & $\mathrm{H}$ & 2.00682400 & 2.98700800 & 3.51140700 \\
\hline $\mathrm{H}$ & 2.14322800 & 1.96885200 & 4.95836200 & C & -0.62589300 & 1.82348700 & 5.45329100 \\
\hline $\mathrm{H}$ & -0.80158000 & 2.78644800 & 5.94571600 & $\mathrm{H}$ & -0.03962500 & 1.20590600 & 6.14517400 \\
\hline $\mathrm{H}$ & -1.60533300 & 1.35270800 & 5.30609400 & C & -0.94536300 & -0.01230400 & 2.87636800 \\
\hline $\mathrm{O}$ & -1.43382400 & 0.32605200 & 1.78750500 & $\mathrm{C}$ & -1.16620900 & -1.54676500 & 4.91161600 \\
\hline C & -2.76140500 & -1.64437800 & 3.08202400 & C & -2.53695900 & -1.83785800 & 5.53048400 \\
\hline $\mathrm{H}$ & -0.56911600 & -2.46080100 & 4.79958700 & $\mathrm{H}$ & -0.58362500 & -0.83239400 & 5.48735000 \\
\hline $\mathrm{H}$ & -3.40577300 & -0.83452600 & 2.72948800 & $\mathrm{H}$ & -2.96408800 & -0.90899200 & 5.92653700 \\
\hline $\mathrm{H}$ & -2.48177200 & -2.56059600 & 6.35016200 & $\mathrm{~N}$ & -1.52519400 & -1.01345200 & 3.58959100 \\
\hline C & -2.52054200 & -2.62587700 & 1.93822900 & C & -1.29185800 & -3.19115900 & 1.75197700 \\
\hline C & -3.62826400 & -3.01478000 & 1.08254000 & C & -1.03833600 & -4.17057900 & 0.73475500 \\
\hline $\mathrm{H}$ & -0.45181400 & -2.88235900 & 2.36875300 & C & -3.39619500 & -3.97686900 & 0.04404700 \\
\hline C & -4.92731600 & -2.49028800 & 1.22068000 & C & 0.23334800 & -4.74240600 & 0.55428100 \\
\hline C & -2.10384100 & -4.55604200 & -0.12917200 & C & -4.46285000 & -4.36924600 & -0.82509200 \\
\hline $\mathrm{H}$ & -5.14237800 & -1.77373800 & 2.00575500 & C & -5.96282000 & -2.88389500 & 0.37131900 \\
\hline C & 0.45851800 & -5.66891000 & -0.46368500 & $\mathrm{H}$ & 1.04618000 & -4.44535800 & 1.21173600 \\
\hline C & -1.86787800 & -5.51338200 & -1.16266300 & C & -5.73764600 & -3.80840700 & -0.64521500 \\
\hline C & -4.19801200 & -5.33647000 & -1.85601100 & $\mathrm{H}$ & -6.95907000 & -2.47403000 & 0.51813500 \\
\hline C & -0.57958700 & -6.05295100 & -1.31159700 & $\mathrm{H}$ & 1.45479700 & -6.07692200 & -0.60110100 \\
\hline C & -2.96141000 & -5.88227300 & -2.01796100 & $\mathrm{H}$ & -6.55162700 & -4.11818000 & -1.29655300 \\
\hline $\mathrm{H}$ & -5.01816200 & -5.62959700 & -2.50733400 & $\mathrm{H}$ & -0.39737800 & -6.77595600 & -2.10301600 \\
\hline $\mathrm{H}$ & -2.78105500 & -6.61406000 & -2.80182400 & $\mathrm{C}$ & 2.18581600 & -0.25476400 & 2.04670300 \\
\hline
\end{tabular}




\begin{tabular}{|c|c|c|c|c|c|c|c|}
\hline $\mathrm{S}$ & 2.28945700 & -1.68236600 & 2.96905600 & $\mathrm{~N}$ & 1.30011900 & 0.73622300 & 2.32435600 \\
\hline $\mathrm{H}$ & 1.20575600 & 1.51243900 & 1.66205700 & S & 2.18649000 & 3.46690100 & -0.46104300 \\
\hline $\mathrm{O}$ & 1.69527700 & 3.84953300 & -1.80105000 & 0 & 1.11780200 & 3.07333500 & 0.50352300 \\
\hline $\mathrm{O}$ & 3.35836200 & 2.55444900 & -0.42758800 & C & 2.84491400 & 5.05243300 & 0.25596800 \\
\hline $\mathrm{F}$ & 3.90347200 & 5.48550500 & -0.43615800 & $\mathrm{~F}$ & 3.21201000 & 4.87004100 & 1.53352300 \\
\hline $\mathrm{F}$ & 1.89975600 & 6.01215900 & 0.22379200 & C & -2.17376700 & 3.29551500 & -0.14032300 \\
\hline $\mathrm{O}$ & -2.84365600 & 3.41785100 & 0.86742400 & $\mathrm{O}$ & -1.90817200 & 4.24852700 & -1.04935800 \\
\hline C & -2.18056300 & 5.68266100 & -0.78207300 & C & -1.56716200 & 6.10551000 & 0.55522000 \\
\hline $\mathrm{H}$ & -1.64344900 & 7.19486000 & 0.64780700 & $\mathrm{H}$ & -0.50967700 & 5.83299800 & 0.59405000 \\
\hline $\mathrm{H}$ & -2.08499200 & 5.64575800 & 1.39772500 & C & -3.69200400 & 5.91782500 & -0.82828200 \\
\hline $\mathrm{H}$ & -4.10347500 & 5.60478400 & -1.79530700 & $\mathrm{H}$ & -3.90323300 & 6.98580400 & -0.70477500 \\
\hline $\mathrm{H}$ & -4.19446800 & 5.36966800 & -0.02801000 & C & -1.45954700 & 6.36552100 & -1.94586500 \\
\hline $\mathrm{H}$ & -1.57590100 & 7.45178100 & -1.87143600 & $\mathrm{H}$ & -1.87377400 & 6.04085700 & -2.90691800 \\
\hline $\mathrm{H}$ & -0.39326300 & 6.12082100 & -1.92509400 & C & -0.97214300 & 1.87717200 & -1.74895500 \\
\hline $\mathrm{H}$ & -0.48246100 & 2.79118000 & -2.08513200 & C & -0.07907900 & 0.68977500 & -1.90663300 \\
\hline $\mathrm{C}$ & 0.96928800 & 0.79276800 & -2.83672600 & C & -0.27838200 & -0.52664300 & -1.23376000 \\
\hline C & 1.79050200 & -0.30040500 & -3.10402900 & $\mathrm{H}$ & 1.16262100 & 1.74680700 & -3.31754200 \\
\hline C & 0.54888400 & -1.61765000 & -1.50679200 & $\mathrm{H}$ & -1.05640200 & -0.62903300 & -0.48275100 \\
\hline $\mathrm{C}$ & 1.57553700 & -1.51340700 & -2.44748900 & $\mathrm{H}$ & 2.60786700 & -0.19762400 & -3.81185700 \\
\hline $\mathrm{H}$ & 0.39125600 & -2.55127000 & -0.97876500 & $\mathrm{H}$ & 2.21656600 & -2.36801100 & -2.64097800 \\
\hline $\mathrm{N}$ & -1.62964900 & 2.07155300 & -0.52860600 & $\mathrm{H}$ & -1.55477800 & 1.39429700 & 0.23839600 \\
\hline C & -2.21118500 & 1.85657500 & -3.06652700 & $\mathrm{H}$ & -1.62547400 & 1.94274600 & -3.98058200 \\
\hline $\mathrm{H}$ & -2.71706700 & 2.78432700 & -2.80461300 & C & -2.97833200 & 0.66504700 & -2.94071100 \\
\hline C & -3.98022500 & 0.53976200 & -1.90479000 & $\mathrm{H}$ & -3.77012000 & 1.20898700 & -1.05883700 \\
\hline $\bar{H}$ & -4.10262400 & -0.49468500 & -1.56004000 & C & -2.63148400 & -0.52715000 & -3.76407800 \\
\hline $\mathrm{H}$ & -3.45322300 & -1.24597900 & -3.82165500 & $\mathrm{H}$ & -1.79029000 & -1.03216900 & -3.25808700 \\
\hline $\mathrm{H}$ & -2.28802800 & -0.25993800 & -4.76720800 & C & -6.79844700 & 0.99963700 & -0.86565800 \\
\hline $\bar{H}$ & -6.74302800 & 0.00871300 & -0.40147700 & $\mathrm{H}$ & -7.85421700 & 1.21951800 & -1.06584700 \\
\hline $\bar{H}$ & -6.43573300 & 1.73383000 & -0.13734600 & C & -6.41615700 & -0.19923000 & -3.71655600 \\
\hline $\mathrm{H}$ & -6.37927200 & -1.21846200 & -3.31432800 & $\mathrm{H}$ & -5.83587800 & -0.17968600 & -4.64623900 \\
\hline $\bar{H}$ & -7.45992100 & 0.01248000 & -3.97951400 & $\mathrm{C}$ & -5.76415600 & 2.80052300 & -3.18519500 \\
\hline $\mathrm{H}$ & -5.17812900 & 2.85669300 & -4.10936600 & $\mathrm{H}$ & -5.35313300 & 3.52904600 & -2.47693100 \\
\hline $\mathrm{H}$ & -6.78574600 & 3.12038200 & -3.42510300 & $\mathrm{Si}$ & -5.79503000 & 1.06185700 & -2.45829800 \\
\hline C & -3.36367900 & -2.35161500 & 4.33792900 & $\mathrm{H}$ & -3.24949600 & -3.43521700 & 4.23616300 \\
\hline $\bar{H}$ & -4.43236500 & -2.14469900 & 4.44610200 & & & & \\
\hline
\end{tabular}

\section{TS-R-4f-nd-6}

Imaginary frequency: $-171.12 \mathrm{~cm}^{-1}$

E[B3LYP/6-31G(d)]: -4788.685900 Hartree

E[PCM(Et $2 \mathrm{O})-B 3 L Y P / 6-311 G(d, p)-D 3(B J)]:-4790.221811$ Hartree

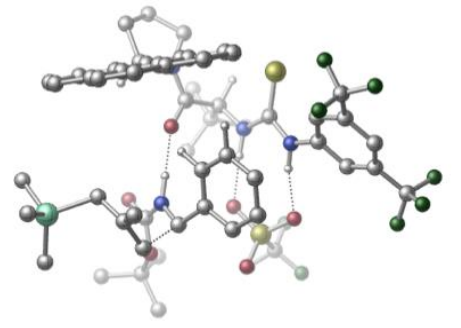

\begin{tabular}{|crrr|rrrr|}
\hline 01 & & & & N & 3.01105600 & 0.06164400 & 0.95294100 \\
\hline H & 2.99360900 & 1.02133700 & 0.58691900 & C & 3.94715100 & -0.75760500 & 0.29615700 \\
\hline C & 5.05381400 & -0.13262500 & -0.29858600 & C & 3.77709500 & -2.13814400 & 0.13072600 \\
\hline C & 5.97867200 & -0.88470500 & -1.02258500 & H & 5.17015900 & 0.94191700 & -0.21396500 \\
\hline C & 4.70716200 & -2.87246200 & -0.60217300 & H & 2.91714200 & -2.62653000 & 0.56268200 \\
\hline C & 5.82057000 & -2.26177500 & -1.17957200 & H & 6.53984800 & -2.84022900 & -1.74641200 \\
\hline C & 7.20099100 & -0.21255900 & -1.59010800 & C & 4.42706800 & -4.32420200 & -0.86923800 \\
\hline F & 8.23138800 & -0.22731500 & -0.71162200 & F & 6.97121200 & 1.07786100 & -1.90621600 \\
\hline F & 7.63802600 & -0.83459300 & -2.71098200 & F & 3.80170200 & -4.93054100 & 0.16823600 \\
\hline F & 3.60456100 & -4.48286900 & -1.94662100 & F & 5.54646800 & -5.02770000 & -1.13494700 \\
\hline
\end{tabular}




\begin{tabular}{|c|c|c|c|c|c|c|c|}
\hline C & 0.35387300 & 0.66802200 & 3.40002100 & $\mathrm{H}$ & 0.82435300 & 0.01185900 & 4.13228100 \\
\hline $\mathrm{C}$ & 0.12118900 & 2.05775600 & 4.09991200 & C & -0.71721400 & 3.03434600 & 3.25863200 \\
\hline $\mathrm{H}$ & -1.71902000 & 2.65123200 & 3.05111100 & $\mathrm{H}$ & -0.24126000 & 3.26358800 & 2.30336300 \\
\hline $\mathrm{H}$ & -0.82512500 & 3.97876500 & 3.80716200 & C & 1.49920200 & 2.68809300 & 4.39372200 \\
\hline $\mathrm{H}$ & 1.36946000 & 3.57490800 & 5.02562400 & $\mathrm{H}$ & 2.00288500 & 3.00670900 & 3.47682500 \\
\hline $\mathrm{H}$ & 2.15919500 & 1.99010200 & 4.92287700 & C & -0.60462000 & 1.82421100 & 5.44113300 \\
\hline $\mathrm{H}$ & -0.78360900 & 2.78606900 & 5.93451900 & $\mathrm{H}$ & -0.00789600 & 1.21153500 & 6.12842400 \\
\hline $\mathrm{H}$ & -1.58162000 & 1.34583800 & 5.30234200 & C & -0.93167500 & -0.01568000 & 2.86868100 \\
\hline 0 & -1.43305900 & 0.31861900 & 1.78433200 & C & -1.12193200 & -1.55154600 & 4.90596500 \\
\hline $\mathrm{C}$ & -2.73164400 & -1.66340900 & 3.08982900 & C & -2.48482600 & -1.85494700 & 5.53616000 \\
\hline $\mathrm{H}$ & -0.51743900 & -2.46008600 & 4.78910200 & $\mathrm{H}$ & -0.54114600 & -0.83179300 & 5.47679100 \\
\hline $\mathrm{H}$ & -3.38630300 & -0.85920100 & 2.74339200 & $\mathrm{H}$ & -2.91716600 & -0.92990800 & 5.93550500 \\
\hline $\mathrm{H}$ & -2.41629400 & -2.57692300 & 6.35549900 & $\mathrm{~N}$ & -1.49667700 & -1.02170000 & 3.58688100 \\
\hline C & -2.49185700 & -2.64209300 & 1.94337500 & C & -1.26018500 & -3.19757500 & 1.74746800 \\
\hline C & -3.60280500 & -3.03903900 & 1.09562600 & C & -1.00649100 & -4.17391200 & 0.72734400 \\
\hline $\mathrm{H}$ & -0.41802100 & -2.88272000 & 2.35828000 & C & -3.37070600 & -3.99772800 & 0.05396900 \\
\hline C & -4.90517000 & -2.52573100 & 1.24458800 & C & 0.26819500 & -4.73585300 & 0.53717600 \\
\hline C & -2.07512800 & -4.56643700 & -0.12948200 & C & -4.44059800 & -4.39749200 & -0.80781500 \\
\hline $\mathrm{H}$ & -5.12026400 & -1.81256300 & 2.03273300 & C & -5.94384600 & -2.92678100 & 0.40257200 \\
\hline C & 0.49318900 & -5.65929800 & -0.48361100 & $\mathrm{H}$ & 1.08336400 & -4.43349400 & 1.18931900 \\
\hline C & -1.83918300 & -5.52053400 & -1.16596400 & C & -5.71865800 & -3.84761100 & -0.61733800 \\
\hline C & -4.17560100 & -5.36097000 & -1.84219600 & $\mathrm{H}$ & -6.94243400 & -2.52582200 & 0.55783700 \\
\hline C & -0.54790700 & -6.05006200 & -1.32474700 & $\mathrm{H}$ & 1.49152300 & -6.05966300 & -0.62844700 \\
\hline C & -2.93591400 & -5.89670500 & -2.01395000 & $\mathrm{H}$ & -6.53504800 & -4.16343100 & -1.26273900 \\
\hline $\mathrm{H}$ & -4.99813200 & -5.65968700 & -2.48794800 & $\mathrm{H}$ & -0.36580400 & -6.77062200 & -2.11841400 \\
\hline $\mathrm{H}$ & -2.75549600 & -6.62590700 & -2.80020200 & C & 2.19663300 & -0.23440500 & 2.01559700 \\
\hline $\mathrm{S}$ & 2.31727500 & -1.66063900 & 2.93839000 & $\mathrm{~N}$ & 1.30373200 & 0.74903100 & 2.29718200 \\
\hline $\mathrm{H}$ & 1.19982400 & 1.52481500 & 1.63572100 & $\mathrm{~S}$ & 2.14776600 & 3.48190500 & -0.49607900 \\
\hline 0 & 1.64375900 & 3.85685500 & -1.83342700 & $\mathrm{O}$ & 1.08929200 & 3.08330600 & 0.47768200 \\
\hline 0 & 3.32682300 & 2.57857400 & -0.46835300 & C & 2.79943900 & 5.07446000 & 0.21145500 \\
\hline $\mathrm{F}$ & 3.84966800 & 5.51350000 & -0.48959100 & $\mathrm{~F}$ & 3.17724400 & 4.89864100 & 1.48684300 \\
\hline $\mathrm{F}$ & 1.84694800 & 6.02708200 & 0.18345800 & C & -2.20803500 & 3.28410300 & -0.14062700 \\
\hline 0 & -2.86896900 & 3.40514700 & 0.87323000 & $\mathrm{O}$ & -1.95978000 & 4.23547100 & -1.05628700 \\
\hline C & -2.24061200 & 5.66843900 & -0.79183400 & C & -3.75419300 & 5.89175000 & -0.82475900 \\
\hline $\mathrm{H}$ & -3.97262100 & 6.95853800 & -0.70349900 & $\mathrm{H}$ & -4.24481000 & 5.34294700 & -0.01760100 \\
\hline $\mathrm{H}$ & -4.17227800 & 5.57157600 & -1.78660900 & C & -1.53574600 & 6.35226600 & -1.96492500 \\
\hline $\mathrm{H}$ & -0.46739600 & 6.11607400 & -1.95290400 & $\mathrm{H}$ & -1.66001100 & 7.43786900 & -1.89380100 \\
\hline $\mathrm{H}$ & -1.95612900 & 6.02038800 & -2.92082600 & C & -1.61796700 & 6.10123200 & 0.53800000 \\
\hline $\mathrm{H}$ & -1.70094200 & 7.19042000 & 0.62667000 & $\mathrm{H}$ & -0.55829900 & 5.83618500 & 0.56830900 \\
\hline $\mathrm{H}$ & -2.12481600 & 5.64154400 & 1.38718600 & C & -1.01494100 & 1.86840700 & -1.75740400 \\
\hline $\mathrm{H}$ & -0.54017500 & 2.78585100 & -2.10517400 & C & -0.11010200 & 0.69074900 & -1.92108600 \\
\hline C & 0.92391700 & 0.80128800 & -2.86618700 & C & -0.28517700 & -0.52414500 & -1.23893200 \\
\hline C & 1.75483300 & -0.28315400 & -3.13861300 & $\mathrm{H}$ & 1.09877500 & 1.75480600 & -3.35499900 \\
\hline C & 0.55213900 & -1.60622400 & -1.51688400 & $\mathrm{H}$ & -1.05183600 & -0.63181700 & -0.47707500 \\
\hline C & 1.56460900 & -1.49475000 & -2.47196100 & $\mathrm{H}$ & 2.56022700 & -0.17459600 & -3.85920200 \\
\hline $\mathrm{H}$ & 0.41309800 & -2.53872800 & -0.98175300 & $\mathrm{H}$ & 2.21366000 & -2.34237800 & -2.66931800 \\
\hline $\mathrm{N}$ & -1.65730800 & 2.06334800 & -0.52913900 & $\mathrm{H}$ & -1.56973200 & 1.38910700 & 0.23923000 \\
\hline C & -2.27158300 & 1.82515000 & -3.05661300 & $\mathrm{H}$ & -1.69978700 & 1.90764600 & -3.97978800 \\
\hline $\mathrm{H}$ & -2.78190600 & 2.75100400 & -2.79642500 & C & -3.02779400 & 0.62875800 & -2.90951300 \\
\hline $\mathrm{C}$ & -4.01424300 & 0.50565300 & -1.85889600 & $\mathrm{H}$ & -3.79750800 & 1.18401300 & -1.02200100 \\
\hline $\mathrm{H}$ & -4.12507600 & -0.52644500 & -1.50341000 & C & -2.68296700 & -0.56822200 & -3.72668000 \\
\hline $\mathrm{H}$ & -3.49834800 & -1.29555800 & -3.76365700 & $\mathrm{H}$ & -1.82861000 & -1.05995400 & -3.22964500 \\
\hline $\mathrm{H}$ & -2.35832500 & -0.30773300 & -4.73784400 & C & -6.81929300 & 0.96287000 & -0.78361100 \\
\hline
\end{tabular}




\begin{tabular}{|c|c|c|c|c|c|c|c|}
\hline $\mathrm{H}$ & -6.75257500 & -0.02236100 & -0.30896700 & $\mathrm{H}$ & -7.87891400 & 1.17594500 & -0.97036000 \\
\hline $\mathrm{H}$ & -6.44877500 & 1.70699000 & -0.06944400 & C & -6.47183400 & -0.27075900 & -3.62429300 \\
\hline $\mathrm{H}$ & -6.42136300 & -1.28429600 & -3.20932600 & $\mathrm{H}$ & -5.90588400 & -0.25930300 & -4.56290800 \\
\hline $\mathrm{H}$ & -7.52095400 & -0.06966100 & -3.87392800 & $\mathrm{C}$ & -5.83103400 & 2.73987300 & -3.14097100 \\
\hline $\mathrm{H}$ & -5.25980500 & 2.78787000 & -4.07481400 & $\mathrm{H}$ & -5.41364600 & 3.47978700 & -2.44839900 \\
\hline $\mathrm{H}$ & -6.85819200 & 3.05055300 & -3.36889200 & $\mathrm{Si}$ & -5.84025200 & 1.01039100 & -2.39183700 \\
\hline $\mathrm{C}$ & -3.31653600 & -2.37656900 & 4.35054200 & $\mathrm{H}$ & -3.19265200 & -3.45899900 & 4.24763200 \\
\hline $\mathrm{H}$ & -4.38624800 & -2.18005400 & 4.46765700 & & & & \\
\hline
\end{tabular}

\section{TS- $R-4 f-n d-7$}

Imaginary frequency: $-290.89 \mathrm{~cm}^{-1}$

E[B3LYP/6-31G(d)]: -4788.685572 Hartree

E[PCM(Et $\left.\left.t_{2} \mathrm{O}\right)-B 3 L Y P / 6-311 G(d, p)-D 3(B J)\right]:-4790.221195$ Hartree

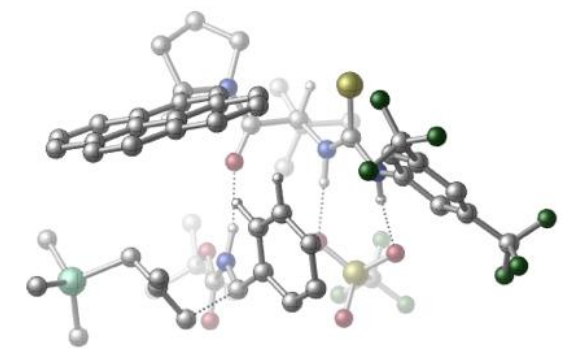

\begin{tabular}{|c|c|c|c|c|c|c|c|}
\hline 01 & & & & $\mathrm{~N}$ & -3.15216000 & -0.60562200 & 0.95506000 \\
\hline $\mathrm{H}$ & -3.04347400 & -1.52500800 & 0.50687500 & C & -4.18274200 & 0.16221200 & 0.38841600 \\
\hline C & -5.19173000 & -0.52555700 & -0.30557700 & $\mathrm{C}$ & -4.20112400 & 1.56297600 & 0.39863600 \\
\hline C & -6.20414300 & 0.18097600 & -0.95281800 & $\mathrm{H}$ & -5.16138200 & -1.60834100 & -0.35781100 \\
\hline C & -5.21285600 & 2.25191100 & -0.26726300 & $\mathrm{H}$ & -3.42389400 & 2.10487400 & 0.91519400 \\
\hline C & -6.22973100 & 1.57612800 & -0.94119900 & $\mathrm{H}$ & -7.01379000 & 2.11979800 & -1.45404400 \\
\hline C & -7.32224900 & -0.57068900 & -1.62641700 & $\mathrm{C}$ & -5.11907100 & 3.74839100 & -0.36260500 \\
\hline $\mathrm{F}$ & -8.35990100 & -0.78027700 & -0.78199700 & $\mathrm{~F}$ & -6.92664100 & -1.77897200 & -2.07351400 \\
\hline $\mathrm{F}$ & -7.81633600 & 0.11413500 & -2.68528700 & $\mathrm{~F}$ & -4.56359000 & 4.30526900 & 0.74019900 \\
\hline $\mathrm{F}$ & -4.33235100 & 4.12831800 & -1.41096600 & $\mathrm{~F}$ & -6.31938400 & 4.33279600 & -0.55183600 \\
\hline $\mathrm{C}$ & -0.32431300 & -1.17657100 & 3.20320200 & $\mathrm{H}$ & -0.80158100 & -0.61214600 & 4.00385500 \\
\hline C & 0.00124700 & -2.60679400 & 3.76572600 & C & 0.77143500 & -3.48556900 & 2.76610200 \\
\hline $\mathrm{H}$ & 1.72796900 & -3.03595900 & 2.48870900 & $\mathrm{H}$ & 0.20777400 & -3.65953500 & 1.84714600 \\
\hline $\mathrm{H}$ & 0.96929900 & -4.46204400 & 3.22713100 & $\mathrm{C}$ & -1.32964800 & -3.29565500 & 4.13156300 \\
\hline $\mathrm{H}$ & -1.12691800 & -4.24095600 & 4.64910200 & $\mathrm{H}$ & -1.92205400 & -3.52407700 & 3.24152200 \\
\hline $\mathrm{H}$ & -1.93828400 & -2.67032300 & 4.79572600 & $\mathrm{C}$ & 0.84791500 & -2.46264200 & 5.04724100 \\
\hline $\mathrm{H}$ & 1.10103500 & -3.45519800 & 5.43666600 & $\mathrm{H}$ & 0.30307600 & -1.93381600 & 5.83889700 \\
\hline $\mathrm{H}$ & 1.79216800 & -1.93609400 & 4.86174600 & C & 0.90365200 & -0.38155800 & 2.69120600 \\
\hline $\mathrm{O}$ & 1.40273200 & -0.61676500 & 1.57930700 & C & 1.04357500 & 0.99841300 & 4.84023100 \\
\hline $\mathrm{C}$ & 2.53539800 & 1.43407500 & 2.98357900 & $\mathrm{C}$ & 2.40794800 & 1.33540500 & 5.45000100 \\
\hline $\mathrm{H}$ & 0.37542900 & 1.86878300 & 4.83384800 & $\mathrm{H}$ & 0.53697500 & 0.18853500 & 5.35813400 \\
\hline $\mathrm{H}$ & 3.22069100 & 0.75165100 & 2.47360200 & $\mathrm{H}$ & 2.91161500 & 0.40906100 & 5.74986300 \\
\hline $\mathrm{H}$ & 2.32465800 & 1.97468100 & 6.33404400 & $\mathrm{~N}$ & 1.39894700 & 0.62368800 & 3.46332600 \\
\hline C & 2.11842800 & 2.54159300 & 2.01755400 & C & 0.81741200 & 2.94738100 & 1.93434300 \\
\hline C & 3.13385100 & 3.21872800 & 1.22926100 & $\mathrm{C}$ & 0.39807600 & 4.03005300 & 1.09072000 \\
\hline $\mathrm{H}$ & 0.04807000 & 2.43254400 & 2.50394200 & $\mathrm{C}$ & 2.73728400 & 4.28956400 & 0.36106800 \\
\hline C & 4.49861100 & 2.87612400 & 1.27811700 & C & -0.94510500 & 4.43835000 & 1.01969500 \\
\hline C & 1.37120800 & 4.69504000 & 0.29097500 & C & 3.71229300 & 4.96860400 & -0.43592800 \\
\hline $\mathrm{H}$ & 4.83487400 & 2.08374800 & 1.93796300 & $\mathrm{C}$ & 5.44432200 & 3.55225300 & 0.50449100 \\
\hline C & -1.33014000 & 5.47121500 & 0.16507800 & $\mathrm{H}$ & -1.68538600 & 3.93248100 & 1.63427300 \\
\hline $\mathrm{C}$ & 0.97161800 & 5.76025600 & -0.57234700 & $\mathrm{C}$ & 5.06046000 & 4.58351000 & -0.34848200 \\
\hline $\mathrm{C}$ & 3.28247300 & 6.03700800 & -1.29745700 & $\mathrm{H}$ & 6.49352500 & 3.27834300 & 0.58239200 \\
\hline C & -0.38369300 & 6.12665300 & -0.62114800 & $\mathrm{H}$ & -2.37725600 & 5.75067900 & 0.10616800 \\
\hline $\mathrm{C}$ & 1.97596000 & 6.41430800 & -1.36385200 & $\mathrm{H}$ & 5.80399900 & 5.11295700 & -0.93961100 \\
\hline $\mathrm{H}$ & 4.03324500 & 6.54624500 & -1.89709400 & $\mathrm{H}$ & -0.69009300 & 6.93157600 & -1.28476700 \\
\hline $\mathrm{H}$ & 1.67053200 & 7.22618000 & -2.01953300 & C & -2.32415300 & -0.32290800 & 2.01107000 \\
\hline
\end{tabular}




\begin{tabular}{|c|c|c|c|c|c|c|c|}
\hline $\mathrm{S}$ & -2.56293700 & 0.96356300 & 3.10090800 & $\mathrm{~N}$ & -1.30595900 & -1.21446300 & 2.12919800 \\
\hline $\mathrm{H}$ & -1.10791800 & -1.83323500 & 1.33313100 & S & -1.80404000 & -3.56457800 & -0.94052100 \\
\hline $\mathrm{O}$ & -1.50970500 & -3.66641400 & -2.37877600 & 0 & -0.72301300 & -2.92628500 & -0.12810500 \\
\hline $\mathrm{O}$ & -3.15856200 & -3.07754600 & -0.56589500 & C & -1.78576200 & -5.31966800 & -0.32226300 \\
\hline $\mathrm{F}$ & -2.73027400 & -6.05402600 & -0.91767600 & $\mathrm{~F}$ & -1.99739200 & -5.34636900 & 1.00651300 \\
\hline $\mathrm{F}$ & -0.58965600 & -5.88792600 & -0.56162700 & C & 2.56770700 & -3.11103200 & -1.20905300 \\
\hline $\mathrm{O}$ & 2.78267900 & -3.66750500 & -2.27547900 & $\mathrm{O}$ & 3.03528700 & -3.47020600 & -0.00981800 \\
\hline C & 3.80303700 & -4.73462700 & 0.14048000 & C & 2.92089100 & -5.92054500 & -0.25908400 \\
\hline $\mathrm{H}$ & 3.42262100 & -6.85421100 & 0.01897900 & $\mathrm{H}$ & 2.73135600 & -5.93312400 & -1.33357700 \\
\hline $\mathrm{H}$ & 1.95973600 & -5.87696000 & 0.26175000 & C & 4.12249900 & -4.76409000 & 1.63564200 \\
\hline $\mathrm{H}$ & 4.67629500 & -3.86688500 & 1.93184300 & $\mathrm{H}$ & 4.73877900 & -5.64053800 & 1.86136300 \\
\hline $\mathrm{H}$ & 3.20862000 & -4.82011600 & 2.23106000 & C & 5.09756900 & -4.66067800 & -0.67684200 \\
\hline $\mathrm{H}$ & 5.71247400 & -5.54067200 & -0.45817600 & $\mathrm{H}$ & 5.67568600 & -3.77132900 & -0.39822600 \\
\hline $\mathrm{H}$ & 4.89281600 & -4.63690000 & -1.74774800 & C & 1.19495900 & -1.48711800 & -2.27891600 \\
\hline $\mathrm{H}$ & 0.96554900 & -2.31843400 & -2.94284800 & C & 0.11059600 & -0.48204000 & -2.20774900 \\
\hline $\mathrm{C}$ & -0.86587500 & -0.52197300 & -3.21836700 & C & 0.04659200 & 0.52593900 & -1.23150300 \\
\hline C & -1.87749800 & 0.43285000 & -3.26395400 & $\mathrm{H}$ & -0.84864400 & -1.32822800 & -3.94541200 \\
\hline C & -0.97188800 & 1.47780900 & -1.28353900 & $\mathrm{H}$ & 0.76977400 & 0.56161800 & -0.42183400 \\
\hline $\mathrm{C}$ & -1.92727400 & 1.44349100 & -2.30146600 & $\mathrm{H}$ & -2.63347300 & 0.37909400 & -4.04182200 \\
\hline $\mathrm{H}$ & -1.01566900 & 2.25251900 & -0.52693700 & $\mathrm{H}$ & -2.71243700 & 2.19293900 & -2.32719600 \\
\hline $\mathrm{N}$ & 1.83207200 & -1.93241000 & -1.12660400 & $\mathrm{H}$ & 1.57553100 & -1.57347600 & -0.20162500 \\
\hline C & 2.51494400 & -0.83391400 & -3.48184000 & $\mathrm{H}$ & 1.93328300 & -0.70296400 & -4.39182200 \\
\hline $\mathrm{H}$ & 3.10586700 & -1.74773500 & -3.45902300 & C & 3.07911200 & 0.32594100 & -2.91950000 \\
\hline C & 4.07514900 & 0.23255100 & -1.86146000 & $\mathrm{H}$ & 3.95916100 & -0.69364900 & -1.28254800 \\
\hline $\bar{H}$ & 4.05486000 & 1.09944200 & -1.18966700 & C & 2.55707400 & 1.67079500 & -3.29795600 \\
\hline $\mathrm{H}$ & 3.27470500 & 2.46930200 & -3.09087600 & $\mathrm{H}$ & 1.66905600 & 1.86377100 & -2.67109200 \\
\hline $\mathrm{H}$ & 2.22812700 & 1.71639700 & -4.33974800 & C & 6.97756200 & -0.07722100 & -0.98375300 \\
\hline $\bar{H}$ & 6.82562500 & 0.74313500 & -0.27356100 & $\mathrm{H}$ & 8.04192200 & -0.09901000 & -1.24760400 \\
\hline $\bar{H}$ & 6.74457100 & -1.01653300 & -0.46881300 & C & 6.32585800 & 1.76729400 & -3.40344800 \\
\hline $\mathrm{H}$ & 6.14903100 & 2.63016400 & -2.75070800 & $\mathrm{H}$ & 5.73191400 & 1.90451900 & -4.31445300 \\
\hline $\bar{H}$ & 7.38257600 & 1.78480700 & -3.69731200 & $\mathrm{C}$ & 6.09546600 & -1.32641600 & -3.69168700 \\
\hline $\mathrm{H}$ & 5.52820300 & -1.19092300 & -4.61920000 & $\mathrm{H}$ & 5.75660600 & -2.25927300 & -3.22630500 \\
\hline $\mathrm{H}$ & 7.14863900 & -1.45986000 & -3.96823000 & $\mathrm{Si}$ & 5.91602600 & 0.14643800 & -2.52808500 \\
\hline C & 3.16468200 & 2.01517100 & 4.29288100 & $\mathrm{H}$ & 3.01555700 & 3.09855500 & 4.32194100 \\
\hline $\bar{H}$ & 4.24251100 & 1.83391800 & 4.33953200 & & & & \\
\hline
\end{tabular}

\section{TS- $R-4 f-n d-8$}

Imaginary frequency: $-204.28 \mathrm{~cm}^{-1}$

E[B3LYP/6-31G(d)]: -4788.681518 Hartree

E[PCM(Et $2 \mathrm{O})-B 3 L Y P / 6-311 G(d, p)-D 3(B J)]:-4790.220456$ Hartree

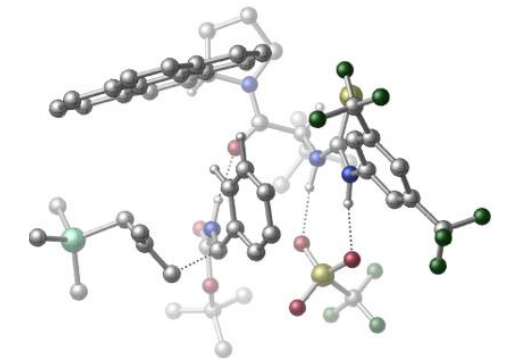

\begin{tabular}{|crrr|crrr|}
\hline 01 & & & & N & 3.08950900 & 0.35634300 & 0.75698000 \\
\hline H & 2.90706700 & 1.28932000 & 0.36750300 & C & 4.03069200 & -0.38431000 & 0.01901200 \\
\hline C & 5.01990600 & 0.31570400 & -0.68493000 & C & 3.95085000 & -1.77608600 & -0.11972700 \\
\hline C & 5.92512100 & -0.37530900 & -1.49213500 & H & 5.06045300 & 1.39707900 & -0.61717000 \\
\hline C & 4.85906200 & -2.44961100 & -0.93247800 & H & 3.17059100 & -2.32045500 & 0.39125400 \\
\hline C & 5.86020300 & -1.76248600 & -1.62107700 & H & 6.56377200 & -2.29245900 & -2.25141400 \\
\hline C & 7.02887300 & 0.38071900 & -2.18342600 & C & 4.66757000 & -3.92304000 & -1.15688700 \\
\hline F & 8.13203700 & 0.47710900 & -1.40445700 & F & 6.66033900 & 1.63857500 & -2.50130600 \\
\hline F & 7.41404300 & -0.23176800 & -3.32819000 & F & 4.21230700 & -4.56083300 & -0.05271700 \\
\hline
\end{tabular}




\begin{tabular}{|c|c|c|c|c|c|c|c|}
\hline $\mathrm{F}$ & 3.74648600 & -4.16289100 & -2.13502900 & $\mathrm{~F}$ & 5.80362000 & -4.53959500 & -1.54154700 \\
\hline C & 0.58506600 & 0.61691600 & 3.42146800 & $\mathrm{H}$ & 1.19188100 & 0.02052500 & 4.10158200 \\
\hline C & 0.25416600 & 1.95656700 & 4.17431300 & C & -0.72845800 & 2.86682200 & 3.41934700 \\
\hline $\mathrm{H}$ & -1.69975100 & 2.39254700 & 3.26315800 & $\mathrm{H}$ & -0.33931800 & 3.16680800 & 2.44389100 \\
\hline $\mathrm{H}$ & -0.88984300 & 3.78143900 & 4.00420700 & C & 1.57870500 & 2.71471200 & 4.40249600 \\
\hline $\mathrm{H}$ & 1.40186900 & 3.57480600 & 5.05937900 & $\mathrm{H}$ & 1.99181400 & 3.09420200 & 3.46341700 \\
\hline $\mathrm{H}$ & 2.33464800 & 2.07692700 & 4.87624700 & C & -0.35711500 & 1.60397900 & 5.54613000 \\
\hline $\mathrm{H}$ & -0.58917600 & 2.52330300 & 6.09546500 & $\mathrm{H}$ & 0.33537300 & 1.01732600 & 6.16200200 \\
\hline $\mathrm{H}$ & -1.29423900 & 1.04287600 & 5.44466700 & C & -0.65821500 & -0.20543100 & 3.00089000 \\
\hline 0 & -1.35420100 & 0.12455100 & 2.02928800 & C & -0.27429500 & -1.84786800 & 4.90856000 \\
\hline C & -2.22396600 & -2.05021000 & 3.41269100 & C & -0.95743100 & -3.19552000 & 5.16982400 \\
\hline $\mathrm{H}$ & 0.79885800 & -1.94121900 & 4.71928700 & $\mathrm{H}$ & -0.42335800 & -1.16938400 & 5.75558900 \\
\hline C & -2.38432900 & -2.98108700 & 4.64683400 & $\mathrm{H}$ & -3.02524300 & -1.30520500 & 3.40281600 \\
\hline $\mathrm{H}$ & -0.92970400 & -3.46405300 & 6.23004000 & $\mathrm{H}$ & -0.45986600 & -3.99407700 & 4.61137700 \\
\hline $\mathrm{H}$ & -2.98713900 & -2.46445600 & 5.40337400 & $\mathrm{H}$ & -2.89671500 & -3.91194500 & 4.39218700 \\
\hline $\mathrm{N}$ & -0.96684100 & -1.32740800 & 3.70675800 & C & -2.35287500 & -2.78991200 & 2.07356800 \\
\hline C & -3.60522600 & -2.76288700 & 1.52255400 & C & -1.31951400 & -3.59253300 & 1.43232100 \\
\hline C & -3.97833600 & -3.51072700 & 0.36004500 & $\mathrm{H}$ & -4.37714600 & -2.15614900 & 1.99257100 \\
\hline C & -1.67021800 & -4.36636500 & 0.27109900 & C & 0.01887400 & -3.63651300 & 1.86500000 \\
\hline C & -5.27968900 & -3.47409300 & -0.17193000 & C & -2.99256700 & -4.32839400 & -0.26459900 \\
\hline C & -0.68466300 & -5.17815800 & -0.37672200 & $\mathrm{H}$ & 0.34968100 & -3.01186500 & 2.68047800 \\
\hline $\mathrm{C}$ & 0.97312100 & -4.42324700 & 1.21932900 & $\mathrm{C}$ & -5.60748300 & -4.22306300 & -1.30236400 \\
\hline $\mathrm{H}$ & -6.03758400 & -2.87264500 & 0.32549400 & $\mathrm{C}$ & -3.33694800 & -5.09175800 & -1.42347900 \\
\hline C & 0.62992400 & -5.19550300 & 0.11559600 & $\mathrm{C}$ & -1.06078400 & -5.94323600 & -1.53421000 \\
\hline $\mathrm{H}$ & 1.99884600 & -4.40625400 & 1.57419100 & $\mathrm{C}$ & -4.64774800 & -5.02048700 & -1.92461900 \\
\hline $\mathrm{H}$ & -6.62209200 & -4.19715200 & -1.69201600 & $\mathrm{C}$ & -2.32487700 & -5.90553800 & -2.03605100 \\
\hline $\mathrm{H}$ & 1.38566300 & -5.79063100 & -0.39052200 & $\mathrm{H}$ & -0.29950500 & -6.55845500 & -2.00751700 \\
\hline $\mathrm{H}$ & -4.91010000 & -5.60528900 & -2.80286000 & $\mathrm{H}$ & -2.58936100 & -6.49104700 & -2.91316700 \\
\hline C & 2.45013200 & -0.00467400 & 1.91501200 & $\mathrm{~S}$ & 2.94344400 & -1.31686500 & 2.88244700 \\
\hline $\mathrm{N}$ & 1.41834500 & 0.81428200 & 2.24141200 & $\mathrm{H}$ & 1.14012600 & 1.54265900 & 1.57705700 \\
\hline S & 1.71737800 & 3.64128400 & -0.58754400 & $\mathrm{O}$ & 1.05886500 & 4.00392200 & -1.86067700 \\
\hline 0 & 0.79408700 & 3.10744900 & 0.45627300 & $\mathrm{O}$ & 2.97585100 & 2.86050200 & -0.70257500 \\
\hline C & 2.27184200 & 5.26957200 & 0.12172200 & $\mathrm{~F}$ & 3.20682800 & 5.83182200 & -0.65049700 \\
\hline $\mathrm{F}$ & 2.77733200 & 5.09457700 & 1.35181800 & $\mathrm{~F}$ & 1.23201400 & 6.12179100 & 0.21208800 \\
\hline C & -2.58225900 & 3.02329800 & 0.20150800 & 0 & -3.17837000 & 3.06646900 & 1.26024900 \\
\hline 0 & -2.46096700 & 4.01534800 & -0.69592600 & C & -2.83252900 & 5.41494600 & -0.36895600 \\
\hline C & -4.35740000 & 5.51858300 & -0.29156900 & $\mathrm{H}$ & -4.64775600 & 6.56145300 & -0.12259400 \\
\hline $\mathrm{H}$ & -4.74691800 & 4.91201900 & 0.52913800 & $\mathrm{H}$ & -4.81600400 & 5.19180200 & -1.23249400 \\
\hline C & -2.26762300 & 6.18285400 & -1.56550400 & $\mathrm{H}$ & -1.18626600 & 6.03219500 & -1.63615400 \\
\hline $\mathrm{H}$ & -2.47019100 & 7.25285600 & -1.45081500 & $\mathrm{H}$ & -2.72885600 & 5.84463100 & -2.50012000 \\
\hline C & -2.15308300 & 5.86039500 & 0.92839800 & $\mathrm{H}$ & -2.31590800 & 6.93626000 & 1.05897600 \\
\hline $\mathrm{H}$ & -1.07631100 & 5.68239900 & 0.87579100 & $\mathrm{H}$ & -2.55978300 & 5.33738300 & 1.79465400 \\
\hline $\mathrm{C}$ & -1.35167300 & 1.75505000 & -1.51008000 & $\mathrm{H}$ & -0.94476100 & 2.71299900 & -1.83451900 \\
\hline C & -0.39096700 & 0.63744200 & -1.74452900 & C & 0.59574800 & 0.82981800 & -2.72717900 \\
\hline C & -0.46668800 & -0.59998200 & -1.08531100 & C & 1.47832800 & -0.19666700 & -3.05453000 \\
\hline $\mathrm{H}$ & 0.69321000 & 1.80179300 & -3.20128900 & $\mathrm{C}$ & 0.42497000 & -1.62227000 & -1.41501100 \\
\hline $\mathrm{H}$ & -1.19929300 & -0.77104100 & -0.30243900 & C & 1.38992800 & -1.42991800 & -2.40563300 \\
\hline $\mathrm{H}$ & 2.24631100 & -0.02565100 & -3.80323400 & $\mathrm{H}$ & 0.36669700 & -2.56974800 & -0.89218300 \\
\hline $\mathrm{H}$ & 2.08284400 & -2.23018100 & -2.64595100 & $\mathrm{~N}$ & -1.97620800 & 1.85496900 & -0.26273200 \\
\hline $\mathrm{H}$ & -1.79977300 & 1.16854400 & 0.47765200 & C & -2.63532200 & 1.70242600 & -2.80506400 \\
\hline $\mathrm{H}$ & -2.07027400 & 1.85985600 & -3.72223600 & $\mathrm{H}$ & -3.18699100 & 2.58612000 & -2.49003000 \\
\hline C & -3.31822200 & 0.46222900 & -2.70539200 & C & -4.31321200 & 0.24731100 & -1.67473500 \\
\hline $\mathrm{H}$ & -4.15661700 & 0.91318200 & -0.81522300 & $\mathrm{H}$ & -4.36129100 & -0.80027800 & -1.35113600 \\
\hline
\end{tabular}




\begin{tabular}{|c|c|c|c|c|c|c|c|}
\hline $\mathrm{C}$ & -2.89626300 & -0.68665600 & -3.55489300 & $\mathrm{H}$ & -3.66492900 & -1.46114400 & -3.62023200 \\
\hline $\mathrm{H}$ & -2.01728500 & -1.14086900 & -3.06514900 & $\mathrm{H}$ & -2.57984400 & -0.37663600 & -4.55463100 \\
\hline $\mathrm{C}$ & -7.19744800 & 0.40610800 & -0.70645500 & $\mathrm{H}$ & -7.12017900 & -0.61774700 & -0.32386600 \\
\hline $\mathrm{H}$ & -8.25445000 & 0.60014400 & -0.92596500 & $\mathrm{H}$ & -6.89276300 & 1.08931200 & 0.09436400 \\
\hline C & -6.63264200 & -0.57762700 & -3.60681500 & $\mathrm{H}$ & -6.49439200 & -1.61466900 & -3.27895000 \\
\hline $\bar{H}$ & -6.05106200 & -0.43229700 & -4.52440300 & $\mathrm{H}$ & -7.69065000 & -0.45276500 & -3.86834900 \\
\hline $\mathrm{C}$ & -6.24604800 & 2.42464400 & -2.87161400 & $\mathrm{H}$ & -5.63742900 & 2.58834800 & -3.76791100 \\
\hline $\mathrm{H}$ & -5.92024300 & 3.13763000 & -2.10551100 & $\mathrm{H}$ & -7.28278500 & 2.67372300 & -3.12959700 \\
\hline Si & -6.14945200 & 0.64619200 & -2.25478100 & & & & \\
\hline
\end{tabular}

\section{TS- $R-4 f-n d-9$}

Imaginary frequency: $-254.00 \mathrm{~cm}^{-1}$

E[B3LYP/6-31G(d)-D3(BJ)]: -4788.690017 Hartree

E[PCM(Et $\left.\left.{ }_{2} \mathrm{O}\right)-\mathrm{B} 3 L Y P / 6-311 \mathrm{G}(\mathrm{d}, \mathrm{p})-\mathrm{D} 3(\mathrm{BJ})\right]:-4790.218917$ Hartree

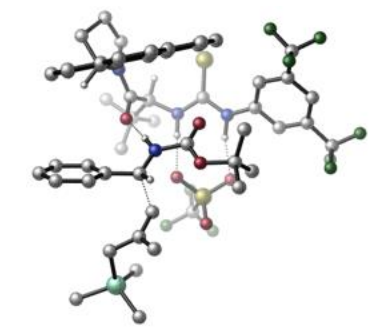

\begin{tabular}{|c|c|c|c|c|c|c|c|}
\hline 01 & & & & $\mathrm{~N}$ & -2.47378400 & -1.48281800 & 1.62380100 \\
\hline $\mathrm{H}$ & -1.85095700 & -2.26531300 & 1.39402700 & C & -3.62946800 & -1.45805800 & 0.82807900 \\
\hline C & -4.05906700 & -2.67860700 & 0.28398600 & C & -4.30165700 & -0.28060000 & 0.46843100 \\
\hline $\mathrm{C}$ & -5.13881900 & -2.71327200 & -0.59828100 & $\mathrm{H}$ & -3.52557600 & -3.59018400 & 0.52831600 \\
\hline C & -5.38047800 & -0.33717000 & -0.40938900 & $\mathrm{H}$ & -3.97164700 & 0.66525400 & 0.86867000 \\
\hline C & -5.81878700 & -1.54844000 & -0.94817300 & $\mathrm{H}$ & -6.66489800 & -1.58238800 & -1.62429500 \\
\hline $\mathrm{C}$ & -5.51185700 & -4.01283800 & -1.25749000 & $\mathrm{C}$ & -6.02264100 & 0.93920400 & -0.87692800 \\
\hline $\mathrm{F}$ & -5.25050700 & -5.07964000 & -0.47532000 & $\mathrm{~F}$ & -4.82070600 & -4.19286800 & -2.41288500 \\
\hline $\mathrm{F}$ & -6.82420200 & -4.05697900 & -1.58251200 & $\mathrm{~F}$ & -5.78944600 & 1.97815300 & -0.04491600 \\
\hline $\mathrm{F}$ & -5.54497900 & 1.31194500 & -2.09872700 & $\mathrm{~F}$ & -7.36061400 & 0.81807800 & -1.01343500 \\
\hline $\mathrm{C}$ & 0.03974900 & 0.12265000 & 3.74489500 & $\mathrm{H}$ & -0.71866300 & 0.55313000 & 4.39810200 \\
\hline C & 1.02375100 & -0.69072100 & 4.66073900 & C & 2.22503100 & -1.29334400 & 3.90643800 \\
\hline $\mathrm{H}$ & 2.87349400 & -0.51434900 & 3.49463000 & $\mathrm{H}$ & 1.91736200 & -1.94268000 & 3.08345300 \\
\hline $\mathrm{H}$ & 2.82013000 & -1.89540100 & 4.60461600 & $\mathrm{C}$ & 0.22165800 & -1.82723700 & 5.32691600 \\
\hline $\mathrm{H}$ & 0.84427000 & -2.33244200 & 6.07504800 & $\mathrm{H}$ & -0.09621000 & -2.57424500 & 4.59513300 \\
\hline $\mathrm{H}$ & -0.67260600 & -1.44469400 & 5.83310700 & $\mathrm{C}$ & 1.55429200 & 0.25205700 & 5.76054100 \\
\hline $\mathrm{H}$ & 2.24406400 & -0.29029300 & 6.41729600 & $\mathrm{H}$ & 0.74160200 & 0.64086300 & 6.38593100 \\
\hline $\mathrm{H}$ & 2.10464800 & 1.10348300 & 5.34127700 & C & 0.72353500 & 1.24505400 & 2.93654500 \\
\hline $\mathrm{O}$ & 1.44293000 & 0.96168800 & 1.96149800 & $\mathrm{~N}$ & 0.51948800 & 2.53334000 & 3.29294700 \\
\hline $\mathrm{C}$ & 0.55174100 & 3.97914100 & 1.22926700 & $\mathrm{C}$ & -0.71522500 & 3.57554600 & 0.92154100 \\
\hline C & 1.29116300 & 4.81089200 & 0.29244000 & C & -1.36993200 & 3.95290800 & -0.29916200 \\
\hline $\mathrm{H}$ & -1.26279900 & 2.93208600 & 1.60483900 & $\mathrm{C}$ & 0.66198500 & 5.19470800 & -0.93670600 \\
\hline $\mathrm{C}$ & 2.60277000 & 5.26025800 & 0.53248000 & $\mathrm{C}$ & -2.67953400 & 3.54407000 & -0.59672000 \\
\hline C & -0.66761600 & 4.76942400 & -1.23164400 & C & 1.36257300 & 6.01239700 & -1.87805400 \\
\hline $\mathrm{H}$ & 3.11119500 & 4.99043500 & 1.45149700 & $\mathrm{C}$ & 3.27875800 & 6.05519900 & -0.39326200 \\
\hline $\mathrm{C}$ & -3.29076500 & 3.92325900 & -1.79118900 & $\mathrm{H}$ & -3.21890600 & 2.92877200 & 0.11671400 \\
\hline C & -1.29911400 & 5.15509700 & -2.45349400 & C & 2.66996000 & 6.43138700 & -1.58545800 \\
\hline C & 0.70298000 & 6.38492800 & -3.10011100 & $\mathrm{H}$ & 4.29123000 & 6.38483400 & -0.17514000 \\
\hline $\mathrm{C}$ & -2.60920200 & 4.71853900 & -2.71099600 & $\mathrm{H}$ & -4.29875100 & 3.58258600 & -2.00471500 \\
\hline C & -0.56625800 & 5.97731300 & -3.37534200 & $\mathrm{H}$ & 3.20025100 & 7.05581600 & -2.30062400 \\
\hline $\mathrm{H}$ & 1.24577800 & 7.00961600 & -3.80580000 & $\mathrm{H}$ & -3.09076500 & 5.01167400 & -3.64104500 \\
\hline $\mathrm{H}$ & -1.05043300 & 6.27163200 & -4.30366000 & C & -2.01215600 & -0.56288500 & 2.52847700 \\
\hline $\mathrm{S}$ & -3.00056800 & 0.59015800 & 3.29071400 & $\mathrm{~N}$ & -0.68824400 & -0.71935300 & 2.80445100 \\
\hline $\mathrm{H}$ & -0.12279600 & -1.25532100 & 2.13835100 & $\mathrm{~S}$ & 0.42165100 & -3.66271200 & 0.45672200 \\
\hline $\mathrm{O}$ & 0.99275900 & -4.14087100 & -0.81766100 & $\mathrm{O}$ & 0.93824000 & -2.33398200 & 0.90701800 \\
\hline $\mathrm{O}$ & -1.04992100 & -3.79552300 & 0.60570600 & C & 1.08534200 & -4.83937300 & 1.73938200 \\
\hline
\end{tabular}




\begin{tabular}{|c|c|c|c|c|c|c|c|}
\hline $\mathrm{F}$ & 0.76708000 & -6.10242100 & 1.44073900 & $\mathrm{~F}$ & 0.57753000 & -4.54472200 & 2.94883900 \\
\hline $\mathrm{F}$ & 2.42526700 & -4.74487000 & 1.82068900 & C & -0.04783400 & 0.13776900 & -1.31296600 \\
\hline 0 & -1.00787000 & 0.24179500 & -0.58351400 & 0 & -0.01892600 & -0.25650800 & -2.59352600 \\
\hline C & -1.25469400 & -0.72253100 & -3.29433300 & C & -2.29753900 & 0.39617000 & -3.31552400 \\
\hline $\mathrm{H}$ & -3.13162100 & 0.09288200 & -3.95775500 & $\mathrm{H}$ & -2.69027000 & 0.60349900 & -2.32066600 \\
\hline $\mathrm{H}$ & -1.86999600 & 1.31800400 & -3.72453000 & $\mathrm{C}$ & -0.73561300 & -1.00932700 & -4.70403000 \\
\hline $\mathrm{H}$ & 0.03668800 & -1.78596400 & -4.68553700 & $\mathrm{H}$ & -1.55774300 & -1.36551700 & -5.33271800 \\
\hline $\mathrm{H}$ & -0.31924400 & -0.10519900 & -5.16113900 & C & -1.75552800 & -1.99775500 & -2.61790900 \\
\hline $\mathrm{H}$ & -2.61515000 & -2.39635600 & -3.16752800 & $\mathrm{H}$ & -0.97565000 & -2.76590700 & -2.59551200 \\
\hline $\mathrm{H}$ & -2.07392000 & -1.80246300 & -1.59431200 & $\mathrm{C}$ & 2.38089500 & 0.35194300 & -1.61749200 \\
\hline $\mathrm{H}$ & 2.18097500 & 0.44307900 & -2.67944800 & C & 3.61581800 & 1.03178500 & -1.15181100 \\
\hline C & 4.40457000 & 1.69794400 & -2.10308800 & C & 4.02345900 & 1.02074000 & 0.19189600 \\
\hline C & 5.57181800 & 2.35670600 & -1.71995300 & $\mathrm{H}$ & 4.08789200 & 1.72227600 & -3.14311000 \\
\hline C & 5.19592200 & 1.67535800 & 0.56752000 & $\mathrm{H}$ & 3.42139600 & 0.52009100 & 0.94233500 \\
\hline C & 5.97144200 & 2.34443000 & -0.38274900 & $\mathrm{H}$ & 6.16328200 & 2.88288100 & -2.46380400 \\
\hline $\mathrm{H}$ & 5.50250800 & 1.66355200 & 1.60974700 & $\mathrm{H}$ & 6.88211100 & 2.85494000 & -0.08182800 \\
\hline $\mathrm{N}$ & 1.24533900 & 0.46326800 & -0.86378400 & $\mathrm{H}$ & 1.29299000 & 0.63885400 & 0.14996200 \\
\hline C & 2.78283300 & -1.60357500 & -1.63351000 & $\mathrm{H}$ & 2.92714600 & -1.75606500 & -0.56786600 \\
\hline $\mathrm{H}$ & 1.80448500 & -1.90338400 & -1.99305000 & C & 3.86431200 & -1.76308200 & -2.49740900 \\
\hline C & 5.23955100 & -1.86113300 & -2.01115600 & $\mathrm{H}$ & 5.96875300 & -1.44957200 & -2.71985200 \\
\hline $\mathrm{H}$ & 5.35917500 & -1.37446500 & -1.03593200 & C & 3.63723900 & -1.75751500 & -3.97972000 \\
\hline $\mathrm{H}$ & 4.00924700 & -0.81013000 & -4.39980500 & $\mathrm{H}$ & 4.20929700 & -2.55231800 & -4.47087800 \\
\hline $\mathrm{H}$ & 2.58147000 & -1.86149100 & -4.24020000 & C & 7.59595200 & -3.56190900 & -1.10754900 \\
\hline $\mathrm{H}$ & 7.67465900 & -2.96245100 & -0.19318300 & $\mathrm{H}$ & 8.01181600 & -4.55282500 & -0.88788600 \\
\hline $\mathrm{H}$ & 8.23256200 & -3.09814300 & -1.87021400 & C & 4.70344900 & -4.47579200 & -0.36591500 \\
\hline $\mathrm{H}$ & 4.74862800 & -3.91697800 & 0.57570700 & $\mathrm{H}$ & 3.65177100 & -4.51706100 & -0.67008400 \\
\hline $\mathrm{H}$ & 5.02665600 & -5.50196900 & -0.15015500 & C & 5.71902900 & -4.70051600 & -3.29634600 \\
\hline $\mathrm{H}$ & 4.68716800 & -4.85238900 & -3.63275900 & $\mathrm{H}$ & 6.27987000 & -4.22309600 & -4.10892800 \\
\hline $\mathrm{H}$ & 6.15546800 & -5.69526100 & -3.14226300 & $\mathrm{Si}$ & 5.80242300 & -3.71704400 & -1.68477000 \\
\hline C & -0.28229400 & 3.03378800 & 4.43444200 & $\mathrm{H}$ & 0.17221600 & 2.71530100 & 5.37909400 \\
\hline $\mathrm{H}$ & -1.30208200 & 2.64269400 & 4.38379000 & C & -0.20895900 & 4.55941700 & 4.28162800 \\
\hline $\mathrm{H}$ & -1.02617900 & 4.91571600 & 3.64601000 & $\mathrm{H}$ & -0.28447400 & 5.07108000 & 5.24537300 \\
\hline C & 1.13874800 & 4.78492300 & 3.58138700 & $\mathrm{H}$ & 1.21671900 & 5.76225300 & 3.09968600 \\
\hline $\mathrm{H}$ & 1.96249900 & 4.69062900 & 4.30051100 & C & 1.20400300 & 3.62053200 & 2.56245900 \\
\hline $\mathrm{H}$ & 2.23075300 & 3.29481400 & 2.37673100 & & & & \\
\hline \multicolumn{8}{|c|}{ TS- $R-4 \mathrm{f}-\mathrm{nd}-10$} \\
\hline \multicolumn{8}{|c|}{$\begin{array}{l}\text { Imaginary frequency: }-248.55 \mathrm{~cm}-1 \\
\text { E[B3LYP/6-31G(d)-D3(BJ)]: }-4788.692958 \text { Hartree } \\
E\left[P C M\left(E t_{2} O\right)-B 3 L Y P / 6-311 G(d, p)-D 3(B J)\right]:-4790.218117 \text { Hartree }\end{array}$} \\
\hline
\end{tabular}

\begin{tabular}{|llll|llll|}
\hline 01 & & & $\mathrm{~N}$ & -1.67573400 & -2.39768200 & 1.21869600 \\
\hline $\mathrm{H}$ & -0.89818400 & -3.02475200 & 0.97825300 & $\mathrm{C}$ & -2.90334100 & -2.79513700 & 0.65988100 \\
\hline $\mathrm{C}$ & -3.00381600 & -4.12344400 & 0.21150500 & $\mathrm{C}$ & -3.97960300 & -1.92464400 & 0.44903100 \\
\hline $\mathrm{C}$ & -4.16675600 & -4.56490200 & -0.41477500 & $\mathrm{H}$ & -2.16536600 & -4.79828800 & 0.34640500 \\
\hline $\mathrm{C}$ & -5.13488800 & -2.38517600 & -0.18307200 & $\mathrm{H}$ & -3.91068700 & -0.89601700 & 0.77002300 \\
\hline $\mathrm{C}$ & -5.24854800 & -3.70478900 & -0.61426700 & $\mathrm{H}$ & -6.15170900 & -4.05511400 & -1.09799100 \\
\hline $\mathrm{C}$ & -4.23835200 & -5.97356600 & -0.94305900 & $\mathrm{C}$ & -6.26773200 & -1.41451300 & -0.37864000 \\
\hline $\mathrm{F}$ & -3.45976800 & -6.82081400 & -0.23924800 & $\mathrm{~F}$ & -3.83367900 & -6.04239500 & -2.23327100 \\
\hline $\mathrm{F}$ & -5.50236400 & -6.45597600 & -0.90630200 & $\mathrm{~F}$ & -5.82000800 & -0.22247500 & -0.86014900 \\
\hline $\mathrm{F}$ & -7.19223500 & -1.87156800 & -1.24982800 & $\mathrm{~F}$ & -6.90998000 & -1.13893900 & 0.77757800 \\
\hline
\end{tabular}




\begin{tabular}{|c|c|c|c|c|c|c|c|}
\hline$C$ & 0.49622700 & -0.23283400 & 3.21278200 & $\mathrm{H}$ & -0.26249800 & -0.08733200 & 3.98151400 \\
\hline C & 1.78424600 & -0.77208100 & 3.93749100 & C & 2.99594800 & -0.93394900 & 3.00120000 \\
\hline $\mathrm{H}$ & 3.34314900 & 0.03506200 & 2.62893600 & $\mathrm{H}$ & 2.77842000 & -1.57125600 & 2.14190100 \\
\hline $\mathrm{H}$ & 3.82259600 & -1.39257000 & 3.55780600 & C & 1.43729000 & -2.13815000 & 4.56571100 \\
\hline $\mathrm{H}$ & 2.27198100 & -2.48220700 & 5.18805400 & $\mathrm{H}$ & 1.25186900 & -2.89920900 & 3.80435200 \\
\hline $\mathrm{H}$ & 0.54708300 & -2.06991800 & 5.20220200 & C & 2.17467600 & 0.19854600 & 5.07046400 \\
\hline $\mathrm{H}$ & 3.07882200 & -0.16446400 & 5.57248900 & $\mathrm{H}$ & 1.38772900 & 0.27363700 & 5.82997400 \\
\hline $\mathrm{H}$ & 2.39245500 & 1.20555400 & 4.69400000 & C & 0.68642100 & 1.09092200 & 2.43320100 \\
\hline 0 & 1.21460000 & 1.07411000 & 1.30799700 & $\mathrm{~N}$ & 0.24370300 & 2.24182200 & 2.99369600 \\
\hline C & -0.69196800 & 3.85137600 & 1.30419800 & C & -1.85132200 & 3.13132800 & 1.28385700 \\
\hline C & -0.53307700 & 4.98696000 & 0.40954200 & C & -2.95333000 & 3.46624100 & 0.42774300 \\
\hline $\mathrm{H}$ & -1.96608200 & 2.26226300 & 1.92586900 & C & -1.62077700 & 5.34371100 & -0.45302400 \\
\hline C & 0.64209700 & 5.75821600 & 0.34708500 & C & -4.14317200 & 2.71940500 & 0.42427200 \\
\hline C & -2.83240300 & 4.58979200 & -0.43967900 & C & -1.50075800 & 6.46183400 & -1.33627400 \\
\hline $\mathrm{H}$ & 1.48703200 & 5.51501500 & 0.98234900 & C & 0.75188800 & 6.84301700 & -0.52334600 \\
\hline C & -5.19806100 & 3.06720800 & -0.41917500 & $\mathrm{H}$ & -4.23058800 & 1.86200400 & 1.08661300 \\
\hline C & -3.92009100 & 4.94528000 & -1.29481300 & C & -0.30528800 & 7.19737700 & -1.35453500 \\
\hline C & -2.61302300 & 6.80085900 & -2.18213400 & $\mathrm{H}$ & 1.67396000 & 7.41794800 & -0.54654400 \\
\hline $\mathrm{C}$ & -5.09103200 & 4.16890100 & -1.26627800 & $\mathrm{H}$ & -6.09974300 & 2.46232700 & -0.42305300 \\
\hline $\mathrm{C}$ & -3.76762400 & 6.08012500 & -2.16235200 & $\mathrm{H}$ & -0.21487800 & 8.04751700 & -2.02626000 \\
\hline $\mathrm{H}$ & -2.50982900 & 7.65569000 & -2.84621500 & $\mathrm{H}$ & -5.91713600 & 4.43512500 & -1.92131300 \\
\hline $\mathrm{H}$ & -4.59767400 & 6.35163300 & -2.81016200 & $\mathrm{C}$ & -1.40067800 & -1.40866400 & 2.12889200 \\
\hline S & -2.57994000 & -0.55997200 & 3.01074400 & $\mathrm{~N}$ & -0.06406400 & -1.21010000 & 2.28941700 \\
\hline $\mathrm{H}$ & 0.56520400 & -1.57966900 & 1.57015200 & $\mathrm{~S}$ & 1.56021300 & -3.85411700 & -0.08668600 \\
\hline 0 & 1.90120900 & -4.23185900 & -1.46895700 & $\mathrm{O}$ & 1.76982600 & -2.40812900 & 0.23849400 \\
\hline $\mathrm{O}$ & 0.28222300 & -4.38343900 & 0.45335900 & $\mathrm{C}$ & 2.85578800 & -4.70617400 & 0.94245100 \\
\hline $\mathrm{F}$ & 2.78691600 & -6.03158400 & 0.80938400 & $\mathrm{~F}$ & 2.71978900 & -4.39631300 & 2.24204700 \\
\hline $\mathrm{F}$ & 4.08697500 & -4.30463900 & 0.55161400 & C & 0.81698900 & 1.63802400 & -2.55187400 \\
\hline 0 & 1.08401900 & 1.94992500 & -3.69884700 & $\mathrm{O}$ & -0.30808200 & 1.13409400 & -2.07148600 \\
\hline C & -1.47107700 & 0.83815900 & -2.96794200 & C & -1.05644700 & -0.18752000 & -4.02508300 \\
\hline $\mathrm{H}$ & -1.94497200 & -0.49076100 & -4.58935000 & $\mathrm{H}$ & -0.32786100 & 0.22538300 & -4.72521000 \\
\hline $\mathrm{H}$ & -0.63968300 & -1.08316000 & -3.55270400 & C & -2.48049200 & 0.24093300 & -1.99221900 \\
\hline $\mathrm{H}$ & -2.72423800 & 0.95534700 & -1.20244200 & $\mathrm{H}$ & -3.40577900 & -0.01519100 & -2.51727300 \\
\hline $\mathrm{H}$ & -2.08110800 & -0.66756200 & -1.53242200 & C & -1.98045600 & 2.14310400 & -3.58046300 \\
\hline $\mathrm{H}$ & -2.90206900 & 1.93937100 & -4.13650900 & $\mathrm{H}$ & -2.21308200 & 2.87097700 & -2.79786500 \\
\hline $\mathrm{H}$ & -1.24916800 & 2.57457800 & -4.26683200 & C & 3.02266800 & 2.18850700 & -1.84343500 \\
\hline $\mathrm{H}$ & 3.01354700 & 2.77973700 & -2.75265300 & C & 3.98764300 & 2.61978700 & -0.81660200 \\
\hline C & 4.89514200 & 3.63763600 & -1.16437800 & C & 4.04808500 & 2.05815900 & 0.46956400 \\
\hline C & 5.83201400 & 4.09671400 & -0.24369700 & $\mathrm{H}$ & 4.85525200 & 4.07433900 & -2.15944000 \\
\hline C & 4.99196200 & 2.52055600 & 1.38663200 & $\mathrm{H}$ & 3.35242700 & 1.27917800 & 0.75837200 \\
\hline C & 5.88321500 & 3.53665800 & 1.03595900 & $\mathrm{H}$ & 6.51958400 & 4.89016600 & -0.52188500 \\
\hline $\mathrm{H}$ & 5.03050500 & 2.08058600 & 2.37911900 & $\mathrm{H}$ & 6.61609700 & 3.89081300 & 1.75553200 \\
\hline $\mathrm{N}$ & 1.76885100 & 1.76124200 & -1.51695100 & $\mathrm{H}$ & 1.50916600 & 1.47396700 & -0.56454700 \\
\hline C & 4.09496900 & 0.77051600 & -2.95023400 & $\mathrm{H}$ & 3.58598100 & 0.98011600 & -3.88684200 \\
\hline $\mathrm{H}$ & 5.07691400 & 1.21914200 & -2.83023200 & C & 3.82554100 & -0.44699000 & -2.32380000 \\
\hline C & 4.68963500 & -0.99926400 & -1.28044200 & $\mathrm{H}$ & 5.27427000 & -0.21897200 & -0.77728100 \\
\hline $\mathrm{H}$ & 4.11099600 & -1.57691400 & -0.54924300 & C & 2.56110000 & -1.16215000 & -2.64693600 \\
\hline $\mathrm{H}$ & 2.67109700 & -2.25032800 & -2.61318000 & $\mathrm{H}$ & 1.83933200 & -0.94916600 & -1.84107800 \\
\hline $\mathrm{H}$ & 2.13325600 & -0.84476800 & -3.60041700 & C & 6.99073500 & -2.80906100 & -0.42363100 \\
\hline $\mathrm{H}$ & 6.33404600 & -3.33739700 & 0.27463200 & $\mathrm{H}$ & 7.79841900 & -3.49533100 & -0.70659700 \\
\hline $\mathrm{H}$ & 7.44526200 & -1.96439800 & 0.10760100 & C & 5.23614600 & -3.68389900 & -2.85439500 \\
\hline $\mathrm{H}$ & 4.44068000 & -4.14415500 & -2.25857000 & $\mathrm{H}$ & 4.79977900 & -3.37597500 & -3.81176500 \\
\hline $\mathrm{H}$ & 5.98544100 & -4.45577000 & -3.07111500 & C & 7.19684700 & -1.28545200 & -3.11467500 \\
\hline
\end{tabular}




\begin{tabular}{|rrrr|lrrr|}
\hline $\mathrm{H}$ & 6.66913900 & -0.90617800 & -3.99734200 & $\mathrm{H}$ & 7.66816100 & -0.43275100 & -2.61097200 \\
\hline $\mathrm{H}$ & 8.00188200 & -1.93978600 & -3.47134500 & $\mathrm{Si}$ & 6.04572400 & -2.24111900 & -1.95641000 \\
\hline $\mathrm{C}$ & -0.65231100 & 3.91185200 & 4.43508100 & $\mathrm{H}$ & -0.56017100 & 4.25754300 & 5.46860800 \\
\hline $\mathrm{H}$ & -1.65708300 & 4.16673700 & 4.08477300 & $\mathrm{C}$ & 0.40070500 & 4.52448800 & 3.49993800 \\
\hline $\mathrm{H}$ & 0.16201700 & 5.54570300 & 3.19497500 & $\mathrm{H}$ & 1.38674700 & 4.53042500 & 3.98148400 \\
\hline $\mathrm{C}$ & -0.41138200 & 2.39726700 & 4.31595600 & $\mathrm{H}$ & 0.25683000 & 2.04936000 & 5.10723200 \\
\hline $\mathrm{H}$ & -1.33418300 & 1.81079500 & 4.35542900 & $\mathrm{C}$ & 0.41627800 & 3.53737200 & 2.30765200 \\
\hline $\mathrm{H}$ & 1.38298800 & 3.51934900 & 1.79873900 & & & & \\
\hline
\end{tabular}

\section{TS- $R-4 f-n d-11$}

Imaginary frequency: $-284.39 \mathrm{~cm}^{-1}$ E[B3LYP/6-31G(d)]: -4788.689850 Hartree E[PCM(Et $\left.\left.{ }_{2} \mathrm{O}\right)-\mathrm{B} 3 L \mathrm{YP} / 6-311 \mathrm{G}(\mathrm{d}, \mathrm{p})-\mathrm{D} 3(\mathrm{BJ})\right]$ : -4790.217344 Hartree

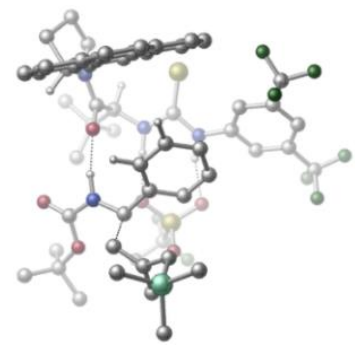

\begin{tabular}{|c|c|c|c|c|c|c|c|}
\hline 01 & & & & $\mathrm{~N}$ & 0.72162900 & 2.44662100 & -1.55292400 \\
\hline $\mathrm{H}$ & 1.68857200 & 2.13085700 & -1.42894000 & C & 0.41629300 & 3.60543500 & -0.81902000 \\
\hline C & 1.47387200 & 4.46318900 & -0.47993700 & C & -0.86949300 & 3.88921100 & -0.33870900 \\
\hline C & 1.23784700 & 5.59010400 & 0.30742800 & $\mathrm{H}$ & 2.47678100 & 4.23749500 & -0.82388200 \\
\hline C & -1.08745300 & 5.02250200 & 0.44231700 & $\mathrm{H}$ & -1.68631800 & 3.22210400 & -0.56978000 \\
\hline C & -0.04381700 & 5.88886600 & 0.76989800 & $\mathrm{H}$ & -0.22455400 & 6.77573400 & 1.36460100 \\
\hline C & 2.39360300 & 6.46639700 & 0.71174500 & $\mathrm{C}$ & -2.46060900 & 5.25271100 & 1.00991600 \\
\hline $\mathrm{F}$ & 3.37335800 & 6.47686000 & -0.21535500 & $\mathrm{~F}$ & 2.95337000 & 6.04550200 & 1.87217300 \\
\hline $\mathrm{F}$ & 2.00647400 & 7.74741200 & 0.91028800 & $\mathrm{~F}$ & -3.44069500 & 4.89683200 & 0.14624300 \\
\hline $\mathrm{F}$ & -2.66649700 & 4.51545600 & 2.13861600 & $\mathrm{~F}$ & -2.66865000 & 6.54091400 & 1.35053300 \\
\hline $\mathrm{C}$ & -0.18711800 & -0.35590100 & -3.73840900 & $\mathrm{H}$ & -0.77694100 & 0.28109300 & -4.39770700 \\
\hline $\mathrm{C}$ & 0.86071600 & -1.10524200 & -4.63954700 & $\mathrm{C}$ & 1.67458500 & -2.16418600 & -3.87735700 \\
\hline $\mathrm{H}$ & 1.04359700 & -2.95560800 & -3.46708000 & $\mathrm{H}$ & 2.24643500 & -1.72730900 & -3.05548800 \\
\hline $\mathrm{H}$ & 2.39280400 & -2.62756100 & -4.56579400 & C & 1.82083600 & -0.05674800 & -5.24040500 \\
\hline $\mathrm{H}$ & 2.46285400 & -0.53309500 & -5.99102500 & $\mathrm{H}$ & 2.47180500 & 0.38083100 & -4.47797200 \\
\hline $\mathrm{H}$ & 1.27444400 & 0.75751500 & -5.73155600 & C & 0.11385100 & -1.80329300 & -5.79437100 \\
\hline $\mathrm{H}$ & 0.83182400 & -2.33310800 & -6.43054100 & $\mathrm{H}$ & -0.41894400 & -1.08407000 & -6.42805100 \\
\hline $\mathrm{H}$ & -0.60442000 & -2.54582500 & -5.42753100 & C & -1.14108500 & -1.28926400 & -2.94840800 \\
\hline $\mathrm{O}$ & -0.76666800 & -1.84988100 & -1.91033500 & C & -3.01978900 & -0.84581900 & -4.61467800 \\
\hline C & -3.33439700 & -2.36842500 & -2.71494200 & C & -4.46148600 & -1.38027000 & -4.60375100 \\
\hline $\mathrm{H}$ & -2.96796600 & 0.24549600 & -4.55664600 & $\mathrm{H}$ & -2.49412400 & -1.17341400 & -5.51607600 \\
\hline C & -4.35190800 & -2.70367300 & -3.83215400 & $\mathrm{H}$ & -2.76276600 & -3.24419500 & -2.39796800 \\
\hline $\mathrm{H}$ & -4.85783600 & -1.50472200 & -5.61564900 & $\mathrm{H}$ & -5.11958100 & -0.68852100 & -4.06833900 \\
\hline $\mathrm{H}$ & -3.94021400 & -3.49070100 & -4.47636600 & $\mathrm{H}$ & -5.30923700 & -3.05114300 & -3.43667300 \\
\hline $\mathrm{N}$ & -2.40730800 & -1.45894600 & -3.41370100 & C & -4.01140600 & -1.74032600 & -1.49762400 \\
\hline C & -3.99083400 & -0.39095200 & -1.29325900 & C & -4.73509700 & -2.59716700 & -0.57166800 \\
\hline C & -4.67130800 & 0.23529200 & -0.19560900 & $\mathrm{H}$ & -3.42533000 & 0.25189800 & -1.96217800 \\
\hline C & -5.42382300 & -1.99672800 & 0.53286200 & C & -4.79092600 & -3.99700300 & -0.70399800 \\
\hline C & -4.63085900 & 1.62472400 & 0.00829600 & C & -5.39432600 & -0.58215400 & 0.72100700 \\
\hline C & -6.14582900 & -2.81263300 & 1.45927900 & $\mathrm{H}$ & -4.27907400 & -4.48607400 & -1.52549300 \\
\hline C & -5.49575200 & -4.78447000 & 0.20655400 & C & -5.28581500 & 2.20346100 & 1.09524300 \\
\hline $\mathrm{H}$ & -4.07983700 & 2.24753600 & -0.69116300 & C & -6.07114700 & 0.01866100 & 1.82673300 \\
\hline C & -6.16944300 & -4.20407800 & 1.27598300 & C & -6.82745400 & -2.18023100 & 2.55622200 \\
\hline $\mathrm{H}$ & -5.51789900 & -5.86296800 & 0.07436000 & C & -6.00062000 & 1.41191500 & 1.99317200 \\
\hline $\mathrm{H}$ & -5.22508200 & 3.27702400 & 1.24266800 & C & -6.79246900 & -0.83099200 & 2.73314400 \\
\hline $\mathrm{H}$ & -6.72036700 & -4.82350100 & 1.97968300 & $\mathrm{H}$ & -7.37761600 & -2.81179300 & 3.24991800 \\
\hline
\end{tabular}




\begin{tabular}{|c|c|c|c|c|c|c|c|}
\hline $\mathrm{H}$ & -6.51108200 & 1.86975100 & 2.83726000 & $\mathrm{H}$ & -7.31319300 & -0.37113900 & 3.56985100 \\
\hline C & -0.06869100 & 1.75414500 & -2.44002300 & S & -1.51623600 & 2.36787000 & -3.08585500 \\
\hline $\mathrm{N}$ & 0.45022500 & 0.55111400 & -2.78902600 & $\mathrm{H}$ & 1.24423300 & 0.19164500 & -2.25459700 \\
\hline $\mathrm{S}$ & 3.91494300 & 0.57314000 & -0.68174100 & 0 & 4.33355100 & 0.12749700 & 0.66899100 \\
\hline $\mathrm{O}$ & 2.96113700 & -0.34845600 & -1.36058800 & 0 & 3.53048500 & 2.00155300 & -0.79284700 \\
\hline C & 5.46286400 & 0.43600900 & -1.70395000 & $\mathrm{~F}$ & 6.40769900 & 1.26312800 & -1.24797700 \\
\hline $\mathrm{F}$ & 5.20207800 & 0.73647000 & -2.98248200 & $\mathrm{~F}$ & 5.94283400 & -0.82284800 & -1.65550700 \\
\hline C & 2.04457700 & -3.66849600 & -0.56842300 & 0 & 1.66418900 & -4.40524200 & -1.45521300 \\
\hline $\mathrm{O}$ & 3.26909600 & -3.63328800 & -0.01256900 & $\mathrm{C}$ & 4.39367100 & -4.41818000 & -0.58165500 \\
\hline $\mathrm{C}$ & 4.11916400 & -5.91090400 & -0.38329400 & $\mathrm{H}$ & 4.98955300 & -6.48909600 & -0.71289700 \\
\hline $\mathrm{H}$ & 3.24893200 & -6.22941800 & -0.95987900 & $\mathrm{H}$ & 3.94663700 & -6.13498600 & 0.67579600 \\
\hline $\mathrm{C}$ & 5.58003100 & -3.94831900 & 0.26259900 & $\mathrm{H}$ & 5.71551900 & -2.86708600 & 0.16609800 \\
\hline $\mathrm{H}$ & 6.49503400 & -4.44693900 & -0.07351500 & $\mathrm{H}$ & 5.42948900 & -4.19425000 & 1.32008500 \\
\hline C & 4.60223100 & -4.04846500 & -2.05235900 & $\mathrm{H}$ & 5.51694800 & -4.53178500 & -2.41386000 \\
\hline $\mathrm{H}$ & 4.72147000 & -2.96699600 & -2.15810000 & $\mathrm{H}$ & 3.76573900 & -4.37916500 & -2.66900900 \\
\hline $\mathrm{C}$ & 1.57924900 & -1.83446300 & 1.01596300 & $\mathrm{H}$ & 2.60371100 & -1.49498300 & 0.90436900 \\
\hline C & 0.59472500 & -0.77672400 & 1.36161400 & C & 1.06623400 & 0.50364900 & 1.69890700 \\
\hline C & -0.78386400 & -1.03825400 & 1.42817600 & C & 0.17202000 & 1.50473000 & 2.07553200 \\
\hline $\mathrm{H}$ & 2.12918200 & 0.71772800 & 1.64208000 & $C$ & -1.67131100 & -0.03641800 & 1.81621900 \\
\hline $\mathrm{H}$ & -1.16971500 & -2.02116000 & 1.17248100 & $\mathrm{C}$ & -1.19712200 & 1.23871000 & 2.13694000 \\
\hline $\mathrm{H}$ & 0.54636000 & 2.49708700 & 2.30796400 & $\mathrm{H}$ & -2.73450800 & -0.24663900 & 1.85618000 \\
\hline $\mathrm{H}$ & -1.89204500 & 2.02681800 & 2.41015700 & $\mathrm{~N}$ & 1.19237300 & -2.75020300 & 0.04539400 \\
\hline $\mathrm{H}$ & 0.32277800 & -2.59521700 & -0.47225000 & $C$ & 1.80249300 & -2.87836400 & 2.60307800 \\
\hline $\mathrm{H}$ & 2.41495500 & -3.66024100 & 2.16441100 & $\mathrm{H}$ & 0.77010600 & -3.15511300 & 2.80265700 \\
\hline $\mathrm{C}$ & 2.43749600 & -2.03797600 & 3.53801400 & $\mathrm{C}$ & 1.67225100 & -1.27911300 & 4.51655100 \\
\hline $\mathrm{H}$ & 0.63660400 & -1.12067100 & 4.19370300 & $\mathrm{H}$ & 2.14104800 & -0.31749400 & 4.75749100 \\
\hline C & 3.90830500 & -1.80612500 & 3.43232100 & $\mathrm{H}$ & 4.33383900 & -1.41669800 & 4.36092700 \\
\hline $\bar{H}$ & 4.08475800 & -1.04898600 & 2.64699300 & $\mathrm{H}$ & 4.44824400 & -2.70409100 & 3.11883400 \\
\hline $\mathrm{C}$ & 0.52986800 & -0.99968100 & 7.32734200 & $\mathrm{H}$ & 1.07109400 & -0.05656700 & 7.46478400 \\
\hline $\mathrm{H}$ & 0.36137800 & -1.43238800 & 8.32103400 & $\mathrm{H}$ & -0.45057100 & -0.76416800 & 6.89795000 \\
\hline $\mathrm{C}$ & 3.20210800 & -2.54814600 & 6.96363700 & $\mathrm{H}$ & 3.79181400 & -1.63096000 & 7.07728400 \\
\hline $\bar{H}$ & 3.78038300 & -3.24707400 & 6.34878100 & $\mathrm{H}$ & 3.10211000 & -2.99582000 & 7.96015200 \\
\hline C & 0.53325700 & -3.80447300 & 5.97566800 & $\mathrm{H}$ & 1.06857400 & -4.50408200 & 5.32400100 \\
\hline $\mathrm{H}$ & -0.45220800 & -3.61862800 & 5.53274400 & $\mathrm{H}$ & 0.37019400 & -4.30921000 & 6.93579000 \\
\hline Si & 1.49345900 & -2.20311000 & 6.24050700 & & & & \\
\hline
\end{tabular}

\section{TS-R-4f-nd-12}

Imaginary frequency: $-271.39 \mathrm{~cm}^{-1}$

E[B3LYP/6-31G(d)]: -4788.690257 Hartree

E[PCM(Et $2 \mathrm{O})-B 3 L Y P / 6-311 G(d, p)-D 3(B J)]:-4790.217258$ Hartree

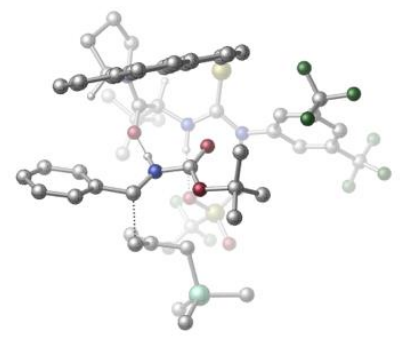

\begin{tabular}{|cccc|cccc|}
\hline 01 & & & & $\mathrm{~N}$ & -2.44263100 & 0.94758800 & 1.71099000 \\
\hline $\mathrm{H}$ & -3.10198600 & 0.17176100 & 1.58386200 & $\mathrm{C}$ & -2.85701100 & 2.16029400 & 1.14228400 \\
\hline $\mathrm{C}$ & -4.23155900 & 2.35366300 & 0.93591400 & $\mathrm{C}$ & -1.95384200 & 3.13274300 & 0.69494500 \\
\hline $\mathrm{C}$ & -4.68887000 & 3.51638900 & 0.31808900 & $\mathrm{H}$ & -4.93172900 & 1.58488500 & 1.24550600 \\
\hline $\mathrm{C}$ & -2.43102200 & 4.29178000 & 0.08366700 & $\mathrm{H}$ & -0.89148200 & 2.96686400 & 0.80530000 \\
\hline $\mathrm{C}$ & -3.79665100 & 4.50191300 & -0.10641600 & $\mathrm{H}$ & -4.15709600 & 5.40418500 & -0.58429300 \\
\hline $\mathrm{C}$ & -6.16924800 & 3.74615000 & 0.16617900 & $\mathrm{C}$ & -1.43118300 & 5.28241600 & -0.44381100 \\
\hline $\mathrm{F}$ & -6.68263700 & 4.41091400 & 1.22827000 & $\mathrm{~F}$ & -6.85426900 & 2.58949000 & 0.05673800 \\
\hline $\mathrm{F}$ & -6.44949000 & 4.49059600 & -0.92955400 & $\mathrm{~F}$ & -1.99254800 & 6.46663000 & -0.76377600 \\
\hline $\mathrm{F}$ & -0.44435500 & 5.53375400 & 0.45162500 & $\mathrm{~F}$ & -0.81465300 & 4.82480700 & -1.56758600 \\
\hline
\end{tabular}




\begin{tabular}{|c|c|c|c|c|c|c|c|}
\hline $\mathrm{C}$ & -0.05708100 & -1.17394900 & 3.48733900 & $\mathrm{H}$ & 0.03497500 & -0.47650800 & 4.32022400 \\
\hline C & -0.47207600 & -2.56552900 & 4.09100400 & C & -0.57129900 & -3.68747600 & 3.03883600 \\
\hline $\mathrm{H}$ & 0.39741100 & -3.88851600 & 2.57197200 & $\mathrm{H}$ & -1.28909800 & -3.45323500 & 2.24941400 \\
\hline $\mathrm{H}$ & -0.91002500 & -4.60887200 & 3.52821200 & C & -1.83891500 & -2.39460400 & 4.78666600 \\
\hline $\mathrm{H}$ & -2.10150800 & -3.31680600 & 5.31848600 & $\mathrm{H}$ & -2.63711600 & -2.18339600 & 4.07076000 \\
\hline $\mathrm{H}$ & -1.81455100 & -1.57845500 & 5.51880200 & C & 0.56811600 & -2.98027500 & 5.15161600 \\
\hline $\mathrm{H}$ & 0.29654600 & -3.95320800 & 5.57653300 & $\mathrm{H}$ & 0.61044600 & -2.26239800 & 5.97935400 \\
\hline $\mathrm{H}$ & 1.57435500 & -3.07944500 & 4.72652600 & C & 1.27096600 & -1.20469300 & 2.69674700 \\
\hline 0 & 1.29421700 & -1.70880400 & 1.55698400 & C & 2.48421800 & -0.06486100 & 4.60741300 \\
\hline C & 3.68744100 & -0.73069300 & 2.57161000 & C & 3.96282700 & 0.33822900 & 4.71547800 \\
\hline $\mathrm{H}$ & 1.79795700 & 0.78291400 & 4.68696700 & $\mathrm{H}$ & 2.22684100 & -0.80148600 & 5.37387100 \\
\hline C & 4.67306500 & -0.63280400 & 3.76185400 & $\mathrm{H}$ & 3.75729700 & -1.69402600 & 2.06059900 \\
\hline $\mathrm{H}$ & 4.32816300 & 0.27362500 & 5.74432600 & $\mathrm{H}$ & 4.10234800 & 1.36830300 & 4.37309000 \\
\hline $\mathrm{H}$ & 4.78103900 & -1.61970500 & 4.22972700 & $\mathrm{H}$ & 5.66329600 & -0.28866000 & 3.45587000 \\
\hline $\mathrm{N}$ & 2.38108200 & -0.68070200 & 3.26136200 & C & 3.90129900 & 0.39954200 & 1.56577500 \\
\hline C & 3.07389800 & 1.48465100 & 1.52990200 & C & 5.05444800 & 0.34181800 & 0.68013600 \\
\hline C & 3.31481500 & 2.60952700 & 0.67108400 & $\mathrm{H}$ & 2.19137400 & 1.52156500 & 2.16243500 \\
\hline C & 5.31692100 & 1.45418000 & -0.18495900 & C & 5.93095200 & -0.75761500 & 0.62798600 \\
\hline C & 2.46592600 & 3.72837100 & 0.66088200 & C & 4.45477100 & 2.59131900 & -0.18270500 \\
\hline C & 6.45048200 & 1.43480100 & -1.05693800 & $\mathrm{H}$ & 5.75893500 & -1.62165100 & 1.26002200 \\
\hline C & 7.02775100 & -0.77153400 & -0.23403400 & C & 2.73602300 & 4.81676400 & -0.16855000 \\
\hline $\mathrm{H}$ & 1.59339100 & 3.73826000 & 1.30800300 & C & 4.72347200 & 3.70834800 & -1.03158600 \\
\hline C & 7.29237000 & 0.31150900 & -1.06505300 & C & 6.69632300 & 2.57233700 & -1.90141000 \\
\hline $\mathrm{H}$ & 7.68198400 & -1.63914900 & -0.25053400 & C & 3.85233100 & 4.81010800 & -1.00336500 \\
\hline $\mathrm{H}$ & 2.05939700 & 5.66526000 & -0.16935400 & C & 5.87562700 & 3.65831400 & -1.88801100 \\
\hline $\mathrm{H}$ & 8.15430700 & 0.29839400 & -1.72791500 & $\mathrm{H}$ & 7.56480500 & 2.54693600 & -2.55551000 \\
\hline $\mathrm{H}$ & 4.05691800 & 5.66212900 & -1.64754600 & $\mathrm{H}$ & 6.08002200 & 4.51136100 & -2.53087400 \\
\hline C & -1.35690300 & 0.70211900 & 2.51351200 & S & -0.50094100 & 1.90859800 & 3.34502500 \\
\hline $\mathrm{N}$ & -1.08981100 & -0.62563600 & 2.62175100 & $\mathrm{H}$ & -1.48372300 & -1.23955900 & 1.90326500 \\
\hline $\mathrm{S}$ & -3.90786900 & -2.14090100 & 0.41846100 & $\mathrm{O}$ & -4.45651900 & -2.19092500 & -0.94929700 \\
\hline $\mathrm{O}$ & -2.41614900 & -2.22885200 & 0.48614900 & $\mathrm{O}$ & -4.46531900 & -1.10029600 & 1.31864800 \\
\hline $\mathrm{C}$ & -4.45856400 & -3.74883400 & 1.17518500 & $\mathrm{~F}$ & -5.79010700 & -3.83751500 & 1.20503500 \\
\hline $\mathrm{F}$ & -3.99050700 & -3.87178100 & 2.42809200 & $\mathrm{~F}$ & -3.97751300 & -4.77950400 & 0.44967300 \\
\hline $\mathrm{C}$ & 0.87811600 & 0.16363700 & -1.58374100 & $\mathrm{O}$ & 0.43586100 & 0.90759800 & -0.73827800 \\
\hline $\mathrm{O}$ & 0.88319800 & 0.33621500 & -2.91534500 & $\mathrm{C}$ & 0.42325100 & 1.61511300 & -3.53286400 \\
\hline C & -1.05634200 & 1.84616800 & -3.22111400 & $\mathrm{H}$ & -1.39691200 & 2.74258800 & -3.75005600 \\
\hline $\mathrm{H}$ & -1.22123200 & 1.99692200 & -2.15445300 & $\mathrm{H}$ & -1.66467100 & 1.00089600 & -3.56132600 \\
\hline $\mathrm{C}$ & 0.63773900 & 1.34617200 & -5.02319600 & $\mathrm{H}$ & 1.69239300 & 1.14205800 & -5.23599700 \\
\hline $\mathrm{H}$ & 0.33390500 & 2.22287100 & -5.60379500 & $\mathrm{H}$ & 0.04105800 & 0.49089900 & -5.35849800 \\
\hline $\mathrm{C}$ & 1.31137000 & 2.75984300 & -3.04735100 & $\mathrm{H}$ & 1.05623400 & 3.67124900 & -3.59835200 \\
\hline $\mathrm{H}$ & 2.36737100 & 2.53453700 & -3.22965600 & $\mathrm{H}$ & 1.16860500 & 2.95775300 & -1.98539300 \\
\hline C & 2.10724000 & -1.89497100 & -2.12057100 & $\mathrm{H}$ & 2.34149500 & -1.39870300 & -3.05404000 \\
\hline C & 3.13833000 & -2.84922300 & -1.64994500 & C & 4.17696700 & -3.17092500 & -2.54107200 \\
\hline $\mathrm{C}$ & 3.11565700 & -3.45135100 & -0.38106500 & C & 5.17566600 & -4.06884100 & -2.17484400 \\
\hline $\mathrm{H}$ & 4.20296300 & -2.70760300 & -3.52451200 & C & 4.11409900 & -4.35690500 & -0.02357200 \\
\hline $\mathrm{H}$ & 2.33770400 & -3.20501300 & 0.33284600 & C & 5.14356800 & -4.66968300 & -0.91430100 \\
\hline $\mathrm{H}$ & 5.97670400 & -4.29892900 & -2.87136500 & $\mathrm{H}$ & 4.08492600 & -4.82175900 & 0.95809100 \\
\hline $\mathrm{H}$ & 5.91669600 & -5.37729600 & -0.62805400 & $\mathrm{~N}$ & 1.49132400 & -1.05485900 & -1.23615800 \\
\hline $\mathrm{H}$ & 1.45548800 & -1.25604200 & -0.22658100 & C & 0.84312000 & -3.17611500 & -3.17303500 \\
\hline $\mathrm{H}$ & 1.49094800 & -4.04566900 & -3.16033300 & $\mathrm{H}$ & 0.82049400 & -2.62996500 & -4.11315200 \\
\hline C & -0.34488700 & -3.22473100 & -2.43331100 & C & -1.44932900 & -2.33037500 & -2.72592300 \\
\hline $\mathrm{H}$ & -1.12125600 & -1.43565700 & -3.26512000 & $\mathrm{H}$ & -2.04027100 & -2.07404300 & -1.84015900 \\
\hline C & -0.44926300 & -4.12229500 & -1.24398600 & $\mathrm{H}$ & -1.45761300 & -4.52919000 & -1.12751800 \\
\hline
\end{tabular}




\begin{tabular}{|c|c|c|c|c|c|c|c|}
\hline $\mathrm{H}$ & -0.29598900 & -3.50315700 & -0.34624500 & $\mathrm{H}$ & 0.28876200 & -4.92801600 & -1.25578600 \\
\hline C & -4.05991900 & -1.78573700 & -4.22084900 & $\mathrm{H}$ & -4.53484200 & -1.53442600 & -3.26776000 \\
\hline $\mathrm{H}$ & -4.83681900 & -2.13528400 & -4.91239500 & $\mathrm{H}$ & -3.62272900 & -0.87483700 & -4.64720900 \\
\hline $\mathrm{C}$ & -3.50165000 & -4.69707800 & -3.22395700 & $\mathrm{H}$ & -3.92581900 & -4.50090000 & -2.23445400 \\
\hline $\mathrm{H}$ & -2.76557700 & -5.50514200 & -3.13914800 & $\mathrm{H}$ & -4.31258100 & -5.06109400 & -3.86754700 \\
\hline $\mathrm{C}$ & -1.87409400 & -3.51640700 & -5.58598100 & $\mathrm{H}$ & -1.09444900 & -4.27697800 & -5.46330700 \\
\hline $\bar{H}$ & -1.40895100 & -2.62361800 & -6.02179400 & $\mathrm{H}$ & -2.59211400 & -3.89920500 & -6.32206000 \\
\hline $\mathrm{Si}$ & -2.76646800 & -3.12762800 & -3.96074800 & & & & \\
\hline
\end{tabular}

\section{TS- $R-4 f-n d-13$}

Imaginary frequency: $-284.76 \mathrm{~cm}^{-1}$

E[B3LYP/6-31G(d)]: -4788.689651 Hartree

E[PCM(Et $\left.\left.{ }_{2} \mathrm{O}\right)-B 3 L Y P / 6-311 G(d, p)-D 3(B J)\right]: ~-4790.217007$ Hartree

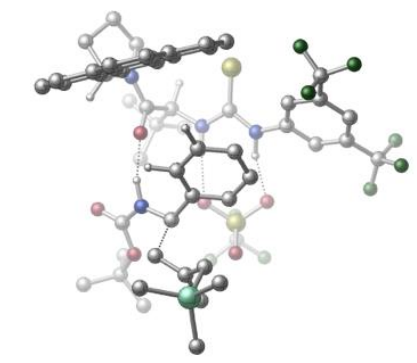

\begin{tabular}{|c|c|c|c|c|c|c|c|}
\hline 01 & & & & $\mathrm{~N}$ & 0.74234300 & 2.47969600 & -1.47660400 \\
\hline $\mathrm{H}$ & 1.70831900 & 2.15928000 & -1.35628300 & C & 0.43704200 & 3.62604100 & -0.72280000 \\
\hline $\mathrm{C}$ & 1.49424000 & 4.47638700 & -0.36489600 & $\mathrm{C}$ & -0.84914200 & 3.89940600 & -0.23747500 \\
\hline $\mathrm{C}$ & 1.25728100 & 5.58728300 & 0.44501600 & $\mathrm{H}$ & 2.49991200 & 4.25036600 & -0.70086500 \\
\hline $\mathrm{C}$ & -1.06751700 & 5.01460600 & 0.56898600 & $\mathrm{H}$ & -1.66482300 & 3.23485000 & -0.47991700 \\
\hline C & -0.02432000 & 5.87421400 & 0.91515000 & $\mathrm{H}$ & -0.20291800 & 6.73957900 & 1.54133900 \\
\hline C & 2.38919400 & 6.52908100 & 0.76098200 & C & -2.43820000 & 5.22564900 & 1.14966800 \\
\hline $\mathrm{F}$ & 2.50283000 & 7.50424200 & -0.17070600 & $\mathrm{~F}$ & 3.57806600 & 5.89358300 & 0.81306400 \\
\hline $\mathrm{F}$ & 2.20691300 & 7.14788200 & 1.95166800 & $\mathrm{~F}$ & -3.42123700 & 4.89213900 & 0.28034200 \\
\hline $\mathrm{F}$ & -2.63778700 & 4.45668900 & 2.25812900 & $\mathrm{~F}$ & -2.64740100 & 6.50334700 & 1.52715200 \\
\hline C & -0.16376700 & -0.26653800 & -3.73382700 & $\mathrm{H}$ & -0.74757900 & 0.38911100 & -4.38004700 \\
\hline C & 0.88740800 & -0.99559200 & -4.64783300 & C & 1.69229200 & -2.07774700 & -3.90905700 \\
\hline $\mathrm{H}$ & 1.05546100 & -2.87748600 & -3.52476800 & $\mathrm{H}$ & 2.25997100 & -1.66549100 & -3.07176500 \\
\hline $\mathrm{H}$ & 2.41350300 & -2.52510300 & -4.60492000 & C & 1.85510600 & 0.06490200 & -5.21441800 \\
\hline $\mathrm{H}$ & 2.50072600 & -0.39380100 & -5.97290400 & $\mathrm{H}$ & 2.50226500 & 0.48014700 & -4.43641900 \\
\hline $\mathrm{H}$ & 1.31481600 & 0.89366100 & -5.68776500 & C & 0.14560300 & -1.65984100 & -5.82564800 \\
\hline $\mathrm{H}$ & 0.86580900 & -2.17527200 & -6.47104100 & $\mathrm{H}$ & -0.38041900 & -0.92203400 & -6.44340700 \\
\hline $\mathrm{H}$ & -0.57770500 & -2.40917200 & -5.48336200 & C & -1.12561500 & -1.21719500 & -2.97464100 \\
\hline $\mathrm{O}$ & -0.75954400 & -1.80665500 & -1.94962000 & C & -2.99220600 & -0.72355500 & -4.64042600 \\
\hline C & -3.32427900 & -2.29390300 & -2.78307800 & C & -4.43558200 & -1.25339500 & -4.65292700 \\
\hline $\mathrm{H}$ & -2.93733500 & 0.36568600 & -4.55344900 & $\mathrm{H}$ & -2.46162000 & -1.02917600 & -5.54664100 \\
\hline C & -4.33520900 & -2.59678500 & -3.91541300 & $\mathrm{H}$ & -2.75767300 & -3.17944500 & -2.48477000 \\
\hline $\mathrm{H}$ & -4.82558200 & -1.35010000 & -5.67030700 & $\mathrm{H}$ & -5.09505500 & -0.57372000 & -4.10392900 \\
\hline $\mathrm{H}$ & -3.92147700 & -3.36807300 & -4.57712000 & $\mathrm{H}$ & -5.29625400 & -2.95140300 & -3.53560000 \\
\hline $\mathrm{N}$ & -2.38951300 & -1.36994400 & -3.45196700 & C & -4.00763800 & -1.69467100 & -1.55478300 \\
\hline C & -3.98313000 & -0.35111300 & -1.31551300 & C & -4.74160200 & -2.57203300 & -0.65655800 \\
\hline C & -4.66927500 & 0.24949800 & -0.20713400 & $\mathrm{H}$ & -3.41019100 & 0.30637900 & -1.96356500 \\
\hline C & -5.43637900 & -1.99732700 & 0.45783200 & C & -4.80186600 & -3.96776300 & -0.82524500 \\
\hline C & -4.62455100 & 1.63300700 & 0.03295900 & C & -5.40268800 & -0.58819000 & 0.68255700 \\
\hline C & -6.16875300 & -2.83376300 & 1.35741300 & $\mathrm{H}$ & -4.28567400 & -4.43760300 & -1.65520300 \\
\hline C & -5.51671500 & -4.77545200 & 0.05941300 & C & -5.28557000 & 2.18631600 & 1.12944300 \\
\hline $\mathrm{H}$ & -4.06544900 & 2.27122600 & -0.64591000 & C & -6.08562800 & -0.01318600 & 1.79819600 \\
\hline C & -6.19638900 & -4.21993900 & 1.13823400 & C & -6.85638700 & -2.22692200 & 2.46499200 \\
\hline $\mathrm{H}$ & -5.54206000 & -5.85010100 & -0.10062000 & C & -6.01069200 & 1.37499800 & 2.00106900 \\
\hline $\mathrm{H}$ & -5.22157300 & 3.25546500 & 1.30495100 & C & -6.81740000 & -0.88283000 & 2.67684400 \\
\hline $\mathrm{H}$ & -6.75516000 & -4.85495500 & 1.82155200 & $\mathrm{H}$ & -7.41444000 & -2.87380300 & 3.13795700 \\
\hline
\end{tabular}




\begin{tabular}{|c|c|c|c|c|c|c|c|}
\hline $\mathrm{H}$ & -6.52586800 & 1.81312700 & 2.85271600 & $\mathrm{H}$ & -7.34277600 & -0.44247200 & 3.52109600 \\
\hline C & -0.04589300 & 1.81056000 & -2.38313900 & S & -1.48820400 & 2.44457400 & -3.02126000 \\
\hline $\mathrm{N}$ & 0.47017000 & 0.61385400 & -2.75740000 & $\mathrm{H}$ & 1.25997500 & 0.23862800 & -2.22761500 \\
\hline $\mathrm{S}$ & 3.92157000 & 0.57052200 & -0.63493800 & 0 & 4.32832200 & 0.09693300 & 0.70989100 \\
\hline $\mathrm{O}$ & 2.96883500 & -0.33374000 & -1.33817100 & 0 & 3.54418100 & 2.00245800 & -0.72124400 \\
\hline C & 5.47621900 & 0.44660900 & -1.64856000 & $\mathrm{~F}$ & 6.42159900 & 1.25976000 & -1.16924600 \\
\hline $\mathrm{F}$ & 5.22608800 & 0.77399400 & -2.92258400 & $\mathrm{~F}$ & 5.95002000 & -0.81530100 & -1.62220200 \\
\hline C & 2.03752500 & -3.67086800 & -0.63972800 & 0 & 1.66039700 & -4.38356200 & -1.54731000 \\
\hline $\mathrm{O}$ & 3.25860800 & -3.65396200 & -0.07546900 & $\mathrm{C}$ & 4.38353600 & -4.42970600 & -0.65637200 \\
\hline $\mathrm{C}$ & 4.10105200 & -5.92563900 & -0.49718500 & $\mathrm{H}$ & 4.97110000 & -6.49939600 & -0.83532100 \\
\hline $\mathrm{H}$ & 3.23336000 & -6.22567700 & -1.08732900 & $\mathrm{H}$ & 3.92038100 & -6.17529100 & 0.55480700 \\
\hline $\mathrm{C}$ & 5.56624600 & -3.98675900 & 0.20731800 & $\mathrm{H}$ & 5.70776900 & -2.90419100 & 0.13855300 \\
\hline $\mathrm{H}$ & 6.48113000 & -4.48147200 & -0.13483000 & $\mathrm{H}$ & 5.40723500 & -4.25804100 & 1.25733400 \\
\hline C & 4.60370000 & -4.02436700 & -2.11595700 & $\mathrm{H}$ & 5.51872100 & -4.50263500 & -2.48335200 \\
\hline $\mathrm{H}$ & 4.72833400 & -2.94114700 & -2.19384800 & $\mathrm{H}$ & 3.76994100 & -4.33580500 & -2.74618600 \\
\hline $\mathrm{C}$ & 1.56755200 & -1.87485000 & 0.98655600 & $\mathrm{H}$ & 2.59318500 & -1.53459700 & 0.88923200 \\
\hline C & 0.58250400 & -0.82448700 & 1.35239100 & C & 1.05317400 & 0.44748300 & 1.72101500 \\
\hline C & -0.79654200 & -1.08656400 & 1.40648600 & C & 0.15754400 & 1.44030300 & 2.11574400 \\
\hline $\mathrm{H}$ & 2.11657800 & 0.66181500 & 1.67458500 & $C$ & -1.68533000 & -0.09332500 & 1.81295500 \\
\hline $\mathrm{H}$ & -1.18159900 & -2.06321400 & 1.12678900 & $\mathrm{C}$ & -1.21201600 & 1.17396600 & 2.16453700 \\
\hline $\mathrm{H}$ & 0.53097000 & 2.42704200 & 2.37226400 & $\mathrm{H}$ & -2.74880100 & -0.30377100 & 1.84323200 \\
\hline $\mathrm{H}$ & -1.90762200 & 1.95618400 & 2.45241800 & $\mathrm{~N}$ & 1.18426500 & -2.76562400 & -0.00823900 \\
\hline $\mathrm{H}$ & 0.31860800 & -2.59455400 & -0.52751400 & $C$ & 1.78018700 & -2.95822300 & 2.54936700 \\
\hline $\mathrm{H}$ & 2.39352900 & -3.73019800 & 2.09471900 & $\mathrm{H}$ & 0.74621900 & -3.23768100 & 2.73665300 \\
\hline $\mathrm{C}$ & 2.41159700 & -2.14156100 & 3.50728100 & $\mathrm{C}$ & 1.64228700 & -1.40482700 & 4.49959100 \\
\hline $\mathrm{H}$ & 0.60828900 & -1.23810400 & 4.17565500 & $\mathrm{H}$ & 2.11068000 & -0.44936500 & 4.76465200 \\
\hline C & 3.88330200 & -1.90970000 & 3.41487800 & $\mathrm{H}$ & 4.30456600 & -1.54346900 & 4.35481100 \\
\hline $\bar{H}$ & 4.06513000 & -1.13419400 & 2.64891300 & $\mathrm{H}$ & 4.42347600 & -2.80083200 & 3.08283600 \\
\hline $\mathrm{C}$ & 0.48651700 & -1.18943400 & 7.31064500 & $\mathrm{H}$ & 1.02704100 & -0.24975900 & 7.47227500 \\
\hline $\mathrm{H}$ & 0.31335800 & -1.64482700 & 8.29333400 & $\mathrm{H}$ & -0.49191100 & -0.94407500 & 6.88217500 \\
\hline $\mathrm{C}$ & 3.16067200 & -2.72902800 & 6.92397600 & $\mathrm{H}$ & 3.75011500 & -1.81472900 & 7.06006300 \\
\hline $\bar{H}$ & 3.74135500 & -3.41455200 & 6.29643500 & $\mathrm{H}$ & 3.05636700 & -3.19828400 & 7.91005900 \\
\hline C & 0.49663300 & -3.96282900 & 5.89563900 & $\mathrm{H}$ & 1.03484500 & -4.64692800 & 5.23003600 \\
\hline $\mathrm{H}$ & -0.48719300 & -3.76720200 & 5.45326800 & $\mathrm{H}$ & 0.33009200 & -4.48970700 & 6.84318300 \\
\hline Si & 1.45528200 & -2.36773000 & 6.20107600 & & & & \\
\hline
\end{tabular}

\section{TS- $R-4 f-n d-14$}

Imaginary frequency: $-252.43 \mathrm{~cm}^{-1}$

E[B3LYP/6-31G(d)]: -4788.690725 Hartree

E[PCM(Et $2 \mathrm{O})-B 3 L Y P / 6-311 G(d, p)-D 3(B J)]:-4790.216899$ Hartree

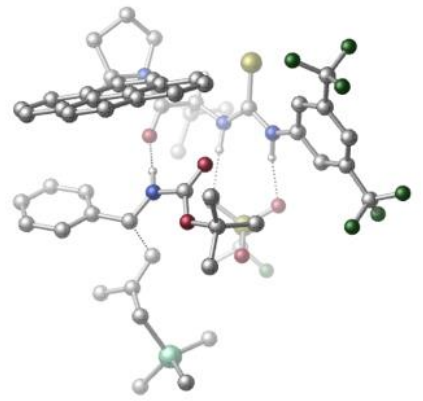

\begin{tabular}{|llll|llll|}
\hline 01 & & & N & -2.01303500 & -1.97204700 & 1.79002300 \\
\hline H & -1.10179800 & -2.43579600 & 1.85802900 & C & -2.87379900 & -2.60323000 & 0.87868000 \\
\hline C & -2.46275900 & -3.86487300 & 0.40767400 & C & -4.05412300 & -2.04622100 & 0.36521800 \\
\hline C & -3.20927000 & -4.53449000 & -0.55738200 & H & -1.54408700 & -4.30211200 & 0.78460400 \\
\hline C & -4.78749600 & -2.73700700 & -0.60007900 & H & -4.39193600 & -1.08444500 & 0.71993200 \\
\hline C & -4.38292400 & -3.98300600 & -1.07340900 & H & -4.95941400 & -4.50802500 & -1.82470800 \\
\hline C & -2.68983700 & -5.82929200 & -1.12053600 & C & -6.06586500 & -2.12483200 & -1.10598800 \\
\hline F & -1.98734000 & -6.53788600 & -0.21391700 & F & -1.86224800 & -5.61384800 & -2.17671800 \\
\hline
\end{tabular}




\begin{tabular}{|c|c|c|c|c|c|c|c|}
\hline $\mathrm{F}$ & -3.68957700 & -6.62134400 & -1.57150300 & $\mathrm{~F}$ & -5.96941000 & -0.77392000 & -1.22007200 \\
\hline $\mathrm{F}$ & -6.40965600 & -2.60039600 & -2.32434700 & $\mathrm{~F}$ & -7.10805800 & -2.36531500 & -0.28061400 \\
\hline C & -0.80696500 & 0.83951100 & 3.80003000 & $\mathrm{H}$ & -1.79479600 & 1.03233900 & 4.21660900 \\
\hline C & 0.17015800 & 0.64733100 & 5.01319700 & C & 1.62582700 & 0.35963700 & 4.59965100 \\
\hline $\mathrm{H}$ & 2.05594000 & 1.19040600 & 4.03364800 & $\mathrm{H}$ & 1.71283700 & -0.54137600 & 3.98803800 \\
\hline $\mathrm{H}$ & 2.23222500 & 0.21004800 & 5.50181700 & C & -0.35646900 & -0.52828300 & 5.86093000 \\
\hline $\mathrm{H}$ & 0.25586000 & -0.64335800 & 6.76321500 & $\mathrm{H}$ & -0.31803100 & -1.46914100 & 5.30438800 \\
\hline $\mathrm{H}$ & -1.39431100 & -0.36424900 & 6.17469600 & C & 0.14028600 & 1.93006600 & 5.86942800 \\
\hline $\mathrm{H}$ & 0.80354700 & 1.81811400 & 6.73473200 & $\mathrm{H}$ & -0.86636200 & 2.13733500 & 6.25220700 \\
\hline $\mathrm{H}$ & 0.48259000 & 2.80759800 & 5.30695800 & C & -0.41523600 & 1.98949400 & 2.84780500 \\
\hline 0 & 0.57226500 & 1.86979400 & 2.10051300 & C & -0.84509400 & 4.20854600 & 1.89216900 \\
\hline $\mathrm{H}$ & 0.24176000 & 4.32406300 & 1.89251900 & $\mathrm{~N}$ & -1.17877800 & 3.10510000 & 2.81669300 \\
\hline C & -1.32211600 & 3.97015900 & 0.46019900 & C & -2.22825200 & 2.99330000 & 0.16439900 \\
\hline C & -0.83207400 & 4.84146900 & -0.59648300 & C & -2.74724700 & 2.79637800 & -1.15908400 \\
\hline $\mathrm{H}$ & -2.58045300 & 2.32175900 & 0.94218200 & C & -1.33496500 & 4.66446200 & -1.92708400 \\
\hline C & 0.11764900 & 5.85626100 & -0.37789500 & C & -3.69209700 & 1.79773200 & -1.44345300 \\
\hline C & -2.29615000 & 3.64791600 & -2.20765200 & C & -0.87829200 & 5.50988000 & -2.98637000 \\
\hline $\mathrm{H}$ & 0.52358900 & 6.01689000 & 0.61477500 & C & 0.56140800 & 6.67173400 & -1.41912900 \\
\hline C & -4.19157500 & 1.63865900 & -2.73556400 & $\mathrm{H}$ & -4.03219500 & 1.14553400 & -0.64505500 \\
\hline C & -2.80738200 & 3.47602600 & -3.53034000 & C & 0.07017600 & 6.50709500 & -2.70955600 \\
\hline C & -1.40854600 & 5.31380900 & -4.30846800 & $\mathrm{H}$ & 1.29701900 & 7.44535100 & -1.21479600 \\
\hline $\mathrm{C}$ & -3.75541600 & 2.46707500 & -3.76865700 & $\mathrm{H}$ & -4.92442200 & 0.86123700 & -2.92868200 \\
\hline C & -2.32961700 & 4.34583000 & -4.56875300 & $\mathrm{H}$ & 0.41516100 & 7.15050800 & -3.51553000 \\
\hline $\mathrm{H}$ & -1.05581900 & 5.96609200 & -5.10407000 & $\mathrm{H}$ & -4.14888600 & 2.33856600 & -4.77438800 \\
\hline $\mathrm{H}$ & -2.72209700 & 4.21503900 & -5.57459000 & C & -2.14724700 & -0.81718600 & 2.51796800 \\
\hline S & -3.63000000 & -0.06714800 & 2.87037200 & $\mathrm{~N}$ & -0.95090200 & -0.36519200 & 2.99087200 \\
\hline $\mathrm{H}$ & -0.10048500 & -0.69833500 & 2.52534300 & $S$ & 1.63251600 & -2.80721300 & 1.36273200 \\
\hline 0 & 2.16027400 & -3.14058200 & 0.02188300 & $\mathrm{O}$ & 1.50375300 & -1.34505700 & 1.62782500 \\
\hline 0 & 0.45734000 & -3.59178900 & 1.81557300 & C & 2.99496900 & -3.32261800 & 2.52233200 \\
\hline $\mathrm{F}$ & 3.27956000 & -4.62142600 & 2.38829500 & $\mathrm{~F}$ & 2.64762900 & -3.08633200 & 3.79548900 \\
\hline $\mathrm{F}$ & 4.11490600 & -2.61433300 & 2.25946800 & C & 0.45968500 & 0.07473200 & -1.13439100 \\
\hline 0 & -0.64621700 & -0.10962800 & -0.67804300 & $\mathrm{O}$ & 0.99275600 & -0.44679600 & -2.24770000 \\
\hline C & 0.19239500 & -1.31889000 & -3.15542600 & C & -0.95357200 & -0.49610600 & -3.74606000 \\
\hline $\mathrm{H}$ & -1.48880400 & -1.10226800 & -4.48537600 & $\mathrm{H}$ & -1.66251400 & -0.19136800 & -2.97440400 \\
\hline $\mathrm{H}$ & -0.57300200 & 0.39909400 & -4.25029400 & C & 1.21758600 & -1.68929700 & -4.22859100 \\
\hline $\mathrm{H}$ & 2.04568100 & -2.25762800 & -3.79111600 & $\mathrm{H}$ & 0.74351100 & -2.31307700 & -4.99296100 \\
\hline $\mathrm{H}$ & 1.61820200 & -0.79342600 & -4.71575400 & C & -0.29124800 & -2.56154200 & -2.40908100 \\
\hline $\mathrm{H}$ & -0.71176200 & -3.27532800 & -3.12542900 & $\mathrm{H}$ & 0.53712600 & -3.04308300 & -1.88142800 \\
\hline $\mathrm{H}$ & -1.06492800 & -2.30958600 & -1.68386300 & C & 2.63039000 & 1.21803900 & -0.96845600 \\
\hline $\mathrm{H}$ & 2.73315900 & 0.99041000 & -2.02390100 & $\mathrm{C}$ & 3.28124800 & 2.49002600 & -0.54925000 \\
\hline $\mathrm{C}$ & 3.97284800 & 3.22813100 & -1.52275000 & $\mathrm{C}$ & 3.22229100 & 2.97684500 & 0.76578600 \\
\hline C & 4.58654000 & 4.43773500 & -1.19442200 & $\mathrm{H}$ & 4.01239200 & 2.86373200 & -2.54671500 \\
\hline $\mathrm{C}$ & 3.84423800 & 4.18142000 & 1.08982600 & $\mathrm{H}$ & 2.67774200 & 2.42990100 & 1.52804000 \\
\hline C & 4.52637400 & 4.91495100 & 0.11519200 & $\mathrm{H}$ & 5.10556900 & 5.00506200 & -1.96180900 \\
\hline $\mathrm{H}$ & 3.79444700 & 4.54940400 & 2.11092700 & $\mathrm{H}$ & 5.00657900 & 5.85411100 & 0.37560300 \\
\hline $\mathrm{N}$ & 1.36506900 & 0.95502200 & -0.51495200 & $\mathrm{H}$ & 1.05089700 & 1.27736000 & 0.40995300 \\
\hline $\mathrm{C}$ & 3.74594700 & -0.25250300 & -0.28583600 & $\mathrm{H}$ & 3.68952100 & -0.03518200 & 0.77701200 \\
\hline $\mathrm{H}$ & 3.08848300 & -1.05684200 & -0.60625000 & C & 4.98719700 & -0.15129400 & -0.92144700 \\
\hline C & 5.21627900 & -0.78320300 & -2.22254900 & $\mathrm{H}$ & 4.27265700 & -1.00652400 & -2.73313600 \\
\hline $\mathrm{H}$ & 5.85785200 & -0.17555100 & -2.87464200 & C & 6.08250300 & 0.68008700 & -0.33014700 \\
\hline $\mathrm{H}$ & 5.95222800 & 0.83576500 & 0.74295500 & $\mathrm{H}$ & 7.06704400 & 0.24204800 & -0.52252100 \\
\hline $\mathrm{H}$ & 6.07639500 & 1.66979300 & -0.81240800 & C & 5.89852700 & -3.24445600 & -3.86696800 \\
\hline $\mathrm{H}$ & 6.29028500 & -2.58173100 & -4.64765100 & $\mathrm{H}$ & 6.42101000 & -4.20471600 & -3.95572000 \\
\hline
\end{tabular}




\begin{tabular}{|llll|llll|}
\hline $\mathrm{H}$ & 4.83865900 & -3.42697600 & -4.07887300 & $\mathrm{C}$ & 7.98058600 & -2.21327000 & -1.81988900 \\
\hline $\mathrm{H}$ & 8.41493200 & -1.50851300 & -2.53895300 & $\mathrm{H}$ & 8.17299500 & -1.83229200 & -0.81034800 \\
\hline $\mathrm{H}$ & 8.53217300 & -3.15709500 & -1.91471300 & $\mathrm{C}$ & 5.39806700 & -3.61769500 & -0.80831200 \\
\hline $\mathrm{H}$ & 5.57779800 & -3.22369200 & 0.19768400 & $\mathrm{H}$ & 4.31356100 & -3.73057200 & -0.90869600 \\
\hline $\mathrm{H}$ & 5.84999700 & -4.61658700 & -0.85831600 & $\mathrm{Si}$ & 6.14131000 & -2.52277200 & -2.13798300 \\
\hline $\mathrm{C}$ & -2.82311800 & 4.78052200 & 3.15304400 & $\mathrm{H}$ & -3.57093200 & 4.62687900 & 2.36838900 \\
\hline $\mathrm{H}$ & -3.27952900 & 5.39005600 & 3.93820200 & $\mathrm{C}$ & -2.33263600 & 3.42798400 & 3.68921600 \\
\hline $\mathrm{H}$ & -1.99837100 & 3.51866300 & 4.72879200 & $\mathrm{H}$ & -3.09023400 & 2.64203500 & 3.62912500 \\
\hline $\mathrm{C}$ & -1.55729800 & 5.41213400 & 2.55631400 & $\mathrm{H}$ & -1.76959100 & 6.21317700 & 1.84469600 \\
\hline $\mathrm{H}$ & -0.92231300 & 5.82050500 & 3.35296400 & & & & \\
\hline
\end{tabular}

\section{TS- $R-4 f-n d-15$}

Imaginary frequency: $-249.96 \mathrm{~cm}^{-1}$

E[B3LYP/6-31G(d)]: -4788.685257 Hartree

E[PCM(Et $\left.\left.{ }_{2} \mathrm{O}\right)-\mathrm{B} 3 L Y P / 6-311 \mathrm{G}(\mathrm{d}, \mathrm{p})-\mathrm{D} 3(\mathrm{BJ})\right]:-4790.216665$ Hartree

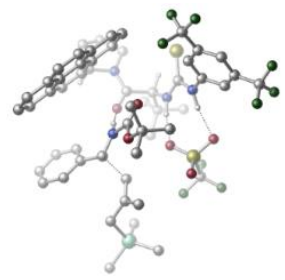

\begin{tabular}{|c|c|c|c|c|c|c|c|}
\hline 01 & & & & $\mathrm{~N}$ & -2.40735700 & -1.63626300 & 1.36666600 \\
\hline $\mathrm{H}$ & -1.75768700 & -2.40634300 & 1.18720200 & $\mathrm{C}$ & -3.58225100 & -1.68103300 & 0.59872400 \\
\hline C & -4.08603300 & -2.93527000 & 0.22604200 & C & -4.20110300 & -0.52118400 & 0.11641100 \\
\hline C & -5.20839500 & -3.01942700 & -0.59753300 & $\mathrm{H}$ & -3.59224100 & -3.83498800 & 0.57598400 \\
\hline C & -5.32540400 & -0.62441600 & -0.70116500 & $\mathrm{H}$ & -3.78702600 & 0.44714400 & 0.36252500 \\
\hline C & -5.84540800 & -1.86809800 & -1.06127900 & $\mathrm{H}$ & -6.72529100 & -1.93899600 & -1.68829700 \\
\hline C & -5.70818600 & -4.36897200 & -1.03908300 & C & -5.92305200 & 0.63820600 & -1.25756300 \\
\hline $\mathrm{F}$ & -7.04501800 & -4.36371700 & -1.25059700 & $\mathrm{~F}$ & -5.44550600 & -5.33139900 & -0.13007100 \\
\hline $\mathrm{F}$ & -5.13310400 & -4.75800700 & -2.20218700 & $\mathrm{~F}$ & -5.12980800 & 1.18450200 & -2.21852400 \\
\hline $\mathrm{F}$ & -7.13313900 & 0.43507100 & -1.81947400 & $\mathrm{~F}$ & -6.07610500 & 1.59428700 & -0.30934000 \\
\hline C & -0.09133700 & 0.09045800 & 3.61274900 & $\mathrm{H}$ & -0.89672000 & 0.33486200 & 4.30474300 \\
\hline C & 0.99219200 & -0.71069900 & 4.41877700 & C & 2.20834600 & -1.13472100 & 3.57136300 \\
\hline $\mathrm{H}$ & 2.75713600 & -0.26938800 & 3.19110800 & $\mathrm{H}$ & 1.91833900 & -1.75596700 & 2.72010900 \\
\hline $\mathrm{H}$ & 2.88937800 & -1.72798400 & 4.19444300 & C & 0.31895800 & -1.97310300 & 4.99590500 \\
\hline $\mathrm{H}$ & 1.01510200 & -2.49460900 & 5.66361700 & $\mathrm{H}$ & 0.02933500 & -2.67012200 & 4.20455300 \\
\hline $\mathrm{H}$ & -0.57930000 & -1.72244600 & 5.57263200 & C & 1.48450900 & 0.16791600 & 5.58736500 \\
\hline $\mathrm{H}$ & 2.24940100 & -0.36735800 & 6.16143200 & $\mathrm{H}$ & 0.67059200 & 0.41391600 & 6.28042100 \\
\hline $\mathrm{H}$ & 1.93585300 & 1.10475000 & 5.23764800 & C & 0.44888300 & 1.39306000 & 2.97311300 \\
\hline $\mathrm{O}$ & 1.27454700 & 1.33775800 & 2.04383400 & C & -0.82162200 & 2.81490200 & 4.64051400 \\
\hline C & 0.48566600 & 3.86378800 & 2.83790800 & C & -0.48012100 & 4.25603700 & 5.03511300 \\
\hline $\mathrm{H}$ & -1.88471900 & 2.68582300 & 4.40888700 & $\mathrm{H}$ & -0.56238600 & 2.10401100 & 5.42459000 \\
\hline $\mathrm{H}$ & 1.56359500 & 3.91015400 & 3.03839100 & $\mathrm{H}$ & 0.43156600 & 4.27241000 & 5.64502000 \\
\hline $\mathrm{H}$ & -1.28112700 & 4.72276600 & 5.61582400 & $\mathrm{~N}$ & -0.00382600 & 2.58438600 & 3.42708000 \\
\hline C & 0.31833800 & 4.08728800 & 1.33879800 & C & 1.40463400 & 4.58020800 & 0.67125300 \\
\hline C & -0.94689900 & 3.94933400 & 0.63432900 & C & 1.36859300 & 4.98453200 & -0.70295400 \\
\hline $\mathrm{H}$ & 2.35105000 & 4.68603800 & 1.19729900 & C & -1.01517300 & 4.35832900 & -0.73968800 \\
\hline C & -2.11191600 & 3.43393600 & 1.22715300 & C & 2.49951800 & 5.48672100 & -1.36722100 \\
\hline C & 0.13497200 & 4.87557800 & -1.40761500 & C & -2.24631600 & 4.24789700 & -1.45917300 \\
\hline $\mathrm{H}$ & -2.09189000 & 3.06658600 & 2.24341200 & C & -3.30581800 & 3.32802300 & 0.51494200 \\
\hline C & 2.42149800 & 5.88160700 & -2.70155100 & $\mathrm{H}$ & 3.44108100 & 5.56077800 & -0.82887000 \\
\hline C & 0.06422000 & 5.28209600 & -2.77616800 & C & -3.37946000 & 3.73068500 & -0.81345000 \\
\hline $\mathrm{C}$ & -2.29033600 & 4.66723000 & -2.83347900 & $\mathrm{H}$ & -4.17882700 & 2.90605700 & 1.00247000 \\
\hline C & 1.21918000 & 5.78261500 & -3.39953300 & $\mathrm{H}$ & 3.30459700 & 6.27112800 & -3.20138800 \\
\hline C & -1.19035100 & 5.16139200 & -3.46490800 & $\mathrm{H}$ & -4.30844800 & 3.62319300 & -1.36546800 \\
\hline $\mathrm{H}$ & -3.23581200 & 4.57915600 & -3.36320800 & $\mathrm{H}$ & 1.16460500 & 6.09450000 & -4.43992200 \\
\hline $\mathrm{H}$ & -1.24401700 & 5.47608400 & -4.50448300 & C & -2.05620100 & -0.71266700 & 2.31893000 \\
\hline
\end{tabular}




\begin{tabular}{|c|c|c|c|c|c|c|c|}
\hline $\mathrm{S}$ & -3.18269100 & 0.24942600 & 3.14326500 & $\mathrm{~N}$ & -0.71965900 & -0.71090400 & 2.57329900 \\
\hline $\mathrm{H}$ & -0.11585100 & -1.16649900 & 1.88544500 & S & 0.42168500 & -3.66174000 & 0.13819800 \\
\hline 0 & 0.73782400 & -3.99425100 & -1.26425100 & 0 & 0.78101100 & -2.26252200 & 0.53252300 \\
\hline 0 & -0.90432200 & -4.07840400 & 0.64751300 & $\mathrm{C}$ & 1.63258700 & -4.68639900 & 1.11346000 \\
\hline $\mathrm{F}$ & 1.43350800 & -5.99130500 & 0.91752100 & $\mathrm{~F}$ & 1.53253400 & -4.42701300 & 2.42601000 \\
\hline $\mathrm{F}$ & 2.89745800 & -4.38998700 & 0.72970900 & $\mathrm{C}$ & -0.23483800 & 4.93917600 & 3.68608300 \\
\hline $\mathrm{H}$ & -1.18522300 & 5.20767600 & 3.21409600 & $\mathrm{H}$ & 0.36805800 & 5.84876000 & 3.75714200 \\
\hline C & 0.24544100 & 0.42571100 & -1.33489000 & 0 & -0.78674200 & 0.54303300 & -0.71442600 \\
\hline $\mathrm{O}$ & 0.41251400 & -0.00546400 & -2.58933500 & C & -0.75907900 & -0.37761600 & -3.43786200 \\
\hline C & -1.68657700 & 0.82912900 & -3.59640100 & $\mathrm{H}$ & -2.44774400 & 0.59394500 & -4.34870500 \\
\hline $\mathrm{H}$ & -2.19451900 & 1.07411600 & -2.66302400 & $\mathrm{H}$ & -1.12963800 & 1.70788100 & -3.93905700 \\
\hline C & -0.09181900 & -0.73138400 & -4.76805100 & $\mathrm{H}$ & 0.59178000 & -1.57792100 & -4.64793600 \\
\hline $\mathrm{H}$ & -0.85569000 & -1.01308600 & -5.49961100 & $\mathrm{H}$ & 0.46637800 & 0.12326100 & -5.16577700 \\
\hline C & -1.46579200 & -1.59094800 & -2.83516400 & $\mathrm{H}$ & -2.23850700 & -1.93689500 & -3.53093200 \\
\hline $\mathrm{H}$ & -0.76319300 & -2.41177500 & -2.66363300 & $\mathrm{H}$ & -1.94690800 & -1.33612100 & -1.89011300 \\
\hline C & 2.69921900 & 0.64275200 & -1.34592800 & $\mathrm{H}$ & 2.61160700 & 0.45953000 & -2.40979300 \\
\hline C & 3.83529400 & 1.51653000 & -0.95691200 & C & 4.80366300 & 1.80789200 & -1.93173600 \\
\hline C & 3.98073400 & 2.05152100 & 0.33439100 & C & 5.89434000 & 2.62279800 & -1.63047800 \\
\hline $\mathrm{H}$ & 4.69134000 & 1.40711800 & -2.93606900 & C & 5.07774700 & 2.85795200 & 0.63114600 \\
\hline $\mathrm{H}$ & 3.23834100 & 1.85149700 & 1.10098900 & $\mathrm{C}$ & 6.03644000 & 3.14645100 & -0.34474000 \\
\hline $\mathrm{H}$ & 6.62752300 & 2.84972600 & -2.39927000 & $\mathrm{H}$ & 5.18448600 & 3.26543500 & 1.63281100 \\
\hline $\mathrm{H}$ & 6.88768900 & 3.77717100 & -0.10382200 & $\mathrm{~N}$ & 1.47702500 & 0.80550900 & -0.76048800 \\
\hline $\mathrm{H}$ & 1.38653500 & 1.05646900 & 0.23293700 & C & 3.36683100 & -1.17933700 & -0.75643600 \\
\hline $\mathrm{H}$ & 3.97420100 & -0.86138700 & 0.08714900 & $\mathrm{H}$ & 2.38694000 & -1.56901500 & -0.49407000 \\
\hline C & 4.02771700 & -1.74699500 & -1.84682000 & C & 5.48490000 & -1.68719000 & -1.98230400 \\
\hline $\mathrm{H}$ & 5.81387700 & -1.71724700 & -3.02819900 & $\mathrm{H}$ & 5.91379600 & -0.81043400 & -1.48150900 \\
\hline C & 3.23683300 & -2.41672100 & -2.93080700 & $\mathrm{H}$ & 2.93141700 & -1.65981800 & -3.67006700 \\
\hline $\mathrm{H}$ & 3.83959600 & -3.15492900 & -3.46804600 & $\mathrm{H}$ & 2.32915400 & -2.88864600 & -2.54186300 \\
\hline C & 8.16540900 & -3.10102700 & -1.62306700 & $\mathrm{H}$ & 8.61450500 & -2.14531200 & -1.32832800 \\
\hline $\mathrm{H}$ & 8.75024700 & -3.89991200 & -1.15104800 & $\mathrm{H}$ & 8.28003900 & -3.20594900 & -2.70830200 \\
\hline $\mathrm{C}$ & 6.17249400 & -3.01039300 & 0.76779700 & $\mathrm{H}$ & 6.52328700 & -2.03105600 & 1.11402200 \\
\hline $\mathrm{H}$ & 5.13339200 & -3.13772500 & 1.08887200 & $\mathrm{H}$ & 6.76897600 & -3.77318600 & 1.28372900 \\
\hline C & 5.57457900 & -4.83349600 & -1.66715300 & $\mathrm{H}$ & 4.53699600 & -4.92158000 & -1.32646600 \\
\hline $\mathrm{H}$ & 5.58977700 & -4.94868300 & -2.75716500 & $\mathrm{H}$ & 6.13004000 & -5.67945600 & -1.24327300 \\
\hline Si & 6.35260300 & -3.21330000 & -1.09802000 & & & & \\
\hline
\end{tabular}

\section{TS-R-4f-nd-16}

Imaginary frequency: $-247.63 \mathrm{~cm}^{-1}$

E[B3LYP/6-31G(d)]: -4788.685970 Hartree

E[PCM(Et $2 \mathrm{O})-B 3 L Y P / 6-311 G(d, p)-D 3(B J)]:-4790.216372$ Hartree

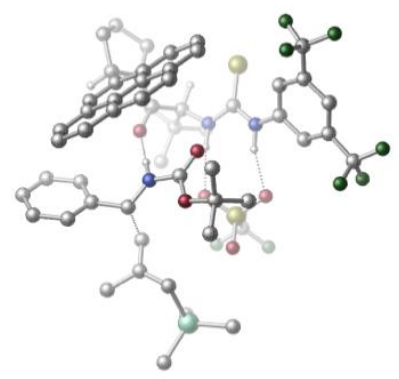

\begin{tabular}{|llll|llll|}
\hline 01 & & & $\mathrm{~N}$ & -1.90710900 & -1.85504200 & 1.52109400 \\
\hline $\mathrm{H}$ & -0.98178500 & -2.26885500 & 1.37977400 & $\mathrm{C}$ & -2.88200900 & -2.39961900 & 0.67004400 \\
\hline $\mathrm{C}$ & -2.58550600 & -3.63434600 & 0.06956600 & $\mathrm{C}$ & -4.07620800 & -1.74933000 & 0.32364600 \\
\hline $\mathrm{C}$ & -3.46255600 & -4.19437900 & -0.85866900 & $\mathrm{H}$ & -1.65961300 & -4.14108900 & 0.31771100 \\
\hline $\mathrm{C}$ & -4.94576800 & -2.33376000 & -0.59368900 & $\mathrm{H}$ & -4.31611700 & -0.79793100 & 0.77075500 \\
\hline $\mathrm{C}$ & -4.65637600 & -3.55956000 & -1.19559800 & $\mathrm{H}$ & -5.34322400 & -4.00834600 & -1.90293500 \\
\hline $\mathrm{C}$ & -3.06529200 & -5.46418900 & -1.56057200 & $\mathrm{C}$ & -6.18650600 & -1.59253000 & -1.01151600 \\
\hline $\mathrm{F}$ & -2.37833700 & -6.30060700 & -0.75541500 & $\mathrm{~F}$ & -2.26605000 & -5.20875400 & -2.63003800 \\
\hline $\mathrm{F}$ & -4.13634500 & -6.14125200 & -2.03263400 & $\mathrm{~F}$ & -5.99508800 & -0.91180600 & -2.17514400 \\
\hline
\end{tabular}




\begin{tabular}{|c|c|c|c|c|c|c|c|}
\hline $\mathrm{F}$ & -7.22638800 & -2.42764100 & -1.22786600 & $\mathrm{~F}$ & -6.58216600 & -0.68111900 & -0.09510700 \\
\hline $\mathrm{C}$ & -0.58790700 & 0.79240900 & 3.68484800 & $\mathrm{H}$ & -1.53730000 & 0.87477200 & 4.21255300 \\
\hline C & 0.51912400 & 0.55362500 & 4.76958700 & C & 1.94992400 & 0.46290000 & 4.20446300 \\
\hline $\mathrm{H}$ & 2.26158000 & 1.40367200 & 3.74231500 & $\mathrm{H}$ & 2.05007900 & -0.32960800 & 3.45903800 \\
\hline $\mathrm{H}$ & 2.64424100 & 0.24032100 & 5.02468200 & C & 0.18414300 & -0.75932600 & 5.50623800 \\
\hline $\mathrm{H}$ & 0.86054000 & -0.89105000 & 6.35934200 & $\mathrm{H}$ & 0.30093900 & -1.62511200 & 4.84936200 \\
\hline $\mathrm{H}$ & -0.84450200 & -0.75718800 & 5.88548800 & C & 0.46135100 & 1.71909300 & 5.77957700 \\
\hline $\mathrm{H}$ & 1.23323900 & 1.58873900 & 6.54663700 & $\mathrm{H}$ & -0.50618000 & 1.76195100 & 6.29487000 \\
\hline $\mathrm{H}$ & 0.64111300 & 2.68948700 & 5.29930400 & C & -0.37264000 & 2.05420600 & 2.81540400 \\
\hline 0 & 0.58002000 & 2.11873500 & 2.01908100 & C & -2.28727400 & 3.25603000 & 3.95995800 \\
\hline C & -1.14201600 & 4.26679000 & 2.03016600 & C & -2.46807800 & 4.77778200 & 4.00586800 \\
\hline $\mathrm{H}$ & -3.21584600 & 2.73522900 & 3.70060300 & $\mathrm{H}$ & -1.93587300 & 2.85522100 & 4.91012000 \\
\hline $\mathrm{H}$ & -0.16690200 & 4.71539200 & 2.25598100 & $\mathrm{H}$ & -1.69840700 & 5.22685100 & 4.64565300 \\
\hline $\mathrm{H}$ & -3.44565500 & 5.06344700 & 4.40529600 & $\mathrm{~N}$ & -1.27269400 & 3.06319600 & 2.89880600 \\
\hline C & -1.18216400 & 4.06718100 & 0.51820400 & C & -0.21586200 & 4.70569300 & -0.20738400 \\
\hline C & -2.25096400 & 3.36880000 & -0.18045700 & C & -0.19959100 & 4.74013300 & -1.63970800 \\
\hline $\mathrm{H}$ & 0.58574600 & 5.22491300 & 0.31294200 & C & -2.26465100 & 3.39512000 & -1.61553500 \\
\hline C & -3.28022600 & 2.67011800 & 0.47381900 & C & 0.80265400 & 5.40846000 & -2.36194900 \\
\hline C & -1.24618500 & 4.07869800 & -2.34473800 & C & -3.30803800 & 2.73182200 & -2.33503700 \\
\hline $\mathrm{H}$ & -3.28289600 & 2.58682200 & 1.55133200 & C & -4.29305300 & 2.02874900 & -0.23812600 \\
\hline C & 0.77765700 & 5.43093200 & -3.75540500 & $\mathrm{H}$ & 1.60183900 & 5.90834500 & -1.82066500 \\
\hline C & -1.26351200 & 4.10620900 & -3.77390100 & C & -4.31206200 & 2.05402600 & -1.62752500 \\
\hline C & -3.30231800 & 2.77680900 & -3.77184800 & $\mathrm{H}$ & -5.06737500 & 1.49287800 & 0.30166800 \\
\hline C & -0.24223000 & 4.78874500 & -4.45541000 & $\mathrm{H}$ & 1.55780100 & 5.95598400 & -4.30070300 \\
\hline C & -2.32944300 & 3.43260300 & -4.46169100 & $\mathrm{H}$ & -5.09202400 & 1.52907200 & -2.17160300 \\
\hline $\mathrm{H}$ & -4.10554800 & 2.26958400 & -4.30083200 & $\mathrm{H}$ & -0.25747900 & 4.81254600 & -5.54256500 \\
\hline $\mathrm{H}$ & -2.34431600 & 3.45918900 & -5.54875200 & C & -1.99246500 & -0.83371100 & 2.43342700 \\
\hline S & -3.44502800 & -0.30654200 & 3.13585300 & $\mathrm{~N}$ & -0.77047500 & -0.33240300 & 2.77397600 \\
\hline $\mathrm{H}$ & 0.03355000 & -0.59852300 & 2.20145500 & $\mathrm{~S}$ & 1.78538100 & -2.87131000 & 1.08466400 \\
\hline 0 & 2.74268000 & -3.18401300 & 0.00077200 & $\mathrm{O}$ & 1.70071200 & -1.41766700 & 1.41370300 \\
\hline 0 & 0.46439600 & -3.53472600 & 1.00204500 & C & 2.55985800 & -3.62116900 & 2.60291700 \\
\hline $\mathrm{F}$ & 2.76087000 & -4.93143300 & 2.43549100 & $\mathrm{~F}$ & 1.77040900 & -3.43972700 & 3.67188500 \\
\hline $\mathrm{F}$ & 3.74682300 & -3.03639900 & 2.85704100 & C & -2.26521000 & 5.19905900 & 2.54629500 \\
\hline $\mathrm{H}$ & -3.18058000 & 5.03399000 & 1.96958700 & $\mathrm{H}$ & -1.98569800 & 6.24972200 & 2.42811800 \\
\hline C & 0.67581100 & 0.27455900 & -1.13528400 & $\mathrm{O}$ & -0.41550900 & 0.05395000 & -0.65897000 \\
\hline 0 & 1.19768700 & -0.22126500 & -2.26486400 & C & 0.38682500 & -1.09401000 & -3.16602600 \\
\hline C & -0.84338000 & -0.32210200 & -3.64660800 & $\mathrm{H}$ & -1.36836000 & -0.91994900 & -4.39988900 \\
\hline $\mathrm{H}$ & -1.53255500 & -0.11872300 & -2.82588200 & $\mathrm{H}$ & -0.55276400 & 0.62793300 & -4.10761300 \\
\hline C & 1.35488600 & -1.35370000 & -4.32121800 & $\mathrm{H}$ & 2.25283600 & -1.87139000 & -3.96873500 \\
\hline $\mathrm{H}$ & 0.86970300 & -1.98520300 & -5.07222500 & $\mathrm{H}$ & 1.65362800 & -0.41528400 & -4.80097100 \\
\hline C & 0.02799300 & -2.39145400 & -2.44315400 & $\mathrm{H}$ & -0.46971100 & -3.07533700 & -3.13927100 \\
\hline $\mathrm{H}$ & 0.92436200 & -2.88416700 & -2.05497800 & $\mathrm{H}$ & -0.65099500 & -2.20346000 & -1.61186900 \\
\hline C & 2.81492700 & 1.48894900 & -0.97093400 & $\mathrm{H}$ & 2.94413500 & 1.18552200 & -2.00267800 \\
\hline C & 3.38027300 & 2.83210700 & -0.65456900 & C & 4.13040900 & 3.48525500 & -1.64460600 \\
\hline C & 3.18807300 & 3.46353300 & 0.58546800 & C & 4.68411000 & 4.74360700 & -1.40497700 \\
\hline $\mathrm{H}$ & 4.26739400 & 3.01253500 & -2.61407600 & C & 3.74710700 & 4.71796600 & 0.82146400 \\
\hline $\mathrm{H}$ & 2.58928600 & 2.98969600 & 1.35707600 & C & 4.49800700 & 5.36088700 & -0.16764500 \\
\hline $\mathrm{H}$ & 5.25561600 & 5.23903200 & -2.18477500 & $\mathrm{H}$ & 3.59512300 & 5.19709800 & 1.78473000 \\
\hline $\mathrm{H}$ & 4.93265000 & 6.33764700 & 0.02573700 & $\mathrm{~N}$ & 1.56076500 & 1.17506900 & -0.51746700 \\
\hline $\mathrm{H}$ & 1.21857300 & 1.50426400 & 0.39425300 & C & 4.08013700 & 0.24485600 & -0.06147700 \\
\hline $\mathrm{H}$ & 4.43997600 & 0.91269100 & 0.71750300 & $\mathrm{H}$ & 3.35599100 & -0.49542800 & 0.26948200 \\
\hline C & 4.98918000 & -0.12573100 & -1.06328000 & C & 4.76545600 & -1.30250400 & -1.89326000 \\
\hline $\mathrm{H}$ & 3.75718500 & -1.70549800 & -1.77165300 & $\mathrm{H}$ & 4.99418600 & -1.11584400 & -2.95095500 \\
\hline
\end{tabular}




\begin{tabular}{|llll|llll|}
\hline $\mathrm{C}$ & 6.21751800 & 0.70554200 & -1.29610700 & $\mathrm{H}$ & 7.03501300 & 0.31035500 & -0.67525500 \\
\hline $\mathrm{H}$ & 6.55575700 & 0.66767600 & -2.33643000 & $\mathrm{H}$ & 6.06684800 & 1.74592100 & -0.99509200 \\
\hline $\mathrm{C}$ & 5.08113300 & -4.34432300 & -2.09087000 & $\mathrm{H}$ & 4.86863300 & -4.27086100 & -3.16416600 \\
\hline $\mathrm{H}$ & 5.70115700 & -5.23608600 & -1.93614900 & $\mathrm{H}$ & 4.13606200 & -4.48351200 & -1.55679700 \\
\hline $\mathrm{C}$ & 7.58758700 & -2.53720900 & -2.38451400 & $\mathrm{H}$ & 7.42452700 & -2.45902000 & -3.46618600 \\
\hline $\mathrm{H}$ & 8.12087000 & -1.63664900 & -2.05879700 & $\mathrm{H}$ & 8.25962800 & -3.38832900 & -2.21716200 \\
\hline $\mathrm{C}$ & 6.28863800 & -2.89057000 & 0.40432800 & $\mathrm{H}$ & 6.82177500 & -2.00626900 & 0.77406500 \\
\hline $\mathrm{H}$ & 5.35665000 & -2.98990200 & 0.96679100 & $\mathrm{H}$ & 6.91270900 & -3.76378000 & 0.63251800 \\
\hline $\mathrm{Si}$ & 5.96275100 & -2.81186200 & -1.44787700 & & & & \\
\hline
\end{tabular}

\section{TS- $R$-4f-nd-17}

Imaginary frequency: $-245.56 \mathrm{~cm}^{-1}$

E[B3LYP/6-31G(d)-D3(BJ)]: -4788.688517 Hartree

$\mathrm{E}\left[\mathrm{PCM}\left(\mathrm{Et}_{2} \mathrm{O}\right)-\mathrm{B} 3 \mathrm{LYP} / 6-311 \mathrm{G}(\mathrm{d}, \mathrm{p})-\mathrm{D} 3(\mathrm{BJ})\right]$ : -4790.216230 Hartree

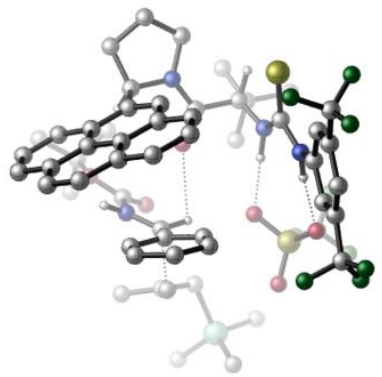

\begin{tabular}{|c|c|c|c|c|c|c|c|}
\hline 01 & & & & $\mathrm{~N}$ & -2.06347600 & -1.92981000 & 1.65641100 \\
\hline $\mathrm{H}$ & -1.30739000 & -2.52828300 & 1.30930400 & C & -3.28129800 & -2.17538600 & 0.99136000 \\
\hline C & -3.21571700 & -3.04937700 & -0.11232700 & C & -4.52420500 & -1.61795900 & 1.32622100 \\
\hline C & -4.34714600 & -3.30574800 & -0.88019800 & $\mathrm{H}$ & -2.27340900 & -3.52593600 & -0.36260300 \\
\hline C & -5.64373500 & -1.88391900 & 0.53399600 & $\mathrm{H}$ & -4.61161900 & -0.98189200 & 2.19408500 \\
\hline C & -5.57652500 & -2.71816200 & -0.57698100 & $\mathrm{H}$ & -6.45231900 & -2.91617500 & -1.18135300 \\
\hline $\mathrm{C}$ & -4.20277500 & -4.15164100 & -2.11579700 & $\mathrm{C}$ & -6.93092800 & -1.18869500 & 0.88614100 \\
\hline $\mathrm{F}$ & -3.35827300 & -5.18484200 & -1.93447900 & $\mathrm{~F}$ & -3.70622200 & -3.41900700 & -3.15159800 \\
\hline $\mathrm{F}$ & -5.38517500 & -4.65488800 & -2.53162900 & $\mathrm{~F}$ & -7.20818700 & -1.26825500 & 2.20365000 \\
\hline $\mathrm{F}$ & -6.87186600 & 0.13947700 & 0.58749400 & $\mathrm{~F}$ & -7.98895200 & -1.69137100 & 0.21507100 \\
\hline C & 0.29885200 & 0.23681500 & 3.44634200 & $\mathrm{H}$ & -0.44212900 & 0.54874200 & 4.18118600 \\
\hline C & 1.55380700 & -0.25465500 & 4.24628500 & C & 2.72193500 & -0.71708600 & 3.35537400 \\
\hline $\mathrm{H}$ & 3.06153200 & 0.06968000 & 2.67766000 & $\mathrm{H}$ & 2.45518800 & -1.57940700 & 2.74152500 \\
\hline $\mathrm{H}$ & 3.56369900 & -1.01305100 & 3.99436200 & C & 1.11451600 & -1.42186500 & 5.15340300 \\
\hline $\mathrm{H}$ & 1.94670300 & -1.72744800 & 5.79885600 & $\mathrm{H}$ & 0.81130200 & -2.29089400 & 4.56304800 \\
\hline $\mathrm{H}$ & 0.27347400 & -1.13790200 & 5.79748900 & $\mathrm{C}$ & 2.03669800 & 0.91023900 & 5.13532300 \\
\hline $\mathrm{H}$ & 2.90296700 & 0.59415900 & 5.72752400 & $\mathrm{H}$ & 1.25851200 & 1.23415400 & 5.83718600 \\
\hline $\mathrm{H}$ & 2.34665300 & 1.77815700 & 4.54013200 & C & 0.58065900 & 1.39706400 & 2.46338900 \\
\hline $\mathrm{C}$ & 0.28126700 & 3.72068500 & 1.76298100 & $\mathrm{H}$ & 1.30193000 & 3.64800700 & 1.38109300 \\
\hline $\mathrm{N}$ & 0.09209600 & 2.63651500 & 2.74476100 & C & -0.69555600 & 3.65216400 & 0.58825400 \\
\hline $\mathrm{C}$ & -1.85761900 & 2.94089000 & 0.67506900 & $\mathrm{C}$ & -0.40237600 & 4.39747000 & -0.62555800 \\
\hline $\mathrm{C}$ & -2.82983200 & 2.91126700 & -0.37999900 & $\mathrm{H}$ & -2.06885400 & 2.35267100 & 1.56370100 \\
\hline C & -1.36176800 & 4.39252000 & -1.69194400 & C & 0.78953700 & 5.12450800 & -0.81324300 \\
\hline $\mathrm{C}$ & -4.01991300 & 2.17313400 & -0.27265400 & $\mathrm{C}$ & -2.57737700 & 3.65692800 & -1.56688500 \\
\hline $\mathrm{C}$ & -1.10343400 & 5.12149600 & -2.89550900 & $\mathrm{H}$ & 1.53551400 & 5.15692600 & -0.02768400 \\
\hline C & 1.03675100 & 5.82295800 & -1.99700100 & C & -4.95089900 & 2.17087300 & -1.31070300 \\
\hline $\mathrm{H}$ & -4.20976300 & 1.59688100 & 0.62851800 & C & -3.53564500 & 3.65073100 & -2.62553800 \\
\hline $\mathrm{C}$ & 0.10269900 & 5.82847200 & -3.02754100 & $\mathrm{C}$ & -2.08966700 & 5.10601300 & -3.94214800 \\
\hline $\mathrm{H}$ & 1.96611500 & 6.37620800 & -2.10596900 & $\mathrm{C}$ & -4.71506300 & 2.90289200 & -2.47313100 \\
\hline $\mathrm{H}$ & -5.86150700 & 1.58971300 & -1.20506200 & C & -3.25109000 & 4.40669500 & -3.81309600 \\
\hline $\mathrm{H}$ & 0.29673500 & 6.38295800 & -3.94259500 & $\mathrm{H}$ & -1.88684200 & 5.67480700 & -4.84667600 \\
\hline $\mathrm{H}$ & -5.44666800 & 2.89968500 & -3.27788400 & $\mathrm{H}$ & -3.98698200 & 4.40937600 & -4.61394800 \\
\hline C & -1.69215600 & -0.93867100 & 2.53588700 & $\mathrm{~S}$ & -2.77809900 & 0.03481300 & 3.40858300 \\
\hline $\mathrm{N}$ & -0.33993900 & -0.82681100 & 2.66851500 & $\mathrm{H}$ & 0.25232900 & -1.25632100 & 1.95098800 \\
\hline $\mathrm{S}$ & 1.18407100 & -3.54332800 & 0.21687600 & $\mathrm{O}$ & 1.86168100 & -3.97316900 & -1.01965000 \\
\hline
\end{tabular}




\begin{tabular}{|c|c|c|c|c|c|c|c|}
\hline 0 & 1.43272200 & -2.11704200 & 0.59041300 & 0 & -0.24300700 & -3.92418500 & 0.36506900 \\
\hline C & 2.03674600 & -4.49668600 & 1.56972100 & $\mathrm{~F}$ & 1.94376700 & -5.81200500 & 1.36105400 \\
\hline $\mathrm{F}$ & 1.49006300 & -4.20957100 & 2.76195200 & $F$ & 3.34063700 & -4.16181000 & 1.61855300 \\
\hline$C$ & 3.09389500 & -0.82709500 & -2.68354800 & $\mathrm{H}$ & 2.43074600 & -1.67458500 & -2.53660600 \\
\hline $\mathrm{H}$ & 2.92191900 & -0.22516300 & -3.57163900 & C & 4.37796500 & -0.89363200 & -2.16115700 \\
\hline $\mathrm{C}$ & 4.77847400 & -1.94446500 & -1.21908000 & $\mathrm{H}$ & 3.91468600 & -2.35104300 & -0.68383300 \\
\hline $\mathrm{H}$ & 5.51303200 & -1.57685900 & -0.49435000 & $\mathrm{C}$ & 5.40144300 & 0.10976800 & -2.61764500 \\
\hline $\mathrm{H}$ & 5.89124100 & -0.25427500 & -3.53143200 & $\mathrm{H}$ & 6.17880800 & 0.27558900 & -1.86826500 \\
\hline $\mathrm{H}$ & 4.93232700 & 1.06691800 & -2.87106900 & C & 5.95310900 & -4.70173800 & -0.64620200 \\
\hline $\mathrm{H}$ & 6.70871200 & -4.29153300 & 0.03455000 & $\mathrm{H}$ & 6.33674500 & -5.65366300 & -1.03328300 \\
\hline $\mathrm{H}$ & 5.05331800 & -4.91314900 & -0.06047700 & C & 7.19832400 & -3.02407000 & -2.92430300 \\
\hline $\mathrm{H}$ & 7.88510000 & -2.50352100 & -2.24605300 & $\mathrm{H}$ & 7.03382100 & -2.38090100 & -3.79680100 \\
\hline $\mathrm{H}$ & 7.71391000 & -3.92422600 & -3.28212300 & C & 4.37399700 & -4.25807200 & -3.30189800 \\
\hline $\mathrm{H}$ & 4.17309800 & -3.57925700 & -4.13926100 & $\mathrm{H}$ & 3.42078000 & -4.48998700 & -2.81582500 \\
\hline $\mathrm{H}$ & 4.78138000 & -5.18660700 & -3.72091100 & $\mathrm{Si}$ & 5.58036400 & -3.51206500 & -2.06345000 \\
\hline C & -0.57821500 & 3.09545000 & 3.97975800 & $\mathrm{H}$ & 0.12121600 & 3.06350700 & 4.82355000 \\
\hline $\mathrm{H}$ & -1.43832500 & 2.46143700 & 4.21227600 & C & -0.97716300 & 4.54351000 & 3.65491300 \\
\hline $\mathrm{H}$ & -0.99923500 & 5.17362300 & 4.54880100 & $\mathrm{H}$ & -1.97396100 & 4.56725900 & 3.20261400 \\
\hline C & 0.07596600 & 4.98750000 & 2.62830900 & $\mathrm{H}$ & -0.23595000 & 5.85110900 & 2.03542600 \\
\hline $\mathrm{H}$ & 1.01615600 & 5.24244200 & 3.13451000 & 0 & 1.19224700 & 1.18421300 & 1.41075600 \\
\hline C & 3.62314100 & 1.81832000 & -0.27292400 & $\mathrm{O}$ & 4.22630500 & 0.88591200 & 0.21328400 \\
\hline $\mathrm{O}$ & 3.83873000 & 3.12929500 & -0.09406400 & C & 4.94946900 & 3.61298000 & 0.76476400 \\
\hline C & 6.28802700 & 3.13563000 & 0.19440000 & $\mathrm{H}$ & 7.10634100 & 3.60217000 & 0.75336600 \\
\hline $\mathrm{H}$ & 6.38956000 & 2.05207300 & 0.27413900 & $\mathrm{H}$ & 6.38568100 & 3.43028500 & -0.85627300 \\
\hline C & 4.82004700 & 5.13247600 & 0.64674700 & $\mathrm{H}$ & 3.85314800 & 5.47324400 & 1.03056500 \\
\hline $\mathrm{H}$ & 5.60970600 & 5.61861400 & 1.22844900 & $\mathrm{H}$ & 4.91272400 & 5.45159200 & -0.39632200 \\
\hline C & 4.73135100 & 3.15100000 & 2.20719700 & $\mathrm{H}$ & 5.48914100 & 3.61022400 & 2.85167800 \\
\hline $\mathrm{H}$ & 3.74495800 & 3.46355400 & 2.56533900 & $\mathrm{H}$ & 4.80864100 & 2.06638800 & 2.29491800 \\
\hline C & 1.95765000 & 0.45636900 & -1.38316300 & $\mathrm{H}$ & 2.05695900 & -0.25224900 & -0.56706700 \\
\hline C & 0.71492900 & 0.45422500 & -2.16834900 & C & -0.24530700 & -0.53980900 & -1.90836500 \\
\hline $\mathrm{C}$ & 0.47258800 & 1.39265800 & -3.18953900 & $C$ & -1.42252600 & -0.59027000 & -2.65112300 \\
\hline $\mathrm{H}$ & -0.06077200 & -1.25676700 & -1.11683500 & $\mathrm{C}$ & -0.70808800 & 1.34366000 & -3.92193100 \\
\hline $\mathrm{H}$ & 1.21031600 & 2.15359000 & -3.43330200 & $\mathrm{C}$ & -1.65530400 & 0.35001700 & -3.65631500 \\
\hline $\mathrm{H}$ & -2.15272100 & -1.36930000 & -2.45722500 & $\mathrm{H}$ & -0.89335000 & 2.08161600 & -4.69610000 \\
\hline $\mathrm{H}$ & -2.57502200 & 0.31116100 & -4.23241000 & $\mathrm{~N}$ & 2.58509700 & 1.65504900 & -1.19525100 \\
\hline $\mathrm{H}$ & 2.16625100 & 2.50242000 & -1.56296900 & & & & \\
\hline
\end{tabular}

\section{TS- $R-4 f-n d-18$}

Imaginary frequency: $-246.30 \mathrm{~cm}^{-1}$ E[B3LYP/6-31G(d)]: -4788.687269 Hartree

$\mathrm{E}\left[\mathrm{PCM}\left(\mathrm{Et}_{2} \mathrm{O}\right)-\mathrm{B} 3 \mathrm{LYP} / 6-311 \mathrm{G}(\mathrm{d}, \mathrm{p})-\mathrm{D} 3(\mathrm{BJ})\right]$ : -4790.215926 Hartree

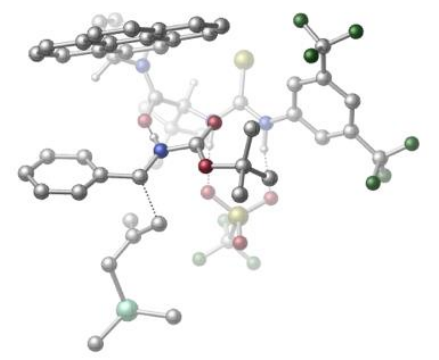

\begin{tabular}{|lrrr|rrrr|}
\hline 01 & & & & $\mathrm{~N}$ & 2.72901700 & 0.82190200 & 1.61031100 \\
\hline $\mathrm{H}$ & 2.37906800 & 1.76681800 & 1.42196800 & $\mathrm{C}$ & 3.86732000 & 0.49231600 & 0.85881100 \\
\hline $\mathrm{C}$ & 4.65021600 & 1.55232200 & 0.37568200 & $\mathrm{C}$ & 4.19452700 & -0.81955800 & 0.48673700 \\
\hline $\mathrm{C}$ & 5.73899200 & 1.29649700 & -0.45763600 & $\mathrm{H}$ & 4.38546400 & 2.57266800 & 0.62987000 \\
\hline $\mathrm{C}$ & 5.28892900 & -1.05427200 & -0.34086300 & $\mathrm{H}$ & 3.58838200 & -1.64005100 & 0.83726500 \\
\hline $\mathrm{C}$ & 6.07867600 & -0.00635800 & -0.81767600 & $\mathrm{H}$ & 6.93402400 & -0.19997600 & -1.45395000 \\
\hline $\mathrm{C}$ & 6.50075800 & 2.44999100 & -1.05174800 & $\mathrm{C}$ & 5.56570100 & -2.45312800 & -0.81777000 \\
\hline
\end{tabular}




\begin{tabular}{|c|c|c|c|c|c|c|c|}
\hline $\mathrm{F}$ & 6.51441000 & 3.52753000 & -0.24081400 & $\mathrm{~F}$ & 5.95069200 & 2.84723900 & -2.22684400 \\
\hline $\mathrm{F}$ & 7.78644400 & 2.12306800 & -1.31696800 & $\mathrm{~F}$ & 5.04958000 & -3.39541900 & 0.00231900 \\
\hline $\mathrm{F}$ & 5.01764500 & -2.67452000 & -2.04649100 & $\mathrm{~F}$ & 6.88758500 & -2.69886800 & -0.94088500 \\
\hline C & -0.17842900 & -0.02723800 & 3.65530000 & $\mathrm{H}$ & 0.44433200 & -0.61018800 & 4.33186100 \\
\hline C & -0.92343200 & 1.04757100 & 4.52474400 & C & -1.89173800 & 1.93006600 & 3.71633100 \\
\hline $\mathrm{H}$ & -2.70035700 & 1.34098500 & 3.27449000 & $\mathrm{H}$ & -1.37950200 & 2.46423500 & 2.91261900 \\
\hline $\bar{H}$ & -2.33865700 & 2.67964100 & 4.38135900 & C & 0.14153500 & 1.94466400 & 5.18867600 \\
\hline $\mathrm{H}$ & -0.33799500 & 2.62593700 & 5.90166600 & $\mathrm{H}$ & 0.67142800 & 2.55161700 & 4.44950300 \\
\hline $\mathrm{H}$ & 0.88380300 & 1.35073300 & 5.73503800 & C & -1.71985800 & 0.32382200 & 5.63031600 \\
\hline $\bar{H}$ & -2.25938200 & 1.05618700 & 6.24152400 & $\mathrm{H}$ & -1.06111600 & -0.23871400 & 6.30313600 \\
\hline $\mathrm{H}$ & -2.46450200 & -0.36788900 & 5.21749900 & C & -1.11919300 & -0.97994500 & 2.88090600 \\
\hline 0 & -1.73473000 & -0.56851400 & 1.87818800 & C & -0.68891400 & -2.89191700 & 4.50602200 \\
\hline C & -2.15249700 & -3.19408300 & 2.58354600 & C & -1.81677800 & -3.83083200 & 4.93948100 \\
\hline $\mathrm{H}$ & 0.22737900 & -3.43843900 & 4.25376200 & $\mathrm{H}$ & -0.44185800 & -2.15152900 & 5.26276500 \\
\hline $\mathrm{H}$ & -3.08571200 & -2.65028400 & 2.40666500 & $\mathrm{H}$ & -2.58198400 & -3.26120100 & 5.48054900 \\
\hline $\mathrm{H}$ & -1.46381800 & -4.63104200 & 5.59686000 & $\mathrm{~N}$ & -1.24481000 & -2.26290200 & 3.29375500 \\
\hline C & -1.61024700 & -3.70630900 & 1.25317300 & C & -0.28229500 & -3.62145500 & 0.95041200 \\
\hline C & -2.52085900 & -4.37256500 & 0.33656500 & C & 0.26945000 & -4.17998400 & -0.25123700 \\
\hline $\mathrm{H}$ & 0.40042900 & -3.11221200 & 1.62558400 & C & -1.99558600 & -4.92482900 & -0.87738200 \\
\hline C & -3.89965800 & -4.50709500 & 0.58178700 & C & 1.64106900 & -4.10791100 & -0.54158100 \\
\hline C & -0.60244400 & -4.83204000 & -1.16999300 & C & -2.86507700 & -5.58432800 & -1.80165300 \\
\hline $\mathrm{H}$ & -4.33108600 & -4.10863400 & 1.49355100 & C & -4.73979500 & -5.15130900 & -0.32629800 \\
\hline $\mathrm{C}$ & 2.15058500 & -4.65716200 & -1.71734300 & $\mathrm{H}$ & 2.30749700 & -3.62164900 & 0.16377200 \\
\hline C & -0.07474200 & -5.39189900 & -2.37366100 & C & -4.23360100 & -5.68506200 & -1.50618600 \\
\hline $\mathrm{C}$ & -2.30807400 & -6.13261600 & -3.00838100 & $\mathrm{H}$ & -5.80036000 & -5.23768000 & -0.10488200 \\
\hline C & 1.30323200 & -5.28960200 & -2.62586100 & $\mathrm{H}$ & 3.21271800 & -4.57655900 & -1.92550200 \\
\hline C & -0.97773800 & -6.04199900 & -3.28183800 & $\mathrm{H}$ & -4.89303900 & -6.18905900 & -2.20866000 \\
\hline $\mathrm{H}$ & -2.98028200 & -6.63094000 & -3.70300700 & $\mathrm{H}$ & 1.70590400 & -5.71553800 & -3.54194500 \\
\hline $\mathrm{H}$ & -0.57342300 & -6.46667700 & -4.19777300 & C & 1.99911700 & 0.04983300 & 2.47679700 \\
\hline $\mathrm{S}$ & 2.59727200 & -1.33835400 & 3.24819900 & $\mathrm{~N}$ & 0.75653400 & 0.55995000 & 2.70721800 \\
\hline $\mathrm{H}$ & 0.38461700 & 1.22660900 & 2.02480000 & $S$ & 0.70009900 & 3.72733400 & 0.33759400 \\
\hline $\mathrm{O}$ & 0.50197200 & 4.22537200 & -1.03539600 & $\mathrm{O}$ & -0.16547700 & 2.56230400 & 0.70163100 \\
\hline $\mathrm{O}$ & 2.10059400 & 3.57027000 & 0.79970100 & C & 0.03628900 & 5.09613800 & 1.41295100 \\
\hline $\mathrm{F}$ & 0.72800500 & 6.22404700 & 1.24586400 & $\mathrm{~F}$ & 0.08033300 & 4.74961600 & 2.70920000 \\
\hline $\mathrm{F}$ & -1.25890100 & 5.34782300 & 1.09986800 & C & -2.36821600 & -4.35524200 & 3.60497300 \\
\hline $\mathrm{H}$ & -1.80053200 & -5.23212300 & 3.27890500 & $\mathrm{H}$ & -3.41878200 & -4.65186500 & 3.66731100 \\
\hline C & 0.00899900 & -0.28404400 & -1.38232700 & $\mathrm{O}$ & 0.87373900 & -0.64182900 & -0.61474200 \\
\hline $\mathrm{O}$ & 0.13880000 & 0.14919900 & -2.63964000 & C & 1.47191700 & 0.29478400 & -3.29913500 \\
\hline C & 2.17112300 & -1.06381400 & -3.35213000 & $\mathrm{H}$ & 3.07957500 & -0.97447300 & -3.95771800 \\
\hline $\mathrm{H}$ & 2.45654200 & -1.41109800 & -2.35941000 & $\mathrm{H}$ & 1.52381400 & -1.81582100 & -3.81627600 \\
\hline $\mathrm{C}$ & 1.08281400 & 0.76919800 & -4.69990300 & $\mathrm{H}$ & 0.53732400 & 1.71725300 & -4.65135800 \\
\hline $\mathrm{H}$ & 1.98574000 & 0.92517800 & -5.29864000 & $\mathrm{H}$ & 0.45770700 & 0.02592400 & -5.20659100 \\
\hline $\mathrm{C}$ & 2.28243300 & 1.35739700 & -2.56004400 & $\mathrm{H}$ & 3.22266300 & 1.53961400 & -3.09215300 \\
\hline $\mathrm{H}$ & 1.73325000 & 2.30214500 & -2.49942200 & $\mathrm{H}$ & 2.52723700 & 1.02968400 & -1.54985000 \\
\hline C & -2.36033200 & 0.15547400 & -1.80700700 & $\mathrm{H}$ & -2.08182700 & 0.15376100 & -2.85356400 \\
\hline $\mathrm{C}$ & -3.76287300 & -0.25172800 & -1.55108200 & $\mathrm{C}$ & -4.64788400 & -0.25404500 & -2.64307800 \\
\hline $\mathrm{C}$ & -4.23306500 & -0.64843800 & -0.28895800 & $\mathrm{C}$ & -5.97644400 & -0.63890200 & -2.48158500 \\
\hline $\mathrm{H}$ & -4.28566000 & 0.03587100 & -3.62676500 & C & -5.56543400 & -1.03022100 & -0.13406400 \\
\hline $\mathrm{H}$ & -3.56671900 & -0.67312800 & 0.56763700 & C & -6.43995700 & -1.02560600 & -1.22241400 \\
\hline $\mathrm{H}$ & -6.64526300 & -0.64494500 & -3.33759000 & $\mathrm{H}$ & -5.92012600 & -1.33959400 & 0.84509000 \\
\hline $\mathrm{H}$ & -7.47509100 & -1.32887700 & -1.09229300 & $\mathrm{~N}$ & -1.35319100 & -0.30766000 & -1.01013200 \\
\hline $\mathrm{H}$ & -1.49625300 & -0.51757000 & -0.01487100 & C & -2.19868700 & 2.20220200 & -1.82250300 \\
\hline $\mathrm{H}$ & -1.24622100 & 2.21754500 & -1.30519600 & $\mathrm{H}$ & -2.15339200 & 2.39640800 & -2.89229700 \\
\hline
\end{tabular}




\begin{tabular}{|c|c|c|c|c|c|c|c|}
\hline C & -3.31100000 & 2.68348700 & -1.13263600 & $\mathrm{C}$ & -4.46353900 & 3.23549700 & -1.84367600 \\
\hline $\mathrm{H}$ & -4.52380900 & 2.87619100 & -2.87745600 & $\mathrm{H}$ & -5.41429900 & 3.04690700 & -1.33201400 \\
\hline C & -3.32724500 & 2.66009800 & 0.36224500 & $\mathrm{H}$ & -4.34610100 & 2.62720500 & 0.75915500 \\
\hline $\mathrm{H}$ & -2.73839900 & 1.83102000 & 0.76020000 & $\mathrm{H}$ & -2.83732500 & 3.56599000 & 0.74044400 \\
\hline C & -5.77367200 & 5.65533000 & -3.14599600 & $\mathrm{H}$ & -6.73500900 & 5.37135300 & -2.70186800 \\
\hline $\mathrm{H}$ & -5.79482600 & 6.73919000 & -3.31291600 & $\mathrm{H}$ & -5.70078300 & 5.17242600 & -4.12746400 \\
\hline C & -4.50496300 & 6.01834300 & -0.34107100 & $\mathrm{H}$ & -5.38049600 & 5.65250300 & 0.20794700 \\
\hline $\mathrm{H}$ & -3.62061800 & 5.87665000 & 0.28811700 & $\mathrm{H}$ & -4.63350400 & 7.09950900 & -0.47800600 \\
\hline C & -2.67003700 & 5.61902100 & -2.82396500 & $\mathrm{H}$ & -1.81575100 & 5.31247700 & -2.20891700 \\
\hline $\mathrm{H}$ & -2.55923200 & 5.15009900 & -3.80926700 & $\mathrm{H}$ & -2.59808100 & 6.70386800 & -2.97195500 \\
\hline $\mathrm{Si}$ & -4.32115100 & 5.19076200 & -2.02699200 & & & & \\
\hline
\end{tabular}

\section{TS-R-4f-nd-19}

Imaginary frequency: $-245.02 \mathrm{~cm}^{-1}$

E[B3LYP/6-31G(d)]: -4788.690098 Hartree

E[PCM(Et $2 \mathrm{O})-B 3 L Y P / 6-311 G(d, p)-D 3(B J)]:-4790.215557$ Hartree

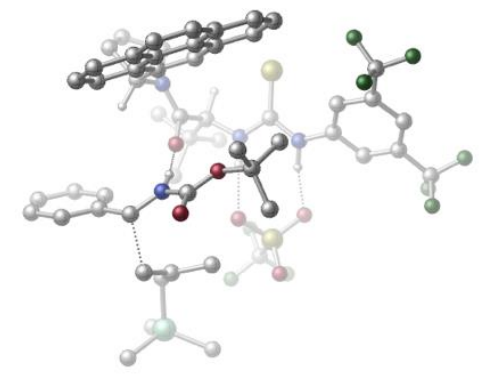

\begin{tabular}{|c|c|c|c|c|c|c|c|}
\hline 01 & & & & $\mathrm{~N}$ & 1.35054000 & 2.55461400 & 1.25766100 \\
\hline $\mathrm{H}$ & 0.51361100 & 3.09256100 & 1.00042800 & C & 2.53785300 & 3.06944100 & 0.70909500 \\
\hline C & 2.52298500 & 4.40379100 & 0.27192600 & C & 3.68923700 & 2.29844700 & 0.50230700 \\
\hline C & 3.64923400 & 4.95244800 & -0.33906300 & $\mathrm{H}$ & 1.62778500 & 5.00125300 & 0.40744200 \\
\hline $\mathrm{C}$ & 4.80406700 & 2.86354500 & -0.11428400 & $\mathrm{H}$ & 3.70995500 & 1.26772000 & 0.82213500 \\
\hline C & 4.80370900 & 4.19335800 & -0.53407300 & $\mathrm{H}$ & 5.68057700 & 4.62953900 & -0.99650500 \\
\hline $\mathrm{C}$ & 3.60171400 & 6.36962100 & -0.84729300 & $C$ & 5.98844500 & 1.98349100 & -0.40667600 \\
\hline $\mathrm{F}$ & 3.17727000 & 6.42346800 & -2.13168900 & $\mathrm{~F}$ & 4.82272100 & 6.95222500 & -0.81625700 \\
\hline $\mathrm{F}$ & 2.76515900 & 7.14122000 & -0.12285700 & $\mathrm{~F}$ & 5.82107300 & 1.28883500 & -1.56362700 \\
\hline $\mathrm{F}$ & 7.13154100 & 2.68800600 & -0.53958600 & $\mathrm{~F}$ & 6.19232900 & 1.05898400 & 0.56316200 \\
\hline $\mathrm{C}$ & -0.58503500 & 0.15172800 & 3.21766300 & $\mathrm{H}$ & 0.18204700 & 0.09657200 & 3.98932800 \\
\hline C & -1.93694600 & 0.52159800 & 3.92981500 & C & -3.14637800 & 0.54125500 & 2.97678200 \\
\hline $\mathrm{H}$ & -3.36645900 & -0.45968000 & 2.59193800 & $\mathrm{H}$ & -2.99596600 & 1.20845500 & 2.12558200 \\
\hline $\mathrm{H}$ & -4.03145500 & 0.88950600 & 3.52342900 & $\mathrm{C}$ & -1.76971500 & 1.91508000 & 4.57101600 \\
\hline $\mathrm{H}$ & -2.64731800 & 2.14787700 & 5.18588000 & $\mathrm{H}$ & -1.67228400 & 2.69857200 & 3.81612500 \\
\hline $\mathrm{H}$ & -0.88455700 & 1.95347500 & 5.21694100 & $\mathrm{C}$ & -2.21896700 & -0.49945800 & 5.05099400 \\
\hline $\mathrm{H}$ & -3.17250700 & -0.26144800 & 5.53606200 & $\mathrm{H}$ & -1.44584000 & -0.47552100 & 5.82833600 \\
\hline $\mathrm{H}$ & -2.29472100 & -1.52436200 & 4.66765200 & C & -0.59973600 & -1.17684800 & 2.42068900 \\
\hline 0 & -1.08807700 & -1.20037600 & 1.27810900 & $\mathrm{C}$ & 0.52206100 & -2.46646300 & 4.31599500 \\
\hline $\mathrm{C}$ & -0.01826600 & -3.56609600 & 2.23816900 & $\mathrm{C}$ & 0.01093800 & -3.85814900 & 4.70178800 \\
\hline $\mathrm{H}$ & 1.61718100 & -2.43789700 & 4.26399600 & $\mathrm{H}$ & 0.19595100 & -1.68757000 & 4.99952800 \\
\hline $\mathrm{H}$ & -0.94785300 & -3.62876600 & 1.66657200 & $\mathrm{H}$ & -1.01194600 & -3.77682800 & 5.08707300 \\
\hline $\mathrm{H}$ & 0.62427500 & -4.32492800 & 5.47821500 & $\mathrm{~N}$ & -0.04383100 & -2.28441400 & 2.97068900 \\
\hline C & 1.17333500 & -3.69352300 & 1.29063600 & C & 2.21421300 & -2.81279400 & 1.33971500 \\
\hline C & 1.22329000 & -4.82012500 & 0.37393400 & C & 3.39159100 & -2.96494600 & 0.53276900 \\
\hline $\mathrm{H}$ & 2.17286900 & -1.95135100 & 2.00060700 & C & 2.38790800 & -4.99399200 & -0.44323500 \\
\hline C & 0.17851000 & -5.75331500 & 0.24639700 & C & 4.45967000 & -2.05443200 & 0.59900200 \\
\hline C & 3.47397700 & -4.07203000 & -0.36025200 & C & 2.47107900 & -6.09667900 & -1.34964900 \\
\hline $\mathrm{H}$ & -0.71836100 & -5.64987800 & 0.84767300 & $\mathrm{C}$ & 0.26662900 & -6.82263600 & -0.64543700 \\
\hline C & 5.58836600 & -2.22326200 & -0.20302300 & $\mathrm{H}$ & 4.39588300 & -1.21144100 & 1.28206500 \\
\hline C & 4.63736400 & -4.24415600 & -1.17119000 & C & 1.39856300 & -6.99865500 & -1.43398800 \\
\hline C & 3.65582100 & -6.24893300 & -2.15010400 & $\mathrm{H}$ & -0.55846500 & -7.52623600 & -0.71958400 \\
\hline C & 5.67944100 & -3.30646800 & -1.07529500 & $\mathrm{H}$ & 6.39045700 & -1.49367700 & -0.15246500 \\
\hline
\end{tabular}




\begin{tabular}{|c|c|c|c|c|c|c|c|}
\hline $\mathrm{C}$ & 4.68978600 & -5.36750200 & -2.06513700 & $\mathrm{H}$ & 1.46277300 & -7.83708700 & -2.12322900 \\
\hline $\mathrm{H}$ & 3.70786200 & -7.09338100 & -2.83319800 & $\mathrm{H}$ & 6.56220700 & -3.43249700 & -1.69759800 \\
\hline $\mathrm{H}$ & 5.57730500 & -5.49883400 & -2.67952200 & $\mathrm{C}$ & 1.17389400 & 1.54923800 & 2.17445400 \\
\hline $\mathrm{S}$ & 2.42308900 & 0.85543500 & 3.09271200 & $\mathrm{~N}$ & -0.13423200 & 1.19602100 & 2.30922500 \\
\hline $\mathrm{H}$ & -0.78536500 & 1.48020600 & 1.57096100 & $S$ & -2.00932900 & 3.63735000 & -0.09393900 \\
\hline $\mathrm{O}$ & -2.37910200 & 3.97362600 & -1.47965500 & 0 & -2.06218200 & 2.17761200 & 0.23279100 \\
\hline $\mathrm{O}$ & -0.80144600 & 4.30525400 & 0.45364700 & $\mathrm{C}$ & -3.39933600 & 4.34224700 & 0.92287500 \\
\hline $\mathrm{F}$ & -3.47017800 & 5.66820100 & 0.79570000 & $\mathrm{~F}$ & -3.24548000 & 4.04213400 & 2.22290900 \\
\hline $\mathrm{F}$ & -4.57624900 & 3.81425800 & 0.51622500 & $\mathrm{C}$ & 0.03139300 & -4.64446000 & 3.37391100 \\
\hline $\mathrm{H}$ & 0.94872700 & -5.23197900 & 3.28354600 & $\mathrm{H}$ & -0.80830100 & -5.34181600 & 3.30170900 \\
\hline C & -0.60056500 & -1.73382000 & -2.58104800 & 0 & -0.81901300 & -2.08070200 & -3.72807600 \\
\hline $\mathrm{O}$ & 0.45234000 & -1.09974300 & -2.09322200 & $C$ & 1.58403300 & -0.67567000 & -2.97949100 \\
\hline C & 1.06489000 & 0.28526600 & -4.05113100 & $\mathrm{H}$ & 1.91893200 & 0.68640600 & -4.60746300 \\
\hline $\mathrm{H}$ & 0.39885000 & -0.21593700 & -4.75589100 & $\mathrm{H}$ & 0.53987800 & 1.12954700 & -3.59219900 \\
\hline $\mathrm{C}$ & 2.50213500 & 0.04436300 & -1.99690800 & $\mathrm{H}$ & 2.81720300 & -0.63092500 & -1.19771000 \\
\hline $\mathrm{H}$ & 3.39759100 & 0.40721400 & -2.51013700 & $\mathrm{H}$ & 1.98986900 & 0.90102400 & -1.54946200 \\
\hline C & 2.25021700 & -1.91710300 & -3.57277200 & $\mathrm{H}$ & 3.14847400 & -1.61162200 & -4.12032600 \\
\hline $\mathrm{H}$ & 2.55665500 & -2.60566500 & -2.78007200 & $\mathrm{H}$ & 1.58294400 & -2.43729500 & -4.26293100 \\
\hline $\mathrm{C}$ & -2.73607900 & -2.52777900 & -1.88924100 & $\mathrm{H}$ & -2.65393900 & -3.11035000 & -2.80028300 \\
\hline $\mathrm{C}$ & -3.65712000 & -3.06498300 & -0.87278400 & $\mathrm{C}$ & -4.44459500 & -4.17489800 & -1.23149100 \\
\hline C & -3.79009800 & -2.51783200 & 0.41420000 & C & -5.33360500 & -4.73704600 & -0.32062700 \\
\hline $\mathrm{H}$ & -4.34841700 & -4.60091900 & -2.22732800 & C & -4.68590900 & -3.08384200 & 1.32123200 \\
\hline $\mathrm{H}$ & -3.18710700 & -1.66772700 & 0.71096200 & $C$ & -5.45724500 & -4.19030800 & 0.95984500 \\
\hline $\mathrm{H}$ & -5.92770000 & -5.59998400 & -0.60711000 & $\mathrm{H}$ & -4.78182800 & -2.65390800 & 2.31421400 \\
\hline $\bar{H}$ & -6.15348800 & -4.62491100 & 1.67166300 & $\mathrm{~N}$ & -1.54213000 & -1.96206900 & -1.55311600 \\
\hline $\mathrm{H}$ & -1.32536700 & -1.64170900 & -0.60070400 & C & -3.95351500 & -1.23232100 & -3.00964900 \\
\hline $\mathrm{H}$ & -3.40858000 & -1.37976000 & -3.93785500 & $\mathrm{H}$ & -4.87862900 & -1.79178900 & -2.90487600 \\
\hline $\mathrm{C}$ & -3.83188000 & 0.00282900 & -2.37328000 & $\mathrm{C}$ & -4.77031400 & 0.44967400 & -1.34323400 \\
\hline $\bar{H}$ & -5.27190700 & -0.39383600 & -0.85257100 & $\mathrm{H}$ & -4.27270000 & 1.08488300 & -0.60029400 \\
\hline C & -2.64936500 & 0.85620700 & -2.67130700 & $\mathrm{H}$ & -2.17276900 & 0.59409100 & -3.61852300 \\
\hline $\bar{H}$ & -2.87999000 & 1.92527100 & -2.63534700 & $\mathrm{H}$ & -1.92188200 & 0.71986000 & -1.85411600 \\
\hline $\mathrm{C}$ & -7.27319600 & 1.99246300 & -0.52573900 & $\mathrm{H}$ & -6.69098100 & 2.58975200 & 0.18297200 \\
\hline $\mathrm{H}$ & -8.14703900 & 2.58534100 & -0.82289500 & $\mathrm{H}$ & -7.64039600 & 1.10234200 & -0.00124300 \\
\hline $\mathrm{C}$ & -5.58480900 & 3.05817600 & -2.92705500 & $\bar{H}$ & -4.85726100 & 3.60371700 & -2.31657100 \\
\hline $\bar{H}$ & -5.09764400 & 2.80004800 & -3.87463500 & $\bar{H}$ & -6.41076600 & 3.74184900 & -3.16058300 \\
\hline C & -7.26315000 & 0.45737000 & -3.21852000 & $\mathrm{H}$ & -6.68009200 & 0.13665600 & -4.08949000 \\
\hline $\mathrm{H}$ & -7.64866500 & -0.44095800 & -2.72117500 & $\mathrm{H}$ & -8.12742800 & 1.01977500 & -3.59290100 \\
\hline $\mathrm{Si}$ & -6.24524700 & 1.53409500 & -2.04184300 & & & & \\
\hline
\end{tabular}

\section{TS- $R-4 f-n d-20$}

Imaginary frequency: $-230.44 \mathrm{~cm}^{-1}$ E[B3LYP/6-31G(d)]: -4788.680117 Hartree E[PCM(Et $\left.\left.{ }_{2} \mathrm{O}\right)-\mathrm{B} 3 \mathrm{LYP} / 6-311 \mathrm{G}(\mathrm{d}, \mathrm{p})-\mathrm{D} 3(\mathrm{BJ})\right]$ : -4790.215442 Hartree

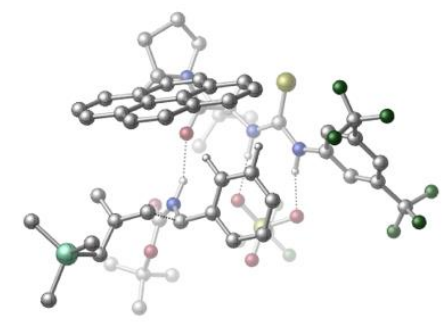

\begin{tabular}{|lrrr|rrrr|}
\hline O 1 & & & $\mathrm{N}$ & 3.34496100 & 0.36230200 & 0.70132300 \\
\hline $\mathrm{H}$ & 3.15059000 & 1.31539300 & 0.36825900 & $\mathrm{C}$ & 4.20223700 & -0.34352500 & -0.16068600 \\
\hline $\mathrm{C}$ & 5.07021000 & 0.41258400 & -0.96556500 & $\mathrm{C}$ & 4.16258500 & -1.73373400 & -0.32836900 \\
\hline $\mathrm{C}$ & 5.88713700 & -0.21847200 & -1.90216300 & $\mathrm{H}$ & 5.07692300 & 1.49280100 & -0.87546000 \\
\hline $\mathrm{C}$ & 4.97117500 & -2.34456500 & -1.28475700 & $\mathrm{H}$ & 3.49677000 & -2.32743500 & 0.27889000 \\
\hline $\mathrm{C}$ & 5.84834900 & -1.60257700 & -2.07519600 & $\mathrm{H}$ & 6.47545800 & -2.08625600 & -2.81436700 \\
\hline $\mathrm{C}$ & 6.86964000 & 0.59476200 & -2.70332600 & $\mathrm{C}$ & 4.77970800 & -3.80950400 & -1.55695900 \\
\hline
\end{tabular}




\begin{tabular}{|c|c|c|c|c|c|c|c|}
\hline $\mathrm{F}$ & 8.08164900 & 0.64432700 & -2.10075000 & $\mathrm{~F}$ & 6.46078300 & 1.86724600 & -2.87363500 \\
\hline $\mathrm{F}$ & 7.07066800 & 0.06300500 & -3.93297100 & $\mathrm{~F}$ & 4.42220300 & -4.50320000 & -0.45009200 \\
\hline $\mathrm{F}$ & 3.78237100 & -4.01241100 & -2.46547500 & $\mathrm{~F}$ & 5.88565500 & -4.39296700 & -2.06251000 \\
\hline C & 1.06488800 & 0.66333400 & 3.55172300 & $\mathrm{H}$ & 1.67056600 & 0.00882400 & 4.17867900 \\
\hline C & 0.86333900 & 2.00405800 & 4.34899700 & C & -0.09141800 & 2.99447900 & 3.66087200 \\
\hline $\mathrm{H}$ & -1.09686700 & 2.58493000 & 3.53913000 & $\mathrm{H}$ & 0.25800000 & 3.29545500 & 2.67120900 \\
\hline $\mathrm{H}$ & -0.16996700 & 3.90100700 & 4.27424500 & C & 2.24571300 & 2.66554700 & 4.52718500 \\
\hline $\mathrm{H}$ & 2.15836300 & 3.52460600 & 5.20308700 & $\mathrm{H}$ & 2.64212900 & 3.03086900 & 3.57598500 \\
\hline $\mathrm{H}$ & 2.97574100 & 1.96925900 & 4.95764600 & C & 0.29764700 & 1.66430500 & 5.74331300 \\
\hline $\mathrm{H}$ & 0.15667800 & 2.58586600 & 6.31918400 & $\mathrm{H}$ & 0.97841400 & 1.02045400 & 6.31363900 \\
\hline $\mathrm{H}$ & -0.67919000 & 1.16952600 & 5.67964900 & C & -0.24686600 & -0.06705700 & 3.18305400 \\
\hline 0 & -0.94188700 & 0.30156400 & 2.22215900 & C & 0.07574700 & -1.75836200 & 5.06728900 \\
\hline C & -1.87050000 & -1.85565600 & 3.56940700 & C & -0.75456800 & -3.01609700 & 5.37017100 \\
\hline $\mathrm{H}$ & 1.11578200 & -1.98385500 & 4.81354800 & $\mathrm{H}$ & 0.06259400 & -1.07006800 & 5.91809100 \\
\hline C & -2.15907700 & -2.65505600 & 4.86251100 & $\mathrm{H}$ & -2.63205700 & -1.09655700 & 3.37741400 \\
\hline $\mathrm{H}$ & -0.73606200 & -3.27157600 & 6.43350800 & $\mathrm{H}$ & -0.36279500 & -3.87344100 & 4.81324500 \\
\hline $\mathrm{H}$ & -2.67158500 & -2.00798800 & 5.58529500 & $\mathrm{H}$ & -2.78971800 & -3.52919100 & 4.68037800 \\
\hline $\mathrm{N}$ & -0.61957400 & -1.15270700 & 3.90940100 & C & -1.74560600 & -2.75624800 & 2.34141400 \\
\hline C & -0.52738100 & -3.21228300 & 1.92438200 & C & -2.94555700 & -3.16437100 & 1.62925800 \\
\hline C & -0.37198700 & -4.09116800 & 0.80109000 & $\mathrm{H}$ & 0.37776100 & -2.88124100 & 2.42667900 \\
\hline C & -2.81532600 & -4.02247500 & 0.48684900 & C & -4.24003000 & -2.75488800 & 2.00499000 \\
\hline $\mathrm{C}$ & 0.88956200 & -4.55330600 & 0.38859400 & $\mathrm{C}$ & -1.53092900 & -4.48744000 & 0.07424400 \\
\hline C & -3.97593700 & -4.42578700 & -0.24681000 & $\mathrm{H}$ & -4.37917100 & -2.12839200 & 2.87925600 \\
\hline C & -5.36685200 & -3.15845200 & 1.28545400 & C & 1.01416700 & -5.38061800 & -0.72716200 \\
\hline $\mathrm{H}$ & 1.77307600 & -4.25100500 & 0.94503900 & $\mathrm{C}$ & -1.39660800 & -5.34212100 & -1.06190400 \\
\hline C & -5.24139200 & -3.97996900 & 0.16846300 & $\mathrm{C}$ & -3.81173800 & -5.28721100 & -1.38672100 \\
\hline $\mathrm{H}$ & -6.35294100 & -2.84016400 & 1.61509900 & C & -0.11535300 & -5.77260300 & -1.44410000 \\
\hline $\mathrm{H}$ & 2.00093900 & -5.70220500 & -1.04450700 & $\mathrm{C}$ & -2.58260100 & -5.72441900 & -1.77601100 \\
\hline $\mathrm{H}$ & -6.12404000 & -4.30036000 & -0.38016000 & $\mathrm{H}$ & -4.70183400 & -5.59034900 & -1.93316300 \\
\hline $\mathrm{H}$ & -0.01174700 & -6.41673800 & -2.31388100 & $\mathrm{H}$ & -2.47877000 & -6.37729100 & -2.63935200 \\
\hline C & 2.74941100 & -0.03621600 & 1.87079300 & $\mathrm{~S}$ & 3.15513700 & -1.46514500 & 2.70781500 \\
\hline $\mathrm{N}$ & 1.82921800 & 0.85122000 & 2.32518200 & $\mathrm{H}$ & 1.50629300 & 1.58486100 & 1.68194900 \\
\hline S & 1.74283100 & 3.55270800 & -0.52243400 & $\mathrm{O}$ & 1.04394200 & 3.98835500 & -1.74335300 \\
\hline 0 & 0.88105600 & 2.83755400 & 0.46657000 & $\mathrm{O}$ & 3.06423700 & 2.89589000 & -0.70404900 \\
\hline C & 2.17150300 & 5.13061000 & 0.36678400 & $\mathrm{~F}$ & 2.97353500 & 5.89902200 & -0.37916500 \\
\hline $\mathrm{F}$ & 2.80147300 & 4.85693900 & 1.52371900 & $\mathrm{~F}$ & 1.06118400 & 5.83167100 & 0.65580700 \\
\hline C & -2.37340800 & 3.13669100 & 0.27204500 & $\mathrm{O}$ & -2.34665100 & 3.59694000 & 1.39224200 \\
\hline 0 & -2.91728000 & 3.70939400 & -0.82096200 & C & -3.22735700 & 5.16224100 & -0.83814300 \\
\hline C & -4.38712700 & 5.45274800 & 0.11799200 & $\mathrm{H}$ & -4.67907000 & 6.50497200 & 0.02772500 \\
\hline $\mathrm{H}$ & -4.10046400 & 5.25501500 & 1.15167400 & $\mathrm{H}$ & -5.25972800 & 4.83815000 & -0.13422600 \\
\hline C & -3.64198100 & 5.39100200 & -2.29294100 & $\mathrm{H}$ & -2.81748100 & 5.15192600 & -2.97187800 \\
\hline $\mathrm{H}$ & -3.91570100 & 6.44061100 & -2.44053300 & $\mathrm{H}$ & -4.50672900 & 4.77149300 & -2.55702900 \\
\hline C & -1.96404800 & 5.95651600 & -0.50113200 & $\mathrm{H}$ & -2.15353600 & 7.02239900 & -0.67176000 \\
\hline $\mathrm{H}$ & -1.13132100 & 5.64309600 & -1.13813800 & $\mathrm{H}$ & -1.67046500 & 5.81836100 & 0.54018100 \\
\hline C & -1.82400700 & 1.34942200 & -1.31458700 & $\mathrm{H}$ & -2.08758400 & 2.10659000 & -2.04466900 \\
\hline C & -0.64576100 & 0.53285100 & -1.73926400 & C & -0.05932300 & 0.83538700 & -2.97712700 \\
\hline C & -0.15933600 & -0.54517500 & -0.98726700 & C & 1.00263400 & 0.07074200 & -3.45850100 \\
\hline $\mathrm{H}$ & -0.41233600 & 1.69036300 & -3.54688200 & C & 0.88848000 & -1.31530100 & -1.48299500 \\
\hline $\mathrm{H}$ & -0.59277100 & -0.78277800 & -0.01969100 & C & 1.46881700 & -1.01593000 & -2.71881500 \\
\hline $\mathrm{H}$ & 1.46390700 & 0.32809400 & -4.40741700 & $\mathrm{H}$ & 1.24903000 & -2.15994200 & -0.90797800 \\
\hline $\mathrm{H}$ & 2.28388800 & -1.63022000 & -3.09003400 & $\mathrm{~N}$ & -1.86949600 & 1.86809900 & -0.03075700 \\
\hline $\mathrm{H}$ & -1.33377300 & 1.43726800 & 0.73242900 & C & -3.19549500 & 0.10937100 & -1.58871700 \\
\hline $\mathrm{H}$ & -2.88483000 & -0.65409000 & -0.87855500 & $\mathrm{H}$ & -3.00870700 & -0.15923800 & -2.62618500 \\
\hline
\end{tabular}




\begin{tabular}{|c|c|c|c|c|c|c|c|}
\hline C & -4.42951000 & 0.73894500 & -1.31627600 & $\mathrm{C}$ & -5.22999900 & 1.34897300 & -2.36926500 \\
\hline $\mathrm{H}$ & -4.63350800 & 1.60583900 & -3.25242100 & $\mathrm{H}$ & -5.78166800 & 2.22952800 & -2.02108700 \\
\hline C & -4.87324700 & 0.80827400 & 0.10200000 & $\mathrm{H}$ & -5.79113300 & 1.38223300 & 0.24468700 \\
\hline $\mathrm{H}$ & -4.06037300 & 1.25884400 & 0.69599000 & $\mathrm{H}$ & -4.99578900 & -0.20144900 & 0.51629300 \\
\hline C & -7.53643600 & 1.12582500 & -4.35181500 & $\mathrm{H}$ & -8.01388900 & 2.00805400 & -3.91009800 \\
\hline $\mathrm{H}$ & -8.32571900 & 0.51848700 & -4.81128800 & $\mathrm{H}$ & -6.87428300 & 1.46842700 & -5.15521400 \\
\hline C & -7.74394800 & -0.41587600 & -1.65535000 & $\mathrm{H}$ & -8.18933500 & 0.44052300 & -1.13591600 \\
\hline $\mathrm{H}$ & -7.22264900 & -1.03078000 & -0.91299900 & $\mathrm{H}$ & -8.56855800 & -1.01806300 & -2.05648600 \\
\hline C & -5.74381800 & -1.37228400 & -3.83219900 & $\mathrm{H}$ & -5.19822600 & -1.96526500 & -3.08944000 \\
\hline $\mathrm{H}$ & -5.03572500 & -1.07772800 & -4.61562500 & $\mathrm{H}$ & -6.48662900 & -2.03234000 & -4.29700400 \\
\hline $\mathrm{Si}$ & -6.60221400 & 0.11704900 & -3.05932100 & & & & \\
\hline
\end{tabular}




\section{TS- $R-4 f-n d-21$}

Imaginary frequency: $-255.83 \mathrm{~cm}^{-1}$ E[B3LYP/6-31G(d)]: -4788.684738 Hartree

E[PCM(Et $\left.\left.{ }_{2} \mathrm{O}\right)-B 3 L Y P / 6-311 G(d, p)-D 3(B J)\right]:-4790.215368$ Hartree

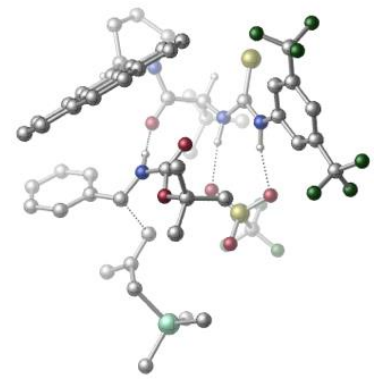

\begin{tabular}{|c|c|c|c|c|c|c|c|}
\hline 01 & & & & $\mathrm{~N}$ & -2.34211300 & -1.45331200 & 1.54859100 \\
\hline $\mathrm{H}$ & -1.50561800 & -2.04283400 & 1.51723700 & $\mathrm{C}$ & -3.35280600 & -1.89473100 & 0.68054300 \\
\hline C & -3.21529800 & -3.19639100 & 0.16507200 & $\mathrm{C}$ & -4.42234100 & -1.10355300 & 0.23684100 \\
\hline C & -4.12110400 & -3.68031800 & -0.77540200 & $\mathrm{H}$ & -2.38353500 & -3.81333600 & 0.48767100 \\
\hline C & -5.32681100 & -1.61411300 & -0.69416800 & $\mathrm{H}$ & -4.53736500 & -0.09898500 & 0.61360900 \\
\hline $\mathrm{C}$ & -5.19279300 & -2.90118500 & -1.21217700 & $\mathrm{H}$ & -5.89735600 & -3.28470600 & -1.93925900 \\
\hline C & -3.88266500 & -5.03074400 & -1.39361100 & $\mathrm{C}$ & -6.49080000 & -0.75734700 & -1.11428100 \\
\hline $\mathrm{F}$ & -3.31566300 & -5.89718500 & -0.52987500 & $\mathrm{~F}$ & -3.04936900 & -4.94498300 & -2.46473100 \\
\hline $\mathrm{F}$ & -5.02797200 & -5.59280200 & -1.84242900 & $\mathrm{~F}$ & -6.13908000 & 0.54865700 & -1.24873200 \\
\hline $\mathrm{F}$ & -7.00924100 & -1.14914300 & -2.30003100 & $\mathrm{~F}$ & -7.49749100 & -0.79069900 & -0.21351000 \\
\hline C & -0.66440300 & 1.04379600 & 3.63715200 & $\mathrm{H}$ & -1.60622300 & 1.33359700 & 4.10159400 \\
\hline C & 0.31727100 & 0.65026100 & 4.79669200 & C & 1.73670000 & 0.28204200 & 4.32306000 \\
\hline $\mathrm{H}$ & 2.24541400 & 1.13758700 & 3.86988100 & $\mathrm{H}$ & 1.72949200 & -0.53225700 & 3.59508700 \\
\hline $\mathrm{H}$ & 2.32793700 & -0.04603100 & 5.18735800 & $\mathrm{C}$ & -0.29215200 & -0.55300800 & 5.54505600 \\
\hline $\mathrm{H}$ & 0.29613300 & -0.76834400 & 6.44511100 & $\mathrm{H}$ & -0.29247000 & -1.45156900 & 4.92228600 \\
\hline $\mathrm{H}$ & -1.32503700 & -0.35385100 & 5.85396800 & $\mathrm{C}$ & 0.41666200 & 1.84265900 & 5.77109100 \\
\hline $\mathrm{H}$ & 1.10988100 & 1.60399200 & 6.58577600 & $\mathrm{H}$ & -0.55366100 & 2.07618800 & 6.22643100 \\
\hline $\mathrm{H}$ & 0.79431600 & 2.74738400 & 5.27766900 & C & -0.15918400 & 2.19358600 & 2.73398900 \\
\hline $\mathrm{O}$ & 0.81365400 & 2.02050300 & 1.97939900 & C & -1.84351500 & 3.80434400 & 3.72916600 \\
\hline $\mathrm{C}$ & -0.40249900 & 4.49074000 & 1.85559900 & $C$ & -1.72595200 & 5.33271200 & 3.71542800 \\
\hline $\mathrm{H}$ & -2.83878100 & 3.46230600 & 3.42332800 & $\mathrm{H}$ & -1.63508100 & 3.38497400 & 4.71280900 \\
\hline $\mathrm{H}$ & 0.62380800 & 4.75191800 & 2.14191900 & $\mathrm{H}$ & -0.92422900 & 5.65289400 & 4.39228000 \\
\hline $\mathrm{H}$ & -2.65164700 & 5.81871100 & 4.03732200 & $\mathrm{~N}$ & -0.82509000 & 3.37253500 & 2.74469100 \\
\hline C & -0.37913400 & 4.24804600 & 0.35001700 & C & 0.72879700 & 4.68524200 & -0.32072800 \\
\hline $\mathrm{C}$ & -1.50742200 & 3.72420300 & -0.40476100 & C & 0.83845400 & 4.67620400 & -1.74936600 \\
\hline $\mathrm{H}$ & 1.57583100 & 5.07093800 & 0.24259100 & C & -1.42925300 & 3.71402100 & -1.83771000 \\
\hline C & -2.67646400 & 3.22415900 & 0.19399800 & C & 1.98550300 & 5.13737500 & -2.41609600 \\
\hline C & -0.26455100 & 4.19115700 & -2.51020000 & C & -2.52669600 & 3.22110500 & -2.61191300 \\
\hline $\mathrm{H}$ & -2.75772000 & 3.16919400 & 1.27023700 & C & -3.73647600 & 2.73934300 & -0.57007100 \\
\hline C & 2.04874800 & 5.12840100 & -3.80853200 & $\mathrm{H}$ & 2.82679500 & 5.50219700 & -1.83214700 \\
\hline C & -0.19076200 & 4.18517400 & -3.93779000 & C & -3.66970900 & 2.73703200 & -1.95800700 \\
\hline C & -2.42805600 & 3.23214000 & -4.04604000 & $\mathrm{H}$ & -4.61683000 & 2.34828100 & -0.07112800 \\
\hline C & 0.97450200 & 4.65940400 & -4.56271300 & $\mathrm{H}$ & 2.94106400 & 5.49352700 & -4.31062600 \\
\hline C & -1.31604100 & 3.69227700 & -4.68204100 & $\mathrm{H}$ & -4.49786000 & 2.34395400 & -2.54160200 \\
\hline $\mathrm{H}$ & -3.27462200 & 2.86031800 & -4.61834900 & $\mathrm{H}$ & 1.02898000 & 4.65829400 & -5.64886100 \\
\hline $\mathrm{H}$ & -1.26178000 & 3.69585600 & -5.76817400 & C & -2.28389200 & -0.35986700 & 2.37668500 \\
\hline$S$ & -3.64313300 & 0.49147200 & 2.93317300 & $\mathrm{~N}$ & -1.00932800 & -0.07151000 & 2.76442600 \\
\hline $\mathrm{H}$ & -0.23994300 & -0.51556200 & 2.25587000 & $S$ & 1.13945900 & -3.02764100 & 1.27597300 \\
\hline $\mathrm{O}$ & 1.94090800 & -3.52458700 & 0.13638100 & $\mathrm{O}$ & 1.23838300 & -1.55513800 & 1.49708600 \\
\hline 0 & -0.24684800 & -3.54177800 & 1.37506000 & C & 1.99466200 & -3.74491000 & 2.76534700 \\
\hline $\mathrm{F}$ & 1.98777000 & -5.08005400 & 2.72639500 & $\mathrm{~F}$ & 1.39242100 & -3.34311900 & 3.89544700 \\
\hline $\mathrm{F}$ & 3.27586900 & -3.32585000 & 2.80684400 & C & -1.35536500 & 5.64148500 & 2.26098300 \\
\hline $\mathrm{H}$ & -2.24772700 & 5.62454800 & 1.62723900 & $\mathrm{H}$ & -0.87322100 & 6.61389900 & 2.12740600 \\
\hline C & 0.59916100 & 0.16497100 & -1.17348900 & $\mathrm{O}$ & -0.53431700 & 0.20333600 & -0.75254200 \\
\hline
\end{tabular}




\begin{tabular}{|c|c|c|c|c|c|c|c|}
\hline $\mathrm{O}$ & 1.06701400 & -0.50056600 & -2.23626900 & C & 0.15120700 & -1.28524200 & -3.11872900 \\
\hline C & -0.86918200 & -0.33738500 & -3.75058500 & $\mathrm{H}$ & -1.46733400 & -0.89221000 & -4.48195000 \\
\hline $\mathrm{H}$ & -1.54178800 & 0.07978800 & -2.99956000 & $\mathrm{H}$ & -0.36885400 & 0.48545700 & -4.27222600 \\
\hline $\mathrm{C}$ & 1.11384400 & -1.83956100 & -4.16955900 & $\mathrm{H}$ & 1.85944400 & -2.49368900 & -3.70535200 \\
\hline $\mathrm{H}$ & 0.55632500 & -2.42839200 & -4.90484700 & $\mathrm{H}$ & 1.62905800 & -1.02914400 & -4.69681000 \\
\hline C & -0.49961000 & -2.41211900 & -2.31855000 & $\mathrm{H}$ & -1.06620100 & -3.06276100 & -2.99327200 \\
\hline $\mathrm{H}$ & 0.25832800 & -3.01564900 & -1.81065500 & $\mathrm{H}$ & -1.18949500 & -2.01636800 & -1.57315800 \\
\hline C & 2.93089900 & 0.91208900 & -0.94954300 & $\mathrm{H}$ & 3.04775200 & 0.59416300 & -1.97919200 \\
\hline C & 3.80633800 & 2.03841000 & -0.53720000 & C & 4.74402000 & 2.51740300 & -1.46665700 \\
\hline C & 3.72493700 & 2.64308500 & 0.72750200 & C & 5.58274300 & 3.58486000 & -1.14482300 \\
\hline $\mathrm{H}$ & 4.80143400 & 2.06430200 & -2.45353200 & C & 4.57141700 & 3.70365000 & 1.04631200 \\
\hline $\mathrm{H}$ & 2.99368800 & 2.30296500 & 1.45370200 & $\mathrm{C}$ & 5.50073500 & 4.17799000 & 0.11596100 \\
\hline $\mathrm{H}$ & 6.29455100 & 3.95240000 & -1.87861100 & $\mathrm{H}$ & 4.50281600 & 4.16392600 & 2.02807600 \\
\hline $\mathrm{H}$ & 6.15574200 & 5.00593200 & 0.37259800 & $\mathrm{~N}$ & 1.62599400 & 0.90484300 & -0.55272300 \\
\hline $\mathrm{H}$ & 1.32931100 & 1.32886000 & 0.33619800 & C & 3.72861800 & -0.73748900 & -0.11579700 \\
\hline $\mathrm{H}$ & 3.68210300 & -0.41619500 & 0.92062300 & $\mathrm{H}$ & 2.92215700 & -1.39863900 & -0.41588800 \\
\hline C & 4.97904400 & -0.95252200 & -0.69223800 & C & 5.12046500 & -1.73852600 & -1.92094900 \\
\hline $\mathrm{H}$ & 4.18929800 & -1.76251500 & -2.49677200 & $\mathrm{H}$ & 5.94078200 & -1.37653700 & -2.55230400 \\
\hline C & 6.21127400 & -0.33717200 & -0.10341700 & $\mathrm{H}$ & 6.07612300 & -0.05392300 & 0.94279700 \\
\hline $\mathrm{H}$ & 7.07295700 & -1.00780600 & -0.19106800 & $\mathrm{H}$ & 6.46105300 & 0.57498400 & -0.66662800 \\
\hline C & 7.14171400 & -3.99189600 & -2.51103700 & $\mathrm{H}$ & 7.97410900 & -3.38146000 & -2.14008300 \\
\hline $\mathrm{H}$ & 7.43204100 & -5.04390000 & -2.40042900 & $\mathrm{H}$ & 7.03136200 & -3.79198700 & -3.58361700 \\
\hline C & 5.76861900 & -3.97191100 & 0.27302000 & $\mathrm{H}$ & 6.58925000 & -3.39063000 & 0.70945300 \\
\hline $\mathrm{H}$ & 4.85458000 & -3.75977900 & 0.83647600 & $\mathrm{H}$ & 6.00710100 & -5.03224800 & 0.42433900 \\
\hline $\mathrm{C}$ & 4.10533300 & -4.65695000 & -2.25658300 & $\mathrm{H}$ & 3.19025200 & -4.45881900 & -1.68772900 \\
\hline $\mathrm{H}$ & 3.92034200 & -4.43669500 & -3.31497100 & $\mathrm{H}$ & 4.32261900 & -5.72939500 & -2.17571400 \\
\hline $\mathrm{Si}$ & 5.53619700 & -3.64845800 & -1.56867500 & & & & \\
\hline
\end{tabular}

\section{TS-R-4f-nd-22}

Imaginary frequency: $-282.73 \mathrm{~cm}^{-1}$ E[B3LYP/6-31G(d)]: -4788.688161 Hartree $\mathrm{E}\left[\mathrm{PCM}\left(\mathrm{Et}_{2} \mathrm{O}\right)-\mathrm{B} 3 \mathrm{LYP} / 6-311 \mathrm{G}(\mathrm{d}, \mathrm{p})-\mathrm{D} 3(\mathrm{BJ})\right]$ : -4790.215072 Hartree

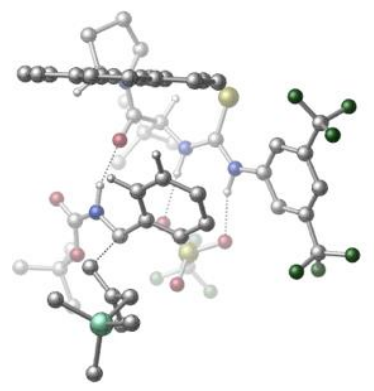

\begin{tabular}{|crrr|crcc|}
\hline 01 & & & & $\mathrm{~N}$ & -0.18080100 & 2.42382400 & -1.92877500 \\
\hline $\mathrm{H}$ & 0.83333900 & 2.34357500 & -1.81045800 & $\mathrm{C}$ & -0.72659200 & 3.48662500 & -1.18880900 \\
\hline $\mathrm{C}$ & 0.19392000 & 4.32170300 & -0.52817200 & $\mathrm{C}$ & -2.09804000 & 3.72788500 & -1.00638200 \\
\hline $\mathrm{C}$ & -0.25221500 & 5.33902500 & 0.31128000 & $\mathrm{H}$ & 1.25773200 & 4.15032100 & -0.65334600 \\
\hline $\mathrm{C}$ & -2.52052400 & 4.75985400 & -0.16908900 & $\mathrm{H}$ & -2.82219700 & 3.11187100 & -1.51580000 \\
\hline $\mathrm{C}$ & -1.61322300 & 5.57522300 & 0.50473100 & $\mathrm{H}$ & -1.95281700 & 6.36552200 & 1.16278900 \\
\hline $\mathrm{C}$ & 0.75814700 & 6.10818300 & 1.11589800 & $\mathrm{C}$ & -3.99560500 & 5.02350400 & -0.02244300 \\
\hline $\mathrm{F}$ & 1.90880300 & 6.30887500 & 0.44548300 & $\mathrm{~F}$ & 1.08361200 & 5.43480100 & 2.25652600 \\
\hline $\mathrm{F}$ & 0.29283400 & 7.31566700 & 1.50378700 & $\mathrm{~F}$ & -4.44157900 & 5.93018800 & -0.91897000 \\
\hline $\mathrm{F}$ & -4.73716800 & 3.90105200 & -0.20293900 & $\mathrm{~F}$ & -4.29943800 & 5.50503200 & 1.20572900 \\
\hline $\mathrm{C}$ & -0.37177400 & -0.75822300 & -3.74680700 & $\mathrm{H}$ & -1.16245400 & -0.39903200 & -4.40489600 \\
\hline $\mathrm{C}$ & 0.77300900 & -1.31122500 & -4.67119500 & $\mathrm{C}$ & 1.89209900 & -2.03178000 & -3.90124500 \\
\hline $\mathrm{H}$ & 1.52491300 & -2.90775200 & -3.36149300 & $\mathrm{H}$ & 2.38205500 & -1.37340000 & -3.18090100 \\
\hline $\mathrm{H}$ & 2.65583600 & -2.36921400 & -4.61367300 & $\mathrm{C}$ & 1.37245100 & -0.12771900 & -5.45922400 \\
\hline $\mathrm{H}$ & 2.06139900 & -0.50590700 & -6.22404200 & $\mathrm{H}$ & 1.93701500 & 0.54660000 & -4.80897200 \\
\hline $\mathrm{H}$ & 0.59473000 & 0.45715900 & -5.96503500 & $\mathrm{C}$ & 0.15560900 & -2.30653900 & -5.67498600 \\
\hline $\mathrm{H}$ & 0.93844200 & -2.69999300 & -6.33324800 & $\mathrm{H}$ & -0.60001700 & -1.82864700 & -6.31019800 \\
\hline
\end{tabular}




\begin{tabular}{|c|c|c|c|c|c|c|c|}
\hline$\overline{\mathrm{H}}$ & -0.30395200 & -3.16390200 & -5.16930100 & C & -0.96433900 & -1.79602900 & -2.75840400 \\
\hline 0 & -0.37116600 & -2.07901800 & -1.71011500 & C & -3.01453300 & -2.11419800 & -4.24210800 \\
\hline C & -2.74320900 & -3.37501400 & -2.15210400 & C & -4.25726300 & -2.98199900 & -3.98777800 \\
\hline $\mathrm{H}$ & -3.24826800 & -1.04914700 & -4.32935500 & $\mathrm{H}$ & -2.49918300 & -2.43539400 & -5.15212700 \\
\hline C & -3.73164400 & -4.11078600 & -3.08911200 & $\mathrm{H}$ & -1.93060200 & -4.02421700 & -1.81611700 \\
\hline $\mathrm{H}$ & -4.69926200 & -3.34518900 & -4.92011500 & $\mathrm{H}$ & -5.02102700 & -2.40541300 & -3.45605200 \\
\hline $\mathrm{H}$ & -3.18597600 & -4.85297300 & -3.68521100 & $\mathrm{H}$ & -4.52274400 & -4.62736900 & -2.54067300 \\
\hline $\mathrm{N}$ & -2.16335400 & -2.36385200 & -3.05627500 & C & -3.44640700 & -2.77938100 & -0.93296100 \\
\hline C & -3.75938200 & -1.45235900 & -0.87208200 & C & -3.83102000 & -3.65943100 & 0.15948300 \\
\hline C & -4.47260900 & -0.87558900 & 0.23181800 & $\mathrm{H}$ & -3.45073300 & -0.78365300 & -1.67073600 \\
\hline C & -4.54918200 & -3.10957900 & 1.27170800 & C & -3.52962900 & -5.03382400 & 0.18128800 \\
\hline C & -4.77351300 & 0.49529200 & 0.28623700 & C & -4.87238200 & -1.71982200 & 1.30808800 \\
\hline C & -4.94555400 & -3.95049400 & 2.35845400 & $\mathrm{H}$ & -2.98429600 & -5.48368800 & -0.64105000 \\
\hline C & -3.91786300 & -5.84516500 & 1.24763100 & C & -5.45838000 & 1.03023500 & 1.37701200 \\
\hline $\mathrm{H}$ & -4.45983100 & 1.13973000 & -0.52982500 & C & -5.57917700 & -1.16616900 & 2.41970800 \\
\hline C & -4.62054000 & -5.31562100 & 2.32475000 & C & -5.66656200 & -3.36910200 & 3.45847700 \\
\hline $\mathrm{H}$ & -3.66843300 & -6.90283800 & 1.23180800 & C & -5.86013200 & 0.21055300 & 2.43101700 \\
\hline $\mathrm{H}$ & -5.67305500 & 2.09437500 & 1.39910500 & C & -5.96901000 & -2.04221900 & 3.48950500 \\
\hline $\mathrm{H}$ & -4.92402800 & -5.95471800 & 3.15049500 & $\mathrm{H}$ & -5.96779100 & -4.02103700 & 4.27518500 \\
\hline $\mathrm{H}$ & -6.39694500 & 0.63326100 & 3.27714100 & $\mathrm{H}$ & -6.51406300 & -1.62074500 & 4.33093800 \\
\hline C & -0.77655500 & 1.45699000 & -2.70764200 & S & -2.35066100 & 1.56568300 & -3.34053400 \\
\hline $\mathrm{N}$ & 0.04477800 & 0.40706700 & -2.96959100 & $\mathrm{H}$ & 0.91207300 & 0.31705400 & -2.43458100 \\
\hline C & 2.80903100 & -3.06544900 & -0.47434200 & 0 & 2.52974000 & -3.95738400 & -1.24906600 \\
\hline 0 & 4.03951300 & -2.69544800 & -0.07385300 & C & 5.25611100 & -3.26987100 & -0.70106800 \\
\hline $\mathrm{C}$ & 5.23226600 & -3.03693400 & -2.21382400 & $\mathrm{H}$ & 6.19678700 & -3.34244000 & -2.63505300 \\
\hline $\mathrm{H}$ & 4.44236200 & -3.61514600 & -2.69416000 & $\mathrm{H}$ & 5.08405300 & -1.97615300 & -2.43208400 \\
\hline C & 6.37501300 & -2.45263100 & -0.05204600 & $\mathrm{H}$ & 6.38879600 & -2.60060000 & 1.03392000 \\
\hline $\mathrm{H}$ & 7.34482400 & -2.77011800 & -0.44884300 & $\mathrm{H}$ & 6.24299800 & -1.38718400 & -0.26279400 \\
\hline C & 5.35866700 & -4.75296300 & -0.33651800 & $\mathrm{H}$ & 6.30319800 & -5.15830800 & -0.71607300 \\
\hline $\mathrm{H}$ & 5.34751400 & -4.88566100 & 0.75158600 & $\mathrm{H}$ & 4.53450300 & -5.32086300 & -0.77192600 \\
\hline C & 2.08158400 & -1.23142100 & 1.00736900 & $\mathrm{H}$ & 2.98113700 & -0.68452000 & 0.74545300 \\
\hline $\mathrm{C}$ & 0.91678800 & -0.38691300 & 1.38235300 & C & 1.11979200 & 0.98740300 & 1.59810300 \\
\hline C & -0.35829800 & -0.93254800 & 1.60372600 & C & 0.06765200 & 1.80040700 & 2.01872800 \\
\hline $\mathrm{H}$ & 2.09891700 & 1.41978300 & 1.41523400 & C & -1.40658500 & -0.11739100 & 2.02681800 \\
\hline $\mathrm{H}$ & -0.53945700 & -1.99198700 & 1.44515000 & C & -1.19642000 & 1.24846200 & 2.23474300 \\
\hline $\mathrm{H}$ & 0.23962100 & 2.86200700 & 2.16751700 & $\mathrm{H}$ & -2.38938500 & -0.54676400 & 2.18778400 \\
\hline $\mathrm{H}$ & -2.02093500 & 1.87864400 & 2.55512400 & $\mathrm{~N}$ & 1.82804600 & -2.30834300 & 0.16574600 \\
\hline $\mathrm{H}$ & 0.90524000 & -2.40287900 & -0.26572900 & $S$ & 3.51451200 & 1.42754100 & -1.17969400 \\
\hline 0 & 2.76692600 & 2.69399300 & -1.37557700 & $\mathrm{O}$ & 4.19033400 & 1.27680000 & 0.13111200 \\
\hline $\mathrm{O}$ & 2.75234800 & 0.22574000 & -1.61836900 & C & 4.91488400 & 1.53479200 & -2.39945600 \\
\hline $\mathrm{F}$ & 4.43868900 & 1.58629800 & -3.65036800 & $\mathrm{~F}$ & 5.70731900 & 0.44933100 & -2.29394800 \\
\hline $\mathrm{F}$ & 5.65907700 & 2.62088400 & -2.17417600 & C & 3.20963500 & -0.96896400 & 3.41082600 \\
\hline C & 2.69631200 & -2.02225700 & 2.62748900 & $\mathrm{H}$ & 1.78535800 & -2.50261600 & 2.97668100 \\
\hline $\mathrm{H}$ & 3.42836500 & -2.68898400 & 2.18179600 & C & 2.39658900 & -0.31130200 & 4.42214100 \\
\hline $\mathrm{H}$ & 1.32423400 & -0.43039000 & 4.22855300 & $\mathrm{H}$ & 2.64294900 & 0.75170400 & 4.53046900 \\
\hline C & 4.56213600 & -0.41960800 & 3.09888700 & $\mathrm{H}$ & 4.46738100 & 0.26642600 & 2.23766300 \\
\hline $\mathrm{H}$ & 4.97757800 & 0.15676500 & 3.92958700 & $\mathrm{H}$ & 5.26569100 & -1.19900400 & 2.79270600 \\
\hline $\mathrm{Si}$ & 2.63876000 & -1.07220100 & 6.21786900 & C & 4.44901200 & -0.93498000 & 6.73267200 \\
\hline $\mathrm{H}$ & 4.80762700 & 0.10039000 & 6.69683400 & $\mathrm{H}$ & 4.57116200 & -1.28573800 & 7.76489300 \\
\hline $\mathrm{H}$ & 5.10776900 & -1.54238900 & 6.10177400 & C & 1.53708100 & -0.02042100 & 7.33033500 \\
\hline $\mathrm{H}$ & 1.84406900 & 1.03170700 & 7.32530700 & $\mathrm{H}$ & 0.48760700 & -0.06509700 & 7.01776800 \\
\hline $\mathrm{H}$ & 1.58983600 & -0.37573200 & 8.36668700 & C & 2.07282400 & -2.87093600 & 6.19720500 \\
\hline $\mathrm{H}$ & 1.03008200 & -2.96502200 & 5.87208600 & $\mathrm{H}$ & 2.68875700 & -3.49011100 & 5.53545100 \\
\hline
\end{tabular}




\section{\begin{tabular}{llll}
\hline $\mathrm{H}$ & 2.14093600 & -3.29890300 & 7.20487500
\end{tabular}}

\section{TS- $R-4 f-n d-23$}

Imaginary frequency: $-289.88 \mathrm{~cm}-1$

E[B3LYP/6-31G(d)]: -4788.685567 Hartree

E[PCM(Et $\left.\left.{ }_{2} \mathrm{O}\right)-B 3 L Y P / 6-311 G(d, p)-D 3(B J)\right]:-4790.214553$ Hartree

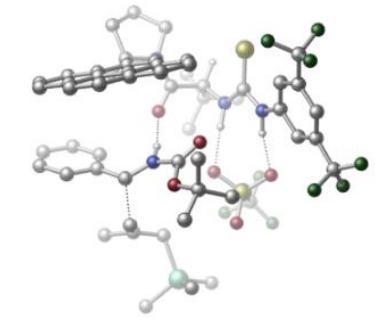

\begin{tabular}{|c|c|c|c|c|c|c|c|}
\hline 01 & & & & $\mathrm{~N}$ & 2.64073300 & 0.56012200 & 1.70614000 \\
\hline $\mathrm{H}$ & 2.25921200 & 1.48941100 & 1.50398200 & $\mathrm{C}$ & 3.78563400 & 0.27644200 & 0.94647300 \\
\hline $\mathrm{C}$ & 4.31280700 & 1.35014600 & 0.19989200 & $\mathrm{C}$ & 4.40043400 & -0.97907300 & 0.83776100 \\
\hline $\mathrm{C}$ & 5.40533500 & 1.15818200 & -0.63892500 & $\mathrm{H}$ & 3.85086700 & 2.32895500 & 0.27439300 \\
\hline C & 5.49571500 & -1.14757800 & -0.01223100 & $\mathrm{H}$ & 4.02736500 & -1.81038400 & 1.41724200 \\
\hline C & 6.01391000 & -0.09328000 & -0.75818500 & $\mathrm{H}$ & 6.86495800 & -0.23752100 & -1.41167500 \\
\hline C & 5.88746700 & 2.29652000 & -1.49681800 & C & 6.13514700 & -2.50798700 & -0.08203200 \\
\hline $\mathrm{F}$ & 5.65862400 & 3.49869200 & -0.93299000 & $\mathrm{~F}$ & 5.26577700 & 2.30524900 & -2.70669500 \\
\hline $\mathrm{F}$ & 7.21326900 & 2.20957600 & -1.75066100 & $\mathrm{~F}$ & 5.20355900 & -3.49598400 & -0.16318100 \\
\hline $\mathrm{F}$ & 6.94360400 & -2.63973100 & -1.15698200 & $\mathrm{~F}$ & 6.88027100 & -2.77754800 & 1.01157500 \\
\hline C & -0.31091100 & -0.27688000 & 3.70055700 & $\mathrm{H}$ & 0.27313700 & -0.92730800 & 4.34998800 \\
\hline C & -1.04126400 & 0.76078500 & 4.62463400 & C & -2.02749100 & 1.68234400 & 3.88072400 \\
\hline $\mathrm{H}$ & -2.86549800 & 1.11787700 & 3.46082900 & $\mathrm{H}$ & -1.55070600 & 2.23023600 & 3.06519400 \\
\hline $\mathrm{H}$ & -2.43422400 & 2.41592400 & 4.58830100 & C & 0.03321600 & 1.62143500 & 5.31995200 \\
\hline $\mathrm{H}$ & -0.43502300 & 2.25667800 & 6.08144000 & $\mathrm{H}$ & 0.54687700 & 2.27358700 & 4.60905300 \\
\hline $\mathrm{H}$ & 0.78749100 & 0.99883700 & 5.81573800 & C & -1.82093300 & -0.02210500 & 5.70239400 \\
\hline $\mathrm{H}$ & -2.35827500 & 0.67458900 & 6.35591000 & $\mathrm{H}$ & -1.15099700 & -0.61508100 & 6.33728200 \\
\hline $\mathrm{H}$ & -2.56653700 & -0.69644200 & 5.26236300 & C & -1.26059300 & -1.13159500 & 2.83190400 \\
\hline $\mathrm{O}$ & -1.85511000 & -0.62100900 & 1.86768800 & C & -0.92495500 & -3.20251000 & 4.27658100 \\
\hline C & -2.24804000 & -3.29926800 & 2.23283400 & C & -2.07209000 & -4.18631700 & 4.52385400 \\
\hline $\mathrm{H}$ & 0.01385000 & -3.71720100 & 4.04004000 & $\mathrm{H}$ & -0.74463000 & -2.54246200 & 5.12247300 \\
\hline $\mathrm{H}$ & -3.16908000 & -2.74012400 & 2.04353100 & $\mathrm{H}$ & -2.88004100 & -3.67992300 & 5.06583800 \\
\hline $\mathrm{H}$ & -1.75972500 & -5.05205100 & 5.11563300 & $\mathrm{~N}$ & -1.40011500 & -2.45223900 & 3.10181600 \\
\hline C & -1.59609300 & -3.65759400 & 0.89986600 & C & -0.24924100 & -3.53469100 & 0.71885200 \\
\hline C & -2.41800000 & -4.21917500 & -0.15910000 & C & 0.40842800 & -3.95546100 & -0.48522400 \\
\hline $\mathrm{H}$ & 0.37002900 & -3.10618400 & 1.50225000 & $\mathrm{C}$ & -1.78561800 & -4.63903300 & -1.37571600 \\
\hline C & -3.81146400 & -4.37770700 & -0.04962800 & $\mathrm{C}$ & 1.79952000 & -3.85034200 & -0.64616000 \\
\hline C & -0.37387700 & -4.51232400 & -1.53756300 & C & -2.56588400 & -5.20129500 & -2.43441300 \\
\hline $\mathrm{H}$ & -4.32369800 & -4.06889300 & 0.85471200 & $\mathrm{C}$ & -4.56397600 & -4.92501100 & -1.08876800 \\
\hline $\mathrm{C}$ & 2.41442700 & -4.28290200 & -1.82051800 & $\mathrm{H}$ & 2.39662600 & -3.43001300 & 0.15724300 \\
\hline $\mathrm{C}$ & 0.26167100 & -4.94629600 & -2.74123000 & $\mathrm{C}$ & -3.95354900 & -5.33479200 & -2.26929500 \\
\hline $\mathrm{C}$ & -1.90155600 & -5.62136400 & -3.63850800 & $\mathrm{H}$ & -5.63872600 & -5.03373000 & -0.96924100 \\
\hline $\mathrm{C}$ & 1.65610100 & -4.82202100 & -2.85871900 & $\mathrm{H}$ & 3.49226400 & -4.19722900 & -1.91674400 \\
\hline $\mathrm{C}$ & -0.55351600 & -5.50144000 & -3.78550700 & $\mathrm{H}$ & -4.54487600 & -5.76745600 & -3.07295300 \\
\hline $\mathrm{H}$ & -2.50620600 & -6.04817900 & -4.43546800 & $\mathrm{H}$ & 2.14172800 & -5.15838900 & -3.77186500 \\
\hline $\mathrm{H}$ & -0.06782600 & -5.83098600 & -4.70112300 & $\mathrm{C}$ & 1.89402500 & -0.21019700 & 2.56003300 \\
\hline$S$ & 2.43615200 & -1.63853200 & 3.30375800 & $\mathrm{~N}$ & 0.66572500 & 0.33134600 & 2.80486000 \\
\hline $\mathrm{H}$ & 0.30698000 & 1.02638000 & 2.14303800 & $S$ & 0.51752100 & 3.60011500 & 0.71236000 \\
\hline $\mathrm{O}$ & 0.27262000 & 4.23437100 & -0.60048100 & $\mathrm{O}$ & -0.39831800 & 2.46678700 & 1.02358000 \\
\hline $\mathrm{O}$ & 1.93589800 & 3.33413700 & 1.05426600 & $\mathrm{C}$ & 0.00174500 & 4.89871000 & 1.94508900 \\
\hline $\mathrm{F}$ & 0.59858900 & 6.06982800 & 1.69226000 & $\mathrm{~F}$ & 0.30986700 & 4.52012900 & 3.19404400 \\
\hline $\mathrm{F}$ & -1.33348100 & 5.08413000 & 1.88494500 & $\mathrm{C}$ & -2.52283100 & -4.56523300 & 3.10393700 \\
\hline $\mathrm{H}$ & -1.92438300 & -5.40058300 & 2.72742000 & $\mathrm{H}$ & -3.57220400 & -4.86983100 & 3.05937700 \\
\hline $\mathrm{C}$ & -0.38369900 & 0.18847000 & -1.36852100 & $\mathrm{O}$ & 0.54746000 & -0.03424900 & -0.63024700 \\
\hline
\end{tabular}




\begin{tabular}{|c|c|c|c|c|c|c|c|}
\hline 0 & -0.34907500 & 0.46975500 & -2.67920300 & C & 0.94955500 & 0.53796000 & -3.41546000 \\
\hline C & 1.60195800 & -0.84465000 & -3.40944900 & $\mathrm{H}$ & 2.50202000 & -0.81960700 & -4.03360600 \\
\hline $\mathrm{H}$ & 1.89065700 & -1.14486700 & -2.40134400 & $\mathrm{H}$ & 0.92302800 & -1.59928600 & -3.82075300 \\
\hline C & 0.50108300 & 0.93172300 & -4.82343200 & $\mathrm{H}$ & 0.00806300 & 1.90968500 & -4.81794300 \\
\hline $\mathrm{H}$ & 1.37261200 & 0.99364700 & -5.48272400 & $\mathrm{H}$ & -0.19069700 & 0.18982900 & -5.23680700 \\
\hline C & 1.83925900 & 1.61653700 & -2.79735900 & $\mathrm{H}$ & 2.72273600 & 1.76531500 & -3.42653900 \\
\hline $\mathrm{H}$ & 1.30511300 & 2.56854700 & -2.71724600 & $\mathrm{H}$ & 2.17808000 & 1.32880900 & -1.80238300 \\
\hline C & -2.81752200 & 0.13743400 & -1.70211000 & $\mathrm{H}$ & -2.58667700 & -0.22926500 & -2.69718500 \\
\hline C & -4.10722700 & -0.35628200 & -1.15672800 & $\mathrm{C}$ & -5.01074100 & -0.95714900 & -2.04869200 \\
\hline $\mathrm{C}$ & -4.46239000 & -0.23934700 & 0.19745500 & C & -6.24094900 & -1.43332400 & -1.60317100 \\
\hline $\mathrm{H}$ & -4.73988000 & -1.06395800 & -3.09656100 & C & -5.69534300 & -0.72130400 & 0.63965700 \\
\hline $\mathrm{H}$ & -3.77612900 & 0.19936100 & 0.91262700 & $\mathrm{C}$ & -6.58819600 & -1.31387900 & -0.25546100 \\
\hline $\mathrm{H}$ & -6.92351700 & -1.90367800 & -2.30501200 & $\mathrm{H}$ & -5.95658200 & -0.63014600 & 1.69031400 \\
\hline $\mathrm{H}$ & -7.54782300 & -1.68357300 & 0.09512200 & $\mathrm{~N}$ & -1.71135100 & 0.17087200 & -0.89976100 \\
\hline $\mathrm{H}$ & -1.77069600 & 0.06019200 & 0.11993000 & $\mathrm{C}$ & -3.21095100 & 1.90758900 & -2.55344200 \\
\hline $\mathrm{H}$ & -2.19027000 & 2.09212200 & -2.87592300 & $\mathrm{H}$ & -3.87056900 & 1.51299400 & -3.32325900 \\
\hline C & -3.78147300 & 2.81633100 & -1.64743900 & C & -2.97712600 & 3.77619500 & -0.91844300 \\
\hline $\mathrm{H}$ & -3.42098500 & 4.07912300 & 0.03629200 & $\mathrm{H}$ & -1.93682000 & 3.46548300 & -0.77756100 \\
\hline $\mathrm{C}$ & -5.25543100 & 2.77408500 & -1.38462000 & $\mathrm{H}$ & -5.42597200 & 2.16013500 & -0.48698600 \\
\hline $\mathrm{H}$ & -5.65336800 & 3.77007000 & -1.17061600 & $\mathrm{H}$ & -5.82026900 & 2.31910700 & -2.20199900 \\
\hline C & -2.10331700 & 6.76058000 & -0.78601600 & $\mathrm{H}$ & -1.08802500 & 6.51422100 & -0.46588300 \\
\hline $\mathrm{H}$ & -2.07736600 & 7.72913400 & -1.30167400 & $\mathrm{H}$ & -2.72599100 & 6.87431600 & 0.10801900 \\
\hline C & -1.58196600 & 5.11740400 & -3.37952100 & $\mathrm{H}$ & -0.62073200 & 4.77417800 & -2.98304400 \\
\hline $\mathrm{H}$ & -1.96271000 & 4.36944200 & -4.08447700 & $\mathrm{H}$ & -1.40431100 & 6.04069100 & -3.94488300 \\
\hline $\mathrm{C}$ & -4.46393400 & 5.99094000 & -2.62097000 & $\mathrm{H}$ & -4.90051600 & 5.26435800 & -3.31602900 \\
\hline $\mathrm{H}$ & -5.18840400 & 6.16986600 & -1.81719300 & $\mathrm{H}$ & -4.35221500 & 6.93594100 & -3.16741000 \\
\hline $\mathrm{Si}$ & -2.77246500 & 5.44996000 & -1.95891900 & & & & \\
\hline
\end{tabular}

\section{TS-R-4f-nd-24}

Imaginary frequency: $-283.98 \mathrm{~cm}^{-1}$ E[B3LYP/6-31G(d)]: -4788.686365 Hartree

$\mathrm{E}\left[\mathrm{PCM}\left(\mathrm{Et}_{2} \mathrm{O}\right)-\mathrm{B} 3 \mathrm{LYP} / 6-311 \mathrm{G}(\mathrm{d}, \mathrm{p})-\mathrm{D} 3(\mathrm{BJ})\right]$ : -4790.214359 Hartree

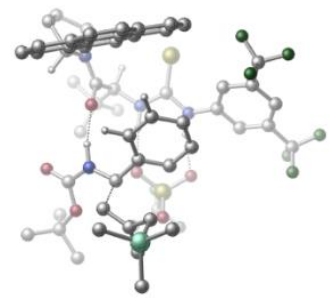

\begin{tabular}{|crrr|rrrr|}
\hline 01 & & & & N & 0.76222000 & 2.37304900 & -1.66145800 \\
\hline $\mathrm{H}$ & 1.72130700 & 2.03968700 & -1.52379800 & $\mathrm{C}$ & 0.48558500 & 3.56816100 & -0.97710900 \\
\hline $\mathrm{C}$ & 1.56768700 & 4.40263600 & -0.65690100 & $\mathrm{C}$ & -0.79568000 & 3.91411300 & -0.52573100 \\
\hline $\mathrm{C}$ & 1.36102000 & 5.56661900 & 0.08330800 & $\mathrm{H}$ & 2.56652400 & 4.13008100 & -0.97824400 \\
\hline $\mathrm{C}$ & -0.98357700 & 5.08345600 & 0.20846600 & $\mathrm{H}$ & -1.63218600 & 3.26723600 & -0.74293400 \\
\hline $\mathrm{C}$ & 0.08494300 & 5.92634100 & 0.51679500 & $\mathrm{H}$ & -0.07253200 & 6.84123600 & 1.07454600 \\
\hline $\mathrm{C}$ & 2.54161400 & 6.41739500 & 0.46969100 & $\mathrm{C}$ & -2.35369300 & 5.38231500 & 0.75089900 \\
\hline $\mathrm{F}$ & 3.53058200 & 6.36232500 & -0.44598500 & $\mathrm{~F}$ & 3.07518500 & 6.01877500 & 1.65037100 \\
\hline $\mathrm{F}$ & 2.19650000 & 7.71688800 & 0.61935200 & $\mathrm{~F}$ & -3.33772100 & 5.00484700 & -0.09847700 \\
\hline $\mathrm{F}$ & -2.58663800 & 4.71662700 & 1.91842800 & $\mathrm{~F}$ & -2.52805000 & 6.69307400 & 1.01684200 \\
\hline $\mathrm{C}$ & -0.20165200 & -0.49309700 & -3.73228200 & $\mathrm{H}$ & -0.77233900 & 0.13824000 & -4.41273000 \\
\hline $\mathrm{C}$ & 0.82849500 & -1.30597500 & -4.59793900 & $\mathrm{C}$ & 1.59850300 & -2.36481100 & -3.79064100 \\
\hline $\mathrm{H}$ & 0.93763800 & -3.12067400 & -3.36021700 & $\mathrm{H}$ & 2.17464700 & -1.91824100 & -2.97710800 \\
\hline $\mathrm{H}$ & 2.30873800 & -2.87482100 & -4.45390100 & $\mathrm{C}$ & 1.82994500 & -0.31151200 & -5.22281300 \\
\hline $\mathrm{H}$ & 2.45950100 & -0.83352100 & -5.95339600 & $\mathrm{H}$ & 2.49094500 & 0.12587900 & -4.46911300 \\
\hline $\mathrm{H}$ & 1.31651800 & 0.50621100 & -5.74291900 & $\mathrm{C}$ & 0.07199200 & -2.01781700 & -5.73843300 \\
\hline $\mathrm{H}$ & 0.77773100 & -2.60172700 & -6.33991900 & $\mathrm{H}$ & -0.41743600 & -1.30370100 & -6.41225700 \\
\hline $\mathrm{H}$ & -0.68380100 & -2.71475900 & -5.35793600 & $\mathrm{C}$ & -1.18038400 & -1.36839700 & -2.90580300 \\
\hline
\end{tabular}




\begin{tabular}{|c|c|c|c|c|c|c|c|}
\hline $\mathrm{O}$ & -0.82413800 & -1.86915900 & -1.83217900 & C & -3.06313500 & -1.09362300 & -4.61772800 \\
\hline C & -3.38207100 & -2.41566700 & -2.61380100 & C & -3.92418000 & -2.29362400 & -5.02365700 \\
\hline $\mathrm{H}$ & -3.67514600 & -0.20272600 & -4.42676900 & $\mathrm{H}$ & -2.31633800 & -0.83506000 & -5.36391600 \\
\hline $\mathrm{H}$ & -2.80779400 & -3.27660300 & -2.26022200 & $\mathrm{H}$ & -3.29588300 & -3.04162300 & -5.52117100 \\
\hline $\mathrm{H}$ & -4.72861800 & -2.01786200 & -5.71237300 & $\mathrm{~N}$ & -2.44660800 & -1.55724700 & -3.36722600 \\
\hline C & -4.02450300 & -1.71818100 & -1.41745900 & C & -3.98118800 & -0.36088600 & -1.28547700 \\
\hline C & -4.75020100 & -2.51480800 & -0.44250200 & C & -4.63906000 & 0.33123700 & -0.21413900 \\
\hline $\mathrm{H}$ & -3.41730200 & 0.23788500 & -1.99557600 & C & -5.41506500 & -1.84813100 & 0.63842800 \\
\hline C & -4.83044500 & -3.91802100 & -0.50425600 & C & -4.57689800 & 1.72869600 & -0.08316400 \\
\hline C & -5.36182600 & -0.42660300 & 0.75284400 & C & -6.13773700 & -2.60455100 & 1.61333900 \\
\hline $\mathrm{H}$ & -4.33674300 & -4.45466000 & -1.30689100 & C & -5.53602100 & -4.64731500 & 0.45285200 \\
\hline C & -5.20867400 & 2.37238900 & 0.98074500 & $\mathrm{H}$ & -4.02629400 & 2.30599700 & -0.82094600 \\
\hline C & -6.01512200 & 0.24045900 & 1.83455900 & C & -6.18589100 & -4.00289900 & 1.50025900 \\
\hline C & -6.79489700 & -1.90643400 & 2.68507000 & $\mathrm{H}$ & -5.57772900 & -5.73052600 & 0.37506900 \\
\hline C & -5.92215100 & 1.63919700 & 1.92802300 & $\mathrm{H}$ & -5.13072000 & 3.45109900 & 1.07170600 \\
\hline C & -6.73679800 & -0.55062400 & 2.79232600 & $\mathrm{H}$ & -6.73754900 & -4.57715300 & 2.24075700 \\
\hline $\mathrm{H}$ & -7.34565000 & -2.49291100 & 3.41685700 & $\mathrm{H}$ & -6.41438200 & 2.14766500 & 2.75376500 \\
\hline $\mathrm{H}$ & -7.23939500 & -0.04054500 & 3.61083200 & C & -0.04228400 & 1.66083600 & -2.52017100 \\
\hline$S$ & -1.47750000 & 2.26908500 & -3.19533100 & $\mathrm{~N}$ & 0.45493700 & 0.43404400 & -2.81785200 \\
\hline $\mathrm{H}$ & 1.23456400 & 0.07626600 & -2.26171200 & $S$ & 3.90883000 & 0.44386000 & -0.70974200 \\
\hline $\mathrm{O}$ & 4.32268400 & 0.05014000 & 0.65850400 & $\mathrm{O}$ & 2.92735400 & -0.48225300 & -1.34141400 \\
\hline $\mathrm{O}$ & 3.56247000 & 1.87549600 & -0.88555800 & $\mathrm{C}$ & 5.44696600 & 0.21690800 & -1.73069400 \\
\hline $\mathrm{F}$ & 6.41729600 & 1.03650200 & -1.31664100 & $\mathrm{~F}$ & 5.18819900 & 0.46667600 & -3.02069900 \\
\hline $\mathrm{F}$ & 5.89121400 & -1.05165100 & -1.62716300 & C & 1.89854400 & -3.71009700 & -0.38569600 \\
\hline 0 & 1.48912800 & -4.47102800 & -1.23848100 & $\mathrm{O}$ & 3.12577500 & -3.69304000 & 0.16495000 \\
\hline C & 4.22027200 & -4.54132100 & -0.37045800 & C & 4.43554400 & -4.24789000 & -1.85729300 \\
\hline $\mathrm{H}$ & 5.33267600 & -4.77750000 & -2.19742200 & $\mathrm{H}$ & 4.58989200 & -3.17708500 & -2.01387800 \\
\hline $\mathrm{H}$ & 3.58657200 & -4.57930500 & -2.45621700 & C & 3.89539700 & -6.01284400 & -0.10212500 \\
\hline $\mathrm{H}$ & 3.72078800 & -6.18171700 & 0.96681800 & $\mathrm{H}$ & 4.74334600 & -6.63573900 & -0.40790100 \\
\hline $\mathrm{H}$ & 3.01154200 & -6.32699400 & -0.66013700 & C & 5.42572900 & -4.07380400 & 0.44766500 \\
\hline $\mathrm{H}$ & 6.32136400 & -4.61980600 & 0.13373800 & $\mathrm{H}$ & 5.27078300 & -4.26345300 & 1.51604900 \\
\hline $\mathrm{H}$ & 5.59857300 & -3.00381200 & 0.29915500 & C & 1.50521400 & -1.79123900 & 1.11558800 \\
\hline $\mathrm{H}$ & 2.54039300 & -1.49423100 & 0.98349000 & C & 0.56119400 & -0.68344700 & 1.41455400 \\
\hline C & 1.08058000 & 0.59379200 & 1.68739900 & C & -0.82536500 & -0.89159400 & 1.50108300 \\
\hline C & 0.22551100 & 1.64352200 & 2.01979900 & $\mathrm{H}$ & 2.15006400 & 0.76644500 & 1.61475300 \\
\hline C & -1.67358300 & 0.15919100 & 1.84518200 & $\mathrm{H}$ & -1.24808100 & -1.87133700 & 1.29617600 \\
\hline C & -1.15178700 & 1.43029500 & 2.10118200 & $\mathrm{H}$ & 0.63694700 & 2.63180500 & 2.20153700 \\
\hline $\mathrm{H}$ & -2.74325100 & -0.01031200 & 1.90125500 & $\mathrm{H}$ & -1.81643900 & 2.25519300 & 2.33868300 \\
\hline $\mathrm{N}$ & 1.08117500 & -2.73737000 & 0.19047300 & $\mathrm{H}$ & 0.21481300 & -2.57733900 & -0.33023100 \\
\hline C & 1.69989800 & -2.76607700 & 2.74827400 & $\mathrm{H}$ & 2.28272800 & -3.58893200 & 2.34563100 \\
\hline $\mathrm{H}$ & 0.65955400 & -2.99698500 & 2.96414000 & C & 2.36799200 & -1.90511800 & 3.64066000 \\
\hline C & 1.63385400 & -1.07383400 & 4.58333800 & $\mathrm{H}$ & 0.60338600 & -0.89394000 & 4.25515100 \\
\hline $\mathrm{H}$ & 2.13747400 & -0.11921200 & 4.77724000 & C & 3.84556200 & -1.73051100 & 3.52031900 \\
\hline $\mathrm{H}$ & 4.28808700 & -1.31144400 & 4.42783500 & $\mathrm{H}$ & 4.04509700 & -1.01925400 & 2.69847200 \\
\hline $\mathrm{H}$ & 4.35249800 & -2.66111300 & 3.24984700 & C & 0.51101000 & -0.61909900 & 7.37869000 \\
\hline $\mathrm{H}$ & 1.08613600 & 0.30939800 & 7.47036900 & $\mathrm{H}$ & 0.32948900 & -0.99737200 & 8.39210200 \\
\hline $\mathrm{H}$ & -0.46135600 & -0.36921200 & 6.93918400 & C & 3.12574700 & -2.27690500 & 7.08667800 \\
\hline $\mathrm{H}$ & 3.74718200 & -1.37640100 & 7.15610300 & $\mathrm{H}$ & 3.67780300 & -3.02379600 & 6.50499200 \\
\hline $\mathrm{H}$ & 3.01303600 & -2.67319300 & 8.10339200 & C & 0.41086700 & -3.48427300 & 6.16475800 \\
\hline $\mathrm{H}$ & 0.92027300 & -4.23485900 & 5.55011800 & $\mathrm{H}$ & -0.56745100 & -3.28570600 & 5.71164000 \\
\hline $\mathrm{H}$ & 0.23010300 & -3.93355100 & 7.14896900 & $\mathrm{Si}$ & 1.42804700 & -1.90707300 & 6.35005200 \\
\hline C & -4.44955600 & -2.83230600 & -3.67945400 & $\mathrm{H}$ & -5.41132900 & -2.37295300 & -3.43282300 \\
\hline $\mathrm{H}$ & -4.60172500 & -3.91523600 & -3.69791800 & & & & \\
\hline
\end{tabular}




\section{TS- $R-4 f-n d-25$}

Imaginary frequency: $-252.68 \mathrm{~cm}^{-1}$

E[B3LYP/6-31G(d)]: -4788.687297 Hartree

E[PCM(Et2O)-B3LYP/6-311G(d,p)-D3(BJ)]: -4790.214309 Hartree

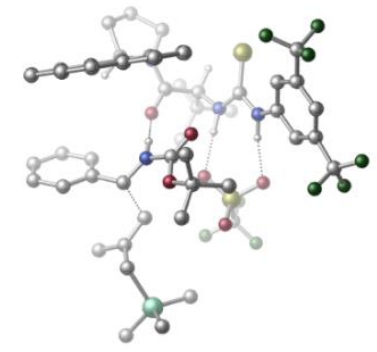

\begin{tabular}{|c|c|c|c|c|c|c|c|}
\hline 01 & & & & $\mathrm{~N}$ & -2.35898600 & -1.50123200 & 1.76130900 \\
\hline $\mathrm{H}$ & -1.55677500 & -2.13618700 & 1.81714800 & C & -3.35081700 & -1.96020100 & 0.88174600 \\
\hline $\mathrm{C}$ & -3.20062800 & -3.27964200 & 0.41229100 & C & -4.42169500 & -1.19386000 & 0.39970300 \\
\hline C & -4.09065600 & -3.80176300 & -0.52120800 & $\mathrm{H}$ & -2.37112500 & -3.88159200 & 0.76861300 \\
\hline C & -5.30215200 & -1.73908100 & -0.53592300 & $\mathrm{H}$ & -4.56251500 & -0.18484100 & 0.75694000 \\
\hline C & -5.15521300 & -3.04099800 & -1.00777000 & $\mathrm{H}$ & -5.84478100 & -3.45179200 & -1.73432800 \\
\hline C & -3.84551900 & -5.17633200 & -1.08152300 & C & -6.45306800 & -0.89329600 & -1.01009400 \\
\hline $\mathrm{F}$ & -3.26538100 & -5.99961300 & -0.18522100 & $F$ & -3.02312800 & -5.13253600 & -2.16285600 \\
\hline $\mathrm{F}$ & -4.99109900 & -5.76535800 & -1.49462000 & $\mathrm{~F}$ & -6.07779100 & 0.39687600 & -1.21739500 \\
\hline $\mathrm{F}$ & -6.97285900 & -1.34487800 & -2.17349900 & $\mathrm{~F}$ & -7.46256000 & -0.85715500 & -0.11314500 \\
\hline C & -0.58142700 & 1.00497400 & 3.74435100 & $\mathrm{H}$ & -1.50668600 & 1.35144000 & 4.20272200 \\
\hline C & 0.39646100 & 0.61679800 & 4.90954100 & C & 1.79975000 & 0.19324400 & 4.43427600 \\
\hline $\mathrm{H}$ & 2.32514400 & 1.01735600 & 3.94305200 & $\mathrm{H}$ & 1.76331400 & -0.64653000 & 3.73679200 \\
\hline $\mathrm{H}$ & 2.39325200 & -0.11782100 & 5.30321400 & $\mathrm{C}$ & -0.24004800 & -0.54552300 & 5.69874500 \\
\hline $\mathrm{H}$ & 0.35232400 & -0.75384900 & 6.59771800 & $\mathrm{H}$ & -0.27807500 & -1.46089400 & 5.10144800 \\
\hline $\mathrm{H}$ & -1.26218000 & -0.30519400 & 6.01477500 & C & 0.53940100 & 1.83413200 & 5.84684900 \\
\hline $\mathrm{H}$ & 1.22889000 & 1.59803800 & 6.66540100 & $\mathrm{H}$ & -0.42057300 & 2.11304600 & 6.29868400 \\
\hline $\mathrm{H}$ & 0.94246300 & 2.71058400 & 5.32392400 & C & -0.03583300 & 2.09231200 & 2.79227100 \\
\hline $\mathrm{O}$ & 0.87684200 & 1.81709400 & 1.99392100 & C & -1.55701300 & 3.89056800 & 3.75834500 \\
\hline C & -0.10257700 & 4.35940100 & 1.85657800 & C & -1.08939900 & 5.34335000 & 3.88491600 \\
\hline $\mathrm{H}$ & -2.57209200 & 3.81956600 & 3.34990100 & $\mathrm{H}$ & -1.53543000 & 3.35550900 & 4.70559900 \\
\hline $\mathrm{H}$ & 0.99070400 & 4.32660400 & 1.88712900 & $\mathrm{H}$ & -0.24301900 & 5.39816600 & 4.58039300 \\
\hline $\mathrm{H}$ & -1.87702400 & 6.00435500 & 4.25900500 & $\mathrm{~N}$ & -0.58807700 & 3.32798200 & 2.80232300 \\
\hline $\mathrm{C}$ & -0.56882100 & 4.14956000 & 0.41909400 & $\mathrm{C}$ & -1.63882100 & 3.35185900 & 0.13530100 \\
\hline C & 0.09067200 & 4.88100200 & -0.64954800 & C & -2.16404300 & 3.21105300 & -1.19240600 \\
\hline $\mathrm{H}$ & -2.12653200 & 2.79060100 & 0.92787500 & $\mathrm{C}$ & -0.41332600 & 4.75391000 & -1.98579200 \\
\hline $\mathrm{C}$ & 1.20409500 & 5.71482500 & -0.43936800 & $\mathrm{C}$ & -3.27839900 & 2.40233800 & -1.46722800 \\
\hline C & -1.54291300 & 3.92564100 & -2.25663800 & C & 0.20936000 & 5.46519100 & -3.05902400 \\
\hline $\mathrm{H}$ & 1.61266800 & 5.83462300 & 0.55797300 & C & 1.80784700 & 6.39992300 & -1.49358900 \\
\hline$C$ & -3.77914500 & 2.29814100 & -2.76422600 & $\mathrm{H}$ & -3.75012000 & 1.85528300 & -0.65697900 \\
\hline C & -2.05674300 & 3.80896400 & -3.58454100 & C & 1.31901000 & 6.28176900 & -2.79015600 \\
\hline $\mathrm{C}$ & -0.32541400 & 5.32548700 & -4.38644200 & $\mathrm{H}$ & 2.66745600 & 7.03482200 & -1.29520900 \\
\hline C & -3.17616200 & 2.99170200 & -3.81281500 & $\mathrm{H}$ & -4.64570500 & 1.67064500 & -2.94889400 \\
\hline C & -1.40666400 & 4.53759900 & -4.63791600 & $\mathrm{H}$ & 1.79005400 & 6.82373900 & -3.60687600 \\
\hline $\mathrm{H}$ & 0.15581000 & 5.87359800 & -5.19324500 & $\mathrm{H}$ & -3.57168100 & 2.90685800 & -4.82238600 \\
\hline $\mathrm{H}$ & -1.80020000 & 4.44831100 & -5.64791100 & C & -2.24327000 & -0.33761300 & 2.47841500 \\
\hline$S$ & -3.53075300 & 0.70443200 & 2.85145200 & $\mathrm{~N}$ & -0.96988500 & -0.13437000 & 2.92332600 \\
\hline $\mathrm{H}$ & -0.21195200 & -0.63226500 & 2.44605100 & $\mathrm{~S}$ & 1.06675400 & -3.06692100 & 1.37247600 \\
\hline $\mathrm{O}$ & 1.57522300 & -3.56324200 & 0.07587200 & $\mathrm{O}$ & 1.22452400 & -1.59633900 & 1.57130100 \\
\hline $\mathrm{O}$ & -0.26152800 & -3.57653300 & 1.79313500 & C & 2.24093900 & -3.78312300 & 2.62685600 \\
\hline$F$ & 2.25489800 & -5.11797000 & 2.57109500 & $\mathrm{~F}$ & 1.89146900 & -3.40631500 & 3.86606400 \\
\hline $\mathrm{F}$ & 3.49253700 & -3.33461200 & 2.39420900 & C & -0.64431100 & 5.69354800 & 2.45633300 \\
\hline $\mathrm{H}$ & -1.50152400 & 6.03254100 & 1.86624600 & $\mathrm{H}$ & 0.10700100 & 6.48757000 & 2.42662600 \\
\hline C & 0.45600400 & -0.05646400 & -1.18404600 & $\mathrm{O}$ & -0.66663800 & -0.02553100 & -0.73281100 \\
\hline $\mathrm{O}$ & 0.89121400 & -0.70286600 & -2.27480500 & C & -0.05087700 & -1.44488900 & -3.16175300 \\
\hline C & -1.03382700 & -0.45026000 & -3.78123800 & $\mathrm{H}$ & -1.66614900 & -0.97335700 & -4.50720600 \\
\hline
\end{tabular}




\begin{tabular}{|c|c|c|c|c|c|c|c|}
\hline $\mathrm{H}$ & -1.67880600 & -0.00389500 & -3.02257700 & $\mathrm{H}$ & -0.50080500 & 0.34961100 & -4.30712000 \\
\hline C & 0.89178000 & -2.02158200 & -4.21942900 & $\mathrm{H}$ & 1.60812000 & -2.71302800 & -3.76237100 \\
\hline $\mathrm{H}$ & 0.31426300 & -2.57529500 & -4.96641700 & $\mathrm{H}$ & 1.44264300 & -1.22509300 & -4.73182200 \\
\hline C & -0.74561600 & -2.56019300 & -2.38191600 & $\mathrm{H}$ & -1.29825400 & -3.20106700 & -3.07702700 \\
\hline $\mathrm{H}$ & -0.01344400 & -3.17336200 & -1.84830700 & $\mathrm{H}$ & -1.45223400 & -2.15366000 & -1.65839100 \\
\hline C & 2.79642200 & 0.68285400 & -1.03513800 & $\mathrm{H}$ & 2.85793600 & 0.40773200 & -2.08247900 \\
\hline C & 3.66274900 & 1.83264200 & -0.65310400 & C & 4.47654500 & 2.40265300 & -1.64483900 \\
\hline C & 3.68928900 & 2.36582000 & 0.64469200 & C & 5.29644900 & 3.49308800 & -1.35123100 \\
\hline $\mathrm{H}$ & 4.45155700 & 2.00353300 & -2.65626300 & C & 4.51667200 & 3.44940100 & 0.93438500 \\
\hline $\mathrm{H}$ & 3.05303000 & 1.95179600 & 1.41961700 & C & 5.32092900 & 4.01613000 & -0.05809200 \\
\hline $\mathrm{H}$ & 5.90960700 & 3.93293000 & -2.13271200 & $\mathrm{H}$ & 4.53151500 & 3.85439700 & 1.94251400 \\
\hline $\mathrm{H}$ & 5.96126700 & 4.86221100 & 0.17548700 & $\mathrm{~N}$ & 1.50201600 & 0.66760800 & -0.58496400 \\
\hline $\mathrm{H}$ & 1.24725800 & 1.07225600 & 0.32526000 & C & 3.62584000 & -0.93482900 & -0.29855500 \\
\hline $\mathrm{H}$ & 3.60817300 & -0.67595700 & 0.75631700 & $\mathrm{H}$ & 2.83700200 & -1.62115100 & -0.59574400 \\
\hline C & 4.86764200 & -1.08115900 & -0.92626600 & C & 4.98258300 & -1.79306700 & -2.20004800 \\
\hline $\mathrm{H}$ & 4.01431900 & -1.87019600 & -2.70712500 & $\mathrm{H}$ & 5.72026400 & -1.33352300 & -2.87132000 \\
\hline $\mathrm{C}$ & 6.09215700 & -0.43710900 & -0.35521300 & $\mathrm{H}$ & 5.98914100 & -0.22128900 & 0.71040200 \\
\hline $\mathrm{H}$ & 6.98353600 & -1.04989500 & -0.52305400 & $\mathrm{H}$ & 6.26339800 & 0.51960900 & -0.87237800 \\
\hline C & 5.17228000 & -4.42222000 & -3.71362800 & $\mathrm{H}$ & 5.63990300 & -3.87702000 & -4.54200600 \\
\hline $\mathrm{H}$ & 5.52389600 & -5.46007500 & -3.76062500 & $\mathrm{H}$ & 4.08969500 & -4.43464500 & -3.88485100 \\
\hline C & 7.47025100 & -3.65704100 & -1.78471200 & $\mathrm{H}$ & 7.99220500 & -3.07744600 & -2.55541200 \\
\hline $\mathrm{H}$ & 7.76168000 & -3.26116800 & -0.80507200 & $\mathrm{H}$ & 7.84885400 & -4.68558700 & -1.83782200 \\
\hline C & 4.72126600 & -4.53847100 & -0.62749500 & $\mathrm{H}$ & 4.99450700 & -4.12463900 & 0.34934700 \\
\hline $\mathrm{H}$ & 3.63062500 & -4.47305000 & -0.70091200 & $\mathrm{H}$ & 4.99908800 & -5.60025300 & -0.62560300 \\
\hline $\mathrm{Si}$ & 5.59498400 & -3.66181400 & -2.03698500 & & & & \\
\hline
\end{tabular}

\section{TS-R-4f-nd-26}

Imaginary frequency: $-261.58 \mathrm{~cm}^{-1}$ E[B3LYP/6-31G(d)]: -4788.682385 Hartree E[PCM(Et $\left.\left.{ }_{2} \mathrm{O}\right)-\mathrm{B} 3 \mathrm{LYP} / 6-311 \mathrm{G}(\mathrm{d}, \mathrm{p})-\mathrm{D} 3(\mathrm{BJ})\right]$ : -4790.214289 Hartree

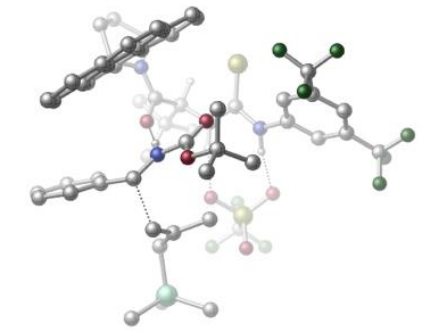

\begin{tabular}{|cccc|cccc|}
\hline 01 & & & & $\mathrm{~N}$ & -2.21472600 & 1.58998800 & 1.34643100 \\
\hline $\mathrm{H}$ & -2.99838500 & 0.96388000 & 1.13037500 & $\mathrm{C}$ & -2.28014900 & 2.84157000 & 0.71157200 \\
\hline $\mathrm{C}$ & -3.53516800 & 3.32666100 & 0.32423400 & $\mathrm{C}$ & -1.12678000 & 3.56895900 & 0.37857400 \\
\hline $\mathrm{C}$ & -3.63409600 & 4.54053800 & -0.36010600 & $\mathrm{H}$ & -4.42643100 & 2.75382400 & 0.55697800 \\
\hline $\mathrm{C}$ & -1.24643700 & 4.77901500 & -0.29795400 & $\mathrm{H}$ & -0.15280500 & 3.17777200 & 0.63206700 \\
\hline $\mathrm{C}$ & -2.49701000 & 5.28407600 & -0.66643400 & $\mathrm{H}$ & -2.58134200 & 6.23582300 & -1.17770200 \\
\hline $\mathrm{C}$ & -4.98495800 & 5.02426000 & -0.81569800 & $\mathrm{C}$ & -0.00999800 & 5.52557200 & -0.71637700 \\
\hline $\mathrm{F}$ & -5.96874300 & 4.65338300 & 0.03069600 & $\mathrm{~F}$ & -5.30712600 & 4.52799200 & -2.03428000 \\
\hline $\mathrm{F}$ & -5.02680200 & 6.37264000 & -0.92164400 & $\mathrm{~F}$ & 1.09089400 & 5.13831100 & -0.03567900 \\
\hline $\mathrm{F}$ & 0.26652100 & 5.33357100 & -2.03749200 & $\mathrm{~F}$ & -0.14293300 & 6.86026900 & -0.55035400 \\
\hline $\mathrm{C}$ & -0.48592600 & -0.81017500 & 3.49521600 & $\mathrm{H}$ & -0.40640900 & -0.11309300 & 4.32906300 \\
\hline $\mathrm{C}$ & -1.11737000 & -2.13965100 & 4.05242600 & $\mathrm{C}$ & -1.19897000 & -3.26985800 & 3.00648600 \\
\hline $\mathrm{H}$ & -0.20285200 & -3.58281800 & 2.67776900 & $\mathrm{H}$ & -1.78040800 & -2.98329100 & 2.12681400 \\
\hline $\mathrm{H}$ & -1.69334700 & -4.13909100 & 3.45780400 & $\mathrm{C}$ & -2.53425700 & -1.81744700 & 4.57063300 \\
\hline $\mathrm{H}$ & -2.94474900 & -2.69066800 & 5.09177600 & $\mathrm{H}$ & -3.21882500 & -1.56392300 & 3.75913100 \\
\hline $\mathrm{H}$ & -2.51840800 & -0.97961700 & 5.27792600 & $\mathrm{C}$ & -0.27351400 & -2.64450000 & 5.24181600 \\
\hline $\mathrm{H}$ & -0.69599800 & -3.58229500 & 5.61983000 & $\mathrm{H}$ & -0.27524400 & -1.93068400 & 6.07425700 \\
\hline $\mathrm{H}$ & 0.76633700 & -2.84692300 & 4.95870100 & $\mathrm{C}$ & 0.91195400 & -1.01558700 & 2.85724800 \\
\hline $\mathrm{O}$ & 1.00220400 & -1.50210800 & 1.71746200 & $\mathrm{C}$ & 2.08728100 & -0.14676000 & 4.93219900 \\
\hline $\mathrm{C}$ & 3.37167000 & -1.10711500 & 3.07337900 & $\mathrm{C}$ & 3.39499500 & -0.71983600 & 5.48399000 \\
\hline
\end{tabular}




\begin{tabular}{|c|c|c|c|c|c|c|c|}
\hline $\mathrm{H}$ & 2.10446400 & 0.94899900 & 4.89687000 & $\mathrm{H}$ & 1.22237100 & -0.44508000 & 5.51897900 \\
\hline $\mathrm{H}$ & 3.33604900 & -2.19807900 & 2.96860500 & $\mathrm{H}$ & 3.23057100 & -1.73977700 & 5.85321300 \\
\hline $\mathrm{H}$ & 3.79314700 & -0.12210900 & 6.30932100 & $\mathrm{~N}$ & 2.02479100 & -0.69447100 & 3.56075200 \\
\hline C & 3.91290000 & -0.57362700 & 1.75063400 & $\mathrm{C}$ & 4.54986700 & -1.48413700 & 0.95354200 \\
\hline C & 3.98026100 & 0.83920000 & 1.40872700 & C & 5.34117300 & -1.11647300 & -0.18383200 \\
\hline $\mathrm{H}$ & 4.49349600 & -2.54198400 & 1.20229000 & C & 4.80769100 & 1.23931600 & 0.30683400 \\
\hline C & 3.27087300 & 1.83662600 & 2.09892100 & C & 5.99856700 & -2.06847800 & -0.98078000 \\
\hline C & 5.49227200 & 0.26846700 & -0.48438100 & C & 4.94817900 & 2.62548800 & -0.01566700 \\
\hline $\mathrm{H}$ & 2.57235900 & 1.56567900 & 2.87826400 & C & 3.38984900 & 3.18291100 & 1.75447800 \\
\hline C & 6.79888600 & -1.66883800 & -2.05028300 & $\mathrm{H}$ & 5.88133500 & -3.12408700 & -0.74880500 \\
\hline C & 6.31963300 & 0.66959400 & -1.57866100 & C & 4.23377300 & 3.58045800 & 0.72396400 \\
\hline C & 5.80700000 & 3.00248600 & -1.10527900 & $\mathrm{H}$ & 2.80172100 & 3.92057600 & 2.29183700 \\
\hline C & 6.96171100 & -0.31653700 & -2.34603700 & $\mathrm{H}$ & 7.30653900 & -2.41702100 & -2.65364800 \\
\hline C & 6.46224200 & 2.07244600 & -1.85325100 & $\mathrm{H}$ & 4.32764700 & 4.63247500 & 0.46649600 \\
\hline $\mathrm{H}$ & 5.91691500 & 4.06163200 & -1.32592600 & $\mathrm{H}$ & 7.59268800 & -0.01163200 & -3.17751900 \\
\hline $\mathrm{H}$ & 7.10499800 & 2.37753700 & -2.67562800 & C & -1.33544700 & 1.18900100 & 2.32336300 \\
\hline$S$ & -0.38975300 & 2.25456500 & 3.24085500 & $\mathrm{~N}$ & -1.34574200 & -0.15576100 & 2.52195400 \\
\hline $\mathrm{H}$ & -1.82489800 & -0.73841500 & 1.83162200 & $\mathrm{~S}$ & -4.33615800 & -1.28297400 & 0.17308200 \\
\hline 0 & -4.69929000 & -1.63407900 & -1.21412900 & $\mathrm{O}$ & -2.97704500 & -1.75553300 & 0.58638400 \\
\hline $\mathrm{O}$ & -4.61455400 & 0.11106000 & 0.59640900 & C & -5.49330500 & -2.31580700 & 1.20200200 \\
\hline $\mathrm{F}$ & -6.76474200 & -2.05148600 & 0.89004100 & $\mathrm{~F}$ & -5.31428800 & -2.07197700 & 2.50971400 \\
\hline $\mathrm{F}$ & -5.26177600 & -3.62387700 & 0.98572100 & C & 4.30584400 & -0.72922300 & 4.25255000 \\
\hline $\mathrm{H}$ & 4.72293200 & 0.26895700 & 4.08376300 & $\mathrm{H}$ & 5.14267300 & -1.42918000 & 4.32672700 \\
\hline C & 1.24628900 & 0.37748000 & -1.50820700 & $\mathrm{O}$ & 0.94476100 & 1.23285000 & -0.70740300 \\
\hline 0 & 1.52047300 & 0.52323700 & -2.81119100 & C & 1.47158400 & 1.86491900 & -3.47025900 \\
\hline $\mathrm{C}$ & 2.44728300 & 2.82404800 & -2.78970000 & $\mathrm{H}$ & 2.48809000 & 3.75300100 & -3.36871500 \\
\hline $\mathrm{H}$ & 2.13586100 & 3.07003900 & -1.77494200 & $\mathrm{H}$ & 3.45322300 & 2.39476400 & -2.76112600 \\
\hline C & 1.93042300 & 1.53886000 & -4.89217100 & $\mathrm{H}$ & 1.26107400 & 0.81192300 & -5.36489800 \\
\hline $\mathrm{H}$ & 1.92602500 & 2.45028900 & -5.49832200 & $\mathrm{H}$ & 2.94658000 & 1.13123400 & -4.89027800 \\
\hline $\mathrm{C}$ & 0.03250700 & 2.38135600 & -3.45785300 & $\mathrm{H}$ & -0.00703800 & 3.35201600 & -3.96164700 \\
\hline $\mathrm{H}$ & -0.63317300 & 1.69499300 & -3.99215300 & $\mathrm{H}$ & -0.33376100 & 2.51868500 & -2.43975300 \\
\hline $\mathrm{C}$ & 1.66587800 & -2.00263400 & -1.96495200 & $\mathrm{H}$ & 2.26003900 & -1.67733100 & -2.81060400 \\
\hline C & 2.02771900 & -3.32391500 & -1.41287800 & C & 2.96941700 & -4.09265500 & -2.12103700 \\
\hline C & 1.46612500 & -3.84345600 & -0.23435000 & C & 3.35279600 & -5.34758600 & -1.65736200 \\
\hline $\mathrm{H}$ & 3.40978800 & -3.69502300 & -3.03211200 & $\mathrm{C}$ & 1.85312300 & -5.10296600 & 0.22354700 \\
\hline $\mathrm{H}$ & 0.74814800 & -3.26631700 & 0.33588800 & C & 2.79281100 & -5.85692400 & -0.48206000 \\
\hline $\mathrm{H}$ & 4.08687200 & -5.92702200 & -2.20992000 & $\mathrm{H}$ & 1.41446600 & -5.49443900 & 1.13690800 \\
\hline $\mathrm{H}$ & 3.08763200 & -6.83787000 & -0.11981000 & $\mathrm{~N}$ & 1.32911100 & -0.97716300 & -1.12912800 \\
\hline $\mathrm{H}$ & 1.13569300 & -1.13848000 & -0.13120600 & C & 0.17625000 & -2.48672600 & -3.34820200 \\
\hline $\mathrm{H}$ & 0.39777600 & -1.67575400 & -4.03596800 & $\mathrm{H}$ & 0.56380900 & -3.46174200 & -3.62901000 \\
\hline C & -1.01816300 & -2.41544900 & -2.62756000 & C & -1.61350100 & -3.57283300 & -1.96920800 \\
\hline $\mathrm{H}$ & -0.87276800 & -4.35062600 & -1.74685800 & $\mathrm{H}$ & -2.15863700 & -3.27867100 & -1.06450500 \\
\hline C & -1.65933700 & -1.08658500 & -2.42388700 & $\mathrm{H}$ & -2.74982300 & -1.16020600 & -2.38568500 \\
\hline $\mathrm{H}$ & -1.36643600 & -0.72283600 & -1.42526700 & $\mathrm{H}$ & -1.34440700 & -0.35046800 & -3.16568100 \\
\hline C & -3.96264800 & -5.52617700 & -1.87990700 & $\mathrm{H}$ & -4.48711800 & -4.87704600 & -1.17083700 \\
\hline $\mathrm{H}$ & -4.71513400 & -6.11623400 & -2.41751800 & $\mathrm{H}$ & -3.34035800 & -6.22394900 & -1.30706400 \\
\hline $\mathrm{C}$ & -4.01054600 & -3.29790600 & -4.05637300 & $\mathrm{H}$ & -4.54905800 & -2.62613000 & -3.38087700 \\
\hline $\mathrm{H}$ & -3.42531500 & -2.69231000 & -4.75861600 & $\mathrm{H}$ & -4.75139800 & -3.85513800 & -4.64370900 \\
\hline C & -1.96559100 & -5.61800600 & -4.26873700 & $\mathrm{H}$ & -1.31710800 & -5.03858000 & -4.93693200 \\
\hline $\mathrm{H}$ & -1.33727700 & -6.33641500 & -3.72869300 & $\mathrm{H}$ & -2.65301800 & -6.19265400 & -4.90178700 \\
\hline $\mathrm{Si}$ & -2.93815600 & -4.50272800 & -3.08676700 & & & & \\
\hline
\end{tabular}

TS- $R-4 f-n d-27$ 
Imaginary frequency: $-235.56 \mathrm{~cm}^{-1}$

E[B3LYP/6-31G(d)]: -4788.685263 Hartree

$\mathrm{E}\left[\mathrm{PCM}\left(\mathrm{Et}_{2} \mathrm{O}\right)-\mathrm{B} 3 \mathrm{LYP} / 6-311 \mathrm{G}(\mathrm{d}, \mathrm{p})-\mathrm{D} 3(\mathrm{BJ})\right]$ : -4790.214199 Hartree

\begin{tabular}{|c|c|c|c|c|c|c|c|}
\hline 01 & & & & $\mathrm{~N}$ & 2.50477700 & -1.63957700 & 1.33703200 \\
\hline $\mathrm{H}$ & 3.03743000 & -0.76265400 & 1.34050500 & $\mathrm{C}$ & 3.11610600 & -2.65024800 & 0.57917000 \\
\hline $\mathrm{C}$ & 4.41858900 & -2.38281800 & 0.11403100 & $\mathrm{C}$ & 2.49992800 & -3.85199900 & 0.20487000 \\
\hline C & 5.07330000 & -3.29499900 & -0.70717900 & $\mathrm{H}$ & 4.90771000 & -1.45680400 & 0.39873200 \\
\hline $\mathrm{C}$ & 3.16951800 & -4.74427400 & -0.63472700 & $\mathrm{H}$ & 1.51489100 & -4.09254700 & 0.57572900 \\
\hline $\mathrm{C}$ & 4.45673800 & -4.48599100 & -1.09749900 & $\mathrm{H}$ & 4.97168900 & -5.19394700 & -1.73467100 \\
\hline C & 6.44358600 & -2.96654500 & -1.23954700 & C & 2.43067300 & -5.97131000 & -1.09481300 \\
\hline $\mathrm{F}$ & 7.12945400 & -2.15024700 & -0.41419700 & $\mathrm{~F}$ & 6.37368400 & -2.34696500 & -2.44210600 \\
\hline $\mathrm{F}$ & 7.18805600 & -4.08210300 & -1.42108500 & $\mathrm{~F}$ & 1.51776900 & -5.66413600 & -2.06183800 \\
\hline $\mathrm{F}$ & 3.25265900 & -6.90157600 & -1.62359100 & $\mathrm{~F}$ & 1.73902900 & -6.55721300 & -0.09574700 \\
\hline $\mathrm{C}$ & -0.06477200 & -0.09509200 & 3.41601300 & $\mathrm{H}$ & -0.12513700 & -0.98386700 & 4.04350700 \\
\hline $\mathrm{C}$ & 0.34544700 & 1.11920900 & 4.34074100 & $\mathrm{C}$ & 0.33023900 & 2.47130100 & 3.60120500 \\
\hline $\mathrm{H}$ & -0.68407800 & 2.73830800 & 3.28653700 & $\mathrm{H}$ & 0.97421100 & 2.47325100 & 2.71954400 \\
\hline $\mathrm{H}$ & 0.69231600 & 3.25548000 & 4.27732900 & $\mathrm{C}$ & 1.76089000 & 0.84078300 & 4.89018900 \\
\hline $\mathrm{H}$ & 2.02760300 & 1.60813600 & 5.62650900 & $\mathrm{H}$ & 2.51966700 & 0.85599900 & 4.10510000 \\
\hline $\mathrm{H}$ & 1.80739100 & -0.13506000 & 5.38832300 & C & -0.61712800 & 1.22419000 & 5.54128300 \\
\hline $\mathrm{H}$ & -0.32894100 & 2.07801200 & 6.16491300 & $\mathrm{H}$ & -0.57735100 & 0.33252800 & 6.17818800 \\
\hline $\mathrm{H}$ & -1.65520600 & 1.38798800 & 5.22987200 & $\mathrm{C}$ & -1.41060400 & 0.11405900 & 2.67665200 \\
\hline $\mathrm{O}$ & -1.44352900 & 0.79406200 & 1.63991900 & C & -2.69146600 & -1.18254000 & 4.44882200 \\
\hline $\mathrm{C}$ & -3.87647500 & 0.08000400 & 2.71139900 & $\mathrm{C}$ & -4.00952900 & -0.67191800 & 5.03882200 \\
\hline $\mathrm{H}$ & -2.73569700 & -2.25672500 & 4.23131300 & $\mathrm{H}$ & -1.84664500 & -1.02300500 & 5.11182400 \\
\hline $\mathrm{H}$ & -3.81481000 & 1.17341000 & 2.74491100 & $\mathrm{H}$ & -3.83310000 & 0.26080700 & 5.58795600 \\
\hline $\mathrm{H}$ & -4.46015800 & -1.38923500 & 5.73135800 & $\mathrm{~N}$ & -2.55307100 & -0.41301200 & 3.19631200 \\
\hline C & -4.33947000 & -0.26443700 & 1.29866000 & C & -4.75836200 & 0.78061600 & 0.52324200 \\
\hline C & -4.52860700 & -1.62388800 & 0.81520600 & C & -5.40793700 & 0.61199600 & -0.74298300 \\
\hline $\mathrm{H}$ & -4.61634700 & 1.79791400 & 0.88132900 & $\mathrm{C}$ & -5.20985600 & -1.82396700 & -0.43146800 \\
\hline $\mathrm{C}$ & -4.09123200 & -2.76151700 & 1.51489800 & $\mathrm{C}$ & -5.84055200 & 1.70308300 & -1.51536500 \\
\hline C & -5.64614500 & -0.71184400 & -1.21206600 & C & -5.46328600 & -3.14959800 & -0.90665200 \\
\hline $\mathrm{H}$ & -3.52194400 & -2.64612500 & 2.42606500 & $\mathrm{C}$ & -4.34358900 & -4.05007100 & 1.04460100 \\
\hline $\mathrm{C}$ & -6.49294200 & 1.49889600 & -2.73060400 & $\mathrm{H}$ & -5.66916100 & 2.71175600 & -1.14756600 \\
\hline C & -6.32081500 & -0.91211200 & -2.45535300 & C & -5.03156400 & -4.24803200 & -0.14744200 \\
\hline $\mathrm{C}$ & -6.15143700 & -3.32232200 & -2.15756100 & $\mathrm{H}$ & -3.98831900 & -4.90386700 & 1.61458900 \\
\hline $\mathrm{C}$ & -6.73090900 & 0.20752300 & -3.19743400 & $\mathrm{H}$ & -6.82531600 & 2.35237700 & -3.31570000 \\
\hline C & -6.55855800 & -2.25688000 & -2.89988100 & $\mathrm{H}$ & -5.22854600 & -5.25444300 & -0.50824400 \\
\hline $\mathrm{H}$ & -6.33800200 & -4.33635000 & -2.50271500 & $\mathrm{H}$ & -7.24214100 & 0.05540800 & -4.14473900 \\
\hline $\bar{H}$ & -7.07409300 & -2.40724600 & -3.84515600 & C & 1.38430000 & -1.64958600 & 2.13507100 \\
\hline $\mathrm{S}$ & 0.61848800 & -3.03828900 & 2.73115100 & $\mathrm{~N}$ & 0.96952900 & -0.38891300 & 2.43873100 \\
\hline $\bar{H}$ & 1.32984000 & 0.38954000 & 1.88142600 & $\mathrm{~S}$ & 3.62115200 & 1.89919700 & 0.72789400 \\
\hline $\mathrm{O}$ & 4.16737300 & 2.33421400 & -0.57024900 & $\mathrm{O}$ & 2.12627700 & 1.87413200 & 0.79759000 \\
\hline $\mathrm{O}$ & 4.24271700 & 0.69625700 & 1.33986200 & $\mathrm{C}$ & 4.08592200 & 3.27954900 & 1.88807400 \\
\hline $\mathrm{F}$ & 5.40980000 & 3.44257500 & 1.92979000 & $\mathrm{~F}$ & 3.64415400 & 3.02961600 & 3.13120100 \\
\hline$F$ & 3.52458300 & 4.43378700 & 1.47487300 & $\mathrm{C}$ & -4.87145600 & -0.41514000 & 3.79582700 \\
\hline $\mathrm{H}$ & -5.35249800 & -1.34152500 & 3.46894100 & $\mathrm{H}$ & -5.66200400 & 0.32245400 & 3.96022400 \\
\hline C & -1.58138900 & 0.00073000 & -2.20106900 & $\mathrm{O}$ & -1.68843000 & 0.14846900 & -3.40486800 \\
\hline $\mathrm{O}$ & -1.39630000 & -1.11346700 & -1.51340800 & $\mathrm{C}$ & -1.07484700 & -2.41094000 & -2.19469700 \\
\hline $\mathrm{C}$ & 0.31464800 & -2.28074800 & -2.82041200 & $\mathrm{H}$ & 0.63740400 & -3.25768900 & -3.19406300 \\
\hline $\mathrm{H}$ & 0.31134900 & -1.57357500 & -3.65440200 & $\mathrm{H}$ & 1.04452700 & -1.95548100 & -2.07230400 \\
\hline $\mathrm{C}$ & -1.07707200 & -3.39741000 & -1.03038500 & $\mathrm{H}$ & -2.08676500 & -3.53109000 & -0.63390000 \\
\hline
\end{tabular}




\begin{tabular}{|llll|lrll|}
\hline $\mathrm{H}$ & -0.69986300 & -4.36647400 & -1.37090800 & $\mathrm{H}$ & -0.43841200 & -3.04411400 & -0.21737100 \\
\hline $\mathrm{C}$ & -2.15154000 & -2.77253800 & -3.21943100 & $\mathrm{H}$ & -1.97221300 & -3.79908400 & -3.55873800 \\
\hline $\mathrm{H}$ & -3.14524600 & -2.73850300 & -2.76313400 & $\mathrm{H}$ & -2.13455000 & -2.10838200 & -4.08382400 \\
\hline $\mathrm{C}$ & -1.95367600 & 2.33095400 & -1.81177600 & $\mathrm{H}$ & -2.57215600 & 2.27475500 & -2.70063000 \\
\hline $\mathrm{C}$ & -2.19540200 & 3.46924400 & -0.91501800 & $\mathrm{C}$ & -3.11653800 & 4.44890100 & -1.33269300 \\
\hline $\mathrm{C}$ & -1.53635900 & 3.62643700 & 0.31679500 & $\mathrm{C}$ & -3.39349200 & 5.54958500 & -0.52789100 \\
\hline $\mathrm{H}$ & -3.61988400 & 4.33601800 & -2.28986300 & $\mathrm{C}$ & -1.81632900 & 4.73434200 & 1.11611500 \\
\hline $\mathrm{H}$ & -0.81452500 & 2.89192300 & 0.65540500 & $\mathrm{C}$ & -2.74241300 & 5.69349700 & 0.70102100 \\
\hline $\mathrm{H}$ & -4.11290500 & 6.29380700 & -0.85710300 & $\mathrm{H}$ & -1.30041900 & 4.84839200 & 2.06461400 \\
\hline $\mathrm{H}$ & -2.95272000 & 6.55445000 & 1.32943700 & $\mathrm{~N}$ & -1.63101600 & 1.10426900 & -1.32028600 \\
\hline $\mathrm{H}$ & -1.46111500 & 0.94572000 & -0.31915200 & $\mathrm{C}$ & -0.48844400 & 3.07424100 & -3.18920600 \\
\hline $\mathrm{H}$ & -0.71169900 & 2.31429200 & -3.93119600 & $\mathrm{H}$ & -0.95022900 & 4.04729900 & -3.33444000 \\
\hline $\mathrm{C}$ & 0.70164700 & 2.98871200 & -2.47803800 & $\mathrm{C}$ & 1.23798700 & 4.12012000 & -1.71796300 \\
\hline $\mathrm{H}$ & 0.45455700 & 4.84155800 & -1.45553800 & $\mathrm{H}$ & 1.75843000 & 3.78393000 & -0.81521200 \\
\hline $\mathrm{C}$ & 1.40774300 & 1.67623100 & -2.40377000 & $\mathrm{H}$ & 1.16829900 & 1.02716900 & -3.24939800 \\
\hline $\mathrm{H}$ & 2.48803200 & 1.79566900 & -2.29225300 & $\mathrm{H}$ & 1.08416700 & 1.17582900 & -1.47605600 \\
\hline $\mathrm{C}$ & 3.26466600 & 6.38547100 & -1.44548200 & $\mathrm{H}$ & 3.72763400 & 5.84635700 & -0.61281800 \\
\hline $\mathrm{H}$ & 4.03232500 & 7.01872300 & -1.90684500 & $\mathrm{H}$ & 2.49329500 & 7.04664300 & -1.03267900 \\
\hline $\mathrm{C}$ & 3.89010000 & 4.09991900 & -3.46485000 & $\mathrm{H}$ & 4.37923600 & 3.49308800 & -2.69622200 \\
\hline $\mathrm{H}$ & 3.49507600 & 3.42724700 & -4.23522900 & $\mathrm{H}$ & 4.65303200 & 4.72933500 & -3.94021300 \\
\hline $\mathrm{C}$ & 1.61254000 & 6.12279400 & -4.06306100 & $\mathrm{H}$ & 1.14144300 & 5.44270000 & -4.78252000 \\
\hline $\mathrm{H}$ & 0.82903800 & 6.76667100 & -3.64523600 & $\mathrm{H}$ & 2.30063200 & 6.76652100 & -4.62485400 \\
\hline $\mathrm{Si}$ & 2.54752900 & 5.18269300 & -2.71081000 & & & & \\
\hline
\end{tabular}

\section{TS- $R-4 f-n d-28$}

Imaginary frequency: $-284.48 \mathrm{~cm}^{-1}$ E[B3LYP/6-31G(d)]: -4788.686185 Hartree $\mathrm{E}\left[\mathrm{PCM}\left(\mathrm{Et}_{2} \mathrm{O}\right)-\mathrm{B} 3 \mathrm{LYP} / 6-311 \mathrm{G}(\mathrm{d}, \mathrm{p})-\mathrm{D} 3(\mathrm{BJ})\right]$ : -4790.214031 Hartree

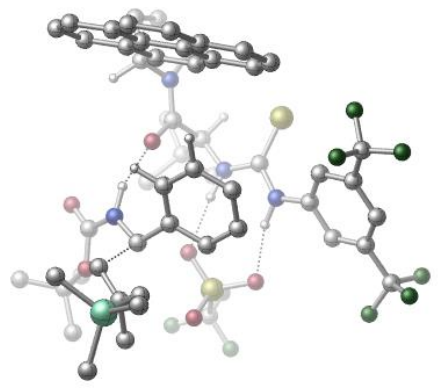

\begin{tabular}{|c|c|c|c|c|c|c|c|}
\hline 01 & & & & $\mathrm{~N}$ & 0.79493800 & 2.41451500 & -1.56968300 \\
\hline $\mathrm{H}$ & 1.75140600 & 2.07176200 & -1.43633200 & C & 0.52244400 & 3.59609800 & -0.85990100 \\
\hline $\mathrm{C}$ & 1.60670000 & 4.41903800 & -0.51835100 & $\mathrm{C}$ & -0.75812800 & 3.93429200 & -0.40083800 \\
\hline C & 1.40257200 & 5.56583200 & 0.24928500 & $\mathrm{H}$ & 2.60702000 & 4.14428900 & -0.83352700 \\
\hline $\mathrm{C}$ & -0.94311100 & 5.08438800 & 0.36379700 & $\mathrm{H}$ & -1.59558700 & 3.29319400 & -0.63129600 \\
\hline $\mathrm{C}$ & 0.12747800 & 5.91655900 & 0.69310700 & $\mathrm{H}$ & -0.02565000 & 6.80965000 & 1.28631200 \\
\hline C & 2.56531500 & 6.47565500 & 0.54618400 & C & -2.31043600 & 5.36687300 & 0.92165500 \\
\hline $\mathrm{F}$ & 2.72434300 & 7.41399900 & -0.41615200 & $\mathrm{~F}$ & 3.72940500 & 5.79923300 & 0.63295800 \\
\hline $\mathrm{F}$ & 2.39422900 & 7.14068200 & 1.71344600 & $\mathrm{~F}$ & -3.29792100 & 5.01817100 & 0.06388400 \\
\hline $\mathrm{F}$ & -2.54000700 & 4.66408600 & 2.06781300 & $\mathrm{~F}$ & -2.48232200 & 6.66853200 & 1.23039600 \\
\hline C & -0.17478800 & -0.38193700 & -3.73133400 & $\mathrm{H}$ & -0.73561300 & 0.27466100 & -4.39589200 \\
\hline C & 0.85598600 & -1.17477500 & -4.61472700 & C & 1.61198000 & -2.26466400 & -3.83613100 \\
\hline $\mathrm{H}$ & 0.94223700 & -3.02913800 & -3.43575500 & $\mathrm{H}$ & 2.18466900 & -1.84894600 & -3.00407800 \\
\hline $\mathrm{H}$ & 2.32385600 & -2.75824500 & -4.50998100 & C & 1.86931400 & -0.16877600 & -5.20080700 \\
\hline $\mathrm{H}$ & 2.50015500 & -0.67229600 & -5.94316400 & $\mathrm{H}$ & 2.52822200 & 0.23951200 & -4.42917000 \\
\hline $\mathrm{H}$ & 1.36579900 & 0.66889300 & -5.69837900 & C & 0.10266300 & -1.84441000 & -5.78262000 \\
\hline $\mathrm{H}$ & 0.80851900 & -2.41431400 & -6.39725300 & $\mathrm{H}$ & -0.37651300 & -1.10564900 & -6.43693900 \\
\hline $\mathrm{H}$ & -0.66099700 & -2.54731900 & -5.42985200 & C & -1.16638100 & -1.27602000 & -2.94107500 \\
\hline $\mathrm{O}$ & -0.82245300 & -1.81454200 & -1.88172100 & $\mathrm{C}$ & -3.03434000 & -0.92980800 & -4.65639700 \\
\hline C & -3.37807700 & -2.31519200 & -2.70031400 & C & -3.90107900 & -2.10887000 & -5.10901400 \\
\hline
\end{tabular}




\begin{tabular}{|c|c|c|c|c|c|c|c|}
\hline $\mathrm{H}$ & -3.64113900 & -0.04131600 & -4.43946200 & $\mathrm{H}$ & -2.28031200 & -0.65165400 & -5.38812300 \\
\hline $\mathrm{H}$ & -2.81282200 & -3.19081700 & -2.36899000 & $\mathrm{H}$ & -3.27489300 & -2.84349300 & -5.62860100 \\
\hline $\mathrm{H}$ & -4.69916500 & -1.80349900 & -5.79260400 & $\mathrm{~N}$ & -2.43023600 & -1.44017300 & -3.41812000 \\
\hline C & -4.02637400 & -1.65096400 & -1.48827700 & C & -3.97222400 & -0.29917800 & -1.31153800 \\
\hline C & -4.76889100 & -2.47221900 & -0.54698800 & C & -4.63487600 & 0.36371400 & -0.22475500 \\
\hline $\mathrm{H}$ & -3.39586300 & 0.31719600 & -1.99608500 & C & -5.43908300 & -1.83499100 & 0.54830600 \\
\hline C & -4.86058300 & -3.87191700 & -0.65512800 & C & -4.56135900 & 1.75549000 & -0.04741700 \\
\hline C & -5.37448400 & -0.41847800 & 0.70954400 & C & -6.17849600 & -2.61606900 & 1.49066700 \\
\hline $\mathrm{H}$ & -4.36316000 & -4.38665600 & -1.46972400 & C & -5.58239700 & -4.62547900 & 0.27056200 \\
\hline C & -5.19844000 & 2.36999600 & 1.03051600 & $\mathrm{H}$ & -3.99782700 & 2.35138500 & -0.76024600 \\
\hline C & -6.03310900 & 0.21900700 & 1.80576400 & C & -6.23762800 & -4.00952500 & 1.33166300 \\
\hline C & -6.84074400 & -1.94716700 & 2.57778700 & $\mathrm{H}$ & -5.63265700 & -5.70518300 & 0.15720700 \\
\hline C & -5.92852600 & 1.61302300 & 1.94590000 & $\mathrm{H}$ & -5.11166900 & 3.44441100 & 1.15742500 \\
\hline C & -6.77181700 & -0.59614400 & 2.72977000 & $\mathrm{H}$ & -6.80199600 & -4.60249400 & 2.04741800 \\
\hline $\mathrm{H}$ & -7.40430500 & -2.55205400 & 3.28440400 & $\mathrm{H}$ & -6.42487100 & 2.09886400 & 2.78272900 \\
\hline $\mathrm{H}$ & -7.27845500 & -0.10836300 & 3.55930200 & C & -0.00908700 & 1.73279000 & -2.45304500 \\
\hline$S$ & -1.43624900 & 2.37057600 & -3.11824300 & $\mathrm{~N}$ & 0.48103800 & 0.51144800 & -2.78333700 \\
\hline $\mathrm{H}$ & 1.25516900 & 0.13190800 & -2.23401700 & $S$ & 3.91889800 & 0.43240600 & -0.65559700 \\
\hline $\mathrm{O}$ & 4.31668100 & -0.00012800 & 0.70568100 & $\mathrm{O}$ & 2.93698700 & -0.46976900 & -1.32042500 \\
\hline $\mathrm{O}$ & 3.58452300 & 1.87062500 & -0.79699100 & C & 5.46494500 & 0.22116300 & -1.66787200 \\
\hline $\mathrm{F}$ & 6.43732000 & 1.02199100 & -1.22310100 & $\mathrm{~F}$ & 5.22014000 & 0.50716800 & -2.95304000 \\
\hline $\mathrm{F}$ & 5.89893800 & -1.05307800 & -1.59425700 & C & 1.87914700 & -3.72104000 & -0.47787400 \\
\hline $\mathrm{O}$ & 1.47186400 & -4.45197900 & -1.35745900 & $\mathrm{O}$ & 3.10177800 & -3.72967100 & 0.08306500 \\
\hline C & 4.19489000 & -4.56858400 & -0.46988000 & C & 3.85724100 & -6.04553500 & -0.25179400 \\
\hline $\mathrm{H}$ & 4.70374400 & -6.66441300 & -0.56950000 & $\mathrm{H}$ & 2.97655200 & -6.33538900 & -0.82764900 \\
\hline $\mathrm{H}$ & 3.67143900 & -6.24714800 & 0.80956100 & C & 5.39648100 & -4.13678100 & 0.37317100 \\
\hline $\mathrm{H}$ & 5.57853600 & -3.06392600 & 0.26081400 & $\mathrm{H}$ & 6.29082500 & -4.67907400 & 0.04930200 \\
\hline $\mathrm{H}$ & 5.23093600 & -4.35972700 & 1.43350000 & C & 4.42517200 & -4.22898400 & -1.94457800 \\
\hline $\mathrm{H}$ & 5.32113400 & -4.75433800 & -2.29424200 & $\mathrm{H}$ & 4.58890800 & -3.15491300 & -2.06510900 \\
\hline $\mathrm{H}$ & 3.57885600 & -4.53421900 & -2.56092700 & C & 1.48528600 & -1.84657200 & 1.07844800 \\
\hline $\mathrm{H}$ & 2.52305000 & -1.55134800 & 0.96394800 & C & 0.54402100 & -0.74431100 & 1.40477400 \\
\hline $\mathrm{C}$ & 1.06619600 & 0.52280900 & 1.71636900 & C & -0.84366800 & -0.95021600 & 1.47837600 \\
\hline $\mathrm{C}$ & 0.21255000 & 1.56550900 & 2.07371800 & $\mathrm{H}$ & 2.13665100 & 0.69361900 & 1.65459800 \\
\hline $\mathrm{C}$ & -1.69029600 & 0.09301400 & 1.84813000 & $\mathrm{H}$ & -1.26822800 & -1.92239800 & 1.24345200 \\
\hline $\mathrm{C}$ & -1.16576100 & 1.35471100 & 2.14236500 & $\mathrm{H}$ & 0.62581200 & 2.54706200 & 2.28518500 \\
\hline $\mathrm{H}$ & -2.76074000 & -0.07450300 & 1.89431100 & $\mathrm{H}$ & -1.82879400 & 2.17475000 & 2.40024200 \\
\hline $\mathrm{N}$ & 1.06316000 & -2.76130700 & 0.12176000 & $\mathrm{H}$ & 0.20263300 & -2.57840000 & -0.40133000 \\
\hline $\mathrm{C}$ & 1.66261700 & -2.87326600 & 2.68323400 & $\mathrm{H}$ & 2.24365100 & -3.68644900 & 2.25898900 \\
\hline $\mathrm{H}$ & 0.61931600 & -3.10431300 & 2.88410000 & $\mathrm{C}$ & 2.32899200 & -2.04316000 & 3.60522600 \\
\hline $\mathrm{C}$ & 1.59284400 & -1.23634900 & 4.56768900 & $\mathrm{H}$ & 0.56527000 & -1.04188600 & 4.23872300 \\
\hline $\mathrm{H}$ & 2.09971800 & -0.29021700 & 4.79233900 & $\mathrm{C}$ & 3.80825200 & -1.87286700 & 3.50003900 \\
\hline $\mathrm{H}$ & 4.24704600 & -1.48685000 & 4.42391900 & $\mathrm{H}$ & 4.01697700 & -1.13558100 & 2.70380000 \\
\hline $\mathrm{H}$ & 4.31199500 & -2.79672600 & 3.20198000 & $\mathrm{C}$ & 0.45515000 & -0.85886200 & 7.36872200 \\
\hline $\mathrm{H}$ & 1.03378600 & 0.06393100 & 7.49088600 & $\mathrm{H}$ & 0.26623900 & -1.26591500 & 8.36955400 \\
\hline $\mathrm{H}$ & -0.51362200 & -0.59177800 & 6.93140000 & C & 3.06385600 & -2.51988600 & 7.04302900 \\
\hline $\mathrm{H}$ & 3.68954900 & -1.62504800 & 7.14145400 & $\mathrm{H}$ & 3.61528000 & -3.25283200 & 6.44328700 \\
\hline $\mathrm{H}$ & 2.94338700 & -2.94432800 & 8.04740700 & $\mathrm{C}$ & 0.34907500 & -3.68623900 & 6.06938300 \\
\hline $\mathrm{H}$ & 0.85843200 & -4.41990600 & 5.43459100 & $\mathrm{H}$ & -0.62598400 & -3.46945200 & 5.61757000 \\
\hline $\mathrm{H}$ & 0.16120400 & -4.16463000 & 7.03842200 & $\mathrm{Si}$ & 1.37239400 & -2.12015400 & 6.30767300 \\
\hline C & -4.43893700 & -2.69071500 & -3.78778000 & $\mathrm{H}$ & -5.40096900 & -2.23652400 & -3.53285000 \\
\hline $\mathrm{H}$ & -4.59481700 & -3.77179300 & -3.84455600 & & & & \\
\hline
\end{tabular}




\section{TS- $R-4 f-n d-29$}

Imaginary frequency: $-244.94 \mathrm{~cm}^{-1}$ E[B3LYP/6-31G(d)]: -4788.688687 Hartree E[PCM(Et $\left.\left.{ }_{2} \mathrm{O}\right)-\mathrm{B} 3 L Y P / 6-311 \mathrm{G}(\mathrm{d}, \mathrm{p})-\mathrm{D} 3(\mathrm{BJ})\right]:-4790.213984$ Hartree

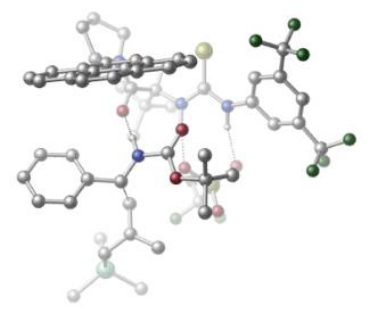

\begin{tabular}{|c|c|c|c|c|c|c|c|}
\hline 01 & & & & $\mathrm{~N}$ & -2.23710100 & -1.76345000 & 1.65024700 \\
\hline $\mathrm{H}$ & -1.51995200 & -2.47426000 & 1.48767500 & $\mathrm{C}$ & -3.41288100 & -1.99715900 & 0.92060600 \\
\hline C & -3.51437400 & -3.24947100 & 0.28542800 & C & -4.43656400 & -1.05895400 & 0.72642500 \\
\hline $\mathrm{C}$ & -4.60259000 & -3.53759600 & -0.53277500 & $\mathrm{H}$ & -2.72514700 & -3.98228200 & 0.41927200 \\
\hline $\mathrm{C}$ & -5.52127900 & -1.37242400 & -0.09354500 & $\mathrm{H}$ & -4.38328600 & -0.09716900 & 1.21386700 \\
\hline C & -5.62289400 & -2.60617800 & -0.73175100 & $\mathrm{H}$ & -6.46746900 & -2.83603400 & -1.36896300 \\
\hline $\mathrm{C}$ & -4.62817600 & -4.83623700 & -1.29223900 & $\mathrm{C}$ & -6.61489700 & -0.35109200 & -0.25462800 \\
\hline $\mathrm{F}$ & -4.01427600 & -5.83332900 & -0.62467000 & $\mathrm{~F}$ & -3.99871300 & -4.71907800 & -2.49071000 \\
\hline $\mathrm{F}$ & -5.89124900 & -5.24049300 & -1.55914600 & $\mathrm{~F}$ & -6.11565300 & 0.90849400 & -0.36417600 \\
\hline $\mathrm{F}$ & -7.36441200 & -0.57609900 & -1.35680100 & $\mathrm{~F}$ & -7.45759400 & -0.33701800 & 0.80108000 \\
\hline $\mathrm{C}$ & 0.12621700 & 0.26842900 & 3.56359000 & $\bar{H}$ & -0.63608800 & 0.50963500 & 4.30316300 \\
\hline $\mathrm{C}$ & 1.33121200 & -0.38159100 & 4.33268200 & $\mathrm{C}$ & 2.53124400 & -0.72725100 & 3.42893600 \\
\hline $\mathrm{H}$ & 2.96607800 & 0.16779800 & 2.97618000 & $\mathrm{H}$ & 2.25462700 & -1.41408600 & 2.62568400 \\
\hline $\mathrm{H}$ & 3.30460800 & -1.21828000 & 4.03279900 & $\mathrm{C}$ & 0.82492400 & -1.66818400 & 5.01692200 \\
\hline $\mathrm{H}$ & 1.60816700 & -2.07670600 & 5.66656300 & $\mathrm{H}$ & 0.56235600 & -2.43763000 & 4.28591800 \\
\hline $\mathrm{H}$ & -0.05891000 & -1.47247300 & 5.63581200 & C & 1.80194200 & 0.60522700 & 5.42159600 \\
\hline $\mathrm{H}$ & 2.65116800 & 0.18041600 & 5.96871100 & $\mathrm{H}$ & 1.01101200 & 0.80819500 & 6.15411100 \\
\hline $\mathrm{H}$ & 2.13289100 & 1.56102300 & 4.99696400 & $\mathrm{C}$ & 0.50885800 & 1.54927300 & 2.78495000 \\
\hline 0 & 1.21987600 & 1.46923200 & 1.76655400 & C & -0.67222000 & 3.05471700 & 4.46120200 \\
\hline C & 0.39124100 & 3.98394600 & 2.46660400 & C & -0.15260600 & 4.45049100 & 4.81423700 \\
\hline $\mathrm{H}$ & -1.75286500 & 3.04994500 & 4.27868100 & $\overline{\mathrm{H}}$ & -0.45644300 & 2.31921200 & 5.23366700 \\
\hline $\mathrm{H}$ & 1.47628600 & 3.97348900 & 2.31826900 & $\mathrm{H}$ & 0.82542200 & 4.36891900 & 5.30422300 \\
\hline $\mathrm{H}$ & -0.82562600 & 4.98637500 & 5.49021800 & $\mathrm{~N}$ & 0.04959100 & 2.74817800 & 3.21185400 \\
\hline C & -0.29720400 & 4.11336900 & 1.11231900 & C & -1.41841000 & 3.39296800 & 0.81963000 \\
\hline C & 0.21036500 & 5.08264400 & 0.15574000 & C & -2.13766800 & 3.55519400 & -0.41093800 \\
\hline $\mathrm{H}$ & -1.79701000 & 2.66069300 & 1.52797300 & C & -0.48684600 & 5.25675300 & -1.08498500 \\
\hline C & 1.35473400 & 5.86851000 & 0.38621300 & C & -3.29995500 & 2.81973000 & -0.69239300 \\
\hline C & -1.66162600 & 4.49805500 & -1.36654400 & C & -0.01407600 & 6.20228200 & -2.04828600 \\
\hline $\mathrm{H}$ & 1.90149700 & 5.77278500 & 1.31815000 & C & 1.80896800 & 6.78657600 & -0.56052900 \\
\hline C & -3.98665500 & 3.00608900 & -1.89119200 & $\mathrm{H}$ & -3.66210100 & 2.10054800 & 0.03516100 \\
\hline C & -2.36595900 & 4.67891200 & -2.59619800 & C & 1.13651000 & 6.95502900 & -1.76595400 \\
\hline C & -0.73853500 & 6.35908900 & -3.28031900 & $\mathrm{H}$ & 2.69594900 & 7.37819200 & -0.34895700 \\
\hline C & -3.52533800 & 3.92289000 & -2.83503000 & $\mathrm{H}$ & -4.88784600 & 2.43090800 & -2.08083600 \\
\hline C & -1.86053700 & 5.63421100 & -3.54210900 & $\mathrm{H}$ & 1.49348500 & 7.67498500 & -2.49848300 \\
\hline $\mathrm{H}$ & -0.36826300 & 7.08129100 & -4.00424200 & $\mathrm{H}$ & -4.06537500 & 4.06405700 & -3.76843700 \\
\hline $\mathrm{H}$ & -2.39814100 & 5.76996600 & -4.47773900 & C & -1.87069400 & -0.72368300 & 2.46910000 \\
\hline$S$ & -2.95688500 & 0.31994600 & 3.24863400 & $\mathrm{~N}$ & -0.52114700 & -0.65841600 & 2.64542600 \\
\hline $\mathrm{H}$ & 0.06794300 & -1.14529800 & 1.96504800 & $S$ & 0.76458300 & -3.67123500 & 0.42982900 \\
\hline $\mathrm{O}$ & 0.97945000 & -4.12567900 & -0.95663500 & $\mathrm{O}$ & 1.01552400 & -2.21050800 & 0.64530700 \\
\hline $\mathrm{O}$ & -0.46170600 & -4.14259900 & 1.11361000 & C & 2.15639300 & -4.47313200 & 1.37309000 \\
\hline$F$ & 2.09199700 & -5.80328500 & 1.29524800 & $\mathrm{~F}$ & 2.13136600 & -4.11453700 & 2.66532800 \\
\hline $\mathrm{F}$ & 3.34501300 & -4.07605100 & 0.85773600 & $\mathrm{C}$ & -0.02015400 & 5.12605500 & 3.44270800 \\
\hline $\mathrm{H}$ & -0.98586900 & 5.53541200 & 3.12925000 & $\mathrm{H}$ & 0.70144600 & 5.94744800 & 3.43534400 \\
\hline C & 0.09911100 & 0.09382600 & -1.47621600 & $\mathrm{O}$ & -0.92450400 & 0.17041400 & -0.83479100 \\
\hline $\mathrm{O}$ & 0.28702000 & -0.47195900 & -2.67322200 & C & -0.85833200 & -1.03191800 & -3.45189400 \\
\hline C & -1.83726600 & 0.09820000 & -3.77540700 & $\mathrm{H}$ & -2.62660100 & -0.28757600 & -4.43000300 \\
\hline
\end{tabular}




\begin{tabular}{|c|c|c|c|c|c|c|c|}
\hline $\mathrm{H}$ & -2.30239200 & 0.49504200 & -2.87157600 & $\mathrm{H}$ & -1.32901300 & 0.91505700 & -4.29985700 \\
\hline C & -0.16967100 & -1.54310400 & -4.71797900 & $\mathrm{H}$ & 0.54914300 & -2.33271000 & -4.47713000 \\
\hline $\mathrm{H}$ & -0.91740000 & -1.96042900 & -5.39982900 & $\mathrm{H}$ & 0.35254900 & -0.73117100 & -5.23616800 \\
\hline $\mathrm{C}$ & -1.50686000 & -2.17837500 & -2.67826300 & $\mathrm{H}$ & -2.24916800 & -2.67476900 & -3.31255800 \\
\hline $\mathrm{H}$ & -0.75829500 & -2.91916900 & -2.38148000 & $\mathrm{H}$ & -2.01321500 & -1.81145500 & -1.78542300 \\
\hline C & 2.50008000 & 0.61338200 & -1.63070900 & $\mathrm{H}$ & 2.39921700 & 0.25698100 & -2.64832800 \\
\hline $\mathrm{C}$ & 3.50150600 & 1.69400400 & -1.45128400 & $\mathrm{C}$ & 4.40617000 & 1.93046400 & -2.50032700 \\
\hline C & 3.56860300 & 2.48943300 & -0.29538800 & C & 5.35784300 & 2.94421900 & -2.40329000 \\
\hline $\mathrm{H}$ & 4.34955200 & 1.32818300 & -3.40371800 & C & 4.52480300 & 3.49838900 & -0.20406000 \\
\hline $\mathrm{H}$ & 2.87419000 & 2.32983600 & 0.52400700 & C & 5.42120600 & 3.72903400 & -1.25095000 \\
\hline $\mathrm{H}$ & 6.04143700 & 3.12456900 & -3.22808100 & $\mathrm{H}$ & 4.56579300 & 4.11427500 & 0.68969500 \\
\hline $\mathrm{H}$ & 6.16072700 & 4.52093000 & -1.17133900 & $\mathrm{~N}$ & 1.28842000 & 0.68733200 & -1.00489500 \\
\hline $\mathrm{H}$ & 1.20387700 & 1.03458300 & -0.04104100 & C & 3.45045000 & -0.98199000 & -0.81624000 \\
\hline $\mathrm{H}$ & 4.04602900 & -0.45807600 & -0.07284200 & $\mathrm{H}$ & 2.54592800 & -1.44985200 & -0.43664300 \\
\hline C & 4.13001000 & -1.62019600 & -1.85442900 & C & 5.55975300 & -1.40023900 & -2.08897200 \\
\hline $\mathrm{H}$ & 5.84291100 & -1.54101300 & -3.13945300 & $\mathrm{H}$ & 5.88868500 & -0.41180100 & -1.74471200 \\
\hline $\mathrm{C}$ & 3.38911000 & -2.53518700 & -2.78341600 & $\mathrm{H}$ & 3.03711700 & -1.95462900 & -3.65073100 \\
\hline $\mathrm{H}$ & 4.04755200 & -3.31417500 & -3.18036000 & $\mathrm{H}$ & 2.51735700 & -2.99472400 & -2.30862200 \\
\hline C & 8.43340300 & -2.31872300 & -1.63811100 & $\mathrm{H}$ & 8.72795400 & -1.27886700 & -1.45417900 \\
\hline $\mathrm{H}$ & 9.14080500 & -2.96202800 & -1.10074200 & $\mathrm{H}$ & 8.55502000 & -2.51809600 & -2.70925600 \\
\hline C & 6.46433200 & -2.28247300 & 0.77623300 & $\mathrm{H}$ & 6.65432600 & -1.22805700 & 1.00875400 \\
\hline $\mathrm{H}$ & 5.45977700 & -2.53696400 & 1.13043000 & $\mathrm{H}$ & 7.17752100 & -2.87971900 & 1.35797500 \\
\hline C & 6.15562500 & -4.43418500 & -1.43123600 & $\mathrm{H}$ & 5.15116000 & -4.65153900 & -1.05150500 \\
\hline $\mathrm{H}$ & 6.17574600 & -4.66373000 & -2.50301800 & $\mathrm{H}$ & 6.84775400 & -5.12787500 & -0.93763300 \\
\hline $\mathrm{Si}$ & 6.66530500 & -2.65670900 & -1.06063300 & & & & \\
\hline
\end{tabular}

\section{TS- $R-4 f-n d-30$}

Imaginary frequency: $-200.00 \mathrm{~cm}^{-1}$

E[B3LYP/6-31G(d)]: -4788.672810 Hartree

$\mathrm{E}\left[\mathrm{PCM}\left(\mathrm{Et}_{2} \mathrm{O}\right)-\mathrm{B} 3 \mathrm{LYP} / 6-311 \mathrm{G}(\mathrm{d}, \mathrm{p})-\mathrm{D} 3(\mathrm{BJ})\right]$ : -4790.213232 Hartree

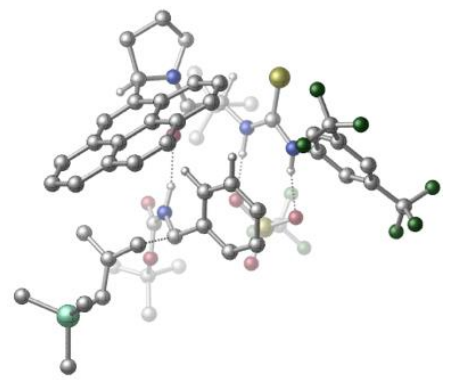

\begin{tabular}{|crrr|lrrr|}
\hline 01 & & & & $\mathrm{~N}$ & 3.27677500 & 0.82143000 & 0.42499700 \\
\hline $\mathrm{H}$ & 2.82570700 & 1.68460600 & 0.09600000 & $\mathrm{C}$ & 4.12855700 & 0.24500200 & -0.53280800 \\
\hline $\mathrm{C}$ & 4.75770700 & 1.09729100 & -1.45235700 & $\mathrm{C}$ & 4.28239200 & -1.13945400 & -0.67522800 \\
\hline $\mathrm{C}$ & 5.53763300 & 0.56527000 & -2.47947300 & $\mathrm{H}$ & 4.60853700 & 2.16851700 & -1.37636400 \\
\hline $\mathrm{C}$ & 5.05246700 & -1.65426100 & -1.71508200 & $\mathrm{H}$ & 3.78271800 & -1.80625200 & 0.01162900 \\
\hline $\mathrm{C}$ & 5.69587600 & -0.81386700 & -2.62390900 & $\mathrm{H}$ & 6.29255400 & -1.22110300 & -3.43087500 \\
\hline $\mathrm{C}$ & 6.26800300 & 1.49227400 & -3.41478600 & $\mathrm{C}$ & 5.05666500 & -3.14254700 & -1.91904200 \\
\hline $\mathrm{F}$ & 7.51107300 & 1.78062000 & -2.96168300 & $\mathrm{~F}$ & 5.62433600 & 2.66637700 & -3.57364800 \\
\hline $\mathrm{F}$ & 6.42142200 & 0.94323100 & -4.64365500 & $\mathrm{~F}$ & 5.11435800 & -3.82519200 & -0.75189000 \\
\hline $\mathrm{F}$ & 3.91197400 & -3.55580000 & -2.53811000 & $\mathrm{~F}$ & 6.08398900 & -3.56089900 & -2.68575000 \\
\hline $\mathrm{C}$ & 1.34358000 & 0.64231000 & 3.53137500 & $\mathrm{H}$ & 2.14569600 & 0.11914900 & 4.04995300 \\
\hline $\mathrm{C}$ & 0.98688300 & 1.89745500 & 4.40764300 & $\mathrm{C}$ & -0.22518000 & 2.69274200 & 3.89496000 \\
\hline $\mathrm{H}$ & -1.14017700 & 2.09687400 & 3.88573700 & $\mathrm{H}$ & -0.07411000 & 3.07353900 & 2.88261400 \\
\hline $\mathrm{H}$ & -0.39099300 & 3.55542100 & 4.55297100 & $\mathrm{C}$ & 2.22417100 & 2.81852000 & 4.43978600 \\
\hline $\mathrm{H}$ & 2.06219300 & 3.62835700 & 5.16125800 & $\mathrm{H}$ & 2.40814100 & 3.27537500 & 3.46324200 \\
\hline $\mathrm{H}$ & 3.12825300 & 2.27421100 & 4.73837600 & $\mathrm{C}$ & 0.69029700 & 1.41485000 & 5.84273400 \\
\hline $\mathrm{H}$ & 0.44940700 & 2.27288300 & 6.48038700 & $\mathrm{H}$ & 1.55332900 & 0.90457700 & 6.28786500 \\
\hline $\mathrm{H}$ & -0.17072100 & 0.73557400 & 5.87644500 & $\mathrm{C}$ & 0.16621000 & -0.33373900 & 3.30005500 \\
\hline $\mathrm{O}$ & -0.74547000 & -0.07088800 & 2.50135900 & $\mathrm{C}$ & 1.13048800 & -1.97823200 & 4.98813300 \\
\hline
\end{tabular}




\begin{tabular}{|c|c|c|c|c|c|c|c|}
\hline $\mathrm{C}$ & -1.01810400 & -2.40783400 & 3.85763200 & $\mathrm{C}$ & 0.72386000 & -3.43275300 & 5.25518400 \\
\hline $\mathrm{H}$ & 2.15269800 & -1.88388100 & 4.61085000 & $\mathrm{H}$ & 1.03585700 & -1.37656200 & 5.89919800 \\
\hline $\mathrm{C}$ & -0.78971300 & -3.44200100 & 4.99606300 & $\bar{H}$ & -1.89865000 & -1.79930100 & 4.08619500 \\
\hline $\mathrm{H}$ & 0.98017500 & -3.74310100 & 6.27250700 & $\mathrm{H}$ & 1.23609900 & -4.10896600 & 4.56452600 \\
\hline $\mathrm{H}$ & -1.32571600 & -3.10532300 & 5.89128600 & $\mathrm{H}$ & -1.17507000 & -4.42997500 & 4.73223000 \\
\hline $\mathrm{N}$ & 0.16014100 & -1.52016500 & 3.96792000 & C & -1.32215400 & -3.04338300 & 2.49505500 \\
\hline C & -2.64988600 & -3.10717500 & 2.17091100 & C & -0.34916300 & -3.66253800 & 1.60441900 \\
\hline C & -3.15309100 & -3.77436000 & 1.00863000 & $\mathrm{H}$ & -3.37793400 & -2.63625400 & 2.82890000 \\
\hline C & -0.82774200 & -4.35747100 & 0.43943200 & C & 1.04331900 & -3.59835300 & 1.79976900 \\
\hline C & -4.52584500 & -3.82736700 & 0.70722900 & C & -2.22197800 & -4.41276700 & 0.13860800 \\
\hline C & 0.09703000 & -4.99095300 & -0.45037800 & $\mathrm{H}$ & 1.45295300 & -3.01791400 & 2.61256700 \\
\hline C & 1.93572300 & -4.21592600 & 0.92224800 & C & -4.97794600 & -4.49145000 & -0.43326400 \\
\hline $\mathrm{H}$ & -5.23587900 & -3.36669600 & 1.39111800 & C & -2.69445800 & -5.09190200 & -1.02737000 \\
\hline C & 1.47361800 & -4.91438400 & -0.18750700 & C & -0.40615900 & -5.67660900 & -1.60902600 \\
\hline $\mathrm{H}$ & 3.00310000 & -4.12440600 & 1.09954100 & C & -4.07434400 & -5.11468800 & -1.29330100 \\
\hline $\mathrm{H}$ & -6.04338600 & -4.53846500 & -0.64466800 & C & -1.73693900 & -5.72722500 & -1.88789600 \\
\hline $\mathrm{H}$ & 2.17821700 & -5.36993700 & -0.87831700 & $\mathrm{H}$ & 0.31241300 & -6.15580900 & -2.26930500 \\
\hline $\mathrm{H}$ & -4.43305200 & -5.63712100 & -2.17681600 & $\mathrm{H}$ & -2.09744600 & -6.24873200 & -2.77114500 \\
\hline C & 2.95774200 & 0.35558400 & 1.67542300 & $S$ & 3.84941100 & -0.85319400 & 2.48059600 \\
\hline $\mathrm{N}$ & 1.89322600 & 0.98913300 & 2.22729500 & $\mathrm{H}$ & 1.34184100 & 1.62448700 & 1.63852700 \\
\hline S & 0.95524800 & 3.67927600 & -0.50023900 & 0 & -0.02015100 & 4.05608300 & -1.54086700 \\
\hline $\mathrm{O}$ & 0.38478900 & 2.86861500 & 0.61634100 & $\mathrm{O}$ & 2.26691200 & 3.16499200 & -0.97391200 \\
\hline C & 1.39985900 & 5.28241300 & 0.33511200 & $F$ & 1.93878400 & 6.14313700 & -0.53605900 \\
\hline $\mathrm{F}$ & 2.28895500 & 5.06091600 & 1.31937200 & $\mathrm{~F}$ & 0.31177000 & 5.85463800 & 0.87926400 \\
\hline C & -2.86738800 & 2.50313100 & 0.86842600 & 0 & -2.87455600 & 2.80573200 & 2.04194100 \\
\hline $\mathrm{O}$ & -3.52016500 & 3.13713300 & -0.12901700 & C & -4.02909400 & 4.52037000 & 0.06150900 \\
\hline C & -2.87885300 & 5.43378300 & 0.48777800 & $\mathrm{H}$ & -3.23075300 & 6.47170500 & 0.49938300 \\
\hline $\mathrm{H}$ & -2.04808500 & 5.35456700 & -0.21866900 & $\mathrm{H}$ & -2.51457800 & 5.18044600 & 1.48446500 \\
\hline $\mathrm{C}$ & -5.18647300 & 4.50998600 & 1.06353500 & $\mathrm{H}$ & -5.97185600 & 3.81556000 & 0.74112100 \\
\hline $\mathrm{H}$ & -5.62685100 & 5.51159500 & 1.12223000 & $\mathrm{H}$ & -4.84147500 & 4.21842400 & 2.05633700 \\
\hline C & -4.51488100 & 4.89061400 & -1.34118300 & $\mathrm{H}$ & -4.91454700 & 5.90979200 & -1.33753100 \\
\hline $\mathrm{H}$ & -5.30988200 & 4.21459600 & -1.67604200 & $\mathrm{H}$ & -3.68888100 & 4.84680200 & -2.05769200 \\
\hline C & -2.09194900 & 1.07601200 & -0.96911600 & $\mathrm{H}$ & -2.37558800 & 1.92961200 & -1.57562400 \\
\hline $\mathrm{C}$ & -0.84778000 & 0.41340000 & -1.46751500 & $\mathrm{C}$ & -0.31111700 & 0.86521900 & -2.68284700 \\
\hline $\mathrm{C}$ & -0.26818600 & -0.68590100 & -0.82003400 & $\mathrm{C}$ & 0.78774500 & 0.22074700 & -3.24807800 \\
\hline $\mathrm{H}$ & -0.72954000 & 1.74537300 & -3.16192400 & C & 0.82293100 & -1.33131800 & -1.39550100 \\
\hline $\mathrm{H}$ & -0.66169100 & -1.03777000 & 0.12910800 & $\mathrm{C}$ & 1.34558100 & -0.88991300 & -2.61444800 \\
\hline $\mathrm{H}$ & 1.20911200 & 0.59177200 & -4.17769100 & $\mathrm{H}$ & 1.26536600 & -2.18466200 & -0.89481800 \\
\hline $\mathrm{H}$ & 2.19188200 & -1.40954100 & -3.05318100 & $\mathrm{~N}$ & -2.20901700 & 1.36965500 & 0.38712700 \\
\hline $\mathrm{H}$ & -1.58777500 & 0.93119000 & 1.07733300 & $\mathrm{C}$ & -3.34445500 & -0.17077900 & -1.48483500 \\
\hline $\mathrm{H}$ & -3.03286700 & -1.01399200 & -0.87095000 & $\bar{H}$ & -3.09093900 & -0.28859900 & -2.53616300 \\
\hline C & -4.64149000 & 0.33171500 & -1.21987200 & C & -5.41438200 & 1.02049400 & -2.24045200 \\
\hline $\mathrm{H}$ & -4.78199900 & 1.42818400 & -3.03741700 & $\overline{\mathrm{H}}$ & -6.05369400 & 1.80845900 & -1.82739000 \\
\hline C & -5.18866700 & 0.14950700 & 0.15213600 & $\mathrm{H}$ & -6.12709500 & 0.68059300 & 0.32344900 \\
\hline $\mathrm{H}$ & -4.43330600 & 0.48219800 & 0.88085800 & $\mathrm{H}$ & -5.32661600 & -0.92148900 & 0.35592300 \\
\hline C & -7.40617400 & 0.87658300 & -4.54097900 & $\mathrm{H}$ & -7.93036700 & 1.74744100 & -4.13091400 \\
\hline $\mathrm{H}$ & -8.13368200 & 0.29621900 & -5.12137900 & $\mathrm{H}$ & -6.64304800 & 1.24086300 & -5.23816900 \\
\hline C & -7.97805000 & -0.78217200 & -1.97334200 & $\mathrm{H}$ & -8.51256600 & 0.05490100 & -1.50930200 \\
\hline $\mathrm{H}$ & -7.56383400 & -1.40483800 & -1.17261100 & $\mathrm{H}$ & -8.72134800 & -1.38871000 & -2.50536700 \\
\hline C & -5.68326700 & -1.64051800 & -3.89296600 & $\mathrm{H}$ & -5.25209900 & -2.27776200 & -3.11234700 \\
\hline $\mathrm{H}$ & -4.86708400 & -1.30675300 & -4.54438100 & $\mathrm{H}$ & -6.34664000 & -2.26868600 & -4.50037800 \\
\hline $\mathrm{Si}$ & -6.65344600 & -0.19115100 & -3.17987600 & & & & \\
\hline
\end{tabular}




\section{TS- $R-4 f-n d-31$}

Imaginary frequency: $-277.00 \mathrm{~cm}^{-1}$ E[B3LYP/6-31G(d)]: -4788.681108 Hartree E[PCM(Et2O)-B3LYP/6-311G(d,p)-D3(BJ)]: -4790.212950 Hartree

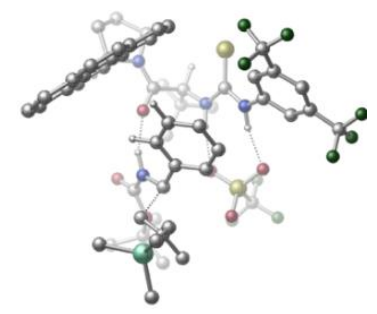

\begin{tabular}{|c|c|c|c|c|c|c|c|}
\hline 01 & & & & $\mathrm{~N}$ & 0.38929800 & 2.79713800 & -1.14649100 \\
\hline $\mathrm{H}$ & 1.37129700 & 2.54932300 & -0.99119900 & C & -0.10560600 & 3.74481600 & -0.23673800 \\
\hline $\mathrm{C}$ & 0.81303800 & 4.60437800 & 0.38247400 & $\mathrm{C}$ & -1.45354100 & 3.79539200 & 0.14948600 \\
\hline C & 0.38172100 & 5.50660900 & 1.35633400 & $\mathrm{H}$ & 1.86135100 & 4.55285500 & 0.10992800 \\
\hline C & -1.86617500 & 4.70657100 & 1.11791200 & $\mathrm{H}$ & -2.16575400 & 3.11710300 & -0.29678800 \\
\hline C & -0.95998600 & 5.57656000 & 1.72841500 & $\mathrm{H}$ & -1.29144600 & 6.29140800 & 2.47162000 \\
\hline C & 1.39390700 & 6.36881100 & 2.06174200 & C & -3.29395300 & 4.67426800 & 1.58889600 \\
\hline $\mathrm{F}$ & 2.42643000 & 6.70257100 & 1.26042200 & $\mathrm{~F}$ & 1.92096100 & 5.73209900 & 3.13727600 \\
\hline $\mathrm{F}$ & 0.84636700 & 7.51722700 & 2.52082900 & $\mathrm{~F}$ & -4.15300700 & 4.28283700 & 0.62143200 \\
\hline $\mathrm{F}$ & -3.45833100 & 3.79786600 & 2.62178500 & $\mathrm{~F}$ & -3.71110700 & 5.87548500 & 2.04126700 \\
\hline C & -0.11833300 & 0.33196000 & -3.81180000 & $\mathrm{H}$ & -0.73555500 & 1.02536400 & -4.38179900 \\
\hline C & 1.03649500 & -0.15293600 & -4.76156800 & C & 1.91472400 & -1.25395600 & -4.14437200 \\
\hline $\mathrm{H}$ & 1.34794400 & -2.15980900 & -3.91891800 & $\mathrm{H}$ & 2.39862800 & -0.92011000 & -3.22378000 \\
\hline $\mathrm{H}$ & 2.70864300 & -1.51824800 & -4.85466200 & C & 1.91687700 & 1.06545000 & -5.11172000 \\
\hline $\mathrm{H}$ & 2.63171000 & 0.78989400 & -5.89644700 & $\mathrm{H}$ & 2.49131300 & 1.41081500 & -4.24745600 \\
\hline $\mathrm{H}$ & 1.31668600 & 1.90556000 & -5.48146600 & C & 0.41631900 & -0.69881800 & -6.06466100 \\
\hline $\mathrm{H}$ & 1.21033100 & -1.06546100 & -6.72494600 & $\mathrm{H}$ & -0.13031500 & 0.07784800 & -6.61371700 \\
\hline $\mathrm{H}$ & -0.26254300 & -1.53957600 & -5.87762500 & C & -1.01238300 & -0.80760000 & -3.25097700 \\
\hline 0 & -0.61852600 & -1.53097500 & -2.32880800 & C & -2.84633500 & -0.26785200 & -4.93432400 \\
\hline $\mathrm{C}$ & -3.09560900 & -2.12897400 & -3.35477700 & $\mathrm{C}$ & -3.80200100 & -1.30236600 & -5.53594100 \\
\hline $\mathrm{H}$ & -3.37920400 & 0.63018200 & -4.59798100 & $\mathrm{H}$ & -2.08106400 & 0.04573900 & -5.64021900 \\
\hline $\mathrm{H}$ & -2.52046700 & -3.03519000 & -3.57940700 & $\mathrm{H}$ & -3.24858500 & -1.98618000 & -6.19136400 \\
\hline $\bar{H}$ & -4.59713900 & -0.83749600 & -6.12658500 & $\mathrm{~N}$ & -2.25620700 & -0.97756300 & -3.77985500 \\
\hline $\mathrm{C}$ & -3.52824600 & -2.25243800 & -1.89655200 & $\mathrm{C}$ & -3.48845100 & -3.51068000 & -1.36302300 \\
\hline C & -4.13966900 & -1.17663000 & -1.12971000 & C & -4.06358200 & -3.84776600 & -0.09407400 \\
\hline $\mathrm{H}$ & -3.02323300 & -4.31418500 & -1.93073000 & $\mathrm{C}$ & -4.74912200 & -1.49095100 & 0.13192800 \\
\hline $\mathrm{C}$ & -4.14974100 & 0.16497500 & -1.54973000 & $\mathrm{C}$ & -4.02229300 & -5.15180300 & 0.42632300 \\
\hline C & -4.71888000 & -2.82194600 & 0.64752500 & C & -5.37649100 & -0.45996100 & 0.90223000 \\
\hline $\mathrm{H}$ & -3.64674500 & 0.45269900 & -2.46183100 & $\mathrm{C}$ & -4.74093100 & 1.16593900 & -0.77850100 \\
\hline $\mathrm{C}$ & -4.61803100 & -5.44736400 & 1.65136400 & $\overline{\mathrm{H}}$ & -3.52380600 & -5.93329900 & -0.14217900 \\
\hline C & -5.32399700 & -3.13271500 & 1.90440600 & C & -5.35769000 & 0.86199500 & 0.42970800 \\
\hline $\mathrm{C}$ & -5.98794000 & -0.80370500 & 2.15762600 & $\mathrm{H}$ & -4.69893800 & 2.19438700 & -1.12363800 \\
\hline $\mathrm{C}$ & -5.26260500 & -4.45178400 & 2.38352700 & $\mathrm{H}$ & -4.58186300 & -6.46274100 & 2.03734600 \\
\hline C & -5.96750100 & -2.07760500 & 2.63634300 & $\mathrm{H}$ & -5.80788700 & 1.65095800 & 1.02690600 \\
\hline $\mathrm{H}$ & -6.47031200 & -0.01080500 & 2.72429300 & $\mathrm{H}$ & -5.72640700 & -4.68938800 & 3.33797700 \\
\hline $\mathrm{H}$ & -6.43516000 & -2.31727100 & 3.58839200 & C & -0.25836800 & 2.21779400 & -2.21373800 \\
\hline $\mathrm{S}$ & -1.66709000 & 2.85754900 & -2.91288300 & $\mathrm{~N}$ & 0.37424800 & 1.11364300 & -2.68399900 \\
\hline $\mathrm{H}$ & 1.15356100 & 0.73071800 & -2.14482800 & $\mathrm{~S}$ & 3.76050500 & 1.11666300 & -0.45934600 \\
\hline 0 & 4.25384700 & 0.48105000 & 0.78647700 & 0 & 2.90302600 & 0.23264100 & -1.29811500 \\
\hline 0 & 3.21625600 & 2.48805200 & -0.30622100 & C & 5.29425900 & 1.33860800 & -1.48787600 \\
\hline $\mathrm{F}$ & 6.15326600 & 2.16812300 & -0.88868300 & $\mathrm{~F}$ & 4.97884000 & 1.83587800 & -2.69111800 \\
\hline $\mathrm{F}$ & 5.90713700 & 0.15192100 & -1.66860000 & C & 2.30029100 & -3.22558900 & -1.09901200 \\
\hline 0 & 2.00036000 & -3.84182900 & -2.10094700 & 0 & 3.50825800 & -3.17951200 & -0.50701600 \\
\hline C & 4.70909800 & -3.75492400 & -1.16234600 & C & 4.90321200 & -3.13304700 & -2.54754000 \\
\hline $\mathrm{H}$ & 5.86401300 & -3.46728200 & -2.95516000 & $\mathrm{H}$ & 4.92294800 & -2.04239300 & -2.47450500 \\
\hline $\mathrm{H}$ & 4.11015200 & -3.43217400 & -3.23375800 & C & 4.57385300 & -5.27865500 & -1.21541600 \\
\hline
\end{tabular}




\begin{tabular}{|c|c|c|c|c|c|c|c|}
\hline $\mathrm{H}$ & 4.40985400 & -5.68746700 & -0.21159100 & $\mathrm{H}$ & 5.49845300 & -5.71434400 & -1.61017000 \\
\hline $\mathrm{H}$ & 3.74419100 & -5.57558600 & -1.85945300 & C & 5.83430500 & -3.32223800 & -0.22006300 \\
\hline $\mathrm{H}$ & 6.79698100 & -3.67348900 & -0.60570900 & $\mathrm{H}$ & 5.69387100 & -3.74958400 & 0.77948000 \\
\hline $\mathrm{H}$ & 5.86723400 & -2.23172800 & -0.13739100 & C & 1.66471800 & -1.72340500 & 0.74934500 \\
\hline $\mathrm{H}$ & 2.66631900 & -1.30731000 & 0.72387500 & C & 0.61210400 & -0.79074200 & 1.23120400 \\
\hline C & 1.00734400 & 0.42586500 & 1.81550200 & C & -0.75280300 & -1.11680400 & 1.18746300 \\
\hline C & 0.05470300 & 1.30340300 & 2.32959700 & $\mathrm{H}$ & 2.05909300 & 0.69297700 & 1.84187100 \\
\hline C & -1.69974400 & -0.23806400 & 1.71144700 & $\mathrm{H}$ & -1.08422700 & -2.05101600 & 0.74231100 \\
\hline C & -1.30082500 & 0.97295300 & 2.28193800 & $\mathrm{H}$ & 0.37317900 & 2.25211200 & 2.75148100 \\
\hline $\mathrm{H}$ & -2.75147200 & -0.49535200 & 1.66581400 & $\mathrm{H}$ & -2.04470100 & 1.66623400 & 2.66208400 \\
\hline $\mathrm{N}$ & 1.36505400 & -2.48663900 & -0.37431900 & $\mathrm{H}$ & 0.49299000 & -2.32491500 & -0.88442200 \\
\hline C & 1.91796300 & -2.99305800 & 2.13826300 & $\mathrm{H}$ & 2.59634800 & -3.65037300 & 1.60251800 \\
\hline $\mathrm{H}$ & 0.90487200 & -3.37084400 & 2.25489600 & C & 2.46924800 & -2.28588100 & 3.22650300 \\
\hline C & 1.62929400 & -1.76462600 & 4.29334700 & $\mathrm{H}$ & 0.59177600 & -1.62464400 & 3.96813000 \\
\hline $\mathrm{H}$ & 2.02039900 & -0.83141800 & 4.71575300 & C & 3.92056400 & -1.93766100 & 3.21060000 \\
\hline $\mathrm{H}$ & 4.29548800 & -1.67683000 & 4.20373200 & $\mathrm{H}$ & 4.05700300 & -1.05164600 & 2.56414300 \\
\hline $\mathrm{H}$ & 4.53279200 & -2.73173800 & 2.77374100 & C & 0.41244500 & -2.06393500 & 7.06998600 \\
\hline $\mathrm{H}$ & 0.88220100 & -1.12609000 & 7.38781100 & $\mathrm{H}$ & 0.25437800 & -2.67551900 & 7.96667100 \\
\hline $\mathrm{H}$ & -0.57296100 & -1.82208100 & 6.65579700 & C & 3.19582700 & -3.34230100 & 6.52842500 \\
\hline $\mathrm{H}$ & 3.71639400 & -2.42204200 & 6.81830400 & $\mathrm{H}$ & 3.83408200 & -3.88215600 & 5.81978300 \\
\hline $\mathrm{H}$ & 3.10875900 & -3.96375600 & 7.42822800 & C & 0.64310100 & -4.57942100 & 5.24968800 \\
\hline $\mathrm{H}$ & 1.24366000 & -5.12016800 & 4.50970300 & $\mathrm{H}$ & -0.34014500 & -4.38284500 & 4.80653200 \\
\hline $\mathrm{H}$ & 0.48905800 & -5.25210300 & 6.10234500 & $\mathrm{Si}$ & 1.48148200 & -2.99044800 & 5.82249600 \\
\hline C & -4.32169900 & -2.04263800 & -4.29988700 & $\mathrm{H}$ & -5.11721900 & -1.46359300 & -3.81864800 \\
\hline $\mathrm{H}$ & -4.72100400 & -3.03762100 & -4.51537800 & & & & \\
\hline
\end{tabular}

\section{TS-R-4f-nd-32}

Imaginary frequency: $-230.40 \mathrm{~cm}^{-1}$ E[B3LYP/6-31G(d)]: -4788.688878 Hartree E[PCM(Et $\left.\left.{ }_{2} \mathrm{O}\right)-\mathrm{B} 3 L \mathrm{YP} / 6-311 \mathrm{G}(\mathrm{d}, \mathrm{p})-\mathrm{D} 3(\mathrm{BJ})\right]$ : -4790.212405 Hartree

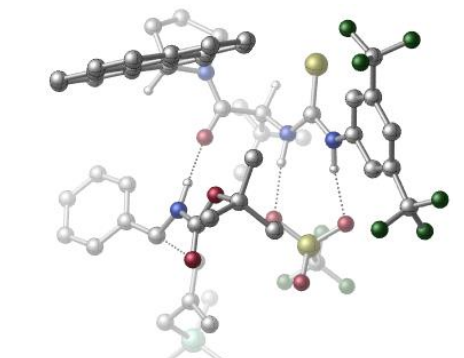

\begin{tabular}{|crrr|lrrr|}
\hline 01 & & & & $\mathrm{~N}$ & -1.59725300 & -2.25931700 & 1.63001200 \\
\hline $\mathrm{H}$ & -0.74207500 & -2.73531900 & 1.32316400 & $\mathrm{C}$ & -2.75590300 & -2.78716400 & 1.03262500 \\
\hline $\mathrm{C}$ & -2.57052800 & -3.90083600 & 0.19307100 & $\mathrm{C}$ & -4.04529200 & -2.24413600 & 1.15574800 \\
\hline $\mathrm{C}$ & -3.64026000 & -4.42360700 & -0.53026200 & $\mathrm{H}$ & -1.58290800 & -4.34011300 & 0.09524200 \\
\hline $\mathrm{C}$ & -5.09913900 & -2.78388800 & 0.42174900 & $\mathrm{H}$ & -4.21521300 & -1.41026200 & 1.81809700 \\
\hline $\mathrm{C}$ & -4.91674500 & -3.87187000 & -0.43137900 & $\mathrm{H}$ & -5.74307000 & -4.27913100 & -1.00210400 \\
\hline $\mathrm{C}$ & -3.38475600 & -5.53764800 & -1.51030700 & $\mathrm{C}$ & -6.47762700 & -2.19241400 & 0.55028100 \\
\hline $\mathrm{F}$ & -2.41248200 & -6.37193500 & -1.09340000 & $\mathrm{~F}$ & -2.99615700 & -5.05066700 & -2.71751700 \\
\hline $\mathrm{F}$ & -4.49366500 & -6.27905000 & -1.73188900 & $\mathrm{~F}$ & -6.47651200 & -1.00825600 & 1.20678800 \\
\hline $\mathrm{F}$ & -7.03348600 & -1.96546500 & -0.66692100 & $\mathrm{~F}$ & -7.32298000 & -3.01251000 & 1.21223500 \\
\hline $\mathrm{C}$ & 0.32947000 & 0.39447900 & 3.25930300 & $\mathrm{H}$ & -0.39080200 & 0.47114200 & 4.07245700 \\
\hline $\mathrm{C}$ & 1.73925400 & 0.16573800 & 3.90979700 & $\mathrm{C}$ & 2.88794700 & 0.09491400 & 2.88612400 \\
\hline $\mathrm{H}$ & 3.01153900 & 1.04157300 & 2.35200800 & $\mathrm{H}$ & 2.72950300 & -0.69337700 & 2.14775100 \\
\hline $\mathrm{H}$ & 3.82582400 & -0.12287600 & 3.41230100 & $\mathrm{C}$ & 1.68635900 & -1.15306600 & 4.70809400 \\
\hline $\mathrm{H}$ & 2.61889200 & -1.28660400 & 5.26931300 & $\mathrm{H}$ & 1.56428600 & -2.01668700 & 4.04980700 \\
\hline $\mathrm{H}$ & 0.85725200 & -1.15321500 & 5.42565400 & $\mathrm{C}$ & 2.02488200 & 1.32299300 & 4.88949500 \\
\hline $\mathrm{H}$ & 3.01467600 & 1.19236900 & 5.34148100 & $\mathrm{H}$ & 1.29543900 & 1.35192200 & 5.70801900 \\
\hline $\mathrm{H}$ & 2.02150900 & 2.29903200 & 4.38849700 & $\mathrm{C}$ & 0.23176900 & 1.65634100 & 2.36803000 \\
\hline $\mathrm{O}$ & 0.76059400 & 1.66542600 & 1.24317900 & $\mathrm{C}$ & -1.03119000 & 2.96499900 & 4.15359600 \\
\hline
\end{tabular}




\begin{tabular}{|c|c|c|c|c|c|c|c|}
\hline C & -0.62218900 & 3.92600200 & 1.95701200 & $\mathrm{C}$ & -0.76809300 & 4.45953600 & 4.35984100 \\
\hline $\mathrm{H}$ & -2.10329900 & 2.73453900 & 4.15726500 & $\mathrm{H}$ & -0.54931400 & 2.34294400 & 4.90422700 \\
\hline $\mathrm{H}$ & 0.35301100 & 4.11915600 & 1.50195100 & $\mathrm{H}$ & 0.27214500 & 4.60855000 & 4.67381700 \\
\hline $\mathrm{H}$ & -1.41681500 & 4.89592700 & 5.12526200 & $\mathrm{~N}$ & -0.46919400 & 2.72750900 & 2.81327500 \\
\hline C & -1.66706400 & 3.76472500 & 0.85718000 & C & -2.64095600 & 2.81348400 & 0.95041400 \\
\hline $\mathrm{C}$ & -1.67081300 & 4.69701300 & -0.25692100 & $\mathrm{C}$ & -3.69461600 & 2.69138500 & -0.01585800 \\
\hline $\mathrm{H}$ & -2.63318100 & 2.10329800 & 1.77320700 & C & -2.70542700 & 4.58706500 & -1.24324600 \\
\hline C & -0.70597900 & 5.70778400 & -0.41966800 & C & -4.69857100 & 1.71579900 & 0.09831800 \\
\hline C & -3.71711700 & 3.58835000 & -1.12259900 & C & -2.73502900 & 5.48476800 & -2.35586300 \\
\hline $\bar{H}$ & 0.08862800 & 5.82461800 & 0.30940900 & $\mathrm{C}$ & -0.74238400 & 6.57739100 & -1.50957200 \\
\hline C & -5.70435700 & 1.61367300 & -0.86207900 & $\mathrm{H}$ & -4.68130500 & 1.03289600 & 0.94236700 \\
\hline C & -4.75221400 & 3.48018500 & -2.10130200 & C & -1.74307900 & 6.47140700 & -2.46941800 \\
\hline $\mathrm{C}$ & -3.78653500 & 5.35169300 & -3.32751600 & $\mathrm{H}$ & 0.02008300 & 7.34591200 & -1.60586700 \\
\hline C & -5.73179500 & 2.48460100 & -1.95027000 & $\mathrm{H}$ & -6.46059600 & 0.84101100 & -0.76233300 \\
\hline $\mathrm{C}$ & -4.74892100 & 4.39667600 & -3.20733900 & $\mathrm{H}$ & -1.76703900 & 7.15382000 & -3.31557700 \\
\hline $\bar{H}$ & -3.79667600 & 6.04023700 & -4.16915900 & $\mathrm{H}$ & -6.51762600 & 2.39929600 & -2.69708600 \\
\hline $\bar{H}$ & -5.53733700 & 4.31224600 & -3.95153000 & $\mathrm{C}$ & -1.41366700 & -1.18228600 & 2.46470800 \\
\hline $\mathrm{S}$ & -2.61749900 & -0.49453800 & 3.44353500 & $\mathrm{~N}$ & -0.12260700 & -0.74076200 & 2.46279800 \\
\hline $\mathrm{H}$ & 0.48119800 & -1.03262700 & 1.68799300 & $\mathrm{~S}$ & 1.73878700 & -3.22430700 & 0.04775100 \\
\hline $\mathrm{O}$ & 2.11360500 & -3.62133500 & -1.31779200 & $\mathrm{O}$ & 1.63228600 & -1.74593000 & 0.26566400 \\
\hline $\mathrm{O}$ & 0.62171900 & -3.96541800 & 0.68523700 & C & 3.22418800 & -3.70411400 & 1.06634800 \\
\hline $\mathrm{F}$ & 3.45005400 & -5.01687300 & 1.00196800 & $F$ & 3.06426600 & -3.35796000 & 2.35377300 \\
\hline $\mathrm{F}$ & 4.32539600 & -3.06296600 & 0.60432700 & $\mathrm{C}$ & -1.00802700 & 5.05667000 & 2.96302000 \\
\hline $\mathrm{H}$ & -2.06482500 & 5.30922200 & 2.83504600 & $\mathrm{H}$ & -0.43158500 & 5.96953300 & 2.78805600 \\
\hline $\mathrm{C}$ & 1.02933600 & 0.33524400 & -2.44021100 & $\mathrm{O}$ & 1.57947500 & -0.12966000 & -3.42033400 \\
\hline $\mathrm{O}$ & -0.23263200 & 0.22721200 & -2.06657800 & $\mathrm{C}$ & -1.20319800 & -0.60723800 & -2.84573700 \\
\hline C & -0.77261500 & -2.07047000 & -2.76144200 & $\mathrm{H}$ & -1.54038000 & -2.70638500 & -3.21495400 \\
\hline $\mathrm{H}$ & 0.17677300 & -2.24700800 & -3.26977800 & $\mathrm{H}$ & -0.66564800 & -2.37246300 & -1.71703500 \\
\hline $\mathrm{C}$ & -2.50882100 & -0.36164800 & -2.09468900 & $\mathrm{H}$ & -2.79498600 & 0.69258200 & -2.13856100 \\
\hline $\mathrm{H}$ & -3.30923900 & -0.95809600 & -2.54373600 & $\mathrm{H}$ & -2.41280500 & -0.65084800 & -1.04512900 \\
\hline $\mathrm{C}$ & -1.29520100 & -0.08896500 & -4.28268800 & $\mathrm{H}$ & -2.11098300 & -0.61249700 & -4.79320600 \\
\hline $\mathrm{H}$ & -1.52506600 & 0.98213800 & -4.29085300 & $\mathrm{H}$ & -0.37077900 & -0.26175300 & -4.83525600 \\
\hline C & 3.03210500 & 1.43992600 & -1.76228500 & $\mathrm{H}$ & 3.29429100 & 1.19462900 & -2.78442300 \\
\hline $\mathrm{C}$ & 3.69271600 & 2.64030800 & -1.21090300 & $\mathrm{C}$ & 4.76707400 & 3.18385700 & -1.93751900 \\
\hline $\mathrm{C}$ & 3.28612800 & 3.26357600 & -0.01896900 & $\mathrm{C}$ & 5.42307900 & 4.32736000 & -1.48585900 \\
\hline $\mathrm{H}$ & 5.07463900 & 2.71658300 & -2.86936800 & $\mathrm{C}$ & 3.94918300 & 4.40417300 & 0.42817200 \\
\hline $\mathrm{H}$ & 2.45360400 & 2.86163700 & 0.55043900 & $\mathrm{C}$ & 5.01727600 & 4.93853100 & -0.29825500 \\
\hline $\mathrm{H}$ & 6.24361800 & 4.74321800 & -2.06351400 & $\mathrm{H}$ & 3.62936900 & 4.88112400 & 1.35059100 \\
\hline $\mathrm{H}$ & 5.52709700 & 5.82947600 & 0.05753500 & $\mathrm{~N}$ & 1.72281700 & 1.16357700 & -1.52305500 \\
\hline $\mathrm{H}$ & 1.27365900 & 1.35922900 & -0.62199600 & $\mathrm{C}$ & 4.11978600 & -0.09028700 & -0.82446100 \\
\hline $\mathrm{H}$ & 4.55861400 & 0.49757800 & -0.02325900 & $\mathrm{H}$ & 3.23794900 & -0.66485700 & -0.55732200 \\
\hline C & 4.96953000 & -0.58311100 & -1.80952300 & C & 6.36436000 & -0.12692500 & -1.90964500 \\
\hline $\mathrm{H}$ & 6.74260400 & -0.16547300 & -2.93891200 & $\mathrm{H}$ & 6.49666400 & 0.88553900 & -1.50677100 \\
\hline $\mathrm{C}$ & 4.47795700 & -1.57868800 & -2.81760100 & $\mathrm{H}$ & 4.06511100 & -1.04213800 & -3.68394700 \\
\hline $\mathrm{H}$ & 5.29823000 & -2.20249800 & -3.18592200 & $\mathrm{H}$ & 3.67660300 & -2.21075500 & -2.42419700 \\
\hline $\mathrm{C}$ & 9.31158700 & -0.57580700 & -1.25910500 & $\mathrm{H}$ & 9.41298400 & 0.48862600 & -1.01658400 \\
\hline $\mathrm{H}$ & 10.07429300 & -1.11824600 & -0.68721600 & $\mathrm{H}$ & 9.54612600 & -0.70162500 & -2.32267100 \\
\hline C & 7.19031000 & -0.97456700 & 0.98436600 & $\mathrm{H}$ & 7.19915100 & 0.08461600 & 1.26769700 \\
\hline $\mathrm{H}$ & 6.21075400 & -1.39075400 & 1.24212300 & $\mathrm{H}$ & 7.93777400 & -1.48583400 & 1.60367500 \\
\hline $\mathrm{C}$ & 7.41865200 & -3.04718100 & -1.30734400 & $\mathrm{H}$ & 6.43667900 & -3.44430900 & -1.02790200 \\
\hline $\mathrm{H}$ & 7.56676500 & -3.22236600 & -2.37927900 & $\mathrm{H}$ & 8.17427900 & -3.63706500 & -0.77351100 \\
\hline Si & 7.58540400 & -1.22686900 & -0.84294900 & & & & \\
\hline
\end{tabular}




\section{TS-R-4f-nd-33}

Imaginary frequency: $-151.17 \mathrm{~cm}^{-1}$

E[B3LYP/6-31G(d)]: -4788.685264 Hartree

E[PCM(Et2O)-B3LYP/6-311G(d,p)-D3(BJ)]: -4790.212127 Hartree

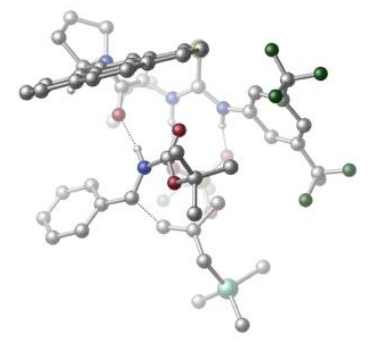

\begin{tabular}{|c|c|c|c|c|c|c|c|}
\hline 01 & & & & $\mathrm{~N}$ & 1.21876900 & 0.24677100 & -2.68863100 \\
\hline $\mathrm{H}$ & 2.06921300 & -0.28640700 & -2.47001100 & C & 1.38482100 & 1.63258000 & -2.57250700 \\
\hline C & 2.68115800 & 2.08309200 & -2.26037200 & C & 0.35010000 & 2.57685100 & -2.67305500 \\
\hline C & 2.91987700 & 3.43583900 & -2.02870400 & $\mathrm{H}$ & 3.49227200 & 1.36525300 & -2.18720500 \\
\hline C & 0.61738300 & 3.92685600 & -2.45697500 & $\mathrm{H}$ & -0.64686600 & 2.25251400 & -2.92618400 \\
\hline C & 1.89641800 & 4.37817600 & -2.13077700 & $\mathrm{H}$ & 2.09234500 & 5.43116400 & -1.96712800 \\
\hline C & 4.27928300 & 3.86926800 & -1.55344900 & C & -0.51507700 & 4.91786500 & -2.49614100 \\
\hline $\mathrm{F}$ & 5.26829100 & 3.08807000 & -2.02534000 & $F$ & 4.35444200 & 3.81623000 & -0.19055300 \\
\hline $\mathrm{F}$ & 4.55940400 & 5.14421000 & -1.89946200 & $\mathrm{~F}$ & -1.58459300 & 4.46207700 & -3.18566800 \\
\hline $\mathrm{F}$ & -0.96217000 & 5.21409100 & -1.24528400 & $\mathrm{~F}$ & -0.14129600 & 6.09035100 & -3.05378600 \\
\hline C & -0.83654100 & -2.77473400 & -2.65467600 & $\mathrm{H}$ & -1.33973900 & -2.52309000 & -3.58747200 \\
\hline C & -0.27107500 & -4.23398800 & -2.80588200 & C & 0.39540100 & -4.76617200 & -1.52228400 \\
\hline $\mathrm{H}$ & -0.33039000 & -4.85447600 & -0.70677400 & $\mathrm{H}$ & 1.21855000 & -4.13355600 & -1.18215300 \\
\hline $\mathrm{H}$ & 0.80529700 & -5.76481000 & -1.71641800 & C & 0.75105800 & -4.22912900 & -3.96143800 \\
\hline $\mathrm{H}$ & 1.09446400 & -5.25181500 & -4.15716200 & $\mathrm{H}$ & 1.62751800 & -3.62099500 & -3.72939000 \\
\hline $\mathrm{H}$ & 0.30317100 & -3.84129500 & -4.88434100 & C & -1.42177200 & -5.18973000 & -3.18298000 \\
\hline $\mathrm{H}$ & -1.03390200 & -6.20961000 & -3.28381900 & $\mathrm{H}$ & -1.87799200 & -4.92035800 & -4.14313400 \\
\hline $\mathrm{H}$ & -2.20712300 & -5.21359900 & -2.41865200 & C & -1.82147300 & -2.58972400 & -1.47223600 \\
\hline $\mathrm{O}$ & -1.38058300 & -2.43426100 & -0.31945200 & C & -3.87182200 & -2.78069700 & -2.97557400 \\
\hline C & -4.12328100 & -2.50874400 & -0.59184000 & C & -5.03317500 & -3.69581500 & -2.57687300 \\
\hline $\mathrm{H}$ & -4.22734300 & -1.80656900 & -3.33275000 & $\mathrm{H}$ & -3.23384400 & -3.20905300 & -3.74362600 \\
\hline $\mathrm{H}$ & -3.68555600 & -3.04265000 & 0.25640400 & $\mathrm{H}$ & -4.69405200 & -4.73777600 & -2.57859300 \\
\hline $\mathrm{H}$ & -5.87752200 & -3.61865000 & -3.26829700 & $\mathrm{~N}$ & -3.15505300 & -2.60295300 & -1.70453600 \\
\hline C & -4.43994500 & -1.07304500 & -0.17537400 & C & -3.98299800 & -0.00579200 & -0.89071500 \\
\hline C & -5.29844400 & -0.85419700 & 0.97780100 & C & -4.34911200 & 1.34627000 & -0.57589900 \\
\hline $\mathrm{H}$ & -3.31338000 & -0.15649300 & -1.73238000 & C & -5.69332600 & 0.48507600 & 1.30110600 \\
\hline C & -5.76333300 & -1.89749100 & 1.79881800 & C & -3.86618800 & 2.43622900 & -1.31775000 \\
\hline C & -5.22821100 & 1.58419300 & 0.51895400 & C & -6.55508100 & 0.73089500 & 2.41533300 \\
\hline $\mathrm{H}$ & -5.47370200 & -2.92174900 & 1.59075700 & C & -6.59471600 & -1.64669000 & 2.89084900 \\
\hline C & -4.24711100 & 3.73899200 & -0.99794900 & $\mathrm{H}$ & -3.18444600 & 2.25339600 & -2.14302000 \\
\hline C & -5.62241300 & 2.92026800 & 0.83582300 & C & -6.99453200 & -0.35017800 & 3.19591500 \\
\hline C & -6.94561500 & 2.08434000 & 2.70456800 & $\mathrm{H}$ & -6.93479400 & -2.47632200 & 3.50512500 \\
\hline C & -5.12159600 & 3.97994800 & 0.06086200 & $\mathrm{H}$ & -3.84516200 & 4.56694200 & -1.57385500 \\
\hline C & -6.50429300 & 3.12848000 & 1.95062900 & $\mathrm{H}$ & -7.64970300 & -0.16225100 & 4.04315300 \\
\hline $\mathrm{H}$ & -7.60896500 & 2.25701100 & 3.54889900 & $\mathrm{H}$ & -5.41799800 & 4.99795700 & 0.30316000 \\
\hline $\mathrm{H}$ & -6.81058200 & 4.14532400 & 2.18533500 & C & 0.10368800 & -0.50950300 & -2.97134200 \\
\hline$S$ & -1.23868100 & 0.02601800 & -3.85598500 & $\mathrm{~N}$ & 0.22099500 & -1.78431300 & -2.50783600 \\
\hline $\mathrm{H}$ & 0.93864400 & -1.96827000 & -1.80124600 & $S$ & 3.81140900 & -1.89248400 & -0.97395200 \\
\hline $\mathrm{O}$ & 4.73829200 & -1.43901500 & 0.07917200 & $\mathrm{O}$ & 2.43736700 & -2.24699900 & -0.49357100 \\
\hline $\mathrm{O}$ & 3.77690400 & -1.06733700 & -2.21199400 & C & 4.52531600 & -3.51877500 & -1.53113100 \\
\hline $\mathrm{F}$ & 5.75259200 & -3.34614300 & -2.02987500 & $\mathrm{~F}$ & 3.75157200 & -4.07349700 & -2.48094500 \\
\hline $\mathrm{F}$ & 4.59420100 & -4.37044600 & -0.49845000 & C & -5.39332900 & -3.24224500 & -1.14598000 \\
\hline $\mathrm{H}$ & -6.24123100 & -2.55283700 & -1.15719000 & $\mathrm{H}$ & -5.67392700 & -4.08947300 & -0.51355700 \\
\hline C & -0.62805200 & 0.73634800 & 1.47682900 & $\mathrm{O}$ & -1.08508900 & 1.10179500 & 0.42019500 \\
\hline $\mathrm{O}$ & -0.33766200 & 1.46365600 & 2.55864500 & C & -0.74264400 & 2.89841700 & 2.66990800 \\
\hline
\end{tabular}




\begin{tabular}{|c|c|c|c|c|c|c|c|}
\hline $\mathrm{C}$ & -2.26625500 & 2.98656800 & 2.59705100 & $\mathrm{H}$ & -2.57716700 & 4.01792400 & 2.79435600 \\
\hline $\mathrm{H}$ & -2.63591700 & 2.70427900 & 1.61059700 & $\mathrm{H}$ & -2.72945100 & 2.34202700 & 3.35180000 \\
\hline $\mathrm{C}$ & -0.22961200 & 3.26687200 & 4.06220400 & $\mathrm{H}$ & 0.85631500 & 3.14132500 & 4.12511900 \\
\hline $\mathrm{H}$ & -0.46770400 & 4.31327600 & 4.27749400 & $\mathrm{H}$ & -0.69867800 & 2.64267800 & 4.82995600 \\
\hline C & -0.05809700 & 3.73065900 & 1.58687000 & $\mathrm{H}$ & -0.28163300 & 4.78921100 & 1.75844300 \\
\hline $\mathrm{H}$ & 1.02871900 & 3.60595700 & 1.62685300 & $\mathrm{H}$ & -0.41220700 & 3.46669300 & 0.59128100 \\
\hline C & 0.21989700 & -1.11256900 & 2.83544800 & $\mathrm{H}$ & 0.26942400 & -0.39208600 & 3.63983400 \\
\hline C & 0.12806300 & -2.52516300 & 3.21532600 & C & 0.12310800 & -2.82768300 & 4.58975000 \\
\hline $\mathrm{C}$ & 0.03955500 & -3.56766800 & 2.27627500 & C & 0.00930200 & -4.14558200 & 5.02082100 \\
\hline $\mathrm{H}$ & 0.19936200 & -2.02316500 & 5.31730500 & C & -0.06564900 & -4.88387600 & 2.71610200 \\
\hline $\mathrm{H}$ & 0.06041100 & -3.35375700 & 1.21383100 & C & -0.08391400 & -5.17690700 & 4.08279300 \\
\hline $\mathrm{H}$ & -0.00421700 & -4.36853900 & 6.08365000 & $\mathrm{H}$ & -0.12656200 & -5.68588600 & 1.98647800 \\
\hline $\mathrm{H}$ & -0.16450000 & -6.20772300 & 4.41633900 & $\mathrm{~N}$ & -0.33447600 & -0.63340200 & 1.70617900 \\
\hline $\mathrm{H}$ & -0.61055500 & -1.25070700 & 0.92732300 & C & 2.47115000 & -1.11977500 & 2.60415200 \\
\hline $\mathrm{H}$ & 2.69136000 & -1.65682800 & 3.52302500 & $\mathrm{H}$ & 2.35346800 & -1.71375800 & 1.70273100 \\
\hline $\mathrm{C}$ & 2.85519200 & 0.19890700 & 2.48947300 & C & 3.47053000 & 0.93633800 & 3.61209700 \\
\hline $\mathrm{H}$ & 3.20893000 & 2.00171000 & 3.59996600 & $\mathrm{H}$ & 3.19068000 & 0.51265600 & 4.58503400 \\
\hline C & 2.74976300 & 0.89334500 & 1.16587300 & $\mathrm{H}$ & 1.92230900 & 0.50272600 & 0.56701800 \\
\hline $\mathrm{H}$ & 2.66186000 & 1.97818000 & 1.26190700 & $\mathrm{H}$ & 3.66037800 & 0.67974600 & 0.59320500 \\
\hline $\mathrm{C}$ & 5.98085200 & 1.97449000 & 4.99575200 & $\mathrm{H}$ & 5.56004700 & 1.65872400 & 5.95794500 \\
\hline $\mathrm{H}$ & 7.07329100 & 1.95918500 & 5.09284500 & $\mathrm{H}$ & 5.68131200 & 3.01527000 & 4.82384100 \\
\hline $\mathrm{C}$ & 5.93575200 & -0.94171000 & 3.89217700 & $\mathrm{H}$ & 5.51707400 & -1.34010600 & 4.82432500 \\
\hline $\mathrm{H}$ & 5.61318100 & -1.58420200 & 3.06504100 & $\mathrm{H}$ & 7.02776300 & -1.01940500 & 3.96205100 \\
\hline C & 6.12264600 & 1.43591200 & 1.92882100 & $\mathrm{H}$ & 5.97727300 & 0.67502700 & 1.15356200 \\
\hline $\mathrm{H}$ & 5.67690800 & 2.37091000 & 1.57200900 & $\mathrm{H}$ & 7.20306600 & 1.60295500 & 2.02627600 \\
\hline $\mathrm{Si}$ & 5.42509300 & 0.84777200 & 3.57816200 & & & & \\
\hline
\end{tabular}

\section{TS-R-4f-nd-34}

Imaginary frequency: $-303.43 \mathrm{~cm}^{-1}$ E[B3LYP/6-31G(d)]: -4788.687135 Hartree E[PCM(Et $2 \mathrm{O})-B 3 L Y P / 6-311 G(d, p)-D 3(B J)]:-4790.212067$ Hartree

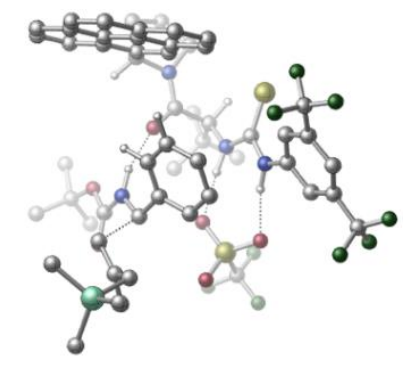

\begin{tabular}{|lrll|lrrr|}
\hline 01 & & & & $\mathrm{~N}$ & 1.85543600 & 1.28490800 & -2.00640200 \\
\hline $\mathrm{H}$ & 2.41857900 & 0.43250300 & -1.91443100 & $\mathrm{C}$ & 2.49712800 & 2.43001400 & -1.50664600 \\
\hline $\mathrm{C}$ & 3.89888100 & 2.41238700 & -1.43573000 & $\mathrm{C}$ & 1.80497200 & 3.53692500 & -0.99670800 \\
\hline $\mathrm{C}$ & 4.58657900 & 3.49208300 & -0.88214700 & $\mathrm{H}$ & 4.44182300 & 1.54858800 & -1.80249600 \\
\hline $\mathrm{C}$ & 2.50950900 & 4.60794900 & -0.45047700 & $\mathrm{H}$ & 0.72582700 & 3.55110200 & -1.02213400 \\
\hline $\mathrm{C}$ & 3.90356500 & 4.60502400 & -0.39153800 & $\mathrm{H}$ & 4.44368100 & 5.44840600 & 0.02065700 \\
\hline $\mathrm{C}$ & 6.08580200 & 3.42929000 & -0.75760500 & $\mathrm{C}$ & 1.73634700 & 5.73898300 & 0.16861100 \\
\hline $\mathrm{F}$ & 6.64426500 & 4.66105800 & -0.79137000 & $\mathrm{~F}$ & 6.64876700 & 2.70052400 & -1.74304200 \\
\hline $\mathrm{F}$ & 6.46237300 & 2.86392100 & 0.41542500 & $\mathrm{~F}$ & 0.59285400 & 6.01043100 & -0.50327500 \\
\hline $\mathrm{F}$ & 1.36182000 & 5.44837700 & 1.44784400 & $\mathrm{~F}$ & 2.45184700 & 6.88052300 & 0.22952000 \\
\hline $\mathrm{C}$ & -0.99961100 & -0.47655200 & -3.47487900 & $\mathrm{H}$ & -1.16624500 & 0.32052200 & -4.19891200 \\
\hline $\mathrm{C}$ & -0.84661900 & -1.81598800 & -4.28287700 & $\mathrm{C}$ & -0.76298400 & -3.06375900 & -3.38576200 \\
\hline $\mathrm{H}$ & -1.66392900 & -3.18624300 & -2.77950800 & $\mathrm{H}$ & 0.09711800 & -3.03309000 & -2.71261600 \\
\hline $\mathrm{H}$ & -0.65238400 & -3.95202500 & -4.02133200 & $\mathrm{C}$ & 0.42823500 & -1.72316900 & -5.14755800 \\
\hline $\mathrm{H}$ & 0.46613900 & -2.57284200 & -5.83988200 & $\mathrm{H}$ & 1.33567900 & -1.75333100 & -4.53854800 \\
\hline $\mathrm{H}$ & 0.44516300 & -0.80239300 & -5.74284900 & $\mathrm{C}$ & -2.06236100 & -1.97071500 & -5.22036600 \\
\hline $\mathrm{H}$ & -1.98132700 & -2.91012400 & -5.77884000 & $\mathrm{H}$ & -2.11537300 & -1.15880400 & -5.95595000 \\
\hline $\mathrm{H}$ & -3.00850700 & -2.00097300 & -4.66681400 & $\mathrm{C}$ & -2.16150900 & -0.47988700 & -2.44658300 \\
\hline
\end{tabular}




\begin{tabular}{|c|c|c|c|c|c|c|c|}
\hline $\mathrm{O}$ & -2.03596000 & -1.04288200 & -1.35047500 & C & -3.70224000 & 0.84045300 & -4.00452600 \\
\hline C & -4.44590100 & 0.15201200 & -1.80317300 & C & -5.17844700 & 0.45692300 & -4.14449600 \\
\hline $\mathrm{H}$ & -3.57277800 & 1.92489700 & -3.89840200 & $\mathrm{H}$ & -3.09687100 & 0.51017000 & -4.84474200 \\
\hline $\mathrm{H}$ & -4.49523600 & -0.85698100 & -1.38420800 & $\mathrm{H}$ & -5.25506000 & -0.54859900 & -4.57482300 \\
\hline $\mathrm{H}$ & -5.72934000 & 1.14370200 & -4.79427100 & $\mathrm{~N}$ & -3.31811100 & 0.16425700 & -2.75717400 \\
\hline C & -4.29621600 & 1.15792800 & -0.66505200 & C & -3.39833000 & 2.18227400 & -0.74393800 \\
\hline C & -5.17590900 & 1.05593000 & 0.48699500 & C & -3.28419100 & 3.18733500 & 0.27413700 \\
\hline $\mathrm{H}$ & -2.71976300 & 2.24931700 & -1.59008700 & C & -5.07767100 & 2.04383000 & 1.52104900 \\
\hline C & -6.12321700 & 0.02742000 & 0.64203800 & C & -2.34917000 & 4.23236600 & 0.18507300 \\
\hline C & -4.13403900 & 3.10914000 & 1.41568500 & C & -5.92707100 & 1.96930300 & 2.66894100 \\
\hline $\mathrm{H}$ & -6.22454700 & -0.73629300 & -0.12129200 & C & -6.94670200 & -0.03733700 & 1.76603400 \\
\hline C & -2.24406200 & 5.18119700 & 1.20185900 & $\mathrm{H}$ & -1.70313100 & 4.29262100 & -0.68656100 \\
\hline C & -4.02765500 & 4.09056100 & 2.44867100 & C & -6.85472100 & 0.92066600 & 2.77018300 \\
\hline C & -5.80438600 & 2.97515600 & 3.68921700 & $\mathrm{H}$ & -7.66827000 & -0.84530800 & 1.85409400 \\
\hline C & -3.07399500 & 5.11411900 & 2.32019900 & $\mathrm{H}$ & -1.50087100 & 5.96790500 & 1.12063300 \\
\hline C & -4.89898600 & 3.98635000 & 3.58607700 & $\mathrm{H}$ & -7.50167900 & 0.86598500 & 3.64240900 \\
\hline $\mathrm{H}$ & -6.45863600 & 2.90889600 & 4.55537700 & $\mathrm{H}$ & -2.98790300 & 5.85816400 & 3.10859700 \\
\hline $\mathrm{H}$ & -4.82008100 & 4.73728000 & 4.36870400 & C & 0.64385000 & 1.17952500 & -2.64623000 \\
\hline$S$ & -0.21909900 & 2.50453700 & -3.26865200 & $\mathrm{~N}$ & 0.22520900 & -0.10479200 & -2.77888400 \\
\hline $\mathrm{H}$ & 0.71964800 & -0.83539600 & -2.25983900 & S & 3.25452700 & -2.13646300 & -1.14174700 \\
\hline 0 & 3.64351400 & -2.65260300 & 0.19046600 & 0 & 1.79375800 & -2.23614400 & -1.42979600 \\
\hline $\mathrm{O}$ & 3.83050800 & -0.81875200 & -1.51091700 & C & 4.02098400 & -3.32354500 & -2.35119900 \\
\hline $\mathrm{F}$ & 5.35262500 & -3.32551400 & -2.22981900 & $\mathrm{~F}$ & 3.70411500 & -2.96612600 & -3.60601400 \\
\hline $\mathrm{F}$ & 3.56662500 & -4.56547400 & -2.13603800 & C & -0.87818800 & -4.13006200 & 0.35030700 \\
\hline 0 & -0.10453300 & -4.95785100 & 0.80592900 & $\mathrm{O}$ & -1.97031700 & -4.36554700 & -0.38661100 \\
\hline C & -2.40164700 & -5.74989800 & -0.69293300 & C & -1.32939800 & -6.47353300 & -1.51201800 \\
\hline $\mathrm{H}$ & -1.71480600 & -7.45011200 & -1.82628200 & $\mathrm{H}$ & -0.41991400 & -6.62690000 & -0.92962000 \\
\hline $\mathrm{H}$ & -1.08089700 & -5.89999900 & -2.41010900 & C & -3.66656900 & -5.52232700 & -1.52316300 \\
\hline $\mathrm{H}$ & -4.40881100 & -4.95631100 & -0.95115100 & $\mathrm{H}$ & -4.10506700 & -6.48541700 & -1.80428800 \\
\hline $\mathrm{H}$ & -3.43554100 & -4.96687100 & -2.43704100 & C & -2.73263500 & -6.48149100 & 0.61191100 \\
\hline $\mathrm{H}$ & -3.17008500 & -7.45865900 & 0.37969500 & $\mathrm{H}$ & -3.46508900 & -5.91234200 & 1.19508300 \\
\hline $\mathrm{H}$ & -1.83775500 & -6.63773400 & 1.21674100 & C & 0.35732200 & -2.27376900 & 1.25978100 \\
\hline $\mathrm{H}$ & 1.27890100 & -2.76910400 & 0.97776000 & C & 0.43035500 & -0.81366100 & 1.46555900 \\
\hline C & 1.69522200 & -0.19983200 & 1.53512100 & C & -0.72239400 & -0.03195300 & 1.66154100 \\
\hline C & 1.79905900 & 1.16867600 & 1.77775400 & $\mathrm{H}$ & 2.58903300 & -0.79370700 & 1.37074000 \\
\hline C & -0.61000300 & 1.33204700 & 1.91424300 & $\mathrm{H}$ & -1.70792800 & -0.48549400 & 1.61617800 \\
\hline C & 0.64939200 & 1.93718900 & 1.97069800 & $\mathrm{H}$ & 2.77920700 & 1.63554700 & 1.80049400 \\
\hline $\mathrm{H}$ & -1.50470700 & 1.92717300 & 2.05895600 & $\mathrm{H}$ & 0.73156900 & 3.00662000 & 2.13936000 \\
\hline $\mathrm{N}$ & -0.76196000 & -2.77056200 & 0.60651500 & $\mathrm{H}$ & -1.36873100 & -2.12930300 & 0.08490900 \\
\hline C & 0.23112800 & -3.00952800 & 3.10742200 & $\mathrm{H}$ & -0.24032000 & -3.94290800 & 2.81999900 \\
\hline $\mathrm{H}$ & -0.40998700 & -2.26629900 & 3.57341100 & C & 1.56645300 & -3.09192600 & 3.52335900 \\
\hline C & 2.18480400 & -2.04256600 & 4.33230100 & $\mathrm{H}$ & 1.69113100 & -1.07410900 & 4.18505700 \\
\hline $\mathrm{H}$ & 3.25699600 & -1.94215100 & 4.12319100 & C & 2.42740100 & -4.20091700 & 3.01453100 \\
\hline $\mathrm{H}$ & 3.18422200 & -4.49399700 & 3.74941300 & $\mathrm{H}$ & 2.97449900 & -3.82545400 & 2.13125900 \\
\hline $\mathrm{H}$ & 1.84695100 & -5.06652200 & 2.69014300 & C & 2.98281500 & -0.91422700 & 7.05024000 \\
\hline $\mathrm{H}$ & 4.03564400 & -0.89521700 & 6.74587200 & $\mathrm{H}$ & 2.95603500 & -0.99352300 & 8.14386300 \\
\hline $\mathrm{H}$ & 2.53810500 & 0.04968500 & 6.77730400 & C & 2.87155200 & -4.00048500 & 6.70076300 \\
\hline $\mathrm{H}$ & 3.91619200 & -4.04088400 & 6.37043800 & $\mathrm{H}$ & 2.34539900 & -4.85487500 & 6.26002500 \\
\hline $\mathrm{H}$ & 2.86846700 & -4.14068300 & 7.78879300 & C & 0.23775600 & -2.35047700 & 6.76197900 \\
\hline $\mathrm{H}$ & -0.32089200 & -3.16730500 & 6.29138700 & $\mathrm{H}$ & -0.25427400 & -1.40818700 & 6.49359000 \\
\hline $\mathrm{H}$ & 0.14443700 & -2.47099400 & 7.84821800 & $\mathrm{Si}$ & 2.05653900 & -2.35584200 & 6.25917900 \\
\hline C & -5.69314000 & 0.46824000 & -2.69293100 & $\mathrm{H}$ & -6.08513200 & 1.45624300 & -2.43415400 \\
\hline $\mathrm{H}$ & -6.49923300 & -0.25333100 & -2.53196600 & & & & \\
\hline
\end{tabular}




\section{TS- $R-4 f-n d-35$}

Imaginary frequency: $-195.19 \mathrm{~cm}^{-1}$

E[B3LYP/6-31G(d)]: -4788.679338 Hartree

E[PCM(Et2O)-B3LYP/6-311G(d,p)-D3(BJ)]: -4790.211996 Hartree

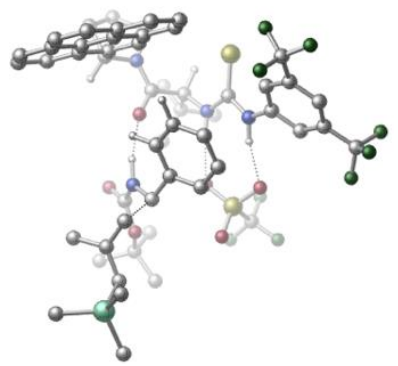

\begin{tabular}{|c|c|c|c|c|c|c|c|}
\hline 01 & & & & $\mathrm{~N}$ & 1.14673100 & 2.60094700 & 0.96628000 \\
\hline $\mathrm{H}$ & 0.14295100 & 2.80630600 & 0.91366000 & C & 1.91536500 & 3.37051200 & 0.07471200 \\
\hline C & 1.41121600 & 4.62080500 & -0.31303500 & C & 3.10158500 & 2.90558100 & -0.50792400 \\
\hline C & 2.09813300 & 5.39423600 & -1.24927800 & $\mathrm{H}$ & 0.47200500 & 4.97015000 & 0.10076900 \\
\hline C & 3.77324700 & 3.69025800 & -1.44302900 & $\mathrm{H}$ & 3.48242400 & 1.93164600 & -0.24024200 \\
\hline C & 3.28823000 & 4.94300800 & -1.81997900 & $\mathrm{H}$ & 3.81721900 & 5.54717000 & -2.54670300 \\
\hline C & 1.58273600 & 6.76509300 & -1.59895500 & C & 4.98031100 & 3.11439400 & -2.12897000 \\
\hline $\mathrm{F}$ & 2.07756000 & 7.71246000 & -0.76781000 & $F$ & 0.23781100 & 6.83697200 & -1.52039000 \\
\hline $\mathrm{F}$ & 1.93605600 & 7.12750800 & -2.85505500 & $\mathrm{~F}$ & 5.73293500 & 2.35660600 & -1.29645500 \\
\hline $\mathrm{F}$ & 4.62640600 & 2.29906200 & -3.16332000 & $\mathrm{~F}$ & 5.78313000 & 4.06605700 & -2.64791400 \\
\hline C & 0.75932400 & 0.01732200 & 3.53610200 & $\mathrm{H}$ & 1.70273200 & 0.29621100 & 4.00438200 \\
\hline C & -0.34944400 & 0.07493500 & 4.64912600 & $\mathrm{C}$ & -1.70680400 & -0.47465000 & 4.18233100 \\
\hline $\mathrm{H}$ & -1.65239900 & -1.52700600 & 3.89426200 & $\mathrm{H}$ & -2.10131100 & 0.08583100 & 3.33272000 \\
\hline $\mathrm{H}$ & -2.43223200 & -0.38529700 & 5.00111900 & $\mathrm{C}$ & -0.51659900 & 1.54392000 & 5.09223500 \\
\hline $\mathrm{H}$ & -1.16533000 & 1.58976000 & 5.97531700 & $\mathrm{H}$ & -0.97850500 & 2.15307700 & 4.31016200 \\
\hline $\mathrm{H}$ & 0.44585500 & 1.99848900 & 5.35637000 & C & 0.11870000 & -0.75138900 & 5.86482800 \\
\hline $\mathrm{H}$ & -0.65344600 & -0.73208900 & 6.64221200 & $\mathrm{H}$ & 1.03657400 & -0.34453800 & 6.30706300 \\
\hline $\mathrm{H}$ & 0.29061900 & -1.80275900 & 5.60558300 & C & 0.92422100 & -1.36511300 & 2.85233700 \\
\hline 0 & 0.15448400 & -1.72473200 & 1.95092500 & C & 2.94387400 & -1.97275000 & 4.30312400 \\
\hline $\mathrm{C}$ & 2.13710500 & -3.48727300 & 2.60004900 & C & 3.07407600 & -3.38244200 & 4.88859200 \\
\hline $\mathrm{H}$ & 3.89287100 & -1.62293700 & 3.87722500 & $\mathrm{H}$ & 2.61111800 & -1.23864200 & 5.03185400 \\
\hline $\mathrm{H}$ & 1.13940500 & -3.90835300 & 2.44982300 & $\mathrm{H}$ & 2.24874200 & -3.56457500 & 5.58665200 \\
\hline $\mathrm{H}$ & 4.01251200 & -3.52363800 & 5.43342600 & $\mathrm{~N}$ & 1.95503300 & -2.16710900 & 3.23375400 \\
\hline C & 2.86142300 & -3.41957400 & 1.25707000 & C & 3.53570000 & -2.29607300 & 0.87555500 \\
\hline $\mathrm{C}$ & 2.88467900 & -4.59765500 & 0.40647600 & C & 4.29032600 & -2.22560300 & -0.34358500 \\
\hline $\mathrm{H}$ & 3.50011300 & -1.40216800 & 1.49237800 & C & 3.62601600 & -4.55114500 & -0.81954900 \\
\hline C & 2.20753200 & -5.78846800 & 0.72662900 & C & 4.97381500 & -1.05909700 & -0.72773300 \\
\hline C & 4.32950100 & -3.36780100 & -1.19485300 & C & 3.66595600 & -5.69353300 & -1.67856100 \\
\hline $\mathrm{H}$ & 1.63687500 & -5.85701300 & 1.64632700 & C & 2.25157500 & -6.89737200 & -0.11858900 \\
\hline C & 5.67884100 & -1.01166300 & -1.93020800 & $\mathrm{H}$ & 4.94277300 & -0.18864200 & -0.07775200 \\
\hline C & 5.06308700 & -3.31528900 & -2.41947400 & C & 2.97217600 & -6.85621600 & -1.30764100 \\
\hline C & 4.41871900 & -5.61588500 & -2.90131400 & $\mathrm{H}$ & 1.71811900 & -7.80262200 & 0.15946900 \\
\hline C & 5.72688200 & -2.12667100 & -2.76582300 & $\mathrm{H}$ & 6.18057400 & -0.09278200 & -2.21661600 \\
\hline C & 5.08467600 & -4.48287200 & -3.25605500 & $\mathrm{H}$ & 3.00560900 & -7.72553900 & -1.96006700 \\
\hline $\mathrm{H}$ & 4.44422800 & -6.49287700 & -3.54396800 & $\mathrm{H}$ & 6.27919400 & -2.08324400 & -3.70156700 \\
\hline $\mathrm{H}$ & 5.64743700 & -4.44279200 & -4.18574900 & C & 1.57105300 & 1.70183600 & 1.91240900 \\
\hline$S$ & 3.19829100 & 1.50737100 & 2.36933500 & $\mathrm{~N}$ & 0.55423300 & 1.01143100 & 2.48919800 \\
\hline $\mathrm{H}$ & -0.38266000 & 1.08860100 & 2.08589100 & S & -2.67743300 & 2.64105500 & 0.70625400 \\
\hline $\mathrm{O}$ & -3.60773100 & 2.33452700 & -0.40359500 & 0 & -2.25690800 & 1.45434300 & 1.50618500 \\
\hline $\mathrm{O}$ & -1.55120000 & 3.54995200 & 0.37450800 & C & -3.70329200 & 3.63080600 & 1.90246300 \\
\hline $\mathrm{F}$ & -4.08811500 & 4.78793000 & 1.35520400 & $\mathrm{~F}$ & -3.00069900 & 3.89235700 & 3.01418300 \\
\hline $\mathrm{F}$ & -4.80786300 & 2.94361900 & 2.25572100 & C & -3.36930400 & -1.73466900 & 1.12488200 \\
\hline $\mathrm{O}$ & -3.30857900 & -2.41906300 & 2.12640100 & 0 & -4.46243400 & -1.11654500 & 0.63885500 \\
\hline C & -5.68235700 & -0.95497700 & 1.47828900 & C & -6.39416600 & -2.30589800 & 1.58160200 \\
\hline $\mathrm{H}$ & -7.32465100 & -2.18883900 & 2.14804800 & $\mathrm{H}$ & -5.76524900 & -3.03735100 & 2.09378400 \\
\hline
\end{tabular}




\begin{tabular}{|c|c|c|c|c|c|c|c|}
\hline $\mathrm{H}$ & -6.65508700 & -2.68997800 & 0.58813900 & C & -6.50039400 & 0.06618000 & 0.68504100 \\
\hline $\mathrm{H}$ & -5.93318100 & 0.99221500 & 0.55585800 & $\mathrm{H}$ & -7.42793500 & 0.29100800 & 1.22185100 \\
\hline $\mathrm{H}$ & -6.76782600 & -0.32012500 & -0.30417100 & $\mathrm{C}$ & -5.32653400 & -0.38724800 & 2.85496400 \\
\hline $\mathrm{H}$ & -6.25621600 & -0.12494700 & 3.37273400 & $\mathrm{H}$ & -4.72740700 & 0.51970100 & 2.75033500 \\
\hline $\mathrm{H}$ & -4.78016300 & -1.10963500 & 3.46103400 & C & -2.28641600 & -0.76590400 & -0.87249200 \\
\hline $\mathrm{H}$ & -3.06375500 & -0.00524100 & -0.84802600 & C & -0.97082100 & -0.24880500 & -1.36528600 \\
\hline C & -0.95708600 & 0.98996700 & -2.02841700 & C & 0.20870600 & -1.00454300 & -1.29260900 \\
\hline C & 0.21933800 & 1.46497800 & -2.60347300 & $\mathrm{H}$ & -1.86161300 & 1.59022600 & -2.06111500 \\
\hline C & 1.38022200 & -0.52634900 & -1.87627100 & $\mathrm{H}$ & 0.22749300 & -1.95967000 & -0.77613300 \\
\hline C & 1.38826000 & 0.70353200 & -2.53922600 & $\mathrm{H}$ & 0.22409700 & 2.43551900 & -3.09071500 \\
\hline $\mathrm{H}$ & 2.28915700 & -1.11345900 & -1.81199100 & $\mathrm{H}$ & 2.30827400 & 1.07452100 & -2.98061300 \\
\hline $\mathrm{N}$ & -2.26974800 & -1.55227900 & 0.28453000 & $\mathrm{H}$ & -1.37930500 & -1.75028600 & 0.75253600 \\
\hline C & -2.79442400 & -1.74594100 & -2.32611600 & $\mathrm{H}$ & -1.99080700 & -2.47947000 & -2.36119500 \\
\hline $\mathrm{H}$ & -2.71306000 & -0.98704900 & -3.10096200 & C & -4.09651300 & -2.28197100 & -2.14587900 \\
\hline C & -5.27565000 & -1.56409200 & -2.59200800 & $\mathrm{H}$ & -5.10876100 & -0.48121500 & -2.63617800 \\
\hline $\mathrm{H}$ & -6.15731200 & -1.78322400 & -1.98186300 & C & -4.21976700 & -3.61537500 & -1.48769700 \\
\hline $\mathrm{H}$ & -5.24098600 & -3.85917800 & -1.18947700 & $\mathrm{H}$ & -3.55854500 & -3.65613300 & -0.61132400 \\
\hline $\mathrm{H}$ & -3.85257700 & -4.39613600 & -2.16889100 & C & -7.36573500 & -0.98766600 & -4.72539700 \\
\hline $\mathrm{H}$ & -8.16520700 & -1.23961500 & -4.01932400 & $\mathrm{H}$ & -7.75168000 & -1.16003300 & -5.73747900 \\
\hline $\mathrm{H}$ & -7.15713900 & 0.08382600 & -4.62886300 & C & -6.20961800 & -3.86926100 & -4.52650900 \\
\hline $\mathrm{H}$ & -6.96387200 & -4.16782800 & -3.78920700 & $\mathrm{H}$ & -5.32205900 & -4.49371100 & -4.37399100 \\
\hline $\mathrm{H}$ & -6.60966500 & -4.11275800 & -5.51848200 & $\mathrm{C}$ & -4.43534700 & -1.55499800 & -5.61854500 \\
\hline $\mathrm{H}$ & -3.52337700 & -2.13858800 & -5.45025500 & $\mathrm{H}$ & -4.17505800 & -0.49293100 & -5.54134700 \\
\hline $\mathrm{H}$ & -4.75367900 & -1.73778400 & -6.65228600 & $\mathrm{Si}$ & -5.81972900 & -2.02564600 & -4.43006500 \\
\hline $\mathrm{C}$ & 2.95645000 & -4.30161200 & 3.65663500 & $\overline{\mathrm{H}}$ & 3.94657900 & -4.53379700 & 3.25398600 \\
\hline $\mathrm{H}$ & 2.47248000 & -5.25262300 & 3.89717900 & & & & \\
\hline
\end{tabular}

\section{TS-R-4f-nd-36}

Imaginary frequency: $-275.58 \mathrm{~cm}^{-1}$ E[B3LYP/6-31G(d)]: -4788.680831 Hartree E[PCM(Et $2 \mathrm{O})-B 3 L Y P / 6-311 G(d, p)-D 3(B J)]:-4790.211443$ Hartree

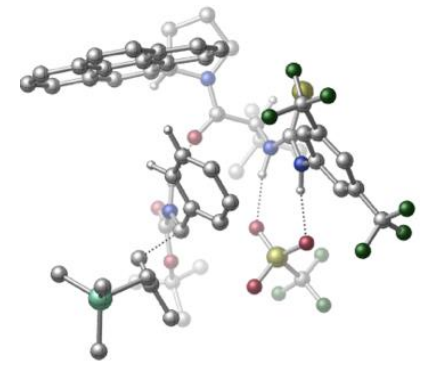

\begin{tabular}{|c|c|c|c|c|c|c|c|}
\hline 01 & & & & $\mathrm{~N}$ & 0.86202600 & 2.70233800 & -1.09277100 \\
\hline $\mathrm{H}$ & 1.79174700 & 2.30165800 & -0.93259700 & C & 0.52124400 & 3.72217300 & -0.18830700 \\
\hline C & 1.55730600 & 4.46695600 & 0.39179500 & C & -0.79860600 & 3.95039500 & 0.22674300 \\
\hline C & 1.26821000 & 5.43573000 & 1.35451000 & $\mathrm{H}$ & 2.58299200 & 4.27738200 & 0.09595300 \\
\hline C & -1.06925600 & 4.92413300 & 1.18448800 & $\mathrm{H}$ & -1.60062700 & 3.35841300 & -0.18925500 \\
\hline C & -0.04445900 & 5.68263400 & 1.75450200 & $\mathrm{H}$ & -0.26388200 & 6.44836900 & 2.48843800 \\
\hline C & 2.39699400 & 6.18316100 & 2.01312300 & C & -2.47936500 & 5.08144900 & 1.68256200 \\
\hline $\mathrm{F}$ & 3.44862300 & 6.35021900 & 1.18484400 & $\mathrm{~F}$ & 2.85704200 & 5.52469400 & 3.10527200 \\
\hline $\mathrm{F}$ & 2.01057900 & 7.40937400 & 2.43373600 & $\mathrm{~F}$ & -3.39971500 & 4.83685500 & 0.72227300 \\
\hline $\mathrm{F}$ & -2.74935400 & 4.21138800 & 2.69807000 & $\mathrm{~F}$ & -2.71728900 & 6.31777300 & 2.16755000 \\
\hline C & -0.03685600 & 0.35852700 & -3.76803500 & $\mathrm{H}$ & -0.56693200 & 1.13184000 & -4.32255900 \\
\hline C & 1.02262100 & -0.25781600 & -4.75183600 & C & 1.77901000 & -1.46196900 & -4.16653600 \\
\hline $\mathrm{H}$ & 1.11318300 & -2.29297400 & -3.92434500 & $\mathrm{H}$ & 2.33101200 & -1.19602600 & -3.26181100 \\
\hline $\mathrm{H}$ & 2.51027400 & -1.81747100 & -4.90376000 & $\mathrm{C}$ & 2.03340700 & 0.84828200 & -5.12110800 \\
\hline $\bar{H}$ & 2.69483400 & 0.49140700 & -5.91965000 & $\mathrm{H}$ & 2.66111600 & 1.12149300 & -4.26779700 \\
\hline $\mathrm{H}$ & 1.52977700 & 1.75440500 & -5.47880400 & C & 0.29605100 & -0.71032100 & -6.03495800 \\
\hline $\mathrm{H}$ & 1.01864800 & -1.14232700 & -6.73646800 & $\mathrm{H}$ & -0.19410700 & 0.12939600 & -6.54231700 \\
\hline $\mathrm{H}$ & -0.45664300 & -1.48091700 & -5.82946600 & C & -1.06118400 & -0.66359600 & -3.21164500 \\
\hline
\end{tabular}




\begin{tabular}{|c|c|c|c|c|c|c|c|}
\hline $\mathrm{O}$ & -0.75106100 & -1.46107000 & -2.31811200 & C & -2.84920700 & 0.22271300 & -4.79292600 \\
\hline C & -3.27585100 & -1.72061100 & -3.33656900 & C & -4.33802600 & -0.13661600 & -4.85970900 \\
\hline $\mathrm{H}$ & -2.67132700 & 1.27428900 & -4.55002100 & $\mathrm{H}$ & -2.35630600 & -0.00391300 & -5.74443400 \\
\hline C & -4.36083100 & -1.61261300 & -4.44303500 & $\mathrm{H}$ & -2.74166800 & -2.67118800 & -3.42389200 \\
\hline $\mathrm{H}$ & -4.75200800 & 0.03304700 & -5.85817700 & $\mathrm{H}$ & -4.91093700 & 0.46964600 & -4.15119900 \\
\hline $\mathrm{H}$ & -4.06912600 & -2.24576600 & -5.29013700 & $\mathrm{H}$ & -5.33819600 & -1.95149600 & -4.09196800 \\
\hline $\mathrm{N}$ & -2.32417600 & -0.65436300 & -3.71994200 & C & -3.87568900 & -1.72225500 & -1.92240500 \\
\hline C & -4.15295600 & -2.96769000 & -1.42675700 & C & -4.29215400 & -0.55320800 & -1.15933800 \\
\hline C & -4.89088900 & -3.19771800 & -0.22147100 & $\mathrm{H}$ & -3.82828300 & -3.84428100 & -1.98476700 \\
\hline C & -5.05248400 & -0.75420600 & 0.04577100 & C & -3.96587500 & 0.76967400 & -1.51075300 \\
\hline C & -5.17896800 & -4.49019500 & 0.24775500 & C & -5.36094200 & -2.06865600 & 0.50922200 \\
\hline C & -5.49229400 & 0.37153900 & 0.81471600 & $\mathrm{H}$ & -3.33591500 & 0.96819600 & -2.36426300 \\
\hline C & -4.38048200 & 1.85658700 & -0.74148400 & C & -5.92195200 & -4.67356200 & 1.41228000 \\
\hline $\mathrm{H}$ & -4.81997000 & -5.34966400 & -0.31337800 & C & -6.11786600 & -2.26607600 & 1.70575300 \\
\hline C & -5.14483500 & 1.66777700 & 0.40364500 & C & -6.26333500 & 0.14420100 & 2.00706100 \\
\hline $\mathrm{H}$ & -4.08311600 & 2.85713400 & -1.03987500 & C & -6.38774600 & -3.57597800 & 2.13441900 \\
\hline $\mathrm{H}$ & -6.14136600 & -5.67986800 & 1.75930200 & C & -6.56723200 & -1.11196000 & 2.43244600 \\
\hline $\mathrm{H}$ & -5.45719500 & 2.52162800 & 0.99963600 & $\mathrm{H}$ & -6.59945700 & 1.01209300 & 2.56942100 \\
\hline $\mathrm{H}$ & -6.96662800 & -3.72575200 & 3.04266700 & $\mathrm{H}$ & -7.15099600 & -1.26311700 & 3.33734800 \\
\hline C & 0.13060400 & 2.23804400 & -2.16046400 & $S$ & -1.16150000 & 3.10274100 & -2.84738000 \\
\hline $\mathrm{N}$ & 0.57695300 & 1.05094600 & -2.64047300 & $\mathrm{H}$ & 1.29716200 & 0.55252500 & -2.11340700 \\
\hline$S$ & 3.92489200 & 0.54388700 & -0.39677000 & 0 & 4.29131600 & -0.18355900 & 0.84264100 \\
\hline 0 & 2.97264300 & -0.18910600 & -1.27770300 & $\mathrm{O}$ & 3.57612200 & 1.97478600 & -0.21745400 \\
\hline C & 5.50108200 & 0.57419300 & -1.38390300 & $\mathrm{~F}$ & 6.44811600 & 1.26718100 & -0.74513000 \\
\hline $\mathrm{F}$ & 5.28742400 & 1.13296800 & -2.58199900 & $\mathrm{~F}$ & 5.95237300 & -0.68095400 & -1.57721100 \\
\hline C & 1.92157800 & -3.54642700 & -1.13868700 & 0 & 1.56963600 & -4.09641700 & -2.16224800 \\
\hline 0 & 3.11036200 & -3.66903900 & -0.52023000 & C & 4.24271000 & -4.37938800 & -1.16553500 \\
\hline $\mathrm{C}$ & 3.91631600 & -5.87221300 & -1.25373000 & $\mathrm{H}$ & 4.78628800 & -6.41461400 & -1.64038400 \\
\hline $\mathrm{H}$ & 3.06962400 & -6.04892700 & -1.91951300 & $\mathrm{H}$ & 3.68036400 & -6.27568400 & -0.26216200 \\
\hline C & 5.39283900 & -4.11100600 & -0.19276700 & $\mathrm{H}$ & 5.56042000 & -3.03524100 & -0.08486600 \\
\hline $\mathrm{H}$ & 6.31186000 & -4.57308300 & -0.56787900 & $\mathrm{H}$ & 5.17807100 & -4.53708600 & 0.79406200 \\
\hline C & 4.54301500 & -3.76130700 & -2.53346800 & $\mathrm{H}$ & 5.46380200 & -4.20493700 & -2.92889900 \\
\hline $\mathrm{H}$ & 4.69628600 & -2.68321500 & -2.43742200 & $\mathrm{H}$ & 3.73438900 & -3.94680700 & -3.24128900 \\
\hline C & 1.43662000 & -2.00534600 & 0.72437700 & $\mathrm{H}$ & 2.48271700 & -1.71731000 & 0.72880200 \\
\hline C & 0.49554200 & -0.95402800 & 1.19349300 & C & 1.02279500 & 0.19883200 & 1.80176900 \\
\hline C & -0.89795700 & -1.10601300 & 1.10872400 & C & 0.17215600 & 1.18409100 & 2.29968800 \\
\hline $\mathrm{H}$ & 2.09858500 & 0.33262900 & 1.85909000 & C & -1.74315600 & -0.12006500 & 1.61563400 \\
\hline $\mathrm{H}$ & -1.33023800 & -1.98613700 & 0.64087100 & C & -1.21220200 & 1.02671300 & 2.21125700 \\
\hline $\mathrm{H}$ & 0.59370600 & 2.08184600 & 2.74193800 & $\mathrm{H}$ & -2.81743600 & -0.24270000 & 1.53623200 \\
\hline $\mathrm{H}$ & -1.87259500 & 1.80507200 & 2.58090500 & $\mathrm{~N}$ & 1.07015100 & -2.71026200 & -0.41690500 \\
\hline $\mathrm{H}$ & 0.23867700 & -2.42788800 & -0.94264800 & C & 1.49502800 & -3.31389300 & 2.09942600 \\
\hline $\mathrm{H}$ & 2.09541200 & -4.04621800 & 1.56778800 & $\mathrm{H}$ & 0.43954200 & -3.55993600 & 2.18954700 \\
\hline C & 2.10791400 & -2.69589500 & 3.20874800 & C & 1.31792300 & -2.08045900 & 4.26370200 \\
\hline $\mathrm{H}$ & 0.31472600 & -1.80514500 & 3.91803400 & $\mathrm{H}$ & 1.81614300 & -1.20888400 & 4.70473400 \\
\hline C & 3.59216700 & -2.53969000 & 3.22929300 & $\mathrm{H}$ & 3.97441600 & -2.34090500 & 4.23391400 \\
\hline $\mathrm{H}$ & 3.85810700 & -1.67235200 & 2.59813000 & $\mathrm{H}$ & 4.10568200 & -3.40219000 & 2.79490200 \\
\hline C & 0.01318200 & -2.23871100 & 7.01175900 & $\mathrm{H}$ & 0.59439000 & -1.37254200 & 7.34822000 \\
\hline $\mathrm{H}$ & -0.24205500 & -2.83088200 & 7.89903100 & $\mathrm{H}$ & -0.92342300 & -1.86728900 & 6.58060700 \\
\hline C & 2.61653600 & -3.86616400 & 6.51402700 & $\mathrm{H}$ & 3.24633400 & -3.02419500 & 6.82438700 \\
\hline $\mathrm{H}$ & 3.19418900 & -4.47924700 & 5.81303200 & $\mathrm{H}$ & 2.42947600 & -4.47793500 & 7.40519500 \\
\hline C & -0.04756500 & -4.74909200 & 5.17062800 & $\mathrm{H}$ & 0.49120800 & -5.35496100 & 4.43351600 \\
\hline $\mathrm{H}$ & -0.98946700 & -4.42264200 & 4.71444500 & $\mathrm{H}$ & -0.30193100 & -5.40517200 & 6.01210300 \\
\hline Si & 0.97854600 & -3.28777400 & 5.77679100 & & & & \\
\hline
\end{tabular}




\section{TS-R-4f-nd-37}

Imaginary frequency: $-255.88 \mathrm{~cm}^{-1}$

E[B3LYP/6-31G(d)]: -4788.684553 Hartree

$\mathrm{E}\left[\mathrm{PCM}\left(\mathrm{Et}_{2} \mathrm{O}\right)-\mathrm{B} 3 \mathrm{LYP} / 6-311 \mathrm{G}(\mathrm{d}, \mathrm{p})-\mathrm{D} 3(\mathrm{BJ})\right]:-4790.211418$ Hartree

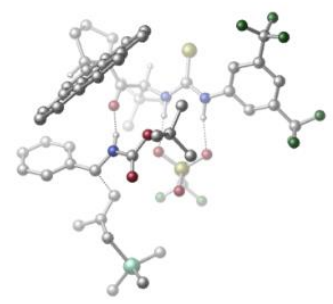

\begin{tabular}{|c|c|c|c|c|c|c|c|}
\hline 01 & & & & $\mathrm{~N}$ & 2.95816500 & 0.83769100 & 1.06135300 \\
\hline $\mathrm{H}$ & 2.28792200 & 1.60558000 & 0.94349300 & $\mathrm{C}$ & 4.17680900 & 1.07127000 & 0.39507800 \\
\hline C & 4.45919700 & 2.40045500 & 0.03953300 & C & 5.06499900 & 0.05865300 & 0.00617200 \\
\hline C & 5.61839800 & 2.70651800 & -0.67016400 & $\mathrm{H}$ & 3.76445300 & 3.18691200 & 0.31309400 \\
\hline C & 6.22548900 & 0.38758100 & -0.69460600 & $\mathrm{H}$ & 4.85073300 & -0.97114700 & 0.24999000 \\
\hline C & 6.51916500 & 1.70682900 & -1.03670500 & $\mathrm{H}$ & 7.42373000 & 1.94994800 & -1.58053200 \\
\hline C & 5.87197100 & 4.12730500 & -1.09860700 & $\mathrm{C}$ & 7.19995300 & -0.70477400 & -1.04656300 \\
\hline$F$ & 5.34332300 & 5.02044400 & -0.23558900 & $\mathrm{~F}$ & 5.33177200 & 4.38629500 & -2.31339400 \\
\hline$F$ & 7.19527100 & 4.39228800 & -1.19764900 & $\mathrm{~F}$ & 6.57703700 & -1.88274700 & -1.28064200 \\
\hline $\mathrm{F}$ & 7.91631800 & -0.40249100 & -2.15450100 & $\mathrm{~F}$ & 8.09167400 & -0.92466900 & -0.05366100 \\
\hline C & 0.70689000 & -0.92059200 & 3.34908200 & $\mathrm{H}$ & 1.53925600 & -1.48439300 & 3.76870100 \\
\hline C & 0.03622800 & -0.13761000 & 4.53830200 & C & -1.25408900 & 0.60780400 & 4.14854300 \\
\hline $\mathrm{H}$ & -2.05894800 & -0.08767700 & 3.89082100 & $\mathrm{H}$ & -1.10299900 & 1.28032100 & 3.30165000 \\
\hline $\mathrm{H}$ & -1.59326100 & 1.20824900 & 5.00208900 & C & 1.06241600 & 0.88285900 & 5.07285100 \\
\hline $\mathrm{H}$ & 0.68950400 & 1.33272000 & 6.00083500 & $\mathrm{H}$ & 1.23277900 & 1.69013100 & 4.35507500 \\
\hline $\mathrm{H}$ & 2.02758700 & 0.40979800 & 5.28703200 & C & -0.29779300 & -1.13839300 & 5.66393200 \\
\hline $\mathrm{H}$ & -0.78955300 & -0.61733600 & 6.49348100 & $\mathrm{H}$ & 0.60541000 & -1.61267100 & 6.06683400 \\
\hline $\mathrm{H}$ & -0.98226200 & -1.92652100 & 5.32475600 & C & -0.24002800 & -1.87634500 & 2.59139700 \\
\hline $\mathrm{O}$ & -1.10770900 & -1.42927200 & 1.82591600 & C & 0.79725600 & -3.89777400 & 3.72693900 \\
\hline C & -0.99780500 & -4.16664600 & 2.06743700 & C & 0.07699800 & -5.22716900 & 3.97844100 \\
\hline $\mathrm{H}$ & 1.79977500 & -4.04794400 & 3.30817800 & $\mathrm{H}$ & 0.90393300 & -3.30907100 & 4.63599400 \\
\hline $\mathrm{H}$ & -2.00769500 & -3.93881300 & 2.42978100 & $\mathrm{H}$ & -0.69998000 & -5.09135000 & 4.74091800 \\
\hline $\mathrm{H}$ & 0.75987900 & -6.00634000 & 4.32967100 & $\mathrm{~N}$ & -0.07631600 & -3.21511400 & 2.74731000 \\
\hline C & -1.07720700 & -4.14217300 & 0.54525000 & C & -2.32994500 & -4.14702600 & -0.00088800 \\
\hline C & 0.07964900 & -4.27587100 & -0.32708700 & C & -2.57660200 & -4.31250700 & -1.40231600 \\
\hline $\mathrm{H}$ & -3.19484900 & -4.03608700 & 0.64958200 & C & -0.13949800 & -4.47702600 & -1.73100400 \\
\hline C & 1.40803600 & -4.22065900 & 0.13114800 & C & -3.87433400 & -4.31967500 & -1.93939200 \\
\hline C & -1.46130700 & -4.49848200 & -2.26900800 & C & 0.97347100 & -4.65731700 & -2.61174700 \\
\hline $\mathrm{H}$ & 1.61417900 & -4.01272700 & 1.17178900 & C & 2.48642900 & -4.38761800 & -0.73735200 \\
\hline C & -4.07617500 & -4.51244200 & -3.30500000 & $\mathrm{H}$ & -4.72270900 & -4.17397500 & -1.27497900 \\
\hline C & -1.67749300 & -4.69805400 & -3.66722100 & C & 2.27690400 & -4.61686800 & -2.09267900 \\
\hline C & 0.72678900 & -4.86675900 & -4.01276900 & $\mathrm{H}$ & 3.49742600 & -4.32663500 & -0.34512300 \\
\hline C & -2.99246000 & -4.70158900 & -4.16071100 & $\mathrm{H}$ & -5.08629300 & -4.51848500 & -3.70616200 \\
\hline C & -0.53636600 & -4.88620100 & -4.51921600 & $\mathrm{H}$ & 3.12126900 & -4.75140100 & -2.76418600 \\
\hline $\mathrm{H}$ & 1.58377600 & -5.00733800 & -4.66710100 & $\mathrm{H}$ & -3.15704800 & -4.85298400 & -5.22476100 \\
\hline $\mathrm{H}$ & -0.70289400 & -5.04319000 & -5.58216700 & C & 2.61863100 & -0.13474900 & 1.97076600 \\
\hline$S$ & 3.69646400 & -1.29426000 & 2.58303500 & $\mathrm{~N}$ & 1.31618200 & -0.04441600 & 2.35919900 \\
\hline $\mathrm{H}$ & 0.67948200 & 0.56581000 & 1.83669300 & S & -0.13787100 & 3.06831300 & 0.52309400 \\
\hline $\mathrm{O}$ & -0.84870000 & 3.55034300 & -0.67604000 & $\mathrm{O}$ & -0.60885800 & 1.74498200 & 1.02796400 \\
\hline $\mathrm{O}$ & 1.34141000 & 3.19354000 & 0.49953500 & C & -0.66228900 & 4.23953300 & 1.87239900 \\
\hline $\mathrm{F}$ & -0.33566800 & 5.49992000 & 1.57434800 & $\mathrm{~F}$ & -0.08225300 & 3.90713200 & 3.03688600 \\
\hline $\mathrm{F}$ & -2.00013600 & 4.17705200 & 2.04414700 & C & -0.55401500 & -5.54460100 & 2.61822900 \\
\hline $\mathrm{H}$ & 0.18814500 & -5.99086000 & 1.94871300 & $\mathrm{H}$ & -1.40131200 & -6.23345900 & 2.67736200 \\
\hline$C$ & -1.63656400 & 0.20775500 & -1.80353900 & $\mathrm{O}$ & -2.19360200 & 0.81558700 & -2.70120300 \\
\hline
\end{tabular}




\begin{tabular}{|c|c|c|c|c|c|c|c|}
\hline 0 & -0.37762500 & -0.18803200 & -1.72993100 & $\mathrm{C}$ & 0.57835900 & 0.05815300 & -2.85442400 \\
\hline C & 0.77815700 & 1.56225200 & -3.04426900 & $\mathrm{H}$ & 1.52309100 & 1.71936300 & -3.83273400 \\
\hline $\bar{H}$ & -0.14871700 & 2.05789100 & -3.33632400 & $\bar{H}$ & 1.14843500 & 2.03030900 & -2.12863100 \\
\hline C & 1.85256900 & -0.60989100 & -2.34299300 & $\bar{H}$ & 1.70082000 & -1.68191100 & -2.19004500 \\
\hline $\mathrm{H}$ & 2.65703100 & -0.47255600 & -3.07302500 & $\mathrm{H}$ & 2.16634100 & -0.16361100 & -1.39596200 \\
\hline $\mathrm{C}$ & 0.06115100 & -0.63322300 & -4.11836000 & $\bar{H}$ & 0.82876100 & -0.56595900 & -4.89729500 \\
\hline $\bar{H}$ & -0.13394400 & -1.69349100 & -3.92806100 & $\mathrm{H}$ & -0.85050100 & -0.16123400 & -4.48894400 \\
\hline C & -3.66930600 & 0.09427700 & -0.57199000 & $\mathrm{H}$ & -4.07181000 & 0.29394000 & -1.55946900 \\
\hline $\mathrm{C}$ & -4.56527800 & -0.67759000 & 0.32334000 & $\mathrm{C}$ & -5.82523500 & -1.04965600 & -0.17568200 \\
\hline $\mathrm{C}$ & -4.20446900 & -1.05919100 & 1.62447500 & $\mathrm{C}$ & -6.70656200 & -1.79761400 & 0.60610700 \\
\hline $\mathrm{H}$ & -6.10479900 & -0.77136700 & -1.18903700 & C & -5.09214900 & -1.79834000 & 2.40431900 \\
\hline $\bar{H}$ & -3.22707600 & -0.79932500 & 2.01495600 & $\mathrm{C}$ & -6.34236200 & -2.16992700 & 1.90093600 \\
\hline $\bar{H}$ & -7.67285100 & -2.08785900 & 0.20332000 & $\bar{H}$ & -4.80480400 & -2.08676700 & 3.41168500 \\
\hline $\mathrm{H}$ & -7.02804700 & -2.74719600 & 2.51493500 & $\mathrm{~N}$ & -2.34151000 & -0.21412200 & -0.65498700 \\
\hline $\mathrm{H}$ & -1.80799500 & -0.57007700 & 0.14506500 & $\mathrm{C}$ & -3.71279000 & 1.99509200 & 0.08378300 \\
\hline $\bar{H}$ & -3.54021900 & 1.82514800 & 1.14261700 & $\bar{H}$ & -2.83897700 & 2.31835100 & -0.47488500 \\
\hline $\mathrm{C}$ & -4.95114600 & 2.48655100 & -0.32817500 & $\mathrm{C}$ & -5.11569200 & 3.05361600 & -1.66972500 \\
\hline $\mathrm{H}$ & -4.32584100 & 2.70808400 & -2.34650100 & $\mathrm{H}$ & -6.10109700 & 2.82975400 & -2.09964600 \\
\hline $\mathrm{C}$ & -6.15607100 & 2.36312700 & 0.55306000 & $\mathrm{H}$ & -5.89024000 & 2.22846800 & 1.60406700 \\
\hline $\bar{H}$ & -6.81690200 & 3.22978800 & 0.44948700 & $\mathrm{H}$ & -6.74003200 & 1.48258800 & 0.24403700 \\
\hline C & -4.86020600 & 5.39807000 & -3.58702900 & $\mathrm{H}$ & -5.71169600 & 5.00046900 & -4.15185300 \\
\hline $\mathrm{H}$ & -4.83031600 & 6.48200100 & -3.75214300 & $\mathrm{H}$ & -3.94406100 & 4.97367000 & -4.01315200 \\
\hline $\mathrm{C}$ & -6.59533700 & 5.74744600 & -1.04365600 & $\mathrm{H}$ & -7.48763900 & 5.33823900 & -1.53248100 \\
\hline $\mathrm{H}$ & -6.69335800 & 5.58429900 & 0.03590700 & $\mathrm{H}$ & -6.60491400 & 6.83286100 & -1.20427500 \\
\hline $\mathrm{C}$ & -3.49995400 & 5.63277600 & -0.78886200 & $\mathrm{H}$ & -3.58539800 & 5.43706200 & 0.28569500 \\
\hline $\mathrm{H}$ & -2.56909600 & 5.16160900 & -1.12142500 & $\mathrm{H}$ & -3.39882500 & 6.71762800 & -0.92061700 \\
\hline $\mathrm{Si}$ & -4.99468200 & 5.01642900 & -1.74236200 & & & & \\
\hline
\end{tabular}

TS- $R-4 f-n d-38$

Imaginary frequency: $-289.62 \mathrm{~cm}^{-1}$ E[B3LYP/6-31G(d)]: -4788.685155 Hartree

E[PCM(Et $\left.\left.{ }_{2} \mathrm{O}\right)-B 3 L Y P / 6-311 G(d, p)-D 3(B J)\right]:-4790.211206$ Hartree

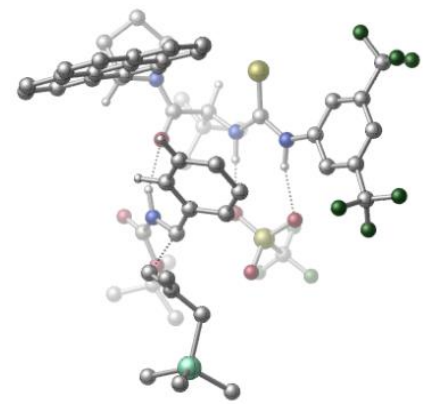

\begin{tabular}{|crrr|rrrr|}
\hline 01 & & & & $\mathrm{~N}$ & 0.51531600 & 2.64695100 & 1.34638600 \\
\hline $\mathrm{H}$ & -0.48990700 & 2.45575800 & 1.30739000 & $\mathrm{C}$ & 0.87765000 & 3.76490500 & 0.56662000 \\
\hline $\mathrm{C}$ & -0.02010500 & 4.14238200 & -0.44439700 & $\mathrm{C}$ & 2.03146600 & 4.53944300 & 0.76685200 \\
\hline $\mathrm{C}$ & 0.26217600 & 5.22644800 & -1.27324800 & $\mathrm{H}$ & -0.94524000 & 3.59117000 & -0.56783100 \\
\hline $\mathrm{C}$ & 2.29236400 & 5.62438700 & -0.07063900 & $\mathrm{H}$ & 2.72079100 & 4.28605800 & 1.55788600 \\
\hline $\mathrm{C}$ & 1.42572100 & 5.97433800 & -1.10580100 & $\mathrm{H}$ & 1.64829300 & 6.80950400 & -1.75801500 \\
\hline $\mathrm{C}$ & -0.67790100 & 5.51855100 & -2.40889800 & $\mathrm{C}$ & 3.50270700 & 6.48108800 & 0.19452000 \\
\hline $\mathrm{F}$ & -1.97048100 & 5.47400100 & -2.02659600 & $\mathrm{~F}$ & -0.54409500 & 4.59923500 & -3.40930300 \\
\hline $\mathrm{F}$ & -0.45661000 & 6.72744000 & -2.96581700 & $\mathrm{~F}$ & 3.21808700 & 7.49833300 & 1.04219500 \\
\hline $\mathrm{F}$ & 4.51346400 & 5.77988200 & 0.74824700 & $\mathrm{~F}$ & 3.97541100 & 7.04451100 & -0.94264000 \\
\hline $\mathrm{C}$ & 1.18891700 & -0.32957000 & 3.38584900 & $\mathrm{H}$ & 2.07711100 & 0.13037800 & 3.81940300 \\
\hline $\mathrm{C}$ & 0.24862200 & -0.72752900 & 4.58481700 & $\mathrm{C}$ & -0.96657200 & -1.56713500 & 4.15789700 \\
\hline $\mathrm{H}$ & -0.67421500 & -2.52087200 & 3.71311500 & $\mathrm{H}$ & -1.59778200 & -1.04038500 & 3.43963200 \\
\hline $\mathrm{H}$ & -1.58136900 & -1.78139700 & 5.04145800 & $\mathrm{C}$ & -0.23734800 & 0.56645900 & 5.27192100 \\
\hline $\mathrm{H}$ & -0.76448400 & 0.31401200 & 6.19976200 & $\mathrm{H}$ & -0.93161900 & 1.12711900 & 4.63930100 \\
\hline $\mathrm{H}$ & 0.59974900 & 1.22693800 & 5.52885500 & $\mathrm{C}$ & 1.06481800 & -1.54402700 & 5.60771700 \\
\hline
\end{tabular}




\begin{tabular}{|c|c|c|c|c|c|c|c|}
\hline $\mathrm{H}$ & 0.42138600 & -1.82951700 & 6.44763000 & $\mathrm{H}$ & 1.90199500 & -0.96547000 & 6.01655400 \\
\hline $\mathrm{H}$ & 1.45804400 & -2.46962000 & 5.17158800 & C & 1.62361800 & -1.52127500 & 2.49468900 \\
\hline 0 & 0.85983700 & -1.98833700 & 1.64081700 & C & 3.91063600 & -1.57830100 & 3.62314600 \\
\hline C & 3.30917300 & -3.20237200 & 1.88601200 & C & 5.08953700 & -2.53224400 & 3.36624300 \\
\hline $\mathrm{H}$ & 4.16185100 & -0.52895700 & 3.44219500 & $\mathrm{H}$ & 3.55194800 & -1.67839100 & 4.65091200 \\
\hline C & 4.42139300 & -3.78885700 & 2.78884300 & $\mathrm{H}$ & 2.45422400 & -3.87608900 & 1.78861500 \\
\hline $\mathrm{H}$ & 5.65952600 & -2.73025700 & 4.27862700 & $\mathrm{H}$ & 5.77497900 & -2.10119300 & 2.62971500 \\
\hline $\mathrm{H}$ & 3.96431500 & -4.38364000 & 3.58956500 & $\mathrm{H}$ & 5.11467800 & -4.42976000 & 2.23928700 \\
\hline $\mathrm{N}$ & 2.87127700 & -2.03407200 & 2.67127400 & C & 3.83886200 & -2.84667400 & 0.49748000 \\
\hline C & 4.13477000 & -1.55702000 & 0.16258800 & C & 4.08916200 & -3.91364600 & -0.45874300 \\
\hline C & 4.71855300 & -1.20098300 & -1.09922600 & $\mathrm{H}$ & 3.91602100 & -0.75047800 & 0.85674900 \\
\hline C & 4.67602000 & -3.58515000 & -1.72471600 & C & 3.78002400 & -5.26248300 & -0.20311100 \\
\hline C & 5.01727500 & 0.13207100 & -1.42720000 & C & 4.99829800 & -2.23150400 & -2.04301700 \\
\hline C & 4.94339100 & -4.61348300 & -2.68196400 & $\mathrm{H}$ & 3.33013300 & -5.54687400 & 0.74180200 \\
\hline C & 4.03819900 & -6.25739100 & -1.14626500 & C & 5.59118500 & 0.44594600 & -2.65908700 \\
\hline $\mathrm{H}$ & 4.78879200 & 0.91549700 & -0.70913900 & C & 5.58405100 & -1.90072700 & -3.30345800 \\
\hline C & 4.61679500 & -5.94279500 & -2.37133600 & C & 5.54084300 & -4.25282600 & -3.93927700 \\
\hline $\mathrm{H}$ & 3.78575600 & -7.28916500 & -0.91639900 & C & 5.87389500 & -0.55582900 & -3.58700600 \\
\hline $\mathrm{H}$ & 5.82084000 & 1.48114100 & -2.89680400 & $\mathrm{C}$ & 5.84813800 & -2.96051000 & -4.23665300 \\
\hline $\mathrm{H}$ & 4.82085800 & -6.72419500 & -3.09941400 & $\mathrm{H}$ & 5.74434200 & -5.04525700 & -4.65573500 \\
\hline $\mathrm{H}$ & 6.32225800 & -0.30217300 & -4.54473100 & $\mathrm{H}$ & 6.29967500 & -2.70750200 & -5.19307300 \\
\hline C & 1.29981600 & 1.72703300 & 1.99911400 & $S$ & 2.99235700 & 1.83514000 & 2.13322000 \\
\hline $\mathrm{N}$ & 0.59214300 & 0.70281000 & 2.54224700 & $\mathrm{H}$ & -0.38972700 & 0.58468600 & 2.28217400 \\
\hline C & -2.55107200 & -3.06697700 & 1.21107900 & 0 & -2.17528900 & -3.82951600 & 2.07808600 \\
\hline $\mathrm{O}$ & -3.81614900 & -2.69033400 & 0.95606400 & C & -4.92462800 & -3.05722300 & 1.87440200 \\
\hline C & -4.62134400 & -2.56958500 & 3.29338500 & $\mathrm{H}$ & -5.50769000 & -2.72770300 & 3.91829800 \\
\hline $\mathrm{H}$ & -3.78374500 & -3.11286100 & 3.73183100 & $\mathrm{H}$ & -4.39294400 & -1.50071300 & 3.28697000 \\
\hline C & -6.10591100 & -2.28359500 & 1.28596800 & $\mathrm{H}$ & -6.32354400 & -2.62204600 & 0.26663500 \\
\hline $\mathrm{H}$ & -6.99954100 & -2.45081900 & 1.89614200 & $\mathrm{H}$ & -5.88977700 & -1.21176700 & 1.26343400 \\
\hline C & -5.15186900 & -4.56942800 & 1.80841500 & $\mathrm{H}$ & -6.03118700 & -4.83332700 & 2.40652400 \\
\hline $\mathrm{H}$ & -5.33725700 & -4.88806600 & 0.77615100 & $\mathrm{H}$ & -4.28868700 & -5.11233700 & 2.19775900 \\
\hline C & -1.99763000 & -1.57476400 & -0.68545900 & $\mathrm{H}$ & -2.82215200 & -0.92098100 & -0.41417500 \\
\hline C & -0.85477500 & -0.89511700 & -1.35396700 & C & -1.00380900 & 0.44455400 & -1.75342300 \\
\hline C & 0.33660400 & -1.57148400 & -1.65814500 & C & 0.02525000 & 1.09584800 & -2.43625800 \\
\hline $\mathrm{H}$ & -1.91918400 & 0.97672100 & -1.51157600 & C & 1.35971500 & -0.91913500 & -2.34256900 \\
\hline $\mathrm{H}$ & 0.47151600 & -2.60762200 & -1.36162600 & C & 1.20758500 & 0.41381700 & -2.73134900 \\
\hline $\mathrm{H}$ & -0.09663200 & 2.13364500 & -2.73100700 & $\mathrm{H}$ & 2.27828700 & -1.44972700 & -2.56741500 \\
\hline $\mathrm{H}$ & 2.01405100 & 0.91861500 & -3.25505400 & $\mathrm{~N}$ & -1.66155400 & -2.49453000 & 0.30121600 \\
\hline $\mathrm{H}$ & -0.68473900 & -2.56595200 & 0.59771800 & $S$ & -3.18790400 & 1.40986000 & 1.17922100 \\
\hline $\mathrm{O}$ & -2.43986600 & 2.65136100 & 0.85932600 & $\mathrm{O}$ & -4.05235800 & 0.89291000 & 0.09118000 \\
\hline $\mathrm{O}$ & -2.34751000 & 0.37708400 & 1.84608500 & C & -4.38780500 & 1.92652900 & 2.50364300 \\
\hline $\mathrm{F}$ & -3.72826800 & 2.34655700 & 3.59032300 & $\mathrm{~F}$ & -5.16517400 & 0.88403200 & 2.85771000 \\
\hline $\mathrm{F}$ & -5.17942900 & 2.91137600 & 2.06873300 & C & -3.23391400 & -1.71101700 & -3.05699700 \\
\hline C & -2.93311700 & -2.58137900 & -1.98924400 & $\mathrm{H}$ & -2.24510300 & -3.39692700 & -2.20163500 \\
\hline $\mathrm{H}$ & -3.75435300 & -2.84333000 & -1.32640300 & C & -4.31544500 & -0.74155500 & -2.96095400 \\
\hline $\mathrm{H}$ & -4.07987200 & 0.18378500 & -3.50224800 & $\mathrm{H}$ & -4.57613300 & -0.49875900 & -1.92364100 \\
\hline C & -2.33779700 & -1.66736500 & -4.25017000 & $\mathrm{H}$ & -2.84977700 & -1.28285300 & -5.13654100 \\
\hline $\mathrm{H}$ & -1.52070700 & -0.96471500 & -4.01819300 & $\mathrm{H}$ & -1.88158000 & -2.63610100 & -4.46981800 \\
\hline $\mathrm{Si}$ & -6.00009700 & -1.33057700 & -3.78190400 & C & -7.22751200 & 0.03758000 & -3.36749500 \\
\hline $\mathrm{H}$ & -6.91359200 & 1.00022200 & -3.78691400 & $\mathrm{H}$ & -8.21775700 & -0.19657700 & -3.77698200 \\
\hline $\mathrm{H}$ & -7.33382100 & 0.16625000 & -2.28463200 & C & -5.76291700 & -1.48691800 & -5.64935900 \\
\hline $\mathrm{H}$ & -5.38755600 & -0.55799900 & -6.09467600 & $\mathrm{H}$ & -5.07269900 & -2.29503300 & -5.91730300 \\
\hline $\mathrm{H}$ & -6.72459000 & -1.71043700 & -6.12780200 & C & -6.52730100 & -2.98483200 & -3.04593400 \\
\hline
\end{tabular}




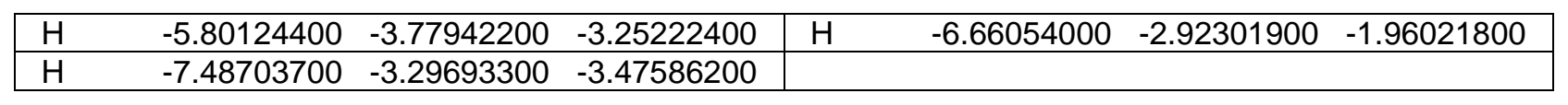

\section{TS- $R-4 f-n d-39$}

Imaginary frequency: $-187.00 \mathrm{~cm}^{-1}$

E[B3LYP/6-31G(d)]: -4788.674348 Hartree

E[PCM(Et $2 \mathrm{O})-B 3 L Y P / 6-311 G(d, p)-D 3(B J)]:-4790.210228$ Hartree

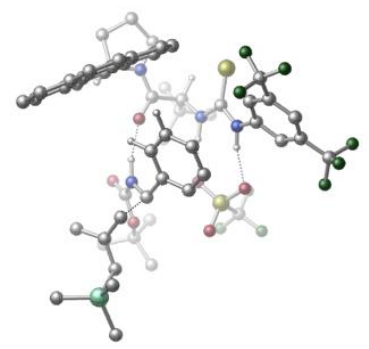

\begin{tabular}{|c|c|c|c|c|c|c|c|}
\hline 01 & & & & $\mathrm{~N}$ & -1.76257700 & -2.41709300 & 0.71304300 \\
\hline $\mathrm{H}$ & -0.81256600 & -2.79116900 & 0.60721900 & C & -2.65996800 & -2.87444300 & -0.26892700 \\
\hline C & -2.44672200 & -4.14262100 & -0.82472800 & C & -3.68694500 & -2.06903900 & -0.77949500 \\
\hline C & -3.26715200 & -4.60049300 & -1.85782600 & $\mathrm{H}$ & -1.62709100 & -4.75382500 & -0.46372200 \\
\hline C & -4.49417400 & -2.53968700 & -1.81154700 & $\mathrm{H}$ & -3.83415100 & -1.07519000 & -0.38299700 \\
\hline $\mathrm{C}$ & -4.30163300 & -3.81045300 & -2.35745700 & $\mathrm{H}$ & -4.93389800 & -4.17050900 & -3.15976300 \\
\hline C & -3.07163200 & -5.99381300 & -2.39395600 & C & -5.50586000 & -1.60869900 & -2.41905600 \\
\hline $\mathrm{F}$ & -3.76851700 & -6.90782600 & -1.67861400 & $\mathrm{~F}$ & -1.77681200 & -6.37247800 & -2.35900700 \\
\hline $\mathrm{F}$ & -3.49136100 & -6.10148700 & -3.67682600 & $\mathrm{~F}$ & -6.05357800 & -0.77195600 & -1.50748600 \\
\hline $\mathrm{F}$ & -4.94092800 & -0.81282100 & -3.37178400 & $\mathrm{~F}$ & -6.51624500 & -2.26867900 & -3.02240400 \\
\hline C & -0.94406000 & -0.23115200 & 3.54122000 & $\mathrm{H}$ & -1.93954800 & -0.35085900 & 3.96652400 \\
\hline C & 0.07607400 & -0.62983300 & 4.66805000 & $\mathrm{C}$ & 1.54389800 & -0.38274200 & 4.28527500 \\
\hline $\mathrm{H}$ & 1.75073900 & 0.67135700 & 4.08855100 & $\mathrm{H}$ & 1.83436300 & -0.95327300 & 3.40048300 \\
\hline $\mathrm{H}$ & 2.18933800 & -0.70620600 & 5.11212300 & $\mathrm{C}$ & -0.12314900 & -2.12694200 & 4.98486200 \\
\hline $\mathrm{H}$ & 0.46526300 & -2.39772500 & 5.86982700 & $\mathrm{H}$ & 0.21008100 & -2.76081300 & 4.15788800 \\
\hline $\mathrm{H}$ & -1.17421100 & -2.36079700 & 5.19260500 & $\mathrm{C}$ & -0.24667500 & 0.19048000 & 5.93380500 \\
\hline $\mathrm{H}$ & 0.45275400 & -0.07434900 & 6.73484900 & $\mathrm{H}$ & -1.26027600 & -0.01021800 & 6.30144500 \\
\hline $\mathrm{H}$ & -0.14603500 & 1.26869500 & 5.75955300 & $\mathrm{C}$ & -0.78814700 & 1.21776500 & 3.01451800 \\
\hline $\mathrm{O}$ & 0.12600700 & 1.52752100 & 2.23851100 & $\mathrm{C}$ & -2.82840100 & 1.95248600 & 4.34948100 \\
\hline $\mathrm{C}$ & -1.51001300 & 3.56639600 & 3.03193800 & $\mathrm{C}$ & -3.56484800 & 3.29707600 & 4.32310200 \\
\hline $\mathrm{H}$ & -3.45486300 & 1.11379500 & 4.03202200 & $\mathrm{H}$ & -2.44767300 & 1.74211800 & 5.35449900 \\
\hline $\mathrm{C}$ & -2.44757800 & 4.30522500 & 4.02558700 & $\mathrm{H}$ & -0.46595700 & 3.81348000 & 3.24483100 \\
\hline $\mathrm{H}$ & -4.07938100 & 3.49558200 & 5.26807200 & $\mathrm{H}$ & -4.31349900 & 3.30925600 & 3.52507500 \\
\hline $\mathrm{H}$ & -1.88861100 & 4.52701600 & 4.94312100 & $\mathrm{H}$ & -2.81159600 & 5.25118200 & 3.61795700 \\
\hline $\mathrm{N}$ & -1.70258900 & 2.14785100 & 3.40504200 & $\mathrm{C}$ & -1.73703600 & 3.99303300 & 1.57375000 \\
\hline $\mathrm{C}$ & -0.93681600 & 5.02025600 & 1.15176600 & $\mathrm{C}$ & -2.78759000 & 3.51013300 & 0.68651700 \\
\hline $\mathrm{C}$ & -1.10436700 & 5.69732600 & -0.09860100 & $\mathrm{H}$ & -0.14122100 & 5.37416500 & 1.80532400 \\
\hline $\mathrm{C}$ & -2.98791600 & 4.18164900 & -0.57056300 & $\mathrm{C}$ & -3.60397900 & 2.39896700 & 0.96874600 \\
\hline $\mathrm{C}$ & -0.27957500 & 6.76476400 & -0.49095000 & $\mathrm{C}$ & -2.15829000 & 5.27608300 & -0.95936800 \\
\hline $\mathrm{C}$ & -4.01905900 & 3.74413300 & -1.46326900 & $\mathrm{H}$ & -3.44289600 & 1.81536600 & 1.86191900 \\
\hline $\mathrm{C}$ & -4.59265000 & 1.97079900 & 0.08296900 & $\mathrm{C}$ & -0.48748500 & 7.41282100 & -1.70692900 \\
\hline $\mathrm{H}$ & 0.52123800 & 7.08628300 & 0.17093300 & $\mathrm{C}$ & -2.36105000 & 5.94304400 & -2.20722900 \\
\hline$C$ & -4.80988700 & 2.63726300 & -1.11714600 & $\mathrm{C}$ & -4.20743300 & 4.43969100 & -2.70750000 \\
\hline $\mathrm{H}$ & -5.18056900 & 1.09325700 & 0.33367500 & $\mathrm{C}$ & -1.51560400 & 7.00854600 & -2.55699100 \\
\hline $\mathrm{H}$ & 0.15501000 & 8.24119200 & -1.99396500 & $\mathrm{C}$ & -3.42133700 & 5.49127300 & -3.06366300 \\
\hline $\mathrm{H}$ & -5.57577500 & 2.28841000 & -1.80520900 & $\mathrm{H}$ & -5.00187700 & 4.09815700 & -3.36665900 \\
\hline $\mathrm{H}$ & -1.67218800 & 7.51755200 & -3.50502100 & $\mathrm{H}$ & -3.57885000 & 6.00394900 & -4.00955000 \\
\hline $\mathrm{C}$ & -2.02788300 & -1.59705400 & 1.78025000 & $S$ & -3.60572200 & -1.24825100 & 2.31521800 \\
\hline $\mathrm{N}$ & -0.91088500 & -1.13431200 & 2.39668300 & $\mathrm{H}$ & -0.00062500 & -1.32856600 & 1.97232400 \\
\hline$S$ & 1.97298000 & -3.19101100 & 0.45375800 & $\mathrm{O}$ & 3.00159900 & -2.98056700 & -0.58968500 \\
\hline $\mathrm{O}$ & 1.77663000 & -2.03116600 & 1.37093100 & $\mathrm{O}$ & 0.69649700 & -3.79314500 & -0.00839400 \\
\hline C & 2.70185300 & -4.50044600 & 1.55699200 & $\mathrm{~F}$ & 2.84856500 & -5.65246800 & 0.89511900 \\
\hline
\end{tabular}




\begin{tabular}{|c|c|c|c|c|c|c|c|}
\hline $\mathrm{F}$ & 1.90951000 & -4.71068400 & 2.61801900 & $F$ & 3.91302100 & -4.11599700 & 2.00685500 \\
\hline C & 3.57380500 & 0.85148500 & 1.42511600 & $\mathrm{O}$ & 3.62496900 & 1.40898600 & 2.50294000 \\
\hline 0 & 4.52674700 & 0.07418700 & 0.87639100 & C & 5.65374700 & -0.44458700 & 1.70021600 \\
\hline C & 6.63247500 & 0.69935400 & 1.97566300 & $\mathrm{H}$ & 7.49758500 & 0.31793000 & 2.52927200 \\
\hline $\mathrm{H}$ & 6.15665000 & 1.48192900 & 2.57068200 & $\mathrm{H}$ & 7.00162600 & 1.13488200 & 1.03934300 \\
\hline C & 6.26329000 & -1.51620800 & 0.79389000 & $\mathrm{H}$ & 5.51620700 & -2.27488600 & 0.54408400 \\
\hline $\mathrm{H}$ & 7.10090500 & -1.99957500 & 1.30751900 & $\mathrm{H}$ & 6.64352300 & -1.08098600 & -0.13646800 \\
\hline C & 5.13686500 & -1.08139300 & 2.99311900 & $\mathrm{H}$ & 5.97055400 & -1.59701400 & 3.48331900 \\
\hline $\mathrm{H}$ & 4.36292700 & -1.81936100 & 2.77206800 & $\mathrm{H}$ & 4.73511800 & -0.33536100 & 3.67849400 \\
\hline C & 2.38039200 & 0.37902800 & -0.68502400 & $\mathrm{H}$ & 2.98232400 & -0.52501600 & -0.74515700 \\
\hline C & 1.00175000 & 0.20360100 & -1.24248900 & C & 0.76089100 & -0.91534300 & -2.05779800 \\
\hline C & -0.00697700 & 1.16342300 & -1.07387400 & C & -0.47083600 & -1.07229700 & -2.68851000 \\
\hline $\mathrm{H}$ & 1.52942900 & -1.67501400 & -2.16613900 & C & -1.23608500 & 1.00121600 & -1.71068700 \\
\hline $\mathrm{H}$ & 0.15084000 & 2.03395500 & -0.44386800 & C & -1.46871300 & -0.10893700 & -2.52607800 \\
\hline $\mathrm{H}$ & -0.65402200 & -1.95377400 & -3.29581200 & $\mathrm{H}$ & -2.01269400 & 1.74441500 & -1.57237000 \\
\hline $\mathrm{H}$ & -2.43171500 & -0.23104200 & -3.01221000 & $\mathrm{~N}$ & 2.49181000 & 1.01193800 & 0.55828800 \\
\hline $\mathrm{H}$ & 1.64993800 & 1.34466600 & 1.03894400 & C & 3.12184600 & 1.39166000 & -2.00465400 \\
\hline $\mathrm{H}$ & 2.49277400 & 2.27884600 & -1.95431300 & $\mathrm{H}$ & 2.90660200 & 0.76550800 & -2.86751100 \\
\hline C & 4.50217900 & 1.61198700 & -1.75403700 & C & 5.51543400 & 0.71597200 & -2.27811400 \\
\hline $\mathrm{H}$ & 5.12439000 & -0.29303600 & -2.45543000 & $\mathrm{H}$ & 6.40450300 & 0.66749300 & -1.64184400 \\
\hline C & 4.88418500 & 2.79949000 & -0.93581500 & $\mathrm{H}$ & 5.92405400 & 2.78194900 & -0.60464700 \\
\hline $\mathrm{H}$ & 4.21917500 & 2.87461900 & -0.06451700 & $\mathrm{H}$ & 4.71111400 & 3.71656800 & -1.51670900 \\
\hline C & 7.50351700 & -0.03351800 & -4.45497500 & $\mathrm{H}$ & 8.31611400 & -0.03985700 & -3.71957300 \\
\hline $\mathrm{H}$ & 7.94743300 & 0.17081900 & -5.43689000 & $\mathrm{H}$ & 7.07326200 & -1.04079600 & -4.48943700 \\
\hline C & 6.96806400 & 2.98344100 & -3.91535300 & $\mathrm{H}$ & 7.74582000 & 3.02808400 & -3.14415500 \\
\hline $\mathrm{H}$ & 6.22597000 & 3.75840300 & -3.69245000 & $\mathrm{H}$ & 7.43929500 & 3.25176500 & -4.86892500 \\
\hline C & 4.78410100 & 1.23923900 & -5.28753200 & $\mathrm{H}$ & 4.00942300 & 1.97823200 & -5.05439500 \\
\hline $\mathrm{H}$ & 4.30557600 & 0.25441700 & -5.34064900 & $\mathrm{H}$ & 5.16476700 & 1.47065700 & -6.28998800 \\
\hline Si & 6.19965100 & 1.26495300 & -4.04426700 & & & & \\
\hline
\end{tabular}

\section{TS- $R-4 f-n d-40$}

Imaginary frequency: $-243.11 \mathrm{~cm}^{-1}$ E[B3LYP/6-31G(d)]: -4788.688599 Hartree

E[PCM(Et $\left.\left.{ }_{2} \mathrm{O}\right)-\mathrm{B} 3 L Y P / 6-311 \mathrm{G}(\mathrm{d}, \mathrm{p})-\mathrm{D} 3(\mathrm{BJ})\right]$ : -4790.210036 Hartree

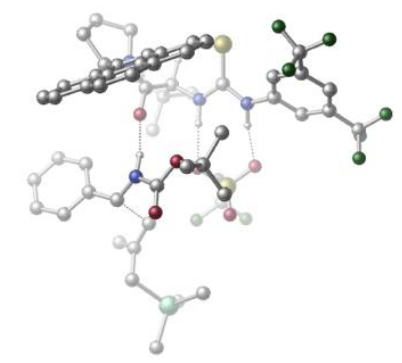

\begin{tabular}{|cccc|cccc|}
\hline 01 & & & & $\mathrm{~N}$ & -1.79937100 & -2.14089600 & 1.52891900 \\
\hline $\mathrm{H}$ & -0.90958900 & -2.63504800 & 1.39247700 & $\mathrm{C}$ & -2.88974400 & -2.76702200 & 0.89932800 \\
\hline $\mathrm{C}$ & -2.79229900 & -4.14373100 & 0.64835800 & $\mathrm{C}$ & -4.00949700 & -2.06614600 & 0.43349400 \\
\hline $\mathrm{C}$ & -3.81146900 & -4.80411200 & -0.03709800 & $\mathrm{H}$ & -1.91616100 & -4.68815700 & 0.98315400 \\
\hline $\mathrm{C}$ & -5.01461500 & -2.74088500 & -0.25555000 & $\mathrm{H}$ & -4.08509200 & -1.00279600 & 0.60376300 \\
\hline $\mathrm{C}$ & -4.93520400 & -4.11383500 & -0.49093400 & $\mathrm{H}$ & -5.72992200 & -4.63344700 & -1.01219700 \\
\hline $\mathrm{C}$ & -3.67090900 & -6.27241500 & -0.34111200 & $\mathrm{C}$ & -6.14981400 & -1.94690900 & -0.84126700 \\
\hline $\mathrm{F}$ & -4.87509700 & -6.88486500 & -0.41655200 & $\mathrm{~F}$ & -2.94589900 & -6.91750000 & 0.59715500 \\
\hline $\mathrm{F}$ & -3.05364700 & -6.47915200 & -1.52784700 & $\mathrm{~F}$ & -5.83582100 & -1.46879800 & -2.07467100 \\
\hline $\mathrm{F}$ & -7.27142900 & -2.68400600 & -0.98439700 & $\mathrm{~F}$ & -6.46620600 & -0.86750100 & -0.08658800 \\
\hline $\mathrm{C}$ & -0.21030900 & 0.51212500 & 3.47931000 & $\mathrm{H}$ & -1.09377200 & 0.63267500 & 4.10546200 \\
\hline $\mathrm{C}$ & 1.00224400 & 0.21916600 & 4.43464400 & $\mathrm{C}$ & 2.35049000 & 0.08641000 & 3.70185400 \\
\hline $\mathrm{H}$ & 2.65281100 & 1.02712100 & 3.23277300 & $\mathrm{H}$ & 2.32497000 & -0.68482100 & 2.92903800 \\
\hline $\mathrm{H}$ & 3.12665500 & -0.19053700 & 4.42632400 & $\mathrm{C}$ & 0.70847200 & -1.09721300 & 5.18377900 \\
\hline $\mathrm{H}$ & 1.47005100 & -1.26504500 & 5.95467800 & $\mathrm{H}$ & 0.72231600 & -1.95485200 & 4.50543500 \\
\hline
\end{tabular}




\begin{tabular}{|c|c|c|c|c|c|c|c|}
\hline $\mathrm{H}$ & -0.27116000 & -1.07017800 & 5.67539200 & $C$ & 1.11286200 & 1.36011000 & 5.46667800 \\
\hline $\mathrm{H}$ & 1.96949800 & 1.18383600 & 6.12722200 & $\mathrm{H}$ & 0.22069100 & 1.42183500 & 6.10159700 \\
\hline $\mathrm{H}$ & 1.26519200 & 2.33576300 & 4.98835500 & C & -0.04728500 & 1.75851400 & 2.57821300 \\
\hline 0 & 0.68460800 & 1.71124700 & 1.57543900 & C & -1.55926800 & 3.17888800 & 4.06243400 \\
\hline C & -0.66107700 & 4.06433200 & 1.98781400 & C & -1.23135500 & 4.65345400 & 4.31711900 \\
\hline $\mathrm{H}$ & -2.62389200 & 3.03072500 & 3.84346800 & $\mathrm{H}$ & -1.29293200 & 2.53465300 & 4.89682600 \\
\hline $\mathrm{H}$ & 0.38869800 & 4.16773200 & 1.70089000 & $\mathrm{H}$ & -0.27246100 & 4.72832500 & 4.84391600 \\
\hline $\mathrm{H}$ & -1.99059800 & 5.15088400 & 4.92808100 & $\mathrm{~N}$ & -0.75242800 & 2.87984000 & 2.86877700 \\
\hline C & -1.51787000 & 3.95205400 & 0.73008800 & C & -2.54875700 & 3.06068300 & 0.66074900 \\
\hline C & -1.27733500 & 4.86647800 & -0.37258000 & C & -3.43035800 & 2.98819500 & -0.46914000 \\
\hline $\mathrm{H}$ & -2.72055500 & 2.35898600 & 1.47288000 & C & -2.13409800 & 4.80309400 & -1.52004600 \\
\hline C & -0.23907300 & 5.81554800 & -0.37275300 & C & -4.49858500 & 2.07694500 & -0.52214400 \\
\hline C & -3.21024600 & 3.86759900 & -1.56860400 & C & -1.91994300 & 5.68449000 & -2.62546000 \\
\hline $\mathrm{H}$ & 0.42551000 & 5.89469600 & 0.48081900 & C & -0.03738200 & 6.67001300 & -1.45662900 \\
\hline C & -5.32983500 & 2.01874900 & -1.64051500 & $\mathrm{H}$ & -4.67207400 & 1.41357900 & 0.32100600 \\
\hline C & -4.06891200 & 3.80597200 & -2.70868800 & C & -0.86523600 & 6.60924200 & -2.57246700 \\
\hline C & -2.79714900 & 5.59881100 & -3.76148900 & $\mathrm{H}$ & 0.77534900 & 7.39091800 & -1.42489500 \\
\hline C & -5.11838800 & 2.87215000 & -2.72197700 & $\mathrm{H}$ & -6.13473600 & 1.29150700 & -1.66887600 \\
\hline C & -3.82201200 & 4.70389600 & -3.80260900 & $\mathrm{H}$ & -0.70386300 & 7.27952700 & -3.41325400 \\
\hline $\mathrm{H}$ & -2.62248100 & 6.27384600 & -4.59596500 & $\mathrm{H}$ & -5.76874700 & 2.82075300 & -3.59195100 \\
\hline $\mathrm{H}$ & -4.47609200 & 4.65472100 & -4.66993000 & C & -1.78145900 & -1.04646000 & 2.35679100 \\
\hline S & -3.16713300 & -0.34606300 & 3.04363800 & $\mathrm{~N}$ & -0.51545200 & -0.61180000 & 2.60625100 \\
\hline $\mathrm{H}$ & 0.22727800 & -0.90947200 & 1.96467900 & $S$ & 1.68559900 & -2.95177300 & 0.40754900 \\
\hline 0 & 2.11559300 & -3.20472500 & -0.97873600 & 0 & 1.60605300 & -1.50647600 & 0.78148800 \\
\hline $\mathrm{O}$ & 0.53024000 & -3.74235000 & 0.90083200 & C & 3.10494400 & -3.57038800 & 1.44440400 \\
\hline $\mathrm{F}$ & 3.32583400 & -4.87099700 & 1.24224800 & $\mathrm{~F}$ & 2.86156100 & -3.37139200 & 2.74908700 \\
\hline $\mathrm{F}$ & 4.24053400 & -2.89801100 & 1.13361400 & C & -1.12305100 & 5.24624800 & 2.90056200 \\
\hline $\mathrm{H}$ & -2.09936600 & 5.60579500 & 2.56263500 & $\mathrm{H}$ & -0.43033700 & 6.09127900 & 2.85140200 \\
\hline C & 1.37647600 & 0.77754700 & -2.24525200 & 0 & 1.97460200 & 0.60275100 & -3.29194400 \\
\hline 0 & 0.12482100 & 0.48766800 & -1.94812900 & C & -0.77053000 & -0.17864400 & -2.94822900 \\
\hline C & -0.13817900 & -1.48409100 & -3.43732200 & $\mathrm{H}$ & -0.89999700 & -2.05876700 & -3.97581100 \\
\hline $\mathrm{H}$ & 0.70016800 & -1.30123300 & -4.11089800 & $\mathrm{H}$ & 0.21518200 & -2.08982100 & -2.59701300 \\
\hline C & -2.02053200 & -0.45062500 & -2.11692300 & $\mathrm{H}$ & -2.42275000 & 0.47788300 & -1.70365200 \\
\hline $\mathrm{H}$ & -2.79374400 & -0.91407000 & -2.73791100 & $\mathrm{H}$ & -1.78771100 & -1.12870600 & -1.29178600 \\
\hline C & -1.04848600 & 0.80970000 & -4.08151900 & $\mathrm{H}$ & -1.76873600 & 0.36466000 & -4.77682000 \\
\hline $\mathrm{H}$ & -1.48229100 & 1.73572400 & -3.69061800 & $\mathrm{H}$ & -0.13601400 & 1.04405500 & -4.63485200 \\
\hline C & 3.35881000 & 1.56631900 & -1.19642400 & $\mathrm{H}$ & 3.69741300 & 1.57347100 & -2.22718600 \\
\hline C & 4.05712800 & 2.50536700 & -0.29781600 & C & 5.23078700 & 3.11267300 & -0.78158200 \\
\hline C & 3.59022900 & 2.84506100 & 0.98178000 & C & 5.92047900 & 4.04386900 & -0.00817000 \\
\hline $\mathrm{H}$ & 5.59282200 & 2.86221800 & -1.77597500 & C & 4.28811200 & 3.77360000 & 1.75289800 \\
\hline $\mathrm{H}$ & 2.67767100 & 2.40210000 & 1.36631500 & C & 5.45047100 & 4.37499200 & 1.26487900 \\
\hline $\mathrm{H}$ & 6.81751200 & 4.51413500 & -0.40078700 & $\mathrm{H}$ & 3.91836300 & 4.03032200 & 2.74172600 \\
\hline $\mathrm{H}$ & 5.98590800 & 5.10029300 & 1.87115900 & $\mathrm{~N}$ & 2.01187400 & 1.37156400 & -1.12772200 \\
\hline $\mathrm{H}$ & 1.49459100 & 1.39987200 & -0.24236600 & C & 4.06648000 & -0.33779900 & -0.85935100 \\
\hline $\mathrm{H}$ & 3.57082800 & -0.48186200 & 0.09443800 & $\mathrm{H}$ & 3.53101300 & -0.73650900 & -1.71664200 \\
\hline C & 5.45409300 & -0.34568100 & -0.90827200 & C & 6.18089800 & -0.55319200 & -2.16647800 \\
\hline $\mathrm{H}$ & 5.60873000 & -0.20910700 & -3.03689300 & $\mathrm{H}$ & 7.16911800 & -0.07794000 & -2.16262000 \\
\hline C & 6.24980600 & -0.10306000 & 0.33695400 & $\mathrm{H}$ & 5.69115700 & -0.36571500 & 1.23824600 \\
\hline $\mathrm{H}$ & 7.19674900 & -0.65178800 & 0.32167800 & $\mathrm{H}$ & 6.50031700 & 0.96813800 & 0.39232400 \\
\hline C & 7.80366800 & -2.45895000 & -3.90810600 & $\mathrm{H}$ & 8.74566900 & -2.01662400 & -3.56281100 \\
\hline $\mathrm{H}$ & 8.01933200 & -3.48467000 & -4.23141900 & $\mathrm{H}$ & 7.47257300 & -1.89962300 & -4.79107900 \\
\hline C & 7.11865700 & -3.34764400 & -1.00927000 & $\mathrm{H}$ & 8.03420300 & -2.89661000 & -0.60813000 \\
\hline $\mathrm{H}$ & 6.36426000 & -3.36445900 & -0.21536500 & $\mathrm{H}$ & 7.35307600 & -4.39039300 & -1.25718700 \\
\hline
\end{tabular}




\begin{tabular}{|llll|llll|}
\hline $\mathrm{C}$ & 4.87732300 & -3.21570300 & -3.16404100 & $\mathrm{H}$ & 4.08783000 & -3.21709500 & -2.40391300 \\
\hline $\mathrm{H}$ & 4.49579200 & -2.68745400 & -4.04627400 & $\mathrm{H}$ & 5.04873100 & -4.25911800 & -3.45839400 \\
\hline $\mathrm{Si}$ & 6.48574400 & -2.46095100 & -2.55079900 & & & & \\
\hline
\end{tabular}

\section{TS- $R-4 f-n d-41$}

Imaginary frequency: $-266.69 \mathrm{~cm}^{-1}$ E[B3LYP/6-31G(d)]: -4788.691300 Hartree E[PCM(Et $\left.\left.{ }_{2} \mathrm{O}\right)-B 3 L Y P / 6-311 G(d, p)-D 3(B J)\right]:-4790.209692$ Hartree

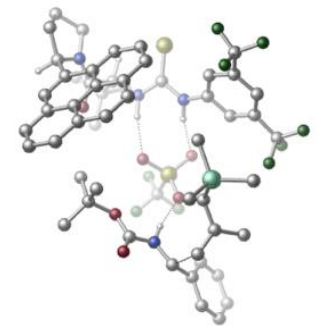

\begin{tabular}{|c|c|c|c|c|c|c|c|}
\hline 01 & & & & $\mathrm{~N}$ & -0.70869500 & 1.59817900 & -2.44319200 \\
\hline $\mathrm{H}$ & 0.07091900 & 0.98659900 & -2.69761200 & $\mathrm{C}$ & -0.31106300 & 2.79221100 & -1.83799400 \\
\hline C & 1.04770300 & 3.14016700 & -1.93151700 & C & -1.16371100 & 3.60338300 & -1.06964700 \\
\hline C & 1.53060300 & 4.27124100 & -1.27374300 & $\mathrm{H}$ & 1.71972900 & 2.51126000 & -2.50606800 \\
\hline C & -0.66104200 & 4.73347100 & -0.43019100 & $\mathrm{H}$ & -2.20616700 & 3.34140200 & -0.97295500 \\
\hline C & 0.68602300 & 5.08960300 & -0.52490200 & $\mathrm{H}$ & 1.06391200 & 5.97482000 & -0.02829100 \\
\hline C & 3.00624000 & 4.54519800 & -1.30434800 & C & -1.56365300 & 5.53070600 & 0.47230900 \\
\hline $\mathrm{F}$ & 3.56881900 & 4.25056000 & -2.49037700 & $\mathrm{~F}$ & 3.66259400 & 3.77722200 & -0.37176600 \\
\hline $\mathrm{F}$ & 3.30473000 & 5.82657100 & -1.01134400 & $\mathrm{~F}$ & -2.86602100 & 5.41816800 & 0.13756200 \\
\hline $\mathrm{F}$ & -1.46035500 & 5.11680100 & 1.76783400 & $\mathrm{~F}$ & -1.25240400 & 6.84479900 & 0.46839400 \\
\hline C & -3.18839900 & -0.98235000 & -3.25075700 & $\mathrm{H}$ & -3.87933900 & -0.25487900 & -3.67514500 \\
\hline $\mathrm{C}$ & -2.89248200 & -2.05657900 & -4.35359500 & C & -1.84649800 & -3.10276600 & -3.92151300 \\
\hline $\mathrm{H}$ & -2.16012400 & -3.63966100 & -3.02371500 & $\mathrm{H}$ & -0.87283600 & -2.64999500 & -3.71548300 \\
\hline $\mathrm{H}$ & -1.70488000 & -3.82703700 & -4.73361800 & C & -2.38737900 & -1.32297000 & -5.61223500 \\
\hline $\mathrm{H}$ & -2.21716300 & -2.04077400 & -6.42353800 & $\mathrm{H}$ & -1.44268700 & -0.80530100 & -5.41898900 \\
\hline $\mathrm{H}$ & -3.11308600 & -0.57992000 & -5.96460900 & C & -4.21074900 & -2.78158700 & -4.69418500 \\
\hline $\mathrm{H}$ & -4.03554600 & -3.52714000 & -5.47824100 & $\mathrm{H}$ & -4.97162700 & -2.08632700 & -5.07058300 \\
\hline $\mathrm{H}$ & -4.62311200 & -3.31019800 & -3.82654300 & $\mathrm{C}$ & -3.77081300 & -1.57452100 & -1.94342500 \\
\hline $\mathrm{O}$ & -3.08293200 & -2.30210300 & -1.22727800 & $\mathrm{C}$ & -6.05239600 & -0.54527900 & -2.41889100 \\
\hline C & -5.62285800 & -1.76462800 & -0.32909800 & C & -7.38543100 & -1.01566900 & -1.82178100 \\
\hline $\mathrm{H}$ & -5.93706700 & 0.54409000 & -2.35934900 & $\mathrm{H}$ & -5.95197200 & -0.83135000 & -3.46683600 \\
\hline $\mathrm{H}$ & -5.66805700 & -2.85608200 & -0.43676600 & $\mathrm{H}$ & -7.67559900 & -1.97592000 & -2.26545400 \\
\hline $\mathrm{H}$ & -8.19476400 & -0.30261100 & -2.00565200 & $\mathrm{~N}$ & -5.04096200 & -1.22339400 & -1.58288700 \\
\hline C & -4.87439500 & -1.49998100 & 0.97278500 & C & -4.71125900 & -2.56454500 & 1.81318700 \\
\hline C & -4.44081500 & -0.18056700 & 1.40618000 & $\mathrm{C}$ & -4.13678400 & -2.45522600 & 3.12042200 \\
\hline $\mathrm{H}$ & -5.02561200 & -3.55323900 & 1.48524000 & C & -3.86031700 & -0.04095400 & 2.71160300 \\
\hline C & -4.55736900 & 0.97418900 & 0.61180300 & C & -3.98393300 & -3.56532600 & 3.96832700 \\
\hline C & -3.71033400 & -1.17165700 & 3.56924600 & $\mathrm{C}$ & -3.42296900 & 1.24221700 & 3.16936700 \\
\hline $\mathrm{H}$ & -4.95324100 & 0.90508400 & -0.39166300 & C & -4.13079700 & 2.22031300 & 1.07034500 \\
\hline $\mathrm{C}$ & -3.42397300 & -3.41983600 & 5.23692700 & $\mathrm{H}$ & -4.31308400 & -4.54319300 & 3.62480800 \\
\hline $\mathrm{C}$ & -3.13477200 & -1.03175400 & 4.86994000 & C & -3.56598600 & 2.35969100 & 2.33296000 \\
\hline C & -2.84639500 & 1.35404200 & 4.48182200 & $\mathrm{H}$ & -4.23373600 & 3.08846000 & 0.42647400 \\
\hline $\mathrm{C}$ & -3.00315300 & -2.16842100 & 5.68485300 & $\mathrm{H}$ & -3.31642100 & -4.28785500 & 5.88210100 \\
\hline $\mathrm{C}$ & -2.70883000 & 0.27209400 & 5.29590500 & $\mathrm{H}$ & -3.21611900 & 3.33158800 & 2.67012500 \\
\hline $\mathrm{H}$ & -2.52428300 & 2.33798100 & 4.81485600 & $\mathrm{H}$ & -2.56755700 & -2.06138200 & 6.67545700 \\
\hline $\mathrm{H}$ & -2.27484200 & 0.37738500 & 6.28754200 & C & -1.97350700 & 1.13697100 & -2.76036900 \\
\hline $\mathrm{S}$ & -3.30194000 & 2.14820800 & -3.03515000 & $\mathrm{~N}$ & -2.00014300 & -0.21433700 & -2.88617400 \\
\hline $\mathrm{H}$ & -1.22372200 & -0.74179400 & -2.48035500 & $\mathrm{~S}$ & 1.87745100 & -1.00262600 & -2.27058700 \\
\hline $\mathrm{O}$ & 2.92767100 & -0.93772500 & -1.20959900 & 0 & 0.56210200 & -1.49916700 & -1.81364900 \\
\hline $\mathrm{O}$ & 1.81318400 & 0.20782800 & -3.12421200 & C & 2.52707400 & -2.33182300 & -3.39689000 \\
\hline $\mathrm{F}$ & 3.75216700 & -2.00302900 & -3.84097000 & $\mathrm{~F}$ & 1.72081500 & -2.49937700 & -4.45065500 \\
\hline $\mathrm{F}$ & 2.61851200 & -3.49328000 & -2.73337500 & C & 2.83050000 & -3.05441600 & 1.83181300 \\
\hline
\end{tabular}




\begin{tabular}{|c|c|c|c|c|c|c|c|}
\hline $\mathrm{O}$ & 3.09644500 & -3.32204800 & 2.99128500 & $\mathrm{O}$ & 1.69158800 & -3.20695600 & 1.17667500 \\
\hline C & 0.44744500 & -3.70876400 & 1.83676700 & C & 0.69706800 & -5.11418600 & 2.38788700 \\
\hline $\mathrm{H}$ & -0.25010500 & -5.52360400 & 2.75550600 & $\mathrm{H}$ & 1.41646400 & -5.10603700 & 3.20892500 \\
\hline $\mathrm{H}$ & 1.06526700 & -5.77497700 & 1.59571800 & $\mathrm{C}$ & -0.55254200 & -3.72898900 & 0.68323200 \\
\hline $\mathrm{H}$ & -0.64202000 & -2.74937600 & 0.20976800 & $\mathrm{H}$ & -1.53918500 & -4.02080600 & 1.05562600 \\
\hline $\mathrm{H}$ & -0.24245800 & -4.44567600 & -0.08386500 & C & 0.03297500 & -2.71533700 & 2.92264800 \\
\hline $\mathrm{H}$ & -0.91736100 & -3.03293500 & 3.36262000 & $\mathrm{H}$ & -0.11684500 & -1.72005300 & 2.49068000 \\
\hline $\mathrm{H}$ & 0.77968600 & -2.66005300 & 3.71892400 & C & 5.04565700 & -2.24852800 & 1.40030100 \\
\hline $\mathrm{H}$ & 5.27036700 & -2.83680500 & 2.28568100 & C & 6.17529300 & -2.08306300 & 0.46078500 \\
\hline C & 7.46942600 & -2.32973900 & 0.95530500 & C & 6.01612200 & -1.73511500 & -0.89264000 \\
\hline C & 8.57899800 & -2.23614900 & 0.12156800 & $\mathrm{H}$ & 7.60062900 & -2.60955200 & 1.99823600 \\
\hline C & 7.13314900 & -1.64766800 & -1.72405000 & $\mathrm{H}$ & 5.03187500 & -1.54016900 & -1.30569800 \\
\hline C & 8.41250300 & -1.89411000 & -1.22356700 & $\mathrm{H}$ & 9.57041600 & -2.43866400 & 0.51637400 \\
\hline $\mathrm{H}$ & 6.99503200 & -1.38840000 & -2.76960300 & $\mathrm{H}$ & 9.27673200 & -1.82512000 & -1.87819200 \\
\hline $\mathrm{N}$ & 3.78224300 & -2.49486200 & 0.95532300 & $\mathrm{H}$ & 3.42697800 & -2.10525800 & 0.06834100 \\
\hline C & 5.03062200 & -0.60603400 & 2.62257900 & $\mathrm{H}$ & 6.06429500 & -0.63806700 & 2.95700200 \\
\hline $\mathrm{H}$ & 4.30602500 & -1.04608000 & 3.30353600 & C & 4.62897500 & 0.45890900 & 1.81512100 \\
\hline C & 3.21869900 & 0.75024400 & 1.58442700 & $\mathrm{H}$ & 2.58971600 & -0.14474300 & 1.66676500 \\
\hline $\mathrm{H}$ & 3.04989200 & 1.22319300 & 0.61212800 & C & 5.64653800 & 1.26607900 & 1.07244300 \\
\hline $\mathrm{H}$ & 5.30449700 & 2.28894000 & 0.89445200 & $\mathrm{H}$ & 5.77803200 & 0.80618400 & 0.07946300 \\
\hline $\mathrm{H}$ & 6.62253700 & 1.27431000 & 1.56462000 & C & 0.69625500 & 2.30072200 & 2.29224000 \\
\hline $\mathrm{H}$ & 0.69185400 & 2.52987100 & 1.22182100 & $\mathrm{H}$ & 0.24616600 & 3.15785000 & 2.80645000 \\
\hline $\mathrm{H}$ & 0.03962500 & 1.43773700 & 2.45108600 & C & 3.46558600 & 3.54999300 & 3.02722000 \\
\hline $\mathrm{H}$ & 3.55746700 & 4.04356600 & 2.05491400 & $\mathrm{H}$ & 4.47532200 & 3.36056600 & 3.41050900 \\
\hline $\mathrm{H}$ & 2.98313900 & 4.25603500 & 3.71454000 & $\mathrm{C}$ & 2.41814100 & 1.08804100 & 4.58762900 \\
\hline $\mathrm{H}$ & 3.42652100 & 0.88041500 & 4.96344800 & $\mathrm{H}$ & 1.87186100 & 0.13874800 & 4.53965300 \\
\hline $\mathrm{H}$ & 1.91453000 & 1.71670200 & 5.33249300 & $\mathrm{Si}$ & 2.44127100 & 1.96834100 & 2.91611500 \\
\hline $\mathrm{C}$ & -7.06079900 & -1.19317000 & -0.33388700 & $\mathrm{H}$ & -7.08173000 & -0.22710400 & 0.18108700 \\
\hline $\mathrm{H}$ & -7.75154600 & -1.86105100 & 0.18868500 & & & & \\
\hline
\end{tabular}

\section{TS-R-4f-nd-42}

Imaginary frequency: $-142.55 \mathrm{~cm}^{-1}$ E[B3LYP/6-31G(d)]: -4788.685029 Hartree

E[PCM(Et $\left.\left.t_{2} \mathrm{O}\right)-B 3 L Y P / 6-311 G(d, p)-D 3(B J)\right]:-4790.209123$ Hartree

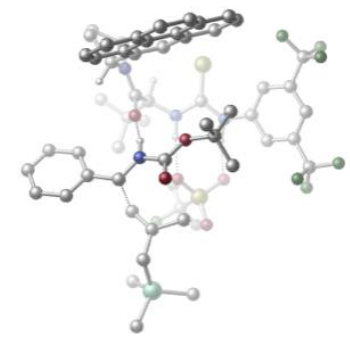

\begin{tabular}{|crrr|rrrr|}
\hline 01 & & & & $\mathrm{~N}$ & 0.21917900 & 2.52416400 & 1.94919100 \\
\hline $\mathrm{H}$ & -0.79857800 & 2.57729100 & 1.82707500 & $\mathrm{C}$ & 0.90442100 & 3.49255500 & 1.19394500 \\
\hline $\mathrm{C}$ & 0.12032300 & 4.24303200 & 0.29670200 & $\mathrm{C}$ & 2.28750200 & 3.72954100 & 1.23800500 \\
\hline $\mathrm{C}$ & 0.71731200 & 5.15734700 & -0.56669100 & $\mathrm{H}$ & -0.95559800 & 4.09883000 & 0.27754100 \\
\hline $\mathrm{C}$ & 2.86297000 & 4.64990100 & 0.36138200 & $\mathrm{H}$ & 2.90060000 & 3.18831100 & 1.94231600 \\
\hline $\mathrm{C}$ & 2.09651600 & 5.36819500 & -0.55422400 & $\mathrm{H}$ & 2.55632200 & 6.07186100 & -1.23694800 \\
\hline $\mathrm{C}$ & -0.13850000 & 5.84036200 & -1.59963900 & $\mathrm{C}$ & 4.34496800 & 4.90402800 & 0.44471300 \\
\hline $\mathrm{F}$ & -1.35184100 & 6.17267100 & -1.11813000 & $\mathrm{~F}$ & -0.35042300 & 5.03040900 & -2.67101300 \\
\hline $\mathrm{F}$ & 0.43795300 & 6.96727500 & -2.07332000 & $\mathrm{~F}$ & 5.02843500 & 3.80726600 & 0.85981400 \\
\hline $\mathrm{F}$ & 4.86168200 & 5.26306800 & -0.75313000 & $\mathrm{~F}$ & 4.64215700 & 5.89587900 & 1.31297200 \\
\hline $\mathrm{C}$ & -0.01028300 & -0.80275700 & 3.45440600 & $\mathrm{H}$ & 0.75616500 & -0.58302700 & 4.19627200 \\
\hline $\mathrm{C}$ & -1.26447500 & -1.34561600 & 4.23014300 & $\mathrm{C}$ & -2.44682500 & -1.71235800 & 3.31326000 \\
\hline $\mathrm{H}$ & -2.19544700 & -2.53476100 & 2.63685400 & $\mathrm{H}$ & -2.78455900 & -0.86949000 & 2.70729300 \\
\hline $\mathrm{H}$ & -3.29342600 & -2.03534300 & 3.93144100 & $\mathrm{C}$ & -1.70541400 & -0.25531500 & 5.22852200 \\
\hline $\mathrm{H}$ & -2.52946100 & -0.62846600 & 5.84803700 & $\mathrm{H}$ & -2.05173200 & 0.64434900 & 4.71622300 \\
\hline $\mathrm{H}$ & -0.88291600 & 0.02841900 & 5.89622300 & $\mathrm{C}$ & -0.86272900 & -2.60202800 & 5.03043600 \\
\hline
\end{tabular}




\begin{tabular}{|c|c|c|c|c|c|c|c|}
\hline $\mathrm{H}$ & -1.73624100 & -2.99293200 & 5.56418600 & $\mathrm{H}$ & -0.09697200 & -2.38010500 & 5.78332700 \\
\hline $\mathrm{H}$ & -0.48961200 & -3.40497900 & 4.38310300 & $\mathrm{C}$ & 0.57040400 & -1.78040400 & 2.40072300 \\
\hline $\mathrm{O}$ & 0.01267600 & -1.89231500 & 1.29411400 & C & 2.43829000 & -2.54694500 & 3.95828300 \\
\hline C & 2.26453700 & -3.43923100 & 1.71943000 & C & 2.77849100 & -4.03585500 & 4.06970700 \\
\hline $\mathrm{H}$ & 3.34059900 & -1.92849900 & 3.88148400 & $\mathrm{H}$ & 1.84850100 & -2.18059000 & 4.79399800 \\
\hline $\mathrm{H}$ & 1.42789900 & -3.91899600 & 1.20483300 & $\mathrm{H}$ & 1.91774800 & -4.57663900 & 4.47984400 \\
\hline $\mathrm{H}$ & 3.63348700 & -4.21919400 & 4.72718400 & $\mathrm{~N}$ & 1.68848200 & -2.48774500 & 2.69373400 \\
\hline C & 3.17986500 & -2.78009700 & 0.69031900 & C & 3.63228900 & -1.50442600 & 0.86062000 \\
\hline C & 3.63455900 & -3.56313900 & -0.44646500 & C & 4.57562500 & -0.89540300 & -0.03395300 \\
\hline $\mathrm{H}$ & 3.27540000 & -0.90336000 & 1.69226900 & C & 4.57298500 & -2.97572300 & -1.35713000 \\
\hline C & 3.19645900 & -4.87522600 & -0.70143400 & C & 5.03834400 & 0.41741500 & 0.15704100 \\
\hline C & 5.04854200 & -1.64641100 & -1.14867300 & C & 5.04335800 & -3.72089500 & -2.48337400 \\
\hline $\mathrm{H}$ & 2.48590300 & -5.35050200 & -0.03372400 & C & 3.65677300 & -5.59201600 & -1.80629000 \\
\hline C & 5.95246300 & 0.98406000 & -0.73091500 & $\mathrm{H}$ & 4.66352400 & 0.99554100 & 0.99688300 \\
\hline C & 5.98787300 & -1.06055500 & -2.05141300 & C & 4.57141500 & -5.02711500 & -2.68839600 \\
\hline C & 5.99060000 & -3.10750400 & -3.37455900 & $\mathrm{H}$ & 3.29664300 & -6.60341900 & -1.97509700 \\
\hline C & 6.42546900 & 0.25441000 & -1.82031500 & $\mathrm{H}$ & 6.28306500 & 2.00601000 & -0.57261100 \\
\hline C & 6.44280700 & -1.84003100 & -3.16902900 & $\mathrm{H}$ & 4.92941200 & -5.59285700 & -3.54506700 \\
\hline $\mathrm{H}$ & 6.34218400 & -3.68573700 & -4.22571400 & $\mathrm{H}$ & 7.13813400 & 0.70242400 & -2.50876900 \\
\hline $\mathrm{H}$ & 7.15977400 & -1.39337100 & -3.85382300 & C & 0.67960000 & 1.43255600 & 2.64552100 \\
\hline $\mathrm{S}$ & 2.23196500 & 1.28461100 & 3.31687300 & $\mathrm{~N}$ & -0.27227700 & 0.46628500 & 2.78685700 \\
\hline $\mathrm{H}$ & -1.08082300 & 0.50205600 & 2.15808400 & S & -3.45904600 & 2.02059000 & 1.09233900 \\
\hline 0 & -4.29418100 & 2.31286300 & -0.08637600 & $\mathrm{O}$ & -2.67665100 & 0.74752100 & 1.01809500 \\
\hline 0 & -2.64630700 & 3.14601100 & 1.62178900 & C & -4.71333300 & 1.69296100 & 2.42841700 \\
\hline $\mathrm{F}$ & -5.53681200 & 2.73353100 & 2.57482100 & $\mathrm{~F}$ & -4.11027300 & 1.45634400 & 3.60633600 \\
\hline $\mathrm{F}$ & -5.44553800 & 0.60659700 & 2.11609700 & C & 3.04670000 & -4.46140400 & 2.61231500 \\
\hline $\mathrm{H}$ & 4.11415800 & -4.40442100 & 2.38313800 & $\mathrm{H}$ & 2.72887700 & -5.49022300 & 2.42000400 \\
\hline C & -0.35548500 & -1.19426700 & -2.57996200 & $\mathrm{O}$ & -0.75133200 & -1.32508600 & -3.72081700 \\
\hline 0 & 0.52870300 & -0.34220900 & -2.09547200 & C & 1.26023200 & 0.61362600 & -2.99539100 \\
\hline C & 0.26531700 & 1.54534900 & -3.68950000 & $\mathrm{H}$ & 0.82815600 & 2.28290600 & -4.27190700 \\
\hline $\mathrm{H}$ & -0.39451600 & 1.00167900 & -4.36745600 & $\mathrm{H}$ & -0.33361700 & 2.09665400 & -2.95783300 \\
\hline C & 2.12940000 & 1.38366600 & -2.00745600 & $\mathrm{H}$ & 2.80443200 & 0.71295400 & -1.47245900 \\
\hline $\mathrm{H}$ & 2.73121700 & 2.12164700 & -2.54719100 & $\mathrm{H}$ & 1.51060900 & 1.91301900 & -1.27776800 \\
\hline C & 2.10563200 & -0.19378500 & -3.98137200 & $\mathrm{H}$ & 2.73204600 & 0.49386800 & -4.55961600 \\
\hline $\mathrm{H}$ & 2.76690200 & -0.88309900 & -3.44689100 & $\mathrm{H}$ & 1.48135100 & -0.75906700 & -4.67664600 \\
\hline C & -1.91884000 & -2.79531400 & -1.77161900 & $\mathrm{H}$ & -2.20581100 & -2.76787600 & -2.81605500 \\
\hline C & -2.23958200 & -4.02912400 & -1.04918100 & C & -3.06545700 & -4.96017900 & -1.70850100 \\
\hline C & -1.73987700 & -4.33680800 & 0.22799700 & C & -3.38348400 & -6.17357400 & -1.10777000 \\
\hline $\mathrm{H}$ & -3.45024600 & -4.72750700 & -2.69834500 & C & -2.06448500 & -5.55327800 & 0.82361700 \\
\hline $\mathrm{H}$ & -1.10494300 & -3.62710400 & 0.74796800 & C & -2.88434800 & -6.47215100 & 0.16292300 \\
\hline $\mathrm{H}$ & -4.01548900 & -6.88640700 & -1.62917700 & $\mathrm{H}$ & -1.67733600 & -5.78477700 & 1.81183700 \\
\hline $\mathrm{H}$ & -3.13286700 & -7.41815800 & 0.63567100 & $\mathrm{~N}$ & -0.84342600 & -2.02157100 & -1.52732600 \\
\hline $\mathrm{H}$ & -0.43666400 & -1.91559600 & -0.58873800 & C & -3.70311800 & -1.60980400 & -1.01332800 \\
\hline $\mathrm{H}$ & -4.36685000 & -2.43413400 & -0.76938000 & $\mathrm{H}$ & -3.11087200 & -1.19927800 & -0.20130400 \\
\hline C & -3.98048700 & -0.82418400 & -2.11257700 & C & -5.08693800 & -1.15423700 & -3.03659900 \\
\hline $\mathrm{H}$ & -4.85190900 & -0.88208100 & -4.07328500 & $\mathrm{H}$ & -5.33866600 & -2.22211100 & -3.00071000 \\
\hline C & -3.19447100 & 0.42371000 & -2.37118500 & $\mathrm{H}$ & -2.27803700 & 0.45498600 & -1.77909700 \\
\hline $\mathrm{H}$ & -2.96521500 & 0.54004400 & -3.43469800 & $\mathrm{H}$ & -3.77781100 & 1.29242400 & -2.04158000 \\
\hline C & -8.01036900 & -0.93550200 & -3.84516100 & $\mathrm{H}$ & -8.11791500 & -2.02316200 & -3.75561300 \\
\hline $\mathrm{H}$ & -9.00040000 & -0.49223100 & -3.68261600 & $\mathrm{H}$ & -7.71620700 & -0.71198500 & -4.87765900 \\
\hline C & -7.26149100 & -0.69024600 & -0.83520100 & $\mathrm{H}$ & -7.29681400 & -1.77474100 & -0.67490200 \\
\hline $\mathrm{H}$ & -6.57322900 & -0.25806000 & -0.10089900 & $\mathrm{H}$ & -8.26179100 & -0.29524900 & -0.61798500 \\
\hline C & -6.59647900 & 1.62241900 & -2.79353600 & $\mathrm{H}$ & -5.98595400 & 2.05997500 & -1.99622700 \\
\hline
\end{tabular}




\begin{tabular}{|c|c|c|c|c|c|c|}
\hline 1 & -6.15918100 & 1.90356600 & -3.75896300 & $\mathrm{H}$ & -7.58950300 & 2.08661600 \\
\hline $\mathrm{Si}$ & -6.76150400 & -0.2451440 & -2.59922000 & & & \\
\hline
\end{tabular}

\section{TS- $R-4 f-n d-43$}

Imaginary frequency: $-279.81 \mathrm{~cm}^{-1}$

E[B3LYP/6-31G(d)]: -4788.679777 Hartree

E[PCM(Et $\left.\left.{ }_{2} \mathrm{O}\right)-\mathrm{B} 3 L \mathrm{YP} / 6-311 \mathrm{G}(\mathrm{d}, \mathrm{p})-\mathrm{D} 3(\mathrm{BJ})\right]$ : -4790.208605 Hartree

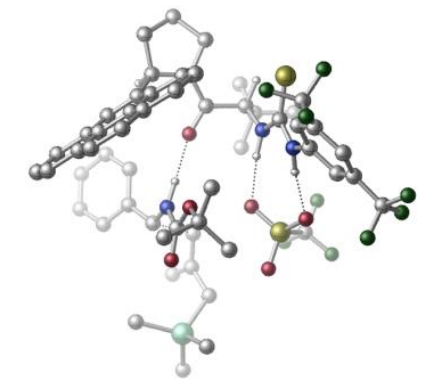

\begin{tabular}{|c|c|c|c|c|c|c|c|}
\hline 01 & & & & $\mathrm{~N}$ & -2.61155500 & -1.24318100 & 1.17393600 \\
\hline $\mathrm{H}$ & -1.93885900 & -1.99462900 & 0.98362600 & C & -3.83442700 & -1.39951900 & 0.49221800 \\
\hline C & -4.20403500 & -2.69322800 & 0.09949400 & C & -4.63508400 & -0.31330300 & 0.11273100 \\
\hline C & -5.36955600 & -2.89210700 & -0.64148300 & $\mathrm{H}$ & -3.57355200 & -3.53472200 & 0.36458700 \\
\hline $\mathrm{C}$ & -5.80041100 & -0.53168800 & -0.61826300 & $\mathrm{H}$ & -4.33958100 & 0.69054500 & 0.37859600 \\
\hline C & -6.18395700 & -1.81874000 & -0.99905200 & $\mathrm{H}$ & -7.09153700 & -1.98043400 & -1.56797400 \\
\hline C & -5.71983500 & -4.27889700 & -1.11206700 & C & -6.68047900 & 0.63814100 & -0.96790800 \\
\hline $\mathrm{F}$ & -5.29211900 & -5.22838200 & -0.25345500 & $\mathrm{~F}$ & -5.16046100 & -4.55365800 & -2.31420600 \\
\hline $\mathrm{F}$ & -7.05566700 & -4.43574400 & -1.25943200 & $\mathrm{~F}$ & -5.97375800 & 1.79098400 & -1.08458200 \\
\hline $\mathrm{F}$ & -7.32803400 & 0.44811700 & -2.14008000 & $\mathrm{~F}$ & -7.62800500 & 0.85810100 & -0.02946100 \\
\hline $\mathrm{C}$ & -0.35839500 & 0.53417900 & 3.45089000 & $\mathrm{H}$ & -1.20144600 & 0.93132500 & 4.01474500 \\
\hline $\mathrm{C}$ & 0.57130100 & -0.20477900 & 4.47696400 & C & 1.83451200 & -0.81732000 & 3.84393600 \\
\hline $\mathrm{H}$ & 2.48018700 & -0.04930100 & 3.40828100 & $\mathrm{H}$ & 1.58985100 & -1.53981900 & 3.06248200 \\
\hline $\mathrm{H}$ & 2.40574900 & -1.34371400 & 4.61896400 & $\mathrm{C}$ & -0.25438000 & -1.32599300 & 5.14050600 \\
\hline $\mathrm{H}$ & 0.32678700 & -1.78999800 & 5.94639700 & $\mathrm{H}$ & -0.51469400 & -2.10766800 & 4.42237900 \\
\hline $\mathrm{H}$ & -1.18427900 & -0.93806700 & 5.57248300 & $\mathrm{C}$ & 1.00298000 & 0.80120900 & 5.56431400 \\
\hline $\mathrm{H}$ & 1.67038900 & 0.31078100 & 6.28204500 & $\mathrm{H}$ & 0.14362600 & 1.18235900 & 6.12918100 \\
\hline $\mathrm{H}$ & 1.54857900 & 1.65572800 & 5.14433200 & C & 0.32496100 & 1.68385000 & 2.67468100 \\
\hline $\mathrm{O}$ & 1.18349500 & 1.43895000 & 1.80997800 & $\mathrm{C}$ & -0.91258800 & 3.41913300 & 4.04868000 \\
\hline $\mathrm{C}$ & 0.53987300 & 4.09824500 & 2.18324600 & C & -0.44787200 & 4.86211100 & 4.27303600 \\
\hline $\mathrm{H}$ & -1.97316300 & 3.36389800 & 3.77768300 & $\mathrm{H}$ & -0.76204600 & 2.79768100 & 4.92983900 \\
\hline $\mathrm{H}$ & 1.61488600 & 4.08067900 & 2.40195800 & $\mathrm{H}$ & 0.44096300 & 4.87323500 & 4.91595200 \\
\hline $\mathrm{H}$ & -1.21819300 & 5.47200900 & 4.75397800 & $\mathrm{~N}$ & -0.06806300 & 2.95721500 & 2.92309000 \\
\hline C & 0.41117700 & 4.13067800 & 0.66428500 & $\mathrm{C}$ & 1.54788600 & 4.41471900 & -0.03921200 \\
\hline $\mathrm{C}$ & -0.85860500 & 4.03930100 & -0.04173000 & C & 1.55953800 & 4.66154100 & -1.45046300 \\
\hline $\mathrm{H}$ & 2.49927800 & 4.47523800 & 0.48552900 & C & -0.88061400 & 4.31107200 & -1.45106900 \\
\hline $\mathrm{C}$ & -2.07118400 & 3.69464300 & 0.58126300 & $\mathrm{C}$ & 2.74087500 & 4.95588000 & -2.15019700 \\
\hline $\mathrm{C}$ & 0.32156700 & 4.62288900 & -2.15454000 & C & -2.11611600 & 4.26211700 & -2.17179500 \\
\hline $\mathrm{H}$ & -2.08887300 & 3.42664700 & 1.62824400 & C & -3.26733700 & 3.63577500 & -0.13390100 \\
\hline $\mathrm{C}$ & 2.70972600 & 5.21195300 & -3.51983300 & $\mathrm{H}$ & 3.68395100 & 4.98316300 & -1.60960100 \\
\hline $\mathrm{C}$ & 0.29815900 & 4.88789300 & -3.55869900 & C & -3.29731000 & 3.92380300 & -1.49345300 \\
\hline $\mathrm{C}$ & -2.11184100 & 4.54340400 & -3.58182800 & $\mathrm{H}$ & -4.18041400 & 3.34863200 & 0.37847300 \\
\hline $\mathrm{C}$ & 1.50385700 & 5.17997100 & -4.21781500 & $\mathrm{H}$ & 3.63209800 & 5.43922600 & -4.04762500 \\
\hline $\mathrm{C}$ & -0.96245300 & 4.84242200 & -4.24603400 & $\mathrm{H}$ & -4.23313600 & 3.86543600 & -2.04301100 \\
\hline $\mathrm{H}$ & -3.06023700 & 4.50692700 & -4.11234200 & $\mathrm{H}$ & 1.48628300 & 5.38142900 & -5.28614600 \\
\hline $\mathrm{H}$ & -0.97892800 & 5.04973200 & -5.31327400 & C & -2.28498000 & -0.33247600 & 2.14827800 \\
\hline S & -3.41440200 & 0.67665300 & 2.91450100 & $\mathrm{~N}$ & -0.96025300 & -0.35747600 & 2.46744600 \\
\hline $\mathrm{H}$ & -0.32367100 & -0.88068700 & 1.85908400 & S & 0.41273000 & -3.45466600 & 0.42989600 \\
\hline $\mathrm{O}$ & 1.14451100 & -4.01225000 & -0.72005900 & $\mathrm{O}$ & 0.85858400 & -2.08749300 & 0.84842200 \\
\hline $\mathrm{O}$ & -1.06537200 & -3.58876500 & 0.40765300 & C & 0.94335800 & -4.52430700 & 1.85796500 \\
\hline $\mathrm{F}$ & 0.63011500 & -5.80448800 & 1.64339600 & $\mathrm{~F}$ & 0.35457100 & -4.12482500 & 2.99798700 \\
\hline
\end{tabular}




\begin{tabular}{|c|c|c|c|c|c|c|c|}
\hline $\mathrm{F}$ & 2.27745400 & -4.43282100 & 2.02548600 & C & -0.09565700 & 5.33789100 & 2.85988300 \\
\hline $\mathrm{H}$ & -1.00090800 & 5.63212800 & 2.31959200 & $\mathrm{H}$ & 0.59140800 & 6.18843700 & 2.84074600 \\
\hline $\mathrm{C}$ & 1.86460300 & -0.26425500 & -1.67570300 & $\mathrm{O}$ & 2.45136100 & -0.90639400 & -2.52924500 \\
\hline 0 & 0.58873800 & 0.07536700 & -1.63169200 & $\mathrm{C}$ & -0.33982200 & -0.23353600 & -2.76391900 \\
\hline C & -0.49216600 & -1.74602800 & -2.92270500 & $\mathrm{H}$ & -1.20935700 & -1.94171300 & -3.72807800 \\
\hline $\mathrm{H}$ & 0.45356100 & -2.22603600 & -3.17621200 & $\mathrm{H}$ & -0.88062900 & -2.20169700 & -2.00858100 \\
\hline C & -1.64334600 & 0.40207100 & -2.28840300 & $\mathrm{H}$ & -1.52967500 & 1.48069600 & -2.15585400 \\
\hline $\mathrm{H}$ & -2.43079900 & 0.22288400 & -3.02770000 & $\mathrm{H}$ & -1.95687900 & -0.03568200 & -1.33747200 \\
\hline C & 0.17786800 & 0.44758200 & -4.03332800 & $\mathrm{H}$ & -0.57157400 & 0.33934900 & -4.82493800 \\
\hline $\mathrm{H}$ & 0.33452100 & 1.51764400 & -3.86093100 & $\mathrm{H}$ & 1.11138100 & -0.00156100 & -4.37772000 \\
\hline C & 3.87885800 & 0.06343200 & -0.42965100 & $\mathrm{H}$ & 4.28730500 & -0.34780900 & -1.34324900 \\
\hline C & 4.75835300 & 1.04647500 & 0.25028400 & C & 6.09035000 & 1.13919600 & -0.18775400 \\
\hline C & 4.31681700 & 1.89119800 & 1.28204200 & C & 6.96751800 & 2.05530100 & 0.39052700 \\
\hline $\mathrm{H}$ & 6.43314400 & 0.50239900 & -0.99933400 & C & 5.20048900 & 2.80198900 & 1.85874600 \\
\hline $\mathrm{H}$ & 3.28724900 & 1.84701800 & 1.62383500 & C & 6.52487600 & 2.88707900 & 1.42028900 \\
\hline $\mathrm{H}$ & 7.99117300 & 2.12250100 & 0.03325500 & $\mathrm{H}$ & 4.85184200 & 3.45121200 & 2.65743900 \\
\hline $\mathrm{H}$ & 7.20603900 & 3.60024200 & 1.87604200 & $\mathrm{~N}$ & 2.54046000 & 0.29018600 & -0.56702700 \\
\hline $\mathrm{H}$ & 1.97350500 & 0.70348200 & 0.18117400 & C & 4.04578500 & -1.58985800 & 0.75510100 \\
\hline $\mathrm{H}$ & 4.38393500 & -1.05994500 & 1.64126400 & $\mathrm{H}$ & 2.99028800 & -1.84805200 & 0.74113200 \\
\hline $\mathrm{C}$ & 4.94529400 & -2.50055700 & 0.19393800 & C & 4.51258500 & -3.57565900 & -0.70772700 \\
\hline $\mathrm{H}$ & 4.95837100 & -4.51757600 & -0.35155200 & $\mathrm{H}$ & 3.42357600 & -3.68626100 & -0.71405300 \\
\hline C & 6.39884400 & -2.42515000 & 0.56860500 & $\mathrm{H}$ & 7.05901700 & -2.71186200 & -0.25653200 \\
\hline $\mathrm{H}$ & 6.67864400 & -1.43316700 & 0.93503600 & $\mathrm{H}$ & 6.58511800 & -3.13929000 & 1.38444500 \\
\hline C & 3.61394200 & -4.21519600 & -3.56190600 & $\mathrm{H}$ & 2.78010400 & -3.51317200 & -3.46057100 \\
\hline $\mathrm{H}$ & 3.85805100 & -4.31291100 & -4.62689900 & $\mathrm{H}$ & 3.27114100 & -5.19126200 & -3.20081900 \\
\hline C & 5.66923200 & -1.91079900 & -3.21743400 & $\mathrm{H}$ & 4.82696900 & -1.21552900 & -3.28491100 \\
\hline $\mathrm{H}$ & 6.45758300 & -1.46565400 & -2.59765700 & $\mathrm{H}$ & 6.08448100 & -2.02663800 & -4.22664700 \\
\hline C & 6.54309600 & -4.81670100 & -2.69591400 & $\mathrm{H}$ & 7.40859700 & -4.49614900 & -2.10254900 \\
\hline $\mathrm{H}$ & 6.25458000 & -5.81628900 & -2.34908100 & $\mathrm{H}$ & 6.88062500 & -4.91530500 & -3.73508200 \\
\hline $\mathrm{Si}$ & 5.09556000 & -3.59801700 & -2.58277600 & & & & \\
\hline
\end{tabular}

\section{TS- $R-4 f-n d-44$}

Imaginary frequency: $-276.38 \mathrm{~cm}^{-1}$ E[B3LYP/6-31G(d)]: -4788.682036 Hartree E[PCM(Et $\left.\left.{ }_{2} \mathrm{O}\right)-B 3 L Y P / 6-311 G(d, p)-D 3(B J)\right]:-4790.207466$ Hartree

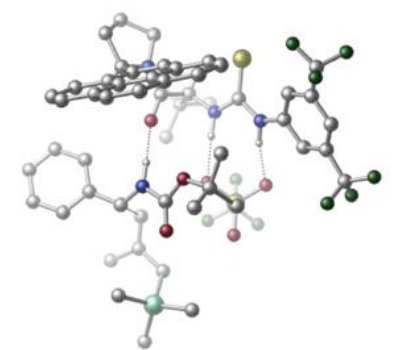

\begin{tabular}{|c|c|c|c|c|c|c|c|}
\hline 01 & & & & $\mathrm{~N}$ & -1.92468600 & -1.92630300 & 1.76179700 \\
\hline $\mathrm{H}$ & -1.06919200 & -2.47635500 & 1.62679200 & $\mathrm{C}$ & -3.01357600 & -2.43121800 & 1.02784800 \\
\hline $\mathrm{C}$ & -2.79866500 & -3.64364500 & 0.34804700 & $\mathrm{C}$ & -4.24715400 & -1.77903100 & 0.86660600 \\
\hline $\mathrm{C}$ & -3.77800500 & -4.16108400 & -0.49745000 & $\mathrm{H}$ & -1.85410300 & -4.16430300 & 0.46571700 \\
\hline C & -5.21139800 & -2.31643100 & 0.01724400 & $\mathrm{H}$ & -4.44410200 & -0.86387900 & 1.40182600 \\
\hline C & -4.99469500 & -3.50579400 & -0.67852100 & $\mathrm{H}$ & -5.74983800 & -3.91067800 & -1.34203200 \\
\hline C & -3.46723400 & -5.39504800 & -1.30202000 & $\mathrm{C}$ & -6.52661500 & -1.60662800 & -0.16359300 \\
\hline $\mathrm{F}$ & -2.68387900 & -6.25998400 & -0.62791700 & $\mathrm{~F}$ & -2.81091600 & -5.08132600 & -2.44929500 \\
\hline $\mathrm{F}$ & -4.58856500 & -6.05678200 & -1.66639900 & $\mathrm{~F}$ & -6.53152100 & -0.37067600 & 0.38811400 \\
\hline $\mathrm{F}$ & -6.82605600 & -1.44983900 & -1.47874000 & $\mathrm{~F}$ & -7.55371500 & -2.29172000 & 0.38478200 \\
\hline C & -0.10292300 & 0.71895700 & 3.52334100 & $\mathrm{H}$ & -0.95770400 & 0.92940000 & 4.16419100 \\
\hline $\mathrm{C}$ & 1.12442800 & 0.45471800 & 4.46448800 & $\mathrm{C}$ & 2.43935300 & 0.18797200 & 3.70853600 \\
\hline $\mathrm{H}$ & 2.74860800 & 1.05638600 & 3.11925800 & $\mathrm{H}$ & 2.35838500 & -0.66743400 & 3.03523700 \\
\hline $\mathrm{H}$ & 3.23332600 & -0.03077700 & 4.43344800 & C & 0.78989700 & -0.76351500 & 5.34933100 \\
\hline $\mathrm{H}$ & 1.57888300 & -0.90955100 & 6.09669000 & $\mathrm{H}$ & 0.71406800 & -1.67919300 & 4.75799600 \\
\hline
\end{tabular}




\begin{tabular}{|c|c|c|c|c|c|c|c|}
\hline $\mathrm{H}$ & -0.15842100 & -0.62314000 & 5.88156700 & $C$ & 1.32120800 & 1.68540200 & 5.37416200 \\
\hline $\mathrm{H}$ & 2.18508000 & 1.52903200 & 6.03010200 & $\mathrm{H}$ & 0.45092500 & 1.85745200 & 6.01908100 \\
\hline $\mathrm{H}$ & 1.51091400 & 2.59955800 & 4.79789100 & C & 0.08873100 & 1.88690900 & 2.52678400 \\
\hline 0 & 0.82825400 & 1.74702500 & 1.53755400 & C & -1.38138100 & 3.45501400 & 3.89703800 \\
\hline C & -0.47793000 & 4.14993600 & 1.74902500 & C & -1.03214600 & 4.94129700 & 4.01757700 \\
\hline $\mathrm{H}$ & -2.45035400 & 3.30105800 & 3.70691000 & $\mathrm{H}$ & -1.10954000 & 2.88291100 & 4.78098900 \\
\hline $\mathrm{H}$ & 0.57861800 & 4.22601100 & 1.47881700 & $\mathrm{H}$ & -0.06230700 & 5.05021700 & 4.51791700 \\
\hline $\mathrm{H}$ & -1.77447400 & 5.49904900 & 4.59637200 & $\mathrm{~N}$ & -0.59545700 & 3.04065600 & 2.72256600 \\
\hline C & -1.30701200 & 3.94450900 & 0.48476900 & C & -2.36159300 & 3.07853400 & 0.47157900 \\
\hline C & -1.01810200 & 4.75002100 & -0.68915500 & C & -3.22113000 & 2.92728800 & -0.66741500 \\
\hline $\mathrm{H}$ & -2.57201400 & 2.46184400 & 1.34162600 & C & -1.85337800 & 4.60665900 & -1.84542600 \\
\hline C & 0.04566500 & 5.66844700 & -0.75075300 & C & -4.31258100 & 2.04333400 & -0.66339400 \\
\hline C & -2.95433300 & 3.69930600 & -1.83487600 & C & -1.59265300 & 5.37976300 & -3.01974800 \\
\hline $\mathrm{H}$ & 0.69538500 & 5.80712300 & 0.10661500 & C & 0.29294600 & 6.41661900 & -1.90159200 \\
\hline C & -5.12429100 & 1.90894600 & -1.78918900 & $\mathrm{H}$ & -4.51814700 & 1.45772900 & 0.22753200 \\
\hline C & -3.79173400 & 3.55902500 & -2.98384100 & C & -0.51373100 & 6.27765000 & -3.02582600 \\
\hline C & -2.44941100 & 5.21637800 & -4.16286600 & $\mathrm{H}$ & 1.12491000 & 7.11579300 & -1.91605000 \\
\hline C & -4.86734400 & 2.65660100 & -2.93737900 & $\mathrm{H}$ & -5.95267200 & 1.20733200 & -1.76903500 \\
\hline C & -3.49817700 & 4.34889200 & -4.14711500 & $\mathrm{H}$ & -0.31695700 & 6.86553100 & -3.91909800 \\
\hline $\mathrm{H}$ & -2.23942800 & 5.80977600 & -5.04963500 & $\mathrm{H}$ & -5.50273600 & 2.54663300 & -3.81300400 \\
\hline $\mathrm{H}$ & -4.13640800 & 4.24011900 & -5.02083100 & C & -1.80024700 & -0.80562200 & 2.54814200 \\
\hline S & -3.09673600 & 0.03169600 & 3.25483500 & $\mathrm{~N}$ & -0.49699700 & -0.45266300 & 2.74762300 \\
\hline $\mathrm{H}$ & 0.21021000 & -0.84860400 & 2.12050100 & $S$ & 1.58392000 & -3.23576600 & 0.89631600 \\
\hline 0 & 2.25640000 & -3.75435500 & -0.30613800 & 0 & 1.58544700 & -1.74283900 & 1.02069200 \\
\hline $\mathrm{O}$ & 0.26418400 & -3.82543300 & 1.23550300 & C & 2.68843300 & -3.78337500 & 2.29180400 \\
\hline $\mathrm{F}$ & 2.80543200 & -5.11302700 & 2.31745300 & $\mathrm{~F}$ & 2.20418600 & -3.37359200 & 3.47653200 \\
\hline $\mathrm{F}$ & 3.91683000 & -3.24913100 & 2.13989700 & C & -0.94637100 & 5.40426800 & 2.55329600 \\
\hline $\mathrm{H}$ & -1.93226700 & 5.71587600 & 2.19565700 & $\mathrm{H}$ & -0.26764100 & 6.25089000 & 2.41613300 \\
\hline C & 1.67744500 & -0.06977000 & -1.82742100 & 0 & 2.35999900 & -0.69682300 & -2.61822500 \\
\hline 0 & 0.36241700 & -0.04626000 & -1.70776100 & C & -0.52220700 & -0.85221100 & -2.60791100 \\
\hline C & -0.30485100 & -2.33675900 & -2.32264300 & $\mathrm{H}$ & -1.02649900 & -2.92916700 & -2.89546800 \\
\hline $\mathrm{H}$ & 0.70380800 & -2.66029500 & -2.58453900 & $\mathrm{H}$ & -0.46937800 & -2.54981100 & -1.26404900 \\
\hline C & -1.91362700 & -0.39654000 & -2.17623900 & $\mathrm{H}$ & -2.05367500 & 0.67127200 & -2.36485300 \\
\hline $\mathrm{H}$ & -2.67305000 & -0.95123000 & -2.73629700 & $\mathrm{H}$ & -2.06837600 & -0.58305300 & -1.11068400 \\
\hline C & -0.25647200 & -0.47656000 & -4.06767400 & $\mathrm{H}$ & -1.01138000 & -0.95998400 & -4.69732100 \\
\hline $\mathrm{H}$ & -0.34431900 & 0.60638600 & -4.20812900 & $\mathrm{H}$ & 0.73123600 & -0.80172300 & -4.39704600 \\
\hline C & 3.59963700 & 1.03131800 & -0.92640100 & $\mathrm{H}$ & 4.02655300 & 0.64722700 & -1.84309600 \\
\hline C & 4.19064400 & 2.30578500 & -0.45182000 & C & 5.36630300 & 2.75277000 & -1.07804500 \\
\hline C & 3.62219100 & 3.08140300 & 0.57181200 & C & 5.96438900 & 3.95161300 & -0.69334200 \\
\hline $\mathrm{H}$ & 5.80169300 & 2.16606600 & -1.88301700 & C & 4.22785200 & 4.27633600 & 0.95485700 \\
\hline $\mathrm{H}$ & 2.70816200 & 2.75661400 & 1.05974600 & C & 5.39785400 & 4.71498000 & 0.32863200 \\
\hline $\mathrm{H}$ & 6.86677200 & 4.29013300 & -1.19440700 & $\mathrm{H}$ & 3.78305800 & 4.87038500 & 1.74859000 \\
\hline $\mathrm{H}$ & 5.86300900 & 5.64819900 & 0.63359300 & $\mathrm{~N}$ & 2.25015400 & 0.83310400 & -0.90481000 \\
\hline $\mathrm{H}$ & 1.65741800 & 1.16941400 & -0.13855000 & C & 4.39683300 & -0.33966700 & 0.36624700 \\
\hline $\mathrm{H}$ & 4.66075400 & 0.37258500 & 1.14297200 & $\mathrm{H}$ & 3.47217600 & -0.88640200 & 0.52898300 \\
\hline C & 5.45521900 & -1.01594400 & -0.24456700 & C & 5.26115900 & -2.26708100 & -0.99018700 \\
\hline $\mathrm{H}$ & 5.99044300 & -3.00159600 & -0.61332500 & $\mathrm{H}$ & 4.25575600 & -2.67396900 & -0.84408800 \\
\hline C & 6.85310100 & -0.48598700 & -0.08875000 & $\mathrm{H}$ & 7.47008800 & -0.66385900 & -0.97552600 \\
\hline $\mathrm{H}$ & 6.86336600 & 0.57986800 & 0.15756500 & $\mathrm{H}$ & 7.33656400 & -1.01833800 & 0.74380500 \\
\hline C & 4.34312700 & -3.49582600 & -3.63947000 & $\mathrm{H}$ & 3.34702500 & -3.05778400 & -3.52010300 \\
\hline $\mathrm{H}$ & 4.52128500 & -3.66603500 & -4.70845500 & $\mathrm{H}$ & 4.34174400 & -4.46962400 & -3.13698600 \\
\hline C & 5.59584100 & -0.65555000 & -3.76321000 & $\mathrm{H}$ & 4.57630000 & -0.25852900 & -3.77796700 \\
\hline $\mathrm{H}$ & 6.26229700 & 0.07846000 & -3.29321900 & $\mathrm{H}$ & 5.92465900 & -0.76397000 & -4.80452300 \\
\hline
\end{tabular}




\begin{tabular}{|llll|llll|}
\hline $\mathrm{C}$ & 7.37905500 & -3.07827400 & -3.10715600 & $\mathrm{H}$ & 8.15588600 & -2.44359400 & -2.66271300 \\
\hline $\mathrm{H}$ & 7.45154700 & -4.06775100 & -2.63978400 & $\mathrm{H}$ & 7.62709900 & -3.19881700 & -4.16900100 \\
\hline $\mathrm{Si}$ & 5.64136500 & -2.34540500 & -2.91284300 & & & & \\
\hline
\end{tabular}

\section{TS- $R-4 f-n d-45$}

Imaginary frequency: $-261.09 \mathrm{~cm}^{-1}$ E[B3LYP/6-31G(d)]: -4788.688893 Hartree

E[PCM(Et $\left.\left.t_{2} \mathrm{O}\right)-B 3 L Y P / 6-311 G(d, p)-D 3(B J)\right]:-4790.206109$ Hartree

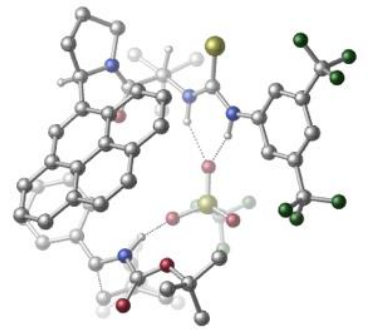

\begin{tabular}{|c|c|c|c|c|c|c|c|}
\hline 01 & & & & $\mathrm{~N}$ & 3.03102800 & -0.33401500 & -1.64875300 \\
\hline $\mathrm{H}$ & 2.09309200 & -0.72869000 & -1.74828300 & $\mathrm{C}$ & 3.93773400 & -1.23269600 & -1.06522600 \\
\hline C & 3.36919200 & -2.40867100 & -0.53640500 & C & 5.32766600 & -1.06050000 & -0.99402700 \\
\hline C & 4.17404700 & -3.37215800 & 0.06139500 & $\mathrm{H}$ & 2.29560200 & -2.56665800 & -0.59341700 \\
\hline C & 6.11482100 & -2.04315100 & -0.38867000 & $\mathrm{H}$ & 5.78022600 & -0.16751900 & -1.39980700 \\
\hline C & 5.55764300 & -3.20149400 & 0.14559900 & $\mathrm{H}$ & 6.18153200 & -3.95380500 & 0.61238600 \\
\hline C & 3.54342700 & -4.58117300 & 0.69831700 & C & 7.61132500 & -1.86841400 & -0.38249300 \\
\hline $\mathrm{F}$ & 2.35428500 & -4.89455400 & 0.14713400 & $\mathrm{~F}$ & 3.32115300 & -4.38252700 & 2.02545300 \\
\hline $\mathrm{F}$ & 4.33841500 & -5.67154000 & 0.60387300 & $\mathrm{~F}$ & 7.97001200 & -0.57428500 & -0.24404900 \\
\hline $\mathrm{F}$ & 8.19494700 & -2.55963800 & 0.62434700 & $\mathrm{~F}$ & 8.17009700 & -2.30953600 & -1.53414400 \\
\hline C & 1.64961600 & 2.85089600 & -2.81930700 & $\mathrm{H}$ & 2.62725400 & 3.32112400 & -2.91717500 \\
\hline C & 0.93467100 & 2.94286300 & -4.20995500 & C & -0.36718700 & 2.11891000 & -4.27600300 \\
\hline $\mathrm{H}$ & -1.09440900 & 2.45802300 & -3.53516200 & $\mathrm{H}$ & -0.18551900 & 1.05273100 & -4.10396300 \\
\hline $\mathrm{H}$ & -0.81005400 & 2.21898000 & -5.27491000 & C & 1.91456800 & 2.42368500 & -5.28097200 \\
\hline $\mathrm{H}$ & 1.45456000 & 2.48158500 & -6.27481100 & $\mathrm{H}$ & 2.18658400 & 1.37983300 & -5.09567000 \\
\hline $\mathrm{H}$ & 2.83937200 & 3.01224000 & -5.30071900 & C & 0.61097000 & 4.42274800 & -4.49727000 \\
\hline $\mathrm{H}$ & 0.15629700 & 4.52355100 & -5.48962400 & $\mathrm{H}$ & 1.51539800 & 5.04444800 & -4.48906400 \\
\hline $\mathrm{H}$ & -0.09696400 & 4.83287200 & -3.76750600 & $\mathrm{C}$ & 0.85784000 & 3.49431400 & -1.65630700 \\
\hline $\mathrm{O}$ & -0.25142400 & 3.05693500 & -1.33618000 & $\mathrm{C}$ & 2.64353700 & 5.27515600 & -1.36527800 \\
\hline C & 0.81689100 & 5.04597200 & 0.26628000 & C & 2.49590700 & 6.58388500 & -0.57852900 \\
\hline $\mathrm{H}$ & 3.57173400 & 4.74759400 & -1.11444400 & $\mathrm{H}$ & 2.63785100 & 5.44298100 & -2.44412700 \\
\hline $\mathrm{H}$ & -0.11935600 & 5.52407700 & -0.05004100 & $\mathrm{H}$ & 1.84923400 & 7.28018900 & -1.12638700 \\
\hline $\mathrm{H}$ & 3.45890500 & 7.07689100 & -0.41569000 & $\mathrm{~N}$ & 1.46319900 & 4.48729900 & -0.94779400 \\
\hline C & 0.44725200 & 4.07837800 & 1.38593200 & C & -0.78007600 & 4.25042400 & 1.96072900 \\
\hline C & 1.36300200 & 3.09600100 & 1.94722800 & $\mathrm{C}$ & -1.22021700 & 3.52192600 & 3.11289800 \\
\hline $\mathrm{H}$ & -1.47000100 & 4.97613900 & 1.53534500 & C & 0.95085400 & 2.35640700 & 3.10650000 \\
\hline C & 2.63531800 & 2.82440000 & 1.41288400 & C & -2.48562900 & 3.72169400 & 3.68840700 \\
\hline C & -0.33283800 & 2.56999600 & 3.69239700 & $\mathrm{C}$ & 1.83043500 & 1.39187000 & 3.69174500 \\
\hline $\mathrm{H}$ & 2.97101800 & 3.33228700 & 0.51989100 & C & 3.48051600 & 1.87373700 & 1.98461200 \\
\hline $\mathrm{C}$ & -2.87443300 & 3.00256800 & 4.81720900 & $\mathrm{H}$ & -3.16323100 & 4.44334100 & 3.23926000 \\
\hline $\mathrm{C}$ & -0.73897800 & 1.83798100 & 4.85052500 & C & 3.08970800 & 1.16672100 & 3.11555400 \\
\hline C & 1.39999200 & 0.67506800 & 4.86133600 & $\mathrm{H}$ & 4.44534500 & 1.68357400 & 1.52398600 \\
\hline $\mathrm{C}$ & -2.01252700 & 2.07170800 & 5.39471100 & $\mathrm{H}$ & -3.85580200 & 3.17141000 & 5.25316000 \\
\hline $\mathrm{C}$ & 0.17583600 & 0.88710600 & 5.41809000 & $\mathrm{H}$ & 3.75282400 & 0.42783700 & 3.55888400 \\
\hline $\mathrm{H}$ & 2.08649400 & -0.04545000 & 5.29989200 & $\mathrm{H}$ & -2.32064300 & 1.51523500 & 6.27668000 \\
\hline $\mathrm{H}$ & -0.12917100 & 0.34117800 & 6.30781600 & C & 3.12453500 & 0.98597300 & -2.01662400 \\
\hline $\mathrm{S}$ & 4.54038600 & 1.91736500 & -1.99628100 & $\mathrm{~N}$ & 1.91762000 & 1.47382100 & -2.42456300 \\
\hline $\mathrm{H}$ & 1.09415200 & 0.89723900 & -2.25946400 & $\mathrm{~S}$ & -0.73841300 & -1.87512200 & -1.58484100 \\
\hline $\mathrm{O}$ & -0.16470900 & -3.07151100 & -0.95218800 & 0 & -1.84887500 & -1.21927300 & -0.83419000 \\
\hline $\mathrm{O}$ & 0.24582800 & -0.91960200 & -2.15860900 & C & -1.61036200 & -2.50960200 & -3.10794800 \\
\hline $\mathrm{F}$ & -0.74442000 & -3.00787800 & -3.98827400 & $\mathrm{~F}$ & -2.30752900 & -1.51955600 & -3.70308400 \\
\hline $\mathrm{F}$ & -2.49043400 & -3.48067600 & -2.77920900 & C & 1.81739500 & 6.13559700 & 0.72048000 \\
\hline
\end{tabular}




\begin{tabular}{|c|c|c|c|c|c|c|c|}
\hline $\mathrm{H}$ & 2.55241100 & 5.70118600 & 1.40621100 & $\mathrm{H}$ & 1.30600500 & 6.94365400 & 1.25112700 \\
\hline C & -2.81407600 & -1.84617600 & 2.75147600 & $\mathrm{O}$ & -3.65264600 & -2.13139800 & 3.58942900 \\
\hline 0 & -1.60613200 & -2.36874200 & 2.56718700 & C & -1.08118200 & -3.48428700 & 3.39672800 \\
\hline C & -1.96851200 & -4.71571500 & 3.19983100 & $\mathrm{H}$ & -1.53244200 & -5.56523400 & 3.73649800 \\
\hline $\mathrm{H}$ & -2.97706600 & -4.54543600 & 3.58354900 & $\mathrm{H}$ & -2.02249700 & -4.98028600 & 2.13834900 \\
\hline $\mathrm{C}$ & 0.30959900 & -3.70115600 & 2.80285400 & $\mathrm{H}$ & 0.93069900 & -2.81047500 & 2.93857100 \\
\hline $\mathrm{H}$ & 0.80310400 & -4.54152300 & 3.30064400 & $\mathrm{H}$ & 0.24885500 & -3.91539500 & 1.73227000 \\
\hline C & -1.00460400 & -3.04005000 & 4.85884400 & $\mathrm{H}$ & -0.48967800 & -3.81109900 & 5.44266200 \\
\hline $\mathrm{H}$ & -0.43271100 & -2.10992600 & 4.94129500 & $\mathrm{H}$ & -1.99881100 & -2.88528900 & 5.28300800 \\
\hline $\mathrm{C}$ & -4.22098100 & -0.18378500 & 1.71983300 & $\mathrm{H}$ & -4.70648400 & -0.20320300 & 2.69332700 \\
\hline C & -4.27000000 & 1.14239500 & 1.04665000 & C & -5.34956400 & 1.99129800 & 1.35664400 \\
\hline $\mathrm{C}$ & -3.27608900 & 1.59980700 & 0.16697800 & $\mathrm{C}$ & -5.43961200 & 3.26068900 & 0.79648600 \\
\hline $\mathrm{H}$ & -6.11507700 & 1.65502600 & 2.05311700 & $\mathrm{C}$ & -3.35978200 & 2.88136600 & -0.38188700 \\
\hline $\mathrm{H}$ & -2.42432200 & 0.98191000 & -0.09518100 & C & -4.44144500 & 3.70781600 & -0.07605400 \\
\hline $\mathrm{H}$ & -6.27734500 & 3.90493600 & 1.04909100 & $\mathrm{H}$ & -2.55232600 & 3.22370100 & -1.02048800 \\
\hline $\mathrm{H}$ & -4.50539200 & 4.70330600 & -0.50768400 & $\mathrm{~N}$ & -3.02235300 & -0.86055800 & 1.77975500 \\
\hline $\mathrm{H}$ & -2.36257700 & -0.84083000 & 0.99694400 & $\mathrm{C}$ & -5.65422900 & -1.24763600 & 0.96249000 \\
\hline $\mathrm{H}$ & -5.82854600 & -1.92646100 & 1.79443200 & $\mathrm{H}$ & -6.39810000 & -0.46423500 & 0.84769500 \\
\hline C & -5.10980100 & -1.80469900 & -0.20968200 & $\mathrm{C}$ & -5.11837800 & -1.08571800 & -1.47601100 \\
\hline $\mathrm{H}$ & -5.35148500 & -0.02370800 & -1.33792800 & $\mathrm{H}$ & -4.14979200 & -1.18405800 & -1.98091600 \\
\hline C & -4.35056400 & -3.07977700 & -0.09902200 & $\mathrm{H}$ & -4.70504900 & -3.70828200 & 0.72156300 \\
\hline $\mathrm{H}$ & -4.33747600 & -3.64084800 & -1.03489500 & $\mathrm{H}$ & -3.29900000 & -2.81216000 & 0.09709500 \\
\hline $\mathrm{C}$ & -6.03356900 & -0.61600400 & -4.32089200 & $\mathrm{H}$ & -4.99514300 & -0.73549500 & -4.65065300 \\
\hline $\mathrm{H}$ & -6.68499200 & -0.88379900 & -5.16156900 & $\mathrm{H}$ & -6.19492300 & 0.44543300 & -4.10026400 \\
\hline $\mathrm{C}$ & -6.08145200 & -3.52006500 & -3.23797800 & $\mathrm{H}$ & -5.02920400 & -3.70283700 & -3.48245700 \\
\hline $\mathrm{H}$ & -6.36190400 & -4.19221100 & -2.41909400 & $\mathrm{H}$ & -6.67806500 & -3.80710800 & -4.11284800 \\
\hline C & -8.14483300 & -1.44450100 & -2.17299200 & $\mathrm{H}$ & -8.34063100 & -2.04394500 & -1.27614900 \\
\hline $\mathrm{H}$ & -8.33067600 & -0.39342600 & -1.92224600 & $\mathrm{H}$ & -8.88284400 & -1.73625000 & -2.93010800 \\
\hline $\mathrm{Si}$ & -6.39158500 & -1.70583700 & -2.82423000 & & & & \\
\hline
\end{tabular}

\section{TS-R-4f-nd-46}

Imaginary frequency: $-220.48 \mathrm{~cm}^{-1}$ E[B3LYP/6-31G(d)]: -4788.690073 Hartree E[PCM(Et $\left.\left.{ }_{2} \mathrm{O}\right)-\mathrm{B} 3 L Y P / 6-311 \mathrm{G}(\mathrm{d}, \mathrm{p})-\mathrm{D} 3(\mathrm{BJ})\right]$ : -4790.204949 Hartree

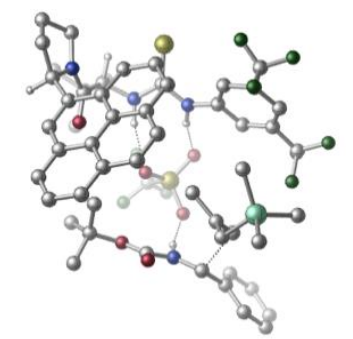

\begin{tabular}{|c|c|c|c|c|c|c|c|}
\hline 01 & & & & $\mathrm{~N}$ & 0.30925200 & 0.89069400 & -2.68453400 \\
\hline $\mathrm{H}$ & -0.31850000 & 1.62297100 & -2.34324100 & C & -0.34902300 & -0.26704200 & -3.10503600 \\
\hline C & -1.74985500 & -0.20569100 & -3.21989600 & C & 0.29265400 & -1.49670300 & -3.33270100 \\
\hline $\mathrm{C}$ & -2.47916700 & -1.34651300 & -3.55241600 & $\mathrm{H}$ & -2.25735100 & 0.73775000 & -3.04474600 \\
\hline $\mathrm{C}$ & -0.45489000 & -2.61827300 & -3.68325100 & $\mathrm{H}$ & 1.36592400 & -1.56519300 & -3.24350600 \\
\hline C & -1.84538000 & -2.56416400 & -3.79947000 & $\mathrm{H}$ & -2.41511900 & -3.43984400 & -4.08512200 \\
\hline $\mathrm{C}$ & -3.98038800 & -1.27017600 & -3.56792200 & $\mathrm{C}$ & 0.24568200 & -3.93830400 & -3.86563300 \\
\hline $\mathrm{F}$ & -4.43354100 & -0.08190300 & -4.01006500 & $\mathrm{~F}$ & -4.49246100 & -1.43164400 & -2.30967600 \\
\hline $\mathrm{F}$ & -4.53664800 & -2.23389700 & -4.33116100 & $\mathrm{~F}$ & 1.53556600 & -3.79468800 & -4.23455600 \\
\hline $\mathrm{F}$ & 0.25354100 & -4.66276100 & -2.71056700 & $\mathrm{~F}$ & -0.36170000 & -4.70932500 & -4.79301300 \\
\hline $\mathrm{C}$ & 3.30918000 & 2.69538700 & -1.58619700 & $\mathrm{H}$ & 3.85773200 & 2.44090600 & -2.49229200 \\
\hline $\mathrm{C}$ & 3.33454400 & 4.25573400 & -1.43762200 & $\mathrm{C}$ & 2.59357200 & 4.76045200 & -0.18412300 \\
\hline $\mathrm{H}$ & 3.05363200 & 4.38831900 & 0.73398800 & $\mathrm{H}$ & 1.54453700 & 4.45577700 & -0.17835100 \\
\hline $\mathrm{H}$ & 2.61686600 & 5.85720100 & -0.16834200 & $\mathrm{C}$ & 2.68990000 & 4.86903200 & -2.69729600 \\
\hline $\mathrm{H}$ & 2.77493400 & 5.96186300 & -2.66702400 & $\mathrm{H}$ & 1.62792600 & 4.61858100 & -2.76727500 \\
\hline $\mathrm{H}$ & 3.18230300 & 4.51683700 & -3.61177700 & $\mathrm{C}$ & 4.80509600 & 4.71538400 & -1.36016300 \\
\hline
\end{tabular}




\begin{tabular}{|c|c|c|c|c|c|c|c|}
\hline $\mathrm{H}$ & 4.85001300 & 5.80439400 & -1.24518900 & $\mathrm{H}$ & 5.35801900 & 4.46120200 & -2.27299900 \\
\hline $\mathrm{H}$ & 5.32890700 & 4.27488300 & -0.50316200 & C & 3.90321700 & 1.94443000 & -0.36853800 \\
\hline 0 & 3.30654800 & 1.94282800 & 0.70909500 & C & 5.98497100 & 1.30700500 & -1.69015000 \\
\hline C & 5.66672300 & 0.53637300 & 0.62162400 & C & 7.34853800 & 0.95474200 & -1.08196600 \\
\hline $\mathrm{H}$ & 5.68088300 & 0.58623700 & -2.45945600 & $\mathrm{H}$ & 5.98454700 & 2.29734500 & -2.14606600 \\
\hline $\mathrm{H}$ & 5.89786400 & 1.29177500 & 1.38348500 & $\mathrm{H}$ & 7.81666000 & 1.85600800 & -0.66744700 \\
\hline $\mathrm{H}$ & 8.03378100 & 0.53005600 & -1.82177900 & $\mathrm{~N}$ & 5.06779800 & 1.25196900 & -0.53383600 \\
\hline C & 4.82595200 & -0.52563800 & 1.32229500 & C & 4.80577100 & -0.48760400 & 2.68779700 \\
\hline C & 4.17183100 & -1.62790100 & 0.63370700 & C & 4.18409900 & -1.49404400 & 3.49535400 \\
\hline $\mathrm{H}$ & 5.28590300 & 0.34184600 & 3.20304900 & C & 3.55189000 & -2.65836200 & 1.41764700 \\
\hline C & 4.11015700 & -1.74037900 & -0.76683600 & C & 4.18145500 & -1.43472200 & 4.89876400 \\
\hline C & 3.55862700 & -2.59595000 & 2.84348400 & C & 2.91583500 & -3.76556000 & 0.77244100 \\
\hline $\mathrm{H}$ & 4.52455200 & -0.96202400 & -1.39202700 & C & 3.47909900 & -2.82011800 & -1.38444600 \\
\hline C & 3.57799900 & -2.43979200 & 5.65238900 & $\mathrm{H}$ & 4.66064800 & -0.59347100 & 5.39393200 \\
\hline C & 2.94375100 & -3.62318600 & 3.62383300 & C & 2.88863700 & -3.82677100 & -0.62901500 \\
\hline C & 2.31605800 & -4.79181600 & 1.58197600 & $\mathrm{H}$ & 3.44158900 & -2.86624900 & -2.46870300 \\
\hline C & 2.96618500 & -3.52328800 & 5.02460600 & $\mathrm{H}$ & 3.58614200 & -2.37925300 & 6.73745500 \\
\hline C & 2.32615500 & -4.72607100 & 2.94194100 & $\mathrm{H}$ & 2.38397600 & -4.65397700 & -1.12038900 \\
\hline $\mathrm{H}$ & 1.85583400 & -5.63675800 & 1.07507900 & $\mathrm{H}$ & 2.49944900 & -4.30586100 & 5.61818200 \\
\hline $\mathrm{H}$ & 1.87199900 & -5.51501700 & 3.53719000 & C & 1.65620700 & 1.20795900 & -2.70357900 \\
\hline S & 2.77275500 & 0.53120700 & -3.77887800 & $\mathrm{~N}$ & 1.96085500 & 2.17221600 & -1.79705300 \\
\hline $\mathrm{H}$ & 1.27815300 & 2.35624600 & -1.05975200 & $S$ & -1.70205400 & 3.25550200 & -0.62329700 \\
\hline $\mathrm{O}$ & -2.95493600 & 3.04388100 & 0.15636400 & $\mathrm{O}$ & -0.47293900 & 2.75770400 & 0.04485000 \\
\hline $\mathrm{O}$ & -1.79907600 & 2.88293900 & -2.05457100 & C & -1.53145300 & 5.10897600 & -0.63074000 \\
\hline $\mathrm{F}$ & -2.64646100 & 5.67832700 & -1.09870400 & $\mathrm{~F}$ & -0.50258100 & 5.47826100 & -1.40811300 \\
\hline $\mathrm{F}$ & -1.30626100 & 5.55415500 & 0.61269500 & C & -2.08072600 & 1.23492600 & 3.24665800 \\
\hline 0 & -1.94703500 & 0.16994000 & 3.82268000 & $\mathrm{O}$ & -1.28436900 & 2.28714000 & 3.22243000 \\
\hline C & 0.07234000 & 2.29047600 & 3.85030800 & $\mathrm{C}$ & -0.07486200 & 2.07245900 & 5.35734500 \\
\hline $\mathrm{H}$ & 0.90293100 & 2.20236400 & 5.83387400 & $\mathrm{H}$ & -0.44022400 & 1.06864800 & 5.58289800 \\
\hline $\mathrm{H}$ & -0.76220900 & 2.80860200 & 5.78866800 & C & 0.56086300 & 3.70244300 & 3.52976800 \\
\hline $\mathrm{H}$ & 0.55082000 & 3.86954400 & 2.44982300 & $\mathrm{H}$ & 1.58450400 & 3.82891400 & 3.89652000 \\
\hline $\mathrm{H}$ & -0.07672600 & 4.45304500 & 4.00899600 & C & 0.94502500 & 1.23400000 & 3.17575400 \\
\hline $\mathrm{H}$ & 1.96132000 & 1.29535600 & 3.57742400 & $\mathrm{H}$ & 1.01062500 & 1.41372300 & 2.09985500 \\
\hline $\mathrm{H}$ & 0.56729900 & 0.22645000 & 3.36610500 & C & -4.24619300 & 0.64034300 & 2.41138500 \\
\hline $\mathrm{H}$ & -4.18910200 & -0.10554900 & 3.19766500 & C & -5.60433000 & 1.06586300 & 2.01692800 \\
\hline C & -6.68755700 & 0.39581300 & 2.61322600 & C & -5.85371300 & 2.10546700 & 1.10410400 \\
\hline C & -7.99669700 & 0.76639600 & 2.31928700 & $\mathrm{H}$ & -6.49847800 & -0.40894000 & 3.31990600 \\
\hline C & -7.16679600 & 2.46664200 & 0.81165800 & $\mathrm{H}$ & -5.03305000 & 2.61933100 & 0.61378600 \\
\hline C & -8.23842300 & 1.80367400 & 1.41570300 & $\mathrm{H}$ & -8.82499800 & 0.24935400 & 2.79495200 \\
\hline $\mathrm{H}$ & -7.35256500 & 3.26952400 & 0.10437900 & $\mathrm{H}$ & -9.25892800 & 2.09315400 & 1.18121000 \\
\hline $\mathrm{N}$ & -3.22719600 & 1.52687400 & 2.47297000 & $\mathrm{H}$ & -3.14443900 & 2.29692100 & 1.79171800 \\
\hline C & -3.91383400 & -0.74664900 & 0.83010600 & $\mathrm{H}$ & -3.79951100 & -0.00072300 & 0.04851900 \\
\hline $\mathrm{H}$ & -4.89313000 & -1.20997900 & 0.89981300 & C & -2.80702700 & -1.46712400 & 1.25263300 \\
\hline C & -2.95560500 & -2.68135100 & 2.07491600 & $\mathrm{H}$ & -3.90824700 & -2.67711200 & 2.62027400 \\
\hline $\mathrm{H}$ & -2.13944700 & -2.77278800 & 2.80037100 & C & -1.43404200 & -1.02384100 & 0.84522800 \\
\hline $\mathrm{H}$ & -0.66667700 & -1.33006300 & 1.55945100 & $\mathrm{H}$ & -1.38082500 & 0.05987400 & 0.69507300 \\
\hline $\mathrm{H}$ & -1.18567800 & -1.47800500 & -0.12523000 & C & -3.25221600 & -5.68978600 & 2.39256100 \\
\hline $\mathrm{H}$ & -2.42632700 & -5.70582800 & 3.11340400 & $\mathrm{H}$ & -3.30518500 & -6.68263700 & 1.92946900 \\
\hline $\mathrm{H}$ & -4.18260200 & -5.53741400 & 2.95231700 & C & -1.37099600 & -4.63473100 & 0.16919600 \\
\hline $\mathrm{H}$ & -0.50993400 & -4.53585300 & 0.84020000 & $\mathrm{H}$ & -1.22396300 & -3.94528700 & -0.66775300 \\
\hline $\mathrm{H}$ & -1.34652500 & -5.64839500 & -0.25046200 & C & -4.45747900 & -4.31804600 & -0.12129000 \\
\hline $\mathrm{H}$ & -4.37479200 & -3.50883100 & -0.85471400 & $\mathrm{H}$ & -5.41105400 & -4.19493000 & 0.40686400 \\
\hline $\mathrm{H}$ & -4.51427300 & -5.26230300 & -0.67696400 & $\mathrm{Si}$ & -2.99769900 & -4.35616100 & 1.07520800 \\
\hline
\end{tabular}




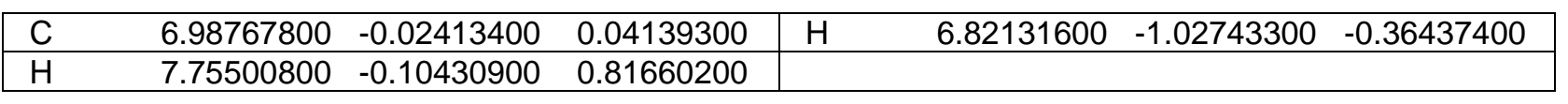

\section{TS- $R-4 f-n d-47$}

Imaginary frequency: $-232.15 \mathrm{~cm}^{-1}$ E[B3LYP/6-31G(d)]: -4788.683279 Hartree

E[PCM(Et $\left.\left.{ }_{2} \mathrm{O}\right)-\mathrm{B} 3 L \mathrm{YP} / 6-311 \mathrm{G}(\mathrm{d}, \mathrm{p})-\mathrm{D} 3(\mathrm{BJ})\right]$ : -4790.204911 Hartree

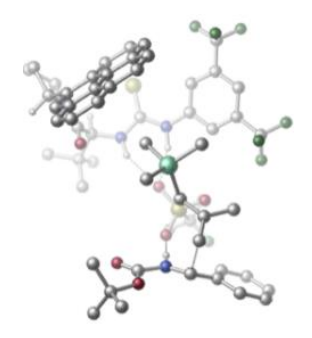

\begin{tabular}{|c|c|c|c|c|c|c|c|}
\hline 01 & & & & $\mathrm{~N}$ & -1.21914300 & -2.27292800 & 1.55268500 \\
\hline $\mathrm{H}$ & -0.25536100 & -1.97664400 & 1.72759500 & C & -1.36336200 & -3.12082600 & 0.44787800 \\
\hline C & -0.17634700 & -3.51692900 & -0.19775500 & C & -2.59367300 & -3.53903600 & -0.08578500 \\
\hline $\mathrm{C}$ & -0.23159800 & -4.29545100 & -1.35223700 & $\mathrm{H}$ & 0.78820500 & -3.20410000 & 0.19338500 \\
\hline $\mathrm{C}$ & -2.62157800 & -4.31349100 & -1.24276000 & $\mathrm{H}$ & -3.51176500 & -3.25891600 & 0.40688100 \\
\hline $\mathrm{C}$ & -1.45104000 & -4.70685800 & -1.89057200 & $\mathrm{H}$ & -1.48635100 & -5.31798100 & -2.78513300 \\
\hline C & 1.03758700 & -4.62267300 & -2.08822000 & C & -3.94420700 & -4.69397100 & -1.85370300 \\
\hline $\mathrm{F}$ & 2.12478100 & -4.60038500 & -1.29589300 & $F$ & 1.27091300 & -3.72066500 & -3.09187500 \\
\hline $\mathrm{F}$ & 0.98417300 & -5.83392400 & -2.68246000 & $\mathrm{~F}$ & -4.98980500 & -4.43654700 & -1.04087600 \\
\hline $\mathrm{F}$ & -4.16762600 & -4.01105100 & -3.00941000 & $F$ & -3.99027200 & -6.00601600 & -2.17510800 \\
\hline C & -2.38742000 & 0.46010500 & 3.69863700 & $\mathrm{H}$ & -3.23572800 & -0.08980300 & 4.10483500 \\
\hline C & -1.54032000 & 1.00485200 & 4.89985000 & C & -0.22916400 & 1.68573000 & 4.45522900 \\
\hline $\mathrm{H}$ & -0.41635400 & 2.51498200 & 3.76954300 & $\mathrm{H}$ & 0.45352500 & 0.98401900 & 3.96441500 \\
\hline $\mathrm{H}$ & 0.29321300 & 2.07284000 & 5.33871700 & C & -1.20450500 & -0.18302000 & 5.82412500 \\
\hline $\mathrm{H}$ & -0.64458000 & 0.16767100 & 6.69924000 & $\mathrm{H}$ & -0.58902700 & -0.92558100 & 5.30712800 \\
\hline $\mathrm{H}$ & -2.11220300 & -0.68413700 & 6.18223900 & C & -2.39253300 & 2.02479700 & 5.68153100 \\
\hline $\mathrm{H}$ & -1.82698700 & 2.40236900 & 6.54099400 & $\mathrm{H}$ & -3.31286200 & 1.57213600 & 6.07150100 \\
\hline $\mathrm{H}$ & -2.66554900 & 2.88866500 & 5.06409500 & C & -2.87474600 & 1.56720600 & 2.72567700 \\
\hline 0 & -2.06378900 & 2.18068100 & 2.03034000 & C & -5.27682200 & 1.28988500 & 3.51769900 \\
\hline C & -4.71582400 & 2.86687200 & 1.71823800 & $\mathrm{C}$ & -6.38016100 & 2.35042800 & 3.41093500 \\
\hline $\mathrm{H}$ & -5.62022500 & 0.30425700 & 3.17972700 & $\mathrm{H}$ & -4.91427300 & 1.18146300 & 4.54068700 \\
\hline $\mathrm{H}$ & -4.29905900 & 3.81706500 & 2.07604700 & $\mathrm{H}$ & -6.18584100 & 3.16444800 & 4.11999300 \\
\hline $\mathrm{H}$ & -7.37004100 & 1.94044600 & 3.63302000 & $\mathrm{~N}$ & -4.21570400 & 1.80298700 & 2.62753600 \\
\hline $\mathrm{C}$ & -4.35320500 & 2.77933500 & 0.23920200 & $\mathrm{C}$ & -3.89871900 & 3.92652800 & -0.34723700 \\
\hline C & -4.57400900 & 1.60065600 & -0.58506600 & C & -3.62942400 & 4.04244200 & -1.74902500 \\
\hline $\mathrm{H}$ & -3.71984200 & 4.80407400 & 0.27050700 & $\mathrm{C}$ & -4.31514200 & 1.68806100 & -1.99489500 \\
\hline $\mathrm{C}$ & -5.01251900 & 0.36659200 & -0.07325400 & $\mathrm{C}$ & -3.15968000 & 5.23337900 & -2.32687500 \\
\hline C & -3.84466900 & 2.90332900 & -2.57767100 & C & -4.50710100 & 0.54359500 & -2.83225800 \\
\hline $\mathrm{H}$ & -5.17533900 & 0.24695300 & 0.98866500 & $\mathrm{C}$ & -5.19459200 & -0.74250500 & -0.89941100 \\
\hline $\mathrm{C}$ & -2.90185900 & 5.30705400 & -3.69449300 & $\mathrm{H}$ & -2.99694600 & 6.10115400 & -1.69217900 \\
\hline $\mathrm{C}$ & -3.57243900 & 2.98499000 & -3.97862800 & C & -4.94663500 & -0.66189700 & -2.26468100 \\
\hline $\mathrm{C}$ & -4.22848600 & 0.65440400 & -4.23845100 & $\mathrm{H}$ & -5.51414400 & -1.68425000 & -0.46379500 \\
\hline C & -3.10338300 & 4.19676300 & -4.51345100 & $\mathrm{H}$ & -2.54091400 & 6.23693200 & -4.12623600 \\
\hline $\mathrm{C}$ & -3.78392000 & 1.81754200 & -4.78883700 & $\mathrm{H}$ & -5.06355600 & -1.54003000 & -2.89380600 \\
\hline $\mathrm{H}$ & -4.38486500 & -0.22408700 & -4.85999900 & $\mathrm{H}$ & -2.90134600 & 4.26069600 & -5.58013600 \\
\hline $\mathrm{H}$ & -3.58194900 & 1.88267000 & -5.85549400 & C & -2.15682300 & -1.62030100 & 2.33222100 \\
\hline $\mathrm{S}$ & -3.73472600 & -2.15796900 & 2.61473400 & $\mathrm{~N}$ & -1.63596100 & -0.50480900 & 2.90513200 \\
\hline $\mathrm{H}$ & -0.72185500 & -0.19813800 & 2.58643000 & $\mathrm{~S}$ & 2.67477200 & -1.29733300 & 2.01943700 \\
\hline $\mathrm{O}$ & 3.55173400 & -0.09761700 & 2.08381900 & $\mathrm{O}$ & 1.28007100 & -1.06491100 & 2.47777700 \\
\hline $\mathrm{O}$ & 2.78363200 & -2.08201700 & 0.76749800 & C & 3.40679500 & -2.41337000 & 3.31579400 \\
\hline $\mathrm{F}$ & 4.68856400 & -2.68734300 & 3.00273400 & $\mathrm{~F}$ & 2.73026000 & -3.56470500 & 3.37610200 \\
\hline $\mathrm{F}$ & 3.37986900 & -1.83114700 & 4.51709200 & C & 4.51407800 & 3.01049500 & 0.47982400 \\
\hline 0 & 3.80636800 & 3.34173800 & 1.40214500 & $\mathrm{O}$ & 5.05252500 & 3.79689600 & -0.46640300 \\
\hline
\end{tabular}




\begin{tabular}{|c|c|c|c|c|c|c|c|}
\hline $\mathrm{C}$ & 4.85700100 & 5.27227000 & -0.43351000 & C & 3.36921000 & 5.59796400 & -0.58367900 \\
\hline $\mathrm{H}$ & 3.24446900 & 6.68275400 & -0.67150300 & $\mathrm{H}$ & 2.79674600 & 5.25024800 & 0.27751900 \\
\hline $\mathrm{H}$ & 2.96379500 & 5.13808400 & -1.49181900 & C & 5.64907000 & 5.73347400 & -1.65766000 \\
\hline $\mathrm{H}$ & 6.70388900 & 5.45403000 & -1.56792500 & $\mathrm{H}$ & 5.58856100 & 6.82252800 & -1.74937800 \\
\hline $\mathrm{H}$ & 5.24632700 & 5.28941500 & -2.57397300 & C & 5.45718600 & 5.83795800 & 0.85575600 \\
\hline $\mathrm{H}$ & 5.41642500 & 6.93208700 & 0.82244600 & $\mathrm{H}$ & 6.50703600 & 5.54097200 & 0.95314000 \\
\hline $\mathrm{H}$ & 4.90750500 & 5.49605900 & 1.73402900 & C & 5.63485500 & 1.21442400 & -0.75495800 \\
\hline $\mathrm{H}$ & 6.13861200 & 2.01349900 & -1.28452500 & C & 6.37476900 & -0.05651600 & -0.68757100 \\
\hline C & 7.39010300 & -0.27335700 & -1.63931100 & C & 6.14393500 & -1.02956500 & 0.29954800 \\
\hline C & 8.14975700 & -1.43718200 & -1.61113900 & $\mathrm{H}$ & 7.58365800 & 0.47954000 & -2.40021600 \\
\hline C & 6.90867300 & -2.19478100 & 0.32220500 & $\mathrm{H}$ & 5.38745600 & -0.87947100 & 1.05993200 \\
\hline C & 7.90675600 & -2.40457200 & -0.63012800 & $\mathrm{H}$ & 8.93314000 & -1.58986600 & -2.34784300 \\
\hline $\mathrm{H}$ & 6.71158000 & -2.93673200 & 1.08962400 & $\mathrm{H}$ & 8.49816100 & -3.31557700 & -0.60824000 \\
\hline $\mathrm{N}$ & 4.88128900 & 1.66451000 & 0.28303100 & $\mathrm{H}$ & 4.42216000 & 1.01521100 & 0.94266700 \\
\hline $\mathrm{C}$ & 4.37857700 & 1.10178100 & -2.48605600 & $\mathrm{H}$ & 5.14267800 & 0.86962400 & -3.22281300 \\
\hline $\bar{H}$ & 4.09479500 & 2.14816300 & -2.41233700 & $\mathrm{C}$ & 3.45937700 & 0.12176300 & -2.13303200 \\
\hline $\mathrm{C}$ & 2.26677900 & 0.43758800 & -1.34924200 & $\mathrm{H}$ & 2.40920400 & 1.32659800 & -0.72095400 \\
\hline $\mathrm{H}$ & 1.98068500 & -0.40978600 & -0.71912100 & C & 3.72698800 & -1.31610300 & -2.44566300 \\
\hline$\overline{\mathrm{H}}$ & 2.84368200 & -1.79827500 & -2.87774400 & $\mathrm{H}$ & 3.91556000 & -1.84939400 & -1.50245100 \\
\hline $\bar{H}$ & 4.57832600 & -1.45169300 & -3.11678200 & C & -0.73865900 & 0.75896200 & -1.10591900 \\
\hline $\mathrm{H}$ & -0.82487500 & -0.26502900 & -0.72455800 & $\mathrm{H}$ & -1.69680100 & 1.01334900 & -1.57079400 \\
\hline $\mathrm{H}$ & -0.60840800 & 1.42609400 & -0.24631900 & C & 0.39866300 & -0.32330300 & -3.79126200 \\
\hline $\mathrm{H}$ & 0.37698600 & -1.36620800 & -3.45746400 & $\mathrm{H}$ & 1.17916200 & -0.22919700 & -4.55591400 \\
\hline $\mathrm{H}$ & -0.56392200 & -0.11440500 & -4.27485700 & C & 0.85244700 & 2.64545800 & -3.02464400 \\
\hline $\mathrm{H}$ & 1.68839800 & 2.72792300 & -3.73001500 & $\mathrm{H}$ & 1.01966300 & 3.35975700 & -2.20941200 \\
\hline $\mathrm{H}$ & -0.05729200 & 2.96097500 & -3.54977400 & $\mathrm{Si}$ & 0.65017700 & 0.88567500 & -2.36326000 \\
\hline C & -6.24267300 & 2.85465300 & 1.96988000 & $\mathrm{H}$ & -6.73094400 & 2.16443600 & 1.27413900 \\
\hline $\mathrm{H}$ & -6.67521900 & 3.84629700 & 1.80929500 & & & & \\
\hline
\end{tabular}

\section{TS- $R-4 f-n d-48$}

Imaginary frequency: $-268.34 \mathrm{~cm}^{-1}$ E[B3LYP/6-31G(d)]: -4788.687991 Hartree

$\mathrm{E}\left[\mathrm{PCM}\left(\mathrm{Et}_{2} \mathrm{O}\right)-\mathrm{B} 3 \mathrm{LYP} / 6-311 \mathrm{G}(\mathrm{d}, \mathrm{p})-\mathrm{D} 3(\mathrm{BJ})\right]$ : -4790.201599 Hartree

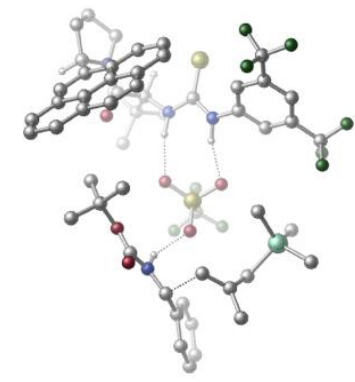

\begin{tabular}{|crrr|rrrr|}
\hline 01 & & & & $\mathrm{~N}$ & 0.76067500 & 2.09548300 & 1.27962100 \\
\hline $\mathrm{H}$ & -0.12289100 & 1.60312600 & 1.42697500 & $\mathrm{C}$ & 0.65672600 & 3.22376500 & 0.44637500 \\
\hline $\mathrm{C}$ & -0.54118200 & 3.95030400 & 0.43865500 & $\mathrm{C}$ & 1.68704200 & 3.59382500 & -0.42672000 \\
\hline $\mathrm{C}$ & -0.68893600 & 5.04376700 & -0.41714000 & $\mathrm{H}$ & -1.34533600 & 3.65971100 & 1.10457300 \\
\hline $\mathrm{C}$ & 1.52853000 & 4.69455900 & -1.26624200 & $\mathrm{H}$ & 2.59910700 & 3.01410600 & -0.44973200 \\
\hline $\mathrm{C}$ & 0.34533000 & 5.43416600 & -1.26832000 & $\mathrm{H}$ & 0.23542400 & 6.29743600 & -1.91321700 \\
\hline $\mathrm{C}$ & -1.98946900 & 5.79558900 & -0.45520500 & $\mathrm{C}$ & 2.63585500 & 5.03415500 & -2.22757600 \\
\hline $\mathrm{F}$ & -2.68701900 & 5.68228200 & 0.69734000 & $\mathrm{~F}$ & -2.81237300 & 5.33647500 & -1.44484100 \\
\hline $\mathrm{F}$ & -1.81222000 & 7.11080600 & -0.69671100 & $\mathrm{~F}$ & 3.85719600 & 4.90902200 & -1.66024800 \\
\hline $\mathrm{F}$ & 2.63033200 & 4.20989500 & -3.30817900 & $\mathrm{~F}$ & 2.53532700 & 6.29534600 & -2.69875900 \\
\hline $\mathrm{C}$ & 2.72454500 & -0.15116000 & 3.42473000 & $\mathrm{H}$ & 3.38642200 & 0.65684200 & 3.73421100 \\
\hline $\mathrm{C}$ & 2.13544100 & -0.80065900 & 4.72400300 & $\mathrm{C}$ & 1.16230300 & -1.96345500 & 4.44727800 \\
\hline $\mathrm{H}$ & 1.64571600 & -2.77414800 & 3.89761900 & $\mathrm{H}$ & 0.29300300 & -1.64194700 & 3.86768200 \\
\hline $\mathrm{H}$ & 0.79520100 & -2.35887300 & 5.40310300 & $\mathrm{C}$ & 1.39948200 & 0.29681900 & 5.51921900 \\
\hline $\mathrm{H}$ & 1.06377000 & -0.10140600 & 6.48439500 & $\mathrm{H}$ & 0.51724400 & 0.65484600 & 4.98046800 \\
\hline $\mathrm{H}$ & 2.04924200 & 1.15817400 & 5.71539300 & $\mathrm{C}$ & 3.30964700 & -1.32810600 & 5.57457700 \\
\hline
\end{tabular}




\begin{tabular}{|c|c|c|c|c|c|c|c|}
\hline$\overline{\mathrm{H}}$ & 2.92869600 & -1.79289200 & 6.49119700 & $\mathrm{H}$ & 3.98762500 & -0.51985700 & 5.87614700 \\
\hline $\mathrm{H}$ & 3.89269200 & -2.08856300 & 5.04110200 & C & 3.48440400 & -1.14611200 & 2.51306500 \\
\hline 0 & 2.87420900 & -2.03410900 & 1.91718500 & C & 5.72596100 & -0.10599500 & 3.13096800 \\
\hline C & 5.57995700 & -1.89630800 & 1.45376400 & C & 7.09122400 & -0.79493400 & 3.00480400 \\
\hline $\mathrm{H}$ & 5.73999700 & 0.91209600 & 2.72221500 & $\mathrm{H}$ & 5.39642400 & -0.04067700 & 4.16854900 \\
\hline $\mathrm{H}$ & 5.48516800 & -2.90390100 & 1.87841300 & $\mathrm{H}$ & 7.18543200 & -1.57915500 & 3.76597000 \\
\hline $\mathrm{H}$ & 7.92142500 & -0.09490900 & 3.13925700 & $\mathrm{~N}$ & 4.82869100 & -0.97346300 & 2.34164100 \\
\hline C & 5.12586800 & -2.00561100 & 0.00197200 & C & 5.00204300 & -3.26636000 & -0.50939900 \\
\hline C & 4.93144200 & -0.86132800 & -0.87536300 & C & 4.68755200 & -3.53258300 & -1.88129800 \\
\hline $\mathrm{H}$ & 5.13742800 & -4.12222300 & 0.14866600 & C & 4.61636600 & -1.09742800 & -2.25580000 \\
\hline C & 5.03265700 & 0.47322300 & -0.44377300 & C & 4.56209500 & -4.83929600 & -2.38287000 \\
\hline C & 4.49426000 & -2.42702400 & -2.75991900 & C & 4.41640700 & 0.00477600 & -3.14586600 \\
\hline $\mathrm{H}$ & 5.22929400 & 0.69465300 & 0.59556800 & C & 4.84230100 & 1.54003800 & -1.32207300 \\
\hline C & 4.25218000 & -5.05938500 & -3.72440800 & $\mathrm{H}$ & 4.71316400 & -5.68103300 & -1.71122800 \\
\hline C & 4.17614600 & -2.66156800 & -4.13380100 & C & 4.53296300 & 1.31543600 & -2.65899500 \\
\hline C & 4.09749800 & -0.25988500 & -4.52237200 & $\mathrm{H}$ & 4.92487700 & 2.55683500 & -0.95001500 \\
\hline C & 4.06051600 & -3.98468200 & -4.59164700 & $\mathrm{H}$ & 4.16274300 & -6.07623200 & -4.09768500 \\
\hline C & 3.98309400 & -1.52992000 & -4.99715700 & $\mathrm{H}$ & 4.36029600 & 2.15420800 & -3.32788600 \\
\hline $\mathrm{H}$ & 3.94857000 & 0.59030800 & -5.18378600 & $\mathrm{H}$ & 3.81934700 & -4.16299400 & -5.63684500 \\
\hline $\mathrm{H}$ & 3.74215100 & -1.71019800 & -6.04213800 & C & 1.84489400 & 1.72529100 & 2.04682400 \\
\hline $\mathrm{S}$ & 3.17742500 & 2.73885400 & 2.30993400 & $\mathrm{~N}$ & 1.70355300 & 0.49296100 & 2.59988400 \\
\hline $\mathrm{H}$ & 0.96093500 & -0.11070600 & 2.24483200 & $S$ & -2.16237000 & -0.30430100 & 1.67861800 \\
\hline $\mathrm{O}$ & -3.17269000 & -0.99700800 & 0.82426900 & $\mathrm{O}$ & -0.87641000 & -1.01604400 & 1.82125300 \\
\hline $\mathrm{O}$ & -2.03878700 & 1.15024000 & 1.40631400 & C & -2.95629600 & -0.37266900 & 3.36044300 \\
\hline $\mathrm{F}$ & -4.06638200 & 0.38164300 & 3.37610700 & $\mathrm{~F}$ & -2.11790700 & 0.07584400 & 4.30142900 \\
\hline$F$ & -3.30278800 & -1.63563000 & 3.65908400 & C & -2.47374000 & -3.68899900 & -1.52455700 \\
\hline 0 & -2.61219100 & -3.89712100 & -2.71593300 & $\mathrm{O}$ & -1.38264900 & -3.73491400 & -0.77900800 \\
\hline C & -0.01341700 & -3.89802500 & -1.35941400 & $\mathrm{C}$ & 0.08036800 & -5.24606300 & -2.07548800 \\
\hline $\mathrm{H}$ & 1.11515800 & -5.40216600 & -2.39914800 & $\mathrm{H}$ & -0.57053900 & -5.28302600 & -2.95131900 \\
\hline $\mathrm{H}$ & -0.19027000 & -6.06080200 & -1.39492500 & C & 0.87041700 & -3.85574200 & -0.11582500 \\
\hline $\mathrm{H}$ & 0.72887500 & -2.92829100 & 0.44383300 & $\mathrm{H}$ & 1.92293200 & -3.91807300 & -0.40602400 \\
\hline $\mathrm{H}$ & 0.64171800 & -4.69749900 & 0.54668800 & C & 0.26560400 & -2.71475400 & -2.28666500 \\
\hline $\mathrm{H}$ & 1.29826100 & -2.77261200 & -2.64466700 & $\mathrm{H}$ & 0.14909300 & -1.77153400 & -1.74320200 \\
\hline $\mathrm{H}$ & -0.40073600 & -2.71918700 & -3.15297000 & C & -4.79217300 & -3.11301600 & -1.23544100 \\
\hline $\mathrm{H}$ & -4.92067400 & -3.64865300 & -2.17054300 & C & -5.98108400 & -3.10289900 & -0.35199400 \\
\hline C & -7.19524200 & -3.56798600 & -0.88815300 & C & -5.93833600 & -2.70392500 & 0.99513900 \\
\hline C & -8.34155500 & -3.63776500 & -0.09852000 & $\mathrm{H}$ & -7.23139600 & -3.90072800 & -1.92281000 \\
\hline C & -7.08870300 & -2.77525900 & 1.77904100 & $\mathrm{H}$ & -5.01807400 & -2.33400500 & 1.43405000 \\
\hline C & -8.29013100 & -3.23965100 & 1.23873600 & $\mathrm{H}$ & -9.26797100 & -4.01346500 & -0.52350400 \\
\hline $\mathrm{H}$ & -7.04263100 & -2.46784200 & 2.81974700 & $\mathrm{H}$ & -9.18046700 & -3.29670100 & 1.85858300 \\
\hline $\mathrm{N}$ & -3.56140500 & -3.34956200 & -0.68909100 & $\mathrm{H}$ & -3.30531500 & -2.86809700 & 0.17611700 \\
\hline C & -4.54013100 & -1.31644600 & -2.17576700 & $\mathrm{H}$ & -4.35078000 & -1.72257300 & -3.16600600 \\
\hline $\mathrm{H}$ & -3.65984400 & -1.03053500 & -1.61078800 & C & -5.68531400 & -0.53917900 & -1.97394300 \\
\hline C & -5.78134200 & 0.36625800 & -0.84063900 & $\mathrm{H}$ & -5.15184600 & 0.03952000 & -0.00527200 \\
\hline $\mathrm{H}$ & -6.81344000 & 0.53687600 & -0.51389800 & C & -6.86612900 & -0.67558600 & -2.88549400 \\
\hline $\mathrm{H}$ & -7.28026800 & 0.30796000 & -3.13501500 & $\mathrm{H}$ & -7.66164500 & -1.22366500 & -2.35929000 \\
\hline $\mathrm{H}$ & -6.62756800 & -1.21059700 & -3.80849900 & C & -5.08453600 & 3.00211900 & 0.49052900 \\
\hline $\mathrm{H}$ & -6.10921700 & 3.10248400 & 0.86769900 & $\mathrm{H}$ & -4.64720300 & 4.00489900 & 0.43068700 \\
\hline $\mathrm{H}$ & -4.49901100 & 2.43124700 & 1.21955300 & C & -6.15571700 & 3.05416300 & -2.43516900 \\
\hline $\mathrm{H}$ & -7.19750900 & 3.10013500 & -2.09582100 & $\mathrm{H}$ & -6.13986400 & 2.58450700 & -3.42593600 \\
\hline $\mathrm{H}$ & -5.80794000 & 4.08687300 & -2.56247800 & C & -3.29170500 & 1.98259000 & -1.86658500 \\
\hline $\mathrm{H}$ & -3.26327800 & 1.44031600 & -2.81841300 & $\mathrm{H}$ & -2.65308200 & 1.46344900 & -1.14442800 \\
\hline $\mathrm{H}$ & -2.86064000 & 2.97523700 & -2.03249100 & $\mathrm{Si}$ & -5.03725300 & 2.16483000 & -1.19558700 \\
\hline
\end{tabular}




\begin{tabular}{|c|c|c|c|c|c|c|c|}
\hline C & 1.06381200 & 1.50590100 & 0.57786800 & $\mathrm{H}$ & -0.60220400 & 0.20534100 & 0.25497000 \\
\hline C & 1.77545500 & 2.29643300 & 1.47846600 & $\mathrm{H}$ & 1.85841600 & 3.04739600 & 3.49978200 \\
\hline $\mathrm{H}$ & 1.38666200 & 1.44053300 & -0.44925400 & $\mathrm{H}$ & 2.65876800 & 2.83047300 & 1.14725400 \\
\hline $\mathrm{N}$ & -2.70076000 & -0.16273300 & 1.90032600 & $\mathrm{H}$ & -2.51948600 & -0.27091000 & 0.89215900 \\
\hline $\mathrm{C}$ & -1.07868600 & -1.35660600 & 3.85505500 & $\mathrm{H}$ & -0.51032800 & -0.83459600 & 4.62011900 \\
\hline $\mathrm{H}$ & -2.06716500 & -1.69650000 & 4.15029700 & $\mathrm{C}$ & -0.38137100 & -2.03450100 & 2.85505200 \\
\hline C & 1.05106400 & -1.85729700 & 2.68588400 & $\mathrm{H}$ & 1.40104200 & -0.89484900 & 3.07605000 \\
\hline $\mathrm{H}$ & 1.38036800 & -1.99562800 & 1.65383800 & C & -1.12434900 & -2.87106200 & 1.87183800 \\
\hline $\mathrm{H}$ & -0.54909500 & -3.74795000 & 1.56249200 & $\mathrm{H}$ & -1.25645300 & -2.28355100 & 0.95009100 \\
\hline $\mathrm{H}$ & -2.11003700 & -3.16953800 & 2.23739800 & $\mathrm{C}$ & 3.81093400 & -3.05227000 & 3.29379300 \\
\hline $\mathrm{H}$ & 3.94130100 & -3.34365300 & 2.24811800 & $\mathrm{H}$ & 4.41480400 & -3.72181600 & 3.91888600 \\
\hline $\mathrm{H}$ & 4.20018700 & -2.03753600 & 3.41478800 & $\mathrm{C}$ & 1.30962600 & -4.89366600 & 3.49321600 \\
\hline $\mathrm{H}$ & 1.35133800 & -5.17116800 & 2.43532200 & $\mathrm{H}$ & 0.26888600 & -4.97339200 & 3.82855900 \\
\hline $\mathrm{H}$ & 1.89605700 & -5.62653400 & 4.06094600 & C & 1.75063600 & -2.65232800 & 5.57695600 \\
\hline $\mathrm{H}$ & 0.69941400 & -2.71398900 & 5.87960100 & $\mathrm{H}$ & 2.09634900 & -1.62648100 & 5.75208100 \\
\hline $\mathrm{H}$ & 2.32338200 & -3.30925600 & 6.24261500 & $\mathrm{Si}$ & 2.00677800 & -3.17340100 & 3.77912100 \\
\hline $\mathrm{C}$ & -4.07888300 & 0.72022500 & -3.59956500 & $\mathrm{H}$ & -4.76589000 & -0.06507600 & -3.93208200 \\
\hline $\mathrm{H}$ & -3.26346300 & 0.82212300 & -4.31720800 & C & -4.82496500 & 2.03856400 & -3.36008400 \\
\hline $\mathrm{H}$ & -5.64652000 & 2.17784100 & -4.06735300 & $\mathrm{H}$ & -4.13608400 & 2.88169500 & -3.46987300 \\
\hline $\mathrm{C}$ & -5.29131000 & 1.92794800 & -1.90265600 & $\mathrm{H}$ & -5.54274900 & 2.89062600 & -1.45201300 \\
\hline $\mathrm{H}$ & -6.16977400 & 1.27393200 & -1.83118400 & $\mathrm{O}$ & -2.28391600 & -0.75960100 & -0.76942500 \\
\hline C & 0.14574000 & -0.38737900 & -3.20128100 & $\mathrm{C}$ & 1.43089800 & 0.10131000 & -2.87141700 \\
\hline $\mathrm{C}$ & -0.12711200 & 0.76341200 & -4.09227300 & $\mathrm{C}$ & 1.27538200 & 1.28004800 & -3.77563500 \\
\hline 0 & 1.92413300 & 2.26637300 & -4.07672900 & $\mathrm{O}$ & -1.09186700 & 1.16140100 & -4.72960900 \\
\hline $\mathrm{H}$ & 2.05732200 & -1.24849500 & -1.52611100 & $\mathrm{~N}$ & 2.34711500 & -0.38827900 & -2.00590000 \\
\hline $\mathrm{C}$ & 3.42591300 & 0.28377400 & -1.41765300 & $\mathrm{C}$ & 4.18177600 & -0.42050500 & -0.46643200 \\
\hline C & 3.70910900 & 1.63157800 & -1.67877200 & C & 5.19631900 & 0.23695500 & 0.22288100 \\
\hline $\mathrm{H}$ & 3.96953400 & -1.46755300 & -0.26747400 & $\mathrm{C}$ & 4.71418600 & 2.26747500 & -0.95440500 \\
\hline $\mathrm{H}$ & 3.15614600 & 2.17765400 & -2.43469200 & $\mathrm{C}$ & 5.46856100 & 1.58663600 & -0.00138900 \\
\hline $\mathrm{H}$ & 6.25382100 & 2.09116300 & 0.54824100 & C & 4.90728100 & 3.74947900 & -1.10986200 \\
\hline $\mathrm{C}$ & 5.97638200 & -0.48335500 & 1.28751400 & $\mathrm{~F}$ & 4.46760700 & 4.21204700 & -2.29663300 \\
\hline $\mathrm{F}$ & 6.19983700 & 4.11247100 & -0.97804600 & $\mathrm{~F}$ & 4.21689800 & 4.42784300 & -0.14684000 \\
\hline $\mathrm{F}$ & 5.49056200 & -0.19414400 & 2.52541900 & $\mathrm{~F}$ & 7.27519200 & -0.10941000 & 1.29285100 \\
\hline $\mathrm{F}$ & 5.93490600 & -1.81974500 & 1.14461800 & & & & \\
\hline
\end{tabular}

\section{TS-S-6f-2}

Imaginary frequency: $-335.85 \mathrm{~cm}^{-1}$

E[B3LYP/6-31G(d)-D3(BJ)]: -4655.654548 Hartree

E[PCM(Et $\left.\left.t_{2} \mathrm{O}\right)-\mathrm{B} 3 L Y P / 6-311 \mathrm{G}(\mathrm{d}, \mathrm{p})-\mathrm{D} 3(\mathrm{BJ})\right]:-4656.839866$ Hartree

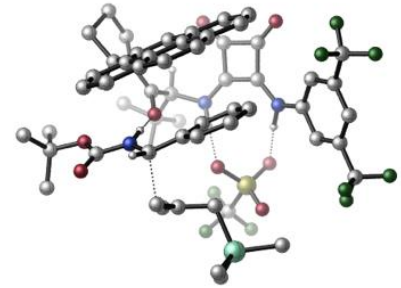

\begin{tabular}{|c|c|c|c|c|c|c|c|}
\hline 01 & & & & C & 2.03340800 & 1.01038000 & -3.20189800 \\
\hline $\mathrm{H}$ & 2.19941400 & 0.46581000 & -4.13119600 & $\mathrm{C}$ & 2.65642300 & 2.43109600 & -3.36451800 \\
\hline $\mathrm{C}$ & 2.62695900 & 3.25011100 & -2.06070100 & $\mathrm{H}$ & 3.25948600 & 2.80044800 & -1.29008900 \\
\hline $\mathrm{H}$ & 1.62066900 & 3.34009900 & -1.65041400 & $\mathrm{H}$ & 2.99775000 & 4.26086200 & -2.26607000 \\
\hline C & 1.87364700 & 3.17273400 & -4.46160200 & $\mathrm{H}$ & 2.35292500 & 4.13491600 & -4.67391600 \\
\hline $\mathrm{H}$ & 0.84302700 & 3.36772600 & -4.15667200 & $\mathrm{H}$ & 1.84980600 & 2.59286700 & -5.39192200 \\
\hline C & 4.12027600 & 2.26423200 & -3.80880300 & $\mathrm{H}$ & 4.58309900 & 3.24759400 & -3.94440700 \\
\hline $\mathrm{H}$ & 4.19388000 & 1.72677100 & -4.76137300 & $\mathrm{H}$ & 4.70989200 & 1.72286300 & -3.05985300 \\
\hline $\mathrm{C}$ & 2.63031600 & 0.23726700 & -2.01809500 & C & 4.02495600 & -1.53222600 & -1.08387900 \\
\hline $\mathrm{H}$ & 4.36324200 & -0.82447300 & -0.32861300 & $\mathrm{~N}$ & 3.51592900 & -0.75102100 & -2.23057300 \\
\hline C & 2.96560200 & -2.44275300 & -0.48104200 & C & 1.88277200 & -2.84681400 & -1.20528900 \\
\hline $\mathrm{C}$ & 3.12080900 & -2.88257500 & 0.89090200 & $\mathrm{C}$ & 0.85236500 & -3.67324000 & -0.65087300 \\
\hline
\end{tabular}




\begin{tabular}{|c|c|c|c|c|c|c|c|}
\hline$\overline{\mathrm{H}}$ & 1.76219400 & -2.51695100 & -2.23158200 & C & 2.09085600 & -3.68448400 & 1.47713700 \\
\hline C & 4.22349500 & -2.52824400 & 1.68727000 & C & -0.27423000 & -4.05256300 & -1.39661500 \\
\hline C & 0.95827300 & -4.07891400 & 0.70903700 & C & 2.18275400 & -4.07751100 & 2.84807200 \\
\hline $\mathrm{H}$ & 5.02985400 & -1.93946600 & 1.26682000 & C & 4.29711400 & -2.90030400 & 3.02803400 \\
\hline C & -1.29677000 & -4.79309800 & -0.80772200 & $\mathrm{H}$ & -0.35474800 & -3.76424200 & -2.43851000 \\
\hline C & -0.07985200 & -4.86069400 & 1.29678300 & C & 3.28699500 & -3.66250200 & 3.60800200 \\
\hline C & 1.13124200 & -4.87820700 & 3.41035200 & $\mathrm{H}$ & 5.15084400 & -2.58849200 & 3.62231400 \\
\hline C & -1.20279600 & -5.19367800 & 0.52360900 & $\mathrm{H}$ & -2.17649700 & -5.04632200 & -1.38943800 \\
\hline C & 0.05258500 & -5.25360500 & 2.67003400 & $\mathrm{H}$ & 3.35098200 & -3.95357100 & 4.65330000 \\
\hline $\mathrm{H}$ & 1.21739500 & -5.17921800 & 4.45139900 & $\mathrm{H}$ & -2.01053200 & -5.76135500 & 0.97746100 \\
\hline $\mathrm{H}$ & -0.73694500 & -5.85588500 & 3.11228900 & $\mathrm{~N}$ & 0.58023600 & 1.06144200 & -3.00202000 \\
\hline $\mathrm{H}$ & 0.23974200 & 1.65658500 & -2.23713800 & S & -1.66303800 & 3.41168200 & -1.02457200 \\
\hline 0 & -2.53072800 & 3.61935200 & 0.14780100 & $\mathrm{O}$ & -0.46974100 & 2.55170300 & -0.76644300 \\
\hline $\mathrm{O}$ & -2.35717700 & 3.06548200 & -2.29098200 & C & -0.94060800 & 5.08998800 & -1.35668600 \\
\hline $\mathrm{F}$ & -1.90230000 & 5.98456700 & -1.58302800 & $\mathrm{~F}$ & -0.12279200 & 5.05298200 & -2.42034100 \\
\hline $\mathrm{F}$ & -0.22046200 & 5.49401900 & -0.29028000 & C & 3.89893100 & 0.67095900 & 2.23820600 \\
\hline 0 & 4.23593700 & 0.76452700 & 3.40529600 & $\mathrm{O}$ & 4.63239700 & 0.96407200 & 1.15189400 \\
\hline C & 5.98928000 & 1.52558500 & 1.26862600 & C & 5.92382800 & 2.86658900 & 1.99982600 \\
\hline $\mathrm{H}$ & 6.90711000 & 3.34737000 & 1.97026600 & $\mathrm{H}$ & 5.63045700 & 2.73275700 & 3.04203600 \\
\hline $\mathrm{H}$ & 5.20478300 & 3.52941700 & 1.50671300 & C & 6.38801200 & 1.71727600 & -0.19388300 \\
\hline $\mathrm{H}$ & 6.37724000 & 0.75998700 & -0.72545900 & $\mathrm{H}$ & 7.39732700 & 2.13572900 & -0.25476500 \\
\hline $\mathrm{H}$ & 5.69561600 & 2.39922600 & -0.69645800 & C & 6.90769000 & 0.52061900 & 1.96396100 \\
\hline $\mathrm{H}$ & 7.93188500 & 0.90799600 & 1.97273000 & $\mathrm{H}$ & 6.91154400 & -0.43156400 & 1.42250600 \\
\hline $\mathrm{H}$ & 6.58936600 & 0.34469400 & 2.99231800 & C & 1.70895500 & -0.15015600 & 2.74337800 \\
\hline $\mathrm{H}$ & 2.15258200 & -0.54002000 & 3.65503100 & C & 0.48384200 & -0.81468700 & 2.28581500 \\
\hline C & -0.23419500 & -1.58623200 & 3.21564700 & C & -0.02452700 & -0.65772000 & 0.98674900 \\
\hline C & -1.42951800 & -2.19443700 & 2.85315000 & $\mathrm{H}$ & 0.16212800 & -1.71850700 & 4.21882700 \\
\hline $\mathrm{C}$ & -1.20921300 & -1.29153400 & 0.62678800 & $\mathrm{H}$ & 0.49098000 & -0.05260500 & 0.25162300 \\
\hline $\mathrm{C}$ & -1.91990600 & -2.05347500 & 1.55206900 & $\mathrm{H}$ & -1.96544100 & -2.80201700 & 3.57530100 \\
\hline $\mathrm{H}$ & -1.57505400 & -1.19170300 & -0.38486400 & $\mathrm{H}$ & -2.83543500 & -2.55151000 & 1.25614700 \\
\hline $\mathrm{N}$ & 2.65227100 & 0.23941700 & 1.82697500 & $\mathrm{H}$ & 2.46546900 & 0.25214600 & 0.81329700 \\
\hline C & 1.10159300 & 1.48069600 & 3.80089000 & $\mathrm{H}$ & 0.50297200 & 0.97028600 & 4.55060300 \\
\hline $\mathrm{H}$ & 2.08973300 & 1.79847800 & 4.12108200 & C & 0.43713400 & 2.19317700 & 2.79972500 \\
\hline C & -0.98258100 & 2.01914000 & 2.57022200 & $\mathrm{H}$ & -1.35307200 & 1.06743500 & 2.96608100 \\
\hline $\mathrm{H}$ & -1.26442300 & 2.15269700 & 1.52368400 & C & 1.22767800 & 3.03748800 & 1.85933800 \\
\hline $\mathrm{H}$ & 0.64946100 & 3.88110100 & 1.47941000 & $\mathrm{H}$ & 1.46167000 & 2.42711200 & 0.97326100 \\
\hline $\mathrm{H}$ & 2.16640500 & 3.38354400 & 2.29972900 & C & -3.81798600 & 3.01744500 & 3.08080400 \\
\hline $\mathrm{H}$ & -3.97969000 & 3.20288600 & 2.01643900 & $\mathrm{H}$ & -4.48961100 & 3.66964400 & 3.65243900 \\
\hline $\mathrm{H}$ & -4.10335800 & 1.98103700 & 3.28887200 & C & -1.47289600 & 5.08374200 & 3.05245100 \\
\hline $\mathrm{H}$ & -1.52259900 & 5.21729400 & 1.96849600 & $\mathrm{H}$ & -0.45006100 & 5.29384400 & 3.38586000 \\
\hline $\mathrm{H}$ & -2.13002300 & 5.83086100 & 3.51385800 & C & -1.72849700 & 3.05756300 & 5.37501900 \\
\hline $\mathrm{H}$ & -0.67999200 & 3.22000300 & 5.64944500 & $\mathrm{H}$ & -1.99995000 & 2.03590400 & 5.66685100 \\
\hline $\mathrm{H}$ & -2.33777800 & 3.74276900 & 5.97656800 & $\mathrm{Si}$ & -2.03018800 & 3.35391200 & 3.53367300 \\
\hline C & 4.05941000 & -1.22235200 & -3.52330200 & $\mathrm{H}$ & 4.78452100 & -0.49627400 & -3.90671300 \\
\hline $\mathrm{H}$ & 3.25585100 & -1.35706600 & -4.24780300 & C & 4.74605300 & -2.54114500 & -3.15029900 \\
\hline $\mathrm{H}$ & 5.56627100 & -2.78341800 & -3.83092800 & $\mathrm{H}$ & 4.02099300 & -3.36014200 & -3.18604500 \\
\hline C & 5.20480200 & -2.30816800 & -1.70503900 & $\mathrm{H}$ & 5.41520800 & -3.23142500 & -1.16066500 \\
\hline $\mathrm{H}$ & 6.10794100 & -1.68463700 & -1.68731900 & O & 2.24020900 & 0.53590400 & -0.87519100 \\
\hline C & -0.16777000 & 0.00413300 & -3.33524200 & C & -1.50013600 & -0.39573300 & -3.08233200 \\
\hline C & 0.13023000 & -1.26348400 & -4.05081700 & C & -1.30389900 & -1.70682800 & -3.75980300 \\
\hline 0 & -1.94780000 & -2.72700100 & -3.93357800 & $\mathrm{O}$ & 1.11300700 & -1.75096700 & -4.58844700 \\
\hline $\mathrm{H}$ & -2.40217600 & 1.27055800 & -2.35544200 & $\mathrm{~N}$ & -2.50427700 & 0.25223100 & -2.44617300 \\
\hline C & -3.57377300 & -0.30859000 & -1.74246500 & C & -4.35001200 & 0.55481500 & -0.94981300 \\
\hline
\end{tabular}




\begin{tabular}{|c|c|c|c|c|c|c|c|}
\hline C & -3.83671400 & -1.68560200 & -1.73540500 & C & -5.35854100 & 0.03234400 & -0.14974800 \\
\hline $\mathrm{H}$ & -4.14298900 & 1.61878800 & -0.94179800 & C & -4.84790100 & -2.18099400 & -0.91379000 \\
\hline $\mathrm{H}$ & -3.27165000 & -2.35844400 & -2.37081700 & $\mathrm{C}$ & -5.61748800 & -1.33920900 & -0.11426100 \\
\hline $\mathrm{H}$ & -6.40237000 & -1.73781600 & 0.51751300 & $\mathrm{C}$ & -5.05053600 & -3.66686700 & -0.81098400 \\
\hline C & -6.17612400 & 0.93208700 & 0.73561300 & $F$ & -4.60862600 & -4.32892600 & -1.89872500 \\
\hline $\mathrm{F}$ & -6.34762500 & -3.99128200 & -0.62986600 & $\mathrm{~F}$ & -4.37421000 & -4.18022200 & 0.25769400 \\
\hline $\mathrm{F}$ & -5.95516100 & 0.65608100 & 2.04678300 & $F$ & -7.50090500 & 0.75735900 & 0.52850500 \\
\hline $\mathrm{F}$ & -5.90290800 & 2.23667000 & 0.54654000 & & & & \\
\hline
\end{tabular}

\section{TS-S-6f-3}

Imaginary frequency: $-251.00 \mathrm{~cm}^{-1}$

E[B3LYP/6-31G(d)-D3(BJ)]: -4655.649623 Hartree

$\mathrm{E}\left[\mathrm{PCM}\left(\mathrm{Et}_{2} \mathrm{O}\right)-\mathrm{B} 3 \mathrm{LYP} / 6-311 \mathrm{G}(\mathrm{d}, \mathrm{p})-\mathrm{D} 3(\mathrm{BJ})\right]$ : -4656.838857 Hartree

\begin{tabular}{|c|c|c|c|c|c|c|c|}
\hline 01 & & & & C & 1.31942800 & -1.29982200 & 3.62572700 \\
\hline $\mathrm{H}$ & 1.34809600 & -0.74814100 & 4.56505200 & $\mathrm{C}$ & 1.73762900 & -2.77150300 & 3.91983400 \\
\hline $\mathrm{C}$ & 1.64489700 & -3.67028000 & 2.67338800 & $\mathrm{H}$ & 2.30398900 & -3.31884100 & 1.87586500 \\
\hline $\mathrm{H}$ & 0.62986300 & -3.73492600 & 2.27417900 & $\mathrm{H}$ & 1.94605300 & -4.68840000 & 2.94404200 \\
\hline $\mathrm{C}$ & 0.80910600 & -3.31156600 & 5.02085100 & $\mathrm{H}$ & 1.09330100 & -4.33731500 & 5.28054500 \\
\hline $\mathrm{H}$ & -0.23221800 & -3.32117700 & 4.69273900 & $\mathrm{H}$ & 0.87758300 & -2.70107300 & 5.92941000 \\
\hline C & 3.18798200 & -2.77707900 & 4.43291200 & $\mathrm{H}$ & 3.49421100 & -3.80195400 & 4.66729900 \\
\hline $\mathrm{H}$ & 3.29107100 & -2.18408100 & 5.34891500 & $\mathrm{H}$ & 3.88724200 & -2.38634200 & 3.68483100 \\
\hline $\mathrm{C}$ & 2.21456300 & -0.60795000 & 2.58751600 & $\mathrm{C}$ & 3.91311000 & 1.03926600 & 1.97898500 \\
\hline $\mathrm{H}$ & 4.32617200 & 0.30512400 & 1.28674000 & $\mathrm{~N}$ & 3.11092300 & 0.30892300 & 2.98372000 \\
\hline C & 3.08109700 & 2.06480100 & 1.22081400 & C & 1.92479500 & 2.56151500 & 1.74830600 \\
\hline C & 3.53557900 & 2.52539600 & -0.07633300 & C & 1.10046300 & 3.50092900 & 1.04895300 \\
\hline $\mathrm{H}$ & 1.57998400 & 2.22177500 & 2.71914500 & C & 2.70857700 & 3.43237000 & -0.81301900 \\
\hline C & 4.74386900 & 2.10194900 & -0.65756300 & C & -0.10450000 & 3.97477700 & 1.59053400 \\
\hline C & 1.49197800 & 3.91750600 & -0.25390200 & C & 3.09167900 & 3.84753600 & -2.12573000 \\
\hline $\mathrm{H}$ & 5.40099400 & 1.43004800 & -0.11822800 & C & 5.10916300 & 2.51508100 & -1.93756200 \\
\hline C & -0.93233500 & 4.81452400 & 0.84874700 & $\mathrm{H}$ & -0.39902400 & 3.67922500 & 2.59106700 \\
\hline C & 0.65261800 & 4.79998300 & -0.99621600 & C & 4.29114500 & 3.36827200 & -2.67230700 \\
\hline C & 2.23313600 & 4.74440000 & -2.84794400 & $\mathrm{H}$ & 6.04961000 & 2.17435700 & -2.36032900 \\
\hline C & -0.56049100 & 5.22256400 & -0.43101800 & $\mathrm{H}$ & -1.87803300 & 5.14022600 & 1.26839800 \\
\hline C & 1.06927400 & 5.19945900 & -2.30939400 & $\mathrm{H}$ & 4.58345400 & 3.68557400 & -3.67006800 \\
\hline $\mathrm{H}$ & 2.53844400 & 5.05471400 & -3.84407600 & $\mathrm{H}$ & -1.21888400 & 5.86710100 & -1.00659100 \\
\hline $\mathrm{H}$ & 0.42845400 & 5.87475300 & -2.87054800 & $\mathrm{~N}$ & -0.05743700 & -1.21866900 & 3.13079700 \\
\hline $\mathrm{H}$ & -0.30129300 & -1.80899400 & 2.33213300 & $\mathrm{~S}$ & -2.14199500 & -3.62987000 & 0.63465000 \\
\hline $\mathrm{O}$ & -1.30532800 & -4.79153500 & 0.29200200 & $\mathrm{O}$ & -1.35113100 & -2.37126500 & 0.89292900 \\
\hline $\mathrm{O}$ & -3.33005300 & -3.37641500 & -0.20112900 & $\mathrm{C}$ & -2.82549400 & -4.01218300 & 2.32045000 \\
\hline $\mathrm{F}$ & -3.67188000 & -5.04071700 & 2.26891800 & $\mathrm{~F}$ & -3.47165200 & -2.93833600 & 2.80317900 \\
\hline $\mathrm{F}$ & -1.82632300 & -4.31797600 & 3.16646100 & $\mathrm{C}$ & 4.32140900 & -1.05726500 & -1.24768600 \\
\hline $\mathrm{O}$ & 4.96476800 & -1.34477700 & -0.25459500 & $\mathrm{O}$ & 4.71751400 & -1.17487700 & -2.52405100 \\
\hline C & 6.04035000 & -1.74360400 & -2.86121800 & C & 7.15035100 & -0.87730400 & -2.26736600 \\
\hline $\mathrm{H}$ & 8.12042200 & -1.24421200 & -2.61858300 & $\mathrm{H}$ & 7.13567200 & -0.90885800 & -1.17770400 \\
\hline $\mathrm{H}$ & 7.03907900 & 0.15978600 & -2.59797300 & $\mathrm{C}$ & 6.04574300 & -1.66977000 & -4.38630100 \\
\hline $\mathrm{H}$ & 5.22577300 & -2.26079200 & -4.80658300 & $\mathrm{H}$ & 6.98980600 & -2.06335700 & -4.77504600 \\
\hline $\mathrm{H}$ & 5.93656900 & -0.63370600 & -4.72184300 & C & 6.10456700 & -3.19171600 & -2.37662200 \\
\hline $\mathrm{H}$ & 7.03622500 & -3.65037700 & -2.72378400 & $\mathrm{H}$ & 5.26890300 & -3.76873600 & -2.78729000 \\
\hline $\mathrm{H}$ & 6.07246400 & -3.24401600 & -1.28754400 & C & 2.28042300 & -0.15552500 & -2.24052300 \\
\hline $\mathrm{H}$ & 2.88093400 & 0.11117400 & -3.10287300 & C & 1.07499900 & 0.65645800 & -2.03859300 \\
\hline
\end{tabular}




\begin{tabular}{|c|c|c|c|c|c|c|c|}
\hline $\mathrm{C}$ & 0.61162600 & 1.42174000 & -3.12286200 & $\mathrm{C}$ & 0.35461600 & 0.66799300 & -0.83461700 \\
\hline C & -0.54280700 & 2.18732600 & -3.00598200 & $\mathrm{H}$ & 1.17301100 & 1.42281900 & -4.05375100 \\
\hline $\mathrm{C}$ & -0.78415400 & 1.45707300 & -0.72062700 & $\mathrm{H}$ & 0.68190800 & 0.08642900 & 0.01847600 \\
\hline C & -1.24542500 & 2.21020000 & -1.79867400 & $\mathrm{H}$ & -0.88067500 & 2.78520700 & -3.84645200 \\
\hline $\mathrm{H}$ & -1.30198200 & 1.50125100 & 0.22468100 & $\mathrm{H}$ & -2.12947600 & 2.82769600 & -1.68729700 \\
\hline $\mathrm{N}$ & 3.03531100 & -0.54730600 & -1.16653600 & $\mathrm{H}$ & 2.65991000 & -0.54295800 & -0.20529000 \\
\hline C & 1.65748400 & -1.83568200 & -3.26130600 & $\mathrm{H}$ & 1.29073500 & -1.35737100 & -4.16551900 \\
\hline $\mathrm{H}$ & 2.63934700 & -2.29224800 & -3.33457100 & C & 0.72703400 & -2.35176500 & -2.35728600 \\
\hline C & -0.68581500 & -2.04048900 & -2.47925800 & $\mathrm{H}$ & -0.86179700 & -1.09590500 & -3.00625900 \\
\hline $\mathrm{H}$ & -1.21170000 & -2.05271000 & -1.52222600 & C & 1.19473000 & -3.14177700 & -1.18545600 \\
\hline $\mathrm{H}$ & 0.48767800 & -3.92993500 & -0.91207400 & $\mathrm{H}$ & 1.22249000 & -2.47330100 & -0.31078000 \\
\hline $\mathrm{H}$ & 2.19464900 & -3.55750400 & -1.33226000 & $\mathrm{C}$ & -3.38993400 & -3.04080100 & -3.52091900 \\
\hline $\mathrm{H}$ & -3.74581700 & -3.22706300 & -2.50418700 & $\mathrm{H}$ & -3.93369700 & -3.70345000 & -4.20568000 \\
\hline $\mathrm{H}$ & -3.63848500 & -2.00890500 & -3.78477300 & C & -1.10001600 & -5.10853100 & -3.08393400 \\
\hline $\mathrm{H}$ & -1.36305500 & -5.27913100 & -2.03543200 & $\mathrm{H}$ & -0.02897100 & -5.30884900 & -3.20573100 \\
\hline $\mathrm{H}$ & -1.64397200 & -5.83918400 & -3.69515200 & C & -0.90847600 & -3.05046700 & -5.38039700 \\
\hline $\mathrm{H}$ & 0.16583900 & -3.24708300 & -5.46829200 & $\mathrm{H}$ & -1.09340300 & -2.01680300 & -5.69649500 \\
\hline $\mathrm{H}$ & -1.42194400 & -3.70754800 & -6.09266500 & $\mathrm{Si}$ & -1.55074500 & -3.37023400 & -3.63201000 \\
\hline C & 3.41356800 & 0.73668700 & 4.36835700 & $\mathrm{H}$ & 3.95793300 & -0.05890700 & 4.88760800 \\
\hline $\mathrm{H}$ & 2.49308900 & 0.96431300 & 4.90808600 & C & 4.30163800 & 1.97056800 & 4.16818400 \\
\hline $\mathrm{H}$ & 4.98824900 & 2.11937500 & 5.00550900 & $\mathrm{H}$ & 3.67949300 & 2.86618600 & 4.07631700 \\
\hline $\mathrm{C}$ & 5.01687600 & 1.68659600 & 2.84111400 & $\mathrm{H}$ & 5.42741300 & 2.58249500 & 2.36986600 \\
\hline $\mathrm{H}$ & 5.83294100 & 0.96955800 & 2.98987700 & $\mathrm{O}$ & 2.04347000 & -0.88812600 & 1.38435000 \\
\hline $\mathrm{C}$ & -0.75860700 & -0.08920200 & 3.28112300 & C & -1.92280100 & 0.44882200 & 2.68546000 \\
\hline $\mathrm{C}$ & -0.55593100 & 1.12074300 & 4.11192200 & $\mathrm{C}$ & -1.83629400 & 1.69339200 & 3.50451900 \\
\hline $\mathrm{O}$ & -2.44799200 & 2.74291700 & 3.60057900 & $\mathrm{O}$ & 0.31108400 & 1.51395500 & 4.87885500 \\
\hline $\mathrm{H}$ & -2.41778400 & -0.98229000 & 1.36870600 & $\mathrm{~N}$ & -2.71065900 & -0.05957500 & 1.71049800 \\
\hline $\mathrm{C}$ & -3.60389200 & 0.62444200 & 0.87734100 & $\mathrm{C}$ & -4.24984300 & -0.12019200 & -0.12349800 \\
\hline $\mathrm{C}$ & -3.80023300 & 2.01012500 & 0.95550900 & $\mathrm{C}$ & -5.06773200 & 0.53246600 & -1.04048000 \\
\hline $\mathrm{H}$ & -4.10555100 & -1.19557300 & -0.18212100 & C & -4.60485700 & 2.63727300 & 0.00713800 \\
\hline $\mathrm{H}$ & -3.33795700 & 2.59108100 & 1.74541500 & $\mathrm{C}$ & -5.24712300 & 1.91553500 & -0.99626600 \\
\hline $\mathrm{H}$ & -5.87837300 & 2.41477400 & -1.72126700 & $\mathrm{C}$ & -4.68292300 & 4.13763200 & -0.01289600 \\
\hline C & -5.72802600 & -0.23709300 & -2.15066800 & $\mathrm{~F}$ & -3.76506300 & 4.65556000 & -0.88111600 \\
\hline $\mathrm{F}$ & -4.42940100 & 4.69001100 & 1.18982300 & $\mathrm{~F}$ & -5.88850700 & 4.58100100 & -0.42495500 \\
\hline $\mathrm{F}$ & -5.04843600 & -0.08435200 & -3.31977400 & $\mathrm{~F}$ & -6.98436500 & 0.20437800 & -2.38285400 \\
\hline $\mathrm{F}$ & -5.79714900 & -1.55595000 & -1.89793200 & & & & \\
\hline
\end{tabular}

\section{TS-S-6f-4}

Imaginary frequency: $-342.77 \mathrm{~cm}^{-1}$

E[B3LYP/6-31G(d)-D3(BJ)]: -4655.651620 Hartree

E[PCM(Et $\left.\left.{ }_{2} \mathrm{O}\right)-\mathrm{B} 3 \mathrm{LYP} / 6-311 \mathrm{G}(\mathrm{d}, \mathrm{p})-\mathrm{D} 3(\mathrm{BJ})\right]$ : -4656.837448 Hartree

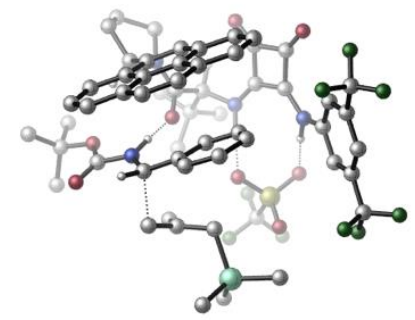

\begin{tabular}{|c|c|c|c|c|c|c|c|}
\hline 01 & & & & C & 2.05543300 & 1.05667700 & -3.17585700 \\
\hline $\mathrm{H}$ & 2.21982000 & 0.54806100 & -4.12572300 & C & 2.68851900 & 2.47962300 & -3.26524900 \\
\hline C & 2.64744100 & 3.23651100 & -1.92435900 & $\mathrm{H}$ & 3.26749600 & 2.74678300 & -1.16816900 \\
\hline $\mathrm{H}$ & 1.63665700 & 3.31431000 & -1.52284600 & $\mathrm{H}$ & 3.02680400 & 4.25333000 & -2.07771900 \\
\hline C & 1.92258800 & 3.27620500 & -4.33574600 & $\mathrm{H}$ & 2.40711200 & 4.24602900 & -4.49492900 \\
\hline $\mathrm{H}$ & 0.88830000 & 3.45934000 & -4.03722400 & $\mathrm{H}$ & 1.91080100 & 2.74136800 & -5.29287700 \\
\hline$C$ & 4.15777100 & 2.33564300 & -3.70054700 & $\mathrm{H}$ & 4.62979200 & 3.32292600 & -3.74622500 \\
\hline $\mathrm{H}$ & 4.24238700 & 1.88470400 & -4.69635400 & $\mathrm{H}$ & 4.73410100 & 1.72788500 & -2.99322900 \\
\hline
\end{tabular}




\begin{tabular}{|c|c|c|c|c|c|c|c|}
\hline $\mathrm{C}$ & 2.62899200 & 0.21984000 & -2.02166600 & $\mathrm{O}$ & 2.23626300 & 0.47376300 & -0.86966700 \\
\hline C & 4.11759300 & -1.15463100 & -3.54887200 & C & 3.99889300 & -1.60822100 & -1.15197700 \\
\hline C & 5.47997900 & -1.68214500 & -3.09551600 & $\mathrm{H}$ & 3.51721700 & -1.92262000 & -4.04541400 \\
\hline $\mathrm{H}$ & 4.19285800 & -0.29720500 & -4.21377800 & $\mathrm{H}$ & 4.39366400 & -0.91566700 & -0.40739500 \\
\hline $\mathrm{H}$ & 6.15193200 & -0.83963600 & -2.89036000 & $\mathrm{H}$ & 5.95206800 & -2.31934100 & -3.84826000 \\
\hline $\mathrm{N}$ & 3.49005400 & -0.78133500 & -2.27036300 & C & 2.92853400 & -2.47751800 & -0.51509200 \\
\hline C & 1.83863300 & -2.87599500 & -1.23154600 & C & 3.09063400 & -2.91012300 & 0.85679300 \\
\hline C & 0.80439700 & -3.68873000 & -0.66563100 & $\mathrm{H}$ & 1.72420500 & -2.55911200 & -2.26325000 \\
\hline C & 2.05773500 & -3.70028200 & 1.45422200 & C & 4.20442100 & -2.56598600 & 1.64207200 \\
\hline C & -0.32901800 & -4.06666100 & -1.40237400 & C & 0.91753000 & -4.09006600 & 0.69550100 \\
\hline C & 2.15806100 & -4.08978100 & 2.82573500 & $\mathrm{H}$ & 5.01291600 & -1.98799100 & 1.21123500 \\
\hline C & 4.28556900 & -2.93282900 & 2.98338000 & C & -1.34818800 & -4.80438200 & -0.80467900 \\
\hline $\mathrm{H}$ & -0.41748100 & -3.77855500 & -2.44389000 & C & -0.11941200 & -4.86650600 & 1.29276400 \\
\hline C & 3.27205700 & -3.68191200 & 3.57506500 & C & 1.10565600 & -4.88175800 & 3.39853200 \\
\hline $\mathrm{H}$ & 5.14751700 & -2.62787300 & 3.56926200 & C & -1.24703500 & -5.20082400 & 0.52747400 \\
\hline $\mathrm{H}$ & -2.23128800 & -5.05913500 & -1.38029800 & C & 0.02038100 & -5.25461700 & 2.66664800 \\
\hline $\mathrm{H}$ & 3.34214200 & -3.96989700 & 4.62082700 & $\mathrm{H}$ & 1.19740900 & -5.17968200 & 4.44000300 \\
\hline $\mathrm{H}$ & -2.05228600 & -5.76694900 & 0.98779700 & $\mathrm{H}$ & -0.76891900 & -5.85172200 & 3.11631400 \\
\hline $\mathrm{N}$ & 0.60071300 & 1.11328200 & -2.98794000 & $\mathrm{H}$ & 0.25560600 & 1.69254900 & -2.21329100 \\
\hline$S$ & -1.65581000 & 3.41828400 & -0.98484300 & 0 & -2.54297800 & 3.60415500 & 0.17645200 \\
\hline 0 & -0.46925800 & 2.54966600 & -0.72407200 & $\mathrm{O}$ & -2.32786200 & 3.10100200 & -2.27043000 \\
\hline $\mathrm{C}$ & -0.92318000 & 5.10126800 & -1.26916600 & $\mathrm{~F}$ & -1.87795400 & 6.00169300 & -1.50107800 \\
\hline $\mathrm{F}$ & -0.08055000 & 5.08270900 & -2.31380700 & $\mathrm{~F}$ & -0.22680700 & 5.48503800 & -0.17945300 \\
\hline C & 3.86949300 & 0.59236000 & 2.25488100 & 0 & 4.19948500 & 0.68592000 & 3.42407600 \\
\hline $\mathrm{O}$ & 4.61575900 & 0.87173300 & 1.17315800 & C & 5.97214000 & 1.43154500 & 1.30238000 \\
\hline C & 5.90010600 & 2.77844700 & 2.02225400 & $\mathrm{H}$ & 6.88445600 & 3.25748500 & 2.00126200 \\
\hline $\mathrm{H}$ & 5.59377900 & 2.65316500 & 3.06179800 & $\mathrm{H}$ & 5.18819400 & 3.43847400 & 1.51521600 \\
\hline $\mathrm{C}$ & 6.39029400 & 1.61237300 & -0.15612700 & $\mathrm{H}$ & 6.38599800 & 0.65133100 & -0.68094000 \\
\hline $\mathrm{H}$ & 7.40074900 & 2.02944700 & -0.20663100 & $\mathrm{H}$ & 5.70547000 & 2.29141200 & -0.67260400 \\
\hline C & 6.88150500 & 0.43131600 & 2.01637800 & $\mathrm{H}$ & 7.90589100 & 0.81794400 & 2.03367700 \\
\hline $\mathrm{H}$ & 6.89076200 & -0.52530300 & 1.48287700 & $\mathrm{H}$ & 6.55101100 & 0.26367800 & 3.04216500 \\
\hline C & 1.66979800 & -0.21235300 & 2.74766900 & $\mathrm{H}$ & 2.10659200 & -0.61902500 & 3.65534600 \\
\hline C & 0.43920800 & -0.85777100 & 2.27718700 & C & -0.29494600 & -1.62757600 & 3.19579400 \\
\hline C & -0.05963700 & -0.68161200 & 0.97698800 & C & -1.49622400 & -2.21610700 & 2.82072000 \\
\hline $\mathrm{H}$ & 0.09332700 & -1.77434800 & 4.20007700 & C & -1.25005400 & -1.29683800 & 0.60398100 \\
\hline $\mathrm{H}$ & 0.46746000 & -0.07616500 & 0.25049000 & C & -1.97638500 & -2.05776300 & 1.51782800 \\
\hline $\mathrm{H}$ & -2.04465400 & -2.82277500 & 3.53426100 & $\mathrm{H}$ & -1.60816500 & -1.18292700 & -0.40887800 \\
\hline $\mathrm{H}$ & -2.89594700 & -2.54195400 & 1.21138000 & $\mathrm{~N}$ & 2.61970800 & 0.17751400 & 1.83742100 \\
\hline $\mathrm{H}$ & 2.44113700 & 0.18497900 & 0.82292300 & C & 1.07461000 & 1.40681600 & 3.82532900 \\
\hline $\mathrm{H}$ & 0.46983100 & 0.88979800 & 4.56549000 & $\mathrm{H}$ & 2.06379600 & 1.71300400 & 4.15337200 \\
\hline C & 0.41829200 & 2.13947100 & 2.83319700 & C & -1.00100700 & 1.97651600 & 2.59366800 \\
\hline $\mathrm{H}$ & -1.37788000 & 1.02043600 & 2.97256800 & $\mathrm{H}$ & -1.27603700 & 2.12684900 & 1.54757500 \\
\hline C & 1.21966300 & 2.99182100 & 1.90975100 & $\mathrm{H}$ & 0.64699000 & 3.83878700 & 1.52943000 \\
\hline $\mathrm{H}$ & 1.46447200 & 2.38668300 & 1.02285200 & $\mathrm{H}$ & 2.15384600 & 3.33253800 & 2.36384500 \\
\hline C & -3.83717700 & 2.97024600 & 3.10172200 & $\mathrm{H}$ & -3.99174100 & 3.17169900 & 2.03917700 \\
\hline $\mathrm{H}$ & -4.51201000 & 3.61433900 & 3.67873600 & $\mathrm{H}$ & -4.12477800 & 1.93099300 & 3.29202400 \\
\hline C & -1.48910700 & 5.03358300 & 3.11966200 & $\mathrm{H}$ & -1.53493400 & 5.18486600 & 2.03794900 \\
\hline $\mathrm{H}$ & -0.46647100 & 5.23509600 & 3.45902400 & $\mathrm{H}$ & -2.14524300 & 5.77514500 & 3.59130000 \\
\hline C & -1.76125100 & 2.97260800 & 5.40943700 & $\mathrm{H}$ & -0.71411900 & 3.12971600 & 5.69215500 \\
\hline $\mathrm{H}$ & -2.03519800 & 1.94678400 & 5.68380200 & $\mathrm{H}$ & -2.37324700 & 3.64896500 & 6.01819900 \\
\hline $\mathrm{Si}$ & -2.05187400 & 3.29763800 & 3.57116700 & C & 5.14118300 & -2.43326200 & -1.80037200 \\
\hline $\mathrm{H}$ & 4.77282600 & -3.43719300 & -2.03210200 & $\mathrm{H}$ & 5.99380300 & -2.54121500 & -1.12446000 \\
\hline C & -0.14001900 & 0.05558600 & -3.33740700 & C & -1.47171600 & -0.35407600 & -3.10583500 \\
\hline
\end{tabular}




\begin{tabular}{|c|c|c|c|c|c|c|c|}
\hline C & 0.17996400 & -1.20339400 & -4.05812000 & C & -1.25679700 & -1.66042700 & -3.78831400 \\
\hline 0 & -1.89299500 & -2.68305200 & -3.97556400 & $\mathrm{O}$ & 1.17773500 & -1.66950800 & -4.58492700 \\
\hline $\mathrm{H}$ & -2.38488900 & 1.30367200 & -2.37581900 & $\mathrm{~N}$ & -2.48802900 & 0.28702000 & -2.48086700 \\
\hline $\mathrm{C}$ & -3.56583300 & -0.27887900 & -1.79450200 & C & -4.34497600 & 0.57792100 & -0.99741400 \\
\hline C & -3.83542100 & -1.65472600 & -1.80812100 & C & -5.36282500 & 0.05054200 & -0.21274500 \\
\hline $\mathrm{H}$ & -4.13225200 & 1.64043900 & -0.97268400 & C & -4.85673400 & -2.15505900 & -1.00195800 \\
\hline $\mathrm{H}$ & -3.26566700 & -2.32223500 & -2.44530200 & C & -5.62923600 & -1.31981800 & -0.19820800 \\
\hline $\mathrm{H}$ & -6.42141600 & -1.72289200 & 0.42157000 & C & -5.07045300 & -3.64045900 & -0.91799200 \\
\hline C & -6.18151600 & 0.94277300 & 0.67918100 & $\mathrm{~F}$ & -4.42693900 & -4.16648900 & 0.16453500 \\
\hline $\mathrm{F}$ & -4.60331500 & -4.29610000 & -1.99911300 & $\mathrm{~F}$ & -6.37400100 & -3.95853700 & -0.77497300 \\
\hline $\mathrm{F}$ & -5.89680100 & 2.24796400 & 0.51292300 & $\mathrm{~F}$ & -5.97423600 & 0.64478400 & 1.98775000 \\
\hline $\mathrm{F}$ & -7.50575800 & 0.78143900 & 0.45777400 & & & & \\
\hline
\end{tabular}

\section{TS-S-6f-5}

Imaginary frequency: $-378.70 \mathrm{~cm}^{-1}$

E[B3LYP/6-31G(d)-D3(BJ)]: -4655.646702 Hartree

$\mathrm{E}\left[\mathrm{PCM}\left(\mathrm{Et}_{2} \mathrm{O}\right)-\mathrm{B} 3 \mathrm{LYP} / 6-311 \mathrm{G}(\mathrm{d}, \mathrm{p})-\mathrm{D} 3(\mathrm{BJ})\right]$ : -4656.837110 Hartree

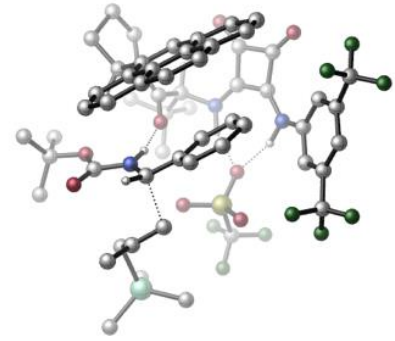

\begin{tabular}{|c|c|c|c|c|c|c|c|}
\hline 01 & & & & C & -1.12773500 & -1.62966500 & 3.53129500 \\
\hline $\mathrm{H}$ & -0.66545300 & -2.41330700 & 4.13243200 & C & -2.48651900 & -1.24350300 & 4.18873500 \\
\hline C & -3.17775200 & -0.08125600 & 3.45355000 & $\mathrm{H}$ & -3.33807200 & -0.29826200 & 2.39534200 \\
\hline $\mathrm{H}$ & -2.59851100 & 0.84309100 & 3.51119600 & $\mathrm{H}$ & -4.14804300 & 0.11499900 & 3.92470200 \\
\hline $\mathrm{C}$ & -2.20825900 & -0.83617900 & 5.64545200 & $\mathrm{H}$ & -3.14595700 & -0.57676400 & 6.14964700 \\
\hline $\mathrm{H}$ & -1.54387700 & 0.03117000 & 5.69177300 & $\mathrm{H}$ & -1.73808700 & -1.65458600 & 6.20405700 \\
\hline $\mathrm{C}$ & -3.40957400 & -2.47342200 & 4.17785100 & $\mathrm{H}$ & -4.36804700 & -2.22421000 & 4.64574900 \\
\hline $\mathrm{H}$ & -2.97386400 & -3.30646200 & 4.74140600 & $\mathrm{H}$ & -3.61734600 & -2.81642100 & 3.15883800 \\
\hline C & -1.26469000 & -2.09197000 & 2.07179700 & C & -0.98675200 & -4.53458400 & 2.70731200 \\
\hline C & -1.20765500 & -3.82783500 & 0.36459100 & $\mathrm{H}$ & -0.14143000 & -4.34413400 & 3.37247900 \\
\hline $\mathrm{H}$ & -1.89147700 & -4.68116800 & 3.30604400 & $\mathrm{H}$ & -2.02360800 & -3.29769700 & -0.12277700 \\
\hline $\mathrm{N}$ & -1.18058800 & -3.40302900 & 1.77575500 & C & 0.10077000 & -3.53505200 & -0.35469100 \\
\hline C & 1.26486900 & -3.38147600 & 0.33982800 & C & 0.10772600 & -3.44513500 & -1.80089400 \\
\hline $\mathrm{C}$ & 2.51583200 & -3.11571800 & -0.30470900 & $\mathrm{H}$ & 1.26376700 & -3.42688800 & 1.42367200 \\
\hline C & 1.34665100 & -3.18630200 & -2.46974100 & C & -1.05473100 & -3.57205500 & -2.58017100 \\
\hline C & 3.68997000 & -2.88484000 & 0.42843000 & C & 2.54918500 & -3.02569500 & -1.72443400 \\
\hline C & 1.37934500 & -3.05530200 & -3.89205700 & $\mathrm{H}$ & -2.00589900 & -3.78491600 & -2.10788300 \\
\hline C & -1.01860300 & -3.41739800 & -3.96494400 & C & 4.87360000 & -2.53972100 & -0.22103100 \\
\hline $\mathrm{H}$ & 3.67200300 & -2.95735800 & 1.50986500 & C & 3.77718600 & -2.72539700 & -2.38480300 \\
\hline C & 0.18377400 & -3.16355900 & -4.61817500 & C & 2.63730500 & -2.79647700 & -4.53520900 \\
\hline $\mathrm{H}$ & -1.94118800 & -3.49598200 & -4.53250000 & C & 4.92164200 & -2.47269700 & -1.61235400 \\
\hline $\mathrm{H}$ & 5.75660500 & -2.30677300 & 0.36535400 & C & 3.78230800 & -2.64010500 & -3.81675600 \\
\hline $\mathrm{H}$ & 0.20834300 & -3.05161600 & -5.69913700 & $\mathrm{H}$ & 2.65100800 & -2.71580800 & -5.61921900 \\
\hline $\mathrm{H}$ & 5.84731600 & -2.19858900 & -2.11047600 & $\mathrm{H}$ & 4.72360000 & -2.42855400 & -4.31760900 \\
\hline $\mathrm{N}$ & -0.20256000 & -0.49349400 & 3.51969100 & $\mathrm{H}$ & -0.54699900 & 0.35035600 & 3.05186700 \\
\hline $\mathrm{S}$ & -1.25171000 & 2.70491500 & 1.26939700 & $\mathrm{O}$ & -0.44794000 & 3.45347000 & 0.28498900 \\
\hline $\mathrm{O}$ & -2.33594700 & 1.87009200 & 0.71191300 & 0 & -0.44220900 & 2.03114600 & 2.33363300 \\
\hline $\mathrm{C}$ & -2.13377300 & 3.99996800 & 2.27401200 & $\mathrm{~F}$ & -1.25719800 & 4.72710600 & 2.97145600 \\
\hline $\mathrm{F}$ & -2.98766500 & 3.41268100 & 3.12965700 & $\mathrm{~F}$ & -2.83927600 & 4.82682400 & 1.48477400 \\
\hline C & -3.29683600 & -1.25896500 & -1.81691900 & 0 & -3.76542800 & -1.24510800 & -2.94379000 \\
\hline $\mathrm{O}$ & -3.82003900 & -1.84339600 & -0.72986400 & $\mathrm{C}$ & -5.12560400 & -2.52233600 & -0.77226800 \\
\hline C & -6.21229200 & -1.52193300 & -1.16696900 & $\mathrm{H}$ & -7.19633100 & -1.98868600 & -1.05432000 \\
\hline
\end{tabular}




\begin{tabular}{|c|c|c|c|c|c|c|c|}
\hline $\mathrm{H}$ & -6.09362500 & -1.20158500 & -2.20319000 & $\mathrm{H}$ & -6.17240800 & -0.64502000 & -0.51234900 \\
\hline C & -5.29810200 & -2.97645500 & 0.67574200 & $\mathrm{H}$ & -4.48600700 & -3.65129200 & 0.96471100 \\
\hline $\mathrm{H}$ & -6.24876500 & -3.50566000 & 0.79316700 & $\mathrm{H}$ & -5.28741000 & -2.11679300 & 1.35205500 \\
\hline C & -5.05684700 & -3.71928300 & -1.72083700 & $\mathrm{H}$ & -6.00273800 & -4.26980800 & -1.68316100 \\
\hline $\mathrm{H}$ & -4.25680300 & -4.40244000 & -1.41561100 & $\mathrm{H}$ & -4.87870400 & -3.39729100 & -2.74780100 \\
\hline C & -1.32770200 & -0.05471100 & -2.42932600 & $\mathrm{H}$ & -1.61278800 & -0.35504600 & -3.43170200 \\
\hline C & 0.11834100 & 0.09162900 & -2.20033600 & C & 0.93751500 & 0.32185500 & -3.31799800 \\
\hline C & 0.69904200 & -0.00720600 & -0.92771700 & C & 2.31152000 & 0.45799800 & -3.17032400 \\
\hline $\mathrm{H}$ & 0.48728100 & 0.38095300 & -4.30564000 & C & 2.07932700 & 0.08306800 & -0.79837700 \\
\hline $\mathrm{H}$ & 0.09594000 & -0.18667100 & -0.04613800 & C & 2.88904500 & 0.33378200 & -1.90541800 \\
\hline $\mathrm{H}$ & 2.93514800 & 0.62956600 & -4.04136900 & $\mathrm{H}$ & 2.53165000 & -0.07095500 & 0.16920800 \\
\hline $\mathrm{H}$ & 3.96286200 & 0.40325300 & -1.78178400 & $\mathrm{~N}$ & -2.09923900 & -0.66521000 & -1.47237100 \\
\hline $\mathrm{H}$ & -1.80689700 & -0.74708500 & -0.49080300 & C & -1.82752400 & 1.89019100 & -2.59244400 \\
\hline $\mathrm{H}$ & -1.53739500 & 2.18177200 & -1.58856700 & $\mathrm{H}$ & -1.13876400 & 2.17863000 & -3.38066800 \\
\hline $\mathrm{C}$ & -3.18616300 & 1.80936900 & -2.90364400 & C & -4.17957500 & 1.75258900 & -1.84541300 \\
\hline $\mathrm{H}$ & -3.75506600 & 1.37719900 & -0.90968800 & $\mathrm{H}$ & -5.08343200 & 1.20833900 & -2.13273600 \\
\hline $\mathrm{C}$ & -3.63770400 & 1.71153500 & -4.32802100 & $\mathrm{H}$ & -4.51648500 & 2.34272200 & -4.50003500 \\
\hline $\mathrm{H}$ & -3.94233400 & 0.67627400 & -4.52455000 & $\mathrm{H}$ & -2.85127400 & 1.99117500 & -5.03365100 \\
\hline $\mathrm{C}$ & -5.45060700 & 3.37255400 & 0.39325500 & $\mathrm{H}$ & -6.33930600 & 2.72984100 & 0.38715200 \\
\hline $\mathrm{H}$ & -5.74382800 & 4.34653800 & 0.80131300 & $\mathrm{H}$ & -4.70985100 & 2.93073400 & 1.06380100 \\
\hline C & -6.14302000 & 4.03928500 & -2.54146900 & $\mathrm{H}$ & -6.96084800 & 3.30931100 & -2.53564400 \\
\hline $\mathrm{H}$ & -5.78331500 & 4.13694100 & -3.57243900 & $\mathrm{H}$ & -6.56509800 & 5.01003500 & -2.25364000 \\
\hline $\mathrm{C}$ & -3.33912700 & 4.76735800 & -1.51877800 & $\mathrm{H}$ & -3.09546800 & 4.91822800 & -2.57719800 \\
\hline $\mathrm{H}$ & -2.43037200 & 4.44407600 & -1.00572200 & $\mathrm{H}$ & -3.62555200 & 5.74030600 & -1.10284100 \\
\hline $\mathrm{Si}$ & -4.76066400 & 3.55246800 & -1.34707300 & C & -1.49218900 & -5.33834100 & 0.48520200 \\
\hline $\mathrm{H}$ & -1.15603100 & -5.89022600 & -0.39552600 & $\mathrm{H}$ & -2.57121000 & -5.50115200 & 0.60383500 \\
\hline C & -0.74669400 & -5.72762600 & 1.76969000 & $\mathrm{H}$ & -1.09815600 & -6.66670900 & 2.20483800 \\
\hline $\mathrm{H}$ & 0.32252000 & -5.82999500 & 1.56195100 & 0 & -1.40014300 & -1.22866800 & 1.19124800 \\
\hline C & 1.11478700 & -0.71472500 & 3.42718900 & C & 2.22121200 & 0.05591800 & 3.00501600 \\
\hline C & 1.96069700 & -1.90604500 & 3.66435300 & C & 3.17649200 & -1.05730400 & 3.28254600 \\
\hline 0 & 4.37587400 & -1.25030900 & 3.19028600 & $\mathrm{O}$ & 1.75061800 & -3.07969200 & 3.93667000 \\
\hline $\mathrm{H}$ & 1.34045900 & 1.76864600 & 2.43639000 & $\mathrm{~N}$ & 2.25699200 & 1.31121000 & 2.49494300 \\
\hline C & 3.22063900 & 1.84743200 & 1.62887500 & C & 2.83768800 & 2.95267100 & 0.85608100 \\
\hline C & 4.47713900 & 1.25661800 & 1.43413600 & C & 3.70155400 & 3.44407400 & -0.11737900 \\
\hline $\mathrm{H}$ & 1.84584800 & 3.38034600 & 0.96167400 & C & 5.31300100 & 1.76038300 & 0.44170800 \\
\hline $\mathrm{H}$ & 4.77914400 & 0.39310000 & 2.01715700 & C & 4.94565100 & 2.85710000 & -0.33872400 \\
\hline $\mathrm{H}$ & 5.60474500 & 3.23357800 & -1.11153500 & C & 6.58726000 & 1.03941200 & 0.10392200 \\
\hline C & 3.22312800 & 4.53170200 & -1.03938900 & $\mathrm{~F}$ & 6.44840100 & 0.34012100 & -1.06003000 \\
\hline $\mathrm{F}$ & 6.95683900 & 0.15318800 & 1.04727800 & $\mathrm{~F}$ & 7.61705900 & 1.89188500 & -0.08900100 \\
\hline $\mathrm{F}$ & 2.59342300 & 4.01021800 & -2.11912300 & $F$ & 4.25131400 & 5.27650700 & -1.51029700 \\
\hline $\mathrm{F}$ & 2.35632400 & 5.36787300 & -0.43749200 & & & & \\
\hline
\end{tabular}

\section{TS-S-6f-6}

Imaginary frequency: $-337.03 \mathrm{~cm}^{-1}$

E[B3LYP/6-31G(d)-D3(BJ)]: -4655.644310 Hartree

E[PCM(Et $\left.\left.{ }_{2} \mathrm{O}\right)-\mathrm{B} 3 \mathrm{LYP} / 6-311 \mathrm{G}(\mathrm{d}, \mathrm{p})-\mathrm{D} 3(\mathrm{BJ})\right]$ : -4656.835274 Hartree

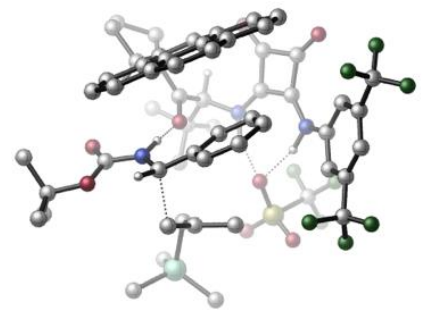

\begin{tabular}{|llll|llll|}
\hline 01 & & & & $\mathrm{C}$ & -1.27720700 & 1.18377700 & 3.49593000 \\
\hline $\mathrm{H}$ & -1.04813400 & 0.84163900 & 4.50569300 & $\mathrm{C}$ & -2.28888000 & 2.36695700 & 3.58579200 \\
\hline $\mathrm{C}$ & -2.68462900 & 2.91099600 & 2.20087900 & $\mathrm{H}$ & -3.16951700 & 2.14591500 & 1.58930300 \\
\hline $\mathrm{H}$ & -1.83063800 & 3.30548900 & 1.64559500 & $\mathrm{H}$ & -3.38616700 & 3.74215100 & 2.33250900 \\
\hline $\mathrm{C}$ & -1.63456500 & 3.49242500 & 4.40600700 & $\mathrm{H}$ & -2.35110900 & 4.30736900 & 4.55714200 \\
\hline
\end{tabular}




\begin{tabular}{|c|c|c|c|c|c|c|c|}
\hline $\mathrm{H}$ & -0.75992600 & 3.90174900 & 3.89496700 & $\mathrm{H}$ & -1.31707600 & 3.13234700 & 5.39205800 \\
\hline $\mathrm{C}$ & -3.55865500 & 1.88464700 & 4.30871000 & $\mathrm{H}$ & -4.28605500 & 2.70142600 & 4.36144300 \\
\hline $\mathrm{H}$ & -3.34407100 & 1.57165400 & 5.33730800 & $\mathrm{H}$ & -4.03763400 & 1.05122700 & 3.78187400 \\
\hline C & -1.79125800 & 0.00940400 & 2.64461100 & 0 & -1.74330800 & 0.12379700 & 1.40603900 \\
\hline C & -2.39917700 & -1.37597000 & 4.67619800 & C & -2.67774500 & -2.26092600 & 2.41822500 \\
\hline C & -3.60746400 & -2.31408100 & 4.68484500 & $\mathrm{H}$ & -1.49825400 & -1.84982900 & 5.08029100 \\
\hline $\bar{H}$ & -2.57514400 & -0.45369600 & 5.22443800 & $\bar{H}$ & -3.39969200 & -1.86471800 & 1.70185000 \\
\hline $\mathrm{H}$ & -4.52649200 & -1.72708600 & 4.57290300 & $\mathrm{H}$ & -3.68035100 & -2.89146200 & 5.61057300 \\
\hline $\mathrm{N}$ & -2.22217300 & -1.11470900 & 3.23895800 & $\mathrm{C}$ & -1.53576600 & -2.95449600 & 1.69265300 \\
\hline $\mathrm{C}$ & -0.26946600 & -2.90500100 & 2.19809900 & $\mathrm{C}$ & -1.80398600 & -3.71913000 & 0.49224300 \\
\hline C & 0.83790200 & -3.55122300 & 1.56132100 & $\mathrm{H}$ & -0.06746900 & -2.33770200 & 3.10095700 \\
\hline C & -0.71060700 & -4.36539200 & -0.16997200 & C & -3.08897500 & -3.84528900 & -0.06501400 \\
\hline $\mathrm{C}$ & 2.14694800 & -3.43251600 & 2.05473000 & $\mathrm{C}$ & 0.60734600 & -4.28196600 & 0.36203700 \\
\hline C & -0.93141100 & -5.08352700 & -1.38565600 & $\mathrm{H}$ & -3.94016200 & -3.38459100 & 0.42250700 \\
\hline C & -3.29198700 & -4.54460500 & -1.25310900 & C & 3.21831900 & -3.99000700 & 1.36024500 \\
\hline $\mathrm{H}$ & 2.32895100 & -2.88489700 & 2.97223000 & $\mathrm{C}$ & 1.70432900 & -4.89044500 & -0.31724600 \\
\hline $\mathrm{C}$ & -2.22821700 & -5.15173900 & -1.91437500 & $\mathrm{C}$ & 0.18611000 & -5.71074700 & -2.03451500 \\
\hline $\mathrm{H}$ & -4.29554800 & -4.62728500 & -1.65997800 & C & 3.00082100 & -4.71779200 & 0.19128800 \\
\hline $\mathrm{H}$ & 4.22877600 & -3.84002700 & 1.72664600 & $\mathrm{C}$ & 1.44421900 & -5.61927800 & -1.52503600 \\
\hline $\mathrm{H}$ & -2.39590900 & -5.69761100 & -2.83932100 & $\mathrm{H}$ & 0.00163000 & -6.26067800 & -2.95389500 \\
\hline $\mathrm{H}$ & 3.84282800 & -5.14331100 & -0.34739100 & $\mathrm{H}$ & 2.28149700 & -6.09009700 & -2.03382500 \\
\hline $\mathrm{N}$ & -0.00880400 & 1.61434100 & 2.90427300 & $\mathrm{H}$ & -0.06369400 & 2.14601600 & 2.03174700 \\
\hline $\mathrm{S}$ & 0.65153700 & 4.33936500 & 0.02693600 & 0 & -0.61635300 & 5.06992600 & 0.18012800 \\
\hline 0 & 0.54495200 & 2.88676300 & 0.42865100 & 0 & 1.39470400 & 4.52419800 & -1.23225100 \\
\hline $\mathrm{C}$ & 1.76143100 & 5.02632000 & 1.35118100 & $\mathrm{~F}$ & 1.90516100 & 6.34438300 & 1.21431500 \\
\hline $\mathrm{F}$ & 2.96955100 & 4.44436300 & 1.28024600 & $\mathrm{~F}$ & 1.24363900 & 4.76830800 & 2.56513800 \\
\hline C & -4.08579400 & -0.86175700 & -0.98073900 & 0 & -4.71715700 & -0.70330900 & 0.04906700 \\
\hline 0 & -4.58740200 & -1.09715700 & -2.20294300 & C & -6.04729900 & -1.20195400 & -2.41963900 \\
\hline $\mathrm{C}$ & -6.61444900 & -2.36005100 & -1.59962700 & $\mathrm{H}$ & -7.66900400 & -2.50160100 & -1.85800700 \\
\hline $\mathrm{H}$ & -6.53849100 & -2.16138400 & -0.53024600 & $\mathrm{H}$ & -6.08069900 & -3.28664400 & -1.83151500 \\
\hline $\mathrm{C}$ & -6.13257700 & -1.49550300 & -3.91545200 & $\mathrm{H}$ & -5.68581700 & -0.68197400 & -4.49578300 \\
\hline $\mathrm{H}$ & -7.17970800 & -1.59929300 & -4.21552200 & $\bar{H}$ & -5.60818800 & -2.42549500 & -4.15645100 \\
\hline C & -6.70891800 & 0.13282100 & -2.08003000 & $\mathrm{H}$ & -7.77392200 & 0.08411000 & -2.32964300 \\
\hline $\mathrm{H}$ & -6.26050300 & 0.93992400 & -2.66826800 & $\mathrm{H}$ & -6.60725500 & 0.36360600 & -1.01883000 \\
\hline $\mathrm{C}$ & -1.92593900 & -1.05466300 & -2.11172800 & $\mathrm{H}$ & -2.44427300 & -1.63672400 & -2.86554200 \\
\hline C & -0.50191300 & -1.37609600 & -1.95246600 & C & 0.13488200 & -2.05088400 & -3.00704500 \\
\hline $\mathrm{C}$ & 0.23895400 & -1.02516700 & -0.81323600 & $\mathrm{C}$ & 1.48446500 & -2.36924300 & -2.92841600 \\
\hline $\mathrm{H}$ & -0.44122100 & -2.33478500 & -3.88424000 & $\mathrm{C}$ & 1.58249400 & -1.37518000 & -0.73247700 \\
\hline $\mathrm{H}$ & -0.22444300 & -0.50403300 & 0.01611100 & C & 2.21387000 & -2.03369600 & -1.78576700 \\
\hline $\mathrm{H}$ & 1.96310400 & -2.89980300 & -3.74527300 & $\mathrm{H}$ & 2.13338300 & -1.15739500 & 0.17029100 \\
\hline $\mathrm{H}$ & 3.25841300 & -2.30569000 & -1.70208300 & $\mathrm{~N}$ & -2.70188500 & -0.80935800 & -1.00680400 \\
\hline $\mathrm{H}$ & -2.28829700 & -0.55573600 & -0.09593200 & C & -2.02094000 & 0.56389500 & -3.34266400 \\
\hline $\mathrm{H}$ & -1.50804200 & 0.17298600 & -4.21715900 & $\mathrm{H}$ & -3.10545600 & 0.57281100 & -3.39789000 \\
\hline $\mathrm{C}$ & -1.37781600 & 1.53672000 & -2.57116700 & $C$ & -2.10828900 & 2.26914200 & -1.55175300 \\
\hline $\mathrm{H}$ & -1.47351400 & 2.57525400 & -0.71767900 & $\mathrm{H}$ & -2.98051400 & 1.71430400 & -1.18685600 \\
\hline $\mathrm{C}$ & 0.08943200 & 1.74770400 & -2.69774800 & $\mathrm{H}$ & 0.35526500 & 2.80752300 & -2.63755900 \\
\hline $\mathrm{H}$ & 0.51301400 & 1.29905600 & -3.59704900 & $\mathrm{H}$ & 0.57094100 & 1.28484200 & -1.82390400 \\
\hline C & -3.70167400 & 4.77490700 & -0.83391500 & $\mathrm{H}$ & -4.40657000 & 4.11899000 & -0.31018600 \\
\hline $\mathrm{H}$ & -4.25368800 & 5.65796100 & -1.17814800 & $\mathrm{H}$ & -2.93163000 & 5.09832600 & -0.12930900 \\
\hline $\mathrm{C}$ & -4.22640000 & 3.33889400 & -3.51683700 & $\mathrm{H}$ & -4.97372100 & 2.69826400 & -3.03408600 \\
\hline $\mathrm{H}$ & -3.80197000 & 2.79007600 & -4.36465000 & $\mathrm{H}$ & -4.75464100 & 4.21081400 & -3.92087100 \\
\hline $\mathrm{C}$ & -1.60558900 & 4.92926300 & -3.16566700 & $\mathrm{H}$ & -1.22207500 & 4.42085200 & -4.05798300 \\
\hline $\mathrm{H}$ & -0.76142500 & 5.16115000 & -2.51099500 & $\mathrm{H}$ & -2.04879600 & 5.87809700 & -3.49210400 \\
\hline
\end{tabular}




\begin{tabular}{|c|c|c|c|c|c|c|c|}
\hline $\mathrm{Si}$ & -2.90233200 & 3.89607200 & -2.28736300 & C & -3.37344700 & -3.19336600 & 3.44770700 \\
\hline $\mathrm{H}$ & -2.70230900 & -4.02143300 & 3.69446700 & $\mathrm{H}$ & -4.29443100 & -3.62084600 & 3.04306100 \\
\hline C & 1.08894400 & 0.86959700 & 3.07747600 & C & 2.31568000 & 0.74976300 & 2.39182700 \\
\hline $\mathrm{C}$ & 1.44447000 & -0.21723400 & 4.02185100 & C & 2.80694800 & -0.30718000 & 3.31838100 \\
\hline 0 & 3.80606400 & -0.99553000 & 3.43236000 & $\mathrm{O}$ & 0.85239100 & -0.82904400 & 4.89730700 \\
\hline $\mathrm{H}$ & 2.04912500 & 2.06026700 & 0.90212500 & $\mathrm{~N}$ & 2.72910900 & 1.38602800 & 1.27016700 \\
\hline C & 3.64746200 & 0.93828700 & 0.31805600 & C & 3.59398400 & 1.54604000 & -0.94467100 \\
\hline C & 4.52088700 & -0.13606200 & 0.54381700 & C & 4.36263500 & 1.03655500 & -1.98631700 \\
\hline $\mathrm{H}$ & 2.93140100 & 2.38886500 & -1.11857800 & C & 5.28439500 & -0.61733100 & -0.51552800 \\
\hline $\mathrm{H}$ & 4.57451700 & -0.60654700 & 1.52042000 & C & 5.21422700 & -0.04862000 & -1.78894700 \\
\hline $\mathrm{H}$ & 5.80191800 & -0.44626000 & -2.60724400 & C & 6.11803400 & -1.85452100 & -0.33020600 \\
\hline $\mathrm{C}$ & 4.15397900 & 1.58396900 & -3.37049300 & $\mathrm{~F}$ & 5.52046900 & -2.93241400 & -0.91776500 \\
\hline $\mathrm{F}$ & 6.30376500 & -2.17276400 & 0.96425600 & $\mathrm{~F}$ & 7.33287300 & -1.73653200 & -0.90676200 \\
\hline $\mathrm{F}$ & 3.10565800 & 0.96209700 & -3.97785700 & $\mathrm{~F}$ & 5.22997400 & 1.39043300 & -4.16295700 \\
\hline $\mathrm{F}$ & 3.87438900 & 2.89999400 & -3.36476600 & & & & \\
\hline
\end{tabular}

\section{TS-S-6f-7}

Imaginary frequency: $-340.86 \mathrm{~cm}^{-1}$

E[B3LYP/6-31G(d)-D3(BJ)]: -4655.647423 Hartree

$\mathrm{E}\left[\mathrm{PCM}\left(\mathrm{Et}_{2} \mathrm{O}\right)-\mathrm{B} 3 \mathrm{LYP} / 6-311 \mathrm{G}(\mathrm{d}, \mathrm{p})-\mathrm{D} 3(\mathrm{BJ})\right]$ : -4656.835256 Hartree

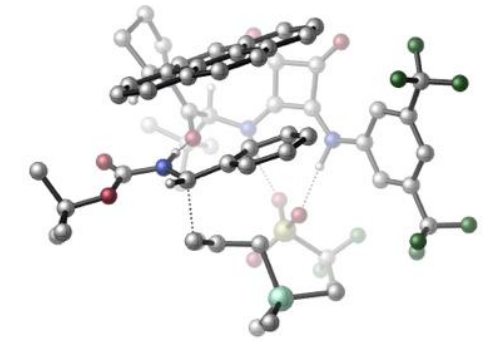

\begin{tabular}{|c|c|c|c|c|c|c|c|}
\hline 01 & & & & $\mathrm{C}$ & 1.31410900 & -1.21417800 & 3.72248700 \\
\hline $\mathrm{H}$ & 1.43801200 & -0.64129900 & 4.64225500 & $\mathrm{C}$ & 1.63919800 & -2.70503400 & 4.04595700 \\
\hline C & 1.58598700 & -3.61437700 & 2.80435100 & $\mathrm{H}$ & 2.32998900 & -3.32257200 & 2.05882300 \\
\hline $\mathrm{H}$ & 0.60290800 & -3.62112400 & 2.32953200 & $\mathrm{H}$ & 1.79599500 & -4.64480900 & 3.11258800 \\
\hline $\mathrm{C}$ & 0.62661700 & -3.20159600 & 5.09413500 & $\mathrm{H}$ & 0.90791600 & -4.20633500 & 5.42879700 \\
\hline $\mathrm{H}$ & -0.38461300 & -3.25683900 & 4.68430000 & $\mathrm{H}$ & 0.61155700 & -2.54473700 & 5.97227500 \\
\hline $\mathrm{C}$ & 3.05627000 & -2.76823500 & 4.64268300 & $\mathrm{H}$ & 3.30602600 & -3.80489600 & 4.89132500 \\
\hline $\mathrm{H}$ & 3.13086900 & -2.17839000 & 5.56357900 & $\mathrm{H}$ & 3.81191500 & -2.40792000 & 3.93529500 \\
\hline C & 2.22122700 & -0.63287100 & 2.62332800 & C & 4.07298800 & 0.79542100 & 1.90215600 \\
\hline $\mathrm{H}$ & 4.39944200 & -0.01300000 & 1.24756500 & $\mathrm{~N}$ & 3.22003600 & 0.20164800 & 2.95582200 \\
\hline $\mathrm{C}$ & 3.34215400 & 1.85868100 & 1.09334400 & C & 2.24070500 & 2.49052700 & 1.59168200 \\
\hline C & 3.83712700 & 2.20845500 & -0.22371600 & C & 1.50441100 & 3.46263200 & 0.84010900 \\
\hline $\mathrm{H}$ & 1.87178000 & 2.23866200 & 2.57944000 & C & 3.09775700 & 3.14967800 & -1.00923200 \\
\hline $\mathrm{C}$ & 5.00044600 & 1.64578900 & -0.77795800 & $\mathrm{C}$ & 0.34897000 & 4.07322700 & 1.35198300 \\
\hline C & 1.92993900 & 3.77144100 & -0.48182200 & C & 3.51803200 & 3.46096100 & -2.33925400 \\
\hline $\mathrm{H}$ & 5.59241200 & 0.94395800 & -0.20219900 & C & 5.40277900 & 1.95812200 & -2.07538600 \\
\hline $\mathrm{C}$ & -0.40102900 & 4.94288200 & 0.56299700 & $\mathrm{H}$ & 0.03126600 & 3.86125300 & 2.36637600 \\
\hline C & 1.17390500 & 4.68655100 & -1.27262600 & C & 4.66806000 & 2.84584500 & -2.85571500 \\
\hline C & 2.74523500 & 4.39440300 & -3.11021100 & $\mathrm{H}$ & 6.30679200 & 1.50983400 & -2.47686200 \\
\hline $\mathrm{C}$ & 0.00433400 & 5.24646700 & -0.73534000 & $\mathrm{H}$ & -1.31349800 & 5.37396400 & 0.96121400 \\
\hline C & 1.62636500 & 4.97915700 & -2.60193600 & $\mathrm{H}$ & 4.98869800 & 3.08424700 & -3.86668000 \\
\hline $\mathrm{H}$ & 3.07868900 & 4.62552600 & -4.11881600 & $\mathrm{H}$ & -0.59279800 & 5.91717500 & -1.34717700 \\
\hline $\mathrm{H}$ & 1.05027200 & 5.68056400 & -3.20014000 & $\mathrm{~N}$ & -0.07369300 & -1.03926400 & 3.29065600 \\
\hline $\mathrm{H}$ & -0.55701800 & -1.81409300 & 2.83436300 & S & -2.18238700 & -3.40247200 & 1.22833100 \\
\hline $\mathrm{O}$ & -1.40499100 & -4.49013300 & 0.61030800 & $\mathrm{O}$ & -1.90421600 & -3.12452700 & 2.65836100 \\
\hline 0 & -2.25779200 & -2.14496600 & 0.41892700 & C & -3.93709100 & -4.00814300 & 1.20439600 \\
\hline $\mathrm{F}$ & -4.28104600 & -4.35040800 & -0.04851900 & $\mathrm{~F}$ & -4.76422500 & -3.03366700 & 1.61764900 \\
\hline $\mathrm{F}$ & -4.08860400 & -5.06913500 & 1.99750200 & C & 4.24223100 & -1.47188700 & -1.16482500 \\
\hline 0 & 4.83340900 & -1.81143400 & -0.15518700 & 0 & 4.64732100 & -1.65506400 & -2.43151000 \\
\hline
\end{tabular}




\begin{tabular}{|c|c|c|c|c|c|c|c|}
\hline C & 5.90263600 & -2.37612100 & -2.73018300 & C & 7.09272000 & -1.63775100 & -2.11831800 \\
\hline $\mathrm{H}$ & 8.02111800 & -2.11629100 & -2.44718500 & $\mathrm{H}$ & 7.05229600 & -1.65780700 & -1.02917000 \\
\hline $\mathrm{H}$ & 7.10772300 & -0.59753200 & -2.45725800 & C & 5.95271100 & -2.32249300 & -4.25538800 \\
\hline $\mathrm{H}$ & 5.08017700 & -2.82021000 & -4.69057300 & $\mathrm{H}$ & 6.85392300 & -2.82697800 & -4.61683100 \\
\hline $\mathrm{H}$ & 5.97160900 & -1.28491400 & -4.60323000 & C & 5.78661500 & -3.81558400 & -2.22956700 \\
\hline $\mathrm{H}$ & 6.66779200 & -4.38363900 & -2.54537700 & $\mathrm{H}$ & 4.90089500 & -4.29787200 & -2.65737300 \\
\hline $\mathrm{H}$ & 5.71948000 & -3.84858500 & -1.14129200 & C & 2.31973500 & -0.38705000 & -2.21549200 \\
\hline $\mathrm{H}$ & 2.95984800 & -0.17617800 & -3.06547600 & C & 1.18910500 & 0.53526500 & -2.03943300 \\
\hline C & 0.80210900 & 1.31435000 & -3.14348600 & C & 0.44929900 & 0.61965900 & -0.84994500 \\
\hline $\mathrm{C}$ & -0.29791900 & 2.15943300 & -3.06136000 & $\mathrm{H}$ & 1.38002100 & 1.26168900 & -4.06260500 \\
\hline C & -0.63725500 & 1.48460600 & -0.77124200 & $\mathrm{H}$ & 0.71579800 & 0.03009500 & 0.01870900 \\
\hline $\mathrm{C}$ & -1.02327500 & 2.24884300 & -1.87013500 & $\mathrm{H}$ & -0.57528100 & 2.76748500 & -3.91659200 \\
\hline $\mathrm{H}$ & -1.17790100 & 1.56932600 & 0.15970600 & $\mathrm{H}$ & -1.86621900 & 2.92528800 & -1.78983000 \\
\hline $\mathrm{N}$ & 3.02011000 & -0.82443600 & -1.11762300 & $\mathrm{H}$ & 2.64069600 & -0.73833900 & -0.16156500 \\
\hline $\mathrm{C}$ & 1.56615200 & -1.99378600 & -3.21216400 & $\mathrm{H}$ & 1.20873600 & -1.48867300 & -4.10596000 \\
\hline $\mathrm{H}$ & 2.50073800 & -2.53525000 & -3.32019400 & C & 0.62095200 & -2.44044100 & -2.28254300 \\
\hline C & -0.75025700 & -1.98166200 & -2.32988000 & $\mathrm{H}$ & -0.85902400 & -1.04287900 & -2.88360900 \\
\hline $\mathrm{H}$ & -1.21468300 & -1.92040600 & -1.34041600 & C & 1.05885700 & -3.27487400 & -1.13022900 \\
\hline $\mathrm{H}$ & 0.26942100 & -3.94389800 & -0.77874100 & $\mathrm{H}$ & 1.25873300 & -2.59820200 & -0.28275200 \\
\hline $\mathrm{H}$ & 1.97752300 & -3.83046400 & -1.33542900 & $\mathrm{C}$ & -3.64182500 & -2.60568800 & -2.98196900 \\
\hline $\mathrm{H}$ & -3.89537700 & -2.72625900 & -1.92571100 & $\mathrm{H}$ & -4.37135700 & -3.17582500 & -3.56966900 \\
\hline $\mathrm{H}$ & -3.76195700 & -1.54913600 & -3.23968200 & C & -1.65163300 & -4.98208300 & -2.72031500 \\
\hline $\mathrm{H}$ & -1.84021800 & -5.07314000 & -1.64635300 & $\mathrm{H}$ & -0.63518700 & -5.34091600 & -2.91983000 \\
\hline $\mathrm{H}$ & -2.34601500 & -5.64768500 & -3.24785600 & C & -1.43453900 & -3.05168500 & -5.13209700 \\
\hline $\mathrm{H}$ & -0.39682700 & -3.35526800 & -5.31311700 & $\mathrm{H}$ & -1.55222400 & -2.02003900 & -5.48428100 \\
\hline $\mathrm{H}$ & -2.07695900 & -3.68774800 & -5.75261100 & $\mathrm{Si}$ & -1.89889900 & -3.21697500 & -3.30936900 \\
\hline C & 3.60319600 & 0.65529500 & 4.31223700 & $\mathrm{H}$ & 4.08730300 & -0.16471100 & 4.85244800 \\
\hline $\mathrm{H}$ & 2.72357300 & 0.99208000 & 4.86119100 & C & 4.59468800 & 1.79018500 & 4.03434000 \\
\hline $\mathrm{H}$ & 5.30916100 & 1.91808400 & 4.85166500 & $\mathrm{H}$ & 4.05397800 & 2.73280700 & 3.90423300 \\
\hline C & 5.25233600 & 1.37222900 & 2.71356300 & $\mathrm{H}$ & 5.73895500 & 2.19862000 & 2.19061500 \\
\hline $\mathrm{H}$ & 5.99728800 & 0.58653600 & 2.88722700 & $\mathrm{O}$ & 1.97905100 & -0.93267400 & 1.43792700 \\
\hline C & -0.65574600 & 0.15827100 & 3.32845900 & C & -1.79838100 & 0.75047000 & 2.73748000 \\
\hline C & -0.28039600 & 1.43017900 & 3.99388700 & C & -1.53904000 & 2.05677500 & 3.40755500 \\
\hline 0 & -2.05073000 & 3.16344600 & 3.44177900 & $\mathrm{O}$ & 0.66754600 & 1.81861900 & 4.66142000 \\
\hline $\mathrm{H}$ & -2.54886800 & -0.71837000 & 1.54898000 & $\mathrm{~N}$ & -2.69926000 & 0.24786500 & 1.86071700 \\
\hline C & -3.56442700 & 0.96540000 & 1.02274000 & C & -4.39687300 & 0.21981300 & 0.17123100 \\
\hline $\mathrm{C}$ & -3.57309000 & 2.36442600 & 0.94678900 & C & -5.19597200 & 0.87210700 & -0.75908600 \\
\hline $\mathrm{H}$ & -4.40649700 & -0.85968900 & 0.24193000 & $\mathrm{C}$ & -4.37583900 & 2.99212200 & -0.00462400 \\
\hline $\mathrm{H}$ & -2.97805000 & 2.95978400 & 1.62889900 & C & -5.18940800 & 2.26421600 & -0.86888400 \\
\hline $\mathrm{H}$ & -5.81057200 & 2.76530500 & -1.60149800 & $\mathrm{C}$ & -4.26970500 & 4.48209600 & -0.17368500 \\
\hline C & -6.05867300 & 0.08487200 & -1.70525300 & $\mathrm{~F}$ & -3.31406600 & 4.80146400 & -1.09483500 \\
\hline $\mathrm{F}$ & -3.92511900 & 5.11044900 & 0.96762500 & $\mathrm{~F}$ & -5.42146200 & 5.02871100 & -0.61468600 \\
\hline $\mathrm{F}$ & -5.58119100 & 0.15576000 & -2.97551300 & $\mathrm{~F}$ & -7.32037400 & 0.56657500 & -1.74625600 \\
\hline $\mathrm{F}$ & -6.13058500 & -1.22095900 & -1.37877600 & & & & \\
\hline
\end{tabular}

\section{TS-S-6f-8}

Imaginary frequency: $-320.92 \mathrm{~cm}^{-1}$

E[B3LYP/6-31G(d)-D3(BJ)]: -4655.647519 Hartree

E[PCM(Et $\left.\left.{ }_{2} \mathrm{O}\right)-B 3 L Y P / 6-311 G(d, p)-D 3(B J)\right]: ~-4656.833697$ Hartree

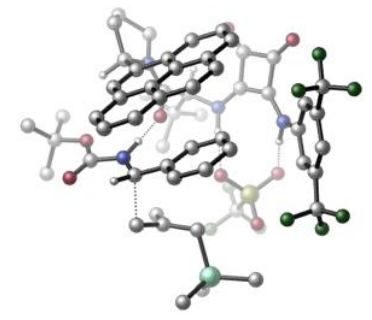




\begin{tabular}{|c|c|c|c|c|c|c|c|}
\hline 01 & & & & C & 2.38810200 & 0.21580900 & -3.05531900 \\
\hline $\mathrm{H}$ & 2.56409200 & -0.44096200 & -3.90710900 & C & 3.21724300 & 1.52059300 & -3.26941700 \\
\hline C & 3.12471400 & 2.48724600 & -2.07447800 & $\mathrm{H}$ & 3.57178200 & 2.05632700 & -1.17418600 \\
\hline $\mathrm{H}$ & 2.09422700 & 2.75586800 & -1.83972900 & $\mathrm{H}$ & 3.66257200 & 3.41028500 & -2.32011000 \\
\hline C & 2.69142200 & 2.20925000 & -4.54056000 & $\mathrm{H}$ & 3.30270900 & 3.08982700 & -4.76762900 \\
\hline $\mathrm{H}$ & 1.65695100 & 2.53596200 & -4.42062600 & $\mathrm{H}$ & 2.73750000 & 1.53272600 & -5.40237000 \\
\hline C & 4.69437600 & 1.14399500 & -3.48062300 & $\mathrm{H}$ & 5.29493300 & 2.05272100 & -3.59631400 \\
\hline $\mathrm{H}$ & 4.83281800 & 0.54328500 & -4.38708400 & $\mathrm{H}$ & 5.10028600 & 0.58786000 & -2.62787600 \\
\hline C & 2.73634500 & -0.51412100 & -1.74417100 & $\mathrm{O}$ & 2.30341900 & -0.05138400 & -0.67658200 \\
\hline $\mathrm{C}$ & 4.07632600 & -2.24520800 & -3.00206000 & $\mathrm{C}$ & 3.79490600 & -2.41344400 & -0.56603300 \\
\hline C & 5.15622200 & -3.16232400 & -2.42415100 & $\mathrm{H}$ & 3.32661200 & -2.79524800 & -3.57834500 \\
\hline $\mathrm{H}$ & 4.49291300 & -1.47118500 & -3.64341300 & $\mathrm{H}$ & 4.49610200 & -1.79557000 & 0.00507100 \\
\hline $\mathrm{H}$ & 6.06630700 & -2.58589100 & -2.21705200 & $\mathrm{H}$ & 5.41661500 & -3.97173400 & -3.11112800 \\
\hline $\mathrm{N}$ & 3.45586200 & -1.65052600 & -1.79656200 & C & 2.66345100 & -2.76982200 & 0.37896400 \\
\hline $\mathrm{C}$ & 2.91185000 & -2.60520800 & 1.71067800 & $\mathrm{C}$ & 1.41508700 & -3.37312500 & -0.04744600 \\
\hline $\mathrm{C}$ & 1.97941200 & -2.97183100 & 2.73062900 & $\mathrm{H}$ & 3.85414900 & -2.16427700 & 2.02524100 \\
\hline$C$ & 0.46766000 & -3.76209700 & 0.95459700 & $\mathrm{C}$ & 1.08486400 & -3.60091600 & -1.39316800 \\
\hline C & 2.23198300 & -2.73642100 & 4.09119100 & C & 0.74721200 & -3.56609400 & 2.33761100 \\
\hline C & -0.78367000 & -4.33556300 & 0.57189800 & $\mathrm{H}$ & 1.77190800 & -3.32459500 & -2.17827600 \\
\hline $\mathrm{C}$ & -0.13677800 & -4.16593100 & -1.75655600 & $\mathrm{C}$ & 1.29208600 & -3.09657100 & 5.05576800 \\
\hline $\mathrm{H}$ & 3.16725200 & -2.26449900 & 4.38261500 & C & -0.20466300 & -3.94075200 & 3.33229400 \\
\hline C & -1.07232300 & -4.51449300 & -0.78887300 & $\mathrm{C}$ & -1.72166600 & -4.70921400 & 1.59241400 \\
\hline $\mathrm{H}$ & -0.36472500 & -4.31652000 & -2.80536600 & $\mathrm{C}$ & 0.09186300 & -3.69846200 & 4.68296400 \\
\hline $\mathrm{H}$ & 1.49949400 & -2.91173200 & 6.10609900 & C & -1.44408300 & -4.52669600 & 2.91171100 \\
\hline $\mathrm{H}$ & -2.03541500 & -4.92148600 & -1.08126300 & $\mathrm{H}$ & -2.67363800 & -5.12616600 & 1.28062300 \\
\hline $\mathrm{H}$ & -0.63527800 & -3.98002600 & 5.44016600 & $\mathrm{H}$ & -2.16767600 & -4.80811500 & 3.67231000 \\
\hline $\mathrm{N}$ & 0.94551900 & 0.47669500 & -3.03693900 & $\mathrm{H}$ & 0.60202900 & 1.17119600 & -2.36328900 \\
\hline $\mathrm{S}$ & -1.17907600 & 3.36423900 & -1.70672100 & 0 & -2.07603300 & 3.91694200 & -0.67621100 \\
\hline $\mathrm{O}$ & -0.14496700 & 2.42269800 & -1.18295600 & 0 & -1.83710200 & 2.87003300 & -2.94091800 \\
\hline C & -0.20194400 & 4.83438400 & -2.28650300 & $\mathrm{~F}$ & -1.00825400 & 5.77411400 & -2.77917800 \\
\hline $\mathrm{F}$ & 0.67924600 & 4.47766200 & -3.23370300 & $\mathrm{~F}$ & 0.49078900 & 5.36250600 & -1.25548100 \\
\hline $\mathrm{C}$ & 3.71317500 & 0.62078900 & 2.50840200 & $\mathrm{O}$ & 3.99825800 & 0.86745200 & 3.66800200 \\
\hline $\mathrm{O}$ & 4.54706400 & 0.54608700 & 1.46025800 & C & 5.98632500 & 0.82859700 & 1.61103600 \\
\hline $\mathrm{C}$ & 6.17978200 & 2.26115900 & 2.10922700 & $\mathrm{H}$ & 7.24572200 & 2.51104800 & 2.09381200 \\
\hline $\mathrm{H}$ & 5.80652000 & 2.37916100 & 3.12722600 & $\mathrm{H}$ & 5.65575900 & 2.96257000 & 1.45120000 \\
\hline C & 6.50233500 & 0.67693500 & 0.18121100 & $\mathrm{H}$ & 6.31678800 & -0.33491100 & -0.19343900 \\
\hline $\mathrm{H}$ & 7.58011200 & 0.86423500 & 0.15180500 & $\mathrm{H}$ & 6.00565800 & 1.38832600 & -0.48479000 \\
\hline $\mathrm{C}$ & 6.61682700 & -0.20925100 & 2.53945300 & $\mathrm{H}$ & 7.70154200 & -0.06238100 & 2.56955600 \\
\hline $\mathrm{H}$ & 6.42180700 & -1.22094400 & 2.16692600 & $\mathrm{H}$ & 6.22259300 & -0.12061700 & 3.55276300 \\
\hline $\mathrm{C}$ & 1.37823600 & 0.35564700 & 2.96155900 & $\mathrm{H}$ & 1.69624100 & 0.12980300 & 3.97420600 \\
\hline $\mathrm{C}$ & 0.08998900 & -0.20989700 & 2.55233700 & $\mathrm{C}$ & -0.82476000 & -0.56180700 & 3.55971000 \\
\hline C & -0.26336800 & -0.39737500 & 1.20695000 & C & -2.05542500 & -1.11598800 & 3.23051800 \\
\hline $\mathrm{H}$ & -0.55126600 & -0.42351600 & 4.60188100 & $C$ & -1.48182300 & -0.98756300 & 0.88913500 \\
\hline $\mathrm{H}$ & 0.40654300 & -0.11634600 & 0.40387900 & $C$ & -2.38109500 & -1.34750900 & 1.89232400 \\
\hline $\mathrm{H}$ & -2.74701300 & -1.40111800 & 4.01716400 & $\mathrm{H}$ & -1.72108500 & -1.18437800 & -0.14702200 \\
\hline $\mathrm{H}$ & -3.31659900 & -1.82753900 & 1.63089600 & $\mathrm{~N}$ & 2.42486300 & 0.36946300 & 2.07395300 \\
\hline $\mathrm{H}$ & 2.31082200 & 0.14959700 & 1.07668000 & $C$ & 1.01242300 & 2.26245400 & 3.59915400 \\
\hline $\mathrm{H}$ & 0.31890200 & 2.03237500 & 4.40334400 & $\mathrm{H}$ & 2.02971000 & 2.49018700 & 3.90462500 \\
\hline$C$ & 0.50329700 & 2.80752100 & 2.42022100 & $C$ & -0.91924000 & 2.79859100 & 2.13928600 \\
\hline $\mathrm{H}$ & -1.45142800 & 2.02843200 & 2.70848600 & $\mathrm{H}$ & -1.14333700 & 2.72813700 & 1.07321000 \\
\hline C & 1.45328000 & 3.27858500 & 1.37304300 & $\mathrm{H}$ & 1.03782100 & 4.09115600 & 0.77619800 \\
\hline $\mathrm{H}$ & 1.61005700 & 2.45119300 & 0.66484200 & $\mathrm{H}$ & 2.42183000 & 3.56683900 & 1.78977000 \\
\hline C & -3.56053000 & 4.34315900 & 2.20857700 & $\mathrm{H}$ & -3.64372400 & 4.27186800 & 1.12127400 \\
\hline
\end{tabular}




\begin{tabular}{|c|c|c|c|c|c|c|c|}
\hline $\mathrm{H}$ & -4.11014800 & 5.23094100 & 2.54482500 & $\mathrm{H}$ & -4.04284900 & 3.46084900 & 2.63949300 \\
\hline C & -0.89065000 & 5.93045300 & 1.91741800 & $\mathrm{H}$ & -0.87972500 & 5.83139200 & 0.82879400 \\
\hline $\mathrm{H}$ & 0.14223700 & 6.03742600 & 2.26841900 & $\mathrm{H}$ & -1.42087900 & 6.85790200 & 2.16553200 \\
\hline $\mathrm{C}$ & -1.58521500 & 4.54354000 & 4.59126300 & $\mathrm{H}$ & -0.53615100 & 4.59110500 & 4.90474100 \\
\hline $\mathrm{H}$ & -2.04311500 & 3.67130000 & 5.07317300 & $\mathrm{H}$ & -2.08812000 & 5.43579000 & 4.98314200 \\
\hline $\mathrm{Si}$ & -1.75870000 & 4.46334300 & 2.71133000 & C & 4.52784700 & -3.65379200 & -1.11809300 \\
\hline $\mathrm{H}$ & 3.80375300 & -4.45016400 & -1.31611000 & $\mathrm{H}$ & 5.25200400 & -4.03355700 & -0.39302400 \\
\hline C & 0.09827600 & -0.47785900 & -3.43401400 & C & -1.28715100 & -0.69431700 & -3.29826500 \\
\hline $\mathrm{C}$ & 0.28959300 & -1.79237400 & -4.10793200 & $\mathrm{C}$ & -1.21721500 & -2.03472500 & -3.93462900 \\
\hline 0 & -1.98156500 & -2.95902300 & -4.14511600 & $\mathrm{O}$ & 1.24436400 & -2.40635400 & -4.55419600 \\
\hline $\mathrm{H}$ & -2.05091600 & 1.10124300 & -2.73612000 & $\mathrm{~N}$ & -2.24850700 & 0.09405900 & -2.75133300 \\
\hline $\mathrm{C}$ & -3.33150100 & -0.32297800 & -1.97995000 & $\mathrm{C}$ & -3.97653900 & 0.64206400 & -1.18964100 \\
\hline $\mathrm{C}$ & -3.71591100 & -1.67119800 & -1.88628200 & $\mathrm{C}$ & -4.96771300 & 0.25031400 & -0.29598600 \\
\hline $\mathrm{H}$ & -3.67034500 & 1.68140200 & -1.24072700 & C & -4.71168800 & -2.03038900 & -0.98341000 \\
\hline $\mathrm{H}$ & -3.24425800 & -2.42220300 & -2.50961400 & $\mathrm{C}$ & -5.35141500 & -1.08446400 & -0.18142500 \\
\hline $\mathrm{H}$ & -6.11717600 & -1.38137700 & 0.52520700 & $\mathrm{C}$ & -5.06155200 & -3.47688900 & -0.77915700 \\
\hline $\mathrm{C}$ & -5.53114900 & 1.25447900 & 0.66906900 & $\mathrm{~F}$ & -4.67471000 & -3.89017500 & 0.46341100 \\
\hline $\mathrm{F}$ & -4.46407000 & -4.29630300 & -1.66611900 & $\mathrm{~F}$ & -6.39140400 & -3.69280600 & -0.85078700 \\
\hline $\mathrm{F}$ & -4.82431600 & 1.24458900 & 1.83285500 & $\mathrm{~F}$ & -6.81287900 & 0.98026200 & 0.99639800 \\
\hline $\mathrm{F}$ & -5.48907800 & 2.51229200 & 0.19136300 & & & & \\
\hline
\end{tabular}

\section{TS-S-6f-9}

Imaginary frequency: $-357.30 \mathrm{~cm}^{-1}$

E[B3LYP/6-31G(d)-D3(BJ)]: -4655.621711 Hartree

$\mathrm{E}\left[\mathrm{PCM}\left(\mathrm{Et}_{2} \mathrm{O}\right)-\mathrm{B} 3 \mathrm{LYP} / 6-311 \mathrm{G}(\mathrm{d}, \mathrm{p})-\mathrm{D} 3(\mathrm{BJ})\right]$ : -4656.821113 Hartree

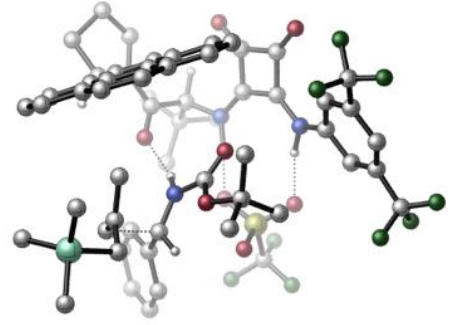

\begin{tabular}{|c|c|c|c|c|c|c|c|}
\hline 01 & & & & C & -0.35836900 & 2.95825500 & 3.06207400 \\
\hline $\mathrm{H}$ & -0.21265400 & 2.70860400 & 4.11177200 & C & -0.40886700 & 4.51407500 & 2.97179400 \\
\hline C & -0.78796600 & 5.03032500 & 1.57548100 & $\mathrm{H}$ & -1.81128100 & 4.74567300 & 1.31773400 \\
\hline $\mathrm{H}$ & -0.12544100 & 4.65457400 & 0.79377600 & $\mathrm{H}$ & -0.73184100 & 6.12554200 & 1.57011400 \\
\hline C & 0.97579300 & 5.05721000 & 3.36292300 & $\mathrm{H}$ & 0.94179700 & 6.15050800 & 3.42854200 \\
\hline $\mathrm{H}$ & 1.73195500 & 4.78409400 & 2.62211300 & $\mathrm{H}$ & 1.29706300 & 4.66806400 & 4.33654300 \\
\hline C & -1.45507500 & 5.01352500 & 3.98405900 & $\mathrm{H}$ & -1.51009200 & 6.10715200 & 3.95659900 \\
\hline $\mathrm{H}$ & -1.20046000 & 4.71668900 & 5.00810000 & $\mathrm{H}$ & -2.45513000 & 4.62841200 & 3.75123500 \\
\hline C & -1.62614400 & 2.25173700 & 2.55030000 & $\mathrm{O}$ & -1.84030700 & 2.13249900 & 1.33191600 \\
\hline C & -2.44334400 & 1.80497000 & 4.92303100 & C & -3.57194600 & 0.84481600 & 2.96685700 \\
\hline C & -3.48840400 & 0.77616400 & 5.36888100 & $\mathrm{H}$ & -1.44753200 & 1.58319200 & 5.30773300 \\
\hline $\mathrm{H}$ & -2.73690200 & 2.81669300 & 5.22256600 & $\mathrm{C}$ & -4.48806600 & 0.75431800 & 4.20570300 \\
\hline $\mathrm{H}$ & -4.05397500 & 1.35203500 & 2.12818500 & $\mathrm{H}$ & -3.94452600 & 1.04332200 & 6.32563000 \\
\hline $\mathrm{H}$ & -3.01911600 & -0.20650000 & 5.47861100 & $\mathrm{H}$ & -5.14186600 & 1.63412100 & 4.24519900 \\
\hline $\mathrm{H}$ & -5.11486700 & -0.13990500 & 4.18980200 & $\mathrm{~N}$ & -2.46991500 & 1.69659100 & 3.44698400 \\
\hline C & -3.08422900 & -0.52557900 & 2.51093300 & C & -1.84339600 & -0.98393500 & 2.84451500 \\
\hline$C$ & -3.97970500 & -1.37092900 & 1.74585000 & $\mathrm{C}$ & -1.36268400 & -2.27297000 & 2.44446400 \\
\hline $\mathrm{H}$ & -1.17789500 & -0.36295300 & 3.43222600 & C & -3.52576500 & -2.66694700 & 1.33549200 \\
\hline C & -5.27181000 & -0.96752400 & 1.36444400 & C & -0.08420700 & -2.73124200 & 2.79661800 \\
\hline C & -2.21170400 & -3.10831200 & 1.66531300 & C & -4.37788700 & -3.51413200 & 0.56072700 \\
\hline $\mathrm{H}$ & -5.64450700 & 0.00699300 & 1.65819900 & C & -6.09701200 & -1.80189800 & 0.60906200 \\
\hline C & 0.37101600 & -3.97324400 & 2.35844200 & $\mathrm{H}$ & 0.55184300 & -2.12297600 & 3.42830000 \\
\hline C & -1.73989700 & -4.38086700 & 1.22260800 & C & -5.66145900 & -3.06339300 & 0.21193100 \\
\hline C & -3.87905400 & -4.79030600 & 0.12820900 & $\mathrm{H}$ & -7.09218900 & -1.46304000 & 0.33456500 \\
\hline $\mathrm{C}$ & -0.44152800 & -4.78586400 & 1.57095100 & $\mathrm{H}$ & 1.36717900 & -4.30408100 & 2.62945500 \\
\hline
\end{tabular}




\begin{tabular}{|c|c|c|c|c|c|c|c|}
\hline C & -2.61740200 & -5.20003200 & 0.43660500 & $\mathrm{H}$ & -6.30986400 & -3.70948200 & -0.37409100 \\
\hline $\mathrm{H}$ & -4.53692100 & -5.42591900 & -0.45887600 & $\mathrm{H}$ & -0.07328600 & -5.74671500 & 1.22164700 \\
\hline $\mathrm{H}$ & -2.25498200 & -6.16744700 & 0.09854700 & $\mathrm{~N}$ & 0.78863500 & 2.38043600 & 2.35450400 \\
\hline $\mathrm{H}$ & 0.91024300 & 2.60389700 & 1.35986600 & C & -0.00783600 & 0.00785200 & -0.92731200 \\
\hline 0 & 0.64308300 & -0.14941100 & 0.08471100 & $\mathrm{O}$ & -0.04053800 & -0.77950500 & -2.01343200 \\
\hline C & 0.98686900 & -1.83352200 & -2.21768400 & C & 0.97033500 & -2.82846400 & -1.06034700 \\
\hline $\mathrm{H}$ & 1.63402400 & -3.66584500 & -1.29419800 & $\mathrm{H}$ & 1.30790300 & -2.37303400 & -0.13249600 \\
\hline $\mathrm{H}$ & -0.03860200 & -3.22422100 & -0.90514700 & C & 0.52519300 & -2.50728200 & -3.50767700 \\
\hline $\mathrm{H}$ & 0.41898800 & -1.77428700 & -4.31364600 & $\mathrm{H}$ & 1.26569200 & -3.25189500 & -3.81476400 \\
\hline $\mathrm{H}$ & -0.43045200 & -3.02333900 & -3.36064400 & C & 2.33430200 & -1.15125800 & -2.41138800 \\
\hline $\mathrm{H}$ & 3.10646200 & -1.90082900 & -2.60453400 & $\mathrm{H}$ & 2.29858900 & -0.44992800 & -3.24841500 \\
\hline $\mathrm{H}$ & 2.61654800 & -0.59199900 & -1.52220200 & C & -1.35500300 & 1.52795700 & -2.27416200 \\
\hline $\mathrm{H}$ & -0.85482800 & 1.05242200 & -3.10828800 & C & -1.55057600 & 2.99172900 & -2.44974200 \\
\hline C & -1.15586500 & 3.56284900 & -3.66522800 & C & -2.10918200 & 3.79641300 & -1.45035000 \\
\hline C & -1.30579300 & 4.93060500 & -3.87682100 & $\mathrm{H}$ & -0.69134000 & 2.94002000 & -4.42346300 \\
\hline C & -2.25638400 & 5.16315100 & -1.66679900 & $\mathrm{H}$ & -2.40111700 & 3.36189600 & -0.50033900 \\
\hline C & -1.85794800 & 5.73300200 & -2.87803900 & $\mathrm{H}$ & -0.97445100 & 5.37117700 & -4.81219400 \\
\hline $\mathrm{H}$ & -2.67269600 & 5.78747900 & -0.88226000 & $\mathrm{H}$ & -1.96974100 & 6.80131500 & -3.03885400 \\
\hline $\mathrm{N}$ & -0.89049900 & 1.07379400 & -1.06493800 & $\mathrm{H}$ & -0.97112500 & 1.65689100 & -0.22644500 \\
\hline$S$ & 2.07359200 & 2.80514300 & -1.37439600 & $\mathrm{O}$ & 1.57876500 & 2.06050500 & -2.54865000 \\
\hline $\mathrm{O}$ & 1.03200200 & 3.15450300 & -0.36825000 & 0 & 3.32719600 & 2.28693800 & -0.76450200 \\
\hline $\mathrm{C}$ & 2.57294700 & 4.46322500 & -2.04146700 & $\mathrm{~F}$ & 3.52826000 & 4.32787700 & -2.96891700 \\
\hline $\mathrm{F}$ & 3.03796900 & 5.23821700 & -1.05260700 & $\mathrm{~F}$ & 1.51817000 & 5.07613200 & -2.59999500 \\
\hline C & -3.16363400 & 0.93927300 & -2.53025100 & $\mathrm{H}$ & -3.57291200 & 1.31232000 & -1.59469900 \\
\hline $\mathrm{H}$ & -3.44974500 & 1.50576200 & -3.41214600 & C & -3.01274700 & -0.44547100 & -2.66732800 \\
\hline C & -2.92839600 & -1.07646000 & -3.97836200 & $\mathrm{H}$ & -2.78248900 & -0.35034700 & -4.78462300 \\
\hline $\mathrm{H}$ & -2.14647500 & -1.84070100 & -4.01854100 & C & -2.95926300 & -1.27373200 & -1.43935900 \\
\hline $\mathrm{H}$ & -2.47508800 & -2.23924400 & -1.58572600 & $\mathrm{H}$ & -2.47568200 & -0.72764800 & -0.62400100 \\
\hline $\mathrm{H}$ & -3.98874400 & -1.44912700 & -1.10258000 & C & -4.67241200 & -2.20316300 & -6.21902100 \\
\hline $\mathrm{H}$ & -3.78924400 & -2.71903500 & -6.61274600 & $\mathrm{H}$ & -5.55531800 & -2.78140400 & -6.51602100 \\
\hline $\mathrm{H}$ & -4.73592200 & -1.22382700 & -6.70665300 & C & -4.48856600 & -3.72093500 & -3.51352700 \\
\hline $\mathrm{H}$ & -3.62356900 & -4.29196100 & -3.87006900 & $\mathrm{H}$ & -4.41031300 & -3.63653600 & -2.42561200 \\
\hline $\mathrm{H}$ & -5.38767200 & -4.30792500 & -3.73726800 & C & -6.03873700 & -1.03974700 & -3.67504600 \\
\hline $\mathrm{H}$ & -6.02834900 & -1.00168900 & -2.57979100 & $\mathrm{H}$ & -6.03308800 & -0.00975700 & -4.04946800 \\
\hline $\mathrm{H}$ & -6.98756600 & -1.49707000 & -3.97947100 & $\mathrm{Si}$ & -4.58855800 & -2.03663900 & -4.34563400 \\
\hline C & 1.24942800 & 0.58850900 & 4.14714300 & $\mathrm{C}$ & 2.34987200 & -0.35965600 & 3.69832700 \\
\hline 0 & 0.50122400 & 0.67925300 & 5.11329300 & $\mathrm{O}$ & 2.83496100 & -1.39232300 & 4.13187100 \\
\hline C & 2.46797100 & 0.46267100 & 2.46225600 & C & 1.42705600 & 1.32933400 & 2.87518400 \\
\hline $\mathrm{N}$ & 3.25617800 & 0.45368500 & 1.36964400 & $\mathrm{H}$ & 3.17775200 & 1.23902600 & 0.71110600 \\
\hline C & 3.95949200 & -0.64359300 & 0.85928400 & C & 3.75436500 & -1.95378200 & 1.31194500 \\
\hline C & 4.84975200 & -0.41249800 & -0.20160800 & C & 4.42376400 & -3.00773100 & 0.69431700 \\
\hline $\mathrm{H}$ & 3.10469300 & -2.14870800 & 2.15297400 & C & 5.50116100 & -1.48269000 & -0.80467000 \\
\hline $\mathrm{H}$ & 4.98751800 & 0.59531800 & -0.57425100 & C & 5.29759600 & -2.79256600 & -0.36940400 \\
\hline $\mathrm{H}$ & 5.81130700 & -3.61954900 & -0.84289200 & C & 4.11316500 & -4.41272100 & 1.12709300 \\
\hline C & 6.35352100 & -1.23816000 & -2.01717900 & $\mathrm{~F}$ & 3.01446400 & -4.90126600 & 0.48512400 \\
\hline $\mathrm{F}$ & 3.84595500 & -4.49651300 & 2.44870900 & $\mathrm{~F}$ & 5.12415100 & -5.26600700 & 0.85986500 \\
\hline $\mathrm{F}$ & 6.89609600 & -0.00488800 & -2.02424200 & $\mathrm{~F}$ & 5.62598000 & -1.35668600 & -3.15832600 \\
\hline $\mathrm{F}$ & 7.36459000 & -2.13164000 & -2.11114200 & & & & \\
\hline
\end{tabular}




\section{TS-S-6f-10}

Imaginary frequency: $-312.42 \mathrm{~cm}^{-1}$

E[B3LYP/6-31G(d)-D3(BJ)]: -4655.625061 Hartree

E[PCM(Et $2 \mathrm{O})-B 3 L Y P / 6-311 G(d, p)-D 3(B J)]:-4656.820696$ Hartree

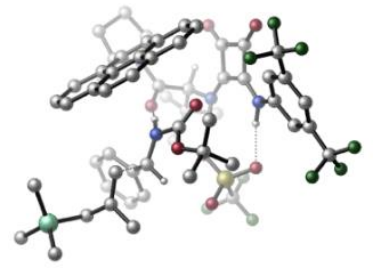

\begin{tabular}{|c|c|c|c|c|c|c|c|}
\hline 01 & & & & $\mathrm{C}$ & 0.67657400 & 3.79557000 & 1.67728800 \\
\hline $\mathrm{H}$ & 0.23516900 & 4.76559200 & 1.44742600 & C & 1.46845100 & 3.93889400 & 3.01256700 \\
\hline C & 2.23513000 & 2.65915200 & 3.38790700 & $\mathrm{H}$ & 3.00135400 & 2.41307700 & 2.65037100 \\
\hline $\mathrm{H}$ & 1.58024700 & 1.79165300 & 3.47613200 & $\mathrm{H}$ & 2.72678600 & 2.80697500 & 4.35678600 \\
\hline C & 0.46864300 & 4.28400900 & 4.12930300 & $\mathrm{H}$ & 1.00864200 & 4.50383800 & 5.05736000 \\
\hline $\mathrm{H}$ & -0.21164700 & 3.45080000 & 4.32222700 & $\mathrm{H}$ & -0.13303500 & 5.16342200 & 3.86986300 \\
\hline C & 2.47200800 & 5.09404000 & 2.85205900 & $\mathrm{H}$ & 3.03179300 & 5.23547800 & 3.78290600 \\
\hline $\mathrm{H}$ & 1.96530600 & 6.03823600 & 2.61960800 & $\mathrm{H}$ & 3.19854000 & 4.88823800 & 2.05758500 \\
\hline C & 1.55439900 & 3.32782000 & 0.51046800 & C & 1.72908000 & 5.65252400 & -0.52250800 \\
\hline C & 2.72535300 & 3.66483400 & -1.57832300 & C & 2.05091400 & 5.95592000 & -1.99172000 \\
\hline $\mathrm{H}$ & 0.69052000 & 5.87156800 & -0.27283200 & $\mathrm{H}$ & 2.39677000 & 6.20331000 & 0.15067800 \\
\hline C & 3.14789500 & 4.93844200 & -2.34087900 & $\mathrm{H}$ & 3.59109600 & 3.12334400 & -1.19400400 \\
\hline $\mathrm{H}$ & 2.36665000 & 6.99230600 & -2.13864300 & $\mathrm{H}$ & 1.16303200 & 5.78403000 & -2.60746400 \\
\hline $\mathrm{H}$ & 4.11839600 & 5.28634800 & -1.96668800 & $\mathrm{H}$ & 3.24223200 & 4.75596200 & -3.41405600 \\
\hline $\mathrm{N}$ & 1.98733400 & 4.20555800 & -0.41834100 & C & 1.85810500 & 2.70320000 & -2.37939100 \\
\hline C & 0.51831600 & 2.93272800 & -2.49985900 & C & 2.43756700 & 1.51078600 & -2.96157500 \\
\hline C & -0.36682800 & 2.01442100 & -3.14551200 & $\mathrm{H}$ & 0.07079800 & 3.80304200 & -2.03228700 \\
\hline C & 1.56447500 & 0.54700000 & -3.56507900 & C & 3.81727000 & 1.23666400 & -2.94610300 \\
\hline C & -1.74484900 & 2.25655600 & -3.23596400 & C & 0.16519000 & 0.79706800 & -3.65456800 \\
\hline C & 2.09326200 & -0.67734600 & -4.08026900 & $\mathrm{H}$ & 4.51084800 & 1.96370700 & -2.53848400 \\
\hline C & 4.32571800 & 0.04770700 & -3.47047600 & C & -2.59398800 & 1.30665000 & -3.79916000 \\
\hline $\mathrm{H}$ & -2.15415600 & 3.18156000 & -2.84417700 & C & -0.70985500 & -0.16513400 & -4.23894800 \\
\hline C & 3.47688000 & -0.90713600 & -4.02145900 & C & 1.18961200 & -1.63497300 & -4.65417100 \\
\hline $\mathrm{H}$ & 5.39875500 & -0.12540400 & -3.45710400 & C & -2.08501100 & 0.10867300 & -4.29517100 \\
\hline $\mathrm{H}$ & -3.66102700 & 1.49658400 & -3.83507800 & C & -0.14756300 & -1.38891700 & -4.73272100 \\
\hline $\mathrm{H}$ & 3.87970200 & -1.83065300 & -4.42848300 & $\mathrm{H}$ & 1.60338600 & -2.56342000 & -5.04008300 \\
\hline $\mathrm{H}$ & -2.75911800 & -0.63213500 & -4.71413300 & $\mathrm{H}$ & -0.81621600 & -2.12000500 & -5.18014300 \\
\hline $\mathrm{N}$ & -0.43447600 & 2.84829800 & 1.80082800 & $\mathrm{H}$ & -0.19881000 & 1.90428300 & 2.12402800 \\
\hline C & -0.17839500 & -0.88037700 & -0.61042300 & $\mathrm{O}$ & -1.07495400 & -0.08693100 & -0.79389400 \\
\hline $\mathrm{O}$ & -0.22712200 & -2.22173400 & -0.71631700 & C & -1.50193400 & -2.97655000 & -0.78539600 \\
\hline C & -2.36194500 & -2.49454400 & -1.94812400 & $\mathrm{H}$ & -3.26022600 & -3.11701600 & -2.00974900 \\
\hline $\mathrm{H}$ & -2.66476800 & -1.45896500 & -1.81934500 & $\mathrm{H}$ & -1.81214700 & -2.58294700 & -2.88908800 \\
\hline C & -1.02326900 & -4.40630800 & -1.02721100 & $\mathrm{H}$ & -0.40546100 & -4.75333900 & -0.19291000 \\
\hline $\mathrm{H}$ & -1.88667900 & -5.07354500 & -1.10970700 & $\mathrm{H}$ & -0.44566500 & -4.47133900 & -1.95584700 \\
\hline C & -2.19118400 & -2.84828400 & 0.56437400 & $\mathrm{H}$ & -3.12667800 & -3.41446900 & 0.56906900 \\
\hline $\mathrm{H}$ & -1.54869400 & -3.21515400 & 1.36817800 & $\mathrm{H}$ & -2.42918400 & -1.80790600 & 0.77271900 \\
\hline C & 1.94285600 & -1.30640500 & 0.47713800 & $\mathrm{H}$ & 1.38307500 & -2.02657800 & 1.06630000 \\
\hline C & 3.05128700 & -0.65056800 & 1.21836800 & C & 3.22368600 & -0.95810500 & 2.57192900 \\
\hline C & 3.96181700 & 0.19885600 & 0.57771400 & C & 4.28993500 & -0.40789100 & 3.28260600 \\
\hline $\mathrm{H}$ & 2.49916300 & -1.59179900 & 3.07118100 & C & 5.03338900 & 0.73476300 & 1.28738600 \\
\hline $\mathrm{H}$ & 3.82703500 & 0.44160100 & -0.47046000 & C & 5.20038500 & 0.43261100 & 2.64174100 \\
\hline $\mathrm{H}$ & 4.39896600 & -0.62921300 & 4.34003200 & $\mathrm{H}$ & 5.73387700 & 1.39667300 & 0.78548500 \\
\hline $\mathrm{H}$ & 6.03210400 & 0.85871500 & 3.19564000 & $\mathrm{~N}$ & 1.11888600 & -0.48911700 & -0.27859300 \\
\hline $\mathrm{H}$ & 1.26864500 & 0.52363000 & -0.20469500 & $S$ & -0.50231800 & -0.87903500 & 3.24168200 \\
\hline $\mathrm{O}$ & 0.05727800 & -2.23915200 & 3.07489300 & $\mathrm{O}$ & 0.15452200 & 0.14752200 & 2.38919400 \\
\hline $\mathrm{O}$ & -1.98088500 & -0.76552200 & 3.26505200 & C & 0.00057900 & -0.38551900 & 4.96134700 \\
\hline $\mathrm{F}$ & -0.54160100 & -1.19496600 & 5.87440200 & $\mathrm{~F}$ & -0.39546200 & 0.87417800 & 5.21078400 \\
\hline
\end{tabular}




\begin{tabular}{|c|c|c|c|c|c|c|c|}
\hline $\mathrm{F}$ & 1.34007000 & -0.43286600 & 5.08911000 & C & 2.76182200 & -2.46373800 & -0.75408600 \\
\hline $\mathrm{H}$ & 1.86104200 & -2.80219100 & -1.25543300 & $\mathrm{H}$ & 3.37320500 & -1.77569300 & -1.33111100 \\
\hline C & 3.41562600 & -3.37468800 & 0.09005600 & $\mathrm{C}$ & 4.80999500 & -3.20474600 & 0.46012200 \\
\hline $\mathrm{H}$ & 5.13365400 & -2.16198300 & 0.36923200 & $\mathrm{H}$ & 5.03107900 & -3.58335600 & 1.46404300 \\
\hline C & 2.62087600 & -4.44310800 & 0.76142200 & $\mathrm{H}$ & 3.24593500 & -5.27484600 & 1.09573000 \\
\hline $\mathrm{H}$ & 2.15767900 & -4.00271400 & 1.66157300 & $\mathrm{H}$ & 1.80474700 & -4.80934600 & 0.13494100 \\
\hline C & 7.71371500 & -3.87184900 & -0.13845200 & $\mathrm{H}$ & 7.84635800 & -4.25198400 & 0.88066600 \\
\hline $\mathrm{H}$ & 8.44832600 & -4.37304200 & -0.77970900 & $\mathrm{H}$ & 7.95032300 & -2.80193400 & -0.13212500 \\
\hline C & 5.52955900 & -6.01044400 & -0.70975300 & $\mathrm{H}$ & 5.60361400 & -6.41494500 & 0.30635100 \\
\hline $\mathrm{H}$ & 4.51343100 & -6.19725800 & -1.07479100 & $\mathrm{H}$ & 6.21656700 & -6.58516700 & -1.34210900 \\
\hline C & 5.71660600 & -3.47540400 & -2.49054000 & $\mathrm{H}$ & 4.70141300 & -3.66028800 & -2.85668800 \\
\hline $\mathrm{H}$ & 5.87928100 & -2.39236700 & -2.50683200 & $\mathrm{H}$ & 6.41790000 & -3.93139000 & -3.19909500 \\
\hline $\mathrm{Si}$ & 5.96651400 & -4.18022700 & -0.76608000 & $\mathrm{O}$ & 1.83783000 & 2.12560800 & 0.41258000 \\
\hline C & -1.48832000 & 2.96223800 & 0.98101000 & C & -2.63534400 & 2.18311500 & 0.71299000 \\
\hline C & -1.87918400 & 3.98351300 & -0.01637500 & C & -3.12619600 & 3.15690100 & -0.30747400 \\
\hline $\mathrm{O}$ & -1.38496600 & 5.01873000 & -0.44774100 & $\mathrm{O}$ & -4.06440900 & 3.23915500 & -1.08015600 \\
\hline $\mathrm{N}$ & -3.09472400 & 1.03438200 & 1.24794300 & $\mathrm{H}$ & -2.57402600 & 0.60732800 & 2.01986100 \\
\hline C & -4.10075300 & 0.21759200 & 0.71252500 & C & -4.66124900 & -0.76238600 & 1.54538700 \\
\hline C & -4.50670100 & 0.30240400 & -0.62473400 & C & -5.60445400 & -1.64736100 & 1.03467900 \\
\hline $\mathrm{H}$ & -4.32182700 & -0.85318100 & 2.57000400 & $\mathrm{C}$ & -5.46860500 & -0.58256800 & -1.10738000 \\
\hline $\mathrm{H}$ & -4.08568200 & 1.05131300 & -1.27822200 & C & -6.02394500 & -1.56761100 & -0.29356600 \\
\hline $\mathrm{H}$ & -6.76643800 & -2.25353800 & -0.68094700 & $\mathrm{C}$ & -5.83573600 & -0.52825300 & -2.56389000 \\
\hline C & -6.10967300 & -2.77064600 & 1.89387900 & $\mathrm{~F}$ & -5.87855900 & 0.73595200 & -3.03522300 \\
\hline $\mathrm{F}$ & -4.92960900 & -1.19505000 & -3.33265900 & $\mathrm{~F}$ & -7.03632700 & -1.09496500 & -2.80872600 \\
\hline $\mathrm{F}$ & -6.04923400 & -2.48131800 & 3.20845100 & $\mathrm{~F}$ & -5.37767700 & -3.90081900 & 1.70397500 \\
\hline $\mathrm{F}$ & -7.39043900 & -3.09167800 & 1.60266100 & & & & \\
\hline
\end{tabular}

\section{TS-S-6f-11}

Imaginary frequency: $-408.51 \mathrm{~cm}^{-1}$

E[B3LYP/6-31G(d)-D3(BJ)]: -4655.625309 Hartree

$\mathrm{E}\left[\mathrm{PCM}\left(\mathrm{Et}_{2} \mathrm{O}\right)-\mathrm{B} 3 \mathrm{LYP} / 6-311 \mathrm{G}(\mathrm{d}, \mathrm{p})-\mathrm{D} 3(\mathrm{BJ})\right]:-4656.819804$ Hartree

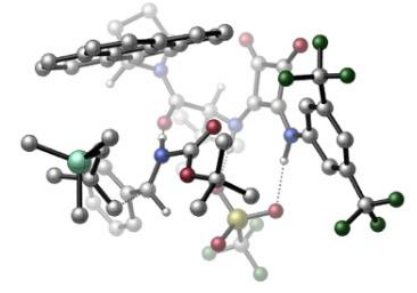

\begin{tabular}{|c|c|c|c|c|c|c|c|}
\hline 01 & & & & C & -1.25129700 & 3.70581000 & 1.81967400 \\
\hline $\mathrm{H}$ & -1.16583200 & 3.94720000 & 2.87929800 & C & -1.71468200 & 4.99483400 & 1.07546200 \\
\hline C & -1.98119000 & 4.75464100 & -0.42002900 & $\mathrm{H}$ & -2.79773500 & 4.04816100 & -0.57842400 \\
\hline $\mathrm{H}$ & -1.10696900 & 4.36333400 & -0.94105700 & $\mathrm{H}$ & -2.25442700 & 5.70566300 & -0.89244900 \\
\hline C & -0.61741600 & 6.06067000 & 1.23603400 & $\mathrm{H}$ & -0.96114200 & 7.01432100 & 0.81947400 \\
\hline $\mathrm{H}$ & 0.29520300 & 5.76975100 & 0.70969100 & $\mathrm{H}$ & -0.36674300 & 6.22146700 & 2.29150100 \\
\hline C & -3.01035600 & 5.49194900 & 1.73973700 & $\mathrm{H}$ & -3.35222500 & 6.41084200 & 1.25135600 \\
\hline $\mathrm{H}$ & -2.85859900 & 5.71472700 & 2.80252200 & $\mathrm{H}$ & -3.81571500 & 4.75326700 & 1.65353600 \\
\hline C & -2.21018700 & 2.52482200 & 1.62529100 & $\mathrm{O}$ & -2.16920800 & 1.88075700 & 0.56897300 \\
\hline C & -3.24061600 & 2.77432700 & 3.94398200 & $\mathrm{C}$ & -3.92572600 & 0.99749700 & 2.39192100 \\
\hline C & -4.03021700 & 1.69855400 & 4.69783700 & $\mathrm{H}$ & -2.27076000 & 2.97829900 & 4.39804200 \\
\hline $\mathrm{H}$ & -3.81694300 & 3.70363000 & 3.86462000 & $\mathrm{C}$ & -4.88712300 & 1.05361100 & 3.59978400 \\
\hline $\mathrm{H}$ & -4.45239800 & 1.14221000 & 1.44549200 & $\mathrm{H}$ & -4.62150800 & 2.11647200 & 5.51681200 \\
\hline $\mathrm{H}$ & -3.33861400 & 0.96126100 & 5.11805500 & $\mathrm{H}$ & -5.74390200 & 1.69531000 & 3.36047800 \\
\hline $\mathrm{H}$ & -5.26477100 & 0.06407400 & 3.86724900 & $\bar{N}$ & -3.06540200 & 2.17243700 & 2.60824800 \\
\hline C & -3.13898100 & -0.30329000 & 2.32251400 & C & -1.88801800 & -0.40383600 & 2.85662200 \\
\hline C & -3.75544700 & -1.46294000 & 1.71084200 & C & -1.12446000 & -1.61379400 & 2.80755800 \\
\hline $\mathrm{H}$ & -1.43021600 & 0.46049300 & 3.32463700 & $\mathrm{C}$ & -3.02770700 & -2.69689000 & 1.68106500 \\
\hline C & -5.04868300 & -1.44102500 & 1.15607300 & C & 0.17391000 & -1.69454900 & 3.33252500 \\
\hline C & -1.70350000 & -2.76423400 & 2.20279800 & C & -3.62965500 & -3.87190400 & 1.13145500 \\
\hline
\end{tabular}




\begin{tabular}{|c|c|c|c|c|c|c|c|}
\hline $\mathrm{H}$ & -5.62854700 & -0.52525600 & 1.16680400 & C & -5.62347900 & -2.59050100 & 0.61256200 \\
\hline $\mathrm{C}$ & 0.90866200 & -2.87371900 & 3.23178100 & $\mathrm{H}$ & 0.61134900 & -0.83280000 & 3.82343900 \\
\hline C & -0.95795300 & -3.97947800 & 2.13584500 & C & -4.93087000 & -3.79760800 & 0.60800600 \\
\hline $\mathrm{C}$ & -2.87205900 & -5.09260700 & 1.11387600 & $\mathrm{H}$ & -6.63078700 & -2.54405600 & 0.20769200 \\
\hline C & 0.35346900 & -4.00406600 & 2.63533500 & $\mathrm{H}$ & 1.91827000 & -2.91028800 & 3.62471000 \\
\hline C & -1.59278100 & -5.13982100 & 1.58046900 & $\mathrm{H}$ & -5.39357000 & -4.69402200 & 0.20377200 \\
\hline $\mathrm{H}$ & -3.34535900 & -5.98537000 & 0.71247100 & $\overline{\mathrm{H}}$ & 0.93195700 & -4.92137300 & 2.56502400 \\
\hline $\mathrm{H}$ & -1.03236100 & -6.07114300 & 1.55515000 & C & 0.12207400 & -0.65728700 & -0.80262600 \\
\hline $\mathrm{O}$ & 0.66504200 & -0.53344200 & 0.27276800 & $\mathrm{O}$ & 0.54397600 & -1.37798100 & -1.86085000 \\
\hline $\mathrm{C}$ & 1.88405200 & -2.00618700 & -1.91312800 & $\mathrm{C}$ & 2.08134500 & -2.93293700 & -0.71516500 \\
\hline $\mathrm{H}$ & 3.01849300 & -3.48510100 & -0.83633500 & $\mathrm{H}$ & 2.12679900 & -2.37695300 & 0.21846600 \\
\hline $\mathrm{H}$ & 1.26168200 & -3.65764400 & -0.64626300 & C & 1.82502100 & -2.79618800 & -3.21892400 \\
\hline $\mathrm{H}$ & 1.62176100 & -2.12905700 & -4.06290100 & $\overline{\mathrm{H}}$ & 2.78660100 & -3.28761000 & -3.39521000 \\
\hline $\mathrm{H}$ & 1.04862700 & -3.56731000 & -3.18037900 & C & 2.92049500 & -0.89737200 & -1.99761900 \\
\hline $\mathrm{H}$ & 3.92606000 & -1.31989900 & -2.07581200 & $\mathrm{H}$ & 2.73380800 & -0.25895900 & -2.86432300 \\
\hline $\mathrm{H}$ & 2.88069100 & -0.27818900 & -1.10585000 & $\mathrm{C}$ & -1.44231700 & 0.33681900 & -2.37433900 \\
\hline $\mathrm{H}$ & -0.57200900 & 0.57069000 & -2.98378000 & $\mathrm{C}$ & -2.53792200 & 1.32531300 & -2.48022200 \\
\hline C & -2.37603700 & 2.39581600 & -3.36845700 & C & -3.71825700 & 1.21631700 & -1.73220700 \\
\hline $\mathrm{C}$ & -3.38156100 & 3.35048000 & -3.50240800 & $\mathrm{H}$ & -1.44641300 & 2.49355800 & -3.91923100 \\
\hline $\mathrm{C}$ & -4.72469600 & 2.16642300 & -1.87559800 & $\mathrm{H}$ & -3.84028900 & 0.39622900 & -1.03206200 \\
\hline C & -4.55912400 & 3.23437500 & -2.76179000 & $\mathrm{H}$ & -3.23946400 & 4.18999400 & -4.17594800 \\
\hline $\mathrm{H}$ & -5.63669800 & 2.07846000 & -1.29176200 & $\mathrm{H}$ & -5.34394000 & 3.97778000 & -2.86824700 \\
\hline $\mathrm{N}$ & -1.12196900 & -0.10038300 & -1.09711000 & $\mathrm{H}$ & -1.51768800 & 0.45033500 & -0.32646000 \\
\hline $\mathrm{S}$ & 1.47023600 & 2.59080600 & -2.27008700 & 0 & 1.29971200 & 1.78091400 & -3.49442000 \\
\hline 0 & 0.36060900 & 2.43177900 & -1.29095100 & 0 & 2.81616500 & 2.57660200 & -1.64445200 \\
\hline C & 1.27538500 & 4.34716700 & -2.84324500 & $\mathrm{~F}$ & 2.22504500 & 4.67902800 & -3.72055000 \\
\hline $\mathrm{F}$ & 1.34600900 & 5.18280200 & -1.79246700 & $\mathrm{~F}$ & 0.07398200 & 4.51568400 & -3.42706100 \\
\hline C & -1.92526700 & -1.08864600 & -3.58912900 & $\mathrm{H}$ & -2.31367300 & -0.50055900 & -4.41641700 \\
\hline $\mathrm{H}$ & -0.91971900 & -1.47201300 & -3.71985700 & $\mathrm{C}$ & -2.81821700 & -1.90645900 & -2.88767000 \\
\hline C & -2.31787600 & -2.93229400 & -1.99558200 & $\mathrm{H}$ & -1.28223500 & -2.75059800 & -1.70264800 \\
\hline $\mathrm{H}$ & -2.95285000 & -3.09574500 & -1.12243200 & $\mathrm{C}$ & -4.29389500 & -1.71660500 & -3.02887400 \\
\hline $\mathrm{H}$ & -4.53777100 & -0.69612600 & -3.33417100 & $\bar{H}$ & -4.66186800 & -2.38922300 & -3.81787300 \\
\hline $\mathrm{H}$ & -4.82507800 & -1.96842500 & -2.10624400 & C & -0.98854300 & -5.70926700 & -2.05806800 \\
\hline $\mathrm{H}$ & 0.01744500 & -5.29097100 & -2.16777800 & $\mathrm{H}$ & -1.20507000 & -5.78426300 & -0.98938000 \\
\hline $\mathrm{H}$ & -0.97864700 & -6.72078500 & -2.48171400 & $\mathrm{C}$ & -3.98683200 & -5.41662600 & -2.81556300 \\
\hline $\mathrm{H}$ & -4.27832800 & -5.56059600 & -1.77030900 & $\mathrm{H}$ & -4.75232800 & -4.79513700 & -3.29307900 \\
\hline $\mathrm{H}$ & -4.00255600 & -6.39590600 & -3.30840200 & $\mathrm{C}$ & -1.78309300 & -4.36663400 & -4.72779700 \\
\hline $\mathrm{H}$ & -2.52296100 & -3.76805300 & -5.27073400 & $\mathrm{H}$ & -0.81758600 & -3.85522500 & -4.80702800 \\
\hline $\mathrm{H}$ & -1.68972900 & -5.32827700 & -5.24625600 & $\mathrm{Si}$ & -2.27088800 & -4.64858800 & -2.93029000 \\
\hline $\bar{H}$ & 0.18467600 & 3.08103000 & 0.37672600 & $\mathrm{~N}$ & 0.07652400 & 3.26714800 & 1.38040800 \\
\hline $\mathrm{C}$ & 0.67198600 & 2.15710300 & 3.63683600 & $\mathrm{C}$ & 1.96195600 & 1.35461100 & 3.51642200 \\
\hline 0 & -0.16112000 & 2.36650100 & 4.50979500 & $\mathrm{O}$ & 2.59667600 & 0.61134100 & 4.24506400 \\
\hline $\mathrm{C}$ & 2.02584900 & 1.79710700 & 2.09305000 & $\mathrm{C}$ & 0.82556900 & 2.53183100 & 2.21210400 \\
\hline $\mathrm{N}$ & 2.89104000 & 1.63232800 & 1.07398700 & $\overline{\mathrm{H}}$ & 2.72514700 & 2.13592000 & 0.19564900 \\
\hline C & 3.89900700 & 0.66062600 & 0.99889700 & C & 3.87391000 & -0.50666200 & 1.77092500 \\
\hline $\mathrm{C}$ & 4.91659600 & 0.84222400 & 0.05048100 & $\mathrm{C}$ & 4.86334000 & -1.47085900 & 1.59131700 \\
\hline $\bar{H}$ & 3.10085100 & -0.65789900 & 2.50877700 & $\mathrm{C}$ & 5.88144800 & -0.14350000 & -0.12457000 \\
\hline $\mathrm{H}$ & 4.91455700 & 1.72671100 & -0.57491900 & C & 5.87138700 & -1.30847000 & 0.64369800 \\
\hline $\mathrm{H}$ & 6.63196700 & -2.06662200 & 0.50737400 & $\mathrm{C}$ & 4.77523800 & -2.74738000 & 2.38005300 \\
\hline $\mathrm{C}$ & 6.88861500 & -0.00846100 & -1.23052100 & $\mathrm{~F}$ & 4.36161200 & -2.53469600 & 3.64870400 \\
\hline $\mathrm{F}$ & 5.95779300 & -3.39479000 & 2.43953200 & $\mathrm{~F}$ & 3.88293200 & -3.61619400 & 1.82943500 \\
\hline $\mathrm{F}$ & 7.12613000 & 1.27700000 & -1.55676600 & $\mathrm{~F}$ & 6.45870400 & -0.63124700 & -2.35905800 \\
\hline $\mathrm{F}$ & 8.07256400 & -0.57534800 & -0.90616200 & & & & \\
\hline
\end{tabular}




\section{TS-S-6f-12}

Imaginary frequency: $-307.56 \mathrm{~cm}^{-1}$

E[B3LYP/6-31G(d)-D3(BJ)]: -4655.623497 Hartree

E[PCM(Et2O)-B3LYP/6-311G(d,p)-D3(BJ)]: -4656.819658 Hartree

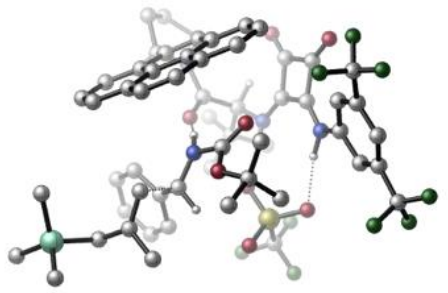

\begin{tabular}{|c|c|c|c|c|c|c|c|}
\hline 01 & & & & $\mathrm{C}$ & 0.76518800 & 3.65197200 & 1.86497700 \\
\hline $\mathrm{H}$ & 0.32815200 & 4.64363900 & 1.73813600 & C & 1.57807800 & 3.64427300 & 3.19658800 \\
\hline C & 2.33599800 & 2.32463100 & 3.41915000 & $\mathrm{H}$ & 3.09686700 & 2.16158400 & 2.65387100 \\
\hline $\mathrm{H}$ & 1.67692300 & 1.45639200 & 3.41239000 & $\mathrm{H}$ & 2.83422000 & 2.35776000 & 4.39528700 \\
\hline C & 0.59804600 & 3.87654600 & 4.35969500 & $\mathrm{H}$ & 1.15487900 & 3.99720800 & 5.29601300 \\
\hline $\mathrm{H}$ & -0.08419100 & 3.03183700 & 4.47910200 & $\overline{\mathrm{H}}$ & -0.00184800 & 4.78099300 & 4.20222000 \\
\hline C & 2.59747300 & 4.79603300 & 3.16075000 & $\mathrm{H}$ & 3.19524100 & 4.79138300 & 4.07861900 \\
\hline $\mathrm{H}$ & 2.10261500 & 5.77237300 & 3.09497100 & $\mathrm{H}$ & 3.29021100 & 4.69801100 & 2.31665200 \\
\hline$C$ & 1.60835000 & 3.29940900 & 0.62924400 & $\mathrm{O}$ & 1.89640300 & 2.11602200 & 0.39917800 \\
\hline C & 1.79582900 & 5.72645400 & -0.11604700 & C & 2.65526100 & 3.88233600 & -1.49001300 \\
\hline $\mathrm{C}$ & 2.87177200 & 6.26894500 & -1.06147000 & $\mathrm{H}$ & 0.78377400 & 5.99405600 & -0.43378500 \\
\hline $\mathrm{H}$ & 1.93585200 & 6.06023600 & 0.91120400 & $\mathrm{H}$ & 3.60924200 & 3.42400100 & -1.21902600 \\
\hline $\mathrm{H}$ & 3.84210500 & 6.28788900 & -0.55059300 & $\mathrm{H}$ & 2.64850100 & 7.28255500 & -1.40558500 \\
\hline $\mathrm{N}$ & 1.98535700 & 4.27083700 & -0.22558300 & C & 1.81468700 & 2.89876900 & -2.28778300 \\
\hline $\mathrm{C}$ & 0.47629100 & 3.13682700 & -2.40739200 & C & 2.39257100 & 1.71320300 & -2.88129800 \\
\hline C & -0.41307900 & 2.22880100 & -3.05860600 & $\mathrm{H}$ & 0.03950700 & 4.01555300 & -1.94187200 \\
\hline C & 1.51785400 & 0.77061700 & -3.51666200 & C & 3.76994300 & 1.42839700 & -2.86110400 \\
\hline $\mathrm{C}$ & -1.79223700 & 2.47104500 & -3.13692200 & C & 0.11926000 & 1.02579700 & -3.60199700 \\
\hline C & 2.04541800 & -0.43823400 & -4.06921800 & $\mathrm{H}$ & 4.46393700 & 2.13561700 & -2.42081900 \\
\hline C & 4.27649900 & 0.25510700 & -3.42011500 & C & -2.64102400 & 1.53821300 & -3.72783000 \\
\hline $\mathrm{H}$ & -2.20218100 & 3.38121700 & -2.71211100 & C & -0.75597600 & 0.08357800 & -4.21829100 \\
\hline C & 3.42741100 & -0.67545100 & -4.01131500 & C & 1.14221500 & -1.37393600 & -4.67859900 \\
\hline $\mathrm{H}$ & 5.34839400 & 0.07490100 & -3.40149800 & C & -2.13094300 & 0.35872200 & -4.26557000 \\
\hline $\mathrm{H}$ & -3.70848800 & 1.72687600 & -3.75499500 & $\mathrm{C}$ & -0.19417400 & -1.12316500 & -4.75265900 \\
\hline $\mathrm{H}$ & 3.82872000 & -1.58561100 & -4.44857300 & $\mathrm{H}$ & 1.55586400 & -2.28939500 & -5.09461300 \\
\hline $\mathrm{H}$ & -2.80459400 & -0.36760400 & -4.70981000 & $\mathrm{H}$ & -0.86254700 & -1.83724400 & -5.22712200 \\
\hline $\mathrm{N}$ & -0.35480200 & 2.70903800 & 1.91740300 & $\mathrm{H}$ & -0.13687600 & 1.74383200 & 2.18315100 \\
\hline C & -0.19858700 & -0.83969000 & -0.67004300 & $\mathrm{O}$ & -1.08290500 & -0.02363400 & -0.80789000 \\
\hline $\mathrm{O}$ & -0.26931300 & -2.17288400 & -0.84572300 & $\mathrm{C}$ & -1.55442600 & -2.90709200 & -0.94494000 \\
\hline $\mathrm{C}$ & -2.42474200 & -2.34720100 & -2.06412900 & $\mathrm{H}$ & -3.33271900 & -2.95305000 & -2.14627500 \\
\hline $\mathrm{H}$ & -2.71051000 & -1.31675100 & -1.87174200 & $\mathrm{H}$ & -1.89130300 & -2.38941800 & -3.01733600 \\
\hline $\mathrm{C}$ & -1.09626400 & -4.32492800 & -1.27906600 & $\bar{H}$ & -0.47258500 & -4.72902200 & -0.47545300 \\
\hline $\mathrm{H}$ & -1.96876300 & -4.97593400 & -1.39060400 & $\mathrm{H}$ & -0.53093000 & -4.33996000 & -2.21734500 \\
\hline $\mathrm{C}$ & -2.22513400 & -2.85283700 & 0.41888500 & $\mathrm{H}$ & -3.17078200 & -3.40162400 & 0.40029400 \\
\hline $\mathrm{H}$ & -1.58008300 & -3.28052400 & 1.18975000 & $\mathrm{H}$ & -2.44186200 & -1.82364000 & 0.69493200 \\
\hline C & 1.92091600 & -1.35895400 & 0.37885400 & $\mathrm{H}$ & 1.35157600 & -2.10059400 & 0.93091100 \\
\hline $\mathrm{C}$ & 3.04772300 & -0.76897900 & 1.14752000 & $\mathrm{C}$ & 3.24062500 & -1.18120200 & 2.47048600 \\
\hline$C$ & 3.96214500 & 0.10991900 & 0.55391500 & $\mathrm{C}$ & 4.33317300 & -0.70924700 & 3.19718800 \\
\hline $\mathrm{H}$ & 2.51328300 & -1.83725800 & 2.93584800 & C & 5.05961800 & 0.56781000 & 1.27901200 \\
\hline $\mathrm{H}$ & 3.81066000 & 0.43771300 & -0.46825600 & C & 5.24921400 & 0.15816100 & 2.60165300 \\
\hline $\mathrm{H}$ & 4.45932900 & -1.01332900 & 4.23194100 & $\mathrm{H}$ & 5.76275400 & 1.25326000 & 0.81383700 \\
\hline $\mathrm{H}$ & 6.10192700 & 0.52282700 & 3.16738000 & $\mathrm{~N}$ & 1.10612700 & -0.48686600 & -0.32397500 \\
\hline $\mathrm{H}$ & 1.27528400 & 0.51858000 & -0.20447600 & $S$ & -0.49099200 & -1.09284900 & 3.18160200 \\
\hline $\mathrm{O}$ & 0.04945800 & -2.44826000 & 2.93341000 & $\mathrm{O}$ & 0.16923700 & -0.03040100 & 2.37594700 \\
\hline $\mathrm{O}$ & -1.96660500 & -0.95944300 & 3.22950800 & C & 0.04165800 & -0.70300600 & 4.91890100 \\
\hline $\mathrm{F}$ & -0.48616600 & -1.56715500 & 5.78928500 & $\mathrm{~F}$ & -0.34912500 & 0.53844300 & 5.25222400 \\
\hline
\end{tabular}




\begin{tabular}{|c|c|c|c|c|c|c|c|}
\hline $\mathrm{F}$ & 1.38295600 & -0.75841800 & 5.02072400 & C & 2.71163500 & -2.45811700 & -0.91731300 \\
\hline $\mathrm{H}$ & 1.80359700 & -2.75342000 & -1.43278400 & $\mathrm{H}$ & 3.33068100 & -1.74761000 & -1.45761900 \\
\hline C & 3.35647000 & -3.42827200 & -0.13371300 & C & 4.75542100 & -3.30139900 & 0.23555100 \\
\hline $\mathrm{H}$ & 5.09480800 & -2.26014500 & 0.20432700 & $\mathrm{H}$ & 4.97783800 & -3.74310700 & 1.21297800 \\
\hline C & 2.54947800 & -4.52386700 & 0.47631000 & $\mathrm{H}$ & 3.16558400 & -5.37683700 & 0.77117000 \\
\hline $\mathrm{H}$ & 2.08159600 & -4.12691800 & 1.39412400 & $\mathrm{H}$ & 1.73523400 & -4.85046900 & -0.17424400 \\
\hline $\mathrm{C}$ & 7.64523500 & -3.96769400 & -0.42675000 & $\mathrm{H}$ & 7.78089900 & -4.40678200 & 0.56798000 \\
\hline $\mathrm{H}$ & 8.36813900 & -4.44081200 & -1.10175100 & $\mathrm{H}$ & 7.89576100 & -2.90291400 & -0.36176100 \\
\hline C & 5.42872800 & -6.04163500 & -1.10549800 & $\bar{H}$ & 5.50498700 & -6.50700500 & -0.11597500 \\
\hline $\mathrm{H}$ & 4.40740700 & -6.19308800 & -1.47244900 & $\mathrm{H}$ & 6.10311300 & -6.58635400 & -1.77679600 \\
\hline C & 5.63488100 & -3.40741800 & -2.73339000 & $\mathrm{H}$ & 4.61581500 & -3.56259900 & -3.10259800 \\
\hline $\mathrm{H}$ & 5.80674500 & -2.32665500 & -2.68541100 & $\mathrm{H}$ & 6.32764000 & -3.82533700 & -3.47310000 \\
\hline Si & 5.88933700 & -4.21715400 & -1.05614300 & $\mathrm{C}$ & 2.88117000 & 5.23741200 & -2.19724500 \\
\hline $\mathrm{H}$ & 2.04991400 & 5.42696200 & -2.88348400 & $\mathrm{H}$ & 3.80392500 & 5.23761900 & -2.78409400 \\
\hline $\mathrm{C}$ & -1.81311400 & 3.98974000 & 0.20923800 & C & -3.07802500 & 3.20032300 & -0.11357800 \\
\hline $\mathrm{O}$ & -1.31375600 & 5.04770100 & -0.15138200 & $\mathrm{O}$ & -4.02794300 & 3.34494700 & -0.86247900 \\
\hline C & -2.58740000 & 2.15774400 & 0.83604200 & $\mathrm{C}$ & -1.42080900 & 2.89656000 & 1.12810700 \\
\hline $\mathrm{N}$ & -3.06172900 & 0.98861400 & 1.31325400 & $\mathrm{H}$ & -2.54081500 & 0.51167800 & 2.05437300 \\
\hline $\mathrm{C}$ & -4.09002900 & 0.22200600 & 0.74792300 & C & -4.51349100 & 0.39075700 & -0.57600600 \\
\hline $\mathrm{C}$ & -4.65652800 & -0.79453200 & 1.53165500 & $\mathrm{C}$ & -5.49721800 & -0.44927000 & -1.09395100 \\
\hline $\mathrm{H}$ & -4.08841400 & 1.16890900 & -1.19185500 & C & -5.62169700 & -1.63308600 & 0.98520800 \\
\hline $\mathrm{H}$ & -4.30452000 & -0.94993800 & 2.54419000 & $\mathrm{C}$ & -6.05803000 & -1.47040300 & -0.32996900 \\
\hline $\mathrm{H}$ & -6.81709800 & -2.12094300 & -0.74517900 & $\mathrm{C}$ & -5.88210800 & -0.30668000 & -2.53973200 \\
\hline C & -6.13315400 & -2.79597700 & 1.78594700 & $F$ & -4.99257000 & -0.93682100 & -3.35730500 \\
\hline $\mathrm{F}$ & -5.91725100 & 0.98316100 & -2.93650600 & $\mathrm{~F}$ & -7.09166500 & -0.84606600 & -2.80167800 \\
\hline $\mathrm{F}$ & -6.05203800 & -2.58292600 & 3.11393500 & $\mathrm{~F}$ & -5.42055300 & -3.92390200 & 1.52258400 \\
\hline $\mathrm{F}$ & -7.42212100 & -3.08171700 & 1.49406800 & & & & \\
\hline
\end{tabular}

\section{TS-S-6f-13}

Imaginary frequency: $-359.66 \mathrm{~cm}^{-1}$

E[B3LYP/6-31G(d)-D3(BJ)]: -4655.621650 Hartree

E[PCM(Et $\left.\left.{ }_{2} \mathrm{O}\right)-\mathrm{B} 3 \mathrm{LYP} / 6-311 \mathrm{G}(\mathrm{d}, \mathrm{p})-\mathrm{D} 3(\mathrm{BJ})\right]$ : -4656.817910 Hartree

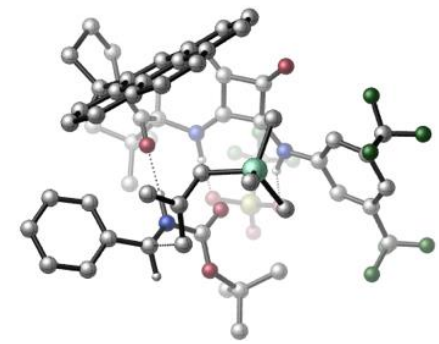

\begin{tabular}{|c|c|c|c|c|c|c|c|}
\hline 01 & & & & C & -0.54245800 & -3.14643900 & -1.91959800 \\
\hline $\mathrm{H}$ & -0.68269800 & -3.22381800 & -2.99986200 & C & -0.35833200 & -4.59881600 & -1.36383100 \\
\hline C & -0.13387800 & -4.61504300 & 0.15970400 & $\mathrm{H}$ & -1.00326700 & -4.22239000 & 0.69566500 \\
\hline $\mathrm{H}$ & 0.75260400 & -4.05361500 & 0.45738700 & $\mathrm{H}$ & 0.02070600 & -5.65022300 & 0.48334300 \\
\hline C & 0.83709700 & -5.24436700 & -2.08922500 & $\mathrm{H}$ & 0.92049800 & -6.29512100 & -1.78922500 \\
\hline $\mathrm{H}$ & 1.78030600 & -4.75754000 & -1.84615800 & $\mathrm{H}$ & 0.69278100 & -5.21557300 & -3.17612600 \\
\hline$C$ & -1.61248200 & -5.43495700 & -1.67608800 & $\mathrm{H}$ & -1.48045800 & -6.44393100 & -1.27225500 \\
\hline $\mathrm{H}$ & -1.77072800 & -5.53453600 & -2.75530800 & $\mathrm{H}$ & -2.51892600 & -5.01520000 & -1.22596400 \\
\hline C & -1.77477100 & -2.44891000 & -1.31114800 & $C$ & -3.18319100 & -2.93794100 & -3.36940400 \\
\hline$C$ & -4.15139300 & -1.89554900 & -1.36822700 & $\mathrm{C}$ & -4.62168500 & -2.48459700 & -3.65264900 \\
\hline $\mathrm{H}$ & -2.45369500 & -2.49534500 & -4.04874300 & $\mathrm{H}$ & -3.10775500 & -4.02756500 & -3.40453800 \\
\hline C & -5.27148100 & -2.46775900 & -2.26295800 & $\mathrm{H}$ & -4.21195200 & -2.28173800 & -0.34981500 \\
\hline $\mathrm{H}$ & -5.12864500 & -3.15058100 & -4.35554700 & $\mathrm{H}$ & -4.62253900 & -1.47699200 & -4.07864700 \\
\hline $\mathrm{H}$ & -5.51492100 & -3.48805200 & -1.94205200 & $\mathrm{H}$ & -6.18084800 & -1.86439300 & -2.21530000 \\
\hline $\mathrm{N}$ & -2.93780100 & -2.45678400 & -1.99174700 & C & -4.16848200 & -0.37596600 & -1.34014100 \\
\hline C & -3.42083900 & 0.34171600 & -2.22567600 & $\mathrm{C}$ & -5.04674400 & 0.30985700 & -0.41370800 \\
\hline C & -3.47127600 & 1.77119300 & -2.29589700 & $\mathrm{H}$ & -2.75301000 & -0.16301600 & -2.91246100 \\
\hline C & -5.08034500 & 1.74238400 & -0.43078300 & $\mathrm{C}$ & -5.87420700 & -0.36194600 & 0.50317600 \\
\hline
\end{tabular}




\begin{tabular}{|c|c|c|c|c|c|c|c|}
\hline $\mathrm{C}$ & -2.72702700 & 2.48824000 & -3.24573300 & C & -4.30603600 & 2.47175400 & -1.38014400 \\
\hline C & -5.91005000 & 2.45142500 & 0.49257000 & $\mathrm{H}$ & -5.89570300 & -1.44434800 & 0.52146300 \\
\hline C & -6.68237500 & 0.33850300 & 1.39849100 & C & -2.81972800 & 3.87741100 & -3.30496400 \\
\hline $\mathrm{H}$ & -2.05973300 & 1.95245500 & -3.91299400 & C & -4.37111300 & 3.89634600 & -1.42989500 \\
\hline C & -6.69717900 & 1.72912900 & 1.40368900 & C & -5.91844400 & 3.88835300 & 0.45318100 \\
\hline $\mathrm{H}$ & -7.30335800 & -0.21208000 & 2.09933900 & C & -3.63019000 & 4.57539800 & -2.41096600 \\
\hline $\mathrm{H}$ & -2.23355800 & 4.42035600 & -4.03958600 & C & -5.18961100 & 4.57802300 & -0.46772600 \\
\hline $\mathrm{H}$ & -7.32771200 & 2.26958800 & 2.10462900 & $\mathrm{H}$ & -6.54063800 & 4.41686900 & 1.17069600 \\
\hline $\mathrm{H}$ & -3.68233100 & 5.66013700 & -2.45407000 & $\mathrm{H}$ & -5.22184900 & 5.66411900 & -0.49359100 \\
\hline $\mathrm{N}$ & 0.64832400 & -2.32674200 & -1.68071100 & $\mathrm{H}$ & 1.32279200 & -2.61604800 & -0.96721300 \\
\hline C & 0.97036000 & -1.41617700 & 2.18770800 & 0 & 1.51357600 & -1.29754300 & 1.11163300 \\
\hline $\mathrm{O}$ & 1.50287900 & -1.28956000 & 3.40340000 & C & 2.98713700 & -1.28772700 & 3.60729600 \\
\hline C & 3.62815600 & -0.14013300 & 2.83798600 & $\mathrm{H}$ & 4.66988400 & -0.03350200 & 3.15360000 \\
\hline $\mathrm{H}$ & 3.61384500 & -0.33614300 & 1.76943500 & $\mathrm{H}$ & 3.11715500 & 0.80510200 & 3.04719200 \\
\hline C & 3.09891300 & -1.07795100 & 5.11367300 & $\mathrm{H}$ & 2.56428200 & -1.86303100 & 5.65808800 \\
\hline $\mathrm{H}$ & 4.15233100 & -1.11066500 & 5.40722200 & $\mathrm{H}$ & 2.69110300 & -0.10391400 & 5.40452700 \\
\hline C & 3.51000800 & -2.65498000 & 3.18038500 & $\mathrm{H}$ & 4.60013000 & -2.67788300 & 3.26918900 \\
\hline $\mathrm{H}$ & 3.09216100 & -3.44327200 & 3.81571900 & $\mathrm{H}$ & 3.25881200 & -2.86551600 & 2.14124600 \\
\hline C & -1.12691700 & -1.50120000 & 3.41635100 & $\mathrm{H}$ & -0.51222000 & -1.58556900 & 4.30559400 \\
\hline C & -2.49111300 & -2.04704000 & 3.56523900 & C & -3.00100400 & -2.18288900 & 4.86676700 \\
\hline C & -3.28455700 & -2.43064800 & 2.47500400 & C & -4.27562400 & -2.69728200 & 5.07835800 \\
\hline $\mathrm{H}$ & -2.38819300 & -1.88788000 & 5.71501400 & C & -4.55466400 & -2.96184100 & 2.69457800 \\
\hline $\mathrm{H}$ & -2.91288500 & -2.31373300 & 1.46338300 & C & -5.05623500 & -3.09340100 & 3.98925300 \\
\hline $\mathrm{H}$ & -4.65589800 & -2.80094600 & 6.09000000 & $\mathrm{H}$ & -5.15416400 & -3.27925700 & 1.84653200 \\
\hline $\mathrm{H}$ & -6.04736000 & -3.50633100 & 4.15170000 & $\mathrm{~N}$ & -0.40567200 & -1.69307700 & 2.27277300 \\
\hline $\mathrm{H}$ & -0.84911900 & -1.80647700 & 1.34891000 & $S$ & 4.26055800 & -3.08868100 & -0.44462400 \\
\hline 0 & 5.31170800 & -3.94472100 & 0.10847400 & $\mathrm{O}$ & 2.86037400 & -3.54971800 & -0.22773700 \\
\hline 0 & 4.42270800 & -1.62390400 & -0.20425900 & $\mathrm{C}$ & 4.43333500 & -3.19802900 & -2.29161500 \\
\hline $\mathrm{F}$ & 5.65150700 & -2.82226500 & -2.69040700 & $\mathrm{~F}$ & 3.52642300 & -2.38774100 & -2.88047300 \\
\hline $\mathrm{F}$ & 4.20731700 & -4.44918600 & -2.72012100 & C & -1.23729900 & 0.49501500 & 3.72531800 \\
\hline $\mathrm{H}$ & -1.64982000 & 0.54432700 & 4.72960800 & $\mathrm{H}$ & -0.16317400 & 0.64000900 & 3.66043000 \\
\hline C & -2.04486900 & 0.91024400 & 2.66140400 & C & -1.50760100 & 1.13385300 & 1.33451000 \\
\hline $\mathrm{H}$ & -0.64877500 & 0.49736300 & 1.09659000 & $\mathrm{H}$ & -2.26096100 & 1.06456300 & 0.54631400 \\
\hline C & -3.51458400 & 1.04699700 & 2.85724900 & $\mathrm{H}$ & -3.90268800 & 1.92470600 & 2.33309000 \\
\hline $\mathrm{H}$ & -4.00990600 & 0.18208500 & 2.39388600 & $\mathrm{H}$ & -3.80139900 & 1.08567600 & 3.91010900 \\
\hline C & -0.74713900 & 3.46646900 & -0.51670700 & $\mathrm{H}$ & -1.77187400 & 3.70548400 & -0.80535700 \\
\hline $\mathrm{H}$ & -0.14502300 & 4.36940000 & -0.65126600 & $\mathrm{H}$ & -0.37422600 & 2.71393500 & -1.21329700 \\
\hline C & -1.77093000 & 4.11054300 & 2.29572700 & $\mathrm{H}$ & -2.79056600 & 4.17487000 & 1.89666200 \\
\hline $\mathrm{H}$ & -1.83138800 & 3.81637400 & 3.34960600 & $\mathrm{H}$ & -1.34196000 & 5.11896200 & 2.25693000 \\
\hline C & 1.02334700 & 2.76752900 & 1.97716400 & $\mathrm{H}$ & 1.01895000 & 2.57892300 & 3.05656000 \\
\hline $\mathrm{H}$ & 1.57257500 & 1.95674600 & 1.48702100 & $\mathrm{H}$ & 1.58361600 & 3.69145500 & 1.80035400 \\
\hline $\mathrm{Si}$ & -0.71204800 & 2.93542800 & 1.26911500 & 0 & -1.71466900 & -1.90377900 & -0.19282400 \\
\hline $\mathrm{C}$ & 0.73494300 & -1.10610400 & -2.20758800 & C & 1.54887400 & 0.04776500 & -2.02819500 \\
\hline C & -0.08656000 & -0.42367200 & -3.22826100 & C & 0.70472400 & 0.84054600 & -2.97120800 \\
\hline 0 & -1.01192500 & -0.74503900 & -3.96846200 & $\mathrm{O}$ & 0.61756000 & 2.00190600 & -3.34411000 \\
\hline $\mathrm{N}$ & 2.65221300 & 0.25454300 & -1.28434800 & $\mathrm{H}$ & 3.10812500 & -0.56810700 & -0.86240900 \\
\hline C & 3.24807100 & 1.47070700 & -0.92774600 & C & 4.42282000 & 1.40578000 & -0.15969500 \\
\hline C & 2.70538200 & 2.72442100 & -1.24963300 & C & 4.99617000 & 2.57186700 & 0.33515700 \\
\hline $\mathrm{H}$ & 4.87559200 & 0.44330000 & 0.04348300 & C & 3.30824900 & 3.87623700 & -0.74763200 \\
\hline $\mathrm{H}$ & 1.84608400 & 2.80229400 & -1.90511100 & C & 4.44409400 & 3.82089000 & 0.05934600 \\
\hline $\mathrm{H}$ & 4.89691000 & 4.72566700 & 0.44490300 & C & 2.67320700 & 5.21444200 & -0.99386500 \\
\hline C & 6.18356200 & 2.46380600 & 1.25062500 & $\mathrm{~F}$ & 1.87207500 & 5.22437800 & -2.07558200 \\
\hline $\mathrm{F}$ & 1.89530100 & 5.58865600 & 0.06859700 & $\mathrm{~F}$ & 3.58887100 & 6.19358900 & -1.14883100 \\
\hline
\end{tabular}




\begin{tabular}{|c|c|c|c|c|c|c|}
\hline $\mathrm{F}$ & 6.98653500 & 1.43284600 & 0.92670800 & $\mathrm{~F}$ & 5.79081800 & 2.26598600 \\
\hline $\mathrm{F}$ & 6.93238300 & 3.58849900 & 1.24470100 & & & \\
\hline
\end{tabular}

\section{TS-S-6f-14}

Imaginary frequency: $-395.71 \mathrm{~cm}^{-1}$

E[B3LYP/6-31G(d)-D3(BJ)]: -4655.617779 Hartree

$\mathrm{E}\left[\mathrm{PCM}\left(\mathrm{Et}_{2} \mathrm{O}\right)-\mathrm{B} 3 \mathrm{LYP} / 6-311 \mathrm{G}(\mathrm{d}, \mathrm{p})-\mathrm{D} 3(\mathrm{BJ})\right]$ : -4656.816844 Hartree

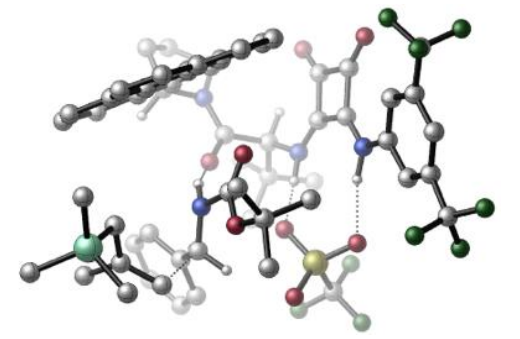

\begin{tabular}{|c|c|c|c|c|c|c|c|}
\hline 01 & & & & C & 0.97943400 & -3.85238600 & 1.27699800 \\
\hline $\mathrm{H}$ & 0.63106300 & -4.34139900 & 2.18667800 & C & 1.63178700 & -4.93629400 & 0.37054700 \\
\hline $\mathrm{C}$ & 2.23504900 & -4.35526700 & -0.91741500 & $\mathrm{H}$ & 3.05599500 & -3.67059400 & -0.70258300 \\
\hline $\mathrm{H}$ & 1.50065700 & -3.81093900 & -1.51085100 & $\mathrm{H}$ & 2.62365500 & -5.17410300 & -1.53438800 \\
\hline $\mathrm{C}$ & 0.55096700 & -5.96942500 & 0.00953300 & $\mathrm{H}$ & 1.00233600 & -6.80742300 & -0.53377800 \\
\hline $\mathrm{H}$ & -0.21817700 & -5.52718200 & -0.62851700 & $\mathrm{H}$ & 0.06321900 & -6.36942100 & 0.90668100 \\
\hline C & 2.74809900 & -5.62707600 & 1.17352500 & $\mathrm{H}$ & 3.24289100 & -6.38183700 & 0.55274400 \\
\hline $\mathrm{H}$ & 2.35232700 & -6.13692200 & 2.06004500 & $\mathrm{H}$ & 3.51523100 & -4.91279900 & 1.49669600 \\
\hline $\mathrm{C}$ & 1.91399900 & -2.69094500 & 1.64897100 & $\mathrm{O}$ & 2.19834700 & -1.83167500 & 0.80336400 \\
\hline C & 2.27554900 & -3.54180400 & 4.02553900 & C & 3.08572900 & -1.35019600 & 3.29708900 \\
\hline $\mathrm{C}$ & 3.50838700 & -3.17420700 & 4.85654100 & $\mathrm{H}$ & 1.34299700 & -3.41527500 & 4.58283900 \\
\hline $\mathrm{H}$ & 2.30991100 & -4.56201500 & 3.64615600 & $\mathrm{H}$ & 3.94021500 & -1.27322900 & 2.61929100 \\
\hline $\mathrm{H}$ & 4.40511200 & -3.61918700 & 4.40843700 & $\mathrm{H}$ & 3.43317900 & -3.52512700 & 5.88946000 \\
\hline $\mathrm{N}$ & 2.34468700 & -2.56991400 & 2.92202800 & $\mathrm{C}$ & 2.23038400 & -0.09915000 & 3.18417300 \\
\hline $\mathrm{C}$ & 0.89012200 & -0.17111100 & 3.43083300 & $\mathrm{C}$ & 2.83963500 & 1.17622100 & 2.87540200 \\
\hline C & 0.03012200 & 0.96818200 & 3.35408400 & $\mathrm{H}$ & 0.43871400 & -1.12938600 & 3.66977700 \\
\hline $\mathrm{C}$ & 2.00372400 & 2.33963700 & 2.81657500 & $\mathrm{C}$ & 4.21945000 & 1.33702200 & 2.65003100 \\
\hline $\mathrm{C}$ & -1.35521100 & 0.86930100 & 3.54883600 & $\mathrm{C}$ & 0.60110200 & 2.23192600 & 3.03609100 \\
\hline C & 2.57797900 & 3.62214900 & 2.55240900 & $\mathrm{H}$ & 4.88162300 & 0.48009600 & 2.70251300 \\
\hline C & 4.77042000 & 2.59303800 & 2.39723600 & C & -2.17500300 & 1.98650300 & 3.40209600 \\
\hline $\mathrm{H}$ & -1.79605600 & -0.08519900 & 3.81329400 & C & -0.23893400 & 3.37957200 & 2.93127200 \\
\hline C & 3.96417600 & 3.72735600 & 2.35523400 & C & 1.71531300 & 4.76983400 & 2.50693200 \\
\hline $\mathrm{H}$ & 5.84332300 & 2.68608400 & 2.24995000 & C & -1.62424700 & 3.22979200 & 3.09946800 \\
\hline $\mathrm{H}$ & -3.24794900 & 1.88470000 & 3.52449400 & C & 0.36866700 & 4.65158100 & 2.66781700 \\
\hline $\mathrm{H}$ & 4.40311900 & 4.70696300 & 2.18254800 & $\mathrm{H}$ & 2.16450000 & 5.74385000 & 2.33984500 \\
\hline $\mathrm{H}$ & -2.26571600 & 4.10188300 & 3.00099800 & $\mathrm{H}$ & -0.27089900 & 5.52926200 & 2.61752400 \\
\hline $\mathrm{N}$ & -0.20926600 & -3.25273200 & 0.65807100 & $\mathrm{H}$ & -0.07968800 & -2.79493900 & -0.25070200 \\
\hline C & 0.49161300 & 1.01451100 & -0.63663000 & $\mathrm{O}$ & -0.13940000 & 0.93479600 & 0.39307200 \\
\hline $\mathrm{O}$ & 0.17074100 & 1.70880900 & -1.73999400 & $\mathrm{C}$ & -1.08704400 & 2.48459800 & -1.83012200 \\
\hline $\mathrm{C}$ & -0.95250200 & 3.70171400 & -0.91691500 & $\mathrm{H}$ & -1.84115800 & 4.33434000 & -1.01382200 \\
\hline $\mathrm{H}$ & -0.85922100 & 3.39389900 & 0.12675500 & $\mathrm{H}$ & -0.07697700 & 4.30136000 & -1.19327600 \\
\hline C & -1.12593700 & 2.86023500 & -3.30950300 & $\mathrm{H}$ & -1.13977500 & 1.95311100 & -3.92074100 \\
\hline $\mathrm{H}$ & -2.02627100 & 3.44472700 & -3.52155600 & $\mathrm{H}$ & -0.25269900 & 3.45906900 & -3.59014500 \\
\hline $\mathrm{C}$ & -2.29169300 & 1.62266800 & -1.47575700 & $\mathrm{H}$ & -3.20556600 & 2.14988300 & -1.76374200 \\
\hline $\mathrm{H}$ & -2.25920500 & 0.68692800 & -2.03746400 & $\mathrm{H}$ & -2.33008400 & 1.39862900 & -0.41092900 \\
\hline C & 2.25178700 & 0.08479300 & -2.04478100 & $\mathrm{H}$ & 1.49060100 & -0.04476600 & -2.81050000 \\
\hline $\mathrm{C}$ & 3.32816500 & -0.92690700 & -2.08040000 & C & 3.39839500 & -1.77248600 & -3.19596400 \\
\hline $\mathrm{C}$ & 4.28102200 & -1.05332400 & -1.05740300 & C & 4.40505600 & -2.72903500 & -3.28908700 \\
\hline $\mathrm{H}$ & 2.63794000 & -1.69683100 & -3.96671900 & C & 5.28798800 & -2.01188700 & -1.15517300 \\
\hline $\mathrm{H}$ & 4.21970300 & -0.42090000 & -0.17778200 & $\mathrm{C}$ & 5.35560000 & -2.84643100 & -2.27226500 \\
\hline $\mathrm{H}$ & 4.43971100 & -3.39216100 & -4.14801200 & $\mathrm{H}$ & 6.01566000 & -2.11113500 & -0.35494400 \\
\hline $\mathrm{H}$ & 6.13940900 & -3.59479800 & -2.34437500 & $\mathrm{~N}$ & 1.74114500 & 0.41566600 & -0.79990600 \\
\hline $\mathrm{H}$ & 1.97922600 & -0.21062900 & -0.02404100 & $S$ & -0.71811400 & -1.76298000 & -2.97869400 \\
\hline
\end{tabular}




\begin{tabular}{|c|c|c|c|c|c|c|c|}
\hline 0 & -0.24521700 & -0.76995000 & -3.96406800 & $\mathrm{O}$ & 0.13563600 & -1.85513100 & -1.76208800 \\
\hline 0 & -2.16974400 & -1.75628100 & -2.67225200 & C & -0.45568500 & -3.40443000 & -3.80774700 \\
\hline $\mathrm{F}$ & -1.17902700 & -3.50097200 & -4.92472700 & $\mathrm{~F}$ & -0.80522300 & -4.40044000 & -2.97673500 \\
\hline $\mathrm{F}$ & 0.84394100 & -3.56153600 & -4.12247600 & C & 2.92106900 & 1.61033900 & -2.99977900 \\
\hline $\mathrm{H}$ & 3.29439100 & 1.12432200 & -3.89735100 & $\mathrm{H}$ & 1.95455900 & 2.09155400 & -3.10240700 \\
\hline C & 3.85032100 & 2.22657200 & -2.15336900 & C & 3.40279100 & 3.14361400 & -1.12330400 \\
\hline $\mathrm{H}$ & 2.35004400 & 3.00243400 & -0.86326900 & $\mathrm{H}$ & 4.02343600 & 3.13130700 & -0.22133900 \\
\hline C & 5.29630200 & 1.87496800 & -2.24482600 & $\mathrm{H}$ & 5.92657900 & 2.55036500 & -1.66314600 \\
\hline $\mathrm{H}$ & 5.42408100 & 0.85624000 & -1.84527400 & $\mathrm{H}$ & 5.64210200 & 1.84198700 & -3.28289600 \\
\hline C & 2.83487400 & 6.10372400 & -0.49765100 & $\mathrm{H}$ & 3.50833200 & 6.14296500 & 0.36390200 \\
\hline $\mathrm{H}$ & 2.73287000 & 7.12145400 & -0.89310400 & $\mathrm{H}$ & 1.85434800 & 5.77514700 & -0.13984200 \\
\hline C & 5.30283700 & 5.37000800 & -2.21356800 & $\mathrm{H}$ & 5.94634200 & 5.22338100 & -1.33825600 \\
\hline $\mathrm{H}$ & 5.70081300 & 4.76129100 & -3.03262400 & $\mathrm{H}$ & 5.39158900 & 6.42113400 & -2.51307600 \\
\hline C & 2.44996800 & 5.07146500 & -3.38407200 & $\mathrm{H}$ & 2.80299500 & 4.40165200 & -4.17495600 \\
\hline $\mathrm{H}$ & 1.40449900 & 4.82253500 & -3.17414500 & $\mathrm{H}$ & 2.47150600 & 6.09386100 & -3.77967200 \\
\hline $\mathrm{Si}$ & 3.50339200 & 4.96248800 & -1.83059600 & C & 3.55209800 & -1.64410500 & 4.74394700 \\
\hline $\mathrm{H}$ & 2.84282800 & -1.19493900 & 5.44619300 & $\mathrm{H}$ & 4.53767700 & -1.21647900 & 4.94724700 \\
\hline C & -1.46475600 & -2.99528600 & 2.88803400 & C & -2.75023200 & -2.20361800 & 2.70690000 \\
\hline 0 & -0.86540100 & -3.50863400 & 3.82281900 & $\mathrm{O}$ & -3.60932100 & -1.77522700 & 3.45972700 \\
\hline $\mathrm{C}$ & -2.41792900 & -2.11793300 & 1.25076300 & C & -1.21611300 & -2.84309000 & 1.43659900 \\
\hline $\mathrm{N}$ & -2.98881000 & -1.60674900 & 0.13731800 & $\mathrm{H}$ & -2.53331100 & -1.78408400 & -0.76277400 \\
\hline $\mathrm{C}$ & -4.11180000 & -0.76959600 & 0.05578900 & $\mathrm{C}$ & -4.69176000 & -0.16236700 & 1.17880900 \\
\hline $\mathrm{C}$ & -4.62893900 & -0.49868500 & -1.21961400 & $\mathrm{C}$ & -5.75210400 & 0.72298400 & 1.00620300 \\
\hline $\mathrm{H}$ & -4.33843000 & -0.39329000 & 2.17589900 & C & -5.67474900 & 0.40750900 & -1.36476700 \\
\hline $\mathrm{H}$ & -4.18306500 & -0.96166800 & -2.09121900 & $\mathrm{C}$ & -6.24892500 & 1.03193700 & -0.25948400 \\
\hline $\mathrm{H}$ & -7.05855600 & 1.74057100 & -0.38125600 & $\mathrm{C}$ & -6.41098600 & 1.33474500 & 2.21152400 \\
\hline C & -6.10828900 & 0.80595400 & -2.74625100 & $\mathrm{~F}$ & -5.59525400 & 1.35549600 & 3.29150400 \\
\hline $\mathrm{F}$ & -7.52245600 & 0.65718700 & 2.57349200 & $\mathrm{~F}$ & -6.79302300 & 2.61218000 & 1.97404300 \\
\hline $\mathrm{F}$ & -5.94975200 & -0.18872900 & -3.64156000 & $\mathrm{~F}$ & -5.38140200 & 1.86268700 & -3.19982500 \\
\hline $\mathrm{F}$ & -7.40429900 & 1.18773500 & -2.78193500 & & & & \\
\hline
\end{tabular}

\section{TS-S-6f-15}

Imaginary frequency: $-434.82 \mathrm{~cm}^{-1}$

E[B3LYP/6-31G(d)-D3(BJ)]: -4655.619167 Hartree

E[PCM(Et $\left.\left.{ }_{2} \mathrm{O}\right)-B 3 L Y P / 6-311 G(d, p)-D 3(B J)\right]:-4656.816636$ Hartree

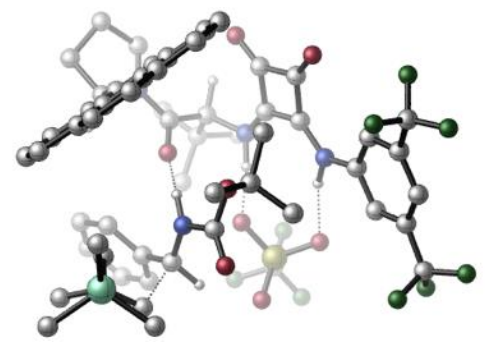

\begin{tabular}{|c|c|c|c|c|c|c|c|}
\hline 01 & & & & C & 1.05872100 & 2.85540600 & -2.77633800 \\
\hline $\mathrm{H}$ & 0.98850900 & 2.72811900 & -3.85811100 & $\mathrm{C}$ & 1.49803800 & 4.32891000 & -2.49351500 \\
\hline C & 1.81824300 & 4.55884300 & -1.00898200 & $\mathrm{H}$ & 2.68858900 & 3.97732200 & -0.69758400 \\
\hline $\mathrm{H}$ & 0.99442900 & 4.28577300 & -0.35219700 & $\mathrm{H}$ & 2.04482400 & 5.61852200 & -0.84478500 \\
\hline C & 0.35925300 & 5.26285200 & -2.93962900 & $\mathrm{H}$ & 0.68277400 & 6.30621800 & -2.85131300 \\
\hline $\mathrm{H}$ & -0.53243200 & 5.13294700 & -2.32397000 & $\mathrm{H}$ & 0.08213400 & 5.08042600 & -3.98484000 \\
\hline C & 2.75539900 & 4.65798600 & -3.31468800 & $\mathrm{H}$ & 3.04323000 & 5.70040300 & -3.14042600 \\
\hline $\mathrm{H}$ & 2.58208000 & 4.53658500 & -4.39020700 & $\mathrm{H}$ & 3.60316300 & 4.02996800 & -3.02136500 \\
\hline C & 2.01799400 & 1.80114000 & -2.19774200 & $\mathrm{C}$ & 3.26170200 & 1.51918200 & -4.40762300 \\
\hline C & 3.93121200 & 0.32764500 & -2.36998400 & $\mathrm{C}$ & 4.31549600 & 0.45114300 & -4.74319100 \\
\hline $\mathrm{H}$ & 2.32770000 & 1.37886400 & -4.95639100 & $\mathrm{H}$ & 3.64876100 & 2.52170200 & -4.60602800 \\
\hline C & 5.06105500 & 0.24502400 & -3.41652400 & $\mathrm{H}$ & 4.28516700 & 0.71958900 & -1.41549900 \\
\hline $\mathrm{H}$ & 4.97173100 & 0.76690500 & -5.55844100 & $\mathrm{H}$ & 3.82632200 & -0.47948500 & -5.04472100 \\
\hline $\mathrm{H}$ & 5.77710500 & 1.05883300 & -3.24916900 & $\mathrm{H}$ & 5.59974500 & -0.70420800 & -3.36529900 \\
\hline $\mathrm{N}$ & 3.03401200 & 1.33288100 & -2.95929100 & C & 3.24476300 & -1.01213700 & -2.15618100 \\
\hline
\end{tabular}




\begin{tabular}{|c|c|c|c|c|c|c|c|}
\hline $\mathrm{C}$ & 2.18214400 & -1.36990400 & -2.93214000 & C & 3.76246400 & -1.93756900 & -1.17050100 \\
\hline C & 1.55277700 & -2.65073400 & -2.83567300 & $\mathrm{H}$ & 1.76745400 & -0.67200400 & -3.64950400 \\
\hline C & 3.13293400 & -3.21796600 & -1.03214900 & C & 4.86574100 & -1.64626100 & -0.34767400 \\
\hline C & 0.48022200 & -3.00998800 & -3.66775200 & C & 2.03581400 & -3.57749300 & -1.86907500 \\
\hline C & 3.62097500 & -4.15754300 & -0.07238500 & $\mathrm{H}$ & 5.37845200 & -0.69573200 & -0.44329700 \\
\hline C & 5.34083300 & -2.57430100 & 0.57943700 & C & -0.10298400 & -4.27084800 & -3.55556100 \\
\hline $\mathrm{H}$ & 0.09361800 & -2.28616900 & -4.37862200 & C & 1.42325800 & -4.86085400 & -1.75436000 \\
\hline C & 4.72838800 & -3.81596200 & 0.72039500 & C & 2.97282500 & -5.43579100 & 0.03647500 \\
\hline $\mathrm{H}$ & 6.20703000 & -2.32675900 & 1.18759900 & C & 0.35988200 & -5.18602100 & -2.61174900 \\
\hline $\mathrm{H}$ & -0.94069700 & -4.52989400 & -4.19526800 & C & 1.92408800 & -5.77122700 & -0.76446200 \\
\hline $\mathrm{H}$ & 5.11532500 & -4.54071300 & 1.43176000 & $\mathrm{H}$ & 3.34717500 & -6.13926200 & 0.77496100 \\
\hline $\mathrm{H}$ & -0.10810200 & -6.16301100 & -2.52379700 & $\mathrm{H}$ & 1.45055500 & -6.74511500 & -0.67129800 \\
\hline $\mathrm{N}$ & -0.27459800 & 2.59056700 & -2.22855100 & $\mathrm{H}$ & -0.40152700 & 2.81838000 & -1.23517200 \\
\hline C & -0.17976000 & -0.49637400 & 1.69264000 & 0 & -0.67671000 & -0.61687300 & 2.80179100 \\
\hline 0 & -0.51068000 & -1.15546800 & 0.58832200 & C & -1.35202100 & -2.38576100 & 0.67725500 \\
\hline C & -2.67515500 & -2.12710900 & 1.39808900 & $\mathrm{H}$ & -3.38014700 & -2.91859000 & 1.12517600 \\
\hline $\mathrm{H}$ & -2.55835800 & -2.10077700 & 2.48074800 & $\mathrm{H}$ & -3.10424300 & -1.17727700 & 1.07841900 \\
\hline C & -1.57490100 & -2.72155400 & -0.78737400 & $\mathrm{H}$ & -0.62260900 & -2.85823500 & -1.29489000 \\
\hline $\mathrm{H}$ & -2.16335800 & -3.63803100 & -0.87994800 & $\mathrm{H}$ & -2.11937400 & -1.92419400 & -1.28945400 \\
\hline C & -0.50996000 & -3.45990700 & 1.36296300 & $\mathrm{H}$ & -1.04871200 & -4.41293200 & 1.34422300 \\
\hline $\mathrm{H}$ & 0.44024800 & -3.59628000 & 0.83719100 & $\mathrm{H}$ & -0.31903200 & -3.20163600 & 2.40757000 \\
\hline C & 1.24860900 & 1.30538000 & 2.38440000 & $\mathrm{H}$ & 0.39385000 & 1.74703200 & 2.89473700 \\
\hline C & 2.33352600 & 2.24184000 & 2.03712200 & C & 2.24685000 & 3.55967400 & 2.50345800 \\
\hline C & 3.46302400 & 1.83865000 & 1.30802500 & C & 3.27388500 & 4.46323500 & 2.24253600 \\
\hline $\mathrm{H}$ & 1.35625200 & 3.87497800 & 3.03686400 & C & 4.48898400 & 2.74476800 & 1.05121600 \\
\hline $\mathrm{H}$ & 3.53210300 & 0.82084800 & 0.93890600 & C & 4.39915100 & 4.05691700 & 1.52262800 \\
\hline $\mathrm{H}$ & 3.18952100 & 5.48780000 & 2.59136700 & $\mathrm{H}$ & 5.35845300 & 2.42775400 & 0.48164900 \\
\hline $\mathrm{H}$ & 5.19936800 & 4.76275900 & 1.31985300 & $\mathrm{~N}$ & 0.90375900 & 0.34466800 & 1.45415000 \\
\hline $\mathrm{H}$ & 1.24372000 & 0.45621000 & 0.49422300 & $S$ & -1.56048800 & 3.36382900 & 1.41821900 \\
\hline 0 & -1.37772600 & 3.09007900 & 2.85522700 & O & -0.48218400 & 2.80097800 & 0.55602000 \\
\hline 0 & -2.91356800 & 3.14820900 & 0.84861500 & C & -1.32701500 & 5.20138700 & 1.27002800 \\
\hline $\mathrm{F}$ & -2.23833800 & 5.85992000 & 1.98831100 & $\mathrm{~F}$ & -1.43387100 & 5.58204100 & -0.01506100 \\
\hline $\mathrm{F}$ & -0.10214500 & 5.55417000 & 1.70516100 & C & 1.78472300 & 0.44833500 & 4.06279900 \\
\hline $\mathrm{H}$ & 1.91055000 & 1.32231200 & 4.69675400 & $\mathrm{H}$ & 0.82850700 & -0.06090300 & 4.14318900 \\
\hline C & 2.91730900 & -0.31981300 & 3.78561100 & C & 2.80794000 & -1.61013700 & 3.12146200 \\
\hline $\mathrm{H}$ & 1.94935300 & -1.66039900 & 2.44428600 & $\mathrm{H}$ & 3.71773500 & -1.89657000 & 2.58539800 \\
\hline C & 4.27894200 & 0.21325800 & 4.08340400 & $\mathrm{H}$ & 5.00548100 & -0.58993000 & 4.23336000 \\
\hline $\mathrm{H}$ & 4.60719000 & 0.79943100 & 3.21019900 & $\mathrm{H}$ & 4.28358700 & 0.88764600 & 4.94351700 \\
\hline C & 2.44473700 & -4.63883500 & 3.50834300 & $\mathrm{H}$ & 3.44153600 & -4.88004700 & 3.12727300 \\
\hline $\mathrm{H}$ & 2.13488500 & -5.44825700 & 4.18036800 & $\mathrm{H}$ & 1.75874600 & -4.62366600 & 2.65708000 \\
\hline C & 3.87345800 & -2.99124200 & 5.69150400 & $\mathrm{H}$ & 4.84757200 & -3.12745500 & 5.20685500 \\
\hline $\mathrm{H}$ & 3.90569100 & -2.05498800 & 6.26006800 & $\mathrm{H}$ & 3.74931200 & -3.80831600 & 6.41211700 \\
\hline C & 0.81918900 & -2.65591800 & 5.28222600 & $\mathrm{H}$ & 0.90223100 & -1.82936900 & 5.99610100 \\
\hline $\mathrm{H}$ & 0.03454700 & -2.39312200 & 4.56542800 & $\mathrm{H}$ & 0.48852000 & -3.54047500 & 5.83950700 \\
\hline $\mathrm{Si}$ & 2.46285500 & -3.00515400 & 4.43958800 & 0 & 1.85412900 & 1.38676900 & -1.04423600 \\
\hline $\mathrm{C}$ & -0.95610200 & 1.53299900 & -2.68445300 & C & -2.13403200 & 0.85275600 & -2.28937400 \\
\hline C & -0.73108400 & 0.61591300 & -3.81786300 & C & -1.96925100 & -0.15294200 & -3.39134800 \\
\hline 0 & 0.12179400 & 0.49245800 & -4.69114100 & $\mathrm{O}$ & -2.51250400 & -1.17837800 & -3.76625600 \\
\hline $\mathrm{N}$ & -2.99082400 & 1.10399200 & -1.27485900 & $\mathrm{H}$ & -2.78822300 & 1.90393400 & -0.66612300 \\
\hline C & -4.08019700 & 0.32842300 & -0.84480100 & C & -4.76308800 & 0.75159400 & 0.30792700 \\
\hline C & -4.47048800 & -0.85448900 & -1.48436700 & C & -5.78985800 & -0.02927700 & 0.82751700 \\
\hline $\mathrm{H}$ & -4.46829700 & 1.66927000 & 0.80296100 & C & -5.48217300 & -1.63085900 & -0.92284600 \\
\hline $\mathrm{H}$ & -3.97610100 & -1.18866000 & -2.39051200 & C & -6.15362700 & -1.23625500 & 0.23110800 \\
\hline
\end{tabular}




\begin{tabular}{|c|c|c|c|c|c|c|c|}
\hline $\mathrm{H}$ & -6.94043100 & -1.84839500 & 0.65303600 & C & -5.75274700 & -2.98619100 & -1.51150300 \\
\hline C & -6.44857900 & 0.38329400 & 2.11543300 & $\mathrm{~F}$ & -5.60483200 & -3.00801200 & -2.84933600 \\
\hline$F$ & -4.88775600 & -3.91037600 & -1.00580200 & $\mathrm{~F}$ & -6.99709700 & -3.42889300 & -1.22759300 \\
\hline $\mathrm{F}$ & -6.44522000 & 1.72002100 & 2.28432100 & $\mathrm{~F}$ & -5.81580400 & -0.15636500 & 3.18367500 \\
\hline $\mathrm{F}$ & -7.73508900 & -0.03333700 & 2.17296100 & & & & \\
\hline
\end{tabular}

\section{TS-S-6f-16}

Imaginary frequency: $-423.84 \mathrm{~cm}^{-1}$

E[B3LYP/6-31G(d)-D3(BJ)]: -4655.616509 Hartree

$\mathrm{E}\left[\mathrm{PCM}\left(\mathrm{Et}_{2} \mathrm{O}\right)-\mathrm{B} 3 \mathrm{LYP} / 6-311 \mathrm{G}(\mathrm{d}, \mathrm{p})-\mathrm{D} 3(\mathrm{BJ})\right]$ : -4656.815557 Hartree

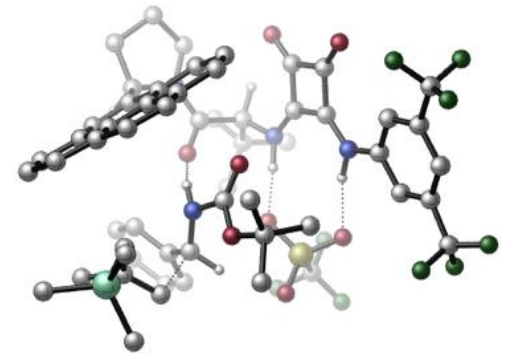

\begin{tabular}{|c|c|c|c|c|c|c|c|}
\hline 01 & & & & C & -0.42273200 & 3.93020100 & 1.01916300 \\
\hline $\mathrm{H}$ & -0.10156400 & 4.39538700 & 1.94947100 & $C$ & -0.71175300 & 5.08281100 & 0.00636300 \\
\hline$C$ & -1.28431900 & 4.58521100 & -1.33165700 & $\mathrm{H}$ & -2.26070400 & 4.11357400 & -1.20589500 \\
\hline $\mathrm{H}$ & -0.63121900 & 3.86029200 & -1.81774900 & $\mathrm{H}$ & -1.40137700 & 5.43941500 & -2.00912100 \\
\hline C & 0.60742800 & 5.83185700 & -0.24829000 & $\mathrm{H}$ & 0.41783900 & 6.72185200 & -0.85891000 \\
\hline $\mathrm{H}$ & 1.32305900 & 5.20191500 & -0.78235700 & $\mathrm{H}$ & 1.07144900 & 6.15690900 & 0.69076300 \\
\hline C & -1.72420700 & 6.04220900 & 0.65685800 & $\mathrm{H}$ & -1.93462100 & 6.87801200 & -0.01904300 \\
\hline $\mathrm{H}$ & -1.33962500 & 6.45945400 & 1.59500900 & $\mathrm{H}$ & -2.67672600 & 5.54161200 & 0.86719200 \\
\hline C & -1.65051300 & 3.04694800 & 1.27257400 & $\mathrm{C}$ & -2.09196100 & 4.10081400 & 3.54603300 \\
\hline C & -3.53305400 & 2.33207600 & 2.64249300 & C & -2.81990600 & 3.43689200 & 4.71626500 \\
\hline $\mathrm{H}$ & -1.02344700 & 4.20760800 & 3.73026200 & $\mathrm{H}$ & -2.52863200 & 5.08026300 & 3.31388300 \\
\hline C & -4.02759700 & 2.76180900 & 4.05237800 & $\mathrm{H}$ & -4.27113900 & 2.62378400 & 1.88732700 \\
\hline $\mathrm{H}$ & -3.10688600 & 4.16097300 & 5.48357000 & $\mathrm{H}$ & -2.16972800 & 2.69212500 & 5.18392500 \\
\hline $\mathrm{H}$ & -4.84764800 & 3.47971800 & 3.94026800 & $\mathrm{H}$ & -4.40776900 & 1.90877300 & 4.61838200 \\
\hline $\mathrm{N}$ & -2.33358200 & 3.16467700 & 2.43167800 & C & -3.37488500 & 0.82215200 & 2.49164200 \\
\hline C & -4.46152200 & 0.17555900 & 1.96992100 & C & -2.24215400 & 0.04638300 & 2.96273500 \\
\hline C & -4.57329800 & -1.24845900 & 1.90730000 & $\mathrm{H}$ & -5.30994300 & 0.76052700 & 1.61932200 \\
\hline C & -2.30649000 & -1.38355400 & 2.86112000 & C & -1.08148000 & 0.61293400 & 3.51642600 \\
\hline C & -5.73536700 & -1.89117600 & 1.44761200 & C & -3.47343400 & -2.03223500 & 2.36193400 \\
\hline C & -1.20485700 & -2.18076100 & 3.29958500 & $\mathrm{H}$ & -0.98454100 & 1.68214500 & 3.60434500 \\
\hline C & -0.01357200 & -0.17401400 & 3.94172800 & C & -5.82708500 & -3.28222500 & 1.45750400 \\
\hline $\mathrm{H}$ & -6.57625100 & -1.28893500 & 1.11284400 & C & -3.56439000 & -3.45873400 & 2.33756700 \\
\hline C & -0.06629000 & -1.55660900 & 3.82672500 & C & -1.30377400 & -3.61046100 & 3.21999400 \\
\hline $\mathrm{H}$ & 0.86639000 & 0.30235800 & 4.36066800 & C & -4.75486600 & -4.05955400 & 1.89436400 \\
\hline $\mathrm{H}$ & -6.74356500 & -3.76376200 & 1.12791100 & C & -2.43292100 & -4.22559300 & 2.77445500 \\
\hline $\mathrm{H}$ & 0.77201000 & -2.16588500 & 4.15350700 & $\mathrm{H}$ & -0.45157500 & -4.19864800 & 3.54989400 \\
\hline $\mathrm{H}$ & -4.83402500 & -5.14353700 & 1.90087300 & $\mathrm{H}$ & -2.49976500 & -5.31011700 & 2.75333000 \\
\hline $\mathrm{N}$ & 0.68452700 & 3.07530400 & 0.58750000 & $\mathrm{H}$ & 0.61236500 & 2.63455800 & -0.33577200 \\
\hline C & -0.41026000 & -0.90648300 & -0.43317400 & 0 & 0.14983900 & -0.67299200 & 0.61555000 \\
\hline $\mathrm{O}$ & -0.10896400 & -1.86902400 & -1.32464300 & C & 1.05238500 & -2.76570900 & -1.13287100 \\
\hline $\mathrm{C}$ & 0.88188900 & -3.55240400 & 0.16691900 & $\mathrm{H}$ & 1.67139900 & -4.30838800 & 0.23548400 \\
\hline $\mathrm{H}$ & 0.94646100 & -2.89431600 & 1.03375000 & $\mathrm{H}$ & -0.08598500 & -4.06499400 & 0.19195900 \\
\hline C & 0.96470300 & -3.67538500 & -2.35538900 & $\mathrm{H}$ & 1.00640400 & -3.08050800 & -3.27310000 \\
\hline $\mathrm{H}$ & 1.80487000 & -4.37616100 & -2.35792800 & $\mathrm{H}$ & 0.03492600 & -4.25486500 & -2.34676300 \\
\hline $\mathrm{C}$ & 2.33200400 & -1.94355100 & -1.15494500 & $\mathrm{H}$ & 3.20334900 & -2.60528600 & -1.15664900 \\
\hline $\mathrm{H}$ & 2.37345400 & -1.32379300 & -2.05416600 & $\mathrm{H}$ & 2.38363500 & -1.30110000 & -0.27832400 \\
\hline C & -1.83293800 & -0.00217700 & -2.19218300 & $\mathrm{H}$ & -0.96693000 & -0.06758900 & -2.84471000 \\
\hline C & -2.74657400 & 1.11656400 & -2.49887600 & $C$ & -2.51974800 & 1.84718200 & -3.67348500 \\
\hline C & -3.81700400 & 1.46652600 & -1.66173100 & $\mathrm{C}$ & -3.34226000 & 2.92098400 & -4.00021800 \\
\hline $\mathrm{H}$ & -1.67400400 & 1.59043700 & -4.30243300 & $\mathrm{C}$ & -4.64269100 & 2.53700500 & -1.99720700 \\
\hline
\end{tabular}




\begin{tabular}{|c|c|c|c|c|c|c|c|}
\hline $\mathrm{H}$ & -3.98415700 & 0.92274600 & -0.73809400 & C & -4.40828800 & 3.26504000 & -3.16544200 \\
\hline $\mathrm{H}$ & -3.14387500 & 3.49663400 & -4.89906500 & $\mathrm{H}$ & -5.46392300 & 2.80914200 & -1.34028500 \\
\hline $\mathrm{H}$ & -5.04963700 & 4.10371500 & -3.42059800 & $\mathrm{~N}$ & -1.54683600 & -0.21842800 & -0.85275800 \\
\hline $\mathrm{H}$ & -1.80175100 & 0.54215600 & -0.21335400 & $S$ & 1.40265200 & 1.44676000 & -2.93385200 \\
\hline 0 & 0.95992800 & 0.39249100 & -3.86770500 & $\mathrm{O}$ & 0.41056600 & 1.75089000 & -1.86555000 \\
\hline 0 & 2.79311700 & 1.34515100 & -2.42852800 & C & 1.42969000 & 3.00417600 & -3.94670700 \\
\hline $\mathrm{F}$ & 2.30016700 & 2.90579400 & -4.95321000 & $F$ & 1.77558500 & 4.04664700 & -3.17364700 \\
\hline $\mathrm{F}$ & 0.20912100 & 3.25189200 & -4.45691200 & C & -2.59172800 & -1.54516300 & -3.11185900 \\
\hline $\mathrm{H}$ & -2.90272000 & -1.06304500 & -4.03458300 & $\mathrm{H}$ & -1.66745500 & -2.10898300 & -3.17005800 \\
\hline $\mathrm{C}$ & -3.57966000 & -2.04232000 & -2.25666000 & $\mathrm{C}$ & -3.22606700 & -2.96323400 & -1.19729200 \\
\hline $\mathrm{H}$ & -2.16402500 & -2.91922800 & -0.94824500 & $\mathrm{H}$ & -3.84942700 & -2.86416700 & -0.30753100 \\
\hline $\mathrm{C}$ & -4.99647500 & -1.59134300 & -2.39945100 & $\mathrm{H}$ & -5.61966000 & -1.92035400 & -1.56549500 \\
\hline $\mathrm{H}$ & -5.02766200 & -0.49569300 & -2.46510400 & $\mathrm{H}$ & -5.42106700 & -1.96658000 & -3.33984500 \\
\hline C & -2.55841900 & -5.89910800 & -0.64087800 & $\mathrm{H}$ & -2.83129300 & -5.69762300 & 0.39808900 \\
\hline $\mathrm{H}$ & -2.76414100 & -6.95510300 & -0.85260400 & $\mathrm{H}$ & -1.47888800 & -5.74185000 & -0.73939700 \\
\hline $\mathrm{C}$ & -5.35769800 & -5.15732600 & -1.79638400 & $\mathrm{H}$ & -5.77332000 & -5.01079600 & -0.79520600 \\
\hline $\mathrm{H}$ & -5.90981000 & -4.51264800 & -2.48932300 & $\mathrm{H}$ & -5.54462400 & -6.19607500 & -2.09361800 \\
\hline C & -2.84535200 & -4.96626200 & -3.57692400 & $\mathrm{H}$ & -3.40541600 & -4.35245000 & -4.29092300 \\
\hline $\mathrm{H}$ & -1.79061100 & -4.68020100 & -3.64815900 & $\mathrm{H}$ & -2.92562200 & -6.01009500 & -3.90335700 \\
\hline $\mathrm{Si}$ & -3.51157300 & -4.79116900 & -1.82392700 & $\mathrm{O}$ & -2.00051100 & 2.22032100 & 0.42028100 \\
\hline C & 1.50591500 & 2.54000100 & 1.49603000 & C & 2.62605200 & 1.67266500 & 1.46486100 \\
\hline C & 1.57040700 & 2.65928500 & 2.96729200 & $\mathrm{C}$ & 2.72391200 & 1.67343100 & 2.95752200 \\
\hline $\mathrm{O}$ & 0.93764600 & 3.26240700 & 3.82642400 & $\mathrm{O}$ & 3.36430000 & 1.09716400 & 3.82116300 \\
\hline $\mathrm{N}$ & 3.29096800 & 1.12407300 & 0.42515500 & $\mathrm{H}$ & 2.96834400 & 1.35143900 & -0.52073600 \\
\hline $\mathrm{C}$ & 4.36560700 & 0.22093800 & 0.46004600 & $\mathrm{C}$ & 4.94707400 & -0.13437400 & -0.76735900 \\
\hline C & 4.83310400 & -0.36803000 & 1.64171400 & $\mathrm{C}$ & 5.95096100 & -1.09499600 & -0.80497300 \\
\hline $\mathrm{H}$ & 4.57993300 & 0.30762500 & -1.68541400 & C & 5.86055100 & -1.30830000 & 1.57602900 \\
\hline $\mathrm{H}$ & 4.40211500 & -0.10342500 & 2.60195400 & $\mathrm{C}$ & 6.42535600 & -1.69252500 & 0.36222000 \\
\hline $\mathrm{H}$ & 7.20631900 & -2.44071000 & 0.32465000 & $\mathrm{C}$ & 6.38604600 & -1.88937400 & 2.86074300 \\
\hline C & 6.43592100 & -1.58228500 & -2.13991400 & $\mathrm{~F}$ & 7.28656900 & -1.06931700 & 3.44773700 \\
\hline $\mathrm{F}$ & 5.39973000 & -2.09919600 & 3.75845300 & $\mathrm{~F}$ & 7.00718500 & -3.07576900 & 2.65974700 \\
\hline $\mathrm{F}$ & 6.42847600 & -0.61106400 & -3.07487700 & $\mathrm{~F}$ & 5.64254300 & -2.58284200 & -2.60636100 \\
\hline $\mathrm{F}$ & 7.69069200 & -2.08039400 & -2.07632500 & & & & \\
\hline
\end{tabular}

\section{TS-S-6f-17}

Imaginary frequency: $-323.44 \mathrm{~cm}-1$

E[B3LYP/6-31G(d)-D3(BJ)]: -4655.614905 Hartree

$\mathrm{E}\left[\mathrm{PCM}\left(\mathrm{Et}_{2} \mathrm{O}\right)-\mathrm{B} 3 \mathrm{LYP} / 6-311 \mathrm{G}(\mathrm{d}, \mathrm{p})-\mathrm{D} 3(\mathrm{BJ})\right]:-4656.815038$ Hartree

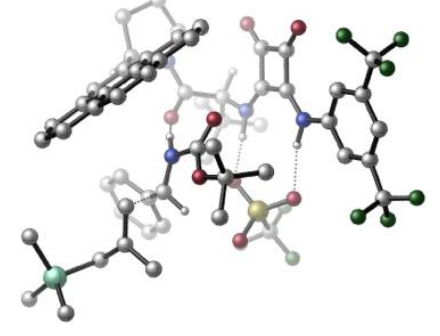

\begin{tabular}{|crrr|lrll|}
\hline 01 & & & & $\mathrm{C}$ & 0.08107600 & 3.00168000 & 2.64921000 \\
\hline $\mathrm{H}$ & -0.48500700 & 3.92857800 & 2.71986700 & $\mathrm{C}$ & 0.68296500 & 2.72245500 & 4.06115500 \\
\hline $\mathrm{C}$ & 1.57690200 & 1.47144400 & 4.10341700 & $\mathrm{H}$ & 2.45090700 & 1.57309000 & 3.45785300 \\
\hline $\mathrm{H}$ & 1.04493100 & 0.57136800 & 3.79366700 & $\mathrm{H}$ & 1.92451500 & 1.31666600 & 5.13188500 \\
\hline $\mathrm{C}$ & -0.48315000 & 2.54917200 & 5.04948100 & $\mathrm{H}$ & -0.09617800 & 2.46724400 & 6.07150300 \\
\hline $\mathrm{H}$ & -1.05493200 & 1.64357900 & 4.83119300 & $\mathrm{H}$ & -1.16943100 & 3.40390100 & 5.01389000 \\
\hline $\mathrm{C}$ & 1.51942300 & 3.94802700 & 4.46956900 & $\mathrm{H}$ & 1.93399800 & 3.80040500 & 5.47258300 \\
\hline $\mathrm{H}$ & 0.91445400 & 4.86216500 & 4.48878900 & $\mathrm{H}$ & 2.36172100 & 4.10708100 & 3.78591300 \\
\hline $\mathrm{C}$ & 1.15600700 & 3.12114000 & 1.56033200 & $\mathrm{C}$ & 0.99682300 & 5.65298900 & 1.48480600 \\
\hline $\mathrm{C}$ & 2.50416600 & 4.44258900 & -0.00379200 & $\mathrm{C}$ & 1.19257000 & 6.53138600 & 0.24771300 \\
\hline $\mathrm{H}$ & -0.04753500 & 5.60977700 & 1.78687600 & $\mathrm{H}$ & 1.61450200 & 6.00150100 & 2.32288500 \\
\hline $\mathrm{C}$ & 2.41607100 & 5.92762900 & -0.46639600 & $\mathrm{H}$ & 3.47092500 & 4.27192000 & 0.48465600 \\
\hline
\end{tabular}




\begin{tabular}{|c|c|c|c|c|c|c|c|}
\hline$\overline{\mathrm{H}}$ & 1.33825400 & 7.58155800 & 0.51502500 & $\mathrm{H}$ & 0.30324300 & 6.47135400 & -0.38480600 \\
\hline $\mathrm{H}$ & 3.33388800 & 6.44910700 & -0.17786500 & $\mathrm{H}$ & 2.33088400 & 5.99303600 & -1.55335500 \\
\hline $\mathrm{N}$ & 1.45983400 & 4.32977700 & 1.03895700 & C & 2.42938300 & 3.43516400 & -1.13923500 \\
\hline C & 3.59474600 & 2.81414100 & -1.48697300 & C & 1.22481900 & 3.17800500 & -1.90437000 \\
\hline C & 3.68894700 & 1.87301500 & -2.56024900 & $\mathrm{H}$ & 4.49771500 & 3.01872500 & -0.91507000 \\
\hline C & 1.29742600 & 2.26456900 & -3.00530700 & C & -0.01544500 & 3.76923200 & -1.61549700 \\
\hline C & 4.88706500 & 1.21271700 & -2.87842700 & C & 2.51717900 & 1.60045100 & -3.32456900 \\
\hline C & 0.13440900 & 2.00400900 & -3.79210700 & $\mathrm{H}$ & -0.12178900 & 4.42184900 & -0.76377600 \\
\hline C & -1.14749700 & 3.50013800 & -2.38232400 & C & 4.93693800 & 0.30379700 & -3.93476000 \\
\hline $\mathrm{H}$ & 5.78179200 & 1.43186300 & -2.30036800 & C & 2.57919000 & 0.67030500 & -4.40672300 \\
\hline C & -1.07450900 & 2.63447300 & -3.46619600 & C & 0.23202300 & 1.08569800 & -4.89035500 \\
\hline $\mathrm{H}$ & -2.09406800 & 3.95764600 & -2.11271100 & C & 3.79719500 & 0.02961100 & -4.68767300 \\
\hline $\mathrm{H}$ & 5.87422300 & -0.18610400 & -4.17978500 & C & 1.39397300 & 0.43970900 & -5.18157100 \\
\hline $\mathrm{H}$ & -1.96087800 & 2.42249800 & -4.05797600 & $\mathrm{H}$ & -0.66005300 & 0.90480000 & -5.48401200 \\
\hline $\mathrm{H}$ & 3.84529500 & -0.67238800 & -5.51624000 & $\mathrm{H}$ & 1.44603200 & -0.25760300 & -6.01381200 \\
\hline $\mathrm{N}$ & -0.87731600 & 1.97100800 & 2.24416900 & $\mathrm{H}$ & -0.56688300 & 0.99524200 & 2.26837700 \\
\hline C & 0.16621100 & -0.45279100 & -1.02672500 & $\mathrm{O}$ & -0.69380300 & 0.38558000 & -1.18963100 \\
\hline $\mathrm{O}$ & 0.13736600 & -1.74349600 & -1.40646900 & C & -1.03794400 & -2.34767700 & -2.07131200 \\
\hline $\mathrm{C}$ & -1.22066800 & -1.69741900 & -3.43901400 & $\mathrm{H}$ & -2.02591400 & -2.20713600 & -3.97888100 \\
\hline $\mathrm{H}$ & -1.48231900 & -0.64394500 & -3.33064000 & $\mathrm{H}$ & -0.30278500 & -1.76987100 & -4.03030700 \\
\hline C & -0.62543300 & -3.81252200 & -2.18936900 & $\mathrm{H}$ & -0.44280800 & -4.23173800 & -1.19525800 \\
\hline $\mathrm{H}$ & -1.42576800 & -4.38563800 & -2.66702300 & $\mathrm{H}$ & 0.28027000 & -3.91568400 & -2.79830600 \\
\hline C & -2.27215400 & -2.20439600 & -1.19403000 & $\mathrm{H}$ & -3.09196000 & -2.79898500 & -1.60934300 \\
\hline $\mathrm{H}$ & -2.07290700 & -2.56602600 & -0.18278700 & $\mathrm{H}$ & -2.58565700 & -1.16440600 & -1.14395100 \\
\hline C & 2.13648500 & -1.17487500 & 0.18170600 & $\mathrm{H}$ & 1.53435600 & -2.03404400 & 0.45317800 \\
\hline $\mathrm{C}$ & 3.10670400 & -0.76006500 & 1.22467600 & C & 3.15571600 & -1.49058700 & 2.41709300 \\
\hline C & 4.00532300 & 0.29330900 & 1.01935300 & C & 4.08086800 & -1.15616200 & 3.40457600 \\
\hline $\mathrm{H}$ & 2.44796600 & -2.29503000 & 2.57895800 & C & 4.93720700 & 0.61630900 & 2.00214700 \\
\hline $\mathrm{H}$ & 3.96354500 & 0.86301400 & 0.09853500 & C & 4.97619100 & -0.10612900 & 3.19793600 \\
\hline $\mathrm{H}$ & 4.09255400 & -1.71111800 & 4.33781500 & $\mathrm{H}$ & 5.62685900 & 1.44004500 & 1.83994200 \\
\hline $\mathrm{H}$ & 5.69814000 & 0.15367300 & 3.96695200 & $\mathrm{~N}$ & 1.38782700 & -0.17540100 & -0.41779400 \\
\hline $\mathrm{H}$ & 1.47823800 & 0.77413700 & -0.03963300 & $\mathrm{~S}$ & -0.62665200 & -2.01806400 & 2.42222800 \\
\hline 0 & 0.07980300 & -3.18892100 & 1.85807600 & O & 0.00499100 & -0.71414800 & 2.08544300 \\
\hline 0 & -2.10174000 & -2.00669200 & 2.28179700 & C & -0.37273300 & -2.15535000 & 4.25851200 \\
\hline $\mathrm{F}$ & -0.92491800 & -3.27273400 & 4.73589300 & $\mathrm{~F}$ & -0.92730600 & -1.09893400 & 4.87459400 \\
\hline $\mathrm{F}$ & 0.94058600 & -2.16701700 & 4.55406300 & C & 3.15999300 & -1.92948700 & -1.21087300 \\
\hline $\mathrm{H}$ & 2.36282900 & -2.05253100 & -1.93617700 & $\mathrm{H}$ & 3.86196300 & -1.12942400 & -1.43117300 \\
\hline C & 3.64732200 & -3.08811900 & -0.58567400 & C & 4.93817000 & -3.10698100 & 0.08143400 \\
\hline $\mathrm{H}$ & 5.27691300 & -2.10153600 & 0.35344300 & $\mathrm{H}$ & 4.94549600 & -3.75907000 & 0.96197200 \\
\hline C & 2.75029900 & -4.27047200 & -0.43986400 & $\mathrm{H}$ & 3.30816400 & -5.18804200 & -0.23874600 \\
\hline $\mathrm{H}$ & 2.08776700 & -4.09311700 & 0.42543500 & $\mathrm{H}$ & 2.09659400 & -4.40462100 & -1.30409000 \\
\hline C & 7.88017000 & -3.85449900 & -0.05041500 & $\mathrm{H}$ & 7.76138200 & -4.54176400 & 0.79475400 \\
\hline $\mathrm{H}$ & 8.73431500 & -4.20076300 & -0.64425400 & $\mathrm{H}$ & 8.13147700 & -2.86654400 & 0.35151200 \\
\hline C & 5.84309300 & -5.49714300 & -1.72692200 & $\mathrm{H}$ & 5.66477100 & -6.19547100 & -0.90113700 \\
\hline $\mathrm{H}$ & 4.93908800 & -5.46697200 & -2.34518700 & $\mathrm{H}$ & 6.64847000 & -5.91600600 & -2.34189500 \\
\hline C & 6.50550000 & -2.57227100 & -2.52830700 & $\mathrm{H}$ & 5.58451200 & -2.49543800 & -3.11501800 \\
\hline $\mathrm{H}$ & 6.74372100 & -1.56747300 & -2.16221800 & $\mathrm{H}$ & 7.31131900 & -2.88275900 & -3.20383500 \\
\hline $\mathrm{Si}$ & 6.32390800 & -3.78624400 & -1.10673200 & O & 1.71513200 & 2.09480900 & 1.15120200 \\
\hline C & -1.89783400 & 2.29949400 & 1.44194500 & C & -2.98508300 & 1.61055100 & 0.85445800 \\
\hline C & -2.32088600 & 3.58750700 & 0.85407600 & C & -3.44275400 & 2.84837600 & 0.14633200 \\
\hline 0 & -1.92342000 & 4.74547800 & 0.90075100 & $\mathrm{O}$ & -4.28299200 & 3.15702100 & -0.68140200 \\
\hline $\mathrm{N}$ & -3.38550200 & 0.32541200 & 0.97475200 & $\mathrm{H}$ & -2.80698900 & -0.30342200 & 1.53763500 \\
\hline C & -4.48577900 & -0.30143200 & 0.36820600 & C & -4.75230600 & -1.63002800 & 0.73412500 \\
\hline
\end{tabular}




\begin{tabular}{|lrrr|lrrr|}
\hline $\mathrm{C}$ & -5.28082600 & 0.31977600 & -0.60337400 & $\mathrm{C}$ & -5.77278200 & -2.33073100 & 0.10197300 \\
\hline $\mathrm{H}$ & -4.13148200 & -2.12090700 & 1.47298900 & $\mathrm{C}$ & -6.31612300 & -0.39887200 & -1.19982400 \\
\hline $\mathrm{H}$ & -5.09106100 & 1.34549800 & -0.90344200 & $\mathrm{C}$ & -6.57182200 & -1.72729600 & -0.86830500 \\
\hline $\mathrm{H}$ & -7.36415900 & -2.27938400 & -1.35662300 & $\mathrm{C}$ & -7.19514200 & 0.30189100 & -2.20016600 \\
\hline $\mathrm{C}$ & -5.92643400 & -3.79700300 & 0.38644000 & $\mathrm{~F}$ & -8.16585200 & 1.02132100 & -1.59230400 \\
\hline $\mathrm{F}$ & -6.49530200 & 1.15808800 & -2.97358500 & $\mathrm{~F}$ & -7.81492100 & -0.57412300 & -3.02623000 \\
\hline $\mathrm{F}$ & -5.62105400 & -4.10689000 & 1.66304100 & $\mathrm{~F}$ & -5.10140800 & -4.53243100 & -0.40416100 \\
\hline $\mathrm{F}$ & -7.18313200 & -4.23430900 & 0.14887300 & & & & \\
\hline
\end{tabular}

\section{TS-S-6f-18}

Imaginary frequency: $-410.80 \mathrm{~cm}^{-1}$ E[B3LYP/6-31G(d)-D3(BJ)]: -4655.615188 Hartree E[PCM(Et $\left.\left.{ }_{2} \mathrm{O}\right)-B 3 L Y P / 6-311 \mathrm{G}(\mathrm{d}, \mathrm{p})-\mathrm{D} 3(\mathrm{BJ})\right]$ : -4656.812186 Hartree

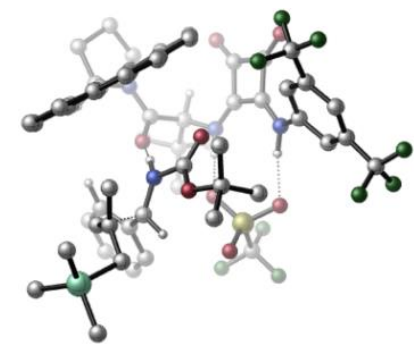

\begin{tabular}{|c|c|c|c|c|c|c|c|}
\hline 01 & & & & C & 0.32784900 & 4.35937500 & 0.05386800 \\
\hline $\mathrm{H}$ & -0.15645300 & 5.07632400 & -0.60689100 & C & 1.00884400 & 5.16253200 & 1.20430300 \\
\hline $\mathrm{C}$ & 1.46139700 & 4.25408700 & 2.35871800 & $\mathrm{H}$ & 2.07721000 & 3.42663200 & 2.00665200 \\
\hline $\mathrm{H}$ & 0.61009100 & 3.83523400 & 2.89705500 & $\mathrm{H}$ & 2.04711500 & 4.84210700 & 3.07481200 \\
\hline C & -0.01674000 & 6.18127700 & 1.72765700 & $\mathrm{H}$ & 0.40270800 & 6.74466000 & 2.56886200 \\
\hline $\mathrm{H}$ & -0.92204000 & 5.67467900 & 2.07588400 & $\mathrm{H}$ & -0.30354700 & 6.89780700 & 0.94813000 \\
\hline $\mathrm{C}$ & 2.22879200 & 5.90721500 & 0.63553100 & $\mathrm{H}$ & 2.66802300 & 6.54608100 & 1.40928000 \\
\hline $\mathrm{H}$ & 1.95458400 & 6.55142100 & -0.20816000 & $\mathrm{H}$ & 3.00631600 & 5.21002500 & 0.30260400 \\
\hline C & 1.31841500 & 3.50627700 & -0.74863800 & C & 1.25308200 & 5.09397100 & -2.73331800 \\
\hline C & 2.63356700 & 3.06355100 & -2.75017700 & C & 1.56945200 & 4.74549700 & -4.18924900 \\
\hline $\mathrm{H}$ & 0.19534500 & 5.30648700 & -2.57642700 & $\bar{H}$ & 1.85463000 & 5.94728100 & -2.39476700 \\
\hline $\mathrm{C}$ & 2.80573700 & 3.84246900 & -4.08418400 & $\overline{\mathrm{H}}$ & 3.57317400 & 3.08475400 & -2.18749500 \\
\hline $\mathrm{H}$ & 1.74666800 & 5.63939800 & -4.79334600 & $\mathrm{H}$ & 0.73124300 & 4.20185300 & -4.63392500 \\
\hline $\mathrm{H}$ & 3.71469100 & 4.45161500 & -4.02723000 & $\bar{H}$ & 2.91393200 & 3.16233500 & -4.93164300 \\
\hline $\mathrm{N}$ & 1.65365800 & 3.87569900 & -2.00342100 & $\mathrm{C}$ & 2.31188500 & 1.58520100 & -2.94904900 \\
\hline C & 3.39321300 & 0.74793300 & -2.93857400 & C & 0.99986100 & 1.05098800 & -3.26693300 \\
\hline C & 3.31776400 & -0.63864400 & -3.28305600 & $\overline{\mathrm{H}}$ & 4.37578000 & 1.15270200 & -2.70241900 \\
\hline $\mathrm{C}$ & 0.88661200 & -0.35026600 & -3.55867900 & $\mathrm{C}$ & -0.17043200 & 1.82687500 & -3.31891500 \\
\hline C & 4.45587200 & -1.45901400 & -3.35564500 & C & 2.03975100 & -1.18728000 & -3.59466100 \\
\hline C & -0.38962400 & -0.91817900 & -3.85813700 & $\mathrm{H}$ & -0.13603500 & 2.88089100 & -3.09849900 \\
\hline C & -1.40601000 & 1.26590900 & -3.63610500 & $\mathrm{C}$ & 4.34577300 & -2.79110800 & -3.75339200 \\
\hline $\mathrm{H}$ & 5.43027500 & -1.03389300 & -3.12806600 & C & 1.93126800 & -2.56545600 & -3.95971600 \\
\hline C & -1.52330400 & -0.09466100 & -3.88858100 & $\mathrm{C}$ & -0.48072800 & -2.31964400 & -4.15572200 \\
\hline $\mathrm{H}$ & -2.28477900 & 1.90011900 & -3.67855300 & $\mathrm{C}$ & 3.09879800 & -3.34133000 & -4.04831300 \\
\hline $\mathrm{H}$ & 5.23873900 & -3.40544000 & -3.83293200 & C & 0.62762700 & -3.10725300 & -4.21734500 \\
\hline $\mathrm{H}$ & -2.49000700 & -0.52633800 & -4.12412800 & $\mathrm{H}$ & -1.46777400 & -2.73512300 & -4.33585800 \\
\hline $\mathrm{H}$ & 3.01779600 & -4.38322100 & -4.34731600 & $\mathrm{H}$ & 0.54439800 & -4.16202400 & -4.46621800 \\
\hline $\mathrm{N}$ & -0.72618000 & 3.47449800 & 0.55368800 & $\mathrm{H}$ & -0.44159200 & 2.75030300 & 1.22277700 \\
\hline C & 0.27257300 & -0.87903900 & 0.15457900 & $\mathrm{O}$ & -0.61886600 & -0.34111400 & -0.46455100 \\
\hline $\mathrm{O}$ & 0.28282700 & -2.11066700 & 0.68938800 & $\mathrm{C}$ & -0.93859300 & -2.96082200 & 0.68076300 \\
\hline C & -1.44160900 & -3.14916700 & -0.74813400 & $\mathrm{H}$ & -2.25594200 & -3.88086700 & -0.74628300 \\
\hline $\mathrm{H}$ & -1.81677800 & -2.21856100 & -1.16632000 & $\mathrm{H}$ & -0.64342900 & -3.52463800 & -1.39709000 \\
\hline $\mathrm{C}$ & -0.45217700 & -4.28455000 & 1.26339900 & $\mathrm{H}$ & 0.00671700 & -4.12604900 & 2.24378000 \\
\hline $\mathrm{H}$ & -1.30745100 & -4.95499100 & 1.39063000 & $\mathrm{H}$ & 0.26512700 & -4.77732500 & 0.59871000 \\
\hline C & -1.96651400 & -2.32787700 & 1.60323200 & $\mathrm{H}$ & -2.86971500 & -2.94349000 & 1.63605500 \\
\hline $\mathrm{H}$ & -1.56682000 & -2.22363200 & 2.61353300 & $\mathrm{H}$ & -2.23989200 & -1.34090900 & 1.24343000 \\
\hline
\end{tabular}




\begin{tabular}{|c|c|c|c|c|c|c|c|}
\hline C & 2.32971400 & -0.54935400 & 1.42216000 & $\mathrm{H}$ & 1.86268300 & -1.19590100 & 2.15718700 \\
\hline $\mathrm{C}$ & 3.14311800 & 0.55380900 & 2.00351500 & $\mathrm{C}$ & 3.08144600 & 0.74938600 & 3.38769800 \\
\hline $\mathrm{C}$ & 3.96674300 & 1.37252800 & 1.22013100 & $\mathrm{C}$ & 3.82565100 & 1.76801900 & 3.98068700 \\
\hline $\mathrm{H}$ & 2.41121300 & 0.13848800 & 3.98256800 & C & 4.71935200 & 2.37722700 & 1.81858900 \\
\hline $\mathrm{H}$ & 3.99850700 & 1.23765800 & 0.14448800 & C & 4.65233500 & 2.57592500 & 3.20058300 \\
\hline $\mathrm{H}$ & 3.74718200 & 1.93416700 & 5.05049100 & $\mathrm{H}$ & 5.35181500 & 3.01369300 & 1.20643400 \\
\hline $\mathrm{H}$ & 5.23560000 & 3.36647200 & 3.66396800 & $\mathrm{~N}$ & 1.50560000 & -0.24731000 & 0.36450800 \\
\hline $\mathrm{H}$ & 1.54074400 & 0.71165500 & -0.00252400 & $\mathrm{~S}$ & -0.69810000 & 0.62311700 & 3.38914300 \\
\hline $\mathrm{O}$ & -0.03269300 & -0.63561900 & 3.78623000 & $\mathrm{O}$ & -0.00698600 & 1.34870800 & 2.29146300 \\
\hline $\mathrm{O}$ & -2.17384600 & 0.56727300 & 3.22543000 & C & -0.48246500 & 1.74046500 & 4.85873800 \\
\hline $\mathrm{F}$ & -1.08541100 & 1.23150800 & 5.93725200 & $\mathrm{~F}$ & -1.01255600 & 2.94824600 & 4.60014300 \\
\hline $\mathrm{F}$ & 0.82186500 & 1.91086300 & 5.14029900 & C & 3.80308000 & -1.69407000 & 0.83162300 \\
\hline $\mathrm{H}$ & 4.10627300 & -1.12384900 & -0.04213500 & $\mathrm{H}$ & 4.45153100 & -1.59696300 & 1.69679900 \\
\hline C & 3.19510500 & -2.93292000 & 0.61871300 & C & 3.13332200 & -3.92750600 & 1.68496100 \\
\hline $\mathrm{H}$ & 3.26902100 & -3.48116600 & 2.67667300 & $\mathrm{H}$ & 2.20584300 & -4.50433500 & 1.66432500 \\
\hline C & 2.63991700 & -3.23138400 & -0.72494400 & $\mathrm{H}$ & 1.94755900 & -4.07293000 & -0.73017000 \\
\hline $\mathrm{H}$ & 2.14824400 & -2.34552200 & -1.13681400 & $\mathrm{H}$ & 3.46336400 & -3.44540400 & -1.41641100 \\
\hline C & 4.44277700 & -6.33458000 & 3.01086000 & $\mathrm{H}$ & 3.46941600 & -6.83568800 & 3.05977100 \\
\hline $\mathrm{H}$ & 5.21630800 & -7.11116600 & 2.98686200 & $\mathrm{H}$ & 4.57310300 & -5.76263000 & 3.93637400 \\
\hline $\mathrm{C}$ & 4.30462800 & -6.21744200 & -0.08584500 & $\mathrm{H}$ & 3.30991700 & -6.67699200 & -0.11109400 \\
\hline $\mathrm{H}$ & 4.41240900 & -5.60153600 & -0.98473800 & $\mathrm{H}$ & 5.04262600 & -7.02595500 & -0.14980700 \\
\hline $\mathrm{C}$ & 6.20494500 & -4.30035500 & 1.44607900 & $\mathrm{H}$ & 6.26812500 & -3.63975500 & 0.57419100 \\
\hline $\mathrm{H}$ & 6.34573900 & -3.68536500 & 2.34250400 & $\mathrm{H}$ & 7.04468800 & -5.00300100 & 1.39260900 \\
\hline $\mathrm{Si}$ & 4.56714400 & -5.22662100 & 1.49416100 & $\mathrm{O}$ & 1.78755800 & 2.47077300 & -0.25604700 \\
\hline C & -1.78189000 & 3.20805800 & -0.22670000 & C & -2.83354500 & 2.27092100 & -0.20029500 \\
\hline C & -2.26242800 & 3.79345800 & -1.50267600 & C & -3.40850900 & 2.78344000 & -1.47591600 \\
\hline $\mathrm{O}$ & -1.86867900 & 4.67170000 & -2.25978000 & $\mathrm{O}$ & -4.33088600 & 2.47798200 & -2.20910500 \\
\hline $\mathrm{N}$ & -3.19982100 & 1.30477800 & 0.66369300 & $\mathrm{H}$ & -2.73296700 & 1.22685400 & 1.57548600 \\
\hline $\mathrm{C}$ & -4.01630500 & 0.21761800 & 0.31971900 & $\mathrm{C}$ & -4.71068100 & -0.44993500 & 1.33716500 \\
\hline C & -4.07698700 & -0.26504200 & -0.99267900 & C & -5.45150400 & -1.58789700 & 1.03414500 \\
\hline $\mathrm{H}$ & -4.62917300 & -0.09906100 & 2.35912100 & $\mathrm{C}$ & -4.84206600 & -1.39317100 & -1.27746200 \\
\hline $\mathrm{H}$ & -3.51242300 & 0.21908000 & -1.77486100 & $\mathrm{C}$ & -5.53374900 & -2.06844700 & -0.27353700 \\
\hline $\mathrm{H}$ & -6.12229900 & -2.94785000 & -0.50193400 & $\mathrm{C}$ & -4.83337300 & -1.93576800 & -2.67838700 \\
\hline C & -6.08915200 & -2.37652900 & 2.14162200 & $\mathrm{~F}$ & -4.85549700 & -0.95508200 & -3.60681700 \\
\hline $\mathrm{F}$ & -3.70598800 & -2.66522700 & -2.92589800 & $\mathrm{~F}$ & -5.87862600 & -2.75233500 & -2.92031400 \\
\hline $\mathrm{F}$ & -6.37417700 & -1.61651100 & 3.21706100 & $\mathrm{~F}$ & -5.26687800 & -3.37200900 & 2.56744800 \\
\hline $\mathrm{F}$ & -7.23598400 & -2.97103500 & 1.74299400 & & & & \\
\hline
\end{tabular}

\section{TS- $R-6 f-1$}

Imaginary frequency: $-371.97 \mathrm{~cm}^{-1}$

E[B3LYP/6-31G(d)-D3(BJ)]: -4655.638776 Hartree

$\mathrm{E}\left[\mathrm{PCM}\left(\mathrm{Et}_{2} \mathrm{O}\right)-\mathrm{B} 3 \mathrm{LYP} / 6-311 \mathrm{G}(\mathrm{d}, \mathrm{p})-\mathrm{D} 3(\mathrm{BJ})\right]$ : -4656.835192 Hartree

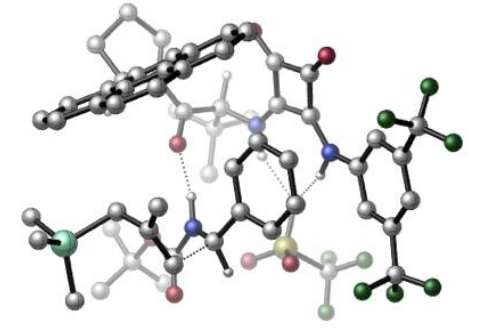

\begin{tabular}{|crrr|llll|}
\hline 01 & & & $\mathrm{C}$ & -1.02190400 & 1.12530300 & 3.53158500 \\
\hline $\mathrm{H}$ & -1.01090600 & 0.54603300 & 4.45617400 & $\mathrm{C}$ & -1.32317100 & 2.61187800 & 3.89987200 \\
\hline $\mathrm{C}$ & -1.23487100 & 3.52241900 & 2.66644900 & $\mathrm{H}$ & -1.88218900 & 3.17486900 & 1.86126400 \\
\hline $\mathrm{H}$ & -0.22323700 & 3.59983500 & 2.26451300 & $\mathrm{H}$ & -1.54129200 & 4.53774700 & 2.94434500 \\
\hline $\mathrm{C}$ & -0.28866100 & 3.06093200 & 4.94642700 & $\mathrm{H}$ & -0.48650300 & 4.09616500 & 5.24580700 \\
\hline $\mathrm{H}$ & 0.72712200 & 3.01437100 & 4.54442500 & $\mathrm{H}$ & -0.33171000 & 2.43370500 & 5.84525100 \\
\hline $\mathrm{C}$ & -2.73172600 & 2.72006300 & 4.50623700 & $\mathrm{H}$ & -2.94411100 & 3.76504900 & 4.75544500 \\
\hline $\mathrm{H}$ & -2.81887300 & 2.13919200 & 5.43109400 & $\mathrm{H}$ & -3.50586000 & 2.38210700 & 3.80812000 \\
\hline
\end{tabular}




\begin{tabular}{|c|c|c|c|c|c|c|c|}
\hline C & -2.04621300 & 0.49440200 & 2.56674100 & 0 & -1.97879400 & 0.68290400 & 1.34089000 \\
\hline $\mathrm{C}$ & -3.19696600 & -0.70544000 & 4.49360900 & $\mathrm{C}$ & -3.94930700 & -0.98070700 & 2.17587100 \\
\hline C & -4.29160800 & -1.78121900 & 4.41709900 & $\mathrm{H}$ & -2.26221300 & -1.08964100 & 4.90819500 \\
\hline $\mathrm{H}$ & -3.53175200 & 0.15178500 & 5.08388100 & C & -5.06659200 & -1.42587300 & 3.14063900 \\
\hline $\mathrm{H}$ & -4.29565200 & -0.25040600 & 1.44142000 & $\mathrm{H}$ & -4.91904300 & -1.78894000 & 5.31201400 \\
\hline $\mathrm{H}$ & -3.83741100 & -2.77100400 & 4.31431900 & $\mathrm{H}$ & -5.74923300 & -0.58759400 & 3.32472200 \\
\hline $\bar{H}$ & -5.64604500 & -2.26227700 & 2.74284300 & $\mathrm{~N}$ & -3.00663900 & -0.30308700 & 3.08285200 \\
\hline C & -3.29878600 & -2.15083600 & 1.45302800 & C & -2.17828500 & -2.74133900 & 1.95801100 \\
\hline $\mathrm{C}$ & -3.89231100 & -2.65959200 & 0.23476200 & $\mathrm{C}$ & -1.50743700 & -3.81691300 & 1.29266200 \\
\hline $\bar{H}$ & -1.72960100 & -2.37585300 & 2.87331300 & $\mathrm{C}$ & -3.20231600 & -3.68590600 & -0.48778100 \\
\hline C & -5.11896900 & -2.19555300 & -0.27350100 & C & -0.35296400 & -4.41124500 & 1.82705800 \\
\hline C & -1.99693500 & -4.24551100 & 0.02721400 & C & -3.72902200 & -4.17108000 & -1.72418200 \\
\hline $\mathrm{H}$ & -5.67621200 & -1.43689000 & 0.26473400 & $\mathrm{C}$ & -5.64719100 & -2.70841600 & -1.45840000 \\
\hline C & 0.33978700 & -5.37859900 & 1.09991500 & $\mathrm{H}$ & 0.01872500 & -4.08285200 & 2.79193800 \\
\hline C & -1.28788100 & -5.24307100 & -0.70449500 & C & -4.95719000 & -3.67282500 & -2.18711600 \\
\hline $\mathrm{C}$ & -2.98539300 & -5.16284400 & -2.45155300 & $\mathrm{H}$ & -6.60621900 & -2.34593600 & -1.81757900 \\
\hline $\mathrm{C}$ & -0.11383000 & -5.78339900 & -0.15438600 & $\bar{H}$ & 1.25146700 & -5.79940200 & 1.51169200 \\
\hline C & -1.81443300 & -5.66533400 & -1.97133700 & $\mathrm{H}$ & -5.37157700 & -4.06171400 & -3.11357000 \\
\hline $\mathrm{H}$ & -3.38598500 & -5.51049000 & -3.40032100 & $\mathrm{H}$ & 0.43577500 & -6.53309900 & -0.71742500 \\
\hline $\mathrm{H}$ & -1.26605100 & -6.41594100 & -2.53441800 & $\mathrm{~S}$ & 1.87511400 & 3.49523200 & 0.08739500 \\
\hline 0 & 2.27438300 & 2.73178100 & -1.11408500 & 0 & 0.68124300 & 4.34666300 & -0.02488600 \\
\hline 0 & 1.91354400 & 2.67175100 & 1.34867800 & $\mathrm{C}$ & 3.29296900 & 4.66208600 & 0.35423200 \\
\hline $\mathrm{F}$ & 4.43187800 & 3.96467500 & 0.50109200 & $\mathrm{~F}$ & 3.09872000 & 5.39853900 & 1.45531700 \\
\hline $\mathrm{F}$ & 3.42377100 & 5.48107900 & -0.69393500 & C & -1.52663100 & 3.03033300 & -1.41206000 \\
\hline 0 & -1.27393000 & 3.60217900 & -2.45728000 & 0 & -2.45093400 & 3.37024400 & -0.50720000 \\
\hline C & -3.13924000 & 4.68025600 & -0.58591800 & C & -2.11542400 & 5.79718300 & -0.40045200 \\
\hline $\mathrm{H}$ & -2.63789200 & 6.75936800 & -0.35846000 & $\mathrm{H}$ & -1.40205200 & 5.81659600 & -1.22539000 \\
\hline $\mathrm{H}$ & -1.55358800 & 5.65521100 & 0.52490500 & C & -4.12094800 & 4.61212700 & 0.58320500 \\
\hline $\mathrm{H}$ & -4.79089600 & 3.75255500 & 0.47259200 & $\mathrm{H}$ & -4.72694000 & 5.52318600 & 0.60634500 \\
\hline $\mathrm{H}$ & -3.59415600 & 4.52196400 & 1.53489300 & C & -3.90581600 & 4.80800900 & -1.90377300 \\
\hline $\mathrm{H}$ & -4.53328100 & 5.70475100 & -1.86313600 & $\mathrm{H}$ & -4.56380200 & 3.94503300 & -2.05235400 \\
\hline $\mathrm{H}$ & -3.22843800 & 4.89258300 & -2.75268000 & $\mathrm{C}$ & -0.06754700 & 1.21708500 & -1.94732600 \\
\hline $\mathrm{H}$ & 0.51278900 & 1.95880900 & -2.48849200 & C & 0.70476700 & 0.01895400 & -1.55524000 \\
\hline $\mathrm{C}$ & 1.86334100 & -0.25787300 & -2.29961700 & $\mathrm{C}$ & 0.27785200 & -0.89646200 & -0.58325000 \\
\hline $\mathrm{C}$ & 2.56043700 & -1.44402800 & -2.10981700 & $\mathrm{H}$ & 2.22959500 & 0.47558600 & -3.01114300 \\
\hline C & 1.00411700 & -2.07057600 & -0.37533100 & $\mathrm{H}$ & -0.61183500 & -0.70636100 & 0.01006500 \\
\hline $\mathrm{C}$ & 2.12996200 & -2.35772200 & -1.14552200 & $\mathrm{H}$ & 3.46161300 & -1.63149800 & -2.68300400 \\
\hline $\mathrm{H}$ & 0.68788100 & -2.76790400 & 0.38625200 & $\mathrm{H}$ & 2.68915400 & -3.27037800 & -0.96988100 \\
\hline $\mathrm{N}$ & -0.94294300 & 1.81543600 & -1.06062900 & $\mathrm{H}$ & -1.20029900 & 1.39691800 & -0.15993200 \\
\hline $\mathrm{C}$ & -1.02331000 & 0.73109500 & -3.51911000 & $\mathrm{H}$ & -0.21860300 & 0.45785400 & -4.19645000 \\
\hline $\mathrm{H}$ & -1.42983100 & 1.72577300 & -3.68044000 & $\mathrm{C}$ & -1.89511700 & -0.29157300 & -3.11956000 \\
\hline C & -3.10303900 & 0.01600900 & -2.37244900 & $\mathrm{H}$ & -2.95737700 & 0.88171400 & -1.71235800 \\
\hline $\mathrm{H}$ & -3.46934000 & -0.83472600 & -1.79215000 & $\mathrm{C}$ & -1.53269400 & -1.72145700 & -3.31447900 \\
\hline $\mathrm{H}$ & -2.41891000 & -2.33612900 & -3.49731900 & $\mathrm{H}$ & -1.09643700 & -2.08503400 & -2.37003800 \\
\hline $\mathrm{H}$ & -0.79231700 & -1.86926100 & -4.10308900 & C & -5.95142100 & 1.03668400 & -2.34036100 \\
\hline $\mathrm{H}$ & -6.24584100 & 0.17680900 & -1.73063700 & $\mathrm{H}$ & -6.83547300 & 1.38522500 & -2.88682200 \\
\hline $\mathrm{H}$ & -5.63883800 & 1.83847700 & -1.66143500 & $\mathrm{C}$ & -5.02947200 & -0.83879700 & -4.66601100 \\
\hline $\mathrm{H}$ & -5.26727200 & -1.74586600 & -4.10372800 & $\mathrm{H}$ & -4.21065100 & -1.07335700 & -5.35637600 \\
\hline $\mathrm{H}$ & -5.90399100 & -0.57330900 & -5.27187300 & $\mathrm{C}$ & -4.01829900 & 2.06473300 & -4.54818100 \\
\hline $\mathrm{H}$ & -3.33639300 & 1.76680600 & -5.35194200 & $\mathrm{H}$ & -3.51564900 & 2.82948100 & -3.94960200 \\
\hline $\mathrm{H}$ & -4.89364700 & 2.53086200 & -5.01671400 & $\mathrm{Si}$ & -4.55870800 & 0.58143900 & -3.52438400 \\
\hline $\mathrm{C}$ & 0.75555700 & -1.45042700 & 3.58548600 & $\mathrm{C}$ & 2.00855800 & -1.95759100 & 2.87707100 \\
\hline 0 & -0.11238200 & -1.93615400 & 4.30578600 & $\mathrm{O}$ & 2.56316300 & -3.03794700 & 2.76248700 \\
\hline
\end{tabular}




\begin{tabular}{|c|c|c|c|c|c|c|c|}
\hline $\mathrm{C}$ & 2.17613300 & -0.57552400 & 2.33258400 & $\mathrm{C}$ & 0.98324900 & -0.13828400 & 2.95506900 \\
\hline $\mathrm{N}$ & 3.00351200 & 0.10732500 & 1.52100000 & $\mathrm{H}$ & 2.73242700 & 1.08534900 & 1.34621400 \\
\hline $\mathrm{C}$ & 4.04068900 & -0.38274200 & 0.72289800 & $\mathrm{C}$ & 4.55065100 & -1.67925000 & 0.84928200 \\
\hline C & 4.52680600 & 0.46717900 & -0.28017300 & C & 5.51217400 & -2.12073400 & -0.05771000 \\
\hline $\mathrm{H}$ & 4.16307100 & -2.35514100 & 1.60465000 & C & 5.48285100 & -0.00116500 & -1.17266800 \\
\hline $\mathrm{H}$ & 4.11045200 & 1.45997100 & -0.40553200 & C & 5.98816800 & -1.29836700 & -1.07686900 \\
\hline $\mathrm{H}$ & 6.72655300 & -1.65873300 & -1.78157400 & C & 5.93798900 & -3.56007500 & 0.00104000 \\
\hline C & 5.84762500 & 0.85373300 & -2.35192500 & $F$ & 5.02803500 & -4.36034300 & -0.62163200 \\
\hline $\mathrm{F}$ & 6.04786000 & -4.01136300 & 1.26532400 & $F$ & 7.12145500 & -3.77039800 & -0.61503200 \\
\hline $\mathrm{F}$ & 5.82303700 & 2.16738700 & -2.06515200 & $\mathrm{~F}$ & 4.96927200 & 0.65527000 & -3.37577500 \\
\hline $\mathrm{F}$ & 7.07395700 & 0.55820300 & -2.83654900 & $\mathrm{~N}$ & 0.30652500 & 1.01176900 & 2.94255100 \\
\hline $\mathrm{H}$ & 0.62893200 & 1.74255500 & 2.30405100 & & & & \\
\hline
\end{tabular}

\section{TS- $R-6 f-2$}

Imaginary frequency: $-361.39 \mathrm{~cm}-1$

E[B3LYP/6-31G(d)-D3(BJ)]: -4655.636393 Hartree

E[PCM(Et $\left.\left.{ }_{2} \mathrm{O}\right)-\mathrm{B} 3 \mathrm{LYP} / 6-311 \mathrm{G}(\mathrm{d}, \mathrm{p})-\mathrm{D} 3(\mathrm{BJ})\right]$ : -4656.828048 Hartree

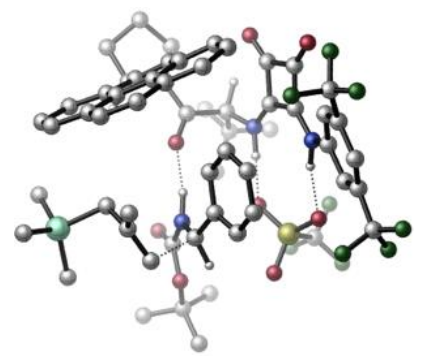

\begin{tabular}{|c|c|c|c|c|c|c|c|}
\hline 01 & & & & $\mathrm{C}$ & -1.29066600 & 0.41711700 & 3.54433000 \\
\hline $\mathrm{H}$ & -0.92344000 & -0.09156700 & 4.43486300 & C & -2.20359600 & 1.58533500 & 4.02882900 \\
\hline C & -2.90477000 & 2.31864700 & 2.87719500 & $\mathrm{H}$ & -3.54718200 & 1.65541000 & 2.29606900 \\
\hline $\mathrm{H}$ & -2.19579600 & 2.78261100 & 2.18901900 & $\mathrm{H}$ & -3.53226300 & 3.11706000 & 3.29119200 \\
\hline C & -1.32886000 & 2.57716400 & 4.81559100 & $\mathrm{H}$ & -1.96332700 & 3.34127200 & 5.27860600 \\
\hline $\mathrm{H}$ & -0.61485300 & 3.08582900 & 4.16267500 & $\mathrm{H}$ & -0.76760200 & 2.07439600 & 5.61266400 \\
\hline C & -3.27378400 & 0.99925800 & 4.96601200 & $\mathrm{H}$ & -3.91960400 & 1.80152200 & 5.33802000 \\
\hline $\mathrm{H}$ & -2.82464200 & 0.50252300 & 5.83397400 & $\mathrm{H}$ & -3.91416900 & 0.27881700 & 4.44399000 \\
\hline C & -1.98620700 & -0.61702700 & 2.63739000 & 0 & -2.13872600 & -0.40392300 & 1.42219400 \\
\hline C & -2.27391500 & -2.25807600 & 4.57041900 & C & -2.83170300 & -2.86765600 & 2.25883700 \\
\hline C & -2.54882000 & -3.76518400 & 4.47511300 & $\mathrm{H}$ & -1.28904300 & -2.04063600 & 4.98655800 \\
\hline $\mathrm{H}$ & -3.04174900 & -1.75652800 & 5.16724500 & C & -3.44238900 & -3.89377900 & 3.23374700 \\
\hline $\mathrm{H}$ & -3.57771600 & -2.43546600 & 1.58918400 & $\mathrm{H}$ & -3.01705500 & -4.15308400 & 5.38343400 \\
\hline $\mathrm{H}$ & -1.61115000 & -4.30760300 & 4.32089200 & $\mathrm{H}$ & -4.47348700 & -3.60627700 & 3.47192700 \\
\hline $\mathrm{H}$ & -3.45470800 & -4.90230400 & 2.81326200 & $\mathrm{~N}$ & -2.35715700 & -1.80458400 & 3.16504600 \\
\hline C & -1.68988900 & -3.43564300 & 1.42568700 & C & -0.40099900 & -3.36381600 & 1.86693300 \\
\hline C & -1.97505100 & -4.05217800 & 0.14690000 & C & 0.71019400 & -3.79558400 & 1.07260900 \\
\hline $\mathrm{H}$ & -0.17650800 & -2.91215700 & 2.82631400 & C & -0.87948500 & -4.41325700 & -0.70192400 \\
\hline C & -3.28088000 & -4.31173600 & -0.30480100 & C & 2.03366800 & -3.68787200 & 1.52883300 \\
\hline C & 0.46288300 & -4.26070200 & -0.24982800 & C & -1.12576200 & -4.93918200 & -2.00742000 \\
\hline $\mathrm{H}$ & -4.12806900 & -4.08549900 & 0.33278800 & C & -3.51014100 & -4.87478200 & -1.56079800 \\
\hline C & 3.10078000 & -3.96676000 & 0.67514100 & $\mathrm{H}$ & 2.22784200 & -3.35687900 & 2.54272600 \\
\hline C & 1.55917100 & -4.56538500 & -1.10979500 & C & -2.45061100 & -5.16424200 & -2.41589200 \\
\hline C & -0.00441600 & -5.22557300 & -2.85948900 & $\mathrm{H}$ & -4.52901300 & -5.08220300 & -1.87563700 \\
\hline C & 2.86871600 & -4.38860200 & -0.63338000 & $\mathrm{H}$ & 4.11740200 & -3.83062100 & 1.02825700 \\
\hline C & 1.27582800 & -5.03458100 & -2.43637600 & $\mathrm{H}$ & -2.63820800 & -5.58918800 & -3.39870600 \\
\hline $\mathrm{H}$ & -0.20310400 & -5.60592600 & -3.85823200 & $\mathrm{H}$ & 3.70757200 & -4.58103200 & -1.29554700 \\
\hline $\mathrm{H}$ & 2.11170800 & -5.25373000 & -3.09541400 & $\mathrm{~N}$ & -0.10148400 & 0.89568100 & 2.83340200 \\
\hline $\mathrm{H}$ & -0.22427100 & 1.57877500 & 2.07784700 & $\mathrm{~S}$ & 0.35922900 & 3.82668400 & 0.17371900 \\
\hline 0 & -0.16322400 & 4.32287400 & -1.11121500 & 0 & -0.43832100 & 2.72167400 & 0.77340500 \\
\hline $\mathrm{O}$ & 1.82187400 & 3.58532600 & 0.24673400 & $\mathrm{C}$ & 0.09263800 & 5.20749000 & 1.38656000 \\
\hline $\mathrm{F}$ & 0.69896900 & 6.32759300 & 0.98727500 & $F$ & 0.58219400 & 4.85466300 & 2.58783000 \\
\hline
\end{tabular}




\begin{tabular}{|c|c|c|c|c|c|c|c|}
\hline $\mathrm{F}$ & -1.22079100 & 5.45658700 & 1.53292500 & C & -3.32489700 & 2.17068500 & -0.78193600 \\
\hline 0 & -4.22668700 & 2.03471900 & 0.02282800 & 0 & -3.25557200 & 3.07860400 & -1.76466200 \\
\hline $\mathrm{C}$ & -4.07497800 & 4.31114100 & -1.74064700 & C & -5.53430800 & 3.95634000 & -2.01642900 \\
\hline $\mathrm{H}$ & -6.12593300 & 4.87552500 & -2.08479100 & $\mathrm{H}$ & -5.94055000 & 3.33638700 & -1.21513800 \\
\hline $\mathrm{H}$ & -5.62736300 & 3.42155600 & -2.96762100 & C & -3.45871500 & 5.12040300 & -2.87897400 \\
\hline $\mathrm{H}$ & -2.39860200 & 5.29868600 & -2.67716300 & $\mathrm{H}$ & -3.97069400 & 6.08336600 & -2.97172100 \\
\hline $\mathrm{H}$ & -3.55404600 & 4.58569200 & -3.83025600 & $\mathrm{C}$ & -3.88250300 & 5.02374200 & -0.40415100 \\
\hline $\mathrm{H}$ & -4.38985100 & 5.99369300 & -0.44185900 & $\mathrm{H}$ & -2.82042800 & 5.19538800 & -0.21938700 \\
\hline $\mathrm{H}$ & -4.30034900 & 4.44461300 & 0.42096400 & C & -1.28010700 & 1.37914800 & -1.82961600 \\
\hline $\mathrm{H}$ & -1.20568800 & 2.37669800 & -2.25277200 & $\mathrm{C}$ & 0.03260200 & 0.71406300 & -1.67493400 \\
\hline C & 1.05626900 & 1.14457200 & -2.53410900 & C & 0.26533700 & -0.36914400 & -0.81628700 \\
\hline C & 2.26865900 & 0.46601100 & -2.59165000 & $\mathrm{H}$ & 0.89213700 & 2.01935500 & -3.15731200 \\
\hline C & 1.49485100 & -1.02655200 & -0.85982100 & $\mathrm{H}$ & -0.49699800 & -0.70512700 & -0.12055500 \\
\hline C & 2.48008000 & -0.64015800 & -1.76734300 & $\mathrm{H}$ & 3.05585900 & 0.81863400 & -3.24868400 \\
\hline $\mathrm{H}$ & 1.68577800 & -1.84282600 & -0.17958800 & $\mathrm{H}$ & 3.41837300 & -1.18141600 & -1.80335000 \\
\hline $\mathrm{N}$ & -2.23027800 & 1.31337400 & -0.82977800 & $\mathrm{H}$ & -2.10083800 & 0.74176200 & 0.01321800 \\
\hline C & -2.08038800 & 0.66438900 & -3.38572900 & $\mathrm{H}$ & -1.39802000 & 0.95302800 & -4.18138800 \\
\hline $\mathrm{H}$ & -2.97628200 & 1.27481700 & -3.33228900 & C & -2.19190700 & -0.71140200 & -3.11165100 \\
\hline C & -3.18452300 & -1.20229900 & -2.17597600 & $\mathrm{H}$ & -3.35137500 & -0.46949900 & -1.37176900 \\
\hline $\mathrm{H}$ & -2.91640900 & -2.17312600 & -1.75231800 & C & -1.17107300 & -1.66688000 & -3.62041600 \\
\hline $\mathrm{H}$ & -1.61605900 & -2.64526600 & -3.82476700 & $\mathrm{H}$ & -0.43649100 & -1.82793200 & -2.81537200 \\
\hline $\mathrm{H}$ & -0.63676600 & -1.29552100 & -4.49698600 & C & -6.03369700 & -1.98748600 & -1.49069500 \\
\hline $\mathrm{H}$ & -5.70127800 & -2.95948100 & -1.11578200 & $\mathrm{H}$ & -7.07997600 & -2.08365000 & -1.80382400 \\
\hline $\mathrm{H}$ & -5.99621100 & -1.27194500 & -0.66105900 & C & -4.89105900 & -2.62543800 & -4.33665100 \\
\hline $\mathrm{H}$ & -4.43746900 & -3.56831000 & -4.01415300 & $\mathrm{H}$ & -4.29686400 & -2.23373900 & -5.17064600 \\
\hline $\mathrm{H}$ & -5.89427500 & -2.84581000 & -4.71968700 & C & -5.57050400 & 0.29097400 & -3.50402800 \\
\hline $\mathrm{H}$ & -5.00465700 & 0.66758300 & -4.36262100 & $\mathrm{H}$ & -5.51072300 & 1.03223100 & -2.70004400 \\
\hline $\mathrm{H}$ & -6.62169200 & 0.22121500 & -3.80885600 & $\mathrm{Si}$ & -4.96274500 & -1.39059600 & -2.91739300 \\
\hline C & 1.45720100 & -0.92421800 & 3.75876900 & C & 2.83187200 & -0.85007100 & 3.12195000 \\
\hline 0 & 0.89884300 & -1.66445800 & 4.56042900 & 0 & 3.85102200 & -1.51638100 & 3.20355900 \\
\hline $\mathrm{C}$ & 2.33287600 & 0.31171500 & 2.32468300 & C & 1.05332300 & 0.23981300 & 2.93722400 \\
\hline $\mathrm{N}$ & 2.83822600 & 1.14562600 & 1.38766200 & $\mathrm{H}$ & 2.28255100 & 1.96125900 & 1.10498300 \\
\hline C & 3.96498400 & 0.92168300 & 0.58334500 & C & 4.67881500 & -0.28302700 & 0.60463400 \\
\hline C & 4.32015600 & 1.91750500 & -0.34023300 & C & 5.69714100 & -0.49437900 & -0.32109700 \\
\hline $\mathrm{H}$ & 4.44903200 & -1.05046200 & 1.33179600 & $\mathrm{C}$ & 5.34791800 & 1.68293600 & -1.24712400 \\
\hline $\mathrm{H}$ & 3.76164200 & 2.84453400 & -0.37305200 & C & 6.04454900 & 0.47398300 & -1.26016300 \\
\hline $\bar{H}$ & 6.83327500 & 0.29462400 & -1.98013300 & $\mathrm{C}$ & 6.31308400 & -1.86061100 & -0.40820600 \\
\hline $\mathrm{C}$ & 5.64051200 & 2.70369900 & -2.31022900 & $\mathrm{~F}$ & 5.58883700 & -2.65928400 & -1.24946800 \\
\hline $\mathrm{F}$ & 6.34798600 & -2.49620900 & 0.78069400 & $\mathrm{~F}$ & 7.56943400 & -1.83152000 & -0.89726800 \\
\hline $\mathrm{F}$ & 5.29884100 & 3.95050300 & -1.93744800 & $\mathrm{~F}$ & 4.95243900 & 2.43145000 & -3.45441200 \\
\hline $\mathrm{F}$ & 6.94882500 & 2.71755100 & -2.64880300 & & & & \\
\hline
\end{tabular}

\section{TS- $R-6 f-3$}

Imaginary frequency: $-111.98 \mathrm{~cm}^{-1}$

E[B3LYP/6-31G(d)-D3(BJ)]: -4655.628776 Hartree

$\mathrm{E}\left[\mathrm{PCM}\left(\mathrm{Et}_{2} \mathrm{O}\right)-\mathrm{B} 3 \mathrm{LYP} / 6-311 \mathrm{G}(\mathrm{d}, \mathrm{p})-\mathrm{D} 3(\mathrm{BJ})\right]$ : -4656.826141 Hartree

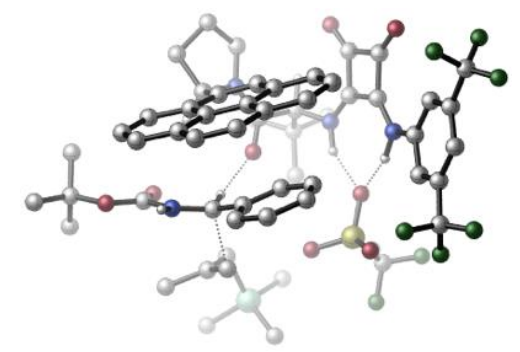

\begin{tabular}{|llll|llll|}
\hline 01 & & & & $\mathrm{C}$ & -0.74775000 & 1.05281400 & 3.61458600 \\
\hline $\mathrm{H}$ & -0.45079900 & 0.57385900 & 4.54619500 & $\mathrm{C}$ & -1.60451500 & 2.29552200 & 3.97924000 \\
\hline $\mathrm{C}$ & -1.90750700 & 3.18092900 & 2.75963000 & $\mathrm{H}$ & -2.45644300 & 2.62913900 & 1.99544800 \\
\hline
\end{tabular}




\begin{tabular}{|c|c|c|c|c|c|c|c|}
\hline$\overline{\mathrm{H}}$ & -0.99676500 & 3.57729300 & 2.30064900 & $\mathrm{H}$ & -2.51195000 & 4.03852100 & 3.07581900 \\
\hline C & -0.82310000 & 3.11212100 & 5.02186600 & $\mathrm{H}$ & -1.39484500 & 3.99959700 & 5.31549600 \\
\hline $\mathrm{H}$ & 0.13735000 & 3.44486800 & 4.61667600 & $\mathrm{H}$ & -0.62496400 & 2.52145000 & 5.92436000 \\
\hline C & -2.92516500 & 1.80044000 & 4.59350200 & $\mathrm{H}$ & -3.53146700 & 2.65162600 & 4.92197500 \\
\hline $\mathrm{H}$ & -2.74620300 & 1.16353100 & 5.46837000 & $\mathrm{H}$ & -3.51671300 & 1.23091100 & 3.86739600 \\
\hline C & -1.46399500 & 0.04127600 & 2.70277400 & C & -2.20002100 & -2.25365100 & 2.28758900 \\
\hline $\mathrm{H}$ & -3.01961900 & -1.82793300 & 1.70250700 & $\mathrm{~N}$ & -1.68168700 & -1.20524000 & 3.18287500 \\
\hline C & -1.13429600 & -2.77960200 & 1.33297300 & C & 0.19049000 & -2.51044400 & 1.51383400 \\
\hline C & -1.55120000 & -3.59051500 & 0.20606000 & C & 1.20164000 & -2.95597300 & 0.60031300 \\
\hline $\mathrm{H}$ & 0.50564200 & -1.91135700 & 2.36142400 & C & -0.57012400 & -4.00310000 & -0.75047200 \\
\hline C & -2.88386600 & -3.98617500 & 0.00406800 & C & 2.56297700 & -2.67539700 & 0.79505500 \\
\hline C & 0.80311100 & -3.68202300 & -0.55747600 & C & -0.96321900 & -4.74522200 & -1.90654800 \\
\hline $\mathrm{H}$ & -3.64458300 & -3.70530900 & 0.72189500 & C & -3.25604700 & -4.72897300 & -1.11597500 \\
\hline C & 3.51383900 & -3.07307300 & -0.14279500 & $\mathrm{H}$ & 2.88316700 & -2.16051100 & 1.69308900 \\
\hline C & 1.78077500 & -4.08869900 & -1.51211800 & C & -2.31434000 & -5.08906000 & -2.07425100 \\
\hline C & 0.03942100 & -5.12829600 & -2.85980100 & $\mathrm{H}$ & -4.29379200 & -5.01890200 & -1.24477600 \\
\hline C & 3.12754700 & -3.76444100 & -1.28841800 & $\mathrm{H}$ & 4.56112200 & -2.85138500 & 0.02624300 \\
\hline C & 1.35035700 & -4.81564800 & -2.67075200 & $\mathrm{H}$ & -2.61086700 & -5.66347900 & -2.94817200 \\
\hline $\mathrm{H}$ & -0.27384700 & -5.68776800 & -3.73758300 & $\mathrm{H}$ & 3.87372400 & -4.07064400 & -2.01695300 \\
\hline $\mathrm{H}$ & 2.09984100 & -5.11771200 & -3.39775200 & $\mathrm{~N}$ & 0.49152100 & 1.41864700 & 2.92488900 \\
\hline $\mathrm{H}$ & 0.40241000 & 1.92322500 & 2.03928700 & S & 0.65100300 & 3.29931900 & -0.74171700 \\
\hline 0 & 1.58121400 & 3.00433900 & -1.84076100 & $\mathrm{O}$ & -0.78541700 & 3.04858100 & -0.98997800 \\
\hline $\mathrm{O}$ & 1.11574200 & 2.80119000 & 0.59473300 & C & 0.72367500 & 5.13929900 & -0.50790100 \\
\hline $\mathrm{F}$ & 0.21202200 & 5.76457000 & -1.57828900 & $\mathrm{~F}$ & 1.98269500 & 5.54776600 & -0.32995900 \\
\hline $\mathrm{F}$ & 0.00394400 & 5.49755700 & 0.57297900 & C & -2.78397700 & 1.23873200 & -2.48836200 \\
\hline $\mathrm{H}$ & -1.84394200 & 1.72721600 & -2.25506000 & $\mathrm{H}$ & -2.87313200 & 0.77731100 & -3.46912400 \\
\hline C & -3.93149700 & 1.67825600 & -1.84201400 & C & -3.85086100 & 2.52878300 & -0.65736800 \\
\hline $\mathrm{H}$ & -2.86296800 & 2.47692600 & -0.19381100 & $\mathrm{H}$ & -4.63333300 & 2.29315500 & 0.07153300 \\
\hline C & -5.27608500 & 1.30972600 & -2.39526000 & $\mathrm{H}$ & -5.54314300 & 2.02654200 & -3.18429000 \\
\hline $\mathrm{H}$ & -6.05978400 & 1.33433100 & -1.63641800 & $\mathrm{H}$ & -5.25355700 & 0.32210600 & -2.86828800 \\
\hline C & -3.48414600 & 5.39815600 & 0.35024600 & $\mathrm{H}$ & -3.99823800 & 5.09623700 & 1.26931100 \\
\hline $\mathrm{H}$ & -3.67529500 & 6.46669100 & 0.19348300 & $\mathrm{H}$ & -2.40962200 & 5.26464500 & 0.49957700 \\
\hline C & -5.91912100 & 4.70964700 & -1.42315000 & $\mathrm{H}$ & -6.51731400 & 4.42396600 & -0.54975300 \\
\hline $\mathrm{H}$ & -6.30366500 & 4.15773700 & -2.28818400 & $\mathrm{H}$ & -6.09880700 & 5.77509300 & -1.61148900 \\
\hline C & -3.08642800 & 4.80069500 & -2.67602900 & $\mathrm{H}$ & -3.39401200 & 4.18711000 & -3.53074000 \\
\hline $\mathrm{H}$ & -2.02185500 & 4.62739300 & -2.49762200 & $\mathrm{H}$ & -3.22287600 & 5.85226400 & -2.95562500 \\
\hline $\mathrm{Si}$ & -4.07571300 & 4.40246100 & -1.12986200 & C & -1.52342800 & -1.67573800 & 4.57650000 \\
\hline $\mathrm{H}$ & -2.29996200 & -1.22563700 & 5.20782000 & $\mathrm{H}$ & -0.54274400 & -1.41447200 & 4.97707800 \\
\hline C & -1.72131600 & -3.18934100 & 4.45604000 & $\mathrm{H}$ & -2.09418900 & -3.63120600 & 5.38364200 \\
\hline $\mathrm{H}$ & -0.76803400 & -3.66890100 & 4.21075700 & C & -2.69528100 & -3.32925700 & 3.28049300 \\
\hline $\mathrm{H}$ & -2.69604600 & -4.32855800 & 2.84062700 & $\mathrm{H}$ & -3.71765400 & -3.09121200 & 3.59888300 \\
\hline 0 & -1.74114000 & 0.34917000 & 1.53558300 & C & -4.57783700 & -1.45962700 & -1.00570700 \\
\hline 0 & -4.81372900 & -0.72627600 & -0.06445000 & $\mathrm{O}$ & -5.38019800 & -2.38140800 & -1.54918700 \\
\hline C & -6.71559400 & -2.65787100 & -0.97199100 & C & -7.58141000 & -1.40005600 & -1.04609800 \\
\hline $\mathrm{H}$ & -8.60647100 & -1.65094800 & -0.75442400 & $\mathrm{H}$ & -7.20762900 & -0.62320900 & -0.37833100 \\
\hline $\mathrm{H}$ & -7.60501800 & -1.01328500 & -2.07006600 & C & -7.25369800 & -3.74582400 & -1.89802100 \\
\hline $\mathrm{H}$ & -6.60516100 & -4.62689100 & -1.88098200 & $\mathrm{H}$ & -8.25465700 & -4.04674800 & -1.57434300 \\
\hline $\mathrm{H}$ & -7.31602900 & -3.38082500 & -2.92769200 & C & -6.56504600 & -3.17433200 & 0.45882000 \\
\hline $\mathrm{H}$ & -7.55244100 & -3.43706100 & 0.85233100 & $\mathrm{H}$ & -5.94310500 & -4.07439500 & 0.48138500 \\
\hline $\mathrm{H}$ & -6.12423000 & -2.41553400 & 1.10738500 & C & -2.39686500 & -0.51592100 & -1.39908700 \\
\hline $\mathrm{H}$ & -2.49646500 & -0.08130200 & -0.40946300 & C & -1.03295500 & -0.74210500 & -1.87639500 \\
\hline C & 0.01481700 & -0.21655700 & -1.10329800 & C & -0.74280500 & -1.40911400 & -3.07976700 \\
\hline C & 1.32844800 & -0.35913900 & -1.53133300 & $\mathrm{H}$ & -0.21789400 & 0.29785200 & -0.17887600 \\
\hline
\end{tabular}




\begin{tabular}{|lrrr|rrrr|}
\hline $\mathrm{C}$ & 0.57339500 & -1.53610400 & -3.50363900 & $\mathrm{H}$ & -1.54112800 & -1.81148500 & -3.69878100 \\
\hline $\mathrm{C}$ & 1.61303600 & -1.00275800 & -2.73368300 & $\mathrm{H}$ & 2.13247800 & 0.03235000 & -0.92633700 \\
\hline $\mathrm{H}$ & 0.79283000 & -2.05061200 & -4.43330300 & $\mathrm{H}$ & 2.64010500 & -1.07596400 & -3.07317300 \\
\hline $\mathrm{N}$ & -3.38095700 & -1.42079300 & -1.71146200 & $\mathrm{H}$ & -3.21245400 & -2.15913300 & -2.38344800 \\
\hline $\mathrm{C}$ & 2.84383900 & 0.59606900 & 2.43934100 & $\mathrm{C}$ & 1.62077600 & 0.73381600 & 3.13385200 \\
\hline $\mathrm{C}$ & 2.04101200 & -0.22190800 & 4.18626900 & $\mathrm{C}$ & 3.36866000 & -0.37972100 & 3.44034900 \\
\hline $\mathrm{O}$ & 1.51316900 & -0.70736700 & 5.17517000 & $\mathrm{O}$ & 4.36914500 & -1.06203300 & 3.57526900 \\
\hline $\mathrm{N}$ & 3.23641800 & 1.14894300 & 1.26886100 & $\mathrm{H}$ & 2.57120400 & 1.82070300 & 0.86539400 \\
\hline $\mathrm{C}$ & 4.30853200 & 0.79036100 & 0.44485900 & $\mathrm{C}$ & 4.30319700 & 1.30094800 & -0.86472500 \\
\hline $\mathrm{C}$ & 5.33712800 & -0.06800700 & 0.85421200 & $\mathrm{C}$ & 5.29570800 & 0.91225400 & -1.75824300 \\
\hline $\mathrm{H}$ & 3.51211000 & 1.97116400 & -1.19133900 & $\mathrm{C}$ & 6.32934400 & -0.42197100 & -0.05875100 \\
\hline $\mathrm{H}$ & 5.35387100 & -0.46773700 & 1.86349200 & $\mathrm{C}$ & 6.32047600 & 0.04938900 & -1.36986800 \\
\hline $\mathrm{H}$ & 7.09522900 & -0.23949000 & -2.06845400 & $\mathrm{C}$ & 7.38440700 & -1.40622800 & 0.36435500 \\
\hline $\mathrm{C}$ & 5.20148800 & 1.34673400 & -3.19415700 & $\mathrm{~F}$ & 7.70307800 & -1.28694600 & 1.66593000 \\
\hline $\mathrm{F}$ & 6.96273800 & -2.68775900 & 0.17804200 & $\mathrm{~F}$ & 8.52110700 & -1.26980400 & -0.35304200 \\
\hline $\mathrm{F}$ & 6.41921900 & 1.43900700 & -3.77423900 & $\mathrm{~F}$ & 4.58676700 & 2.53291100 & -3.33500900 \\
\hline $\mathrm{F}$ & 4.49438900 & 0.44227300 & -3.92716300 & & & & \\
\hline
\end{tabular}

\section{TS- $R-6 f-4$}

Imaginary frequency: $-362.43 \mathrm{~cm}-1$

E[B3LYP/6-31G(d)-D3(BJ)]: -4655.631432 Hartree

$\mathrm{E}\left[\mathrm{PCM}\left(\mathrm{Et}_{2} \mathrm{O}\right)-\mathrm{B} 3 \mathrm{LYP} / 6-311 \mathrm{G}(\mathrm{d}, \mathrm{p})-\mathrm{D} 3(\mathrm{BJ})\right]$ : -4656.825889 Hartree

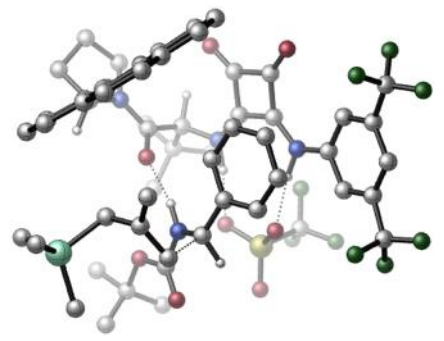

\begin{tabular}{|c|c|c|c|c|c|c|c|}
\hline 01 & & & & C & -1.17706500 & 1.34947900 & 3.30084100 \\
\hline $\mathrm{H}$ & -0.98209000 & 0.81053900 & 4.22819800 & C & -1.69851200 & 2.77479600 & 3.68632500 \\
\hline C & -2.39537500 & 3.47115400 & 2.51133300 & $\mathrm{H}$ & -3.29003900 & 2.91927600 & 2.20659100 \\
\hline $\mathrm{H}$ & -1.74797600 & 3.57267000 & 1.64287400 & $\mathrm{H}$ & -2.70745200 & 4.47465200 & 2.82499600 \\
\hline C & -0.50916500 & 3.61895100 & 4.18041900 & $\mathrm{H}$ & -0.88024200 & 4.57271500 & 4.57227100 \\
\hline $\mathrm{H}$ & 0.19998100 & 3.83956800 & 3.38089500 & $\mathrm{H}$ & 0.03074700 & 3.10921900 & 4.98711600 \\
\hline C & -2.71768200 & 2.65143100 & 4.83234100 & $\mathrm{H}$ & -3.01984700 & 3.65185000 & 5.16008700 \\
\hline $\mathrm{H}$ & -2.30000100 & 2.12742000 & 5.69980600 & $\mathrm{H}$ & -3.62457400 & 2.13099700 & 4.50789300 \\
\hline C & -2.15523300 & 0.52128800 & 2.44389300 & $\mathrm{O}$ & -2.13838000 & 0.60062900 & 1.20346200 \\
\hline C & -3.09638700 & -0.66482400 & 4.50106000 & C & -3.85497000 & -1.20585900 & 2.23626200 \\
\hline C & -4.04237500 & -1.87660100 & 4.53487300 & $\mathrm{H}$ & -2.10484300 & -0.89112600 & 4.89907000 \\
\hline $\mathrm{H}$ & -3.52230000 & 0.17117700 & 5.05784700 & C & -4.88430300 & -1.72157700 & 3.26037200 \\
\hline $\mathrm{H}$ & -4.30803100 & -0.59934700 & 1.45016600 & $\mathrm{H}$ & -4.64536200 & -1.89481500 & 5.44635000 \\
\hline $\mathrm{H}$ & -3.46858200 & -2.80660000 & 4.49218200 & $\mathrm{H}$ & -5.66830600 & -0.96856600 & 3.40432700 \\
\hline $\mathrm{H}$ & -5.35500200 & -2.65389500 & 2.93918300 & $\mathrm{~N}$ & -3.00836500 & -0.32730600 & 3.06108500 \\
\hline C & -3.05075200 & -2.33601800 & 1.61018100 & C & -1.87339900 & -2.73857100 & 2.16915600 \\
\hline C & -3.55839300 & -3.01565000 & 0.43783400 & C & -1.07634500 & -3.79013200 & 1.61566200 \\
\hline $\mathrm{H}$ & -1.47757400 & -2.23473000 & 3.04217800 & C & -2.74486200 & -4.01956600 & -0.18016800 \\
\hline C & -4.81638300 & -2.73595300 & -0.12615300 & C & 0.13078100 & -4.19254200 & 2.21011400 \\
\hline C & -1.49655900 & -4.39333600 & 0.39732800 & C & -3.18830600 & -4.66748300 & -1.37399600 \\
\hline $\mathrm{H}$ & -5.46666200 & -2.00242900 & 0.33724400 & $\mathrm{C}$ & -5.25646000 & -3.40151900 & -1.27017900 \\
\hline C & 0.93462500 & -5.14750500 & 1.58858300 & $\mathrm{H}$ & 0.45567400 & -3.71956500 & 3.13141200 \\
\hline C & -0.66976800 & -5.37199500 & -0.22857400 & C & -4.44947500 & -4.34493100 & -1.90015900 \\
\hline C & -2.32756500 & -5.63616500 & -1.99567700 & $\mathrm{H}$ & -6.23976300 & -3.17681900 & -1.67377300 \\
\hline C & 0.54617400 & -5.72403100 & 0.38008100 & $\mathrm{H}$ & 1.88158000 & -5.42157500 & 2.04245400 \\
\hline C & -1.12191500 & -5.96524300 & -1.45504800 & $\mathrm{H}$ & -4.79635300 & -4.85363300 & -2.79584700 \\
\hline $\mathrm{H}$ & -2.66703600 & -6.10964300 & -2.91330500 & $\mathrm{H}$ & 1.18337100 & -6.46062100 & -0.10211100 \\
\hline
\end{tabular}




\begin{tabular}{|c|c|c|c|c|c|c|c|}
\hline$\overline{\mathrm{H}}$ & -0.48514200 & -6.70158000 & -1.93826000 & $\mathrm{~S}$ & 1.35513300 & 3.86638500 & -0.11091900 \\
\hline $\mathrm{O}$ & 1.22274800 & 4.95016200 & -1.08467500 & 0 & 0.14773300 & 3.58057900 & 0.71727700 \\
\hline $\mathrm{O}$ & 1.99825600 & 2.61066200 & -0.60562400 & C & 2.56664400 & 4.45878300 & 1.16540600 \\
\hline $\mathrm{F}$ & 3.77736000 & 4.64831000 & 0.63226100 & $\mathrm{~F}$ & 2.67372600 & 3.52552300 & 2.13898200 \\
\hline $\mathrm{F}$ & 2.15196400 & 5.59841400 & 1.72738900 & C & -1.64165700 & 2.83251600 & -1.74087900 \\
\hline 0 & -1.29156600 & 3.34079200 & -2.79238500 & 0 & -2.63753300 & 3.22991500 & -0.94769100 \\
\hline C & -3.26491000 & 4.56008800 & -1.17716200 & C & -2.21407100 & 5.64193100 & -0.93910200 \\
\hline $\mathrm{H}$ & -2.68902100 & 6.62671100 & -1.00997600 & $\mathrm{H}$ & -1.40668000 & 5.58638400 & -1.67061700 \\
\hline $\mathrm{H}$ & -1.77435000 & 5.53210600 & 0.05498300 & C & -4.37056100 & 4.60544500 & -0.12579800 \\
\hline $\mathrm{H}$ & -5.04639300 & 3.75085600 & -0.24038100 & $\mathrm{H}$ & -4.95206400 & 5.52414900 & -0.25164300 \\
\hline $\mathrm{H}$ & -3.95946200 & 4.59251600 & 0.88297500 & C & -3.88694500 & 4.63210400 & -2.57358400 \\
\hline $\mathrm{H}$ & -4.45480000 & 5.56488200 & -2.65685000 & $\mathrm{H}$ & -4.58650200 & 3.80190000 & -2.72264100 \\
\hline $\mathrm{H}$ & -3.12954900 & 4.61095500 & -3.35547800 & C & -0.15328700 & 1.02312800 & -2.09971400 \\
\hline $\mathrm{H}$ & 0.37821200 & 1.75260100 & -2.70519000 & C & 0.69734300 & -0.08876200 & -1.62881700 \\
\hline C & 1.84975800 & -0.35815700 & -2.38641800 & C & 0.35924900 & -0.93799100 & -0.56756600 \\
\hline C & 2.63571400 & -1.47024200 & -2.11462700 & $\mathrm{H}$ & 2.13988600 & 0.32225000 & -3.18213900 \\
\hline C & 1.17567300 & -2.03312600 & -0.27836400 & $\mathrm{H}$ & -0.52474800 & -0.75101500 & 0.03598400 \\
\hline C & 2.29961200 & -2.31295600 & -1.05371500 & $\mathrm{H}$ & 3.53240800 & -1.64649600 & -2.69843800 \\
\hline $\mathrm{H}$ & 0.93702300 & -2.67105600 & 0.55839400 & $\mathrm{H}$ & 2.93097000 & -3.15961100 & -0.80623600 \\
\hline $\mathrm{N}$ & -1.07007700 & 1.64837500 & -1.27561200 & $\mathrm{H}$ & -1.32926000 & 1.30669200 & -0.34280800 \\
\hline C & -1.08025700 & 0.36192000 & -3.61881600 & $\mathrm{H}$ & -0.27104100 & 0.09207500 & -4.29225100 \\
\hline $\mathrm{H}$ & -1.55853800 & 1.30847700 & -3.85535700 & C & -1.87189200 & -0.68548100 & -3.12208700 \\
\hline C & -3.08145200 & -0.40328000 & -2.37245000 & $\mathrm{H}$ & -2.99280200 & 0.53336700 & -1.80496300 \\
\hline $\mathrm{H}$ & -3.36762900 & -1.22104000 & -1.70532100 & C & -1.40954400 & -2.09666500 & -3.21189900 \\
\hline $\mathrm{H}$ & -2.25262600 & -2.78548700 & -3.31770700 & $\mathrm{H}$ & -0.92584900 & -2.34873000 & -2.25432800 \\
\hline $\mathrm{H}$ & -0.67933000 & -2.25604300 & -4.00763200 & C & -5.99721500 & 0.37790700 & -2.34430400 \\
\hline $\mathrm{H}$ & -6.18473900 & -0.43673300 & -1.63690800 & $\mathrm{H}$ & -6.92886700 & 0.57910800 & -2.88563100 \\
\hline $\mathrm{H}$ & -5.74309300 & 1.27409300 & -1.76597900 & C & -4.97051900 & -1.61056100 & -4.53464200 \\
\hline $\mathrm{H}$ & -5.09958100 & -2.48675000 & -3.89328000 & $\mathrm{H}$ & -4.15629200 & -1.82324300 & -5.23739800 \\
\hline $\mathrm{H}$ & -5.88663900 & -1.48028600 & -5.12287800 & C & -4.20445500 & 1.36775300 & -4.68402300 \\
\hline $\mathrm{H}$ & -3.49698400 & 1.06833200 & -5.46476300 & $\mathrm{H}$ & -3.77828800 & 2.22709400 & -4.15803300 \\
\hline $\mathrm{H}$ & -5.11960200 & 1.70755000 & -5.18406600 & $\mathrm{Si}$ & -4.60181600 & -0.06330000 & -3.52875400 \\
\hline $\mathrm{C}$ & 0.90545300 & -0.84704200 & 3.52766900 & C & 2.19226400 & -1.29975100 & 2.86776900 \\
\hline 0 & 0.12136600 & -1.32936100 & 4.34071100 & $\mathrm{O}$ & 2.86902900 & -2.31437800 & 2.91709800 \\
\hline $\mathrm{C}$ & 2.17682600 & -0.01575500 & 2.09971700 & C & 0.94907100 & 0.37530100 & 2.69686400 \\
\hline $\mathrm{N}$ & 2.91585200 & 0.58574900 & 1.14746000 & $\mathrm{H}$ & 2.51140000 & 1.41585000 & 0.69837400 \\
\hline $\mathrm{C}$ & 4.02837400 & 0.07814500 & 0.46301600 & C & 4.65045200 & -1.12970000 & 0.79686600 \\
\hline $\mathrm{C}$ & 4.47701500 & 0.80641100 & -0.64865400 & C & 5.66575600 & -1.62131000 & -0.02119500 \\
\hline $\mathrm{H}$ & 4.30963200 & -1.71365600 & 1.64515700 & C & 5.48426700 & 0.28671800 & -1.45246400 \\
\hline $\mathrm{H}$ & 3.99216100 & 1.73962100 & -0.90994400 & C & 6.09012500 & -0.93430800 & -1.15644400 \\
\hline $\mathrm{H}$ & 6.86625800 & -1.33835300 & -1.79352200 & $\mathrm{C}$ & 6.20726100 & -2.99249000 & 0.26813700 \\
\hline C & 5.79357200 & 0.97802400 & -2.74805900 & $\mathrm{~F}$ & 5.37269500 & -3.95460700 & -0.21598200 \\
\hline $\mathrm{F}$ & 6.33942300 & -3.22432500 & 1.58800800 & $\mathrm{~F}$ & 7.41088200 & -3.20064400 & -0.30872600 \\
\hline $\mathrm{F}$ & 5.69650600 & 2.31738800 & -2.65613500 & $\mathrm{~F}$ & 4.91458000 & 0.58729100 & -3.71652100 \\
\hline $\mathrm{F}$ & 7.02805500 & 0.68266400 & -3.20752900 & $\mathrm{~N}$ & 0.10145400 & 1.39992400 & 2.58552600 \\
\hline $\mathrm{H}$ & 0.20594900 & 2.10334700 & 1.84541400 & & & & \\
\hline
\end{tabular}

\section{TS- $R-6 f-5$}

Imaginary frequency: $-214.10 \mathrm{~cm}^{-1}$

E[B3LYP/6-31G(d)-D3(BJ)]: -4655.632268 Hartree

E[PCM(Et $\left.\left.{ }_{2} \mathrm{O}\right)-B 3 L Y P / 6-311 G(d, p)-D 3(B J)\right]:-4656.825173$ Hartree

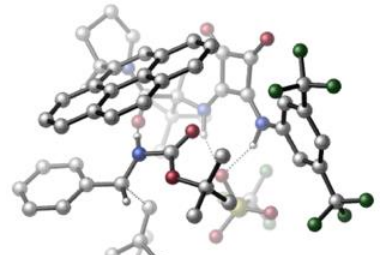




\begin{tabular}{|c|c|c|c|c|c|c|c|}
\hline 01 & & & & C & -1.67897800 & -0.58298100 & 3.44104500 \\
\hline $\mathrm{H}$ & -1.33810300 & -1.14725500 & 4.30867200 & C & -2.77753200 & 0.40896000 & 3.92148800 \\
\hline $\mathrm{C}$ & -3.37294100 & 1.22825800 & 2.76422100 & $\overline{\mathrm{H}}$ & -3.93343400 & 0.59639500 & 2.07068000 \\
\hline $\mathrm{H}$ & -2.60452300 & 1.75392100 & 2.19461700 & $\bar{H}$ & -4.05313200 & 1.98649700 & 3.16715800 \\
\hline C & -2.14464700 & 1.36157400 & 4.95075000 & $\mathrm{H}$ & -2.91638400 & 2.00828500 & 5.38308300 \\
\hline $\mathrm{H}$ & -1.38572100 & 1.99973800 & 4.49262600 & $\mathrm{H}$ & -1.67261000 & 0.80374900 & 5.76824200 \\
\hline $\mathrm{C}$ & -3.89672700 & -0.40315700 & 4.59504800 & $\mathrm{H}$ & -4.68570400 & 0.26728000 & 4.95232800 \\
\hline $\mathrm{H}$ & -3.51977700 & -0.96487800 & 5.45769900 & $\mathrm{H}$ & -4.35702300 & -1.11324000 & 3.89750100 \\
\hline C & -2.16477500 & -1.54325800 & 2.34948800 & $\mathrm{O}$ & -2.31540800 & -1.10016800 & 1.19362700 \\
\hline $\mathrm{N}$ & -2.36585700 & -2.84165800 & 2.62773500 & $\mathrm{C}$ & -1.41220800 & -3.91747800 & 0.61478400 \\
\hline C & -0.15842400 & -3.83055500 & 1.14816100 & C & -1.57719500 & -4.08504300 & -0.81492700 \\
\hline $\mathrm{C}$ & 1.02483900 & -3.85820100 & 0.34502700 & $\bar{H}$ & -0.02631300 & -3.67023500 & 2.21260200 \\
\hline $\mathrm{C}$ & -0.41098600 & -4.04053800 & -1.64731800 & $\mathrm{C}$ & -2.82932600 & -4.23275100 & -1.43434500 \\
\hline C & 2.30329000 & -3.74870000 & 0.90933000 & C & 0.88596400 & -3.92673200 & -1.06962700 \\
\hline $\mathrm{C}$ & -0.54540400 & -4.07859800 & -3.06913500 & $\overline{\mathrm{H}}$ & -3.72911900 & -4.30335800 & -0.83505200 \\
\hline $\mathrm{C}$ & -2.94949400 & -4.27931400 & -2.82226600 & $\mathrm{C}$ & 3.43136300 & -3.67372200 & 0.09494800 \\
\hline $\mathrm{H}$ & 2.41236800 & -3.70290900 & 1.98733900 & C & 2.04632400 & -3.85439400 & -1.89582300 \\
\hline $\mathrm{C}$ & -1.82541900 & -4.18802400 & -3.63454800 & $\mathrm{C}$ & 0.63804900 & -3.99685300 & -3.87893700 \\
\hline $\mathrm{H}$ & -3.93580900 & -4.36689700 & -3.26799500 & C & 3.30628400 & -3.72122000 & -1.29164400 \\
\hline $\mathrm{H}$ & 4.41116500 & -3.55981800 & 0.54567000 & C & 1.87529100 & -3.89378200 & -3.31993300 \\
\hline $\mathrm{H}$ & -1.92619700 & -4.21134800 & -4.71664300 & $\mathrm{H}$ & 0.52213200 & -4.02567700 & -4.95945300 \\
\hline $\mathrm{H}$ & 4.19005400 & -3.63700900 & -1.91652500 & $\mathrm{H}$ & 2.76128200 & -3.83916100 & -3.94757900 \\
\hline $\mathrm{N}$ & -0.50584100 & 0.11470000 & 2.91594100 & $\mathrm{H}$ & -0.64686500 & 0.80332300 & 2.17652400 \\
\hline $\mathrm{S}$ & -0.05280500 & 3.66740400 & 0.69491300 & $\mathrm{O}$ & 1.30650700 & 4.18779600 & 0.48091800 \\
\hline $\mathrm{O}$ & -1.10264100 & 4.13722300 & -0.23367200 & $\mathrm{O}$ & -0.08420100 & 2.18426700 & 0.94239900 \\
\hline C & -0.56631900 & 4.35067400 & 2.34588900 & $\mathrm{~F}$ & -0.42155500 & 5.67635700 & 2.38160900 \\
\hline $\mathrm{F}$ & 0.17925300 & 3.80368500 & 3.32037300 & $\mathrm{~F}$ & -1.85716700 & 4.05694200 & 2.59788100 \\
\hline $\mathrm{C}$ & 0.17364300 & -0.60814300 & -1.37486500 & $\mathrm{O}$ & 0.87455300 & -0.73392700 & -0.39633700 \\
\hline $\mathrm{O}$ & 0.51027400 & -0.17396000 & -2.59190800 & C & 1.79543700 & 0.52861600 & -2.85110200 \\
\hline $\mathrm{C}$ & 2.98026100 & -0.35830400 & -2.48286400 & $\mathrm{H}$ & 3.90815000 & 0.16257600 & -2.73854200 \\
\hline $\mathrm{H}$ & 2.99477200 & -0.59046100 & -1.42092000 & $\mathrm{H}$ & 2.93991000 & -1.29528300 & -3.04390200 \\
\hline C & 1.73941700 & 0.75490500 & -4.35937900 & $\mathrm{H}$ & 0.87035900 & 1.36244000 & -4.63439200 \\
\hline $\mathrm{H}$ & 2.64137500 & 1.28236300 & -4.68422400 & $\mathrm{H}$ & 1.68158300 & -0.20093500 & -4.88943300 \\
\hline $\mathrm{C}$ & 1.76643400 & 1.84689700 & -2.08758600 & $\mathrm{H}$ & 2.68548800 & 2.40757300 & -2.26938400 \\
\hline $\mathrm{H}$ & 0.92309100 & 2.46503600 & -2.40914100 & $\mathrm{H}$ & 1.67742400 & 1.67796800 & -1.01644700 \\
\hline $\mathrm{C}$ & -2.09257100 & -0.35162100 & -2.18461800 & $\mathrm{H}$ & -1.63182400 & -0.02576600 & -3.10817600 \\
\hline $\mathrm{C}$ & -3.47347800 & -0.84936400 & -2.32525500 & $\mathrm{C}$ & -4.03091000 & -0.85132400 & -3.61155900 \\
\hline C & -4.24583200 & -1.29095600 & -1.24083000 & C & -5.32655800 & -1.31881100 & -3.82147700 \\
\hline $\mathrm{H}$ & -3.43748900 & -0.50205900 & -4.45225200 & $\mathrm{C}$ & -5.54489300 & -1.74361300 & -1.45314500 \\
\hline $\mathrm{H}$ & -3.82386800 & -1.27881700 & -0.24216800 & $\mathrm{C}$ & -6.08700700 & -1.76735700 & -2.74098800 \\
\hline $\mathrm{H}$ & -5.74111300 & -1.32964800 & -4.82501600 & $\mathrm{H}$ & -6.13751600 & -2.08376200 & -0.60855000 \\
\hline $\mathrm{H}$ & -7.09971500 & -2.12627500 & -2.89981500 & $\mathrm{~N}$ & -1.19450000 & -0.94543700 & -1.34609600 \\
\hline $\mathrm{H}$ & -1.51109200 & -1.25938300 & -0.42078300 & $\mathrm{C}$ & -2.32171900 & 1.47771800 & -1.25303000 \\
\hline $\mathrm{H}$ & -2.96570100 & 1.12505400 & -0.45344700 & $\mathrm{H}$ & -1.28883000 & 1.62769200 & -0.96718000 \\
\hline C & -2.85210100 & 2.27390900 & -2.25720600 & C & -4.29046800 & 2.43489100 & -2.42475000 \\
\hline $\mathrm{H}$ & -4.58799300 & 2.60802800 & -3.46452800 & $\mathrm{H}$ & -4.85916100 & 1.59951600 & -2.00178100 \\
\hline$C$ & -1.91906200 & 3.00422500 & -3.17497100 & $\mathrm{H}$ & -1.19378400 & 2.30874100 & -3.61369300 \\
\hline $\mathrm{H}$ & -2.44266300 & 3.52415200 & -3.98008200 & $\mathrm{H}$ & -1.34346700 & 3.72627400 & -2.58285200 \\
\hline C & -6.70153600 & 4.18263900 & -1.84801300 & $\mathrm{H}$ & -7.27016700 & 3.28008700 & -1.59596700 \\
\hline $\mathrm{H}$ & -7.14670800 & 5.02071400 & -1.29861200 & $\mathrm{H}$ & -6.83544800 & 4.37854200 & -2.91808800 \\
\hline $\mathrm{C}$ & -4.64350800 & 3.61873800 & 0.42279400 & $\mathrm{H}$ & -5.05731200 & 2.64347600 & 0.70203800 \\
\hline
\end{tabular}




\begin{tabular}{|lrrr|lrrr|}
\hline $\mathrm{H}$ & -3.58126700 & 3.63443500 & 0.68285200 & $\mathrm{H}$ & -5.14540500 & 4.37873800 & 1.03396000 \\
\hline $\mathrm{C}$ & -3.88199800 & 5.51065600 & -1.89609100 & $\mathrm{H}$ & -2.85445400 & 5.42819500 & -1.52679600 \\
\hline $\mathrm{H}$ & -3.85743900 & 5.66369400 & -2.98098500 & $\mathrm{H}$ & -4.32364500 & 6.40867600 & -1.44712800 \\
\hline $\mathrm{Si}$ & -4.87758900 & 3.99691300 & -1.39985200 & $\mathrm{C}$ & -2.28255700 & -3.52193900 & 3.93711700 \\
\hline $\mathrm{H}$ & -3.17242600 & -3.28418600 & 4.53136800 & $\mathrm{H}$ & -1.38554500 & -3.20954100 & 4.47309100 \\
\hline $\mathrm{C}$ & -2.24640400 & -5.00859100 & 3.55923200 & $\mathrm{H}$ & -1.21204100 & -5.32364500 & 3.39372500 \\
\hline $\mathrm{H}$ & -2.66857100 & -5.64062900 & 4.34481600 & $\mathrm{C}$ & -3.03863000 & -5.07278800 & 2.24539300 \\
\hline $\mathrm{H}$ & -2.81562100 & -5.96020800 & 1.64859800 & $\mathrm{H}$ & -4.11603500 & -5.05903500 & 2.45042400 \\
\hline $\mathrm{C}$ & -2.63429500 & -3.77734500 & 1.51289400 & $\mathrm{H}$ & -3.46333600 & -3.37484700 & 0.93029100 \\
\hline $\mathrm{C}$ & 1.98511000 & -0.12351700 & 2.50251700 & $\mathrm{C}$ & 0.70922000 & -0.44210500 & 3.01188300 \\
\hline $\mathrm{C}$ & 1.21587200 & -1.65103200 & 3.69431100 & $\mathrm{C}$ & 2.60582500 & -1.31127700 & 3.16181900 \\
\hline $\mathrm{O}$ & 0.71715600 & -2.55668800 & 4.35233100 & $\mathrm{O}$ & 3.70660600 & -1.82974800 & 3.20807400 \\
\hline $\mathrm{N}$ & 2.38948000 & 0.91237400 & 1.74277300 & $\mathrm{H}$ & 1.65041300 & 1.56836300 & 1.47247400 \\
\hline $\mathrm{C}$ & 3.59217400 & 1.01742300 & 1.03015300 & $\mathrm{C}$ & 3.90485600 & 2.25899500 & 0.45834800 \\
\hline $\mathrm{C}$ & 4.44032900 & -0.07769000 & 0.82285600 & $\mathrm{C}$ & 5.04445200 & 2.38574700 & -0.33029000 \\
\hline $\mathrm{H}$ & 3.24121000 & 3.10501700 & 0.60588500 & $\mathrm{C}$ & 5.57409400 & 0.07740100 & 0.02931200 \\
\hline $\mathrm{H}$ & 4.22528000 & -1.03453200 & 1.27743700 & $\mathrm{C}$ & 5.89056600 & 1.30076600 & -0.55812800 \\
\hline $\mathrm{H}$ & 6.77382200 & 1.40730700 & -1.17516600 & $\mathrm{C}$ & 6.39228100 & -1.13415800 & -0.31459700 \\
\hline $\mathrm{C}$ & 5.31867900 & 3.68904600 & -1.02587500 & $\mathrm{~F}$ & 6.40083500 & -2.05320000 & 0.67330200 \\
\hline $\mathrm{F}$ & 5.89551200 & -1.75594700 & -1.42087100 & $\mathrm{~F}$ & 7.67570700 & -0.82233800 & -0.59366100 \\
\hline $\mathrm{F}$ & 6.64177300 & 3.89074900 & -1.21618600 & $\mathrm{~F}$ & 4.83424300 & 4.74595400 & -0.35076000 \\
\hline $\mathrm{F}$ & 4.74683800 & 3.71254400 & -2.26254900 & & & & \\
\hline
\end{tabular}

\section{TS- $R-6 \mathrm{f}-6$}

Imaginary frequency: $-370.48 \mathrm{~cm}^{-1}$

E[B3LYP/6-31G(d)-D3(BJ)]: -4655.632809 Hartree

E[PCM(Et $\left.\left.{ }_{2} \mathrm{O}\right)-\mathrm{B} 3 \mathrm{LYP} / 6-311 \mathrm{G}(\mathrm{d}, \mathrm{p})-\mathrm{D} 3(\mathrm{BJ})\right]$ : -4656.824841 Hartree

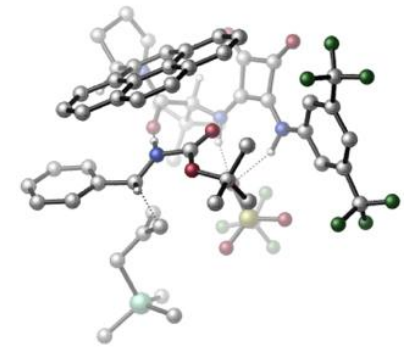

\begin{tabular}{|c|c|c|c|c|c|c|c|}
\hline 01 & & & & C & -1.45239200 & -1.10492600 & 3.57724500 \\
\hline $\mathrm{H}$ & -0.99179600 & -1.81035400 & 4.26761400 & C & -2.51032000 & -0.29495800 & 4.38011300 \\
\hline $\mathrm{C}$ & -3.26754700 & 0.71190900 & 3.50288100 & $\mathrm{H}$ & -3.84875200 & 0.21009200 & 2.72574900 \\
\hline $\mathrm{H}$ & -2.58995400 & 1.41665300 & 3.01828200 & $\mathrm{H}$ & -3.95445800 & 1.29613000 & 4.12584600 \\
\hline C & -1.77989800 & 0.45971100 & 5.50393600 & $\mathrm{H}$ & -2.50832500 & 0.95479600 & 6.15600800 \\
\hline $\mathrm{H}$ & -1.11334600 & 1.22403400 & 5.09639900 & $\mathrm{H}$ & -1.18230700 & -0.22292700 & 6.11956600 \\
\hline $\mathrm{C}$ & -3.51244700 & -1.28760900 & 4.99305700 & $\mathrm{H}$ & -4.26748400 & -0.74879100 & 5.57546900 \\
\hline $\mathrm{H}$ & -3.01413100 & -1.99485000 & 5.66600400 & $\mathrm{H}$ & -4.03825400 & -1.86017900 & 4.21953400 \\
\hline $\mathrm{C}$ & -2.01992700 & -1.86475200 & 2.37190200 & $\mathrm{O}$ & -2.28804000 & -1.22862300 & 1.33263200 \\
\hline $\mathrm{N}$ & -2.14952000 & -3.20094700 & 2.42170500 & C & -1.25170200 & -3.91353700 & 0.23264900 \\
\hline C & 0.01625600 & -3.83978600 & 0.73284300 & C & -1.46037400 & -3.93732800 & -1.20058600 \\
\hline $\mathrm{C}$ & 1.17274500 & -3.75919700 & -0.10500000 & $\mathrm{H}$ & 0.18175600 & -3.79058500 & 1.80334800 \\
\hline C & -0.32477000 & -3.77519800 & -2.05952100 & C & -2.72768100 & -4.06792000 & -1.79149600 \\
\hline C & 2.46748000 & -3.68684100 & 0.42699900 & C & 0.98880800 & -3.69315000 & -1.51430300 \\
\hline $\mathrm{C}$ & -0.50551300 & -3.67919200 & -3.47325000 & $\mathrm{H}$ & -3.60416400 & -4.22308900 & -1.17398300 \\
\hline C & -2.89302900 & -3.98461800 & -3.17303800 & C & 3.56831800 & -3.52387500 & -0.41163100 \\
\hline $\mathrm{H}$ & 2.61107100 & -3.74613000 & 1.50026100 & C & 2.12079600 & -3.52995100 & -2.36591200 \\
\hline C & -1.80073600 & -3.77423400 & -4.00672700 & C & 0.64919400 & -3.49502500 & -4.30775600 \\
\hline $\mathrm{H}$ & -3.89044800 & -4.06365200 & -3.59459600 & C & 3.39885500 & -3.44313200 & -1.79185900 \\
\hline $\mathrm{H}$ & 4.56124600 & -3.44195100 & 0.01685200 & C & 1.90311200 & -3.43146000 & -3.78086300 \\
\hline $\mathrm{H}$ & -1.93723000 & -3.69857200 & -5.08255800 & $\mathrm{H}$ & 0.49839600 & -3.42033000 & -5.38177400 \\
\hline $\mathrm{H}$ & 4.26126400 & -3.29055900 & -2.43352800 & $\mathrm{H}$ & 2.76715800 & -3.30324100 & -4.42807000 \\
\hline
\end{tabular}




\begin{tabular}{|c|c|c|c|c|c|c|c|}
\hline $\mathrm{N}$ & -0.36459000 & -0.26366300 & 3.07383800 & $\mathrm{H}$ & -0.59568100 & 0.49152000 & 2.42701000 \\
\hline S & -0.21812800 & 3.44551300 & 1.05051200 & $\mathrm{O}$ & -1.15455600 & 3.98233100 & 0.04110900 \\
\hline $\mathrm{O}$ & -0.26410100 & 1.95155600 & 1.18421600 & $\mathrm{O}$ & 1.15031500 & 3.98619900 & 1.04764300 \\
\hline C & -0.93845200 & 4.02860200 & 2.66488200 & $\mathrm{~F}$ & -0.74164300 & 5.33617100 & 2.83437300 \\
\hline $\mathrm{F}$ & -0.38260500 & 3.36709200 & 3.69316700 & $\mathrm{~F}$ & -2.27112500 & 3.79716700 & 2.69662800 \\
\hline C & 0.07709900 & -0.40970400 & -1.31277800 & $\mathrm{O}$ & 0.79682700 & -0.59636400 & -0.35760500 \\
\hline $\mathrm{O}$ & 0.40080600 & 0.06925100 & -2.51898600 & $\mathrm{C}$ & 1.65412300 & 0.82873700 & -2.76725100 \\
\hline C & 2.87488400 & -0.03812500 & -2.48464000 & $\mathrm{H}$ & 3.78109800 & 0.53999100 & -2.68937600 \\
\hline $\mathrm{H}$ & 2.89947600 & -0.36647400 & -1.44848300 & $\mathrm{H}$ & 2.87055900 & -0.91938200 & -3.12922000 \\
\hline C & 1.54500700 & 1.14476100 & -4.25617700 & $\mathrm{H}$ & 0.65060800 & 1.74177600 & -4.46386200 \\
\hline $\mathrm{H}$ & 2.42199900 & 1.71607300 & -4.57554000 & $\mathrm{H}$ & 1.49682500 & 0.22022900 & -4.84030000 \\
\hline C & 1.60996600 & 2.09787300 & -1.92394200 & $\mathrm{H}$ & 2.50965200 & 2.69279300 & -2.09483800 \\
\hline $\mathrm{H}$ & 0.74261300 & 2.71319600 & -2.18278900 & $\mathrm{H}$ & 1.55947000 & 1.86052300 & -0.86288800 \\
\hline C & -2.16823100 & -0.22228700 & -2.21287100 & $\mathrm{H}$ & -1.69535600 & -0.10362900 & -3.17983500 \\
\hline C & -3.55540200 & -0.73409100 & -2.28195400 & C & -4.18821100 & -0.72141000 & -3.53211500 \\
\hline C & -4.25332300 & -1.21429900 & -1.16528400 & C & -5.49228000 & -1.19048100 & -3.67317500 \\
\hline $\mathrm{H}$ & -3.64595800 & -0.35767300 & -4.40110800 & C & -5.55824000 & -1.68050000 & -1.31019100 \\
\hline $\mathrm{H}$ & -3.77730100 & -1.23990000 & -0.19205000 & C & -6.18133500 & -1.67429700 & -2.55996800 \\
\hline $\mathrm{H}$ & -5.96617900 & -1.18439800 & -4.65027800 & $\mathrm{H}$ & -6.08947700 & -2.05693500 & -0.44041000 \\
\hline $\mathrm{H}$ & -7.19721700 & -2.04356500 & -2.66579100 & $\mathrm{~N}$ & -1.29671100 & -0.70271100 & -1.27263500 \\
\hline $\mathrm{H}$ & -1.62033000 & -1.04319400 & -0.36064300 & C & -2.20149000 & 1.77929100 & -1.96972700 \\
\hline $\mathrm{H}$ & -1.27233300 & 1.85725600 & -1.42173600 & $\mathrm{H}$ & -2.17052100 & 2.14654300 & -2.99335900 \\
\hline C & -3.38305300 & 1.96978800 & -1.25022300 & C & -4.59026300 & 2.44678500 & -1.90135900 \\
\hline $\mathrm{H}$ & -4.59809900 & 2.25671000 & -2.97970800 & $\mathrm{H}$ & -5.51015200 & 2.07181800 & -1.44223200 \\
\hline C & -3.41404400 & 1.69671700 & 0.20981400 & $\mathrm{H}$ & -3.13718200 & 2.61536900 & 0.73647700 \\
\hline $\mathrm{H}$ & -4.40987100 & 1.39329000 & 0.54278100 & $\mathrm{H}$ & -2.67444900 & 0.95229700 & 0.50698900 \\
\hline C & -6.20829200 & 4.89707800 & -2.65110400 & $\mathrm{H}$ & -7.09451500 & 4.45997100 & -2.17698000 \\
\hline $\mathrm{H}$ & -6.32911300 & 5.98687000 & -2.64089800 & $\mathrm{H}$ & -6.19089400 & 4.57447500 & -3.69837100 \\
\hline $\mathrm{C}$ & -4.69137100 & 4.91627000 & 0.06033500 & $\mathrm{H}$ & -5.44122100 & 4.35314600 & 0.62752400 \\
\hline $\mathrm{H}$ & -3.71830500 & 4.78830600 & 0.54418900 & $\mathrm{H}$ & -4.95589000 & 5.97860600 & 0.12982600 \\
\hline $\mathrm{C}$ & -3.09227800 & 5.08909800 & -2.58550400 & $\mathrm{H}$ & -2.19789600 & 4.83867700 & -2.00531100 \\
\hline $\mathrm{H}$ & -2.97137500 & 4.70779700 & -3.60635800 & $\mathrm{H}$ & -3.15406700 & 6.18227400 & -2.64814100 \\
\hline $\mathrm{Si}$ & -4.62630800 & 4.40585400 & -1.74752200 & C & -1.93667200 & -4.10076800 & 3.57660000 \\
\hline $\mathrm{H}$ & -2.77013100 & -3.99569400 & 4.27957100 & $\mathrm{H}$ & -0.99957100 & -3.86243600 & 4.08268700 \\
\hline $\mathrm{C}$ & -1.92025100 & -5.49812600 & 2.94062900 & $\mathrm{H}$ & -0.89973000 & -5.76284900 & 2.64933400 \\
\hline $\mathrm{H}$ & -2.28234500 & -6.26432900 & 3.63094500 & C & -2.80312700 & -5.34561800 & 1.69376200 \\
\hline $\mathrm{H}$ & -2.62133300 & -6.11351200 & 0.93826400 & $\mathrm{H}$ & -3.86396900 & -5.37892300 & 1.97048600 \\
\hline C & -2.44756700 & -3.93839400 & 1.17560700 & $\mathrm{H}$ & -3.30024900 & -3.45918500 & 0.69405100 \\
\hline C & 2.10550700 & -0.32461500 & 2.47418900 & C & 0.87993600 & -0.75956600 & 3.02067400 \\
\hline C & 1.47931600 & -2.01212900 & 3.52550200 & C & 2.81848200 & -1.54261000 & 2.96870200 \\
\hline $\mathrm{O}$ & 1.05795500 & -3.01688500 & 4.08771400 & $\mathrm{O}$ & 3.94319500 & -2.00473000 & 2.90636500 \\
\hline $\mathrm{N}$ & 2.42070900 & 0.79840600 & 1.80068100 & $\mathrm{H}$ & 1.64000900 & 1.43193300 & 1.61464300 \\
\hline C & 3.58750900 & 1.03009600 & 1.05469800 & C & 3.82888200 & 2.33578800 & 0.60498900 \\
\hline C & 4.46852600 & 0.00024800 & 0.70201000 & C & 4.93020500 & 2.59203700 & -0.20645400 \\
\hline $\mathrm{H}$ & 3.14004700 & 3.13143500 & 0.86691100 & C & 5.56425300 & 0.28475700 & -0.10878600 \\
\hline $\mathrm{H}$ & 4.31035800 & -1.00755500 & 1.05806100 & C & 5.80931500 & 1.57441300 & -0.57567100 \\
\hline $\mathrm{H}$ & 6.66313100 & 1.78231600 & -1.20818800 & $\mathrm{C}$ & 6.41536300 & -0.84914900 & -0.60439300 \\
\hline C & 5.12590900 & 3.97081700 & -0.77062800 & $\mathrm{~F}$ & 6.49574400 & -1.86409700 & 0.28104500 \\
\hline $\mathrm{F}$ & 5.90317700 & -1.37406700 & -1.75283400 & $\mathrm{~F}$ & 7.67471200 & -0.45572700 & -0.89062900 \\
\hline $\mathrm{F}$ & 6.43238800 & 4.25020800 & -0.97762500 & $\mathrm{~F}$ & 4.62145000 & 4.93018100 & 0.02532000 \\
\hline $\mathrm{F}$ & 4.51199800 & 4.09604700 & -1.98027400 & & & & \\
\hline
\end{tabular}


Imaginary frequency: $-343.09 \mathrm{~cm}^{-1}$

E[B3LYP/6-31G(d)-D3(BJ)]: -4655.633485 Hartree

E[PCM(Et $\left.\left.{ }_{2} \mathrm{O}\right)-B 3 L Y P / 6-311 G(d, p)-D 3(B J)\right]:-4656.824583$ Hartree

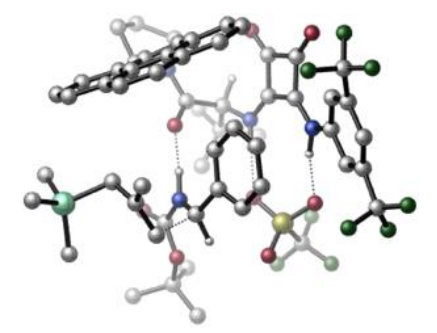

\begin{tabular}{|c|c|c|c|c|c|c|c|}
\hline 01 & & & & $\mathrm{C}$ & -1.38460900 & 0.40905500 & 3.47249600 \\
\hline $\mathrm{H}$ & -1.03466300 & -0.05457200 & 4.39480400 & C & -2.31626300 & 1.59753600 & 3.86696700 \\
\hline $\mathrm{C}$ & -3.04503100 & 2.20774800 & 2.66347900 & $\mathrm{H}$ & -3.69979300 & 1.48789300 & 2.16983700 \\
\hline $\mathrm{H}$ & -2.34701500 & 2.59824100 & 1.92154300 & $\mathrm{H}$ & -3.66335800 & 3.04630600 & 3.00522700 \\
\hline $\mathrm{C}$ & -1.45714400 & 2.67795600 & 4.54897600 & $\overline{\mathrm{H}}$ & -2.10879200 & 3.44817200 & 4.97690900 \\
\hline $\mathrm{H}$ & -0.78688700 & 3.16692500 & 3.83727000 & $\mathrm{H}$ & -0.85132700 & 2.25776500 & 5.36073900 \\
\hline $\mathrm{C}$ & -3.36855700 & 1.08660200 & 4.86655000 & $\mathrm{H}$ & -4.04198200 & 1.90492500 & 5.14220000 \\
\hline $\mathrm{H}$ & -2.90674600 & 0.71499600 & 5.78914900 & $\mathrm{H}$ & -3.98468700 & 0.28927100 & 4.43452100 \\
\hline $\mathrm{C}$ & -2.04621200 & -0.68041500 & 2.60187500 & $\mathrm{O}$ & -2.17616100 & -0.53207300 & 1.37441900 \\
\hline C & -2.43474100 & -2.22865800 & 4.59379600 & C & -2.88373500 & -2.94943800 & 2.30719300 \\
\hline $\mathrm{C}$ & -3.58983700 & -3.23154300 & 4.63278600 & $\mathrm{H}$ & -1.47996300 & -2.68189200 & 4.87704800 \\
\hline $\mathrm{H}$ & -2.60299300 & -1.36332700 & 5.22957700 & $\mathrm{H}$ & -3.68264500 & -2.54511200 & 1.68102000 \\
\hline $\mathrm{H}$ & -4.54505200 & -2.69357700 & 4.64817600 & $\mathrm{H}$ & -3.54870300 & -3.88058900 & 5.51181800 \\
\hline $\mathrm{N}$ & -2.40959500 & -1.84725600 & 3.17320200 & C & -1.76981700 & -3.48254300 & 1.41924300 \\
\hline $\mathrm{C}$ & -0.48250500 & -3.47088700 & 1.87062700 & C & -2.06697300 & -4.01828400 & 0.10901500 \\
\hline C & 0.62122300 & -3.88552900 & 1.05886900 & $\mathrm{H}$ & -0.25629000 & -3.08507500 & 2.85883700 \\
\hline C & -0.97650700 & -4.37631000 & -0.74887300 & C & -3.37684800 & -4.21604000 & -0.36203800 \\
\hline $\mathrm{C}$ & 1.94579800 & -3.82989400 & 1.52269400 & C & 0.36701900 & -4.28810100 & -0.28331200 \\
\hline C & -1.23045600 & -4.83971600 & -2.07682700 & $\mathrm{H}$ & -4.22158900 & -3.99173700 & 0.27969500 \\
\hline C & -3.61453900 & -4.72100700 & -1.64032400 & C & 3.00826800 & -4.10919500 & 0.66363200 \\
\hline $\mathrm{H}$ & 2.14540900 & -3.53284100 & 2.54630900 & C & 1.45892100 & -4.59467700 & -1.14893900 \\
\hline C & -2.55827800 & -5.00853700 & -2.50068300 & C & -0.11306800 & -5.12847500 & -2.93354000 \\
\hline $\mathrm{H}$ & -4.63710400 & -4.88341100 & -1.96914100 & C & 2.77039100 & -4.47898400 & -0.65976700 \\
\hline $\mathrm{H}$ & 4.02699800 & -4.01179700 & 1.02366700 & C & 1.16967200 & -4.99921200 & -2.49542100 \\
\hline $\mathrm{H}$ & -2.75191400 & -5.38812700 & -3.50073500 & $\mathrm{H}$ & -0.31739200 & -5.46133200 & -3.94806800 \\
\hline $\mathrm{H}$ & 3.60654200 & -4.67348400 & -1.32499600 & $\mathrm{H}$ & 2.00251700 & -5.21968900 & -3.15788300 \\
\hline $\mathrm{N}$ & -0.17605000 & 0.85796800 & 2.77096300 & $\mathrm{H}$ & -0.26249100 & 1.56088400 & 2.02926800 \\
\hline S & 0.49655500 & 3.84229700 & 0.16574500 & 0 & 0.22454500 & 4.12200100 & -1.25577500 \\
\hline 0 & -0.46517000 & 2.90907200 & 0.81590100 & 0 & 1.91099000 & 3.55359400 & 0.51819600 \\
\hline C & 0.16194200 & 5.44982400 & 1.03129800 & $\mathrm{~F}$ & 1.05256100 & 6.37775600 & 0.67431700 \\
\hline $\mathrm{F}$ & 0.22022100 & 5.28133100 & 2.36090300 & $\mathrm{~F}$ & -1.06849300 & 5.90144000 & 0.71888300 \\
\hline C & -3.28580600 & 2.05186800 & -0.76960600 & 0 & -4.29592800 & 1.74431300 & -0.16178200 \\
\hline 0 & -3.10357700 & 3.13907800 & -1.52463400 & C & -3.99704900 & 4.31514500 & -1.42591600 \\
\hline C & -4.15509700 & 4.72573900 & 0.03792100 & $\mathrm{H}$ & -4.67312100 & 5.68980900 & 0.07963800 \\
\hline $\mathrm{H}$ & -3.17505600 & 4.84302900 & 0.50518400 & $\mathrm{H}$ & -4.73432400 & 3.99169300 & 0.59888500 \\
\hline $\mathrm{C}$ & -5.33365400 & 3.98532200 & -2.08597900 & $\mathrm{H}$ & -5.18255900 & 3.66204800 & -3.12139400 \\
\hline $\mathrm{H}$ & -5.96623700 & 4.87930900 & -2.09876900 & $\mathrm{H}$ & -5.85371300 & 3.19836800 & -1.53565100 \\
\hline $\mathrm{C}$ & -3.21458500 & 5.36873900 & -2.20636400 & $\mathrm{H}$ & -3.76561900 & 6.31455500 & -2.21013900 \\
\hline $\mathrm{H}$ & -3.06681800 & 5.04932400 & -3.24326800 & $\mathrm{H}$ & -2.23434200 & 5.52546700 & -1.74864600 \\
\hline C & -1.16743600 & 1.37679800 & -1.75189300 & $\mathrm{H}$ & -1.06121500 & 2.40577400 & -2.08511700 \\
\hline $\mathrm{C}$ & 0.13252000 & 0.68535000 & -1.59094200 & C & 1.18679000 & 1.13754700 & -2.40196300 \\
\hline C & 0.32259300 & -0.44203300 & -0.78109700 & $C$ & 2.38452500 & 0.43477500 & -2.46622600 \\
\hline $\mathrm{H}$ & 1.05775500 & 2.04979100 & -2.97621600 & C & 1.54113400 & -1.12061800 & -0.82536300 \\
\hline $\mathrm{H}$ & -0.46268000 & -0.79946400 & -0.12258800 & C & 2.55404500 & -0.71422500 & -1.69161200 \\
\hline $\mathrm{H}$ & 3.19388200 & 0.80128400 & -3.08797900 & $\mathrm{H}$ & 1.70017800 & -1.96941000 & -0.17866300 \\
\hline
\end{tabular}




\begin{tabular}{|c|c|c|c|c|c|c|c|}
\hline $\mathrm{H}$ & 3.48167600 & -1.27312200 & -1.73162100 & $\mathrm{~N}$ & -2.15884200 & 1.23547600 & -0.80029300 \\
\hline $\mathrm{H}$ & -2.09143800 & 0.57328200 & -0.01751800 & C & -1.89823200 & 0.79344700 & -3.38091100 \\
\hline $\mathrm{H}$ & -1.18081300 & 1.13284400 & -4.12385200 & $\mathrm{H}$ & -2.79543800 & 1.40307200 & -3.33296000 \\
\hline C & -2.03548500 & -0.60017400 & -3.22047200 & C & -3.07006700 & -1.14648300 & -2.36772800 \\
\hline $\mathrm{H}$ & -3.25831300 & -0.47525200 & -1.51659400 & $\mathrm{H}$ & -2.83493800 & -2.15213800 & -2.01166700 \\
\hline C & -0.99889500 & -1.52663100 & -3.75166900 & $\mathrm{H}$ & -1.44125900 & -2.48381800 & -4.04398500 \\
\hline $\mathrm{H}$ & -0.30123900 & -1.75094700 & -2.92907600 & $\mathrm{H}$ & -0.42450700 & -1.09988200 & -4.57604900 \\
\hline C & -5.96594700 & -1.71230800 & -1.72446500 & $\mathrm{H}$ & -5.67149800 & -2.66610900 & -1.27730700 \\
\hline $\mathrm{H}$ & -7.00822100 & -1.79615800 & -2.05370000 & $\mathrm{H}$ & -5.91611300 & -0.94211600 & -0.94569500 \\
\hline C & -4.80929800 & -2.52119500 & -4.53272600 & $\mathrm{H}$ & -4.43766700 & -3.48425000 & -4.16915800 \\
\hline $\mathrm{H}$ & -4.16055700 & -2.19863400 & -5.35573800 & $\mathrm{H}$ & -5.81287600 & -2.67834200 & -4.94508700 \\
\hline C & -5.33095600 & 0.44954700 & -3.81796500 & $\mathrm{H}$ & -4.70725600 & 0.77094800 & -4.65878200 \\
\hline $\bar{H}$ & -5.27676800 & 1.21150700 & -3.03379600 & $\mathrm{H}$ & -6.36875400 & 0.41813900 & -4.17145200 \\
\hline $\mathrm{Si}$ & -4.83857300 & -1.24018800 & -3.15320400 & C & -3.42304700 & -4.00031100 & 3.31322500 \\
\hline $\mathrm{H}$ & -2.68256500 & -4.79688500 & 3.43290000 & $\mathrm{H}$ & -4.35036600 & -4.45988700 & 2.96036200 \\
\hline C & 0.95982900 & 0.17415400 & 2.91661300 & C & 2.26540300 & 0.21424300 & 2.36329500 \\
\hline C & 1.29463500 & -1.00498700 & 3.75022100 & C & 2.69229300 & -0.97683400 & 3.15862000 \\
\hline $\mathrm{O}$ & 0.68706600 & -1.71329100 & 4.54309300 & 0 & 3.68381500 & -1.68020300 & 3.26467000 \\
\hline $\bar{N}$ & 2.83608200 & 1.05583400 & 1.47388200 & $\mathrm{H}$ & 2.32400000 & 1.90787700 & 1.21299000 \\
\hline $\mathrm{C}$ & 3.98484700 & 0.82006200 & 0.70833300 & C & 4.39510500 & 1.82466000 & -0.18228100 \\
\hline C & 4.66929200 & -0.40153700 & 0.73256300 & C & 5.44742900 & 1.58155400 & -1.05819600 \\
\hline $\mathrm{H}$ & 3.85964100 & 2.76608100 & -0.21318100 & C & 5.71263300 & -0.62102400 & -0.16271300 \\
\hline $\mathrm{H}$ & 4.39432000 & -1.17658300 & 1.43615000 & C & 6.11440800 & 0.35567300 & -1.07119000 \\
\hline $\mathrm{H}$ & 6.92190400 & 0.16996100 & -1.76833900 & C & 6.29637700 & -2.00112100 & -0.25440500 \\
\hline C & 5.79781500 & 2.61290400 & -2.09311000 & $\mathrm{~F}$ & 6.28424800 & -2.65386100 & 0.92572900 \\
\hline $\mathrm{F}$ & 5.57444100 & -2.77019700 & -1.12488800 & $\mathrm{~F}$ & 7.56534900 & -1.99726100 & -0.71068800 \\
\hline $\mathrm{F}$ & 5.47955600 & 3.86175200 & -1.70615400 & $\mathrm{~F}$ & 5.13457300 & 2.38042800 & -3.26036000 \\
\hline $\mathrm{F}$ & 7.11496400 & 2.59704600 & -2.39525300 & & & & \\
\hline
\end{tabular}

\section{TS- $R-6 f-8$}

Imaginary frequency: $-343.15 \mathrm{~cm}^{-1}$

E[B3LYP/6-31G(d)-D3(BJ)]: -4655.633485 Hartree

$\mathrm{E}\left[\mathrm{PCM}\left(\mathrm{Et}_{2} \mathrm{O}\right)-\mathrm{B} 3 \mathrm{LYP} / 6-311 \mathrm{G}(\mathrm{d}, \mathrm{p})-\mathrm{D} 3(\mathrm{BJ})\right]$ : -4656.824582 Hartree

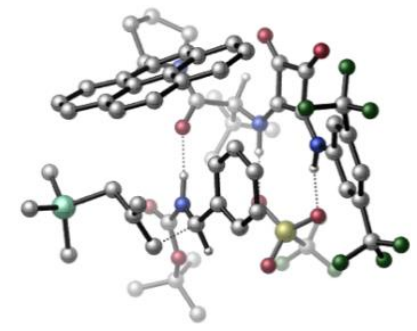

\begin{tabular}{|c|c|c|c|c|c|c|c|}
\hline 01 & & & & $\mathrm{C}$ & -1.38460300 & 0.40907000 & 3.47250100 \\
\hline $\mathrm{H}$ & -1.03465500 & -0.05455600 & 4.39480800 & $\mathrm{C}$ & -2.31625700 & 1.59755000 & 3.86697300 \\
\hline C & -3.04501900 & 2.20776900 & 2.66348400 & $\mathrm{H}$ & -3.69978000 & 1.48791700 & 2.16983700 \\
\hline $\mathrm{H}$ & -2.34699800 & 2.59826300 & 1.92155100 & $\mathrm{H}$ & -3.66334500 & 3.04632700 & 3.00523200 \\
\hline C & -1.45713900 & 2.67796500 & 4.54899100 & $\mathrm{H}$ & -2.10878700 & 3.44818300 & 4.97692000 \\
\hline $\mathrm{H}$ & -0.78687400 & 3.16693200 & 3.83729100 & $\mathrm{H}$ & -0.85133000 & 2.25777000 & 5.36075700 \\
\hline C & -3.36855600 & 1.08661400 & 4.86654900 & $\mathrm{H}$ & -4.04198400 & 1.90493600 & 5.14219600 \\
\hline $\mathrm{H}$ & -2.90675100 & 0.71500800 & 5.78915000 & $\mathrm{H}$ & -3.98468300 & 0.28928300 & 4.43451500 \\
\hline C & -2.04620900 & -0.68040200 & 2.60188300 & $\mathrm{O}$ & -2.17616900 & -0.53206200 & 1.37442800 \\
\hline C & -2.43472700 & -2.22864400 & 4.59380800 & C & -2.88372800 & -2.94942600 & 2.30720800 \\
\hline C & -3.58982400 & -3.23152700 & 4.63280400 & $\mathrm{H}$ & -1.47994900 & -2.68187800 & 4.87705800 \\
\hline $\mathrm{H}$ & -2.60297600 & -1.36331200 & 5.22958800 & $\mathrm{H}$ & -3.68263800 & -2.54509900 & 1.68103400 \\
\hline $\mathrm{H}$ & -4.54503900 & -2.69356000 & 4.64819800 & $\mathrm{H}$ & -3.54868600 & -3.88057300 & 5.51183600 \\
\hline $\mathrm{N}$ & -2.40958600 & -1.84724300 & 3.17321300 & C & -1.76981200 & -3.48253600 & 1.41925900 \\
\hline C & -0.48249900 & -3.47087600 & 1.87064000 & $\mathrm{C}$ & -2.06697000 & -4.01828500 & 0.10903500 \\
\hline $\mathrm{C}$ & 0.62122800 & -3.88552500 & 1.05888400 & $\mathrm{H}$ & -0.25628200 & -3.08505700 & 2.85884800 \\
\hline C & -0.97650600 & -4.37632000 & -0.74885100 & C & -3.37684500 & -4.21603900 & -0.36201600 \\
\hline
\end{tabular}




\begin{tabular}{|c|c|c|c|c|c|c|c|}
\hline $\mathrm{C}$ & 1.94580300 & -3.82988600 & 1.52270600 & C & 0.36702100 & -4.28810900 & -0.28329300 \\
\hline C & -1.23045600 & -4.83973700 & -2.07680100 & $\mathrm{H}$ & -4.22158600 & -3.99172800 & 0.27971500 \\
\hline C & -3.61453900 & -4.72101500 & -1.64029900 & C & 3.00827200 & -4.10919500 & 0.66364500 \\
\hline $\mathrm{H}$ & 2.14541700 & -3.53282500 & 2.54631900 & C & 1.45892200 & -4.59469300 & -1.14891800 \\
\hline C & -2.55827900 & -5.00855600 & -2.50065500 & C & -0.11307000 & -5.12850600 & -2.93351300 \\
\hline $\mathrm{H}$ & -4.63710500 & -4.88341700 & -1.96911500 & C & 2.77039300 & -4.47899700 & -0.65975000 \\
\hline $\mathrm{H}$ & 4.02700300 & -4.01179500 & 1.02367800 & C & 1.16967100 & -4.99924100 & -2.49539600 \\
\hline $\mathrm{H}$ & -2.75191700 & -5.38815200 & -3.50070400 & $\mathrm{H}$ & -0.31739600 & -5.46137100 & -3.94803800 \\
\hline $\mathrm{H}$ & 3.60654300 & -4.67350300 & -1.32497900 & $\mathrm{H}$ & 2.00251500 & -5.21972500 & -3.15785800 \\
\hline $\mathrm{N}$ & -0.17604500 & 0.85798200 & 2.77096600 & $\mathrm{H}$ & -0.26248900 & 1.56089500 & 2.02926600 \\
\hline S & 0.49655400 & 3.84229500 & 0.16572600 & 0 & 0.22453000 & 4.12201900 & -1.25578700 \\
\hline 0 & -0.46515700 & 2.90905000 & 0.81587300 & $\mathrm{O}$ & 1.91099500 & 3.55359900 & 0.51816000 \\
\hline C & 0.16193500 & 5.44980400 & 1.03130800 & $\mathrm{~F}$ & 1.05253400 & 6.37775400 & 0.67432400 \\
\hline $\mathrm{F}$ & 0.22024200 & 5.28129200 & 2.36091000 & $\mathrm{~F}$ & -1.06851200 & 5.90140800 & 0.71892500 \\
\hline C & -3.28580200 & 2.05187100 & -0.76960000 & $\mathrm{O}$ & -4.29592300 & 1.74431100 & -0.16177800 \\
\hline 0 & -3.10356900 & 3.13908700 & -1.52461500 & C & -3.99706200 & 4.31514100 & -1.42591900 \\
\hline C & -5.33365200 & 3.98529000 & -2.08599900 & $\mathrm{H}$ & -5.96624800 & 4.87926800 & -2.09880600 \\
\hline $\mathrm{H}$ & -5.85370700 & 3.19833300 & -1.53567200 & $\mathrm{H}$ & -5.18253600 & 3.66201100 & -3.12141000 \\
\hline C & -3.21460300 & 5.36874100 & -2.20636300 & $\mathrm{H}$ & -2.23436600 & 5.52548300 & -1.74863700 \\
\hline $\mathrm{H}$ & -3.76564900 & 6.31455000 & -2.21014800 & $\mathrm{H}$ & -3.06682200 & 5.04932300 & -3.24326500 \\
\hline C & -4.15513800 & 4.72574200 & 0.03791300 & $\mathrm{H}$ & -4.67318600 & 5.68979900 & 0.07961600 \\
\hline $\mathrm{H}$ & -3.17510600 & 4.84305900 & 0.50518700 & $\mathrm{H}$ & -4.73435400 & 3.99168600 & 0.59887500 \\
\hline C & -1.16743100 & 1.37680200 & -1.75188300 & $\mathrm{H}$ & -1.06122200 & 2.40577200 & -2.08512700 \\
\hline C & 0.13252000 & 0.68535200 & -1.59094000 & C & 1.18679000 & 1.13755000 & -2.40196200 \\
\hline $\mathrm{C}$ & 0.32259500 & -0.44202800 & -0.78109300 & C & 2.38452700 & 0.43478200 & -2.46621900 \\
\hline $\mathrm{H}$ & 1.05775300 & 2.04979200 & -2.97621900 & C & 1.54113800 & -1.12061000 & -0.82535400 \\
\hline $\mathrm{H}$ & -0.46267900 & -0.79946000 & -0.12258400 & C & 2.55405000 & -0.71421500 & -1.69160100 \\
\hline $\mathrm{H}$ & 3.19388500 & 0.80129200 & -3.08797100 & $\mathrm{H}$ & 1.70018200 & -1.96940100 & -0.17865200 \\
\hline $\mathrm{H}$ & 3.48168200 & -1.27310900 & -1.73160600 & $\mathrm{~N}$ & -2.15883800 & 1.23547400 & -0.80029300 \\
\hline $\mathrm{H}$ & -2.09143200 & 0.57328300 & -0.01751600 & C & -1.89824300 & 0.79344500 & -3.38093600 \\
\hline $\mathrm{H}$ & -1.18081900 & 1.13285000 & -4.12386700 & $\mathrm{H}$ & -2.79544200 & 1.40307800 & -3.33296200 \\
\hline C & -2.03548800 & -0.60016900 & -3.22048600 & C & -3.07007300 & -1.14648100 & -2.36774000 \\
\hline $\mathrm{H}$ & -3.25832200 & -0.47524500 & -1.51661100 & $\mathrm{H}$ & -2.83493100 & -2.15212800 & -2.01166600 \\
\hline C & -0.99888700 & -1.52662100 & -3.75167000 & $\mathrm{H}$ & -1.44123100 & -2.48383000 & -4.04394400 \\
\hline $\mathrm{H}$ & -0.30120300 & -1.75088600 & -2.92908800 & $\mathrm{H}$ & -0.42453000 & -1.09988700 & -4.57608100 \\
\hline C & -5.96595900 & -1.71233900 & -1.72450400 & $\mathrm{H}$ & -5.67151400 & -2.66614200 & -1.27734600 \\
\hline $\mathrm{H}$ & -7.00823000 & -1.79619000 & -2.05375200 & $\mathrm{H}$ & -5.91613800 & -0.94215200 & -0.94572800 \\
\hline C & -4.80927200 & -2.52121600 & -4.53275100 & $\mathrm{H}$ & -4.43766400 & -3.48427600 & -4.16917500 \\
\hline $\mathrm{H}$ & -4.16050400 & -2.19866200 & -5.35574400 & $\mathrm{H}$ & -5.81284100 & -2.67834800 & -4.94514000 \\
\hline C & -5.33096200 & 0.44951900 & -3.81799900 & $\mathrm{H}$ & -4.70724400 & 0.77093000 & -4.65879800 \\
\hline $\mathrm{H}$ & -5.27680300 & 1.21147900 & -3.03382700 & $\mathrm{H}$ & -6.36875000 & 0.41809800 & -4.17151300 \\
\hline $\mathrm{Si}$ & -4.83856900 & -1.24021000 & -3.15322700 & C & -3.42304200 & -4.00029600 & 3.31324200 \\
\hline $\mathrm{H}$ & -2.68256400 & -4.79687400 & 3.43291500 & $\mathrm{H}$ & -4.35036500 & -4.45986700 & 2.96038300 \\
\hline C & 1.29464300 & -1.00496900 & 3.75022500 & C & 2.69229900 & -0.97681800 & 3.15861900 \\
\hline 0 & 0.68707700 & -1.71326800 & 4.54310300 & $\mathrm{O}$ & 3.68382100 & -1.68018600 & 3.26466900 \\
\hline C & 2.26540600 & 0.21425600 & 2.36329100 & C & 0.95983400 & 0.17416900 & 2.91661300 \\
\hline $\mathrm{N}$ & 2.83608300 & 1.05584300 & 1.47387300 & $\mathrm{H}$ & 2.32400500 & 1.90789100 & 1.21298800 \\
\hline C & 3.98484400 & 0.82006900 & 0.70832100 & C & 4.66928700 & -0.40153300 & 0.73255100 \\
\hline C & 4.39510300 & 1.82466400 & -0.18229600 & C & 5.71262700 & -0.62102200 & -0.16272600 \\
\hline $\mathrm{H}$ & 4.39431500 & -1.17657700 & 1.43613900 & C & 5.44742600 & 1.58155400 & -1.05821200 \\
\hline $\mathrm{H}$ & 3.85964200 & 2.76608700 & -0.21319600 & C & 6.11440300 & 0.35567300 & -1.07120500 \\
\hline $\mathrm{H}$ & 6.92189900 & 0.16995900 & -1.76835400 & C & 6.29637000 & -2.00112000 & -0.25441600 \\
\hline C & 5.79781000 & 2.61290600 & -2.09312600 & $\mathrm{~F}$ & 5.57443200 & -2.77019700 & -1.12489500 \\
\hline
\end{tabular}




\begin{tabular}{|rrrr|lrrr|}
\hline F & 6.28424300 & -2.65385700 & 0.92572100 & $F$ & 7.56534100 & -1.99726200 & -0.71070100 \\
\hline$F$ & 5.47960700 & 3.86175900 & -1.70614100 & $F$ & 5.13451800 & 2.38046900 & -3.26035400 \\
\hline$F$ & 7.11494800 & 2.59700900 & -2.39531600 & & & & \\
\hline
\end{tabular}

\section{TS- $R-6 f-9$}

Imaginary frequency: $-204.05 \mathrm{~cm}^{-1}$

E[B3LYP/6-31G(d)-D3(BJ)]: -4655,639362 Hartree

$\mathrm{E}\left[\mathrm{PCM}\left(\mathrm{Et}_{2} \mathrm{O}\right)-\mathrm{B} 3 \mathrm{LYP} / 6-311 \mathrm{G}(\mathrm{d}, \mathrm{p})-\mathrm{D} 3(\mathrm{BJ})\right]$ : -4656.823502 Hartree

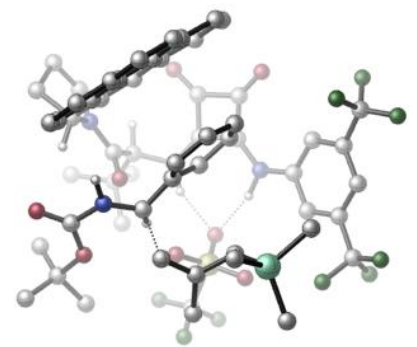

\begin{tabular}{|c|c|c|c|c|c|c|c|}
\hline 01 & & & & $\mathrm{C}$ & -2.73289800 & -1.18492900 & -2.98329600 \\
\hline $\mathrm{H}$ & -3.06084200 & -0.53000400 & -3.79124700 & C & -3.33449900 & -2.60455500 & -3.22365900 \\
\hline $\mathrm{C}$ & -2.66597300 & -3.64939900 & -2.31314300 & $\mathrm{H}$ & -2.60330800 & -3.30534400 & -1.27911500 \\
\hline $\mathrm{H}$ & -1.65242200 & -3.88215200 & -2.64584600 & $\mathrm{H}$ & -3.23612100 & -4.58435700 & -2.33790400 \\
\hline C & -3.09027000 & -2.98172700 & -4.69463700 & $\mathrm{H}$ & -3.45676300 & -3.99544400 & -4.89202100 \\
\hline $\mathrm{H}$ & -2.02272500 & -2.95473500 & -4.93366400 & $\mathrm{H}$ & -3.61060400 & -2.29526100 & -5.37357200 \\
\hline C & -4.84844300 & -2.58443000 & -2.95509800 & $\mathrm{H}$ & -5.26393600 & -3.58360900 & -3.12331800 \\
\hline $\mathrm{H}$ & -5.36836600 & -1.89756600 & -3.63129300 & $\mathrm{H}$ & -5.08168600 & -2.29752200 & -1.92437200 \\
\hline C & -3.12672000 & -0.56206400 & -1.62614000 & $\mathrm{O}$ & -2.55693400 & -0.92142200 & -0.58951800 \\
\hline C & -4.86164200 & 0.91523400 & -2.76346300 & C & -4.40862500 & 1.09459700 & -0.36337700 \\
\hline C & -5.72092100 & 2.03072800 & -2.14489500 & $\mathrm{H}$ & -4.20041700 & 1.28264600 & -3.55211200 \\
\hline $\mathrm{H}$ & -5.49271400 & 0.11955600 & -3.16917000 & $\mathrm{C}$ & -5.81944000 & 1.64059700 & -0.66334100 \\
\hline $\mathrm{H}$ & -4.41113900 & 0.36144300 & 0.44370100 & $\mathrm{H}$ & -6.69513800 & 2.11250400 & -2.63394400 \\
\hline $\mathrm{H}$ & -5.21165000 & 2.99376000 & -2.24306000 & $\mathrm{H}$ & -6.55657900 & 0.84170800 & -0.52026900 \\
\hline $\mathrm{H}$ & -6.08676000 & 2.47745400 & -0.01430600 & $\mathrm{~N}$ & -4.07801200 & 0.40204900 & -1.61952000 \\
\hline C & -3.42740300 & 2.21931700 & -0.05680600 & C & -2.60838500 & 2.71135400 & -1.02938100 \\
\hline C & -3.40940100 & 2.81695100 & 1.26312600 & $\mathrm{C}$ & -1.71779600 & 3.81016600 & -0.80869500 \\
\hline $\mathrm{H}$ & -2.60663400 & 2.26566700 & -2.01576700 & C & -2.52393700 & 3.91419500 & 1.51338500 \\
\hline C & -4.20545400 & 2.34637600 & 2.32435300 & C & -0.86801800 & 4.28541500 & -1.81883200 \\
\hline C & -1.68601500 & 4.41674000 & 0.47732500 & $\mathrm{C}$ & -2.45400000 & 4.49560900 & 2.81679600 \\
\hline $\mathrm{H}$ & -4.87946400 & 1.51197800 & 2.17595200 & C & -4.11525300 & 2.91119700 & 3.59850700 \\
\hline C & 0.01263100 & 5.33455300 & -1.56279700 & $\mathrm{H}$ & -0.89265700 & 3.82612000 & -2.80062100 \\
\hline C & -0.79136600 & 5.49834100 & 0.72682200 & C & -3.25259000 & 3.97425100 & 3.84641300 \\
\hline C & -1.55179600 & 5.59182800 & 3.03916400 & $\mathrm{H}$ & -4.73168900 & 2.51248500 & 4.39899200 \\
\hline C & 0.04919900 & 5.93923600 & -0.30786200 & $\mathrm{H}$ & 0.67852700 & 5.67344700 & -2.35030900 \\
\hline C & -0.76251900 & 6.07498900 & 2.04057900 & $\mathrm{H}$ & -3.19230400 & 4.41345900 & 4.83879400 \\
\hline $\mathrm{H}$ & -1.51608000 & 6.03228100 & 4.03234800 & $\mathrm{H}$ & 0.73831100 & 6.75772300 & -0.11523500 \\
\hline $\mathrm{H}$ & -0.08581500 & 6.90545700 & 2.22589200 & $\mathrm{~N}$ & -1.26889000 & -1.23708900 & -3.05067400 \\
\hline $\mathrm{H}$ & -0.79714500 & -1.97165800 & -2.51928500 & $\mathrm{~S}$ & 0.92703000 & -3.40856700 & -0.60051500 \\
\hline $\mathrm{O}$ & 2.33167200 & -3.32117100 & -0.15089900 & 0 & -0.07954100 & -2.80158000 & 0.29382500 \\
\hline $\mathrm{O}$ & 0.73606100 & -3.06313100 & -2.04616800 & C & 0.55986900 & -5.23012000 & -0.52312400 \\
\hline $\mathrm{F}$ & 1.40802200 & -5.91559600 & -1.29174600 & $\mathrm{~F}$ & -0.69376700 & -5.49074400 & -0.92838300 \\
\hline $\mathrm{F}$ & 0.68222700 & -5.65420900 & 0.74551200 & C & -3.28247900 & -1.17457000 & 2.62542600 \\
\hline $\mathrm{O}$ & -4.43619900 & -0.87907500 & 2.87818400 & 0 & -2.76926200 & -2.38317200 & 2.41994600 \\
\hline C & -3.64245500 & -3.56279500 & 2.21552300 & C & -4.32133600 & -3.90693200 & 3.53928900 \\
\hline $\mathrm{H}$ & -4.90309800 & -4.82743400 & 3.42157900 & $\mathrm{H}$ & -4.99241900 & -3.10547600 & 3.85369800 \\
\hline $\mathrm{H}$ & -3.57242200 & -4.07476000 & 4.32058800 & C & -2.64219400 & -4.63603500 & 1.79556300 \\
\hline $\mathrm{H}$ & -2.10817000 & -4.33573100 & 0.89318400 & $\mathrm{H}$ & -3.16756700 & -5.57722200 & 1.60525600 \\
\hline $\mathrm{H}$ & -1.90638300 & -4.80744300 & 2.58803100 & C & -4.64279700 & -3.27227600 & 1.09647900 \\
\hline $\mathrm{H}$ & -5.11382700 & -4.20950500 & 0.78254900 & $\mathrm{H}$ & -4.12037100 & -2.83878500 & 0.24118300 \\
\hline $\mathrm{H}$ & -5.41960400 & -2.58003300 & 1.42453400 & $\mathrm{C}$ & -1.06400600 & -0.40306800 & 1.94603300 \\
\hline
\end{tabular}




\begin{tabular}{|c|c|c|c|c|c|c|c|}
\hline $\mathrm{H}$ & -1.01188600 & -1.27018800 & 1.30164100 & C & -0.29563100 & 0.78454100 & 1.53720900 \\
\hline C & 0.28671900 & 0.79338200 & 0.26241600 & C & -0.06862400 & 1.86488800 & 2.40732800 \\
\hline $\mathrm{C}$ & 1.09709900 & 1.86098100 & -0.12176900 & $\mathrm{H}$ & 0.09246500 & -0.03589400 & -0.40729400 \\
\hline $\mathrm{C}$ & 0.78233700 & 2.89875300 & 2.03605400 & $\mathrm{H}$ & -0.53414800 & 1.88265100 & 3.38948900 \\
\hline C & 1.37742200 & 2.89255800 & 0.77074700 & $\mathrm{H}$ & 1.52277300 & 1.89156500 & -1.11356600 \\
\hline $\mathrm{H}$ & 0.95824400 & 3.72207200 & 2.71952100 & $\mathrm{H}$ & 2.03832500 & 3.69637900 & 0.46696200 \\
\hline $\mathrm{N}$ & -2.28168600 & -0.20440000 & 2.54057200 & $\mathrm{H}$ & -2.59182700 & 0.73760300 & 2.76378400 \\
\hline C & 0.10340200 & -1.15756900 & 3.49970000 & $\mathrm{H}$ & -0.62565900 & -1.78794000 & 4.00057500 \\
\hline $\mathrm{H}$ & 0.22853100 & -0.16435900 & 3.91049000 & C & 1.21354700 & -1.77898100 & 2.92375600 \\
\hline C & 2.30509300 & -1.01288000 & 2.32908400 & $\mathrm{H}$ & 1.95261300 & -0.05845800 & 1.92818700 \\
\hline $\mathrm{H}$ & 2.81151400 & -1.57822800 & 1.54263100 & C & 1.30995000 & -3.27207700 & 2.94925600 \\
\hline $\mathrm{H}$ & 1.95004600 & -3.65645600 & 2.15627300 & $\mathrm{H}$ & 0.32126900 & -3.73045700 & 2.87806500 \\
\hline $\mathrm{H}$ & 1.74386300 & -3.56452600 & 3.91796500 & C & 4.72950600 & 0.81067400 & 2.66996500 \\
\hline $\mathrm{H}$ & 5.41322700 & 0.32064700 & 1.97326400 & $\mathrm{H}$ & 5.33611100 & 1.38317800 & 3.38213300 \\
\hline $\mathrm{H}$ & 4.11657900 & 1.52090300 & 2.10334700 & C & 4.65384400 & -1.92402400 & 4.16350000 \\
\hline $\mathrm{H}$ & 5.11377700 & -2.42634400 & 3.30607300 & $\mathrm{H}$ & 4.03163800 & -2.65488600 & 4.69274100 \\
\hline $\mathrm{H}$ & 5.45614700 & -1.61679600 & 4.84487500 & C & 2.79629100 & 0.41912900 & 5.03160300 \\
\hline $\mathrm{H}$ & 2.15520100 & -0.26384400 & 5.59928900 & $\mathrm{H}$ & 2.17677200 & 1.25241200 & 4.67869600 \\
\hline $\mathrm{H}$ & 3.53973700 & 0.83125300 & 5.72422500 & $\mathrm{Si}$ & 3.65558000 & -0.43689000 & 3.58607800 \\
\hline C & -0.90835700 & 1.19015200 & -3.86440900 & C & 0.55773100 & 1.58831300 & -3.70386900 \\
\hline 0 & -1.94285200 & 1.74957600 & -4.19880200 & 0 & 1.19522500 & 2.61971900 & -3.83764000 \\
\hline C & 0.81071000 & 0.20241900 & -3.21476300 & C & -0.55374500 & -0.14882200 & -3.34111600 \\
\hline $\mathrm{N}$ & 1.84751200 & -0.53015600 & -2.74158800 & $\mathrm{H}$ & 1.62063900 & -1.50630100 & -2.51991600 \\
\hline $\mathrm{C}$ & 3.08572700 & -0.07751800 & -2.26702800 & C & 3.52448100 & 1.24438600 & -2.42266700 \\
\hline C & 3.87176600 & -0.98835400 & -1.54745900 & $\mathrm{C}$ & 4.70062500 & 1.64932900 & -1.79630100 \\
\hline $\mathrm{H}$ & 2.93911400 & 1.95898600 & -2.99428800 & $\mathrm{C}$ & 5.05763400 & -0.56212900 & -0.95576600 \\
\hline $\mathrm{H}$ & 3.52965900 & -2.00680100 & -1.39919100 & $\mathrm{C}$ & 5.48399600 & 0.76086900 & -1.06125500 \\
\hline $\bar{H}$ & 6.39306200 & 1.09301500 & -0.57855400 & $\mathrm{C}$ & 5.05138100 & 3.11067300 & -1.82398500 \\
\hline $\mathrm{C}$ & 5.86495700 & -1.56853400 & -0.18417200 & $\mathrm{~F}$ & 4.33975500 & 3.79402900 & -0.88546300 \\
\hline $\mathrm{F}$ & 4.77449900 & 3.68115000 & -3.01162400 & $\mathrm{~F}$ & 6.35653700 & 3.32931700 & -1.55543400 \\
\hline $\mathrm{F}$ & 6.40582600 & -2.50902200 & -0.98275200 & $\mathrm{~F}$ & 5.10008900 & -2.22714800 & 0.72728700 \\
\hline $\mathrm{F}$ & 6.87883300 & -0.99152200 & 0.50362200 & & & & \\
\hline
\end{tabular}

\section{TS- $R-6 \mathrm{f}-10$}

Imaginary frequency: $-311.98 \mathrm{~cm}-1$

E[B3LYP/6-31G(d)-D3(BJ)]: -4655.629957 Hartree

E[PCM(Et $\left.\left.{ }_{2} \mathrm{O}\right)-\mathrm{B} 3 L Y P / 6-311 \mathrm{G}(\mathrm{d}, \mathrm{p})-\mathrm{D} 3(\mathrm{BJ})\right]$ : -4656.823325 Hartree

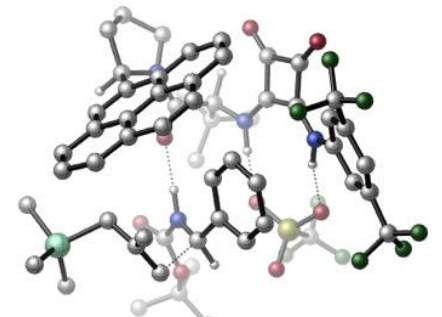

\begin{tabular}{|c|c|c|c|c|c|c|c|}
\hline 01 & & & & C & -1.29483200 & 0.31766000 & 3.50288800 \\
\hline $\mathrm{H}$ & -0.81586400 & -0.00809200 & 4.42549200 & $\mathrm{C}$ & -2.42435400 & 1.31631500 & 3.89833300 \\
\hline C & -3.29646300 & 1.74165600 & 2.71161400 & $\mathrm{H}$ & -3.82888600 & 0.89893000 & 2.26932600 \\
\hline $\mathrm{H}$ & -2.70824200 & 2.22597200 & 1.92981900 & $\mathrm{H}$ & -4.04297200 & 2.46725000 & 3.05616100 \\
\hline C & -1.76188900 & 2.56077400 & 4.51695700 & $\mathrm{H}$ & -2.53150200 & 3.21127600 & 4.94764000 \\
\hline $\mathrm{H}$ & -1.21843300 & 3.13873300 & 3.76449400 & $\mathrm{H}$ & -1.06143500 & 2.28969900 & 5.31613300 \\
\hline C & -3.32096700 & 0.64197700 & 4.95156100 & $\mathrm{H}$ & -4.13817200 & 1.31614200 & 5.22893900 \\
\hline $\mathrm{H}$ & -2.76465200 & 0.40396900 & 5.86605200 & $\mathrm{H}$ & -3.77575700 & -0.27912200 & 4.56678000 \\
\hline C & -1.75435600 & -0.93427600 & 2.72174600 & $\mathrm{O}$ & -2.08558900 & -0.87303600 & 1.52556900 \\
\hline C & -1.53646000 & -2.37390200 & 4.79951600 & C & -1.98839500 & -3.37978300 & 2.60557600 \\
\hline C & -2.18573300 & -3.74644500 & 4.99223000 & $\mathrm{H}$ & -0.47907900 & -2.37029000 & 5.07724200 \\
\hline $\mathrm{H}$ & -2.03591000 & -1.59612900 & 5.37313600 & $\mathrm{H}$ & -3.03159800 & -3.31868200 & 2.27429900 \\
\hline $\mathrm{H}$ & -3.27167700 & -3.63730700 & 5.09803700 & $\mathrm{H}$ & -1.80687900 & -4.25758300 & 5.88122800 \\
\hline
\end{tabular}




\begin{tabular}{|c|c|c|c|c|c|c|c|}
\hline $\bar{N}$ & -1.68945400 & -2.13085700 & 3.34704100 & C & -1.15487000 & -3.67787100 & 1.36958300 \\
\hline C & -1.82274200 & -4.17543200 & 0.28746100 & C & 0.29514200 & -3.59389700 & 1.33563000 \\
\hline C & -1.16456900 & -4.60347900 & -0.91032100 & $\mathrm{H}$ & -2.90760300 & -4.25082400 & 0.32243100 \\
\hline C & 0.97811200 & -3.99364900 & 0.13979800 & C & 1.07081400 & -3.13699200 & 2.41452200 \\
\hline C & -1.86632800 & -5.12859500 & -2.00827100 & C & 0.25202500 & -4.47238300 & -0.98853200 \\
\hline C & 2.40297600 & -3.89774600 & 0.06119600 & $\mathrm{H}$ & 0.59687500 & -2.80998700 & 3.32638600 \\
\hline C & 2.46161900 & -3.06554400 & 2.33552600 & C & -1.19450100 & -5.48777600 & -3.17582200 \\
\hline $\mathrm{H}$ & -2.94290000 & -5.25923000 & -1.93556200 & C & 0.93194400 & -4.83148400 & -2.19253400 \\
\hline C & 3.12616400 & -3.43192200 & 1.16979100 & C & 3.05880500 & -4.23365700 & -1.17140800 \\
\hline $\mathrm{H}$ & 3.02450000 & -2.69324200 & 3.18448900 & C & 0.18691400 & -5.32857700 & -3.27424800 \\
\hline $\mathrm{H}$ & -1.75081300 & -5.89922600 & -4.01367400 & C & 2.35760200 & -4.67540200 & -2.25033800 \\
\hline $\mathrm{H}$ & 4.20551400 & -3.33268000 & 1.10059500 & $\mathrm{H}$ & 4.13568600 & -4.10672300 & -1.22437000 \\
\hline $\mathrm{H}$ & 0.70472900 & -5.60460800 & -4.18883400 & $\mathrm{H}$ & 2.86813200 & -4.92471800 & -3.17661000 \\
\hline $\mathrm{N}$ & -0.23103100 & 0.94895000 & 2.71589200 & $\mathrm{H}$ & -0.47699900 & 1.53510000 & 1.91191300 \\
\hline $\mathrm{S}$ & -0.22678800 & 3.86955400 & -0.07501000 & $\mathrm{O}$ & -0.68330000 & 4.04712600 & -1.46612700 \\
\hline 0 & -0.94108600 & 2.80881300 & 0.68828600 & $\mathrm{O}$ & 1.24457700 & 3.84547600 & 0.12993800 \\
\hline C & -0.76417500 & 5.42076200 & 0.79103000 & $\mathrm{~F}$ & -0.09293100 & 6.48091600 & 0.33521300 \\
\hline $\mathrm{F}$ & -0.55566300 & 5.31046600 & 2.11174800 & $\mathrm{~F}$ & -2.07942000 & 5.63349300 & 0.58977600 \\
\hline $\mathrm{C}$ & -3.78702500 & 1.46031700 & -0.64297700 & $\mathrm{O}$ & -4.70130100 & 1.01567800 & 0.02848300 \\
\hline $\mathrm{O}$ & -3.82415500 & 2.53059500 & -1.44235100 & C & -4.89789800 & 3.54243700 & -1.33951200 \\
\hline C & -5.03864800 & 3.99816100 & 0.11276100 & $\mathrm{H}$ & -5.71736500 & 4.85688100 & 0.15053000 \\
\hline $\mathrm{H}$ & -4.06801100 & 4.31021200 & 0.50524700 & $\mathrm{H}$ & -5.44192500 & 3.20454400 & 0.74278400 \\
\hline C & -6.19286200 & 2.95729600 & -1.89844900 & $\mathrm{H}$ & -6.03855200 & 2.58672000 & -2.91759600 \\
\hline $\mathrm{H}$ & -6.96217300 & 3.73609700 & -1.93258900 & $\mathrm{H}$ & -6.55148800 & 2.13904200 & -1.27083800 \\
\hline C & -4.35012800 & 4.66889100 & -2.21290800 & $\mathrm{H}$ & -5.04867500 & 5.51161300 & -2.21577000 \\
\hline $\mathrm{H}$ & -4.21530900 & 4.32661000 & -3.24431900 & $\mathrm{H}$ & -3.38260400 & 5.00458200 & -1.82982100 \\
\hline C & -1.63595200 & 1.11347000 & -1.71991400 & $\mathrm{H}$ & -1.71810900 & 2.13612200 & -2.08007500 \\
\hline $\mathrm{C}$ & -0.22993300 & 0.66136900 & -1.59652900 & C & 0.69334700 & 1.20551300 & -2.50414300 \\
\hline $\mathrm{C}$ & 0.18863200 & -0.33076800 & -0.70291600 & C & 1.99058500 & 0.71086200 & -2.57625600 \\
\hline $\mathrm{H}$ & 0.38508000 & 2.02432900 & -3.14693500 & C & 1.50550600 & -0.78678100 & -0.74845400 \\
\hline $\mathrm{H}$ & -0.49280200 & -0.75366800 & 0.02649400 & C & 2.39272200 & -0.30763200 & -1.70973100 \\
\hline $\mathrm{H}$ & 2.69768500 & 1.14348100 & -3.27559300 & $\mathrm{H}$ & 1.83632100 & -1.51876800 & -0.02770400 \\
\hline $\mathrm{H}$ & 3.39995800 & -0.70370800 & -1.75836200 & $\mathrm{~N}$ & -2.54238500 & 0.84354200 & -0.70900700 \\
\hline $\mathrm{H}$ & -2.33284800 & 0.21419000 & 0.07354600 & C & -2.33050000 & 0.36879200 & -3.28080400 \\
\hline $\mathrm{H}$ & -1.73250800 & 0.81148800 & -4.07339500 & $\mathrm{H}$ & -3.32687700 & 0.79401100 & -3.19942800 \\
\hline C & -2.18212800 & -1.01756700 & -3.07267500 & C & -3.05910300 & -1.72439400 & -2.16295300 \\
\hline $\mathrm{H}$ & -3.39668500 & -1.06782100 & -1.34935700 & $\mathrm{H}$ & -2.58791000 & -2.62202200 & -1.75917900 \\
\hline C & -1.00132000 & -1.74157900 & -3.61380300 & $\mathrm{H}$ & -1.25870300 & -2.76890700 & -3.88933600 \\
\hline $\mathrm{H}$ & -0.26272800 & -1.82366400 & -2.80051900 & $\mathrm{H}$ & -0.52874400 & -1.22460900 & -4.45055200 \\
\hline C & -5.57256000 & -3.29366100 & -1.58944100 & $\mathrm{H}$ & -4.99119600 & -4.18690200 & -1.33568000 \\
\hline $\mathrm{H}$ & -6.56949800 & -3.62332700 & -1.90446200 & $\mathrm{H}$ & -5.68897100 & -2.69533900 & -0.67859700 \\
\hline C & -4.39207900 & -3.30358200 & -4.48082600 & $\mathrm{H}$ & -3.70916900 & -4.13080500 & -4.25864600 \\
\hline $\mathrm{H}$ & -3.93291100 & -2.69325900 & -5.26720900 & $\mathrm{H}$ & -5.31577200 & -3.73002700 & -4.88890800 \\
\hline C & -5.75534500 & -0.74428400 & -3.32930700 & $\mathrm{H}$ & -5.31285500 & -0.14692800 & -4.13391400 \\
\hline $\mathrm{H}$ & -5.84978700 & -0.10901900 & -2.44164000 & $\mathrm{H}$ & -6.76640500 & -1.02449900 & -3.64789000 \\
\hline $\mathrm{Si}$ & -4.74133600 & -2.27976900 & -2.94009500 & C & -1.84773900 & -4.47255100 & 3.68780300 \\
\hline $\mathrm{H}$ & -0.81624900 & -4.83824900 & 3.70991500 & $\mathrm{H}$ & -2.49868100 & -5.32345200 & 3.47201800 \\
\hline C & 1.67268000 & -0.24968800 & 3.96783500 & C & 3.01589200 & 0.02281200 & 3.30626300 \\
\hline 0 & 1.27054300 & -0.91509500 & 4.91177800 & $\mathrm{O}$ & 4.15629500 & -0.37629500 & 3.46649600 \\
\hline C & 2.29119600 & 0.90314400 & 2.34417000 & C & 1.04255300 & 0.63043600 & 2.94872100 \\
\hline $\mathrm{N}$ & 2.64222000 & 1.68529700 & 1.29813500 & $\mathrm{H}$ & 1.97335100 & 2.38766100 & 0.95776300 \\
\hline C & 3.76940000 & 1.51193300 & 0.49071800 & C & 4.57802800 & 0.37040000 & 0.57428400 \\
\hline C & 4.02015900 & 2.46379600 & -0.50951000 & C & 5.59210200 & 0.17620700 & -0.35792600 \\
\hline
\end{tabular}




\begin{tabular}{|c|c|c|c|c|c|c|c|}
\hline $\mathrm{H}$ & 4.41795100 & -0.36536500 & 1.34858300 & C & 5.04675900 & 2.24913500 & -1.42332300 \\
\hline $\mathrm{H}$ & 3.38042900 & 3.33470300 & -0.59187100 & C & 5.84196300 & 1.10329700 & -1.36828700 \\
\hline $\mathrm{H}$ & 6.62782800 & 0.93733500 & -2.09462800 & C & 6.29879900 & -1.14754900 & -0.37989600 \\
\hline C & 5.23149500 & 3.20942400 & -2.56414800 & $\mathrm{~F}$ & 5.63140300 & -2.02908400 & -1.18826800 \\
\hline $\mathrm{F}$ & 6.36461700 & -1.72934300 & 0.83493000 & $\mathrm{~F}$ & 7.55254700 & -1.05972500 & -0.86594300 \\
\hline $\mathrm{F}$ & 4.79816400 & 4.44918900 & -2.27058700 & $\mathrm{~F}$ & 4.54133300 & 2.80072100 & -3.66540900 \\
\hline $\mathrm{F}$ & 6.52598900 & 3.30263700 & -2.94048800 & & & & \\
\hline
\end{tabular}

\section{TS-R-6f-11}

Imaginary frequency: $-346.60 \mathrm{~cm}^{-1}$

E[B3LYP/6-31G(d)-D3(BJ)]: -4655.628528 Hartree

$\mathrm{E}\left[\mathrm{PCM}\left(\mathrm{Et}_{2} \mathrm{O}\right)-\mathrm{B} 3 \mathrm{LYP} / 6-311 \mathrm{G}(\mathrm{d}, \mathrm{p})-\mathrm{D} 3(\mathrm{BJ})\right]$ : -4656.822292 Hartree

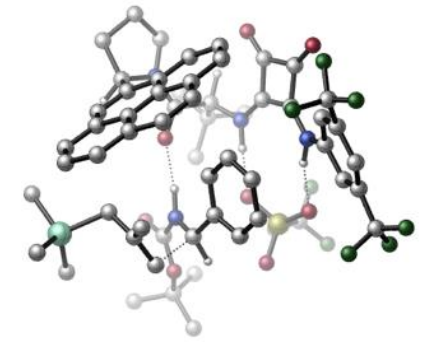

\begin{tabular}{|c|c|c|c|c|c|c|c|}
\hline 01 & & & & C & -1.19803300 & 0.46557100 & 3.55973800 \\
\hline $\mathrm{H}$ & -0.70599800 & 0.11829500 & 4.46599400 & C & -2.30212400 & 1.46785900 & 4.01002700 \\
\hline C & -3.16124800 & 1.99124400 & 2.85172700 & $\mathrm{H}$ & -3.69987200 & 1.19130800 & 2.34262200 \\
\hline $\mathrm{H}$ & -2.56644900 & 2.52451600 & 2.10676000 & $\mathrm{H}$ & -3.90269000 & 2.69637900 & 3.24654200 \\
\hline C & -1.60420100 & 2.65033600 & 4.70485300 & $\mathrm{H}$ & -2.35475600 & 3.31372800 & 5.14899600 \\
\hline $\mathrm{H}$ & -1.01606700 & 3.23700700 & 3.99363700 & $\mathrm{H}$ & -0.93582500 & 2.31030500 & 5.50537300 \\
\hline C & -3.20989500 & 0.74333500 & 5.01961400 & $\mathrm{H}$ & -3.99298700 & 1.42280800 & 5.37206500 \\
\hline $\mathrm{H}$ & -2.64711800 & 0.39891000 & 5.89526500 & $\mathrm{H}$ & -3.70826000 & -0.12133000 & 4.56448600 \\
\hline C & -1.70753900 & -0.75551600 & 2.76985400 & $\mathrm{O}$ & -2.06489500 & -0.65888200 & 1.58293600 \\
\hline C & -1.42570800 & -2.25872500 & 4.80178300 & C & -2.08982700 & -3.16394800 & 2.61319800 \\
\hline C & -1.29599100 & -3.78382500 & 4.83909800 & $\mathrm{H}$ & -0.52406900 & -1.75270800 & 5.15003500 \\
\hline $\mathrm{H}$ & -2.27995600 & -1.92433900 & 5.40050900 & C & -2.22230700 & -4.25029000 & 3.71024500 \\
\hline $\mathrm{H}$ & -3.06883000 & -2.95067000 & 2.17504700 & $\mathrm{H}$ & -1.57668600 & -4.18896900 & 5.81493500 \\
\hline $\mathrm{H}$ & -0.26373700 & -4.08354300 & 4.63787000 & $\mathrm{H}$ & -3.26003700 & -4.27452400 & 4.06210400 \\
\hline $\mathrm{H}$ & -1.97415600 & -5.24221500 & 3.32688300 & $\mathrm{~N}$ & -1.67661100 & -1.96694700 & 3.37142000 \\
\hline C & -1.20176200 & -3.58073300 & 1.43847100 & C & -1.86866600 & -4.15894200 & 0.39495300 \\
\hline C & 0.24913600 & -3.49673200 & 1.39106900 & C & -1.22113100 & -4.66347300 & -0.77643800 \\
\hline $\mathrm{H}$ & -2.95329000 & -4.23433100 & 0.43945000 & C & 0.92426700 & -3.97833000 & 0.21689000 \\
\hline C & 1.04165500 & -2.96350300 & 2.42333300 & C & -1.93346700 & -5.24811600 & -1.83680500 \\
\hline C & 0.19344800 & -4.53289200 & -0.87323900 & C & 2.34892700 & -3.88988900 & 0.11878100 \\
\hline $\mathrm{H}$ & 0.58364600 & -2.55931600 & 3.30971300 & C & 2.43246300 & -2.91299200 & 2.33125900 \\
\hline C & -1.27165100 & -5.67212200 & -2.98793400 & $\mathrm{H}$ & -3.00952700 & -5.37221700 & -1.74761200 \\
\hline C & 0.86336300 & -4.96360600 & -2.05970300 & C & 3.08568100 & -3.36118900 & 1.18897700 \\
\hline C & 2.99595100 & -4.30473500 & -1.09429400 & $\mathrm{H}$ & 3.00450000 & -2.49611800 & 3.15280100 \\
\hline C & 0.10938200 & -5.52117200 & -3.10496500 & $\mathrm{H}$ & -1.83505900 & -6.12704200 & -3.79805800 \\
\hline C & 2.28803900 & -4.81253400 & -2.13875600 & $\mathrm{H}$ & 4.16533000 & -3.27795800 & 1.10602700 \\
\hline $\mathrm{H}$ & 4.07261700 & -4.18281500 & -1.16116100 & $\mathrm{H}$ & 0.61996700 & -5.84885300 & -4.00644000 \\
\hline $\mathrm{H}$ & 2.79172800 & -5.11925600 & -3.05141900 & $\mathrm{~N}$ & -0.14714600 & 1.09310300 & 2.75671200 \\
\hline $\mathrm{H}$ & -0.41034900 & 1.63598000 & 1.92732200 & $S$ & -0.25837100 & 3.83704900 & -0.13585700 \\
\hline $\mathrm{O}$ & -0.92507100 & 4.16146000 & -1.40944600 & $\mathrm{O}$ & -0.86370900 & 2.69270200 & 0.59950400 \\
\hline $\mathrm{O}$ & 1.22557600 & 3.79145800 & -0.16188700 & $\mathrm{C}$ & -0.63349200 & 5.26045800 & 0.99521200 \\
\hline $\mathrm{F}$ & -0.17788400 & 6.41193700 & 0.49662000 & $\mathrm{~F}$ & -0.05996900 & 5.04939900 & 2.19246400 \\
\hline$F$ & -1.96045800 & 5.36839700 & 1.18582200 & C & -3.78053100 & 1.60836300 & -0.70678100 \\
\hline $\mathrm{O}$ & -4.62483200 & 1.36469500 & 0.13451300 & $\mathrm{O}$ & -3.88097300 & 2.48001300 & -1.71795100 \\
\hline C & -4.88876000 & 3.56292400 & -1.71129300 & C & -6.27758700 & 2.96380100 & -1.92162300 \\
\hline $\mathrm{H}$ & -7.01020000 & 3.77066000 & -2.03051100 & $\mathrm{H}$ & -6.56455100 & 2.34074800 & -1.07273600 \\
\hline $\mathrm{H}$ & -6.30019400 & 2.35798900 & -2.83388800 & C & -4.44494700 & 4.41116800 & -2.90052100 \\
\hline
\end{tabular}




\begin{tabular}{|c|c|c|c|c|c|c|c|}
\hline $\mathrm{H}$ & -3.41845800 & 4.75694400 & -2.74807200 & $\mathrm{H}$ & -5.10267500 & 5.27964300 & -3.00586100 \\
\hline $\mathrm{H}$ & -4.48850600 & 3.83037800 & -3.82828300 & C & -4.77521300 & 4.35287800 & -0.40915100 \\
\hline $\mathrm{H}$ & -5.43275700 & 5.22707700 & -0.46398300 & $\mathrm{H}$ & -3.74938400 & 4.69983000 & -0.26689100 \\
\hline $\mathrm{H}$ & -5.07087000 & 3.74874800 & 0.45018700 & $\mathrm{C}$ & -1.67832400 & 1.09317700 & -1.81424100 \\
\hline $\mathrm{H}$ & -1.76709000 & 2.07352100 & -2.27416900 & C & -0.27715600 & 0.63577400 & -1.68201400 \\
\hline C & 0.63889100 & 1.11476900 & -2.63133200 & C & 0.14343800 & -0.30424100 & -0.73381200 \\
\hline C & 1.93292700 & 0.60844100 & -2.68507500 & $\mathrm{H}$ & 0.32728500 & 1.88881400 & -3.32720300 \\
\hline C & 1.45493100 & -0.77409500 & -0.76580800 & $\mathrm{H}$ & -0.53417500 & -0.67385600 & 0.02733300 \\
\hline C & 2.33630700 & -0.35847800 & -1.76242100 & $\mathrm{H}$ & 2.63583900 & 0.99213400 & -3.41626400 \\
\hline $\mathrm{H}$ & 1.78744900 & -1.46636900 & -0.00693900 & $\mathrm{H}$ & 3.33984900 & -0.76509400 & -1.79782700 \\
\hline $\mathrm{N}$ & -2.56803500 & 0.92959000 & -0.76819700 & $\mathrm{H}$ & -2.32178800 & 0.41809800 & 0.08584700 \\
\hline $\mathrm{C}$ & -2.42152100 & 0.20824800 & -3.30175600 & $\mathrm{H}$ & -1.84239400 & 0.58217200 & -4.14239000 \\
\hline $\mathrm{H}$ & -3.40961900 & 0.65024900 & -3.21835000 & C & -2.26341000 & -1.15089200 & -2.97435900 \\
\hline C & -3.10786600 & -1.77349100 & -1.97361400 & $\mathrm{H}$ & -3.39159800 & -1.04713800 & -1.19784200 \\
\hline $\mathrm{H}$ & -2.62521500 & -2.63989800 & -1.51942300 & C & -1.10277100 & -1.92405100 & -3.49101900 \\
\hline $\mathrm{H}$ & -1.37889500 & -2.96410800 & -3.69153500 & $\mathrm{H}$ & -0.34616100 & -1.96209900 & -2.69159200 \\
\hline $\mathrm{H}$ & -0.64560100 & -1.47149100 & -4.37248500 & C & -5.58191700 & -3.33850700 & -1.21064300 \\
\hline $\mathrm{H}$ & -5.00538000 & -4.24406200 & -0.99252300 & $\mathrm{H}$ & -6.60684500 & -3.64608800 & -1.44879900 \\
\hline $\mathrm{H}$ & -5.61819800 & -2.73210800 & -0.29832200 & $\mathrm{C}$ & -4.57104900 & -3.42318900 & -4.16103800 \\
\hline $\mathrm{H}$ & -3.87100400 & -4.24118500 & -3.95833200 & $\mathrm{H}$ & -4.16422800 & -2.83327900 & -4.99061900 \\
\hline $\mathrm{H}$ & -5.51554600 & -3.86455500 & -4.49962300 & C & -5.86983500 & -0.83410200 & -3.00018700 \\
\hline $\mathrm{H}$ & -5.47679600 & -0.25847700 & -3.84527500 & $\mathrm{H}$ & -5.91469300 & -0.17235900 & -2.12784600 \\
\hline $\mathrm{H}$ & -6.89698900 & -1.12390900 & -3.25170000 & $\mathrm{Si}$ & -4.82891700 & -2.35741100 & -2.63059200 \\
\hline C & 1.79079300 & -0.08320900 & 3.97633800 & C & 3.11365800 & 0.17412800 & 3.26983500 \\
\hline 0 & 1.41548700 & -0.74371400 & 4.93620300 & $\mathrm{O}$ & 4.25937600 & -0.21722000 & 3.40952600 \\
\hline $\mathrm{C}$ & 2.35681600 & 1.01547600 & 2.29743200 & $\mathrm{C}$ & 1.12952800 & 0.77065600 & 2.95703400 \\
\hline $\mathrm{N}$ & 2.67118100 & 1.74081600 & 1.19939700 & $\mathrm{H}$ & 1.98490500 & 2.40939900 & 0.82866500 \\
\hline $\mathrm{C}$ & 3.77313000 & 1.52213100 & 0.36629500 & $\mathrm{C}$ & 4.57294700 & 0.37658400 & 0.47351100 \\
\hline $\mathrm{C}$ & 4.00394000 & 2.42927400 & -0.67921300 & $\mathrm{C}$ & 5.56129400 & 0.13592000 & -0.47553000 \\
\hline $\mathrm{H}$ & 4.42661500 & -0.32721600 & 1.27919800 & C & 5.00480700 & 2.16899500 & -1.60955000 \\
\hline $\mathrm{H}$ & 3.36870200 & 3.30076100 & -0.78229200 & $\mathrm{C}$ & 5.79287900 & 1.01986700 & -1.52783000 \\
\hline $\mathrm{H}$ & 6.55923100 & 0.81838000 & -2.26601200 & $\mathrm{C}$ & 6.25623400 & -1.19420500 & -0.46254700 \\
\hline C & 5.17113100 & 3.08185400 & -2.79150900 & $\mathrm{~F}$ & 5.56075700 & -2.10070100 & -1.21808000 \\
\hline $\mathrm{F}$ & 6.34802400 & -1.72795300 & 0.77232500 & $\mathrm{~F}$ & 7.49764100 & -1.13731600 & -0.98331800 \\
\hline $\mathrm{F}$ & 4.73812000 & 4.33157900 & -2.54376300 & $\mathrm{~F}$ & 4.46838200 & 2.62642600 & -3.86623900 \\
\hline $\mathrm{F}$ & 6.46039400 & 3.16230000 & -3.18816800 & & & & \\
\hline
\end{tabular}

\section{TS-R-6f-12}

Imaginary frequency: $-304.04 \mathrm{~cm}^{-1}$

E[B3LYP/6-31G(d)-D3(BJ)]: -4655.625269 Hartree

E[PCM(Et $\left.\left.t_{2} \mathrm{O}\right)-B 3 L Y P / 6-311 G(d, p)-D 3(B J)\right]:-4656.821816$ Hartree

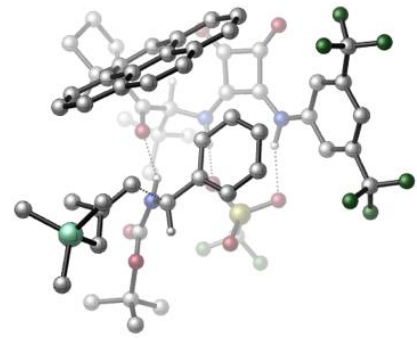

\begin{tabular}{|lrll|lrll|}
\hline 01 & & & & $\mathrm{C}$ & -0.03600900 & 1.38006100 & 3.99226400 \\
\hline $\mathrm{H}$ & 0.56713400 & 1.04836200 & 4.83711200 & $\mathrm{C}$ & -0.85441200 & 2.62194500 & 4.45761900 \\
\hline $\mathrm{C}$ & -1.84348900 & 3.11152200 & 3.39109300 & $\mathrm{H}$ & -2.59415000 & 2.35862100 & 3.14586100 \\
\hline $\mathrm{H}$ & -1.35163100 & 3.38438000 & 2.45784800 & $\mathrm{H}$ & -2.36465200 & 4.00171200 & 3.76205600 \\
\hline $\mathrm{C}$ & 0.13716300 & 3.74866900 & 4.79564300 & $\mathrm{H}$ & -0.39994700 & 4.59288100 & 5.24244300 \\
\hline $\mathrm{H}$ & 0.64572600 & 4.11041900 & 3.89803400 & $\mathrm{H}$ & 0.89892100 & 3.41385300 & 5.51026800 \\
\hline $\mathrm{C}$ & -1.63546600 & 2.23509400 & 5.72532400 & $\mathrm{H}$ & -2.21011400 & 3.09530300 & 6.08475700 \\
\hline $\mathrm{H}$ & -0.96494300 & 1.91953200 & 6.53350400 & $\mathrm{H}$ & -2.34681000 & 1.42469200 & 5.52787200 \\
\hline $\mathrm{C}$ & -0.90376000 & 0.21033700 & 3.49536900 & $\mathrm{O}$ & -1.37590600 & 0.21218900 & 2.34795900 \\
\hline
\end{tabular}




\begin{tabular}{|c|c|c|c|c|c|c|c|}
\hline $\mathrm{C}$ & -0.61753000 & -1.07497500 & 5.68441200 & C & -1.78254100 & -2.03540700 & 3.74683500 \\
\hline C & -0.85483500 & -2.57541000 & 5.90765400 & $\mathrm{H}$ & 0.43512000 & -0.80258600 & 5.77560100 \\
\hline $\mathrm{H}$ & -1.21180900 & -0.47462200 & 6.38123600 & C & -2.05066700 & -2.89484600 & 4.99869600 \\
\hline $\mathrm{H}$ & -2.70578500 & -1.69152600 & 3.27754700 & $\mathrm{H}$ & -1.04152700 & -2.80856800 & 6.95921300 \\
\hline $\mathrm{H}$ & 0.02356500 & -3.14409300 & 5.58794700 & $\mathrm{H}$ & -2.98603700 & -2.57244600 & 5.47179600 \\
\hline $\mathrm{H}$ & -2.13753200 & -3.95678100 & 4.75567400 & $\mathrm{~N}$ & -1.09182400 & -0.85861500 & 4.30258400 \\
\hline C & -0.92779900 & -2.73503700 & 2.69858500 & C & 0.43424200 & -2.68282400 & 2.76652100 \\
\hline C & -1.56404500 & -3.45409700 & 1.61501000 & C & 1.27897200 & -3.25861400 & 1.76385900 \\
\hline $\mathrm{H}$ & 0.91974700 & -2.13820600 & 3.56877500 & C & -0.74527500 & -3.98178300 & 0.56398900 \\
\hline C & -2.95370700 & -3.66372100 & 1.54578700 & C & 2.67849200 & -3.16764200 & 1.83336300 \\
\hline C & 0.67324900 & -3.86775900 & 0.62899400 & C & -1.34726700 & -4.63621200 & -0.55526300 \\
\hline $\mathrm{H}$ & -3.59477000 & -3.31476000 & 2.34754700 & C & -3.52538200 & -4.35443400 & 0.47708300 \\
\hline C & 3.46962600 & -3.61783700 & 0.77672700 & $\mathrm{H}$ & 3.14492500 & -2.71222900 & 2.70014500 \\
\hline C & 1.49034800 & -4.34536000 & -0.43744600 & C & -2.73942300 & -4.82112800 & -0.57264800 \\
\hline $\mathrm{C}$ & -0.50259900 & -5.09657300 & -1.62270200 & $\mathrm{H}$ & -4.59621400 & -4.54047300 & 0.47428900 \\
\hline C & 2.88368800 & -4.19321500 & -0.34989700 & $\mathrm{H}$ & 4.54619100 & -3.49112900 & 0.82247600 \\
\hline C & 0.84987500 & -4.94798200 & -1.57128600 & $\mathrm{H}$ & -3.19375800 & -5.35664300 & -1.40184600 \\
\hline $\mathrm{H}$ & -0.97238300 & -5.57352300 & -2.47922700 & $\mathrm{H}$ & 3.50793700 & -4.51802200 & -1.17683200 \\
\hline $\mathrm{H}$ & 1.47332800 & -5.29692600 & -2.39015800 & $\mathrm{~N}$ & 0.90469600 & 1.71199700 & 2.91789800 \\
\hline $\bar{H}$ & 0.51814500 & 2.16004700 & 2.07860700 & $\mathrm{~S}$ & 0.18100800 & 3.48754300 & -0.56533400 \\
\hline $\mathrm{O}$ & -0.69141000 & 3.36893300 & -1.74636000 & 0 & -0.12436800 & 2.51695900 & 0.52375000 \\
\hline $\mathrm{O}$ & 1.63872800 & 3.61446600 & -0.81005300 & C & -0.27103900 & 5.11450500 & 0.20819600 \\
\hline $\mathrm{F}$ & -0.01220900 & 6.13107300 & -0.61990200 & $\mathrm{~F}$ & 0.43274300 & 5.29052200 & 1.34127400 \\
\hline $\mathrm{F}$ & -1.57606900 & 5.13776200 & 0.52263700 & C & -3.32176400 & 2.04800000 & -0.21935700 \\
\hline 0 & -3.57832500 & 2.72780500 & 0.75067800 & $\mathrm{O}$ & -3.94202400 & 2.09200800 & -1.41655600 \\
\hline $\mathrm{C}$ & -4.79286600 & 3.24679800 & -1.77016600 & C & -6.07299300 & 3.19903300 & -0.93684600 \\
\hline $\mathrm{H}$ & -6.75052800 & 3.99800400 & -1.25656600 & $\mathrm{H}$ & -5.84754600 & 3.32953900 & 0.12227200 \\
\hline $\mathrm{H}$ & -6.58663600 & 2.24065800 & -1.07775900 & C & -5.08430600 & 3.00093800 & -3.24939900 \\
\hline $\mathrm{H}$ & -4.15409400 & 2.98891900 & -3.82571300 & $\mathrm{H}$ & -5.72360500 & 3.79870400 & -3.63962800 \\
\hline $\mathrm{H}$ & -5.60021400 & 2.04604000 & -3.39472400 & C & -4.00985200 & 4.54643500 & -1.58472000 \\
\hline $\mathrm{H}$ & -4.56903500 & 5.36822200 & -2.04517200 & $\mathrm{H}$ & -3.03049600 & 4.46879700 & -2.06527000 \\
\hline $\mathrm{H}$ & -3.85560700 & 4.77248700 & -0.53053300 & C & -1.93135600 & 0.41162200 & -1.35856200 \\
\hline $\mathrm{H}$ & -2.33518400 & 0.88681300 & -2.24405400 & C & -0.50906900 & 0.02052200 & -1.51708400 \\
\hline C & 0.03943600 & 0.13426100 & -2.80281100 & C & 0.25031500 & -0.53433800 & -0.48051500 \\
\hline C & 1.32570500 & -0.32720900 & -3.06359700 & $\mathrm{H}$ & -0.54237800 & 0.60343100 & -3.59130800 \\
\hline C & 1.53477000 & -0.99174900 & -0.74959100 & $\mathrm{H}$ & -0.15925600 & -0.61483600 & 0.52136700 \\
\hline C & 2.06450500 & -0.91652000 & -2.03854300 & $\mathrm{H}$ & 1.75936300 & -0.20708700 & -4.05039100 \\
\hline $\mathrm{H}$ & 2.12992300 & -1.41437600 & 0.04763900 & $\mathrm{H}$ & 3.05767300 & -1.30479500 & -2.23181500 \\
\hline $\mathrm{N}$ & -2.35590900 & 1.05011200 & -0.20986700 & $\mathrm{H}$ & -1.79276300 & 1.01526800 & 0.64431600 \\
\hline C & -2.70833500 & -1.29113200 & -1.47865000 & $\mathrm{H}$ & -2.32824200 & -1.73595400 & -0.56303800 \\
\hline $\mathrm{H}$ & -2.22953300 & -1.65601900 & -2.38194200 & C & -4.08762500 & -1.04061900 & -1.54449700 \\
\hline C & -4.76786200 & -0.86460900 & -2.82125800 & $\mathrm{H}$ & -4.09435500 & -0.48616900 & -3.59895000 \\
\hline $\mathrm{H}$ & -5.64297500 & -0.21339600 & -2.74239000 & C & -4.83021000 & -0.90116200 & -0.26611200 \\
\hline $\mathrm{H}$ & -5.85675100 & -0.55349000 & -0.39506600 & $\mathrm{H}$ & -4.27687300 & -0.20345500 & 0.38159100 \\
\hline $\mathrm{H}$ & -4.81958800 & -1.85612200 & 0.27284200 & C & -6.46597500 & -2.15741600 & -4.99560400 \\
\hline $\mathrm{H}$ & -7.33871000 & -1.56386300 & -4.70074900 & $\mathrm{H}$ & -6.83243300 & -3.07207600 & -5.47641800 \\
\hline $\mathrm{H}$ & -5.90865700 & -1.58739700 & -5.74753600 & C & -6.36934800 & -3.46567200 & -2.17648300 \\
\hline $\mathrm{H}$ & -7.19563800 & -2.85055900 & -1.80236600 & $\mathrm{H}$ & -5.73114400 & -3.72972500 & -1.32742800 \\
\hline $\mathrm{H}$ & -6.80004700 & -4.39394300 & -2.56964100 & C & -3.87362200 & -3.57372700 & -4.02310500 \\
\hline $\mathrm{H}$ & -3.20974900 & -3.76366300 & -3.17398600 & $\mathrm{H}$ & -3.29513300 & -3.05680600 & -4.79745600 \\
\hline $\mathrm{H}$ & -4.17682600 & -4.54500400 & -4.43216500 & $\mathrm{Si}$ & -5.38560900 & -2.57838100 & -3.51227200 \\
\hline C & 2.68913900 & 0.00929600 & 3.64483700 & $\mathrm{C}$ & 3.79654200 & -0.10033000 & 2.61385000 \\
\hline 0 & 2.41525500 & -0.54924500 & 4.70052400 & $\mathrm{O}$ & 4.80607500 & -0.77915200 & 2.51447800 \\
\hline
\end{tabular}




\begin{tabular}{|c|c|c|c|c|c|c|c|}
\hline C & 3.04766900 & 0.90209900 & 1.79266200 & C & 2.03392700 & 1.01674400 & 2.78127500 \\
\hline $\mathrm{N}$ & 3.19750100 & 1.50225300 & 0.58768600 & $\mathrm{H}$ & 2.51614900 & 2.21851700 & 0.31769300 \\
\hline $\mathrm{C}$ & 4.02703600 & 1.08763000 & -0.46625200 & C & 4.84391300 & -0.04749600 & -0.38225300 \\
\hline$C$ & 3.95599800 & 1.80018300 & -1.67397200 & $\mathrm{C}$ & 5.53753400 & -0.47685700 & -1.51064700 \\
\hline $\mathrm{H}$ & 4.93973400 & -0.59645700 & 0.54653000 & C & 4.67307400 & 1.35679800 & -2.77998700 \\
\hline $\mathrm{H}$ & 3.30863100 & 2.66518700 & -1.75077300 & C & 5.46657900 & 0.21128100 & -2.72013600 \\
\hline $\mathrm{H}$ & 6.00884100 & -0.13571600 & -3.59116300 & C & 6.24196700 & -1.80193500 & -1.46699100 \\
\hline C & 4.50565300 & 2.05581000 & -4.09982200 & $F$ & 5.41609100 & -2.79522900 & -1.91254800 \\
\hline $\mathrm{F}$ & 6.62537900 & -2.15352900 & -0.22357200 & $\mathrm{~F}$ & 7.33395700 & -1.83123300 & -2.25942600 \\
\hline $\mathrm{F}$ & 4.12701000 & 3.33904300 & -3.96137400 & $\mathrm{~F}$ & 3.56042900 & 1.44318300 & -4.86361200 \\
\hline $\mathrm{F}$ & 5.64800100 & 2.03885400 & -4.82411800 & & & & \\
\hline
\end{tabular}

\section{TS- $R-6 f-13$}

Imaginary frequency: $-282.69 \mathrm{~cm}^{-1}$

E[B3LYP/6-31G(d)-D3(BJ)]: -4655.633825 Hartree

$\mathrm{E}\left[\mathrm{PCM}\left(\mathrm{Et}_{2} \mathrm{O}\right)-\mathrm{B} 3 \mathrm{LYP} / 6-311 \mathrm{G}(\mathrm{d}, \mathrm{p})-\mathrm{D} 3(\mathrm{BJ})\right]$ : -4656.820110 Hartree

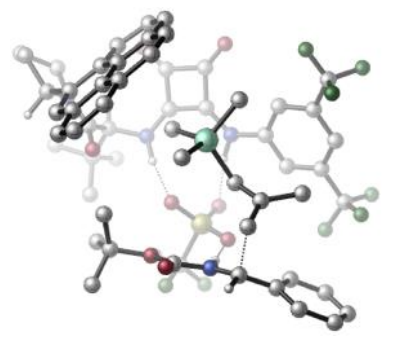

\begin{tabular}{|c|c|c|c|c|c|c|}
\hline 01 & & & C & -3.21749000 & -2.34878800 & -2.25675000 \\
\hline $\mathrm{H}$ & -3.75498800 & $-1.98025200-3.13101100$ & C & -3.21467600 & -3.90761100 & -2.31122700 \\
\hline C & -2.53267400 & $-4.53282800-1.08384600$ & $\mathrm{H}$ & -3.07580700 & -4.30547700 & -0.16443400 \\
\hline $\mathrm{H}$ & -1.50732700 & -4.18275500 & $\mathrm{H}$ & -2.49557700 & -5.62147500 & -1.20700300 \\
\hline C & -2.47074100 & -4.34235800 & $\mathrm{H}$ & -2.53538900 & -5.42990600 & -3.70411800 \\
\hline $\mathrm{H}$ & -1.41314000 & -4.06749600 & $\mathrm{H}$ & -2.90639500 & -3.87803600 & -4.47938000 \\
\hline C & -4.66598500 & -4.41294600 & $\mathrm{H}$ & -4.67780700 & -5.50820800 & -2.37450900 \\
\hline $\mathrm{H}$ & -5.16667700 & -4.08359400 & $\mathrm{H}$ & -5.25511000 & -4.07427700 & -1.51861800 \\
\hline C & -3.84275600 & $-1.77110400-0.96915100$ & $\mathrm{O}$ & -3.22191100 & -1.80481600 & 0.09474200 \\
\hline C & -5.98662900 & -1.19941500 & C & -5.69371300 & -0.61630600 & 0.15162900 \\
\hline C & -7.36188900 & -1.03897400 & $\mathrm{H}$ & -5.75603900 & -0.37769300 & -2.89990200 \\
\hline $\mathrm{H}$ & -5.90327100 & $-2.13119000 \quad-2.76997000$ & $\mathrm{H}$ & -5.83065700 & -1.43917700 & 0.86350600 \\
\hline $\mathrm{H}$ & -7.72899500 & $-2.01513100 \quad-1.22061700$ & $\mathrm{H}$ & -8.10029400 & -0.61881800 & -2.24837700 \\
\hline $\mathrm{N}$ & -5.06538100 & $-1.18632200-1.06140900$ & C & -4.93773100 & 0.47461700 & 0.88902800 \\
\hline C & -4.85395800 & 0.36477600 & C & -4.43017600 & 1.66847200 & 0.23818000 \\
\hline C & -4.34088400 & 3.08443400 & $\mathrm{H}$ & -5.20624900 & -0.54374000 & 2.72940500 \\
\hline C & -3.92520700 & 2.73275700 & C & -4.36964200 & 1.81958400 & -1.15595900 \\
\hline C & -4.26878900 & 1.27926300 & C & -3.90747100 & 2.61718900 & 2.47262900 \\
\hline C & -3.40705600 & 3.91629800 & $\mathrm{H}$ & -4.70416200 & 1.02499300 & -1.80192100 \\
\hline $\mathrm{C}$ & -3.81899300 & -1.74481500 & $\mathrm{C}$ & -3.80075700 & 2.33020900 & 5.26610800 \\
\hline $\mathrm{H}$ & -4.58925900 & 0.34986700 & C & -3.42929100 & 3.68970600 & 3.28512400 \\
\hline C & -3.34725100 & -0.95703500 & C & -2.94443900 & 4.98550800 & 1.28309100 \\
\hline $\mathrm{H}$ & -3.74193900 & -2.82553600 & $\mathrm{C}$ & -3.39327600 & 3.52611300 & 4.67880700 \\
\hline $\mathrm{H}$ & -3.75349300 & 2.21652100 & $\mathrm{C}$ & -2.96339300 & 4.88447800 & 2.64005700 \\
\hline $\mathrm{H}$ & -2.91998200 & -1.41912700 & $\mathrm{H}$ & -2.57473100 & 5.88811800 & 0.80317600 \\
\hline $\mathrm{H}$ & -3.02627800 & 4.34146200 & $\mathrm{H}$ & -2.60791600 & 5.70279000 & 3.26074000 \\
\hline $\mathrm{N}$ & -1.85689700 & -1.81595500 & $\mathrm{H}$ & -1.17411100 & -2.12893300 & -1.67549300 \\
\hline $\mathrm{S}$ & 1.58688400 & $-2.73895600 \quad-0.78035700$ & $\mathrm{O}$ & 2.69573400 & -2.12365000 & -0.00113400 \\
\hline $\mathrm{O}$ & 0.23141700 & $-2.31165600-0.36012100$ & $\mathrm{O}$ & 1.79064400 & -2.71603000 & -2.25052000 \\
\hline $\mathrm{C}$ & 1.70882400 & $-4.53698300 \quad-0.32885900$ & $\mathrm{~F}$ & 2.87026400 & -5.03724700 & -0.75875400 \\
\hline $\mathrm{F}$ & 0.70674300 & $-5.22977000 \quad-0.88427400$ & $\mathrm{~F}$ & 1.64651600 & -4.68021100 & 1.00316300 \\
\hline $\mathrm{C}$ & 1.45946000 & -1.69079200 & $\mathrm{O}$ & 1.28279700 & -1.15211500 & 4.55283500 \\
\hline $\mathrm{O}$ & 0.62718700 & -2.45215500 & $\mathrm{C}$ & -0.73708200 & -2.77589000 & 3.29881300 \\
\hline
\end{tabular}




\begin{tabular}{|c|c|c|c|c|c|c|c|}
\hline $\mathrm{C}$ & -0.59802100 & -3.52492300 & 4.62283000 & $\mathrm{H}$ & -1.58830700 & -3.86426300 & 4.94427000 \\
\hline $\mathrm{H}$ & -0.17651900 & -2.88904100 & 5.40290100 & $\mathrm{H}$ & 0.03768900 & -4.40743900 & 4.49493000 \\
\hline $\mathrm{C}$ & -1.30642500 & -3.67073000 & 2.20499800 & $\mathrm{H}$ & -1.40977300 & -3.11658100 & 1.27297400 \\
\hline $\mathrm{H}$ & -2.29723300 & -4.02136900 & 2.51144700 & $\mathrm{H}$ & -0.66153300 & -4.53757400 & 2.03958500 \\
\hline C & -1.55279000 & -1.49077700 & 3.42065200 & $\mathrm{H}$ & -2.58930800 & -1.75039000 & 3.65893800 \\
\hline $\mathrm{H}$ & -1.56482800 & -0.95953300 & 2.46623800 & $\mathrm{H}$ & -1.17082600 & -0.83858300 & 4.20761000 \\
\hline $\mathrm{C}$ & 3.64234600 & -0.74163000 & 3.26601500 & $\mathrm{H}$ & 3.56928800 & -0.66092800 & 4.34613600 \\
\hline C & 5.01277100 & -0.75381200 & 2.72889700 & C & 6.04272100 & -0.37201400 & 3.60608200 \\
\hline $\mathrm{C}$ & 5.33071100 & -1.10866100 & 1.40762100 & C & 7.36656100 & -0.35900600 & 3.18200000 \\
\hline $\mathrm{H}$ & 5.79705700 & -0.09258100 & 4.62765700 & C & 6.66139600 & -1.10001000 & 0.99192100 \\
\hline $\mathrm{H}$ & 4.55750100 & -1.39493400 & 0.70342400 & C & 7.67818700 & -0.72733700 & 1.87134400 \\
\hline $\mathrm{H}$ & 8.15306100 & -0.06885900 & 3.87214900 & $\mathrm{H}$ & 6.89632700 & -1.37435100 & -0.02899500 \\
\hline $\mathrm{H}$ & 8.71099500 & -0.72089600 & 1.53573100 & $\mathrm{~N}$ & 2.66058100 & -1.54078200 & 2.75635600 \\
\hline $\mathrm{H}$ & 2.68434100 & -1.88952100 & 1.78688400 & C & 2.98733300 & 1.20329300 & 3.17404700 \\
\hline $\mathrm{H}$ & 3.75549000 & 1.71363800 & 3.74905200 & $\mathrm{H}$ & 2.08507200 & 0.95078400 & 3.72040100 \\
\hline $\mathrm{C}$ & 2.91636100 & 1.44811300 & 1.80333700 & C & 1.82059500 & 0.93789900 & 1.00018700 \\
\hline $\mathrm{H}$ & 1.46662200 & -0.03775800 & 1.35289000 & $\mathrm{H}$ & 2.06866900 & 0.86799800 & -0.06122400 \\
\hline C & 4.03057100 & 2.16585200 & 1.11568200 & $\mathrm{H}$ & 3.64189500 & 2.88246200 & 0.38467700 \\
\hline $\mathrm{H}$ & 4.63010600 & 1.44023800 & 0.54876300 & $\mathrm{H}$ & 4.69265100 & 2.67931500 & 1.81662800 \\
\hline $\mathrm{C}$ & -1.09651400 & 0.93563300 & 0.22178900 & $\mathrm{H}$ & -0.64622100 & 0.02154100 & -0.17404600 \\
\hline $\mathrm{H}$ & -1.52735700 & 1.50189700 & -0.60829800 & $\mathrm{H}$ & -1.92279500 & 0.62658500 & 0.86601900 \\
\hline $\mathrm{C}$ & 0.40975000 & 3.68273000 & 0.42152900 & $\mathrm{H}$ & 0.40123100 & 3.66595500 & -0.67111300 \\
\hline $\mathrm{H}$ & 1.34752600 & 4.14812600 & 0.74288900 & $\mathrm{H}$ & -0.41279900 & 4.32785600 & 0.74836400 \\
\hline C & -0.22562800 & 2.10239900 & 2.99151700 & $\mathrm{H}$ & 0.46128900 & 2.78288500 & 3.50641400 \\
\hline $\mathrm{H}$ & -0.18557800 & 1.13129700 & 3.49446700 & $\mathrm{H}$ & -1.23696900 & 2.49382800 & 3.12301600 \\
\hline $\mathrm{Si}$ & 0.16388700 & 1.96928700 & 1.15651400 & $\mathrm{C}$ & -7.06909400 & -0.13266300 & -0.36044500 \\
\hline $\mathrm{H}$ & -6.99457600 & 0.91092100 & -0.68179900 & $\mathrm{H}$ & -7.82359400 & -0.18570200 & 0.42849300 \\
\hline $\mathrm{C}$ & -1.65041100 & -0.62394700 & -2.93732400 & $\mathrm{C}$ & -0.51685600 & 0.19332300 & -3.10743700 \\
\hline $\mathrm{C}$ & -2.53596400 & 0.37768400 & -3.58845600 & $\mathrm{C}$ & -1.32656900 & 1.32351200 & -3.63901300 \\
\hline 0 & -3.70334800 & 0.41053500 & -3.93928900 & $\mathrm{O}$ & -1.14773400 & 2.49919700 & -3.90416100 \\
\hline $\mathrm{N}$ & 0.78591800 & -0.09264100 & -2.87033000 & $\mathrm{H}$ & 1.01924200 & -1.08065800 & -2.73107300 \\
\hline $\mathrm{C}$ & 1.86718300 & 0.77871100 & -2.77211300 & $\mathrm{C}$ & 3.14132100 & 0.20676400 & -2.60893200 \\
\hline C & 1.72516300 & 2.17524400 & -2.77533600 & C & 4.25029300 & 1.02728800 & -2.44750200 \\
\hline $\mathrm{H}$ & 3.24621700 & -0.87090400 & -2.58828000 & $\mathrm{C}$ & 2.85493900 & 2.96936800 & -2.59285900 \\
\hline $\mathrm{H}$ & 0.75401800 & 2.62931700 & -2.94207000 & $\mathrm{C}$ & 4.12524900 & 2.41621700 & -2.43552500 \\
\hline $\mathrm{H}$ & 4.99141500 & 3.05083600 & -2.28846600 & C & 2.72545100 & 4.46284000 & -2.48158500 \\
\hline C & 5.61657600 & 0.42253100 & -2.30002600 & $\mathrm{~F}$ & 3.55337200 & 5.10552500 & -3.33241800 \\
\hline $\mathrm{F}$ & 1.47534600 & 4.89966300 & -2.71251100 & $\mathrm{~F}$ & 3.07074800 & 4.87673100 & -1.22816400 \\
\hline $\mathrm{F}$ & 5.56665500 & -0.89702000 & -2.00872800 & $\mathrm{~F}$ & 6.31440200 & 1.02615600 & -1.30067200 \\
\hline $\mathrm{F}$ & 6.35733800 & 0.55781400 & -3.41933700 & & & & \\
\hline
\end{tabular}

\section{TS- $R-6 f-14$}

Imaginary frequency: $-306.71 \mathrm{~cm}^{-1}$

E[B3LYP/6-31G(d)-D3(BJ)]: -4655.622296 Hartree

E[PCM(Et $\left.\left.t_{2} \mathrm{O}\right)-B 3 L Y P / 6-311 G(d, p)-D 3(B J)\right]:-4656.819420$ Hartree

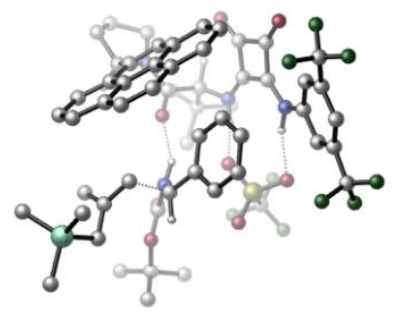

\begin{tabular}{|crrr|crrr|}
\hline 01 & & & & $\mathrm{C}$ & -0.08255300 & 1.85820700 & 3.79923100 \\
\hline $\mathrm{H}$ & 0.51569500 & 1.65391000 & 4.68750700 & $\mathrm{C}$ & -0.92207400 & 3.14511100 & 4.06480100 \\
\hline $\mathrm{C}$ & -1.92013000 & 3.44097100 & 2.93707700 & $\mathrm{H}$ & -2.66974000 & 2.65481700 & 2.83339300 \\
\hline $\mathrm{H}$ & -1.43807000 & 3.54945300 & 1.96623900 & $\mathrm{H}$ & -2.44288200 & 4.38001900 & 3.15268100 \\
\hline $\mathrm{C}$ & 0.04923400 & 4.32915200 & 4.21580700 & $\mathrm{H}$ & -0.50163400 & 5.22010600 & 4.53774700 \\
\hline
\end{tabular}




\begin{tabular}{|c|c|c|c|c|c|c|c|}
\hline$\overline{\mathrm{H}}$ & 0.54075800 & 4.56278400 & 3.26799200 & $\mathrm{H}$ & 0.82506100 & 4.12071800 & 4.96252200 \\
\hline C & -1.70144500 & 2.96330900 & 5.37893200 & $\mathrm{H}$ & -2.31840500 & 3.84816800 & 5.56799000 \\
\hline $\mathrm{H}$ & -1.02923100 & 2.84091500 & 6.23690800 & $\mathrm{H}$ & -2.37490700 & 2.09909500 & 5.33479600 \\
\hline C & -0.92259700 & 0.61137900 & 3.45891000 & 0 & -1.39413900 & 0.44821900 & 2.32286100 \\
\hline C & -0.68753000 & -0.33317400 & 5.81863900 & C & -1.74170600 & -1.61023600 & 4.02539900 \\
\hline C & -1.73865000 & -1.25355300 & 6.44420600 & $\mathrm{H}$ & 0.32991200 & -0.71918300 & 5.93800900 \\
\hline $\mathrm{H}$ & -0.71820400 & 0.67631300 & 6.22194600 & $\mathrm{H}$ & -2.70532000 & -1.34413600 & 3.58445700 \\
\hline $\mathrm{H}$ & -2.67323000 & -0.69911700 & 6.59031200 & $\mathrm{H}$ & -1.42232000 & -1.65094300 & 7.41260400 \\
\hline $\mathrm{N}$ & -1.07228700 & -0.34811700 & 4.39935200 & C & -0.92535000 & -2.40151300 & 3.01603700 \\
\hline C & 0.43745500 & -2.36645600 & 3.07947400 & C & -1.57783300 & -3.21046400 & 2.01061800 \\
\hline C & 1.26976600 & -3.04492700 & 2.13386700 & $\mathrm{H}$ & 0.92978400 & -1.76641500 & 3.83818500 \\
\hline C & -0.76989700 & -3.85819400 & 1.01930200 & C & -2.97052800 & -3.40618300 & 1.96665700 \\
\hline C & 2.67108700 & -2.96791100 & 2.18947500 & C & 0.65017600 & -3.75857300 & 1.06879000 \\
\hline C & -1.38546800 & -4.62188400 & -0.02082900 & $\mathrm{H}$ & -3.60361700 & -2.96236500 & 2.72679200 \\
\hline C & -3.55514400 & -4.20197300 & 0.98201800 & C & 3.45080200 & -3.53727200 & 1.18344900 \\
\hline $\mathrm{H}$ & 3.14788700 & -2.42910200 & 3.00112400 & C & 1.45631200 & -4.35753700 & 0.05637900 \\
\hline C & -2.77954400 & -4.79069500 & -0.01307900 & C & -0.55150900 & -5.20391600 & -1.03615600 \\
\hline $\mathrm{H}$ & -4.62839800 & -4.37284000 & 1.00257500 & C & 2.85190300 & -4.21837300 & 0.12440900 \\
\hline $\mathrm{H}$ & 4.52928300 & -3.42336100 & 1.21301200 & $\mathrm{C}$ & 0.80289900 & -5.06737000 & -1.00573100 \\
\hline $\mathrm{H}$ & -3.24441100 & -5.40702900 & -0.77779400 & $\mathrm{H}$ & -1.03091400 & -5.76427500 & -1.83492500 \\
\hline $\mathrm{H}$ & 3.46811200 & -4.63823500 & -0.66501900 & $\mathrm{H}$ & 1.41808400 & -5.50910100 & -1.78512000 \\
\hline $\mathrm{N}$ & 0.87063600 & 2.04788800 & 2.70136200 & $\mathrm{H}$ & 0.49650400 & 2.38862000 & 1.80799600 \\
\hline S & 0.19337400 & 3.38319700 & -0.98042700 & 0 & -0.66096100 & 3.11701200 & -2.15062700 \\
\hline 0 & -0.12782600 & 2.55278900 & 0.21501200 & $\mathrm{O}$ & 1.65426300 & 3.48470200 & -1.21632400 \\
\hline $\mathrm{C}$ & -0.27594900 & 5.09069900 & -0.42001500 & $\mathrm{~F}$ & -0.02644400 & 5.99613700 & -1.37081800 \\
\hline $\mathrm{F}$ & 0.42349500 & 5.41803000 & 0.68157500 & $\mathrm{~F}$ & -1.58207600 & 5.13960800 & -0.11207400 \\
\hline C & -3.32591500 & 1.98320200 & -0.49397300 & 0 & -3.60892400 & 2.76805300 & 0.38511100 \\
\hline $\mathrm{O}$ & -3.91866000 & 1.88525000 & -1.70194200 & $\mathrm{C}$ & -4.76313200 & 2.98738100 & -2.20695400 \\
\hline $\mathrm{C}$ & -6.06097700 & 3.03417300 & -1.40158800 & $\mathrm{H}$ & -6.73194100 & 3.78734800 & -1.82840700 \\
\hline $\mathrm{H}$ & -5.85892500 & 3.28982600 & -0.36077200 & $\mathrm{H}$ & -6.57068900 & 2.06415800 & -1.43914200 \\
\hline C & -5.02214200 & 2.56776000 & -3.65272100 & $\mathrm{H}$ & -4.07963400 & 2.49157000 & -4.20355200 \\
\hline $\mathrm{H}$ & -5.65407000 & 3.31133300 & -4.14787300 & $\mathrm{H}$ & -5.53333500 & 1.60030000 & -3.69510500 \\
\hline C & -3.98492600 & 4.30213900 & -2.15967900 & $\mathrm{H}$ & -4.53648700 & 5.06322000 & -2.72232500 \\
\hline $\mathrm{H}$ & -2.99706700 & 4.17224100 & -2.61065100 & $\mathrm{H}$ & -3.85019100 & 4.64984400 & -1.13633400 \\
\hline C & -1.90593500 & 0.23775600 & -1.40942900 & $\mathrm{H}$ & -2.29639500 & 0.60555000 & -2.35004200 \\
\hline C & -0.48146500 & -0.16340900 & -1.50143500 & C & 0.08114300 & -0.20440500 & -2.78578700 \\
\hline C & 0.26761100 & -0.58551800 & -0.39723300 & C & 1.37076500 & -0.68997000 & -2.97527300 \\
\hline $\mathrm{H}$ & -0.49280100 & 0.16456500 & -3.63123000 & C & 1.55567400 & -1.06775100 & -0.59571800 \\
\hline $\mathrm{H}$ & -0.15186300 & -0.54506300 & 0.60295100 & C & 2.09912100 & -1.14872900 & -1.87847800 \\
\hline $\mathrm{H}$ & 1.81545200 & -0.68830400 & -3.96445700 & $\mathrm{H}$ & 2.14270400 & -1.38887700 & 0.25306900 \\
\hline $\mathrm{H}$ & 3.09481400 & -1.55511000 & -2.01257700 & $\mathrm{~N}$ & -2.35487300 & 1.00076300 & -0.34926700 \\
\hline $\mathrm{H}$ & -1.81221500 & 1.06157000 & 0.51656800 & C & -2.67791600 & -1.47263000 & -1.33808200 \\
\hline $\mathrm{H}$ & -2.33347600 & -1.78874600 & -0.35734700 & $\mathrm{H}$ & -2.16374300 & -1.95061100 & -2.16577100 \\
\hline C & -4.05223700 & -1.23704400 & -1.49125400 & C & -4.67956500 & -1.23248100 & -2.80732800 \\
\hline $\mathrm{H}$ & -3.97559600 & -0.94807800 & -3.59817800 & $\mathrm{H}$ & -5.56015500 & -0.58526700 & -2.84820300 \\
\hline C & -4.84942900 & -0.94045900 & -0.27323300 & $\mathrm{H}$ & -5.85787200 & -0.58422500 & -0.49104200 \\
\hline $\mathrm{H}$ & -4.30907200 & -0.19355000 & 0.32736400 & $\mathrm{H}$ & -4.89567000 & -1.83438600 & 0.36054100 \\
\hline C & -6.31022000 & -2.80177900 & -4.84781600 & $\mathrm{H}$ & -7.19938800 & -2.19558100 & -4.64081100 \\
\hline $\mathrm{H}$ & -6.65025000 & -3.77290300 & -5.22646800 & $\mathrm{H}$ & -5.74685000 & -2.31144500 & -5.64976500 \\
\hline C & -6.25272200 & -3.77279300 & -1.89493400 & $\mathrm{H}$ & -7.09867000 & -3.13612700 & -1.61183300 \\
\hline $\mathrm{H}$ & -5.62858900 & -3.92247900 & -1.00853000 & $\mathrm{H}$ & -6.65686500 & -4.74940400 & -2.18587900 \\
\hline C & -3.71553400 & -4.04421600 & -3.66698900 & $\mathrm{H}$ & -3.06142200 & -4.11305900 & -2.79231600 \\
\hline $\mathrm{H}$ & -3.13517400 & -3.61371100 & -4.49117000 & $\mathrm{H}$ & -3.99199800 & -5.06507000 & -3.95690500 \\
\hline
\end{tabular}




\begin{tabular}{|c|c|c|c|c|c|c|c|}
\hline $\mathrm{Si}$ & -5.25531100 & -3.02629100 & -3.30453000 & C & -1.91916200 & -2.34572900 & 5.37933200 \\
\hline $\mathrm{H}$ & -1.13666400 & -3.10372500 & 5.48171600 & $\mathrm{H}$ & -2.88452000 & -2.85589800 & 5.44007300 \\
\hline $\mathrm{C}$ & 1.99754000 & 1.33632200 & 2.66760200 & C & 3.02597200 & 1.09955000 & 1.71834300 \\
\hline $\mathrm{C}$ & 2.63049700 & 0.43769400 & 3.65932300 & C & 3.75260500 & 0.19570800 & 2.66471800 \\
\hline 0 & 2.33395800 & 0.02243700 & 4.77202400 & $\mathrm{O}$ & 4.75703400 & -0.49792200 & 2.66152400 \\
\hline $\mathrm{N}$ & 3.20023300 & 1.55606900 & 0.45414500 & $\mathrm{H}$ & 2.52512600 & 2.23598700 & 0.09142100 \\
\hline C & 4.04432300 & 1.01992200 & -0.53052100 & C & 3.98986800 & 1.58269700 & -1.81581600 \\
\hline C & 4.86033500 & -0.09550400 & -0.29994500 & C & 4.72209500 & 1.01159000 & -2.85097900 \\
\hline $\mathrm{H}$ & 3.34257100 & 2.43036500 & -2.00478700 & C & 5.56941500 & -0.65529100 & -1.35954700 \\
\hline $\mathrm{H}$ & 4.94167400 & -0.53028700 & 0.68900100 & C & 5.51513700 & -0.11690200 & -2.64355900 \\
\hline $\mathrm{H}$ & 6.06919800 & -0.56483900 & -3.45935300 & C & 6.27344000 & -1.96452000 & -1.14950400 \\
\hline C & 4.57045200 & 1.54553400 & -4.24755100 & $\mathrm{~F}$ & 6.63568700 & -2.16785000 & 0.13271700 \\
\hline $\mathrm{F}$ & 5.45562400 & -3.00488600 & -1.49010000 & $\mathrm{~F}$ & 7.37889700 & -2.08368200 & -1.91452400 \\
\hline $\mathrm{F}$ & 4.18795800 & 2.83489400 & -4.27005300 & $\mathrm{~F}$ & 3.63589300 & 0.84201800 & -4.94341400 \\
\hline $\mathrm{F}$ & 5.72185900 & 1.44472000 & -4.95039500 & & & & \\
\hline
\end{tabular}

\section{TS- $R-6 f-15$}

Imaginary frequency: $-200.40 \mathrm{~cm}^{-1}$

E[B3LYP/6-31G(d)-D3(BJ)]: -4655.616989 Hartree

$\mathrm{E}\left[\mathrm{PCM}\left(\mathrm{Et}_{2} \mathrm{O}\right)-\mathrm{B} 3 \mathrm{LYP} / 6-311 \mathrm{G}(\mathrm{d}, \mathrm{p})-\mathrm{D} 3(\mathrm{BJ})\right]$ : -4656.816777 Hartree

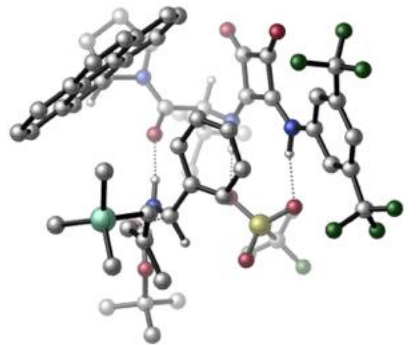

\begin{tabular}{|c|c|c|c|c|c|c|c|}
\hline 01 & & & & C & 1.62772800 & 2.01783400 & -3.29248200 \\
\hline $\mathrm{H}$ & 1.52575000 & 1.73105200 & -4.33864200 & $\mathrm{C}$ & 2.15376800 & 3.48814200 & -3.26662800 \\
\hline C & 2.48141800 & 3.99113900 & -1.85239600 & $\mathrm{H}$ & 3.26056200 & 3.39923600 & -1.36956600 \\
\hline $\mathrm{H}$ & 1.61247800 & 3.98025500 & -1.19321300 & $\mathrm{H}$ & 2.83488800 & 5.02716300 & -1.91744800 \\
\hline C & 1.07590100 & 4.38824600 & -3.89604100 & $\mathrm{H}$ & 1.46760500 & 5.40485100 & -4.01419800 \\
\hline $\mathrm{H}$ & 0.18531200 & 4.44361000 & -3.26514400 & $\mathrm{H}$ & 0.77406900 & 4.02349500 & -4.88544600 \\
\hline $\mathrm{C}$ & 3.43057300 & 3.55667100 & -4.12260700 & $\mathrm{H}$ & 3.80693300 & 4.58481800 & -4.14336000 \\
\hline $\mathrm{H}$ & 3.24240000 & 3.24872700 & -5.15788600 & $\mathrm{H}$ & 4.22655300 & 2.92600100 & -3.71018500 \\
\hline C & 2.55537100 & 1.01965200 & -2.57999400 & 0 & 2.55083800 & 0.91827900 & -1.34605200 \\
\hline $\mathrm{C}$ & 3.53951800 & 0.19343400 & -4.77957700 & $\mathrm{C}$ & 4.28565200 & -0.68813700 & -2.60583800 \\
\hline C & 4.30334800 & -1.11189500 & -5.02300800 & $\mathrm{H}$ & 2.57175200 & 0.21474700 & -5.28218800 \\
\hline $\mathrm{H}$ & 4.13438500 & 1.05648600 & -5.09730400 & $\mathrm{C}$ & 5.14647400 & -1.28289600 & -3.75181600 \\
\hline $\mathrm{H}$ & 4.90798900 & -0.06797200 & -1.95384600 & $\mathrm{H}$ & 4.91219300 & -1.06028300 & -5.92981600 \\
\hline $\mathrm{H}$ & 3.60521300 & -1.94520900 & -5.13635700 & $\mathrm{H}$ & 6.07443400 & -0.70615900 & -3.83434900 \\
\hline $\mathrm{H}$ & 5.41689400 & -2.32220000 & -3.55287500 & $\mathrm{~N}$ & 3.35763500 & 0.21565500 & -3.31421300 \\
\hline $\mathrm{C}$ & 3.67269100 & -1.74744400 & -1.69420500 & $\mathrm{C}$ & 4.32745300 & -1.96025500 & -0.51409200 \\
\hline C & 2.55523200 & -2.60592900 & -2.04877800 & $\mathrm{C}$ & 4.01238700 & -3.03374000 & 0.37727300 \\
\hline $\mathrm{H}$ & 5.14953600 & -1.30423800 & -0.23744700 & $\mathrm{C}$ & 2.23326700 & -3.70604900 & -1.18517300 \\
\hline $\mathrm{C}$ & 1.74385600 & -2.40108200 & -3.17730100 & $\mathrm{C}$ & 4.73023800 & -3.25694200 & 1.56380900 \\
\hline $\mathrm{C}$ & 2.95974700 & -3.92478200 & 0.02078200 & $\mathrm{C}$ & 1.15985000 & -4.58953000 & -1.52106700 \\
\hline $\mathrm{H}$ & 1.90015500 & -1.54538800 & -3.81111100 & $\mathrm{C}$ & 0.68251500 & -3.25188400 & -3.48435800 \\
\hline $\mathrm{C}$ & 4.43157700 & -4.34773600 & 2.37810200 & $\mathrm{H}$ & 5.53907400 & -2.58073800 & 1.82790900 \\
\hline $\mathrm{C}$ & 2.64422200 & -5.02595700 & 0.87423400 & $\mathrm{C}$ & 0.40233600 & -4.34874600 & -2.67741200 \\
\hline $\mathrm{C}$ & 0.85888400 & -5.68441300 & -0.64180000 & $\mathrm{H}$ & 0.05901300 & -3.02740500 & -4.34320200 \\
\hline $\mathrm{C}$ & 3.39765400 & -5.22057900 & 2.04309300 & $\mathrm{H}$ & 5.01006800 & -4.52001600 & 3.28120900 \\
\hline C & 1.56727000 & -5.89842300 & 0.49995400 & $\mathrm{H}$ & -0.42656700 & -5.00879300 & -2.91773000 \\
\hline $\mathrm{H}$ & 0.04042700 & -6.34561000 & -0.91384000 & $\mathrm{H}$ & 3.16921600 & -6.06818100 & 2.68375200 \\
\hline $\mathrm{H}$ & 1.32963500 & -6.73686700 & 1.14962000 & $\mathrm{~N}$ & 0.28991900 & 1.89848700 & -2.70586600 \\
\hline $\mathrm{H}$ & 0.14077200 & 2.29945300 & -1.77303500 & $\mathrm{~S}$ & -1.21046200 & 3.50632700 & 0.59079200 \\
\hline
\end{tabular}




\begin{tabular}{|c|c|c|c|c|c|c|c|}
\hline 0 & -1.10162900 & 3.51695300 & 2.06326300 & 0 & -0.05318500 & 2.88355800 & -0.10495700 \\
\hline 0 & -2.51981400 & 3.07766500 & 0.03558100 & $\mathrm{C}$ & -1.09513600 & 5.28626100 & 0.07161200 \\
\hline $\mathrm{F}$ & -2.03132200 & 6.02437900 & 0.67391100 & $\mathrm{~F}$ & -1.26043000 & 5.37967800 & -1.25846000 \\
\hline $\mathrm{F}$ & 0.11259500 & 5.78768300 & 0.37715000 & $\mathrm{C}$ & 2.65119400 & 2.62286000 & 1.78968300 \\
\hline 0 & 3.47686600 & 3.22568000 & 1.13412700 & 0 & 2.28311600 & 2.89139900 & 3.06189400 \\
\hline $\mathrm{C}$ & 2.60356700 & 4.18542700 & 3.69003000 & $\mathrm{C}$ & 4.11329600 & 4.29745200 & 3.89795500 \\
\hline $\mathrm{H}$ & 4.33762800 & 5.20748600 & 4.46493600 & $\mathrm{H}$ & 4.63385700 & 4.33757200 & 2.94061000 \\
\hline $\mathrm{H}$ & 4.48489000 & 3.43910100 & 4.46901000 & C & 1.86268300 & 4.08748700 & 5.02275300 \\
\hline $\mathrm{H}$ & 0.79049200 & 3.95368700 & 4.84899100 & $\mathrm{H}$ & 2.01037900 & 5.00367900 & 5.60283100 \\
\hline $\mathrm{H}$ & 2.23774200 & 3.24373900 & 5.61365500 & C & 2.03136400 & 5.31613700 & 2.83833200 \\
\hline $\mathrm{H}$ & 2.16730800 & 6.26883700 & 3.36165200 & $\mathrm{H}$ & 0.96387200 & 5.15389900 & 2.67352200 \\
\hline $\mathrm{H}$ & 2.53236500 & 5.37415700 & 1.87102400 & C & 0.90569900 & 0.94731600 & 2.01273400 \\
\hline $\mathrm{H}$ & 0.39663900 & 1.68483700 & 2.62637800 & $\mathrm{C}$ & -0.03332400 & 0.06886000 & 1.26405100 \\
\hline C & -1.37984400 & 0.06695600 & 1.66431400 & C & 0.39702900 & -0.85034000 & 0.30122500 \\
\hline C & -2.26595300 & -0.88162200 & 1.16305900 & $\mathrm{H}$ & -1.73200400 & 0.82746400 & 2.35487900 \\
\hline C & -0.50816600 & -1.76986300 & -0.23258700 & $\mathrm{H}$ & 1.42771200 & -0.85269200 & -0.03677800 \\
\hline C & -1.82743500 & -1.81200700 & 0.21844000 & $\mathrm{H}$ & -3.29953200 & -0.87246900 & 1.49140400 \\
\hline $\mathrm{H}$ & -0.18008700 & -2.46110800 & -0.99575500 & $\mathrm{H}$ & -2.51928900 & -2.54331800 & -0.18668300 \\
\hline $\mathrm{N}$ & 1.98024000 & 1.50599300 & 1.32734600 & $\mathrm{H}$ & 2.13913500 & 1.28878500 & 0.33755600 \\
\hline C & 1.69032100 & -0.09804900 & 3.27885600 & $\mathrm{H}$ & 2.31674700 & 0.64902700 & 3.75841800 \\
\hline $\mathrm{H}$ & 2.23013100 & -0.82125400 & 2.67064000 & C & 0.62163500 & -0.60461000 & 4.05358900 \\
\hline C & 0.04715600 & -1.89746200 & 3.77471300 & $\mathrm{H}$ & 0.28750700 & -2.24942000 & 2.76630700 \\
\hline $\mathrm{H}$ & -1.03182700 & -1.94068800 & 3.95401500 & C & -0.02429100 & 0.28798700 & 5.05668500 \\
\hline $\mathrm{H}$ & -0.52772100 & -0.27404200 & 5.84735600 & $\mathrm{H}$ & -0.80416300 & 0.86264800 & 4.53069100 \\
\hline $\mathrm{H}$ & 0.67307400 & 1.01249800 & 5.48393800 & C & 0.36947100 & -4.88407200 & 4.14536300 \\
\hline $\mathrm{H}$ & -0.71714500 & -5.01352700 & 4.08776800 & $\mathrm{H}$ & 0.77831000 & -5.72836300 & 4.71321500 \\
\hline $\mathrm{H}$ & 0.77278900 & -4.93179100 & 3.12934800 & C & -0.01965500 & -3.08726100 & 6.65170100 \\
\hline $\mathrm{H}$ & -1.11172700 & -3.13507800 & 6.57328400 & $\mathrm{H}$ & 0.24576600 & -2.14950700 & 7.15242600 \\
\hline $\mathrm{H}$ & 0.29858200 & -3.90623900 & 7.30819600 & C & 2.66099200 & -2.97013400 & 5.11247700 \\
\hline $\mathrm{H}$ & 2.87918100 & -2.04006800 & 5.64965900 & $\mathrm{H}$ & 3.13851700 & -2.91978900 & 4.12980900 \\
\hline $\mathrm{H}$ & 3.13194700 & -3.79172800 & 5.66517400 & $\mathrm{Si}$ & 0.81112600 & -3.25481400 & 4.96955500 \\
\hline C & -0.52708400 & 0.93197500 & -3.13066400 & C & -1.79602400 & 0.42957500 & -2.74541000 \\
\hline C & -0.41413900 & -0.04824000 & -4.23243700 & C & -1.71411200 & -0.66983800 & -3.75944800 \\
\hline $\mathrm{O}$ & 0.38957400 & -0.27256000 & -5.12992900 & 0 & -2.34177600 & -1.67194200 & -4.06125200 \\
\hline $\mathrm{N}$ & -2.67104400 & 0.86720900 & -1.81266400 & $\overline{\mathrm{H}}$ & -2.42328200 & 1.70609000 & -1.27586900 \\
\hline C & -3.83882500 & 0.23517300 & -1.36658500 & C & -4.56283500 & 0.85753700 & -0.33704300 \\
\hline $\mathrm{C}$ & -4.25692700 & -1.01260600 & -1.84368800 & $\mathrm{C}$ & -5.64515000 & 0.20287600 & 0.23993600 \\
\hline $\mathrm{H}$ & -4.23893100 & 1.81827400 & 0.04360800 & $\mathrm{C}$ & -5.34368600 & -1.64567000 & -1.24398000 \\
\hline $\mathrm{H}$ & -3.72257600 & -1.50695800 & -2.64924200 & C & -6.04678100 & -1.06079800 & -0.19392700 \\
\hline $\mathrm{H}$ & -6.87765500 & -1.57233300 & 0.27397000 & $\mathrm{C}$ & -5.65474600 & -3.05178900 & -1.67164800 \\
\hline $\mathrm{C}$ & -6.27833700 & 0.80208600 & 1.46172000 & $\mathrm{~F}$ & -5.62344500 & -3.20103000 & -3.00926100 \\
\hline $\mathrm{F}$ & -4.73793500 & -3.92018200 & -1.16017800 & $F$ & -6.86583900 & -3.46642800 & -1.24017400 \\
\hline $\mathrm{F}$ & -6.29936200 & 2.14798000 & 1.42727000 & $\mathrm{~F}$ & -5.58333900 & 0.44845500 & 2.57924200 \\
\hline $\mathrm{F}$ & -7.54672700 & 0.37484200 & 1.64480900 & & & & \\
\hline
\end{tabular}

\section{TS- $R-6 f-16$}

Imaginary frequency: $-291.64 \mathrm{~cm}^{-1}$

E[B3LYP/6-31G(d)-D3(BJ)]: -4655.616948 Hartree

$\mathrm{E}\left[\mathrm{PCM}\left(\mathrm{Et}_{2} \mathrm{O}\right)-\mathrm{B} 3 \mathrm{LYP} / 6-311 \mathrm{G}(\mathrm{d}, \mathrm{p})-\mathrm{D} 3(\mathrm{BJ})\right]$ : -4656.816359 Hartree

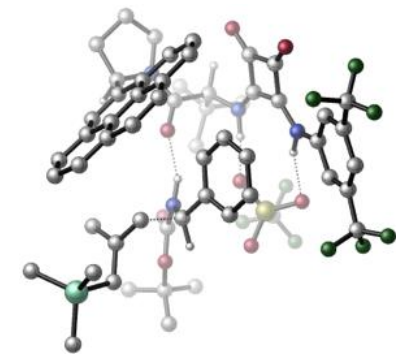




\begin{tabular}{|c|c|c|c|c|c|c|c|}
\hline 01 & & & & $C$ & -0.47111600 & 0.97234800 & 4.09266600 \\
\hline $\mathrm{H}$ & -1.10544800 & 1.71718700 & 4.57198100 & C & 0.16887500 & 0.10259600 & 5.22029600 \\
\hline C & 1.19315800 & -0.90714600 & 4.68194700 & $\mathrm{H}$ & 2.05109800 & -0.41940200 & 4.21633600 \\
\hline $\mathrm{H}$ & 0.76822500 & -1.57817500 & 3.93619100 & $\mathrm{H}$ & 1.56031800 & -1.52402600 & 5.51040900 \\
\hline C & -0.96383200 & -0.64979000 & 5.94096500 & $\mathrm{H}$ & -0.56314300 & -1.16427400 & 6.82164900 \\
\hline $\mathrm{H}$ & -1.41793700 & -1.40166200 & 5.29056700 & $\mathrm{H}$ & -1.75207300 & 0.03500900 & 6.27644200 \\
\hline C & 0.86903100 & 1.03859200 & 6.22019400 & $\mathrm{H}$ & 1.31627400 & 0.44969100 & 7.02798000 \\
\hline $\mathrm{H}$ & 0.16532000 & 1.74685000 & 6.67325900 & $\mathrm{H}$ & 1.67675600 & 1.60660000 & 5.74406900 \\
\hline C & 0.57424400 & 1.67721900 & 3.20895000 & $\mathrm{O}$ & 1.18007300 & 1.05992700 & 2.32017700 \\
\hline C & 0.20680700 & 3.89312900 & 4.40558300 & C & 1.86377800 & 3.65405100 & 2.60132900 \\
\hline C & 0.60717200 & 5.29256500 & 3.92725100 & $\mathrm{H}$ & -0.87497800 & 3.75493600 & 4.43989200 \\
\hline $\mathrm{H}$ & 0.63129200 & 3.68300500 & 5.39312100 & C & 1.95600300 & 5.06940100 & 3.22999400 \\
\hline $\mathrm{H}$ & 2.79347400 & 3.10581800 & 2.78232300 & $\mathrm{H}$ & 0.67402500 & 5.99912100 & 4.75900800 \\
\hline $\mathrm{H}$ & -0.13148700 & 5.67977800 & 3.22075600 & $\mathrm{H}$ & 2.76679400 & 5.07769200 & 3.96697600 \\
\hline $\mathrm{H}$ & 2.18146300 & 5.82864800 & 2.47797000 & $\mathrm{~N}$ & 0.80759900 & 2.99730900 & 3.39534100 \\
\hline C & 1.68933200 & 3.66105400 & 1.08478000 & C & 2.83757700 & 3.49491600 & 0.36249200 \\
\hline C & 0.44458400 & 3.93252100 & 0.38737900 & C & 2.88831500 & 3.57368700 & -1.06450100 \\
\hline $\mathrm{H}$ & 3.76942100 & 3.29552700 & 0.88774600 & C & 0.46413800 & 4.00757200 & -1.04546000 \\
\hline C & -0.79133200 & 4.10605800 & 1.03377800 & C & 4.08663600 & 3.43342600 & -1.78531500 \\
\hline C & 1.67338700 & 3.80703700 & -1.77195700 & C & -0.73979500 & 4.28309900 & -1.76502400 \\
\hline $\mathrm{H}$ & -0.87112200 & 3.98854100 & 2.10036200 & C & -1.95848700 & 4.38925400 & 0.32517600 \\
\hline C & 4.08753900 & 3.48143300 & -3.17792400 & $\mathrm{H}$ & 5.02053200 & 3.31365300 & -1.24104700 \\
\hline $\mathrm{C}$ & 1.68312500 & 3.85110400 & -3.19985800 & C & -1.93486400 & 4.48944700 & -1.06090100 \\
\hline C & -0.70389100 & 4.30983900 & -3.19984600 & $\mathrm{H}$ & -2.89312500 & 4.48824800 & 0.86637900 \\
\hline $\mathrm{C}$ & 2.89851900 & 3.67311600 & -3.88005000 & $\mathrm{H}$ & 5.02253500 & 3.38096900 & -3.72168700 \\
\hline $\mathrm{C}$ & 0.44894200 & 4.09545800 & -3.88893300 & $\mathrm{H}$ & -2.85034000 & 4.68804700 & -1.61114700 \\
\hline $\mathrm{H}$ & -1.63366700 & 4.50161200 & -3.72852400 & $\mathrm{H}$ & 2.90587400 & 3.70991800 & -4.96611300 \\
\hline $\mathrm{H}$ & 0.45707000 & 4.11643600 & -4.97534400 & $\mathrm{~N}$ & -1.34625900 & 0.17949400 & 3.22858800 \\
\hline $\mathrm{H}$ & -0.95633800 & -0.67595800 & 2.81851600 & $S$ & -0.62090100 & -3.27256000 & 1.37689300 \\
\hline 0 & 0.32381000 & -3.85608400 & 0.40730700 & O & -0.29600300 & -1.87435900 & 1.77517500 \\
\hline $\mathrm{O}$ & -2.06635200 & -3.46040100 & 1.10567700 & C & -0.35955800 & -4.22045000 & 2.95333500 \\
\hline $\mathrm{F}$ & -0.60144400 & -5.52290200 & 2.77570600 & $\mathrm{~F}$ & -1.18435700 & -3.75295400 & 3.90667600 \\
\hline $\mathrm{F}$ & 0.90229300 & -4.07440200 & 3.38875400 & C & 2.90640800 & -1.98876100 & 1.16727700 \\
\hline $\mathrm{O}$ & 3.15459200 & -1.99021300 & 2.35433100 & $\mathrm{O}$ & 3.43013900 & -2.80948600 & 0.23510200 \\
\hline C & 4.19150800 & -4.01181900 & 0.63475700 & C & 3.38504500 & -4.85584800 & 1.62031500 \\
\hline $\mathrm{H}$ & 3.88794400 & -5.82058600 & 1.74975300 & $\mathrm{H}$ & 2.37987700 & -5.02875600 & 1.22851200 \\
\hline $\mathrm{H}$ & 3.30169200 & -4.36817700 & 2.59004700 & C & 5.54045700 & -3.57078700 & 1.20132000 \\
\hline $\mathrm{H}$ & 6.08741100 & -2.96425500 & 0.46959400 & $\mathrm{H}$ & 6.14933700 & -4.45082900 & 1.43403300 \\
\hline $\mathrm{H}$ & 5.40069200 & -2.98866200 & 2.11372600 & C & 4.35274300 & -4.75930000 & -0.68768300 \\
\hline $\mathrm{H}$ & 4.90465800 & -5.68955800 & -0.52173400 & $\mathrm{H}$ & 4.90417700 & -4.16054100 & -1.41909200 \\
\hline $\mathrm{H}$ & 3.37131800 & -5.00622900 & -1.10340800 & C & 1.66237400 & -1.14291000 & -0.74721400 \\
\hline $\mathrm{H}$ & 1.88011500 & -2.11904500 & -1.16451400 & C & 0.32866600 & -0.62248100 & -1.14564300 \\
\hline C & -0.32364900 & -1.26790700 & -2.20727300 & C & -0.22654400 & 0.53756500 & -0.59435900 \\
\hline C & -1.49337000 & -0.73787700 & -2.74307200 & $\mathrm{H}$ & 0.08764600 & -2.19173900 & -2.60520600 \\
\hline C & -1.40383900 & 1.05532100 & -1.12570700 & $\mathrm{H}$ & 0.25733400 & 1.03900700 & 0.23638100 \\
\hline C & -2.02124600 & 0.44325800 & -2.21807900 & $\mathrm{H}$ & -2.00235100 & -1.25817700 & -3.54668900 \\
\hline $\mathrm{H}$ & -1.83864300 & 1.94478100 & -0.69084300 & $\mathrm{H}$ & -2.92631900 & 0.86947300 & -2.63691500 \\
\hline $\mathrm{N}$ & 2.06722500 & -1.05448100 & 0.57290800 & $\mathrm{H}$ & 1.56657500 & -0.45856900 & 1.23833100 \\
\hline C & 2.72515400 & -0.05497900 & -1.82696600 & $\mathrm{H}$ & 2.47065600 & 0.91715100 & -1.41334200 \\
\hline $\mathrm{H}$ & 2.28311600 & -0.24713800 & -2.80051000 & C & 4.05046200 & -0.48893300 & -1.64123200 \\
\hline C & 4.66460900 & -1.46330500 & -2.52793300 & $\mathrm{H}$ & 3.92555500 & -2.11426800 & -3.00834000 \\
\hline $\mathrm{H}$ & 5.42516700 & -2.06754800 & -2.02598800 & C & 4.80697700 & 0.06319800 & -0.48844200 \\
\hline $\mathrm{H}$ & 5.74923400 & -0.45383000 & -0.29979900 & $\mathrm{H}$ & 4.16905900 & 0.02234600 & 0.40670600 \\
\hline
\end{tabular}




\begin{tabular}{|c|c|c|c|c|c|c|c|}
\hline $\mathrm{H}$ & 4.99610200 & 1.12974600 & -0.65685700 & C & 6.45542100 & -1.94634900 & -4.93552200 \\
\hline $\mathrm{H}$ & 7.19753000 & -2.44279700 & -4.30014700 & $\mathrm{H}$ & 6.98113100 & -1.54427900 & -5.80964400 \\
\hline $\mathrm{H}$ & 5.75269400 & -2.70783700 & -5.29206800 & $\mathrm{C}$ & 6.78629100 & 0.68531200 & -3.32000900 \\
\hline $\mathrm{H}$ & 7.49834700 & 0.22063700 & -2.62852100 & $\mathrm{H}$ & 6.27275400 & 1.49253800 & -2.78871400 \\
\hline $\mathrm{H}$ & 7.36526100 & 1.14011300 & -4.13249800 & C & 4.25879700 & 0.25132800 & -5.08371100 \\
\hline $\mathrm{H}$ & 3.71910900 & 1.03393300 & -4.54126600 & $\mathrm{H}$ & 3.52772700 & -0.47740200 & -5.45273800 \\
\hline $\mathrm{H}$ & 4.72658600 & 0.71834500 & -5.95890500 & $\mathrm{Si}$ & 5.56483800 & -0.56901500 & -4.01085300 \\
\hline C & -2.35426100 & 0.76786600 & 2.58082300 & C & -3.24314000 & 0.39653700 & 1.53873200 \\
\hline $\mathrm{C}$ & -2.94691200 & 2.11653100 & 2.68685200 & $\mathrm{C}$ & -3.85130200 & 1.76694000 & 1.51943300 \\
\hline $\mathrm{O}$ & -2.75384700 & 3.10638800 & 3.38359700 & $\mathrm{O}$ & -4.65081900 & 2.39270900 & 0.84217100 \\
\hline $\mathrm{N}$ & -3.38030600 & -0.76802800 & 0.86385500 & $\mathrm{H}$ & -2.77551300 & -1.54793300 & 1.13328600 \\
\hline $\mathrm{C}$ & -4.11263000 & -0.99959300 & -0.31174900 & $\mathrm{C}$ & -3.92687700 & -2.23034100 & -0.96229800 \\
\hline $\mathrm{C}$ & -4.95376500 & -0.03842700 & -0.88265900 & $\mathrm{C}$ & -4.53850400 & -2.46206800 & -2.18819000 \\
\hline $\mathrm{H}$ & -3.27746700 & -2.97790900 & -0.52300100 & C & -5.54796700 & -0.29669200 & -2.11721600 \\
\hline $\mathrm{H}$ & -5.11039300 & 0.92172400 & -0.40177000 & $\mathrm{C}$ & -5.34888300 & -1.49845200 & -2.78993600 \\
\hline $\mathrm{H}$ & -5.81032200 & -1.68092000 & -3.75194000 & $\mathrm{C}$ & -6.29988500 & 0.81852700 & -2.78494400 \\
\hline $\mathrm{C}$ & -4.21497300 & -3.72061300 & -2.94151000 & $\mathrm{~F}$ & -7.04498400 & 1.53028900 & -1.91839000 \\
\hline $\mathrm{F}$ & -5.43509200 & 1.69457800 & -3.36928100 & $\mathrm{~F}$ & -7.11968800 & 0.37387500 & -3.76263600 \\
\hline $\mathrm{F}$ & -3.90729600 & -4.74693000 & -2.12828200 & $\mathrm{~F}$ & -3.14339200 & -3.53513900 & -3.76364100 \\
\hline $\mathrm{F}$ & -5.23772900 & -4.11376800 & -3.73363200 & & & & \\
\hline
\end{tabular}

\section{TS-R-6f-17}

Imaginary frequency: $-287.01 \mathrm{~cm}^{-1}$

E[B3LYP/6-31G(d)-D3(BJ)]: -4655.629077 Hartree

E[PCM(Et $\left.\left.{ }_{2} \mathrm{O}\right)-\mathrm{B} 3 \mathrm{LYP} / 6-311 \mathrm{G}(\mathrm{d}, \mathrm{p})-\mathrm{D} 3(\mathrm{BJ})\right]$ : -4656.815657 Hartree

\begin{tabular}{|lrll|lrrr|}
\hline $\mathrm{O}_{\mathrm{H}}$ & & & $\mathrm{C}$ & 3.33871200 & 1.05598500 & -2.67439200 \\
\hline $\mathrm{H}$ & 3.62642800 & 0.45570300 & -3.53853100 & $\mathrm{C}$ & 3.90388200 & 2.49939400 & -2.86197000 \\
\hline $\mathrm{C}$ & 3.68835500 & 3.37108700 & -1.61118200 & $\mathrm{H}$ & 4.31667500 & 3.03460200 & -0.78209800 \\
\hline $\mathrm{H}$ & 2.65724500 & 3.35282100 & -1.26053400 & $\mathrm{H}$ & 3.95054300 & 4.40967100 & -1.84196600 \\
\hline $\mathrm{C}$ & 3.20582100 & 3.13373300 & -4.07784600 & $\mathrm{H}$ & 3.64694600 & 4.11406400 & -4.29040400 \\
\hline $\mathrm{H}$ & 2.13760600 & 3.27335800 & -3.90305300 & $\mathrm{H}$ & 3.32440700 & 2.50725200 & -4.97004800 \\
\hline $\mathrm{C}$ & 5.41434000 & 2.42603000 & -3.14328600 & $\mathrm{H}$ & 5.81845500 & 3.43848800 & -3.25080000 \\
\hline $\mathrm{H}$ & 5.62742400 & 1.88662100 & -4.07370000 & $\mathrm{H}$ & 5.95727500 & 1.94191600 & -2.32362800 \\
\hline $\mathrm{C}$ & 3.80976900 & 0.39113700 & -1.37000900 & $\mathrm{O}$ & 3.25281300 & 0.68521600 & -0.31069300 \\
\hline $\mathrm{C}$ & 5.58793800 & -0.96456500 & -2.58414000 & $\mathrm{C}$ & 5.29554300 & -1.12114600 & -0.14976000 \\
\hline $\mathrm{C}$ & 6.87537600 & -1.50438600 & -1.95377700 & $\mathrm{H}$ & 5.04210600 & -1.73556200 & -3.13826200 \\
\hline $\mathrm{H}$ & 5.77628700 & -0.13686400 & -3.26495500 & $\mathrm{H}$ & 5.75112800 & -0.31314400 & 0.43550100 \\
\hline $\mathrm{H}$ & 7.57321000 & -0.67871500 & -1.76928300 & $\mathrm{H}$ & 7.37583800 & -2.23410200 & -2.59614500 \\
\hline $\mathrm{N}$ & 4.80887600 & -0.52252800 & -1.41397100 & $\mathrm{C}$ & 4.25934500 & -1.77332400 & 0.74729900 \\
\hline $\mathrm{C}$ & 4.25588900 & -1.42238000 & 2.06515400 & $\mathrm{C}$ & 3.37389500 & -2.82626800 & 0.28711000 \\
\hline $\mathrm{C}$ & 3.43137000 & -2.06704100 & 3.04178800 & $\mathrm{H}$ & 4.89917400 & -0.61415500 & 2.40219300 \\
\hline $\mathrm{C}$ & 2.60753100 & -3.54955700 & 1.25627200 & $\mathrm{C}$ & 3.21369000 & -3.16450500 & -1.06642800 \\
\hline $\mathrm{C}$ & 3.39567800 & -1.65836800 & 4.38379700 & $\mathrm{C}$ & 2.63157800 & -3.17173600 & 2.63002400 \\
\hline $\mathrm{C}$ & 1.78849400 & -4.64524000 & 0.84422300 & $\mathrm{H}$ & 3.71439400 & -2.59392100 & -1.83108800 \\
\hline $\mathrm{C}$ & 2.37278300 & -4.20198800 & -1.46561400 & $\mathrm{C}$ & 2.60054900 & -2.32965200 & 5.31021600 \\
\hline $\mathrm{H}$ & 3.99463000 & -0.80629300 & 4.69269300 & $\mathrm{C}$ & 1.84148700 & -3.87151800 & 3.59084600 \\
\hline $\mathrm{C}$ & 1.69309900 & -4.96112800 & -0.51992900 & $\mathrm{C}$ & 1.05261900 & -5.37091000 & 1.84129300 \\
\hline $\mathrm{H}$ & 2.24305600 & -4.39536700 & -2.52533500 & $\mathrm{C}$ & 1.83901100 & -3.43035100 & 4.92381300 \\
\hline $\mathrm{H}$ & 2.57846700 & -1.99463800 & 6.34300800 & $\mathrm{C}$ & 1.07280700 & -5.00163500 & 3.15162700 \\
\hline $\mathrm{H}$ & 1.05399600 & -5.78287600 & -0.83157800 & $\mathrm{H}$ & 0.45911200 & -6.22220000 & 1.51813300 \\
\hline $\mathrm{H}$ & 1.23140400 & -3.95877600 & 5.65388200 & $\mathrm{H}$ & 0.49834900 & -5.55475500 & 3.89027800 \\
\hline
\end{tabular}




\begin{tabular}{|c|c|c|c|c|c|c|c|}
\hline $\mathrm{N}$ & 1.87120000 & 1.06378700 & -2.64675900 & $\mathrm{H}$ & 1.44045600 & 1.67058800 & -1.94255700 \\
\hline$S$ & -0.58046300 & 3.49536500 & -0.87092800 & $\mathrm{O}$ & -1.43283100 & 3.87475400 & 0.28446000 \\
\hline 0 & 0.51217600 & 2.54850900 & -0.52456000 & 0 & -1.31974600 & 3.11706200 & -2.09984100 \\
\hline $\mathrm{C}$ & 0.26787700 & 5.08825000 & -1.31505100 & $\mathrm{~F}$ & -0.63287000 & 6.05511100 & -1.49748900 \\
\hline $\mathrm{F}$ & 0.97451300 & 4.93737200 & -2.44537100 & $\mathrm{~F}$ & 1.10533500 & 5.45105800 & -0.33419200 \\
\hline C & 0.51148800 & 2.23348400 & 3.06680600 & $\mathrm{O}$ & 0.81309200 & 1.28203200 & 3.76486800 \\
\hline 0 & 1.28450900 & 3.21137500 & 2.61391900 & $\mathrm{C}$ & 2.71418000 & 3.30255700 & 2.98983300 \\
\hline C & 2.79640600 & 3.60748100 & 4.48492800 & $\mathrm{H}$ & 3.84199200 & 3.77112400 & 4.76691900 \\
\hline $\mathrm{H}$ & 2.40542600 & 2.77422200 & 5.07349500 & $\mathrm{H}$ & 2.23049200 & 4.51345600 & 4.72579100 \\
\hline $\mathrm{C}$ & 3.18711300 & 4.48160600 & 2.14385800 & $\mathrm{H}$ & 3.04688900 & 4.26410800 & 1.08267900 \\
\hline $\mathrm{H}$ & 4.24894500 & 4.67038600 & 2.33120600 & $\mathrm{H}$ & 2.62158000 & 5.38596900 & 2.38902400 \\
\hline C & 3.45357500 & 2.02166700 & 2.61242500 & $\mathrm{H}$ & 4.52940100 & 2.18959500 & 2.74446600 \\
\hline $\mathrm{H}$ & 3.27347900 & 1.75376000 & 1.56967500 & $\mathrm{H}$ & 3.14926800 & 1.18815000 & 3.24599000 \\
\hline C & -1.76121200 & 1.52782900 & 2.87749600 & $\mathrm{H}$ & -1.43263900 & 0.80736900 & 3.61943300 \\
\hline C & -3.18628600 & 1.91060100 & 2.96520800 & C & -4.00987100 & 1.14577800 & 3.80764900 \\
\hline C & -3.73931200 & 2.97978200 & 2.24434400 & C & -5.36007600 & 1.45291400 & 3.94250700 \\
\hline $\mathrm{H}$ & -3.58186100 & 0.31686200 & 4.36593600 & C & -5.08989100 & 3.28354300 & 2.38785400 \\
\hline $\mathrm{H}$ & -3.12832700 & 3.55528200 & 1.55926300 & C & -5.90218100 & 2.52620900 & 3.23342200 \\
\hline $\mathrm{H}$ & -5.98647100 & 0.85959500 & 4.60181500 & $\mathrm{H}$ & -5.51277000 & 4.10567100 & 1.81934800 \\
\hline $\mathrm{H}$ & -6.95662500 & 2.76594400 & 3.33340400 & $\mathrm{~N}$ & -0.80109800 & 2.46681800 & 2.63912600 \\
\hline $\mathrm{H}$ & -0.94633700 & 3.18145500 & 1.91623900 & C & -1.85220800 & 0.31238300 & 1.23215000 \\
\hline $\mathrm{H}$ & -1.56951700 & 1.05099100 & 0.49181600 & $\mathrm{H}$ & -2.90995500 & 0.09353100 & 1.29583000 \\
\hline C & -0.93768000 & -0.69591100 & 1.54115000 & C & -1.35216700 & -1.86487500 & 2.32744100 \\
\hline $\mathrm{H}$ & -2.05263700 & -1.58457800 & 3.12471100 & $\mathrm{H}$ & -0.50193900 & -2.39994200 & 2.75957600 \\
\hline C & 0.47388500 & -0.60731700 & 1.06404700 & $\mathrm{H}$ & 1.17607800 & -0.75916500 & 1.88851600 \\
\hline $\mathrm{H}$ & 0.69557700 & 0.33522500 & 0.56154300 & $\mathrm{H}$ & 0.65894300 & -1.42549700 & 0.35302400 \\
\hline C & -2.55300100 & -4.69353700 & 2.26784300 & $\mathrm{H}$ & -3.05352700 & -4.50179800 & 3.22437000 \\
\hline $\mathrm{H}$ & -1.57594000 & -5.13489400 & 2.47521300 & $\mathrm{H}$ & -3.15410800 & -5.43047300 & 1.72148700 \\
\hline $\mathrm{C}$ & -1.54056700 & -3.37193200 & -0.38722400 & $\mathrm{H}$ & -0.56155400 & -3.84099900 & -0.26751200 \\
\hline $\mathrm{H}$ & -1.38726800 & -2.41794500 & -0.89814900 & $\mathrm{H}$ & -2.14082700 & -3.99545400 & -1.05617100 \\
\hline C & -4.12798800 & -2.39055900 & 1.04622300 & $\mathrm{H}$ & -4.14253400 & -1.45674200 & 0.48294700 \\
\hline $\mathrm{H}$ & -4.60511400 & -2.20630400 & 2.01622400 & $\mathrm{H}$ & -4.74935400 & -3.10247600 & 0.49195800 \\
\hline $\mathrm{Si}$ & -2.40247800 & -3.11321300 & 1.25337300 & C & 6.39098000 & -2.10031400 & -0.62673500 \\
\hline $\mathrm{H}$ & 5.95481000 & -3.09020500 & -0.79118100 & $\mathrm{H}$ & 7.17995400 & -2.20378500 & 0.12246100 \\
\hline $\mathrm{C}$ & 1.20894600 & -0.05331800 & -2.96857600 & $\mathrm{C}$ & -0.14202100 & -0.45387300 & -2.90960600 \\
\hline C & 1.63409700 & -1.35720200 & -3.53517300 & $\mathrm{C}$ & 0.18470500 & -1.82962600 & -3.39906500 \\
\hline $\mathrm{O}$ & 2.68769600 & -1.83372900 & -3.92661900 & $\mathrm{O}$ & -0.40582500 & -2.88084700 & -3.57116700 \\
\hline $\mathrm{N}$ & -1.23071000 & 0.25234900 & -2.51964200 & $\mathrm{H}$ & -1.09582100 & 1.25260900 & -2.34098800 \\
\hline C & -2.55219200 & -0.18315100 & -2.37971600 & C & -3.45398800 & 0.69271500 & -1.75233700 \\
\hline $\mathrm{C}$ & -2.99446000 & -1.44329100 & -2.80561600 & $\mathrm{C}$ & -4.76799800 & 0.29475600 & -1.53785600 \\
\hline $\mathrm{H}$ & -3.12137400 & 1.67437500 & -1.43571100 & $\mathrm{C}$ & -4.32151300 & -1.80960000 & -2.58736600 \\
\hline $\mathrm{H}$ & -2.30790800 & -2.13932400 & -3.27686200 & C & -5.22356600 & -0.95478900 & -1.95882100 \\
\hline $\mathrm{H}$ & -6.24762000 & -1.25878300 & -1.78318600 & $\mathrm{C}$ & -4.75905500 & -3.20222400 & -2.94741000 \\
\hline $\mathrm{C}$ & -5.66261100 & 1.14964900 & -0.68850400 & $\mathrm{~F}$ & -6.08192100 & -3.26517500 & -3.21090600 \\
\hline $\mathrm{F}$ & -4.10143000 & -3.68915500 & -4.01408800 & $\mathrm{~F}$ & -4.53090000 & -4.06809700 & -1.91735900 \\
\hline $\mathrm{F}$ & -5.32100200 & 2.45344700 & -0.71854500 & $\mathrm{~F}$ & -5.58913900 & 0.75214500 & 0.61253600 \\
\hline $\mathrm{F}$ & -6.95970600 & 1.05418500 & -1.04796700 & & & & \\
\hline
\end{tabular}

\section{TS- $R-6 \mathrm{f}-18$}

Imaginary frequency: $-220.61 \mathrm{~cm}-1$

E[B3LYP/6-31G(d)-D3(BJ)]: -4655.630472 Hartree

$\mathrm{E}\left[\mathrm{PCM}\left(\mathrm{Et}_{2} \mathrm{O}\right)-\mathrm{B} 3 \mathrm{LYP} / 6-311 \mathrm{G}(\mathrm{d}, \mathrm{p})-\mathrm{D} 3(\mathrm{BJ})\right]$ : -4656.815418 Hartree

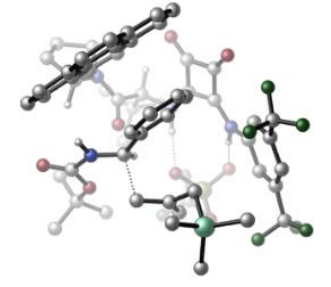




\begin{tabular}{|c|c|c|c|c|c|c|c|}
\hline 01 & & & & $\mathrm{C}$ & 2.13362100 & 3.01563200 & -1.95718100 \\
\hline $\mathrm{H}$ & 2.62318100 & 2.77669600 & -2.90260500 & C & 2.25725100 & 4.55340100 & -1.71569800 \\
\hline $\mathrm{C}$ & 1.70192200 & 4.96972800 & -0.34452000 & $\mathrm{H}$ & 2.34867600 & 4.62191400 & 0.46278700 \\
\hline $\mathrm{H}$ & 0.70139500 & 4.58227100 & -0.15926400 & $\mathrm{H}$ & 1.65207900 & 6.06271100 & -0.28857700 \\
\hline C & 1.47900900 & 5.27038300 & -2.83437600 & $\mathrm{H}$ & 1.63409000 & 6.35230800 & -2.75700500 \\
\hline $\mathrm{H}$ & 0.40650900 & 5.07746700 & -2.76724500 & $\mathrm{H}$ & 1.82270900 & 4.94816000 & -3.82470200 \\
\hline C & 3.73226900 & 4.98178900 & -1.78732600 & $\mathrm{H}$ & 3.81040300 & 6.05549400 & -1.58568200 \\
\hline $\mathrm{H}$ & 4.16136000 & 4.80433800 & -2.78043500 & $\mathrm{H}$ & 4.34416400 & 4.46375700 & -1.04042500 \\
\hline C & 2.71362900 & 2.11839000 & -0.83741600 & 0 & 2.01519500 & 1.83060200 & 0.14237000 \\
\hline C & 4.95933100 & 1.85356600 & -2.02906900 & C & 4.50670200 & 0.68928900 & 0.02538600 \\
\hline C & 6.26015500 & 1.91013900 & -1.22255700 & $\mathrm{H}$ & 4.95928800 & 1.02005400 & -2.74095600 \\
\hline $\mathrm{H}$ & 4.75386100 & 2.76748700 & -2.57774600 & $\mathrm{H}$ & 4.14233600 & 1.01155800 & 1.00192200 \\
\hline $\mathrm{H}$ & 6.37502600 & 2.91185700 & -0.79378400 & $\mathrm{H}$ & 7.14069600 & 1.70333400 & -1.83704200 \\
\hline $\mathrm{N}$ & 3.96339700 & 1.61546300 & -0.98044300 & C & 4.08980000 & -0.75204400 & -0.23882700 \\
\hline C & 3.55303100 & -1.11454600 & -1.43799700 & C & 4.34390600 & -1.76230400 & 0.76736400 \\
\hline C & 3.25177800 & -2.47433700 & -1.77036600 & $\mathrm{H}$ & 3.34161600 & -0.36616100 & -2.19014600 \\
\hline C & 4.03876200 & -3.12866500 & 0.46688600 & C & 4.84726700 & -1.45538500 & 2.04433900 \\
\hline C & 2.70482000 & -2.82591000 & -3.01430200 & C & 3.51291800 & -3.48773400 & -0.80700800 \\
\hline C & 4.24781100 & -4.14245000 & 1.45197000 & $\mathrm{H}$ & 5.07110600 & -0.43054900 & 2.31191700 \\
\hline C & 5.01894000 & -2.44954500 & 3.00943800 & $\mathrm{C}$ & 2.42438200 & -4.15944200 & -3.30616700 \\
\hline $\mathrm{H}$ & 2.49007400 & -2.04851200 & -3.73993500 & C & 3.22370500 & -4.84902700 & -1.11504100 \\
\hline $\mathrm{C}$ & 4.72831400 & -3.77949100 & 2.71960500 & $C$ & 3.94746000 & -5.50655400 & 1.11578300 \\
\hline $\mathrm{H}$ & 5.38721800 & -2.17441300 & 3.99329700 & $\mathrm{C}$ & 2.68454100 & -5.16125100 & -2.37330800 \\
\hline $\mathrm{H}$ & 1.99060100 & -4.41457400 & -4.26821400 & C & 3.46824800 & -5.84571200 & -0.11259600 \\
\hline $\mathrm{H}$ & 4.87575300 & -4.55024100 & 3.47167200 & $\mathrm{H}$ & 4.12039200 & -6.26995000 & 1.87016400 \\
\hline $\mathrm{H}$ & 2.46145500 & -6.19896300 & -2.60868800 & $\mathrm{H}$ & 3.25099900 & -6.88377000 & -0.35178100 \\
\hline $\mathrm{N}$ & 0.72568300 & 2.63214300 & -2.11428200 & $\mathrm{H}$ & 0.09120100 & 2.89225500 & -1.34756600 \\
\hline $\mathrm{S}$ & -2.44096300 & 3.70136700 & -0.25737400 & 0 & -3.49483200 & 3.40709900 & 0.72352500 \\
\hline $\mathrm{O}$ & -1.11090600 & 3.07767800 & 0.03776600 & 0 & -2.81880800 & 3.53901200 & -1.68927100 \\
\hline C & -2.12113100 & 5.52405000 & 0.08062500 & $\mathrm{~F}$ & -3.25998200 & 6.21324500 & -0.17115300 \\
\hline $\mathrm{F}$ & -1.28396200 & 5.95064800 & -1.04205600 & $\mathrm{~F}$ & -1.55430400 & 5.77456800 & 1.11040900 \\
\hline $\mathrm{C}$ & 2.29324100 & 0.73509900 & 3.28879700 & 0 & 3.37208300 & 0.81043800 & 3.84500700 \\
\hline $\mathrm{O}$ & 1.33580500 & 1.65099600 & 3.21063200 & C & 1.56579000 & 3.05498100 & 3.64173000 \\
\hline $\mathrm{C}$ & 2.85656500 & 3.58479300 & 3.01768700 & $\mathrm{H}$ & 2.87286200 & 4.67580200 & 3.10682400 \\
\hline $\mathrm{H}$ & 2.88074000 & 3.31937100 & 1.95916400 & $\mathrm{H}$ & 3.74020900 & 3.17611900 & 3.50886400 \\
\hline C & 1.59981000 & 3.08295200 & 5.16725900 & $\mathrm{H}$ & 0.67213300 & 2.67308900 & 5.58049700 \\
\hline $\mathrm{H}$ & 1.69609300 & 4.11862400 & 5.51018200 & $\mathrm{H}$ & 2.44743500 & 2.50900900 & 5.54884300 \\
\hline $\mathrm{C}$ & 0.33971000 & 3.76462500 & 3.07574600 & $\mathrm{H}$ & 0.40206000 & 4.83819200 & 3.27838600 \\
\hline $\mathrm{H}$ & -0.57640000 & 3.38330900 & 3.53876900 & $\mathrm{H}$ & 0.26306500 & 3.60885500 & 1.99843500 \\
\hline $\mathrm{C}$ & 0.80896600 & -0.54661400 & 1.80427900 & $\mathrm{H}$ & 0.52935300 & 0.38828500 & 1.33064400 \\
\hline $\mathrm{C}$ & 0.66535100 & -1.78426100 & 1.02523500 & C & 0.23087200 & -1.70553800 & -0.30378200 \\
\hline $\mathrm{C}$ & 0.86133000 & -3.04654600 & 1.61306800 & $\mathrm{C}$ & -0.00710600 & -2.86861200 & -1.03471700 \\
\hline $\mathrm{H}$ & 0.10060800 & -0.73044900 & -0.76070000 & C & 0.58197600 & -4.20291800 & 0.89532400 \\
\hline $\mathrm{H}$ & 1.19982100 & -3.12329200 & 2.64291600 & C & 0.14008300 & -4.11386900 & -0.42788500 \\
\hline $\mathrm{H}$ & -0.28951800 & -2.80385500 & -2.07682700 & $\mathrm{H}$ & 0.72860900 & -5.17252900 & 1.35862300 \\
\hline $\mathrm{H}$ & -0.07630300 & -5.01360900 & -0.99251800 & $\mathrm{~N}$ & 1.89059400 & -0.43715900 & 2.63943100 \\
\hline $\mathrm{H}$ & 2.54419000 & -1.20886800 & 2.72858000 & C & -0.77757400 & -0.67791100 & 3.13615400 \\
\hline $\mathrm{H}$ & -0.48424500 & 0.15121100 & 3.77034200 & $\mathrm{H}$ & -0.52760900 & -1.67105300 & 3.49236500 \\
\hline $\mathrm{C}$ & -1.89343000 & -0.51351600 & 2.31601100 & C & -2.54445000 & -1.64075300 & 1.65611500 \\
\hline $\mathrm{H}$ & -1.84784300 & -2.44492400 & 1.39906400 & $\mathrm{H}$ & -3.10907200 & -1.33533700 & 0.77356000 \\
\hline C & -2.39270300 & 0.85409500 & 2.02097100 & $\mathrm{H}$ & -3.45017400 & 0.86525100 & 1.75418000 \\
\hline $\mathrm{H}$ & -1.85210900 & 1.24794600 & 1.14618100 & $\mathrm{H}$ & -2.20450600 & 1.56235900 & 2.83052700 \\
\hline C & -5.05226400 & -3.44064800 & 1.89479000 & $\mathrm{H}$ & -5.79757100 & -2.75878600 & 1.47455200 \\
\hline
\end{tabular}




\begin{tabular}{|lrrr|rrrr|}
\hline $\mathrm{H}$ & -5.58005000 & -4.15677100 & 2.53610800 & $\mathrm{H}$ & -4.58823100 & -3.99602800 & 1.07407700 \\
\hline $\mathrm{C}$ & -4.58755900 & -1.22577100 & 4.01949500 & $\mathrm{H}$ & -5.18361000 & -0.51359800 & 3.44140600 \\
\hline $\mathrm{H}$ & -3.84728500 & -0.66166900 & 4.59797000 & $\mathrm{H}$ & -5.25789300 & -1.72146100 & 4.73187200 \\
\hline $\mathrm{C}$ & -2.72115700 & -3.69567300 & 3.91652300 & $\mathrm{H}$ & -2.00972300 & -3.16798000 & 4.56141300 \\
\hline $\mathrm{H}$ & -2.15194900 & -4.37104300 & 3.26713900 & $\mathrm{H}$ & -3.35648300 & -4.31013100 & 4.56532900 \\
\hline $\mathrm{Si}$ & -3.77160900 & -2.50024400 & 2.90184900 & $\mathrm{C}$ & 6.05372000 & 0.86512500 & -0.10413100 \\
\hline $\mathrm{H}$ & 6.51071000 & -0.09035200 & -0.37313700 & $\mathrm{H}$ & 6.49823400 & 1.18605600 & 0.84158600 \\
\hline $\mathrm{C}$ & 0.42351100 & 1.52060900 & -2.78224200 & $\mathrm{C}$ & -0.73562500 & 0.71955900 & -2.89820100 \\
\hline $\mathrm{C}$ & 1.22056600 & 0.58360900 & -3.61251900 & $\mathrm{C}$ & -0.00820700 & -0.32547900 & -3.67189100 \\
\hline $\mathrm{O}$ & 2.37265000 & 0.54655300 & -4.01765000 & $\mathrm{O}$ & -0.23156300 & -1.45394700 & -4.08319800 \\
\hline $\mathrm{N}$ & -1.98224900 & 0.93824500 & -2.41388200 & $\mathrm{H}$ & -2.20593000 & 1.90606900 & -2.14153500 \\
\hline $\mathrm{C}$ & -2.95101300 & -0.00912000 & -2.08633800 & $\mathrm{C}$ & -4.08415100 & 0.42413900 & -1.37887200 \\
\hline $\mathrm{C}$ & -2.79605700 & -1.37331600 & -2.37910300 & $\mathrm{C}$ & -5.03323300 & -0.50780400 & -0.96359300 \\
\hline $\mathrm{H}$ & -4.20598000 & 1.47469800 & -1.13733200 & $\mathrm{C}$ & -3.74791100 & -2.28141900 & -1.93124900 \\
\hline $\mathrm{H}$ & -1.95267600 & -1.71122500 & -2.96905900 & $\mathrm{C}$ & -4.88330100 & -1.86622100 & -1.23478300 \\
\hline $\mathrm{H}$ & -5.62054700 & -2.58386500 & -0.90257600 & $\mathrm{C}$ & -3.50096100 & -3.75537400 & -2.08368700 \\
\hline $\mathrm{C}$ & -6.16821100 & -0.03913600 & -0.09871700 & $\mathrm{~F}$ & -2.56371700 & -4.03890600 & -3.00738200 \\
\hline $\mathrm{F}$ & -3.05986500 & -4.28880100 & -0.90516200 & $\mathrm{~F}$ & -4.62318200 & -4.43042500 & -2.40810600 \\
\hline $\mathrm{F}$ & -6.70828200 & 1.11108800 & -0.52825600 & $\mathrm{~F}$ & -5.73509100 & 0.18319300 & 1.17975500 \\
\hline $\mathrm{F}$ & -7.15581200 & -0.95739400 & -0.00638100 & & & & \\
\hline
\end{tabular}

\section{TS- $R-6 f-19$}

Imaginary frequency: $-384.94 \mathrm{~cm}^{-1}$

E[B3LYP/6-31G(d)-D3(BJ)]: -4655.609778 Hartree

$\mathrm{E}\left[\mathrm{PCM}\left(\mathrm{Et}_{2} \mathrm{O}\right)-\mathrm{B} 3 \mathrm{LYP} / 6-311 \mathrm{G}(\mathrm{d}, \mathrm{p})-\mathrm{D} 3(\mathrm{BJ})\right]$ : -4656.801409 Hartree

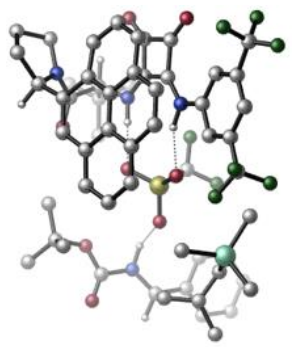

\begin{tabular}{|c|c|c|c|c|c|c|c|}
\hline 01 & & & & C & -1.57144400 & -3.96852600 & -1.53402800 \\
\hline $\mathrm{H}$ & -2.52971600 & -4.16388000 & -2.01480800 & C & -0.50223800 & -4.78228400 & -2.29808900 \\
\hline C & 0.93030600 & -4.57490400 & -1.78179400 & $\mathrm{H}$ & 1.02362700 & -4.85868100 & -0.73310400 \\
\hline $\mathrm{H}$ & 1.24406900 & -3.53278300 & -1.86974500 & $\mathrm{H}$ & 1.61987000 & -5.18334500 & -2.38006500 \\
\hline C & -0.57253300 & -4.36393100 & -3.77720100 & $\mathrm{H}$ & 0.10848900 & -4.97669000 & -4.37900100 \\
\hline $\mathrm{H}$ & -0.28204300 & -3.31497900 & -3.89524500 & $\mathrm{H}$ & -1.58583800 & -4.48140700 & -4.17964800 \\
\hline C & -0.88700200 & -6.26564200 & -2.15599800 & $\mathrm{H}$ & -0.18872900 & -6.89749800 & -2.71594800 \\
\hline $\mathrm{H}$ & -1.89489500 & -6.45705800 & -2.54519400 & $\mathrm{H}$ & -0.85537900 & -6.58363700 & -1.10706200 \\
\hline C & -1.68891200 & -4.20613700 & -0.02224800 & $\mathrm{O}$ & -0.71735600 & -4.08465400 & 0.72344400 \\
\hline C & -4.08438100 & -5.02205000 & -0.14679400 & C & -3.18664500 & -3.96769900 & 1.88462300 \\
\hline C & -4.79958600 & -5.61598900 & 1.07847700 & $\mathrm{H}$ & -4.72768900 & -4.35501000 & -0.72997800 \\
\hline $\mathrm{H}$ & -3.71726700 & -5.79471600 & -0.82653300 & $\mathrm{H}$ & -2.41340000 & -4.47356100 & 2.47018400 \\
\hline $\mathrm{H}$ & -4.33076900 & -6.57062300 & 1.34259300 & $\mathrm{H}$ & -5.85979900 & -5.80481000 & 0.88657500 \\
\hline $\mathrm{N}$ & -2.96107400 & -4.29864900 & 0.46288900 & C & -3.00577000 & -2.47153700 & 2.11090100 \\
\hline $\mathrm{C}$ & -1.88536400 & -2.06911700 & 2.78034200 & C & -3.91992400 & -1.46827800 & 1.58638000 \\
\hline C & -1.58226200 & -0.69408500 & 3.03995200 & $\mathrm{H}$ & -1.17132300 & -2.81755000 & 3.11015300 \\
\hline $\mathrm{C}$ & -3.68803600 & -0.09222100 & 1.91565900 & C & -5.01172600 & -1.75781200 & 0.74822900 \\
\hline $\mathrm{C}$ & -0.39918800 & -0.29626300 & 3.68101900 & C & -2.52216900 & 0.29602800 & 2.63794200 \\
\hline C & -4.60330800 & 0.91706400 & 1.48271000 & $\mathrm{H}$ & -5.18611700 & -2.76779400 & 0.41200500 \\
\hline $\mathrm{C}$ & -5.87314000 & -0.76092900 & 0.29433700 & C & -0.14328100 & 1.05078000 & 3.92803300 \\
\hline $\mathrm{H}$ & 0.31655500 & -1.05468200 & 3.98111700 & C & -2.27400300 & 1.67036200 & 2.93452300 \\
\hline C & -5.68937600 & 0.56349900 & 0.67121200 & C & -4.36832400 & 2.28263400 & 1.86195100 \\
\hline $\mathrm{H}$ & -6.67880700 & -1.02286400 & -0.38365800 & $\mathrm{C}$ & -1.07341500 & 2.02477200 & 3.56898700 \\
\hline $\mathrm{H}$ & 0.78449800 & 1.34361800 & 4.41150500 & C & -3.25761400 & 2.64440100 & 2.55600900 \\
\hline
\end{tabular}




\begin{tabular}{|c|c|c|c|c|c|c|c|}
\hline $\bar{H}$ & -6.35186300 & 1.34107500 & 0.30385200 & $\mathrm{H}$ & -5.08584900 & 3.02915200 & 1.53836100 \\
\hline $\mathrm{H}$ & -0.87386500 & 3.07396600 & 3.76736300 & $\mathrm{H}$ & -3.07294800 & 3.68696900 & 2.79710600 \\
\hline $\mathrm{N}$ & -1.37500400 & -2.50581800 & -1.60674300 & $\mathrm{H}$ & -0.59360100 & -2.15057000 & -1.05165200 \\
\hline S & 1.63166300 & -0.36887300 & -0.58133000 & $\mathrm{O}$ & 3.04642800 & -0.18518500 & -0.16114400 \\
\hline 0 & 1.02599100 & -1.65485900 & -0.19405300 & $\mathrm{O}$ & 0.77117900 & 0.81825400 & -0.36696000 \\
\hline C & 1.78124600 & -0.44330000 & -2.43282500 & $\mathrm{~F}$ & 2.35019600 & 0.68411200 & -2.89712600 \\
\hline $\mathrm{F}$ & 0.58050400 & -0.58885200 & -3.00348900 & $\mathrm{~F}$ & 2.56147100 & -1.47662500 & -2.79915500 \\
\hline C & 5.16994300 & -2.11406900 & 1.60708000 & $\mathrm{O}$ & 5.85344500 & -1.86897100 & 2.58375200 \\
\hline 0 & 4.11299700 & -2.90694200 & 1.49697800 & C & 3.37677900 & -3.39056300 & 2.69406400 \\
\hline C & 4.27307900 & -4.32878500 & 3.49889300 & $\mathrm{H}$ & 3.69115700 & -4.76600400 & 4.31729900 \\
\hline $\mathrm{H}$ & 5.13052600 & -3.79995400 & 3.91830400 & $\mathrm{H}$ & 4.63242500 & -5.14546400 & 2.86430500 \\
\hline $\mathrm{C}$ & 2.18984300 & -4.12168100 & 2.07839500 & $\mathrm{H}$ & 1.58826000 & -3.45647400 & 1.45688200 \\
\hline $\mathrm{H}$ & 1.55073800 & -4.52282600 & 2.87141500 & $\mathrm{H}$ & 2.53326100 & -4.95801800 & 1.46093000 \\
\hline C & 2.91657100 & -2.17244100 & 3.49351800 & $\mathrm{H}$ & 2.26956700 & -2.50388800 & 4.31241300 \\
\hline $\mathrm{H}$ & 2.34013200 & -1.50218800 & 2.84873900 & $\mathrm{H}$ & 3.76195400 & -1.62922600 & 3.92181700 \\
\hline $\mathrm{C}$ & 6.31635100 & -0.50767500 & 0.23088200 & $\mathrm{H}$ & 7.17223300 & -0.63759200 & 0.88530300 \\
\hline $\mathrm{C}$ & 6.62783100 & -0.03325700 & -1.13564700 & C & 7.91218900 & 0.46870100 & -1.39683800 \\
\hline C & 5.68185900 & -0.05746300 & -2.17107700 & C & 8.24351300 & 0.93892000 & -2.66602200 \\
\hline $\mathrm{H}$ & 8.65599300 & 0.47386400 & -0.60424500 & C & 6.01449500 & 0.41680700 & -3.43705100 \\
\hline $\mathrm{H}$ & 4.68402700 & -0.43621100 & -1.99645200 & C & 7.29274600 & 0.91794500 & -3.68859000 \\
\hline $\mathrm{H}$ & 9.24477100 & 1.31265800 & -2.85819300 & $\mathrm{H}$ & 5.26583600 & 0.39622700 & -4.22294500 \\
\hline $\mathrm{H}$ & 7.54971700 & 1.28498900 & -4.67769500 & $\mathrm{~N}$ & 5.41625400 & -1.54092000 & 0.34888400 \\
\hline $\mathrm{H}$ & 4.58733900 & -1.51578600 & -0.24156000 & C & 5.52037400 & 0.96538000 & 1.32825300 \\
\hline $\mathrm{H}$ & 6.10650700 & 0.84136000 & 2.23425300 & $\mathrm{H}$ & 4.51518200 & 0.56235900 & 1.36375200 \\
\hline C & 5.68718500 & 2.13998400 & 0.57784700 & C & 4.70082600 & 2.54923800 & -0.39075600 \\
\hline $\mathrm{H}$ & 4.11321500 & 1.71074300 & -0.77314800 & $\mathrm{H}$ & 5.11106100 & 3.16244800 & -1.19873900 \\
\hline C & 6.93277700 & 2.95463100 & 0.71190200 & $\mathrm{H}$ & 6.68670800 & 4.02045700 & 0.77618600 \\
\hline $\mathrm{H}$ & 7.53634600 & 2.82702700 & -0.19760500 & $\mathrm{H}$ & 7.53410600 & 2.66797900 & 1.57780800 \\
\hline C & 2.10674700 & 3.88052500 & -1.04192700 & $\mathrm{H}$ & 2.65798000 & 4.25293000 & -1.91354700 \\
\hline $\mathrm{H}$ & 1.32439800 & 4.60674200 & -0.80806200 & $\mathrm{H}$ & 1.62983700 & 2.93365100 & -1.31001000 \\
\hline C & 4.01577100 & 5.27589900 & 0.97376000 & $\mathrm{H}$ & 4.55012300 & 5.78333500 & 0.16203200 \\
\hline $\mathrm{H}$ & 4.70766000 & 5.14973400 & 1.81467600 & $\mathrm{H}$ & 3.21591300 & 5.94761800 & 1.30835600 \\
\hline C & 2.55458700 & 2.69977700 & 1.87342800 & $\mathrm{H}$ & 3.32489800 & 2.48430500 & 2.62248900 \\
\hline $\mathrm{H}$ & 2.10175400 & 1.75944600 & 1.55140500 & $\mathrm{H}$ & 1.77249400 & 3.29149800 & 2.35620100 \\
\hline $\mathrm{Si}$ & 3.26559500 & 3.63827500 & 0.41465100 & $\mathrm{C}$ & -4.56733000 & -4.58760800 & 2.20393200 \\
\hline $\mathrm{H}$ & -5.34982200 & -3.82753300 & 2.21237900 & $\mathrm{H}$ & -4.56182200 & -5.05267800 & 3.19340800 \\
\hline C & -4.07268200 & -0.53254200 & -2.39259600 & C & -3.74236800 & -2.01867500 & -2.47283600 \\
\hline C & -2.72695000 & -0.33711100 & -1.76348900 & C & -2.41731700 & -1.71105500 & -1.87944500 \\
\hline 0 & -4.31691900 & -3.03672700 & -2.82319400 & $\mathrm{O}$ & -5.02692500 & 0.15506000 & -2.70700500 \\
\hline $\mathrm{N}$ & -2.01832600 & 0.69086100 & -1.22799700 & $\mathrm{H}$ & -1.08872300 & 0.48594300 & -0.86106200 \\
\hline C & -2.43017900 & 2.01023700 & -1.00834700 & C & -1.56948800 & 2.85043600 & -0.28038600 \\
\hline C & -3.66280600 & 2.50615300 & -1.45040900 & C & -1.95340800 & 4.15608700 & -0.00002000 \\
\hline $\mathrm{H}$ & -0.63237600 & 2.46370800 & 0.09572200 & C & -4.02296200 & 3.81710300 & -1.14597500 \\
\hline $\mathrm{H}$ & -4.34868600 & 1.87044700 & -2.00148800 & C & -3.18246600 & 4.66037200 & -0.42640300 \\
\hline $\mathrm{H}$ & -3.48670000 & 5.67011700 & -0.18076200 & C & -5.40985000 & 4.27548600 & -1.49836800 \\
\hline C & -1.06682100 & 5.04901100 & 0.81365000 & $\mathrm{~F}$ & -6.31045600 & 3.86584100 & -0.55927200 \\
\hline $\mathrm{F}$ & -5.83078400 & 3.78486800 & -2.67763800 & $\mathrm{~F}$ & -5.50289700 & 5.62278100 & -1.55543200 \\
\hline $\mathrm{F}$ & -0.09257500 & 4.36566800 & 1.46429700 & $\mathrm{~F}$ & -1.76334300 & 5.72962300 & 1.75462800 \\
\hline$F$ & -0.43748400 & 5.98480600 & 0.05566500 & & & & \\
\hline
\end{tabular}




\section{Starting Materials}

\section{NMR data}

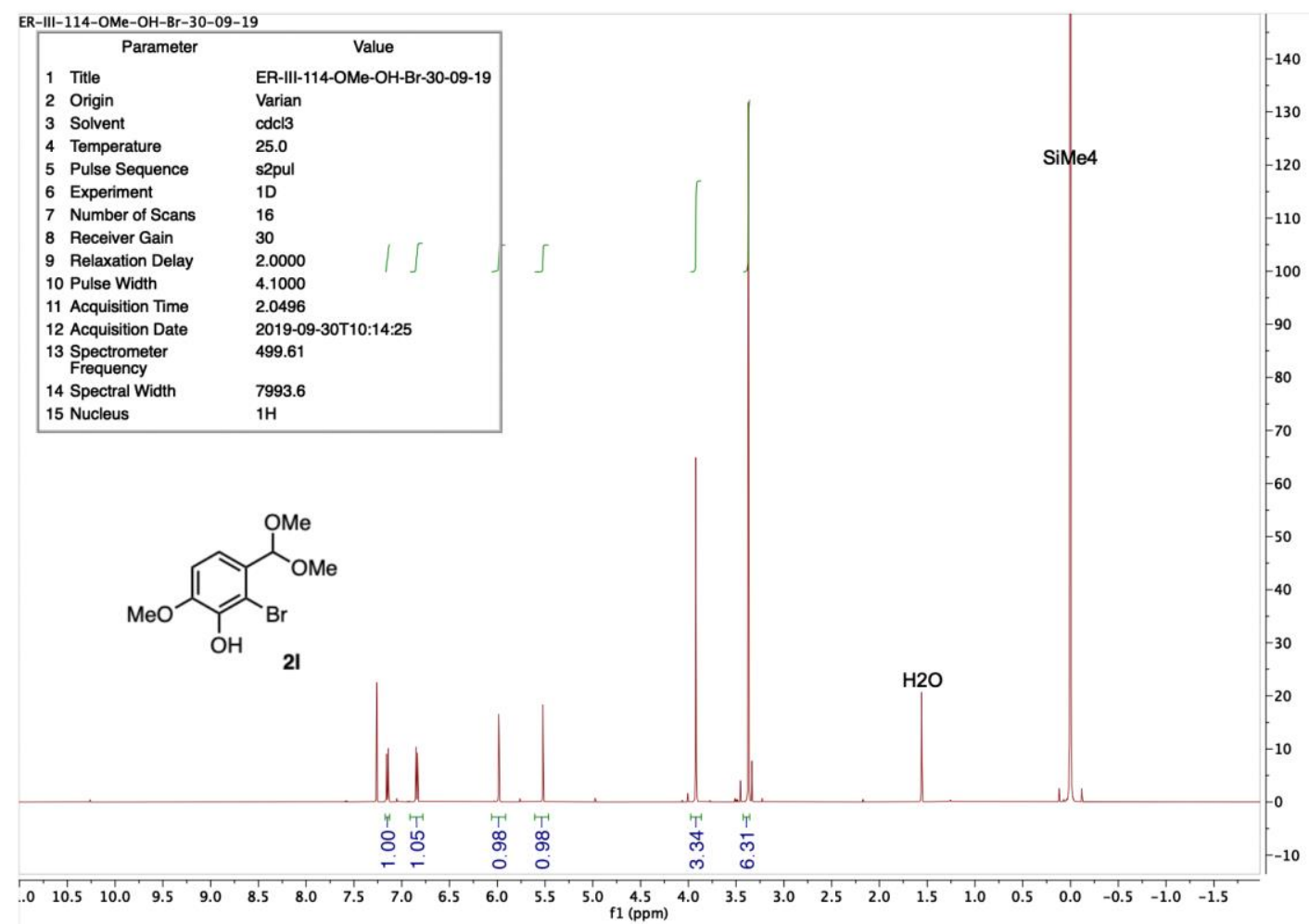

SMP-V-091-13C
\begin{tabular}{|lll|}
\hline \multicolumn{1}{|c}{ Parameter } & \multicolumn{1}{c}{ Value } \\
1 & Title & SMP-V-091-13C \\
2 & Origin & Varian \\
3 & Solvent & cdcl3 \\
4 & Temperature & 25.0 \\
5 & Pulse Sequence & s2pul \\
6 & Experiment & $1 \mathrm{D}$ \\
7 & Number of Scans & 801 \\
8 & Receiver Gain & 60 \\
9 & Relaxation Delay & 0.3000 \\
10 & Pulse Width & 8.4000 \\
11 & Acquisition Time & 1.0428 \\
12 & Acquisition Date & $2018-02-01 \mathrm{~T} 10: 37: 15$ \\
13 & Spectrometer & 125.71 \\
Frequency & \\
14 & Spectral Width & 31421.8 \\
15 & Nucleus & $13 \mathrm{C}$ \\
\hline
\end{tabular}
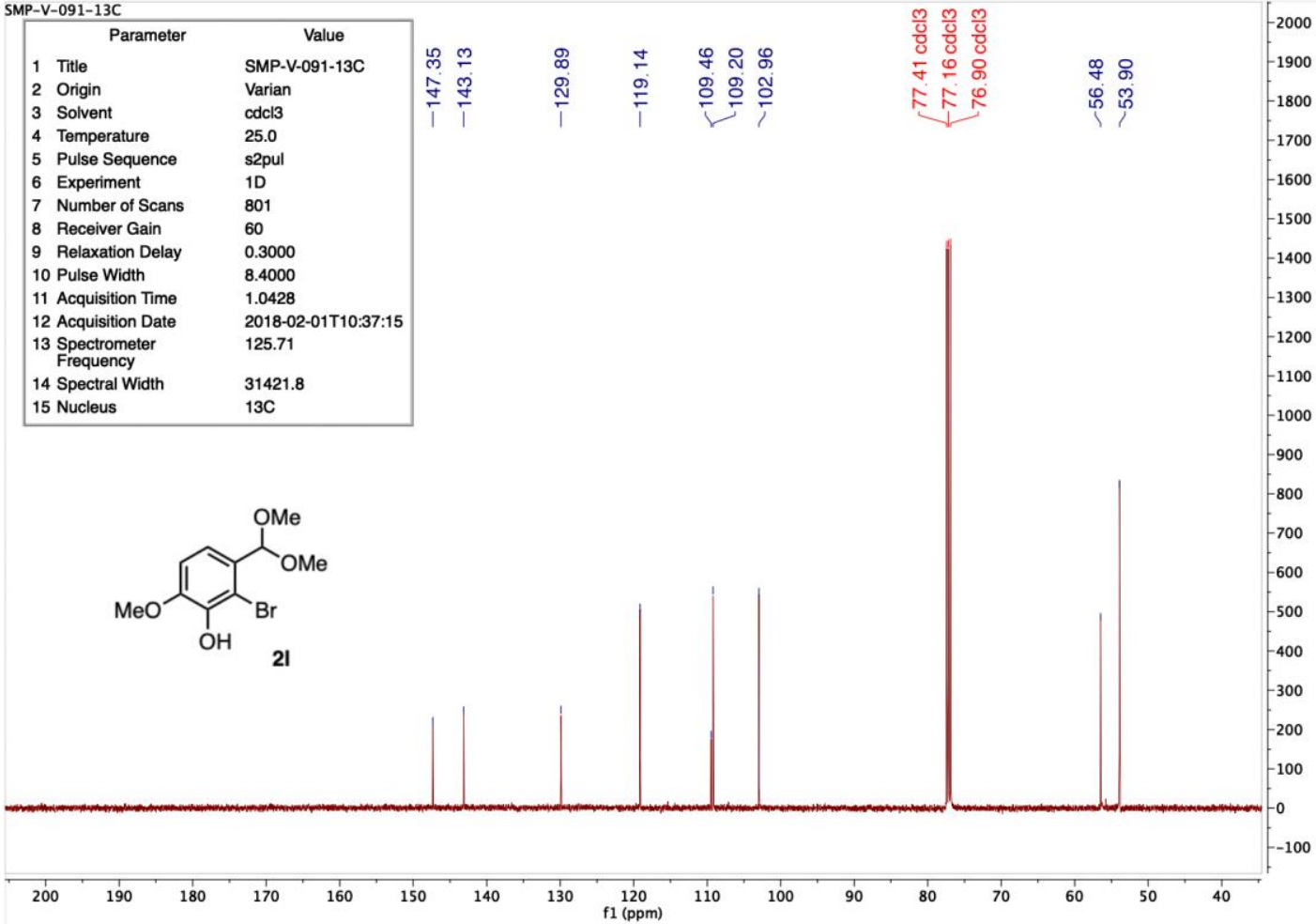


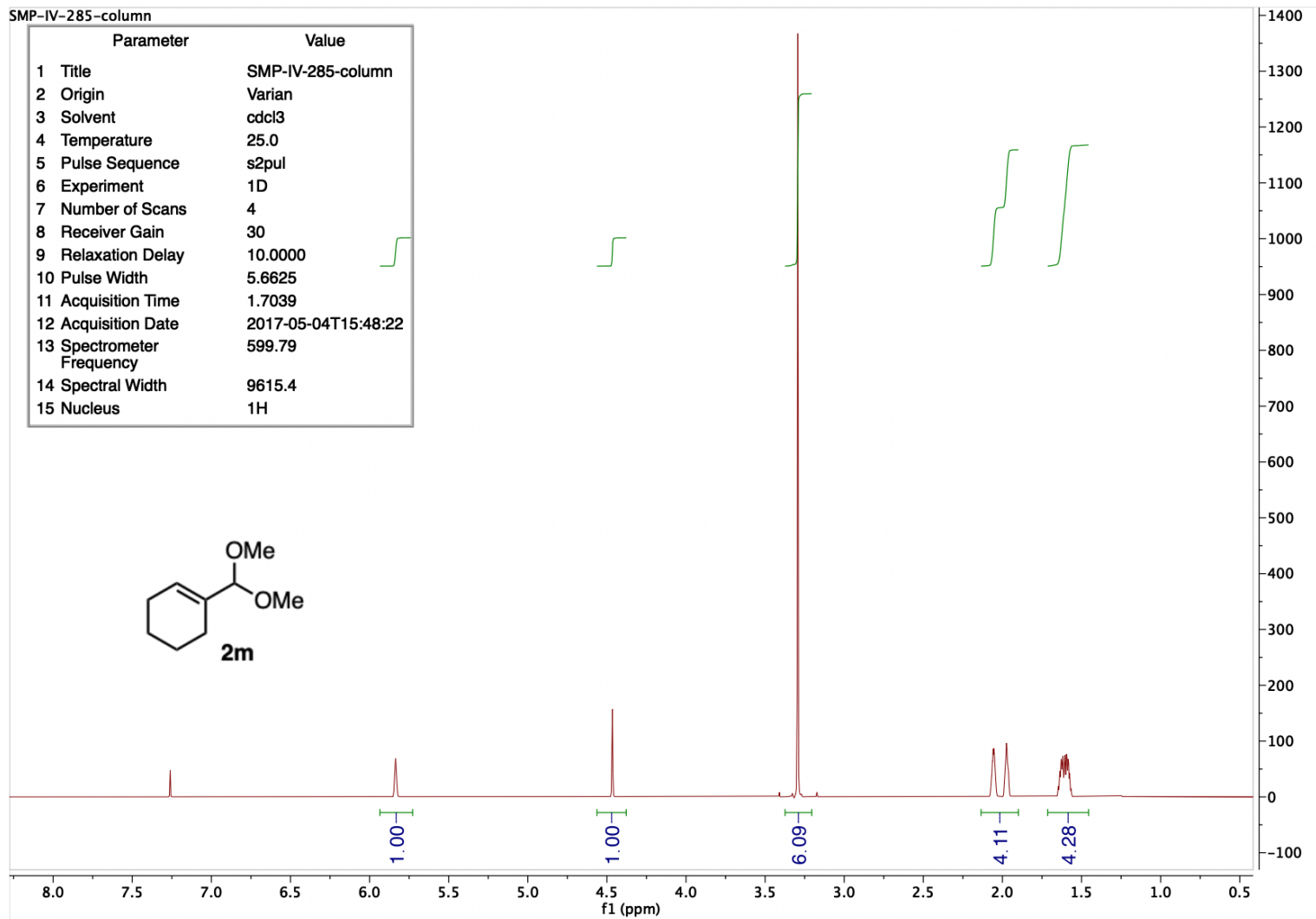

SMP-V-180-13C

\begin{tabular}{|lll|}
\hline \multicolumn{1}{|c|}{ Parameter } & \multicolumn{1}{c|}{ Value } \\
1 & Title & SMP-V-180-13C \\
2 & Origin & Varian \\
3 & Solvent & cdcl3 \\
4 & Temperature & 25.0 \\
5 & Pulse Sequence & s2pul \\
6 & Experiment & $1 \mathrm{D}$ \\
7 & Number of Scans & 385 \\
8 & Receiver Gain & 60 \\
9 & Relaxation Delay & 0.3000 \\
10 & Pulse Width & 8.4000 \\
11 & Acquisition Time & 1.0428 \\
12 Acquisition Date & $2018-02-04 T 15: 58: 13$ \\
13 Spectrometer & 125.71 \\
Frequency & \\
14 Spectral Width & 31421.8 \\
15 Nucleus & $13 \mathrm{C}$ \\
\hline
\end{tabular}<smiles>COC(OC)C1=CCCCC1</smiles>

$\begin{array}{lllllllllllllllllllllllllllllllll}230 & 220 & 210 & 200 & 190 & 180 & 170 & 160 & 150 & 140 & 130 & 120 & 110 & 100 & 90 & 80 & 70 & 60 & 50 & 40 & 30 & 20 & 10 & 0 & -10\end{array}$ 

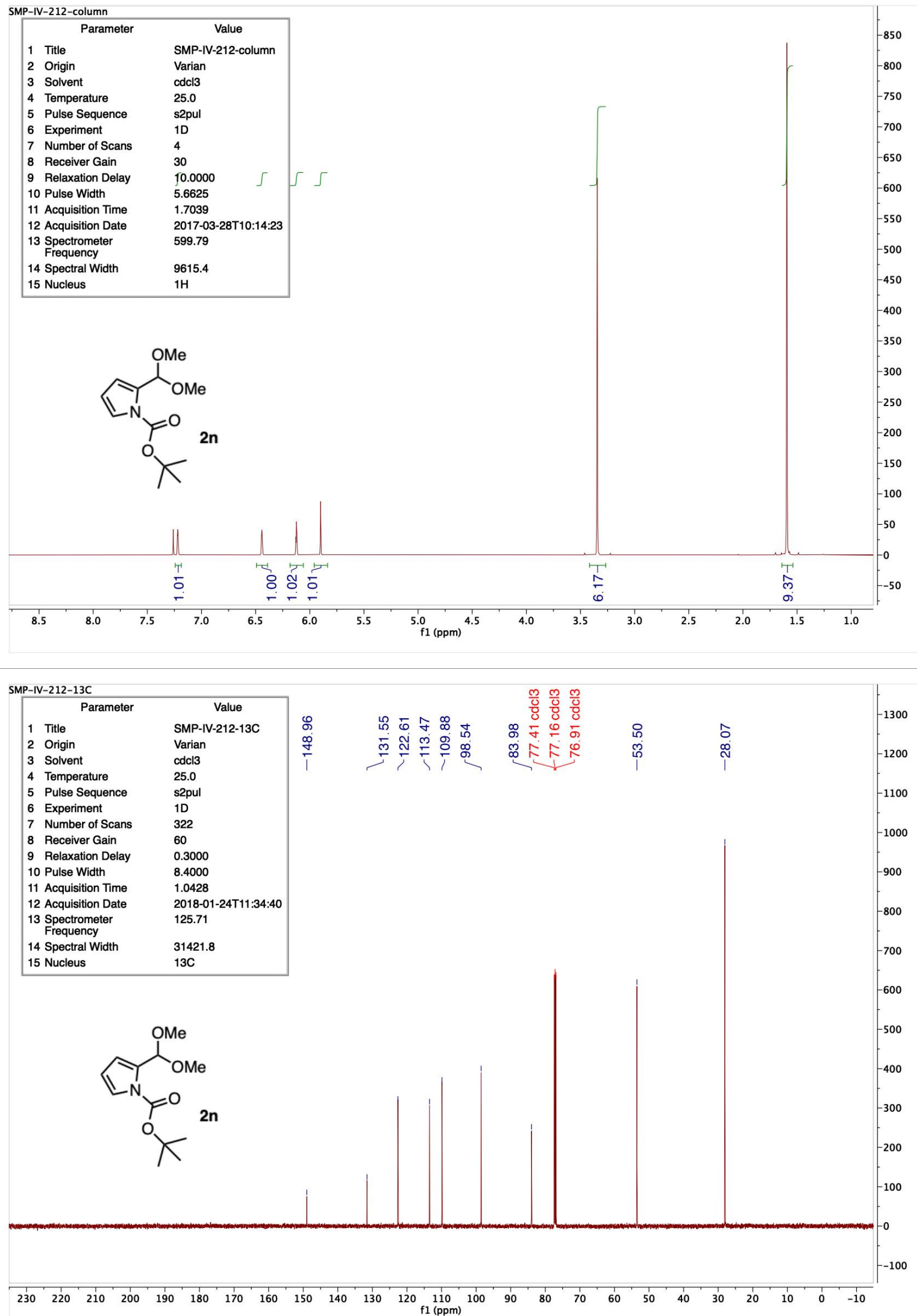

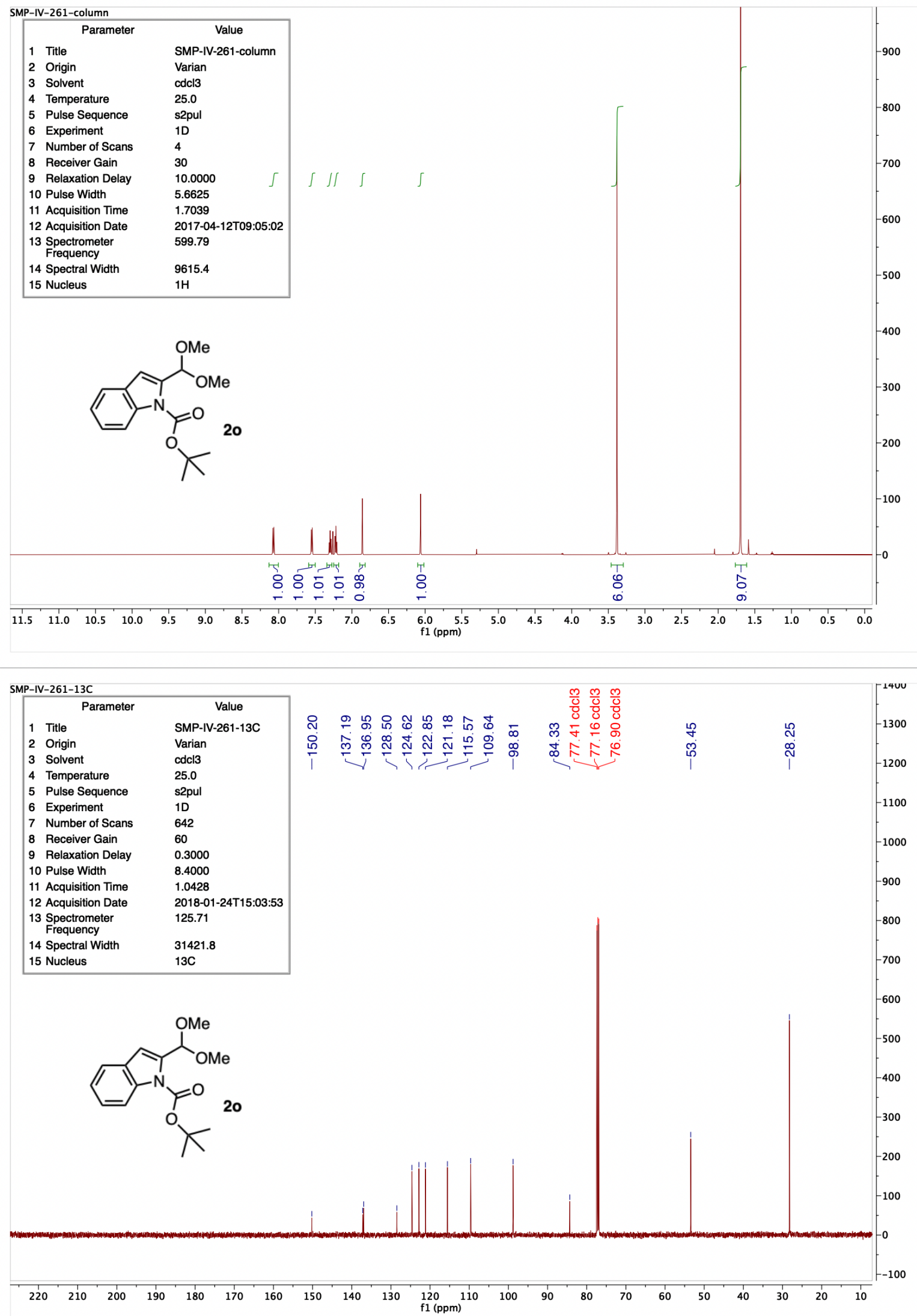

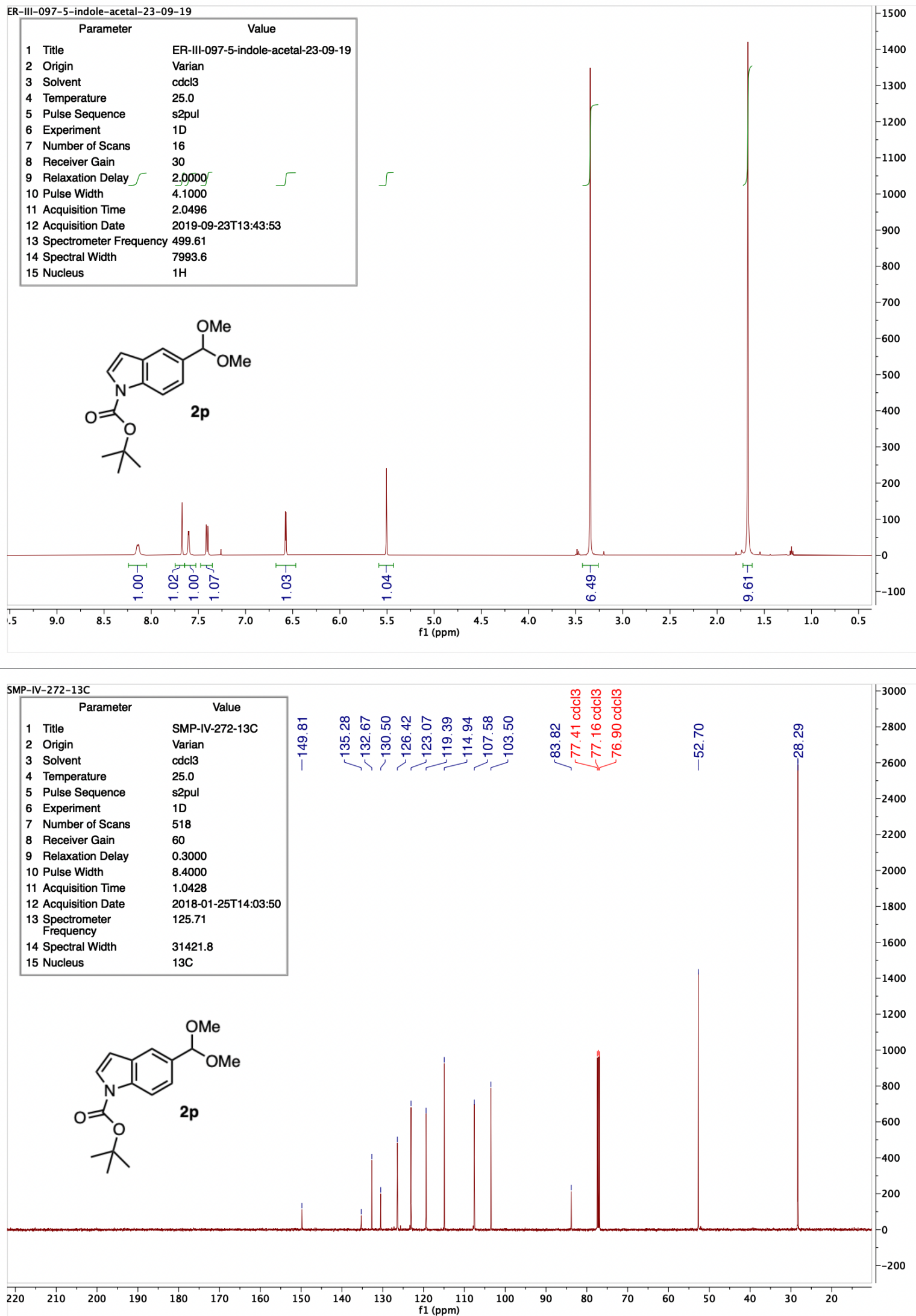

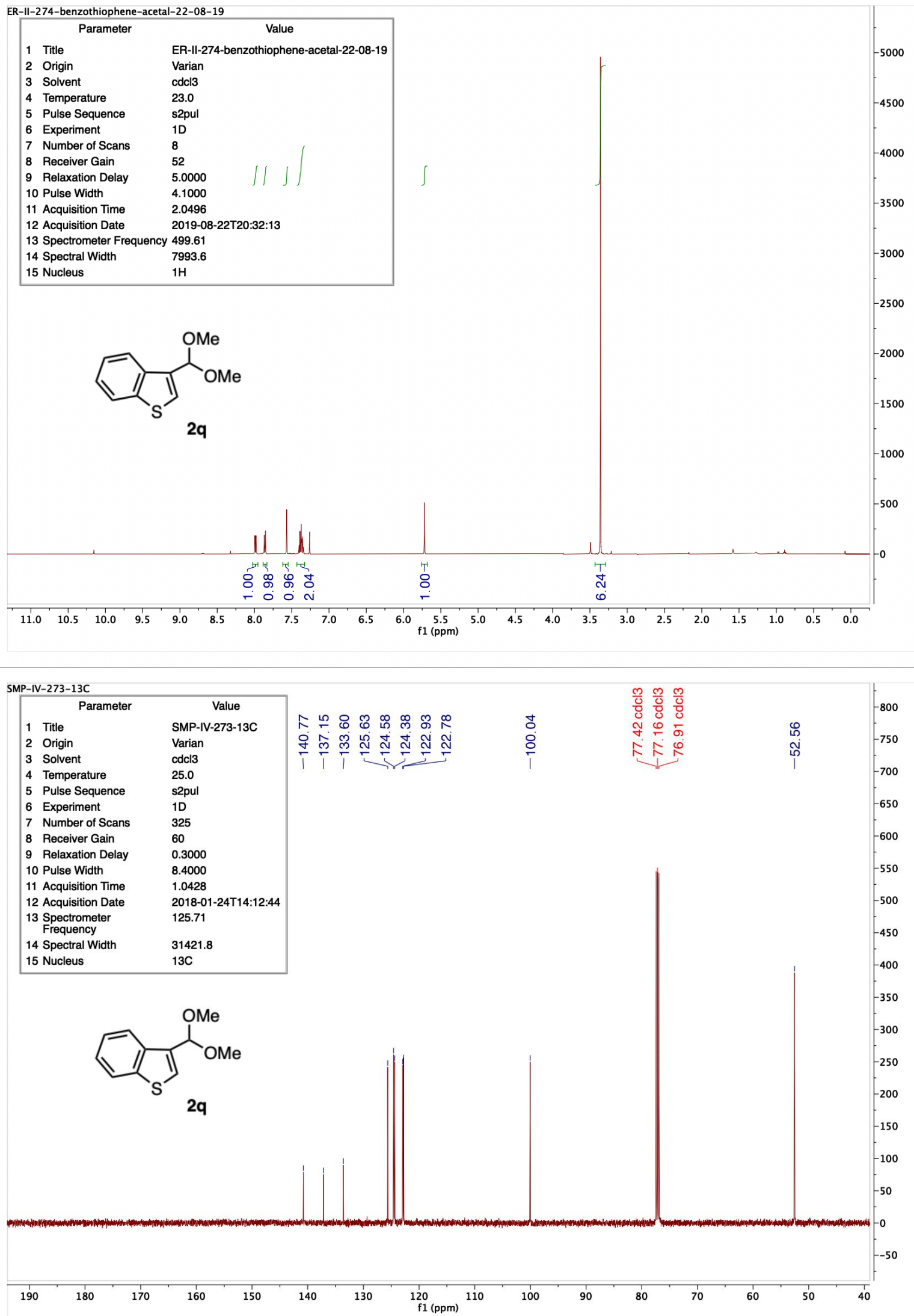


\section{Products}
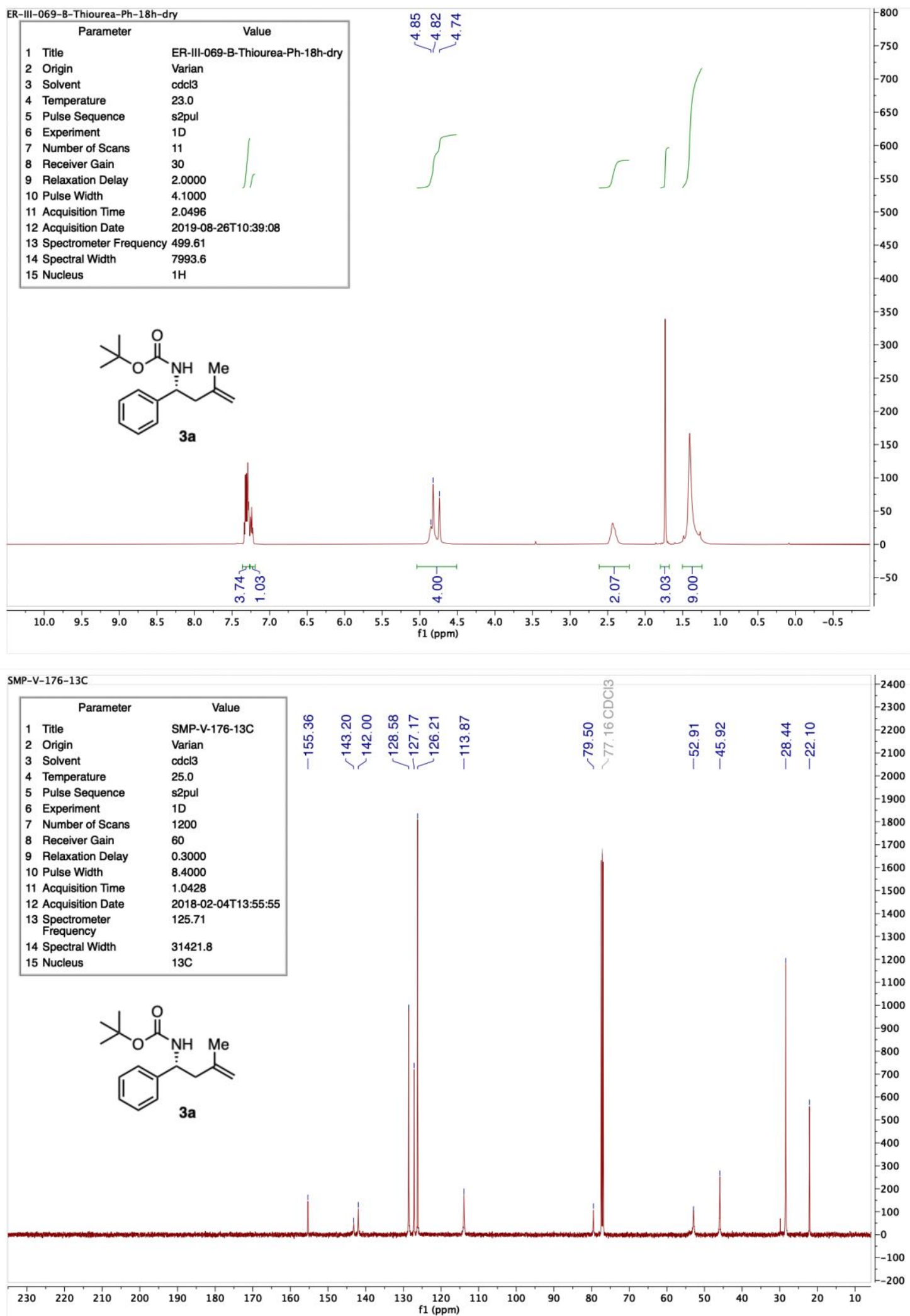

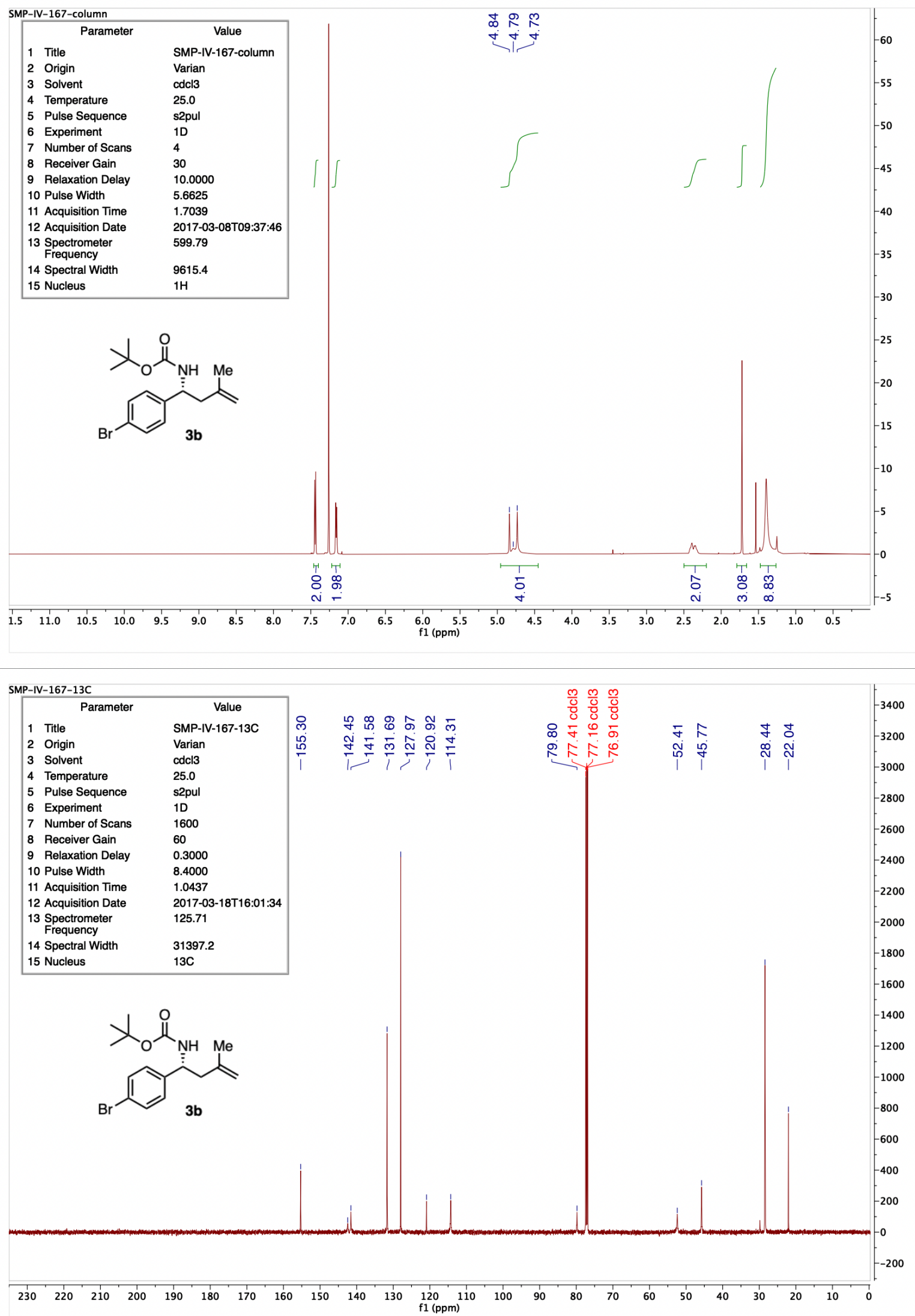


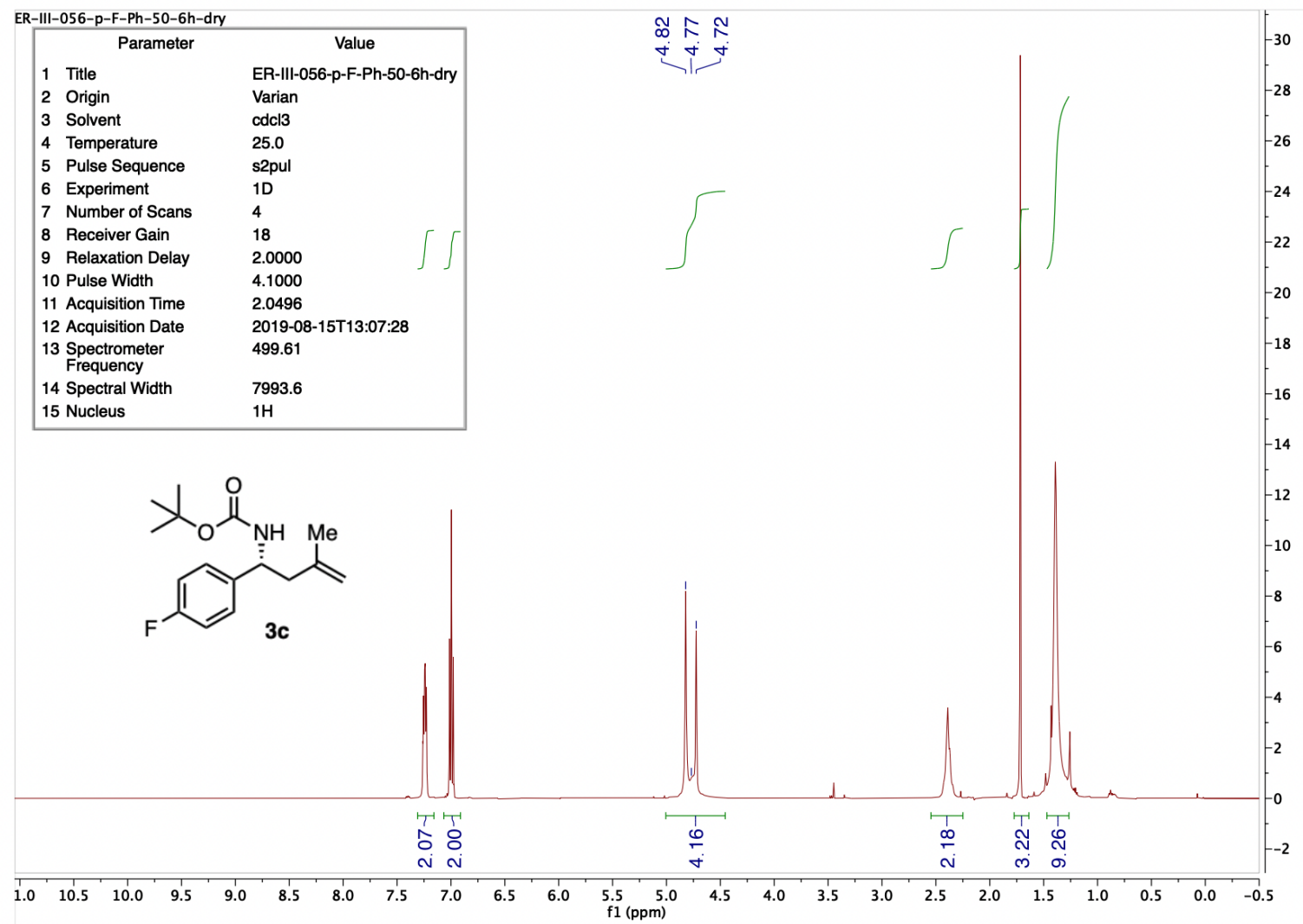

SMP-V-074-13C

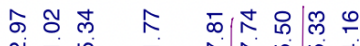

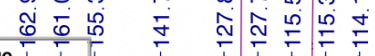

\begin{tabular}{|lll|}
\hline \multicolumn{1}{|c}{ Parameter } & \multicolumn{2}{c}{ Value } \\
1 & Title & SMP-V-074-13C \\
2 & Origin & Varian \\
3 & Solvent & cdcl3 \\
4 & Temperature & 25.0 \\
5 & Pulse Sequence & s2pul \\
6 & Experiment & $1 \mathrm{D}$ \\
7 & Number of Scans & 1600 \\
8 & Receiver Gain & 60 \\
9 & Relaxation Delay & 0.3000 \\
10 & Pulse Width & 8.4000 \\
11 Acquisition Time & 1.0428 \\
12 Acquisition Date & $2018-01-24 T 17: 06: 55$ \\
13 Spectrometer & 125.71 \\
$\quad$ Frequency & \\
14 Spectral Width & 31421.8 \\
15 Nucleus & $13 \mathrm{C}$ \\
\hline
\end{tabular}

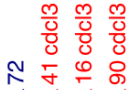

소슈요

एल

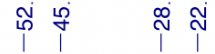

$>_{\mathrm{O}} \stackrel{\mathrm{O}}{\mathrm{NH}} \mathrm{Me}$
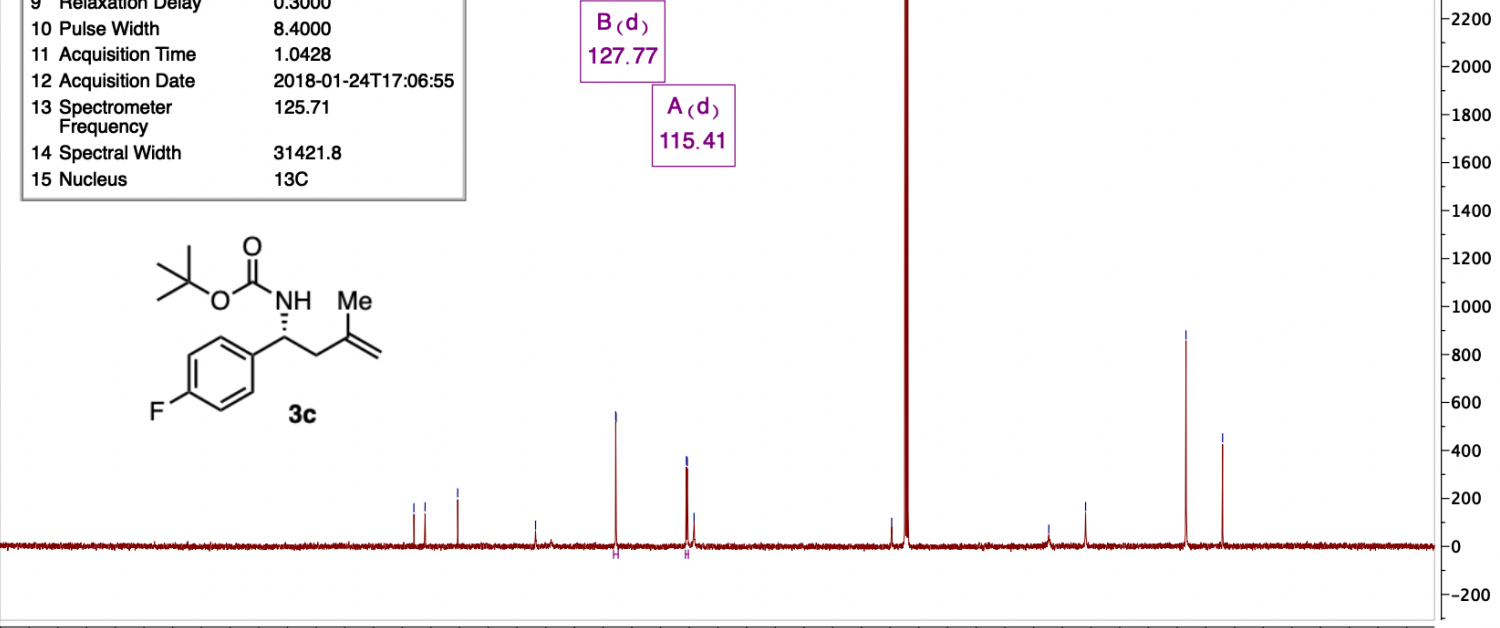

$\begin{array}{llllllllllllllllllllllllllllll}230 & 220 & 210 & 200 & 190 & 180 & 170 & 160 & 150 & 140 & 130 & 120 & 110 & 100 & 90 & 80 & 70 & 60 & 50 & 40 & 30 & 20 & 10 & 0 & -10\end{array}$ 

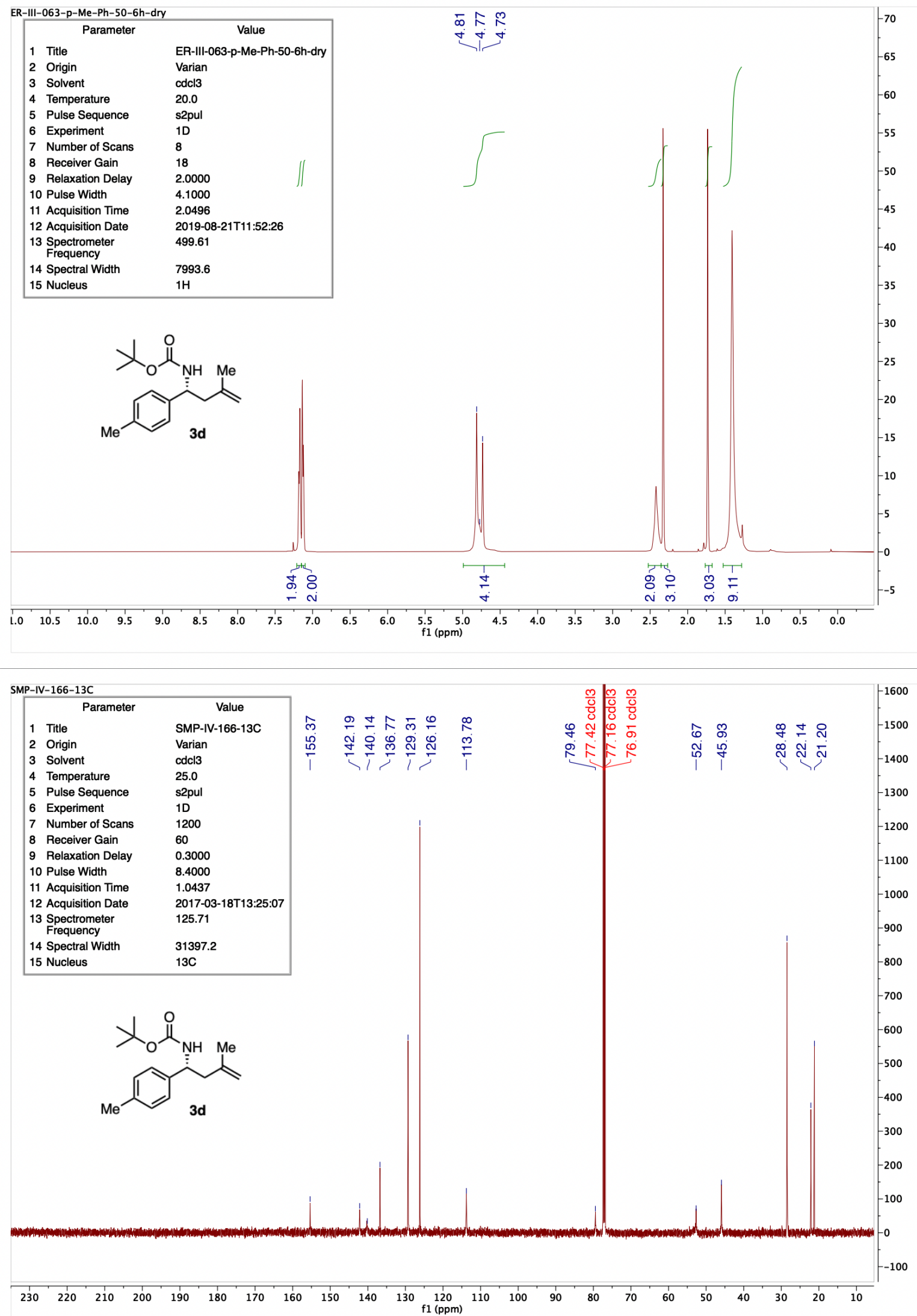


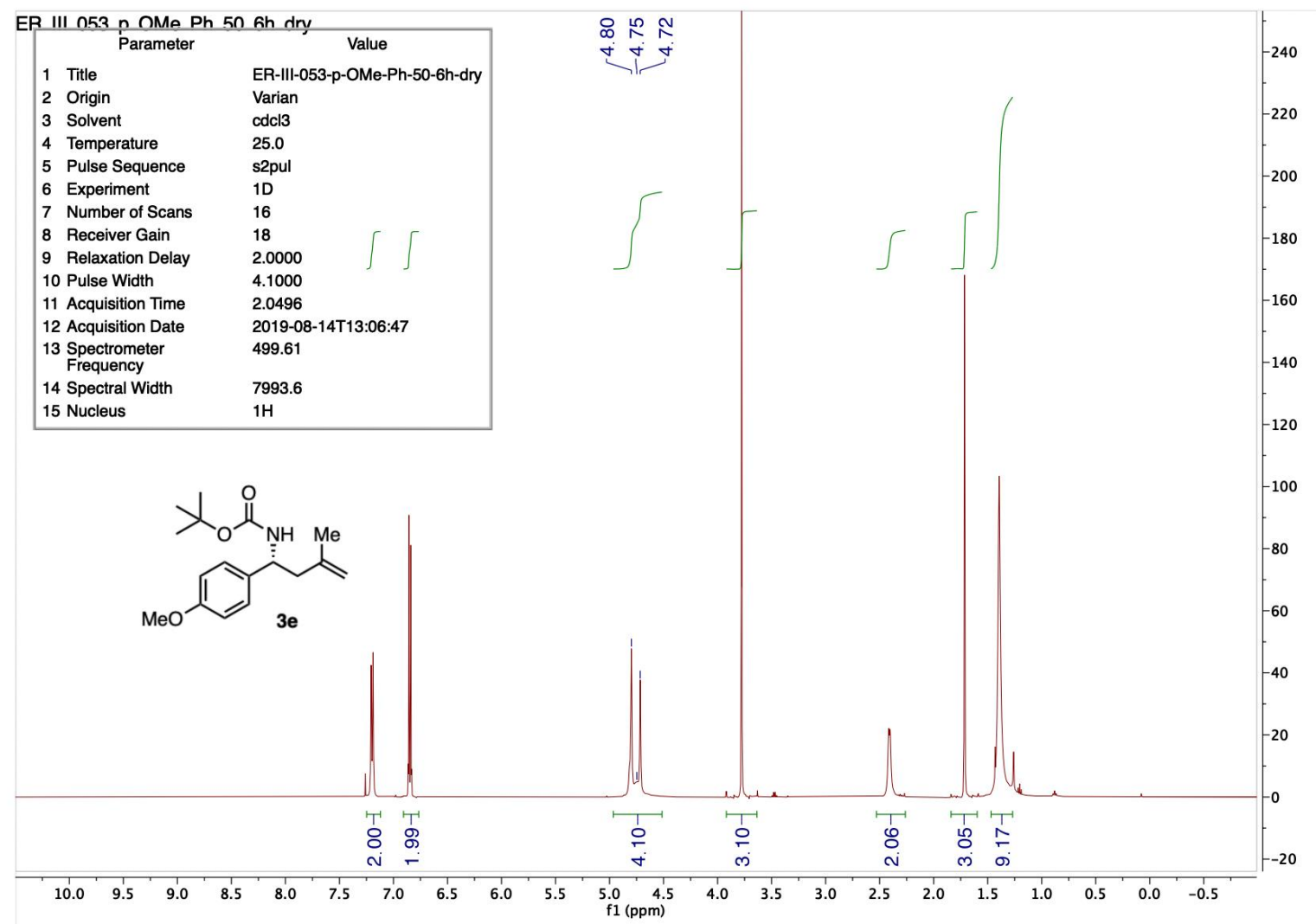

SMP-IV-185-13C

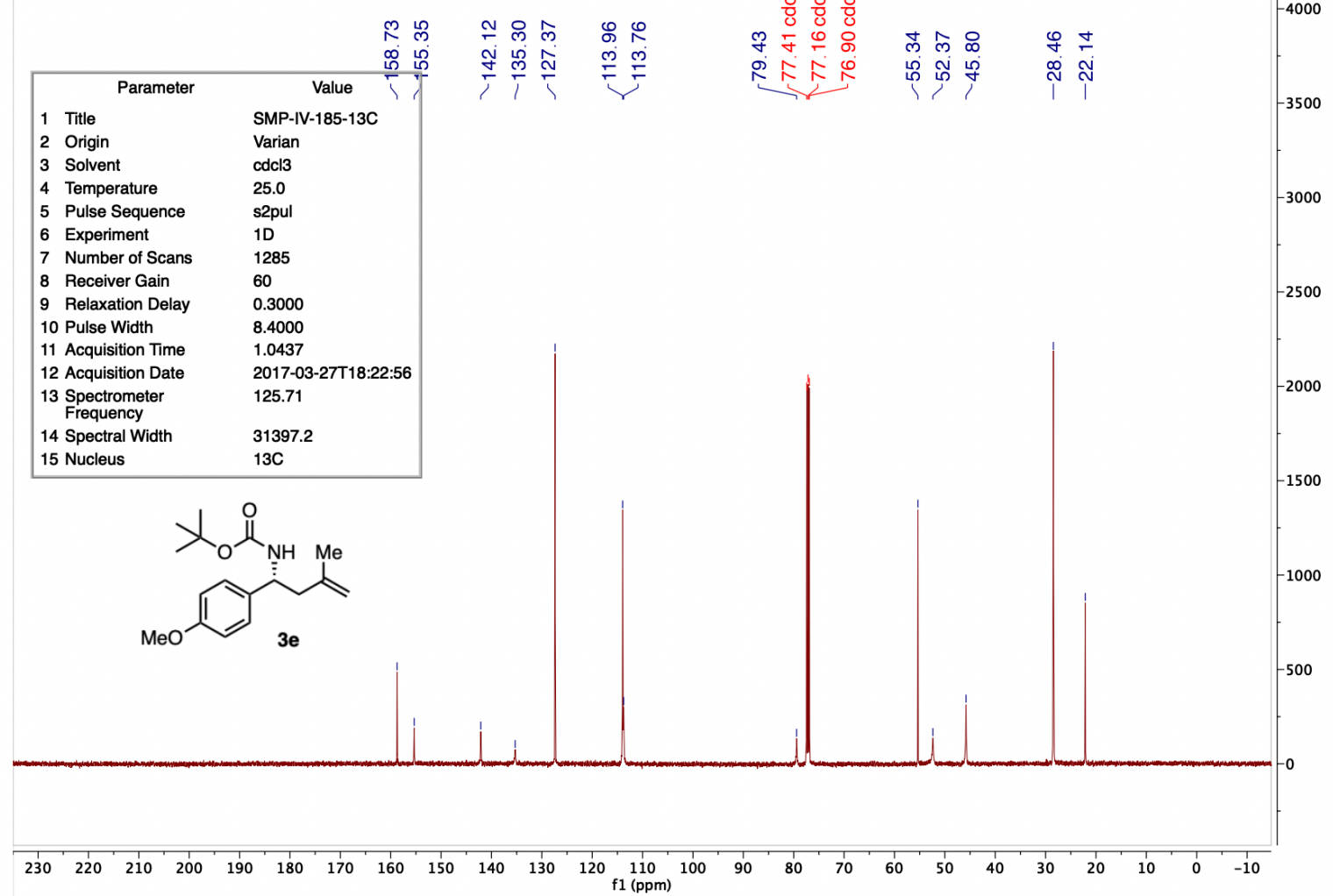




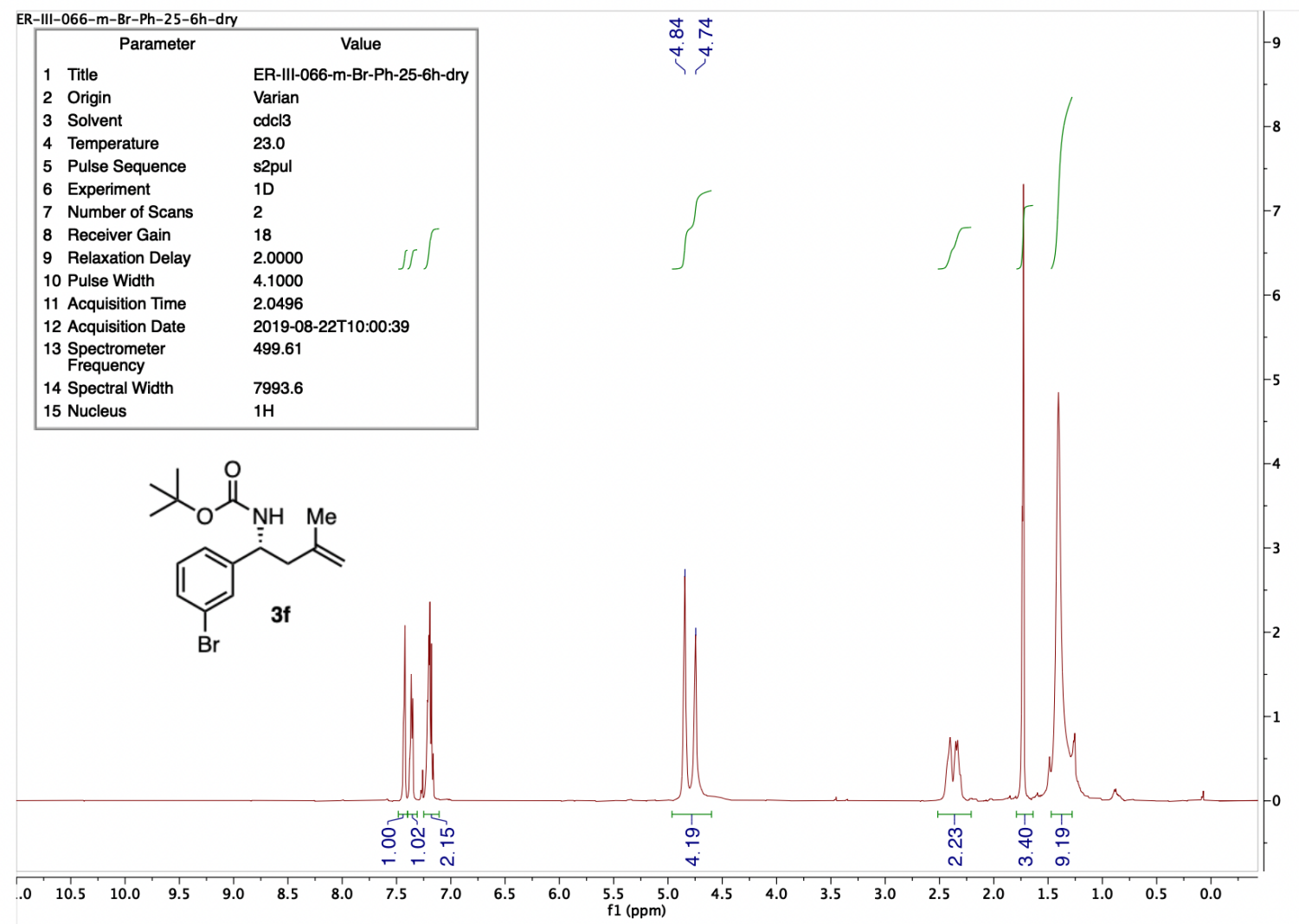

SMP-IV-190-13C

లి ఇ

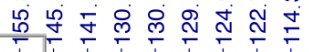

\begin{tabular}{|lll|}
\hline \multicolumn{1}{|c}{ Parameter } & \multicolumn{1}{c}{ Value } \\
1 & Title & SMP-IV-190-13C \\
2 & Origin & Varian \\
3 & Solvent & cdcl3 \\
4 & Temperature & 25.0 \\
5 & Pulse Sequence & s2pul \\
6 & Experiment & $1 \mathrm{D}$ \\
7 & Number of Scans & 1283 \\
8 & Receiver Gain & 60 \\
9 & Relaxation Delay & 0.3000 \\
10 Pulse Width & 8.4000 \\
11 Acquisition Time & 1.0437 \\
12 Acquisition Date & $2017-03-28 T 13: 54: 32$ \\
13 Spectrometer & 125.71 \\
$\quad$ Frequency & \\
14 Spectral Width & 31397.2 \\
15 Nucleus & $13 \mathrm{C}$ \\
\hline
\end{tabular}

\begin{tabular}{rr|rr}
$\frac{m}{0}$ & $\frac{m}{0}$ & $\frac{m}{0}$ \\
$\frac{0}{0}$ & $\frac{0}{0}$ & 0 \\
0 & 8
\end{tabular}

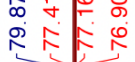

ᄂ? $\infty$

के 8

กิ

$\sim_{0}^{\circ}$
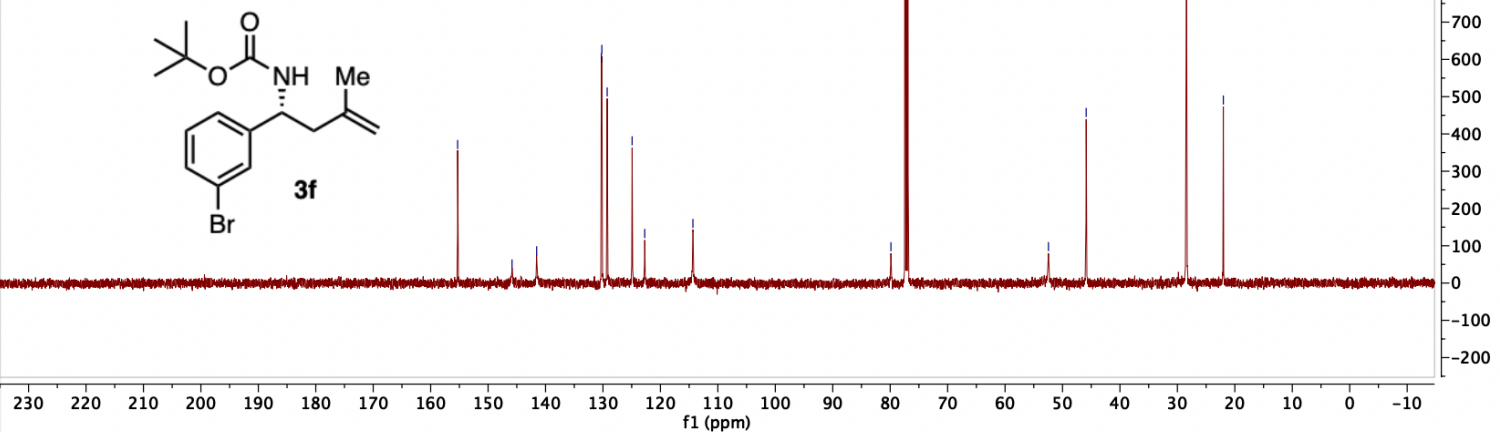

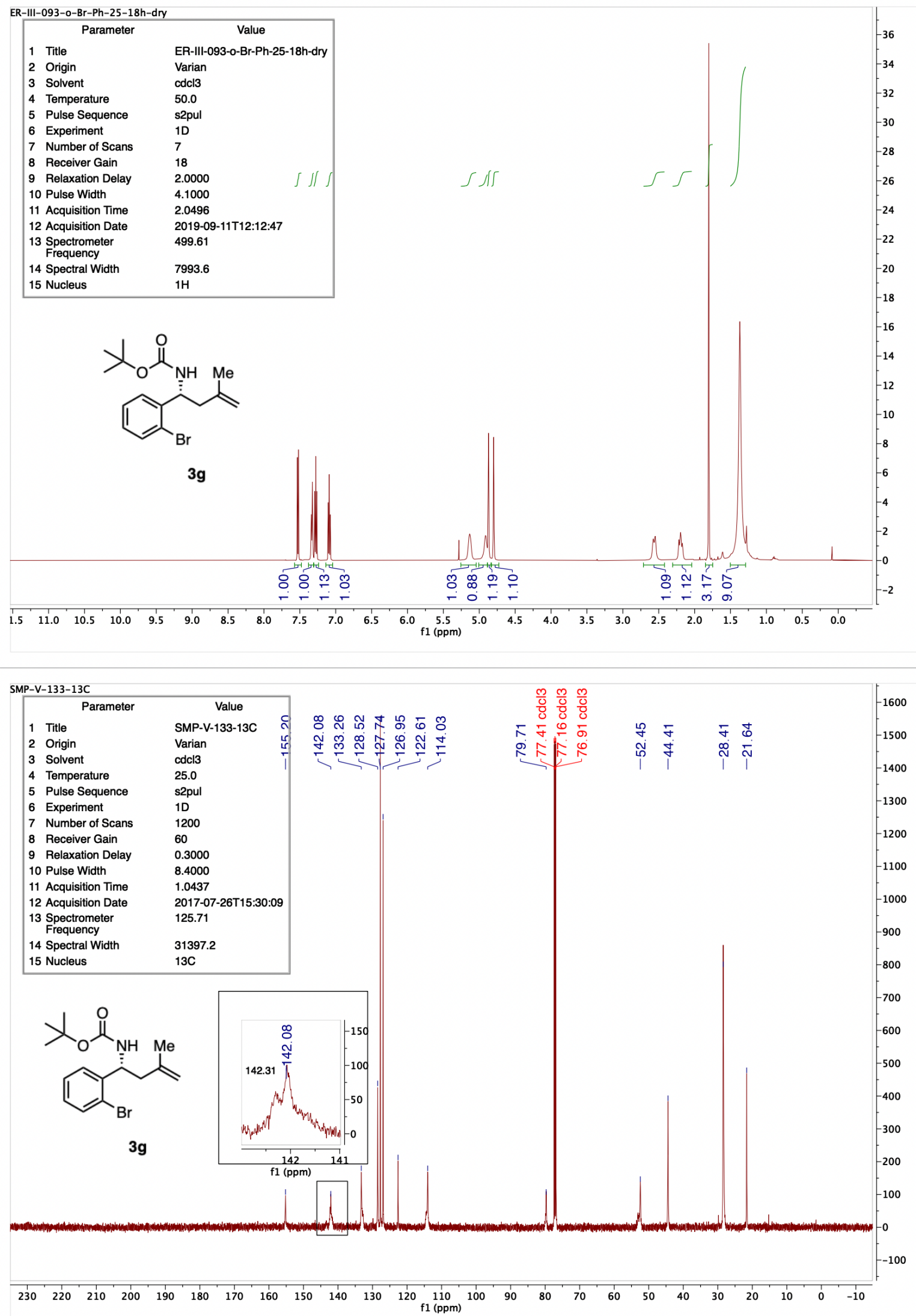


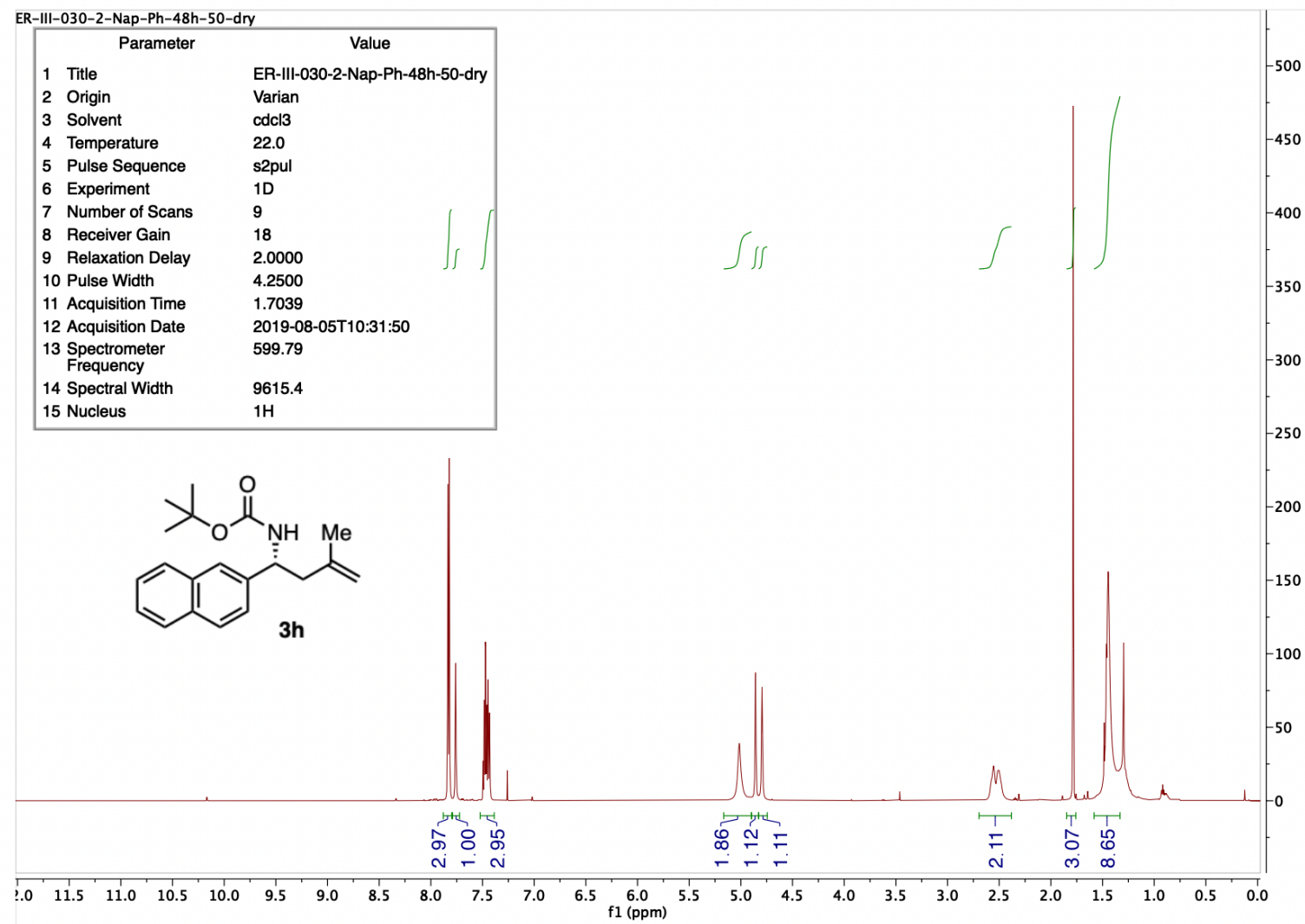

SMP-V-162-13C
\begin{tabular}{|lll|}
\hline \multicolumn{1}{|c|}{ Parameter } & \multicolumn{1}{c|}{ Value } \\
1 & Title & SMP-V-162-13C \\
2 & Origin & Varian \\
3 & Solvent & cdcl3 \\
4 & Temperature & 25.0 \\
5 & Pulse Sequence & s2pul \\
6 & Experiment & $1 \mathrm{D}$ \\
7 & Number of Scans & 993 \\
8 & Receiver Gain & 60 \\
9 & Relaxation Delay & 0.3000 \\
10 & Pulse Width & 8.4000 \\
11 & Acquisition Time & 1.0428 \\
12 & Acquisition Date & $2018-01-26 T 14: 25: 28$ \\
13 & Spectrometer & 125.71 \\
Frequency & \\
14 Spectral Width & 31421.8 \\
15 & Nucleus & $13 \mathrm{C}$ \\
\hline
\end{tabular}

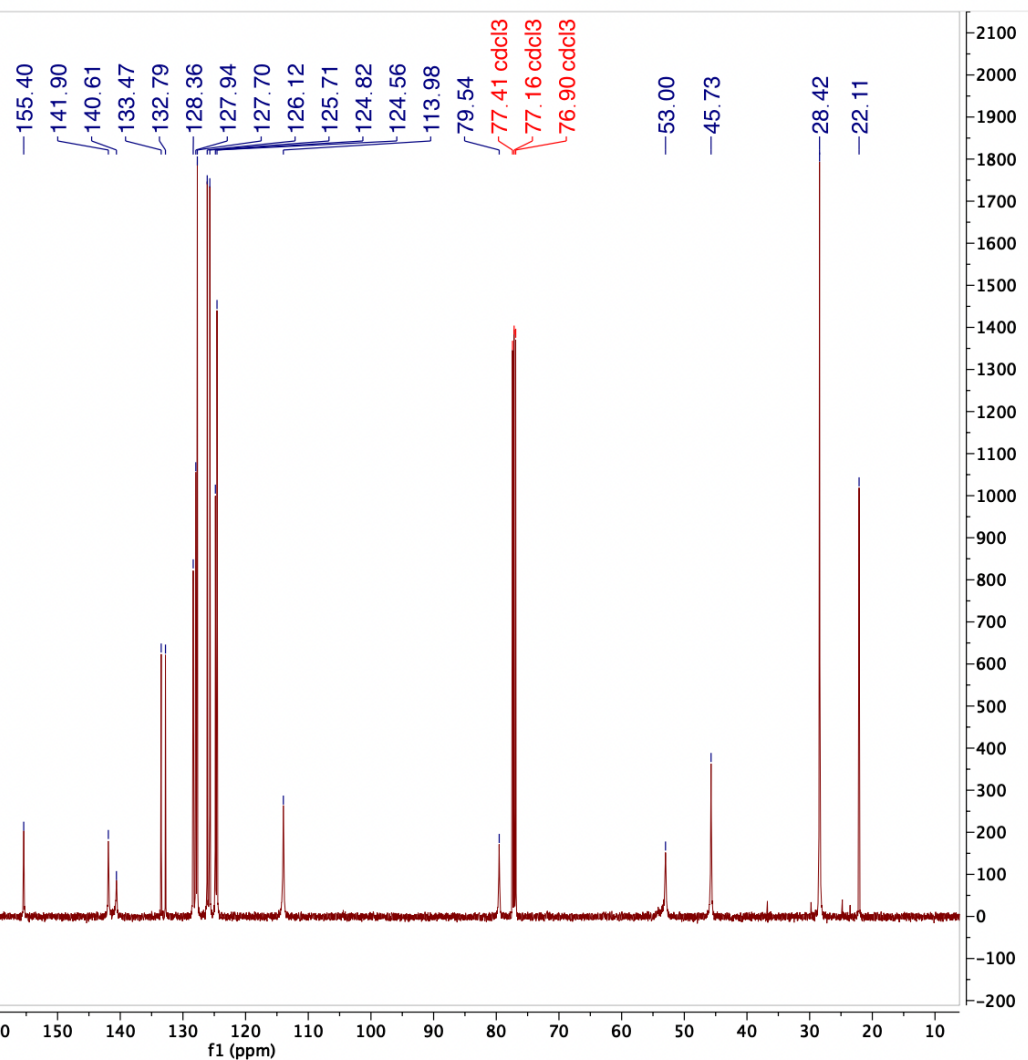




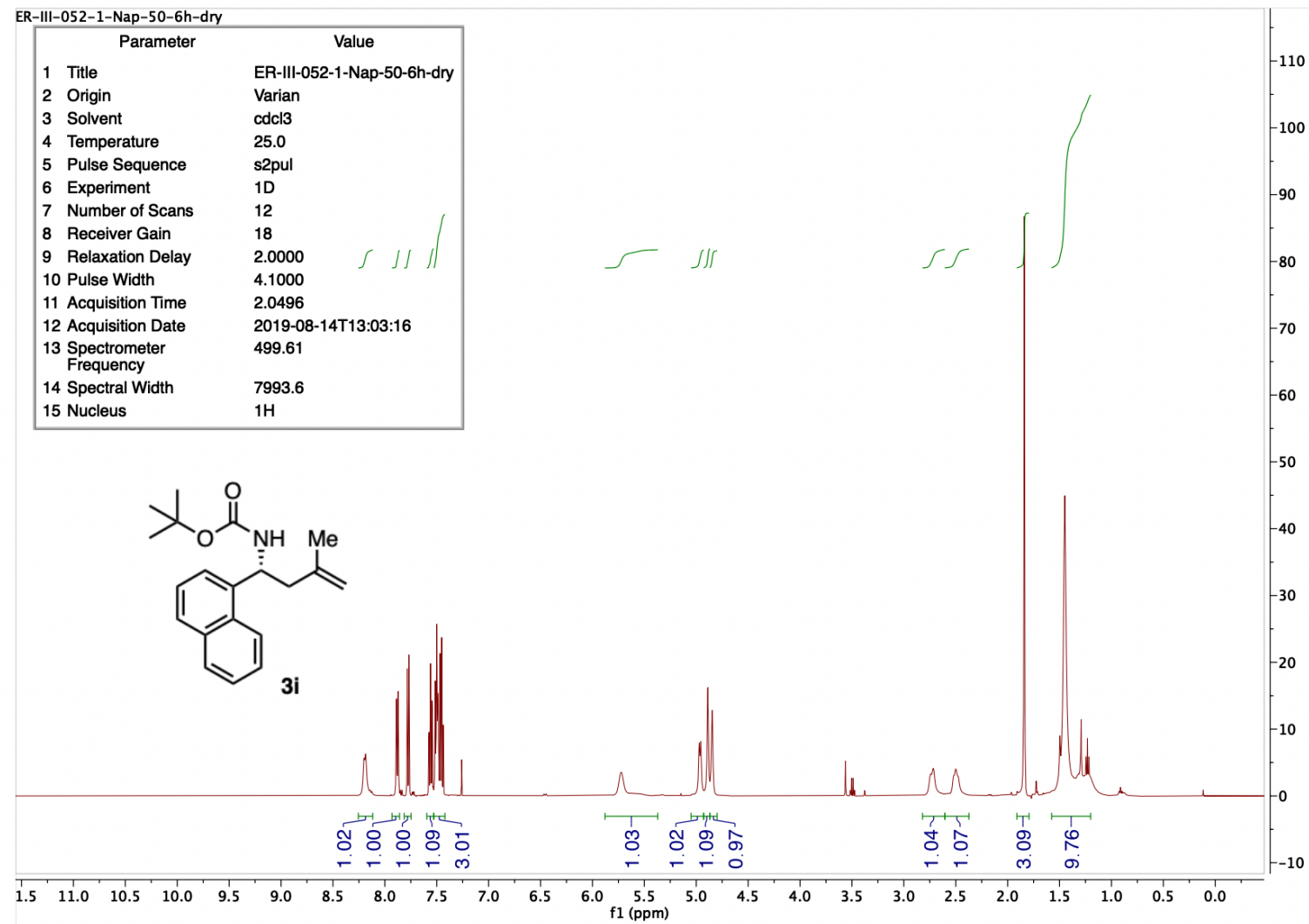

SMP-IV-218-13C
\begin{tabular}{|lll|}
\hline \multicolumn{1}{|c|}{ Parameter } & \multicolumn{1}{c|}{ Value } \\
1 & Title & SMP-IV-218-13C \\
2 & Origin & Varian \\
3 & Solvent & cdcl3 \\
4 & Temperature & 25.0 \\
5 & Pulse Sequence & s2pul \\
6 & Experiment & $1 \mathrm{D}$ \\
7 & Number of Scans & 1203 \\
8 & Receiver Gain & 60 \\
9 & Relaxation Delay & 0.3000 \\
10 & Pulse Width & 8.4000 \\
11 & Acquisition Time & 1.0437 \\
12 & Acquisition Date & $2017-04-07 T 12: 55: 01$ \\
13 & Spectrometer & 125.71 \\
Frequency & \\
14 Spectral Width & 31397.2 \\
15 Nucleus & $13 \mathrm{C}$ \\
\hline
\end{tabular}

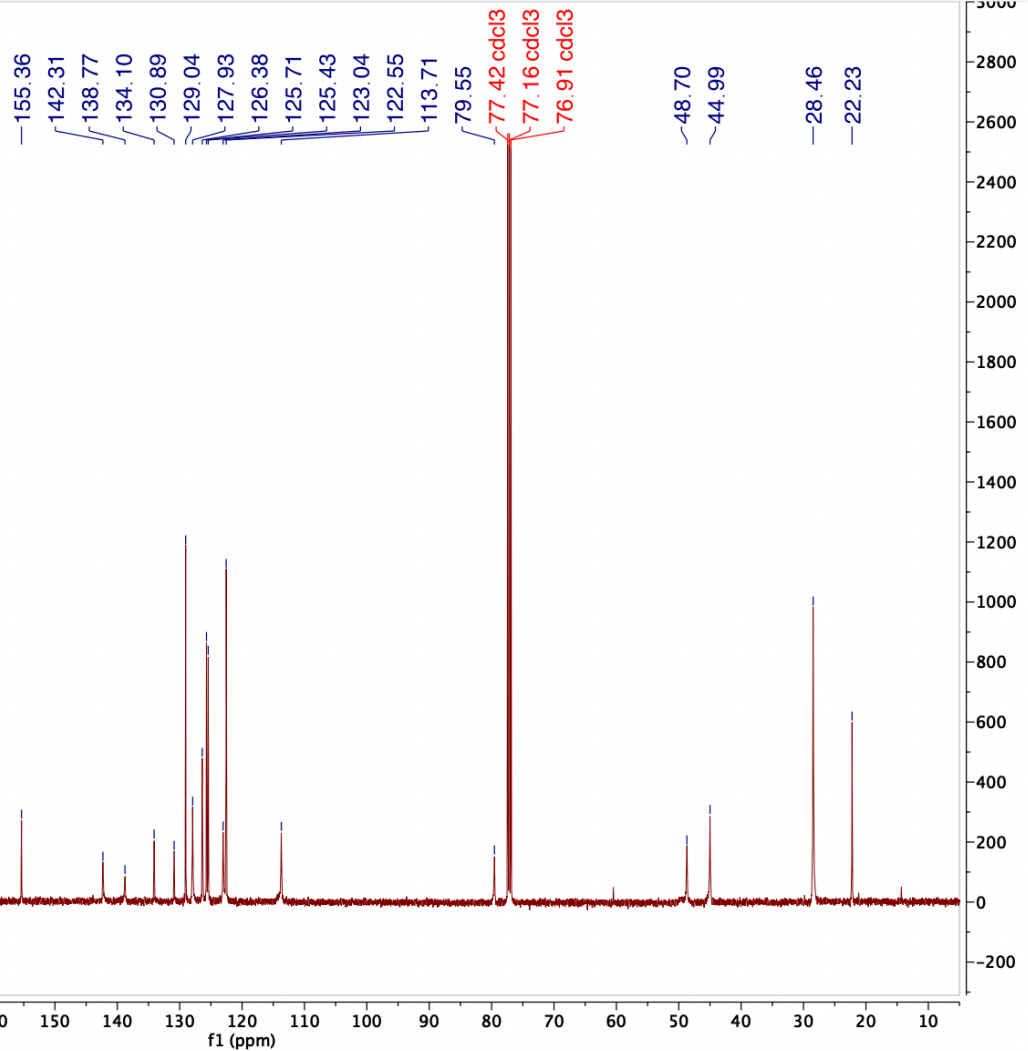




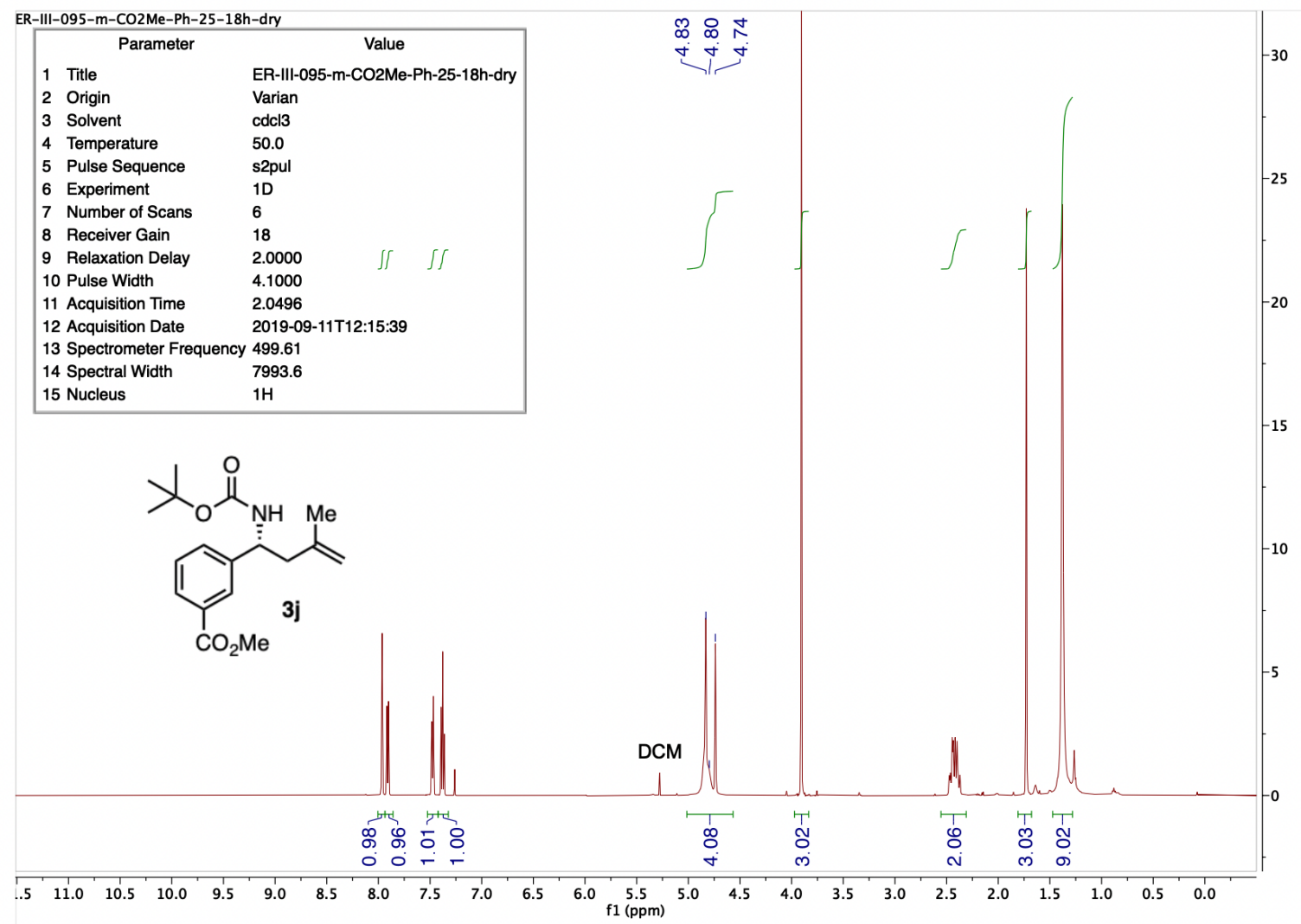

SMP-V-070-13C Gradient Shimming

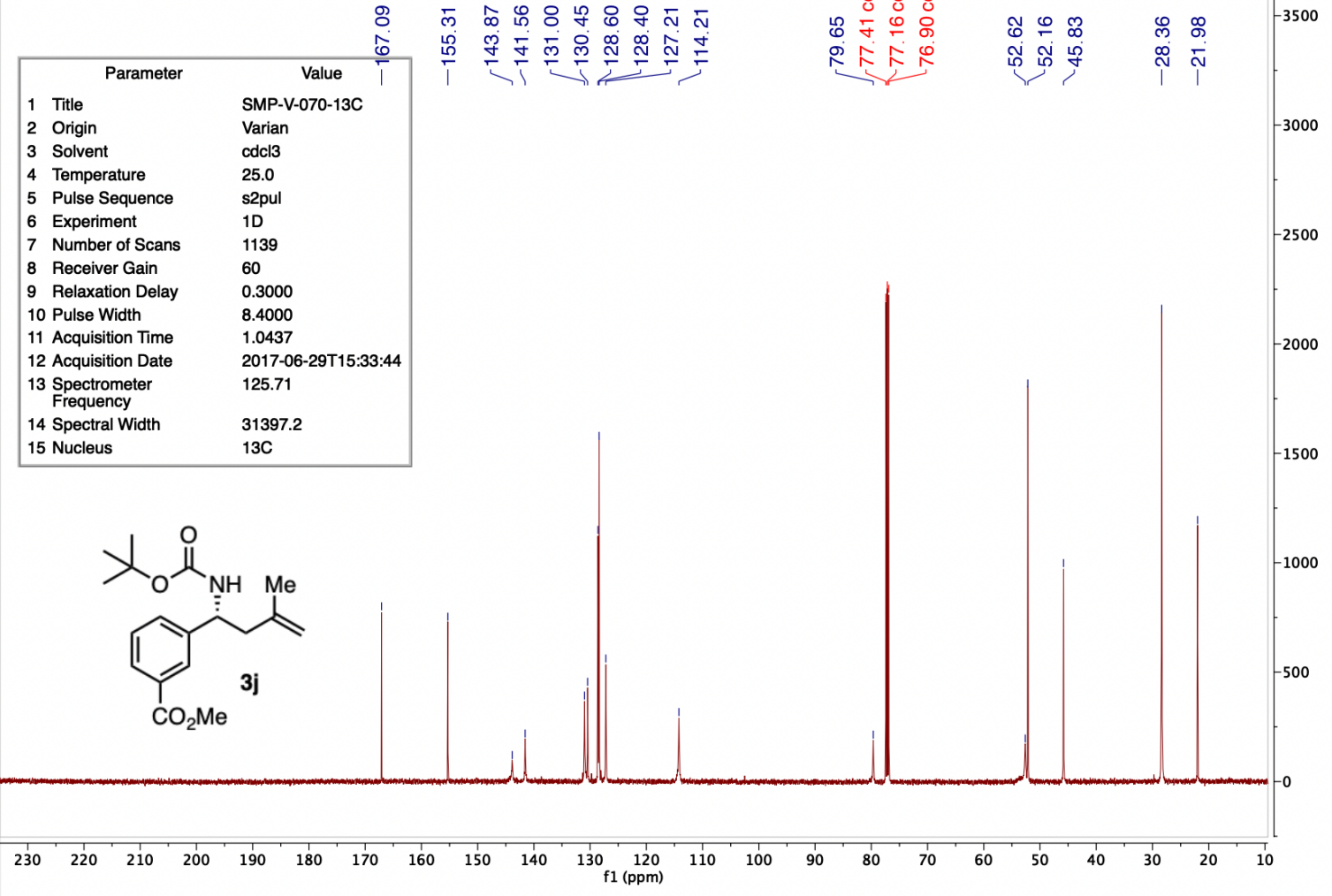



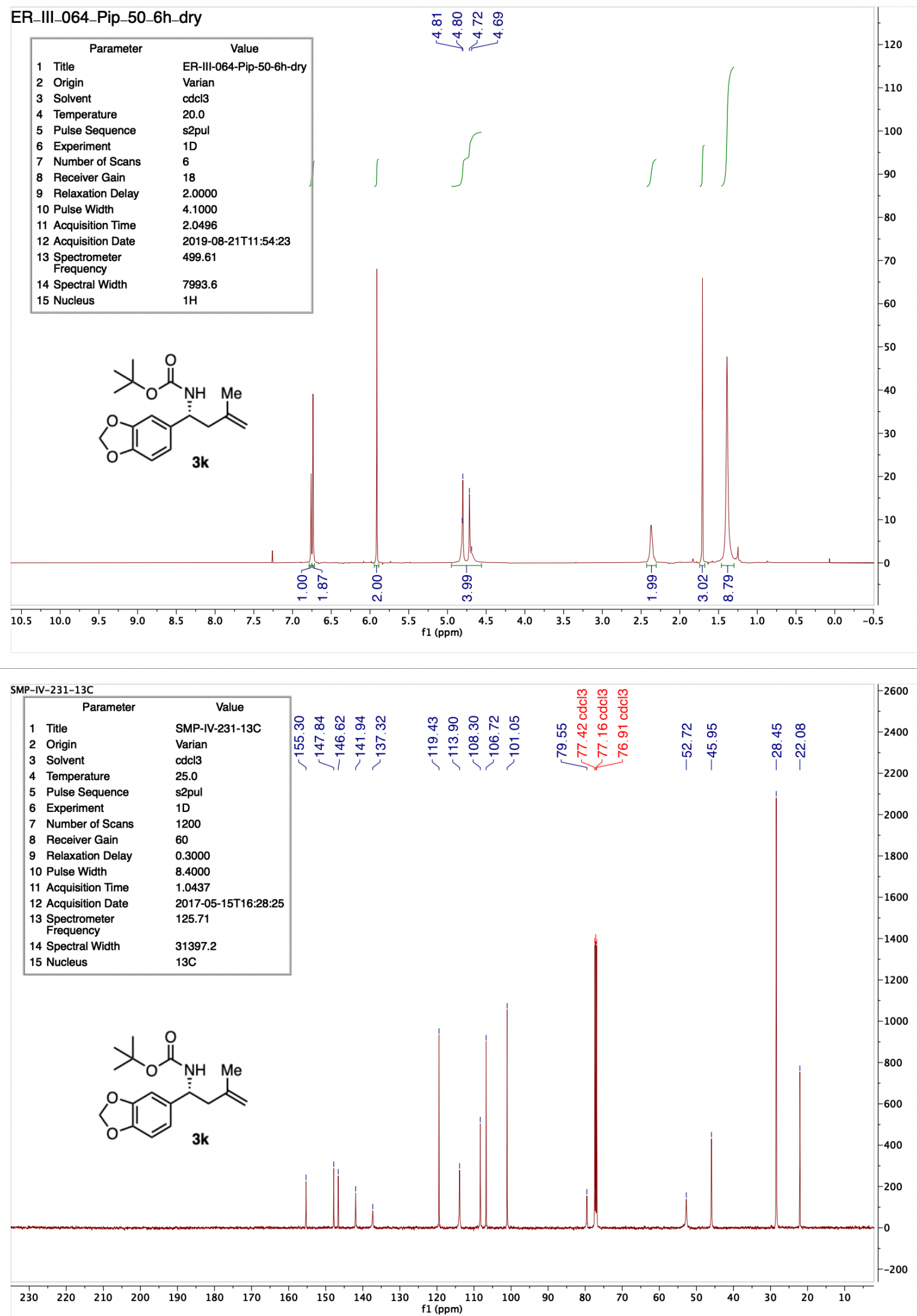

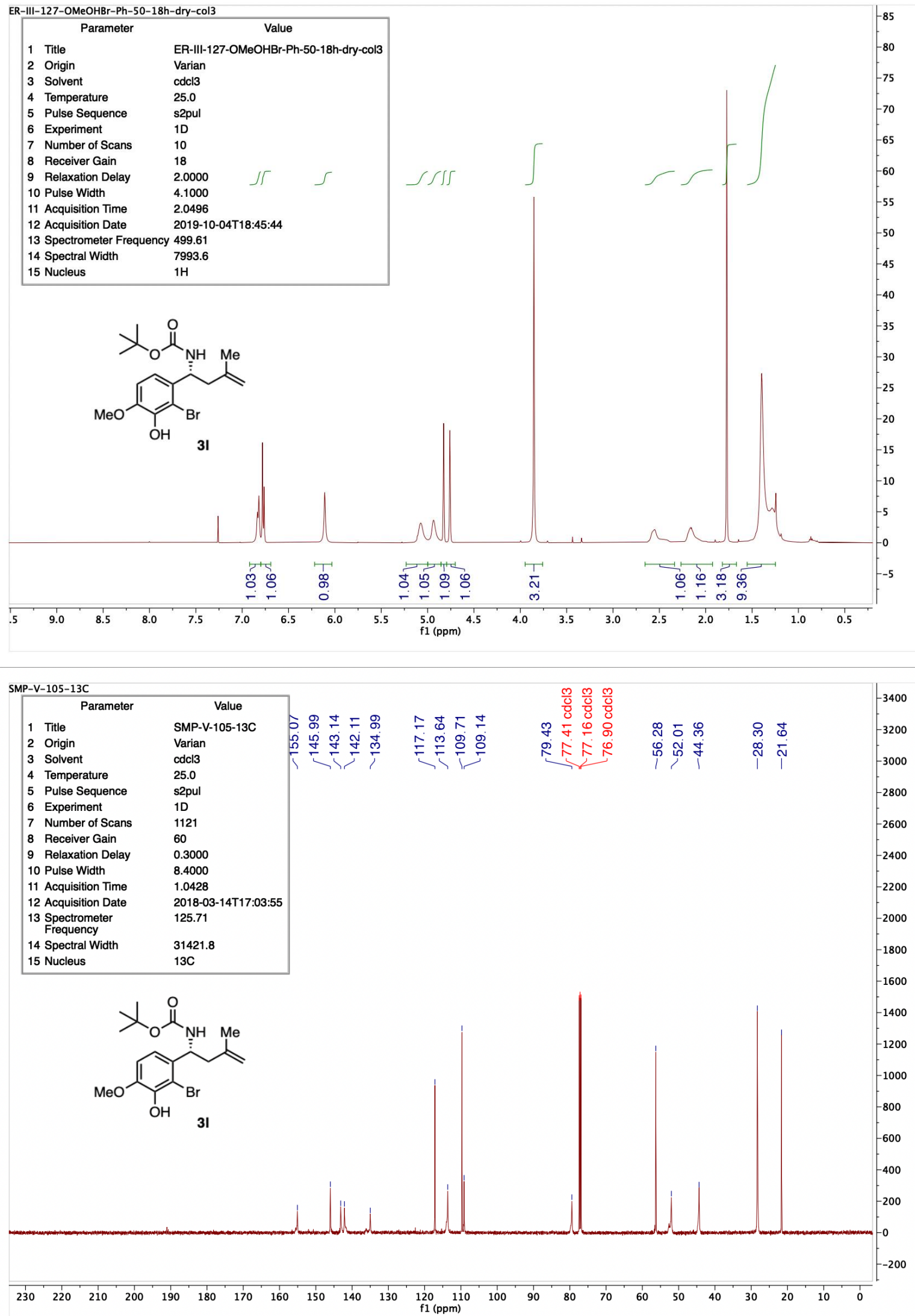

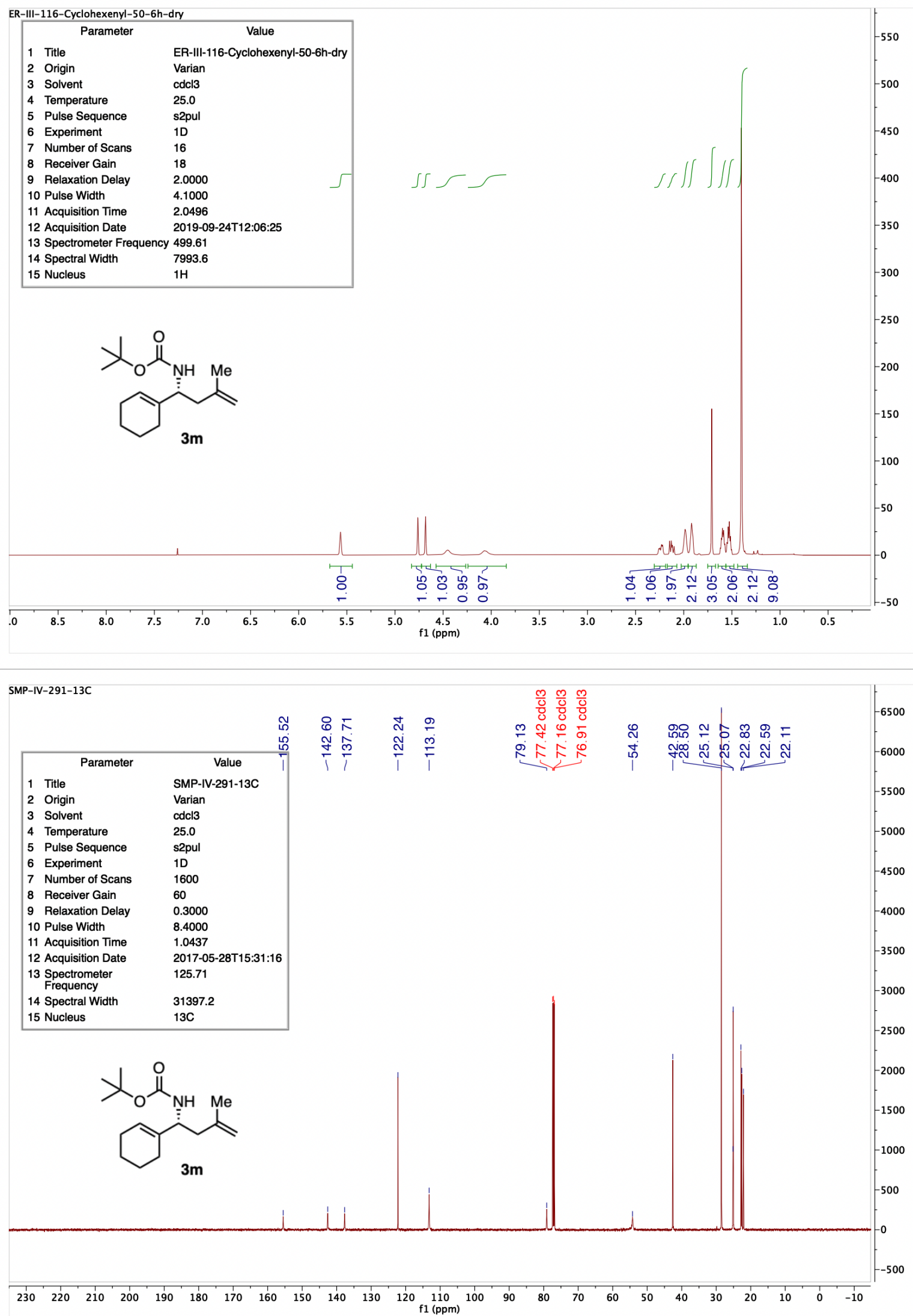

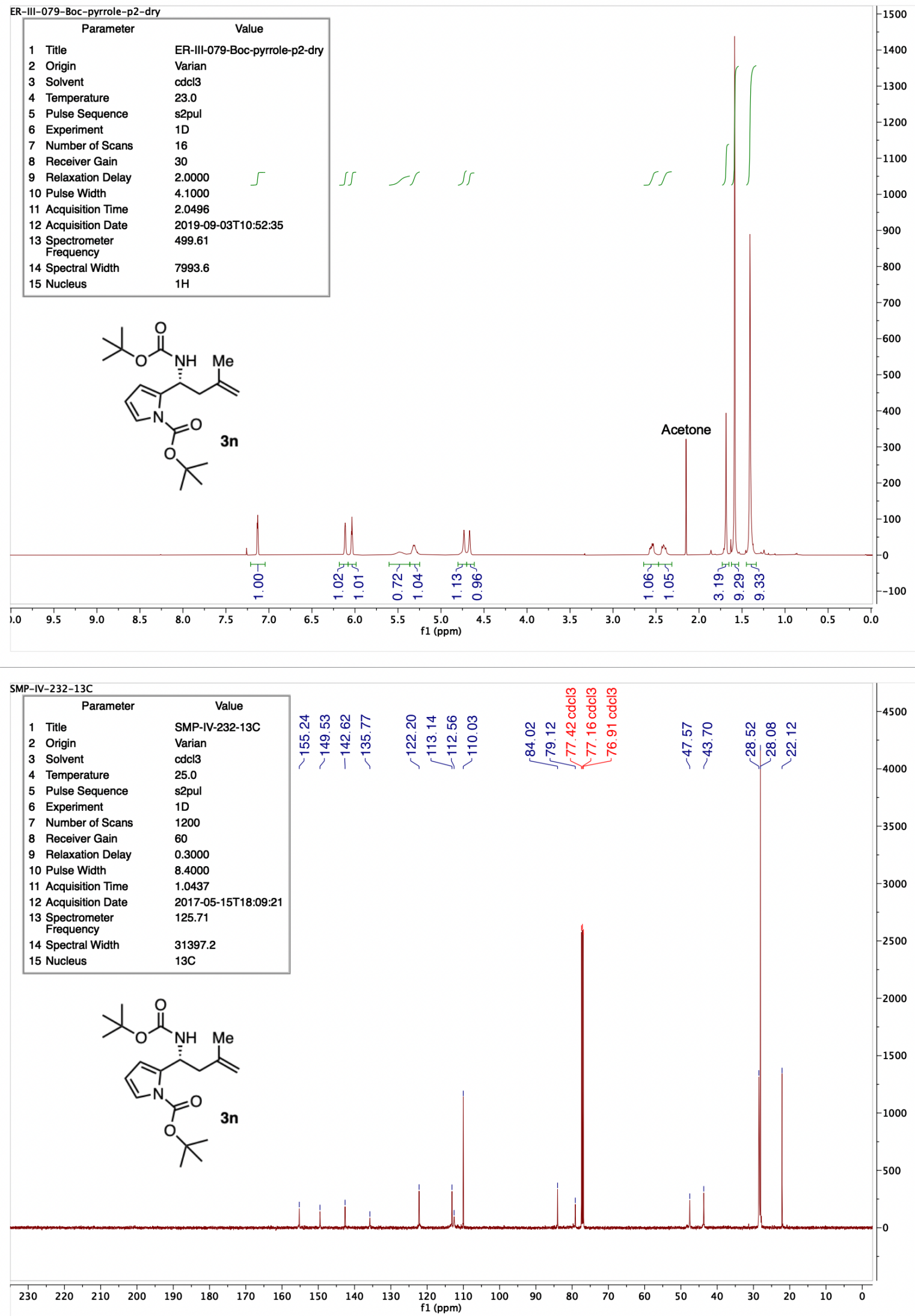

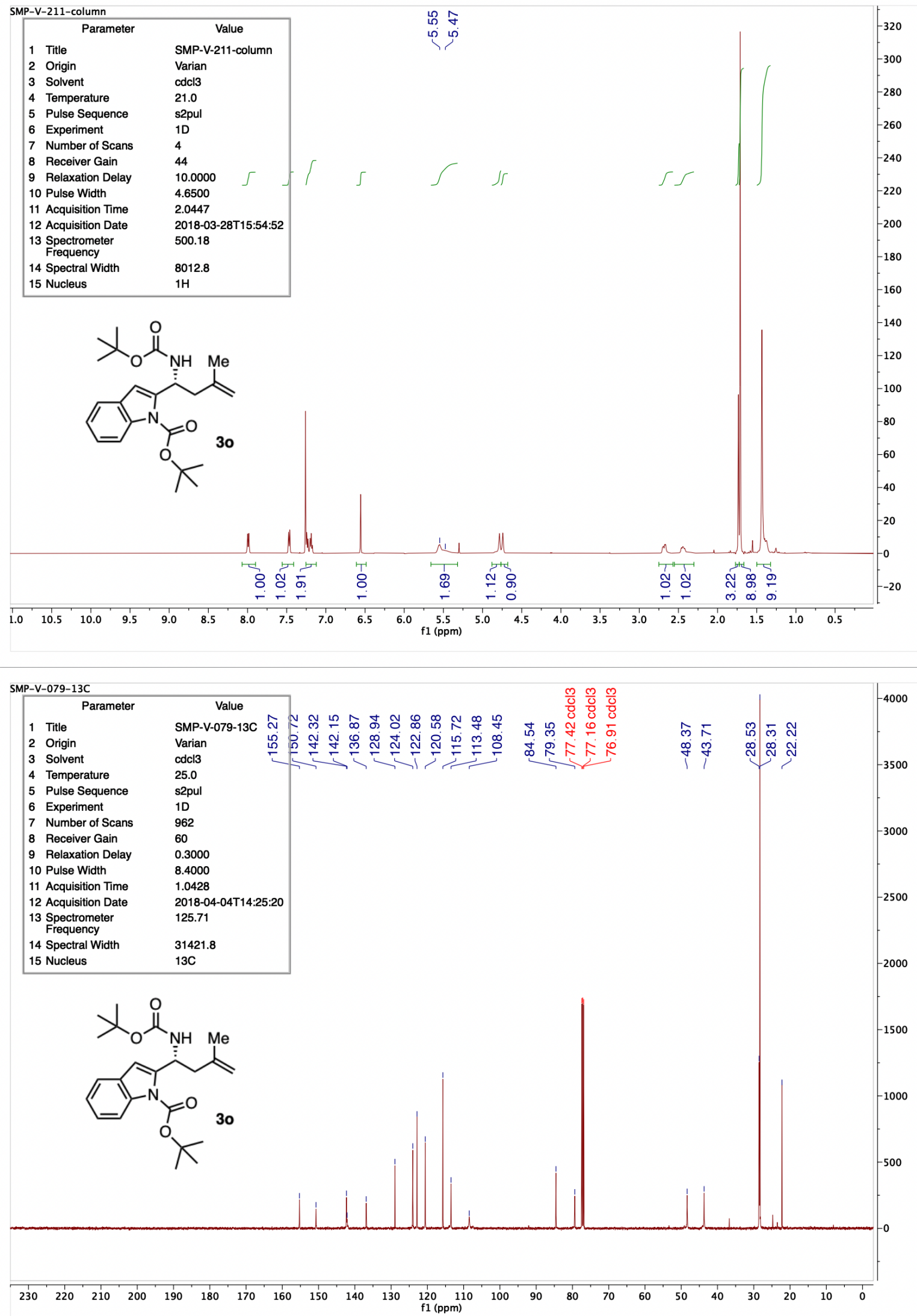

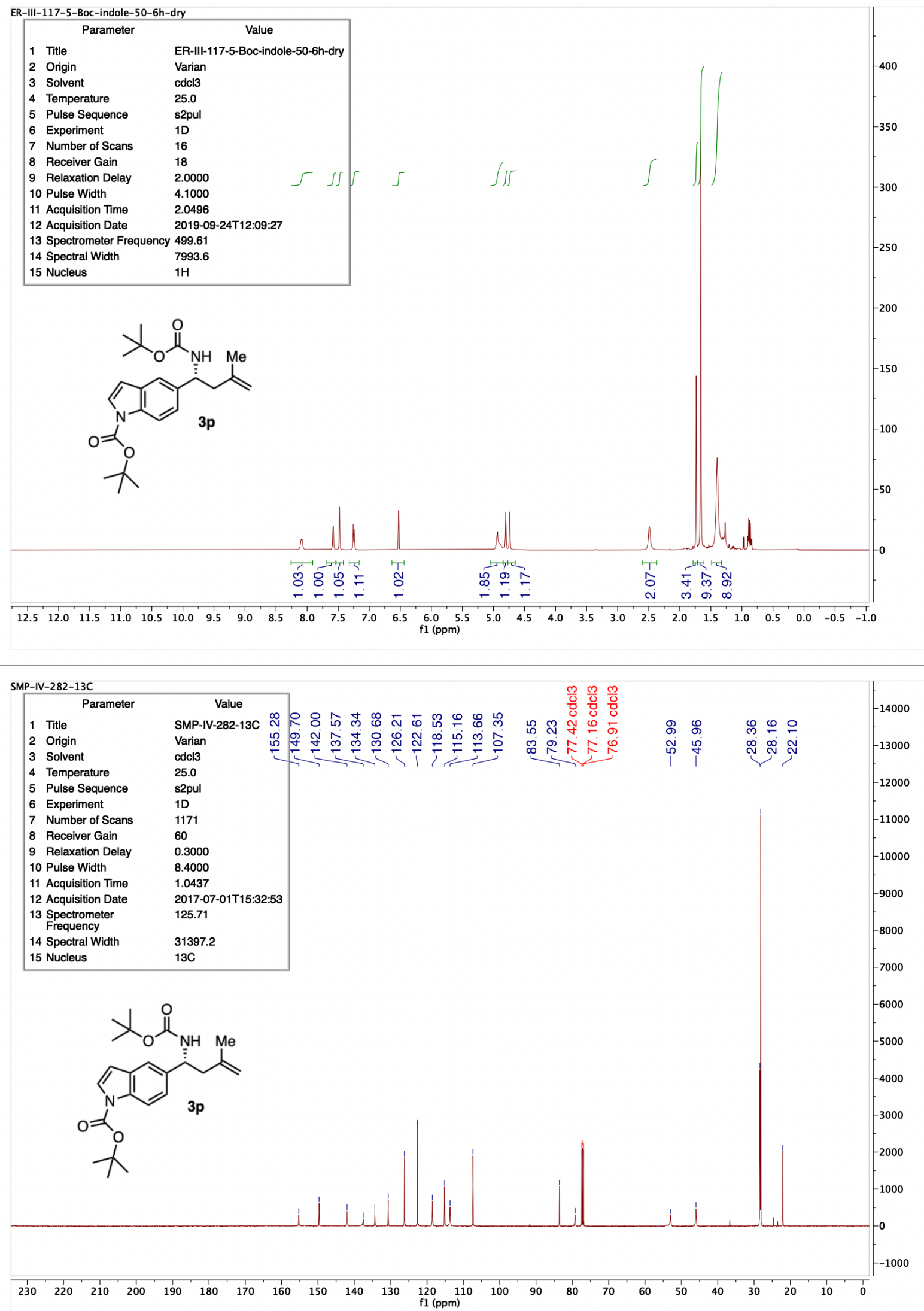


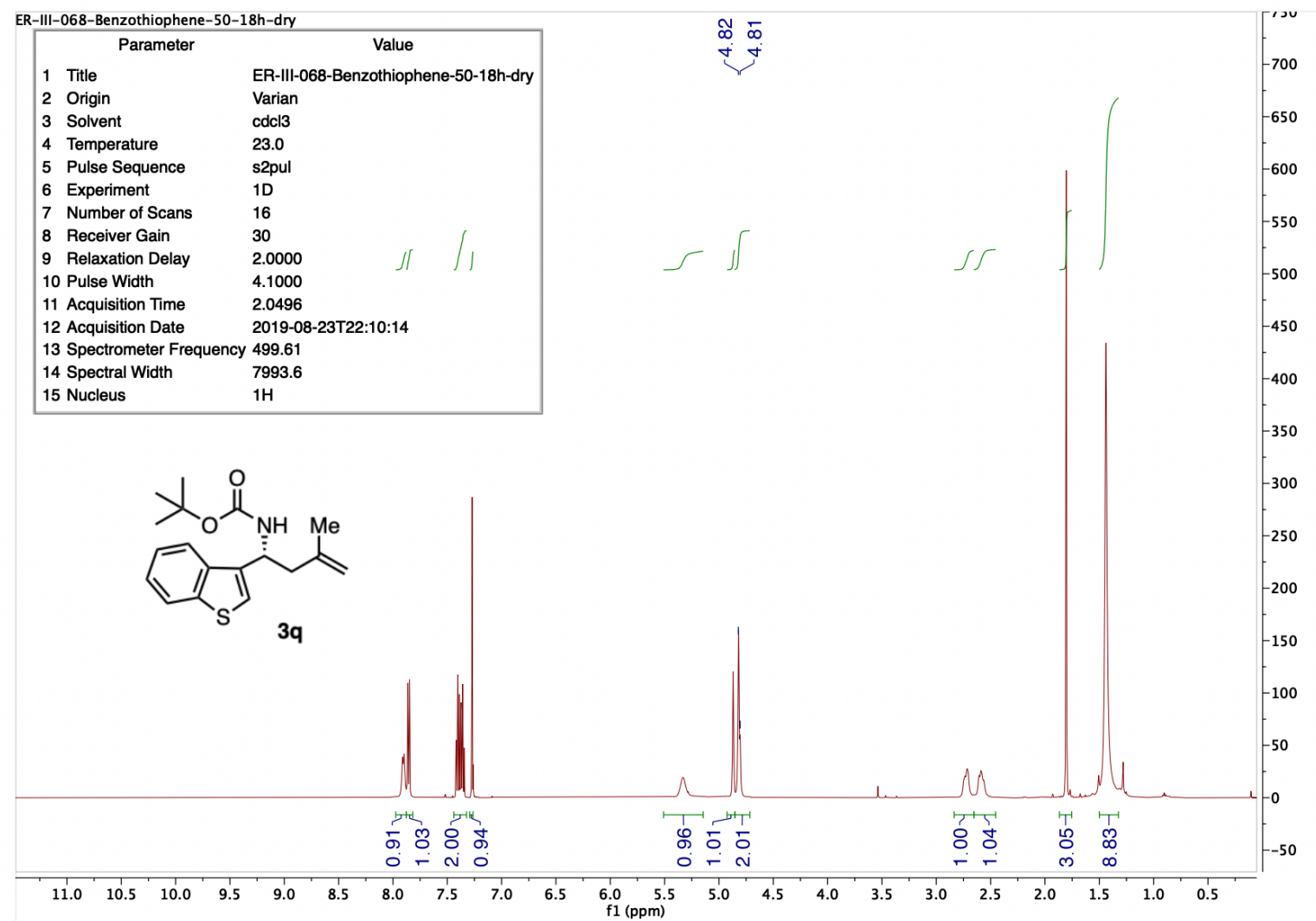

SMP-IV-283-13C

$\frac{m}{0} \frac{m}{0} \frac{m}{0}$

ช บ

弪令

守 学

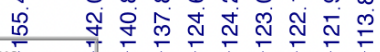

\begin{tabular}{|lll|}
\hline \multicolumn{1}{|c}{ Parameter } & \multicolumn{1}{c|}{ Value } \\
1 & Title & SMP-IV-283-13C \\
2 & Origin & Varian \\
3 & Solvent & cdcl3 \\
4 & Temperature & 25.0 \\
5 & Pulse Sequence & s2pul \\
6 & Experiment & $1 \mathrm{D}$ \\
7 Number of Scans & 1320 \\
8 Receiver Gain & 60 \\
9 Relaxation Delay & 0.3000 \\
10 Pulse Width & 8.4000 \\
11 Acquisition Time & 1.0437 \\
12 Acquisition Date & $2017-05-28 \mathrm{~T} 13: 30: 29$ \\
13 Spectrometer & 125.71 \\
$\quad$ Frequency & \\
14 Spectral Width & 31397.2 \\
15 Nucleus & $13 \mathrm{C}$ \\
\hline
\end{tabular}

$>_{0} \stackrel{ }{N}$

作

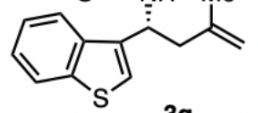

$3 q$

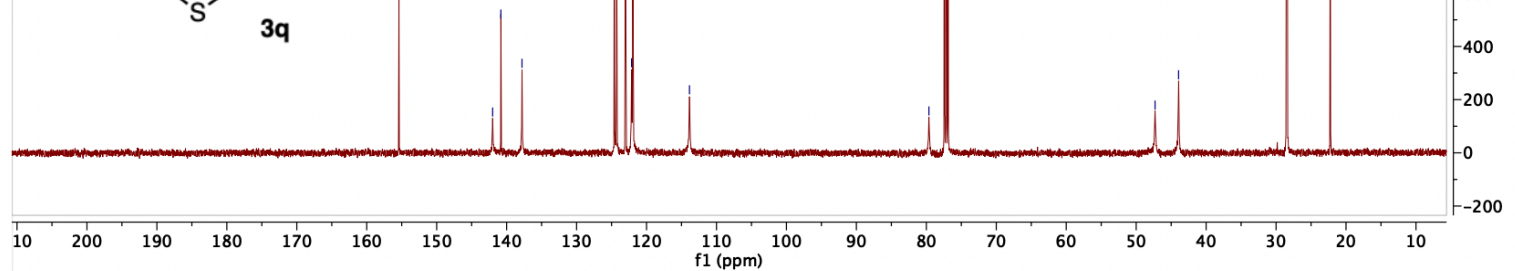




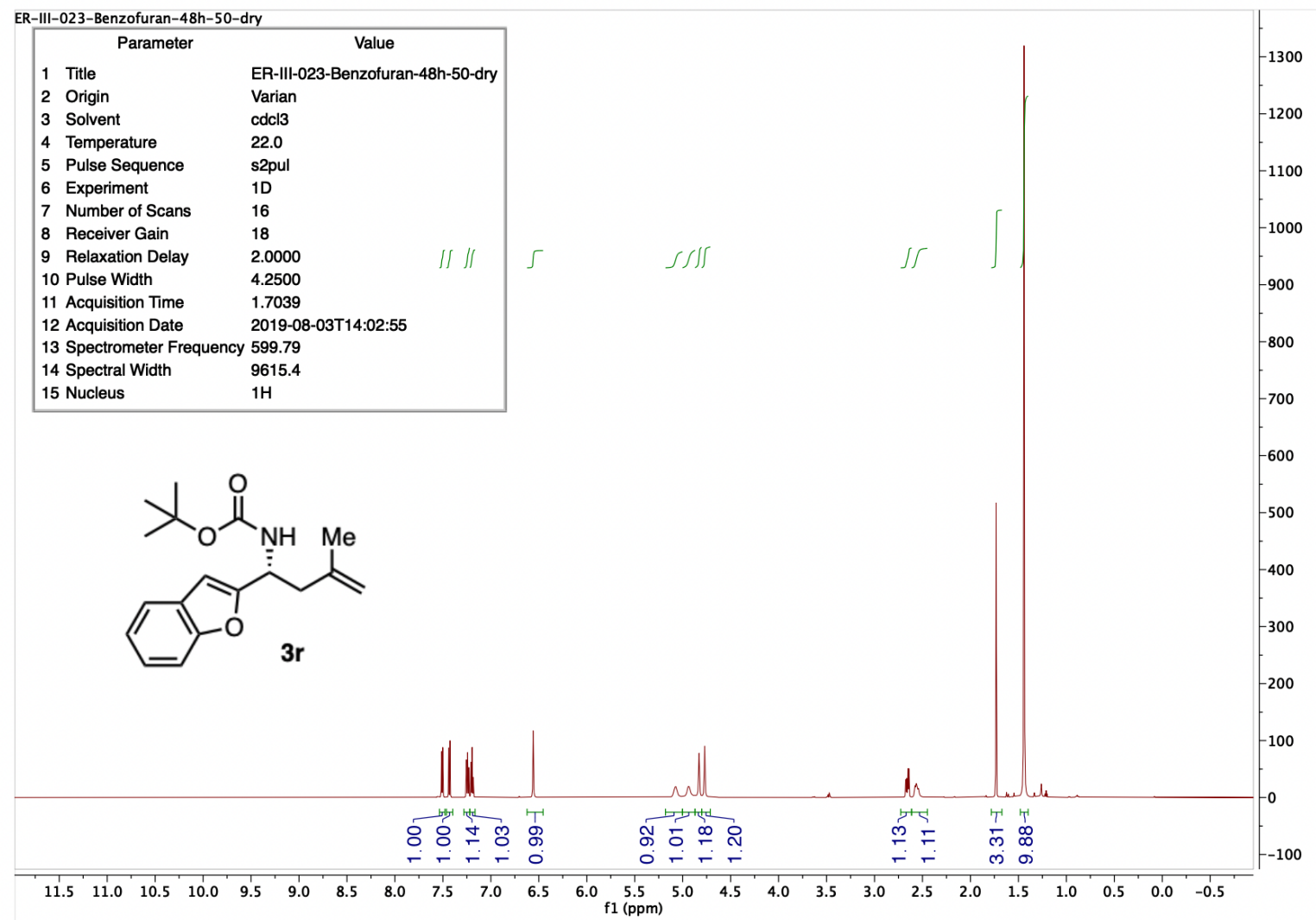

SMP-IV-290-13C

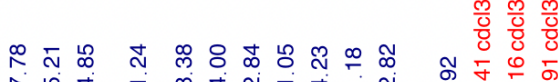

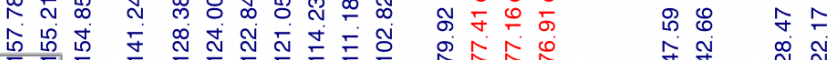

\begin{tabular}{|lll|}
\hline \multicolumn{1}{|c}{ Parameter } & \multicolumn{2}{c}{ Value } \\
1 & Title & SMP-IV-290-13C \\
2 & Origin & Varian \\
3 & Solvent & cdcl3 \\
4 & Temperature & 25.0 \\
5 & Pulse Sequence & s2pul \\
6 & Experiment & $1 \mathrm{D}$ \\
7 & Number of Scans & 1373 \\
8 & Receiver Gain & 60 \\
9 & Relaxation Delay & 0.3000 \\
10 & Pulse Width & 8.4000 \\
11 & Acquisition Time & 1.0437 \\
12 Acquisition Date & $2017-05-28 T 14: 22: 04$ \\
13 Spectrometer & 125.71 \\
Frequency & \\
14 Spectral Width & 31397.2 \\
15 Nucleus & $13 \mathrm{C}$ \\
\hline
\end{tabular}

ค พ $\stackrel{1}{1}$ ก

6000

埩

000

500

000

3500

000

500
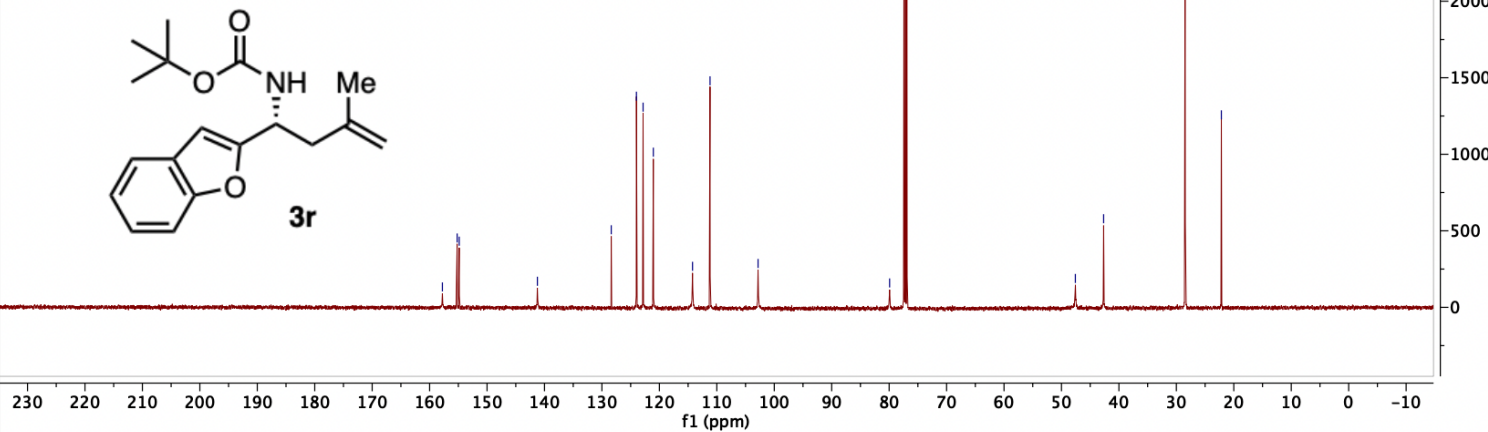


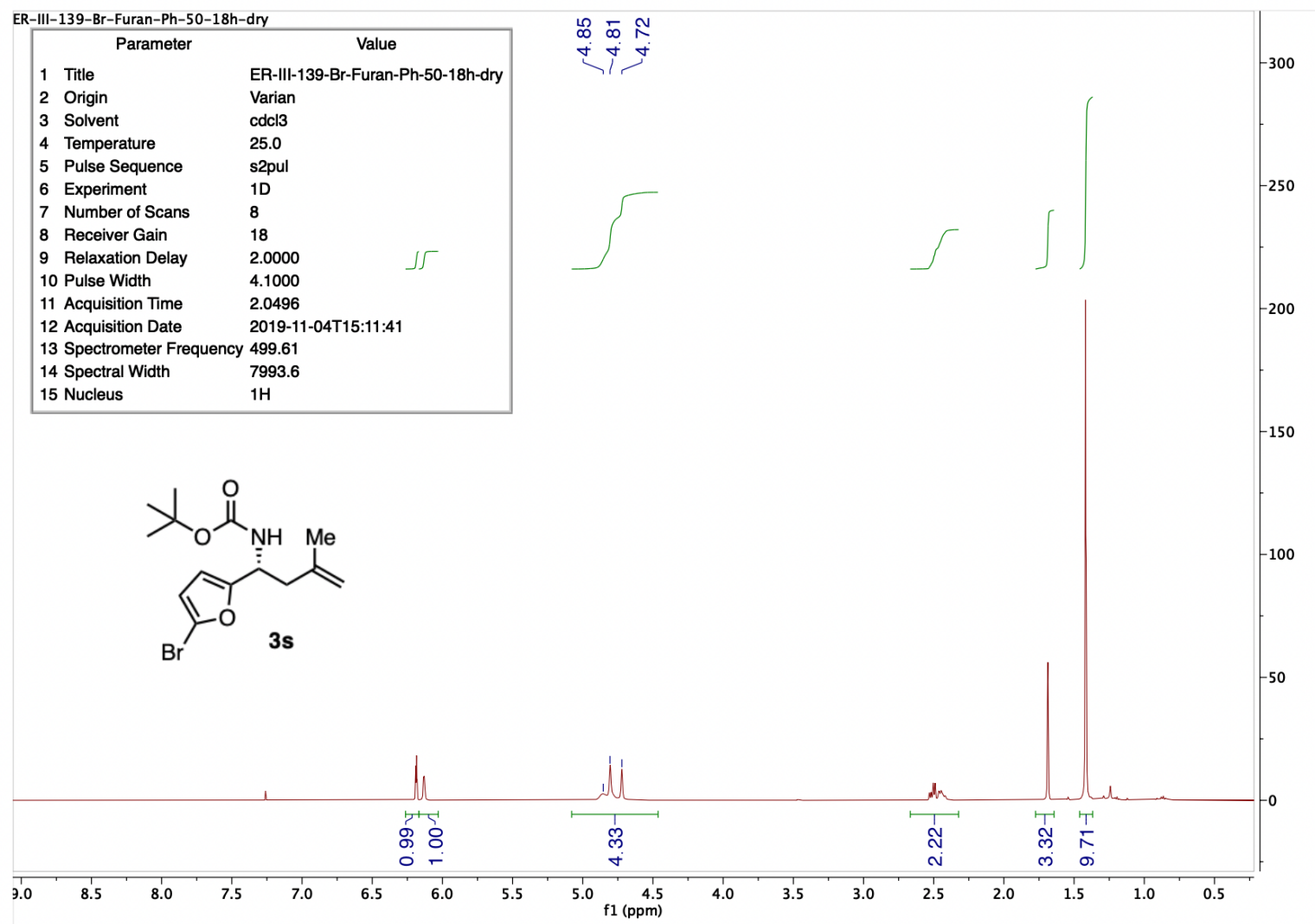

SMP-V-089-13C Gradient Shimming

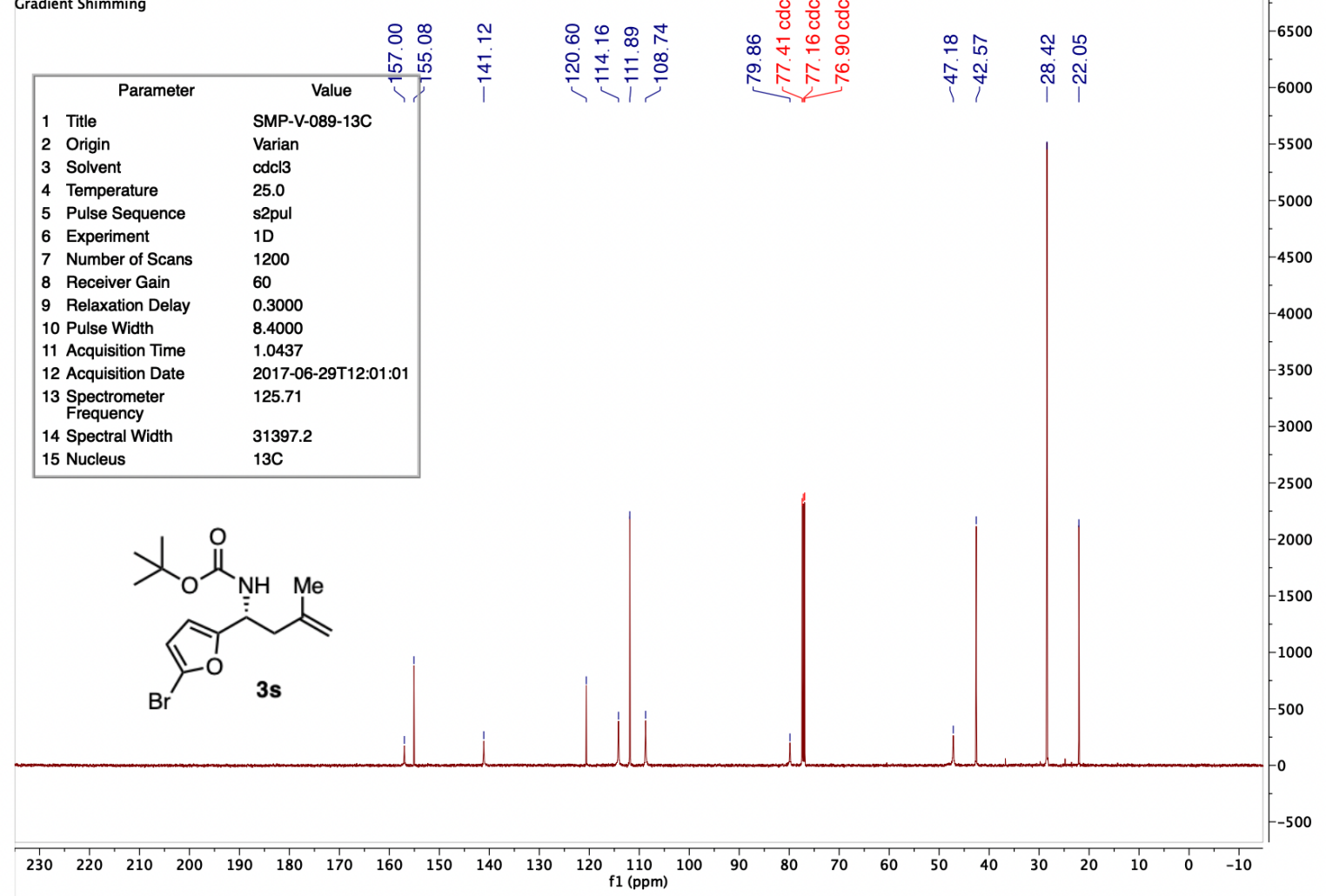



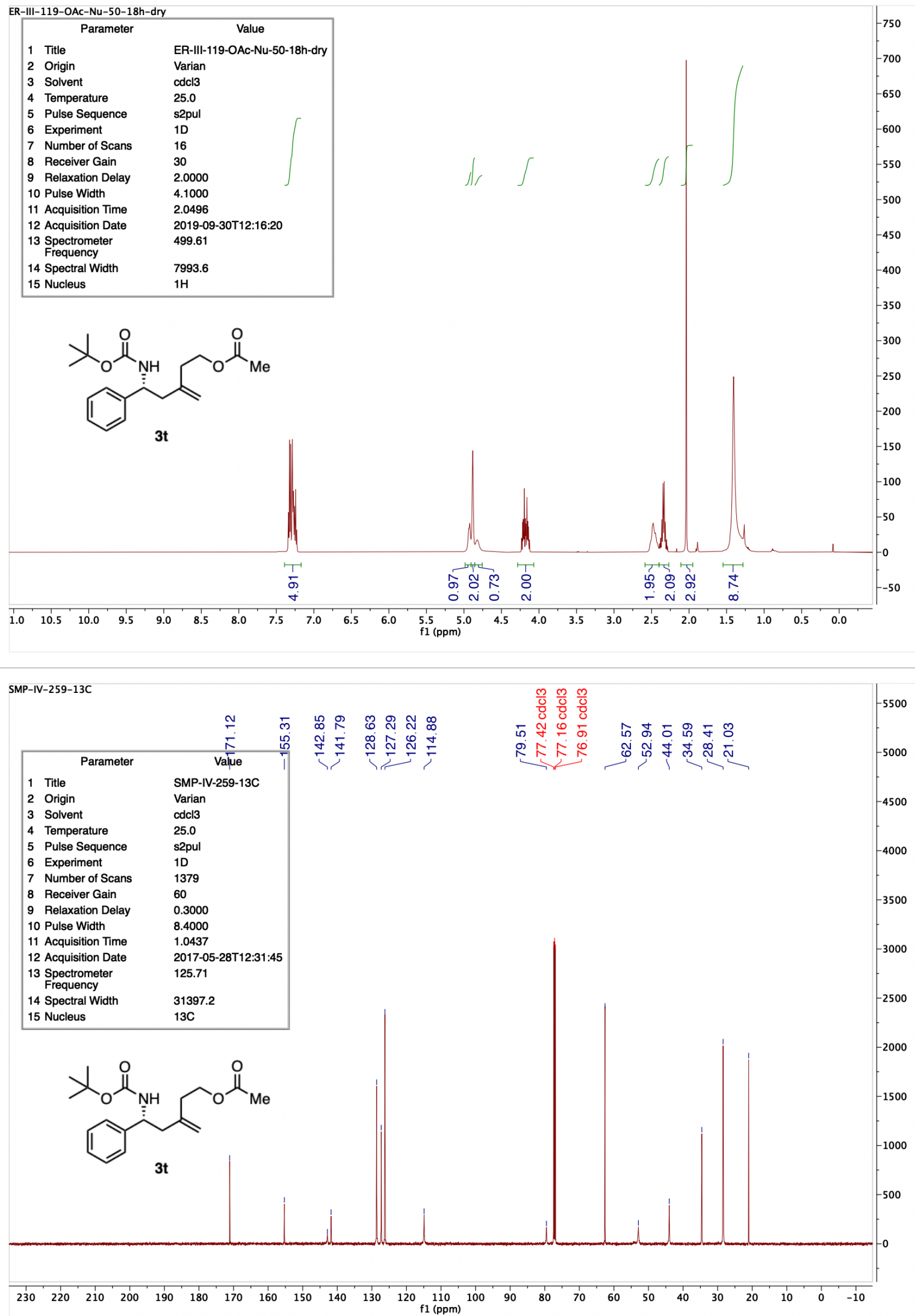


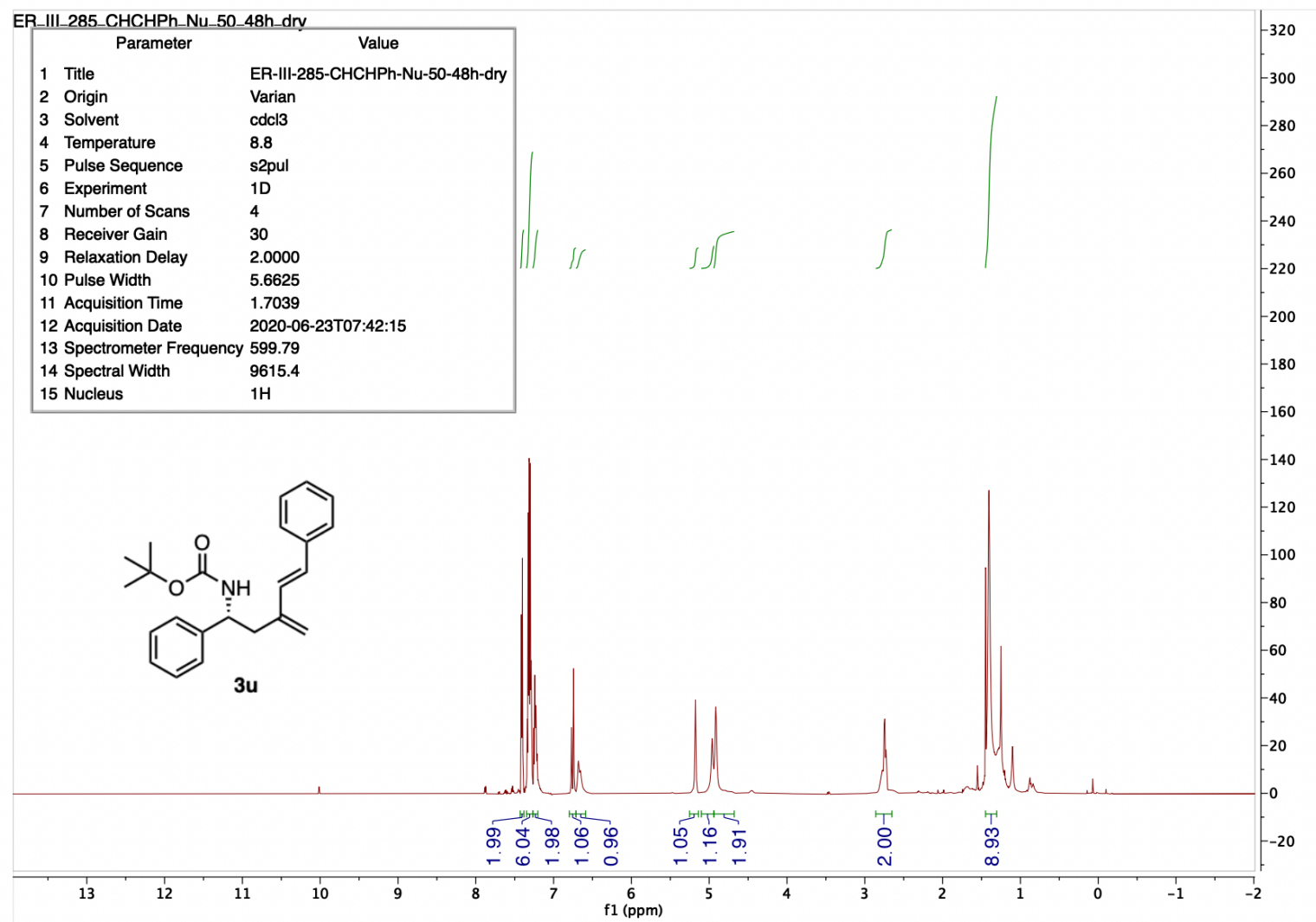

SMP-V-041-13C
\begin{tabular}{|lll|}
\hline \multicolumn{1}{|c|}{ Parameter } & \multicolumn{1}{c|}{ Value } \\
1 & Title & SMP-V-041-13C \\
2 & Origin & Varian \\
3 & Solvent & cdcl3 \\
4 & Temperature & 25.0 \\
5 & Pulse Sequence & s2pul \\
6 & Experiment & $1 \mathrm{D}$ \\
7 & Number of Scans & 1200 \\
8 & Receiver Gain & 60 \\
9 & Relaxation Delay & 0.3000 \\
10 & Pulse Width & 8.4000 \\
11 & Acquisition Time & 1.0428 \\
12 & Acquisition Date & $2018-02-04 T 15: 30: 50$ \\
13 & Spectrometer & 125.71 \\
Frequency & \\
14 Spectral Width & 31421.8 \\
15 & Nucleus & $13 \mathrm{C}$ \\
\hline
\end{tabular}

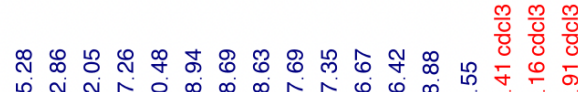

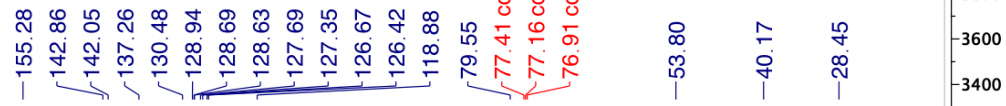

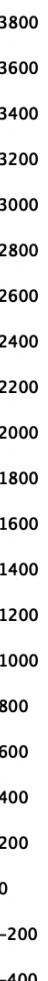

$\begin{array}{lllllllllll}230 & 220 & 210 & 200 & 190 & 180 & 170 & 160 & 150 & 140 & 130 \underset{\mathrm{f} 1(\mathrm{ppm})}{120}\end{array}$ 


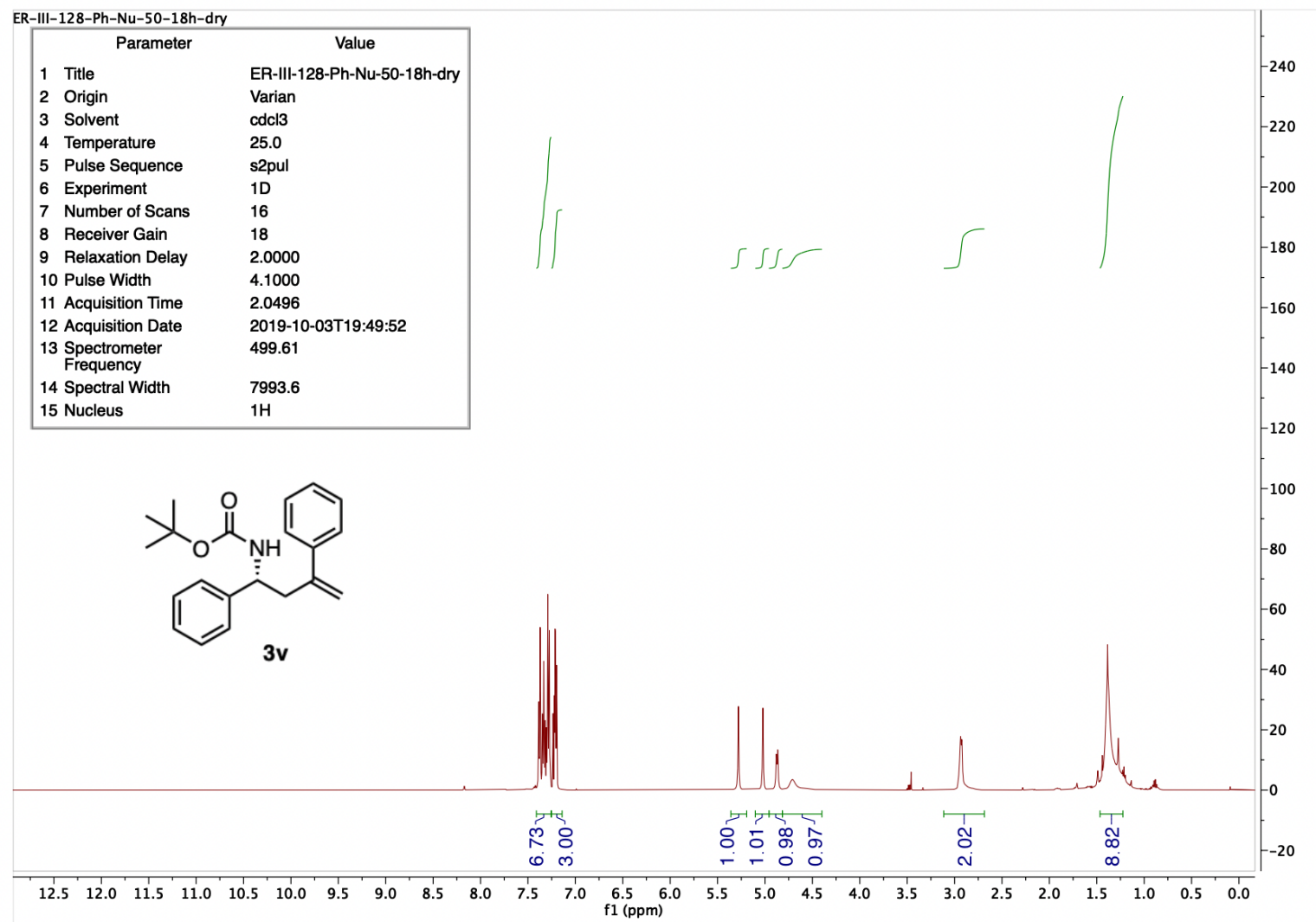

SMP-V-183-13C
\begin{tabular}{|lll|}
\hline \multicolumn{1}{|c|}{ Parameter } & \multicolumn{1}{c|}{ Value } \\
1 & Title & SMP-V-183-13C \\
2 & Origin & Varian \\
3 & Solvent & cdcl3 \\
4 & Temperature & 25.0 \\
5 & Pulse Sequence & s2pul \\
6 & Experiment & $1 \mathrm{D}$ \\
7 & Number of Scans & 1200 \\
8 & Receiver Gain & 60 \\
9 & Relaxation Delay & 0.3000 \\
10 & Pulse Width & 8.4000 \\
11 & Acquisition Time & 1.0428 \\
12 & Acquisition Date & $2018-02-23 T 12: 15: 18$ \\
13 & Spectrometer & 125.71 \\
Frequency & 31421.8 \\
14 & Spectral Width & $314 \mathrm{C}$ \\
15 & Nucleus & $13 \mathrm{C}$ \\
\hline
\end{tabular}

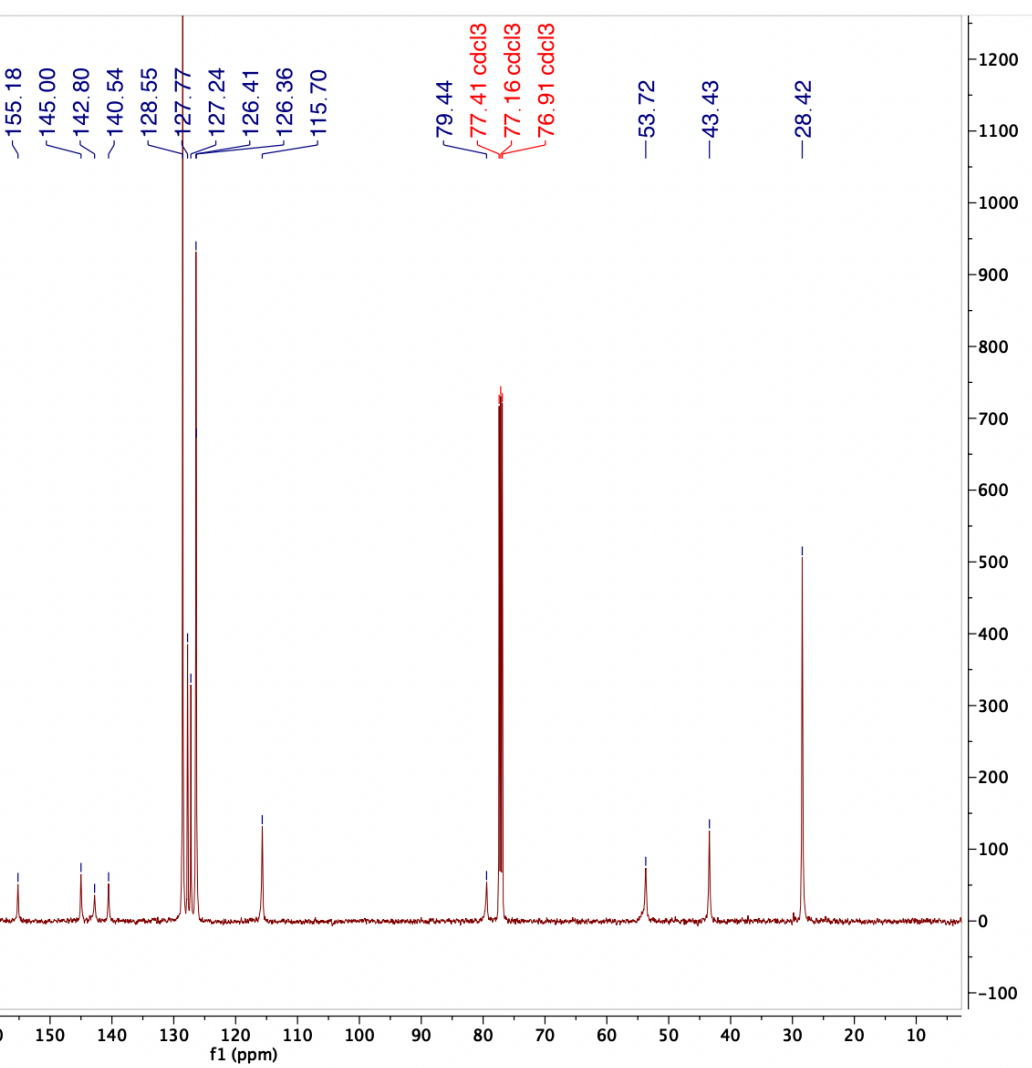



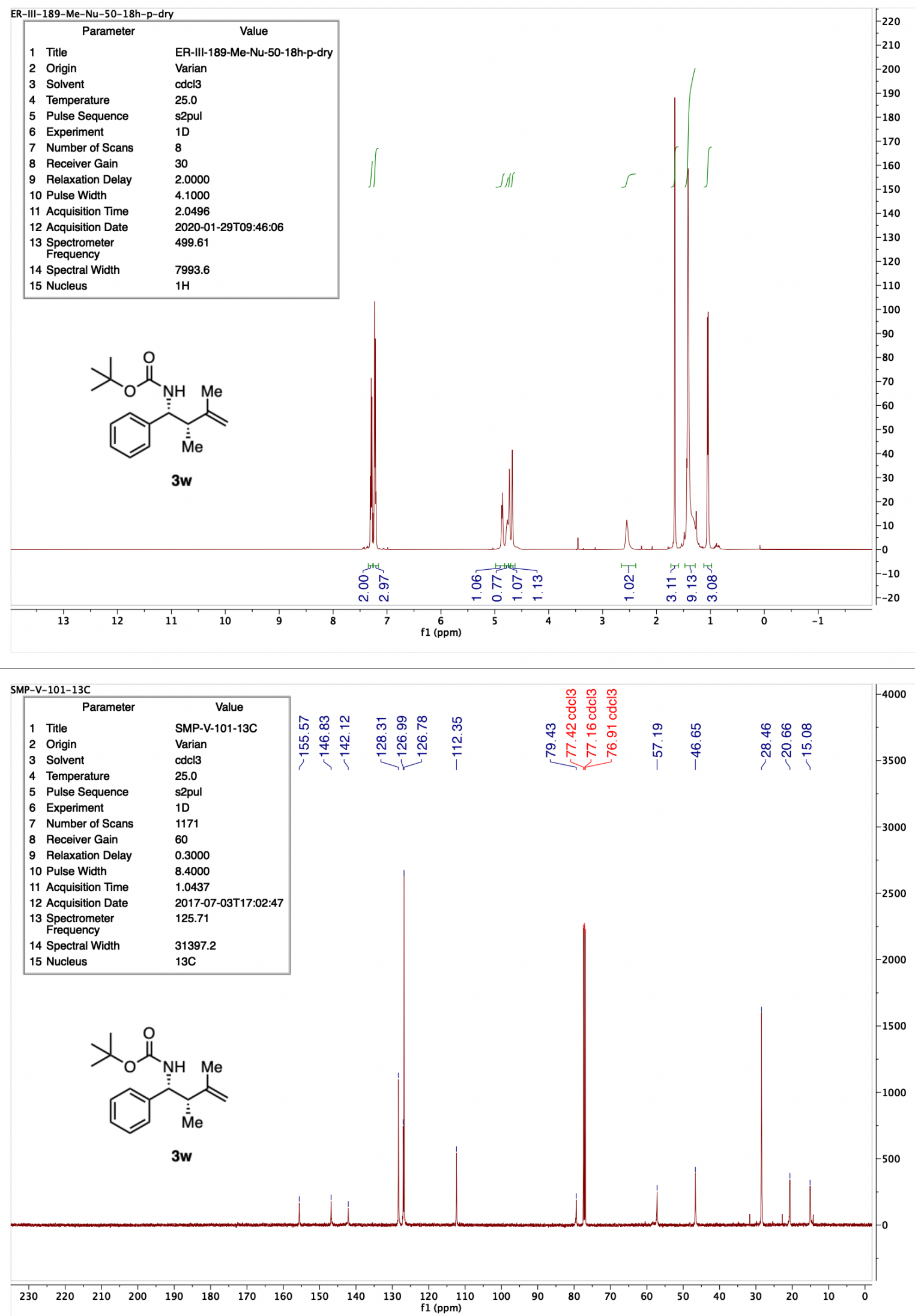

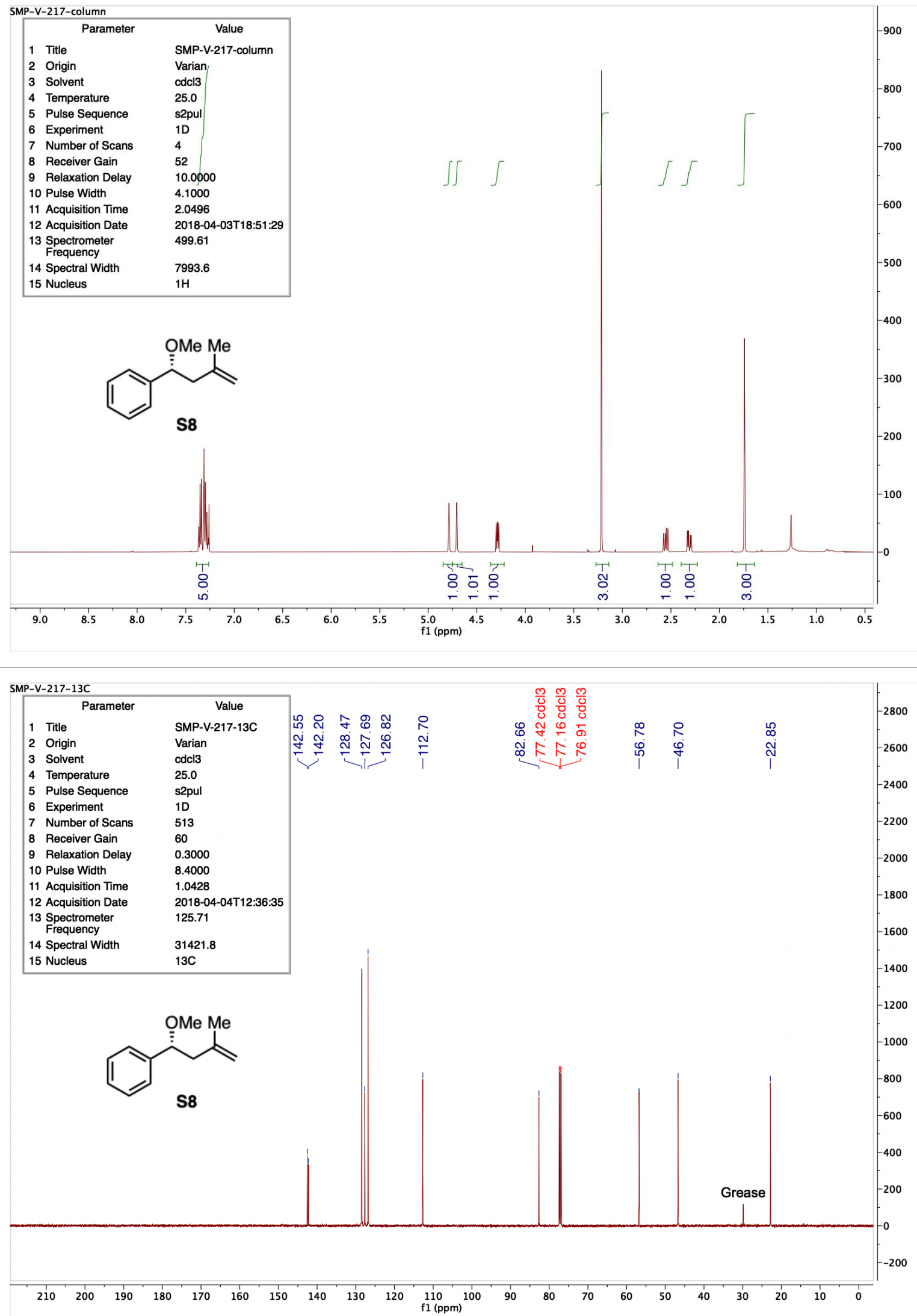
Catalysts

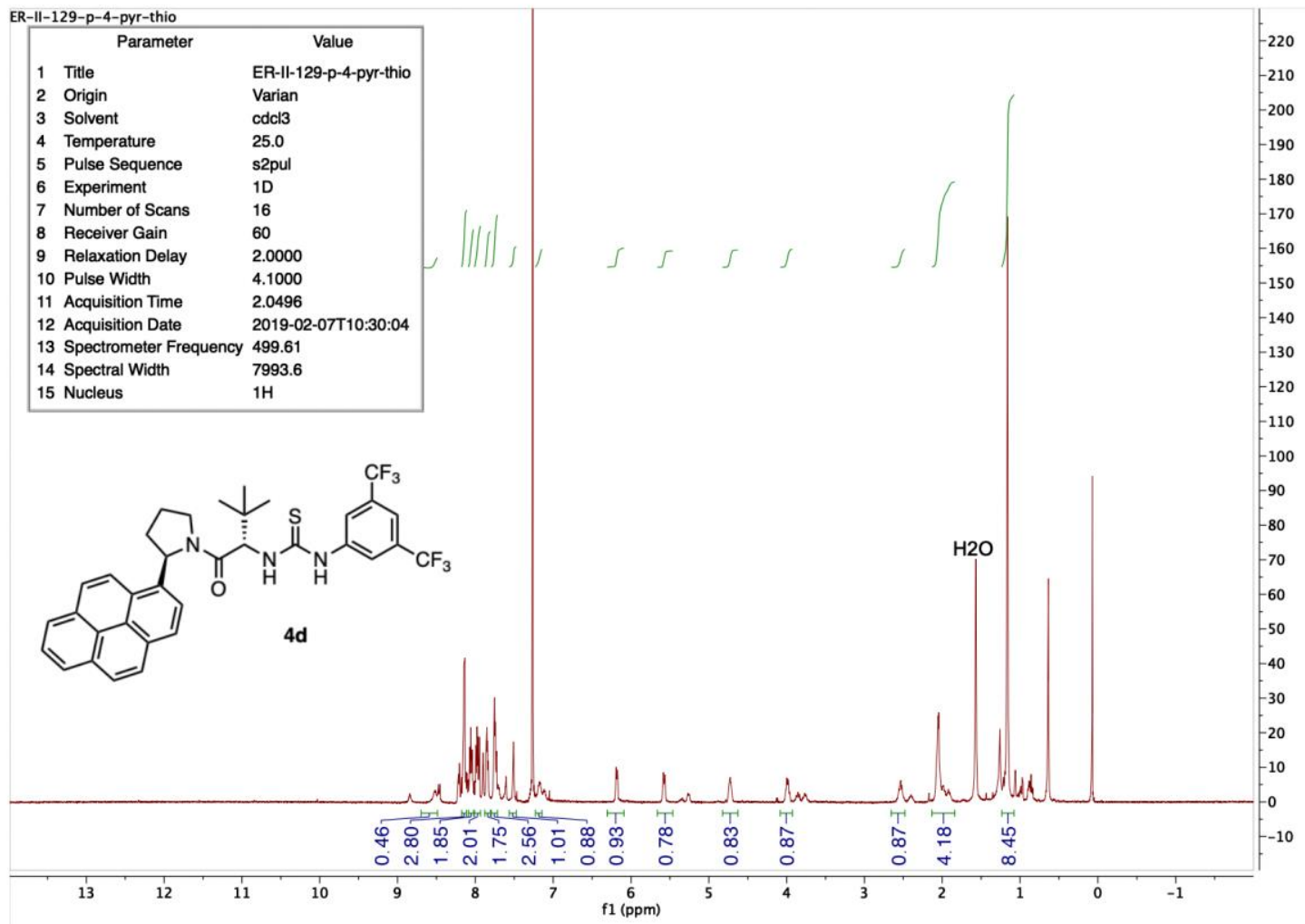

\begin{tabular}{|c|c|}
\hline \multicolumn{2}{|l|}{ ER-II-129-1-pyr-thio-13c } \\
\hline Parameter & Value \\
\hline 1 Title & ER-11-129-1-pyr-thio-13c \\
\hline 2 Origin & Varian \\
\hline 3 Solvent & cdcl3 \\
\hline 4 Temperature & 25.0 \\
\hline 5 Pulse Sequence & s2pul \\
\hline 6 Experiment & 1D \\
\hline 7 Number of Scans & 2352 \\
\hline 8 Receiver Gain & 60 \\
\hline 9 Relaxation Delay & 1.0000 \\
\hline 10 Pulse Width & 7.0000 \\
\hline 11 Acquisition Time & 0.9478 \\
\hline 12 Acquisition Date & 2020-01-02T16:27:40 \\
\hline $\begin{array}{l}13 \text { Spectrometer } \\
\text { Frequency }\end{array}$ & 125.71 \\
\hline 14 Spectral Width & 34572.2 \\
\hline 15 Nucleus & $13 \mathrm{C}$ \\
\hline
\end{tabular}
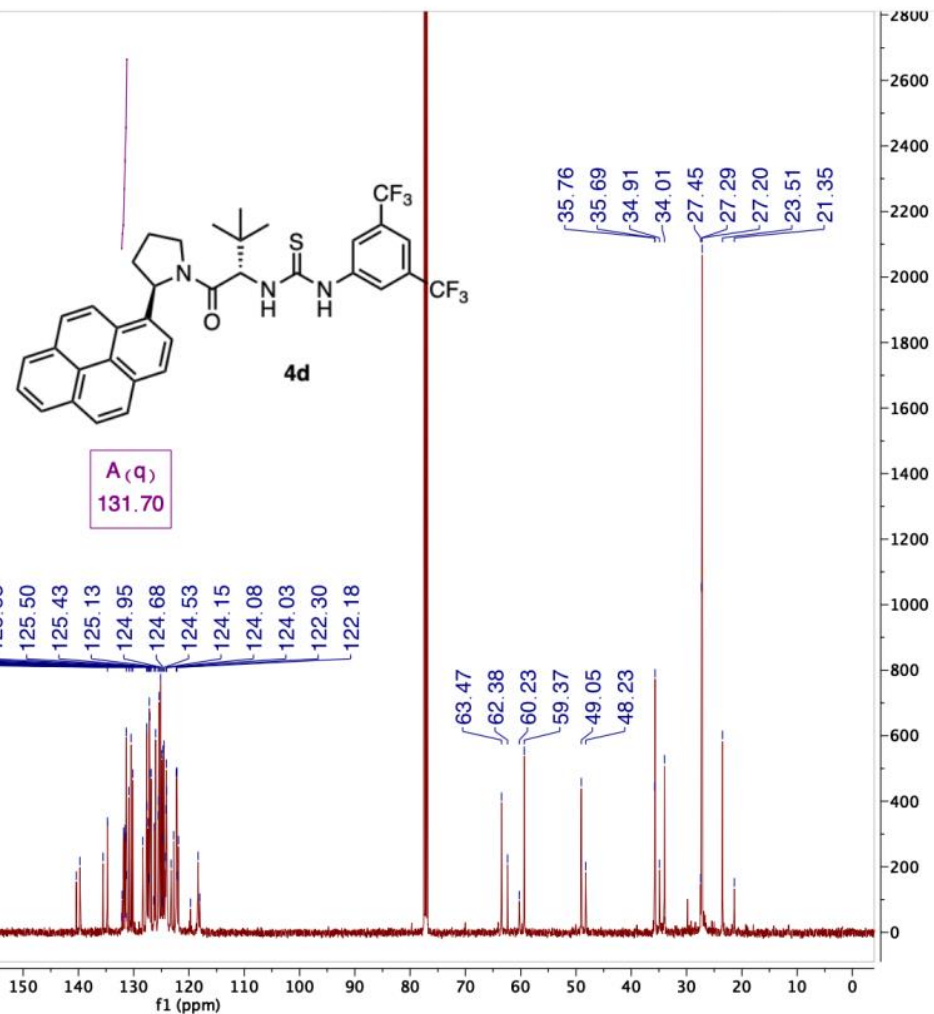

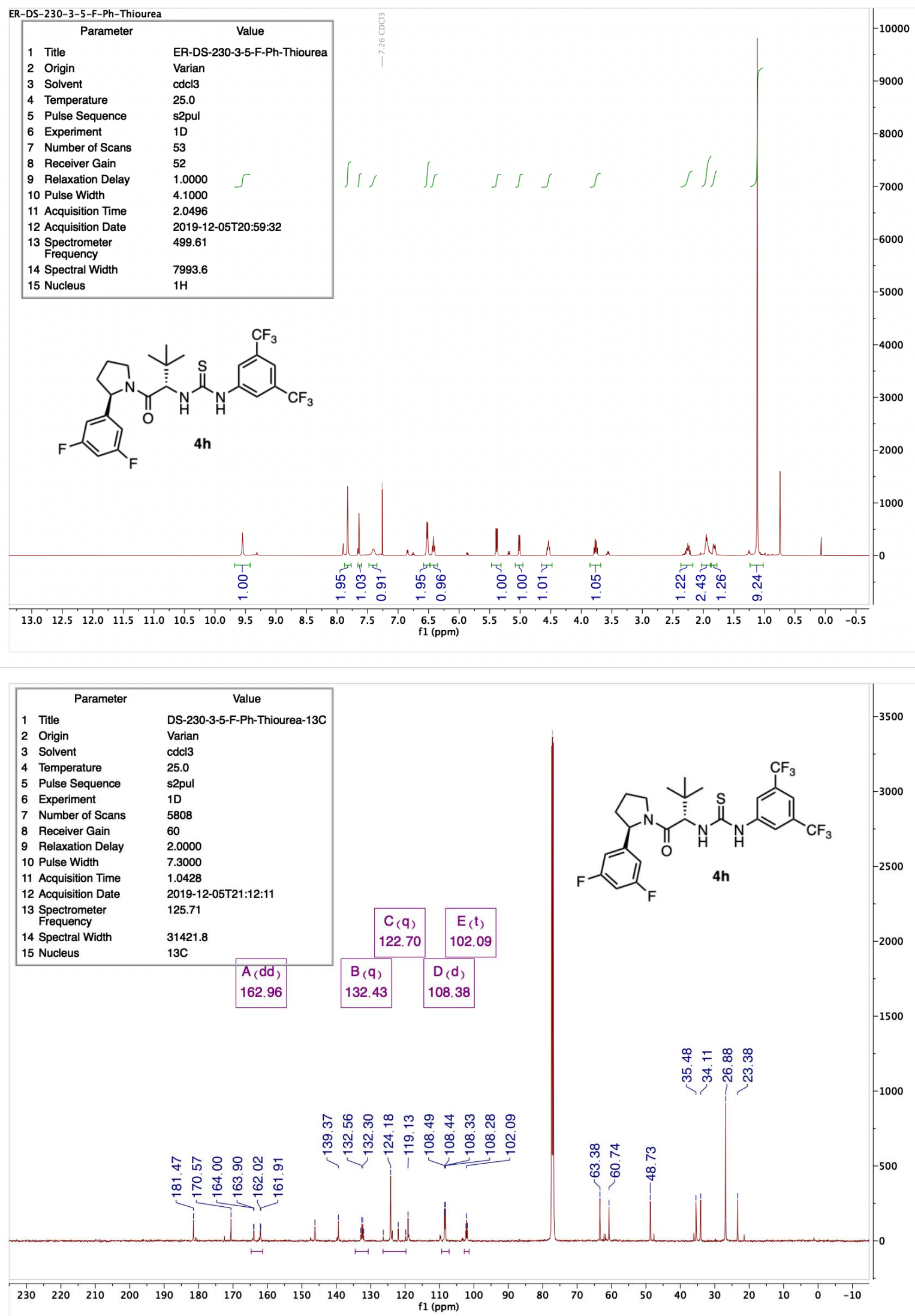

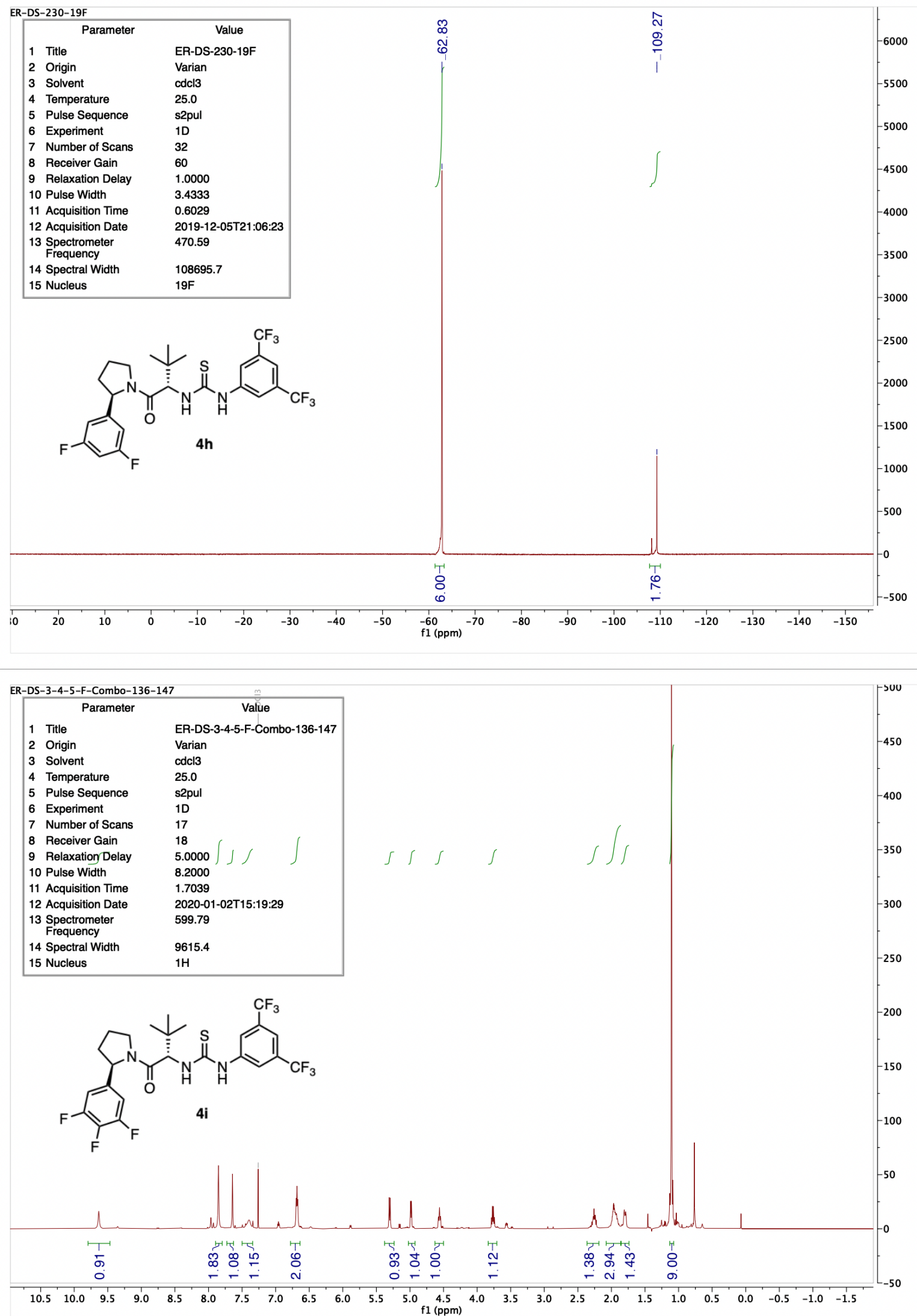

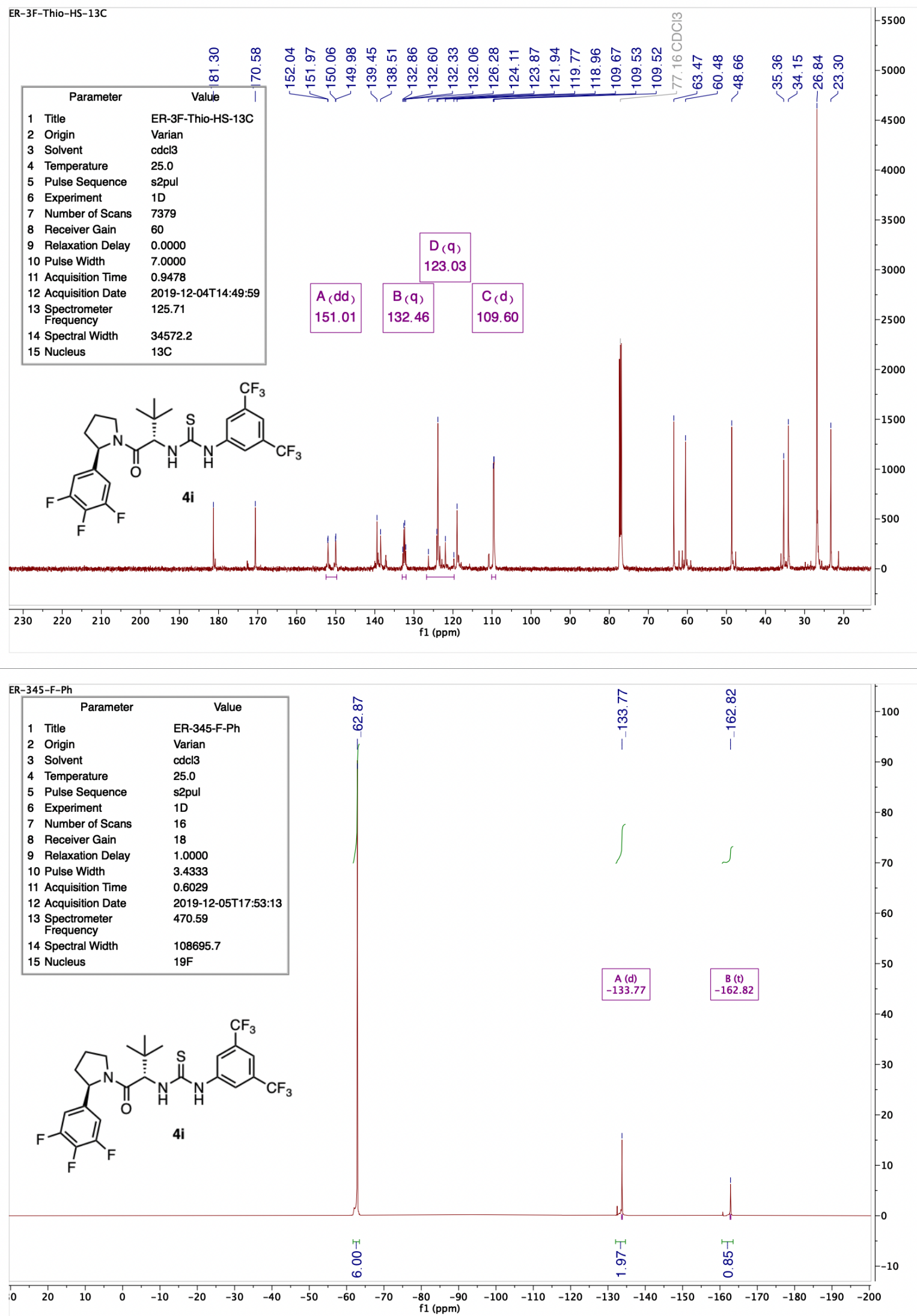

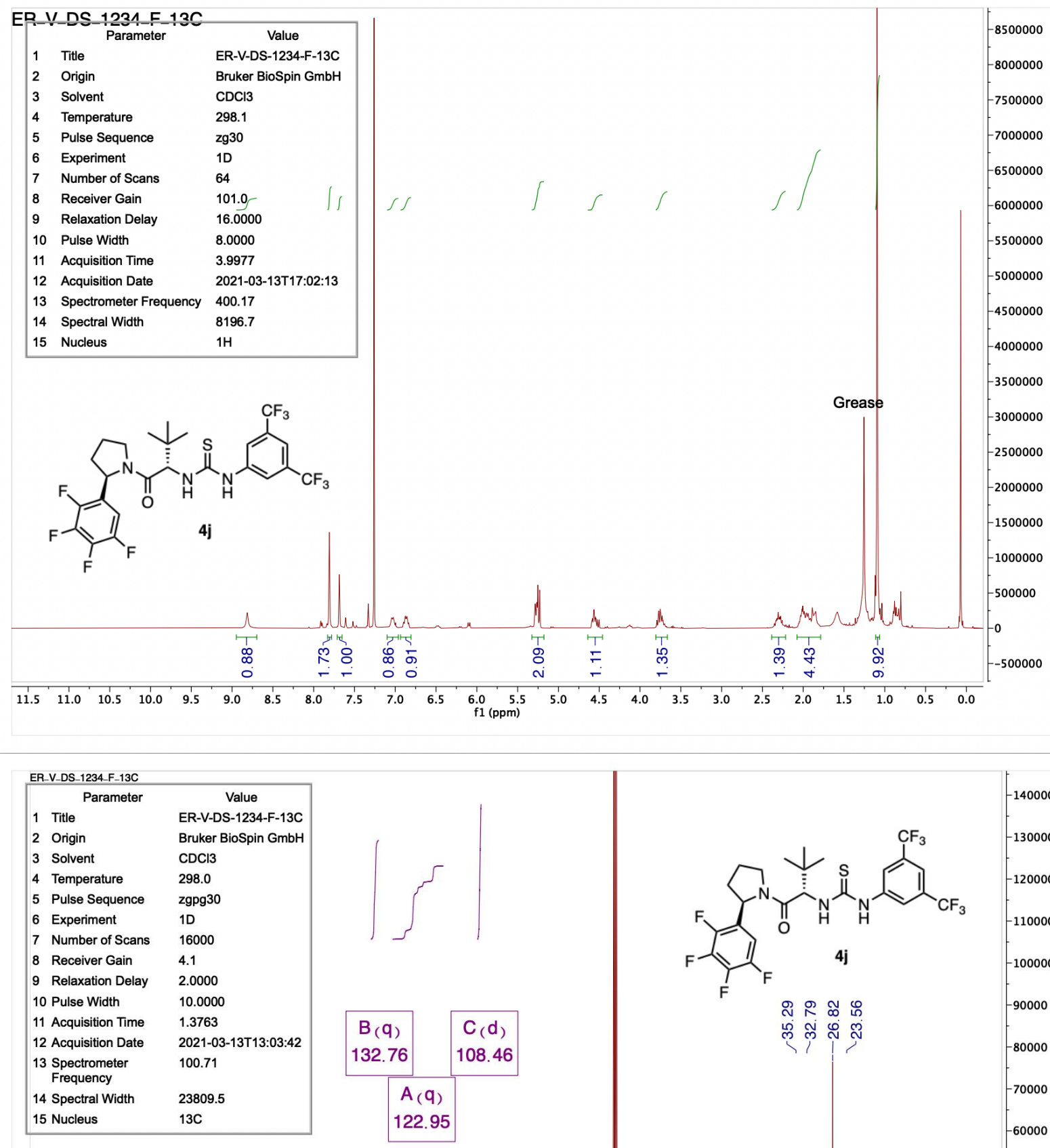

ชู

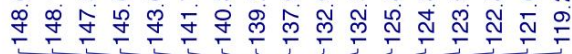
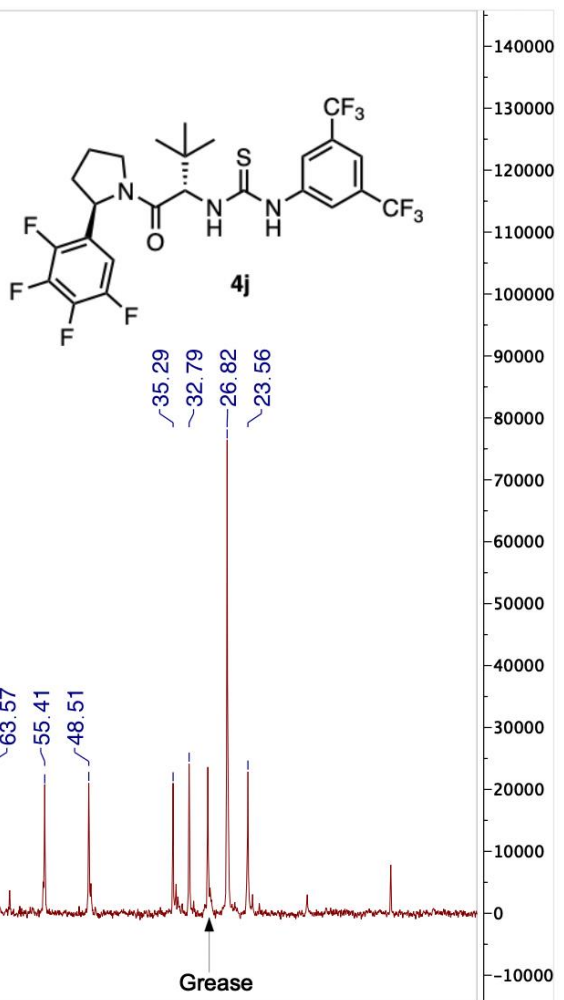

\begin{tabular}{lllllllllllllllllllllllll}
\hline 210 & 200 & 190 & 180 & 170 & 160 & 150 & 140 & 130 & 120 & 110 & 100 & 90 & 80 & 70 & 60 & 50 & 40 & 30 & 20 & 10 & 0 & -10
\end{tabular} 

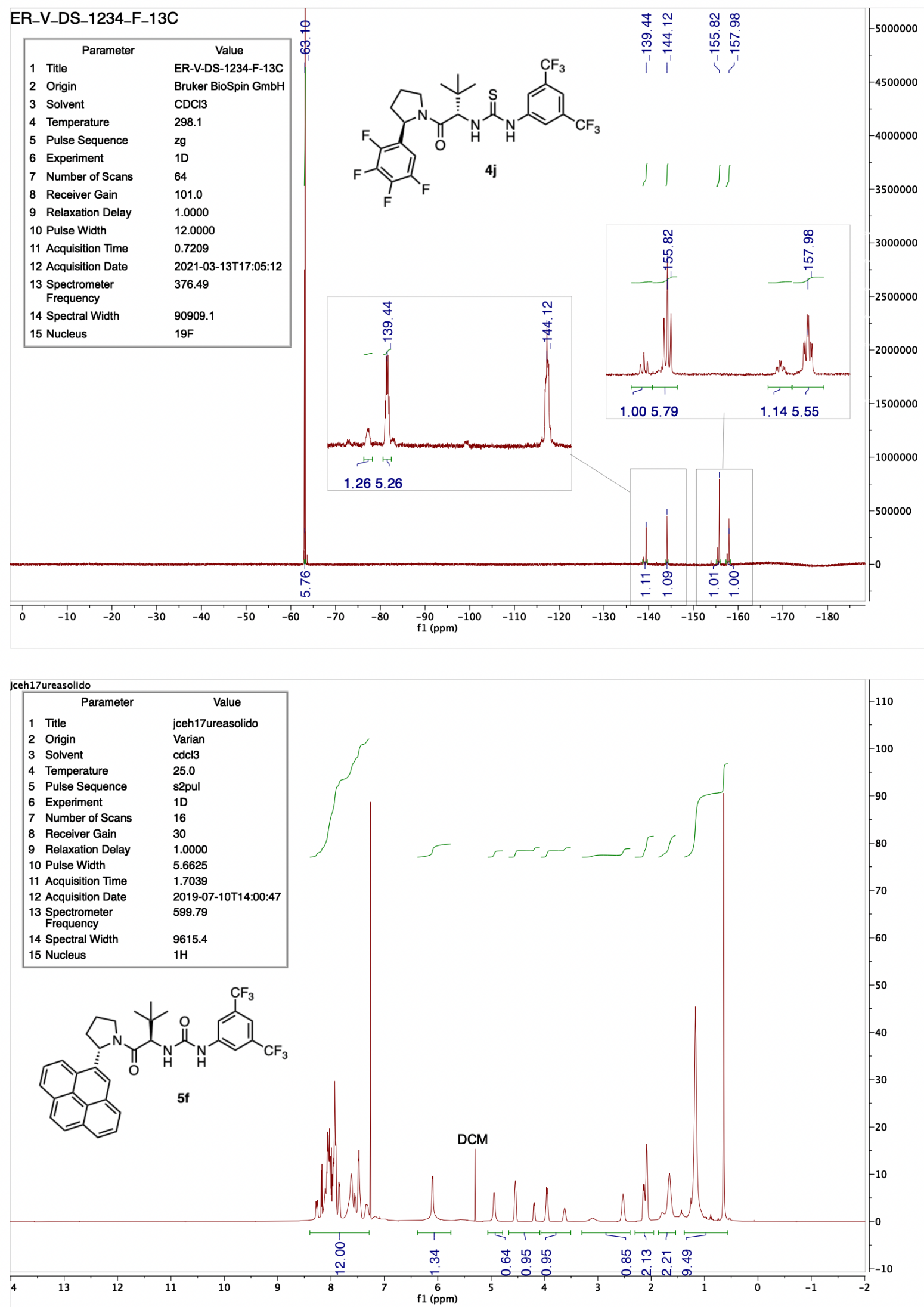


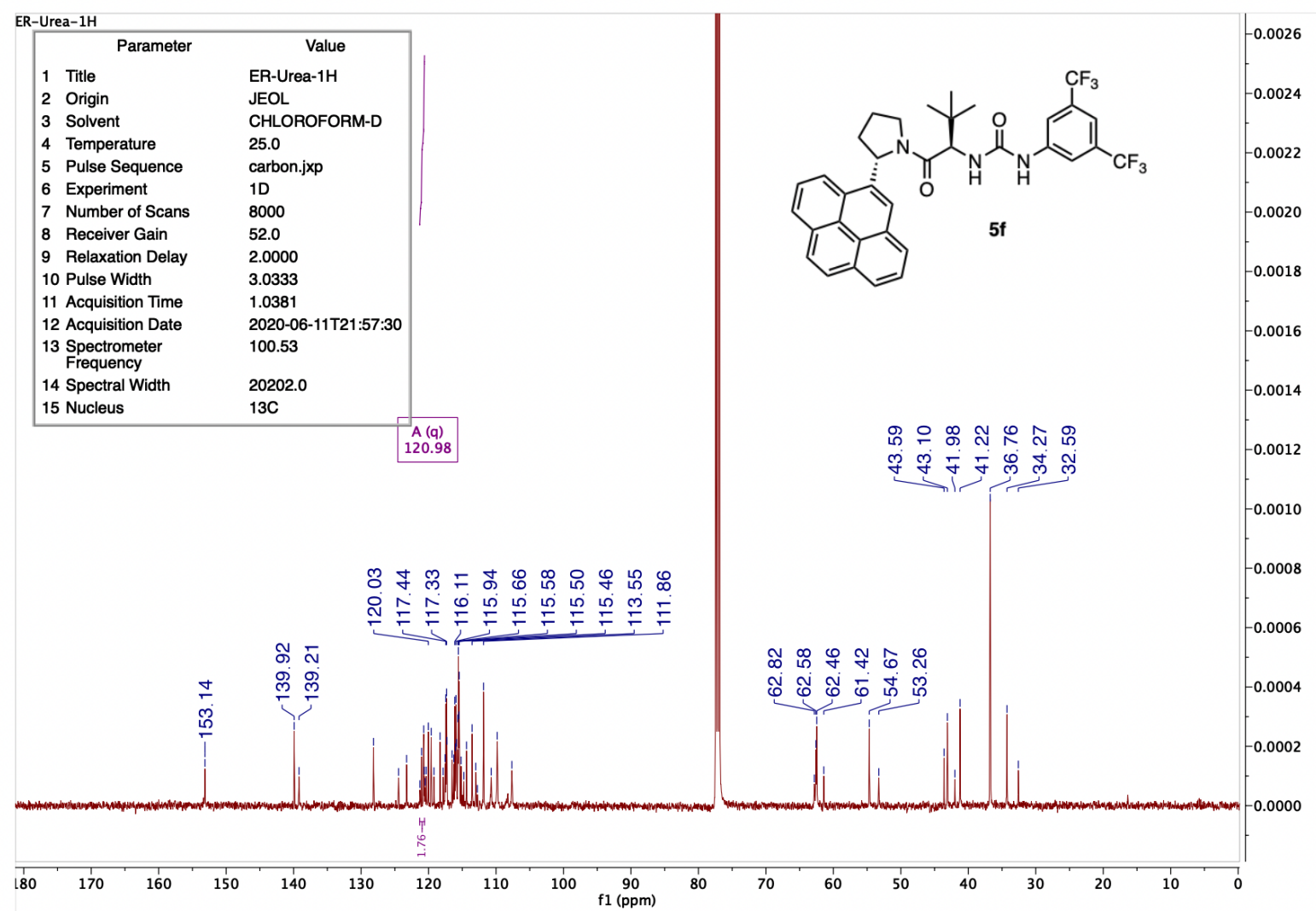

ER-II-240-Oct29
\begin{tabular}{|lll|}
\hline \multicolumn{1}{|c|}{ Parameter } & \multicolumn{1}{c|}{ Value } \\
1 & Title & ER-II-240-Oct29 \\
2 & Origin & Varian \\
3 & Solvent & cdc13 \\
4 & Temperature & 25.0 \\
5 & Pulse Sequence & s2pul \\
6 & Experiment & $1 \mathrm{D}$ \\
7 & Number of Scans & 12 \\
8 & Receiver Gain & 50 \\
9 & Relaxation Delay & $2.0000 / \int \mid$ \\
10 & Pulse Width & 4.1000 \\
11 & Acquisition Time & 2.0496 \\
12 Acquisition Date & $2019-10-29 T 11: 08: 15$ \\
13 Spectrometer & 499.61 \\
Frequency & \\
14 Spectral Width & 7993.6 \\
15 Nucleus & $1 \mathrm{H}$ \\
\hline
\end{tabular}<smiles>CC(C)(C)OC(=O)N[C@H](C(=O)Nc1cc([N+](=O)[O-])ccc1N)C(C)(C)C</smiles>

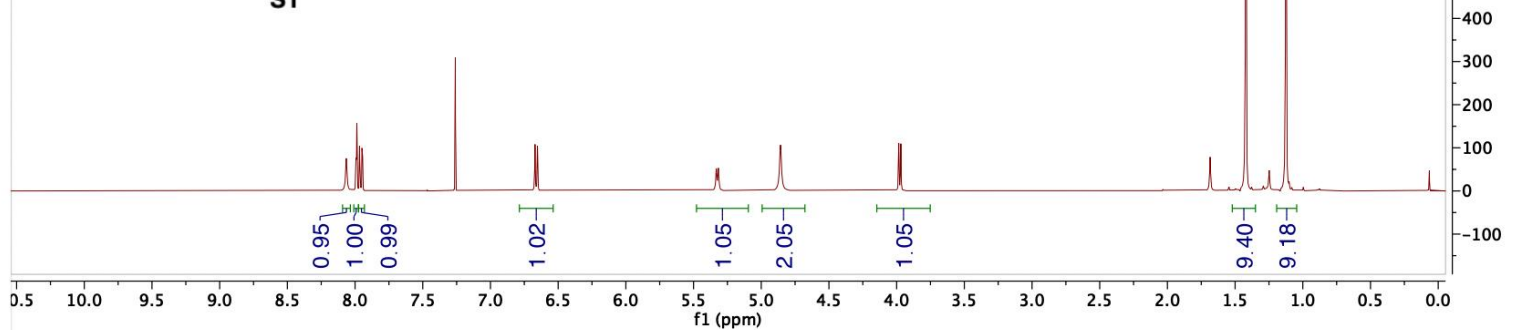




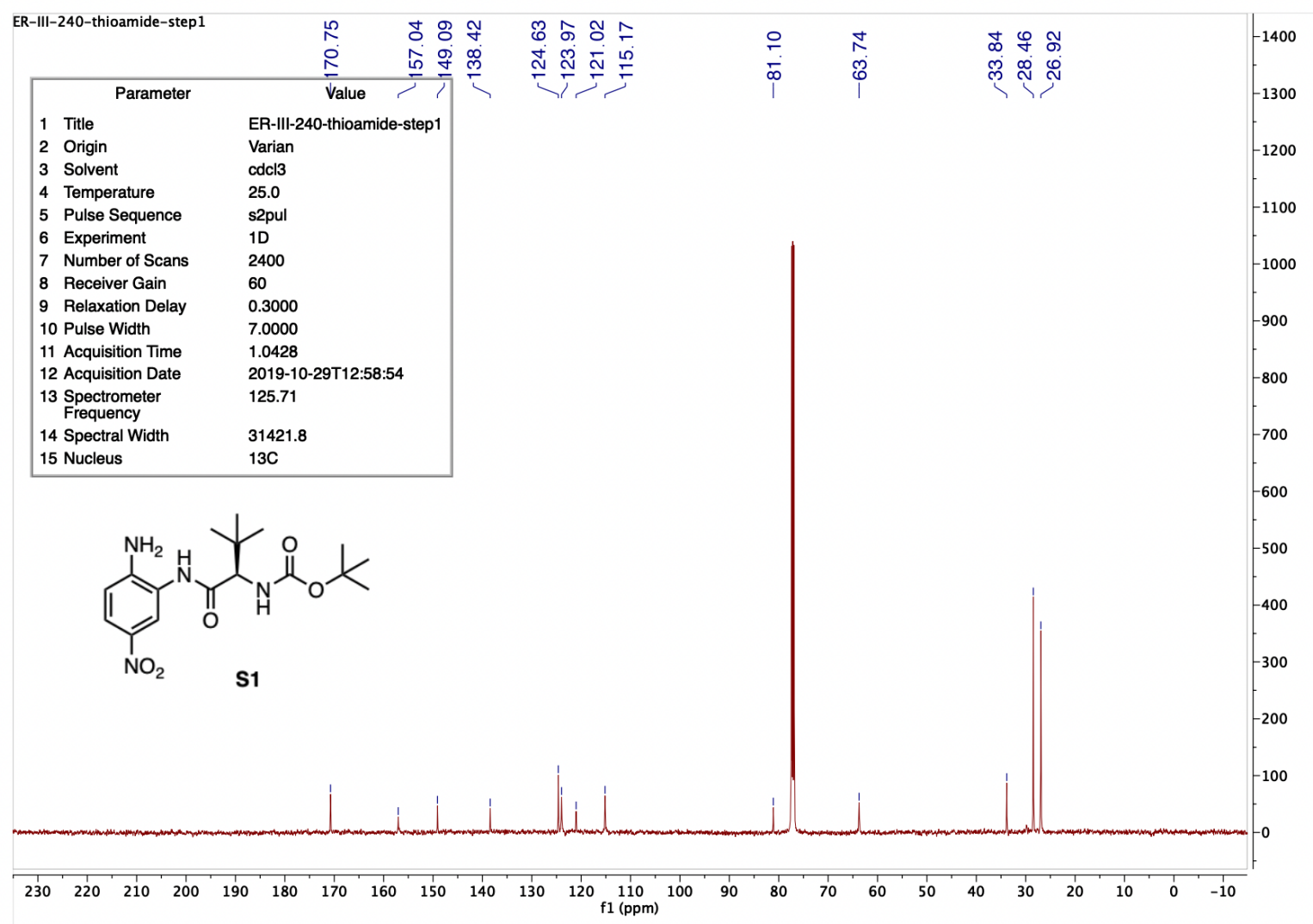

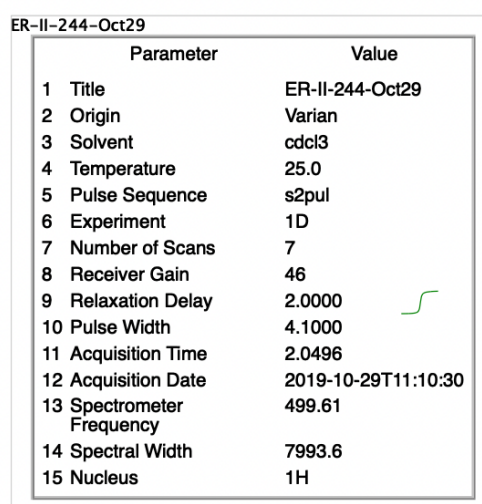<smiles>CC(C)(C)OC(=O)N[C@H](C(=S)Nc1cc([N+](=O)[O-])ccc1N)C(C)(C)C</smiles>

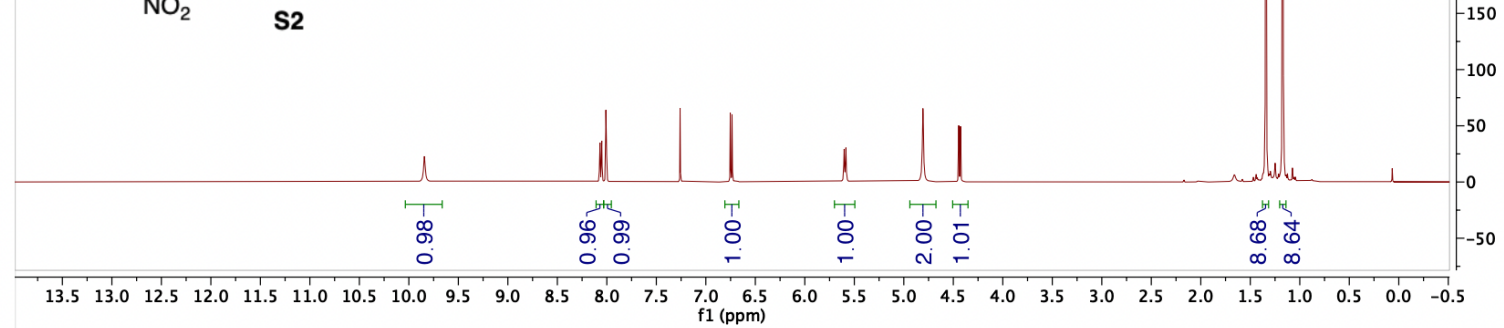



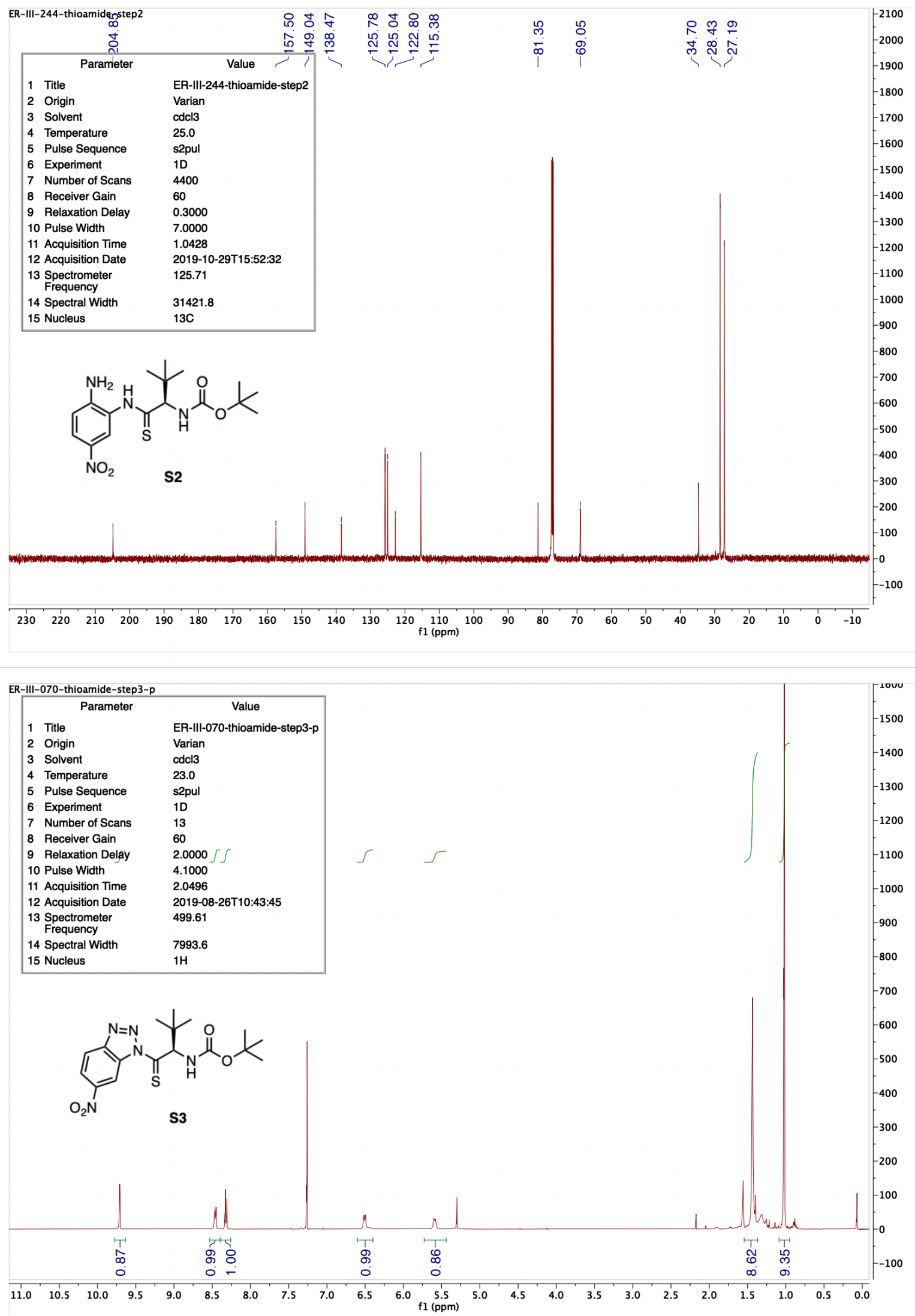

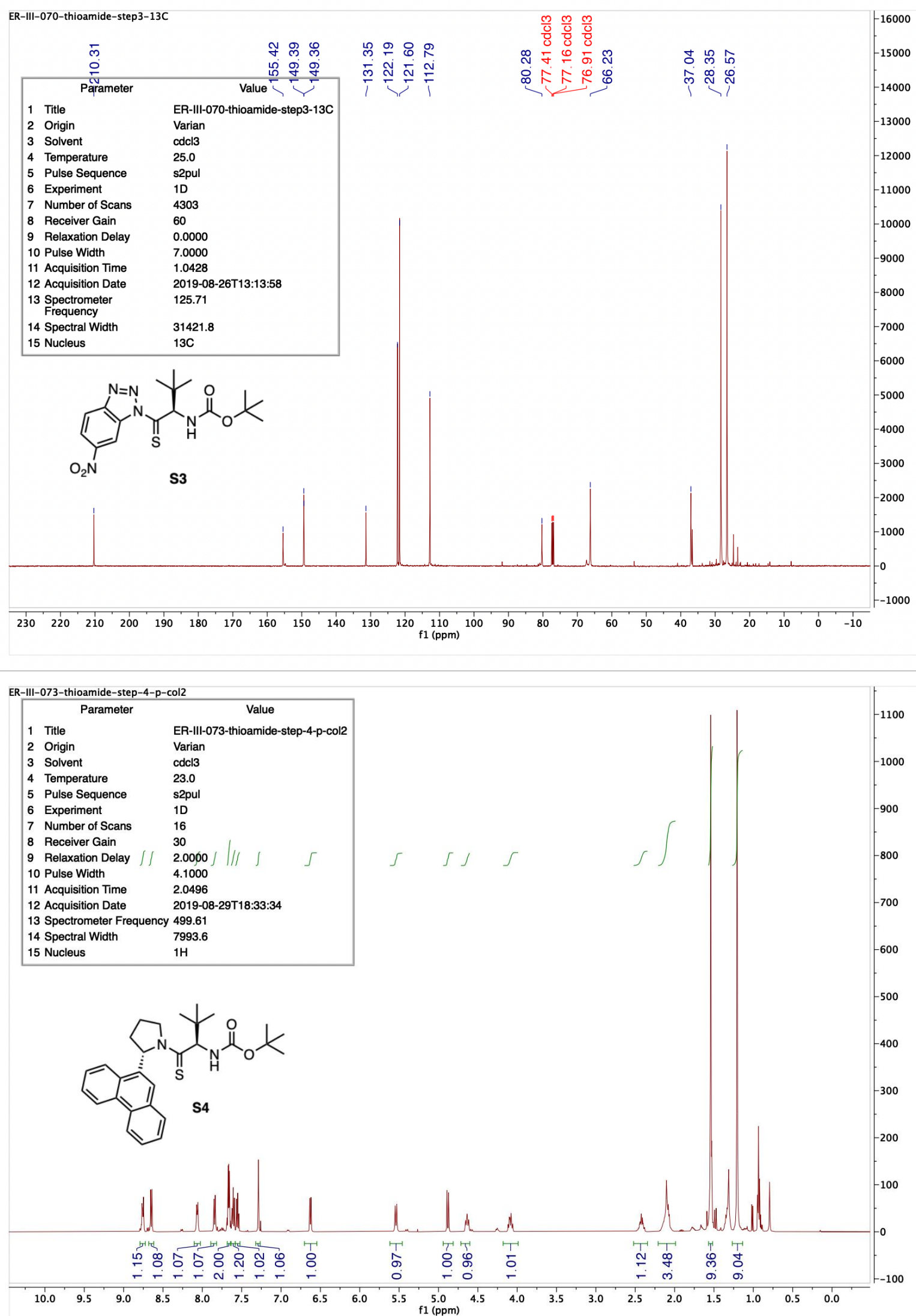

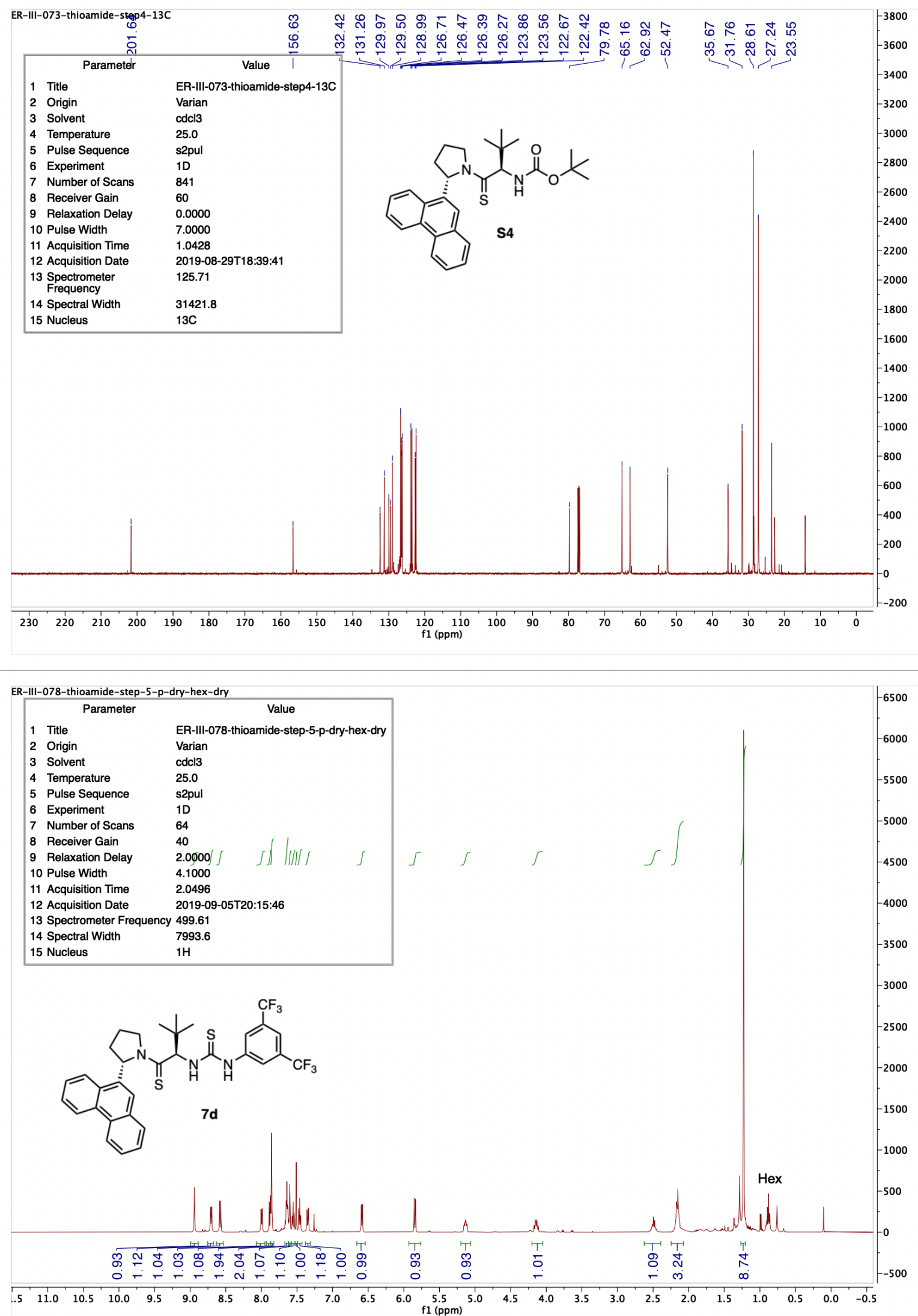

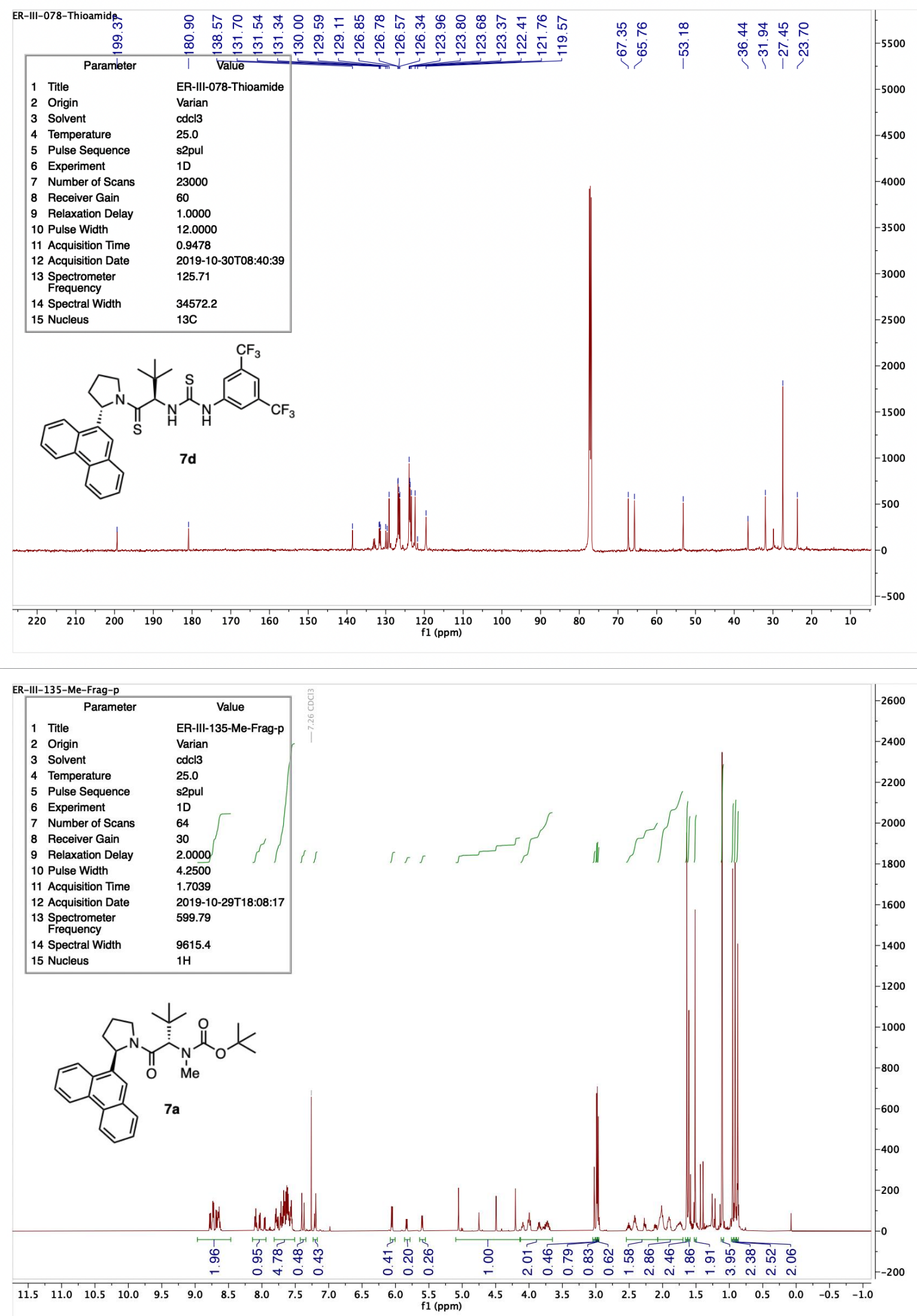


\begin{tabular}{|} 
ER-III-036-Me-Fragment-13C \\
\begin{tabular}{|lll|}
\hline \multicolumn{3}{|c|}{ Parameter } \\
1 & Title & VR-III-036-Me-Fragment-13C \\
2 & Origin & Varian \\
3 & Solvent & cdcl3 \\
4 & Temperature & 25.0 \\
5 & Pulse Sequence & s2pul \\
6 & Experiment & $1 \mathrm{D}$ \\
7 & Number of Scans & 1921 \\
8 & Receiver Gain & 60 \\
9 & Relaxation Delay & 2.0000 \\
10 & Pulse Width & 12.0000 \\
11 Acquisition Time & 0.9478 \\
12 Acquisition Date & $2019-09-25 T 12: 06: 19$ \\
13 & Spectrometer & 125.71 \\
Frequency & \\
14 Spectral Width & 34572.2 \\
15 Nucleus & $13 \mathrm{C}$ \\
\hline
\end{tabular}
\end{tabular}

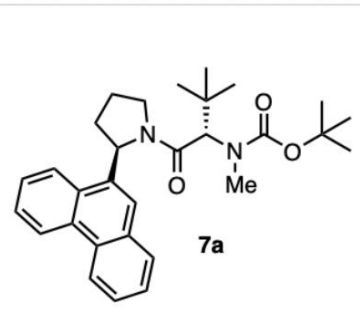

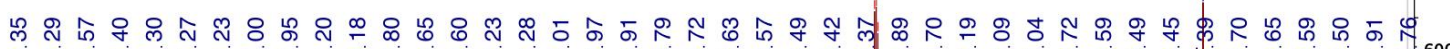
চ心
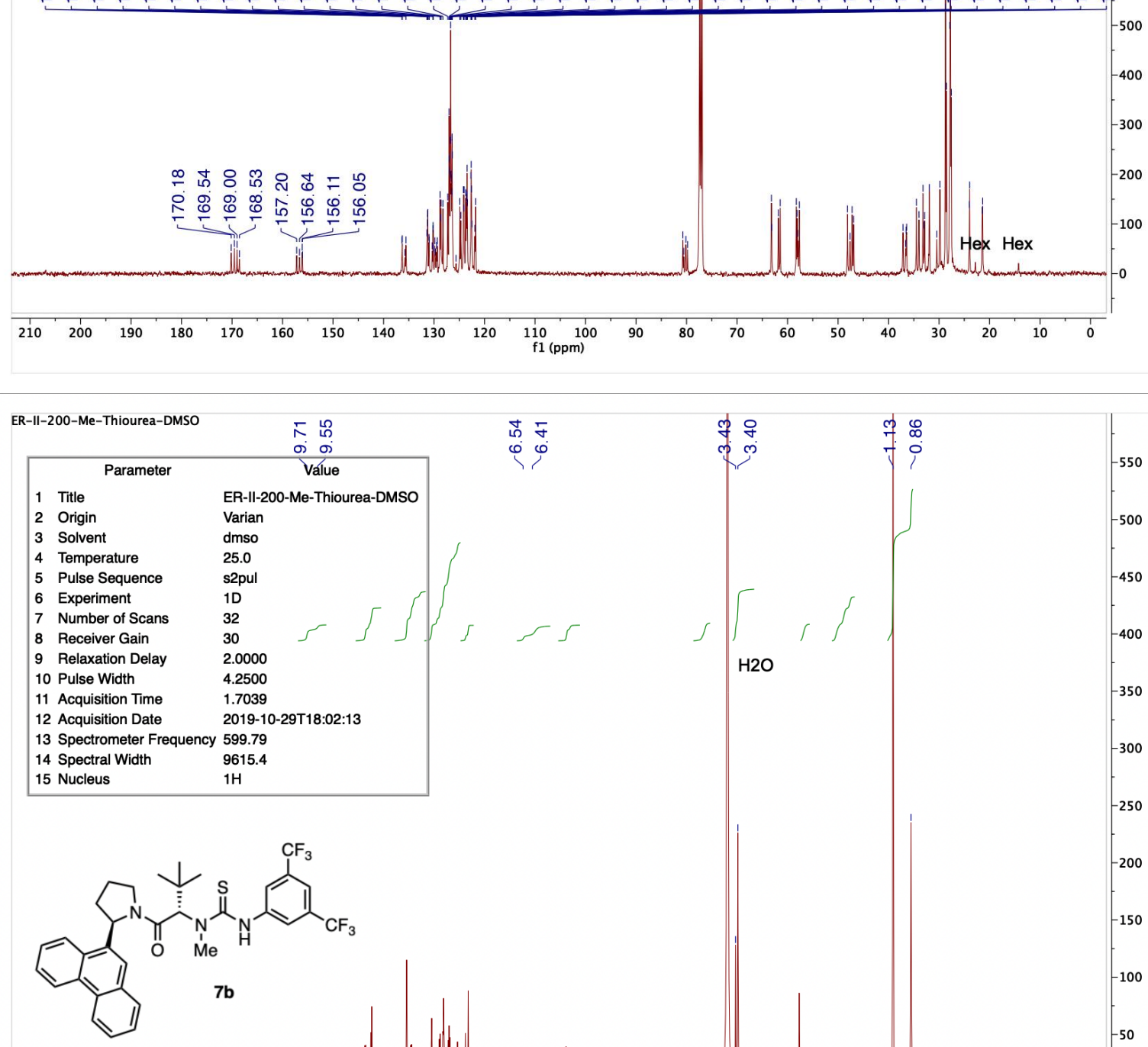

Integration of both rotamers
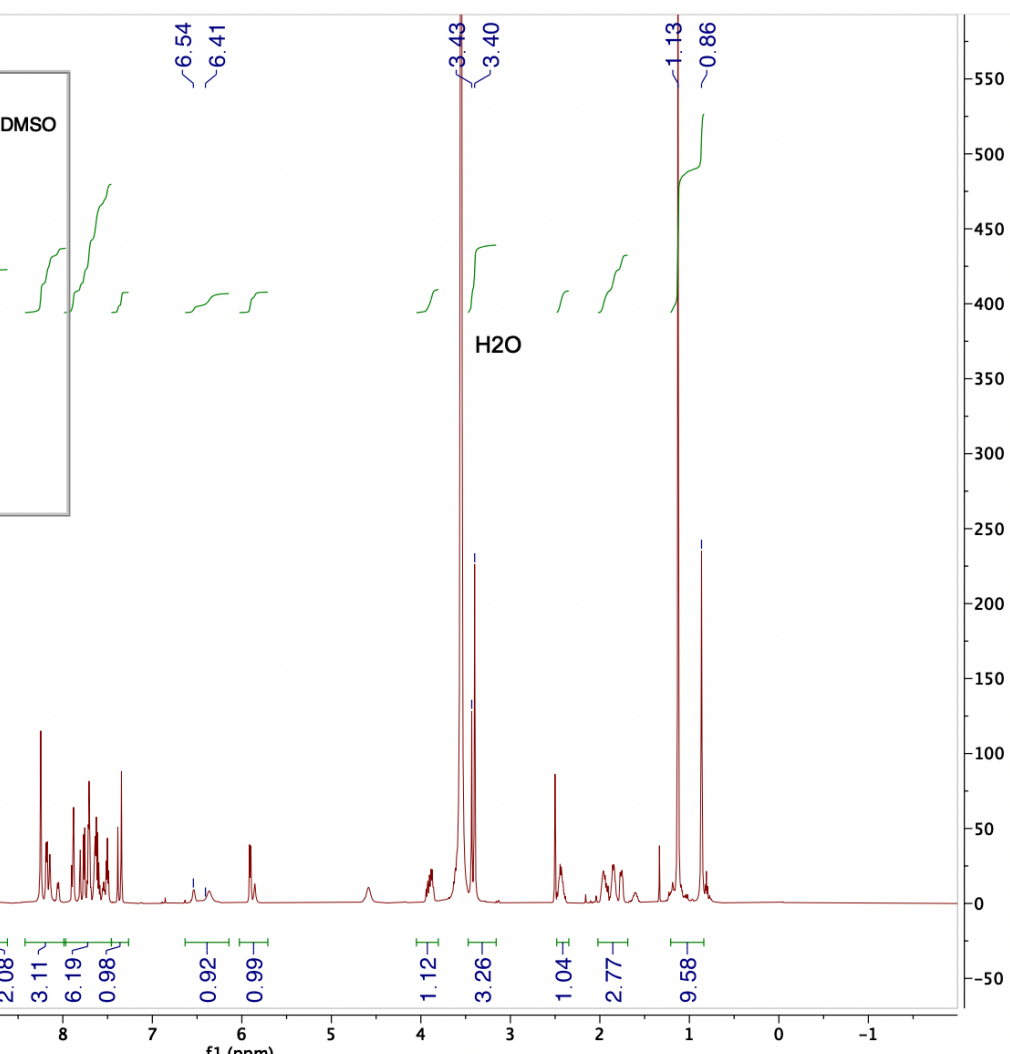


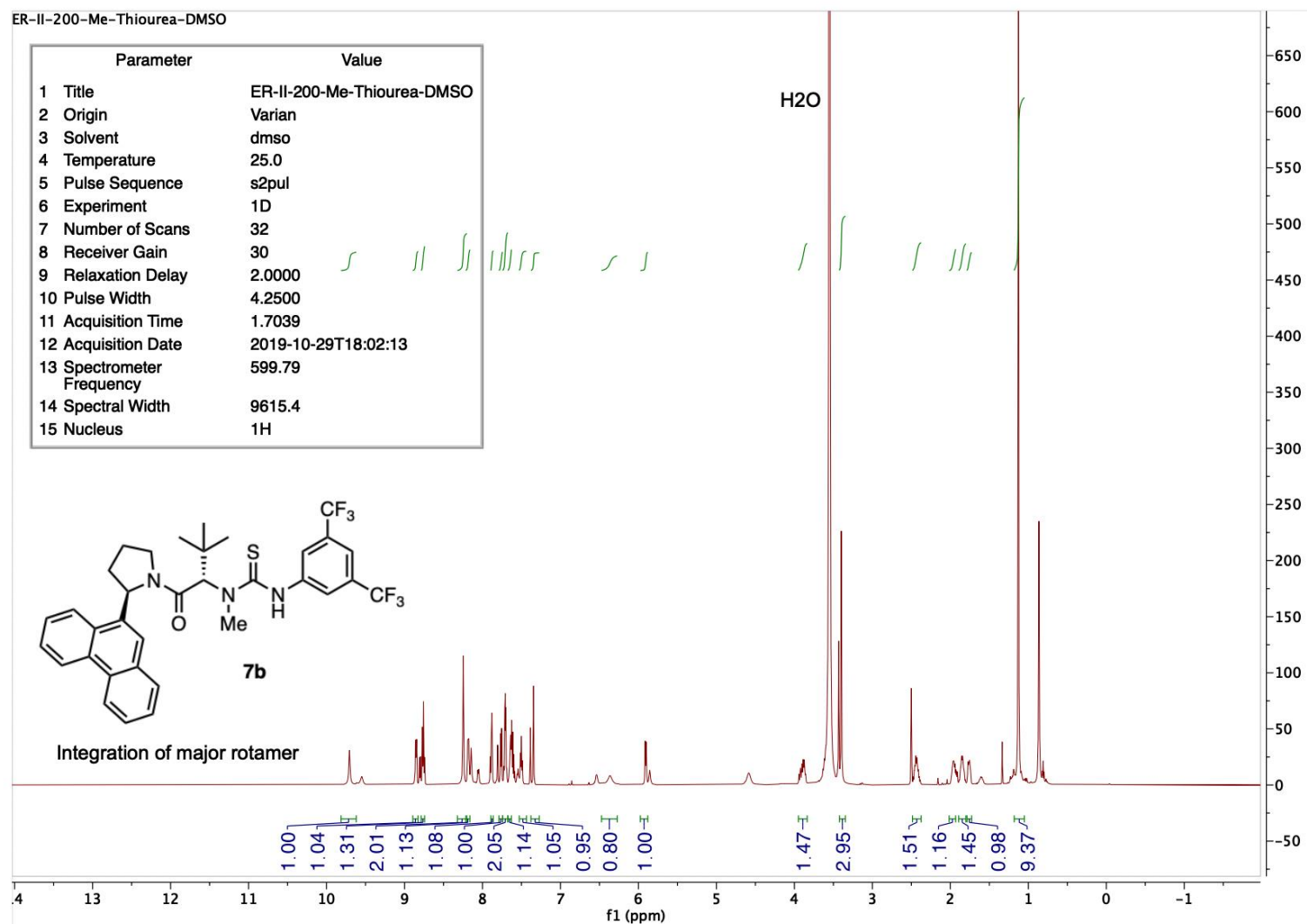

\begin{tabular}{|c|c|c|}
\hline \multicolumn{3}{|c|}{ ER-II-200-Me-Thiourea-dmso } \\
\hline & Parameter & Value \\
\hline 1 & Title & ER-II-200-Me-Thiourea-dmso \\
\hline 2 & Origin & Varian \\
\hline 3 & Solvent & dmso \\
\hline 4 & Temperature & 25.0 \\
\hline 5 & Pulse Sequence & s2pul \\
\hline 6 & Experiment & 1D \\
\hline 7 & Number of Scans & 5000 \\
\hline 8 & Receiver Gain & 60 \\
\hline 9 & Relaxation Delay & 0.0000 \\
\hline & Pulse Width & 12.0000 \\
\hline 11 & Acquisition Time & 0.9478 \\
\hline & Acquisition Date & 2019-10-29T19:50:02 \\
\hline & Spectrometer Frequency & 125.71 \\
\hline & Spectral Width & 34572.2 \\
\hline & Nucleus & $13 \mathrm{C}$ \\
\hline
\end{tabular}

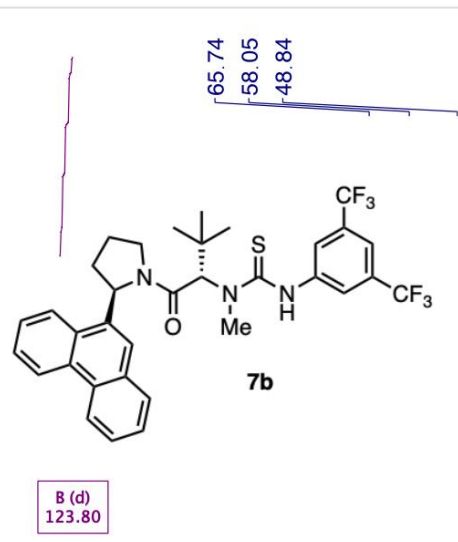

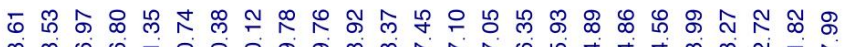

守守宛品

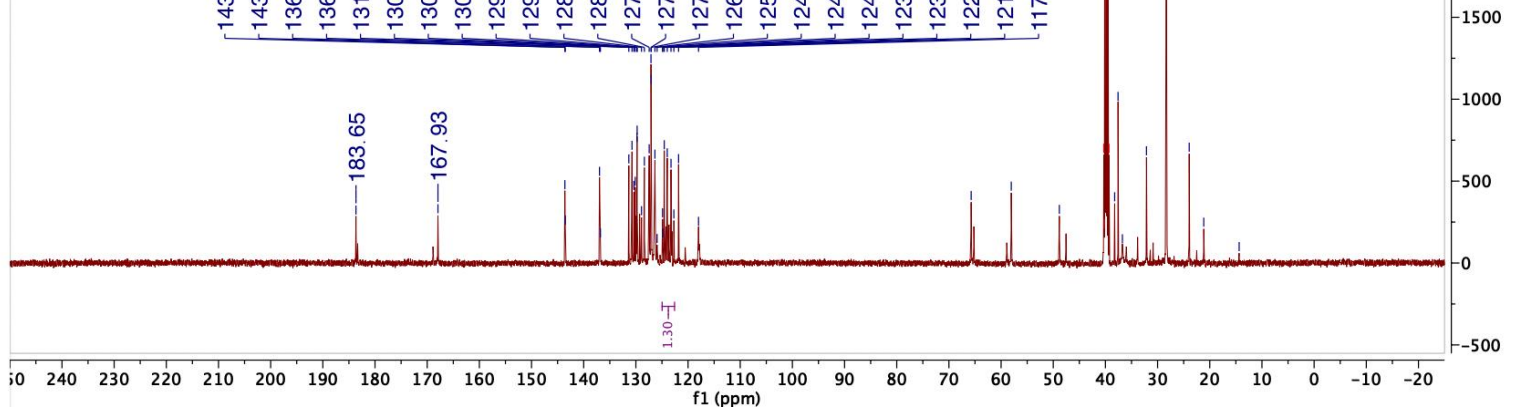


1 Gregg, B.T.; Golden, K.C.; Quinn, J.F. Tetrahedron 2008, 64, 3287-3295.

2 Sakai, N.; Moritaka, K; Konakahara, T. Eur. J. Org. Chem. 2009, 4123-4127.

3 Wiles, C.; Watts, P.; Haswell, S.J. Tetrahedron 2005, 61, 5209-5217.

4 Bourget, C.; Kotera, M.; Lhomme, J.; Trevisiol, E.; Laayoun, A.; Tora, C.; Sothier, I. WO 2002090584, 2002. (Compound 39).

${ }^{5}$ Napolitano, E.; Giannone, E.; Fiaschi, R.; Marsili, A. J. Org. Chem. 1983, 48, 3653-3657.

${ }^{6}$ Liu, Y.; Huang, Y.; Song, H.; Liu, Y.; Wang, Q. Chem. Eur. J. 2015, 21, 5337-5340.

7 Schneider, U.; Dao, H.T.; Kobayashi, S. Org. Lett. 2010, 12, 2488-2491.

${ }^{8}$ Farahat, A.A.; Kumar, A.; Barghash, A.E-D.M.; Goda, F.E.; Eisa, H.M.; Boykin, D.W. J. Het. Chem. 2010, 47, 167-170.

${ }^{9}$ Trost, B.M.; Chan, D.M.T.; Nanninga, T.N. Org Synth 1984, 62, 58.

10 Ochiai, M.; Fujita, E.; Arimoto, M.; Yamaguchi, H. Chem. Pharm. Bull. 1983, 31, 86-93.

${ }^{11}$ Lam, Y-H.; Bobbio, C.; Cooper, I.R.; Gouverneur, V. Angew. Chem. Int. Ed. 2007, 46, 5106-5110.

12 Guo, L.; Leiendecker, M.; Hsiao, C-C.; Baumann, C.; Rueping, M. Chem. Commun. 2015, 51, 19371940.

${ }_{13}^{13}$ Andreini, B.P.; Carpita, A.; Rossi, R.; Scamuzzi, B. Tetrahedron 1989, 45, 5621-5640.

${ }^{14}$ Reisman, S. E.; Doyle, A. G., Jacobsen, E. N. J. Am. Chem. Soc. 2008, 130, 23, 7198-7199.

15 Knowles, R. R.; Lin, S.; Jacobsen, E. N. J. Am. Chem. Soc. 2010, 132, 5030-5032.

${ }^{16}$ Bergonzini, G.; Schindler, C.S.; Wallentin, C.-J.; Jacobsen, E.N.; Stephenson, C. R. J. Chem. Sci., 2014, 5, 112-116.

17 Liu, R.Y.; Wasa, M.; Jacobsen, E.N. Tet. Lett. 2015, 56, 3428-3430.

18 Shalaby, M. A., Grote, C. W., Rapoport, H. J. Org. Chem. 1996, 61, 9045-9048.

19 Soares, P., Lucas, X., Ciulli, A. Bioorg Med Chem, 2018, 26, 11, 2992-2995.

${ }^{20}$ Ramachandran, P. V., Burghardt, T. E., Bland-Berry L. J. Org. Chem., 2005, 70, 20, 7911-7918. For ${ }^{1} \mathrm{H}$ NMR shifts of related crotyl amine compounds see 22a (anti, $\delta 0.83(\mathrm{~d}, J=6.7 \mathrm{~Hz}, 3 \mathrm{H})$ ) and 23a (syn, $\delta$ $1.06(\mathrm{~d}, J=7.2 \mathrm{~Hz}, 3 \mathrm{H}))$.

21 Delaye, P.-O.; Vasse, J.-L.; Szymoniak, J. Org. Biomol. Chem. 2010, 8, 3635-3637.

22 Hatano, M.; Moriyama, K.; Maki, T.; Ishihara, K. Angew. Chem. Int. Ed. 2010, 49, 3823-3826.

23 Song, J.; Wang, Y.; Deng, L. J. Am. Chem. Soc. 2006, 128, 6048-6049.

24 (a) Wenzel, A.G.; Jacobsen, E.N. J. Am. Chem. Soc. 2002, 124, 12964-12965. (b) Yang, J.W.; Pan, S.C.; List, B. Org. Synth. 2009, 86, 11-17.

${ }^{25}$ Gizecki, P.; Youcef, R.A.; Poulard, C.; Dhal, R.; Dujardin, G. Tet. Lett. 2004, 45, 9589-9592.

${ }^{26}$ Mayr, H.; Bug, T.; Gotta M.F.; Hering, N.; Irrgang, B.; Janker, B.; Kempf, B.; Loos, R.; Ofial, A. R.;

Remennikov, G.; Schimmel, H. J. Am. Chem. Soc. 2001, 123, 9500-9512.

27 For $R$ enantiomer, see: (a) Tillman, A. L.; Dixon, D. J. Org. Biomol. Chem. 2007, 5, 606-609; (b) Kashikura, W.; Mori, K.; Akiyama, T. Org. Lett. 2011, 13, 1860-1863.

For S enantiomer, see: (c) Shang, M.; Cao, M.; Wang, Q.; Wasa, M. Angew. Chem. Int. Ed. 2017, 56, 13338-13341.

${ }^{28}$ For full characterization of racemic product, see: (a) Downey, C.W.; Johnson, M.W.; Tracy, K.J. J. Org. Chem. 2008, 73, 3299-3302. For $S$ enantiomer, see: (b) Zhou, P.; Cai, Y.; Zhong, X.; Luo, W.; Kang, T.; Li, J.; Liu, X.; Lin, L.; Feng, X. ACS Cat. 2016, 6, 7778-7783.

${ }^{29}$ Hatano, M.; Takagi, E.; Ishihara, K. Org. Lett. 2007, 9, 4527-4530.

30 Onodera, G.; Toeda, T.; Toda, N.-N.; Shibagishi, D.; Takeuchi, R. Tetrahedron 2010, 66, 9021-9031.

${ }^{31}$ Gaussian 16, Revision A.03, Frisch, M. J.; Trucks, G. W.; Schlegel, H. B.; Scuseria, G. E.; Robb, M. A.; Cheeseman, J. R.; Scalmani, G.; Barone, V.; Petersson, G. A.; Nakatsuji, H.; Li, X.; Caricato, M.; Marenich, A. V.; Bloino, J.; Janesko, B. G.; Gomperts, R.; Mennucci, B.; Hratchian, H. P.; Ortiz, J. V.; 
Izmaylov, A. F.; Sonnenberg, J. L.; Williams-Young, D.; Ding, F.; Lipparini, F.; Egidi, F.; Goings, J.; Peng, B.; Petrone, A.; Henderson, T.; Ranasinghe, D.; Zakrzewski, V. G.; Gao, J.; Rega, N.; Zheng, G.; Liang, W.; Hada, M.; Ehara, M.; Toyota, K.; Fukuda, R.; Hasegawa, J.; Ishida, M.; Nakajima, T.; Honda, Y.; Kitao, O.; Nakai, H.; Vreven, T.; Throssell, K.; Montgomery, J. A., Jr.; Peralta, J. E.; Ogliaro, F.; Bearpark, M. J.; Heyd, J. J.; Brothers, E. N.; Kudin, K. N.; Staroverov, V. N.; Keith, T. A.; Kobayashi, R.; Normand, J.; Raghavachari, K.; Rendell, A. P.; Burant, J. C.; Iyengar, S. S.; Tomasi, J.; Cossi, M.; Millam, J. M.; Klene, M.; Adamo, C.; Cammi, R.; Ochterski, J. W.; Martin, R. L.; Morokuma, K.; Farkas, O.; Foresman, J. B.; Fox, D. J. Gaussian, Inc., Wallingford CT, 2016.,

32 Becke, A.D. Phys. Rev. A 1988, 38, 3098-3100; Lee, C.; Yang, W.; Parr, R.G. Phys. Rev. B 1988, 37, 785; Vosko, S.H.; Wilk, L.; Nusair, M. Can. J. Phys. 1980, 58, 1200-1211; Stephens, P. J.; Devlin, F.J.; Chabalowski, C.F.; Frisch, M.J. J. Phys. Chem. 1994, 98, 11623-11627.

33 Ditchfield, R.; Hehre, W. J.; Pople, J. A. J. Chem. Phys. 1971, 54, 724; Hehre, W. J.; Ditchfield, R.; Pople, J. A. J. Chem. Phys. 1972, 56, 2257; Gordon, M.S. Chem. Phys. Lett. 1980, 76, 163-168; Francl, M.M.; Pietro, W.J.; Hehre, W.J.; Binkley, J.S.; DeFrees, D.J.; Pople, J.A.; Gordon, M.S. J. Chem. Phys. 1982, 77, 3654-3665.

${ }^{34}$ Frisch, M.J.; Pople, J.A.; Binkley, J.S. J. Chem. Phys.1984, 80, 3265-3269.

35 Grimme, S.; Antony, J.; Ehrlich, S.; Krieg, H. J. Chem. Phys. 2010, 132, 154104; Grimme, S.; Ehrlich, S.; Goerigk, L. J. Comp. Chem. 2011, 32 1456; Becke, A.D.; Johnson, E.R. J Chem Phys 2005, 122, 154101; Johnson, E.R.; Becke, A.D. J Chem Phys 2005, 123, 24101; Johnson, E.R.; Becke, A.D. J Chem Phys 2006, 124, 174104.

36 Kruse, H., Goerigk, L., Grimme, S., J. Org. Chem. 2012, 77, 23, 10824-10834; Peng, Q., Duarte, F., Paton, R. S., Chem. Soc. Rev. 2016, 45, 6093-6107.

${ }^{37}$ Raghavachari, K.; Binkley, J.S.; Seeger, R.; Pople, J.A. J. Chem. Phys. 1980, 72, 650-654; McLean, A.D.; Chandler, G.S. J. Chem. Phys., 1980, 72, 5639-5648.

38 Mennucci, B.; Cances, E.; Tomasi, J. J. Phys. Chem. B. 1997, 101, 10506-10517.

${ }^{39}$ CYLview, 1.0b; Legault, C. Y., Université de Sherbrooke, 2009 (http://www.cylview.org)

40 Zhao, Y.; Truhlar, D.G. Theoretical Chemistry Accounts, 2008, 120, 215-241.

41 Johnson, E.R.; Keinan, S.; Mori-Sánchez, P.; Contreras-García, J.; Cohen, A.J.; Yang, W. J. Am. Chem. Soc. 2010, 132, 18, 6498-6506. Lu, T.; Chen, F. J. Comput. Chem., 2012, 33, 580-592. Multiwfn manual (http://sobereva.com/multiwfn/Multiwfn_manual.html).

42 Humphrey, W., Dalke, A., Schulten, K. J. Molec. Graphics, 1996, 14, 33-38. (http.//www.ks.uiuc.edu/Research/vmd/). 